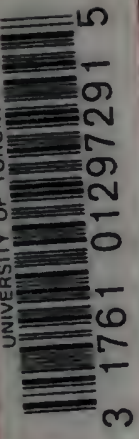


Digitized by the Internet Archive in 2008 with funding from Microsoft Corporation 

NORTH AMERICAN

Geology and Paleontology

FOR THE USE OF

\section{AMATELRS, STUDENTS, AND SCIENTISTS}

BY

S. A. M I L L E R 



\section{PREFACE.}

A General knowledge of Geology is probably of greater importance to the people of the United States than a like amount of information in any other department of natural science; but every one will admit the state of learning in this branch is not of a very high grade. There is a common complaint among well-informed people who lave given Geology no special study that the language used is technical, the names long, difficult to understand, and not unfrequently bear upon their face the evidence of affectation, as if those coining, the words had attempted to make them as obscure as possible. It is a fact, however, that technical names are absolutely essential to a correct understanding of every branch of Natural History; and when the system of nomenclature is once learned the names are readily understood, and much more easily remembered, than the arbitrary names of individual things possibly can be. In this work an effort has been made to popularize the rules of nomenclature, and to define the technical words in the text or in the Glossary.

Generic and specific names, which have been used by authors where the fossils are not known to occur in the Palaozoic rocks of North America, are printed in italics. Synonyms, names not described as required by the rules of nomenclature, preoccupied names, and those condemned for any other reason, are also printed in italies. When an anthor has referred his species to a genus to which it does not belong, the specific name will be found in italics under such generic name, and referred to the genus to which it belongs, and at the latter place the original erroneous generic reference will be found in parenthesis.

An attempt has been made to define all genera known from the Palæozoic rocks of North America; the name of the author of each genus is given, the date of coining the word, and an abbreviated reference to the book and page where published, and the etymology of the word and name of the type species. The names of all the species, arranged in alphabetical order, will be found under the genera to which they belong; and also the authors of them, the dates and places of publication, and very frequently 
references to two places of publication, especially where, in the first instance, the species was defined without illustration, as has been too frequently done in society publications, the place above and beyond all others where no species should be described unless accompanied by proper illustrations.

An attempt has also been made to correct the misspelling of words so as to perfect the nomenclature, and we call special attention to the Index of Genera, where a few corrections are made that were overlooked in the text, and where the gender of eaeh genus is indicated.

After the author had commenced the preparation of this work, which was several years ago, knowing the great expense attending the making of illustrations, he applied to several State Geologists and others for the privilege of taking electrotypes from the wood-cuts belonging to the State Governments and to the individuals; and he has now to express his acknowledgments to Alfred R. C. Selwyn, F. R. S, F. G. S., Director of the Geological Survey of Canada, who placed at his disposal all belonging to the Canadian Survey, and he availed himself of about one hundred and sixty of the original figures used by the late Prof. Billings; and also to express his obligations to the late Prof. A. H. Worthen, from whom he obtained nearly all those used in the Geological Survey of Illinois. After a very large number of figures had been made by the expensive proeess of wood-engraving, he learned of the much cheaper electrotype process, and engaged the services of the Kline Photo-engraving Company, of Cincinnati, and for the accuracy and faithfulness with which many figures have been reproduced he is indebted to the skill of the artists in that company.

Cincinkati, November, 1889. 


\section{NORTH AMERICAN GEOLOGY.}

\section{CHAPTER I. \\ DEFINITIONS AND LAWS OF GEOLOGY.}

$\S 1$. GEOLOGY is the science which comprehends the structure of the earth and investigates its history. It does not extend to the beginning, nor throw any light upon the astronomical theory that the world was, at one time, in a gaseous state, and later in a condition of fluidity. It commences at the most ancient rocks found upon the surface of the earth. These had their origin, in sedimentary deposition, at the bottom of an ocean. The world was then as large as it is now, and beyond the fact that these rocks were once merely sedimentary layers at the bed of a sea, the previous history of the earth is unknown, and all prior time is impenetrable darkness. Geology commences at the lowest discovered rocks, and investigates the overlying strata, the changes that have taken place, the lapse of time, and the development of organic life, to the present moment. If the strata of rocks on the surface of the earth were horizontal, the science would extend over a short period of time, and might be learned as rapidly as we progress in zoölogy, auatomy, or other branches of Natural History ; but the rocks are inclined at various angles, and form synclinal troughs and anticlinal ridges, and expose, in the order of sedimentary deposition, at the maximum more than forty miles in thickness. Mountain regions rarely afford so good opportunities for the study of Geology as a country unbroken, except by the exposures in stone-quarries and the banks of streams. In some States the dip of the strata is quite uniform for a hundred miles or more, without any folds or flexures. It is in these areas the student will find the most inviting fields for the study of the science.

$\$ 2$. The laws of the science have been ascertained, from observation and investigation of the changes now taking place, from a knowledge of those which have occurred within the historical period, from the evidence of change in more remote ages, from the study of the skeletons and harder parts of animals and plants, and the process of infiltration of mineral matter into these organisms, which fills up the cavities and produces petrifactions, and from the study and determination of the characters of the petrifactions found in the rocks of nearly all ages. Neither plants nor animals turn to stone; flesh can not petrify. When a body is sufficiently firm to preserve its form until water, holding lime or silica in chemical solution, can penetrate the cavities, saturate it, and deposit the stony matter as the organism decays, we have a fossil or petrifaction. The laws of nature are uniform in their operation. The diversified character of the rocks has resulted from general causes, and the uplifting and inclination of sediments did not occur in one period of time, but are distributed through and belong to all geological ages. We do not assume 
the intensity of any forces exceeded, in times past, those which are now in activity.

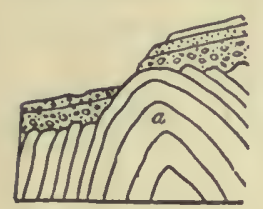

Fro. 1. Antlellnalaxis at $a$. Strata dlsturbed, folded, and denuded, and afterward unconformahle strata depos-
Ited upon them, followed by conformable layers. directions from a given point, which is the anticlinal axis. A symclinal axis is the reverse of an anticlinal axis. Rocks are called stratified whether the planes of the beds are parallel to each other, or rest unconformably. Conformable strata have the planes of the beds parallel to each other, and unconformable strata have the planes of the strata of one bed resting upon the edges of the strata of another. This must necessarily mark an interval of time between the two which is not represented by a deposit. A fault is a dis-

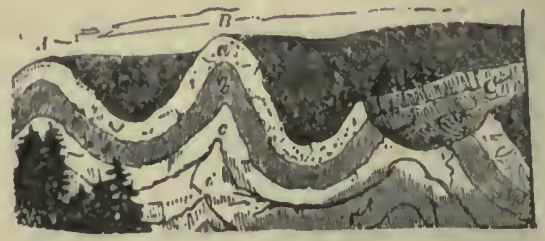

Fia. 2. Sectlon of the Jura Mointalns, jllustratIng tie folding of struta, antielins! axis nt $A$ and $B$. Syncllinal axls between $A$ and $B$ and between
$B$ and $C$. Strata unbroken at $A$ and $B$, but broken at $C, a, b, c$, and $d$. Strata conformable, though at $C, a, b, c$, and $d$. Strata contorm

location of stratr so that the continuity of the mass is destroyed by one side of the

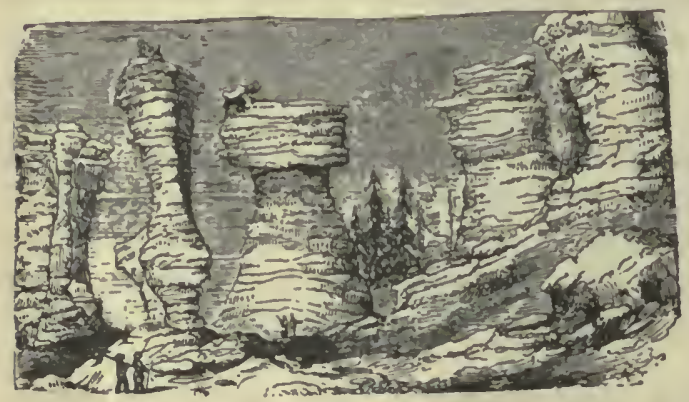

FIg. 3. Esearpment ou the right, absuts representing the slope from the escarpment. Ontliers, lone rocks, or standing columns in the center and to the left of the illustration. The central figure is a form sometimes called a cheese rock. fracture being elevated higher than the other. A dyke is a wall of rock between the two sides of a fault or fracture, interrupting the continuity of the beds on either side. Sometimes a dyke shows an overflow at the top. When strata terminate abruptly, they terminate in an escarpment. An outlier is a lone rock in place, or a hill detached by erosion from the surrounding mass of similar beds, of which it evidently once formed a part.

$\S 4$. The erosion of the earth never ceases. Decomposed and disintegrated substances are being constantly removed by rain and superficial waters to a lower level than they previously occupied. The erosion or denudation must be followed by the deposition of the materials. The deposit at one place can only progress at the rate with which it is transported from another. All strata consist of transported matter, and, as Lyell said, the evidence of the work of denudation is defective, because it is the tendency of every destroying

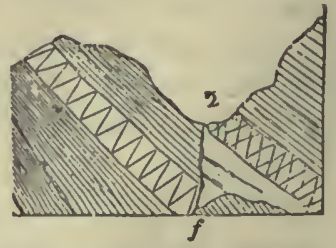

Frg. 4. Strata inellned but

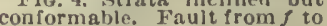

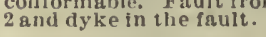
cause to obliterate in great part the signs of its own agency. Stratified rocks, therefore, indicate only part of the erosion which the earth's surface has undergone, 
because the same materials in a multitude of cases have been broken up again and again, and restratified, presenting for our observation only the last of the many forms through which they have passed. The oldest rocks, as well as the most recent, were formed from the waste of older rocks than themselves, therefore we can never see any part of the primitive earth or original solidified matter.

$\S 5$. The atmospheric forces, in activity and disturbing the surface of the earth, are generally combined with the aqueous, as in frost; or the chemical, as in the union with carbonic acid; but the effects of air and wind are, by no means, inconsiderable. The surface of all exposed rocks and earthy materials bear the evidences of disintegration and denudation. The sun dries up the mud and cracks the earth and soils, while the winds sweep the dust from roads and barren places. Grains of sand, driven by the wind, will groove and polish the hardest rocks and minerals, and sometimes fairly dissolve and carry away limestone and more friable substances. Sand blowing is used in the arts for etching hard materials. All soils have resulted from the disintegration of rocks, and when not transported, the quality depends upon the character of the parent rock immediately below; and the penetration of the soil to the unaltered parent rock will reveal the different stages of the change effected by atmospheric agencies, aided more or less by the effects of frost and water. The winds, blowing inland from large bodies of water, carry sand from the beaches, and pile it in mounds and ridges, called sand-dunes; and the same effects are produced upon the deserts, and to a greater or less extent wherever light or loose materials are exposed to its action. A wind-storm blew a standing locomotive off the railroad track at East St. Louis, and other storms have been known to move bodies weighing several tons. The geological effects of the wind therefore are conspicuous in some parts of the world, while in others they are so slight as to be quite overlooked.

$\S 6$. Water is an active solvent of rocky substances, and the solvent power increases with heat and pressure. It is also a powerful mechancial agent. It will enter the minute openings in the hardest rocks, freeze, and chip up minute scales; and so it will enter larger cracks and orifices, freeze and break open large rocks, or burst from ledges immense masses. Ice, freezing at the margin of lakes and ponds, by expansion, crowds the loose rocks on the shores in the form of ridges of bowlders, and freezing around the free rocks at the bottom in shallow water or near the shore, will, when broken up by partial thawing, and assisted by the force of waves and winds, transport such rocks to distant places. Mud, sand, gravel, and pieces of rock are transported down stream by all rivers, and the transportation is aided by the ice in the temperate and colder latitudes. On the shores of the St. Lawrence transported bowlders are found weighing many tons.

$\S 7$. The eapacity of the atmosphere to take up aqueous vapor in suspension, increases with the temperature, and when saturated the least interference with the currents of the air will precipitate rain. Hence there is more rain in warmer than in colder latitudes. Clouds drifting against mountains and high lands will discharge rain. The rain falls upon the ground, disintegrates earthy substances, and transports the disintegrated materials resulting from its own action, and from atmospheric agencies, down the valleys to the ocean. It is said the Ganges annually carries to the sea $6,368,000,000$ cubic feet of sediment, which, being spread over the whole basin of the river, comprehending 400,000 square miles, would make a layer 1-1751 of a foot thick. The Ganges, therefore, erodes its basin one foot in 1,751 years. 
The area of the Mississippi basin is 1,244,000 square miles, and the annual discharge of sediment by the river is estimated at $7,471,411,200$ cubic feet, an amount sufficient to cover the whole basin 1-4640 of a foot. Therefore the Mississippi River removes from its basin a thickness of one foot in 4,640 years.

$\S 8$. The greater number of valleys in North America have been carved out by the streams flowing in them at substantially the same rate of excavation that is now in progress. All the valleys in Ohio, Indiana, and Illinois, have been excavated by the slow process of the action of rain and the rivers. The Mississippi and all its tributaries have excavated their own valleys, with the exception of a few in the mountain regions. Not only have the valleys been thus excavated, but much of the intervening land has been denuded of many feet of surface rocks. While the beds of the older streams sink extremely slow, if at all, the valleys are gradually widening by the wear and tear of rain and storm. This erosion has taken place since the close of Palieozoic time. The hills are usually terraced because the strata are of different degrees of hardness and durability, the softer and more easily disintegrated are gradually removed by atmospleric influences and the transporting power of rains and springs, leaving the harder and more solid standing out in more or less abrupt slopes and cliffs.

$\$ 9$. The lower limit of perpetual snow under the equator is 16,000 feet above the sea, in the Swiss $A \mathrm{lps}$, in latitude $46 \mathrm{~N}$., it is 8,500 feet, and in the arctic and antarctic regions it reaches the level of the sea. The isothermal lines, around the earth, being affected by the distribution of the land and water surface and the ocean currents, do not follow the degrees of latitude; therefore, in ages past, when the land and water occupied different areas, and the ocean currents moved in other routes, the isothermal lines were correspondingly changed. Above the line of perpetual snow there is an augmentation from year to year, and below it, during the colder seasons, the snow falls many feet in thickness. An equilibrium is preserved by the melting of the snow in sunshine, by occasional rains to which it is subjected, and by the natural tendency to creep down the mountain side by the force of its own gravity. This movement gives rise to glaciers, which follow the depressions or ravines on the sides of the mountains to a considerable distance below the perpetual line of snow. They move very slowly, but transport sand, gravel, and masses of rock, and smooth, polish, and groove their rocky cliannels, because fragments of rock get interposed between the glacier and the rocks of the valley. The stones carried along on the ice are called the "moraines" of the glacier. "There is always one line of blocks on each side, these are called the "lateral moraines." Where there are confluent glaciers the lateral moraines of the tributary glacier are carried into the larger stream of ice, and are called "medial moraines."

$\$ 10$. The effects of glaciers upon the face of the earth are not important, notwithstanding so much has been said about them, and it is evident they have not been much more imposing in past geological ages than they are now. There are probably no evidences of glacial action upon the continent of North America where they do not now exist, except in a few places in the Rocky Mountain region, where they have departed on account of the drainage of adjacent lakes, and some indications in the New England Mountains where they are unknown now, either because that region is somewhat depressed, or because the Arctic Current does not hug the shore as far south as it did in the Pliocene or Post-pliocene period. 
$\S 11$. In high northern and southern latitudes glaciers descend into the sea, where fragments are broken off, which are called "icebergs." Icebergs bear all the earth and rocks they did when constituting part of a glacier, and they soon fall in with ocean currrents, and are drifted great distances before they are dissolved, and let the "moraines" fall to the bottom of the sea. In this manner the submarine surface is strewn with foreign mud, sand, gravel, bowlders, and fragments of rock. Coast-ice acts in the same manner when blown out into the sea by off-shore winds. The ice sinks into the ocean eight times as deep as it projects above the surface, and when in shallow water it impinges upon the submarine bottom, the force of the current or the winds may cause it to' polish or groove the rocks, if fragments intervene, in the same manner that glaciers will polish or groove their valleys. Icebergs drift from Baffin's Bay to the latitude of the Azores, from Greenland to the mouth of the Gulf of St. Lawrence, from the antarctic regions to the Cape of Good Hope, and also to Chili, in South America. Darwin saw one in the southern seas bearing a rock visible twelve feet above the surface, 1,400 miles distant from any known land. Icebergs have a transporting power more than a thousand-fold greater than glaciers, and an eroding power but little inferior, and yet the action of icebergs is inconspicuous now, and has been, so far as we know, in all the ages gone by.

$\S 12$. A large part of the rain sinks into the ground, takes up mineral matter in chemical solution, flows out in springs, and transports its load to the ocean. In this manner many caves and caverns are excavated. The waves produced by storms and tides beat down the shores of large bodies of water, and deposit the materials at other places. The ocean currents have a drifting and denuding action where the water is shallow. The wear and tear of the earth by the action of water never ceases, and the more we contemplate the subject, the better able we are to realize the magnitude of the never-euding destruction.

$\S 13$. The violence of earthquakes, and the fires of the volcanoes, the elevations and depressions of land with respect to the sea, seem to have operated within the historical period on as grand a scale as we are warranted in believing they did in past geological ages. Earthquakes and volcanic fires are intimately connected, and neither penetrate the earth to any great depth. Earthquakes have been felt upon the surface of the earth when miners, at a depth of 1,000 feet or more, have not experienced the sensation. The transmission of the vibration is more distinct, and phenomena more apparent where the strata are hard rocks than where they consist of sand and gravel, or softer material. All volcanoes are near large bodies of water, and observation has shown that water gains access to the volcanic foci, and that steam is a powerful agent in all eruptions. The pressure or force of gravity of the layers of the surface of the earth develops the latent heat, so there is an increase of temperature at the rate of about one degree for every sixty feet penetrated for the first 2,000 or 3,000 feet. The deeper borings have not shown the regular continuing increase of the heat, nor is the increase uniform through different kinds of rock, or at different places. The better opinion seems to be that neither this increase of heat, nor the volcanic fires afford any evidence of the internal fluidity of the earth, but, on the contrary, the earth is probably solid, with exception of local caverns near the surface, and local masses of melted matter resulting from chemical causes which are in operation at no great depth.

$\S 14$. All are more or less familiar with the story of the buried cities of Hercu- 
laneum and Pompeii, and the great eruptions of Vesuvius. In 1669 a current of lava flowed from Etna, having a width of 600 yards, and a depth of 40 feet when it reached the sea at the distance of fifteen miles. In 1783 Skapter Jokul, in Iceland, sent forth two currents of lava in opposite directious, one of which extended fifty miles, and the other forty-five. The extreme breadth of the one in Skapter valley was fifteen miles, and the other had a breadth of seven miles. The ordinary height of the current was 100 feet, but in narrow defiles it sometimes amounted to 600 feet. There is no evidence of a volcanic eruption on the continent of North America in past geological ages that surpassed this in volume.

About midnight, August 11, 1772, a luminous cloud appeared to envelop Papandayang, a volcano on the island of Java, and in a short time it actually fell $\cdot$ in with a great noise. Immense quantities of volcanic substances were thrown out and distributed for many miles around. It is estimated the mountain for fifteen miles in length and six in breadth was swallowed up in the earth by this commotion. Forty villages were ingulfed or destroyed, and 2,957 inhabitants perished. It seems in this instance the eruptions had formed a corresponding cavity beneath the surface, and when the weight above overcame the resistance, the volcano suddenly fell into the abyss beneath.

A voleano forced its way from beneath the sea into the atmosphere off St. Michael's, Azores, in 1811. It was first seen above the sea on June 13th. 'The appearances were exceedingly beautiful, the volcano shooting up columus of the blackest cinders to the height of between 700 and 800 feet above the surface of the water. When not ejecting ashes, an immense body of vapor or smoke revolved almost horizontally on the sea. The bursts were accompanied by explosions resembling a mixed discharge of cannon and musketry, and a great abundance of lightning. By the 4 th of July an island was formed a mile in circumference and 300 feet high. In the center there was a crater full of hot water, which discharged itself through an opening facing St. Michael's. The island subsequently disappeared beneath the water.

Twelve islands constitute the Hawaiian Group, four of these are mere barren rocks; the remaining eight have an area of about 6,000 square miles. All of these islands are volcanic, and no other rocks than volcanic are found upon them save a few remuants of sea-beaches. They are all mountainous, and the deep sea surroundings have shown the islands are only the summits of gigantic mountain masses. Mauna Kea, on Hawaii, is 13,900 feet above the sea, and Mauna Loa 13,700 feet. If the ocean were driven away, it is said these mountain peaks would stand 30,000 feet above the foot of the mountain range. On Hawaii the volcanic forces are still in operation. On Maui they rested at a recent epoch, or within a few hundred years. On the other islands they have long been extinct, and the piles built up have been greatly eroded. On Hawaii there are two grand foci of volcanic eruption where the fires are now raging, Mauna Loa and Kilauea. Mauna Loa is the largest volcano in the world, and none approach it in the magnitude of its eruptions. A moderate eruption represents more material than Vesuvius has emitted since the days of Pompeii, and the flow of 1855 would have nearly built Vesuvius. On the whole, it appears there are as many active volcanoes, and some as vast and frightful in eruptive power as seem to have existed at any other single period in geological time. 
$\$ 15$. An earthquake in New Zealand in 1856 raised a tract of land comprising 4,600 square miles, from one to nine feet. In 1822, and again in 1835, the coast of Chili for several hundred miles was elevated from one to three or four feet or more. The estimated area raised in 1822 amounted to 100,000 square miles. In 1819 an earthquake at Cutch, in the delta of the Indus, raised an extent of country' about fifty miles long and sixteen miles wide, ten feet, while a considerable tract in the delta of the Indus sank down. Such are a few of the effects produced by earthquakes in the present century; they are similar to those which have occurred in every century during the historical period, and are quite as extensive as any we are warranted in believing occurred in any of the earlier geological ages.

$\S 1$. It is said large tracts of land are elevated and depressed without the intervention of earthquakes. It is said there has been an elevation of land bordering the Baltic, duriug the historic period, of about three feet in a century. The whole coast of Scandinavia is said to be gradually rising at a very slow pace. A large area in Greenland is reported as slowly subsiding. At Fort Lawrence, in the Bay of Fundy, there is a pine and beach forest covered at high tide by about thirty feet of water. And it is claimed there is some evidence of subsidence on part of the New England Coast, where we have the most indubitable evidence of an elevation of several hundred feet since the beginning of the Post-pliocene period, but these elevations and depressions may have been accompanied with earthquakes.

$\S 17$. Earthquakes and volcanoes have a common origin, the former always accompany the eruption of the latter, and it is not likely any great areas of land rise or fall without the intervention of the same energies. The proximate cause of volcanic and earthquake phenomena is not fully known, and it is much easier to show the improbability of the many theories offered for their explanation than to present one free from objections. Volcanoes are intermittent in their eruptions; they act by spasms of activity, separated by intervals of repose. If they were vents to internal fluidity of the earth, the streams of flowing fire would be constant, not intermittent explosions. If they were vents to any great mass of melted matter pent up until strength enough were obtained to force a passage way to the surface of the earth, when the vents would open the reservoirs would exhaust themselves and close forever. Volcanoes are not to be attributed to the remains or residue of enormous heat contained in the globe, at some remote period of its physical evolution, or considered as lending any support to the nebular hypothesis, or the theory that the earth was at one time in a gaseous or fluid condition.

Geyser (from the Icelandic word geysa, to gush,) is a periodically eruptive or intermittent hot spring, from which the water is projected in a fountain-like column. The analogy between it and a volcano is so striking that it might be called a volcano erupting hot water instead of melted lava. In the case of a geyser, cold water is supposed to sink from the surface to heated rocks; it starts as a passive liquid, and by its molecular absorption of heat is converted in the depths into an elastic, explosive gas, which ejects it through another orifice to the surface. The gas forces out the column of water and escapes; then quiet ensues until a new supply of water is furnished. This accounts for the intermitting flows. Grant the local heated condition of the rocks below, and all the phenomena of the geysers may be accounted for.

The melted lavas of volcanoes bring up with them great quantities of the vapor 
of water, having an enormous expansive power which is given off as steam at the moment of eruption. Lava is generally a sponge-like mass of myriads of visible vesicles formed by the sudden exclusion of the water-vapor in the act of solidification. There is abundant evidence of the participation of water and its constituent gases in volcanic phenomena. Frotn the proximity of volcanoes to or occurrence in the sea, it has been supposed their active state is produced by the percolation of seawater to metallic bases of the earths, or alkalies, at various depths, which bases become inflamed and chemical action ensues, producing the cruption. The oxygen of the water is supposed to unite with the metallic base, the hydrogen to unite with sulphur, forming sulphureted hydrogen gas, and with the chlorine forming muriatic acid gas, etc. The gases evolved from volcanoes are muriatic acid gas, sulphur combined with oxygen or hydrogen, carbonic acid gas, nitrogen, and aqueous vapor. Electricity is a factor in all earthquakes and volcanic eruptions. Its action is manifest in the atmospheric disturbances, in the undulatory movement on the surface of the earth, and in the speed with which the earthquake wave travels. An earthquake moves in the direction of the wave at a rate frequently exceeding fifty miles in a minute, and when the movement is communicated to the waters of the ocean, the waves follow at a pace hundreds of times slower. Suppose a powerful current of electricity near the surface of the earth, to be broken, and suddenly restored, the shock may be supposed to resemble that of an earthquake. Fusion might result in consequence of such restoration. The crystallization of stratified rocks might break such electrical currents, if any exist in the earth, or it might disturb the equanimity of the electricity if it exists in a passive state, to the same extent as if it were a broken and restored current. In other words, subterranean electric currents, if once excited, may melt the rocks and produce the heat necessary, when assisted by the presence of a sufficient quantity of water, to produce volcanic eruptions. Such are some of the theories to account for the instigating or proximate causes of earthquakes and volcanoes.

The mouth of a volcano is called a crater, though the pit on Kilauea has been called a caldera. If steam alone escapes through a vent, it is called a fumarole; but if sulphurous vapors also escape, it is called a solfatara. When hot springs deposit lime, it is called tufa; but if the deposit is silicious, it is called sinter or geyserite. Lava consists of silica, alumina, lime, magnesia, soda, potash, and iron oxide. If the silica is in excess, it is trachyte, and belongs to what lithologists call the acidic group, from the large quantity of silicic acid it contains; but if there is a large proportion of soda or potash and lime or magnesia, and not more than 50 per cent of silica, it is a basalt, and belongs to the basic group, from the larger quantity of alkaline and earthy bases it contains. Trachyte is a grayish igneous rock, of rough fracture owing to the grains of glassy feldspar which mainly constitute it. Basalt may be light-colored crystalline or granitoid, or dark colored, compact, massive, like dolerite; but in addition to labradorite and pyrnxene, it contains chrysolite in disseminated grains. When lava becomes glassy, it is called obsidian.

$\S 18$. The most important change taking place upon the earth is in constant operation at the bed of the ocean. Near the shore it is a littoral deposit; farther away it is a chalky deposit, consisting of foraminifera and shells, and in deeper water it is a red, silicious clay. The character of the deposit is dependent upon the depth of the ocean, except where washings from land affect it. The depth of the pure 
globigerina ooze, or chalky deposit, is limited to about 2,250 fathoms, and at greater depths the deposit gradually passes into fine pure clay, and below 2,500 fathoms it consists almost wholly of a silicate of the red oxide of iron and alumina. At moderate depths shells fall upon the bottom, in perfect condition; as the depth increases they become more and more brittle, and finally break up and disappear by the chemical action which affects them, until, having passed through 2,500 fathoms of water, nothing is left save an insoluble residue, which constitutes the red clay. The simple fact of the increasing depth of the ocean gives variety to the character of the deposits. But at the greatest depth to which the dredge has descended, which exceeds five miles, the silicious shells of Radiolarians exist as abundantly as they do in the shallower depths of the ocean. Such deposits, in the process of induration, become stratitied and laminated, and form calcareous, argillaceous, and arenaceous or silicious rocks.

$\S 19$. Animals, secreting carbonate of lime, have played an important part in modifying the surface of the earth. The coral-making polyp has wrought great changes, because the reef-forming genera continue the accumulation, on the same spot, for centuries, and the influence of the Bryozoa, which produce only delicate corals, is everywhere conspicuously engraved. There are other agents, inferior in operation, affecting the surface of the earth, and all combined have served in times past to deposit in water all the rocks constituting the continent of North America, and to elevate the land above the seas and lakes, after such deposition, and again to denude it and present it to us with its mountains and valleys as they now exist.

$\S 20$. Every part of the surface of the earth has been covered with water, and much of what is now dry land has been several times inundated; and it is supposed a large part, if not the whole area covered by the oceans, has, at some period of time, been above the water line. The elevations and depressions have been in the form of ridges, with intervening basins, in different ages of the world; and basins, existing in the same age, have been filled with deposits of different kinds and in different degrees of rapidity, - some being filled with drifted materials, and others with the secretions of animal and vegetable organisms. Consequently there is a great diversity in the structure of the land of different continents, and they must be separately investigated. The most recent deposits may be made on the most ancient rocks. Cretaceous deposits may occur upon the Silurian, or Jurassic on the Devonian; hence, many difficulties are encountered in ascertaining the chronological order of the strata upon each continent; and this would be utterly impossible were it not for the animal and vegetable remains, which have followed the progress of time in evolutions of type and structure in different oceanic basins, so as to furnish the means of approximately parallelizing the strata. Different kinds of rocks are forming at unequal depths of the ocean, at the same time; conglomerates and sandstones in shallow water and near the shores; chalky, and slaty or shaly in deeper water, and silicious farther from land and at still greater depths. Strata of the same kind are not continuous over large areas; but change within short distances from sandstone to shale or limestone; hence, it is never safe to trust to the character of the rock for the testimony to prove its age. We must go to the fossils for the evidence, because it has been ascertained that species did not generally live beyond a geological period, and characterized different Groups of rocks, and thus become infallible guides to the order of superposition. No two periods are represented by like assemblages of fossil 
forms, and this dissimilarity furnishes the facts upon which the Groups of rocks are distinguished from each other. Comparison of the fossils shows a progression in development along an ascending scale toward the higher and more enduring plants and auimals, and the extinction of lower or less highly organized forms.

$\$ 21$. Sandstone is a rock made of sand derived from a silicious rock. When pure it is used for making glass. Iron usually colors it red or yellowish, and often cements it into good buildiug stone. When a little clay is intermixed it is called freestone, and if it contains gravel it is conglomerate, or if loosely cemented in the air and not under water a pudding-stone. When sandstone is subjected to heat and pressure it is metamorphosed and becomes quartzite.

Shale is a soft, fine-grained, aluminous rock, in layers. If it is pure it is clay shale; if it contains sand it is sandy shale; if bituminous matter, bituminous shale. When the shale is hardened it becomes slate. Slate rocks among the metamorphic series are called schists. The clay slate used in North Carolina for making slatepencils is called pyrophyllite.

Limestone is ordiuarily composed of lime and earbonic acid, with impurities of clay, sand, and iron. Hydraulic limestone eontains clay aud maguesia. Magnesian limestone is called dolomite, after Dolomieu, a mineralogist. . Lithographic stone is a very even-grained, compact limestone, usually of buff or drab color. Chalk is a soft limestune, and marble is a hard crystalline limestone. Gypsum, alabaster, calcite, dogtooth spar and satin spar are names given to crystalline limestone.

$\S 22$. The general order of superposition of the rocks of North America has been ascertained, and they have been divided into Systems and Groups. Another division has been made, founded on the orgauisms that occur in the rocks, viz: Eozoic, Palæozoic, Mesozoic and Cænozoic. Some use the word Archæan instead of Eozoic. The Eozoic includes the Laurentian and Taconic Systerns. The Palrozoic includes the Lower Silurian, Upper Silurian, Devonian, Subcarboniferous and Carboniferous Systems. The Mesozoic includes the 'Triassic, Jurassic and Cretaceous Systems. The Crenozoic is synonymous with the Tertiary System. These Systems may be very closely parallelized with the strata of Europe and other parts of the world. The words "System" and "formation" are in use with this nomenclature, as Devonian "System" or Devonian "formation," but more generally they are both omitted as unuecessary appendages to the names of the divisions.

The Taconic is iutroduced in many places with conglomerate layers resting unconformably upon the Laurentian ; the Lower Siluriau commences with the Potsdam sandstone, the Upper Silurian with the Medina saudstone, the Devonian with the Oriskany sandstone, the Subcarboniferous with the Waverly sandstone, and the Coal Measures with the Carboniferous Conglomerate. Each of these great divisions commences with drifted materials, and importaut changes of the fauna. They are each capable of subdivision into Groups, and they are not only convenient in the discussion of the science, but they are, to a certain extent, founded in nature.

$\S 23$. For the purpose of more definite classification these larger divisions are subdivided. Each subdivision is called a "Group," and it generally bears the name of the place where first studied and described; as, the Potsdam Group, so named because the strata were first studied and described at Potsdam, New York. This method is preferred to any other, because the geographical name, when combined with the word "Group," is sufficiently technical. It can not be used for any other 
purpose; it can never mislead as to the mineral structure or relative position of the strata, and it indicates the typical locality of the exposure. Sandstones, conglomerates, limestones, and shales, occur in nearly every Group, and for this reason geological subdivisions can not be established upon the mineral or chemical characters of the rocks. The rocks which form these Groups are composed of a few simple minerals, which are repeated over and over again in the different layers, but not in chronological succession, nor in any other way indicative of age or position. To speak of a rock as a limestone, sandstone, shale, slate, or clay, conveys no idea of its geological age or place. It is merely the expression of a mineralogical character.

$\S 24$. Prof. Rogers conceived the idea of improving the nomenclature of the palreozoic rocks by dividing them into fifteen parts, and giving them names significant of their relative ages. This he did by using words suggesting metaphorically different parts of a day, as follows: Primal, Auroral, Matinal, Levant, Surgent, Scalent, Premeridian, Meridian, Post Meridian, Cadent, Vergent, Pouent, Vespertine, Umbral, and Seral, meaning respectively the formations of the Dawn, Daybreak, Morning, Sunrise, Mounting Day, Climbing Day, Forenoon, Noon, Afternoon, Declining Day, Descending Day, Sunset, Evening, Dusk, and Nightfall. Unfortunately for his attempt to substitute another for the geographical nomenclature then quite well established and susceptible of indefinite expansion, without the use of conflicting terms or words that could mislead the student, there were several extensive Groups of rocks full of the remains of animal life, then unexplored, and consequently quite unknown to his system. For obvious reasons the nomenclature suggested by Mr. Rogers has not been adopted.

$\S 25$. The words series, strata, layer, deposit, zone, bed, horizon, period, age, epoch, and era are not technical names, but are used in geological descriptions, because expressive and convenient. Each Group must, in all cases, depend upon the palsentological characters, and cau never rest upon the structure of the rocks. When properly defined, it is established, and no one has a right to substitute another name for it, nor to propose a name, simply because of inability to properly distinguish it at a particular locality. For example, the Trenton, Utica, and Hudson River Groups bad been long established, when some one, being unable to distinguish the Utica in the vicinity of Cincinnati, and not knowing whether the rocks are Trenton or Hudson River, proposed to call the exposure the "Cincinnati Group." The black slate, which characterizes the Utica in New York, does not exist at Cincinnati, though calcareous slates and shales of the same age do, and they contain Triarthrus becki, Leptobolus lepis, and other characteristic fossils, while the Hudson River is plainly distinguishable above, and the Trenton as readily determined below. If the Utica had thinned out in its extension westward from New York before reaching Cincinnati, there would have been no excuse for calling the Trenton or Hudson River, or both of them together, by a new name, nor is there any excuse for so doing when the Utica is easily distinguished.

$\$ 26$. Another kind of synonymy to be deplored exists where a Group has been named and thoroughly defined, and for some trivial reason, the geologists of another locality use another name for rocks of the same age without regard to priority in nomenclature. For example, the Calciferous Group was established and defined so as to include rocks other than Calciferous sandrock, and ten years after- 
ward rocks of the same age on the Mississippi were called the "Lower Magnesian Limestone," and geologists of that locality persist in the use of the latter name, because they say the word Calciferous is not admissible from the lithological character of the rock. It is to be regretted that the name Calciferous has come down to this generation as the name of a Group of rocks, but it is as well established as the name of any other Group, and like the word Tertiary, which has no application to the rocks to which it is applied, is fastened upon the science, and so interlocked with it that it can not be eradicated even were it desirable so to do. With how much less reason should we encourage the use of another mineralogical name, having more limited application, in its stead!

$\$ 27$. The rule is, the law of priority should be rigorously enforced where a Group has been named, and the fossils have been so described and illustrated that it may. be identified by a palacontologist elsewhere than at the typical locality. Synonymy is always the result of ignorance, and much of it has come from those whose work has been absolutely worthless.

$\$ 28$. Experience has shown the impracticability of making lesser subdivisions for the purpose of geological nomenclature, than Groups, especially in the present state of the science, though it is eminently fit and proper to speak of the marl-beds or sandstone layers in any Group, or of the Glyptocrinus or Orthis beds at any locality. Minute and careful definition and description of the characters of each and every part of a Group is one thing, and the suggestion of a geological subdivision, founded upon a marked peculiarity at one locality, which can not be distinguished at another, is quite a different thing. It must not be supposed none of the Groups will be subdivided, but proposing a name is not establishing a Group. The Coal Measures ought to be divided into Groups because of the great thickness of the fossiliferous rocks, and a temporary division in some localities is indicated by the use of the words Upper, Middle, and Lower Coal Measures, but great palæontological information must be acquired before any practicable subdivision can be made.

$\S 29$. The stratigraphical division of the rocks of North America into Groups bearing geographical names, with an approximate thickness in ascending order, is as follows:

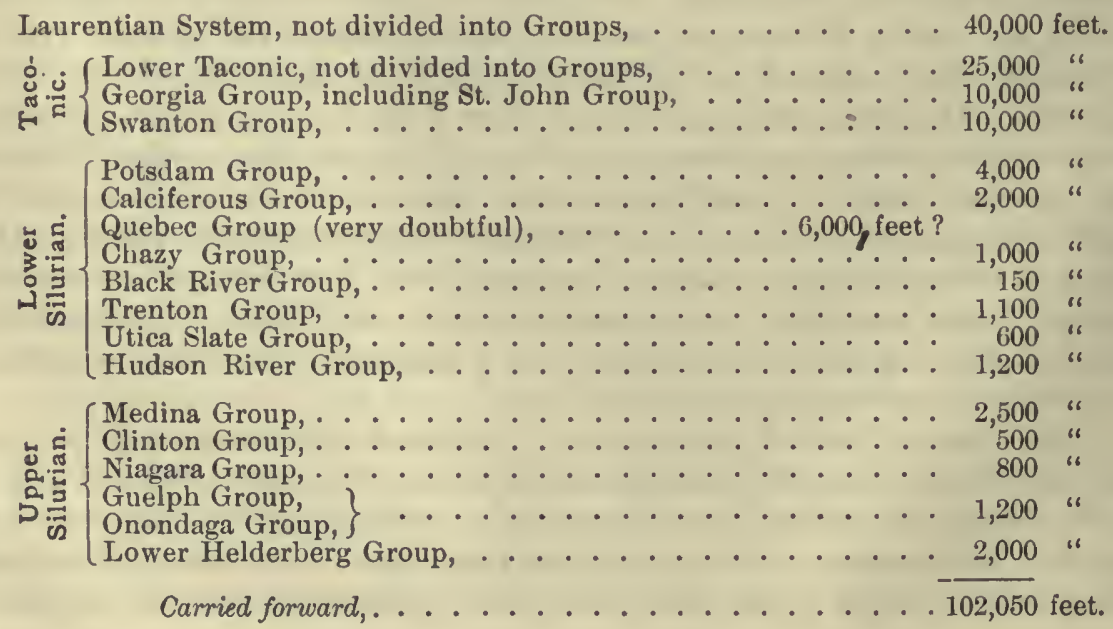


Brought forward, . . . . . . . . . . . 102,050 feet.

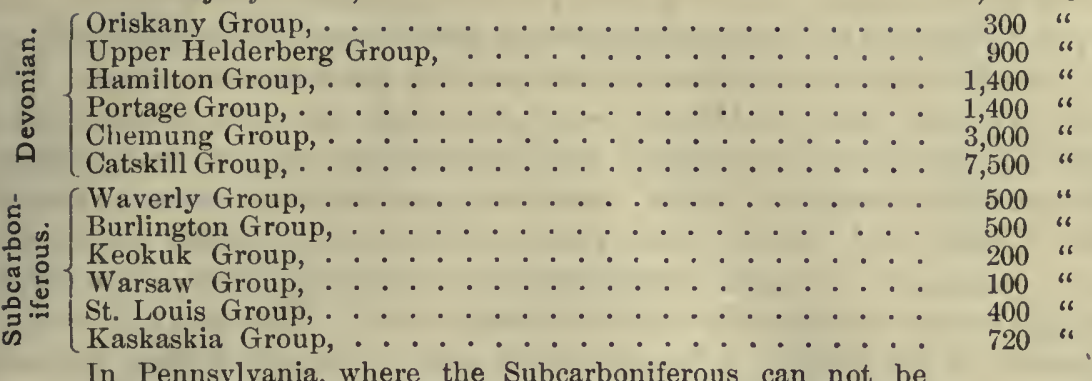

separated into Groups, there is a thickness of 5,000 feet, and in Nova Scotia 6,000 feet.

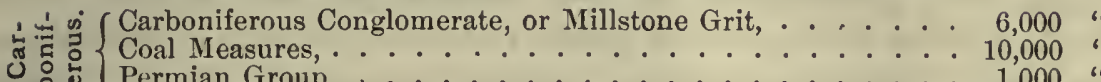

¿ Permian Group, . . . . . . . . . . . . . 1,000

Triassic not divided into Groups, . . . . . . . . . . 25,000

Jurassic not divided into Groups, . . . . . . . . . . . 10,000

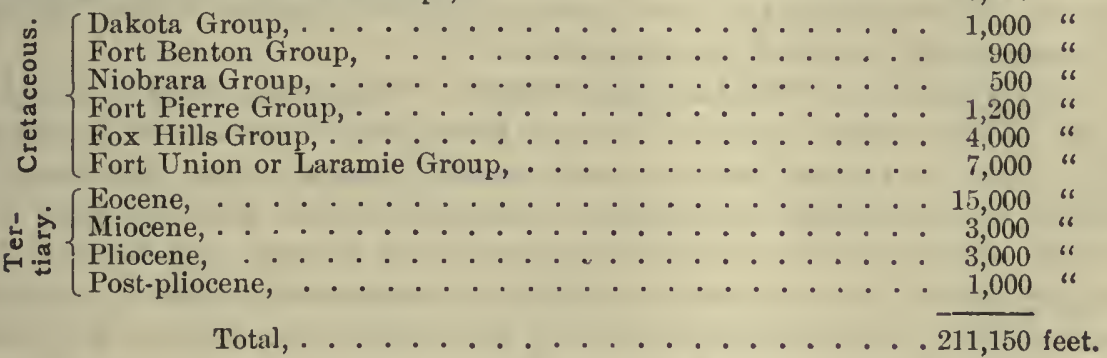

\section{CHAP'TER II.}

\section{LAURENTIAN SYSTEM.}

$\S 30$. The Laurentian System was so named from the Laurentian Mountains, and not from the St. Lawrence River. The name was applied to the metamorphic rocks of Canada as a scientific term, by Sir William Logan, in the Report of Progress of the Geological Survey of Canada for the years 1852-53. His special study of these rocks began as early as 1846 . He applied the name to all rocks lower than the Potsdam; but Emmons had preceded him in defining the Taconic System, which rests uncomformably upon the rocks that comprise nearly all which Logan studied; and hence the Laurentian is confined to the rocks below the Taconic. The rocks consist of sedimentary strata altered to a highly crystalline condition - great vertical thicknesses of gneiss and granitoid rocks, separated by masses of crystalline limestone and quartzite. Previous to this geographical name they were called azoic, metamorphic, or primary rocks.

Granite is a word derived from the granular texture of the rock to which it is applied. It is crystalline and composed of quartz, felspar, and mica. The felspar usually gives the predominating color. When the granite is stratified, the laminx being separated by thin scales of mica, it is gneiss or granite schist; if mica is in excess, it is mica schist; when hornblende displaces the mica, it is syenite (named 
from Syene, in Egypt); and if it only partially displaces it, it is syenitic granite. Many of the granites and syenites are intrusive, while others, not distinguishable from these, take the place of sedimentation and pass into gneiss or mica schist. Felspar signifies rock-spar from the German word fels, a rock, though it is usually spelled feldspar from the German word feld, a field, and therefore made to signify field-spar. There are several species of felspar, dependent upon the potash, soda, or line they contain. That which usually enters granite is orthoclase, or potash felspar, and is compact laminated, or compact crypto-crystalline, consisting. of about the following substances: silica 64.6, alumina 18.5, and potash 16.9. When soda enters into the composition of the felspar, it becomes albite, and the granite is then disposed to undergo spontaneous disintegration, which sometimes takes place below direct atmospheric influences at great deptlss in the earth. The kaolin of the Chinese is derived from felspar from the disintegration of granitic rocks, and porcelain clay is often from the same source. Garnets are common in gneiss and mica schist. The most common mica, and that which generally enters into granite, gneiss, and related rocks, is called muscovite. Other species in the mica group are called phlogopite, biotite, lepidomelane, astrophyilite, lepidolite, and cryophyllite.

$\$ 31$. Logan said of the Laurentian System: "Stretching on the north side of the St. Lawrence from Labrador to Lake Huron, this series occupies by far the larger portion of Canada, and its strata probably possess a great thickness. To determine the superposition of the various members of such an ancient series of rocks is a task which has never yet been accomplished in geology, and the difficulties attending it arise from the absence of fossils to characterize its different members. Bands of the crystalline limestone are easily distinguished from bands of the gneiss; but it is scarcely possible to know from local inspection whether any mass of limestone in one part is equivalent to a certain mass in another. They all resemble one another lithologically, and although masses dipping in the same direction are met with, running for considerable distances rudely parellel with one another, it is scarcely ever safe to take for granted that they are stratigraphically distinct. The dips avail but little in tracing out the structure; for in the numerous folds of the series the dips are frequently overturned, and the only reliable mode of pursuing the investigation and working out the physical structure, is patiently and continuously to follow the outcrop of each important mass in all its windings as far as it can be traced, until it becomes cevered up by superior, unconformable strata; is cut off by a great dislocation, or disappears by thinning out."

$\$ 32$. The surface area accupied by the Laurentian series in Canada and British America, exclusive of any exposure that may exist in the Cordillera or Rocky Mountains, is not less than 250,000 square miles. The northern limit is the Arctic Ocean; from here it may be traced south upon the western side of Hudson's Bay, and appearing upon its eastern side it spreads over the greater part of Labrador, and extends to the Gulf of St. Lawrence. The southern limit is the St. Lawrence from Labrador to Cape Tourmente, a distance of 600 miles, except a narrow border of Taconic on the Strait of Belle Isle; another at the mouth of the Mingan River; a third near the Seven Islands, and two on Murray Bay River, and the Gouffre. Extending westwardly it occurs 30 miles north of Montreal, and follows up the Ottawa River for a distance. It then strikes off to the Thousand Islands, and crosses over: into New York, where it exposes an area of 10,000 square miles. From there it 
extends north-westerly a short distance north of Lake Huron, and bordering upon Lake Superior, a great part of its length, it appears at Lake of the Woods, north of Rainy River, though an arm extends south of Lake Superior into Michigan and Wisconsin. The western boundary of this great area extends from Lake of the Woods in a sinuous northerly direction among the lakes, and following the highlands that divide the waters which flow into Hudson's Bay from those flowing in other directions, to the Arctic Ocean. There are some patches within this general outline covered with Taconic rocks, or those of Post-pliocene age.

$\$ 33$. The arm of this great exposure, which appears in the Upper Peninsula of Michigan, has an area of about 1,839 square miles, consisting of several tracts, one of which touches Lake Superior west of Marquette. The rocks are chiefly granite, gneiss, syenite, and crystalline limestone, which thus far have afforded no useful minerals. The surface area in Wisconsin is somewhat greater. Other exposures in the United States are confined to irregular areas in the mountain regions. North Carolina exposes about 20,000 square miles, or nearly half the State. One belt from twenty to twenty-five miles wide, crosses the northern part of the subeastern section of the State upon which the capital is situated. It extends northward into Virginia, and southward beyond Cape Fear River. It consists generally of gneiss, which passes into granite or mica schist. Another belt extends from the southern border of the State at Catawba River in a north-east direction, almost to the Virginia line near Roxboro, and reappears eight or ten miles to the eastward and crosses the northern border about midway of Granville County. There is another small area in the southern part of Orange County. Limited areas are found in Georgia, Virginia, Pennsylvania, New Jersey, Vermont, New Hampshire, and at other places in the Appalachian chain. A small area occurs in Missouri near Iron Mountain, and another in Arkansas. In the Rocky Mountain region there are many exposures, some of which are quite large. They generally trend in the direction of the mountain chain, and are found in Mexico, New Mexico, Arizona, Nevada, Utah, Colorado, Idaho, Nebraska, Wyoming, and Montana.

$\S 34$. A section taken by Logan in the region where he studied the rocks, is as follows:

1. Orthoclase gneiss, composing Trembling Mountain, . . . . . . 5,000 feet.

2. Crystalline limestone of Trembling Lake, .......... . 1,500"

3. Orthoclase gneiss, . . . . . . . . . . . . . . . . 4,000 "

4. Crystalline limestone of Great Beaver and Green Lakes, with interstratified garnetiferous rock and hornblendic orthoclase gneiss, 2,500 "

5. Orthoclase gneiss, garnetiferous gneiss and quartzite below the Grenville limestone, ....... 3,500 "

6. Crystalline limestone of Grenville, with "interstratified gneiss, . . 750 "

7. Orthoclase gneiss, ................. 1,580 "

8. Proctor's Lake limestone, .................... 20 "

9. Orthoclase gneiss, passing gradually into Anorthosite between Proctor's Lake and Morin band, .......... 3,400 "

10. Anorthosite above the Morin band, . . . . . . . . 10,000 "

Total, ..................... 32,250 feet.

This is about the thickness in New Hampshire, and not equal to the estimated thickness in Wisconsin. Enough is known, however, to show this section of Logan's by no means represents the total thickness in Canada. 'The better opinion seems to be that the Laurentian series has a thickness in Canada of more than 40,000 feet, 
and probably more than eight miles, or quite as great as it is in Bohemia or any other European country.

$\S 35$. While these are the oldest rocks known, they were, in their unmetamorphosed condition, ordinary sediment in water derived from materials that pre. ceded them. They were formed by the disintegration, denudation, and redeposition of older rocks, which in their turn preceded others, in how many cycles of change we have no means of knowing. Their upheaval above the surface of the sea was the beginning of the North American continent. The trend of the range in this upheaval is as nearly east and west as the later elevations of the Appalachian and Rocky Mountain chains are north and south. These rocks were until recently supposed to have preceded the existence of both vegetable and animal organisms, and were, therefore, called azoic, but in addition to the fossil Eozoon cauadense there are other evidences of organic life, as follows:

1. The iron ore evidences organic life, because all the accumulations of iron now in progress are formed by the agency of organic matter. The peroxide of iron existing in the rocks is not soluble in water alone, but the addition of decomposing organic matter deoxidizes it, and carbonate of iron is formed, which is soluble and may be precipitated. Peroxide of iron being insoluble, the infiltrating waters which take up soda, lime, and magnesia from sediments, can not remove this metal unless they contain organic matter. The evidence of the reducing and dissolving action of organic matter is, in the great thickness of sediments, almost destitute of iron and in the extensive beds of iron ore.

2. The masses of limestone tend to prove the existence of organic matter, because limestone in process of formation is almost wholly composed of shells, corals, tests of forminifera, and other animal secretions, and nearly all the unmetamorphosed limestones of past ages are largely composed of organic relics.

3. Graphite occurs in beds, imbedded masses, and in scales; in granite, gneiss, mica schist, and crystalline limestones; it results from the alteration by heat of coal in the Coal Measures, and is a common product of furnaces. Its presence is, therefore, an evidence of organic matter, because we know of no other source for its derivation, and are able to trace its origin to vegetable matter in rocks of a less remote date. It is inferred the carbon was collected by marine vegetation at that early period.

4. In the lowest non-metamorphosed rocks, and in the shales and limestones of the Taconic System, several classes of the animal subkingdom are represented, which indicates, if we judge by analogy with subsequent changes and progress of life, that the seas in much earlier times must have teemed with life. This is the only view consistent with the modern theory of evolution and the present state of knowledge concerning the development of animals and vegetables.

5. The Eozoon canadense, a fossil rhizopod, is found in the Grenville band of limestone near the middle of the series. The limestone is thus described: "The general character of the rock connected with the fossil produces the impression that it is a great foraminiferal reef, in which the pyroxene masses represent a more ancient portion, which, having died and become much broken up and worn into cavities and deep recesses, afforded a seat for a new growth of foraminifera, represented by the calcareo-serpentinous part. This in its turn became broken up, leaving, however, in some places, uninjured portions of the organic structure. The main difference 
between this foraminiferal reef and more recent coral reefs seems to be, that while with the latter are usually associated many shells and other organic remains, in the more ancient one the only remains yet found are those of the animal which built the reef.".

6. The relatively large amount of potash in the Laurentian series indicates an abundant marine vegetable life, because later fossil fucoidal layers frequently abound in potash, and living algæ secrete potash from the ocean in such form as to retain it in the sediments now accumulating, and in which they are buried.

7. And, negatively, we have no good reason to think the Laurentian Age was lifeless; beside, the actual elements composing the Laurentian rocks are not different from those in succeeding formations; indeed, oxygen, hydrogen, silicon, aluminum, magnesium, calcium, potassium, sodium, iron, and carbon constitute .99 of all the rocks in the world.

$\S 36$. The change which sedimentary strata of sands and clays, composed of silica, alumina, and potash, underwent to form granite, gneiss, and mica schist; the transformation of sand into quartzite, and all other changes caused by crystallization and new combinations, are supposed to be owing to chemical and molecular forces, acting under the conditions of pressure, heat, and moisture. The pressure of a deep sea would develop a high degree of heat. The mountain ranges have undergone volcanic and earthquake upheavals which may have accompanied the metamorphism as active agencies. It would seem to be a law that mountain upheavals follow great sedimentary deposits, and the chemical action is most powerful under the grandest accumulations; but the idea that such accumulations bend the crust of the earth, or the crust of the earth contracts and wrinkles up mountain chains in the act of cooling, is too chimerical for consideration.

$\S 37$. Sedinentation ceased when the beds were forced above the ocean, but continued elsewhere. When the beds were elevated, the wear and wash from atmospheric and aqueous forces began, and deposits ensued upon the margin of the land and in the depths of the ocean. The denudation of the anticlinal heights has furnished many geological sections, but the older rocks remain hidden from view, and will forever remain unknown. There is absolute nonconformability of the Laurentian rocks with overlying Groups at every locality which has been examined. The Taconic is introduced by total nonconformability, and frequently with a conglomerate containing pebbles derived from the adjacent Laurentian. Here is an unrevealed chapter of geological history, one that has not been reached and read, and never can be unless some region is unearthed where the Taconic rests conformably upon the Laurentian. The Laurentian is the home of granite, marble, gneiss, and other valuable building rocks, and the best mica quarries; but the precious metals have been found only in the intrusive, altered, or sedimentary rocks of later times. 


\section{CHAPTER III.}

\section{TACONIC SYSTEM.}

$\S 38$. In 1842, Ebenezer Emmons, in his Report on the Second Geological District of New York, described the rocks lying on the sides of the Taconic Mountains, parallel with the boundary line between New York and Vermont, under the name of the Taconic System. He found the belt on the western border of the mountains more than fifteen miles wide, and on the easteru side nearly twenty-five miles, making a total width of nearly forty miles. The rocks occur in Westchester, Columbia, Rensselner, and Washington Counties, and stretching the whole length of Vermont, enter Canada, and extend beyond Quebec. He mentioned a typical locality in Berkshire, Massachusetts. The general character of the rocks was given as follows:

1. A coarse, granular limestone of various colors called Stockbridge limestone from the quarries at that place.

2. Granular quartz rock, generally fine-grained, in firm, tough crystalline masses of a brown color, but sometimes white, granular, and friable.

3. Magnesian slate.

4. Sparry limestone.

5. Taconic slate, which is extremely fine-grained and only slightly coherent.

He traced the rocks in a north and south course for 150 or 200 miles, and observed the fact that they underlie the Potsdam sandstone wherever it does not rest upon the gneissoid strata.

§39. In 1844 he published the "Taconic System," reviewed his former work, furnished numerous evidences in support of the existence of these rocks below the Potsdam and above the gneissoid rocks, or what are now known as Laurentian, and ascertained they had a thickness, as shown by a single section, of more than two miles. He said, taking one broad view of the whole system, it might be described as consisting of fine and coarse slates, with subordinate beds of chert, fine and coarse limestones, and gray, brown, and white saudstone; these admitting, however, of further divisions. The leading divisions recognized were:

1. Granular quartz, or brown sandstone, resting unconformably upon the older gneiss. It is the least regular in its continuation of any of the rocks of the Taconic System, and generally appears in insulated mountain masses, as at Oak Hill between Adams and Williamstown, Mass., at Monument Mountain, in the south part of Berkshire, in the east part of Bennington, Vt., and in Dutchess, Putnam, and Westchester Counties, New York.

2. Stockbridge limestone, generally known as Stockbridge marble, and occurring in New York, Vermont, Massachusetts, and Connecticut. Commencing at Sing Sing, it runs a northerly course through Westchester, Dutchess, and Columbia Counties, and extends into Connecticut. It passes up the valley of the Housatonic into the upper valleys of the Hoosic, and onward into Vermont, and is well represented at Williamstown, Massachusetts.

3. Magnesian slate, which composes the highest mountains in the Taconic ranges. The range of mountains composed of this slate, extending along the western 
border of Massachusetts and through Vermont, often rising to the height of fifteen hundred feet, known as the Taconic range, furnished the name to this System. It crosses the Hudson about thirty miles above New York City, and passing south through New Jersey, enters Pennsylvania.

4. Sparry limestone, a name given to it many years before by Prof. Amos Eaton. It occupies a belt of country in the eastern part of Dutchess, Columbia, Rensselaer, and Washington Counties, and passing north strikes the west line of Arlington, Vermont.

5. Taconic slate, with its subordinate beds of roofing-slate and coarse brecciated layers, occupies almost the whole of Columbia, Rensselaer, and Washington Counties, and extends to the base of the Taconic range, which separates New York from Vermont and Massachusetts, and has an immense thickness. It crosses the Hudson above Newburg, and passes through Orange County into New Jersey. From the roofing-slate he defined Diplograptus simplex, and from the Taconic slate in Washington County Bythotrephis flexuosa, B. rigida, Palaochorda marina, Nemapodia tenuissima, Nereites deweyi, $N$. gracilis, $N$. jacksoni, $N$. lanceolatus, $N$. loomisi, $N$. pugnus, Myrianites murchisoni, and $M$. sillimani.

6. Black slate, forming, so far as he knew, the highest member of the Taconic System, and from which he defined Elliptocephala asaphoides and Atops trilineatus.

$\S 40$. He identified the Smithfield limestone in Rhode Island with the Stockbridge limestone, and an accompanying slate with the Magnesian Slate, and in Blackstone Valley found the brown sandstone and fine granular quartz. He recognized in the slates at Waterville, Maine, the Taconic Slate of. New York, and found the Nereites at Kennebec. The fine roofing-slates on the Piscataqua he found subordinate to the Taconic Slate, in like manner as they exist in New York. And, jointly with Douglas Houghton, the Taconic System was found largely developed in the Upper Peninsula of Michigan; the slates of the formation with their fucoidal impressions and the granular quartz were both recognized. In 1846, he reproduced his work on the Taconic System in a book on the Agriculture of New York, with an appendix describing a conglomerate at the base, resting unconformably upon granite rocks.

$\S 41$. In this manner this geological subdivision was first determined, defined, and established, and it should have been recognized from that time forward. But others, much less informed, disputed the existence of the rocks, erroneously referred his fossils to more recent genera; and some, finding the same rocks, gave them differerent names, which added to the confusion, and seriously retarded the progress of knowledge respecting them. It may be later researches have not, in every respect, sustained his determinations, but Ford's work near Albany, New York, where the position taken by Emmons was most violently assaulted, has not only corroborated him, but has forever set the questions at rest in that locality. Wing, Dale, Marcou, and Dwight have sustained his assertions respecting the want of conformability of the Hudson River Slates with the Taconic. All the surveys of Michigan and Wisconsin have sustained him, though the geologists apply the later name, Huronian, to the Strata. His determinations of the rocks in North Carolina have been most fully confirmed by later geologists, though some use the word Huronian when referring to them.

$\S 42$. In 1849, Alexander Murray, an assistant on the Geological Survey of 
Canada, in the Report of Progress for the year 1847, described the rocks on the north side of Lake Huron, and constituting many of the adjacent islauds, under the name of "quartz rocks and sandstones, conglomerates, slates, and limestones," and correctly identified them as resting unconformably upon the older granite and syenitic gneiss, and succeeded unconformably by the Potsdam, but he did not call them by any geological name. If he had read Emmons's "Taconic System," it is difficult to conceive why he should have hesitated in referring the rocks to that System. In the Report of Progress of 1856, he redescribed the rocks, under the name of the "Huronian Series," which was adopted by the officers of the Canadian Survey, without once mentioning the Taconic System. From that time forward authors have generally used the name Huronian, and have almost annihilated the name Taconic. The word Taconic, however, has priority over Huronian. It is equally appropriate, and the definition of the fossils in the Upper Slates at once furnished the means of tracing it and determining it at different and distant places. The word "Huronian" is, therefore, a synonym for Taconic, and comprehended, as used originally by the Canadian Geologists, substantially the same series of rocks, though not ascending quite so high.

$\S 43$. A section of the so-called Huronian, but more properly called the Lower Taconic, between Missisquoi and St. Mary's Rivers, in ascending order, is as follows:

1. Gray quartzite, ............ 500 feet.

2. Greenish, red-weathering chloritic and epidotic slates, . . . 2,000

3. White quartzite, etc., .............. 1,000

4. Slate conglomerate, ................. 1,280

5. Limestone, . . . . . . . . . . . . . . . . 300

6. Slate conglomerate, etc., . . . . . . . . . . 3,000 “

7. Red quartzite, etc., . . . . . . . . . . . 2,300

8. Red jasper conglomerates, etc., . . . . . . . . 2,150

9. White quartzite, etc., . . . . . . . . . . 2,970

10. Yellowish chert, etc., . . . . . . . . . . . 400 "

11. White quartzite, etc., ............. . . . 1,500 "

12. Yellowish chert, etc., . . . . . . . . . 200 "

13. White quartzite, $\therefore \therefore . \therefore . \therefore . \therefore . \therefore 400$

Total,

18,000

Another section adds to this one 4,000 feet, and even then the maximum thickness of the series in that locality has not been reached.

$\S 44$. Throughout the Huronian region, the whole series bears evidence of great disturbance, and is frequently cut with intrusive masses of greenstone, granite, or other igneous rocks. The more recent disturbances frequently bear metalliferous veins, which give to the country its value as a mineral region. Copper and iron are the chief minerals, and abound in nearly every section. Gold and silver sometimes occur. The Taconic of Michigan contains vast beds of iron ore. The ores are magnetic, red specular hematite and soft hematite resembling the brown hematite of other States. The magnetic and specular ores are the most prized, and usually contain from 60 to 70 per cent of iron, and hardly a trace of phosphorus or sulphur. (Phosphorus makes iron brittle when it is cold, and is therefore called cold-short, though it is malleable when hot, while sulphur makes it brittle when it is hot, and it is therefore called red-short.) The Lake Superior region is the chief locality of the world for native copper. It is so pure the aborigines manufactured it into implements. The copper-bearing rocks extend eastward 
along the south shore of the lake for more than forty miles, then forming a narrow belt stretch in a north-east direction for about a hundred miles to the extremity of Keweenaw Point. The copper occurs in a rock called melaphyre, associated with beds of conglomerate, and appears to be interstratified with them. Sometimes bands of slate separate beds of melaphyre. The native copper exists in sheets, strings, and masses, and is sometimes associated with silver. In Ashland County, Wisconsin, the copper-bearing series has a thickness of more than four miles, though not very rich in the ore. The Taconic area in Minnesota is large. It extends across the northern border, and forming an elbow in the north-east extends diagonally through the State to the south-west corner. Here there is a hard, reddish, metamorphic sandstone, called the Sioux quartzite, interstratified with which is a layer of red indurated clay or pipestone, one foot thick, called Catlinite, largely used for the manufacture of pipes. The quarry is thirty miles north of the south-west corner of the State, and four miles east of the west line. The Sioux quartzite occurs in the north-west corner of Iowa.

$\S 45$. The geographical extent of these rocks in Canada is very great. They may be traced from near Lake Temiscaming 80 miles north-west of Lake Nipissing, south-westward to Lake Huron, and from thence westward on the north shore of the lake and the north shore of Lake Superior, and on beyond Lake of the Woods, a distance in all of about 800 miles. They pass beneath the lakes and expose a large area in the Upper Peninsula of Michigan at Marquette and Menominee, and a great thickness, extending from the lowest to the highest Taconic, as first ascertained by Houghton; thence they pass into Wisconsin, exposing a large area and quite as complete a representation of the series, while another arm extends from Duluth into Minnesota. The thickness in Michigan is about four miles; but in Wisconsin, including the copper-bearing series, which is three-fourths of igneous material, the thickness is much greater; and even excluding the igneous material the thickness exceeds four miles. The upper part of the Taconic System in Wisconsin, formerly called the "Copper-bearing series," has received the unattractive name of the Keweenawan formation, from the Keweenaw Point; but as it is part of the Taconic System the preferable name is the older one of the "Copper-bearing series." The rocks appear between Scoresby Bay and Cape Cresswell, in Lat. $82^{\circ} 40^{\prime} \mathrm{N}$., where Nares and Feilden called them Cape Rawson beds.

§ 46. In 1856 Emmons divided the System into Upper and Lower Taconic. The Canadian Geologists in 1863 placed his Upper Taconic in the Silurian System and called it "Lower Potsdam," which name therefore became a synonym. The only geographical names which have been used to subdivide the Upper Taconic into Groups, which seem in the present state of learning to be worthy of retention, are, in descending order, the Swanton Group, the Georgia Group, and the St. John Group-if in fact the latter is below the Georgia, and therefore not a synonym. Emmons placed the Stockbridge limestone in the Lower Taconic; but it would seem from the examinations made by others, that his division would have been more clearly marked if the Stockbridge limestone had been retained in the Upper Taconic. The Paradoxides beds at Braintree, Mass., in Newfoundland and New Brunswick, and wherever found on the continent, belong to the Upper Taconic. The same difficulty exists in the West, in separating the Upper Taconic from the overlying rocks of the Potsdam, that has led to so much discussion in the East; and the confusion is 
increased by the addition of numerous symonyms-the ready weapon to which ignorance resorts.

$\S 47$. In 1863 G. F. Matthew named the rocks exposed at St. John, New Brunswick, the "St. John Group." He described them as arenaceous, argillaceous, and carbonaceous shales, and clay slates; often sandy, with sandstone and quartzite, having a thickness of 4,500 feet, and having an exposure about 30 miles long and 4 miles wide. He collected Paradoxides, Conocoryphe, Obolella, Orthis, Orthisina, Stenotheca, Hyolithes, and Lingula. In 1865 he and Bailey and Hartt correlated these rocks with the slates of Vermont having Elliptocephala asaphoides, and the schistose beds at Braintree, Mass., holding Paradoxides harlani, and thus proved their "St. John Group" to be a synonym for Emmons's "Black Slate," in the Upper Taconic System. Furthermore, they identified the slates with some found in Newfoundland containing Paradoxides and Conocoryphe. Later they divided the Lower Taconic of New Brunswick, which they called Huroniau, into the "Coldbrook Group," the "Coastal Group," and the "Kingston Group," and estimated the thickness as exceeding 10,000 feet.

$\S$ 48. The Vermont Geologists iu 1861 called the Black Slate, Taconic Slate, and Roofing-slate of Emmons the "Georgia Group." The name has priority over the "St. John Group," and if the Taconic System is to be divided into Groups with geographical names, and these three divisions of Emmons are to be thrown together in one Group, then ihey must under the laws of nomenclature bear the name of the Georgia Group. The Black Slate has, however, been called the Swanton Group, and if this name should become desiruble then the Upper Taconic would be divided into the Swanton Group and the Georgia Group, and their maximum thickness in Vermont exceeds two miles. This division is that adopted by Perry, who has shown the Potsdam sandstone rests directly upon the Swanton Group, or Black Slate, as originally asserted by Emmons, and that both the Swanton Group and the Georgia Group are fossiliferous.

$\$ 49$. The Taconic rocks extend from Canada East and Maine to Gcorgia and Alabama, flanking almost continuously the ranges of mountaius upon both the eastern and western slopes. Their thickness in New Hampshire is over four miles, and in Vermont the maximum must exceed five miles. The slate belts of York and Lancaster Counties, $\mathrm{Pr}$., and the rocks containing the valuable ores of nickel and copper belong to this System. There are five extensive ontcrops in North Carolina, and three or four subordinate ones. They rest unconformably upon the belts of the exposed Laurentian, and very much resemble in their character the subdivisions in Vermont and New York. The largest outerop is from twenty to forty miles wide, and extends quite across the State. The maximum thickness exceeds five miles. There are large outcrops in Virginia, South Carolina, Georgia, and Tennessee, and limited outcrops in Alabama. Gold, silver, copper, lead, iron, and other valuable minerals, occur in these rocks not only in veins, fissures, and dykes, but in seams following the stratification and as part of the sedimentary materials. In Northern Georgia gold exists in seams, with milky quartz, following the stratification of hornblende schists, and constituting as truly sedimentary rocks as the schists themselves do. The seams are stratified within the slaty sediments, and are of the same age as the Taconic System. These seams are so constant they characterize the slates or schists in the Appalachian System. They are metalliferous, 
and frequently auriferous, or cupriferous. 'The magnetic and specular iron ores also occur with the material of the slates as a deposit of the same age, and constituting part of the same system. This mineral wealth is so distributed it is practically inexhaustible. The Taconic appears in Missouri, Arkansas, and Texas. The iron ore district about Iron Mountain and Pilot Knob containing porphyry rocks is of this age, but the granite to the east is Laurentian. The ore is found in very thick veins in Iron and Shepard Mountain, and Pilot Knob. It is specular ore, containing between sixty and seventy-five per cent of iron, free from sulphur and bearing no more than a mere trace of phosphorus. The rocks appear in numerous places in the Rocky Mountain ranges from Mexico to British Columbia, often exposing great geographical areas and an immense thickness, and they are usually metalliferous.

$\$ 50$. The genera, regarded as typical of the Taconic fauna, and which do not pass up into Silurian rocks, are Paradoxides, Microdiscus, Atops, Elliptocephala, Conocoryphe, Anopolenus, Bathynotus, Solenopleura, Acrothele, Salterella, Scenella, Iphidea, Hyolithellus, Archococyathus, and Ethmophyllum. There are some others peculiar to these rocks, but they are either obscure or limited in their distribution. Some genera closed their existence in Silurian time, others reached the Devonian age, and some from this remote period, as Orthis, Orthisina, Orthoceras, and Leperditia, continued to live to the Carboniferous, though Orthoceras reached its most remarkable development in the Black River Group, and Orthis in the Hudson River. Not a single species belonging to the Upper Taconic system crossed over the line that separates it from the Potsdam Group of the Lower Silurian, so far as any reliable determination has thus far been made. This, supported as it is by a want of conformability, indicates a vast lapse of time between the deposit of the Upper Taconic and the commencement of the Potsdam period. The Taconic is composed in part of the disintegrated materials of prior Laurentian rocks, while the Potsdam represents the washings of the Laurentian and Taconic. The order Graptolida appeared in this system, and reached its maximum development of genera, species, and numbers (if the Point Levis beds referred by the Canadian Geologists to the Quebec Group belong to the Upper Taconic, as claimed by Marcou and others, and as the author believes), and became extinct in the Upper Silurian System. This is the first order of animal life to reach the highest stage of its existence, and the first to become extinct. It is referred to the class Hydrozoa, but if more was known of it, very likely it would form a distinct class.

$\S 51$. The Cupriferous series of the lake region, called also the Keweenaw, Keweenian, Keweenawan, and Nipigon series, is supposed to underlie nearly the whole basin of Lake Superior, or an area of about 28,000 square miles, and a surface area upon the borders of the lakes and their immediate vicinity of about 18,000 square miles. This series has been divided into an upper and lower division, with an estimated maximum thickness of 15,000 feet for the upper division, and 35,000 feet for the lower, which rests upon the slates and quartzites of the Taconic System, the last having a variable thickness that reaches a maximum of at least 22,000 feet. The Cupriferous series consists of eruptive flows and detrital rocks, with massive dykes. The region was, in Taconic days, represented by a volcano, which has sunk beneath the waters of the lake. The flows were followed by detrital rocks, representing the intervals of time between them; but these detrital rocks are com. 
posed largely of conglomerate layers and large-sized pebbles, indicating strong currents of water. The flows visible upon the borders of the lakes were forced through fissures by volcanic energies. The copper which occurs in the conglomerates, amygdaloids, epidote veins, and otherwise, is supposed to have been precipitated from water holding it in solution, or leached from detrital rocks where it was originally deposited in a sulphureted form. R. D. Irving, who has studied closely the copper-bearing rocks of this region, says the explorer for transverse veins should bear in mind that epidote, prehnite, and chlorite are favorite associates of copper, while laumonitic veins, and those bearing a predominating quantity of calcite, are not so rich; that a wide vein in amygdaloidal or other soft rock will pinch to a mere seam within the massive and compact layers; and in sandstone and conglomerate deposits the valuable belts have been found where the conglomerate is overlaid with trap, or in sandstone very rich in basic detritus. Any of the conglomerate seams from Keweenaw Point to Minnesota may be cupriferous. All of the upper division of the series is noncupriferous, except the Nonesuch sandstone belt in the Porcupine Mountains; and all the belts and areas of acid rocks, such as the central area of the Porcupine Mountains, and the great spread of red rock in the Brule Lake country in Minnesota, and all belts and areas of coarse-grained basic rocks, such as the great area of coarse gabbro in the Bad River region in Wisconsin, and the similar area which occupies the belt of country from Duluth to Brulé Lake, are also noncupriferous. The slates and quartzites of the Taconic System which lie below the Cupriferous series on the north shore of Lake Superior, have been called the Animikie Group. About three-fourths of the great thickuess of the rocks is referred to volcanic overflows, and does not, therefore, belong to the geological column, the whole of which is the result of sedimentary deposition.

\section{CHAPTER IV.}

\section{SILURIAN SYSTEM.}

$\S 52$. IN 1833, Sir R. I. Murchison, in a memoir read before the Geological Society of London, divided the fossiliferous rocks below the Devonian into six Groups. He founded this subdivision upon the fossils, and mentioned such species as were then defined. This was followed in the succeeding year by other memoirs, and in 1835 he concluded all these Groups might be placed in one System, and in honor of the ancient tribe of Silures, who inhabited Wales, he named it the Silurian. He placed three Groups in the Upper Silurian, and three in the Lower Silurian. Before this time no knowledge of the order of the strata had been ascertained, and hence he is entitled to the credit of the name. Subsequently palæontologists found his discoveries were world-wide in their application, and it was not long until the distinction between the Upper and the Lower Silurian had been observed in North America, and the base of the Lower Silurian had been recognized in the Potsdam sandstone, and the base of the Upper Silurian in the Medina sandstone.

$\S 53$. Some years later, the word Cambrian was applied in England to the rocks which belong to the Lower Silurian, and to inferior strata which are the equiva- 
lent in part of the Taconic System. It was not used in the sense of a "Group," but in the higher sense of a "System," as these words were then understood. It was never well defined, and it crossed one of the grandest and most important breaks in geological time- that which separates the Taconic and Silurian Systems. No careful geologist or palæontologist uses the word in the nomenclature of American strata, though it occasionally occurs in incoherent geological papers, and sometimes we see such monsters in nomenclature as Cambro-Silurian and Siluro- Cambrian.

\$54. The Lower Silurian in North America is divided, in ascending order, into the following Groups: viz., Potsdam, Calciferous, Quebec, Chazy, Black River, Trenton, Utica Slate, and Hudson River. The Upper Silurian is divided, in ascending order, into the Medina, Clinton, Niagara, Onondaga, Guelph, and Lower Helderberg.

\section{POTSDAM GROUP.}

$\S 55$. Prof. Ebenezer Emmons, in the Annual Report of the Geological Survey of New York for 1838, described the sandstone at Potsdam in St. Lawrence County, and proposed for it the designation "Potsdam Sandstone." It was subsequently described quite fully in the New York Reports, and finally the Canadian Geologists in 1863 called the rocks the Potsdam Group. The lowest portion at Potsdam is a granitic conglomerate, in which large masses of quartz, the size of a peck measure, are sometimes inclosed. These were water-worn and rounded before being enveloped in the deposit. The sandstone is quite variable in texture and color, but its composition is uniformly silicious. At some places it is an even-grained mass in compact layers, and at others it is traversed by joints. In some localities a dark, slaty sandstone, about ten feet in thickness, intervenes between the Potsdam and Calciferous, at others a coarse brecciated rock, and at others the passage is very gradual into the Calciferous sand-rock. The thickness in New York is from 100 to 200 feet. The exposure is narrow, but extends from near the Thousand Islands to Lake Champlain, and enters Vermont with a thickness of about fifty feet.

$\S 56$. It extends from New York into Canada, where it attains a thickness ranging from 300 to 700 feet, and at the summit the sandstone is interstratified with magnesian limestone that constitutes a passage to the Calciferous. There is more diversity in the rocks in Canada than in New York, and limestones and slate sometimes occur with the sandstone. It rests unconformably upon the Laurentian, and fills up inequalities where the Taconic System does not intervene, and it also rests unconformably upon the Taconic when it is present. The sandstone appears to have been deposited in shallow water along the margin of a sea. The tracks and wind marks support that view. In its extension westerly, by the way of Lake Huron and Lake Superior, across Wisconsin and into Minnesota, the same variations in thickness occur. Sometimes it attains a thickness of 3,000 feet, and again thins out to 40 or 50 feet. For several miles in distance near Beauharnois, Canada, the strata are marked by the tracks of Protichnites. The surfaces on which the tracks are inpressed are sometimes smooth, and sometimes beautifully ripple-marked. On the latter the tracks have often beaten down the ripplemarks, and the sand of the ridge has been dragged into the furrow, in such a way as to show the direction in which the animal was progressing. Fucoids are abundant in the upper part of the Group, and Scolithus so common as to be quite 
characteristic, and near St. Genevieve the rock is completely honey-combed with it to the depth of three feet.

$\$ 57$. It is largely distributed in Northern Michigan, and striking into Wisconsin north of Green Bay gradually widens southerly as a surface rock, until it reaches the central part of the State, where it has a width of 100 miles. It then curves northwardly and enters Minnesota, forming the high hills on the Mississippi River. It is unconformable with the rocks below, and rests upon an exceedingly irregular surface, sometimes filling depressions in the quartzite or metamorphic rocks of several hundred feet. Its upper surface is uniform, and graduates in to the Calciferous Group or the Lower Magnesian limestone, as the rocks in these States are called. The exposed area in Wisconsin is about 12,000 square miles, the thickness very irregular by reason of the great depressions and elevations at the base, and the maximum thickness is fully 1,000 feet. The rock is chiefly composed of cemented grains of silicious sand, but presents several varieties, as the calcareous, argillaceous, ferruginous, and green sand, and the waters issuing from it in places contain a small percentage of lime salts. In the argillaceous class the clayey material becomes so abundant as to render the rock shaly, and so impervious to water that valuable springs occur at its upper exposed surface. In the calcareous class the lime becomes so great in some layers that they are more properly limestones than sandstones, and so associated with magnesia that they become arenaceous dolomites. In the ferruginous class, at one extreme, the amount of iron oxide is barely sufficient to color or cement the mass, and at the other so great as to make an iron ore. In the green sand there are two classes, one in which the grains are colored by iron, and the other consisting of deep green grains of glauconite. The green sand is not restricted to the Potsdam in Wiscousin, for it also occurs in the Calciferous and St. Peter's Sandstone. It is almost identical with the Cretaceous green sand of New Jersey, and similar deposits in existing seas. The surface area in Michigan, Wisconsin, Iowa, and Miunesota is estimated at 25,000 square miles, which is about half the surface area on the continent; but it is generally believed to exist under many of the more recent deposits, and, therefore, to cover several hundred thousand square miles. Springs and streams of soft water are abundant where it forms the surface rock, and a good supply of soft water has been found wherever it has been penetrated with the drill ; its existence, therefore, becomes a question of much economical interest where a supply of good water is desired from artesian boring. The drill has never reached it in Ohio, though a supply of good water is imperatively demanded in some parts of the State; and it is to be hoped an effort will be made to determine whether it exists below the Calciferous, which has been reached with the drill many times.

$\$ 58$. It is exposed at numerous places in the Appalachian System from New York to Tennessee. In New Jersey it reaches a thickness of 3,000 feet, and if both the Chilhowee sandstone and Knox Group in Tennessee belong to it, it has a thickness of 9,000 feet, but probably 5,000 feet of this belongs to the Taconic. In the southern and south-eastern counties of Missouri it has a thickness of 700 feet. It appears in several counties in Northern Texas, along the margins of the Big Horn, Laramie, and Wind River ranges, at the Black Hills, and in other regions of the Rocky Mountain System from Mexico to British America. The erosion by water and weathering has left picturesque scenery in the sandstone at many places. The 
"Pictured Rocks" of Lake Superior, the "Dalles" of the Wisconsin, and the "Chasm of the Au Sable" in New York are examples.

§5. Everywhere it is essentially an accumulation of sandstone and pebbles from the adjacent Laurentian gneisses, granites and syenites, and Taconic quartzites and schists, resulting from the disintegrating influences of air and water. It contains ripple-marks, wave-lines, mud cracks, animal tracks, and worm burrows, which evidence shallow seas and shore lines. The continent at the time of its deposit did not have one twentieth its present area. There is nothing known to iudicate the climate was different then from what it is now, except so far as the relative difference of land and water surface would necessarily change it. Some species of fossils prevailed over great areas, as Hyolithes primordialis, Lingulepis pinniformis, L. prima, Dicellocephalus minnesotensis, $D$. osceola, and Ptychaspis minuta, and therefore become somewhat characteristic of the Group. Though composed almost wholly of sand it was slowly deposited. The sandstone is frequently charged with fossils to its full capacity, indicating a formation almost as slow as marine limestone is now made. There is no doubt that Calcareous mud was forming in the depths of the ocean at the same time the sand was deposited nearer the shore, but no limestone group of the Potsdam age has been found, unless it exists in the Eureka district of Nevada.

\section{CHAPTER V. \\ CALCIFEROUS GROUP.}

$\S 60$. THis name was first applied by Prof. Eaton to a gray rock consisting of lime and fine grains of sand, so intimately blended as to appear homogeneous. It contains calcite and a sparkling surface, but passes into a carbonate of lime, containing beds of magnesian limestone and a small amount of iron. The Group was defined by Vanuxem in 1842, in the Geology of the Third District of New York. He united the silicious layers above the Potsdam, the calciferous sand-rock, and the fucoidal layers in one Group. The rocks consist in general of three varieties-silicious, magnesian, and carbonate of lime, with intermediate grades of composition. They pass from compact to granular, and granular to porous, the latter having cavities lined with crystals of quartz, calcareous spar; or, instead of being lined, pussessed of a single beautiful perfect crystal of limpid quartz, nearly filling the space. Middleville and Little Falls are noted localities for these crystals, some of which contain a fluid or anthracite, which enhances their value as cabinet specimens. The structure of the rock is often oolitic, passing into thick layers having a concretionary structure, as in agate. The typical localities are in Montgomery and Herkimer Counties.

At Chazy the following ascending section occurs:

1. Silico-calcareous beds, more or less interspersed with sparry masses, 30 to 35 feet; fossils rare and cherty.

2. Limestone, in which the plates of Cystideans abound, 20 feet.

3. Dull, gray, earthy mass, without fossils, and passing into oolitic beds, 10 feet.

4. Cystidean limestone, similar to the first though of a brighter red color, 15 feet. 
5. Massive earthy and silicious limestone containing trilobites, 20 feet, followed by beds of similar character of greater thickness containing brachiopods.

6. Red Cystidean limestone, susceptible of a fine polish, 15 feet.

7. Drab-colored, thin-bedded, earthy magnesian beds, suitable for hydraulic lime, of considerable thickness; fossils rare, except fucoids. Toward the top of the rock it is blue and frequently cherty, oolitic, and concretionary, the upper masses from 20 to 30 feet thick.

$\S 61$. The Group is persistent, and surrounds the irregular dome of Laurentian rocks, which form the northeru highlands of New York, in a belt, overlying the Potsdam. It is chiefly a hard calcareous sandstone or arenaceous limestone, resting upon the margin of the Potsdam sandstone, from Lake Ontario eastwardly to Vermont, and from New Jersey north, near the line of New York and Vermont, into Canada. It forms a narrow belt of surface exposure, with a variable thickness from 50 to 350 feet. Lake Champlain has cut a channel through it for twenty miles. In Canada, adjacent to New York and Vermont, it is, in the lower part, a dark, bluish-gray, crystalline, strougly coherent dolomite or magnesian limestone, and in the upper part a bluish-gray, calcareous argillite, but its characters are different in different localities. It is usually found as a narrow belt following the sinuosities of the Potsdam Sandstone, from west of Lake of the Woods to the Atlantic sea-board; but where the rocks bave been disturbed by volcanic energies it may be absent or difficult of detection. The surface area of its distribution in Canada is several thousand square miles, and in its undisturbed condition the maximum thickness rarely exceeds 450 feet, though in Newfoundland, where it is a definitely stratified limestone, it has a thickness of more than 2,000 feet. In the region of the Mingan Islands, in the Gulf of St. Lawrence, the fossil casts and shells are in a good state of preservation.

$\S 62$. In Northern Michigan and on the Menominee and Escanaba it preserves its New York characters in a remarkable degree, although its thickness may not exceed 50 feet. The upper portions are highly calcareous, and on fresh fracture show the peculiar granular structure so characteristic in New York. It is thinbedded, and contains small cavities lined with crystals of calc-spar, quartz, or hornblende. The surfaces of the layers are often covered with fucoidal impressions. From St. Mary's River westerly to the Wisconsin and the Mississippi there is a gradual augmentation in the thickness of the rocks and a material change in their composition. The Group enters Wisconsin from Michigan a few miles from Green Bay, and striking south-west upon the border of the Potsdam it forms a serrated margin from five to fifteen miles in width, until it reaches the streams that flow in to the Mississippi in the south-western part of the State, where it is exposed upon some of the streams for a distance of 75 or 100 miles. It crosses the Mississippi and the north-eastern corner of Iowa, appearing in the bluffs and hills more conspicuous than the Potsdam, though not so thick, because it is a much firmer rock. It is a buff-colored dolomite, without uniformity of texture or stratification, and weathers into rough, bold, and often picturesque fronts along the valleys, and has a thickness of about 250 feet.

$\S 63$. From Iowa the area of exposure is a little west of north through Minnesota, reaching as far west as the second tier of counties from the Mississippi and following the bluffs with limited outcrops in Wisconsin to Lake Pepin, north of 
which and east of the St. Croix it forms the surface of nearly two large counties of Wisconsin. It follows the Mississippi north of Minneapolis for several miles before it is covered with later formations. The conspicuous perpendicular walls of rock, cropping out from the hills and bluffs along the Mississippi from the St. Croix to the mouth of the Wisconsin, belong to this Group. Throughout the exposures in Wisconsin, Iowa, and Minnesota, it is conformable with the underlying Potsdam, and unconformable with the overlying rocks. The lower surface is plane, while the upper surface is undulating, and in some instances the undulations are said to swell in short distances into elliptical domes, rising 100 feet above their bases, like billows on the sea. These undulations are the work of denudation during the interval that elapsed before the deposition of superimposed strata. The Group in Wisconsin is frequently called the Lower Magnesian limestone, and some one in Minnesota has called it the Shakopee Group, because the stone has been quarried at a village bearing that Indian name. The Magnesian limestone is usually sufficiently pure to burn to a serviceable quicklime. The chief impurities are quartz, clay, iron, and green sand. The dolomite occurs in the earthy, granular, crystalline, and crypto-crystalline forms, and chert is irregularly distributed. Argillaceous material is not abundant, except in shaly bands, where it may constitute 20 per cent of the whole; and the amount of silica disseminated through the rock varies from 1 to 10 per cent. The difference in the composition and hardness of the layers causes the surface rocks to present great irregularities, which are much enhanced and exaggerated by weathering, and hence outliers have a rough and often grotesque exterior.

$\$ 64$. The Group is displayed in grand proportions in the southern counties of Missouri, where it consists of an upper and lower division of magnesian limestone with an intermediate division of sandstone. These received the names, in descending order, of the "Second Magnesian limestone," the "Second Sandstone," and the "Third Magnesian limestone." The upper division is generally composed of beds of earthy magnesian limestone, interstratified with shale-beds and layers of white chert, with occasionally thin beds of white sandstone, and near the lower part thick, cellular, silico-magnesian limestone-beds. It constitutes many of the bluffs of the Osage and its tributaries, and also of the Missouri from Osage to Jefferson City. It is often a lead-bearing rock, as in Cole County. The thickness rarely exceeds 200 feet, though on the Meramec it is 300 feet. The middle division is usually a brownish sandstone, stratified in firm, regular beds from 2 inches to 3 feet in thickness, though sometimes friable. The surfaces are often ripple-marked. The thickness rarely exceeds 150 feet. The upper part often occurs in thin strata with beds of intercalated chert abounding in fossils. The third division is generally a thickbedded, coarsely crystalline, bluish-gray magnesian limestone, with occasional thick chert-beds. It is the chief lead-bearing rock of South-east and Southern Missouri, and is frequently exposed along the streams in bold escarpments from 200 to 300 feet high. The ores of lead, zinc, copper, nickel, and cobalt, occur in fissures and caves, or disseminated in small masses in the limestone itself. The lead occurs sometimes in masses of galena accompanied with copper pyrites disseminated through layers of limestone, while the ores of nickel and cobalt occur in clay slate. At other places bands of red clay inclose calamine (silicate of zinc), galena, and heavy spar (sulphate of baryta). The maximum thickness is about 600 feet, though it seldom exceeds 300 feet. The maximum thickness of the three divisions is more 
than 1,000 feet, but the Group at no single locality displays so great a thickness. From Missouri the Group extends southerly across Arkansas into San Saba, Llano, McCulloch, Menard, Mason, and Lampasas Counties, in Texas, where the maximum thickness is more than 400 feet. It is exposed in narrow belts in the Appalachian chain from New York and New Jersey to Tennessee and Georgia, but has not been very clearly distinguished in the mountain regions of the West.

$\S 65$. It is said this Group in some localities graduates into the Quebec; but on the other hand it is claimed the Quebec belongs to the Taconic System, and is below the Potsdam. It is certain many of the rocks referred to the Quebec Group belong to the Taconic, and some of them may belong to the Calciferous or the Chazy, or may form passage beds from one to the other. The oldest known Lamellibranchiata are found in this Group. Among the fossils having the greatest distribution, and which are most characteristic, we may mention Ophileta complanata, O. uniangularis, Holopca turgida, H. dilicula, and Orthoceras primigenium. Pleurotomaria canadensis and Leptena barabuensis occur in this Group and in the Potsdam. Pleurotomaria calcifera, P. postumia, Holopea dilicula, Helicotoma perstriata, Machurea matutina, M. sordida, Eccyliomphalus canadensis, Camarella calcifera, Lingulella mantelli, L. irene, Amphion salteri, Buthyurus cordai, B. conicus, and Asaphus canalis have been described from this Group and from the Quebec. These identifications may well be doubted, uuless the rocks containing all these species really belong to the Calciferous.

\section{CHAPTER VI.}

\section{QUEBEO GROUP.}

$\S 66$. The Quebec Group was first characterized and its position between the Calciferous aud Chazy asserted, upon palæontolngical evidence, in 1862, by Prof. Billings. His position was supported by the Cauadian Geology in 1863, and in Decade 2 of a later date. The limits of the Group are still a subject of discussion, and part of the rocks originally referred to it belong to the Upper Taconic; but another part of them may form passage beds from the Calciferous to the Chazy, and occupy a position which warrants the name of an iudependent Group. The name was derived from the city of Quebec, where it was subdivided into the Levis, Lauzon, and Sillery divisions. The Levis was named from Point Levis, where it is fossiliferous, and has a thickness of 6,145 feet; the Lauzon from Lauzon, where its thickness is 4,000 feet, and it is non-fossiliferous; and the Sillery from Sillery Cove, where it is 2,000 feet thick and almost barren of fossils. The Lauzon division is below the Levis, and, from fragments of fossils found in pebbles, it has been since ascertained that it belongs to the Upper Taconic. A great fault at the Island of New Orleans and auother near the Falls of Montmorency, with lesser faults, are said to account for the erroneous reference of this division to the Quebec. It was supposed at one time that the Sillery and Lauzon were upper members of the Quebec Group; but both of them belong to the Upper Taconic.

$\S 67$. The Group has been traced from Vermont to Newfoundland, a distance of 1,000 miles, and the Levis division noted at several localities, where it consists of a variety of shales, with some sandstones and conglomerates, distinguished by 
the general black or dark color. In Newfoundland the Levis division consists of graptolitic shales, having a thickness of 4,000 feet, which are followed by about 1,000 feet of serpentines and diorites referred to the Lauzon division; and these by black slates and limestones, having a thickness of 4,000 feet, referred to the Sillery division. Serpentines, diorites, and slates sound like Upper Taconic, and it may be undiscovered faults have given rise to an erroneous determination of the order, and therefore the so-called Lauzon and Sillery may be below the Levis; or it may be an erroneous identification of the Lauzon and Sillery; and yet the true solution may be found in the fact that all three divisions belong to the Upper Taconic, for the trilobites described by Billings, from these rocks in Newfoundland, have a primordial or Taconic aspect. The author has never had an opportunity to examine the rocks of the Quebec Group, but an examination of the present state of the learning respecting it, makes it very doubtful whether or not the name should be retained. If the Group belongs to the Taconic System, as most of it undoubtedly does, possibly the name should be retained. If that part of it in the East from which Calciferous fossils have been obtained, constitutes all of it except that which belongs to the Taconic, then probably the name should be stricken from Lower Silurian nomenclature, and the part containing such fossils should be included in the Calciferous Group, in which event the Chazy Group would include some of the rocks referred to the Quebec in the Western mountains, and the rest would belong to the Upper Taconic.

$\S 68$. The Quebec Group has been recognized in the Wahsatch Range, in Utah, at Pogonip Mountain, Nevada, and other places in the Western mountain chains, where the Calciferous and Chazy have not been distinguished from it. In the Pogonip mountain-beds the following species are said to pass from clearly distinguished beds of the Potsdam Group up three or four thousand feet into as certainly determined beds of the Quebec Group, viz.: Iingulepis maera, L. minuta, L. manticula, Acrotreta gemma, Agnostus communis, A. bidens, A. neon, Crepicephalus haguei, and C. unisulcatus.

$\S 69$. In this Group we find the first illustration of an important branch of the animal kingdom reaching its highest stage of development, and subsequently declining, and finally becoming extinct. The first known Graptolites appear in slates of the Upper Taconic System, and reach the climax of evolution in the Quebec Group, and become extinct in the Upper Silurian era. The development of these forms seems to have been wonderful. About thirty genera have been distinguished in America, and to these have been referred about 170 species. The Group is said to be connected specifically with higher Groups by Maclurea atlantica and Asaphus canalis, that occur in the Chazy, and by Leptoena sericea, which is common to all the Groups in some of its varietal forms as high as the Clinton.

$\S 70$. This Group is said to graduate up into the Chazy without lithological lines of separation, and without an abrupt break in the chain of fossils. Clear passage-beds occur where the Groups are well developed, and even where there is nonconformability some fossil species are said to be common to the two Groups. The geographical surface distribution is confined to limited areas east of the Appalachian System, and to small exposures among the Western chains; but it must represent a vast period of time, as evidenced by the great development and evolution of its animals, and by the erosion of the Calciferous where it does not exist. 
$\S 71$. Bitumen, or mineral pitch, is a product resulting from the distillation of vegetable and animal matter within the earth. It has a pitch-like odor, and burns with a bright flame without any ash, and varies from. liquid naphtha to solid as phaltum. Naphtha is a nearly colorless fluid, having a pungent smell, that issues from the rocks in Persia. Its specific gravity is about $7-10$, and by exposurc it loses its transparency and odor, and acquires a yellowish or brown color, becomes thicker and heavier, and approaches petroleum. Petroleum is so called from exuding as an oil from the rocks. Its specific gravity is $87-100$, and by exposure to the air and the application of heat it may be converted into asphaltum. Asphaltum was so named from a lake iu Judea, where it rises in a liquid form to the surface of the water and then hardens. Its specific gravity varies from 1.07 to 1.65. It is quite brittle and electric, though coal is not. Bituminous matter occurs in the limestones and dolomites of the Quebec Group, and the odor may be detected in many places by striking or heating the rocks. A black, combustible, coal-like matter is found with crystals of bitter spar and quartz, sometimes coating the crystals or the walls of cavities, and at other times in the form of buttons or drops, evidently having been introduced in a liquid state and subsequently hardened. It fills veins and fissures in limestones, shales, and sandstones, and even in the trap-rocks which traverse these. It is very pulverulent, brittle, of a shining black color, and yields from ten to twenty per cent of volatile matter. It approaches anthracite in its characters. The volatile matter is a hydrocarbon gas. It has resulted from the slow alteration of liquid bitumen in the fissures of the strata. The bitumen was derived from marine vegetation or marine animals, which underwent a special mineralization, producing the bituminous matter instead of coal. It is due to chemical reactions, by which it retained a greater proportion of hydrogen in its combination than would have been retained if it had been converted into coal.

\section{CHAPTER VII. \\ CHAZY GROUP.}

$\S 72$. The Chazy Group was first defined in the Report of the Second District of New York in 1842, by Prof. Emmons, under the name of the Chazy limestone. The name was derived from the town of Chazy, where it has a thickness of 130 feet, reposes unconformably upon the Calciferous, and is succeeded by the Birdseye limestone. It is a dark, irregular, thick-bedded limestone, containing many rough, flinty, or cherty masses, and extends as a belt into Vermont, where it exposes more surface area than any other Group of the Iower Silurian, and has a maximum thickness of 300 feet. It was called the "Chazy Formation" in the Geology of Canada for 1863, because shales and sandstones are there associated with the limestone. It occupies a narrow area about the Ottawa and Montreal, and extends to the Mingan Islands and Newfoundland, its thickness not exceeding 300 feet. The western extension of the belt appears in cliffs on the coast of Lake Winnipeg, in the region of Lakes Huron and Superior, in Michigan, Wisconsin, , Iowa, and Minnesota. In the lake region it consists of arenaceous and arenaceo- 
calcareous beds, sometimes difficult to distinguish from the Calciferous layers, followed by beds of argillo-calcareous composition.

\$73. In 1852 David Dale Owen called it the "St. Peter's Sandstone," after the river of that name, now sometimes called the Minnesota River, where it is usually made up of grains of limpid and colorless quartz, remarkable for whiteness. It occupies part of the slope between the first and second terrace at Prairie du Chien, forns the base of the bluffs at the St. Peter's, and the lower nineteen feet at the Falls of St. Anthony. It rests upon the billowy surface of the Calciferous, fills up the depressions, and is followed conformably by the Trenton. In the lower part there is some shaly material and conglomerate matter washed from the Calciferous and older rocks, but above this it is a remarkably uniform, white or yellow, friable quartzose sandstone, substantially free from silt and calcareous or ferruginous cement. There are oblique and discordant lines of stratification, supposed to be due to the shifting of the waves during deposition, and near the upper surface there is more or less argillaceous material. In some localities it is tinged yellow or red by the oxides of iron, and cemented in streaks, and weathers irregularly. The outliers and standing rocks are brightly colored, and are called pictured or painted rocks. The thickness will exceed 200 feet where filling a depression in the Calciferous; but the average thickness does not exceed 100 feet. Occasionally ripple-marks, fucoidal impressions, and tubes of Scolithus occur in the harder layers, but the only fossil yet described from this region is Lingulepis morsei. The absence of fossils is due to want of preservation.

\$ 74. Prof. T. C. Chamberlin says the constituent grains of sand in this Group are derived in the main from granitoid and schistose rocks, which are composed of particles of quartz intermixed with a variety of softer and more decomposable crystalline minerals. In the metamorphism the quartz was usually last in crystallization, and occupied the angular interstitial spaces between the crystals that had already taken shape, and hence while crystalline in internal structure it molded itself about the crystals of the previously formed minerals. It was thus angular, but not in its own crystalline form. Upon decomposition the associated minerals were mainly reduced to earths and clays, while the undecomposable quartz remained in angular grains. By the action of streams in carrying these down to the sea, and by the agency of the waves in distributing them, the grains were sifted, assorted, rolled, rounded, and finally deposited in the forms in which we now find them. The majority are worn into somewhat spherical grains; others less acted upon remain quite angular. The angularity, however, is not what is characteristic of freely forming quartz crystals, but is due to the circumstances under which it was formed. In the original crystalline rock occasional cracks and cavities occurred filled with secondary quartz, which in such a situation assumed its own crystalline form; and in the sandstone itself secondary crystals might have been formed after deposition, just as they have been in adjacent limestone-beds where their secondary origin is unquestionable, and the degradation of the rock inclosing these would furnish points and fragments of true crystals of quartz, which might not be so far worn as to lose their characteristic form.

$\S 75$. It occupies a narrow area fringing the Calciferous, or exposed in river banks, stretching in an irregular course from the Lower Menominee River on the north-eastern border of Wisconsin to the mouth of the Wisconsin River. It occurs 
in North-eastern Iowa and the enstern part of Minnesota, where its dip is westerly. It occurs in Illinois, at Oregon, on Rock River, and at La Salle, on the Illinois, caused by a local uplift. The unevenness of the Calciferous bed, as proven by artesian boring, is greater near the margin or shore-line of the oceanic deposit than elsewhere. It is known, by artesian boring, in Minnesota more than 100 miles from the Mississippi, and in Illinois more than 100 miles from its exposure in Wisconsin. In some places the sand mingles with the calcareous materials and forms passage beds to the Trenton, and at other places the transition, while conformable, is abrupt. In Missouri the upper part of the Group received the name of the "First Magnesian Limestone," and the lower part the "First Sandstone" and the "Saccharoidal Sandstone." The latter presents very few characters not found in the exposures in Illinois and Wisconsin; while the former is limited in its distribution, and indicates local changes in the deposition of the upper part of the Group. It is usually a gray or buff crystalline, cherty, magnesian limestone, filled with silicious patches, breaking readily with the hammer, and extremely variable in thickness. In New Jersey it consists of a fine, even-grained limestone, sometimes a pure dolomite, except near the base, where there are sandy and calcareous layers. It occurs in long, narrow belts, in a north-east and south-west direction, corresponding to synclinal and anticlinal axes. From this State and from Pennsylvania it is exposed in numcrous places within the Appalachian System as far south as Alabama, and may generally be detected by the presence of Maclurea magna. In Tennessee the lower part is an argillaceous limestone, varying in thickness from 50 to 600 feet; and if the marble of Knox County is referred to it, its upper part will have a thickness of more than 400 feet. It occurs in the Wahsatch Range in Utah, in the White Pine district of Nevada, in the Wind River Mountains of Wyoming, and in numerous other localitics in the great system of mountain ranges of the West, where it also bears the name of the Quebec Group. It has been identified in the Arctic regions, on King William's Island, North Devon, and Depot Bay, in Bellotis Strait, where it is a dolomitic limestone. It graduates into the Black River wherever the latter is separable from the Trenton, and especially where the Birdseye limestone is present. Numerous fossil species connect it intimately with the overlying rocks, many of which occur as high as the Hudson River, viz.: Strophomena alternata, S. incrassata, Orthis perveta, Leperditia canadensis, L. louckana, L. amygdalinx, Orthoceras multicameratum, O. bilineatum, and Modiolopsis nasuta. The most characteristic fossil is Maclurea magna. 


\section{CHAP'TER VIII.}

\section{BLACK RIVER GROUP.}

$\S 76$. THE Black River Group was defined by Lardner Vanuxem, in the Geological Report for the Third District of New York in 1842, and named from its exposures on Black River. The name "Black River limestone" was applied to the cliff extending from Boonville through Lewis into Jefferson County, the cliff being composed of the Birdseye limestone of the Mohawk and the rocks upon which the well-characterized Trenton limestone is placed. We find the name Birdseye limestone applied to rocks in the report of 1838 , but not in the sense of the name of a Group of rocks, as the term Black River was used in 1842, and if the name had been so used it would necessarily give way to the geographical name. The Birdseye limestone was distinguished on the Mohawk by its light dove-color, thick layers, and the presence of crystalline particles representing Phytopsis tubulos $a$ or other organisms, which caused the rock to break readily or possess a kind of brittleness, and when broken to clearly show the crystalline spots. This character is not persistent in geographical distribution, and the greatest thickness of the rucks is only about 30 feet. The Black River limestone is distinguished by the abundance of Cephalopoda, and especially by remarkably large Orthoceras, some of which are 10 feet in length and a foot in diameter; beside, it has quite an extensive distribution. The thickness on Black River is about 50 feet.

$\$ 77$. From New York it extends into Vermont, where about 12 or 14 feet in thickness becomes a black, finely granular mass, susceptible of a high polish, and has received the name of the Black Marble of Isle La Motte. In Vermont it rarely exceeds 20 feet in thickness; but it outcrops in Pennis Valley, Pennsylvania, with greater thickness than it possesses in New York. It crosses into Canada, and forms a belt upon the margin of the Chazy, but rarely attains any great thickness, though on the St. Lawrence, 90 miles below Quebec, it has a thickness of 130 feet. It has been identified by the presence of gigantic Orthoceras on the north-west side of Lake IVinnipeg; and its existence has been noted in the Lake Superior region, on St. Mary's, Escanaba, and Menominee Rivers, and on St. Joseph and Sugar Islands. It has been identified at various places in the Appalachian System, but it thins out westwardly and has a limited area of surface distribution. By some it is regarded as a local and peculiar phase of the lower part of the Trenton, or as constituting merely beds of passage from the Chazy to the Trenton, but there are palieontological reasons for retaining the name as a geological subdivision. It contains many species unknown in the Trenton, though others pass up, as the two Groups are conformable, and both represent the deeper oceanic deposits of limestone. But the strongest reason for holding to the geological separation of so small a thickness of limestone from other Groups is that the family Orthoceratida, which commenced its existence in the Upper Taconic, increased in genera and species in succeeding ages until it reached its maximum development in this Group. Subsequently, it diminished in number of species and size of specimens, though it found a home in every Group, until it became extinct in the latest Carboniferous epoch. The Cyrtoceratidoe and Endoceratidee were highly developed, and the Gomphoceratidoe, 
Phragmoceratida, and Gyroceratido here first developed their essential characters. In the Birdseye limestone at Montmorency, Canada, petroleum exudes in drops from fossil corals, supposed to have its origin either in the marine animals or fucoidal vegetation.

\section{CHAP'TER IX.}

\section{TRENTON GROUP.}

$\S 78$. Tre Trenton Group was named from Trenton, Oneida County, New York. The limestone at the Falls, where it is more than 100 feet thick, was called the Trenton limestone long prior to the use of the words in a geological sense. In 1838 Vanuxem referred to the Trenton limestone, but it was not until 1842 that he and Prof. Emmons so described the Group as to establish it. At Trenton Falls there are two kinds of stone-one a dark, fine-grained limestone, in thin layers, separated by black shale, and abounding in fossils; the other a gray, coarse-grained limestone, in thick layers, forming the top of the mass, and much less fossiliferous. The Group has quite an extensive surface distribution in belts upon the margin of the older rocks in New York, and varies somewhat in its characters, but seems at all times to be a limestone, with the exception of shaly partings. It is 400 feet thick at Chazy, the greatest exposed thickness, and from here it thins toward the east.

$\S 79$. It enters Vermont from New York in three narrow outcrops, consisting of black layers and seams of limestone and occasional argillaceous matter, with a maximum thickness of about 400 feet. It enters New Jersey, and crosses the counties of Warren and Sussex, with a maximum thickness of about 200 feet. It is frequently exposed in the broken-up hills and mountains of Pennsylvania, showing a thickness from 300 to 700 feet. The exposures continue to occur southerly in the Appalachian Mountains in crossing Virginia, North Carolina, and Tennessee, where, in the eastern part of the latter State, there is a thickness of 1,100 feet, and in the middle part of about 500 feet. It is exposed by an ancient uplift in the central part of Kentucky over several counties, forming a large part of what is called the Blue-grass Region, and reaches as far north as the Ohio River. The thickness is about 700 feet.

$\$ 80$. It has an extensive geographical distribution in Canada. The Montreal and Ottawa sections bave each a thickness of 600 feet. The sections in TVestern Canada, on the Trent River and at Collingwood, have a thickness of 750 feet, but it thins westerly, and in following the outcrops around Lakes Huron and Michigan the exposures rarely exceed 50 feet in thickness. In passing south of Lake Superior it crosses Sulphur, St. Joseph's, and Great Encampment Islands, and thence stretches west and south-west near Little Bay de Noquet and Green Bay, and enters Wisconsin near the mouth of the Menominee River. From here the exposure extends south-west across the State, displaying a large area in the south-western part, and, entering the State of Illinois, occupies more or less of the surface in four or five of the north-western counties. From here the exposures bear north-west and north, occupying several counties in North-eastern Iowa, with a continuing belt across 
Minnesota to St. Paul and the Falls of St. Anthony. In Wisconsin, Illinois, and Iowa, the Group is separable into two divisions, the lower one being a blue limestone called the Trenton limestone, and the upper a dolomite called the Galena limestone; which is the lead and zinc bearing rock of that region. The thickness of these divisions is variable, but where most persistent, as in South-western Wisconsin, the upper or Galena has a thickness of 250 feet, aud the lower or blue limestone 120 feet. It thins northward through Minnesota, and the Galena division gradually disappears. The layers of limestone become thin and shaly, with sometimes ferruginous oolitic layers. The Galena appears as a lenticular mass or as thinning toward the east and north, and was apparently derived, so far as detrital matter occurs, from western sources, though a great part, like that of other limestones, was a deposit from the harder parts of animal organisms. Erratics and bowlders found in Northern Dakota indicate the existence of Galena limestone in the mountains of British America. The Galena contains about 86.6 lead and 13.4 sulphur, and occurs in fissures and crevices in the limestone, and not in true veins. The ore is supposed to have been precipitated from an aqueous solution. It was called the Galena limestone from the lead or galena, and from its typical exposure at Galena, Illinois. The lead area is about 4,000 square miles, two-thirds of which is in Wisconsin and the rest about equally divided between Illinois and Iowa.

$\S 81$. It forms some large surface exposures in Southern Missouri, where outcrops occur 400 feet in thickness. Numerous outcrops occur among the Western mountain ranges and in the Arctic regions, on King William's Island, at North Somerset, Boothia, and other places. It was found by the artesian boring at Louisville, $\mathrm{Ky}$, at Columbus, Ohio, and it is expected it will be found by boring at almost any place upon the continent, save where the rocks of older date are exposed upon the surface. It was not formed upon the margin of an island or continent, but is a regular sea deposit of general distribution where the depth did not exceed 2,000 fathoms. The materials are marine, the mass being remains of organic secretions, with little detrital matter. The fauna was abundant, and embraced representatives of nearly all the great subdivisions of invertebrate life that now have an existence in the ocean, and several orders and classes, as the Graptolites, Cystideans, and Trilobites, which have become extinct. The Graptolites and Trilobites were then on the decline, while Crinoids, Cystideans, Brachiopods, Corals, Gasteropods, and Lamellibranchs were on the increase.

\$82. Receptaculites oweni is peculiar to and characteristic of the Galena division of this Group, and it is usually accompanied with Lingula quadrata, Murchisonia major, Fusispira elongata, and other characteristic species. The species most characteristic of the Trenton Group, and which may be relied upon as determining its age wherever they occur, are Orthis tricenaria, found in New York, Canada, Kentucky, Missouri, and Nevada; Orthis pectinella, found in New York, Canada, and Kentucky; Cyrtolites compressus, found in New York, Canada, Wisconsin, and Minnesota; Hybocrinus tumidus, H. conicus, Amygdalocystites florealis, A. radiatus, Blastoidocrinus carcharidens, found at Ottawa, Canada, and High Bridge, Kentucky; Leperditia fabulites and Conularia quadrata, found in New York, Canada, and Kentucky; and Orthis borealis, found in Canada, Wisconsin, Minnesota, and Kentucky. The genus Amygdalocystites has a wide geographical distribution, though a rare fossil in every locality, and, so far as known, is confined to this Group. Other char- 
acteristic species are Bythotrephis succulens, Monticulipora lycoperdon, Schizocrinus nodosus, Stictopora elegantula, Orthis bellarugosa, O. aqquivalvis, Trochonema umbilicatum, Subulites elongatus, and Helicotoma planulata.

$\S 83$. There are numerous species which continued to live until the Hudson River age, and are therefore cominon to three Groups, as Strophomena alternata, S. rhomboidalis, Leptena sericea, Zygospira modesta, Rhymchonella capax, Calymene callicephala, Asaphus gigas, and Ceraurus pleurexanthemus. Such species are usually quite variable in form and size, and seem to have changed to suit the conditions of their habitat, and also, in accordance with the theory of evolution, to have reached the climax of development, and subsequently gradually declined. Strophomena rhomboidalis occurs in Trenton, Utica Slate, Hudson River, Clinton, Niagara, Lower Helderberg, Upper Helderberg, Hamilton, Chemung, Waverly, Burlington, and Keokuk Groups. Its vertical range exceeds that of any other species in any of the rocks of the known world, and its geographical distribution is common to every continent where strata of these ages have been studied and described. The varietal forms have been called $S$. tenuistriata from the Lower Silurian, S. depressa from the Upper Siluriau, and $S$. rhomboidalis from the Devonian and Subcarboniferous. The Lower Silurian specimens are usually smaller, and have fewer concentric wriukles over the visceral region, than those frum the Upper Silurian and Devonian, while the length of the front and lateral margins from the geniculation is usually greater in the Upper Silurian than it is in the Lower Silurian, Devonian, or Subcarboniferous specimens; but these differences are not so constant as to form inflexible characters, and hence it is that many of the learned and better palæontologists have classed them all together under the first and oldest specific name. The various forms which Strophomena alternata assume in the same Group of rocks are wonderful; the radiating striæ differ in size and number; the hinge line is sometimes longer and at other times shorter than the greatest width of the shell. The shells are sometimes much longer than wide, and at other times as much shorter. The lateral sides are sometimes straight, and at other times rounded. Some sliclls are nearly flat, others are deeply concave on the dorsal side and highly convex on the ventral. Age in some specimens appears to have materially thickened the shells, and preserved strong imbricating lines of growth, while in other cases we have much larger shells that are very thin and destitute of imbrications. Like differences may be distinguished in other species having great vertical distribution, as in Rhynchonella capax and Zygospira modesta.

$\$ 84$. The rocks of this Group are composed almost entirely of remains of the hard parts of animals that swarmed in the seas of that age. Some shells are preserved in good condition, but generally the comminuted fragments are held together by lime cement, forming the limestone strata, leaving well-preserved specimens to be found only in the shaly partings. It is common to find that one animal bas grown upon another, as a Lichenocrinus upon a brachiopod, and a bryozoan upon the former, under such circumstances as to show the shell was at the bottom of the ocean during the growth of the Lichenocrinus, and that the latter must have ceased to grow before the bryozoan attached. From this we infer the clearness of the water, for otherwise mud would have intervened; and we also infer a slow deposition of materials, for the lives of two animals transpired before the deposit was 
sufficient to cover a thin shell. There is no evidence of any difference between the temperature of the water then and now, nor between the climate then and now.

$\S 85$. Wherever the Black River limestone exists, the Trenton is conformable with it ; and where the Black River is not distinguished, the Trenton is usually conformable with the Chazy. The Trenton is conformable with the Utica Slate above, in New York and Canada; but there is an abrupt change in the character of the rocks, and a marked difference in the fauna, while in Kentucky it graduates up into calcareous shales of the age of the Utica Slate by imperceptible grades, so the line of separation can not be determined, except as based upon a slowly changing fauna.

$\S 86$. Light carbureted hydrogen gas is often the product of the transformation of organic matter at ordinary temperatures, and is abundant in the palæozoic rocks from the Chazy to the Permian. A spring at Caledonia, Canada, issuing from the Trenton Group, evolves 300 cubic inches of carbureted hydrogen gas per minute. It is saline water. Another discharges somewhat less, and another discharges large quantities of sulphureted hydrogen gas. This is not considered surprising when it is remembered the Chazy Group in the Ottawa Valley includes a considerable thickness of shales and argillaceous limestones, and the Quebec Group offers successions of limestones and shales, whose slow decomposition from infiltrating waters will furnish such gases. In higher strata, however, the carbureted hydrogen gas escapes in much greater quantities, as at the burning spring near Niagara Falls, and in the region of the oil-wells. Carbureted hydrogen gas is the well-known "fire-damp" of the coal-mines. It collects in ill-ventilated galleries of collieries, and when sufficiently mixed with the atmosphere, if it comes in contact with an unprotected flame, it explodes with great violence. It exudes from all rocks charged with petroleum or naphtha, and was known and used for fuel before the Christian era on the Caspian Sea, where it is evidently inexhaustible. Petroleum occurs in the cavities of fossils, Orthoceras sometimes holding serveral ounces of it, at Pakenham and Lancaster, Canada. While both carbureted hydrogen and petroleum occur in the rocks of the Quebec and all succeeding Groups, yet none has been found of commercial value as low as the Trenton. The reasons are, absence of porous strata and cavities for its collection, and because the animal and vegetable matter was not collected in sufficient quantity at any single locality. It has been asserted the gas in Western Ohio and Northern Indiana is from this Group, but the author thinks all the evidence is against such conclusion. 


\section{CHAPTER X. \\ UTICA SLATE GROUP.}

$\S 87$. This Group was named the Utica Slate from Utica, New York, and quite fully defined as a geological subdivision in 1842 by both Vanuxem and Emmons in their respective reports. It is in typical localities a dark-colored slate or shaly mass, highly charged with carbon, and agreeing in its composition with the dark layers that separate the limestone strata in the Trenton Group. The surface exposure forms a belt resting upon the Trenton, extending from New Jersey across New York into Vermont, passing under Lake Champlain and entering Canada. The greatest thickness in New York is about 600 feet, and in Vermont about 100 feet. It exposes considerable surface in Canada, never exceeding 500 feet in thickness, and extends from Lake Huron, where it thins out, to the eastern shores of the continent, appearing on the Saguenay, in Newfoundland, and the Island of Anticosti. It is very fossiliferous, and everywhere characterized by the presence of Triarlhrus becki; and in the vicinity of Ottawa Triarthus spinosus is abundant, and the Scotch fossil, Siphonotreta scotica, occurs. It is often interstratified with thin bands of limestone.

$\$ 88$. It is exposed in numerous places in the Appalachian System, and attains a thickness in Huntingdon County, Pennsylvania, of more than 1,000 feet. It thins out westerly, and loses its character as a black slate before reaching the Ohio River, where it is composed of blue calcareous shales and marls with interstratified thin limestones, apparently forming beds of passage from the Trenton to the Hudson River without any want of conformability. The change in its lithological characters would have prevented forever its identification in the banks of the Ohio, had it not beeu for the tell-tale fossils. The abundance of Triarthrus becki and Leptobolus lepis and associate fossils settled the question of its identity. It is unknown farther west, but exists in the Arctic regions as a more or less calcareous slate. The fossils of the greatest geographical distribution, and by which it may generally be recognized, are Triarthrus becki, Leptobolus lepis, Asaphus canadensis, - Lingula progne, and Graptolithus quadrimucronatus. The rocks are composed in part of mechanical sediment, derived from sources east of the Appalachian System, and not almost wholly of shells and the harder parts of animals, as the Trenton is below and the Hudson River above. It thins westerly, and as the mechanical sediment disappears the marine deposits form continuous passage beds from the Trenton to the Hudson River. The strongest reason for its retention as a geological subdivision is found in the fauna with which it abounds; for at many localities, e.g., Cincinnati, Ohio, and Jefferson County, New York, it can only be separared from the Hudson River Group by an arbitrary line; and at other localities, e. g., Deerfield, New York, and in Kentucky, the Trenton Group is so blended with it that the line of demarcation is wholly obscured. The Galena limestone of Northern Illinois, Eastern Iowa, and South-western Wisconsin occupies substantially the same geological position, though its affinities are more closely allied with the Trenton, while the relations of this Group are nearer the Hudson River; beside, none of the characteristic fossils of this Group are found in the Galena, and none of the 
characteristic fossils of the Galena occur in this Group. A petroleum spring rises from this Group on the Grand Manitoulin Island, and saline springs at Varennes evolve large volumes of carbureted hydrogen gas. At one of these springs the gas has been collected in a holder, and employed in lighting a house. The black shales of this Group contain variable amounts of combustible matter, and when distilled they give, beside inflammable gases, portions of oily matter, which in the shales of Collingwood are equal to four or five per cent.

\section{CHAPTER XI.}

\section{HUDSON RIVER GROUP.}

§ 89. The Hudson River Group was named from an exposure near Hudson River in New York, and first defined in the geological report by Vanuxem in 1842. At the typical locality it consists of shales, shaly sandstones, slates, and thick-bedded grits, stratified and conformable, alternating many times without any regular order of alternation. It was called the Lorraine Shales by Emmons, who mentions, as occurring at one place in New York, that structure called "Cone within Cone," which is so common in the Devonian and later formations. Its maximum thickness in New York is about 800 feet.

$\$ 90$. The Group is largely exposed in Pennsylvania and other States in the Appalachian System, as far south as Tennessee, and has a thickness in some places of 1,200 feet. In the latter State it has been called the Nashville Group. It is the surface rock of many counties in Kentucky, extending from above Maysville on the Ohio, to near Louisville. In the south-eastern part of Indiana and the southwestern part of Ohio, it consists of alternating layers of blue calcareous clay and limestone, and has a thickness of about 800 feet. It has been called in this section the Blue limestone. It occurs in the northern part of Illinois, southern part of Wisconsin, and north-eastern part of Iowa. Its thickness in these States does not exceed 240 feet. In the south-eastern part of Missouri its thickness is about 250 feet, and it appears in Texas and New Mexico. It has a wide geographical range in Canada, extending from the Island of Anticosti and the eastern border west, by way of the Great Lakes, to the Red River of the north, and again appearing in the mountain ranges bordering the Pacific. In the vicinity of Toronto its thickness is about 1,100 feet, but it is much thinner in its western extension, and in the region of the Great Lakes rarely exceeds $\mathbf{1 0 0}$ feet. Its greatest thickness in Eastern Canada is about 2,000 feet.

§91. This Group is persistent and of almost universal distribution, except upon the older rocks that were dry land before its deposition. We would expect to find it almost anywhere on the continent by boring through more recent deposits. It is the equivalent, to some extent, of the Caradoc sandstone, or Bala Group, of England and Wales, and is represented in different European exposures. Like the Trenton and all earlier Groups, it is a marine deposit made in water of considerable depth, not a littoral or shore-line deposit as the Potsdam Group was, though the sandstone occurring in many of the northern exposures was evidently mechanical and derived from land at no great distance to the north. 
$\$ 92$. The seas swarmed with animal life and fucoidal organisms, and the rocks are composed almost wholly of their remains. It is literally a graveyard of invertebrate life. The Brachiopoda and Bryozoa reached in this age the stage of their greatest varietal development, and possibly the highest state of their existence.

$\S 93$. As the exposure in Ohio, Indiana, and Kentucky is very large and quite characteristic of it in other places, it may be fit and proper to further define it. To go from the Ohio River, at Cincinnati, west 51 miles to Osgood, Indiana, or north to Dayton, or north-east to Xenia, Ohio, one will pass across the upturued edges of this Group, and reach the Niagara. The rocks dip westerly and northerly at the rate of about ten feet in a mile. The hills at Cincinnati expose about 400 feet in thickness, constituting the lower half of the Group; and the upper half, or about 400 feet, occurs between the top of these hills and the bordering Niagara Group, about 50 miles distant to the north and west. The area of its exposure in Ohio is all of Hamilton, Butler, Warren, Clermont, and Brown Counties, and part of eight counties that border upon these. The exposure in Indiana is about half as great, reaching as far north as Richmond, and bordering the Ohio nearly half-way from Madison to Jeffersonville. The exposure in Kentucky is greater than in Ohio, for it surrounds the Trenton Group in that State. Throughout the whole area it is composed of alternate layers of calcareous clay and limestone of varying thickness. In some places calcareous clay is 6 or 8 feet thick, without a layer of stone. At other places one layer of stone, 4, 6, 8, or 10 inclies in thickness, follows another, with intervening layers of calcareous clay of much less thickness, for 40 or 50 feet. It is rare to find a layer of limestone more than a foot in thickness. All the layers are broken into small, irregular pieces of suitable size for cellar and other light stone-work, for which they are used. The blue calcareous clay exposed to the action of the weather for a few years loses its color and becomes of a dull gray hue. The sulphuret of iron occurs in the blue rocks, but instead of this we find iron oxide and sulphate of lime in the gray. 'The silicious matter prevails over the carbonate of lime in the layers of calcareous clay, while the carbonate of lime is much in excess of the silicious matter in the stone, due, in part at least, to the fact that the stones are a mass of more or less comminuted shells, corals, and crinoids. There is nothing in the general character and appearance of the rocks and calcareous clays to indicate the changes which the fossils undergo; that is, the changes are not to be attributed to surrounding conditions without the aid of that law of animal evolution which the science of palæontology teaches us has taken place in all past geological ages.

$\$$ 94. Some fossils, as Calymene callicephala, Asaphus megistus, A. gigas, Beyrichia chambersi, Leptcena sericea, Bellerophon bilobatus, Zygospiva modesta, Strophomena alternata, and Orthis testudinaria, pass from the extreme lower part to the extreme upper part of the Group; and all of them save Beyrichia chambersi are known from lower rocks, and Leptcena sericea occurs in higher ones. Streptorhynchus hallianum has a limited range in the lower part, $S$. planoconvexum and $S$. sinuatum a limited range below the middle of the Group, $S$. nutans and $S$. sulcatum in the middle of the upper half of the Group, and S. subtentum and S. filitextum in the upper part. Lichenocrinus crateriformis, $L$. dyeri, and $L$. pattersoni are confined to the lower half, and $L$. tuberculatus and $L$. affinis to the upper part. Acidaspis crossotus occurs in the lower part, $A$. anchoralis and $A$. cincinnatiensis in the middle part, while $A$. onealli occurs in the 
upper part. Rhynchonella capax, $R$. dentata, Streptelasma corniculum, Favistella stellata, Tetradium fibratum, Cypricardites haynesi, etc., are confined to the upper part. Such are a few illustrations of the changing fauna at different elevations. To completely present the subject would require the enumeration of all the species. Crinoids, as a rule, are limited vertically, and hence each species is sought in its particular range. Species having a wide geographical distribution, and characteristic of the Group are Aulopora arachnoidea, Stomatopora inflata, Orthis occidentalis, O. subquadrata, O. retrorsa, Pterinea demissa, $P$. insueta, Cyclonema bilix, and Glyptocrinus decadactylus.

$\$ 95$. With this Group the Lower Silurian closes, because at its top we have the greatest break stratigraphically and palæontologically that occurs from the base of the Potsdam to the top of the Lower Helderberg, and because it approaches nearer the line of division established by Murchison, between his Lower and Upper Silurian, than any other line, if, indeed, it is not identical with it. Wherever the Hudson River has been examined on the continent, the superimposed rocks are unconformable with it, no passage-beds are found, and the palæontological break is almost complete. In the Western States the Niagara Group succeeds it, and rests unconformably upon it. In the Eastern States it is succeeded by the Medina and Cliuton Groups before the Niagara is reached, but the Medina rests unconformably upon it. On the Island of Anticosti it has a thickness of 950 feet, and is followed by rocks apparently conformable with it, although there is an abrupt palæontological break. Of 121 species known to Prof. Billings from Anticosti, 80 disappear at once below the dividing line, and 41 only appear above it, where they are joined by 45 species that are not found below. This palæontological break is less than it is at any other known place on the continent; but it is so great as to show that probably the strata are not strictly conformable.

$\$ 96$. There is an important period of time indicated by this want of conformability and palæontological change. Vast ages must have intervened, which are not represented by any known rocks on the continent. More than 400 genera bave been idescribed as existing previous to this time, more than three-fourths of which had become extinct. Or, in other words, less tban one-fourth of the genera which had come into existence prior to the close of the Lower Silurian Age continued to have an existence afterward. No evidence of the existence of land-plants has ever been discovered in Lower Silurian rocks. WVe are convinced, however, that land had existed above water for ages; that it was necessarily refreshed by sun and rain, by warmth and air, and that it may have sustained some kind of land vegetation. If the land vegetation did not possess hard parts capable of preservation, of course none will ever be found. Neither has any evidence of the existence of land or fresh-water animals ofthis era ever been discovered. 


\section{CHAPTER XII.}

\section{UPPER SILURIAN.}

$\S 97$. AlL the rocks of the Upper Silurian System are marine; but landplauts, or such as may have existed in marshes, and received support from sunlight and air, have been found within them. No remains of land or fresh-water animals, or marine vertebrates, have been discovered in North America. There is no radical difference in the general character of the Lower Silurian and Upper Silurian fossils, because vertebrates had not made their appearance, and the same orders of invertebrates were represented in each era; but the separation into two Systems is very convenient, because both are introduced with sandstone Groups, and the Trenton in the Lower Silurian, and Niagara in the Upper Silurian, are alike extensive in geographical distribution, and some analogy may be traced between the upper Groups in each System. On the whole, the calling of one System Lower Silurian, and the other Upper Silurian, was a happy hit in nomenclature as well as correct in science.

\section{MEDINA GROUP.}

§98. Turs Group took its name from Medina, New York. The rocks were described by Vanuxem in 1842, under the names Oneida Conglomerate, Gray Sandstone of Oswego, and Mediua Sandstone. At the typical localities they are conglomerate, and gray and red sandstone. The conglomerate is hard and gritty, and composerl of quartz pebbles and sand so firmly cemented as to be used for millstones. The sundstone is argillaceous, thinly laminated, and of red, gray, and mottled colors. Where it is not fragile, but firmly cemented, it makes a good building stone, and has been largely used for paving streets, as it readily breaks into stoncs of regulation size. The Group borders Lake Ontario on the south, and extencls in an east and west line of exposure about three-fourths the length of the State, and, entering Canada at the Niagara River, continues to Lake Huron. In Oneida and Oswego Counties the thickness is from 500 to 600 feet; at the west end of Lake Ontario 614 feet, and at Lake Huron 100 feet. It thins so rapidly that few, if any, traces have been discovered west of this lake. A small surface area in New Jersey has a thickness of 900 feet, and a larger one in Pennsylvania has a thickness of 2,500 feet. It occurs in patches among the broken ranges of the Appalachian System in Maryland and other States, as far south as Tennessee; but is unknown in the Western States.

$\S 99$. The conglomerate is 500 feet thick in the Shawangunk Mountains, and 700 feet in the Kittatinny Valley in Pennsylvania. It graduates into the gray sandstone, and then into the red sandstone, so they can scarcely be distinguished except by color; and the gray sandstone in like manner graduates into the conglomerate by enlarging and increasing the number of its pebbles; so there is no reason, stratigraphical or palæontological, for subdividing the Group, as was done in early work on the New York Survey. It always rests unconformably upon the Hudson River Group, and bears the internal evidence of having been derived from land immediately north and east, and of having been deposited in shallow water, subject 
to waves and currents which transported the materials only short distances. The conglomerate indicates a shore-line and rapid deposition, and is almost non-fossiliferous, though a few fragments of fucoids and shells, generally too imperfect for definition, have been found in it. The sandstone, too, bears the evidence of having been deposited near the land in shallow water, not only in wave-lines, rill-marks about shells, and ripple-marked slabs, but in mud-cracks produced by sun-drying. In all these respects it compares with the Potsdam, which separates the Taconic from the Lower Silurian.

$\S 100$. In the more argillaceous part of the sandstone, fossils are sometimes fairly well preserved. The characteristic fossils are Arthrophycus harlani, both genus and species being confined to this Group, and having a wide distribution, and Lingulella cuneata, a strongly marked species. Saline springs are common throughout the whole extent of these rocks, and brine is universally found by boring. The brine is frequently impure from the presence of muriate of lime and iron. Carbureted hydrogen gas rises in many places on the Erie Canal east of Lockport, and at Gasport it was collected and used for illuminating purposes a half century ago.

\section{CHAPTER XIII.}

\section{CLINTON GROUP.}

$\S 101$. This Group was named from the town of Clinton, in New York, and defined by Vanuxem in 1842; and re-defined by $\mathrm{Hall}$ in 1843 iu the Geological Survey of that State. The rocks have no uniformity in color or composition. At the typical locality there is green and black-blue shale; green, gray, and red sandstone, often laminated; calcareous sandstone and red fossiliferous iron ore beds; at other places, it consists of shaly sandstones and shales of various colors, impure limestones, conglomerates, and oolitic iron ore, with concretions. It occupies a narrow belt of country in New York, commencing near Canajoharie, and stretching westward south of Lake Ontario, resting on the Medina Group, with the greatest width in Wayne County, and, entering Canada at Hamilton,extends west to Lake Huron, appearing on Drummond, Manitoulin, Cockburn, and other islands, and probably enters the Peninsula of Michigan with a thickness of less than 50 feet, and rapidly thins out. The maximum thickness in New York is about 400 feet. The two npper bands of limestone included by the New York geologists in the Clinton Group, are now generally classed with the Niagara, as they possess no fossils peculiar to the Clinton, and the shales which separate them thin out in their extension into Canada. In its easterly extension from New York, outcrops occur as far as Anticosti Island and Newfoundland. On Anticosti it is described as one of the divisions of the Anticosti Group, which there includes the rocks from the Hudson River to the Niagara, and has a maximum thickness of about 500 feet. It occurs in the Appalachian chain as far south as Georgia and Tennessee, and in crossing Pennsylvania develops a thickness of more than 2,000 feet. The Group thins out before reaching the IVestern States, and is unknown except upon the borders of the Appalachian and Laurentian elevations. It appears to have resulted from the mechanical deposition of materials derived from land lying north and east 
of it, and to represent a border-land and shallow water deposit, that extended only a short distance from the primitive source of its materials.

$\$ 102$. In Western New York the dividing line between the Medina and Clinton is sharply defined, and the materials of which each are composed are quite distinct; but in the central part they graduate into each other, the Clinton being largely composed of sandstone. There is strong resemblance between the marine vegetation which abounds in the two periods. Westerly the Clinton is more calcareous and more fossiliferous, and graduates up into the Niagara in its lithological and fossil characteristics. The Mediua, Cliuton, and Niagara are elearly defined in some localities; but in others the Medina graduates into the Clinton, and in others the Clinton blends with the Niagnra. There is no want of conformability between them where best developed, and the lines of separation show only a changed condition or altered circumstances under which the deposition was continued from one Group to the other. Pentamerus oblongus, Spirifera radiatu, Meristella cylindrica, and Lingulella lamellata are among the species accredited both to the Clinton and Niagara, and which show the intimate relation between the Groups. The Clinton abounds in fucoids, tracks, and trails, the former being more abundant than iu. any earlier Group. 'The fossils having the greater distribution and being most characteristic are Ichnophycus tridactylus, Graptolithus clintonensis, Helopora fragilis, Athyris naviformis, Leptocolia hemispherica, Triplesia congesta, Cyelonema cancellatum, and Comulites distans. The iron ore beds are frequently thick enough to be valuable, and are worked successfully. They are sometimes very fossiliferous, and the quantity of iron is decisive proof of the vegetable character of the fucoids of that age, and the absence of land-plants among the fossils is almost conclusive against their existence at that period.

\section{CHAPTER XIV. \\ NIAGARA GROUP.}

$\$ 103$. Tnis Group was named from its development at Niagara Falls, where the rock over which the water is precipitated belongs to it. It was defined by Vanuxem in 1842 , and by Hall in 1843 . It is the most persistent in its geographical distribution of any Upper Silurian Group; indeed, wherever the Upper Silurian is found it is present, except with the exposed belts of the lower Groups, and not unfrequently it constitutes the whole formation. It generally consists of limestone and slaales, but sometimes becomes arenaceous, argillaceous, or highly ferruginous. In New York it exposes an east and west belt almost the entire length of the State, a short distance south of Lake Ontario, with a maximum thickness of 300 feet. Near Niagara Falls there are 165 feet of limestone (directly at the falls 85 feet) overlying 80 feet of shale. In its western extension it crosses the Niagara River into Canada, appears at Lake Huron, on Manitoulin and Drummond Islands, occupies the southern part of the northern peninsula of Michigan, spreads over the south-eastern part of Wisconsin and the northern part of Illinois. Keeping south of the Lower Silurian area in the north-western part of Illinois, it enters Iowa below Dubuque, and presents a surface exposure 160 miles in length by 40 or 50 in 
breadth. In Wisconsin, Illinois, and Iowa it is principally a magnesian limestone, sometimes too porous or friable for building purposes, but suitable for lime, as at Chicago and Racine; at other places having a good reputation for buildings, as at Joliet. It sometimes occurs more or less saturated with petroleum, as at Chicago, where it indicates the presence of shales immediately below it, and in some localities near its base it contains beds of hematite in small lenticular concretions, as at Iron Ridge, in Dodge County, Wisconsin. The maximum thickness in Illinois is 640 feet, in Wisconsin 800 feet, and in Illinois and Iowa 600 feet. It occurs in southeastern Canada, in New Brunswick, Newfoundland, and Anticosti, where its maximum thickness is 800 feet. It occurs in nearly all the States to which the Appalachian System extends. In crossing Pennsylvania, where it consists mostly of shales, it has a maximum thickness of 1,600 feet. It occupies extensive areas in Tennessee and Alabama; and in the latter State that part of it which was originally a porous magnesian limestone, subsequently became infiltrated with iron in solution, and now constitutes the celebrated fossiliferous iron ore of Alabama. It forms a sub-circular belt of exposures from 5 to 60 miles in width surrounding the great Lower Silurian area in the middle part of Kentucky, South-western Ohio, and South-eastern Indiana, where it consists of hard, blue and gray limestone, yellowish and whitish-yellow magnesian limestone, and shales, variously alternating and combining, with a maximum thickness of about 600 feet. In some places near the base there is iron-stained chert. At Cedarville, near the top, the pcrous magnesian limestone is used for the manufacture of lime, and the harder limestone at Dayton, St. Paul, and other places is used for building and other economic purposes. It surrounds the Lower Silurian and Taconic uplift in the southern part of Missouri, and frequently occurs in the Rocky Mountain ranges. It outcrops far to the north, in the Arctic regions north of British America. Fossils have been described from its exposures on Beechy, Cornwallis, Griffiths, Seal, Napoleon, and Offley Islands, from Capes Hilgard, Hotham, Louis, and other points. It is substantially the equivalent of the Wenlock in England, and has its representative in Scandinavia, Russia, Germany, and other European countries. Several species of fossils occurring in the upper part of the Group at Waldron, Indiana, are identical with those occurring at the equally celebrated locality on the Island of Gottland, in the Baltic Sea. It is so constantly present where the rocks from the Lower Silurian to the Devonian are exposed, that it is regarded as a universal Group underlying nearly all the more recent rocks on this continent.

$\S 104$. It is a deep-sea deposit, as distinguished from all mechanical, littoral, shore-line, and marsh deposits, and, like most other undisturbed marine sediments, is generally limestone. The ocean must have swarmed with invertebrate life during the entire age, as the rocks are almost wholly constituted of their harder parts. It is so thoroughly characterized by its fossils that a palæontologist has little difficulty in recognizing it wherever it exists. It is in this Group the earliest landplants occur-Psilophyton and Glyptodendron. The latter was founded upon an impression of uncertain value in a magnesian limestone. Psilophyton is supposed to have been a marsh-plant that drifted in the ocean and became imbedded in the mud, which preserved its characters. Psilophyton princeps is the oldest fossil landplant in America. Fucoids are scarce; in striking contrast with their abundance in the Clinton. Sponges were more numerous than in any preceding age. Coral- 
reefs were formed, which may now be traced for many miles; single masses were several feet in diameter, and the beauty of their structure is not surpassed by any of the corals which now abound in the ocean. Some of the species, too, were almost world-wide in their distribution, as Halysites catenulatus, Heliolites pyriformis, and Favosites forbesi. It is famous, too, for its Echinoderms. The Cystideans commenced their existence in Taconic times, as evidenced by the plate called Eocystites, and reached the climax of their evolution and development in this Group, and almost suddenly disappeared from the face of the earth, a few small species only being found in the Lower Helderberg and Lower Devonian, where the entire order became extinct. Cystideans were marine animals, related to the Crinoidea. Some were sessile; others possessed a column and roots, by which they attached to other objects; and others were free, and possessed a flexible column tapering to a point, which could be used for attaching purposes. The head was globular, oval, pyriform, conical, cylindrical, or of any other shape, but always covered with an external skeletou composed of polygonal calcareous plates, which are sometimes very richly ornamented. The fracture of the plates presents the same crystalline structure as crinoidal plates do. In some species the number of plates and order of arrangement remained coustant throughout the life of the animal, the size of the animal increasing by the growth of the original plates, which enlarged throughout, instead of by addition to the edges. In other species the plates are not limited in number, and have no order of arrangement; they increase in size, or new plates are introduced, so as to destroy uniformity in different specimens in the same species. In other species the dorsal side has a definite number of plates and regular order of arrangement without any increase, while the ventral side has no order of arrangement of the plates, and they increase in number to cover the increased growth of the animal. There are usually two principal apertures, and often many smaller ones through which the most important functions of the animal economy were exercised. One of these is called the mouth, and is found on the side near the base or near the apex. It is a curious fact that so important an organ as the mouth occurs almost anywhere on the body of a Cystidean, but, of course, always occupying the same position in each species. Another aperture, ealled the ambulacral orifice, occurs near the center of the upper part of the body, and between the bases of the arms, when the species possessed such organs. The other apertures are called calycine pores and pectinated rhombs. The calycine pores served in some manner to introduce water into the interior of the animal, but they bear little resemblance to each other in different species, and one ean form no adequate idea of the system of circulation. Pectinated rhombs differ in number and position in different species, and sometimes do not occur at all. Their function, too, is an absolute mystery, except they furnished another medium of communication from the exterior to the interior of the body. The Blastoidea commenced existence in this Group by the appearance of Stephanocrinus, and became extinct in Carboniferous times. The order Myelodactyloidea, another Echinoderm of very uncertain affinity, seems to have been confined to this age. The development of the Crinoidea was wonderful, no less than 15 genera making their first appearance, eight of which are unknown in later rocks. The Graptolitidæ herè became extinct.

$\$ 105$. Holocystites occurs in Ohio, Indiana, Illinois, New York, and other States. Twenty-five species have been defined, and none are known from higher 
or lower rocks, and it may therefore be considered a characteristic genus. Eucalyptocrinus has a wider geographical distribution, and is more abundant, and for the same reason may be called characteristic. Orthis elegantula, O. flabellum, O. hybrida, Calymene blumenbachi, and Illanus barriensis have almost world-wide distribution, and are characteristic of rocks of this age.

$\$ 106$. The dolomites of this Group in Canada are more or less bituminous. In some parts of Western New York they contain so much solid bitumen that it exudes from the rocks when heated. The escape of carbureted hydrogen from these rocks is of common nccurrence. Lyell described in 1841 a " burning spring" on the river just above Niagara Falls, where the light hydro-carbon gas rose from beneath the water out of the limestone rock. The invisible gas makes its way in countless bubbles through the clear, transpareut water, and on the application of a lighted candle it plays about with a lambent, flickering flame, which seldom touches the water, the gas being at first too pure to be inflammable, and only obtaining sufficient oxygen after mingling with the atmosphere at the height of several inches above the surface of the river. This gas had its origin in the shale, which forms the lower part of the Falls, and has found its way up through 85 feet or more of quite compact limestone. Petroleum occurs in Niagara limestone at Chicago, which had its origin in some shaly strata beneath, but artesian boring failed to discover it in commercial quantities. Where gas or oil escapes from surface limestone there is little prospect of finding accumulations of commercial importance by artesian boring, because so much has escaped in the ages which have passed away since the elevation of the limestone above the water of the sea. There must be an impervious covering of clay or stone to retain such volatile substances in valuable quantities.

\section{CHAPTER XV.}

\section{GUELPH GROUP.}

$\$ 107$. This Group was named from the town of Guelph in Canada, and defined by Logan in 1863. It appears as a lenticular mass, resting upon the Niagara, and having a maximum thickness of 160 feet. It is a limestone dolomite, particularly distinguished for having no fossil Echinoderms, while it is rich in other fossils closely allied to those in the Niagara, some of the species being identical. It may have been a brackish water-deposit in an arm of the sea. It occurs in the north-western part of Ohio with all the fossils and characteristics pertaining to it in Canada, but is unknown elsewhere. It is doubtless of the same age as the Onondaga Group, and probably should not bear a distinct name, as among the very few fossils found in the latter, Murchisonia boydi and Cyclonema sulcatum occur in the Guelph. Megalomus canadensis, the most common species, and Trimerella grandis are found in the Niagara. The characteristic fossils are Pentamerus occidentalis, Murchisonia bivittata, M. longispira, Subulites ventricosus, Pleurotomaria solarioides, and Dinobolus galtensis. 


\section{CHAPTER XVI. \\ ONONDAGA GROÚP.}

$\$ 108$. Tnis Group was named the Onondaga Salt Group, hy the New York Geologists, from Onondaga County, New. York, in 1839, and re-defined by Vanuxem in 1842 , and by Hall in 1843. The Canadian Geologists very properly dropped the word "salt" from the name. It consists, on Oneida Creek and Cayuga Lake, in the lower part, of clayey deposits and red shale, showing green spots, followed by gypseous shales and impure limestones, which at the commencement alternate with the red shale, and this is followed by the gypseous deposit, which embraces the great lenticular masses quarried for plaster, and this by a magnesian rock having groups of needle-form cavities caused by the crystallization of sulphate of magnesia, and the upper member is the Waterlime. It rests upon the Niagara from the western line of New York, east to the middle part of Herkimer County, where the Niagara thins out; it then rests upon the Clinton until it disappears, and then upon older rocks until it reaches the Hudson River. It is therefore unconformable with the underlying rocks in midale and Eastern New York. The red shale loses its color west of the Genesee, becomes a bluish green, and gradually thins out, showing the unconformability in Western New York. The passage from the Niagara to the Onondaga is abrupt, offering no gradation in character of products or in continuation of fossil species. The great mass of gypseous deposits consists of yellowish or drab, and brownish colored argillaceous, and calcareous shale and slate, or of hard and compact slate, which weathers as if hacked by an instrument. The dark color of the gypsum, and brownish color of other rocks, is due to carbonaceous matter. An important member is called the vermicular limerock, which is gray or blue, and perforated with holes and cells, once filled with soluble saline material, which subsequently dissolved, leaving the cavities, some of which are hopper-shaped, and were produced by common salt, as no other common soluble mineral presents similar ones. The sulphate of magnesia cavities are lined with carbon, showing the liquid that held the salt in solution, contained bituminous matter, the salt ejecting its particles in the act of assuming form, as oceurs in the purification of acetic acid when obtained from the distillation of wood. This Group is celebrated for its salines, and formerly furnished nearly all the salt consumed in New York; for this reason it has been called the Salina and Saliferous Group. Sulphate of Strontian and sulphurets of lead and zinc occur in small quantities. Sulphuric acid escapes with the water from the earth in many localities, giving rise to acid springs, and sometimes destroying the water in wells for culinary purposes, as the sulphuric acid becomes strong enough to coagulate milk.

$\S 109$. The Group attains its greatest thickness at about 1,000 fect in Wayne County, and gradually diminishes westerly, so that on Graud River, Canada, it does not exceed 300 feet, which belongs chiefly to the upper portions, from the summit to a little below the gypsum-beds. The beds of gypsum are never continnous for long distances, but appear as detached lenticular or dome-like masses; the strata above them being arched over and often broken, while those below constitute an even, undisturbed floor. The Group is continued through Lake Huron to 
the Straits of Mackinac, where it forms the island and the points of the main land. The thickness on the peninsula of Michigan does not exceed 50 feet. It is broken up in a ridge extending west from the west end of Lake Erie near the southern line of Michigan, where it is much thicker, and again at Put-in Bay Island, and at Sandusky and other places in Ottawa County, Ohio, and may be seen on the western and south-west anticlinals, which pass through Wood County, and as far south as Delaware and Pike. The thickness in Ohio has not been accurately ascertained, but including the Waterlime, which is not separable, the thickness is several hundred feet. It has been identified in Missouri, varying from 10 to 75 feet in thickness. It does not occur in Wisconsin or Iowa, and is unknown south of Pennsylvania in the Appalachian system. The composition of the rocks indicates shallow water; but as there is no conglomerate, it does not appear as a shore deposit.

$\$ 110$. It is not very fossiliferous at any locality, and generally fossils are extremely rare. In addition to the two species mentioned as common to the lower part of it and the Guelph, Orthoceras sublove, Euomphalus sulcatus, and Avicula triquetra were early described from Wayne County ; but the indistinct forms of Spirifera, Atrypa, and Cornulites remain without specific names.

$\$ 111$. The Waterlime takes its name from the earthy, drab-colored limestone used for making hydraulic cement, and is regarded by some as a distinct Group, while the Canadian Geologists regard it as the lower member of the Lower Helderberg. It has its characteristic minerals and fossils; but, following the New York Geologists, it is here treated as the upper member of the Onondaga. In New York and Pennsylvania its thickness is from 30 to 300 feet, and is well-defined and recognized by its mineral nature, its fossils and position. In Eastern New York a brownish limestone, oftell mottled, containing corals, fragments of crinoids, and small Orthoceras forms the base of it. All the species of Pterygotus belong to the Waterlime, while Eurypterus remipes and Pterinea rugosa are characteristic of it in New York. The species which has the greatest geographical distribution in the Onondaga, is that peculiar form called Pleurodictyum problematicum.

$\$ 112$. The whole Group contains more or less carbonaceous matter, and the quarries usually smell of petroleum, and the limestone generally gives up the odor when struck with a hammer. This Group is the source of a large part of the gas supplied by the gas-wells of Ohio and Indiana. It is the chief source of the salt manufactured in New York and in Michigan. On the St. Clair River, at Marine City, rock-salt occurs in a mass, extending from 1,633 feet to 1,748 feet below the surface, which is mined by forcing fresh water down into it to take up the salt, and afterward pumping the brine and evaporating it. Thick masses of rock-salt have been formed at various other places in this Group within the salt districts of New York, Michigan, and Ontario. 


\section{CHAPTER XVII.}

\section{LOWER HELDETRERG GROUP.}

$\$ 113$. Thrs Group was named from the Helderberg Mountains, and defined by Hall in 1859, in the third volume of the Palwontology of New York. The lower member is a thin-bedded, often thinly laminated, dark-blue limestone, resting on the Waterlime-beds called Teutaculite limestone. The second member is a thin limestone full of Stromatopora, followed by a dark-gray concretionary limestone, in irregular layers, charged with Pentamerus galeatus and other fossils, which has a maximum thickness in Otsego County of 80 feet, and is called the Pentamerus limestone. The third is a blue, drab-weathering, calcareous shale and blue limestone, full of Spirifera macropleura and other fossils, having a maxinum thickness in Albany County of 70 feet, called the Delthyris or Catskill Shaly limestone, from Catskill Creek, near Madison, Greene County. The fourth member is a light-gray limestone, full of broken Encrinites, having a thickness of 25 feet. And above this there is a bluish-gray limestone, charged with Brachiopoda, ealled the Upper Pentamerus limestone. These local subdivisons are not recognized at any distance from the Helderberg Mountains, nor does the Group occur in Western New York or Western Canada. Strata of this age occur in two or three small outliers in the great basin near Montreal, at the distance of 200 miles from the nearest exposure of the Group in Now York. 'The most important of these is on the Island of St. Helen's, opposite Montreal. The Group, however, is quite largely developed in the Eastern Provinces, where it includes part of the Gaspe limestones. It is exposed on both sides of the Hudson River, and forms the outlier known as Becrafts Mountain, aud appears in Maine and New Hampshire. Its maximum thickness in New York is about 400 feet, and nearly as much in Maine, while at Gaspe it is 2,000 feet. It extends southwardly to Tennessee, having a thickuess in Pemusylvania of 1,400 feet, in Virginia 1,000 feet, in New Jersey 150 feet, and in Temessee 100 feet. It has been identified at Cape Frazier in latitude $80^{\circ}$.

$\$ 114$. This is an important Group on the eastern part of the continent, but does not occur west of the Appalachian system, which is in striking contrast with the Onondaga, that spreads out westerly from New York instend of southerly. It abounds in limestone strata, and the evidences of marine life, the latter apparently succeeding that of the Ningara age, by gradual change and development.

Crinoids, Corals, Bryozoans, Brachiopods, Gasteropods, Lamellibranchs, and Crustaceans were abundant, but we have no evidence that a vertebrate land or fresh-water animal had yet made its appearance on this continent. The evidence of swamp or air vegetation is on the increase, and here we discover the genus Annularia, which subsequently became so abundant in the Coal Measures. The characteristic fossils are: Tentaculites gyracanthus, Spirifera macropleura, S. vanuxemi, Eatonia singularis, E. medialis, Pentamerus galeatus, P. pseudogaleatus, Streptoryhnchus radiatum, Strophonella punctulifera, Meristella lavis, Rhynchonella semiplicata, R. ventricosa, Strophodonta varistriata, Avicula naviformis, A. manticula, Beyrichia granulata, and $B$. notata.

$\$ 115$. Petroleum springs occur on the St. John's River and on Silver Brook, 
in the Gaspe series, and in cavities of an amygdaloidal greenstone at Tar Point, which has hardened in some instances to the consistency of pitch, and from its peculiar odor the name Tar Point was given to the locality. The source of this oil is from the fossiliferous rocks or shales beneath, and exudes from an anticlinal. No good well has, however, been discovered by boring in these rocks.

$\S 116$. With this Group the Upper Silurian closes, because we have another stratigraphical and palæontological chasm, and have arrived at the top of the System as established by Murchison. The absolute want of conformability, with the overlying rocks, is everywhere apparent, and an age of time is therefore "unrepresented in the geological column.

\section{CHAPTER XVIII.}

\section{DEVONIAN SYSTEM.}

$\$ 117$. The Devonian was named in 1837, by Murchison, from Devonshire, in England. It has greater thickness, and is capable of more subdivisions based upon its fossils in this country than in any other part of the world. It is subdivided in ascending order as follows: Oriskany Group, Upper Helderberg Group, Hamilton Group, Portage Group, Chemung Group, and Catskill Group.

$\S 118$. It commences with a sandstone formation, after which it consists principally of limestone and shales. It is unconformable with the Upper Silurian at all places, except possibly Gaspe, Canada, where the sediment seems to have been regularly deposited from one age to the other. Its greatest development is in New York and Pennsylvania, where mechanical detritus accompanies the marine deposits. During this era land-plants became abundant, and fish swarmed within the seas, while the Archipelago, which had existed in the Silurian era, began to assume somewhat the outlines of a coutinent, though by no means such as we now behold. Corals, Crinoids, Brachiopods, Gasteropods, Cephalopods, Lamellibranchs, and Crustaceans were abundant, while Cystideans became extinct. It was a long and glorious era, marked by more progress in animal and vegetable organisms than characterized earlier ages. The plants increased in number of genera and species from the Lower to the Upper Devonian, until the flora presented a strong resemblance to that of the Subcarboniferous, especially in the prevalence of Gymnosperms and Cryptogams, though very few species are identical in the two Systems. It is everywhere unconformable with the Subcarboniferous. The masses and dykes of intrusive granite in Nova Scotia, which penetrate all the rocks older than the Subcarboniferous, belong to the close of the Devonian. The carbonaceous shales of this System exceed in thickness those of any other System of rocks, and, as a result, they are the chief oil and gas producing rocks on the continent. Very valuable iron ores and manganese ores occur in this System in different States. In Virginia luge masses of manganese are found imbedded in exposed sandstone ledges, where the supply seems to be practically inexhaustible. 


\section{CHAPTER XIX. \\ ORISKANY GROUP.}

$\$ 119$. This Group was defined as the Oriskany sandstone by Vanuxem, in 1839, and named from the white sandstoue oceurring at the Falls of the Oriskany, in Oneida County, N. Y., where it is about 20 feet in thickness. It forms a narrow belt of rough sandstone from the Hudson to Cayuga Lake, charged with peculiar fossils, and varying from a few inches to 30 feet in thickness. It stretches south in the Appalachian region through Pennsylvania, Marylaud, and Virginia, and has a thickness in Pennsylvania of 300 feet. It appears in New Jersey with a thickness of 130 feet. In Maine there is a large exposure between Parlin Pond and Aroostook, and it exists at Gaspe and in Nova Scotia. It is known in Canada at but few places, one of the principal exposures being at North Cayuga, and covering ouly 230 aeres. In Southern Illinois it is underlaid with silicious limestune, ealled the Clear Creek limestone, which constitutes incomplete passage-beds from the Upper Silurian. It is also known in Missouri.

$\$ 120$. It appears as a belt deposited upon the shores of the islands which then existed, and to mark their outlines in a greater or less degree. Like other arenaceous deposits, it indicates the presence of land and shallow water. It abounds in the easts of Brachiopods and Gasteropods in New York, Marylund, and Virginin, and in some places Crinoids occur. The characteristic specics are Spirifera arenosa, S. arrecta, S. pyxidata, Rensselaeriu ovoides, Orthis proximus, O. musculosa, Strophodontu magniventra, S. magnifica, Cyrtina rostrata, Fatonia peculiuris, Leptocoelia fabellites, and Platystoma ventricosum. In some places in Virginin the shells are silicified and quite free from adhering matter, and the exterior markings and intermal structure are well preserved, even the internal coils of Brachiopoda are beatifully represented. Near Cumberland, Md., a few elegant crinoids have been found, and one Cystidean, Anomalocystites disparilis, which is the latest known representative of that order, except Strobilocystites calvini.

$\$ 121$. The Brachiopods are Devonian in their character rather than Siluriau, and there is graduation to the sueceeding rocks through the Cauda galli grit, which is a dark, gritty slate, bearing few fossils. 'The rocks are not such as to have preserved land-plants very well; but they should have preserved fish-teeth if any then existed, but no trace of them has been discovered. 


\section{CHAPTER XX.}

\section{UPPER HELDERBERG GROUP.}

$\$ 122$. This Group was named from the Helderberg Mountains, where it was divided into the Cauda-galli grit, Schoharie grit, Onondaga limestone, and Corniferous limestone. The Corniferous limestone being the only one which has any great geographical distribution, the Canadian Geologists in 1863 used "Corniferous formation" instead of Upper Helderberg; but as Corniferous is a mineralogical word, Upper Helderberg is to be preferred. The Cauda-galli grit is a dark gritty slate covered with Taonurus cauda-galli, and graduates into the Schoharie grit, which is an arenaceous limestone weathering to a brownish color. These occur in the eastern counties of New York, Albany, Greene, and Schoharie, but soon thin out and are not found west as far as the center of the State. The scales and bony plates of fish are first found in the Schoharie grit. The Onondaga is a gray subcrystalline, coralline limestone. It is followed by the Corniferous limestone, which bears dark-colored, cherty beds, that break with a horny fracture, which suggested the name Corniferous; but the cherty beds occur in various places in these two divisions, and there is no real line of separation between them. The chert, or hornstone, is largely composed of microscopic, silicious forms of plants or protophytes, spiculie of sponges, fragments of the dental apparatus of Gasteropods, and other organisms. The aggregate thickness of the Group in New York is about 300 feet.

$\$$ 123. From New York the Group extends in a belt west across the peninsula of Canada to Mackinac Island, where it is 250 feet thick, and from thence into Michigan where its thickness is 354 feet. It appears at Sandusky and Northwestern Ohio, at Columbus, and on the Ohio a few miles below the mouth of the Scioto, resting upon the Waterlime Group, which has great thickness in this State. It crosses into Northern Indiana, and striking south-westerly, crosses the Ohio River at Louisville. It appears in Illinois, Iowa, Missouri, and Tennessee, resting on the Oriskany, or the Waterlime, or the Niagara, and everywhere preserving the character of the great coral-reef period of the Devonian, but never exceeding a thickness of about 300 feet. In New Jersey, however, the Caudagalli grit has a thickness of 400 feet, and the Corniferous limestone 500 feet, making a total thickness of 900 feet. It occurs in the western mountain ranges, and is one of the most persistent and generally distributed Groups.

$\$ 124$. It is a marine limestone, distinguished for the remarkable abundance of corals, and coral reefs, the variety in form, number, and size of species, some specimens being several feet in diameter, and larger than any belonging to any earlier period. It is distinguished also for its fish remains, which consist of teeth, or the outer bony covering, sometimes so abundant as to constitute the major part of layers, 3 or 4 inches, or even more, in thickness. Some were very large and singularly constructed. The Macropetalichthys sullivanti had a head 15 inches in length composed of hard, bony plates, covered with a thick skin dotted with tubercles. Cephalopods are abundant and quite characteristic, and in a few places drifterl land-plants have been found, but they are not of general occurrence. The 
most characteristic species among the invertebrates, and those by which the rocks may be readily identified are Cyathophyllum rugosum, Favosites goldfussi, Syringopora maclurii, Phillipsastrea vernenili, Nucleocrinus verneuili, Spirifera acuminata, $S$. gregaria, Pentamemes knighti, P. aratus, Stricklandinia elongata, Paracyclas occidentalis, Conocardium subtrigonale, Platyceras dumosum, Tentaculites scalariformis, and Dalmanites selenurus. In the vicinity of Davenport, Iowa, it furnishes an abundance of durable and massive building material and contains cavernous openings, as if worn out by the action of water, and filled up subsequently with material derived from higher rocks, and especially those of the Hamilton Group. The quarries at Columbus, Ohio, and North Vernon, Indiana, are in this Group. The strata in the vieinity of the Straits of Mackinac have been eroded and excavated so as to produce the Island of Mackinac, and large masses of the materials have been transported and distributed over Southern Michigan and Ohio.

$\$ 125$. The limestones of this Group in Canada are usually bituminous, and petroleum frequently fills the cells of corals and other fossils. 'The corals often prevail in distinet bands, some of which will be saturated with the oil, while others will not. Petroleum springs rise from this Group at Tilsonburg, and other places along an auticlinal which runs through the Western Peninsula. The oil being lighter than water, and permeating the strata, naturally rises to the lighest part of the anticlinal between the impervious layers of rock, and escapes to the surface. In other localities the bitumen is solid, and takes the form of asphaltum or minerul pitch, as at Kincardine, where slaty beds contain from 10 to 15 per cent of bitumen soluble in benzole. No good well, however, has been discovered in Canada by boring in these rocks, though it has been contended the oil at Enniskillen and on the Thames has its source here. Where the oil has been found in this Group, it has had its source in the Waterlime or in the shales below.

\section{CHAP'TER XXI.}

HAMILTON GROUP.

$\$$ 126. Thus Group was named from Hamilton, Madison County, New York, and defined by Vanuxem in 1842, though he did not include within it the Marcellus Shale, Tully Limestone, and Genesee Slate. 'The divisions made for it in New York are Marcellus Shale, Ludlowville Shale, Encrinal Limestone, Moscow Shale, Tully Limestone, and Genesee Slate. The rocks are not suseeptible of this division, exeept locally, and they all belong to a single Group. The Marcellus Shale was named from Marcellus, where it is an argillaceous slaty rock, bearing much carbonaceous matter, and sometimes small pieces of coal, and has a thickness of about 200 feet. It contains layers of impure limestone, and abounds in fossils. In many places it contains so much bitumen as to give out flame when thrown into the fire, which led the early settlers to explore it throughout its whole extent for coal, only, of course, to suffer disappointment. It is not separable from the Ludlowville Shale by any well-defined characters. The Ludlowville Shales were named from the town of that name, and separated from the Moscow Shale by a layer of limestone 3 or 4 feet thick, called the Encrinal limestone; but such 
division is scarcely worthy of recognition. The three have a thickness varying from 300 to 900 feet, extend from Lake Erie to the Hudson, and abound in fossils. The Tully limestone was named from Tully, where it is burnt for lime, and has a thickness of 14 to 20 feet. The Genesee slate, named from the opening of the gorge of the Genesee River at Mount Morris, where it is a black, argillaceous fissile mass, attains a thickness of 150 feet and closes the era of the Hamilton Group in New York.

$\S 127$. The Group extends from the Hudson to Lake Erie, occupying a belt of variable width in the central part of the State, and attaining a maximum thickness in the eastern part of 1,200 to 1,400 feet, and diminishing to about 300 feet in the western part. The valleys of Seneca and Cayuga Lakes are excavated for more than half their length in these rocks, and the banks and ravines afford the best facilities for examination. It is an olive shale, with slates and sandstones in the eastern, and calcareous shale and limestone in the western part of the State. The bedded rocks are remarkable for the abundance of ripple-marks, and wave-lines, and the shales abound in carbonaceous material, due to vegetation. Fucoids and marine plants are common, and coniferous trees and ferns grew to a good size, and drifted into the ocean, where they were imbedded and preserved, so as to show much of their form and structure. The New York subdivisions are lost in the extension across the peninsula of Canada from Lake Erie to Lake Huron, and the Group becomes a limestone in Michigan. It occurs at only one place in Wisconsin, which consists of a strip about 10 miles long and 5 or 6 wide, near Milwaukee, where it is an impure limestone, quite fossiliferous, and largely mined for the manufacture of hydraulic cement. It occurs iu Ohio, resting on the Upper Helderberg as far south as Columbus, and the upper part of the limestone at the Falls of the Ohio, is referred to it. It occurs at Davenport and New Buffalo, in Iowa, and also in Illinois and Missouri. It appears among the western mountains, on the Mackenzie River, in Alaska, and in the Arctic regions. It has greater thickness in Pennsylvania, New Jersey, Virginia, and other States in the Appalachian chain, than it has in the West, and contains much more mechanical sediment. In the East it is a mud rock supplied with drift materials and marine remains, while more westerly it is exclusively a marine calcareous rock.

$\S 128$. It is of quite general distribution and usually readily determined by its invertebrate fossils, which exceed in number almost all earlier Groups. Lepidodendron, which became so common in the Coal Measures, is found in the shales. The remains of fish are much like those of the Upper Helderberg, though species are distinct. The characteristic fossils, and those by which the Group may usually be determined, are Heliophyllum halli, Spirifera pennata, S. granulifera, Tropidoleptus carinatus, Rhynchonella venustula, Athyris spiriferoides, Leiorhynchus limitare, L. quadricostatum, Orthonoti undulata, Cypricardella bellistriuta, Cimitaria recurva, Pterinea flabellum, Modiomorpha concentrica, Bellerophon patulus, Pleurotomuria sulcomarginata, Styliola fissurella, Homalonotus dekayi, and Phacops bufo.

$\S 129$. The oil-springs of Enniskillen and of the Thames, in Canada, were known to the Indians and to the settlers from an early period. The oil floated upon the surface of the waters, and formed by its drying beds of tarry bitumen. On sinking through the clay from 40 to 60 feet, a bed of gravel is reached, from which considerable supplies of petroleum are obtained. Such are called surface-wells, 
and are less productive than the deeper ones. Below the gravel thin limestones, shales, and clays occur for a distance of about 230 feet before the Upper Helderberg limestones are reached. One of these wells, when sunk to a depth of 200 feet below the surface, yielded, when first opened, 2,000 barrels of oil in twenty-four hours. In some of the wells bored in this vieinity, both oil and water flowed to the surfince, and in some of the deeper ones the water is saline. Wells bored into the Upper Helderberg limestone sometimes reached small quantities of oil, but no valuable wells have thus far been discovered in Canada by boring below the Hanilton Group. The flowing wells soon become intermittent, and within a year cease to flow altogether; they continue, however, to furnish oil by pumping for a limited period, and then appear to be exhausted. The petroleum differs in volatility; the less volatile contains paraftine in solution, and is suited for lubricating machinery, while the more volatile is best suited for light. The alliaceous odor of some of the unrefined oil is due to the presence of a little sulphureted hydrogen. Petroleum is modified on exposure to the air by volatilization and oxidation, and eventually assumes a solid form. Thus near Oil C'reek, in Enniskillen, the thickened oil formed two layers, called gum-beds, of a viscid, tarry consistence, covering two or three acres with a thickness from a few inches to two feet. In sinking a well, a bed of this asphaltum, from 2 to 4 inches thick, was met with at a depth of 10 feet, upon a layer of gravel. It contained the remains of leaves and inseets, which were imbedded in it during its slow accumulation and solidification. In boring the oilwells there is always a greater or less disengagement of inflammable carburetel hydrogen-gas, and sometimes it is liberated with explosive violence. The strata almost everywhere in that region hold in a condensed state portions of light carbureted hydrogen, which is discharged wherever a natural fissure or an artifieial boring furnishes a vent. The shale on Sulphur Island, at the mouth of Thunder Bay in Lake Huron, is so highly eharged with bituminous matter that it has been set on fire and burned for months. The bitumen burns out and leaves the shale with a reddened appearance.

\section{CHAP'TER XXII.}

\section{PORTAGE GROUP.}

$\S 130$. This Group was named from Portage, New York, and defined by Hall in 1843. It consists of variable shales and sandstones, forming in New York an east and west band, resting upon the Hamilton Group, and dipping south about 25 feet in a mile. 'The sandstones produce falls in the streams, beautiful caseades, and grand and striking.scenery. The highest perpendicular fall of water and decpest canons and'gorges in the State exist in this Group. It thickens westerly aud thins easterly, and does not extend to the extreme eastern part of the State. Sandstones greatly predominate in the eastern part, while shales increase westerly, until the whole Group becomes a mass of black, bituminous shale. The thickness on the Genesee is 1,000 feet, on Lake Erie 1,400 feet. A considerable part of Lake Erie is excavated out of this Group, which shows a belt on the south side extending nearly to Sandusky; and from here it bends southerly across Ohio, leaving Columbus to the west, and, reaching the Ohio River below the mouth of the Scioto, 
it crosses into Kentucky, and is soon broken up in the spurs of the mountain ranges. It crosses Lake Erie, and occupies a small part of the Canadian peninsula, and enters the southern peninsula of Michigan, where Winchell called it the Huron Group. From Michigan it crosses the north-western corner of Ohio, and enters Indiana, forming a belt across that State by way of Indianapolis, and, reaching the Ohio River at New Albany, crosses into Kentucky, and extends far toward Tennessee. It was called the Black Shales in the Geological Survey of Ohio for 1838, and in that of Indiana for 1839, and in later surveys of Kentucky, Indiana, and Tennessee. The thickness in Ohio is from 200 to 1,000 feet or more, in Indiana from 100 to 200, and in Tennessee from 10 to 150 feet. It has never been recognized west of these States, and is therefore classed as a Group belonging to the Appalachian mountain system.

$\$ 131$. Fucoids, wave-lines, and ripple-marks are numerous, and occur throughout its distribution. The paucity of fossils in this Group, when compared with those above and below it, is one of its striking characters. Whole days may be spent in some parts of it without finding a shell, though fucoids are in the greatest abundance. Land-plants occur in profusion in New Brunswick, some of which are of gigantic size. Gomiatites complanatus, Panenka speciosa, and Spirifera levis occur in New York and in Ohio, and may therefore be considered characteristic. Fish of large size, covered with thick heavy plates, and having jaws and teeth strong enough to crush a body the size of a man, occur in it. Cladodus, a carnivorous fish, became abundant in this period, and flourished until the Permian. It was world-wide in its distribution, and its vertical range exceeds that of any other genus of fishes. The Group seems to have been deposited in internal seas or arms of the ocean, and is the last Group of the Devonian System, having a large geographical distribution, for the Chemung and Catskill are comparatively local in their extension. In Ohio there are large concretionary balls of impure limestone, some of them several feet in diameter, and it was in one of these the monster Dinichthys was discovered.

$\$ 132$. The Group is distinguished as the great seat of petroleum, and is supposed to be the source from which the chief supply in this country is derived. In New York, Pennsylvania, and Ohio the wells are bored through the overlying rocks until the Portage is reached, or the saturated sands that overlic it furnish the supply. Ten per cent of the shales is bituminous and carbonaceous matter. The shale yields oil by distillation, and gas and oil springs abound in its sandstones, and in those which overlie it. The great oil-sands in the oil regions of Pennsylvania belong to the Chemung, and have doubtless been fed as well from the shales of this Group as from those of the Chemung, which furnish the same products. The gas at Fredonia, New York, in this Group, was used for lighting houses in 1820. Lyell described it in his travels in 1841 , and it has been in constant use, with little variation in the supply, ever since. 


\section{CHAPTER XXIII.}

\section{OHEMUNG GROUP.}

5 133. This Group was named from the exposure at the Chemung Upper Narrows, at Chemung, New York, and defined by Vanuxem and Hall in 1842 and 1843. The shale and sandstone at Ithaca, having a thickness at Hectur's Fulls of 400 feet, was called the Ithaca Group, bint it is only part of the Chemung. The Chemung consists of a highly fossiliferous series of shales and thin-bedded sandstones and impure limestones, and an infinite variety formed from admixture of these. Except in a few localities there is no marked line between it and the Portage below. The two are distiuguished by their fossils. The shales vary in color from a leep black to olive-green, with every grade of intermixture; the sandstones are gray, olive, or green, and almost the whole series weathers to a brownish olive. The Group forms an cast aud west belt across the southern part of New York, having a thickness in the eastern part of 2,000 feet, dipping southerly at 25 feet or more to the mile, and thinning westwardly, so as not to be determined a short distance from where it crosses the line of Ohio. It is unknown farther west. In its extension from Lastern New York into Penusylvania the thickness increases until it exceeds 3,000 feet. It occurs at New Brunswick aud at Gaspe, Canada, but has not been satisfactorily determined at many other places, though it probably occurs in

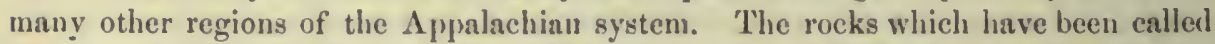
Chemung in Ohio, Indiuna, Illinois, Missouri, Iowa, and Michignn belong to the Waverly, except the thin, tapering belt in North-eastern Ohio, already mentioned.

$\$ 134$. The alternations and interlaninations of shales and sandstones show deposition under similar circumstances to those under which the Portage was deposited. The source of the materials was to the enst or south-east of New York, as evidenced by the thiuning of the deposits and diminution of sandy strata toward the west. 'The land-plants occur in Instern New York, and disapjear westerly, proving the land existed in that direction. The marine and land plants are abundant in the sandstones, while marine shells increase with the decline of the sandstones and augnentation of the shales westerly, though fucoids continue in abundance wherever the Group exists. The plants foreshadow the approaching Curboniferous System by the presence of Archuopteris, Cyclopteris, Sigillaria, Lepidodeudron, and Trigonocarpon. The fauna has more of a Carboniferous aspect than any which preceded it, and there is a diminution of the types which characterized the earlier Devonian. The species having the greater distribution and most characteristic are Lepidodendron chemungense, Archeopteris laxu, Asterophyllites parvulus, Orthis impressa, Orthix tioga, Streptorhynchus chemungense, S. pectinaceum, Strophodonta cayuta, S. mucronata, Choneter muricatus, Productella hirsuta, Spirifera disjuncta, S. mesacostalis, Atrypa dumosa, A. hystrix, Aviculopecten duplicatus, A. megistriatus, Leptodesma longispinum, L. spinigerum, Leiopteria chemungensis, Pterinopecten dispandus, $P$. crenicostatus, P. suborbicularis, Pterinea consimilis, Crenipecten crenulatus, Mytilarca chemungensis, and Phacops nupera.

$\$ 135$. Springs, evolving carbureted hydrogen-gas, or gas accompanied with petroleum, are common throughout nearly all that part of New York and Pennsyl- 
vania covered with the Chemung. The rocks in nearly all localities emit a bituminous odor on percussion, and petroleum often exudes from the crevices. The oil and gas products are the same in the Chemung as in the Portage. The gas and the oil had the same origin. They are both hydrocarbons. They were both derived from vegetable and animal organisms. Wherever shales are found containing carbonaceous matter, evidence of these products may be obtained. It is possible the gas was first produced, and from it the petroleum has been derived, making the latter a secondary product; but the evidence seems to prove they were both formed at the same period of time, and during the decomposition of the organisms, and before the mud had indurated or hardened into rock. And the evidence also seems to prove they were derived almost wholly from marine plants, for the shales bearing the greater number of fucoids are those to which we ascribe the greater supplies of hydrocarbons. The sandstones which overlie these shales are porous and capable of holding from one-eighth to one-tenth their bulk of petroleum, which is sufficient to account for the flowing wells of Pennsylvania which are bored until they penetrate the sandstone. Many of the wells penetrate only the Chemung sandstone, though the oil is derived from the shales of the Portage as well as from the Chemung. The supposed connection of petroleum and gas with anticlinal axes, or synclinal ones, has not been verified by observation, nor supported with reason, neither are they dependent upon faults or crevices, and much less has the depth of the well any connection with the level of the sea. Wells are as valuable when bored below the sea level as they are when the proper rock is struck above that horizon.

\section{CHAPTER XXIV.}

\section{CATSIKILL GROUP.}

$\S 136$. This Group was named by Emmons from the Catskill Mountaius, and quite fully defined by Vanuxem in 1842. It consists of sandstones, shales, slates, conglomerates, and impure limestones. The prevailing color of the arenaceous portion is brick-red, though all of it is more or less colored with iron, and the shales are gray, olive-red, or green. It exists only in a few counties in Southeastern New York, in the Catskill Mountains, where it has a thickness of 3,000 feet, and dips rapidly toward Pennsylvania, where it reaches a thickness of 7,500 feet, and soon disappears. It does not extend west of the :Genesee Valley in New York, and is wholly unknown on any part of the continent west of that State. It is conformable with the Chemung, and is distinguished only by the change in lithology, and by the fossils. No Corals, Crinoids, Brachiopods, or Trilobites have been described from it, and only a few Lamellibranchs. The land-plants are generally very poorly preserved. The fish remains are relied upon to really prove the rocks belong to the Devonian rather than to the Subcarboniferous age, and though these are rare and poorly preserved, they show it is the equivalent of the Old Red Sandstone of England, and therefore Devonian. In some places the sand is cemented and forms a grindstone grit, and there are hard concretionary masses, and strata unequally hardened, that weather into picturesque rocks. The Group is 
almost wholly a mechanical deposit of very limited distribution and enormous thickness. There are ripple-marks and other evidences of shallow water in different strata. The fossils charactéristic of it are Aneimites obtussus, Amnigenia castskillensis, Holoptychius americanus, H. taylori, and Dipterus shemoodi.

\$137. The total maximum thickness of the several Groups belonging to the Devonian as given above is 14,500 feet, though no single section would furuish such a depth. The greatest thickness is in Pennsylvania, and next in New York. The thickness at Gaspe, Canada, is 7,036 feet, and the divisions into Groups are not well defined. In the Western States several Groups are missing, and the thickness of the rest is only a few hundred feet. All the strata are marive; no laud or fresh-water shells have been found within them, and the land-plants are fairly supposed to have drifted to the places where they oceur. The Devonian is everywhere unconformable with the superimposed Subearboniferous, which always begins with a conglomerate or sandstone. The great reef-forming Corals so conspicuous in the Upper Helderberg and Hamilton, did not survive the era. Cystideans becane extinct. The family Spiriferida, which commenced in the Upper Silurian, became most prosperous in this age, and lived until the Jurassic. The three most notable steps in the progress of development are found in the growth and abundance of land-plants, the appearance of insects, and in the introduction and diversity of fish. The Devonian fish belong to the Selachians or cartilaginous fislies, the Ganoids, or fishes covered with plates or bony seales, and the Placoderms. There is nothing known in connection with plants or animals indieating the temperature of the sea, or elimate on land, was different then from what it is now.

\section{- chAPTER XXV.}

\section{SUBCARBONIFEIROUS SYSTEM.}

$\$$ 138. Tuns System was named and defined by David Dale Owen in 1838, in the Geological Survey of Indiana. He found it to consist of massive sandstones, limestones, and shales, lying between the Devonian and the Coal Measures, to be characterized by Pentremites and other peculiar fossils, and to be eapahle of subdivision into Groups. The name Subearboniferous indicates its position is below the Coal Measures. In the great valley of the Mississippi it is divided, in ascending order, into Waverly, Burlington, Keokuk, Warsaw, St. Louis, and Kaskaskia Groups. These Groups have been fully defined in Illinois, Iowa, Missouri, Arkansas, Indiana, Ohio, Kentucky, and Tennessee, and ean be determined with more or less satisfaction beneath the Coal Measures in the four larger eoal-basins, though not throughout their whole extent. For example, while the Groups are not distinctly marked in Pennsylvania, they can be readily determined on the opposite side of the basin in Kentucky and Tennessee. This is beeause the rocks consist largely of sandstones and shales in the east, which did not preserve well the fossils, while in the west they are principally limestones, containing fossils in great profusion and perfection. In Pennsylvania the sandstones and shales have a thickness of 5,000 feet, which thin westerly and southerly, and gradually give way to limestones and deep marine deposits. 
$\S 139$. In Nova Scotia the lower part is called Lower Coal Measures, and the upper part Lower Carboniferous Marine Formation, or more generally the whole is called Lower Carboniferous, even where its thickness is 6,000 feet. It consists of sandstones, shales, conglomerates, and limestones, with beds of gypsum. The limestones bear Brachiopods specifically identical with those of corresponding age in the Illinois basin. In Penusylvania and in Nova Scotia thin seams of coal occur in the strata, which is not the case farther west. On Cape Breton the thickness is 4,600 feet. In the Rocky Mountain region there is a thickness of 4,000 to 7,000 feet or more, and the several Groups may be determined at different places. The System has been divided in the west into the Lodore Group, Tonto Group, Red Wall Group, Lower Aubrey Group, and Upper Aubrey Group. Prof. Dawson found no palæontological or stratigraphical reason for regarding the Subcarboniferous as a System distinct from the Carboniferous, but as it is generally capable of subdivision into Groups, is always unconformable with the Devonian, begins with a sandstone, and is followed by a conglomerate or sandstone unconformable with it, there is good reason for retaining the name, though if the lines were not better defined elsewhere than in Nova Scotia, we might join Prof. Dawson in discarding it.

$\$ 140$. There are some fossils in this System almost world-wide in distribution, and belonging alike to all the Groups into which it has been subdivided; viz., Spirifera striata, Athyris lamellosa, A. planosulcata, Orthis michelini, O. resupinata, and Productus semireticulatus. There are some that occur in the rocks of this age in each of the Coal-basins on this continent; as, Athyris subtilita and Productus cora. It is in this Sytem at Hillsborough, New Brunswick, the bituminous mineral Albertite is so abundant. The rocks are thin-bedded shales, composed of fine, indurated clay, with much bituminous matter, and are full of fossil fishes in a good state of preservation. The shales have been disturbed and contorted,--and contain the vein of asphaltic mineral called Albertite. The theory of its creation is as follows: The argillaceous mud which formed the indurated shales, was charged with finely comminuted vegetable matter, which in its decomposition furnished the petroleum that at some later age escaped into a vein or fissure in the rocks, and by losing its more volatile parts and"partial oxidation, it hardened into the coaly or asphaltic substance. No extra heat for such transformation was necessarily required. Springs yielding petroleum flow from these rocks in various places. Peroxide of manganese, used in bleaching and in gas manufacture, occurs in limestone near the base of the System, and wad or black manganese ore is abundant at different places. Alum frequently occurs from the spontaneous weathering of pyritous shales, and is sometimes manufactured from them. Saline springs are not uncommon; indeed, they are numerous from the commencement of the Upper Silurian rocks to the close of this System, and occur occasionally both above and below such range. The conglomerate on the Stewiacke, Musquodoboit, and St. Mary's Rivers, is auriferous. It was formed from auriferous quartz-veins, derived from the Taconic System, and gold occurs in it exactly as in modern auriferous gravels, being found in the lower part of the conglomerate and in the hollows and crevices of the underlying unconformable rocks. The rocks of the age of this System in Europe are commonly known as the Mountain Limestone. 


\section{CHAP'TER XXVI. \\ WAVERLY GROUP.}

$\$$ 141. Thrs Group was named in 1838 , by Mr. C. Briggs, an assistant geologist on the Ohio Survey, from Waverly, Ohio, where it consists of a fine-grained sandstone, about 300 feet in thickness, superimposed upon a black argillaceous slate 200 or 300 feet thick, and is followed by from 40 to 80 feet of conglomerate. He identified the rocks at Portsmouth, Piketown, and Chillicothe. Mr. J. W. Foster, another assistant, followed them through Licking and Fairfield Counties. In 1839 David Dale Owen, after having examined the rocks in Ohio, found them in Indiana, Illinois, and Kentucky, and described the freestone knobs displayed back of New Albany as the Waverly Sandstone series, and referred them to the base of his Subcarboniferous System. Owen established this Group as a geological subdivision by a fair definition. Owen, Norwood, Pratten, and other Western geologists recognized the Group from that time forward. In 1841 Hubbard recognized the Group in the geological survey of Michigan. Hall and some Eastern geologists erroneously asserted the rocks were of Devonian age. In 1861, Meek and Worthen, having ascertained, upon palieontological evidence, the limestones at Rockford, Indiana, at Chotean, Missouri, and at Kinderbook, in Pike County, Illinois, belong to the base of the Subcarboniferous rocks, proposed to call them the Kinderhook Group. They understood they were making a synonym, but supposed they were including less in their Group than is included in the Waverly. In the same year Alexander Winchell described the Marshall Group of Michigan, and afterward thoroughly defined it, and proved its identity with the Waverly Group, the Kinderhook, the Yellow sandstone series of Iowa, and Choteau limestone, Vermicular sandstone and shale, and Lithographic limestone of Missouri.

$\$$ 142. The Group in Ohio forms a belt from 10 to 20 miles in width, commencing near the mouth of the Scioto, and bearing north and north-east toward Cleveland, but widening as it approaches Lake Erie, until its width exceers 40 miles. It rests upon the Portage Group, and has been called in its northern extension the Cuyahoga shale, Berea Grit, Bedford and Cleveland Shales. It crosses the Ohio from the Scioto, and entering Kentucky is soon broken up anong the mountain ranges. In Indiana it forms a belt extending from New Albany north, by way of Rockford, and south across the Ohio River, by way of Danville and Knob Lick, Kentucky. The fossiliferous, greenish, mottled limestone at Rockford, so famous for its Goniatites is at the base of the Group. The maximum thickness in Indiana is 500 feet, in Kentucky 200 feet. In Michigan, at Marshall, Hillsdale, and other places, it consists of reddish, yellowish, and greenish sandstones, having a thickness of 160 feet, and the Napoleon sandstone, 123 feet in thickness. It furnishes large quantities of salt and gypsum. The brine is obtained by boring and pumping, and very large salt-works are established on the Lower Saginaw River. Salt has been largely manufactured from brine obtained from the rocks in Ohin. The celebrated Ohio freestone, so much used for building purposes, is from this Group.

$\S 143$. In Missouri, the Lithographic limestone has a thickness of 55 feet; is a fine-grained, compact limestone, breaking with a free, conchoidal fracture, and is 
especially characterized by Pentremites roemeri. The Vermicular sandstone has a thickness of 75 feet, and is ramified with irregular perforations resembling wormburrows. The Choteau limestone has a thickness of 100 feet, and was named from Choteau Springs, in Cooper County. It has an extensive geographical distribution. At Burlington, Iowa, the Group has a thickness of 77 feet, and consists of shales and sandstones, capped by a four-feet bed of oolitic rock. It thins northerly until it disappears. It has a thickness in Illinois of 200 feet, and at Kinderhook it consists of grit-stones, sandy and argillaceous shales, with thin beds of fine-grained and oolitic limestone. It has been identified in the Wahsatch Range, in Utah, and at other places in the great West.

$\$ 144$. The fauna, on the whole, has assumed a Carboniferous aspect, noticeable in the species which pass to higher Groups, and more strongly in the genera of fish remains. Fossils having a wide distribution and characteristic species are Productella concentrica, Productus cooperensis, Spirifera carteri, S. extenuata, S. peculiaris, Syringothyris halli, Athyris hannibalensis, Rynchonella hubbardi, R. missouriensis, Centronella allii, Bellerophon cyrtolites, Grammysia hannibalensis, Orthoceras indianense, Goniatites oweni, G. marshallensis, and Phillipsia doris.

\section{CHAPTER XXVII.}

\section{BUTLINGTON GROUP.}

$\$ 145$. This Group was named from Burlington, Iowa, where it was called the Burlington limestone before it was described as a geological subdivision. No single geologist seems to have established the Group, or to have introduced the name to science, though the first full definition is in the geological survey of Iowa for $\mathbf{1 8 5 8}$. The limestone at Burlington is subcrystalline, often friable, and largely composed of crinoidal remains, has a thickness of 100 feet, and thins out northwardly. It increases in silicious matter toward the top, until the limestone merges into silicious beds, which, without evidence of unconformability, separate it from the Keokuk Group. Hall referrerl these cherty layers to the Keokuk, but White, Wachsmuth, and others refer them to the Burlington. In its southern extension, the Group dips below the bed of the Mississippi, and rises again at Quincy, and furnishes a fine exposure at Hannibal, Missouri. It exists in nearly every county on the Mississippi, from St. Louis to Iowa, and west from St. Charles to Howard County, and at Sedalia. The thickness varies from 100 to 500 feet. From a collection of fossils received from Prof. Cope, the author identified the Group in the Lake Valley Mining District of New Mexico; and it doubtless exists at other places in the great West.

$\$ 146$. The separation of the Burlington from the Keokuk could not be maintained were it not for the great change in the specific characters, of the Crinoids, and this resulted probably from the deeper, or clearer, or less disturbed water in the western localities during the Burlington period, than existed in the eastern localities. The detrital material may have prevented the recognition of the Group in the Appalachian system, and rocks of the same age in Ohio, Kentucky, Indiana, and other States may be referred to the Waverly or the Keokuk. In no other 
period did the harder parts of Crinoids so completely form the limestone, and hence it is pre-eminently the age of Crinoids. As the Graptolida reached the height of development in the Quebec or Upper Taconic, the Orthoceratida in the Black River, and the Cystidea in the Niagara; so did the Crinoidea in the Burlington. The bed of the ocean was eovered with a dense growth of Crinoids, one generation after another, while the superineumbent water swarmed with fish and invertebrate life. About 400 species of Crinoids, or one-fourth of all known, are from this Group. Among those having the greater distribution and being most characteristic are Dorycrinus missouriensis, D. parvus, D. unicornis, Batocrinus christyi, B. pyriformis, B. rotundus, Actinocrinus proboscidialis, Platycrinus planus, Amphoracrinus divergens, Belemnocrinus typus, Strotocrinus regalis, Steganocrinus comcinnus, and Physetocrinus ventricosus.

\section{CHAPTER XXVIII.}

\section{INEOKUIE GROUP.}

\$147. This Group was named from Keokuk, Iowa, where it was extensively quarried, and known as the Keokuk limestone, before it was known as a geological subdivision. It was first defined by Owen in 1852, and afterward by Hall in 1858 . As defined by Hall, it consisted of fifty feet of fossiliferous limestone capped by 40 feet of shale, abounding in geodes of quartz, called the geode bed. Others refer the chert layers, which separate it from the Burlington, to this Group. It rapidly thins out to the north, but maintains its thickness southerly to the mouth of the Illinois River, and appears in the south-western part of Missouri, with a thickness of 200 feet, where it is a lead-bearing rock. It crops out in Indiana, 40 or 50 miles north-west of Crawfordsville, and extends southerly, erossing into Kentucky a short distance below New Albany. The thickness does not mueh exceed 100 feet. It is celebrated at Crawfordsville for the abundance and perfection of the Crinoids; entire specimens-roots, column, head, arıns, and pinnules-have been collected. It is well displayed in Southern Kentucky, at King's Mountain tunnel, and in Tennessee, where the thickness is 200 feet. It occurs in Richland County, Ohio, and at other places on the western border of the Appalachian coal basin, but has not been described on the eastern border. It has been identified at numerous places in the western mountain ranges.

$\$ 148$. Ores of lead and zine occur in South-western Missouri in pockets and fissures associated with limestone and chert, and some of the mines are very rich and have been largely worked. In New Mexico and south of there, in Mexico, silver and lead occur in veins and fissures, some of the mines being very valuable. Some of the fossils having an extensive distribution, and being characteristic, are Dorycrinus mississippiensis, Cyathocrinus multibrachiatus, Barycrinus hoveyi, Forbesiocrinus wortheni, Platycrinus hemisphericus, Agaricocrinus americams, A. wortheni, Actinocrinus lowei, A. pernodosus, Batocrinus biturbinatus, B. indianensis, Goniasteroidocrinus tuberosus, Cyathocrinus subtumidus, Palcacis compressus, Amplexus fragilis, Productus vittatus, Orthis keokuk, Spirifera kcokuk, S. suborbicularis, Platyceras fissurellum, $P$. equilaterale, and Iithophaga illinoisensis. 


\section{CHAPTER XXIX.}

\section{WARSAW GROUP.}

$\S 149$. This Group was named from Warsaw, Illinois, by Hall, in 1856, and more fully defined in 1858. At the typical locality, near Warsaw, it consists of maguesian, arenaceous, and shaly limestones, abounding in Bryozoa. It is conformable with the Keokuk, only a few feet in thickness, and generally considered as a member of the Keokuk. I have retained it, because so many small fossils have been described from it, which have been the means of identifying it, at great distances from the typical locality. It occurs below the limestone of the cliffs at Alton, Illinois; at Bloomington and Spergen Hill, Indiana; and in St. Genevieve County, Missouri, where it attains its maximum thickness of 100 feet. It should probably be regarded as a mere member of the Keokuk Group. Some of the fossils having great distribution, and therefore characteristic, are Endothyra baileyi, Dichocrinus simplex, Alloprosallocrinus conicus, Batocrinus icosidactylus, Pentremites koninckanus, Productus biseriatus, Spiriferina norwoodana, Athyris hirsuta, Rhynchonella grosvenori, $R$. mutata, Terebratula turgida, T. formosa, Cypricardinia indianensis, Bellerophon sublovis, Naticopsis carleyana, Holopea proutana, Cyclonema leavenworthanum, Pleurtomaria subglobusa, and Spirorbis annulatus.

\section{CHAPTER XXX.}

\section{ST. LOUIS GROUP.}

$\$ 150$. THIs Group was named and described by Dr. Shumard in the Geological Survey of Missouri, in 1855. In St. Louis County it is celebrated for its splendid quarries, and consists of hard crystalline limestone, sometimes cherty, with thin layers of argillaceous shales, and has a maximum thickness of 250 feet. It forms bluffs below St. Louis as far as Carondelet, where it dips beneath the Mississippi, but soon rises again, and forms bluffs as far as the Meramec, some of which are 175 feet high. It is exposed in the western part of Illinois and eastern part of Missouri and Iowa, thinning out a short distance north of Keokuk. It forms a band of red clay, chert, and limestone bordering the Indiana coal-fields, aud crosses Kentucky and Tennessee, south, by way of Clarksville. It borders the Appalachian coal-field in Southern Kentucky, and may be seen at Burnside, on the Cincinnati Southern Railroad and in Eastern Kentucky. In Indiana it consists of limestones, more or less argillaceous, with beds of red clay, sometimes containing geodes, and having a thickness of 200 to 300 feet. It does not lose its thickness in Kentucky or T'ennessee, but becomes more cherty and silicious. It is everywhere cavernous, and abounds in sunken rivers, lost or subterranean streams, and in surface, funnel-shaped siuk-holes. The Mammoth Cave of Kentucky, and the Wyandotte Cave of Indiana, which has been explored 23 miles, and has a room 240 feet high, are in this Group. 
$\$ 151$. These underground avenues have resulted from percolating water, without the intervention of earthquakes or other extraordinary agency. Surfice water from ordinary rain-storms, fuding its way through the ground as it does, to supply common springs, will take up carbonate of lime in ehenical solution in limestone countries, and by so doing the fissures through which it passes will be enlarged. In massive limestones with thin, shaly partings, the constant action for ages of percolating water, aided by disengaged earbonic-acid gas, will enlarge the fissures into rivulets, which will culminate in a subterranean river, finding nn outlet in some open stream at a lower level. Such is the process by which the sink-holes, caverns, and subterranean streams in this Group of rocks have been formed. Slight projections on the walls record the different stages of the streams as they were slowly eutting their way to greater depths in the limestone. At the bottom of caverns where little or no water is now flowing, rounded pebloles that have played their part in grinding out the channels oceur, as well as sand and clay.

$\$ 152$. When water, holding bicarbonate of lime in solution, slowly drops from the ceiling of a eavern, exposed to the air long enough to allow one equivalent of carbonic-ncid gas to escaje, the lime is crystallized. If the deposit takes place from above dowuward, in the form of an icicle, it constitutes stalactite; but if it forms on the floor, from below upwarl, it is stnlagmite. These two sometimes meet and form columns. If the solution which forms the stalnetites is free from oxide of iron and other impurities, they will he trnnslucent or milk-white. The presence of iron gives them a dirty yellow, rel, or brown eolor. The ehambers in which gypsum oecurs are dry, and when rosettes of alabaster or translucent lime are formed the caverns must be dry, as they will not form in a damp atmosphere.

$\$ 153$. The fossils having the greatest distribution, and which are most characteristic of this Group are Irithostrotion canadense, L. proliferum. Productus ovalus, P. marginicinctus, Melonites multiporus, Myalina st hudovici, Temnocheilus coxanum, and Solenocheilus collectum. Ores of lead and zinc occur in pockets and fissures in Livingston, Crittenden, and Caldwell Counties, Kentucky, and at Rosiclare, Illinois. The ores are associated with fluor spar and calc spar. 'The principal gangue with which the lead is associated in Hardin County, Illinois, is fluor spar, and it is thoroughly disseminated through it. The fluor spar is used for the manuficture of hydro-fluoric acid, and as a flux for smelting ores, where sulphuret of rinc is associated with galena. Lead occurs associnted with different minerals and in many Groups of rocks, but never appears to have had an igneous origin. 


\section{CHAPTER XXXI.}

\section{KASKASKIA GROUP.}

$\S$ 154. Dr. Geo. G. ANd B. F. Shumard were acquainted with this Group, in Kentucky, Indiana, Illinois, Missouri, and Arkansas, in 1852, but did not name it. Iu 1856 Hall named it, from Kaskaskia, Illinois, and more fully defined it in 1858. In 1866 Prof. Worthen called it the Chester Group, because he had proposed the name in 1853 , and had so informed Prof. Hall while acting as his assistant in 1855; but the latter published the information, and instead of using the name Chester used Kaskaskia. Chester is the shortest and best name, but Kaskaskia has priority of publication. At the typical locality it consists of a compact, arenaceous, and coarse-textured limestone, with shaly partings, in the lower part, heavybedded sandstone and limestone, with shaly partings, in the central part, followed by a mass of green shale, succeeded by heavy-bedded limestone. The thickness at Chester is 198 feet, at Huntsville, Alabama, 635 feet, on the southern line of Tennessee 720 feet, at the northern line 400 feet, and in Indiana 300 feet. It forms a belt surrounding the Illinois and Indiana Coal-basin, exists upon the western and south-western border of the Appalachian Coal-basin, and upon the eastern border of the Missouri and Arkansas Coal-basin. It consists everywhere of fossiliferous limestones and sandstones, and is followed by rocks unconformable with it.

$\$ 155$. The fossils having the greatest distribution and most characteristic are Acrocrinus shumardi, Agassizocrinus conicus, Hydreionocrinus depressus, Pentremites godoni, P. sulcatus, P. cervinus, P. obesus, P. pyriformis, Pterotocrinus capitalis, Talarocrinus comigerus, Zeacrinus maniformis, Athyris sublamellosa, A. subquadrata, Spirifera increbescens, Spiriferina spinosa, Euomphalus planidorsatus, and Temnocheilus spectabile.

\section{CHAPTER XXXII.}

\section{CARBONIFEROUS SYSTEM.}

$\$ 156$. This system is divided into the Carboniferous Conglomerate, Coal Measures, and Permian Group. The Carboniferous Conglomerate rests unconformably upon the Subcarboniferous rocks, and forms a belt around all the coalbasius. It is a massive sandstone or conglomerate, almost nonfossiliferous, except the occasional presence of Stigmaria, Calamites, and Lepidodendron. In Indiana the thickness is about 200 feet, in Illinois about 300 feet, in Kentucky 500 feet, in Ohio 200 feet, in Michigan 100 feet, in Pennsylvania 1,500 feet, in Virginia 1,000 feet, and in Nova Scotia, where it is called the Millstone grit, 6,000 feet. The pebbles are well rounded, showing the fragments of rock were rolled for a long time on the beaches by the action of the winds and waves, before they were cemented into rock. A similar conglomerate separates the Subcarboniferous and Coal Measures in Europe, where it is called the Millstone Grit. It bears the marks everywhere of a shore-line deposit that surrounded the basins of internal seas. It does not underlie the whole of the Coal Measures-the central parts of the basins are free from it, as is shown by artesian boring. 


\section{CHAPTER XXXIII.}

\section{COA L MEASURES.}

$\$ 157$. TuE name "Coal Measures" originated among the miners of England before Geology became a seience. It is familiarly used in the earliest text-books on Geology, as a scientific term, which was understood without a definition. It is applied to part of the Carboniferous System, and not to Cretaceous or Tertiary Coal regions. The Coal Measures consist of beds of sandstone, shale, slate, limestone, elay, and coal, which are variable in their geographical distribution. The area eovered in North Ameriea is estimated at about 210,000 square miles, nearly all of which is included in five fields, funr of which are in the United States and one in Nova Seotia. Canada and British America are destitute of this important leposit, as well as many States in the Union, among which are Maine, New Hampshire, Vermont, Connecticut, New York, New Jersey, Delaware, South Carolina, Florida, Mississippi, Louisiana, Minnesota, and Wisconsin.

$\$ 158$. The Coal Measures of Novia Scotia rest upon Subearboniferous rocks, and are divided into the Millstone Grit, Middle Coal Formation, and Upper Coal Formation. A section of the Millstone Grit is as follows: 1. Reddish shales and red and gray sandstones, having a thickness of 2,082 feet, containing no coal, and poor in fossils, exeept a few drifted trunks of trees. 2. Sandstones, red shales, and a few dark-colored shales, with nine small or rudimentary coal-beds, with a total thickness of 3,240 feet. The underelays abound in Sigillaria, and some strata are quite fossiliferous, containing plants, crustaceans, and fish. 3. Red and gray sandstones, red and chocolate shales, arenaceous conglomerates, and thin beds of concretionary limestones, huving a thickness of 700 feet, making a total thickness of 6,000 feet. The Middle Coal Formation includes the productive coal-bels, and contains no marine limestones or conglomerates. It consists of shales and sandstones, and has a thickness of 4,000 feet. The Upper Coal Formation consists of shales, sandstones, conglomerutes, limestone, and eoal, and has a thickness of 3,000 feet. On Cape Breton, the last two divisions have a thickness of 10,000 feet, making the maximum thickness of the Measures, 16,000 fect. From Nova Scotia the Measures dip south-west, and reappear in the form of a subtriangular basin in New Brunswick. The area in Nova Scotia and New Brunswick is 18,000 square miles. The coal is all hituminous. There are 72 seams and numerous dark bands containing more or less carbonaceous material. A coal-bed at Pictou is $37 \frac{1}{2}$ feet thick, and another 221 feet. A large part of the coal-basin is beneath the waters of the Atlantic and the Gulf of St. Lawrence.

$\$ 159$. The first coal-field in the United States is the Appalachian, which extends over important parts of Pennsylvania, Virginia, West Virginia, Maryland, Ohio, Kentucky, Tennessee, and Alabama. Its length is 875 miles, and width from 30 to 200 miles. The anthracite region is in the north-eastern part of Pennsylvania, and does not cover 500 square miles. The coal-beds form synclinals, anticlinals, or stand highly tilted on their edges, but are never horizontal. All the other parts of this great area, estimated at 60,000 square miles, produce only bituminous coal, and the beds may be horizontal or possessed of a slight dip, to which all the strata 
are subjected. The aggregate thickness of the coal-beds in the Pottsville district is 120 feet, in the Wilkesbarre district 62 feet, and in the Pittsburg district $25 \frac{1}{2}$ feet. The thickest vein at Wilkesbarre is $29 \frac{1}{2}$ feet, and at Pittshurg 8 feet. The best seam in Ohio is from 6 to 12 feet in thickness, and is called the Hocking River Coal-bed.

$\$ 160$. The second coal-field in importance covers nearly two-thirds of Illinois, the western part of Indiana, and the western part of Kentucky, and has an area of 47,000 square miles. The coal is bituminous, and the aggregate thickness of the coal-beds is about 40 feet. Indiana is celebrated for her block coal. There are ten seams of coal in a vertical thickness of 600 feet in Illinois, and six of them are from 2 and one-half to 6 feet each in thickness.

$\$ 161$. The third coal-field in importance is the larger one, and occupies parts of Iowa, Missouri, Kansas, Nebraska, Arkansas, and Texas, and has an area of 80,000 square miles. The coal is all bituminous. The western part of Missouri and eastern part of Kansas bear coal in abundance. The Coal Measures are the lowest Group of rocks exposed in Kansas, and have a thickness of 2,000 feet. There are 22 seams of coal, varying in thickness from a few inches to seven feet. Ten of them are more than a foot each in thickness. The coal in Arkansas is excellent.

$\$ 162$. The fourth coal-field is in Michigan, and occupies about 6,700 square miles, with a thickness of about 125 feet. The coal is bituminous, and consists of one bed from 3 to 5 feet in thickness throughout the whole shallow basin, being thinnest near the border. Toward the central axis of the basin there are 2 or 3 thin seams in close proximity to the main seam. The shales are well stocked with fern-leaves and other terrestrial vegetation. There is a small area in Rhode Island and Massachusetts of about 1,000 square miles, having a thickness of 6,500 feet, but possessing no valuable coal-seam. The basin has suffered by the metamorphism of the rocks and plication of the strata. The coal-seams have been changed to anthracite, and are often somewhat wedge-shaped or of irregular thickness.

$\$ 163$. The Coal Measures were deposited in basius, and must necessarily vary much in thickness, the Group in Nova Scotia being thicker than elsewhere, and the Group in Michigan thinner. The maximum thickness in Peunsylvania is 8,00u feet; Ohio, 2,500 feet; Tennessee, 2,500 feet; Western Kentucky, 3,500 feet; Indiana, 1,000 feet, and Missouri, 2,000 feet. The Group is frequently separated into an upper and lower series by the intervention of a conglomerate, and sometimes more than one conglomerate exists in the Group. Marine vegetation abounds at some localities, and land or marsh plants are distributed throughout the shales, sandstones, and coal. Coal was formed from plants which grew in swamps, narshes, and open seas, and, where valuable, it is quite free from sediment, such as would have accompanied much disturbance of the water.- The beds usually rest on clay, bearing Stigmaria and stumps of trees, and are followed by rocks bearing the leaves of the vegetation of that era. The clay beneath the coal-beds is usually an argillaceous sediment, almost devoid of alkalies, and represents the ancient soil in which the coal vegetation flourished, and apparently deprived it of the greater part of its potash. This clay is usually excellent fire-clay. From the coal, as from modern peat, the alkalies were almost entirely removed by the action of water. The waters were fresh, brackish, and salt at different times and at different places. The 
marshes were subject to overflows, as shown by the remains of fish and heds of sand and shale, while land-shells, air-breathing reptiles, and trees show the presence of land. The bark of the trees was the imost durable part, and it is not unusual in sandstone to find only a cast of the tree, covered with a thin film of coal, retaining the original markings of the bark. Some blocks of coal are composed of thin layers formed from the bark of trees and nothing else. Beds vary in purity, from coal with less than one per cent of earthy matter to dark-colored shales, with only a trace of coal.

$\$ 164$. When bituminous coal has lost part of its hydrocarbon gas, it is semibituminous, as at Blossburg and Broad Top Mountain coal-fields in Pennsylvauia ; but if the bitumen is all driven off, it is converted into anthracite. $\Lambda$ t gnsworks bituminous coal is put in a retort, and by the application of heat the gas is driven off, leaving a residue of coke; but if the gas is driven off under great pressure, the residuum is anthracite. When coal melts and runs together in the fire, forming a crust which must be broken to give vent to the draft, it is coking coal. Splint-coal or block-coal does not melt and run together, and is therefore dry-burning coal. Cammel-coal burns with a bright flame like that of a candle, from which circumstance it derived its name. Cannel was the pronunciation of caudle in Scotland and England, where this coal received its name. Coal containing sulphur is unfit for smelting iron ores in a blast-furnace, and is not suitable for the manufacture of illuminating gas.

$\$ 165$. Bituminous shales frequently contain iron ore disseminated tlırough them, either as a carbonate or sesquioxide, and sometimes forming black-bands. The same layer of shale which constitutes black-band ore at one place will have the ore gathered in balls, arranged in rows, at another place. By chemical affinity the disseminated particles were brought together, nud formed into balls or clises; and hence the iron exists in all stages, from fine distribution through the shales to layers of kidney ores, with whitened shales intervening. The iron ores of the Coal Measures are generally hardened mul, charged with iron, or clay-iron stone, and rarely yield more than 40 per cent of iron, and they are not of much value except as they exist around the margin of the Appalachian coal-field in the Lower Coal Measures. No good iron-mines are found in the other coal-basins. The greater part of iron manufactured from these ores has been obtained in Penusylvania.

$\$ 166$. The first trace of reptiles observed in the Carboniferous System consisted of foot-prints, found in 1841, in the Lower Coal Measures of Horton Bluff, in Nova Scotia. This was followed in 1844 by the discovery of reptilian bones at Saarbruck, and in 1851 to 1853 , bones in Nova Scotia, and the land-suail, Pupa vetusta. Since that time the discoveries have been numerous. There is no reason to suppose the atmosphere was charged then with any more carbonic acid than it is now; on the contrary, the air-breathing animals prove it was not. The life of plants and animals is controlled by oxygen, and the adaptation of organs is in accordance with its properties. If there was less oxygen in the atmosphere, the membranous reptile lung could not supply the demands of its system, and analogy proves these animals could not have existed in the coal period with a less proportion of oxygen than is required now.

$\$ 167$. The coal-beds and the vegetation of the coal period are usually suffi- 
cieut to determine the age of the rocks, but some of the invertebrate characteristic fossils of wide geographical distribution are: Fusulina cylindrica, Lophophyllum proliferum, Spirifera camerata, Productus rogersi, $P$. nebraskensis, $P$. longispinus, Chonetes mesolobus, Athyris subtilite, Spiriferina kentuckiensis, Macrodon carbonarius, Allorisma subcuneatum, Aviculopecten rectilaterarius, Pernopecten aviculatus, Pinna peracuta, Crenipecten retiferus, Myalina subquadrata, Bellerophon carbonarius, Pleurotomaria tabulata, $P$. sphcrulata, Macrochilina gracilis, M. primigenia, M. kansasensis, M. carinata, Nautilus missouriensis, Phillipsia missouriensis, and $P$. sangamonensis.

\section{CHAPTER XXXIV.}

\section{PERMIAN GROUP.}

$\S 168$. This Group was described by Murchison in 1845, in Russia and the Ural Mountains, and named from Perm, in Russia. It was first ascertained in this country by Swallow in 1858, in Kansas, where it has a thickness of 320 feet. Norwood announced its existence in Illinois, and Shumard described it in the Guadalupe Mountains of New Mexico, where it consists of white limestone, having a thickness of 1,000 feet. In Kansas it consists of magnesian limestone, marls, shales, conglomerates, and gypsum; the magnesian character increases southerly to New Mexico. Fossils are abundant on the Cottonwond, with sun-cracks and ripple marks, and sometimes small piles of fossils and fragments appear, as if washed together. It is conformable with the Coal Measures. In Pennsylvania the Upper Barren Measures, having a thickness of 1,000 feet, are referred to it. It is claimed the reptilian remains in Illinois and Texas have shown its existence in those States. It is always unconformable with the rocks above, in this country and elsewhere. Characteristic species are Pseudomonotis hawni, Myalina permiana, Bakevellia parva, Monotis hall, and Pleurophomus subcuneatus.

$\S 169$. This Group closes the Palæozoic series, to which this work is chiefly devoted. All the Groups exist in New York and Pennsylvania, except the subdivisions of the Subearboniferous can not be distinguished, and the doubtful Quebee Group has no existence there. The maximum thickness in these States is about 38,000 feet. 'Some of the Groups in the Lower Silurian have greater thickness in other States than they have in these two, and the Coal Measures are much thicker in Nova Scotia than they are in Pennsylvania. The whole Palæozoic series in the western ranges of mountains has an estimated thickness of about 40,000 feet. 


\section{CHAP'TER XXXV.}

\section{TRIASSIC SYSTEM.}

$\S 170$. Th: Mesozoic era is divided into three grand ages-Triassic, Jurassic, and Cretaceous. The name Triassic was applied to the rocks in Germany, in allusion to a threefold 'division which they present in that country; but no such division exists in America. Indeed, notwithstanding the vast thickness of the rocks, they have thus far baftled all attempts to divide them into Groups, and, on account of the similarity of the rocks with the Jurassic, and the barrenness of fossils in the eastern exposures, these Systems have not been satisfactorily defined and separated. On the eastern part of the continent they fill synclinal troughs, and have bcen very much disturbed by intrusive rocks and volcanic action. 'They generally rest on Laurentian or Taconic strata, and, of course, the bed is always unconformable. But on the westeru part of the coutinent they are frequently undisturbed, and spread over great areas of country, resting on unconformable rocks. The Triassic in the Connecticut Valley extends from Northficld, in the morthern part of Massachusetts, across the latter State and Connecticut to New Haven, on Long Island Sound, a distance of 105 miles. It fills a synclinal trough, and has its greatest width at the mouth of the Farmington River, which is about 20 uniles. The rocks cousist of red sandstones, conglomerates, shales, and oecasionally impure limestone. The naximum thickness is about 20,000 fect, but the upper 8,500 feet is referred to the Jurnssic, leaving 11,500 fect for the Triassic. A great many reptilian tracks, some fish and a few laud-plants and fucoids, have been described from these rocks. Much excellent building-stone has been quarried from the sandstone. About 15 miles west of the exposure, on Long Island Sound, there is another exhibit, about 6 or 7 miles long and 2 miles wide.

$\$ 171$. A long trough and great exposure begins at Stony Point, on the Hudson, and cxtends across New Jersey, Pennsylvania, and Maryland to Culpeper County, Virginia. It has a length of about 350 miles, and, though frequently narrowing to a breadth of 4 or 5 miles, expands in New Jersey to a wilth of ahont 36 miles. The general character of the rocks is like those in the Connecticut Valley, and the total thickness on the Delaware River is 27,000 feet, part of which is probably Jurassic. Another range crosses the Potomac near Washington City, and extends 25 or 30 miles beyond Richmond, and another exists 25 miles west of this one. There is a valuable coal-ficld in this System in Virginia, which is about 26 miles long and 4 to 12 wide. The James River flows through the middle of it, about 15 miles from the northern extremity, while the Appomattox traverses it near its southern border, and on its eastern side it is distant from Richmond about 13 miles. A great many fossil plants have been described from this locality. There are two basins in North Carolina. One begins at Lakeville, and extends about 30 miles south-west to Germantown, bcing from 4 to 6 miles wide; and the other commences in Granville County, six miles south of Oxford, and extends south-west about 120 miles, reaching 6 miles into South Carolina. Its width is generally about 6 miles, but at the widest part 18 miles. The thickness in some places exceeds 25,000 feet; the area is about 1,000 square miles, nearly one-third 
of which contains coal-beds. Very valuable beds of coal and beds of good argillaceous iron ore are distributed through it. Many fossils have been described from these rocks, and among them Dromatherium sylvestre, the earliest fossil mammal yet discovered in America. The rocks occur in Nova Scotia, on the north and south sides of Cobequid Bay, from Moose River to the month of North River, and on the south side of the Bay of Fundy. Prince Edward's Island, which stretches for 125 miles along the northern coast of Nova Scotia and New Brunswick, consists of rocks of this age.

$\S 172$. The red beds of the Triassic, consisting of every texture of sandstone and all varieties of red, are distributed almost throughout the Rocky Mountain system from Mexico to the Arctic regions, covering hundreds of thousands of square miles. Fossils have been collected and described from every territory and from nearly every mountain range throughout this vast extent of country. Over extensive areas of country the Triassic rocks are more than a mile in thickness, and bear internal evidence of having been deposited in the depths of the ocean without any mechanical sediment. Not a single species of any organism found in rocks earlier or later than the Triassic have ever been found within it, and very. few genera are common to it and rocks of earlier or more recent date.

$\S 173$. In Colorado and Utah the lower part of the Triassic has been called the Shinarump Group, and the upper part the Vermilion Cliff Group. The rocks of the Shinarump are persistent in their characters for hundreds of miles, and the coloring is strong and deep. They weather into striking architectural forms and terraced buttes. The rocks of the Vermilion Cliff Group are colored a brilliant red, approximating vermilion, or sometines inclining to orange, and constitute the great cliff-forming series of the West. The Group consists of massive layers of homogeneous sandstone, from 100 to 300 feet in thickness, with shaly layers intervening; the shales disintegrate, and thereby the sandrock is undermined and breaks off vertically. This process, in time, has presented a series of perpendicular walls and sloping taluses. In the West Humboldt Range of Mountains the lower part has been called the Koipato Group, and the upper part the Star Peak Group. 'The maximum thickness in this region has been estimated at 16,000 feet. The fantastic columns in the "Garden of the Gods" and in Pleasant Park, Colorado, have been weathered out of the sandstones of this System. 


\section{CHAPTER XXXVI.}

\section{JURASSIC SYSTEM.}

$\$ 174$. The Jurassic System was named from the Jura Mountains, of Switzerland. No Trigonia, Belemnites, Ammonites, or specially eharacteristic fossils of the Jurassic, have been found on the Atlantic side of the continent, notwithstanding the upper part of the rocks deseribed in the last chapter may be Jurassic. The Jurassic fossils, however, oceur in the Rocky Mountain Ranges from Mexico to the Aretic regions. The rocks exist in every State and Territory throughout that vast extent of country, varying in thickness from a few hundred feet to 10,000 feet. They follow the Triassic, and generally rest upon it. Fossils have been described from California, Arizona, New Mexico, Idaho, Colorado, Nevada, Montana Dakota, British Columbia, Cook's Inlet, Alaska, Point Wilkie on Prince Patrick's Land, and the islands north of Grimell I Land. In some parts of its grand geo- graphical distribution it is composed of sunclstones and clays, resembling, in appearance, the Triassic; but in others it consists of limestones, sandstones, shales, and clays, indicating shallow water, and bearing no resemblance to the Triassic. The limestones are frequently fossiliferous, and show the progress animal lite had made in the ocean, and vegetation had made on the land. Of 50 genera of vertebrates deseribed from the Jurassic, none of them are Palcozoic, and only two have been doubtfully identified in the C'retaceous. Ammonites, Ceratites, and Belemnites made their first appearance in the Jurassic, and became extinet in the Cretaceous. The genus Sprivifera, so abundant in the Devonian and Carboniferous, hecame extinct in the Jurassic. Several genera of mammalian remains have been defined from the Jurassic, but they are all peeuliar to it. No single species of plant or animal is common to the Jurassic and any other formation. 'T'en genera of Carboniferous plants have been identified in the Jurassic, and four genera occurring in the Jurassic have been identitied in the Cretaceous. There is a general progress anong the invertebrates towarl succeding ages, but the evolution of the vertebrates is very much more marked. There is almost universal unconformability with the overlying Cretaceous, and hence there is an era of time not represented by the rocks. It has been called the Reptilian age, because of the gigantic sauriaus which then infested the seas. Some of the rocks belonging to this System in California, and, especially about Mariposa, are said to be gold-bearing, but minerals are generally very scarce. 


\section{CHAPTER XXXVII.}

\section{CRETACEOUS SYSTEM.}

$\$$ 175. The name Cretaceous is from the Latin Creta, chalk, and was applied to the rocks in Europe long before its use as a geological term. The existence of the Cretaceous on this continent was first ascertained, in 1827, by Morton and Vanuxem. The Cretaceous is found either exposed upon the surface, or covered by the Tertiary, forming a border of variable width on the Atlantic Coast, from New York to Florida. In like manner it occurs everywhere south of the $33 \mathrm{~d}$ parallel, with the exception of limited areas in the mountain regions. It covers nearly all Mississippi, extends into Tennessee and Arkansas, and reaches Southern Illinois. West of the 97 th Meridian, from the $33 \mathrm{~d}$ parallel to the Aretic Ocean, the whole country is covered with this formation, with the exception of the areas in the mountain regions, exposing older rocks and inconsiderable extensions of land, where it has been swept away, and an area of some magnitude north and west of Hudson's Bay. This includes, of course, the whole extent covered by the Tertiary and more recent deposits. It is found east of the $97 \mathrm{th}$ Meridian, extending into Iowa, Minnesota, and some parts of British America. Or, approximately stated, the Cretaceous forms the surfacerock, or is overlaid with the Tertiary and recent strata over nearly half the North American continent, and from the extensive denudation it has suffered, we may fairly presume, at the commencement of the deposit, the land surface was not half its present dimension. In the east and south the formation is exclusively marine, but in the west the marine is succeded by a brackish-water deposit.

$\$ 176$. Meek and Hayden divided the marine Cretaceous of Kansas, Nebraska, and the great West, in 1861, in ascending order, into the Dakota Group, Fort Bentou Group, Niobrara Group, Fort Pierre Group, and Fox Hills Group. The Dakota Group was named from Dakota County, where it consists of sandstones, with alternations of various colored clays, and beds, and seams of impure lignite, silicified wood, and great numbers of leaves of the higher types of dicotyledonous trees, with casts of Pharella dakotensis, Axincea siouxensis, and Cyrena arenaria. The thickness in that locality is 400 feet, in North-western Colorado 600 feet, and in the San Juan region 1,000 feet. It is the supposed equivalent of the Eutaw Group of Alabama and Mississippi, which has a thickness of about 400 feet and contains beds of lignite.

$\$ 177$. The Fort Benton Group was named from Fort Benton on the Upper Missouri, where it consists of dark-gray, laminated clays, sometimes alternating with seams of limestone. It abounds in Inoceramus, Ammonites, Scaphites, Nautilus, and other fossils, and has a thickness of 800 feet.

$\$ 178$. The Niobrara Group was named from Niobrara, in Nebraska, where it consists of marls and limestones, and abounds in Inoceramus, Ostrea, and remains of fish, and has a thickness of 200 feet. It has an extensive geographical distribution, but rarely exceeds 500 feet in thickness.

$\S 179$. The Fort Pierre Group was named from Fort Pierre, in Dakota, where it consists of clays containing carbonaceous matter, seams of gypsum, and masses of sulphuret of iron, and abounds in the shells of Cephalopods, Lamellibranchs, 
remains of fish and saurians, and has a thickness of 700 feet. In Northern Colorado it is 800 feet thick, and in Alabama and Mississippi it is known as the Rotten limestone, and reaches a thickness of 1,200 feet.

$\$ 180$. The Fox Hills Group was named from Fox Hills, in Dakota, where it consists of gray, ferruginous, and yellowish sandstones, and arenaceous clays, abounding with shells of Cephalopods, Lamellibranchs, Gasteropods, remains of fish and saurians, and has a thickness of 500 feet. East of the Colorado Range its thickness is 1,500 feet, in the valley of Bitter Creek 3,000 feet, and in that of the North Platte 4,000 feet. It is the same as the Ripley Group of North Carolina, Alabama, and Mississippi, which has a thickness of about 400 feet.

$\S 181$. The thickness of the marine Cretaceous in New Jersey is about 700 feet. It is valued in that State for its fertile marl, and beds of kaolin in its lower part. In Louisiana its thickness is more than 1,000 feet, in the Uintah Mountain region 7,000 feet, and in New Mexico and British America more than a mile at many places. The eañon of San Carlos, on the Rio Grande, exposes a elear perpendicular height above the river level of 1,500 feet of Cretaceous strata. The Cretaceous is the Coal-bearing formation at Vancouver's Island and other points on the Pacific Coast.

$\S 182$. There is in the West, superimposerl upon the marine Cretaceous strata, rocks which were deposited in brackish water, and form transition-beds from the strictly. marine condition of the Cretaceous to the epoch of numerous fresh-water lakes, which were scattered all over the country west of the Mississippi, and north in British America to the Arctic regions. These rocks were named in 1861, by Meek and Hayden, the Fort Union Group. They consist of beds of clay and sand, with numerous seams and local deposits of lignite and beds of coal. The passage from the marine to the brackish-water deposits, and from the latter to the freshwater deposits, is without abrupt change in the sediment, and with complete conformability. There is no evidence of any important physical or climatic change, beyond the gradual filling up of the basins of the sea and the recession of the salt and brackish water, appearance of fresh-water lakes, and their gradual disappearance. The Fort Union Group has been called the Judith River Group, the Bitter Creek Group, the Bear River Group, the Laramie Group, and by divers other names. It has a thickness, in Bitter Creek Valley, Wyoming, of 6,000 feet, and in Bear River Valley, in Utah, of 7,000 feet. Its geographical distribution extends for a thousand miles in length, and a maximum width of 500 miles or more, with a varying thickness from 100 feet or less, to 7,000 feet or more. It abounds in plants belonging to Eocene genera, which connect the Cretaceous and Tertiary flora by insensible degrees, while the Dinosaurian remains demonstrate its Cretaceous age.

$\$ 183$. Before the diseovery of this Group, absolute nonconformability was supposed to exist between Cretaceous and Tertiary rocks, and this is the ease where marine Tertiary follows the marine Cretaceous, wherever known in the world. But here, where the marine Cretaceous is as recent as elsewhere, and the eontinuance of the period is represented by brackish-water deposits, and then fresh-wrater deposits in lakes eut off from the ocean, the rocks are conformable, and the vegetable and animal kingdoms show the slow progress of advancing ages. About one-third of the genera of plants belonging to that period have become extinct, but the living plants; Corylus americana, C. rostrata, Davallia tenuifolia, and Onoclea sensibilis, have 
been identified from the Fort Union Group, thus specifically uniting the Cretaceous era with the present time. It is possible, too much confidence in this identification may lead to error, and better specimens may show specific distinctions; but it is an important fact, they so closely resemble the living forms as to be mistaken for them, and show how closely the living are connected with the ancient dead. Among the Cretaceous genera of invertebrates, about one-third survive; three genera of reptiles, Crocodilus, Trionyx, and Emys survive; but no genus of birds or mammals has come down from that age to the present. There is no great break or chasm discoverable in vegetable or animal life in passing back to the Cretaceous era. No sudden physica! change has taken place over which some deposit may not furnish a connecting bridge. No evidence of any great climatic change is furnished, either in the animal or vegetable world, but on every hand we are encouraged to look at uniformity in the organisms, subject only to a constant, almost imperceptible evolution. Seams of productive coal occur at different places in this Group.

\section{CHAPTER XXXVIII.}

\section{TERTIARY SYSTEM.}

$\S 184$. THE organic remains of the Tertiary are so completely blended with the living, that no Quaternary age or period can be distinguished. The words Primary and Secondary have become quite obsolete in Geology, while Tertiary is so interwoven with the science as to be permanently fastened to the nomenclature, notwithstanding its definition, as the third age, has no application to the period to which it relates. The subdivision of the Tertiary, with reference to the survival of conchological species into Eocene, Miocene, Pliocene, and Post-pliocene, brings us to the living species as gradually as the species change within any of the subdivisions of geological time, or within any division of the strata into Groups. The Tertiary rocks generally consist of marls, clays, sands, or other friable material, filling depressious in the underlying rocks, and, though widely distributed, seldom form hard, continuous strata. This condition of the rocks made it difficult to determine the order of superposition, until a comparison of the shells had been made with living species. This comparison led to the naming of the rocks containing about 3 or 4 per cent of living species, the Eocene, which signifies the dawn of the present state of things; those containing 15 to 20 per cent of living species, the Miocene, which implies less recent; and those containing 90 to 95 per cent of living species, the Pliocene, which means more recent; and those having all the imbedded fossil shells identical with living species, though containing extinct mammalian remains, Postpliocene. Instead of determining the rocks by the per cent of living species, the contrary course is now adopted, and the age is determined by the extinct species. Certain species are regarded as types of Eocene age, or Miocene, as the case may be, and from the presence of these the rocks are referred to the proper Group. This subdivision of the Tertiary, with "reference to the survival of conchological species and the division into geographical Groups, have made a double system of nomenclature. 
$\$ 185$. The marine Eocene, commencing in New Jersey with a thickness of 37 feet, and exposing only a narrow surface area, crosses Maryland by way of Fort Washington; Virginia, by way of Fredericksburg, Richmond, and Petcrsburg; North Carolina, by way of Newbern and Wilmington; South Carolina, by way of Charleston and Shell Bluff, on the Savannah River; Georgia, by way of Milledgeville; Alabama, by way of Claiborne; and Mississippi, by way of Jackson and Vicksburg. In South Carolina it consists of loose sand, clay, gravel, sandstone, limestone, aud marl, covers a large area, aud has a thickness of 1,100 feet. It is divided into the Buhrstone Group, Santee beds, and Ashley and Cooper beds. It is exposed in Florida, and reaches up into Tennessee, where it is called the Porter's Creek Group. Conrad subdivided it in Alabama and Mississippi, where it has a thickness of about 900 feet, into the Claiborne Group, Jackson Group, St. Stephen's Group, and Vickshurg Group. It crosses Louisiaua, appears in Arkansas, and offers numerous exposures in Texas, Mexico, and California. It is extremely fossiliferous at many places, and nowhere conformable with the underlying rocks.

$\$ 186$. The gradual elevation of the western ranges of mountains through Cretaceous and Tertiary time, the formation of bays and arms of the sea, and lakes which have drained themselves in continuing succession, have linked the Tertiary with the Cretaceous, aud bound the Eocene, Miocene, Pliocene, and Post-pliocene with the present, almost as one connected age. In these lake regions the Eocene is divided into the Wahsatch Group, Green River Group, Bridger Group, and Brown's Park Group, and there are uumerous synonyms for each oue of them. The Wahsatch is characterized by its brick-red color, and has a thickness of 8,000 feet; the Green River Group is quite fossiliferous, aud has a thickness of 7,500 feet; the Bridger Group rests conformably on the Green River, consists of Bad Land sandstones, limestones, shells, and marls, and has a thickness of 2,000 feet; and the Brown's Park Group has a thickness of 2,500 feet. 'The combined thickness of the Eocene in the Western Territories is therefore 20,000 feet.

$\$ 187$. The marine Miocene beginning at Martha's Vineyarl, though it may exist as far north as Maine, crosses New Jersey through Cumberland County, and forms a border upon the east and south of the Eocene exposure a large part of the way to the Mississippi River, and west across Louisiana, Texas, and Mexico. It is not conformable with the Eocene, and in some parts does not interveue between it and later deposits. It has its greatest thickness in California, where it exceeds 3,000 feet. The Coast Range of mountaius is composed in large part of strata of this age, and hence its elevation has been siuce the Miocene period. It is highly fossiliferous, and the shells generally belong to living genera, and many of the species still survive in the waters bordering the adjacent coast, thus indicating no material change in the climate since that period. The Miocene lake deposits, like the Eocene, cover great extensions of Territory and reach an enormous thickness. In Nebraska it has been divided into the Wind River Group, which has a thickness of 2,000 feet, and the White River Group, which has a thickness of 1,000 feet. On the divide between the Arkansas and South Platte, where the thickness is from 1,500 to 2,000 feet, it is called the Monument Creek Group, and in Oregon it is called the Truckee Group.

$\S 188$. The marine Pliocene strata are found in Maryland, superimposed upon the Miocene, in South Carolina, upon the Eocene, and generally forming a narrow border at the east of these outcrops on the Atlantic coast, and a wider border on 
the south adjoining the Gulf Coast. Fossil shells of species now living on the adjacent coast, abound at every point, and demonstrate beyond reasonable doubt the climate and the waters on the eastern and southern coast of the United States, and in California, were then the same that now prevails. There is no palæontological evidence, so far known, that the Pliocene climate was different from the present on this continent, and as the outlines of the continent were then nearly as they are now, no material difference can be inferred. The Pliocene graduates into the Post-pliocene, so that separation of the strata frequently becomes impracticable, and an arbitrary approximating line for separation is assumed. The Pliocene lake deposits in Nebraska, are called the Loup Fork Group, and have a thickness of 400 feet and cover a great extent of territory, and in North-western Kansas have a thickness of 500 feet. In Wyoming they have a thickness of 1,500 feet, and are called the Niobrara Pliocene. In Bear River Valley they are called the Salt Lake Group and the Cache Valley Group, and the thickness is from 500 to 1,500 feet.

$\$ 189$. The Post-pliocene is represented by marine deposits on the coast, and by drift, sand, and gravel, in the middle part of the continent. In South Carolina it is confined to a belt along the coast 8 or 9 miles wide, and the fossil shells are those of species inhabiting the coast. In Los Angeles Valley, in California, the thickness is 500 feet; but where depressions upon the coast have been filled the thickness may be 1,000 or 1,500 feet, and so at the mouths of rivers where a delta has been formed, as at the mouth of the Mississippi, the Past-pliocene becomes of very great thickness. There are some Lake deposits of this age in the great West, which have a thickness of 500 feet or more. The marine Post-pliocene is usually conformable with the Pliocene, and graduates into the present deposits without disturbance. In South Carolina the bones of horses, hogs, dogs, rabbits, beavers, tapirs, and other mammals occur in the layers of blue mud and sand throughout the period. At some time during this age, man made bis appearance on this continent, for none of his work is found preceding it, nor preceding the drift; but his stone implements are associated with the remains of the mastodon and mammoth, and such animals as survived the drift period in such condition as to show they lived at the same time.

$\S 190$. During the Post-pliocene era, a portion of the country about Hudson's Bay was submerged by the ocean, as shown by the fossiliferous marine sands and clays occurring at 300 or 400 feet above the present level of the ocean. The rocks, too, are striated in all directions, as if done by icebergs or shore-ice holding angular fragments of rock. The New England States and New Brunswick, and that portion of Canada south of the St. Lawrence River and east of the vicinity of Montreal, was submerged, with the exception of the mountain elevations. Several beaches are shown at Murray Bay 90 miles below Quebec, varying from 30 to 326 feet above the bay; like beaches occur at Montreal and at various other places in this part of Canada. All these deposits abound in marine fossils belonging to living species in the Gulf of St. Lawrence and on the near coast of the Atlantic. The surface of the rocks below these deposits is polished and striated in the direction of the St. Lawrence Valley. Like phenomena occur over New Brunswick and the New England States, and extending as far south as the mouth of the Hudson; but they appear on no other part of the continent. These deposits contain no terrestrial or fresh-water fauna, and, so far as the marine life is concerned, connect the lowest of the clays with the present time by an unbroken chain of animal existence. 
$\S 191$. South of the Laurentian Mountains the surface of the rocks beneath the bowlder clay is striated in the direction of the valleys, but there is no connection between these and those occurring north of the mountains in the Hudson's Bay region. The force which produced the scratches did not cross the mountains nor exist upon them. Prof. Dawson has proven the bodies which produced them came from the Atlantic Ocean, and following up the St. Lawrence drifted to the south, at various angles, some floating over New Brunswick, and others over Maine, and others through Lake Champlain, and re-entering the Atlantic Ocean by the Hudson River, while others were driven beyond Montreal into the mouth of the Ottawa River. In New Brunswick the strix are related to the contour of the surface of the land, and conform to the direction of the river valleys. A south-easterly course prevails in the western part of Charlotte County, and a south-western course in the valleys east and north-east of St. Jnhn. A map of Maine showing the course of the rivers will show the course of the stria. The appearance of the surface geology of this State early suggested the fact that a great rush of waters poured over it from a northerly source, and transported by its power the surface débris which had accumulated in earlier ages by subaerial forces, and large masses of rock from parent ledges, and deposited them in regions more or less distant firom the several sources; and as they passed along they striated and grooved the rocks against which they impinged, or over which they rubbed in the traveled course. The strix conform to the valleys as a rule, and therefore have their courses in all directions, though some are found deflected at right angles to their original course. The Katahdin Mountains formed an obstruction around which the striating agency operated, but it did not cross the summit. The strix occur on the north side of the mountains, but not upon the south side. In Vermont, New Hampshire, Massachusetts, and Connecticut, beneath the drift, sand, gravel, bowlders, and clay, the surface of the rocks is grooved and furrowed in a general southern direction, though varying with the contour and course of the valleys. At the Island of New York the current swept from the north-west to the south-east, and the furrows are most strongly marked on the north-western slopes of the hills, and least on the south-eastern. In many instances they are very distinct on the western and north-western slopes, extending to the highest point of the rocks; but no traces exist on the eastern and south-eastern slopes, although both slopes are equally exposed. The strix are most numerous in the middle part of the island, somewhat less in the western, and least in the eastern, showing the current was deflected southward in the middle part of the island. Throughout all this area south of the Gulf of St. Lawrence and the St. Lawrence Valley, we have, in the strixe and furrows and in the distribution of clay, bowlders, gravel, sand, and fossils, the evidence of an overflow of the whole country, except the higher hills and mountains, the overflow resulting from subsidence of the coast, and the evidence that the Arctic current, instead of leaving the coast on approaching the mouth of the gulf, as it does now, flowed into the gulf and across the depressed New England area, transporting its fields of ice, which grounded upon the northern slopes of hills and mountains, and rubbed the rocks in the valleys and plains wherever the surface soil and subaerial accumulations were swept off by the grinding weight of a mass, driven by a current through water too shallow to float it. In the Gaspe Peninsula, ocean-terraces and stratified clay, containing marine testacea, occur at the height of 600 feet above the sea. In the Champlain region of Ver- 
mont, and the triangular area of 9,000 square miles extending from there to the Ottawa Valley, the marine fossiliferous clays and sand occur at all elevations, as high as 500 feet. They form a coating for New Brunswick, and a continuous belt on the coast of Maine 150 feet above the ocean. The marine species in these clays and sand are such as live at moderate depths, or varying from the littoral zone to 200 fathoms. The submergence must therefore have been much more than 600 feet, because the shells and bones must have had some depth of water, as well as the clay, to protect them, in order to produce the fossilization, and they received a covering of drift materials sufficient to protect them from the ocean currents, which then swept over that region, and the disintegrating and denuding agencies which have prevailed during the long train of centuries that have since elapsed.

$\S 192$. The fresh-water drift surrounds the great central lakes of the continent, spreads out over a large country in British America, and overspreads part of each of the States in the Valley of the Mississippi. This drift consists of clays, gravel, bowlders, and sand, containing no marine organisms, but bearing land vegetation which now flourishes in the same latitude, and fresh-water shells and the bones of terrestrial animals of the Post-pliocene age. There are beaches surrounding the lakes which show the lakes have occupied much higher levels than they now do, and were stationary for a time at each of these beaches. The terraces and lake deposits of sand and clay in Wisconsin show that Lake Superior stood 600 feet higher than it does now, at one time, in the Post-pliocene age, at which time it could have overflowed nearly the whole country south of it to the Gulf of Mexico. These terraces and lake deposits occur at different elevations surrounding Lakes Michigan, Huron, Erie, and Ontario, showing they were elevated as high as Lake Superior during this period. They have been noticed 750 feet higher than Lake Ontario. Here was then one grand central Post-pliocene lake, several times as large as all of them combined are now. Upon the shores of this lake angular rocks were rolled into bowlders and beaten down to gravel and sand, that formed beaches and terraces, which were subsequently swept south by the overflowing lake, and spread over Western Ohio, Western Kentucky, nearly all of Michigan, Indiana, Illinois, and Mississippi, and the eastern part of the States bordering the Mississipi River on the west. Large bowlders are spread over these States south as far as the Ohio River, though they gradually diminish in size in that direction, and soon the gravel disappears, and only the finer materials are spread over Mississippi and reach to the Gulf. Beneath these clays and sands, where the rocks were denuded of their subaerial débris, the surface is frequently scratched and furrowed. This is especially the case where the higher lands were overflowed. The scratches and furrows appear to have been made by shore-ice on the margin of the lake or lakes when occupying different elevations, and by ice carrying angular rocks and bowlders, that were driven against the shores or shallow places. They bear in all directions, and frequently cross each other, which proves they could not have been made by one body, or by any number of bodies moving in the same direction.

$\S 193$. Commencing in the lower tier of counties in New York, where the hills are from 600 to 800 feet above the level of the narrow valleys, and extending south over all the highlands of Pennsylvania, Virginia, West Virginia, the Carolinas, Georgia, Alabama, Eastern Kentucky, and Tennessee, and south to the Gulf of Mexico, there is an absolutely driftless area, and the surface rocks are free from 
scratches and furrows. It was dry land, and much of it high and mountainous, when the marine clays and sands were strewn over the territory adjacent to the Gulf of St. Lawrence and the New England States, and dry land during the period of the drift of the central part of the continent, and for geological ages antecedent thereto. The precipitous ledges and profound valleys of deuudation, the overhanging rocks and castellated outliers, furnish incontestable evidence of the ordinary eroding agencies through a period of time commencing anterior to the Tertiary epoch. There are extensive driftless areas in Eastern and Southern Ohio free from scratches and furrows on the surface rocks, and from drift, sand, gravel, and bowlders, and they are characterized by outliers, monument rocks, sharp ridges, and rugged scenery. The drift materials extend from the lakes to the sources of the rivers that flow into the Ohio, and over more or less of the land intervening between the head-waters; but below this they occur only in the valleys of the larger rivers. Wherever the valley was large enough to carry off the flow of water from the north, the adjacent land was not overflowed, and the height of the water in the valley is marked by river terraces. In Eastern Ohio, only those rivers having their sources in the central and northern part of the State have river terraces, as the Scioto, Hocking, and Muskingum, while the smaller tributaries, such as Raccoon, Shade, and little Muskingum, have not a vestige of drift, or scratch, or furrow, from their sources to the Ohio. The Ohio River Valley was large enough to carry off the water that flowed across Ohio and Iudiana, and hence no drift crossed the valley until it reached the western part of Kentucky. 'Throughout the drift area of Ohio, Indiana, and Illinois, it is common in excavations below the drift to fud an ancient soil of vegetable mold resting upon stratified rocks in place. Beech, sycamore, hickory, and cedar have been found where they grew prior to the drift; but beneath the ancient soil no striated or furrowed rock has ever been discovered.

$\$ 194$. There is a driftless area in the south-western part of Wisconsin, covering about 13,000 square miles, or nearly one-fourth of the State, and which extends into Northern Illinois, North-eastern Iowa, and Eastern Minnesnta. 'There is no drift, sand, clay, or gravel, and, as in all cases where these do not occur, there are no scratches or furrows on the surface of the rocks. This area was not overflowed by the lake, and is a region of narrow, ramifying valleys, narrow, steep-sided, dividing ridges, whose directions are toward every point of the compass, and whose perfectly coinciding horizontal strata prove conclusively their subaerial erosion. The ravines are all in direct proportion to the relative sizes of the streams in them. North and east of this driftless area, from 25 to 75 miles, there is a scantiuess of drift and numerous outliers, attesting the ordinary effects of erosion. The "Stand Rock," in the dells of the Wisconsin, the isolated ridges and peaks in the central part of the State, rising from 100 to 300 feet abruptly from the low ground around them, and composed of horizontaily stratified sandstone, or of saudstones capped with limestone, prove the regular erosion for ages, and are quite inconsistent with any single mechanical eroding power that must have operated upon the whole country alike. In Dakota County, Minnesota, there is an outlier of the St. Peter's sandstone known as "Lone Rock," whose summit is 100 feet higher than the surrounding country, and from which many other outliers are in view; and yet in the valleys the drift prevails and bowlders abound. In Wabasha County, the "Twin Mounds," and in Olmsted County the "Sugar Loaf Mound" and the "Lone Mound," attest in like manner 
the continuing erosion since Silurian times. The two lonely towers in the valley of the south branch of Root River, in Fillmore County, known as "Eagle Rocks," rise as high as the rocky walls of the valley, and evidence subaerial erosion, but are inconsistent with the idea that any large body of ice ever passed down the valley or across it.

$\S 195$. There is no drift in California, nor on the Pacific Coast as far worth as British Columbia and Alaska. There are no indications of it in the Rocky Mountain regions, or upon the great plains of the West. There are no such exhibitions of scratched and grooved rocks succeeded by fossiliferous marine clays and sands, with bowlders, as occur in the New England States and St. Lawrence region, nor of scratched rocks and ancient soils succeeded by clay, sand, and gravel, with bowlders, as occur in the central part of the continent; but, on the contrary, the whole country west of the Mississippi Valley is absolutely driftless, except as to local drift produced upon the shores of Tertiary lakes, and more or less distributed by the rivers that, in the course of time, cut out the canons which drained them. On the borders of the ancient lakes and rivers there are terraces, marking shore-lines at various places from Mexico to Alaska, but they are standing monuments to disprove the existence of a continental ice-sheet; for no one can conceive of the movement of a heavy body of ice across a valley without disturbing the graveled terraces that border upon both sides at different elevations. The natural towers that stand as evidence of erosion from the Wahsatch times to the present; from the Green River Eocene to the present; from the Bridger Eocene to the present; from the White River Miocene to the present; the columnar masses, irregular pyramids, sandstone towers, and turreted outliers of the Bad Lands of Colorado, Wyoming, Montana, Dakota, and British Columbia; the monuments on Monument Creek; the Garden of the Gods; the buttes in all the mountain chains; the transverse ridges, lone mountains, and exalted peaks; and the whole array of cañons from Texas and Mexico to Alaska, - all alike tell us, in language unmistakable, that no glacial sheet ever moved south upon the western plains or mountain ranges.

$\S 196$. Indeed, there is no evidence a glacial sheet ever existed on any part of the continent; none that gives any warrant to the hypothesis of a glacial period. On account of the valleys, hills, and mountains, no glacial sheet could move; and if one had ever existed, the waters flowing from it would have cut out channels of such dimensions they could have been not only traced, but their dimensions would have been such they could not be mistaken for any of the valleys now existing. Had there been a glacial period, northern plants and shells would be found occupying their places as far south as Florida, Louisiana, and Texas. But, on the contrary, no such flora or fauna is found farther south than it now exists, while the present flora and fauna occur in the same latitude throughout the Post-pliocene age, and passing back through earlier ages, unmolested by any visible climatic changes. The scratches and furrows so often cited as evidence of the glacial period do not exist upon the mountains, but occur only in the valleys and lower lands that were overflowed by water; and in these valleys there are now standing lone rocks and outliers that a glacier moving in the valleys would necessarily have swept away. The scratches and furrows are readily accounted for without the hypothesis of a glacial period; and on account of their position on the northern side of the higher elevations of land and not upon the southern, and their universal course up the 
valleys from the lakes without regard to the direction of the valleys, they can not be accounted for as glacial phenomena, for they are wholly inconsistent with it. The glacial epoch is a theoretical blunder, not supported by scientific facts or intelligent reasoning, and contrary to all geographical, geological, and palron tological information. There is no such geological period, and no gap into which it can possibly be injected.

\section{CHAPTER XXXIX.}

\section{NOMENCLATURE.}

THE rules of nomenclature are, with few exceptions, firmly established. They have resulted from years of experience and reflection, and tend to secure fixity and convenience in the designation of animals and plants. *Each animal and each plant has a name consisting of two words-the first generic, and the second specific. This is called the binomial system, or Linnsan method of nomenclature. The genera are arranged in families, the families in orders, the orders in classes, and the classes in subkingdoms. These divisions are sometimes further separated into sections or intermediate groups, often distinguished by the prefixes sub and super.

Linuæus first consistently applied the binomial system of nomenclature to all classes of organisms in 1758, in the 10th edition of Systema Nature; but he applied it to botany in Species Plantarum, published in 1753. It had bcen used intermittingly by earlier authors. Naturalists have generally adopted 1753 as the starting-point for the binomial system in botany, and 1758 for zoology, or, without reason, the 12 th edition of Systema Naturce, published in 1766. It can make no difference in palrontology which is regarded as the starting-point, for the last precedes the science. The names in the binomial system assume the Latin form by taking a Latin termination.

\section{DENOMINATION OF HIGHER GROUPS THAN GENERA.}

The names of groups higher than genera are usually taken from some of the principal characters. They are expressed by siugle words of Greek or Latin origin, in which a certain harmony of form and termination is preserved for groups of similar nature; as, Phanerogamæ, Cryptogamæ; Cephalnpoda, Gasteropoda.

Compounds of Greek and Latin words are not allowable. In cryptogamic botany, ancient names of families, such as Musci and Filices, have been employed as names of classes or sub-classes. Botanical cohorts or sub-cohorts are designated by the name of one of their principal families, with the termination ales.

The families in botany are designated by the name of one of their principal genera, with the termination acere, as Rosa, Rosacex; Ranunculas, Ranunculacece. To which there are the following exceptions: 1. When the genus from which the

*Note.-See Report of the 12th Meeting of the British Association for the advancement of Science, held at Manchester in June, 1842, Reprinted Cin. Quar. Jour. Sci., Vol. I, p. 351 ; Report of the British Association at Birmingham, in 1865, and Report of the Committee (W. H. Dall) on Zoological Nomenclature, to section B. of the American Association for the Advancement of Science, at the Nashville Meeting in 1877. The authorities are quite fully cited in the latter report. 
name of the family is taken ends in Latin with $i x$ or is (genitive icis, idis, or iscis), the termination icece, idece, or inece is permitted; as, Salix, Salicinece; Berberis, Berberideø; Tamarix, Tamariscinea. 2. When the genus from whence the name of the family is derived has a name of inconvenient length, and there is not a tribal name in the family formed from the same generic name, the termination ea is admitted; as, Dipterocarpece, from Dipterocarpus. 3. For some very large families universally known under their exceptional names, the ancient designation is preserved; as, Cruciferce, Compositce, and Graminece. 4. An old generic name no longer preserving that rank, but applied only to a section, or even a species, may be maintained as the base of a family name; as, Hippocastanece, from Aesculus hippocastanum.

Botanical sub-families are formed from the name of one of the genera contained in them, with the termiuation $e x$ or inece, and also the names of tribes and subtribes which take the termination exe; as, Rosece, from $R o s a$.

The names of zoological families are formed by adding the termination $i d a$ to the earliest known, or most characteristic genus contained in them; and of subfamilies by adding the termination ince; as, Terbebratula, Terebratulidae; Strix, Strigida, not Strixida; Buceros, Bucerotida, not Bucerosida or Bucerida. The $i$ in ida is short; but in ince it is long.

Names of higher rank than genera are not rigidly subject to the law of priority, because their limits fluctuate with the advancement of science, and changes are therefore allowable when newly discovered facts have made the name erroneous. And when a geuus from whose name a family name has been taken, is removed to another family, the family name may be dropped, and a new one may be coined for the remaining genera.

\section{ORTHOGRAPHY.}

The rules of Latin orthography must be adhered to. Greek names are Latinized by substituting for the Greek letters their Latin equivalents, according to the following table:

\begin{tabular}{|c|c|c|c|c|}
\hline$\alpha$ & $=$ & $a ;$ & $(\beta \bar{\gamma} \tau \alpha)$ & Beta. \\
\hline$\beta$ & $=$ & b ; & $\left(\beta \rho \alpha \chi_{i \omega \nu}\right)$ & Brachium. \\
\hline$\gamma$ & $=$ & $g$; & $(\gamma \lambda \tilde{\omega} \sigma \sigma \alpha)$ & Glossa. \\
\hline$\delta$ & $=$ & $d$; & $(\delta \iota \psi(\dot{a}))$ & Dipsas. \\
\hline & $=$ & e; & $\left(\dot{u} \alpha \lambda \varepsilon \varepsilon^{\prime}(\varsigma)\right.$ & Hyalea, not Hyalæa. \\
\hline$\zeta$ & $=$ & $\mathbf{z}$ & $\left(\zeta i^{\prime} \cup \varphi \omega_{\nu}\right)$ & Zizyphus, Zizyphinus. \\
\hline 8 & $=$ & e ; & $\left(\pi \varepsilon\left\llcorner\rho \eta^{\prime} \nu \eta\right)\right.$ & Pirena, not Pirina. \\
\hline final & $=$ & $a ;$ & 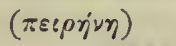 & Pirena, not Pirene. \\
\hline $9, \theta$ & $=$ & th ; & 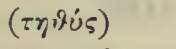 & Tethys; $(\theta \varepsilon \tau \iota \varsigma)$ Thetis. \\
\hline : & $=$ & $\mathrm{i}$ & $\left(\beta a \lambda_{\ell} 0_{5}\right)$ & Balia, not Balea. \\
\hline$x$ & $=$ & $\mathrm{c} ;$ & $\left(\left\{\pi \pi 0 \times \rho \eta^{\prime} \nu \eta\right)\right.$ & Hippocrena, not Hippochrenes. \\
\hline$\lambda$ & $=$ & 1 & $\left(\varphi \cup \lambda \lambda i_{5}\right)$ & Phyllis. \\
\hline$\mu$ & $=$ & $\mathrm{m}$ & $\left(\mu \varepsilon \lambda \alpha_{5}\right)$ & Melas. \\
\hline v & $=$ & $\mathbf{n}$ & $\left(\pi \varepsilon \varepsilon \rho \eta^{\prime} \nu \eta\right)$ & Pirena. \\
\hline$\xi$ & $=$ & $\mathrm{x}$; & 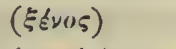 & Xenus, Xenophora. \\
\hline$o, \omega$ & $=$ & 0 & 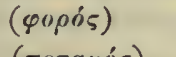 & Phorus ; $(\pi \tilde{\omega} \mu \alpha)$ Poma. \\
\hline & $=$ & $\mathrm{p}$ & ( & Potamus. \\
\hline
\end{tabular}




\begin{tabular}{|c|c|c|c|}
\hline$\rho$ & $\mathbf{r}$ & $(\pi \tau \varepsilon \rho \dot{\nu} \nu)$ & Pterum. \\
\hline$\rho \rho$ & $=\mathrm{rrh}$ & $(\varphi \cup \lambda \lambda i \rho-\delta o \eta \dot{\eta})$ & Phyllirrhoa, not Phyllirhoe. \\
\hline$\sigma, \varsigma$ & $=\mathrm{s} ;$ & $\left(\gamma^{\lambda} \omega \sigma \sigma \delta \varsigma\right)$ & Glossus, \\
\hline$\tau$ & $t$ & $(\pi \tau \varepsilon \rho \dot{\nu} \nu)$ & Pterum. . \\
\hline$v$ & $=$ & (úpiós) & Hybolithus, not Hibolites. \\
\hline$\varphi$ & $=\mathrm{ph}$ & 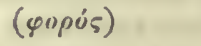 & Phorus. \\
\hline$x$ & $=\mathrm{ch}$ & $(x o y \lambda i a s)$ & Cochlias. \\
\hline$\psi$ & $=\mathrm{ps}$ & $\left(\zeta^{\prime} \alpha \mu \mu \rho\right)$ & Psammus. \\
\hline$\alpha \iota$ & $=. \mathfrak{x}$ & $(\lambda \iota \mu \nu \tilde{a} \iota 0 \varsigma)$ & Limnæa, not Limnea. \\
\hline av & $=\mathrm{au}$ & 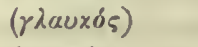 & Glaucus. \\
\hline E८ & $=$ & $(\tau \in i \nu \omega)$ & Exotenobranchia. \\
\hline$\hat{\varepsilon} \iota$ & $=$ & $(\chi \tilde{\varepsilon} \tilde{\varepsilon} \lambda ! \xi)$ & Chilostoma, not Cheilostoma. \\
\hline$\varepsilon v$ & $=\mathrm{eu}$ & 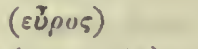 & Eurus. \\
\hline$\varphi$, ot & $=\mathrm{oe}$ & $(\delta i \zeta, 0 i x \in \omega)$ & Dioeca, not Dioica. \\
\hline$o v$ final & $=\mathrm{um}$ & $(\varepsilon \varphi i \pi \pi \iota \nu)$ & Ephippium, not Ephippion. \\
\hline os final & $=$ us & 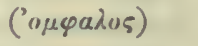 & Euomphalus, not Euomphalos. \\
\hline ov & $=\mathbf{u}$ & 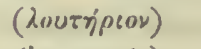 & Lutcrium, not Lotorium. \\
\hline$r$ & $=\mathrm{ng}$ & ('arrapsia) & Angaria. \\
\hline$r x$ & $=\mathrm{nch}$ & $(\breve{\gamma} \gamma \chi \omega \sigma \tau \delta / \mu \alpha)$ & Anchistoma, not Angistoma. \\
\hline$\gamma x$ & $=\mathrm{nc}$ & 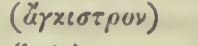 & Ancistrodon, not Agkistrodon. \\
\hline$\rho$ & $=\mathrm{rh}$ & $\left({ }^{\circ} \rho \varepsilon \alpha\right)$ & Rhea. \\
\hline c & h; & 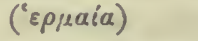 & Hermaa, not Ermæa. \\
\hline
\end{tabular}

It follows therefore, that Buthotreplis must, according to the laws of etymology, be spelt Bythotrephis; Xenophasia, instead of Zenophasia; Pcocephala, instead of Poiocephala. In Latinizing modern words where the rules of classic usage do not apply, the etymology must be preserved, even though it includes letters and combinations unknown in Latin; thus, wooduardi, instead of vudvardi; knighti, instead of cnichti; bullocki, instead of bullocci; eschscholtzi, instead of essolzi; nebraskensis, instead of nebrascensis. But words of barbarous origin should be rendered as classical in appearance as is consistent with the preservation of their original sound; as, tocous, instead of tockus; ansure, instead of ansuree; argunda, instead of argoondat.

In Latinizing proper names and converting them into specific ones, they assume a distinctive character, which they did not before possess. The rule is to use the termination $u s$, genitive $i$, when the name ends with a consonant; as, Miller, milleri. But when it ends in a vowel, ius, genitive $i i$; as, Moore, moorii. This rule * is often violated, but it would be much better strictly to adhere to it.

\section{PRIORITY.}

It is of the highest importance that we retain the first defined and illustrated names of genera and species. The British Association said:

"It being admitted on all hands that words are only the conventional signs of ideas, it is evident that lauguage can only attain its end effectually by being permanently established and generally recognized. This consideration ought, it would seem, to have checked those who are continually attempting to subvert the established language of Zoology, by substituting terms of their own coinage. But, forgetting the true value of language, they persist in confounding the name of a species or 
group with its definition; and because the former always falls short of the fullness of expression found in the latter, they cancel it without hesitation, and introduce some new term which appears to them more characteristic, but which is utterly unknown to the science, and is therefore devoid of all authority. If those persons were to object to such names of men as Long, Little, Armstrong, Golightly, etc., in cases where they fail to apply to the individuals who bear them, or should complain of the names of Gough, Laurence, or Harvey, that they were devoid of meaning, and should hence propose to change them for more characteristic appellations, they would not act more unphilosophically or inconsiderately than they do in the case before us; for, in truth, it matters not, in the least, by what conventional sound we agree to designate an individual object, provided the sign to be employed be stamped with such an authority as will suffice to make it pass current. Now, in Zoology, no one person can subsequently claim an authority equal to that possessed by the person who is the first to define a new genus or describe a new species; and hence it is that the name originally given, even though it may be inferior in point of elegance or expressiveness to those subsequently proposed, ought as a general principle to be permanently retained. To this cousideration we ought to add, the injustice of erasing the name originally selected by the person to whose labors we owe our first knowledge of the object; and we should reflect how much the permission of such a practice opens a door to obscure pretenders for dragging themselves into notice at the expense of original observers."

"The name originally given by the founder of a group, or the describer of a species, should be permanently retained to the exclusion of all subsequent synonyms."

"As the number of known species which form the ground-work of zoological science is always increasing, and our knowledge of their structure becomes more complete, fresh generalizations continually occur to the naturalist, and the number of genera and other groups requiring appellations is ever becoming more extensive. It thus becomes necessary to subdivide the contents of old groups, and to make their definitions continually more restricted. In carrying out this process, it is an act of justice to the original author that his generic name should never be lost sight of, and it is no less essential to the welfare of the science, that all which is sound in its nomenclature should'remain unaltered amid the additions which are continually being made to it."

"A generic name, when once established, should never be canceled in any subsequent subdivision of the group, but retained in a restricted sense for one of the constituent portions."

"When a genus is subdivided into other genera, the original name should be retained for that portion of it which exhibits in the greatest degree its essential characters as at first defined. Authors frequently indicate this by selecting some one species as a fixed point of reference, which they term the 'type of the genus.' When they omit doing so, it may still in many cases be correctly inferred that the first species mentioned on their list, if found accurately to agree with their definition, was regarded by them as the type. A specific name or its synonyms will also often serve to point out the particular species, which by implication must be regarded as the original type of a genus. In such cases we are justified in restoring the name of the old genus to its typical signification, even when later authors have done otherwise." 
"The generic name should always be retained for that portion of the original genus which was considered typical by the author."

"Example.-The genus Picumnus was established by Temminck, and included two groups, one with four toes, the other with three, the former of which was regarded by the author as typical. Swainson, however, in raising these groups at a later period to the rank of genera, gave a new name, Asthenurus, to the former group, and retained Picumnus for the latter. In this case we have no choice but to restore the name Picumnus Tem., to its correct sense, canceling the name Asthenurus Sw., and imposing a new name on the three-toed group which Swainson had called Picumnus."

"When no type is indicated, then the original name is to be kept for that subsequent subdivision which first received it."

"When the evidence as to the original type of a genus is not perfectly clear and indisputable, then the person who first subdivides the genus may affix the original name to any portion of it at his discretion, and no later auther has a right to transfer that name to any part of the original genus."

"When an author infringes the law of priority by giving a new name to a genus, which has already been properly defined and named, the only penalty which can be attached to this act of negligence or injustice, is to expel the name so introduced from the pale of science."

"When two authors define and name the same genus, both making it exactly of the same extent, the later name should be canceled in toto, and not retained in a modified sense."

"No special rule is required for the eases in which the later of two generie names is so clefined as to be less extensive in signification than the earlier; for if the later includes the type of the earlier genus, it would be canceled by the operation of the rule that the generic name should always be retained for that portion of the original genus which was considered typical by the author."

"If the later name be so defined as to be equal in extent to two or more previously published genera, it must be canceled, in toto."

"A genus compounded of two or more previously proposed genera, whose characters are now deened insufficient, should retain the name of one of them. If these original generic names differ in date, the oldest one should be the one adopted."

The committee on zoological nomenclature, appointed by the American Association for the Advancement of Science, said:

"A change in the diagnostic characters, or a revision which carries with it the exclusion of certain elements of a group, or the inclusion of new elements, does not authorize the change of the name or names of a group."

"When a group or genus is divided into two or more groups, the original name must be preserved and given to one of the principal divisions. The division including the typical species of the primitive genus, if any type had been specified, or the oldest, best known, or most characteristic of the species originally included when the primitive genus was first described by its author, is the portion for which the original name is to be preserved. If there is no section specially so distinguished, that which retains the larger number of species should retain the old name, but the latter can not be applied to a restricted group containing none of the 
species referred to the primitive group by its author at the time when it was described, or when he enumerated the species contained in it."

The rule that a subsequent author can not revise a genus and substitute as its type a species different from that relied upon by the founder of the genus seems to be well settled in England and America. The instances of strictly adhering to it under circumstances where it would have seemed to accommodate the author to violate it, are numerous. For instance, Professor Hall, mistaking the type of the genus Retzia, proposed and defined the genus Rhynchospira; afterward ascertain. ing that Rhynchospira was a synonym for Retzia, he abandoned it and proposed Rhynchotreta for the form which he had originally mistaken for Retzia. Had it not been for this rule he might have abaudoned Retzia evax as the type of his geuus Rhynchospira, and substituted Rhynchonella cuneata, which became the type of Rhynchotreta. If you can substitute another than the origiual species as the type of a genus, I can substitute another, and so we destroy all fixity in the type and designated characters, throw the science into confusion, and seriously impair the value and reliability of generic characters.

When an author has specified no type, the first species defined is to be taken as the type, or if the genus is to be divided, no type having yet been selected, a species may be chosen from among those originally specified as belonging to the genus, due regard being paid to the necessity of retaining as . many of the original species as possible in the division which is to retain the old name.

In dividing a genus of which there are already synonyms, if the synonyms are typified by the same species or group of species selected as types of the primitive genus, they should not be again used. When, however, the so-called synonyms are founded on species belonging to different sections of the genus, although the names may have been considered coextensive in their application, and the genus is to be divided accordingly, the so-called synonyms become the proper designations for which other names can not be applied.

In case of the consolidation of two or more groups of the same nature, the oldest name must be retained for the whole. If both, or all, are of the same date, the reviser may select the one to be retained. If a name be so defined as to be equal in extent to two or more previously described, it must be canceled. When it is necessary to divide a species, the form which received the old specific name must retain it.

A generic name must have a single neaning, and therefore two genera can not bear the same name, even though belonging to distinct subkingdoms.

\section{AS TO PUBLICATION.}

Publication consists of the insertion of a distinct exposition of essential characters in a printed book which is kept for sale, or which has been generally distributed among those conversant with the subject. Where figures are necessary to an understanding of the character of the organism, they must accompany the definition or it will be invalid. The tendency of the science of palæontology is to demand in all cases both definition and illustration before the publication is to be recognized. There are many species whose characters are so complicated and parts so minute, that an exposition of the essential ones, so they may be understood by those conversant with the fossils in the class, can only be made by illustration 
accompanied by proper definition; the science therefore demands the rule shall be co-extensive with its necessities, and good authors refuse to recognize names unless the publication is such that their meaning may be readily comprehended.

A communication in a public assembly or learned society, or the reading of a paper containing new names at such meeting, printing of the names in a catalogue, labeling the fossils in a collection, printing the names and description in a newspaper, either one or all these attempts to introduce the names, does not constitute a publication within the rule, and hence give the names no place in science. Nor does the printing of the names with brief definitions in an obscure pamphlet, or even in the Journal of a learned society, where the definition will not enable an ordinary palæontologist to identify or distinguish the species at another locality than the typical one, give them any right to claim recognition. Occasional pamphlets independently issued, and insufficiently advertised and distributed, or very small editions that can not reach the students of the science generally, are not publications within the rule.

The date borne by a publication will be presumed to be accurate, though this presumption is only prima facie, and may always be contested, and the true date shown, from which time alone do names have any validity.

A species is not to be considered as named unless both generic and specific names are simultaneously applied to it.

Where a genus or species is announced in a publication, and subsequently described in another publication, the latter only is entitled to recognition. It is essential in establishing a genus that some species be referred to it.

\section{NAMES TO BE REJECTFD, CHANGED, OR MODIFIED.}

A generic name should be rejected when it has been previously applied to another valic genus of organisms, even if it has received general currency. It should also be rejected when it expresses a positively fulse character in the genus, and is therefore liable to propagate crror, and especially is this the case where the definition is so erroneous as not to entitle it to recognition; but where the name has received general circulation, and the error is not such as to seriously misleal, the name is retained; as, Athyris aud Atrypa. So a specific name should be rejected when it is already applied to another species or subdivision in the same genus, or when a geographical name of a country entirely removed from the habitat of the species is used.

A name should be rejected when it is formed of two words belonging to different languages, as en put before a Latin name, sub before a Greek name, oides, opsis suffixed to a Latin name; or when it is identical if properly spelled, according to its true derivation, with a prior valid name, as Platystoma of Conrad, being preoccupied, can not be retained simply because he misspelled it Platyostoma.

A name should always be rejected when it outrages decency.

It is inelegant and tautological to derive a generic name from the specific name of its typical species. For example, Corvus pyrrhocorax, Linn., was afterward advanced to a genus under the name of Pyrrhocorax. The name therefore became Pyrrhocorax pyrrhocorax. The rule is now to reject all such generic names, except those which, from long usage, have imbedded themselves into science; none of which, however, can claim a place in palæontology. 
When a species is transferred from one genus to another in which there is a species of the same name, the older specific name is retained, and the oldest tenable synonym is adopted for the other form, if there be one; and if not, a new specific name is proposed. But if the form bearing the prior specific name is transferred to another genus, the original specific name of the later species must be restored, and the new specific name must fall into synonymy. This is the necessary result of the law of priority.

When a name is published, the author has no more control over it than any other one. He has the same rights, no more and no less, than other naturalists.

\section{SELECTION OF NAMES AND MODIFICATION.}

The best names are derived from Latin and Greek, and express some distinguishing characteristic of the object to which they are applied. In palæontology it is more consistent with practice and uniformity to derive the generic names from Greek and the specific names from Latin; and if the name as proposed exhibits a faulty construction, any naturalist is authorized to correct it. When a wrong gender is given to a species by its termination, not agreeing with a genus, it is the duty of a naturalist to correct it.

When a name derived from a person has not been written according to the real orthography of his name, it may be changed, provided it does not involve the first syllable and thereby disturb the arrangement of indices, tables, catalogues, and dictionaries, in alphabetical order, or interfere with long-established usage. The botanical congress at London, in 1866, refused to change the name Cinchona, named after the Countess Chincon, because of established usage. In 1866 Hall described Glyptocrinus nealli in honor of O'Neall; but the name must stand as described, not only because its change would interfere with indices, tables, catalogues, and dictionaries, but Hall had the right to construct the specific name nealli as he did, and the fixity of nomenclature will not allow another to change it. Scalaria turtoni, named after Miss Turton, may be changed to S. turtona; and Viviparus being inconsistent with itself may be changed to Vivipara, because the change is in the end of the name to conform to the rules of grammar.

Names of persons are Latinized and not adopted in Greek form, but where en is prefixed or oides or opsis suffixed, one may not be authorized to change it, because the name is not of Latin origin, though it is in very bad taste. Buffoonery has no place in science; hence Latin puns on names, as faba after Mr. Bean, should be rejected in all cases as a poor joke.

The name of a person must have the termination Latinized, but the specific name can not be composed of the Christian and surname, because it would not be binomial, and can not be made to conform to the rules. Geographical names are eminently fit and suitable when they indicate the locality from which the type was collected. Barbarous names are not in good taste in Palæontology, though they have been defended in other departments of Natural History. Names expressive of trades and professions are not in good taste. Mythological and historical names are generally in bad taste for specific names, though they have been largely used; but mythological names for genera have usually been defended. The right to use both is conceded. Names expressive of something else than a character of the fossil, as centennialis for a Hyolithes are in very bad taste, and sometimes even absurd. Com- 
parative names are often appropriate; but those expressive of size, as maximus, minor, and minimus, are too frequently rendered inaccurate by after discoveries, and are therefore objectionable.

Both generic and specific names derived from persons engaged in palseontological pursuits are very appropriate. Names of harsh and inelegant pronuneiation ought to be avoided, as also words of too great length or having more than five syllables.

Generic names may be compounded from other genera to express the position of the genus as intermediate to or allied with two other genera, care being taken not to adopt such as are of too great length, and not to corrupt them in trying to render them shorter. Aviculopecten and Aviculopinna are examples of the appropriate use of compound words, notwithstanding their length, while Tellinomya is more fanciful than real, and yet not to be discarded.

In compounding words all the radical or essential parts of the constituent mem. bers must be retained, and no change made except in the variable terminations. Words coined at random, or without any derivation or meaning, will not be recognized.

The names of genera are in all eases essentially substantive, and hence adjective terms can not be employed for them without doing violence to grammar; for instance, Anomaloides proposed as a generic name must be disregarded. The same may be said of names in the genitive case, which are wholly inadmissible, without reformation.

\section{FORMATION OF NAMES.}

The generic name always begins with a capital letter, the specific nane with a small initial letter, even when derived from person or place. The generic name is a noun, while the specific name has the force of an adjective. The specific name is in no instance a proper noun, but all species are equal, and should therefore be written alike. It is a violation of a plain rule of grammar to write a specific name with a capital letter; beside, there is an advantage in obeying the rule, for by so doing the eye at a glance distinguishes specific from generic names.

The generic name retains the gender which belongs to it in the language from which it is taken. Where no change is marle in the termination of the last word in a generic name, the gender of that word determines the gender of the genus. Thus cercas, nema, stoma, and desma are in the Greek of the neuter gender, and consequently all genera ending with these words, such as Urthoceras, Loxonema, Phragmostoma, Lyrodesma, are neuter.

In defining a new genus the etymology should be given, and a species should be selected as the type. There is no excuse for neglecting these rules, except that the author is incapable of giving the etymology of his proposed generic name, and is not sufficiently confident of his definition to dare venture to rely upon one of his species as the type.

When a generic name is derived from the name of a person, it is stripped of all titles and preliminary particles, reducer to the genitive case, and the letter $a$ is appended, thus taking on a feminiue form. The following examples illustrate the method, viz.: Names, Brun, Bruni, Bruno, Brunus, Bruna, Brune, Bruny. Generic form, Brunia, Bruniia, Brunoia, Brunusia, Brunæa, Brunia, Brunyia. $Y$ at the end of a word of one syllable is treated as a consonant, as Quoy, Quoyia; Gay, Gayia; and mute $e$ final becomes $i$, or is dropped entirely, as Perouse, Perousia. 
Every specific name agrees in gender with the genus to which it belongs, and if an adjective, its termination must show it. If the specific name is a substantive, the termination is not necessarily changed. The rule is not to change the ending of a common noun or mythological name, but to make an adjective, and the name of a person or place, indicate the gender of the genus to which it belongs.

The following rules govern the use of these terminations:

-alis. This Latin termination, implying resemblance, is seldom used, lexcept in words already compounded.in Greek and Latin; and when otherwise, it must be annexed to the stem of the word, as rectilateralis, quadrilateralis.

-anus. This Latin termination implies resemblance or association, and may be added to proper names, personal or local; though in science its use is almost confined to the former. If the word is capable of taking a classic form, the termination should be simply annexed to the stem as Linnceus, linnceanus; Lesquereux (lescuria), lescurianus; in conformity with classic usage; pagus, paganus; Claudius, claudianus; Neapolis, neapolitanus. In other cases, the addition of this termination must follow the same rule as those for ensis, as America, anericanus; Geinitz, geinitzanus; Meek, meekanus ; Erie, erianus; Italy (ia), italianus.

-atus. This Latin termination strictly implies the possession of the thing to the name of which it is added. I It is therefore affixed to the stem of common names only ; as, costa, costatus; galea, galeatus ; fornix, fornicatus; sinus, sinuatus ; stria, striatus; lobus, lobatus; rostrum, rostratus. It is worthy of remark here that this termination sometimes loses its at, to shorten the word. The practice is not commendable from a linguistic stand-point, but some of the terms so made have become fixed in the nomenclature; as, Orthis biloba.

-formis. This Latin termination implies resemblance of shape, and should be confined to Latin words, to the stem of which it is joined by the connecting vowel $i$; as, laterna, laterniformis; ] pistillum, pistilliformis. In forming terms, such as the first given above from Latin words ending in $\alpha$, the error of using $\alpha$ as the connecting vowel should be avoided; being inconsistent with classic usage, as well as more awkward and lengthy, thus we have from terra, terricola; gemma, gemmifer; squama, squamiger; tuba, tubiformis; etc.

-ensis. This is a Latin termination, expressive of locality, and can not therefore be correctly employed, except as an affix to the name of a place. This rule has been traversed in few real, but in many apparent instances. Lingula morsensis is an illustration of the former. In accordance with law, this has been changed to $L$. morsii, being given in honor of Mr. Morse. Zygospira cincinnatiensis, Pupa vermilionensis, Cardium napoleonense, Athyris hannibalensis are apparent exceptions; but these terms are formed from words which, though originally personal or trivial, have now become local names, and consequently no valid objection can be raised against them. In using this termination the following rules have been generally followed: 1st. If the name of the place ends in a consonant, the termination is annexed to the word; as, Clinton clintonensis. $2 \mathrm{~d}$. If the name ends in $a$ or $e$, these letters are dropped, and the termination then annexed; as, Canada, canadensis; Minnesota, min. nesotensis; Iowa, iowensis; Indiana, indianensis; Lasalle, lasallensis; Erie, eriensis. $3 \mathrm{~d}$. If the name ends in $i, o$, or $u$, that vowel is retained; as, Mississippi, mississippiensis; Missouri, missouriensis ; Chicago, chicagoensis ; Colorado, coloradoensis ; Chouteau, chouteauensis. 4th. If the name ends in $y$, that letter becomes $i$ upon the addition 
of the termination; as, Kentucky, kentuckiensis; Alleghany, alleghaniensis; in accordance with classic usage, as Sicily, siciliensis.

- $i$. The terminination $i$ is to be considered a mere indication of the Latin genitive case, and custom rather than correctness has, in some sense, legalized its addition to any name. In practice, however, it is almost restricted to proper names. Thus we have knighti, littoni, flemingi, ivesi.

-icus. This Greek termiuation implies resemblance, and may be added to common names under the same rules as those given for - ensis, except that, in forming the word, a vowel is suppressed if it would precede the termination; thus, Macedon, macedonicus; Italy (ia), italicus. It is little used, except as an affix to the name of a river or country; as, euphraticus, anglicus, or in such words as ellipticus.

-eus. This Latin termination has been occasionally employed; but as it implies "made of," it is evidently seldom, if ever, admissible in palæontology. The term eboraceus, from eboracum, the Latin name for York, is a misnomer and should have been eboracensis.

-inus. This termination is applied $t$ () both common and proper names. Latin usage restricted its application more than modern scientific practice has done, and applied it mainly to proper names, local terms, and living beings; as, caninus, alpinus; but did not sanction such words as rugatinus, sulcatinus, secalinus, taximus, aud velutimus. The termination is used subject to the same laws as -ensis.

-ites. This termination expresses the fossil nature of the specineu.) It is a contraction of the Greek word lithos, a stone. In most instances it coulesces with the last vowel of the root. This and long usage in many words, such as Aramoniites, Belemnites, Pyrites, have conpletely established the long $i$, while the gender is determined by that of the Greek word to be masculine. All specific terms in the genus must, therefore, be of this gender.

—oides. This Greek terminatjon, signifying "like," should be added only to the stems of words of Greek origin. No connecting vowel is necessury. Thus we have dactylos, dactyloides; discos, discoides. The Latin form -oideus obeys the same laws, except the Greek termination is alike in all genders, while the Latin is inflected as Isatin adjectives of similar termination.

Compound terms. In forming compound terms care should be taken to connect them rightly. If an adjective of three terminations, or a noun of the second Latin declension, composes the first part of the word, either $i$ or o may be employed as a connecting vowel, the choice being largely determined by the ear. 'Thus sulcomarginatus is better than sulcimarginatus, and crassicaulis than crassocantis. If the adjective has but one or two terminations, or the noun be of the first, third, or fourth Latin declension, the connecting vowel $i$ should always be employed; as, tenuistriatus, pinniformis, ilicifolius, retiformis, cornifer. The connecting vowel 0 is admissible by Greek usage in all declensions; as, Ulodendron, Oycloconcha, Syringodendron, Alethopteris, Dictyonema, Dictyopteris, except that where the first part of the word is an adjective ending in -ys, it is shorter, and at the same time consonant with classic usage to employ no connecting vowel at all; thus, pachyderma, euryteines, Platystoma, etc., are better than pachyoderma, euryoteines, Platyostoma, etc. 


\section{NORTH AMERICAN}

\section{PALAOZOIC FOSSILS.}

By the little words plants and animals we include all the organisms in the world. But science, demanding technical words and controlling characteristics, has added the word "Kingdom" to these common names; and hence all organisms and all which have existed in the past are divided between the "Vegetable Kingdom" and the "Animal Kingdom."

\section{VEGETABLE KINGDOM.}

The Palæozoic Fossil plants are divided into seven classes; viz., Fucoides, Fungi, Equisetacex, Filicacex, Lycopodiacex, Cordaitex, and Coniferæ. The Fucoides are also called Sargassites and Thalassophites. They are supposed to have some affinity with the leathery marine vegetation called Fucus or the Sargassum. The fossils are merely casts, showing, as a rule, no structure whatever. Lesquereux says marine vegetation readily disintegrates and passes into a gelatinous, half-fluid matter, which penetrates the sand, so that the lowest strata of the great heaps thrown up by the waves and exposed to atmospheric action, do not generally preserve traces of their organisms for more than a year. The fossil forms may have been harder, and contained less gelatinous matter in their cells, and probably had only a remote resemblance to the living Fucus or Sargassum, though there ean be no reasonable doubt they are representatives of extinct marine cryptogamous plants.

The fossils referred to this Class have never been distributed into Orders and Families. The genera are as follows: Archæophyton, Arthraria, Arthrophycus, Asterophycus, Astropolithon (Graptolite?), Blastophycus, Bythotrephis, Calamophycus, Chondrites, Conostichus, Cruziana, Dactylophycus, Dendrophycus, Discophycus, Dystactophycus, Eophyton, Heliophycus, Hippodophycus, Ichnophycus, Licrophycus, Palæophycus, Phytopsis, Protostigma, Rusophycus, Sphenothallus, Taonurus, Trichophycus.

The Fungi are cellular cryptogamus plants (kruptos, hidden; gamos, marriage). They are flowerless plants, in which the fructifying organs are so minute as to escape detection without a microscope. The spores are sometimes naked, and in other cases inclosed in a theca. The evidence of the existence of this Class in Palæozoic rocks is extremely meager, though Lesquereux refers a species of Rhizomorpha to it.

The vascular cryptogamous plants flourished to such an extent in the Carboniferous era, that it has been called the "Age of Acrogens," and the "Age of Coal- 
plants." The Classes and Orders have been named as Latin adjectives in the feminine plural, to agree with plantre (plants), which is said to be always understood. Thus from Equisetum, by prolonging the termination into acea, we have Equisetacer; from Filices, Filicacex, etc.

The Equisetacex are either cellular or vascular flowerless plants, producing spores instead of seeds. The Palrozoic fossils are all referred to one Order, the Calamariæ. The genera are as follows: Anarthrocanna, Annularia, Arthrostigma, Asterophyllites, Bechera, Bornia, Calamites, Calamodendron, Calamostachys, Equisetites, Macrostachya, Nematophyllum, Sphenophyllum, Volkmanuia.

The Filicacex, or ferns, are too common among existing plants to have escaped the notice of any one. The Palrozoic ferns are divided into Orders as follows:

I. ORDER, NEUROPTERIDEA.

Cyclopteris, Dictyopteris, Lesleya, Neuropteris, Odontopteris. UNCERTAIN RFLATION TO THE ORDER.

Baiera, Cardiopteris, Danæites, Idiophyllum, Megalopteris, Neriopteris, Orthogoniopteris, Trniopteris.

2. Order Alethopterideas.

Alethopteris, Callipteridium, Callipteris, Icscuropteris, Protoblechnum.

3. Order, Pseudopecopthideas.

Pseudopecopteris.

4. Order, PecopterideAi.

Beinertia, Cymoglossa, Lonchopteris, Oligocarpia, Pecopteris, Phyllopteris.

5. ORDER, SPHENOPTERIDEA.

Eremopteris, Hymenophyllites, Sphenopteris.

6. Order, Adiantites.

Aneimites, Archæoptéris, Triphyllopteris.

FERNS OF UNCERTAIN AFFINITY.

Asteropteris, Crematopteris, Pachypteris, Rhacophyllum.

SEPARATE FRUCTIFICATION OF FERNS.

Sorocladus.

RACHIS OF FERNS.

Rhachiopteris.

RHIZOMA OF FERNS.

Stigmarioides.

STEMS OR TRUNKS OF FERNS.

Caulopteris, Megaphytum, Psaronius, Stemmatopteris. 
The existing Lycopodiaceæ inhabit the deep shade of the forests, the surface of bogs, or the slopes of mountains, where there is a high degree of humidity, except a few species, which have the power of closing the leaves under the heat of the sun and opening them to receive the rain or fog. Some of them, like the "Ground Pine," are evergreens, and none of them grow beyond a few feet in length. Many Carboniferous plants of this Class, however, were grand and stately trees, two feet or more in diameter, and fifty feet or more in length. Lesquereux says, in speaking of Carboniferous plants:

"The leaves of the Lycopodiaceæ are generally in a spiral order, modified sometimes in their relative disposition, even in the same species. They are narrow, linear-lanceolate, of various length, according to species, all with a strong midrib. Their point of attachment upon the stems is marked by scars of divers forms, which greatly vary in size, according to the age of the fragments, or rather of the part of the tree from which the fragments of bark are derived. It is essentially from the characters of these leaf-scars that species of the Lepidodendræ have been established."

"The fructifications, rarely found attached to their support, are in cylindrical . or ovate spikes, sessile or pedicellate, composed of sporanges attached to the anterior base of leaves or blades of various forms, which, curved upward and imbricated, cover the outside of the cones. The sporanges contain organisms of two kinds, either very small ones (microspores), which are like powder, or agglutinated globules of matter, distinct only with microscopes of great power. They may represent the male fertilizing pollen. Or, and more generally, they contain macrospores, large, true globular seeds, angular on one side, and rounded on the other."

The class may be divided into three orders, as follows:

\section{ORDER, LEPIDODENDREF.}

Acanthophyton (?), Cyclostigma, Dechenia, Diplostegium, Glyptodendron, Halonia, Knorria, Lepidocystis, Lepidodendron, Lepidophlœum, Lepidophloios, Lepidophyllum, Lepidostrobus, Leptophloeum, Lycopodites, Plumalina, Psilophyton, Sporangites, Sporocystis, Ulodendron.

2. ORDER, TENIOPHYLLE王.

Tæniophyllum.

3. Order, Sigillariat.

Didymophyllum, Pinnularia, Sigillaria, Sigillarioides, Sigillariostrobus, Spirangium, Stigmaria, Syringodendron.

\section{Order, Noeggerathia..}

Noeggerathia, Whittleseya.

The Cordaitex, an extinct class, are represented in the Coal Measures, generally by fragments of ribbon-like leaves, and most rarely by stems bearing leaves and flowers. They belong to the Gymnosperms, and occupy a position somewhat intermediate between the Noeggerathiæ and Coniferæ. The genera are as follows: 
Antholithes, Asterocarpus, Cardiocarpou, Carpolithes, Cordaianthus, Cordaicarpus, Cordaistrobus, Cordaites, Desmiophyllum, Dicranophyllum, Lepidoxylon, Rhabdocarpus, Trigonocarpum.

\section{FRUIT OF UNCFRTAIN AFFINITY.}

\section{Gulielmites.}

The Conifera are exogenous evergreen trees and shrubs, with branching truuks containing a resinous juice. They have a strobile cone or solitary seed. Three Palæozoic genera have been referred to the Conifers: viz., Dadoxylon, Saportiea, and Walchia, but there must be doubt about the reference of Dadoxylon to this Class.

\section{WOOD OF UNCERTAIN AFFINITY.}

Celluloxylon, Nematoxylon, Ormoxylon, Prototaxites, Sternbergia, Syringoxylon.

Acanthopityton, Dawson, 1862, Quar. Jour. Geo. Soc., vol. 18. p. 324. [Ety. akartha, thorn; phyton., plant.] Cylindrical branches, ramifying in alternate man. ner, striated, with scattered tubereles, on which are borne short spines. Type A. spinosum.

spinosum, Dawson, 1862, Quar. Jour. Geo. Soc., vol. 18. p. 324 , Chemung Gr.

Alethopreris, Sternberg, 1825, Vers. Darst. Flora der Vorwelt. p. 21. [Ety. alethos, true; pleris, fern.] Fronds polypinnate; pinnules coriaceous, simple, mostly entire, enlarged at the base, connate or free, borders reflexed; midrib distinct, imniersed into the epidermis, marked by a groove on the upper surface; prominent on the lower; lateral veins simple or forking once, open, often in riglit angle to the rachis; fructifications marginal. Type A. lonchitica.

acuta, see Pecopteris acuta.

ambigua, Lesquereux, 1880, Coal Flora of Pa., p. 182, Coal Meas.

aquilina, Schlotheim, 1820, (Filicites aquilinus,) Petrefaktenkunde, p. 405, and Coal Flora of Pa., p. 181, Coal Meas. bunburyi, Andrews, 1875, Ohio Pal., vol. 2 , p. 421, Coal Meas.

coxana, Lesquereux, 1861, Geo. Sur. Ky., vol. 4, p 433, Coal. Meas.

crassa, Lesquereux, 1884, Coal Flora of Pa., p. 748, Coal Meas.

crenulala, Brongniart, as identified by Lesquereux, in Geo. Sur. Ill., vol. 2, p. 439, is Psendopecopteris suberenulata.

cristata, see Pecopteris cristata.

discrepans, Dawson, 1862, Jour. Geo. Soc., vol. 18, p. 222 , Devonian.

distans, Lesquereux, 1858, Geo. Sur. Pa., vol. 2, p. 865 , is a variety of $A$. lonchitica.

emarginala, see Pecopteris emarginata. erosa, see Pecopteris erosa.

evansi, Lesquereux, 1884, Coal Flora of Pa., p. 834, Coal Meas. falcata, Lesquercux, 1870, Geo. Sur. Ill., vol. 4, p. 396, Coal Meas.

gibsoni, Lesquereux, 1850, Coal Flora of Pa., p. 183, Coal Mleas.

grandifolia, Newberry, 1873, Ohio I'al., vol. 1, p. 384 , Coal Meas.

grandis, Dawson, 1863, Can. Nat. \& Geol., vol. 8 , and Acad. Geol. p. 484, Coal Meas. halli, see Pecopteris halli.

helenx, Lesquereux, 1850, Coa] Flora of Pa. p. 179, Coal Meas.

heterophylla, Lindley \& Hutton, 1833, (Pecopteris heterophylla,) Foss. Flora, vol. 1, p. 113, Coal Meas.

holdeni, see Protoblechnum holdeni.

hymenophylloides, see I'seudopecopteris hymenophylloides.

inflata, see Callipteridium inflatiim.

ingens, Dawson, 868, Acad. Geol. p. 553, Devoทiลn.

lævis, Lesquereux, 1858, Geo. Sur. Pa., vol. 2, 1. 865, Coal Meas.

lanceolata, see Pecopteris lanceolata.

lonchitica, Schlotheim, 1820, (Filicites lonchiticus,) Nachtrage zur Petrefuktenkunde, "p. 411, and Coal Flora of Pa., p. 177, Coal Meas.

longifolia, see $\mathrm{Pe}-$ copteris longi-

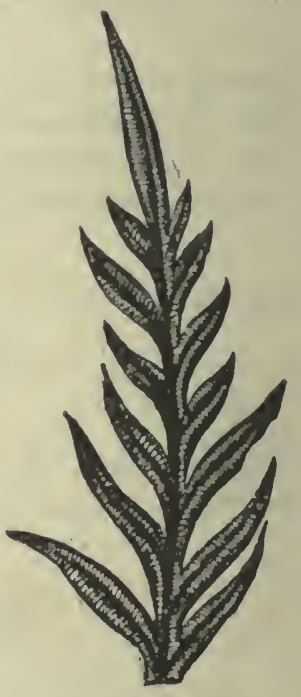

Fra. 5.-A lethopteris lonchltiea. folia. massillionis, see Callipteridium massilloneum. 
maxima, Andrews, 1875, Ohio Pal., vol. 2, p. 421 , Coal Meas.

mazonana, see Pseudopecopteris mazonana.

muricata, see Pseudopecopteris muricata. nervosa, see Pseudopecopteris nervosa.

obscura, Lesquereux, 1858, Geo. Sur. Pa., vol. 2 , p. 865 , syn. for Callipteridium rugosum.

oweni, see Callipteridium oweni.

pectinata, Lesquereux, 1866, Geo. Sur. Ill., vol. 2, p. 469, Coal Meas.

pennsylvanica, Lesquereux, 1858, Geo. Sur. Pa., vol. 2, p. 864, Coal Meas.

perleyi, Hart, 1868, Acad. Geol. p. 554, Devonian.

pluckeneti, see Pseudopecopteris pluckeneti.

preciosa, see Pecopteris preciosa.

pteroides, see Pecopteris pteroides.

robusta, Lesquereux, 1884, Coal Flora of Pa., p. 835, Coal Meas.

migosa, see Callipteridium rugosum.

serlii, Brongniart, 1828, (Pecopteris serlii,)

Hist. d. Veg. Foss. p. 292, and Coal

Flora of Pa., p. 176, Coal Meas.

sermula, see Pecopteris serrula.

serrulata, see Pecopteris serrulata.

sheaferi, see Pseudopecopteris sheaferi.

solida, see Pecopteris solida.

spinulosa, see Pseudopecopteris spinulosa.

stellata, see Pecopteris stellata.

tæniopteroides, see Pecopteris tæniopteroides.

urophylla, Brongniart, 1828, (Pecopteris urophylla,) Hist. d. Veg. Foss. Coal Meas.

virginiana, Fontaine \& White, 1880, Perm. or Up. Carb. Flora, p. 88, Coal Meas. or Permian.

Anarthrocanna, Gœppert, 1845, in Tchih. Voy. [Ety. an, without; arthron joint; canna, a plant.] Cylindrical stems, more or less swelling at the nodes, with ribs flattened and continuous instead of forming joints as in Calamites.

perryana, Dawson, 1863, Quar. Jour. Geo. Soc. vol. 19 , p. 461 , and Foss. plants of Dev. and Up. Sil. formations, p. 27, Catskill Gr.

Aneimites, Dawson, 1861, Quar. Jour. Geo. Soc. vol. 17, p. 5. [Ety. from Aneimia, a genus.] Pinnules clustered, petiolate or attached by a narrow base, with flabellate venation. Type $A$. acadicus, closely related to Cyclopteris.

acadicus, Dawson, 1861, Quar. Jour. Geo. Soc., vol. 17, p. 5 , and vol. 21 , p. 153 , Low. Coal Meas.

bockshii, Gœeppert, 1836, (Adiantites bockshii,) Syst. Filic. Foss. p. 384, and Foss. plants of Dev. and Up. Sil. of Can. p. 46, Chemung Gr.

obtusus, Lesquereux, 1858, (Noeggerathia obtusa,) Geo. Sur. Pa., vol. 2, p. 854, and Foss. plants of Dev. and Up. Sil. of Can., p. 46, Catskill Gr.

validus, Dawson, 1862,(Cyclopteris valida,)
Quar. Jour. Geo. Soc., vol. 18, p. 319, and Foss. plants of Dev. and Up. Sil. of Can., p. 46, Ham. Gr.

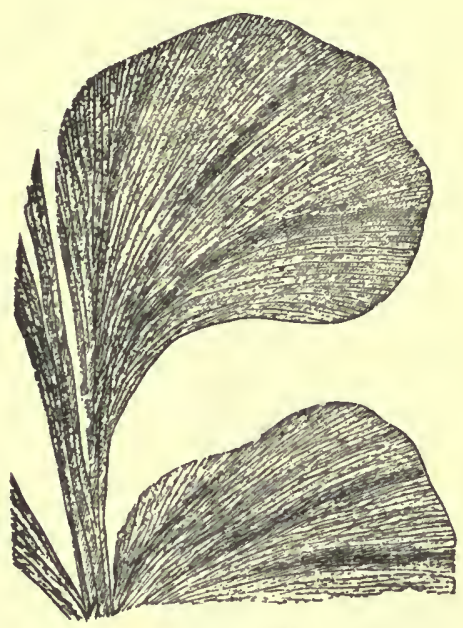

FIG. 6.-Aneimites obtusus.

Annularia, Sternberg, 1820, Essai d'un exposé Geognostico-botanique d. l. Flore. du monde primitif. 2d Cahier, p. 36. [Ety. annulus, a ring.] Stem articulate, striate, with a strong diaphragm traversing it at the articulations; branches opposite, nearly in right angles from the articulations; leaves verticillate, lanceolate, spathulate, or lingulate, abruptly or gradually acuminate, or obtuse, even emarginate at the apex; fructifications in long cylindrical spikes, with close articulations, and narrowly lanceolate bracts, bearing round sporanges in the axils of the leaves, or double, oval ones, pedicellate and attached in the middle of the internodes. Type A. spinulosa. This name is preoccupied in the subkingdom Mollusca, and Wood in 1860 proposed to substitute Trochophyllum; but Trochophyllum was preoccupied for a genus of corals in 1851, by Edwards and Haine.

acuminata, see Sporangites acuminatus.

antiqua, Dawson, 1861, Can. Nat. and Geol. vol. 6, p. 170, Devonian.

calamitoidea, Schimper, 1869, Pal. Veget., vol. 1, p. 349, and Coal Flora of Pa., p. 48, Coal Meas.

clavata, Lesquereux, 1880, (Trochophyllum clavatum,) Coal Flora of Pa., p. 65, Coal Meas.

cuspidata, Lesquereux, 1884, Coal Flora of $\mathrm{Pa}$, p. 725 , Subcarboniferous.

dawsoni, Schimper, 1869, Palæontologie Vegetale, vol. 1, p. 350, and Coal Flora of Pa., p. 51, Devonian. Proposed for Asterophyllites latilolius, of Dawson, because that name was preoccupied; but I have retained Asterophyllites latifolius because it is doubtful whether it is an Annularia. 
emersoni, Lesquereux, 1880, Coal Flora of Pa., p. 50, Coal Meas.

fertilis, Sternb., 1824, Vers. Darst. Flora der Vorwelt, p. 31, Coal Meas.

inflata, Lesquereux, 1870, Geo. Sur. Ill., vol. 4, p. 423, Coal Meas.

laxa, Dawson, 1871, Foss. Plants Canada, p. 31, Devonian.

longifolia, Brongniart, 1828, Prodrome Hist. Veg. Foss., p. 156, and Coal Flora of Pa., p. 45, Coal Meas.

minuta, Bronginart, 1828, Prodr. Hist. Veg. Foss., p. 155, and Coal Flora of Pa., p. 49, Coal Meas.

radiata, Brongniart, 1822, Class. d. Veg. Foss., p. 35 in Mus. d. Hist. Nat., vol. 8, pl. 13, fig. 7, and Coal Flora of Pa., p. 50 , Subconglomerate.

romingeri, Lesquereux, 1877, Trans. Am. Phil. Soc., p. 166, Low. Held. Gr.

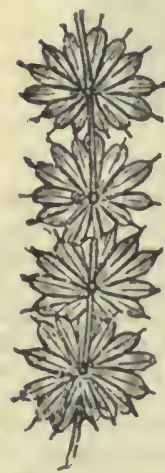

FIa. 7.-A nnulariasphe nophyliolues. sphenophylloides, Zenker. 1833, (Galium sphenophylloides, ) in Leonls. v. Bronn's Jahrb., p. 398, and Coal Flora of Pa., p. 48, Coal Meas.

tuberculata, Sternberg, 1823, (Bruckmannia tuberculata) Vers. Darst. Flor. d. Vorwelt. Fasc. 4, p. 29, and Coral Flora of $\mathbf{P a}$, p. 723, Coal Meas.

Antiolitues, Brongniart, 1822, Mem. du Mus. d'Hist. Nat., vol. 8, p. 203. [Ety. anthos, flower; lithos, stone.] Supposed to be the flowers of Cordaites or other trees. The characters are not very definite. Type A. lilincea. devonicus, Dawson, 1868, Acad. Geol., p. 566, Devonian.

floridus, Dawson, 1871, Foss. Plants Can., p. 63, Devonian.

piteairnix, L ind le y and Hutton, 1835 , Foss. Flora of Great Britain, vol. 2, p. 82 , Coal Measure.

priscus, Newberry, 1873, Ohio Pal. vol. 1, p. 363, Coal Meas. pygmeus, Daw son, 1863, Can. Nat., vol. 8 , and Acad. Geol., p. 477. Coal Meas. rhabdocarpus, Daw son, 1863, Can. Nat., vol. 8, and Acad. Geol., p. 477. Coal Meas.

squamosus, Dawson, 1863 , Can. Nat., vol. 8, and Aead. Geol., p. 477, Coal Meas.

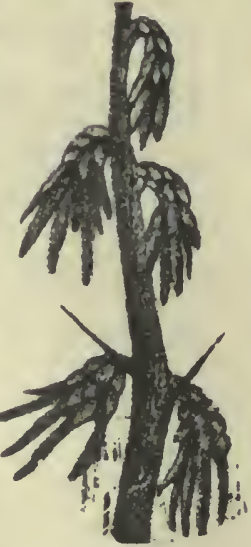

FIG. 8.

Antholithes priscus. spinosus, Dawson, 1868, Acad. Geol., p. 477, Coal Meas. Aphlebia adnascens, see Rhacophyllum adnascens. fabellata, see Rhacophyllum flabellatum. irregularis, see Rhacophyllum irregulare. Araucarites gracilis, see WValchia gracilis. Arcitaopryon, Britton, 1S8S, Ann. N. Y. Acad. Sci. vol. 4, p. 123. [Ety. archaios, alleient; phyton, plant.] Founded unon black films of graphite in erystalline limestone. Type A. newberryanum. Probably a Graptolite.

newberryanum, Britton, 1888, Ann. N. Y. Acad. Sci. vol. 4, p. 123, Taconic.

Archjopteris, Dawson, 1863 , Can. Nat. vol. $\mathrm{S}$, and Foss. iplants of Dev. and Up. Sil. Can. pp. 48, 98. [Ety. archaios, ancient; pteris, fern.] Frond bipinuate; pinnules obovate, inequilateral, narrowing to the base and decurrent on the partial petioles, the main petiole often having accessory pinnules, at the bases of the pinnæ. Veins spreading from the base, curved or straight, dividing dichotomously into fine veinlets; fertilo piunze bearing groups of oval sporecases instead of pinnules. Type A. hibernica.

acadica, see Aneimites acadicus.

alleyhaniensis, syn. for A. rogersi.

bockschiana, see Aneimites bockschii.

browni, see Cyclopteris browni.

denticulata, Lesquereux, 1884, Coal Flora of Pa., p. 774, Subcarboniferous.

gaspensis, Dawson, 1881, Can. Nat. and Geol., vol. 10, p. 8, Devonian.

hallana, Goeppert, 1852, (Cyclopteris hallana,) Die fossil Flora des Uebergangsgebirges, p. 145. Proposed as a sub. stitute for Sphenopteris laxa, which latter name was preoccupied in that genus; but when referred to this genus, laxa inust be restored.

harti, Dawson, -1863 (Palreopteris hartti, Can. Nat., vol. 8, and Acad. Geol., p. 485, Coal Meas. hibernica, Furbes, 1852, (Cyclopteris hibernica,) Proc. Brit. Ass'n, and Coal Flora of Pa., p. 305, Chemung Gr.

jacksoni, Dawson, 1861 ,

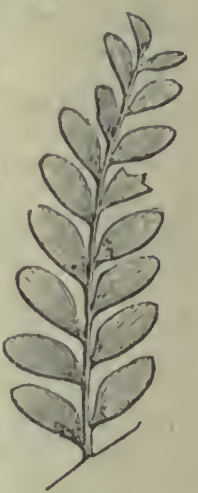

FIG. 9.

Archeopleris hlbertifea.
(Cyclopteris jacksoni,) Can. Nat. and Geo., vol. 6, p. 173, Catskill Gr.

laxa, Hall, 1843, (Splenopteris laxa,) Geo. Rep. 4th Dist. N. Y., p. 275, Chemung Gr. This species has also been named A. hallana.

macilenta, Lesquereux, 1884, Coal Flora of Pa., p. 775, Catski'l G'r.

minor, Lesquereux, 1858, (Noeggerathia minor,) Geo. Sur. Pa., vol. 2, p. 854 , Catskill Gr.

obliqua, Lesquereux, 1880, Coal Flora of Pa., pp. 300, 774, Catskill Gr. oblusa, see Aneimites obtusus. rogersi, Dawson, 1863, (Cyclopteris rog- 
ersi,) Quar. Jour. Geo. Soc., vol. 19, p. 463, and Coal Flora of Pa., pp. 307, 776, Catskill Gr.

sphenophyllifolia Lesquereux, 1884, Coal Flora of Pa., p. 775, Catskill Gr.

stricta, Andrews, 1875, Ohio Pal. vol. 2, p. 418, Coal Meas.

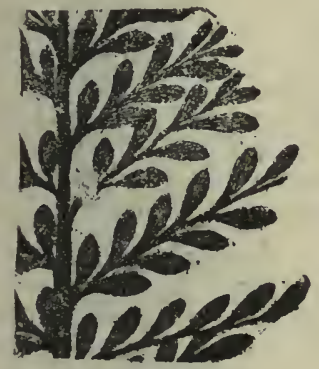

FIG. 10.-A rchæopteris stricta.
Aristophycus, Miller and Dyer, 1878, Cont. to Pal. No. 2, p. 3. Probably inorganic, and, if fucoidal, too irregular and too little known to be retained as ă genus.

ramosum, $\mathrm{Mill}$ e $\mathrm{r}$ and Dyer, 1878 , Cont. to Pal. No. 2, p.4. Hud. Riv. Gr. Probably inorganic.

ramosum var germanum, Miller and Dyer, 1878, Cont. to Pal. No. 2, p. 4, Hud. Riv. Gr. Probably inorganic.

Artirraria, Billings, 1874 , Pal. Foss., vol. 2, p. 66 . [Ety. arthron, a joint.] Cylindrical stems with an expansion at each end in the form of a dumb-bell. Type A. antiquata.

antiquata, Billings, 1874 ,

Pal. Foss., vol. 2, p. 66,

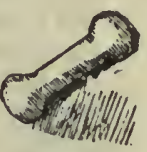

Fre. 11.

Arthraria antiquata.
Upper Taconic.

biclavata, S. A. Miller, 1875, Cin. Quar. Jour. Sci., vol. 2, p. 354, Hud. Riv. Gr. Arthropir ycus, Hall, 1852, Pal. N. Y., vol. 2, p. 4. [Ety. arthron, joint; phykos, seaplant.] Simple or branching, rounded or subangular, flexuous, transversely ridged or furrowed. Type A. harlani.

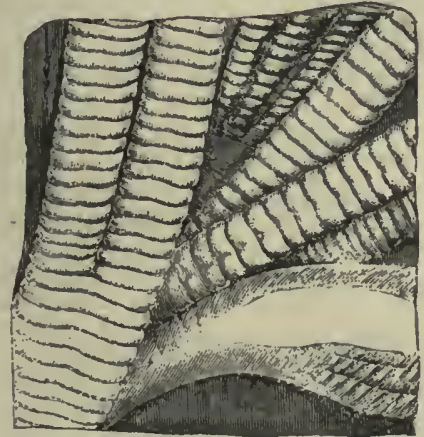

FIG. 12,-Arthrophycus harlani.

harlani, Conrad, 1838, (Fucoides harlani,) Ann. Rep. N. Y., p. 113, and Pal. N. Y., vol. 2, p. 5, Medina sandstone.

montalto, Simpson, 1888, Dict. Foss., found in Pa. Medina (?) Gr.

Arthrostigma, Dawson, 1871, Foss. Plants Canada, p. 41. [Ety. arthron, joint; stigma, a dot or puncture.] Stems elongated, cylindrical, bifurcating, and giving off lateral branches; irregularly furrowed longitudinally, with circular leaf scars arranged in whorls, and bearing linear rigid leaves with circular bases, structure apparently cellular, with a slender vascular axis. Type $\mathbf{A}$. gracile.

gracile, Dawson, 1871, Foss. Plants Can., p. 41, Devonian.

Artisia, Sternberg, syn. for Sternbergia.

transversa, see Sternbergia transversa.

Asolanus, Wood, 1860, syn. for Sigillaria.

camptotznia, syn. for Sigillaria monostigma. manephleus, a doubtful species of Sigillaria.

ornithicnoides.see Sigillaria ornithicnoides. Asplenites, Gœppert, 1836, Systema Filicum Fossilium. [Ety. Asplenium, a genus of ferns.]

elegans, see Eremopteris elegans.

ruber, Lesquereux, 1858, Geo. Sur. Pa., vol. 2, p. 864, Coal Meas. This species does not seem to be recognized by Lesquereux in his later work.

Asterocarpus, Gœppert, 1836, Syst. Fil. Foss., p. 188. [Ety. aster, star; karpos, fruit.] Fructification on lanceolate pinnules, marked by large star-like sori. Type A. sternbergi.

grandis, Lesquereux, 1870, Geo. Sur. IIl., vol. 4, p. 469, Coal Meas.

sternbergi, Gœppert, 1836, Syst. Filic. Foss., p. 188, Coal Meas.

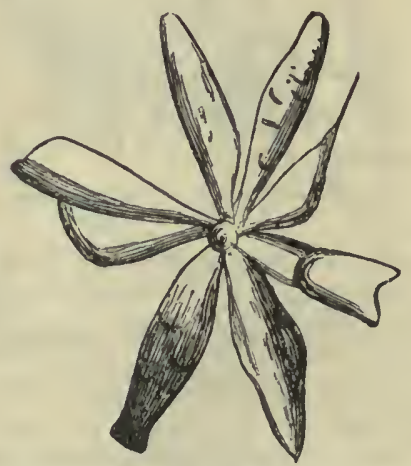

FIG. 13.-Asterophycus Simplex.

Asterophycus, Lesquereux, 1876, 7th Ann. Rep. Geol. Sur. Ind., p. 139. [Ety. aster, star; phykos, a sea-weed.] Stem short, cylindrical; frond expanded and divided star-like from the top of the central axis; segments flattened or inflated. Type A. coxi.

coxi, Lesquereux, 1876, 7th Ann. Rep. Geol. Sur. Ind., p. 139, Low. and Up. Coal Meas.

simplex, Lesquereux, 1880, Coal Flora of Pa., p. 13, Coal Meas.

Asterophyllites. Brongniart, 1822, Mem. du Mus. t. 8, p, 203. [Ety. aster, star; 
phyllon, leaf; lithos, stone.] Stems articulate; branches opposite; central axis hollow or solid; leaves verticillate, free to the base, linear, acuminate, simple nerved; fructifications in elongated ears, bearing round sporanges in the axils of the leaves. Typo A. equisetiformis.

acicularis, Dawson. 1862, Quar. Jour. Geol. Soc., vol. 18, p. 310, Devonian.

anthracinus, Heer, 1877, Fl. Foss. Helv., vol. 4, p. 50, and Coal Flora of Pa., p. 36 , Coal Meas.

apertus, see Macrostachya aperta.

brardi, Brongniart, 1828, Prodr. Hist, Veg. Foss., p. 159, Coal Meas.

crassicaulis, Lesquereux, 1858, Geo. Sur.

Pa., vol. 2, p. 851, Coal Meas.

curtus, see Bechera curta.

equisetiformis, Schlotheim, 1804, (Casuarinites equisetiformis,) Beitrag Zur. Flora der Vorwelt, tab. 1, fig. 1, and Coal Flora of Pa., p. 35, Coal Mieas. erectifolius, Andrews, 1875, Ohio Pal., vol. 2, p. 425, Coal Mens.

fasciculatus, Iesquereux. 1850, Coal Flora of Pa., p. 41, Coal Meas.

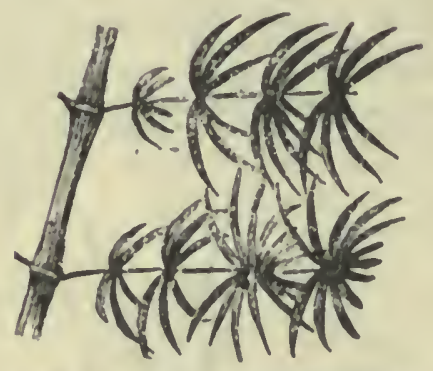

Fro. 14.-Asterphyllites follosus.

foliosus, Lindley \& Hutton, 1833, Foss. Flora, vol. 1, p. 77, and Coal Flora of Pa., p. 38, Coal Meas.

gracilis, Lesquereux, 1860, Geo. Sur. Ark., vol. 2, p. 310, Coal Meas.

grandis, see Bechera grandis.

lanceolalus, see Macrostachya lanceolata. latifolius, Dawson, 1862, Quar. Jour. Geo.

Soc., vol. 18, 1). 311, Devonian. The same form was called by Schimper Annularia duwsoni.

laxus, Dawson, 1868, Acad. Geol., p. 539, Devonian.

lentus, Dawson, 1871, Foss. Plants Can., p. 29 , Devonian.

longifolius, Sternberg, 1823, (Bruckmannia longifolia,) Vers. Darst. Flora der Vorwelt fasc. 4, p. 58, Coal Meas.

minutus, Andrews, 1875, Ohio Pal., vol. 2, p. 424, Coal Meas.

ovalis, see Calamostachys ovalis.

parvulus, Dawson, 1861, Can. Nat. and Geo., vol. 6, p. 168, and Acad. Geol. p. 539, Chemung Gr.

radialus, see Annularia radiata. rigidus, Stern berg, 1824, (Bruck mannia rigida,) Vers. Darst. Flor. derVorwelt, i. 29, and Coal Flora of Pa., p. 37, Coul Meas. scutigerus, Dawson, 1862, Quar. Jour. Geo. Soc., vol. 18, p. 311 , Devonian. stachioides, W ood, 1860, (Lepidostrob u s stachioides, ) Proe. Acad. Nat. Sci. Phil., vol. 12, p. 240, Coal Meas. sublevis, Lesquereux, 1858, Geo. Sur. Pa., vol. 2, p. 851, Coal Meas.

trinervis, Dawson, 1863, Cun. Nat., vol. 8, and Acad. Geol. p. 479, Coal Meas.

tuberculatus, sce Annularia $t u$ bercu lata.

Asteromteris, Daw son, 1851, Quar. Jour. Geo. Soc., vol. 37, p. 299 . [1:ty, aster, star; pteris, fern.] Stenis of ferns having the axial portion composed of vertical radiating plates of scalariform tissue FIa. 15.-Astropolithon imbedded in pa-

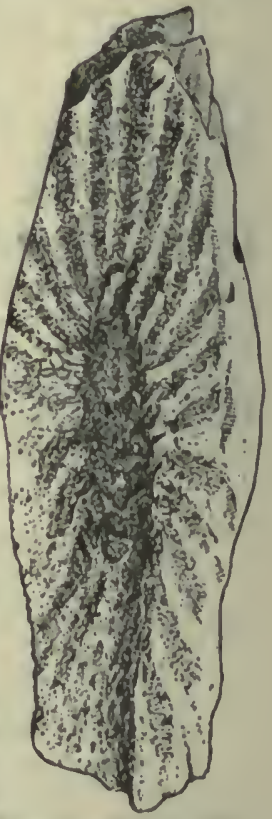
renchyma, and having the outer eylinder composed of elongated cells traversed by leaf-bundles similar to those of Zygopteris. Type A. novoboracensis. novoboracensis, Dawkon, 1881, Quar. Jour. Geo. Soc., vol. 37, p. 299, Portilge Gr. Astrololition, Dawson, 1888, Geo. Hist. Plants, p. 31. A peculiar impression, supposed by Prof. Dawson to be fucoidal. Judiging from the illustration, I would refer it to the Graptolicla. Tyjo A. hindii.

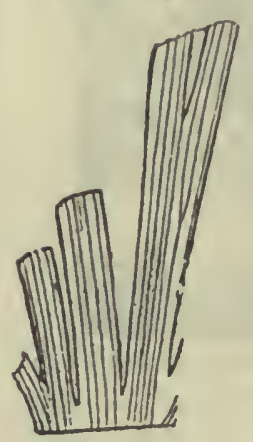

Fig. 16.-Baiera virginlana. lindii, Dawson, 1888, Geo. Hist. plants, p. 31, Up. Taconic. BAiera, Fr. Braun, 1840 , Die Petrefukten d. Naturalien Samml. [Ety. proper name.] Leaves petiolate, flabelliform, dichotomous, many parted; nerves in o ach lacinia, several, dichotonous, and proceeding parallel with each other; leai substance leathery. Type B. treniata.

virginiana, Fontaine \& White, I880, Perm or Up. Carb. Flora, p. 103, Coal Meas. or Permian. 
Becilera, Sternberg, 1824, Vers. Darst. Flora der Vorwelt, p. 30. [Ety. proper name.] Like Asterophyllites in its verticillate leaves, but distinguished by its tumid joints and deeply and widely furrowed stems. Type B. grandis.

grandis, Sternberg, 1S24, Vers. Darst. Flora der Vorwelt, fasc. 4, p. 30, and Coal Flora of Pa., p. 41. Coal Meas.

tenuis, Bunbury, 1846, Am. Jour. Sci., 2d series, vol. 2, p. 232, Coal Meas.

Beinerta, Gœppert, 1836, Syst. Filic. Foss. p. 273. [Ety. proper name.] Distinguished from Pecopteris by the treble flexuous nerves; sometimes anastomosing, and may have its actual representative in the Gymnogramme. Type B. gymnogrammoides.

goepperti, Dawson, 1863, Can. Nat., vol. 8, and Acad. Geol. p. 485, Coal Meas.

Bergeria marginata, see Lepidodendron marginatum.

Bergeria rhombica, see Lepidodendron rhombicum.

Blastopirycus, Miller \& Dyer, 1878, Jour.Cin. Soc. Nat. Hist., vol. 1, p. 24. [Ety. blastos, bud; phukos, sea-weed.] Plant bilobate with a button-like protuberance at the junction. Type B. diadematum.

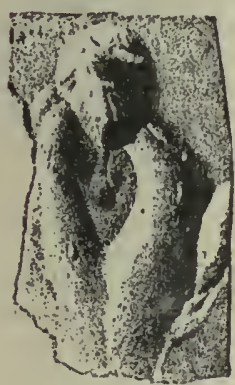
Blastophyeus diadematum.
FIG. 17. diadematum, Miller \& Dyer, 1878, Jour. Ciu. Soc. Nat. Hist., vol. 1, p. 24, Utica Slate Gr.

Borvia, F. A. Roemer, 1854, Palieontographica, vol. 3. [Ety. proper name.] Stenis cylindrical, articulate and furrowed as in Calamites; articulations scarcely contracted; ribs cut square or obtuse at the articulations, continuous, not alternating, thinly striate; cortical cylinder thick; leaves verticillate, free, linearlanceolate. Type B. radiata.

inornata, Dawson, 1862, (Calamites inornatus,) Quar. Jour. Geo. Soc., vol. 18, p. 310 , Genessee Slate.

radiata, Brongniart, 1828, (Calamites radiatus,) Hist. d. Veg. Foss., p. 122, and Coal Flora of Pa., p. 30, Subconglomerate.

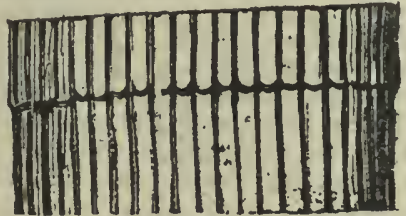

FIG. 18.-Bornia transitionis.

transitionis, Gceppert, 1852, (Calamites transitionis,) Foss. Fl. d. Uebergsg., p. 116, and Quar. Jour. Geo. Soc., vol. 18, p. 309, Ham. Gr.
Bothrodendron punctatum, see Ulodendron punctatum.

Brachyphyllum obtusum, see Lepidocystis obtusus.

Bruckmannia longifolia, see Asterophyllites longifolius.

rigida, see Asterophyllites rigidus.

tuberculata, see Annularia tnberculata.

Bythotrephis, Hall, 1847, Pal. N. Y., vol. 1, p. 8. [Ety. buthos, depth of the sea; trephos, to grow.] Stems subcylindrical or compressed ; branches numerous, divaricating, sometimes leaf-like. Type B. antiquata.

antiquata, Hall, 1847, Pal. N. Y., vol. 1, p. 8, Calcif. Gr.

asteroides, Fitch, 1849, Trans, Ag. Soc., and Emmons Ám. Geol., p. 101, Upper Taconic.

cæspitosa, Hall, 1850, 3d Rep. N. Y. St., Mus. Nat. Hist., p. 178, Trenton Gr.

flexuosa, Emmons, 1844, (Fucoides flexuosa,) Taconic system, p. 69, Upper Taconic.

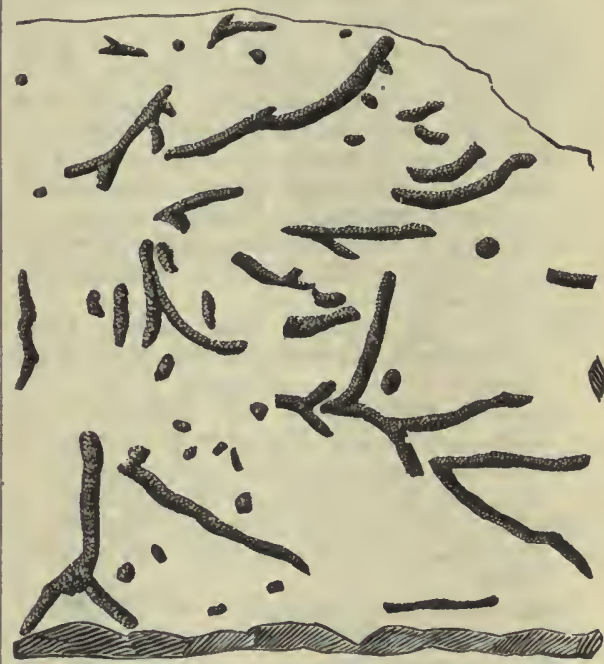

Fig. 19.-Bythotrephis ramulosa, showing the ends, and branehes as they appear on a nodule.

gracilis, Hall, 1843, Geo. Rep., 4th Dist. N. Y., p. 69, and Pal. N. Y., vol. 1, p. 62, Trenton to Clinton Gr.

gracilis var. crassa, Hall, 1852, Pal. N. Y., vol. 2, p. 19, Clinton Gr.

gracilis var. intermedia, Hall, 1852, Pal. N. Y., vol. 2, p. 19, Trenton to Clinton Gr.

granti, Dawson, 1888, Geo. Hist. of Plants, p. 37, Clinton Gr.

gregaria, Ringueberg, 1888, Proc. Acad. Nat. Sci. Phil., p. 131, Niagara Gr.

impudica, Hall, 1852, Pal. N. Y., vol. 2, p. 20, Clinton Gr.

lesquereuxi, Grote \& Pitt, 1876, Bull. Buff. Soc. Nat. Hist., vol. 3, p. 88, Waterlime Gr. palmata, Hall, 1852, Pal. N. Y., vol. 2, p. 20, Clinton Gr. 
ramosa, Hall, 1852, Pal. N. Y., vol. 2, p. 21, Clinton Gr.

ramulosa, S. A. Miller, 1874, Cin. Quar. Jour. Sci., vol.1, p. 235, Utica Slate Gr. rigida, Emmons, 1844, (Fucoides rigidus,) Taconic System, p. 69, Upper Taconic.

subnodosa, Hall, 1817, Pal. N. Y., vol. 1, p. 262, Hud. Riv. Gr.

succulens, Hall, 1847, Pal. N. Y., vol. 1, p. 62, Trenton Gr.

tenuis, Hall, 1852, Pal. N. Y., vol. 2, p. 1S, Trenton Gr. The Trenton form of $B$. gracilis.

Calanites, Guettard, 1751, Mem. Ac. Sci., Paris. [Ety. calamus, \& reed.] Plants arborescent; trunks cylindrical, articulate; artieulations variable in distance, rapidly closer toward the narrowed obconical base; surface narrowly ribbed lengthwise; ribs equal, simple, parallel, contracted or rounded at the articulations; branches nearly at right angles, verticillate like the leaves, which aro lanceolate, acuminate, simple nerved. Type C. suckovi.

approximatus, Sternberg, 1820, Essai d'un exposé Geognostico-botanique d. l. Fl. d. Monde primitif 2d Cahier, p. 36, and Coal Flora of Pa. p. 26, Coal Meas.

bistriatus, Lesquereux, 1858, Geo. Sur. Pa., vol. 2 , p. 850 , Coal Meas. This namo was preoccupied by Sternberg.

canniformis, Schlotheim, 1820, Petrefactenkunde, p. 398, and Coal Flora of Pa., p. 24, Coal Meas.

cisti, Brongniart, 1828, Hist. d. Veg. Foss. p. 129, and Coal Flora of Pa., p. 27, Coal Meas.

cruciatus, Brongniart, 1828, Hist. d. Veg. Foss. t. 1. p. 128, Coal Meas.

disjunctus, Iesquereux, 1858, Geo. Sur. P̊., vol. 2, p. 850 , Coal Meas.

dubius, Artis, 1825, Antedil. Phytology, pl. 13, and Coal Flora of Pa., p. 27, Coal Meas.

gigas, Brongniart, 1828, Hist. d. Veg. Foss., 1 , p. 136, and Coal Flora of Pa., p. 25, Coal Meas.

gracilis, Lesquereux, 1861, Geo. Sur. Ky., vol. 4, p. 436, Coal Meas.

inornatus, see Bornia inornata.

major, Weiss, 1872, Fossil Flora d. jungsten Steinkolen formation, p. 119, and Coal Flora of Pa., p. 21, Coal Meas.

nodosus, Sternberg, 1820, Essai d'un Exp. Geog.-Botan, d. 1. Fl. d. Monde primitif $2 \mathrm{~d}$ Cahier, p. 36, Coal Meas.

nova-scoticus, Dawson, 1863, Can. Nat. \& Geol., vol. 8, and Acad. Geol. p. 479, Coal Meas.

pachyderma, Brongniart, 1828, Hist. d. Veg. Foss., 1, p. 132, and Coal Flora of Pa., p. 28, Coal Meas.

radiatus, see Bornia radiata.

ramifer, Stur, 1875, Culm Flora d. Mährisch-Sehlesischen Dachschiefers, p. 82 , and Coal Flora of Pa., pp. 23, 703, Coal Meas.

ramosus, Artis, 1825, Antedil. Phytology, pl. 2, and Coal Flora of Pa., pp. 22, 702, Coal Meas.

suckovi, Brongniart,1828, Hist. d. Veg. Foss., t. 1. p. 124 , aid Coal Flor. of Pa., p.20, Coal Meas.

transitionis, se e Bornia transitionis.

undulatus, Brongniart, 1828 , Hist. d. Veg. Foss. 1. p. 127, Coal Meas. voltzi, Brongni-

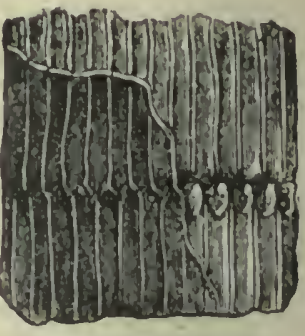

Frg. 20.-Calnmltes Suckovi. art, 182S, Hist. d. Veg. Foss. 1, p. 135, and Acad. Geol. p. 194, Coal Meas.

Calamocladus, Schimper. 1869, Pal. Veget, vol. 1, p. 423. Not clearly distinguislied from Asterophyllites and founded upon $A$. longifolius as the type, and including A. equisetiformis, A. foliosus, A. rigidus, and Bechera grandis.

Calamodendros, Brongniart, 1829, Hist. d. Veg. Foss. vol. 1, p. 133. [Ety.calamus, reed; dendron tree.] Central cylinder striate lengthwise and articulate surrounded ly a tlick, woody cylinder or bark, with outside surface smooth. The strueture is allied to Sigillaria, but the appearance is like Calamites. Type

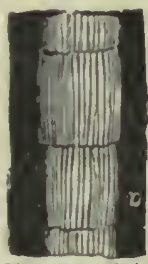

Fia. 21-Cirlamodendron npproxiinatuin. C. approximatum.

antiquum, Dawson, 1871 , Foss. Plants Canada, p. 24. Devonian.

approximatum, Brongniart, 1828, Hist. d. Veg. Foss., vol. 1, p. 133, Coal Meas.

obscurum. Dawson, 1863 , Can. Nat., vol. 8 , and Acad. Geol. p. 476, Coal Meas.

tenuistriatum, Dawson, 1871, Foss. Plants Canada, p. 25, Devonian.

Calamopiryeus, Lesquereux, 1877 ; Proc. Am. Phil. Soe., p. 165. [Eiv. calamus, reed; phukos, sea-plant.] Fronds simple, elongated, gradually tapering to a point; cavity divirled by transverse membranes, either passing through the whole diameter, or connected in the middle to vertical subdivisions. Type C. septum.

septum, Lesquereux, 1877, Proc. Am. Phil. Soc. p. 165, Low. Held. Gr.

Calanostacirys, Schimper, 1869, Traité de Paleontologie Vegetale, vol. 1, p. 328. [Ety. calamus, reed; stachys, plant.] Spikes doubtifully considered as fruetifications of Asterophyllites. Type C. typicus.

brevifolius, Lesquereux, 1884, Coal Flora of $\mathrm{Pa}$., p. 718 , Coal Meas.

lanceolatus, Lesquereux, 1884, Coal Flora of Pa., p. 715, Subconglomerate. 
ovalis, Lesquereux, 1858, (Asterophyllites ovalis), Geo. of Pa., p. 851, and Coal Flora of Pa., p. 717, Coal Meas.

prælongus, see Volkmannia prælonga.

Callipteridium, Weiss, 1872, Foss. Flora d. jungsten Steinkohlen formation. [Ety. from the genus Callipteris.] Fronds large, polypinnate; pinnules attached to the rachis by the whole base, often decurrent, and the lower descending to the main rachis, connate or disjointed at the base; primary nerve strong, dis solved below the apex; lateral veins oblique, curved in passing to the borders, dichotomous, the basilar attached to the rachis. Type C. sullivanti.

aldrichi, Lesquereux, 1880, Coal Flora of Pa., p. 171, Coal Meas.

dournaisi, Brongniart, 1828, (Pecopteris dournaisii), Hist. d. Veg. Foss., p. 282, and Coal Flora of $\mathrm{Pa} .$, p. 747, Coal Meas.

dawsonauum, Fontaine \& White, 1880, Perm. or Up. Carb. Flora., p. 56, Coal Meas. or Permian.

grandifolium, Fontaine \& White, 1880 , Perm. or Up. Carb. Flora., p. 58, Coal Meas. or Permian.

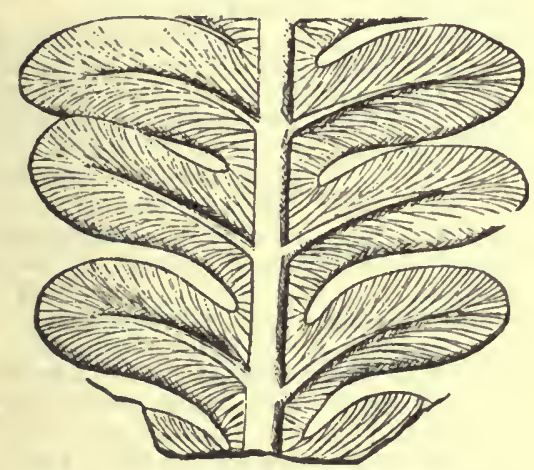

FIG.22.-Callipterldium sullivauti.

grandini, Brongniart, 1828, (Pecopteris grandini,) Hist. d. Veg. Foss., p. 286, and Coal Flora of Pa., p. 748, Coal Meas.

inflatum, Lesquereux, 1870, (Alethopteris inflata,) Geo. Sur. Ill., vol. 4, p. 393, Coal Meas.

inæquale, Lesquereux, 1880, Coal Flora of Pa., p. 168, Coal Meas.

mansfieldi, Lesquereux, 1880, Coal Flora of Pa., p. 166, Coal Meas.

massilloneum, Lesquereux, 1866, (Alethopteris massillionis,) Geo. Sur. Ill.; vol. 2, p. 438, Low. Coal Meas.

membranaceum, Lesquereux, 1880, Coal Flora of Pa., p. 172, Coal Meas.

neuropteroides, Lesquereux, 1880, Coal Flora of Pa., p. 166, Coal Meas.

oblongifolium, Fontaine \& White, 1880, Perm. or Up. Carb. Flora, p. 56, Coal Meas. or Permian.

odontopteroides, Fontaine \& White, 1880 , Perm. or Up. Carb. Flora, p. 59, Coal Meas. or Permian. oweni, Lesquereux, 1860, (Alethopteris oweni,) Geo. Rep. of Arkansas, vol. 2, p. 309, Coal Meas.

pardeei, Lesquereux, 1880, Coal Flora of Pa., p. 169, Coal Meas.

rigidum, Lesquereux, 1884, Coal Flora of Pa., p. 746, Coal Meas.

rugosum, Lesquereux, 1858, (Alethopteris rugosa,) Catal. Potts. Ass'n, p. 11, and Coal Flora of Pa., p. 169, Coal Meas.

sinuatum, Brongniart, 1828, (Pecopteris sinuata,) Hist. d. Veg. Foss., p. 296, and Coal Flora of Pa., p. 745, Coal Meas.

sullivanti, Lesquereux, 1854, (Callipteris sullivanti,) Bost. Jour. Nat. Hist., vol. 6, p. 423, and Geo. Sur. Pa., vol. 2, p. 866, Coal Meas.

unitum, Fontaine \& White, 1880, Perm. or Up. Carb. Flora, p. 60, Coal Meas.

Callipteris, Brongniart, 1828, Hist. d. Veg. Foss., p. 249. [Ety. kallos, beautiful; pteris, fern.] Fronds polypinnate; pinnules sessile and sometimes occurring on the principal rachis, thick; parenchyma dense, nerves immersed, showing creases in the leaf-substance, simple or forking once. Type C, conferta.

conferta, Sternberg, 1824, (Sphenopteris conferta,) Vers. Darst. Flor. d. Vorwelt and Perm. or Up. Carb. Flora of Pa., p. 54, Coal Meas. or Permian.

sullivanti, see Callipteridium sullivanti.

Cardocarpon, Brongniart, 1828, Prodr. Hist. Veg. Foss., p. 87. [Ety. kardia, heart; karpos, fruit.] Seeds of various forms, composed of a conipressed, generally cordiform or oval nucleus, surrounded by a flattened, fibrous border, or a membranaceous wing. Type C. majum.

affine, Lesquereux, 1860, Geo. of Ark., vol. 2, p. 311, Coal Meas.

annulare, Sternberg, 1824, (Carpolithes annularis,) Vers. Darst. Flor. d. Vorwelt and Coal Flora of Pa., p. 814, Subconglomerate.

annulatum, Newberry, 1873, Ohio Pal., vol. 1, p. 374, Coal Meas.

apiculatum, Gœppert \& Berger, 1848, De fructibus et seminibus, p. 23 , and Coal Flora of Pa., p. 571, Subconglomerate.

baileyi, Dawson, 1868, Acad. Geol., p. 554, Devonian.

bicornutum, Lesquereux, 1870, (Ptilocarpus bicornutus,) Geo. Sur. Ill., vol. 4, p. 443 , Coal Meas:

bicuspidatum, Sternberg, 1820, (Carpolithes bicuspidatus,) Flora der Vorwelt, and Coal Flora of Pa., p.573, Coal Meas. bisectum, Dawson, 1863, Can. Nat. and Geol., vol. 8, and Acad. Geol., p. 491, Coal Meas.

circulare, Lesquereux, 1884, Coal Flora of Pa., p. 812, Coal Meas.

conglobatum, Lesquereux, 1884, Coal Flora of Pa., p. 810, Coal Meas.

congruens, Grand Eury, 1877, Flore Carbonifere, p. 236, and Coal Flora of Pa., p. 573, Coal Meas. 
cornutum, Dawson, 1862, Quar. Jour. Geo. Soc., vol. 18, p. 324, Devonian.

crampi, Hartt, 1S68, Acad. Geol., p. 55t, Devonian.

crassum, Lesquereux, 1SS4, Coal Flora of Pa., p. 812, Coal Meas.

dilatatum, Lesquereux, 1884, Coal Flora of Pa., p. 806, Subcarboniferons.

diminutivum, Lesquereux, 1880, Coal Flora of Pa., p. 570, Coal Meas.

diplotesta, Lesquereux. 18St, Coal Flora of Pa., p. 812 , Coal Meas.

divergens, I,esquereux, 1SS4, Coal Flora of Pa., p. S11, Coal Meas.

ellipticum, Sternberg, 1820, (Carpolithes ellipticus,) Flor. d. Vorw., p. 40, and Coal Flora of Pa., p. 814, Coal Meas.

elongatum, Newberry, 1873, Ohio Pal., vol. 1, p. 324 , Coal Meas.

fasciculatum, Lesquereux, 1580, Coal Floru of Pa., p. 570, Coal Meas.

fluitans, Dawson, 1863, Can. Nat. and Geol., vol. 8, and A cad. Geol., p. 491, Coal Meas.

harveyi, Lesquereux, 18S4, 13th Rep. Ind. Geol., p. 10\%, and Coal Flora of Pa., p. 808, Coal Meas.

ingens, Lesquereux, 1560, Geo. of Ark., vol. 2, p. 311, Coal Meas.

late-alatum, Lesquereux, 1880, Coal Flora of Pa., p. 565, Coal Meas.

latior, Ifesquereux, 1884, Coal Meas. of Pa., p. 811, Coal Meas.

latum. Newberry, 18\%", Ohio Pal., vol. 1, p. $3 \pi 2$, Coal Meas.

lescurianum, n. sp. Coal Meas. Proposed instead of C. ovale Lesquereux, in Coal Flora of Pa., p. 810 , which name was preoccupied.

longicollis, Lesquereux, 1884, Coal Flora of Pa., p. 808, Coal Meas.

mamillaum, see Rhabdocarpus mamillatus. marginatum, Artis, 182S, Antedil. Phytol., pl. 22, Coal Meas.

minus, Newberry, 1873, Ohio Pal., vol. 1, p. 372, Coal Meas.

newberrvi, Andrews, 1875, Ohio Pal., vol. 2, p. 425 , Coal Meas.

obliquum, Dnwson, 1862, Quar. Jour. Geo. Soc., vol. 18, p. 324, Devonian.

orbiculare, Newberry, 1853, Ann. of Sci., vol. 1, p. 374, Coal Mleas.

ovale, Dawson, 1871, Foss. Plants Can., p. 60, Devonian.

male, Lesquereux, 18S4, Coal Flora of Pa., p. 810 , Coal Meas. The namo was preoccupied. See C. lescurianum.

ovatum, Grand 'Eury, 1877, Flore Carbonifere, p. 236, Coal Meas.

pachytesta, Lesquereux, 1880, Coal Flora of Pa., p. 565, Coal Meas.

patens, Lesquereux, 1884, Coal Flora of Pa., p. 807, Coal Meas.

plicatum, Lesquereux, 1858, Geo. Sur. Pa., vol. 2, p. S76, Coal Meas.

punctatum, Gœp pert, 1836, Syst. Filic. Foss., p. 24, and Coal Flora of Pa., 597, Coal Meas.

pusillum, Lesquereux, 1854, Coal Flora of Pa., p. 815 , Coal Meas. regulare, Sternberg, 1820, (Carpolithes regularis, ) Flor.d. Vorw., and Coal Flora of Pa., p. 572, Coal Meas.

retusun), Sternberg, 1520, (Carpolithes retusus, Flora der Vorwelt, and Ohio Pal., vol. 1, p. 374, Coal Mists.

samariforme, New berry, 1873, Ohỉn Pal., vol. 1 , p. 375 , Coal Meas.

simplex, Lesqu $u$ reu $x$ 1850, Coal Flora of $\mathrm{Pa}$. p. 569, Coal Meas.

spcciosus, Lesq u ere $u x$, 18S4, Coal

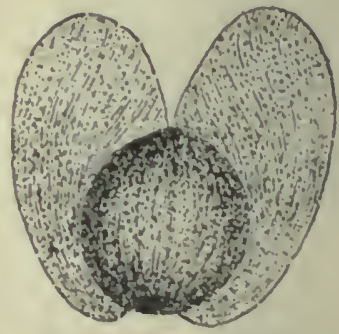

FıG. 23.-Cardlocarpon snmarlfortne.
Flora of Pa., p. 807, Coal Meas.

tenellum, Dawson, 1873, Rep. Foss. Plants, p. 28, Subcarboniferous.

trevortoni, Lesquereux, 1858, Geo. Sur. Pa., vol.2, p. 876, Coal Meas.

zonulatum, Lesquereux, 18S0, Coal Flora of Pa., p. 568, Coal Meas.

Cardioltriss, Schimper, 1S69, Traité de Paléontologie Vegetale, vol. 1, p. 457. [Ety. kardia, heart; pleris, fern.] L.eaves simple, pinnate; stipe striated, rounded, base spoon-like, dilated; pinnæ perpendicular, opposite close, imbricated, corlato-ovate, leathery, margins reflexed; primary nerves numerous, equal, dichotomous. 'Typo C. polymorpha.

eriana, Dawson, 1881, Quar. Jour. Geo. soc. Lond., vol. 37, p. 305, Devonian.

Carpolitiles, Schlotheim, 1820, Petrefactenkunde. [Ety. karpos, fruit; lithos, stone.] Seeds of uncertain relation not referable by" their characters to other genera. acuminatus, Sternberg, 1821, Flor. d. Vorw. and Coal Flora of P'a., p. 596, Coal Meas.

biearpus, Fontaine \& White, 1880, Perm. or Up. Carb. Flora, p. 98, Coal Meas. or Permian.

bicuspidalus, see Cardiocarpon Vbicuspidatum.

bifidus, Lesquereux, 1858, Geo. Sur. Pa., vol. 2, p. 877, Coal. Meas.

bullatus, see Lepidocystis bullatus.

butleranus, Lesquereux, 1884, Coal Flora of $\mathrm{Pa} ., \mathrm{p} .824$, Coal Meas.

cerasiformis, Sternbery, Flor. d. Vorw., vol. 2, p. 208, and Coal Flora of P'a., p. 824 , Coal Meas.

cistula, Lesquereux, 1866, Geo. Sur. Ill., vol. 2, p. 451, Coal Meas.

clavalus, see Rhabdocarpus clavatus.

compactus, Dawson, 1871, Foss. Plants Canada, p. 63, Devonian.

conicus, Lesquereux, 1884, Coal Flora of Pa., p. 824, Coal Meas.

corticosus, Lesquereux, 1870, Geo. Sur. Ill., vol. 4, p. 462, Coal Meas. 
disjunctus, Lesquereux, 1858, Geo. Sinr. Pa., vol. 2, p. 877, syn. for Trigonocarpon dawesi.

fasciculatus, Lesquereux, 1866, Geo. Sur. Ill., vol. 2, p. 457, Coal Meas.

fragarioides, Newberry, 1873, Ohio Pal., vol. 1 , p. 370, Coal Meas.

granularis, Sternberg, 1820, Flora der Torwelt, and Coal Flora of Pa. p. 825, Coal Meas.

jacksonensis, seeRhabdocarpus jacksonensis.

latior, Lesquerenx, 1884, Coal Flora of Pa., p. 826 , Coal Meas.

lunatus, Dawson, 1863, Quar. Jour. Geo. Soc., vol. 19 , p. 464, Devonian.

marginatus, Fontaine \& Wlite, 1880, Perm. or Up. Carb. Flora, p. 98. The name was preoccupied by Artis in 1825. See C. whitianus.

minimus, Sternberg, 1820, Flora der Vorwelt and Coal Flora of Pa., p. 825, Coal Meas.

multistriatus, see Rhabdocarpus multistriatus.

perpusillus, Lesquereux, 1884, Coal Flora of Pa., p. 825 , Coal Meas.

persicaria, Lesquereux, 1870, Geo. Sur. Ill., vol. 4, p. 462, Coal Meas.

platimarginatus, see Rhabdocarpus platimarginatus.

retusus, see Cardiocarpon retusum.

siliqua, Dawson, 1863, Quar. Jour. Geo. Soc. Lond., vol. 19, p. 465, Devonian.

spicatus, Dawson, 186:, Quar. Jour. Geo. Soc. Lond., vol. 19, p. 461 , Devonian.

transsectus, Lesquereux, 1884, Coal Flora of Pa., p. 826, Coal Meas.

trilocularis, see Trigonocarpon triloculare. umbonatus, Sternberg, 1820, Vers. Darst. Flora der Vorwelt, Coal Meas.

venosus, see Rhabdocarpus venosus.

vesicularis, see Lepidocystis vesicularis. whitianus n. sp. Coal Meas. or Perm. Proposed instead of C. marginatus, Fontaine \& White, 1880, in Perm. or Up. Carb. Flora, p. 98, which name was preoccupied.

Casuarinites equisetiformis, see Asterophyllites equisetiformis.

Caulerpites, Brongniart, 1828, Prodr. d. Hist. d. eg. Foss., p. 21. [Ety. kaulos, stem; erpo, creep.] Stem simple or ramose, covered with short branches, in form of leaves doubled or imbricated. Type C. lycopodioides.

marginatus, see Taonurus marginatus.

Caulopteris, Lindley \& Hutton, 1833, Foss. Flora, vol. 1, p. 121. [Ety. kaulos, stem; pteris, a fern.] Scars with the inside disk either marked by linear bands, remains of vessels passing from the trunk to the base of the rachis, or covered by impressions of rootlets obliterating its shape, or merely ovate or elliptical, without traces of horseshoe-shaped vascular lines. These lines may have been, in some cases, effaced by abrasion of the surface or covered by rootlets. Type C. primieva. acanthophora, Lesquereux, 1870 , Geo. Sur. Ill., vol.4, p.458, Coal Meas. It may be a synonym for Ülodendro $\mathrm{I}$ punctatum. antiqua, Newberry, 1871, Quar. Jour. Geo. Soc. vol. $27, \mathrm{p}$. 271, Up. Held. Gr. cisti, Brongniart, 1828 , (Sigilla ri a cisti,) Hist. Veg. Foss. p, 418 , and Coal Flora of Pa., p. 345, Coal Meas.

elliptica, Fontaine \&

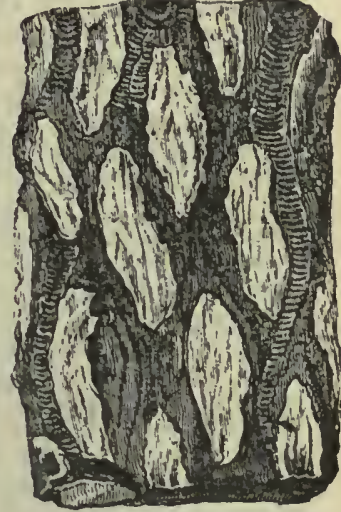

FIG. 24.-Caulopteris primava.
White, 1880 , Perm. or Up. Carb. Flora, p. 95, Coal Meas. or Permian.

giffordi, Lesquereux, 1880, Coal Flora of Pa., p. 343, Coal Meas.

gigantea, see Stemmatopteris gigantea. gigantea, Fontaine \& White, 1880 , Perm. or Up. Carb. Flora, p. 95, Coal Meas. or Permian.

insignis, see Stemmatopteris insignis.

intermedia, Lesquereux, 1870, Geo. Sur. Ill., vol. 4, p. 459, Coal Meas.

lacoi, Lesquereux, 1880, Coal Flora of Pa., p. 344, Coal Meas.

lockwoodi, Dawson, 1871, Quar. Jour. Geo. Soc., vol. 27, p. 270, Chemung Gr. mansfieldi, Lesquereux, 1880, Coal Flora of Pa., p. 346, Coal Meas.

obtecta, Lesquereux, 1870, Geo. Sur. Ill., vol. 4 , p. 457 , Coal Meas.

peregrina, Newberry, 1871, Quar. Jour. Geo. Soc., vol. 27, p. 272 , Up. Held. Gr.

punctata, see Stemmatopteris punctata.

wortheni, see Stemmatopteris wortheni.

Celleloxylon, Dawson, 1881, Lond. Quar.

Jour. Geo. Soc., vol. 37, p. 302. [Ety. cellula, a small apartment; xylon, wood.] Trunk showing in cross section, large and somewhat unequal cells disposed in narrow concentric bands, between wider bands of fine fibrous tissue; no medullary rays; longitudinal section shows either cells superimposed in vertical rows, or a sort of banded prosenchymatous tissue. The structure appears to have been of exogenous growth. Type C. primævum.

primævum, Dawson, 1881, Quar. Jour. Geo. Soc., vol. 37, p. 302, Ham. Gr. Penhallow says this is an Algæ, and belongs to the genus Nematophycus.

Chloephycus, Miller \& Dyer, 1878, Cont. to Pal., No. 2, p. 3. Probably inorganic.

plumosum, Miller \& Dyer, Cont. to Pal., No. 2, p. 3, Hud. Riv. Gr. Probably inorganic. 
Chondrites, Sternberg, 1833, Vers. Darst. Flora der Vorwelt, p. 25. [Ety. from its resemblance to Chondrus crispus, or Irish moss.] Fronds cartilaginous, filiform or robust stems, dichotomous, branchy; rounded or compressed. Type C. antiquus.

antiquus, Brongniart, 182S, (Fucoides antiquus,) Hist. d. Veg. Foss., vol. 1, p. 63, Devonian.

colletti, see Taonurus colletti.

targioni, Brongniart, 1828, (Fucoides targioni,) Hist. d. Veg. Voss., t. 1, p. 56 , Coal Meas.

Cososticius, Lesquereux, 1876, 7 th Ann. Rep. Geo. Sur. Ind., p. 142. [Ety. konos, cone; stichos; row.] Stipe cyliudrical, continuous; frond enlarging from the base upward in the shape of a plate, or of a cup, or incrensing by successive superposed layers or concentrical laminr; top concave, cup-shaped. Type C. ornatus.

broadheadi, Lesquereux, 1850, Coal Flora of Pa., p. 15. Coal Meas.

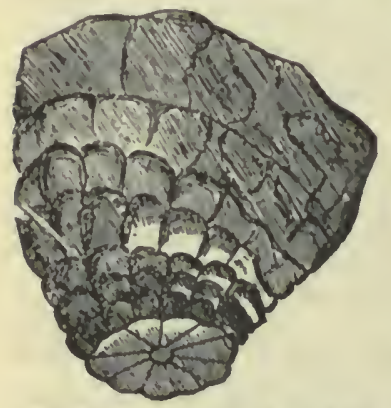

FIก. 25.-Conostichus ornatus. ornatus, Lesquereux. $15 i 6,7 \mathrm{cli}$ Ann. Rep. Gieo. Sur. Ind., p.142, Coal Meas. prolifer, Les. quoreux. 1880 , Coal Flora of Pa., p. 16 , Coal Mens. Cordaiantius, Grand 'Eury, 1877 , Flore Car. bonifere, p. 22S. [Ety. Cordaites, a genus; anthos, flower.] Flowers and fruits of Cordaites, found isolated or in fragments where their relation to stem-bearing leaves is unknown. A provisional name only. Type C. gemmifer.

bracteatus, Lesquereux, 1870, (Schultzia bracteata,) Geo. Sur. Ill., vol. 4, p. 427, Coal Meas.

dichotomus, Lesquereux, 1850, Coal Flora of Pa., p. 546, Conl Meas.

ebracteatus, Lesquereux, 1884, Coal Flora of Pa., p. S44, Coal Meas.

flexuosus, Lesquereux, 1884, Coal Flora of Pa., p. 802, Coal Meas.

gemmifer, Grand 'Eury, 1877, Flore Carbonifere, p. 22S, and Coal Florn of Pa., p. 545, Coal Meas.

ovatus, Lesquereux, 1850, Coal Flora of Pa., p. 545, Coal Meas.

rugosus, Lesquereux, 1884, Coal Flora of Pa., p. 803, Coal Meas.

scaber, Lesquereux, 1884, Coal Flora of Pa., p. 844, Coal Meas.

simplex, Lesquereux, 1880, Coal Flora of Pa., p. 535, Coal Meas. spicatus, Lesquereux, 1854, Coal Flora of Pa., p. 802, Coal Meas.

Cordaicanpus, Grand 'Eury, 1877, Flore Carbonifere, p. 236. [Eity. Cordaites, a genus ; karpos, fruit.] Seeds of variable size and shape. Type C. ovatus.

apiculatus, Lesquereux, 1850 , Coal Flora of Pa., ऍ. 551, Coal Meas.

cinctus, Lesquereux, 1854 , Coal Flora of Pa., p. 804 Coal Meas.

costatus, Lesquerenx, 1880 (Cordaites costatus, Coal Fio. 26. Flora of Pal. 1. 540, Coal apleulatus. Meas.

gutbieri, Grand 'Eury, 1877, Flore Carbonifere, p. 236, Coal Meas.

lineatus, Lesquereux, 1884, Coal Flora of Pa., p. S05. Conl Meas.

ovatus, Grand 'Eury, 1877, Flore Carbonifere, p. 236, and Coal Flora of Pa., p. 550 , Coal Meas.

stabilis, Lesquereux, 1834, Coal Flora of Pa., p. 805, Coal Mens.

Cordaistroizus, Leequerenx, 1850, Coal Flora of Pa., p. 551. [Ety. Cordaites, a genus; strobus, cone.] Strobile cylindrical, tapering to a blunt acumen, coverel by transversely rhomboidal scars placed in spiral, bearing narrow, linear leaves, with tho characters, form, and nervation of leaves of Corlaites. Type C. grandeuryi.

grandeuryi, Iesquereux, 1880, Coal Flora of Pa., p. 552, Coal Meas.

Condaites, Unger, 1850, Gen. et sp., p. 277. [Ety. proper name.] Trunks of large size, irregularly branching, formed of a large medullar canal or pitb; marked on the outer surface by trnnsverse, narrow, parallel, simple ribs, rarely joined by divisions, covered by double or triple layers of wood and bark, converted by fossilization into thin layers of coal; leaves in spiral order, more or less distant, ribbon.like, of various length and width, linear, or more generally gradually enlarging upward, obtuse, entire or undulate, and split at the apex; borders curving to the sessile, or semi-embracing, somewhat inflated base; surface marked lengthwise by primary and secondary parallel simple nerves, generally more rlistant in the middle of the leaves, and slightly inflated toward the base; flowers in racemes from the axils of the leaves; fruits generally oval, sessile, of various size. Type $C$. borassifolius.

angustifolius, Dawson, 1861, Can. Nat., vol. 6 , p. 10, Ham. Gr.

angustifolius, Lesquereux, see C. diversifolius.

borassifolius, Sternberg, 1820, (Flabellaria borassifolia,) Essai d. Exp. Geogn-botan. d. 1. Flora d. monde primitif, 2 d Cahier., p. 36, and Coal Flora of Pa., p. 532, Coal Meas. 
communis, Lesquereux, 1878, Proc. Am. Phil. Soc., p. 320, and Coal Flora of Pa., p. 534, Coal Meas.

costatus, Lesquereux, 1878, Proc. Am.

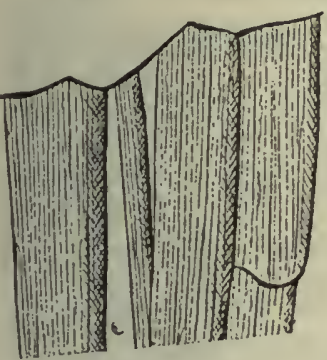

Frg. 27.-Cordaites costatus.

Coal Flora of Pa., p. 530, Coal Meas.

diversifolius, Lesquereux, 1880, Coal Flora of Pa., p. 535, Coal Meas. Proposed instead of C. angustifolius Lesquereux, which was preoccupied.

flexuosus, Dawson, 1863, Quar. Jour. Geo. Soc., vol. 19, p. 462, Catskill Gr.

gracilis, Lesquereux, 1878, Proc. Am. Phil. Soc., p. 322, and Coal Flora of Pa., p. 539, Coal Meas.

grandifolius, Lesquereux, 1878, Proc. Am. Phil. Soc., p. 318, and Coal Flora of Pa:, p. 530, Coal Meas.

lacoei, Lesquereux, 1880, Coal Flora of Pa., p. 535, Coal Meas.

lingulatus, Grand 'Eury., 1877, Flore Carbonifere, p. 218, and Coal Flora of Pa., p. 533, Coal Meas.

mansfieldi, Lesquereux, 1878, Proc. Am. Phil. Soc., p. 321, and Coal Flora of Pa., p. 537, Coal Meas.

radiatus, Lesquereux, 1880, Coal Flora of Pa., p. 540, Coal Meas.

robbi, Dawson, 1861, Can. Nat., vol. 6, p. 8, Ham. Gr.

serpens, Lesquereux, 1880, Coal Flora of Pa., p. 324, Coal Meas.

simplex, Dawson, 1863, Can. Nat., vol. 8, and Acad. Geol., p. 490, Coal Meas.

validus, Lesquereux, 1878, Proc. Am. Phil. Soc., p. 317, and Coal Elora of Pa., p. 529, Coal Meas.

Crematopteris, Schimper, 1869, Traite de Paleontologie Vegetale, vol. 1, p. 596. [Ety. krematos, hanging; pteris, fern.] Rachis thick; pinnules sessile, ovateoblong, contracted at the base, and subauriculate. Type C. typica.

pennsylvanica, 'Lesquereux, 1880, Coal Flora of Pa., p. 307, Coal Meas.

Cruziana, D'Orbigny, 1842, Voy. dans l'Amer. Merid. t. 3, pt. 2, p. 30. [Ety. proper name.] A transversely wrinkled fucoid, much like Rusophycus. Type C. rugosa.

linnarsoni, White, 1874, Rep. Invert. Foss., p. 5, and Geo. Sur. W. 100th Merid., vol. 4, p. 32 , Upper Taconic. rustica, White, 1874, Rep. Invert. Foss., p. 5, and Geo. Sur. W. 100th Merid., vol. 4 , p. 3\%, Up. Taconic.

similis, Billings, 1874, Pal. Foss., vol. 2, p. 68 , Up. Taconic.

Cyclopteris, Brongniart, 1828, Prodr. Hist. Veg. Foss., p. 51. [Ety. kuklos, circle; pteris, fern.] Leaflets orbicular or reniform, large, veins numerous, and not positively referable to other genera. Type C. orbicularis.

acadica, see Aneimites acadicus.

alleghaniensis, Meek, 1876, Desc. Foss. Plants Va. Syn. for Archæopteris rogersi.

antiqua, Dawson, 1863, Can. Nat. and Geo., vol. 8, and Acad. Geol., p. 481, Coal Meas.

bockshii, see Aneimites bockshii.

browni, see Rhacophyllum browni.

crispa, Germ. \& Kaulf, 1831, (Filicites crispus,) Nova. Acta. Acad., vol. 15, p. 229, Coal Meas.

elegans, Lesquereux, 1858, Bost. Jour. Nat. Hist., vol. 6, p. 416, and Geo. Sur. Pa., vol. 2, p. 856, Coal Meas.

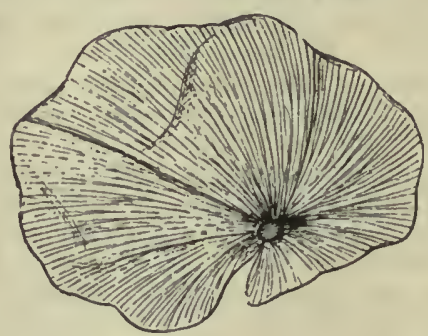

FIG. 28,-Cyclopteris elegans.

fimbriata, see Neuropteris fimbriata.

flabellata, Brongniart, 1828, Prodr. Hist.

Veg. Foss., p. 52, Coal Meas.

germari, see Neuropteris germari.

hallana, see Archæopteris hallana.

hispida, Dawson, 1863, Can. Nat. and Geol., vol. 8, and A cad. Geol., p. 481, Coal Meas.

hirsuta, Lesquereux, 1858, Geo. Sur. Pa., vol. 2 , p. 856 , Coal Meas.

incerta, Dawson, 1862, Quar. Jour. Geo. Soc., vol. 18, p. 320, Ham. Gr.

jacksoni, see Archæopteris jacksoni.

laciniata, see Neuropteris laciniata.

lescuriana, see Triphyllopteris lescuriana.

oblata, Lindley \& Hutton, 1837, Foss.

Flora, vol. 3, pl. 217, Coal Meas.

obliqua, Brongniart, 1828, Prodr. Hist.

Veg. Foss., p. 52, Coal Meas.

orbicularis, Brongniart, 1828, Prodr. Hist.

Veg. Foss., p. 52, Coal Meas.

problematica, Dawson, 1871, Foss. Plants

Dev. and Up. Sil., p. 47, Devonian.

rogersi, see Archæopteris rogersi.

trichomanoides; see Neuropteris trichomanoides.

undans, Lesquereux, 1858, Geo. Sur. Pa., vol. 2, p. 855 , Coal Meas. 
valida, see Aneimites validus.

varia, Dawson, 1S62, Quar. Jour. Geo. Soc., vol. 18, p. 319 , Devonian.

virginiana, see Pseudopecopteris virginiana.

wilsoni, Wood, 1860, Proc. Acad. Nat. Sci., p. 519, Coal Meas.

Crclostigas, Haughton, 1560, Ann. and Mag. Nat. Hist., 3d ser., vol. 5, p. 444. [Ety. kuklos, circle; stigma, a dot or puncture.] Stems arborescent, surface tuberculate, rugose lengthwise; tubercles in regular spiral order, small, subglobose, more generally conical, acute, topped with a vascular terminal and prominent point, or more rarely flattened at the top into small, round areoles, with the vaseular point in the middle; decorticated surface smooth or obscurely striate lengthwise by the series of tubercles, which are oval, elevated or prominent, and gradually effaced downward or decurring, preserving the impressions of the central vascular sears. Type C. kiltorkense.

afline, Dawson, 1851, Quar. Jour. Geo. Soc., vol. 37, p. 301, Chemung Gr.

densifolium, Dawson, 1871, Foss. Plants Can., p. 43, Devonia.

kiltorkense, Haughton, 1860, Ann. and Mag. Nat. Hist., 3d ser., vol. 5, p. 444, Subcarboniferous.

Cramoolossa, Sehimper, 1869, Traite de Paleontologie Vegetale, vol. 1, p. 553. [Ety. kumo, wavy; glossu, tongue.] Frond pinnate, or bipinnate; punna oblong, sessile, alternate, margin lohed; nerves simple or branching, reaching the margin and leaving triangular spaces without nerves. Type C. goeppertana.

breviloba, Fontaine \& White, 1850, Perm. and Up. Carb. Flora, p. S6, Coal Meas. or Permian.

formosa, Fontaine \& White, 1850, Perm. and Up. Carb. Flora, p. 86, Coal Meas. or Permian.

lobata, Fontaine \& White, 1880, Perm. and Up. Carb. Flora, p. 87, Coal Meas. or Permian.

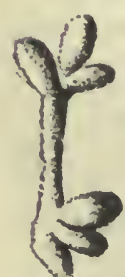

FIG. 29.Dactylophycus irjaigitatum.

Utica Slate Gr.

obtusifolia, Fontaine \& White, 1880, Perm. and Up. Carb. Flora, p. 85, Coal Meas. or Permian.

DACTYLOPIycus, Miller \& Dyer, 1878, Cont. to Pal., No. 2, p. 1. [Ety. dactylos, finger; phukos, sea-plant.] Stem divided at one or both ends into three or more short subequal branches, and closely related to Ichnophycus. 'Type D. tridigitatum.

quadripartitum, Miller \& Dyer, 1878, Cont. to Pal., No. 2, p. 2 ,

tridigitatum, Miller \& Dyer,1878, Cont. to Pal., No. 2, p. 1, Utica Slate Gr.
Dadoxylox, Endlicher, 1840, Syn. Con. [Sig. pine or torch-wood.] Branching trunks, with distinct zones of growth and a pith-of Sternbergia type; wood-cells, with rows of areoles witl oval pores; medullary rays with series of cells.

acadianum, Dawson, 1863, Can. Nat., vol. 8 , and Acad. Geol., p. 473, Coal Meas.

annulatum, Dawson, 1863, Can. Nat., vol. 8 , and Acad.Geol. p. 473, Coal Meas.

antiquum, Dawson, 1863, Can. Nat., vol. S, and Acad. Geol. p. 473, Coul Meas.

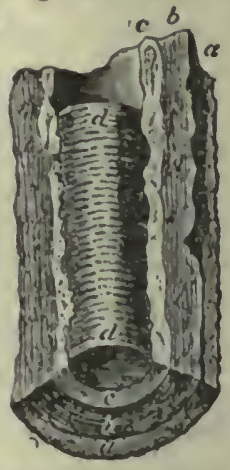

elarkii, Dawison, 1852, Foss. Ilants Erian and Up. Sil. Formations, pt. 2, p. 124, Genesce slunles.

Fia. आ. - Dutloxylon. $a$, baik ; $b$ woody z on e or Illser $(p$ leurencliy in a) ; c, inedulla or pilli; $d$. cast of linilow pith or sternberhalli, Dawson, 1862 , gla.

Quar. Jour. Gieo. Soc., vol. 18, p. 306 , Him. Gr.

materiarium, Dawson, 1863, Can. Nat. vol. 8, and Acad. Geol., p. 473, U1. Coal Meas.

newberryi, Dawson, 1871, Foss. Plants Can., p. 14, Portage Gr.

ouangondianum, Dawson, 1S61, Can. Nat.,

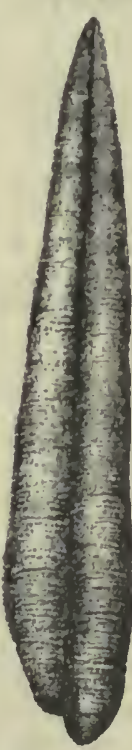

Fia. 31.Danaltes
maerophyl. lus. vol. 6. and Acad. Geol, p. 534, U\}. Devonian.

DAvжiтs, Goppert, 1836, Syst. Filic. Foss., p. 380. [Ety. from the genus Danaxa.] Fronds pinnate; secondary veins conning out in right angles from the primary straight nerve, simple or dichotomous; sporanges, on the lower side of the lamina, placed in rows from the medial nerve to near the borders along the lateral veins, oval or linear exannulate. Type $\mathrm{D}$. asplenioides.

asplenioides, var. major, Bunbury, 1846, Quar. Jour. Geol. Soc., vol. 2, p. 85. Coal Meas.

emersoni, Lesquereux, 1880 , Coal Flora of Pa., p. 157, Coal Meas.

macrophyllus, Newberry, 1873 (Alethopteris macrophylla, Ohio Pal., vol. 1, p. 3S3, Low. Coal Meas.

Dechenia, Goppert, 1841, Die Gattungen der fossilen Pflanzen, p). 43. [Ety. proper name.] Stems arborescent; leaf-scars in continuous spiral lines; bolsters oblong, rounded, marked by obscure concen trical striæ, on the middle 
of which were attached leaves, probably cylindrical. Type D. euphorbioides.

striata, Lesquereux, 1880, Coal Flora of Pa., p. 431, Coal Meas.

Dendrophycus, Lesquereux, 1884, Coal Flora of Pa., vol. 3, p. 699. [Ety. den. dron, tree; phukos, sea-weed.] Root composed of tubulose flattened filaments, irregularly branching and widely spreading from the base of the rhizoma ; rhizoma cylindrical, simple, long and thick; fronds at first top-shaped, very large, tree-like, and many times divided when opened; primary and secondary branches thick and somewhat flat on one side, dichotomous; ultimate divisions cylindrical, narrow and pointed. Type $\mathrm{D}$. desori.

desori, Lesquereux 1884, Coal Flora of Pa., vol. 3., p. 699, Devonian.

Desmiophyluum, Lesquereux, 1880, Coal Flora of Pa., p. 556. [Ety. desmos, band; phyllon, leaf.] Stems slender; leaves narrow, sublinear, gradually enlarged from the base, single and sparse or joined 3 or 4 together and fasciculate at the base; surface of stem and leaves irregularly ribbed lengthwise by prominent large bundles of nerves buried under the epidermis, which is thick, irregularly granulose, by splitting of the coaly layer. Type D. gracile.

gracile, Lesquereux, 1880, Coal Flora of Pa., p. 557, Coal Meas.

Dicranophyllum, Grand 'Eury, 1877, Flore Carbonifere, pl. 30. [Ety. dikranos, twopointed; phyllon, leaf.] Stems slender, leaves narrow, linear, subcoriaceous, of various length, forking, or dividing in filaments in the upper part, marked with a few thick primary nerves, and intermediate nervilles, more or less immersed into the epidermis.

dichotomum, Lesquereux, 1880, Coal Flora of Pa., p. 553, Coal Meas.

dimorphum, Lesquereux, 1879, Proc. Am. Phil. Soc., p. 329, and Coal Flora of Pa., p. 554, Coal Meas.

Dictuolites, see Dictyophyton in the class Protozoa.

becki see Dictyophyton becki.

Dictyopteris, Gutbier, 1835, Verst. Zwick. Schwarzk, p. 63. [Nty. dictyon, net; pteris, fern.] Frond bipinnate; pinnules cordate, truncate or rounded at the base, sessile or short pedicelled, oblong, obtuse or lanceolate, entire; veins flexuous, connected by flexures and intersections, forming a more or less distinct and close reticulation of polygonal meslies. Type D. brongniarti.

cordata, Roemer, in Pflanzen d. prod. Steinkohlengeb. am Harz und Piesberg in Palæontographica, vol. 9, p. 186, and Coal Fiora of Pa., p. 833, Coal Meas.

neuropteroidea, Gutbier, 1852, Verst. Stein Sachs., p. 23, and Coal Flora of Pa., p. 833, Coal Meas.

obliqua, Bunbury, 1847, Quar. Jour. Geo. Soc., vol. 3, p. 427, and Coal Flora of Pa., p. 116, Coal Meas.

rubella, L e sque reux, 1870, Geo. Sur. Ill., vol. 4 , p. 388, Coal Meas. scheuchziri, $\mathrm{H}$ of $\mathrm{f}$ man, in Roem. Pflanz. d. Kohlengeb. am Harz in Paleont. IX, $\mathrm{p}$. 186, and Coal

Flora of Pa., p. 832, Coal Meas.

Dinymophylum, Goppert, 1841, Gatt. der Foss. Pflanzen, p. 35. [Ety. didymos, double; phyllon, a leaf.] Trunk arborescent, cylindrical; leaves double, united at the base, disposed in spiral order, appressed; areoles prominent, reniform, eacli resembling a pair of small areoles attached to each other. Type D. schottini.

oweni, Lesquereux, 1870, (Sigillaria oweni,) Geo. Sur. Ill., vol. 4, p. 498, Coal Meas.

reniforme, Dawson, 1862, Quar. Jour. Geo. Soc., vol. 18, p. 309, Ham. Gr.

Diplazites emarginatus, see Pecopteris emarginata.

Diplostegium, Corda, 1845, Beitrage zur Flora der Vorwelt, p. 112. [Ety. diplos, double; stege, a covering; but spelled by Corda Diplotegium.] Thick trunks of trees longitudinally furrowed; bark thick, and imbricated in short cylindrical overlaps. Type D. brownanum.

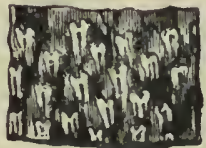

FIG. 39.

Diplostegium retusum. brownanum, Corda, 1845, Beitrage zur Flora der Vorwelt, p. 112, Coal Meas.

retusum, Dawson, 1863, Can. Nat., vol. 8, and Acad. Geol., p. 490, Coal Meas.

truncatum, Lesquereux, 1860, Geo. Sur. Ark., vol. 2. Syn. for Knorria imbricata.

Discophycus, Walcott, 1879, Trans. Alb. Inst., vol. 10, p. 19. [Ety. diskos, disk; phukos, sea-plant.] Frond discoid, slightly convex, and substance coria ceous. Type D. typicale.

typicale, Walcott, 1879, Trans. Alb. Inst., vol. 10, p. 19, Utica slate.

Dystactophycus, Miller \& Dyer, 1878, Cont. to Pal., No. 2, p. 2. [Ety. dustaletos, hard to arrange; phukos, sea-plant.] Frond mammiform, expanded and concentrically wrinkled. Type D. mammillanum. 
mammillanum, Miller \& Dyer, 1878, Cont. to Pal., No. 2, p. 2, Hud. Riv. Gr.

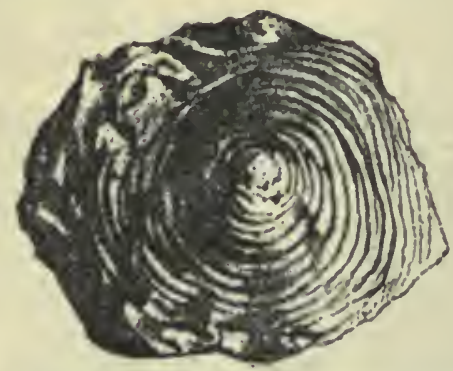

Fiu. 34.-Dystuctophycus mammilanum.

Eорнгтом, Torell, 1868, Bidr. t. Sparagm. geogn. och. paleont., p. 36 . [Ety. e08, dawn; phytom, a plant.] Slender, cylindrical, reed-like fucoids, longitudinally striated. Type E. linneanum.

explanatum, Dawson, 1870, Can. Nat. and Geol., Low. Arenig rocks.

jukesi, Billings, 1874, Pal. Foss., vol. 2, p. 65 , Up. Taconic.

linneanum (?), Torell, 186s, Bidr. t. Sparagm. geogn. och. paleont., ए. $36, \mathrm{Up}$. Taconic.

Equisetites, Stemberg, 1833, Ver. Darst. Flora der Vorwelt, vol. 2, p. 43. [Ety. equus, a horse; seta, a hair or bristle; in allusion to the resemblance to a horse-tail.] Arborescent ; stems articulate; articulations surrounded with costate sheaths, dentate on the border. Type E. gigantea.

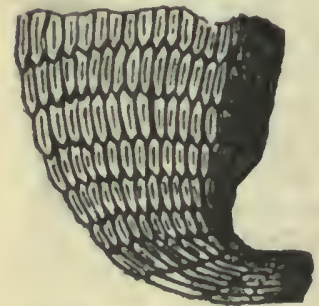

Frg. 35.-Equisetites curtus. col u m a $r$ is, Brong n i r t. 1828, ( Kiqu i setum columnare,) Hist. l eg. Foss., t. 1, p. 115, Coal Meas.

curtus, Dawson, 1863, Syn. Carb. Flora in Can. Nat., vol. 8 , and Acad. Geol., p. 443, Coal Meas. elongatus, Fontaine \& White, 1850, Perm. or Up. Carb. Flora, p. 33, Coal Meas. or Permian. gracilis, Lesquerenx, 1884, Coal Flora of Pa., p. 729, Coal Meas.

macrodontus, IVood, 1860, Proc. Acad. Nat. Sci. Not satisfactorily defined.

occidentalis, Lesquereux, 1870, Geo. Sur. Ill., vol. 4, p. 425, Coal Meas.

stellifolius, Harlan, 1835, (Equisctum stellifolium,) Trans. Geo. Soc. Pa., vol. 1, p. 261, Coal Meas. Syn. for Annularia longifolia?

striatus, Fontaine \& White, 1880, Perm. or Up. Carb. Flora, p. 3t, Coal Meas. or Permian.

wrightanus, see Echinocaris wrightana.

Equisetum, see Equisetites. columnare, see Equisetites columnaris. stellifolium, see Equisetites stellifolius.

Eremopteris, Schimper, 1869, Traite de Palæontologie Vegetale, vol. 1, p. 416. [Ety: eremos, isolated; pteris, fern.] Upper part of fronds dichotomous; pinnre open or oblique, irregularly pinnatifid; laciniae long, obovate or wedge-form, the lower ones deeply cut; the lateral veins enter the lobes in acute angles of divergence from the midrib, and passing up to the borders are flabellate, dichotomous, parallel, and close. Type $1 \%$ artemisiifolia.

artemisiifolia, Sternberg, 1824, (Sphenopteris artemisiafolia, ) Vers. Darst. Flora der Vorwelt, p. 4t, and Coal Flora of Pa., p. 293, Coal Meas.

cheathami, Lesquereux, 1854, Coal Flora of $\mathrm{Pa} ., \mathrm{p}$. 770, Coal Meas.

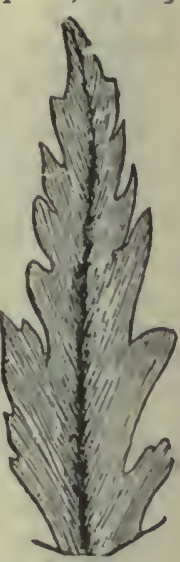

F'ro. 36 . Eremopteris margliuas. crenulata, Iesquereux, 1876, Geo. Rep. of A labama, p. 75, and Coal Flora of Pa., p. 292, Coal Meas.

dissecta, Iesquereux, 1876, Geo. Rep. of Alahama, p. 75, and Coal Flora of $\mathrm{Pa}$., p. 293, Coal Meas.

elegans, Ettingshausen, 1852, (Asplenites elegans,) Die Steinkohlen flora v. Stradonitr in Bolimen, p. 15, and Coal Flora of l'a., p. 294, Coal Meas.

flexuosa, Iesquereux, 1876, Geo. Rep. of Alabana, p. 75, and Coal Flora of Pa., p. 293, Coal Meas.

marginata, Andrews, 1875, Ohio Pal., vol. 2, ए. 422, Coal Meas.

microphylla, Lesquereux, 1880, Coal Flora of Pa., p. 296, Coal Meas.

missouriensis, Iesquereux, 1880, Coal Flora of Pa., p. 295, Coal Meas.

Ficoidites scabrosus, Hildreth, 1837, Am. Jour. Sci. and Arts, vol. 31, p. 30, Low. Coal Meas. Not recognized, but probably a Sigillaria.

Filicites, Schlotheim, 1820, Nachtr. zur Petref. It was used for all fossil ferms, and hence is not of generic value. acuminatus, sce Neuropteris acuminata. aquilinus, see Alethopteris aquilina. arborescens, see Pecopteris arborescens. crispus, see Cyclopteris crispa. gracilis, see Plumalina gracilis. lonchiticus, see Alethopteris lonchitica. milloni, see Pecopteris miltoni. penniformis, see Pecopteris penniformis. pluckeneti, see Pseudopecopteris pluckeneti. plumosus, see Pecopteris plumosa. oreopteridis, see Pecopteris oreopteridis. trifoliatus, see'Pseudopecopteris trifoliats. Flabellaria borassifolia, see Cordaites borassifolius.

Fucoides, Brongniart, 1822, in Mem. d. Hist. 
Nat. and Hist. d. Veg. Foss., t. 1., p. 50 It was used to comprehend the Sargassites or Thalassophytes, and hence is of more than generic value.

alleghaniensis, see Arthrophycus harlani. auriformis, Hall, 1843. Not organic.

bilobatus, see Rusophycus bilobatum. caudagalli, see Taonurus caudagalli.

demissa, Conrad probably phytopsis tubulosa.

dentatus, Brongniart probably Diplograptus pristiniformis.

filiciformis, see Rhacophyllum filiciforme. flexuosus, see Bythotrephis flexuosa.

gracilis, see Bythotrephis gracilis.

graphica. Not defined so as to be determined.

harlani, see Arthrophycus harlani.

heterophyllus, Hall. Not defined so as to be determined.

retort, see Taonurus retortus.

rigidus, see Bythotrephis rigida.

secalinus, Hall syn. for Diplograptus simplex.

serra, Brongniart, see Graptolithus bryonoides.

simplex, see Diplograptus simplex. velum, see Taonurus velum.

verticalis, see Scolithus verticalis.

Galium sphenophylloides, see Annularia sphenophylloides.

Gly ptodendron, Claypole, 1878, Am. Jour. Sci. and Arts, $3 u^{\prime}$ ser., vol. 15 , p. 302.

[Ety. glyptos sculptured; dendron, tree.] Stem thick, covered with rhomboidal areoles, the lower portions of which are depressed. Type G. eatonense.

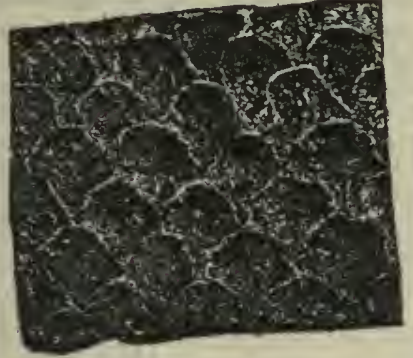

FrG. 37.-Glyptodendron eatonense.

eatonense, Claypole, 1878, Am. Jour. Sci. and Arts, 3d. ser., vol. 15, p. 302, Niagara Gr.

Goniopteris newberryana, see Pecopteris newberryana.

oblonga, see Pecopteris oblonga.

Gordia marina, see Palæochorda marina.

Gulielmites, C einitz, 1858, Leithpflanzen d.

Rothleig. u. d. Zechstein, Sachsen, p. 19. [Ety. from the genus Gulieima.] A kind of fruit, of uncertain affinity.

orbicularis, Fontaine \& White, 1880 ,

Perm. and Up. Carb. Flora, p. 99, Coal Meas. or Permian.

permianus, Geinitz, 1858, Leithpflanzen d. Rothleig. u. d. Zechstein, Sachsen, p. 19, Permian.
Halonia, Lindley \& Hutton, 1835, Foss. Flora, vol. 2, 户. 11. [Ety. from its close affinity with Halonia.] Stems of medium size, dichotomous; cortex tuberculate; spaces intermediate to the tubercles marked with rhomboidal scars; decorticated surface, covered with punctiform round or oval papillæ, obtuse or perforated in the center, placed in spiral order. Type H. tortuosa.

flexuosa, Goldenberg, 1855, (Ulodendron flexuosum, Flora Sarræpontana fossilis, vol. 1, pl. 2, fig. 10, and Coal Flora of Pa., p. 416, Coal Meas.

mans fi e l di,

Lesquereux,

1880, Coal

Flora of $\mathrm{Pa}$.

p. 414, Coal Meas.

pulchella, Ies-

q u e re ux,

1860, Geo.

Sur. Ark.,

vol. 2 , p. 311 ,

Coal Meas.

secreta, Jes-

$q u$ e $r$ e u $x$,

1880, Coal

Flora of Pa.,

p. 417, Coal

Meas.

tortuosa, Lind-

ley \& Hut- Fig. 38.-Halonia Hexuosa.

ton, 1835, Foss. Flora, vol. 2, p. 11, Coal Meas.

tuberculata, Brongniart, 1838, Hist. d.

Veg. Foss., vol. 2. pl. 28, and Coal Flora of Pa., p. 411, Coal Meas.

Harlania, syn. for Arthrophycus.

halli, syn. for Arthrophycus harlani.

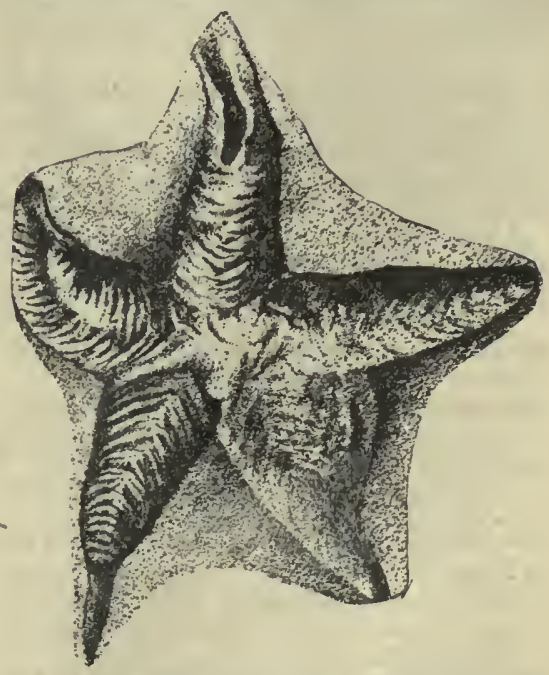

Fra 39.-Heliophycus stelliforme.

Heliophycus, Miller \& Dyer, 1878, Cont. to Pal. No.2, p. 2. [Ety. helios, the sun; 
phukos, sea-plant.] 'Star-like frond, having five rays; transversely wrinkled. Type H. stelliforme.

stelliforme, Miller \& Dyer, 1878, Cont. to Pal. No. 2, p. 2, Hud. Riv. Gr.

Hipponopirycus, Hall \& Whitfieln, 1872, 24th Rep. N. Y. St. Mus. Nat. Hist., p. 203. [Ety. hippodos, horse-foot; phukos, seaplant.] Founded upon cavities in sand. stone, having a form similar to that which a putty ball will assume, when pressed between thumb and finger, leaving a rounded rim on three sides of the disc, the compressed margin being truncate. Type H. cowlesi.

cowlesi, Hall \& IVhitfield, 1872, 24th Rep. N. Y. St. Mus. Nat. Hist., p. 203, Chem. ung Gr.

HYMENOPIIYLLITES, Goppert, 1836, Syst. Filic. Foss. [Ety. from the genus Hymenophyllum.] Frond membranaceous, many times regularly pinnately divicled or irregularly cut, lobed with pinnatifid or dichotomous divisions, decurring on a broad common rachis, which is sometimes indistinct; veins pinnate, percurrent, solitary in each division. Type H. gersdorfi.

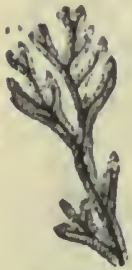

Fio. 40.

Hymenophyllites curtilobus. adnascens, see Rhacophyllum adnascens.

alatus, see Splienopteris alata.

arborescens, see Rliacophyllum arborescens.

ballantini, see Sphenopteris ballantini.

capilluris, Lesquereux, 1858, Geo. Sur. Pa., vol. 2, p. $\$ 63$. Coal Meas.

clurki,see Rhacoyhyllum clarki. curtilobus, Dawson, 1862, Quar. Jour. Geo. Soc., vol. 18, p. 321 , and Acad. Geol., p. 552; Devonian.

delicatulus, Brongnıart, 1828, Hist. d. veg. Foss, p. 185, Coal Meas.

flexicaulis, see Sphenopteris flexicaulis.

furcutus, see Splienopteris furcata.

gersdorfi, Gœppert, 1836, Syst. Filic. Foss.

Devonian.

giganteus, see Rhacopbyllum lactuca.

gutbieranus, Unger, 1850, Gen. et. sp., p. 132, Coal Meas.

hildrethi, see Sphenopteris hildrethi. inflatus, see Rhacophyllum inflatum.

lacluca, see Rhacophyllum lactuca.

mollis, see Rhacophyllum molle.

my riophyllus, Brongniart, 1828, (Sphenopteris myriophylla,) Hist. d. Veg. Foss., p. 184, Coal Meas.

obtusilobus, Goeppert, 1836, Syst. Filic. Foss., Devonian.

pentadactylus, Dawson, 1863, Can. Nat. and Geol., vol. 8, and Acad. Geol., p. 485, Coal Meas.

pinnatifidus, Lesquereux, 1866, Geo. Sur. Ill., vol. 2, p. 436, Coal Meas.

schlotheimi, Brongniart, 1828, (Sphenopteris schlotheimi,) Hist. d. Veg. Foss., p. 193, Coal Meas. spinosus, see Sphenopteris spinosa. splendens, see Sphenopteris splendens. strongi, see Rhacophyllum strongi.

subfurcatus, Dawson, 1868, Acad. Geol., p. 55, Devonian.

tenuifolius, Brongniart, 1828, (Sphenoy)teris tenuifolia,) Hist. d. Veg. Foss., p. 190 , Coal Meas.

thalliformis, see Rhacophyllum tlıalliforme. trichomanoides, see Splienopteris trichomanoides.

tridactylites, see Sphenopteris tridactylites. Icisopirycus, Hall, 1852 ,

Pal. N. I., vol. 2, p. 26. [Ety. ichnos, a footprint; phukos, a sea. weed.] Tridactyle impressions somewhat resembling a foot-track, the middle stem being the longer. Type I. Fis. 41.-Ichnotridactylum. plycus trldacty. tridactylum, Hall, 1852 ,

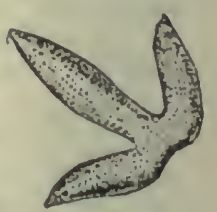
Pal. N. Y., vol. 2, p. 26, Clinton Gr.

Inioliyusu, Lesquereux, 1880, Coal Flora of Pa., p. 159. [Ety. idios, peculiar; phyllon, leaf ] Leaves small, round, or broadly obovate; medial nerve thick, gradually narrowed and effacing in joining the borders; lateral secondary veins sub-opposite, thick, passing in an inside curve toward the borders, gradually eflaced in the reticulation; venules more or less continuous; sometimes crossing each other in contrary directions, and forming, by intersections, quadrate or rhomboidal meshes. Type I. rotundifolium.

rotundifolium, Lesquereux, 1880, Coal Flora of Pa., 1). 160, Coal Meas.

Konorra, Sternberg, 1825, lissai d. Fixp. (ieogn-botan. d.l. Flor, du Monde primitif fasc. 4, p. 37. [Ety. proper name.] Trunks covered witl elongated semiconical or truncate t u bercles placed in spiral order more or less imbricated. leaving, after falling off, round convex marks, witlı a single, vascular scar in the mid. dle; leaves long, linear,

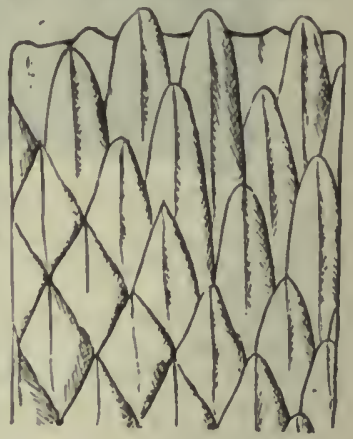

FIG, 42.

Knorria Imbricata. more or less inflated at the base, with a flat medial nerve. Type $K$. imbricata.

compact\&, Lesquereux, 1884, Coal Flora of Pa., p. 839, Coal Meas.

imbricata, Sternberg, 1825, Flor. d. monde primitif fasc. 4, p. 37 , Kaskaskia Gr. 
selloni, Sternberg, 1825, Flor. d. monde primitif fasc. 4, p. 37-50, Coal Meas.

taxina, Lindley \& Hutton, 1833-5, Foss. Flora, vol. 2, p. 37, Coal Meas.

Lepidocystis, Lesquereux, 1880,Coal Flora of Pa., p. 454. [Ety. lepis, scale; kustis, blad(ler.] Spore cases long, naked, attached in right angle and opposite to a broad rachis; or short, placed in spiral order upon long, flexuous axes; or isolated sporanges, detached from strobiles of unknown character. Type L. pectinatus.

angularis, Lesquereux, 1880, Coal Flora of Pa., p. 456, Coal Meas.

bullatus, Lesquereux, 1870, (Carpolithes bullatus,) Geo. Sur. Ill., vol. 4, p. 463, Coal Meas.

fraxiniformis, Goeppert \& Berger, 1848, (Carpolitlıe 3 fraxiniformis,) De Fruct. et Sem., p. 26, Coal Meas.

lineatus, Lesquereıx, 1880, Coal Flora of Pa., p. 454, Coal Meas.

obtusus, Lesquereux, 1858, (Brachyphyllum obtusum,) Geo. of Pa., vol. 2, p. 876, Coal Meas.

pectinatus, Lesquereux, 1880, Coal Flora of Pa., p. 454, Coal Meas.

quadrangularis, Lesquereux, 1880, Coal Flora of Pa., p. 455, Coal Meas.

vesicularis, Lesquereux, 1870, (Carpolithes vesicularis,) Geo. Sur. Ill., vol. 4, p. 462, Coal Meas.

Lepidodendron, Sternberg, 1820, Essai d'un expose Geognostico-botanique de la flore du monde primitif, 1st Cahier, p. 25. [Ety. lepis, scale; dendron, tree.] Surfuce of the stem marked by scars, points of leaf attachments; leaf scars (bolsters) rhomboidal, oblong, upon the bark of large trees or small branches, variable in size according to their position, often disfigured; central cicatrices (inside scars) rhomboidal, transversely dotted by three points (vascular scars) bearing, generally, under the lower margin two oval small tubercles, scars of bundles of vessels (appendages) placed on each side of a medial line (cauda), which, like the appendages, is more or less listinct, sometimes deep and wrinkled across, sometimes obsolete. Type L. dichotomum.

aculeatum, Sternberg, 1820, Essai'd. 'Exp. Geogul-botan. d. 1. flor. d. monde primitif, 1st Cahier, p. 25, and Coal Flora of Pa., p. 371, Coal Meas.

acuminatum, Goppert, 1852, Foss. Fl. d. Uebergangsgebirge, p. 185, Subcarboniferous.

andrewsi, Lesquerenx, 1880, Coal Flora of Pu., p. 389, Coal Meas.

alveolare, see Sigillaria alveolaris.

binerve, Bunbury, 1847, Quar. Jour. Geo. Soc.. vol. 3, p. 431, Coal Meas.

bordre, Wood, 1860, Proc. Acad. Nat. Sci., p. 239. Coal Meas.

brittsi, Lesquereux, 1880, Coal Flora of Pa., p. 368, Coal Meas. carinatum, Lesquereux, 1858, Geo. Sur. Pa., vol. 2, p. 875, Coal Meas.

chemungense, Hall, 1843, (Sigillaria chemungensis,) Geo. Rep. 4th Dist. N. Y., p. 275, Chemung Gr.

chilalloeum, Syn. for L. distans.

clypeatum, Lesquereux, 1858, Geo. Sur. Pa., vol. 2, p. 875, Coal Meas.

conicum, Lesquereux, 1858, Geo. Sur. Pa., vol. 2, p. 874, Coal Meas.

corrugatum, Dawson, 1860, Quar. Jour. Geo. Soc., vol. 15, p. 313, and Acad. Geol., p. 253, Waverly Gr.

costatum, Lesquereux, 1866, Geo. Sur. Ill., vol. 2, p. 453, Kaskaskia Gr.

crenatum, Sternberg, 1820, Flor. d. monde primitif, 1st Cahier, p. 25, and Coal Flora of Pa., p. 394, Coal Meas.

cruciatum, Lesquereux, 1870, Geo. Sur. Ill., vol. 4, p. 432, Coal Meas.

cuspidatum, Lesquereux, 1880, Coal Flora of Pa., p. 388, Coal Meas.

cyclostigma, Lesquereux, 1880, Coal Flora of Pa., p. 394, Coal Meas.

decurtatum, Dawson, 1863, Can. Nat., vol. 8, and Acad. Geol., p. 487, Coal Meas.

dichotomum, Sternberg, 1820, Flor. d. monde primitif, 1st Cabier, p. 25, and Coal Flora of Pa., p. 384, Coal Meas.

dikrocheilum, Wood, 1860, Proc. Acad. Nat. Sci., p. 239, Coal Meas.

dilatatum, Lindley \& Hutton, 1831, Foss. Flora, vol. 1., p. 27, Coal Meas.

diplostrgiodes, Lesquereux, 1860, Geo. Sur. Ark.. vol. 2., p. 311, Coal Meas.

distans, Lesquereux, 1858, Geo. Sur. Pa., vol. 2, p. 874, Coal Meas.

drepanaspis, Wood, 1860, Proc. Acad. Nat. Sci., Phil., vol. 12 , p. 240 , Coal Meas.

dubium, Wood, syn. for L. rimosum.

elegans, Sternberg, 1824, (Lycopodiolithes elegans,)

Vers. Darst.Flor.

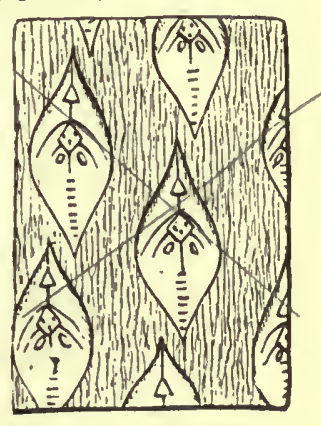

d. Vorwelt 4 fasc., FIG. 43.-Lepidodendron p. 8, Coal Meas.

forulatum, Lesquereux, 1870, Geo. Sur. Ill., vol. 4, p. 431, Cosl Meas.

gaspanum Dawson, 1859, Qnar. Jonr. Geo. Soc., vol. 15, p. 484, and Acad. Geol., p. 541, Catskill (ir. Probably the same as Vanuxem's Sigillaria simplicitas.

giganteum, Lesquereux, 1858, Gen. Sur. Pa., vol. 2., p. 874, Coal Mras.

gracile, Lindley \& Hutton, 1831, Foss. Flora, vol. 1, p. 30, Coal Meas.

greeni, Lesquereux, 1870, Geo. Sur. Ill., vol. 4, p. 43, Coal Meas.

harcourti, Witham, 1832, Trans. Nat. Hist. Soc., New, upon Tyne, p. 51, Coal Meas. ichthyolepis, Wood, 1860, (Lepidophloios ichthyolepis,) Proc. Acad. Nat. Sci. Phil., p. 240, Coal Meas. 
ingens, Wood, syn. for L. aculentum.

lanceolatum, Lesquereux, 1880, Coal Flora of Pa., p. 369 , Coal Meas.

latifolium, Lesquereux, 1850, Coal Flora of Pa., p. 370, Coal Meas.

lesquereuxi, Wood, syn. for L. clypeatum. longifolium, Brongniart, 1828, Prodr. Hist. Veg. Foss., p. 85, and Coal Flora of Pa., p. 373, Coal Meas.

magnum, Wood, 1860 , Proc. Acad. Nat. Sei., Phil., p. 239, Coal Meas.

mammillalum, Lesquereux, syn. for L. veltlıeimanum.

marginatum, Presl, 1826, (Bergeria marginata, in Sternberg Flor. d. Vorw., p. 134 , and Coal Flora of Pa., p. 784 , Coal Mens.

mekiston, Wood, syn. for L. modulatum. mirleki, Grepert, 1836, Syst. Filic. Foss., n. 465, and Coal Flora of Pa., p. 395 , Coal Meas.

modulatum, Lesquereux, 1558, Geo. Sur. Pu., vol. 2, p. 874. Coal Meas.

morrisanum, Lesquereux, 1870, Geo. Sur. Ill., vol. 4, p. 430, Coal Meas.

obovatum Sternberg, 1820, Flor. 1. moude primitif, Ist Cahier, p. 25, Coal Meas.

obscurum, Lesquereux, 1866, Geo. Sur. 111., vol 2, p. 453, Kaskaskia Gr.

obtusum, Lasquerenx, 1858, Geo. Sur. Pa., vol. 2, p. 875, Coal Meas.-

oculatum. Lesquereux, syn. for L. distans. onirni, Wood, syn. for L. vestitum.

personatum, Dawson, 1863, Can. Nat. and Geo.; vol. 8, and Acad. Geol., p. 48., Coal Meas.

pictoense, Dawson, 1563, Can. Nat. and Geo., vol. 8, and Acad. Geol. p. 487, Coal' Meas.

plicatum, Dawson, 1863, Can. Nat. and Geo. vol. 8, and Acad. Geol., p. 488, Coal Meas.

plumarium, Lindley \& Hutton, 1835, Foss. Flora, vol. 3, p. 151, Coal Meas.

politum, syn. for. L. modulatum.

primevuin, Rogers, 1858, Geo. Sur. Pa., vol. 2, p. 675 , Ham. Gr.

quadrangulatum, Schlotheim, 1820, (Palmacites quadrangulatus,) Petrefactenknnde, p. 395, and Coal Flora of Pa., p. 383 , Coal Meas.

quadrilaterale, Lesquereux, 1880, Coal Flora of Pa., p. 389, Coal Meas.

radiato-plicatum, Dawson, 1873, Rep. Foss. Plants, p. 32, Subcarboniferous.

radicans, Lesquereux, 1866, Geo. Sur. Ill., vol. 2, p. 454 , Coal Meas.

rectangulum, Wood, 1860, Proc. Aead. Nat. Sei. Phil., vol. 12 , p. 519 , Coal Meas. rhombieum, Presl, 1833, (Bergeria rbombica,)in Sternberg's Flor. d. Vorw., vol. 2, p. 184, Coal Meas.

rigens, Lesquerewx, 1870, Geo. Sur. Ill.; vol. 4, p. 429, Coal Mers.

rigidum, Lesquereux, 1854, Coal Flora of Pa., p. 839, Coal Meas.

rimosum, Sternberg, 1820, Flor. d. monde primitif, 1st Cahier, p. 25, and Coal Flora of Pa., p. 392, Coal Meas. rugosum, syn. for L. dichotomuus.

rushvillense, Andrews, 1875, Ohio Pal., vol. 2 ; p. 423 , Coal Meas.

salebrosum, Wood, 1860, Proc. Acad. Nat. Sei., Phil., p. 520, Coal Meas.

scobiniforme, Meek, 1876, Buli. Phil. Soc. Wash., p. 13, Waverly Gr. Probably a syn. for L. currugatim.

scutatum,

Les que -

reux, 1880 ,

Coal Flora of Pa., p. 369, Coal Meas.

selaginoides, Sternberg, 1820, Flor. d. monde primitif, 2d Calier, p. 35, Coal Meas.

8 igillarioides, Le 8 q u e reux, 1858 . fo undod upon a dec o rticated speeimen of L. restitum or L. latifolium. $\mathrm{s}$ i m p] ex ,

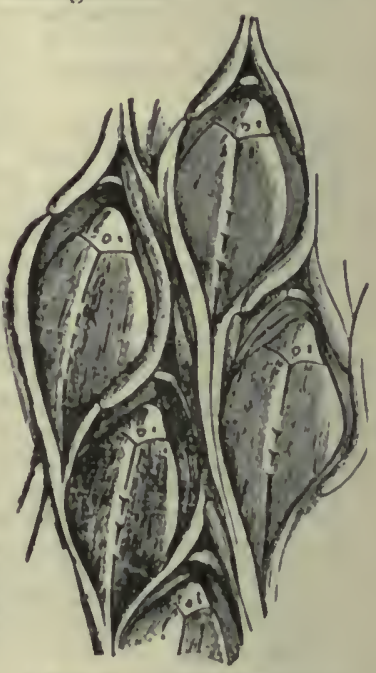

Fsg. Af, - Lepliodendron sternbergi.
Lesquereux, 1566, Geo. Sur. Ill., vol. 2, p. 4ذ่4, Coal Meas.

squamiferum, Isesquereux, 1880, Coal Flora of Pa., p. 376, Coal Meas.

sternbergi, Brongniart, 1828, Prodr. Hist. Veg. Foss., n. 85, Coal Meas.

tetragonum, Sternberg, 1821, Flor. d. monde primitif, 2d Cahier, p. 35 , Coal Meas.

tijoui, Lesquereux, 1870, Geo. Sur. Ill., vol. 4, p. 431, Coal Meas.

tumidum, Bunbury, 184\%, i Lepidophloios tumidum,) Quar. Jour. Geo. Soe., vol. 3 , p. 432, Coal Mers.

turbinatum, Lesquereux, 1866, Geo. Sur. Ill., vol 2, p. 453, Kaskaskia $\mathrm{Gr}$.

undulatum, Sternberg, 1820, Flor. d. monde primitif, 1st Cahier, p. 25, Coal Meas.

uræum, Wood, 1S60, Proc. Acad. Nat. Sci., p. 240, Coal Meas.

veltheimanum, Sternberg, 1823, Vers. Darst. Flora der Vorwelt, vol. 1, p. 12 , Kaskaskia Gr.

venustum, Wood, syn. for I. obtusum.

vestitum, Lesquereux, 1858, Geo. Sur. Pa., vol. 2; p. S74, Coal Meas.

wortheni, I,esquereux, 1866, Geo. Sur. Ill., vol. 2, p. 452, Coal Meas.

LEPIDOPHLOIOs, Sternberg, 1823, Vers. Darst. Flora der Vorwelt. [Ety. lepis, scale; phloios, the bark.] Stems arborescent, erect, with four ranked branches disposed in spiral order; leaves coriaceous, linear, long, narrow, with a thick medial 
nerve, bearing at base thick, suberect or recurved bolsters, inflated in the upper part and dotted with small vascular points; leaf-scars transversely rhoinboidal, marked horizontally by three vascular scars, minutely papillose under the cortex. Type L. laricinus.

acadianus, Dawson, 1863, Can. Nat. and Geo., vol. 8, and Acad. Geol. p. 489, Coal Meas.

antiquus, Dawson, 1871, Foss. Plants Canada, p. 36, Devonian.

auriculatus, Lesquereux, 1870, Geo. Sur. Ill., vol. 4, p. 439, Coal Meas.

crassicaulis, Corda, 1845, Beitrage zur Flora der Vorwelt, p. 18, Coal Meas.

dilatatus, Lesquereux, 1884, Coal Flora of Pa., p. 781, Coal Meas.

ichthyoderma, Lesquereux, 1880, Coal Flora of Pa., p. 426, Coal Meas.

ichthyolepis, see Lepidodendron ichthyolepis.

irregularis, Lesquereux, 1860, Geo. Sur. Ark. vol. 2, p. 311, Coal Meas.

laricinus, Sternberg, 1820, (Lepidodendron laricinum,) Flor. d. monde primitif, 1st Cahier, p. 25, Coal Meas.

lesquereuxi, Andrews, 1875, Ohio Pal., vol. 2, p. 423, Coal Meas.

macrolepidotus, Goldenberg, 1862, Flora sarræpontana fossilis, vol. 3, p. 37, Coal Meas.

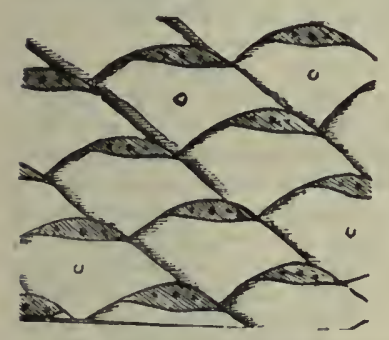

FIG. 45.-Fepldophloios macrolepidotus. obcord a tus,

L e s q u e reux, 1866 , Geo. Sur. Ill., vol. 2, p. 457 , Coal Meas.

parvus, Dawson, 1863, Call. Nat. and Geo., vol. 8 , and Acad.Geol., p. 490 , Coal Meas.

platystigma, Dawson, 1863, Can. Nat. and Geo., vol. 8, and Acad. Geol., p. 490, Coal Meas.

prominulus, Dawson, 1863, Can. Nat. and Geo., vol. 8, and Acad. Geol., p. 489, Coal Meas.

protuberans, Lesquereux, 1870, Geo. Sur. Ill., vol. 4, p. 440, Coal Meas.

sigillarioides, Lesquereux, 1880, Coal Flora of Pa., p. 425, Coal Meas.

tetragonum, Dawson, 1863, Can. Nat. and Geo., vol. 8, and Acad. Geol., p. 490, Coal Meas.

tumidus, Lesquereux, see Lepidodendron tumidum.

Lepidophyllum, Brongniart, 1828, Prodr. d. Hist. Veg. Foss., p. 87. [Ety. lepis, scale; phyllon, leaf.] Blades or bracts, either joined to sporanges, or sporangiophores of Lepidostrobus, or isolated. Type L. majum. acuminatum, Lesquereux, 1858, Geo. Sur. $\mathrm{Pa}$. , vol. 2, p. 875 . The name was preoccupied by Gutbier in 1843 , but as it is a Lepidostrobus the name may be retained.

affine, Lesquereux, 1858, Geo. Sur. Pa., vol. 2 , p. 875 , Coal Meas.

auriculatum, Lesquereux, 1866, Geo. Sur. Ill., vol. 2, p. 457, Coal Meas.

brevifolium, Lesquereux, 1858, Geo. Sur. Pa., vol. 2, p. 876, Coal Meas.

campbellanum, Lesquereux, 1884, Coal Flora of Pa., p. 786, Coal Meas.

coriaceum, Lesquereux, 1884, Coal Flora of Pa., p. 787, Coal Meas.

cultriforme, Lesquereux, 1884, Coal Flora of Pa., p. 785, Coal Meas.

elegans, Lesquereux, 1884, Coal Flora of Pa., p. 787, Coal Meas.

fallax, Lesquereux, 1884, Coal Flora of Pa., p. 786, Coal Meas.

foliaceum, see Lepidostrobus foliaceus.

gracile, Lesquereux, 1884, Coal Flora of Pa., p. 786, Coal Meas.

hastatum, see Lepidostrobus hastatus.

intermedium, Lindley \& Hutton, 1831, Foss. Flora, vol. 1, p. 125, Coal Meas.

lanceolatum, see Lepidostrobus lanceolatus.

linearifolium, Lesquereux, 1880, Coal Flora of Pa., p. 452, Coal Meas.

majum, Brongniart, 1828, Prodrome d'une Hist. Veg. Foss., p. 87, and Coal Flora of Pa., p. 449, Coal Meas.

mansfieldi, Lesquereux, 1880, Coal Flora of Pa., p. 449, Coal Meas.

minutum, Lesquereux, 1884, Coal Flora of Pa., p. 787, Coal Meas.

morrisanum, Lesquereux, 1880, Coal Flora of Pa., p. 448, Coal Meas.

obtusum, Lesquereux, 1858, Geo. Sur. Pa., vol. 2, p. 875, Coal Meas.

plicatum, Lesquereux, 1858, Geo. Sur. Pa., vol. 2, p. 876, Coal Meas.

rostellatum, Lesquereux, 1870, Geo. Sur. Ill., vol. 4, p. 443, Coal Meas.

striatum, Lesquereux, 1870, Geo. Sur. Ill., vol. 4, p. 443, Coal Meas.

trinerve, Brongniart, 1828, Prodr. Hist. Veg. Foss., p. 87, and Lindley \& Hutton's Foss. Flora, vol. 2, p. 195, Coal Meas.

truncatum, see Lepidostrobus truncatus. tumidum, Lesquereux, 1880, Coal Flora of Pa., p. 448, Coal Meas.

Lepidostrobus, Brongniart, 1828, Prodr. d. Hist.Veg. Foss., p. 87. [Ety. lepis, scale ; strobus, cone.] Strobiles cylindrical or ovate, oblong, conical ; composed of sporanges (spore-cases) subcylind rical or clavate, emarginate at the apex, supported in the middle lengthwise by bracts formed of a pedicel, attached like the sporanges in right angle to the axis, linear or oblanceolate, either simple, not longer than the sporanges, or prolonged into lanceolate, obtuse or acuminate laminæ, curved upward on the outside of the strobiles and imbricated on their sides, or merely inflated 
at the outer end, and covering the apex of the sporanges by a rhomboidal small shield; spores, triquetre on one side, half globular on the other, like those of the Iycopods, homomorphous or dimorphous. Type L. ornatus.

acuminatus, Lespuereux, 1858, (Lepidophyllum acuminatum,) Geo. Pa., vol. 2, p. 875, Coal Meas.

aldrichi, Lesquertux, 1850, Coal Flora of I'a., p: 441, Coal Meas.

butleri, Lesquereux, 1884, Coal Flora of Pa., p. 840, Coal Meas.

connivens, Lesquereux, 1870, Geo. Sur. Ill., vol. 4, p. 442, Coal Meas.

foliaceus, Lesquereux, 1870, (Lepidopliyllum foliaceum,) Geo. Sur. Ill., vol. 4, p. 444, Coal Meas.

globosus, Daweon, 1861, Can. Nat. and Geo., vol. 6, p. 174, Devonian.

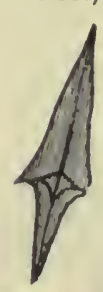

Fig. 46. Lepidostro bus hustalus. goldenbergi, Schimper, 187\%, Traite de Palcontologio Vegetale, vol. 2, p. 61, Coal Meas.

hastatus, Iesquereux, 1858, (Lepidophyllum hastatum,) Geo. Sur. Pa., vol. 2, p. 876, Coal Meas.

incertus, Lesquereux, 1880 , Coal Flora of P'a., p. 442 , Coal Meas.

lacoei, Lesquereux, 1850, Coal Flora of Pa., p. $\$ 39$, Coal Meas.

lanceolatus, Brongniart, 1828, (Iepidophyllum lanceolatum,) Prodr. d. Hist. d. Veg. Foss., p. 87, and Coal Flora of Pa., p. 436 , Coal Meas.

lancifolius, Lesquereux, 1870, Geo. Sur. Ill., vol. 4, p. 442, Coal Meas.

latus, Lesquereux, 1884, Coal Flora of Pa., p. 841, Coal Meas.

Jongifolius, Dawson, 1863, Can. Nat. and Geo., vol. 8, and Acad. Geol. p. 489, Coal Meas.

mantfieldi, Lesquereux, 1880, Coal Flora of Pa., p. 444, Coal Meas.

miratilis, Newberry, 1873, (Polysporin mirubilis,) Ohio Pal., vol. 1, p. 362, Low. Coal Meas.

oblongifolius, Lesquereux, 1870, Geo. Sur. Ill., vol. 4, p. 441, Coal Mers.

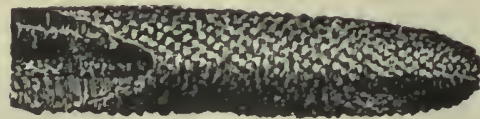

FIG. 47,-Lepldostrobus ornatus, Cone $1 / 2$ size.

ornatus, Parkinson, 1811, Organic Remaink, vol. 1, pl. 9, fig. 1, and Coal Flora of Pa., p. 440, Coal Meas.

ovatifolius, Lesquereux, 1870, Geo. Sur. Il]., vol. 4, p. 441, Coal Meas.

pinaster, Lindley \& Hutton, 1837, Foss. Flora, vol. 3, p. 129, Coal Meas.

prælonyus, Lesquereux, 1880, Coal Flora of Pa., p. 433, Coal Meas. princeps, Lesquereux, 1866, Gco. Sur. Ill., vol. 2. p. 455, Coal Meas.

quadratus, Lesquereux, 1880, Coal Flora of Pa., p. 44t, Coal Meas.

richardsoni, Dawson, 1861, Can. Nat. and Geo., vol. 6, p. 174, Devonian.

salisburyi, Lesquereux, 1850, Coal Flora of Pa., p. 443 , Coal Meas.

spectabilis, Lesquereux, 1S50, Coal Flora of Pa., p. 435, Coal Meas.

squamosus, Dawson, 1563, Can. Nat. and Geo., vol. \&, and Acad. Geol., p. 489, Coal Meas.

stachioides, sec Asterophyllites stachioides. trigonolepis, Bunbury, 1847, Quar. Jour. Geo. Soc., vol. 3, 1. 432 , Coal Mens.

truncatus, Iesquereux, 1870, Geo. Sur. IIl., vol. 4, p. 442, Coal Meas.

variabilis, Lindley \& Hutton, 1833, Foss. Flora, vol. 1, 1). 31, and Coal Flora of Pa., p. 434, Coal Meas.

Lei'idoxylox, Lesquereux, 1878, Proc. Am. Phil. Soc., p. 334 , and Coal Flora of Pa., p. 557. [lity. lepis, scale; xylon, wood.] Stems large, tapering to a point; bark thin, covered with leafy scales; leaves variable, sublinear, narrowed or enlarged to the point of attachment, forking upward in two or more lnciniae; nervation distinct with the gluss; primary nerve's parallel, buried in the epidermis, inflated or half round; intermediate veinlets thin, visible on the dccorticated face. Type L. anomalum. anomalum, Lesquereux, 1880, Coal Flora of $\mathrm{Pa} .$, p. 557, Coal neas.

Lei'tollifof.u, Dawson, 1862, Quar. Jonr. Geo. Soc., vol. 18, p. 316. [Lity. leptos, slender; phlois, the bark of a tree.] Stein covered with continuous rhombic areoles, each with a single sinall scar a little above its center, and above this a very slight furrow; decorticaterl stems, with spiral punctiform scars in slight depressions; bärk thin, pith cylinder very large, with transverse markings of the character of Sternbergia. Type L. rhombicum.

rhombicum, Dawson, 1862, Quar. Jour. Geo. Soc., vol. 18, p. 316, Devonian.

Lescuroptrais, Schimper, 1869, Pulcontologie Vegetalp, vol. 1, p. 465. [Eiy. proper name; pleris, a fern.] Fronds larg', bi

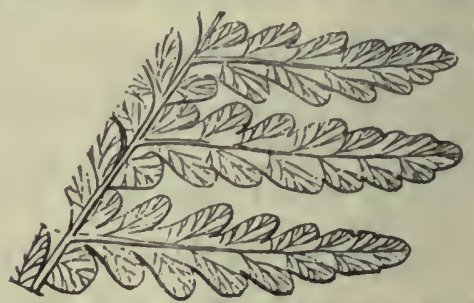

FIG. 48.-Lescuropteris adiantites.

tripinnate; rachis broad; foliate; pinnæ pinnatifid, close, oblique; divisions ovate, acute, inclined outside, connate 
to the middle, decurrent to the rachis, primary nerve thin, dichotomous; lower pairs of lateral veins emerging from the rachis, the other alternately from the midrib, forking twice, the upper forking once or simple. Type L. moorii. adiantites, Lesquereux, 1854, (Neuropteris adiantites,) Bost. Jour. Nat. Hist., vol. 6, p. 419, and Coal Flora of Pa., p. 163, Coal Meas.

moorii, Lesquereux, 1858, (Neuropteris inoori,) Geo. Sur. Pa., vol 2, p. 860, Coal Meas.

Lesleya, Lesquereux, 1880, Coal Flora of Pa., p. 142. [Ety. proper name.] Pinnæe simple, very entire, sublanceolate, gradually narrowing toward the base, traversed by a thick costa effaced under the apex; veins oblique, curved, equal, repeatedly dichotomous. Type L. grandis.

grandis, Lesquereux, 1880, Cual Flora of Pa., p. 143, Coal Meas.

microphylla, Lesquereux, 1884, Coal Flora of Pa., p. 831, Coal Meas.

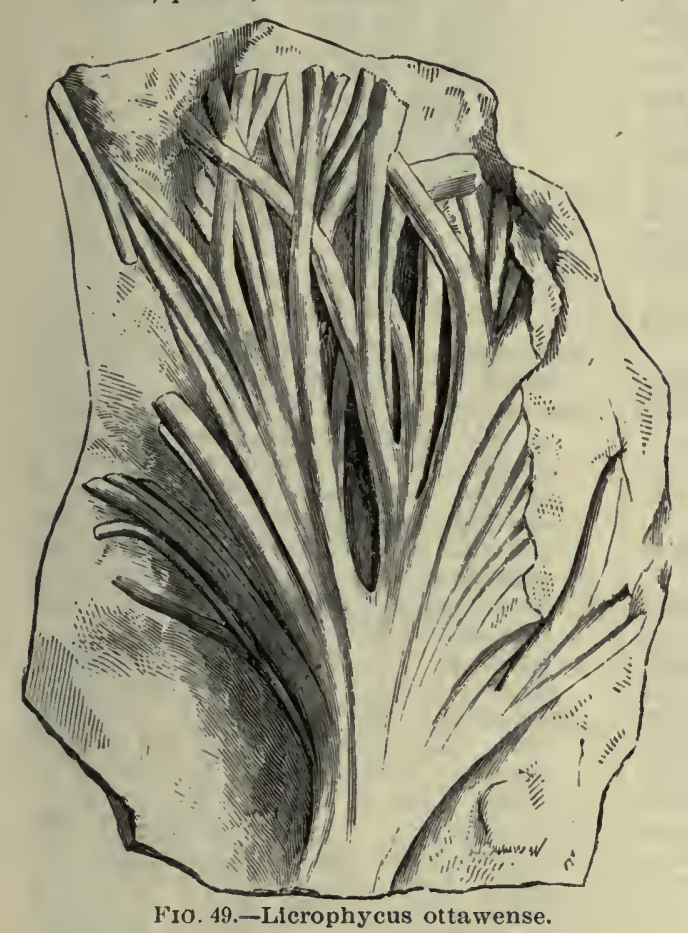

Licrophycus, Billings, 1862, Pal. Foss., vol. 1, p. 99. [Ety. likros, a fan; phykos, sea-weed.] Composed of numerous, elongated, subcylindrical stems, radiating from a common root, and remaining single, or branching at an acute anyle. 'Type $\mathrm{L}$. ottawense.

flabellum, Miller \& Dyer, 1878, Jour. Cin. Soc. Nat. Hist., vol. 1, p. 25, Hud. Riv. Gr. formosum, Billings, 1866, Catal. Sil. Foss. Antic., p. 72, Hud. Riv. Gr.

hiltonense, Billings, 1862, Pal. Foss., vol. 1, p. 101, Black Riv. and Trenton Gr. hudsonicum, Billings, 1862, Pal. Foss., vol. 1, p. 101, Hud. Riv. Gr.

minor, Billings, 1862, Pal. Foss., vol. 1, p. 100, Trenton Gr.

ottawense, Billings, 1862, Pal. Foss., vol. 1, p. 99, Trenton $\mathrm{Gr}$.

robust um, Billings, 1866, Catal. Sil. Foss. Antic., p. 72, Hud. Riv. Gr.

vagans, Billings, 1866, Catal. Sil. Foss. Antic., p. 72, Hud. Riv. Gr.

Lithodictuon becki, Conrad. Not properly defined; but see Dictyophyton becki.

Lonchoptrers, Brongniart, 1828, Prodr. Hist.

Veg. Foss., p. 59. [Ety. lonche, spear; pteris, fern.] Pinnate or bipinnate; pinnules contiguous at the base, nearly at right angles to petiole, oblong-elongate, obtuse, middle-sized veins reticulated with finer ones. Type L. bricei. tenuis, Dawson, 1863, Can. Nat. and Geol.; vol. 8, and Acad. Geol., p. 483, Coal Meas.

Lycopodiolithes elegans, see Lepidodendron elegans.

LycopoDites, Brongniart, 1822, Mem. du Mus. d'Hist. Nat. de Paris, and Lycopodiolithis of Schlotheim and Sternberg. [Ety. from Lycopodium, the club moss.] Plants herbaceous; leaves of the same or of two different forms upon the same branches, distichous or in spiral order; fructifications in small cylindrical spikes. Type L. pinniformis.

annulariifolins, Lesquereux, 1870 , Geo. Sur. Ill., vol. 4, p. 426, Coal Meas.

arborescens, Lesquereux, 1884, Coal Flora of Pa., p. 778, Coal Meas.

asterophyllitifolius, Lesquereux, 1866, reo. Sur. Ill., vol. 2, p. 447, Coal Meas.

cavifolius, Lesquereux, 1861, Geo. Sur. Ky., vol. 4, p. 437, Coal Meas.

comosus, Dawson, 1863, Quar. Jour. Geo. Soc., vol. 19, p. 462, Devonian.

flexifolins, Lesquereux, 1884, Coal Flora of Pa., p. 779, Coal Meas.

lacoei, Lesquere ux, 1884, Coal Flora of Pa., p. 780, Coal Meas.

matthewi, D aw son, 1861, Can. Nat. and Geo., vol. 6, p. 171, and Acad. Geol., p. 540, Devonian.

meeki, Lesquereux, 1870, Fig. 50.-IycoGeo. Sur. Ill., vol. 4, p. Thewi; $a$, branch 426, Coal Meas.

ortoni, Lesquereux, 1880 , leaves.

Coal Flora of Pa., p. 357, Coal Meas. pendulus, Lesquereux, 1880, Coal Flura of Pa., p. 357, Coal Meas. 
plumula, see Plumalina plumula.

richardsoni, Dawson, 1863, Quar. Jour. Geo. Soc., vol. 19, 1). 461, Devonian.

simplex, Lesquereux. 1884, Coal Flora of Pa., p. 779, Coal Meas.

strictus, Lesquereux, 1880, Coal Flora of Pa., p. 360, Coal Meas.

uncinatus, Lesquereux, 1866, (Selaginites uncinatus,) Geo. Sur. Ill., vol. 2, p. 446, Coal Meas.

vanuxemi, Dawson, 1862, Quar. Jour. Geo. Soc., vol. 18, p. 314, Syn. for Plumalina plumula.

Macrostachya, Schimper, 1869, Traite de Paléontologie Vegetale, vol. 1, p. 332. [Ety. makros, long; stachys, a plant.] Plants arborescent, articulate; articulations close; cortex thin, smooth or striate; impressions of the internal surface plano-costate; furrows narrow, alternating at the articulations; leaves appressed, linear, cariuate or marked with a medial nerve, acuminate, finely truncate; leaf scars marked upon the articulations by transversely oval rings, like the links of a chain; sears of branches verticiliate, large, round, umbonate, with a stigmarioid eentral mamilla; spikes very large, cylindrical; bracts lanceolate, costate in the middle, imbricate, searcely longer than the internodes. Type M. infundibuliformis. aperta, Lesquereux, 1858, (Asterophyllites apertus,) Geo. Sur. Pa., vol. 2, p. 852, Coal Meas.

communis, Lesquereux, 1884, Coal Flora of Pa., p. S28, Coal Meas.

infundibuliformis, Brongniart, 1828 , (Equisetum infundibuliforme, Hist. Veg. Foss., t. 1, p. 119, Coal Meas.

lanceolata, Lesquereux, 1858, (Asterophyllites lanceolatus,) Geo. Sur. Pa., vol. 2 , p. 852 , Coal Meas.

minor, Lesquereux, 1884, Coal Flora of Pa., p. 829, Coal Meas.

Megalol'teris, Dawson, 1871, Foss. Plants Dev, and Up., Sil. Formations, p. 51. [Ety. megale, great ; pteris, fern.] Fronds very large, pinnate, ultimate pinna oblique, sublinear or lanceolate, entire, the lower side broadly decurrent on the rachis, which thus becomes alate, the upper narrowed in a curve, confluent ; midrib thick, canaliculate on the upper surface, half cylindrical on the lower, gradually narrowed, but distinct to the apex of the leaves; veins open, emerging from the rachis in a more open angle of divergence, curving upward in reaching the borders, close dichotomous. Type M. dawsoni.

abbreviata, Lesquereux, 1880, Coal Flora of Pa., p. 151, Coal Meas.

dawsoni, Hartt, 1868, (Neuropteris dawsoni,) Acad. Geol., p. 550, Devonian.

dentata, Lesquereux, 1884, Coal Flora of Pa., p. 833, Coal Meas.

fasciculata, Lesquereux, 1880, Coal Flora of Pa., p. 150, Coal Meas. hartti, Andrews, 18ij, Ohio Pal., vol. 2, p. 416, Coal Meas.

lata, Andrews, 1875, Ohio Pal., vol. 2, p. 417, Coal Meas.

marginata, Lesquereux, 1850, Coal Flora of Pa., p. 152, Coal Meas.

minima, Andrews, 1875, Ohio Pal., vol. 2, p. 416, Coal Meas.

ovata, Andrews, 1875, Ohio Pal., vol. 2, p. 417 , Coal Meas.

rectinervis, Lesquereux, 1884, Coal Flora of Pa., p. 744, Coal Meas.

serrata, Lesquereux, 1884, Coal Flora of Pa., p. 834, Coal Meas.

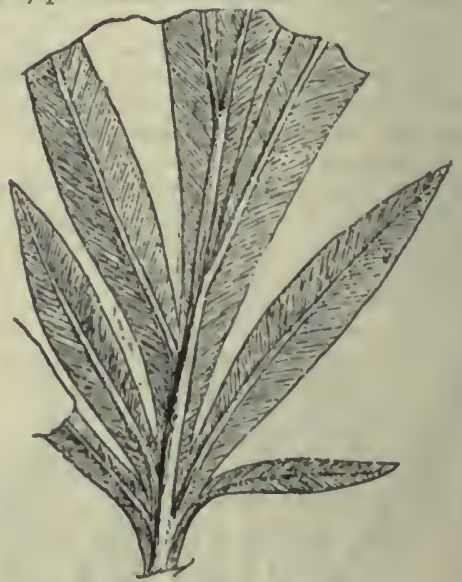

lig. B1.-Megalopteris south welli.

southwelli, Lesquerenx, 1880, Coal Flora of Pa., p. 148, Coal Meas.

Megapiytos, Artis, 1828, Antedil. Phytol., p. 20. [Ety. megas, grent; phylon, a plant.] Sears large, round-quadrate in outline, mostly contiguous, placed in opposite biserial rows; internal disks convex, with central or vaseular impressions in the form of a horseshoe, or a medial band dividing the disks into twolobes, joined in the middle. Type M. frondosum.

goldenbergi, Weiss, 1860, Zeitsch d. deutsch Geo. Gesellsh. XII, p. 510, Coal Meas.

grandeuryi, Lesque. reux, 1880, Coal Flora of Pa., p. 350, Coal Meas.

humile, Dawson, 1863 , Can. Nat. and Geol., vol. 8, and Acad. Geol. p. 486, Coal Meas.

maclayi, Lesquereux, Fig. 52.-Megaphy1866, Geo. Sur. Ill.,

vol. 2 , p. 458 , Coal Meas.

magnificum, Dawson, 1863, Can. Nat. and Geol., vol. 8, and Acad. Geol., p. 486, Coal Meas. 
protuberans, Lesquereux, 1866, Geo. Sur. Ill., vol. 2, p. 458 , Kaskaskia Gr.

Nematophycus, Carruthers, 1872, Month. Micro. Jour. Syn. for Prototaxites. logani, see Prototaxites logani.

Nematophyluum, Fontaine \&.White, 1880, Perm. or Up. Carb. Flora, p. 35. [Ety. nema, thread; phyllon, leaf.] Stem covered with a thick, very finely striate epidermis, internodes remote, swollen ; leaves verticillate, numerous, very long and thread-like, of equal width throughout, finely striate, without nerves, united at the base in a narrow annular band. Type N. angustum.

angustum, Fontaine \&. White, 1880 , Perm. or Up. Carb. Flora, p. 35, Coal Meas. or Permian.

Nematoxylon, Dawson, 1863, Quar. Jour. Geo. Soc., vol. 19, p. 466. [Ety. nema, a thread; xylon, wood.] Carruthers, Penhallow, and others say this genus belongs to the Algæ, and is a syn. for Nematophycus. Fragments of wood, with a smooth bark and a tissue wholly composed of elongated cylindrical cells, with irregular pores or markings; no

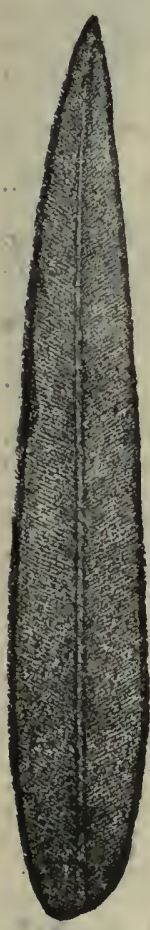

FIG. 53.-Neriopteris lauceolata. Single pin. nule. pith, medullary rays or rings of growth. Type N. crassum.

crassum, Dawson, 1863, Quar. Jour. Geo. Soc., vol. 19, p. 466, Devouian.

tenue, Dawson, 1863, Quar. Jour. Geo. Soc., vol. 19, p. 467, Devonian.

Nephropteris, Brongniart, 1828, Tab. des gener.

elegans, see Cyclopteris elegans.

fimbriata, see Neuropteris fimbriata.

germari, see Cyclopteris germari.

hirsuta, see Cyclopteris hirsuta.

laciniata, see Cyclopteris laciniata.

orbicularis, see Cyclopteris orbicularis.

trichomanoides, see Cyclopteris trichomanoides.

undans, see Cyclopteris undans.

Nerlopteris, Newberry, 1873, Ohio Pal., vol. 1, p. 378. [Ety. nerion, the oleander; pteris, a fern.] Frond pinnate or bipinnate; rachis strong, punctate; pinnules lanceolate, simple, entire ; medial nerve strong, extending from base to summit; secondary nerves given off at an acute angle, numerous simple or forked at base, parallel, equal; fructifications marginal. Type N. lanceolata.

lanceolata, Newberry, 1873, Ohio Pal., vol. 1, p. 381, Coal Meas.
Neuropteris, Brongniart, 1822, Mem. du Mus. d'Hist. Nat. de Paris, t. 8, p. 203, and Prodr. d. Hist. d. Veg. Foss, p. 52. [Ety. neuron, nerve; pteris, fern.] Fronds simple, bi, tri-pinnate; pinnules varying from round to ovate, obtuse, or obtusely acuminate, mostly entire, rounded, cordate, or auricled at the base, attached to the rachis by the middle ; sessile, or rarely short pediceled; veins either from the base of the pinnules or from a costa, diverging fanlike and arched backward, in passing toward the borders, many times dichotomous; costa generally dissolved at or below the middle; basilar veins simple or in fascicles. Type $\mathrm{N}$. acuminata.

acuminata, Schlotheim, 1820, (Filicites acuminatus,) Petrefactenkunde, p. 412 , and Coal Flora of $\mathrm{Pa} .$, p. 123, Coal Meas.

acutifolia, Brongniart, 1828, Hist. d. Veg. Foss., p. 229, Coal Meas.

adiantites, see Lescuropteris adiantites.

agassizi, Lesquereux, 1880, Coal Flora of Pa., p. 117, Coal Meas.

angustifolia, Brongniart, 1828, Hist. d. Veg. Foss., p. 231, and Coal Flora of Pa., p. 89, Coal Meas.

anomala, Lesquereux, 1880, Coal Flora of Pa., p. 118, Coal Meas.

aspera, Lesquereux, 1880, Coal Flora of Pa., p. 121, Coal Meas.

attenuata, Lindley \& Hutton, 1837, Foss. Flora, vol..3, p. 65, Coal Meas.

auriculata, Brongniart, 1828, Hist. d. Veg. Foss., p. 236, Coal Meas.

biformis, Lesquerenx, 1880, Coal Flora of Pa., p. 121, Coal Meas.

blissi, Lesquereux, 1884, Coal Flora of Pa., p. 737, Coal Meas.

callosa, Lesquereux, 1880, Coal Flora of Pa., p. 115, Coal Meas.

capitata, Lesquereux, 1870, Geo. Sur. Ill., vol. 4, p. 383, Coal Meas.

carri, Lesquereux, 1884, Coal Flora of Pa., p. 731 , Coal Meas.

cisti, Brongniart, 1828, Hist. d. Veg. Foss., p. 238, Coal Meas.

clarksoni, Lesquereux, 1858, Geo. Sur. Pa., vol. 2, p. 857, Coal Meas.

collinsi, Lesquereux, 1870, Geo. Sur. Ill., vol. 4, p. 382 , Coal Meas.

cordata, Brongniart, 1828, Hist. Veg. Foss., p. 229, and Coal Flora of Pa., p. 91, Coal Meas.

cordato-ovata, see Pseudopecopteris cordato-ovata.

coriacea, Lesquereux, 1870, Geo. Sur. Ill., vol. 4, p. 387, Coal Meas.

crassa, Dawson, 1868, Acad. Geol., p. 551, Devonian.

crenulata: Brongniart, 1828, Hist. Veg. Foss., p. 234, and Coal Flora of Pa., p. 116, Coal Meas.

cyclopteroides, Dawson, 1863, Can. Nat. and Geol., vol. 8, and Acad. Geol. p. 482, Coal Meas.

dawsoni, see Megalopteris dawsoni. 
decipiens, Lesquereux, 1880, Coal Flora of Pa., p. 93, Coal Meas.

delicatula, Lesquereux, 1858, Geo. Sur. Pa., vol. 2, p. 858, Coal Meas.

dentata, Lesquereux, 1858, Geo. Sur. Pa., vol. 2 , p. 859 , Coal Meas.

desori, Lesquereux, 1854, Bost. Jour. Nat. Hist., vol. 6, p. 418, and Geo. Sur. Pa., vol. 2 , p. 859 , Coal Mras.

dictyopteroides, Fontaine \& White, 1880 , Perm. or Up. Carb. Flora, p. 49, Coal Meas. or Permian.

dilatata, Lindley \& Hutton, 1835, (Cyelopteris dilatata,) Foss. Flora, vol.2, p. 29, Coal Meas.

elrodi, Lesquereux, 1850, Coal Flora of Pa., p. 107, Coal Meas.

eveni, Lesquereux, 1866, Geo. Sur. Ill., vol. 2, p. 430, Coal Meas.

faseiculata, Iesquereux, 1870, Geo. Sur. Ill., vol. 4, p. 381, Coal Meas.

fimbriata, Lesquereux, 1854, (Cyclopteris fimbriata, ) Jour. Bost. Soc. Nat. Hist., p. 416, and Coal Flora of Pa., p. 81, Coal Meas.

fissa, Lesquereux, 1858, Geo. Sur. Pa., vol. 2, p. 857 , Coal Meas.

flexuosa, Sternberg, 1825, Vers. Darst. Flora der Vorwelt, p. 16, Coal Meas.

germari, Gappert, 1836, (Adiantites germari,) Systema Filicum Fossilium, p. 21s, and Coal Flora of Pa., p. 113, Coal Meas.

gibbosa, Lesquereux, 1858, Geo. Sur. Pa., vol. 2, n. 858 , Coal Meas.

gigantea, Stornberg, 1825, Vers. Darst. Flora der Vorwelt, p. 16, Coal Meas.

grangeri, Brongniart, 1828, Hist. Veg. Fnss.. p. 237, and Coal Flora of Pa., p. 105, Coal Meas.

griffithi, Issquereux, 1884, Coal Flora of Pa., p. 737, Coal Mías.

heterophylla, Brongniart, 1822, (Filicites heterophylla) Mem. du Mus. d'Hist. Nat. de Paris, t. 8, p. 203, Coal Meas.

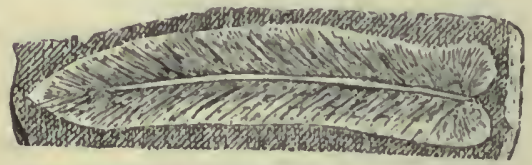

Fig, 54.-Neuropteris hirsuta.

hirsuta, Lesquereux, 1854, Bost. Jour. Nat. Hist., vol. 6, p. 417, and Coal Flora of Pa., p. 85, Coal Meas.

inflata, Lesquereux, 1866, Geo. Sur. III., vol. 2, p. 431, Coal Meas.

ingens, Lindley \& Hutton, 1833, Foss. Flora, vol. 2., p. 29, Coal Meas.

lacerata, syn. for Neuropteris fimbriata.

laciniata, Lesquereux, 1858, (Cyclopteris laciniata,) Geo. Sur. Pa., vol. 2, p. 855 , Coal Meas.

loshi, Brongniart, 1828, Hist. d. Veg. Foss., p. 242, and Coal Flora of Pa., p. 98, Coal Meas.

micropbylla, Brongniart, 1828, Hist. Veg. Foss., p. 245, Coal Meas. minor, Lesquereux, 1858, Geo. Sur. Pa., vol. 2 , p. 859 , Coal Meas.

missouriensis, Lesquereux, 1880, Coal Flora of Pa., p. 104, Coal Meas.

moori, see Lescuropteris moorii.

oblongifolia, Lesquereux, 1854, Coal Flora of Pa., p. 732, Coal Meas.

obscura, Lesquereux, 1880, Coal Flora of Pa., p. 108, Coal Meas.

odontopteroides, Fontaine \& White, 1850 , Perm. or Up. Carb. Flora, p. 50, Coal Meas. or Permian.

pachyderma, Lesquereux, 1866, Geo. Sur. Ill., vol. 2, p. 430, Coal Meas.

perelegans, Dawson, 1863, Can. Nat. and Geol., vol. 8, and Aead. Geol., p. 482, Coal Meas.

platynervis, Fontaine \& White, 1880 , Perm. or Up. Carb. Flora, pl. 8, fig. 2, Coal Meas. or Permian.

plicata, Sternberg, 1825, Vers. Darst. Flora der Vorwelt, p. 74, and Coal Flora of Pa., p. 96, Coal Meas.

polymorpha, Dawson, 1862, Quar. Jour. Geo. Soc., vol. 18, p. 320 , Devonian.

rarinervis, Bunbury, 1847, Quar. Jour. Geo. Soc., vol. 3, p. 425, and Coal Flora of Pa., p. 109, Coal Meas.

reniformis, Brongniart, 1828, (Cyelopteris reniformis,) Hist. d. Veg. Foss., p. 216, and Coal Flora of Pa., p. 77, Coal Meas.

retorquata, Dawson, 1871, Foss. Plants Canada, p. 50, Devonian.

rogersi, Lesquuereux, 1858, Geo. Sur. Pa., vol. 2, p. 556 , Coal Meas.

rotundifolia, Brongniart, 1828, Hist. Veg. Foss, p. 238, and Coal Flora of Pa., p. 97, Coal Meas.

selwyni, Dawson, 1871, Foss. Plants Cañada, p. 50, Devoninn.

serrulata, Dawson, 1562, Quar. Jour. Geo. Soc., vol. 18, p. 320, Devonian.

smilacifolia, Sternberg, 1824, Vers. Darst. Flora der Vorwelt, vol. 2, p. 29, Coal Meas.

smithsi, Lesquereux, 1876, Geo. Rep. of Alabama, p. 76, and Coal Flora of Pa., v. 106, Coal Meas.

soreti, Brongniart, 1828, Prodr. Hist. Veg. Foss., p. 53, and Hist. d. Veg. Foss., t. 1, p. 244, Coal Meas.

speciosa, Lesquereux syn. for $\mathrm{N}$. rogersi. subfalcata, Lesquereux, 1880, Coal Flora of Pa., p. 102, Coal Meas.

tenuifolia, Sternberg, 1825, Vers. Darst. Flora der Vorwelt, p. 17, and Coal Flora of Pa., p. 100, Coal Meas.

tenuinervis, see Odontopteris tenuinervis. trichomanoides, Brongniart, 1828, (Cyclopteris trichomanoides,) Hist. d. Veg. Foss., p. 217, and Coal Flora of Pa., p. 79, Coal Meas.

undans, Lesquereux, 1854, Bost. Jour. Nat. Hist., vol. 6, p. 418, and Geo. Sur. Pa., vol. 2, p. 859 , Coal Meas.

verbenifolia, Lesquereux, 1866, Geo. Sur. Ill., vol. 2, p. 431, Coal Meas. 
vermicularis, Lesquereux, 1861, Geo. Sur. Ky., vol. 4, p. 434, Coal Meas.

villiersi, Brongniart, 1828, Prodr. Hist. Veg. Foss, p. 53, Coal Meas.

Neggerratila, Sternberg, 1828, Essai d'un expose Geognostico-botanique de la Flore du monde primitif, $2 d$ Cahier, p. 37. [Ety. proper name.] Branch with a slender rachis bearing pinnate leaves attached to the stem by a semi-twisted base, dilated upward, veins flabellate and dichotomous. Type N. foliosa.

beinertiana, Gœppert, 1842, Gatt. d. Foss. Pflanzen, Coal Meas.

bockschii, see Aneimites bockschii.

bockschiana, syn. for Aneimites bockschii. dispar, Dawson, 1863, Can. Nat. and Geol., vol. 8, and Acad. Geol., p. 480, Coal Meas.

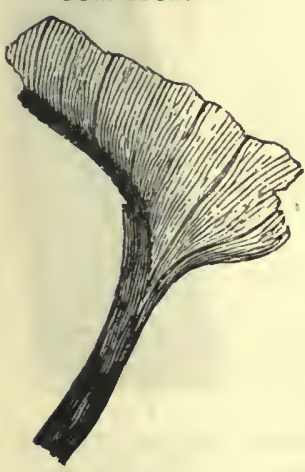

FIG. 55.

Noggerathia dispar. flabellata, Lindley \& Hutton, 1832,Foss. Flora, vol. 1,p. 89, Coal Meas.

gilboensis, Dawson, 1871, Quar. Jour. Geo. Soc., vol. 27, p. 273 , Chemung Gr. minor, see Archæopteris minor. obliqua, see A rchæopteris obliqua. obtusa, see Aneimites obtusus.

ODONTOPTERIS, Brongniart,1822, Mem. du Mus. d'Hist. Nat. de Paris, t. 8, p. 203. [Ety. odous, tooth; pteris, fern.] Fronds large, bipinnate; pinnæ opposite or subalternate; pinnules of various forms, generally oblong, obtuse, joined to the rachis by their whole base, sometimes decurrent, either disjointed and separate to the base or connate to the middle, generally becoming confluent toward the top of the pinnæ, and gradually effaced in passing to a terminal leaflet; lower pinnules sometimes attached to the main rachis and difform; veins emerging from the rachis, more rarely from a midrib; veinlets thin, dichotomous, diverging straight or in curve, in passing to the borders. Type o. brardi.

abbreviata, Lesquereux, 1880, Coal Flora of Pa., p. 138, Coal Meas.

æqualis, Lesquereux, 1866, Geo. Sur. Ill., vol. 2 , p. 434, Coal Meas.

affinis, Lesquereux, 1884, Coal Flora of Pa., p. 742, Coal Meas.

alata, Lesquereux, 1858, Catal.'Pottsville Foss., p. 6, and Coal Flora of Pa., p. 131, Coal Meas.

alpina, Sternberg, 1825, (Neuropteris alpina,) Flora d. Vorwelt, vol. 2, p. 76, and Coal Flora of Pa., p. 126, Coal Meas. antiqua, Dawson, 1863, Can. Nat. and Geo., Coal Meas.

bradleyi, Lesquereux, 1870, Geo. Sur. III., vol. 4, p. 390, Coal Meas.

brardi, Brongniart, 1822, Mem. du. Mus. d'Hist. Nat. de Paris, t. 8, p. 205, tah. 2, fig. 5, and Coal Flora of Pa., p. 132, Coal Meas.

britannica, Gutbier, 1842, Abdrucke u. Verst. d. Zwick. Schwarzk. u. sei. Um. geb. Zwick., p. 68, and Coal Flora of Pa., p. 830, Coal Meas.

cornuta, Lesquereux, 1880, Coal Flora of Pa., p. 128, Coal Meas.

crenulata, of Brongniart, as indentified by Lesquereux in Gen. Sur. Pa., vol. 2, p. 860 , is $\mathrm{O}$. subcrenulata.

dawsonana, n. sp. Devonian. Proposed instead of 0 . squamosa, in Quar. Jour. Geo. Soc. Lond., vol. 37, p. 305, which was preoccupied.

deformata, Lesquereux, 1880, Coal Flora of Pa., p. 141, Coal Meas.

densifolia, Fontaine \& White, 1880, Perm. or Up. Carb. Flora, p. 54, Coal Meas. or Permian.

dilatata, Lesquereux, 1884, Coal Flora of Pa., p. 831, Coal Meas.

dubia, Lesquereux, 1858, Geo. Sur. Penn., vol. 2, p. 860 , Coal Meas.

gracillima, Newbe r ry, 1873 Ohio Pal., vol. 1, p. 382, Coal Meas.

heteropbylla,Lesquereux, 1866 , Geo. Sur. Ill., vol. 2, p. 433, Coal Meas.

intermedia, Lesquereux, 1860, Geo. Sur. Ark., vol. 2, p. 313, Coal Meas.

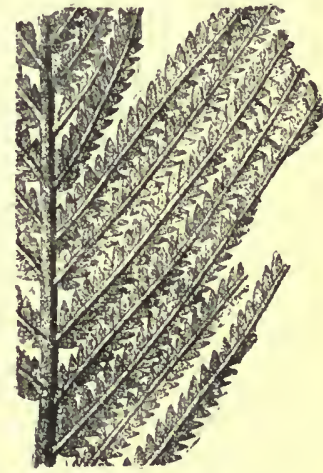

FIG. 56.

Odontopteris gracillima. lescurei, Wood, 1860, Trans. Am. Phil. Soc., vol. 13, p. 348, Coal Meas.

monstruosa, Lesquereux, 1884, Coal Flora of Pa. p. 741, Coal Meas.

nervosa, Fontaine \& White, 1880, Perm. or Up. Carb. Flora, p. 52, Coal Meas. or Permian.

neuropteroides, Newberry, 1873, Ohio Pal., vol. 1, p. 381. The name was preoccupied by Roemer, and the species has been named $O$. newberryi.

newberryi, Lesquereux, 1880, Coal Flora of Pa., p. 127, Low. Coal Meas.

obtusiloba var rarinervis, Fontaine \& White, 1880, Perm. or Up. Carb. Flora, p. 52, Coal Meas. or Permian.

pachyderma, Fontaine \& White, 1880, Perm, or Up. Carb. Flora, p. 53, Coal Meas. or Permian.

patens, Lesquereux, 1884, Coal Flora of Pa., p. 740, Coal Meas. 
reichiana, Lesquereux, 1884, Coal Flora of Pa., p. 831, Coal Meas.

rotundifolia, Wood, 1866, Trans. Am. Phil. Soc., vol. 13, p. 348, Coal Meas.

schlotheimi, Brongniart, 1828, Hist. d.Veg.

Foss., p. 256, and Coal Flora of Pa., p. 136, Coal Meas.

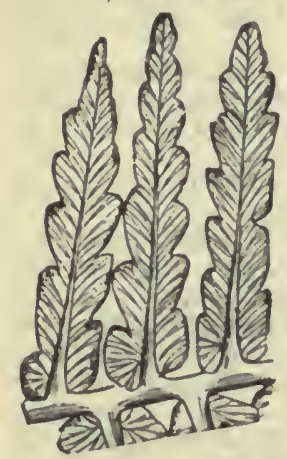

Fra. 57.-Odontopteris schlothelmi. sphenopteroides, Lesquereux, 1880, Coal Flora of Pa., p. 139, Coal Mtas. squamosa, Lesquereux, 1854, Bist. Jour. Nat. Hist., vol. 6 , p. 419 , and Geo. Sur. Pa., vol. 2, p. 560 , Coul Meas.

squamosa, Dawson, 1881, Quar. Jour. Geo. Soc. I,ond., vol. 37 , p. 305 , Devonian. The name was preoccupied. See $\mathrm{O}$. dawsonana.

subcrenulata, Lesquereux, 1880, Coal Flora of Pa., p. 137, Coal Meas.

subcuneata, Bunbury, 1847, Quar. Geo. Jour., vol. 3 , p. 427 , and Coal Flora of Pa., p. 134, Coal Meas.

tenuinervis, Lesquereux, 1858, (Neuropteris tenuinervis, Geo. Sur. P'a., vol.?, p. 859 , Coal Mens.

wortheni, Lesquereux, 1866, Geo. Sur. Ill., vol. 2, p. 432, Coal Meas.

Oligocari'ia, Goppert, 1841-48, Die Gattungen der fossilen Pflanzen, p. 3. [Ety. oligos, few; karpos, fruit.] Fronds bipinnate or tripinnatifid; primary pinnse oblong-lanceolate; secondary divisions, open, linear, pinnately divided in oblong or half round lobes or leaflets, connate at the base, crenulate; primary and secondary veins nearly of the same size, thin but distinct; lateral veins curved to the borders, simple or forked. Type O. gutbieri.

alabameusis, Lesquereux, 1875, Geo. Rep. Ala., p. 76, and Coal Flora of Pa., p. 266, Coal Meas.

flagellaris, Lesquereux, 1858, (Splienopteris flagellaris,) Geo. Sur. Pa., vol. 2, p. 862, Coal Meas.

gutbieri, Gceppert, 1841-48, Die Gattungen der fossilen Planzen, p. 3, Coal Meas.

Ormoxylon, Dawson, 1871, Foss. Plants Canada, p. 14. [Ety.ormos, a chain, a cord; xylon, wood.] Woody stems, witls cells of the character of those of Dadoxylon, very thick walled, with thrte rows of hexagonal areoles, having oval pores and medullary rays of one row of cells. Pith cavity composed of a series of spherical chambers, separated by thick, transverse eellular partitions. Type $\mathrm{O}$. erianum.

elianım, Dawson, 1871, Foss. Plants Canada, p. 14, Portage Gr.
Orthogoniopteris, Andrews, 1875, Ohio Pal., vol. 2, p. 418. [Ety.orthogoniopteris, rectangular-fern.] Frond simply pinnate; pinnules alternate, lanceolate or oblong-linear, rounded and tapering to an acute point, enlarged and decurrent on the lower side to an auricle rounded in the upper part in joining the lamina a little above its point of attacliment to the rachis; merlial nerve thick, ascending to the apex; nervules fine and numerous, uniform, at right angle to the midrib, decurring to it at the point of attachment, forking unce near the base. Type 0 . clara.

clara,. Andrews, 1875 , Ohio Pal, vol. 2, p. 419, Coal Meas.

gilberti, Andrews, 1875 , Ohio Pal., vol. 2, $\mu$. 420 , Coal Mr as.

Pachyphyllum, Lesquereux, 1858, Gieo. Sur. Pa.,. vol. 2. [lity. pachys, thick; phyllon, a leaf.] This name was preoccupied in the class Polypi. See Rhacophyllum.

affine, see Rhacophyllum affine.

fimbriatum, see Rhacophyllum fimbriatum.

hirsutum, see Rliacophyllum hirsutum.

laceratum, see Rhaeophyllum laceratum.

lactuca, see Rliaco.

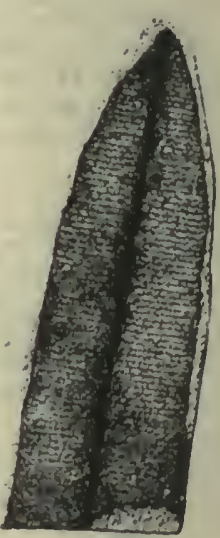

Frg. 58

Orthogoviopteris clara, part of a plnutule. pliyllum lactuca.

PacuYpreis, Brongniart, 1828, Prodr. Hist. Veg. Foss., 1). 49. [Ety. pachys, thick; pteris, fern.] Frond pinnate or bipinnate, bearing opposite coriaceous jinnules, with a medial nerve or without nervation, narrowed toward the base, not joined to the rachis. Type P. lanceolata.

gracillima, Lesquereux, 1870, Geo. Sur. Ill., vol. 4, p. 419, Coal Meas.

Pa deor'll ycus, Hall, 1847, Pal. N. Y., vol. 1, p. 7. [Ety.palaios, ancient ; phykos, seaweed.] Stems simple or dichotomous, branches cylindrical or slightly flattened, obtuse, surface smootl or dotted. Type $\mathrm{P}$. tubulare.

articulatum, Wincliell, 1S64, Anı. Jonr. Sci. and Arts, $2 d$ series, vol. 37, p. 231, Potsdam Gr.

beauliarnoisense, Billings, 1862, Pal. Foss., vol. 1, p. 98, Calcif. Gr.

beverleyense, Billings, 1862, Pal. Foss., vol. 1. p. 97, Potsdain Gr.

congregatum, Billings, 1861, Pal. Foss., vol. 1 ; p. 3 , Potsdam Gr.

divaricatum, Lesquereux, 1876, 7 th Ann. Rep. Geol. Sur. Ind., p. 138, Cual Meas.

funiculus, Billings, 1862, Pal. Foss., vol. 1, p, 98, Calcif. Gr. 
gracile, Lesquereux, 1876, 7th Ann. Rep. Geol. Sur. Ind., p. 137, Coal Meas.

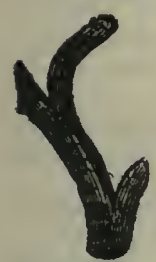

FIG. 59.

Palaophycusgracile. incipiens, Billings, 1861, Pal. Foss., vol.1, p. 2, Potsdam Gr. informe, Winchell, 1864, Am. Jour. Sci. and A rts, vol. 37, p. 232, Potsdam Gr.

irregulare, Hall, 1847, Pal. N. Y., vol. 1, p. 8, Calcif. Gr. milleri, Lesquereux, 1876,7 th Ann. Rep. Geol. Sur. Ind., p. 136 , Coal Meas. Foss.,vol. 1, p. 98, Trenton Gr. occidentale, Whitfield, 1877, Rep. Pal. Black Hills, p. 7, and Geol. Black Hills, p. 332, Potsdam Gr.

plumosum. Whitfield, 1878, Ann. Rep. Geo. Sur. Wis., p. 50, and Geo. Wis., vol. 4, p. 169, Potsdam Gr.

rugosum, Hall, 1847, Pal. N. Y., vol. 1, p. 63, Trenton Gr.

simplex, Hall, 1847, Pal. N. Y., vol. 1, p. 63, Trenton Gr.

striatum, Hall, 1852, Pal. N. Y., vol. 2, p. 22, Clinton Gr.

tortuosum, Hall, 1852, Pal. N. Y., vol. 2, p. 6, Medina sandstone.

tubulare, Hall, 1847, Pal. N. Y., vol. 1, p. 7, Calcif. Gr.

virgatum, Hall, 1847, Pal. N. Y., vol. 1, p. 263, Hud. Riv. Gr.

Palaopteris, Schimper, being preoccunied by Geinitz, see Archæopteris.

acadica, see Aneimites acadicus.

hartii, see Archæopteris harti.

Palroxyris, Brongniart, 1828, Prodr. d. Hist. d. Veg. Foss., p. 137. [Ety. palaios, ancient; xyris, plant.] An inflorescence. Type P. regularis. The fossils which have been referred to this genus in the American palreozoic rocks are now referred to Spirangium.

appendiculata, see Spirangium appendiculatum.

corrugata, see Spirangium corrugatum. prendeli, see Spirangium prendeli.

Palmacites oculatus, see Sigillaria oculata. noggerathi, see Trigonocarpum noggerathi.

Pecopteris, Brongniart, 1822, Class d. Veg. Foss. in Mem. du Mus, d'Hist. Nat. d. Paris, tom. 8, p. 203. [Ety. peko, comb; pteris, fern.] Fronds, bi, tripinnate; pinnæ long, pinnatifid; pinnules adhering to the rachis by the whole base, often more or less deeply connate, not decurring; borders generally contiguous, or nearly so; secondary veins derived from the medial nerve of the pinnules, simple, bi or trifurcate. Type, $P$. longifolia is the first species mentioned in the Prodr. d. Hist. d. Veg. Foss., and the first mentioned in the Coal Flora of Pa. is $\mathrm{P}$. unita, while $\mathrm{P}$. penniformis is a representative species. abbreviata, Brongniart, 1828, Hist. d. Veg. Foss., p. 337, and Coal Flora of Pa., p. 248 , Coal Meas. acuta, Brongniart, 1828, Hist. d. Veg. Foss., p. 350, and Coal Flora of Pa., p. 241, Coal Meas.

æqualis, Brongniart, 1828, Prodr. d. Hist. d. Teg. Foss., p. 58, Coal Meas.

alata, see Sphenopteris alata.

alata, Schimper, 1869, Pal. Veg., t. 1, p. 531, syn. for Pseudopecopteris decurrens

angustipinna, Fontaine \& White, 1880 , Perm. or Up Carb. Flora, p. 76, Coal Meas, or Permian.

angustissima, Sternberg, 1820, Vers. Darst. Flor. d. Vorw., p. 18, and Coal Flora of Pa., p. 257, Coal Meas.

aquilina, see Alethopteris aquilina.

arborescens, Schlotheim, 1820, (Filicites arborescens,) Petrefaktenkunde, p. 404, and Coal Flora of Pa., p. 230, Coal Meas.

arguta, Siernberg, 1820, Vers. Darst. Flor. d. Vorw., i. 19, and Coal Flora of Pa., p. 227, Coal Meas.

aspera, Brongniart, 1828, Hist. d. Veg. Foss., p. 339, and Coal Flora of Pa., p. 242, Coal Meas.

aspidioides, Brongniart, 1828, Hist. d. Veg. Foss., p. 311, and Coal Flora of Pa., p. 756, Coal Meas.

asplenioides, Fontaine \& TVhite, 1880, Perm. ol' Up. Carb. Flora, p. 72, Coal Meas. or Permian.

bucklandi, Brongniart, 1828, Hist. d. Veg. Foss., p. 319, and Coal Flora of Pa., p. 244, Coal Meas.

callosa, see Pseudopecopteris callosa.

candollana, Brongniart, 1828, Hist. d. Veg.

Foss, p. 305, Coal Flora of $\mathrm{Pa}$. p. 243, Coal Meas.

carri, Lesquereux, 1884, Coal Flora of Pa., p. 758, Coal Meas.

chærophylloides, see Sphenopteris chærophylloides.

cisti, Brongniart, 1828, Hist. d. Veg. Foss., p. 330, and Coal Flora of Pa., p. 243, Coal Meas.

clarki, Lesquereux, 1880, Coal Flora of Pa., p. 261, Coal Meas.

clintoni, Lesquereux, 1880, Coal Flora of Pa., p. 251, Coal Meas.

concinna, Lesquereux, 1854, Bost. Jour. Soc. Nat. Hist., vol. 6, p. 424 , and Ger. Sur. Pa., vol. 2, p. 867, Coal Meas. But the name was preoccupied by Presl in 1833.

crenulata, Brongniart, not American. The form sometimes referred to it is Pseudopecopteris subcrenulata.

cristata, Gutbier, 1843, Gaea von Sachsen, p. 80, and Coal Flor. of Pa., p. 256, Coal Meas.

cristata, see Sphenopteris cristata.

cyathea, Schlotheim, 1820, (Filicites cyatheus,) Petrefaktenkunde, p. 403, Coal Meas.

decurrens, see Pseudopecopteris decurrens. decurrens, Dawson, 1862. The name being preoccupied, it was changed to $\mathrm{P}$. discrepans. 
densifolia, Dawson, 1874, Foss. Plants of Canada, p. 56, Devoniau.

dentata, Brongniart, 1828, Hist. d. Veg.

Foss., p. 346, and Coal Flora of Pa., p. 240, Coal Meas.

distans, Lesquereux, 1854, Bost. Jour. Soc. Nat. Hist., vol. 6, p. 423, and Geo. Sur. Pa., vol. 2, p. 866, Coal Meas. The name was preoccupied by Rost in 1839 .

dournaisi, see Callipteridium dournaisi.

dubia, Sternberg, 1820, Tent. Flor. Primord, p. 19, and Gutbier in Gaea von Sachsen, Coal Meas.

elegans, Gœppert, 1836, (l'ulypodites elegans, ) Syst. Filic. Foss., $\mathrm{l}$. 3H, and Coal Flora of Pa., p. 228, Cual Meas.

elliptica, Bumbury, 1846, Quar. Jour. Geo. Soc., vol. 2, p. 82 , and Coal Flora of Pa., p. 245, Coal Meas.

elliptica, Fontaine \& White, 1880, (Goniopteris elliptica,) Pern. or Up. Carb. Flora, p. 83, Coal Meas. or Permian. The name was preoccupied.

emarginata, Gceppert, 1836, (Diplazites emarginatus,) Syst. Filic. Foss., p. 274, and Conl Flora of Pa., ט. 225, Coal Meas.

erosa, Gutbier, 1843, Grea. von Sachsen, p. 81, and Coal Flora of Pa., p. 255, Coal Meas.

flavicans, Presl, 1833, in Sternberg, Vers. Darst. Flor. d. Vorw., vol. 2, p. 127. Probably not American.

georgiana, Lesquereux, 1884, Coal Flora of Pa., p. 759, Coal Meas.

germari, Weiss, 1869, (Cyatheites germari,) Foss. Flora d. Jungsten Steink. Form., Up. Coal Meas. or Permian.

germari var. crassinervis, Fontaine \& White, 1850, Perm. or Up Carb. Flora, p. 70, Conl Meas. or Permian.

germari var. cuspidata, Fontaine \& White, 1880, Perm. or Up. Carb. Flora, p. 70, Coal Meas. or Permian.

goniopteroides, Fontaine \& Wlite, 1880 , Perm. or Up. Carb. Flora, p. 80, Coal Meas. or Permian.

halli, Lesquereux, 1870, (Aletlopteris halli,) Geo. Sur. Ill., vol. 4, p. 394, Coal Meas.

beerana, Fontaine \& White, 1880, Perm. or Up. Carb. Flora, p. 77, Coal Meas. or Permian.

hemiteloides, Brongniart, 1828, Hist. d. Veg. Foss., p. 314, Coal Meas.

heterophylla, see Alethopteris heterophylla. imbricata, Fontaine \& White, 1880, Perm. or Up. Carb. Flora, p. 72, Coal Meas. or Permian.

inclinata, Fontaine \& White, 1880, Perm. or Up. Carb. Flora, p. 80, Coal Meas. or Permian.

incompleta, Lesquereux, 1858, Geo. Sur. Pa., vol. 2, p. 868, Coal Meas.

ingens, Dawson, 1862, Quar. Jour. Geo. Soc., Lond., vol. 18, p. 322, Devonian.

lanceolata, Lesquereux, 1870, (Alethopteris lanceolata,) Geo. Sur. Ill., vol. 4, p. 398, Coal Meas. lanceoluta, Fontaine \& White, 1880, Perm. or Up. Carb. Flora, p. 79, Coal Meas. or Permian. The name was preoccupied; beside, it is probably a syn. for P. unita.

latifolia, Fontaine \& White, 1850, Perm. or Up. Carb. Flora, p. 79, Coal Meas. or Permian.

lepidorachis, Brongniart, 1S:S, Hist. d. Veg. Foss., p. 313, Coal Meas.

lescuriana, n. sp. Coal Meas. Proposed instead of P. obsoleta, Lesquereux, 18.4, Coal Flora of Pa., p. 758, which name was preoccupjed.

longifolia, Brongniart, 1828, Hist. d. Veg. Foss. p. 273, and Coal Flora of $\mathrm{Pa}, \mathrm{p}$. 226, Coal Meas.

loschi, Brongniart, 182S, Hist. d. Veg. Foss. p. 355, Coal Meas.

lyratifolia, Goeppert, 1841, (Sphenopteris lyratifolia,) Die Gattungen d. Foss. Pflanzen, p. 71, and Coal Flora of Pa., p. 259, Coal Meas.

mantelli, Brongmiart, 1828, Prodr. d. Hist. d. Veg. Foss., p. 57, Coal Meas.

marginata, see Alethoptcris marginata.

merianopteroides, Fontaine of White, 1850, Perm. or Up. Carb. Flora, 1. 78, Coal Meas. or Permian.

microphylla, Brongniart, 1828, Ilist. d. Veg. Foss. p. 340, and Coal Flora of Pa., v. 263, Corl Meas.

milleri, Harlan, 1835, Trans. Geo. Soc. Pa., Coal Mea

miltoni, Artis, 1825, (Filicites miltoni,) Anted. Pliytol. pl. 4, and Coal Flora of Pa., p. 247, Coal Meas.

muricala, see Pseudopecopteris muricata. murrayana, Brongniart, as identified by Lesquereux in Geo. Sur. Ill., vol. 2, p. 443, see Sphenopteris pseudo-murrayana.

neriosa, see I'seudopecopteris nervosa. newberryana, Fontaine \& White, 1880, (Gonjopteris newberryana,) Perm. or Up. Carb. Flora, p. 84, Coal Meas. or Permian.

newberryi, see Psendopecopteris newberryi. nodosa, Gœppert, 1836, (Aspidites nodosus,) Systema Filicum Fossilium, p. 372, and Coal Flora of Pa., p. 233, Coal Meas.

notata, Lesquerenx, 1854, Bost. Jour. Soc. Nat. Hist., vol. 6, p. 424, and Geo. Sur. Pa., vol. 2, p. 866, Coal Meas.

oblonga, Fontaine \& White, 1880, Perm. or Up. Carb. Flora, p. 83, Coal. Meas. or Permian.

obsoleta, Harlan, 1835, Trans. Geo. Soc. Pa., Coal Meas.

obsoleta, Lesquereux, 1884, Coal Flora of Pa., p. 758. The name was preoccupied. See P. lescuriana.

oreopteroidea, Schlotheim, 1820, (Filicites oreopteridius,) Petrefaktenkunde, p. 407, and Coal Flora of Pa., p. 238, Coal Meas.

ornata, Lesquereux, 1884, Coal Flora of Pa., p. 760, Coal Meas. 
ovata, Brongniart, 1828, Prodr. d. Hist. d. Veg. Foss, p. 58, Coal Meas.

ovoides, Fontaine \& White, 1880, Coal Flora of Pa., p. 79, Coal Meas. ur Permian.

páchypteroides, Fontaine \& White, 1880 , Perm. or Up. Carb. Flora, p. 76, Coal Meas. or Permian.

penniformis, Biongniart, 1822, (Filicites pennæeformis, Class des Veg. Foss., in Mem. du Mus. d'Hist. Nat. de Paris, tom. 8, p. 203, and Coal Flora of Pa., p. 23?, Coal Meas.

platynervis, Fontaine \& White, 1880 , Perm. or Up. Carb. Flora, p. 73, Coal Meas. or Permian.

platyrachis, Brongniart, 1828, Hist. d. Teg. Foss. p. 312, and Coal Flora of Pa., p. 232, Coal Meas.

pluckeneti, see Pseudopecopteris pluckeneti.

plumosa, Artis, 1825, (Filicites plumosus,) Anted. Phytol., pl. 17, Coal Meas.

polymorpha, Brongniart, 1828, Prodr. d. Hist. d. Veg. Foss., p. 56, Coal Meas.

preciosa, Hartt, 1868, Acad. Geol., p. 553, Deronian.

pteroides, Schlotheim, 1820, (Filicites pteridius,) Petrefaktenkunde, p. 406, and Coal Flora of Pa., p. 249, Coal Meas.

pusilla, see Pseudopecopteris pusilla.

quadratifolia, Lesquereux, 1880, Coal Flora of Pa., p. 234, Coal Meas.

rarinervis, Fontaine \& White, 1880 , Perm. or Up. Carb. Flora, p. 71, Coal Meas. or Permian.

rigida, Dawson, 1863, Can. Nat. and Geo., vol. 8, and Acad. Geol., p. 485, Coal Meas.

robusta, Lesquereux, 1880, Coal Flora of Pa., p. 229, Coal Meas.

rotundifolia, Fontaine \& White, 1880, Perm. or Up. Carb. Flora, p. 73, Coal Meas. or Permian.

rotundiloba, Fontaine \& White, 1880 , Perm. or Up. Carb. Flora, p. 74, Coal Meas. or Permian.

schimperana, Fontaine \& White, 1880 , Perm. or Up. Carb. Flora, p. 75, Coal Meas. or Permian.

schimperi, Lesquereux, 1884, Coal Flora of Pa., p. 835, Coal Meas.

serlii, see Alethopteris serlii.

serpillifolia, Lesquereux, 1880, Coal Flora of Pa., p. 237, Coal Meas.

serrula, Lesquereux, 1858, (Alethopteris serrula,) Geo. Sur. Pa., vol. 2, p. 865, Coal Meas.

serrulata, Hart, 1868, Acad. Geol., p. 553, Devonian.

sheaferi, see Pseudopecopteris sheaferi.

sillimani, see Pseudopecopteris sillimani. sinuata, see Callipteridium sinuatum.

solida, Lesquereux, 1870, (Alethopteris solida,) Geo. Sur. Ill., vol. 4, p. 397, Coal Meas.

squamosa, Lesquereux, 1870, Geo. Sur. Ill., vol. 4. p. 400, Coal Meas. stellata, Isesquereux, 1866, (Alethopteris stellata,) Geo. Sur. Ill., vol. 2, p. 440 , Low. Coal Meas.

strongi, Lesquereux, 1870, Geo. Sur. Ill, vol. 4, p. 399, Coal Meas.

subialcata, Fontaine \& White, 1880 , Perm. or Up. Carb. Flora, p. 70, Coal Meas. or Permian.

tæniopteroides, Bunbury, 1847, Quar. Jour. Geo. Soc., vol. 3, p. 428 , Coal Meas.

tenuinervis, Fontaine \& White, 1880, Perm. or Up. Carb. Flora, p. 77, Coal Meas. or Permian.

tenuis, Brongniart, 1828, Prodr. d. Hist. d. Veg. Foss., p. 57, Coal Meas.

unita, Brongniart, 1828, Hist. d. Veg. Foss., p. 342, Coal Meas.

urophylla, see Alethopteris urophylla.

vellutina, Lesquereux, 1854 , Bost. Jour. Soc. Nat. Hist., vol. 6 , p. 423, and Geo. Sur. Pa., vol. 2, p. 866, Coal Meas. venulosa, Lesquereux, 1880 , Coal Flora of Pa., p. 230, Coal Meas.

vestita, Lesquereux, 1880, Coal Flora of Pa., p. 252, Coal vis. 60. Meas.

villosa, Brongniart, 1828, Hist.

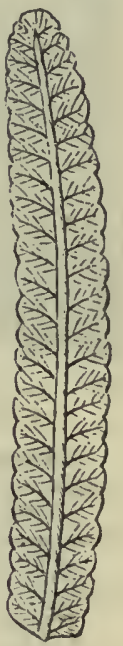

Pecopteris d. Veg. Foss., p. 316, and Coal Flora of Pa., p. 253, Coal Meas.

Phyllopteris, Brongniart, 1849, Table d. Gen. d. Veget. Foss., pp. 22, 103. [Ety. phyllon, leaf; pteris, fern.] Pinnate,

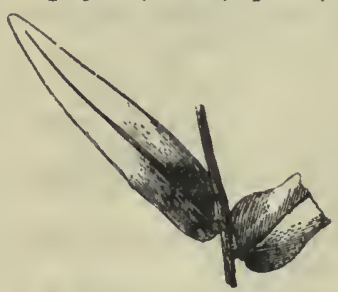

FiG. 61.-Phyllopteris untiqua. pinnules oblong or lanceolate, pointed, attached by the middle of the base; midrib strong, extending to the point,giving off oblique nerves, which have

obliquely pinnate nervules not anastomosing. Type P. phillipsi.

antiqua, Dawson, 1863, Can. Nat., vol. 8, and Ácad. Geol., p. 484, Coal Meas.

Physophyius, Schimper, 1869. Syn. for Taonurus.

marginatus, See Taonurus marginatus.

Phytolithus, Martin, 1809, Petrificata Derbiensia. [Ety. phyton, plant; lithos, stone.] Applied indiscriminately to fossil wood.

cancellatus, syn. for Lepidodendron cancellatum.

notatus, see Sigillaria notata.

tessellatus, see Sigillaria tessellata.

transversus, see Sternbergia transversa. 
Phytopsis, Hall, 1847, Pal. N. Y., vol. 1, p. 38. [Ety. phyton, plant; opsis, resem-

Fia. 62.-Phytopsls tubulosa.

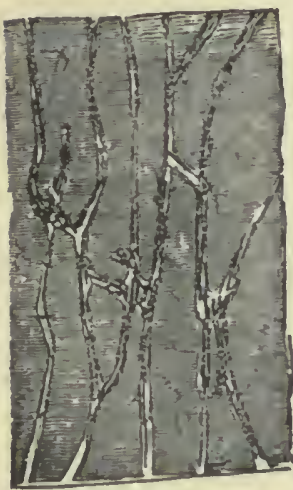

blance.]. Stems cylindrical or subeylind rical, straight or flexuous, erect or procumbent, br a n c hed: branches diverging and anastomosing; structure cellular, consisting apparently of thin lamina, with transverse divisions, or having a reticulated structure. Thisstructure is too obscure for satis. factory determination. Type P.tubulosi.

cellulosa, Hall, 1847, Pal. N. Y., vol. 1, p. 39, Birdseye Gr.

tubulosa, Hall, 1847, Pal. N. Y., vol. 1, p. 38, Birdseye Gr.

Pinnularia, Lindley \& Hutton, 1835, Foss. Flora, vol. 2, p. 81. [Ety. pinna, a feather.] Roots or rootlets divided in filaments of variablo length and thickness, and generally possessing few definable vharacters. Type $P$. capillacea.

calamitarum, Lesquereux, 1858, Geo. Sur. Pa., vol. 2, p. 878, Coal Meas.

capillacea, Lindley \& Hutton, 1835, Foss. Flora, vol. 2, p. 81, Coal Meas.

confervoides, Lesquereux, 1858, Geo. Sur. Pa., vol. 2, n. 878, Coal Mleas.

crassa, Dawson, 1863, Can. Nat., vol. 8, and Acad. Geol., p. 480, Coal Meas.

dispalans, Dawson, 1862, Quar. Jour. Geo. Soc., vol. 18, p. 312, Devonian.

elongata, Dawson, 1871, Foss. Plants Can., p. 33 , Devonian.

ficoides, Lesquereux, 1868, Geo. Sur. Pa., vol. 2, p. 878 , Coal Meas.

horizontalis, Lesquereux, 1858, Geo. Sur. Pa., vol. 2, p. 878, Coal Meas.

nodosa, Dawson, 1871. Foss. Plants Can., p. 33, Devonian.

palmatifida, Lesquereux, 1860, (Rhizolithes palmatifidus,) Geo. Sur. Ark., vol. 2 , p. 313 , Coal Mleas.

pinnata, Lesquereux,, 1858, Geo. Sur. Pa., vol. '2, p. 878, Coal Meas.

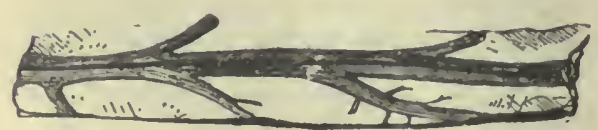

FIG. 63.-PInnularla ramoslssima.

ramosissima, Dawson, 1863, Can. Nat., vol. 8, and Acad. Geol., p. 480, Coal Meas.

Plumalina, Hall, 1858, Can. Nat. and Geo., vol. 3, p. 175. [Ety. pluma, a smal] feather.] Simple fronds, with linear pinnules diverging, from each side, in the same plane, and more or less ascend. ing. It is a peculiar plant, described, originally, as a Grap. tolite, to which opin. ion Prof. Hall still adheres. On the other hand, Prof. Dawson claims the characters prove it is a vegetable, and in this to is supported by the fact that all Graptolites had become extinct, as shown by their absence in several groups of rocks before the appearance

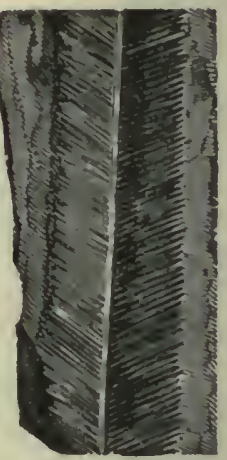

FI'i. 64. of this form. Type P. plumaria.

densa, Hall, 1879, 30th Rep. N. Y. St. Mus. Nat. Hist., pl. 4, fig. 6, Ham. Gr. gracilis, Shumard, 1855, (Filicites gracilis,) Geo. Rep. Mo., p. 208, Waverly Gr. in Lithographic limestone.

linearis, Lesquereux, 1880, ('Trochophyllum lineare,) Coal Flora of Pa., p. (i4, Waverly Gr.

plunaria, Hall, 1843, (Filicites?) Geo. Rej, p. 273, and 4th Dist. N. Y.

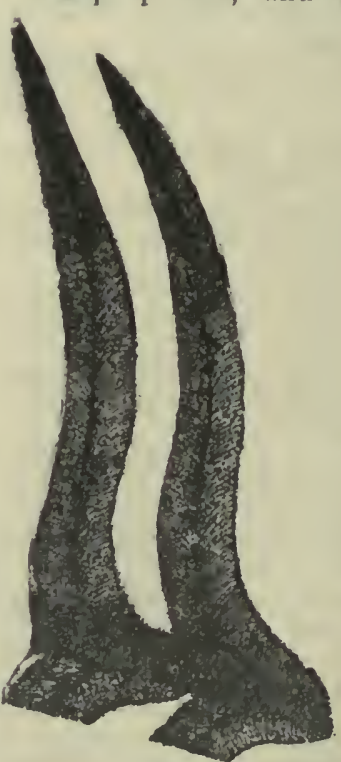

FIG. 65.

Protoblechnum holdeul.
Can. Nat.and Geo. vol. :3, p. 175, Chemung Gr.

pluinula, Dawson, 1873 , (Lycopodites plin $\mathrm{ln}(\mathrm{a}$, ) Rep. Fos 8 . plants Low. $\mathrm{Carb}$. and Millstone Grit, p. 24, Su bearboniferolls.

Polyporites, Lindley \& Hutton, 1833, Foss, Flora, vol. 1, p. 181. This $y \in \mathrm{n}$ us was founded upon a fisliseale, and the form referred to it in Geo. Sur. Pa., vol. 2 , p. 847 , is quite a different thing.

Polysporia, Newberry, syn. for Lepidostrobus. mirabilis, see Lepidostrobus mirabilis.

Protoblechnum, Lesquerenx, 1880, Coal Flora of Pa., p. 188. [Ety. protos, first; 
Blechnum, a genus.] Fronds large, pinnate; rachis thick, scaly toward the base; pinnæ long, narrow linear-lanceolate, acuminate, entire, enlarged at base on the lower side to a decurring auricle, generally free; medial nerve percurrent; lateral veins open, curving to the borders, forking twice. Type P. holdeni.

holdeni, Andrews, 1875, (Alethopteris holdeni,) Ohio Pal., vol. 2, 420, Coal Meas.

Protostigma, Lesquereux, 1877, Proc. Am. Phil. Soc., p. 169. [Ety. protos, first; stigma, a brand or dot.] Stems with rhomboidal scars as in Sigillaria, but without vascular scars in the middle. Not a land-plant, but a fucoid. Type P. sigillarioides.

sigillarioides, Lesquereux, 1877, Proc. Am. Phil. Soc., p. 169, Hud. Riv. Gr.

Prototaxites Dawson, 1859, Quar. Jour. Geo. Soc., vol. 15, p. 484. [Ety. protos, first; taxus, yew-tree; so named from the spirally marked cells characteristic of the genus Taxites.] Woody and branching trunks, with concentric rings of growth and medullary rays; cells of pleurenchyma not in regular lines, cylindrical, thick-walled, with a double series of spiral fibers; discs or bordered pores few, circular and indistinct. The specimens found are usually silicified, with the bark in a coaly state. Type P. logani.

logani, Dawson, 1859, Quar. Jour. Geo. Soc., vol. 15, p. 484, Devonian. This is the oldest known exogenous tree in America, according to Dawson, but Carruthers says it is a huge sea-weed and has named it Nematophycus logani.

Psaronius, Cotta, 1832, Dendrol in Beziehung, p. 27. [Ety. psaros, speckled.] Stems of tree-ferns, covered below by adventive roots, increasing by their superposition the conical base of the trunks; cortex thick, parenchymatous ; woody cylinder, subdivided into branches composed of fascicles of ressels either half cylindrical or diversely plicate, immersed in cellular medullar tissue. Type P. helmintholithus.

erianus, Dawson, 1871, Foss. Plants Can., p. $58, \mathrm{Ham}$. Gr.

textilis, Dawson, 1871, Foss. Plants Can., p. 59, Ham. Gr.

Pseudopecopteris, Lesquereux, 1880, Coal Flora of Pa., p. 189. [Ety. pseudo, false; Pecopteris, a genus.] Primary rachis forking near the base in diverging brancbes of equal size, or divaricate and dichotomous; branches polypinnate, ultimate divisions sometimes forked; pinnules connate or separated to the base, of various shape, oblong-obtuse or ovate-lanceolate, oblique or in right angle, decurring to the rachis and bordering it by a narrow wing; lateral veins oblique, generally forking once, the lowest pair twice. Type P. mazonana. abbreviata, Lesquereux, 1854, (Sphenopteris abbreviata,) Bost. Jour. Soc. Nat. Hist., vol. 6, p. 419, and Geo. Sur. Pa., vol. 2, p. 861, Coal Meas.

acuta, Brongniart, 1828, (Sphenopteris acuta,) Hist. d. Veg. Foss., p. 207, and Coal Flora of Pa., p. 215, Coal Meas.

anceps, Lesquereux, 1880, Coal Flora of Pa., p. 207, Coul Meas.

andræana, Roehl, 1868, (Sphenopteris andræana,) Fossile Flora der. Steinkohlen formation Westphalens, p. 62, and Coal Flora of Pa., p. 754, Coal Meas.

callosa, Lesquereux, 1866, (Pecopteris callosa,) Geo. Sur. Ill., vol. 2, p. 442, Low. Coal Meas.

cordato-ovata. Weiss, 1869, (Neuropteris cordato-ovata,) Foss. Flor. d. jungst. Steink. form., p. 28, and Coal Flora of Pa., p. 205, Coal Meas.

decipiens, Lesquereux, 1854, (Sphenopteris decipiens,) Bost. Jour. Soc. Nat. Hist., vol. 6, p. 420, and Geo. Sur. Pa., vol. 2, p. 862, Coal Meas.

decurrens, Lesquereux, 1854, (Pecopteris decurrens,) Bost. Jour. Soc. Nat. Hist., vol. 6, p. 424, and Geo. Sur. Pa., vol. 2, p. 867 , Coal Meas.

denudata, Lesquereux, 1880, Coal Flora of Pa., p. 212, Coal Meas.

dimorpha, Lesquereux, 1880, Coal Flora of Pa., p. 201, Coal Meas.

glandulosa, Lesquereux, 1854, (Sphenopteris glandulosa,) Bost. Jour. Soc. Nat. Hist., vol. 6, p. 420, and Geo. Sur. Pa., vol. 2, p. 862 , Coal Meas.

hispida, Lesquereux, 1884, Coal Flora of Pa., p. 755 , Coal Meas.

hymenophylloides, Lesquereux, 1870 , (Alethopteris hymenophylloides,) Geo. Sur. Ill., vol. 4, p. 393, Coal Meas.

irregularis, Sternberg, 1833, (Sphenopteris irregularis,) Vers. Geog. Darst. Flor.d.Vorw., vol. 2, p. 68 , Coal Meas.

latifolia, Brongniart,' 1828, (Sphenopteris latifolia,) Hist. d. Veg. Foss., p. 205 , and Coal Flora of Pa., p. 215, Coal Meas.

macilenta, Lindley \& Hutton, 1835, Foss. Flora, vol. 2, pl. 151, and Coal Flora of Pa., p. 219, Coal Meas. mazonana, Lesquereux, 1870, (Alethopteris mazonana,) Geo. Sur. Ill., vol. 4, p. 391, Low. Coal Meas.

muricata, Brongniart, 1828, (Pecopteris muricata,) Hist. d. Veg. Foss., p. 352, and Coal Flora of Pa., p. 203, Coal Meas.

Frg. 66. ervosa, Brongniart, 1828, Pseudopecopd. Veg. Foss., p. 297, and nana. Coal Flora of Pa., p. 197, Coal Meas. newlerryi, Lesquereux, 1854, (Sphenopteris newberryi,) Bost. Jour. Soc. Nat. 
Hist., vol. 6, p. 420, and Geo. Sur. Pa., vol. 2, p. 862 , Coal Meas.

numunularia, Gutbier, 1842, Abdrucke u. Verst. d. Zwick. Schwarzk. u. Seiner. Umgebungen, p. 43, and Coal Flora of Pa., p. 752, Coal Meas.

obtusiloba, Brongniart, 1828, (Sphenopteris obtusiloba,) Hist. d. Veg. Foss., p. 204, and Coal Flora of Pu., p. 753, Coul Meas.

pluckeneti, Sclılotheim, 1830, (Filicites pluckeneti, ) Petrefaktenkunde, p. 410, and Coal Flora of Pa., p. 199, Coal Meas.

polyphylla, Lindley \& Hutton, 1835 , (Sphenopteris polyphylla,) Foss. Flora, vol. 2, pl: 147, and Coal Flora of Pa., p. 218, Coal Meas.

pusilla, Lesquereux, 1854, (I'ecopteris pusillı,) Bost. Jour. Soc. Nat. Iist., vol. 6, p. 424, and Geo. Sur. Pa., vol. 2, p. 866, Coal Meis.

sheaferi, Lesquercux, 1858, (Y'ecopteris sheaferi,) Catal. Potts. Ass'n, p. 11, and Coal Flora of $\mathbf{P a}$, p. 194, Cosl Meas.

sillimani, Brongniart, 1825, (l'ecopteris sillimani,) Hist. d. Veg. Foss., p. 353, and Coal Flora of P'a., p. 206, Coal Meas.

speciosa, Lesquereux, 18s0, Coal Flora of Pa., p. 216, Coal Meas.

spinulosi, Iesquereux, 1870, (Alethopteris spinulosa,) Geo. Sur. Ill., vol. 4, p. 396, Coal Meas.

subcrenulata, Lesquereux, 18s0, Coal Flora of Ha., p. 193, Coal Meas.

subnervosa, Roemer, F. A., 1860, Paleontographica, vol. 9, p. 192 , and Coal Florn of P'a., 1). 198, Coal Meas.

trifoliata, Artis, 1825, (Filicites trifoliatus,) A uted. Phytol., pl. 2, and Coal Flora of Pa., p. 217, Coal Meas.

virginiana, Meek, 1875, (Cyclopteris virginiana,) Bull. Phil. Soc. of Washington, p. 18, and Coal Flora of Pa., p. 217, Waverly Gr.

Psilopiyton, Dilwson, 1859, Quar. Jour. Geo. Soc., vol. 15, p. 478. [Ety. psilon, smooth; phyton, stem.] Stems dichotomous; young branches carinate; rhizomes cylindrical, villous or scaly; marked with round scars, points of attachment of cylindrical rootlets; leaves in spiral order, small or rudimentary, acicular, squarrose, open ; fructifications in small, naked sporanges, spindleshaped or clavate, axillary, or in pairs at the extremity of the branches. Type P. princeps.

cornutum, I,esquereux, 1877, Proc. Am. Phil. Soc., p. 165, Low. Held. Gr.

elegans, Dawson, 1862, Quar. Jour. Geo. Soc., vol. 18, p. 315, Devonian.

glabrum, Dawson, 1862, Quar. Jour. Geo. Soc., vol. 18, p. 315 , Devonian. gracillimum, see Dendrograptus gracillimus.

princeps, Dawson, 1859, Quar. Jour. Geo. Soc., vol. 15 , p. 479 , Upper Silurian and
Devonian. This is the oldest known plant in America. It is supposed to have grown in a marsh.

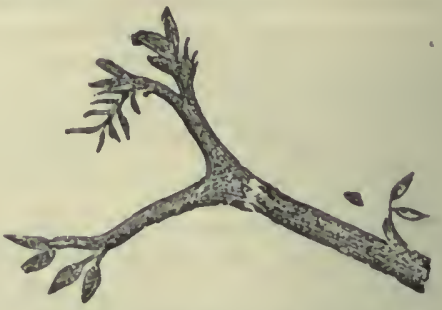

Fig. 67.-1'sllophytou prluceps.

princeps var. ornatım, Dawson, 1871, Foss. Plants, p. 38, Devonian.

robustium, Dawson, 1859, Quar. Jour. Geo. Soc., vol. 15,1$) .479$, Devonian.

Ptilocarpus, Lesquereux, 1870, Geo. Sur. III., vol. 4, p. 493, Syn. for Cardiocarpon.

bicornutus, see Cardiocarpon bicornutum. Ptilophyton, Dawson, 1878, Scottish Devonian Plants in Can. Nat., vol, 8. This is founded upon Lycopodites vanuxemi as the type, which is the same as P'umalina plumula, and falls therefore as a synonym.

gracile, sec Plumaliua grucilis.

lineare, Lesquereux, see Plumalina liuearis. plumula, see Lycopodites plumula.

vanuxemi, syn. for l'lumalina plumula.

Rila здоca mpus, (ioppert \& Berger, 1848, De Fruct. et Sem., p. 20. [H.ty. rhabdos, stria; karpos, fruit.] Seeds ovate or oblong, costate or striate, acute or acuminate, surrounded by a putamen sometimes deficient. Type R. tumicatus.

abnormalis, Lesquereux, 1884, Coal Flora of Pa., p. 818, Coal Meas.

acuminatus, Newberry, 1873, Ohio Pal., vol. 1, p. 378, Coal Meas.

amygdalilormis, Goppert \& Berger, 1848, de Fruct et Sem., p. 21, Coal Meas.

apiculatus, Newberry, 1873, Ohio Pal., vol. 1, p. 377, Coal Meas.

a rcuatus, Lesquereux, 1861, Geo. Sur. Ky., vol. 4, p. 434, Coal Mens. beinertianus, Goeppert \& Berger 1848 , De Fruct. et. Semin., p. 20, and Coal Flora of Pa., p. 844, Coal Meas. bockshianus, Goeppert \& Berger, 1848, De Fruct. et Semin. p. 21, and Coal Flora of $\mathrm{Pa}$. p. 844, Coal Meas. Fra. 68.-Rhabdocarpus carinatus, New-

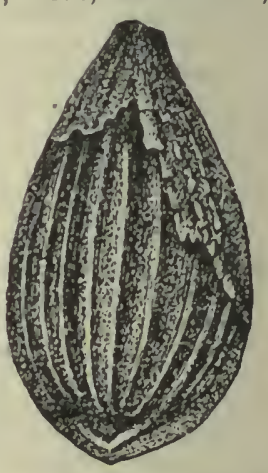
berry, 1873, Ohio Pal., vol. 1, p. 376, Coal Meas.

clavatus, Nternberg, 1820, (Carpolithes clavatus,) Vers. Darst. Flora der Vor- 
welt, and Coal Flora of Pa., p. 581, Coal Meas.

cornutus, Lesquereux, 1880, Coal Flora of Pa., p. 583, Coal Meas.

costatus, Newberry, 1873, Ohio Pal., vol. 1, p. 378, Coal Meas., syn. ? for R. acuminatus.

danai, Foster, 1854, Ann. of Sci., vol. 1, p. 129, and Ohio Pal., vol. 1, p. 376, Coal Meas.

emarginatus, Lesquereux, 1884, Coal Flora of Pa., p. 818, Coal Meas.

howardi, Lesquereux, 1880, Coal Flora of Pa., p. 575, Coal Meas.

inflatus, Lesquereux, 1884, Coal Flora of Pa., p. 815, Coal Mieas.

insignis, Dawson, 1863, Can. Nat. and Geo., vol. 8, and Acad. Geol., p. 478, Coal Meas.

insignis, Lesquereux, 1880, Coal Flora of Pa., p. 575. The name being preoccupied, it has been called $R$. lescurianus.

jacksonensis, Lesquereux, 1866, (Carpolithes jacksonensis,) Geo. Sur. Ill., vol. 2, p. 461, Low. Coal Meas.

lævis, Newberry, 1873, Ohio Pal., vol. 1, p. 377, Coal Meas.

laticostatus, Lesquereux, 1884, Coal Flora of Pa., p. 815 , Coal Meas.

lescurianus, S. A. Miller, 1883, 2d. Ed. Am. Pal. Foss., p. 256, Coal Meas. Proposed instead of $R$. insignis, Lesquereux, which was preoccupied.

mammillatus, Lesquerenx, 1870, Geo. Sur. Ill., vol. 4, p. 461, Coal Meas.

minutus, Lesquereux, 1860, Geo. Sur. Ark., vol. 2, p. 313, Coal Meas.

multistriatus, P'resl, 1833, (Carpolithes multistriatus,) in Steruberg's Flor. d. Vorw., vol. 2, p. 208, and Coal Flora of Pa., p. 578, Coal Meas.

oblongus, Fontaine \& White, 1880, Perm. or Up. Carb. Flora, p. 98, Coal Meas. or Permian.

pachytesta, Lesquereux, 1884, Coal Flora of Pa., $\mu .816$, Coal Meas.

platimarginatus, Lesquereux, 1860, (Carpolithes platimarginatus,) Geo. Sur. Ark., vol. 2, p. 312, Low. Coal Meas.

subglobosus, Lesquereux, 1884, Coal Flora of Pi., p. 817 , Coal Meas.

tenax, Lesquereux, 1884, Coal Flora of Pa. p, 818, Coal Meas.

venosus, Sternberg, as identified by Lesquereux, 1858, Gieo. Sur. Pa., vol. 2, p. 870 , Coal Meass. Not noticed in Coal Flora of $\mathrm{Pa}$., and probably not American.

Rhachiopteris, Dilwson, 1862, Quali. Jour. Geo. Soc., vol. 18, p. 323. [Ety. rachis, a stalk; pteris, fern.] Detached leaf-stalks of ferns; stipes half an inch wide or less; unevenly striate, giving off opposite branches, which are abruptly broken off at short distances from the stipe. Type R. pinnata.

affinis, Lesquereux, 1870, (Stigmarioides affinis,) Geo. Sur. Ill., vol. 4, p. 455, Coal. Meas. cyclopteroides, Dawson, 1862, Quar. Jour. Geo. Soc., vol. 18, p. 323, Catskill Gr. gigantea, Dawson, 1871, Foss. Plants Can., p. 57, Ham. Gr.

palmata, Dawson, 1871, Foss. Plants of Canada, p. 57, Ham. Gr.

pinnata, Dawson, 1862, Quar. Jour. Geo. Soc., vol. 18, p. 323, Catskill Gr.

punctata, Dawson, 1862, Quar. Jour. Geo. Soc., vol. 18, p. 323 , Catskill Gr.

selago, Lesquereux, 1870, (Stigmarioides selago,) Geo. Sur. Ill., vol. 4, p. 456, Coal Meas.

squamosa, Lesquereux, 1884, Coal Flora of Pa., p. 838, Coal Meas.

striata, Dawson, 1862, Quar. Jour. Geo. Soc., vol. 18 , p. 323 , Chemung Gr.

tenuistriata, Diwsen, 1862, Quar. Jour. Geo. Soc., vol. 18, p. 323, Ham. Gr.

Rhacophyllum, Schimper, 1869, Palæontologie Vegetale, vol. 1, p. 684. [Ety. rakos, rugged; phyllon, leal.] Fronds either flabelliform, many times subdivided or pinnate, irregularly pinnatifid, bipinnatifid; rachis flat, often much dilated, scarcely thicker than the foliaceous lamina, which is very variable in the size and the mode of its divisions; veins numerous, more or less indistinct, following the rachis in parallel bundles, dichotomous in the ioliaceous divisions. Type, R. flabellatum.

adnascens, Lindley \& Hutton, 1835, (Scbizopteris adnascens, ) Foss. Flora, vol. 2, p. 57, and Coal Flora of Pa., p. 321, Coal Ieas.

affine, Lesquereux, 1858, (P'achyphyllum affine,) Geo. Sur. Pa., vol. 2, p. 863, Coal Meas.

arborescens, Lesquereux, 1870, (Hymenophyllites arborescens,) Geo. Sur. Ill., vol. 4, p. 415, Coal Meas.

browni, Dawson, 1861, (Cyclopteris browni, Quar. Jour. Geo Soc., vol. 17, p. 32, Portage Gr.

clarki, Lesquereux, 1866, (Hymenophyllites clarki,) Geo. Sur. Ill., vol. 2, p. 438, Coal Meas.

corallinum, Lesquereux, 1880, Coal Flora of Pa., p. 317, Coal Meas. Misspelled corralum in the text.

cornutum, Lesquereux, 1880, Coal Flora of Pa., p. 317, Coal Meas.

expansum, Lesquer ux, 1880, Coal Flora of Pa., p. 313, Coal Meas.

filiciforme, Gutbier, 1842, (Fucoides filiciformis,) Abdr. u. Verst. d. /wwick. Schwarzk. u. sein. Umg., p. 11, and Coal Flora of Pa., p. 316, Coal Meas.

filiforme, Gutbier, 1842, (Fucoides filiformis,) Abdr. u. Verst. d. Zwwick. Schwarzk. u. sein. Umg., p. 12, and Coal Flora of Pa., p. 838, Coal Meas.

fimbriatum, Lesquerenx, 1858, (Pachyphyllum fimbriatum, ) Geo. Sur. Pa., vol. 2 , p. 863 , Coal Meas.

flabellatum, Sternberg, 1833, (Aphlebia flabellata,) Flor. d. Vorw., vol. 2, p. 11.2, and Coal Flora of Pa., p. 311, Coal Meas. 
fucoideum, Lesquereux, 1880, Coal Flora of Pa., p. 325, Coal Meas.

haınulosum, Lesquereix, 1880, Coal Flora of Pal., p. 321, Coal Meas.

hirsutum, Lesquereux, 1858, (PachyphylJum hirsutum,) Geo. Sur. Pa., vol. 2, p. 863, Coal Meas.

inflatum, Lesquereux, 1870, (Hymenophyllites inflatus,) Geo. Sur. Ill., vol. 4, p. 414, Coal Meas.

irregulare, Germar, 1844, (Aphlebia irregularis,) Verst. d. Steink. v. Wettin u. Löbejün, p. 57, and Coal Flora of Pa., p. 326, Coal Meas.

laceratum, Lesquereux, 1858, ( P'achyphy]lum laceratum, Geo. Sur. P'a., vol. 2, p. 863, Coal Meas.

laciniatum, Fontaine of White, 1880, Perm. or Up. Carb. Flora, p. 94, Coal Meas, or Permian.

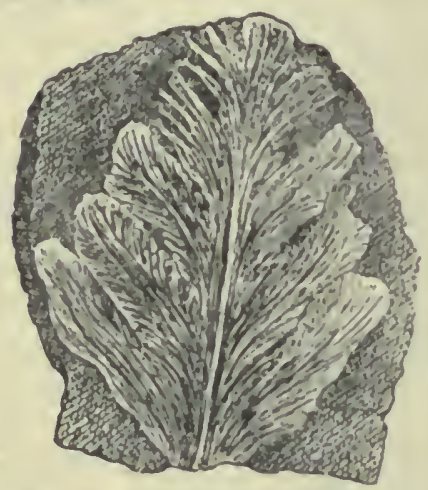

Fic. 69.- Rhacophyllum lactues.

lactuca, Sternberg, 1833, (Schizopteris lactuca, Flor. d. Vorw. vol. 2, p. 112, and Coal Flora of P'a., p. 315, Coal Meas.

membranaceum, Lesquereux, 1880, Coal Flora of $\mathrm{Pa} .$, p. 312 , Coal Meas.

molle, Lesquereux, 1870, (Hymenoplıyllites mollis,) Geo. Sur. Ill., vol. 4, p.418, Coal Meas.

scolopendrites, Lesquereux, 1\$58, (Scolopendrites dentatus,) Geo. Sur. Pa., vol. 2, p. 868 , Coal Meis.

spinosum, Lesquereux, 1880, Coal Flora of $\mathrm{Pa}$, p. 320, (Yoal Meas.

strongi, Lesquereux, 1870, (Hymenophyllites strongi,) Geo. Sur. Ill., vol. 4, p. 417, Coal Meas.

thalliforme, Lesquerenx, 1870, (Hymenophyllites tballiformis, Geo. Sur. Ill., vol. 4, p. 417, Coal Meas.

trichoideum. Iesquerenx, 1880, Coal Flora of Pa., p. 322, Coal Meas.

truncatun, Lesquereux, 1880, Coal Flora of Pal., p. 311, Coil Meas.

Rhizolithes, F. Braun, 1847, in Flora, etc. [Ety. rhiza, root; lithos, stone.]

palmatifidus, see Pinnularia palmatifidus.

Rhizomopteris, Schimper, 1869, Traits de Paleontologie Vegetale, vol. 1, p. 699. [Sig. the rlizomas of ferns.] Thisgenus, ss the name indicates, comprehends the rhizonas of ferns. Type, R. lycopodioides. Some of the species of Lycodites as L. uncinatus have been referred to it.

Rurzomorpira, Roth, as iclentified by Lesquereux, Coal Flora of Pa., p. 3. [Ety. rhiza, root; morpha, form.] Fungous filaments of hard substance, disposed in branches abnormally divided, and often anastomosing; generally living under the deenying bark of trees.

sigillarix, Iesquereux, 1877, Proc. Am. Phil. Soc., p. 174, and Coal Flora of $\mathrm{Pa}_{\text {., }}$ p. 3, Coal Meas.

Rotularia longifolia, see Sphenophyllum lon. gifolium.

Rusopirycus, Hall, 1852, Pal. N. Y., vol. 2, p. 23. [Ety. rusos, rugose; phykos, seaplant.] Simple or branehed steins, transversely wrinkled, and often possessing a central longitudinal depression. 'lype R. clavatum.

asperum, Miller \& Dyer, 1878, Jour. Cin. Soc. Nat. Ilist., vol. 1., p. 25, Utica Slate.

bilobatum, Vanuxem, 1842, (Fucoidles biInbatus, Geo. Rep. x. Y., p. 79, Hud. Riv. and Clintor, Gr.

clavatum, Hall, 1852, Pal. N. Y., vol. 2, p. 23, Clinton Gr. grenvillense, $\mathrm{Bi}$. lings, 186;, Pal. Foss., vol. 1, p. 101, Chazy Gr.

pudieum, Hall, 1852, Pal. N. Y., vol. ?, p. 24, Hud. Kiv and Clinton Gr.

subangulatun, Hall, 1852, Pal. N. Y., Fic. 70--Kusophyeus vol. 2, p. 23, clin- bilobatum. ton $\mathrm{Gr}$.

Sagenaria veltheimianu, see lepidodendron veltheimianum.

SAPORT NA, Fontaine \& White, 1880, Perm. or Up. Carb. Flora, p. 99. [Ety. proper name.] Leaves siuple, subreniform, flabellate or suborbicular, cuneate, bordered at the base with a woody rim, terminal margin incised; petiole long, slender, and grooved on the upper surface; nerves parting flabellately from the summit of the petiole and the woody bisal margin, all passing into the laminse; leaf substance thin. Type S. grandifolia.

grandifolia, Fontaine \& White, 1880, Perm. or Up. Carb. Flora, p. 101, Coal Mens. or Permian.

salisburioides, Fontaine \& White, 1880 P'erm. or U p. Carb. Flora, p. 102, Coal Meas. or Permian.

Schizopteris, Brongniart, 1828, Prodr. d. Hist. d. Veg. Foss., p. 63. [Lty. schizo, I cleave; pteris, fern.] Frond laciniate, 
or cut in linear erect or curved divisions, sometimes enlarged at the top, marked with thin parallel veins without branching, being split in fascicles with the divisions. Type S. anomala.

adnascens, see Rhacophyllum adnascens.

lacluca, see Rhacophyllum lactuca.

Schutzia, Gœppert, 1848, Permian Flora, p. 161. [Ety. proper name.] Stems either single or branching, bearing on short alternate pedicels small cones or strobiles of an ovate, truncate form, a compound of imbricate, broadly linear pointed scales, united at the base. Type S. anomala.

bracteata, see Cordaianthus bracteatus.

Scolopendrites, Lesquereux, 1858, Geo. Sur. Pa., vol. 2, p. 868. This name is abandoned.

dentatus, see Rhacophyllum scolopendrites.

Selaginites Brongniart, 1828, Prodr. d. Hist. d. Veg. Foss., p. 84. Stems dichotomous; leaves small, numerous, imbricated, sometimes enlarged at the base, scarcely leaving any visible scars. Type S. patens. The genus is regarded as synonymous with Lycopodites.

cavifolius, see Iycopodites cavifolius.

crassus, Lesquereux, 1866, Geo. Sur. Ill., vol. 2, p. 446, syn. for Lycopodites cavifolius.

formosus, Dawson, 1861, Can. Nat., vol. 6, p. 176. Not a plant, but a fragment of a crustacean.

uncinatus, see Lycopodites uncinatus.

Sigilla ria, Brongniart, 1822, Class. des Veg. Foss. in Mem. du Mus. d'Hist. Nat. de Paris, tom. 8, p. 203. [Ety. sigillum, a seal; from the seal-like scars of fallen leaves stamped upon the bark.] Trunks large, simple or dichotomous near the apex, marked by leaf-scars in vertical series, separated by furrows or placed in spiral order, either contiguous or more or less distant, very variable in size and shape, round, oval, truncate, or emarginate, hexagonal, transversely rhomboidal, witl three vascular scars, one simple, medial, punctiform, the two others lateral of semi-lunar or linear shape. Leaves linear, long, triplicate, carinate, or plane, with a distinct medial nerve. Type S. punctata.

acuminata, Newberry, 1874, Proc. Cleveland Acad. Sci., p. 164, and Coal Flora of Pa., p. 496, Coal Meas.

alternans, Sternberg, 1833, Flor. der Vorw., vol. 2 , p. 50, Coal Meas.

alveolaris, Sternberg, 1820, (Lepidodendron alveolare,) Essai d'un exposé Geognostico-botanique de la Flore du monde primitif, 1st Cahier, p. 25, Coal Meas.

angusta, Brongniart, 1828, Hist. d. Veg. Foss.; Coal Meas.

approximata, Fontaine \& White, 1880 , Perm. or Up. Carb. Flora, p. 96, Coal Meas. attenuata, Lesquereux, 1858, Catal. Potts. Foss., p. 17, and Coal Flora of Pa., p. 488, Coal Meas.

biercei, syn. for S. ichthyolepis.

brardi, Brongniart, 1822, Class. des Veg. Foss. tab. 1, fig. 5, and Coal Flora of Pa., p. 477, Coal Meas.

bretonensis, Dawson, 1865, Quar. Jour. Geo. Soc., vol. 20, p. 148, and Acad. Geol., p. 475, Coal Meas.

brochanti, Brongniart, 1828, Hist. d. Veg. Foss., p. 442, and Coal Flora of Pa., p. 842, Coal Meas.

brongniarti, Geinitz, 1855, Die Verst. d. Steink. form. Sachsen, p. 47, Coal Meas.

browni, Dawson, 1861, Quar. Jour. Geo. Soc., vol. 17, and Acad. Geol., p. 180, Coal Meas.

catenoides, Dawson, 1865, Quar. Jour. Geo. Soc.,

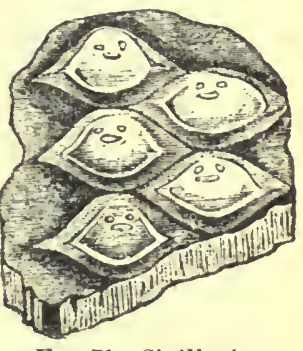

Fra. 71.-Siglllaria brardi. vol. 20, p. 147, and Acad. Geol., p. 474, Coal Meas.

catenulata, Lindley \& Hutton, 1831, Foss. Flora, vol. 1, p. 163, Coal Meas.

chemungensis, see Lepidodendron chemungense.

cisti, see Caulopteris cisti.

corrugata, Lesquereux, 1861, Geo. Sur. $\mathrm{Ky}$., vol. 4, p. 437 ; redefined 1870 , Geo. Sur. Ill., vol. 4, p. 445. Coal Meas.

cortei, Brongniart, 1828, Prodr. d. Hist. d. Veg. Foss., p. 64, and Coal Flora of Pa., p. 495, Coal Meas.

cuspidata, Brongniart, 1828, Prodr. d. Hist. d. Veg. Foss., p. 65, and Coal Flora of Pa., p. 486, Coal Meas.

cymatoides, Wood, 1860, Proc. Acad. Nat. Sci., vol. 12, p. 520, Coal Meas.

defrancii, Brongniart, 1828, Prodr. d. Hist. d. Veg. Foss., p. 66, Coal Meas.

dentata, Newberry, 1874, Proc. Cleveland Acad. Sci., p. 165, Coal Meas.

dilatata, Lesquereux, 1858, Geo. Sur. Pa., vol. 2, p. 871 , Coal Meas.

discoidea, Lesquereux, 1858, Geo. Sur. Pa., vol. 2, p. 873, Coal Meas.

dournaisi, Brongniart, 1828, Hist. d. Veg. Foss., p. 441, Coal Meas.

dubia, Lesquereux, 1858, Geo. Sur. Pa., vol. 2, p. 872 , syn. for S. cortei.

elegans, Sternberg, 1826, (Favularia elegans,) Tent. flor. primord., p. 14, Coal Meas.

elliptica, Brongniart, 1828, Hist. d. Veg. Foss., p. 447, and Coal Flora of Pa., p. 494, Coal Meas

elongata, Brongniart, 1822, Ann. des Sci. Nat., tom. 4, p. 23, Coal Meas.

eminens, Dawson, 1863, Can. Nat., vol. 8, and Acad. Geol. p. 475, Coal Meas. 
fissa, Lesquereux, 1858, Geo. Sur. Pa., vol. 2, p. 871 , Coal Meas.

flexuosa, Lindley \& Hutton, 1837, Foss.

Flora, vol. 3, p. 147, Coal Meas.

grandeuryi, Lesquereux, 1884, Coal Flora of Pa., p. 795, Coal Meas.

hexagona, Schlotheim, 1820, (Palmacites hexagonus,) Petrefaktenkunde, p. 394, and Coal Flora of Pa., p. 483, Coal Meas. ichthyolepis, Sternberg, 1833, Flora d. Vorw., vol. 2, p. 38, and Coal Flora of Pa., p. 482, Coal Meas.

intermedia, Brongniart, 1828, Hist. d. Veg. Foss., p. 474, Coal Meas.

knorri, Brongniart, 1828, Prodr. d. Hist. d. Veg. Foss., p. 65, Coal Meas.

lacoei, Lesquereux. 1880, Coal Flora of Pa., p. 499, Coal Meas.

læviga1a, Brongniart, 1828, Prodr. d. Hist. d. Veg. Foss., p. 64, and Coal Flora of Pa., p. 500, Coal Meas.

leioderma, Brongniart, 1828, Hist. d. Veg. Foss., p. 422, and Coal Flora of Pa., p. 476, Coal Meas.

lepidoclendrifolia, Brongniart, 1828 , Prodr. d. Hist. (l. Veg. Foss., p. 426, and Coal Flora of Pa., p. 477, Coal Meas.

leptoderma, Lesquereux, 1880, Coal Flora of Pu., r. 489, Coal Meas.

lescurii, Schimper, 1S69, 'Trait. de Paléontologie Vegetale, vol. 2, p. 85, Coal Meas. leveretti, Lesquereux, 1854, Coal Flora of Pa., p. 800, Coal Meas.

lorenzi, Lesquereux, 1880, Coal Flora of Pa., p. 473, Coal Meas.

lorwayana, Dawson, 1873, Rep. on Foss. Plants, p. 43 , Subcarboniferous.

mammillaris, Brongniart, 1\$28, Hist. d Veg. Foss., p. 451, and Coal Florn of Pa., p. 483, Coal Meas.

marginata, Lesquereux, 1850, Coal Flora of Pa., p. 498, Coal Jieas.

marineria, Hillreth, 1837, Am. Jour. Sci. and Arts, vol. 31, p. 30, L.ow. Coal Meas.

maseiliensis, Lesquereux, 1870, Geo. Sur. 11l., vol. 4, \%. 446, Coal Meas.

menardi, Brongniart. 1828. Hist. d. Veg. Foss., p. 430, and Coal Flora of Pa., p. 479, Coal Meas.

monostigma, Lesquereux, 1866, Geo. Sur. Ill., vol. 2, p, 449, Coal Meas.

notata, Steinhaur, 1818, (Phytolithus notatus,) Trans. Am. Phil. Assoc., vol. 1, p. 294, and Coal Flora of Pa., p. 486 , Coal Meas.

obliqua, Brongniart, 1828, Hist. Veg. Foss., p. 429, and Coal Flora of Pa., p. 470 , Coal Meas.

obovata, Lesquereux, 1858, Geo. Sur. Pa., vol. 2 , p. 872, Coal Meas.

oculata, Schlotheim, 1820, (Palmacites oculatus,) Petrefaktenkunde, p. 394, Coal Meas.

orbicularis, Brongniart, 1828, Prodr. d. Hist. d. Veg. Foss., p. 65, Coal Meas.

organum, Sternberg, 1820, (Syringodendron organum,) Flor. der Vorw., p. 23, and Lindley \& Hutton, 1831, Foss. Flora, Vol. 1, p. 199, Coal Meas. ornithicnoides, Wood, 1860, Proc. Acad. Nat. Sci., vol. 12, p. 238, und Trans. Am. Phil. Soc., vol. 13, p. 348, Coal Meas.

ovalis, Lesquereux. 18S0, Coal Flora of Pa., p. 495̄, Coal Meas.

oveni, see Didymophyllum oweni.

pachyderma, see Syringodendron pachy. derma.

palpebra, Dawson, 1862, Quar. Jour. Geo. Soc., vol. 18, p. 307, and Acad. Geol. p. 536, Devonian.

perplexa, Wood, 1866, Proc. Arad. Nat. Sci. Phil. vol. 12. p. 237, Coal Meas.

pittstonana, Lesquereux, 1850, Coal Flora of Pa., p. 493, Coal Meas.

planicosta, Dawson, 1863, Can. Nat. and Geo., vol. 8, and Acad. Geol., p. 474, Coal Meas.

polita, Lesquereux, 1858, Geo. Sur. Pa., vol. 2, p. 872, Coal Meus.

pulchra, Newberry, 1874, Proc. Cleveland Acad. Sci. p. 165, Coal Meas.

pyriformis, Brongniart, 1828, Prodr. d. Hist. d. Veg. Foss., p. 65, and Coal Flosa of Pa., p. 799, Cnul Meas.

reniformis, Brongniart, 1822, A nu. des Sei. Nut., t. 4, p. 32, and Conl Flora of Pa., p. 501, Coal Meas.

reticulata, Lesquereux, 1860, Geo. Sur. Ark., vol. 2, 1\% 310, Coal Mleas.

rugosn, Brongniart, 1828, Prndr. I ist. Veg. Foss., p. 64, and Coal lilora of Pa., p. 497, Coal Meas.

saulli, Brongniart, 182s, Hist. Veg. loss., vol. 1, p. 456, and Coal Flora of Pa., p. 842, Coal Mear.

schimperi, Jesumereux, 1858, Geo. Sur. I'a., vol. 2, p. 871, Coa] Me:s.

schlotheimana, Brongniat. 1828, Hist. Veg. Foss., p. 469, Conl Jeas. Ameriean Sp. (?)

sculuta, Lesquerenx, 1858, Geo. Sur. l'a., vol. 2, p. 871, Coal Mens. Syn. or S. obliqua?

scutellata, Brongniart, 1822, Class. des Veg. Foss., tab. 1, fig. 4. Coal Mleas.

semina, Lesquereux, 1870, Geo. Sur. 111., vol. 4. v. 463, Coal Meas.

serlii, Brongniart, 1828, Hist. l. Veg. Foss., p. 43i3, and Coal Flora of P'a., p. 480 , Crial Mreas.

sillimani, Brongniart, 1828, Hist. Veg. Foss., p. 459, and Coal Flora of Pa., p. 493, Coal Meas.

simplicitas, Vanuxem, 1843, Geo. Rep. 3d Dist. N. Y., p. 190, Catskill Gr.

solanus, Wood, 1860, Proc. Acad. Nat. Sci., Coal Meas. [Solanus in text; solenotus on plate; solena in Trans. Am. Phil. Soc., vol. 13.]

spinulosa, Germ., 1844, Vers. v. Wettin, etc., p. 58, Coal Meas.

stellata, Lesquereux, 1858, Geo. Sur. Pa., vol. 2, p. 871, Coal Meas.

striata, Dawson, 1863, Can. Nat. and Geol., vol. 8, and Quar. Jour. Geo. Soc., vol. 15, p. 147, Coal Meas.

sydenensis, Dawson, 1863, Can. Nat. and 
Geol., vol. 8, and Acad. Geol., p. 475, Coal Meas.

tessellata, Steinhaur, 1818, (Phytolithus tessellatus,) Trans. Am. Phil. Assoc., vol. 1, p. 295, and Coal Flora of Pa., p. 481, Coal Meas.

vanuxemi, Goppert, 1852, Die fossile Flora des Uebergangsgebirges, p. 546, and Coal Flora of Pa., p. 505, Coal Meas. venosa, Brongniart, 1828, Hist. d. Veg. Foss., p. 424, and Coal Flora of Pa., p. 842 , Coal Meas.

voltzi, Brongniart, 1828, Prodr. d. Hist. d. Veg. Foss., p. 65, and Coal Flora of Pa., p. 492, Coal Meas.

williamsi, Lesquereux, 1880, Coal Flora of Pa., p. 488, Coal Meas.

yardleyi, Lesquereux, 1858, Catal. Potts. Foss., p. 17, and Coal Flora of Pa., p. 491, Coal Meas.

Sigllla Rioides, Lesquereux, 1870, Geo. Sur. Ill., vol. 4, p. 449. [Ety. from its resemblance to the genus Sigillaria.] Fragments of roots bearing stigmariod leaves attached to sigillarioid rhomboidal scars. Type S. radicans.

radicans, Lesquereux, 1870, Geo. Sur. Ill., vol. 4, p. 449, Coal Meas.

stellaris, see Stigmaria stellaris.

Sigillariostronus, Lesquereux, 1884, Coal Flora of Pa., p. 794. [Ety. the genus, Sigillaria ; strobus, cone.] Sporanges attached in horizontal rows to a vertical axis, supported by persistent sporangiophores, with lanceolate scales, turned up and imbricate. Type S. laurencianus.

laurencianus, Lesquereux, 1884, Coal Flora of Pa., p. 794, Coal Meas.

Solenoula, Wood, 1860, Proc. Acad. Nat. Sci., vol. 12 , p. 238 . [Ety. solen, a channel; ou'os, entire.] Probably a decorticated Syringodendron. Type S. psilophlous. psilophloeus, WVood, 1860, Proc. Acad. Nat. Sci., p. 238, Coal Meas.

Sorocladid, Lesquereux, 1880, Coal Flora of Pa., p. 327. [Ety. soros, a heap: one of the fruit dots on the back of the frond; klado, I break in pieces.] $\mathrm{A}$ name proposed for fruiting fragments not well understood. Type S. stellatus.

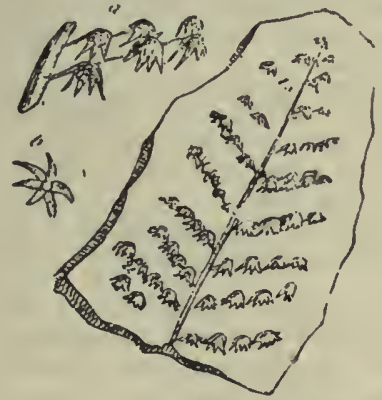

Fig. 72. - Sorocladus asteroides.

asteroides, Lesquereux, 1870, (Staphylopteris asteroides,) Geo. Sur. Ill., vol. 4, p. 406, Coal Meas. ophioglossoides, Lesquereux, 1850, Coal Flora of Pa., p. 329, Coal Meas.

sagittatus, Lesquereux, 1870, (Staphylopteris sagittatus,) Geo. Sur. Ill., vol. 4, p. 407, Coal Meas.

stellatus, Lesquereux, 1860, (Staphylopteris stellata,) Geo. Sur. Ark., vol. 2, p. 309, Coal Meas.

wortbeni, Lesquereux, 1870, (Staphylopteris wortheni,) Geo. Sur. Ill., vol. 4, p. 405, Coal Meas.

SPHenophyllum, Brongniart, 1828, Prodr. $d$. Hist. Veg. Foss., p. 68. [Ety. sphen, a wedge; phyllon, a leaf.] It was called Sphenophyllites by Brongniart in 1822. Plant herbaceous; stems articulate, inflated at the articulations, pinnately, bipinnately divided; leaves verticillate, sessile, wedge-form, with lateral borders entire, crenulate, dentate, or laciniate-lobate at the upper margin; medial nerve none; veins straight dichotomous; fructifications in cylindrical spikes, with bracts curved upward in a sharp flexure from near the base; sporanges globular in the axils of the bracts. Type S. schlotheimi.

angustifolium, Germar, 1844, Verst. d. Steink. v. Wett., u. Löbejün, and Coal Flora of Pa., p. 726, Coal Meas.

antiquum, Dawson, 1861, Can. Nat., vol. 6 , p. 170, Devonian.

bifurcatum, Lesquereux, 1860, Geo. Sur. Ark., vol. 2, p. 309, Coal Meas.

brevifolium, Newberry, not defined.

cornutum, Lesquereix, 1870, Geo. Sur. Ill., vol. 4, p. 421, Coal Meas.

densifoliatum, Foutaine \& White, 1880 , Perm. or Up. Carb. Flora, p. 37, Coal Meas. or Permian. Syn. (?) for S. angustifolium.

emarginatum, Brongniart, 1828, Prodr. d. Hist. Veg. Foss., p. 68, and Coal Flora of Pa., p. 53, Coal Meas.

erosum, Lindley \& Hutton, 1833, Foss. Flora, vol. 1, p. 43, and Coal Flora of Pa., p. 55. Coal Meas.

filiculme, Lesquereux, 1858, Geo. Rep. Pa., vol. 2, p. 853, Coal Meas.

fontainianum, S. A. Miller, 1883, 2d. Ed. Am. Pal. Foss., p. 258, Up. Coal Meas. Proposed instead of $\mathrm{S}$. latifolium, in Perm. or Up. Carb. Flora, p. 36, which was preoccupied.

latifolium, Wood, 1866, Trans. Am. Phil. Soc., vol. 13, p. 347, Coal Meas.

latifolium, Fontaine \& White, 1880. The name was preoccupied. See S. fontainianum.

longifolium, Germar, 1831, (Rotularia longifolia,) Isis, p. 426, and Coal Flora of Pa., p. 53, Coal Meas.

oblongifolium, Germar, 1844, Verst. d. Steink. v. Wett., u. Löbejün, p. 12, and Coal Flora of Pa., p. 57, Coal Meas.

primæerum, Lesquereux, 1877, Proc. Am. Phil. Soc., p. 167, Hud. Riv. Gr. I think this is not a plant. 
saxifragifolium, Sternberg, 1825, (Rotularia saxifragifolia,) Vers. Darst. Flora

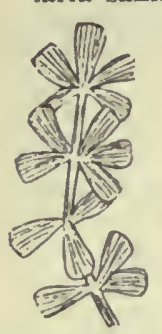

Fig. 73.

Sphenophyllum schlotheimt. der Vorwelt, and Coal Flora of Pa., p. 726, Coal Meas.

schlotheimi, Brongniart, 1828, Prodr. Hist. Veg. Foss., p. 68, and Coal Flora of Pa., p. 52, Coal Meas.

tenerrimum, Stur, 1877, Culm. Flora, p. 108, and Coal Flora of $\mathrm{Pa}$., p. 728, Coal Meas.

trifoliatum, Lesquereux, 185 S, Geo. Sur. Pa., vol. 2 , p. 853, Coal Meas.

Spilenol'teris, Brongniart, 1822, Mem. du Mus. d'Hist. Nat. de Paris, tom. 8, p. 203. [Ety. sphen, wedge; pteris, fern.] Fronds bi, tri, polypinnate; divisions open or in right angles; pinnules narrowed at base, often deeurring or cuneiform, pinnately lobed; lobes rarely entire, crenulate, dentate, or laciniate; primary nerve slender, alternately dichotomous, simple, branches entering the bas? of each lobe to pass by branchlets into the subdivisions of the lamina. Type S. elegans.

abbreviala, see P'seudopecopteris abbreviata.

acrocarpa, Fontaine \& White, 1880, Perm. or Up. Carb. Flora, p. 40, Coal Meas. or Permian.

acula, See Pseudopecopteris acuta.

adiantoides, Lindley \& Hutton, 1835, Foss. Flora, vol. 2, p. 91, Coal Meas.

alata, Brongniart, 1828, (Pecopteris alata, ) Hist. d. Veg. Foss., 1. 361, Coal Meas. alabamensis, see Oligocarpia alabamensis.

artemesiaefolia see Eremopteris artemesiifolia.

auriculata, Fontaine \& White, 1880 , Perm. or Up. Carb. Flora, p. 42, Coal Meas. or Permian.

ballantini, Andrews, 1875, (Hymenophyllites ballantini,) Ohio Pal., vol. 2, p. 422, Coal Meas.

brittsi, lesquereux, 1880, Coal Flora of Pal., p. 277, Coal Meas.

canadensis, Dawson, 1863, Can. Nat. and Geol., vol. 8, and Acad. Geol. p. 243, Coul Meas.

chierophylloides, Brongniart, 1828, (Pecopteris chærophylloides,) Hist. d. Veg. Foss., p. 357, and Coal Flora of l'a., p. 270, Coal Meas.

communis, Lesquereux, 1884, Coal Flora of Pa., p. 762, Coal Meas.

coriacea, Fontaine \& White, 1880, P'erm. or Up. Carb. Flora, p. 41, Coal Meas. or Permian.

crenata, Lindley \& Hutton, 1835, looss. Flora, vol. 2, pl. C., and Coal Flora of Pa., p. 835, Coal Meas.

cristata, Brongniart, 1828, (l'ecopteris cristata,) Hist. d. Veg. Foss.. p. 356, and Coal Flora of Pa., p. 273, Coal Meas.

davallana, Goppert, 1841, Gatt. d. Foss. Pflanzen, Cual Meas.

decipiens, see Pseudopecopteris decipiens. delicatula, see Hymenophyllites delicatulus.

dentara, Fontaine \& White, 18sio, P'erm. or Up. Carb. lilora, p. 42, Coal Meas. or Permian.

dilatala, as identified by Lesquereux. Syn. for Pseudopecopteris decipiens.

dissecta, Brongniart, 1528. Hist. $\mathrm{l}$. Veg. Foss., p. 183, and Coal Flora of l'a., p. 836, Cual Meas.

divaricata, Gopppert, 1836, (Cheilanthes divaricatus, ) Syst. Filic. Foss., p. 238, and Coal Flora of Pa., p. 767, Coal Mers.

dubuissoni, Brougniart, 1828, Hist. d. Veg. Finss., p. 195, and Coal Flora of Pa., p. 275, Coal Meas.

elequus, Broneniart, 1822, Class. d. Veg. Foss. pl. 2, fig. 2, and Coal Flora of Pa., p. 287, Coal Miens.

fascicularis, Roemer, 1866, heitr. in Paleont., vol. 9 , p. 179 , and Coal Flora of Pu., p. 837, Coal Meas.

flaccida, Crepin, 1874, Bull. Acall. Roy. of Belgium, p. 7 , and Coal Flora of P'a., p. 291, Coal Meas.

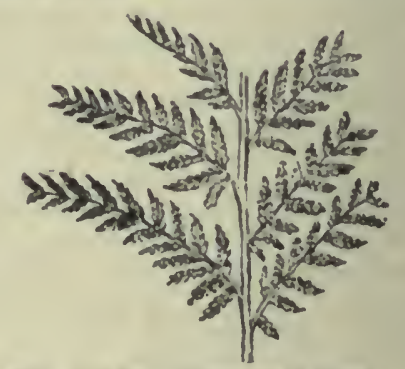

Fin. 74. - Splienopteris crenath.

flagellaris, see Oligocurpia flugellaris.

flexicaulis, Iesquereux, 1860, (Hymenophyllites flexicaulis,) Geo. Sur. Ark., vol. 2 , p. 309 , Coal Meas.

folinsa, Fontaine \& White, 1880, Perm. or Up. Carb. Flora, p. 44, Coal Meas. or Permian.

fnciformis, Lesquereux, 1884, Am. Naturalist, vol. 18, p. 921, Carboniferous.

fureata, Brongniart, 1828, Hist. d. Veg. Fos8., p..179, and Coal Flora of Pa., p. 282, Coal Meas.

gersdorfii, see IIymenophyllites gersdorfii. glandulosa, see Pseudopecopteris glandulosa.

goniopteroides, Lesquereux, 18s0, Coal Flora of $\mathrm{Pa}$., p. 269, Coal Meas.

gracilis, Brônguiart, 1828, Hist. d. Veq. Fnss., p. 197, and Coal Flora of Pia., p. 276, Coal Mras.

gravenhorsti, Brongniart, 1828, IIist. Veg. d. Fnss., p. 191, and Coal Flora of Pa., p. 274, Cual Meas. 
hartti, Dawson, 1862, Quar. Jour. Geol. Soc., vol. 18, p. 321, Devonian.

harveyi, Lesquereux, 1884, Coal Flora of Pa., p. 766, Coal Meas.

bastata, Fontaine \& White, 1880, Perm. or Up. Carb. Flora, p. 46, Coal Meas. or Permian.

hildrethi, Lesquereux, 1858, (Hymenophyllites hildrethi,) Geo. Sur. of $\mathrm{Pa}$., vol. 2, p. 863 , Coal Meas.

hitchcockana, Dawson, 1862, Quar. Jour. Geol. Soc., vol. 1S, p. 321, Devonian.

hœeninghausi, Brongniart, 1828, Hist. Veg. d. Foss., p. 199, and Coal Flora of $\mathrm{Pa}$., p. 288, Coal Meas.

hymenophylloides, Brongniart, 1828, Prodr. d. Hist. d. Veg. Foss., p. 51, and Coal Flora of Pa., p. 764, Coal Meas.

inæquilateralis, Lesquereux, 1884, Coal Flora of Pa., p. 765, Coal Meas.

intermedia, Lesquereux, 1858, Geo. Sur. Pa., vol. 2. The name was preoccupied in 1852 by Ettingshausen. It is now $\mathrm{S}$. mediana.

irregularis, see Pseudopecopteris irregularis.

larischii, Stur, 1877, (Calymmotheca larischii,) Culm Flora, p. 168, and Coal Flora of Pa., p. 288, Coal Meas.

latifolia, see Pseudopecopteris latifolia.

latior, Dawson, 1863, Can. Nat., vol. 8, and Acad. Geol., p. 483, Coal Meas.

laxa, Hall, 1843, Geo. Rep. 4th Dist. N. Y., Chemung Gr. This name was preoccupied by Sternberg. See A rchæopteris hallana.

lescuriana, Fontaine \& White, 1880, Perm. or Up. Carb. Flora, p. 44, Coal Meas. or Permian.

lesquereuxi, Newberry, 1858, Geo. Sur. Pa., vol. 2, p. 862 , Coal Meas.

linearis, Sternberg, 1820, Vers. Darst. Flor. d. Vorw., p. 15, Low. Coal Meas.

lyratifolia, see Pecopteris lyratifolia.

macilenta, see Pseudopecopteris macilenta.

marginata, Dawson, 1862, Quar. Jour. Geo. Soc., vol. 18, p. 321, Devonian.

mediana, Lesquereux, 1880, Coal Flora of Pa., p. 271, Coal Meas.

microcarpa, Lesquereux, 1880, Coal Flora of Pa., p. 280, Loal Meas.

microloba, Gœeppert, 1836, Syst. Filic. Foss., p. 238, Coal Meas.

minutisecta, Fontaine \& White, 1880, Perm. or Up. Carb. Flora, p. 43, Coal Meas. or Permian.

mixta, Schimper, 1869, -Traité de Paléontologie Vegetale, p. 382, and Coal Flora of Pa., p. 276, Coal Meas.

munda, Dawson, 1863, Can. Nat. and Geo., vol. 8, and Acad. Geol., p. 483, Coal Meas.

myriophylla, see Hymenophyllites myriophyllus.

newberryi, see Pseudopecopteris newberryi. obovata, Lindley \& Hutton, 1835, Foss. Flora, vol. 2, p. 75, and Coal Flora of Pa., p. 769, Coal Meas.

obtusiloba, see Pseudopecopteris obtusiloba.

pachynervis, Fontaine \& White, 1880, Perm. or Up. Carb. Flora, p. 46, Coal Meas. or Permian.

paupercula, Lesquereux, 1866, Geo. Sur. Ill., vol. 2, p. 435, Coal Meas.

pilosa, see Callipteris pilosa.

plicata, Lesquereux, 1858, Geo. Sur. Pa., vol. 2 , p. 862 , Coal Meas.

poluphylla, see Pseudopecopteris polyphylla.

pseudomurrayana, Lesquereux, 1880, Coal Flora of Pa., p. 271, Coal Méas.

pterota, Wood, 1866, Trans. Am. Phil. Soc. vol. 13, p. 348 , Coal Meas.

quercifolia, Gœeppert, 1836, Syst. Filic. Foss., p. 252, and Coal Flora of Pa., p. 286, Coal Meas.

recurva, Dawson, 1863, Quar. Jour. Geo. Soc., vol. 19 , p. 464 , Devonian.

rigida, Brongnif rt, 1828, Hist. Veg. Foss., p. 201, Coal Meas.

royi, Lesquereux, 1884, Coal Flora of Pa., p. 768, Coal Meas.

scaberrima, Lesquereux, 1870, Geo. Sur. Ill., vol. 4, p. 408, Coal Meas.

schlotheimi, see Hymenophyllites schlotheimi.

solida, Lesquereux, 1884, Coal Flora of Pa., p. 769, Coal Meas.

spinosa, Goppert, 1841, Gatt. Foss. Pflanzen, p. 70, aud Coal Flora of Pa., p. 281, Coal Meas.

splendens, Dawson, 1871, Foss. Plants Canada, p. 53, Devonian.

splendens, Lesquereux, 1870, (Hymenophyllites splendens,) Geo. Sur. Ill., vol. 4, p. 413, Coal Meas.

squamosa, Lesquereux, 1858, Gen. Sur. Pa., vol. 2, p. 862, Coal Meas.

subalata, Weiss, 1869, Foss. Flora d. jungst. Steink. form., p. 57, and Coal Flora of Pa., p. 272, Coal Meas.

tenella, Brongniart, 1828, Hist. Veg. Foss., p. 186, and Coal Flora of Pa., p. 836, Coal Meas.

tenuifolia, see Hymenophyllites tenuifolius.

tracyana, Lesquereux, 1884, Coal Flora of Pa., p. 766, Coal Meas.

trichomanoides, Brongniart, 1828, Hist. d. Veg. Foss., p. 182, and Coal Flora of Pa., p. 286, Coal Meas.

tridactylites, Brongniart, 1828, Hist. d. Veg. Foss. p. 181, and Coal Flora of Pa., p. 284, Coal Meas.

trifoliata, see. Pseudopecopteris trifoliata.

Sphenothallus, Hall, 1847, Pal. N. Y., vol. 1, p. 261. [Ety. sphen, a wedge ; thallos, a branch or frond.] Stem with diverging wedge-formed leaves, thickened, and sometimes subcoriaceous. T'ype S. angustifolius. 
angustifolius, Hall, 1847, Pal. N. Y., vol. 1, p. 261, Hud. Riv. Gr.

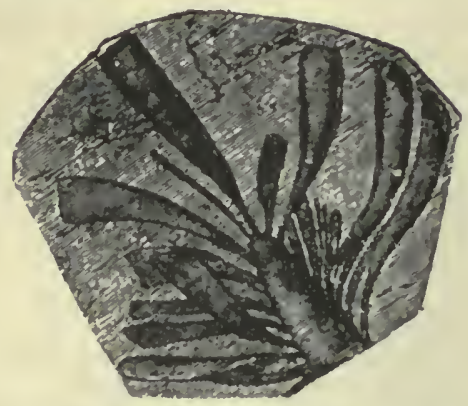

Fia. 75.--Sphenothallus angustifollus.

latifolius, Hall, 1s47, Pal. N. Y., vol. 1, p. 262, Hud. Riv. Gr.

Sprancium, Schimper, 1s74, Traiti de Paleontologie Vegetale, vol. 2, p.514. [lity. speira, that which is twisted; from the coiled marking arounil the pod.] Oblong or spindle-shaped bodies formed of narrow linear leaves, interwoven or twisted in spiral, with the ends united into a pedicel, which joins them horizontally or in umbels. Type S. carbonarium.

appendiculatum, Lesquerewx, 1870, (Palaeoxyris appeudiculata, ) Geo. Sur. III., vol. 4 , p. 465 , Coal Meas.

corrugatum, 1, squereux, 1870, (Palicoxyris corruguta, ) Geo. Sur. Ill., vol. 4, p. 466, Coal Meas.

intermedium, Lesquereux, 1840, Coal Flora of Pa., p. 521, Coal Mens.

multiplicatum, Lesquereux, 1sso, Coal Flora of Pa., 1. 520, Conl Meas.

prendeli, Lesquereux, 1870 , Geo. Sur. Ill., vol. 4, p. 464, Coal Mens.

Spirophyton, Hall, 1 1 ij3, 16th Rep. N. Y. St. Mus. Nat. Hist. p. 78. [Eiy. speira, a coil; phyton, a plant.] Syı. for Tronurus.

cauda-galli, see Taonurus caudagalli.

crassum, see Taonurus crassus.

typus, see Taonurus typus.

velum, see Taonurus velum.

Sporanoitrs, Dawsun, 1863 , Can. Nat. and Geol., vol. 8, and Proc. Geo. Soe. I.ond., vol. 15, p. 164. [Sig. seed-vessel.] Spores and spore-cases of Lepidodendron, Calamites, and similar plants, which can not be otherwise referred. Type S. papillatus.

acuminatus, Dawson, 1861, (Annularia acuminata, ) Can. Nat., vol. 6, and Acad. Geol., p. 540, Portage Gr.

bilobatus, Dawson, 1883, Proc. Am. Ass. Ad. Sci., vol. 32, p. 260, Marcellus Shale.

glaber, Dawson, 1863, Can. Nat., vol. 8, and Acad. Geol., p. 491, Coal Meas.

huronensis, Dawson, 1871, Am. Jour. Sci. and Arts, p. 257, Ham. Gr.

papillatus, Dawsou, 1863, Can. Nat., vol. 8, and Acad. Geol., p. 491, Coal Meas.
Sporocystis, Lesquereux, 1880, Coal Flora of Pa., p. 458. [Ety. sporos, seed; kustis, bladder.] Agglomerations of macrospliores grouped togethe or cohering or agglutinate by the borders, more gen. erally without cases, and therefore of uncertain reference. TypeS. planus.

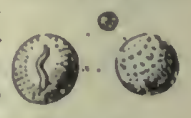

Fเง. วิง.

Sporangltes paplliatus. planus, Lesquereux, 1850, Conl Flora of Pr., p. 458, Coal Meas.

Staphylupteris, 1838, Presl, in Sternb. Vers. Darst. Flora der Vorwelt. [Ety. stuphyle, bunch of grapes; pleris, fern.] Not an American jalæozole genus.

asteroides, see Sorocladus usteroides. sagittata, see Soroeladus sngittatus. stellata, see Sorocladus stellatus. urrtheni, see Sorocladus wortlieni.

Sтеммлтоютеแs, Corda, 1845, Beiträge zur Flora der Vorwelt, p. Fit. [1ity. stemmatos, a wreath; pteris, fern.] Trunks, erect, cylindrical; sears large, disciform, oval, round, or ovate, not contiguous. disposed in fuincuncial or spiral order; outside borters or rings flat; internal disk formed by impressions of fascicles of vaseular tissnes, shaped like a horseshoe, the horns curving inwurd in the upper part of the sears, either short and looked, or descending below the middle of the scars, and there united. 'Types. peltigera.

aneeps, Lesquereux, 1884, Coal Flurn of Pa., p. 838, Coal Mers.

angustuta, Lesquereux, 1880, Coal Fiora of Pa., p. 339, Coal Меяs.

cyelostigna, Lesquerenx, 1880, Coal Flora of $\mathrm{Pa}, \mathrm{p} .341$, Coal Meas.

emarginata, Lesquereux, 1880, Coal Flora of Pu., p. 337, Coal Meas.

gigantea, Lesquereux, 1858, (Caulopteris gigantea,) Geo. of P'a., vol. 2, 1). 869, Coal Meas.

hirsuta, Lef(yuereux, 1880, Coal Flora of Pa., p. 337, Coal Meas.

insignis, Lesquerenx, 1870, (Caulopteris insignis,) Geo. Sur. III., vol. 4, p. 459,

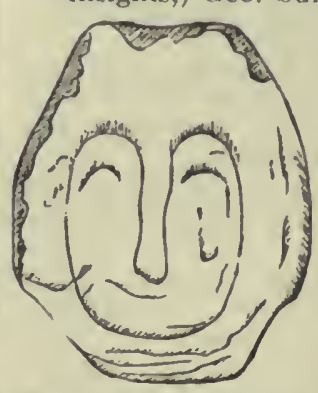

FIG. 77.-sternmatopteris minica, leaf scar. Coal Meas.

microstigma, Lesquereux, 1884, Coal Flora of I'u., I). 8:38, Coit Meas. mimica, Lespuereux, 1880, Ćal Flora of $\mathrm{P}_{\mathrm{z}}$., $\mathrm{p}$. 341 , Coal Meas. polita, Lestuereux, 1880, Ćal Flora of $I^{2} a, p$. 342 , Coal Meas. punctata, Lesquerenx, 1858,

(Caulopteris punctata,) Gro. Sur. Pa., vol. 2 , p. 869 , Cual Meas.

schimper, Lesquereux, 1880, Coal Flora of Y., p. 338, Coal Meas. 
squamosa, Lesquereux, 1880, Coal Flora of Pa., p. 339, Coal Meas.

wortheni, Lesquereux, 1866, (Caulopteris wortheni,) Geo. Sur. Ill., vol. 2, p. 459, Coal Meas.

Strernbergia, Artis, 1825, Antediluvian Phytology, p. ४. [Ety. proper name.] The pitlis of Dadoxylon, Sigillaria, and other plants usually preserved as casts in sandstone, retaining more or less perfectly the transverse partitions into which the pith cylinders were divided in the process of growth. Type $\mathrm{S}$. transversa.

transversa, Steinhaur, 1818, (Phytolithus transversus,) Trans. Am. Phil. Ass'n., vol. 1 , p. 295 , Coal Meas.

var. angularis, Dawson, 1865, Quar. Jour. Geo. Soc., vol. 22, p. 165, Coal Meas.

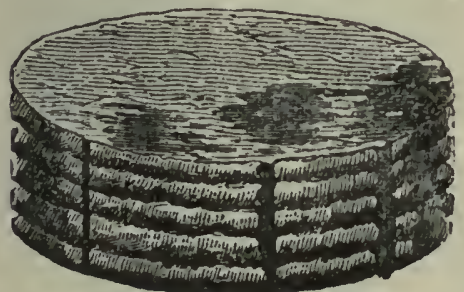

FIG. 78. Sterubergla augularis, pith of Dadoxylon.

var. approximata, Dawson, 1865, Quar. Jour. Geo. Soc., vol. 22, p. 165, Coal Meas.

var. distans, Dawson, 1865, Quar. Jour. Geo. Soc., vol. 22, p. 165, Coal Meas. var. obscura, Dawson, 1865, Quar. Jour. Geo. Soc., vol. 22, p. 165, Coal Meas. Stigmaria, Brongniart, 1822, Class. d. Veg. Foss. in Mem. du. Mus. d'Hist. Nat. d. Paris, tom. 8, p. 203. [Ety. stigma, a dot or puncture.] Floating stems or roots, generally growing horizontally, distantly dichotomous; branches long, scarcely variable in size in their whole length, subcylindrical or compressed; pith, a woody cylinder, often eccentrical, composed of fascicles of vessels disposed star-like ; leaves long, tubulose, linear when flattened, leaving after disruption, on the surface of the stems, round scars composed of two concentrical rings, with a central umbonate mammilla, pitted in the middle by a punctiform vascular scar. Type $\mathrm{S}$. ficoides.

amœna, Lesquereux, 1880, Coal Flora of Pa., p. 516, Coal Meas.

anabathra, Corda, 1845, Beiträge zur Flora der Vorwelt, p. 34, Coal Meas.

areolata, Dawson, 1871, Foss. Plants Canada, p. 23, Devonian.

costata, Lesquereux, 1858, Geo. Sur. Pa., vol. 2, p. 870 , Coal Meas.

elliptica, Lesquereux, 1870, Geo. Sur. 11l., vol. 4, p. 451 , Coal Meas.

eveni, see Stigmarioides eveni. exigua, Dawson, 1862, Quar. Jour. Geo. Soc., vol. 18, p. 308, Chemung Gr.

ficoides, Brogniart, 1822, Mem. du. Mus. d'Hist. Nat. de Paris, tom. 8, p. 203, Coal Meas.

ficoides var. a, b, c, d, e, f, g, h, i, k, l, Dawson, 1865, Quar. Jour. Geo. Soc., vol. 22, p. 148, Coal Meas.

ficoides var. reticulata, Gœppert, 1841, Gatt. d. Foss. Pflanzen, p. 13, Coal Meas.

ficoides var. stellata, Gœppert, 1841, Gatt. d. Foss. Pflanzen, p. 13, Coal Meas.

ficoides var. undulata, Gœppert, 1841 , Gatt. d. Foss. Pflanzen, p. 13, Coal Meas.

irregularis, Lesquereux, 1858, Geo. Sur. Pa., vol. 2, p. 870, Coal Meas. [Ety. from the irregularity of the scars.]

minor, Gœeppert, 1841, Gatt. d. Foss. Pflanzen, p. 13, Coal Meas.

minuta, Lesquereux, 1858, Geo. Sur. Pa., vol. 2, p. S71, Coal Meas.

minutissima, Dawson, 1871, Foss. Plauts Can., p. 23, Uevonian.

perlata, Dawson, 1871, Foss. Plants Canada, page 22, Devonian.

pusilla, Dawson, 1863, Quar. Jour. Geo. Soc., vol. 19 , p. 460 , Devonian.

radicans, Lesquereux, 1858, Geo. Sur. Pa., vol. 2 , p. 870 , Coal Meas.

stellaris, Lesquereıx, 1870, (Sigillarioides stellaris,) Geo. Sur. Ill., vol. 4, p. 450 , Coal Meas.

umbonata, Lesquereux, 1858, Geo. Sur. Pa., vol. 2, p. 870, Coal Meas.

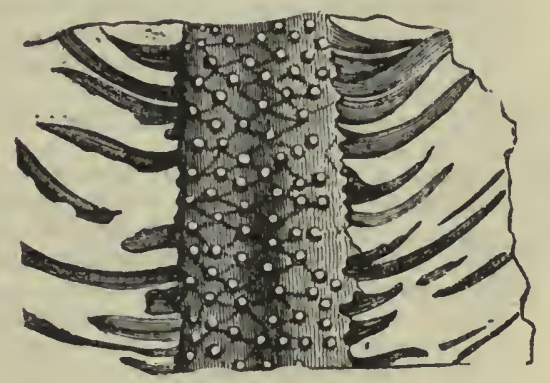

FIG. 79.-Stigmaria ficoides, 1/4 diam.

Stigmarioines, Lesquereux, 1870, Geo. Sur. Ill., vol. 4, p. 452. [Ety. from its resemblance to Stigmaria.] Fragments of rhizomas, with surface marked by small round impressions, irregularly disposed and without central vascular points, base of detached radicles or filaments. Type S. eveni.

affinis, see Rachiopteris affinis.

eveni, Lesquereux, 1866, (Stigmaria eveni,) Geo. Sur. Ill., vol. 4, p.448, Coal Meas.

linearis, Lesguereux, 1870, Geo. Sur. Ill., vul. 4, p. 455 , Coal Meas.

rugosus, Lesquereux, 1870, Geo. Sur. Ill., vol. 4, p. 470 , Coal Meas.

selago see Rachiopteris selago. 
truncatus, Lesquereux, 1870, Geo. Sur. Ill., vol. 4 , p. 453 , Coal Meas.

tuberosus, Lesquereux, 1870, Geo.Sur. Ill., vol. 4 , p. 453 , Coal Meas.

villosus, Lesquerenx, 1870, Geo. Sur. Ill., vol. 4, p. 454 , Coal Meas.

Strobilus caryophyllus, Hildreth, 1837, Am. Jour. Sei. and Arts, vol. 31 , p. 32, Coal Meas. Possibly a Stigmaria.

Syrungodendron, Sternberg, 1820, Essai d'un exposé Geognostico-botanique de la Flore du monde primitif, 1st Cahier, p. 26. [Ety. syrinx, a pipe; dendron tree.] Cortex costate; vaseular scars united in one; resembles decorticated stems of Sigillaria. Type S. pes capreoli.

bistriatum, Wood, 1860, Proc. Acad. Nat. Sei., vol. 12, p. 521, Coal Meas.

brongniarti, Geinitz, 1855,(Sigillaria brongniarti,) Verst. d. Steink form. in Sachsen, p. 47, and Coal Flora of l'a., p. 504, Coal Meas.

cyclostigma, Brongniart, 1828, Hist. d. Veg. Foss., p. 480, and Coal Flora of Pa., p. 505, Coal Meas.

gracile, Dawson, 1862, Quar. Jour. Geo. Soc., vol. 18 , p. 308 , Waverly Gr.

kirtlandium, Hildreth, 1837, Am. Jour. Sci. \& Arts, vol. 31, p. 29, Coal Meas.

magnificum, Wood, 1866, Trans. Am. Phil. Soc., vol. 13, p. 352, Coal Meas.

organum, see Sigillaria organum.

pachyderma, Brongniart, 1828, (Sigillaria pachyderma,) Prodr. d. Hist. d. Veg. Foss., p. 65, and Coal Flora of Pa., p. 503, Coal Meas.

peseapreoli, Sternberg, 1820, Essai d'un expose Geognostico-botanique de la Flure du monde primitif, 1st Cahier, p. 26, Coal Meas.

porteri, Lesquereux, 1870, Geo. Sur. Ill., vol. 4, p. 448, Coal Meas.

Syringoxylon, Dawson, 1862, Quar. Jour. Geo. Soc., vol. 18, p. 305. [Ety. syrinx, a pipe; xylon, wood.] Woody tissue close, thick-walled; ducts many times the diameter of the wood-cells, thin walled, with transverse pores in several series; medullary rays of two or more series of muriform cells; growth rings, distinct. Type S. mirabile.

mirabile, Dawson, 1862, Quar. Jour. Geo. Soc., vol. 18 , p. 305 , Ham. Gr.

Tantophylum, Lesquereux, 1878, I'roc. Am. PLil. Snc., p. 330. [Ety. tainia, ribbon; phyllon, leaf.] Stems large, leaves crowded, fistular, flat by compression, thick, exactly linear, decurring at the base ; surface smooth, opaque, or shining. Type $\mathrm{T}$. decurrens.

brevifolium, Lesquereux, 1880, Coal Flora of Pa., p. 788, Coal Meas.

contextum, Lesquerenx, 1878, Proc. Am. Phil. Soc., p. 332, Coal Meas.

decurrens, Lesquereux, 1878, Proc. Am. Phil. Soc., p. 331, and Coal Flora of Pa., p. 464, Coal Meas.

deflexum, Lesquereux, 1878, Proc. Am. Phil. Soc., p. 331, Coal Meas.
Teniopteris, Brongniart, 128, Prodr. Hist. d. Veg. Foss., p. 61. [Ety. tainia, ribbon; pteris, fern.] Fronds simple, large, linear; medial nerve canaliculate, strong; veins open, or in right angle, thin, forking a little above the base or more generally simple, parallel, sometimes joined to a marginal nerve. Ty pe T. vittata.

lescuriana, Fontaine \& White, 1850, Perm. or Up. Carb. Flora, p. 91, Coal IIeas. or Permian.

newberryana, lontaine \& White, 1880 Perm. or Up. Carb. Flora, p. 91, Coal Meas. or Permian.

smithi, Lesquereux, 1875, Geo. Rep. Ala., p. 78, and Coal Flora of Pa., p. 153, Coal Meas.

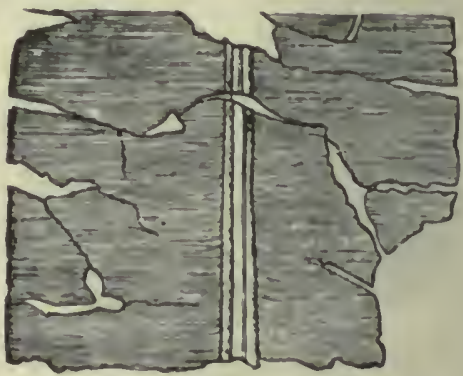

Fio. 80,-Trenlopterls smithl.

truncata, Lesquereux, 1884, Coal Flora of Pa., p. 743, Coal Mens.

Taonurus, Fisher-Ooster, 1858, Foss. Fucoiden d. Schweizer Alpen, p. 41. [Ety. taon, peacock; oura, tril.] Frond nembranaceous, derived from utricules attached to a lateral or central axis erected or twisted in spiral, flattened in various ways, ribbed; ribs or strixe curved, seythe-shaped, converging to the borders, which are either free, naked or attached on one side or all around to the axis or its branches. Type T. eaudagalli.

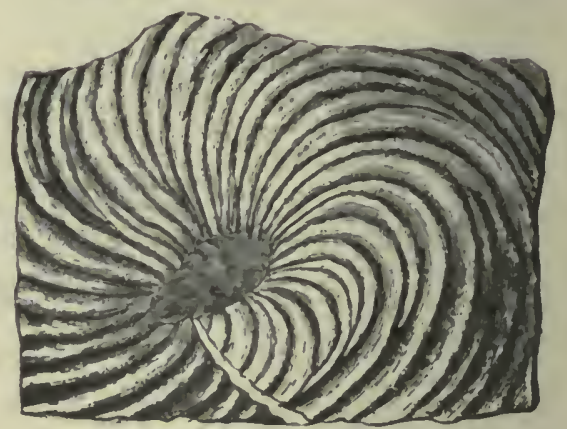

Fig.81.-Tnonurus crudugnll1.

archimedes, Ringueberg, 1884, (Spirophyton archimedes,) Proc. Acad. Nat. Sci. Phil., p. 144, Medina Gr.

caudagalli, Vanuxem, 1842, (Fucoides 
caudagalli,) Geo. Rep. 3d Dist. N. Y., p. 128, Devonian.

colletti, Lesqueruux, 1870, (Chondrites colletti,) Geo. Sur. Ill., vol. 4, p. 379 , Coal Meas.

crassus, Hall, 1863, (Spirophyton crassum,) 16 Rep. N. Y. St. Mus. Nat. Hist., p. 83 , Waverly Gr.

marginatus, Lesquereux, 1866, (Caulerpites marginatus,) Trans. Am. Phil. Soc., vol. 13, p. 314 , Subcarb.

retortus, Vanuxem, 1842, (Retort fucoid,) Geo. Rep. 3d Dist., N. Y., p. 176, Portage Gr.

typus, Hall, 1863, (Spirophyton typus,) 16 Rep. N. Y. St. Mus. Nat. Hist., p. 80, Ham. or Chemung Gr.

velum, Vanuxem, 1842, (Fucoides velum,) Geo. Rep. 3d Dist. N. Y., p. 176, Ham. Gr.

Trichomanites, Gœppert, 1836, Syst. Filic. Foss. [Ety. from the plant Trichomanes.] This genus is only known in America by fragments of slender pinnules attached to long petioles, which are of doubtful generic affinity.

filicula, Dawson, 1863, Quar. Jour. Geo. Soc., vol. 19, p. 464, Devonian.

Trichophycus, Miller \& Dyer, 1878, Jour. Cin. Soc. Nat. Hist., vol. 1, p. 24. [Ety. trichos, hair; phukos, sea-weed.] Simple branching stems having markings as if by the folding down of tilaments. Type T. lanosum.

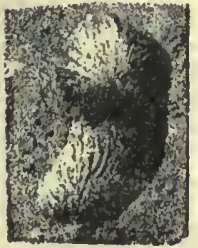

FrG. 82.-Tr1chophycus lanosum, Miller \& Dyer, 1878, Jour. Cin. Soc. Nat. Hist., vol. 1, p. 24, Hud. Riv. Gr.

sulcatum, Miller \& Dyer, 1878, Cont. to Pal., No. 2, p. 4, Hud. Riv. Gr. venosum, S. A. Miller, 1879, Jour. Cin. Soc. Nat. Hist., vol. 2, p. 112, Hud. Riv. Gr. karpos, fruit.] Fruits ovoid, compressed at the base point of insertion, three or six costate, the ribs more distinct and prominent toward the base, sometimes disappearing above; apex pitted by a small, round or triquetre mammillate cavity. 'I'ype T. parkinsoni.

adamsi, Lesquereux, 1884, Coal Flora of Pa., p. 820, Coal Meas.

ampulliforme, Lesquereux, 1884, Coal Flora of Pa., p. 823, Coal Meas.

avellanum, Dawson, 1863, Can. Nat. and Geo., vol. 8, and Acad. Geol., p. 478, Coal Meas.

bertholletiforme, Foster, 1853, Ann. of of Sci., vol. 1, and Ohio Pal., vol. 1, p. 369, Coal Meas.

carbonarium, King, 1854, Proc. Acad. Nat. Sci., vol. 7,.p. 66, Coal Meas.

dawsi, Lindley \& Hutton, 1837, Foss. Flora, vol. 3, p. 321, and Coal Flora of Pa., p. 586, Coal Meas. giffordi, Lesquereux, 1880, Coal Flora of Pa., p. 592, Coal Meas.

grande, Lesquereux, 1884, Coa] Flora of Pa., p. 821 , Coal Meas.

hildrethi, Lesquereux, 1858, Geo. Sur. Pa., vol. 2, p. 877, Coal Meas.

hildrethi, Dawson, syn. (?) for Trigonocarpon triloculare.

hookeri, Dawson, 1861, Quar. Jour. Geol. Soc., vol. 17, p. 525, Coal Meas.

intermedium, Dawson, 1863, Can. Nat. vol. 8, and Acad. Geol., p. 478, Coal Meas.

juglans, Lesquereux, 1866, Geo. Sur. Ill., vol. 2, p. 460, Low. Coal Meas.

kansaseanum, Lesquereux, 1884, Coal Flora of Pa., p. 822, Coal Meas.

magnum, Newberry, 1873, Ohio Pal., vol. 1, p. 369 , Coal Meas.

mentzelianum, Gœppert. \& Berger, 1848, De Fruct. et. Sem., p. 19, and Coal Flora of $\mathrm{Pa}$. . p. 590, Coal Meas.

minus, Dawson, 1863, Can. Nat. and Geol., vol. 8, and Acad. Geol., p. 478, Coal Meas.

multicarinatum, Newberry, 1873, Ohio Pal., vol. 1, p. 478, Carb. Conglomerate.

multistriatum, Lesquereux, 1884, Coal Flora of Pa., p. 823, Coal Meas.

noggerathi, Sternberg, 1820, (Palmacites noeggerathi,) Flor. d. Vorw., p. 55, and Coal Flora of Pa., p. 584, Coal Meas. oblongum, Lindley \& Hutton, 1837, Foss. Flora, vol. 3, p. 193, Coal Meas.

oliviforme, Lindley \& Hutton, 1837, Foss. Flora, vol. 3, p. 222, and Coal Flora of Pa., p. 590, Coal Meas.

ornatum, Newberry, 1873 , Ohio Pal., vol. 1, p. 368, Carb. Conglomerate.

parkinsoni, Brongniart, 1828, Prodr. Hist. Veg. Foss., p. 137, and Coal Flora of Pa., p. 589, Coal Meas.

perantiquum, Dawson, 1871 , Foss. Plants Canada, p. 62, Devonian.

perpusillum, Lesquere $\mathrm{u}$, Trigonocar1884, Coal Flora of Pa., p. oliviforme. 820 , Coal Meas.

racenosum, Dawson, 1862, Quar. Jour. Geo. Soc., vol. 18, p. 324, Devonian.

rostellatum, Lesquereux, 1866, Geo. Sur. Ill., vol. 2, p. 460, Up. Coal Meas.

rotundum, Dawson, 1863, Can. Nat., vol. 8, and Acad. Geol., p. 478, Coal Meas. saffordi, Lesquereux, 1880, Coal Flora of Pa., p. 587, Coal Meas.

schultzanum, Gœppert \& Berger, 1848, De Fruct., etc., p. 19, and Coal Flora of Pa., p. 819, Coal Meas.

sigillarix, Dawson, 1863. Can. Nat., vol. 8, and Acad. Geol., p. 477, Coal Meas.

tricuspidatum, Newberry, 1873, Ohio Pal., vol. 1, p. 368, Coal Meas.

triloculare, Hildreth, 1837, (Carpolitbes trilocularis,) Am. Jour. Sci., vol. 31, p. 29, Conglomerate and Low. Coal Meas. 
woodruffi, Moss, 1852, Proc. Acad. Nat. Sci., vol. 5, Coal Meas.

Triphyllopteris, Schimper, 1874, Traite de Paléontologie Vegetale, vol. 2, p. 40. [Ety. tria, three; phyllon, a leaf ; pteris, a fern.] Lower pinnules subopposite, tripartite or trifoliate, upper ones simple, all narrowed or contracted to a flat, slightly decurring pedicel; veins all equal, simple or dichotomous, diverging fan-like. Type T. lescuriana.

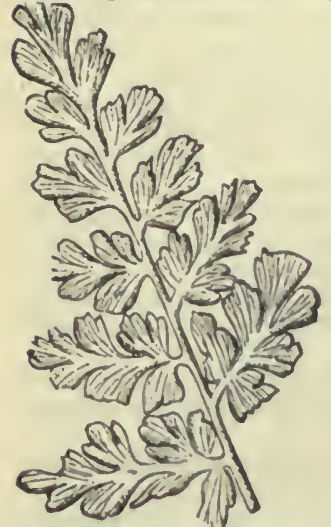

FIG. 81.-Tripliyllopteris chenthami. cheathami, Lesquereux, 1884, 13th Rep. Geo. Sur. Ind., p. 70, Coal Meas.

lescuriana, Meek, 1875, (Cyclop)teris lescuriana,) Bull. Phil. Soc. Wash., p. 16. and Coal Flora of Pa., $p$. 297, Coal Meas. Trochophyllum, Wood, 1860, Proc. Acad. Nat. Sci. This name was proposed as a substitute for $A n$ nularia,Sternb. because the latter was preoccupied as a generic name in the subkingdom Mollusca; but Trochophyllum was preoccupied for a genus of fossil corals by Edwards of Haine, in 1851.

clavatum, see Annularia clavata.

lineare, see Plumalina linearis.

Uiodendron, Rlıode, 1823, Beitrüge z. Pllanz. d. Vorwelt. [Ety. ule, wood; dendron, tree.] Arborescent; rarely branching; bearing in two opposite rows round or oval scars, im. pressions of the base of strob. iles, marked with concen. trical scales and a central mam. milla; leaves short lanceolate, leaf scars disposed in spiral, small, rhom. boidal or subrhomboidal; fructifications in long, cylindrical strobiles. Type U. majus. c o m m u t at u m. Schimper, 1874, Pal. Veg., vol. 2, p. 40 , Coal Meas.

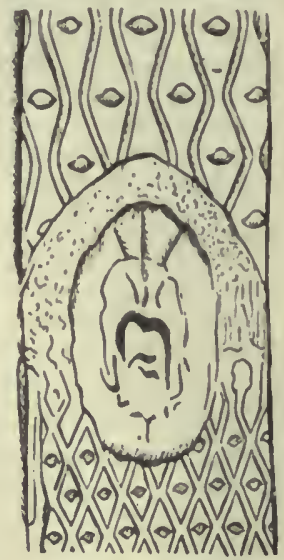

FrG. 85.-Ulodendron elongatum. ellipticum, Sternberg, 1838, Vers. Darst. Flora der Vorwelt, vol. 2, p. 186, and Coal Flora of Pa., p. 405, Coal Meas. elongatum, Lesquereux, 1870, Geo. Sur. Ill., vol. 4, p. 437 , Coal Meas.

flexuosum, see Halonia flexuosa.

lindleyanum, Presl, 1833, in Sternberg. Vers. Darst. Flora der Vorwelt, p. 185, Coal Meas. majum, Rhode, 1823 , Beitr. z. l'flanz. d. Vorw., pl. 3, fig. 1, and Coal Flora of Pa., p. 401, Coal Meas.

minus, Lindley \& Hutton, 1831, Foss. Flora, vol. 1, p. 6, Coal Meas. punctatuin, Lindley \& Hutton, 1833, (Bothrodendron punctatum, ) Foss. Flora, vol. 2, p. 80, aud Coal Flora of Pa., p. 405, Coal Meas.

Volkmania, Sternberg, 1823, Tent. Flor.

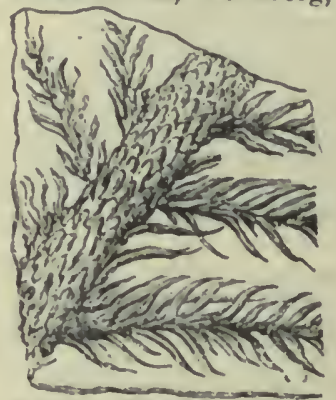

Fug, 87.-Wrichla p!nn!tormls.

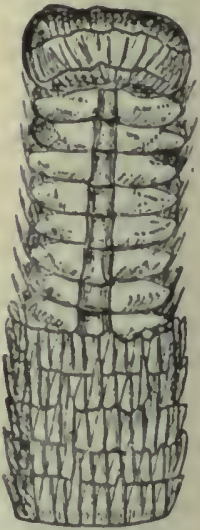

lis. 86.-VolkPrimerd. Flor. [Ety. proper namie.] Stems striated, articulated, and the in fl or escence spiked. Closely related to As to rophyllites. Type V. polystachya.

crassil, Lesquere ux, 1884 Coal Flora of Pa., p. 719, Coal Meas. fertilis, Lesquereux, 1884, Coal Flora of Pa., p. 720, Coal Meas.

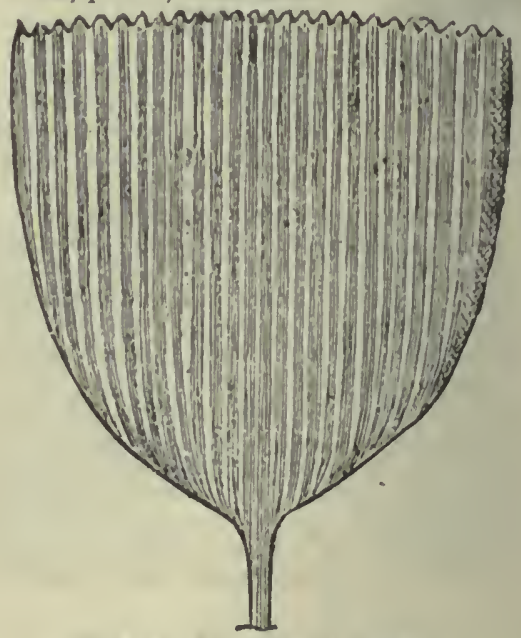

FIG. 88.-Whlttleseya elegans.

prælonga, Lesquereux, 1880, (Calainostachys prolongus,) Coal Flora of l'a., p. 59, Coal Meas. 
Walchia, Sternberg, 1825, Vers. Darst. Flora der Vorwelt, p. 22. [Ety. proper name.] Arborescent, like Araucaria; branches with feathery foliage; strobiles oblong, cylindrical, or elongated, with ovate scales, sharp or lanceolate; seed minute, ovate. Type W. pinniformis.

gracilis, Dawson, 1863, (Araucarites gracili s,) Can. Nat., vol. 8, and Acad. Geol. p. 474 , Coal Meas.

robusta, Dawson, 1871, Rep. on Prince Edward Island, p. 43, Coal Meas.
Whittleseya, Newberry, 1874, Proc. Cleveland Acad. Sci., p. 43. [Ety. proper name.] Frond simple or pinnate, nerves fasciculate, confluent to the base, not dichotomous. Type W. elegans.

elegans, Newberry, 1874, Proc. Cleveland Acad. Sci., p. 43, Coal Meas.

integrifolia, Lesquereux, 1880, Coal Flora, of Pa., p. 524, Coal Meas.

microphylla, Lesquereux, 1884, Coal Flora of Pa., p. 843, Coal Meas.

undulata, Lesquereux, 1880, Coal Flora of Pa., p. 525, Coal Meas.

\section{AnIMAL KingDOM.}

The Animal Kingdom is divided into seven Subkingdoms, viz.: Protozoa or Protista, Coelenterata, Echinodermata, Molluscoidea, Mollusca, Articulata, and Vertebrata. ?

\section{SUBKINGDOM PROTOZOA.}

\section{(protos, first ; zoon, animal).}

The Palæozoic Protozoa are included in two Classes, viz.: Rhizopoda and Porifera.

\section{CLASS RHIZOPODA (riza, root; pous, foot).}

The Rhizopoda are the simplest and lowest forms of animal life. They are generally microscopic, though some of them are more or less conspicuous to the naked eye. They abound in fresh-water ponds, where each consists of a shapeless mass, constantly changing its form, and shooting out and withdrawing finger-like processes, but visible only under the magnifying power of a microscope. They occur in marshes, ponds, lakes, and seas, and wherever dampness existś, from the greatest depths to the snow-line of the mountains. The greater portion are marine, and have tiny shells that enter into the composition of the ocean mud, and abound in the sands of every ocean shore. The simplest kinds are not provided with a shell or investing membrane, but consist of a fluid, viscid, albuminoid jelly, having an extensile and contractile power, which is regarded as the elementary basis of organic bodies in general. This jelly is called protoplasm (protos, first; plasso, I mold), and resembles in motive power the flesh of higher animals, from which character it is called sarcode (sarx, flesh; eidos, form). The protoplasm has no fixed organs of any kind, internal or external. Dr. Carpenter, speaking of the Rhizopoda, says:

"If the views which $I$ have expressed as to the nature and relations of their living substance be correct, that substance does not present any such differentiation as is necessary to constitute what is commonly understood as 'organization' even 
of the lowest degree and simplest kind; so that the physiologist has here a case in which those vital operations which he is accustomed to see carried on by an elaborate apparatus are performed without any special instruments whatever-a little particle of apparently homogeneous jelly changing itself into a greater variety of form than the fabled Proteus, laying hold of its food without members, swallowing it without a mouth, digesting it without a stomach, appropriating its nutritious material without absorbent vessels or a circulating system, moving from place to place without muscles, feeling (if it bas any power to do so) without nerves, propagating itself without genital apparatus; and not only this, but in many instances forming shelly coverings of a symmetry and complexity not surpassed by those of any testaceous animals."

The fresh-water, shapeless, gelatinous mass is called the Amoba, and it shows a voracious disposition by seizing upon minute substances and appropriating them to the nutrition of its own jelly. This it does by surrounding and inclosing the food supply, which is retained until it is dissolved or the desired part appropriated.

The lowest Order of Rhizopoda has received the name of Monera (moneres, simple), of which Prof. Haeckel says:

"In a state of rest most Monera appear as small globules of slime, invisilble, or barely visible, to the naked eye, and at most about the size of a pin-head. When the Moner moves, there are prodnced on the surface of the little slime-ball fingerlike processes, or very fine radiating threads, the so-called false feet, or pseudopods. The latter are simple continuous processes of the structureless, albumen-like mass of which the body consists. We are unable to perceive different parts in it, and we can obtain direct proof of the absolute simplicity of the semi-fluid mass of albumen; for, with the aid of the microscope, we can follow the Moner as it receives its nourishment. When minute bodies suitable for food, as, for instance, small particles of decayed organic bodies or microscopic plants and infusoria, accidentally . come into contact with the Mouer, they remain hanging to the sticky surface of the semi-fluid mass of slime, and here produce an irritation, which is followed by a strong afflux from the slimy mass of the body, and they become finally completely inclosed by it, or they are drawn into the body of the Moner by displacement of the several albuminous particles, and there digested, being absorbed by simple diffusion (endosmosis).

"Just as simple as is the nourishment is the mode of reproduction of these primitive beings, which one can not positively call animal or plant. All Monera propagate themselves only in an asexual manner by self-division. When such a speck-for example, a Protamœba or a Protogenes-has attained a certain size by the assimilation of foreign albuminous matter, it falls into two pieces; there is formed a constriction around the middle, which finally leads to the separation of the two halves. Each half becomes rounded, and then appears as an independent individual, which commences anew the simple play of the vital phenomena of nutrition and propagation. In other Monera (Vampyrella) the body, in the process of propagation, instead of two, falls into four equal parts; and in others again (Protomonas, Protomyxa, Myxastrum), at once into a large number of small globules of slime, each of which again, by simple growth, becomes like the parent body."

The marine Rhizopoda are usually furnished with a horny shell, and live, singly or socially, in shells having a series of chambers. The Sub-class Monothalamia 
(monos, one; thalamos, chamber), includes those Rhizopoda which are inclosed in a single shell, and have a minute opening for the extrusion of the filamentous processes by which motion is effected. The Sub-class Polythalamia includes those having calcareous shells, consisting of a series of distinct chambers, which sometimes communicate with each other, and at other times appear to be completely closed up. Each chamber is supposed to contain an independent animal, though the individual animals may be so connected, through the openings communicating between the cells, as to constitute a common mass. In some genera each chamber presents only a single external opening, but in most genera the substance of the shell is pierced by minute pores, like a sieve, through which delicate filaments. are protruded.

The Order Radiolaria (radiolus, a litle ray,) includes many beautiful forms, living and swimming in vast multitudes near the surface of the ocean. Most of them have a complex silicious skeletou of great beauty of form and symmetry, and after death the skeletons sink to the bottom of the ocean, where they often furnish the chief part of the mud. On the island of Barbadoes, Tertiary strata 1,100 feet in thickness, consisting of marls, tripoli, and ferruginous sandstone, are largely composed of the silicious skeletons of Radiolaria. The Nicobar Islands of the Indian Archipelago, consisting of clays, marls, and arenaceous marls, to the extent of 2,000 feet in thickness of Tertiary age, are largely composed of the remains of this Order.

The Order Foraminifera (foramen, an aperture; fero, I bear,) includes all the families of Palæozoic Rhizopoda noticed in this work. They are marine shellbearing animals, living at the bottom of oceans and seas, attached, free, or pelagic, and swimming on the surface of the water, from whence their dead shells form an incessant rain to the bottom of the ocean. They are generally microscopic, though a few are several inches in diameter. Some extinct genera are much larger than any of the living forms. Prof. Leidy obtained 18,700 shells of a single species of Nonionina from an ounce of mud scraped from the surface, between tides, at Atlantic City. In another sample, from Cape May, he obtained 38,400 shells; and in an ounce from the bathing beach at Newport, Rhode Island, he estimated there were 280,000 shells of several genera and species. The sediment of the Atlantic Ocean is so largely constituted of one kind of foraminiferous shell, that it is generally called Globigerina ooze. Common chalk is almost wholly composed of the shells of Foraminifera. The building stone of the city of Paris is almost wholly made of the shells of Foraminifera belonging to the Sub-order Miliola. The Nummulite limestone of different countries is composed of foraminiferous shells, and so is the Fusulina limestone of Carboniferous age. The microscopic genera and species of the Palæozoic rocks have not been much studied. The classification of the Palæozoic Foraminifera, so far as they have been investigated, is as follows:

Family CalCispherride.-Calcisphæra.

Family Eozoonide.-Eozoon.

Famisy Fusulinide.-Fusulina, Loftusia, Mœllerina.

Family Globigerinide.-Calcarina.

Famil y Lituolider.-Endothyra, Nodosinella, Valvulina.

Family Affinity, uncertain.-Rhabdaria. 


\section{CLASS PORIFERA (poros, canal; phero, I bear).}

The Porifera include the Sponges, and are not to be regarded as any more highly organized than the Rhizopoda. A sponge consists of a congeries of horny filaments, interlaced in every direction so as to form an intricate network of intercommunicating cells. Imbedded in these filaments, in the majority of sponges, are a number of minute needle-shaped, or forked, or radiated silicious, or calcareous particles of various forms, called spicula. The spiculæ may be acicular and pointed at both ends, or have a small knob at one end, while the opposite end is pointed; or one end may be a fork, with two or three prongs. The horny filaments, with their contained spiculæ, constitute the skeleton which supports the living sponge. The living sponge consists of a mere coating of gelatinous matter spread over all the filaments, of the consistence of the white of an egg, which runs frcely away from the skeleton or framework of the sponge when taken out of the water. Under the microscope this gelatinous matter is found to consist of an aggregation of sarcode cells, and each cell appears to possess an indepeudent existence; and cven when detached from its fellows it has the power to move by the exteusion of its substance in various directions. In a living sponge there is an infinite number of minute holes, and a lesser number of larger openings. The water is imbibed through the smaller pores, aud thrown out from the larger ones. The circulation results from the action of cilia, in much the same way motion is effected by the Rhizopoda.

Sponges attrch themselves to all kinds of objects, whether fixed or floating. Some cover rocks and shells with a spongy incrustation; others hang from floating sea-weeds, and others shoot up branched stems, or a massive, globular framework. The Cliona is a boring sponge, that imbeds itself in shells or other calcareous substances. Sponges of the same species assume very differeut forms. In fact, there are no animals in which the variations are as great in a single species. They attain their greatest development in tropical seas, but occur in the most northern latitudes.

The genera and species of living sponges are largely founded upon the framework and spiculæ, and of course the same characters are sought in fossil sponges for the purpose of classification. Among the Palrozoic sponges, form is of much more importance than it is among living sponges, as we may believe, because we find so many specimens of the same form and size in a given species, not only at one locality, but at distant places, even hundreds or thousands of miles apart, in the same Group of rocks; as, for instance, Astylospongia prcemorsa, on the Island of Gottland, in the Baltic Sea, and in Tennessee and Indiana. When Silurian sponges are silicified, the surface is generally very poorly preserved, and the spiculac perfectly preserved; but calcareous and unsilicified specimens of the same species will show a well-preserved exterior and no spiculæ. It is therefore impossible to determine whether the sponge in its living state had calcareous or silicified spiculx. In the fossilization of sponges and other bodies, and even long after fossilization has taken place, silica will be taken up, and lime will be deposited in its place in some waters; while in other waters lime will be taken up, and silica will be deposited in its stead. An original calcareous sponge, when converted into a silicious fossil, will preserve the spiculæ; but if a sponge bears silicious spiculæ, and is converted 
into a calcareous fossil, the spiculæ will disappear in the coarser lime materials. A possible exception might exist if, in the process of change, the interior of the sponge were converted into calcspar. The spiculæ, therefore, are of importance in the determination of genera and species among Palrozoic sponges, only when silicified specimens can be obtained.

The arrangement of the Palæozoic sponges into families is as follows:

Family Anthaspideldids.-Anthaspidella, Climacospongia, Edriospongia, Streptosolen, Zittelella.

Famili Arch жsocyathide.-Archæocyathus, Ethmophyllum.

Family Astreospongide.-Astræospongia.

Family Astylosponfidel-Astylospongia, Aulocopina, Calathium, Conopterium, Cyathospongia, Eospongia, Palæomanon, Palæospongia, Trachyum, Trichospongia.

Family Beatricide.-Beatricea.

Family Brachiospongide.-Brachiospongia, Chirospongia.

Family Dictyospongide.-Cleodictya, Cyathophycus, Dictyophyton, Ectenodictya, Lyriodictya, Phragmodictya, Physospongia, Protospongia, Rauffella, Rhombodictyon, Thamnodictya, Uphantæenia.

Family Dystactospongide.-Dystactospongia, Heterospongia, Saccospongia.

Family Leptonitide.-Leptonitus.

Family Microspongidze.-Hindia, Microspongia.

Family Paldeacidas.-Palæacis.

Family Pasceolide. -Pasceolus.

Family Pattersonidde.-Pattersonia.

Family Pharetrones.-Batospongia, Camarocladia, Cylindrocolia, Streptospongia.

Family Receptaculitide.-Cerionites, Receptaculites.

Family Stromatoporid w.-Caunopora, Cœnostroma, Cryptozoon, Dictyostroma, Megastroma, Strephochetus, Stromatocerium, Stromatopora, Syringostroma.

Family affinity uncertain.-Astroconia, Fungispongia, Lepidolites, Leptomitus.

Anthaspidella, Ulrich \& Everett, (in press,) Geo. Sur. Ill., vol. 8, p. 256. [Ety. anthos, flower; aspis, shield; ellus, diminutive.] Saucer or funnel-shaped, supported by a short, subcylindrical stem; inosculating, radiating channels numerous, and those on the upper surface form radical canals that pass through the sponge-wall, and open into the channels of the lower surface; radiating canals closely arranged in vertical series, separated by vertical sheets of spicules; oscula on the upper surface; spicules bifid at each end, the bifurcations directed nearly at right angle, and slightly curving, and so arranged as to leave minute canals of triangular, quadrate, or polygonal form; surface sometimes covered with a dermal layer. Type A. mammulata. fenestrata, Ulrich \& Everett, (in press,) Geo. Sur. Ill., vol. 8, p. 264. Trenton Gr. firma, Ulrich \& Everett, (in press,) Geo. Sur. Ill., vol. 8, p, 263, Trenton Gr.

florifera, Ulrich \& Everett, (in press,) Geo. Sur. Ill., vol. 8, p. 259, Trenton Gr. grandis, Ulrich \& Everett, (in press,) Geo. Sur. Ill., vol. 8, p. 262, Trenton Gr. magnifica, Ulrich \& Everett, (in press,) Geo. Sur. Ill., vol. 8, p. 265, Trenton $\mathrm{Gr}$.

mammulata, Ulrich \& Everett, (in press, ) Geo. Sur. Ill., vol. 8, p. 258, Trenton Gr. obliqua, Ulrich \& Everett, (in press,) Geo. Sur. Ill., vol. 8, p. 265, Trenton Gr. parvistellata, Ulrich \& Everett, (ir press,) Geo. Sur. Ill., vol. 8, p. 260, Trenton Gr.

scutula, Ulrich \& Everett, (in press,) Geo. Sur. Ill., vol. 8, p. 261, Trenton Gr. 
Archæocyathellus, Ford, 1873, Am. Jour. Sci. and Arts, 3d ser., vol. 6, p. 135, syn. for Ethunophyllum.

Arch жocy a tu us, Billings, 1861. Pal. Foss., vol. 1, p. 3 , and 354 . [Ety. arche, beginning; cyathus, c'up.] An elongated, cylindrical, sponge-like body ; lange end open; central ravity lined by an endotheca and external surface by an epitheca; intervening space being filled with poriferous and cellular tissue; walls perforated. Type A. atlanticus.
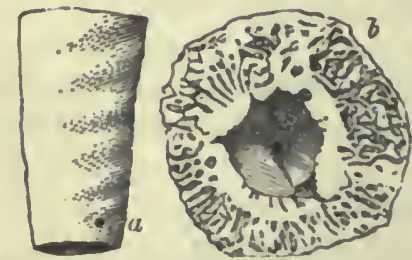

Fic. 89.-Archroncyaihus atlantlcus. $a$, reduced ; $b$, trussverse sectlou.

atlanticus, Billings, 1861, Pal. Foss., vol. 1, p. 5, Up. Tuconic.

billingsi, Wulcott, 1886, Bull. U. S. Geo. Sur., No. 30 , p. 74 , Up. Taconic.

minganensis, see Ftlinıpliyllum minganense, though Hincle lias mude it the type of a new genus, Archaoscy phis. profundus, see lithmophyllum profundum. rensselaricus, see Ethmopliyllum rensse]aricum.

Astracospongia, Roemer, 1860, Si]. Fauna d. West Tenn., p. 13. [Ety. aster, star; spongia, sponge,] Glot,ular or disk-like, free sponge conposed of regular sturshrped spicules, witbout order, no epitueca or canals. Type A. meniscus.

hamiltonensis, Meek \& Worthen, 1866, Proc. Chi. Acad. Sci., vol.1, p. 12, Ham. Gr.

meniscus, Roemer, 1848, (Blunenbachinm meniscus,) Leonh. and Bronn's Jahrb., p. 683 , Niagara Gr.

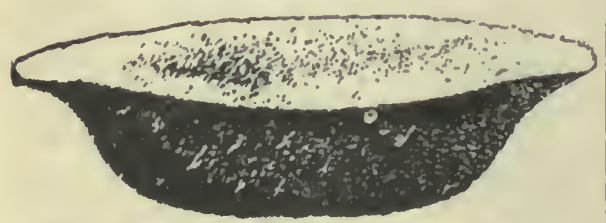

FIG. 90.-Astræospongla meniscus.

Astroconia, Sollas, 1881, Quar. Jnur. Geo. Suc. Lond., vol. 37, p. 254. [Ety. aster, star; kınia, dust.] Fuunded upon the appearance of various spicula in a grayish silicious dolomite. Characters not distinct. Type A. granti. The name was preoccupied by Edwards \& Haime in 1848.

granti, Sollas, 1881, Quar. Jour. Geo. Soc. Lond., vol. 37, p. 254, Niagara Gr.
Astylospongia, Roemer, 1860, Sil. Fauna d. West Tenn., y. 7. [Ety. astylos, without a pillar; spongia, sponge.] Globular or disk-like, free sponge; inner texture formed of small, regular, star-shaped spicules, connected by their rays; canals running from the center to the surface crossed by concentric canals. Type A. premorsa.

bursa, Hall, 1876, 2sth Rep. N. Y. St. Mus. Nat. Hist., p. 105, Niazara Gr.

christiana, Meek \& Worthen, 1868, Geo. Sur. Ill., vol. 3 , p. 34t, Niagara Gr.

imbricato-articulata, Roemer, 1848, (Siphonia imbricato-articulata,) Leoul. and Bronn's Jahrb., p. 685 , and Sil. Fauna d. West Tenu., p. 12, Niagara Gr.

inciso-lobata, Roeiner, 1848, (Spongia inciso-lobata, Leonh. and Bronn's Jahrb., p. 685, and Sil. Fauna d. West Tenn., p. 11, Niagara (ir.

inornata, Hall, 1863, 16th Rep. N. Y. St. Mus. Nat. Hist., p. 70, syn. for Hindia fibrosa.

parvula, Billings, 1861, Pal. Foss., vol. 1, p. 20, Trenton Gr.

perryi, Billings, 1861, Geo. Vermont, p. 957, Black Riv. Gr.

premorsa, Goldfuss, 1826, (Siphonia presmorsa,) l'etref. Germ., 1. 17, and Sil. Fuuna. d. West Tenn., p. 8, Niagara Gr.

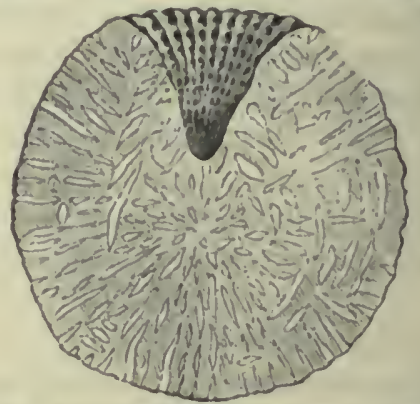

FIG. 91.-Astylospongla premorsa. Vertical section, showing cup.

stellatim-sulcata, Roemer, 1848, (Spongia stellatim-sulcata,) l,onh. and Bronn's Jahrb., p. 686 , and Sil. Fauna West Tenn., ). 11, Niagara Gr.

A ulocorrina, Billings, 1875, Can. Nat. and Geul., vol. 7, p. 230. [Ety. aulokopeo, cut into pipes.] Elongate, ovate, or pyriform; upler face concave, with an osculum in the center, from which ridges radiate over the surface and descend to the base; the osculum is the opening of a central cavity, from which sinaller branching canals rudiate. Type A. granti.

granti, Billings, 1875, Can. Nat. and Geol., vol. 7, p. 231, Niagara Gr.

Batospongia, Ulrich, (in press,) Geo. Sur. III., vol. 8. p. 246. [Ety. balos, prickly bush; spongia, sponge.] Subhemispherical or subglobose, consisting of small, inosculating, subcylindrical or flattened 
branches, which arise from a reticulated base ; base covered with a dermal layer, which exhibits on its inner side a network of subst+llate or irregularly branched spicule fiber; spicules acerate, bifid, trifid, or four-rayed. Type $B$. spicata.

spicata, Ulrich, (in press,) Geo. Sur. Ill., vol. 8, p. 246, Coal Meas.

Beatricea, Billings, 1875, Rtp. of Progr. Geo. Sur. Can., p. 343. [Ety. proper name.] This genus was supposed by Hyatt (Am. Jour. Sci. and Arıs, 1865,) to belong to the class Cephalopoda, and he proposed a new order for the genus, tu-wit: Ceriolites, from kerion, a honey-comb; lithos, a stone; and a family Ceriolida. They are, however, long, cylindrical spongeoid bodies. Type B. nodulosa.

nodulosa, Billings, 1857, Rep. of Progr. Geo. Sur. Can., p. 344, Trenton and Hud. Riv. Gr.

undulata, Billings, 1857, Rep. of Progr. Geo. Sur. Can., p. 344, Trenton Gr.

Belemospongia, Ulrich, (in press,) Geo. Sur. Ill., vol. 8, p. 248. [Ety. belemnos, a dart; spongia, sponge.] Composed of elongate acerate spicules, which radiate upward and outward from a pointed base; spicules large, and joined to each other by short processes. Type B. fascicularis.

fascicularis, Ulrich, (in press,) Geo. Sur. Ill., vol. 8, p. 248, Burlington Gr.

Blumenbachium, Konig, 1820, Icones fossiles, sectiles.

meniscus, see Astraeospongia meniscus.

Bracilospongia, Marsh, 1867, Am. Jour. Sci. and Arts, $2 \mathrm{l}$ ser., vol. 44, p. 88. [Ety. brachium, arm; spongia, spunge.] A short vase or hollow central nucleus, throwing out large, hollow arms, which are closed at the distal extremities; skeleton comparatively thin and bearing a network of spicules; all observed specimens are silicious, and outer surface therefore destroyed. Type B. digitata.

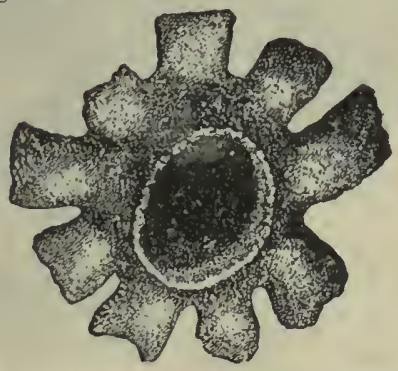

FIG. 92,-Brachiospongla digitata. 1/4 dam., showing large gastrul cavity.

digitata, Owen, 1857, (Scyphia digitata,) Geo. of $\mathrm{Ky}$., vol. 2, p. 111, Trenton Gr. hoveyi, Marsh, 1874, Trans. Kansas Acad. Sci., p. 344, syn. for B. digitata, but founded on a specimen having twelve arms.

lyoni, Marsh, 1867, Am. Jour. Sci. and Arts, $2 \mathrm{~d}$ ser., vol. 44, p. 88, syn. for B. digitata, but founded on a specimen having eleven arms.

roemerann, Marsh, 1867, Am. Jour. Sci. and Arts, $2 d$ ser., vol. 44, p. 88, syn. for B. digitata.

Calathium, Billings, 1865, Pal. Foss., vol. 1, p. 208. [Ety. kalathos, a small wicker basket.] Cylindroturbinate in form, perforated by small canals arranged in longitudinal and transverse rows; apertures round, oval, or quadrangular; cup deer. Type C. formosum.

affine, Billings, 1S65,

Pal. Fuss. vol. 1, p. 209, Qnebec Gr.

anstedi, Billings. 1865, Prl. Foss.. vol. 1, p. 201 and 337, Quebec Gr.

canarlense, Billings, 1865, Pal. Foss., vol. 1, p. 377 , Cliazy Gr.

fittoni, Billings, 1865, Fug. 93. - Calathlum Pal. Foss., vol. 1, p. canudense. Vertiformosum, Bill ing $\mathrm{s}$, calsection, showing
cup.

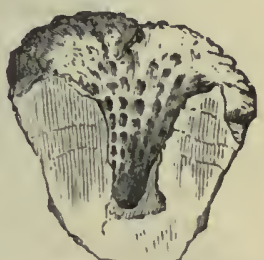

1565, Pal. Foss., vol. 1, p. 209, Quebec Gr. infelix, Ulrich \& Everett, (in press,) Geo. Sur. Ill., vol, 8, p. 27t, Trenton Gr.

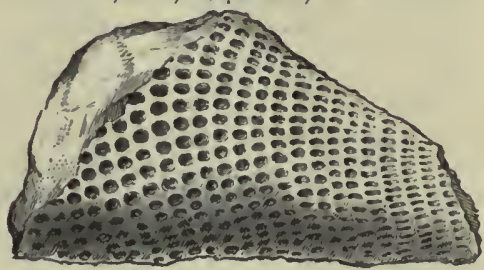

FIG. 94.-Calathium formosum.

pannosum, Billings, 1865, Pal. Foss., vol. 1, p. 335, Quebec Gr.

paradoxicum, Billings, 1865, Pal. Foss., vol. 1, p. 358, Calcif. Gr. Hinde, in 1859, Quar. Jour. Geo. Soc., p. 144, made this species the type of a new genus, Nipterella.

Calcarina, D'Orbieny, 1826, Tableau Methodique de la Classe des Cephalopodes, in Annales des Sciences Naturelles, tome 7. [Ety. calcis, limestone.] Free, convoluted, depressed, spire-coiled, supplemental growths of the interior shell, aperture slit in the terminal chamber close to the penultimate convolution. A living genus in tropical seas.

ambigua, Brady, 1878, Monograph of Carboniferous and Permian foraminifera, p. 141, Carboniferous.

Calcisphera, Williamson, 1880, Mem. Org. of the plants of the Coal Mras., pt. 10. [Ety. calcis, limestone; sphrra, sphere.] A minute globular test, having an aperture; wall composed of minute calcareous grains. Type C. robusta. 
robusta, Williamson, 1880 , Mem. Org. of the Coal Meas., pt. 10, Up. Held Gr.
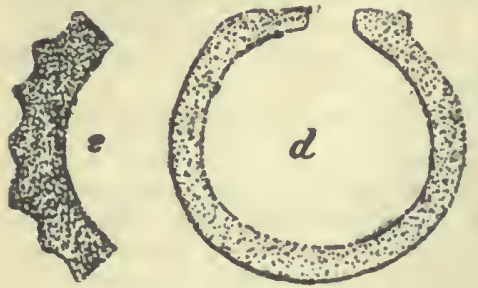

Fig. 05-Calclsphera robusta u, untural slze. $b$, magnifled, showing sculpture; $c$, showing aperture; $d$, magnifled. showlng ajerture; $e_{\text {, }}$ section of wall magnifed.

Camarochadia, Ulrich \& Everett, (in press, ) Geo. Sur. Ill., vol. 8, p. 280 . [Ety. kamara, arehing cbamber; klados, twig. Small, subcylindrical branching stems; interior canuls irregular, separated by thin, cribrose walls; spicules threerayed. Type C. dichotoma.

dichotoma, Ulrich \& Everett, (in press, ) Geo. Sur. Ill., vol. 8, p. 281, Trenton Gr.

Caunopora, Phillips, 1841, Pal. Foss., Cornwall and Devon. and W. Somerset, p. 18. [Ety. chaunos, loose; poros, perforation.] Amorphous, composed of con. centric or nearly plain masses, perforated by flexuous or vermiform small tubuli, and by larger, straight, subparallel or radiating onen tubes, persistent through the mass. Type C. placenta.

hudsonica, Dawson, 1879, Quar. Jour. Geo. Soc., vol. 35, p. 52, Niagara Gr.

incrustans, Hall \& Whitfield, 1873, (Stromatopora incrustans,) 23d Rep. N. Y. St. Mus. Nat. Hist., p. 227, Chemung Gr.

mirabilis, Spencer, 1S84, Bull. No, 1 Univ. St. Mo., p. 47, Niagara Gr.

planulata, Hall \& Whitfield, 1873, 23d Rep. N. Y. St. Mus. Nat. Hist., p. 228, Chemung Gr.

walkeri, Spencer, 1884, I3ull. No. 1, Mus. Univ. St. Mo., 1). 46, Niagara Gr.

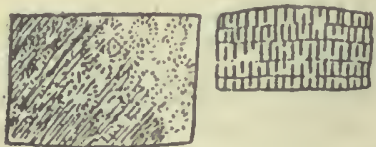

FIG. 96.-Cuunopora wulkeri, vertieal and jorl- zontal secllon enlarged.

Cerionites, Meek \& Worthen, 1868, Geo. Sur. Ill., vol. 3, p. 346. [Ety. kerion, honey-coinb; lithos, stone.] Founded upon casts apparently holding an intermediate position between Pasceolus and Receptaculites. The pits are bexagonal and upon the convex side, perforated in the center by a minute circular opening, while those upon the under side are imperforate. 'Iype C. dactyloides.

dactyloides, Owen, 1844, (Lunulites dact y loides,) Rep. on Min. Lands, p. 69), Niagara Gr.

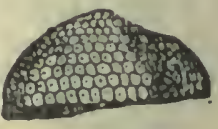

Cimrospoxgia : n. gen.Fra. 97.-Cerionites [Ety. cheir, hand; duetyloises. spongia, s p o n ge.]

General form liand-like, or somewhat like a compressed goblet; composed of internal filamentous or fibrous substance, which is covered with a thin, lobed, vesicular parenehyma; it was firmly fastened by an cxpanded base to a solid rock or the sea-botton; above the base it is a flattened obconoiclal cup, with a deep sulcus down the middle of each side, bringing the sides nearly together; on each side of the sulcus the interior of the sponge is hollow, showing a large gastral cavity; the whole skeleton is openly vesicular or porous. The type species is silicified, and does not show the surface markings, but a calcarcous specimen, supposed to belong to the same genus, is finely papil. lated. No microscopie sections have been made to ascertain the character of the spicules, but doubtless both parenchyma and fibrous substance bear spicules similar to those of Brachiospongia. In the surface lobes and filaments it resembles Pattersonia, but is distinguished by its vesicular and porous substance and coarser filaments. In its large gastral cavity, thin skeleton, and vesicular parencliyma, it resembles Brachiospongia. Type C. wenti.

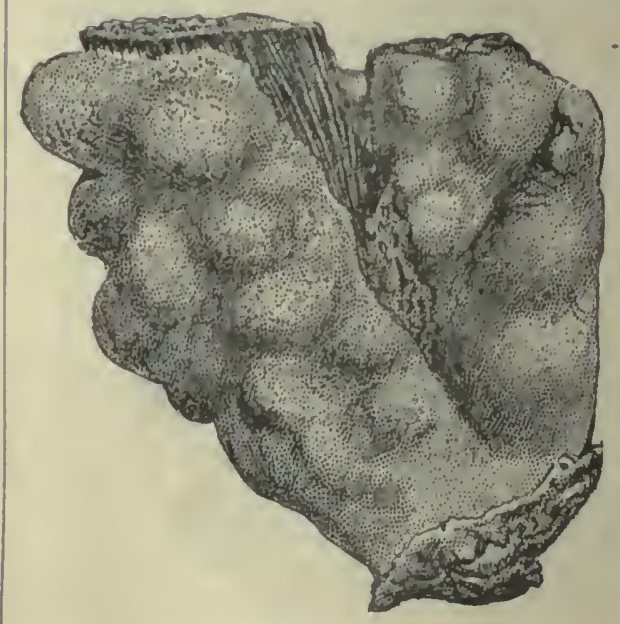

FiG. 98.-Chirospongla wents.

faberi, n. sp. This species is founded upon a calcareous fragment of the parenchyma, about one-third of which is shown in the figure. It is thin, and 
belonged to the side of a large, hollow specimen. The semi-elliptical lobes are nearly equal in size, and regularly distributed in rows over the surface. The surface is reticulated with fine papillæ, presenting to the naked eye the appearance of a bryozoum. The illustration shows rhomboidal depressions instead of papillæ. Found associated with Pattersonia and fragments of sponge filaments near the middle of the Hud. Riv. Gr., about 350 feet above low-water mark at Cincinnati. Collector, Charles Faber.

wenti, n. sp. This species is founded upon a silicified specimen having the characters above ascribed to the genus, and being well illustrated in the figure. The fibrous substance shown in the sulcus formed the basal attachment, as the parenchymatous surface tissue does not appear at the bottom. The lobes are large, somewhat semi-elliptical in outline, of unequal size, and irregularly disposed, but not pendent as in Pattersonia. The substance of the filaments and parenchyma, as shown, where broken off and weathered at the top and bottom of the specimen, is openly vesiculose or irregularly porous, resembling to the naked eye somewhat the appearance of Alveolites goldfussi. The species is named in honor of Mr. C. E. Went, of Frankfort, $\mathrm{Ky}$., who found it in the Trenton Group near that city.

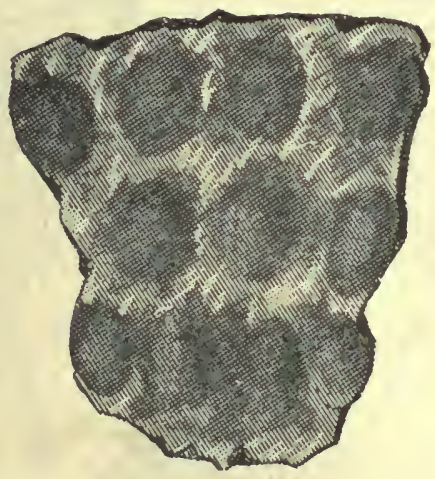

FIG. 99.-Chirospongia faberi ; reticulated depressions should indicate papillæ.

Cleodicty A, Hall, 1884, 35th Rep. N. Y. St. Mus. Nat. Hist., p. 467. [Ety. kleo, closed up ; dictuon, net.] Frond rapidly expanding from the base to a subglobose or hemispherical form, bearing a row of large, rounded nodes on the periphery; tube abruptly contracted above, and extending in a cylindrical or slightly expanded form. Substance composed of regular lattice-work of sixrayed spicules and bundles of acicular rods. Type C. gloriosa. gloriosa, Hall, 1884, 35th Rep. N. Y. St. Mus. Nat. Hist., p. 479, Keokuk Gr. mohri, Hall, 1884, 35th Rep. N. Y. St. Mus. Nat. Hist., p. 479, Keokuk, Gr. Cnemidium, Goldfuss, 1826, Petref. Germ., p. 15. [Ety. knemidos, armor for the legs, a sort of boot.] Type C. lamellosum. trentonensis, see Palæospongia trentonensis. Canostroma, Winchell, 1867, Proc. Am. Ass. Ad. Sci., p. 91. [Ety. koinos, shared in common; stroma, layer.] Distinguished from Stromatopora by the absence of central, simple, radiating tubes, which in this genus is represented by a group of more or less divergent ascending tubuli, so that the surface of the last layer presents eminences, not with a single large pore at the summit, but with several small pores diverging from their sides. Type C. monticuliferum.

botryoideum, Spencer, 1884, Bull. No. 1, Mus. Univ. St. Mo., p. 50, Niagara Gr. constellatum, Hall, 1852 ,

(Stromatopora constellata,) Pal. N. Y., vol. 2, p. 324, Coralline limestone, Niagara Gr.

galtense, Dawson, 1879, Quar. Jour. Geo. Soc., vol. 35, p. 52, Guelph Gr. monticuliferum, Winclsell, 1866, (Stromatopora monticulifera,) Rep. Low. Fig. 100.-ConPenin. Mich., p. 91, ostroma conHam. Gr. pustuliferum, Winchell, section.

1866, (Stromatopora pustulifera,) Rep. Low. Penin. Mich., p. 90, Ham. Gr.

ristigouchense, Spencer, 1884, Bull. No. 1, Univ. St. Mo., p. 49, Low. Held. Gr. solidulum, Hall \& Whitfield, 1873, (Stromatopora solidula,) $23 \mathrm{~d}$ Rep. N. Y. St. Mus. Nat, Hist., p. 227, Chemung Gr.

Conopteizium, Winchell, 1865, Proc. Acad. Nat. Sci., p. 110. [Ety. lonos, cone; poterion, cup.] Cells crowded, inseparable, rapidly enlarging, walls marked by vertical striæe, and a few pores communicate between the cells; epitheca exterior. Type C. effusum.

effusum, Winchell, 1865, Proc. Acal. Nat. Sci., p. 111, Waverly Gr. or Lithographic limestone.

Coscinopora infundibuliformis, see Receptaculites infundibuliformis.

Coscinopora sulcata, Owen, 1844, see Receptaculites oweni.

Cryptozoon, Hall, 1884, 36th Rep N. Y. St. Mus. Nat. Hist., p. 95. [Ety. kruptos, hidden; zoon, animal.] Composed of irregular, concentric laminæ, resembling Stromatopora, substance traversed by minute canals, which branch and anastomose irregularly. Type C. proliferum. minnesotense, Wincheli, 1886, 14th Ann. Rep. Geo. Minn., p. 313, Calciferous Gr. proliferum, Hall, 1884, 36th Rep. N. Y. St. Mus. Nat. Hist., p. 95, Calciferous 
Cyatiopirycus, Walcott, 1879, Trans. Alb. Inst., vol. 10, p. 18. [Ety. kuathos, cup ; phukos, sea-weed,] Hollow, ('yatliform, with a reticulated strueture. Type C. reticulatum.

reticulutnn, Walcott, 1879, Trans. Alb. Inst., vul. 10, p. 18, Utica Slate.

subsphericum, Wulcott, 1879, Trans. Alb. Inst, vol. 10, p. 19, Utica Slate.

Cratiosponeia, Hall, 1882, Foss. Corals Niagara and U1). He]d. Grs., p. 15. [Eıy. krathos, cup; spongia, sponge.] Body solir, turbinate, cyathiform: structure similar to Astylospongia. Type C. excrefcens.

excrescens, Hall, 1882, Foss. Corals Niagara and Up. Held. Grs., p. 15, and 35 th Rep. N. Y. St. Mus. Nat. Hist., p. 419 , Niagara Gr.

Cycindrocokis, Ulrich, 1899, Am. Gen., vol. 3. 1. 245. [Ety. kulindros, cylinder; koilos, belly.] Cylindrieal; central cloaca; walls thick, radiating eanals. Ty po C. endoceroidea.

covingtonensis, Ulrich, 1889, Am. Geo., vol. 3, j. 247, Hud. Riv. Gr.

endoceroidea, Ulrich, 1889, Am. Geo., vol. 3, p. 246, Trenton Gr.

minnesotensis, Ulrich, 1889, Am. Geo., vol. 3, p. 248, Trenton Gr.

minor, Uirich, 1889, Am. Geo., vol. 3, p. 248, Trenton Gr.

Dentalina, D’Orbigny, 1826, Anı. Des. Sci. Nat., t. 7, p. 89. [Jity. dentale, looth; inus, implying resemblance.]

prisculla, see Nodosinella priscilla.

Dictyopuyton, Hull, 1863, l6th Rep. N. Y. St. Mus., p. \$7. [ [ly . dictyon, net; phytom, plunt.] Turbinate or infundibuliform, with nodose or conical protuberances or hollow stems externally, and marked by minute rectaugular spaces, and consisting of a reticulate envelope. Type D. filitextile.

abseus, Hall, 1884, 35th Rep. N. Y. St. Mus. Nat. Hint., p. 474 , Waverly Gr. annulatum. Hall, 1863, 16th Rep. N. Y. St. Mus. Nat. Hist., p. 90, Chenumg Gr. baculum, Hall, 1884, 35ıh Rep. N. Y. St. Mus. Nat. Hist., p. 471, Chemung Gr.

becki, Conrad, 1837, (Lithodictuon becki) Ann. Rep. N. Y., p. 167, and Pul. N. Y., vol. 2, p.6, Medina Sandstone.

catilliforme, see l'bragmodictya catilliformis.

cinctum, Hall, 1884, 35th Rep. N. Y. St. Mus. Nat. Hist., p. 472, Ch+mung Gr. conradi, Hall, 1863, 16th Rep. N. Y. St. Mus. Nat. Hist., p. 89 , Chemung Gr.

cylindricum, Whitfield, 1881, Bull., No. 1, A m. Mus. Nat. Hist., p. 19, Keokuk Gr. fenestratun, Hall, 1863, 16th Rep. N. Y. St. Mus. Nat. Hist., 1).90, Chemung Gr. filitextile, Hull, 1863, 16th Rep. N. Y. St. Mus. Nat. Hist., p. 88, Chemung Gr.

hamiltonense, Hall, $1884,35 \mathrm{tl}$, Rep. N. Y. St. Mus. Nat. Hist., p. $468, \mathrm{Ham}$. Gr. irregulare, Hall, 1884, 35th Rep. N. Y. St. Mus. Nat. Hist., p. 470 , Chemung Gr. neuberryi, see Thamnodictya newberryi. nodosum, Hall, 1S63, ]6th Rep. N. I. St. Mus. Nat. Hist., p. 91, Chemung Gr. parallellum, Hall, 1S\$4, 35th Rep. N. Y. St. Mus. Nat. Hist., p. 471, Chemung Gr. patulum, Hall, 1884, 35th Rep. N. Y. St. Mus. Nat. Hist., p. 469, Chem?ng Gr. prismaticum, Hull, 1884,35 th $\left.\mathbf{R}_{\boldsymbol{\prime}}\right\} . \mathrm{N}$. Y. St. Mus. Nat. Hist., p. 469, Chemung, Gr. rainosum, Lefquereux, 18st, Coal Flora of Ps., p. 827, Up. Chemmuy Gr.

redfieldi. Hull, $1863,16 \mathrm{th}$ Rep. N. Y. St. Mus. Nat. Hist., p. 88, Wuverly Gr.

rude, Hall, 1863 , 16 h h $\mathrm{K}$ p. N. Y. St. Mus. Nat. Hist., p. 90, Clıemung Gr.

sacculus, Hall, 1894, 351 h Rep. N. Y. St. Mus. Nint. Hist., p. 473 , Waverly Gr. telum, Hall, 18st, 35th Rep. N. Y. St. Mus. Nut. Hist., 1. 470, Chemung Gr.

tenue, Hall, 1 sist, 35 th Rep. N. Y. St. Mus. Nat. Hist., p. 474, Waverly Gr.

tuberosun), Conrad, 1842, (IIylnoreras tuherosum,) Jour. Acall. Nat. Sci. Phil., vol. 8. p. 267, Chemung Gr.

Dictyostroma, Nicholson, 1875, Ohio Pal., vol. 2, p. 254. [Ety. dictyon, net; stroma, layer.] Allied to Stromatopura, but the upper surface of each lamina is devel. oped into conical puints, which support the lamina above instead of pillars. The lamine? have liorizontal canals, and are jrobably unimutely perforate. Ty $\mathrm{P}_{0} \mathrm{D}$. undulatum.

reticulatum, Spencer, Fir. 101.-Diclyo1884, Bull. Mus.

Univ. St. Mo., p. 51, Niagara Gr. undulatum, Nicholson, 1875, Olıin Yal., vol. 2, 1). '254, Niagara Gr.

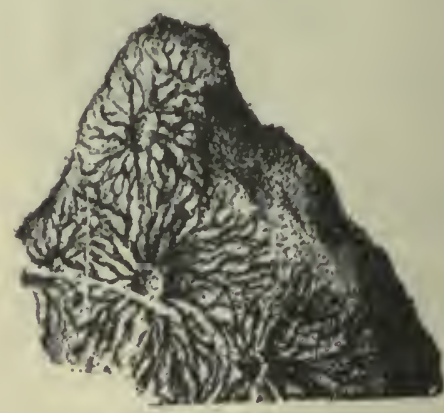

Fig. 102.

Dystactospongia insolens.

Dystactospongia, S. A. Miller, 1852, Jour. Cin. Soc. Nat. Hist., vol. 5, p. 42. [Ety. dystaklos, hard to arrange ; spongia, 
sponge.] Massive, hemispherical, attached with a strong radiating framework. Structure vesicular. Type D. insolens.

insolens, S. A. Miller, 1882, Jour. Cin. Soc. Nut. Hist., vol. 5, p. 43, Hud. Riv. Gr.

minima, Ulrich, 1889, Am. Geol., vol. 3, p. 243, Hıd. Riv. Gr.

minor, Ulrich \& Everett, (in press,) Geo. Sur. Ill., vol. 8, p. 278, Trenton Gr.

ruris, Ulrich \& Everett, (in press,) Geo. Sur. Ill., vol. 8, p. 279, Trenton Gr.

Ectenodictya, Hall, 18S4, 35th Rep. N. Y. St. Mus. Nat. Hist., p. 466. [Ety. eltense, stretched out; dictuon, net.] A reticulate frond irregularly expanded or explanate; reticulation irregular presenting radiating and concentric striae. Type E. implexa.

burlingtonensis, Hall, 1884, 35th Rep. N. Y. St. Mus. Nat. Hist., p. 476, Waverly Gr.

excentrica, Hall, 1884, 35th Rep. N. Y. St. Mus. Nat. Hist., p. 476, Keokuk Gr.

expansa, Hall, 1884, 35th Rep. N. Y. St. Mus. Nat. Hist., p. 475, Waverly (ir.

implexa, Hall, 1884, 35th Rep. N. Y. St. Mus. Nat. Hist., p. 475, Waverly Gr.

Edriospongia, Ulrich \& Everett, (in press,) Gro. Sur. Ill., vol. 8, p. 271. [Ety. edrion, a seat; spongia, sponge.] Massive, lobate, attached by a broad base; sides irrogularly dented; radiating canals, connected by tortuous, vertical ones; minute canals formed by spicules; sides covered with a dermal layer. Type E. basalis.

basalis, Ulrich \& Everett, (in press,) Geo. Sur. Ill., vol. 8, p. 272, Trenton Gr.

Endotiryra, Phillips, 18t5, Proc. Geol. and Pulvtech. Soc. IV. Riding Yorks., vol. 2, p. 279. [Ety. endos, within; thura, door.] Free, spiral, rotaliform, segments numerous, texture subarenaceous, imperforate, aperture simple. Type E. bowmani.

baileyi, Hall, 1858, (Rotalia baileyi,) Trans.

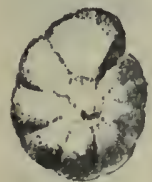

Frg. 103. balleyi. magnifled.
Endothyra Alb. Ins., vol. 4, p. 34, and 1882, Bull. Mus. Nat. Hist., p. 42, Warsaw Gr.

Eospongia, Billings, 1861, Pal. Foss., vol. 1, p. 18. [Ety. eos, dawn ; spongia, sponge.] Subglobular, pyriform ol - sulihemispherical, not free, pores radiating.irregularly from the central axis; cup of variable depth. Type I:. roemeri.

roemeri, Billings, 1861, Pal. Foss., vol. 1, p. 19, Chazy Gr.

varians, Billings, 1861, Pal. Foss, vol. 1, p. 19, Chazy Gr.

Eozoon, Dawson, 1865, Can. Nat. and Geo., $2 \mathrm{~d}$ ser., vol. 2, p. 54. [Ety. eos, dawn; zoon, animal.] Massive, in large sessilé patches or irregular cylinders, growing at the surface, by the addition of suc- cessive laminæ, internally, the chambers are flattened, irregular, with numerous rounded extensinis, and separated by walls of variable thickness, penetrated by septal orifices irregularly disposed; thicker parts of the walls with fine branching tubuli; the apprarance to the naked eye is something like Stromatopora. Type E. canadense.

canadense, Dawson, 1865, Can. Nat. and Geo., $2 \mathrm{~d}$ ser., vol. 2, p. 54, Laurentian. The most ancient organism.

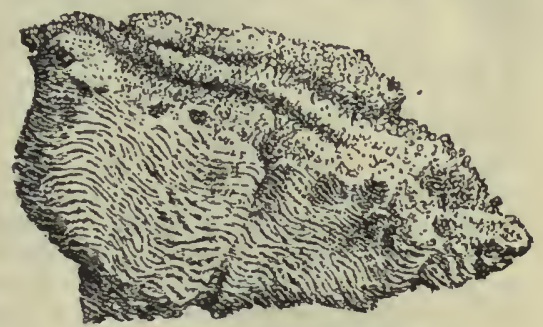

Fra. 104.-Ezoon canadense.

Eтнморir ylum, Meek, 1868, Am. Jour. Sci. and Arts, $2 \mathrm{~d}$ ser., vol. 45, p. 62. [Ety. thmns, sieve; phyllon, plant.] Body simple, elongate, turbinate, cup-shaped, clavate or cylindro-conical, curved or straight, corrugated, lobed, or ribbed, penetrated by round or oval pores, in vertical or horizontal rows; vertical septa numerous, originating at the outer wall, and extending to the inner one, poriferous; inner wall with or without vesicular tissue, extending into the central cup; series of septa and walls sometimes repeated; spiculie branching. Type E. whitneyi. gracile, Meek, syn. for E. whitneyi.

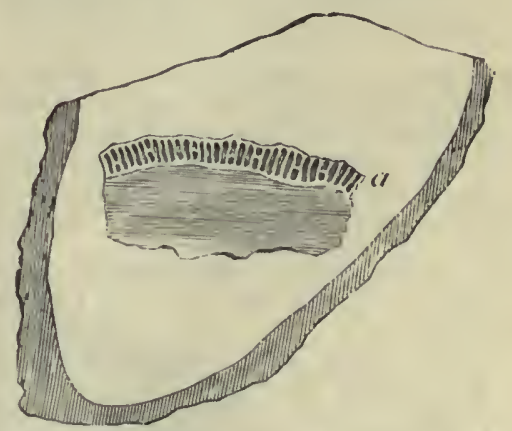

FIG $10 \bar{s}-$ Eihinophyllum profundum. Longitudinal and transverse section of a fragment.

minganense, Billings, 1859, (Petraia mingauensis,) Can. Nat. and Geol., vol. 4, p. 346, and Pal. Foss., vol. 1, p. 354. Calciferous Gr. Hinde, in 1889, Quar. Jour. Geo. Soc., p. 142, proposed this species as the type of a new genus Archreoscy phia. 
profundum, Billings, 1861, (A rchrocyathus profundus,) Pal. Foss., vol. 1, p. 4,

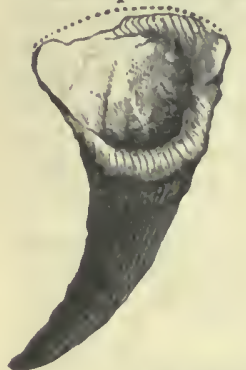

FIG. 106.-Ethmophyllum pro-

fundum. Base of at. tachment.
Up. Taconic.

rarum, Ford, 1878,

(Protocyathus rarus,)

Am. Jour. Sci. and

Arts, 3d ser., vol. 15,

p. 124, Up. Taconic. rensselrericum, Ford,

1873, (Archrecyяthellus rensselæricus,) Am. Jour. Sci. and $\mathrm{Arts}, 3 \mathrm{~d}$ ser., vol. 5, p. 211,Up. Taconic. whitneyi, Meek, 1868, Am. Jour. Sei. and Arts, 2d ser., vol. 45 , p. 62, Up. Taconic.

Fungispongia, Ringueberg, 188t, Proc. Acad. Nat. Sci., p. 147. [Ety. fungus, a musliroom; spongia, a sponge.] Definition very poor. Type $\mathrm{F}$. irregularis. irregularis, Ringueberg, 1884, 1'roc. Acad. Nat. Sci., p. 147, Clinton Gr. Very poorly defined.

Fusulisa, Fischer, 1837, Oryet. du Gouv. de Moscou., p. 126. [Ety. fusus, spindle; inus, little.] Shell fusiform, symmetrically involute, surface furrowed coincident with the septa witbin; aperture a narrow slit in the middlo part, foramina passing through the walls; septa widening toward the extremities. Type F. cylindrica.

cylindrica, Fisclser, 1837, Oryct. du. Gouv. de Moscou., p. 126, Coal Meas.
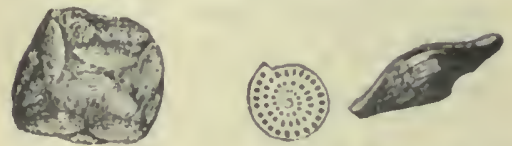

Fig. 107.-Fusulina cyllndrica. Nalurul slze, magnifled, and trunsverse sectlon.

cylindrica var. ventricosa, see $F$. ventricosa. depressa, Fischer, 1837, Oryct. du Gouv. de Moscou., p. 127, Coal Meas.

elongata, Shumard, 1858, Trans. St. Louis Acad. Sci., vol. 1, p. 297. Termian Gr.

gracilis, Meek, 186t, Pal. of California, vol. 1, p. 4, Coal Meas.

hyperborea, Salter, 1855, Belchur's Last Arctic Voyage, vol. 2, p. 380, Carboniferous.

robusta, Meek, 1864, Pal. California, vol. 1, p. 3, Coal Meas.

ventricosa, Meek \& Hayden, 1864, Pal. Upper Mo., p. 14, Coal Meas.

Heterosponora, Ulrich, 1889, Am. Geol., vol. 3, p. 239. [Ety. heteros irregular; spongia, sponge.] Sublobate, compressed branches, covered with mouths of tortuous canals; skeleton composed of loosely interwoven spicule fibers. Type H. subramosa.

aspera, Ulrich, 1889, Am. Geol., vol. 3, p. 241, Hud. Riv. Gr. knotti, Ulrich, 18s9, Am. Geol., vol. 3, p. 241, IIud. Riv. Gr.

subramosa, Ulrich, iss9, An. Geol., vol. 3, p. 240 , Hud. Riv. Gr.

Hrxia, Duncan, 1879, Ann. and Mag. Nat. Hist., 5th ser., vol. 4, p. 91. [Iity. proper name.] Free, spheroidal, without involution of texture; stnall central space oceupied by spicules which form \& series of bifurcating, long, straight canals, that open at the surface ; spicules more or less in shape of a stemmed tripod, with four limbs, and swollen or fringed at the ends. Typo H. fibrosa. This may be a synonym for Microspongia; but as the latter is calcareous, and the spicules have not been determined, both generic names are retained.

fibrosa, Roemer, 1860,(Calamopora fibrosa,) Sil. Faunณ W. Tenn., p. 20, Niagara Gr. inxequalis, Ulrich \& Fverett, (in press, ) Geo. Sur. Ill., vol., 8, p. 275, Trenton Gr.

spheriodalis, Duncan, 1879, Ann. and Mag. Nat. Hist., 5th ser., vol. 4, p. 91, syn. for H. fibrosa.

parea, sce Microspongia parva.

Hystrisionala, Ulrich, (in press,) Geo. Sur. 11l., vol. 8, p. 245. [lity. hystrix, jorcupine; spongia, sponge.] Subglobular or ovoid; spicules arranged radiately from the base, most of them biacerate and taper each way to pointed ends, sone trific at one end, and others fourrayed. Type H. carbonaria.

carbonaria, Ulrich, (in press,) Geo. Sur. IIl., vol. 8, 1), 245, Coal Meas.

Ischadiles tessellatus, see Receptaculites tessellatus.

Lastocta Dia, Hinde, 1884. [Ety. lasios, shaggy; klados, twig.] Skelet on composed of elongate, slender, straight, ncrerate spicules, pointed at, botlı ends. Type L. compressa.

hindii, Ulrich, (in press,) Gico. Sur. Ill., vol. 8 , p. 249 , Keokuk Gr.

Lepidoutres, Ulrich, 1879, Jour. Ciu. Soc. Nat. Hist., vol. 2, p. 20. [ [ity. lepis, seale; lithos, stone.] Subspherical or subcylindrical bodies, hollow within and consisting of exteriorly imbricating scales. 'Type L. dickliauti. 'The name was preoccupicd in mineralogy. dickhanti, Ulrich, 1879, Jour. Cín. Soc. Nat. Hist., val. 2. j. 2I, Hud. Riv. Gr.

elongatus, Uĺrich, 1879, Jour. Cin. Soc. Nat. Hist., vol. 2, p. 22, Hud. Riv. $\mathrm{Gr}$. This is not distinet from I. dickhauti.

Leptomitus, Walcott, 1886, Bull. U. S. Geo. Sur. No. 30, p. 89. [Ety. leptos, fine; mitos, thread.] Elongate bodies, formed of fine, thread-like, longitudinal lines, apparently imbedded in a delicate membrane, slowly expanding from a narrow base. Type L. zitteli.

zitteli, Walcott, 1886, Bull. U. S. Geo. Sur. No. 30 , p. 89 , Georgia Gr. 
Leptopterion, Ulrich, 1889, Am. Geol., vol. 3, p. 239. [Ety. leptos, thin; poterion, cup.] Obconical, annulated free sponge; wall thin, outer surface reticulated. Type L. mammiferum. Not well defined. mammiferum, Ulrich, 1889, Am. Geol., vol. 3, p. 239, Hud. Riv. Gr.

Lomusia, Carpenter \& Brady, 1869, Trans. Roy. Soc., p. 742. [Ety. proper name.] Small foraminifer, with oval or elliptical test, consisting, primarily, of a continuous lamina coiled upon itself, with interspaces divided in to chambers. Type L. persica.

columbiana, Dawson, 1879, Quar. Jour. Geo. Soc., vol. 35, p. 74, Coal Meas.

Lunulites? dactyloides, see Cerionites dactyloides.

Lyrodictya, Hall, 1884, 35th Rep. N. Y. St. Mus. Nat. Hist., p. 466. [Ety. lyra, lyre; dictuon, net.] Cyathiform, reticulate fronds composed of stellate spicules, with broad,'strong, longitudinal bands of acicular spicules, showing an alternating bifurcation. Type L. romingeri.

romingeri, Hall, 1884, 35th Rep. N. Y. St. Mus. Nat. Hist., p. 476, Keokuk Gr. Megastroma, Dawson, 1883, Report on Redpath Mus. No. 2, p. 12. [Ety. megas, great; stroma, layer.] Somewhat like Stromatopora; layers consisting of two membranes, beset with spicules, pointing in wards like two brushes facing each other; membranes porous or reticulate. Type M. laminosun.

laminosum, Dawson, 1883, Rep. on Redpath Mus. No. 2, p. 12, Subcarboniferous. Microspongia, Miller \& Dyer, 1878, Jour. Cin. Soc. Nat. Hist., vol. 1, p. 37. [Ety. micros, small; spongia, sponge.] Free, no epitheca; compact, without large openings; structure radiate. Type II. gregaria.

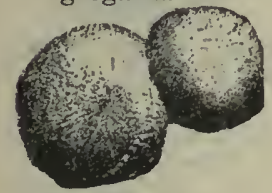

FIG. 108.-Micro-

spongla gregaria, gregaria, Miller \& Dyer, 1878, Jour. Cin. Soc. Nat. Hist., vol. 1, p. 37 , Hud. Riv. Gr.

parva, Ulrich, 1889, (Hindia parva,) Am. Geol., vol. 3, p. 244, Trenton Gr.
Mallerina, Ulrich, 1886, Cont. to Am. Pal., p.34. [Ety. proper name.] Consisting of two suborbicular, thin-walled chambers, outer one with spiral ridges, inner one smooth; at the ends of the outer chamber there is a round opening, surrounded by an elevated border, where the ridges terminate. Type M'. greenei.

greenei, Ulrich, 1886, Cont. Am. Pal., p. 35, Up. Held. Gr.

Nodosinella, Brady, 1876, Monograph Carb. and Perm. Foraminifera, p. 102. [Ety. nodus, knot; ellus, diminutive.] Free, straight, or arcuate, not spiral; constricted at intervals, test imperfo- rate, texture finely arenaceous, aperture simple or compound. Type $\mathrm{N}$. digitata.

priscilla, Dawson, 1868 , (Dentalina priscilla,) Acadian Geology, 1). 285, Carboniferous.

Nullipora, Iamarck, 1801, Śstéme des Auin. sans Vert. [Ety. nullus, no; poros, pore.] Not American Palre. ozoic.

?obtexta, White, 1862, Proc. Bost. Soc. Nat. Hist., vol. 9, p. 33, Burlington Gr.

Orbiculites? reticulata, see Receptaculites reticulatus.

PALEACIS, Edwards \& FIG. 109.-NodoHaime, 1860, Hist. Nat. sinella priscilla. des Coralliaires, vol. 3, Natural size and p. 171. [Ety. palaios, ancient; akis, barb.] Skeleton cuneate or turbinate, adherent, cups 1 to 12 , cell-like, margins crenulate, separated by depressions; substance pierced by microscopic tubuli. Type $P$. cuneiformis.

compressus, Meek \& Worthen, 1860, (Sphenopterium compressum,) Proc. Acad. Nat. Sci. Phil., p. 448, and Geo. Sur. Ill., vol. 2, p. 234, Keokuk Gr.

cuneatus, Meek \& Worthen, 1860, (Sphenopoterium cuneatum,) Proc. Acad. Nat. Sci., p. 448, syn. for P. cuneiformis. cuneiformis, M. Edwards, 1860, Hist. Nat. d. Corollairs, tome 3, p. 171, Warsaw Gr.

enormis, Meek, \& Worthen, 1860, (Sphenopoterium enorme,) Proc. Acad. Nat.

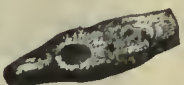

FIG. 110. Palracls cuneiformis.

Sci., p. 448, and Geo. Sur. Ill., vol. 2, p. 146, Kinderhook Gr.

enormis, var. depressus, Meek \&Worthen, 1866, (Sphenopoterium enorme var. depressum,) Geo. Sur. Ill., vol. 2, p. 146, Kinderhook Gr.

obtusus, Meek \& Worthen, 1860, (Sphenopoterium obtusum,) Proc. Acad. Nat. Sci., p. 448, and Geo. Sur. Ill., vol. 2, p. 233 , Keokuk Gr.

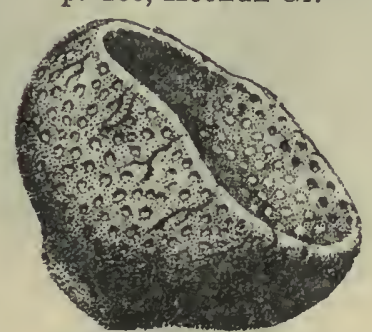

FiG. 111.-Palæomanon cratera.

PALAOMAN ON, Roemer, 1860 , Sil. Fauna West Tenn., p. 12. [Ety. palaios, ancient; Manon, a genus of sponges.] $\mathrm{Cy}$ lindrical or irregular, cupshaped, free, upper surface d is play ing

large, dispersed openings, with inter- 


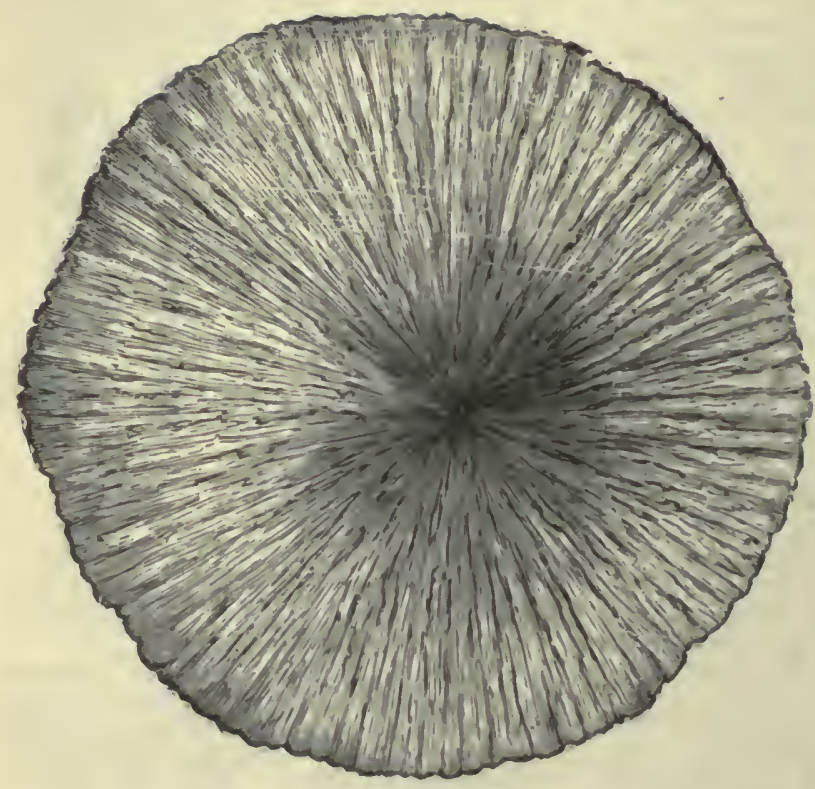

Fig. 112.-Palrospongia trentonensts. Vlew of callee. trentonensis, Worthen, 1875, (Cnemidium trentonense,) Geo. Sur. Ill., vol. 6, p. 491, Trenton Gr.

Pascnolus, Billings, 1857, Rep. of Progr. Geo. Sur. Can., p 342. [Eiy. pasceolus, leather mon. eybug.] Subglobularbod. jes marked picisilit. on the cast clnuuli. as if by polygonal plater, and with i sear or ileprtssion for an a'taching stem. 'Type P. globosus.

claurlii, S. A. Miller, 1874, Cin. Quar. Jour. Sei., vol. 1, 1. 6, Hud. Riv. Gr.

darwini, S. A. Miller, 1874, Cin. Quar. Jour. Sici., vol. 1, p. 5, Hud. Riv. Gr.

globosus, Billings, 1857, Rep. of I'royr. Geo. Sur. Can., p. 343, Trenton Gr.

vening space minutely porous. Type P. cratera.

cratera, Ruemer, 1848, (Siphonia cratera,) Leonb. und Bronn's Jahrb., p. 685, Niagara Gr.

roemeri, Walcott, 1885, Monog. U. S. Geo. Sur., vol. 8, p. 99, Devonian.

Paldzofponata, D’Orhigny, 1850, P'rodr. $\mathrm{d}$. Palcont., t. 1, p. 26. [F.ty. palaios, ancient; spongia, spongr.] Cyathifurm, irregular, surface reticulated irregularly, hv concentric and transverse lines. Type P. eyathiformis.

cyathiformis, Hall, 1847, (-_ cyathiformis, ) Pal. N. Y., vol. 1, p. 72, Trenton $\mathrm{Gr}$.

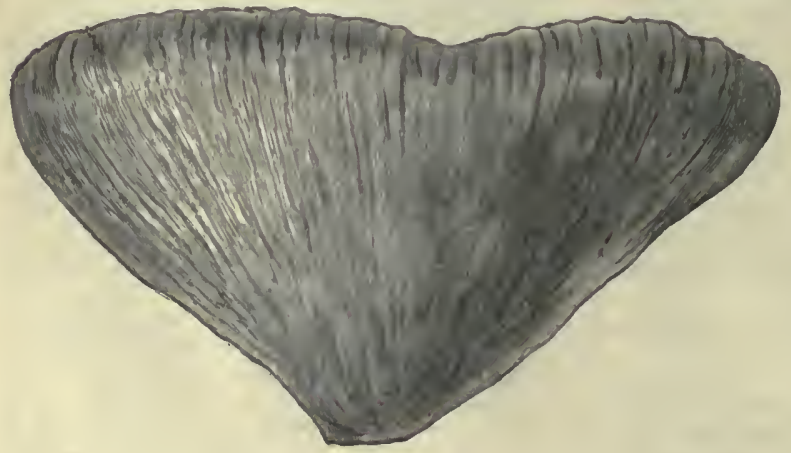

Fig. 113.-Palæospongla trentoneusis. Side view. gregarius, Billings, 1866. Catal. Sil. Poss. Antic., 1). 72, Anticosti Gr.

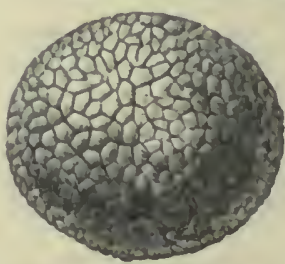

Fra. 115. - Pasceolus darwlul. Upper surface.

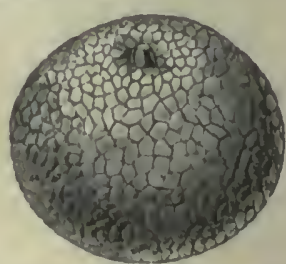

Fıc. 116- Pnsceolus darwinl. Under surface. balli, Billings, 1857, Rep. of Progr. Geo. Sur. Can., p. 342, Anticosti (ir.

intermedius, Billings, 1866, Catal. Sil. Fu4s. Antic., p., 72, Anticosti Gr.

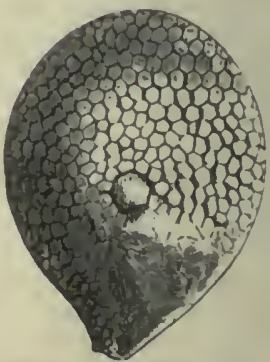

Fra. 117.-Pasceolus halli. 
Pattersonia, S. A. Milller, 1882, Jour. Cin. Soc. Nat. Hist., vol. 5, p. 43. [Ety. proper name.] Solid, amorphous, no large openings; lobed, pendent ex. pansions on the upper surface, and bundles of fine filaments at the base and in the interior, which do not merge into the parenchyma of the sponge; spicules unknown. Trpe P. difficilis. aurita, Beecher, 1889, (Strubilospongia aurita,) Mem. Pea. Mus., vol. 2, p. 28, Trenton Gr.

difficilis, S. A. Miller, 1882, Jour. Cin. Soc. Nat. Hist., vol. 5, p. 43, Hud. Riv. Gr.

tuberosa, Bepcher, 1889, (Strobilospongia tuberosa,) Mem. Pea. Mus., vol. 2, p. 28, Trenton Gr.

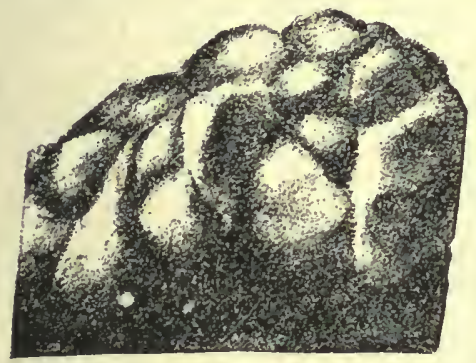

Fig. 118.-Pattersonia difficlis. Fragment of upper surface.

Phragmodictya, Hall, 188t, 35th Rep. N. Y. St. Mus. Nat. Hist., p. 466. [Ety. phragmns, a partition; dictuon, a net.] Cyliudrical or cup.shaped frunds, with a concave diaphragm near the broadly expanded base. Substance composed of a reticulate tissue of six and three rayed spiculis and long cylindrical rods. Type $\mathbf{P}$. catillifornis.

catilliformis, Whitfield, 1881, (Dictyophyton catillifurmis,) Bull. No. 1, Am. Mus. Nat. Hist., p. 18, Kenkuk Gr.

lineata, Hall, 1884, 35th Rнp. N. Y. St. Mus. Nat. Hist., p. 478, Krokık Gr.

patelliformis, Hall, 1884, 35th kej. N. Y. St. Mus. Nut. Hist., p. 478, Keokuk Gr.

Physospongla, Hall, 1884, Abstr. 35th Rep. N. Y. St. Mus. Nat. Hist., p. 467. [Ety. physa, blarder; spongia, sponge.] Frond cylindrical, expanding from the base; surface divided into from 8 to 24 lnngitudinal areas by bands of tubular spicules, and int, regular quadrules by concentric bands of spicules; surface bullate; spicules anchor-shaped. Interzonate tissue finely reticulated. Type $P$. dawsoni.

alternata, Hall, 1884, 35th Rep. N. Y. St. Mus. Nat. Hist., p. 481, Keokuk Gr.

colletti, Hall, 1884, 35th Rep. N. Y. St. Mus. Nat. Hist., p. 480, Kerokuk Gr.

dawsoni, Whitfitd, 1881, (Uphantæenia dawsoni,) Bull. No. 1, Am. Mus. Nat. Hist., p. 16, Keokuk Gr.
Protocyathus, Ford, 1878, Am. Jour. Sci. and Arts, $3 \mathrm{~d}$ ser., vol. 15, p. 124, syn. for Ethmophyllum.

Protospongia, Salter, 1864, Quar. Jour. Geo. Soc., vol. 20, p. 238. [Ety. protos, first; spongia, sponge.] Skeleton loose, reticulate formed of cruciform spiculæ in one plane. Typ “ P. fenestrata.

fenestrata, Salter, 1864, Qnar. Jour. Geo. Soc., vol. 20, p. 238, and Mon. U. S. Geo. Sur., vol. 8. p. 11, Up. Taconic.

rarus, sre Ethmophyllum rarum.

RAufFella, Ulrich, 1859, Am. Geol., vol. 3, p. 235. [Ety. proper nume.] Hollow cylindrical stems or radiully arranged leaves; wall thin, composed of two layers of spicule tissue, inner one porous, outer one composed of large spicules app aring as threads interwoven. Type R. filosa.

filısн, Ulri.h, 1889, Am. Geol., vol. 3, p. 237, Trenton Gr.

palmipes, Ulrich, 1889, Am. Geol., vol. 3, p. 238, Trenton Gr.

Receptaculitks, DeFrance, 1827, Dict. Sci. Nat., tome 45. p. 5. [Ety. receptaculum, receptacle ; lithos, stont.] Subylobular, discoid, or infundiliulitorm ; composed of cylindrical columns, connected at their upper and luwer ends loy transverse stolons. Typ $\mathbf{R}$. neptunei.

arcticus, Etheridge, 1878, Quar. Jour. Geo. Soc., vul. 34, p. 576, L"wer Silurian.

bursiformis, Hall, 1883. Rep. St. Geol., pl. 23, fig. 12-14, Schohнrie Grit.

calciferus, Billings, 1865, Pal. Foss., vol. 1, p. 351 , Calcif. Grr.

canadensis, Billings, 1863, (Ischadites canadensis,) Geo. of Can., p. 309, Anticosti Gr.

circularis, Emmons, 1856, Am. Geol., p. 230. Hud. Riv. Gr.

dactyloides, see Cerionites dactyloides.

devonicus, Whitfield, 1882, Ann. N. Y. Acad. Sci., vol. 2, p. 198, Up. Held. Gr.

eatoni, Hall, 1863, 16th Rep. N. Y. St. Mus. Nat. Hist., p. 68-26, Schoharie Grit.

elegantulus, Billings, 1865, Pal. Foss., vol. 1, p. 360, Calcif. Grr.

ellipticur, Walcott, 1885, Monog. U. S. Geo. Sur., vol. 8, p. 67, Chazy Gr.

elongatus, Walcott, 1885, Monog. U. S. Geo. Sur., vol. 8, p. 66, Chazy Gir.

formosus, Meek \& Worthen, 1870, Proc. Acad. Nat. Sci., p. 23, and Geo. Sur. Ill., vol. 6, p. 500, Niagara Gr.

fungusus, Hall, 1861, Geo. Rep. Wis., p. 15, Galena Gr.

globularis, Hall, 1861, Supp. Geo. Sur. Wis., p. 16, and Geo. Sur. Ill., vol. 3, p. 301, Galena Gr.

hemisphericus, Hall, 1861, Geo. Rep. Wis., p. 16, and Geo. Wis., vol. 4, p. 269, Niagara $G r$.

infundibuliformis, Eaton, 1832, (Coscinopora infundibuliformis,) Geo. Text Book, p. 44, Low. Held. Gr. 
infundibulum, Hall, 1S61, Geo. Rep. Wis., p. 16, Niagara Gr.

insularis, Billings, 1866, Catal. Sil. Foss. Antic., p. 29, Anticosti Gr.

iowensis, Owen, 1852, (Selenoides iowensis,) Geo. Sur. Wis., Iowa, and Minn., p. 587, Trenton Gr.

jonesi, Billings, 1865, Pal. Foss., vol. 1, p.

389, Low. Held. Gr.

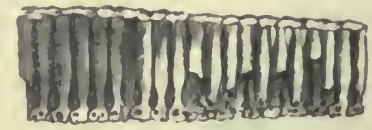

Frc. 119.-Receptaculltes occldentalls, showing the tubes.

mammillaris, Walcott, 18s5, Monog. U. S. Geo. Sur., vol. 8, p. 65, Chazy Gr.

monticulatus, Hall, 1883, Rep. St. Geol., pl. 23, fig. 3-11, Low. Held. Gr.

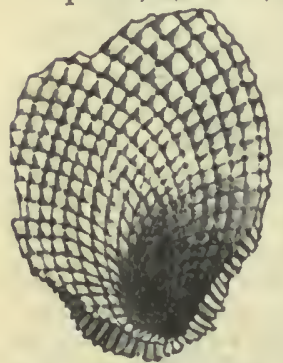

Fra. 120.- Receptrcu11 tes occldentalis, showing the endorhiln, the pores at the angles of the plates, and deeply concave nucleus.

1814, (Orbituloides reticulata, Rep. on

Minn. Lands, p. 70, Niagara Gr.

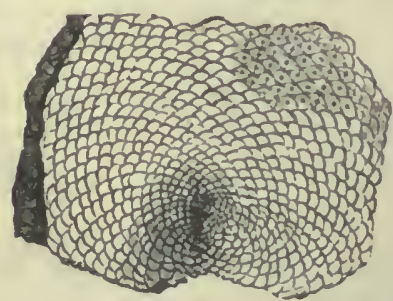

Fig. 121.-Receptaculites occldentalls, showlng the in ucleus and ectorhin.

sacculus, Hall, 1879, Desc. New Species Foss. from Waldron, Ind., 1. 1, and 11th Rep. Geo., and Nat. Hist. Ind., p. 222, Niagara Gr.

squamifer, Hall, 1859, (Dictyocrinus squamifer, $\mathrm{Pul}$. N. Y., vol. 3, p. 135, Low. Held. Gr.

subturbinatus, Hall, 1863, Trans. Alb. Inst., vol. 4, p. 224, Niagara Gr.

sulcatus, Owen, 1844. This name was preoccupied by Goldfuss, and the species is now named $R$. oweni. tessellatus, Winchell \& Marcy, 1865, Mem. Bost. Soc. Nat. Hist., vol. 1, 1. 85, Niagara Gr.

Ris bDaria, Billings, Pal. Foss., vol. 1, p. 357. [Ety, rhabdos, rod.] Sinall, cylindrical bodies, with a rough surface and a perforation in the center. 'Type $\mathrm{R}$. fragilis.

fragilis, Billings, 1565, Pal. Foss., vol. 1, j. 357 , Calciferous Gr.

furcata, Billings, 1S65, Pal. Foss., vol. 1, p. 358 , Calciferous Gr.

Rhomвonctyon, Whitfeld, 1586, Bull. Am. Mus. Nat. Hist., vol. 1, p. 347. [lity. rhombos, rhomb; dichyon, net.] Globular, discoid, or cyathiform, composed of two or more sets of rods crossing each other at various angles, but not clividing, and leaving rhombic spaces filled with another substance. Type R. reniforme.

discum, Whitfield, 1856, Bull. Am. Mns. Nat. Hist., vol. 1, p. 348, Utica Slate.

reniforme, Whitfield, 1886, Bull. Am. Nat. Hist., vol. 1, p. 347, Utica Slate.

reniforme var. rhombiforme, Wlitfield, 1S56, Bull. Am. Mus. Nat. Hist., vol. 1, p. 348 , Utica Slate.

Rotalia, Lamarck, 1504, Ann. Mus. [Lty. rota, wheel.] Not Palacozoic.

baileyi, seo Endothyra baileyi.

Saccammina, Sarg, 1868, Vitlensk-Selsk. Forhandl., p. 248. [Ety. diminutive of saktos, a bag.] Not American Palieozoic.

eriana, Dawson, 1881, Can. Nat., vol. 10, syn. for Calcispliara robusta.

Saccosiongia, Ulrich, 1879, Am. Geo., vol. 34, p. 242. [Ety. sakkos, hag; spongia, sponge.] Subeylindrieal, with a central cloacal cavity extending through it; walls porous, traversed witl, tortuous branching canals intercommunicating with each other. Type S. ruclis.

danvillensis, Ulrich, 1859, Am. Geol., vol. 3 , p. 243, I'renton Gr.

rudis, Ulrich, 1859, Am. Geol., vol. 3, p. 242, Trenton Gr.

Scyphia, Oken, 1815. Not American Palsozoic.

digitata, see Brachiospongia digitata.

stellala, Troost, 1840, not properly defined.

Selenoides, Owen, 1852, syn. for Receptaculites.

iowensis, see Receptaculites iowensis.

Siphonia, Parkinson, 1820, Organ. Rein. Not American Palæozoic.

cratera, see Palcomanon cratera.

imbricato-articulata, see Astylospongia imbricato-articulata.

premorsa, see Astylospongia præmorsa.

Sphenopterium, Meek \& Worthen, 1860, syn. for Palxeacis.

compressum, see Palacacis compressus. cuneatum, see Palreacis cuneiformis.

enorme, see Palseacis enormis.

enorme var. depressum, see Palæacis enormis var. depressus.

obtusum, see Palæacis obtusus. 
Spongia, Linnæus. Not American Palæozoic.

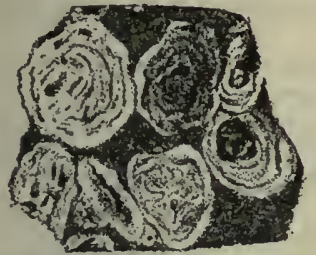

inciso-lobata, see Astylospong i a incisolobata.

stellatim-su le a ta, see Astylospongia stellatimsulcata.

STREPHOCHETUS, Seely, 1885, Am. Jour. Sci. and Arts, 3d ser., vol. 30, p. 355 . [Ety. strepho, I ensis, showill slab.

twine; ochetos, canal.] A free calcareous sponge, showing concentric layers composed of minute twining canals. Type S. ocellatus.

atratus, Seely, 1885, Am. Jour. Sci. and Arts, vol. 32 ; p. 32 , Black Riv. Gr.

brainerdi, Seely, 1885, Am. Jour. Sci. and Arts, 3d ser., vol. 32 , p. 32 , Chazy Gr.

ocellatus, Seely, 1885, Am. Jour.

Sci. and Arts, vol. 30 , p. 357 , Chazy Gr.

richmondensis, S. A. Miller, 1882, (Stromatocerium richmondense,) Jour. Cin. Soc.

、 Nat. Hist., vol. 5, p. 41, Hud. Riv. Gr.

Streptosolen, Ulrich \& Everett, (in press, ) Geo. Sur. Ill., vol. 8, p. 273. [Ety. streptos, twisted; solen, a channel.] Obconical, pedunculate ; central oscula having thin walled tubes extending to the base; oscula surrounded with radiating canals, between which there are vertical ones. Type S. obconicus. obconicus, Ulrich \& Everett, (in press, Geo. Sur. Ill., vol. 8, p. 274, Trenton Gr. Streptospongia, Ulrich, 1889, Am. Geo., vol. 3, p. 244. [Ety. streptos, twisted ; spongia, sponge.] Massive, composed of intertwining vertical lamellæ, separated by tortuous linear interspaces. Type S. labyrinthica. Poorly defined.

labyrinthica, Ulrich, 1889, Am. Geo., vol. 3, p. 244, Hud. Riv. Gr.

Strobilospongia, Beecher, 1889, Mem. Pea. Mus., vol. 2, p. 14, syn. for Pattersonia. aurita, see Pattersonia aurita.

tuberosa, see Pattersonia tuberosa.

Stromatocerium, Hall, 1847, Pal. N. Y., vol. 1, p. 48. [Ety. stroma, layer; kerion, honey-comb.] Hemispherical, composed of numerous concentric vesicular layers, more or less wrinkled, without the pores that characterize Stromatopora. Type S. rugosum.

richmondense, see Strephochetus richmondensis.

rugosum, Hall, 1847, Pal. N. Y., vol. 1, p. 48, Birdseye and Black Riv. Gr.
Stromatopora, Goldfuss, 1826 , Petref.Germ., p. 22. [Ety. stroma, stratum; poros, pore.] Dimorphous masses or extended sheets composed of delicate calcareous laminx, in successive layers, separated by minute, vertical pillars, dividing the interval into minute subquadrangular cavities; the whole is perforated by canals irregularly disposed and possessed of exhalant apertures. Type S. concentrica. cæspitosa, Winchell, 1866, Rep. Low. Penin. Mich., p. 91, Ham. Gr.

compacta, Billings, 1862, Pal. Foss., vol. 1, p. 55, Black Riv. Gr. There is some doubt about the reference of this species to this genus. Possibly it is a bryozoan. concentrica, Goldfuss, 1826, Germ. Petref., 
incrustans, see Caunopora incrustans.

mammillata, Nichnlson, 1873, Ann. and Mag. Nat. Hist., 4 th ser., vol. 12, p. 92, Corniferous $\mathrm{Gr}$.

monticulifera, see Cœnostroma monticuliferum.

nodulata, Nicholson, 1875, Ohio Pal., vol. 2, p. 249, Corniferous Gr.

nulliporoides, Nicholson, 1875, Pal. Prov. Ont., p. 78, Ham. Gr.

nux, Winchell, 1866, Rep. Low. Penin. Mich., p. 91, Ham. Gr.

ostiolata, Nicholson, 1873, Ann. and Mag. Nat. Hist., 4th ser., vol. 12, p. 90, Guelph Gr.

perforata, Nicholson, 1874, Ann. and Mag. Nat. Hist., 4th ser., vol. 13, and Pal. Prov. of Ont., p. 15, Corniferous Gr.

ponderosa, Nicholson, 1875, Ohio Pal., vol. 2, p. 246, Corniferous Gr.

pustulifera, see Canostroma pustuliferum. pustulosa, Safford. Not defined.

solidula, see Coenostroma solidulum.

subcylindrica, James, 1855, Jour. Cin. Soc. Nat. Hist., vol. 7, p. 20, Hud. Riv, Gr. Poorly defined. Not a Stromntopora. None have been found in Lower Silurian rocks.

substriatella, Nicholson, 1875, Ohio Pal., vol. 2, p. 248, Corniferous Gr.

tuberculata, Nicholson, 1873, Ann. and Mag. Nat. Hist., 4th ser., vol. 12, p. 90 , Corniferous Gir.

verrucosa, Troost, 1840, 5th Geo. Rep. Tenn., p. 66, Devonian? Not recognized.

Strotospongra, Ulrich \& Wverett, (in press,) Geo. Sur. Ill., vol. 8, 1). 276. [Ety. strotos, twisted; spongia, sponge.] Funnel-shaped, composed of thin, intricately intertwined vertical leaves, arranged radiately around oscula; cloacal depressions, having apertures of vertical tubes in them; sponge-wall traversed by intertwined canals, having perforated thin walls; spicules minute, three-rayed. 'Type S. maculosa.

maculosa, Ulrich \& Everett, (in press,) Geo. Sur. 11l., vol. 8, p. 277, Trenton Gr. Syringophyllum, Ulricl, (in press,) Geo. Sur. Ill., vol. 8, p. 250. The name was preoc'upied by Edwards of Haime.

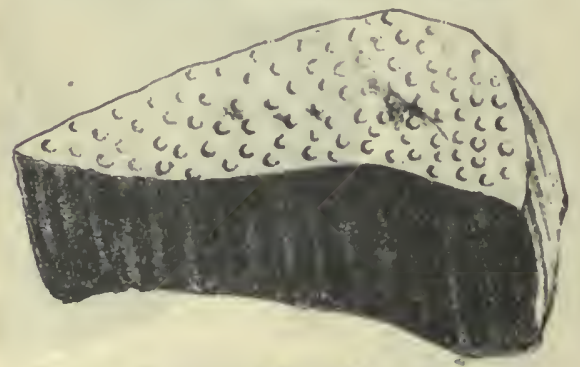

Fro. 125.-Syringostroma columnare.

Syringostroma, Nicholson, 1875, Ohio Pal., vol. 2, p. 251. [Ety. syrinx, pipe; stroma, layer.] Massive, composed of con- centric laming, and vertical villars firmly amalgamated. It is intiuntely related to Stromatopora. Tyje S. columnare.

columnare, Nicholson, 1875, Ohir, Y'al., vol. 2, p. 253, Corniferous Gr.

densum, Nicholson, 1875, Ohio Pal., vol. 2, p. 251, Corniferous Gr.

Textularia palrotrochus, see Valvulina palieotrochus.

Tilamodicty A, Hall, 18S4, 35th Rep. N. I. St. Mus. Nat. Hist. p 466. [Fty. thamnos, shrub; dictuon, net.] Fronds tubular below, rapidly expanding and cyathiform or infundibuliform above, with twelve strong, longitudinal ridges dividing the surface into twelva ureas. Substance reticulate. Type $T$. newberryi.

newber ryi, Hall, 1863, (D i c t.y o. phyton newberryi,) 16th Ren. $\mathrm{N}$. Y. St. Mus. Nat. Hist., p. 87, $\mathrm{WV}_{\mathrm{\Omega}-}$ verly Gr.

Tracryus, Billings, 1865, Pal. Foss., vol. 1, p.211. [Ety. trachus, ró ug h, rugged.] Turbinate or cylindrical, with a cup on the upper surface. It has a

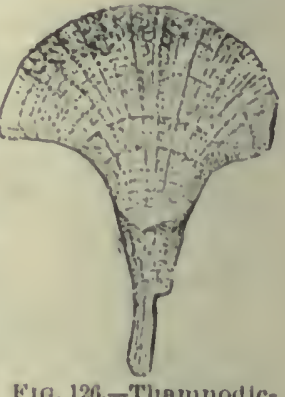

19. 126.-Thamuodlc 'yn newberry! close texlure, without large camuls. 'Tyue 'T'. cyathiforme.
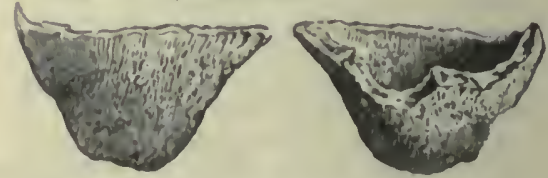

Fit: 127.-Trachyum cyuthlforme.

cyathiforme, Billings, 1865, Pal. Foss., vol. 1, p. 211, Quebec Gir.

rugosum, Billings, 1865, Pnl. 'Poss., vol. 1, j. 212, Quebec Gr.

Trichosrosata, Billings, 1865, l'al. Foss., vol. 1, 1. 357. [Ety. trichias, to show hairs; spongia, sponge.] Large, rudely hemispheric, minutely fibrous, and full of wlongate cylindrical or acerate spicules, just visible to the naked eye. There are also numerous irregular branching canals. Type T. sericea.

sericea, Billings, 1865, Pul. Foss, vol. 1, p. 257, Calciferous $\mathrm{Gr}$.

Upuantasia, Vanuxem, 1842, Geo. Rep. 3d Dist. N. Y., J. 183. [Fiy. uphantos, "loven; tainia, ribbon.] Composed of ligulate radiating and concentric bands, the reticulations being produced by the substance of the frond, and not by superficial strix. For many ytars it was supposed to represent a marine plant. Type U. chemungensis. 
chemungensis, Vanuxem, 1842, Geo. Rep. 3 d Dist. N. Y., p. 183, Chemung Gr.

dawsmi, see Physospongia dawsoni.

Valvulina, D'Orbigny, 1826, Tabl. Method. d. 1. Classe d. Cephalopodes. [Ety. valva, door; inus, implying resemblance.] Free or adherent, spirally trochoid, turbinoid, planoconvex or sub. cylindrical, chambers spirally arranged, sometimes terminating in a rectilinear series. Aperture in the umbilical angle, on the inferior surface, protected by a valvular tongue. Type $\mathrm{V}$. triangularis.

bulloides, Brady, 1876, Monog. Carb. and Perr. Foraminifera, p. 89, Carbouiferous.

decurre is, Brady, 1873, Mem. Geo. Sur. Scotland, pp. 63-95, Carboniferous.

palieotrochus, Ehrenberg, 1854, (Textnlaria palæotrochus,) Mikrogeologie, Carboniferous?

plicata, Brady, 1873, Mem. Geo. Sur., Scotland, pp. 66-95, Carboniferous. rudis, Brady, 1876, Monog.Carb. and Perm. Foraminifera, p. 90, Carboniferous.

Zitrelella, Ulrich \& Everett, (in press,) Geo. Sur. Ill., vol. 8, p. 267. [Ety. proper name.] Pedunculate, attached, variable in shape; upper surface with a shallow, central depression, with thin walled, vertical tubes extending to the base; radiating, inosculating canals, separated by spicular tissue, giving the appearance of vertical fissures. Type Z. typicalis. Ulrich \& Everett refer Palæospongia trentonensis to this genus, so probably this genus is a synonym for Palæospongia.

inosculata, Ulrich \& Everett, (in press, ) Geo. Sur. Ill., vol. 8, p. 271, Trenton Gr. lobata, Ulrich \& Everett, (in press,) Geo. Sur. Ill., vol. 8, p. 270, Trenton Gr.

typicalis, Ulrich \& Everett, (in press,) Geo. Sur. Ill., vol. 8, p. 268, Trenton Gr. They have also made the varieties pistilliformis, subrotunda, and turbinata.

\section{SUBKINGDOM CELENTERATA.}

THE Colenterata (koilos, hollow; entera, intestines) are divided into three Classes: viz., Anthozoa, Hydrozoa, and Ctenophora; the first two of which include the palæozoic fossils of this Subkingdom. The Anthozoa (anthos, flower; zoon, animal) are nore generally known by the name Polypi (polys, many; pous, foot). They are all aquatic, usually cylindrical, organized for sedentary life, have no locomotive organs, and are provided with a circle of retractile tentaculæ around the mouth, which is destitute of any masticating apparatus, and they have a central gastric cavity. There are no special organs of sense, and they increase by budding, dividing, and by means of ova.

The skeleton which the polyps secrete is technically called the corallum. The secretions take place at the sides and lower part of the polyp, but not in the disk or stomach. Each septum is secreted between a pair of radiating, fleshy partitions or septa of the polyp, and hence the radiate structure of ordinary corals is an expression of the internal radiate structure of the polyp. The corallum is essentially a skeleton of carbonate of lime, the open spaces in which show the structure of the polyp animal. The bottom of the calyx, or calycle, in the corallum may be made by the meeting of the septa, or by the twisting of them together, with the addition of a point or columella at the center; or the bottom may be a porous or vesicular mass; or it may be solid, because the coral secretions of the polyp may fill up the pores, or because there are formed periodically, as the polyp grows upward, solid horizontal plates across the bottom, called tabulæ.

Wherever a tabula cuts off the connection of the polyp with the coral below, the tissues below the tabula dry and wither, and we have dead coral below the 
tabula, and the living polyp above. In this way massive corals are formed; the secretions take place at the top, and the animal euts itself off from the coral skeleton below. Prof. Dana says :

"It is not more surprising, nor a matter of more difficult comprehension, that a polyp should form structures of stone (earbonate of lime) ealled coral, than that the quadruped should form its bones, or the mollusk its shell. The processes are similar, and so the result. In each case it is a simple auinal secretion; a secretion of stony matter from the aliment which the animal receives, produced by the parts of the animal fitted for this secreting process; and in each, carbonate of lime is a constituent or one of the constituents of the secretion."

Ordinary corals of the present seas have a hardness a little greater than common marble, or about equal to aragonite, and give a ringing sound when struck with a hammer.

The Anthozoa are divided into three Subclasses, two of which, Zoantharia (zoon, animal; anthos, flower) and Alcyonaria (alkuoneion, a zoopicyte, like the kingfisher's nest), oecur in palrozoic rocks. The Zoantharia are divided into seven orders, four of which are said to be palæozoic: viz., Perforata, Tabulata, Rugosa, and Tubulosa. To the Perforata the genera Protarea and Pleurodictyun have been very doubtfully referred, and the Auloporida have been elassed with the Tubulosa. All other palrozoic corals are referred to the Tabulata and Rugosa. The family Favositidæ is typical of the Tabulata, and the family Cyathophyllida of the Rugosa. Authors are not in accord respecting the ordinal relations of all the families, and hence we will simply arrange them alphabetically.

\section{CLASS ANTHOZOA.}

\section{SUBCIASS ZOANTHARIA.}

FAMLY AULOPORDA.-Aulopora, Romingeria.

Family Chetetide.-Chetetes, Dania, Ptychonema.

Family Columaridde.-Calapoeia, Columnaria, Favistella.

Family Cyathophylude.-Acervularia, Aerophyllum, Amplexus, Anisophyllum, Arachnophyllum, Astræophyllum, Aulacophyllum, Aulophyllum, Axophyllum, Baryphyllum, Blothrophyllum, Bucanophyllum, Campophyllum, Chonophyllum, Clisiophyllum, Coleophyllum, Craspedophyllum, Crepidophyllum, Cyathophyllum, Diphyphyllum, Dunctuella, Elasmophyllum, Eridophyllum, Hadrophyllum, Hallia, Heliophyllum, Heterophrentis, Lithostrotion, Lophophyllum, Omphyma, Pachypliyllum, Palæophyllum, Phillipsastrea, Ptychophyllum, Pyenostylus, Streptelasma, Strombodes, Stylastrea, Trochophyllum, Zaphrentis.

Family Cycloditidx.-Combophyllum, Discophyllum, Microcylus, Palieoeyclus.

Faym Cystiphyldide.-Cystiphorolites, Cystiphyllum, Cystostylus.

Family Favositidd.-Alveolites, Chonostegites, Cladopora, Cœenites, Dendropora, Emmonsia, Favosites, Leptopora, Lunatipora, Michelinia, Pleurodietyum, Pachypora, Sphrerolites, Striatopora, Syringolites, 'Trachypora, Vermipora.

Family Halysitide. - Halysites.

Family Poritide.-Protarea. 
Family Syringoporidæ.-Cannapora, Syringopora, Thecostegites.

Fanily Tetradirde.-Tetradium.

FAMILY TheCIDæ.-Thecia.

\section{Subclass Alcyonaria.}

Family Bolboporitide.-Bolboṕorites.

Family Helioporide.-Heliolites, Lyellia, Plasmopora.

Family Monticuliporidæ.-Dekayella, Dekayia, Diplotrypa, Monotrypa,

Monotrypella, Monticulipora, Nebulipora, Nyctopora, Prasopora.

FAMily Stelliporide.-Stellipora.

\section{CLASS HYDROZOA.}

This class is represented in palæozoic rocks by carbonaceous horny skeletons, called Graptolites. They are usually flattened, forming a thin film between shaly or slaty layers, and generally, in whatever rocks they occur, they are more or less compressed. Specimens are found in clay nodules and in calcareous clay beds, at Cincinnati and vicinity, which are cylindrical branching bodies, or have subquadrate stipes, covered with a thin, carbonaceous coating. The interior of one species is divided by longitudinal partitions of thin, carbonaceous films, into three departments, one of which is only about half the capacity of either of the other two. The denticulated edges on flattened films become projecting cells on more perfect specimens. The projecting cells may be subcircular or angular, and lead directly to the interior. When the interior substance is absent, and the cells are pressed together, instead of being pressed into the stipe, there is presented a diagrammatic side view of the cells, which furnishes the usual saw or denticulated aspect, but which gives a very imperfect, and frequently a very erroneous, idea of the form of the animal. This was the first Order of organisms to reach a high state of development, and the first to become extinct.

\section{ORDER GRAP'TOLIDA.}

Family Callograptide.-Acanthograptus, Callograptus, Cyclograptus, Dendrograptus.

Family Dictyonemide.-Calyptograptus, Dictyonema, Rhizograptus.

FAMILY Graptolitide.-Cladograptus, Climacograptus, Clonograptus, Dicranograptus, Didymograptus, Diplograptus, Graptolithus.

Family Glossograptide.-Glossograptus, Retiograptus.

Family Nemagrap'Tid As.-Nemagraptus.

Family Monograptide,-Monograptus.

FaMily Inocaulide.-Inocaulus.

Family Megalograptide.-Megalograptus.

Family Oldhaminde.-Oldhamia.

Family Phyllograptide.-Phyllograptus.

Family Ptilograptide.-Ptilograptus.

FAMILY RASTRITIDA.-Rastrites.

Family Retiolitide.-Retiolites. 
Family Staurograptida.-Staurograptus.

Fammy Thamnograptids.-Bythograptus, Thamnograptus. Family Uncertain.-Dawsonia.

Acantiograptus, Spencer, 1878, Can. Nat.,

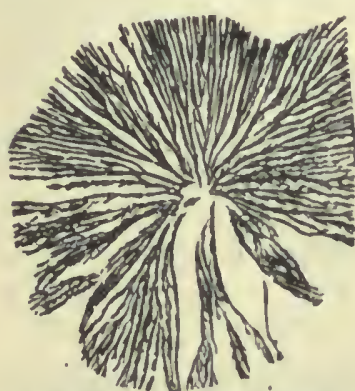

F1G. 128.-A canthograptus pulcher.

Can. Nat, vol, Can. Nat., vol. 8, p. 463, and Bull. No.1, pulcher, Spencer, 1884, Bull. No. 1, Mus.

Univ. St. Mo., p. 32, Niagara Gr.

A cervularia, Scliweigger, 1820, Handb. der

Naturg., p. 418. [Ety. acerrus, a heap; considered as a body.] Compound, massive, cells presenting two separated walls, as in Aulophyllum; septa well developed between the walls, but much less in the central area; no columella tabulx little develo ped; increasing by gemmation. Type A. baltica.

a d j u n c t i va, White, 1880, Proc. U. S. Nat. Mus., vol. 2, p. 255, and Cont. to $\mathrm{Pal}$. No. 6 , p. 120, Carboniferous.

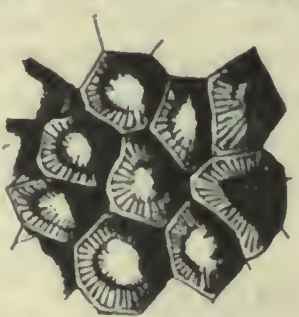

F10. 129.-A cervularia clintonensis.

clintonensis, Nicholson, 1875, Ohio Pal., vol. 2, p. 227, Niagara Gr.

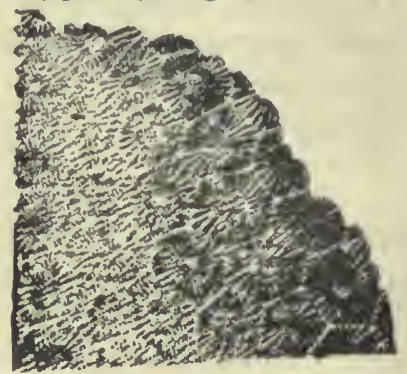

FIG. I30.-Acervularla davidsoni.

davidsoni, Edwards \& Haime, 1851, Pol. Foss. d. Terr. Pal., p. 418, Up. Held. and Ham. Gr. inequalis, Hall \& Whitfield, 1573, 2id Rep. N. Y. St. Mus. Nat. Hist., p. 23:3, Chemung $\mathrm{Gr}$.

pentagona, Goldfuss, 1826, (Cyathopliyllum pentagonum,) Petref. Germ., p. 60 , Devonian.

profunda, Hall, 1858, Geo. Sur. Iowa, p., 477, Ham. Gr.

AcropnyLuU, Thomson \& Nicholson, 1876, Ann. and Mag. Nat. Hist. 4th ser., vol. 17, p. 455 . [Ety. akros, summit? phyllon, leaf.] Corallum sinuple, turbinate, or sub-cylindrical, straight, or curved; septa numerous, well-developed, coalescing, and curving as thiy reach the tabulie,

forming proninent, tortuous ridges on the central, elevated portion, and becoming complicated with the tabula to form the conspicuous, central p rominon ce, which often forms a central axis ; fossette reaches from the base of the elevation to the margin of the calyx; exterior usually constricted. Type A. oneidaense.

oneidaense, Billings, 1859, (Clisiopliyllum oneidaense, Can. Jour., p. 128, Up. Held. Gr.

agaricia, Lamarck, 1801, Syst. des A nim. sans Vert. Not Palæozoic.

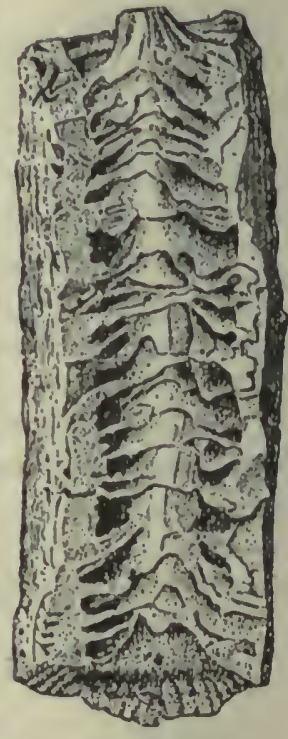

Fin. 181-AcropliyiIum oneldnense. swinderniana, see Thecis swinderniana. A lveolitus, Iamarck, 1801, Syst. des Anim. sans Vert., p. 375. [Ety. alveus, cavity; lithos, stone.) Dendroid, massive, or inerusting; corallites short, prismatic, or cylindrical; walls united; tabuluc complete; mural pores large, usually near the angles of the tubes, few in number; calices oblique, lower lip most prominent; septa absent, or forming toothlike projections. Type A. escharoides.

aretica, Woodward, 1879, Lond. Geo. Mag. n. s., vol 5, Devonian.

billingsi, Nicholson, 1874, Geo. Mag. n. s., vol. 1, p. 55, Up. Held. Gr.

confertus, Nicholson, 1874, Geo. Mag. n. s., vol. 1, p. 54, Up. Held. Gr. 
cryptodens, Billings, 1859, Can. Jour., vol. 4, p. 115, Up. Held. Gr.

distans, Nicholson, 1874, Geo. Mag. n. s., vol. 1, p. 54, Up. Held. Gr. dubia, see Favosites dubius.

explanatus, Hall, 1883, Rep. St. Geol., pl. 13, fig. 16, and Pal. N. Y., vol. 6, p. 11, Low. Held. Gr.

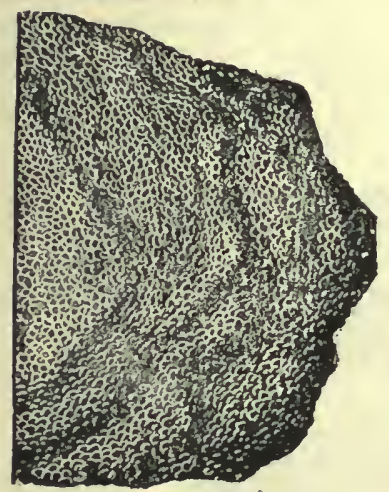

Fra. 132.-Alveolites gold fussi. exsul, see Callopora exsul.

fischeri, see Pachypora fischeri.

frondosus, see Pachypora frondosa. goldfussi, Billings, 1860 , Can. Jour., vol. 5 , p. 255, Ham. $\mathrm{Gr}$.

granulosus, $\mathrm{J}$ a $\mathrm{m}$ e $\mathrm{s}$, 1875, Catal. Cin. Foss., p. 2

Not defined so as to be recognized.

hemisphericus, D’Orbigny, 1850, Prodr. d. Paléont.. t. 1, p. 49 . Not defined so as to be recognized.

irregularis, Whitfield, 1878, Ann. Rep. Geo. Sur. Wis., p. 72 , and Geo. Wis., vol. 4, p. 251, Hud. Riv. Gr.

labechi, Edwards \& Haime, 1851, Pol. Foss. d. Terr. Pal., p. 257, Anticosti Gr. labiosus, Billings, 1859, Can. Jour., vol. 4, p. 114, Up. Held. Gr.

megastoma, Winchell, 1866, Rep. Low. Penin. Mich., p. 89, Ham. Gr.

multilamella, Meek, 1877, U. S. Geo. Sur. 40th Parallel, vol. 4, p. 25, Devonian.

niagarensis, Nicholson \& Hinde, 1874, Can. Jour., vol. 14, p. 150, Niagara Gr. niagarensis, Rominger, see $\mathrm{A}$. undosus. ramulosus, Nicholson, 1874, Geo. Mag. n. s., vol. 1, p. 55, Up. Held. Gr.

repens, Fought, 1749, (Millepora repens,) Amaen. Acad., vol. 1, p. 99, Niagara Gr. reticulata, see Fayosites reticulatus.

rockfordensis, Hall \& Whitfield, 1873, 23d Rep. N. Y. St. Mus. Nat. Hist., p. 229, Chemung Gr.

roemeri, Billings, 1860, Can. Jour., vol. 5, p. 255, Ham. Gr.

selwyni, Nicholson, 1874, Geo. Mag. n. s., vol. 1, p. 15, Up. Held. Gr.

squamosus, Billings, 1860, Can. Jour., vol. 5, p. 25i, Up. Held. Gr.

strigillatus, Winchell, 1866, Rep. Low. Peninsula Mich., p. 89, Ham. Gr.

subramosus, Rominger, 1876, Foss. Corals, p. 43, Ham. Gr.

undosus, S. A. Miller, 1883, Am. Pal. Foss., $2 d$ ed., p. 262, Niagara Gr. Proposed for the species described by Rominger in 1876 , in Foss. Corals, p. 40 , under the preoccupied name of A. niagarensis. vallorum, Meek, 1868, Trans. Chi. Acad. Sci., p. 86, Devonian.

Amplexus, Sowerby, 1814, Mineral Conchology, vol. 1, p. 165. [Ety. amplexus, encircling.] Resembles Zaphrentis, éxcept the septa do not extend to the center, they leave the upper surface of the tabulæe exposed in that part; septal fossula bighly developed in the upper portion of the corallum; tabulæ well developed; surface usually constricted. Type A. coralloides.

annulatus, Whitfield, 1878, Ann. Rep. Geo. Sur. Wis., p. 80 , and Geo. Wis., vol. 4, p. 314, Niagara Gr.

cingulatus, Billings, 1862, Pal. Foss., vol. 1, p. 106, Mid. Sil.

coralloides, Sowerby, 1814, Min. Conch., vol. 1, p. 165, Warsaw Gr.

exilis, Billings, 1875, Can. Nat. and Geol., vol. 7 , p. 232 , Up. Held. Gr.

fieldeni, Etheridge, 1878, Quar. Jour. Geo. Soc., vol. 34, p. 589, Niagara Gr.

fenestratus, Whitfield, 1878, Ann. Rep. Geo. Sur. Wis., p. 80, and Geo. Wis., vol. 4, p. 278, Niagara Gr.

fragilis, White \& St. John, 1868, Trans. Chi. Acad. Sci., p. 116, Keokuk Gr.

hamiltoniæ, Hall, 1876, Illust. Dev. Foss., pl. 19, Ham. Gr.

intermittens, Hall, 1876, Illust. Dev. Foss., pl. 32, Ham. Gr.

junctus, Hall, 1882, Foss. Corals Niagara and Up. Held. Groups, p. 11, and 35th

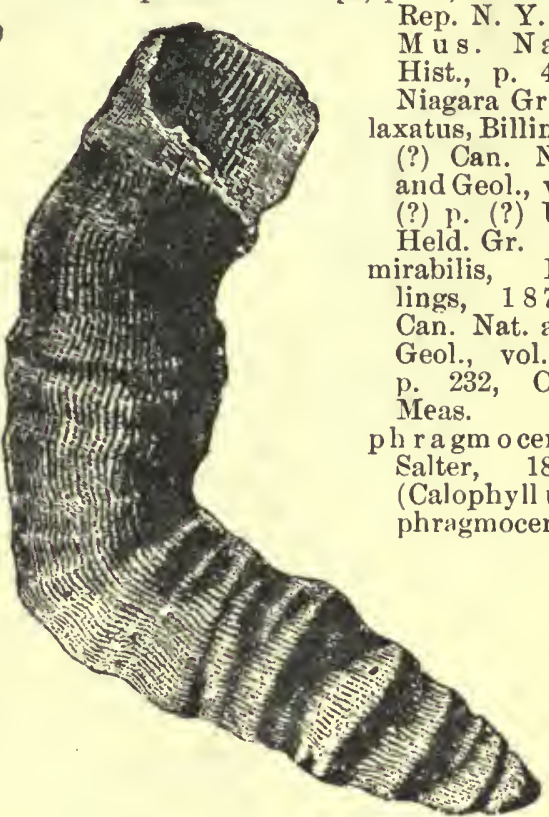

FIG. 133.-Amplexus yandelli

Sutherland's Jour., vol. 2, p. ccxxx, Niagara Gr.

shumardi, Edwards \& Haime, 1851, (Cya. 
thophyllum shumardi,) Pol. Foss. Terr. Pal., p. 370, Niagara Gr.

uniformis, Hall, 188\%, Foss. Corals Niagara \& Up. Held. Grs., p. 11, and 35th Rep. N. Y. St. Mus. Nat. Hist., p. 415, Niagara Gr.

yandelli, Edwards \& Haime, 1851, Pol. Foss. d. Terr, Pal., p. 344. Up. Held. Gr. zaphrentiformis, White, 1876, Geo. of Uinta Mountains, p. 107, and Cont. to Pal. No. 6, p. 120, Low. Aubrey Gr.

Anisopiyludu, Edwards \& Haime, 1851, Pol. Foss. d. Terr. Pal., p. 351. [Ety. anisos, unequal ; phyllon, leaf.] Distin. guished from Zaphrentis by the great development of three primary septa, one of which faces the septal fossula; t?is fossula extends to the center of the visceral chamber, and there ceases to be distinct from the bottom of the calycle. Type A. agassizi.

agassizi, Edwards \&', Haime, 1851, Pol. Foss. d. Terr. Pal., p. 351, Low. Held. Gr. bilamellatum, Hall, 1852, Foss. Corals Niagara and Up. Held. Grs., p. 9, and 35 th Rep. N. Y. St. Mus. Nat. Hist., p. 413, Niagara Gr.

trifureatum, Hall, 1882, Foss. Corals Niagara and Up. Held. Grs., p. 9, and 12 th Rep. Ind. Geol. \& Nat. Hist., p. 273, Niagara Gr.

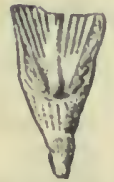

Fro. 134.

A 11180 phyll u in u n $11 \mathrm{ar}$ gum. unilargum, Hall, 1882, Foss. Corals Niagara and Up. Held. Grs., p. 8, and 12th Rep. Ind. Geol. \& Nat. Hist., p. 272, Niagara Gr.

Inthophyllum, Schweigger, 1820, Handb. der. Naturg., p. 41\%, Not a Palieozoic genus.

denticulatum, Goldfuss, 1826 , Petref. Germ., p. 46, Niagara Gr. Not determined.

expansum, Owen, 1840, Rep. on

Mineral Lands, 1). 69. Not defined so as to be recognized.

Arachnopirylum, Dana, 1848, Koophytes U. S. Expl. Exped., vol. 8, p. 360. [Ety. arachne, spider; phyllon, leaf.] Massive, encrusting, having obtusely defined polygonal scars, with a depressed, flattened center, in which the septa ineet; septa thin, perforated; buds marginal, structure vesicular, arranged in transverse undulations, corresponding to the form of the cells; no defining walls to the center or between the stars; center marked by a few vertical strix, resulting from the twisted edges of the septs. Type A. baltica. (Acervularia baltica of authors.)

richardsoni, Salter, 1852, Sutherland's Jour., vol. 2., p. cexxxii, Up. Sil.

Astræa, Lamarck, 1816, Hist. Nat. d. Anim. sans Vert., vol. 2, p. 257. Not a Palæozoic genus.

gigas, see Phillipsastrea gigas.

hennahi, see Smithia hennahi.

helianthoides, see Heliophyllum halli.

mammillaris, see Strombodes mammillaris. mammillaris, see Lithostrotion mammillare. rugosa, see Cyathopliyllum rugosun.

tessellata, Troost. Not defined.

Astreopirylum, Nicholson \& Hinde, 1874, Can., Jour., vol. 14, p. 152. [Ety. aster, star; phyllon, leaf.] Corallumagregate; corallites cylindrical and united by numerous mural expansions, which form complete floors; septa meeting in the center, forming a columella; costal radii prolonged over the successive exothecal floors; tabulx rudimentary or absent (?). 'Type $A$. gracile.

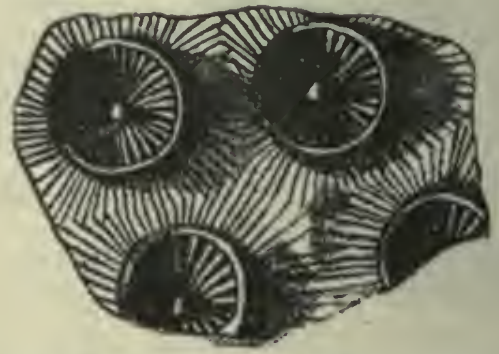

Fio. 185.-Astriophyllum graclle, grently en. linged, showlng cullces, contluent intral expanslons, und costal radil.

gracile, Nicholson \& Minde, 1874, Can. Jour., vol. 14, p. 153 and Pal. Ontario, p. 57, Niagara Gr.

Astrocerium, Hall, 1852, Pal. N. Y., vol. 2, p. 120. [Ety. aster, star; kerion, honeycomb.] It was supposed to be distinguislied from Favosites by the presence of twelve or more slender spiniform rays, but it is a synonym. Type $\boldsymbol{A}$. venustum.

constrictum, see Favosites constrictus. parasiticum, see Favosites parasiticus. pyriforme, see Favosites pyriformis. venuatum, see Favosites venustus.

A ulacolirllum, Edwards \& IIaime, 1850, Brit. Foss. Corals, p. Ixvii. [Fty. aulos, furrow; phyllon, leaf.] Resembles Hallia, though the septal fossula is not re. placed by a primary septum, lut forms a narrow groove at the bottom where the adjoining septa meet. Type $\mathbf{A}$. sulcatum.

bilaterale, Hall, 1882, Foss. Corals Niagara and Up. Held. Grs., p. 25, and 35th Rep. N. Y. St. Mus. Nat. Hist., p. 429, Up. Held. Gr.

convergens, Hall, 1882, Foss. Corals Niagara and Up. Held. Grs., p. 22, and 12th Rep. Ind. Geo., p. 281, Up. Held. Gr. cruciforme, Hall, 1882, Foss. Corals Niagara and Up. Held. Grs., p. 24, and 12 th Rep. Ind. Geo., p. 283, Up. Held. Gr. pinnatum, Hall, 1882, Foss. Corals Niagara and Up. Held. Grs., p. 23, and 12 th Rep. Ind. Geo., p. 284, Up. Held. Gr. poculum, Hall, 1882, Foss. Corals Niagara and Up. Held. Grs., p. 25 and 1'th Rep. Ind. Geo., p. 283, Up. Held. Gr. 
præciptum, Hall, 1882, Foss. Corals Niagara and Up. Held. Grs., p. 24, and 12th Rep. Ind. Geo., p. 280, Up. Held. Gr. prateriforme, Hall, 1882, Foss. Corals Niagara and Up. Held. Grs., p. 23, and 12th Rep. Ind. Geo., p. 282, Up. Held. Gr.

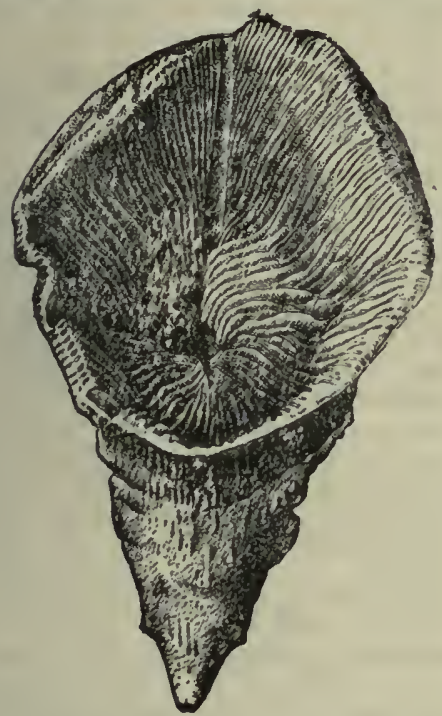

Fig. 136.-Aulacophyllum prlnceps.

princeps, Hall, 1882, Foss. Corala Niagara and Up. Held Grs., p. 23, and 12th Rep. Ind. Geo., p. 281, Up. Held. Gr.

reflexum, Hall, 1882, Foss. Corals Niagara and Up. Held. Grs., p. 24, and 12th Rep. Ind. Geo., p. 284, Up. Held. Gr.

sulcatum, D'Orbigny, 1850, (Caninia sulcata,) Prodr. d. Pal.t. 1, p. 105, and 12 th Rep. Ind. Geo., p. 279, Up. Held. Gr.

tripinnatum, Hall, 1882, Foss. Corals Niagara and Up. Held. Grs., p. 25, and 12 th Rep. Ind. Geo., p. 285, Up. Held. Gr. trisculcatum, Hall, 1882, Foss. Corals Niagara and Up. Held. Grs., p. 25, and 12 th Rep. Ind. Geo., p. 279, Up. Held. Gr.

Aulophyllum, Edwards \& Haime, 1850, Brit. Foss. Corals, p. Ixx. [Ety. aulos, pipe; phyllon, leaf.] Corallum simple; septa well-developed; mural investments double, the interior dividing the visceral chamber into two parts-one central and colummar, the other external and annular; no columella; tabulæe not well developed. Type A. proliferum.

richardsoni, Meek, 1868, Trans. Chi. Acad. Sci., p. 81, Devonian.

Aulopora, Goldfuss, 1826, Petref. Germ., p. 82. [Ety, aulos, pipe; poros, pore.] Creeping, increasing by latero-basal gemmation; corallites pyriform, trumpet-shaped, the cavity of each communicating with the one from which it springs; no pores; septa absent or rudimentary. Type A. serpens.

annectans, Clarke, 1885, Bull, 16. U. S. Geo. Sur., p. 63, Genesee shales.

aperta, Winchell, 1866, Rep. Low. Penin. Mich., p. 91, Ham. Gr.

arachnoidea, Hall, 1847, Pal. N. Y., vol. 1, p. 76, Trenton and Hud. Riv. Gr.

canadensis, see Hederella canadensis.

conferta, Winchell, 1866, Rep. Low. Penin. Mich., p. 91, and Rominger's Foss. Corals, p. 88, Ham. Gr.

cornulites, Hall, 1883, Rep. St. Geo., pl. 2, figs. 21 and 22, Low. Held. Gr.

cornuta, see Romingeria cornuta.

cyclopora, Winchell, 1866. Rep. Low. Penin. Micl., p. 92, Ham. Gr.

elongata, Hall, 1887, Pal. N. Y., vol. 6., p. 5, Low. Held. Gr.

erecta, Rominger, 1876, Foss. Corals, p. 88, Ham. Gr.

filiformis, see Hederella filiformis.

iowensis, Hall \& Whitfield, 1873, 23d Rep. N. Y. St. Mus. Nat. Hist., p. 235, Chemung Gr.

precius, Hall, 1876, 28th Rep. N. Y., St. Mus. Nat. Hist., p. 107, Niagara Gr.

repens, Walch, et Knorr, 1775, (Milleporites repens,) Sammlung von Merkw., vol. 3 , p. 179, and Sil. Fauna W. Tenn., p. 28 , Niagara Gr.

saxivada, Hall \& Whitfield, 1873, 23d Rep.

N. Y. St. Mus. Nat. Hist., p. 235, Chemung Gr.

schoharie, Hall, 1874, 26th Rep. N. Y. St. Mus. Nat. Hist., p. 110, Low. Held. Gr. serpens, Goldfuss, 1826, Germ. Petref., p. 82, and Rominger's Foss. Corals, p. 87, Ham. Gr.

serpuloides, Winchell, 1866, Rep. Low. Penin. Mich., p. 91, Ham. Gr. subtenuis, Hall,

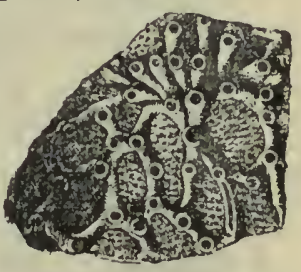

FIG. 137.-Aulopora serpens. 1883, Rep. St. Geo., pl. 2, fig. 9-20, Low. Held. Gr.

tubiformis, Goldfuss, 1826, Germ. Petref., p. 82, and Murch. Sil. Syst., Up. Held. and Ham. Gr.

tubula, Hall, 1883, Rep. St. Geo., pl. 2, fig. 7-8, Low. Held. Gr.

umbellifera, see Romingeria umbellifera.

vanclevii, Hall, 1883, 12th Rep. Ind. Geo., p. 255, Niagara Gr.

Axinura, Castlenau, syn. for Lithostrotion.

canadense, see Lithostrotion canadense.
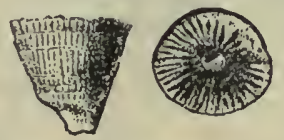

FIG. 138.-Axophyllum rude.
Axopiiyllum, Edwards \& Haime, 1850 , B rit. Foss. Corals, p. Ixxii. [Ety. axon, a $x$ is; phyllon, leaf.] Corallum simple, trochoid, and in structure resembling Lithostrotion. Type A. expansum. 
infundibulum, Worthen, 1875, Geo. Sur. Ill., vol. 6 , p. 525, Coal Meas.

rude, White \& St. John, 1868, Trans. Chi. Acad. Sei., p. 115, Coal Mleas.

Baryphyludi, Edwards \& Haime, 1850, Brit. Foss. Corals, p. lxvi. [Ety. barys, heavy; phyllon, leaf.] Corallum short; calice superficial; slight septal fossula corresponding to one of the branches of a cross, the other three of which are primary septa; younger septa inclined toward the primary ones. Type B. verneuilanum.

arenarium, Meek \& Worthen, 1868, Geo. Sur. Ill., vol. 3, p. 409, Onondaga $\mathrm{Gr}$.

fungulus, White, 1878, P'roc. Acad. Nat. Sci. Phil., p. 29, Niagara Gr.

verneuilanum, Edwards \& Haime, 1551, Pol. Foss. d. Terr. Pal., p. 352, Niagara $\mathrm{Gr}$.

Blotiropiy luua, Billings, 1859, Can. Jour., vol. 4, p. 130. [Ety. blothros, tall-growing; phyllon, leaf.] Corallum simple, turbinate, or eylindrical, having the central region occupied by flat, transverse diaphragms; an intermediato area, with strong radiating septa, and an outer area, in which there are imperfect diaphragms, projecting upward, and having on their upper surface rudimentary septa; a thin, complete epitheca, and a septal fossette. Type B. decorticatum.

approximatum, Nicholson, 1873, Can. Nat.

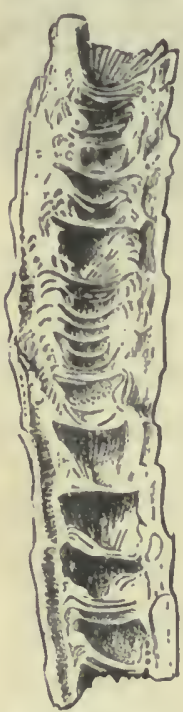

FIG. 139. - Blothropbylium promissum. and Geo., vol. 7, p. 140, Up. Held. Gr.

exspitosum, Rominger, 1876, Foss. Corals, p. 114, Niagara Gr.

decorticatum, Billings, 1859, Can. Jour., vol. 4. p. 130 , Up. Held. Gr.

multicalicatum, Hall, 1582, Foss. Corals Niagara and Up. "Held. Grs., p. 44, and 35th Rep. N. Y. St. Mus. Nat. Hist., p. 448, Up. Held. Gr.

papulosum, Hall, 1882, Foss. Corals Niagara and Up. Held. Grs., p. 44 , and 35 th Rep. N. Y. St. Mus. Nat. Hist., p. 448, U1). Held. Gr.

promissum, Hall, 1882, Foss. Corals Niagara and Up. Held. Grs., p. 45 , and 12 th Rep. Ind. Geo., p. 304, Up. Held. Gr.

sinuosum, Hall, 1882, Foss. Corals Niagara and Up. Held. Grs., p. 45, and 35th Rep. N. Y. St. Mus. Nat. Hist., 1. 449, Up. Held. Gr.
Bolboporites, Pander, 1830, Beitr. zur Geognosie Russlands, p. 106. [Ely. bolbos, bulb ; poros, pore.] Small, globular, showing basal attacliment; structure dense. The type of the genus is said to be neither a coral nor bryozoan, but to belong to the Echinodermata. The form which Billings referred to the genus is probably a coral.

americanus, Billings, 1859, Fit. 140,-BolljoCan. Nat. and Geo, corles ainerivol. 4, p. 429, Chazy of buse ; $b, c, d$, Gr.

Buca Noph yluum, Ulrich, 1856, Cont. to $\mathrm{Am}$. Pal., p. 31. [Ety. bucane, trumpet; phyllon, leaf.] Corallum trumpet-shaped, consisting of a long, slender, cylindrical stem, with the upper end abruptly dilated into a cup, which becomes oblique in older specimens; interior of cup with numerous septal strix, which become obsolete at the bottoin. Type B. gracile.

gracile, Ulrich, 1886, Cont. to An. Pal., p. 31, Up. Held. Gr.

Bytuogurtus, Hall, 1861, Geo. Rep. Wis., p. 18. [Ety. buthos, in tile deep; gropho, I write.] Frond consisting of a central stipe, with closely arranged lateral branches, flexuous or recurved; celluliferous on one side; substance corneous brown or black. Type B. laxus.

laxus, Hall, 1861, Geo. kep. Wis., p. 19, Trenton Gr.

Calamopora. Goldfuss, syn. for Favosites.

bazallicu, see Fuvosites basalticus.

cellulala, Castelnau, 1843. Not recognized.

cristata, see Favosites cristatus.

cumberlandica, see Favosites eumberland. icus.

farosa, see Favosites favosus.

fibrosa, see Monticulipora fibrosa.

fibrosa, Roemer, see Hindia fibrosa.

forbesi var. discoidea, see Favosites forbesi var. discoideus.

goldfussi, see Favosites goldfussi.

gothlandica, see liavosites gothlandicus.

heliolitiformis, see Favosites heliolitiformis.

hemispherica, see Favosites hemispherieus.

infundibulifomnis, Goldfuss, identified by D'A rehiac und Verneuil. Not an Ameriean species.

mackrothi, see Chetetes mackrothi.

maxima, see Favosites maximus. minula, Castelnau. Not recognized. minutissima, Castelnau. Not recognized. radians, Castelnau. Not recognized. tumida, see Chetetes tumidus.

verneuili, Castelnau, syn. for Monticulipora fibrosa.

winchelli, see Favosites winchelli.

Cala Pecia, Billings, 1865, Can. Nat. and Geo., $2 d$ ser., vol. 2, p. 425 . [Ety. kalos, 
beautiful ; poikilos, spotted.] Composite, hemispherical or subspherical, corallites slencler, tubular, perforated as in Favosites, outside striated by imperfectly developed costæ; septa about 24 ; tabulæ thin; when corallites are not in contact the space is filled with vesicular tissue. Type C. canadensis.

anticostiensis, Billings, 1866, Catal. Sil. Foss. Antic., p. 32, Hud. Riv. Gr.

canadensis, Billings, 1865, Can. Nat. and Geo., $2 \mathrm{~d}$ ser., vol. 2, p. 426, Black Riv. Gr.

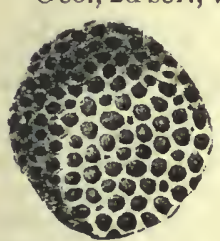

FIG. 141.-Cala poecla crlbriformis. cribriformis, $\mathrm{Nich}$ ol son, 1874, (Columnopor a cribriformis,) Geo. Mag., vol. 1, p. 253, and Pal. Ohio, vol. 2 , p. 186, Hud. Riv. Gr.

huronensis, Billings, 1865, Can. Nat. and Geo., 2d ser., vol. 2, p. 426 , Hud. Riv. Gr.

Calceola, Lamarck, 1801, Syst. des Anim. sans Vert., p. 139. [Ety. calceola, a slipper.] Corallum simple, operculated, subtriangular, pyramidal; calice deep; septa narrow; structure dense. Type C. sandalina.

americana, Safford, syn. for C. tennesseensis.

attenuala, Lyon, 1879, Proc. Acad. Nat. S.'i. Phil., p. 45, Niagara Gr. Lindstrom referred this species to his genus Rhizopliyllum.

corniculum, Lyon, 1879, Proc. Acad. Nat. Sci. Phil., p. 43, Niagara Gr. Syn. (?) for C. tennesseensis.

coxi, Lyon, 1879 ,

Proc. A c a d

Nat. Sci. Phil., p. 44, Niagara Gr. Syn. (?) for C. tennesseensis.

plicata, Conrad, 1840 , Ann. Rep. N. Y., p. 207, Low. Fig. 142. - Calceola sandaHeld. Gr.

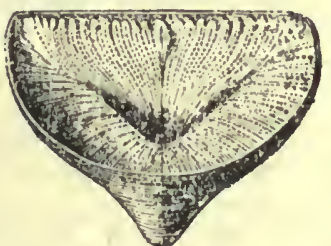

pusilla, Hall, 1882, Foss. Corals Niagara and Up. Held. Grs., p. 15, and 35th Rep. N. Y. St. Mus. Nat. Hist., p. 419 , Niagara $\mathrm{Gr}$.

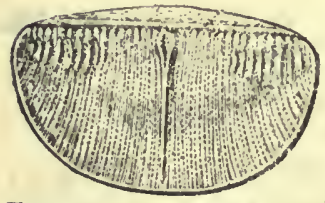

FIG. 143.-Calceola sandalina, operculum. sandalina, Iamarck. Not American.

ten n e s seen sis, Roemer, 1852, Lethæ Geognost., p. 385 , and Sil. Fauna W. Tenn.,p. 73, Niagara Gr. Lindstrom referred this species to his genus Rhizophyllum.

Callograptus, Hall, 1865, Can. Org. Rem. Decade 2, p. 133 . [Ety. kallos, beautiful; grapho, I write.] Flabellate fronds, with numerous slender, bifurcating branches proceeding from a strong stem ; branches and divisions celluliferous on one side, striate on the other; sometimes distantly and irregularly united by transverse dissepiments. Type C. elegans.

elegans, Hall, 1865, Can. Org. Rem. Decade 2, p. 134, Quebec Gr. or Up. Taconic.

granti, Spencer, 1884, Buil. No. 1, Mus. Univ. St. Mo., p. 21, Niagara Gr. minutus, Spencer, 1884, Bull. No. 1, Mus. Univ. St. Mo., p. 22, Niagara Gr. multicaulis, Spencer, 1884, Bull. No. I, Mus. Univ. st. Mo. p. 22. Niagara Gr.

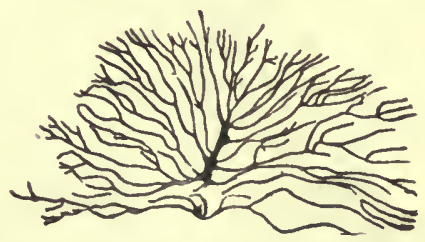

Fia. 141.-Cullograptus uiagarensis.

niagarensis, Spencer, 1878, Can. Nat., vol. 8, and Bull. No. 1, Mus. Univ. St. Mo., p. 21, Niagara Gr.

salteri, Hall, 1865, Can. Org. Rem. Decade 2, p. 135, Quebec Gr., or Up. Taconic.

Calophyllum, Dana, 1846, Am. Jour. Sci., p. 183, syn. for Amplexus.

phragmoceras, see Amplexus phragmoceras.

Calyptograptus, Spencer, 1878, Can. Nat., vol. 8, p. 459. [Ety. kalyptos, covered; grapho, I write.] Cyathiform, bifurcating branches, not connecting laterally; resembles Dic'yonema. Type $\mathrm{C}$. cyathiformis.

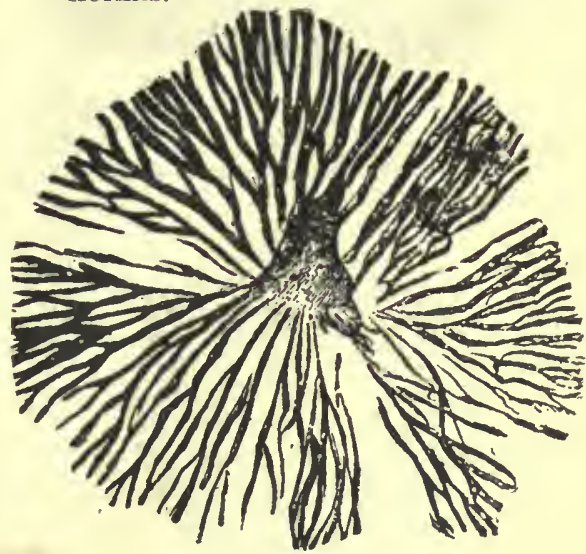

FIG. 145.-Caly ptograptus cyathiformis.

cyathiformis, Spencer, 1878, Can. Nat., vol. 8, p. 459, Niagara Gr.

subretiformis, Spencer, 1878, Can. Nat. vol. 8, p. 460, Niagara Gr. 
Campophyllum, Edwards \& Haime, 1850, British Foss. Corals, p. Ixviii. [Ety. kampto, I bend; phyllon, leaf.] Simple, tall, protected by an epitheca; septa well developed; tabula very large and smooth toward the center; interseptal area vesicular. Type C. flexuosum.

nanum, Hall \& Whitfield, 1873 , 23d Rep. N. Y. St. Mus. Nat. Hist., p. 232, Chemung Gr.

texanum, Shumard, 1859, Trans. St. Louis Acad. Sci., vol. 1, p. 388 , Permian.

torquium, Owen, 1S52, (Cyathophyllum tor q u i u m, )

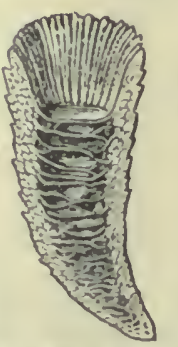

Fı, 146.-Cam. po p h y llum hexuosu m. tion $1 / 2$ dinm. s li o w I n g snioolh central tabulne.

Geo. Rep. Wis., Iowa, and Minn., pl.

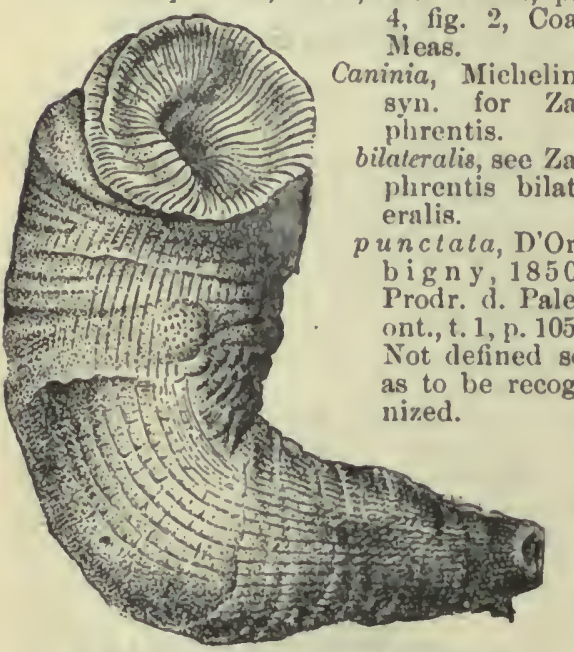

Fig. 147.-Campopliyllum torqulum.

sulcata, D’Orbigny, 1850, Prodr. d. Paleont., t. 1, p. 105 . Not defined so as to be recognized.

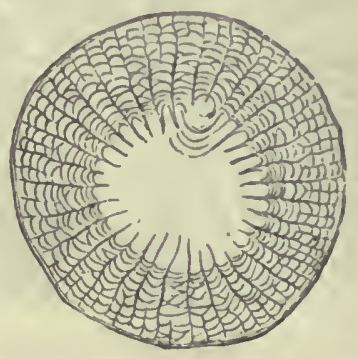

FIG. 148.-Campophyllum torqulum. Trausverse section.

Cannapora, Hall, 1852, Pal. N. Y., vol. 2, p. 43. [Ety. kanna, reed; poros, pore.] Massive, tubular, united externally by tabulæ; distinguished from Syringopora, by the regular transverse external tabula and by the internal structure of the corallites. Type C. junciformis. annulata, Nicholson \& Hinde, 1874, Can. Jour., p. 154, and Pal. Prov. of Ontario, p. 58 , Niagara Gr.

junciformis, Hall, 1852, Pal. N. Y., vol. 2, p. 43, Clinton Gr.

Caryophyllia, Lamarck, 1816. Not Palæozoic. cornicula, see Zaphrentis cornicula. gigantea, see Zaphrentis gigantea. pulmonea, see Zaphrentis pulmonea. Catenipora, Lamarck, 1816, syn. for Halysites. michelini, Castlenau, syn for Halysites catenulatus.

Cinetetes, Fisclier, 1837. Oryet. du Gouv. Moscou, p. $159 . \quad$ [Fty. chaite, hair.] Corallum conglomerate; corallites very long, basaltiform; calyces polygonal; tabula not connected or on the same plane in diflerent corallites; walls aunalgamated, imperforate; growth fissiparous. Type C. radians.

abmiptus, see Ilonotry pella abrupta.

requidistans, Hall, 1881, Bryozoans Up. Held Gr., p. 4, Up. Held. Gr.

approximatus, Nicholson, syn. for Monticulipora dalii.

arbusculus, see Monotrypella arbuscula. areticus, Haughton, 1857, Jour. Roy. Dub. Soc., vol. 1, Silurian.

attritus, Nicholson, syn. for Dekayia aspera. barrandii, see Montículipora barrandii.

briareus, see Monotrypella briareus.

calicula, see Aspirlopora caliculus.

carbonarius, Worthen, 1875, Geo. Sur. Ill., vol. 6 , p. 526, Coal Meas.

cincinnatiensis, seo Monticulipora cincinnatiensis.

clathratulus, James \& Nicholson, syn. for Monticulipora pavonia.

clavacoideus, see Leptopora clavacoidea. colliculatus, Hall, 1883, Rep. St. Geo., pl. 8, fig. 1-4, and Pal. N. Y., vol. 6, p. 11, Low. Held. Gr.

columnaris, see Tetradium columnare. compressus, see Peronopora compressa. consimilis, see Monotrypella consimilis. corticans, Nicholson, syn. for Spatiopora tuberculata.

corticosa, see Trematopora corticosa.

crassus, Lonsdale, 1845, (Stenopora erassa,)

Russ. and Ural Mts., vol. 1, p. 631, Coal

Meas.

crebrirama, Hall, 1881, Bryozoans Up. Held. Gr., p. 4, Up. Held. Gr. dalei, see Monticulipora dalii.

decipiens, see. Monticulipora decipiens. delicatulus, see Monticulipora delicatula. discoideus, see Amplexopora discoidea. egenus, Hall, 1881, Bryozoans Up. Held. Gr., p. 4, Up. Feld. Gr.

elegans, sce Discotry pa elegans.

exilis, Dawson, 1868, Acad. Geo., p. 287, (Stenopora exilis,) Subcarb.

expansus, Ringueberg, 1886, Bull. Buf. Soc. Nat. Sci., vol. 5, p. 20 . Not properly defined. 
fibrosus see Monticulipora fibrosa.

fliasa, see Monticulipora filiasa.

fletcheri, Edwards \& Haime, as identified in Ohio Pal., vol. 2, p. 197, is Monticulipora ulrichi.

frondosus, see Monticulipora frondosa.

fruticosus, Hall, 1876, Illust. Foss., pl. 38, Hanl. Gr.

fruticosus, Hall, 1883, see Monotrypella arbuscula.

furcatus, Hall, 1876, Illust. Devon. Foss., pl. 37, Ham. Gr.

fusiformis, Whitfield, 1878, Ann. Rep. Geo. Sur. Wis., p. 70, and Geo. Wis., vol. 4, p. 248 , Hud. Riv. Gr. Not a Chetetes; probably a Bryozoan.

gracilis, see Batostomella gracilis.

granuliferus, see Homotrypella granulifera.

hamiltonensis, Winchell, 1866, Rep. Low. Penin. Mich., p. 89, Ham. Gr.

helderbergix, see Ptychonema helderbergire.

humilis, Hall, 1876, Illust. Devon. Foss., pl. 37, Up: Held. Gr.

internascens, Hall, 1881, Bryozoans Up. Held. Gr., p. 4, Up. Held. Gr.

irregularis, see Monticulipora irregularis.

jamesi, see Batostoma jamesi.

lycoperdon, see Monticulipora lycoperdon.

mackrothi, Geinitz, 1846, (Calamopora mackrothi,) Grund, p. 586, Permian, American (?)

mammulatus, see Monticulipora mamuiata.

microscopica, Winchell, 1866, Rep. Low. Penin. Mich., p. 90, Ham. Gr.

milleporaceus, Edwards \& Haime, 1851, Mon: d. Pol. Fuss. d. Terr. Pal., p. 272, Coal Meas.

moniliformis, see Monticulipora moniliformis.

monticulatus, Hall, 1883, Rep. St. Geo., pl. 8 , fig. 5-7, and Pal. N. Y., vol. 6, p. 12 , Low. Held. Gr.

muscatinensis, White, 1876, Proc. Acad.

Nat. Sci. Phil., p. 27, Devonian.

newberryi, see Prasopora newberryi.

nodulusus, see Callopora nodulosa.

onealli, see Callopora onealli.

ortoni, see Atactoporella ortoni.

pavonia, see Ptilodictya pavonia.

petechialis, see Petigopora petechialis.

petropolitanus, Pander, 1830, Russ. reiche, p. 105. Not an American species.

pulchellus, Edwards \& Haime, as identi-

fied in Ohio Pal., vol. 2, p. 195, is Monticulipora andrewsi.

quadrangularis; see Paleschara quadrangularis.

quadratus, see Monotrypella quadrata.

ramosus, see Monticulipora ramosa.

rhombicus, Nicholson, syn. for Monotrypella quadrata.

migosus, see Monticulipora rugosa.

rugosus, Edwards \& Haime, is a variety of Monticulipora ramosa.

sigillarioides, see Callopora sigillarioides.

sphæricus, see Favosites spbæricus.

spinigerus, Lonsdale, 1845, (Stenopora spinigera,) Geo. Russ. and Ural Mts., vol. 1, p. 631, Coal Meas.

subglobosus, see Monticulipora subglobosa. subpulchellus, see Monticulipora subpulchella.

tabulatus, see Ptychonema tabulatum. tuberculatus, see Spatiopora tuherculata. tumidus, Phil-

lips, 1836 ,

(Calamopora

tumida,)Geo

Yorkshire, p.

200 , Subcarb.

undulatus, s e e

Mon ti c u-

lipora un-

dulata.

venustus, see

M o n ticulip-

ora venusta.
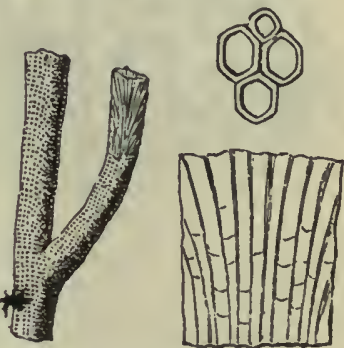

Chо Nophyllum, Fig. 149.-Chetetes tumidus. Ed wards \&

Haime, 1850, Brit. Foss. corals, p. Ixix.

[Ety. chonos, funnel; phyllon, leaf.] Corallum simple, constituted, principally, by a series of infundibuliform tabulæ, superposed and invaginated, upon the surface of which, equally developed septal radii extend from center to circumference; no walls or columella. Type C. perfoliatum.

belli, Billings, 1865, Can. Nat. and Geo. vol. 2 , p. 431 Clinton Gr.

capax, Hall, 1882, Foss. Corals Niagara \& Up. Held. Grs., p. 6, and 35th Rep. N. Y. St. Mus. Nat. Hist., p. 410, Niagara Gr.

ellipticum, Hall \& Whitfield, 1873, 23d Rep. N. Y. St. Mus. Nat. Hist., p. 233, Chemung Gr.

magnificum, Billings, 1860, Can. Jour., vol. 5 , p. 264 , Up. Held. Gr.

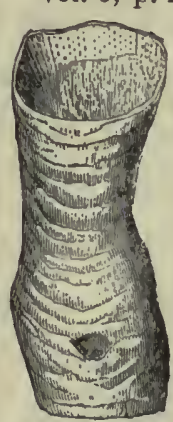

FIG, 150,- Cho nophyllum niagarense. niagarense, Hall, 1852 , (Conophyllum niagarense,) Pal. N. Y., vol.2, p. 114, Niagara Gr.

ponderosum, Rominger, 1876, Foss. Corals, p. 117, Ham. Gr.

sedaliense, White, 1880,12 th Rep. U.S. Geo. Sur. Terr. p. 157, Choteau limestone. vadum, $H$ all, 1884, 35 th Rep. N. Y. St. Mus. Nat. Hist., p. 410, Niagara Gr. validum, Hall, 1882, Foss. Corals Niagara and Up. Held. Grs., p. 6, and 12th Rep. Geo. Ind., p. 272 , Niagara Gr.

Chonostegites, Edwards \& Haime, 1851, Pol. Foss. d. Terr. Pal., p. 299. [Ety. konos, cone; stege, covering.] Subhemispheric; corallites cylindrical, annulated, connected at the expansions, imperforate at the constrictions; mural pores where the icorallites; are contiguous; tabulæ 
numerous; septa consisting of sbort spines; growtu by gemmation. Type C. clappi.

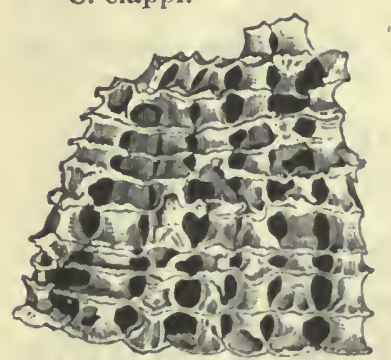

clappi, Ed$\mathrm{w}$ ards \& $\mathrm{H}$ a i $\mathrm{me}$, 1851, Pol. Foss. d. Terr. Pal., p. $299, \mathrm{Up}$. Held. Gr.

ordinatus, Billings, 1859, (Haimeoph y llum ordin a $t$ u $m$,) Can. Jour., vol. 4, p. 139, Up. Held. Gr.

Clanograltés, Gejuitz, 1852, (Cladograp)sus,) Verst. Grauw. Sachs. and Emmons, Am. Geo., p. 107. [Ety. klados, twig; grapho, I write.] Serrations, or cells, arranged on tho outer sides of branching stipes; no axis.

dissimilaris, Emmons, 1856, Am. Geo., p. 107, Upper 'l'uconic.

inæqualis, Emmons, 1856, Am. Geo., p. 107, Upper Taconic.

Cladopora, Hall, 1852, Pal. N. Y., vol. 2, p. 137. [Ety. klados, twig; poros, pore.] Ramuse or reticulate; branches cylindrical or compressed; terminations terete; corallites radiating from the axis, and opening upon the surface in rounded or subangular expanied moulhs; tabulx and septal crests usu. ally obsolcte, sometimes present; corallites connected by mural pores. Type C. seriata.

alpenensis, Rominger, 1876, Foss. Corals, p. 51, Ham. Gr.

aspera, Rominger, 1876, Foss. Corals, p. 56 , Up. Held. Gr.

cespitosa, Hall, 1852, Pal. N. Y., vol. 2, p. 138 , Niagara Gr.

canadensis, Rominger, 1876, Foss. Corals, p. 49, syn. for Pachypora frondosa.

cervicornis, Hall, 1852, Pal. N. Y., vol. 2, p. 139 , Niagara Gr.

dichotoma, Hall, 1858, Geo. Sur. Iowa, p. 478, Ham. Gr.

expatiata, Rominger, 1876, Foss. Corals, p. 57, Up. Held. Gr.

fibrosa, Hall, 1852, Pal. N. Y., vol. 2, p. 139, Niagara Gr.

imbricata, Rominger, 1876, Foss. Corals, p. 56, Up. Held. Gr.

labiosa, Billings, 1859, Can. Jour., vol. 4, p. 138 , Up. Held. Gr.

laquenta, Rominger, 1876, Foss. Corals, p. 46, Niugara Gr,

lichenoides, Winchel] \& Marcy, 1865 , Bost. Soc. Nat. Hist., vol. 1, p. 84 , Niagara $\mathrm{Gr}$.

lichenoides, Rominger, 1876, see C. winchellana.

macrophora, Hall, 1852, Pal. N. Y., vol. 2, p. 140 , Niagara Gr.

magna, Hall \& Whitfield, 1873, 23d Rep. N. Y. St. Mus. Nat. Hist., p. 230, Up. Held. Gr.

multipora, Hall, 1852, Pal. N. Y., rol. 2, p. 140 , Niagara Gr.

palmata, Hall, 1873, 23 d Rep. N. I. St. Mus. Nat. Hist., p.

231, Up. Held. Gr.

pinguis, Rominger, 1S76. Foss. Corals, p. 53, Up. Held. Gr. prolifica, Hall \& Whitfield, 1873, 23d Rep. N. Y. St. Mus. Nat. Hist., p. 230, Up. Held. Gr.

pulchra, Rominger, 1876, Foss. Corals, p. 54, Up. Held. Gr. reticulata, Hall, 1852 , 141, Niagara Gr.

rimosa, Rominger, 1876, Foss. Corals, p. 53, Up. Held. Gr.

rolulista, Rominger, 1876, Foss. Corals, p. 55, Up. Held. and Ham. Gr.

sarmentosa, Hall, 1876, Desc. New Spec. Foss., p. 3, and 11 th Geo. Sur. Ind., p. 230, Níagara Gr.

seriata, Hall, 1S52, Pul. N. Y., vol. 2, p. 137. Niagara Gr.

turgida, Rominger, 1876, Foss. Corals, p. 49 , Up. Held. Gr.

verticillata, Winchell \& Marcy, 1865, Bost. Suc. Nat. Hist., vol. 1, p. 84, Niagura Gr. winchellana, S. A. Miller, 1883, $2 \mathrm{~d}$ Ed. Am. Pal. Foss., p. 265, Up. Held. Gr. Proposed for the species described by Roininger under the preoccupied name of C. lichenoides, in Foss. Corals, p. 47 .

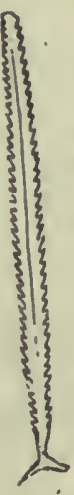

Fro. 153.-Cl1macog $\mathrm{r}$ a $\mathrm{p}$ tus bjcornis.
Cuimacograitus, Hall, 1865 , Can. Org. Rem. Decade 2, p. 111. [Ety. klimax, ladder; grapho, I write.] Simple stipes, with sub. parallel margins, liaving a range of cells on eacls side; axis subquadrate; apertures transversely oval or subquadrate; denticles on the upper side of the apertures. Type C. bicornis.

antennarius, Hall, 1863, (Graptolithus autennarius,) Geo. of Can., p. 955, and Can. Org. Rens. Decade 2, p. 112, Quebec Gr. bicornis, Hall, 1847, (Graptolithus bicornis, Pal. N. Y., vol. 1, p. 268, Hud. Riv. Gr.

emmonsi, Walcott, 1886, Bull. U. S. Geo. Sur. No. 30, p. 93, Upper Taconic. parvus, Hall, 1865, Can. Org. Rem. Decade 2, p. 57, Hud. Riv. Gr. Not defined. typicalis, Hall, 1865, Can. Org. Rem. Decade 2, p. 57, Hud. Riv. Gr. 
Clisiophyllum, Dana, 1848, Explor. Exped., vol. 8, p. 361. [Ety. klision, tent; phyllon, leaf.] Simple, branched or aggregate,

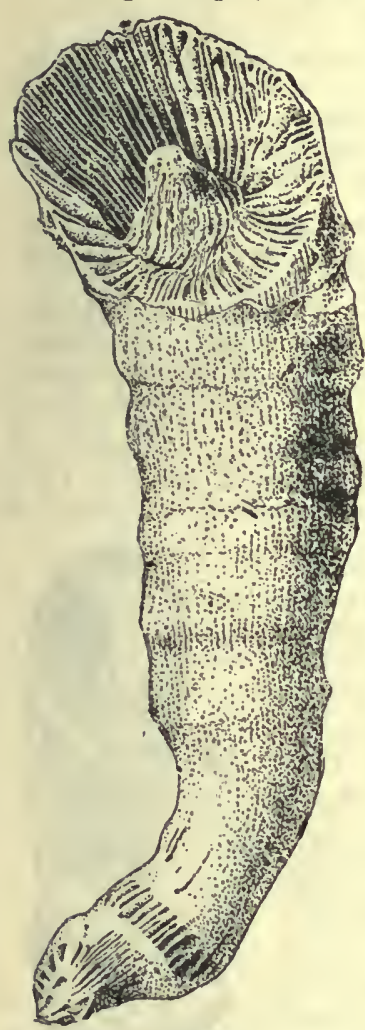

Fre. 154.-Clislophyllum con1geruin.

with vertical radiating lamellæ or septa ; central area vesicular a n d form ing a conical boss or colu. mella, exterior to which the vesicular p lates incline o u t ward a $n d$ upward;calycle deep. 'T'y p e C. danianum. austini, Salter, 1852, (Strephodes austini,) Sutherland's Jour., vol. 2, p. c c x x x , Devonian.

con i ger u m, Rominger, 1876 , (Zaphrentis conigera, ) Foss.Corals, p. 40, Up. Held. Gr.

d a n i a n $\mathrm{m}$, Edwards \& Haime, 1854, Pol. Foss. d. Terr. Pal., p. 412, Low. Held. Gr.

gabbi, Meek, 1864, Pal. California, vol. 1 , p. 8, Carboniferous.

oneidaense, see Acrophyllum oneidaense. pluridiale, Nicholson, 1874, Pal. Prov. Ont., p. 21, Up. Held. Gr.

tumulus, Salter, 1855, Belcher's Last Arctic Voyage, vol. 2, p. 383, Carb.

Clonograptus, Hall, 1873, Ann. and Mag. Nat. Hist., 4th ser., vol, 13. [Ety. klon, twig; grapho, I write.] Composed of numerous slender, regular branching, cylindrical stipes; cells small; forming small denticulations on one side. Type C. rigidus.

flexilis, Hall, 1858, (Graptolithus flexilis,) Geo. Sur. Can., p. 119, and Can. Org. Rem. Decade 2, p. 103, Quebec Gr.

rigidus, Hall, 1857, (Graptolithus rigidus,) Geo. Sur. Can., p. 121, and Can. Org. Rem. Decade 2, p. 105, Quebec Gr.

Canites, Eichwald, 1829, Zoologia specialis, vol. 1, p. 186. [Ety. koinos, living together.] Corallum incrusting, massive, or ramose; corallites vertical or oblique to the surface, remote, imbedded in a conenchyma; calices irregular, prominent, triangular, quincuncially arranged; lower margin most prominent; interstices increasing by age, and reducing the cavity of the cell-tubes; no septa; taubulæ distinct; mural pores large and few. Type C. clathrata.

crassus, Rominger, 1876, (Limaria crassa,) Foss. Corals, p. 45, Niagara Gr.

falcatus, Piout, 1859, (Limaria falcata) Trans. St. Louis Acad. Sci., vol. 1, p. 445, Up. Held. Gr.

fruticosus, Steininger, 1834, (Limaria fruticosa,) Bull. Soc. Geo. France, vol. 1, p. 339, and Pal. N. Y., vol. 2, p. 143, Niagara Gr.

laminatus, Hall, 1852, (Limaria laminata,) Pal. N. Y., vol. 2, p. 143, Niagara Gr.

lunatus, Nicholson \& Hinde, 1874, Can. Jour., p. 149, and Pal. Prov. Ont., p. 55, Niagara Gr.

ramulosus, Hall, 1852, (Limaria ramulosa,) Pal. N. Y., vol. 2,

FIG. 155.- p. 142, Niagara Gr.
Coenites ColEopHyLlim, Hall, 1883, 12th
lunatus. lunatus. Rep. Geo. Sur. Ind., p. 317. [Ety. koleos, sheath; phyllon, leaf.] Corallum simple; substance composed of closely arranged, invaginated tabulæ, more or less oblique to the axis; rays obscure; calices oblique. Type C. rom ingeri.

pyriforme, Hall, 1883, 12th Rep. Geo. Sur. Ind., p.318, Up.Held. Gr.

romingeri, Hall, 1883, 12th Rep. Geo. Sur. Ind., p. 317, Up. Held. Gr.

Columnaria, Goldfuss, 1826 , Germ. Petref., p. 72. [Ety. columnarius, formed of columns.] Aggregate, corallites poly gon a l. longitudina $11 \mathrm{y}$ sulcated, b u t readily separable; no mural pores; tabulæ n u merous; septa rudimentary; in-

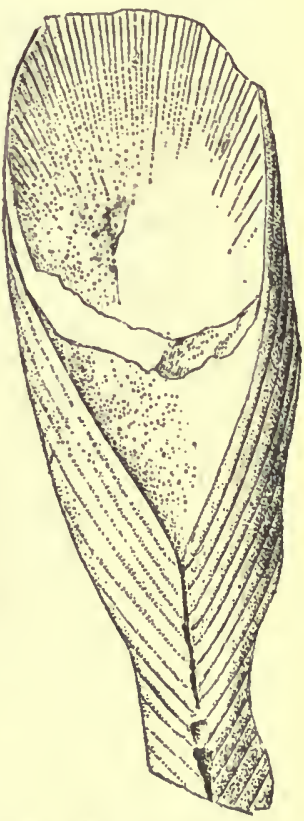

FIG. 156.-Coleophyllum romingeri. crease by fission. Type C. alveolata. alveolata, Goldfuss, 1826, Germ. Petref., p. 72, and Pal. N. Y., vol. 1, p. 47, Black Riv. Gr. 
blainvilli, Billings, 1858, Can. Nat. and Geo., vol. 3, and Rep. of Progr. Geo. Sur. Can., p. 166, Hud. Riv. Gr.

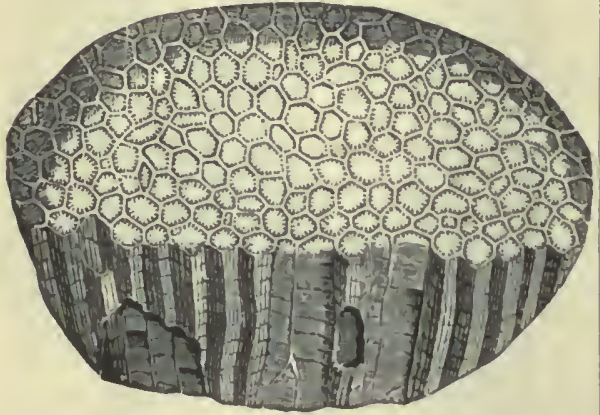

Fia. 157.-Columnaria alveolatu.

carterenis, Safford, 1869, Geo. of Temn., p. 285, Trenton Gr.

divergens, Troost, 1840, 5th Geo. Rep. Tenn., y. 72, Devonian.

erratica, Billings, 1858, Can. Nat. and Geo., vol. 3, and Rep. of Progr. Geo. Sur. Can., p. 167, Trenton Gr.

goldfussi, Billings, 1858, Can. Nat, and Geo., vol. 3, and Rep. of Progr. Geo. Sur. Can., p. 166, Hud. Riv. Gr.

halli, Nicholson, 1879, Tabulate corals, syn. for C. alveolata.

herzeri, Romiuger, 1876, syn. for Favistella stellata.

incerta, Billings, 1859, Can. Nat. and Geo., vol. 4, p. 128, Chazy Gr.

inrequalis, Hall, 1852, Pal. N. Y., vol. 2, p. 223, Coralline limestone.

inlermedia, Eaton, 1832, Gieo. Text-book, p. 41. Not recognized.

mammillaris, Castelnau. Not recognized. multiradiata, Castelnau, 1843, Syst. Sil., p. 44. Not recognized. Probably same as Favistella stellata.

parva, Billings, 1859, Can. Nat. and Geo., vol. 4, p. 428, Chazy Gr.

rigida, Billings, 1858, Can. Nat. and Geo., vol. 3, and Rep. of Progr. Geo. Sur. Can., p. 167, Hud. Riv. Gr.

sutherlaadi, Salter, 1852, Sutherland's Jour., vol. 2, p. cexxxii, Devonian.

troosti, Castelnau, 1843, Syst. Sil., p. 44, syn. for Lonsdaleia papillata.

Columnopora, Nicholson, 1874, London Geo. Mag. N. S., vol. 1, p. 253, and Ohio Pal., vol. 2, p. 186, syn. for Calapocia. cribriformis, see Calapoecia cribriformis.

Combophyllum, Edwards \& Haine, 1850 , Brit. Foss. Corals, p. Ixvii. [Ety. kombos, strip of cloth ; phyllon, leaf.] Curallum, in form like Cyclolites; single septal fossula; septa exsert and regularly radiate. Type C. osismorum.

multiradiatum, Meek, 1868, Trans. Chi. Acad. Sci., p. 84, Devonian.

Conophyllum, Hall, 1852, Pal. N. Y., vol. 2, syn. for Chonophyllum.

niagarense, see Chonophyllum niagarense. Constellaria, Dana, 1848, syn. for Stellipora. constellata, syn. for Stellipora antlieloidea. fischeri, see Stellipora fischeri.

florida, see Stellipora florida.

polystomella, see Stellipora polystomella.

Craspedophylum, Dybowski, 1873, Beschreibung neuen aus Nordanerika, Stammenden, Devonisclien art der Zoantharia rugosa, p. 153 . [Ely. kraspedos, an edge; phyllon leaf.] Probably a syn. for Heliophyllum. Type C. americanum.

americanum, Dybowski, 1873, Beschr. n. a. Nord. Stamm. Dev. a. d. Yoanth. rugosa, p. 153, Up. Held. Gr.

Creipopiylued, Nicholson \& Thompson, 1877, Proc. Roy. Soc. Edinburgh, vol. 9, p. 149. [Ety. krepis, horseshoe; phyllon, leaf.] Distinguislied from Heliopliyllum, by the centrul part of the tabulate area being shut off from the rest of the visceral chamber by a secondary investment, in the form of a central pipe, which is crossed, by tabulie; this pipe is sometimes open or h o r s e shoe-slınped. Type C. archiací.

arehiaci, Billings, 1860, (D i phy phyllum arehinci,) Can. Jour., vol. $\overline{5}$, ]). 260 , Ham. Gr.

subciespitosum, Nicholson, 1874, (Heliophyllum suberespitosum,) Isond. Geo. Mag. n. ser., vol. 1, p. $5 \mathrm{~S}$, Ham. Gr.

Cyatuaxonia, Michelin, 1846, Icon. Zooph., j. 258. [Ety. huathos, cup ; axones, a tablet made to turn on its axis.] Simple; calice deep; columella styliform, strong and prominent; septa extend- Crepldophyllum sul)ing to the columella;

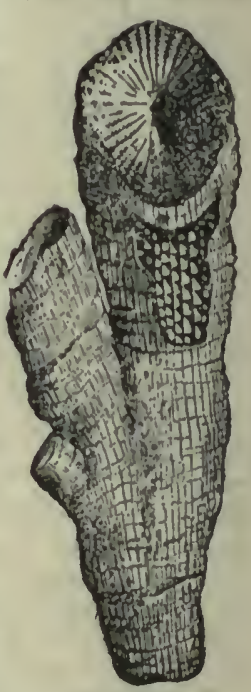

F10. 15\%. the place of one of them occupied, by a deep depression or septal fossula. Type C. cornu.

columellata, Hall, 1882, Foss. Corals Niagara and Up. Held. Gr., and 35 th Rep. N. Y. Mns. Nat. Hist., p. 415, Niagara Gr.

cyuodon, Rafinesque \& Clifford, 1820, (Turbinolia eynodon,) Monog. d. Turbinolides in Ann. d. Phys. d. Brux., t. 5, p. 234, Waverly Gr.

distorta, Worthen, 1875, Geo. Sur. Ill., vol. 6 , p. 526, Coal Meas.

herzeri, Hall, 1882, Foss. Corals Niagara and Up. Held. Gr., p. 11, and $12 t h$ Rep. Ind. Geo., p. 275, Niagara Gr. profunda, Edwards \& Haime, 1851, Pol. Foss. d. Terr. Pal., p. 323, Carboniferous. 
prolifera, see Lophophyllum proliferum. wisconsinensis, Whitfield, 1878, Ann.

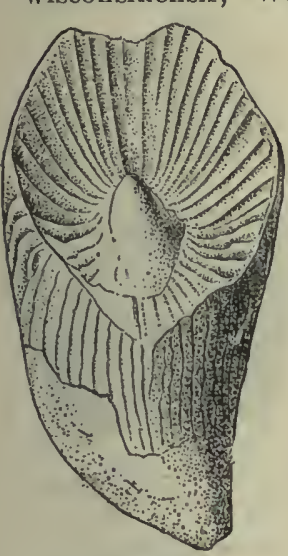

FiG. 159.

Cyathaxnuia herzeri. Geo. Sur. Wis. p. 79 , and Geo. Sur. Wis., vol. 4, p. 277, Niagara $\mathrm{Gr}$.

C Y A T H О Р Y Y L U M, Goldfuss, 1826 , Petref. Germ., p. 54. [Ety, kuathos, cup ; phyllon,leaf.] Simple or composite; no costx; septa extending to the center and twisting together, giving the appearance of a columella; tabulæ only in the center of the visceral chamber, the outer area

being filled with vesicular dissepiments; exterior wall provided with an epitheca. Type C. crspitosum.

agglomeratum, Castelnau, 1843. Not recognized.

ammoris, Castlenau, 1843. Not recognized.

anticostiense, Billings, 1862, Pal. Foss., vol. 1, p. 109, Anticosti Gr., Div. 4.

arborescens, Castelnau, 1843, Syst. Sil., p. 48. Not recognized.

arcticum, Meek, 1868, Trans. Chi. Acad. Sci., p. 79, Devonian.

articulatum, Wahlenberg, (Madreporites articulatus,) Nov. Act. Upsal., vol. 8, p. 97, Up. Sil.

arctifossa, Hall, 1882, Foss. Corals Niagara, und Up. Held. Gr., p. 40, and 12th Rep. Ind. Geo., p. 297, Up. Held Gr.

atlas, Castelnau, 1843, Syst. Sil., p. 47. Not recognized.

billingsi, Dawson, 1868, Acad. Geol., p. 287. Subcarboniferous.

bullatum, Hall, 1882, Foss. Corals Niagara and Up. Held. Grs., p. 41, and 35th Rep. N. Y. St. Mus. Nat. Hist., p. 445 , Up. Held. Gr.

bullulatum, Hall, 1882, Foss. Corals Niagara and Up. Held. Grs., p. 12, and 35 th Rep. N. Y. St. Mus. Nat. Hist., p. 412, Niagara Gr.

crespitosum, Goldfuss, 1826, Petref. Germ., p. 60 , Up. Held. Gr.

calyculare, Owen, 18t0, Rep. on Mineral Lands, p. 69, Devonian.

canaliculatum, Hall, 1882, Foss. Corals

Niagara and Up. Held. Grs., p. 39, and 35th Rep. N. Y. Mus. Nat. Hist., p. 443, Up. Held. Gr.

ceratiles, Goldfuss. Not American.

coalitum, Rominger, 1876, Foss. Corals, p. 108, Up. Held. Gr.

cohærens, Hall, 1882, Foss. Corals Ni- agara and Up. Held Grs., p. 41, and 35th Rep. N. Y. St. Mus. Nat. Hist., p. 445, Up. Held. Gr. c on a tum, Hall, 1876 ,'Illust. Dev, Foss., pl. 31, Ham. Gr. con centricum, Hall, 1882, Foss. Corals Niagara and Up. Held. Gr., p. 42 , and 12 th Rep. Geo. Sur. Ind.,p.316, Up. Held. Gr. conicum, Cas telnau, 1843 , Syst. Sil., p. 48 . Not recognized.

c orinthiu m,

Ow e n, 1840 , Fra. 160.-Cyathophyllum Rep. on Minu. caspitosum. b. transLands, p. 69, section.

Devonian.

cristatum, Rominger, 1876, Foss. Corals, p. 108 , Ham. Gr.

depressum, Hall, 1882, Foss. Corals Niagara and Up. Held. Gr., p. 40, and 12th Rep. Ind. Geol., p. 298. Up. Held. Gr.

dianthus, Goldfuss, 1826, Germ. Petref., p. 54, Onondaga $\mathrm{Gr}$.

dilatatum, Castelnau, 1843, Syst. Sil., p. 48. Not recognized.

distinctum, Castelnau, 1843, Syst. Sil., p. 49. Not recognized.

d'orbignyi, Castelnau, 1843, Syst. Sil., p. 49. Not recognized.

eriphyle, Billings, 1862, Pal. Foss., vol. 1, p. 111, Anticosti Gr., Div. 4.

euryone, Billings, 1862, Pal. Foss., vol. 1, p. 110, Anticosti Gr., Div. 4.

excentricum, Goldfuss. Not American.

exfoliatum, Hall, 1882, Foss. Corals Niagara and Up. Held. Grs., p. 39, and 35th Rep. N. Y. St. Mus. Nat. Hist., p. 443, Up. Held. Gr.

flexuosum, Owen, syn. for Campophyllum torquium.

galerum, Hall, 1876, Illust. Dev. Foss., pl. 32, Ham. Gr.

geniculatuı, Rominger, 1876, Foss. Corals, p. 103, Ham. Gr.

gigas, Yandell \& Shumard, syn. for Zaphrentis gigantea.

goldfussi, Castelnau, 1843, Syst. Sil., p. 47. Not recognized.

goliath, Castelnau, 1843, Syst. Sil., p. 47. Not recognized.

gracile, Troost, 5th Rep. Tenn., Subcarb. Not recognized.

gradatum, Hall, 1876, Illust. Dev. Foss., pl. 31, Ham. Gr.

helianthoides, Goldfuss, see Heliophyllum halli.

houghtoni, Rominger, 1876, Foss. Corals, p. 104, Ham. Gr.

impositum, Hall, 1882, Foss. Corals Niagara and Up. Held. Grs., p. 40, and 
12th Rep. Geo. Sur. Ind., p. 299, Up. Held Gr.

interruptum, Billings, 1862, Pal. Foss., vol. 1 , p. 109 , Mid. Sil.

intertrium, Hall, 1884, 35th Rep. N. Y. St. Mus. Nat. Hist., p. 416 , Niagara Gr. intervesicula, Hall, 1882, Foss. Corals Niagara and Up. Held. Grs., p. 38, and 35th Rep. N. Y. St. Mus. Nat. Hist., p. 442, Up. Held. Gr.

juvene, Rominger, 1876, Foss. Corals, p. 101, Up. Held. Gr.

leseuri, Fdwards \& Haime, 1851, Pol. Foss. d. Terr. Pal., p. 371, Onondaga Gr.

michelini, Castelnau, 1843, Syst. Sil., p. 48. Not recognized.

nanum, Hall, 18i6, Illust. Dev. Foss., pl. 22, Ham. Gr.

nepos, Hall, 1876, Illust. Dev. Foss, pl. 22, Ham, Gr.

nevadense, Meck, 1877, U. S. Geo. Sur. 40tl. Parallel, vol.4, p. 60, Carboniferous. nymphale, Billings, 1862, Pal. Foss., vol. 1, p. 111, Mid. Sil.

palmeri, Meek, 1877, U. S. Geo. Sur. 40th Parallel, vol. 4, p. 33, Devonian.

palum, Hall, 1876, Illust. Dev. Foss., pl. 31, Ham. Gr.

panicum, Winchell, 1866, Rep. Low Penin. Mich., p. 90 , Ham. Gr.

partitum, Wincliell, 1866, Rep. Iow.

Penin. Mich., p. 90, Ham. Gr.

pasithea, Billing8, 1862, Pal. Foss., vol. 1, p. 112, Mirl. Sil.

pelagicum, Billings, 1862, Pal. Foss., vol. 1, p. 108, Anticosti Gr., Div. 2.

pennanti, Billings, 1862, Pal. Foss., vol. 1, p. 107, Mid. Sil.

perfossulatum, Hall, 1882, Foss. Corals, Niagara and Up. Held. Gr., p. 42 and 35th Rep. N. Y. St. Mus. Nat. Hist., p. 446, Up. Held. Gr.

perlamellosum, Hall, 1876, Illust. Devon.

Foss., pl. 39, Up. Held. Gr.

perplicatum, Hall, 1882, Foss. Corals Niagara and Up. Held Grs., p. 42, and 35th Rep. N. Y. St. Mus. Nat. Hist., p. 447, Up. Held. Gr.

picthorni, Salter, 1852, (Strephodes picthorni,) Sutherland's Jour., vol. 2, p. ccxxx, Devonian.

plicatulum, Castelnau, 1843, Syst. Sil., p. 48. Not recognized.

plicatum, Goldfuss, 1826, Germ. Petref. Not American.

profundum, see Streptelasma profundum. puslulatum, Conrad, 1848. Not properly defined.

quadrigeminum, Goldfuss. Not American. radicula, Rominger, 1876, Foss. Corals, p. 109, Niagara Gr.

robustum, Hall, 1876, Illust. Devon. Foss., pl. 22, Ham. Gr.

robustum, Hall, 1882, Foss. Corals Niagara and Up. Held. Grs., p. 43, Up. Held.

Gr. The name was preoccupied.

rollini, Castelnau, 1843, Syst. Sil., p. 49. Not recognized.

rugosum, Hall, 1843, (Astrea rugosa,) (ieo. Sur. 4th Dist. N. Y., p. 159, Up. Held. Gr. scalenum, Hall, 1852, Foss. Corals Niagara and Up. Held. Grs., p. 42 , and $35 \mathrm{th}$ Rep. N. Y. St. Mus. Nat. Hist., p. 446, Up. IIeld. Gr.

scyphus, Rominger, 1876, Foss. Corals, p. 103, Ham. Gr.

septatum, Hall, 1852, Foss. Corals Niagara and Up. Held. Grs., Fig. 101,-Cyatholshyllum p. 41 , and $35 \mathrm{tl}^{\mathrm{s}}$ Rep. N. Y. St.
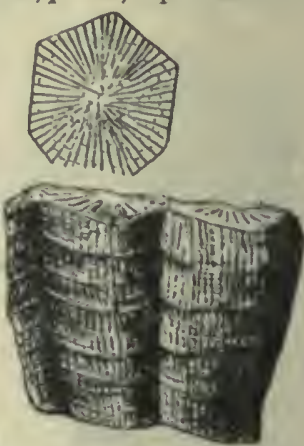

Mus. Nat. Hist., p. 44i, Up. Held. Gr. shumardi, see Amplexus shumardi.

solitarium, Billings, 1866, Catal. Sil. Foss. Antic., p. 93, Clinton and Niagara Grs.

strialulum, Castelnau, Syst. Sil., p. 48. Not recognized.

subcrespitosum, Meek, 1872, 6th Rep. Huyden's Geo. Sur. Terr., p. 470, and U. S. Geo. 40 Parallel, vol. 4, p. 60, Sul)carboniferous.

torquium, see Campophyllum torquium.

turbinalum, Goldfuss. Not American.

undulatum et multiplicatum, Owen, 1840, Rep. on Min. Lands. Not binomial.

validum, Hall, 1876, Illust. Devon. Foss., pl. 39, Up. Held. Gr.

vanuxemi, Hall, 1559, figured without specific name in 1843, Geo. Rep. 4tl Dist. N. I., Tab. 49, fig. 3, 3a, Hanı. Gr. vermiculare, Owen, syn. for Campopliyllum torquium.

vesiculatum, Hall, 18S2, Foss. Corals Niagara and Up. Held. Grs., p. 41, and 12 th Geo. Sur. Ind., p. 297, Up. Held. Gr. resiculosum, see Cystiphyllum vesiculosum. vicinum, Castelnau, 1843, Syst. Sil., p. 48. Not recoguized.

wahlenbergi, Billings, 1862, Pal. Foss., vol. 1, p. 108, Anticosti Gr., Div. 3.

zenkeri, Billings, 1860, Can. Jour., vol. 5, p. 262, Up. Held. Gr.

Cyathopora iowensis, Owen, sce Striatopora iowensis. 'There is no genus Cyathopora, and if Dr. Owen did not intend to refer his species to Cyatliophora, then he failed to establish a genus, by neglecting to define it.

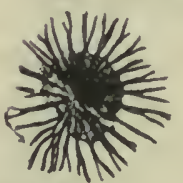

Fig.162.-Cyclograptus rotadentatus.
Crclograprus, Spencer, 1884, Bull. No. 1, Mus. Univ. St. Mo., p. 42. [Ety. kuklos, disk; grapho, I write.] A circular disk, with stipes radiating from the radicle to the margin and in a free manner beyond. Type C. rotadentatus.

rotadentatus, Spencer, 1884, Bull. No. 1, Mus. Univ. St. Mo., p. 42, Niagara Gr. 
Cyclolites, Lamarck, 180l, Syst. Anim. sans Vert., p. 369 . Not a Palæozoic genus. rotuloides, see Palæocyclus rotuloides.

Cystiphorolites. Recently proposed genus but the reference mislaid. [Ety. kustis, a small cavity; phoros, bearing; lithos, stone.] Corallum compound, formed of superimposed series of cups, which in vertical sections appear as layers of unequal,vesiculose plates, reseubling Cystiphyllum; layers radiated, margins of cells broad, expanded, and confluent. Type C. major. Proposed instead of Vesicularia, Rominger, which was preoccupied.

major, Rominger, 1876, iVesicularia major,) Foss. Corals, p. 135, Niagara Gr.

minor, Rominger, 1876, (Vesicularia minor,) Foss. Corals, p. 136, Niagara Gr. variolosus, Rominger, 1876, (Vesicularia variolosa,) Foss. Corals, p. 136, Niagara Gr.

Cystiphyluum, Lonsdale, 1839, Murch. Sil. Syst., p. 691. [Ety. kustis, cavity; phyllon, leaf.] Simple, turbinate, or cylindrical, rarely aggregate ; interior filled with vesicular tissue; septa rudimentary or absent. Type C. siluriense.

aggregatum, Billings, 1859, Can. Jour., vol. 3 , p. 136, Ham. Gr.

americanum, Edwards \& Haime, 1851, Pol. Foss. d. Terr. Pal., p. 464, Ham. Gr.

americanum var. arcticum, Meek, 1868, Trans. Chi. Acad. Sci., p. 80, Ham. Gr.

bifurcatum, Hall, 1882, Foss. Corals Niagara and Up. Held. Grs., p. 55, and 35th Rep. N. Y. St. Mus. Nat. Hist., p. 459, Up. Held. Gr.

bipartitum, Hall, 1882, Foss. Corals Niagara and Up. Held. Grs., p. 55, and 35 th Rep. N. Y. St. Mus. Nat. Hist., p. 459, Up. Held. Gr.

conifollis, Hall, 1876, Illust. Dev. Foss., pl. 30, Ham. Gr.

corrugatum, Hall, 1876, Illust. Devon. Foss., pl. 29, Ham. Gr.

crateriforme, Hall, 1882, Foss. Corals Niagara and Up. Held. Grs., p. 57, and 35 th Rep. N. Y. St. Mus. Nat. Hist., p. 461, Up. Held. Gr.

cylindricum, Lonsdale. Not American.

fruticosum, Nicholson, 1875, Greo. Mag., vol. 2, N. S., p. 32, Corniferous Gr.

grande, Billings, 1859, Can. Jour., vol. 4, p. 138, Corniferous Gr.

granilineatum, Hall, 1882, Foss. Corals Niagara and Up. Held. Grs., p. 14, and 12th Rep. Ind. Geo. p. 274, Niagara Gr.

huronense, Billings, 1866, Catal. Sil. Foss. Antic., p. 92, Clinton and Niagara Grs.

infuudibulum, Hall. 1882, Foss. Corals Niagard and Up. Held. Grs., p. 58, and 35th Rep. N. Y. St. Mus. Nat. Hist., p. 462, Up. Held. Gr.

latiradius, Hall, 1882, Foss. Corals Niagara and Up. Held. Grs., p. 57, and 12 th Rep. Geo. Sur. Ind., p. 304, Up. Held. Gr.

maritimum, Billings, 1862, Pal. Foss., vol. 1, p. 112, Mid. Sil. mundulum, Hall \& Whitfield, 1873, 23d Rep. N. Y. St. Mus. Nat. Hist., p. 234, Chemung Gr.

muricatum, Hall, 1882 Foss. Corals, Niagara and Up. Held. Grs., p. 56, and 35 th Rep. N. Y. St. Mus. Nat. Hist., p. 460, Up. Held. Gr.

nanum, Hall, 1882, Foss. Corals Niagara and Up. Held. Grs., p. 56, and 35 th Rep. N. Y. St. Mus. Nat. Hist., p. 460, Up. Held. Gr.

obliquum, Hall, 1882, Foss. Corals Niagara and Up. Held. Grs.. p. 58, and 35th Rep. N. Y. St. Mus. Nat. Hist., p. 462, Up. Held. Gr.

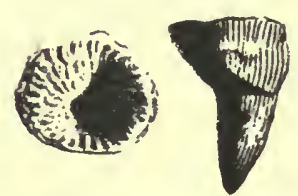

Fı. 163.-Cystiphyllum ohloense. ohioense, Nicholson, 1875, Ohio Pal., vol. 2, p. 234, Corniferous Gr.

pustulatum, Hall, 1882, Foss. Corals, Niagara and Up. Held. Grs., p. 58, and 12th Rep. Geo. Sur. Ind., p. 262, Uj. Held. Gr. quadrangulare, Hall, 1882, Foss. Corals Niagara and Up. Held. Grs., p. 56, and 35th Rep. N. Y. St. Mus. Nat. Hist., p. 460, Up. Held. Gr.

scalatum, Hall, 1882, Foss. Corals Niagara and Up. Held. Grs., p. 59, and 35th Rep. N. Y. St. Mus. Nat. Hist., p. 463, Up. Held. Gr.

senecaense, Billings, 1859, Can. Jour., vol. 4, p. 137, Devonian. squamosum, Nicholson, 1875, Geo. Mag. N. S., vol. 2 , p. 31 , Corniferous Gr.

striatura, Hall, 1882,

Foss. Corals Niaga r a and Up. Held. Grs., p. 59, and 35 th Rep. N. Y. St. Mus.

Nat. Hist., p. $463, \mathrm{Up}$. Held. Gr. sulcatum, Billings, 1859, Can.

Nat. and

Geo., vol. 3, p. 136, Corn ifer- FIG. 164.-Cystiphyllum vesicuous Gr.

s u p erbum,

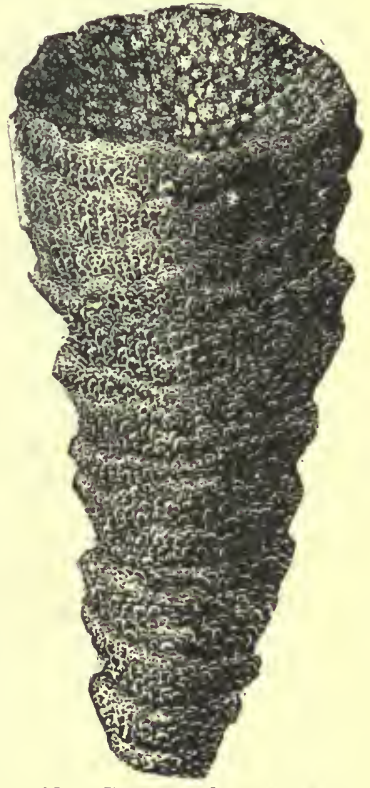

Nicholson, 1875, Geo. Mag. vol. 2, N.S., p. 33, Ham. Gr.

supraplanum, Hall, 1882, Foss. Corals Ni- 
agara and Up. Held. Grs., p. 57, and 35 th Rep. N. Y. St. Mus. Nat. Hist., p. 461, Up. Held. Gr.

tenuiradius, HaIl, 1882, Foss. Corals Niagara and Up. Held. Grs., p. 56, and 35th Rep. N. Y. St. Mus. Nat. Hist., p. 460 , Up. Held. Gr.

varians, Hall, 1876, Illust. Devon. Foss., pl. 29; Ham. Gr.

vesiculosum, Goldfuss, 1826, (Cyathophyllum vesiculosum,) Germ. Petref., p. 58, Devonian.

Cystostylus, Whitfield, 1580, Ann. Rep. Geo. Sur. Wis. and Geo. Wis., vol. 4, p. 273. [Ety. kustis, cavity; stylos, stalk.] Aggregate, cylindrical, corallites in contact or united by transverse filaments; increase by bifurcation, structure cystose as in Cystiphyllum; formed by imperfect transverse plates arranged in circular, funnel-formed order; septa and tabulæ obso!ete. Type C. typicus.

infundibulum, Whitficld, 1878, (Syringopora infundibulum,) Ann. Rep. Geo. Sur. Wis., p. 79, and Geo. Wis., vol. 4, p. 274, Niagara Gr.

typicus, Whitfield, 1880, Ann. Rep. Geo. Sur. Wis. and Geo. Wis., vol. 4, p. 274, Niagara Gr.

Dania, Edwards \& Haime, 1849, Comp. Rend., t. 29, p. 261. [Ety. proper name.] Corallum having most of tho characters of Chetetes, but with the tabulse connected through the corallites so as to divide the mass into parallel strata. Type D. huronica.

huronica, Edwards \& Haime, 1849, Comp. Rend., t. 29, p. 261, Up. Sil.

Dawsoxia, Nicholson, 1873, Ann. Mag. Nat. Hist., ftl ser., vol. 12. [Ity. proper name.] Supposed to be the ovarian vesicles of Graptolites. 'Type D. campanulata.

acuminata, Nicholson, 1873, Ann. Mag. Nat. Hist., 4th ser., vol. 12, Quebec Gr. campanulata, Nicholson, 1873, Ann. Mag. Nat. Hist., vol. 12, Quebec Gr.

rotunda, Nicholson, 1873, Aun. Mag. Nat. Hist., vol. 12, Quebec Gr.

tenuistriata, Nicholson, 1873, Ann. Mag. Nat. Hist., vol. 12, Quebec Gr.

Dek a yelia, Ulrich, 1882, Jour. Cin. Soc. Nat. Hist., vol. 5, p. 155. [Ety. diminutive of Dekayia.] Ramose, interstitial cells; spiuiform tubuli of two kinds, larger ones arranged as in Dekayia, others more numerous; diaphragms in both sets of tubes. Type D. obscura.

obscura, Ulrich, 1883, Jour. Cin. Soc. Nat. Hist., vol. 6, p. 89, Hud. Riv. Gr.

robusta, Foord, 1884, Ann. and Mag. Nat. Hist., 5th ser., vol. 14, p. 341, Hud. Riv. Gr.

DEkA YiA, Edwards \& Haime, 1851, Mon.d. Pol. Foss. d. Terr. Pal., p. 277. [Ety. proper name.] Distinguished from Monticulipora by having little protuberances on the surface between the angles of the corallites. Type D. aswera. appressa, Ulrich, 1883, Jour. Cin. Soc. Nat. Hist., vol. 6, p. 15:, Hud. Riv. Gr.

aspera, Ed. iv a r d s \& $\mathrm{H}$ a i m e, 1851, Pol. Fosi. d. Terr. Pal., p. 278 . Hud. Riv. Gr.

altrita, syn. for D. aspera.

multispi-Fig. 165.-Dekayla aspera, natnosa, Ul- ural size, and nuginlfled. rich, 1883, Jour. Cin. Soc. Nat. Hist., vol. 6, p. 154, Hud. Riv. Gr.

paupera, Ulrich, 1883, Jour. Cin. Soc. Nat. Hist., vol. 6, p. 153, Hud. Riv. Cir. pelliculata, Ulrich, 1883, Jour. Cin. Soc. Nat. IIist., vol. 6, p. 150, Hurl. Riv. Gr. trentonensis, Ulrich, 1883, Jour. Cin. Soc. Nat. Hist., vol. 6, p. 151, Trenton Gr.

Dendrogra l’tus, Hall, 1865, Can. Org. Rem., Decalle 2, p. 126. [Kty. dendron, tree, grapho, I write.] Simple or aggregate; foot-stalk strong, sometimes with a rootlike bulb; ramified above into slightly divergent branches, celluliferous on one sicle. Type D. ballanus.

compactus, Walcott, 1879, Utica Slatc and related formations, p. 21, Utica Slate.

dawsoni, Spencer, 1884, Bull. No. 1, Mus. Univ. St. Mo., ». 18, Niagara Gr.

diffusus, Hall, 1865, Can. Org. Rem., Decade 2, p. 132, Quebec Gr.

divergens, Hall, 1865, Can. Org. Rem., Decade 2, p. 129, Quebec Gr.

dubius, n. s]). Proposed instead of D. simplex, Spencer, in Bull. No. 1, Mus. Univ. St. Mo., p. 17, which was ureoccupied. Niagara Gr.

erectus. IIall, 1865, Can. Org. Rem., Decade 2, p. 130, Quebec Gr.

flexuosus, Hall, 1865, Can. Org. Rem., Decade' 2, p. 127, Quebec Gr.

frondosus, Spencer, 1884, Bull. No. 1, Mus. Univ. St. Mo., p. 18, Niagara Gr.

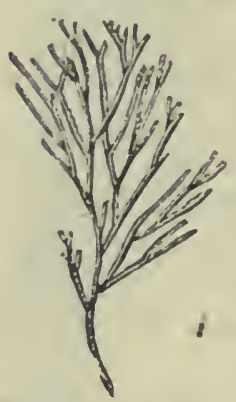

FiG. 166, - Dendrograptus hallauus. fruticosus, Hall, 1865 , Can. Org. Rem., Decade 2, p. 131, Quebec Gr.

gracilis, Hall, 1865, Can. Org. Rem., Decade 2, p. 132. Quebec Gr. gracillimus, Lesquereux, 1877, Proc. Am. Phil. Soc. p. 164, (Psilophy. ton gracillimum.) Hud. Riv. Gr.

hallanus, Prout, 1851, (Graptolithus ha l: lanus,) Am. Jour. Sci. 2d ser., vol. 11, p. 187, Potsdam sandstone. novellus, Hall, 1879, Desc. New Spec. Foss., p. 2, and 11th Rep. Geo. Sur. Ind., p. 225, Niagara Gr. 
prægracilis, Spencer, 1884, Bull. No. 1, Mus. Univ. St. Mo., p. 19, Niagara Gr. primordialis, Matthew, 1885, Trans. Roy. Soc. Can., p. 31, St. John Gr.

ramosus, Spencer, 1884, Bull. No. 1, Mus. Univ. St. Mo., p. 17, Niagara Gr.

simplex, Walcott, 1879, Utica Slate and related formations, p. 20 , Utica Slate.

simplex, Spencer, 1984, Bull. No. 1, Mus. Univ. St. Mo., p. 17. The name was preoccupied. See D. dubius.

spinosus, Spencer, 1884, Bull. No. 1, Mus. Univ. St. Mo., p. 19, Niagara Gr.

striatus, Hall, 1865, Can. Org. Rem., Decade 2, p. 129, Quebec Gr.

tenuiramosus, Walcott, 1879, Utica Slate and related formations, p. 21, Utica Slate.

Dexdropor , Michelin, 1846, Icon. Zooph., p. 187. [Ety. dendron, tree; poros, pore.] Corallum arborescent, witb very delicate, smooth branches; calices distant, and surrounded by a narrow, obtuse margin ; septa small, but distinct. Type D. explicita.

alternans, Rominger, 1876, Foss. Corals, p. 64, Ham. Gr.

neglecta, Rominger, 1876, Foss. Corals, p. 63, Up. Held. Gr.

ornata, see Trachypora ornata.

proboscidialis, Rominger, 1876, Foss. Corals, p. 65, Ham. Gr.

reticulata, Rominger, 1876, Foss. Corals, p. 65 , Ham. Gr.

Dichograptus, syn. for Graptolithus.

Dicranograptus, Hall, 1865, Can. Org. Rem., Decade 2, p. 46. [Ety. dikranos, two pointed; grapho, I write.] The lower part of the stipe has a row of cells on each side, but above, the stipe bifurcates, and has cells only on the outer side of each bifurcation. Type D. ramosus.

divaricatus, Hall, 1859, (Graptolithus divaricatus,) Pal. N. Y., vol. 3, p. 513, Hud. Riv. Gr.

furcatus, Hall, 1847, (Graptolithus furcatus,) Pal. N. Y., vol. 1, p. 273, Utica Slate.

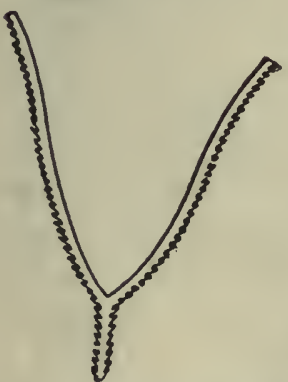

FIG. 167.-Dicranograptus ramosus. ramosus, $\mathrm{Hall}$, 1847, (Grapto. lithus $\mathrm{ramo-}$ sus,) Pal. N. Y., vol. 1 , p. 270 , Utica Slate. sextans, $\mathrm{H}$ a 11 , 1847, (Graptolithus sextans, Pal. N. Y., vol. 1, p. 273, Utica Slate.

DictryonkM , Hall, 1852, Pal, N. Y. vol. 2 , p. 174 . [Ety. dictyon, uet; nema, thread.] Fronds consisting of flabelliform or funnel-shaped expansions, composed of slender, radiating branches, which frequently bifurcate as they recede irom the base; branches united laterally by transverse dissepiments; exterior striated; interior surface celluliferous or serrate. Type D. retiforme.

expansum, Spencer, 1884, Bull. No. 1, Mus. Univ. St. Mo., p. 25, Niagara Gr. fenestratum, Hall, 1851, in Foster and Whitney's Rep. on Lake Superior Land Dist., p. 223, U.p. Held. Gr.

gracile, Hall, 1852, Pal. N. Y., vol. 2, p. 175, Niagara Gr.

grande, Nicholson, 1873, Ann. Mag. Nat. Hist., 4th ser., vol. 12, Quebec Gr.

irregulare, Hall, 1865, Can. Org. Rem., Decade 2, p. 136, Quebec Gr.

murrayi, Hall, 1865, Can. Org. Rem., Decade 2, p. 138, Quebec Gr.

neenah, Hall, 1S61, Geo. Rep. Wis., p. 17, Trenton Gr.

pergracile, Hall \& Whitfield, 1872, 24th Rep. N. Y. St. Mus. Nat. Hist., p. 181, and Acad. Geo., p. 563, Niagara Gr. quarlrangulare, Hall, 1865, Can. Org. Rem.,

Decade 2, p. 138, Q'1 sec Gr.

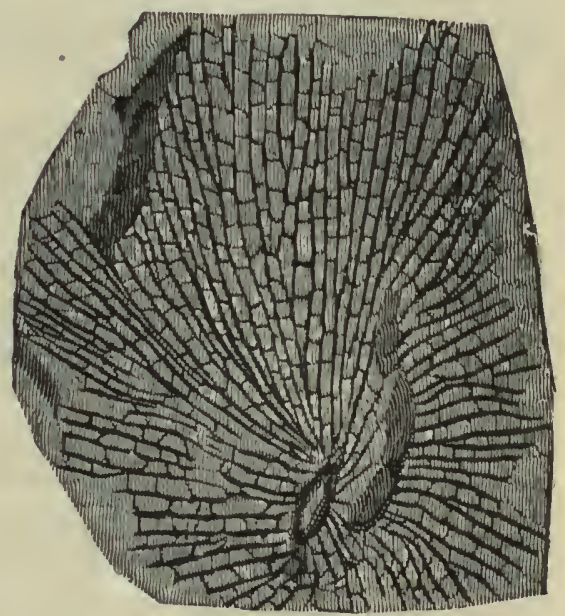

FIG. 168.-Dietyonema retiforme.

retiforme, Hall, 1843, (Gorgonia retiformis,) Geo. Rep, 4th Dist. N. Y., p. 115, and Pal. N. Y., vol. 2, p. 174, Niagara Gr.

robustum, Hall, 1865, Can. Org. Rem., Decade 2, p. 137, Quebec Gr.

splendens, Billings, 1874, Pal. Foss., vol. 2, p. 12, Gaspe limestone No. 1, Up. Sil. tenellum, Spencer, 1878, Can. Nat., vol. 8, and Bull. No. 1, Mus. Univ. St. Mo., p. 26, Niagara Gr.

websteri, Dawson, 1860, Can. Nat. and Geo., vol. 5, and Acad. Geo., p. 563, Niagara Gr.

Didymograptus, McCoy, 1851, Brit. Pal. Foss., p. 3-9. [Ety. didymos, double; grapho, I write.] Consisting of forked stipes, straight or curved; one celluliferous side. Type D. murchisoni. 
caduceus, Salter, 1853, (Graptolithus cadnceus,) Quar. Jour. Geo. Soc., vol. 9, p. 87, Quebec Gr.

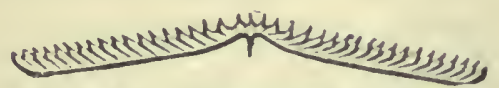

Fio. 169.-Didy mograptus geminus.

geminus, Hisinger, 1840, (Prionotus geminus,) Leth Suecia, Supp. 2, p. 5, pl. 38, Quebec Gr.

Diphy PH Yllum, Lonsdale, 1845, Russ. and Ural Mts., vol. 1, p.624. [Ety. diphyia, division; phyllon, leaf.] Corallum simple, composite, increasing by lateral gemmation; corallites tall, cylindrical, connected by epithecal or radiciform expansions, with each other; central area occupied by tabule; circumscribed by an inner wall; exterior vesicular zone occupied by septa, which are confined between the outer and inner mural investment; no colımella. Type D. conicum.

adnatum, Hall, 1882, Foss. Corals Niagara and Up. Held Grs., p. 54, and 12th Rep. Geo. Sur. Ind., p. 303, Up. Held. Gr.

apertun, Hall, 18S2, Foss. Corals Niagara and Up. Held. Grs., p. 54, and 12th Rep. Geo. Sur. Ind., p. 303, Up. Held. Gr.

archinci, see Crepidophyllum archiaci.

arundinaceum, Billings, 1859, Can. Jour., vol. 4, p. 134, Corniferous limestone.

breve, Hall, 1882, Foss. Corals Niagara and Up. Held. Grs., p. 55, and 35th Rep. N. Y. St. Mus. Nat. Hist., p. 459, Up. Held. Gr.

crspitosum, Hall, 1852, (Diplophyllum (cespitosum,) Pal. N. Y., vol. 2, p. 116, Niagara $\mathrm{Gr}$.

coralliferum, Hall, 1852, (Diplophyllum coralliferum,) Pal. N. Y., vol. 2, p. 322, Coralline limestone.

cylindraceum, Hall, 1882, Foss. Corals Niagara and Up. Held. Grs., p. 54, and 35 th Rep. N. Y. St. Mus. Nat. Hist., p. 458, Up. Held. Gr.

fasciculum, Meek, 1877, U. S. Geo. Sur. 40th Parallel, vol. 4, p. 29, Devonian.

gigas, Rominger, 1876 , Foss. Corals, p. 125, Niagara Gr.

gracile, McCoy, 1854, Brit. Pal. Foss., p. 88, Up. Held Gr.

huronicum, Rominger, 1876, Foss. Corals, p. 121, Niagara Gr.

rectiseptatum, Rominger, 1876, Foss. Corals, p. 124, Ham. Gr.

rugosum, Edwards \& Haime, 1851, (Eridophyllum rugosum,) Pol. Foss. des Terr. Pal., p. 424, Niagara Gr.

simcoense, Bíllings, 1859, (Eridophyllum simcoense,) Can. Jour.. vol. 4, p. 131, Up. Held. Gr.

stramineum, Billings, 1859, Can. Jour., vol. 4, p. 135, Corniferous Gr.

strictum, Edwards \& Haime, 1851, (Eri- dophyllum strictum,) Poll. Foss. des Terr. Pal., p. 424, Up. II leld. Gr.

tumidulum, Hall, 188., Foss. Corals Niagara and Up. Held. Grs., p. 55, and 12th Rep. Geo. Sur. Ind., p. 303, Up. Held. Gr.

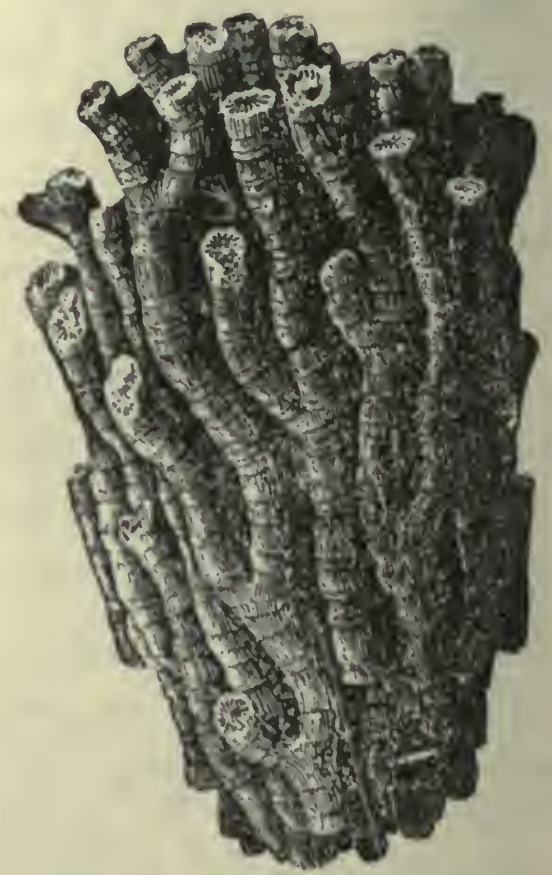

Fra. 170.-DIphyphyllum stramlueum.

vennori, Billings, 1865, (Eridophyllum vennori,) Can. Nat. and Geo., 2d ser., vol. 2, p. 431, Clinton Gr.

verneuilanum, Edwards \& IIaime, 1850, (Eridophyllum vernenilanum,) Brit. Foss. Corals, p. lxxi, and Iol. Foss. des Terr. Pal., p. 424, Up. Held Gr.

Dirlograptus, AicCoy, 1854, (Diplograpsus,) Brit. Pal. Rocks, p. 3. [lity. diploos, duplex; grapho, I write.) Stipes simple, flattened, or quadrangular ; cellules, in single series, on the two sides of a double central axis; cellules oblique, opening toward the apex; cell denticles prominent, often mucronate. Type D. foliaceus.

amplexicaulis, Hall, 1847, (Graptolithus amplexicaulis,) Pal. N. Y., vol. 1, p. 79, Trenton Gr.

angustifolius, Hull, 1859, (Graptolithus angustifolius,) Pal. N. Y., vol. 3, p. 515, Hud. Riv. Gr.

ciliatus, Emmons, 1856, Am. Geo., p. 105, Up. Taconic.

dissimilaris, Emmons, 1856, Am. Geo., p. 105, Up. Taconic.

foliaceous, (?) Murch, 1839, (Graptolites foliaceus,) Murch. Sil. Syst., p. 695, Hud. Riv. Gr. 
foliosus, Emmons, 1856, Am. Geo., p. 105, Up. Taconic.

folium, Hisinger, 1837, (Prionotus folium, Leth. Suec., p. 113, Hud. Riv. Gr.

hudsonicus, Nicholson, 1875, Pal. Proc. Ont., p. 38, Hud. Riv. Gr. hypniformis, White, 1874, (Graptolithus hypniformis,) Rep. Invert. Foss., p. 12, and Geo. Sur. W. 100th Mer., vol. 4, p. 63, Trenton Gr.

FIG. 171.

Diplo-

graptus

folium.

inutilis, Hall, 1865, Can. Org. Rem., Decade 2, p. 111, Quebec Gr.

laciniatus, Emmons, 1856, Am. Geo., p. 236, Up. Taconic.

marcidus, Hall, 1859, (Graptolithus marcidus,) Pal. N. Y., vol. 3, p. 514, Hud. Riv. Gr.

mucronatus, Hall, 1847, (Graptolithus mucronatus,) Pal. N. Y., vol. 1, p. 263, Hud. Riv. Gr.

obliquus, Emmons, 1856, Am. Geo., p. 106, Up. Taconic.

peosta, Hall, 1861, (Graptolithus peosta,) Geo. Rep. Wis., p. 17, Trenton Gr.

pristiniformis, Hall, 1858, (Graptolithus pristiniformis,) Geo. Sur. Can., p. 133, and Can. Org. Rem., Decade 2, p. 110, Quebec Gr.

pristis, (?) Hisinger, 1837, (Prionotus pristis,) Leth Suec., p. 114, and Pal. N. Y., vol. 1, p. 265, Hud. Riv. Gr.

putillus, Hall, 1865, Can. Org. Rem., Decade 2, p. 44, Hud. Riv. Gr.

rugosus, Emmons, 1856, Am. Geo., p. 105, Up. Taconic.

rectangularis, McCoy, 1851, Brit. Pal. Rocks, p. 3, Low Sil.

secalinus, Hall, 1847, (Fucoides secalinus,) Pal. N. Y., vol. 1, p. 267, syn. for D. simplex.

simplex, Emmons, 1844, (Fucoides simplex,) Taconic system, p. 27, and Pal. N. Y., vol. 1, p. 267, Up. Taconic.

spinulosus, Hall, 1859, (Graptolithus spinulosus,) Pal. N. Y., vol. 3, p. 517, Hud. Riv. Gr.

whitfieldi, Hall, 1859, (Graptolithus whitfieldi,) Pal. N. Y., vol. 3, p. 516, Hud. Riv. Gr.

Diplophyllum, Hall, 1852, Pal. N. Y., vol. 2, p. 115, syn. for Diphyphyllum.

cxspitosum, see Diphyphyllum cxespitosum. coralliferum, see Diphyphyllum coralliferum.

DiplotryPa, Nicholson, 1879, Pal. Tab. Corals, p. 292 . [Ety. diploos, double; trypa, hole.] Corallites of two kinds, the larger thin walled, polygonal, tabulæ remote; often aggregated in clusters, (monticules); smaller corallites angular, thin walled, never completely isolating the larger ones; tabulæ numerous. Type D. petropolitana.

infida, Ulrich, 1886, 14th Rep. Geo. Sur. Minn., p. 88, Trenton Gr.

milleri, Ulrich, 1882, Jour. Cin. Soc. Nat. Hist., vol. 5, p. 245, Niagara Gr. regularis, Foord, 1883, Micropalæontology, p. 13, Trenton Gr.

DiscophylluM, Hall, 1847, Pal. N. Y., vol. 1, p. 277. [Ety. diskos, disk; phyllon, leaf.] Discoid flattened, rays numerous, proceeding from the center and terminating in a thickened border. Type D. peltatum.

peltatum, Hall, 1817, Pal. N. Y., vol. 1, p. 277, Up. Taconic.

Duncanella, Nicholson, 1874, Ann. Mag. Nat. Hist., 4th ser., vol. 13, p. 333. [Ety. proper name.] Corallum simple, obconical; calycle deep, circular; rays strong, exsert; epitheca striated vertically; closely allied to Streptelasma. Type D. borealis.

borealis, Nicholson, 1874, Ann. Mag. Nat. Hist., 4th ser., vol. 13, p. 333 , Niagara Gr.

ElasmophylLUM, Hall, 1882, Foss. Corals Niagara and Up. Held Grs., p. 38. [Ety. elasma, lamellæ; phyllon,

elasma, lamellæ; phyllon, FrG. 172.
leaf.] Simple, turbinate, Du canella lamellæ extending to the borealis center, twisted or not; in-

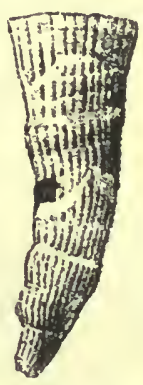
magnified. terlamellar cysts continuing to the center; no tabulæ. Type E. attenuatum.

attenuatum, Hall, 1882, Foss. Corals Niagara and Up. Held. Grs., p. 38, and 35 th Rep. N. Y. St. Mus. Nat. Hist., p. 442, Up. Held. Gr.

Emmonsis, Edwards \& Haime, 1851, Monographie des Polyp., Foss. des Terr. Palæoz., p. 246. [Ety. proper name.] Distinguished from Favosites by the compound character of the diaphragms, but generally regarded as a synonym. Type F. hemispherica.

hemispherica, Troost, 1840, (Calamopora hemispherica,) 5th Rep. Geo. Tenn., p. 72, Up. Held. Gr.

hemispherica, Yandell \& Shumard, 1847, (Favosites hemisphericus,) Contrib. to Geo. of Ky., p. 7. Same species described by Troost.

Eridophyllum, Edwards \& Haime, 1850, Brit. Foss Corals, p. Ixxi, syn. for Diphyphyllum.

rugosum, see Diphyphyllum rugosum.

simcoense, see Diphyphyllum simcoense.

strictum, see Diphyphyllum strictum.

vennori, see Diphyphyllum vennori.

verneuilanum, see Diphyphyllum, verneuilanum.

Favastrea, DeBlainville, 1830, Man. d.Actinol, p. 374. Not an American palæozoic genus.

striata, D'Orbigny, 1850, Prodr. d. Paléont., t. 1, p. 48. Not defined so as to be recognized.

Faviphyllum, as used by Hall, 1852, Stans. Exped. to Great Salt Lake, p. 407. Not defined, and founded upon a silicified, indeterminate fragment. 
Favistella, Hall, 1847, Pal. N. Y., vol. 1, p. 275. [Ety. favus, boney-comb; stella, star.] Massive, hemispherical, corallites polygonal, increasing by lateral development; walls not separable as in Favosites, nor perforated by pores; tabula close; septa of alternately larger and smaller size, the larger reaching the center: twelve or more in each corallite. Type F. stellata.

ealicina, Nicholson, 1874, Rep. Brit. Ass'n. and Pal. Tab. Corals, p. 197, Hud. Riv. Gr.

favosidea, Hall, 1852, Pal. N. Y., vol. 2, p. 41, Clinton Gr.

franklini, Salter, 1852, Sutherland's Jour., vol. 2, p. ccxxxi, Up. Sil.

reticulata, Salter, 1852, Sutherland's Jour., vol. 2 , p. ccxxix, Up. Sil.
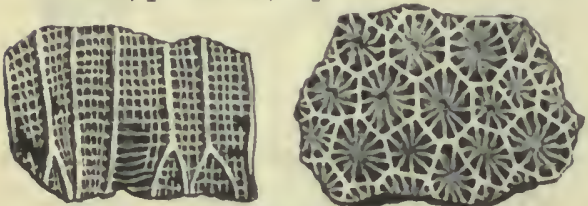

F1G. 173.-Favistelln stellatu.

stellata, IIall, 1847, Pal. N. Y., vol. 1, p. 275, Hud. Riv. Gr.

Favosites, Lamarck, 1812, Cours. de Zool. du Mus. d'Hist. Nat. and Hist. des An. sans Vert., vol. 2, p. 204. [Ety. favus, honey-comb.] Massive or branched, composed of numerous more or less polygonal corallites; tabulxe present; septa absent or rudimentary ; walls perforated by one or more rows of inural pores, connecting the corallites. Type F. alveolatis.

alpenensis, Winchell, 1866, Rep. Low. Penin. Mich., p. 88, Ham. Gr.

alveolaris, DeBlainville. Not American.

arbuscula, Hall, 1876, Illust. Devon. Foss., pl. 36, Ham. Gr.

argus, Hall, 1876, Illust. Dev. Foss., pl. 13, Ham. Gr.

asper, D’Orbigny, 1849, Prodr. de Paleont., t. 1, p. 49, Clinton Gr.

basalticus, Goldfuss, 1826, Germ. Petref., p. 78, (Calamopora basaltica,) Devonian. billingsi, Rominger, 1876, Foss. Corals, p. 29, Ham. Gr.

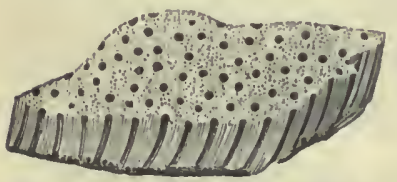

canadensi s,

Bill ing 8 , 1858, (Fistulipora canaden sis,) Can. Nat. and Geol., vol. 4, p. 98, Up. Held. Gr.

capax, Billings, 1866, Catal. Sil. Foss. Antic., p. 6, Hud. Riv. Gr.

cervicornis, DeBlainville, 1830, (Alveololites cervicornis,) Dict., vol. 60, p. 369, Devonian.

chapmani, Nicholson, 1874, Pal. Prov. Ont., p. 52, Up. Held. Gr.

clausus, Rominger, 1876, Foss. Corals, p. 37, Up.Held. and Ham. Gir.

conicus, Hall, 1874, 26th Rep. N. Y. St. Mus. Nat. Hist., p. 112, and Pal. N. Y. vol. 6, p. 9, Low. Held. Gr.

constrictus, Ilall, 1852, (Astrocerium constrictum,) Pal. N. Y., vol. 2, p. 123, Niagara $\mathrm{Gr}$.

cristatus, Edwards \& Haime, 1851, Pol. Foss. Terr. Palæoz., p. 242, Niagara Gr. cumberlandicus, Troost, 1840, (Calamopora cumberlandica,) 5th Geo. Rep. Tenn., p. 70, Kaskaskia Gr.

digitatus, Rominger, 1876, Foss. Corals, p. 39, Ham. Cir.

divergens, Winchell, 1862, Proc. Acad. Nat. Sci., p. 112, and Geo. Sur. W. 100th Merid., vol. 4, p. 79, Subcarb.

dubius, DeBlainville, 1830, (Alveolites dubius,) Dict., vol. 60, p. 370, Corniferous Gr.

dumosus, Winchell, 1866, Rep. Low. Penin. Mich., p. 89, Ilan. Gr.

emmonsi, Roninger, 1876, Foss. Corals, p. 27, Up. IIeld. Fr. Syn. (?) for F. heliolitiformis.

emmonsi, Hall, 1876, Illust. Dev. Foss., pl. 9. The namo was preoccupied.

epidermatus, Rominger, 1862, Anı. JourSci. and Art8, vol. 34, p. 396, Corniferolls Gr.

epidermatus var. biloculi, Hall, 1876 , Illust. Dev. Foss., pl. 7, Up. Held. Gr. epidermatus var. corticosus, Hall, 1876, Illust. Dev. Foss., pl. 10, Up. Held. (ir. excretus, Hall, 1876, 28th Rep. N. Y. St. Mus. Nat. Hist., syn. for H. spinigerus. explanatus, IIall, 1876 , Illust. Dev. Fos s., pl. 14, Ham. Gr.

favosus, Goldfuss, 1826, Germ: Petref., p. 77, and Pal. N. Y., vol. 2, p. 126, (Calamopora favosa,) $\mathrm{Ni}$ agara Gr.

flabelliformis, Troost, 1813. Not satis- FIG. 175.-Favosites forfactorily defined. besi var. occldentalis. forbesi, Edwards \& Iraine, 1854, Brit. Foss. Corals, p. 258, Niagara Gr.

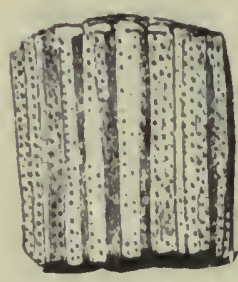

Fio, 176. - Favosites gold fussi.

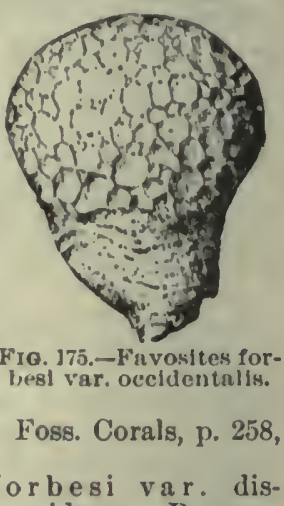
coideus, Roemer, 1860, (Calamopora forbesi var. discoidea,) Sil. Fauna IV. Tenn., p. 19, Niagara Gr.

forbesi var. occidentalis, Hall, 1876 , 28th Rep. N. Y. St. Mus. Nat. Hist., p. 109, Niagara Gr.

forbesi var. waldronensis, Nicholson, 1879, syn. for F. forbesi var. occidentalis. 
goldfussi, Castelnau, 1843, (Calamopora goldfussi,) Syst. Sil., p. 47, Up. Sil.

goldfussi, D’Orbigny, 1850, Prodr. de Paléont., p. 107, Devonian. The name was preoccupied.

gothlandicus, Lamarck, 1816, Hist. An. sans Vert., vol. 2, p. 206, Up. Held. and Haın. Grs.

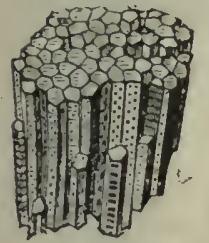

hamiltonensis, Rominger, 1876, Foss. Corals, syn. for F. dumosus.

bamiltonice, Hall, 1876, Illust. Dev. Foss., p]. 34, Ham. Gr.

helderbergix, Hall, 1874 , 26th Rep. N. Y. St. Mus. Nat. Hist., p. 111, and Pal. N. Y., vol. 6 , p. 8 , Low. He]d. Gir.

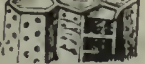

heliolitiformis, Rominger, 1862, (Calamopora heliolitiformis,) Am. Jour. Sci., vol. $34,2 \mathrm{~d}$ series, p. 397, Devonian.

Fig. 177.-Favosites gothlandicus.

hemisphericus, Troost, 1840, (Calamopora hemispherica,) 5th Geo. Rep. Tenn., p. 72, Up. Held. Gr. Same as Emmonsia hemispherica.

hemisphericus var. distortus, Hall, 1876, Illust. Dev. Foss., pl. 5, Up. Held. Gr.

hemisphericus var. rectus, Hall, 1876, Illust. Dev. Foss., pl. 2C, Up. Held. Gr.

hisingeri, Edwards \& Haime, 1851, Pol. Foss. des Terr. Palæoz., p. 240, Niagara $\mathrm{Gr}$.

hispidus, Rominger, 1876, Foss. Corals, p. 23, Niagara Gr.

infundibuliformis, as identified by D'Archiac \& Verneuil. Not American.

intertextus, Roininger, 1876, Foss. Corals, p. 38, Ham. Gr.

invaginatus, Nicholson, 1875, Ohio Pal., vol. 2, p. 232, Corniferous Gr.

limitaris, Rominger, 1876, Foss. Corals, p. 36 , Corniferous Gr.

lycoperdon, see Monticulipora lycoperdon. mammillaris, Castelnau, 1843. Not recognized.

mancus, Winchell, 1865, Proc. Acad. Nat. Sci., p. 112, Kinderhook Gr.

maximus, Troost, 1840, (Calamopora maxima,) 5th Rep. Geo. Tenn., p. 73, Devonian.

minimus, Hall, 1874, 26th Rep. N. Y. St. Mus. Nat. Hist., p. 113, Low. Held. Gr. niagarensis, Hall, 1852, Pal. N. Y., vol.2, p. 125 , Niagara Gr.

niagarensis var spinigerus, see $\mathrm{F}$. spinigerus. nitellus, Winchell, 1866, Rep. Low. Pen. Mich., p. 89. Ham. Gr.

obliquus, Rominger, 1876, Foss. Corals, p. 24, Niagara Gr

occidens. Whitfield, 1878, Ann. Rep. Geo. Sur. Wis., p. 78, and Geo. Wis., vol. 4, p. 313, Niagara Gr.

parasiticus, Hall, 1852, (Astrocerium parasiticum, Pal. N. Y., vol. 2, p. 122, Niagara Gr. This name was preoccupied by Phillips in his Geol. of Yorkshire. placenta, Rominger, 1876, Foss. Corals, p. 34, Ham. Gr.

pleurodictyoides, Nicholson, 1875, Ohio Pal., vol. 2, p. 231, Corniferous Gr.

polymorphus, Goldfuss, 1826, Germ. Petref., p. 79, Corniferous Gr.

prolificus, Billings, 1865, Can. Nat. and Geol., 2d ser., vol. 2, p. 429, Hud. Riv. Gr.

proximus, Hall, 1883, Rep. St. Geol., pl. 7, fig. 13-15, and Pal. N.Y., vol. 6, p. 10, Low. Held. Gr.

pyriformis, Hall, 1852, (Astrocerium pyriforme,) Pal. N. Y., vol. 2, p. 123, Niagara $\mathrm{Gr}$.

radiatus, Rominger, 1876, Foss. Corals, p. 33, Ham. Gr.

radiciformis, Rominger, 1876, Foss. Corals, p. 34, Devonian.

reticulatus, DeBlainville, 1840, (Alveolites reticulatus,) Dict., vol. 60, p. $369, \mathrm{Ni}-$ agara Gr.

sphericus, Hall, 1874,(Chetetes sphericus,) 26th Rep. N. Y. St. Mus. Nat. Hist., p. 111, and Pal. N. Y., vol. 6, p. 9, Low. Held. Gr.

spinigerus, Hall, 1876, (F. niagarensis val. spinigerus,) 28th Rep. N. Y. St. Mus. Nat. Hist., p. 108, Niagara Gr.

spongilla, Rominger, 1876, Foss. Corals, p. 24, syn. for $\mathrm{F}$. spinigerus.

striatus, Say, 1818, Am. Jour. Sci., vol. 1, p. 381, Niagara Gr.

troosti, Edwards \& Haime, 1851, Mon. d. Pol. Foss. d. Terr. Pal, p. 238, Devonian.

tuberosus, Rominger, 1876, Foss. Corals, p. 31, Corniferous Gr.

turbinatus, Billings, 1859, Can. Jour., vol. 4, p. 109, Up. Held. \& Ham. Gr.

venustus, Hall, 1852, [Astrocerium venustum, Pal. N. Y., vol. 2, p. $120, \mathrm{Ni}$ agara Gr.

verneuili, Castelnau, 1813, syn. for Monticulipora fibrosa.

whitfieldi, White, 1874, Rep. Invert. Foss., syn. for $\mathrm{F}$. divergens.

winchelli, Rominger, 1862, (Calamopora winchelli,) Am. Jour. Sci., vol. 34, 2d ser., p. 397, Devonian.

Favosilopora, Kent, 1870, Ann. and Mag. Nat. Hist., 3d ser., vol. 6, p. 384.

palxozoica, Kent, 1870, Ann. and Mag. Nat. Hist., 3d ser., vol. 6, p. 384. Not recognized.

Filicites gracilis, see Plumalina gracilis.

Geoporites americanus, D'Orbigny, 1850. Not defined so as to be recognized.

Glossograptus, Emmons, (Glossograpsus,) 1856, Am. Geo., p. 108. [Ety. glosse, tongue; grapho, I write.] Stipe free; thin, membranaceous, ligulate, extremities rounded, axis distinct. Type G. ciliatus.

ciliatus, Emmons, 1856, Am. Geo., pt. 2, p. 108, Up. Taconic.

setaceus, Emmons, 1856, Am. Geo., pt. 2, p. 236, Up. Taconic. 
Graptolithus, Linnzus, 1736, Syst. Nat., 1st Ed., but it was not until 1767, in the 12 th Ed., that any species were defined. [Ety.grapho, I write; lithos, stone.] Stipes elongated, slender, flattened, or quadrangular; they may be simple or bifurcating; the cells enter the central canal and open their mouths upward, so as to form denticles on the margins when compressea. Type $\mathrm{G}$. scalaris.

abnormis, Hall, 1858, Geo. Sur. Can., p. 117, and Can. Org. Rem., Decade 2, p. 106, Quebec Gr.

alatus, Hall, 1858, Gieo. Sur. Can., p. 127, and Can. Org. Rem., Decade 2, p. 93, Quebec Gr.

amplexicaulis, see Diplograptus amplexicaulis.

angustifolius, see Diplograptus angustifolius.

annectans, Walcott, 1879, Utica Slate and related formations, p. 20, Utica Slate.

antennarius, see Climacograptus antennarius.

approximatus, Nicholson, 1873,(Tetragraptus approximatus,) Ann. and Mag. Nat. Hist., 4th ser., vol. 12, Quebec (ir.

arcuatus, Hall, 1565. Can. Org. Rem., Decade 2, p. 79, Quebec Gr.

bicornis, see Climacograptus bicornis.

bifidus, Hall, 1858, Can. Nat. and Geo., vol. 3, p. 73, Quebec (ir.

bigsbyi, Hall, 1865, Can. Org. Rem., Decade 2, p. 86, Quebec Gr.

bryonoides, Hall, 1858, Geo. Sur. Can., p. 126, and Can. Org. Rem., Decade 2, p. 84, Quebec Gr.

caduceus, see Didymograptus caduceus.

elintonensis, Hall, 1843, Geo. Rep. 4th Dist. N. Y., p. 74, and' Pal. N. Y., vol. 2, p. 39, Clinton Gr.

constrictus, Hall, 1865, Can. Org. Reın., Decade 2, p. 76, Quchec Gr.

crucifer, Hall, 1858, Geo. Sur. Can., p. 125, and Can. Org. Rem., Decade ", p. 92, Quebec Cir.

dentatus, Einmons, 18 12 , Geo. Rep. N. Y., p. 279, Utica Slate.

denticulatus, Hall, 1858, (ieo. Sur. Can., p. 132, and Can. Org. Rem., Decade 2, p. 88, Quebee Gr.

divaricatus, Hall, 1859, Pal. N. Y., vol. 3, p. 513, Hud. Riv. Gr. See Dicranograptus divaricatus.

divergens, Hall, 1859, Pal. N. Y., vol. 3, p. 509, Hud. Riv. Gr.

ensiformis, see Retiolites ensiformis.

extensus, Hall, 1858, Geo. Sur. Can., p. 132, and Can. Org. Rem., Decade 2, p. 80, Quebec Gr.

extenuatus, Hall, 1865, Can. Org. Rem., Decade 2, p. 75, Quebec Gr.

flaccidus, Hall, 1865, Can. Org. Rem., Decade 2, p. 143, Utica Slate.

flexilis, see Clonograptus flexilis.

foliaceus, see Diplograptus foliaceus.

folium, see Diplograptus folium. fruticosus, Hall, 1858, Geo. Sur. Can., p. 128 and Can. Org. Rem., Decade 2, p. 90 , Quebec Gr.

furcatus, see Dicranograptus furcatus.

gracilis, Ilall, 1847, Pal. N. Y., vol. 1, p. 274, Utica Slate.

hallanus, see Dendrograptus hallanus.

headi, Hall, 1858, Geo. Sur. Can., p. 127, and Can. Org. Rem., Decade 2, p. 94, Quebec Gr.

hypniformis, see Diplograptus hypuiformis. indentus, Hall, 1858, Gen. Sir. Can., p. 128, and Can. Org. Rem. Decade 2, r. 74, Quebec Cir.

lavis, Hall, 1847, I'nl. N. Y., vol. 1, p. 274, Utica Slate.

$\log$ a $\mathrm{i}, 1858$, Geo.Sur.Can). p. 115, and Can. Org. Rem., Decade 2, p. 100 , Quebec (ir.

marcidus, sce $\mathrm{D}$ i plograptus marcidus.

mi lesi, II a I1, 1861 , Geo. Sur. Vermont, vol. 1. p. 37\%,

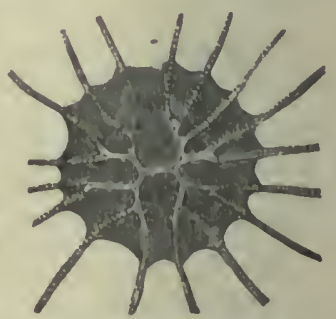

Fรa. 178.-Graptollthus logusl.
Quebec Gr.

mucronatus, see Diplograptus murronatus. multifasciatus, Hull, 1859, Pal. N. Y., vol. 3, p. 50s, and Can. Org. Rem., Deeade 2, p. 10, Hud. Riv. Gir.

nitidus, Hall, 1858, Geo. Sur. Can., p. 129, and Can. Org. Rem., Decade 2, p. 69, Quebec Gir.

oetobrachiatus, Hall, 1858, Geo. Sur. Can., p. 122, and Cann. Org. K(*m., Decarle 2, p. 96 , Quebec Gr.

octonarius, Hall, 1858, Cieo. Sur. Can., r. 124, and Can. Org. Rem., Decarle 2, 1. 95, Quebec (ir.

patulus, Hall, 1858, Geo. Sur. Can., p. 131, and Can. Org. Rem., Decade ", 1). 71, Quebec Gr.

pennatulus, Hall, 1865, Can. Org. Rem., Decarle 2, \%. 82, Quebec Gr.

peosta, see Diplograptus peosta.

pristis, sce Diplograptus pristis.

putillus, see Diplograptus putillus.

pristiniformis, see Diplograjtus pristiniformis.

quadribrachiatus, Hall, 1858, Geo. Sur. Can., p. 125, and Can. Org. Rem., Decarle 2, p. 91, Quebec Gr.

quadrimucronatus, Hall, 1865, Can. Org. Rem., Decade 2, 1. 144, Utica Slate. ramosus, see Dicranograptus ramosus. ramulus, Hall, 1865, Can. Org. Rem., Decade 2, p. 108, Quebec Gr.

ramulus, White. The name was preoccupied. See G. whitianus.

richardsoni, Hall, 1865, Can. Org. Rem., Decade 2, p. 107, Quebec Gr.

rigidus, see Clonograptus rigidus.

scalaris, Linnzus, as identified by Hall in Pal. N. Y., vol. 1, p. 271, Utica Slate. 
secalinus, see Diplograptus secalinus.

serratulus, Hall, 1847, Pal. N. Y., vol. 1, p. 274, Utica Slate.

sagittarius, Linnæus, 1767, Syst. Nat., as identified by Hall in Pal. N. $Y$., vol. 1 , p. 272, Utica Slate.

sextans, see Dicranograptus sextans.

similis, Hall, 1865, Can. Org. Rem., Decade 2, p. 78, Quebec Gr.

spinulosus, see Diplograptus spinulosus.

subtenuis, Hall, 1877, Am. Pal. Foss., p. 244, Hud. Riv. Gr.

tentaculatus, see Retiograptus tentaculatus.

tenuis, Hall, 1847, Pal. N. Y., vol. 1, p. 272. The name was preoccupied by Portlock in 1843. See G. subtenuis. venosus, see Retiolites venosus.

whitfieldi, see Diplograptus whitfieldi.

whitianus, S. A. Miller, 1883, Am. Pal. Foss., p. 269, Hud. Riv. Gr. Proposed instead of G. ramulus, White, 1874, which was preoccupied. See Geo. Sur. W. 100th Mer., vol. 4, p. 62.

Hadroph yllum, Edwards \& Haime, 1850, Brit. Foss. Corals, p. lxvii. [Ety. hadros, mighty; phyllon, leaf.] Corallum short; calicle super-

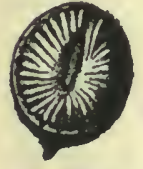

Fig. 179

$\mathrm{H}$ a drophyl-

lum glans.

\begin{abstract}
ficial ; one very large septal
\end{abstract} fossula and three small ones representing a cross; radiate arrangement of the septa somewhat irregular. Type H. orbignyi.

glans, White, 1862, (Zaphrentis glans,) Proc. Bost. Soc. Nat. Hist., vol. 9 , p. 32, and Cont. to Pal., No. 8, p. 156, Burlington $\mathrm{Gr}$.

orbignyi, Edwards \& Haime, 1850, Brit. Foss. Corals, p. lxvii, Up. Held Gr.

Haimeophyllum, Billings, 1859, Can. Jour., vol. 4, p. 139 , syn. for Chonostegites.

ordinatum, see Chonostegites ordinatus. Halli, Edwards \& Haime, 1851, Mon. d. Pol. Foss. d. Terr. Pal., p. 353. [Eıy, proper name.] Corallum tall, turbinate; septa extending to the center; one large septum occupying the place of the septal fossula, and the neighboring septa directed toward it, so as to assume a pinnate arrangement; no columella. Type H. insignis.

divergens, Hall, 1882, Foss. Corals Niagara and Up. Held. Grs., p. 8, and 35th Rep. N. Y. Mus. Nat. Hist., p. 412, Niagara Gr.

divisa, Hall, 1882, Foss. Corals Niagara and Up. Held. Grs., p. 8, and 35th Rep. N. Y. St. Mus. Nat. Hist., p. 412, Niagara $\mathrm{Gr}$.

insignis, Edwards \& Haime, 1851, Mon. d. Pol. Foss. d. Terr. Pal., p. 353, Up. Held. Gr.

pluma, Hall, 1882, Foss. Corals Niagara and Up. Held. Grs., p. 8, and 35th Rep. N. Y. St. Mus. Nat. Hist., p. 412, Niagara Gr. scitula, Hall, 1882, Foss. Corals Niagara and Up. Held. Grs., p. 7, and 35th Rep. N. Y. St. Mus. Nat. Hist., p. 411, Niagara Gr.

Halysites, Fischer, 1813, Zoognosia, vol. 1, p. 387. [Ety. halyson, a small chain.] Corallites long, arranged in single series, united laterally in the form of elliptical expansions, presenting a chain-like arrangement; epitheca thick; septa usually absent or rudimentary, but, in perfect specimens, extending to the center of the visceral chamber; tabulæe horizontal. Type $\mathbf{H}$. catenulatus.

agglomeratus, Hall, 1843, (Catenipora agglomerata,) Geo. Rep. 4th Dist. N. Y., Tab. Foss. No. 22, fig. 2, and Pal. N. Y., vol. 2 , p. 129 , Niagara Gr.

catenulatus, Linnæus, 1767, (Tubi pora catenulata,) Syst. Nat. 12th Ed., p. 1270, Niagara Gr.

catenulatus

fieldeni Fth e ridge, 1878 , Quar. Jour. Geo. Soc., vol. 34, p. 582, Up. Sil.

c a t en ulatus var. harti, Etheridge, 1878, Quar. Jour. Geo. Suc., vol. 34, p. 583, Up. Sil.

catenulatus var.

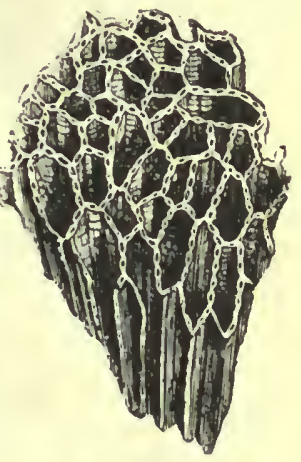

FIa. 180.-Halysites catenulatus. $\mathrm{m}$ i c r o p o r s,

Whitfield, 1882, Geo. Wis., vol. 4, p. 272, Niagara, Gr.

compartus, Rominger, 1876, Foss. Corals, syn. for $H$. agglomeratus.

escharoides, Lamarck, 1816, (Catenivora escharoides,) Hist. des Anim. sans Vert., vol. 2, p. 207, Niagara Gr.

gracilis, Hall, 1851, (Catenipora gracilis,) Geo. Lake Sup. Land Dist., vol. 2, p. 212, Hud. Riv. Gr.

labyrinthicus, Goldíuss, 1826, (Catenipora labyrinthica,) Petref. Germ., p. 71, Niagara Gr.

meandrina, Troost, 1840, (Catenipora meandrina,) 5th Geo. Rep. Tenn., Niagara Gr. The definition is too meagre for identification.

parryi, König, 1824, (Catenipora parryi,) Supp. to App. of Capt. Parry's Voyage for the Discovery of a North-west Passage, Up. Sil.

sexto-attenuatus, Owen, 1862, Geo. Sur. Ind., p. 362, Niagara Gr.

Harmodites rugosus, D'Orbigny, 1850 , Prodr. de Paleont., t. 1, p. 50. Not defined so as to be recognized.

Heliolites, Guettard, 1770, Mem. 3, p. 454. [Ety. helios, sun; lithos, stone.] Coralfum spheroidal, hemisnherical or ramose; corallites of larger and smaller size, the 
larger ones cylindrical, with twelve infoldings of the wall or septa, not reaching the center, the smaller ones polygonal, investing the larger ones; walls amalgamated; tabulæe numerous; no columella. Type H. interstinctus.

affinis, Billings, 1865, Can. Nat. and Geo., 2d ser., vol. 2, p. 427, Hud. Riv. and Mid. Sil.

elegans, Hall, 1852, Pal. N. Y., vol. 2, p. 130, Niagara Gr.

exiguus, Billings, 1865, Can. Nat. and Geo., $2 \mathrm{~d}$ ser., vol. 2, p. 428, Mid. Sil.

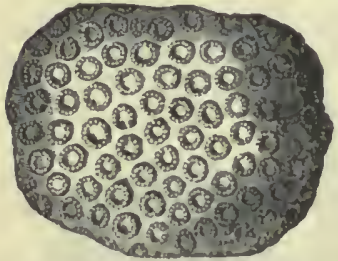

FIG. 181.- Heliolites Interstinctus. in ters tinctus,

L in $\mathbf{n}$ : u s, 1767, Madrepora interstincta,) Syst. Nat..12th El., p. 1276 , Niagara cir.

macrost ylus, H a 1 1, 1852, Pal. N. Y., vol. 2, p. 135 , Niagara Gr.

megastoma, McCoy, 1846, Sil. Foss. of Ireland, p..62, Niagara (ir."

pyriformis, Guettard, 1770, Mem. 3, p. 454, and Pal. N. Y., vol. 2, p. I33, Niagara Gir.

sparsus, Billings, 1865, Can. Nat. and Geo., 2d ser., vol. 2, p. 428, Mid. Sil.

speciosus, Billings, 1865, Can. Nat. and Geo., 2d ser., vol. 2, p. 426, Mid. Sil.

spiniporus, Hall, 1852, Pal. N. Y., vol. 2, p. 131, Niagara (ir.

subtubulatus, McCoy, as identified by Rominger, 1876, Foss. Corals, p. 13, Niagara Gr.

tenuis, Billings, 1865, Can. Nat. and Geo., $2 \mathrm{~d}$ ser., vol. 2, p. 428, Mid. Sil.

Heliopil yluds, Hall, 1848, in Dana. Zooph., p. 356 . [Ety. helios, sun; phyllon, leaf.] Corallum simple; septa well developed and producing lateral lamellar prolongations, which extend from the wall toward the center of the visceral chainber, so as to represent ascending arches and to constitu te irregular central tabula, and which are united toward the circumference by means of vertical dissepiments. Type H. Jalli.

acuminatum, Hall, 1882, Foss. Corals Niagara and Up. Held. Grs., p. 46, and 12th Rep. Ind. Geo., p. 310, Up. Held. Gr.

rquale, Hall, 1882, Foss. Corals Niagara and Up. Held. Grs., p. 47, and 35th Rep. N. Y. St. Mus. Nat. Hist., p. 451 , Up. Held. Gr.

æquum,-Hall, 1882, Foss. Corals Niagara and Up. Held. Grs., p. 51, and 12th Rep. Ind. Geo., p. 314, Up. Held. Gr.

alteruatum, Hall, 1882, Foss. Corals Niagara and Up. Held. Grs., p. 45, and 12 th Rep. Ind. Geo., p. 305, Up. Held. Gr. annulatum, Hall, 1882, Foss. Corals Niagara and Up. Held. Grs., p. 48, and 12th Rep. Ind. Geo., p. 307, Üp. Held. Gr.

arachne, Hall, 1876, Illust. Dev. Foss., pl. 24, Ham. Gr.

campaniforme, Hall, 1882, Foss. Corals Niagara and Up. Held. Grs., p. 53, and 35 th Rep. N. Y. St. Mus. Nat. Hist., y. 457, Up. Held. Gr.

canadense, Billings, 1859, Can. Jour., vol., 4. p. 125 , Up. Ifeld. Gr.

cancellatum, Hall, 1852, Foss. Corals Niagara and Up. Held. Grs., 1. 53, and 35tl Rep. N. Y. St. Mus. Nat. Hist., p. 457, Up. Held. Cir.

cayugaense, Billings, 1859, Cau. Jour., vol. 4, p. 124, Up. Held. Gr.

colbornense, Nicholson, 1875, Can. Nat. and Geo., vol. 7, p. 143, Up. Held. Gr.

colligatum, Billings, 1859, Can. Jour., vol. 4, p. 126, Up. Held. Gr.

compactum, Hall, 1882, Foss. Corals Niagara and Up. Held. Grs., p. 48, and 12th Rep. Ind. Geo., p. 30s, Up. Held. Gr.

confluons, Hall, 1876, Illust. Dev. Foss., pl. 26 and 27, II am. Gr.

degener, Ilall, 1876, Illust. Dev. Foss., pl. 25, Ham. Gr.

dentatum, Ilall, 1852, Foss. Corals Niagara and Up. Held. Grs., r). 48, and 35 th Rep. N. Y. St. Mus. Nat. Hist., p. 452, Up. Held. Gr.

denticulatum, Hall, 1882, Foss. Corals Niagara and Up. Meld. Grs., p. 52, and 12 tlı lep. Ind. Geo., p. 313, Up. IIeld. Gr.

dentilineatum, Hall, 1882, Foss. Corals Niagara and Up. Held. Grs., p. 13, and 35th IRep. X. Y. St. Mus. Nat. Hist., p. 417, Niagara Gr.

distans, IIall, 1882, Foss. Corals Niagara and $U$. Held. Grs., p. 50, and 12 th Rep. Ind. Geo., p. 308, Up. Held. Gr.

eriense, Billings, 1859, Cun. Jour., vol. 4, p. 124, Corniferous Gr.

exiguum, Billings, 1860, Can. Jour., vol. 5 , p. 26l, Cornifernus Gr.

fasciculatum, Hall, 1882, Foss. Corals Ni. agara and Up. Held. Grs., p. 48, and 35th Rep. N. Y. St. Mus. Nat. Mist., p. 452, Up. Held. Gr.

fecundum, IIall, 1882, Foss. Corals Niagara and Up. Held. Grs., p. 49, and 12th Rep. Geo. Ind., p. 309, Up. Held Gr.

fissuratum, Hall, 1882, Foss. Corals $\mathrm{Ni}$ agara and Up. Held. Grs., p. 53, and 35 tl $_{1}$ Rep. N. Y. St. Mus. Nat. Hist., p. 457, Up. Held. Gr.

gemmatum, Hall, 1882, Foss. Corals Niagara and Up. IIeld. Grs., p. 49, and 12th Rep. Geo. Ind., 1) 310, Up. Held Gr.

gemmiferum, Hall, 1882, Foss. Corals Niagara and Up. Held. Grs., p. 13, and 35 th Rep. N. Y. St. Mus. Nat. Hist., p. 417, Niagara Gr. 
halli, Edwards \& Haime, 1850, Brit. Foss. Corals, p. 235, Ham. Gr.

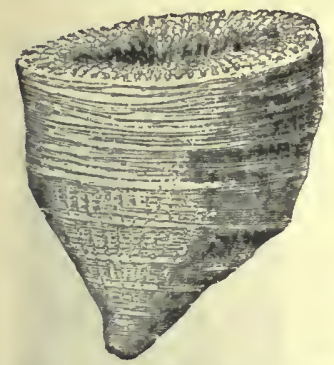

halli var. obconicum, $\mathrm{H}$ a $\mathrm{ll}$, 1876, Ill u st. Dev. Foss., p]. 25, Ham. Gr.

halli var. reflexum, Hall, 1876, Illust. Dev. Foss., pl. 23, Ham. Gr.

imbricatum, Hall, 1882 , Foss. Corals Niagara and Up. Held. Grrs., p. 46 , and 35 th Rep. N. Y. St.

FIG. 182. - Heliophyllum Mus. Nat. Hist., p. 450, Up. Held. Gr. incrassatum, Hall, 1882, Foss. Corals $\mathrm{Ni}$ agara and Up. Held. Grs., p. 46, and 12th Rep. Geo. Ind., p. 309, Up. Held Gr. infundibulum, Hall, 1883, 12th Rep. Geo. Ind., p. 305, Up. Held. Gr.

invaginatum, Hall, 1882, Foss. Corals $\mathrm{Ni}$ agara and Up. Held. Grs., p. 47, and 12th Rep. Geo. Ind., p. 306, Up. Held. Gr.

irregulare, Hall, 1876, Illust. Dev. Foss., pl. 24, Ham. Gr.

latericrescens, Hall, 1882, Foss. Corals Niagara and Up. Held. Cirs., p. 49, and 12th Rep. Geo. Ind., p. 314, Up. Held Gr. lineolatum, Hall, 1882, Foss. Corals Niagara and Up. Held. Grs., p. 50, and 35 th Rep. N. Y. St. Mus. Nat. Hist., p. 454, Up. Held. Gr.

mitella, Hall, 1882, Foss. Corals Niagara and Up. Held. Grs., p. 14, and 35th Rep. N.'Y. St. Mus. Nat. Hist., p. 418, Niagara Gr. nettlerothi, Hall, 1882, Foss. Corals Niagara and Up. Held. Grs. p. 51, and 12 th Rep. Geo. Ind., p. 312, Up. Held. Gr.

pocillatum, Hall, 1884, Foss. Corals Niagara and Up. Held. Grs., p. 50, and 35th Rep. N. Y. St. Mus. Nat. Hist., p. 454, Up. Held. Gr.

pravum, Hall, 1882, Foss. Corals Niagara and Up. Held. Grs., p. 13, and 12th Rep. Geo. Ind., p. 274, Niagara Gr.

proliferum, Nicholson, 1874, Rep. Pal. Ont. Can., p. 27, Up. Held. Gr.

proliferum, Hall, 1876, Illust. Dev. Foss., pl. 26 , is probably a syn. for H. proliferum, Nicholson.

puteatum, Hall, 1882, Foss. Corals Niagara and Up. Held. Grs., p. 14, and 35th Rep. N. Y. St. Mus. Nat. Hist., p. 418, Niagara Gr.

scyphulus, Hall, 1882, Foss. Corals Niagara and Up. Held. Grs., p. 51, and 12th Rep. Geo. Ind., p. 306, Up. Held. Gr. sordidum, Hall, 1882, Foss. Corals Niagara and Up. Held. Grs., p. 52, and 12th Rep. Geo. Ind., p. 311, Up. Held. Gr.

subcæspitosum, see Crepidophyllum subcæspitosum.

tenuimurale, Hall, 1882, Foss. Corals Niagara and Up. Held. Grs., p. 51, and 12tl'Rep. Geo. Ind., p. 307, Up. Held. Gr. tenuiseptatum, Billings, 1859, Can. Jour., vol. 4, p. 126, Ham. Gr.

venatum, Hall, 1882, Foss. Corals Niagara and Up. Held. Grs., p. 46, and 35th Rep. N. X. St. Mus. Nat. Hist., p. 450, Up. Held. Gr.

verticale, Hall, 1882, Foss. Corals Niagara and Up. Held. Grs., p. 47, and 35th Rep. N. Y. St. Mus. Nat. Hist., p. 451, Up. Held. Gr.

Heterophrentis, Billings, 1875, Can. Nat. and Geo., vol. 7, p. 235. [Ety. heteros, irregular; phren, midriff or lamella.] Corallum simple, turbinate, calice large, septal fossette well-defined, bottom smooth or with a pseudo-columella, septa, below the calice, sharp edged; often with their inner edges twisted together, usually rounded on approaching the margin; apparently only a single transverse diapliraym, which forms the floor of the cup. Type H. spatiosa. compta, Billings, 1875, Can. Nat. and Geo., vol. 7, p. 236, Corniferous Gr.

excellens, Billings, 1875, Can. Nat. and Geo., vol. 7, p. 236, Corniferous Gr.

prolifica, Billings, 1875, Can. Nat. and Geo., vol. 7, p. 236, Corniferous Gr.

spatiosa, Billings, 1858, (Zaphrentis spatiosa,) Can. Nat. and (reo., vol. 3, p. 430, Onondaga and Corniferous Gr.

Heterotrypa, Nicholson, 1879, Pal. Tab. Cor., p. 291. Proposed as a subgenus of Monticulipora, making M. mammulata the type which is the type of Monticulipora. This is a violation of the elementary principles of nomenclature.

Houghtonia, syn. for Calapocia.

huronica, see Calapoecia huronica.

INOCAULIS, Hall, 1852, Pal. N. Y., vol. 2, p. 176. [Ety. inos, small sprouts; kaulos, stem.] Expanded, bifurcating, fenestrate, and usually indicated by simple black rays connected by small cross bars. Type I. plumulosus.

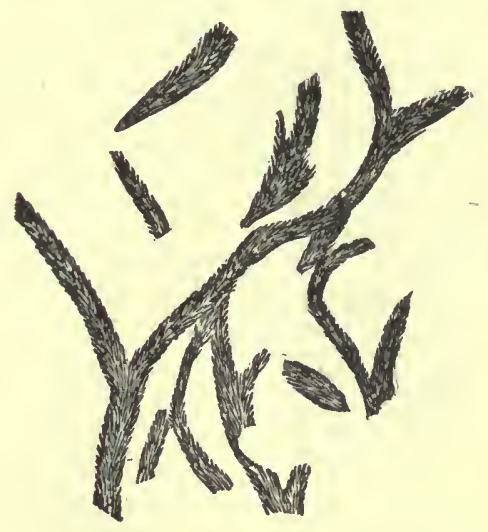

FIG. 183.-Inocaulis plumulosus.

anastomica, Ringueberg, 1888, Proc. Acad. Nat. Sci. Phil., p. 131, Niagara Gr. 
arbuscula, Ulrich, 1879, Jour. Cin. Soc. Nat. Hist., vol. 2, p. 28, Hud. Riv. Gr. bellus, Hall \& Whitfield, 1875, Ohio Pal., vol. 2, p. 122, Niagara Gr.

cervicornis, Spencer, 1884, Bull. No. 1, Mus. Univ. St. Mo., p. 37, Niagara Gr. diffusus, Spencer, 1884, Bull. No. 1, Mus. Univ. St. Mo., p. 36, Niagara Gr.

divaricatus, Hall, 1879, Desc. New sp. Foss., p. 2, and 11th Rep. Geo. Ind., p. 225 , Niagara Gr.

phycoides, Spencer, 1884, Bull. No. 1. Mus. Univ. St. Mo., p. 38, Niagara Gir. plumulosus, Hall, 1851, Pal. N. Y., vol. 2, p. 176, Niagara Gr.

problematicus, Spencer, 1878, Can. Nat. vol. 8, and Bull. No. 1, Mus. Univ. St. Mo., p. 36, Niagara Gr.

ramulosus, Spencer, 1884, Bull. No. 1, Mus. Univ. St. Mo., p. 38, Niagara Gr. walkeri, Spencer, 1854, Bull No. 1, Mus.

Univ. St. Mo., p. 35, Ningara Gr.

Lamellopora, Oren, 1840, Rep. on Minn. Lands, p. 70. Verly poorly defined, but a syn. for Stromatapora.

infundibularia, Owen, 1840, Rep. on Minn. Lands, p. 70. A species of Stromatapora, poorly defined.

Lepropora, Winchell, 1863, Proc. Acad. Nat. Sci. Phil., p. 2. [Fty. leptos, shallow; poros, cell.] Discoidal, cclls shallow: walls vertically striated; interior vesicular; cups elevated in the center, and displaying radial septa. 'Type $\mathrm{H}$. typus. typus, Winchell, 1863, Proc. Acad. Wat. Sci. Phil. p. 3, Marshall Gr.

winchelli, White, 1879, Bull. U. S. Sur. vol. 5, p. 211, and Cont. to Pal. No. 6, p. 121, Carboniferous.

Limaria, Steininger, 1834, Bull. Soc. Geo. France, vol. 1, p. 339. The name was preoccupied by Link in 1807, and by Rafinesque in 1815. See Conites. crassa, see Conites crassus. falcata, see Conites falcutus. fruticosa, see Conites fruticosus.

laminata, see Conites laminatus.

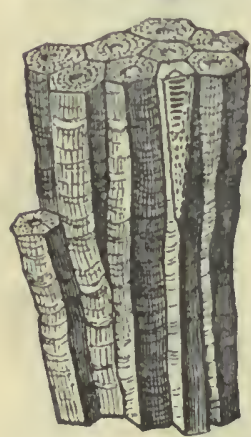

FIG. 18\%. - Lithostrotion basaltiforme. ramulosa, see Cunites ramulosus.

Lisostrosia, Nicholson \& Thompson, 1877, Proc. Roy. Soc. Edinb.,vol. 9, p. 149. [Ety. proper name.] Type L. columnaris. columnaris, Nicholson \& Thompson, 1877 , Proc. Roy. Soc. Edinb., vol. 9, ग. 149, Devonjan.

Linipora rotunda, Troost, not defined.

Lituostrotion, Lbwyd, 1869, Lithophyl. Britann. Ichnographia, Epistola 5, tab.xxiii.

[Ety. lithos, stone; strotion, little rafter.] Corallum composite, astreiform; corallites loosely approximated, and circu-

lar or intimately united, and polygonal; septa numerous; calices unequal; structure as in Clisiophyllum. Type L. basaltiforme.

californiense, Meek, 1864, Pal. California, vol. 1, p. 6. Carb. canadense, Castelnau, 18t3, (Axin u r a canadensis,) Syst. Sil., p. 49, St. Louis Gr. harmodites, Ed wards \& Haime, 1851 , Mon. d. Pol. Foss: d.Terr. Pal., p. 440 , Carboniferous.

junceum. Fleming, 1828, (Caryo. pliyllaa juncea,) Brit. Anim., p. 509, Subcarb.

mammillare, Castel-

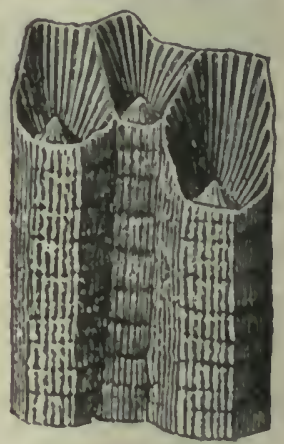

Fyo. 185, - Idthostrotion cnundense.

nau, 18t3, (As-

trea mammillaris,) Syst. Sil., p. 50, syn. for I. canadense.

microstylum, White, 1880,12 th Rep. U.S. Geo. Sur. Terr., p. 158, Kinderhook or Waverly Gr.

pictoense, Billings, 1868, Acad. Geo., p. 285, Carb.

proliferum, Hall, 1858, Geo. Rep. Iowa, p. 668, St. Louis Gr.

stokesi, Edwards \& Haime, 1851, Mon. d. Pol. Foss. d. Terr., Pal., p. 440, Carboniferous.

whitneyi, Meek, 1875, Wheeler's Sur. W. 100 Mer., vol. 4, p. 103 Coal Meas.

Loxsdalia, McCoy, 1849, Ann. \& Mag. Nat. Hist.2d ser., vol. 3, p. 10. [Ety. proper name.] Corallum aggregate; corallites circular, not laterally.united; septa and tabulie numerous; visccral chamber separated into two zones, the outer one

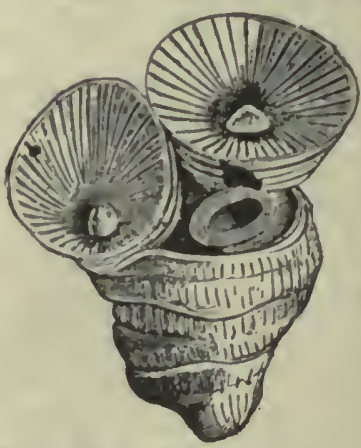

F16. 186.-Lonsdalla floriformis, typical of the genus. 1837, (Cyathophyllum papillatum, Oryct. de Moscou., p. 155, Carboniferous. American. (?)

LophopHYLLU, Edwards \& Haime, 1850 , Brit. Foss. Corals, p. Ixvi. [Ety. lophos, ridge; phyllon, leaf.] Corallum resembling Zaphrentis, excepting that a cres- 
centiform columella occupies the center of the calice, and is in continuity by one of its ends with a small septum placed in the middle of the septal fossula, and by the other end with the opposite primary septum. Type L. konincki.

calceola, see Zaphrentis calceola.

expansum, White, 1876, Proc. Acad. Nat. Sci. Phil., p. 27, and Cont. to. Pal., No. 6. p. 157, Keokuk Gr.

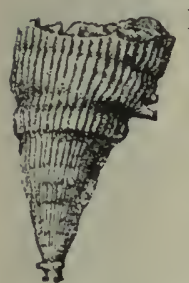

rol i ferum,

$\mathrm{McCh}$ es-

ney, 1860 ,

(Cyathaxonia prolifera, ) New Pal. Foss., $p$. 75, and Pal. E. Neb., p. 144, Coal Meas.

Fig. 187.-Lo-LunATIPORA, Winchell, 1866, pliophyllum Rep. Low. Penin. Mich. proliferum. p. 89. [Ety. lunatus, crescent-formed; poros, pore.] Massive or with corallites consolidated; corallites long, curving outward from an imaginary axis; walls double ; tabulæ present; no mural pores. Type L. michiganensis. michiganensis, Winchell, 1866, Rep. Low. Penin. Mich., p. 89, Ham. Gr.

Lyellia, Edwards \& Haime, 1851, Mon. Pol. Foss. Terr. Pal., p. 226. [Ety. proper name.] Corallum massive; corallites cylindrical; walls thick, costulated, free toward their terminations, and united by vesicular conenchyma; septa 12 ; tabulæ irregular. 'Type L. americana.

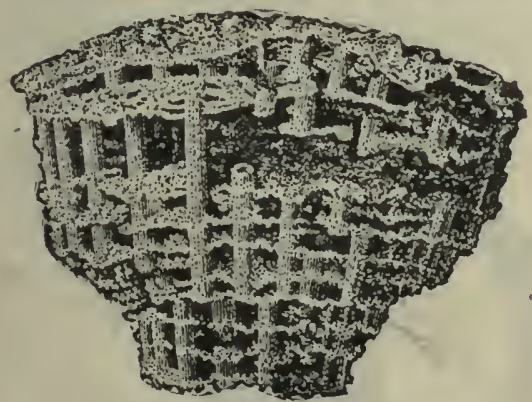

FIG. 188.-Lyellia americana.

americana, Edwards \& Haime, 1851, Mon. Pol. Foss. Terr. Pal., p. 226, Up. Held. Gr. decipiens, Rominger, 1876, Foss. Corals, p. 17, Niagara Gr.

glabra, Owen, 1840, (Sarcinula glabra,)

Rep. on Minn. Lands, p. 70, Niagara Gr. papillata, Rominger, 1876, Foss. Corals, p. $16, \mathrm{Ni}-$ agara Gr.

parvitu ba,

Rominger,

1876, Pal.

Foss. Corals, p. 17, $\mathrm{N}$ i a gar Gr.

Madrepora $r$ epens, Troost. Not satisfactorily defined.

MEGALOGRAPTUs, S. A. $\mathrm{M}$ i l l e r, 1874, Cin. Quar.Jour. Sci., vol.1, p. 343 [Ety. megale, large: grapho, I w rite.] Very large cylin $\mathrm{d} \mathrm{ri}$ cal, bearing fronds with spinous processes,and covered with cellular openings. Type Fia. 190.-Megalograptus welchl. M. welchi. Frond, showing cells and spiwelc h i, S. nous processes.

A. Miller, 1874, Cin. Quar. Jour. Sci., vol. 1, p. 343 , Hud. Riv. Gr. 
Michelinia, DeKoninck, 1842, Descrides Anim. Foss. Belg., p. 29. [Ety. proper name.] Corallum composite, forming hemispherical, depressed, or pyriform masses of prismatic or subeylindrical corallites; mural pores; tabulae; tubes having stria or ridges; epitheca concentrically wrinkled, with root-like prolongations. Type M. favosa.

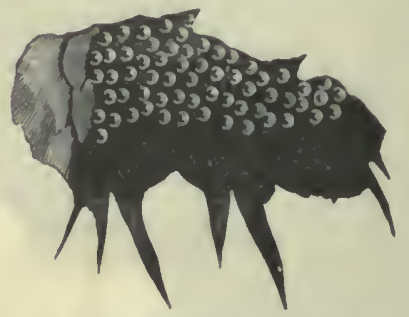

Fin. 191.-Megrlogrnptus welchi. Frond, showcelis und splnous processes.

convexa, D'Orbigny, 1850, Prodr. de Paleont., t. 1, p. 107, Onondaga and Corniferous Girs.

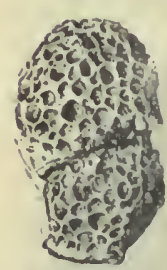

Fig. 192.-

Miselinia eugenere. dividua, Hall, 1876 , Illust. Dev. Foss., pl. 18, llam. Gir.

eugener, White, 18st, 13th, Rep. Geo. Ind., p. 119, Cosl Meas.

expansa, White, 1880, 12th Rep. U. S. Geo. Sur. Terr., p. 158, Waverly Gr. favositoidea, Billings, 1858 , Rep. of P'rogr. Can. Geo. Sur., p. 175 , Up. Held. Gr.

insignis, Rominger, 1876, Foss. Corals, p. 75 , Up. Held. and Ham. Gr.

intermittens, Billings, 1859, Can. Nat. and Geo. Sur., vol. 4 , p. 113, Corniferous Gr.

lenticularis, Hall, 1874, 26th Rep. N. Y. St. Mus. Nat. Hist., p. 113, Low. Held. Gr.

placenta, White, 1880 , 12 th Rep. U. S. Geo. Sur. Terr., p. 157, Waverly Gr.

stylopora, Eaton, 1832 ,

(Astrea styloporn,) Geo.

Text book, p. 40, and

Illust. Dev. Foss., pl. 18,

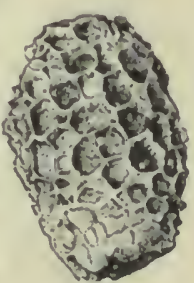

FIG. 193.-

Mle hell $\mathrm{n}$ । cugener. Anotller specimen, with men, wher corml. lifes.
Ham. Gr.

trochiscus, Rominger, 1876, Pal. Foss., p. 76, syn. for Pleurodietyum americanum. Microcycuus, Meek \& Worthen, 1868, Geo. Sur. Ill., vol. 3, p. 420. [Ety. mikros, small; kuklos, circle.] Corallum free or with a minute central point of attach. ment, discoidal ; no columella ; calice shallow, with a single fossula; septa short, radiating regularly, or those nearest the fossette converging a little toward its sides; epitheea well developed. Type M. discus.

discus, Meek \& Worthen, 1S68, Geo. Sur. Ill., vol. 3, p. 420 , Ham. Gr.

Millepora repens, see Alveolites repens.

Mosograptes, E m mons, 1856 , (Monograpsus,) Am. Geo, p. 106. [Lity. FiG. 194-Mreromonos, one; grapho, I cyclus discus. write.] Serrations confined to one edge of the stipe; axis none. Type M. elegans.

convolutus var. coppingeri, Etheridge, 1878, Quar. Jour. Geo. Soc., vol. 34, p. 577 , Silurian.

eleg:nns, Emmons, 1556, Am. Geo., p. 106, Up. Taconic.

rectus, Fmmons, 1856, Am. Geo., p. 107, Up. Taconic.

MoNotkyi'A, Nicholson, 1879, Pal. Tab. Corals, p. 320. [Ety. monos, one; trupa, hole.] Corallites of two kinds; the larger aggregnted into elusters (monticules); the smaller occupying the space between the monticults; both larger and smaller thin-walled, polygonal trb. ula remote. Type M. undulata. 'This was proposed as a subgenus for Monticulipora; upon microseopial examinntion and upon such a state of facts, I prefer, at present, to leave the species under the genus Montieulipora.

(?) spinulosa, Hall, 1887, P’al. N. Y., vol. 1, p. 67, Low. Held. Gr.

MoNothypella, Ulrich, 1882, Jour. Cin. Soc. Nut. Hist., vol. 5, p. 153. [lity. monotrypa; and illus, diminutive.] Ramose, smooth or tuberculated, cells of one kind only; walls thin, in the axiul region, and thicker townrd the periphery ; diaphragms straight; no spiniform tubuli. Type M. requalis.

abrupta, Hull, 1879, (Chetetes abruptus, $32 d$ Rep. N. Y. St. Mus. Nat. Hist., p. 148, Low. Held. Gr.

requalis, Ulrich, 1882, Jour. Cin. Soc. Nat. Hist., vol. 5, p. 247, Hud. Riv. Gr.

arbusculn, Hall, 1887, Pal. N. Y., vol. 6, p. 12, Lriw. Hela. Gr.

briareus, Nielıolson, 1875, (Chetetes bri-

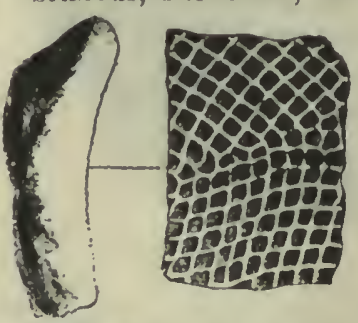

Fiส. 105.--Monotry pelln quadratn, natural slze and magnlfled. areus,) Olıio Pal., vol. 2. 1). 202, Uticus Siate.

consimilia, Hall, 1876, (Clietetes consimilis, ) 28th Rep. N. Y. St. Mus. Nat. Hist., 1). 110, Niagara Gr. densn, Iall, 187 , ('Trematopora denea,) 26th Rep. N. Y. St. Mus. Nat. Hist., p. 105, Low. Held. Gr. 
quadrata, Rominger, 1866, (Chetetes quadratus,) Proc. Acad. Nat. Sci. Phil., p. 3, and Ohio Pal., vol. 2, p. 201, under the name of Chetetes rhombicus, Hud. Riv. Gr.

subquadrata, Ulrich, 1882, Jour. Cin. Soc. Nat. Hist., vol. 5, p. 249 , Hud. Riv. Gr.

Monticulipora, D'Orbigny, 1850, Prodr. de Paléont., t. 1, p. 25. [Ety. monticulus, hillock; poros, pore.] Corallum of every form and shape; corallites usually of two kinds, one minute ; tabulæ numerous; walls separable, thickened toward the mouths of the tubes; corallites often aggregated, upon the surface, in numerous monticules; no septa; no mural pores: increase by gemmation. Lindstrom, Ulrich, and otbers, class this genus with the Bryozoa, while Nicholson, Edwards \& Haime, and others, class it with the Polypi, where it seems to belong. Type M. mammulata.

adherens, Billings, 1859, (Stenopora adherens,) Can. Nat. and Geo., vol. 4, p. 427 , Chazy Gr.

andrewsi, Nicholson, 1881, Struct. and Affin. of Montic., p. 128, Hud. Riv. Gr. Ulrich refers it to Callopora.

barrandi, Nicholson, 1874, (Chetetes barrandi,) Quar.Jour. Geo. Soc., vol. 30, and Pal. of Ontario, p. 60, Ham. Gr.

bulbosa, Billings, 1865, (Stenopora bulbosa,) Can. Nat. and Geo., 2d ser., vol. 2, p. 429, Mid. Sil.

calceolus, Miller \& Dyer, 1878, Jour. Cin. Soc. Nat. Hist., vol. 1, p. 26, Hud. Riv. Gr.

cincinnatiensis, James, 1875, (Chetetes cincinnatiensis,) Int. Catal. Cin. Foss., p. 2, and Nicholson, Struct. and Affin. Montic., p. 226, Hud. Riv. Gr.

compressa, Ulrich, 1882, (Peronopora compressa) Jour. Cin. Soc. Nat. Hist., vol. 5, p. 244, Hud. Riv. Gr.

consimilis, Ulrich, 1882, Jour. Cin. Soc. Nat. Hist., vol. 5, p. 238, Hud. Riv. Gr.

dalii, Edwards \& Haime, 1851, (Chetetes dalii,) Pol. Foss. d. Terr. Pal., p. 266, Hud. Riv. Gr. Ulrich refers it to Callopora.

dawsoni, Nicholson, 1881, Struct. and Affin. Montic., p. 141, Hud. Riv. Gr.

decipiens, Rominger, 1866, (Chetetes decipiens,) Proc. Acad. Nat. Sci. Phil., p. 3, Hud. Riv. Gr.

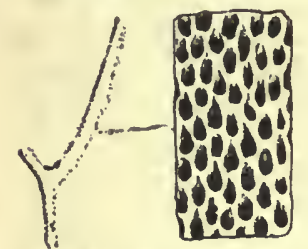

FIG. 196,-Montlculipora dellcatula. delicatula, Nicholson, 1874, (Chetetes delicatulus) Quar. Jour. Geo. Soc., vol. 30 , and Ohio Pal., vol. 2, p. 199, Hud. Riv. Gr. Probably a Bryozoum and not a Monticulipora.

dychei, James, 1883, Jour. Cin. Soc. Nat. Hist., vol. 6, p. 235, Hud. Riv. Gr. fibrosa, Goldfuss, 1826, (Calamapora fibrosa,) Germ. Petref., p. 82, Hud. Riv. and Clinton Grs.

filiasa, D'Orbigny, 1850, Prodr. d. Pal., t. 1, p. 25, and Edwards \& Haime, Pol. Foss. d. Terr. Pal., p. 266, Hud. River Gr. frondosa, D'Orbigny, 1850, Prodr. d. Pal., t. 1, p. 25, and Ohio Pal., vol. 2, p. 208. Hud. Riv. Gr.

gracilis, see Batostomella gracilis.

grandis, Ulrich, 1886, 14th Rep. Geo. Sur. Minn., p. 78, Trenton Gr.

implicata, see Batostoma implicata.

irregularis, Ulrich, 1879, (Chetetes irregu-

laris,) Jour. Cin. Soc. Nat. Hist., vol. 2, p. 129 , Hud. Riv. Gr.

lævis, Ulrich, 1882, Jour. Cin. Soc. Nat. Hist., vol. 5 , p. 236, Hud. Riv. $\mathrm{Gr}$.

1 y c operdon, S a y, 1847, ( $\mathrm{F}$ a vosites 1 y c o p e r-Fro. 197.-Monticulipora lyeodon,) Hall,

Pal. N. Y., vol. 1, p. 64, Trenton Gr. mammulata, D'Orbigny, 1850, Prodr. de Paléont., t. 1, p. 25, and Ohio Pal., vol. 2, p. 207, Hud. Riv. Gr.

molesta, Nicholson, 1881, Struct. and Affin. of Montic., p. 224, Hud. Riv. Gr. moniliformis, Nicholson, 1874, (Chetetes moniliformis,) Geo. Mag., vol. 1, p. 57, and Pal. of Ont., p. 60, Ham. Gr.

monticula, White, 1876, Proc. Acad. Nat. Sci. Phil., p. 27, Devonian.

multituberculata, Whitfield, 1878, Ann.

Rep. Geo. Sur. Wis., p. 71, and Geo.

Wis:, vol. 4, p. 250 , Hud. Riv. Gr.

parasitica, Ulrich, 1882, Jour. Cin. Soc. Nat. Hist., vol. 5, p. 238, Hud. Riv. Gr. patulus, Billings, 1859, (Stenopora patula,)

Can. Nat. and Geo., vol. 4, p. 427, Chazy Gr.

pavonia, see Ptilodictya pavonia.

petasiformis, Nicholson, 1881, Struct. and Affin. of Montic., p. 190, Hud. Riv. Gr. punctata, Whitfield, 1878, Ann. Rep. Geo. Sur. Wis., p. 71, and Geo. Wis., vol. 4, p. 249, Hud. Riv. Gr.
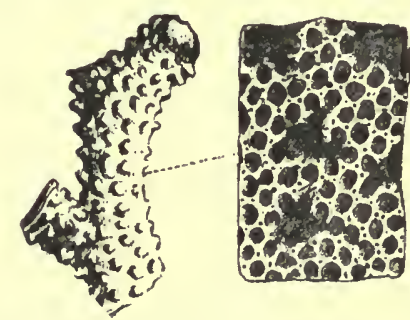

FrG. 198.-Monticulipora ramosa, naturai size and magnifled.

ramosa, D’Orbigny, 1850, Prodr. d. Pal., t. 1, p. 25, and Edwards \& Haime, Pol. Foss. de Terr. Pal., p. 266, and Ohio Pal., 
vol. 2 , under the name of Chetetes dalii, Hud. Riv. Gr. Ulrich refers it to Callopora.

rectangularis, Whitfield, 1878, Ann. Rep. Geo. Sur. Wis., p. 70, and Geo. Wis., vol. 4 , p. 249 , Hud. Riv. Gr.

rugosa, Hall, 1847, (Chetetes rugosus,) Pal. N. Y., vol. 1, p. 67, Trenton Gr.

rugosa, Edwards \& Haime, 1851, Pol. Foss. de Terr. Pal., is merely a variety or form of M. ramosa, and associated with it in the Hud. Riv. Gr.

selwyni, see Prasopora selwyni.

seluyni var. hospitalis, see Prasopora selwyni var. hospitalis.

solitaria, Ulrich, 1883, (Heterotrypa solitaria,) Jour. Cin. Soc. Nat. Hist., vol.6, p. 88 , Hud. Riv. Gr.

subglobosa, Ulrich, 1879, (Chetetes subglobosus,) Jour. Cin. Soc. Nat. Hist., vol. 2, p. 129, Hud. Riv. Gr.

subpulchella, Nicholson, 1875, (Chetetes subpulchellus,) Ohio Pal., vol. 2, p. 196, Hud. Riv. Gr.

trentonensis, Nicholson, 1581, Struct. and Aftin. Montic., p. 149, Trenton Gr.

tuberculata, see Spationora tuberculata.

ulrichi, Nicholson, 1881, Struct. and Affin. Montic., p. 131, Hud. Riv. Gr.

undulata, Nicliolson. 1875, (Chetetes undulatus,) Pal. of Ont., p. 10, and Struct. and A ffin. Montic., p. 170, Trenton and Hud. Riv. Gr.

uniformis, Ulrich, 1882, (Peronopora uniformis,) Jour. Cin. Soc. Nat. Hist., vol. 5, p. 244, Hud. Riv. Gr.

vaupeli, Ulrich, 1853, (Heterotryna vaupeli,) Jour. Cin. Soc. Nat. Hist., vol. 6, p. 85, Hud. Riv. Gr.

venusta, Ulrich, 1878, (Chetetes venustus,) Jour. Cin. Soc. Nat. Hist., vol. 1, p. 93, Utica Slate.

westoni, Foord, 1883, Cont. to Micro-I'nl., p. 7, T'renton Gr.

wetherbyi, Ulrich, 1882, Jour. Cin. Soc. Nat. History, vol. 5, p. 239, Trenton Gr. whiteavesi, Niclsolson, 1879, Pal. Tab. Corals, p. 316, and Struct. and Affin. of Montic., p. 160, Trenton Gr.

Nebulipora, McCoy, 1850, Ann. and Mag. Nat. Hist., 2d ser., vol. 6, p. 284. [Ety. nebula, thick mist; poros, pore. $]$ Incrusting or forming lenticular masses, with a concentrically wrinkled epitleca below, composed of small prismatic corallites perpendicular to the upper surface, with clusters of rather larger size, all in contact ; tabulse at regular distances; no septa. Type N. explanata.

papillata, McCoy, 1850, Ann. and Mag. Nat. Hist., vol. 6, p. 284, Hudson Riv. Gr.

NEMAGRAPTU8, Emmons, (Nemagrapsus,) 1856, Am. Geo., pt. 2, p. 109. The termination graptus is preferred because grapsus is used in the nomenclature of crustacea. [Ety. nema, thread; grapho, I write.] Axis elongated and thread-like, simple or com- pound branches, round at the base, and flattened at the extremities; cells arranged on the flattened part of the axis instead of the margin. Type $N$. elegans. capillaris, Emmons, 1856, Am. Geo., pt. 2, p. 109, Up. Taconic.

elegans, Emmons, 1856, Am. Geo., pt. 2, p. 109 , Up. Taconic.

NyctoporA, Nicholson, 1879, Pal. Tab. Corals, p. 182. [1.ty. nuktos, night; poros, pore.] Corallum composite, massive; corallites polygonal, in contact; walls thin, amalgamated; mural pores numerous, small; septa, in the form of marginal vertical ridges; 10 to 15 in each corallite; tabula numerous, complete, horizontal. Ty pe $\mathbf{N}$. billingsi.

billingsi, Nicholson, 1879, Pal. T'ab. Corals, p. 184, Trenton Gr.

Ordinama, Forbes, 1850, Dub. Geo. Jour. [Ety. proper name.] Strong stems, with branches arranged in whorls; substance corneous; cellules undetermined. Type 0 . antiqua.

antiqua, Forbes, 1850, Dublin Geo. Jour., Potsdsm Gr.

fruticosa, Hall, 1865, Can. Org. Rem. Decade 2, p. 50, Trenton Gr.

OMPIYMA, Rafinesque, 1820 , Anu. des Sci. Pliys. d Bruxclles, vol. 5, 1). 234. [Ety. omphax, precious stone.] Simple, turbinate, wall with rudi-Fra. 109.-- Olımentary epitheca, pro- ham is anlucing radiciform ap- tiqua.

pendages; septa numerous, equally developed and divided into four groups by an equal number of sliallow fossulce; tabule "smooth toward the center. Type 0 . turbinata. congregata, Billings, 1866 ,

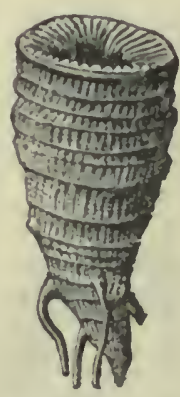

Fig. $200 .-\mathrm{Om}$ ph y ma lurbluata. Catal. Sil. Foss. Antic., p. 93, Clinton and $\mathrm{Ni}$ agara $\mathrm{Gr}$.

drummondi, Billings, 1866, Catal. Sil. Foss. Intic., p. 93, Clinton and $\mathrm{Ni}$ agara Grs.

stokesi, Edwards \& Haime, 1851, (Ptychophyllum stokesi,) Polyp. Foss. Pal., p. 407, and Geo. Wis., vol. 4, p. $279, \mathrm{Ni}$ agara $\mathrm{Gr}$.

verrucosa, Rafinesque \& Clifford, 1820, Monog. d. Turbinolides in Ann.d. Phys. d. Brux., t. 5, p. 235, Niagara Gr.

Pacitypiyludu, Edwards \& Haime, 1850, Brit. Foss. Corals, p. lxviii. [Ety. pachys, thick; phyllon, leaf.] Corallum, composite, increasing by lateral gemmation; corallites united by the development of the costr and exotheca; tabulæe abundant. Type P. bouchardi. 
solitarium, Hall \& Whitfield, 1873, 23d Rep. N. Y. St. Mus. Nat. Hist., p. 232, Chemung Gr.

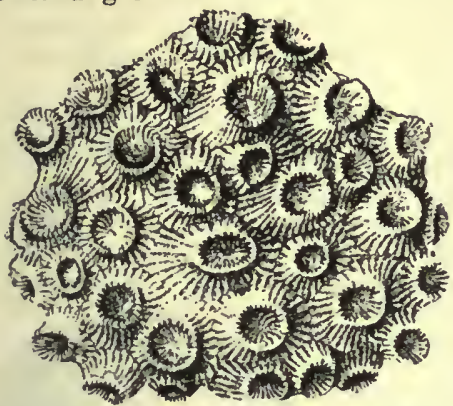

FiG. 201.-Pachyphyllum woodmani.

woodmani, White, 1870, (Smithia woodmani,) Geo. Sur. Iowa, vol. 1, p. 188, Chemung Gr.

PACHYPORA, Lindstrom, 1873, Ofversight af K. Vetensk Akad. Forhandl., p. 14. [Ety, pachys, thick; poros, pore.] Dendroid or frondescent; corallites polygonal or subcylindrical, walls thickened toward their mouths, by concentric layers of sclerenchyma; calices annular; oblique, or semilunar; septa obsolete or mere spiniform projections ; tabulæ complete, remote; mural pores few, irregular, and often large. Type P. lamellicornis.

fischeri, Billings, 1860, (Alveolites fischeri,) Can. Jour. n. s., vol. 5, p. 256, Up. Held. Gr.

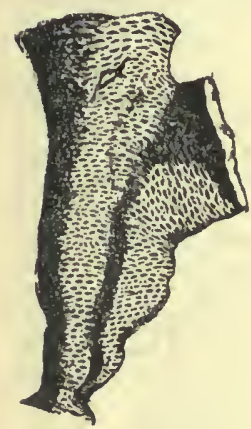

Fig. 202,-Pachy pora frondosa. frondosa, Nicholson, 1874 , (Alveolites frondosus,) Geo. Mag., vol. 1, p. 15, and Rep. Pal. Ontario, p. 57, Ham. Gr. ornata, Rominger, 1876, (Dendropora ornata,) Pal. Foss. Corals, p. 62, Ham. Gr.

Palmocyclus, Edwards \& Haime, 1849 , Comptus rendus, t. 29 , p. 71. [Ety. palaios, ancien t; kuklos, circle.] Corallum circular; fossula deep, broad, circular; septa thick, not numerous or cemented together. Type P. porpita.

kirbyi, Meek, 1868, Trans. Chi. Sci., p. 85, Devonian.

rotuloides, Hall, 1852, (Cyclolites rot uloides,) Pal. N. Y., vol. 2, p. 42 , Clinton Gr.

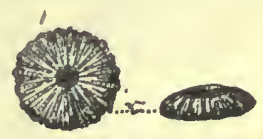

Paldophyllum, B i l- Fig. 203.-Palæocyclus lings, 1858, Rep. of

Prog. Geo. Sur. Can., p. 168. [Ety. palaios, ancient; phyllon, leaf.] Fascicu- late or aggregate; corallites surrounded by a thick wall; septa extending the whole length; tabulæ absent or rudimentary ; increase by lateral budding. Distinguished from Streptelasma by form. ing aggregate masses. Type P. rugosum.

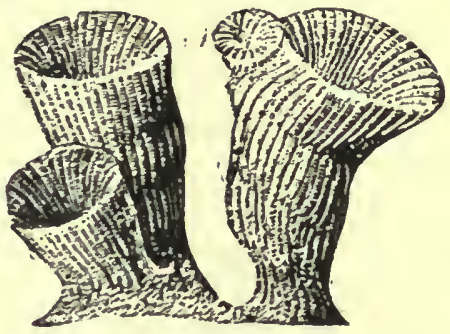

FIG. 204.-Palcophyllum divaricaus.

divaricans, Nicholson, 1875, Pal. Ohio, vol. 2, p. 220 , Hud. Riv. Gr.

rugosum, Billings, 1858, Rep. of Progr. Can. Geo. Sur., p. 168, Trenton Gr.

Palxotrochis, Emmons, 1856, Geo. Rep. Midland counties of North Carolina. Two species were mentioned, P. major and $P$. minor, both of which are supposed to be concretions, and therefore inorganic.

Peronopora, Nicholson, syn. for Monticulipora.

Petraia, Munster, 1839, Beitrage zur Petrefaktenkunde, vol. 1, p. 42. [Ety. petraios, that grows among rocks.] Simple, turbinate; septa of one or two sizes, the larger extending from the walls to the center, where they are more or less twisted; no tabulæ or connecting vesicular plates. Type $\mathrm{P}$. decussata. Streptelasma is by some regarded as a synonym, by others as a subgenus, and by others as quite distinct. The forms in this country which have been referred to Petraia are all, probably, Streptelasma, and for that reason I have so referred them.

angulata, see Streptelasma angulatum. aperta, see Streptelasma apertum.

fanningana, see Streptelasma fanninganum.

forresteri, Honeyman, 1868, Acadian Geology, p. 594. A catalogue name. latuscula, see Streptelasma latuscula. logani, see Streptelasma logani.

minganensis, see Archæocyathus minganensis.

ottauensis, see Streptelasma ottawense. pulchella, see Streptelasma pulchellum. pygmæa, see Streptelasma pygmæum. rustica, see Streptelasma rusticum. selecta, see Streptelasma selectum. waynensis, see Streptelasma waynense.

Phillipsastrea, D'Orbigny, 1849, Note Sur. des Polypiers Fossiles, p. 12. [Ety. proper name; aster, star.] Composite, resembling Strombodes, but differing in the septa of neighboring corallites 
being confluent, and consequently the calices are not definitely cireumscribed; no exterior walls; interior mural investment well characterized; center of tabulic presenting a columellar tubercle. Type P. hennabi.

affinis, Billings, 1874, Pal. Foss., vol. 2, p. 11, Gaspe limestone No. S, Devonian. gigas, Owen, 1840, (Astraea gigas,) Rep. on Mineral lands, p. 70, Devonian.

hennahi, Lonsdale, 1840, (Astrrea hennabi,) Geo. Trans., vol. 5, p. 697, Devonian.

johanni, Hall \& Whitfield, 1S73, (Smithia johanni,) 23d,Rep. N. Y'. St. Mus. Nat. Hist., p. 234, Chemung Gir.

mammillaris, see Strombodes mammillaris. multiradiata, Hall \& Whitfield, 1873

(Smithia multiradiata,) 23d Rep. N. Y.

St. Mus. Nat. IIist., p. 234, Chemung Gr.

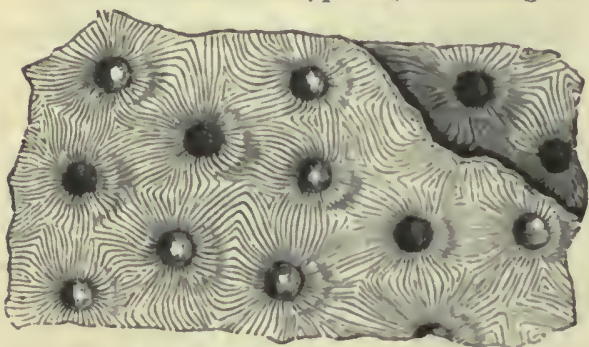

Fra. 205.-Phlllipsastrea vermeul\}.

verneuli. Edwards \& Haime, 1S51, Polypiers Foss. des Terr. Pal., p. 447, Ham. Gr.

verrilli, Meek, 1868, (Smithia verrilli,) Trans. Chi. Acad. Sci., p. 83, Devonian. yandelli, Rominger, 1876, Foss. Corals, p. 130, Up. Held. Gr. Not well defined.

Phyllograitus, Hall, 1858, Rep. of l'rogr. Can. Geo. Sur. p. 135. [Ety. phyllon,

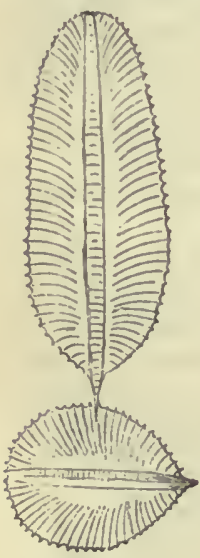

Frg. 206.-Phyllograptus typus. leaf; grapho, 1 write. Frond consisting of simple or compound foliiform stipes, which aro celluliferous on the two opposite sides, the margins having a mueronate extension from each cellule; supported on a slender radiele, or com. bired in groups. Type P. typus.

angustifolius, Hall, 1858 , Rep. of Progr. Can. Geo. Sur., p. 139, and Dec. 2. Org. Rem., p. 125 , Quebec Gr.

anna, Hall, 1S65, Can. Org. Rem., Decade 2, p. 124, Quebec Gr.

dubius, Spencer, 1884, Bull.

No. 1, Mus. Univ. St. Mo., p. 15, Niagara Gr. illicifolius, Hall, 1858, Rep. of Progr. Can. Geo. Sur., p. 139, and Dec. 2, Org. Rem., p. 121, Quebec Gr.

loringi, White, 1874, Rep. Invertebrate Foss., p. 9, and Geo. Sur. IV. 100th Mer., vol. 4, p. 51, Quebee Gr.

similis, Hall, 1858. Can. Nat. and Geo. vol., 4, syn. for Graptolithus bigsbyi.

typus, Hall, 1S5S, Rep. of Progr. Can. Geo. Sur., p. 137, and Dec. 2, Org. Rem., p. 118, Quebec Gr.

Plasmopora, Edwards \& IIaime, 184? Comptes rend., t. 29, 1. 262. [Ety. plasma, cast; poros, pore.] Free, sul). hemispleric; basal epitheca, concentrically folded; cali. ces i m. mersed; septa rudime ut a ry; tabulie hori zon t a l; walls thin: conencliy: mat com posed of vertieal radiate laminse unito d b v sina 11 e horizontal pla tes. Type P. petaliformis.

follis, Ed-

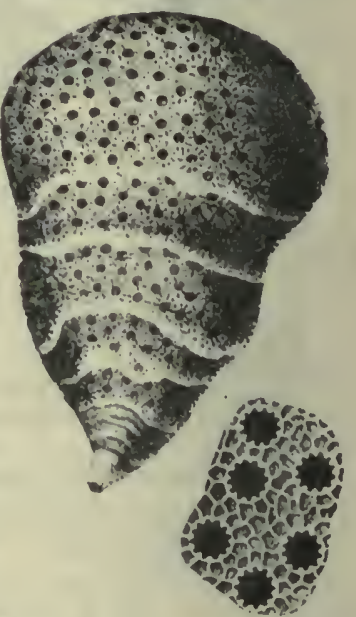
w a rds \& Fro. 207. - Plasinoporn follik. H a i m e, Fra. 207. - Plasinopora follik. 1851, Mon.

Pol. Foss. de Terr. l'al., p. 220, Niagara $\mathrm{Gr}$.

Pleunodiction, Goldfuss, 1826, Petref. Germ., vol, 1, p. 209. [Ety pleura, sidle; ditfyon, net. Corallum discoidal, upper surface convex; corallites diverging from the center of the base, polygonal or subeylindrical; walls thick; mural pores irregular; tabulie not numerous, but sometimes inosculating; septa rudimentary, in the form of marginal ridges. There is usually a vermiform body at the central part of the base. Type P. problematicum.

americanum, Roemer, 1876, Lethe Pale. ozoica, pl. 33, figs. $2 a$ and $2 b$. Ham. Gr.

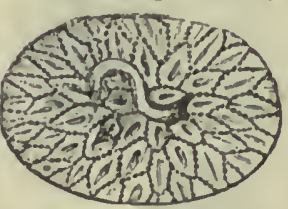

Frg. 208-Pleurodictyum problematicum. Under side, showing serpula llke body. problem a ti e u $\mathrm{m}$, Goldfuss, 1826 , Petref. Germ., vol. 1, p. 113, Onondaga Gr.

Polydilasma, Hall, 1852, Pal. N. Y., vol. 2 , syn. for Zaphrentis.

turbinat $u m$, see Zaphrentis turbinata.

Porites, Lamarck, 1816, Hist. des Anim. Eans Vert., t. 2, p. 267. Not an American Palæozoic genus. 
astrseiformis, Owen, 1840, Rep. on Mineral lands, Devonian. This may be the same species subsequently described as Pachyphyllum woodmani.

puriformis, as identified by d'Archiac \& Verneuil, not American.

vetustus, see Protarea vetusta.

Prasopora, Nicholson \& Etheridge, 1877 , Ann. and Mag. Nat. Hist., 4tl ser., vol. 20, p. 38 . [Ety. prason, sea-plant; poros, pore.] Corallum compound, concavoconvex or hemispheric; corallites radiating from a wrinkled basal epitheca; larger and smaller corallites intermingled throughout the colony; no monticules; corallites thin-walled, prismatic; large ones with an exterior zone of vesicular tabula surrounding a vacant central tube, which may be crossed by an occasional tabula; smaller ones arranged in a zone around the larger ones, and crossed by numerous, close-set, complete, horizontal tabulæ. 'Type P.gravæ. affinis, Foord, 1883, Cont. to Micropalieontology, p. 12, Trenton Gr.

conoidea, Ulrich, 1886, 14th Rep. Geo. Sur. Minn., p. 87, Trenton Gr.

contigua, Ulrich, 1886,14 th Rep. Geo. Sur. Minn.. p. 87, Trenton Gr.

newberryi, Nicholson, 1875, (Chetetes newberryi,) Ohio Pal., vol. 2, p. 212, Hud. Riv. Gr.

nodosa, Ulrich, 1882, Jour. Cin. Soc. Nat. Hist., vol. 5 , p. 245 , Hud. Riv. Gr,

oculata, Foord, 1883, Cont. to Micropalaenntology, p. 11, Trenton Gr.

selwyni, Nicholson, 1881, (Monticulipora selwyni,) Struct. and Affin. of Montic., p. 206, Trenton Gr.

stwyni var. hospitalis, Nicholson, 1881, (Monticulipora selwyni var. hospitalis,) Struct, and Aftin. of Montic., p. 206, Hud. Riv. Gr.

simulatrix, Ulrich, 1886, 14th Rep. Geo. Sur. Minn,, p. 85, Trenton Gr.

Prionotus, Nilsson, 1835 , Leth. Suec. folium, see Diplograptus folium.

pristis, see Diplograptus pristis.

Protarea, Edwards \& Haime, 1851, Pol. Foss. des Terr. Pal., p. 208. [Ety.

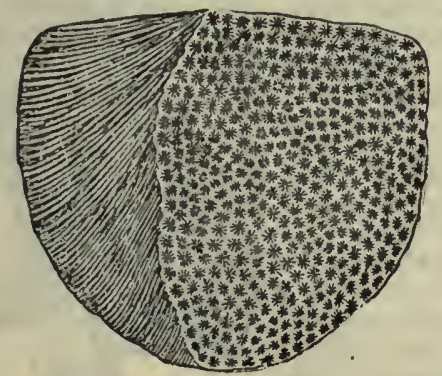

Fug. 209.--Protarea vetusta, on Strophomena alternata.

protos, first; araios, porous.] Thin, incrusting; calices equal, hexagonal, shallow; septa 12, extending but slightly into the visceral chamber; walls thick. Type $P$. vetusta.

vetusta, Hall, 1847, (Porites vetustus,) Pal. N. Y., vol. 1, p. 71, Trenton \& Hud. Riv. Grs.

verneuili, Edwards \& Haime, 1851, Pol. Foss. des Terr. Pal., p. 209, Silurian. (?)

Protograptus, Matthew, 1885, Trans. Roy. Soc. Can., p. 31. [Ety. protos, first; grapho, I write.] Stipes thin, flat, elongate, dichotomously branched; having a central axis, and being alate on each side; pores arranged along the axis of the stipe; axis and margin of the stipe connected by delicate nervules. Type P. alatus.

alatus, Matthew, 1885, Trans. Roy. Soc. Can., p. 32, St. John Gr.

Prilograptus, Hall, 1865, Can. Org. Rem., Decade 2, p. 139. (Ety. ptilon, feather; grapho, I write.] Plant-like, rooted, simple or branching ; branches plumose, pinnules alternate on opposite sicles; celluliferous on one face only; branches cylindrical or flattened. Type P. plumosus.

foliaceus, Spencer, 1878, Can. Nat., vol. 8, and Bull. No. 1, Mus. Univ. St. Mo., p. 41, Niagara Gr.

geinitzanus, IIall, 1865,

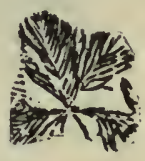

Can. Org. Rem., De- FrG. 210.--Ptllograpcade 2, p. 140, Quebec tus follaceus. Gr.

plumosus, Hall, 1865, Can. Org. Rem., Decade 2, p. 140, Quebec Gr.

Prychonema, Hall, 1887, Pal. N. Y., vol. 6, p. xiv. [Ety. ptyche, wrinkle; nema, thread.] Massive or ramose, composed of thin-walled, strongly corrugated cells, which are apparently without diaphragms. Type P. tabulatum.

helderbergix, Hall, 1874, (Chetetes helder. bergiæ, ) 26th Rep. N. Y. St. MIs. Nat. Hist., p. 110, Low. Held. Gr.

tábulatum, Hall, 1876, (Chetetes tabulatum,) Illus. Der. Foss., pl. 37, and figs. 16-19, and Pal. N. Y., vol. 6, p. 14, Up. Held. Gr.

Pтүслорнy lum, Lonsiale, 1839, Sil. Syst., p. 691, and E. \& H. Brit. Foss. Corals, p. Ixix. [Ety. ptyche, ridye; pliyllon, leaf.] Corallum simple, having infundibuliform tabulae superposed and invaginated; septastrongly twisted toward the center of the tabulir so as to constitute a spurious columella. Type P. stokesi.

canadense, Billings, 1862, Pal. Foss., vol. 1, p. 107, Mid. Sil. Anticosti Gr., Division 4.

floriforme, Hall, 1882, Foss. Corals Niagara and Up. Held. Grs., p. 5, and 35 th Rep. N. Y. St. Mus. Nat. Hist., p. 409, Niagara frr. 
fuleratum, Hall, 1882, Foss. Corals Niagara and Up. Held. Grs., p. 6, and 35th Rep. N. Y. St. Mus. Nat. Hist., p. $410, \mathrm{Ni}-$ agara Gr.

infundibilum, Meek, 1877, U. S. Geo. Sur. 40th Parallel, vol. 4, p. 28, Devonian.

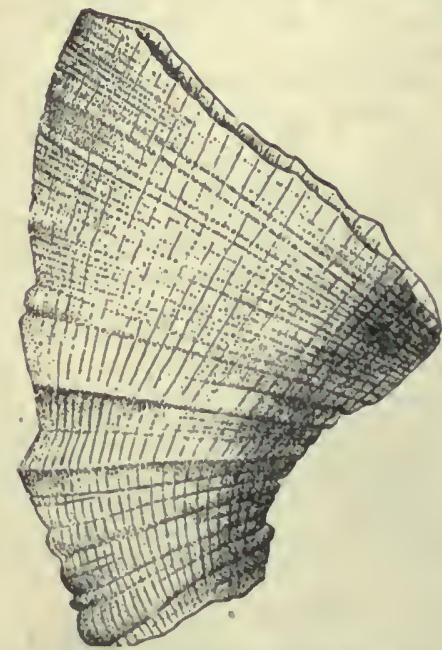

FI(:. 211.-Ptychoplÿllum knappl.

knappi, Hall, 1883, 12th Rep. Geo. Ind., p. 278 , Up. Held. Gr.

stokesi, Elwards \& Haine, 1851, Brit. Foss. Corals, p. Ixix, Niagara Gr.

striatum, Hall, 1882, Foss. Corals Niagara and Up. Held. Grs., p. 22, and 35th Rep. N. Y. St. Mus. Nat. Hist., p. 426, Up. Held. Gr.

versiforme, Hall, 1882, Foss. Corals Ni. agara and Up. Held. Grs., p. 22, and 35 th Rep. N. Y. St. Mus. Nat. Hist., p. 426, Up. Held. Gr.

Prcnostruus, Whiteaves, 1884, I'al. Foss., vol. 3, p. 2. [Ety. puknos, dense; stylos, column.] Corallum aggregate; corallites slender, divided by calicular gemmation, at distant intervals, into sets of three or more ascending flexnous branches; structure similar to Amplexus, but tabula horizontal and not embracing. Type I'. guelphensis.

elegans, Whiteaves, 1884, Pal. Foss.,

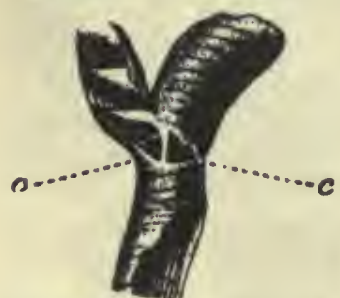

Fig. 212. - Pycnost y $!$ us guelphensls; two brunches are brokeu off at $C, C$.

proposed Romingeria. vol. 3, p. 4, Guelph Gr.

gu e l p h e nsis, Wh i teaves, $1884, \mathrm{P}$ al. Foss., vol, 3, p. 3, Guelph Gr.

Quenstedtia, Rominger, 1876, Foss. Corals, p. 71. Being preoccupied, $\mathrm{Nicholson}$ niagarensis, see Romingeria niagarensis.
Rastrites, Barrande, 1850, Graptolites de Boheme, p. 64. [Sig. a rake.] Suall, almost linear. very long, stipe slightly curved ; interior $\mathrm{c}$ a $\mathrm{n}$ a $\mathbf{l}$ connecting the cellules, which are on the convex side and isolated from FIG. 213. - Rastrites peree a $\mathrm{eh}$ other.

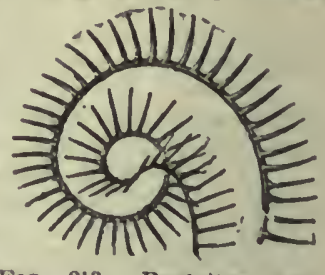
Type R. peregrinus.

barrandi, Hall, 1S59, Pal. N. Y., vol. 3, p. 521, Hud. Riv. Gr.

Retiogiza Ptus, Hall, 1865, Dec. 2, Org. Rem. p. 115. [ Ety. rete, net; gr $\iota p h o$, I write.] Frond simple or compound; stipes numerous arranged bi:aterally on an axis, elongate, oval or lanceolate with longitulinal axis and reticulate structure; margins with mucronate points. Type R. tentaculatus.

barrandi, Hall, 1860, 13th Rep. N. Y. St. Mus. Nat. Hist., p. 61, Ind. Riv. Gr.

eucharis, II 11,1865 , Can. Org. Rem., Deeade 2, p. 146, Utica Slate.

geinitzanus, Hall, 1S5?, I'al. N. Y., vol. 3, p. 518, Hud. Riv. Gr.

tentaculatus, Hall, 1858, (Gruptolithus tentaculatus,) Rep. of Prog. Can. Geo. Sur., p. 134, and Dec. 2, Org. Rem., p. 116, Quebec Gr.

ReTiol.ITES, Barrande, 1850, Graptolites de 4 Boheme, p. 68. [lity. rete, 7 net; lithos, stone.] Stipes thin, flat, elongate, triangular, composed of two series of cellules symmetrically arranged, in regard to the axis; orifices on the sides of the triangle. Type $R$. geinitzanus.

ensiformis, Hall, 1858, (Graptolithus ensiformis,) Rep. of Prog. Can. Geo. Sur., p. 133, and Decade 2, Org. Rem., p. 114, Quebec Gr.

tlolltes vosus.

venosus, Hall, 1852, (Graptolithus venosus,) Pal. N. Y., vol. 2, p. 40, Clinton Gr.

RIIZOGR AITUS, Spencer, 1878 Can. Nat. vol. 8, p. 460 . [Ety. rhiza, root; grapho I write.] $\mathrm{Cy}$ athiform, bif u reating branches with dichotomous terninations and more or less Fra. 215.-Rhizograptus bul. reticulate; stem terminating in a bulb. Type $\mathbf{R}$. bulbosus. 
bulbosus, Spencer, 1878, Can. Nat., vol. 8 , p. 460, and Bull. No. 1, Mus. Univ. St. Mo., p. 30, Niagara Gr.

Romingeria, Nicholson, 1879, Tab. Corals, p. 114. [Ety. proper name.] Corallum lax, spreading; corallites cylindrical, annulated, multiplying by lateral gemmation, and typically producing new tubes, in umbellate whorls or verticils, at short intervals; where the walls are in contact with the visceral chambers they are connected by mural pores; tabulæ complete, remote; septa represented by vertical rows of spinules. It resembles Aulopora, but is only attached basally, and is therefore free throughout the greater part of its extent. Type R. umbellifera.

cornuta, Billings, 1859, (Aulopora cornuta,) Can. Jour., vol. 4, p. 119, Up. Held. and Ham. Grs.

niagarensis, Rominger, 1876, (Quenstedtia niagarensis,) Foss. Corals, p. 72, Niagara Gr.

umbellifera, Billings, 1859, (Aulopora umbellifera,) Can. Jour., vol. 4, p. 119, Up. Held. Gr.

Sarcinula, Lamarck, 1816, Hist. des Anim. sans Vert., t. 2, p. 222. Not an American Palaeozoic genus.

glabra, Owen, 1840, Rep. on Mineral Lands. See Lyellia glabra.

(?)obsoleta, Hall, 1857, Geo. Lake Sup. Land Dist., vol. 2, Hud. Riv. Gr. Not recognized.

ramosa, Eaton, 1832, Geo. Text Book, p. 41. Not properly defined.

Smithia, Edwards \& Haime, 1851, Pol. Foss. des Terr. Pal. The name was preoccupied for a genus in botany, and is a syn. for Phillipsastrea.

johanni, see Phillipsastrea johanni.

multiradiata, see Phillipsastrea multiradiata.

woodmani, see Pachyphyllum woodmani. verrilli, see Phillipsastrea verrilli.

Spharolites, Hinde, 1875, Proc. Geo. Soc. Lond., vol. 31, p. 514. [Ety. from the spheroidal form.] Type S. nicholsoni. nicholsoni, Hinde, 1875, Proc. Geo. Soc. Lond., vol. 31, p. 514, Low. Held. Gr.

StaUROGra prus, Emmons, 1856, (Staurograp-

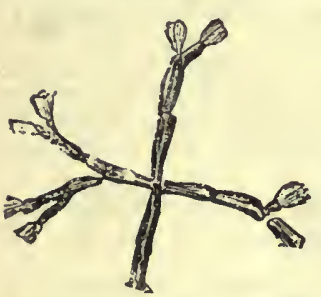

F'1G. 216. - Staurograptus dichotomus. sus,) Am. Geo., pt. 2, p. 108 . [Eiy. stauros, cross; grapho, I w r i te.] Disk free, cruciform, arms four dic h o tomous cells terminal, substancemem bran aceous. Type S. dichotonus.

dichotomus, Emmons, 1856, Am. Geo. p. 109, Up. Taconic.
StelliporA, Hall, 1847, Pal. N. Y., vol. 1, p. 79. [Ety. stella, star; poros, pore.] Corallum dendroid or incrusting; corallites dimorphic; apertures subcircular ; no septa; tabulæ abundant; surface covered with conspicuous star-shaped elevations and depressions. Type S. antheloidea.

antheloidea, Hall, 1847, Pal. N. Y., vol. 1, p. 79. Trenton and Hud. Riv. Grs.

fischeri, Ulrich, 1883,(Constellaria fischeri,) Jour. Cin. Soc. Nat. Hist., vol. 6, p. 270, Hud. Riv. Gr.

florida, Ulrich, 1882, (Constellaria florida,) Jour. Cin. Soc. Nat. Hist., vol. 2, p. 257, Hud. Riv. Gr.

limitaris, Ulrich, 18 9, Jour. Cin. Soc. Nat. Hist., vol. 2, p. 126, Hud. Riv. Gr. Syn. (?) for S. polystomella.

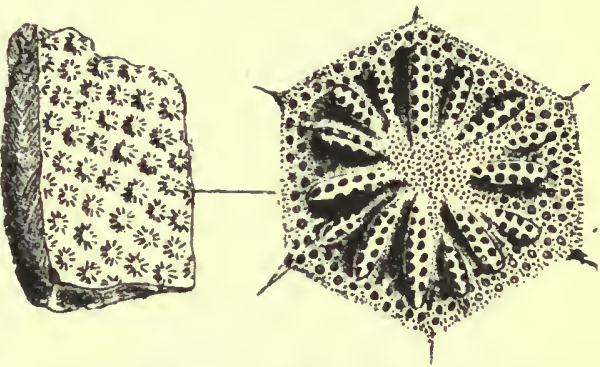

FIG. 217.-Stelilpora polystomella, natural size and maguified star.

polystomella, Nicholson, 1873, (Constellaria polystomella,) Ohio Pal., vol. 2, p. 215, Hud. Riv. Gr.

Stenopora, Lonsdale, 1844, App. to Darwin's Volcanic Islands, p. 161, and Geo. Russ. and Ural Mts., vol.1, p. 631. [Ety. stenos, narrow; poros, pore.] Corallum very similiar to Chetetes, but having small styliform processes at the angles of the calices, as understood by Edwards \& Haime. Nicholson defines the genus, and restricts it to specimens from Australia and Van Diemen's Land, which, as in the type, have constricted corallites and minute mural pores. Type S. ovata. bulbosa, see Monticulipora bulbosa.

adhertns, see Monticulipora adherens. crassa, see Chetetes crassus.

fibrosa, see Monticulipora fibrosa.

exilis, Dawson, 1868 , Acad. Geo., p. 287, Subcarboniferous.

huronensis,see Tetradium huronense.

lib a $n a$, Safford, 1869 , Geo. of Tenn. Notdefined. patula, see Mon-

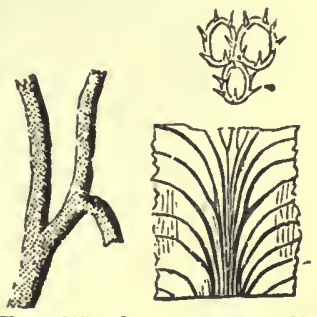
FrG. 218.-Stenopora exills. ticulipora patula.

spinigera, see Chetetes spinigerus. 
Strephodes, McCoy, 1849, syn. for Cyathophyllum.

austini, see Clisiophyllum austini.

pickthorni, see Cyathophyllum pickthorni.

Streptelasma, Hall, 1847, Pal., N. Y., vol. 1, p. 17. [Ety. streptos, twisted; elasma, lamella.] Turbinate, gradually or abruptly expanding; cup deep; lamellæ or septa longitudinal, spirally twisted toward the center; no tabulæ or fos. sette. Type S. expansum.

atuidistans, Hall, 1852, Foss. Corals Niagara and Up. Held. Grs., p. 20, and 35 th Rep. N. Y. St. Mus. Nat. Hist., p. 424, Up. Held. Gr.

ampliatum, Hall, 1882, Foss. Corals Niagara and Up. Held. Grs., p. 19, and 35th Rep. N. Y. St. Mus. Nat. Hist., p. 423, Up. Held. Gr.

angulatum, Billings, 1862, (Petraia angulata,) Pal. Foss., vol. 1, p. 103, Hud. Riv. Gr.

apertum, Billings, 1862, (Petraia aperta,) Pal. Foss., vol. 1, p. 102, Black Riv. Gr.

calyculus, Hall, 1852, Pal. N. Y., vol. 2, p. 111, Niagara Gr.

coarctatum, Hall, 1852, Foss. Corals $\mathrm{Ni}$ agara and Up. Held. Grs; p. 21, and 12th Rep. Geo. Sur. Ind., p. 275, Up. Held. Gr.

conspicuum, Hall, 1852, Foss. Corals Niagara and Up. Held. Grs., p. 19, and 35 th Rep. N. Y. St. Mus. Nat. Hist., p. 423, Up. Held. Gr.

conulus, Rominger, 1876, Foss. Coruls, p. 144, Niagara Gr.

corniculum, Hall, 1847, Pal. N. Y., vol. 1, p. 69, Trenton and Hud. Riv. Grs.

crassum, Hall, 1847, Pal. N. Y., vol. 1, p. 70 , Trenton $\mathrm{Gr}$.

crateriforme, Hall, 18s2, Foss. Corals Niagara and Up. Held. Grs., p. 20, and 35̃th Rep. N. Y. St. Mus. Nat. Hist., p. 424, Up. Held. Gr.

dissimile, Hall, 1882, Foss. Corals Niagara and Up. Held. Grs., p. 17, and 35th Rep. N. Y. St. Mus. Nat. Hist., p. 421, Up. Held. Gr.

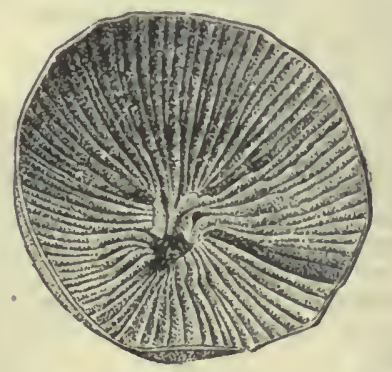

FiG. 219.-Streptelasma inflatum, transverse sectlon.

expansum, Hall, 1847, Pal. N. Y., vol. 1, p. 17, Chazy Gr. extans. Hall, 1882, Foss. Corals Niagara and Up. Held. Grs., p. 5, and 35th Rep. N. Y. St. Mus. Nat. Hist., p. $409, \mathrm{Ni}$ agara $\mathrm{Gr}$.

fanningana, Safford, 1869, (Petraia fanningana,) (ieo. Tenn., p. 320, Low. Held. (ir.

fossula, Hall, 1882, Foss. Corals Niagara and Up. Held. Grs., p. 19, and 35th RRp. N. Y. St. Mus. Nat. Hist., P. 423, Up. Held. Gr.

inflatum, Hall, 1852, Foss. Corals Niagara and Up. Held. Grs., p. 18, and 12th Rep. Geo. Ind., p. 276, Up. Held. (ir.

involutum, Hall, 1882, Foss. Corals $\mathrm{Ni}$ agara and Up. Held. Firs., p. 20, and 35 th Rep. N. Y. St. Mus. Nat. Hist., p. 424, Up. Held. Gr.

lamellatum, Hall, 1Ss, Foss. Corals Niagura and Up. Held. (irs., p. 1T, and $3 \bar{t}$ th Rep. N. Y. St. Mus. Nat. Hist, p. 421, U. Held. Gr.

laterariun, Hall, 1882 , Foss. Cornls $\mathrm{Nj}$ agara and Up. Held. (irs., p. 18, and 35th Rep. N. Y. St. Mus. Nat. Hist., p. 422, Coruiferous limestone.

latuscula, 13illings, 1862, (P'etraia latuscula,) Pal., Foss., vol. 1, p. 104, Mid. Sil. Anticosti, Div. 4.

limitàre, Hall, 1852, Foss. Corals Niagrara and Up. Held. (irs., 1). 5, and 35th Rep. N. Y. St. Mus. Nat. Hist., p. 409, Niagara (ir.

logani, Nicholson, 1875, (l'etraia logani,) Can. Nat., vol. 7, p. 143, Up. Held. Gir.

mammiferum, Hall, 1882, Foss. Corals Niagara und Up. Held. (irs., p. 21, and 35 th Rep. N. Y. St. Mus. Nat. Hist., p. 425, Up. Held. (ir.

minimum, Hall, 1876, 28th Rep. N. Y. St. Mus. Nat. Hist., p. 106, syn. for Duncanella borealis.

multilamellosum, Hall, 1847, Pal. X. Y., vol. 1, 1. 70, Trenton Gr.

ottawensis, Billings, 1865, (Petraia ottawensis,) Can. Nat. and Geo., $2 d$ ser., vol. 2, Trenton Gr.

papillatum, Hall, 1882, Foss. Corals Niagara and $U_{p}$. Held. Grs., p. 21, and 12th Rep. (ieo. Ind., p. 276, Up. Held. Gir.

parvulum, Hall, 1847, Pal. N. Y., vol. 1, p. 71, Trenton Gir.

patuluen, Rominger, 1876, Foss. Corals, p. 143, Niagara Gr.

profundum, Conrad, 1843, Proc. Acad. Nat. Sci. 1'hil., 1. 335, (Cyathophyllum profundum,) and Hall, 1847, Pal. N. Y., vol. 1, 1. 49, Birdseye, Black Riv. and Trenton Grs.

fulchellum, Billings, 1865, (Petraia pulchella, Can. Nat. and Geo., $2 \mathrm{~d}$ ser., vol. 2 , p. 424 , Mid. Sil.

pygmateum, Billings, 1862, (Petraia pygmaa,) Pal. Foss., vol. 1, 1. 103, Mid. Sil. Anticosti, Div. 4.

radicans, Hall, 1876, 28th Rep. N. Y. St. Mus. Nat. Hist., p. 106, Niagara Gr. 
rectum, Hall, 1843, (Strombodes rectus,) Geo. Rep. 4th Dist. N. Y., p. 200, and

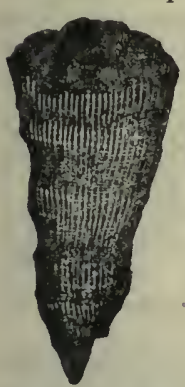

FIG. 220. Strep. telasma rectum.
Illust. Dev. Foss., pl. 19 , Ham. Gr.

rusticum, Billings, 1858 , (Petraia rustica,) Rep. of Progr. Geo. Sur. Can., p. 168, Hud. Riv. Gr.

selectum, Billings, 1865, (Petruia selecta,) Can. Nat. and Geo., 2d ser., vol. 2 , p. 429, Mid. Sil.

simplex, Hall, 1882, Foss. Corals Niagara and $\mathrm{U} p$. Held. Grs., p. 18, and 12 th Rep. Geo. Ind., p. 277, Up. Held. Gr.

spongiaxis, Rominger, 1876, Foss. Corals, p. 144, Niagara Gr. strictum, Hall, 1874, 26th Rep. N. Y. St. Mus. Nat. Hist., p. 114, Low. Held. Gr. tenue, Hall, 1882, Foss. Corals Niagara and Up. Held. Grs., 1. 17. and 12 th Rep. Geo. Ind., p. 278, Up. Held. Gr.

ungula, Hall, 1876, Illust. Dev. Foss., pl. 19, Ham. Gr.

waynensis, Safford, 1869, (Petraia waynensis,) Geo. of Telln., p. 314, Low. Held. Gr.

Striatopora, Hall, 1852, Pal. N. Y., vol. 2, p. 156. [Ety. striatus, striated; poros, pore.] Ramose; corallites thick-walled, angular, conical; cells opening upon the surface in expanded, angular, cuplike depressions, which are longitudinally striated, and between the strix the bands may bear spinules; tabula and mural pores common. Type 's. fl xuosa. carbonaria, White, 1862, Proc. Bost. Soc. Nat. Hist., vol. 9, Burlington Gr.

cavernosa, Rominger, 1876, Foss. Corals, p. 60, Corniferus Gr.

flexuosa, Hall, 1852, Pal. N. Y., vol. 2, p. 156, Niagara Gr.

formosa, Billings, 1860, Can. Jour., vol. 5, p. 254 , Up. Held. Gr.

huronensis, Rominger, 1876, Foss. Corals, p. 58 , Niagara Gr.

iowensis, Owen, 1840, (Cyathopora jowensis,) Rep. on Min. Lands of Iowa, etc., p. 69 , Ham. Gr.

issa, Hall, 1874, 26th Rep. N. Y. St. Mus. Nat. Hist., p. 114, Lnw. Held. Gr.

limbata, Eaton, 1832, (Madrepora limbata,) Geo. Text Bonk, p. 30, and Illust. Dev. Foss., pl. 33, Ham. Gr.

linnæeana, Billings, 1860, Can. Jour., vol. 5 , p. 253 , Ham. Gr.

miscouriensis, M e e k \& Worthen, 1868, Geo. Sur. Ill., vol. 3 , p. 369 , Low.

Held. Gr.
rugosa, Hall, 1858, Geo. of Fra: 221.-StriIowa, y. 479 , syn. for s. næeuna. iowensis.

Strombodes, Schweigger, 1820, Handb. der Naturg., p. 418. [Ety. strombos, twisting.]
Composite, increasing by calicular gemmation; corallites constituted principally by a series of superposed, invaginated, infundibuliform tabulæ, united by ascending trabiculæ, so as to form a colnmnar mass; calices pentagonal, well circumscribed, and completely covered with the septal radii; outer walls not well developed, and inner mural investment rudimentary. Type S. pentagonus.

alpenensis, Rominger, 1876, Foss. Corals, p. 133, Ham. Gr. Is this a syn. for $\mathrm{S}$. mammillaris?

diffluens, Edwards \& Haime, 1851, Pol. Fos. des Terr. Pal., p. 431, Anticosti Gr. distortus, Hall, 1843, Geo. Rep. 4th Dist. N. Y., p. 209, Ham. Gr. . Too imperfectly described for recognition. Probably a Ieliopbyllum.

eximius, Billings, 1866, Catal, Sil. Foss. Antic., p. 93, Clinton and Niagara Girs. gracilis, Billings, 1862, Pal. Foss., vol. 1, p. 113, Mid. Sil.

helianthoides, (?) Heliopbyllum halli.

mammillaris, Owen, 1840, (Astrea mammillaris,) Rep. on Min. Lands, p. 70, and Rominger, in Pal. Foss., p. 133, Niagara Gr.

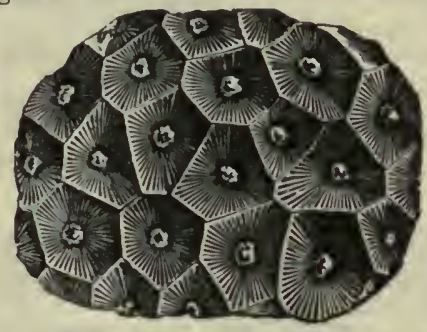

FIG. 222.-Strombodes pentagonus.

pentagonus, Goldfuss, 1826, Germ. Petref. p. 62, Niagara Gr.

pygmaeus, Rominger, 1876, Foss. Corals, p. 132, Niagara Gr.

(?) rectus, see Streptelasma rectum.

separatus, Ulrich, 1886, Cont. to Am. Pal., p. 32, Niagara Gr.

simplex, see Zaphrentis simplex.

striatus, D’Orbigny, 1850, Prodr. de Paléont., p. 48 Niagara Gr.

Stylastrea, Lonsdale, 1845, Geo. and Pal. of Russia, and Ural Mts., p. 621. [Ety. sty'os, pillar; aster, star.] Composite, large; corallites prismatic; easily separable; walls thick, striated longitudinally, and wrinkled transversely; within the walls there is a narrow, vesicular, perithecal zone, and within it a lamelliferous area; septa numerous, not reaching the center; tabulæ abundant. Type S. inconferta.

anna, Whitfirld, 1882, Ann. N. Y. Acad. Sci., vol. 2, p. 199, Up. Held. Gr.

Syringolites, Hinde, 1879, Geo. Mag., vol. 6, p. 244. [Ety. syrinx, pipe; lithos, stone.] Composite; large with basal epitheca; corallites polygonal, thin- 
walled, with mural pores, and a cylindrical tube in the center of each corallite. Type S. huronensis.

huronensis, Hinde, 1879, Geo. Mag., vol. 6 , p. 246, Niagara Gr.

Syringopora, Goldfuss, 1826, Germ. Petref., p. 75. [Ety. syrinx, pipe; poros, pore.] Corallum aggregating, at first creeping aiter the manner of Aulopora, then sending up numerous vertical, cylindrical corrallites, usually flexuous, subparallel, and connected laterally by more or less transverse processes; septa rudimentary; tabulie close set, infundibuliform; epitheca well developed. Type S. reticulata.

alectiformis, Winchell, 1866, liep. Low. Penin. Mich., p. 90 , Ham. Gr.

annulata, Rominger, 1876, Foss. Corals, p. 81, Niagara Gr.

aulopora, Salter, 1855, Belcher's Last of the Arctic Voyages, vol. 2, p. 385, Carboniferous.

cleviana, Edwards \& Haine, 1851, Pol. Foss. d. Terr. Pal., p. 295, Corniferous Gr:

compacta, Billings, 1858, Can. Nat. and Geo., vol. 3, p. 422, Up. Sil.

crassata, Winchell, 1866, Rep. Iow. Penin. Mich., p. 90, Ham. Gr.

dalmani, Billings, 1858, Can. Nat. and Geo., vol. 3, p. 423, Up. Sil.

debilis, Billings, 1858, Can. Nat. and Geo., vol. 3, p. $423, \mathrm{Up}$. Sil.

elegans, Billings, 1858, Can. Nat. and Geo., vol. 3, p. 425, Corniferous Gr.

fencstrata, Winchell, 1866, Rep. Low. Penin Mich., p. 90, Ham. Gr.

fibrata, Rominger, 1876, Foss. Corals, p. 82, Niagara Gr.

harveyi, White, 1862, Proc. Bost. Soc. Nat. Hist., vol. 9, p. 32, Waverly or Kinderhook Gr.

hisingeri, Billings, 1859, Can. Nat. and Geo., vol. 4, p. 116, Corniferous Gr.

infundibulum, see Cystostylus infundibulum.

intermedia, Nicholson, 1874, Rep. Pal. Prov. Ont. Can., p. 126, Ham. Gr.

laxata, Billings, 1859, Can. Jour., vol. 4, p. 118, Corniferous Gr.

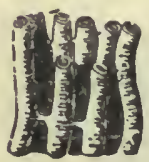

maclurii, Billings, 1860, Can. Jour., vol. 5, p. 258, Corniferous $\mathrm{Gr}$.

multattenuata, McChesney, 1860, New Pal. Foss., p. 75, and Pal. F. Neb., p. 144, Coal Meas.

Fig. 223.Sy ringopore macluril.

multicaulis, Hall, 1852, $\mathrm{Pal}$. N. Y., vol. 2, p. 119 , Niagara Gr.

nobilis, Billings, 1859, Can. Nat. and Geo., vol. 4, p. 118 , Up. Held. Gr.

parallela, Etheridge, 1878, Quar. Jour. Geo. Soc., vol. 34, p. 583, Up. Sil.

perelegans, Billings, 1859, Can. Jour., vol. 4, p. 117, Up. Held. Gr.

retiformis, Billings, 1858, Can. Nat. and Geo., vol. 3 , p. 424, Up. Sil. reticulata, Goldfuss, 1826, Petref. Germ., p. 76 , Devonian.

tabulata, Edwarls \& Haime, 1851, Pol. Foss. des Terr. Pal., p. 288, Up. Helı. Gr. tenella, Rominger, 1876, Foss. Corals, p. 81, Niagara Gr.

tubiporoides, Yandell \& Shumard, 1847, Cont. to Geo of Ky., p. 8, Curnifernus Gr. tubiporoides, Billings, see $\mathrm{S}$. maclurii.

verneuli, Elwards \& Haime, 1851 , Polyp. Foss. de Terr. Pal., p. 289, Corniferous $\mathrm{Gr}$.

verticillata, Goldfuss, 1826, Petref. Germ., p. 76, Niagara Gr.

Tetradium, Dana, 1848, Wilkes, Expl. Exped. Zooph., vol. 8, p. 701. [Ety. tetra, four.] Aggregate, massive, subhemispheric; corallites long, prismatic, in close contact; septa few, not reacling the center of the visceral chamber (typically four); tabulie numerous, coniplete; calices generally petaloid; no mural pores; increase by fission. Type. 'T. fibratum.

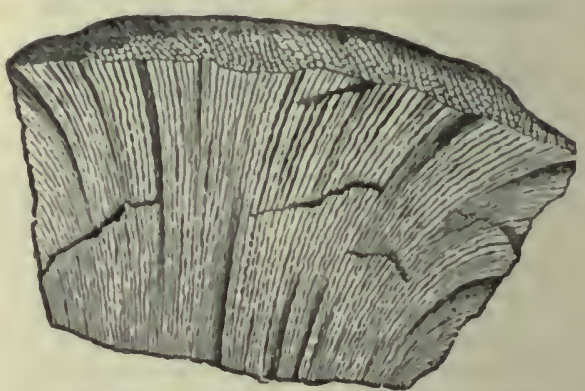

Fig. 224.-Tetradium fibratum.

columnare, Hall, 1847, (Chetetes columnaris,) Pal. N. Y., vol. 1, p. 68, Trenton $\mathrm{Gr}$.

fibratum, Safford, 1856, Am. Jour. Sci. vol. 22, p. 237, Hud. Riv. Gr.

fibratum var. apertum, Safford, 1856 , Am. Jour. Sci., vol. 22 . p. 237, Hud. Riv. Gr.

fibratum var. $m$ i nus, Safford, 1856 , Aw. Jour. Sci., vol. 22 , p. 238, Hud. Riv. Gr.

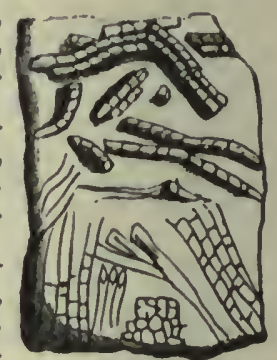

huronense, Billings, 1865, (Stenopora huronensis,) Pal.

FIG. 225.-Ted radlum flbralum. Corallites senttered through the rock. Foss., vol. 1, p. 185, Hud. Riv. Gr. peachi var. canadense, Foord, 1883, Cont. to Micro. Pal., p. 24, Trenton Gr.

Tetragraptus, Salter, 1863, Quar. Jour. Geo. Soc., vol. 19. [Ety. tetra, four ; grapho, I write.] This genus is not regarded with much favor. Graptolithus bryonoides is made the typical species. $G$. quadribrachiatus is also placed in it. 
approximatus, see Graptolithus approximatus.

Thamnograptus, Hall, 1859, Pal. N. Y., vol. 3, p. 519. [Ety. thamnus, shrub; grapho, I write.] Fronds consisting of straight or flexuous stipes, with alternating or widely diverging branches; branches long, simple, or ramose, in the same mauner as the stipe; the main stipe and branches are marked by a central longitudinal, depressed line, indicating the axis. Type T. typus.

anna, Hall, 1865, Can. Org. Rem., Decade 2, p. 141, Quebec Gr.

bartonensis, Spencer, 1878, Can. Nat., vol. 8 , and Bull. No. 1, Mus. St. Univ. Mo., p. 39, Niagara Gr.

capillaris, Hall, 1859, Pal. N. Y., vol. 3,

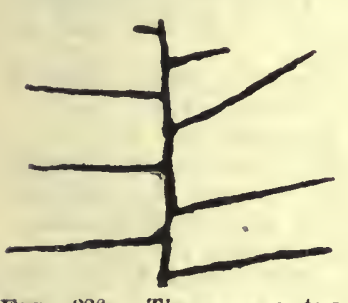

F1G. 226. - Thamıograptus typus. p. 520, Hud. Riv. Gr.

$\mathrm{multi}$ or $\mathrm{m}$ is Spencer, 1884 Bull. No. 1, Mus. Univ. St. Mo., p. $40, \mathrm{Ni}$ agara $\mathrm{Gr}$.

typus, H a ll , 1859 , Pal. N. Y., vol. 3, p. 519, Hud. Riv. Gr.

Thecia, Edwards \& Haime, 1849, Comptes rend., t. 29, p. 263. [Ety. theke, sheath.] Corallum massive, with an abundant, compact, spurious conenchyma, produced by the septa becoming cemented together laterally; septal system highly developed; tabulæ numerous; calices shallow, with a small deep fossula. Type T. swindernana.

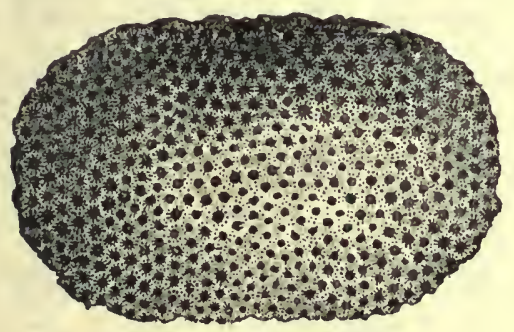

FIG. 227.-Thecia major.

major, Rominger, 1876, Foss. Corals, p. 67, Niagara Gr.

minor, Rominger, 1876, Foss. Corals, p. 68, Niagara Gr.

ramosa, Rominger, 1876, Foss. Corals, p. 69, Up. Held. Gr.

swindernana, Goldfuss, 1829, (Agaricia swindernana,) Petref. Germ., p. 109, Niagara Gr.

Thecostegites, Edwards \& Haime, 1849, Comptes rend., t. 29, p. 261. [Ety. theke, sheath; stege, covering.] Corallites cylindrical, short and united by short mural expansions situated at various heights: tabulæ horizontal. Type T. bouchardi.

bouchardi, Michelin, 1845, (Harmodites bouchardi,) Icon. Zooph., p. 185. This species was described from France, and is probably not American.

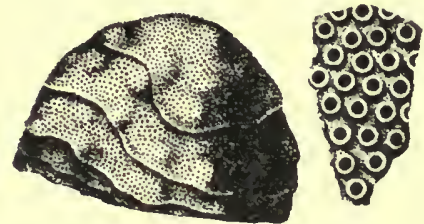

FIG. 228.-Thecostegites hemispherlcus, natural size and magnified.

hemispherious, Roemer, 1860, Sil. Fauna W. Tenn., p. 25, Niagara Gr.

Trachypora, Edwards \& Haime, 1851, Pol. Foss. d. Terr. Pal., p. 305. [Ety. trachys, rough; poros, pore.] Dendroid; calices slightly salient; no septa; cœnenchyma abundant, solid, and surface marked by strong, irregular, vermicular, subechinulated striæ. Type T. davidsoni.

austini, Worthen, (in press,) Geo. Sur. Ill., vol. 8 , p. 81 , Coal Meas.

elegantula, Billings, 1860, Can. Jour., vol. 5, p. 254, Ham. Gr.

ornata, Rominger, 1876, (Dendropora ornata,) Foss. Corals, p. 62, Ham. Gr.
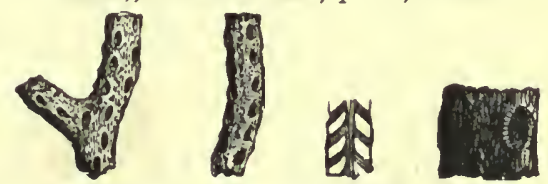

FiG. 229.-Trachypora elegantula. Portion of two corallites - a longitudinal section and a corallite eularged.

Trochophyllum, Edwards \& Haime, 1851, Mon. d. Pol. Foss. de Terr. Pal., p. 356. [Ety. trochos, wheel; phyllon, leaf.] Simple, trochoid; calice shallow; septa thick, not denticulate, extending almost to the center of the visceral chamber, where a small tabula is visible; fossula rudimentary and occupied by a small septum. Type T. verneuilanum.

verneuilanum, Edwards \& Haime, 1851, Mon. d. Pol. Foss. d. Terr. Pal., p. 357, Subcarloniferous.

Tubipora, Linnæus, 1758, Syst. Nat., 10th Ed., p. 789. Not American Palæozoic.

lamellosa, Owen, 1840, Rep. on Min. Lands, p. 78. Not defined. Probably a Syringopora.

Vermipora, Hall, 1874, 26th Rep. N. Y. St. Mus. Nat. Hist., p. 109. [Ety. vermis, worm; poros, pore.] Ramose; corallites cylindrical, close, increasing by lateral gemmation, and projecting at the surface; tabulæ remote; no mural pores connecting corallites. Type V. serpuloides. 
fasciculata, Rominger, 1876, Foss. Corals, p. 70, Ham. Gr.

niagarensis, Rominger, 1876, Foss. Corals, p. 70 , Niagara Gr.

robusta, Hall, 1883, Rep. St. Geo., pl. 2, figs. 32,33 , Low. Held. Gr.

serpuloides, Hall, 1874, 26th Rep. N. Y. St. Mus. Nat. Hist., p. 110, Low. Held. Gr.

tortuosa, Hall, 1883, Rep. St. Geo., pl. 2, fig. 23, Low. Held. Gr.

Vesicularia, Rominger, 1876, Foss. Corals, p. 135. This name was preoceupied among the Bryozon. See Cystiphorolites.

major, see Cystiphorolites мнјоr.

minor, see Cystiphorolites minor.

variolosa, see Cystiphorolites variolosus.

Zaphrentis, Rafinesque, 1820, Ann. des Sci. Phys. Brux., vol. 5, p. 234. [Ety. $z a$, very ; phrentis, diaphragm.] Simple, turbinate; lamellæ simple, alternate, extending from the epitheca to the center of the visceral chamber; tabula well developed, extending from wall to wall, and deflected downward around the periphery; no columella; calice deep, with a single strongly developed fossula occupying the place of one of the lamellae. Type Z. phrygia.

acuta, White \& Whitfield, 1862, Proc. Bost. Soc. Nat. Hist., vol. 8, p. 306, Waverly or Chotean Gr.

affinis, Billings, 1865, Can. Nat. and Geo., 2d ser., vol. 2, p. 430, Hud. Riv. Gr.

ampla, Hall, 1876, Illust. Dev. Foss., pl. 21, Ham. Gr.

annulata, Hall, 1882, Foss. Corals Niagara and Up. Held. Grs., p. 33, and 35th Rep. N. Y. St. Mus. Nat. Hist., p. 437, Up. Held. Gr.

bellistriata, Billings, 1865, Can. Nat. and Geo., 2d ser., vol. 2, p. 430, Hud. Riv. Gr.

bigsbyi, Billingz, 1866, Catal. Sil. Foss. Antic., p. 92, Clinton and Niagara Grs.

bilateralis, Hall, 1852, (Caninia bilateralis, ) Pal. N. Y., vol. 2, p. 41, Clinton and Niagarn Grs.

calcariformis, Hall, 1882, Foss. Corals Niagara and Up. Held. Grs., p. 33, and 12 th Geo. Ind., p. 293, Up. Held. Gr.

calceola, White \& Whitfield, 1862, (Lophophyllum calceola.) Proc. Bost. Soc. Nat. Hist., vol. 8, p. 305, and 1880 , Cont. to Pal. No. 6, p. 156, Waverly or Choteau Gr.

eanadensis, Billings, 1862, Pal. Foss., vol. 1, p. 105, Hud. Riv. Gr.

cannonensis, Winchell, 1869, Geo. of Tenn., p. 442, Waverly or Kinderhook Gr.

carniatas, Worthen, (in press,) Geo, Sur. Ill., vol. 8, p. 75, Keokuk Gr.

cassedayi, M. Edwards, 1860, Hist. d Corallaires, t. 3, Warsaw Gr.

celator, Hall, 1876, 28th Rep. N. Y. St. Mus. Nat. Hist., p. 107, Niagara Gr. centralis, Fdwards of Haime, 1851, Mnn. d. Pol. Foss. d. Terr. Pal., p. 328, Up. Held. Gr.

chesterensis, Worthen, (in press,) Geo.Sur. Ill.; vol. 8, p. 73, Kaskaskia Gr.

cinctosa, Billings, 1866, Catal. Sil. Foss. Antic., ¡. 92, Clinton and Niagara Grs. cingulosa, Billings, 1874, Pal. Foss., vol. 2, p. 10, Gaspe linestone No. 8, Devonian.

clappi, syn. for Z. gigantea.

cliffordana, Edwards \& Haime, 1851, Mon. d. Pol. Foss. d. Terr. Pal., p. 329, Subcarboniferous.

colletti, Hall, 1852, Foss. Corals Niagara and Up. Held. Grs., p. 28, and 12th Rep. Geo. Ind., p. 315, Up. Held. Gr.

complanata, Hall, 18S2, Foss. Corals $\mathrm{Ni}$ agara and Up. Held. Grs., p. 26, and 35th Rep. N. Y. St. Mus. Nat. Hist., p. 430, Up. Held. Gr.

compressa, M. Ellwarls, 1860, Hist. d. Corallaires, t. 3, IV arsaw Gr.

compressa, see \%. davisana.

concava, Hall, 1852, Foss. Corals Niagara and Up. Held. Grs., p. 35, and 12th Rep. Geo. Ind., p. 291, Up. Held. Gr. conigera, gee Clisiophyllum conigerum. constricta, Hall, 185:, Foss. Corals Niagara and Up. Held. Grs., p. 33, and 35 th Rep. N. Y. St. Mus. Nat. Hist., p. 437. Up. Held. Gr.

contorta, Hall, 1882, Foss. Corals Niagara and Up. Held. Grs., p. 37, and 35th Rep. N. Y. St. Mus. Nat. Hist., p. 441, Up. Held. Gr.

convoluta, Hall, 1882, Foes. Corals Niagara and Up. Held. Grs., p. 37, and 12 th Rep. Geo. Ind. p. 294, Up. Held. Gr.

cornicula, Leslleur, 1820, (Caryophyllia cornicula,) Mem. du Mus., vol. 6, p. 297, Up. Held. Gr.

corrugata, Hall, 1882, Foss. Corals Niagara and Up. Held. Grs., p. 27, and 35 th Rep. N. Y. St. Mus. Nat. Hist., p. 431, Schoharie Gr.

corticata, Billings, 1874, Pal. Foss., vol. 2, 1). 9, Low. Devonian.

cristulata, Hall, 1882, Foss. Corals Niagara and Up. Held. Grs., p. 10, and $35 t h$ Rep. N. Y. St. Mus. Nat. Hist., p. 414, Niagara Gr.

cruciformis, Hall, 1883, 12th Rep. Geo. Ind., p. 315, Up. Held. Gr.

curvata, Hall, 1882, Foss. Corals Niagara and Up. Held. Grs., p. 35, and 35th Rep. N. Y. St. Mus. Nat. Hist., p. 439, Up. Held Gr.

eyathiformis, Hall, 1882, Foss. Corals Niagara and Up. Held. Grs., p. 27, and 12th Rep. Geo. Ind., p. 290, Up. Held. Gr.

cylindraceas, Worthen, (in press,) Geo. Sur. Ill., vol. 8, p. 78, Kaskaskia Gr. cystica, Winchell, 1866, Rep. Low. Penin. Mich., p. 90, Ham. Gr.

dalei, Edwards \& Haime, 1851, Mon.d. Pol. Foss. d. Terr. Pal., p. 329, Warsaw Gr 
davisana, n. sp. Up. Held. Gr. Proposed instead of $Z$. compressa of Rominger, 1876, Foss. Corals, p. 151, pl. 53, which was preoccupied.

deformis, Hall, 1883, 12th Rep. Geo. Ind., p. 290, Up. Held. Gr.

denticulata, Eichwald, 1857. Probably not American.

desori, Edwards \& Haime, 1851, Mon. d. Pol. Foss. d. Terr. Pal., p. 333, Low. Held. Gr.

duplicata, Hall, 1882, Foss. Corals Niagara and Up.-Held. Grs., p. 32, and $12 \mathrm{th}$ Rep. Geo. Ind., p. 293, Up. Held. Gr.

edwardsi, Nicholson, 1875, Ohio Pal., vol. 2, p. 235, Up. Held. Gr.

egeria, Billings, 1875, Can. Nat. and Geo., vol. 7, p. 234, Up. Held. Gr.

elegans, Hall, 1882, Foss. Corals Niagara and Up. Held. Grs., p. 37, and 12th Rep. Geo. Ind., p. 287, Up. Held. Gr.

elliptica, White, 1862, Proc. Bost. Soc. Nat. Hist., vol. 9, p. 31 , and 1880 , Cont. to Pal., No. 6, p. 155, Burlington Gr.

eriphyle, Billings, 1875, Can. Nat. and Geo. vol. 7, p. 233, Up. Held. Gr.

excentrica, Meek, 1873, Haydens, 6th Rep. U. S. Geo. Sur. Terr., p. 495, and Geo. Sur. W. 100th Mer., vol. 4, p. 101, Coal Meas.

fastigata, Hall, 1882, Foss. Corals Niagara and Up. Held. Grs., p. 30, and 35th Rep. N. Y. St. Mus. Nat. Hist., p. 434, Up. Held. Gr.

fenestrata, Nicholson, 1875, Can. Nat. and Geo., vol. 7, p.' 138, Up. Held. Gr.

foliata, Hall, 1882, Foss. Corals Niagara and Up. Held. Grs., p. 34, and 12th Rep. Geo. Ind., p. 286, Up. Held. Gr.

frequentata, Hall, 1882, Foss. Corals Niagara and Up. Held. Grs., p. 31, and 35th Rep. N. Y. St. Mus. Nat. Hist., p. 435, Up. Held. Gr.

fusiformis, Hall, 1882, Foss. Corals Niagara and Up. Held. Grs., p. 29, and 12th Rep. Geo. Ind., p. 296, Up. Held. Gr.

genitiva, Billings, 1875, Can. Nat. and Geo., vol. 7, p. 235, Up. Held. Gr.

gibsoni, White, 1884, 13th Rep. Geo. Ind., p. 117, Coal Meas.

gigantea, Lesueur, 1820, Mem. du. Mus., vol. 6, Up. Held. Gr.

glans, see Hadrophyllum glans.

gravis, Hall, 1882, Foss. Corals Nigara and Up. Held. Grs., p. 36, and 35th Rep. N. Y. St. Mus. Nat. Hist., p. 440, Up. Held. Gr.

gregaria, Rominger, 1876, Foss. Corals, p. 149, Niagara Gr.

halli, Edwards \& Haime, 1851, Mon. d. Pol. Foss. d. Terr. Pal., p. 341, Ham. Gr.

haysi, Meek, 1865, Am. Jour. Sci. and Arts, $2 \mathrm{~d}$ ser., vol. 40, p. 32, Low. Held. Gr.

hecuba, Billings, 1875, Can. Nat. and Geo., vol. 7, p. 234 , Up. Held. Gr.

herzeri, Hall, 1882, Foss. Corals Niagara and Up. Held. Grs., p. 35, and 12th Rep. Geo. Ind., p. 292, Up. Held. Gr. ida, Winchell. 1865, Proc. Acad. Nat. Sci. Phil., p. 117, Waverly or Kinderbook Gr.

illinoisensis, Worthen, (in press,) Geo. Sur. Ill. vol. 8, p. 77, Keokuk Gr.

inxqualis, Hall, 1882, Foss. Corals Niagara and Up. Held. Grs., syn. for Palæopbyllum divaricans.

inclinata, Hall, 1882, Foss. Corals Niagara and Up. Held. Grs., p. 34, syn. for Cyathophyllum angustatum.

incondita, Billings, 1874, Pal. Foss., vol. 2, p. 7, Devonian.

invenusta, Billings, 1875, Can. Nat. vol. 7, p. 233 , Up. Held. Gr.

irregularis, Hall, 1882, Foss. Corals Niagara and Up. Held. Grs., p. 34, and 35 th Rep. N. Y. St. Mus. Nat. Hist., p. 438, Up. Held. Gr.

knappi, Hall, 1882, Foss. Corals Niagara and Up. Held. Grs., p. 34, and 35 th Rep. N. Y. St. Mus. Nat. Hist., p. 438, Up. Held. Gr.

lanceolata, Worthen, (in press,) Geo. Sur. Ill., vol. 8, p. 76, Warsaw Gr.

latisinus, Hall, 1882, Foss. Corals Niagara and Up. Held. Grs., p. 10, and 35 th Rep. N. Y. St. Mus. Nat. Hist., p. 414, Niagara Gr.

macfarlani, Meek, 1868, Trans. Chi. Acad. Sci., p. 83, Devonian.

marcoui, Edwards \& Haime, 1851, Mon. d. Pol. Foss. d. Terr. Pal., p. 337, Niagra Gr.

minas, Dawson, 1868, Acad. Geo., p. 286, Subcarboniferous.

multilamella, Hall, 1852, Stans. Fx. to Gt. Salt Lake, p. 408, Coal Meas.

multilamellata, Nicholson, 1875, Ohio Pal., vol. 2, p. 236 . The name was preoccupied, and the definition is very imperfect.

nitida, Hall, 1882, Foss. Corals Niagara and Up. Held. Grs., p. 31, and 12th Rep. Geo. Ind., p. 288, Up. Held Gr.

nodulosa, Rominger, 1876, Foss. Corals, p. 148, Corniferous Gr.

offleyensis, Etheridge, 1878, Quar. Jour. Geo. Soc., vol. 34 , p. 588, Up. Sil.

ovalis, Hall, 1882, Foss. Corals Niagara and Up. Held. Grs., p. 29, and 12th Rep. Geo. Ind., p. 294, Up. Held. Gr.

ovibus, Salter, 1855, Belcher's Last of the Arctic Voyages, vol. 2, p. 382, Carboniferous.

parasitica, Worthen, (in press,) Geo. Sur. Ill., vol. 8, p. 79, Kinderhook Gr.

patens, Billings, 1865, Can. Nat. and Geo. 2d. ser., vol. 2, p. 430, Mid. Sil.

pellaensis, Worthen, (in press,) Geo. Sur. Ill., vol. 8, p. 74, St. Louis Gr.

planima, Hall, 1882, Foss. Corals Niagara and Up. Held. Grs., p. 29, and 12 th Rep. Geo. Ind., p. 292, Up. Held. Gr.

ponderosa, Hall, 1882, Foss. Corals Niagara and Up. Held. Grs., p. 27, and 12 th Rep. Geo. Ind., p. 288, Up. Held. $\mathrm{Gr}$. 
pressula, Hall, 1882, Foss. Corals Niagara and Up. Held. Grs., p. 10, and 35 th Rep. N. Y. St. Mus. Nat. Hist., p. 414, Niagara Gr.

profunda, Hall, 1882, Foss. Corals Niagara and Up. Held. Grs., p. 31, and 12th Rep. Geo. Ind., p. 287, Up. Held. Gr. prolifica, Billings, 1858, Can. Jour. N. S., vol. 4, p. 121, Up. Held. and Ham. Grs. prona, M. Edwards, 1860, Hist. d. Corallaires, t. 3, Warsaw Gr.

pulmonea, Lesueur, 1820, (Caryophyllia pulmonea,) Mem. du. Mus., vol. 6, Carboniferous.

racinensis, Whitfield, 1880, Ann. Rep. Geo. Sur. Wis. and Geo. Wis., vol. 4, p. 277, Niagara Gr.

rafinesquii, Edwards \& Haime, 1851, Mon. d. Pol. Foss. d. Terr. Pal., p. 329, Up. Held. Gr.

recta, Meek, 1S68, Trans. Chi. Acad. Sci., p. 82 , Devonian.

reversa, Worthen, (in press,) Geo. Sur. Ill., vol. 8, p. 78, Warsaw Gr.

rigida, Hall, 1882, Foss. Corals Niagara and Up. Held. Grs., p. 9, and 35th Rep. N. Y. St. Mus. Nat. Hist., p. 413, Niagara $\mathrm{Gr}$.

roemeri, Edwards \& Haime, 1851, Mon. d. Pol. Foss. d. Terr. Pal., p. 341, Delthyris Shale, Low. Held. Gr.

rugatula, Billings, 1874, Pal. Foss., vol. 2, p. 8, Gaspe limestone No. 1, U1). Sil.

sentosa, Hall, 1882, Foss. Corals Niagara and Up. Held. Grs., p. 32, and 35 th Rep. N. Y. St. Mus. Nat. Hist., p. 436, Up. Held. Gr.

simplex, Hall, 1843, (Strombodes simplex,) Geo, Rep. 4th Dist. N. Y., 1. 200, and Illust. Dev. Foss., pl. 21, Ham. Gr. solida, Hall \& Whitfield, 1873, 23d Rep. N. Y. St. Mus. Nat. Hist., p. 231, Chemung Gr.

spatiosa, see Heterophrentis spatiosa.

spergenensis, Worthen, (in press,) Geo. Sur. 1ll., vol. 8, p. 77, W arsaw Gr.

spinulifera, Hall, 1858, Geo. Sur. Iowa, p. 650, Warsaw Gr.

spinulosa, Edwards \& Haime, 1851, Pol. Foss. d. Terr. Pal., p. 334, Kiaskaskia Gr.

spissa, Hall, 1882, Foss. Corals Niagara and Up. Held. Grs., p. 30, and 12th Rep. Geo. Ind., p. 289, Curniferous limestone. stansburyi, Hall, 1852, Stans. Ex. to Gt. Salt Lake, p. 408, Coal Meas.

stokesi, Edwards \& Haime, 1851, Pol. Foss. d. Terr. Pal., p. 330, Niagara Gr. subcompressa, Hall, 1882, Foss. Corals Niagara and Up. Held. Grs., p. 28, and 12 th Rep. Geo. Ind., p. 286, Up. Held. Gr. subrecta, Billings, 1875, Can. Nat. and Geo., vol. 7, p. 235, Up. Held. Gr.

subvada, Hall, 1882, Foss. Corals Niagara and Up. Held. Grs., p. 11, and 35th Rep. N. F. St. Mus. Nat. Hist., p. 415, Niagara Gr.

subvesicularis, IIall, 1882, Foss. Corals Niagara and Up. Held. Grs., p. 10, and 35th Rep. N. Y. St. Mus. Nat. Hist., p. 414, Niagara Gr.

tabulata, Hall, 1882, Foss. Corals Niagara and Up. Held. Grs., p. 27, and 35th Rep. N. Y. St. Mus. Nat. Hist., p. 431, Up. Held. Gr.

terebrata, Hall, 1883, 12th Rep. Geo. Ind., p. 316 , Up. Held. Gr.

torta, Hall, 1882, Foss. Corals Niagara and Up. Held. Girs., p. 30, and 12th Rep. Geo. Ind., p. 285, Up. Held. Gr.

transversa, Hall, 1882, Foss. Corals $\mathrm{Ni}$. agara and Up. Held. Grs., p. 36, and 35th Rep. N. Y. St. Mus. Nat. Hist., p. 440, Up. Held. Gr.

transversensis, Winchell, 1866, Rep. Low. Penin. Mich., p. 00, Ham. Gr.

trisutura, Hall, 1882, Foss. Corals Niagara and Up. Held. Grs., p. 30, and 12th Rep. Geo. Ind, p. 289, Up. Held. Gr.

turbinata, Hall, 1852, (Polydilasma turbinatum, Pal. $\mathbf{N}$. Y., vol. 2, p. $112, \mathrm{Ni}$ agara $\mathrm{Gr}$.

ulrichi, Worthen, (in press,) Geo. Sur. Ill., vol. 8, p. 76 , Warsaw Gr.

umbonata, Rominger, 1876, Foss. Corals, p. 146, Ham. Gr.

undata, Hall, 1883, 12tb Rep. Geo. Ind., p. 291, Up. Held. Gr.

ungula, Rominger, 1876, Foss. Corals, p. 151, Up. Held. Gr.

varsoviensis, Worthen, (in press,) Geo. Sur. Ill., vol. 8, p. 78, Keokuk Gr.

venusta, Hall, 188:, Foss. Corals Niagara and Up. Held. Grs., p. 38, and 35th Rep. N. Y. St. Mus. Nat. Hist., p. 442 , Up. Helrl. Gr.
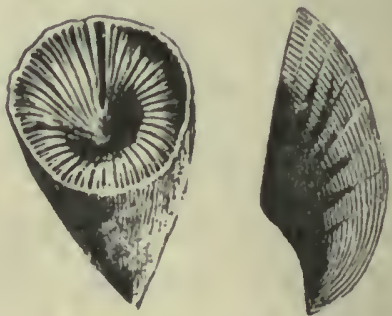

Fig. 230.-Zaphrentis worthent.

wortheni, Nicholson, 1875, Ohio Pal., vol. 2. p. 235 , lorniferus $\mathrm{Gr}$. 


\section{SUBKINGDOM ECHINODERMATA.}

Tris Subkingdom is represented, in the Palæozoic rocks, by the Classes Crinoidea, Stellerida, and Echinida.

The word "Crinoidea" was first used in 1821, by J. S. Miller, who published a book entitled "A Natural History of the Crinoidea." He used it as a family name, but later investigations raised it to the rank of a Class. The Palæozoic Orders, into which the Class is divided, are Palæocrinoidea, Blastoidea, Cystoidea, Lichenocrinoidea, Agelacrinoidea, Cyclocystoidea and Myelodactyloidea. The Stellerida are represented by the Orders Asteroidea and Ophiuroidea, and the Echinida by the Order Perischoechinida.

The fossils consist of plates, variously arranged and connected, all of which are composed of peculiar crystalline lime. The principal parts of the Palæocrinoidea are the calyx or body, arms, pinnules, column, and base or root. The Agelacrinoidea and Lichenocrinoidea were attached, by one side, to some foreign substance. The Cyclocystoidea were free or attached in like manner. Whether the Myelodactyloidea were free or attached to other bódies is unknown. Some of the Cystoidea were sessile, others possessed columns tapering to a point, and others had bases or roots for attachment. The Blastoidea possessed columns, but whether or not any of them attached by bases or roots is unknown. All Palæocrinoidea had columns, but some did not have bases or roots. The Orders bearing pinnules are the Blastoidea, Palæocrinoidea, and part of the Cystoidea.

Prof. Wachsmuth has claimed the construction of the vault affords good characters for the separation of the Palæocrinoidea into families, and has distinguished three plans upon which the summit is constructed, viz.:

1. The summit composed of a more or less pliable, sometimes perhaps squamous integument, yielding to motion, in the body and arms.

2. The summit composed of solid plates, with a porous ventral sac, located posteriorly, on the disk, and closed at the top. Anal opening rarely observed, but, probably, lateral.

3. The summit composed of heavy immovable plates, closely joining and forming a dome arching the entire oral side. Anal opening directly through the wall of the dome or at the extremity of a tube, the so-called proboscis.

Without underestimating his work, a single illustration will show that families can not always be distinguished by the construction of the vault; for in the family Heterocrinida, there is no resemblance between the vaults of Ectenocrinus, Heterocrinus, Iocrinus, and Ohiocrinus. Ectenocrinus has no tube or proboscis, Ohiocrinus has a large spiral tube, and Iocrinus has a long cylindrical one, extending beyond the ends of the arms and flowing pinnules.

We believe the separation of the Palæocrinoidea into families must be based upon the construction of the calyx and vault, but chiefly upon the former. Probably no family should be made to include genera, some of which have subradials 
and others do not. It is therefore of the first importance to ascertain whether the crinoid has one or two circles of plates below the radials. Those having only one circle have been called monocyclic, and those having two circles dicyclic. The circle at the base is composed of what we call the "basal plates," and the second circle, whenever it has an existence, is composed of "subradial plates." In this we follow Billings, Meek, Agassiz, and most other standard authors. Carpenter and Wachsmuth call the "subradials" the "basals" in all cases where they occur, and the lower plates "underbasals;" but where there are no "subradials," they follow the well-established nomenclature in calling the first circle of plates " basals."

The presence or absence of regular interradials, it seems, should always be regarded as of family importance.

The number of basal plates should also be regarded as of fanily importance. If not in all cases, then in connection with the general structure of the calyx and vault the families will be sufficiently well-defined. Those characters upon which genera are founded, when combined, in certain associations will form families; and under this head several inportant fnmilies have been created.

Generic characters, as a matter of course, are to a certain exteut included in the family characters; but the form and construction of the column is of generic importance. The general form of the calyx and vault, and the number of primary radials, and the construction of the azygous aren, are always of gencric importance. Beside, certain "combinations and associations of what are usually regarded as specific characters have been made the basis for establishing genera.

Wachsnuth, speaking from experience, says in young crinoids the basals are the most perfectly developed parts; they attain nearly their full size in young individuals, greater in proportion than the subradials and radials, which are comparatively early developed, and at a time when the interradial and anal plates have scarcely made their appearance. The latter develop the slowest, and in some genera increase continually, both in size and number, during the growth of the individual. Abnormal growths, or sudden modifications of specific characters, almost always take place in the interradial and azygous areas, the azygous rays and dome. His experience is corroborated by others, and the author never saw a small specimen that did not have its basals or first circle of plates as distinctly marked as they occur in large specimens of the same species.

The columns of crinoids very frequently show injuries received by the animal in its life-time. The column is sometimes much swollen on one side and depressed on the other; sometimes a parasite that attached to the column is found imbedded or enveloped in the crinoid column. The animal could also repair its arms and other parts of its body by secretions of lime in the same way.

Some of the Cystoidea may be arranged into families, upon characters similar to those upon which families are founded in the Palaeocrinoidea; but, generally, this is not the case. Some of the Cystoidea possessed an ambulacral opening and two other orifices, the purposes of which may not be fully understood; in others, the two openings referred to are absent. In addition to these, many bear openings called "pectinated rhombs," and all have pores passing through the plates. These pores passed to organs called "hydrospires," which were largely developed within the calyx of the Cystoidea and Blastoidea. The communication, through the test, with the outside water is supposed to show the hydrospires belonged to the respira- 
tory system. The number and position of the larger orifices and the pectinated rhombs constitute the principal basis for family classification. The Blastoidea have orifices at the summit of the calyx which are important in classification. Some have fissures at the summit, others have slits along each side of the ambulacra, and others have five pairs surrounding an oral center. These openings connect with hydrospires situated beneath the ambulacra. These orifices are of family importance, and some have regarded the number of hydrospires as of generic importance.

In the nomenclature of the Blastoidea the calyx consists of the basals, radials or forked plates, and orals or deltoid plates. The suture between the basals and radials is the basi-radial suture. The ridge at the median line of an oral is an oral or interradial ridge. In the forked plates the lower part is the body of the radial, and the two prongs are the limbs. Between the limbs is the radial sinus, which is occupied by the ambulacrum, consisting of a lancet-piece, which is excavated lengthwise by the food-groove or ambulacrum, and against it rest side plates or pore pieces, marked by pinnule pits or sockets, and there are also side plates. Beneath the ambulacra there are interradial systems of lamellar tubes or hydrospires. The openings of these tubes on the ventral surface of the calyx, as in Codaster, are called hydrospire slits; if they are concentrated beneath the ambulacra, as in Codonites, the gap between the edge of the lancet-plate and the sides of the radial sinus is the hydrospire cleft, which leads downward into the hydrospire canal. The canals open externally by spiracles, sometimes called ovarian openings. The spiracles of the anal interradius may be confluent with the anal opening to form the anal spiracle. The plates covering the mouth and peristome, and which are sometimes coutinued down the ambulacra covering the food-grooves, are the summit plates or the vault.

The Cyclocystoidea have tubes radiating from the center of the disk, which connect with a circular tube in the rim. It is evident there was both a circular and radiate system of circulation in this order of animals. The Myelodactyloidea also had a compound internal system of both circular and radiate circulation. The Lichenocrinoidea attached by a base that appears to have been a single solid plate. Internally there are numerous thin, upright septa radiating from the center, which supported the very small external plates, and the sarcode between which must have been connected with the tube in the column to have given support to it, and to have maintained it in an upright position. The column tapered to a point, and no evidence has been found of any external opening of these animals. The affluent and effluent openings that abound in all other Echinoderms, and even among the sponges, have thus far never been discovered in the Lichenocrinoidea. The notice of this order in Wachsmuth's Palæocrinoidea seems to be wholly erroneous. The three orders-Cyclocystoidea, Myelodactyloidea and Lichenocrinoidea-are unknown in rocks later than the Upper Silurian.

The Class Stellerida is composed of animals with a flattened and more or less pentagonal body and central disk. The mouth opens in the center of the lower surface of the disk; the skin is coriaceous, the whole body more or less flexible, and along the lower surface of each arm or prolonged ray from the central disk, there is a more or less distinct furrow from which the ambulacra are protruded. The Palæozoic orders, Asteroidea and Ophiuroidea, are exceedingly abundant in all existing seas. In the common starfish the arms are mere prolongations of the disk, and the plates from which the ambulacra are exserted are in deep furrows along the lower 
surface of the arms. The mouth is in the center of the lisk, and the ramifications of the stomach extend a greater or less distance iuto the arm-furrows. In the Ophiuroidea [Ophis, snake; oura, tail] there are usually five simple curving or flowing arms with undefined furrows and furnished with cirri, which give them a ragged and tangled exterior.

The class Echinida is composed of animals having a complete exterior calcareous shell of closely-fitting plates, which prevents all flexion of the body. The anima] has no arms, but the holes, through which the sucking feet are protruded, are arranged upon five rows of plates running from the center of the top of the shell to the angles of the mouth at the bottom; or, when they are confined to the dorsal surface, they form a distinct five-rayed star surrounding the apex of the shell. A striking character in this class is the manner in which spines are articulated upon tubercles on the surface of the shell; the base of the spines being hollowerl for the reception of the convex surface of the tubercle, and, being sustained in place by a ligament, the spines are movable, and serve economical purposes. The Palsozoic order Perischoechineda is extinct, but some of them had an internal masticatory apparatus that will compare with any that exists in the living representatives.

\section{CLASS CRINOIDEA.}

\section{ORDER PALASOCRINOIDEA.}

Family Acrocrinid A:-Acrocrimus.

Family Actinocrinid - Actinocrinus, Agaricocriuus, Alloprosallocrinus, Amphoracrinus, Batocrinus, Dorycrinus, Eretmocrinus, Gennæocrinus, Megistocrinus, Melocrinus, Physetocrinus, Saccocrinus, Siphonocrinus, (?) Steganocrinus, Stereocrinus, Strotocrinus, Teleiocrinus.

Family Agassizocrinida.-Agassizocrinus.

Family Alangerinids.-Allagecrinus.

Family Ancyrocrinide.-Ancyrocrinus.

Family Arthracantumd:- Arthracantha.

Family Beidem nocrinid a:-Belemnocrinus.

Family Calceocrinidw-Calceocrinus, Deltacrinus.

Family Camarocrininje.-Camarocrinus.

Family Catillocrinide.e-Catillocrinus.

Family Cupressocrinida.-Aspidocrinus.

Famil Cyathocinidos-Ampheristocrinus, Arachnocrinus, Atelestocrinus,

Barycrinus, Carabocrinus, Cyathocrinus, Erisocrinus, Eupachycrinus, Euspirocrinus, Menocrinus, Palaocrinus, Vasocrinus.

FAmil, Drchocrinide. - Cotyledonocrinus, Dichocrinus, Pterotocrinus, Talarocrinus.

Family Dimetocirinidas.-Coronocrinus, Cytocrinus.

Family Edrocrinida.-Edriocrinus.

Family EuCalyptocinidis,-Eucalyptocrinus.

Family Gasterocomid . -Myrtillocrinus.

Family Gaurockinide.-Gaurocrinus, Retiocrinus, Rhaphanocrinus, Thysanocrinus.

Family Glyptasterids.-Gly ptaster, Lampterocriuus. 
Family Glyptochinides.-A rchæocrinus, Compsocrinus, Glyptocrinus, Pyenocrinus, Schizocrinus.

Family Haplocrinide.-Coccocrinus, Haplocrinus.

Family Heterocrinid d.-Ectenocrinus, Heterocrinus, Iocrinus, Ohiocrinus.

Fanily Hybocrinides-Anomalocrinus, Hybocrinus.

FamiLY IChTH yocRinid «.-Cleiocrinus, Ichthyocrinus, Lecanocrinus, Mespilocrinus, Nipterocrinus, Onychocrinus, Taxocrinus.

Family Melocrinidz:-Allocrinus, Dolatocrinus, Macrostylocrinus, Mariacrinus, Technocrinus.

Family Pisocrinide.-Pisocrinus.

Family Platycrinida.-Cordylocrinus, Eucladocrinus, Marsupiocrinus, Platycrinus.

Family Poteriocrinide.-Bursacrinus, Coliocrinus, Dendrocrinus, Graphiocrinus, Homocrinus, Hydreionocrinus, Merocrinus, Ottawacrinus, Poteriocrinus, Stemmatocrinus, Zeacrinus.

FAMILY RHODOCRINID\&.-Goniasteroidocrinus, Hadrocrinus, Lyriocrinus, Rhodocrinus.

Family Synbathocrinide:-Synbathocrinus.

Family Taxocrinida:-Cupulocrinus, Forbesiocrinus, Taxocrinus.

Family Xenocrinide.-Xenocrinus.

Family affinity uncertain.-Brachiocrinus, Closterocrinus, Cystocrinus.

\section{Order Cystoidea.}

Family Amygdalocystide.-Amygdalocystites, Palæocystites.

Famity Anomalocystide.-Anomalocystites.

Family Caryocrinidel.-Caryocrinus.

Family Comarocystida.-Comarocystites.

Fanily Echinocystid ג.-Echinocystites.

Family Eocystid d:-Eocystites.

Fanily Gonphocystid ж.-Gomphocystites, Hemicosmites.

FaniL y HoLocystid d.-Allocystites, Crinocystites, Holocystites.

Family Hyвоcystid ж)-Hybocystites.

Family Leíadocrinid).-Apiocystites, Callocystites, Glyptocystites, Lepadocrinus, Pleurocystites, Sphaerocystites, Strobilocystites.

Family Platycystide.-Platycystites.

Family uncertain.-Heterocystites, Lysocystites, Malocystites, Porocrinus.

\section{ORDER BLASTOIDEA.}

FAMIL Y BLASTOIDOCRINIDE.-Blastoidocrinus.

Family Codasteridæ.-Codaster, Heteroschisma.

Fanily Codonitide.-Codonites.

Family Eleutherocrinidd:- Eleutherocrinus.

Family Granatocrinide.-Granatocrinus, Schizoblastus.

Family Nucleocrinide. - Nucleocrinus.

Family Pentremitide.-Pentremites, Pentremitidea.

Family Stepilanocrinide.,-Stephanocrinus.

Family Troostocrinide.-Troostocrinus, Tricelocrinus. 


\section{ORDER AGELACRINOIDEA.}

Famils Agrlackinima-Agelacrinus, Echinodiscus, Edrioaster, Lepidodiscus. Famiry Hemicystina.-Hemicystites.

Order Myel,odactyi,oidea.

Family Mylonactyıin.k.-Myelodactylus.

Order Cyclocystoidea.

Famiy Cyciocystomin.t.-Cyclocystoides.

\section{ORIDER LICHENOCRINOIDEA.}

Family Lichexo(rinins:- - I jichenocrinus.

\section{CI,ASS STELLERIDA.}

\section{ORDER AstiroIDLA.}

Famid Onychasthimin.r-Onychaster.

Family Paldastinidx-Cholaster, Compsaster, Palaaster, Palaasterina, Petraster, Schoenaster, Stenaster, Tremataster.

\section{ORDER OPHIUROIDEA.}

Family Protastirins:-Eugaster, Palcocoma, Protaster, 'Twniaster.

\section{CIAASS ECHINIDA.}

\section{ORDIER PERISCHOECHINIDA.}

Family Archaocidarins:-A rchaocidaris, Eocidaris, Lepidocidaris, Perischodomus, Pholidocidaris.

Family Lepidechinid k.-Hybochinus, Lepidechinus.

Famiry Palechinidж:-Lepidesthes, Melonites, Oligoporus, Palechinus.

Acrocrinus, Yandell, 1855, Am. Jour. Sci. and Arts, 2d ser., vol. 20, p. 135. [Ety. akros, extreme, from the great number of plates covering the body; krinon, lily.] Body goblet or urn-shaped, consisting of many series of plates; two basals, the suture from the anterior to the posterior side, followed by a series of small plates, and these again by another and another, so that the plates reach the 5 th to 10 th series before the arms become free; the size of the plates increase as they approach the arms; arms 20, long, composed of two series of plates bearing pinnules; column round. Type A. shumardi.

shumardi, Yandell, 1855, Am. Jour. Sci. and Arts, 2d ser., vol. 20, p. 135, Kaskaskia Gr.

urniformis, Hall, 1858, Geo. Rep. Iowa, p. 690, Kaskaskia Gr. wortheni, Wachsmutl, 1882, Bull. No. 1, Ill. St. Mus. Nat. Hist., p. 4 , and Geo. Sur. Ill., vol. 7 , p. 343 , Coal Meas.

Actinocrinus, Miller, 1821, Nat. Ilist. Crinoidea, p. 95. [Ety. aktin, ray; krinon, lily.] Body turbinate, plates sculptured; basals 3 ; primary radials $3 \times 5$; secondary $1 \times 10$, axillary; succeeding radials having a single series to each division, one axillary, the other simple; arms 20 to 50 or more; pinnules; regular interradials, one in the first series, two in the second, and one or two in the third; azygous interradials, one in line with the first primary radials, and ot the same size, two in the second series, and one, two, or three in succeeling series; vault variable, plates nodose; tube or proboscis large, subcentral; column long. Type A. triacontadactylus. 
abnormis, see Megistocrinus abnormis. ægilops, see Teleiocrinus ægilops. æqualis, see Batocrinus æqualis.

æquibrachiıtus, see Batocrinus æquibrachiatus.

xquibrachiatus var. alatus, syn. for Batocrinus æquibrachiatus.

agassizi, Troost, 1850, Catal. ' Not defined. althea, see Teleiocrinus althea.

amplus, see Saccocrinus amplus.

andrewsianus, see Batocrinus andrewsanus. araneolus; see Steganocrinus araneolus.

arnoldi, Wachsmuth \& Springer, (in press,) Geo. Sur. Ill., vol. 8, p. 168, Kinderhook Gr. asterias, McChesney, 1860 , Desc. New. Pal. Foss. Syn. for Batocrinus verrucosus.

asteriscus, see Batocrinus asteriscus. biturbinatus, see Batocrinus biturbinatus.

brevicornis, see Megistocrinus brevicornis.

brevis, see Agaricocrinus brevis.

brontes, Hall, 1860, Sup. to Geo. Sur. Iowa, p. 47, and Geo. Sur. Ill., vol. 5, p. 341, Warsaw Gr.

cxlatus, Hall, 1858 , Geo. Sur. Iowa, p. 585 , and Geo. Sur. Ill., vol. 5, p. 341, Burlington Gr.

calyculoides, see Eretmocrinus calyculoides.

calyculus, see Batocriuus calyculus. calypso, see Gennæocrinus calypso.

cassedayi, see Gennæocrinus cassedayi. curica, see Eretmocriuus carica.

caroli, see Batocrinus caroli.

cauliculus, see Gennzeocrinus cauliculus.

chloris, Hall, 1861, Desc. New Crinoidea, syn. for A. tenuisculptus.

christyi, Shumard, 1855, see Batocrinus christyi.

christyi, Hall, see Saccocrinus christyi.

clarus, Hall, 1861, Desc. New Crinoidea,

p. 2, and Geo. Sur. Ill., vol. 5, p. 341,

Burlington Gr.

clavigerus, see Batocrinus clavigerus.

clio, see Eretmocrinus clio.

clivosus, see Teleiocrinus clivosus.

cloelia, see Eretmocrinus clcelia.

clypeatus, see Batocrinus clypeatus.

concavus, see Dorycrinus concavus. concinnus, see Steganocrinus concinnus.

copei, see Physetocrinus copei.

corbulis, see Eretmocrinus corbulis.

coreyi, Lyon \& Casseday, 1859, Am. Jour.

Sci. and Arts, $2 d$ ser., vol. 29 , p. 76 ,

Keokuk Gr.

corniculum, Hall, 1858, Geo. Rep. Iowa, p. 566, Burlington Gr. Wachsmuth says it is a syn. for Agaricocrinus brevis.

cornigerus, Hall, see Dorycrinus cornigerus.

cornigerus, Lyon \& Casseday, see Gennæocrinus cornigerus.

cormutus, Troost, 1850, Catal. Not defined. 
doris, see Batocrinus doris. erodus, see Teleiocrinus erodus. eryx, Hall, 1861, Desc. New Crinoidea, p.

12, Burlington Gr.

eucharis, see Gennæocrinus eucharis. evansi, see Megistocrinus evansi. excerptus, Hall, 1861, Desc. New Cri-
Foss., p. 24, and Trans. Chi. Acad. Sci., p. 17, Burlington Gr. icosidactylus, see Batocrinus icosidactylus. indianensis, see Batocrinus indianensis. inflatus, see Amphoracrinus inflatus. infrequens, Hall, 1861, Desc. New Crinoidea, ए. 14, Burlington Gr.

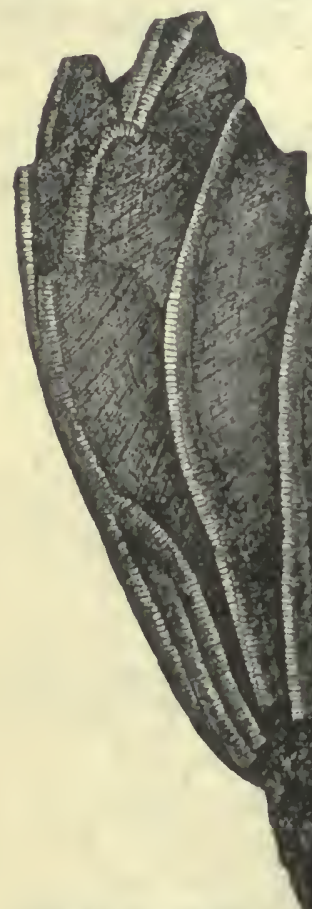

noidea, p. 3 , and Geo. Sur. Ill., vol. 5, p. 341, Burling. ton $\mathrm{Gr}$.

fibula, 'Troost, 1850 , Catal. Not defined. frecellus, see Agaricocrinus fiscellus.

fosteri, McChesney, 1860, Desc. New Päl.

Foss., p. 19, and

Trans. Chi. Acad. Sci., p. 14, Burlington Gr.

formosus, see Batocrinus formosus. gemmiformis, see Eret-

mocrinus gemmiformis.

gibbosus, Troost, 1850 . Not detined.

glans, Hall, 1860, Sup. to Geo. Sur. Iowa, p. 16, Burlington Gr.

glyptus, see Strotocrinus glyptus. gouldi, see Dorycrinus gouldi. hageri, see Batocrinus hageri. helice, see Agaricocrinus helice. helice var, eris, see Agaricocrinus eris. humboldti, Troost. Not defined.

hurdanus, McChesney, 1860. New Pal. 
matuta var. attenuatus, see Eretmocrinus attenuatus.

meeki, see Macrostylocrinus meeki.

minor, Hall, 1858, Geo. Rep. Iowa, p.

573, Burlington Gr.

mississippiensis, see Dorycrinus misissippiensis.

mississippiensis var. spiniger, see Dorycrinus mississippiensis var. spiniger.

missouriensis, see Dorycrinus missouriensis. moniliformis, Miller, cited by Tronst. Not American.

mortoni, Troost, 1850. Not defined.

multibrachiatus, Hall, 1858, Geo. Rep.

Iowa, p. 580, Burlington Gr.

multibrachiatus var. echinatus, Hall, 1861,

Desc. New Crinoidea, p. 10, Warsaw Gr. multicornis, see Centrocrinus multicornis. mundulus, see Batocrinus mundulus.

multiradiatus, Shumard, 1857, Trans. St.

Louis Acad. Sci., p. 75, and Geo. Rep.

Iowa, p. 579, Burlington Gr.

nashvillx, see Batocrinus nashvillæ.

nnshvillx var. subtractus, see Batocrinus

nashvillæ var. subtractus.

novoluachiatus, Wachsmuth \& Springer,

(in press,) Geo. Sur. Ill., vol. 8, p. 165,

Kinderhook Gr.

nyssa, see Gennæocrinus nyssa.

oblatus, see Batocrinus oblatus.

obpyramidalis, see Melocrinus obpyramidalis.

olla, McCoy, 1849. Not American.

olliculus, syn. for Megistocrinus whitii. opusculum, Hall, 1861,

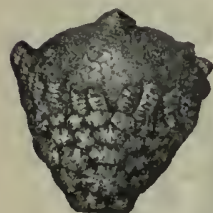

Fig. 233. - Actinocrinus ornatissimus. Bost. Jour. Nat. Hist., p. 264, Burlington Gr. ornatissimus, W a c b smuth \& Springer, (in press), Geo. Sur. Ill., vol. 8, p. 163 , Kinderhook Gr.

ornatus, see Physetocrinus ornatus.

ovatus, Hall, 1861, Desc. New Crinoidea, p. 19, Burlington Gr.

papillatus, see Batocrinus papillatus. parves, see Dorycrinus parvus.

pendens, see Dorycrinus pendens.

penicillus, Meek \& Worthen, 1869, Proc

Acad. Nat. Sci. Pliil., p. 155, and Geo.

Sur. Ill., vol. 5, p. 342, Burlington Gr. pentagonus, see Steganocrinus pentagonus. pentaspinus, see Centrocrinus pentaspinus. pernodosus, Hall, 1858, Geo. Rep. Iowa, p. 608, Keoluk Gr.

perumbrosus, see Strotocrinus perumbrosus. pistilliformis, see Batocrinus pistilliformis. pistillus, see Batocrinus pistillus.

planobasalis, see Amphoracrinus planobasalis.

planodiscus, see Batocrinus planodiscus. plumosus, Hall, 1843, Geo. Rep. 4th Dist. N. Y., p. 72, and Pal. N. Y., vol. 2, p. 180, Clinton Gr. Not an Actinocrinus, and the fragments too poor for determination.

pocillum, see Gennæocrinus pocillum. polydactylus, see Mariacrinus polydactylus. precursor, see Dorycrinus præcursor.

proboscidialis, Hall, 1858, Geo. Rep. lowa, p. 584, Burlington Gr.

pyriformis, see Batocrinus pyriformis.

pyriformis, var. rudis, Meek \& Worthen, see Batocrinus pistilliformis.

pyramidatus, see Agaricocrinus pyramidatus.

quadrispinus, see Amphoracrinus quadrispinus.

quaternarius, Hall, 1860, Frg.234.-ActinoSupp. Geo. Rep. Iowa, crinus probosp. 22, Burlington Gr. cldialis.

quaternarius var. spiniferus, Hall, 1861,

Desc. New Crinoidea, p. 11, Burlington Gr.

quinquelobus, see Dorycrinus quinquelobus.

ramulosus, see Eretmocrinus ramulosus. regalis, see Strotocrin us regalis.

remibrachiatus, see Eretmocrinus remibrachiatus.

reticulatus, see Physetocrinus reticulatus. rotundus, see Batocrinus rotundus.

'rudis, see Teleiocrinus rudis.

rusticus, Hall, 1861, Desc. New Crinoidea, p. 2, syn. for A. scitulus.

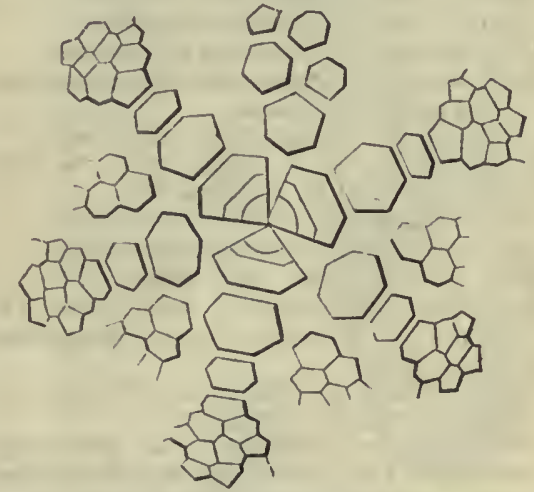

FIG. 235.-Actinocrinus scitulus. Dlagram x 2 .

scitulus, Meek \& Worthen, 1860, Proc.

Acad. Nat. Sci. Phil., p. 386, and Greo.

Sur. Ill., vol. 2, p. 202, Burlington Gr. sculptus, see Steganocrinus sculptus. securis, Hall, 1861, Desc. New Crinoidea, p. 14, Burlington Gr.

semiradiatus, see Saccocrinus semiradiatus. senarius, see Physetocrinus senarius.

sexarmatus, Hall, 1860, Supp. Geo. Rep. Iowa, p. 21, Burlington Gr.

sillimani, Meek \& Worthen, syn. for $\mathbf{A}$. scitulus.

similis, see Batocrinus similis.

sinuosus, see Batocrinus sinuosus.

speciosus, Meek \& Worthen, syn. for Strotocrinus regalis.

spinobruchiatus, see Amphoracrinus spinobrachiatus. 
spinotentaculus, Hall, 1860, Supp. Geo.

Rep. Iowa, p. 86, Burlington Gr. spinulosus, see Dorycrinus spinulosus. steropes, see Batocrinus steropes. subaculealus, sce Dorycrinus subaculeatus, subrqualis, see Batocrinus subxqualis. subturbinatus, see Dorycrinus subturbinatus.

subumbrosus, Hall, syn. for Teleiocrinus liratus.

subventricosus, see Physetocrinus subventricosus.

superlahus, see Megistocrinus superlatus. symmetricus, see Dorycrinus symmetricus. tenuidiscus, Hall, 1861, Desc. New Crinoidea, p. 14, Burlington Gr.

tenuiradialus, Hall, 1847, see Palæocystites tenuiradiatus.

tenuirudiutus, Hall, 1861, see Teleiocrinus tenuiradiatus.

tenuisculptus, McChesney, 1860, Desc. New Pal. Foss., p. 15, and Trans. Chi. Acad. Sci., pl. 5, fig. 11, Burlington Gr. thalia, Hall, 1861, Desc. New Crinoidea, p. 13, Burlington Gr.

themis, Hall, 1861, Desc. New Crinoidea, p. 11, Burlington Gr.

thetis, Hall, 1861, Desc. New Crinoidea, p. 11, Burlington Gr.

thoas, Hall, syn. for A. reticulatus. tholus, syn. for A. glans.

tricornis, see Dorycrinus tricornis.

trinodus, see Dorycrinus trinodus. turbinalus, see Batocrinus turbinatus.

turbinatus var. elegans, see Batocrinus turbinatus var. elegans.

umbrosus, see Telejocrinus umbrosus. uni.arinatus, Hall, 1860, Supp. to Geo.

Sur. Iowa, p. 48, Keokuk Gr.

unicornis, see Doryerinus unicornis. unispinus, see Dorycrinus unispinus. urna, Troost, 1850. Not defined.

urniformis, IcChesney, 1860, Now Pal. Foss., p. 23, вyn. for Eretmocrinus konincki.

validus, Meek \& Worthen, 1860, syn. for Steganocrinus concinnus.

ventricosus, see Physetocrinus ventricosus. ventricosus var. cancellatus, see Physetocri-

nus ventricosus var. caucellatus. ventricosus var. internodus, see Physetocrinus ventricosus var. internodus. verneuili, see Melocrin us verneuili. verneuilianus, see Eretmocrinus verneuilanus.

verrucosus, Hall, 1858, Geo. Rep. Iowa, p. 578, Burlington Gr.

viaticus, White, 1874, Rep. Invert. Foss., p. 16, and Geo. Sur. W. 100th Merid., vol. 4 , p. 82 , Subcarboniferous.

viminalis, sec Amphoracrinus viminalis. wachsmuthi, White, 1862, Proc. Bost. Soc. Nat. Hist., vol. 9, p. 17, syn. for A. scitulus.

wachsmuthi, White, 1880, see Batocrinus wachsmuthi.

whitfieldi, see Saccocrinus whitfeldi. whitii, see Megistocrinus whitii. yandelli, see Batorinus yandelli.
Agaricocrinus, Troost, 1850, Catal. in Proc. Am. Ass'n, and Hall in Geo. Sur. Iowa, p. 560. [Ety. Agaricus, mushroom; krinon, lily.] The form of the calyx is that of an inverted basin or mushroom; plates smooth; dome composed of large nodose plates and smaller convex ones, the central plate being the largest in the body; basals 3, small; priniary radials $3 \times 5$; sccondary radials 1 or $2 \times 10$, which are succeeded by shorter arm-plates; regular interradials $3 ; \Omega z y$ gous plates 4 to 7 , or more: aperture, at the upper part, directed laterally; arins long, constructed of two rows of plates bearing pinnule; columns round. Type A. americanus.

americanus, Roemer, 1854, ( $A$ mphoracrinus americanus,) Bronn's Leth. Geog., vol. 2, p. 250 , and Geo. Sur. Iowa, 1י 617, Keokuk Gr.

bellitrema, Hall, 1861, Bost. Jour. Nat. Hist., p. 281, Burlington Gr. Wachsmuth says it is a syn. for $\mathrm{A}$. ornotrema. brevis, Hall, 1858, (Actinocrinus brevis,) Geo. Sur. Iowa, p. 567, Burlington Gr.

bullatus, Hall, 1858, Geo. Sur. Iowa, p. 562, Burlington Gir. Wachsmuth says it is a syn. for $\mathrm{A}$. americanus.

convexus, Ifall, 1860, (A. pentagonus var. convexus,) Supp. to (ieo. Sur. Iowa, p. 58 , Burlington Gr.

Fyo. 236.-A garicocrinus crassus, azygous vlew of
caly x.

corrugatus, Hall, 1861, Desc. New Spec. Crin., p. 4, and Bost. Jour. Nat. Hist., p. 283, Burlington Gr. Wachsmuth says it is a syn. for A. pyramidatus founded upon a mature specimen.

crassus, Wetherby, 1881, Jour. Cin. Soc. Nat. Hist., vol. 4, p. 178 , Keokuk Gr.

elegans, Wetherby, 1881, Jour. Cin. Soc. Nat. Hist., vol. 4, 1 . 179, Keok uk Gr.

eris, Hall, 1864,

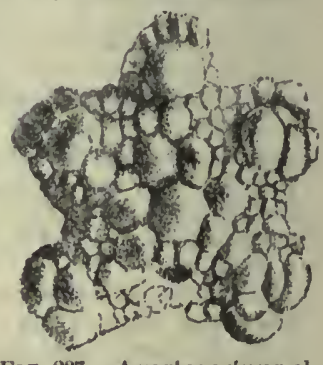

Fio. 237.-Agarlcocrinus elegans, vlew of the vault. Rep. N. Y. St. Mus. Nat. Ilist., p. 53, 
and Ohio Pal., vol. 2, p. 164, Waverly $\mathrm{Gr}$.

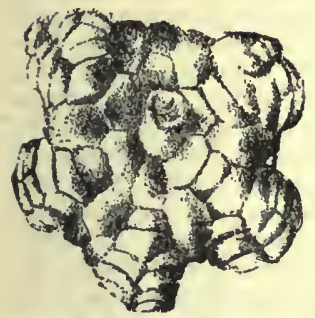

FIG. 238.-Agaricocrinus elegans, basal view.

excav at us, Hall, 1861, (Actinocrinus excavatus,) Desc. New Spec. Crin., p. 3, and Bost. Jour. Nat. Hist., p. 282, Burling to $\mathrm{n} \mathrm{G} \mathrm{r.}$ Wachsmuth says it is a syn. for A. americanus.

fiscellus, Hall, 1861, (Actinocrinus fiscellus,) Desc. New Spec. Crin., p. 2, and Bost. Jour. Nat. Hist., p. 272, Burlington Gr. geometricus, Hall, 1860, Supp. to Geo. Sur. Iowa, p. 56, Burlington Gr. gracilis, Meek \& Worthen, 1861, Proc. Acad. Nat. Sci. Phil., p. 135, Burlington Gr.

helice, Hall, 1864, (Actinocrinus helice, ) 17th Rep. N. Y. St. Mus. Nat. Hist., p. 53, and Ohio Pal., vol. 2 , p. 163 , Waverly Gr.

inflatus, Hall, 1861, Desc. New Criniodea, p. 4, and Bost. Jour. Nat. Hist., p. 284, Burlington Gr.

macadamsi, Worthen, (in press,) Geo. Sur. IIl., vol. 8, p. 94, Keokuk Gr.

nodosus, Meek \&

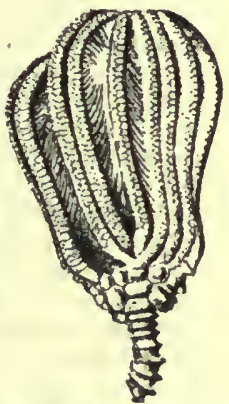

FIg. 239-Agaricocrinus helice.
Worthen, 1869, Proc. Acad. Nat. Sci. Phil., p. 167, and Geo. Sur. Ill., vol. 5, p. 387, Burlington Gr. Wachsmuth says it is a syn. for A. americanus.

nodulosus, Worthen, (in press,) Geo. Sur. Ill., vol. 8, p. 94, Keokuk Gr.

ornotrema, Hall, 1861, Desc. New Crinoidea, p. 3, Burlington Gr.

pentagonus, Hall, 1860, Supp. Geo. Rep. Iowa, p. 57, Burlington Gr.

pentagorus var. convexus, see A. convexus. planoconvexus, Hall, 1861, Desc. New Crinoidea, p. 3, and Bost. Jour. Nat. Hist., p. 280, Burlington Gr.

pyramidatus, Hall, 1858, (Actinocrinus pyramiclatus, Geo. Rep. Iowa, p. 565, Burlington Gr.

springeri, White, 1882, 11th Rep. Geo. and Nat. Hist. Indiana, p. 363, Keokuk Gr.

stellatus, Hall, 1858, Geo. Rep. Iowa, p. 564, Burlington Gr.

tuberosus, Troost, 1850, Catal. Hall. 1858, Geo. Rep. Iowa, p. 617, syn. for A. americanus.

whitfieldi, Hall, 1858, Geo. Rep. Iowa, p. 621, Keokuk Gr.

wortheni, Hall, 1858, Geo. Rep. Iowa, p. 619, Keokuk Gr.
Agassizocrinus, Troost, 1850 Mss., Shumard, 1853, Marcy's Rep. Red Riv., and Hall, 1s58, Geo. Rep. Iowa, p. 684. [Ety. proper name; krinon, lily.] Calyx conical or semielliptical; not ornamental; basals 5, usually anchylosed, very small inner cavity; subradials 5 , thick, usually anchylosed; radials $2 \times 5$; arms 10 ; azygous plates 3 or 4 ; column evidenced by a small cylindrical tube extending from a minute cicatrix at the center of the basals to the interior of the cup. Type A. dactyliformis.

carbonarius, Worthen, 1873, Geo. Sur. Ill., vol. 5, p. 566, Up. Coal Meas.

chesterensis, Worthen, 1873, Geo. Sur. Ill., vol. 5, p. 558, Kaskaskia Gr.

conicus, Owen \& Shumard, 1851, Jour. Acad. Nat. Sci, Phil., 2d. ser., vol. 2, p. 93, and Geo. Sur. Ill., vol. 5, p. 557, Kaskaskia Gr.

constrictus, Hall, 1858, Geo. Rep. Iowa, p. 687 , Kaskaskia Gr.

dacty liform is,

Troost, 1850 , described by Shumard, 1853, Marcy's R e p. Red. Riv., p. 199, Kaskaskia Gr.

gibbosus, $\mathrm{H}$ all, 1858, Geo. Rep. Iowa, $p, 686^{\circ}$, Kaskaskia Gr. globosus, Worthen, 1873, Geo. Sur. Ill., vol. 5 , p. 557, Kaskaskia Gr.

gracilis, T roost, 1850. Not defined.

hemisphericus, Worthen, 1882, Bull. No. 1, Ill. St. Mus. Nat. Hist., p. 37, and Geo. Sur. Ill.,

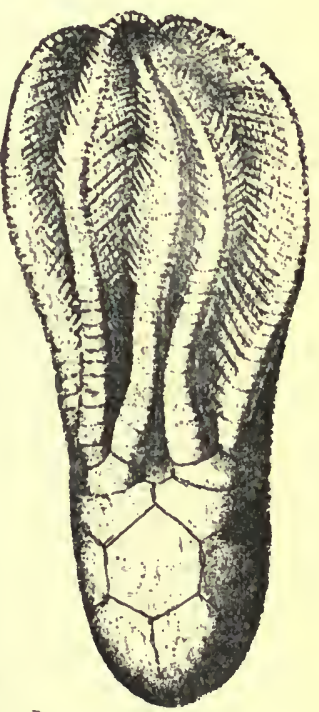
vol. 7 , p. 316, Fig. 240. - A gassizocrinus Kaskaskia Gr.

occidentalis,

Owen \& Shumard, 1852, (Poteriocrinus occidentalis,) Jour. Acad. Nat. Sci. Phil., vol. 2, p. 92, Kaskaskia Gr.

papillatus, Worthen, 1882, Bull. No. 1, III. St. Mus. Nat. Hist., p. 36, and Geo. Sur. Ill., vol, 7, p. 315, Kaskaskia Gr. pentagonus, Worthen, 1873, Geo. Sur. Ill., vol. 5, p. 556, Kaskaskia Gir.

tumidus, Owen \& Shumard, 1852, (Poteriocrinus tumidus, ) Jour. Acad. Nat. Sci. Phil., vol. 2, p. 90, Kaskaskia Gr.

Agelacrinus, Vanuxem, 1842, (Agelacrinites,) Geo. Rep. 3d Dist. N. Y., p. 158. [Ety. agele, herd; krinon, lily.] A thin, circular, parasitic disk ; upper face more or less convex, and composed of thin imbricating plates; ambuls cra consist- 
ing of a double series of alternating plates, forming convex ridges, constituting part of the upper face, and bearing two or more rows of ainbulacral pores; ovarian or anal aperture is situated within the azygous interambulacral area, surrounded by cuneiform plates. Type A. hamiltonensis.

billingsi, Chapman, 1860, Can. Jour., vol. 5, p. 358, Trenton Gr.

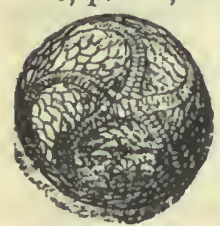

FIG. 241.-Agelnerinus cincinnatlensis.

hamiltonensis, Vanuxem, 1842, Geo. Rep. 3d Dist. N. Y., p. 158, Ham. Gir.

holbrooki, James, 1887. Jour. Cin. Soc. Nat. Ilist., vol. IO, p. 25. Hud. Riv. Gr.

kaskaskiensis, see Fehinodiscus kaskaskiensis.

pileus, Hall, 1866, Adv, sheets, 24th Rep. N. Y. St. Mus. Nat. Hist., 1. 214, and Ohio Pal., vol. 1, p. 56, Hud. Riv. Gr.

septembrachiatus, Miller \& Dyer, 1878 , Jour. Ciu. Soc. Nat. Hist., vol. 1, p. 27, Hud. Riv. Gir.

squamosus, Meek \& Worthen, 1868, Proc. Acad. Nat. Sci. Phil., 1). 35\%, and Geo. Sur. Ill., vol. 5, p. 573, Keokuk (ir.

stellatus, see Hemicystites stcllatus.

vorticellatus, 1 all, 1866, Adv. sheets, 24 th Rep. N. Y. St. Mus. Nut. Hist., p. 215, and Ohio Pal., vol. 1, p. 57, Hud. Riv. (ir.

Allagecrinus, Etheridge \& Carpenter, 188!, Ann. and Mag. Nat. Hist., p. 2Sl. [Ety. allage, change; krinon, lily.] Calyx minute, pyriform, without ormamentation; basals 5 , anchylosed ; radials $1 \times 5$; arms 10; interradials none; column round. Type $A$. austini.

carpenteri, Wachsmuth, 1882, Bull. No. 1, Ill. St. Mus. Nat. Hist., p. 40, and Geo. Sur. Ill., vol. 7, p. 341, Kaskaskia Gr.

Atcocrixus, Wachswuth and Springer, (in press,) Geo. Sur. Ill.,. vol. 8, p. 206. [Ety, allos, another; krinon, lily.] Calyx small; arms stout; basals 3 , small ; primary radials $3 \times 5$, first large, others smaller; secondary radials 2 or 3 , rounded, quadrangular ; arms composed of transverse plates; interradials two or more deeply impressed; column small; canal pentangular. Type A. typus.

typus, Wachsmuth \& Springer, (in press,) Geo. Sur. Ill., vol. 8, p. 207 , $\mathrm{Ni}$. agara Gr.

Allocystites, n. gen. [Ety. allos, another; kustis, bladder.] Small, irregular]y subelliptical, tapering below to a small column; plates polygonal, without definite order of arrangement and of very unequal size; all the plates poriferous; mouth near the margin of the summit; the plates which form it cover part of the body, and on approaching the orifice curve up so as to form part of the opening. The collector says when found it projected an eighth of an inch, and the plates forming the projection were accicieutally broken off. The ambulacral opening is upon the extreme height of the summit, and projects above the body, where it is cuvered by minute plates forming a pentagonal star. Type A. hammelli.

hammelli, n. sp., Niagara Gr. In addition to the characters above ascribed to the genus, the ranges of plitles, if in regulur series, would forni about six series; the first series are anchylosed so that two plates only ean be distinguished; in the second rauge there are seven plates; above this the plates are exiremely variable in form nnd size, no two of them being alike; only four plates are distinguished as forming the moutl, but there is no reasonable doubt there are five, and that one is narrow, and siturted between the mouth and umbulueral orifice, as is usual in this Iamily of Cystidians. The projecting mouth-plates and elevated imbulacrul opening specially characterize this genus and species. The specitic name is in honor of Mr. J. F. Hammell, of Matison, Indiana, who collected it in Jefferson County.
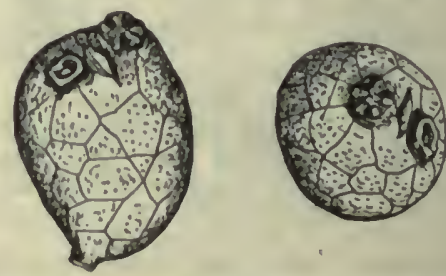

Fir. 242.-Allocystles hanuell. Slie and simmit view.

Allopiosallocizinus, Casseday \& Lyon, 1860, Proe. Am. Acad. Arts and Sci., vol. 5, p. 29.] Ety. alloprosallos, inclin. ing first to one side and then to anpther ; krinon, lily.] Turbinate; basals 3 ; primary radials $3 \times 5$; secondary radials $2 \times 10$; regular interradials 1 ; azygous plates 3 ; vault elevated, bearing a central tube or proboseis; arns 11 to 13 ; distinguished from Agaricocrinus by general form and fewer interradials. Type A. conicus.

conicus, Casseday \& Lyon, 1860, Proc. Am. Acad. Arts and Sci., vol. 5, p. 29, Warsaw Gr.

euconus, see Batocrinus euconus.

depressus, Casseday \& Lyon, 1860, Proc. Am. Acar. Arts and Sci., vol. 5, p. 31, Warsaw Gr. 
Ampheristocrinus, Hall, 1879, Desc. New Spec. Foss., p. 11, and 11th Rep. Geo. and Nat. Hist. Indiana, p. 278. [Ety. ampheristos, disputed; krinon, lily.] Turbinate, base attenuate; azygous area, large ; plates 6 ; basals 3 ; subradials 5 ; radials $1 \times 5$, with a narrow cicatrix on the middle of the top of each for the attachment of the arms; no regular interradials. Type A. typus.
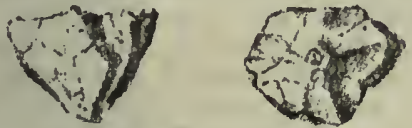

FIG. 243.-Ampheristocrlnus typus. Basal and side view of calyx.

typus, Hall, 1879, Desc. New Spec. Foss., p. 11, and 11th Rep. Geo. and Nat. Hist. Indiana, p. 278, Niagara Gr.

Amphoracrinus, Austin, 1848, Quar. Jour. Geo. Soc. Lond., vol. 4, p. 292, and Geo. Sur. Ill., vol. 5, p. 386. [Ety. umphora, cup; krinon, lily.] Body short, lobed, dome elevated, with tube or proboscis excentric on the azygous side; basals 3 ; primary radials $3 \times 5$; secondary radials $1 \times 10$; arıns numerous, variable, composed of a double series of plates; regular interradials 3 ; azygous interradials, 3 or 4 large ones and a few smaller ones; column round. Type A gilbertsoni.

americanus, see Agaricocrinus americanus. bellatrema, see Agaricocrinus bellitrema.

divergens, Hall, 1860, (Actinocrinus divergens,) Supp. Geo. Rep. Iowa, p. 36, and Geo. Sur. Ill., vol. 5, p. 388, Burlington Gr.

excarntus, see Agaricocrinus excavatus. inflatus, eee Agaricocrinus inflatus.

jerseyensis, Worthen, (in press,) Geo. Sur. Ill., vol. 8, p. 96, Kinderbook Gr.

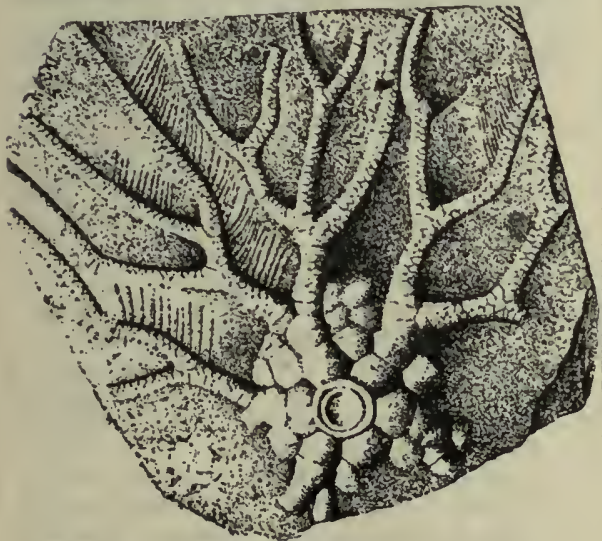

Fia. 244.-Amphoracrinus viminalis.

planobasalis, Hall, 1858, (Actinocrinus planobasalis,) Geo. Rep. Iowa, p. 19, Burlington Gr. Wachsmuth says it is a syn. for $A$. divergens. quadrispinus, White, 1862, (Actinocrinus quadrispinus,) Proc. Bost. Soc. Nat. Hist., vol. 9, p. 15, Burlington Gr. Wachsmuth says it is a syn. for $A$. divergens.

spinobrachiatus, Hall, 1860, (Actinocrinus spinobrachiatus,) Supp. Geo. Rep. Iowa, p. 6, and Geo. Sur. Ill., vol, 5, p. 389, Burlington $\mathrm{Gr}$.

viminalis, Hall, 1864, (Actinocrinus viminalis,) 17th Rep. N. Y. St. Mus. Nat. Hist., p. 54, and Ohio Pal., vol. 2. p. 165, Waverly Gr.

Amygdalocystites, Billings, 1854, Can. Jour., vol. 2, p. 270, and Can. Org. Rem., Decade 3, p. 63. [Ety. amygdalos, almond; kustis, bladder.] Body flattened, ovate, covered with nonporiferous plates arranged without order; ambulacral opening at the apex, mouth near by; arms recumbent, composed of a double series of plates; column round. Type A. florealis.

florealis, Billings, 1854, Can. Jour., vol. 2, p. 270, and Can. Org. Rem., Decade 3, p. 63, Trenton Gr.

florealis var. lævis, W. R. Billings, 1883, Trans. No. 4, Ottawa Field Nat. Club, p. 52, Trenton Gr.

huntingtoni. Wetherby, 1881, Jour. Cin. Soc. Nat. Hist., vol. 4, p. 177, Trenton Gr. radiatus, Billings, 1854, Can. Jour., vol. 2, p. 271 , and Can. Org. Rem., Decade 3, p. 65, Trenton Gr.

tenuistriatus, Billings, 1854, Can. Jour., vol. 2, p. 271, and Can. Org. Rem., Decade 3,

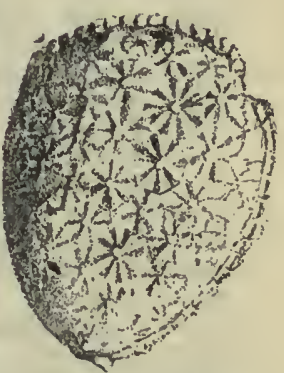

FIG. 245.-Amygdalocystites huntingtoul. p. 64, Trenton Gr.

ANCYRocrinus, Hall, 1862, 15th Rep. N. Y. St. Mus. Nat. Hist., p. 89. [Ety. ankura, grapnel; krinon, lily.] A bulb witl lateral ascending processes and a central column. But little is known of this genus. Type A. bulbosus.

bulbosus, Hall, 1862, 15th Rep. N. Y. St. Mus. Nat. Hist., p. 90, Ham. Gr.

spinosus, Hall, 1862, 15th Rep. N. Y. St. Mus. Nat. Hist., p. 90, Up. Held. Gr.

Anomalocrinus, Meek \& Worthen, 1868, Geo. of Ill., vol. 3, p. 327. [Ety. anomalos, irregular; krinon, lily.] Calyx depressed, irregularly saucer-shaped; basals 5 ; sometimes a 6 th intercalated one ; radials $1 \times 3$ and $2 \times 2$, the last ones truncated in the central part for the free arms, and curving over on the vault on either side, thus widely separating the arms; arms irregular, frequently bifurcating, composed of a single series of plates, round on the exterior; pinnules strong; vault convex 
and supposed to possess a tube or proboscis; column large and longitudinally from 5 to 20 partite. Type A. incurvus.

caponiformis, Lyon, 1869, (Ataxocrinus caponiformis,) Trans. Am. Phil. Soc., vol. 13, p. 464, and Jour. Cin. Soc. Nat. Hist., vol. 2, p. 109, Hud. Riv. Gr.

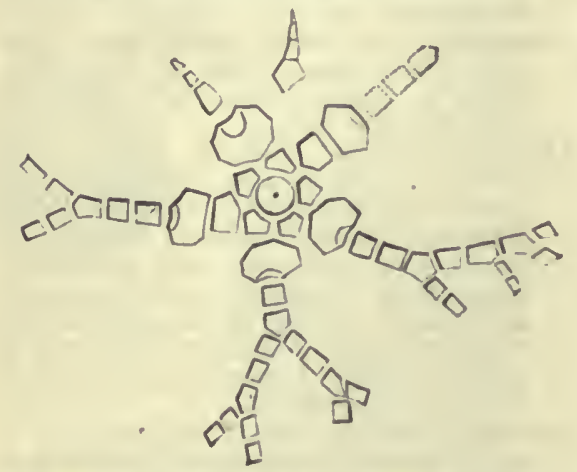

Fig. 240.-Anomalocrinus Incụrvus. Dagrau.

incurvus, Meek \& Worthen, 1865, (Heterocrinus incurvus,) Proc. Acad. Nat. Sci. Phil., p. 148, and Geo. Sur. Ill., vol. 3, p. 327, Hud. Riv. Gr.

A Nomalocystites, Hall, 1859, Pal. N. Y. vol. 3, p. 132. [Ety. anomalos, irregular; knstix, bladder.] Somewhat semielliptical, sides unequal, vertical oulline oval or ovoid; first series of plates 3 on the convex and 2 on the flat or concave side; second series 4 or $j$ on the convex side and 2 on the concave sicle; third series 4 on the convex and 1 on the other; succeeding series have smaller plates and the ajex is unknown; column large at the body and very rapidly tapering; no pores or pecti. nated rhombs. Type $A$. cornutus. Wetherby supposed this to be a Crustacean and gave it the name of Enoploura.
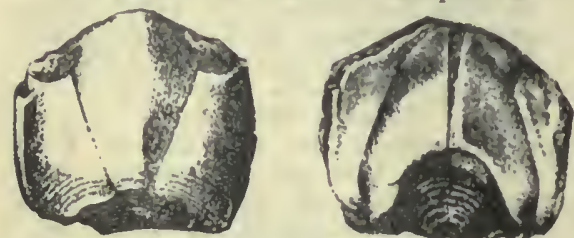

Fig. 217,-Anomalocystites brlanolies. Convex and flitlened sides.

balanoides, Meek, 1872, Am. Jour. Sci., 3 d ser., vol. 3 , p. $42 \%$, and Ohio Pal., vol. 1, p. 41. Hud. Riv. Gr.

cornutus, Hall, 1859, Pal. N. Y., vol. 3, p. 133 , Low. Held. Gr.

disparilis, Hall, 1859, Pal. N. Y., vol. 3, p. 145 , Oriskany sandstone.

huxleyi, Billings, 1858, (Ateleocystites huxleyi,) Can. Org. Rem., Decade 3, p. 72, Trenton Gr.
Anomaloides, Ulrich, 1878, Jour. Cin. Soc. Nat. Hist., vol. 1, p. 92. A word constructed of adjectives making it meaningless, contrary to the rul+ 8 of nomenclature, and the attempt to found a genus was made on a fossil fragment not understood.

reticulalus, Ulrich, 1878, Jour. Cin. Soc. Nat. Hist., vol. 1, p. 92, Hud. Riv. Gr. A fossil fragment not undersiood.

Aplocrstites, Forbes, 1848, Mem. Geo. Sir. Great Brit., vol. 2, p. 502. [Ety. apuim, pear; kustix, bladder.] Body ovoii, or oblong oval, angular and covered by four series of plates; first series has 4 plates: second series 5 ; third series 5 or 6 ; fourth series 5 or more; arms 4 , recumbent and filling sliallow grooves at the angles of the body, column rapidly tapering; ovarian sperture near the summit, on the anterior side; all the plates bearing calycine pores: a pectinated rhomb upon each sisle in the $3 d$ and fth series, and one on the anterior side in the $2 d$ series, but these may be variable. Type $A$. pentremitoides.

canadensis, Billings, 1566 , Catal. Sil. Foss. Antic., p. 90, Niagara Gr.

elegans, Hall, 1852, Pal. N. Y., vol. 2, p. 243 , Niagara $(i r$.

huronensis, Billings, 1866, Catal. Sil. loss. Antic., p. 91, Ningara lir.

imago, Hall, 1867, 20th Rep. $\mathbf{N}$. Y. St. Mus. Nat. Hist., p. 358, Niagara lir.

tecumseth, Billings, 1866, Catal. Sil. Foss. Antic., p. 91, Niagara $(i r$.

Aracisocust8, Meek \& Worthen, 1866, rieo. Sur. Ill., vol. 2, 1). 177. [1;y. arachne, spider; krinon, lily.] Calyx swall, resembling Cyathocrinus, but more depressed; basils 5; subradials 5 ; radials : $\times 55$; arms long, robust, spreading, furrow der.); no pinnules; azygous plate supporting a laterul tube ; column round. Type $\AA$. bulbosus.

bulbosus, Hull, 1860, (Cynthorrinus bulbosus,) 15th Rep. N. Y. St. Mis. Nat. Hist., p. 123, Up. Held. (ir.

extensus, Wachsmuth \& Springer, 1879, Revis. Palwoerinoidea, p. 93, I Iam. (ir. knappi, Wachsmuth \& Springer, 1879 , Revis. Palrocrinoidea, p. Y:), Hain. (ir. pisiformis, Roemer, 1860 , (Poteriocrinus pisiformis,) Sil. Fauna W. Tenn., p. 54, Niagara lir. Wachsmuth says it is a Lecanocrinus.

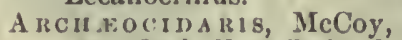
1844, Carb. Foss. Ireland, p. 173. [Ety. archaios, ancient; ridaris, turban.] Spherical; ambulacra narrow, each composed of two ranges of plates, with two pores in each plate; interambulacral plates large, thin, eacli with a large, perforated, central tubercle, surrounded, at its base, by a smooth ring, and rounded, at the base, for the artic- 
ulation of a primary spine, and the whole surrounded by smaller tubercles for the articulation of secondary spines; mouth surrounded by numerous imbricating plates; jaws strong with mesial suture; primary spines large, variously ornamented. Type A. urii.

aculeata, Shumard, 1858, Trans. St. Louis Acad. Sci., vol. 1, p. 223, Permian Gr.

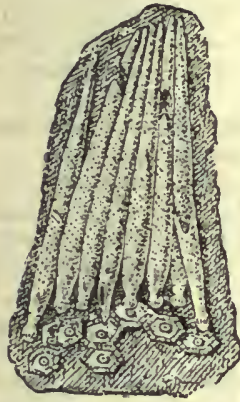

FIG. 249. - Archæocidaris agassizi, showing spines. agassizi, Hall, 1858, Geo. Rep. Iowa, p. 698 , Burlington Gr. biangulata, Shumard, 1858, Trans. St. Louis Acad. Sci. vol. 1 , p. 224, Coal Meas.

cratis, White, 1876 , Geol. of Uinta Mountains, p. 109, and Cont. to Pal., No. 6, p. 130 , Lower Aubrey Gr.

dininni, White, 1880 , Proc. U. S. Nat. Mus., vol. 2, p. 260, and Cont. to Pal.,

No. 6, p. 131, Up. Coal Meas.

edgarensis, Worthen \& Miller, 1883, Geo. Sur. Ill., vol. 7, p. 337, Up. Coal Meas.

gracilis, Newberry, 1861, Ives Col. Ex. Ex., p. 117, Up. Carb.

illinoisensis, Worthen \& Miller, 1883, Geo. Sur. Ill., vol. 7, p. 338, St. Louis Gr.

keokuk, Hall, 1858, Geo. Rep. Iowa, p. 699, Keokuk Gr.

longispina, Newberry, 1861, Ives Col. Ex. Ex., p. 116, Up. Carb.

megastylus, Shumard, 1858, Trans. St. Louis Acad. Sci., vol. 1, p. 225, Up. Coal Meas.

mucronata, Meek \& Worthen, 1860, Proc. Acad. Nat. Sci. Phil., p. 395, and Geo. Sur. Ill., vol. 2, p. 295, Kaskaskia Gr.

newberryi, Hambach, 1884, Trans. St. Louis Acad. Sci., vol. 4, p. 548, Kaskaskia Gr.

norwoodi, Hall, 1858, Geo. Rep. Iowa, p. 701, Kaskaskia Gr.

ornata, Newberry, 1861, Ives Col. Ex. Ex., p. 116, Up. Carb.

shumardana, Hall, 1858, Geo. Rep. Iowa, p. 699, Warsaw Gr.

spinoclavata, Worthen \& Miller, 1883, Geo. Sur. Ill., vol. 7, p. 337, Coal Meas. triplex, White, 1882, Rep. Carb. Invert. Foss. New Mex., p. xxii, Coal Meas.

triserrata, Meek, 1872, Pal. E. Neb., p. 151, Up. Coal Meas.

trudifera, White, 1874, Rep. Invert. Foss., p. 17, and Geo. Sur. W. 100 th Mer., vol. 4, p. 104 , Carb.

verneuiliana, Swallow, 1858, Trans. St. Louis Acad. Sci. This name was preoccupid by King. The species is A. aculeata.

wortheni, Hall, 1858, Geo. Rep. Iowa, p. 700 , St. Louis Gr.
Archeochinus, Wachsmuth \& Springer, 1881, Proc. Acad. Nat. Sci. and Rev. Palæocrinoidea, p. 189. [Ety. urchaios, ancient; krinon, lily.] Basals 5; subradials 5 ; primary radials $3 \times 5$; secondary radials 3 or $4 \times 10$; median line of radial plates keeled as in Glyptocrinus ; interradial areas wide; arms composed of a double series of plates; column round. Tyne A. lacunosus.

desideratus, Billings, 1885, Trans. Ottawa Field Nat. Club, p. 248, Trenton Gr.

lacunosus, Billings, 1857, (Glyptocrinus lacunosus,) Rep. of Prog. Geo. Sur. Can., p. 261, and Org. Rem., Decade 4, p. 61, Trenton Gr.

marginatus, Billings, 1857, (Glyptocrinus marginatus,) Rep. of Prog. (reo. Sur. Can., p. 260, and Org. Rem., Decade 4, p. 59, Trenton Gr.

microbasalis, Billings, 1857, (Rhodocrinus microbasalis,) Rep. of Progr. Geo. Sur. Can., p. 264, and Org. Rem., Decade 4, p. 63 , Trenton Gr.

pyriformis, Billings, 1857, (Rhodocrinus pyriformis,) Rep. of Prog. Geo. Sur. Can., p. 262, and Org. Rem., Decade 4, p. 61, Trenton Gr.
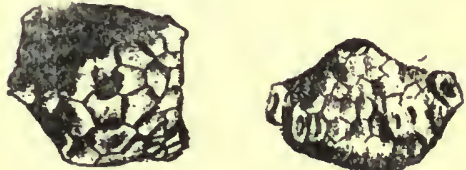

FIG. 250.-A rehæocrinus sculptus.

sculptus, S. A. Miller, 1882, Jour. Cin. Soc. Nat. Hist., vol. 5, p. 83 and 117 , Trenton Gir.

Arthracantha, Williams, 1883, Proc. Am. Phil. Soc., p. 84. [Ety. arthron, joint; akantha, spine.] Calyx bowl-shaped; plates of body and arms covered with spine-bearing tubercles; basals 3 ; primary radials $3 \times 5$, the lower one large, the others small; an azygous interradial as large as the primary radials rests upon the basals, and is followed by numerous small plates; regular interradials small; arms 10 , bearing pinnules; column round. Type A. ithacensis.

carpenteri, Hinde, 1885, (Hystricrinus carpenteri,) Ann. and Mag. Nat. Hist., p. 162, Ham. Gr. Probably a syn. for A. punctobrachiata.

ithacensis, Williams, 1883, Proc. Am. Phil. Soc., p. 83, Ham. Gr.

punctobrachiata, Williams, 1883, Proc. Am. Phil. Soc., p. 83, Ham. Gr.

Aspidocrinus, Hall, 1859. Pal. N. Y., vol. 3, p. 122. [Ety. aspis, shield; krinon, lily.] Calyx broadly circular, depressed, bemispheric or scutelliform ; upper margin plain or plicate exteriorly; articulating edges irregular; point for attachment of column small. Type A. scutelliformis. 
callosus, Hall, 1859, Pal. N. Y., vol. 3, p. 123. Low. Held. Gr.

digitatus, Hall, 1859, Pal. N. Y., vol. 3, p. 123, Low. Held. Gr.

scutelliformis, Hall, 1859 , Pal. N. Y., vol. 3, p. 122, I.ow. Held. Gr.

Asterias, Lamarck, 1815, Hist. Nat. Anim. sans Vert. Not Palæozoic. anthonyi, see Palreaster jamesi. antiqua, see Palæaster antiqua. antiquata, see Palaeaster antiquata. matutina, see Palæaster matutina.

Asterocrinus, Lyon, 1857, Geo. Sur. Ky., vol. 3. This name was preoccupied by Munster. See Pterotocrinus. capitalis, see Pterotocrinus capitalis. coronarius, see Pterotocrinus coronarins.

Astrios, Troost, 1850, Catalogue. Not defined.

tennesseex, Troost, 1850. Not defined. basals and subradials; basals 5 ; subradials 5 ; long, narrow, irregular; 3 hexagonal, 2 heptagonal; azygous radial, non-arm bearing, the other four supporting estch from 2 to 5 brachials; the eight arms give off branching armlets; azygous plate large, resting upon two subradials; column pentangmar. Type A. delicatus.

delicatus, Wachsmuth \& Springer, 1886 , Rev. Pal., pt. 3, p. 22:, Burlington Gr. robustus, Wachsmuth \& Springer, 1856, Rev. Pal., pt. 3, p. 223, Burlington or base of Keokuk Gr.

Balanocrinus, Troost, 1850 . This name was preoccupied. See Lampterocrinus.

inflatus, see Lampterocrinus inflatus.

Barycrinus, Wachsmuth, 1868, Proc. Acad. Nat. Sci., p. 338. [Ety. barus, heavy; krinon, lijy.] Distinguished from Cyathocrinus by being more robust, having thicker plates, and a shisl. lower cup; there are usually two azygous plates, while in Cyathocrinus there is never more than one; there are never more than two brachials, and these are shorter and wider than in Cyathocrinus; the arms are shorter, lieavier, and have II arrower grooves; the column is stouter; subpentagonal and longitudinally five partite, with a highly or. ganized central canal. Type B. angulatus.

angulatus, Meek \&

F1a. "51.-Barycrinus wnchkmuthl. Dtagram.
Astrocrinites, Conrad in Catalogue Ann. Geo. Rep., 1840-41. This name was proposed, but not defined; moreover it was preoccupied.

pachydactylus, see Mariacrinus pachydactylus.

Astylocrinus, Roener, 1854, Leth. Geo., p. 229 , syn. for Agassizocrinus.

lacvis, syn. for Agassizocrinus dactyliformis.

Ataxocrinus, I.yon, 1869, syn. for Anomalocrinus.

caponiformis, see Anomalocrinus caponiformis.

Ateleocystites, Billings, 1858, Can. Org. Rem., Decade 3, p. 72, syn. for Anomalocystites.

huxleyi, see Anomalocystites huxleyi.

Atelestocrinus, Wachsmuth \& Springer, 1886, Rev. Pal., pt. 3, p. 221. [Ety. atelestos, incomplete; krinon, lily.] Calyx elongate, bell-shaped, sides concave, restricted along the suture between
Worthen, 1860, (Cyathocrinus angulatus,) Proc. Acad. Nat. Sci. Phil., p. 391, and Geo. Sur. 1ll., vol. 2, p. 234, Keokuk Gr.

bullatus, Hall, 1858, (Cyathocrinus bullatus,) Geo. Sur. Iowa, p. 624, Keokuk Gr.

cornutus, Owen \& Slummard, 1850, (Cyathocrinus cornutus,) Jour. Acad. Nat. Sci., $2 d$ ser., vol. 2, p. 63, and Geo. Sur. Wis., Iowa, and Minn., p. 591, Burlington Gr.

crassibrachiatus, Hall, 1860, (Cyathocrinus crassibrachiatus,) Sup. to Geo. Sur.

Iowa, p. 60, Keokuk Gr.

geometricus, Meek \& Worthen, 1873, Geo. Sur. Ill., vol. 5, p. 485, Keokuk Gr.

herculens, Meek \& Worthen, 1868, Proc. Acad. Nat. Sci. Phil., p. 341, and Geo. Sur. Ill., vol. 5, p. 485, Keokuk Gr. hoveyi, Hall, 1861, (Cyathocrinus hoveyi,) Dese. New Crin., p. 5, and Geo. Sur. Il]., vol. 5, p. 486, Keokuk Gr. 
kelloggi, White, 1862, (Cyathocrinus kelloggi,) Proc. Bost. Soc. Nat. Hist., p. 8, Krokuk Gr.

magister, Hall, 1858, (Cyathocrinus magister, ) Geo. Sur. Iowa, p. 628, Keokuk Gr.

magnificus, Meek \& Worthen, 1868, Proc. Acad. Nat. Sci. Phil., p. 340, and Geo. Sur. Ill., vol. 5, p. 483, Keokuk Gr.

mammatus, Worthen, 1873, Geo. Sur. Ill., vol. 5 , p. 486, Keokuk Gr.

pentagonus, Worthen, 1873, Geo. Sur. Ill., vol. 5, p. 487, Keokuk Gr.

protuberans, Hall, 1858, (Cyathocrinus protuberans,) Geo. Sur. Iowa, p. 626, Keokuk Gr. Wachsmuth says it is a syn. for B. bullatus.

rhombiferus, Owen \& Shumard, 1850, (Poteriocrinus rhombiferus, Jour. Acad. Nat. Sci. Phil., 2d ser., vol. 2, and Geo. Wis., Iowa, and Minn., p. 595, Burlington Gr.

sculptilis, Hall, 1860, (Cyathocrinus sculptilis,) Supp. Geo. Sur. Iowa, p. 59, Burlington $\mathrm{Gr}$.

solidus, Hall, 1861, (Cyathocrinus solidus,) Desc. New Crin., p. 5, and Bost. Jour. Nat. Hist., vol. 7, p. 293, Burlington Gr.

spectabilis, Meek \& Worthen, 1869, Proc. Acad. Nat. Sci. Phil. and Geo. Sur. Ill., vol. 5 , p. 530 , St. Louis Gr.

spurius, Hall, 1858, (Cyathocrinus spurius,) Geo. Sur. Iowa, p. 625, $\mathrm{Ke}$ okuk Gr.

stellatus, Hall, 1858, (Cyathocrinus stellatus,) Geo. Sur. Iowa, p. 623, Keokuk Gr.

striatus, Worthen, 1875, Geo. Sur. Ill., vol. 6 , p. 515 , Keokuk Gr.

thomae, Hall, 1860, (Cyathocrinus thomae) Supp. Geo. Sur. Iowa, p. 61, Warsaw Gr.

tumidus, Hall, 1858, (Cyathocrinus tumidus,) Geo. Sur. Iowa, p. 624, Keokuk Gr.

wacbsmuthi, Meek \& Worthen, 1861, (Cyathocrinus wachsmuthi,) Proc. A cad. Nat. Sci. Phil., p. 136, and Geo. Sur. Ill., vol. 3, p. 482, Burlington Gr.

Batocrinus, Casseday, 1854, Deutsche Zeitschr, d. Geol. Gesellsch, vol.6, p.237, and Geo. Sur. Ill., vol. 2, p. 150. [Ety. batos, prickly bush; krinon, lily.] Calyx biturbinate or globose; basals 3 ; primary radials $3 \times 5$; secondary radials $2 \times 10$; tertiary radials $2 \times 2 \times 10$; regular interradials 1 to 5 ; azygous plates 6 to 12 or more; tertiary radials meet so as to cut off the connection of the interradials with the dome plates; vaultelevated; tube or proboscis nearly central ; arms 18 to 26 or 36 to 40 ; pinnules ; column round, distinguished from Actinocrinus by the quadrangular second radial instead of hexagonal; by the number of plates in the interradial areas; by the number of secondary radials; and by having a double series of plates in each arm from the beginning. Type B. icosidactylus. æqualis, Hall, 1858, (Actinocrinus æqualis, ) Geo. Rep. Iowa, p. 592, Burlington Gr.

æquibrachiatus, McChesney, 1860, (Actinocrinus æquibrachiatus,) New Pal. Foss., p. 25, and Trans. Chi. Acad. Sci., p. 18, Burlington Gr.

æquibrachiatus, var. alatus, Hall, 1861, (Actinocrinus æquibrachiatus var. alatus, Bost. Jour. Nat. Hist., vol. 7, p. 263. Wachsmuth says it is a syn. for $B$. æquibrachiatus.

andrewsanus, McChesney, 1859, (Actinocrinus andrewsanus,) New Pal. Foss., p. 27, and Trans. Chi. Acad. Sci., p. 20, Burlington $\mathrm{Gr}$.

asteriscus, Meek \& Worthen, 1860, (Actinocrinus asteriscus,) Proc. Acad. Nat. Sci. Phil., p. 385, and Geo. Sur. Ill., vol. 2, p. 207, Burlington Gr.

biturbinatus, Hall, 1858, (Actinocrinus biturbinatus,) Geo. Sur. Iowa, p. 616, Keokuk Gr.

calyculus, Hall, 1860, (Actinocrinus, calyculus,) Supp. Geo. Sur. Iowa, p. 55, Warsaw Gr.

calyculus var. hardinensis, Meek \& Worthen, 1866, (Actinocrinus caylculus var. hardinensis,) Proc. Acad. Nat. Sci. Phil., p. 253, Warsaw Gr.

caroli, Hall, 1860, (Actinocrinus caroli,) Supp. Geo. Rep. Iowa, p. 54, Warsaw Gr.

cassedayanus, Meek \& Worthen, 1868, Proc. Acad. Nat. Sci. Phil., p. 353, and Geo. Sur. Ill., vol. 5, p. 370, Burlington $\mathrm{Gr}$.

christyi, Shumard, 1855, (Actinocrinus christyi,) Geo. Sur. Mo., p. 191, Burlington Gr.

clavigerus, Hall, 1860, (Actinocrinus clavigerus,) Supp. Geo. Sur. Iowa, p. 44, Burlington Gr. Wachsmuth says it is a syn. for B. similis.

clypeatus, Hall, 1860, (Actinocrinus clypeatus,) Supp. Geo. Sur. Iowa, p. 12, and Geo. Sur. Ill., vol. 2, p. 150, Burlington Gr.

discoideus, Hall, 1858, (Actinocrinus discoideus,) Geo. Rep. Iowa, p. 594, Burlington Gr.

dodecadactylus, Meek \& Worthen, 1861, (A c tin o crinus dodecadactylus,) Proc. Acad. Nat. Sci. Phil., p. 13, and Geo. Sur. Ill., vol. 2, p. 205, Burlington $\mathrm{Gr}$.

doris, Hall, 1861 , (A) Tin , 1861 FIG. 252.- Batocrinus (Actinocrinus dodecadactylus. Dladoris,) Desc. New gram.

Crinoidea, p. 15,

Burlington Gir. Wachsmuth says it is a syn. for B. æqualis.

euconus, Meek \& Worthen, 1860, (Alloprosallocrinus euconus,) Proc. Acad. Nat. Sci. Phil., p. 164, Warsaw Gr. 
formosus, Hall, 1860, (Actinocrinus formosus,) Supp. to Geo. Sur. Iowa, p. 30, Burlington Gr. Wachsmuth says it is a syn. for B. discoideus.

hageri, McChesney, 1860, (Actinocrinus hageri,) New Pal. Foss., p. 28, and Trans. Cbi. Acad. Sci., p. 21, Burlington $\mathrm{Gr}$.

icosidactylus, Casseday, 1854, (Actino. crinus icosidactylus,) Weitsch. Deutsch. Geol. Gesellsch, vol. 6, p. 238, Warsaw Gr.

indianensis, Casseday \& Lyon, 1859, Am. Jour. Sci. and Arts, 2d ser., vol. 29, p. 75, Keokuk G̈r.

inornatus, Hall, 1860, (Actinocrinus inornatus,) Supp. to Geo. Sur. Iowa, p. 3t, Bnrlington Gr. Wachsmuth says it is a syn. for B. clypeatus.

irregularis, Casseday, 185ّ, Zeitsch. Deutsch. Geol. Gesell., vol. 6. p. 238, Warsaw Gr.

lagunculus, Hall, 1860, (Actinocrinus lagunculus,) Supp. to Geo. Sur. Iowa, p. 41, Warsaw Gr.

laura, Hall, 1861, (Actinocrinus laura, Desc. New Crinoldea, p. 15, Burlington Gr.

lepidus, Hall, 1860, (Actinocrinus lepidus, ) Supp. to Geo. Sur. Iowa, p. 32, Burlington Gr.

longirostrib, Hall, 1858, (Actinocrinus longirostris,) Geo. Sur. Iowa, p. 589, Burlington Gr.

lovii, Wachsmuth \& Springer, 1881, Proc. Acad. Nat. Sci., p. 342, Burlington $\mathrm{Gr}$.

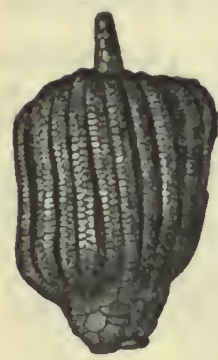

macbridii, Wachsmuth \& Springer, (in press,) Geo. Sur. Ill., vol. 8, p. 172, Kinderhook Gr.

montgomeryensis, Worthen, 1854, Bull. No. 2, Ill. St. Mus. Nat. Hist., [. 35, and Geo.

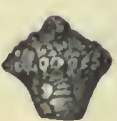

Sur. III., vol. $8, \mathrm{p}$. 83, Keokuk Gr.

mun $d u l u s$, Hall, 1860, (Actinocri: nus mun-

FIG. 253.-Batocrinus macbridil.

Iowa, p. 39 ,

dulus, Supp.
Warsaw Gr.

nashvillae, Troost, Hall, 1858, (Actinocrinus nashvilla,) Geo. Sur. Iowa, p. 609, Keokuk Gr.

nashvillae var. subtractus, White, 1863 , (Actinocrinus nashvillæe var. subtractus,) Proc. Bost. Soc. Nat. Hist., vol. 9, p. 16, Keokuk Gr.

neglectus, see Eretmocrinus neglectus.

oblatus, Hall, 1860, (Actinocrinus oblatus,) Supp. to Geo. Sur. Iowa, p. 38, Burlington Gr. Wachsmuth says it is a syn. for $B$. rotundus. papillatus, Hall, 1S60, (Actinocrinus papillatus,) Supp. to Geo. Sur. Iowa, p. 29, Burlington Gr. Wachsmuth says it is a syn. for B. clypeatus.

pistilliformis, Meek \& Wortlen, 1865, Proc. Acad. Nat. Sci. Phil., p. 153, and Geo. Sur. 1ll., vol. 2, p. 151 Waverly or Kinderhook Gr.

pistillum, Meek \& Worthen, 1865, (Actinocrinus pistillus,) Proc. Acad. Nat. Sci. Phil., p. 152, and Geo. Sur. III., vol. 3 , p. 472 , Burlington $\mathrm{Gr}$.

planoliscus, Hall, 1860, (Actinocrinus planodiscus, Supp. to Geo. Sur. Iowa, p. 45, Warsaw Gr.

pyriformis, Shumard, 1855, (Actinocrinus pyriformis,) Geo. Sur. Mo., p. 192, Burlington Gr.

quasillus, Meek \& Worthen, 1869, Proc. Acad. Nat. Sci. Phil., p. 352, and Geo. Sur. Ill., vol. 5. p. 369, Burling. ton Gr.

rotundus, Yandell \& Shumard, 1855, (Actinocrinus rotundus,) Geo. Sur. Mo. p. 191, Burlington Gr.

similis, Hall, 1860, (Actinocrinus similis,) Supp. to Geo. Sur. Iowa, p. 40, Keokuk Gr. sinuosus, Hall, 1860, (Acti- dus. nocrinus sinuosus, ) Supp. to Geo. Sur. Iowa, p. 26 Burlington Gr.

steropes, Hall, 1860, (Actinocrinus steropes,) Supp. to Geo. Sur. Iowa, p. 43, Keokuk Gr.

subacqualis, McClıesney, 1860, (Actino. crinus subrequalis,) New Pal. Foss. p. 17, and Trans. Chi. Acad. Sci., p. 13, Burlington Gr. Wachsmuth says it is a $8 y n$. for B. discoideus.

subconicus, Worthen, 1884, Bull. No. '2, III. St. Mus. Nat. Hist., J, 26, and Geo. Sur. Ill., vol. 8 , p. 8t, Keokuk Gr.

trochiscus, Meek \& Worthen, 1868, Proc. Acad. Nat. Sci. Phil., p. 354 , and Geo. Sur. Ill., vol. 5, p. 372, Burlington Gr.

turbinatus Hall, 1858, (Actinocrinus turbinatus,) Geo. Rep. Iowa, p. 587, Burlington Gr.

turbinatus var. elegans, (Actinocrinus turbinatus var elegans,) Geo. Rep. Iowa, p. 588, Burlington Gr.

unionensis, Worthen, (in press,) Geo. Sur. Ill., vol. 8, p. 84, St. Lau is Gr.

wachsmuthi, White, 1880, (Actinocrinus wachsmuthi,) 12 th Rep. U. S. Geo. Sur. Terr., p. 162, and $2 d$ Rep. Ind. Geo. Sur., p. 510 Keokuk Gr.

whitii, Wachsmuth \& Springer, 1881, Proc. Acad. Nat. Sci., Phil., p. 343, Keokuk Gr.

yandelli, Shumard, 1857, (Actinocrinus yandelli,) Trans. St. Louis Acad. Sci., vol. 1, p. 76 , and Geo. Sur. Ill., vol. 5, p. 341, Keokuk Gr. 
Belem nocrinus, White, 1862 , Proc. Bost. Soc.

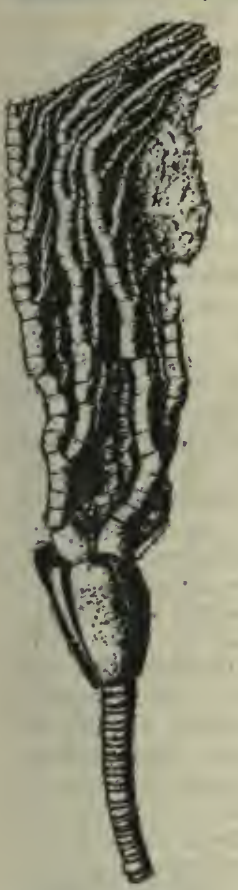

FiG. 255.-Belemnocrinus ty pus.

Nat. Hist., vol. 9, p. 14. [Ety. belemnon, dart, javelin ; krinon, lily.] Basals 5, large, long, narrow and ofirregular shape; radials 5 ; arms 10 , with one or two syzygies in each joint; pinnules long, arm. like, and bifurcating; azygous plate rests between radials, and upon one of the basals, and supports a large ventral sac composed of numerous hexago. nal plates; column pentagonal, with or without lateral cirrhi. Type B. typus.

florifer, Wachsmuth \& Springer, 1877, Am. Jour. Sci. and Arts, $3 d$ ser., vol. 13, p. 256 , Burlington Gr.

pourtalesi, Wachsmuth Jour. Sci. and Arts, 3 d ser., vol. 13, p. 258 , Burlington Gr.

typus, White, 1862 , Proc. Bost. Soc. Nat. Hist., vol. 9, p. 14, \& Springer, 1877, Am. Burlington Gr.

whitii, Meek \& Wortlen, 1866, Proc. Acad.

Nat. Sci. Phil., p. 251, and Geo. Sur.

Ill., vol. 3 , p. 463 , Burlington Gr.

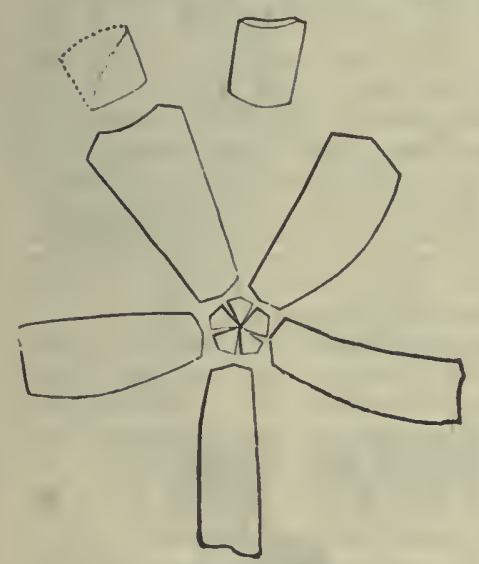

Frg. 256.-Belemnocrinus whitil.

Diagram $\times 2$.

Blastoidocrinus, Billings, 1859, Can. Org. Rem., Decade 4, p. 18. [Ety. blastos, a bud ; eidos, form ; krinon, lily.] 'The general form is like a Pentremites; basals do not rest upon the top of the column, but have their inner edges turned upward, and the column passes on into the visceral cavity; the deltoids occupy the whole space between the pseudambulacra; the orifices unknown; column round. Type B. carcharidens.

carcharidens, Bill ings, 1859, Geo. Sur. of Can., Dec. ade 4, p. 18, Chazy Gr.

Brachiocrinus, Hall, 1859, Pal. N. Y., vol. 3, p. 118. [Ety. brachium, an arm; krinon, 1 i $1 \mathrm{y}$.]

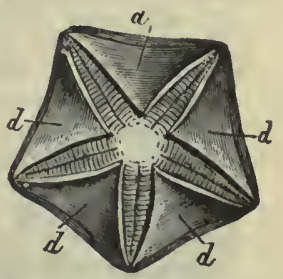
Founded upon Fig. 257.-Blastoidocr. arms rounded at nus varcharidens. $d$, the base, com- deltoid plates extending the whole posed of single ar- length of the pseudticulating plates ambulacra.

having thickened, node-like joints, and bearing pinnules. Type B. nodusarius. nodosarius, Hall, 1859, Pal. N. Y., vol. 3, p. 118, Low. Held. Gr.

Bursacrinus, Meek \& Wortlien, 1861, Proc. Acad. Nat. Sci. Phil., p. 136. [Ety. bursa, purse; krinon, lily.] Calyx somewhat like Graphiocrinus, but arms widely different; basals 5 ; subradials 5 ; radials $2 \times 5$; regular interradials 0 ; azvgous interradial 1 ; arms wide, flat, jointing below, in compact series, and bifurcating above. Type B. wachsmuthi.

confirıatus, White, 1862 , Proc. Bost. Soc. Nat. Hist., vol. 9, p. 11, Burlington Gr. wachsmuthi, Meek \& Worthen, 1861, Proc. Acad. Nat. Sci. Phil., p. 137, and Geo. Sur. Ill., vol. 3, p. 479, Burlington Gr.

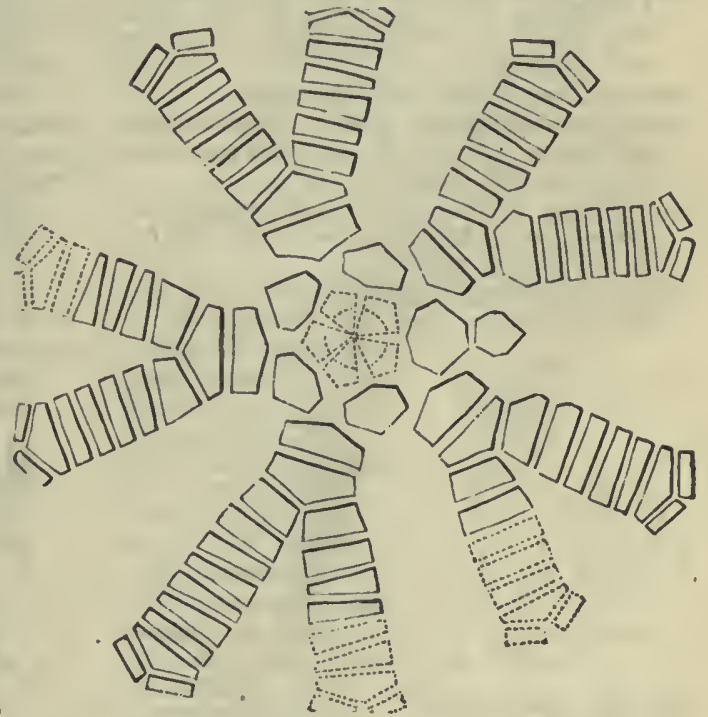

F1G. 258.-Bursacrinus wachsmuthi. Dlagram.

Cacabocrinus, Troost, 1850. Never desuribed. The fossils referred to it belong to Dolatocrinus. 
Calathocrinus, Hall, 1861. The name was preoccupied by Von Meyer in 1848. See Teleiocrinus.

Calchocrinus, Hall, 1852, Pal. N. Y., vol. 2, p. 352 , and 13 th Rep. N. Y. St. Mus. Nat. Hist., p. 122. [Ety. culceus, shoe; krinon, lily.] Base, a single subtriangular or semioval plate, composed of four anchylosed pieces, with cicatrix, for columnar attachment at lower angle; body, above the base, consisting of 5 or 7 plates, of which two are much the larger; a central, elongated plate separates the two large lateral radial plates, and bears an arm; lateral radial plates, each, support brachials that bear bifurcating arms;" azygous side arched and composed of 4 or more plates, after which a free arm arises. Type C. chrysalis.

articulosus, Billings, 1859, (Heterocrinus articulosus,) Can. Org. Rens., Decade 4, p. 51, Trenton Gr.

barrandii, Walcott, 1883, 35th Rep. N. Y. St. Mus. Nat. Hist., p. 212, Trenton Gr. barrisi, see Deltacrinus barrisi.

bradleyi, see Deltacrinus bradleyi.

chrysalis, Hall, 1860, (Cheirocrinus chrysalis,) 13th Rep. Y. Y. St. Mus. Nat. Hist., p. 123, Niagara Gr.

clarus, see Deltacrinus clarus.

dactylus, see Deltacrinus dactylus.

furcillatus, Billings, 1887, Trans. Ottawa Field Nat. Club, vol. 3, p. 51, Trentou Gr. inxqualis, Billings, 1859, (Heterocrinus insequalis,) Can. Org. Rem., Decade 4, p. 51, Trenton Gr.

lamellosus, Hall, 1860, (Cheirocrinus lamellosus,) 13th Rep. N. Y. St. Mus. Nat. Hist., p. 123, Burlington Gr. Not well defined.

nodosus, see Deltacrinus nodosus.

perplexus, Sliumard, 1866, (Cheirocrinus perplexus,) Trans. St. Iouis Acad. Sci., vol. 2 , p. 358 , Keokuk Gr.

punctatus, Ulrich, 1856, (Cremacrinus punctatus,) 14th Rep. Geo. Sur. Minn., p. 107, Trenton Gr.

radicula, Ringueberg, 1882, Jour. Cin. Soc. Nat. Hist., vol. 5, p. 120, Niagara Gr.

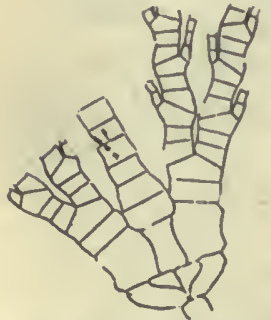

Fig. 259.-Calceocrinus rugusus. robustus, Worthen,

(in press, Geo. Sur. Ill., vol. 8, p. 92 , Keokuk Gr. rugosus, Billing 8 , 1887, Trans. Ottawa Field Nat. Club, vol. 3, p. 53, Trenton $\mathrm{Gr}$.

stigmatus, see Deltacrinus stigmatus. tunicatus, $\mathrm{H}$ a ll, 1860, (Cheirocrimus tunicatus, ) 13th Rep. N. Y. St. Mus. Nat. Hist., p. 124, Keokuk Gr. ventricosus, Hall, 1860, (Cheirocrinus ventricosus,) 13th Rep. N. Y. St. Mus. Nat. Hist., p. 123, Burlington Gr. wachsmuthi, see Deltacrinus wachsmuthi. Callocystites, Hall, 1S52, Pal. N. Y., vol. 2, p. 238. [Ety. kallos, beautiful; kustis, b ladder. ] Ovoid; 1st series of plates 4 ; $2 \mathrm{~d}$ series 8 ; $3 \mathrm{~d}$ series about the same number; small plates at the apex; arms rfcumbent, resting in a small shallow groove; pectinated rhombs in three pairs; oral, ovarian and anal apertures. Type C. jewetti.

jewetti, Hall, 1852 Pal $\mathrm{Y}$ Y Fro.260.CalN.Y. 100 y slltes

tripectinutus, Ringueberg, 1886, Bull. Buf. Soc. Nat. Sei., vol. 5, p. 12, Niagara Gr. Camanocirixus, Hall, 1879, 28uh Rev. N. Y. St. Mus, Nat. Hist., p. 205. [Ety. kamara, arching chambers; krincm, lily.] Body large, unsymmetrical, externally lobed, chambesed within and bearing no arms; wall of the dome composed of two layers, the infolding of the inner one forming the partitions dividing the chambers; subcircular area in the basal portion composed of spreading, radiciform, bifurcating rays, composed of plates resembling those of a crinoid column, and connected ly irrt gular polygonal plates; ambulacral openings between bifureations near the outer rim of the area; column eylindrical, jnterual canal fiverayed. Type C. stellatus.

clarki, Няll, 1879, 28th Rep. X. Y. St. Mus. Nat. Ilist., p. 209, Low. Held. Cir.

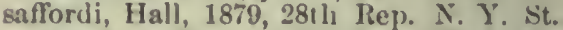
Mus. Nut. Hist., p. 208, Low. Held. Gr. stellatus, Ilall, 1879, 28th Rep. N. Y. St. Mus. Nat. Hist., p. 207, Low. Held. Gr. Campanulites, Troost, 1850. Not defined. tessellatus, Troost, 1850. Not defined.

Canistrocrinus, Wachsmutl \& Springer, 1885, Palaeocrinida, vol. 1, pt. "3, p. 94. Founded upon Glyptocrinus richardsoni and $\mathrm{G}$. pattersoni, two widely different species. 'l'he generic characters are not satisfactorily pointed out.

Carabocrinus, Billings, 1857, Rep. of Progr. Geo. Sur. Can., ए. 275, and Can. Ory. Rem., Dreade 4, p. 30. [Ety. karabos,

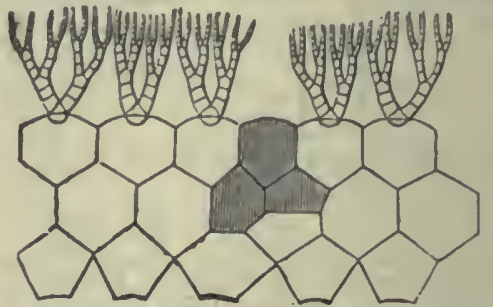

Fia. 261.-Carabocrinus radiatus. Dlagram.

a crab; krinon, lily.] Calyx globular or ovoid ; basals 5 ; subradials 5 ;'primary radials $5 ;$ arms five, and frequently 
dividing; regular interradials 0 ; azygous interradials 3 , the first one resting on a basal plate; five calycinal, ambulacral grooves on the dome; opening in the margin over the azygous plates. Type C. radiatus.

radiatus, Billings, 1857, Rep. of Progr. Geo. Sur. Can., p. 276, and Can. Org. Rem., Decade 4, p. 31, Trenton Gr.

tuberculatus, Billings, 1859, Can. Org. Rem., Decade 4, p. 33, Hud. Riv. Gr.

vancortlandti, Billings, 1859, Can. Org. Rem., Decade 4, p. 32, Trenton Gr.

Caryocrines, Say, 1825, Jour. Acad. Nat. Sci., vol. 4, p, 289. [Ety. karyon, a nut; krinon, lily.] Body ovoid or subglobuse; 1 st series of plates $4 ; 2 \mathrm{~d}$ series $6 ; 3 \mathrm{~d}$ series 6 , which bear 9 to 13 arms more or less; vault covered by polygonal plates of moderate size; upon the azygous side, near the outer edge of the vault, 6 triangular plates, forming a conical elevation, represent the mouth or anal orifice; calycine pores numerous, and also in double rows radiating from the center of the body plates; no pectinated rhombs; column round. Type C. ornatus.

ylobosus, Troost, 1850 . Not defined. granulatus, Troost, 1850 . Not defined. hexagonus, Troost. Not defined. insculptus, Troost. Not defined.

loricatus, Say, 1825, Jour. Acad. Nat. Sci., vol. 4, syn. for C. ornatus.

meconoideus, Troost. Not defined.

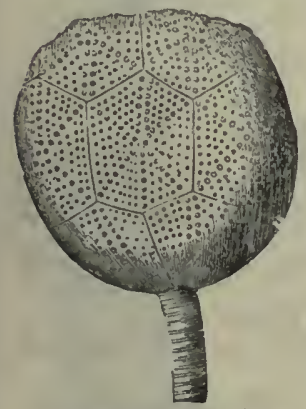
Fig. 262.-Caryocrinus
ornatus. ornatus, Say, 1825, Jour. Acad. Nat. Sci., vol. 4, p. 289 , and Pal. N. Y., vol. 2, p. 216, Clinton and $\mathrm{Ni}$ agara Gr.

Cary ocystites, Von Buch, as cited by Hall in 1861, in Geo. Rep. Wis. See Holocystites.

alternatus, see Holocystites alternatus. ocystites cylindricus.

cylindricus, see Hol-

Catillicrinus, Troost, 1850, Cat. Foss. described by Shumard, 1866, Trans. St. Louis Acad. Sci., vol. 2, p. 357. [Ety. catillus, a small bowl; krinon, lily.] Calyx hemispherical concave at the bottom; basals 5 ; primary radials $1 \times 5$; secondary radials 1 x 5 , very irregular; arms numerous, rising directly from the summit of the radials; column round. Type C. tennesseer.

bradleyi, Meek \& Worthen, 1868, Proc. Acad. Nat. Sci. Phil., p. 342, and Geo. Sur. Ill., vol. 5, p. 504, Keokuk Gr.

tennesseer, Troost, 1850, Catalogue, but described by Shumard, in 1866, in Trans.
St. Louis Acad. Nat. Sci., vol. 2, p. 358, Warsaw Gr.

wachsmuthi, Meek \& Worthen, 1866, (Synbathocrinus wachsmuthi,) Proc. Acad. Nat. Sci. Phil., p. 251, and Geo. Sur. Ill., vol. 3, p. 465, Burlington Gr. Centrocrinus, Wachsmuth \& Springer, 1881, Proc. A cad. Nat. Sci. Proposed as a subgenus under Actinocrinus, to include A. multicornis and A. pentaspinus, but the name was preoccupied by Âustin in 1843.

tennesseensis, Worthen, (in press,) Geo. Sur. Ill., vol. 8 , p. 95 , Niagara Gr.

Ceriocrinus, Wh ite, 1880 , proposed as a subgenus of Erisocrinus, but the name was pre- FiG. 263.-Catillocrinus occupied.

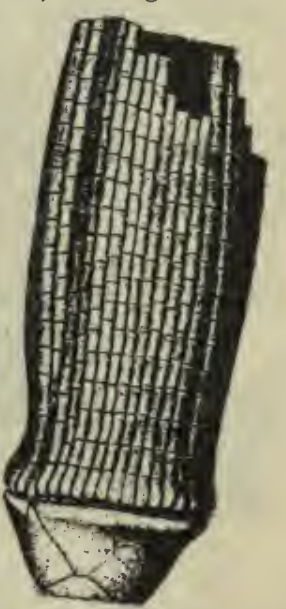

Cheirocrinus, Hall, 1860, 13th Rep. N. Y. St. Mus. Nat. Hist., p. 122 . This name was preoccupied by Eichwald in 1856, and is a syn. for Calceocrinus.

chrysalis, see Calceocrinus chrysalis. clarus, see Deltacrinus clarus.

dactylus, see Deltacrinus dactylus.

lamellosus, see Calceocrinus lamellosus. nodosus, see Deltacrinus nodosus. perplexus, see Calceocrinus perplexus. stigmatus, see Deltacrinus stigmatus. tunicatus, see Deltacrinus tunicatus. ventricosus, see Calceocrinus ventricosus.

Cholaster, Worthen \& Miller, 1883, Geo. Sur. Ill., vol. 7, p. 328. Ety. cholos, defective; aster, star.] Body truncated pentagonal; central area circular, large, deep; rays distant, small, short, truncated; centro-dorsal plate large, surrounded by five plates in the position of radials. Type C. peculiaris.

peculiaris, Worthen \& Miller, 1883, Geo. Sur. Ill., vol. 7, p. 328, Kaskaskia Gr.

Cleiocrinus, Billings, 1857, Rep. of Progr. Geo. Sur. Can., p. 276, and Can. Org. Rem., Decade 4, p. 52. [Ety. kleio, I close; krinon, lily.] Calyx conical or pyriform; basals 5 ; primary radials $3 \times 5$; secondary radials $4 \times 10$; tertiary radials numerous; azygous interradials forming a single series from the base to the top of the calyx; regular interradials none; arms numerous and compact. Type C. regius.

grandis, Billings, 1869, Can. Org. Rem., Decade 4, p. 54, Trenton Gr.

libanus, Safford, 1869, Geo. of Tenn. Not defined. 
magnificus, Billings, 1859, Can. Org. Rem., Decade 4, p. 54, Trenton Gr.

regius, Billings, 1857, Rep. of Prog. Geo.

Sur. Can., p. 277, and Can. Org. Rem.,

Decade 4, p. 53, Trenton Gr.

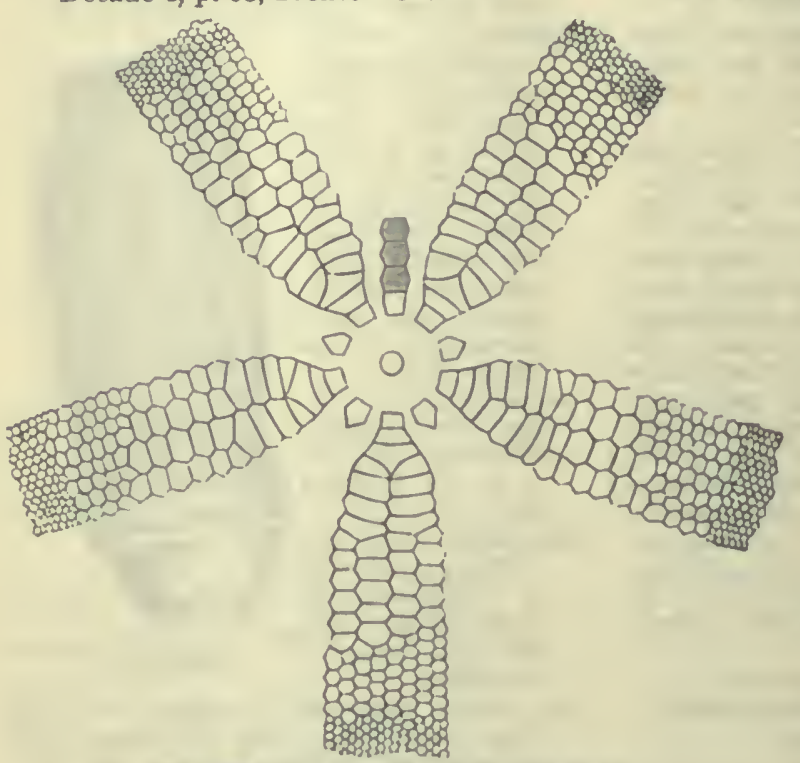

FIG. 201.-Clelocrinus regius. Diagram. tetragonal and two pentagonal, each having its inner apex notclied to form part of the round columnar canal; radials $1 \times 5$, large, equal, reashing to the truncated summit, to which, from their mesial gibbosity, they give a pentagonal outline; deltoid plates on the summit; mouth central, and from it five prominent ambulacra diverge, one to each angle, each being on a thick tapering ridge, divided by a mesial sulcus; from the re-entering angles of these interradial ridges four otlier ridges extend to the midlile of the four straight sides, the fiftl space having no ridge, but, instead, a lirge ovate opening; liydrospire slits in four interradial areas, but no liydrospire canals, and no pores. 'l'ype C. acutus.

alternalus, Lyon, 1857, Geo. Sur. Ky., vol. 3, p. 493 . A misprint for $\mathrm{C}$ aitenuatus. americanus, Shumard, 18.58 , Trans. St. Jouls A(rad. Sci., vol. 1, p. 239, Up. Held. Gr. Syn. (?) for C. pyranidatus.

Crosterocrinus, Hall, 1852, Pal. N. Y., vol. 2, p. 79. [Ety. kloster, a spindle; krinon, lily.] Body obconic; basals 3 ; subradials $1 \times 5$; number of radials unknown; azygous interradials present; arms composed of a siugle series of plates; column round. Type C. elongatus.

elongatus, Hall, 1852, Pal. N. Y., vol. 2, p. 179, Clinton Gr.

Coccocrisus, Muller, 1855 , Verliand, Naturhist. Vereins Rhein und Westph., Jahr. 12, p. 20. [Ety. kokkos, a berry; krinon, lily.] Basals 3 ; radials $2 \times 5$; interradials 1 ; column round; distinguished from Haplocrinus by the characters of the first radials and the oral plates, and from Platycrinus by the character of the vault which is composed of five oral plates resting upon the five interradials, and by the character of the column. Type C. rosaceus.
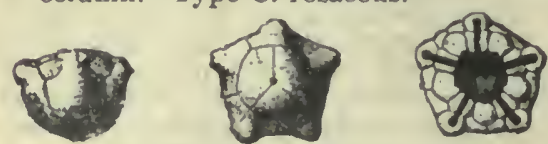

FIG. 265.- Coccocrinus bacea.

bacca, Roemer, 1860, Sil. Fauna West Tenn., p. 57, Niagara Gr.

Conaster, MrcCoy, 1849, Ann. \& Mag. Nat. Hist., 2d ser., vol. 3, p. 250. [Ety. kodon, a bell; aster, star.] Calyx inverted conical; summit broad; basals 3 , one attenuatus, Lyom, 1857 ,

Geo. Sur. Ky., vol.

3. p. 493-498, Up.

Held Gr.

can adensis, Billings.

Not defined.

gratiosus, S. A. Miller, 1880, Jour. Cin. Soc.

Nat. Hist., vol. 2, p. H'IG. 2bB.-Co d n ster 257, Keokuk Gr. The grallosus. Summit hydrospire slits are cusi, tlie lat $\mathrm{a}$ er visible on the casts showing nu nperbut are very fine.

hindii, Etheridge \& Carpenter, 1882, Ann. and Mag. Nat. Hist., p. 235, Ham. Gr. C. canndensis. (?)

kentuckiensis, Shumard, 1858, Trans. St. Louis Acad. Sci., vol. 1, p. 239, Burlington $\mathrm{Gr}$.

pentalobus, see Stephanocrinus pentalobus.
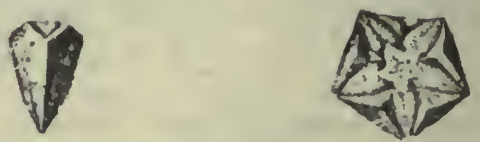

FIG. 2157.-Codaster pulchellus. Summit and side views.

pulchellus, Miller \& Dyer, 1878, Jour. Cin. Soc. Nat. Hist.; vol. 1, p. 35, Niagara Gr. Possibly a Stephanocrinus.

pyramidatus, Śhumard, 1858, Trans. St. Louis Acad. Sci., vol. 1, p. 238, Up. Held. Gr. 
whitii, Hall, 1861, Desc. New Crinoidea, p. 10, and Bost. Jour. Nat. Hist., vol. 7, p. 237, Burlington Gr.

Codonites, Meek \& Worthen, 1869, Proc. Acad. Nat. Sci. Phil., p. 84, and Geo. Sur. Ill., vol. 5, p. 463. [Ety. kodon, bell; lithos, stone.] Calyx truncateobpyramidal, elongate below, which distinguishes it, in form, from Pentremites and allied genera; deltoid plates constricted in the middle; anal opening large, remote from the center; ambulacra narrow, without marginal pores; side plates large, their apposed edges having pinnule sockets; ten spiracles parallel or subparallel to the ambulacra ; the slits are equally developed in all the interradial areas, while they are absent in the azygous interradius of Codaster. Type C. stelliformis.

campanulatus, Hambach, 1884, Trans. St.

Louis Acad. Sci., vol. 4, p. 548,

Burlington Gr.
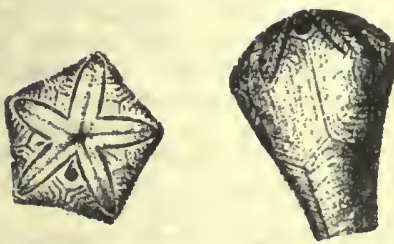

FIG. 268.-Codonites conicus.

conicus, Wachsmuth \& Springer, (in press,) (Orophocrinus conicus,) Geo. Sur. Ill., vol. 8, p. 201, Waverly or Kinderhook Gr.

gracilis, Meek \& Worthen, 1869, Proc. Acad. Nat. Sci. and Geo. Sur. Ill., vol. 5, p. 467, Burlington Gr.

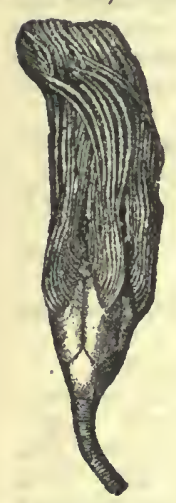

Fra. 269.- Codonites fusiformis. fusiformis, Wachsmuth \&Springer, (in press,) (Orophocrinus fusiformis, (reo.Sur.Ill., vol. 8 , p. 203, Waverly or Kinderhook Gr. stelliformis, Owen \& Sh u m a rd, 1850. (Pentremites stelliformis,) Jour. Acad. Nat. Sci. Phil., 2d ser., vol. 2, p. 67, Burlington Gr.

Camocrinus, IV hite, 1863, Jour. Bost. Soc. Nat. Hist., vol. 7, p. 499. [Ety. koilia, belly; krinon, lily.] Distinguished from Hydreionocrinus and Zeacrinus by its balloonshaped ventral sac or proboscis, and from the former, also, by the less robust body and comparatively longer arms. Type C. dilatatus.

cariniferus, Worthen, 1873, (Zeacrinus wortheni,) Geo. Sur. Ill., vol. 5, p. 535, St. Louis Gr. dilatatus, Hall, 1861, (Poteriocrinus dilatatus,) Desc. New Crinoidea, p. 6, and Bost. Jour. Nat. Hist., p. 300, Burlington Gr.

lyra, Meek \& Worthen, 1869, (Zeacrinus lyra,) Proc. Acad. Nat. Sci., p. 152, and Geo. Sur. Ill., vol. 5, p. 432, Burlington Gr.

subspinosus, White, 1863, Jour. Bost. Soc. Nat. Hist., vol. 7, p. 501, Burlington Gr.

ventricosus, Hall, 1861, (Poteriocrinus ventricosus,) Desc. New Crinoidea, p. 6 , and Bost. Jour. Nat. Hist., p. 301, Burlington Gr.

Colocrinus, Meek \& Worthen, 1865, Proc. Acad. Nat. Sci. Phil., and Geo. Sur. Ill., vol. 2 , p. 214 , syn. for Dorycrinus.

concavus, see Dorycrinus concavus.

Comarocystites, Billings, 1854, Can. Jour., vol. 2, p. 269, and Can. Org. Rem.,

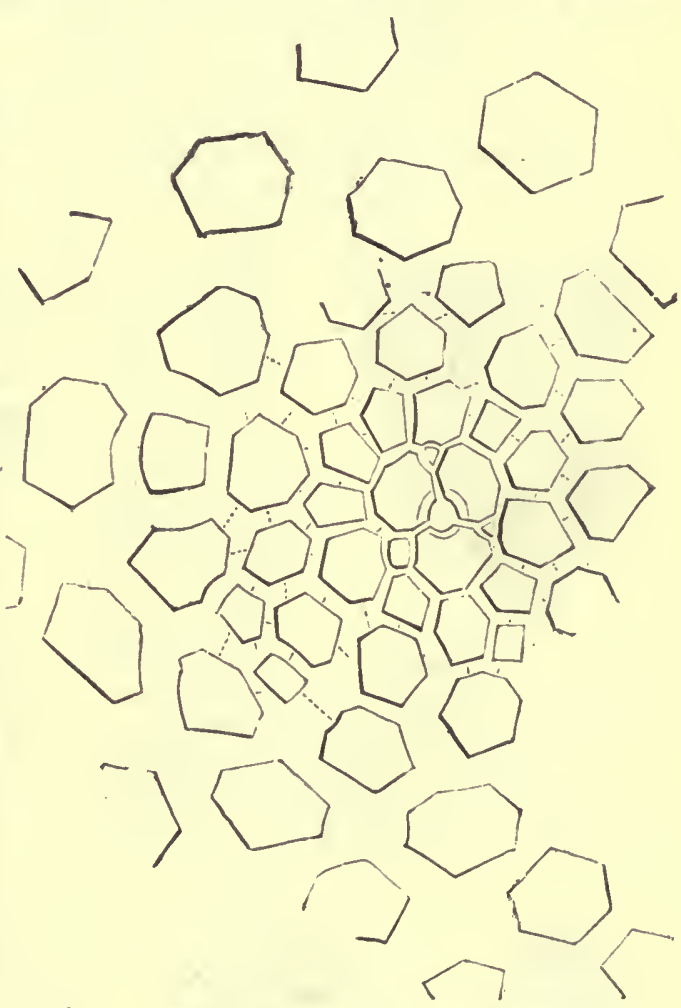

Frg. 270.-Comarocystites shumard1. Diagram of part of it.

Decade 3, p, 61. [Ety. komaron, strawberry; kustis, bladder.] Body ovate; 1st series of plates 3 , above which there are from 5 to 11 series, in irregular order; mouth or valvular orifice near the summit; arms free, grooved, bearing pinnules; ambulacral orifice at the apex; column round; all the plates poriferous. Type C. punctatus. 
obconicus, Meek \& Worthen, 1865, (C. shumardi var. obconicus,) Proc. Acad. Nat. Sci. Phil., p. 144, and Geo. Sur. Ill., vol. 3, p. 294, Trenton Gr.

punctatus, Billings, 1854, Can. Jour., vol. 2, p. 270, and Can. Org. Rem., Decade 3 , p. 61, Trenton Gr.

shumardi, Meek \& Worthen, 1865, Proc. Acad. Nat. Sci. Phil., p. 143, and Geo. Sur. Ill., vol. 3, p. 292, Trenton Gr.

Compsaster, Worthen \& Miller, 1853, Geo. Sur. Ill., vol. 7, p. 327. [Ety. kompsos, elegant; aster, star.] Central disk small; rays large, long, fusiform; grooves deep, bordered by numerous adambulacral plates; several rows of diskplates upon each side of the ambulacral furrows. Type C. formosus.

formosus, Worthen \& Miller, 1853, Geo. Sur. Ill., vol. 7, p. 327, Kaskaskia Gr.

Compsocinus, S. A. Miller, 1883, Jour. Cin. Soc. Nat. Hist., vol. 6, p. 233. [Ety. kompsos, elegant; krinon, lily.] Basals 4 ; primary radials, $3 \times 5$; secondary radials 2 or more by 10 ; tertiary radials more or less numerous; median line of radials keeled; interradials numerous; column four-sided. Type C. harrisi.

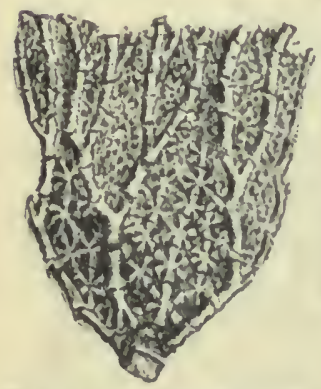

F10. 271. - Compsocrinus harrisl. Mag.2 diam. harrisi, S. A. Miller, 1881 , (Glyptocrinus harrisi,) Jour. Cin. Soc. Nat. Hist., vol. 4, p. 74, Hud. Riv. $\mathrm{Gr}$.

Conocrinus, Troost. Not defined.

Co DYLOCRINUS, Angelin, 1878, I con. Crin. Suec., p. 3 . [Ety. kordyle, a cudgel; krinon, lily.] Body resembling Platycrinus; basals 3 ; un. equal; radials $3 \times 5$; arms single or branching; pinnules long; interradials between the upper edges of the first radials; followed by 3 or 4 more; column cylindrical. Type C. comtus.
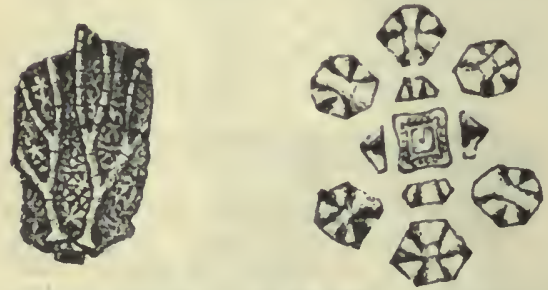

Fro. 272.-Compsocrinus harrisl. Diagram of basa! part and a specimen flattened, natural size.

parvus, Hall, 1861, (Platycrinus parvus,) Pal. N. Y., vol. 3, p. 114, Low Held. Gr. plumosus, Hall, 1861, (Platycrinus plumosus,) Pal. N. Y., vol 3, p. 113, Low. Held. Gr.

ramulosus, Hall, 1861, (Platycrinus ram. ulosus,) Pal. N. Y., vol. 3, p. 115, Low. Held. Gr.

Coronocrinus, Hall, 18џ9, Pal. N. Y., vol. 3, p. 124. [Ety. korone, a crown; krinon, fily.] Founded upon the fragment of the upper part of the calyx showing great breadth, probably hemispheric form, and as many as 40 arm openings in the circumference. Wacusmuth says it is a syn. for Dolatocrinus, but as that genus is not known, in rocks, so low as this is found, there is great doubt about the synonymy. Type C. polydactylus.

polydactylus, Hall, 1859, Pal. N. Y., vol. 3, ر. 124, Low. Held. Gr.

Cotyledonocrinus, Casseday \& Lyon, 1860, Proc. Am. Acud. Arts and Sci., vol. 5, p. 26. [Ety. kotyledon, any cup-shaped cavity; krinon, lily.] Basals 2; radials $3 \times 5 ;$ sccondary radials $2 \times 10 ;$ arms 10 ; interradials $3 \times 5$. Distinguished from Dichocrinus by having no azygous plate in line with the first radials, and belioved, by Wachsmuth, to have been founded upon an abnormal Dichocrinus. Typo C. pentalobus.

pentalobus, Casseday \& Iyon, 1860, Proc. Am. Acad. Arts and Sei., vol. 5, p. 26, Kaskaskia Gr.

Cremacrinus, Ulrich, syn. for Calceocrinus. punctatus, see Calceocrinus punctatus.

Crinocystites, Hall, 1864, 20th Rep. N. Y. St. Mus. Nat. Hist., p. 361. [Ety. krinon, lily ; kustis, bladder.] Elongate, swelling in the upper third of the azygous side, and contracting below the arms; covered by five or more ranges of irregularly disposed plates; central and submarginal apertures. Type C. chrysalis.

chrysalis, Hall, 1864, 20th Rep. N. Y. St. Mus. Nat. Hist., p. 362. Niagara Gr.

(?) rectus, Hall, 1864, see Rloodocrinus (?) rectus.

Crinosoma antiqua, Castelnau, 1843, Syst. Sil. Probably a fucoid.

Cromyocrinus, Trautschold, 1867, syn. for Eupachycrinus.

gracilis, seo Eupachycrinus gracilis.

Crumenxcriniles, Troost, 1850 . Not defined. ovalis, Troost, 1850. Not defined.

Cryptoblastus, Ftheridge \& Carpenter, 1886, Catalogue of Blastoidea, p. 229. This genus is founded upon Granatocrinus melo, and distinguished from Granatocrinus, by a slight difference, in the hydrospires. They also referred to it G. pisum, and two other species, about which they had very little information.

Clenocrinus, Bronn, 1840, Leonh. und Bronn. Jahrb, syn. for Melocrinus.

bainbridgensis, see Melocrinus bainbridgensis. 
breviradiatus, see Melocrinus breviradiatus.

Cupellxcrinus, Troost, 1850. Not defined.

Cupulocrinus, D'Orbigny, 1850, Prodr. d. Pal., t. 1, p. 23. Proposed instead of Scyphocrinus, Hall, Pal. N. Y., vol. 1 , p. 85 , that was preoccupied, by Zenker. Basals 5 ; radials $4 \times 5$, regular interradials 3 ; azygous interradials 4 ; arms 10 ; column round. Type C. heterocostalis. Wachsmuth regards it as a syn. for Taxocrinus.

heterocostalis, Hall., 1847, (Scyphocrinus heterocostalis,) Pal. N. Y., vol. 1, p. 85, Trenton Gr.

Uyathocrinus, Miller, 1821, Nat. Hist. Crinoidea, p. 85. [Ety. cyathos, cup or goblet; krinon, lily.] Calyx saucershaped; basals 5 ; subradials 5 ; radials $1 \times 5$; as large or larger than the basals, with articulating facet occupying only part of the width of a plate; brachials irregular in number; arms long, branching; column round; no regular interradials; azygous interradial 1 , which is followed, in the ventral sac or proboscis, by other plates. Type C. planus.

æmulus, Hall, 1879, Desc. new spec. foss., p. 10, and 11th Rep. Geo. and Nat. Hist., Indiana, p. 266, Niagara Gr. angulatus, see Barycrinus angulatus.

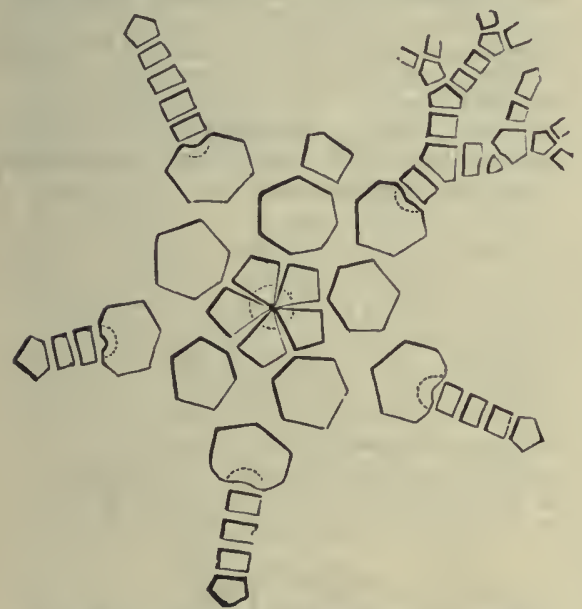

FIG. 273.-Cyathocrinus arboreus. Dlagram.

arboreus, Meek \& Worthen, 1865, Proc. Acad. Nat. Sci. Phil., p. 160, and Geo. Sur. Ill., vol. 3, p. 520, Keokuk Gr.

barrisi, Hall, 1861, (Poteriocrinus barrisi,)

Desc. New Crin., p. 5, and Bost. Jour.

Nat. Hist., p. 303, Burlington Gr.

barydactylus, Wachsmuth \& Springer, 1878, Proc. Acad. Nat. Sci., p. 257, Burlington Gr.

bulbosus, see Arachnocrinus bulbosus.

bullatus, see Barycrinus bullatus.

conglobatus, Troost. Not defined. cora, Hall, 1864, 20th Rep. N. Y. St. Mus.

Nat. Hist., p. 366, Niagara Gr.

cornutus, see Barycrinus cornutus.

corrugatus, Troost. Not defined.

crassibrachiatus, see Barycrinus crassibrachiatus.

crassus, see Eupachycrinus crassus. crateriformis, Troost. Not defined.

crawfordsvillensis, S. A. Miller, 1882,

Jour. Cin. Soc. Nat. Hist., vol. 5, p. 79, Keokuk Gr.

decadactylus, Lyon \& Casseday, 1860, Am. Jour. Sci. and Arts, vol. 29, p. 73, Keokuk Gr.

depressus, Troost, see Zeacrinus depressus. divaricatus, Hall, 1858, Geo. Sur. Iowa, p. 554 , Burlington Gr.

enormis, Meek \& Worthen, 1865, (Poteriocrinus enormis,) Proc. Acad. Nat. Sci. Phil., p. 152, and Geo. Sur. Ill., vol. 3, p. 481, Burlington Gr.

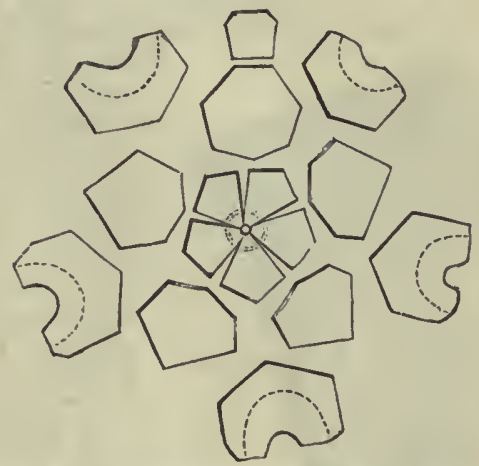

FIG.274.-Cyathocrinus farleyi. Diagram.

farleyi, Meek \& Worthen, 1866, Proc. Acad. Nat. Sci. Phil., p. 252, and Geo. Sur. Ill., vol. 3, p. 517, Keokuk Gr. fasciatus, see Macrostylocrinus fasciatus. florealis, see Zeacrinus florealis.

fragilis, Meek \& Worthen, 1868, Proc. Acad. Nat. Sci. Phil., p. 237, and Geo. Sur. 1ll., vol. 5, p. 401, Burlington Gr. globosus, Troost. Not defined.

gilesi, Wachsmuth \& Springer, 1878, Proc. Acad. Nat. Sci., p. 259, Burlington Gr.

granuliferus, Shumard, 1854, Red Riv. Expl. Louisiana, p. 199, Kaskaskia Gr. hamiltonensis, Worthen, 1882, Bull. No. 1, Ill. St. Mus. Nat. Hist., p. 32, syn. for C. parvibrachiatus.

harrisi, S. A. Miller, 1880, Jour. Cin. Soc. Nat. Hist., vol. 2, p. 255, Keokuk $\mathrm{Gr}$. As suggested at the time of describing this species, it may become the type of a new genus.

harrodi, Wachsmuth \& Springer, 1879, Proc. Acad. Nat. Sci., p. 87, Keokuk Gr. hexadactylus, Lyon \& Casseday, 1860, Am. Jour. Sci., vol. 29, p. 74, syn. for Vasocrinus lyoni. The name was essentially incorrect and definition wrong. hoveyi, see Barycrinus hoveyi. 
inæquidactylus, Whitfield, 1882, Ann. N. Y. Acad. Sci., vol. 2, p. 219, Kaskaskia Gr.

incipiens, Hall, 1861, Desc. New Crinoidea, p. 5 , and Bost. Jour. Nat. Hist., vol. 7 , p. 296 , Burlington Gr.

inflatus, Troost. Not defined.

inflexus, see Erisocrinus inflexus.

inspiratus, Lyon, 1860, Trans. Am. Phil. Soc., vol. 13, p. 457, Keokuk (ir.

intermedius, Hall, 1858, (ieo. Rep. Iowa, p. 627, Keoknk Gr.

iowensis, Owen \& Shumard, 1850, Jour. Acarl. Nat. Sci., 2d ser., vol. 2, p. 63, and Geo. Sur. Wis., Iowa, and Minn., p. 591, Burlington Gr.

kelloggi, see Barycrinus kelloggi.

læviculus, Lyon, 186l, Proc. Acad. Nat. Sci. Phil., p. 409, Up. Held. Gr.

latus, Hall, 1861, Desc. New Crinoidea, p. 5 , syn. for Barycrinus sculptilis.

lamellosus, White, 1863, Jour. Bost. Soc. Nat. Hist., vol. 7, p. 504, Burlington Gr.

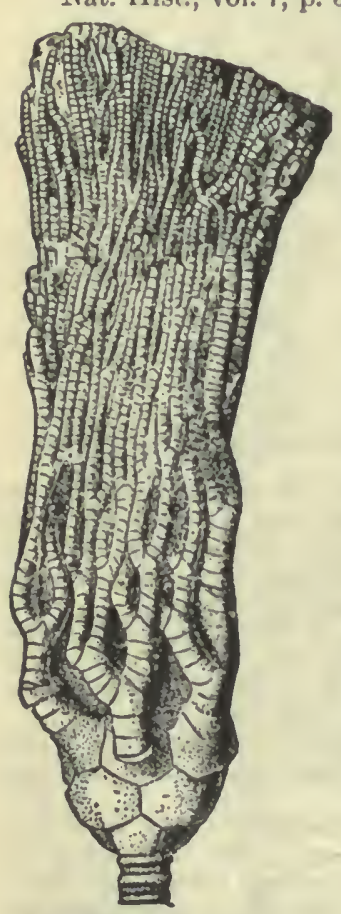

FIG. 275.-Cyathocrinus multibrachiatus. lyoni, see Vasocrinus lyoni.

macropleurus, sce Vasocrinus macropleurus.

magixter, see lBarycrinus magister.

magn oliiformis, see Zeacrinus $\mathrm{m}$ a $\mathrm{g} \mathrm{n}$ o $1 \mathrm{i} \mathrm{i}$. formis.

malvaceus, Hall, 1858, Geo. Sur. Iowa, p. 554, Burlington $\mathrm{Gr}$. Wa $\mathrm{cl} 8 \mathrm{muth}$ says it is founded on a depressed specimen of $\mathrm{C}$. iowensis.

maniformis, see Zeacrinus maniformis.

m a r s h allensis, Worthen,1882, I3ull. No. 1, I1]. St. Mus. Nat. Hist., p. 33, and Geo. Sur. Ill., vol. 7, p. 310 , W averly or $\mathrm{K}$ ind e rhook Gr.

multibrachiatus, Lyon \& Casseday, 1859, Am. Jour. Sci., vol. 28 , p. 245 , Keokuk Gr.

nucleus, Hall, 1876, (Dendrocrinus nucleus,) 28th Rep. N. Y. St. Mus. Nat. Hist., p. 136, Niagara Gr.

ornatissimus, Hall, 1843, Geo. Rep. 4th Dist. N Y., p. 247, Portage Gr.

parvibrachiatus, Hall, 1861, Desc. New Crinoidea, p. 6, and Bost. Jour. Nat. Hist, vol. 7, p. 294, Keokuk Gr. pentalobus, see Eupachycrinus pentalobus. planus, Troost. Not defined.

polyxo, Hall, 1863, Trans. Alb. Inst., vol. 4, p. 199, and 28th Rep. N. Y. St. Mus. Nat.-Hist., p. 135, Niagara Gr.

poterium, Meek \& Worthen, 1870, Proc. Acad. Nat. Sci. Pliil., p. 24, and Geo.

Sur. Ill., vol. 5, p. 489, Keokuk Gr. protuberans, see Barycrinus protuberans. pusillus, see Lecanocrinus pusillus.

pyriformis, Murchison as identified by Hull. See Ichthyocrinus lrevis

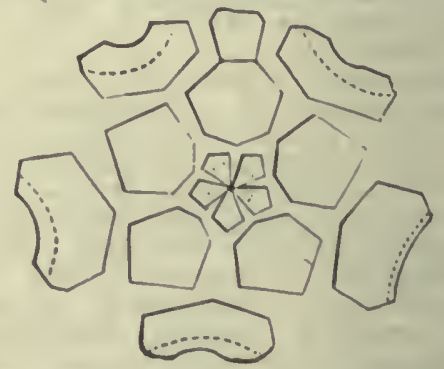

Fxo. 276.-Cyathocrinus quinqueiobus.

quinquelobus, Meek \& Worthen, 1865

Proc. Acad. Nat. Sci. 1'hil., p. 150, and Geo. Sur. Ill., vol. 3, p. 519, Keokuk Gr.

rarus, Iyon, 1869, Trans. Am. Phil. Soc., vol. 13, p. 453, Up. Held. Gr.

rigidus, White, $186^{\circ}$, Proc. Bost. Soc. Nat.

Hist., vol. 9, p. 8, Burlington Gr.

robustus, Troost. Not defined.

roemeri, Troost. Not defined.

rotundatus, Hall, 1858, Geo. Rep. Iowa, p. 555, Burlington Gr.

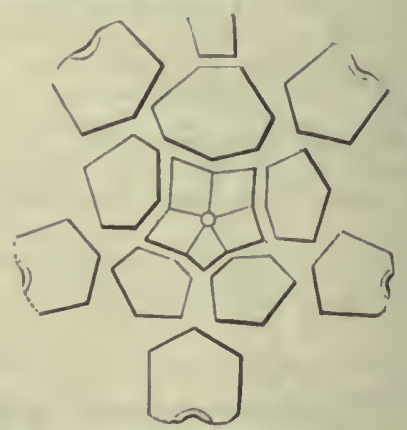

FIG. 277.-Cyathocrinus safford1. Diagram.

saffordi, Meek \& Worthen, 1860, Proc. Acad. Nat. Sci. Phil., p. 371, and Geo. Sur. Ill., vol. 2, p. 336, Keokuk Gr. sangamonensis, see Eupachycrinus sangamonensis.

scitulus, Meek \& Worthen, syn. for Barycrinus scitulus.

sculptilis, see Barycrinus sculptilis. Not defined.

sculptus, Troost. Not defined.

solidus, see Barycrinus solidus. 
somersi, Whitfield, 1882, Ann. N. Y. Acad. Sci., vol. 2, p. 226, Coal Meas.

spurius, see Barycrinus spurius.

stellatus, see Barycrinus stellatus.

stillativus, White, 1880 , Proc. U. S. Nat. Mus., vol. 2, p. 258, and Cont. to Pal. No. 6, p. 125 , Up. Coal. Meas.

subtumidus, Meek \& Worthen, 1869, Proc. Acad. Nat. Sci. Phil., p. 151, and Geo. Sur. Ill., vol. 5, p. 487, Keokuk Gr.

tennesseex, Troost. Not defined.

tenuibrachiatus, Lyon, 1869, Trans. Am. Phil. Soc., vol. 13, p. 460, Up. Held. Gr.

tenuidactylus, Meek \& Worthen, 1868, Proc. Acad. Nat. Sci. Phil., p. 238, and Geo. Sur. Ill., vol. 5, p. 403, Burlington $\mathrm{Gr}$.

thomæ, see Barycrinus thomæ.

tiaræformis, see Ichthyocrinus tiariformis. tumidus, see Barycrinus tumidus.

vanhornii, S. A. Miller, 1881, Jour. Cin. Soc. Nat. Hist., vol. 4, p. 261, Niagara Gr.

viminalis, Hall, 1861, Desc. New Crin., p. 5, syn. for C. iowensis.

wachsmuthi, see Barycrinus wachsmuthi. waldronensis, Miller \& Dyer, 1878, Cont. to Pal. No. 2, p. 6, Niagara Gr. Wachsmuth refers it to Macrostylocrinus.

waukoma, Hall, 1864, 20th Rep. N. Y. St. Mus. Nat. Hist., p. 367, Niagara Gr.

wortheni, Lyon, 1861, Proc. Acad. Nat. Sci. Phil., p. 410, Up. Held. Gr.

Cyclaster, Billings, 1857, Rep. of Progr. This name was preoccupied. See Edrioaster.

bigsbyi, see Edrioaster bigsbyi.

Cyclocystordes, Billings \& Salter, 1858, Can. Org. Rem., Decade 3, p. 86. [Ety. kuklos, circle ; kustis, bladder; eidos, form.] Body consisting of a circular disk, surrounded by a series of short, cylindrical, perforated, porous plates; the interior is covered by an integument of small plates, with radiating channels, which bifurcate and connect with the channelin the marginal series, which makes a complete circle; mouth supposed to be central; mammillary elevations on the exterior of the rim as if for the attachment of small spines. Type C. halli.

anteceptus, Hall, 1866, 24th Rep. N. Y. St. Mus. Nat. Hist., p. 219, Trenton $\mathrm{Gr}$.
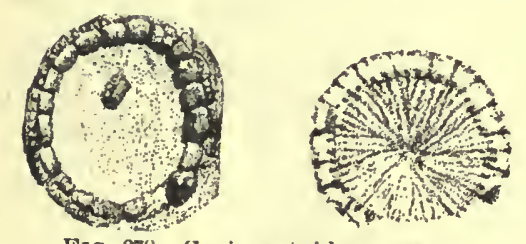

FIG. 278.-Cyclocystoides magnus.

bellulus, Miller \& Dycr, 1878, Jour. Cin. Soc. Nat. Hist., vol. 1, p. 34, Hud. Riv. Gr. halli, Billings, 1858, Can. Org. Rem., Decade 3, p. 86, Trenton Gr.

huronensis, Billings, 1865, Pal. Foss., vol. 1, p. 393, Hud. Riv. Gr.

magnus, Miller \& Dyer, 1878, Jour. Cin. Soc. Nat. Hist., vol. 1, p. 32, and vol. 4, p. 70, Hud. Riv. Gr.

minus, Miller \& Dyer, 1878, Jour. Cin. Soc. Nat. Hist., vol. 1, p. 33, Hud. Riv. Gr.

mundulus, Miller \& Dyer, 1878, Jour. Cin. Soc. Nat. Hist., vol. 1, p. 34, Hud. Riv. Gr.

nitidus, Faber, 1886, Jour. Cin. Soc. Nat. Hist., vol. 9, p. 17, Hud. Riv. Gr.

parvus, Miller \& Dyer, 1878, Jour. Cin. Soc. Nat. Hist., vol. 1, p. 33, Hud. Riv. Gr.

salteri, Hall, 1866, 24th Rep. N. Y. St. Mus. Nat. Hist., p. 218, Trenton Gr.

Cystocrinús, Roemer, 1860, Sil. Fauna West Tenn., p. 56. [Ety. kustis, bladder; krinon, lily.] A cylindrical body, the interior of which looks like a crinoid column, but the external part consists of a compact mass of tubes connecting with the central canal. Wachsmuth has called it a detached column, but it is anomalous, and I retain the genus. Type C. tennesseensis.

tennesseensis, Ro e mer, 1860 , Sil. Fauna West Tenn, p. 56, Niagara Gr. Cytocrinus, Roemer, 1860 , Sil. Fauna West Tenn., p. 46 , syn for Melocrinus.

lævis, see Melocrinus læevis.

$D æ m$ on icri-

nites,'Troost

Not d e fined.

De cadactylo-

crinites,

Owen. Not FIG. 279.-Cystocrinus tennesdefined.

Decadocrinus, Wachsmuth \& Springer, 1879, Proc. Acad. Nat. Sci. Phil. and Revis. Palæocrinoidea, pt. 1, p. 119. It was described as a subgenus of Poteriocrinus, but it hardly arises to that dignity. Their type is Scaphiocrinus scalaris.

Deltacrinus, Ulrich, 1886, 14th Rep. Geo. Sur. Minn., p. 109. [Ety. delta, Greek letter; krinon, lily.] Basal piece triangular, composed of anchylosed plates; four plates form the dorsal side above the base; lower central plate triangular and separated from the upper triangular plate by the union of the two lateral radial plates. Distinguished from Calceocrinus, which has a long plate on the dorsal side between the lateral radials instead of the two triangular plates separated, as above described by the union of the two radials. Type D. clarus. 
barrisi, Worthen, 1875, (Calceocrinus barrisi, Geo.Sur. Ill., vol. 6, p. 510, Ham. Gr.

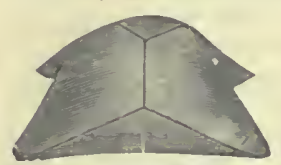

Frg. 280. $\rightarrow$ Deltacrinus barrist. bradleyi, Meek \& Worthen, 1869 , (Calceocrinus bradlevi,) Proc. Acad. Niat. Sci. Phil., p. 73, and Geo.Sur. Ill., vol.5, p. 502 , Keokuk Gr.

clarus, Hall, 1S62, (Cheirocrinus clarus,) 15 th Rep. N. Y. St. Mus. Nat. Hist., p. 88, Niagara Gir.

dactylus, Hall, 1560, (Cheirocrinus dactvlus,) 13th Rep. N. Y. St. Mus. Nat. Hist., p. 123, Burlington (ir.

nodosus, Hall, 1560, (Cheirocrinus nodosus,) 13th Rep. N. Y. St. Mus. Nat. Hist., p. 124, Keokuk (ir.

stigmatus, Hall, 1563, (Chejrocrinus stigmatus, Trans. Alb. Inst., vol. 4, p. 225, Niagara Gr.

tunicatus, Hall, 1660, (Chejrocrinus tunicatus,) 13th Rep. N. Y. St. Mus. Nat. Hist., p. 124, Keokuk (ir. wachsm ut hi, Meek \&
Worthen, 1869 , (Ca) ceocrinus wachsmuthi,) Proc. Acal. Nat. Sci. Phil., p. 74, and Geo. Sur. Ill., vol.

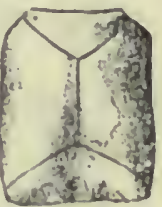
therinusatlgmatus, Dorsal vlew of cal y $x$ enI a r ged 2 diam.
10. $281 .-1$ el5 , p. 444, Burlington Fr.

Dendrocrixus, Hall, 1852, Pal. N. Y., vol. 2 p. 193. [Ety. dendron, tree; krinon, lily.] Calyx obconoidal ; basals 5 ; subradials 5; radials $1 \times 5$, and an additional one caused by a division of the plate on the lelt side of the large azy-

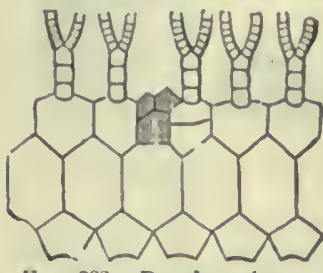

Fs. 282.-Dendrocrinus. Dlagram. gous one ; regular interradials 0 ; azygous interradial 1 ; lirge and long proboscis or ventral sac rises from the azygous interradial; arms long, branching: ambulacral furrow deep; pinnule's wanting; column round or pentagonal; without base or roots for attachment. Type D. longidactylus.

acutidactylus, Billings, 1857, Rep. of Progr. Geo. Sur. Can., p. 266, and Can. Org. Rem., Decade 4, p. 37, Trenton Gr. ahcilla, Hall, 1879, Desc. New Spec. Foss. p. 9, and 11 th Rep. Geo. and Nat. Hist. Indiana, p. 271, Niagara Gr.

alternatus, Hall, 18+7, (Poteriocrinus alternatus, Pal. N. Y., vol. 1, p. 83, Trenton Gr.

angulatus, see Palæocrinus angulatus.

angustatus, Meek \& Worthen, 1870, (Homocrinus angustatus,) Proc. Acad. Nat. Sci. Phil., p. 30, and Geo. Sur. Ill., vol. 6, p. 492, Hud. Riv. Gr. caduceus, Hall, 1866, 2tth Rep. N. Y. St. Mus. Nat. Hist., p. 208, Hud. Riv. Gir. casii, Meek, 1871, Am. Jour. Sei. and Arts, 3 d ser., vol. 2. p. 295, and Ohio Pal., vol. 1, p. 2S, Hud. Riv. Gr.

cincinnatiensis, Meek, 1872, Proc. Acad. Nat. Sci. Phil., p. 312, and Ohio Pal., vol. 1, p. 20, Hud. Riv. Gr.

conjugans, Billings, 1857, Rep. of Progr. Geo. Sur. Can., p. 268, and Can. Org. Rem., Decade 4, p. 41, Trenton Gir. celsus, Ringueberg, 1988, Proc. Acad. Nat. Sci. Phil., p. 132, Niagara Gir.

curtıs, see Derocrinus curtus.

cylindricus, Billings, 1859, Can. Org. Rem., Decado 4, p. 44, Trenton (ir.

dyeri, Mcek, 1872, Proc. Acacl. Nat. Sci. Phil., p. 310, and Ohio, I'al., vol. 1, p. 24. Hud. Riv. Gr.

erraticus, S. A. Miller, 1851, Jour. Cin. Soc. Nat. Hist., vol. 4, p. 316, Hud. IRiv. Gr.

gracilis, Hall, 1847,(Poteriocrinus gracilis,) Pal. N. Y., vol. 1, p. 84, Trenton (ir.

gregarius, Billings, 1857, Rep. of J'rogr. Geo. Sur. Can.. p. 265, and Can. Org. Rem., Decade 4, p. 36, Trenton (ir.

humilis, Billings, 1857, Rep. of I'rogr. Geo. Sur. Can., p. 265, and Can. Org. Rem., Decade 4, p. 39, Trenton Gr.

jewetti, Billings, 1859, Can. Org. Rem., Decade 4, p. 43, Trenton Gir.

latibrachiatus, Bill. ings, 1857, $\mathrm{R} \in \mathrm{p}$. Prog. Can. Gieo, Sur., p. 270, and Can. Org. Rem., Decade 4, p. 39, Hud. Riv. Gr.

longidactylus, Hall, 1852, Pal. N. Y., vol. 2 , p. $193, \mathrm{Ni}$ agara Gr.

modestus, safford. Not defined.

navigiolum, S. A. Miller, 1880, Jour. Cin. Soc. Nat. Hist., vol. 3, p. 235, Utica Slate fir.

nucleus, see Cyathocrinus nucleus.

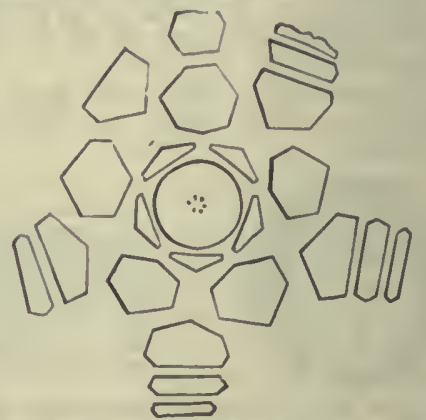

Fic. 284.-Dendrocriuus oswegoensis. Diagram.

oswegoensis, Meek \& Worthen, 1868, Geo. Sur. Ill., vol. 3, p. 333, Hud. Riv. Gr. 
polydactylus, Shumard, 1857, (Homocrinus polydactylus,) Trans. St. Louis Acad. Sci., vol. 1, p. 78, and Ohio Pal., vol. 1, p. 22, Hud. Riv. Gr.

posticus, Hall, 1872, (Poteriocrinus posticus,) 24th Rep. N. Y. St. Mus. Nat. Hist., p. 209, and Ohio Pal., vol. 1, p. 22, Hud. Riv. Gr.

proboscidiatus, Billings, 1857, Rep. of Progr. Can. Geo. Sur., p. 267, and Can. Org. Rem., Decade 4, p. 38, Trenton Gr.

retractilis, Walcott, 1883 , 35th Rep. N. Y. St. Mus. Nat. Hist., p. 211, Trenton $\mathrm{Gr}$.

rusticus, Billings, 1857, Rep. of Prog. Geo. Sur. Can., p. 270, and Can. Org. Rem., Decade 4, p. 41, Trenton Gr.

similis, Billings, 1857, Rep. of Progr. Geo. Sur. Can., p. 267, and Can. Org. Rem., Decade 4, p. 40, Trenton Gr.

tener, Billings, 1866, Catal. Sil. Foss. Antic., p. 9, Hud. Riv. Gr.

Dicirocrinus, Munster, 1839, Beitrag. Zur. Petref., vol. 1, p. 2. [Ety. dicha, in two parts; krinon, lily.] Calyx deep, cup-shaped; plates delicate; basals 2 ; primary radials 5 , large, resting two upon each basal, and the other in a notch at one end of the basal suture, opposite which there is a large azygous plate in line with the first radials; succeeding radials 1 to 3 , in eacl ray, the last supporting arms; arms 10, long, bifurcating and bearing stout pinnules; interradials 4 or 5 , small, situate above the first radials; vault slightly elevated, with a small opening upon the azygous side; column round. Type D. radiatus.

angustus, White, 1862, Proc. Bost. Soc. Nat. Hist., vol. 9, p. 19, Burlington Gr.

chesterensis, see Pterotocrinus chesterensis. constrictus, Meek \& Worthen, 1860 , Proc. Acad. Nat. Sci. Phil., p. 381, and Geo. Sur. Ill., vol. 2, p. 263, Warsaw $\mathrm{Gr}$.

conus, Meek \& Worthen, 1860; Proc. Acad. Nat. Sci. Phil., p. 381, and Geo. Sur. Ill., vol. 2, p. 169 , Burlington Gr. cornigerus, see Talarocrinus cornigerus.

coxanus, Worthen, 1882, Bull. No. 1, Ill.

St. Mus. Nat. Hist., p. 35, and Geo.Sur. Ill., vol. 7, p. 313, Keokuk Gr.

crassitestus, White, 1862, Proc. Bost. Soc.

Nat. Hist., vol. 9, p. 19, Burlington Gr. crassus, see Pterotocrinus crassus.

dichotomus, Hall, 1860, Supp. to Greo. Sur. Iowa, p. 85, IVarsaw Gr.

elegans, Casseday \& Lyon, see Talarocrinus elegans.

expansus, Meek \& Wortben, 1868. The name was preoccupied by DeKoninck \& LeHon, but the name is probably a synonym for $\mathrm{D}$. polydactylus.

ficus, Casseday \& Lyon, 1860, Proc. Am. Acad. Arts and Sci., vol. 5, p. 24, and Geo. Sur. Ill., vol. 5, p. 502, Keokuk Gr. inornatus, Wachsmuth \& Springer, (in press,) Geo. Sur. Ill., vol. 8, p. 190 , Waverly or Kinderhook Gr.

hamiltonensis, Worthen, 1882, Bull. No. 1, Ill. St. Mus. Nat. Hist., p. 35, and Geo. Sur. Ill., vol. 7, p. 313, Keokuk Gr.

lachrymosus, Hall, 1860 , Supp. to Geo. Sur. Iowa, p. 84, Burlington Gr. Wachsmuth says it is a syn. for Platycrinus subspinulosus. lævis, Hall, 1860, Supp. Geo. Sur. Iowa, p. 83, Burlington Gr.

lineatus, Meek \& Worth- FIG. 285. - Dichoén, 1869, Proc. Acad. Crinus inornatus. Nat. Sci. Phil., p. 69 , and Geo. Sur. IIl., vol. 5, p. 440, Burlington $\mathrm{Gr}$.

liratus, Hall, 1861, Desc. New Crinoidea, p. 5, and Jour. Bost. Nat. Hist., vol. 7, p. 290, Burlington Gr.

ornatus, Wachsmuth \& Springer, 1881, Proc. Acad. Nat. Sci. Phil. and Revis. Palxocrin., p. 84, Keokuk. Gr. This name was proposed instead of $\mathrm{D}$. sculptus, Casseday \& Lyon, because the latter was preoccupied.

ovatus, Owen \& Shumard, 1850, Jour. Acad. Nat. Sci., $2 d$ ser., vol. 2, p. 61, and Geo. Sur. Iowa, Wis., and Minn., p. 590 , Burlington Gr.

pisum, Meek \& Worthen, 1869, Proc. Acad. Nat. Sci. Phil., p. 69, and Geo. Sur. Ill,. vol. 5, p. 441, Burlington Gr. plicatus, Hall, 1861, Desc. New Crinoidea, p. 4, and Jour. Bost. Soc. Nat. Hist., vol. 7 , p. 288, Burlington Gr.

pocillum, Hall, 1861, Desc. New Crinoidea, p. 5, and Jour. Bost. Soc. Nat. Hist., vol. 7, p. 291, Burlington Gr.

polydactylus, Casseday \& Lyon, 1860 , Proc. Am. Acad. Arts and Sci., vol. 5, p. 20, Keokuk Gr.

protuberans, Hall, see Pterotocrinus protuberans.

scitulus, Hall, 1861, Desc. New Crinoidea, p. 4, and Jour. Bost. Soc. Nat. Hist., vol. 7, p. 289, Burlington Gr.

sculptus, Casseday \& Lyon, 1860, Proc. Am. Acad. Arts and Sci., vol. 5, p. 25. The name was preoccupied by DeKoninck \& LeHon in 1853 . See D. ornatus.

sexlobatus, see Talarocrinus sexlobatus. simplex, Shumard, 1857, Trans. St. Louis Acad. Sci., p. 74, and Geo. Sur. Iowa, p. 654, Warsaw Gr.

striatus, Owen \& Shumard, 1850, Jour. Acad. Nat. Sci., 2d ser., vol. 2, and Geo. Sur. Iowa, Wis., and Minn., p. 590, Burlington Gr.

symmetricus, see Talarocrinus symmetricus. 
Dictyocrinus, Con rad, 1841, (Dictuocrinites,) Ann. Rep. N. Y. and Pal. N. Y., vol. 3, p. 135, syn. for Receptaculites.

squamifer, Hall, see Receptaculites squamifer.

Dolatocrinus, Lyon, 1857, Geo. Sur. Ky., vol. 3, p. 482. [Ety. dolalus, hewn or tooled; krinon, lily.] Body subspheroidal ; caly x basin-shaped ; vault liemispherical, depressed in the interradial areas; basals anchylosed and probably numbering 5 ; radials $3 \times 5$; secondary radials $2 \times 10$; sometimes tertiary radials $2 \times 20$; arms 20 to 40 , bifurcating and bearing pinnules; interradials 5 or more, the first one large ; aperture subcentral; column round. Type D. lacus. canadensis, Whiteaves, 1887, Cont. to Can. Pal. vol. 1, p. 99, Ham. Gr.

glyptus, Hall., 1862, (Cacabocrinus glyptus,) 15th Rep. N. Y. St. Mus. Nat. Hist., p. 140, Ham. Gr.

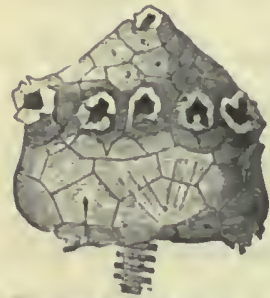

Fyo. 286-Dolatocrimus incus. Side view.

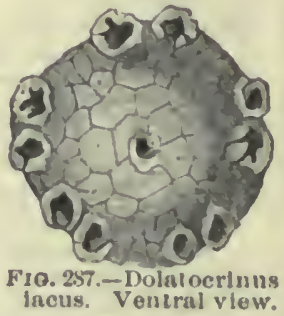

glyptus var. intermedius, Hall, $1862,(\mathrm{Ca}-$ cabocrinus glyptus var. intermedius,) 15th Rep. N. Y.St. Mus. Nat. Hist., p. 141, Ham. Gr.

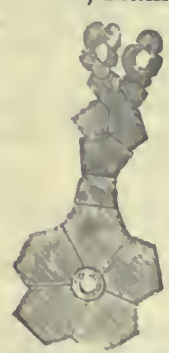

Fro. 285.-Dolalocrinas lacus. Dlagram of a ray.

Iacus, Lyon, 18.57, Geo. Sur. Ky., vol. 3, p. 482 , Up. Held. Gr.

lamellosus, Hall, 1862 , (Cacabocrinus lamellosus,) 15th Rep. N. Y. St. Mus. Nat. Hist., p. 141, Up. Held. Gr.

liratus, Hall, 1862, (Cacabocrinus liratus,) 15th Rep. N. Y. St. Mus. Nat. Hist., p. 139, Ham. Gr.

liratus var. multilira, Hall, 1862, (Cacabocrinus liratus var, multilira,) 15 th Rep. N. Y. St. Mus. Nat. Hist., p. 139, Hum. Gr.

marshi, Lyon, 1869, Trans. Am. Plıil. Soc., vol. 13, p. 461, Up. Held. Gr.

ornatus, Meek, 1871, Proc. Acad. Nat. Sci., p. 57, Up. Held. Gr.

speciosus, Hall, 1862, (Cacabocrinus speciosus,) 15th Rep. N. Y. St. Mus. Nat. Hist., p. 137, Up. Held. Gr.

triadactylus, Barris, 1885, Proc. Dav. Acad. Sci., vol. 4, p. 100 , Ham. Gr.

troosti, Hall, 1862, (Cacabocrinus, troosti,) 15 th Rep. N. Y. St. Mus. Nat. Hist., p. 138, Ham. Gr.
Donacicriniles, Troost. Not defined. simplex, Troost. Not defined.

Dorycrinus, Roemer, 1854, Arcliiv. f. Naturgesch Jahrg. 19, p. 207. [Ety. dory, spear; krinon, lily.] Body turbinnte or subglobose, truncate at the base, depressed in the interradial spaces so as to make it pentalobate; dome convex and usually bearing from 1 to 6 spines; basals 3 ; primary radials $3 \times 5$; seconclary radials $2 \times 2$; or where there are tertiary radials, there are only $1 \times 2$ secondaries; arms 24 to 40 ; interradials 2 or 3 , in two series; azygous area very different from the interralial areas, and having several more plates and an aperture near the top directed laterally; readily distinguished from Batocrinus and Fretmocrinus by the lobed form of the body, by the azygous area and lateral opening, and by the shortness of the arms. Type D. mississippiensis.

canaliculatus, Meek \& Worthen, 1869, Proc. Acad. Nat. Sci., p. 166, and Geo. Sur. Ill., vol. 5, p. 381, Burlington Gr. coneavus, Meek \&

Worthen, 1861. (Actinocrinus coneavus,) Proc. A cad. Nut. Sci. Phil., p. 131 , and Geo. Sur. III., vol. 2 , p. 215 , Low. Burlington Gr.

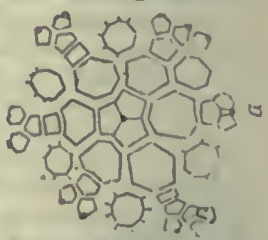
cornigerus, Hall,1858, Fra. 259.-1)oryerinus (Actinocrinus cor-

nigerus,) Geo. Rep. Iowa, p. 576, Burlington Gr.

gouldi, Hall, 1858, (Actinocrinus gouldi,) Geo. Rep. Iowa, p. 613, Keokık Gr.
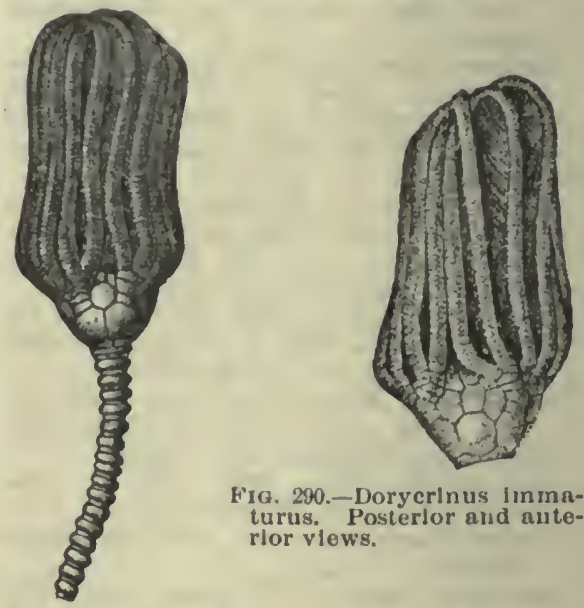

Fia. 290.-Dorycrinus inmaturus. Posterlor and auterlor vlews.

immaturus, Wachsmuth \& Springer, (in press,) Geo. Sur. Ill., vol. 8, p. 175 , Waverly or Kinderhook Gr.

kelloggi, Worthen, 1875, Geo. Sur. III. vol. 6, p. 513, Keokuk Gr. 
lineatus, S. A. Miller, 1881, Jour. Cin. Soc. Nat. Hist., vol. 4, p. 310, Burlington Gr.

mississippiensis, Roemer, 1853, Archiv. fur Nat. Jahr. 19, p. 207, Keokuk Gr.

mississippiensis, var. spiniger, Hall, 1860, Supp. to Geo. Sur. Ioya, p. 53, Keokık Gr.

missouriensis, Shumard, 1858, (Actinocrinus missouriensis,) Geo. Rep. Mo., p. 190, Burlington Gr.

parvibasis, Wachsmuth \& Springer, (in press,) Geo. Sur. Ill., vol. 8, p. 177, Kinderhook Gr.
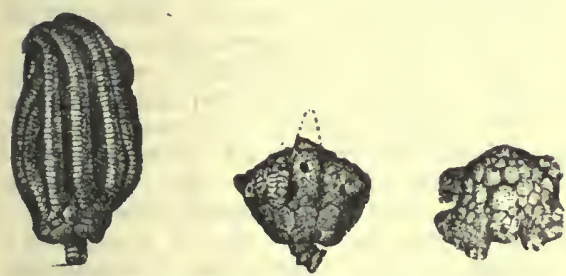

FIG. 291.-Dorycrinus parvibasis. Anterior, posterior, aud ventral views.

parvus, Shumard, 1855, (Actinocrinus parvus,) Geo. Sur. Mo., p. 193, Upper Burlington $\mathrm{Gr}$.

pendens, Hall, 1860, (Actinocrinus pendens,) Supp. to Geo. Sur. Iowa, p. 31, Burlington $\mathrm{Gr}$.

præcursor, Hall, 1862, (Actinocrinus præcursor,) 15 th Rep. N. Y. St. Mus. Nat. Hist., p. 131, Ham. Gr.

quinquelobus, Hall, 1860, (Actinocrinus quinquelobus,) Supp. to Geo. Rep. Iowa, p. 15, Burlington Gr.
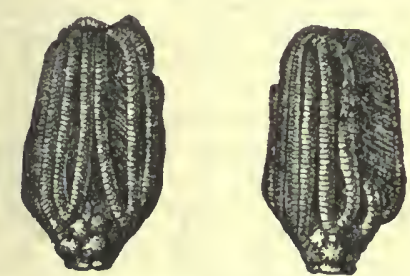

FIG. 292.-Dorycrinus radiatus. Posterior and auterior views.

quinquelobus var. intermedius, Meek \& Worthen, 1868, Proc. Acad. Nat. Sci., p. 346, and Geo. Sur. Ill., vol. 5, p. 385, Burlington Gr.

radiatus, Wachsmuth \& Springer, (in press,) Geo. Sur. Ill., vol. 8, p. 176, Kinderhook Gr.

roemeri, Meek \& Worthen, 1868, Proc. Acad. Nat. Sci. Phil., p. 346 , and Geo. Sur. Ill., vol. 5, p. 383, Burlington Gr.

spinulosus, Hall, 1860, (Actinocrinus spinulosus,) Supp. Geo. Sur. Iowa, p. 52, Keokuk Gr.

subaculeatus, Hall, 1858, (Actinocrinus subaculeatus,) Geo. Rep. Iowa, p. 570, Burlington $\mathrm{Gr}$.

$\mathrm{s} u \mathrm{~b}$ t u rbinatus, Meek \& Worthen, 1860, (Actinocrinus subturbinatus,) Proc. Acad. Nat. Sci. Phil.,p. 388 , and Geo. Sur. Ill., vol. 2, p. 212, Burlington Gr.

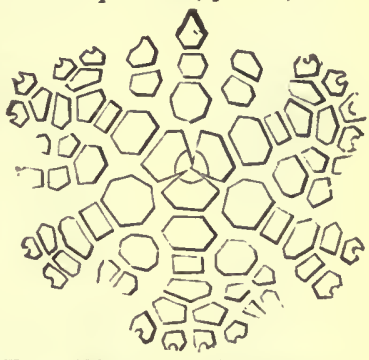

symmetricus, Hall, 1858, (Actinocrinus symmetricus,) Geo. Sur. Iowa, p. 574, Burlington Gr.

trinodus,) Hall, 1858, (Actinocrinus trinodus,) (ieo. Sur. Iowa, p. 575, Burlington Gr.

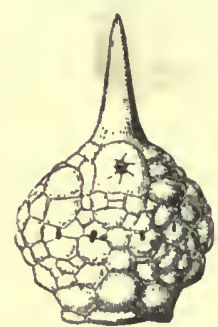

Frg.294-Dorycriuns unicornis. unicornis, Owen \& Shumard, 1850, (Actinocrinus unicornis,) Jour. Acad. Nat. Sci. Phil., vol. 2 , new ser., p. 67, and Geo. Sur. Ill., vol. 5, p. 380, Burlington Gr.

unispinus, Hall, 1861, Actinocrinus unispinus,) Desc. New Crinoidea, p. 2, and Bost. Jour. Nat. Hist., vol. 7, p. 270, Burlington Gr.

Echinocystites, Hall, 1864, 20th Rep. N. Y. St. Mus. Nat. Hist., p. 360 . The name was preoccupied by Wyville Thompson. See Lysocystites.

nodosus, see Lysocystites nodosus.

Ecrinodiscus, Worthen \& Miller, 1883, Geo. Sur. Ill., vol. 7, p. 335. [Ety. echinos, sea urchin;' diskos, quoit.] Body discoid; depressed convex, larger plates in the center of the interradial areas, none imbricating; narrow elongate plates form the border and pass to the under side forming a non-sessile rim; ambulacra 5, connected near the center, and composed of numerous interlocking plates; mouth central or subcentral. Type E. optatus.

kaskaskiensis, Hall, 1858, (Agelacrinus kaskaskiensis,) Geo. Sur. Iowa, p. 696, Kaskaskia Gr.

optatus, Worthen \& Miller, 1883, Geo. Sur. Ill., vol. 7. p. 336, Kaskaskia Gr.

Echino-encrinites, Meyer, 1826, Karst. A rchiv. Nat., vol. 7. [Ety. echinos, sea urchin; krinon, lily.]

anatiformis, see Glyptocystites anatiformis. fenestratus, Troost. Not defined.

Echinus drydenensis, see Eocidaris drydenensis.

gyracanthus, see Tentaculites gyracanthus. 
Ectenocrinus, n. gen. [Ety. ekteino, I stretch out; krinon, lily.] General

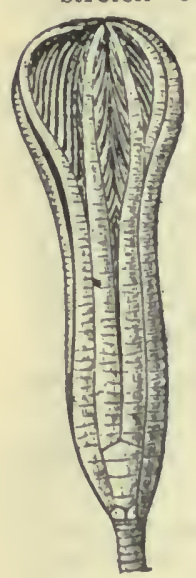

F1G. 295 .

Ectenocrinus grandis. form very elongate; calyx small, subcylindrical, moderately expanding; basals 5, unequal; radials irregular, four plates in three series, before the bifurcation of the free arms, and three in each of the other two series; arms 10, long; pinuules strong; azygous platus 3 , following each other, but not in a direct line; vault unknown; column very long, round, tripartite, and attaching by an expanded base. Type F. simplex. This genus is founded upon Heterocrinus simplex, Hall, as the type, because the genus Heterocrinus was founded upon H. heterodactylus, as the type, which is quite widely removed from H. simplex.

canadensis, Bill-

ings, 1859 , (Heterocrinus can a de n sis,) Can. Org. Rem., Decade 4, p. 48, Trenton Gr. grandis,

1873, (Heterocrinus sim. plex var. grandis, ) Pal. Ohio,

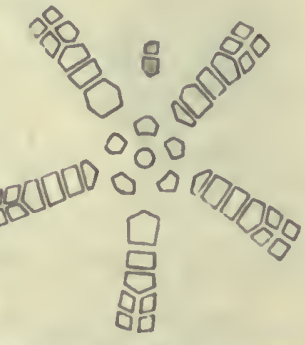

rol. 1, pl. 1, Fia. 296.-Ectenocrinus fig. 7, Hud. simplex. Diagram. Riv. Gr.

simplex, Hall, 1847, (Heterocrinus simplex, ) Pal. N. Y., vol. 1, p. "s0, Trenton and Hud. Riv. Gr.

Enaloaster, Billings, 1858, Can. Org. Rem., Decade 3, p. 82. [Ety. edrion, seat; aster, star.] A substitute for Cyclaster, proposed in 1857 , the latter name having been preoccupied. Body sessile, discoid; plates numerous, irregular, polygonal; ambulacral grooves 5, tapering, composed of two series of oblong ossicles; with four rows of ambulacral pores in each; mouth large, formed of five oral and five internal ossicles. Type E. bigsbyi.

bigsbyi, Billings, 1857, (Cyclaster bigsbyi,) Rep. of Progr. Geo. Sur. Can., p. 293, and Can. Org. Rem., Decade 3, p. 82, Trenton Gr.

Edriocrinus, Hall, 1859, Pal. N. Y., vol. 3, p. 119. [Ety. edrion, seat; krinon, lily.] Body obconic; base solid, without column; radials 5 , resting, in depressions, in the base; azygous plates 2, one large, resting in a basal depres- sion, the other smaller and succeeding the first; arms composed of transversely linear plates and bifurcating. Type E. pocilliformis.

pocilliformis, Hall, 1859, Pal. X. Y., vol. 3, p. 121, Low. Held. Gr.

pyriformis, Hall, 1862, 15th Rep. N. Y. St. Mus. Nat. Hist., p. 88, Up. Held. Gr. sacculus, Hall, 1859, Pal. N. Y., vo!. 3, p. 143 , Oriskany sandstone.

Elwacrinus, Roemer, 1852, syn. for Nucleocrinus.

kirkwoodensis, see Nucleocrinus kirkwood. ensis.

verneuili, see Nucleocrinus verneuili.

Eleutuerocrinus, Shumard \& Yandell, 1856, Proc. Acad. Nat. Sci. Phil., vol. 8 p. 73. [Ety. eleutheros, free; krinon, lily.] Calyx subelliptical, resembling Nueleocrinus in form but depressed on the azygous side; truncaled at the sammit and bulged on one side; subtriangular at the base and prolonged on one of its sides; basals 3 , one small, two irregular and much elongated; radials $1 \times 5$, four-forked, occupying nearly the length of the calyx, one short and not forked; interradials $1 \times 5$; pseudambulacral areas 5 , four linear, extending nearly the entire length of the calyx, one short, subtriangular, situated on the summit plane; apertures 8 (?). Type E. cassedayi.

cassedayi, Shumard \& Yandell, 1856, Proc. Acad. Nat. Sci. I'hil., vol. 8, p. 74, Up. Held. Gr.

whitfeldi, Hall, 1862, 15th Rep. N. Y, St. Mus. Nat. Hist., A pp. C, p. 123, Ham. Gr. Focidaris, Desor, 1858, Synopsis des Eehinides Fossiles. [Ety. eos, dawn; cidaris, turban.] Plates hexagonal; one large tubercle on each plate, sinooth at the base and perforated at the summit; distinguished from Archeocidaris by the absence of a second ring. Type L.

drydenensis, Vanuxem, 1842, (Lchinus (Irydenensis,) Geo. Rep. 31 Dist. N. Y., p. 184, and 20th Rep. N. Y. St. Mus. Nat. Hist., p. 343, Cliemung Gr. hallanus, Geinit\%, 1866, Carb. und Dyas. ill Neb., p. 61, and Pal. E. Neb., p. $152, \mathrm{Up}$. Coal Meas.

squamosus, see Lepidocidaris squamosus.

Eocystites, Billings, 1868, Acad. Geol., p. 643. [Ety. eos, dawn; kustis, bladder.] Plates numerous, varying in size, form, and ornamentation, usually radiately sculptured. Type E. primevus.

longrdactylus, W'alcott, 1886 , Bull. U. S. Geo. Sur., No. 30 , p. 94, Upper Taconic.

primævus, Billings, 1868, Acad. Geol. p. 643, Up. Taconic, St. John's Gr.

Eretmocrinus, Lyon \& Casseday, Fig. 297. 1859, Am. Jour. Sci. and Eocystites Arts, vol. 28, p. 241. [Ety. eretmos, oar; krinon, lily.] Body bitur- 
binate or subglobose, vanlt exceeding the caly $x$ in size; basals 3 , forming a wide rim; primary radials $3 \times 5$; arm openings 12 to 22 ; arms simple or compound, long, flattened in the upper portions; interradials 1 to 3 ; azygous interradials, 8 or more; tube or proboscis excentric and extending beyond the infolding arms; column round. Type E. magnificus.

adultus, Wachsmuth \& Springer, 1881, Proc. Acad. Nat. Sci. Phil., p. 349, Keokuk Gr.

attenuatus, Hall, 1861, (Actinocrinus matuta var. attenuatus,) Desc. New Crin., p. 14, Burlington Gr.

calyculoides, Hall, 1860, (Actinocrinus calyculoides,) Supp. to Geo. Sur. Iowa, p. 17, Burlington Gr.

carica, Hall, 1861, (Actinocrinus carica,) Desc. New Crin., p. 10, Burlington Gr.

clio, Hall, 1861, (Actinocrinus clio,) Desc. New Crinoidea, p. 1, and Bost. Jour. Nat. Hist., vol. 7, p. 262, Burlington Gr.

clœlia, Hall, 1861, (Actinocrinus clœlia,) Desc. New Crinoidea, p. 1, and Bost. Jour. Nat. Hist., vol. 7 , p. 266, Burlington Gr.

corbulis, Hall, 1861, (Actinocrinus corbulis,) Desc. New Crin., p. 1, and Bost. Jour. Nat. Hist., vol. 7, p. 265, Burlington $\mathrm{Gr}$.

coronatus, Hall, 1860, (Actinocrinus coronatus,) Supp. Geo. Sur. Iowa, p. 28, Burlington Gr.

gemmiformis, Hall, 1860, (Actinocrinus gemmiformis,) Supp. Geo. Sur. Iowa, p. 23, Burlington Gr.

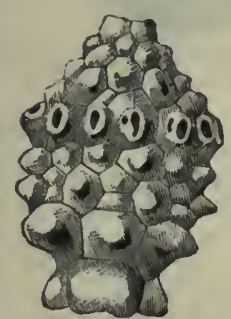

FIG. 298.

Eretmocrinus konincki. intermedius, Waclssmuth \& Springer, 1881, Proc. Acad. Nat. Sci., p. 348, Keokuk Gr.

konincki, Shumard, 1855, (A ctinocrinus konincki,) Geo. Sur. Mo., p. 194, Burlington Gr.

leucosia, Hall, 1861, (Actinocrinus leucosia,) Desc. New Crin., p. 1, and Bost. Jour. Nat. Hist., vol. 7, p. 261, Burlington Gr.

magnificus, Lyon \& Casseday, 1859, Am. Jour. Sci. and Arts, vol. 28, p. 241 , Keokuk Gr.

matuta, Hall, 1861, (Actinocrinus matuta) Desc. New Crinoidea, p. 14, Burlington Gr.

matuta var. attenuata, see E. attenuatus.

neglectus, Meek \& Worthen, 1869, (Batocrinus neglectus,) Proc. Acad. Nat. Sci., $\mu .355$, and Geo. Sur. Ill., vol. 5, p. 377 , Burlington $\mathrm{Gr}$.

originarius, Wachsmuth \& Springer, 1881, Proc. Acad. Nat. Sci., p. 348, Keokuk Gr. ramulosus, Hall, 1858, (Actinocrinus ramulosus,) Geo. Sur. Iowa, p. 615, Keokuk Gr.

remibrachiatus, Hall, 1861, (Actinocrinus remibrachiatus,) Desc. New Crinoidea, p. 11 , Burlington Gr:

varsouviensis, Worthen, 1882, Bull. No. 1, Ill. St. Mus. Nat. Hist., p. 30, and Geo. Sur. IIl., vol. 7, p. 306, Warsaw Gr. verneuilanus, Shumard, 1855, (Actinocrinus verneuilianus,) Geo. Sur. Mo., p. 193, Burlington Eretmocrinus Gr.

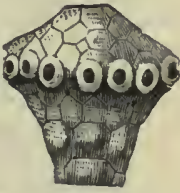

FIG. 299 Eretmocrinus
verneuilauus.

Erisocrinus, Meek \& Worthen, 1865, Am. Jour. Sci. and Arts, vol. 89, p. 174. [Ety. eris, contention; krinon, lily.] Calyx saucer-shaped; basals 5 , small; subradials 5 , large; radials $2 \times 5$, large; no interradials; arms 10 ; column round. Type E. typus.

antiquus, Meek \& Worthen, 1869, Proc. Acad. Nat. Sci. Phil., p. 71, and Geo. Sur. Ill., vol. 5, p. 447, Burlington Gr.

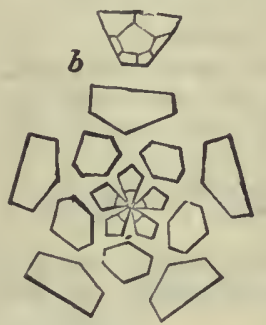

FIG. 300. - Erisocrinus conoideus. Side view, natural size; $b$, diagram, 2 diam. cognatus, Wachsmuth \& Springer, 1887, Note to p. 255, Revis. Palæocrinoidea, syn. for $\mathrm{E}$. planus.

conoideus, Meek \& Worthen, 1865 , Proc. Acad. Nat. Sci. Phil., p. 150 , and Geo. Sur. Ill., vol. 2 , p. 318 , Up. Coal Meas.

inflexus, Geinitz, 1866, (Cy a th o crinus inflexus,

Carb. und Dyas, in Neb., p. 62, and White's Cont. to Pal., No. 6, p. 128 , Coal. Meas.

nebraskensis, Meek \& Worthen, 1865 , Am. Jour. Sci., vol. 89 , p. 174, Up. Coal Meas. Regarded a variety of E. typus. planus, White, 1880, Proc. U. S. Nat. Mus., vol. 2, p. 25\%, and Cont. to Pal., No. 6 , p. 127 , Coal Meas.
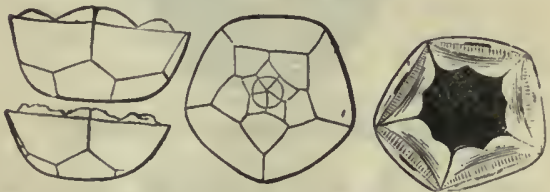

Frg. 301.-Erisocrinus typus. Two side views, basul view, and top view of calyx.

typus, Meek \& Worthen, 1865, Am. Jour. Sci. and Arts, vol. 89, p. 174, and Geo. Sur. Ill., vol. 2, p. 319 , Up. Coal Meas. tuberculatus, see Eupachycrinus tuberculatus.

whitii, Meek \& Worthen, 1869, Proc. Acad. Nat. Sci. Phil., p. 72, and Geo. Sur. Ill., vol. 5, p. 448, Burlington Gr. Eucalyptocrinus, Goldfuss, 1826, Petref. Germ., p. 212. [Ety. eu, well; kalyptos, covered; krinon, lily.] Body turbinate 
or bowl-shaped from base to arms, and with arms and interbrachial plates subovate or subelliptical; batsals 4 , concealed in basal cavity and developed in the interior; primary radials $3 \times 5$, the first large; secoudary radials $2 \times 10$; interradials 3 , one very large; arms 20 , composed of a double series of plates, which fill the interbrachial spaces; interbrachial plates solid, extending from the interradial plates as bigh as the arms reach, and uniting at the summit; proboscis or tube extending to the top, and sometines far beyond; column round; attaching by branching roots. Type E. rosaceus.

armosus, see Siphonocrinus armosus.

crelatus, Hall, 1843, (Hypanthocrinites crelatus,) Geo. Rep. 4tl, Dist. N. Y., p. 113, and 281 l $\operatorname{Rep.~N.~Y.~St.~Mus.~Nat.~}$ Hist., p. 142, Niagara Gr.

chicagoensis, Wincliell \& Marcy, 186., Mem. Bos. Soc. Nat. Hist., p. 90, Niagara $\mathrm{Gr}$.

conicus, Troost. Not defined.

constrictus, Hall, 1879, Trans. Alb. Inst., vol. 10 (Abstract, p. 10), and 11 th Rep. Geol. and Nat. Hist. Ind., 1. 273, Niagara Gr.

cornutus, Hall, 1864, 20th liep. N. Y. St. Mus. Nat. Hist., p. 363, Niagara Gr.

cornutus var. excavatus, Hall, 1864, 20th Rep. N. Y. St. Mus. Nat. Mist., p. 364, Niagara Gr.

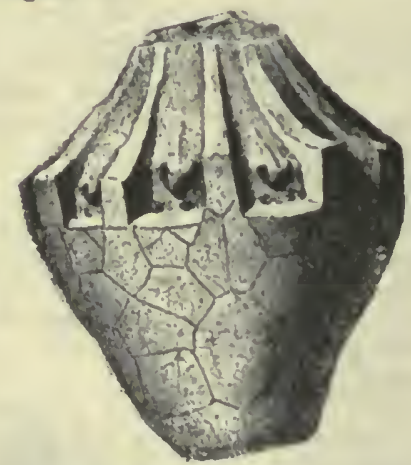

FIG. 302.-Eucaly ptocrinus crassus.

crassu8, Hall, 1863, Trans. Alb. Inst., vol. 4, p. 197, and 28th Rep. N. Y. St. Mus. Nat. Hist., p. 141, Niagara Gr.

decorus, Phillips, 1839, (Hypanthocrinites decorus, ) Murch. Sil. Syst., p. 672 , and Pal. N. Y., vol. 2, p. 207, Niagara $\mathrm{Gr}$.

depressuв, S. A. Miller, 1880, Jour. Cin. Soc. Nat. Hist., vol. 3, p. 232, Niagara Gr.

egani, S. A. Miller, 1850, Jour. Cin. Soc. Nat. Hist., vol. 3, p. 140, Niagara Gr. extensus, Troost. Not defined.

gibbosus, Troost. Not defined. goldfussi, Troost. Not defined.

inconspectus, Ringueberg, 1854, Proc. Acad.

Nat. Sci., p. 148. Not properly defined. levis, Troost. Not defined.

magnus, Worthen, 18\%5, Geo. Sur. Ill., vol. 6, p. 501 , Ningara Gr.

nashvillx, Troost. Not defined.

obconicus, Hall, 1864, 20th Rep. N. I. St. Mus. Nat. Hist., p. 365, Niagara Gr.

ormatus, Hall, 1861, Rep. of Progr. Geo. Sur. of Wis., p. 20, Niagara Gr.

ovalis, Troost, as figured by Hall, 1876 , 2Sth Rep. N. I. St. Mus. Nat. Hist., p. 143, Niagara Gr.

papulosus, Hall, 1852, Pal. N. Y., vol. 2, p. 211, Niagara Gr.

phillipsi, Troost. Not defined.

proboscidalis, S. A. Miller, 1882, Jour. Cin. Soc. Nat. Hist., vol. 5, p. $224, \mathrm{Ni}$ agara $\mathrm{Gr}$.

ramifer, Roemer, 1860, Sil. Fauna West Tenn., 1). 51, Niagaru Gr.

rotundus, S. A. Miller, 1882, Jour. Cin. Soc. Nat. Hist., vol. 5, p. 82 , Niagara Gr.

splendidus, J'roost, Catal. Ilall \& IVhit. field, 1875̄, Ohio Pul., vol. 2, p. 128, Niagara $\mathrm{Gr}$.

tennesseer, Troost. Not defined.

tuberculat us, Miller \& Dyer, 1878, Jour. Cin. Soc. Nat. Hist., vol. 1, p. 36, $\mathrm{Ni}$ agara Gr.

turbinatus, S. A. II i I I e r, 1882, Jour. Cin. Soc. Nat. IIist., vol. 5 , p. 82, Niagara Gr.

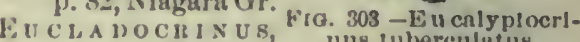
Meek, 1871, U. S.

Geo. Sur. Terr., p. 373. [Ety. eı, very ; klados, branch; krinon, lily.] Calyx like Platycriuns, and distinguished by having the radial series cxtended in the form of tubular free rays, whicl beur arins, altermately, on either side, throughout their length; arms coinposed of a double series of interlocking plates. Type k. montanensis.

millebrachiatus, Wachsmuth \& Springer, 1878, Proc. Acad. Nat. Sci. Phil., p. 245 , Burlington and Keokuk Gr.

montanensis, Meek, 1871, Hayden's Rep. U. S. Geo. Sur. Ter., p. 373, Subcar. boniferous.

pleuroviminus, White, 1862, (Platycrinus pleuroviminus,) Proc. Bost. Soc. Nat. Hist., vol. 9, p. 17, Up. Burlington $\mathrm{Gr}$.

Eugaster, Hall, 1868, 20th Kep. N. Y. St. Mus. Nat. Hist., p. 332. [Ety. euge, pre-eminent, remarkable; aster, star.] A central, alated disk, with five long, slender flexuous rays; disk composed on the ventral side of small polygonal 
plates; rays consisting of a double series of alternating, subquadrate, ambulacral ossicles, with curved ambulacral plates; oral plates 10 ; pores large, two rows in each ray; adambulacral and disk-plates spine-bearing. Type E. logani.

concinnus, Ringueberg, 1886, Bull. Buff. Soc. Nat. Sci., vol. 5, p. 8, Niagara $\mathrm{Gr}$.

logani, Hall, 1868, 20th Rep. N. Y. St. Mus. Nat. Hist., p. 333, Ham. Gr.

EupAcirycrinus, Meek \& Worthem, 1865 , Proc. Acad. Nat. Sci. Phil., p. 159. [Ety. eu, very; pachys, thick ; krinon, lily.] Calyx saucer or bowl shaped; plates heavy, tumid; sutures strongly defined; basals 5 ; subradials 5 ; radials $2 \times 5$, and sometimes there are third radials in some of the rays; the second radials are often spinebearing; azygous interradials 3 ; arms 5 or 10 , composed of a double series of plates, bearing pinnules; column round. Type E. quatuordecembrachalis.

asperatus, Worthen, 1882, Bull. No. 1, Ill. St. Mus. Nat. Hist., p. 34, and Geo. Sur. Ill., vol. 7, p. 311, Kaskaskia Gr.

bassetti, Worthen, 1875, Geo. Sur. Ill., vol. 6, p. 528 , Coal Meas.

boydi, Meek \& Worthen, 1870, Proc. Acad. Nat. Sci. Phil., p. 30, and Geo. Sur. Ill., vol. 5, p. 554, Kaskaskia Gr.

craigi, Worthen, 1875, Geo. Sur. Ill., vol. 6 , p. 527, Coal Meas.

crassus, Meek \& Worthen, 1870, (Cyathocrinus . crassus,) Proc. Acad. Nat. Sci. Phil., p. 392, and Geo. Sur. Ill., vol. 2, p. 314, Low. Coal Meas.

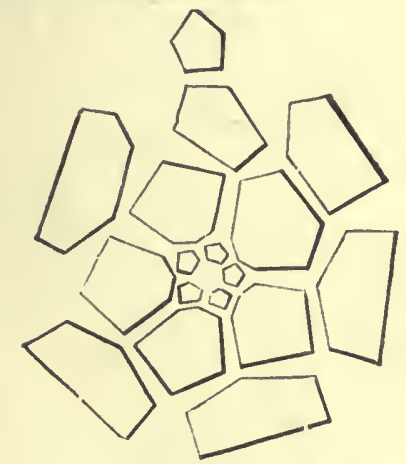

FIG. 304.-Eupachycrinus erassus. Diagram.

fayettensis, Worthen, 1873, Geo. Sur. Ill., vol. 5 , p. 565 , Up. Coal Meas.

formosus, Worthen, 1873, (Zeacrinus formosus,) Geo. Sur. Ill., vol. 5, p. 549, Kaskaskia Gr.

germanus, S A. Miller, 1879, Jour. Cin. Soc. Nat. Hist., vol. 2, p. 40, Kaskaskia Gr. gracilis, Wetherby, 1880, (Cromyocrinus gracilis,) Jour. Cin. Soc. Nat. Hist., vol. 2 , p. 248, Kaskaskia Gr.

hemisphericus, Shumard, 1858, (Poteriocrinus hemisphericus, ) Trans. St. Louis, Acad. Sci., vol. 1, p. 221, and Geo. Sur. Ill., vol. 5, p. 561, Coal Meas.

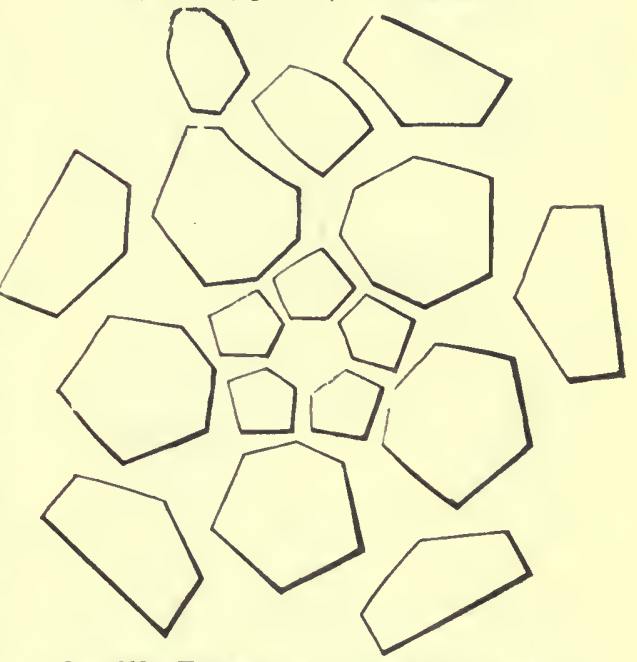

FIG. 305.-Eupachycrinus saugamonensis. Dlagram.

monroensis, Worthen, 1882, Bull. No. 1, St. Mus. Nat. Hist., p. 30, Kaskaskia Gr.

orbicularis, Hall, 1861, (Scaphiocrinus orbicularis,) Bost. Jour. Nat. Hist., p. 311, Keokuk Gr.

pentalobus, Hall, 1858, (Cyathocrinus pentalobus,) Geo. Sur. Iowa, p. 687 , Kaskaskia Gr.

platybasis, White, 1876, Geo. Uinta Mountains, p. 108, and Cont. to Pal., No. 6, p. 124, Low. Aubrey Gr.

quatuordecembrachialis, Lyon, 1857, (Graphiocrinus quatuordecembrachialis,) Geo. Sur. Ky., vol. 3, p. 477, Kaskaskia Gr.

sanctiludovici, Worthen, (in press,) Geo. Sur. Ill., vol. 8, p. 98, St. Louis Gr.

sangamonensis, Meek \& Worthen, 1861, (Cyathocrinus sangamonensis,) Proc. Acad. Nat. Sci. Phil., p. 392, and Geo. Sur. Ill., vol. 2, p. 310, Up. Coal Meas.

spartarius, S. A. Miller, 1879, Jour. Cin. Soc. Nat. Hist., vol. 2, p. 38, Kaskaskia Gr.

subtumidus, Worthen, 1867, (Zeacrinus subtumidus,) Geo. Sur. Ill., vol. 5, p. 548, Kaskaskia Gr.

tuberculatus, Meek \& Worthen, 1865, (Erisocrinus tuberculatus,) Proc. Acall. Nat. Sci. Phil., p. 150, and Geo. Surr. Ill., vol. 2, p. 319, Coal Meas.

verrucosus, White \& St. John, 1869, Trans. Chi. Acad. Sci., p. 117, Coal Meas. 


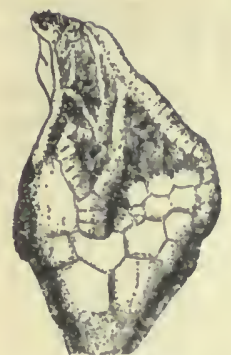

FIG. 306. - Euspirocrinus obconlcus.
EUspirocrinds, Angelin, 1878, Iconogr. Crinoid Suec., p. 24. [Ety. euspeires, winding; krinon, lily.] Calyx cyathiform; basals 5 ; subradials 5 , large; radials $1 \times 5$, wider than high, excavated for the attachment of the arms; azygous area wide, plates large ; ventral tube composed of large plates; vault covered with large plates; arms bifurcate. Type E. spiralis.

obconicus, W. R. Billings, 1885, Ottawa Field Nat. Club, vol. 2, p. 248, Tren. ton Gr.

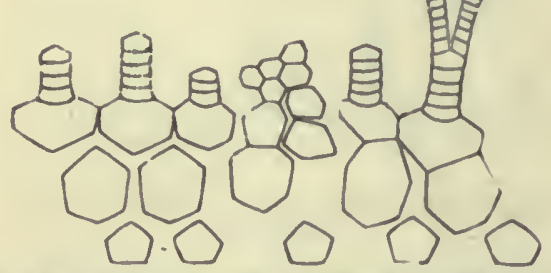

Fia. 307.-Euspirocrinus obconfeus. IMagrnm.

Forbesocrinus, DeKoninck \& LeHon, 1854,

Resch. Crin. Carb. Belg., p. 118. [Ety. proper name; krinom, lily.] Calyx large, plates heavy ; basals 3 ; subradials 5 ; primary radials 3 or $4 \times 5$; secondary radials 2 to $4 \times 10$; tertiary radials 2 to $4 \times 20$; arms 50 to 60 , long and sometimes. dividing; regular interradials 10 to 20 or more; azygous interradials numerous; in teraxillaries 10 to 20 or more. Type F nobilis. agassizi, Hall, 1858 and 1860, Geo. Sur. of Iowa, p. 631, Burlington Gr.

agassizi var. giganteus, Meck \& Worthen, 1861, Proc. Acad. Nat. Sci. Phil. p. 131, and Geo. Sur. Ill., vol. 3 , p. 495 , Burlington $\mathrm{Gr}$.

asteriformis, see Onychocrinus as teriformis.

cestriensis, Hall, 1860, Supp. to Geo. Iowa, p. 68, Kaskaskia Gr.

communis, see Taxocrinus communis.

giddingi, see Taxocrinus giddingi. juvenis, see Taxocrinus juvenis.

kelloggi, see Taxocrinus kelloggi.

lobatus, see Taxocrinus lobatus.

lobatus var. tardus, see Taxocrinus lobatus var. tardus.

meeki, see Taxocrinus meeki. monroensis, see Onychocrinus monroensis. multibrachiatus, see Taxocrinus multibrachiatus.

noruoodi, see Onychocrinus norwoodi. nuntius, see Taxocrinus nuntius.

parvus, Wetherby, 1879, Jour. Cin. Soc.

Nat. Hist., vol. 2, p. 138, Kaskaskia Gr.

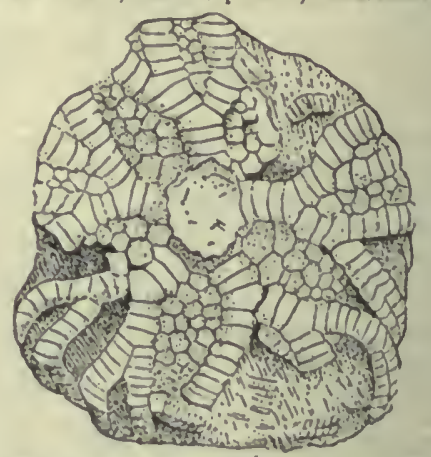

Fra. 308.-Forbesocrinus wortleni.

pratteni, see Melocrinus pratteni.

ramulosus, Lyon \& Casseday, seo Onychocrinus ramulosus.

ramulosus, Hall, see Taxocrinus ramulosus. saffordi, see Taxocrinus saffordi.

semiovatus, see Taxocrinus semiovatus.

shumardanus, see Taxocrin us shumardanus. spiniger, see Taxocrinus spiniger.

subramulosus, Shumard, 1866, syn. for

Taxocrinus ramulnsus.

thiemii, see Taxocrinus thiemii.

whitfieldi, see Taxocrinus whitfieldi.

wortheni, Hall, 1858, Geo. Rep. Iowa, p.

632, Keokuk Gr.

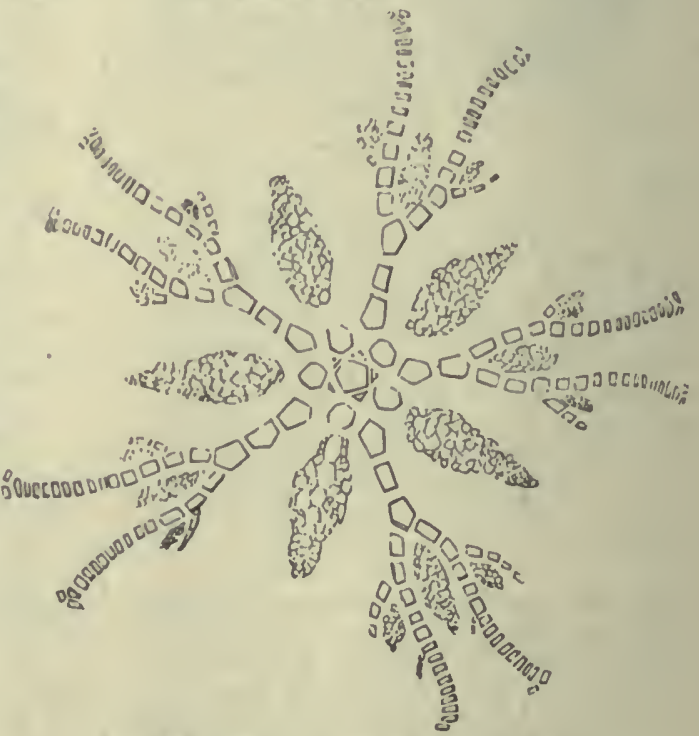

Fra. 309.-Gaurocrinus nealll. Diagram.

Gaurocrinus, S. A. Miller, 1883, Jour. Cin. Soc. Nat. Hist., vol, 6, p. 228. [Ety. gauros, haughty; proud; krinon, lily.] 
Calyx having strong radial ridges and depressed interradial and intersecondary radial areas; basals 5 ; subradials 5 ; primary radials $3 \times 5$, or the left posterior ray only 2 ; secondary radials 10 to $16 \times 10$; interradial plates numerous and small; azygous area supported by a ridge up the middle series of plates; vault covered by small plates, which are continued as a covering over the arm furrows; arms 20 or more, bearing pinnules. Type G. nealli.

angularis, Miller \& Dyer, 1878, (Glyptocrinus angularis, Jour. Cin. Soc. Nat. Hist., vol. 1, p. 28, Hud. Riv. Gr.

cognatus, S. A. Miller, 1881, (Glyptocrinus

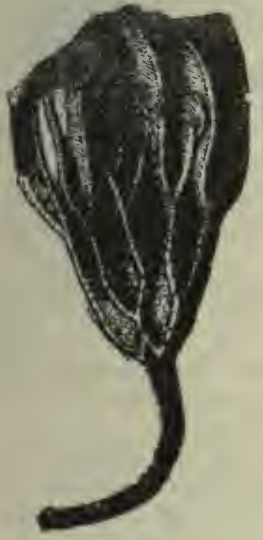

FIG. 310,-Gaurocrinus nealli. cognatus,) 'Jo u r. Cin. Snc. Nat.Hist., vol. 4, p. 75 , Hud. Riv. Gr.

m ag $n$ ifi c us, S. A. Miller, 1883, Jour. Ci n. Soc. Nat. Hist., vol. 6, p. 230, Hud. Riv. Gr. nealli, Hall, 1866 , (G ly p t o c rinus nealli, ) A dv. Sheets 24th Rep. N. Y. St. M u s. Nat. Hist., p. 206 , and Ohio Pal., vol. 1, p. 34, Hud. Riv. Gr.

s plendens, S. A. Miller, 1883, Jour. Cin. Soc. Nat. Hist., vol. 6, p. 230, Trenton Gr.

Genn foorinus, Wachsmuth \& Springer, 1882, Proc. Acad. Nat. Sci., p. 334. [Ety. gennaios, of noble birth ; krinon, lily.] Body wider than high, lobed, striated; vault low; basals 3 ; primary radials $3 \times 5$; secondary radials $1 \times 10$; interradials 5 to 7 ; azygous interradials more numerous, and having three plates in the second row instead of two, as in Actinocrinus; interaxillaries 1 to 3 . Type G. cornigerus.

caly pso, Hall, 1862, (A ctinocrinus calypso,) 15th Rep. N. Y. St. Mus. Nat. Hist., p. 133, Ham. Gr.

cassedayi, Lyon, 1861, (Actinocrinus cassedayi,) Proc. Acad. Nat. Sci. Phil., p. 410, Up. Held. Gr.

cauliculus, Hall, 1862, (Actinocrinus cauliculus,) 15th Rep. N. Y. St. Mus. Nat. Hist., p. 132, Ham. Gr.

cornigerus, Lyon \& Casseday, 1859 , (Actinocrinus cornigerus,) Am. Jour. Sci., vol. 28, p. 238, Ham. Gr.

eucharis, Hall, 1862, (Actinocrinus eucharis,) 15th Rep. N. Y. St. Mus. Nat. Hist., p. 130, Ham. Gr.

kentuckiensis, Shumard, 1860, (Actinocrinus kentuckiensis,) Trans. St. Louis Acad. Sci., p. 345, syn. for G. cornigerus. nyssa, Hall, 1862, (Actinocrinus nyssa,) 15th Rep. N. Y. St. Mus. Nat. Hist., p. 129, Ham. Gr.

pocillum, Hall, 1862, (Actinocrinus pocillum,) 15th Rep. N. Y. St. Mus. Nat. Hist., p. 134, Ham. Gr.

Glyptaster, Hall, 1852, Pal. N. Y., vol. 2, p. 187. [Ety. glyptos, sculptured; aster, star.] Calyx obconical, depressed between the arm bases, radial portion ridged; basals 5 ; subradials 5 ; primary radials $3 \times 5$; secondary radials 2 or more by 10 ; interradials 6 or more; azygous interradials more numerous; arms 10 , composed of double series of plates. Type G. brachiatus.

brachiatus, Hall, 1852, Pal. N. Y., vol. 2, p. 187, Niagara Gr.

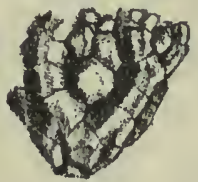

Fig. 311.-Glyptaster egani. Natural size and enlarged.

egani, S. A. Miller, 1881, Jour. Cin. Soc. Nat Hist., vol. 4, p. 261, Niagara Gr.

inornatus, Hall, 1863, Trans. Alb. Inst., vol. 4, p. 205, and 2sth Rep. N. Y. St.

Mus. Nat. Hist., p. 134, Niagara Gr.

o c c ide n t a lis, Hall, 1863 , Trans. Alb. Inst., vol. 4, p. 204, and 28 th Rep. N. Y. St. Mus. Nat. Hist., p. 134, Niagara Gr. occidentalis var. crebescens, Hall, 1879 , 28th Rep. N. Y. St. Mus. Nat. Hist., p. 133, Niagara Gr.

pentangularis, H a 11, FiG. 312.-Glyptaster 1867,20 th Rep.

N. Y. St. Mus. Nat. Hist., p. 369, Niagara $\mathrm{Gr}$.

Gly PTocrinus, Hall, 1847, Pal. N. Y., vol. 1, p. 280 . [Ety. glyptos, sculptured;

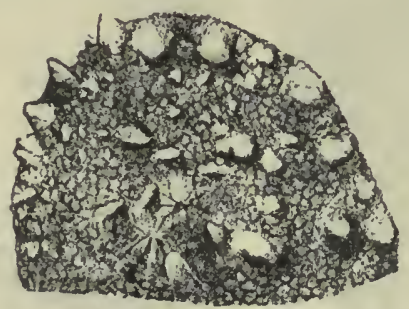

FIG. 313. - Glyptocrinus decadactylus. Part of vauit mag. 6 diam, showing excurrent opening.

krinon, lily.] Calyx obconoidal, interradial areas flattened or depressed; surface sculptured and having radial ridges; basals 5 ; primary radials $3 \times 5$; 
scondary radials 1 or more by 10 ; tertiary radials usually present; arms 10 to 20 or more, bearing pinnules; first interradial resting upon the first

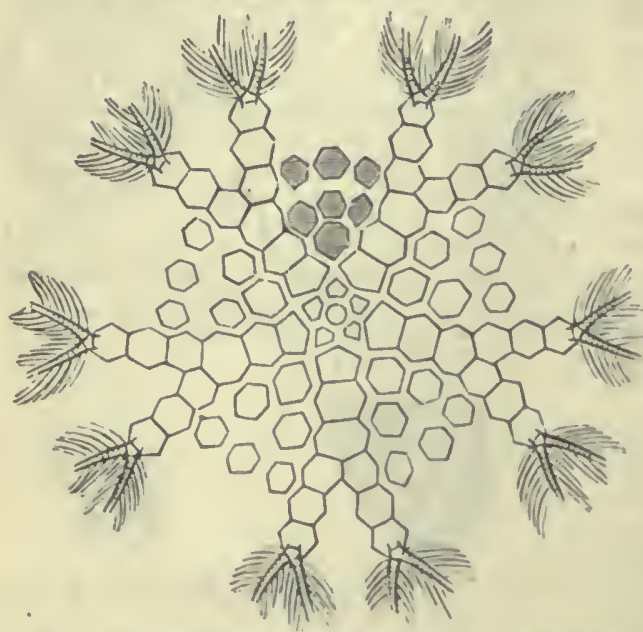

Fit:. 314.-Glyptocrinus decadactylus. Dlagram.

primary radials, and followed by succeeding ranges of two or inore, which graduate into the vault; vault slightly

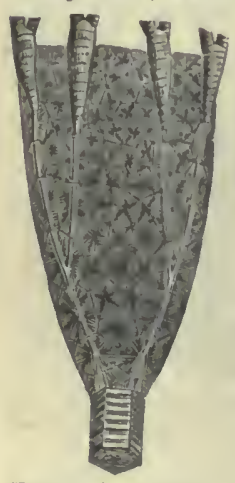

Fra. 316. - Glyptocrinus foriishelli. convex, with sunken interradial areas; plates becoming swaller as they approach the inner face of the arms, and becoming a sonewhat granular continuous cover over the ambulacral furrows; excurrent opening subcentral on the upper face of the vault; column Fia $315,-$-Giypto-
round, without base or crinus deearoots for attachment. dactylus.

Type G. decadaetylus.

angilaris, sce Gaurocrinus ancularis.

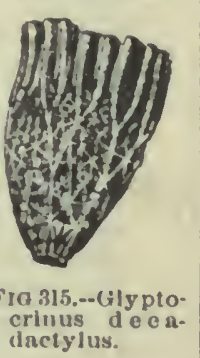

argutus, Walcott, 1883 , 35th Rep. N. Y. St. Mus. Nat. Hist., p. 207 Trenton Gr.

armosus, see Siphonocrinus arinosis.

baeri, see Xenocrinus baeri.

carleyi, see Mariacrinus carleyi.

cognatus, see Gaurocrinus cognatus.

decadactylus, Hall, 1847 , Pal. N. Y., vol. $1, \mathrm{p}$. 281, Hud. Riv. Gr.

dyeri, Meek, 1872, Proc. Acad. Nat. Sci. Pliil.,p. 314 , and Ohio Pal., vol. 1, p. 32, Hud. Riv. Gr. dyeri var. sublaevis, $S$. A. Miller, 1878, Jour. Cin. Soc. Nat. Hist., vol. 1, p. 103, Hud. Riv. Gr. fimbriatus, Sbunard, 1855, Geo. Sur. Mo., p. 194, Trenton Gr.

fornshelli, S. A. Miller, 1874, Cin. Quar. Jour. Sci.. vol. 1, p. 348, Hud. Riv. Gr. gracilis, Wetherby, syn. for Gaurocrinus angularis.

harrisi, see Compsocrinus harrisi.

lacunosus, see Archreocrinus lacunosus. libanus, Safford, 1869, Geo. of Tenn. Not defined.

marginalus, see Archwocrinus marginatus.

miamiensis, S. A. Miller, 1882, Jour. Cin. Sor: Nat. Hist., vol. 5, p. 34, Hud. Riv. Gr.

nealli, see Gauroerinus nealli.

nobilis, see Siphonocrinus nobilis.

ornatus, Billings, 1857, Rep. of Progr.

Geo. Sur. Can., p. 260, and Can. Org. Rem., Decade 4, p. 60, Trenton Gr.

parvus, Hall, 1872, 24 th Rep. N. Y.

St. Mus. Nat. Hist., p. 207, II ud. Riv. Gr.

pattersoni, S. A. Miller, 1882, Jour. Cin. Soc. Nat. Hist., vol. 5, p. 80 , Utica Slate Gr.

plumosus, Hall, 1843, (Actinocrinus plumosus,) Geo. Rep. 4 th Dist. N. Y., p. 72, and Pul. N. Y., vol. 2, p. 180, Clinton Gr. Fonnded upon fragments too poor for even generic determination.

priscus, Billings, 1857, Rep. of Progr. Geo. Sur. Can., p. 257, and Can. Org Rem., Decade 4, p. 56, Black Riv. and Trenton Grs.

quinquepartitus, Billings, 1S59, Can. Org. Rem., Deeade 4, pl. 8, fig. 4a, 4b, Trenton Gr.

ranulosus, Billings, 1856, Can. Nat. Geo., vol. 1, and Can. Org. Rem., Decade 4, P. 57, Trenton Gr.

richardsoni, Wetherby, 1850, Jour. Cin.

Soe. Nat. Hist., vol. 2, p. 245, Hud. Riv. Gr.

sculptus. S. A. Miller, 1882, Jour. Cin. Soe. Nat. Hist., vol. 5, p. 37, Hud. Riv. Gr.

shafferi, see Pyenocrinus shafferi.

shafleri var. germanus, see Pyenocrinus germanus.

siphonatu8, Hall, 1861, syn. for Siphonocrinus armosus.

subglobosus, Meek, 1873, (G. dyeri var subglobosus,) Pal. Obio, vol. 1, p. 34, Hud. Riv. Gr.

subnodosus, sce Rhaphanocrinus subnodosus.

Gry Ptocystites, Billings, 1854, Can. Jour., vol. 2 , p. 215, and Can. Org. Rem., Decade 3, p. 53. [Ety. glyptos, sculptured; kustis, bladder.] Boày elongate, cylindrical; four series of plates, 4 in the basal and 5 in each suecceding series; mouth in one of the plates of the seeond series; ambulacral orifice at the center of the summit where it receives the five ambulacral grooves; arms recumbent upon the apex of the fossil, 
and grooves beset with small plates ; 10 to 13 pectinated rhombs; column short, tapering to a point. Type G. multiporus. anatiformis, Hall, 1847, (Echinoencrinites anatiformis,) Pal. N. Y., vol. 1, p. 89, Trenton Gr.

forbesi, Billings, 1857, Rep. of Progr. Geo. Sur. Can., p. 283, and Can. Org. Rem., Decade 3 , p. 59 , Yhazy Gr.

logani, Billings, 1857, Rep. of Progress, Geo. Sur. Can., p. 282, and Can. Org. Rem., Decade 3, p. 57, Trenton Gr.

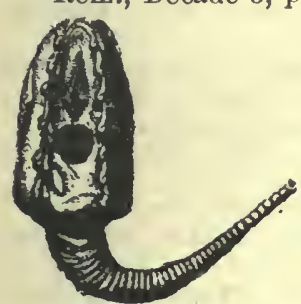
logani var. gracilis, Billings, 1858, Can. Org. Rem., Decade 3, p. 59, Trenton Gr. multiporus, Billings, 1854, Can. Jour., vol. 2, p. 215, and Can. Org. Rem., Decade 3, p. 54, Trenton Gr.

FrG. 317.-Glyptocys- GOMPHOCYSTITES, tites multiporus.

Hall, 1869, 20th

Rep. N. Y. St. Mus. Nat. Hist., p. 351, [Ety. gomphos, nail or rudder; kustis, bladder.] Elongate pyriform, narrow

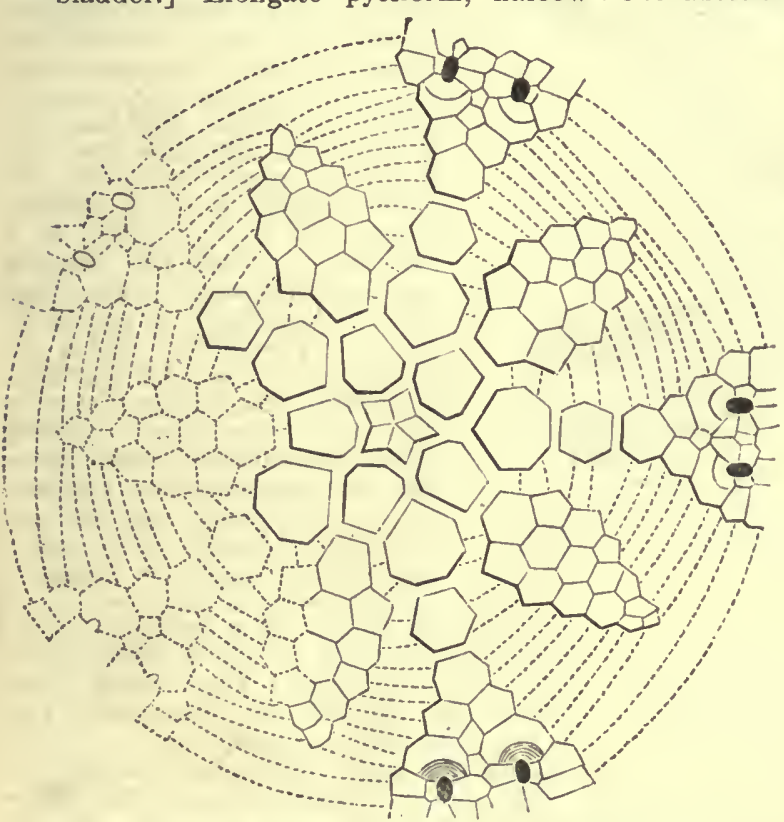

FrG. 318,-Goniasteroidocrinus fiscellus. Diagram 2 diam. indianensis n. sp., Niagara Gr. Upper part elliptical in outline and regularly convex; five ambulacral grooves curve spirally outward from an ambulacral orifice within the groove near the mouth, and extend below the summit; mouth round, situate between two of the ambulacral grooves; each anhbulacral groove has a suture in the bottom of it, but there is no other evidence of the subdivision of the top into plates;

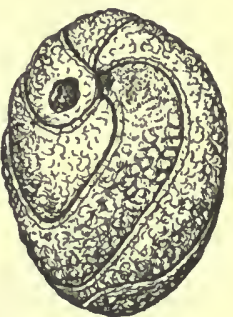

FrG. 319. - Gompliocystitesindianensis. Summit view. even the mouth appears as a hole through a solid test; whole surface tuberculated, and each tubercle pierced with a pair of pores. Collected by J. F. Hammell in Jefferson County, Indiana.

tenax, Hall, 1864, 20th Rep. N. Y. St.

Mus. Nat. Hist., p. 352, Niagara Gr.
Goniasteroldocrinus, Lyon \& Casseday, 1859, Am. Jour, Sci., vol. 28, 2d series, p. 233. [Ety. like the recent genus Goniaster; krmon, lily.] Body short, cylindrical, or subglobose ; basals 5 ; subradials 5 , often protuberant; primary radials $3 \times 5$ : secondary radials 2 to $4 \times 10$; arms numerous, delicate, pend-

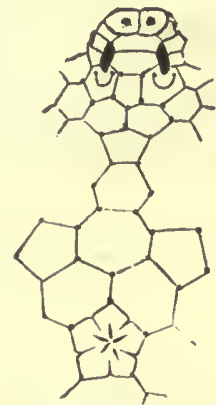

FIG 320. - Dla grammatic view of Gilbertsocrinus bursa to show it is distinct from Gon:asteroidocrinus. below, inflated above; plates spirally arranged; ambulacral orifice central on the upper surface; mouth excentric; arms sessile, and curving from the ambulacral orifice outward to or below the point of greatest diameter. Type G. glans.

clavus, Hall, 1864, 20th Rep. N. Y. St. Mus. Nat. Hist., p. 353, Niagara Gr.

glans, Hall, 1864, 20th Rep. N. Y. St. Mus. Nat. Hist., p. 352, Niagara Gr. ent, supporting pinnules; interradials 10 to $18 \mathrm{in}$ each area; vault depressed and extending in five or six pseudo-brachial appendages star-like, which bifurcate, then spread, curve, and terminate each in a point; these brachial appendages separate the interradial areas from the dome; excurrent orifice sublateral, not protruding. Type S. tuberosus. There are some who nse Ollacrinus as the 
generic name, but it was not defined or established; there are others who use Gilbertsocrinus, but it, probably, is a distinct genus, and, so far, not known in America.

fiscellus, Meek \& Wortben, 1861, (Trematocrinus fiscellus,) Proc. Acad. Nat. Sci. Phil., p. 383, and Geo. Sur. Ill., vol. 2, p. 222, Burlington Gr.

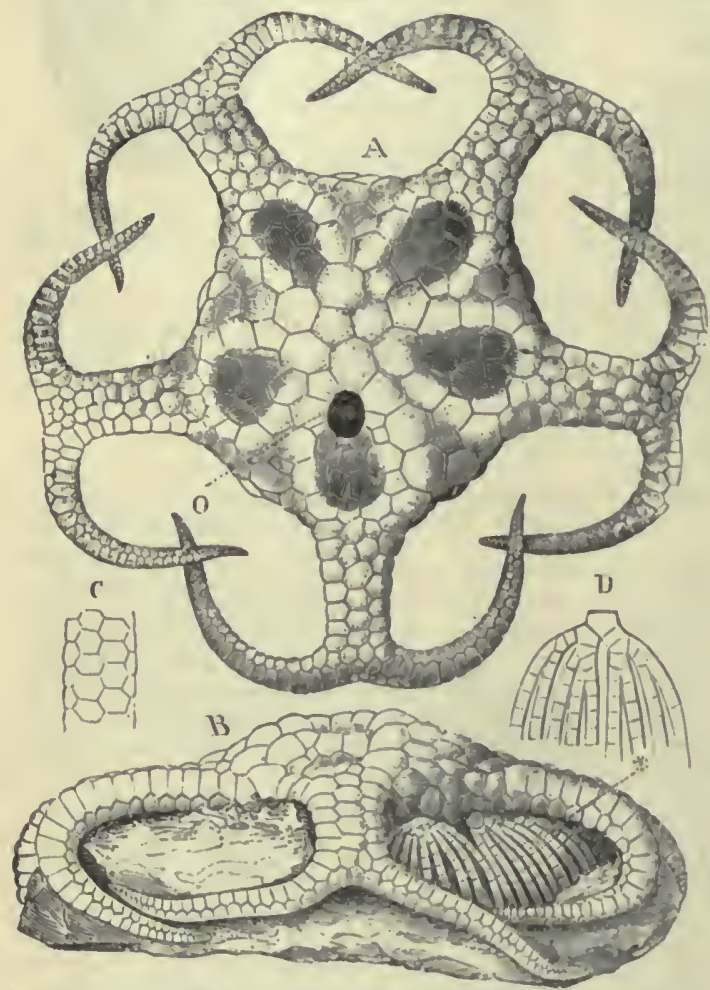

Frg. 321.- - ioulasteroidocrinus tuberosus. A is the vault: $O$, the opeuing; $B$, side view of vault; $C$. under slde of fulse arms; $D$, eulargernent of bast of arins.

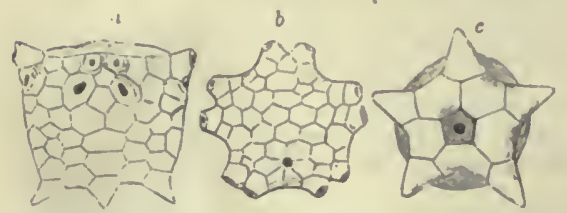

Fra. 322.-Three views of Gilbertsocrinus calcaratus, to show the genus is distluct from Gonlasteroldocrinus.

obovatus, Meek \& Worthen, 1869, Proc: Acad. Nat. Sci., Phil., p. 76, and Geo. Sur. Ill., vol. 5, p. 391, Burlington Gr. papillatus, Hall, 1860, (Trematocrinus papillatus,) Supp. to Geo. Rep. Iowa, p. 76, Burlington $\mathrm{Gr}$.

reticulatus, Hall, 1861, (Trematocrinus reticulatus,) Desc. New Crinoiden, p. 9, and Bost. Jour. Nat. Hist. vol. 7, p. 325, Burlington Gr. robustus, Hall, 1860, (Trematocrinus robustus,) Supp. to Geo. Rep. Iowa, p. 77, Keokuk Gr.

spinigerus, Hall, 1862, (Trematocrinus spinigerus,) 15th Rep. N. Y. St. Mus. Nat. Hist., p. 128, Ham. Gr.

tenuiradiatus, Meek \& Worthen, 1869, Proc. Acad. Nat. Sci. Phil., p. 75, and Geo. Sur. Ill., vol. 5, p. 389, Burlington Gr. tuberculosus, Hall, 1860, (Tremato. crinus tuberculosus,) Supp. to Geo. Rep. Iowa, p. 75, Burlington Gr.

tuherosus, Lyon \& Casseday, 1859, Am. Jour. Sci., vol. 2S, $2 \mathrm{~d}$ ser., p. 233, Kaskaskia Gr.

typus, Hall, 1860, (Trematocrinus typus,) Supp, to Geo. Rep. Iowa, p. 73 , Burlington $\mathrm{Gr}$.

Granatocrinus, Troost, 1850, Cat. Foss. in Am. Jour. Sci., vol. 8, p. 420, and described by Hall, 1862, 15th Rep. N. Y. St. Mus. Nat. Hist., p. 146. [Ety. granatos, granular; krinon, lily.] Calyx subglobose oval or elliptic; the proportions of the plates giving a very different outline to the calyx from that of a Pentremites; summit de pressed convex; base flattened or concave ; ambulacral areas like those in Pentremites, but narrower, and extending nearly or quite the entire length; basals 3 , sunken so as not to be visible in a side view; radials and deltoids similar to those of Pentremites and proportionally as variable; slender, thread-like arms, or pinnules, as in Pentremites; ambulacra and lancet-plates in narrow sinuses; anal opening as in Pentremites; central opening and spiracles often closed by small plates; ten narrow hydrospiral canals open externally by either five or ten apertures. Type G. norwoodi. cidariformis, Troost. Not defined. cornutus, Meek \& Worthen, 1861, (Pentremites cornutus,) Proc. Acad. Nat. Sci. Phil., p. 141, and Geo. Sur. Ill., vol. 2 , p. 276 , St. Louis Gr.

curtus, Shumard, 1855, (Pentremites curtus,) Geo. Rep. Mo., p. 187, Warsaw Gr.

glaber, Meek \& Worthen, 1869, Proc. Acad. Nat. Sci. Phil., p. 91, and Geo. Sur. Ill., vol. 5, p. 537, St. Lnuis Gr. granulatus, Roemer, 1852, (Pentatrematites granulatus,) Monog. Blast., p. 43, Warsaw Gr.

granulosus, Meek \& Worthen, 1865, Proc. Acad. Nat. Sci. Phil., p. 165, and Geo. Sur. Ill., vol. 5, p. 508, Keokuk Gr.

leda, Hall, 1862, (Pentremites leda,) 15th Rep. N. Y. Mus. Nat. Hist., p. 149, Ham. Gr. 
lotoblastus, White, 1874, Rep. Invert. Foss., p. 15, and Geo. Sur. W. 100th Mer., vol. 4, p. 80, Subcarb.

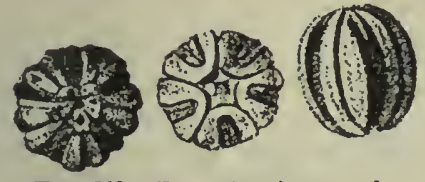

FIG. 323-Granatocrinus melo.

melo, Owen \& Shumard, 1850, (Pentremites melo,) Jour. Acad. Nat. Sci. Phil., 2d ser., vol. 2, p. 65, Burlington Gr. Etheridge \& Carpenter made this species the type of a new genus, Cryptoblastus, and referred to the same genus G. pisum. The generic characters, however, are not apparent.

melo var. projectus, see Granatocrinus projectus.

melonoides, see Schizoblastus melonoides.

missouriensis, Shumard, 1866, Trans. St. Louis Acad.Sci., vol.2, p. 375, Waverly Gr. neglectus, Meek \& Worthen, 1869, Proc. Acad. Nat. Sci. Phil., p. 90, and Geo. Sur. Ill., vol. 5, p. 471, Burlington Gr.

norwoodi, Owen \& Shumard, 1850, (Pentremites norwoodi,) Jour. Acad. Nat. Sci. Phil., 2d ser., vol. 2, p. 64, Burling-

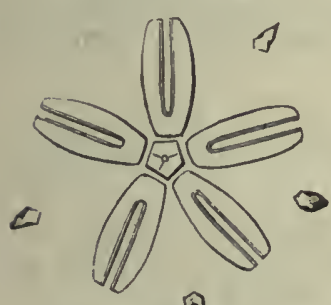

6
Frg. 324.-Granatocrinus projectus. Diagram.

Phil. p. 42, and Geo. Sur. Ill. Nat. Sci. 496, Burlington Gr.

roemeri, Shumard, 1855, (Pentremites roemeri,) Geo. Rep. Mo., p. 186, Waverly Gr. sayi, see Schizoblastus sayi.

shumardi, Meek \& Worthen, 1866, Proc. Acad. Nat. Sci. Phil., p. 257, and Geo. Sur. Ill., vol. 3, p. 498, Burlington Gr.

Graphiocrinus, DeKoninck \& LeHon, 1853, Rech. Crin. Carb. Belg., p. 115. [Ety. graphion, writing instrument; krinon, lily.] The authors described this genus as having only basals and radials, but as re-defined, by Wachsmuth, there are five basals concealed by the column; subradials 5 ; radials $1 \times 5$, upper margins straight; brachials $1 \times 5$; sutures gaping; arms 10 , long, heavy, short joints, parallel sutures; pinnules long; azygous interradial 1, small, but extending above the radials; strong ventral sac or proboscis. Type G. encrinoides.

carbonarius, Meek \& Worthen, 1861, (Scaphiocrinus carbonarius.) Proc. Acad.
Nat. Sci. Phil., p. 140, and Geo. Sur. Ill., vol. 5, p. 562, Coal Meas.

dactylus, Hall, 1860, Supp. to Geo. Rep. Iowa, p. 80, and Geo. Sur. Ill., vol. 5 , p. 559 , St. Louis $\mathrm{Gr}$.

longicirrifer, Wachsmuth \& Springer, (in press,) Geo. Sur., Ill., vol. 8 , p. 193, KinderhookGr. macadamsi, Worthen, 1873, (Scaph iocrinus macadamsi,) Geo. Sur. Ill., vol. 5, p. 495, Keokuk Gr. quatuordecembrachialis, see Eupachycrinus decembrachialis.

rudis, Meek \& Worthen, 1869, (Scaphio crin us rudis, FIG. 325.-GraphiocrProc. Acad. Nat.

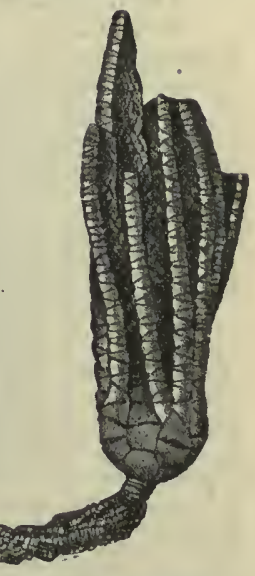

Sci. Phil., p. 39, and Geo. Sur. Ill., vol.

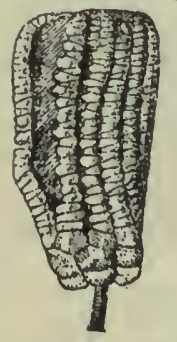

FIG. 326.-Graphiocriuus Itrudis. 5 , p. 412 , Burlington $\mathrm{Gr}$. simplex, Hall, 1858, (Scaphiocrinus simplex,) Geo. Sur. Iowa, p. 551, Burlington Gr.

spinobrachiatus, Hall, 1861, (Scaphiocrinus spinobrachiatus,) New Pal. Crin., p. 8, and Bost. Jour. Nat. Hist., p. 306, Burlington Gr.

striatus, Meek \& Worthen, 1869, (Scaphiocrinus striatus,) Proc. Acad. Nat. Sci. Phil., p. 142, and Geo.Sur. Ill., vol.5, p.418, Burlington Gr.

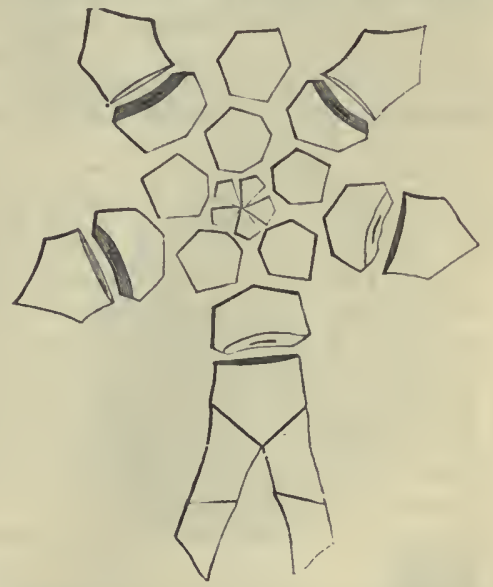

FIG. 327.-Graphiocrinus wachsmuthi. Diagram, 2 diam.

tortuosus, Hall, 1861, (Scaphiocrinus tortuosus,) Desc. New Crin., p. 7, and Bost. Jour. Nat. Hist., p. 309, Burlington Gr. 
wachsmuthi, Meek \& Worthen, 1861, (Scaphiocrinus wacbsmuthi,) Proc. Acad. Nat. Sci. Phil., p. 141, and Geo. Sur. Ill., vol. 3, p. 488, Burlington Gr.

Hadrocrinus, Lyon, 1869, Trans. Am. Phil. Soc., vol. 13, p. 445. [Ety. adros, full grown; krinon, lily.] Calyx broad, low vasiform, dome hemispherical; basals 3 , hidden by the column ; primary radials $2 \times 5$; secondary, tertiary, and higher orders of radials, having 2 in each series; arm-openings numerous, and not separated by interradials; interradials 3 or 4 ; column round. Type H. plenissimus.

discus, Lyon, 1869, Trans. Am. Phil. Soc., vol. 13 , p. 448 , Up. Held. Gr.

pentagonus, Lyon, 1869, Trans. Phil. Soc., vol. 13, p. 446 , Up. Held. Gr.

plenissimus, Lyon, 1869, Trans. Phil. Soc., vol. 13 , p. 445, Up. Held. Gr.

Halysiocrinus, Ulrich, 1856, 14 tl Rep. Geo. Sur. Minn., p. 110, syn. for Deltacrinus.

Ha Plocrinus, Steininger, 1834,Bul. Soc.Geol. France, t. 8, 1st series, p. 232. [Ety. haploos, simple; krinon, lily.] Calyx small subturbinate; basals 5 ; radials $2 \times 3$ plus $1 \times 2$, protruding at the center of the superior face for the attachment of arms; dome convex, composed of 5 plates, having sutures from the center of the arm-openings toward the central part of the dome. Type H.sphreroideus. clio, Hall, 1862, 15th Rep. N. Y. St. Mus. Nat. Hist., App. C., p. 115, Marcellus shale.

granulatus, Troost, Not defined.

hemisphericus, Troost. Not defined.

maximus, Troost. Not defined.

ovalis, Troost. Not defined.

Hemicossites, Von Buch, 1840, Monatsber. d. Berlin Akad., p. 129, and Geol. Russia, vol. 2, p. 31. [Ety. hemi, half; kosmos, sphere.] Body having four series of plates; basals 4; second series 6 ; third series 9 ; ovarian orifice between second and third series; mouth central. Type H. malum.

subglobosus, Hall, 1864, 20th Rep. N. Y. St. Mus. Nat. Hist., p. 359, Niagara Gr. Hemjcystites, Hall, 1852, Pal. N. Y., vol. 2, p. 245. [Ety. hemi, half; kustis, bladder.] Parasitic, circular, more or less convex on the ursper surface and some. times sac-like in form; composed of numerous imbricating plates; ambulacra 5, straight, radiating from the center and composed each of a double series of alternating plates, forming part of the upper surface; aperture excentric. Type H. parasiticus,

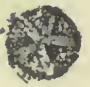

Fra. 328, - Hemicystites stellatus.

N. Y., vol. 2 , p. 246 , Niagara Gr. stellatus, Hall, 1866, Adv. Sheets 24 th Rep. N. Y. St. Mus. Nat. Hist., p. 215, Hud. Riv Gr.

Hetreocrinus, Hall, 1847, Pal. N. Y., vol. 1. p. 278. [Ety. heteros, irregular; krinon, lily.] Calyx, small, slightly expandled; basals 5 ; radials irregular, two or three of the rays having two plates each, and the others only one; four radials supported on the basals; the other is smaller and rests on the azygous plate, and supports the ventral sac on onc side and the braclials on the other; brachials, gencrally, four to each ray, the last one axillary, and supporting two arms, which sometimes branch at irregular distances; pinnules strong; azygous plate pentagonal ; column pentagonal, pentapartite; attaching base small. Type H. heterodactylus. articulosus, see Calccocrinus articulosus.

bellevillensis, W. R. Billings, Trans. No. 4, Ottawa Field Naturalists Club, p. 49, Trenton Gr.

canadensis, see Ectenocrinus canadensis.

constrictus, see Ohiocrinus constrictus.

constrictus var. compactus, seo Ohiocrinus compactus.

crassus, see locrinus crassus.

exilis, Hall, 1866, 24th Rep. N. Y. St. Mus.

Nat. Hist., p. 213, Trenton and Hud.

Riv. Gr.

exiguus, Meek, syn. for H. exilis.

gracilis, Hall, 1847, Pal. N. Y., vol. 1, p. 280, Hud. Riv. Gr. Not properly defincd.

geniculatus, Ulrich, 1879, Jour. Cin. Soc. Nat. Hist., vol. 2, p. 16, Utica Slate Gr. heterolactylus, Hall, 1847, Pal. N. Y., vol. 1, p. 279, Hud. Riv. Gr.

insqualis, see Calceocrinus insequalis. incurvus, see Anomalocrinus incurvus.

isodactylus, syn. for Olıiocrinus compactus.

juvenis, Hall, 1866, 24th

Rep. N. Y. St. Nat. Hist., p. 212, Hud. Riv. Gr.

laxus, sce Ohiocrinus laxus. milleri, Wetherby, 1880 , Jour. Cin. Soc. Nat. Hist., vol. 3, p, 153, 'Trentou Gr.

chanus, see Ohiocrinus cehanus.

pentagonus, Ulrich, 1882 ,

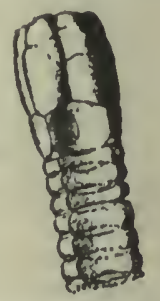

Jour. Cin. Soc. Nat. Hist., FıG. 3:9.-Hetervol. 5 , Hud. Riv. Gr. o e ri i u s jupolyxo, syn. for Iocrinus vents.

subcrassus.

simplex, see Ectenccrinus simplex.

simplex var. grandis, see Ectenocrinus grandis.

subcrassus, see Iocrinus subcrassus.

tenuis, Billings, 1557, Rep. of Progr. Geo.

Sur. Can., p. 273, and Can. Org. Rem.,

Decade 4, p. 50, Trenton Gr.

vaupeli, syn. for $\mathbf{H}$. constrictus. 
Heterocystites, Hall, 1852, Pal. N. Y., vol. 2, p. 229. [Ety. heteros, irregular; kustis, bladder.] Basals 4, irregular in size; second series 10, large; bigher plates numerous, but exact order and number undetermined. Type $\mathrm{H}$. armatus.

armatus, Hall, 1852, - Pal. N. Y., vol. 2, p. 229, Niagara Gr.

Heteroschisma, W achsmuth, 1883, Geo. Sur. Ill., vol. 7, p. 352. [Ety. heteros, irregular; schisma, slit.] It is distinguished from Codaster by the sunken hydro-

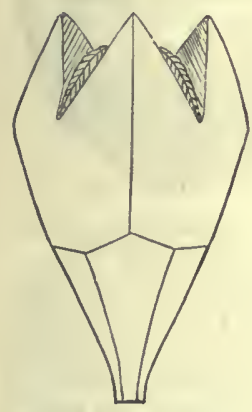

Fig. 330.-He terosc h is m a gracile. Side view, 3 diam. spiral areas and exposure of the orals, only, immediately contiguous to the mouth; the limbs are extended interradially, into pyramidal ridges, which the hydrospires enter obliquely. Type H. gracile.

alternatum var. elongatum, Wachsmuth, 1883, Geo. Sur. Ill., vol. 7, p. 354 . Founded upon a magnified view of Codaster attenuatus. gracile, Wachsmuth, 1883, Geo. Sur. Ill., vol. 7, p. $354, \mathrm{Ham}$. Gr.

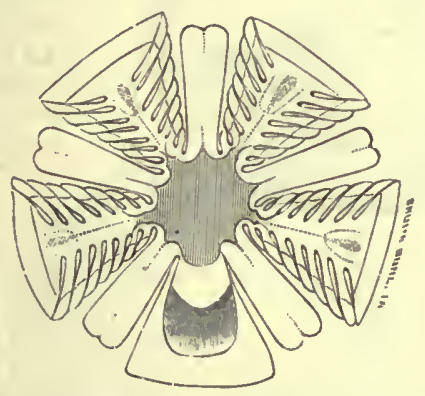

Fig. 33i.-Heteroschisma gracile. Horizontal section of hydrospires, 5 diam.

Holocystites, Hall, 1864, 20th Rep. N. Y. St. Mus. Nat. Hist., p. 353. [Ety. holos, entire; kustis, bladder.] Body cylindrical, subovate or globose, free, sessile, or attaching by roots, and covered by numerous ranges or irregular series of larger and smaller poriferous plates; ambulacral opening central or subcentral ; mouth excentric ; smaller opening between these; arms mere spinous processes. Type H. cylindricus.

abnormis, Hall, 1864, 20th Rep. N. Y. St. Mus. Nat. Hist., p. 355, Niagara Gr.

alternatus, Hall, 1861, (Caryocystites alternatus,) Rep. of Progress Geo. Sur. Wis., p. 23, and 20th Rep. N. Y. St. Mus. Nat. Hist., p. 355, Niagara Gr.

baculus, S. A. Miller, 1879, Jour. Cin. Soc. Nat. Hist., vol. 2, p. 105, Niagara Gr. brauni, S. A. Miller, 1878, Jour. Cin. Soc. Nat. Hist., vol. 1, p. 130, Niagara Gr. canneus n. sp. Niagara Gr. Body long, irregularly subcy 1 in drical; summit prolonged on the flattened side in the direction of the a $\mathrm{m} \mathrm{bu} l$ a cral orifice; plates long, polygonal, of irregu. lar size; eight ranges may be counted in ourspecimen, and one or two have been broken f $r \circ \mathrm{m} \mathrm{th}$ e low er end; the ambulacral orifice is s u r rounded by six plates; below this there is a range of eight plates, three

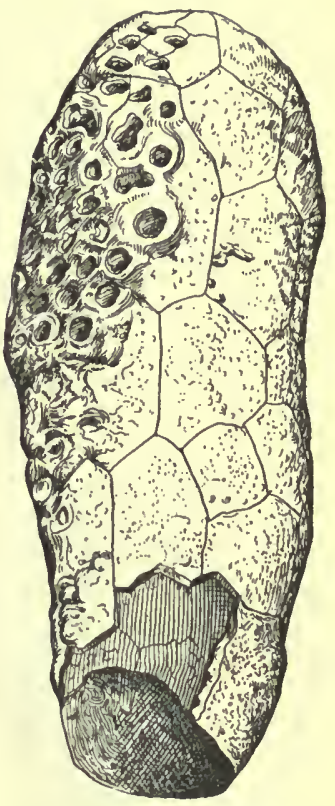

FIG. 332.-Holocystites canneus. of $\mathrm{w} \mathrm{h} \mathrm{i} \mathrm{c} \mathrm{h}$

reach the mouth, and one of which bears the anal orifice; there are no arms, ambulacral spines, or cicatrices; there are eight plates in the next range, two of which join the mouth; the mouth in this genus is generally upon the flattened side of the specimen and opposite the posterior bulge, but not so in this species, for the ambulacral area is prolonged on the flattened side, and the bulge is opposite thereto, while the mouth is on the side of the summit between the bulge and the flattened side;

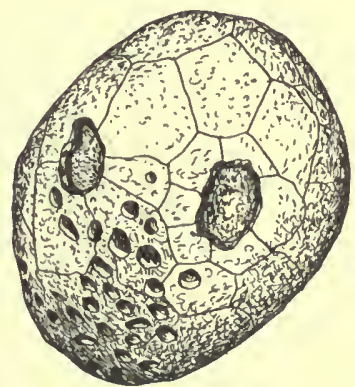

FIG. 333.-Holocystites canneus. summit view.

all the plates are very poriferous, the pores penetrating the plates in clusters of from two to seven instead of by pairs as is usual in this genus; the flattened 
side is covered by numerous pits and a thickening of the plates; these pits do not pass through the plates, though they cover a series of plates, sutures and all; such pits have been found on different species, and it is probable they represent a disease of the test, as they seem to destroy the pores and anchylose the sutures. Collected by J. F. Hammell, of Madison, in Jefferson County, Indiana. cylindricus, Hall, 1861, (Caryocystites eylindricus,) Anı. Rep. Geo. Wis., p. 23, and 20th Rep. N. Y. St. Mus. Nat. Hist., p. 354, Niagara Gr.

dyeri, S. A. Miller, 1879, Jour. Cin. Soc. Nat. Hist., vol. 2, p. 108, Niagara Gr. elegans, S. A. Miller, 1878, Jour. Cin. Soc. Nat. Hist., vol. 1, p. 136, Niagara Gr.

faberi, n. sp. Niagara Gir. Body somewhat

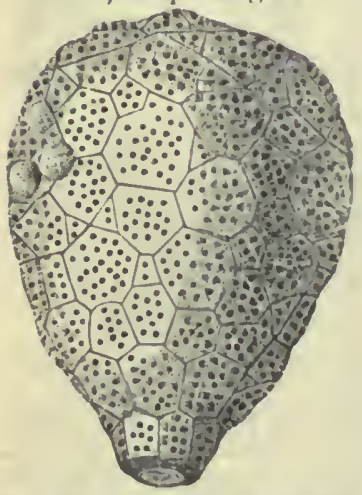

o bo vate: large a $x$ ial canal passes down into the column; seven plates in the first range; two small interc a l a t e d plates on the posterior sidebetween the first and 8 e c o n d $r$ a $n g$ e s; seven plates in the second rallge; above

Fio. 334.-Holocystltes faberl.

the second range the plates are polyg onal, of all sizes, and not disposed in ranges; if they were in ranges there would be about seven below the sum. mit; ambulacral orifice on the posterior side of the summit, elliptical, surrounded by six plates, four of which are protuberant or swollen at the orifice, but no arms ever attached, nor are there cicatrices for spines; moutlı pentagonal, on the anterior side of the summit, separated from the ambulacral orifice by two plates, one of which bears the anal orifice; on the posterior side, below the summit, there are three circular pits, whieh do not seem to have been of any economical use; en-

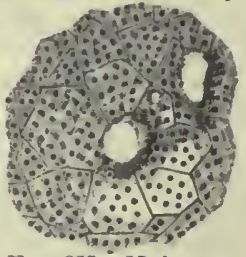
tire surface porifer-FI(3. 335.-Holocysous in pairs, which tltes faberi. open through small tubercles. Collected by Charles Faber, of Cincinnati, (in whose honor I have given the specific name,) in Jefferson County, Indiana.

globosus, S. A. Miller, 1878, Jour. Cin. Soc. Nat. Hist., vol. 1, p. $133, \mathrm{Ni}$ agara Gr.

liammelli n. sp. Niagara Gr. Body subglobose, flattened on the anterior side; sessile, no perforation for a column; basals seven; between the first and second se$r$ ies there a re $\mathrm{five}$ plates in. serted on the posterior side ; ten plates in the second series: ten in the third; nine i $\mathrm{n}$ th $\mathrm{e}$ fourth, and nine in the fifth, which, 336-Holocystltes $1 \mathrm{~mm}$ ries reaches

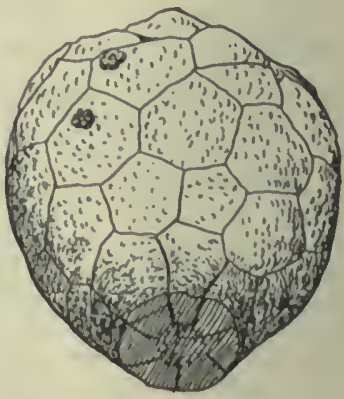
the mouth; above these there are eight plates in the series which bore the ambulacral spines, und some smaller plates surrounding the ambulacral orifice on the summit; four cieatrices for ambulacral spines; mouth at the margin of

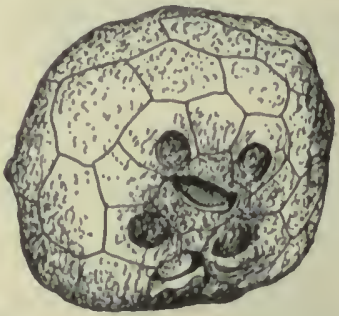

FIo. 337. - Holocystites hammelll. Summit view; mouth on the lower slde. the summit; anal opening in the plate adjoin in $\mathrm{g}$ the inouth and be $t$ ween it and the a $\mathrm{mbula}$. cral orifice; a 11 the plates poriferous in pairs; o n the ante. rior side below the mouth there is one plate in tho third series, and one in the fourth series, each bearing a prominent tubercle, with a circle of pores passing through it, giving it a radiate appearance on top; this character may not be of specific importance, but the tubercles are different from those observed on other specimens. Collected by J.F. Hammell, in Jefferson County, Indiana, in whose bonor I have given the specific name. jolietensis, S. A. Miller, 1882, Jour. Cin. Soc. Nat. Hist., vol. 5, p. 223, Niagara Gr. ornatus, S. A. Miller, 1878, Jour. Cin.

Soc. Nat. Hist., vol. 1, p. 132, Niagarn Gr. ovatus, Hall, 1864, 20 th Rep. N. Y. St.

Mus. Nat. Hist., p. 357, Niagara Gr. perlongus, S. A. Miller, 1878, Jour. Cin. Soc. Nat. Hist., vol. 1, p. 132, Niagara Gr. plenus, S. A. Miller, 1878, Jour. Cin. Soc. Nat. Hist., vol. 1, p. 135 , Niagara Gir. pustulosus, S. A. Miller, 1878, Jour. Cin. Soc. Nat. Hist., vol. 1, p. 134, Niagara Gr. rotundus, S. A. Miller, 1879 , Jour. Cin.

Soc. Nat. Hist., vol. 2, p. 107, Niagara Gr. 
scutellatus, Hall, 1864, 20th Rep.N. Y. St. Mus. Nat. Hist., p. 357, Niagara Gr. sphæricus, Winchell \& Marcy, 1865, Mem. Bost. Soc. Nat. Hist., vol. 1, p. 111, Niagara Gr. Not defined so as to be recognized.

subglobosus n. sp. Niagara Gr. Body globose; no axial canal, hence the species was sessile; seven plates in the first range; above this there are four ranges below the summit of fourteen plates, each with an additional plate in the third range on the posterior side; ambulacral orifice in the center of the summit, elongated transversely, surrounded by six plates, four of which. have cicatrices for attaching spines; anal orifice near a cicatrix and near the oral orifice; the whole surface is poriferous

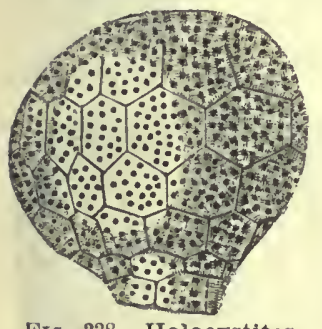

FIG. 338.-Holocystites subglobosus. in pairs which open on the surface in or na mental sculpt u red figures, somewhat like the Greek letter $\omega, \quad(\mathrm{Omega})$. Collected by Charles Faber, in Jefferson County, Indiana.

subrotundus, S. A. Miller, 1879, Jour. Cin. Soc. Nat. Hist., vol. 2, n. 107, Niagara Gr.

tumidus, S. A. Miller, 1879, Jour. Cin. Soc. Nat. Hist., vol. 2, p. 104, Niagara Gr.

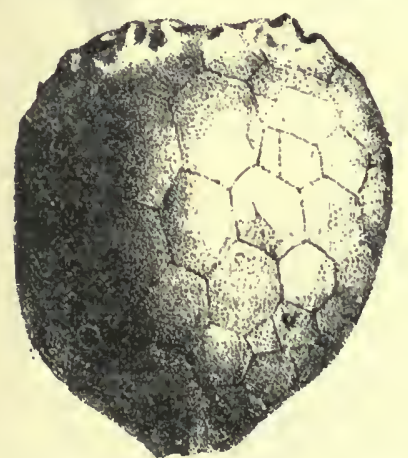

Fio. 339.-Holocystites turbinatus.

turbinatus, S. A. Miller, 1880, Jour. Cin. Soc. Nat. Hist., vol. 2, p. 259, Niagara Gr. ventricosus, S. A. Miller, 1879 , Jour. Cin.

Soc. Nat. Hist., vol. 2, p. 108, Niagar.। Gr. wetherbyi, S. A. Miller, 1878, Jour. Cin.

Soc. Nat. Hist., vol. 1, p. 131 Niagara G'r. winchelli, Hall, 1864, 20th Rep. N. Y. St. Mus. Nat. Hist., p. 356, Niagara Gr.

Homocrinus, Hall, 1852, Pal. N. Y., vol. 2, p. 185. [Ety. homos, like; krinon, lily.] Basals 5; subradials 5 ; radials $1 \times 5$; azygous interradials 2 ; proboscis or ventral sac long and large; arms bifur- cating; pinnules wanting; column round. Distinguished from Dendrocrinus by the proportionally larger basals, and absence of one radial, and from Poteriocrinus by the arrangement of the azygous plates and absence of pinnules. Type $H$. parvus. .

angustatus, see Dendrocrinus angustatus. crassus, Whiteaves, 1887, Cont. to Can. Pal., vol. 1, p. 95, Ham. Gr.

cylindricus, Hall, 1852, Pal.' N. Y., vol. 2, p. 186, Niagara Gr.

parvus, Hall, 1852, Pal. N. Y., vol. 2, p. 185, Niagara Gr.

polydactylus, see Dendrocrinus polydactylus.

proboscidialis, Hall, 1859. Pal. N. Y., vol. 3. p. 38 , Oriskany sandstone. scoparius, Hall, $1859, \mathrm{Pal}$. N. Y., vol. 3, p. 102, Low. Held. Gr.

H Y B O C I N US,

Worthen \& Miller, 1883, Geo. Sur. Ill., vol. 7, p. 331 . [Ety. hubos, humpbacked; echinos, se aurchin.] Test flexible, subs p he roidal, five ambulacral areas, with numerous ranges of in t e rlocking plates imbricating upward, a n d

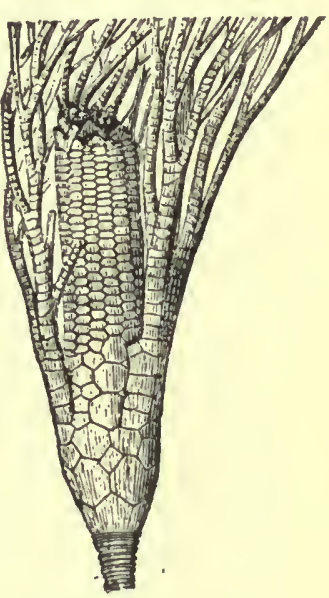

FIG. 340.-Homocriutus scoparius. each perforated with a pair of pores; interambulacral areas narrower; plates imbricate downward; surface granular; jaws strong. Type H. spectabilis.

spectabilis, Worthen \& Miller, 1883, Geo. Sur. Ill., vol. 7, p. 332, Kaskaskia Gr.

Hyвocrinus, Billings, 1857, Rep. of Progr. Geo. Sur. Can,, p. 274, and Can. Org. Rem., Decade 4, p. 23. [Ety. hubos, humpbacked; krinon, lily.] Calyx pro-

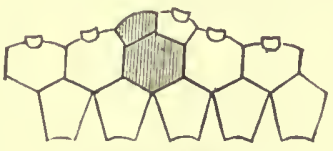

HIG. 341.-Hyboerinus conicus. Diagram. tuberant on the azygous side; basals 5 ; radials $1 \times 5$; azygous interradials 2 ; arms 5; no pinnules; column round. Type $\mathrm{H}$ conicus.

conicus, Billings, 1857, Rep. of Progr. Geo. Sur. Can., p. 274, and Can. Org. Rem., Decade 4, p. 29, Trenton Gr.

pristinus, Billings, 1859, Can. Org. Rem., Decade 4, p. 23, Chazy Gr.

tumidus, Billings, 1857, Rep. of Progr. Geo. Sur. Can., p. 275, and Can. Org. Rem., Decade 4, p. 28, Trenton Gr. 
Hyносуsтiтes, Wetherby, 1880, Jour. Cin. Suc. Nat. Hist., vol. 3, p. 150. [Ety. hubos, humpbacked; kustis, bladder.] Calyx like Hybocrinus, and order of

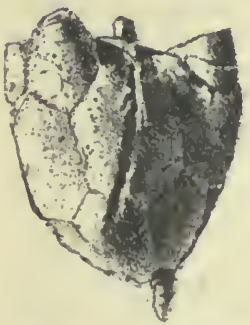

Fic. $342,-\mathrm{Hybocy}$ stites probiematicus. arrangement of the two series of plates is the same, but distinguished by having three arm-like projections, and two or more recumbent arms with ambulacral opening central ; from the peristome, the five ambulacra diverge; three are directed to the arm. like projections, and are supposed to pass over the top of them and extend clownward upon the exterior; valvular opening between the upper azygous plate and the mouth. Type H. problematicus.

problematicus, Wetherby, 1850, Jour. Cin. Soc. Nat. Hist., vol. 3, p. 150, Trenton Gr.

Hydreionocrinus, DeKoninck, 1858, Bull. Acad. Royale Belgique, vol. 8 , pt. 2, p. 13. [Ety. hydreion, water-bucket; krinon, lily.] Calyx short, rounded below; basals 5 ; subradials 5 ; radials $1 \times 5$; brachials $1 \times 4+2 \times 1$; arms as in /eacrinus, and pinnules short; distinguished from Zeacrinus by the ventral sac, which extends beyond the arms and covers them like a roof; the upper plates are convex or spinous; respiratory pores in the sutures of the cylin. drical part of the sac, which is covered, by the arms. Typo H. woodanus.

acanthophorus, Meek \& Worthen, 1870 , (Zeacrinus acanthophorus,) Proc. Acad. Nat. Sci. Phil., p. 28, and Geo. Sur. Ill., vol. 5, p. 563 , Up. Coal Meas.

armiger, Meek \& Wortlien, 1870, (Zeacrinus armiger,) Proc. Acad. Nat. Sci. Phil., p. 27, and Geo. Sur. Ill., vol. 5, p. 547, Kaskaskia Gr.

depressus, Troost, as defined by Hall, 1858, (Zeacrinus depressus,) Geo. Sur. Iowa, p. 546 , Kaskaskia Gr.

discus, Meek \& Worthen, 1860, (Zeacrinus discus,) Proc. Acad. Nat. Sci. Phil., p. 39, and Geo. Sur. Ill., vol. 2, p. 312, Up. Coal Meas.

mucrospinus, McChesney, 1859, (Zeacrinus mucrospinus,) Desc. New Pal. Foss., p. 10, and Trans. Chi. Acad. Sci., p. 7, and Geo. Sur. Ill., vol. 5, p. 563, Coal Meas.

verrur:osus, see Eupachycrinus verrucosus. wetherbyi, Wrchsmuth \& Springer, 1886 ,

Revis. Paleocrinoidea, pt. 3, p. 245, Kaskaskia Gr.

Hypanthocrintes, Phillips, 1839, Murch. Sil. Syst. [Ety. upo, under; anthos, flower; krinon, lily.]

cælatus, see Eucalyptocrinus crelatus.

decorus, see Eucalyptocrinus decorus.

Hystricrinus, Hinde, 1885, Ann. and Mag. Nat. Hist., p. 158, gyn. for Arthracantha. carpenteri, see Arthracantha carpenteri.

IcHThyocrixus, Conrad, 18t2, Jour. Acad. Nat. Sci. Phil., vol. 8, p. 279. [Ety. ichthys, fish; krinon, lily.] (ieveral forn, including incumbent arms, ovoid or pear-shaped; calyx cup-shaped; basals 3 ; subradials 1 x 5 ; primary radials 3 or $4 \times 5$, short and increasing, in willt, upward; sccondary and tertiary radials similar in form to the primaries; arms 40 to 60 or more. 'Type I. lrevis.

burlingtonensis, Hall, 1855 , Geo. Sur. Iowa, p. 5.)7, Burlington $\mathrm{Gr}$.

clintonensis, Hall, 1852, Pal. N. Y., vol. 2, p. 181, Clinton Gr.

F I 0.318 Ichilinoor 1 un us corbls. corbis, Winchell \& Marcy, 1865, Mem. Bost. Soc. Nat. Hist., vol. 1, p. 89 , and Jour. Cin.

Soc. Nat. Hist., vol. $4, \mathrm{p} .175, \mathrm{Ni}$ agara Gr.

I ie $\mathrm{v}$ is, Con. r a d, $18+2$, Jour. Acad. Nat. Sci. vol. $8, p$ 279 , and Pal. N. I. vol. 2,11 $195, \mathrm{Ni}$. agara $\mathrm{Gr}$.

no b i l i s: W a c h. $\sin u t h$ \& Sipringer, 1878, Proc. Acad. Nat. Sci., P. $25 t$, Upper Burlington and $\mathrm{K}$ e o k u k Gr.

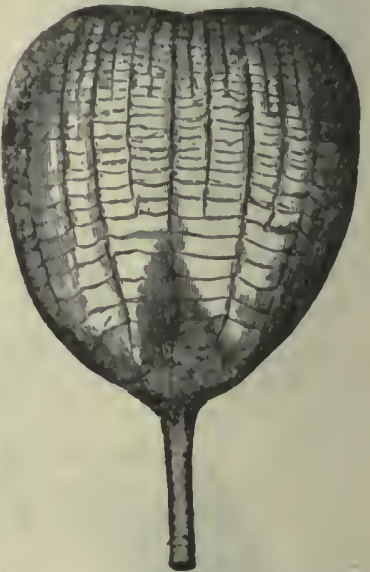

Fja. 3:4-Ichthyocrinus irovis. subangularis, Hall, 1863, Trans. Alb. Inst., vol. 4, p. 207, and 11 th Rep. Geo. Ind., p. 268 , Niagara Gr.

tiariformis, 'Troost, as defined by Hall, 1858, (Cyathocrinus tiariformis,) Geo. Sur. Iowa, p. 558, Sul)tarboniferous. Icosidactylocrinites. Not defined. Iocrivus, Hall, 1866,. Advance sheets, 24th Rep. N. Y. St. Mus. Nat. Hist., p. 212. [Ety. io, in triunph; krinon, lily.]

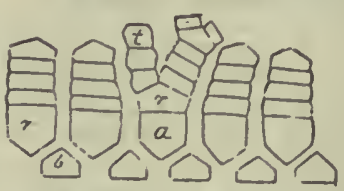

Fig.345--Iocrinus. Diagram: $b$, basuls ; $r$, radiais ; $c, a z y$ gous plate; $t$, plates of tube. ventral sac very long, extending beyond the arms, subcylindrical longi- 
tudinally, five partite, and corrugated transversely; column sharply pentagonal. Type I. subcrassus.

crassus, Meek \&.Worthen, 1865, (Heterocrinus (rassus, ) Proc. Acad. Nat. Sci. Phil., p. 147, and Geo. Sur. Ill., vol. 3, p.

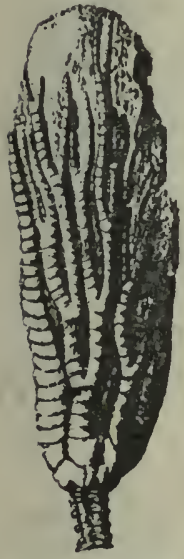
Fig. 316.- Iocri-
nus suberassus. 325, Hud. Riv. Grr. polyxo, syn. for I. sub('rassus.

subcrassus, Meek \& Worthen, 1865, (Heterocrinus, subcrassus,) Proc. Acad. Nat. Sci. Phil., p. 148, and Geo. Sur. Ill., vol. 3 , p. 325 , Hud. Riv. Gr.

trentonensis, IValcott, 1884, 35th Rep. N. Y. St. Mus. Nat. Hist., p. 210, Trenton $\mathrm{Gr}$.

LAMPTEROCRINUS, $R$ oe me $r$, 1860, Sil. Fauna IVest Tenn., p. 37. [Ety. lampter, lamp; krinon, lily. Calyx urn-shaped, contracted between the armbases, and bulged out on the azygous side; basals 5 ; subradials 5 ; primary radials $3 \times 5$; secondary radials, $1 \times 10$; interradials 8 or 10 , graduating into the vault; azygous interradials more numerous; vault unsymmetrical, and bearing a subcentral proboscis or veniral sac; arms unknown. 'Type L. tt nnesseeusis.

inflatus, Hall, 1861, (Balanocrinus inflatus,) Rep. of Progr. Sur. of Wis., p. 22 , and 20 th. Rep. N. Y. St. Mus. Nat. Hist., p. 328 , Niagara Gr.

parvus, Hall, 1879, Desc. New Spec. Foss. p. 9, and 11 th Rep. Geo. Nat. Hist. Ind., p. 272, Niagara Gr.

sculptus, syn. for L. tennesseensis.

te n nesseensis,

$\mathrm{R}$ o e $\mathrm{m}$ e r

1860 , Sil.

Fauna IVest

Tenn., p. 37

Niagara Gr.

L E C A N OCRINUS, $\mathrm{H}$ a 11,1852 , Pal. N. Y. vn]. 2 , p. 199 . [Ety. lekane, basin; krinon, lil y.] Body and arnis subglobose; plates heavy ; basals 3 ; subradials $1 \times 5$; primary radials 2 or $3 \times 5$; secondary $\mathrm{ra}$ -

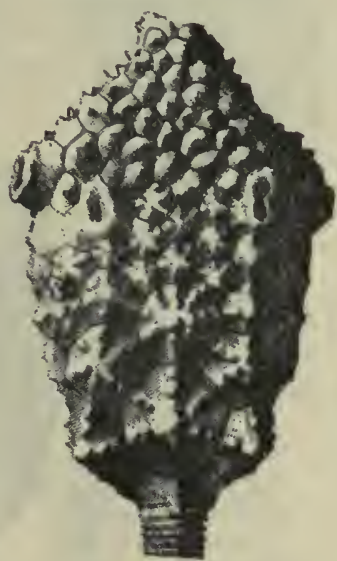

Fig. 317.-Lampterocrinus tennesseensis dials 1 to $3 \times 10$; azygous interradials 2 ; arms as in Ichthyocrinus; column round. Type L. macropetalus.

caliculus, Hall, 1852, Pal. N. Y., vol. 2, p. 203, Niagara Gr.

elegans, see Taxocrinus elegans.

excavatus, Ringueberg, 1886, Bull. Buf. Soc. Nat. Sci., vol. 5, p. 11, Niagara Gr. incisus, Ringueberg, Bull. Buf. Soc.

Nat. Sci., vol. 5, p. 10, Niagara Gr. lævis, see Taxocrinus lievis.

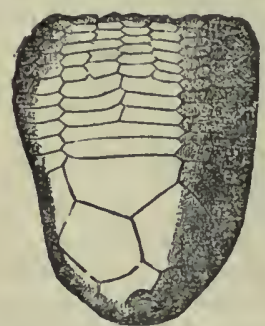

FiG. 348.-Lecanocrinus macropetajus. macropetalus, Hall, 1852, Pal. N. Y., vol. 2 , p. $199, \mathrm{Ni}$ agara $\mathrm{Gr}$.

nitidus, $\mathrm{R}$ in g u e berg, 1886, Bull. Buf. Soc. Nat. Sci., vol. 5, p. 9 , Niagara Gr. ornatus, Hall, 1852 , Pal. N. Y., vol. 2, p. 201, $\mathrm{N}$ i agara $\mathrm{Gr}$.

pusillus, Hall, 1863, (Cy a thocrinus pusillus,) Trans. Alb. Inst., vol. 4, p. 200 , and 11 th Rep. Geo. and Nat. Hist. Ind., p. 267, Niagara Gr.

pusillus, Winchell \& Marcy, syn. for L. pusillus.

puteolus, Ringueberg, 1886, Bull. Buf. Soc. Nat. Sci., vol. 5, p. 11, Niagara Gr. simplex, Hall, 1852, Pal. N. Y., vol. 2, p. 202, Niagara $\mathrm{Gr}$.

solidus, Ringueberg, 1886, Bull. Buf. Soc. Nat. Sci., vol. 5, p. 8, Niagara Gr.

Lecrthiocrinus, White, 1880, Proc. U. S. Nat. Miss., vol. 2, p. 257. This name was preoccupied by Muller in 1858, and by Zittel in 1879. See Menocrinus. adamsi, see Menocrinus adamsi.

olliculiformis, see Menocrinus olliculiformis.

LEPADOCRINus, Conrad, 1840, (Lepocrinites,) Ann. Rep. N. Y., p. 207. [Ety. from the resemblance to the Lepas or Barnacle Anatifa ; krinon, lily.] Body oblong or ovoid, consisting of four series of plates; first series 4 ; second series 5 ; third series 4 ; fourth series 5 ; pectinated rhombs 3 to 5 ; arms 3 or 4 , recumbent, and consisting of a double series of interlocking plates, resting, in shallow grooves; plates poriferous, column tapering. Type L. gebhardi.

gebhardi, Courad, 1810, Fra. 349.-Lepado(Lepocrinites g e b- crinus gebbardi. hardi,) Anu. Rep. N. Y., p. 207, and Pal. N. Y., vol. 3, p. 127, Low. Held. Gr. 
moorii, Meek, 1871, (Lepocrinites moorii,) Am. Jour. Sci., 3d series, vol. 2, p. 296, and Ohio Pal., vol. 1, p. 39, Hud. Riv. Gr.

Lepidecilinus, Hall, 1861, Desc. New Spec. Crinoidea, p. 18. [Ety. lepis, scale; echinus, sea-urchin.] Subspheroidal; ambulacral area having a double row of plates imbricating downward, with two pores in each plate, near the outer end; interambulacral areas wide, and having numerous ranges of plates, imbricating from below upward, and from the center outward. Type L. imbricatus.

imbricatus, Hall, 1861, Desc. New Crinoidea, p. 18, Burlington Gr.

rarispinus, Hall, 1867, 20 th Rer. N. Y. St. Mus. Nat. Hist., p. 340, Waverly Gr. LePIDEstils, Meek \& Worthen, 1868, Geo. Sur. Ill., vol. 3, p. 522. [Ety. lepis, scale; esthes, garment.] Subsplieroidal;

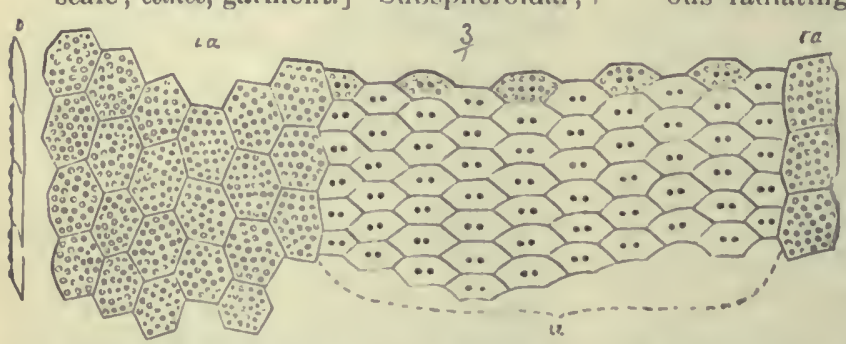

Fir. 350.-Lepilesthes corey1. Dingram 3 tlam.; (a.) mubuincruls; (b.) section of tlicin; position of interninhulucruls. A. squamosus.

squamosus, Meek \& Worthen, 1873, (ieo. Sur. Ill., vol. 5, p. 478 , Burlington Gr.

I.EPINODIsces, Meek \& IVorthen, 1875, Geo. Sur. 'Ill., vol. 5, p. 573. [Ety. lepis, scale; diskos, quoit:] A proposed subgenus for Agelacrinus, founded upon

Lepocrinites, Conrad, 1840. The correct orthography seems to be Lepadocrinus. moorei, Meek, see Lepadocrinus moorii. Licursocrisus, Hall, 1866, Adv. sheets 24 th Rep. N. Y. St. Mus. Nat. Hist., p. 216. [Ety.lichen, tree-moss; krinon, lily.] Body parasitic, discoid, more or less crateriform, from the center of which arises a long tapering column, each ring of which is composed of small interlocking plates; upper surface of body covered with polygonal plates, which are supported in the interior by numerous radiating lamellx. Type I. dyeri. affinis, S. A. Miller, 18.82, Jour. Cin. Soc. Nat. Hist., vol. 5, p. 229, Hud. Riv. Lir. crateriformis, Hall, 1866 , Adv. slieets 24th Rep. N. Y. St. Mus. Nat. Hist., p. 217, Hud. Riv. Gr.

dubius, S. A. Miller, 1880, Jour. Cin. Soc. Nat. Hist., vol. 3. p. 234, Utica Slate Gr.

dyeri, IIall, 1866, dilv. sheets, 24 th Rep. I. Y.

ambulacral areas wide, having numerous plates, and imbricating from above downward, and having two pores in each plate, nearly central; interambulacral areas narrow, plates imbricating from below npward, as well as outward from the midille; jaws well developed; surface granular. Type I. coreyi.

colletti, White, 1878, Proc. Acad. Nat. Sci. Pliil., p. 33, and Cont. to Pal., No. 8 , p. 163, Keokuk Gr.

coreyi, Meek \& Worthen, 1868, Geo. Sur. Ill., vol. 3, p. 525. Keokuk Gr.

formosus, S. A. Miller, 1879, Jour. Cin. Soc. Nat. Hist., vol. 2, p. 41, Keokık Gr.

Lepinocinaris, Meek \& Worthen, 1873, Geo. Sur. Ill., vol. 5, p. 478. Ety. lepis, scale; Cidnris, a genus.] Body large, globose, eight or more rows of imbricating plates in the middle of each interambulacral area, but only two reach the oral apertures; plates hexagonal or pentagonal; tubercles for the support of primary spines smooth and in the center of each plate; pustules near the outer edge of the plates for the secondary spines; ambulacra narrow; plates slightly imbricating in the opposite direction from the interambulacral series, and each pierced by two pores; jaws strong. Type $I_{\text {d. squamosus. }}$
St. Mus. Nat. Hist., p. 216, Hud. Riv. Gr.
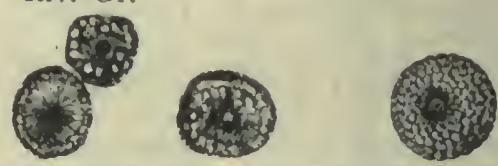

I'ir.351,-Lichenocrinus tuberculatus; oue spect. inen shows the radlathig lasuclla:

pattersoni, S. A. Miller, 1879, Jonr. Cin. Soc. Nat. Hist., vol. 2, p. 118, Itica Slate fir.

tuberculatus, S. A.

Miller, 1874 , Cin. Quar. Jour. Sci., vol. 1, p. 346, Hud. Riv. Gr.

LYuIOCRINL', Hall, 1852, Pal. N. Y., vol. 2 , p. 197 . [Ety. lyrion, small ly re; $k r$ ino $n$, lily.] Calyx hem ispherical; hasals 5 ; subradials 5 ; primary ralials $3 \times 5$; secondary Fin. 352 -Lyriocrinus daeradials $2 \times 10$;

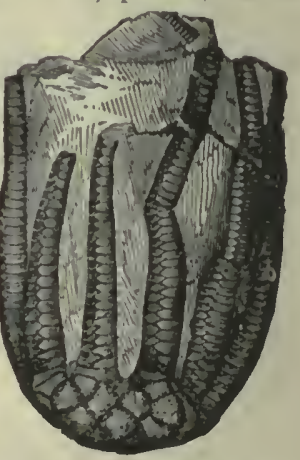
interradial areas 4 or 5 , which are in closed above by the secondary radials; 
azygous area similar to the regular interradial areas; vault almost flat, depressed interradially, composed of small plates; opening excentric; arms 10 , composed of a double series of interlocking plates. Type L. dactylus.

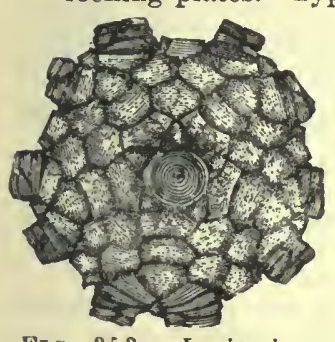

FI . 353. - Lyriocrinus dactylus. Basal view. dactylus, $\mathrm{H}$ all 1843, (M a rsupiocrinites (?) dactylus,) Geo. Rep. 4th Dist. N. Y., p. 114, and Pal. N. Y., vol. 2, p. 197, Niagara Gr.

meli ssa, H a ll, 1863, (Rhodocrinus melissa,) Trans. Alb. Inst., vol. 4, p. 198, and 11th Rep. Gen. and Nat. Hist. Ind., p. 269, Niagara Gr.

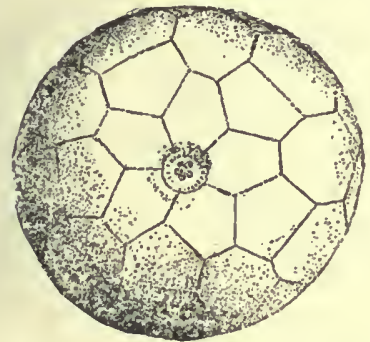

Fia. 354.-Lyrlocrinus melissa. Basal view.

sculptilis, Hall, 1864, 20th Rep. N. Y. St. Mus. Nat. Hist., p. 368, Niagara Gr. sculptus, see Archæocrinus sculptus.

L Y S O CYSTITES, n. $g$ en. [Ety. lysis, setting free; kustis, bladder.] Body subspheroidal, composed of four ranges of plates; basal plates probably 4 , s ucceeded by two ranges of 5 plates each and dome p l a t e s; mouth central; ovarian aperturelateral. Type L. nodosus. This

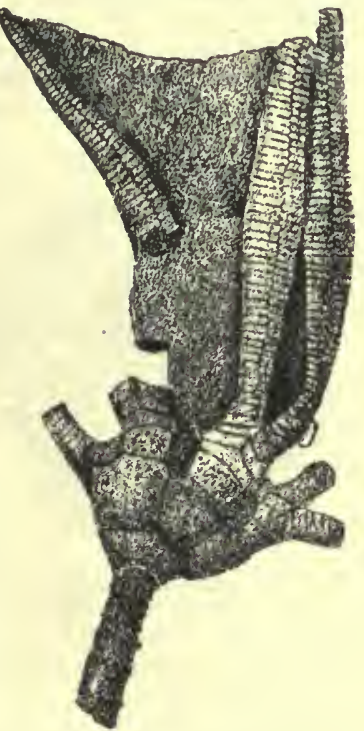

I G. 355.- Macrostylocriuus fusibrachiatus.

generic name is proposed instead of

Echinocystites, Hall, which was preoccupied. The genus is known only from casts.

nodosus, Hall, 1864, (Echinocystites nodosus, 20th Rep. N. Y. Mus. Nat. Hist., p. 360, Niagara Gr.

Macrostylocrinus, Hall, 1852, Pal. N. Y., vol. 2, p. 203. [Ety. makros, long; stylos, an arm ; krinon, lily.] Calyx urnshaped; basals 3 ; primary radials $3 \times 5$; secondary radials 1 or more by 10 ; regular interradials 3 ; azygous interradials 4 ; arms 10 . Type $M$. ornatus.

fasciatus, Hall, 1876, (Cyathocrinus fasciatus,) 28th Rep. N. Y. St. Mus. Nat. Hist., p. 130, Niagara Gr. Probably a syn. for M. meeki.

fusibrachiatus, Ringueberg, 1882, Jour. Cin. Soc. Nat. Hist., vol. 5, p. 119 , Niagara Gr.

meeki, Lyon, 1861, (Actinocrinus meeki, Proc. Acad. Nat. Sci. Phil., p. 411, Niagara Gr.

ornatus, Hall, 1852, Pal. N. Y., vol. 2, p. 204, Niagara Gr.

striatus, Hall, 1868, Trans. Alb. Inst., vol. 4, p. 207, and 20 th Rep. N. Y. St. Mus. Nat. Hist., p. 327, Niagara Gr.

striatus var. granulosus, Hall, 1879, 28th Rep.

N. Y. St. Mus. Nat. Hist., p. 129 Niagara Gr.

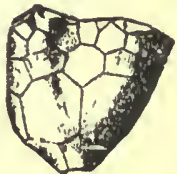
MA L OCY S T T Es, Billings, nus striatus. 1858, Can. Org. Rem.,

Decade 3, p. 66. [Ety. malum, apple ; kustis, bladder.] Body ovate or globular; plates nonporiferous and in very irregular series; first series 3 ; second series 10 or 12 , and in all the series 40 or 50; mouth apical: ambulacral orifice near the upper part; arms recumbent, 8 or more. Type M. murchisoni. barrandi, Billings, 1858, Can. Org. Rem., Decade 3, p. 67, Chazy Gr.

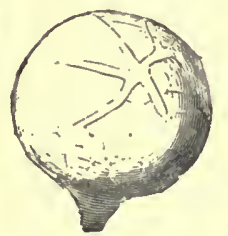

FiG. 357. - Malocystites murchisoni. econdary radials $3 \times 10$; tertiary radials 1 or more by 20 ; interradials 3 to 10 , the first one supported by the first radials; azygous area large and plates numerous; vault inflated, plates small ; arms composed of a double series of interlocking plates, and not unfrequently bearing armlets consisting also of a double series of interlocking plates; column round. Type M. nobilissimus. 
carleyi, Hall, 1862, (Glyptocrinus car-

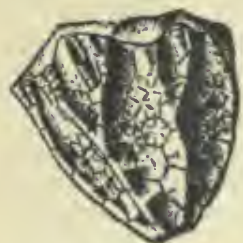

Hif. 358. - Marlacrinus carleyi.

leyi,) Trans. Alb, Inst., vol. 4, p. 203. and 11 th Rep. Geo. and Nat. Hist. Ind., p. 261, Niagara Gr.

macropetalus, $\mathrm{H}$ a ll, 1859, Pal. N. Y., vol. 3 , p. 111, Low. Held. Gr.

nobillissimus, $H$ all, 1859, Pal. N. Y., vol. 3 , p. 105 , Low. Held. Gr.

pachydactylus, Conrad, 1841, (Astrocrinites pachydactylus,) Ann. Rep. N. Y., p. 34, and Pal. N. Y., vol. 3, p. 107, Low. Held. Gr. Syn. (?) for M. polydactylus.

paucidactylus, Hall, 1859, Pal. N. Y., vol. 3, p. 109, Low Held. Gr.

plumosus, Hall, 1859, Pal. N. Y., vol. 3, p. 110, Low. Held. Gr.

polydactylus, Bonny, 1837, (Actinocrinus polydactylus,) Am. Jour., vol. 31, syn. for M. pachydactylus?

ramosus, Hall, 1859, Pal. N. Y., vol. 3, p. 147. Low. Held, Gr.

stoloniferus, Hall, 1859, Pal. N. Y., vol. 3, p. 112, Low. Held. Gr.

warreni, Ringueberg, 1888, Proc. Acad. Nat. Sci. Pliil., p. 133, Niagara (ir.

M arsupiocrinus, Phillips, 1839, Murch. Sil. Syst., p. 672. [Ety. marsups, bag; krinon, lily.] Basals 3 ; primary radials $2 \times 5$; secondary radials $2 \times 5$; arms 20 ; distinguished from Platycrinus by the higher order of radials, by the round column, instead of elliptic, and by liaving a larger canal. Type M. calatus.

dactylus, see Lyriocrinus dactylus.

tennesseensis, Roemer, 1860, (Platycrinus (tennesseensis, ) Sil. Fauna West Tenn., p. 35, Niagara Gr.

tentaculatus, Hall, 1861, (Platycrinus tentaculatus,) Pal. N. Y., vol. 3, p. 116, Low. Held. Gr.

Mraistocrixus, Owen \& Shumard, 1852, Geo. Sur. Wis., Iowa, and Minn., p. 594. [Ety. megislos, very great; krinon, lily.] Body basin-shaped; basals 3 ; primary radials $3 \times 5$; secondary radials $1 \times 10$; tertiary radials 1 or more $\times 20$; interradials numerous; first azygous plate like the first radials, and resting on the basals, succeeded by three plates, and these by numerous smaller ones; arms in double series of short plates, bifurcating and bearing pinnules; vault convex; orifice excentric or lateral; column round. Type M. evansi.

abnormis, Lyon, 1857, (Actinocrinus abnormis,) Geo. Sur. Ky., vol. 3, p. 479, Up. Held. Gr.

concavus, Wachsmuth, 1885, Proc. Dav. Acad. Sci. vol. 4, p. 96, Ham. Gr.

brevicornis, Hall, 1858, (Actinocrinus brevicornis,) Geo. Sur. Iowa, p. 571, Burlington $\mathrm{Gr}$. crassus, White, 1862, Proc. Bost. Soc. Nat. Hist., vol. 9, p. 17, Burlington Gr.

depressus, Hall, 1862, 15th Rep. N. Y. St. Mus. Nat. Hist., p. 134, Hamilton Gr.

evansi, Owen \& Shumard, 1850, (Actinocrinus evansi,) Jour. Acad. Nat. Sci. vol. 1 , pt. 2, p. 68, and Geo. Sur. Wis., Iowa, and Minn., p. 594, Burlingtou Gr.

farnsworthi, White, 1876, Proc. Acad. Nat. Sci., p. 29, Ham. Gr.

infelix, see Saccocrinus infelix.

knappi, Lyon \& Casseday, 1857, I'roc. Acad. Nat. Sci. Phil, p. 412, Up. Held. Gr. latus, Hall, 1858, Geo. Sur. Iowa, vol. 1, pt. 2, p. 480, Ham. Gr.

marcouanus, see Saccocrinus marcouanus. necis, see Stccocrinus necis.

nobilis, Wachsmutlı \& Springer, (in press, ) Geo. Sur. Ill., vol. 8, p. 169, IVaverly or Kinderhook Gr.

nodosus, Barris, I 879 , Proc. Dav: Acad. Nat. Sci., vol. 2 , p. 285, Up. Held. Gr.

nodosus var. multidecoratus, Barris, 1885, Proc. Dav. Acad. Nat. Sci., vol. 4, p. 98 , Ham. Gr.

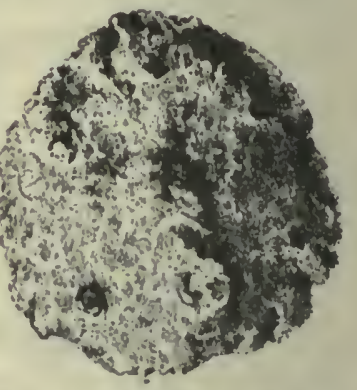

ontario, Hull, Fig. 359.-Megistocrinus pllea1862 , 15th lus. Vlew of vault.

Rep. N. Y. St. 'Mus. Nat. Hist., p. 136, Hail. Gir.

parvirostris, syn. for M. plenus.

parvus, Wachsmuth \& Springer, (in press,) Geo. Sur. III., vol. 8, p. 171, Kinderhook Gr.

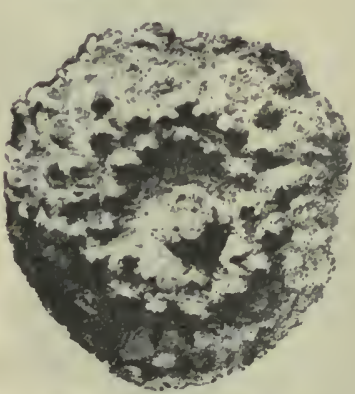

pileatus, S. A. Miller, 1879 , Jour. Cin. Soc. Nat. Hist., vol. 2, I). 114, Up. Held. Gr.

p) lo nus, White, 1862, Proc. Bost. Soc. Nat. Hist., vol. $9, \mathrm{p}$. 16, Bur lington Gr. tu60.-Megistocrinus pilenrugosus, Lyon \& Casseday, 1859, Am.
Jour. Sci., vol. 28, j. 243, Up. Held Gr.

spinulosus, Lyon, 1861, Jour. Acad. Nat. Sci. Phil., p. 413, Up. Held. Gr. whitii, Hall, 1861, Jour. Bost. Soc. Nat. Hist., vol. 7, p. 271, Burlington Gr. 
Melocrinus, Goldfuss, 1826, Petref. Germ.,

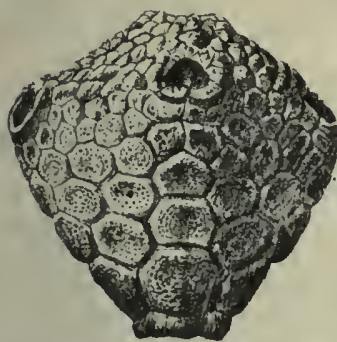

FIG.361.-M elocrinus bain-

p. 197. [lity. melo, melon; krinon, lily.] Body obconoidal; surface ornamente d ; inte $\mathrm{r} r \mathrm{adia} 1$ a $r$ e s depressed; basals 4 ; radials $3 \times 5$; secondary radials 2 or $3 \times 10$; tertiary radials 2 or $3 \times 20$; bridgensis. interradials 8

to 12 ; azygous plates more numerous; vault convex, with orifice excentric. Type M. hieroglyphicus.

bainbridgensis, Hall \& Whitfield, 1875 , (Ctenocrinus b a inbridgensis, Olijo Pal., vol. 2, p. 158 , Purtage Gr.

breviradia t us, Hall \& Whitfield, 1875 , (Ctenocrinus brevir a d i a -

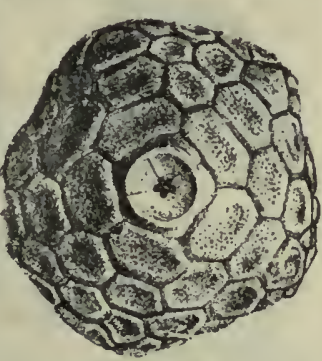
tus, Ohio Pal., Fig. 362.-Melocrinus bainvol. 2 , p. 160 , bridgensis. Basal view. Ham. Gr.

clarkii, Williams, 1882, Proc. Acad. Nat. Sci., p. 31, Chemung Gr.

lævis Roemer, 1860, (Cytocrinus lævis,) Sil. Fuuna W. Tenn., p. 56, Niagara Gr.

nodosus Hall, 1861, Geo. Rep. Wis., p. 19, Devonian.

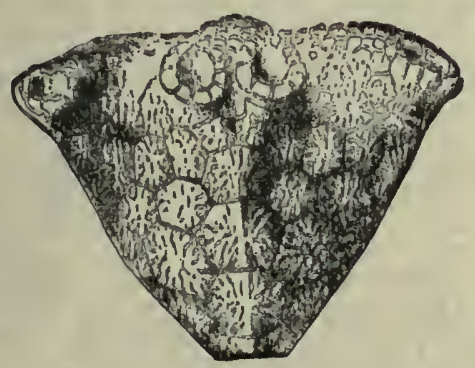

FIG. 363.-Melocrinus obconicus.

obconicus, Hall, 1863, Trans. Alb. Inst., vul. 4, p. 206, Niayara Gr.

obpyramıdalis, Winchell \& Marcy, 1865, (Actinucrinus obpyramidalis,) Mem. Bust. Suc. Nat. Hist., vol. 1, p. 87, Niaydra Gr.

pratteni, McChesney, 1860, (Forbesocrinus

pratteni,) New Pal. Foss., p. 29, and Trans. Chi. Acad. Sci., p. 22, Warsaw $\mathrm{Gr}$.

sculptus, Hall, 1852, Pal. N. Y., vol. 2, p. 228, Niagara Gr.

verneuili, Troost, 1850, (Actinocrinus verneuili,) and Hall, 20th Rep. N. Y. St. Mus. Nat. Hist., p. 327, Niagara Gr.

Melonites, OWen \& Norwood, 18 $16, \mathrm{Am}$. Jour. Sci., 2d series, vol. 2, p. 225. [Ety. melon, melon; lithos, stone.] Test spheroidal, divided into five convex ambulacral and five convex interambulacral areas, resemibling in form a melon, with ten ribs or convex elevations and as many sharply defined depressions; plates of the interambulacral areas large, thick, hexagonal, not overlapping, arranged in series, 8 or 10 of which cover the wider part, but not more than two reach the apical disk; ambulacral areas covered with about 8 or 10 rows of plates, each plate having two pores, so arranged that the pores of the central two ranges are at the ends most distant from the median line; ocular plates, without pores, and

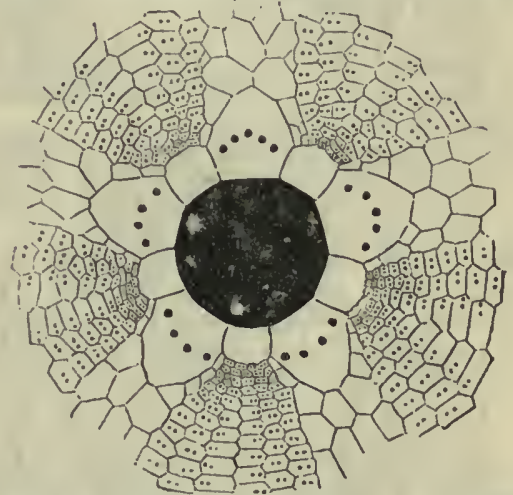

FIG. 364,-Melonites multiporus. Apical disk, genital and ocular pores.

much smaller than the genital, which have inumerous pores that differ, in number, in the same species; jaws very strong. Type M. multiporus.

crassus, Hambach, 1884, Trans. St. Louis Acad. Sci., vol. 4 , p. 548, St. Louis Gr.

danx, see Oligoporus danæ. irregularis, $\mathrm{Hambach}$, 188t, Trans. St. Louis Acad. Sci., vol 4, p. 548, St. Louis Gr. multiporus, Owen \& Nor-

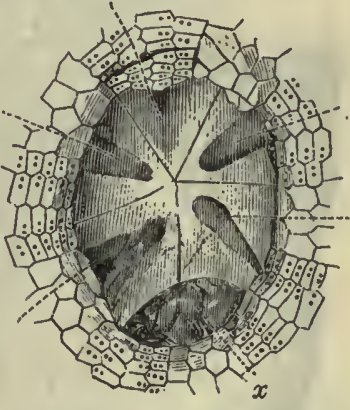

FIs. 365.-Melonites multiporus. Oral opening and jaws, displaced at $x$ so as to leave an opening. wood, 1846 , Am. Jour. Sci., 2d ser., vol. 2, p. 225, St. Louis Gr. 
stewarti, Safford, 1869, Geo. of Tenn., p. 346, St. Louis Gr.

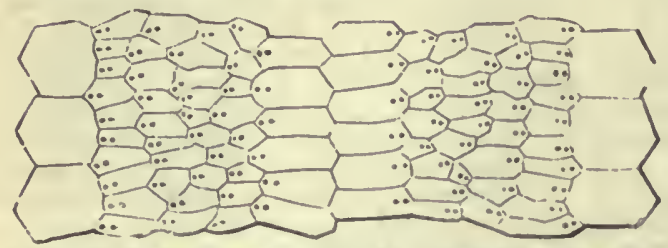

Frg. 366.-Melonites multjporus. Dlagram, showlug pores in anubulacral area, 2 dinm.

Menocrinus, n. gen. Ety. menos, strength of body; krinon, lily.] Calyx sumewhat globular; basals 3 ; subradials $1 \times 5$; radials $1 \times 5$; azygous and interradials 0 ; which distinguishes the genus from Platyerinus. Type M. olliculiformis. This generic name is proposed as a substilute for Lecythiocrinus, White, 1880 , because that name was preoccupied by Mnller in 1858, and by Zittel in 1879.

adansi, Worthen, 1882, (Lecythiocrinus adamsi,) Bull. No. 1, Ill. St. Mus. Nat. Hist., p. 37, and Geo. Sur. Ill., vol. 7, p. 317, Cosl Meas.

olliculiformis, White, 1880, (Ifecythiocrinus olliculiformis,) Proc. U. S. Nut. Mus., vol. 2, p. 257, and Cont. to Pal., No. 6, p. 124, Up. Coal. Meas.

Merocrises, Walcott, 1883, 35th Rep. N. Y. St. Mus. Nat. Hist., p. 208. [Ely. meros, hip-joint; krinon, lily.] Basals 5, pentangular, low, broad; subradials 5 , bexagunal, short, broad; radials pentagonal, four support upon the upper truncate face of each a row of six or seven brachials, and the azyzous plate from the same level, the fifth radial; right fosterior radial like the azygous plate, but having an angular upper side, giving off, on one side, the ventral tube, and on the other a row of brachials; arms long, hifurcating, without pinnules. Type M. typus.

curius, Ulrich, 18\%9. (Den-

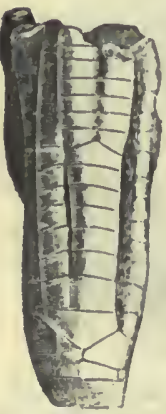

FIG. 367.-Merocriu us curtus. once, taper rapidly, infold and incline obliquely from left to right; azygous plate 1, resting upon the larger basal; column round. Type M. forbesanus.

konincki, Hall, 1860, Supp. to Geo. Sur. Iowa, p. 69, Burlington Gr. scitulus, Hull, 1861, Desc. New Crinoidea, p. 9, Burlington Gr.

MYelodactylus, Hall, 1852, Pai. N. Y., vol. 2, p. 191. [Ety. myelos, the inside pith; daclylus, finyer.] Body consisting of a coil rolled, in the same plane, with finger-like processes, from each coil, overlapping the next inner one; coil and processes perforated so as to form connecting channels. Type M. convolutus. brachiatus, Hall, 1852, Pal. N. Y., vol. 2, p. 232, Niagara Gr.
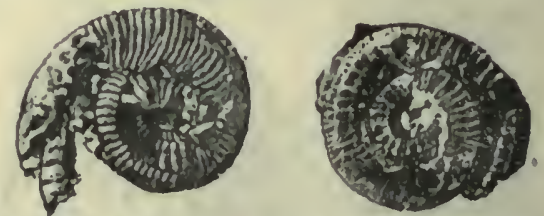

Fiw. 368.-Myelodactylus bridgportensis.

bridgportensis, S. A. Miller, 1880, Jour. Cin. Soe. Nat. Hist., vol. 3, p. 141, Niagara $\mathrm{Gr}$.

convolutus, Hall, 1852, Pal. N. Y., vol. 2, p. 192, Nisgara Gr.

M yrtillocrinus, Sandberger, 1856, Verst. der Rliein. Schi. Syst. in Narsau. [Ety. murtillus, myrtle; krinon, l1ly.] Body subglobose or ovoid; basals 5 ; subradials 5 ; radials $1 \times 5$; dome consisting of 5 plates altemating $\mathrm{u}$ ith the radials; arms 5 ; columnar canal, quudrangular. Type M. elongatus.

americanus, Hall, 1862, 15 th Rep. N. Y. St. Mus. Nat. Hist., App. C., p. 114, Up. Held. Gr.

Nemalocrinus, Meek \& Worthen, 1866, Proc. Acarl. Nat. Sci. Phil., p. 251, syn. for Culillncrinue.

Nipterockinus, Wachsmuth, 1868, Proc. Acarl. Nat. Sei. Phil., p. 341. [Ety. nipter, washing vessel; krinon, lily.] Culyx basin-shaped; basals 3 , nearly hidden by the column; subralials $1 \times 5$; radials 3 or $4 \times 5$, the first one very large; arms bifurcating; column round. Type N. wachsmuthi.

arborens, Worthen, 1863, Geo. Sur. III., vol. 5 , p. 436 , Burlington Gr.

waclismuthi, Meek \& Worthen, 1868, Proc. Acad. Nat. Sci. Pliil., p. 341, and Geo. Sur. Ill., vol. 5, p. 435, Burlington $\mathrm{Gr}$.

Nuci.mocisinus, Conrad, 1842, Jour. Acad. Nat. Sci. Plıil., vol. 8, p. 280, [Ety. nucleus, a little nut: krinon, lily.] C'alyz ellipisoidal; basals 3, small, hidden within the columnar ('avity; radials 5 , forming a small cup, deeply scalloped 
for receiving the bases of the narrow, elongite ambulacra, and having projecting lips forıning a quinquepod; deltuid plates 6, elongate, forming three-fourthis of the calyx; a narrow intercalated plate, on the azygous side, reaclies from the aperture to the radial, and divides the di.ltoil into two narrow curving plates; sinuses narrow, extending the entire length of the calyx; ambulacra narrow; lancet plates long and very narrow; side platos numerous; hydrospires pendent, two tubes or elongated sacs on pach side of an ambulacrum; spiracles in five pairs, which notch the deltoid plates; mouth large, covered normally with plates; azyunus opening large. Types $N$. elegans and $N$. vernenili.

angularia, Lyon, 1857, (Olivanites angularis,) Geo. Sur. Ky., vol. 3, p. 492, Ham. Gr.

canadens s, Montgomery, 1881, Can. Nat. and Geol. Vul. 10, p. 83, Ham. Gr.

con radi, Hall, 1862,15 th $R$. p. N. Y. St. Mus. Nat. Hist., App. C., p. 121, Up. Hold. Gr.

elegans, Conrad, 1842, Jour. Acad. Nat. Sti. Phil., p. 280, Hain. Gr.

hallii, syn. for Nucleocrious elegans.

kirkwoudensis, Shumurt, 1863, (Eleacrinus kirkwoodensis,) Trans. St. Louis Acad. Sici., vol. 2, p. 113, St. Louis Gr. lucina, Hali, 1862, 15th Rep. N. Y. St. Mus. Nat. Hist., App. C., p. 120,

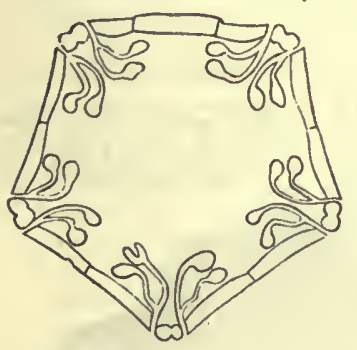

FrG. 369.-Nuclencrinus obovat us. Crons section of hydrospires, 2 dium.
Ham. Gr. $\mathrm{m}+\mathrm{loniformis,}$ Barris, 1883, (El eacrinus $m+1$ o $n$ i firmis,) Geo. Sur. Ill., vol. 7. p. 361 , Huri. Gr. obovatus, Barr i $\mathbf{s}, 1883$, ( E leacrinus obovatus, ) Gron. Sur. Ill., vol. $7, \mathrm{p}$. 358, H a m. Gr.

verneuili, Trnost, 1811, (Pentremites verneuili,) 6th Rep. on the Gen. of Tenn., p. 14, and Geo. Sinr. Ky. vol. 3 , p. 488 , Ujp. Held. Grr.

vern uili var. nomum, Etheridge \& Carpenter, is simply a rounded form very common among other specimens, and without varietal char- FIG. 370.-Nucleoacters.

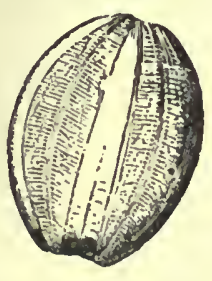

crinus verneulii.

Orlocrinus, Warhsmuth \& Springer, 1885, Palæocrinoidea. pt. 3, p. 208. [Etv. proper name; krinom, lily.] Plates of calyx arranged as in Heterocrınus; arms 10, bearing bifurcating pinnules; ventral tube large, having a spiral form somewliat like the cast of a Murchisonia, and covered with hexagonal plates; column pentagonal and pentapartite. Type O. laxus.

compactus, Meek, 1873, (Heterocrinus constrictus var. compactı1s,) Ohio Pal., vol. 1, pl. 11, Hud. Riv. Gr.

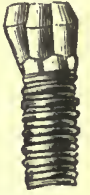

Fra. 371. Ohlocrinus constrictus, Hall, 1866, (Hete- compuctus. rocrinus constrictus,) 24th Rep. N. Y.

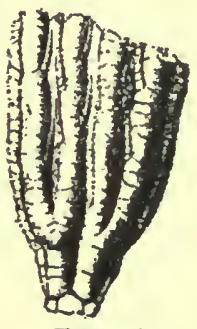

Fig. 372 . Ojilocrinus constrictus. St. Mus. Nat. Hist., p. 210, Hud. Riv. Gr.

laxus, Hall, 1866, (Heterocrinus laxus,) 24th Rep. N. Y. St. Mus. Nat. Hist., p. 211, H u d. Riv. Gr.

œhanus, Ulrich, 1882 , (Heterocrin us œhanus,) Jour. Cin. Soc. Nat. Hist., vol. 5, p. 175, Hud. Riv. Gr.

Oligoporus, Meek \& Worthen, 1860, Proc. Acad. Nat. Sci. Phil., p. 474. [Ety. oligos, few; poros, passage.] In form like Melonites, but distinguished by having only four rows of ambulacral plates, and four double rows of pores in each ambulacral area. Type O. dunæ. Desor used the name Oligopores, in 1858, for a section of the Cidıridæ, and Prof. Meek said if desirable to change Oligoporus, for that reasin he would suggest Melonopsis in its place.

coreyi, Meek \& Worthen, 1870, Proc. Acad. Nat. Sci. Phil., p. 34, Keokuk Gr.

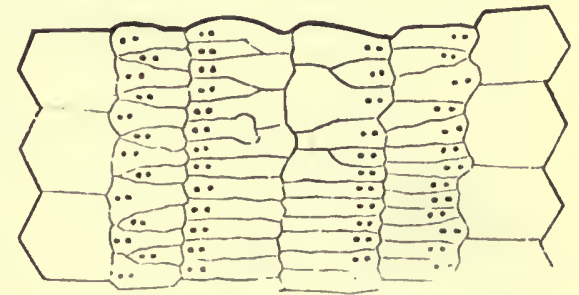

Fig. 373.-Oligoporus danæ; 2 diam.

danæ, Meek \& Worthen, 1860, (Melonites danæ, ) Proc. Acad. Nat. Sci. Phil., n. 397, and Gro. Sur. Ill., vol. 2, p. 249, Keokık Gr.

nohilis, Meek \& Wnrthen, 1868, Proc. Acad. Nat. Sci. Phil., p. 358, and Geo. Sur. Ill., vol. 5, p. 476, Burlington Gr.

parvus. Hambach, 1884, Trans. St. Louis A cad. Sei., vol. 4, p. 548, Keokuk Gr.

Olivanites, syn. for Nucleocrinus.

a)gularis, see Nucleocrinus angularis. verneuili, see Nucleocrinus verneuili. 
Ollacrinus, Cumberland, 1826, Appendix to Reliquiæ Conservata. Figured without description, and subsequently declared by DeKoninck \& LeHon to be a Rhodocrinus. Wachsmuth Springer priority for this name over Gonia s te roidocrinus, without good reason, however, as shown by Meek in Ill. Geo. Sur., vol. 2 , p. 217.

Onychaster, Meek \& Worthen, 1868, Geo. Sur. Ill., vol. 3, p. 526. [Ety. onyx, claw ; aster, star.] A small sub-discoid body, with five long, slender, rounded, flexible rays ; dorsal side of disk composed of an outer circle of five pairs of plates each, pierced with an ovarian pore, and two inner circles of five pairs each, n o n p or iferous, and surrounding a central anal opening ; outside the pore plates, each pair is followed by two or three pairs of interlocking transverse plates, connecting with the dorsal side of the rays; farther there are lanceolate plates, furrowed and having pores between the inner ends. Type $O$. flexilis.

barrisi, Hall, 1861, (Protaster barrisi,) Desc. New. Crinoidea, p. 18, and Geo.

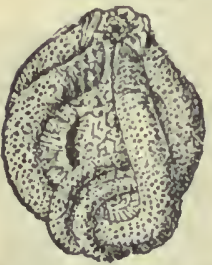

Fra. 374.--Unychaster flexillis. Sur. Ill., vol. 5, p. 476, Burlington Gr.

flexilis, Meek \& Worthen, 1868, Geo. Sur. Ill., vol. 3, p. 526, Keokuk Gr.

ONYCHOCRINUs, Lyon \& Casseday, 1859, Am. Jour. Sci., 2d series, vol. 29, p. 77. [Ety. onyx, claw ; krinon, lily.] Calyx low, arms like the talons of a fowl; barals 3 ; subradials 5; radials 4 to $7 \times 5$; arms short, branching; interradials 3 to 20 ; vault depressed; column large. Type 0 . exculptus.

asteriformis, Hall, 1861, (Forbesiocrinus asteriformis,) Desc. New Crin., p. 9, and Geo. Sur. Ill., vol. 2, p. 243, Keokuk Gr. distensus, Worthen, 1882, Bull. No. 1, Ill. St. Mus. Nat. Hist., p. 31, and Geo. Sur.Ill., vol. 7, p. 307, Kaskaskia Gr. 
magnus, Worthen, 1875, Geo. Sur. Ill., vol. 6, p. 520, St. Louis Gr.

monroensis, Meek \& Worthen, 1861, (Forbesiocrinus monroensis,) Proc. Acad. Nat. Si:i. Phil., p. 130, and Geo. Sur. Ill., vol. 2, p. 244, Keokuk Gr.

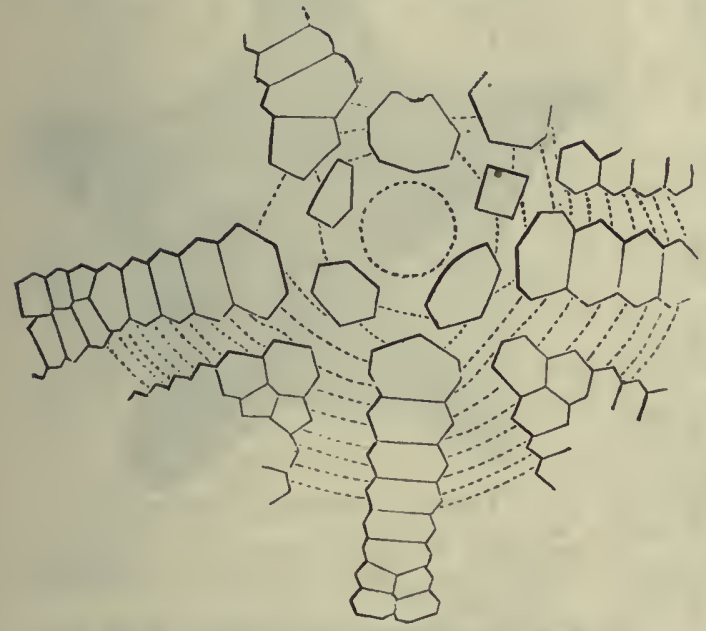

FIG. 377.-Onychocrinus exculptus. Diagram.

norwoodi, Meek \& Worthen, 1860, (Forbesiocrinus norwoodi,) Proc. Acad. Nat. Sci. Phil., p. 389, and Geo. Sur. Ill., vol. 2 , p. 245 , syn for $O$. exculptus.

ramulosus, Lyon \& Casseday, 1859, (Forbesiocrinus ramulosus,) Am. Jour. Sci. and Arts, vol. 28, p. 235, Keokuk Gr.

Orophocrinus was proposed by von Seebach, in 1864, in Nachr. k. Gesellsch. Wis. sench. Gottingen, p. 110, for Pentremites stelliformis, Owen \& Shumard. The definition was very imperfect, and was made in a foreign language, in a foreign country, and in a journal having no circulation in America, where the fossil occurs. The definition was so obscure, its application to the species was not noticed until Ludwig discovered it in 1878 , and probably never would have been, had Meek \& Worthen not described the genus, under the name of Codonites, in 1869 , and illustrated it in their great work on the Geology of Illinois. Neither the publication or definition of von Seebach is such as to allow Orophocrinus to stand in preference to Codonites.

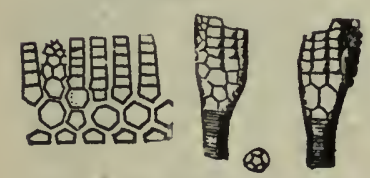

FIG. 378.-Ottawacrinus typus.
OTtawacrinus, W. R. Billings, 1887 , Ottawa Nat. Club, vol. 1 . p. 49. [Ety. propername; krinon, lily.] Calyx obconical, basals 5 ; subradials 1 x 5 ; radials $1 \times 5$; arms 5 ; azygous plate rests on a basal as in Dendrocrinus, and from which it is distinguished only by the arrangement of the plates on the azygous side. Type O. typus.

typus, W. R. Billings, 1887, Ottawa Nat.

Club, vol. 1, p. 49, Trenton Gr.

PACH yCRinus, Billings, 1859, Can. Org. Rem., Decade 4, p. 22. [Ety. pachys, thick; krinon, lily.] Calyx saucer-shaped; basals 1 x 5 ; radials $1 \times 5$. Type $P$. crassibasalis.

crassibasalis, Billings, 1859, Can. Org. Rem., Decade 4, p. 22, Chazy Gr.

Pachylocrin us, Wachsmuth \& Springer, 1879, Proc. Acad. Nat. Sci. Phil., p. 115. Proposed for a division of Poteriocrinus of less than generic importance, but later the same authors referred their type to Woodocrinus.

Palanster, Hall, 1852, Pal. N. Y., vol. 2, p. 247. [Ety. palaios, ancient; aster, star.] Stellate, disk small; two ranges of plates in each ambulacral groove, and two on either side, adambulacral and marginal; four ranges of pores in each groove; oral plates in pairs at the base of the rays; dorsal plates polygonal, sometimes spinous, madreporic tubercle. Type $\mathrm{P}$. niagarensis.

antiqua, Locke, 18t6, (Asterias antiqua,) Proc. Acad. Nat. Sci. Phil., vol. 3, p. 38, Hud. Riv. Gr. Too poorly defined for determination.

antiquus, Troost, 1835, (Asterias antiqua,) Trans.' Geo. Soc. Penn., vol. 1, p. 232, Hud. Riv. Gr.

clarkanus, S. A. Miller, 1880, Jour. Cin. Soc. Nat. Hist., vol. 3, p. 236, Hud. Riv. Gr.

clarki, S. A.

$\mathrm{Miller}$,

Jour. Cin.

Soc. Nat.

Hist., vol.

1. p. 102 , see Palæ-

a s $t$ e $r$ clarkanus.

cra w fords-

villensis,

S. A. Miller, 1880, Fig. 37. Jour. Cin. form tubercle.

Soc. Nat.

Hist, vol. 2, p. 256, Keokuk Gr.

dubius, Miller \& Dyer, 1878, Cont. to Pal., No. 2, p. 256, Utica Slate Gr.

dyeri, Meek, 1872, Am. Jour. Sci., 3d series, vol. 3 , p. 257, and Ohio Pal., vol. 1, p. 58, Hud. Riv. Gr.

eucharis, Hall, 1868, 20th Rep. N. Y. St. Mus. Nat. Hist., p. 330, Ham. Gr. 
exculptus, S. A. Miller, 1881, Jour. Cin. Soc. Nat. Hist., vol. 4, p. 69, Hud. Riv. Gr.

finii, Ulrich, 1879 , Jour. Cin. Soc. Nat. Hist., vol. 2, p. 19, Utica Slate Gr. granti, Spencer, 188t, Bull. No. 1, Mus. Univ. St. Mo., p. 53, Clinton Gr. granulosus, Hall, 1868, 20th Rep. N. Y.St. Mus. Nat. Hist., p. 327, Hud. Riv. Gr.

harrisi, S. A. Miller, 1879, Jour. Cin. Soc. Nat. Hist., vol. 2, p. 117, Hud. Riv. Gr.

incomptus, Meek, 1872, Am. Jour. Sci., 3d series, vol. 3 , p. 275 , and Ohio Pal., vol. 1, p. 64, Hud. Riv. Gr.

jamesi, Dana, 1863, (Pوlæsterina (?) jamesi,) Am. Jour. Sci., 2d series, vol. 35, p. 295 , Hud. Riv. Gr.

longibrachiatus, S. A. Miller, 1878, Jour. Cin. Soc. Nat. Hist., vol. 1, p. 102, Hud. Riv. Gr.

maguificus, S. A. Miller, 1884, Jour. Cin. Soc. Nat. Hist., vol. 7, p. 16, Hud. Riv. Gr.

matutinus, Hall, 1847, (Asterias matutina,) I'al. N. Y., vol. 1, p. 91, Trenton Gr.

L miamiensis, S. A. Miller, 1850, Jour. Cin.

- Soc. Nat. Hist., vol. 3, p. 143, Hud. Riv. Gr.

parviusculus, Billings, 1860, Can.Nat. and

E Geo., vol. 5, p. 69, and Acad. Geol., p. 594, Mid. Sil.

niagarensis, Hall, 1852, Pal. N. Y., vol. 2,

- p. 247, Niagara Gr.

pulchellus, see Stenaster pulchellus.

shafferi, Hall, 1868, 20th Rep. N. Y. St. Mus. Nat. Hist., p. 326, Hud. Riv. Gr.

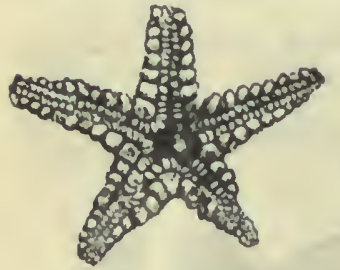

FIG. 380 -Palnaster simplex. simplex, S. A. Miller, 1878, Jour. Cin. Soc. Nat. Hist., vol. 1. p. 29, Hud. Riv. Gr.

spinulosus, Mil. ler \& Dyer, 1878, Jour. Cin. Soc. Nat. Hist., vol. 1 , p. 32 , Hud. Riv. Gr. w i l bera $\|$ a ,

Meek \& Worthen, 1861, (Petraster wilberianus,) Proc. Acad. Nat. Sci. Phil., p. 142, Hud. Riv. Gr.

Paleasterina, McCoy, 1851, Brit. Pal. Fons., p. 59 , but first defined, loy Salter, 1857 , Ann. Mag. Nat. Hist. [Ety. palaios, ancirnt; aster, star; inus, resemblance.] Pentagonal, depresstd, with plated disk that fills up the angles, leaving the ravs but slightly produced; ambulucra stiallow, hordered by subquadrate plates. Type $\mathbf{P}$.

approximata, Miller \& Dyer, 1878, Jour. Cin. Soc. Nat. Hist., vol. 1, p. 30 , Hud.

Riv. Gr.

fimbriata, see Schaenaster fimbriatus. jamesi, see Palæaster jamesi. rigida, see Petraster rigidus. rugosa, Billings, 1857, Rep of Progr. Geo. Sur. Cau., 1. 291, and Can. Org. Rem. Vec. 3 , p. $77, \mathrm{Hud}$. Riv. Gr.

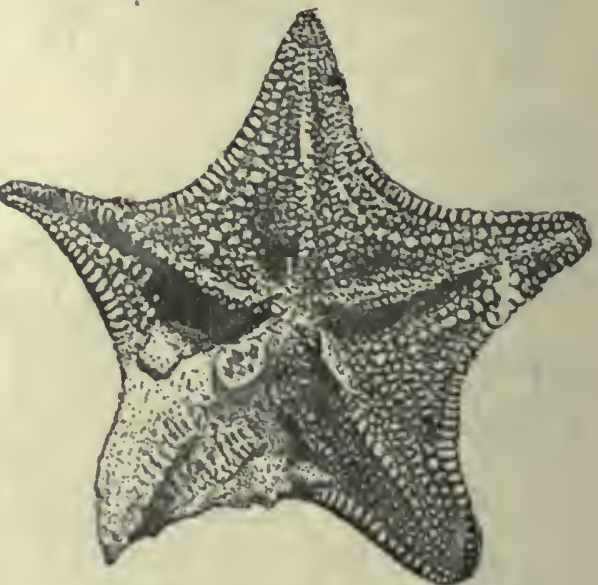

Fig. S81.-Paimasterlna speciusa.

speciosa, Miller \& Dyer, 1878, Jour. Cin. Soc. Nat, Hist., vol. 1, p. 30, Hud. Riv. Gr.

strllata, Billings, 1857, Rep. of Progr. Geo. Sur. Cun., p. 290, and Can. Org. Rem., Derade 3, 1). 76, Trenton Gr.

Palaciunus, Mr.Cuy, 1844, Curb. Foss. Irelani, \%. 172. [lity, prlaios, sucient; echinus, sea-urchin.] Iurgw, "vil or spheruidal; plutes spinous; 5 to 8 ranges of plutes in the interanbulacral areas; 2 ranges in the antunlairul areas, each plate perforated at the outer $\mathrm{n}$ nd by two pores. Type P. kornigi.

burlingtoneusis, Meek \& Worthen, 1860 , Proc. Acad. Nat. Sci. Phil., 1). 396, and Geo. Sur. Ill., vol. 2, p. 230, Burlington Gr.

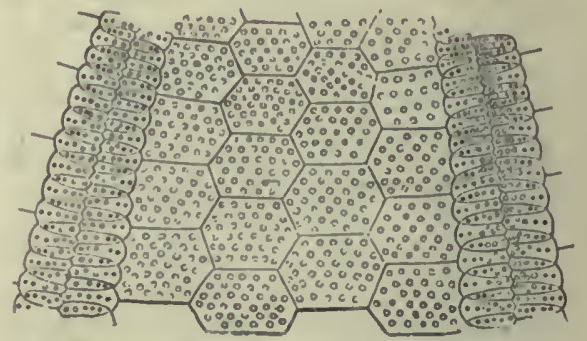

FIG. 382.-Palæchinus buringtonensis, 2 diam.

gracilis, Meek \& IVorthen, 1869, Proc. Acad. Nat. Si:i. Phil., p. 82, and Geo. Sur. Ill., vol. 5, p. 473, Burliugion Gr. Paldencoma, Salter, 1857, Anu. and Mrg. Nat. Hist., 21 series, vol. 17. [Ety. paluios, ancient; coma, hair.] Disk small, plates spinous, rays sliallow, and having ambularral, adambulacral, and marginal ulates, the latter bearing spines, inclined toward the extremity of the ray. Type $P$. marstoni. 
cylindrica, see Tæniaster cylindricus.

princeps, Hall, 1868, (Ptilonaster princeps,) 20th Rep. N. Y. St. Mus. Nat. Hist., p. 334, Chemung Gr.

spinosa, see Tæniaster spinosus.

Paldeocrinus, Billings, 1850, Can. Org. Rem., Decade 4, p. 24. [Ety. palaios, ancient; krinon, lily.] Calyx oval or pyriform; basals 5 ; radials $1 \times 5$; azygous interradials 1 to 3 ; calycinal ambulacra 5 , radiating from the center to the bases of the arms. Type P. striatus.

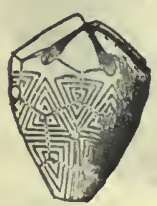

Fig. 383. - Palæocrinus stri. atus.

Can. Org. Rem.

Decade 4 , p. 45

Trenton Gr.

striatus, Billings,

1859, Can. Org.

Rem., Decade 4,

p. 25, Chazy Gr.

sulcalus, Safford. FI . 384. - Palæocrinus Not defined.

Palaocystites, Billings, 1858, Can. Org. Rem., Decade 4, p. 68. [Ety. palaios, ancient; kustis, bladder.] Body oval or pyriform; plates numerous and poriferous at the margins. Type $P$. tenuiradiatus.

chapmani, Billings, 1858, Can. Org. Rem., Decade 3, p.71, Chazy Gr.

dawsoni, Billings, 1858, Can. Org. Rem., Decade 3, p. 70, Chazy Gr.

pulcher, Billings, 1859, Can. Nat. Geo., vol. 4, p. 430 , Chazy Gr.

tenuiradiatus, Hall, 1847, (Actinocrinus tenuiradiatus,) Pal. N. Y., vol. 1, p. 18, Chazy Gr.

Parisocrinus, Wachsmuth \& Springer, 1879, Proc. Ácad. Nat. Sci. Phil., p. 115. [Ety. parisos, resembling; krinon, lily.] A division of Poteriocrinus of less than generic value, with $P$. perplexus as the type, and including $P$. nereus, $\mathbf{P}$. salignoides, $P$. teniubrachiatus, and Cyathocrinus intermedius.

Pentacrinites hamptoni, Emmons, 1842, Geo. Rep. N. Y., Trenton Gr. This is merely the plate of a crinnid column.

Pentagonites, proposed by Rafinesque for a crinoid column.

Pentremites, Sav, 1820, Am. Jour. Sci., vol. 2 , p. 36. [Ety. pente, five; remos, a board or plate.] Calyx globose, ovoid, or pyriform; base never distinctly trilobate, nor excavated in the middle line; section more or less triangular; basals 3 , forming a small cup; radials or fork plates $1 \times 5$, long, forming the greater part of the calyx; limbs long, with flat or concave sides and truncated above; sinus, subpetaloid; deltoid plates 1 ×5, small; ambulacra subpetaloid, resting in the sinuses or forks of the radials; lancet plates resting below on under lancet plates, and forming about a third the width of the ambulacra; side plates numerous and abutting the lancet plates; hydrospire or ambulacral pores partially excavated out of the sides of the sinuses; pinnules attached between the pores; hydrospires from 3 to 9 , pendent, but partially contained within the sub. stance of the radials near their distal ends; spiracles or apertures single or double, partially excavated in the deltoid plates; posterior spiracles confluent with the azygous opening; peristome covered by minute polygonal plates; column round. Type $\mathbf{P}$. godoni.

abbreviatus, Hambach, 1880, Trans. St. Louis Acad. Sci., vol. 4, p. 155 , Kaskaskia Gr.

angularis, Lyon, 1860, Trans. St. Louis Acad. Sci., vol. 1, p. 631, Kaskaskia Gr. basilaris, Hambach, 1880, Trans. St. Louis Acad. Sci., vol. 4, p. 145, Kaskaskia Gr. bipyramidalis, Hall, 1858, see Troostocrinus bipyramidalis.

bradleyi, Meek, 1873, 6th Rep. Geo. Sur. Terr., p. 470 . Not satisfactorily defined.

broadheadi, Hambach, 1880, Trans. St. Louis Acad. Sci., vol. 4, p. 145, Kaskaskia Gr.

burlingtonensis, Meek \& Worthen, 1870 , Proc. Acad. Nat. Sci. Phil., and Geo. Sur. Ill., vol. 5, p. 461, Burlington Gr. calyce, Hall, 1862, 15th Rep. N. Y. St. Mus. Nat. Hist., p. 122, Ham. Gr.

calycinns, Lyon, 1860, Trans. St. Louis Ácad. Sci., vol. 1, p. 628, Kaskaskia Gr. cervinus, Hall, 1858, Geo. Sur. Iowa, p. 690 , Kaskaskia Gr.

cherokeus, Troost, 1850, Catal. Proc. Am. Assoc. Ad. Sui. and Geo. Sur. Iowa, p. 691, Kaskaskia Gr.

chesterenais, Hambach, 1880, Trans. St. Louis Acad. Sci., vol. 4, p. 145, Kaskaskia Gr.

clavatus, Hambach, 1880, Trans. St. Louis Acad. Sci., vol. 4, Kaskaskia Gr.

conoideus, Hall, 1858, Trans. Alb. Inst., vol. 4, p. 5, and Geo. Sur. Iowa, p. 655, Warsaw Gr.

cornulus, see Granatocrinus cornutus.

curtus, see Granatocrinus curtus.

decussatus, Shumard, 1858, Trans. St. Louis Acad. Sci., vol. 1, p. 243, Keokuk Gr.

elegans, Lyon, 1860, Trans. St. Louis Acad. Sci., vol. 1, p. 632, Kaskaskia Gr.

elongatus, Shumard, 1855, Geo. Rep. Mo., p. 187, Burlington Gr.

florealis, Schlotheim, 1820, syn. for $\mathrm{P}$. godoni. 
gemmiformis, Hambacb, 1884, Trans. St. Louis Acad. Sci., vol. 4, p. 548, Kaskaskia Gr.

globosus, Say, as identified by Troost, 1850, probably Pentremites sulcatus.

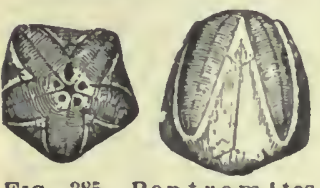

Fig. 385.-Pentrem i tes godoni, DeFrance, 181S, Dict. Sci. Nat., t. 14, p. 467, Kaskaskia $\mathrm{Gr}$.

granulatus, see Granatocrinus granulatus.

grostenori, S h llmard, see Troostocrinus grosvenori.

hemisphericus, Hambach, 1880, Trans. St. Louis Acad. Sci., vol. 4, p. 145, Kaskaskia Gr.

kentuckiensis, see Codaster kentuckiensis.

koninckan us, Hall, 1858, Trans. Alb. Inst., vol. 4, p. 4, and Geo. Sur. Iowa, p. 656, Warsaw Gr.

laterniformis, Owen \& Shumard, 1850, Jour. A cad. Nat. Sci, 2d series, vol. 2, p. 66 , Kaskaskia Gr.

leda, Hall, see Granatocrinus leda.

lineatus, see Trnostocrinus lineatus.

longicostalis, Hall, 1860, Supp. to Geo. Iowa, p. 85 , Wareaw Gr. Not satisfactorily defined.

lycorias, Hall, 1863, 16th Rep. N. Y. St. Mus. Nat. Hist., p. 123, Ham. Gr.

maia, Hall, 1862, 15th Rep. N. Y. St. Mus. Nat. Hist., p. 122, Ham. Gr.

melo, see Granatocrinus melo.

missouriensis, Swallow, 1863, Trans. St. Louis Acad. Sci., vol. 2, p. 81, Kaskaskia $\mathrm{Gr}$.

nodosus, Hambach, 1880, Trans. St. Louis Acad. Sci., vol. 4, p. 145, Kaskaskia $\mathrm{Gr}$.

norwoodi, see Granatocrinus norwoodi.

obesus, Lyon, 1857, Geo. Sur. Ky., vol. 3, p. 469 , Kaskaskia Gr.

obliquatus, see Tricœlocrinus obliquatus. ovalis, Owen. Not de. fined.

potteri. Ham bach, 1880, Trans. St. Louis Acad. Sci., vol. 4, p. 156, Burlington $\mathrm{Gr}$.

pyriformis, Say, 1825 , Jour. Acad. Nat. Sci. Phil., vol. 4 , p.

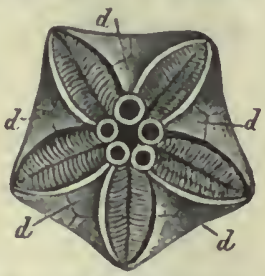

294, Kaskaskia Gr. Fra. 3s6.-Pentremites reinwardti, Troost, pyriformis. Ventral see Troostocrinus surface; $d$, deltold reinwardti.

robustüs, Lyon, 1860, Trans. St. Louis A cad. Sci., vol. 1, p. 629, Kaskaskia Gr.

roemeri, see Granatocrinus roemeri.

sampsoni, Hambach, 1884, Trans. St. Louis Acad. Sci., vol. 4, p. 548, Choteau or Waverly Gr.

sayi, see Schizoblastus sayi.

sirius, White, 1862, Proc. Bost. Soc.

Nat. Hist., vol. 9, p. 20, Burlington Gr. spinosus, Hambach, 1880, Trans. St. Louis Ar'ad. Sci., vol. 4, p. 145, Kaskaskia Gr. stelliformis, see Oroplincrinus stelliformis. subconnidı.us, Met k, 1873, Hayden's Gto. Sur. Trrr, p. 471, Subcarb.
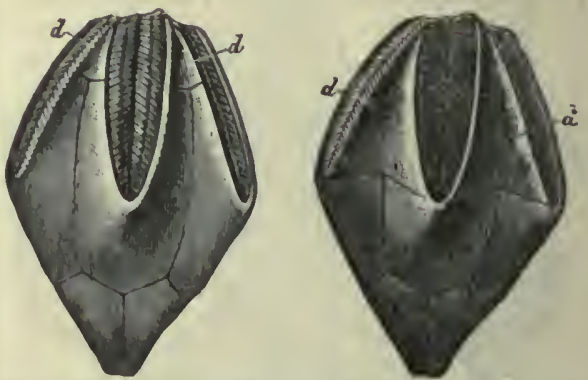

FIG. 387.-Pentrenttes pyriformls. One sluns the deltold plutes extended to tio sintumili.

subcylindricus, see Troostocrinus subcylindricus.

subtruncatus, see Troostocrinus subtruncatus.

sulcatus, Roemer, 1852, Monog. Blastoid., p. 354, Kaskaskia Gir.

symmetricus, Hall, 1858, Geo. Rep. Iowa, p. 694, Kaskarkia Gr.

tennesseex, 'Troost. Not defiued.

troosti, Shumard, 1866, Truns. St. Louis Acad. Sci., vol. 2, p. 386, Kaskaskia Gr. truncatus, Conrad, 1843, l'roc. Acad. Nat. Sci. Phil., vol. 1, p. 334, Watsaw Gr.

varsouviensis, see Tricœlocrinus vursouviensis.

verneuili, see Nucleocrinus vernenili.

whitii, Hall, 1862,15 th Rep. N. Y. St. Mus. Nat. Hist., p. 122, Ham. Gr. voodmani, see Tricœlicrinus woodmani. wortheni, see Tricœlicrinus wortheni.

Pentremitidea D'Orbigny, 1849, Prodr. d. Paléunt., t. 1, p. 102. [Ety. from Pentremites.] Number and disposition of plates as in Pentremites, but the deltoids are inconspicuons, confined to the summit, rarely visible in a side view ; spiracles large. It is also closely connected with Truostocriuus, and is of doubtful generic value. Type $P$. schultzi.

americana, Barris, 1883, Geo. Sur. Ill., vol. 7, p. 363, Ham. Gr.

filosa, Whiteaves, 1887 , Cont. to Cun. Pal., vol. 1, p. 104, Ham. Gr.

Perrscriodomus, M c Coy, 1849, Ann. Nat. Hist., vol. 3, p. 251 . [Ety. perischos, inclosing; domus, house.] Spheroidal, subpentagonal; ambulacra narrow, two rows of small plates imbricat-

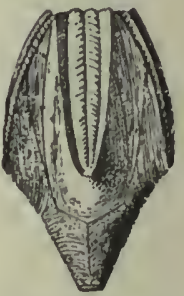
Ig. 388. - Pentrein 1 t 1 dea a merleana. 2 diain. pierced by one pair of simple pores; interambulacra wide, with five rows of 
plates imbricating upward, and from the center outward; primary spines on the rows adjoining the ambulacra, the supporting tubercle being small, perforated, but not crenulated, and surrounded by a double ring; ovarian plates having 6 pores; mouth and anal openings small, central. Type $\mathbf{P}$. biserialis.

illinoisensis, Worthen \& Miller, 1883, Geo. Sur. Ill., vol. 7, p. 333, Kaska-kia Gr.

Pereiochocrinus, Austin, 1843, Ann. and Mag. Nat. Hist., vol. 11, p. 203. Not defined so as to be recognized, though some authors use it instead of Saccocrinus.

Petraster, Billings, 1858, Can. Org. Rem., Decade 3, p. 79. [Ety. petros, stone; aster, star.] Closely related to Palæaster, and having both marginal and adambulacral plates, with a lew diskplates, on the ventral side. Type $P$. rigidus.

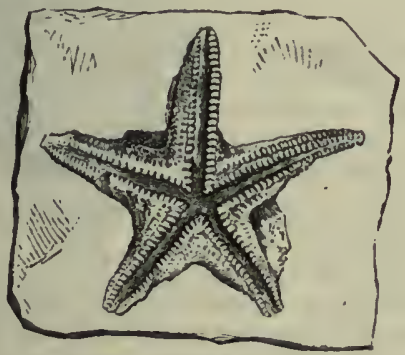

Frg. 389.-Pelruster bellulus.

bellulus, Billings, 1865, Pal. Foss., vol. 1, p. 393, Niagara Gr.

rigidus, Billings, 1857, (Palæasterina rigidus,) Rep. of Progr. Geo. Sur. Can., p. 291, and Can. Org. Rem., Decade 3, p. 80, Trenton Gr.

wilberianus, see Palæaster wilberanus.

Philocrinus, Koninck, 1863. [Ety. philos, favorite ; krinon, lily.]

pelvis, Meek \& Worthen, 1865, Am. Jour. Sci., $2 \mathrm{~d}$ series, vol. 39, syn. for Erisocrinus typus.

Pholidocidaris, Meek \& Worthen, 1869 , Proc. Acad. Nat. Sci. Phil., p. 77. [Eıy. pholidos, scale ; kideris, turban.] Interambulacrals thin, irregular, imbricating upward and laterally; five or more rows; only two reaching the extremities; marginal rows and those on the lower side having primary tubercles, showing a pit in the top, and being surrounded by two rings; ambulacral areas wide, with six or more rows of plates imbricating downward, each plate pierced by two pores, and the larger ones having additional pores. Type P. irregularis.

irregularis, Meek \& Worthen, 1869, Proc. Acad. Nat. Sci. Phil., p. 78, and Geo. Sur. Ill., vol. 5, p. 512, Keokuk Gr.
Physetocrinus, Meek \& Worthen, 1869 , Proc. Acad. Nat. Sci. Phil., p. 158. [Ety. physetos, inflated ; krinon, lily.] Distinguished from Actinocrinus, in the form and construction of the vault, which has no proboscis, and has pores along the radial portions of the dome; and, also, in having no hook-like projections along the pinnules. Type $\mathbf{P}$. ventricosus.

asper, Meek \& Worthen, 1869, Proc. Acad. Nat. Sci. Phil., p. 161, and Geo. Sur. Ill., vol. 5, p. 351, Burlington Gr. c o p e i, S. A. Miller, 1881 , (A c tinocrinus copei,) J our. Cin. Soc. Nat. Hist., vol. 4 , p. 310 , Burrlington Gr.

dilatatus, Meek \& Worthe n, 1869, (Stroto- FIG. 390, - Physetocrinus crinus dilat a- FIG. $390,-$ Physetc tus, ) Proc.

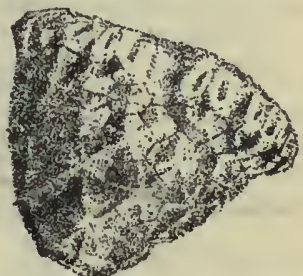
Acad. Nat. Sci. Phil. p. 162, and Geo. Sur. Ill., vol. 5, p. 363, Burlington Gr. ornatus, Hall, 1858, (Áctinocriuus ornatus,) Geo. Sur. Iowa, p. 583, Burlington Gr.

reticulatus, Hall, 1861, (Actinocrinus reticulatus) Desc. New Crin., p. 3, Burlington Gr.

subventricosus, McChesney, 1860, (Actinocrinus subventricosus,) Desc. New Crin. Pal. Foss., p. 21, and Trans. Chi. Acad. Sci., p. 16, Burlington Gr.

ventricosus, Hall, 1858, (Actinocrinus ventricosus,) Geo. Sur. Iowa, p. 595, Burlington Gr.

ventricosus var, cancellatus, Hall, 1861, (Actinocrinus ventricosus var. cancellatus,) Bost. Jour. Nat. Hist., vol. 7, p. 279, Burlington Gr.

ventricosus var. internodus, Hall, 1861, (Actinocrinus ventricosus var. internodus,) Bost. Jour. Nat. Hist., vol. 7, p. 278, Burlington Gr.

Prsocrinus, DeKoninck, 1858, Bull. Acad. Roy. Belgique, $2 \mathrm{me}$ ser., tome 3, p. 24. [Ety. pisos, pea; krinon, lily.] Calyx round globular; basals 5 , forming a triangle; these are followed by three large plates, forming nearly the entire calyx; one of these supports two small plates, and a small plate is supported, in a notch, between the other two larger plates; arms 5; column round. Type P. pilula.

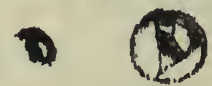
nus gemmiformis. Natural size and magnifled.
Fig. 391.-Plsoerl- gemmiformis, S. A. Miller, 1879, Jour. Cin. Soc. Nat. Hist., vol. 2, p. 113, Niagara Gr.

globosus, Ringueberg, 1884, (Triacrinus globosus,) Proc. Acad. Nat.

Sci. Phil., p. 146, Clinton Gr. 
pyriformis, Ringueberg, (Trincrinus pyriformis,) Proc. Acad. Nat. Sci. Phil., p. 145, Clinton Gr.

Platycrinus, Miller, 1821, Nat. Hist. Crinoiclea, p. 73. [Ety. platys, flat; krinon, lily.] Calyx bowl-shнped; hasals 3 ; primary radials $12 \times 5$; regular interradials $1 \times 4$; azygous interradials, 1 large and 3 small; dume elevated; arms 10 to 35 , beariug pinnules; coluinn large and twisted. Type $P$. lrevis.

aequalis, Hall, 1861, Desc. New. Crin., p. 117 , und Geo. Sur. Ill., vul. 5, p. 456, Burlington Gr.

americanus, Owen \& Shumard, 1850, Jour. Acal. Nut. Sci. Puil., $2 d$ ser., vol. 2, p. 89 , Burlington Gr.

anndixoni, Troost. Not defined.

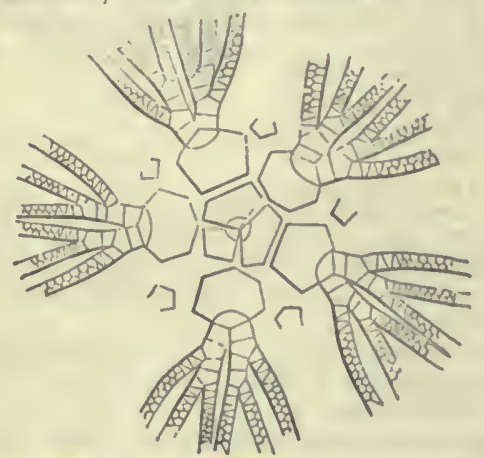

Fig. 392.-Platycrinus asper. Dlagram.

asper, Meek \& Worthen, 1861, Proc. Acad. Nat. Sci. Phil., p. 129, and Geo. Sur. Ill., vol. 3, p. 468, Burliugton Gr. bedfurtensis, Hill \& Whitfilil, 1875, Oliio Pal., vol. 2, p. 161, Erit S'iales.

bloomfeldensis, S. A. Miller, 1880, Juur. Cin. Soc. Nat. Hist., vol. 2, p. 257 , Keokuk Gr.

bonoensis, White, 1878, Proc. Acad. Nat. Sci. Phil., p. 30, and Cont. to Pal., No. 6, p. 160, Keokuk Gr.

brevinodus, Hall, 1861, Desc. New Crinoidea, p. 4, and Bost. Jour. Nat. Hist., vol. 7, p. 286, Krokuk Gr.

burlingtonensis, Owen \& Shumard, 1850, Jour. Acad. Nat. Sì., $2 d$ ser., vol. 2, p. 60 , Burlington Gr.

calyculus, Hall, 1861, Desc. New Crin., p. 16, Burlingtun Gr.

canaliculatus, Hall, 1858, Geo. Sur. Iowa, vol. 1. pt. 2, p. 539, Burlington Gr.

cavus, Hall, 1858, Geo. Sur. Iowa, p. 527, Burlington $\mathrm{Gr}$.

elytis, Hall, 1861, Drsc. New Crin., p. 4, and Bust. Jour. Nat. Hist., vol. 7, p. 285, Burlington $\mathrm{Gr}$.

contritus, Hall, 1863,17 th Rep. N. Y. St. Mus. Nat. Hist., p. 54, and Ohio Pal., vol. 2 , p. 166, Waverly Gr.

corporiculus, Ringueberg, 1886. Bull. Buf. Soc. Nat. Sci., vol. 5 , p. 12 , Niagara Gr. Not properly defined. corrugatus, Owen \& Shumard, 1850, Jour. Acai. Nat. Sei., vol. 2, p. 59, Burling. ton Gr.

depressus, Owen. Not defined.

discoideus, Owen \& Slıumard, 1850, Jour. Acad. Nat. Sici., $2 d$ ser., vol. 2, p. 58, Burlington Gr.

eboraceiss, Hull, 1862, 15th Rep. N. Y. St. Mus. Nat. Hist., p. 119, Ham. Gr.

eleguns, Hall, 18ı1, Desc. New Crin., p. 4, and Bust. Juur. Soc. Nat. Hist., vol. 7, p. 2S5, Burlington Gr.

eninulus, Hall, 1861, Desc. New Crin., p. 17, Burlington Gr.

eriensis, Hall, 1862, 15th Rep. N. Y. St. Mus. Nat Hist., p. 119, Hatu Gr.

ex('avatus, Hall, 1861, Desc. New Crin., p. 4, and Bust. Jour. Nat. Hist., vol. 7, p. 286, Burliugton $\mathrm{Gr}$.

exsertus, Hall, 1858, Geo. Sur. Iowa, p. 539, Burlington Gr.

faberi, n. 8p. Calyx bowl-shaped; subcylindrical above; attaching point for column projecting below; base unarked by three keels, corresponding with the 8 u $\imath$ u res between the basal plates, and along which the Fia. 393.-P'lntyerium sutures may be dis. faberl, $x$. tinknished; raclials

large, width a littlo greater than height; cicatrix for uttachuent of arms in the center of the upper face of each radiul, and occupying about one-thircl of the width of the plate; surface marked by a row of tubercles radiating on each

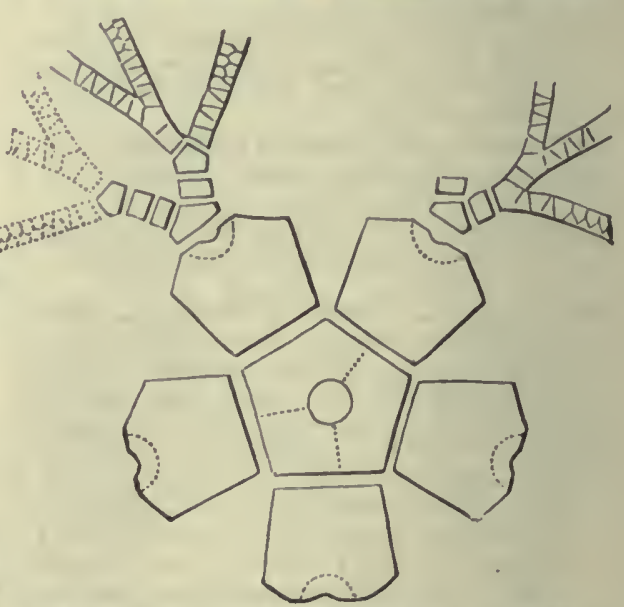

Fro. 394 -Platycrinus hemlspherleus. Dlagram.

plate from the angle of union of basal and radial plates, and also a few scattering tubercles; collected in Scott County, West Va., in the St. Louis or Kaskaskia Gr. 
georgii, Hall, 1860, Supp. to Geo. Sur. Iowa, p. 82, Warsaw Gr.

glyptus, Hall, 1861, Desc. New Crin., p. 16, Burlington Gr.

graphicus, Hall, 1863, 17th Rep. N. Y. St. Mus. Nat. Hist., p. 55, and Otio Pal., vol. 2 , p. 166, Waverly Gr.

halli, Sliumard, 1866, Trans. St. Louis Arad. Sci., vol. 2, p. 388, and Geo. Sur. Ill., vol. 5, p. 454, Burlington Gr.

haydeni, Meek, 1872, Hayden's Geo. Sur. Terr., p. 469, and Cont. to Pal., No. 6, p. 122, Subcarboniferous.

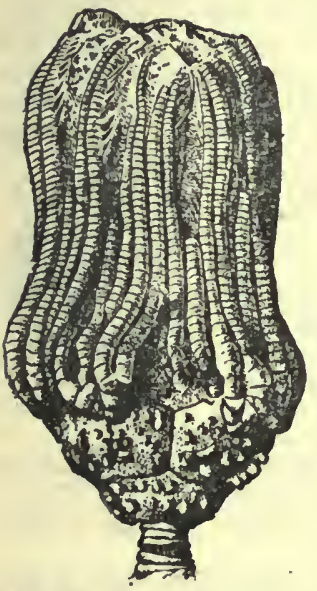

FiG. 395.-P1 a t y c rin us hemlsphericus. he $\mathrm{m}$ is phericus, Meek.\& Worthen, 1865 , Proc. Acad. Nat. Sei. Phil., p. 16, and Geo. sur. Ill., vol. 3, p. 511, Keokuk Gr.

huntsvillæ, Troost. Not defined.

i $\mathrm{n} \mathrm{com} \mathrm{p} \mathrm{t} \mathrm{u} \mathrm{s}$. White, 1863, Jour. Bost. Soc. Nat. Hist., vol. 7 , p. 503, and Geo. Sur. Ill., vol. 5. p. 459 , Burlington Gr. inornalus, syn. for P. burlingtonensis.

insculptus, Troost. Not defined.

leai, Lyon, 1869 , Trans. Am. Phil. Soc., vol. 13, p. 459, Up. Held. Gr.

lodensis, Hall \& Whitfield, 1875, Ohio Pal., vol. 2, p. 168, Waverly Gr.

monroensis, Worthen, 1882, Bull. No. 1, IIl. St. Mus. Nat. Hist., p. 30, and Geo. Sur. Ill., vol. 7 , p. 306, St. Louis Gr.

montanensis, see Eucladocrinus montanen-is.

mulibrachiatus, Meek \& Worthen, 1861, Proc. Acad. Nat. Sci. Phil., p. 135, Warsaw Gr.

nioten is, M ek \& Worthen, 1865, Proc. Acad. Nat. Sci. Phil. p. 162 , and Gro. Sur. Ill., vol. 3, p.513, Keokuk Gr. nodobrachiatus, Hall, 1858, Geo.

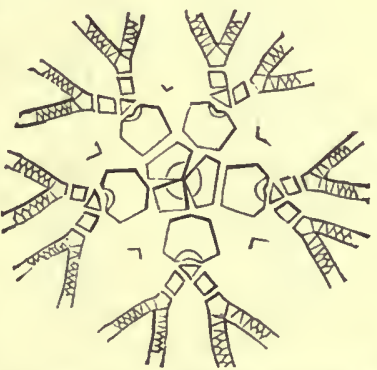

Sur. Inwa, Frg.396.-Platycrinus niotensis. p. 542 , Burlington Gr.

nodulosus, Hall, 1858, Geo. Sur. Iowa, p. 541, Burlington Gr. nucleiformis, Hall, 1858, Geo. Sur. Iowa, p. 540, Burlington $\mathrm{Gr}$.

olla, Hall, 186l, Desc. New Crin. The name was preoccupied. See P. halli.

ornigranulus, McChesney, 1860, Desc. New Pal. Foss., p. 5, and Trans. Chi. Acad. Sci., p. 3, Burlington Gr.

oweni, Meek \& Worthen, 1861, Proc. Acad. Nat. Sci. Phil., p. 120, Burlington Gr.

parvinodus, Hall, 1861, Desc. New Crinoidea, p. 17, Burlington Gr.

parvulus, Meek \& Worthen, 1865, Proc. Acad. Nat. Sci. Phil., p. 163, and Geo. Sur. Ill., vol. 5, p. 555, Kaskaskia Gr. parvus, see Cordylocrinus parvus.

penicillus, Meek \& Worthen, 1860, Proc. Acad. Nat. Sci. Phil., p. 380, and Geo. Sur. Ill., vol. 2, p. 266, St. Lou is Gr.

perasper, Meek \& Worthen, 1865, Proc. Acad. Nat. Sci. Phil., p. 161, Burlington Gr.

pileiformis, Hall, 1858, Geo. Sur. Iowa, p. 529, Burlington Gr.

planus, Owen \& Shumard, 1850, Jour. Acad. Nat. Sci. Phil., $2 d$ ser., vol. 2, p. 57, Burlington Gr.

plenus, Meek \& Worthen, 1860, Proc. Acad. Nat. Sci. Phil., p. 380, and Geo. Sur. Ill., vol. 2, p. 267, St. Lnuis Gr.

pleurovimineus, see Eucladocrinus pleurovimineus.

plumosus, see Cordylocrinus plumosus.

pocilliformis, Hall, 1858, Geo. Sur. Iowa, p. 528, Burlington $\mathrm{Gr}$.

poculum, S. A. Miller, 1881, Jour. Cin. Soc. Nat. Hist., vol. 4, p. 311, Burlington Gr.

polydactylus, Troost. Not defined.

præmaturus, Hall \& Whitfield, 1875, Ohio Pal., vol. 2, p. 124, Niagara Gr.

prænuntius, Wuchsmuth \& Springer, 1878, Proc. Acad. Nat. Sci. Plil., p. 249, Burlington Gr.

prattenanus, Meek \& Wortlien, 1860 , Proc. Acad. Nat. Sci. Phil., p. 379, and Geo. Sur. Ill., vol. 2, p. 264, St. Louis Gr.

pratteni, Worthen, 1860, Trans. St. Louis Acad. Sci., vol. 1. p. 69, Burlington Gr. pumilus, Hall, 1860, Supp. to Geo. Sur. Iowa, p. 82, Warsaw Gr.

quinquenodus, White, 1862, Pror. Bost. Soc. Nat. Hist., vol. 9, p. 18, Burlington Gr.

ramulosus, see Cordylocrinus ramulosus. regalis, Hall, 1861, Desc. New Crinoidea, p. 16, Burlington Gr.

richfieldensis, Hall \& Whitfield, 1875, Ohio Pal., vol. 2, p. 167, Waverly Gr. saffordi, Troost, 1850, Hall, 1858, Geo. Sur. Iowa, p. 634, Keokuk Gr.

saræ, Hall, 1858, Geo. Sur. Iowa, p. 673, St. Louis Gr.

scobina, Meek \& Worthen, 1861, Proc. Acad. Nat. Sci. Phil., p. 129, and Geo. Sur. Ill., vol. 3, p. 466, Burlington Gr. sculptus, Hall, 1858, Geo. Sur. Iowa, p. 536, Burlington $\mathrm{Gr}$. 
shumardanus, Hall, 1858, Geo. Sur. Iowa, vol. 1, pt. 2, p. 532, Burlington Gr.

siluricus, Hall, 1879, Desc. New Spec. Foss., p. 9, and 11th Rep. Geo. Sur. Ind., p. 256, Niagara Gr.

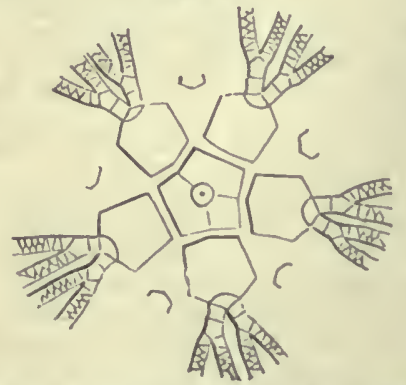

FIG. 397.-Platyerinus scoblna. Dingram.

striobrachiatus, Hall, 1861, Desc. New Crinoidea, p. 4, and Bost. Jour. Nat. Hist., vol. 7, p. 287, Burlington Gr. subspinosus, Hall, 1858, Geo. Sur. Iowa, p. 536, Burlington $\mathrm{Gr}$.

subspinulosus, Hall, 1860, Supp. to Geo. Sur. Iowa, p. 81, Burlington Gr.

symmetricus, Wachsmuth \& Springer, (in press,) Geo. Sur. 1ll., vol. 8, p. 186, Waverly or Kinderhook Gr.

tennesseensis, see Marsupiocrinus tennesseensis.

tentaculatus, see Marsupiocrinus tentaculatus.

tenuibrachiatus, Meek \& Worthen, 1869. Proc. Acad. Nat. Sci. Phil., p. 16, and Geo. Sur. Ill., vol. 5, p. 450, Burlington $\mathrm{Gr}$.

truncatulus, Hall, 1858, Geo. Sur. Iowa, p, 538, Burlington $\mathrm{Gr}$.

truncatus, Hall, 1858, Geo. Sur. Iowa, p. 537, Burlington Gr.

tuberosus, Hall, 1858, Geo. Sur. Iowa, p. 534, Burlington Gr.

verrucosus, White, 1863, Jour. Bost. Soc. Nat. Hist., vol. 7, p. 502, Burlington Gr. vexabilis, White, 1875 , U. S. Sur. W. 100th Meridian, vol. 4, p. 81, Sub. Carb. wortheni, Hall, 1858, Geo. Sur. Iowa, p. 530 , Burlington Gr.

yandelli, Owen \& Shumard, 1850, Jour. Acad. Nat. Sci., 2 d ser., vol. 2, p. 58 , Burlington $\mathrm{Gr}$.

Platrycystites, n. gen. [Ety. platys, flat; kustis, bladder. ] General form compressed elliptical, or like the kernel of a peach-seed, with a narrow rim on the border; axial canal passes down into the column; three plates in the first range, one of them bending around the rim and the other two having the dividing suture in the rim itself; there are five large plates in the second range, and above these there are three large plates, on the azygous side, with six or more smaller ones on the border; the whole surface is granular and every plate full of minute pores. Type P. faberi.

faberi, n. sp. Basal plates hexagonal, longer than wide, one of them bending around the bordered rim, and the other two uniting at the middle of the rim; a large subcentral hexagonal plate in the second range on the azygous side rests upon the upper sicte of the plate, which is separated by a suture in the rim from an adjoining basal plate, but does not reach the other basal; this large hexagonal plate joins two plates in the second range with its under sloping sides; three large plates rest upon the three upper faces of this large liexagonal plate; the one upon the superior face is octagonal, resting between the other two large plates and having five smaller ones joining its upper faces. The specimen is worn at the upper edge so as to destroy the orifices. It was received by Charles Faber among a lot of fossils from the Kaskaskia Group in the southern part of West Viruinia, but as no cystideans have ever been found above the Lower Devonian, and as the specimen is worn as if Fus.34. Plntycys. it had been drifted, the

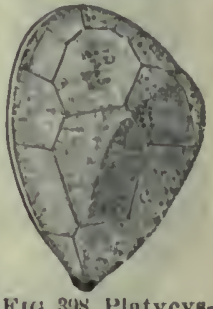
tiles fuberl probability is that it belongs to tho Silurian rocks.

Pleurocystites, Billings, 1854, Can. Jour., vol. 2, p. 250. Ety. pleuron, side; kustis, bladder.] Body oval, flat; doreal side with large plates, ventral with smaller ones; two free arms: mouth at the base on the left side; small orifice near the apex; three pectinated rhoinbs, two in the upper half and one in the lower; column short. Type P. squamosus.

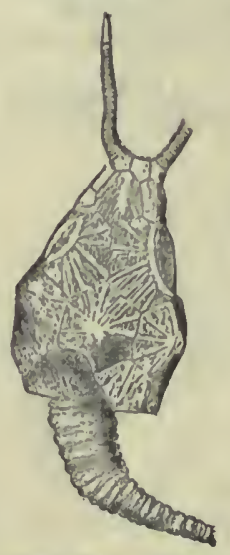

Fig. 399.-Pleurocystites squamosus. anticostiensis, Billings, 1857, Rep. of Progr. Geo. Sur. Can., p. 288, and Cun. Orz. Rem., Decade 3, p. 52 , Hud. Riv. Gr.

elegans, Billings, 1857, Rep. of Progr. Geo. Sur. Can., p. 287, and Can. Org. Rem., Decade 3, p. 5 l, Trenton $\mathrm{Gr}$.

exornatus, Billings, 1857, Rep. of Progr. Geo. Sur. Can., p. 287, Trenton Gr.

filitextus, Billings, 1854, Can. Jour., vol. 2, p. 252, and Can. Org. Rem., Decade 3, p. 48, Trenton Gr.

robustus, Billings, 1854, Can. Jour., vol. 2, p. 252, and Can. Org. Rem., Decade 3, p. 49, Trenton Gr. 
squamosus, Billings, 1854, Can. Jour., vol. 2, p. 251, and Can. Org. Rem., Decade 3,1 . 49, Trenton $\mathrm{G} r$.

Polrocrinus, Billings, 1857, Rep. of Progr. Geo. Sur. Can., p. 279. [Ety. from the poriferous areas similar to pectinated rhombs.] Calyx conical; basals 5 ; subradials $1 \times 5$; radials $1 \times 5$; azygous in-

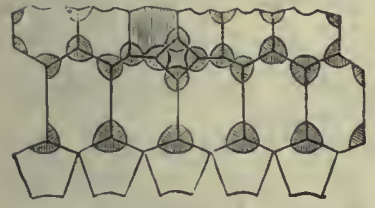

FIG. 400.-Porocrinus conicus. Diagram. terradials 2; pectinated rhombs nu$\mathrm{m}$ e $\mathrm{r}$ ous. Ty pe P. conicus. conicus, Bill. ings, 1857 , $\mathrm{Re} \mathrm{p}$. of Progr. Geo. Sur. Can., p. 279, and Call. Org. Rem., Decade 4, p. 34, Trenton Fr.

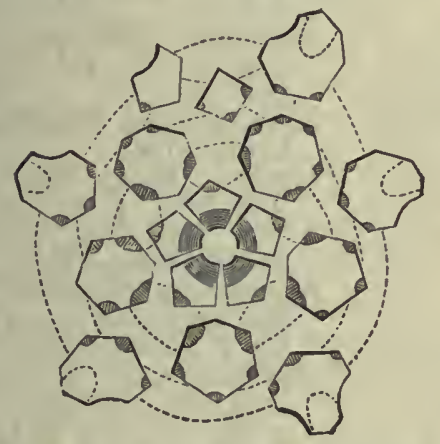

FIG. 401. -Porocrlnus crassus. Dlagram.

crassus, Meek \& Worthen, 1865, Proc. Acad. Nat. Sci. Phil., p. 115, and Geo. Sur. Ill., vol. 3, p. 330, Hud. Riv. Gr. pentagonus, Meek \& Worthen, 1865. Proc. Acad. Nat. Sci. Phil., p. 146, and Geo. Sur. Ill., vol. 3 , p. 332, Trenton Gr. s m ith i, Grant, 1881, Trans. Ot-Fig. 402.-Porocrinus crastawa Field Nat- sus. One basal and two uralists' Club, subradtals enlarged.

No. 2, p. 42, Trenton Gr.

Poteriocrinus, Miller, 1821, Nat. Hist. Crinoidea, p. 68. [Ety. poterion, goblet; krinon, lily.] Caly x obconical; basals 5 ; sub-radials 5 ; radials $1 \times 5$, with a variable number of smaller ones, the azygous ray often having more than the others; azygous plates 3 or 4 , within the calyx, succeeded by smaller ones that form part of the ventral sac; vault produced in a long sac or proboscis; arms simple or branching and bearing pinnules. Type $\mathbf{P}$. crassus.

æqualis, Hall, 1860, Supp. to Geo. Sur. Iowa, p. 63, Burlington Gr.

alternatus, see Dendrocrinus alternatus. anomalos, Wetherby, 1880, Jour. Cin. Soc. Nut. Hist., vol. 3, p. 158, Kaskaskia Gr.

arachniformis, Worthen, 1s82, Bull. No. 1, Ill. St. Mus. Nat. Hist., p. 13, and Geo. Sur. Ill., rol. 7, p. 281, Keokuk Gr.

asper, Worthen, 1882, Bull. No. 1, Ill. St. Mus. Nat. Hist., p. 11, and Geo. Sur. Ill., vol. 7, p. 278, Keokuk Gr. asperatus, Worthen, 1882, Bull. No. 1, Ill. St. Mus. Nat. Hist., p. 12, and Geo. Sur. Ill., vol. 7, p. 280, Keokuk Gr.

barrisi, see Cyathocrinus barrisi.

bayensis, see Scaphiocrinus bayensis. bisselli, Worthen, 1873, Geo. Sur. Ill., vøl. 5 , p. 546, Kaskaskia Gr.

briareus, see Scaphiocrinus briareus.

buffaloensis, Worthen, (in press,) Geo.

Sur. 11l., vol. 8, p. 89, Ham. Gr.

burketi, see Scaphiocrinus burketi.

bursiformis, White, 1862, Proc. Bost. Soc.

Nat. Hist., vol. 9 , p. 10 , Burlington Gr. caduceus, see Dendrocrinus caduceus. calyculus, Hall, 1858, Geo. Sur. Iowa, p. 553, Burlington $\mathrm{Gr}$.

calyx, Hall, 1879, Desc. New Spec. Foss., p. 10, and 11th Rep. Geo. Sur. Ind., p. 266, Niagara Gr.

carbonarius, see Grapbiocrinus carbonarius.

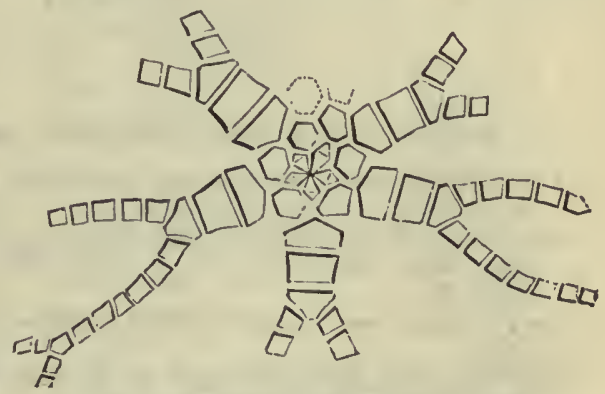

FIG. 403.-Poterlucrinos carinatus. Diagram.

carinatus, Meek \& Worthen, 1861, Proc. Acad. Nat. Sci. Phil., p. 139, and Geo. Sur. Ill., vol. 3, p. 486, Burlington Gr. clarkii, Williams, 1882, Proc. Acad. Nat. Sci. Phil., p. 21, Chemung Gr.

claytonensis, Worthen, 1882, Bull. No. 1, Ill. St. Mus. Nat. Hist., p. 18, and Geo. Sur. Ill., vol. 7, p. 288, Warsaw Gr. clytis, Worthen, 1882, Bull. No. 1, Ill. St. Mus. Nat. Hist., p. 16, and Geo. Sur. Ill., vol. 7 , p. 294 , St. Louis Gr.

columbiensis, Worthen, 1882, Bull. No. 1, Ill. Si. Mus. Nat. Hist., p. 22, and Geo. Sur. Ill., vol. 7, p. 293, Kaskaskia Gr.

concinnus, Meek \& Worthen, 1870, Proc. Acad. Nat. Sci. Phil., p. 26, and Geo. Sur. Ill., vol. 5, p. 490, Keokuk Gr. coreyi, Worthen, 1875, Geo. Sur. Ill., vol. 6, p. 516, Keokuk Gr.

cornellanus, Williams, 1882, Proc. Acad. Nat. Sci. Phil., p. 18, Chemung Gr. 
corycia, Hall, 1863, 17th Rep. N. Y. St. Mus. Nat. Hist., p. 57, and Ohio Pal., vol. 2, p. 173, Waverly Gr.

coxanus, see Scaphiocrinus coxanus. crineus, Hull, 1863, 17 th Rep. N. Y. St. Mus. Nat. Hist., p. 56, and Obio Pal., vol. 2, p. 172, Waverly Gr. cultidaclylus, see Scaphiocrinus cultidactylus.

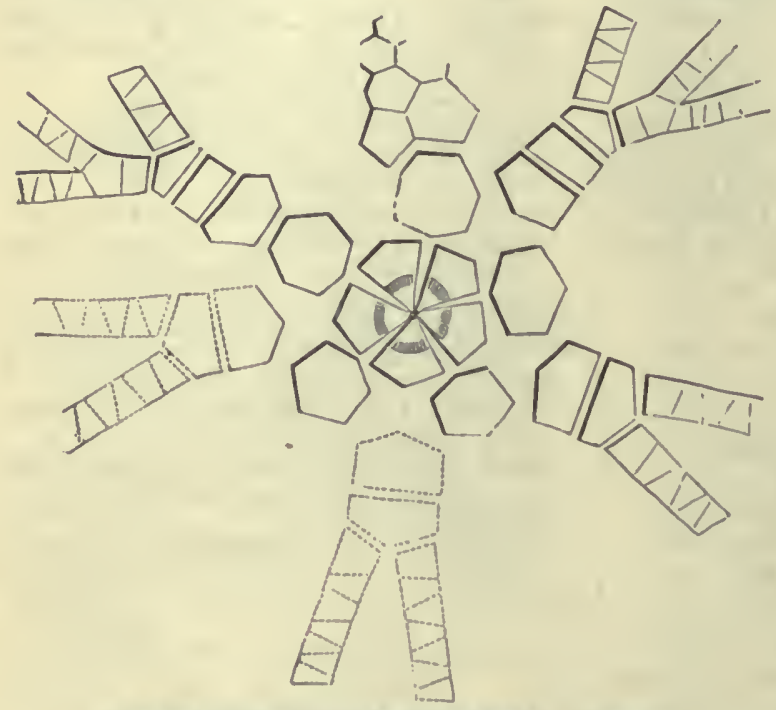

FIG. 404.-Poleriocriuus indianensts. Diagram. hoveyi, Worthen, 1875, Geo. Sur. Ill., vol. 6, p. 516, Keokuk Gr.

illinuisensis, Wurthen, 1882, Bull. No. 1, 11l. St. Mus. Nat. Hist., p. 19, und Geo. Sur. Ill., vol. 7, p. 289, WVarsaw Gr.

indentus, Hall, 1862, 15̄th Rep. N. Y. St. Mus. Nat. Hist., \%. 122, Han. Gr.

indianensis, Meek \& Worthen, 1865, Proc. Acad. Nat. Sci. Phil., p. 155, and Geo. Sur. Ill., vol. 3, p. 515, Keokuk Gr.

iowensis, see Scaphiocrinus jowtusis.

jesupi, Whitfield, 1881, Bull. Nu. 1, Am. Nat. Hist., p. 7, syn. for 1'. swallıvi.

kaskaskiensis, see Scaphiocrinus karkaski+nsis.

keokuk, Hall, 1860, Supy. to Ger. Sur. Iowa, 1. 64, Keokuk Gr.

lasallensis, Worthen, 1875, Geo. Sur. Ill., vol. 6, p. 526, Coal Mras.

Latidactylus, gee Scaphiocrinus lutidactylus.

lepidus, Hall, 1861, Desc. New Crin., p. 6, aud Bust. Jour. Nat. Hist., vol. 7, p. 304, Burlington Gr.

longidactylus, Slıumard, 1855. The name was preoccujied. See P. missourínisis.

macrupinensis, IVorthen, 1873, Geo. Sur. 111., vol. 5, p. 561, Up. Crial Mras.

mammiformis, Worthen, (in

cylindricus, Lyon, 1860, Trans. Am. Phil.

Soc., vol. 13, p. 458, Up. Held. Gr.

davisanus, S. A. Miller, 1882, Jour. Cin.

Soc. Nat. Hist., vol. 5, p. 226, Up.

Held. Gr.

decndactylus, see Scaphiocrinus decadaciylus.

diffusus, Hall, 1862, 15th Rep. N. Y. Mus.

Nat. Hist., p. 121, Ham. Gr.

dilatatus, see Colincrinus dilatatus.

divaricatus, Ha11, 1860, Supp. to Geo. Sur.

Iowa., p. 65, WVarsaw Gr.

elsahensis, Worthen, (iu press,) Geo. Sur.

11l., vol. 8, p. 88, Kinderliook Gr.

enormis, see Cyathocrinus enormis.

floreulis, see Zeacrinus florealis.

fountainensis, Worthen, 1882, Bull. No. 1,

Ill. Mus. Nut. Hist., p. 17, and Geo. Sur.

Ill., vol. 7, p. 286, St. Louis Gr.

fusiformis, Hall, 1861, Desc. New Cri-

noidra, p. 6, and Bost. Jour. Nat. Hist.,

vol. 7 , p. 302 , Burlington Gr.

gracilis, see Dtndrocriuus gracilis.

grtgarius, Williams, 1882, Proc. Acad.

Nat. Sci. Phil., p. 22, Chenung Gr.

hamiltonensis, Worthen, 1882, Bull. No.

1, Ill. St. Mus. Nut. Hist., p. 7, and Geo.

Sur. Ill., vol. 7, p. 273, Keokuk Gr.

hardinensis, Worthen, 1873, Geo. Sur.

Ill., vol. 5, p. 533, St. Louis Gr.

hemisphericus, see Eupachycrinus hemisphericus. press, ) Geo. Sur. 111., vol. 8, p. 91, Warsaw Gr.

maniformis, sce Zeacrinus maniformis. meekanus, Shuınard, 1855, Geo. Rep. Mo., p. 188, Burlington Gr.

milleri, Wetherby, 1880, Jour. Cin. Soc.

Nat. Hist., vol. 3, p. 330, Kaskaskia Gr.

missouriensis, Sh u m ard. 1857 , Trans. St. Louir Acad. Sci., p. 80, and Geo. Sur. Iowa, p. 669, St. Louis Gr.

montrnenvis, see Srapliocrinus montanensis.

municipalis, Troost. Not defined.

nassa, $\mathrm{H}: 11,1862,15 \mathrm{th}$ Rep. N. Y. St. Mus. Nat. Hist., p. 120, Ham. Gr.

nauvooensis, see Sraphiocrinus nauvooensis.

nereus, Hall, 1862, 15th Rep. N. Y. St. Mus. Nat. Hist., p. 121, Ham. Gr. FI G. 405. - Ponet lierothanus, A teriocrinus Miller, 1882, Juur. Cin. Held. Gr.

nodobacal:s, Worthen, (in press,) Geo. Sur. Ill., vol. 8, p. 89, Șt. Louis Gr. Soc. Nat. Hist., vol. 5, p. 227, Up. 
norwoodi, Meek \& Worthen, 1865, Proc. A cad. Nat.Sci.Phil., p. 159, Kaskarkia Gr. nycteus, Hall, 1862, 10̄th Kep. N. Y. St. Mus. Nut. Hist., p. 120, Ham. Gr.

obuncus, White, 1862, Proc. Bost. Soc.

Nat. Hist., p. 10, Burlington Gr.

occidentalis, Owen \& Thumard, see Agassizocriuus oceidentalis.

occidentulis, Worthen, see Scaphiocrinus occidentalis.

okuwensis, see Scaphiocrinus okawensis. orpstes, see Sraphiocrinus orestes.

otterensis, Worthen, 1882, Bull. No. 1,

III. St. Muヶ. Nat. Hist., p. 14, and Geo.

Sur. Ill., vol. 7, p. 283, Krokuk Gr.

peculiaris, Wortlien, 188:, Bıll. No. 1, III. St. Mns. Nat. Hist., p. 25, and Geo. Sur. 11l., vol. 7, p. 298, Karkaskia Gr. penicilliformis, IVorthen, 1882, Bull. No.1, Ill. St. Mus. Nat. Hist., 1. 9, and Geo. Sur. Ill., vol. 7, r. 276, Keokuk Gr.

perplexus, Meek \& Worthen, 1869, Proc.

Acad. Nat. Sci. Phil., p. 138, and Geo.

Sur. Ill., vol. 5, p. 405, Burlington Gr. pis f rmis, see Arachllocrinu pisi'ormis.

pleias, Hall, 1863, 17th Rep. N. Y. St.

Mus. Nat. Hist., p. 57, and Ohio Pal., vol. 2 , p. 173 , Waverly Gr.

popensis, see Ficapliocrinus popensis.

posticus, see Denrirocrinus p inticus.

proboscidinlis, Worthen, 1875, Geo. Sur.

Ill., vol. 6, p. 518, St. Louis (ìr.

propinquis, ste Scaphiocrinus propinquns. rhumbilerus, see Barycrinus rbombiferus. richtirldensis, Wurtlien, 1882, Bull. No. 1,

Ill. St. Mus. Nat. Hisi., p. 15, and Geo.

Sur. Ill., vol. 7, p. 285, Kinderbouk Gr. rowleyi, Worthen, (in press,) Geo. Sur.

Ill., vol. 8, p. 90, Kaskaskia Gr.

rugosua, Slıumard, 1858, Tians. St. Louis

A cad. Sci., vril. 1, p. 223, Coal Meas.

salignoirles, White, 186.), Proc. Bost. Soc.

Nat. Hist., vol. 9, p. 10, Burlingion Gr. salteri, see Scaphio'rinus salteri.

sculptus, TVorthen, 1882, Bull. No. 1, Ill.

St. Mus. Nat. Hist.. p. 21, and Gı o. Sur.

IIl., vol. 7, p. 292, Kaskaskia Gr.

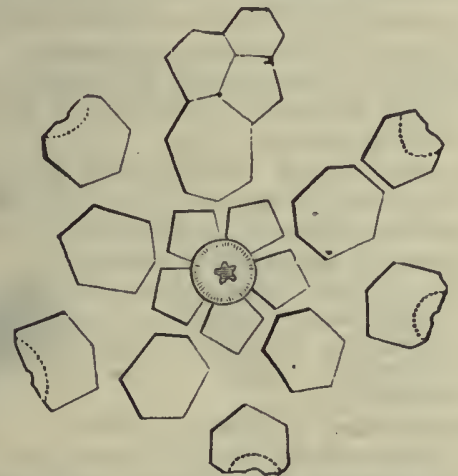

FIG. 406.-Poteriocrinus subimpressus.

similis, Worthen, 1882, Bull. No. 1, Ill. St. Mus. Nut. Hist., p. 23 , and Geo. Sur. III., vol. 7, p. 295 , Keokuk Gr.

simplex, Lyon, 1869, Trans. Am. Phil. Suc., vol. 13, p. 458, Up. Held. Gr.

solidus, Meek \& Wortıen, 1861, Proc. Acril. Nat. Sci. Phil., p. 140, Burlington Gr.

spimbrachiatus, Scaphiocrinus brachiatus. spinulıl rıs, IVurthen, 1884, Bull. No. 2,

I.।. St. Mus. Nat. Hist., p. 27, and Geo. Sur. Ill., vul. 8, ґ. 86, Kaskaskia Gr. spinulijerus, Worthen, (in press,) see Zeaırinus spinuliferus.

spinusus, set Zeacriıus spinnsus.

sub mpres-us, Meek \& Wirtlien, 1861, Pric. Arall. Nat. Sci. Phil., \%. 13, and Geo. Sur. Ill., vol. 3, p. 485, Burlington Gir.

subramılısus, Worthen, 1882, Bull. No. 1,

Ill. St. Mu*. Nat. Hist., ». 14, and Geo.

Sur. IIl., vol. 7. p. 284, K rokuk Gr.

subtunidu*, Merk \& Wirth+11, 1865, Proc.

Acall. Nint. Sci. Phil., p. 159 , Kaskaskia Gr.

swallovi, Metk \& IVorthen, 1860, Proc. Acall. Nat. Sci. Phil., p. 394, and Geo. Sur. Ill., vol. 2, p. 183, Burlingtols Gr.

talboti, Worthen, 1882, Bull. No. 1, Ill.

St. Mus. Nat. Hist., p. 7, alid Geo. Sur. IIl., vol. 7, p. 287, St. Louis Gr.

tentaculalus, Worthen, 1882, Bull. No. 1

Ill. St. Mus. Nat. Hirt., p. 10, and Geo.

Sur. 11l., vil. 7, p. 277, Keı.kuk Gr.

tenuibrailiatus,

Meek \& Wor-

then, 1861, Proc.

Acad. Nut. Sci.

Phil., p. 138 , and

Geo. Sur. IIl.,

vil. 3 , p. 484 ,

Burlington Gr.

tenurfactylus, Meek

\& Worthinn, sti

Sea lhincrinus

ten uillactylus.

te nuilactylus, Firs. 407. - Poteriocriuus Wirthen, 1882, tenuibrachlatus. Di Bull. No. 1, Ill.

St. Mus. Nat. Hist., p. 6, and Geo. Sur. 11l., vol. 7, p. 271, Krokuk Gr. Wachemutlı says this is a Sraphiorrinus, and he has proposed to call it Sce pliverinus chseurus.

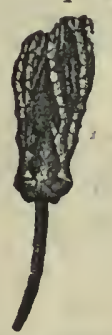

FIG. 408.-Poteriocrius ulriclit. ulriclii, Worthen, (in press,)

Gen. Sur. 1ll., vol. 8, p. 87.

Keokuk Gr.

tumidus, see Agassizocrinus 1 uııjdıs.

validu*, Wurthen, 1882 , Bull.

No. 1, Ill. St. Mus. Nat.

Hist., p. 18, and (ieo. Sur. 111., vil. 7, p. 287, Warsaw Gr.

vanhornei, Worthen, 1875 , Geo. Sur. Ill., vol. 6, p. 517. St. Louis Gr.

vars" ierıis, see Scaphiocrinus varsoviensis.

ventricosus, see Cœliocrinus ventricosus. venustus, see Scaphiocrinus venustus. 
verticillus, Hall, 1862, 15th Rep. N. Y. St. Mus. Nat. Hist., p. 94, Haus. Gr. wachsmulhi, Meek \& Worthen, see Graphiocrinus wachsmuthi.

wachsmuthi, Wetherby, 1880, (Scytafocrius wachsmuthi, Jour. Ciu. Soc. Nat. Hist., vol. 3, p. 155, Kaskaskia Gr. wetherbyi, S. A. Miller, 1879, Jour. Cin.

Soc. Nat. Hist., vol. 2, p. 36, Kaskaskia Gr.

zethus, Williams, 1882, Proc. Acad. Nat. Sei., p. 27, Chemung Gr.

Protaster, l'orbes, 184y, Mem. Geo. Sur. Great Britain, Decaile 1. [Ety. prolos, first; aster, star.] Disk circular, compos'd of squaniform plates; rays flexuous, composed of two series of ambulacral plates, bordered by spinous adambulacral ones; oral plates five. Type P. miltoni.

barrisi, see Onychaster barrisi.

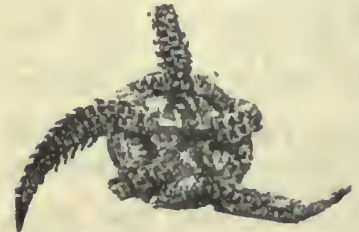

FIG.409.-Protaster flexuosus. fle $x$ u 0 \& $\mathrm{us}$ $\mathrm{Mi}$ il e $\mathrm{r}$ \& Dyer, 1878, Jour. Cin. Soc. $N$ a t. Hist., vol. 1 , p. 31, Utica Slate \& Hud. Riv. Gr. forbesi, H a 11 , 1859, Pal.

N. Y., vol. 3, p. 134, Low. Helı. Gr. granuliferus, Meek, 1872, Am. Jour. Sci., 3d ser., vol. 3, p. 274, and Ohio Pul., vol. 1, p. 68, Hud. Riv. Gr.

gregarius, Merk \& Worthen, 1869, Proc. Acad. Nat. Sci. Phil., p. 169, and Geo. Sur. Ill., vol. 5, p. 509, Keokuk Gr.

miamiensis, S. A. Miller, 1882, Jour. Cin. Soc. Nat. Hist., vol. 5, p. 116, Hud. Riv. Gr.

stellifer, Ringueberg, 1886, Bull. Buff. Soc.

Nat. Sci., vol. 5, p. 7, Niagara Gr.

Protasterina, syn. for Prolaster.

fimbriuta, syn for Protaster flexuosus.

Prerotocrinus, Lyon \& Casseday, 1860, Am. Jour. Sci., vol. 29, p. 68. [Ety. pterotos, feathered; krinon, lily.] Calyx saucershaped, wider thun high; vault high, with five wing like processes that characterize this genus; hasals 2 ; radials 1 or $2 \times 5$, the second being small; secondary radials $1 \times 10$; tertiary radials 2 or $3 \times 20$; azygous in. terradial 1 ; arms 20 , reachingonly to the vault; column round. Type $P$. capitalis.

acutus, Wetherby, 1879, Jour. Cin. Soc. Nat. Hist., vol. 2, p. 134, Kaskaskia Gr.

bifurcatus, Wetherby, 1879 , Jour.

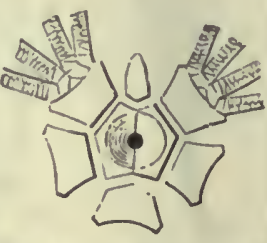

Fia. 410.-Pterotocrinus chentere $\mathrm{n}$ \& 1 \&. Dlagram.
Cin. Soc. Nat. Hist., vol. 2. p. 136, Kaskaskia Gr. capitalis, Lyon, 1857, (Astrocrinus capitalis,) Geo. Sur. Ky., vol. 3, p. 472, Kas. kaskia Gr.

chesterensis, Meek \& Worthen, 1860 , (Actinocrinus chesterensis,) Proc. Arad. Nat. Sci. Phil., 1). 383, and Geo. Sur. Ill., vol. 2, p. 292, Kaskaskid (ir.

coronarius, Lyon, 1857, (Asterocrinus coronarius, Geo. Sur. Ky., vol. 3, p. 476 , Kaskaskia Gr.

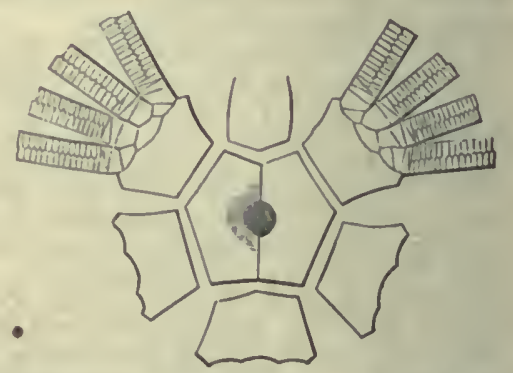

Fig. 411.-Pterotocrinus crassus, Dlagram.

crassus, Meek \& Worthen, 1860. (Dichocrinus crassus,) Proc. Acad. Nut. Sci. Phil., p. 382, and Geo. Sur. Ill., vol. 2, p. 290, Karkaskia Gr.

depressus, I,yon \& Casseday, 1860, Am. Jour. Sci., vol. 29, p. 68, and Geo. Sur. Ill., vol. 5, p. 559, Kaskaskia Gr.

proluherans, Hall, 1858, (Dichoerinus protuberans.) Geo. Sur. Iowa, p. 689 , Kaskrskia Gr.

pyramilalis, Tyon \& Casseday, 1860 , A in. Jour. Sci., vol. 29, p. 69, Kaskaskia Gr.

rugusus, Lyon \& Casseday, 1860, Am. Jour. Sci., vol. 29, p. 71, Kaskaskirt Gr.

sexlobatus, see Talarocrinus sexlobatus.

spatulatus, Wetherby, 1879, Jour. Cin. Sic. Nat. Hist., vol. 2, p. 137, Kaskaskia Gr.

Ptionaster, Hall, 1868, syn. for Palæocoma. princep8, see Pulsocoina princeps.

Ptychocrinus, IVaclismuth \& Springer, 1886 , Revis. Palæocrinoidea, pt. 3, p. 99, syn. for Gaurocrinus.

Prcnocrinus, S. A. Miller, 1883, Jour. Cin. Soc. Nat. Hist., vol. 6, p. 231. [Ety. puknns, deuse; krinon, lily.] Culyx small, cup-sliaped; basals 5 ; radials $3 \times 5$; regular interradials 3 ; urms 10 , sometimes dividing after brenming free. Type $\mathbf{P}$. shafferi.

germanus, S. A. Miller, 1880, (Glyptocrinus shafferi val. gerinunus, Jour Cin So Tour. Cin. Soc. Pyenocrtius Hnd. Riv. Gr.

shaffuri, S. A. Miller, 1875, (Glyptocrinus shafferi,) Cin. Quar. Jour. Sci., vol. 2, 
p. 277, and Jour. Cin. Soc. Nat. Hist., vol. 3 , p. 233, Hud. Riv. Gr.

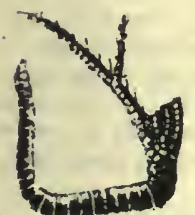

FIG. 413.-Pycnocrinus shafferi.

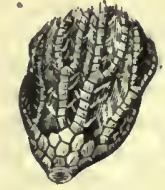

Fig. 414.-Pyc. n o c r i n u s shafferi. Enlarged $2 \frac{1}{2}$
dlam.

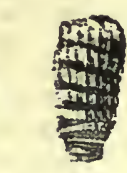

Fig. 415.

Pyenocri nus shafferi. Colunn colled around a column of Glyptocrinus.

Pygorhynchus gouldi. Not recognized.

Retiocrisus, Billings, 1858, Can. Org. Rem., Decade 4, p. 63. [Ety. retium, net; krinon, lily.] Calyx basin-shaped; radial ridges very prominent; basals 5 , large ; sub. ratlials 5 , large; primary radials $4 \times 5$; secondary radials 4 to $6 \times 10$; plates in interradial areas, numerous, with a large central row in the azygous area extending up the side of a ventral tube ; column round. Type $\mathrm{R}$. stellaris.

fimbriatus, Billings, 1859, Can. Org. Rem., Decade 4, p. 65, Hud. Riv. Gr.

gracilis, Wetherby, syn. for Gaurocrinus angularis.

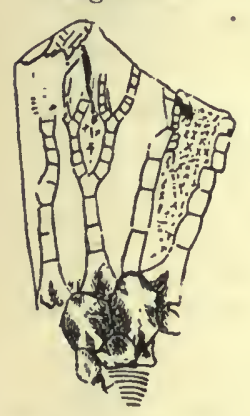

Frg. $416,-$ Retlocrinus stellaris subnodosus, Walcott, Rep. N. Y. St. Y. St. Mus. Nat. Hist., p. 208 Trenton Gr.

Rhodockinus, Miller, 1821, Nat. Hist., Crinoidea, p. 106. [Ety. rhodon, rose; krinon, lily.] Body subglobose, often wider than high, constricted near the arm bases; basals 5, small ; subradials 5 ; primary radials 3 to $4 \times 5$; secondary radials 1 to $3 \times 10$; arms widely separated, and composed of two rows of interlocking plates; interradial areas wide, plates large; vault depressed; orifice excentric and protruding; column round. Type $R$. verus.

asperatus, Billings, 1859, Can. Org. Rem., Decade 4, p. 27, Chazy Gr.

barrisi, Hall, 1861, Desc. New Crin., p. 9, and Jour. Bost. Soc. Nat. Hist., vol. 7 p. 322, Burlington Gr. barrisi var. divergens, Hall, 1861, Desc.

New Crin., p. 9, and Jour. Bost, Soc. Nat. Hist., vol. 7, p. 322, Burlington Gr.

coxanus, Worthen, 1882, Bull. No. 1, 111. St. Mus. Nat. Hist.. p. 29, and Geo. Sur. Ill., vol. 7, P. 305, Keokuk Gr.

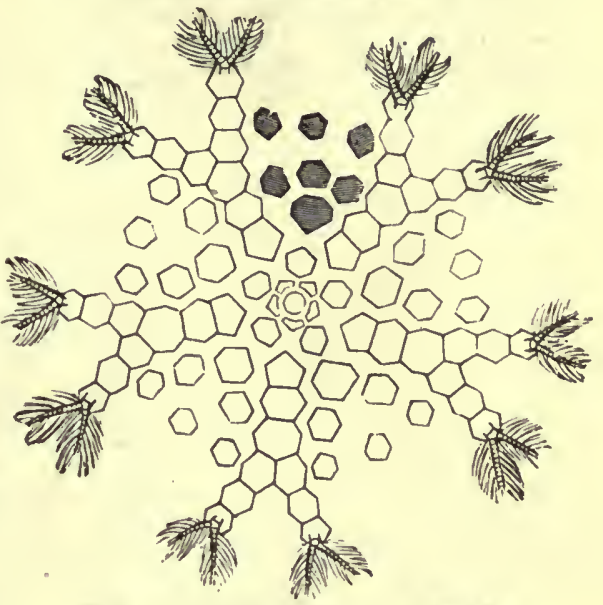

FrG. 417.-Rhbdocrinus. Diagram.

gracilis, Hall, 1862, 15th. Rep. N. Y. Mus. Nat. Hist., p. 127, Ham. Gr. halli, Lyón, 1861, Proc. Acad. Nat. Sci. Phil., p. 412, Low. Held. Gr. kirbyi, Wachsmuth \& Springer, (in press,) (ieo. Sur. Ill., vol. 8, p. 180 , Kinderbook Gr.

melissa, see Lyriocrinus melissa.

microbasalis, see Arch-

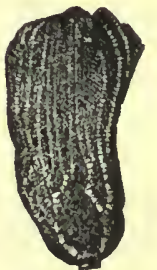

aeocrinus nicroba-FIG.418. -Rhodocrlsalis.

nanus, Meek \& Wor-

then, 1866, Proc. Acad. Nat. Sci. Phil.,

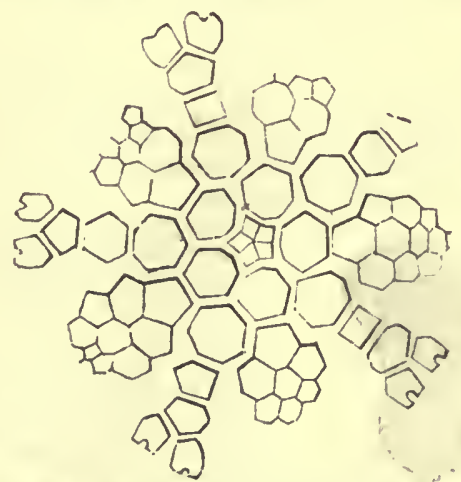

Fig. 419--Rhodocinus nanus. Diagram.

p. 254 , and Geo. Sur. Ill., vol. 3, p. 476 , Burlington Gr. 
nodulosus, Hall, 1862,15 th Rep. N. Y.

Mus. Nat. Hist., p. 126, Ham. Gr. pyriformis, see Archæocrinus pyriformis. rectus, Hall, 1867, 20th Rep. N. Y. Mus.

Nat. Hist., p. 368, Niagara Gr. spinosus, Hall, 1862,15 th Rep. N. Y.

Mus. Nat. Hist., p. 127, Ham. Gr. varsoviensis, Hall, 1860, Supp. to lieo.

Sur. Iowa, p. 80, Warsaw Gr. vesperalis, White, 1880, Proc. U. S. Nat.

wortheni, Hall, 1858, Geo. Sur. Iowa, p. 556, Burlington Gr.

SAccocrixus, Hall, 1852, Pal. N. Y., vol. 2, p. 205. [Ety. sakkos, bag; krinon, lily.] Calyx large, urn-shaped; basals 3 ; primary radials $3 \times 5$; secondary radials 1 to $4 \times 10$; tertiary radials, in some species; regular interradials 10 to 17 ; vault lepressed, opening subcentral; arms 10 to 30 ; column round. Type S. speciosus.

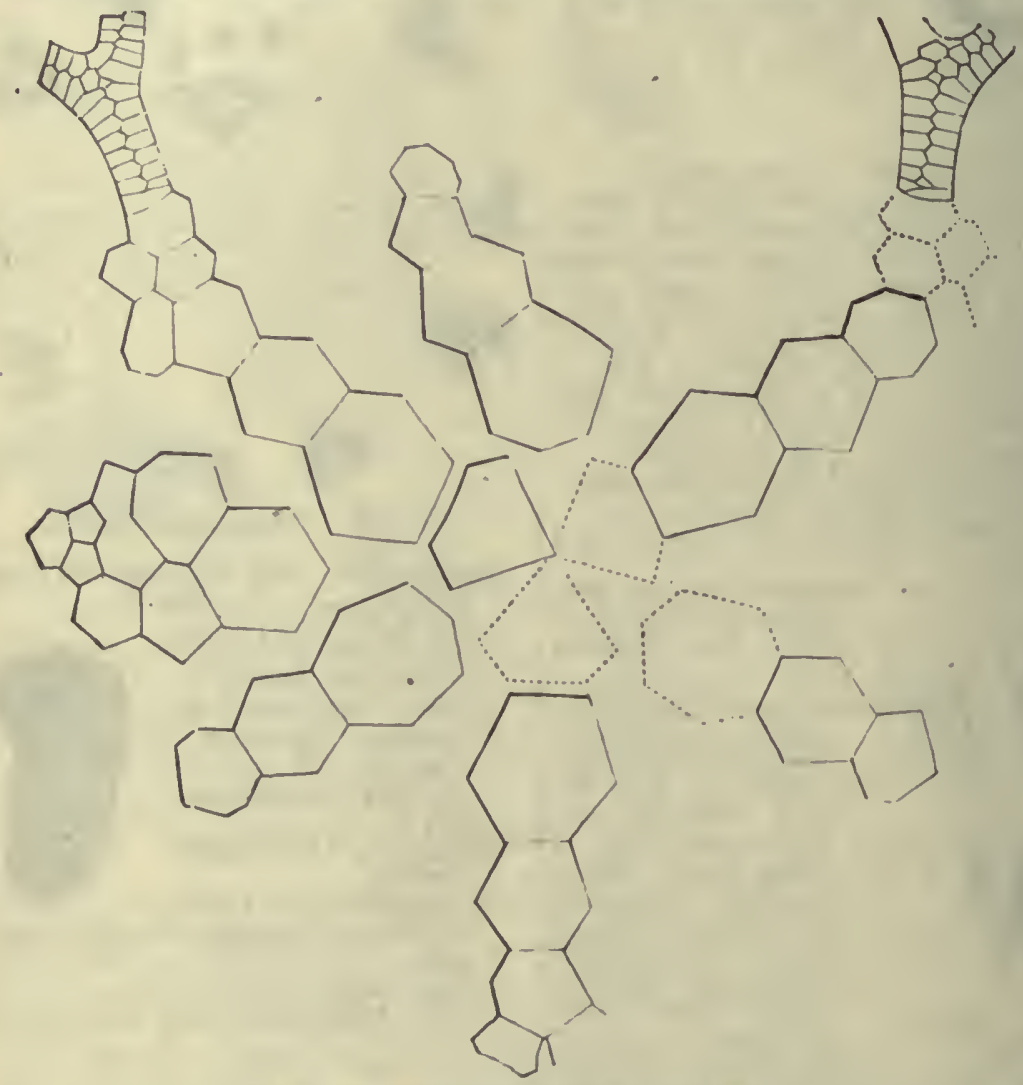

F'Ia. 420. - Saccocrinus ampluw. Dlagran.

Mus., vol. 2, p. 259, and Cont. to. Pal. No. 6, p. 129 . Up. Coal Meas. wachsmuthi, Hall, 1861, Desc. New Crin., p. 18 , Burlington $\mathrm{Gr}$.

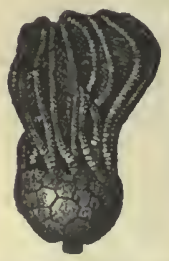

F'IG. 421-Rhodocrinus waterslanus. watersianus, wachsmuth \& Springer, (in press,) Geo. Sur. 1ll., vol. 8, p. 184, Kinderhook, Gr.

whitii, Hall, 1861, Desc. New Crin., p. 9, and Jour. Bost. Soc. Nat. Hist., vol. 7, p. 324, Burlington Gr.

whitii var. burlingtonensis, Hall, 1861, Desc. New Crin.,p. 9, and Jour. Bost. Soc. Nat. Hist., vol. 7, p. 325, Burlington Gr. amplus, Meek \& Worthen, 1861, (Actinocrinus amplus,) Proc. Acal. Nat. Sci. Phil., p. 133, and Geo. Sur. Ill., vol. 3. p. 470, Burlington $\mathrm{Gr}$. cli $\mathrm{r}$ ist y i, Hall, 1863, (Actinocrinus, christyi,) Trans. Alb. Inst., vol. 4, p. 196, and 28th Rep. N. Y. Mus. Nat. Hist., p. 127 , Niagara Gr.

egani, S. A. Miller, 1881, Jour. Cin. Soc. FIG. 422. - Saccocri. Nat. Hist., vol. 4, p. nus christyl. 173, Niagara Gr.

infelix, Winchell \& Marcy, 1865, (Megistocrinus infellx, ) Mem. Bost. Soc. Nat. Hist., p. 110, Niagara Gr. 
marcouanus, Winchell \& Marcy, 1865, (Megistocrinus marcouanus,) Mem. Bost. Soc. Nat. Hist., p. 87, Niagara Gr.

necis, Winchell \& Marcy, 1865, (Megistocrinus necis,) Mem. Bost. Soc. Nat. Hist., p. 110, Niagara Gr.

ornatus, Hall \& Whitfield, 1875, Ohio Pal., vol. 2 , p. 126, Niagara Gr.

pyriformis, S. A. Miller, 1882, Jour. Cin. Soc. Nat. Hist., vol. 5, p. $81, \mathrm{Ni}$ agara Gr.

semiradiatus, Hall, 1867, 20th Rep. N. Y. St. Mus. Nat. Hist., p. 370 , Niagara Gr. speciosus, Hall, 1852, Pal. N. Y., vol. 2, p. 205, Niagara Gr.

tennesseensis, Troost, Ms., Hall \& Whitfield, 1875, Ohio Pal., vol. 2, p. 125, Niagara $\mathrm{Gr}$.

urniformis, S. A. Miller, 1881, Jour. Cin. Soc, Nat. Hist., vol. 4, p. $170, \mathrm{Ni}-$ agara Gr.

whitfieldi, Hall, 1867, synonym for Saccocrinus christyi.

Scaphiocrinus, Hall, 1858, Geo. Sur. Iowa, p. 550. [Ety. scaphion, skiff; krinon, lily.] Calyx obconoidal ; basals 5 ; subradials 5 ; radials $2 \times 5$; regular interradiais 0 ; azygous interradials 1 to 6 ; arms 10, simple or bifurcating, plates projecting laterally; sutures gaping. Type S. simplex. Wachsmuth \& Springer refer the type to Graphiocrinus and substitute, as the type S. dichotomus.

abnormis, Worthen, 1875, Geo. Sur. Ill., vol. 6 , p. 519 , St. Louis Gr.

regina, Hall, 1863, 17th Rep. N. Y. Mus. Nat. Hist., p. 57, Waverly Gr.

rqualis, Hall, 1861, Desc. New Crin. p. 8, and Geo. Sur. Ill., vol. 5, p. 494, Keokuk Gr.

bayensis, Meek \& Worthen, 1865, Proc. Acad. Nat. Sci. Phil., p. 157, and Geo. Sur. Ill., vol. 5, p. 550, Kaskaskia Gr.

briareus, Worthen, 1882, (Poteriocrinus briareus,) Bull. No. 1, Ill. St. Mus. Nat. Hist., p. 12, and Geo. Sur. Ill., vol. 7, p. 279, Keokuk Gr.

burketi, Worthen, 1882, (Poteriocrinus burketi,) Bull. No. 1, Ill. St. Mus. Nat. Hist., p. 5, and Geo. Sur. Ill., vol. 7, p. 270, Keokuk Gr.

carbonarius, see Graphiocrinus carbonarius.

cariuatus, Hall, 1861, Desc. New Crin., p. 8 , and Jour. Bost. Soc. Nat. Hist., vol. 7, p. 310, Burlington Gr.

clio, Meek \& Worthen, 1869, Proc. Acad. Nat. Sci. Phil., p. 144, and Geo. Sur. Ill., vol. 5 , p. 408, Burlington Gr.

coreyi, Meek \& Worthen, 1869, Proc. Acad. Nat. Sci. Phil., p. 148, and Geo. Sur. Ill., vol. 5, p. 494, Keokuk Gr.

coxanus, Worthen, 1882, (Poteriocrinus coxanus,) Bull. No. 1, Ill. St. Mus. Nat. Hist., p. 43, and Geo. Sur. Ill., vol. 7, p. 269, Keokuk Gr. cultidactylus, Hall, 1860, (Poteriocrinus cultidactylus,) Supp. to Geo. Sur. Iowa, p. 62, and Geo. Sur. Ill., vol. 7, p. 301, Burlington Gr.

dactyliformis, Hall, 1858, Geo. Sur. Iowa, p. 670 , St. I.ouis Gr.

decabrachiatus, Hall, 1858, Geo. Sur. Iowa, p. 679 , St. Louis Gr.

decadactylus, Meek \& Worthen, 1860, (Poteriocrinus decadactylus,) Proc. Acad. Nat. Sci. Phil., p. 394, and Geo. Sur. Ill., vol. 2, p. 238 . Keokuk Gr.

delicatus, Meek \& W orthen, 1869 , Proc. Acad. Nat. Sci. Phil., p. 144, and Geo. Sur. Ill., vol. 5 , p. 407 , Burlington $\mathrm{Gr}$.

depressus, Meek \&

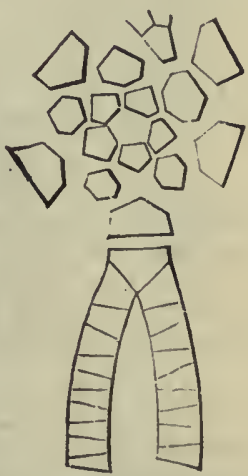

Worthe n, 1870, Fig. 423.-ScaphioeriProc. Acad. Nat. nus decadactylus. Sci. Phil., p. 27, Diagram. and Geo. Sur. Ill., vol. 5, p. 492 , Keokuk Gr.

dichotomus, Hall, 1858, Geo. Sur. Iowa, p. 553, Burlington Gr.

divaricatus, Hall, 1860, Supp, to Geo. Sur. Iowa, p. 65, Burlington Gr.

doris, Hall, 1861, Desc. New Crin., p. 7, and Jour. Bost. Soc. Nat. Hist., vol. 7, p. 312, Burlington Gr.

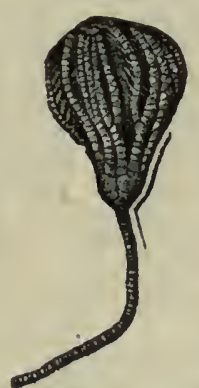

FIG. 424-Seaphlocrinus elegantulus. elegantulus, Wachsmuth \& Springer, (in press) (ito. Sur. Ill., vol. 8, J. 195 , Kinderhook Gr.

extensus, Wachsmuth \& Springer, 1886, Revis. Palreocrinoidea pt. 3 , p. 237. Proposed instead of Poteriocrinus asper, Worthen, but the latter name was not preoccupied.

fiscellus, Meek \& Worthen, 1869, Proc. Acad. Nat. Sci. Phil., p. 146, and Geo. Sur. Ill., vol. 5, p. 424, Burlington (ir.

gibsoni, White, 1878 , Proc. Acad. Nat. Sci. Phil., p. 31, and Cont. to Pal., No. 8, p. 161, Keokuk Gr.

globosus, Wachsmuth \& Springer, (in press) Geo. Sur. Ill., vol. 8, p. 196 , Kinderhook Gr. gurleyi, White, 1878, Proc. Acad. Nat. Sci. Phil., p. 32, and Cont. to Pal., Fig. 425.-ScaphNo. 8, p. 162, KeokukGr. iocrinus globohalli, Hall, 1861, Desc. sus.

New Crin., p. 7, and Jour. Bost. Soc. Nat. Hist., vol. 7, p. 308, Burlington Gr. 
huntsvillæ, Worthen, 1873, Geo..Sur. Ill., vol. 5 , p. 534, St. Louis Gr.

internodius, Hall, 1858, Geo. Sur. Iow a, p. 679, St. Louis Gr.

iowensis, Worthen, 1882, (Poteriocrinus jowensis,) Bull. No. 1, Ill. St. Mus. Nat. Hist., p. 6, and Geo. Sur. Ill., vol. 7, p. 272, Keokuk Gr.

juvenis, Meek \& Worthen, 1869, I'roc. Acad. Nat. Sci. Phil., p. 146, and Geo. Sur. Ill., vol. 5, p. 417, Burlington Gir.

kaskaskiensis, Worthen, 1882, (Puteriocrinus kaskaskiensis,) Bull. No. 1, Ill. St. Mus. Nat. Hist., p. 27, and Geo. Sur. Ill., vol. 7, p. 300, Kaskaskia (ir.

latidactylus, Worthen, 1882, ('Poterioerimus latidactylus, ' Bull. No. 1, Ill. St. Mus. Nat. Hist., p. 8, and Geo. Sur. Ill., vol. 7, p. 275 , Keokuk (ir.

liliiformis, Meek \& Worthen, 1869, Proc. Acad. Nat. Sci. Phil., p. 138, Burlington Gr.

lirione, Hall, 1863, 17th Rep. N. I. Mus. Nat. Hist., p. 58, Waverly (ir.

longidactylus, McChesney, 1860, New Pal. Foss., p. 7, Kaskaskia (ir.

macadamsi, see Graphiocrinus macadamsi.

macrodactylus, Meek \& Worthen, 1869, Proc. Acad. Nat. Sci. Phil., p. 140, and Geo. Sur. Ill., vol. 5, p. 415, Burlington Gr.

montanensis, Meek, 1872, (Poteriocrinus montanensis,) Hayden's Geo. Sur. Terr., p. 469 , and Cont. to Pal., No. 6, p. 128 , Subcarboniferous.

uanus, Meek \& Worthen, 1869, Proc. Acad. Nat. Sci. Pbil., p. 141, and Geo. Sur. Ill., vol. 5, p. 423, Burlington (ir. nauvooensis, Worthen, 1882, (Poteriocrinus nauvooensis,) Bull. No. 1, Ill. St. Mus. Nat. Hist., p. 13, and Geo. Sur. Ill., vol. 7, p. 282, Keokuk Gr.

nodobrachiatus, Hall, 1861, Desc. New Crin., p. 8, and Jour. Bost. Soc. Nat. Hist., vol. 7, p. 314, Keokuk Gr. notabilis, Meek \& Worthen, 1869, Proc. Acad. Nat. Sci. Phil., p. 148, and Geo. Sur. Ill., vol. 5, p. 410, Burlington Gr.

obscurus, Wacbsmuth \& Springer, 1886, Revis. Palacocrinoidea, pt. 3, p. 236, Keokuk Gr.

occidentalis, Wortben, 1882, (Poteriocrinus occidentalis,) Bull. No. 1, Ill. St. Mus. Nat. Hist., p. 10, and Geo. Sur. Ill., vol. 7, p. 278, Keokuk Gr.

okawensis, Worthen, 1882, (Poteriocrinus okawensis,) Bull. No. 1 , Ill. St. Mus. Nat. Hist., p. 24, and Geo. Sur. Ill., vol. 7, p. 296 , Kaskaskia Gr.

orbicularis, see Eupachycrinus orbicularis.

orestes, Worthen, 1882, (Poteriocrinus orestes,) Bull. No. 1, Ill. St. Mus. Nat. Hist., p. 7, and Geo. Sur. Ill., vol. 7, p. 273, Keokuk Gr. penicillus, Meek \& Worthen, 1869, Proc. Acad. Nat. Sci. Phil., p. 142, and Geo..Sur. 111., vol. 5, p. 414, Burlington Gr.

popensis, Worthen, 1852, (Poteriocrinus popensis,) Bull. No. 1, Ill. St. Mus. Nat. Hist., p. 23, and Geo. Sur. Ill., vol. 7 , p. 296 , Kaskaskia Gr.

propinquus, Worthen, 1882, (Poteriocrinus propinquus, ) Bull. No. 1, Ill. St. Mus. Nat. Hist., p. 26, and Geo. Sur. Ill., vol. 7, p. 299, Kaskaskia Gr.

ramulosus, Hall, 18bil, Desc. New I'rin., p. 7, and Jour. Bost. Soc. Nut. Hist., vol. 7, p. 307, Burlington Fr.

randolphensis, Worthen, 1873, Geo. Sur. Ill., vol. 5, p. 551, Kaskaskia Gr.

robustus, Hall, 1861, Desc. New Criı., p). 7, and Jour. Bost. Soc. Nat. 1 Iist., vol. 7, p. 315, Keokuk Gr.

rudis, see Grnphiocrinus rudis.

rusticellus, White, 1863, Proc. Bost. Soc: Nat. Hist., vol. 7 , p. 505, Burlington Gr.

salteri, Worthen, 188:, (Poteriocrinus salteri,) Bull. No. 1, Ill. St. Mus. Nat. Hist., p. 13, and Geo. Sur. Ill., vol. 7, p. 291, Kaskaskia Gr.

scalaris, Mcek \& Worthen, 1869, Proc. Acad. Nat. Sci. Phil., p. 145, and Geo. Sur. Ill., vol. 5, p. 421, Burlington $\mathrm{Gr}$.

scoparius, Hall, 1858, Geo. Sur. Iowa, ). 680 , Kaskaskia Gr.

simplex, see Graphiocrinus simplex.

spinifer, Wetherby, 1880, Jour. Cin. Soc.

Nat. Hist., vol. 3, p. 157, Kaskaskia Gr. spinobraihialus, sce Graphiocrinus spinobrachiatus.

spiuobrachiatus, Worthen, 1882, (I'oteriocrinus spinobrachiatus,) Bull. No. 1, IIl. St. Mus. Nat. Hist., p. 20, und Geo. Sur. Ill., vol. 7, p. 290, Kaskaskia Gr. striatus, see Graphiocrinus striutus. subcarinatus, Hall, 1863, 17 th Rep. N. Y. Mus. Nat. Hist., p. 58, Waverly Gr.

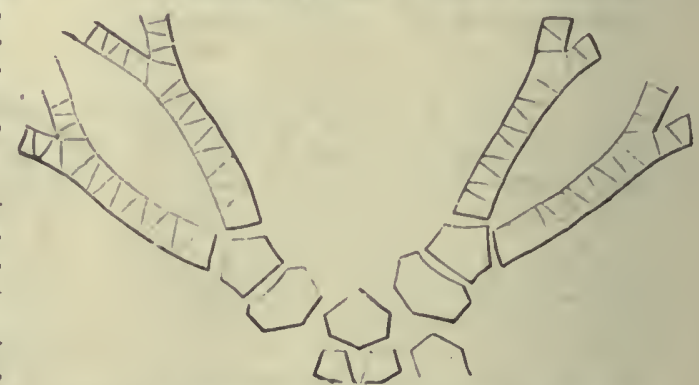

Fia. 426.--Scaphiocrinus tenuldaclyfus. Dlagran.

subtortuosus, Hall, 1863, 17th Rep N. Y. Mus. Nat. Hist., p. 59, Waverly Gr.

tenuidactylus, Meek \& Worthen, 1865, Proc. Acad. Nat. Sci. Phil., p. 156, нnd Geo. Sur. Ill., vol. 3, p. 490 , Burlington Gr. 
'tethys, Meek \& Worthen, 1869, Proc. Acad: Nat. Sci. Phil, p. 143, and Geo. Sur. Ill., vol. 5, p. 419, Burlington Gr.

tortuosus, see Grapliocrinus tortuosus.

unicus, Hall, 1861, Desc. New Crin., p. 8, and Geo. Sur. Ill., vol. 5, p. 493, Keokuk Gr.

varsoviensis, Worthen, 1882, (Poterio(:rinus varsoviensis,) Bull. No. 1, Ill. St. Mus. Nat. Hist., p. 20, and Geo. Sur. Ill., vol. 7, p. 290, Warsaw Gr.

venustus, Worthen, 1882, (Poteriocrinus venustus,) Bull. No. 1, Ill. St. Mus. Nat. Hist., p. 24, and Geo. Sur. III., vol. 7 , p. 297 , Kaskaskia Gr.

wachsmuthi, see Graphiocrinus wachsmuthi.

whitii, Hall, 1861, Desc. New Crin., p. 7, and Jour. Bost. Soc. Nat. Hist., vol. 7, p. 306, Burlington Gr.

Sсніzов _As'tus, Etheridge \& Carpenter, 1882 , Ann. \& Mag. Nat. Hist., vol. 9, p. 243. [Ety. schiza, cleft; blastos, bud.] Calyx in form like Granatocrinus; basals confined to the base, sometimes visible, in a side view; deltoids always visible in a side view; ambulacra narrow and sublinear, extending the height of the calyx; lancet-plates nearly concealed by the side plates; latter from 20 to 80 in number; 1 to 4 hydrospire folds on each side of an ambulacrum; spiracles minute linear slits between the lancet-plate and the deltoid ridges; surface ornamented witlı strix. Type S. sayi.

melonoides, Meek \& Worthen, 1869, (Granatorinu melonoideas,) Proc. Acad. Nat. Sci. Phil., p. 88, and Geo. Sur. Ill., vol. 5, p. 468, Burlington $\mathrm{Gr}$.

sayi, Shumard, 1855, (Pentremites saýi,) Geo. Rep. Mo., p. 185, Burlington Gr.

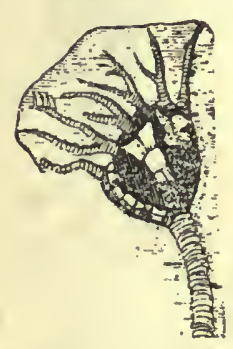

FIG. 427.-Schizocrinus nodosus.
Schizocirnus, Hall, 1847, Pal. N. Y., vol. 1, p. 81. [Ety, schiza, cleft; krinon, lily.] Basals 5; primary radials $3 \times 5$; secondary radials $2 \times 10$; interradials 5 or inore; arms short, brauching, bearing pinnules; colum n round. Type S. nodosus.

nodosus, Hall, 1847, Pal. N. Y., vol. 1, p. 81 . Trenton Gr.

striatus, Hall, 1847, Pal. N. Y., vol. 1, p. 316, Trentou Gr. Probably belongs to another genus.

Schoen aster, Meek \& Worthen, 1860, Proc. Acad. Nat. Sci., p. 449, and Geo. Sur. III., vol. 2, p. 277. [Ety. schoinos, rope; aster, star.] Pentagonal disk, with angles produced into ravs; margius between rays concave und spinons; plates alternating on dorsal sicle of rays, and on ventral side of disk imbricating inward and laterally towaid the ambulacra; furrows wide, detp, bordered with a single row of adambulacrals, which become the marginal plates of the free rays. Type S. fimbriatus.

fimbriatus, Meek \& Worthen, 1860, (Palrasterina fimbriata,) Proc. Acar. Nat. Sci. Phil., p. 449, and Geo. Sur. Ill., vol. 2 , p. 278, St. Louis Gr.

wachsmuthi, Meek \& Worthen, 1866, Proc. Acad. Nat. Sci. Phil., p. 259, and Geo. Sur. Ill., vol. 3, p. 499, Burlington $\mathrm{Gr}$.

Scyphocrinus, Hall, 1847, Рu]. N. Y., vol. 1. Preoccupied, by Tinker, in 1839. See Cupulocrinus.

heterocostalis, see Cupulocrinus heterocostalis.

Scytalocrinus, Wachsmuth \& Springer, 1879, Proc. Acad. Nat. Sci. Phil. A division of Poteriocrinus of less than generic importance, with P. robustus as the type.

wachsmuthi, see Poteriocrinus wachsmuthi.

Siphonocrinus, s. A. Miller, 1888, Am. Geol., vol. 1, p. 263. [Ety. siphon, bent tube; krinon, lily.] Basals 3 (?) small.

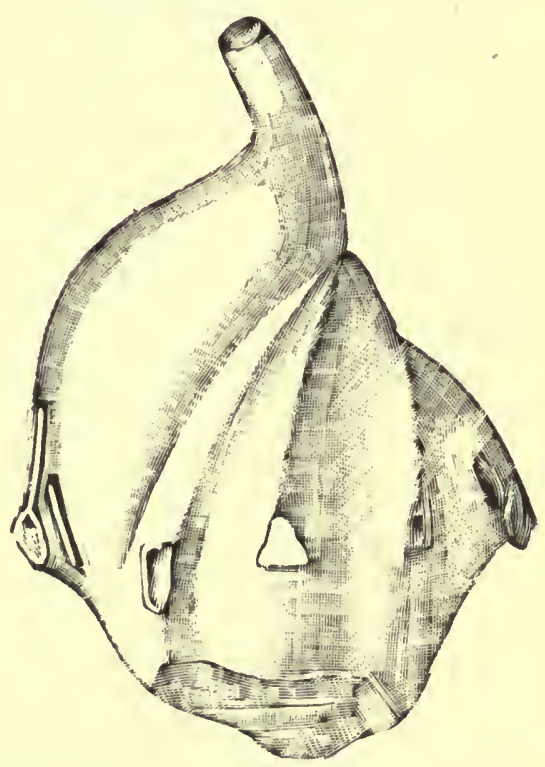

FI(i.428.-Siphonocrinus nobilis; iaternl view of an internal cast.

Wachsmuth says there are 5; primary radials $3 \times 5$; first interradials nearly as large as primary radials, and succeeded by two smaller ones, and 
these by three or more; first azygous plute as large as the primaries; it rests upon the basals and is succeeded by three plates; the following ranges have more plates and cover an expanded azygous side; vault verv large, higb, and bears a proboscis either projected upward or recumbent; surface of plates ornamented. Type S. nobilis.

armosus, McChesney, 1861. (Euealyptocrinus armosus, ) New. Pal. Foss., p. 95, and 20 th Rep. N. Y. St. Mus. Nat. Hist., p: 373 , Niagara Gr.

nobilis, Hall, 1861, (Glyptrerinus nobilis,) Geo. Sur. Wis., p. 21, and 20th Rep. N. Y. St. Mus. Nat. Hist., p. 328, Niagara Gr.

Spharocrinus, Meek \& Wurthen, 186t;. The naine was preoc('upied, by Ruemer. See Coeloerinus.

SpILrocrstres, Hall, 1859, Pal. N. Y., vol. 3. p. 130. [Ety. sphaira, sphere ; kustis, bladder.] Splıeroidal, wider than high; arms, in two principal pairs, witl numerous bifurcations; brichial sulei obliquely lobed; mouth apieal; opening subapieal; ovarian opening on the summit; basal plates 4, others un. known. Type S. multilascialus.

mullifasciatus, Hall, 18.59, I'al. N. I., vol. 3, p. 130, Lıw. Held. Gr.

Squamaster, Ringuelerg, 1s86, Bull. Huf. Soc. Nat. Hist., vol. 5 , p. 5 .

Steqaxocrinus, Meek \& Worthen, 1866, Geo. Sur. Ill., vol. 2, p. 195. [Ety. stegranos, covered; krinon. lily.] General form like Actinocrinus: basals 3; primary

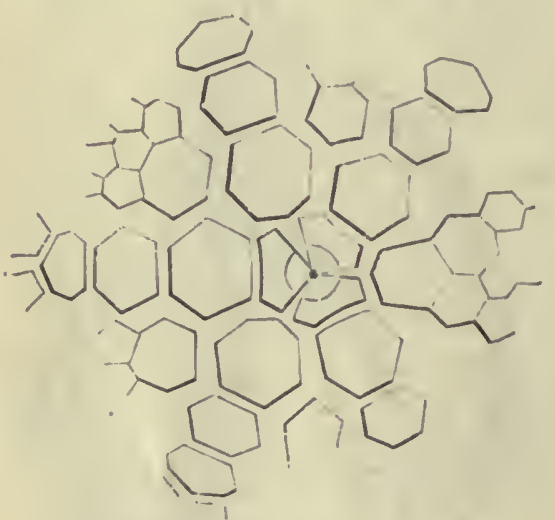

FI(i. 429 -Steganocrinus concinnus.

radials $3 \times 5$; seconclary radials $1 \times 2 \times$ 5 , in each ray; regular interradials 3 to $6 \times 4$; azygous inlerradials 3 to 10 or more; vault elevated, with long subcentral tube; arms bifurcating ; column round. Trpe S. pentagoums.

araneolus, Meek \& Worthen, 1S60. (Actinocrinus araneolus,) Proc. Acad. Nat. Sci. Pbil., p. 387, and (ieo. Sur. Ill., vol. 2, p. 198, Burliugton Gr. concinnus, Shumard, 1855, (Actinoerinus concinnus,) Geo. Sur. Mo., p. 189, Burlington $\mathrm{Gr}$.

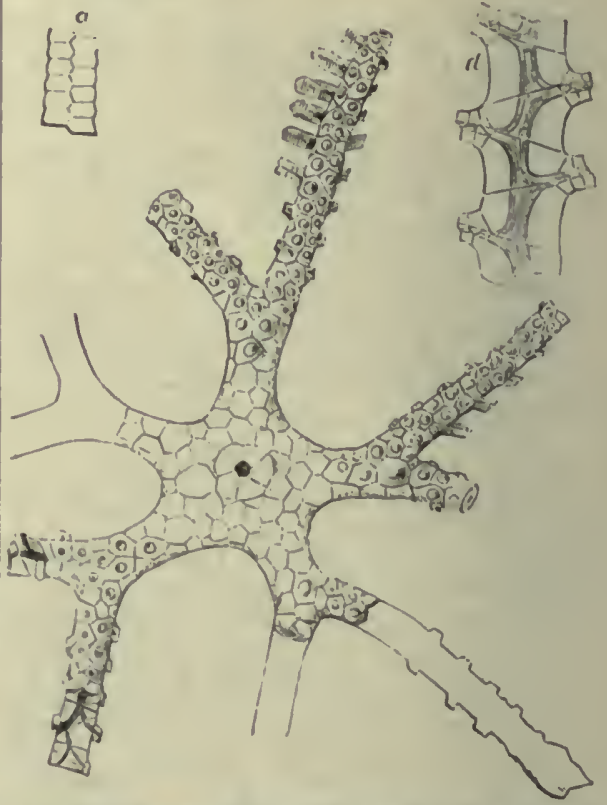

Fia. tiso-siegunocrinus pentugonuk. Vintl nud part of the ruys; $"$ uul $d$ showlug structure of the rays.

pentagonus, Hall, 1858, (Acthocrinus pentugonus,) Geo. Sur. Jowa, p. 577, Burlington fir.

sculptus, Hall, 1858, (Actinocrimus scul]). tus,) (ieo. Sur. Jowa, p. 582, Burling. ton $\mathrm{Gr}$.

Struma tocizinus, Trautschold, 1867, Crin. d. jungeren Bergkalkes b. Moskau, p. 28. [Ety. stemma, wreath ; krivon, lily.] Calyx low, cup-shaped ; basals 5 , anchylused; subradials 5; radials twice as wide as high; brachials $1 \mathrm{x}$ 5 ; urms heary. 'Type s. cornuus. This is . Iosely related to lirisocrinus and Eupachycrinus.

trautscholdi, Wachs. mulh \& Springer, 1886, Revis. Palieocrinuidea, pt. 3 , p.

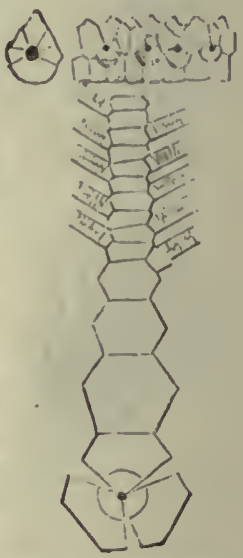

FiG. 431.-Stegunocrinus sculptus. Dingram of a'ray and transverse nectlons. 2506, Keokuk Gr.

Stexaster, Billings, 1858, Can. Org. Rem., Decade 3, p. 77. [Ety. stenos, narrow; aster, star.] Disk small, rays extended, flexible; dorsal side covered with small 
plates; adambulacrals square or oblong; orals 10 . Type S. salteri.

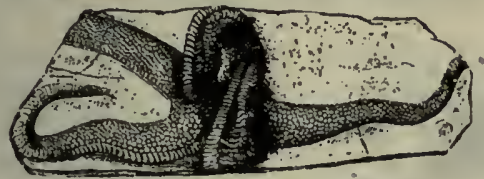

FIG. 432.-Stenaster grandls. pulchellus, Jour. Cin. Soc. Nat. Hist. vol. 1, p. 35, Niagara Gr.

Sterkocrinus, Barris, 1879, Proc. Dav. Acad. Sci., vol. 2, p. 282. [Ety. stereos, firm; krinon, lily. Distinguished from Dolatocrinus by having $2 \times 5$ instead of $3 \times 5$ primary radials; one large interradial succeeded by a smaller one, and this by smaller ones, within the depressions, between the arm bases. Type $\mathrm{S}$. triangulatus.

triangulatus, Barris, 1879, Proc. Dav. Acad. Sci., vol. 2, p. 283, Up. Held. Gr.

grandis, Meek, 1872, Am. Jour. Sci., 3d ser., vol. 3 , p. 258 , and Ohio Pal., vol. 1 , p. 66, Hud. Riv. Gr.

huxleyi, Billings, 1865, Pal. Foss., vol. 1, p. 213, Quebec Gr.

pulchellus, Billings, 1857, (Palæaster pulchellus,) Geo. Sur. Can., p. 292, and Can. Org. Rem., Decade 3, p. 79, Trentou $\mathrm{Gr}$.

salteri, Billings, 1858, Can. Org. Rem., Decade 3, p. 78, Trenton Gr.

Stenocrinus, Wachsmutl। \& Springer, 1885, Palicocrinoidea, pt. 3, p. 207, syn. for Heterocrinus.

StePHANocrinus, Conrad, 1842, Jour. Acad. Nat. Sci., vol. 8, p. 278. [Ety. stephanos, coronet; krinon, lily.] A blastoid, with 3 basals, 5 fork pieces or radials, and 5 orals; aperture subcentral; ambulacral appendages, but thus far the hydrospires are unknown. Type S. angulatus. Some authors refer this genus to the Palæocrinoidea.

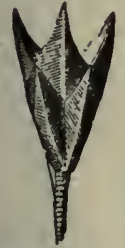

FIG. 433.- Stephanocrinus angulatus. angulatus, Conrad, 1842 , Jour. Acad. Nat. Sci., vol. 8 , p. 279 , and Pal. N. Y., vol. 2 , p. 212, Niagara $\mathrm{Gr}$. gemmiformis, Hall, 1852, Pal. N. Y., vol. 2, p. 215, Niagara $\mathrm{Gr}$.

osgoodensis, S. A. Miller, 1879, Jour. Cin. Soc. Nat. Hist., vol. 2, p. 116, Niagara Gr. Wachsmuth has said this species was described from internal casts, but it was not. pentalobus, Hall, 1879, (Codaster pentalobus,) Desc. New Spec. Foss., p. 13, and 11th Rep. Greo. Sur. Ind., p. 280, Niagara Gr. pulchellus, Miller \& Dyer, 1878, (Codaster

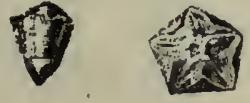

IG. 434. Stephanocrinus puichellus, instead of Codaster pulchellus, as shown by fig. 268 
izontal disk, completely isolating the azygous and interradial areas, from the vault, and supporting the free, ascend- ectypus, Meek \& Worthen, 1869, I'roc. Acad. Nat. Sci. Phil., p. 159, and (ieo. Sur. Ill., vol. 5, p. 353, Burlington Gr.

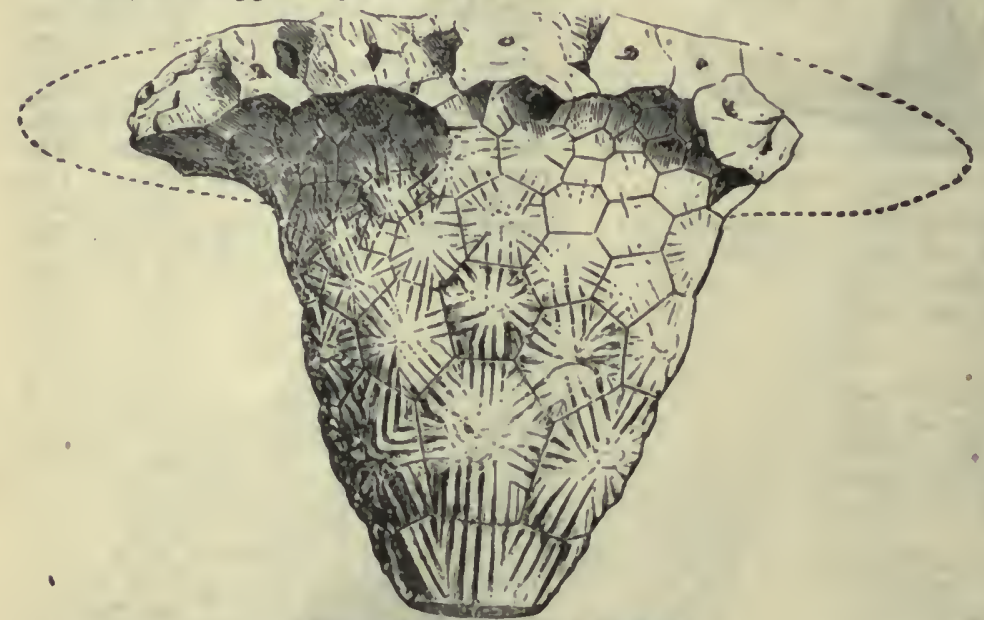

Fis. 436.-Sitrolocrinus regalls.

glyptus, Hall, 1860, (Actinocrinus glyptus, ) Supp. to Gen. Sur. Iown, p. 2, Burlington Gr.

p e r u in brosus, Hall, 1860 , (Actinocrinus peru in bro. sue, ) Supp. to Ge o. sur Iowa, p. 7 , Burlington Gr. regalis, Hall, 1860, (Actinocrinus regalis,) Supp. to Geo. S II . Iока, p. 8, and Geo. Sur.

ing arms around its margin; interradials 9 or 10 or more; azygous interradials 9 to 13 or more, the first one resting on the basals; vault depressed, opening subcentral; arms 30 to 72 or more; col. umn round. Type S. perumbrusus. asperrimus, Meek \& Worthen, 1869, Proc. Acad. Nat. Sci. Phil., p. 160, and Geo. Sur. Ill., vol. 5, p. 349, Burlington (ir.

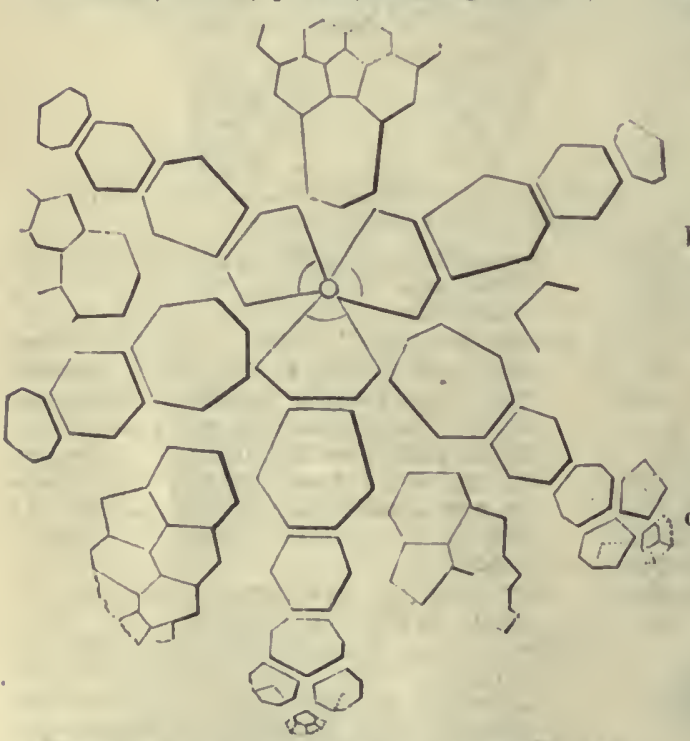

Frg. 437.-Strotocrinus regalis. [Hagram, ${ }^{2} \frac{1}{2}$ diam.

III., vol. 2, p. 192, Burlington Gr. umbrosus, IIall, 1858, (Actinocrinus "umbrosus, ) Geo. Sur. Iowa, p. 590, Burlington $\mathrm{Gr}$.

Synbatiockinus, Phillipg, 1836, Geol. Yorkshire, pt. 2, p. 206. [Ety. sun, together; bathos, depth; krinon, lily.] Calyx small; arms large and of great length. basals 3 ; radials $2 \times 5$; azygous plates 1 ;, 2 or more; arms simple and com. posed of plates in single series. Type S. conicus.

brevis, Meek \& Worthen, 1869, Proc. Acad. Nat. Sci. Phil., p. 68 , and Geo. Sur. Ill., vol. 5 , p. 439 , Burlington $\mathrm{Gr}$.

de $\mathrm{ntatus}$, Owen \&Shumard, 1852, Geo. Sur. Wis., Iowa,

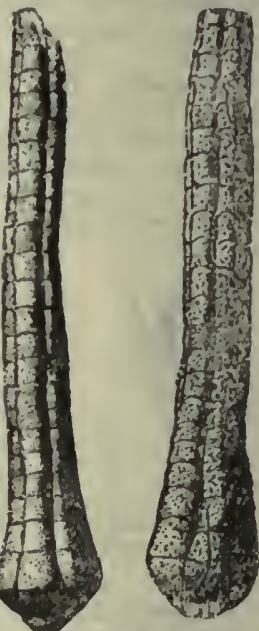
and Minn., Fra. 438. - syubalhocrp. 597, Bur- uus granuliferus. Anlington $\mathbf{G r}$. gr a $n$ ulatus, Troost. Not defined.

bloomfieldensis, S. A. Miller, 1882, Jour. Cin. Soc. Nat. Hist., vol. 2, p. 258, and vol. 4 , p. 76 , Up. Burlington or Keokuk Gr.

dilatatus, see Physetocrinus dilatatus. granuliferus Wetherby, 1880, Jour. Cin. Soc. Nat. Hist., vol. 2, p. 250, Waverly $\mathrm{Gr}$.

matutinus, Hall, 1858, Geo. Sur. Iowa, p. 483, Ham. Gr. 
oweni, Hall, 1860 , 13 th Rep. N. Y. Mus. Nat. Hisi., p. 111, Waverly Gr.

rapillatus; Hall, 1861, Desc. New Crin., p. 18, Burlington Gr.

robustus, Shumard, 1866, Trans. St. Louis Acad. Sci., vol. 2, p. 397, and Geo. Sur. IIl., rol. 6, p. 514, Keokuk Gr.

swallovi, Hall, 1858, Geo. Sur. Iowa, p. 672, St. Louis Gr.

tennesser, Troost. Not defined.

tennesseensis, Roemer, 1860, Sil. Fauna West 'l'enn., p. 55, Niagara Gr.

wrichsmuthi, see Catillocriuns wachsmuthi. wortheni, Hall, 1858, Geo. Sur. Iowa, p. 560 , Burlington Gr.

Syringocrinus, Billings, 1859. Can. Org. Rem., Decarle 4, p. 65. [Ely. syrinx, pipe; krinon, lily.] Founded, possibly, on the frayment of a ventral sac; at all events, not a well-characterized genus. Type S. paralloxicus.

paradoxicus, Billings, 1859, Can. Org. Rem., Decade 4, p. 65, Trenton Gr.

Taniaster, Billings, 1858, Can. Org. Rem,, Decade 3, p. 80. [Ety. tainia, ribbon; aster, star.] No disk or irarginal plates; rays long, flexible, spinous; adambulacral plates elongated; two rows of ambulacral pores; ossicles contracted in the middle. Type T. spinosus.

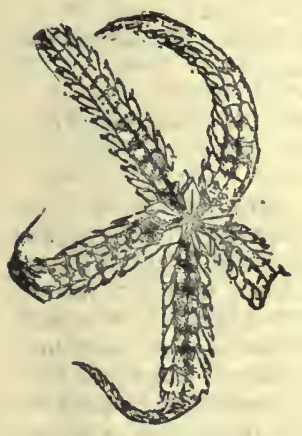

F1G. 439.-Tæenluster spiuosus. cylindricus, Bill-

i n g s, 1857 , ( Palæocom a cylindrica, ) Geo. Sur. Can., p. 292, Trenton Gr.

elegans, S. A. Miller, 1882 , Jour. Cin. Soc. Nat. Hist., vol. 5. p. 41, Hud. Biv. Gr.

spinosus, Billings, 1857 , (Pal:eocoma spinosa,) Geo. Sur. Can., p. 292 , and Can. Org. Rern., Decade 3, p. 80, Trenton Gl.

Talarocrines, Wachsmutl \& Springer, 1881, Proc. Acad. Nat. Sci. Puil., p. 259. [Ety. talarus, basket; krinon, lily.] Calyx sub-conical ; suture lines inpressed; distinguished from Dichocrinus by its higher vault and having the opening through it and not at the end of a tube, aud in having the secondary radials form part of the calyx. Type $\mathbf{T}$. cornigerus.

cornigerus, Shumard, 1857, (Dichocrinus cornigerus,) Trans. St. Louis Acad. Sci., vol. 1, p. 72, Kaskaskia Gr.

elegans, Lyon \& Casseday, 1860, (Dichocrinus elegans,) Proc. Am. Acad. Arts and Sci., vol. 5, p. 22, St. Louis Gr.

ovatus, Worthen, 1882, Bull. No. 1, 111. St. Mus. Nat. Hist., p. 36, and Geo. Sur. Ill., vol. 7, p. 314, Kaskaskia Gr.

sexlobatus, Shumard, 1857, (Dichocrinus sexlobatus,) Trans. St. Loụis Acad. Sci., vol. 1 , p. 73 , Kaskaskia Gr.

symmetricus, Lyou \& Casseday, 1860 , (Dichocrinus symmetricus,) Proc. Am. Acail. Arts and Sci., vol. 5, p. 22, Kaskaskia Gr.

Taxocrinus, Phillips, 1843, Morris Cat. Brit. Foss., p. 90. [Ety. taxus, yew-tree; krinon, lily.] Calyx short, cup-shaped; basals 3 , small, unequal; subradials 5 , one larger than the others; primary radials 3 or 4 by 5 ; secondary radials 3 to six by 10 ; tertiary radials supporting arıns; interradials 0 to 9 ; azygous interradials 2 to 5 ; arms dividing once or twice. Type T. egertoni.

communis, Hall, 1863, (Forbesocrinus communis,) 17th Rep. N. Y. St. Mus. Nat. Hist., p. 55, and Olio Pal., vol. 2, p. 169 , Waverly Gr.

curtus, Williams, 1882, Proc. Acad. Nut. Sci. Phil., p. 30, Chemung Gr.

elegans, Billings, 1857, (Lecanocrinus elegans,) Geo. S u r. Can., p. 278, and Can. Ory. Rem., Decrade 4, p. 47, Trenton Gr.

fletcheri, Worthen, 1882, Bull. No. 1, IIl. St. Mus. Nat. Hist., p. 31 , and Geo. Sur. Ill., vol. 7, p. 308, Keokuk Gr.

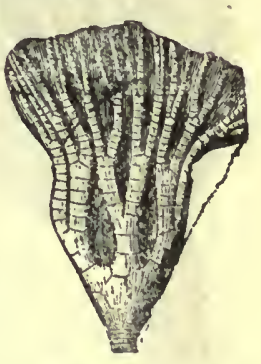
giddiugei, Hall, 1858

FIG. 440.-Taxocrinus (?) elegans.

(Forbesocrinus giddingei,) Geo. Sur. Iowa, p. 633, Keokuk Gr.

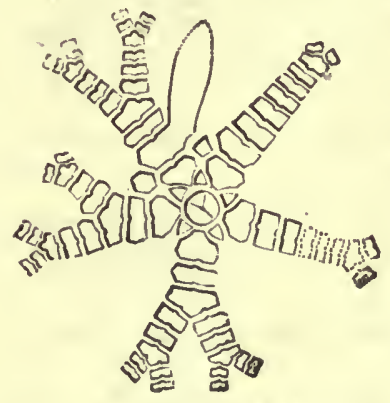

Fig. 441.- laxocrinus gricills. Diagrain. g r a cil is , M e e k \& Worthen, 1865, Proc. Acad. Nat. Sci. Phil., p. 142 , and ieo. Sur. Ill., vol. 3, p. 421 , Ham. Gr.

inter $\mathrm{med} \mathrm{i}$ us, Wachs$\mathrm{muth}$ \& Springer, (iin press,) Geo. Sur. Ill., vol. \&, p. 199, Kinderhook Gr.

interscapularis, Hall, 1858, Geo. Sur. Iowa, p. 482, Ham. Gr.

ithacensis, Williams, 1882, Proc. Acad. Nat. Sci. Phil., p. 28, Chemung Gr.

juvenis, Hall, 1861, (Forbesocrinus juvenis,) Bost. Jour. Nat. Hist., vol. 7, p. 319 , Burlington Gr.

kelloggi, Hall, 1863, (Forbesocrinus kelloggi,) 17th Rep. N. Y. St. Mus. Nat. Hist., p. 56, and Ohio Pal., vol. 2, p. 171, Waverly Gr. 
Javis, Billings, 1857, Geo. Sur. Can., p. 278, and Can. Org. Rem., Decade 4, p. 47, Trenton Gr.

lobatus, Hall, 1862, (Forbesocrinus lobatus,) 15th Rep. N. Y. St. Mus. Nat. Hist., p. 124, Ham. Gr.

lobatus var. tardus, Hall, 1863, (Forbesocrinus lobatus var. tardus, ) 17th Rep. N. Y. St. Mus. Nat. Hist., p. 56 and Ohio Pal., vol. 2, p. 171, Waverly Gr.

meeki, Hall, 1858, (Forbesocrinus meeki,) Geo. Sur. Iowa, p. 631, Keokuk Gr.

multibrachiatus, Lyon \& Casseday, is 5 , (Forbesocrinus multibrachiatus,) Am. Jour. Sci. and Arts, rol. 28, p. 235, Keokuk Gr.

multibrachiatus var. colletti, White, 1881 2d Ann. Rep. Bureau of Statistics of Indiana, p. 506, Keokuk Gir.

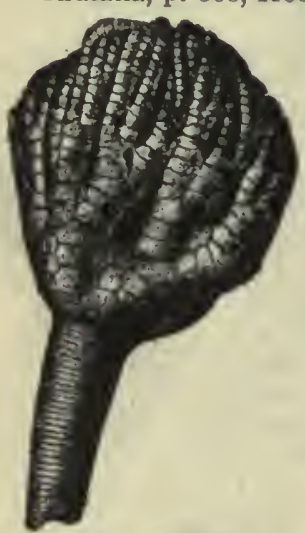

Fia. 442.-Taxocrinus robustus. nuntius, Hall, 1862, (Forbesocrinus nuntius, ) 15th Rep. N. Y. St. Mus. Nat. Hist., p. 124. Hain Gr. rainulosus, Hall, 1860, (Forbesocrinus ramulosus,) Supp. Geo. Sur. Iowa, p. 67. Burlington $\mathrm{Gr}$.

robustus, Wachsmuth, press, Geo. Sur. Ill., vol 8, Kind e r h o o k Gr.

$80 \mathrm{miovat}$ il $\mathrm{s}$ Meek \& Worthen, 1860 , (Forbesocrinus semiovatus,) Proc. Acad. Nat. Sci. Phil., p. 389 , and Geo: Sur. Ill., vol. 2, p. 272, St. Louis Gr.

shumardanus, Hall, 1858, (Forbesocrinus shumardanus,) Geo. Sur. Iowa, p. 671, St. Lovis Gr.

thiemii, Hall, 1861, (Forbesocrinus thiemii,) Desc. New Crin., p. 8, and Geo. Sur. Ill., vol. 5, p. 389, Burlington Gr.

whitfieldi, Hall, 1858, (Forbesocrinus whitfieldi,) Geo. Sur. Iowa, p. 632, Kaskaskia Gr.

Technocunus, Hall, 1859, Pal. N. Y., vol. 3. p. 139. [Ety. "techne, art; krinon, lily.] Basals 4, one larger than the otherg; primary radials $3 \times 5$; secondary radials $1 \times 10$; tertiary radials $2 \times 20$; interradials $3 \times 5$; arms simple, bearing pinnules; column round. Type $T$. andrewsi.

andrewsi, Hall, 1859, Pal. N. Y., vol. 3, p. 141 , Oriskany sandstone.

sculptus, Hall, 1859, Pal. N. Y., vol. 3, p. 143 , Oriskany sandstone.

spinulosus, Hall, 1859, Pal. N. Y., vol 3, p. 140 , Oriskany sandstone. striatus, Hall, 1859, P'al. N. Y., vol. 3, p. 142 , Oriskany saudstone.

Telemocrinus, Wachsmuth \& Springer, 1881, Próc. Acad. Nat. Sci. Phil., U. 320. [Ety. teleios, perfect; kri.on, lily.] Distinguished from Strotocriuus, with which it has generally been classed, by having a long ventral tube, instead of a simple opening through the vault. Type T. umbrosus.

regilops, Hall, 1560, (Actinoerinus rgilops,) Supp. to Geo. Sur. Iowa, p. 5, Up. Burlington Gr.

althea, Hall. 1861, (Actinocrinus althea,) Desc. New Crin., p. 13, Up. Burlington $\mathrm{Gr}$.

clivosus, Hall, 1861, (Aetinocrinus clivogus,) Bost. Jour. Nat. Hist., vol. 7, p. 274, Up. Burlington Gr.

erodus, Hall, 1861, (Actinocrinus erodus,) Desc. New Crin., p. 12, Up. Burlington Gr.

insculptus, Hall, 1861, (Actinocrinus insculptus, ) Desc. New Crin., p. 12, Up. Burlington $\mathrm{Gr}$.

liratus, Hall, 1860, (Aetinoerinus liratus, ) Supp. to Geo. Sur. Iowa, p. 1, and Geo. Sur. Ill., vol. 5, p. 355, Burlington Gr.

rudis, Hall, 1860, (Actinocrinus rudis,) Supp. to Geo. Sur. Iowa, p. 33, Burlington Gr.

tenuiraliatus, Hall, 1861, (Actinocrinus tenuiradiatus,) Desc. New Crin., p. 12, Burlington $\mathrm{Gr}$.

umbrosus, Hall, 1858. (Actinocrinus umbrosus,) Geo. Sur. Iowa, p. 590, Up. Burlington Gr.

Turranocrinus, Hall, 1852, Pal. N. Y., vol. 2, p. 188. [Ety. thysanos, fringed; krinom, lily.] Calyx small, subglobose; basals 5 ; subradials 5 ; jrimary radials $3 \times 5$; ; secondary raclials 2 or more $\times 10$; regular interradials 3 ; azygous area wide, lower plates large, smaller above; arins composed of a double series of plates, with piunules; column round. Type $\mathrm{T}$. liliiformis.

aculeatus, Hall, 1852, I'a]. N. Y., vol. 2, p. 130. Niugara Gr.

canaliculatus, Hall, 1852, Yal. N. Y., vol. 2, p. 189, Niagara Gr.

immaturus, Hall, 1852, Pal. N. Y., vol. 2, p. 191, Niagara Gr.

liliiformis, Hall, 1852, Pal. N. Y., vol. 2, p. 188, Niagara Gr.

microbasalis, see Archacocinus microbasalis.

pyriformis, see Archæocrinus pyriformis. Tremataster, Worthen \& Miller, 1883, Geo. Sur. 1ll., vol. 7, p. 330. [Lity. trema, opening; aster, star.] Central part discoid; rays long, flexuous, a double series of ambulacral plates, with tapering ends directed toward the apices of the rays, upon each side of which there is a series of curved adambulacral plates, which form the margin of the rays; pores large between the con- 
tracting sides of the ambulacral plates, and the concave sides of the curving adambulacrals; four plates border on each pore; orals 10 . Type T. difficilis.

difficilis, Worthen \& Miller, 1883, Geo. Sur. Ill., vol. 7, p. 330, Kaskaskia Gr.

Trematocrinus, syn for Goniasteroidocrinus.

fiscellus, see Goniasteroidocrinus fiscellus.

papillatus, see G. papillatus.

reticulatus, see $G$. reticulatus.

robustucs, see $\mathrm{G}$. robustus.

spinigrmus, see G. spinigerus.

tuberculatus, see G. tuberculatus.

typus, see G. typus.

Triucrinus, Ringueberg, 1887, Proc. Acad. Nat. Sci. Pliil., p. 144. The name was preoccupied; beside it is probably a svin. for Pisocrinus.

globinsus, see Pisocrinus globosus.

pyriformis, see Pisocrinus pyriformis.

Tricolocrinus. Meek \& Worthen, 1868, Proc. Acad. Nat. Sci. Phil., p. 356, and Geo. Sur. Ill., vol. 5, p. 507. [Ety.treis, thiree; koilos, hollow; krinon, lily.] Calyx subpyramidal, or subfusiform ; base short, trihedral, and excavated along the interbasal sutures; summit contracted; radials long and narrow; doltoids small; ambulacra narrow, deeply situated in the sinuses; hydrospires small, three (?) on a side; spiracles and mouth small; anus large ; column circular. Type T. woodinani.

meekanus, Etheridge \& Carpenter, 1886, Catal. of Blastoidea, p. 208, Warsaw Gr.

obliquatus, IRoemer, 1851, (Pentatrematites obliquatus,) Archiv f. Naturgesch., Jahrg. xvii, p. 367, St. Louis Gr.

varsouviensis, Worthen, 1875, Geo. Sur. Ill., vol. 6, p. 521, St. Louis Gr.

woodmani, Meek \& Worthen, 1868, (Pentremites, Troostocrinus) Tricolocrinus woodmani,) Proc. Acad. Nat. Sci. Phil., p. 356, and Geo. Sur. Ill., vol. 5, p. 506, Warsaw Gr.

Troostocrinus, Shumard, 1865, Trans. St. Louis Acad. Sci., vol. 2, p. 384. [Ety. proper name; krinon, lily.] Distinguished from Pentremites by the slender, subfusiform shape, linear ambulacra, lancet plates concealed, triangular base, and simple summit structure; spiracles at the sides of the proximal side plates; hydrospiral canals open into linear spiracular apertures. Type $\mathrm{T}$. reinwardti.

bipyramidalis, Hall, 1858, (Pentremites bipy ramidalis,) Geo. Sur. Iowa, p. 607, Keokuk Gr.

grosvenori, Shumard, 1858, (Pentremites grosvenori, ) Trans. 'St. Louis Acad. Sci., vol. 1 , p. 240 , Warsaw Gr. lineatus, Shumard, 1858, (Pentremites lineatus,) Trans. St. Louis Acad. Sci., vol. 1, p. 241, Burlington Gr. This is made the type of the genus Metablastus by Etheridge \& Carpenter, to which they also refer T. wortheni and Tricœlocrinus varsouviensis.

reinwardti, Troost, 1835, (Pentremites reinwardti,) Trans. Geo. Soc. Pa., vol. 1, p. 224, Niagara Gr. subcylindricus, Hall \& Whitfield, 1875, (Pentremites subcylindricus,) Ohio Pal., vol. 2, p. 129 , Niagara Gr.

subtruncatus, Hall, 1858, (Pentremites subtruncatus,) Geo. Sur. Iowa, p. 485, Ham. Gr.

wortheni, Hall, 1858, (Pentremites wortheni,) Geo. Sur. Iowa, p. 606, Keokuk Gr.

VAsocrinus, Lyon, 1857, Geo. Sur. Ky., vol. 3, p. 485 . [Ety. vas, vessel; krinon, lily.] Calyx low, vase-shaped: basals 5 ; subradials, 5; primary radials, $1 \times 5$; secondary radials $2 \times 5$; arms, 10 or more; azygous interradials 2 or more, first one large; ventral sac. Type V. valens.

lyoni, Hall, 1861, (Cyathocrinus lyoni,) Desc. New Crin., p. 3, and Bost. Jour. Nat. Hist., vol. 7, p. 298, Keokuk Gr.

-macropleurus, Hall, 1861. (Cyatlıocrinus macropleurus,) Desc. New Crin., p. 5, and Bost. Jour. Nat. Hist., vol. 7, p. 295, Burlington Gr.

sculptus, Lyon, 1857, Geo. Sur. Ky., vol. 3 , p. 486, Ham. Gr.

valens, Lyon, 1857, Geo. Sur. Ky., vol. 3, p. 485 , Ham. Gr.

Xexocrinus, S. A. Miller, 1881, Jour. Cin. Soc. Nat. Hist., vol. 4, p. 71 and 176. [Ety. xenos, strange; krinon, lily.] Ba-
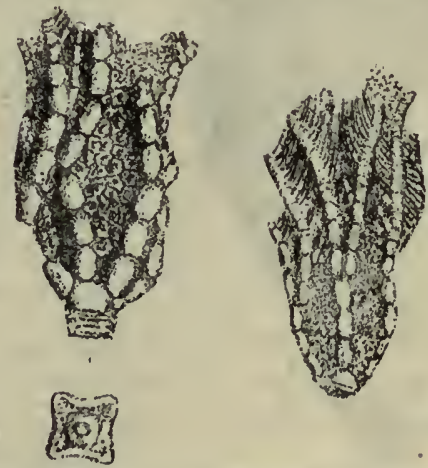

FIG. 444.-Xenocrinus penícillus. Azygons and opposite side vlews and end of column.

sals 4 ; primary radials $3 \times 5$; secondary radials 4 to $6 \times 10$; interradial areas excavated and filled with numerous 
plates; azygous area having a central vertical series of plates which continue up the ventral sac; column square. Type $\mathrm{X}$. penicillus.

baeri, Meek, 1872, (Glyptocrinus baeri,) Am. Jour. Sci. and Arts, 3d ser., vol. 3 , p. 260, and Ohio Pal., vol. 1, p. 37, Hud. Riv. Gr.

penicillus, S. A. Miller, 1881, Jour. Cin. Soc. Nat. Hist., vol. 4 , p. 72 , Hud. Riv. Gr.

Zeacrinus, Troost, Catal. Foss. 1850, and lescriber by Hall, 1858 , Geo. Sur. Iowa, p. 541. [Ety. zea, Indian corn; krinon, lily.] Calyx low, basin-shaped; basals 5 , hidden by the column; subradials 5 ; radials $2 \times 5$, with from 1 to 6 additional in the azygous ray; azygous interradials $f$ to 7 ; $\operatorname{arms} 10$ to 40 , with pinnules; ventral sac subpyramidal, coveral with small plates; column round. Type $\%$. magnoliif, rrmis.

acanthophomes, see Hydreionocrinus acan. thophorus.

arboreus, Worthen, 1873, Geo. Sur. III., vol. 5, p. 53t, Sr. Louis Gr.

(trmiger, see Hydreinnocrinus armiger.

isper, Neek \& Worthen, 1869, Pror. Aead. Nat. Sci. Phil., 1) 150, and Geo. Sur. 111., vol. 5, p. 430, Burling. ton $\mathrm{Gr}$.

bifurcatus, McChesney, 1860, New Pal. Foss., 1. 10, and Trans. Chi. Acad. Sci., vol. 1, 1. 71, Kaskaskia Gr.

ruriniferus, see Coliocrinus cariniferus.

compactilis. Worthen, 1873, Geo. Sur. Ilk., vol. 5 , 1. 536, Kaskaskia Gr.

coxanus, Worthen, 1882, Bull. No. 1, Ill. St. Mus. Nat. Hist., p. 27 , and Geo. Sur. IIl., vol. T, p. 302, Keokuk Gl:

crctssus, see Eupachycrinus crassus. crateriformis, Troost. Not defined.

depressun, see Hyilreionocrinus depressus. discus, see Hydreionocrinus discus.

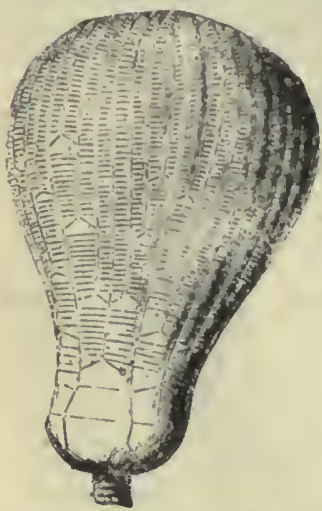

FIg. 44.-Zencrinus: elegans. elegans, Hall, 1858, Geo. Sur. Iоพя, p. 547, Burlington Gr. tlorealis, Yandell of Slinmard, 1847, (Cyathocrinus florealis,) Cont. to Geo. ky., p. 24, Kaskaskia $\mathrm{Gr}$.

formosus, see Eupachyer in us formosus.

intermedi us, Hall, 1858 , Geo.Sur.Iowa, p. 681, Kaskaskia 'Gr. keokık, worthen, 1882, Bull. No. 1, IIl. St. Mus. Nat. Hist., p. $2 S$, and Geo. Sur. Iil., vol. 7, p. 30 , Keokuk Gr.

lyra, see Coliocrimus lyra. magnoliiformis, Owen \& Norwood, 1846, (Cyathocrinus maynoliiformis,) Research Pot. Carb. Rocks Ky., and Geo. Sur. Iowa, p. 68t, Kaskaskiu Gr.

maniformis, Yandell \& Shumard, 18 47 , (Poteriocrinus maniformis,) Cont. 10 Geo. Ky., p. 24, Kaskaskia Gr.

merope, Hall, 1S63, 17 th Kep. N. Y. St. Mus. Nat. Hist., p. 60, and Ohio Pal., vol. 2, p. 178 , Wuverly Gr.

moorii, Whitfeld, 1882, Ann. N. Y. dead. Sci., vol. 2, p. 227, Coul IIpas.

mucrospinus, see Hylreionocrinus mucrospinus.

nodusıs, Waclismuth \& Sprincrer, 1886, Revis. Palneocrinoidea, pt. 3, p. 243, Keokuk Gr.

ovalis, Lvon \& Casserlay, 1858, Am. Jour. Sci., 2d ser., vol. 29, p. 7 l, Kinskaskia Gr. paternua, Hall, 1863, itth Rep. N. Y. St.

Mus. Nat. Hist., p. 59, Wuveriv Gr. perangulatus, White, 1862 , Proc. Bost. Soc: Nat. Hist., vol. 9, p. 11, Burlington Gr. pikensis, IVurthen, 1882. Bull., No. 1, Ill. St. Mus. Nat. Hist., 1. 29, and Geo. Sur. III., vol. 7, p. 304, Burlinuton Gr.

planobrachiutus, Meek \& Worthen, 1860 , Proc. Acad. Nat. Sci. Phill., r. 391, and Geo. Sur. Ill., vol. 2, p. 240, Keokuk Gr. ramosus, Hall, 1858, Geo. Sur. Iowa, p. 548, Burlington $\mathrm{Gr}$. sacculus, White, 1862, Proc. Bost. Soc. Nat.

Hist., vol. 9, ए. 12, Burlington Gr.
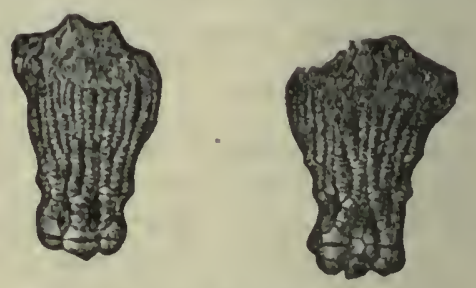

Fia. H16.-\%encrinus splumblerus.

sacenlus var. concinnus, IVliile, 1862, Proc. Bost. Soc. Nat. Hist., vol. 9, p. 12, Burlington Gr. scobina, Meek \& Wortien, 1869, Proc. Acad. Nat. Sci. l'lil., p. 149, and (ieo. Sur. III., vol. 5, p. 426 , Burlington Gr.

scoparius. If all, 1861, Desc. New Crin., p. 6, and Jour. Bost. Soc. Nat. Hist., vol. 7 , I. 305, Burlington Gr.

serratus, Meek \& Wortlien, 1861,

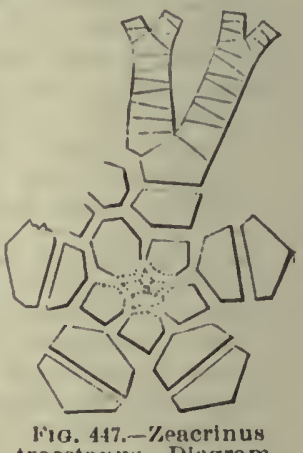

Prortien, 1801, troostums. Diagram.

Proc. Acad. Nat. Sci. Phil., p. 151, and Geo. Sur. Ill., vol. 5, 1. 428, Burlington $\mathrm{Gr}$. 
spinosus, Owen \& Shumard, 1852, (Poteriocrinus spinosus, ) Jour. Acad. Nat. Sci. Phil., vol. 2, p. 91, Kaskaskia Gr.

spinuliferus, Worthen, (in press,) (Poteriocrinus spinuliferus,) Geo. Sur. Ill., vol. 8 , p. 90, Kaskaskia Gr.

stimpsoni, Lyon, 1869, Trans. Am. Phil. Soc., vol. 13, p. 465, Subcarb. subtumidus, see Eupachycrinus subtumidus.

troostanus, Meek \& Worthen, 1860, Proc. Acad. Nat. Sci. Phil., p. 390, and Geo. Sur. Ill., vol. 2, p. 186, Burlington $\mathrm{Gr}$.

wortbeni, Hall, 1858, Geo. Sur. Iowa, p. 683 , Kaskaskia Gr.

\section{SUBKINGDOM MOLLUSCOIDA.}

\section{CLASS BRYOZOA.}

The Bryozoa are small animals that grow in clusters, forming branched or mosslike compound structures. Each animal lives in a separate cell, called a zoœcium, into which it can retract itself, though some connection exists between the animals. -The Flustra or Sea-mats, abundant on the shores of the ocean, and the moss-like encrustations so common on marine shells, are examples. All known Palæozoic Bryozoa were marine, and lived in calcareous cells, forming a mass that is often difficult to distinguish from the true corals.

This calcareous mass or skeleton is called the bryozoum or zoarium. It is found encrusting other objects, or standing on a foot-stalk, with basal attachment, and, in other cases, apparently free. There are rarely any such calcareous partitions in the cell-tubes as abound in the true corals, and the method of reproduction was exclusively gemmiparous, while the true corals were increased by both gemmiparous and fissiparous reproduction.

The animal consists of a bent tube or alimentary canal, having an œsophagus, stomach, and intestine. The two orifices of the canal are situated close together, but the anal opening is beyond the ring of ciliated tentacles that surround the mouth. Thus constituted, the alimentary canal is inclosed in a sac having two openings corresponding to the two extremities of the canal. Generally the upper side of this sac is flexible, and admits of being invaginated, so that when the animal retracts itself into its cell the inverted portion forms a sheath around the tentacles. Ova may be developed in a receptacle attached to the zoccium, called the ocecium, or in an inflation of the surface of the zoarium, called a gonocyst. The gonœcium is a modified zoœcium. The term oœcia is also applied to these-structures. Many Bryozoa have appendicular organs called avicularia and vibracula. The avicularia may be pedunculate, and sway to and fro, or fixed and firmly attached to the zoccium. The vibracula are flexible, bristle-like structures, set in the exeavated summit of a knob-like elevation or blunt spine.

Some naturalists refer the Monticuliporidæ and Stelliporidæ to the Bryozoa, and probably the latter should be so classed on as good grounds as the Fistuliporidæ are referred to the Bryozoa. The Palæozoic Bryozoa are referred to an order called the Gymnolæmata, which are supposed to have had a complete ring of 
tentacles around the mouth. This order has been divided into five suborders, viz.: Chilostomata, Cryptostomata, Trepostomata, Cyclostomata, and Ctenostomata. The families which we recognize are as follows:

Fanily Acanthociadidn.-Acanthocladia, Diplopora, Glauconome, Ichthy. orachis, Ptilopora, Ramipora, Septopora, Synocladia.

Family Amplexolorids.-A mplexopora, A tactopora, Discotry pa, Leptotry pa, Petalotrypa.

Famir, Artirostylins, - Arthroclema, Arthrostylus, Helopora, Nematopora, Nematoporella, Sceptropora.

Family Ascodictronids.-Ascodictyon, Rhopalonaria.

Family Batostomilidos-Anisotrypa, Batostoma (?), Batostomella, Leioclema, Peronopora.

FAmiLy Botrylloporidis-Botryllopora.

FAMiLY BYThoporides.-Rythopora.

Family Ceramoporina. - Aspidopora, Ceramella, Ceramopora, Ceramoporella, Chiloporella, Crepipora, Eridopora, Glossotrypa, Idiotrypa, Lichenalia, Lichenotrypa, Odontotrypa, Petigopora, Phractopora, Pileotrypa, Sagenella, Selenopora, Spatiopora.

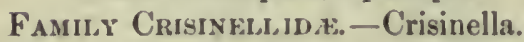

Family Enaldodorida-Diploclema, Enallopora, Protocrisina.

Family Fenestillinds-Archimedes, Clathropora, Coscinella, Coscinium, Coscinotrypa, Evactinopora, Fenestella, Fenestralia, Fenestrapora, Helicopora, Hemitrypa, Isotrypa, Loculipora, Lyropora, Phyllopora, Polypora, Ptilopora, Ptiloporella, Ptiloporina, Reptaria, Reteporina, Seuicoscinium, Semiopora, Tectulipora, Unitrypa.

Family Fistuliporid æ. -Actinotrypa, Buscopora, Callopora, Calloporella, Callotrypa, Chilotrypa, Coelocaulis, Eridopora, Favicella, Fistulipora, Lichenotrypa, Pinacotrypa, Selenopora, Strotopora.

Famil y HeLiotrypid ж-Heliotrypa.

Family Labechindi.-Labechia.

Family Patesciaridid.-Puleschara.

Family Pinaceloporids:-Phacelopora.

Family Ptilonictyoninds-Coscinella, Cyclopora, Cycloporella, Escharoporn, Graptodictya, Heterodictya, Phrenopora, Proutella, I'tiludictya, Ptilotrypa, Streblotrypa, Worthenopora.

Family Riandomisontid.t.-Acanthoclema, Anisotrypa, Bactropora, Cieloconus, Nemataxis, Rhombopora, Tropidopora.

Famili Rhinoporida, - Rhinopora.

Family Sphragioporid Ai-Sphragiopora.

FAmily Stictoporid A.-Acrogenia, Arthropora, Cystodictya, Dichotrypa,

Dicranopora, Eurodictya, Euspilopora, Goniotrypa, Heliotrypa, Intrapora, Pachydictya, Phractopora, Phyllodictya, Prismopora, Rhinidictya, Scalaripora, Stictopora, Stictoporella, Stictoporina, Stictotrypa, Sulcopora, Tæniodictya, Tæniopora, Thamnotrypa.

Family Subretiporid «.-Chainodictyon, Drymotrypa, Subretepora.

Family Thamniscidas-Criscinella, Diplopora, Thamniscus. 
Famil y Theonoide.-Scenellopora.

Family Trematoporid ж.-Acanthoclema, Amplexopora, Atactopora, Atactoporella, Bactropora, Chilotrypa, Diamesopora, Homotrypa, Homotrypella, Nemataxis, Nicholsonella, Orthopora, Trematella, Trematopora, Tropidopora,

Family Tubuliporids.-Berenicea, Clonopora, Cystopora, Hederella, Hernodia, Stomatopora.

Acanthocladia, King, 1849, Ann. and Mag. Nat. Hist., 2d ser., vol. 3, p. 389. [Ety. akantha, spine; klados, branch.] Stem symmetrically and bilaterally branched, more or less on one plane; rarely bifurcating; branches short, simple, occasionally elongated and becoming bilaterally branched; celluliferous on one side only; cell apertures circular and arranged in three or more longitudinal series, separated by dividing ridges. Type $A$. anceps.

americana, Swallow, 1858, Trans. St. Louis Acad. Sci., vol. 1, p. 180, Permian Gr.

fruticosa, Ulrich, (in press,) Geo. Sur. Ill., vol. 8, pl. 65, Up. Coal Meas.

Acanthochema, Hall, 1887, Pal. N. Y., vol. 6, p. 72. [Ety. akantha, spine; klema, twig.] Ramose, solid, cells arising from a central axis; cell apertures oval, in longitudinal parallel rows, about ten on a branch, separated by longitudinal ridges; between the apertures, in the longitudinal direction, there are spiniform nodes. Type A. alternatum.

alternatum, Hall, 1881, (Trematopora alternata,) Bryozoans of the Up. Held. Gr., and Pal. N. Y., vol. 6, p. 72, Up. Held. Gr.

bispinulatum, Hall, 1881, (Callopora bispinulata, ('Trans. Alb. Inst., vol. 10, p. 882, and Pal. N. Y., vol. 6, p. 182, Ham. Gr.

confluens, Ulrich, 1888, (Rhombopora confluens,) Bull. Denison Univ., p. 91, Cuyahoga Shales.

divergens, Hall, 1887. Pal. N. Y., vol. 6, p. 73, Up. Held Gr.

ovatum, Hall, 1887, Pal. N. Y., vol. 6, p. 73, Up. Held. Gr.

scutulatum, Hall, 1881, (Trematopora scutulata,) Trans. Alb. Inst., vol. 10, p. 180, and Pal. N. Y., vol. 6, p. 190, Ham. Gir.

sulcatum, Hall, 1887, Pal. N. Y., vol. 6, p. 192, Ham. Gr.

triseriale, Hall, 1883, (Stictopora triserialis,) Rep. St. Geol. and Pal. N. Y., vol. 6 , p. 74, Up. Held. Gr.

A crogenı, Hall, 1884, Rep. St. Geol. p. 51. [Ety. akros, sharp; genea, growth.] Frond ramose; two branches proceeding from the truncate termination of each preceding one; base of each division obconical, terete above and strongly striated, gradually becoming flattened and celluliferous; margins noncelluliferous; apertures in rows separated by ridges, central range of a pertures the smaller. Type A. prolifera.

prolifera, Hall, 1884, Rep.

St. Geol., p.

52, and Pal.

N. Y., vol.

6, p. 267,

Ham. Gr.

A C T INOTR YPA,

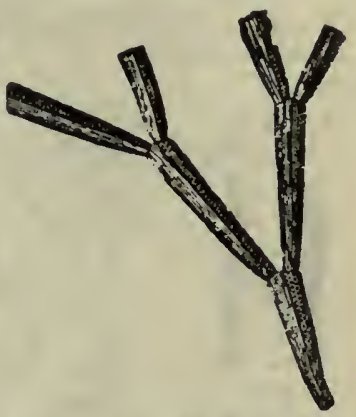

Ulrich, Geo.

Sur. Ill., vol. Fia. 148-Acrogenia prollfera. 8 , p. 386 , (in press.) [Ety. aktin, a ray; tmupa, an opening.] Like Dichotrypa. Cell apertures showing the projecting ends of from eight to ten vertical septalike ridges, that extend down on the inner side of the tubular vestibule nearly or quite to the primitive apertures. Type A. peculiaris.

peculiaris, Rominger, 1866, (Fistulipora peculiaris,) Proc. Acad. Nat. Sci. Phil., p. 10, Keokuk Gr.

Alecto, Lamouroux, 1821, Exposi. Method. It was preoccupied by Leach in the class Echinodermata, when Lamouroux used it, and hence Stomatopora is used in its place.

auloporoides, see Stomatopora auloporoides.

canadensis, see Hederella canadensis.

confusa, see Stomatopora confusa.

frondosa, see Stomatopora frondosa.

inflata, see Stomatopora inflata.

nexilis, see Stomatopora nexilis.

Amplexopora, Ulrich, 1882, Jour. Cin. Soc.

Nat. Hist., vol. 5, p.154. [Ety. amplexus, an encircling; poros, pore.] Ramose; cells of one kind only; walls thin in the axial part of the branches, but thicker in the peripheral region; acanthopores numerous. Type A. cingulata.

affinis, Ulrich, (in press,) Geo. Sur. Ill., vol. 8, pl. 36, Hud. Riv. Gr.

canadensis, Foord, 1883, Cont. to Micropalæontology, p. 17, Trenton Gr. 
cingulata, Ulrich, 1882, Jour. Cin. Soc. Nat. Hist., vol. 5, p. 254, Hud. Riv. Gr.
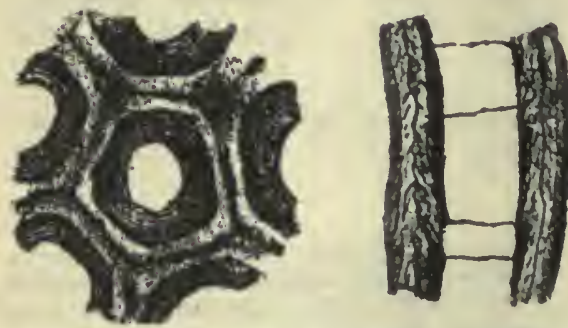

Fı. 449.-Amplexopora clngulatn. Tungentla! sectlou $x 50$ showing wall struclure, und vert]. cial siection $x$ 5n.

discoidea, Nicholson, 1875, (Cluetetes discoidens,) Ohio Pal., vol. 2, p. 206,

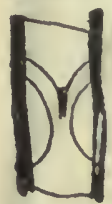

Fig. 450.-Amplex opora robustu $(n)$ nel-sha ped dlaph $\mathrm{r}$ ig m. suplosed (1) be a modifled cystiphrag m.

Hud. Riv, Gr.

pustulosa, Ulrich, (in press,) Geo. Sur. Ill., vol. 8, pl. 36. Hud. Riv. Gr. robusta, Ulrich, 1883, Jour. Cin. Soc. Nat. Hist., vol. 6, p. 82. Hud. Riv. Gr. septoea, Ulrich, 1879, (Atactopora septosa,) Jour. Cin. Soc. Nat. Hist., vol. 2, p. 125, Hud. Riv. Gr.

8 uperba, Foord, 1883, Cont. to Micropalieontology, p. 16, 'I'renton Gr.

winchelli, Ulrich, 1886, 14th Rep. (ieo. Sur. Minn., p. 91, Trenton Gr.

A Nisotry'A, Ulrich, 1883, Jour. Cin. Soc. Nat. Hist., vol. 6, p. 275. [Ety. anisos, unequal; trupa, perforation.] Ramose, hollow, inner side lined with an epitheca; walls of tubes thin in the interior, and thickened exteriorly; no interstitial cells or spiniform tubuli. Type A. nymmetrica.

fistulosa, Ulrich, (in pres*, Geo.Sur, Ill.. vol. 8 , pl. 72, St. Louis Gr.

ramulosa, Ulrich, (in press,) (ieo. Sur. Ill., vol. $\mathrm{S}$, pl. 72, St. Louis Gr.

solida, Ulrich, (in press,) Geo. Sur. Ill., vol. 8 , pl. 72, Kaskaskia Gr.

symmetrica, Ulrich, 1883, Jour. Cin. Soc. Nat. Hist., vol. 6 , p. 76 , Kaskaskia Gr.

Arcinmenes, LeSueur, 1842, (Retepora archimedes,) Am. Jour. Sci., vol. 43, p. 19. [Ety. from its resemblance to the machine for raising water, consisting of a tube rolled in a spiral form around a cylinder, invented by Archimedes. Distinguished from Fenestella by its axis and mode of growth; the tlabelliform expansion acquiring a solid central axis, around which it revolves in an ascending spiral form, spreading equally in all directions. Type A. wortheni. communis, Ulrich, (in press), Genl. Sur. III., rol. 8. pl. 63, Kaskaskia Gr.

compactus, Ulrich,

(ii) press), Geol.

Sur. Ill., vol. 8,

pl. $63, \mathrm{~K}$ askaskia Gr.

distans, Ulriclı. (in press), Geol. Sur. IIl., vol. 8, pl. 63, Kaskaskia G̈r. grandis, Ulrich, (in press), Geol. Sur. Ill., vol. 8, pl. 63, Keokuk Gir.

intermedius, Ul. rich, (in press), Geol. Sur. IIl., vol. $8, \mathrm{pl} .63$,
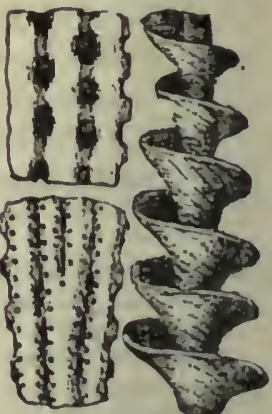

Fia. 451.-Archimedes reversus.

Kaskaskia Gr.

invaginatus, Ulrich, (in press), Geol. Sur. 1ll., vol. 8, pl. 63, Kuskuskia Gr.

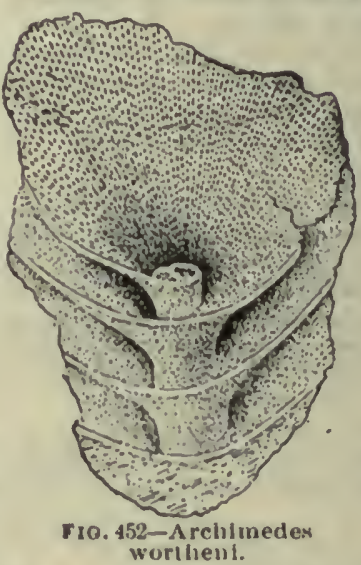

laxis, Hall, 1857, l'roc. A m. Ars'n Ad. Sci., vol, $10, \mathrm{p}$. 176, Kaskaskia Gेr. merk a $n$ us, Hall, 1857. Proc: A in. Ans'n Arl. Sci., vol. 10 , 1. $176, \mathrm{Kas}-$ kaskia Gr. uegligens, U1rich, (in press), Geo. Siur. 111., vul. 8 , pl. 63, Keokuk Gr.

owenanus, Hall, 1857, Proc. Ani. Ak8'1) Ad. Sci., vol. 10, p. 176, Keokuk Gr. perminimus, Ulrich, (in press), Geo. Sur. Ill., vol. 8, pl. 63, Kaskankia Gr.

proutanus, Uİrich, (in press), Geo. Sur. 1ll., vol. 8, l. 63, Kuskaskia Gr.

reversus. Hall, 1858, Geo. Rtp. Iowa, p. 652, Warsaw Gr.

sublaxus, Ulrich, (in press), (ico. Sur. Ill., vol. 8, pl. 63, Kaskaskia Gr.

swallovanus, Hall, 1857, Proc. Am. Ass'n Ad. Sci., vol. 10, p. 176, Kaskaskia $\mathrm{Gr}$.

terebrilormis, Ulrich, (in press), Geo. Sur. Ill., vol. 8, pl. 63, Kaskaskıa Gr.

wortheni, Hall, 1857, Proc. Am. Ass'n Ad. Sci., vol. 10, j. 176, and Geo. Sur. Iowa, p. 651, Warsaw Gir.

Archimedipora, D'Orb., 1850, Prod. de I'al., t. 1 , p. 102 , syn. for Arclimedes. Archimedipora archimedes was too tautological, and by common corsent Archimedes has become the generic name.

archimedes, see Archimedes. 
Arthroclema, Billings, 1862, Pal. Foss., vol. 1, p. 54. [Ety. arthron, joint; klema, twig.] Cylindrical jointed stem, with long, slender-jointed branches; pores oval. Type A. pulchellum.

angulare, Ulrich, (in press), Geo. Sur. Ill., vol. 8, pl. 29, Hud. Riv. Gr.

billingsi, Ulrich, (in press), Geo. Sur. Ill., vol. 8, Trenton Gr.

pulchellum, Billings, 1862, Pal. Foss., vol. 1, p. 54, Trenton Gr. (See p: 329.) spiniforme, see Helopora spiniformis.

Arthronema, Ulrich, 1882, Jour. Cin. Soc. Nat. Hist., vol. 5, p. 160 . The name was preoccupied. See Arthrostylus.

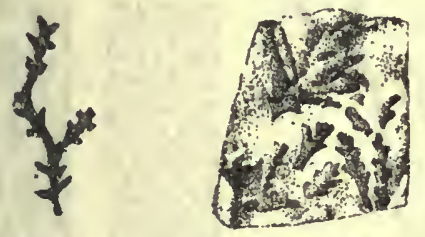

FIG. 453.-Arthropora shafferi.

curtum, see Arthrostylus curtus.

tenue, see Arthrostylus tenuis.

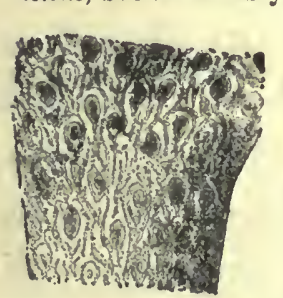

Fig. 454. - Arthropora shafferi. Magnifled section.
A RTIIROPORA, Ulrich, 1882, Jour. Cin. Soc. Nat. Hist., vol. 5, p. 152 . [Ety. arthron, a joint ; poros, perforation.] Zoarium like Stictopora, but in short, jointed, branching segments ; cell apertures subcircular, and surrounded by interstitial pits. Type A. shafferi.

shafferi, Meek, 1872 , (Stictupora shafferi,) Proc. Acad. Nat. Sci., p. 317, and Ohio Pdl., vol. 1, p. 69, Hud. Riv. Gr.

simplex, Ulrich, 1886 , 14th Rep. Geo. Sur. Minn., p. 65, Trenton $\mathrm{Gr}$.

Artirostylus, Ulrich, 1888 Am. Geol, Fra. 45.5.-Arthropora vol. 1, p. 230. [Ety. shatreri. Tangential arthron, joint;

stylos, pillar.] Ramose, composed of subcylindrical segments, swollen at each end, celluliferous on one side, striated on the other; cells hetween elevated lines. Type A. tenuis.

curtus, Ulrich, 1882, (Arthronema curtum,) Jour. Cin. Soc. Nat. Hist., vol. 5, p. 161, Hud. Riv. Gr. tenuis, Ulrich, 1882, (Arthronema tenue,) Jour. Cin. Soc. Nat. Hist., vol. 5, p. 160, Trenton Gr.

Asconlctyon, Nicholson, 1877, Ann. and Mag. Nat. Hist., 4th ser., vol. 19, p. 463. [Fity. askos, leather bottle; dictyon, net.] Organism composite, parasitic, composed of numerous calcareous cells; minutely perforated. Type A. 1usiforme. It is probably a sponge.

fusiforme, Nicholson, 1877, Ann. and Mag. Nat. Hist., 4 th ser., vol. 19 , p. 463 , Ham. Gr.

stellatum, Nicholson, 1877, Ann. and Mag. Nat. Hist., 4th ser., vol. 19, p. 464, Ham. Gr.

Aspidopora, Ulrich, 1882, Jour. Cin. Soc. Nat. Hist., vol. 5, p. 155. [Ety. aspis, shield; poros, perforation.] Thin, free expansions; concentrically wrinkled and striated epitheca on the lower side; cells gradually increasing in size toward the center of the convex expansion; interstitial cells numerous; diaphragms cross both kinds of tubes; spiniform tubuli present. Type A. areolata.

areolata, Ulrich, 1883 , Jour. Cin. Soc. Nat. Hist., vol. 6, p. 164, Utica Slate.

caliculus, James, 1875, (Chetetes caliculus,) Int. Catal. Cin. Foss., p. 1, and Nicholson Struct. and Affin. Montic., p. 165, Utica Slate.

parasitica, Ulrich, 1886, 14th Rep. Geo. Sur. Minn., p. 90, Trenton Gr.

Atactopora, Ulrich, 1879, Jour. Cin. Soc. Nat. Hist., vol. 2, p. 119. [Ety. atactos, without regularity; poros, pore.] Incrusting; surface with monticules or maculæ; cell apertures petaloid, surrounded by rows of blunt spines; interstitial cells in clusters; tube walls inflected; diaphragms present. Type A. hirsuta.

hirsuta, Ulrich, 1879, Jour. Cin. Soc. Nat. Hi-t., vol. 2, p. 120, Hud. Riv. Gr.

maculata, Ulrich, 1879, Jour. Cin. Soc. Nat. Hist., vol. 2, p. 121, Hud. Riv. Gr.

multigranosa, see Atactoporella multigranosa.

mundula, see Atactoporella mundula.

septosa, see Amplexopora septosa.

? subramosa, Ulrich, 1879, Jour. Cin. Soc. Nat. Hist., vol. 2, p. 124, Hud. Riv. Gr.

tenella, see Atactoporella tenella.

Atactoporella, Ulrich, 1883, Jour. Cin. Soc. Nat. Hist., vol. 6, p. 247. [Ety. diminutive of Atactopora.] Incrusting; surface with monticules or maculæ; cell apertures petaloid; interstitial cells numerous; spiniform tubuli and diaphragms. Type A. typicalis.

multigranosa, Ulrich, 1879, (Atactopora multigranosa,) Jour. Cin. Soc. Nat. Hist., vol. 2, p. 122, Hud. Riv. Gr.

mundula, Ulrich, 1879, Atactopora mundula,) Jour Cin. Soc. Nat. Hist., vol. 2, p. 123, Hud. Riv. Gr. 
newportensis, Ulrich, 1883, Jour. Cin. Soc. Nat. Hist., vol. 6, p. 250, Utica Slate.

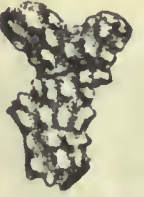

FiG. 456. - Atretoporella newportensis. ortoni, Nicholson, 1874, (Chetetes ortoni,) Quar. Jour. Geo. Soc., vol. 30, p. 513, and Ohio Pal., vol. 2 , p. 211 , Hud. Riv. Gr.

sclucherti, Ulrich, 1883, Jour. Cin. Soc. Nat. Hist., vol. 6, p. 251, Hud. Riv. Gr.

tenella, Ulrich, 1879, (A tactopora tenella,) Jour. Cin. Soc. Nat. Hist., vol. 2, p. 123, Hud. Riv. Gr.

typicalis, Ulrich, 1883, Jour. Cin. Soc. Nat. Hist. vol. 6, p. 248, Utica Slate.

Bactropora, Hall, 1887, Jal. N. Y., vol. 6, p. 193. [Ety. baktron, staff; poros, pore.] Ramose, solid; base tapering, striated ; cells tubular, curved oblique from the center; septa thin, apertures oval, distant near the base, closer above; interspaces granulose. Type B. granistriata. curvata, Hall, 1887, Pal. N. Y., vol. 6, p. 194, Han. Grr.

granistriata, Hall, 1881, ('Trematopora granistriata,) Trans. Alb. Inst., vol. 10, p. 182, and Pal. N. Y., vol. 6, p. 193, Ham. Gr.

simplex, Uirich, (in press,) Geo. Sur. Ill., vol. 8 , pl. 70, Keokuk Gr.

Batostowa, Ulrich, 1852, Jour. Cin. Soc. Nat. Hist., vol. 5, p. 154. [Ety. balos, prickly bush; stoma, mouth.] Rarnose, base expanded; cell apertures ovate or circular, surrounded by a ring-wall; interstitial tubes numerous; spiniform tubuli abundant. Type B. jmplicatum.

fertile, Ulrich, 14th Rep. Geo. Sur. Minn., p. 92 Trenton Gr.

imperfectum, Ulrich, (in press,) Geo. Sur. Ill., vol. 8, pl. 35, Hud. Riv. Gr.

implicatum, Nicholson, 188I, (Monticulipora implicata,) Struct. and Aflin. of Montic., p. 147, Hud. Riv. Gr.

irrasum, Ulrich, 1886, 14th Rep. Geo. Sur. Minn., p. 94, Trenton Gr.

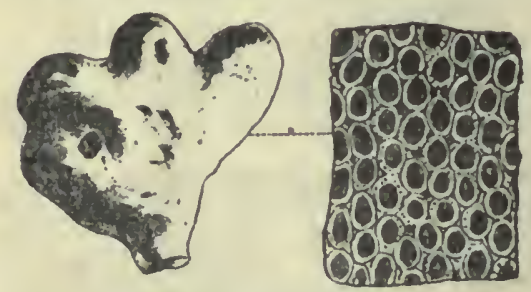

Fig. 457.-Batostoma jamesi. Natural slze and niagnified.

jamesi, Nicholson, 1874, (Chetetes jamesi,) Quar. Jour. Geo. Soc., vol. 30, p. 506, and Ohio Pal., vol. 2, p. 200 Hud. Riv. Gr.

manitobense, Ulrich, (in press,) Micropalæontology, p. 7, Hud. Riv. Gr. (?) ottawense, Foord, 1883, Cont. to Micropalacontology, p. 18, Trenton Gr.

rugosum, Whitfield, 1882, (Fistulipora rugosa,) Geo. Wis., vol. 4, p. 255, Hud. Riv. Gr.

variabile, Ulrich, (in press,) Geo. Sur. Ill., vol. 8, pl. 35. IIud. Riv. Gr.

Batostomblia, Ulrich, 1882, Jour. Cin. Soc. Nat. Hist., vol. 5, p. 154. [Ety. diminutive of Batostoma.] Ramose, smooth, cell apertures small; interstitial cells and spiniform tubuli; walls of tubes in the peripheral region thick. Type B. gracilis.

abrupta, Ulrich, (in press,) Geo. Sur. Ill., vol. 8 , pl. 75, Kaskaskia Gr.
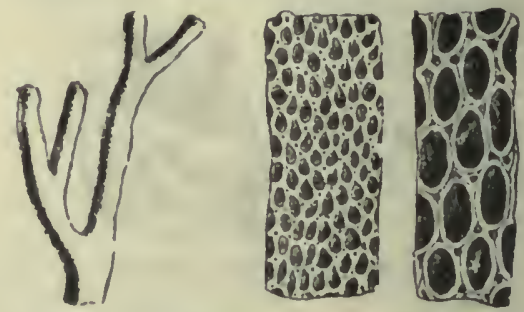

Fro. 458.-Batostomelia grachls. Natural sl\%e and enlarged.

gracilis, Nicholson, 1874, (Chetetes gracilis,) Quar. Jonr. Geo. Soc., vol. 30, p. 504, and Ohio Pal., vol. 2, p. 198, Hud. Riv. Gr.

interstincta, Ulrich, (in press,) Geo. Sur. Ill., vol. 8, pl. 75, St. Louis Gr.

niticlula, Ulrich, (in press,) Geo. Sur. Ill., vol. 8 , pl. 75 , Kaskaskia Gr.

obliqua, Ulrich, (in press,) Geo. Sur. Ill., vol. 8 , pl. 46 , Ham. Gr.

spinulosa, Ulrich, (in press,) Geo. Sur. Ill. vol. 8 , pl. 75 , Kaskaskia Gr.

simulatrix, Ulrich, (in press,) Geo. Sur. Ill., vol. 8, pl. 35, Hud. Riv. Gr.

BereniceA, Lamoureux, 1821, Exp. Meth. des, genres. d. pol., 80. [Fty. mythological name.] Incrusting, composed of a very thin, calcareous, foliaceous base, bearing numerous ovate, distinctly separated cells, not piled; aperture round near the broad anterior end; cells disposed in an obscurely radiated arrangement. Type $B$. diluviama.

insueta, Dawson, 1883, Rep. on Redpath, Mus. No. 2, p. 12, Subcarboniferous.

minnesotensis, Ulrich, 1886, 14th Rep. Geo. Sur. of Minn., p. 58, Trenton (ir. primitiva, Ulrich, 1882, Jouı. Cin. Soc. Nat. Hist., vol. 5, p. 157, Hud. Riv. Gr. vesiculosa, Ulrich, 1882, Jour. Cin. Soc. Nat. Hist., vol. 5, p. 158 , Utica Slate or lower part Hud. Riv. Gr.

Botryllopora, Nicholsun, 1874, Geo. Mag. Lond. n. s., vol. 1, p. 160 . [Ety. botryllos, cluster; poros, pore.]. Incrusting, forming systems of small circular disks, the upper surfaces of which are marked with radiating, cell-bearing ridges; non- 
poriferous space in the center of each disk, round which the radiating ridges occupy a slightly elevated zone. Type B. socialis.
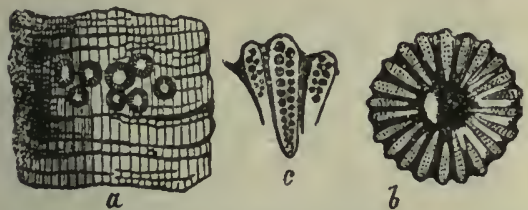

FIG. 459.-Botryllopora socialls. $a$, Group on coral natural size ; $b$, eularged specimen; $c$, enlarged ray to show pores.

socialis, Nicholson, 1874, Geo. Mag. Lond. n. s., vol. 1, p. 160, Ham. Gr.

Buscopora, Ulrich, 1886, Cont. to Am. Pal., p. 22. [Ety. Busk, proper name; poros, perforation.] Zoarium thin, lamellate; incrusting or free; under surface, with a concentrically wrinkled epitheca; zoœeia tubular, short, with subcircular apertures and a faintly elevated border or peristome; posterior margin, with a tooth-like process divided at its termination; accessory cells present; interstitial spaces vesiculose ; zoceial tubes, with diaphragms. Type B. lunata.

dentata, Ulrich, syn. for B. lunata.

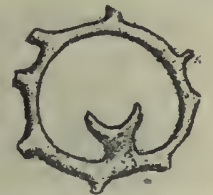

Fig. 460.-Buscopora lunata. Tangenfial sectlon, sloowing aperture or lunarium. lunata, Rominger, 1866, (Fistulipora lunata,) Proc. Acad. Nat. Sci. Phil.,p. 7, and Pal. N. Y., vol. 6, p. 77, Up. Held. Gr.

lunata var. tubulata, Hall, 1887, (Lichenalia lunata var. tubulata,) Rep. St. Geol. for 1885 , pl.

31, and Pal. N. Y., vol. 6. p. 78, Up. Held. Gr.

Bythopora, Miller \& Dyer, 1878, Cont. to Pal. No. 2, p. 6. [Ety. buthos, depths of sea; poros, pore.] Dendroid, branches small, sometimes anastomosing, smooth;

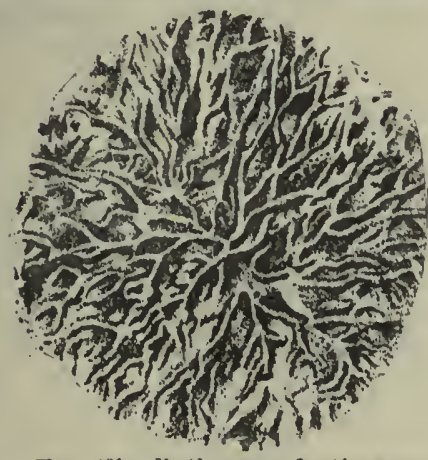

FIG. 461.-Bythopora fruticosa.

cell apertures longer than wide, separated by impressed lines. Type $B$. fruticosa. arctipora, Nicholson, 1875, (Ptilodictya arctipora,) Ann. and Mag., ser. 4, vol. 15 , p. 180 , Utica Slate.

delicatula, (?) instead of Monticulipora delicatula.

fruticosa, Miller \& Dyer, 1878, Cont. to Pal. No. 2, p. 6, Hud. Riv. Gr.

herricki, Ulrich, 1886, 14th Rep. Geo. Sur. Minn., p. 99, Trenton Gr.

nashvillensis, S. A. Miller, 1880, Jour. Cin. Soc. Nat. Hist., vol. 3, p. 143, Trenton Gr. striata, Ulrich, (in press,) MicropaIæontology, p. 10, Hud. Riv. Gr.

C A l L o P O R A, Hall, 1852, Pal. N. Y., vol. 2, p. 144. Fig. 462. - Bythopora [Ety. kallos, beau- nashvlliensis. Magnitiful; poros, pore. $]$ fied.

Ramose, smooth, or tuberculated; cell tubes cylindrical; interstitial cells numerousi; diaphragms numerous, no spiniform tubuli; in tercellular space occupied by septate tubuli. Type C. elegantula. aculeolata, see Colocaulis aculeolata.

? aspera, Hall, 1852, Pal. N. Y., vol. 2, p. 147, Niagara Gr.

bipunctata, Hall, 1884, Rep. St. Geol., p. 15, syn. for Streblotrypa hamiltonensis. bispinulata, see Orthopora bispinulata.

cellulosa, Hall, 1883, Rep. St. Geol., pl. 12, fig. 7-9, Low. Held. Gr.

cervicornis, Hall, 1879, Desc. New Spec. Foss., p. 3, and 11th Rep. Ind. Geo. Sur., p. 238, Niagara Gr.

cincinnatiensis, Ulrich, syn. for Leioclema occidens.

diversa, Hall, 1879, Desc. New. Spec. Foss.,

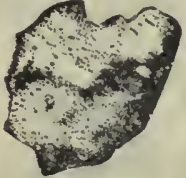

FiG. 463. - Callopora exsul. p. 4, and 11th Rep. Ind. Geo. Sur., p. 239, Niagara Gr.

elegantula, Hall, 1852 , Pal. N. Y., vol. 2, p. 144, Niagara Gr.

? exsul, Hall, 1876, (Alveolites exsul,) 28 th Rep. N. Y. St. Mus. Nat. Hist., p. 115, Niagara Gr.

fistulosa, Hall, 1883, Rep. St. Geol., pl. 12, fig. 1-6, Low. Held. Gr.

florida, Hall, 1852, Pal. N. Y., vol. 2, p. 146, Niagara Gr.

geniculata, Hall, 1887, Pal. N. Y., vol. 6, p. 75, Up. Held, Gr.

hemispherica, see Fistulipora hemispherica. heteropora, see Callotrypa heteropora. hyale, see Colocaulis hyale.

incrassata, see Fistulipora incrassata. incontroversa, Ulrich, 1886, 14th Rep. Geo. Sur. Minn., p. 96, Trenton Gr. internodata, see Callotrypa internodata. irregularis, see Cœlocaulis irregularis. laminata, Hall, 1852, Pal. N. Y., vol. 2, p. 146, Niagara Gr. 
macropora, see Callotrypa macropora. macropora var. signuta, see Callotrypa macropora var. signata.

magnopora, Foerste, 1887, Bull. Denison University, p. 173, Niagara Gr.

minutissima, see Leiclema minutissimum. missouriensis, Rominger, syn. for Leioclema punctatum.

multiseriala, see Callotrypa multiseriata. nodulosa, Nicholson, 1874, (Chetetes nodulosus, Quar. Jour. Geo. Soc., vol. 30 , p. 506, and Ohio Pal., vol. 2, p. 200, Hud. Riv. Gr.

nummiformis, Hall, 1852, Pal. N. Y., vol. 2 , p. 148, Niagara Gr.

oculifera, see Callotrypa oculifera.

olijoensis, Foerste, 1887, Bull. Denison Univ., p. 174, Niagara Gr.

onealli, Jamps, 1875, (Chetetes onealli,)

Int. Catal. Cin. Foss., p. 2, Hud. Riv. Gr. oppleta, Hall, 1887, Pal. N. Y., vol. 6, p. 21, Low. Held. Gr.
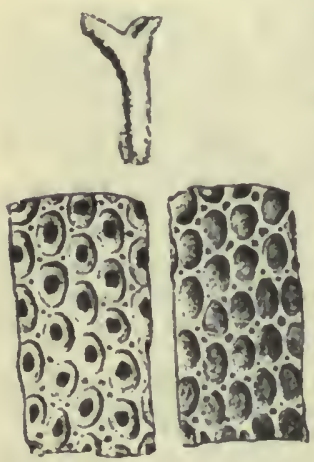

FIG. -464.-Callopors sigll. larloldes. Natural size and magnifled. parasitica, see Fistulipora parasitica.

perelegans, Hall, 1874, 26th Rep. N. Y. St. Mus. Nat.Hist.,p.102, Low. II eld. Gr. ponderosa, $\quad \mathrm{se} \mathrm{e}$ F istull por a ponderosa.

puncta'a, see Leioclema punctatuin.

punctillata, Winchell, 1866, Rep. Low. Penin. Mich., p. 88, Hanı. Gr.

sigillarioides, $\mathrm{Nicholson}$, 1875, (Chetetes sigillarioides,) Ohio Pal., vol. 2, p. 203, Hud. Riv. Gr. singularis, Hall.18i6, 28 th Rep. N. Y. St. Mus. Nat. Hist., p. 115, Niugara Gr. aubnodosa, Ulrich, (in press), Geo. Sirr. Ill., rol. $8, \mathrm{pl}$. 33, Hud. Riv. Gr. subplana, Ulrich, 1882, Jour. Cin. Soc. Nat. Hist. vol. 5, p. 253 , Hud. Riv. Gr. nodulata, 1856, 14th Rep. Geo. Sur. Minı. p. 95, Trenton Gr.

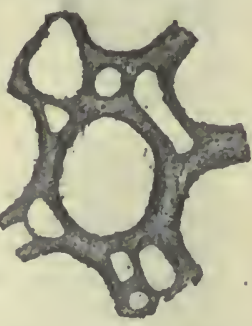

Fio. 465. - Crllopora subnodost. Taugenlul section $\times 50$, showling amalgamation of walls. unispina, ste Cnllotrypa unispina. venusta, see Colocaulis Vнnusta.

Calloporki.la, Uirich, 1882, Jour. Cin. Soc. Nat. Hist., vol. 5, p. 15t. [Ety. diminutive of Callopora.] Thin expansions, epitheca below; tuhes with thick walls containing interstitial cells or angular mesopores; diaphragms and spiniform tubuli. Type C. liarrisi.

harrisi, Ulricl,, 1883, Jour. Cin. Soc. Nat. Hist., vol. 6, p. 91, Hud. Riv. Gr.

nodulosa, Ulrich, (in press,) Geo. Sur. Ill., vol. 8, pl. 33, Hud. Riv. Gr.

Callotrzi'A, Hall, 1857, Pal. N. Y., vol. 6, p. 24. [Ety. kallos, heantiful ; trupa, foramen.] Distinguished from Callopora by having a solid intercellular space, or one occupied witl minute tubuli without septa. Type C. macropora.

heteropora, Hall, 1874, (Callopora heteropora,) 26th Rep. N. Y. St. Mus. Nut. Hist., p. 102, Jow. Helıl. Gr.

internodata, Hall, 1881, (Callopora internodata,) Trans. Alb. Inst., vol. 10, p. 182, and Pal. N. Y., vol. 6, p. 189, Ham. Gr. macropora, Hall, 1874, (Callopora macropora,) 26th Rep. N. Y. St. Mus. Nat. Hist., p. 101, Low. Held. Gr.

macropora var. signata, Hall, 1874, ('Trematopora signata,) 26 th Rep. N. Y. St. Mus. Nat. Hist., p. 104, Low. Held. Gr. multiseriatu, Hall, 1881, (Callopora nuttiseriata,) Bryozoans of the Up. Held. Gr., p. 7, and Pal. N. Y., vol. 6, p. 75, Up. Helil. Gr.

oculifera, Hall, 1879, (Callopora oculifera,) 32d Rep. N. Y. St. Mus. Nat. Hist., p. 155, Low. Held. Gr.

paucipora, Hall, 1887, Pal. N. Y., vol. 6, 1). 23, fig. 21, Low. Ifeld. Gr.

striata, Hall, 1887, Pal. N. Y., vol. 6, p. 26, Low. Held. Gr.

unispina, Hall, 1874, (Callopora unispina,) 26th Rep. N. Y. St. Mus, Nat. Hist., 1). 102, Low. Held. Gr.

Carinopora, Nicholson, 1874, Ann. and Mag. Nat. Hist., 4th ser., vol. 13, and Pal. Prov. Ont., p. 109, synonym for Fenestella.

hindi, Nicholson, 1874, Ann. and Mag. Nat. Hist., 4th ser., vol. 13, and Pal. Prov. Ont., p. 111. Not a good Epecies.

Ceramelua, Hall, 1887, Pal. N. Y., vol. 6, p. 19. [Fity. keramis, imbricated.] Thin, growing from a spreading base, celluliferous on botl sides, tubes oblique; peristomes elevated; maculæ sterile, depressed. 'Type C. seidacea.

scidacea, Hall, 1857, Pal. N. Y., vol. 6, p. 240, Ham. Gr.

Cerajopora, Hall, 1852, Pal. N. Y., vol. 2, p. 168. [Ety. keramis, inbricated like roof tile; poros, pore.] Discoidal, free or attached by the center of the base to foreign bodies; under surface with one or more layers of small, irregular, intercommunicating cells, which do not form tubes; cells large, oblique, imbricating, arranged in a radial manner around the depressed center, communicating with each other and the mesopores by means of remote perforations in their walls; mesopores irregular, short, numerous at the center of the colony, decreasing in number toward the margin. Type C. imbricata. 
agellus, Hall, 1867, 28th Rep. N. Y. Mus. Nat. Hist., p. 120, Niagara Gr.

beani, James, 1885, Jour. Cin. Soc. Nat. Hist., vol. 7, p. 23, Hud. Riv. Gr.

confluens, Hall, 1876,28 th Rep. N. Y. Mus. Nat. Hist., p. 119, Niagara Gr.

explanata, Hall, 1879, Desc. New. Spec. Foss., p. 5, and 11th Rep. Ind. Geo. and Nat. Hist., p. 245, Niagara Gr.

foliacea, Hall, 1852, Pal. N. Y., vol. 2, p. 170, Niagara Gr.

huronensis, Nicholson, 1875, Geo. Mag. n. s., vol. 2 , p. 37 , Ham. Gr.

imbricata, Hall, 1852, Pal. N. Y., vol. 2, p. 169 , Niagara Gr.

incrustans, Hall, 1852, Pal. N. Y., vol. 2, p. 169 , Niagara Gr.

labecula, Hall, 1876, 28th Rep. N. Y. Mus. Nat. Hist., p. 119, Niagara Gr.

labeculoidea, Hall, 1883, Rep. St. Geol., pl. 16, fig. 1-2, and Pal. N. Y., vol. 6, p. 33, Low. Held. Gr.

maculata, Hall, 1874, 26th Rep. N. Y. Mus. Nat. Hist., p. 108, Low. Held. Gr. maxima, Hall, 1874, 26th Rep. N. Y. Mus. Nat. Hist., p. 109, Low. Held. Gr.

nicholsoni, James, 1875, Int. to Catai. Cin. Foss., p. 3, Hud. Riv. Gr.

nothus, Hall, 1879, Desc. New Spec. Foss., p. 6, and 11th Rep. Ind. Geo. and Nat. Hist., p. 244, Niagara Gr.
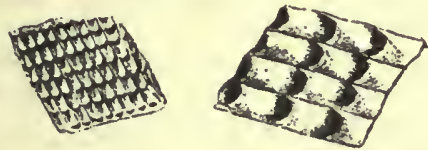

FIG. 466.-Ceramopora ohioensis. Fragment natural size and magnified.

ohioensis, Nicholson, 1875, Ohio Pal., vol. 2, p. 265, Hud. Riv. Gr.

orbiculata, Ringueberg, 1886, Bull. Buf. Soc. Nat. Hist., vol. 5, p. 19. Not properly defined.

parvicella, Hall, 1879, 32d Rep. N. Y. St. Mus. Nat. Hist., p. 158, Low. Held. Gr. raripora, Hall, 1879, Desc. New Spec. Foss., p. 6, and 11th Rep. Ind. Geo. and Nat. Hist., p. 24t, Niagara Gr.

Ceranoporella, Ulrich, 1882, Jour. Cin. Soc. Nat. Hist., vol. 5, p. 156 , and Geo. Sur. Ill., vol. 8, (in press.) [Ety. from Ceramopora.] Incrusting, consisting of one or more thin layers; zoœcial tubes short, apertures rounded, direct or oblique, and more or less nearly isolated by mesopores. Type C. distincta.

distincta, Ulrich, (in press,) Geo. Sur. Ill., vol. 8, pl. 39, Hud. Riv. Gr.

granulosa, Ulrich, (in press,) Geo. Sur. 1ll., vol. 8, pl. 41, Hud. Riv. Gr.

stellata, Ulrich, (in press,) Geo. Sur. Ill., vol. 8, pl. 41, Hud. Riv. Gr.

Ceriopora, Goldfuss, 1826, Germ. Petref. [Ety. kerion, honey-comb; poros, pore.] Not a Palrozoic genus.

hamiltonensis, see Streblotrypa hamiltonensis.
Chainodictyon, Foerste, 1887, Bull. Denison Univ., p. 81. Zoaria flabellate, consisting of narrow inosculating branches, poriferous on one side only, the other with concentric or lunate plications. Fenestrules elliptical; zocecia subtubular in two to four alternating series, their apertures rounded and placed at the bottom of sloping areas. Type C. laxum.

laxum, Foerste, 1887, Bull. Denison Univ., vol. 2, p. 81, Low. Coal Meas.

laxum var. minor, Ulrich, (in press,) Geo. Sur. Ill., vol. 8, pl. 62, Low. Coal Meas. Chiloporella, Ulrich, 1882, Jour. Cin. Soc. Nat. Hist., vol. 5, p. 157. [Ety. cheilos, edge or lip; poros, pore; ella, dim.] Flabellate fronds or compressed branches, from a greatly expanded heavy crust; zoœcial tubes long, very thin-walled, large, and of irregular shape in the axial region; walls much thickened near the surface; apertures ovate, the lunarium conspicuously elevated; mesopores numerous; diaphragms few, generally absent. 'Type C. flabellata.

flabellata, Ulrich, 1879, (Fistulipóra flabellata,) Jour. Cin. Soc. Nat. Hist., vol. 2, p. 28. Hud. Riv. Gr.

Chilotrypa, Ulrich, 1884, Jour. Cin. Soc. Nat. Hist., vol. 7, p. 49. [Ety. cheilos, edge ; trupa, opening.] Ramose, small central tube to which the zoœcia are attached; interstitial spaces vesiculose; diaphragms wanting, or few. Type C. hispida.

lispida, Ulrich, 1884, Jour. Cin. Soc. Nat. Hist., vol. 7, p. 50, Kaskaskia Gr.

ostiolata, Hall, 1852, (Trematopora ostiolata,) Pal. N.Y., vol.2, p. 152, Niagara Gr.

C I A T II R OPORA, Hall, 1852, Pal. N. Y., vol. 2 , p. 159 . [Ety. clathrum, lattice ; poros, pore.] Ret i culate, $\mathrm{u} \mathrm{n}$ iformly poriferous on both sides of the bifoliate fronds; apertures more or less quadr an.gular, regularly arranged in parallel series or obliquely in quincun $\mathrm{x}$ order. Type C. alcicornis. a l cic or $\mathrm{n}$ is, Hall, 1852, Pal. N. Y., vol. 2 , p. 159 , Niagara Gr.

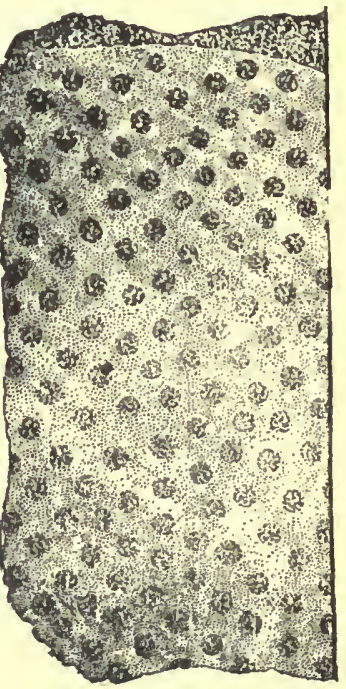

FIG. 467.-Clatliropora frondosa. 
carinata, Hall, syn. for Coscinotrypa cribriformis.

elintonensis, Hall \& Whitfield, 1875, Ohio Pal., vol. 2, p. 113, Niagara Gr.

flabellata, Hall, 1851, Foster \& Whitney's Rep., vol. 2, p. 207, Trenton (Yr.

frondosa, Hall, 1852, Pal. N. Y., vol. 2, p. 160, Niagara Gr.

gracilis, Spencer, 1884, Bull. No. 1, Mus. Univ. St. Mo., p. 54, Niagara Gr.

intermedia, Nicholson \& Hinde, 1874, Can. Jour., p. 156, Niagara Gr.

intertexta, Nicholson, 1874, Geo. Mag. Lond. n. s., vol. 1, p. 125, Corniferous $\mathrm{Gr}$.

strialura, see Coscinium striaturum.

Clonopora, Hall, 1881, Bryzoans of the Up. Held. Gr., p. 20. [Ety. klonos, confusion; poros, pore.] Consisting of an aggregation of elongate, cylindrical, tubular cells, which at intervals become free and turn abruptly outward in an umbelliform expansion, or in alternation; cell apertures expanded or narrowly trumpet-shaped. Type $\mathrm{C}$. semireducta.

fasciculata, Hall, 1887, l'al. N. Y. vol. 6 , p. 289, Ûp. Held. Gr.

incurva, 'Hall, 1881, Bryozoans of Up. Held. Gr., p. 2(1, Up. Held. Gr.

semireducta, Hall, 1881, Bryozoans of Up. Held. Gr., p. 20, Up. Held. (ir.

Cunlocauiss, Hall, 1887, Pal. N. Y., vol. 6, p. 23. [Ety. koilos, hollow; kaulos, stem.] Ramose, structure like Callopora, but growing as hollow stems, the thin expansion lined with a striated epitheca. Type C. venusta.

aculeolata, IIall, 18S1, (Callopora aculeolata,) Bryozoans of Up. Held. Gr., p. 7 , and Pal. N. Y., vol. 6, p. 76, Up. Held. Gr.

hyale, Hall, 1874, (Callopora hyale,) 26th Rep. N. Y. St. Mus. Nat. Híst., p. 100, and Pal. N. Y., vol. 6, p. 76, Up. Held. Gr.

irregularis, Hall, 1S81, (Callopora irregularis,) Bryozoans of the Up. Held. Gr., p. 7, and Pal. N. Y., vol. 6, p. $76, \mathrm{Up}$. Held. Gr.

mediopora, Hall, 1887, Pal. N. Y., vol. 6, p. 23, Jow. Held. Gr.

venusta, Hall, 1874, (Callopora venusta,) 26th Rep. N. Y. Mus. Nat. Hist., p. 101, and Pal. N. Y., vol. 6, p. 23, Low. Held Gr.

Caloconus, Ulrich, Geo. Sur. Ill., vol. 8, p. 402. [Ety. koilos, hollow; konos, cone.] Zoaria simple, hollow, expanding gradually from the striated and sub-acute basal extremity, substance thin; external characters of zocecia as in Rhombopora; primitive portion short; hemisepta well developed. Type C. rhombicus.

granosus, Ulrich, (in press,) Geo. Sur. Ill., vol. 8 , pl. 72, Kaskaskia Gr.

rhombicus, Ulrich, (in press,) Geo. Sur. Ill., vol. 8, pl. 72, St. Louis Gr.
Coscinelua, Hall, 1887, Pal. N. Y., vol. (i, p. 19. [Ety. diminutive of Coscinium.] Distinguished from Coscinium by the presence of minute, angular pits between the cell apertures and around the margins of the fenestrules. Type C. elegantula.

cosciniformis, Nicholson, 1875, (Ptilodictya cosciniformis,) Geo. Mag., vol. 2, p. 35, and Pal. Prov. Ont., p. 80, Ham. Gr.

elegantula, Hall, 1857, Pal. N. Y., vol. 7, p. 239, Ham. Gr.

Cosciniva, Keyserling. 1846, Geognost. beobaclit., p. 192. [Ety. Kookinion, a little sieve.] Lobed, leuf-like expansions, cells on each side, quincuucially arranged; perforated as in Adcona cribriformis; intercellular spaces wide, and permeated with capillary tubuli, which fill up with age; the dividing plate lias a cancellous structure on either side, from the outer cellules of which the large oblique cells, terminating on the free surface, take their rise. Type C. cyclops.

asterium, Prout, 1860, Trans. St. Lonis Acad. Sci., vol. 1, p. 574, Keokuk Gr.

cribriform", see Coscinotrypa cribriformis. cyclops, Keyserling, 1846, Geognost. beobacht., 1. 192, Up. Held. Gr.

elegaus, l'rout, 1860, Trans. St. Louis Acad. Sci., vol. 1, p. 572 , St. Ionis Gr. escharoides, Y'rout, 1860, 'I'rans. St. Louis Acad. Sci., vol. 1, p. 574, (erroneously written eschurense, ) Keokuk Gr.

keyserlingi, Prout, 1858, Trans. St. Louis Acad. Sci., vol. 1, p. 269, Warsa w Gr.

latum, Ulrich, (in press, ) Geo. Sur. Ill., vol. $8, \mathrm{pl}$. 76 , Burlington Gr.

michelini, Prout, 1860, Trans. St. Louis Acad. Sci., vol. 1, p. 573, St. Louis Gr. plumosum, Prout. 1860, Trans. St. Louis Acad. Sci., vol. 1, p. 572 , St. Jouis Gr. saganella, Prout, 1860, Trans. St. Louis Acad. Sci., vol. 1, p. 573, St. Louis Gr. striatum, Hall, 1887, Pal. N. Y., vol. 6, p. 238, Ham. Gr.

striaturum, Hall, 1887, Pal. N. Y., vol. 6, p. $88, \mathrm{Up}$. Held. Gr.

tuberculatum, Prout, 1860, Trans. St. Lou is Acad., vol. 1, p. 573, Keokuk Gr.

wortheni, Prout, 1860, Trans. St. Louis Acad. Sci., vol. 1, p. 571, Keokuk Gr.

CoscinotiyPa, Hall, 1887, Pal. N. Y., vol. 6, p. 19. [Ety. koskinion, a little sieve; trupa, door.] Explanate, celluliferous on botlı sides, with fenestrules at varying distances; surface plicated; cells tubular, arising from a mesotheea; apertures trilobate, denticulated ; intercellular tissue vesiculose. 'lype C. cribriformis.

carinata, Hall, syn. for C. cribriformis.

cribriformis, Prout, 1858, (Coscinium cribriforme,) Trans. St. Louis Acad. Sci., vol. 1, p. 267, Up. Held. Gr.

Crateripora, Ulrich, 1879, Jour. Cin. Soc. Nat. Hist., vol. 2, p. 29, C. erecta, C. lineata, and C. lineata var. expansa, rep- 
resent the basal articulating sockets of Ptilodictya and Arthropora, and are not entitled to rank as species.

Crepipora, Ulrich, (in press,) Geo. Sur. Ill., vol. 8. [Ety. krepis, horseshoe; poros, pore.] Incrusting, lamellate or massive, with a wrinkled epitheca on the lower side, in one case forming regular hollow branches; surface exhibiting, at subregular intervals, maculæ of mesopores, appearing as minutely porous or subsolid elevations or depressions; zocecia very little oblique, the apertures varying from rhomboidal to subpyriform; lunarium well marked in perfect examples; best shown in tangential sections; mesopores usually restricted to the maculæ; diaphragms present. Type C. simulans.

epidermata, UIrich, (in press,) Geo. Sur. Ill., vul. 8, pl. 40, Hud. Riv. Group.

hemispherica, Ulrich, (in press,) Geo. Sur. Ill., vol. 8, pl. 40, Hud. Riv. Gr.

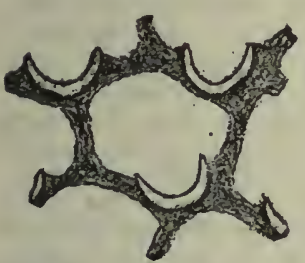

FIG. 468.-Creplpora simulans. Tangential sectlon, showing lunarium. impressa, Ulrich, (in press,) Geo. Sur. Ill., vol. 8 , pl. 40, Hud. Riv. Gr.

simulans, Ulrich, (in press,) Geo. Sur. Ill., vol. 8, pl. 39 and 40 , Hud. Riv. Gr. solida, Ulrich, (in press,) Geo. Sur. Ill., vol. 8 , pl. 40, Hud. Riv. Gr.

Crisina scrobiculata, see Crisinella scrobiculata.

Crisinella, Hall, 1883, Rep. St. Geol. Def., pl. 26. [Ety. from Crisina.] Ramose, solid, celluliferous on one side; cells in oblique, ascendirg rows from the center to the margin of the branch; peristomes prominent; interapertural spaces, with polygonal pits or mesopores. Type C. scrobiculata.

scrobiculata, Hall, 1881, (Crisina scrobiculata,) Bryozoans of the Up. Held. Gr. p. 20, and Pal. N. Y., vol. 6, p. 103, Up. Held Gr.

Cryptopora, Nicholson, 1874, Ann. and Mag. Nat. Hist., 4th ser., vol. 13, and Pal. Prov. Ont., p. 102. Founded upon a cast from the under side of a Fenestella. mirabilis, Nicholson. Not a species.

Cyclorora, Prout, 1860, 'Trans. St. Louis Acad. Sci., vol. 1, p. 574. [Ety. kuklos, circle; poros, pore.] Discoidal, frondescent or incrusting; plates sometimes superposed with subprismatic cells longer than broad, having their sides formed of a minutely porous interstitial network, developed from an epitheca marked by transverse bands more or less concentric, separating the bases of the cells; cells shallow and expanded; interstitial cells. Type C. fungia.

discoidea, see Proutella discoidea. expatiata, Ulrich, (in press,) Geo. Sur. Ill., vol. 8, pl. 68, Keokuk Gr.

fungia, Prout, 1860, Trans. St. Louis Acad. Sci., vol. 1, p. 577, Keokuk Gr.

jamesi, Prout, syn. for Ptilodictya pavonia.

polymorpha, Prout, 1860 , Trans. St. Louis Acad. Sci., vol. 1, p. 578, Kaskaskia Gr.

Cycloporella, Ulrich, (in press,) Geo. Sur. Ill., vol. 8, p. $40 t$. [Ety. dim. of $C y$ clopora.] Thin discoidal expansion; zonecia subtubular, with a succession of superior hemisepta in the vestibular portion ; irregular mesopores abundant; acanthopores of large size, numerous. Type C. spinifera.

perversa, Ulrich, (in press,) Geo. Sur. Ill., vol. 8, pl. 69, Keokuk Gr.

spinifera, Ulrich, (in press,) Geo. Sur. Ill., vol. 8, pl. 69, Keokuk Gr.

Cystodictya, Ulrich, 1882, Jour. Cin. Soc. Nat. Hist. vol. 5, p. 152. [Ety. kuslis, a bladder; dictyon, net.] Zoarium like Stictopora, but with wide interstitial spaces occupied with vesicular tissue. Type C. ocellata

americana, Ulrich, (in press,) Geo. Sur. Ill. vol. 8, pl. 76, Keokuk Gr.

angusta, Ulrich, 1888, Bull. Denison Univ., p. 81, Waverly Gr.

hamiltonensis, Ulrich. (in press), Geo. Sur. Ill., vol. 8 , pl. 43, Ham Gr.

lineata, Ulrich, 1884, Jour. Cin. Soc. Nat. Hist. vol. 7, p. 37, Keokuk Gr.
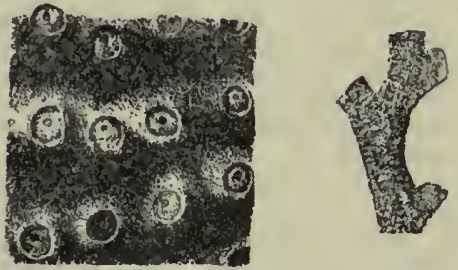

FIG. 469.-Cystodycta ocellata. Natural size and 18 diam.

lineata var. major, Ulrich, (in press,) Geo. Sur. Ill., vol. 8, pl. 76, St. Louis Gr.

lineata, var. stludovici, Ulrich, (in press,) Geo. Sur. Ill., vol. 8, pl. 76, St. Louis Gr.

nitida, Ulrich, (in press,) Geo. Sur. Ill., vol. 8, pl. 76, Keokuk Gr.

ocellata, Ulrich, 1882 , Jour. Cin. Soc. Nat. Hist., vol. 5, p. 170 , Keokuk Gr.

pustulosa, Ulrich, (in press, Geo. Sur. Ill FIG. 470.-Cysto-

vol. 8, pl. 76, Keokuk Tangential secGr.

simulans, Ulrich, 1888 ,

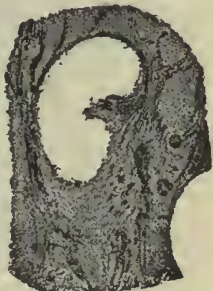
tion slowing lunarium.

Bull. Denison Univ., p. 81, Waverly Gr. zigzag, Ulrich, 1888, Bull. Denison Univ., p. 81 , Cuyahoga Shales. 
Cystopora, Hall, 1881, Bryozoans of Up. Held. Gr., p. 19. [Ety. kustis, bladder; poros, pore.] Simple or branching subcylindrical stipes; cells arising from the axis; circular and subcylindrical below, enlarged ahove the middle and becoming ampullate, turning abruptly outward below the apertures, which are extremely contracted; cell-tubes exposed more than half their lengtb. Type C. geniculata.

geniculata, Hall, 1881, Bryozoans of the Up. Held. Gr., p. 20, and Pal. N. Y., vol. 6, p. 103, Up. Held. Gr.

Di a mesolorA, Hall, 1852, Pal. N. Y., vol. 2, p. 158 , and vol. 6 , p. 19 . [Ety. diame80s, the part between ; poros, opening.] Ramose, hollow, epitheca on inner surface; intercellular space solid; surface like Trematopora. Type D. dichotoma. camerata, Hall, 1883, (Trematopora camerata, Rep. Str. Geol. and P'al. N. Y., vol. 6, p. 72, Up. Held. Gr.

communis, Ulrich, (in press,) Geo. Sur. Il]., vol. 8, pl. 39 and 41 , Utica Slate. constricta, Hall, 1874, (T'rematopora constricta,) 26th Rep. N. Y. St. Mus. Nat. Hist., p. 104, Low. Held. Gr.

dichotoma, Hall, 185:, Pal. N. Y., vol. 2, p. 158, I, w. Held. Gr.

dispersa, Hall, 1879, (Trematopora dispersa,) 32d Rep. N. Y. Nt. MIus. Nat. Hist., p. 150, Low. Held. G̈r.

vaupeli, Ulrich, (in press,) Gen. Sur. Ill., vol. 8, pl. 39 and 41 , Utica Slate.

Dichotryl'A, Ulrich, (in press,) Geo. Sur. IIl., vol. 8, p. 386. [Ety. dicha, double: trupa, opening.] Consisting of large bifoliate expansions; the surface with solid macule; zoncial and minute structure as in Cystodictya. Type D. foliata.

elegans, Ulrich, (in press,) Gco. Sur. Ill., vol. 8 , pl. 76 , St. Ionis Gr.

expatiata, Ulriclı, (in press,) (jeo. Sur. Ill., vol. 8, St. Jouis Gr.

flabellum, Rnminger, 1866, (1)istulipora flabellum,) Proc. Acad. Nat. Sci., I'hil., p. 9, St. Louis Gr.

foliata, Ulrich, (in press,) Geo. Sur. Ill., vol. 8, pl. 42 , Ham. Gr.

grandis, Ulrich, (in press,) Geo. Sur. Ill., vol. 8, pl. 42, Niagara Gr.

intermedia, Ulrich, (in press, Geo. Sur. Ill., vol. 8, pl. 76, St. Louis Gr.

lyroides, Ulrich, (in press,) Geo. Sur. Ill., vol. $8, \mathrm{pl} .77$, St. Louis Gr.

Dicranojolia, Úlrich, 1882, Jour. Cin. Soc. Nat. Hist., vol. 5, p. 166. [Ety, dikranos, two-pointed; poros, pore.]' Zoarium like Stictopora, but distinguished by being composed of ligulate joints, the edges being subparallel to near the upper end, when they diverge and bear two segments; cell-mouths between raised longitudinal lines; no interstitial cells. Type D. internodia.

emacerata, Nicholson, 1875, (Ptilodictya emacerata,) Pal. Obio, vol. 2, p. 26l, Hud. Riv. Gr. fragilis, Billings, 1866,(Ptilodictya fragilis, ) Catal. Sil. Foss. Antic., p. 9, Hud. Riv: Gr.
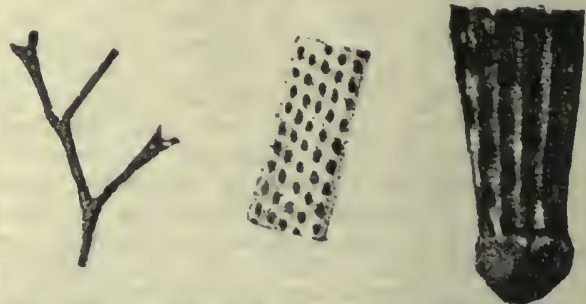

Fro. 471-Dicrauopora Interuodia. Nalural size and magnifiel.

internodia, Miller \& Dyer, 187s, (P'tilodictya internodia,) Cont. to Pal., No. 2, p. 7, Hud. Riv. Gir.

lata, Ulrich, 1882, Jour. Cin. Soc. Nat. Hist., vol. 5, p. 166, Hud. Riv. Gr.

nitidula, Billings, 1866, (Ptilodictya nitidula,) Catal. Sil. Foss. Antic., p. 9, Hud. Riv. Gr.

trentonensis, Ulrich, 1882, Jour. Cin. Soc. Nat. Hist., vol. 5, p. 167, Trenton (ir.

Diploclema, Ulrich, (in press,) (ico. Sur. Ill., vol. 8, p. 368. [isty.diploos, double; klema, twig.] Ranose, ovate in tross section; zoccia tubular, long, appar. ently moniliform proximally; sepurated internally by an axiul lamina, from which they gradually diverge to open on the two sides of the compressed branches; apertures prominent, isolated, somewliat constricted and circular; external wall thin. Type D. trentonense. trentomense, UIrich, (in press,) Geo. Sur. Ill., vol. 8, pl. 53, Trenton Gr.

Dupiopora, Young if Young, 1875, I'roe. Nat. Hist. Soc. Glasgow. [Ety. diploos, double; poros, pore.] Very slender straight stems, throwing ofl a few lateral branches of equal dimensions; obverse or poriferous side, with two ranges of \%oucia apertures, and moderately developed merlium keel; reverse striated. Type D. marginalis.

bifurcata, Ulrich, (in press,) Geo. Sur. Ill., vol. 8, pl. 62, Kaskaskia (ir.

biserialis, Ulrich, (in press,) Geo. Sur. Ill., vol. 8, pl. 62, Low. Coal Meals.

Discotruy' Ulrich, 1882, Jour. Cin. Soc. Nat. Hist., vol. 5, p. 155. [Ety. diskos, quoit; trupa, opening.] Free, thin, circular expansions; celis rhomboidal or hexagonal; low monticules, with clusters of large cells present; no interstitial cells or spiniform tubuli. Type $\mathrm{D}$. elegans.

?devonica, Ulrich, 1856, Cont. to Am. Pal., p. 25, Up. Held. Gr.

elegans, Ulrich, 1879, (Chetetes elegans,) Jour. Cin. Soc. Nat. Hist., vol. 2, p. 130, Hud. Riv. Gr.

ENallopora, D'Orbigny, 1850, I'rodr. d. Paléont., t. 1, p. 22. [Ety. enallos, changed ; poros, pore.] Small bifurcating branclses, without connecting bars; cell-rnouths 
prominent on each side, opening laterally and alternately. Type E. perantiqua.

cinctosá, Ulrich, 1882, (Mitoclema cinctosa, Jour. Cin. Soc. Nat. Hist., vol. 5, p. 159, Trenton Gr.

perantiqua, Hall, 1847, (Gorgonia peràntiqua,) Pal. N. Y., vol. 1, p. 76, Trenton Gr.

Eridopora, Ulrich, 1882, Jour. Cin. Soc. Nat. Hist., vol. 5, p. 137. [Ety. eridos, in dispute; poros, pore.] Zoarium thin, incrusting; cell-mouths oblique, ovate, or subtriangular, one side more prominent than the other, surrounded by angular interstitial cells, which do not form tubes, and may be either open or closed; intertubular spaces vesicular. Type E. macrostoma. Should this genus prove to be founded upon reliable characters, then many of the parasitic species now placed with Fistulipora will be referred to it.

macrostnma, Ulrich, 1882, Jour. Cin. Soc.

Nat. Hist., vol. 5, p. 137, Kaskaskia Gr. minima, Ulrich, 1886, Cont. to Am. Pal., p. 21, Up. Held. Gr.

punctifera, Ulrich, 1882, Jour. Cin. Nat. Hist., vol. 5, p. 138, Kaskaskia Gr.

Eschara, Lamarck, 1801, Syst. An. sans Vert. [Ety.eschara, scar.] Not American Palæozoic.

? concentrica, Prout, Trans. St. Louis Acad. Sci., vol. 1, p. 234, Coal Meas. Not recognized.

ovatipora, Troost, 1840, 5th Geo. Rep. Tenn. Low. Sil. Not recognized.

reticulata, Troost, 1840 , 5th Geo. Rep. Tenn. Low. Sil. Not recngnized.

? tuberculata, Prout, 1858, Trans. St. Louis Acad. Sci., vol. 1, p. 234. Coal Meas. Not recngaizpd.

Escharopora, Hall, 18t7, Pal. N. Y., vol. 1, p. 72. [Ety. eschara, scar ; poros, pore.] Cylindrical, solid, tapering above, expanded and root-like below; cells oval, inclosed in a rhomboid, by elevated oblique lines; tubes radiating from an imaginary axis. Type E. recta.

angusta, Hall, 1879, Desc. New Spec. Foss., p. 6, and 11th Rep. Ind. Geo. and Nat. Hist., p. 245, Niagara Gr.

lirata, see Ptilodictya lirata.

nebulosa, see Ptilodictya nebulosa.

recta, Hall, 1847, Pal. N. Y., vol. 1, p. 73 , Trenton Gr.

recta var. nodosa, Hall, 1847, Pal. N. Y., vol. 1, p. 73, Trenton Gr.

tenuis, see Phænopora tenuis.

Eurydictya, Ulrich, (in press,) Geo. Sur. Ill., vol. 8, p. 389. [Ety. eurys, broad; dictyon, a net.] Broad, simple, or irregularly divided, bifoliate expansions, without nonporiferous parallel margins; surface with more or less conspicuous, small, solid maculæ or monticules; zoœcial structure very much as in Sulcopora, the differences being of small importance, and due to zoarial habit.

Type E. montifera. Syn. (?) for Phænopora.

calhounensis, Ulrich, (in press,) Geo. Sur. Ill., vol. 8, pl. 30, Trenton Gr.

montifera, Ulrich, (in press,) Geo. Sur. Ill., vol. 8, pl. 30, Hud. Riv. Gr.

sterlingensis, Ulrich, (in press,) Geo. Sur. Ill., vol. 8, pl. 30, Hud. Riv. Gr.

Euspilopora, Ulrich, (in press,) Geo. Sur. Ill., vol. 8, p. 389. [Ety. euspilos, full of dots; poros, pore.] Small, bifoliate, lobate or irregularly dividing branches; cell apertures subcircular, arranged between longitudinal spinous ridges at the center of the stipe; at intervals several short oblique rows of cells extend outward from the central rows to near the margins of the frond; these alternate with concave nonporiferous but finely granular spaces, which do not extend out as far as the celluliferous lobes, and which cause the edges of the frond to be serrate; internally a vertical row of shellow vesicles behind the vestibular portion of the zocecia; all the remaiuing interspaces traversed by numerous minute tubuli. Type E. serrata. Syn. (?) for Stictopora.

barrisi, Ulrich, (in press,) Geol. Sur. Ill., vol. 8 , pl. 43, Ham. Gr.

serrata, Ulrich, (in press,) Geo. Sur. Ill., vol. 8 , pl. 43 , Ham. Gr.

Evactinopora, Meek \& Worthen, 1865, Proc. Acad. Nat. Sci. Phil., p. 165. [Ety. evactinos, with beautiful rays; poros, pore.] Free, consisting of four or more vertical leaves which radiate from an imagiuary axis; rays thin, celluliferous on both sides; interstitial spaces occupied by vesicular cells, filled with sclerench y ma, which is traversed by canals. Type E. radiat:. Fig. 472.-Evacgrandis, Meek \& Worthen, tiioporagrau1868, Geo. Sur. Ill., vol. dis. Pores 2 3 , p. 503, Burlington Gr.

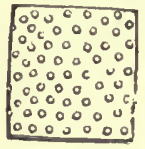

FIG. 473.-Evactinopora radi. a!a. Pores 2 diam.

sexradiata, Meek \& Worthen, 1868, Geo.

Sur. Ill., vol. 3, p. 502, Burlington Gr.

Favicella, Hall, 1887, Pal. N. Y., vol. 6, p. 19. [Ety. favus, honey-comb; ellus, diminutive.] Fiee or incrusting, thin expansion; apertures inclosed in polygonal vestibular areas, similar to selenopora; intercellular surface occupied by minute mesoporfs; structure vesiculose. Type F. inclusa.

inclusa, Hall, 1881, (Thallostigma inclusa,) Trans. Alb. lust., vol. 10, p. 188, and Pal. N. Y., vol. 6, p. 234, Ham. Gr. 
Fenestella, Lonsdale, 1839, Murch, Sil. Syst. [Ety. fenestella, little window.] Zoarium, flattened or infundibuliform, composed of rays radiating from a base and uniting laterally by dissepiments, so as to form a net-work, the meshes of which are usually oblong; inner surface of rays rounded and striated, and without cells; cells on the outer side of the rays in two rows, one on eacl, side of a median ridge; dissepiments without cells. Type F. antiqua. acculis, see Unitrypa acaulis.

acmea, Hall, 1876, 28tlı Rep. N. Y. Mus.

Nat. Hist., p. 124, Niagara Gr.

aculeata, see Poly pora aculeata.

acuticosta, Roerner, 1860, Sil. Fauna West.

Tenn., p. 30, Niagara Gr.

adnatu, see Polypora adnata.

adornata, Hall, 1887, Pal. N. Y., vol. vi, p. 66, Low. Held. Gr,

adraste, Hall, 1883, Rep. St. Geol., pl. 20, fig. 20-22, Low. Held. Gr.

sequalis, Hall, 1881, Bryozoans of the Up. Held. Gr., p. 31, and Pal. N. Y., vol. 6 , p. 112 , Up. Held. Gr.

resyle, Hall, 1883, Rep. St. Geol., pl. 19, tig. $11-13$, and $\mathrm{Pal}$. N. Y., vol. 6, p. 46 , Low. Held. Gr.

albida, Hall, 1887, 6th Ann. Rep. Geo. N. Y., p. 48, Waverly Gr.

albida var. richfieldensis, Ulrich, 1888 , Bull. Denison Univ., p. 66, Waverly $\mathrm{Gr}$.

althrea, Hall, 1883, Rep. St. Geol., pl. 19, fig. 17-19, and Pal. N. Y., vol. 6 , p. 48 , Low. Held. Gr.

ambigua, see Loculipora ambigua.

angulata, Hall, 1881, Bryozoaus of the Up.

Held. Gr., p. 28, Up. Held. Gr.

angustata, Hall, 1884, 36th Rep. N. Y. St.

Mus. Nat. Hist., p. 60, Ham. Gr.

anonyma, Hall, 1881, Bryoznans of the

Up. Held. Gr., p. 34, Up. Held. Gr.

aperta, Hall, 1857, 6th Ann. Rep. St.

Geol. N. Y., p. 58, Waverly Gr.

arctica, Salter, 1855, Belcher's Last Arctic Voyage, vol. 2, p. 385, Carboniferous.

arta, see Polyuora arta.

aspectans, Hall, 1884, 36th Rep. N. Y. St.

Mus. Nat. Hist., p. 59, Ham. Gr.

assita, Hall, 1884, 36th Rep. N. Y. St. Mus. Nat. Hist., n. 64, Ham. Gr.

banyana, Prout, 1859, Trans. St. Louis Acad. Sci., vol. 1, p. 450, Warsaw Gr.

bellistriata, Hall, 1879, Desc. New Spec. loss., p. 7, and $11 \mathrm{th}$ Rep. Ind. Geo. and Nat. Hist., p. 252, Niagara Gr.

bicornis, Spencer, 1884, Bull. No. 1, Mus. Univ. St. Mo., p. 55, Clinton Gr.

bifurca, Ulrich, 1886, Cont. to Am. l'al., p. 6, Up. Held, Gr.

bifurcata, Prout, 1866, Trans. St. Lonis Acad. Si.i., rol. 2, p. 411, Ham. Gr.

bigeneris, Ulrich, 1886, Cont. to Am. Pal., p. 11, Up. Held. Gr.

biimbricata, Hull, 1881, Bryozoans of the Up. Held. Gr., p. 31, and Pal. N. Y., vol. 6, p. 122, Up. Held. Gr. biserialis, see Hemitrypa biserialis.

biseriata, Hall, 1881, Bryozoans of the Up. Held. Gr., p. 25, and Pal. N. Y., vol. 6, p. 113, Up. Held. Gir.

biserrulata, Hall, 1881, Bryozoans of the Up. Held. Gr., p. 30, and I'al. N. Y., vol. 6 , p. 128 , Up. Held. Gr.

brevilinea, Hall, 1854, 36th Rep. N. Y. St. Mus. Nat. Hist., p. 70, Ham. Gr.

brevisulcata, see Polypora brevisulcata.

burlingtonensis, Ulrich, (in press,) Geo. Sur. Ill., vol. 8, pl. 49. Burlington fir.

cavernosa, Ulrich, 1588, Bull. Denison Univ., p. 69, Waverly Gr.

celsipora, see Polypora celsipora.

celsipora var. minima, see Polypora celsipora var. minima.

celsipora var. minor, see Polypora celsipora var. minor.

cestriensis, Ulrich, (in press,) Geo. Sur. Ill., vol. 8, pl. 51, Kaskaskia Gr.

cinctuta, Hall, 1884,36 th Rep. N. Y. St. Mus. Nat. Hist., p. 62, Ham. Gr.

cingulats, Ulrich, (in press,) Geo. Sur. Ill., vol. 8. pl. 52, Keokuk (ir.

clathrata, Hall, 1887, Pal. N. Y., vol. 6, p. 117, Up. Held. Gr.

cleia, Hall, 1883, Rep. St. Geol., pl. 20, fig. 14-15, Low. Held. Gr.

compacta, see Poly pora compacta.

compressa, Ulrich, (in press,) Geo. Sur. Ill., vol. 8, pl. 50, Keokuk Gr.

compressa, var. nododorsalis, Ulrich, (in press.) Geo. Sur. Ill., vol. 8, pl. 50, Keokuk Gr.

compressa, see Polypora compressa.

conferta, Hall, 1879, Desc. New Spec. Foss., p. 7, and 11th Rep. Ind. Cico., and Nat. Hist., p. 252, Niagara Gir.

confertipora, Hall, 1887, Pal. N. Y., vol. 6. p. 108, Up. Held. Gr.

conjunctira, see Isotrypa conjunctiva.

coronis, Hall, 1883, Rep. St. (icol., pl. 21, fig. 10-13, Low. Held. Gr.

corticata, Prout, 1858, Trans. St. Louis Acad. Sci., vol. 1, p. 231, Coal Meas.

crebripora, Hall, 1874, 26th Rep. N. Y. St. Mus. Nat. Hist., p. 25, Low. Held. Gr.

criturosa, Hall, 1852, Pal. N. Y., vol. 2, p. 166, Niagaru Gr.

cribrosa, see Heunitrypa cribrosa.

cultellata, see Polypora cultellata.

cultrata, Hall, 1881, Bryozoans of the Up. Held. Gr., p. 29, and Pal. N. Y., vol. 6, p. 119, Up. Held. Gr.

curvata, Hall, 1884, 36th Rep. N. Y. St. Mus. Nat. Hist., p. 69, Ham. Gr.

curvijunctura, Hall, 1881, Bryozoans of the Up. Held. Gr., p. 29, and Pal. N. Y., vol. 6, p. 107, Up. Held. Gr.

cylindracea, Hall, 1881, Bryozoans of the Up. Held. Gr., p. 2t, Up. Held. Gr.

davidsoni, Nicholson, 1875, Geo. Mag., vol. 2 , n. s., p.36, Ham. Gr. 
delicata, Meek, 1871, Proc. Acad. Nat. Sci. Phil., vol. 23, p. 159, and Ohio

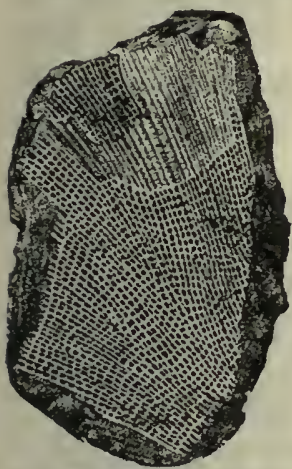
Pal., vol. 1, p. 273, Waverly Gr. de p ressa, Hall, 1881, Bryozoans of the Up. Held. Gr., p. 30, and Pal. N. Y., vol. 6 , p. 111 , Up. Held. Gr.

dilata, Prout., 1866, Trans. St. Louis Acad. Sci., vol. 2, p. 411, Ham. Gr. dispanda, Hall, 1887, Pal. N. Y., vol. 6 , p. 114 , Up. Held. Gr.

FIG. 474.-Fenestella delicata. Part of a frond.

call 1852

distans, see Polypora distans.

\section{4, Niagara Gr.}

elegantissima, Eichwald, 1860, Lethæa Rossica, p. 364, Up. Coal Meas.

elegantissima, Hall, 1881. This name was preoccupied, but see Unitrypa elegantissima.

elevatipora, Ulrich, (in press,) Gieo. Sur. IIl., vol. 8, pl. 51, Kaskaskia Crr.

elongata, see Polypora elongata.

emaciata, Hall, 1884, 36th Rep. N. Y. St.

Mus. Nat. Hist., p. 68, Ham. Gr.

erectipora, Hall, 1881, Bryozoans of the Up. Held. Gr., p. 33, and Pal. N. Y., vol. 6, p. 118, Up. Held. Gr.

eudora, see Polypora eudora.

exigua, Ulrich, (in press,) Geo. Sur. Ill., vol. 8, pl. 51, Warsaw Gr.

eximia, Winchell, 1866, Rep. Low. Penin. Mich., p. 92, Ham. Gr.

exornata, Hall, 1884, 36th Rep. N. Y. St. Mus. Nat. Hist., p. 67, Ham. Gr.

fastigata, see Unitrypa fastigata.

favosa, see Hemitrypa favosa.

filiformis, Nicholson, 1874, Geo. Mag., vol. 1 , n. s., p. 199, Up. Held. Gr. The superficial network of some species of Unitrypa.

filistriata, Ulrich, (in press,) Geo. Sur. Ill., vol. 8, pl. 49, Burlington Gr.

filitexta, Winchell, 1866, Rep. Low. Penin. Mich., p. 92, Ham. Gr.

fistulata, see Polypora fistulata.

flabellata, Phillips, 1836, Geo. York, pt. 2, p. 198, Coal Meas., or Permian. Not American. (?)

flabelliformis, see Polypora flabelliformis.

flexuosa, Ulrich, (in press,) Geo. Sur. Ill., vol. 8, pl. 51, Kaskaskia Gr.

foliata, Ulrich, 1888, Bull. Denison Univ., p. 67 , Waverly Gr.

funicula, Ulrich, (in press,) Geo. Sur. Ill., vol. 8 , pl. 51, Keokuk Gr.

gracilis, see Subretepora gracilis.

granifera, Hall, 1881, Bryozoans of the Up. Held. Gr., p. 33, and Pal. N. Y., vol. 6 , p. 125 , Up. Held. Gr.

granilinea, see Polypora granilinea. granulosa, Whitfield, 1878, Ann. Rep. Geo. Sur. Wis., p. 68, and Geo. Sur. Wis., vol. 4, p. 252, Hud. Riv. Gr.

hemitrypa, Prout, 1859, Trans. St. Louis Acad. Sci., vol. 1, p. 444, Warsaw Gr.

herrickana, Ulrich, 1888, Bull. Denison Univ., p. 63, Waverly Gr.

hestia, Hall, 1883, Rep. St. Geol., pl. 20, fig. 12-13, Low. Held. Gr.

hexagonalis, see Polypora hexagonalis.

hexagonalis var. foraminulosa, see Polypora hexagonalis var. foraminulosa.

idalia, Hall, 1874, 26th Rep. N. Y. St. Mus. Nat. Hist., p. 95, Low. Held. Gr. idothea, see Polypora idothea.

iniequalis, Ulrich, (in press,) Geo. Sur. Ill., vol. 8, pl. 52, Coal Meas.

inflexa, Hall, 1884, 36th Rep. N. Y. St. Mus. Nat. Hist., p. 64, Ham. Gr.

intermedia, Prout, 1858, Trans. St. Louis Acad. Sci., vol. 1, p. 231, Cóal Meas.

interrupta, Hal!, 1881, Bryozoans of the Up. Held. Gr., p. 32, and Pal. N. Y., vol. 6, p. 123, Up. Held. Gr.

junceus, Hall, 1883, Rep. St. Geol., pl. 20, fig. 16-18, Low. Held. Gr.

lavistriata, see Polypora lævistriata.

largissima, see Poly pora largissima.

lata, see Unitrypa lata.

latijunctura, Hall, 1881, Bryozoans of the Up. Held. Gr., p. 31, and Pal. N. Y., vol. 6, p. 128 , Up. Held. Gr.

latitruncata, Hall, 1884, 36th Rep. N. Y. St. Mus. Nat. Hist., p. 58, Ham. Gr.

lavinodata, see Polypora lavinodata.

limbata, Foerste, 1887, Bull. Denison Univ., vol. 2, p. 83, Low. Coal Meas.

lineanoda, Hall, 1881, Bryozoans of the Up. Held. Gr., p. 22, Up. Held. Gr.

lunulata, Hall, 1881, Bryozoans of the Up. Held. Gr., p. 31, and Pal. N. Y., vol. 6, p. 121, Up. Held. Gr.

lyelli, Dawson, 1868, Acad. Geol.; p. 288, Subcarboniferous.

magnifica, Nicholson, 1874, Geo. Mag., vol. 1. n. s., p. 197, Up. Held. Gr.

marcida, Hall, 1884, 36 th Rep. N. Y. St. Mus. Nat. Hist., p. 57, Ham. Gr.

marginalis, Nicholson, 1874, Geo. Mag., vol. 1, n. s., p. 197, Up. Held. Gr.

marginata, McCoy, 1862, Carb. Foss. of Ireland, p. 206, Up. Coal Meas. Not American. (?)

meekana, Ulrich, 1888, Bull. Denison Univ., p. 64, Waverly Gr.

microtrema, D’Orbigny, 1850, Prodr. d. Paléont. Not properly defined.

mimica, Ulrich, (in press,) Geo. Sur. Ill., vol. 8 , pl. 52, Low. Coal. Meas.

modesta, Ulrich, (in press,) Geo. Sur. Ill., vol. 8 , pl. 52, Low Coal Meas.

multiplex, Hall, 1884, 36th Rep. N. Y. St.

Mus. Nat. Hist., p. 57, Ham. Gr.

mulitiporata var. lodiensis, Meek, 1875,

Ohio Pal., vol. 2, p. 274 , Waverly Gr.

multispinosa, Ulrich, (in press,) Geo. Sur.

Ill., vol, 8, pl. 50, Keokuk Gr.

mutabilis, see Polypora mutabilis.

nervata, see Ptiloporella nervata. 
nervia, see Unitrypa nervia.

nervia var. constricla, see Unitrypa nervia var. constricta.

nexa, see Polypora nexa.

nodosa, Prout, 1866, Trans. St. Louis Acad. Sci., vol. 2, p. 410, Ham. Gr.

norwoodana, Prout, 1858, Trans. St. Louis Acad. Sci., vol. 1, p. 233, Coal Meas.

oxfordensis, Ulrich, syn. for F. granulosa. papillata, Hall, syn. for Polypora paxillata.

parallella. Hall, 1881, Bryozoans of the Up. Held. Gr., p. 26, and Pal., N. Y., vol. 6 , p. 107, Up. Held. Gr.

peculiaris, Hall, 1883, Rep. St. Geol., pl. 33, fig. 19-21. Up. Held. Gr.

parvulipora, Hall, 1876, 2Sth Rep. N. Y. St. Mus. Nat. Hist., p. 123, Niagara Gr.

patellifera, Ulrich, 1886, Cont. to Pal., p. 8, Up. Held. Gr.

paxillata, see Polypora paxillata.

perangulata, see Polypora perangulata.

perelegans, Meek, 1872, Pal. E. Nebraska, p. 153, Coal Meas.

perforata, see Loculipora perforata.

permarginata, Hall, 1881, Bryozoans of the Up. Held. Gr., p. 30, and I'al. N. Y., vol. 6, p. 127 , Up. Held. Gr.

perminuta, Ulricl, (in press, Geo. Sur. Hll., vol. 8, pl. 52, Low. Coal Meas.

pernodosa, see Unitrypa pernodosa.

perplexa, Hall, 1881, Bryozoans of the Up. Held. Gr., p. 33, and Pal. N. Y., vol. 6 , p. $130, \mathrm{U}$. Held. (ir.

pertenuis, Hall, 1879, Desc. New Spec. Foss., 13. 6, and 11th Rep. Geo. Ind. and Nat. Hist., p. 25l, Niagara Gir.

pertenuis, Hall, 1881. The name was preoccupied, see F. proutana.

perundata, see l'olypora perundata.

perundulata, see Reteporina perundulata. philia, Hall, 18s3, Rep. St. Geol., pl. 20, fig. 9-11, Low. Held. Gr.

planiramosa, Hall, 1883, Rep. St. Geol., pl. 18, fig. 14-18, syn. for Polypora eompressa.

planiramosa, Hall, 1884,36 th lRep. N. Y. St. Mus. Nat. Hist., p, 65, Ham. Gr.

plebeia, McCoy, 1862, syn. Carb. Foss. Ireland, p. 203, Up. Coal Meas.

plumosa, see Hemitrypa plumosa.

popeana, Prout, 1853, Trans. St. Louis Acad. Sci., vol. 1, p. 229, Permian Gr. porosa, see Polvpora porosa.

pracursor, see Unitrypa praccursor.

prisea, Lonsdale, 1839, Murch. Sil. Syst., p. 178, Clinton Gr.

proceritas, Hall, 1887, Pal. N. Y., vol. 6, p. 115, Up. Held. Gr.

prolixa, Hall, 1879, Dese. New Spec. Foss., p. 8, and 11th Rep. Ind. Geo. and Nat. Hist., p. 253, Niagara Gr.

propria, see Polypora propria.

proutana, S. A. Miller, 1882, 2d Ed. Am. Pal. Foss., p. 291, Up. Held. Gr. Proposed instead of $F$. pertenuis, Hall, 1881, Bryzoans of the Up. Held. Gr., p. 29 , which was preoccupied. pulchella, Ulrich, 1886, Cont. to Am. P'al., p. 9, Up. Held. Gr.

puncto-striata, Hall, 1876, 28th Rep. N. Y. St. Mus. Nat. Hist., p. 68, Niagara Gr.

quadrangula, Hall, 1884, 36th Rep. N. Y. St. Mus. Nat. Hist, p. 68 , Ham. Gr.

guadrangularis, see Polypora quadrangularis.

quadrula, Hall, 18s3, Rep. St. Geol., pl. 21, fig 19-22, Low. Held. Gr.

regalis, Ulrich, 1888, Bull. Denison Univ., p. 70, and Geo. Sur. Ill., vol. 8, pl. 50, Keokuk and Waverly Grs.

remota, Foerste, 1887, Bull. Denison Univ., vol. 2, p. 84 and 87 , Low. Coal Meas.

rhombifera, see Reteporina rhombifera.

rigida, see Polypora rigida.

robusta, see Polypora robusta.

rudis, Ulrich, (in press,) Geo. Sur. Ill., vol. 8, pl. 49, Keokuk Gir.

scalaris, see Unitrypa scalaris.

sculptilis, Ulrich, 18S6, Cont. to Am. Pal., p. 10, Up. Held. Gr.

semirotunda, Hall, 1881, Bryozoans of the Up. Held. (ir., p. 32 , and Pul. N.Y., vol. 6 , p. 125, Up. Held Gr.

separala, see Polypora separuta.

serrata, Hall, 1881, Bryozoans of the Up.

Held. Gr., p. 28, and Pal. N. Y., vol. 6, p. 110, Up. Held. Gr.

serratula, Ulrich, (in press,) (ieo. Sur. Ill., vol. 8, pl. 50, Warsaw, St. Ionis, and Kaskaskia (ir.

sevillensis, Ulrich, (in press,) Geo. Sur. Ill., vol. 8, pl. 52, Low. Coal Meas.

shumardi, Prout, 1858, Trans. St. Louis Acad. Sci., vol. 1, p. 232, Up. Coal Meas. singularitas, Hall, 1881, Bryozoans of the Up. Held. (ir., p. 29, and Pal. N. Y. vol. 6, p. 114, U p. Held. Gr.

sinuosa, Hall, 1887, Pal. N. Y., vol. (;, ). 116, Up. Held. Gr.

spio, Hall, 1887, Pal. N. Y., vol. (i, p. 47, Low Held. Gr.

stellata, Hall, 1881, Bryozoans of the Up.

Held. Gr., p. 29, and Pal. N. Y., vol. 6, p. 109, Up. Held. Gr.

stipata, see Unitrypa stipata.

striata, see Reteporina striata.

striatopora, see Polypora striatopora.

subflexuosa, Ulrich, 1888, Bult. Denison

Univ., p. 68, Waverly Cir.

submutans, see Polypora submutans.

subretiformis, Prout, 1858, Trans. St. Louis Acad. Sci., vol. 1, p. 233, Coal Meas.

substriata, Hall, 1881, Bryozoans of the Up. Held. Gr., p. 35, Up. Iteld. Gr. subtortilis, Hall, 1884, 36 th Rep. N. Y. St. Mus. Nat. Hist., p. 71, Ham. Gr. sylvia, Hall, 1874, 26th Rep. N. Y. St. Mus. Nat. Hist., p. 96, Low. Ifeld. Gr. tantulus, Hall, 1879, Desc. New Spec. Foss., p. 8, and 11th Rep. Ind. Gieo. and Nat. Hist., p. 253, Niagara (ir.

teyulata, see Unitrypa tegulatı.

tenax, Ulrich, (in press,) Geo. Sur. Hll., vol. 8, pl. 51, Keokuk, St. Lonis and Kaskaskia Grs. 
tenella, Hall, 1887, Pal. N. Y., vol. 6, p. 105, Up. Held. Gr.

tenuiceps, Hall, 1852, Pal. N. Y., vol. 2, p. 165, Niagara Gr.

tenuis, Hall, 1852, Pal. N. Y., vol. 2, p. 51, Clinton Gr.

thyene, Hall, 1883, Rep. St. Geol., pl. 21, fig. 1-5, Low. Held. Gr.

torta, Hall, 1881, Bryozoans of the Up. Held. Gr., p. 30, Up. Held. Gr.

trituberculata, Prout, 1858, Trans. St. Louis Acad. Sci., vol. 1, p. 228, Coal Meas.

tuberculata, Hall, 1887, Pal. N. Y., vol. 6, p. 116, Up. Held. Gr.

variabilis, Prout, 1858, Trans. St. Louis Acad. Sci., vol. 1, p. 231, Coal Meas.

variopora, Hall, 1881, Bryozoans of the Up. Held. Gr., p. 28, Up. Held Gr.

vera, Ulrich, (in press,) Geo.Sur. Ill., vol. 8, pl. 44, Ham. Gr.

verrucosa, Hall, 1883, Rep. St. Geol., pl. 33, fig. 11, and Pal. N. Y., vol. 6, p. 110 , Up. Held. Gr.

virgosa, Eichwald, 1860, Lethaea Rossica, p. 358, Up. Coal Meas. Probably not American.

wortheni, Ulrich, (in press,) Geo. Sur. Ill., vol. 8 , pl. 52, Low. Coal Meas.

Fenestralia, Prout, 1858, Trans. St. Louis Acad. Sci., vol. 1, p. 235. [Ety. from genus Fenestella.] Zoarium like Fenestella, from which it is distinguished by having two rows of cells on each side of the median ridge. Type F. stludovici.

stludovici, Pront, 1858, Trans. St. Louis Acad. Sci., vol. 1, p. 235, St. Louis Gr.

stludovici var. compacta, Ulrich, (in press,) Geo. Sur. Ill., vol. 8, pl. 59, St. Louis $\mathrm{Gr}$.

Fenestra pora, Hall, 1885, Rep. St. Geol., p. 36. [Ety. fenestra, opening; poros, pore.] Forms of Fenestellidie liaving the branches connected by dissepiments,

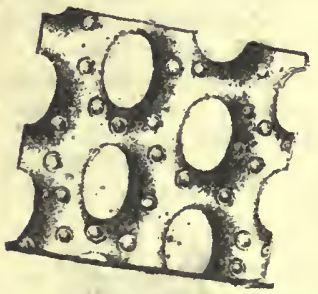

Fig. 475. - Fenestrapora biperforata. Magnified nonporiferous side. two ranges of cell apertures, separated by a carina bearing pores; noncelluliferous side with conspicuous pores. Type F. biperforata.

biperforata, Hall, 1885, Rep. St. Geol., pl. 2, fig. 17, Up. Held. Gr.

infraporosa, Ulrich, 1886, Cont. to Amer. Pal., p. 14, Un. Held Gr.

occidertalis, Ulrich, (in press), Geo. Sur. Ill., vol. 8, pl. 44 and 54, Ham. Gr.

Fistulupora, MeCoy, 1849, Ann. and Mag. Nat. Hist., 2d ser., vol. 3, p. 130. [Ety. fistula, pipe; poros, pore.] Incrusting or massive; corallites long, cylindrical, thick-walled, not in contact; tabulæe numerous ; cells circular, smooth-edged ; intervals between corallites filled with vesicular plates, tabulated. Type F. minor.

acervulosa, Rominger, 1866, Proc. Acad. Nat. Sci., p. 7, Ham. Gr. astricta, Ulrich, (in press,) Geo. Sur. 1ll., vol. 8, pl. 47 and 48 , Ham. Gr. canadensis, see Favosites canadensis.

carbonaria, Ulrich, 1884, Jour. Cin. Soc. Nat. Hist., vol. 7 , p. 45, Up. Coal Frg 476.-FistuMeas.

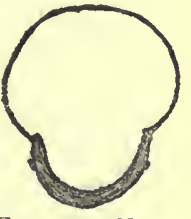
clausa, see Meekopora Tangentiai clausa.

collina, Ulrich, (in press,) ing iunasium.

Geo. Sur. Ill., vol. 8, pl. 47 and 48 , Ham. Gr.

communis, Ulrich, (in press,) Geo. Sur. Ill., vol. 8, pl. 47 and 48, Ham. Gr.

compressa, Rominger, 1866, Proc. Acad. Nat. Sci., p. 10, Keokuk Gr.

confertipora, Hall, 1881, (Thallostigma confertipora,) Trans. Alb. Inst., vol. 10, p. 184, and Pal. N. Y., vol. 6, p. 211, Ham. Gr.

constricta, Hall, 1881, (Lichenalia constricta,) Trans. Alb. Inst., vol. 10, p. 183, and Pal. N. Y., vol. 6, p. 227, Ham. Gr.

corrugata, Ulrich, (in press,) Geo. Sur. Ill., vol. 8, pl. 47 and 48, Ham. Gr.

crassa, Rominger, 1866, Proc. Acad. Nat. Sci., p. 8, Ham. Gr.

decipiens, Hall, 1881, (Thallostigma decipiens,) Trans. Alb. Inst., vol. 10 , p. 187, and Pal. N. Y., vol. 6, p. 232 , Ham. Gr.

densa, Hall, 1881, (Thallostigma densa,) Trans. Alb. Inst., vol. 10, p. 186, and Pal. N. Y., vol. 6, p. 231, Ham. Gr. digitata, Hall, 1881, (Thallostigma digitata) Trans. Alb. Inst., vol. 10, p. 185, and Pal. N. Y., vol. 6, p. 229, Ham. Gr. elegans, see Pinacotry ra elegans.

eriensis, Rominger 1866, Proc. Acad. Nat. Sci., p. 8, Ham. Gr.

excellens, Ulrich, 1884, Jour. Cin. Soc.

Nat. Hist., vol. 7, p. 46, Kaskaskia Gr. flabellata, see Chiloporella flabellata. flabellum, see Dichotrypa flabellum.

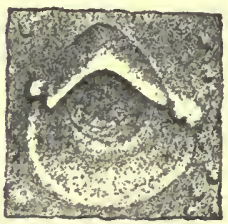

Fia.477.-Fistulipora foordi. Opercular cover $\mathrm{x} 50$. foordi, Ulrich, (in press,) Geo. Sur. IIl., vol. 8 , pl. 47 and 48 , Ham. Gr.

lualli, Rominger, 1866 , Proc. Acad. Nat. Sci., p. 6, Niagara Gr.

helios, Rouinger, 1866 , Proc. Acad. Nat. Sci. p. 7, Corniferous $\mathrm{Gr}$. hemi-pberica, Hall, 1881, (Callopora hemispherica,) Trans. Alb. Inst., vol. 10, p. 183, and Pal. N. Y., vol. 6, p. 226 , Ham. Gr. 
incrassata, Nicholson, 1874, (Callopora incrassata,) Geo. Mag. Lond. n. s., vol. 1, p. 13, and Rep. Pal. Ont., p.61, Ham. Gr. tercellata, Trans. Alb. Inst., vol. $10, p$. 13, and Pal. N. Y., vol. 6, p. 87, Up. Held. Gr.

involvens, Hall, 1887, Pal. N. Y., vol. 6, p. 221, Ham. Gr.

Jabiosa, Winchell, 1866, Rep. Low. P'enin. Mich., p. 88, Ham. Gr.

lamellata, Hall,.1881, (Thallostigma lamellata, Trans. Alb. Inst., vol. 10, p. 13, and Pal. N. Y., vol.6, p. 87, Up. Held. Gr. lens, Whitfield, 1878. Ann. Rep. Geo. Sur. Wis., p. 69, and Geo. Wis., vol. 4 , p. 256, Hud. Riv. Gr. longimacula,) Trans. Alb. Inst., vol. 10, p. 185, and Pal. N. Y., vol. 6, p. 209, Ham. Gr.

lunata, see Buscopora lunata.

micropora, Hall, 18S4, Tluallostigma micropora, Rep. St. Geol., p. 26, Ham. Gir. minuta, Rominger, 1866, Proc. Acad. Nat. Sci., p. 7, Ham. Gr.

monticulata, Ulrich, Geo. Sur. Hll., vol. 8, pl. 47 and 48 , Ham. (ir.

multiculeata, Hall, 1884, (Thallostigma multiculeata,) Rep. St. (ieol., p. 23, Ham. Gr.

neglecta, Rominger, 1866, Proc. Acad. Nat. Sci., p. 6, syn. for Lichenalia concentrica. intercellata, Hall, 1881, (Thallostigma in-

longimacula, Hall, 1881, (Thallostigına

ponderosa, Hall, 1874, (Callopora ponderosa,) 26th Rep. N. Y. St. Mus. Nat. Hist., p. 103, Low. Held Gr.

rugosa, sce Batostoma rugosa.

satfordi, Winchell, 1866, Rep. Low. Penin. Mich., p. 88 , Ham. Gr.

scrobiculata, Hall, 1884, (Thallostigma scrobiculata,) Rep. St. (ieol., p. 20, Ham. (ir.

segregata, Hall, 1884, (Thallustigma segregata,) Rep. St. Cieol., p. 27, Ham. Gr.

serrulata, Hall, 18S4, (Thallostigma serrulata,) Rep. St. Geol., p. 22, Ham. (ír.

solidissima, Whitfield, 1878, Ann. Rep. (ieo. Sur. Wis., p. 69, and Geo. Wis., vol. 4, p. 255, Hud. Riv. (ir.

spergenensis, lRominger, 1866, 'roc. Acad. Nat. Sci., p. 9, Warsaw Gr.

spheroidea, Hall, 1884, (Thallostigma spheroidea,) Rep. St. Geol., p. 31, Ham. (ir.

spinulifera, Rouninger, 1866, Proc. Aral. Nat. Sci., p. 8, Ham. Gr.

stellifera, Kominger, 1866, Proc. Acad. Nat. Sci., p. 7, Ham. (ir.

subtilis, Hall, 1884, (Thallostigma subtilis,) Rep. St. Cicol., p. 30, Ham. (ir.

sulcata, Rominger, 1866, Proc. Acarl. Nat. Sci., p. 7, Ham. Gir.

triangularis, Hall, 1854, (Thallostigma triangularis,) Rep. St. Geol., p. 32, Ilain. (ir.

trifaria, Hall, 1887, Pal. N. Y., vol. 'i, p. 222, Ha!n. (ir.

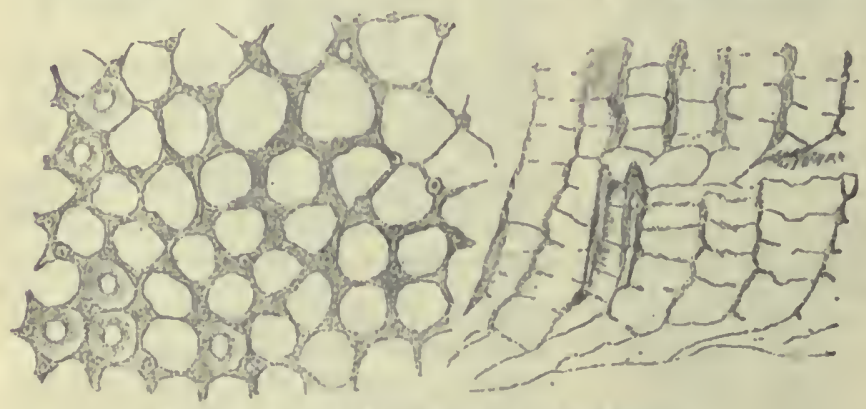

trifolia, Rominger, 1866, Proc. Acad. Nat. Sci., p. 9, Keokuk (ir.

triloba, Hall, 1587, Pal. N. Y., vol. 6 , p. 29 , Low. HIeld. (ir.

umbilicata, Hall, 1884, (Thallostigina $1 \mathrm{~m}$ bilicata,) Rep. St. Geol., p. 23, IJam. Gr.

unilinea, Hall, 1887, Pal. N. Y., vol. 6, p. 217, Ham. Lir.

utriculus, Rominger, 1866. Proc. A cad.

nodulifera, Meek, 1872, Pal. E. Neb., p. 143, Up. Coal Meas.

normalis. Ulrich, 1886, Cont. to Am. Ial., p. 20, Up. Held. Gr.

occidens, Hall \& Whitfield, 1873, 23d Rep. N. Y. St. Mus. Nat. Hist., p. 229, Chemung Gr.

oweni, James, 1885, Jour. Cin. Soc. Nat. Hist., vol. 7 , p. 21, Hud. Riv. Gr. Poorly defined.

parasitica, Hall, 1879, (Callopora parasitica,) 32d Rep. N. Y. St. Mus. Nat. Hist., p. 157, Low. Held Gr.

peculiaris, see Actinotrvpa peculiaris.

prolifica, Ulrich, 1884, Jour. Cin. Soc. Nat.

Hist., vol. 7, p. 45, St. Louis Gr.

proporoides, Nicholson, 1879, Pal. Tab. Corals, p. 310, Ham. Grr.
Nat. Sci., P. 8, Ham. (ir.

variopora, Hall, 1884, (Thallostigma variopora,) Rep. St. (ieol., p. 18, Ham. (ir.

Flustra, Linnseus, 1745, Ammenitates academicre. Not $\mathrm{Pa}$ leozoic.

carbaseoides, Eaton, 1832, Cieo. Text Book, p. 44. Not recognized.

spatulata, see Worthenopora spatulata. Fig, 77 - Flustra (") tutuberculata, Prout, berculata. A perture 1859, Trans. St. x 50.

Louis Acad. Sci., vol. 1, p. 447, Warsaw (ir. Not a flustra. 
Giauconome, Goldfuss, 1826, Germ. Petref., vol. 1 , p. 100 , as emended by Lonsdale in Murch. Sil. Syst., p. 677. [Ety. mythological name.] Narrow central stem, with lateral branches; two rows of cells separated by a keel on the face of each branch, and opposite side striated. Type G. disticha.

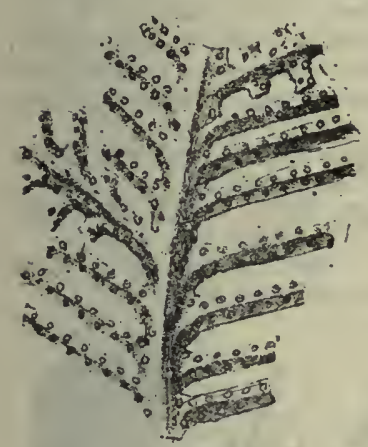

FIG. 480.-Glauconome nereidis. bellula, Ulrich, (Pinnato pora bellula, Geo. Sur. Il!., vol. 8 , pl. 66, Low. Coal Meas. carinata, Hall, 1884, Rep. St. Geol., p. 60, Ham. Gr. curvata, UIrich, 1888, (Pinnatop ora curvata,) Bull. Denison Univ., p. 76, Cuyahoga Shales.

flexuosa, Ulrich, (in press), Geo.Sur. Ill., vol. 8, pl. 66, Keokuk Gr.

intermedia, Ulrich, 1888, (Pinnatopora intermedia,) Bull. Denison Univ., p. 74, Cuyahoga Shales.

minor, Ulrich, 1888, (Pinnatopora minor,) Bull. Denison Univ., p. 77, Cuyahoga Shales.

nereidis, White, 1874, Rep. Invert. Foss., p. 18, and Geo. Sur. W. 100th Mer., vol. $4, \mathrm{p} .105$, Carboniferous.

nodata, Hall, 1881, Bryozoans of the Up. Held. Gr., p. 18, and Pal. N. Y., vol. 6, p. 102, Up. Held Gr.

simulatrix, Ulrich, 1888, (Pinnatopora simulatrix,) Bull. Denison Univ., p. 75, Cuyahoga Shales.

sinuosa, Hall, 1881, Bryozoans of the Up. Held. Gr., p. 18, and Pal. N. Y., vol. 6, p. 101, Up. Held. Gr.

subangulata, Ulrich, 1888, (Pinnatopora subangulata,) Bull. Denison Univ., p. 76, Cuyahoga Shales.

tenuiramosa, Ulrich, 1888, (Pinnatopora tenuiramosa,) Bull. Denison Univ., p. 79, Cuyahoga Shales.

tenuistriata, Hall, 1881, Bryozoans of the Up. Held. (ir., p. 19, and Pal. N. Y., vol. 6, p. 102 , Up. Held. Gr.

trilineata, Meek, 1872, Pal. E. Neb., p. 157, Coal Meas.

vinii, Ulrich, 1888, (Pinnatopora vinii,) Bull. Univ., p. 77, Cuyahoga Shales.

whitii, Foerste, 1887, (Pinnatopora whitii,) Bull. Univ., p. 78, Low. Coal Meas.

youngi, Ulrich, 1888, (Pinnatopora youngi,) Bull. Univ., p. 78, Cuyahoga Shales.

GlossotryPA, Hall, 1887, Pal. N. Y., vol. 6, p. xvii. [Ety. glosse, the tongue; trupa, opening.] Zoarium tubular; cells arising from the epitheca lining the cylin- drical frond, intersected by narrow projections from the cell walls, extending partially across the cell tube; apertures paliform ; intercellular structure vesiculose. Type G. paliformis.

paliformis, Hall, 1881, (Lichenalia paliformis, Trans. Alb. Inst., vol. 10, p. 11, and Pal. N. Y., vol. 6, p. 85, Up. Held. Gr. Glyptotrypa, Ulrich, syn. (?) for Coscinium. GON IOTRYPA,

Ulri ch,

(in press),

Micropalxontology, p. 14. [Ety. gonia, angle; trupa, opening. ] Bifoliate, join te d, segments $\mathrm{s}$ ma l 1, FIG. 481. - Goniotrypa bilatereach face alis." 1 , Transverse section; 2 , with a transverse section lower down; central 3 , tangential section of the upridge ;

cells in longitudinal rows; apertures oval, directed obliquely outward. Type G. bilateralis. Syn. (?) for Dicranopora.

bilateralis, Ulrich, (in press), Micropalæontology, p. 15, Hud. Riv. Gr. (?)

Gorgonia, Linnæus, 1745, Amænitates Acad. [Ety. mythological name.] Not American Palrozoic.

anticorum, Castelnau, 1843, Syst. Sil., p. 50. Not recognized.

(?) aspera, see Subretepora aspera.

dubia, Goldfuss, 1826, Petref. Germ. Permian. Not recognized.

ehrenbergi, see Phyllopora ehrenbergi. infundibuliformis, Eaton, 1832, Geo. Text Book, p. 43. Not recognized. perantiqua, see Enallopora perantiqua. retiformis, see Dictyonema retiforme. siluriana, Castelnau, 1843, Syst. Sil., p. 50. Not recognized.

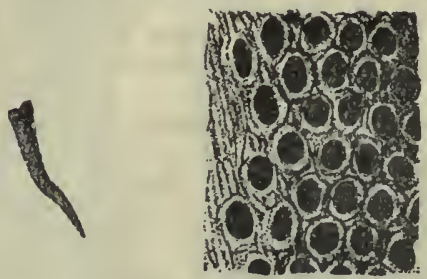

FIG. 482.-Graptodictya nitida. Naturai size and magnified.

Graptodictya, Ulrich, 1882, Jour. Cin. Soc. Nat. Hist., vol. 5, p. 165 . [Ety. grapho, I write ; dictyon, net.] Zoarium pointed below, branching above, cell apertures circular, and separated by interstitial pits or sulci; distinguished from Ptilodictya by the circular cells and surrounding pits. Type G. perelegans. 
nitida, Ulrich, 1882, Jour. Cin. Soc. Nat. Hist., vol. 5, p. 166, Hud. Riv. Gr.

perelegans, Ulrich, 1878, (Ptilodictya perelagans,) Jour. Cin. Soc. Nat. Hist., vol. 1, p. 94, Hud. Riv. Gr.

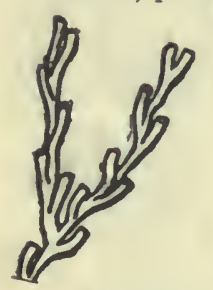

FIg 483.-Hederella canadensls. Magniflet.

Hederelia, Hall, 1884, Rep. densis.

canadensis, Nicholson, densis, Can. Nat. and Geol., vol. 7, p. 14t, and Pal. Prov. Ont., p. 124, Up. Held. and Ham. Gr. cirrhosa, Hall, 1884, Rep. St. Geol., p. 53, Ham. Gr.

conferta, Hall, 1884, (Ptilionella conferta,) Rep. St. Geol., p. 56, Ham. Gr.

filifornis, Billings, 1858, (A ulopora filiformis,) Can. Jonr., vol. 4, p. 119, Hain. Gr.

magna, Hall, 1884, Rep. St. Geol., p. 55, Ham. Gr.

Helicopora, Claypole, 1883, Quar. Jour: Gén. Soc., p. 30. [Ety. helix, spiral; poros, pore.] Bryozoum expanded, fenestrate, and spiral; formed of slender, bifurcating rays, poriferous on one face, connected by nonporiferous bars, forming an open net-work; cells arranged in two rows along the rays, one row on each side of a median keel; axis none, or consisting only of the thickened inner border of the bryozoum, not straight, but forming a spiral, rounded, nonporiferous, or slightly poriferous, inner margin. Type H. latispiralis. Regarded by some as a synonym for Fenestella, and distinguished only by the spiral form.

archimediformis, Claypole, syn. for Ar-

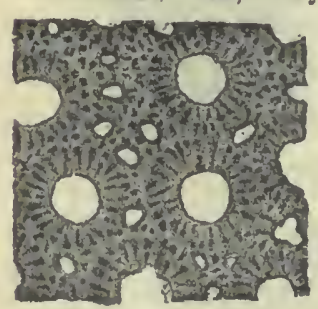

Fig. 481,-Heliotrypa bifolia. Tangenilal section $\times 50$. [Ety, helios, sun;
trupa, opening.] Bifoliate, interstitial cells developed from the prostrate por-

latispiralis, Claypole, 1883, Quar. Jour. Geo. Sci., p.32, Niagara Gr. ulrichi, Claypole, 1883, Quar.Jour. Geo. Soc., p. 33, Up. Held. Gr.

HeLIOTRYPA, Ulrich, 1883, Jour. Cin. Soc. Nat. Hist., vol. 6 p 277 . St. Geol., p. 53. [Ety. hedera, ivy.] Bryozoum parasitic, procumbent, attached the entire length; main axis tubular, from which proceed lateral tubular cells, giving it the general appearance of Stomatopora. Type H. cana1873, (Alecto (?) cana-

tion of the zocecia ; intercommunication by means of radially arranged tubuli. Type H. bifolia.

bifnlla, Uirich, 1883, Jour. Cin. Soc. Nat. Hist., vol. 6, p. 2\%8, Kaskaskia Gr.

Helopora, Hall, 1852, Pal. N. Y., vol. 2, p. 44. [Ety. helos, nail; poros, pore.] Zoarium jointed; segments small, simple, cylindrical, often swollen at the extremities; cells oval or subangular, and arranged between longitudinal elevated lines or in quincunx. Type H. fragilis.

armata, Billings, 1866, Catal. Sil. Foss. Antic., p. 38, Anticosti Gr.

bellula, Hillings, 1866, Crtal. Sil. Foss. Antic., p. 38, Anticosti Gr.

circe, Billings, 1866, Catal. Sil. Foss. Antic., p. 39, Anticosti Gr.
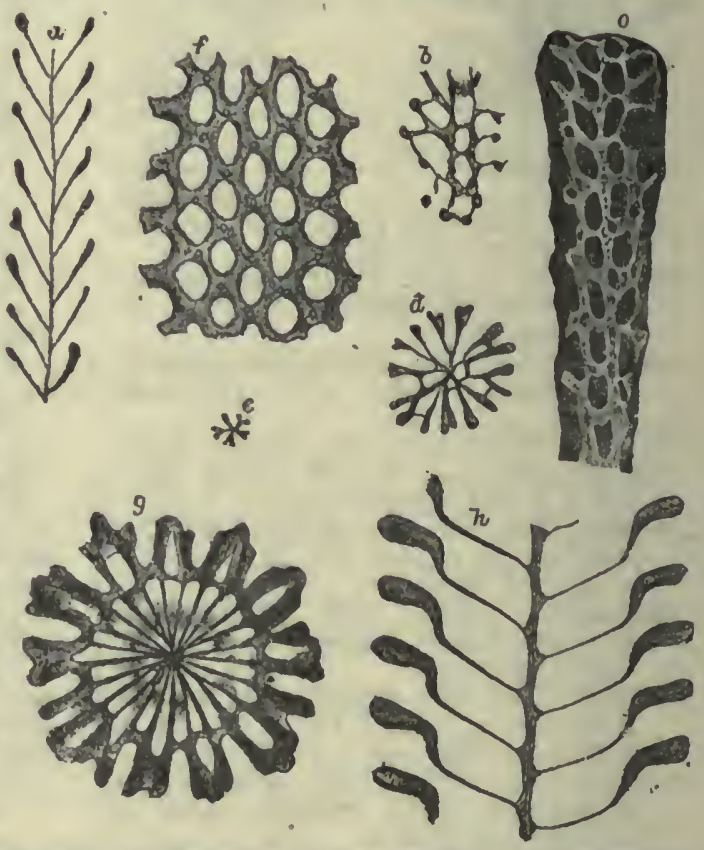

19. 485. $\rightarrow-l$, , Helopora frugllls; $f-h$, Heloporn IIndstroml, sectlous $\mathrm{x}$ 50.

concava, Billings, 1866, Catal. Sil. Foss. Antic., p. 37, Anticosti Gr. divaricata, Ulrich, 1886, 14th Rep. Geo. Sur. Minn., p. 59, Trenton Gr.

formosa, Billings, 1865, Catal. Sil. Foss. Antic., p. 37, Anticosti Gr.

fragilis, Hall, 1852, Pal. N. Y., vol. 2, p. 44, Clinton Gr.

fragilis var. acadiensis, Hall, 1860, Can. Nat. Frg. 486. - Heloand Geo., vol. 5, Anti- pora rragllls. costi Gr.

irregularis, Billings, 1866, and magulfed. Catal. Sil. Foss. Antic., p. 39, Anticosti Gr. imbricata, Ulrich, (in press,) Geo. Sur. Ill., vol. 8, pl. 29, Hud. Riv. Gr. 
lineata, Billings, 1866, Catal. Sil. Foss. Antic., p. 36, Anticosti Gr.

lineopora, Billings, 1866, Catal. Sil. Foss. Antic., p. 38, Anticosti Gr.

nodosa, Billings, 1866, Catal. Sil. Foss. Antic., p. 38, Anticosti Gr.

spiniformis, Ulrich, 1882, (Arthroclema spiniforme,) Jour. Cin. Soc. Nat. Hist., vol. 5, p. 161, Trenton Gr.

striatopora, Billings, 1866, Catal. Sil. Foss. Antic., p. 39, Anticosti Gr.

strigosa, Billings, 1866, Catal. Sil. Foss. Antic., p. 37, Anticosti Gr.

tenuis, see Arthrostylus tenuis.

varipora, Billings, 1866, Catal. Sil. Foss. Antic., p. 40, Anticosti Gr.

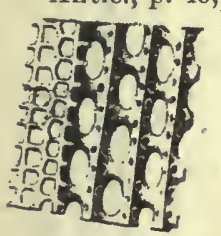

FIG. 487. - Hemitrypa biordo.
Hemitry PA, Phillips, 1841, Pal. Foss. Cornwall, Devon, and W. Somerset, p. 27. [Ety. emisos, half ; trupa, perforation.] Fenestelloid; branches connected by dissepiments; cell apertures in two ranges, separated by carinæ, which are elevated, widened at the summit, and connected by scalæ, which meet midway and coalesce, forming pseudocarinæ. 'Type H. oculata.

aspera, Ulrich, (in press). Geo. Sur. Ill., vol. 8, pl. 57, Keokuk Gr.

biordo, Hall, 1887, Pal. N. Y., vol. 6, p. 149, Up. Held. Gr.

bis rialis, Hall, 1879,32 d Rep. N. Y. St. Mus. Nat. Hist., p. 174, Low. Held. Gr. biserialis var. exilis, Hall, 1887, Pal. N. Y., vol. 6 , p. 57, Low. Held. Gr.

columellata, Hall, 1887, Pal. N. Y., vol. 6, p. $146, \mathrm{Up}$. Held. Gr.

cribrosa, Hall, 1881, (Fenestella cribrosa,) Trans. Alb. Inst., vol. 10, p. 35, Up. Held. Gr.

dubia, syn. for Loculipora ambigua.

favosa, Hall, 1881, Trans. Alb. Inst., vol. 10 , p. 35, and Pal. N. Y., vol. 6, p. 148, Up. Held. Gr.

nodosa, Ulrich, (in press,) Geo. Sur. Ill., vol. 8, pl. 57, Keokuk Gr.

prima, Hall, syn. for Unitrypa nervia.

pateriformis, Ulrich, (in press:) Geo. Sur. Ill., vol. 8, pl. 57, Keokuk Gr.

perstriata, Ulrich, (in press,) Geo. Sur. Ill., vol. 8, pl. 57, Keokuk Gr.

plumosa, Prout, 1858, (Fenestella plumosa,) Trans. St. Louis Acad. Sci., vol. 1, p. 236, Keokuk and Warsaw Gr.

proutana, Ulrich, (in press,) Geo. Sur. Ill., vol. 8, pl. 57, proposed instead of Fenestella hemitrypa of Prout, Keokuk and Warsaw Grs.

proutana var. nodulosa, Ulrich, (in press,) Geo. Sur. Ill., vol. 8, pl. 57, Keokuk Gr. proutana var. vermifera, Ulrich, (in press,) Geo. Sur. Ill., vol. 8, pl. 57, Warsaw Gr.

tenera, Ulrich, (in press,) Geo. Sur. Ill., vol. 8, pl. 44, Hamilton Gr. ulrichi, Foerste, 1887, Bull. Denison Univ., vol. ¿, p. 152, Clinton Gr.

Hernodia, Hall, 1884, Rep. St. Geol., p. 58. [Ety. hernodes, like a young sprout.] Bryozoum parasitic, procumbent, increasing by gemmation like Aulopora; budding lateral, and for some distance in contact and frequently coalescing with the parent cells. Type $H$. humifusa.

humifusa, Hall, 1884, Rep. St. Geol., p. 58, Ham. Gr.

Heterodictya, Nicholson, 1875, Geo. Mag., vol. 2, n. s., p. 33, and Pal. Prov. Ont., p. 79. [Ety. heteros, irregular; dictyon, net.] The correct orthography is $\mathbf{H e}$ terodictyon. Flattened, two-edged frond, with subparallel sides, consisting of two series of cells upon opposite sides of a central membrane; cells are in longitudinal rows; tabulæ present. Type $\mathbf{H}$. gigantea.

gigantea, Nicholson, 1875, Geo. Mag., vol. 2, p. 34, and Pal. Prov. Ont., p. 79, Subcarboniferous.

Hippothoa, Lain- F.I G. 488.-Heterodictya ouroux, 1821 .

Expos method. Not Palæozoic.

inflata, see Stomatopora inflata.

HomotryPa, Ulrich, 1882, Jour. Cin. Soc. Nat. Hist., vol. 5, p. 240. [Ety. homos, similar; trupa, perforation.] Ramose or subfrondescent; surface smooth or bearing monticules; cells, circular, ovate or polygonal, thin-walled; groups of larger-sized cells; mesopores absent or restricted to the macula; spiniform tubuli, diaphragms and cystiphragms present. Type H. curvata.

arbuscula, Ulrich, (in press), Geo. Sur. Ill., vol. 8, pl. 38, Birdseye Gr.

curvata, Ulrich, 1882, Jour. Cin. Soc. Nat. Hist., vol. 5, p. 241, Hud. Riv. Gr.
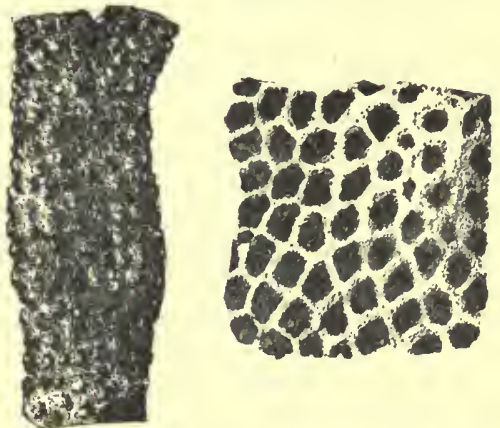

ErG. 489.-Homotry pa obliqua. Natural size and magnified.

exilis, Ulrich, 1886, 14th Rep. Geo. Sur. Minn., p. 80, Trenton Gr.

flabellaris, Ulrich, (in press), Geo. Sur. Ill., vol. 8, pl. 32, Hud. Riv. Gr. 
gelasinosa, Ulrich, (in press), Geo. Sur. Ill., vol. 8, pl. 32 , Hud. Riv. Gr.

insignis, Ulrich, 1886, 14th Rep. Geo.Sur. Minn., p. 82, Trenton Gr.

minnesotensis, Ulrich, 1886, 14th Rep. Geo. Sur. Minn., p. 79, Trenton Gr.

obliqua, Ulrich, 1882, Jour. Cin. Soc. Nat. Hist., vol. 5, p. 243, Hud. Riv. Gr.

subramosa, Uirich, 1886, 14th Rep. Geo. Sur. Minn., p. 81, Trenton Gr.

Homotrypella, Ulrich, 1886, 14th Rep. Geo. Sur. Minn., p. 83. [Ety. homotrypa, a genus; ellus, diminutive.] Zoarium ramose; monticules wanting; interstitial cells present; zoccia sma!l, moderately thick walls, and cystoid diaphragms straight; spiniform tubu'i numerous. Type $H$. instabilis.

contexta, Ulrich, (in press), Geo. Sur. Ill., vol. 8, pl. 32, Hud. Riv. Gr.

granulifera, Ulrich, 1879, (Chetetes granuliferus,) Jnur. Cin. Soc. Nat, Hist., vol. 2, p. 128, Trenton Gr.

instabilis, Ulrich, 1856, 14th Rep. Geo. Sur. Miun., p. 83, Trenton Gr.

Hornera, Lamouroux, 1821, Expos. Method. des genres de L'Ordre des Pol. [Ety. proper name.] Not American Palreozoic.

dichotoma, see Subretepora dichotoma.

Icirnyorachis, MeCoy, 1844, Carb. Foss. Ireland, p. 205. [Ety. ichthys, fish ; rachis, backbone.] Bryozoum plumose, consisting of a rachis, with short lateral branches or pinnules; celluliferous on one side; cell apertures in two ranges on the branches, and in three or more on the main stem. Type I. newenhami.

nereis, Hall, 1874, 26th Rep. N. Y. St. Mus. Nat. Hist., p. 08, Low. Held. Gr.

IdrotryPA, Ulrich, 1883, Jour. Cin. Soc. Nat. Hist., vol. 6, p. 272 . [Ety. idios, peculiar; trupa, opening.] Parasitic, interstilial celts angular, both cells with diaphragıns; spiniform tubuli present. Type I. parasitica.

parasitica, Ulrich, 1883, Jour. Cin. Soc. Nat. Hist., rol. 6, p. 273, Niagara Gr.

Intra PORA, Hall, 1881, Bryozoans of the Up. Held. Gr., p. 16. [Ety. intra, within; poros, pore.] Resembling Stictopora, branches broad; intercellular spaces regularly punctured or pitted, as if by minute cell apertures; cells with rounded mouths and short prostrate portion; intercellular 'space vesiculose. Type I. puteolata.

puteolata, Hall, 1881, Bryozoans of the Up. Held. Gr., p. 16, and Pal. N. Y., vol. 6 , p. 97 , Up. Held. Gr.

Intricaria, Defrance, 1823, Dictionnaire des Eciences Naturelles. Not a Palæozoic genus.

clathrata, see Subretepora clathrata. reticulata, see Sulretepora reticulata.

IsotryPA, Hall, 1885, Rep. St. Geol., p. 37. [Ety. isos, equa] ; trupa, perforation.] Fenestelloid, having the branches con- nected by dissepiments, and with two ranges of cell apertures, separated by carinr, elevated and much thickened above, connected by distinct lateral processes; the reverse face has on or near the dissepiments conspicuons pores larger than the cell apertures. Type I. conjunctiva.
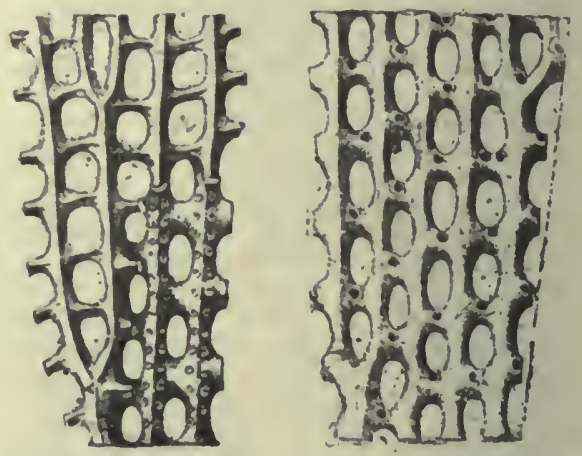

FIG. 400.-Isotrypu conjunctiva. Celluliferons side and uonceliniterous slde, with pores on the dissepiments.

bifaria, syn. for I. conjunctiva.

conjunctiva, Hall, 1881, (Fenestella conjunctiva,) Trans. Alb. Inst., vol. 10, p. 143, and Pal. N. Y., vol. 6, 1. 143, Up. Held. Gr.

consimilis, Hall, 1885, Rep. St. Geol., pl. 2, fig. 14, Up. Held. Gr.

IAABECIIA, Edwards \& Haime, 1851, Pol Foss. des Terr. Pal., p. 297. [Ety. proper name.] Bryozoum laminar, incrusting, or attached by part of the base, and having the remainder covered by an epitheca; surface covered with rounded or elongated, solid, tubercles, separated by an imperforate calcarcous membrane; interually it consists of vertical columns extending from the epitheca below, and terminating above in the surface tubercles, the interepaces between the columns consisting of lenticular vesicles, the uppermost layer of which gives rise to the secmingly imperforate membrane between the tubercles. Type L. conferta. Probably this genus belongs to the Protozoa, and is related to the sponges.

montifera, Ulrich, 1886, Cont. to Am. Pal., p. 33, Hud. Riv. Gr.

Lemoclem A, Ulrich, 1882, Jour. Cin. Soc. Nat. Hist., vol. 5, p. 141. [Ety. leios, smooth ; klema, twig.] Ramose, lamellate, or parasitic; surface even; rellmouths small, rounded, surrounded by interstitial cells; tubes thin-walled; diaphragms remote; acanthopores abundant. Type L. punciatum.

araneum, Ulrich, (in press? Geo. Sur. IIl., vol. 8, pl. 75, Kaskaskia Gr.

foliatum, Ulrich. (in press,) Geo. Sur. Ill., vol, 8, p. 301, Warsaw Gr. 
gracillimum, Ulrich, (in press,) Geo. Sur. Ill., vol. 8, pl. 75, Keokuk Gr.

minutissimum, Nicholson, 1875, (Callopora minutissima.) Pal. Prov. of Ontario, p. 77, Hamilton Gr.
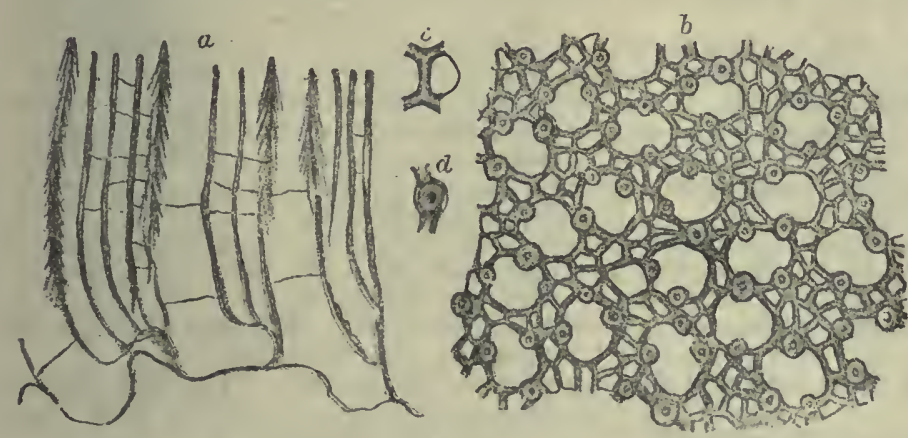

FIG. 491.-Lelociema foliatum. $a$, Vertical section $\mathrm{x} 28$, showing entire thickness of zoarium, tabulation of zoxecia and mesopores, and siructure of the acaniliopores ; $b$, tangential secliou $\times 28$, showlug distribution of acanthopores, inesopores, and zoccla; $c$, small portion of wall $\times 50 ; d$, acauthopore $x$, showling its struclure.

puuctatum, Hall, 1858, Callopora punctata, Geo. Sur. Iowa, p. 653, Keokuk and Warsaw Grs.

subglobosum, Ulrich, (in press,) Geo.Sur. Ill., vol. 8, pl. 75. Kinderhook Gr.

wachsmuthi, Ulrich, (in press, Geo. Sur. Ill., vol. 8, pl. 75, Kinderhook Gr.

wilmingtonense, Ulrich, (in press,) Geo. Sur. Ill., vol. 8, pl. 34, Hud. Riv. Gr.

LeptotryPA, Ulrich, 1883, Jour. Cin. Soc. Nat. Hist., vol. 6, p. 158. [Ety. leptos, tlin; trupa, perforation.] Thin, incrusting ; cells polygonal, thin-walled; surface, with monticules; spiniform tubuli; no diaphragms or rudimentary. Type I. minima.

clavacoidea, James, 1875, (Chetetes clavacoidea,) Int. Catal. Cin. Foss., p. 2, and Nicholson on Struct. and Affin. Montic, p. 182, Hud. Riv. Gr.

clavis, Ulrich, 1883, Jnur. Cin. Soc. Nat. Hist., vol. 6, p. 161, Utica Slate.

cortex, Ulrich, 1883, Jonr. Cin. Soc. Nat. Hist., vol. 6, p. 162, Utica Slate.

hexagonalis, Ulrich, (in press,) Geo. Sur. Ill., vol. 8, pl. 36, Trenton Gr.

minima, Ulrich, 1883, Jour. Cin. Soc. Nat. Hist., vol. 6, p. 159, Hud. Riv. Gr. ornata, Ulrich, 1883, Jonr. Cin. Soc. Nat. Hist., vol. 6, p. 160, Hud. Riv. Gr.

semipilaris, Ulrich, (in press,) Geo. Sur. Ill., vol. 8, pl. 36, Hud. Riv. Gr.

stidhami, Ulrich, (in press,) Geo. Sur. Ill., vol. 8, pl. 36, Hud. Riv. Gr.

Lichenalia, Hall, 1852, Pal. N. Y., vol. 2, p. 171. [Sig.from resemblance to a lichen.] Membranous expansions, growing in circular or fiabellate forms, celluliferous on one side, the other covered with an epitheca; cells septate, arising from the enitheca; apertures circular or trilobate, often denticulate; interaper-
Geol., p. 37, Ham. Gr.

tural space smooth; intercellular space vesiculose. Type L. concentrica. alternata, Hall, 1881, Bryozoans of the Up. Held. Gr., n. 8, and Pal. N. Y., vol. 6 , p. 80 , Up. Held. Gr.

alveata, see Odontotrypa alveata.

bistriata, Hall, 1881, Bryozoans of the Up. Held. Gr., p. 8, and Pal. N. Y., vol. 6 , p. 79, Up. Held. Gr.

bullata, Hall, 1887, Pal. N. Y., vol. 6, p. 205, Ham. Gr.

carinata, Hall, 1881, Bryozoans of the Ur. Held. Gr., p. 9, Up. Held. Gr. circinrta, see Selenopora circincta. clivulata, see Pileotrypa clivulata.

clypeiformis, Hall, 1884, Rep. St. colliculata, Hall, 1884, Rep. St. Geol., p. 36, Ham. Gr.

complexata, see Selenopora complexáta. concentrica, Hall, 1852, Pal. N. Y., vol. 2, p. 171, Niagara Gr.
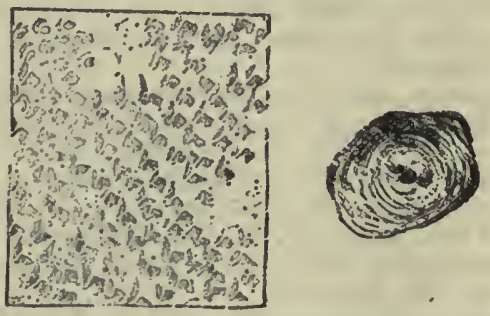

Fio. 492.-Ljchenalia concentrica. Under surface and upper surface inagnifled.

concentrica var. maculata, Hall, 1879, 28 ll $_{1}$ Rep. N. Y. St. Mus. Nat. Hist., p. 117, Niagara Gr.

concentrica var. parvula, Hall, 1876, 28th Rep. N. Y. St. Mus. Nat. Hist., p. 117, Niagara Gr.

confus,, Hall, 1887, Pal. N. Y., vol. 6, p. 204, Ham. Gr.

constricta, see Fistulipora constricta.

conulata, Hall, 1881, Bryozoans of the Up.

Held. Gr., p. 9, and Pal. N. Y., vol. 6, p. 81, Up. Helr. Gr.

cornuta, Hall, 1887, Pal. N. Y., vol. 6, p. 203, Ham. Gr.

crassa, Hall, 1879, (Trematopora crassa,) 32 d Rep. N. Y. St. Mus. Nat. Hist., p. 152, Low Held. Gr.

crustacea, Hall, 1881, Bryozoans of the

Up. Held. Gr., p. 8, Up. Held. Gr.

cultellata, Hall, 1884, Rep. St. Geol., p. 35

Ham. Gr.

denticulata, see Pileotrypa denticulata. 
dissimilis, Hall, 1883, Rep. St. Geol., pl. 15, fig. 10-13, Low. Held. Gr.

distans, Hall, 1883, Rep. St. Geol., pl. 15, fig. 8-9, Low. Held. Gr.

foliacea, Hall, 1884, Rep. St. Geol., p. 35, Ham. Gr.

geometrica, Hall, 1887, Pal. N. Y., vol. 6, p. 79 , Up. Held. Gr.

granifera, see Pileotrypa granifera.

imbricella, Hall, 1884, Rep. St. Geol., p. 35, Ham. Gr.

longispina, see Lichenotrypa longispina.

lunata, see Buscopora lunata.

maculosa, Hall, 1884, (Trematopora mac. nlosa,) 26th Rep. N. Y. St. Mus. Nat. Hist., p. 106, I.ow. Held. Gr.

operculata, Hall, 1887, Pal. N. Y., vol. 6, p. 205, Ham. Gr.

ovata, Hall, 1887, Pal. N. Y., vol. 6, p. 80 , Up. Held. Gr.

paliformis, see Glossotrypa paliformis.

permarginata, Hall, 1881, Bryozoans of the Up. Held. Gr., p. 10, and Rep. St. Geol., 1883, pl. 24, fig. 20, Up. Held. (ir. pustulosa, Hall, 1887, Pal. N. Y., vol. 6, p. 206, Ham. Gr.

pyriformis, see Pileotrypa pyriformis.

radiata, Hall, 1881, Bryozoans of the Up. Held. Gr., p. 10, Up. Held. Gr.

ramosa, Hall, 1887, Pal. N. Y., vol. 6, p. 199, Ham. Gr.

serialis, Hall, 1887, Pal. N. Y., vol. 6, p. 32, Low. Held. Gr.

stellata, Hall, 1884, Rep. St. Geol., p. 33, Ham. Gr.

subcava, Hall, 1881, Bryozoans of the Up. Held. Gr., p. 8, and Rep. St. Geol., 1883, pl. 24, fig. 23-25, Up. Held. Gr.

substellata, Hall, 1881, Bryozoans of the Up. Held. Gr., p. 7, and Rep. St. Geo., 1883, pl. 24, fig. 26, Up. Held. Gr.

subtrigona, Hall, 1887, Pal. N. Y., vol. 6, p. 196 , Ham. Gr.

tessellata, Hall, 1887, Pal. N. Y., vol. 6, p. 207, Ham. Gr.

torta, Hall, 18s3, Rep. St. Geol., pl. 15, fig. 1-7, Low. Held. Gr.

tortuosa, Hall, 1883, Rep. St. Geol., pl. 13, fig. 17-18, Low. Held. Gr.

vesiculata, Hall, 1887, Pal. N. Y., vol. 6. p. 197, Ham. Gr.

LichenotrypA, Ulrich, 1886, Cont. to Am. Pal.. p. 23. [Ety. lichen, a trec-moss; trupa, perforalion.] Zoarium thin, incrusting, in early growth like Fistulipora, with slort, tubular zocecia, wide, concave interspaces, subcircular apertures, posterior margin elevated; in later growth peristomes of adjacent cells unite by thin, irregular walls, which traverse the interstitial spaces, and form an irregular net-work, with spine-like elevations; interstitial cells present. Type L. cavernosa. Syn. (?) for Lichenalia.

eavernosa, Ulrich, 1886, Cont. to Am. Pal., p. 24, Up. Held. Gr.

longispina, Hall, 1881, (Lichenalia longispina,) Trans. Alb. Inst., vol. 10, p. 11, and Pal. N. Y., vol. 6, p. 287, Up. Held. Gr.

Loculipors, Hall, 1887, Pal. N. Y., vol. 6, p. xxiii. [Ety. loculus, cell; poros, pore.] Fenestelloid; branches connected by dissepiments; cell apertures in two ranges, surrounding the fenestrules; branches and dissepiments carinated; carinæ elevated and much thickened above, having the appearance of the branches and dissepiments of the noncelluliferous face of the frond. Type L. perforata.

ambigua, Hall, 1876, (Fenestella ambigua,) 28 th Rep. N. Y. St. Mus. Nat. Hist., p. 123, Niagara Gr.

circumstata, l1all, 1887, Pal. N. Y., vol. 6, p. 144, Up. Held. Gr.

perforata, Hall, 1884, (Fenestella perforata,) 36th Rep. N. Y. St. Mus. Nat. Hist., p. 65, Han. Gr.

Lyropora, Hall, 1857, Proc. Am. Ass. Ad. Sci., vol. 10, ๒. 179. [Ety. lyra, lute; poros, pore.] Zoarium consisting of a reticulated expansion, margined by two strong diverging supports which curve outward and upward; the rays of the expansion carry from two to five rows of cells; but there are none in the dissepiments; fenestrules small, ovate. Type L. lyra.

cinctura, IIall, 1885 , Rep. St. Geol., pl. 1, Ham. Gr.

divergens, Ulrich, (in press,) Geo. Sur. Ill. vol. 8, pl. 58, Kaskaskia Gr.

lyra, Hall, 1857, Fia.493.-Lyroporn cincProc. Am. Ass. turu. Noncellullfer-

Ad. Sci., vol. 10 , p. 179, Kaskaskia Gr.

ovalis, Ulrich, (in press,) Geo. Sur. Ill., vol. 8, pl. 58, Kaskaskia Gr.

quincuncialis, Hall, 1857, Proc. Am. Ass. Ad. Sci., vol. 10, p. 179, Kaskaskia Gr.

ranosculum, Ulrich, (in press,) Geo. Sur. Ill., vol. 8, pl. 58, Kaskaskia Gr.

retrorsa, Meck \& Worthen, 186S, Geo. Sur. 11l., vol. 3, p. 504, Burlington Gir. subquadrans, Hall, 1857, Proc. Am. Ass. Ad. Sci., vol. 10, p. 179, Kaskaskia Gr. MEEKOPORA, Ulrich, (in press,) Geo. Sur. Ill., vol. 8, p. 383. [Ety. proper name.] Bifoliate, sometimes branching; the median laminx thin, flexuous; cells arranged with their oblique apertures directed toward the distal margin of the expansion; lunarium noderate or obsolete; zocecial tubes oblique, the anterior walls thinnest and flexuous; diaphragms numerous, often recurved; oocium a large oval cell, showing as a convex space with a small apical perforation. Type M. eximia.

(?) aperta, UUIrich, (in press,) Geo. Sur. Il]., vol. 8, pl. 76, Keokuk Gr. 
approximata, Ulrich, (in press,) Geo. Sur. 11l., vol. 8, pl. 77, Kaskaskia Gr.

clausa, Ulrich, 1884 ,

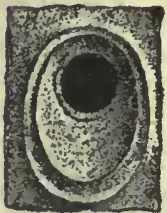

(Fistulipora? clausa,) Jour. Cin. Soc. Nat. Hist., vol. 7, p. 47, Kaskaskia Gr.

eximia, Ulrich, (in press,) Geo. Sur. Ill., vol. 8 , pl. 77, Kaskaskia $\mathrm{Gr}$.

Fia.494.-Meekopora clausa. A percular cover $\times 50$.

Mitoclema, Ulrich, 1882, Jour. Cin. Soc. Nat. Hist., vol. 5, p. 150. Syn. for. Enallopora.

cinctosa, see Enallopora cinctosa.

Nemataxis, Hall, 1887, Pal. N. Y., vol. 6, p. 74. [Ety. nema, thread; axon, axis.] Ramose, solid, bifurcating, cells arising from a filiform axis, apertures oval, in parallel rows, separated by ridges; surface marked with monticules, destitute of cell apertures, and extending across the branch, give it an annulated appearance. Type N. fibrosus.

fibrosus, Hall, 1887, Pal. N. Y., vol. 6, p. 74, Up. Held. Gr.

simplex, Hall, 1887, Pal. N. Y., vol. 6, p. 193, Ham. Gr.

Nematopora, Ulrich, (in press,) Geo. Sur. Ill., vol. 8, p. 401. [Ety. nema, thread; poros, pore.] Slender, ramose, continuous above the pointed basal extremity ; zoœcia subtubular, short, arranged in a radial manner around one or two minute axial tubes; apertures ovate or subcircular, with peristome, generally arranged between longitudinal ridges; one or two diaphragms occasionally present. Type N. quadrata.

alternata, Ulrich, (in press,) Geo. Sur. Ill., vol. 8. pl. 29, Galena Gr.

delicatula, Ulrich, (in press,) Geo. Sur. Ill., vol. 8, pl. 29, Galena Gr.

quadrata, Ulrich, (in press,) Geo. Sur. Ill., vol. 8, pl. 29, Trenton Gr.

retrorsa, Ulrich, (in press,) Geo. Sur. Ill., vol. 8, pl. 29, Galena Gr.

Nicholsonella, Ulrich, (in press,) Geo.Sur. Ill., vol. 8, p. 374. [Ety. proper name.] Irregularly intertwining, flattened branches, sometimes laminated; zoœcia tubular, with diaphragms in the "mature" region; apertures circular, with a granose peristome; interspaces wide, occupied by numerous angular mesopores, that more or less isolate the zoœcia; walls of both the zoœcia and mesopores thin, and in the mature region traversed longitudinally by tubuli; the interzoœcial spaces are filled with a calcareous deposit, into which the tubuli continue, but in which the mesopore walls become unrecognizable ; mesopores with thick and numerous diaphragms. Type N. ponderosa.

cumulata, Ulrich, (in press,) Geo. Sur. Ill., vol. 8, pl. 33, Hud. Riv. Gr. ponderosa, Ulrich, (in press,) Geo. Sur. Ill., vol. 8, pl. 34, Trenton Gr.

Odontotrypa, Hall, 1887, Pal. N. Y., vol. 6, p. xvii. [Ety. odous, tooth; trupa, opening.] Distinguished from Lichenalia, by the oblique trilobate, closely arranged cell apertures, with strongly elevated, denticulated margins, forming a crescentic projection over the aperture. Type 0 . alveata.

alveata, Hall, 1881, (Lichenalia alveata,) Trans. Alb. Inst., vol., 10, p. 10, and Pal. N. Y., vol. 6, p. 85, Up. Held. Gr.

Orthopora, Hall, 1887, Pal. N. Y., vol. 6, p. xiv. [Ety. orthos, straight; poros, pore.] Zoarium ramose, solid; cell apertures arranged in parallel, longitudinal rows; intercellular space solid, or occupied near the surface by minute tubuli; no septa. Type $O$. regularis.

bispinulata, Hall, 1884, (Callopora bispinulata,) Rep. St. Geol., p. 14, Ham. Gr.

ornata, Hall, 1887, Pal. N. Y., vol. 6, p. 184, Ham. Gr.

regularis, Hall, 1874, (Trematopora regularis,) 26th Rep. N. Y. St. Mus. Nat. Hist., p. 105, Up. Held. Gr.

reticulata, Hall, 1887, Pal. N. Y., vol. 6, p. 179, Ham. Gr.

rhombifera, Hall, 1874, ('Trematopora rhombifera,) 26th Rep. N. Y. St. Mus. Nat. Hist., p. 106, Up. Held. Gr.

scutulata, Hall, 1881, (Trematopora scutulata,) Trans. Alb. Inst., vol. 10, p. 6 , and Pal. N. Y., vol.6, p. 70, Up. Helò. Gr. Pachydictya, Ulrich, 1882, Jour. Cin. Soc. Nat. Hist., vol. 5, p. 152. [Ety. pachys, thick; dictyon, net.] Zoarium composed of large, thick, branching fronds; cells ovate, separated by interstitial tubes; diaphragms in both sets of tubes; median epithecal plates perforated by minute foramina. Type $P$. robusta.

conciliatrix, Ulrich, 1886, 14th Rep. Geo. Sur. Minn., p. 76, 'Trenton Gr.

everetti, Ulrich, (in press,) Geo. Sur. Ill., vol. $8, \mathrm{pl}$. 33, Trenton Gr.

fimbriata, Ulrich, 1886, 14th Rep. Geo. Sur. Minn., p. 75, Trenton Gr.

firma, Ulrich, (in press,) Geo. Sur. Ill., vol. 8, pl. 31, Hud. Riv. Gr.

foliata, Ulrich, 1886,14 th Rep. Geo. Sur. Minn., p. 73, Trenton Gr.

gigantea, Ulrich, (in press,) Geo. Sur. Ill., vol. $8, \mathrm{pl} .31$, Hud. Riv Gr.

occidentalis, Ulrich, 1886, 14th Rep. Geo. Sur. Minn., p. 75, Trenton Gr.

robusta, Ulrich, 1882, Jour. Cin. Soc. Nat. Hist., vol. 5, p. 173, Trenton Gr.

splendens, Ulrich, (in press,) Geo. Sur. Ill., vol. 8, pl. 31 and 32, Hud. Riv. Gr. Paleschara, Hall, 1874, 26th Rep. N. Y. St. Mus. Nat. Hist., p. 107. [Sig. ancient Eschara.] Incrusting expansions; cells polygonal, oblong, separated by thin solid walls; macula present; no spiniform tubuli or diaphragms. Type $\mathbf{P}$. incrustans. 
amplectans, Hall, 1884, Rep. St. Geol., p. 7, Ham. Gr.

aspera, Hall, 1876, syn. for P. maculata. bifoliata, syn. for Piilodictya nebulosa.

bilateralis, Hall, 1883, Rep. St. Geo., pl. 16, fiy. 22-25, Low. Held. Gr.

concentrica; Hall, 1887, Pal. N. Y., vol. 6, p. 67, Low. Held. Gr.

foliala, syn. for Ptilodictya nebulosa.

incrassata, Hall, 1879,28 th Rep. N. X.

St. Mus. Nat. Hist., p. 121, Niagara Gr. incrustans, Hall, 1874, 26th Rep. N. Y. St.

Mus. Nat. Hist., p. 107, Low. Held. Gr. intercella, Hall, 1884, Rep. St. Geol., p. 5, Ham. Gr.

maculata, Hall, 1876, 28th Rep. N. Y. St.

Mus. Nat. Hist., p. 121, Niagara Gr.

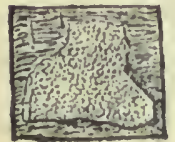

Fig. 495.- Pale. schara offula. offula, Hall, 1876, 28th Rep. N. Y. St. Mus. Nat. Hist., p. 120, Niagara Gr. pertenuis, Hall, 1884, Rep. St. Geol., p. 7. Ham. Gr. radiata, Hiall, 1883, Rep.

St. Geol., p. 16, fig. 13-14, Low. Held. Gr.

reticulata, Hall, 1884, Rep. St. Gcol., p. 6, Ham. Gr.

sphaerion, Hall, 1876, 28th Rep. N. Y. St. Mus. Nat. Hist., p. 121, Niagara Gr.

tenuis, Hall, 1887, Pal. N. Y., vol. 6, p. 36, Low. Held. Gr.

variacella, Hall, 1854, Rep. St. Geol., p. 6, Ham. Gr.

Petalotay 'A, Ulrich, (in press,) Geo. Sur. Ill., vol. 8, p. 377. [Ety. pelalos, spread out; trupa, an opening.] Bifoliate, consisting of irregular, compressed branches or simple fronds, celluliferous on both sides; zoceial tubes prismatic, arising from a strongly flexuous mesial line; apertures subcircular or polygonal ; mesopore-like interspaces, that do not differ in their tabulation from the zoœcia, may occur; very small acanthopores (?) occupy many of the angles of junction. Type P. compressa. compressa, Ulrich, (in press,) Geo. Sur. III., vol. 8, pl. 46, Ham. Gr.

delicata, Ulrich, (in press,) Geo. Sur. III., vol. 8 , pl. 46 , Ham. Gr.

Petigopora, Ulrich, 1882, Journal Cin. Soc. Nat. Ilist., vol. 5, p. 155. [Ety. petigo, scab; poros, pore.] Stnall
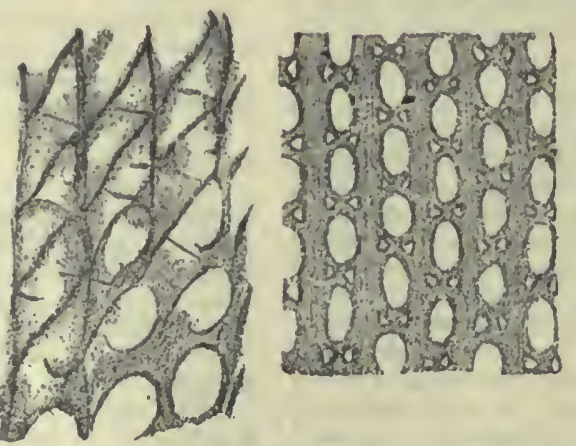

gregaria, Ulrich, 1883, Jour. Cin. Soc. Nat. Hist., vol. 6, p. 155, Hud. Riv. Gr.

petechialis, Nicholson, 1875, (Chetetes petechialis,) Ohio Pal., vol. 2, P. 213, Hud. Riv. Gr.
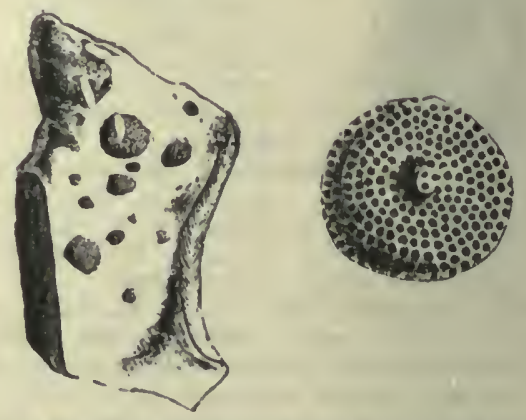

Fia. 496.-1'ellgopora petechinlts on ^ Monticullpora; also, specituen greatly enlarged.

Puacklolora, Ulrich, (in press, Gico. Sur. IIl., vol. 8, p. 36s. [Ety. phakelos, bundle; poros, pore.] Zoarium articulated; scgments short, obconical, consisting of two or more equal, conical zoccia, with slightly contracted circular apertures. Type P. pertenuis.

constricta, Ulrich, (in press,) Geo. Sur. IIl., vol. 8, pl. 29, Trenton (rr.

pertenuis, Úlricl,, (in press.) Geo. Sur. Ill., vol. 8, pl. 29, Galena Gr.

Pil anopora, Hall, 1852, Pal. N. Y., vol. 2, p. 46. [Ety. phaino, to open or make a window; poros, pore.] Zoarium forming thin, broad, or ensiform expansions, celluliferons on both sides; cellules oval and arranged between elevated lines; maculae often developed; distinguished from Ptilodictya and Stictopora by the absence of a nonporiferous, striated edge. Type P. explanata.

patches adhering to forcign objects, with a narrow nonporiferous band or germinating membrane along the outer margin; no interstitial cells; spiniform tubuli present. Type P. gregaria.

asperula, Ulrich, 1883, Jour. Cin. Soc. Nat. Hist., vol. 6, p. 157, Hud. Riv. Gr.

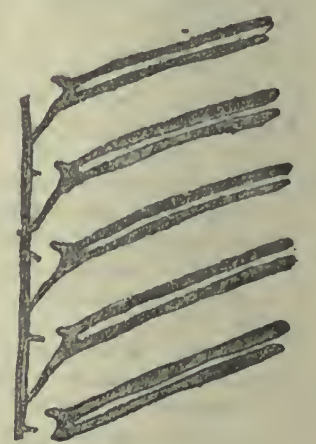

eetlons $\times 50$. 
expansa, Hall \& Whitfield, 1875, Ohio Pal., vol. 2, p. 114, Niagara Gr.

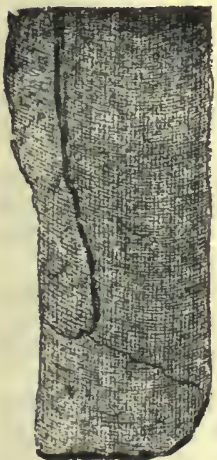

Frg. 498. - Phrenopora expansa. explanata, Hall, 1852, Pal. N. Y., vol. 2, p. 46, Clinton Gr.

multipora, Hall, 1851, Geo. Lake Supp. Land Dist., vol. 2, p. 206, Trenton Gr.

te nu is, Hall, 1874, (Escharopora tenuis, 26th Rep. N. Y. St. Mus. Nat. Hist., p. 99, Low. Held. Gr.

Phractopora, Hall, 1881, Trans. Alb. Inst., vol. 10, p. 12. [Ety. phractos, inclosed; poros, pore.] Zoarium explanate, free or incrusting, frequently contorted, cellulifer-

ous on one or both faces; surface elevated at irregular intervals into prominent crests; cells tubular, without septa ; intercellular structure vesiculose near the base, septate above. Type P. cristata.

cristata, Hall, 1881, Trans. Alb. Inst., vol. 10 , p. 12 , and Pal. N. Y., vol. 6, p. 99, Up. Held. Gr.

cristata var. lineata, Hall, 1887, Pal. N. Y., vol. 6 , p. 99, Up. Held. Gr.

Phyllodictya, Ulrich, 1882, Jour. Cin. Soc. Nat. Hist., vol. 5, 1. 153. [Ety. phyllon, leaf; dictyon, net.] Zoarium forming simple, leaf-like expansions, sometimes branched; cell apertures small, oblique, with the lower $\mathrm{m}$ arg i $\mathrm{n}$ lipped; interstitial spaces min u t e l y granular or p un c tate. Ty p e P. frondosa.

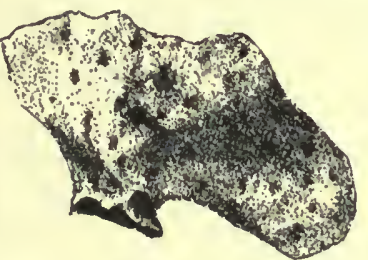

F Ic. 499.-Phyllodicty a frondosa.

frondosa, Ulrich, 1882, Jour. Cin. Soc. Nat. Hist., vol. 5, p. 174, Trenton Gr.

Phyllopora,' King, 1849, Ann. and Mag. Nat. Hist., $2 d$ ser., vol. 3, p. 389. [Ety. phyllon, leaf; poros, perforation.] Zoarium like Fenestella, but having cellulas on the whole of the under surface of the rays in two or more ranges. Type P. ehrenbergi.

aspera, Ulrich, (in press,) Geo. Sur. Ill., vol. 8, pl. 46, Up. Held. Gr.

corticosa, see Subretepora corticosa,

ehrenbergi, Geinitz, 1846, (Gorgonia ehrenbergi,) Grundriss, p. 585, Permian Gr. Very doubtfully identified in America.

superba, Ulrich, (in press,) Geo. Sur. Ill., vol. 8, pl. 46 and 55, Ham. Gr.

variolata, see Subretepora variolata.
Phylloporina, Ulrich, syn. for Subretepora. Pileotry Pa, Hall, 1887, Pal. N. Y., vol. 6, p. xvi. [Ety. pileos, cap; trupa, opening.] Distinguished from Lichenalia by having the posterior portions of the peristomes strongly elevated and arched, with distinct denticulations in the aperture, which, in the course of growth, form two longitudinal striations along the interior of the cell wall. Type P. denticulata.

clivulata, Hall, 1881, (Lichenalia clivulata,) T'rans. Alb. Inst., vol. 10, p. 9, and Pal. N. Y., vol. 6, p. 83, Up. Held. Gr. denticulata, Hall, 1881, (Lichenalia denticulata,) Trans. Alb. Inst., vol. 10, p. 8 , and Pal. N. Y., vol. 6, p. 84, Up. Held. Gr.

granifera, Hall, 1881, (Lichenalia granifera, Trans. Alb. Inst., vol. 10, p. 11, and Pal. N. Y., vol. 6, p. 84, Up. Held. Gr.

pyriformis, Hall, 1881, (Lichenalia pyriformis,) Trans. Alb. Inst., vol. 10, p. 12 , and Pal. N. Y., vol. 6, p. 82, Up. Held. Gr.

Pinacotrypa, Ulrich, (in press,) Geo. Sur. Ill., vol. 8, p, 384. [Ety. pinax, plank; trupa, opening.] Thin, contorted expansions, with a wrinkled epitheca below ; zocecia with subcircular apertures, a well-developed granose peristome, thin walls, and, so far as observed, no lunarium ; interspaces wide, occupied by a single series of very large angular mesopores, which never present the appearance of vesicular tissue; diaphragms horizontal, few in the zoocial tubes, numerous in the mesopores. Type P. elegans.

elegans, Rominger, 1866, (Fistulipora elegans,) Proc. Acad. Nat. Sci. Phil., p. 9, Ham. Gr.

Pinnatopora, syn. for Glauconome.

curvata, see Glauconome curvata.

intermedia, see Glauconome intermedia. minor, see Glauconome minor.

simulatrix, see Glauconome simulatrix. subangulata, see Glauconome subangulata. tenuiramosa, see Glauconome tenuiramosa. vinei, see Glauconome vinii.

whitei, see Glauconome whitii.

youngi, see Glanconome youngi.

Polypora, McCoy, 1845, Carb. Foss. Ireland, p. 206. [Ety. polys, many; poros, pore.] Zoarinm like that of Fenestella, from which it is distinguished by having no median ridge on the celluliferous side of the rays, and in having from three to ten rows of cell openings. Type $P$. dendroides.

aculeata, Hall, 1881, (Fenestella aculeata) Trans. Alb. Inst., vol. 10, p. 21 , and Pal. N. Y., vol. 6, p. 157, Up. Held. Gr. adnata, Hall, 1881, (Fenestella adnata,) Trans. Alb. Inst., vol. 10, p. 25, and Pal. N. Y., vol. 6, p. 152, Up. Held. Gr.

albionensis, Spencer, 1884, Bull. No. 1, Univ. St. Mo., p. 55, Niagara Gr. 
approximata, Ulrich, (in press,) (ieo. Sur. Ill., vol. 8, pl. 61, Kaskaskia Gr.

arkonensis, S. A. Miller, 1883, 2 d ed. Am. Pal. Foss., p. 292, Ham. Gr. Proposed instead of P. tuberculata, Nicholson, in (Feo. Mag. for A pril, 1874, and Rep). Pal. Prov. Ont., p. 100 , tigs. 37 , a, b, c. Found at Arkona, township of Bosanquet, Canada.

arta, Hall, 1879, (Fenestella arta,) 32d Rep. N. Y. St. Mus. Nat. Hist., p. 163, Low. Held. Gr.

biarmica, Keyserling, 1846, Geognost. Beobacht, p. 191. Geinitz referred a form from the Coal Meas., and Prout referred one from the Kaskaskia Gr. to it. Probably not an American species.

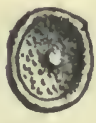

FIg. 500.-Polypora biseriatn. A perture fiaving the central perforatifun of the cover closed, $x$ i 0 . biseriata, Ulrich, (in press,) Geo. Sur. Ill., vol. 8, pl. 60 , Warsaw and St. Louis $\mathrm{Fr}$.

blandida, Ulrich, 1886, Contri. to Amer. Pal., p. 18, Up. Held. Cir.

brevisulcata, IIall, 1881, (Fenestella brevisulcata,) Trans. Alb. Inst. vol. 10, p. 26 , and Pal.

N. Y., vol. 6, p. 168, Up. Held. (ir.

burlingtonensis, Ulrich, (in press,) (ieo. Sur. Ill., vol. 8, pl. 59, Burlington Gr.

(earinella, Hall, 1887, Pal. N. Y., vol. 6, p. $153, \mathrm{Up}$. Held. Gr.

celsipora, Hall, 1881, (Fenestella celsipora, ) Trans. Alb. Inst., vol. 10, p. 24, and Pal. N. Y., vol. 6, p. 150, Up. Held Gr.

celsipora var. uninima, Hall, 1881, (Fenestella celsipora var. minima,) Trans. Alb., Inst., vol. 10, p. 24 , and Pal. N. Y., vol. 6, p. 151, Up. Held. Gr.

celsipora var. minor, Hall, 1881, (Fenestella, celsipora var. minor,) Trans. Alb. Inst., vol. 10, p. 24, and Pal. N. Y., vol. 6, p. 151, Up. Held. Gr.

cestriensis, Ulrich, (in press,) Geo. Sur. IIl., vol. 8, pl. 60, Kaskaskia Gr.

compacta, Hall, 1879, (Fenestella compacta,) 32d Rep. N. Y. St. Mus. Nat. Hist., p. 163, Low. Held. Gr.

complanata, Ulrich, (in press,) Geo. Sur. Ill., vol. 8, pl. 60, Kaskaskia Gr.

compressa, Hall, 1878, (Fenestella Compressa,) 32 d Rep. N. Y. St. Mus. Nat. Hist., p. 164, Low. Held. Gr.

corticosa, Ulrich, (in press,) Gieo. Sur. Ill., vol. 8, pl. 61, Kaskaskia Gr.

crebescens, Hall, 1887, Pal. N. Y., vol. 6, p. 170, Up. Held. Gr.

crassa, Ulrich, (in press,) Geo. Sur. Ill., vol. 8, pl. 61, Up. Coal Meas.

cultellata, Hall, 18S1, (Fenestella cultellata, ) Trans. Alb. Inst., vol. 10, p. 21, and Pal. N. Y., vol. 6, p. 160, Up. Held. Gr. distans, Hall, 1881, (Fenestella distans, ) Trans. Alb. Inst., vol. 10, p. 24, and Pal. N. Y., vol. 6, p. 161, Up. Held. Gr. elegans, Hall, 1974, 26th Rep. N. Y. St. Mus. Nat. Hist., p. 97, Low. Held. Gr. elongata, Hall, 1882, Rep. St. Geol. and Pal. N. Y., vol. 6, p. 153 , Up. Held. Gr.

eudora, Hall, 1887, Pal. N. Y., vol. 6, p. 58, Low. Held. Gr.

fistulata, Hall, 1884, (Fenestella fistulata,) 36 th Rep. N. Y. St. Mus. Nat. Hist., p. 59, Ham. Gr.

flabelliformis, Hall, 1851, (Fenestella flabelliformis,) Trans. Alb. Inst., vol. 10, p. 23, and Pal. N. Y., vol. 6, 1. 161, Up. Ileld. Gr.

gracilis, Prout, 1860, Traus. St. Iouis Acad. Sci., p. 580, Warsaw (ir.

grandis, Toula, 1875, N. Jahrbuch, 1). 230, Carboniferous.

granilinea, Hall, 1881, (Fenestella granilinea,) Trans. Alb. Inst., vol. 10, p. 27, and Pal. N. Y., vol. 6, p. 154, Up. Held. Gr.

hallana, Prout, 1860, Trans. St. Iouis A cad. Sei., vol. 1, p. 580, W arsaw Gr.

hamiltonensis, Prout, 1866, Geo. Sur. Ill., vol. 2, p. 423, Ham. Gr.

hexagonalis, Ilall, 1881, (Fenestella luexagonalis,) Truns. Alb. Inst., vol. 10, p. 27, and Pal. N. Y., vol. 6, 1). 164, U1). Held. Gr.

hexagonalis var. foraminulosa, Hall, 1881, (Fenestella hexagonalis var. foraminulosa,) Trans. Alb. Inst., vol. 10, 1. 27, and Pal. N. Y., vol. (i, p. 165, Up. Held. (ir.

idothea, Hall, 1879, (Fenestella idother,) $32 d$ Rep. N. Y. St. Mus. Nat. Hist, p. 97, Low. Ifeld. Gr.

imbricata, l'rout, 1866, Trans. St. Louis Acad. Sci., vol. 2, ए. 412, Devonian.

impressa, Ulrich, 1888, Bull. Denison Univ., vol. 4, p. 72, Cuyatıoga Sliale.

incepta, Hall, 1852, ['al. N. Y., vol. 2, p. 167, Niagara Gir.

intermedia, Prout, 1858, 'Trans. St. I.ouis Acad. Sci., vol. 1, p. 272, Up. Held Gr.

lrevinodata, Hall, 1881, (Fenestella lævinodata, Trans. Alb. Inst., vol. 10, p. 28, and Pal. N. Y., vol. 6, p. 169, Up. Held. Gr.

lievistriata, Hall, 1883, Rej. St. (ieol. and Pal. N. Y., vol. (i, p. 159, Up. Held. (ir.

largissima, Hall, 1881, (Fenestella largissima), Trans. Alb. Inst., vol. 10, 1. 22, and 1'yl. N. Y., vol. 6, 1. 156, Up. Held. (ir.

lilea, Hall, 1974, 26ith Rel. N. Y. St. Mus. Nat. Hist., p. (i2, Low. He)d. (ir.

maccoyana, Ulrich, (in press, (ieo. Sur. Ill., vol. 8, yl. 59. Keokuk Gr.

megastoma, DeKoninck, 1863, Quar. Jour. fieo. Soc., vol. 19, p. 5, Carboniferous. mexicana, Prout, 1858, Trans. St. Louis Acad. Sei., vol. 1, p. 270 , Permian (ir.

mutabilis, Hall, 1881, (Fenestella mutabilis,) Trans. Alb. Inst., vol. 10, p. 25, and Pal. N. Y., vol. 6, p. 166, Up. Held. (ir.

nexa, Hall, 1881, (lienestella nexa,) Trans. Alb. Inst., vol. 10, p. 25 , and Pal. N. Y., vol. 6, p. $165, U_{p}$. Held. Gir. 
nodocarinata, Ulrich, (in press,) Geo. Sur. Ill., vol. 8, pl. 61, Coal Meas.

obliqua, Hall, 1887, Pal. N. Y., vol. 6, p. 64, Low. Held. Gr.

rapillata, McCoy, 1862, Carb. Foss. of Ireland, p. 226, Up. Coal Meas.

paxillata, Hall, 1879, (Fenestella paxillata,) 32d Rep. N. Y. St. Mus. Nat. Hist., p. 164, Low. Held. Gr.

perangulata, Hall, 1881, (Fenestella perangulata,) Trans. Alb. Inst., vol. 10, $\mathrm{p}$. 23, and Pal. N. Y., vol. 6, p. 162, Up. Held, Gr.

perundata, Hall, 1881, (Fenestella perundata,) Trans. Alb. Inst., vol. 10, p. 27, and Pal. N. Y., vol. 6, p. 163, Up. Held. Gr.

pornsa, Hall, 1881, (Fenestella porosa,) Trans. Alb. Inst., vol. 10, p. 26, aud Pal. N. Y., vol. 6 , p. 163 , Up. Held. Gr.

propria, Hall, 1881, (Fenestella propria,) Trans. Alb. Inst., vol. 10, p. 22, and Pal. N. Y., vol. 6, p. 157, Up. Held. Gr.

(?) psyche, Billings, 1874, Pal. Fcss., vol. 2, j. 11, Gaspe limestone No. 8, Devonian. pulchella, Nicholson, 1874, Geo. Mag. Lond. n. 8., vol. 1, p. 161, Corniferous Gr. yuadrangularis, Hall, 1881, (Fenestella quadrangularis,) Trans. Alb. Inst., vol. 10, p. 21, and Pal. N. Y., vol. 6, p. 158, Up. Held. Gr.

rallialis, Ulrich, (in press,) Geo. Sur. Ill., vol. 8, pl. 60, Keokuk Gr.

retrorsa, Ulrich, (in press,) Geo. Sur. Ill., vol. 8, pl. 59, Keokuk Gr.

rigida, Prout, 1866, Trans. St. Louis Acad. Sci., vol. 2, p. 412, Up. Held. Gr.

rigida, Hall, 1881, (Fenestella rigida,)

Trans. Alb. Inst., vol. 10, p. 22, and Pal.

N. Y., vol. 6 , p. 155 , Up. Held. Gr.

robusta, Hall, 1881, (Fenestella robusta,)

Trans. Alb. Inst., vol. 10, p. 22, and Pal.

N. Y., vol. 6 , p. 156 , Up. Held. Gr.

rustica, Hall, 1887, Pal. N. Y., vol. 6, p. 169, Up. Held. Gr.

separata, Hall, 1882, Rep. St. Geol, and Pal. N. Y., vol. 6, p. 166, Up. Held. Gr.

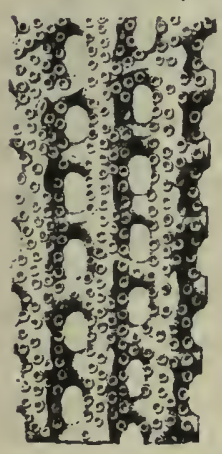

FIG. 501.-Poly pora sh um ardi. Enlaiged. shumardi, Prout, 1858,

Trans. St. Louis Acad.

Sci., vol. 1, p. 271, Up. Held. Gr.

simulatrix, Ulrich, (in press,) Geo. Sur. Ill., vol. 8 , pl. 59, Keokuk Gr.

spinulifera, Ulrich, (in press,) Geo. Sur. Ill., vol. 8 , pl. 61 , Kaskaskia Gr. and Coal Meas.

stragula, White, 1874, Rep. Invert. Foss., p. 19 , and Geo. Sur. W. 100th Mer. vol. 4, p. 108, Coal Meas.

striatopora, Hall, 1881, (Fenestella striatopora,) Trans. Alb. Inst., vol. 10 , p. 23, and Pal. N. Y., vol. 6, p. 168, Up. Held. Gr. stricta, Hall, 1887, Pal. N. Y., vol. 6, p. 59, Low. Held. Gr.

submarginata, Meek, 1872, Pal. E. Neb., p. 154 , Coal Meas.

submutans, Hall, 1881, (Fenestella submutans,) Trans. Alb. Inst., vol. 10, p. 21, and Pal. N. Y., vol. 6, p. 167, U!. Held. Gr.

tenella, Nicholson, 1874, Geo. Mag. Lond. n. s., vol. 1, p. 162 , Corniferous Gr.

transversa, Ulrich, 1886, Cont. to Am. Pal., p. 18, Up. Held. Gr.

tuberculata, Prout, 1859 , Trans. St. Louis Acad. Sci., vol. 1, p. 449, Kaskaskia Gr. tuberculata, Nicholson, see P. arkonensis. varsoviensis, Prout, 1858, Trans. St. Louis Acad. Sci., vol. 1, p. 237, WVarsaw Gr.

varsoviensis var. spininodata, Ulrich, (in press,) Geo. Sur. Ill., vol. 8, pl. 60, Warsaw Gr.

wbitii, Ulrich, (in press,) Geo. Sur. Ill., vol. 8 , pl. 62, Coal Meas.

whitii var. eximia, Ulrich, (in press, ) Geo. Sur. Ill., vol. 8, pl. 62, Coal Meas.

Prismopora, Hall, 1881, Bryozoans of the Up. Held. Gr., p. 17. [Ety. prismos, the hole made by a cylindrical saw; poros, pore.] Ramose, branches triangular, dichotomous, each side celluliferous; tubes radiate from the center to each angle, margins noncelluliferous; interstitial spaces smooth, vesicular. Type $\mathrm{P}$. triquetra.

dilatata, Hall, 1884, Rep. St. G'eol., p. 50, Ham. Gr.

lata, Hall, 1887, Pal. N. Y., vol. 6, p. 266, Ham. Gr.

minima, Ulrich, (in press,) Geo. Sur. Ill., vol. 8, pl. 78, Coal Meas.

paucirama, Hall, 1881, Bryozoans of the Up. Held. Gr., p. 17, and Rep. St. Geol., 1883, pl. 25, fig. 11, Up. Held. Gr.

serrata, Meek, 1875, (Ptilodictya serrata,) Pal. Ohio, vol. 2, p. 327, Coal Meas.

serrulata, Ulrich, 1884, Jour. Cin. Soc. Nat. Hist., vol. 7, p. 41, Kaskaskia Gr. Perhaps the same as $\mathbf{P}$. serrata.

sparsipora, Hall, 1881, (Thallostigma sparsipora,) Trans. Alb. Inst., vol. 10, $p$. 13, and Pal. N. Y., vol. 6, p. 288, Up. Held. Gr.

triquetra, Hall, 1881, Bryozoans of the Up. Held. Gr., p. 17, and Rep. St. Geol. 1883, pl. 25, fig. 8-10, Up. Held. Gr.

Protocrisina, Ulrich, (in press,) Geo. Sur. Ill., vol. 8, p. 369. [Ety. protos, first; Crisina, a genus.] Ramose, celluliferous on one side only; cells subtubular, with prominent circular apertures; reverse finely grano-striate; small pores, apparently communicating with the interior of the zooecia, are rather irregularly distributed over both sides of the branches ; axis thin, cruciform in transverse section; external walls thick. Type P. exigua.

exigua, Ulrich, (in press,) Geo. Sur. Ill., vol. 8, pl. 29 and 53, Trenton and Hud. Riv. Gr. 
Proutella, Ulrich, (in press, ) Geo. Sur. Ill., vol. 8, p. 403. [Ety. proper name.] Discoid, thin, free, lower surface convex and lined with a concentrically wrinkled epitheca; primary zocecia subtubular, the sucreeding ones shorter, all rather thin-walled; aperture broadelliptical, surrounded by a narrow, sloping area, hexagonal in outline; when perfect, with a depressed delicate calcareous plate, that closes a little less than two-thirds of the opening, the orifice left being subtriaugular in form, without thickened margins, and situated at the anterior side; with age, a second, third, and more layers of zoœcia are developed directly over the first, so that they gradually form a zocecial tuhe seemingly baving the cavity intersected by incomplete diapliragins; these appear to spring from the posterior wall, and extend about one-half the distance ncross. Type Cyclopora discoidea. Syn.? for Cyclopora.

discoidea, Pront, 1860, (Cyclopora discoidea,) Trans. St. Louis Acad. Sci., vol. 1, p. 578, Keokuk (ir.

Pteropora duogeneris, Hall, syn. for Tæniopora exigua.

Ptilionella, Hall, 1884, Rep. St. Geol., p. 56, syn. for Reptaria.

nodata, see Reptaria nodata.

penniformis, see Reptaria penniformis.

Ptilodictya, Lonsdale, 1839, Murch. Sil. Syst., p. 676. [Ety. ptilon, feather; dictyon, net.] Tho correct orthography is Ptilodictyon. Zoarium pointed below, articulating into a spreadiog base, above a leaf-like expansion, which is sometimes lobed at the distal extremity, celluliferous on both faces, divided by a mesial lamina; margin without cells; apertures circular or subquadrate; no intercellular tissue. Type $\mathrm{P}$. lanceolata.

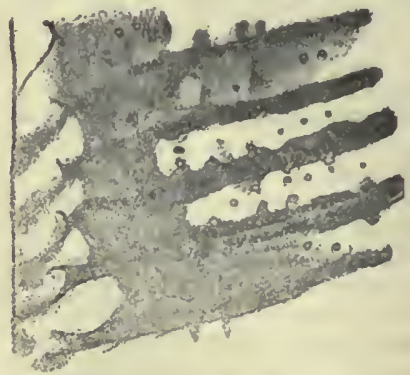

FIG. 502.-Ptllodletya maculata. Vertical scction x 35 , showing splnous process.

acuminata, James, 1876, Int. Catal. Cin. Foss., p. 3, Hud. Riv. Gr. Not well defined.

alcyone, see Pachydictya alcyone. arctipora, see Bythopora arctipora. arguta, see Stictopora arguts.

bipunctata, Van Cleve, 1883, 12th Rep. Ind. Geol. and Nat. Hist., p. 266, Niara Gr.

briareus, Ulrich, 1Ss2, Jour. Cin. Soc. Nat. Hist., vol. 5, p. 164, Trenton Gr.

canadensis, Billings, 1566, Catal. Sil. Foss. Antic., p. 9, Hud. Rıv. Gr.

carbonaria, see Stictopora curbonaria. cosciniformis, see Coscinella cosciniformis. dictyota, Mrek, 1873, Huyden's 6th Rep. Geo. Sur. Terr., p. 465, Subcarboniferous. emacerala, see Dicranopora emacerata. excellens, see Phanopora excellens.

explicans, Sufford, 1869, Geo. of Tenn. Not defined.

falciformis, Nicholson, 1875, Ohio Pal., vol. 2, p. 259, Hud. Riv. Gr.

fenestelliformis, Nicholson, 1875, Ohio Pal., vol. 2, p. 263, Hud. Riv. Gr.
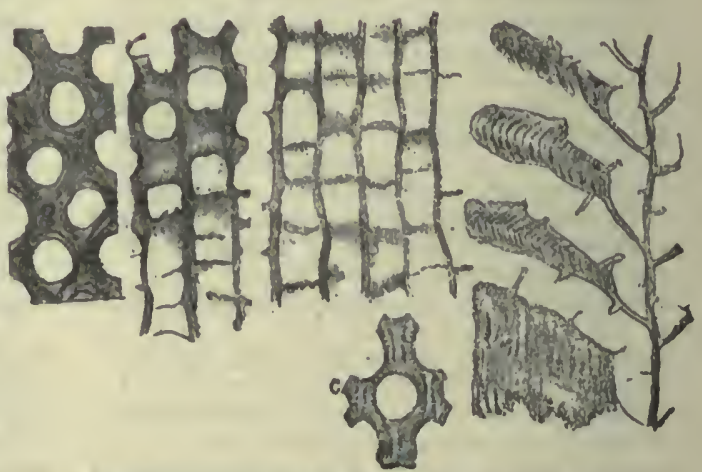

Fio. 503.-Pthodictya maguiten. Sectims $x$ 50.

flagellum, Nicholson, 1875, Ohio I'al., vol. 2 , p. 26?, Hud. Riv. Gr.

fragilis, seo Dicranopora fragilis.

gladiola, Billings, 1866, Catal. Sil. Foss., Antic., p. 10, Anticosti Gr.

hilli, James, 1882, (as tigured by Ulrich,) Jour. Cin. Soc. Nat. Hist., vol. 5, pl. 7, Trenton Gr.

internodia, see Dicranopora internodia.

libana, Safford, 1869, Geo. of Tenn. p. 286, Trenton Gr.

lirata, Hall, 1874, (Escharopora lirata,) 26 th Rep. N. Y. St. Mus. Nat. Hist., p. 100, Low. Held. Gr.

maculata, Ulrich, 1882, Jour Cin. Soc. Nat. Hist., vol. 5, p. 163, Hud. Riv. Gr. magnifica, S. A. Miller, 1878, Jour Cin. Soc. Nat. Hist., rol. 1, p. 100, Hucl. 1Riv. Gr. meeki, Nicholson, 1874, Geo. Mag. n. s., vol. 1, p. 123, Corniferous and Ham. Gr. multiramis, Safford. Not defined.

nebulosa, Hall, 1874, (Escharopora nebulosa,) 26th Rep. N. Y. St. Mus. Nat. Hist., p. 99, Low. Held. Gr.

nilidula, see Dicranopora nitidula.

nodosa, James, 1882, (as figured by U1rich), Jour. Cin. Soc. Nat. Hist., vol..5, pl. 7, Hud. Riv. Gr. The name was preoccupied. Sce P. variabilis. 
obliqua, Ringueberg, 1884, (Stictopora rbliqua,) Proc. Acad. Nat. Sci., p. 146, Clinton Gr. Not well defined.

parallela, Hall, 1887, Pal. N. Y., vol. 6, p. 270, Ham. Gr.
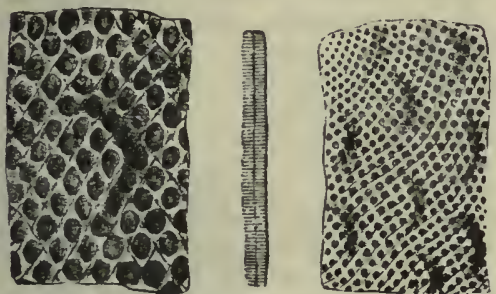

FIG. 504-Ptilodictya pavonia. Natural size, and maguified.

pavonia, D’Orbigny, 1850, Prodr. de Paleont., t. 1, p. 22, Hud. Riv. Gr.

perelegans, see Graptndictya perelegans.

plumaria, James, 1882, (as figured by Ulrich,) Jour. Cin. Soc. Nat. Hist., vol. 5, pl. 7, Hud. Riv. Gr.

plumea, Hall, 1887, Pal. N. Y., vol. 6, p. 271, Ham. Gr.

punctata, Nicholson \& Hinde, 1874, Can. Jour., p. 7, Clinton Gr.

ramosa, Ulrich, 1882, Jour. Cin. Soc. Nat. Hist., vol. 5, p. 164, Trenton Gr.

retiformis, Hall, 1887, Pal. N. Y., vol. 6, p. 272 , Ham. Gr.

rustica, see Stictopora rustica.

scutulata, Hall, 1884, (Stictopora scutulata,) Rep. St. Geol., p. 47, Ham. Gr. serrata, see Prismopora serrata.

subrecta, Ulrich, 1886, 14th Rep. Geo. Sur. Minn., p. 63, Trenton Gr.

sulcata, Billings, 1886, Catal. Sil. Foss.

Antic., p. 35 , Anticosti Gr.

superba, Billinga, 1866, Catal. Sil. Foss.

Antic., p. 35, Anticosti Gr.

symmetra, Saffurd. Not defined.

tarda, Billings, 1874, Pal. Foss., vol. 2, p.

13, Gaspe Limestone No. 8, Devonian.

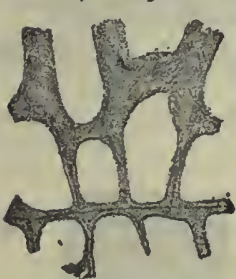

Fig. 505.-Ptilodictya variubijis. 'Trans. verse section $x 50$ showiug tíne basal portion of the iwo layers of zocela, and the duplex character of the median of lamina. Between the plates there is no sertes of median tubuli. tenera, see Stictopora tenera.

tenuis, see Phaenopora tenuis.

triangulata, White, 1878, Proc. Acad. Nat. Sci., p. 35, and Cont. to Pal. No. 6, p. 131, Coal Meas.

variabilis, Ulrich, instead of $P$. nodosa, James, that was preoccupied, Hud. Riv. Gr.

whiteavesi, Ulrich, (in press,) Micropalieontology, p. 18, Hud. Riv. Gr. (?)

Tilopora, McCoy, 1844 , Syn. Carb. Foss. Ireland, p. 200. [Ety. ptilon, plume; poros, pore.] Flabelliform attached by roots, from which a strong midrib arises, giving origin on each side to thin, equidistant rays, connected by regular dissepiments; external face of the rays carinate and bearing two rows of pores. Type P. flustriformis.

acuta, Ulrich, (in press,) Geo. Sur. Ill., vol. 8, pl. 65̃, Burlington and Keokuk Gr.

cylindracea, Ulrich, (in press,) Geo. Sur. Ill., vol. 8, pl. 66, Keokuk Gr.

infrequens, Hall, 1887, Pal. N. Y., vol. 6, p. 28t, Ham. Gr.

nodosa, Hall, 1884, Rep. St. Geol., p. 59, Ham. Gr.

paupera, Ulrich, (in press,) Geo. Sur. Ill., vol. 8, pl. 66, Keokuk Gr.

prouti, Hall, 1858, Geo. Rep. Iowa, p. 653, Warsaw Gr.

striata, Hall, 1884, Rep. St. Geol., p. 58, Ham. Gr.

valida, Ulrich, (in press,) Geo. Sur. Ill., vol. 8, pl. 65 and 66, Keokuk Gr.

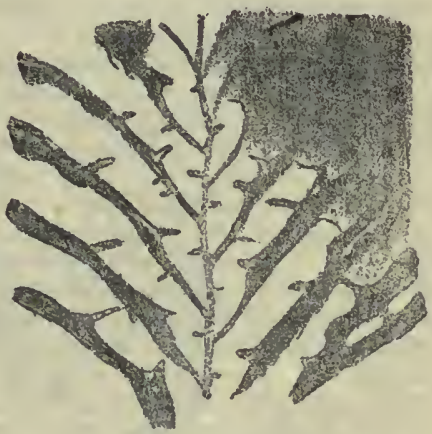

Fra. 506.-Ptllodictya variabilis. Vertical section x 35 , showing hemisepti.

Ptiloporella, Hall, 1887, Pal. N. Y., vol. 6, p. xxiv. [Ety. from the genus Ptilopora.] Bryozoum growing in the same manner as Ptiloporina, but with only two ranges of cell apertures on the branches. Type P. laticrescens.

inæqualis, Hall, 1887, Pal. N. Y., vol.6, p. 171, Up. Held. Gr.

laticrescens, Hall, 1887, Pal. N. Y., vol. 6, p. 171, Up. Held. Gr.

nervata, Nicholson, 1875, (Fenestella nervata,) Obio Pal., vol. 2, p. 264, Niagara Gr.

Ptiloporina, Hall, 1887, Pal. N. Y., vol. 6, p. xxiv. [Ety. from the genus Ptilopora.] Resembling Fenestella, some branches larger than others; ordinary branches originate laterally from one or both sides of the primary branches, not bifurcating as in ordinary forms of Fenestella; cell apertures in three or more ranges. Type $\mathrm{P}$. conica.

conica, Hall, 1887, Pal. N. Y., vol. 6, p. 172, Up. Held. Gr.

disparilis, Hall, 1887, Pal. N. Y., vol. „6, p. 173 , Up. Held. Gr.

pinnata, Hall, 1887, Pal. N. Y., vol. 6, p. 172, Up. Held. Gr. 
sinistralis, Hall, 1887, Pal. N. Y., vol. 6, p. 174, Up. Held. Gr.

Ptilotrypa, Ulrich, (in press,) Geo. Sur. Ill., vol. 8, p. 393. [Ety. ptilon, feather ; trupa, an opening.] Bifoliate, forming large ramose expansions. Zoceial tubes and apertures very oblique; at the upper extremity of the acutely ovate aperture there is a small cell which is best seen in tangential sections; surface with irregular, longitudinally channeled spots. Type P. obliquata.

obliquata, Ulrich, (in press,) Geo. Sur. Ill., vol. 8 , pl. 30 , Hud. Riv. Gr.

Ramipora, Toula, 1875, Permo-CarbonFossilien von der West Küste von Spitzbergen, p. 6. [Ety. ramus, branch; poros, pore.] Staff in cross section, rounded, rhombic, with keel on both sides; branches in pairs, one on each side, and these connected in like manner by rays, upward and downward; pores on one side, on each side of the keel. Type R. hochstetteri.

hochstetteri, 'Toula, 1875, Permo-CarbonFossilien von der West Küste von Spitzbergen, p. 6, Carboniferous.

Reptakia, Rolle, 1851, Leonlard \& Bronn, Neues Jahrb., p. 810. [Ety. repto, to ereep.] Zoarium parasitic, procumbent, attached its entire leugth; consisting of a rachis, from which proceed laterally, at regular intervals, cylindrical cell tubes, and at irregular distances tubes which have the same manner of growth as the primary rachis; cell-tubes turn abruptly outward at their distal extremities, and open in an aperture parallel with the axis of the branch. Type R. stolonifera.

nodata, Hall, 1884, (Ptilionella nodata) Rep. St. Geol., p. 57, Iam. Gr.

penniformis, Hall, 1884, (Ptilionella penniformis,) Rep. St. Geol., p. 56, Hain. Gr. stolonifera, Rolle, 185!, Leonhard \&

Bronn, Neues Jahrb., p. 810, Ham. Gr. Retepora, Lamarck, 1801, Syst. An. sans. Vert. [Ety. rete, net; poros, pore.] Not a Palæozoic genus.

angulata, see Subretepora angulata.

antiqua, as identified by d'Archiac \& Verneuil. Not Americain:

archimedes, see Archimedes.

asperato-striata, see Subretepora asperatostriata.

clintoni, Vanuxem, 1842, Geo. Rep. 3d Dist. N. Y. Not recognized.

diffusa, see Thamniscus diffusa.

fenestrala, see Subretepora fenestrata.

foliacea, Hall, 1847. This name I'rof.

Hall says may be erased from the list. grasilis, see Subretepora gracilis.

hamiltonensis, see Reteporina hamiltonensis.

incepta, see Subretepora incepta.

phillipsi, see Reteporina phillipsi.

prisca, see Reteporina prisca.

irentonensis, see Subretepora trentonensis.
ReteporiNa, D'Orbigny, 1850, Proilr. d. Paléont., t. 1., p. 101. [Ety. from Retepora.] Resembling Retepora, but having on the greater part of the branches only two ranges of cell apertures; branches connected by anastomosis or by dissepiments so short as to be essentially wanting. Type $R$. prisca.

coalescens, Hall, 1887, Pal., N. Y., vol. 6, p. 120, Up. Held. Gr.

hamiltonensis, Prout, 1866, (Retepora hamiltonensis,) Trans. St. Louis, A cad. Sci., vol. 2, p. 412, Ham. Gr.

perundulata, Hall, 18S4, (Fenestella perundulata,) 36tlı Rep. N. Y. St. Mus. Nat. Hist., p. 63, Hain. (ir.

phillipsi, Nieholson, 1874, (Retepora phillipsi,) Geo. Mag. n. s., vol. 1, p. 163, Corniferous $\mathrm{Gr}$.

prisca, Goldfuss, 1831, (Retepora prisea,) Petref. Gern., vol. i, p. 103, IIam. (ir. rhombifera, Ifall, 1881, (Fenestella rhombifera,) Trans. Alb. Inst., vol. 10, p. 32, and Pal. N. Y., vol. 6, p. 120, Up. Held. Gr.

striata, Hall, 1884, (Fenestella striata,) 36th Rep. N. Y. St. Mus. Nat. Hist., p. 72, Ham. Gr.

RHinidictYa, Ulrich, 1882, Jour. Cin. Soc. Nat. Hist., vol. 5, p. 152. [Ety. rhine, file; diclyon, net.]. Zoarium narrow, branching at long intervals; cells surrounded by a close series of small spiniform tubuli; otherwise like Stictopora. Type R. nicholsoni. Syn. for Sulcopora probably.
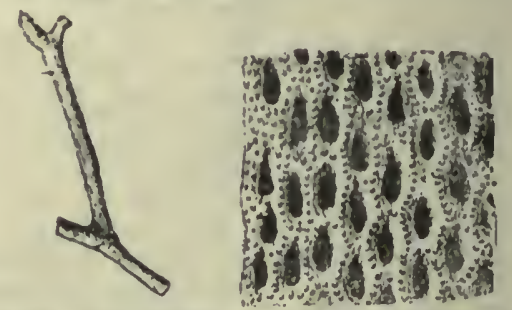

Fia. 507.- Rhinidictya nichoisonl. Natural size and mignifled is dium.

granulosa, Hall, 1887, Pal. N. Y., vol. vi, p. 40, Low. Held. Gr.
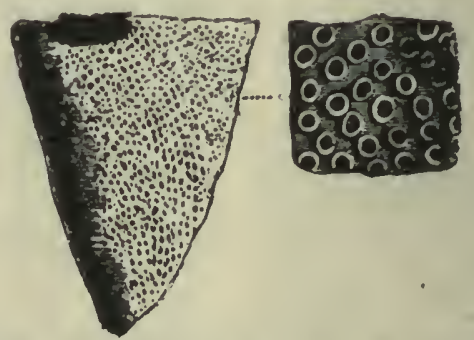
Fig. 508. - Rhinopora verrucosa. Natural size
and enlarged.

nicholsoni, Ulrich, 1882, Jour. Cin. Soc. Nat. Hist., vol. 5, p. 170, Trenton Gr. 
Rhinopora, Hall, 1852, Pal. N. Y., vol. 2, p. 48. [Ety. rhine, file; poros, pore.] Expanded or subcylindrical and hollow; celluliferous on two sides; cells arranged in quincunx order, roundish or oval, and raised in little pustules over the surface. Type $R$. verrucosa.

curvata, Ringueberg, 1886, Bull. Buf. Soc. Nat, Sci., vol. 5, p. 19, Niagara Gr.

frondosa, Hall \& Whitfield, 1875, Ohio Pal., vol. 2, p. 112, Niagara Gr.

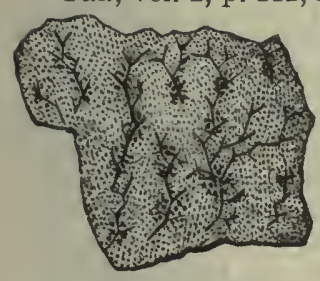

F1G. 509.-Rhinopora frondosa. tuberculosa, Hall, 1852, Pal. N. X., vol. 2, p. 170 , Niagara Ġr.

tubulosa, Hall, 1852, Pal. N.Y., vol. 2 , p. 49 , Clinton Gr.

venosa, Spencer, 1884, Bull. No.

St. Mo., p. 54, Clinton Gr. 1, Mus. Univ.

verrucosa, Hall, 1852, Pal. N. Y., vol. 2, p. 48, Clinton Gr.

RномвороRA, Meek, 1872, Pal. Eastern Nebraska, p. 141. [Ety.rhombos, rhomb; poros, pore.] Ramose, tubular, cells short; septa none; corallites radiating obliquely outward and upward on all sides from an imaginary axis; mouths rhombic or rhombic oval, and arranged in longitudinal and oblique spiral rows; interspaces thick, with minute pores visible in microscopic sections. Type $R$. lepidodendroidea.

armata, Ulrich, 1884, Jour. Cin. Soc. Nat. Hist., vol. 7, p. 31, Kaskaskia Gr.

(?) asperrima, Ulrich, (in press,) Geo. Sur. Ill., vol. 8, pl. 70, Keokuk Gr.

attenuata, Ulrich, (in press,) Geo. Sur. Ill., vol. 8, pl. 70, Keokuk or Warsaw Gr.

confluens, see Acanthoclema confluens.

crassa, Ulrich, 1884, Jour. Cin. Soc. Nat. Hist., vol. 7, p. 25, Up. Coal Meas.

decipiens, Ulrich, (in press,) Geo. Sur. Ill., vol. 8, pl. 71, St. Louis Gr.

dichotoma, Ulrich, (in press,) Geo. Sur. Ill., vol. 8, pl. 70, Burlington Gr.

elegantula, Ulrich, 1884, Jour. Cin. Soc. Nat. Hist., vol. 7, p. 33, Kaskaskia Gr. exigua, Ulrich, (in press,) Geo. Sur. Ill., vol. 8 , pl. 70 , Burling. ton Gr.

gracilis, Ulrich, (in press,) Geo. Sur. III., vol. 8 , pl. 70, Burling. ton Gr.

incrassata, Ulrich, 1888, Bull. Denison Univ., p. 89 , C u y a o g a shales.

lepidodendroidea, Meek

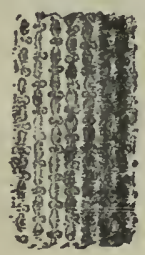

1872, Pal. Eastern Neblaska, $\rho .141$, Up. Coal Meas. olıioensis, Ulrich, 1888, Bull. Denison Univ., p. 90, Cuyahoga Shales.

persimilis, Ulrich, 1884, Jour. Cin. Soc. Nat. Hist., vol. 7, p. 30, Kaskaskia Gr. pulchella, Ulrich, 1884, Jour. Cin. Soc. Nat. Hist., vol. 7, p. 31, Kaskaskia Gr. simulatrix, Ulrich, (in press,) Geo. Sur. Ill., vol. 8, pl. 71, St. Louis Gr.

(?) spiralis, Ulrich, (in press,) Geo. Sur. Ill., vol. 8, pl. 71, Keokuk Gr.

subannulata, Ulrich, (in press,) Geo. Sur. IIl., vol. 8, pl. 45, Ham. Gr.

sulcifera, Ulrich, (in press,) Geo. Sur. Ill., vol. 8, pl. 45, Ham. Gr.

tabulata, Ulrich, (in press,) Geo. Sur. Ill., vol. 8, pl. 70, Kaskaskia Gr.

tenuirama, Ulrich, (il press,) Geo. Sur. Ill., vol. 8, pl. 70, Kaskaskia Gr.

transversalis, Ulrich, (in press,) Geo. Sur. Ill., vol. 8, pl. 71, Keokuk Gr.

varia, Ulrich, (in press,) Geo. Sur. Ill., vol. 8, pl. 71, Keokuk Gr.

wortheni, Ulrich, 1884, Cin. Soc. Nat. Hist., vol. 7, p. 32, Kaskaskia Gr.

Rhopalonaria, Ulrich, 1879, Jour. Cin. Soc. Nat. Hist., vol. 2, p. 26. [Ety. ropalon, a club.] Cells slender, fusiform, in single anastomosing series; cell mouths near the middle of the cells. Type $R$. venosa.

pertenuis, see Stomatopora pertenuis.

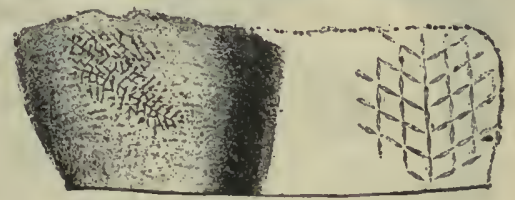

F'1G. 511.-Rhopalonaria venosa.

venosa, Ulrich, 1879, Jour. Cin. Soc."Nat. Hist., vol. 2, p. 26, Hud. Riv. Gr.

Sagenelia, Hall, 1852, Pal. N. Y., vol. 2, p. 172. [Ety. sagenella, a little dragnet.] Membranous net incrusting other bodies; cells in parallel or diverging series, more or less oblongquadrangular, and separated by a thin lamina. Type S. membranacea.

ambigua, Walcott, 1879, Utica Slate and related formations, p. 22, Utica Slate.

elegans, Hall, 1876 , 28th Rep. N. Y. St. Mus. Nat. Hist., p. 118 , Niagara Gr.

me m b ra n cea, Hall, 1852, Pal. N. Y., vol. 2, p. FiG 512,-Sagenella ele172, Niagara Gr.

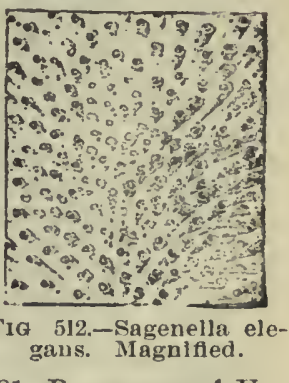

Scalaripora, Hall, 1881, Bryozoans of Up. Held. Gr., p. 17. [Ety., scalare, ladder; porus, pore.] Irregular groups of triangular branches, more or less concave, traversed transversely by sharp, elevated laminæ at regular distances; cel- 
luliferons on each face; cells radiating from the center to each angle of the branch; margins and summit of laminæ noncelluliferous. Type S. scalari(i)rmis.

approximata, Ulrich, (in press,) Geo. Sur. IIl., vol. 8, pl. 43, Ham. Gr.

scalariformis, Hall, 1881, Bryozoans of Up. Held. Gr., p. 18, and Pal. N. Y., vol. 6, p. 100 , U p. Held. Gr.

separata, Ulrich, (in press,) Geo. Sur. Ill., vol. 8 , pl. 43 , Ham. Gr.

subconcava, Hall, 1881, Bryozoans of Up. Held. Gr., p. 18, and Pal. N. Y., vol. 6, p. 100, Up. Held. Gr.

Scenellopora, Ulrich, 18s2, Jour. Cin. Soc. Nat. Hist., vol. 5, p. 150. [Ety. scene, tent; ellus, diminutive; poros. pore.] Zoarium broad, obconical; cell apertures on ridges, which radiate from the subsolid and depressed center of the upper surface. Type S. radiata.

radiata, Ulrich, 1882, Jour. Cin. Soc. Nat. Hist., vol. 5. p. 158, Trenton Gr.

Sceiтtiopora, Ulrich, 1888, Am. Geo., vol. 1, p. 228. [Ety. skeptron, staff; poros.
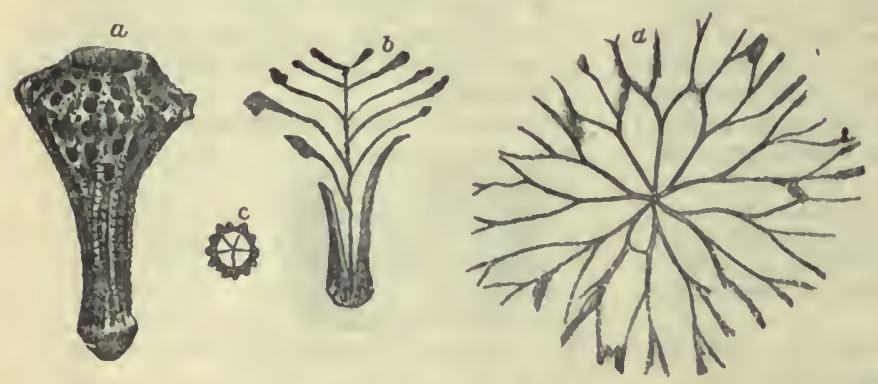

F1G. 513.-Sceptropora facula, $x$ 18. (a, Segment; $\boldsymbol{b}$, vertlcal section; a transverse sectlon; $d$, transverse section of expanded part. S. rhomboideum.

Semicoscinium, Prout, 1859, Trans. St. Louis Acad. Sci., vol. 1, p. 443. [Sig. somewhat like Coscinium.] Laf-like expansion, somewhat penniform, without a shaft; so!e formed of longitudinal and horizontal parallel riolges, surmounted by a cellular tissue, divided perpendicularly by thin, longitudinul septa, corresponding to the ridyes, nud supporting parallel lines of tortuous tubes alternately approximating and receding from eacli other; covered by a deuse, strong crust, divided into a net-work of rays and dissepiments bounding rhomboidal or ovate fenestrules, giving passage to oblique cells; the tortunus tubes give place to quincuncial, oval openings in the fenestrules; ench tortnous tube has a line of cells on each sicle. Type

eriense, Prout, Trans. St. Louis Acad Sci., vol. 1, p. 579, Up. Held. (ìr.

obliquatum, Ulrich, 1886, Cont. to Am. Pal. p. 13, Up. Held. Gr.

planodoreatum, Ulrich, (in press,) Geo. Sur. Ill., vol. 8 , pl. 45, Up. Held. Gr.

rhomboidenm, Prout, 1859, Trans. St. Louis Acad. Sci., vol. 1, p. $443, \mathrm{Up}$. Held. Gr.

rhombicum, Ulrich, (in press,) Geo. Sur. Ill., vol. 8 , pl. $45-54$, Hum. Gr.

tuberculatum, Prout, 1860, Trans. St. Louis Acad. Sci., vol. 1, p. 579 , Up. Held Gr.

Semiopora, Hall, 1884 , Rep. St. Geol., p. 51 . [Ety. semi, half; poros, pore.] Bryo-

pore.] Zoarium articulated; segments short, numerous, club-shaped, lower half striated, noncelluliferous; upper half expanded, celluliferous, and having one or more articulating sockets; zoøcia subtubular, radially arranged about a central axis; apertures subovate. Type S. facula.

facula, Ulrich, 1885, Am. Geo., vol. 1, p. 228, Hud. Riv. Gr.

Selenopora, Hall, 1887, Pal. N. Y., vol. 6, p. xvii. [Fty. selene, moon; poros, pore.] Zoarium explanate, incrusting; apertures subcircular, with an elevated denticulate peristome, and situated within polygonal vestibular areas formed by connecting walls, which traverse the surface; interior structure as in Lichenalia. Type S. circincta.

circincta, Hall, 1881,(Lichenalia circincta,) Trans. Alb. Inst., vol. 10, p. 11, and Pal. N. Y., vol. 6, p. 86, Up. Held. Gr.

complexa, Hall, 1881, (Lichenalia complexata,) Trans. Alb. Inst., vol. 10, p. 11 , and Pal. N. Y., vol. 6, p. 87, Up. zoum ramose; branches infrequent, bifurcating or tribifurcating; margins parallel; celluliferous on both sides; cells arising from a mesial epitheca; apertures in longitudinal parallel rows, separated by ridges; two minute pits on the transverse space between adjacent apertures; apertures near the margin larger and more oblique than the others; margin striated; noncelluliferous. Type S. bistigmata.

bistigmata, Hall, 1881, Rep. St. Geol., p. 57, Ham. Fr.

SeptoporA, Prout, 1859, Trans. St. Louis Acarl. Sci., vol. 1, p. 448. [Ety. septum, partition; porus, pore.] Zoarium like Fenestella, but distinguished by the dis. sepiments, which have from one to four rows of cells. Type S. cestriensis.

cestriensis, Prout, 1859, Trans. St. Louis Acad. Sci., vol. 1, p. 448, Kaskaskia Gr.

decipiens, Ulrich, (in press,) Geo. Sur. Ill., vol. 8 , pl. 66, Kaskaskia Gr.

delicatula, Ulrich, (in press,) Geo. Sur. Ill., vol. 8, pl. 64, Low Coal Meas. 
robusta, Ulrich, (in press,) Geo. Sur. Ill., vol. 8 , pl. 64 , Up. Coal. Meas.

subquadrans, Ulrich, (in press,) Geo. Sur. Ill., vol. 8, pl. 6 t, Kaskaskia Gr.

Spatropora, Ulrich, 1882, Jour. Cin. Soc. Nat. Hist., p. 155. [Ety. spatium, spread out; poros, pore.] 'Thin, incrusting; surface smooth or tuberculated; cells shallow; interstitial cells and spiniform tubuli. Type S. aspera.

areolata, Foord, 18s3, Cont. to Micropalæontology, p. 21, Trenton Gr.

aspera, UJrich, 1853, Jnur. Cin. Soc. Nat. Hist., vol. 6, p. 166, Hud. Riv. Gr.

lineata, Ulrich, 1883, Jour. Cin. Soc. Nat. Hist., vol. 6, p. 167, Hud. Riv. Gr,

maculosa, Ulrich, 1883, Jour. Cin. Soc. Nat. Hist., vol. 6, p. 167, Hud. Riv. Gr. montifera, Ulrich, 1883, Jour. Cin. Soc. Nat. Hist., vol. 6, p. 168, Hud. Riv. Gr.

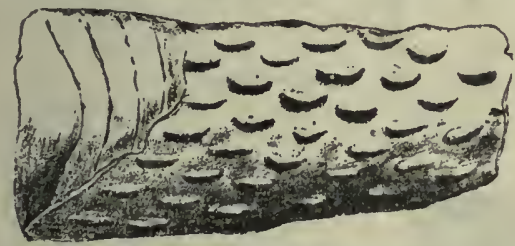

FIG. 514.-Spatiopora tuberculata on an Orthoceras.

tuberculata, Edwards \& Haime, 1851, (Chetetes tuberculatus,) Pol. Foss. d. Terr. Pal., p. 268, Hud. Riv. Gr.

Sphragiopora, (in press,) Ulrich, Geo. Sur. Ill., vol. 8, p. 398. Parasitic, forming very small subhemispheric patclies on foreign bodies; cells with circular apertures and slight peristome, arranged in a subradial manner, in single or double rows. Type S. parasitica.

parasitica, Ulrich, (in press,) Geo. Sur. Ill., vol. 8, pl. 65, Kaskaskia Gr. and Coal Meas.

SrictoporA, Hall, 1847, Pal. N. Y., vol. 1, p. 73. [Ety. sticto3, punctured; poros, pore.] Zoarium attached to foreign objects by an expanded base, ramose, branches thin, furnishing an acutely elliptical transverse section, and composed of two layers of cells, separated by epithecal lamina; cell apertures oval or circular, surrounded by peristome, separated by raised longitudinal lines; no interstitial cells ; maryins nonporiferous and striated. Type S. elegantula. acuta, Hall, 1847, Pal. N. Y., vol. 1, p. 74, Trenton Gr.

alcyone, Billings, 1865, (Ptilodictya alcyone,) Catal. Sil. Foss. Antic., p. 36, Anticosti Gr.

alternata, Hall, 1887, Pal. N. Y., vol. 6, pl. xxiii, A, Low. Held. Gr.

angularis, Hall, 1887, Pal. N. Y., vol. 6, p. 252, Ham. Gr.

arguta, Billings, 1865, (Ptilodictya arguta,) Catal. Sil. Foss. Antic., p. 36, Anticosti Gr.

basalis, Ulrich, 1882, Jour. Cin. Soc. Nat. Hist., vol. 5. p. 169 , Trenton Gr.

bifurcata, VanCleve, 1883, 12th. Rep. Ind. Geo. and Nat. Hist., p. 267, Niagara Gr.

bifurcata, Hall, see S. bristolensis.

bristolensis, n. sp. Ham. Trr. Proposed instead of S. bifurcata, Hall, 1887, Pal. N. Y., vol. 6, p. 254 , which name was preoccupied.

carbonaria, Meek, 1871, Proc. Acad. Nat. Sci. Phil., p. 160, and Ohio Pal., vol. 2, p. 328, Coal. Meas.

c om pressa, VanCleve, 1853, 12th Rep. Ind. Geol., and Nat. Hist., p. 267, Niagara Gr.

crassa, Hall, 1852, Pal. N. Y., vol. 2, p. 45, Clinton $\mathrm{Gr}$.

crescens, Hall, 1887, Pal. N. Y., vol. 6, p. $91, \mathrm{Up}$. Held. Gr.

crenula ta, Hall, 1884, Rep. St. Geol., p. 44, Ham. Gr.

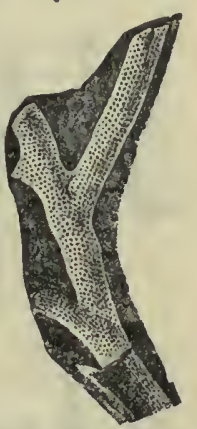

dicholoma, Hall, syn. for Fic. 515.-St1ctoS. subcarinata.

divergens, Hall, 1887, Pal.

N. Y., vol. 6, p. 257, Ham. Gr.

elegantula, Hall, 1847, Pal. N. Y., vol. 1, p. 75, Trenton Gr.

fenestrata, see Sulcopora fenestrata.

fidelis, Ulrich. 1886, 14th Rep. Geo. Sur.

Minn., p. 68, Trenton Gr.

fragilis, see Dicranopora fragilis.

fruticosa, Hall, 1881, Bryozoans of the Up. Held. Gr., p. 14, and Rep. St. Geol., pl. 25, fig. 12, 13, Up. Held. Gr.

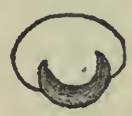

FIG.516.--stictopora gilberti. Tuugentiai section, show-

ing iunarium.
granatula, Hall, 1887, N. Y., vol. 6, p. 38, Low. Held. Gr. granifera, Hall, 1884, Rep. St. Geol., p. 45, Ham. Gr.

graminifolia, Ringueberg, 1884, Proc.

Acad. Nat. Sci., p. 147, Niagara Gr. Very poorly defined.

incisurata, Hall, 1884, Rep. St. Geol., p. 38, Ham. Gr.

incrassata, Hall, 1884, Rep. St. Geol., p. 47, Ham. Gr.

indenta, Hall, syn. for S. incisurata.

interstriata, Hall, 1884, Rep. St. Geol., p. 45, Ham. Gr.

invertis, Hall, 1881, Bryozoans of the Up. Held. Gr., p, 15, and Rep. St. Geol., pl. 25, fig. 24-26, Up. Held. Gr.

labyrinthica, Hall, 1847, Pal. N. Y., vol. 1, p. 50, Birdseye Gr.

lichenoides, Meek, 1873, Ohio Pal., vol. 1, p. 194, Up. Held. Gr. 
limata, Hall, 1887, Pal. N. Y., vol. 6, p. 250, Ham. Gr.

linearis, Hall, 1881, Bryozoans of the Up. Held. Gr., p. 15, and Rep. St. Geol., 1883, pl. 25, fig. 4-5, Up. Held. Gr.

lobata, Hall, 1887, Pal. N. Y., vol. 6, p. 256, Ham. Gr.

magna, Hall \& Whitfield, 1875, Ohio Pal., vol. 2 , p. 112 , Niagara Gr.

multifida, VanCleve, 1883, 12th Rep. Ind. Geol. and Nat. Hist., p. 268, Niagara Gr. multipora, Hall, syn. fo: S. incisurata.
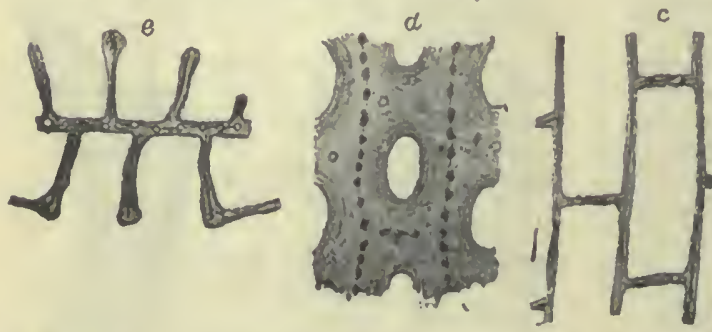

Ham. Gr.

ovatipora, Hall, 1881, Bryozoans of the Un. Held. Gr., p. 14, and Rep. St. Geol., pl. 25, fig. 23, 23a, Up. Held. Gir.

palmipes, Hall, 18s4, Rep. St. Geol., p. 41, Ham. Gr.

papillosa, Hall, 1883, Rep. St. (ieol., pl. 13, fig. 12-13, Low. Held. Gr.

paupera, Ulrich, 1886, 14th Rep. Geo. Sur. Minn., p. 69, Trenton Gr.

perarcta, Hall, 1881, Bryozoans of the Up. Ifeld. Gr., 1. 15, and Pal. c N. Y. vol. 6, p. פG, Up. Held.'Gr.

permarginata, Hall, 1884, Rep. St. Geol., p. 46, Ilam. Gr.

punctipora, IIall, 1852, Pal. N. Y., vol. 2, p. $157, \mathrm{Ni}$ agara $\mathrm{Gr}$.

ramosa, Hall, 1847, Pal. N. $Y$. , vol. 1, p. 51 , Birdseye Gr.

raripora, Hall, 1852, Pal. N. Y., vol. 2, p. 46, Clinton Gr.
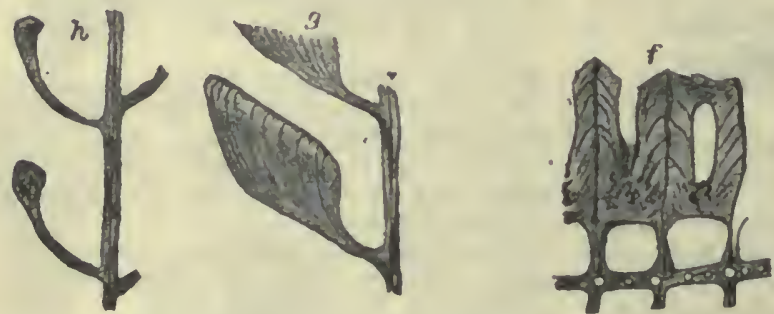

FIG. 517.-Stictopora inutubllis. Deep tangeutial sectlon $\times$ 50, showing the primillve portion of the gocicla and the mediun tubull In their walls; $\boldsymbol{d}$, Inugentlal section $\times 50 ; e$, Iransverse section $x 50$ showing inedian tubuli: $\times 50 ; g$, verileal section $\times 50 ; h$, vertleal section $x 50$.

mutabilis, Ulrich, 1886, 14th Rep. Geo.

Sur. Minn., p. 66, Trenton Gr.

nitidula, see Dicranopora nitidula.

obliqua, syn. for S. incisurata.

obliqua, Ringueberg, see Ptilodictya obliqua.

obsoleta, Hall, 1887, Pal. N. Y., vol. 6, p. - 37, Low. Held. Gr.
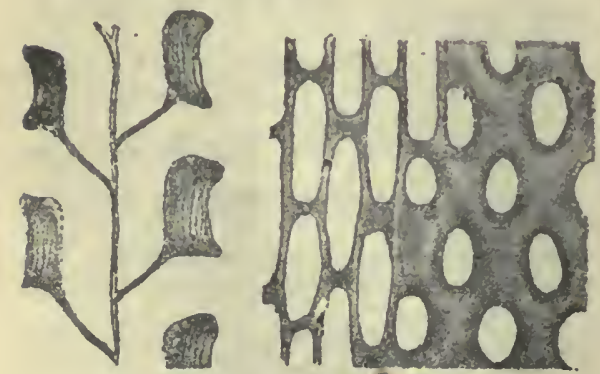

F'ig. 518.-Stietoporn ovatipora.

orbipora, Hall, 1879, Dese. New Spec. Foss, p. 5, and 11 th Rep. Ind. Geo. and Nat. Hist., p. 248, Niagara Gr. rigida, Hall, 1881, Bryozoans of the Up. Ield. Gr., p. 14, and Rep. St. (ieol., 1883, pl. 25, fig. 15-16, Up. Held. Fr.

rustica, Billings, 1865,

(Ptilodietya rustica,) Catal. Sil. Foss. Antic., p. 36 , Anticosti Gr.

seitula, Hall, 1857, Pal. N. Y., vol. 6 , pl. Ixi, Niagara Gr. scutulata, see Ptilodictya scutulata.

semistriata, Hall, 1881, Bryozoans of the Up. Held. Gr., p. 14, and Rep. St. Geol., 1883, pl. 25, fig. 17-20, Up. Fig. 519.-Sisetopors Held. Gr.

serrata, see Prismopora

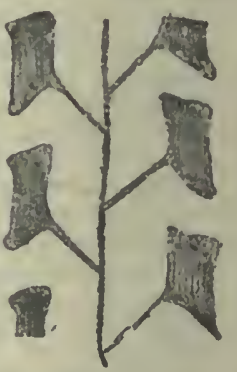
serrata.

shafferi, see Arthropora shafferi.

similis, Hall, 1876, 28th Rep. N. Y. St. Mus.

Nat. Hist., p. 122 Niagara Gr.

sinuosa, Hall, 1884, Rep. St. Geol., p. 4\%, Ham, Gr. 
striata, Hall, 1887, Pal. N. Y., vol. 6, p. 246, Ham. Gr.

subcarinata, see Tæniopora subcarinata.

subrigida, Hall, 1884, Rep. St. Geol., p. 43, Ham. Gr.

sulcata, Winchell, 1866, Rep. Low. Penin. Mich., p. 92, Ham. Gr.

tenera, Billings, 1865, (Ptilodictya tenera,) Catal. Sil. Foss. Antic., p. 36, Anticosti Gr.

trilineata, Hall, 1887, Pal. N. Y., vol. 6, p. 243, Ham. Gr.

triserialis, see Acanthoclema triseriale.

tumulosa, Hall, 1887, Pal. N. Y., vol.6, p. 246, Ham. Gr.

vanclevei, Hall, 1883, 12th Rep. Ind. Geol. and Nat. Hist., p. 268, Niagara Gr.

variabilis, Prout, 1866, Trans. St. Louis Acad. Sci., vol. 2, p. 413, Up. Held. Gr. vermicula, Hall, 1887, Pal. N. Y., vol. 6, p. 93. Up. Held. Gr.

Stictoporelia, Ulrich, 1882, Jour. Cin. Soc. Nat. Hist., vol. 5 , p. 152 . [Ety. diminutive of Stictopora.] Distinguished from Stictopora by interstitial pits between the longer diameters of the cell apertures. Type S. interstincta.

angularis, Ulrich, 1886, 14th Rep. Geo. Sur. Minn., p. 71, Trenton Gr.

? basalis, Ulrich, (in press,) Geo. Sur. Ill., vol. 8, pl. 68, 69, and 75, Keokuk Gr.
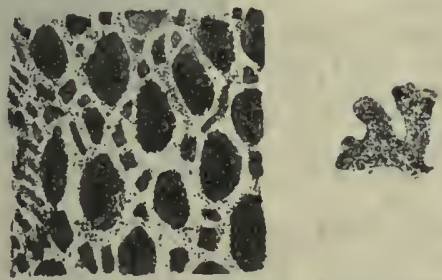

FIG. 520.-Stictoporella interstincta. Natural size and magnified 18 diam.

cribrosa, Ulrich, 1886, 14th Rep. Geo. Sur. Minn., p. 69, Trenton Gr.

frondifera, Ulrich, 1886, 14th Rep. Geo. Sur. Minn., p. 72, Trenton Gr.
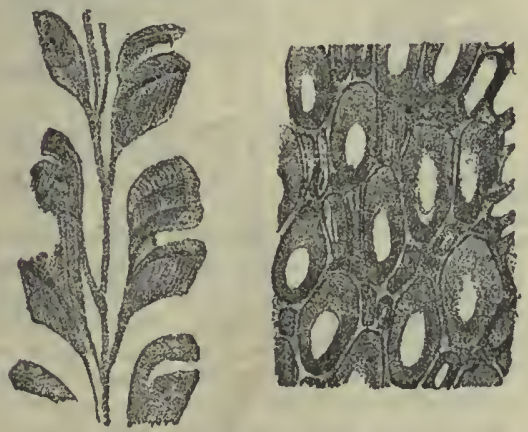

FIG. 521.-Stictoporella in terstincta.

interstincta, Ulrich, 1882, Jour. Cin. Soc. Nat. Hist., vol. 5, p. 169, Utica Slate Gr. ? undulata, Ulrich, (in press,) Geo. Sur. Ill., vol. 8, pl. 69, Kaskaskia Gr.
Strctoporina, Hall, 1887, Pal. N. Y., vol. 6, p. xx. [Ety. diminutive of Stictopora.] Zoarium obtusely pointed at the base, enlarging above and becoming flattened; bifurcations, few ; cells tubular arising from a mesotheca; apertures oval ; interapertural space elevated, angular, inclosing the apertures in rhomboidal or polygonal areas. Type S. claviformis. claviformis, Hall, 1881, (Trematopora claviformis,) Trans. Alb. Inst., vol. 10, p. 181, and Pal. N. Y., vol. 6, p. 269, Ham. Gr.

Stictotrypa, Ulrich, (in press,) Geo. Sur. Ill., vol. 8. Syn. (?) for Stictopora.

Stomatopora, Bronn, 1825, System d. urwetl. Pflanzenthiere. [Ety. stoma, mouth; poros, perforation.] Zoarium adnate; cells in single branching series, mouths elevated, and at the end of the tubular cells. Type S. dichotoma.

alternata, Hall \& Whitfield, 1873, 23d Rep. N. Y. St. Mus. Nat. Hist., p. 235, Chemung Gr.

auloporoides, Nicholson, 1875, (Alecto auloporoides, ) Ohio Pal., vol. 2, p. 267, Hud. Riv. Gr.

confusa, Nicholson, 1875, (Alecto confusa, ) Ohio Pal., vol. 2, p. 267, Hud. Riv. Gr. frondosa, Nicholson, 1875, (Alecto frondosa,) Obio Pal., vol. 2, p. 266, Hud. Riv. Gr.

inflata, Hall, 1847, (Alecot inflata, ) Pal. N. Y., vol. 1, p. 77, Trenton and Hud. Riv. Grs.

nexil is, James, 1875, (Alecto nexilis,) Int. to Catal. Cin. Foss., p. 3, Hud. Riv. Gr.

pertenuis, Ulrich, 1886, (Rhopalonaria pertenuis,) 14th Ann. Rep. Geol. Sur. Minn., p. 59, Fra. 522.-StomaTrenton Gr.

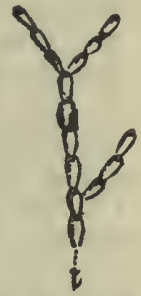
proutana, S. A. Miller, Natural size 1882, Jour. Cin. Soc. Nat.

Hist., vol. 5, p. 39, Hud. Riv. Gr.

Streblotrypa, Ulrich, (in press,) Geo. Sur. Ill., vol. 8, p. 403 . [Ety. streblos, turned about ; trupa, an opening.] Ramose, slender, solid; cells radiating trom an imaginary axis, their primitive portion long, tubular; or from a linear axis when they are somewhat shorter; inferior hemisepta best developed, situated rather far down; apertures regularly elliptical, or somewhat truncated at the posterior margin, surrounded by a slight peristome and, within this, sometimes a narrow sloping area ; arranged usually in rather regular longitudinal series; back of the aperture, occupying the depressed front of the cell, there are from one to twelve or more small pita, which, when numerous, are arranged in two or three rows; small acanthopores occasionally present. Type S. nicklesi. 
denticulata, Ulrich, 1888, (in press,) Bull. Denison Univ., vol. 4, p. 85, Cuyahoga shale.

distincta, Ulrich, Geo. Sur. Ill., vol. 8, pl. 71, Kaskaskia Gr.

hamiltonensis, Nicholson, 1874, (Ceriopora hamiltonensis,) Geo. Mag., vol. 1, p. 161, Ham. Gr.

hertzeri, Ulrich, 1888, Bull. Denison Univ., rol. 4, p. 85 , Cuyahoga shale.

major, Ulrich, (in press,) Geo. Sur. Il]., vol. 8, pl. 71, Keokuk Gr.

multiporata, Ulrich, 1888, Bull. Denison Univ., vol. 4, p. 87, Waverly Gr.

nicklesi, Ulrich, (in press,) Geo. Sur. Ill., vol. 8, pl. 71, Kaskaskia Gr.

obliqua, Ulrich, 1858, Bull. Denison Univ., vol. 4, p. 85 , Cuyahoga shale.

radialis, Ulrich, (in press,) Geo. Sur. Ill., vol. 8, pl. 72, Keokuk Gr.

regularis, Ulrich, 1888, Bull. Denison Univ., vol. 4, p. 88, Waverly Gr.

striata, Ulrich, 1858, Bull. Denison Univ., vol. 4, p. 87, Waverly Gr.

subspinosa, Ulrich, (in press,) Geo. Sur. Ill., vol. 8, pl. 71, Kaskaskia Gr.

Strotopora, Ulrich, (in press,) Geo. Sur. Ill., vol. 8, p. 383. [Ety. strotos, spread; poros, pore.] Ramose, branches large, irregular, solid or hollow ; large, abruptly spreading cells, which are supposed to represent oœcia, are distributed among the ordinary zocecia; when well-preserved they appear on the zoarial surface as strongly convex nodes, about $0.5 \mathrm{~mm}$. in diameter, with an opening on one side. In all other respects like Fistulipora. Type S. foveolata.

dermata, Ulrich, (in press,) Geo. Sur. IIl., vol. 8, pl. 77, Keokuk Gr.

foveolata, Ulrich, (in press,) Geo. Sur. Ill., vol. 8, pl. 77, Keokuk Gr.

perminuta, Ulrich, (in press,) Geo. Sur. Ill., vol. 8, pl. 47, Up. Held. Gr.

Sobretelora, D'Orbigny, 1850 , Prodr. d. Paléont., t. ], p. 22. [Ety. from Retepora.] Filiform, cylindrical branches, irregularly anastomosing; cells in a single row on the upper side of the branches (Ulrich says from 2 to 8 rows); apertures eircular or oval. Type S. reticulata.

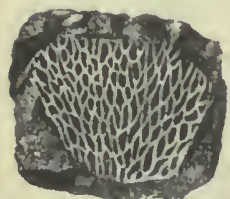

F1G. 523.-Subretepora angulata. angulata, Hall, 1852 . (Petepora angulata,) Pal. N. Y., vol. 2, p. 163, Niagara Gr.

aspera, Hall, 1847 , (Gorgonia (?) aspera,

Pal. N. Y., vol. 1, p. 16, Chazy Gr.

asperatostriata, $\mathrm{H}$ a 11 ,

1852, (Retepora asperatostriata,) Pal. N. Y., vol. 2, p. 161, Niagara Gr.

clathrata, Miller \& Dyer, 1878, (Intricaria clathrata,) Cont. to Pal., No. 2, p. 7, Hud. Riv. Gr.

corticosa, Ulrich, 1886, (Phyllopora corticosa,) 14th Rep. Geo. Sur. Minn., p. 61, Trenton Gr.

dawsoni, Ulrich, (in press,) (Phylloporina dawsoni,) Geo.Sur. Ill., vol. 8 , pl. 54, Trenton Gr.

dichotoma, Hall, 1852, (Hornera dichotoma,) Pal. N. Y., vol. 2, p. 163, Niagara Grr.

fenestrata, Hall, 1850, (Retepora fenestrata,) 3d Rep. N. Y. St. Mus. Nat. Hist., p. 178, Trenton Gr.

gracilis, $\mathrm{H}$ a ll, 1847, (Retopora gracilis,) Pal. N. Y., vol. 1, p. 15 , Chazy Gr. incepta, $\mathrm{Hall}$, 1847, (Retepora incepta,) Pal. N. Y., vol. 1 , p. 15, Chazy Gr. reticulata, Hall, 1847, (Intricaria reticulnta,) Pal. N. Y., vol. 1, p. 77, Trenton Gr.

trentonensis, Nicholson, 1875, (Retepora trentonensis, ) Geo. Mag., vol. 2 , p. 37, Trenton $\mathrm{Gr}$.

variolata, Ulrich, 1882, (Phyllopora variolata,

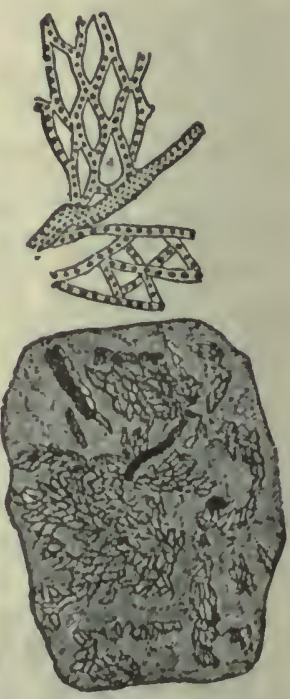

Jour. Cill. Soc. FIg. 524.-Subretepora reNat. Hist., vol. ticulata. Natural $81 \mathrm{ze}$ 5, p. 160 , Hud. and maguifled.

Riv. Gr.

Sulcopora, D'Orbigny, 1850, Prodr, d. Paléont., t. 1, p. 22. [Ety. sulcus, furrow ; poros, pore.] Distinguished from Stictopora by the obtuse extremities of the branches, and by the perpendicular rows of apertures separated by elevated ridges and eross bars. Type $\mathrm{S}$. fenestrata.

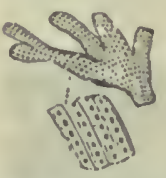

Fio. 525. -Sulcoporn fenestrata. Natu. ral size and magnifled. fenestrata, Hall., 1847, (Stic t o pora fenestrata,) Pal. N. Y., vol, 1, P. 16, Chazy $\mathrm{Gr}$.

Srocladia, King, 1849, Ann. and Mag. Nat. Hist., 2 d ser., vol. 3 , p. 389. [Ety. syn. together; klados, young branch.] Cup-shaped, with a central root-like base ; reticulated, composed of rounded, narrow, often branched interstices, bearing on the inner face from 3 to 5 alternating, longitudinal rows of prominent edged cells, separated by narrow keels, studded with vesicles; dissepiments thin, spur-shaped, extending upward, and meeting those from the adjoining interstice, and bearing two rows of cells. Type S. virgulacea. 
biserialis, Swallow, 1858, Trans. St. Louis Acad. Sci., p. 179, Up. Coal Meas.

rectistyla, Whitfield, 1882 , Ann. N. Y. Acad. Sci., vol. '2, p. 220, Kaskaskia Gr.

Teniodictya, Ulrich, (in press,) Geo. Sur. IIl., vol. 8. [Ety. tainia, ribbon; dictuon, net.] Zoaria growing from a basal expansion into dichotomously divided narrow branches or broad fronds; cell structure very much as in some species of Ptilodictya (P. pavonia, D'Orb.); apertures elliptical or subcircular, surrounded by a sloping area; interspaces ridge-like; both "hemisepta" present. Type T. ramulosa.

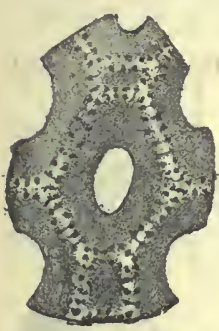

FIG. 526. - Treniodictya cingulata. - Tangential section $\times 50$, showing a transverse inling of tile central reglon of the walls, a character often present among the Ptilodictyonidse. cingulata, Ulrich, (in press,) Geo. Sur. Ill., vol. 8, pl. 67, Keokuk $\mathrm{Gr}$.

frondosa, Ulrich, (in press,) Geo. Sur. Ill., vol. 8, pl. 70, Keokuk Gr.

interpolata, Ulrich, 1888 Bull. Denison Univ., vol. 4, p. 80 , Cuyahoga Shale.

ramulosa, Ulrich, (in press,) Geo. Sur. Ill., vol. 8, pl. 67, Keokuk $\mathrm{Gr}$.

ramulosa var. burlingtonensis, Ulrich, (in press,) Geo. Sur. Ill., vol. 8,"pl. 67, Burlington $\mathrm{Gr}$.

subrecta, Ulrich, (in press,) Geo. Sur. Ill., vol. 8 , pl. 67, St. Louis Gr.

TanioporA, Nicholson, 1874, Geo. Mag. Lond. n. s., vol. 1, p. 120 . [Ety. tainia, ribbon; poros, pore.] Flattened linear expansion; dichotomous; celluliferous on both sides. Distinguished from Ptilodictya and Stictopora by a central, longitudinal keel, which divides the frond into two lateral halves, and by prominent cell-mouths. TypeT. exigua. exigua, Nicholson, 1874, Geo. Mag. Lond. n. s., vol. 1, p. 122, Ham. Gr.

occidentalis, Ulrich, (in press,) Geo. Sur. Ill., vol. 8, pl. 42, Ham. Gr.

penn if or $\mathrm{m}$ is,

$\mathrm{Nicholson}$, $1874, \mathrm{G}$ e o. Mag, Lond. $\mathbf{n}$. s., vol. $1, p$. 123, Ham. Gr.

su bcarinat a, Hall, 1881 , (Stictopora s u bcarinata,) Trans. Al b. Inst., vol. 10 , p. 191, and Pal. N. Y.
vol. 6, p. 261 vol. $6, \mathrm{p}$.
Ham. Gr. Ham. Gr.
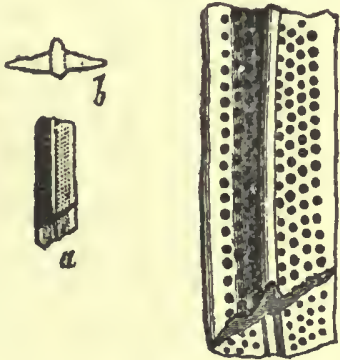

FIG. 527.-Tæniopora penniformis. $a$, Naturai size; $b$, transverse section enlarged; c, fragment enlarged. confertipora, see Fistulipora confertipora. decipiens, see Fistulipora decipiens.

densa, see Fistulipora densa, digitata, see Fistulipora digitata. inclusa, see Favicella inclusa. intercellatum, see Fistulipora intercellata. lamellatum, see Fistulipora lamellata. longimacula, see Fistulipora longimacula. micropora, see Fistulipora micropora. multaculeata, see Fistulipora multaculeata. plana, see Fistulipora plana.

scrobiculata, see Fistulipora scrobiculata. segregata, see Fistulipora segregata. serrulata, see Fistulipora serrulata. sparsipora, see Prismopora sparsipora. spheroidea, see Fistulipora spheroidea. subtilis, see Fistulipora subtilis. triangularis see Fistulipora triangularis. umbilicata, see Fistulipora umbilicata. variopora, see Fistulipora variopora.

Thamniscus, King, 1849, Ann. and Mag.

Nat. Hist., 2d ser., vol. 3, p. 389. [Ety. thamniskos, little shrub.] Stems frequently bifurcating more or less on one plane; celluliferous on the side overlooking the imaginary axis; cellules imbricated and arranged in quincunx; gemmuliferous vesicles overlying the cell apertures. Type T. dubius.

cisseis, Hall, 1883, Rep. St. Geol., pl. 22, fig. 23-30, Low. Held. Gr.

diffusus, Hall, 1852, (Retepora diffusa,) Pal. N. Y., vol. 2, p. 160, Niagara Gr.

fruticella, Hall, 1883, Rep. St. Geol. pl. 22, fig. 33, Low. Held. Gr.

divaricans, Ulrich, (in press,) Geo. Sur. Ill., vol. 8, pl. 62, Keokuk Gr.

furcillatus, Ulrich, (in press,) Geo. Sur. Ill., vol. 8, pl. 62, Kaskaskia Gr.

multiramus, Hall, 1881, Bryozoans of the

Up. Held. Gr., p. 19, and Rep. St. Geol. 1883, pl. 26, fig. 1-5, Up. Held. Gr.

nanus, Hall, 1881, Bryozoans of the Up.

Held. Gr., p. 19, Up. Held. Gr. niagarensis,

Hall, 1876 ,

28th Rep.

N. Y. St.

Mus. Nat.

$\mathrm{H}$ is t., p.

126, Niagara Gr.

nysa, Hall,

1883, Rep.

St. Geol.,

pl. 22, fig.

$47-48$,

L o w e r

Held. Gr.

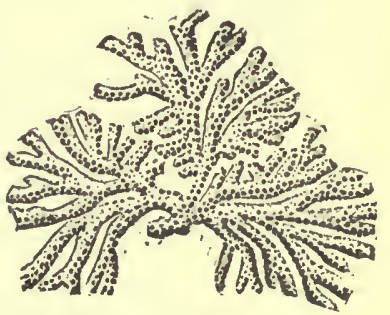

FIG. 528.-Tiramniscus niagarensis. octonarius, Ulrich, (in press,) Geo. Sur. IIl., vol. 8, pl. 62, Up. (ソoal. Meas. pauciramus, Hall, 1884, Rep. St. Geol., p. 60, Ham. Gr.

ramulosus, Ulrich, (in press,) Geo. Sur. Ill., vol. 8, pl. 62, Kaskaskia Gr.

ramulosus var. sevillensis, Ulrich, (in press,) Geo. Sur. Ill., vol. 8, pl. 62, Low. Coal Meas. 
variolata, Hall, 1883, Rep. St. Geol., pl. 22, fig. 34-46, Low. Held. Gr.

sculptilis, Ulrich, (in press, Geo. Sur. III., vol. 8, pl. 62, Keokuk Gr.

Thamnopora, Hall. This name was preoccupied. See Thamnotrypa.

divaricata, see Thamnotrypa divaricata.

TinamotryPa, Hall, 1887, Pal. N. Y., vol. 6, p. 101. [Ety. thamnos, bush ; trupa, perforation.] Narrow, branching stipe, celluliferous on both sides; the divisions are not by bifurcation, as in Stictopora, but by lateral and abrupt divergence from the main stipe. Type T. divaricata.

divaricata, Hall, 1881, Trans. Alb. Inst., vol. 10, p. 16, and Pal. N. Y., vol. 6, p. 101, Up. Held. Gr.

Trematella, Hall, 1886, Rep. St. Geol. and Pal. N. Y., vol. 6, p. xiv. [Ety. trema, hole; ellus, diminutive.] Ramose, solid; cells tubular, in contact below, diverging near the surface, intersected by septa; interapertural surface marked by pseudo-pores. Type T. annulata.

annulata, Hall, 1881, (Trematopora annulata,) Bryozoans of the Up. Held Gr., p. 5, and Pal. N. Y., vol. 6, p. 69, Up. Held. Gr.

arborea, Hall, 1881, (Trematopora arborea,) Bryozoans of the Up. Held. Gr., p. 5, and Pal. N. Y., vol. 6, p. 69, Up. Held. Gr.

glomerata, Hall, 1887, Pal. N. Y., vol. 6, p. 70, Up. Held. Gr.

nodosa, Hall, 1887, Pal. N.Y., vol. 6, p. 176, Ham. Gr.

perspinulata, Hall, 1881, (Trematopora perspinulata,) Trans. Alb. Inst., vol. 10, p. 181, and Pal. N. Y., vol. 6, p. 175, Ham. Gr.

Trematopora, Hall, 1852, Pal. N. Y., vol. 2 , p. 149. [Ety. trema hole ; poros, pore; Ramose, branches solid, tuberculated or smooth; interstitial cells, spiniform tu. buli, and diaphragms present. Type T. tuberculosa.

alternata, see Acanthoclema alternatum.

americana, S. A. Miller, Jour. Cin. Soc. Nat. Hist., p. 312, Burlington Gr.

annulifera, Whitfield, 1878, Ann. Rep. Geo. Sur. Wis., p. 67, and Geo. Wis., vol. 4, p. 254, Hud. Riv. Gr.

annulata, see Tremateila annulata.

annulata var. pronaspina, Hall, 1881, Bryozoans of the Up. Held. Gr., p. 6, Up. Held. Gr.

arborea, see Trematella arborea.

aspera, Hall, 1852, Pal. N. Y., vol. 2, p. 154, Niagara Gr.

calloporoides, Ulrich, (in press,) Geo. Sur. Ill., vol. 8, pl. 38 , Galena Gr.

camerata, see Diamesopora camerata.

canaliculata, Hall, 1883, Rep. St. Geol. pl. 11, fig. 12, Low. Held. Gr.

carinata, Hall, 1887, Pal. N. Y., vol. 6, p. 179, fiam. Gr.

claviformis, see Stictoporina claviformis. coalescens, Hall, 1852, Pal. N. Y., vol. 2, p. 150, Niagara Gr. constricta see Diamesopora eonstricta.

corticosa, Hall, 1874, 26th Rep. N. Y. St. Mus. Nat. Hist., p. 105, and Pal. N. Y., vol. 6, p. 15 , Low. Held. Gr.

crassa, see Lichenalia crassa.

crebipora, Hall, 1879, Desc. New Spec., Foss., p. 3, and 11th Rep. Ind., Geol. and Nat. Hist., p. 236, Niagara Gr.

debilis, Ulrich, (in press,) Geo. Sur. Ill., vol. 8, pl. 34, Galena Gr.

densa, Hall, 1874, 26th Rep. N. Y. St. Mus. Nat. Hist., p. 105, Low. Held. Gr. dispersa, see Diamesopora dispersa.

echinata, Hall, 1876, 26th Rep. N. Y. St. Mus. Nat. Hist., p. 112, Niagara Gr.

elongata, Hall, 1887, Pal. N. Y., vol. 6, p. 183, Ham. Gr.

fragilis, Winchell, 1863, Proc. Acad. Nat. Sci., p. 3, Waverly Gr.

granifera, Hall, 1887, Pal. N. Y., vol. 6, p. 186, Нат. Gr.

granistriata, see Bactropora granistriata.

granulata, Whitfield, 1878, Ann. Rep. Geo. Sur. Wis., vol. 4, p. 253, Hud. Riv. Gr. granulifera, Hall, 1852, Pal. N. Y., vol. 2 , p. 154 , Niagara Gr. The same species is marked "n. sp." in 28th Rep. $N$. Y. St. Mus. Nat. Hist., probably - by mistake.

halli, Ulrich, 1883 , Jour. Cin. Soc. Nat. Hist., vol. 6, p. 261, Niagara Gr.

hexagona, H a ll,

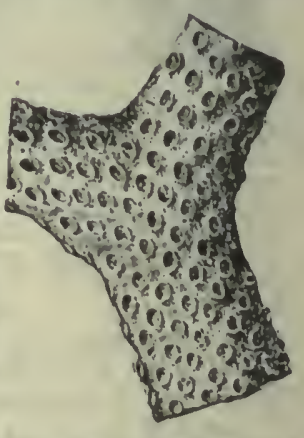
1887, Pal. N. Y., 'Fro. 529. - Trematopora vol. 6 , p. 178, larged.

Ham. Gr.

immersa, Hall, 1887, Pal. N. Y., vol. 6, p. 185, Ham. Gr.

infrequens, Hall, 1876, 28th Rep. N. Y. St. Mus. Nat. Hist., p. 111, Niagara Gr.

interplana, Hall, 1887, Pal. N. Y., vol. 6, p. 186, Ham. Gr.

linenta, Hall, 1887, Pal. N. Y., vol. 6, p. 181, Ham. Gr.

macropora, Hali, 1879, Desc. New Spec. Foss., p. 4, and 11th Rep. Ind Geo. and Nat. Hist., p. 236, Niag. Gr.

maculosa, see Lichenalia maculosa.

minuta, Hall, 1876, 28th Rep. N. Y. St. Mus:' Nat. Hist., p. 113, Niagara Gr.

nitida, Ulrich, (in press,) Geo. Sur. IIl., vol. 8, pl. 34, Hud. Riv. Gr.

nodosa, Hall, 1887, Pal. N. Y., vol. 6, pl. xxiii, Low. Held. Gr.

orbipora, Hall, 1884, Rep. St. Geol., p. 12, Ham. Gr.

ornata, Ulrich, 1886, 14th Rep. Geo. Sur. Minn., p. 98, Trenton Gr.

osculum, Hall, 1876, 28th Rep. N. Y. St.

Mus. Nat. Hist., p. 110, Niagara Gr.

ostiolata, see Chilotrypa ostiolata. 
ovatipora, Hall, 1883, Rep. St. Geo., pl. 11, fig. 13-14, Low. Held. Gr.

parallela, Hall, 1883, Rep. St. Geol., pl. 11, fig. 13-14, Low. Held. Gr.

perspinulata, Hall, 1884, Rep. St. Geol., p. 11, Ham. Gr.

polygona, Hall, 1884, Rep. St. Geol., p. 9, Ham. Gr.

ponderosa, Hall, 1874, 26th Rep. N. Y. St.

Mus. Nat. Hist., p. 106, Low. Held. Gr. punctata, Hall, 1852 , Pal. N. Y., vol. 2, p. 151, Niagara Gr.

primigenia, Ulrich, 1886, 14th Rep. Geo.

Sur. Minn., p. 97, Trenton Gr.

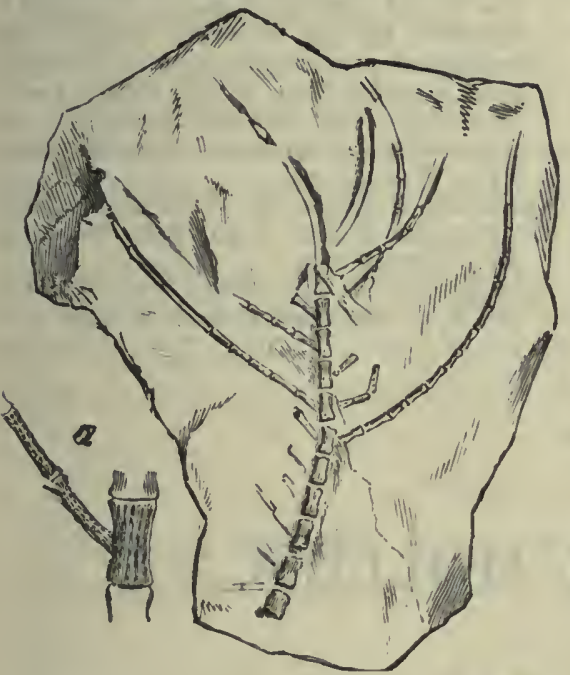

FIG. 530.-Arthroclema pulchellum. $a$, Magnified vlew. (See page 293.)

rectilinea, Hall, 1881, Bryozoans of the Up. Held. Gr., p. 6, Up. Held. Gr. regularis, see Orthopora regularis. rhombifera, see Orthopora rhombifera. scutulata, see Acanthoclema scutulatum. scululata, see Orthopora scutulata.

signata, see Callotrypa macropora var. signata.

solida, Hall, 1852, Pal. N. Y., vol. 2, p. 153, Niagara Gr.

sparsa, Hall, 1852, Pal. N. Y., vol. 2, p. 155, Niagara Gr.

spiculata, Hall, 1877, 1st Ed. Am. Pal. Foss., p. 245, Niagara Gr.

spinulosa, Hall, 1852, Pal. N. Y., vol. 2, p. 155. Niagara Gr.

spinulosa, Hall, 1876, 28th Rep. N. Y. St. Mus. Nat. Hist. The name was preoccupied. See T. spiculata.

striata, Hall, 1852, Pal. N. Y., vol. 2, p. 153, Niagara Gr.

subimbricata, Hall, 1879, Desc. New Spec.

Foss., p. 4, and 11th Rep. Ind. Geo., p. 234, Niagara Gr.

subquadrata, Hall, 1884, Rep. St. Geol., p. 11, Ham. Gr.

superba, Billings, 1866, Catal. Sil. Foss. Antic., p. 93, Clinton and Niagara Grs. tortalinea, Hall, 1884, Rep. St. Geol., p. 10, Ham. Gr.

transversa, Hall, 1884, Rep. St. Geol., p. 8, Ham. Gr.

tuberculosa, Hall, 1852, Pal. N. Y., vol. 2, p. 149 , Niagara Gr.

tubulosa, Hall, 1852, Pal. N. Y., vol. 2, p. 151, Niagara Gr.

varia, Hall, 1876, 28th Rep. N. Y. St. Mus. Nat. Hist., p. 111, Niagara Gr.

variolata, Hall, 1876,28 th Rep. N. Y. St. Mus. Nat. Hist., p. 113, Niagara Gr.

vesiculosa, Winchell, 1863, Proc. Acad. Nat. Sci., p. 3, Burlington Gr.

whitfieldi, Ulrich, 1883, Jour. Cin. Soc. Nat. Hist., vol. 6, p. 262, Niagara Gr.

Tropidopora, Hall, 1887, Pal. N. Y., vol. 6, p. 71. [Ety. tropis, keel; poros, pore.] Ramose, solid, cells in irregular longitudinal rows, separated by sinuous ridges; peristomes thin, slightly elevated. Type T. nana.

nana, Hall, 1887, Pal. N. Y., vol. 6, p. 71, Up. Held. Gr.

Tuberculopora, Ringueberg, 1886, Bull. Buf. Soc. Nat. Sci., vol. 5, p. 21. Not properly defined.

inflata, Ringueberg, 1886, Bull. Bıf. Soc. Nat. Sci., vol. 5, p. 21. Not properly defined.

UnitryPA, Hall, 1885, Rep. St. Geol., p. 36. [Ety. unus, one ; trupa, perforation.] Form like Fenestella, having the branches connected by dissepiments; cell apertures, in two ranges, separated by carinie, which are elevated, widened at the summit, and connected by thin, lateral processes or scalæ more or less numerous. Type U. lata.

acaulis, Hall, 1881,

(Fenestella acan-

lis,) Bryozoans of Up. Held. Gr. p. 33, and Pal. N. Y., vol. 6 , p. 131, Up. Held. Gr. aciulis var. inclinis, Hall, 1887,

Pal. N. Y., vol, 6, p. 132, Up.

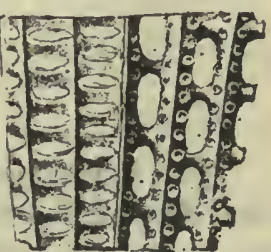
Held. Gr.

acclivis, Hall, 1887, Pal. N. Y., vol. 6, p. 138, Up. Held. Gr.

biserialis, Hall, 1882, (Fenestella biserialis,) Rep. St. Geol. and Pal. N. Y., vol. 6, p. 57, Low. Held. Gr.

conferta, Ulrich, 1886, Cont. to Am. Pal., p. 17, Up. Held. Gr.

consimilis, Hall, 1887, Pal. N. Y., vol. 6, p. 142, Up. Held. Gr.

elegantissima, Hall, 1881, (Fenestella elegantissima,) Trans. Alb. Inst., vol. 10, p. 36, and Pal. N. Y., vol. 6, p. 140, Up. Held. Gr.

fastigata, Hall, 1881, (Fenestella fastigata,) Trans. Alb. Inst., vol. 10, p. 36, and Pal. N. Y., vol. 6, p. 141, Up. Held. Gr ficticius, Hall, 1887, Pal. N. Y., vol. 6, p 137, Up. Held. Fr. 
lata, Hall, 1881, (Fenestella lata,) Trans. Alb. Inst., vol. 10, p. 34, and Pal. N. Y., vol. 6, p. 136, Up. Held. Gr.

nana, Hall, 1887, Pal. N. Y., vol. 6, p. 133, Up. Held. Gr.

nervia, Hall, 1874, (Fenestella nervia,) 26th Rep. N. Y. St. Mus. Nat. Hist., p. 93, Low. Held. Gr.

nervia var. constricta, Hall, 1879, (Fenestella nervia var. constricta,) $32 \mathrm{~d}$ Rep. N. Y. St. Mus. Nat. Hist., p. 174, Low. Held. Gr.

præcursor, Hall, 1874, (Fenestella præecursor,) 26th Rep. N. Y. St. Mus. Nat. Hist., p. 94, Low. Held. Gr.

pernodosa, Hall, 1881, (Fenestella pernodosa, Trans. Alb. Inst., vol. 10 , p. 35, Pal. N. Y, vol. 6, p. 139, Up. Held. Gr. projecta, Hall, 1887, Pal. N. Y., vol. 7, p. 132, Up. Held. Gr.

retrorsa, Ulrich, 1886, Cont. to Am. Pal., p. 15, Up. Held. Gr.

scalaris, Itall, 1884, (Fenestella scalaris,) 36th Rep. N. Y. St. Mus. Nat. Hist., p. 66, Ham. (ir.

spatiosa, Hall, syn. for U. lata. stipata, Hall, 1881, (Fenestella stipata,) Trans. Alb. Inst., vol. 10 , p. 34 , and Pal. N. Y., vol. 6, p. 134, U'p. Held. Gr. tegulata, Hall, 1881, (Fenestella tegulata,) Trans. Alb. Inst., vol. 10, p. 34, and Pal. N. Y., vol. 6, p. 135, Up. Held. Gr. transversa, Hall, 1887, Pal. N. Y., vol. 7, p. $132, \mathrm{U}_{1}$. Held. Gr.

WORTIIENOPORA, Ulrich, (in press,) Geo. Sur. Ill., vol. 8, p. 403. [Ety. proper name.] Bifoliate, branching or palmate; cells regularly arranged, subtubular or elongate rhomboidal, with the aperture semi-elliptical; on the surface the line of junction between the cells is marked by an elevated ridge; the truncated posterior margin of the aperture is raised into a less strong transverse bar; the elongate triangular depressed front appears perfectly plane. Type W. spinosa.

spatulata, Prout, 1859, (Flnstra spatulata,) Trans. St. Louis Acad. Sci., vol. 1, p. 446, Warsaw Gr.

spinosa, Ulrich, (in press,) Geo. Sur. Ill., vol. 8, pl. 68, Keokuk and Warsaw Grs.

\title{
SUBKINGDOM MOLLUSCA.
}

\section{CLASS BRACHIOPODA.}

\author{
[Ety. brachium, arm ; pous, foot.]
}

Tre Brachiopoda are all marine animals, having a bivalve shell and a pair of long, ciliated, and usually spiral arms, with which they produce a current of water that carries the food to the mouth, which is close to the middle of the base of the shell. The valves of the shell, instead of being placed on cach side of the animal, as in the Lamellibranchiata, are placed above and below it; so they are dorsal and ventral valves, instead of right and left valves. The ventral valve is generally larger than the dorsal, and projects beyond it at the beak. The beak is generally perforated, for the passage of a muscular peduncle, for the attachment of the animal; but in the Lingulidx, the peduncle projects from the interior of the shell, between the umbones. When there is no peduncle, the shell attaches by the beak, or by the whole surface of the ventral valve. The dorsal valve is always free and imperforate. There is generally a pair of teeth in the ventral valve, developed from the hinge margin, that lock in corresponding cavities in the dorsal valve. Some genera have no teeth or hinge.

The shells of the living Rhychonellidx and of many fossil genera consist of flattened prisms, parallel with each other, and directed obliquely to the surface of the shells, the interior of which is imbricated by their outcrop. The substance of 
the shell is traversed by small canals from one surface to the other, through which little cœcal processes of the outer layer of the mantle pass, and are covered externally by a thickening of the epidermis.

They have no special branchial apparatus. The respiratory function is performed by the mantle, which is traversed by numerous blood-vessels. The arms are frequently supported upon a calcareous framework on the interior of the dorsal valve, as shown in the illustration of Waldheimia australis. The valves are opened by cardinal muscles, which originate on each side of the center of the ventral valve, and converge toward the hinge margin of the dorsal valve, behind the dental sockets, where there is usually a prominent cardinal process. The valves are closed by adductor muscles, of which there are four in Crania and Discina. In many fossil genera there are spiral processes, or loops, upon which are founded family distinctions.

Shells are sometimes silicified, and become so transparent that they show the coils when held up to the light. Sometimes the coils are preserved in empty shells; and when shells are found wholly filled with spar, both valves may be removed, and the sparry matrix scraped away on either side until the spirals may be clearly seen by holding the specimen up to the light.

The class was divided by King into two orders-the Clistenterata and Tretenterata-which correspond with the Arthropomata and Lyropomata of other authors. These divisions include the families as follows:

\section{ORDER ARTHROPOMATA.}

Athyridæ, Atrypidæ, Orthidæ, Nucleospiridæ, Pentameridæ, Porambonitidæ, Productidæ, Rhynchonellidæ, Spiriferidæ, Strophomenidæ, Terebratulidæ, Triplesiidæ.

\section{ORDER LYOPOMATA.}

Craniidæ, Discinidæ, Lingulidæ, Obolidæ, Pholidopidæ, :Siphonotretidæ, Trimerellidæ.

Family Athyride.-Acambona, Athyris, Eumetria, Merista, Meristella, Whitfieldia.

Family Atrry Zygospira.

Family Cranimda.-Crania, Pseudocrania.

Family Discinid z.-Discina, Orbiculoidea, Schizocrania, Schizobolus, Trematis. Fanıly Lingulid ж.-Dignomia, Lingula, Lingulella, Lingulasma, Lingulepis.

Family Nucleosipirid z.-Hindella, Meristina, Nucleospira, Retzia, „Trematospira.

Family Oвоцгdж.-Dicellomus, Elkania, Leptobolus,jLinnarsonia, Obolella, Obolus.

Fammy Orthide. - Meekella, Orthis, Orthisina, Skenidium, Vitulina.

Family Pentameridz.-Amphigenia, Anastrophia, Gypidula, Pentamerella, Pentamerus, Stenoschisma.

Family Pholidopid a.-Pholidops.

Family Porambonitida.-Porambonites.

Family Productid ze.-Aulosteges, Chonetes, Productella, Productus, Strophalosia. 
Family Rhynchovelidie.-Camarella, Camarophoria, Eatonia, Eichwaldia, Leiorhynchus, Rhynchonella, Rhynchotreta, Stenosehisma.

Famm. Siphonotretids.-Acrothele, Acrotreta, Iphidæ, Kutorgina, Schizambon, Siphonotreta.

Family Spiriferids:-Amboenlia, Cyrtia, Cyrtina, Martinia, Spirifera, Spiriferina, Syntrielasma, Syringothyris, Trigonotreta.

Family Strophomenida.-Leptæna, Streptorhynchus, Stropliodonta, Strophomena, Strophonella.

Fanily Terebratui.id s:-Centronella, Cryptonella, Leptocelia, Rensseleria, Terebratula, Tropidoleptus, Vitulina, Waldheimia.

Family Trimereldidx.-Dinobolus, Lingulops, Monomerella, Trimerella.

Famity Triplesima-Triplesia.

Acambona, White, 1862, Proc. Bost. Soc. Nat. Hist., vol. 9, p. 27. [Ety ake, point; ambon, umbo.] Syn. for Lumetria.

prima, see Eumetria prima.

Acrotiele, Linnarsson, 1876 , Bibangtill $\mathrm{K}$. Vet. A kad. Handl., p. 20, Swed. Acad. Sci. on the Brachiopoda of the Paradoxides beds. [Ety. akros, pointed; thele, nipple; from the ajex of the valve.] Shell thin, corneous, subcircular in outline, depressed, concentrically marked, and sometimes radiated; apex of ventral valve teat-like; subcentral or near the posterior margin, perforated; dorsal valve slightly convex, posterior margin slightly reflexed, and internally a low median longitudinal septum represented by an impression in the cast.

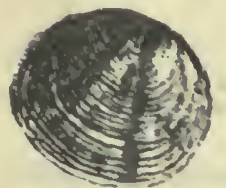

F10. 532,-Acrothele subsldua. In. terlor of lorsal valvo enlarged.
Type A. coriacea.

dichotoma, Walcott, 1855, Monogr. U. S. Geo. Sirr., vol. S, p. 14. Up. Taconic.

matthewi, Hart, 1868, (Lingula matthewi,) Acad. Geol., p. 644, St. Jobn Gr.

subsidua, White, 1874,

(Acrotreta subdsidua,

Rep. Invert. Foss., p. 6, and Geo. Sur. W. 100th Mer., vol. 4, p. 34, Up. Taconie.

Acrotret a, $a$

Kut orga,

1848, Uber die Siphonotretx aus den Verhan d 1 un gen der Kaiserlich en Mineralogisc he $\mathbf{n}$ Gesellscat $t$ fur Jahr., p. 260 , and Davidson's Brachiopoda, vol.

1, p. 133. [Ety. akros, the top or summit; tretos, perforated.] Shell triangu- lar, larger valve conical, false area flat, bent back at right angles to the margin of the valve, lougitudinally grooved along tho center, and perforated at its extremity by a small circular aperture, the lines of growtli encircle tlie shell and pass uninterruptedly over the false area; the smaller valve flat, operculiIorm, smooth, marked by concentric lines of growth; valves unarticuluted. Type A. subcouica.

attcnuata, 1873, 6th Rep. Hayclen's fico. Sur. 'Terr., p. 463, Up. Taconic.

baileyi, Matthew, 1885, Trans. Roy. Soc. Cau., p. 36, st. John Gr.

gemma, Billings, 1865, Pal. Foss., vol. 1. p. 210, Quebec Gr.

gulielma, Matthew, 1885, Trans. Roy. Soc. Can., 1. 37, St. Jolın Gr.

pyxidicula, White, 1874, Rep. Invert. Foss., p. 9, and Geo. Sur. IV. 100th Mer., vol. 4, p. 53, Potsdain Gr.

subsidua, see Acrothele subsidua.

Eigilops, Ilall, 1850, 3d Re]). N. Y. St. Mus. Nat. Hist., p. 179 . Tlie name was preoccupied for a genus in botany; beside it was founded on the cast of a lamellibranch.

subcarinata. Namo not to be retainerl.

Aмвосcela, Hall, 1860, 13th Rep. N. Y. St. Mus. Nat. Hist., p. 71. [Fty. ambon, umbo; koilos, the belly.] Distinguished from Orthis, Spirifera, etc., by the interior markings in the ventral valve, the thickened margins of the fissure are produced in short, strong teetl, but there is scarcely any extension of the dental plates; in the dorsal valve the bases of the crura continue attached to the inner surface of the valve for more than one-third of itslength before becoming free; there is a lateral projection from these crural bases bounding the teeth sockets; the cardinal process is elongate, lying between tlie crura, and is bifurcated at the outer extremity as in Cyrtina; the muscular impressions are below the middle of the valve, often near the front and quadruple; the dorsal valve being concave, flat or de- 
pressed convex, the spires lie in the ventral valve. Type A. umbonata.

fimbriata, Claypole, 1883, Proc. A in. Phil. Soc., p. 232, Portage Gr.

gemmula, syn. for Spirifera planoconvexa.

gregaria, Hall, 1860, 13th Rep. N. Y. St. Mus. Nat. Hist., p. 81 , and Pal. N. Y., vol. 4, p. 261, Chemung Gr.

minuta, WVhite, 1862 , Proc. Bost. Soc.

Nat. Hist., vol. 9, p. 26, Waverly Gr. nucleus, syn for Ambocclia umbonata.

præumbona, Hall, 1857, (Orthis praumbona,) 10th Rep. N. Y. St. Mus. Nat. Hist., p. 167. and Pal. N. Y., vol. 4, p. 262, Ham. $\mathrm{Gr}$.

subumbona, see Spirifera, subumbona.

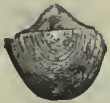

umbonata, Conrad, 1842, Frg.534-Am(Orthis umbonata,) Jour. bocolia umAcad. Nat. Sci., vol. 8, p.

264, and Pal. N. Y., vol. 4, p. 259, Marcellus Shale and Ham. Gr.

unbonata var. gregaria, see A. gregaria.

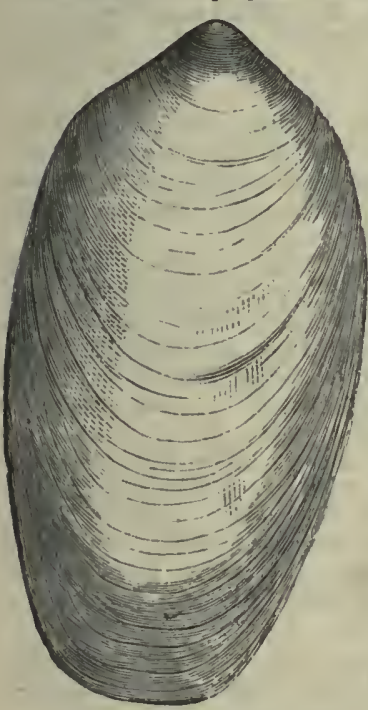

AMPH I GE N I A , Hall, 1867, Pal. N. Y., vol. $4, p$. 382. [Ety. amphi, on both sides; $g$ e $n$ e $a$, growth.] Inequival ve, oval, ovoid, or subtria $\mathrm{ng} \mathrm{u}$ ] a $\mathrm{r}$, more or less con v e $x$ wit hout mesial fold or sinus; valves articula $t$ i $n g$ by teeth and s o c k e ts, w it hou t area; dental lanellæ, in the ventral valve, c on jo ined elongata.

on their dorsal sides, forming an angular pit, which opens by a triangular fissure beneath the beak, and in its anterior extension is supported on a central septum; dorsal valve with a thickened cardinal process bordered by teeth sockets anchylosed to the bottom and supporting the crura, which extend into the cavity of the shell. Type A. elongata.

curta, Meek \& Worthen, 1868, (Stricklandinia elongata var. curta,) Geo. Sur. Ill., vol. 3, p. 402, Oriskany sandstone.

elongata, Vanuxem, 1842, (Pentamerus elongata,) Geo. 3 d Dist. N. Y., p. 132 , and Pal. N. Y., vol. 4, p. 383, Schoharie grit and Up. Held. Gr.

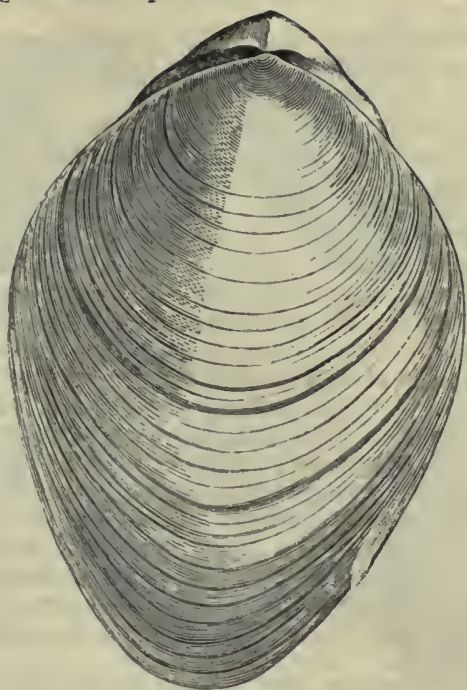

FIG. 536.-A mphigenia elongata.

elongata var. undulata, Hall, 1867, Pal. N. Y., vol. 4, p. 384, Up. Held. Gr. elongata var. subtrigonalis, Hall, 1857, (Meganteris subtrigonalis,) 10th Rep. N. Y. St. Mus. Nat. Hist., p. 123, Up. Held. Gr.

Anastrophia, H a 11 , 1867, Pal. N. Y., vol. 4, p. 373. [Ety. ana, with ; strophe, a turning round; the relation of the valves is the reverse of that of Pentamerus.] Gib-Frg. 537.-Ampligebous; ventral valve nia elongala. the smaller, gibbous in its upper part, depressed or sinuate below, with the $\mathrm{V}$-shaped pit sessile for nearly its entire length; small flattened space on each side of the fissure; dorsal valve ventricose, with promiuent umbo; hingeplate extended in gradually converging vertical lamellæw wich are joined to the shell throughout their length, while the crura are extended into the cavity, in thin free lamellae. Type A. verneuili.
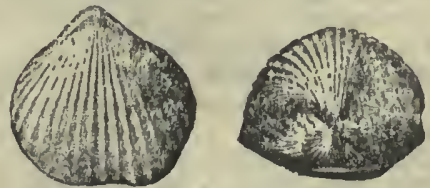

FIG. 538.-A nastrophia in ternascens.

internascens, Hall, 1879, 28th Rep. N. Y. St. Mus. Nat. Hist., p. 168, Niagara Gr. 
interplicata, Hall, 1852, (Atrypa interplicata,) Pal. N. Y., vol. 2, p. 275, Niagara Gr.

reversa, Billings, 1857, (Pentamerus reversus,) Rep. of Prog. Geo. Sur. Can., p. 215 , Mid. Sil.

verneuili, Hall, 1857, 10th Rep. N. Y. St. Mus. Nat. Hist., p. 104, and Pal. N. Y., vol. 3, p. 260, Low. Ileld. Gr.

ANazyg A, Davidson, 1883, Supp. to Brit. Brachiopoda, vol. 5, pt. 1, p. 128. [Ety. ana, upward ; zygos, a connecting band.] Small, longitudinally oval and striated; position of spiral cones as in Zygospira; about four coils in each spiral cone; stems attach to the hinge plate of the dorsal valve, extend parallel for \& short distance, and then, bending at right angles, form two large curves facing the lateral parts of the valve; before reaching their furthest extension in front, they give off a circular band or loop, which is directed upward toward the beak, and is exterior to the spiral cones on their dorsal side. Type $\mathrm{A}$. recurvirostra.

recurvirostra, Hall, 1847, (Atrypa recurvirostra,) Pal. N. Y., vol. 1, p. 140, Trenton Gr.

Anomia, Linnæus, 1767, Syst. Nat., 12th Ed. [Ety. anomios, unequal.] Not Palreozoic.

biloba, see Orthis biloba.

pecten, see Strophomena pecten.

reticularis, see Atrypa reticularis.

Anomites, Wahlenberg, 1821, Act., Unsal.

exporrectus, see Cyrtia exporrecta.

glaber, see Spirifera glabra.

punctatus, see Productus punctatus.

resupinatus, see Ortlis resupinatus.

reticularis, see Atry pa reticularis.

rhomboidalis, sce Stropliomena rhomboidalis.

scabriculus, see Productus scabriculus.

semireticulatus, see Productus semireticulatus.

ATHyRIS, McCoy, 1844, Carb. Foss. Ireland, pp. 128 and 146. [Ety. $a$, without; thuris, a small door; in allusion to the absence of a deltidium or door. But the name is erroncous.] Nearly orbicular or ovate, both valves convex; no cardinal area, foramen, or hinge-line; spiral appendages attached to the linge plate of the dorsal valve, very large, nearly filling the shell; a strong mesial septum in rostral part of dorsal valve; dental lamellæ moderate; pallial and ovarian impressions thick, numerous, dichotomous; tissue of shell fibrous. Type A. spiriferoides.

americana, Swallow, 1863, Trans. St. Louis Acad. Sci., vol. 2, p. 89, Kaskaskia Gr.

angelica, Hall, 1861, 14th Rep. N. Y. St. Mus. Nat. Hist., p. 99, and Pal. N. Y., vol. 4, p. 292, Chemung Gr.

argentea, Shepard, 1838, Am. Jour. Sci. and Arts, vol. 34, p. 152, Up. Coal Meas. biloba, Winchell, 1865, (Spirigera biloba,) Proc. Acad. Nat. Sci., p. 118, Kinderhook Gr.

blancha, Billings, 1863, Proc. Port. Soc. Nat. Hist., vol. 1, p. 115, Low. Held. Gr. caputserpentis, Swallow, 1863, Trans. St. Louis Acad. Sci., vol. 2, p. 90, Up. Coal Mens.

charitonensis, Swallow, 1860, (Spirigera charitonensis,) 'Trans. St. Louis Acad. Sci., vol. 1, 1). 651, Coal Meas.

chloe, Billings, 1860, Can. Jour., vol. 5, p. 282, Ham. Gr.

clara, Billings, 1860, Can. Jour., vol. 5, p. 274, Up. Held. Gr.

claytoni, Hall \& Whitfield, 1877, U.S. Geo. Expl. 40th parallel, vol. 4, p. 256, Waverly Gr.

clintonensis, Swallow, 1863, Trans. St. Louis Acad. Sci., vol. 2, p. 89 , Kaskaskia Gr.

clusia, Billings, 1860, Can. Jour., vol. 5, p. 279, Up. Held. Gr.

cora, Hall, 1860, 13th Rep. N. Y. St. Mus. Nat. Hist., p. 94, and Pal. N. Y., vol. 4, p. 291, Ham. and Chemung Grs.

corpulenta, Winchell, 1S63, (Spirigera corpulenta,) Proc. Acad. Nat. Sci., p. 6, Waverly Gr.

crassicardinalis, IVhite, 1860, Bost. Jour. Nat. Hist., vol. 7, p. 229, Waverly Gr.

eborea, Winchell, 1866, (Spirigera eborea,) Rep. Low. Peninsula Mich., p. 94, Ham. Gr.

euzona, Swallow, 1863, Trans. St. I Louis Acad. Sci., vol. 2, p. 91, Kaskaskia Gr.

differens, McChesney, 1860, New Pal. Foss., p. 47, syn. for A. subtilita.

formosa, Swallow, 1863, Trans. St. Louis Acad. Sci., vol. 2, p. 91, Kaskaskia Gr.

fultonensis, Swallow, 1860, (Spirigera fultonensis,) Trans. St. Louis Acad. Sci., vol. 1, p. 650, Ham, Gr.

hannibalensis, Swallow, 1860, (Spirigera hannibalensis,) Trans. St. Louis Acad. Sci., vol. 1, p. 649, Waverly or Kinderhook Gr.

hawni, Swallow, 1860, (Spirigera hawnii,) Trans. St. Louis Acad. Sci., vol. 1, p. 652, Coal Meas.

headi, see Zygospira lıeadi.

headi var. anticostiensis, see Zygospira headi var. anticostiensis.

headi var. borealis, see Zygospira leadi var, borealis.

harpalyce, Billings, 1863 , Proc. Port. Soc. Nat. Hist., vol. 1, p. 116, Low. Held. Gr. hirsuta, Hall, 1858, Trans. Alb. Inst., vol. 4, p. 8, and Bull. Am. Mus. Nat. Hist., p. 49, Warsaw Gr.

incrassata, Hall, 1858, Geo. Rep. Iowa, p. 600 , Burlington Gr.

intervarica, McChesney, 1860, Pal. Foss., p. 78, Burlington Gr. Not recognized. jacksoni, Swallow, 1860, (Spirigera jacksoni,) Trans. St. Louis Acad. Sci., vol. 1, p. 651, Coal Meas.

julia, see Meristella julia. 
junia, Billings, 1866, Catal. Sil. Foss. Antic., p. 46, Anticosti Gr.

lamellosa, Leveille, 1835, (Spirifer lamellosus,) Mem. Geol. Soc. France, vol. 2 , p. 39 , Waverly Gr.

lara, Billings, 1866, Catal. Sil. Foss. Antic., p. 47, Anticosti Gr.

maconensis, Swallow, 1860, (Spirigera máconensis,) Trans. St. Louis Acad. Sci. vol. 1, p. 651, Coal Meas.

maia, see Spirifera maia.

minima, Swallow, 1860, (Spirigera minima,) Trans. St. Louis Acad. Sci., vol. 1, p. 649 , Ham. Gr.

missouriensis, Swallow, 1860, (Spirigera missouriensis,) Trans. St. Louis Acad. Sci., vol. 1, p. 650, Coal Meas.

missouriensis, Winchell, 1865, (Spirigera missouriensis,) Proc. Acad. Nat. Sci., p. 117, Lithographic limestone. This name was preoccupied.

monticola, White, 1874, (Spirigera monticola,) Rep. Invert. Foss., p. 16, and Geo. Sur. W. 100th Mer., vol. 4, p. 91, Subcarboniferous.

naviformis, Hall, 1843, (Atrypa naviformis,) Geo. 4th Dist. N. Y., p. 71, and Pal. N. Y., vol. 2, p. 76, Clinton Gr.

obmaxima, McChesney, 1860, Desc., New Pal. Foss., p. 80, and Geo. Sur. W. 100th Mer., vol. 4, p. 92, Waverly Gr.

obvia, McChesney, 1860, Pal. Foss, p. 81, Kaskaskia Gr. Not recognized.

ohioensis, Winchell, 1865, Proc. Acad. Nat. Sei., p. 118, Waverly Gr.

orbicularis, McChesney, 1860, New Pal. Foss., Coal Meas. Not recognized.

papilioniformis, McChesney, 1867, Trans. Chi. Acad. Sci., vol. 1, Kaskaskia Gr.

parvirostris, Meek and Worthen, 1860, Proc. Acad. Nat. Sci. Phil., p. 451, Keokuk Gr. Referred later to A. planosulcata.

pectinifera, Swallow, 1863, Trans. St. Louis Acad. Sci., vol. 2, p. 88, Keokuk Gr.

perinflata, McChesney, 1860, Desc. New Pal. Foss., p. 81, Keokuk Gr. Not recognized.

persinuata, Meek, 1877, U. S. Geo. Sur., 40th parallel, p. 81, Carboniferous.

planosulcata, Phillips, 1836, Geo. York., vol. 2, p. 220, Keokuk Gr.

plattensis, Swallow, 1863, Trans. St. Louis Acad. Sci., vol. 2, p. 87, Up. Coal. Meas.

polita, Hall, 1843, (Atrypa polita,) Geo. 4th Dist. N. Y., pl. 65, fig. 5, and Pal. N. Y., vol. 4, p. 293 , Chemung Gr.

prinstana, see Meristella prinstana.

prouti, Swallow, 1860, (Spirigera proutii,) Trans. St. Louis Acad. Sci., vol. 1, p. 649 , Kinderhook or Waverly Gr.

reflexa, Swallow, 1863, Trans. St. Louis Acad. Sci., vol. 2. p. 88, Warsaw Gr.

singletoni, Swallow, 1863, Trans. St. Louis Acad. Sci., vol. 2, p. 87, Low. Coal Meas.

solitaria, Billings, 1866, Catal. Sil. Foss. Antic., p. 48, Anticosti Gr.

spiriferoides, Eaton, 1831, (Terebratula spiriferoides,) Am. Jour. Sci., vol. 21, p.
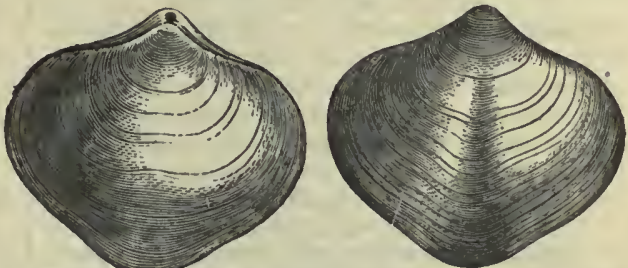

ra. 539.-Athyris spiriferoides. Dorsal and ventral view.

137, and Pal. N. Y., vol. 4, p. 285, Cornif. and Ham. Gr.

squamosa, Worthen, 1884, Bull. No. 2, Ill. St. Mus. Nat. Hist., p. 24, and Geo. Sur. Ill., vol. 8 , p. 103 , St. Louis Gr.

sublamellosa, Hall, 1858, Geo. Rep. Iowa, p. 702, Kaskaskia Gr.

subquadrata, Hall, 1858, Geo. Rep. Iowa, p. 703, Kaskaskia Gr.

subtilita, Hall, 1852, Stans- Athyris spirifbury's Exped. to Great eroides. Side Salt Lake, p. 409, Coal

Meas.

trinuclea, Hall, 1858, (Terebratula trinuclea,) Trans. Alb. Inst., vol. 4, p. 7, and Geo. Sur. Iowa, p. 659, Warsaw Gr.

tumida, Dalman, 1827, (Atrypa tumida.) The fossil usually referred to this species is Whitfieldia maria, which Davidson regarded as a synonym for W. tumida.

tumidula, Billings, 1866, Catal. Sil. Foss. Antic., p. 47, Anticosti Gr.

turgida, Shaler, 1865, Bulletin No. 4, M. C. Z., Anticosti Gr. Not defined so as to be recognized.

ultravarica, McChesney, 1861, Desc. New Pal. Foss., p. 79, Keokuk Gr. Not recognized.

umbonata, see Hindella umbonata.

vittata, Hall, 1860, 13th Rep. N. Y. St. Mus. Nat. Hist., p. 89, and Pal. N. Y., vol. 4, p. 289, Cornif. and Ham. Grs.

A try PA, Dalman, 1827, Vet. Acad. Handl., p. 102. [Ety. a, without; trypa, a hole or perforation. It was supposed the shells had no foramen in the beak. The name is erroneous.] Suborbicular, transverse or elongated; articulating by teeth and sockets; beak of the ventral valve produced and incurved, the apex truncated by a small, round perforation, sometimes separated from the hingeline by a deltidium; valve more or less convex with or without a defined sinus; a strong tooth on each side at the base of the broad fissure is somewhat bilobed at the summit, with a crenulated groove on the back; from the base of the teeth a curving ridge extends forward and partially incloses a broad, muscular 
scar ; dorsal valve convex, with or without a mesial fold; hinge plato divided in the middle with a tooth.like plate on each side, the crura originating outside of these close to the dental sockets, and outside of the latter, close to the shell margins, there is a crenulated fold, which occupied the groove at the base of the tooth; the spires originating from the crura form two hollow cones, directed into the cavity of the dorsal valve, their adjacent sides being flattened and apices brought close together near the center of the bnttom of the cavity; the processes at the base of the crura are directed into the cavity of the dorsal valve, and unite to form a loop; surface smooth, striate, or costate; structure fibrous. Type A. reticularis. acutiplicata, see Leptocolia acutiplicata. aculirostra, see Rlynchonella acutirostra. aquiradiata, Conrad, 1642, Jour. Acad.

Nat. Sci., vol. 8, p. 266, J.ow. Held. Gr. sequiradiata, see Rhynchonella sequiradiata.

affinis, syn. for Atrypa reticularis. altilis, sec Rhynchonella altilis.

ambigua, see Camarella ambigua.

aprinis, see Rhynchonella aprinis.

arata, see Pentamerella arata.

aspera, Schlotheim, 1813, (Terebratula aspera,) Petrefaktenkunde, p. 263, Ham. and Chemung Grs.

aspera var. occidentalis, Hall, 1858, Geo. Rep. Iowa, vol. 1, pt. 2, ]. 515, Ham. Gr. bidens, see Rhynclionella bidens.

bisulcata, see Camarella bisulcata.

borealis, Schlotheim, as identifled by d'Archiac \& V'erneuil. Not American. brevirostris, as identitied by Ilall, Pal. N. I., vol. 2, 1). 278. See Pentamerus brevirostris and Anastrophia verneuili. camura, see Trematospira camura. capax, see Rhynchonella capax.

cassidea, as identified by d'Archiac \& Verneuil. Not American.

chemungensis, Conrad, 1842, Jour. Acad.

Nat. Sci., vol. 8, p. 265, Chenung Gr. circulus see Camarella circulus.

concinna, see Nucleospira concinna. comis, see l'entamerus comis.

concentrica, syn. for Athyris spiriferoides. congesta, see Triplesia congesta.

congregata, see Stenoclisma congregatum. contracta, sce Stenochisina contractum. corallifera, see Eichwaldia corallifera. crassirostra, Hall, 1852, Pal. N. Y., vol. 2, p. 269, Niagara Gir.

crenulata see Terebratula crenulata.

cuboides, as identified by $\mathrm{Hall}$ and others.

See Rhynchonella venustula.

cuneata, see Rhynchonella cuneata. cuspidata, see Triplesia cuspidata. cylindrica, see Meristella cylindrica. deflecta, Hall, 1847, l'al. N. Y., vol. 1, p. 140, Trenton Gr.

dentata, see Rhvnchonella dentata. disparilis, see Colospira disparilis. dubia, see Rhynchonella dubia. dumosa, Hall, 1843, Geo. Rep., 4th Dist. N. Y., p. 272, Chemung Gr.

duplicata, see Stenochisma duplicatum. elongata, syn. for Rensselreria ovoides. emrcerata, see Rhynchonella emacerata. exigua, Hall, 1847, Pal. N. Y., vol. 1, p. 141, Trenton Gr.

eximia, see Stenochisma eximium. extans, see 'Íriplesia extans.

fabella, syn. for Leptocolia hemispherica. Rabellites, see Leptocoelia flabellites. galeala, see Pentamerus galeatus.

gibbosa, Hall, 1852, Pal. N. Y., vol. 2, p. 79, Clinton Gr.

globuliformis, see Leiorhynchus globuliforme.

hemiplicata, see Cnmarella hemiplicata.

hemispherica, see Leptocalia hemisplierica. hirsula, see Trematospira hirsuta.

liystrix, Hall, 1843, Geo. Rep. 4tl, Dist.

N. Y., p. 272, and Pal. N. Y., vol. 4, p. 326, Chemung Gr.

impressa, Hall, 1557, 10th 1Rep. N. Y. St. Mus. Nat. Hist., p. 122, and Pal. N. Y., vol. 4, p. 315, Sclioharie Grit.

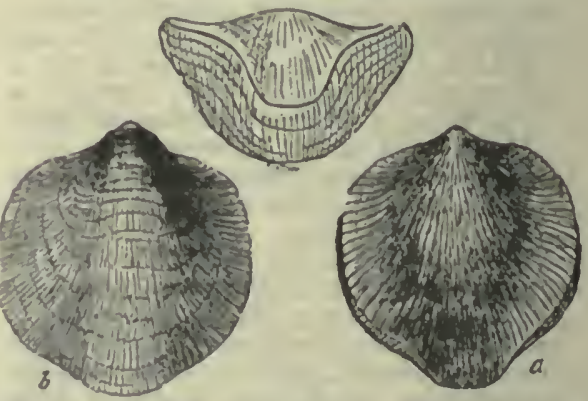

Fra. 541.-Alrypa retlculurls, a, Dorsnl valve; $b$, veutral valve; $c$ anterlor vlew.

impressa, Shaler. The nane was preoccupied.

increbescens, syn. for Rhynchonella capax. inflata. Conrad, 1843, Geo. Rep. 3d Dist. N. Y. Not defined.

intermedia, Hull, 1852, Pal. N. Y., vol. 2, p. 77, Clinton Gr.

interplicata, see Anastrophia interplicata. lavis, see Meristella lavis.

lamellata, see Rhynchonella lamellata.

laticosta, Phillips, 1841, (Terebratula laticosta,) Pal. Foss., Chemung Gr. This species is not clearly identified in America.

lentiformis, syn. for Atrypa reticularis.

limitaris, see Leiorhynchus limitare.

mansoni, Salter, 1852, (Rhynchonella mansoni,) Sutlicrland's Jour., vol. 2, p. cexxi, Devonian.

marginalis, (?) Dalman, 1827, (Terebratula marginalis,) Vet. Acad. Handl., p. 143, Niagara Gr.

medialis, see Fatonia medialis.

mesacostalis, see Leiorhynchus mesacostale. modesta, see Zygospira modesta.

nasuta, see Meristella nasuta. naviformis, see Athyris naviformis. 
neglecta, see Rhynchonella neglecta.

nitida, see Meristina nitida.

nitida var. oblata, see Meristina nitida var. oblata.

nodostriata, Hall, 1852, Pal. N. Y., vol. 2, p. 272, Niagara Gr.

nucleolata, Hall, 1852, Pal. N. Y., vol. 2,

p. 328 , Coralline limestone.

nucleus, see Triplesia nucleus.

nustella, Castelnau, 1843, Syst. Sil., p. 39.

Not recognized.

oblata, Hall, 1852, Pal. N. Y., vol. 2, p. 9, Medina Gr.

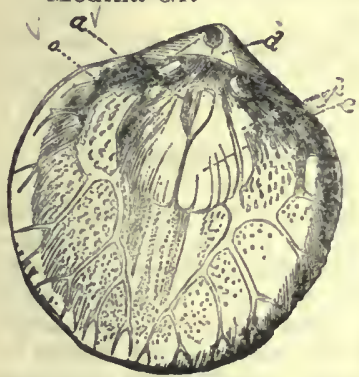

Fra. 542.-Alrypa retleularis. Interior of ventral valve: $a$, Impression of adductor mus. cle; $c$, cardinal nuscle; $p$ pedicle muscle; $o$, ovarian sinus; $d$, deltidium.

obtus iplicata, see Rhynchonella obtusiplicata. octocostata, see Pentam erella arata. peculiaris, see Eatonia peculiaris.

phoca, Salter, 1852, (Rhynchonella p hoc a , ) S u t he r land's Jour., vol. $2, p$. cexxvi, Devonian.

planoconvexa, see Leptocolia planoconvexa. plebeia, Conrad, 1843, Geo. Rep. 3d Dist.

N. Y., Ham. Gr. Preoccupied name.

pleiopleura, see Rhynchonella pleiopleura. plena, see Rhynchonella plena.

plicata, see Rhynchonella plicata.

plicatella, (\&) Linnæus, as identified by Hall, in Pal. N. Y., vol. 2, p. 279. May be stricken from the list as an erroneous identification.

plicatula, see Rhynchonella plicatula.

plicifera, see Rhynchonella plicifera.

polita, see Athyris polita.

prisca, syn. for Atrypa reticularis.

pseudomarginalis, Hall, 1860, 13th Rep.

N. Y. St. Mus. Nat. Hist., p. 84, and

Pal. N. Y., vol. 4, p. 327, Üp. Held. Gr. quadricostata, see Leiorhynchus quadricostatum.

q u adricostata,

Hall, 1852, see Rhynchonella quadricostata.

rectiplicata, Conrad, 1842, Jour. Acad. Nat. Sci., vol. 8, p. 265, Low. Held. Gr.

recurvirostra, see Anazyga recurvirostra. reticularis, Linnæus, 1767, plate.

(Anomia reticularis,) Syst. Nat., ed. 12, p. 1132, and Pal. N. Y., vol. 2, p. 72. It occurs, with its varieties, in all the Groups of the Upper Silurian and Devonian formations, except the Oriskany sandstone. Some of its varieties or synonyms are, Atrypa affinis, A. lentiformis, A. prisca, A. tribulis, Hippariony $x$ consimilis, etc.

robusta, see Rhynchonella robusta.

rostrata, see Meristella rostrata.

rugosa, see Rhynchonella rugosa.

scitula, see Meristella scitula.

semiplicata, see Rhynchonella semiplicata. singularis, see Eatonia singularis.

sordida, see Rhynchonella sordida.

spinosa, Hall, 1843, Geo. 4th Dist. N. Y.,

p. 200, Cornif., Ham., Tully, and Chemung Grs. Equal to Atrypa aspera var. occidentalis.

subcuboides, D'Orbigny, see Rhynchonella venustula.

subtrigonalis, see Rhynchonella subtrigonalis.

sulcata, see Merista sulcata.

tenuilineata, Hall, 1843, Geo. th Dist.

N. Y., p. 272, Chemung Gr.

tribulis, syn. for Atrypa reticularis.

tumida, see A thyris tumida.

unguiformis, syn. for Orthis proximus.

unisulcata, see Meristella unisculcuta.

Aulostegres, Helmerson, 1847, Bull. de la

Classe Physi. Math. Acad. Sci. St. Pe-

tersburg, vol. 6, p. 135. [Ety. aulos, tube; stege, chamber.] Shell subpen-

tagonal; ventral valve most convex,
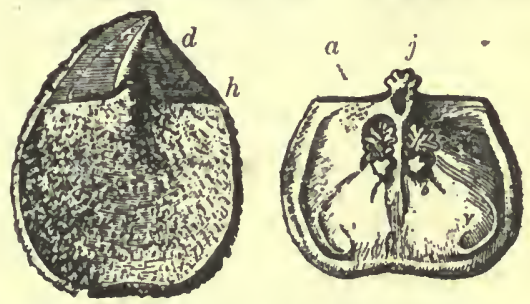

FIG. 541.-Aulosteges waugenheimi. $h$, Triangular hinge area; $d$, convex psendodeltidiuin ; $j$ / cardinal process; $a$, adductor impression.

beak produced, twisted, area triangular, interrupted by a pseudodeltidium not reaching the hinge-line, which is straight and tootbless; dorsal valve convex at the umbo, depressed or concave laterally; cardinal edge more or less developed; surface of valves with short tubular spires; in the interior of the dorsal valve a trifid cardinal process is made to fill the uncovered portion of the fissure, and serve as the point of attachment to the cardinal muscle; under this process a longit udinal mesial ridge extends nearly to the margin, and on either side are elongated, ramified adductor scars; the reniform impressions, after dividing the above named muscle, extend by an outward oblique curve to near the margin, wlien, turning backward and inward, termi- 
nate some distance from their origin; two brachial elevations under the adductor move toward the center of the valve. Type A. wangenheimi.

guadalupensis, Shumard, 1858, Trans. St. Louis Acad. Sci., vol. 1, p. 292, Permian Gr.

spondyliformis, White \& St. Jolın, 1868, Trans. Chi. Acad. Sci., p. 118, Up. Coal. Meas.

Billingsia, Ford, 18S5. The name being preoccupied, see Elkania.

Brachymemu, Shaler. The name was preoccupied for a genus of Coleoptera. See Anastrophia.

Brachyprion, Shaler, syn. for Strophomena. geniculatum, see Strophomena geniculata. leda, see Strophomena leda.

ventricosum, see Strophonsena ventricosa.

Camarella, Billings, August, 1858, Can. Nat. and Geol., vol. 4, p. 301. [Ety. kamara, arching chamber; ellus, diminutive.] Shell ovate or subcircular, beaks small, linge-line short; mesial fold and sinus becoming obsolete in the uiddle part of the shell, below which the radiating strice are more or less numerous, while above concentric striac occur. Type C. volborthi.
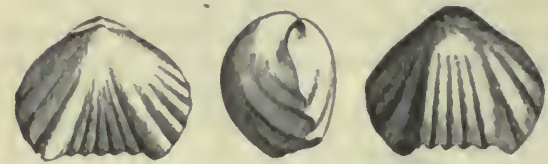

Fia. 5s5.-Camarella liemlplicata. Dorsal, ven. tral, and slde vlews.

ambigua, Hall, 1847, (Atrypa ambigua,) Pal. N. Y., vol. 1, p. 143, 'Trenton Gr. anticuata, Billings, 1861, Pal. Foss., vol. 1, p. 10, Georgia Gr.

bisulcata, Emmons, 1842, (Orthis bisulcata.) Geo. Rep. N. Y., p. 395 , and Pal. X. Y., vol. 1, p. 139 , Trenton Gr.

breviplicata, Billings, 1865, 1al. Foss., vol. 1, p. 304, Quebec Gr.

calcifera, Billings, 1861, Can. Nat. and $\mathrm{G}_{e} \mathrm{O}$., vol. 6 , p. 318 , Calcif. Gr.

circulus, Hall, 1847, (Atry pa circulus,)

Pal. N. Y., vol. 1, p. 142, 'Trenton Gr. congesta, see Triplesia congesta. custata, Billings, 1865, Pal. Foss., vol. 1, p. 305, Quebec Gr.

cuspidata, see Triplesia cuspidata. extans, see Triplesia extuns.

hemiplicata, Hall, 18t7, Atrypa hemiplicata,) Pal. N. Y., vol. 1., p. 144, Trenton Gr.

lenticularis, Billings, 1866, Catal. Sil.

Foss. Antic., p. 45, Anticosti Gr.

longirostra, Billings, 1858, Can. Nat. and Geo., vol. 4, p. 302, Chazy Gr.

nucleus, see Triplesia nucleus.

ops, Billings, 1862, Pal. Foss., vol. 1, p. 148 , Mid. Sil.

ortoni, see Triplesia ortoni.

panderi, Billiugs, 1858, Can. Nat. and Geo., vol. 4, p. 301, Black Riv. Gr. parva, Billings, 1865, Pal. Foss., vol. 1, p. 219, Quebec Gr.

polita, Billings, 1865, Pal. Foss., vol. 1, p. 305, Quebec Gr.

primordialis, see Triplesia primordialis. reversa, see Anastroplia reversa. varians, Billings,

1859, Can. Nat.

and Geo., vol.

4, p. 445 , Cloazy Gr.
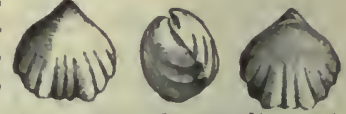

- Clac. 546. - Camarella volin $g$ s, 1859, and side views.

Can. Nat. and

Geo., vol. 4, p. 301, Black Riv. Gr.

waldronensis, see Triplesia waldronensis.

Camarium, Hall, 1859, Pal. N. Y., vol. 3, p. 486 , syn. for Merista.

elongatum, see Merista elongata.

typum, see Merista typus.

Camalomioria, King, 1844, Ann. and Mag. Nat. Ilist., vol. 14, p. 313. [Ety. kamara, an arched chamber; phoreo, I carry.] Subtrigonal, convex longitudinally; mesial fold and sinus; beak acute, inore or less incurved, small fissure beneath; no area or deltidium; plicated, impunctate, articulating by teetlı and sockets; dental plates in the ventral valve, conjoined at their dorsal margins, forming a trougli-shaped process affixed to a low, medio-Iongitudinal plate; the space between the sockets in the dorsal valve is occupjed by a small, cardinal, muscular protuberance, on either side of which two slender processes curve upward; from beneath the cardinal process a vertical mesial septum, a third or more of the length of the valve, supporting along its upper edge a spatula-shaped process, dilated toward its free extremity, and projected with a curve to near the center of the shell. Type C. schlotheimi.

bisulcata, Shumard, 1858, Trans. St. Louis Acad. Sci., vol. 1, p. 296, Permian Gr.

eucharis, Ilall, 1867, Pal. N. Y., vol. 4, p. 368 , Corniferous Gr.
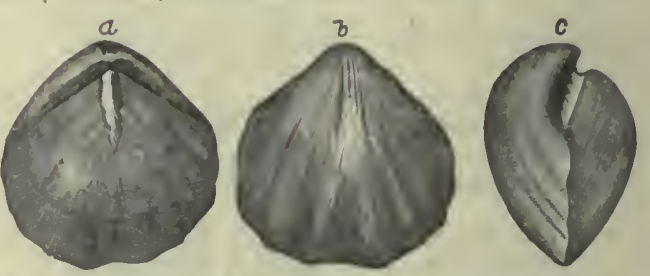

FIa. 517.-Camarophoria giffordi. $a$, Dorsal vlew; $b$, ventral valve; $c$, profle vlew.

giffordi, Worthen, 1882, Bull. No. 1, Ill. St. Mus. Nat. Hist., p. 39, and Geo. Sur. Ill., vol. 7, p. 318, Middle Conl Meas.

globulina, Phillips, 1814, as identified by Geinitz, is Rhynchonella uta.

occidentalis, S. A. Miller, 1881, Jour. Cin. Soc. Nat. Hist., vol. 4, p, 313, Burlington Gr. 
schlotheimi, Von Buch, 1834, (Terebratulites schlotheimi,) Mem. de la Soc. Geol., vol. 3, p. 138, Permian Gr.

subtrigona, Meek \& Worthen, 1860 , (Rhynchonella subtrigona,) Proc. Acad. Nat. Sci. Phil., p. 451, and Geo. Sur. Ill., vol. 2, p. 251, Keokuk Gr.

swallovana, Shumard, 1859, Trans. St. Louis Acad. ści., vol. 1, p. 394, Permian Gr.

wortheni, Hall, 1858, (Rhynchonella wortheni,) Trans. Alb. Inst., vol. 4, p. 11, and Bull. Am. Mus. Nat. Hist., p. 54, Warsaw Gr.

Centronelua, Billings, 1859, Can. Nat. and Geo., vol. 4, p. 131. [Ety. a little point.] General form like Terebratula; dorsal valve with a loop consisting of two ribbon-like lamellæ, which extend about half the length of the shell, at first curving outward and then approaching until their lower extremities meet at an acute angle; here they unite and are reflected backward toward the beak in a thin, flat, vertical plate; near their origin each bears upon the ventral side a single triangular crural process. Type C. glansfagea.

allii, Winchell, 1865, Proc. Acad. Nat. Sci., p. 123, Waverly or Marshall Gr.

alveata, Hall, 1857, (Rhynchonella alveata,) 10th Rep. N. Y. St. Mus. Nat. Hist., p. 124, Onondaga Gr.

anna, Hartt, 1868, Acad. Geol., p. 300, Subcarb.

billingsana, Meek \& Worthen, 1868, Geo. Sur. Ill., vol. 3, p. 352, Niagara Gr.

crassicardinalis, Whitfield, 1882, Bull.Ann. Mus. Nat. Hist., No. 3, p. 55, Warsaw Gr.

flora, Winchell, 1879, Proc. Am. Phil. Soc., vol. 12, p. 254, Marshall Gr.

glansfagea, Hall, 1857, (Rhynchonella glansfagea,) 10th Rep. N. Y. St. Mus. Nat. Hist., p. 125, and Pal. N. Y., vol. 4, p. 399, Schoharie grit, Cornif. Gr. and Oriskany sandstone.

glaucia, Hall, 1867, Pal. N. Y., vol. 4, p. 403, Ham. Gr.

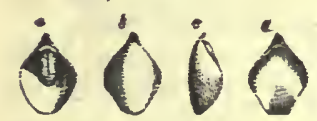

Frg. 518.-Centron e 11 a hecate. $\alpha$, Showing loop; $b$, $c$, and $d$, differeut views. hecate, Bill ing s, 1861, Can. Jour. vol. 6, p. $272, \mathrm{Up}$. Held. Gr.

impressa, Hall, 1861, 14th Rep. N. Y. St. Mus. Nat. Hist., p. 102, and Pal. N. Y., vol. 4, p. 402, Ham. Gr. Prof. Billings said this is a syn. for $C$. hecate.

julia, Winchell, 1862, Proc. Acad. Nat. Sci. vol. 14, p. 405, and Pal. N. Y., vol. 4, p. 419, Marshall Gr.

ovata, Hall, 1867, Pal. N. Y., vol. 4; p. 419, Up. Held. Gr.

Charionella, Billings, 1861, Can. Jour. Ind.

Sci., and Art, p. 148, syn. for Meristella. circe, see Meristella circe.

doris, see Meristella doris.
(?) hyale, see Meristella hyale.

Chonetes, Fischer, 1837, Oryckt. Moscou, p. 134. [Ety. chone, a little cup.] Shell thin, semi-cylindrical, transverse section semi-oval, ventral valve convex, dorsal concave hinge-line straight; external margin of the area of ventral valve bearing a row of tubular spines, foramen distinct but partially closed by a pseudo-deltidium; dorsal valve with a cardinal process, simple at the base, but bifid orgrooved at the extremity; valves articulated by teeth, surface radiately striated, often spinous, interior pustulose or papillose. Type C. arcinulatus. acutiradiatus, Hall, 1843, (Stropbomena acutiradiata,) Geo. Rep. 4th Dist. N. Y., p. 171, and Pal. N. Y., vol. 4, p. 120, Up. Held. Gr.

antiope, Billings, 1874, Pal. Foss., vol. 2, p. 19, Low Devonian.

arcuatus, Hall, 1857, 10th Rep. N. Y. St. Mus. Nat. Hist., p. 116, and Pal. N. Y. vol. 4 , p. 119 , Up. Held. Gr.

armatus, DeKoninck, the specimens referred to this species belong to $C$. pusillus.

canadensis, Billings, 1874, Pal. Foss., vol. 2 , p. 17, Lower Devonian.

carinatus, Conrad, 1842, (Strophomena carinata,) Jour. Acad. Nat. Sci., vol. 8, p. 257, and Pal. N. Y., vol. 4, p. 133, Ham. Gr.

complanatus, Hall, 1857, 10th Rep. N. Y. St. Mus. Nat. Hist., p. 56, and Pal. N. Y. vol. 3, p. 418, Oriskany sandstone.

cornutus, Hall, 1843, (Strophomena cornuta,) Geo. Rep. 4th Dist. N. Y., and Pal. N. Y., vol. 2, p. 64, Clinton Gr.

dawsoni, Billings, 1874, Pal. Foss., vol. 2, p. 18, Low. Devonian.

deflectus, Hall, 1857, 10th Rep. N. Y. St. Mus. Nat. Hist., p. 149, and Pal. N. Y., vol. 4, p. 126, Ham. Gr.

emmeteusis, Winchell, 1866, Rep. Low. Penin. Mich., p. 92, Ham. Gr.

filistriatus, Walcott, 1885, Monogr. U. S. Geo. Sur., vol. 8, p. 127, Devonian.

fischeri, Norwood \& Pratten, 1854, Jour. Acad. Nat. Sci., vol. 3, p. 25, Kinderhook Gr.

flemingi, Norwood \& Pratten, 1854, Jour. Acad. Nat. Sci., vol. 3, p. 26, Permian Gr. geinitzanus, N. Sp., Up. Coal Meas. Proposed instead of C. glabra of Geinitz in Carb. und Dyas "in Neb., p. 60, tab. 4, fig. 15 to 18 , which name was preoccupied.

geniculatus, White, 1862, Proc. Bost. Soc. Nat. Hist., vol. 9, p. 29, Waverly or Marshall Gr.

gibbosa, syn. for C. deflectus.

glaber, Hall, 1857, 10th Rep.N. Y. St. Mus. Nat. Hist., p. 117, Up. Held. Gr.

glabra, Geinitz, 1866, Carb. und Dyas. The name was preoccupied. See C. geinitzanus.

granuliferus, Owen, 1852, Geo. Rep. Wis.. Iowa, and Minn., p. 583, Coal Me as 
hemisphericus, Hall, 1857, 10th Rep. N. Y. St. Mus. Nat. Hist., p. 116, and Pal. N. Y., vol. 4, p. 118, Scholıarie grit and Cornif. Gr.

illinoisensis, Worthen, 1860, Trans. St. Louis Acad. Sci., vol. 1, p. 571, and Geo. Sur. Ill., vol. 3, 户. 505, Kaskaskia Gr.

iowensis, Owen, 1852, Geo. Rep. Iowa, Wis. and Minn., p. 584, Carb.

koniuckanus, Norwood \& Pratten, 1854, Jour. Acad. Nat. Sci., vol. 3, 2 d ser., p. 30 , Devolian.

lævis, Keyes, 1888, Proc. Acad. Nat. Sci. Phil., pl. xii, figs. 3a, 3b, Coal Meas.

laticosta, syn. for C. mucronatus.

lepidus, Hall, 1857, 10th Rep. N. Y. St. Mus. Nat. Hist., p. 148, and Pal. N. Y. vol. 4, p. 132, Marcellus slıale and Ham. Gr.

lineatus, Conrad, 1839, (Stropliomena lineata,) Ann. Geo. Rep. N. Y., p. 64, and Pal. N. Y., vol. 4, p. 121, Up. Ileld. Gir.

littoni, Norwood \& Pratten, 1854, Jour. Acad. Nat. Sci., vol. 3, p. 25 , Ham. Gr.

loganensis, Hall \& Whitficld, 187t, U. S. Geo. Expl. 40th Parallel, vol. 4, p. 253, Waverly Gr.

logani, Norwood \& Pratten, 1854, Jour. Acad. Nat. Sci. vol. 3, p. 30, Burling ton $\mathrm{Gr}$.

logani var. aurora, Hall, 1867, Pal. N. Y. vol. 4, p. 137, Tully limestone and Ham. Gr.

maclurii, Norwood \& Pratten, 1854, Jour. Acad. Nat. Sci., vol. 3, p. 23, Ham. Gr.

macrostriatus, Walcott, 1885, Monogr. U. S. Geo. Sur., vol. 8, p. 126, Devonian.

martini, Norwood \& Pratten, 1854, Jour. Acad. Nat. Sci., vol. 3, p. 29. Ham. Gr. melonicus, Billings, 1874, Pal. Foss., vol. 2, p.15, Gaspe limestone No. 8, Devonian.

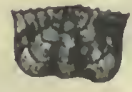

Fra. 549.-Choneles mesolobus. Ventral valve. mesolobus, Norwood \& Pratten, 1854, Jour. Acad. Nat. Sci., vol. 3, p. 27, Coal Meas.

michiganensis, Stevens, 1858, Am. Jour. Sci., vol. 25, p. 262, Marsluall $\mathrm{Gr}$.

millepunctatus, Meek \& Worthen, 1870 , Proc. Acad. Nat. Sci., p. 35, and Geo. Sur. Ill., vol. 5, p. 566, Coal Meas. minimus, Hall. Being preoccupied by Sowerby. See C. undulatus.

mucronatus, Hall, 18t3, Geo. Rep. 4th Dist. N. Y., p. 180, and Pal. N. Y., vol. 4, p. 124, Corniferous and Ham. Grs. mucronata, Meek, \& Hayden, 1858, Proc. Acad. Nat. Sci., p. 262, Coal Meas. This name was preoccupied; moreover it is a svn. for C. granuliferus.

multicosta, Winchell, 1863, Proc. Acad. Nat. Sci., p. 5, Marehall Gr.

muricatus, Hall, 1867, Pal. N. Y., vol. 4, p. 143, Chemung Gr. novascotieus, Hall, 1860, Can. Nat. and Geo., vol. 5, p. 144, Niagari Gr. ornatus, Shumard, 1855, Geo. of Mo., p. 202, Waverly or Kinderhook Gr.

parvus, Shumard, 1855, Geo. of Mo., p. 201, Coal Meas.

permianus, Shumard, 1859, Trans. St. Louis Acad. Sci., vol. 1, p. 390, Permian $\mathrm{Gr}$.

planumbonus, Meek \& Worthen, 1860 , Proc. Acad. Nat. Sci., p. 450, an I Geo. Sur. Ill., vol. 2, p. 253, Keokuk Gr.

platy notus, White, 1874 , Rep. Invert. Eoss., p. 19, and Geo. Sur. IV. 100 Mer., vol. 4, p. 121, Subcarboniferous.

pulchellus, Wincliell, 1862, Proc. Acad. Nat. Sci., p. 410, Marshall Gr.

pusillus, Hall, 1857, 10th Rep. N. Y. St. Mus. Nat. Hist., 1. 149, and Pal. N. Y., vol. 4, p. 128, Ham. Gr.

reversus, Whitfifld, 1882, Desc. New Snec. Foss., from Ohio, p. 213, Marcellus shale.

scitulus, Hall, 1S57, 10th Rep. N. Y. St. Mus. Nat. Hist., p. 147, and Pul. N. Y. vol. 4, p. 130, Ham. Gr.

setigerus, Ilall, 1843, (Strophomena setigeri,) Gen. Rep. 4th Dist. N. Y., p. 180 , and Pal. N. Y., vol. 4, p. 129, Ham. and Chemung Grs.

shumardanus, DeKoniuck, 1847, Recherches sur les Anim. Foss., p. 192, Waverly $\mathrm{Gr}$.

smithi, Norwood \& Pratten, 1854, Jour. Acad. Nat. Sci., vol. 3, p. 24, Coal Meas.

striatellus, Dalman, 1827, (Orthis striatella,) Kongl. Svenska Ak. Handl., p. 111, Up. Sil.

syrtalis, syn. for C. carinata.

tenuistriatus, Hall, 1860 , Can. Nat. and Geo., vol. 5, p. 144, Up. Sil.

tuomeyi, Norwood \& Pratten, 1854, Jour. Acad. Nat. Sci., vol. 3, 2d ser., p. 28, IJa1n. Gr.

undulatus, 11all, 1879, 28th Rep. N. Y. St. Mus. Nat. Hist., p. 155, Niagara Gir.

variolatus, DeKoninck, 1847, Mnnogr. du genre Chonetes, p. 206, Coal Meas.

verneuilanus, Norwood \& Pratten, 1854, Jour. Acad. Nat. Sci., vol. 3, 1). 26, Coal Meas.

verneuilanus var, utahensis, Meek, 1876 , Simpson's Rep. on Cit. Basin of Utah, l. 348 , Carlıoniferous.

yandellanus, Hall, 1857, 10th Rep. N. Y.St. Mus. Nat. Hist., p. 118, and Pal. N. Y., vol. 4, p. 123, Corniferous Gr.

Colosirira, Hall, 1858, Trans. Alb. Inst., vol. 4, p. 146. [Ety. koilos, hollow; speira, spire.] Ovate or suborbicular, concavo-convex, surface finely plicated, usually undefined mesial fold and sinıs, beak small, foramen triangular; internal spires forming two flattened coils connected by a strong loop. Type C. concava. 
concava, Hall, 1857, (Leptocœlia concava,) 10th Rep. N. Y. St. Mus. Nat. Hist., p.

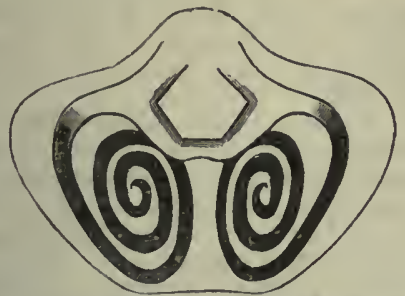

Fig. 550.-Colospira concava. Magnified view of spirals.

107, and Pal. N. Y., vol. 3, p. 245, Corniferous $\mathrm{Gr}$.
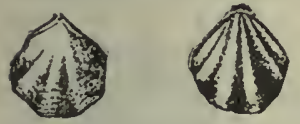

FiG. 551.-Coelospira disparllis. Dorsal and ventral views. dichotoma, II a I l, 1859, (Leptocolia dichotoma,) Pal. N. Y., vol. 3 , p. $452, \mathrm{O}$ r is k a n y sandstone.

disparilis, $\mathrm{H}$ a 11 , 1852, (A t r y p a disparilis,) Pal. N. Y., vol. 2, p. 277 , Niagara Gr.

Crania, Retzius, 1781, Schriften der Berliner Gesellschaft Naturforschende Freund, vol. 2, p. 72. [Ety. kranion, the upper part of a skull.] Shell circular, subquadrate, transverse, or elongated, attached by its ventral valve to some foreign object; upper or dorsal valve more or less convex or conical; apex central or subcentral; surface smooth, spiny, radiated, or concentrically lined, and not unfrequently having the markings of the object to which the lower valve is attached; no articulating linge or ligament, but valves held in place by four muscles; anterior adductor scars approximate and close to the center; posterior pair near the cardinal edge, and widely separated; structure calcareous and tubular. Type C. brattenburgensis.

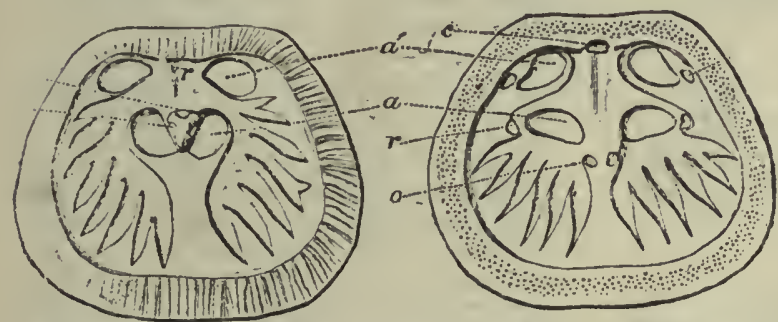

FIG. 552. - Cranla anomala, 2 diam. $a$, Anterior adductors; $a$ posterjor adductors; $c$, protractor sliding muscles; $\epsilon$, cardinal inuscle; $r, 0$, retractor sliding muscles.

acadiensis, Hall, 1860, Can. Nat. and Geo., vol. 5, p. 144, Up. Sil.

anna, Spencer, 1884, Bull. No. 1, Mus.

Univ. St. Mo., p. 57, Niagara Gr.

aurora, Hall, 1863, 16th Rep. N. Y. St.

Mus. Nat. Hist., p. 30, Schoharie Grit. bella, Billings, 1874, Pal. Foss., vol. 2, p. 15 , passage beds between Up. Sil. and Devonian.

bordeni, Hall \& Whitfield, 1872, 24th Rep. N. Y. St. Mus. Nat. Hist., p. 187, Up. Held. Gr.

carbonaria, Whitfield, 1882, Desc. New Spec. Foss., from Ohio, p. 229, Coal Meas.

corrugata, Hall, 1843, (Orbicula corrugatus,) Geo. Rep. N. Y., p. 109, Niagara Gr.

crenistriata, Hall, 1860, 13th Rep. N. Y.

St. Mus. Nat. Hist., p. 78, and Pal. N. Y., vol. 4, p. 28 , Ham. Gr.

deformata, Hall, 1847, (Orbicula deformata,) Pal. N. Y., vol. 1, p. 23, Chazy Gr. Is it a Crania?

dentata, Ringueberg, 1886, Bull. Buf. Soc. Nat. Sci., vol. 5, p. 16, Niagara Gr.

dyeri, S. A. Miller, 1875, Cin. Quar. Jour. Sci., vol. 2, p. 13, Hud. Riv. Gr.

eccentrica, Emmons, 1856, (Orbic- FIG. 553. ula eccentrica,) Am. Geol,, p. dyerl. 112, Up. Taconic.

famelica, Hall \& Whitfield, 1873, 23d Rep. N. Y. St. Mus. Nat. Hist., p. 236, Chemung Gir.

gracilis, Ringueberg, 1886, Bull. Buf. Soc. Nat. Sci., vol. 5 , p. 17, Niagara Gr.

granulosa, Winchell, 1880, 8th Rep. Geo. Sur. Minn., p. 63, Trenton Gr.

gregaria, Hall, 1863, 16th Rep. N. Y. St. Mus. Nat. Hist., p. 31, Ham. Gr.

hamiltonix, Hall, 1860,13 th Rep. N. Y. St. Mus. Nat. Hist., p. 77, and Pal. N. Y., vol. 4, p. 27, Ham. Gr.

lælia, Hall, 1866, 24th Rep. N. Y. St. Mus. Nat. Hist., p. 220, Hud. Riv. Gr.

leoni, Hall, 1860, 13th Rep. N. Y. St. Mus. Nat. Hist., p. 78, and Pal. N. Y., vol. 4, p. 30, Chemung Gr.

modesta, White \& St. John, 1868, Trans. Chi. Acad. Sci., p. 118, Up. Coal Meas.

multipunctata, S. A. Miller, 1875, Cin. Quar. Jour. Sci.,

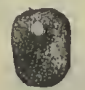
vol. 2 , p. 13 , FiG. 554.-CraHud. Riv, nia muli Gr.

pannosa, Ringueberg, 1886, Bull. Buf. Soc. Nat. Sci., vol. 5, p. 17, Niagara Gr. parallella, Ulrich, 1878, Jour. Cin. Soc. Nat. Hist., vol. 1, p. 98, Hud. Riv. Gr.

percarinata, Ulrich, 1878, Jour. Cin. Soc. Nat. Hist., vol. 1, p. 98, Hud. Riv. Gr.

permiana, Shumard, 1859, Trans. St. Louis Acad. Sci., vol. 1, p. 395, Permian Gr. prima, Owen, 1852, (Orbicula prima,) Geo. Sur. Iowa, Wis., and Minn., p. 583, Potsdam Gr.

radicans, Winchell, 1866, Rep. Low. Peninsula Mich., p. 92, Ham. Gr. 
reposita, White, 1866, Proc. Bost. Soc. Nat. Hist., vol. 9, p. 8, Ham. Gr. reticularis, S. A. Miller, 1875, Cin. Quar. Jour. Sci., vol. 2, p. 280 , Hud. Riv. Gr. rowleyi, Gurley, 1883, New Carb. Foss. Kinderhook (ir. Not defined and published as required by the rules of nomenclature. scabiosa, Hall, 1866, 24th Rep. N. Y. St. Mus. Nat. Hist., p. 220, Hud. Riv. Gr. setifera, Hall, 1863, Trans. Alb. Inst., vol. 4, p. 209, Niagara Gr.

setigera, Hall, 1866, 24 th Rep. N. Y. St. Mus. Nat. Hist., p. 220, Trenton Gr. sheldoni, White, 1862, Proe. Bost. Soc. Nat. Hist., vol. 9, p. 8, Ham. Gr.

siluriana, Hall, 1863, Trans. Alb. Inst., vol. 4, p. 208, Niagara Gr.

socialis, Ulrich, 1878, Jour. Cin. Soc. Nat. Hist., vol. 1, p. 99, Hud. Riv. Gr.

spinigera, Hall, 1879, Desc. New Spec. Foss., p. 13, and 11th Rep. Geo. and Nat. Hist. Ind., p. 283, Niagara lir.

trentonensis, Hall, 1866, 24th Kep. N. Y. St. Mus. Nat. Hist., p. 219, Trenton (ir.

truncata, Emmons, 1556, (Orbicula truncata,) Am. Geol., 1. 200, Trenton Gr.

CryotonnlaA, Hall, 1861, 14th Rep. N. Y. St. Mus. Nat. Hist., p. 102. [Sig. a little eavity.] Equilateral, inequivalve, elongate oval or ovoid; valves unequally convex, no mesial fold or sinus; ventral valve with beak extended or incurved, perforate; foramen terminal; punctate smooth or with concentric stria; articulating by teeth and sockets; dental lamellie of the ventral valve extending downward into the cavity of the sliell; crura extend in a long recurved loop, with long processes into the ventral valve, between which and the apex they are united by a transverse band. Type $\mathrm{C}$. rectirostra.

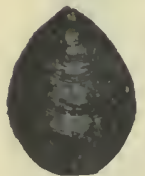

FIG. in6. - Cryptonella IInclklant. Dorsal and profile views.

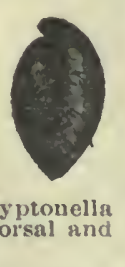
calvini, Hall \& Whitficld, 1870 , 23d Rep. N. Y'. St. Mus. Nat. Hist., p. 239, Chemung Gr.

circula, Walcott, 1885, Monogr. U. S. Geo. Sur., p. 163, Devonian. eudora, Hall, 1867, Hal. N. Y., vol. 4, p. 398, Chemung Gr.

iphis, Hall, 1867, l'al. N. Y., vol. 4, \}. 396, Up. Held. Gr.

lens, Hall, 1860, (Terebratula lens,) 13th Rep. N. Y. St. Mus. Nat. Hist., p. 89, Up. Held. Gr.

lincklæni, Hall, 1860, ('Terebratula lincklæni,) 13th Rep. N. Y. St. Mus. Nat. Hist., p. 88, Ham. Grr.

pinonensis, Walcott, 1885, Monogr. U. S. Geo. Sur., p. 163, Devonian.

planirostra, Hall, 1860, (Terebratula planirostra,) 13th Rep. N. Y. St. Mus. Nat.
Ifist., p. S9, and Pal, N. Y., vol. 4, p. 395 , Ilam. (ir.

rectirostra, Hall, 1860, (Terebratula rectirostra,) 13th Rep. N. Y. St. Mus. Nat. Ilist., p. 88, and Pal. N. Y., vol. 4, p. 394, Ilam. Gr.

Crrtia, Dalman, 1827, Kongl. Vet. Acad. IIandl., p. 93. [Ety. kyrtia, a fishing basket.] Shell somew bat trigonal, valves convex, hinge-line nearly as long as the width of the shell, articulating by teeth and sockets; ventral valve deep, more or less pyramidal, beak straight or slightly recurved, area wide and triangular, fissure covered by a convex pselldodeltidium, generally perforated elose to the beak by a circular foramen, a longitudinal depression in the delticlium sometimes. shows, at the extremity a eircular aperture for the passage of pedicle muscular fibers; dorsal valve less convex; a mesial longitudiual septum, in the ventral valve, extends from llie fissure to near the margin, to the sides of which the dental plates converge, and are united after having formed the fissure walls. Type C. exporrecta.

acutirostris, see Cyrtina acutirostris.

biplicala, see Cyrtina biplicata.

curvilineata, see Cyrtina curvilineata.

dalmani, see Cyrtina dalmani.

exporrecta, Walleuberg, 1S21, Nova. Acta. Regire. Soc. Sei., vol. 8 , v. 64 , and $24 t$ l Rep. N. Y. St. Mus. Nat. Hist., p. 183, Niagara $\mathrm{Gr}$.

exporiecta var. arrecta, Hall \& Whitfield, 1872, 24th His. 5is7.-UyrRep. N. Y. st. Mus. Nat. rectr.

Hist., p. 183, Niagara Gr.

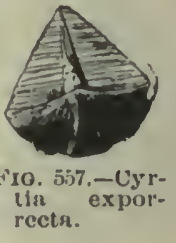
hamillonensis, see Cyrtina hamiltonensis. missouriensis, see Cyrtina missouriensis.

inyrtea, Billings, 1862, Pal. Foss., vol. 1, p. 165, Mid sil.

occidentalis, see Cyrtina oceidentalis.

rostrata, see Cyrtina rostrata.

triquetra, see Cyrtina triquetra.

umlionata, see Cyrtina umbonata.

Crmersa, Davidson, 1858, Monog. Brit. Carb. Braci., p. 66. [Ety. the diminutive of Cyrtia is Cyrtidium, but the author said he preferred bad (ireek to a long name.] Spirifera-like shells; valves very unequal, ventral being extremely clevated, with high area and narrow fissure, elosed by a pseudodeltidium; dental plates converge from the inner margins of the fissure, and, uniting, form a septum to the botton of the internal cavity, thus dividing it into two parts; shell punctate. Type C. heteroclyta.

acutirostris, Shumard, 1855, (Cyrtia acutirostris,) Geo. Rep. Mo., p. 204, Wa verly or Choteau Gr.

affinis, Billings, 1874, Pal. Foss., vol. 2, p. 49, Gaspe No. 8, Devonian. 
billingsi, Meek, 1868, Trans. Chi. Acad. Sci., p. 97 , Ham. Gr.

biplicata, Hall, 1857, (Cyrtia biplicata,) 10 th Rep. N. Y. St. Mus. Nat. Hist., p. 165, Schoharie grit and Cornif. Gr.

crassa, Hall, 1867, Pal. N. Y., vol. 4, p. 267, Up. Held. Gr.

curvilineata, White, 1865, (Cyrtia curvilineata,) Proc. Bost. Soc. Nat. Hist., vol. 9, p. 25, and Pal. N. Y., vol. 4, p. 270, Ham. Gr.

dalmani, Hall, 1857, (Cyrtia dalmani,) 10th Rep. N. Y. St. Mus. Nat. Hist., p. 64, Low. Held. Gr.

davidsoni, Walcott, 1885, Monogr. U. S. Geo. Sur., vol. 8, p. 146, Devonian.

euphemia, Billings, 1863, Can. Nat. and Geol., vol. 8, p. 19, Corniferous Gr.
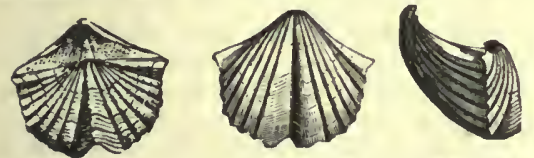

F'1G. 528.-Cyrtina hamiltonensis. Dorsal, ventral, aud side views.

hamiltonensis, Hall, 1857, (Cyrtia hamiltonensis, ) 10th Rep. N. Y. St. Mus. Nat. Hist., p. 166, and Pal. N. Y., vol. 4, p. 268, Schoharie grit, Cornif. and Ham. Grs.

hamiltonensis var. recta, Hall, 1867, Pal. N. Y., vol. 4, p. 270 , Ham. Gr.

missouriensis, Swallow, 1860, (Cyrtia missouriensis,) Trans. St. Louis Acad. Sci., vol. 1, p. 647, Ham. Gr.

occidentalis, Swallow, 1860, (Cyrtia occidentalis,) Trans. St. Louis Acad. Sci., vol. 1, p. 648 , Ham. Gr.

panda, Meek, 1868, Trans. Chi. Acad. Sci., p. 100, Ham. Gr.

pyramidalis, Hall, 1852, (Spirifer pyramidalis,) Pal. N. Y., vol. 2 , p. 266 , Niagara Gr.

rostrata, Hall, 1857, (Cyrtia rostrata,) 10th Rep. N. Y. St. Mus. Nat. Hist., p. 64, Oriskany sandstone.

triquetra, Hall, 1858, (Cyrtia triquetra,) Geo. Rep. Iowa, vol. 1, pt. 2, p. 513, Ham. Gr.

umbonata, Hall, 1858, (Cyrtia umbonata,) Geo. Rep. Iowa, vol. 1, pt. 2, p. 512, Ham. Gr.

Delthyris, Dalman, 1827, syn. for Spirifera.

acanthoptera, syn. for Spirifera disjuncta. acuminata, Conrad, see Spirifera acuminata.

acuminata, Hall, syn. for Spirifera mesacostalis.

acutilirata, see Orthis acutilirata.

arenosa, see Spirifera arenosa.

audacula, see Spirifera audacula.

bialveata, see Spirifera bialveata.

bilobata, see Orthis bilobata.

brachynota, see Spirifera brachynota.

chemungensis, syn. for Spirifera disjuncta. congesta, see Spirifera congesta.

cuspidata, syn. for Spirifera disjuncta.

decemplicata, see Spirifera decemplicata. deltoidea, syn. for Orthis lynx.

disjuncta, see Spirifera disjuncta.

duodenaria, see Spirifera duodenaria.

dupliplicata, see Spirifera dupliplicata.

euruteines, see Spirifera euruteines.

expansa, see Pterotheca expansa.

fimbriata, see Spirifera fimbriata.

granulifera, see Spirifera granulifera.

granulosa, see Spirifera granulosa.

inermis, see Spirifera disjuncta.

lævis, see Spirifera lævis.

macronota, see Spirifera macronota.

macropleura, see Spirifera macropleura. medialis, see Spirifera medialis.

mesacostalis, see Spirifera mesacostalis.

mesastrialis, see Spirifera mesastrialis.

microptera, syn. for Orthis lynx.

mucronata, see Spirifera mucronata.

niagarensis, see Spirifera niagarensis.

pachyptera, see Spirifera pachyptera.

perlata, see Spirifera disjuncta.

prolata, see Spirifera prolata.

prora, see Spirifera prora.

radiata, see Spirifera radiata.

raricosta, see Spirifera raricosta.

rugatina, see Spirifera rugatina.

sculptilis, see Spirifera sculptilis.

staminea, see Spirifera staminea.

triloba, see Spirifera triloba.

undulata, see Spirifera undulata.

varica, see Orthis varica.

ziczac, see Spirifera ziczac.

Dicellomus, Hall, 1873, 23d Rep. N. Y. St. Mus. Nat. IIist., p. 246. A generic name proposed for the reception of Obolella crassa and 0 . polita, without distinguishing the generic characters.

Dicraniscus, Meek, syn, for Triplesia.

ortoni, see Triplesia ortoni.

Dignomia. Hall, 1873, 23d Rep. N. Y. St.

Mus. Nat. Hist., p. 245 . [Ety. di, from; dis, twice; gnoma, a sign.] Lingula-like shells having a longitudinal septum in one or both valves. Type D. alveata. alveata, Hall, 1873, 23d Rep. N. Y. St.

Mus. Nat. Hist., p. 245, Ham. Gr.

Dinobolus, Hall, March, 1871, 23d Rep.

N. Y. St. Mus. Nat. Hist., p. 247. [Ety.

dis, twice; Obolus, a genus of shells.]

Shell subcircular, valves thick; umbo

of the ventral valve slightly prominent; area wider than long; platform sinuated, widely V-shaped; crescent prominently marked in crown and sides; hinge moderately thick, edge rounded, with a pair of subcardinal sears in front of the cardinal facet; umbo of the brachial valve tumid; platform trilobed; outer margins raised; antemedian portion rounded, projecting, and terminating in a median plate; crescent a marked linear scar on the hinge; arching forward in front of the cardinal facet; an indentation on the inner border of its sides near the hinge, another further forward; outer border a fine line; subcardinal scar in the umbonal cavity: rhomboidal, postmedian scar in front of the latter. Type D. conradi. 
canadensis, Billings, 1857, (Obolus canadensis,) Rep. of Progr. Geo. Sur. of Can., p. 189, and Can. Nat., vol. 6, p. 222, Black Riv. Gr.

cnnradi, Hall, 1868, (Obolus conradi,) 20th Rep. N. Y. St. Mus. Nat. Hist., p. 368, Niagara Gr.

galtensis, see Trimerella galtensis.

magnificus, Billings, 1872, (Obolellina magnifica,) Canadian Naturalist, vol. 6, p. 330, Black Riv. Gr.

parvus, Whitfield, 1882, Geo. Wis., vol. 4, p. 347, Galena Gr.

Discin A, Lamarck, 1819, Hist. Nat. A nim. sans Vert., vol. 6, p. 236. [Ety. discus, a flat, round plate; the termination inus, implying resemblance.] Circular, longitudinally or transversely oval; dorsal valve conical, with apex inclined toward the posterior margin; ventral

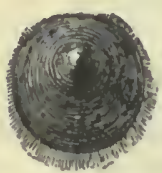

F'IG. 559.-Discina ostreoldes.

valve opercular, flat, or partly convex, perforated by a narrow, oval, longit udi nal slit. reaching to near the posterior wargin, and placed in the

middle of an oval depressed disk ; sur-

face smootl, striated

from the apex to the margin, or having concentric lines of growtl produced in foliacenns expansions; structure horny, and perforated by minute tubuli. Type D. ostreoides.

acadica, see Stenotheca acadica.

alleghania, Hall, 1860, 13th Rep. N. Y. St. Mus. Nat. Hist., p. 77 , and Pal. N. Y., vol. 4 , p. 25 , Chemung Gr.

ampla, Hall, 1867, Pal. N. Y., vol. 4, p. 17, Oriskany sandstone. Proposed instead of $\mathrm{D}$. grandis of Hall.

capax, White, 1862, Froc. Bost Soc. Nat.

Hist., vol.9, p. 30, Waverly or Marshall Gr. capuliformis, McCliesney, syn. for D. nitida. circe, Billings, 1862 ,

Pal. Foss, vol. 1, p. 51, Trenton Gr. See remarks on $\mathrm{D}$. la. mellosa.

clara, Spencer, 1884, Bull. No. 1, Mus. Univ. St. Mo., p. 56, Niagara Gr.

connat a, Walcott. 1885, Monogr. U. S.

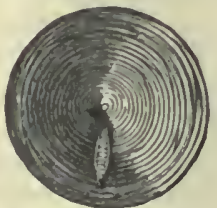

FIG. 560.-Discina clrce.
Geo. Sur., vol. 8, p. 214, Devonian.

con radi, Hall, 1859, Pal. N. Y., vol. 3 , p. 161, Low. Held. Gr. convexa, Shumard, 1858, Trans. St. I,ouis Acad. Sci., vol. 1, p. 221, Coal Meas. discus, Hall, 1859, Pal. N. Y., vol. 3, p. 159, Low. Held. Gr.

doria, Hall, 1863, J6th Rep. N. Y. St. Mus. Nat. Hist., p. 26, Ham. Gr.

elmira, Hall, 1863, 16th Rep. N. Y. St. Mus. Nat. Hist., p. 29, Chemung. Gir. gallaheri, Winchell, 1865, Proc. Acad. Nat. Sci., p. 112, Marshall Gr.

grandis, Vanuxem, 1842, Geo. Rep. Bd Dist. N. Y., p. 152, and Pal. N. Y., vol. 4, p. 17, Cornif. and Ham. Gr.

grandis, Hall, 1859, Pal. N. Y., vol. 3. The name was preoccupied. See D. ampla.

humilis, Hall, 1863, 16th Rep. N. Y. St. Mus. Nat. Hist., p. 25, Marcellus slate and Ham. Gr.

inutilis, Hall, 1S63, 16th Rep. N. Y. St. Mus. Nat. Hist., p. 130, Potsdam Gr.

lamellosa, Hall, 1847, (Orbicula lanellosa.)

The name was preoecupied by Broderick in 1833. Bullings has described it as $D$. circe.

lodensis, Vanuxem, 1842, (Orbicula lodensis,) Geo. Rep. 3d Dist. N. Y., p). 168, and Y'al. N. Y., vol. 4, p. 22, Genesee Slate.

manhattanensis, Meek and IIayden, 1859 , Proc. Acad. Nat. Sci., p. 25, Coal Meas.

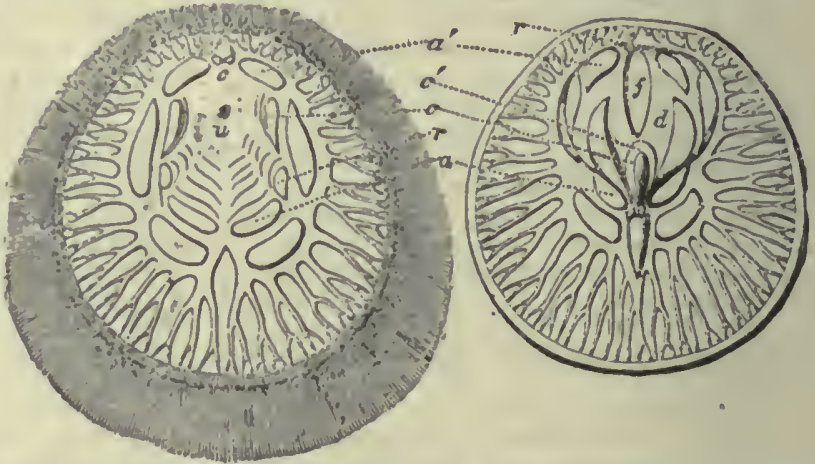

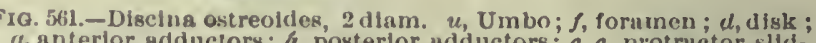
u, anterior adductors; $f$, posterior. udductors; $c, c$, protructor slik-
ing muscles ; $r$, retructor muscles.

marginalis, Whitfield, 1880, Ann. Rep. Geo. Sur. Wis., p. 70, and Geo. Wis., vol. 4, p. 325 , Ham. Gr.

media, Hall, 1863, 16th Rep. N. Y. St. Mus. Nat. Hist., p. 27, Ham. and Chemung Grs.

meekana, Whitfield, 1882, Desc. New Spec. Foss. Irom Ohio, p. 228, Coal Meas. microscopica, Shumard, 1861, Am. Jour. Sci. and Arts, vol. 32, p. 213, Potsdam Gr.

minuta, Hall, 1843, (Orbicula minuta,) Geo. Rep. 4th Dist. N. Y., p. 180, and Pal. N. Y., vol. 4 , p. 16, Marcellus Shale. missouriensis, Shumard, 1858, Trans. St. Louis Acad. Sci., Coal Meas. Syn. for D. nitida. 
neglecta, Hall, 1863, 16th Rep. N. Y. St. Mus. Nat. Hist., p. 29, Chemung Gr.

newberryi, Hall, 1863, 16th Rep. N. Y. St. Mus. Nat. Hist., p. 30, Waverly Gr.

nitida, Phillips, 1836, (Orbicula nitida,) Geo. of York., vol. 2, p. 221, and Geo. Sur. Ill., vol. 5, p. 572, Coal Meas.

patellaris, Winchell, 1863, Proc. Acad. Nat. Sci., p. 4, Waverly or Marshall Gr.

pelopea, Billings, 1862, Pal. Foss., vol. 1, p. 52, Trenton Gr.

pleurites, Meek, 1875, Ohio Pal., vol. 2, p. 278, Waverly $\mathrm{Gr}$.

randalli, Hall, 1863, 16th Rep. N. Y. St. Mus. Nat. Hist., p. 25, Ham. Gr.

saffordi, Winchell, 1869, Geo. of Tenn., and, in 1870, Proc. Am. Phil. Soc., p. 248, Marshall Gr.

seneca, Hall, 1863, 16th Rep. N. Y. St. Mus. Nat. Hist., p. 26, Ham. Gr.

sublamellosa, Ulrich, 1878, Jour. Cin. Soc. Nat. Hist., p. 97. Probably the cast of a Trematis.

subtrigonalis, McChesney, 1865, Desc. New Pal. Foss, Coal Meas. Not recognized. tenuilamellata, $\mathrm{Hall}$, 1852, (Orbicula tenuilamellata,) Pal. N. Y., vol. 2, p. 250, Niagara Gr.

tenuilamellata var. subplana, Hall, 1860, Can. Nat. and Geol., vol. 5, p. 144,Up. Sil. tenuilineata, Meek \& Hayden, 1859, Proc. Acad. Nat. Sci., p. 25, Coal Meas.

tenuistriata, Ulrich, 1878, Jour. Cin. Soc. Nat. Hist., p. 96. Probably the cast of a Trematis.

trigonalis, syn. for D. subtrigonalis. truncata, see Schizobolus truncatus.

tullia, Hall, 1863, 16th Rep. N. Y. St. Mus. Nat. Hist., p. 28, Tully limestone. vanuxemi, Hall, 1859, Pal. N. Y., vol. 3, p. 162, Water-lime or Low. Held. Gr.

varsoviensis, Worthen, 1884, Bull. No. 2, Ill. St. Mus. Nat. Hist., p. 23, and Geo. Sur. Ill., vol. 8, p. 102, Keokuk Gr.

Eatonia, Hall, 1857, 10th Rep. N. Y. St. Mus. Nat. Hist., p. 90, and 12th Rep., p. 35. [Ety. proper name.] Oval, ovoid, subcircular, elongate, or transverse; valves very unequally convex; mesial fold and sinus; beak of ventral valve small, perforate, closely incurved over the umbo of the dorsal valve; two teeth in the ventral, with corresponding sockets in the dorsal valve; a prominent bifurcating cardinal process and four crural processes in the dorsal valve distinguish this genus. Type E. medialis. eminens, Hall, 1857, N. Y. St. Mus. Nat. Hist., p. 92, and Pal. N. Y., vol. 3, p. 242,

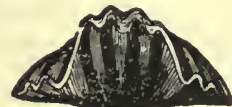

FIg. 562.-Eatoula medialls. Anterior view.

peculiaris eculiaris, Conrad, 1841, (Atrypa pe-
culiaris,) Ann. Rep. N. Y., p. 56, and
Pal. N. Y., vol. 3, p. 244, Oriskany and Low. Held. Gr.

pumila, Hall, 1859, Pal. N. Y., vol. 3, p. 437, Oriskany sandstone.

singularis. Vanuxem, 1842, (Atrypa singularis,) Geo. Rep. 3 d Dist. N. Y., p. 120, and Pal. N. Y., vol. 3 , p. 243, Low. Held. Gr.

sinuata, Hall, 1857, 10th Rep. N. Y. St. Mus. Nat. Hist., p. 91, and Pal. N. Y., vol. 3, Oriskany sandstone.

whitfieldi, Hall, 1859, Pal. N. Y., vol. 3, p. 437 , Oriskany sandstone.

Eichwasia, Billings, 1858, Rep. of Progr. Geo. Sur. Can., p. 190. [Ety. proper name.] Ovate or subtrigonal, with or without mesial fold and sinus; ventral valve obscurely perforate on the umbo; apex acute and entire; space beneath occupied by an imperforate concave plate; interior of the rostral cavity containing a transverse septum; dorsal valve with a slender cardinal process and a very elevated medio-longitudinal septum; valves articulated in a narrow groove in the dorsal valve; surface of the shell reticulate, solid, and fibrous beneath. Type E. subtrigonalis. anticostiensis, Billings, 1866, Catal. Sil. Foss. Antic., p. 10, Hud. Riv. Gr.

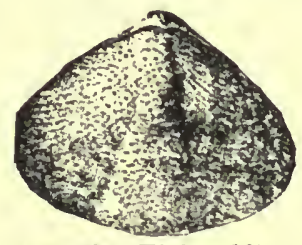

FrG. 564.-Elchwaldia conc in $n$ a, Hall, 1868, 20th Rep. N. Y. St. Mus. Nat. Hist., p. 319, Niagara Gr.

c or a llifera, Hall, 1852, (A try pa corallifera,) Pal. N. Y., vol. 2 , p. 281, Niagara Gr. Prof. Davidson regarded this shell as identical with $\mathbf{E}$. capewelli, which was described in 1848 , in Bull. Soc. Geol. France, vol. 3.

$\mathrm{g}$ i b bos a,

Hall, 1868, 20tb Rep. N. Y. St. Mus. Nat. Hist. p.
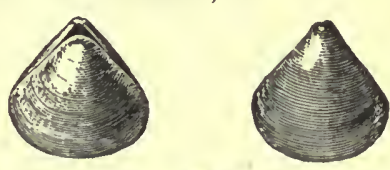
319 , Niagara Gr.

reti c ulata, Hall, 1863,

( $\mathrm{R} \mathrm{h}$ v n chonella(?) reticulata,) Trans.

Alb. Inst. vol. 4 p' FIG. 565.- Echwalda subtrigovol. 4, p. nalis. Dorsul, ventral, side, 217, Niag- front, and apex vlews. a r a G.r.

Davidson said a syn. for E. capewelli.

subtrigonalis, Billings, 1858, Rep. of Progr.

Geo. Sur. Can., p. 192, Black Riv. Gr. 
Elkania, Ford, 1886, Am. Jour. Sci. and Arts, 3d ser., vol. 32, p. 325. [Ety. proper name.] Shell thin, calcareous, inarticulate, longitudinally ovate or subcurcular, convex; ventral valve, with solid beak and minute-grooved area; muscular scars, six in each valve; beneath the rostrum a spoon-shaped pit separates the scars. Type E. desiderata.

desiderata, Billings, 1862, (Obolella desiderata,) Pal. Foss., vol. 1, p. 69, Up. Taconic.

Eumetria, Hall, 1864, 16th Rep. N. Y. St. Mus. Nat. Hist., p. 59. Shell longitudinally suboval; striated, without mesial fold and sinus; structure punctate; beak of the ventral valve ineurved; hinge area contracted ; foramen large; internal spires as in Athyris; dorsn! valve in the form of a pectinoid shell, with diverging lamellae, which extend beneath the cardinal area of the ventral valve on either side of the center; processes extending into the cavity of the dorsal valve, gradually converge, and are united by a transverse concave septum. Type E. vera.

prima, White, 1862, (Acambona prima,) Proc. Bost. Soc. Nat. Ilist., vol. \%, p. 27, Burlington Gr.

vera, Hall, 1858, (Retzia vera,) (ico. Sur. Iowa, p. 704, Kaskaskia Gr.

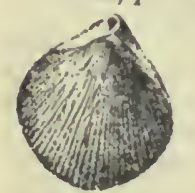

Fig. 566.-Eumetria verneull. ana. Lond. Geo. Mag. vol. 8, p. 11. [Ety. proper name.] Shell ovate; spiral coils in the dorsal valve for the support of the brachial appendages connected by a loop as in Atrypa; lamellac converge downward like the letter $V$, with the extremities turned slightly upward before uniting; principal coils face the lateral margins; ends of the spirals meet in the center of the shell; spirals consist of four or five compressed coils. Type G. obovata.

headi, Meek, 1873, ('/ygospira headi,) Ohio Pal., vol. 1, p. 127, Hud. Riv. Gr. Goniocolia, Hall, syn. for Pentagonia.

Gypidul, A, Hall, 1867, Pal. $\Lambda^{r}$. Y., vol. 4, p. 373. [Ety. gyps, vulture; in allusion to the strongly incurved beak.] Sliort, gibbous or ventricose, ventral valve much|the larger, with or without mesial fold; a large fissure, and elongate, much incurved, trough-shaped pit; dorsal valve depressed in front; area on both valves, that of the ventral striated as in Spirifera; lamellx of dorsal valve separate and diverging. Type $G$. occidentalis. leviuscula, Hall, 1867, Pal. N. Y., vol. 4 , p. 381, Devonian.

munda, Calvin, 1878, Bull. U. S. Geo. Sur., vol. 4 , No. 3 , p. 730, Low. Devonian.

obsolescens, see Pentamerella obsoleseens. occidentalis, Hall, 1858, (Pentanerus occidentalis, ) Geo. Rep. Iowa, vol. 1, pt. 2, p. 514, Ham. Gr.

unguiformis, Vlrich, 1886, Cont. to Am. Pal., p. 28, Niagara Gr.

Hemiproniles, Pander, 1830. This nane, not having been defined, las been superseded by Streptorhynchus, if the two names refer to the same form.

americanus, see Streptorhynchus americanum.

Hindelda, Davidson, 1882, Monogr. Brit. Foss., Brachiopoda, vol. 5, p. 130. [Ety. proper name.] Shell elongate, ovate; about six coils in each spiral; , apices direeted laterally ; stems attuclied to the hinge plate, and extending into the interior, they are abruptly bent back ward, and then form a broad, rounded curve, facing the bottom of the dorsal valve; when they reach the front they give off a semicircular loop, having a spikelike process at the top, directed towurd the beak. Type H. umbonata.

umbonata, Billings, 1865, (Athyris umbonata,) Pal. Foss., vol. 1, p. 144, Micl. Sil., Anticosti Div. 1.

Hipperiony.x, Vanuxem, 184:, Geo. 3d Dist. N. Y., p. 124, syn. for Orthis. 'The genus was founded on a cast.

consinilis, syn. for Atrypa reticularis.

proximus, see Orthis proximus.

similaris, Vanuxem, 1842, Geo. Rep. 3d Dist. N. Y., Oriskany sandstone. Not defined.

I puIDEA, Billings, 1874, Pal. Foss., vol. 2, p. 76. [E.ty. proper name.] Ventral valve conical, elevated at the beak, hinge-line nearly straight, posterior angles rounded, sides and front nearly uniformly rounded; posterior side posterior side with a large lden bella. false area and a convex pseudodeltidium; dorsal valve semicircular, moderately convex, most elevated at the beak; surface concentrically marked. Type I. bella.

bella, Billings, 1872, Can. Nat., vol. 6, p. 477, and Pal. Foss., vol. 2, p. 76, Up. Taconic.

sculptilis, see Kutorgina sculptilis.

Koninckia, Suess, 1853. MS. published by Woodward, 1854, in Manual of Mollusca, p. 231. [Ety. proper name.] Shell circular, inequivalve, compressed; ventral valve convex, with a slight longitudinal depression; beak incurved, with auricular expansions; dorsal valve concave; surface smooth; no area or deltidium ; valves inarticulated; mesial ridge in dorsal valve; oral appendages supported by a 
spiral, calcified lamella. Type K. leonhardi.

americana, Swallow, 1863, Trans. St. Louis Acad. Sci., vol. 2, p. 94 , Kaskaskia Gr. Kutorgin A, Billings, 1861, Pal. Foss., vol. 1, p. 8. [Ety. proper name.] Shell more

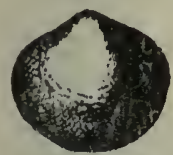

Fig. ล68.-Kutorgina pannula. Enl a rged dlam. or less subquadrate in outline; hinge-line straight; sides slightly convex, anterior angles rounded, front slightly convex ; surface with concentric ridges terminating on the cardinal edges, and the course conforming to the margin of the shell, and sometimes with lines radiating from the beak to the margin; ventral valve tumid, most convex about the middle, beak slightly (lepressed; cardinal edges straight or slightly concave and diverging from the beak at an obtuse angle; dorsal valve less convex, most elevated at the beak, and along the middle there is a shallow concavity extending to the front margin. Type K. cingulata.

cingulata, Billings, 1861, Pal. Foss., vol. 1, p. 8 , Up. Taconic.

labradorica, Billings, 1861, (Obolus labradoricus,) Pal. Foss., vol. 1, p. 6, Up. Taconic.

latourensis, Matthew, 1885, Trans. Roy. Soc. Can., p. 42, St. John Gr.

minutissima, Hall \& Whitfield, 1877, U.S. Geo. Expl. 40th parallel, syn. for $K$. sculptilis.

pannula, White, $187+$,(Trematis pannulus,) Rep. Invert. Foss., p. 6, and Geo. Sur. IV. 100th Mer., vol. 4, p. 36, Up. Taconic.

prospectensis, Walcott, 1885, Monogr. U. S. Geo. Sur. Terr., vol. 8, p. 19, Up. Taconic.

pterineoides, Matthew, 1885, Trans. Roy. Soc. Can., p. 43, St. John Gr.

sculptilis, Meek, 1873, (Iphidea sculptilis,) 6th Ann. Rep. U. S. Geo. Sur. Terr., p. 479, and Monogr. U. S. Geo. Sur. Terr., vol. 8 , p. 20 , Potsdam Gr.

stissingensis, Dwight, 1859, Am. Jour. Sci. and Arts, $3 \mathrm{~d}$ ser., vol. 38, p. 145, Up. Taeonic.

whitfieldi, Walcott, 18s5, Monogr. U. S. Geo. Sur. Terr., vol. 8, p. 18, Up. Taconic.

Leiorhyxcius, Hall, 1860, 13th Rep. N. Y. St. Mus. Nat. Hist., p. 75 . [Ety. leios, smooth; rhynchos, beak.] Ovate, circular or transverse, valves unequally convex; mesial fold and sinus, which are plicated; articulating by teeth and sockets; apex of ventral valve perforate, two cliverging lamella extend into and join the sides or bottom of the rostral cavity ; muscular impressions occupy a narrow triangular cavity below the dental lamellæ; median septum in the dorsal valve extending half the length of the shell; hinge plates, narrow, strong processes, embraced by the curving teeth of the opposite valve; substance fibrous. Type L. quadricostatum. dubium, Hall, 1867, Pal. N. Y., vol. 4, p. 364, Marcellus Shale.

globuliforme, Vanuxem, 1842, (Atrypa globuliformis,) Geo. 3d Dist. N. Y., p. 182, and Pal. N. Y., vol. 4, p. 364, Chemung Gr.

hecate, Clarke, 1885, Bull. U. S. Geo. Sur. No. 16, p. 31, Genesee Shales.

huronense, Nicholson, 1874, Geo. Mag. Lond., n. s., vol. 1, p. 120, Ham. Gr.

iris, Hall, 1867, Pal. N. Y., vol. 4, p. 360 , Chemung Gr.

kelloggi, Hall, 1867, Pal. N. Y., vol. 4, p. 361 , Chemung Gir.

laura, Billings, May, 1860, (Rhynchonella laura,) Can. Jour., vol. 5, p. 273, Ham. Gr. limitare, Vanuxem, 1842, (Orthis limitaris,) Geo. $3 d$ Dist. N. Y., p. 146, and Pal. N. Y., vol. 4, p. 356, (Atrypa limitaris,) 4th Dist. N. Y., Marcellus Shale.

mesacostale, Hall, 1843,

(Atrypa mesacostalis,) FiG. 569.-LeioGeo. 4th Dist. N. Y., pl r h y 11 c h us 64, and Pal. N. Y., vol. tum. 4, p. 362, Chemung Gr.

multicosta, Hall, 1860, 13th Rep. N. Y. St. Mus. Nat. Hist., p. 85, and Pal. N. Y., vol. 4 , p. 358 , Ham. Gr.

mysia, Hall, 1867, Pal. N. Y., vol. 4, p. 357, Marcellus Shale.

nevadense, Walcott, 1885, Monogr. U. S. Geo. Sur., vol. S, p. 157, Devonian.

newberryi, Hall \& Whitfield, 1873, 23d Rep. N. Y. St. Mus. Nat. Hist., p. 240, Waverly Gr.

quadricostatum, Vanuxem, 1812, (Orthis quadricostata,) Geo. $3 d$ Dist. N. Y., p. 168 , and Pal. N. Y., vol. 4, p. 357, Genesee Slate.

sesquiplicatum, Winchell, 1866, Rep. Low. Penin. Mich., p. 95, Ham. Gr.

sinuatum, Hall, 1867, Pal. N. Y., vol. 4, p. 362, Chemung Gr.

Lept AxNA, Dalman, 1827, Kongl. Vet. Acad. Handl., p. 93. [Ety. leptos,thin.] Shell thin, semicircular, transversely elon. gated, smooth or finely striated; hingeline straight, ventral valve convex, fissure partly covered by a deltidium; beak inconspicuous, sometimes perforated ; cardinal area narrow ; muscular scars small, not marginal; adductor scars close to a mesial rirlge, while the cardinal scars are on either side; vascular impressions radiating; dorsal valve concave; socket ridges large, cardinal process small, multifid, connate with their bases; adductor impressions large, produced, elongated, and bordered by ridges; area on both valves. Type L. transversalis.

alternata, see Strophomena alternata. alternistriata, see Strophomena alternistriata. 
anuloga, see Strophomena analoga. aspera, James, syn. for L. sericea. barabuensis, Winchell, 1864, (Orthis barabuensis,) Am. Jour. Sci. and Arts, 2d ser., vol. 37 , p. 229 , and Geo. Wis., vol. 4 , p. 171, Potsdam Gr.

bipartita, see Strophomena bipartita. camerata, see Strophomena camerata. concava, Hall, 1857, 10 th Rep. N. Y. St. Mus. Nat. Hist., p. 47, and Pal. N. Y., vol. 3 , p. 197, Low. Ileld. Gr.

decipiens, Billings, 1562, Pal. Foss., vol. 1, p. 74, Quebec Gr.

deflecta, see Streptorhynchus deflectum. deltoidea, see Strophomena deltoidea. depressa, see Strophomena depressa. fasciata, see Strophomena fasciata. filtexta, see Streptorbyuchus filitextum. fragaria, syn. for Productella subaculeata. incrassata, see Strophomena incrassatı. indenta, see Strophodonta indenta. laticosta, syu. for Tropidoleptus carinatus. melita, Hall \& Whitfield, 1577, U. S. Geo.

Expl. 40th parallel, vol. 4, p. 208, Potsdam Gr.

membranacea, see Productella hirsuta. mesacosta, Shumard, 185.5, (ieo. Rep. Mo., p. 205, Trenton (ir.

nasuta, see Strophonena nasuta.

uncleata, Hall, 1857, 10th Rep. N. Y. St.

Mus. Nat. Hist., 1), 47, and Pa]. N. Y.

vol. 3, p. 419 , Oriskany sandstone.

obscura, see Strophomena obscura.

orthididea, see Strophomena orthididea.

planoconvexa, see Streptorliyuchus planoconvexum.

planumbona, see Streptorhyncluus planumbonum.

plicatella, Urich, 1879, Jour. Cin. Soc.

Nat. Hist., vol. 2, リ. 15, Utica Slate Gr. plicifer $\alpha$, see Strophomena plicifera. profunda, see Strophodonts profunda.

prolongata, Foerste, 1885, Bull. Sci. Lab.

Denison Univ., p. 79, Niugara Gr. punctulifera, see Stroplionella punctuliferu. quadrilatera, syn. for Stroplıomena rhomboidalis.

recta, see Streptorhynchus rectum.

rugosa, see Strophomena rugosa.

semiovalis, syn. for I. sericet.

sericen, Sowerby, 1839, Murch. Sil. Syst.,

p. 636, and Pal. N. Y., vol. 1, p. 110,

Trenton to Clinton Gr.
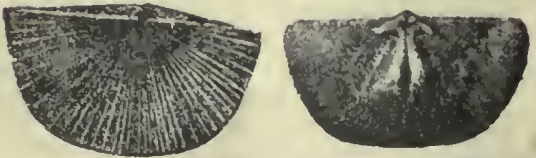

Fig. 570.-Leptrena sericea. Dorsal view, and interlor of dorsal valve.

sordida, Billings, 186:2, Pal. Foss., vol. 1, p. 73, Quebec Gr.

subquadrata, Hall, 1883, Rep. St. Geol, pl. 46 , fig. 32,33 , Jow. Held. Gr.

subtenta, see Streptorhynchus subtentum. tenuilineata, see Strophomena tenuilineata. tenuistriala, see Strophomena tenuistriata. tranversalis, Wablenberg, 1821, (Anomites transversalis,) Act. Soc. Upsal., vol: 8, p. 64, and Pal. N. Y., vol. 2, p. p. 256, Anticosti and Clinton Gr.

trilobata, see Strophomena trilobata.

vicina, Castelnau, 1843, Syst. Sil., p. 39. Not recognized.

Lei'Tonolus, Hall, 1871, 24th Rep. N. Y. St. Mus. Nat. Ilist., p. 226. [Ety. leptos, minute; Obolus, a genus.] Shell small, ovate, fragile, semiphosphatic, concentrically lined; ventral valve with an area and pedicel groove, inuscular sear elevated, subquadrate; dorsal valve with trifid muscular impressions. Type I. lepis.

insignis, Hall, 1\$71, 24th Rep. N. Y. St. Mus. Nat. llist., p. 227, Utica Slate.

lepis, llall, 1871, 2tth Rep. N. Y. St. Mus. Nat. Hist., p. 226, Utica Slate.

occidentalis, llall, 1871, 2tth Rep. N. Y. St. Mus. Nat. Hist, p. 227, Utica Slate. Inptockila, llall, 1857, loth Rep. N. Y. St. Mus. Nat. Hist., p. 107, and 12th Rep., p. 32. [ [ity. leptos, minute; koilia, belly; in allusion to the shallow visceral cavity.] Shell inequivalve, variable in form, plicated, usually mesial fold and sinus, substance lamellose or fibrous; ventral valve convex, beak c xtended, and more or less incurved: foramen terminal, the lower side formed by two deltoid pieces; two strong teeth, denticulated; muscular impressions marking a flabelliform area with a thin median septum, adductor imprints small ; dorsal vulve flat, concavc, or depressed convex; on each side of a strong cardinal process are the decp, oblique, dental fossets, from the inner margins of which the crural processes proceed, supported below by thickened plates, extending obliquely on the border of the muscular impression toward the middle of the chell; muscular impres. sion divided by a low median septum; the crura, in their extension, are united, in a flattened disk, which terminates in an acute point; on the center of the cardinal side a slender process extends downward, and near tho junction of the crura two slender processes extend into the cavify of the ventral valve. Type I. flabellites.

acutiplicata, Conrad, 1841, (Atry )a acutiplicata,) Ann. Rep. N. Y., p. 54, and Pal. N. Y., vol. 4, p. 365, U’p. Held. Gr. concava, see Colospira concava.

dichotoma, see Coelospira dichotoma.

disparilis, see Coelospira disparilis. fimbriata, Hall, 1859, Pal.

N. Y., vol. 3 , p. 451 , Oriskany sandstone.

flabellites, Conrad, 1841, Frs. 571.- I,epto(Atrypa flabellites,) colia fisbelites. Ann. Rep. $\mathbf{N}$. Y., .

55, and P'al. N. Y., vol. 3, p. 449, Oriskany sandstone. 
hemispherica, Sowerby, 1839, (Atrypa hemispherica,) Murch. Sil. Syst., p. 639, and Pal. N. Y., vol. 2, p. 74, Clinton Gr.

imbricata, see Trematospira imbricata.

intermedia, Hall, 1860, Can. Nat. and Geo., vol. 5, p. 144, Up. Sil.

planoconvexa, Hall, 1852, (Atrypa planocon vexa,) Pal. N. Y., vol. 2, p. 75, Clinton Gr.

propria, Hall, syn. for L. flabellites.

Lingula, Bruguiere, 1792, Encyc. Metb., tab. 250. [Ety. lingula, a little tongue.] Shell oblong or ovoid, depressed, thin, gaping at each end, rounded or subtruncate in front, pointed at the beaks, consisting of alternate fibrous, corneous, and tubular testaceous, phosphatic laminæ; valves convex, held together by the action of muscles, beak of ventral valve more pointed and prominent than the other: surface smooth or concentrically lined; peduncle long, thick, cylindrical,fleshy, and flexible; there are twelve muscular impressions in the dorsal, and thirteen in the ventral, valve. Type $\mathrm{L}$. anatina. No Palcozoic

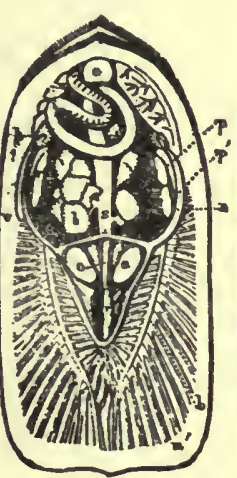

alveata, Hall, 1863, 16th Rep. N. Y. St. Mus. Nat. Hist., p. 23, and Pal. N. Y., vol. 4 , p. 12 , Ham. Gr.

ampla, Owen, 1852, Geo. Sur. Wis., Iowa, and Minn., p. 583, Potsdam Gr.

antiqua, Emmons, 1842, Geo. Rep. N. Y., p. 268, and Pal. N. Y., vol. 1, p. 3, Potsdam Gr.

antiquata, Emmons, 1856, Am. Geol., p. 20', Potsdam Gr.

artemis, Billings, 1874, Pal. Foss., vol. 2, p. 14, passage beds between Up. Sil. and Devonian.
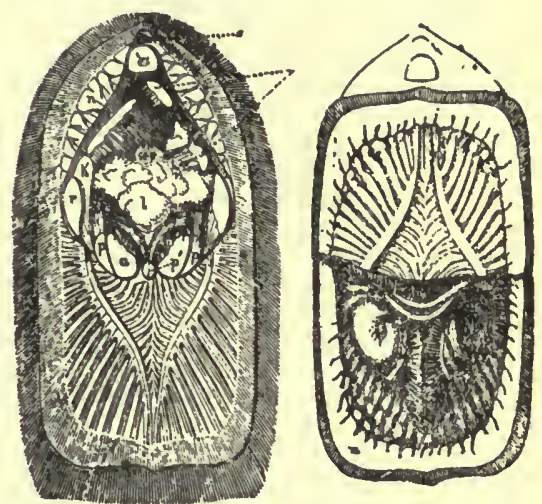

G. 573.-Lingula angtlna, $a a$, Anterlor adductors; $a$, posterior adductor; $p p$, external protractors; $p p$, central protractors; $r r$, anterlor retractors; $r$, posterlor retiractors; $c$, capsule of pedicel; $n$, vlsceral sheath; 0 , nesophagus; $s$, stomach; $l$, liver; $v$, vent; $h$, auricles, etc.

shell is positively known to agree with this genus in its muscular impressions, and probably none belong to it. Many referred to it belong to Lingulella, others to Lingulepis, and others, may be, to undefined genera. The external appearance, however, resembles Lingula, and for want of material to distinguish internal characters, they are left, provisionally, where the authors of the species left them.

acuminata, Conrad, 1839, Ann. Rep. N. Y., p. 64, Calcif. Gr.

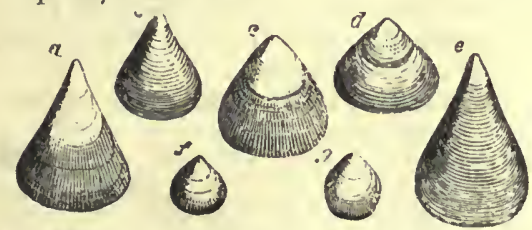

FIG. 572.-Llngula acuminata. Varlous forms; $a, b, c$, and $e$ are ventral valves; $d$, dorsal; and faid $g$ are young shells.

acutangula, Roemer, 1852, Kreid. von Texas, p. 90, Silurian.

acutirostra, Hall, 1843, Geo. Rep. 4th Dist. N. Y., p. 77, and Pal. N. Y., vol. 2, p. 56, Clinton Gr.

æequalis, Hall, 1847, Pal. N. Y., vol. 1, p. 95, Trenton Gr.

albapinensis, Walcott, 1885, Monogr. U.S. Geo. Sur., vol. 8 , p. 108 , Devonian. attenuata, Sowerby. The fossil referred by Hall to this species is described by Billings under the name of $\mathrm{L}$. daphne. aurora, see Lingulella aurora.

belli, Billings, 1859, Can. Nat. Geo., vol. 4, p. 431, Chazy Gr.

bicarinata, Ringueberg, 1884, Proc. Acad. Nat. Sci., p. 149, Niagara Gr. Not defined so as to be recognized.

billingsana, Whiteaves, 1878, Am. Jour. Sci. and Arts, 3d ser., vol. 16, p. 226, St. John's Gr.

bisulcata, Ulrich, 1889, Am. Geol., vol. 3, p. 380, Utica Slate.

briseis, Billings, 1862, Pal. Foss., vol. 1, p. 48, Trenton Gr.

calumet, N. H. Winchell, 1885, 13th Ann. Rep. Geo. Sur. Minn., p. 65, Taconic. Probably an Obolella.

canadensis, Billings, 1862, Pal. Foss., vol. 1, p. 114, Hud. Riv. Gr.

carbonaria, Shumard, 1858, Trans. St. Louis Acad. Sci., vol. 1,p. 215, Coal Meas. centrilineata, Hall, 1859 , Pal. N. Y., vol. 3, p. 155, Low. Held. Gr.

ceryx, Hall, 1863, 16th Rep. N. Y. St. Mus.

Nat. Hist., p. 19, and Pal. N. Y., vol. 4, p. 5 , Schoharie grit.

elintoni, Vanuxem, 1842, Geo. Rep. N.Y., p. 79, and Pal. N. Y., vol. 2, p. 54, Clinton Gr.

cobourgensis, Billings, 1862, Pal. Foss., vol. 1, p. 50, Trention Gr. 
complanata, Williams, 1882, Proc. A. A. A. S., vol. 30 , p. 188 , Chemung Gr. concentrica, Conrad, 1839, Ann. Rep. N. Y., p. 64, and Geo. Rep. 31 Dist. N. Y., p. 168 , Genesee Slate.

covingtonensis, Hall \& Whitfield, 1875, Ohio Pal., vol. 2, p. 67, Utica Slate.

crassa, Hall, 1847, Pal. X. Y., vol. 1, p. 98, Trenton Gr.

craufordsvillensis, Gurley, 1883, New Carb. Foss., p. 2, Keokuk Gr. The publication is not such as to entitle it to recognition.

cuneata, see Lingulella cuneata.

curta, Conrad, 1842, Jour. Arad. Nat. Sei., vol. 8 , p. 266 , and I'al. N. Y., vol. 1, p. 97, Útica Slate.

('uyahoga, Hall, 1863, 16th Rep. N. Y. St. IIus. Nat. Hist., p. 24, and Pal. N. Y., vol. 4 , p. 15, Waverly Gr.

cyane, Billings, 1865, Pal. Foss., vol. 1, p. 216, Quebec Gr.

daphne, Billings, 1862, Pal. Foss., vol. 1, p. 50, Trenton Gr. See L. attenuata.

dawsoni, Mathew, 1S84, Bull. U. S. Feo. Sur., vol. 2, p. 283, St. Jolın Gr.

delia, Hall, 1863, 16th Rep. N. Y. St. Mus. Nat. Hist., p. 20, and Pal. N. Y., vol. 4, p. 12, Ham. Gr.

densa, Hall, 1863, 16th Rep. N. Y. St. Mus. Nat. Ilist., P. 22, and Pal. N. Y., vol. 4, 1). 11, Hain. Gr.

clesiderata, Hall, 1863, 16th Rep. X. I. St. Mus. Nat. IIist., $\mu .19$, and $P_{n 1} . Y_{:}: Y_{\text {., }}$ vol. 1, J. 6, Up. Held. Gr.

elderi, Whifficld, 1880, Am. Jour. Sci. and Arts, 3d ser., vol. 19, p. 472, and Geo. Wis., vol. 4, p. 345, Trenton Gr.

elegantuk, syn. for Lingula yuadrata.

elliptica, Hull, 1843, Geo. Rep. 4th Dist. N. Y. The name was prenceupied by Phillips in 1836. See L. subelliptica.

elliptica, Emmons, 1856, A m. Geol. The nume was preoccupied.

elongata. Ilall, 1847, Pul. N. Y., vol. 1, p. 97, Trenton Gr.

exilis, Hall, 1860, 13th Rep. N. Y. St. Mus. Nat. Mist., p. 77. and Pal. N. Y., vol. 4, p. 7 , Marcellus Shale.

eva, Billings, 1861, Can. Nat. Geo., vol. 6, p. 150, Black Riv. Gr.

forbesi, Billings, 1862, Pal. Foss., vol. 1, p. 115, Hud. Riv. and Mid. Sil. Grs.

gibbosa, Hall, 1879, Desc. New Spec. Foss. p. 13, and 11th Kep. Geo. and Nat. Hist. Ind., p. 284, Niagara Gr.

halli, White, 1562, Proc. Bost. Soc. Nat. Hist., vol. 9, p. 8, Burlington Gr.

hurlbuti, W'inchell, 1880, Geo. Sur. Minn., 8th Rep., p. 62, Galena Gr.

huronensis, Billings, 1859, Can. Nat. Geo., vol. 4, p. 433, Chazy and Black Riv. Grs.

ingens, Spencer, 1884, Bull. No. 1, Mus. Univ. st. Mo., p. 56, Niagara Gr.

insularis, Billings, 1866, Catal. Sil. Foss. Antic., p. 40, Anticosti Gr.

iole, Billings, 1865, Pal. Foss., vol. 1, $\dot{\mathrm{p}}$. 215, Quebec Gr. iouensis, see Lingulella iowensis.

irene, Billings, 1862, Pal. Foss., vol. 1, r. 71, Quebec Gr.

iris, Billings, 1865, Pal. Foss., vol. 1, p. 301, Quebec Gr.

kingstonensis, Billings, 1862, Pal. Foss., vol. 1, p. 48, Black Riv. Gr.

lamellata, Hall, 18t3, Geo. Rep. 4th Dist. N. Y., p. 108, and Pal. N. Y., vol. 2, p. 249, Clinton and Niagara Grs.

leana, Hall, 1863, 16th Rep. N. Y. St. Mus. Nat. Hist., p. 20, and Pal. N. Y., vol. 4, p. 9, Han. Gr.

ligea, Hall, 1860, 13th Rep. N. I. St. Mus. Nat. Hist., p. 76 , and Pal. N. Y., vol. 4, p. 7, Ham. Gr.

ligea var. Hall, 1867, P'al. N. Y., vol. 4, p. 8, Portage $\mathrm{Gr}$.

ligea var. nevadensis, Walcott, $1 \$ 85$, Monogr. U. S. Geo. Sur., vol. 8, (). 107, Devonian.

lonensis, Walcott, 1855, Monogr. U. S. lieo. Sur., vol. 8, p. 108. Devonian.

lucretia, Billings, 1874, I'al. Foss., vol. 2, p. 14, passage berls between $\mathrm{U}_{[\mathrm{l}}$. Sil. and Devonian.

lyelli, IBillings, 1859, Can. Nat. Geo., vol. 4, p. 348, Calcif. and Chatzy Gr.

maida, Hall, 1863, 16 th $\operatorname{Rep.~} \mathrm{N}$. Y. St. Mus. Nat. Hist., p. 20, and Jal. N. Y., vol. 4, p. 9, Hain. Cr.

manni, Iall, 1863, 16th Rep. N. Y. St. Mus. Nat. Hist., p. 20, and I'al. N. Y., vol. 4, p. 6, Up. Held. Gr.

mantelli, Billings, 1859. Can. Nat. (ico., vol. 4, p. 349, Calcif. Cir.

manticula, White, 1864, Rep. Invert. Foss., p. 9, and Cieo. Sur. W. 10uth Mer., vol. 4, p. 5:, Up. Taconic.

matthewi, sce Acrothele matthewi.

melie, IIall, 1863, 16th Rep. N. Y. St. Mus. Nat. Hist., p. 24. and Pal. N. Y., vol. 4, p. 14, Waverly (ir.

membranacea, Winchell, 186:3, Proc. Acad. Nat. Sci. I'hil., vol. 15, p. 3, Marshall Gir.

minuta, Meck, 1868, Trans. Chi. A (ad. Sci., p. 87, Devonian.

mosia, Hall, 186:", I6th Rep. N. Y. St. Mus. Nat. Hist., p. 126, Potsclam (ir.

murnayi, Billings, 187£, l'al. Foss., vol. 2, p. 66, Up. Taconic.

mytiloides, Sowerby, 1812, Min. Conch., p. 55, tab. 19, Coal Meas.

nel)raskensis, Meek, 1872, (L. scotica var. nebraskensis,) P'al. E. Neb., p. 158, Coal Meas.

norwoodi, James, 1875, Cin.

Quar. Jour. Sci., vol. 2, p.

10, Utica Slate Gr.

nuda, Hall, 1863, l6th Rep. Fig. 574,-Lin-

V. Y St Mus Nat. Hist. 1). 22 , Ham. Gr.

nympha, Billings, 1865, Pal. Foss., vol. 1, p. 214, Quebec Gr.

oblata, Hall, 1843, Geo. Rep. 4th Iist. N. Y., p. 77, and Pal. N. Y., vol. ', p. 54, Clinton Gr. 
oblonga, Conrad, 1839, Ann. Rep. N. Y. The name was preoccupied, and afterward it was called $\mathrm{L}$. clintoni.

obtusa, Hall, 1847, Pal. N. Y., vol. 1, p. 98, Trenton Gr.

ovata, McCoy, 1844, Syn. Sil. Foss. Ireland, p. 24. Not clearly identified in America.

paliformis, Hall, 1860, 13th Rep. N. Y. St. Mus. Nat. Hist., p. 76, and Pal. N. Y., vol. 4, p. S, Ham. Gr.

papillosa, Emmons, 1S56, Am. Geol., p. 202, Trenton Gr.

perlata, Hall, 1859, Pal. N. Y., vol. 3, p. 156, Low. Held. Gr.

perovata, Hall, 1852, Pal. N. Y., vol. 2, p. 55, Clinton Gr.

perplexa, Hall, 1877, 1st ed. Am. Pal. Foss., P. 244. Proposed instead of L. elliptica, which was preoccupied, but D'Orbigny had previously proposed L. subelliptica.

perryi, Billings, 1861, Pal. Foss., vol. 1, p. 20, Black Riv. Gr.

philomela, Billings, 1862, Pal. Foss., vol. 1, p. 49, Trenton Gr.

pinniformis, see Lingulepis pinniformis.

polita, see Obolella polita.

prima, see Lingulepis prima.

prima, Emmons, 1856, Am. Geol. This name was preoccupied.

proctori, Ulrich, 1889, Am. Geol., vol. 3, 13. 377, Trenton Gr.

p'rogne, Billings, 1862, Pal. Foss., vol. 1, p. 47, Utica Slate and Trenton Grs.

punctata, Hall, 1863, 16th Rep. N. Y. St. Mus. Nat. Hist., p. 21, and Pal. N. Y., vol. 4, p. 10, Ham. Gr.

quadrata, Eichwald, 1829, (Crania quadrata,) Zool. Specialis, vol. 1, p. 273, and Pal. N. Y., vol. 1, p. 96, Trenton Gr.

quebecensis, Billings, 1862, Pal. Foss., vol. 1, p. 72, Quebec Gr.

reclilatera, Hall, 1859, Pal. $\mathrm{N}$. Y., vol. 3 , p. 156, Low. Held. Gr.

rectilateralis, Emmons, 1842, Geo. Rep. N. Y., p. 399, Utica Slate.

riciniformis, Hall, 1847, Pal. N. Y., vol. 1, p. 95, Trenton Gr.

scotica, Davidson, 1860, Monogr. Scot. Carb. Brach., 1. 62, Waverly Gr.

scotica var. nebraskensis, see L. nebraskensis.

spathata, Hall, 1859, Pal. N. Y., vol. 3, p. 157, Low. Held. Gr.

spatiosa, Hall, 1859,Pal. N. Y., vol. 3, p. 158, Low. Held. Gr.

spatulata, Vanuxem, 184:, Geo. Rep. 3d Dist. N. Y., p. 168, and Pal. N. Y., vol. 4, p. 13, Genesee slate.

stoneana, Whitfield, 1882, Geo. Wis., vol. 4, p. 344, Potsdam Gr.

striata, Emmons, 1856, Am. Geol., p. 112, Up. Taconic.

subelliptica, D’Orbigny, 1850, Prodr. d. Paleont., t. 1, p. 34, Clinton Gr. Proposed instead of $\mathrm{L}$. elliptica, Hall, in 1843, Geo. Rep. 4th Dist. N. Y., p. 77. suboblonga, D'Orbigny, syn. for L. clintoni. subspatulata, Meek \& Worthen, 1868, Geo. Sur. Ill., vol. 3, p. 437, Ham. Gr.

thedfordensis, Whiteaves, 1887, Cont. to Can. Pal., vol. 1, p. 111, Haw. Gr.

trentouensis, Conrad, 1842, Jour. Acad. Nat. Sci., vol. 8, p. 266, Trenton Gr.

triquetra, Clarke, 1885, Bull. U. S. Geo. Sur., vol. 16, p. 62, Portage (ir.

umbonata, Cox, 1857, Geo. Sur. Ky.,vol. 3, p. 576, Coal Meas.

vanhorni, S. A. Miller, 1875, Cin. Quar. Jour. Sci., vol. 2, p. 9, Hud. Riv. Gir.

varsoviensis, Worthen, Fxa. 575.-Llngula 1884 , Bull. No. 2 Ill. Fra. vanhorni.

St. Mus. Nat. IIist., p.

24 , and Geo. Sur. Ill., vol. S, p. 104, Warsaw Gr.

whitii, Walcott, 1885, Monogr. U. S. Geo. Sur., vol. 8, p. 109, Devonian.

whitfieldi, Ulrich, 1889, Am. Geol., vol. 3, p. 381, Utica Slate.

winona, Hall, 1863, 16th Rep. N. Y. St. Mus. Nat. Hist., p. 126, Potsdam Gir.

Lixgulenasma, Ulrich, 1889, Am. Geol., vol. 3, p. 383. [Ety. Lingula, a genus ; clasma, plate.] Form and composition like Lingula; pedicle valve with slightly projecting beak, faintly arched deltidium, no area, small socket on each side of the deltidial borders, and subtriangular scar opposite their anterior ends subtriangular, trilobed platform from base of detidium to middle of valve, with central part produced below in a low median ridge; two muscular scars on the lower lateral sides of the platform; brachial valve, with transverse ridge and swollen ends for sockets on the opposite valve; platform concave, elevated in front, and prolonged in a median plate, subcardinal, umbolateral and postmedian scars. Type L. schucherti.

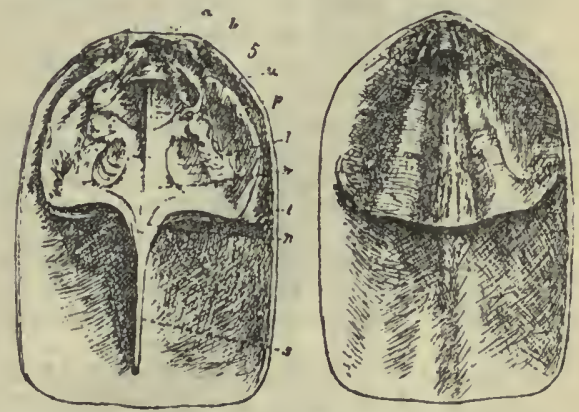

FIG. 576.-Lingulelasma schucherti. $a$, Posterior transverse ridge; $b$, subcardinal scars; $u$, $12 \mathrm{~m}$ bolateral scars; $p$, postmedian scars; $i$, lateral scars; $m$, medlan scars; $n$, anterlor scurs; $t$ transversc scars; $s$, septum; $5 a$, ventral valve.

schucherti, Ulrich, 1889, Am. Geol., vol. 3, p. 389, Hud. Riv. Gr. 
I.INGulelda, Salter, 1861, Mem. Geo. North Wales, and Geo. Sur. Gt. Brit., vol. 3, p. 333. [Ety. diminutive of Lingula.]

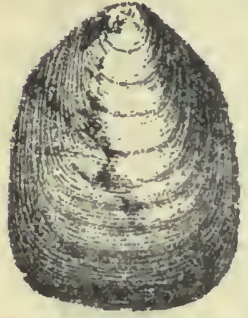

Fig. 577.- Lingulelln chucinuntleusis.
General form like Ling u la ; nearly cquivalve, veutral valve pointed with a pedicle groove; muscular scars as in obolus, but anterior retractors more linear, and sliding muscles small and not quite as much external. Type L. davisi.

?aftinis, Billings, 1874

Pal. Foss., vol. 2, p. 67, Up. Taconic.

aurora, Hall, 1861, (Lingula aurora,) Geo. Rep. Wis., p. 24, Potsdam Gr.

crelata, IIall, 1847, (Orbicula cielata,) P'al. N. Y., vol. 1, p. 290, Georgia (ir.

cincinnatiensis, $\mathrm{Hall}$ \& Whitfield, 1875, Ohio Pal., vol. 2, p. 67, Ilud. Riv. Gr. cuneata, Conrad, 1839,

(lingula cuneata,) Geo.

Rep. N. Y., p. 64, and

Pal. N. Y., vol. 2, p. 8 , Clinton Gr.

dawsoni, Matthew, 1885, T'rans. Roy. Soc. Can., p. 33, St. John Gr.

ella, Hall \& Whitfield, 1877, (Lingulepis ella,) Geo. Expl. 40th Parallel, vol. 4, p. 232, Up. Taconic.

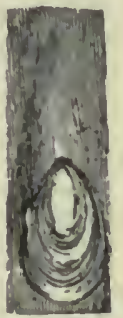

gra 1887 .

and Arts., 3d ser., vol. 34, p. 187, Up. Taconic.

inflata, Matthew, 1885, Trans. Koy. Soc. Can., p. 33, St. John Gr.

iowensis, Owen, 1840, (Lingula iowensis,) Rep. Min. Lands, p. 70, Galena Gr.

lamborni, Meek, 1871, Proc. Acad. Nat. Sci., p. 185, Calciferous or Potsdam Gr.

linguloides, Matthew, 1885, Trans. Roy. Soc. Can., p. 34, St. John Gr.

? spissa, Billings, 1874, Pal. Foss., vol. 2, p. 67, Up. Taconic.

Linguleprs, Hall, 1863, 16th Rep. N. Y. St. Mus. Nat. Hist., p. 126. [Ety. lingula, little tongue; lepis, scale.] Linguloid, inequivalve, equilateral, ovate or spatulate, corneous, phosphatic; visceral impressions in dorsal valve flabelliform, in ventral valve tripartite, the lateral divisions the larger. Type L. pinniformis.

cuneolus, Whitfield, 1877, Prelim. Rep. Pal. Black Hills, p. 8, and Geol. Black IIills, p. 336, Potsdam Gr.

dakotensis, Meek \& Hayden, 1864, Pal. Up. Mo., p. 3, and Geol. Black IIills, p. 337, Potsdam Gr.

ella, See Lingulella ella.

mæra, Hall \& Whitfield, 1877, U. S. Expl. 40th Parallel, vol. 4, p. 206, Potadam Gr. minima, Whitfield, 1884, Bull. Am. Mus. Nat. II ist., vol. 1, p. 139, Up. Taconic. minutr, Hall \& Whitfield, 1877, U. S. Expl. 40th Parallel, vol. 4, 1. 206, Potsdam $\mathrm{Gr}$.

morsii, N. H. Winchell, 1876, (Lingula morsensis,) Geol. Fillmore Co., Minn., v. 31, St. Peters sandstone.

perattenuata, Whitficld, 1877, Prelim. Rep. Pal. Blnck Hills, p. 9, and Geol. Black Hills, p. 337, Potsdam fir.

pin $\mathrm{n}$ iformis, Owen, 1852, (Lingula pinniformis, Geo. Rep. Iowa. Wis., and Minn., p. 583, Potsdam Gr.

prima, Conrad, 1847 , (Lingula prima,) Pal. N. Y., vol. 1, p. 3, Potsdam Gr.

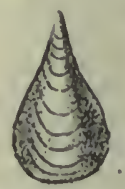

Fig. 579.-I Ingulepls phimlformis.

Lisgulol's, Hall, 1871, 23d Rep. N. Y. St. Mus. Nat. Hist., p. 245. [Fty. Lingula, a genus; opsis, appearance.] In external appearance like Lingula or Lingulella: the ventral valve presents a small area, with a narrow pedicle groove and a large lobed muscular impression, which, in the cast, extends as a narrow groove toward the base of the shell; the ramifications of the vascular lines originate at nearly the same point as in existing Lingula, but do not extend so far backward toward the beak. Type L. whitfieldi.

whitfieldi, Hall, 1871, 23d Rep. N. Y. St. Mus. Nut. Ilist., p. 245, Low. Sil.

Linna kssonia, Walcott, 1885, Am. Jour. Sci. and Arts, $3 \mathrm{~d}$ ser., vol. 29, p. 114. [Ety. proper name.] Ovate or subcircular; inarticulate; apex of ventral valve perforated by a minute foramen; no area; curdinal edge thin; two scars in the interior, on each side of the foramen, close to the posterior margin; dorsal valve, with no area; two scars in the interior, close to the posterior margin, separated by a ridge that extends forward between two small divaricator scars. Type L. transversa.

taconica, Walcott, 1887, Am. Jour. Sci. and Arts, 3d ser., vol. 34, p. 189, Up. Taconic.

transversa, Hartt, 1868, (Obolella transversa, Acad. Geol., 1. 644, St. John Gr.

Martinia, McCoy, 1844, syn. Carb. Foss. Ireland, p. 128. - [Ety. proper name.] General characters the same as Spirifera, for which it is usually regarded as a synonym. It is distinguished by its smooth surface without radiating ribs, and by having smaller spiral appendages. Type M. decora.

athyroides, Winchell, 1866, liep. Low. Penin. Mich., p. 9t, Ham. Gr.

planoconvexa, see Spirifera planoconvexa. subumbonata, Hall, 1867, (Spirifera subumbonata,) Pal. " $i$, voi. 1, p. 234, Ham. Gr. and Tuly isictone. 
Mmekella, White \& St. John, 1868, Trans. Chi. Acad. Sci., vol. 1, p. 120. [Ety. proper name.] Globose, rather longer than wide, plications large, hinge-line shorter than greatest breadth of the valves; dorsal valve most prominent on the umbo, beak incurved, no mesial sinus; cardinal process long, curviug backward in front of the pseudodeltidium, and having upon each side a wing-like expansion, which is curved $u p$ at its outer edge forming an elongated dental fossette; ventral valve more convex, cardinal area high, no median septum; two broad dental lamella, continuous from the cardinal teeth to the beak, pass directly in front of the sutures between the cardinal area and the pseudodeltidium, and thence, slightly diverging, extend forward along the bottom of the valve about half-way to the front, the anterior margins of the lamellæ arching backward and upward to the dental processes; a cross section shows three chambers opening anteriorly into the shell. Type M. striato-costata.

striato-costata, Cox, 1857, (Plicatula striato-costatı,) Geo. Rep. Ky., vol. 3, p. 568 , Coal Meas.
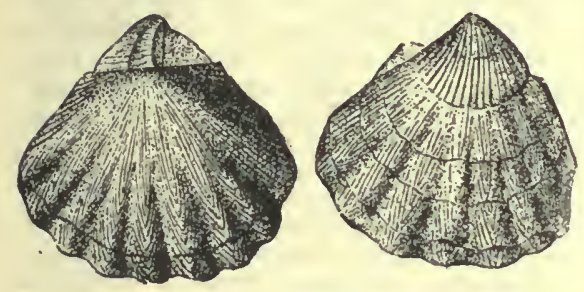

FIG. 580.-Meekella striatocostala. Dorsal and ventral view.

Meganteris xquiradiata, see Rensselæria æquiradiata.

cumberlandiæ, see Rensselæria cumberlandiæ.

elliptica, see Rensselæria elliptica.

elongata, see Amphigenia elongata.

lævis, see Rensselæria lævis.

mutabilis, see Rensselæria mutabilis.

ovalis, see Rensselæri ovalis.

ovoides, see Rensselæria ovoides.

subtrigonalis, see Amphigenia elongata var. subtrigonalis.

suessana, see Rensselæria suessana.

Merista, Suess, 1851, Jahrb. Geol. Reichs. Austalt, vol. 2, p. 150. [Ety. meros, a part.] General form like Athvris, usually mesial fold and sinus poorly defined; the principal stems forming the spirals attach to the hinge plate, incline forward toward the interior of the shell, then abruptly bend backward and make a curve facing the bottom of the dorsal valve, and, after converging to about lalf their length, again diverge toward the front and form the first spiral coil ; there are 10 or 12 whorls in eacl spiral; the genus is distinguished by a shoe-lifter process under the beak of the ventral valve, consisting of two roof-shaped plates, fixed by their lateral margins to the medio-longitudinal region of the valve, and with their narrow end fitting under the extremity of the beak. Type M. herculea. urcuata, see Meristella arcuata.
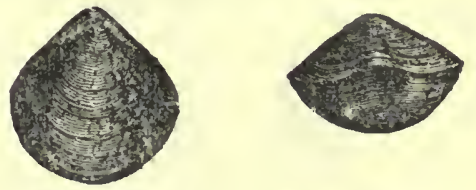

EIg. 581. - Merista bella. Dorsal and anterior view.

bella, Hall, 1857, 10th Rep. N. Y. St. Mus. Nat. Hist., p. 92, and Pal. N. Y., vol. 3, p. 248, Low. Held. Gr.

bisulcata, Vanuxem, 1843, (Atrypa bisulcata,) Geo. Rep. 3d Dist. N. Y., p. 112 , and Pal. N. Y., vol. 3, p. 253, Low. Held. Gr.

elongata, Hall, 1859, (Canırium elougatum,) Pal. N. Y., vol. 3, p. 488, Low. Held. Gr.

houghtoni, Winchell, 1862, Proc. Acad. Nat. Sci., vol. 6, 2d ser., p. 407, Portage $\mathrm{Gr}$.

lavis, see Meristella lævis.

lata, Hall, 1859, Pal. N. Y., vol. 3, p. 431, Oriskany sandstone.

lens, Winchell, 1866, Rep. Low. Penin. Mich., p. 94, Ham. Gr.

meeki, Hall, 1857, 10th Rep. N. Y. St. Mus. Nat. Hist., p. 97, and Pal. N. Y., vol. 3 , p. 252 , Low. Held. Gr.

princeps, see Meristella princeps.

subquadrata, Hall, 1857, 10th Rep. N. Y. St. Mus. Nat. Hist., p. 93, and Pal. N. Y., vol. 3, p. 249, Low. Held. Gr.

sulcata, Vanuxem, 1842, (Atrypa sulcata) Geo. Rep. N. Y., p. 112, Waterlime Gr.

typus, Hall, 1859, (Camarium typus,) Pal. N. Y., vol. 3, p. 487, Low. Held. Gr.

Meristella, Hall, 1860, 13th Rep. N. Y. St. Mus. Nat. Hist., p. 74. [Ety. diminutive of Merista.] Shells variable in form. ovoid or transverse; valves unequally convex, with or without a median fold and sinus; beak of ventral valve imperforate, incurved over the beak of the other valve; no area; valves articulating by teeth and sockets; surface smooth or with fine concentric stria; interior of dorsal valve having a longitudinal septum and the upper part of the ventral valve a deep subtriangular muscular impression, which unites with the rostral cavity; spires are continued from their origin obliquely backward into the cavity of the ventral valve, and then, 
recurving upon themselves, are reunited laterally. Type M. lavis.

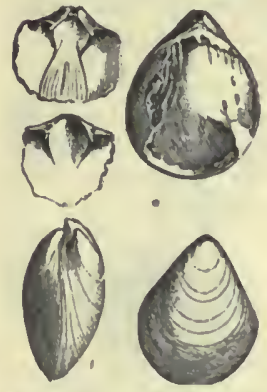

Fig. 582. - Merlstella clrce. Sliowing remuins of splral appendages in lie dorsal valve.

arcuata, Hall, 1857, (Merista areuata,) 10th Rep. N. Y. St. Mus. Nat. Ilist., p. 95 , and Pal. N. Y., vol. 3 , p. 249 , Low. Held. Gir.

barrisi, Hall, 1860, 13th Rep. N. Y. St. Mus. Nat. Hist., p. 84, and Pal. N. Y., vol. 4, p. $304, \mathrm{Mar}$ cellus shale and Ham. Gr.

circe, Billings, 1861, (Charionella circe, Can. Jour., vol. 6, p. 273, Up. Held. (ir.

cylindriea, Hall, 1852, (Atrypa cylindrica.) Pal. N. Y., vol. 2, p. 76 , Clinton and Niagara Gr.

doris, 1860, Hall, 13th Rep. N. Y. St. Mus. Nat. Hist., p. 84, and Pal. N. Y., vol. 4, 1. 303, Schoharie grit and Cor. niferous $\mathrm{Gr}$.

elissa, syn. for Meristella nasuta.

haskinsi, Hall, 1860, 13th Rep. N. Y. St. Mus. Nat. Hist., p. 84, and Pal. N. Y., vol. 4 , p. 306 , II am. Gr.

(?) hyale, Billings, 186\%), (Charionclla (?) liyale, Pal. Foss., vol. 1, p. 166, Guelph Gr.

julia, Billings, 1862, (Athyris julia,) Pal. Foss., vol. 1, p. 146, Mid. Sil.

lævis, Vauuxem, 1843, (Atrypa lavis, Geo. Rep. 3d Dist. N. Y., p. 120, and Pal. N. Y., vol. 3, p. 247, Iow Held. Gr. lenta, Hall, 1867, Pal. N. Y., vol. 4, 13.420, Oriskany sandstone.

maria, see Whitfieldia maria.

meta, Ilall, 1867, P'al. N. Y., vol. 4, p. 308, Ham. Gr.

nasuta, Conrad, 1840, (Atrypa nasuta,) Ann. Rep. N. Y., p. 18, and J'al. N. Y., vol. 4, p. 299, Schobarie grit, Up. Held. and llam. Gr.

princeps, Iall, 1857, (Merista princeps,) 10 th Rep. N. Y. St. Mus. Nat. Hist., p. 95, and Pal. N. Y., vol. 3, p. 252, Low. Held. Gr.

prinstana, Billings, 1862, (Athyris prinstana,) Pal. Foss., vol. 1, p. 145, Mid. Sil. rectirostra, Hall, 1879, Desc. New Spec. Foss., p. 15, and 11th Rep. Geo. and Nat. Ilist. Ind., p. 301, Niagara Gr.

rostrata, Hall, 1843, (Atrypa rostrata,) Geo. Rep. 4th Dist. N. Y., p. 202, and Pal. N. Y., vol. 4, p. 307 , Ham. (ir. and Tully limestone.

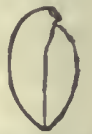

scitula, Hall, 1843, (Atrypa FIG.583.-Merisscitula,) Geo. 4th Dist. Slde view. N. Y., p. 171, and Pal.

N.Y., vol. 4, p.302, Corniferous Gr. Hall regards $M$. circe as a syn. for this species. unisulcata, Conrad, 1841, (Atrypa unisulcata,) Ann. Rep. N. Y., p. 56, and Pal. N. Y., vol. 4, p. 309, Up. Held. and Haus. Gr.

Mrristin A, Hall, 1867, 20th Rep. N. Y. St. Mus. Nat. Ilist., p. 186, and Pal. N. Y., vol. 4, p. 299. [City. Merista, a genus; inus, implying resemblance.] General form like Meristella; apex perforated; lamellie of the spires united by a simple loop; spirals oval, and each contains about nine convolutions; the two principal stems attach to the hinge plate and extend into the interior between the spirals, where they bend backward and give forth converging lamellae, which cross between the spirals to the ventral side, where they unite in an angular point. Type M. nitida.

nitida, Hall, 1843, (Atrypa nitida,) (ieo. Rel). 4th Dist. N. Y., pl. 14, and Pal. N. Y., vol. 2, p. 268, Niagara (ir.

nitida var. oblata, IIall, 1852, (Atrypa nitida var. oblata,) Fro. ixi. - Meristinn Pal. N. Y., vol. 2, p. nitidu. vorsal
269, Niagara Gr.

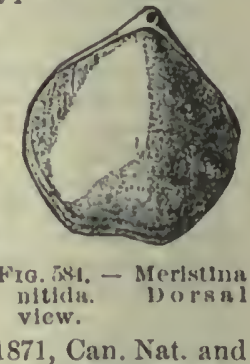

MoNomerralaA, Billings, 1871, Can. Nat. and Geo., vol. 6, p. 220. [Ety. monos, one; meros, a part; ella, diminutive termination.] Shell thick, eircular or transversely oval in its marginal outline; umbo of the pedicle; valve large, projecting, double-chambered; area and deltidium large; linge thick, elevuted, ledge-shaped, concave in the middle portion; cardinal facet a wall-like space behind the ledge or flat of the linge; cardinal buttress strong, lamelliform; platform flat, slightly elevated, widest, highest, and obtusely angulated in front; umbo of the brachial valve rounded; hinge moderately thick; platform trilobed; usmally with a thin margin. Type M. prisca.

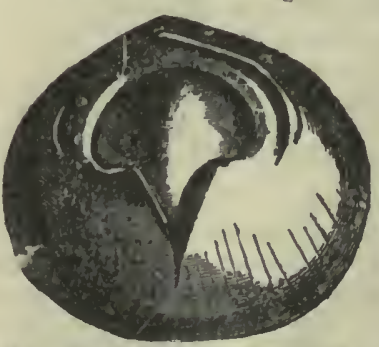
newber $\mathbf{y} \mathrm{i}$, Hall \& Whitfield, 1875, Ohio, l'al., vol. 2, p. 131, Niagara Gr.

orbiculari 8 , Bill in $\mathrm{g} \boldsymbol{\mathrm { s }}$, 1871, Can. Nat., vol. 6, p. 220, Guelph Gr.

FIg. 585.-Monomerella prisca.

Ha. xs.-Monomerella prisca.

l. Foss., vol. 3 , p. 5, Guelph Gr.

ovata var. lata, Whiteaves, 1884, Pal. Foss., vol. 3, p. 6, Guelph Gr.

prisca, Billings, 1871, Can. Nat. and Geol., vol. 6, p. 220 , Guelph Gr. 
Nucleospira, Hall, 1859, Pal. N. Y., vol. 3, p. 219. [Ety. nucleus, kernel; speira, spire.] Shell spheroidal, or transversely elliptical, more or less gibbous, and furnished with spires as in Spirifera; linge-line short, cardinal extremities rounded, valves subequal, articulating by teeth and sockets; surface smootb, structure punctate and covered with minute hair-like spines; ventral valve having the beak extended, with a triangular depression beneath, on each side of which at the base is a strong tooth, a narrow septum from beak to base; dorsal valve with spatulate cardinal process, which bends upward into the cavity of the opposite beak; from the sides of this process the brachial processes originate, which support the spires; muscular imprints confined to a narrow oval space. Type $\mathrm{N}$. ventricosa.

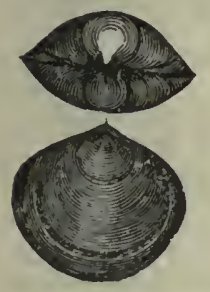

FIG. $586 .-\mathrm{Nu}-$ cleospira conclunit. barrisi, White, 1860, Bost. Jour. Nat. Hist., vol. 7 , p. 227, Kinderhook Gr. concentrica, Hall, 1859, Pal. N. Y., vol. 3, p. 223, Low. Held. Gr. concinna, Hall, 1843, (Atrypa concinna, Geo. 4th Dist. N. Y., p. 200, and Pal. N. Y., vol. 4, p. 279, Hamilton Gr.

elegans, Hall, 1859, Pal. N. Y., vol. 3, p. 222 , Low. Held. Gr.

pisiformis, Hall, 1859, (Orthis pisum, 1852, Pal. N. Y., vol.' 2,) Pal. N. Y., vol. 3 , p. 218 , Niagara Gr.

rotundata, W'hitfield, 1882, Desc. New Spec. Foss. from Ohio, p. 194, Low. Held. Gr.

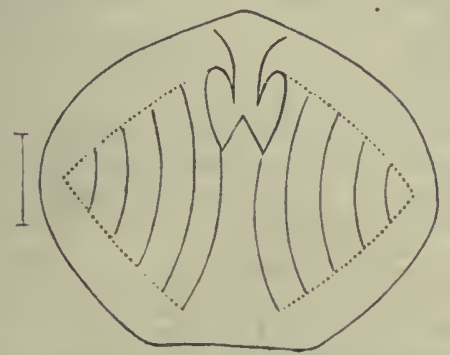

F1(i. 587.-Spiral colls of Nucleosplra pisiformls.

ventricosa, Hall, 1859, Pal. N. Y., vol. 3, p. 220, Low. Held. Gr. This species was first described in 1856, in 9th Reg. Rep., as Spirifera ventricosa.

Obolella, Billings, 1861, Pal. Foss., vol. 1, p. 7. [Ety. diminutive of obolus, a small Greek coin.] Shell ovate, circular or subquadrate, convex or planoconvex; ventral valve with a false area, which is sometimes minute and usually grooved for the passage of the peduncle; dorsal valve with or without an area; muscular impressions in the ventral valve four, one pair in front of the beak near the middle or in the upper half of the shell, and the others situated one on pach side near the cardinal edge; shell calcareous; surface concentrically striated. Type 0 . chromatica.

ambigua, Walcott, 1885, Monogr. U. S. Geo. Sur., vol. 8, p. 67, Cházy Gr.

chromatica, Billings, 1861, Pal. Foss., vol. 1, p. T, Up. Taconic.

cingulata, Billings, 1861, Pal. Foss., vol. 1, p. 8, Up. Taconic.

circe, Billings, 1871, Can. Nat. and Geol., vol. 6 , p. 219 , Up. Taconic.

crassa, Hall, 1847, (Orbicula crassa,) Pal. N. Y., vol. 1, p. 290 , Up. Taconic Gr.

desiderala, see Elkania de-FIg 588-Obolsiderata.

discoider, Hall \& Whitfeld, 1877 ingmuscular 187, C. Seo. Expl. Iimpresslous : 40th parallel, vol. 4, p. $d$, side view. 205, Potsdam Gr.

gemma, Billings, 1871, Can. Nat. and Geol., vol. 6, p. 218, Up. Taconic.

ida, Billings, 1862, Pal. Foss., vol. 1, p. 71, Quebec Gr.

misera, Billings, 1874, Pal. Foss., vol. 2, p. 69 , Up. Taconic.

nana, Meek \& Hayden, 1861, Proc. Acad. Nat. Sci. Phil., p. 435, and Pal. Up. Mo., p. 4, Potsdam Gr.

nitida, Ford, 1873, Am. Jour. Sci. and Arts, $3 \mathrm{~d}$ ser., vol.5, p. '213, Uy. Taconic. polita, Hall, 1861, Geo. Rep. Wis., p. 24, and Geol. Black Hills, p. 339, (Lingula polita,) Potsdam Gr.

pretiosa, Billings, 1862, Pal. Foss., vol. 1, p. 68, Quebec Gr.

transversa, see Linnarssonia transversa.

Obolellina, Billings, Dec., 1871, Can. Nat., vol. 6 , p. 220 , syn. for Dinobolus.

canadensis, see Dinobolus canadensis. gallensis, see Dinobolus galtensis.

magnifica, see Dinobolus magnifieus.

Oвоцus, Eichwald, 1829, Zoologia Specialis, vol. 1, p. 274. [Ety. obolus, a small coin.] Shell orbicular, equilateral, transverse or elongated, depressed ; valves not articulated; larger valve most convex, beak obtuse or pointed, wide flattened cardinal edge or false alea, over which the concentric lines of sulface growth pass uninterruptedly; cardinal edge grooved longitudinally by a semicylindrical furrow; smaller valve shorter, slightly convex, without prominent beak ; hinge-line an arch ; cardinal edge flattened, horizontally striated; surface smooth or having minute undulating wrinkles; interior of larger valve with a mesial ridge, on each side of which are two oval muscular scars, one pair near the cardinal angles, the other toward the center of the valve beyond the mesial ridge; structure calcareocorneous. Type O. apollinis. 
canadensis, see Dinobolus canadensis. conradi, see Dinobolus conradi.

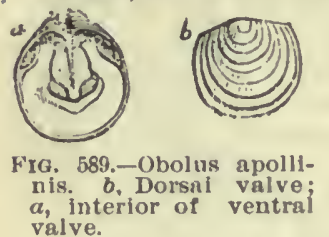

gallensis, see Trimerella galtensis.

labradoricus, se e Kutorgina labradorica.

(?) murrayi, Billings, 1865, Pal. Foss., vol. 1, p.

362, Quebec Gir. or Up. Taconic.

peetinoides, Whitfield, 1875, Ludlow's Rep. Black Hills of Dakota, p. 103, Up. Taconic.

Orbicula, Cuvier, 1808, Tabe. Elem. d'Hist. Nat., p. 435 , syn. for Crania. celata, see Lingulella ciclata. cancellata, see Trematis cancellata. corrugata, see Crania corrugata. crassa, see Obolella crassa. deformata, see Crania deformata. eccentrica, see Crania eccentriea. filosa, see Schizocrania filosa. grandis, see Discina grandis. lamellosa, see Discina lamellosa. lodensis, see Discina lodensis. minuta, see Discina minuta. nitida, see Discina nitida. prima, see Crania prima. squamiformis, see Pholidops squamiformis. subtruncata, see Pholidops subtruncatus. tenuilamellata, sce, Discina tenuilamellata. terminalis, see Trematis terminalis. truncata, see Crania truncata.

Oristculoidfi, D'Orbigny, 1847, Comptes rendus de l'Academie des Seiences, and l'rodr. de Paléont., t. 1, p. 44. [Ety. Orbiula, a genus; oides, like.] Suborbicular, patelliform, longitudiually or transversely oval, upper valve convex, with vertex near the posterior margin; lower valve conical or concave; no pedicle disk; a narrow oval or cireular aperture, more or less confined in its shape, is sitnated in a furrow or depres. sion. Type O. elliptica.

conica, Dwight, 1880, Am. Jour. Sci. and Arts, 3त ser., vol. 19, p. 452, Trenton Gr.

Ortus, Dalman, 1827, Kongl. Vet. Acad. Handl., p. 93. [Ety. orthos, straight, in allusion to the straight hinge-line.] Shell variable in form, hinge-line straight; valves convex or plano-convex, plicated, with or without mesial fold and sinus; cardinal area notched in the center; ventral valve with two prominent diverging teeth, muscular impression saucer-shaped, divided by a median septum on which the central adductor attached; divaricator and pedicle impressions, lateral, fan-like; dorsal valve with a tooth-like, cardinal process between two eurved brachial processes; adductor impression quadruple; vascnlar impressions numerous, spreading; no coiled spiral arms. Type 0 . zonata and $\mathrm{O}$. callactis. aeuminata, Billings, 1859, Can. Nat. (ieo., vol. 4, p. 440, Chazy Gr.

acutiliruta, Conrad, 1842, (Delthyris acntilirate, Jour. Acad. Nat. Sci., vol. \&, p. 260 , Hud. Riv. Gr.

acutiloba, Ringueberg, 1888, Proc. Acad. Nat. Sei. Phil., p. 134, Niagara Gr.

requivalvis, Hall, 1847, Pal. N. Y., vol. 1, p. 120, Trenton Gr.

aequivalva, Shaler. The name was prooccupied.

requivalvis, Hall, see Orthis erynu.

alata, Shaler. The name was prenccupied. alsus, Hall, 1863, 16th Rep. N. Y. St.

Mus. Nat. Hist., p. 33, Sehoharie grit.

alternans, Castelnaı, 1843, Syst. Sil., 1. 38. Not recognized.

amoena, Winchell, 18s0, Geo. Sur. Minn. Sth Rep., p. 65, Hud. Riv. Gr.

anticostienvis, syn. for Orthis porcata.

apicalis, Billings, 1865, Pal. Foss.. vol. 1, p. 301, Quebec Gr.

armanda, Billings, 1865, Pal. Foss., vol. 1, p. 303, Quebec Gr.

assimilis, Hall, 1859, Pal. N. Y., vol. $3, \mathrm{p}$. 175, Low. Held. Gr.

aurelia, Billings, 1874, Pal. Foss., vol. 2, p. 34, Gaspe limestone No. 8, Devonian. barabuensis, see Leptunn barabuensis.

battis, Billings, 1865, Pal. Foss., vol. 1, 1. 185, Quebec Gr.

bellarugosa, Conrad, 1543, Proc. Acal. Nat. Sci. Phil., vol. 1, p. 333, and Pal. N. Y., vol. 1, p. 118, Trenton Gr.

bellula, Meek, 1873, Ohio Pal., vol. 1, !. 103, Hud. Riv. Gr.

biforata, Schlotheim, 1820, ('Terebratulites biforatus,) Petrefaet., p. 265, Trenton and Hud. Riv. Grs.

billingsi, Hartt, 1868, Acad. Geol., p. 644, St. John Gr.

biloba, Linnaws, 1767, (Anomia biloba,) Limne. Syst., ed. 12, p. 1154, Niagara Cir.

bilobata, Conrad, 1838, (Delthyris bilobata,) Ann. Rep. N. Y. The name was preoceupied by Sowerby.

bisulcata, see Camarella bisulcata.

borealis, Billings, 1859, Can. Nat. Geo., vol. 4, p. 436, Chazy and Trenton Grs. carbonaria, Swallow, 1858, syn. for Orthis pecosi.

carinata, Hall, 1843, Geo. Rep. 4th Dist. N. Y., p. 267, and Pal. N. Y., vol. 4, p. 58, Portage and Chemung Grs.

carleyi, Hall, syn. for Orthis retrorsa. centrilineata, Ifall, 1847, Pal. N. Y., vol. 1, p. 289, IIud. Riv. Gr.

centrosa, n. sp., Hud. Riv. Gr. Proposed instead of O. crassa, in Cin. Quar. Jour. Sci., vol. 1, p. 20, and Ohio Pal., vol. 1, p. 117 , pl. 10 , fig. 3 .

charlottae, Winchell, 1880, Geo.Sur. Minn., Sth Rep., p. 67, IIud. Riv. Gr.

cincinnatiensis, S. A. Miller, 1883 , $2 \mathrm{~d}$ lid. Am. Pal. Foss., p. 296, Hud. Riv. Gr., Cincinnati, Ohio. Proposed instead of Orthis costata, Hall, 1845, Am. Jour. Sci. and Arts, vol. 48, p. 294. 
circularis, Winchell, 1880, Geo. Sur. Min. 8th Rep., p. 66, Hud. Riv. Gr.

circulus, Hall, 1843, Geo. Rep. 4th Dist. N. Y., p. 71, and Pal. N. Y., vol. 2, p. 86, Clinton Gr.

clarkensis, Swallow, 1863, Trans. St. Louis Acad. Sci., vol. 2, p. 81, Keokuk Gr.

cleobis, Hall, 1863, 16th Rep. N. Y. St. Mus. Nat. Hist., p. 35, Onondaga limestone, and Up. Held. Gr.

clytie, Hall, 1861, 14th Rep. N. Y. St. Mus. Nat. Hist., p. 90, Trenton Gr.

coloradoensis, Shumard, 1860, Trans. St. Louis Acad. Sci., vol. 1, p. 627, Potsdam Gr.

coloradoensis, Meek, 1870 , see O. desmopleura.

concinna, Hall, 1859, Pal. N. Y., vol. 3, p. 172, Low. Held. Gr.

conradi, Castelnau, 1843, Syst. Sil. p. 37. Not recognized.

conradi, Winchell, 1880, Geo. Sur. Minn. 8th Rep., p. 68, Hud. River. Gr.

cooperensis, Swallow, 1863, Trans. St. Louis Acad. Sci., vol. 2, p. 82, Warsaw Gr.

corinna, Billings, 1865, Pal. Foss., vol. 1, p. 302, Quebec Gr.

costalis, Hall, 1847, Pal. N. Y., vol. 1, p. 20, Chazy Gr.

costata, Hall, 1845. This name was preoccupied by Sowerby in 1839. See O. cincinnatiensis.

crassa, James, 1874. Cin. Quar. Jour. Sci., vol. 1, p. 20. The name was preoccupied by Lindstrom in 1860 . See O. centrosa. crispata, Emmons, 1842, Geo. Rep. N. Y., p. 404, Trenton Gr.

cumberlandia, Hall, 1859, Pal. N. Y., vol. 3 , p. 481 , Oriskany sandstone.

cuneata, Owen, 1852, Geo. Sur. Wis., Iowa, and Minn., p. 585, Devonian.

cyclas, Hall, 1860, 13th Rep. N. Y.St. Mus. Nat. Hist., p. 78, and Pal. N. Y., vol. 4, p. 52, Ham. Gr.

cyclus, James, syn. for Orthis multisecta. cypha, James. Not characterized so as to establish a species.

dalyana, S. A. Miller, 1881, Jour. Cin. Soc. Nat. Hist., vol. 4, p. 313, Burlington Gr. davidsoni, Verneuil, 1840, Bull. Geol. Soc. France, vol. 5, p. 341, Up. Sil.

daytonensis, Foerste, 1885, Bull. Sci., Lab. Denison Univ., p. 87, Niagara Gr.

deflecta, see Streptorhynchus deflectum.

deformis, Hall, 1857, 10th Rep. N. Y. St. Mus. Nat. Hist., p. 44, Pal. N. Y., vol. 3 , p. 174, Low. Held. Gr.

delicatula, Billings, 1865, Pal. Foss., vol. 1, p. 217, Quebec Gr.

dentata, Pander, 1830, (Porambonites dentatus,) Bietr. Geogn. Russl., p. 100, Trent. and Hud. Riv. Gr.

desmopleura, Meek, 1872, Hayden's Geo. Rep. of Wyoming, p. 295, Silurian. Proposed instead of 0 . coloradoensis. dichotoma, syn. for Orthis fissicosta. discus, Hall, 1859, Pal. N. Y., vol. 3, p. 165, Low. Held. Gr. disparilis, Conrad, 1843, Proc. Acad. Nat. Sci., vol. 1, p. 333, and Pal. N. Y., vol. 1, p. 119, Black Riv. and Trenton Gr.

dubia, Hall, 1858, Trans. Alb. Inst., vol. 4, p. 12, and Bull. Am. Mus. Nat. Hist., p. 45, Warsaw Gr.

eboracensis, n. sp., Up. Held. Gr. Proposed instead of $O$. lenticularis of Vanuxem in Rep. 3d Dist. $N$. Y., p. 147 , which was preoccupied. It was redescribed by Hall in Pal. N. Y., vol. 4, p. 35.

electra, Billings, 1862, Pal. Foss. vol. 1, p. 79, Quebec Gr.

elegantula, Dalman, 1827, Kongl. Vet. Acad. Handl., p. 117, and Pal. N. Y., vol. 2, p. 57, and 252, Clinton and $\mathrm{Ni}$ agara $\mathrm{Gr}$.
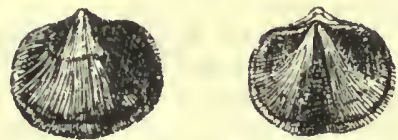

Fra. 590.-Orthls elegantula. Dorsal and ventral vlews.

ella, Hall, 1861, 13th Rep. N. Y. St. Mus. Nat. Hist., p. 121, Hud. Riv. Gr.

emacerata, Hall, 1860, 13th Rep. N. Y.

St. Mus. Nat. Hist., p. 121, Hud. Riv. Gr. emarginata, see Orthis oblata var. emarginata.

eminens, Hall, 1857, 10th Rep. N. Y. St. Mus. Nat. Hist., p. 42, and Pal. N. Y., vol. 3 , p. 167, Low. Held. Gr.

erratica, Hall, 1847, Pal. N. Y., vol. 1, p. 288, Hud. Riv. Gr.

eryna, Hall, 1863, (Corrigenda eryna,) 16th Rep. N. Y. St. Mus. Nat. Hist., p. 35, and Pal. N. Y., vol. 4, p. 42, Cornif. Gr. Named instead of $O$. requivalvis in 10th Rep., p. 102.

eudocia, Billing8, 1862, Pal. Foss., vol. 1, p. 83, Quebec Gr.

eurekensis, Walcott, 1885, Monogr. U. S. Geo. Sur., vol. 8, p. 22, Up. Taconic.

euryone, Billings, 1862, Pal. Foss., vol. 1, p. 78, Quebec Gr.

evadne, Billings, 1862, Pal. Foss., vol. 1, p. 81, Quebec Gr.

fasciata, Hall, 1852, Pal. N. Y., vol. 2, p. 255, Niagara Gr.

fausta, Foerste, 1885, Bull. Sci. Lab. Denison Univ., p. 85, Niagara Gr.

fissicosta, Hall, 1847, Pal. N. Y., vol. 1, p. 121, Hud. Riv. Gr.

fissiplica, Roemer, 1860, Sil. Fauna West Tenn., p. 64, Niagara Gr.

flabellum, Sowerby, 1839, in Murch. Sil. Syst., p. 639, and Pal. N. Y., vol. 2, p. 254, Niagara Gr.

flava, Winchell, 1865, Proc. Acad. Nat. Sci., p. 117, Marshall Gr.

gemmicula, Billings, 1862, Pal. Foss., vol. 1, p. 75, Quebec Gr.

gibbosa, Billings, 1857, Rep. of Progr. Geo. Sur. Can., p. 296, Black Riv. Gr. hamburgensis, Walcott, 1885, Monogr. U. S. Geo. Sur., vol. 8, p. 73, Chazy Gr. 
highlandensis, Walcott, 1886, Bull. U. S. Geo. Sur., No. 30, p. 119, Upper Taconic.

hipparionyx, syn. for O. proximus.

hippolyte, Billings, 1862, Pal. Foss., vol. 1, p. 81, Quebec Gr.

huromensis, Castelnau, 1843, Syst. Sil., p. 37. Not recognized.

hybrida, Sowerby, 1839, Murch. Sil. Syst., p. 630 , Niagara Gr.

idonea, Hall, 1867, Pal. N. Y., vol. 4, p. 52, Ham. Gr.

imperator, Billings, 1859, Can. Nat. Geo., vol. 4 , p. 435 , Chazy Gr.

impressa, Hall, 1843, Geo. Rep. 4th Dist., N. Y., p. 268, and Pal. N. Y., vol. 4, p. 60, Chemung Gr.

inæqualis, Hall, 1858, Geo. of Iowa, p. 490, Ham. Gr.

infera, Calvin, 1878, Bull. U. S. Geo. Sur. Terr., vol. 4, No. 3, p.728, Low. Devonian. insculpta, Hall, 1847, Pal. N. Y., vol. 1, p. 125, Hud. Riv. Gr.

insignis, see Skenidium insigne.

interlineata, Sowerby, see Orthis tioga.

interstrialis, Phillips, 1841, Pal. Foss., Devonian. Thts species is probably foreign to America.

jowensis, Hall, 1858, Geo. of Iowa, p. 488, Ham. Gr.

iowensis var. furnarius, Hall, 1858 , p. 489, Geo. of Iowa, Ham. Gr.

iphigenis, Billings, 1862, I'al. Foss,, vol. 1, p. 133, Trenton Gr.

jamesi, Hall, 1861, 14th Rep. N. Y. St. Mus. Nat. Hist., p. 89, Hud. Riv. Gr.

kankakensis, McChesney, 1860, Desc. New Pal. Foss., p. 77, Hud. Riv. Gr.

kassubr, Winchell, 1880, Geo. Sur. Minu. 8th Rep., p. 65. Hud. Riv. Gr.

kennicolti, McChesney, syn. for O. retrorsa. keokuk, Hall, 1858, Geo. Rep. Iowa, p. 640 , Keokuk Gr. This species was referred to Orthis unbraculum of DeKoninck by $\mathrm{O}$ wen.

lasallensis, McChesney, 1860, New Pal. Foss., p. 32, syn. for Streptorhynchus crassum.

laticosta, Meek, 1873, Pal. Ohio, vol. 1, p. 116, Hud. Riv. Gr.

laurentina, Billings, 1857, Rep. of Geo. Sur. Can., p. 297, Mid. Sil., Anticosti Gr., Div. 1.

lenticularis, Vanuxem, 1842, Geo. Rep. 3d Dist. N. Y., p. 139. The name was preoccupied by Wahlenberg in 1821. See O. eboracensis.

leonensis, Hall, 1867, Pal. N. Y., vol. 4, p. 62, Chemung Gr.

lepida, Hall, 1860, 13th Rep. N. Y. St. Mus. Nat. Hist., p. 78, and Pal. N. Y., vol. 4, p. 46, Ham. Gr.

lepis, as identified by d'Archiac \& Verneuil. Not American.

leptrenoides, Emmons, 1842, Geo. Rep. N. Y., p. 396, Trenton Gr.

leucosia, Hall, 1860, 13th Rep. N. Y. St. Mus. Nat. Hist., p. 80 , and Pal. N. Y., vol. 4, p. 48, Ham. Gr. limitaris, see Leiorby nchus limitare.

livia, Billings, 1860, Can. Jour. Ind., Sci. and Art, vol. 5, p. 267, Up. Held. Gr.

lonensis, Walcott, 1885, Monogr. U. S. Geo. Sur., vol. 8, p. 74, Trenton Gr.

lucia, Billings, 1874, Yal. Foss., vol. 2, p. 35, Gaspe Limestone No. 8, Devonian.

lynx, Eichwald, 1830, (Terebratula lynx, ) Nat. Skizze von Podol., p. 202, and Pal. N. Y., vol. 1, p. 133, Trenton and Hud. Riv. Grs.

maria, Billings, 1862, Pal. Foss., vol. 1, p. 137, Anticosti Gr., Div. 1, Mid. Sil.

macfarlanii, Meek, 1868, Trans. Chi. Acad. Sci., vol. 1, p. 88, Ham. Gr.

macleodi, Whitfield, 1889, Bull. Am. Mus. Nat. Hist., vol. 2, p. 43, Calciferous Gr.

media, Shaler, 1865, Bull. No. 4, M. C. '/., p. 65, Anticosti Gr. This is probably only a variety of $O$. elegantula.

media, Winchell, 1880, Geo. Sur. Minn. 8 th Rep., p. 64, Hud. Riv. Gr. The name was preoccupied.

meeki, S. A. Miller, 1875, Cin. Quar. Jour. Sci., vol. 2, p. 20, Hud. Riv. Gr. A variety of $O$. testudinaria.

merope, Billings, 1862, Pal. Foss., vol. 1, p. 139, Trenton Gr.

michelini, (Terebratula michelini,) I'Eveille, 1835, Mem. Soc. Geol. France, vol. 2, p. 39, Subcarboniferous.

michelini var. burlingtonensis, $\mathrm{Hall}, 1858$, Geo. Rep. Iowa, p. 596, Burlington Gr. minna, Billings, 1865, Pal. Fos8., vol. 1, p. 303, Quebec Gr.

minneapolis, Winchell, 1880, Geo. Sur. Minn., 8th Rep., p. 63, Hud. Riv. Gr.

missouriensis, $1855, \mathrm{Ge} O$. Rep. 205, Up. Sil.

missouriensis, Swallow, 1860 , Trang St Luis This name was preoc- Small Trencupied.

mitis, Hall, 1863, 16th Rep.

N. Y. St. Mus. Nat. Hist., p. 34, Schobarie grit.

morrowensis, James, not defined so as to be recognized.

multisecta, Meek, 1873, Ohio Pal., vol. 1, p. 112, Iud. Riv. Gr.

multistriata, Hall, 1857, 10th Rep. N. Y. St. Mus. Nat. Hist., p. 45, and Pal. N. Y., vol. 3, p. 176, Low. Held. Gr. musculosa, Hall, 1857, 10th Rep. N. Y.St. Mus. Nat. Hist., p. 43 , Oriskany sandstone.

mycale, Billings, 1862, Pal. Foss., vol. 1, p. 82, Quebec Gr.

nisis, Hall \& Whitfield, 1872, 24th Rep. N. Y. St. Mus. Nat. Hist., p. 181, Niagara Gr.

nucleus, Hall, syn. for Ambocolia unbonata.

oblata, Hall, 1857, 10th Rep. N. Y. St. Mus. Nat. Hist., p. 41 , and Pal. N. Y., vol. 3, p. 162, Low. Held. Gr. 
oblata var. emarginata, Hall, 1859, Pal. N. Y., vol. 3, p. 164, Low. Held. Gr.

occasus, Hall, 1860, 13th Rep. N. Y. St. Mus. Nat. Hist., p. 111, Waverly Gr. occidentalis, Hall, 1847, Pal. N. Y., vol. 1, p. 127, Trenton to Hud. Riv. Gr.

orbicularis, Sowerby, 1839, Murch. Sil. Sys., p. 611, Up. Sil.

orthambonites, Eichwald, 1840, Sil. Syst. in Esthl., p. 150, Quebec Gr.

pecosi, Marcou, 1858, Geo. N. America, p. 48, Coal Meas. This species was subsequently described by Swallow under the name of Orthis carbonaria.

pecten, as identified by d'Archiac \& Verneuil. Not American.

pectinella, Emmons, 1842, Geo. Sur. 2d Dist. N. Y., p. 394, and Pal. N. Y., vol. 1, p. 123, Trenton Gr.

pectinella var. semiovalis, Hall, 1847, Pal. N. Y., vol. 1, p. 124, Trenton Gr. Not distinguishable from the type species.

peduncularis, Hall, 1859, Pal. N. Y., vol. 3, p. 174, Low. Held. Gr.

peloris, Hall, 1863, 16th Rep. N. Y. St. Mus. Nat. Hist., p. 32, Schoharie grit. penelope, Hall, 1860,13 th Rep. N. Y. St. Mus. Nat. Hist., p. 79, and Pal. N. Y., vol. 4 , p. 50, Ham. Gr.

pepina, Hall, 1863, 16th Rep. N. Y. St. Mus. Nat. Hist., p. 135, Potsdam Gr.

perelegans, Hall, 1857, 10th Rep. N. Y. St. Mus. Nat. Hist., p. 44, and Pal. N. Y., vol 3 , p. 171, Low. Held. Gr.

perversa, see Streptorhynchus perversum. perveta, Conrad, 1843, Proc. Acad. Nat. Sci., vol. 1, p. 333, and Pal. N. Y., vol. 1, p. 120, Black Riv. and 'Trenton Grs. pigra, Billings, 1859, Can. Nat. Geo., vol. 4, p. 442, Chazy Gr.

pisum, as identified by Hall, see Nucleospira pisiformis.

planoconvexa, Hall, 1859, Pal. N. Y., vol. 3, p. 168, Low. Held. Gr.

platys, Billings, 1859, Can. Nat. Geo., vol. 4, p. 438, Chazy Gr.

plicata, Vanuxem, see Spirifera vanuxemi. plicatella, Hall, 1847, Pal. N. Y., vol. 1, p. 122, Trenton and Hud. Riv. Grs.

pogonipensis, Hall \& Whitfield, 1877, U. S. Geo. Expl. 40th parallel, vol. 4, p. 232, Chazy Gr.

porcata, McCoy, 1844, Sil. Foss. of Ireland, p. 32, Trenton, [Hud. Riv., and Mid. Sil.

porcia, Billings, 1859, Can. Nat. Geo., vol. 4, p. 439, Chazy Gr.

præumbona, see Ambocoelia præumbona.

pratteni, McChesney, 1860, New Pal. Foss., Coal Meas. Not recognized.

prava, Hall, 1858, Geo. of Iowa, p. 490, Ham. Gr.

propinqua, Hall, 1857, 10th Rep. N. Y. St. Mus. Nat, Hist., p. 110, and Pal. N. Y., vol. 4, p. 43, Up. Held. Gr.

proximus, Vanuxem, 1842, (Hipparionyx proximus,) Geo. Rep. 3d Dist. N. Y., p. 124, and Pal. N. Y., vol. 3, p. 407, Oriskany sandstone. punctostriata, Hall, 1852, Pal. N. Y., vol. 2, p. 254, Niagara Gr.

pyramidalis, see Skenidium pyramidale.

quacoensis, Matthew, 1885, Trans. Roy.

Soc. Can., p. 43, St. John Gr.

quadricostata, see Leiorhynchus quadricostatum.

remnicha, Winchell, 1886, 14th Ann. Rep. Geo. Minn., p. 317, Potsdam Gr.

resupinata, Martin, 1809, Petref. Derb., tab. 49, figs. 13 and 14, Subcarb.

resupinoides, Cox, 1857, Geo. Sur. Ky., vol. 3 , p. 570, Coal Meas.

retrorsa, Salter, 1858, Mem. Geo. Sur. of Gt. Brit., vol. 2, p. 373, Trenton and Hud. Riv. Grs.

rhynchonelliformis, Shaler, 1865, Bull. No. 4, M. C. Z., p. 66, Anticosti Gr.

richmondi, McChesney, 1860, New. Pal. Foss., p. 32, syn. for Streptorhynchus crassum.

robusta, Hall, 1858, Geo. Rep. Iowa, p. 713, syn. for Streptorhynchus crassum.

rugiplicata, Hall \& Whitfield, 1872, 24th

Rep. N. Y. St. Mus. Nat. Hist., p. 182, Niagara Gr.

ruida, Billings, 1866, Catal. Sil. Foss. Antic., p. 42, Anticosti Gr.

salemensis, Walcott, 1887, Am. Jour. Sci. and Arts, 3d ser., vol. 34, p. 190, Up. Taconic.

sandbergi, Winchell, 1886, 14th Ann. Rep. Geo. Minn., p. 318, Potsdam Gr.

schohariensis, Castelnau, 1843, Syst. Sil., p. 36. Not recognized.

scovillii, S. A. Miller, 1882, Jour. Cin. Soc. Nat. Hist., vol. 5, p. 40, Hud. Riv. Gr. sectostriata, Ulrich, Jour. Cin. Soc. Nat. Hist., vol. 2 , p. 15, syn. for $\mathrm{O}$. ella.

semele, Hall, 1863, 16th Rep. N. Y. St. Mus. Nat. Hist., p. 34, Onondaga and Up. Held. Grs.

sinuata, Hall, 1847, Pal. N. Y., vol. 1, p. 128, Hud. Riv. Gr.

sola, Billings, 1866, Catal. Sil. Foss. Antic., p. 12, Hud. Riv. Gr.

solitaria, Hall, 1860 , 13th Rep. N. Y. St. Mus. Nat. Hist., p. 80, and Pal. N. Y., vol. 4 , p. 45 , Ham. Gr.

stonensis, Safford, 1869, Geo. of Tenn., p. 286, Trenton and Nashville Grs.

striatella, see Chonetes striatellus.

striatula, Emmons, 1842, Geo. Rep. N. Y. This name was preoccupied by Schlotheim.

strophomenoides, Hall, 1857, 10th Rep. N. Y. St. Mus. Nat. Hist., p. 46 , and Pal. N. Y., vol. 3, p. 177, Low. Held. Gr.

subcarinata, Hall, 1857, 10th Rep. N. Y. St. Mus. Nat. Hist., p. 42 , and Pal. N. Y., vol. 3 , p. 169, Low. Held. Gr.

subrequata, Conrad, 1843, Proc. Acad. Nat. Sci., vol. 1, p. 333, and Pal. N. Y., vol. 1, p. 118, Chazy to Trenton Gr.

subelliptica, White \& Whitfield, 1862 , Proc. Bost. Soc. Nat. Hist., vol. 8, p. 292, Waverly or Kinderhook Gr.

subjugata, syn. for Orthis occidentalis. 
subnorlosa, Hall, 1879, Desc. New Spec. Foss., p. 14, and 11th Rep. Geo. and Nat. Hist. Ind., p. 286, Niagara Gr. suborbicularis, Hall, 1858, Geo. of Iowa, p. $486, \mathrm{Ham}$. Gr.

subquadrata, Hall, 1847, Pa]. N. Y., vol. 1, p. 126, Trenton to Hud. Riv. Gr. subumbona, see Martinia subumbonata. swallovi, Hall, 1858, Geo. Rep. Iowa, p. 597, Burlington Gr.

tenuidens, Hall, 1852, Pal. N. Y., vol. 2, p. 58 , Clinton Gr.

tenuistriata, Hall, 1843, Geo. Rep. 4th

Dist. N. Y., p. 244, Portage Gr. The name was preoccupied by Sowerby.

testudinaria, Dalman, 1827, Vet. Acad. Hand., p. 115, and Pal. N. Y., vol. 1, p. 117, Trenton and Hud. Riv. Grs.

thiemii, White, 1860, Jour. Bost. Soc. Nat. Hist., vol. 7, n. 231 , and Cont. to Pal. No. 8 , p. 164 , Kinderhook Gr. tioga, Hall, 1867, Pal. N. Y., vol. 4 , p. 59, (O. interlineata, Sow.,) Geo. Rep. 4th Dist. N. Y., Portage and Chemung Grs.

tricenaria, Conrad, 1843, Proc. Acad. Nat. Sci., vol. 1, p. 333, and Pal. N. Y., vol. 1, p.121, Trenton Gr.

trinucleus, Hall, 1852, Pal. N.Y., vol.2, p. 58, Clinton Gr.

FIG. 592 . Orthis tri. cenaria. Hait nat uralize. triplicatella, Meek, 1873, Ohio Pal., vol. 1, p. $109, \mathrm{H}$ ud. Riv. Gr.

tritonia, Billings, 1862, Pal. Foss., vol. 1, p. 76. Quebec Gr.

tubulostriata, Hall, 1857, 10th Rep. N. Y. St. Mus. Nat. Hist., p. 42, and l'al. N. Y. vol. 3, p. 166, Low. Ifeld. Gr.

tulliensis, Vanuxem, 1843, Geo. Rep. 3d Dist. N. Y., p. 163, and Pal. N. Y., vol. 4, p. 55, Tully limestone.

uberis, Billings, 1866, Catal. Sil. Foss. Antic., p. 42, Anticosti Gr.

umbonatu, see Ambococlia umbonata.

umbraculum, DeKoninck, see Orthis keo-

kuk and Streptorhynchus umbraculum. unguiculus, Phillips, as identified by Hall in 1843, see Ambocolia gregaria.

unguiformis, Castlenau, 1813, Syst. Sil., p. 37 , syn. for Orthis hipparionyx.

vanuxemi, Hall, 1857, 10 Rep. N. Y. St. Mus. Nat. Hist., p. 135, and Pal. N. Y., vol. 4, p. 47, Ham. Gr.

vanuxemi, Winchell, 1862, Proc. Acad. Nat. Sci., vol. 6, 2 d ser., p. 409, Portage Gr. The name was preoccupied.

varica, Con rad, 1842, (Delthyris varica) Jour. Acad. Nat. Sci., vol. 8, p. 262, and Pal. N. Y., vol. 3, p. 179, Low. Held. Gr.

Orthisina, D'Orbigny, 1850, Prodr. d. Pal., vol. 1, p. 16. [Ety. Orthis, a genus; inus, implying resemblance to.] External characters of Orthis, but the triangular pit in the cardinal area of the ventral valve is closed by a cicatrix with an oval perforation near the apex; interior of ventral valve with two broad, dental lamella bordering the cardinal pit, and converging to a mesial line at the surface of the shell; interior of dorsal valve with a Fio.593-Orthising randtrifid rostral ava. Doral and ventooth, from tral valves.

which a small mesial septuin extends toward the margin; lateral cardinal teeth as in Orthis. "Tpye 0 . verneuili. alternata, see Streptorhynchus perversum. arclostriata, see Streptorhynchus arctostriatum.

crassa, see Streptorlyynchus crassum.

diversa, Shaler, syn. for Orthisina verneuili.

festinata, Billings, 1861, Pal. Foss., vol. 1, p. 10, Georgia Gr.

grandaeva, Billings, 1859, Can. Nat. Geo., vol. 4, p. 349, Calcif. Gr.

missouriensis, Swallow, 1858, syn. for Meekella striatocostata.

occidentalis, Swallow. 1863, Trans. St. Iouis Acad. Sci., vol. 2, p. 82, ITp. Coal Meas.

orientalis, Whitficld, 1884, Bull. Am. Mus. Nat. Hist., vol. 1, p. 139, Georgia Gr. shumardana, Swallow, 185S, Trans. St. Louis Acad. Sci., p. 183, Permian Gir.

transversa, Walcott, 1886, Bull. U.S. (reo. Sur., No. 30, p. 121, Up. Taconic.

verneuili Eichwald, 1842, (Orthis verneuili,) Urwelt Russie, vol. 2, p. 51, Trenton and Anticosti Gir.

Pentagonia, Cozzens, 1846, Ann. N. Y. Lyceum, vol. 4, p. 158. [Ety. pente, five; gonia, an angle.] This genus seems to have been founded upon Conrad's A trypa unisulcata, which is now referrerl to Meristella. The genus is not recognized by authors.

peersi, Cozzens, 1846, Aun. N. Y. Ly ceum, vol. 4, p. 158, syn. for Meristella unisulcata.

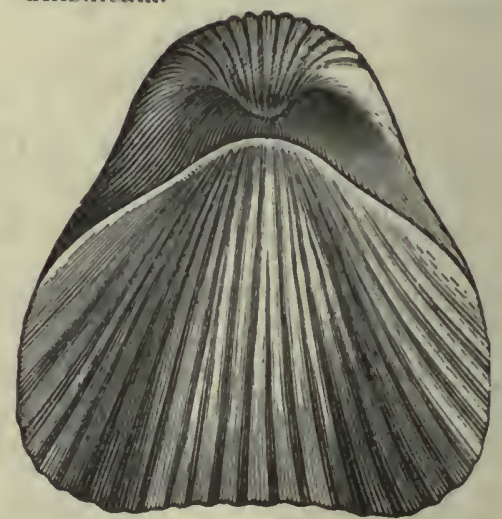

Frg. 594.-Pentamerella arata. Dorsal vlew.

Pentamerella, Hall, 1867, Pal. N. Y., vol. 4, p. 375 . [Ety. diminutive of Penta- 
merus.] Ventral valve gibbous, beak incurved, fissure triangular, area narrow, mesial sinus; in the interior an elongate, spoon-shaped pit, the upper part supported on a central septum; dorsal valve couvex, mesial fold; crura conjoined at their bases, making a V-shaped pit, which is attached to the valve in its upper part, and continues sessile for about half the length of the shell; surface plicated. Type $P$. arata.

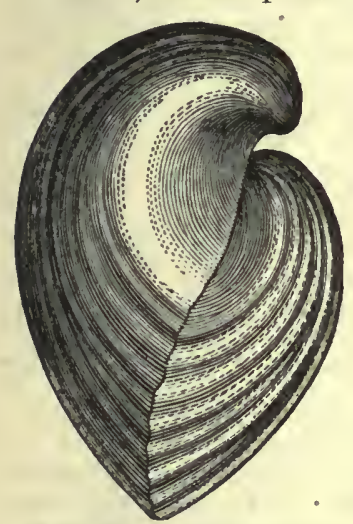

Fre. 595.-Pentamerella arata. Side view. arata, Conrad,

1841, (Atrypa arata and Atrypa octo-costatz,) Ann. Rep. N. Y., p. 55, and Pal. N. Y., vol. 4, p. 375 , Schoharie grit and Up. Held. Gr.

compressa, Ringueberg, 1886,

Bull. Buf. Soc. Nat. Sci., vol. 5 , p. 15, Niagara Gr.

dubia, Hall,1860, (Spirifer dubius,) 13th Rep. N. Y. St. Mus. Nat. Hist., p. 90, Ham. Gr. micula, Hall, 1867, Pal. N. Y., vol. 4, p. 378, Ham. Gr.

obsolescens, Hall, 1867, Pal. N. Y., vol. 4, p. 379, Devonian.

papilionensis, Hall, 1858, (Pentamerus papilionensis,) Geo, Rep. Iowa, vol. 1, pt. 2, p. 514, Ham. Grr.

Pentamerus, Sowerby, 1812, Min. Conch., vol. 1, p. 73. [E.ty. penta, five; meros, apartments.] Shell globose, ovate, receiving valve largest; generally destitute of mesial fold and sinus, but when present, the fold is in the receiving, and the sinus in the entering valve; no hinge-line; area large, undefined, and having a deep, triangular pit in the center, under the beak of the dorsal valve, and into which the beak of the entering valve is strongly incurved; internally the receiving valve has one large bipartite central septum, the walls of which suddenly divaricate as they approach the entering valve, forming the walls of the external triangular opening, and inclosing between them a triangular chamber much smaller than the two lateral ones; in the entering valve the two corresponding plates are subparallel, and separate from their origin, being so curved that internal casts show one of their edges, like the diverging cardinal teeth of Orthis, and the inner edges form the long, subparallel slits, the middle one of the three resulting chambers being much the narrower. Type P. knighti. aratus, see Pentamerella arata.

arcuosus, McChesney, 1861, New Pal. Foss., p. 87, Niagara Gr. Not recognized.

barrandi, Billings, 1857, Rep. of Progr., Geo. Sur. Can., p. 296, Mid Sil.

beaumonti, Castelnau, 1843, Syst. Sil., p. 38. Not recognized.

bisinuatus, McChesney, 1859, New Pal. Foss., p. 85, and Trans. Chi. Acad. Sci., vol. 1, p. 30. Niagara Gr.

borealis, Meek, 1868, Trans. Chi. Acad. Sci., p. 95, Ham. Gr. This name was preoccupied by Eichwald in 1840 .

brevirostris, Sowerby, 1839, (Terebratula brevirostris,) Murch. Sil. Syst., p. 631, and Pal. N. Y., vol. 2, p. 278. Niagara Gr.

chicagoensis, Winchell \& Marcy, 1865, Mem. Bost. Soc. Nat. Hist., p. 94, Niagara Gr.

comis, Owen, 1852, (Atrypa comis,) Geo. Sur. Wis., Iowa and Minn., p. 583, Ham. Gr.

coppingeri, Etheridge, 1878, Quar. Jour. Geo. Soc., vol. 34, p. 593, Up. Silurian. crassiradiatus, McChesney, 1861, New Pal.

Foss., p. 87, Niagara Gr. Not recognized. deshayesi, Castelnau, 1843, Syst. Sil, p. 38. Not recognized. Probably syn. for Amphigenia elongata.

elongatus, see Amphigenia elongata.

fornicatus, Hall, 1852, Pal. N. Y., vol. 2, p. 81 , Clinton Gr.

galeatiformis, Meek \& Worthen, syn. for $P$. galeatus.

galeatus, Dalman, 1827, (A trypa galeatus,) Vet. Acad. Handl., p. 130, and Pal. N. Y., vol. 3, p. 257, Low. Held Gr.

intralineatus, Winchell, 1866, Rep. Low Penin. Mich., p. 94, Ham. Gr.

knappi, Hall \& Whitfield, 1872, 24th Rep. N. Y. St. Mus. Nat. Hist., p. 184, Niagara Gr.

knighti, Sowerby, 1812, Min. Conch., vol. 1, p. 73 , Devonian. laqueatus, Conrad, 1855, Proc. Acad. Nat. Sci., p. 441, Niagara Gr.

lenticularis, White \& Whitfield, 1862, Proc. Bost. Soc. Nat. Hist., vol. 8, p. 295, Kinder-

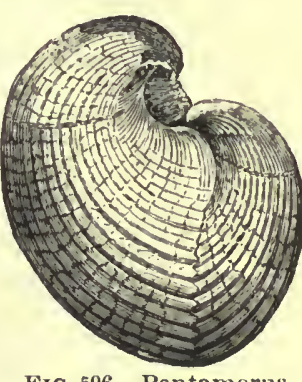

Fig. 596.-Pentamerus knighti. hook Gr.

littoni, Hall, 1859, Pa]. N. Y., vol. 3, p. 262, Low. Held. and Niagara Gr.

lotis, Walcott, 1885, Monogr. U. .S. Geo. Sur., vol. 8 , p. 161, Devonian.

multicostatus, Hall, 1861, Rep. of Progr.

Wis. Sur., p. 1, Niagara Gr.

nucleus, Hall \& Whitfield, 1872, 24t h

Rep. N. Y. St. Mus. Nat. Hist., p. 200 , Niagara Gr. 
nysius, Hall \& Whitfield, 1872, 24th Rep. N. Y. St. Mus. Nat. Hist., p. 184, Niagara Gr. There are two varieties, one having coarse and the other finer radii. These are designated $P$. nysius var. crassicostus and $P$. nysins var. tenuicostus.

oblongus, Sowerby, 1839, Murch. Sil. Syst., p. 641, and Pal. N. Y., vol. 2, p. 79, Clinton and Niagara Gr.

oblongus var. cylindricus, Hall \& Whitfield, 1872, 24th Rep. N. Y. St. Mus. Nat. Hist., p. 183, Niagara Gr.

occidentalis, Hall, 1852, Pal. N. Y., vol. 2, p. 341, Guelph Gr.

occidentalis, see Gypidula occidentalis. ovalis, Hall, 1852, Pal. N. Y., vol. 2, p. 103, Clinton Gr.

papilionensis, see Pentamerella papilionensis.

pergibbosus, Hall \& Whitfield, 1875, Ohio Pal., vol. 2, p. 139, Niagara Gir.

pesovis, Whitfield, 1882, Desc. New Spec. Foss., from Ohio, p. 195, Low. Held. Gr. pseudogaleatus, Hall, 1857, 10th. Rep. N. Y.St. Mus. Nat. Hist., p. 106 , and Pal. N. Y., vol. 3, p. 259, Low. Held. Gr. reverum, see Anastrophia reversa.

salinensis, Swallow, 1860, Trans. St. Louis Acad. Sci., vol. 1, p. 6ว่2, Devonian.

similior, Winchell \& Marcy, 1865, (Spirifera similior,) Mem.

Bost. Soc. Nat. Hist. p. 93, Niagara Gr.

subglobosus, Meek \& Worthen, 1868, Geo. Sur. Ill., vol. 3, p. 429, Ham. Gr.
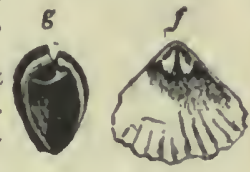

trisinuatus, McChes-

ney, 1861, Desc. New Fıa. 598.-l'orambonites ottawensis, $a, b, c, d$, Different vlews; $e$, inPal. Foss., p. 86, terior of ventral valve; $f$, Interlor of dorsil valve; $g$, sliowing oral Niagara Gr.

ventricosus, Hall, 1861, Rep. Progr. Wis. Sur., p. 2, and 20th Rep. N. Y. St. Mus. Nat. Hist., p. 374. Niagara Gr.

verneuili, see Anastrophia verneuili.

Promidops, Hall, 1859, Pal. N. Y., vol. 3, p. 489. [Ety. pholis, pholidos, a scale.] Small, thin, subelliptical, inequivalve; apex excentric, foramen in front of the apex of the ventral valve; surlace marked by concentric lamellix of growth; dorsal valve marked with bilobed muscular impressions. Type P. squamiformis.

arenaria, Hall, 1867, Pal. N. Y., vol. 4, p. 413, Oriskany sandstone.

areolata, Hall, 1863, 16th Rep. N. Y. Mus. Nat. Hist., p. 31, Schoharie grit.

bellula, Walcott, 1885, Monogr. U. S. Geo.

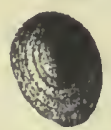

Fra. 597.-Pholidops elncinnatjensis.

Hist., p. 92 linguloides, Hall, 1867, Pal. N. Y., vol. 4, p. 414, Han. Gr.

oblata, Hall, 1867, Pal. N. Y., vol. 4 , p. 414, Ham. Gr.

ovalis, Hall, 1863, Truns. Alb. Inst., vol. 4, p. 209, Niagara Gr.

ovata, Hall, 1859, Pul. N. Y., vol. 3, I. 490, Low. Held. Gr.

quadrangularis, IValcott, 1885, Monogr. U.S.Geo.Sur., vol. S, p. 114, Devonian. squamiformis, Hall, 1843, (Orbicula squsmiformis,) Geo. Rep. 4th Dist. N. Y., p. 10S, and Pal. N. Y., vol. 2, p. 250, Niagara Gr.

subtruncata, Hall, 1847, (Orbicula subtruncata,) Pal. N. Y., vol. 1, 1). 290 , Hud. Riv. Gr.

terminalis, Hall, 1859, Pal. N. Y., vol. 3, p. 490 , Oriskany sandstone.

trentonensis, Hall, 1866, 24th Rep. N. Y.

St. Mus. Nat. Hist., ए. 221, 'Trenton (ir. Platystrophia, King, syn. for Orthis.

regularis, syn. for Urthis lynx.

Plectambonites arca, syn. for Leptana transversalis.

glabra, syn. for Leptana sericea.

tenera, syn. for Leptana transversalis.

Plicatula, L.amarck, $1809 . \quad$ Not 1'alazozoic. striatocostata, see.Mcekella striatocostata. Porannonitws, Pander, 1830, Beitrage zur fieog. des Russichen Reiches, p. 99.
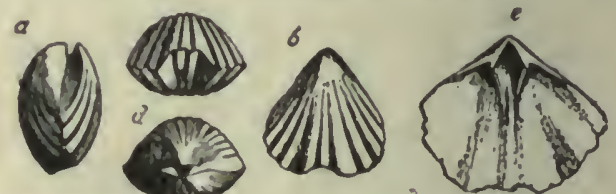
32 , Ham. Gr.

[Ety. poros, oprening; ambon, umbone.] Subglobose, depressed, dorsal valve the larger, beaks obtuse, subequal, seprrated by a small cardinal area in each valve; foramen in each valve small, triangular, reaching the hiuge-line; two long, slightly diverging dental lamellæ in each valve, those of the ventral valve closer together; surface coarsely punctured in lines. Type P. requirostris.

denlatus, see Orthis dentita.

obscurus, Hall \& Whitfield, 1877, U. S. Geo. Expl. 40, h parallel, vol. 4 , p. 234 , Quebec (ir.

ottawensis, Billings, 1862, Pal. Foss., vol. ], p. 140, Black Riv. Gr.

Productella, Hall, 1867, Pal. N. Y., vol. 4, p. 153. [Sig. diminutive of Productus.] Shells having the general form of Productus, but with a narrow area on each valve, a foramen or callosity on the ventral area, small teeth, and more or less distinct teeth sockets. Type P. subaculeata.

arctirostrata, Hall, 1857, (Productus aretirostratus,) 10th Rep. N. Y. St. Mus. 
Nat. Hist., p. 177, and Pal. N. Y., vol. 4, p. 182, Chemung Gr.

bialveata, Hall, 1867, Pal. N. Y., vol. 4, p. 183, Chemung Gr.

boydi, Hall, 1857, (Productus boydii,) 10th Rep. N. Y. St. Mus. Nat. Hist., p. 179, and Pal. N. Y., vol. 4, p. 169, Chemung Gr.

concentrica, Hall, 1857, (Productus concentricus, 10 th Rep. N. Y. St. Mus. Nat. Hist., p. 180, Kinderhook Gr.

costatula, Hall, 1867, Pal. N. Y., vol. 4, p. 180, Chemung Gr.

costatula var. strigata, Hall, 1867, Pal.

N. Y., vol. 4, p. 181, Chemung Gr.

dissimilis, see P. hallana.

dumosa, Hall, 1861, (Productus dumosus,) 14th Rep. N. Y. St. Mus. Nat. Hist., p. 99, and Pal. N. Y., vol. 4, p. 162, Ham. Gr.

eriensis, Nicholson, 1874, Geo. Mag., n. s., vol. 1, p. 118, Cornif. Gr.

exanthemata, Hall, 1857, (Productus exanthematus,) 10 th Rep. N. Y. St. Mus. Nat. Hist., p. 174, and Pal. N. Y., vol. 4, p. 163 , Ham. Gr.

hallana, Walcott, 1885, Monogr. U. S. Geo. Sur., vol. 8, p. 130 , Ham. Gr. Proposed instead of $\mathbf{P}$. dissimilis of Hall, which was preoceupied by DeKoninck.
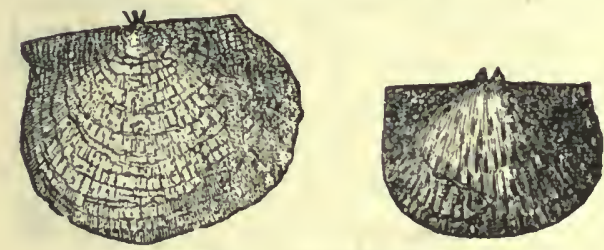

FIG. 599.-Productella hirsuta.

hirsuta, Hall, 1857, (Productus hirsutus,) 10th Rep. N. Y. St. Mus. Nat. Hist., p. 175 , Chemung Gr.

hirsuta var. rectispina, Hall, 1867, Pal. N. Y., vol. 4, p. 168, Chemung Gr.

hystricula, Hall, 1867, Pal. N. Y., vol. 4, p. 178, Chemung Gr.

lachrymosa, Conrad, 1842, (Strophomena lachrymosa,) Jour. Acad. Nat. Sci., vol. 8 , p. 256, and Pal. N. Y., vol. 4, p. 174, Chemung Gr.

Tachrymosa var. lima, Conrad, 1842, (Strophomena lima,) Jour. Acad. Nat. Sci., vol. 8, p. 256, and Pal. N. Y., vol. 4, p. 174, Chemung Gr.

lachrymosa var. stigmata, Hall, 1867, Pal. N. Y., vol. 4, p. 174, Chemung Gr.

navicella, Hall, 1857, (Productus navicella,) 10th Rep. N. Y. St. Mus. Nat. Hist., p. 172, and Pal. N. Y., vol. 4, p. 156, Cornif. and Ham. Grs.

newberryi, Hall, 1857, (Productus newberryi,) 10th Rep. N. Y. St. Mus. Nat. Hist., p. 180, Chemung Gr.

onusta, Hall, 1867, Pal. N. Y., vol. 4, p. 184, Chemung Gr.

pyxidata, Hall, 1858, (Productus pyxidatus,) Geo. of Iowa, p. 498, Ham. Gr. rarispina, Hall, 1857, (Productus rarispinus,) 10th Rep. N. Y. St. Mus. Nat. Hist., p. 178, and Pal. N. Y., vol. 4, p. 170, Chemung Gr.

shumardana, Hall, 1858, (Productus shumardanus,) Geo. Rep. of Iowa, vol. 1, pt. 2, p. 499, and Pal. N. Y., vol. 4, p. 157, Up. Held. Gr., Marcellus shale, Ham. and Chemung Grs.

speciosa, Hall, 1857, (Productus speciosus,) 10th Rep. N. Y. St. Mus. Nat. Hist., p. 176, and Pal. N. Y., vol. 4, p. 175, Chemung Gr.

spinulicosta, Hall, 1857, (Productus spinulicostus,) 10th Rep. N. Y. St. Mus. Nat. Hist., p. 173, and Pal. N. Y., vol. 4, p. 160, Marcellus shales and Ham. Gr.

striatula, Hall, 1867, Pal. N. Y., vol. 4, p. 177, Chemung Gr.

subaculeata, Murchison, 1840, (Productus subaculeatus,) Bul. Soc. Geo. de France, vol. 11, p. 255 , and Pal. N. Y., vol. 4, p. 154, Waverly Gr.

subalata, Hall, 1857, (Productus subala- Fig.600.-Productella tus,) 10th Rep. N.Y. valve. Ventral St. Mus. Nat. Hist.,

p. 174, and Pal. N. Y., vol. 4, p. 165, Ham. Gr.

truncata, Hall, 1857, (Productus truncatus,) 10th Rep. N. Y. St. Mus. Nat. Hist., p. 171, and Pal. N. Y., vol. 4, p. 160, Marcellus shales and Ham Gr.

tullia, Hall, 1867, Pal. N. Y., vol. 4, p. 164, Ham. Gr.

Pronuctus, Sowerby, 1812, Min. Conch., vol. 1, p. 153. [Ety. productus, produced-so named from one valve of the shell being prolonged beyond the other, and often to a great extent.] Shell inequivalve, transverse, or elongated with auricular expansions; ventral valve convex, geniculated, or perpendicularly incurved; hinge-line straight; area narrow, or the cardinal edge thickened; beak incurved; in the interior a narrow mesial ridge separates two elongated, ramified, muscular adductor scars; under and outside these are two deep, longitudinally subquadrateimpressions for cardinal muscles, widely separated by a crest, and lower down toward the center of the shell two deep concave subspiral depressions for spiral or labial appendages; dorsal valve concave, following the other valve; cardinal process for the attachment of muscles prominent, trifid, and below it a mesial ridge, upon each side of which are the ramified adductor scars; outside and in front of these are two reniform impressions; a prominence on each side the mesial ridge indicates the origin of spiral arms; surface of shell striated, more or less concentrically 
wrinkled, and bearing tubular spines. Types $P$. longispinus and $P$. semireticulatus.

requicostatus, Shumard, 1855, Geo. Rep. Mo., p. 201, Coal Meas.

alternatus, Norwood \& Pratten, 1854, Jour. Acad. Nat. Sci., 2d series, vol. 3, p. 20 , Keokuk Gr.

altonensis, Norwood \& Pratten, 1854, Jour. Acad. Nat. Sci., 2d series, vol. 3, p. 7, Kaskaskia Gr.

americanus, Swallow, 1863, Trans. St. Louis Acad. Sci., vol. 2, p. 91, Up. Coal Meas.

arctirostratus, see Productella aretirostrata.

arcuatus, Hall, 1858, Geo. Rep. Iowa, p. 518, Kinderhook Grr.

asper, McChesney, syn. for P. nebraskensis.

auriculatus, Swallow, 1863, Trans. St. Louis Acad. Sci., vol. 2, p. 92, Coal Meas.

biseriatus, Hall, 1858, Trans. Alb. Inst., vol. 4, p. 12, and Bull. Am. Mus. Nat. Hist., p. 46, Warsaw Gr.

boonensis, Swallow, 1858, Trans. St. Louis Acad. Sci., vol. 1, p. 217, Coal Meas.

boydi, see Productella boydi.

ealhounanus, Swallow, 1858, Trans. St. Louis Acad. Sci., vol. 1, p. 215, Coal Meas. Prof. Meek regarded this name as a synonym for $P$. semireticulatus.

callawayensis, Swallow, 1860, Trans. St.

Louis Acad. Sci., vol. 1, 1). 640, Ham. Gr.

cancrini, as identified by (ieinit\%, is $P$. pertenuis of Meek.

capaci, D'Orbigny, 1843, as identified by early authors, is referred to $P$. longispinus.

cestriensis, Worthen, 1860, Trans. St. Louis Acad. Sci., vol. 1, p. 570, Kaskaskia Gr.

clavus, Norwood \& Pratten, 1854, Jour. Acad. Nat. Sci.. "d series, vol. 3, p. 10, Coal Meas.

comoides, as identified by d'Archiac \& Verneuil. Not American.

concentricus, see Productella concentrica. confragosus, Conrad, 1835, Trans. Geo. Soc. Penn., vol. 1, p. 2, p. 267, Coal Meas. This species is not recognized.

cooperensis, Swallow, 1860, Trans. St. Louis Acad. Sci., vol. 1, p. 640, Waverly or Choteau Gr.

cora, D’Orbigny, 1842, Paléont. d. l'Am. Merid., p. 48, Coal Meas.

cora var. mogoyoni, Marcou, 1858, (ieo. N. Amer., p. 45, Subcarboniferons.

coriformis, Swallow, 1863, Trans. St. Louis Acad. Sci., vol. 2, p. 94, Kaskaskia Gr. costatoides, Swallow, 1858, Trans. St. Louis Acad. Sci., vol. 1, p. 217, Up. Coal Meas.

costatus, Sowerby, 1827, Min. Conch., vol., 6, p. 115, Coal Meas. It is doubtful whether this species has been identified in America. curtirostratus, Winchell, 1865, Proc. Acad. Nat. Sci., p. 114, Marshall Gr.

delawari, Marcou, 1858, Geol. N..Amer., p. 45, Subcarb.

depressits, Sowerby, 1825, see Strophomena depressa.

depressus, Swallow, 1863, Trans. St. Louis Acad. Sci., vol. 2, p. 93, Keokuk (ir.

dissimilis, see Productella dissimilis.

dolorosus, Winchell, 1865, Proc. Acad.

Nat. Sci., p. 114, Marshall Gr.

dumosus, see Productella dumosa.

duplicostatus, Winchell, 1865, Proc. Acad. Nat. Sci., p. 113, Marsliall Gr.

elegans, Norwood \& Pratten, 1854. This name was preoccupied, and the fussil is now named $P$. cestricnsis.

exanthematrs, sce Productella exanthemata. fasciculatus, McChesney, 1860, New Pal. Foss. Coal Mers. Not recognized.

fentonensis, Swallow, 1863, Trans. St. Louis Acad. Sci., vol. 2, p. 33, Keokuk Gr.

flemingi, Sowerby, 1812, Min. Conch., vol. 1, p. 155 , Subcarb.

Alemingi var. burlingtonensis, Hall, 1858, Geo. Rep. Iowa, p. 598, Burlington Gr. gracilis, Winchell, 1865, Proc. Acad. Nat. Sci., p. 112, Cuyahoga shale.

gradatus, Swallow, 1863, Trans. St. Lonis Acad. Sci., vol. 2, p. 93, Keokuk (ir.

hepar, Morton, 1836, Am. Jour. Sci. and Arts, vol. 29, p. 149, Coal Meas. Not recognized.

hildrethanus, Norwood \& Pratten, 1854, Jour. Acad. Nat. Sci., 2d ser., vol. 3, p. 18, Coal Meas.

hirsutiformis, Walcott, 1885, Monvgr. U. S. Feo. Sur., vol. 8, p. 13:3, Up. Devonian.

hirsutus, see Productella hirsuta.

horridus, as identified by Geinitz, 1866. Prof. Meck regarded the fossil as l'. longispinus.

incurvatus, Shepard, 1838, Am. Jour. Sci., vol. 34, p. 144. Not recognized. Probably a Streptorhynchus or Strophodonta.

indianensis, Hall, 1858, Trans. Alb. Inst., vol. 4, p. 13, and Bull. Am. Mus. Nat. Hist., vol. 4, p. 47, Warsaw Gr.

inflatus, syn. for $\mathrm{P}$. semircticulatus.

ivesi, Newberry, 1861, Ives's Col. lix. Exped., p. 122, Mid. Carb.

lasallensis, Worthen, 1873, Geo. Sur. Ill., vol. 5, p. 569, Up. Coal Meas.

lavicostus, White, 1860 , Bost. Jour. Nat. Hist., vol. 7, p. 230, Kinderhook Gr.

latissimus, Sowerby, 1822, Min. Conch., vol. 4 , p. 32, Carb.
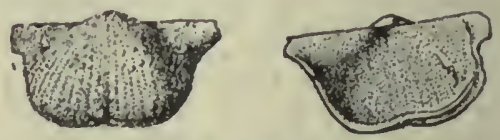

Fig. 601.-Productus longisplnus. Dorsis and ventral vlews.

longispinus, Sowerby, 1812, Min. Conch., vol. 1, p. 154, Coal Meas. 
Lobatus, as identified by d'Archiac \& Verneuil. Not American.

magnicostatus, Swallow, 1860, Trans. St. Louis Acad. Sci., vol. 1, p. 641, Coal Meas.

magnus, Meek \& Worthen, 1861, Proc. Acad. Nat. Sci. Phil., p. 142, and Geo. Sur. Ill., vol. 3, p. 528, Keokuk Gr.

marginicinctus, Prout, 1857, Trans. St. Louis Acad. Sci., vol. 1, p. 43, St. Louis Gr.

mesialis, Hall, 1858, Geo. Rep. Iowa, p. 636, Keokuk Gr.

mexicanus, Shumard, 1858, Trans. St. Louis Acad. Sci., vol. 1, p. 291, Permian Gr.

morbillianus, Winchell, 1865, Proc. Acad. Nat. Sci., p. 113, Burlington Gr.

multistriatus, Meek, 1860, Proc. Acad. Nat. Sci., vol. 12, p. 309, and Simpson's Rep. Gt. Basin of Utah, p. 350, Coal Meas.

muricatus, Norwood \& Pratten, 1854, Jour. Acad. Nat. Sci. Phil., vol. 3, p. 14, Coal Meas. Prof. Meek regarded this as a syn. for $\mathrm{P}$. longispinus.

nanus, Meek \& Worthen, 1860, Proc. Acad. Nat. Sci., p. 450, and Geo. Sur. Ill., vol. 2, p. 320, Coal Meas.

navicella, see Productella navicella.

nebraskensis, Owen, 1852, Geo. Rep. Wis., Iowa, and Minn., p. 584, Coal Meas.

nevadensis, Meek, 1877, U. S. Geo. Sur. 40th parallel, p. 64, Carboniferous.

nodosus, Newberry, 1861, Ives' Col. Ex. Exped., p. 124, Carb.

norwoodi, Swallow, 1858, Trans. St. Louis Acad. Sci., p. 182, Permian Gr.

occidentalis, Newberry, 1861, Ives' Col. Ex. Exped., p. 122, Up. Carb.

orbignyanus, DeKoninck, 1847, Mon. du genre Productus, p. 152, Up. Coal Meas.

ovatus, Hall, 1858, Geo. Rep. Iowa, p. 674. St. Louis Gr.

parvulus, Winchell, 1863, Proc. Acad. Nat. Sci., p. 4, Marshall Gr.

parvus, Meek \& Wortlien, 1860, Proc. Acad. Nat. Sci. Phil., p. 450, and Geo. Sur. Ill., vol. 2, p. 297, Kaskaskia Gr.

pectenoideus, Shepard, 1838, Am. Jour. Sci., vol. 34, p. 150 . Not recognized. Probably a Streptorhynchus.

pertenuis, Meek, 1872, Pal. E. Neb., p. 164, Coal Meas.

phillipsi, Norwood \& Pratten, 1854, Jour. Acad. Nat. Sci., vol. 3, 2 d series, p. 8 , Subcarb.

pileiformis, syn. for Productus cora.

pileolus, Shumard, 1858, Trans. St. Louis Acad. Sci., vol. 1, p. 291, Permian Gr.

pocillum, Morton, 1836, Am. Jour. Sci. and Arts, vol. 29, p. 149, Coal Meas. Not recognized.

popii, Shumard, 1858, Trans. St. Louis Acad. Sci., vol. 1, p. 290, Permian Gr. portlockanus, Norwood \& Pratten, 1854 , Jour. Acad. Nat. Sci., 2 d series, vol. 3, p. 15, Coal Meas.

prattenanus, Norwood, 1854, Jour. Acad. Nat. Sci. Phil., 2d series, vol. 3, p. 17, Coal Meas.

punctatus, Martin, 1809, Petrif. Derb., pl. 37, fig. 6, Low. Carb. and Coal Meas. pyxidatus, see Productella pyxidata.

pyxidiformis, DeKoninck, 1847, Monographie du genre Productus, p. 220, Subcarboniferous.

rarispinus, see Productella rarispina.

rogersi, Norwood \& Pratten, 1854, Jour. Acad. Nat. Sci., vol. 3, p. 9, Coal Meas. Prof. Meek regarded this as a synonym for $P$. nebraskensis.

scabriculus, (Conchyliolithus Anomites scabriculus,) Martin, 1809, Petrif. Derb., p. 8 , tab. 36 , fig. 5 , Carb.

scitulus, Meek \& Worthen, 1860, Proc. Acad. Nat. Sci.. p. 451, and Geo. Sur. Ill., vol. 2, p. 280, St. Louis Gr.

semipunctatus, Shepard, 1838, Am. Jour. Sci., vol. 34, p. 153, Coal Meas.

semipunctatus, Hildreth, 1838, syn. for $\mathrm{P}$. punctatus.

se mi r eticulatus, Martin, 1809, (Conch y liolithus Anomites se mire ticulatus,) Petrif. Derb., p. 7, Keokuk Gr.

se mis t riatus,

Meek, 1860,

Proc. Acad. Nat. Sci., vol. 12, p. reticulatus.

309, and Simp-

son's Rep. Gt. Basin of Utah, p. 349 , Coal Meas.

setigerus, Hall, 1858, Geo. Rep. Iowa, p. 638 , Keoknk Gr.

setigerus var. Keokuk, Hall, 1858, Geo.

Rep. Iowa, p. 639, Keokuk Gr.

shumardanus, see Productella shumardana. speciosus, see Productella speciosa.

spinulicostus, see Productella spinulicosta. spinulosus, Sowerby, 1812, Min. Conch., vol. 1, p. 155, Carb.

splendens, Norwood \& Pratten, 1854, Jour. Acad. Nat. Sci. Phil., vol. 3, p. 11, Coal Meas. Prof. Meek regarded this as a synonym for $\mathbf{P}$. longispinus. subaculeatus, see Productella subaculeata. subalatus, see Productella subalata.

subhorridus, Meek, 1877, U. S. Geo. Sur., 40 th parallel, p. 75 , Carboniferous.

sulcatus, Castelnau, 1843, Syst. Sil., p. 39, Not recognized,

symmetricus, McChesney, 1860, Desc. New Pal. Foss., p. 35, and Pal. E. Neb. p. 167, Coal Meas.

tenuicostus, Hall, 1858, Geo. Rep. Iowa, p. 675, St. Louis Gr.

tenuistriatus, Verneuil, 1845, Geol. Russia and Ural Mountains, vol. 2, p. 260, Carb. 
truncatus, see Productella truncata.

tubulospinus, McChesney. Syn. for. P. semipunctatus.

viminalis, White, 1862 , Proc. Bost. Soc. Nat. Hist., vol. 9, p. 29, Burlington Gr.

vittatus, Hall, 1858, Geo. Rep. Iowa, p. 639, Keokuk Gr.

wabashensis, Norwood \& Pratten, 1854, Jour. Acad. Nat. Sci., vol. 3, p. 13, Coal Meas.

wilberianus, McChesney, syn. for P. nebraskensis.

wortheni, Hall, 1858, Geo. Rep. Iowa, p. (i35, Keokuk Gr.

Pskudocrania, McCoy, 1851, Ann. and Mag. Nat. Hist., 2d series, vol. 8, p. 387. [Fty. pseudo, false; Crania, a genus.] Shell slightly inequivalve, free; each valve depressed, subconical; dorsal valve with or without a small cardinal area ; internally, margin broad, flat, smooth, or minutely striated concentrically; anterior pair of nuscular impressions much larger than the posterior pair; pallial impressions numerous, linear, not interrupted along the middle. Type $P$. divaricata.

anomala, Winchell, 1866, Rep. Low. Pen. Mich., p. 92, Ham. Gr.

Renssel.enia, Hall, 1859, Pal. N. Y., vol. 3, 1. 454. [Ety. proper name.] Inequivalve, oval, ovoid, or suborbicular, elongated, rarely transverse, sometimes subtrigonal, gibbous or ventricose; no mesial fold or sinus; beak prominent , incurved, foramen terminal; articulation by two widely separated teeth and sockets; surface striated; structure punctate. Type R. ovoides.

æquiradiata, Conrad, 1842, (Atrypa sequiradiata,) Jour. Acad. Nat, Sci., vol. 8, p. 266, and Pal. N. Y., vol. 3, p. 255, Low. Held. Gr.

coudoni, McChesney, 1861, New Pal. Foss., p. 85 , Oriskany sandstone.

cumberlandia, Hall, 1857, (Meganteris cumberlandix,) 10th Rep. N. Y. St. Mus. Nat. Hist., p. 101, and Pal. N. Y. vol. 3 , p. 464, Oriskany sandstone.

elliptica, Hall, 1857, (Meganteris elliptica) 10th Rep. N. Y. St. Mus. Nat. Hist., p. 98, Low. Held. Gr.

elongata, see Amphigenia elongata.

intermedia, Hall, 1859, Pal. N. Y., vol. 3, p. 463, Oriskany sandstone.

johanni, Hall, 1867, Pal. N. Y., vol. 4, p. 385, Up. Held. Gr.

lævis, Hall, 1857, (Meganteris lavis,) 10th Rep. N. Y. St. Mus. Nat. Hist., p. 99, Low. Held. Gr.

lavis, Meek, 1868, Trans. Chi. Acad. Sci., p. 108. This name was preoccupied.

marylandica, Hall, 1859, Pal. N. Y., vol. 3, p. 461, Oriskany sandstone.

mutabilis, Hall, 1857, (Meganteris mutabilis,) 10th Rep. N. Y. St. Mus. Nat. Hist., p. 97, Low. Held. Gr.

ovalis, Hall, 1857, (Meganteris ovalis, 10th Rep. N. Y. St. Mus. Nat. Hist., p. 101, and Pal. N. Y., vol. $3, \mathrm{p}$. 458 , Oriskany sandstone.

ovoides, Eaton, 1832 . (TerebratuIa ovoides,) Geo. Textbook, p. 45, and Pal. N. Y., vol. 3, p. 456, Oriskany sandstone.

sues a n a, Hall, 1857 , (Meganteris 8 uessana, Fig. 603,- Rensselneria ovolder. 10th Rep. N. Y. St. Mus. Nat. Hist., p. 100, and Pal. N. Y., vol. 3, p. 459, Oriskany sandstone.

portlandica, Billings, 1863, P'roc. Port. Soc. Nat. Hist., vol. 1, p. 115, Low. Held. Gr.

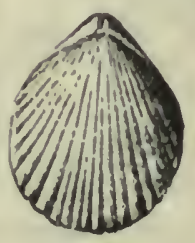

Fig. (601. lRelzia evax.
RexziA, King, 1850, Monograph of Permian Foss., p. 137. [Ety. proper n a $\mathrm{me}$ e. Longitudinally oval, ribbed, with large punctures; foramen in ventral valve; area triangular; fissure closed. Type $R$. adrieni.

altirostris, White, 1862, Proc. Bost. Soc. Nat. Hist., vol. 9, p. 28, Marshall Gr.

compressa, Meck, 1864, Pal. California, vol. 1, p. 14, Coal Meas.

deweyi, Hall, 1857, (Waldheimia deweyi,) 10 th Rep. N. Y. St. Mus. Nat. Hist., p. 89, Low. Held. Gir.

dubia, Billings, 1863, Proc. Port. Soc. Nat. Hist., vol. 1, p. 113, Low. Held. Gr.

eleetra, Billings, 1863, Proc. Port. Soc. Nat. Hist., vol. 1, p. 114, Low. Held. Gr.

eugenia, Billings, 1861, Can. Jour., vol. 6, p. 147, Ham. Gr.

evax, Hall, 1863, (Rhynchospira evax,) Trans. Alb. Inst., vol. 4, p. 213, and Rep. Geol. and Nat. Hist. Ind., Niagara Gr.

formosa, Hall, 1857, (Waldheimia formosa,) 10th Rep. N. Y. St. Mus. Nat. Hist., p. 88, Low. Held. Gr.

hippolyte, Billings, 1863, Proc. Port. Soc. Nat. Hist., vol. 1, p. 112, Low. Held. Gr. lepida, Hall, 1860, (Rhynchospira lepida,) 13th Rep. N. Y. St. Mus. Nat. Hist., p. 83, and Pal. N. Y., vol. 4, p. 275, Ham. Gr.

marcyi, Shumard, 1854, (Terebratula marcyi,) Marcy's Exp. Red Riv., p. 177, Kaskaskia Gr.

maria, Billings, 1863, Proc. Port. Soc. Nat. Hist., vol. 1, p. 112, Low. Held. Gr. 
meekana, Shumard, 1858, Trans. St. Louis Acad. Sci, vol. 1, p. 295, Permian Gr.

mormoni, Marcou, 1858, (Terebratula mormonii,) Geo. N. Amer., p. 51, Coal . Meas. This species (1) was subsequently, though in the same year, described by Shumard under the name R. punctilifera.

FIG. 605.-Retzia mormoni.

osagensis, Swallow, 1860, Trans. St. Louis Acad. Sci., vol. 1, p. 653, Waverly or Choteau Gr.

papillata, Shumard, 1858, Trans. St. Louis Acad. Sci., vol. 1, p. 294, Permian Gr.

popana, Swallow, 1860, Trans. St. Louis Acad. Sci., vol. 1, p. 654, Waverly or Choteau Gr.

punctilifera, Shumard, 1858, syn. for Retzia mormoni.

polypleura, Winchell, 1862, Proc. Acad. Nat. Sci., 2 d ser., vol. 6, p. 406, Portage Gr.

sexplicata, White \& Whitfield, 1862, Proc. Bost. Soc. Nat. Hist., vol. 8, p. 294, Kinderhook Gr.

sinuata, Hall, 1860, (Rhynchospira sinuata,) Can. Nat. and Geol., vol. 5, Up. Sil.

subglobosa, Hall, 1867, (Rhynchospira subglobosa,) Pal. N. Y., vol. 4, p. 421, Up. Held. Gr.

subglobosa, McChesney, syn. for Retzia mormoni.

vera, see Eumetria vera.

vera var. costata, see Eumetria vera var. costata.

verneuilana, see Eumetria verneuilana.

woosteri, White, 1879, Bull. U. S. Sur., vol. 5, No. 2, p. 215, and Cont. to Pal., No. 6, p. 134, Coal Meas.

Rhynchonelua, Fischer, 1809, Mem. Soc. Imp. Mosc., vol. 2, p. 35. [Ety. rhynchos, beak; ella, little.] Shell oval or trigonal, subglobose, with or without mesial fold and sinus; surface plicated; beak of ventral valve acute, entire, prominent, curved; foramen under the beak, by the incurving of which it is sometimes closed, partly surrounded by a deltidium, which is composed of two pieces; two teeth in the ventral valve, supported by dental plates, which extend to the bottom of the valve; two sockets in the dorsal valve; apophyses two, short, flattened, curved, attached to the hinge plate; adductor scars four, separated by a mesial ridge; pedicle scars on the cardinal plates; pedicle muscles of the ventral valve in a saucershaped cavity at the base of the dental plates; shell impunctate. Type R. loxia.

abrupta, Hall, 1857, 10th Rep. N. Y. St. Mus. Nat. Hist., p. 68, and Pal. N. Y., vol. 3. p. 228, Low. Held. Gr. acadiensis, Davidson, 1863, Quar. Jour. Geo. Soc., vol. 19, p. 172, Low Carb. acinus, Hall, 1863, Trans. Alb. Inst., vol. 4, p. 215, Niagara Gr.

acutiplicata, Hall, 1857, N. Y. St. Mus. Nat. Hist., p. 73, and Pal. N. Y., vol. 3, p. 232, Low Held. Gr.

acutirostris, Hall, 1847, (Atrypa acutirostra,) Pal. N. Y., vol. 1, p. 21, Chazy Gr.

æquivalvis, Hall, 1857, 10th Rep. N. Y. St. Mus. Nat. Hist., p. 66, and Pal. N. Y., vol. 3 , p. 224, Low. Held. Gr.

æquiradiata, Hall, 1852, (Atrypa æquiradiata.) Pal. N. Y., vol. 2, p. 70, Clinton Gr.

ainslæi, Winchell, 1886, 14th Ann. Rep. Geo. Minn., p. 315, Trenton Gr.

alyeri, McChesney, 1860, New Pal. Foss. Carb. Not recognized.

altilis, Hall, 1847, (Atrypa altilis,) Pal. N. Y., vol. 1, p. 23, Chazy Gr.

altiplicata, Hall, 1857, 10th Rep. N. Y. St. Mus. Nat. Hist., p. 72, and Pal. N. Y., vol. 3, p. 231, Low Held. Gr.

alveata, see Centronella alveata.

ambigua, Calvin, 1878, Bull. U. S. Geo. Sur., vol. 4, No. 3, p. 729, Low. Devonian.

angulata, Linnæus, as identified by Geinitz, syn. for Syntrielasma hemiplicatum.

anticostiensis, Billings, 1862, Pal. Foss., vol. 1, p. 142, Hud. Riv. Gr.

aprinis, DeVerneuil, 1845, (Terebratula aprinis,) Geo. Russia and Ural Mts., vol. 2 , p. 90, and Pal. N. Y., vol. 2, p. 280, Niagara Gr.

arctirostrata, Swallow, 1863, Trans. St. Louis Acad. Sci., vol. 2, p. 84, Kaskaskia Gr.

argentea, Billings, 1866, Catal. Sil. Foss. Antic., p. 43, Anticosti Gr.

argenturbica, White, 1874, Rep. Invert. Foss., p. 14, and Geo. Sur. W. 100th Mer., vol. 4, p. 75, Hud. Riv. Gr.

aspasia, Billings, 1863, Proc. Port. Soc. Nat. Hist., vol. 1, p. 111, Low. Held. Gr. barquensis, Winchell, 1862, Proc. Acad. Nat. Sci., p. 408, Marshall Gr.

barrandi, Hall, 1857, 10th Rep. N. Y. St. Mus. Nat. Hist., p. 82, and Pal. N. Y., vol. 3 , p. 442 , Oriskany sandstone.

bialveata, Hall, 1857, 10 th Rep. N. Y. St. Mus. Nat. Hist., p. 73, and Pal. N. Y., vol. 3, p. 233, Low. Held. Gr.

bidens, Hall, 1852, (Atrypa bidens,) Pal. N. Y., vol. 2, p. 69, Clinton Gr.

bidentata, Hisinger, 1826, (Terebratula bidentata,) Vet. Acad. Handl., p. 343, and Pal. N. Y., vol. 2, p. 276, Niagara Gr.

billingsi, see Stenoschisma billingsi.

boonensis, Shumard, 1855, Geo. Rep. Mo., p. 205, Burlington Gr.

brevirostris, see Pentamerus brevirostris. camplellana, Hall, 1857, 10th Rep. N. Y. St. Mus. Nat. Hist., p. 79, and Pal. N. Y., vol. 3, p. 239, Low. Held. Gr. 
camerifera, Winchell. 1862, Proc. Acad. Nat. Sci., p. 408, Marshall Gr.

capax, Conrad, 1842, (Atrypa capax,) Jour. Acad. Nat. Sci., vol. 8, p. 264, Hud. Riv. Gr.
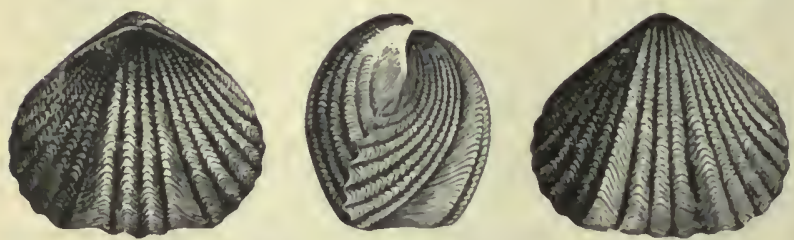

Fig. 606.-Rhynchonella capax.

caput-testudinis, White, 1862, Proc: Bost. Soc. Nat. Hist., vol. 9, p. 23, Burling. ton Gr.

carica, see Stenoschisma carica.

carbonaria, McChesney, 1860, New Pal.

Foss., Coal Meas. Not recognized.

carolina, see Stenoschisma caiolina.

castanea, Meek, 1868, Trans. Chi. Acal. Sci., 1. 93, Devonian.

congregrita, see Stenoschisma congregatum. coniracta, see Stenoschisma contractum. cooperensis, Shumard, 1855, (ieo. Rep.

Mo., p. 204, Waverly or Choteau Gr. corinthia, Billings, 1865, Pal. Foss., vol. 1, p. 220, Quebec Gr.

cuboides, Sowcrby, (Atrypa cuboides,) see $R$. venustula.

curcata, see Rhynchotreta cuneuta var. Americana.

dawsonana, Davidson, 1863, Quar. Jour. Geo. Soc., vol. 19, p. 172, Subcarboniferous.

dentata, Hall, 1847, (Atrypa dentata,) Pal. N. Y., vol. 1, p. 148, Hud. Riv. Gr.

dotis, sce Stenoschisma dotis.

dryope, Billings, 1874, Pal. Foss., vol. 2, p. 37, liaspe limestone No. 8, Devonian.

dubia, Hall, 1847, (Atrypa dubia,) Pal. N. Y., vol. 1, p. 21, Chazy Gr.

duplicata, syn. for Stenoschisma contractum.

eatoniiformis, McChesney, 1860, New l'ul. Foss., syn. for $R$. rockymontana.

emacerata, Hall, 1852, (Atrypa emacerata,) Pal. N. Y., vol. 2, p. 71, Clinton Gr.

eminens, Hall, 1857, 10th Rep. N. Y. St. Mus. Nat. Hist., p. 78 , and $\mathrm{Pal} . \mathrm{N} . \mathrm{Y}$., vol. 3, p. 237, Low. Held. Gr.

emmonsi, Hall \& Whitfield, 1877, U. S. Geo. Expl. 40th Parallel, vol. 4, p. 247, Devonian.

endlichi, Meek, 1876, U. S. Geo. Sur. of Colorado, p. 47, and White's Cont. to Pal. No. 6, p. 133, Up. Devonian.

eurekensis, Walcott, 1885, Monogr. U. S. Geo. Sur., vol. 8, j. 223, Subcarboniferous.

eva, Billings, 1866, Catal. Sil. Foss. Antic., p. 44, Anticosti Gr. evangelina, Hartt, 1868, Acal. Geol., p.
299, Subcarboniferous. excellens, Billings, 1874, Pal. Foss., vol. 2 , p. 36 , Grspe limestone No. 8, Devonian.

eximia, see Stenoscbisma eximiun.

explanata, McChesney, 1860, Desc. New Pal. Foss., Kaskaskia Gr. Not recognized.

titchana, Hall, 1857, 10th Rep. N. Y. St. Mus. Nat. Hist., p. 85, and Pal. N. Y., vol. 3 , p. 441 , Oriskany sandstone.

formosa, sce Stenoschisma formosum.

fringilla, Billings, 1862, Pal. Foss., vol. 1, p. 141, Anticosti Gr., Div. 1., Mid. Sil.

glacialis, Billings, 1S62, Pal. Foss., vol. 1, p. 143, Anticosti Gr., Div. 1, Mid. Sil. glansfagea, see Centronella glausfagen.

greenana, Ulrich, 1886, Cont. to Am. Pal., p. 26, Waverly Gr.

grosvenori, IIal!, 1858, Trans. Alb. Inst., vol. 4, p. 10, and Bull. Am. Mus. Nat. Hist., p. 53, Warsaw Gr.

guadalup:e, Shumard, 1858, 'Trans. St. Iouis Acad. Sci., vol. 1, p. 295, P'ermian Gr.

heteropsis, Winchell, 1865, Proc. Acarl. Nat. Sci., p. 121, Marshall Gr.

horsfordi, see Stenoschismn horsfordi.

hubbardi, Winchell, 1862 , Proc. Acad. Nat. Sci., p. 407. Marshall Gr.

huronensis, Winchell, 1862 , l'roc: Acud. Nat. Sci., 2ul ser., rol. 6, p. 409, Portage (ir. hydraulica, Whitfield, 1882, Deac. New Spec. Foss. from Ohio, p. 194, Low. lleld. Gr.

ida, Hartt, 1868, Acal. Geol., p. 298, Subcarboniferous.

illinoisensis, Worthen, 1884, Bull. No. 2, Ill. St. Mus. Nat. Hist, p. 24, and (ieo. Sur. Ill., vol. 8, p. 104, Coal Meas.

increbescens, syn. for Rhynchonella capax. indentata, Shumard, 1859, Trans. St. Louis Acad. Sci., vol. 1, p. 393, Permian Gir.

indianensis, Halt, is63, Trans. Alb. Inst., vol. 4, p. 215. Niagara Gr.

inrequiplicatu, Hall, 1857, 10th liep. N. Y. St. Mus. Nat. Hist., p. 126, Up. Held. Gr.

intermedia, Barris, 1879, Proc. Davenport Acad. Sci., vol. 2, p. 285, Up. Held. Gr. inutilis, Hall, 1857, 10th Rep. N. Y. St. Mus. Nat. Hist., p. 74, and Pal. N. Y., vol. 3, n. 233, Low. Held. Gr.

janea, Billings, 1866, Catal. Sil. Foss. Antic., p. 43, Anticosti Gr.

lacunnsa. Not an American species.

lamellata, Hall, 1852, (Atrypa lamellata,)

Pal. N. Y., vol. 2, p. 329, Coralline Limestone.

laura, see Leiorhynchus laura.

macra, Hall, 1858, Trans. Alb. Inst., vol. 4, p. 11. and Bull. Am. Mus. Nat. Hist., p. 52, Warsaw Gr.

mainensis, Billings, 1863, Proc. Port. Soc. Nat. Hist., vol. 1, p. 110, Low. Held. (ir. 
marshallensis, Winchell, 1862, Proc. Acad. Nat. Sci., p. 408, Marshall Gr.

medea, Billings, 1860, Can. Jour., vol. 5, p. 271, Corniferous Limestone.

metallica, White, 1874, Rep. Invert. Foss., p. 20, and Geo. Sur. W. 100th Mer., vol. 4, p. 129 , Carb.

inica, Billings, 1866, Catal. Sil. Foss. Antic., p. 44, Anticosti Gr.

micropleura, Winchell, 1865, Proc. Acad. Nat. Sci., p. 122, Marshall G'r.

missouriensis, Shumard, 1855, Geo, of Mo., p. 204, Waverly or Choteau Gr.

multistriata, Hall, 1857, 10th Rep. N. Y. St. Mus. Nat. Hist., p. 85, and Pal. N. Y., vol. 3 , p. 440 , Oriskany sandstone.

mutabilis, Hall, 1857, 10th Rep. N. Y. St. Mus. Nat. Hist., p. 66, and Pal. N. Y., vol. 3, p. 225, Low. Held. Gr.

mutata, Hall, 1858, Trans. Alb. Inst., vol. 4, p. 10, and Geo. Sur. Iowa, p. 658, Warsaw Gr.

neenah, Whitfield, 1880, Ann. Rep. Geo. Sur. Wis., p. 62, and Geo. Wis., vol. 4, p. 265, Trenton Gr.

neglecta, Hall, 1852, (Atrypa neglecta,) Pal. N. Y., vol. 2, p. 274, Niagara Gr.

neglecta var. scobina, Meek, 1872, Am. Jour. Sci. and Arts, 3 d ser., vol. 4, p. 277, and Ohio Pal., vol. 1, p. 179, and vol. 2, p. 116, Niagara Gr.

nobilis, Hall, 1857, 10th Rep. N. Y. St. Mus. Nat. Hist., p. 80, and Pal. N. Y., vol. 3 , p. 240 , Low. Held. Grr.

nucleolata, Hall, 1857, 10th Rep. N. Y. St. Mus. Nat. Hist., p. 68, and Pal. N. Y., vol. 3, p. 227, Low. Held. Gr.

nucula, Sowerby, 1839. (Terebratula nucula,) Murch. Sil. Syst., p. 611, Up. Sil.

nutrix, Billings, 1866, Catal. Sil. Foss. Antic., p. 43 , Anticosti Gr.

oblata, Hall, 1857, 10th Rep. N. Y. St. Mus. Nat. Hist., p. 86, and Pal. N. Y., vol. 3 , p. 439 , Oriskany sandstone.

obsolescens, Hall, 1860, 13th Rep. N. Y. St. Mus, Nat. Hist., p. 111, Waverly $\mathrm{Gr}$.

obtusiplicata, Hall, 1852, (Atrypa obtusiplicata,) Pal. N. Y., vol. 2, p. 279, Niagara Gr.

occidens, Walcott, 1885, Monogr. U. S. Geo. Sur., vol. 8, p. 152, Devonian.

opposita, White \& Whitfield, 1862, Proc. Bost. Soc. Nat. Hist., vol. 8, p. 294, Kinderhook Gr.

orbicularis, see Stenoschisma orbiculare.

orientalis, Billings, 1859, Can. Nat. Geo., vol. 4, p. 443, Chazy Gr.

osagensis, Swallow, 1858, syn. for Rhynchonella uta.

ottumwa, White, 1862, Proc. Bost. Soc. Nat. Hist., vol. 9 , p. 23 , and Cont. to Pal., No. 8, p. 165, St. Louis Gr.

parvini, McChesney, syn. for Camerophoria subtrigona.

perlamellosa, Whitfield, 1878, Ann. Rep. Geo. Sur. Wis., p. 73, and Geo. Sur. Wis., vol. 4, p. 265, Hud. Riv. Gr. perrostellata, Swallow, 1863, Trans. St. Louis Acad. Sci., vol. 2, p. 85, Kaskaskia Gr.

persinuata, Winchell, 1865, Proc. Acad. Nat. Sci., p. 121, Marshall Gr.

phoca, see Atrypa phoca.

pisum, Hall \& Whitfield, 1875, Ohio Pal. vol. '2, p. 135, Niagara Gr.

planoconvexa, Hall, 1857, 10th Rep. N. Y. St. Mus. Nat. Hist., p. 75, and Pal. N. Y., vol. 3, p. 235, Low. Held. Gr.

pleiopleura, Conrad, 1841, (Atrypa pleiopleura,) Ann. Rep. N. Y., p. 55, and Pal. N. Y., vol. 3, p. 440, Oriskany sandstone.

plena, Hall, 1847, (Atrypa plena,) Pal. N. Y., vol. 1, p. 21, Chazy Gr.

plicata, Hall, 1852, (Atrypa plicata,) Pal. N. Y., vol. 2, p. 10, Medina Gr.

plicatula, Hall, 1843, (Atrypa plicatula,) Geo. Rep. 4th Dist. N. Y., p. 71, and Pal. N. Y., vol. 2, p. 74, Clinton Gr.

plicifera, Hall, 1847, (Atrypa plicifera,) Pal. N. Y., vol. 1, p. 22, Chazy Gr.

principalis, Hall, 1857, 10th-Rep. N. Y. St. Mus. Nat. Hist., p. 84, and Pal. N Y., vol. 3, p. 443, Oriskany sandstone.

prolifica, see Stenoschisma prolificum.

pugnus, Martin, 1809, (Conchiliolithus Anomites pugnus,) Petrif. Derb., pl. 22, figs. 4 and 5, Subcarboniferous.

pustulosa, White, 1860, Bost. Jour. Nat. Hist., vol. 7, p. 236, Burlington Gr. py ramidata, Hall, 1857, 10th Rep. N. Y. St. Mus. Nat. Hist, p. 70, and Pal. N. Y. vol. 3, p. 229, Low. Held Gr.

pyrrha, Billings, 1866, Catal. Sil. Foss. Antic., p. 44, Anticosti Gr.

quadricostata, Hall, 1852, (Atrypa quadricostata,) Pal. N. Y., vol. 2, p. 68, Clinton $\mathrm{Gr}$.

ramsayi, Hall, 1859, Pal. N. Y., vol. 3, p. 446, Oriskany sandstone.

raricosta, Whitfield, 1882, Desc. New Spec. Foss., from Ohio, p. 201, Up. Held. Gr. recurvirostra, see Anazyga recurvirostra. reticulata, see Eichwaldia reticulata.

ricinula, Hall, 1858, Trans. Alb. Inst, vol. 4, p. 9, and Bull. Am. Mus. Nat. Hist., p. 53, Warsaw Gr.

ringens, Swallow, 1860, Trans. St. Louis Acad. Sci., vol. 1, p. 653, Burlington Gr. robusta, Hall, 1852, Pal. N. Y., vol. 2, p. 71, (Atrypa robusta,) Clinton Gr.

rockymontana, Marcou, 1858, (Terebratula rockymontana,) Geo. North America, p. 50, Coal Meas.

royana, see Stenoschisma royanum.

ridleyana, Safford, 1869, Geo. of Tenn. Not defined.

rudis, Hall, 1857, 10th Rep. N. Y. St. Mus. Nat. Hist., p. 75, and Pal. N. Y., vol. 3, p. 235, Low. Held. Gr.

rugosa. Hall, 1852, (Atrypa rugosa,) Pal. N. Y., vol. 2, p. 271, Niagara Gr.

saffordi, Hall, 1860, Can. Nat. and Geo., vol. 5, p. 144, Low. Held. Gr.

sageraua, Winchell, 1862, Proc. Acad. Nat. Sci., p. 407, Marshall Gr. 
sappho, see Stenoschisma sappho.

semiulicata, Conrad, 1841, (Atrypa semiplicata, ) Ann. Rep. N. Y., p. 56, and Pal. N. Y., vol. 3, p. 224, Low. Held. Gr. septata, Hall, 1859, Pal. N. Y., vol. 3, p. 443, Oriskany sandstone.

sordida, Hall, 1847, (Atrypa sordida) Pal. N. Y., vol. 1, p. 148, Trenton Gr.

speciosa, Hall, 1857, 10th Rep. N. Y. St. Mus. Nat. Hist., p. 81, and Pal. N. Y., vol. 3, p. 444 , Oriskany sandstone.

stephani, see Stenoschisma stephani.

stricklandi, Sowerby, 1839, (Terebratula stricklandi,) Murch. Sil. Syst., p. 631, Niagara Gr.

subcircularis, Winchell, 1862, Proc. Acad.

Nat. Sci., p. 408, Marshall Gr.

subcuboides. Not an American species.

subcuneata, Hall, 1856, Trans. Alb. Inst., vol. 4, p. 11, and Geo. Sur. Iowa, p. 658, Warsaw Gr.

subtrigona, sce Camerophoria subtrigona. subtrigonalis, Hall, 1847, (Atrypa subtrigonalis,) Pal. N. Y., vol. 1, 1. 145, 'Trenton Gr.

sulcoplicata, Hall, 1857, 10th Rep. N. Y. St. Mus. Nat. Hist., p. 76, and Pal. N. Y., vol. 3, p. 236, Low. Held. Gr.

tennesseensis, Roemer, 1860, Sil. Fanna West Tenn., p. 72, Niagara Gr. tethys, see Stenoschisma tethys.

tetraptyx, Winchell, 1865, Proc. Acad. Nat. Sci., p. 120, Kinderhook Gr.

texana, Shumard, 1859, Trans. St. Louis Acad. Sci., vol. 1, p. 393, Permian Gr. thalia, see Stenoschisma billingsi.

thera, Walcott, 1885, Monogr. U. S. Geo. Sur., vol. 8, p. 223, Subcarboniferous.

transversa, Hall, 1857, 10th Rep. N. Y. St. Mus. Nat. Hist., p. 74, and Pal. N. Y., rol. 3, p. 234, Low. Held. Gr.

tuta, S. A. Miller, 1881, Jour. Cin. Soc., Nat. Hist., vol. 4, p. 315, Burlington Gr. unica, Winchell, 1865, Proc. Acad. Nat. Sci., p. 122, Marshall Gr.

unisulcata, see Meristella unisulcata.

uta, Marcou, 1858, (Terebratula uta,) Geo.

N. Amer., p. 58, Coal Meas. This was subsequently described by Swallow as R. osagensis.

vellicata, Hall, 1857, 10th Rep. N. Y. St. Mus. Nat. Hist., p. 71, and Pul. N. Y., vol. 3, p. 230, Low. Held. Gr.

ventricosa, Hall, 1857, 10th Rep. N. Y. St. Mus. Nat. Hist., p. 78, and Pal. N. Y., vol. 3 , p. 238 , Low. Held. Gr.

venustula, Hall, 1867, Pul. N. Y., vol. 4, p. 346, Tully limestone. This was identified by Vanuxem, 1842, Geo. 3d Dist. N. Y., as Alrypa cuboides of Sowerby.

vicina, Billings, 1866, Catal. Sil. Foss. Antic., p. 44, Anticosti Gr.

warrenensis, Swallow, 1860, Trans. St. Louis Acad. Sci., vol. 1, p. 653, Ham. Gr.

wasatchensis, White, 1874, Rep. Invert. Foss., p. 19, and Geo. Sur. W. 100th Mer., vol. 4. p. 130 , Carb. whitiana, S. A. Miller, 1883, 2d Fd. Am. Pal. Foss., p. 297, Niagara Gr., from Waldron, Indiana. Proposed instead of R. whitii, Hall, 1863, Trans. Alb. Inst., vol. 4, p. 216, and also in 28th Rep. N. Y.St. Mus. Nat. Hist., p. 164, pl. 26, figs. 23-33, and again in the 11th Anu. Rep. Geol. and Nat. Hist. of Indiana, p. 307 , pl. 26 , figs. 23-33.

whitii, Winchell, 1862, Proc. Acad. Nat. Sci., p. 407, Marshall Gr.

uhiti, Hall, see R. whitiana.

wilsoni, Sowerby, 1816, ('Terebratula wilsoni,) Min. Concli., vol. 2. p. 38, Niagara Gr.

wortheni, sce Camarophoria wortheni.

Rhynchospira, Hall, 1859, Pal. N. Y., vol. 3, syn, for Retzia.

deveyi, see Retzia deweyi.

evax, see Retzia evax.

formosa, sce Retzia formosa.

lepida, see Retzia lepida.

nobilis, see Trematospira nobilis.

rectirostra, see Trematospira rectirostra. subglobosa, see Retria subglobosa.

sinuata, see Retzia sinuata.

Rry Nomotreta, Hall, 1879, 28th, Rep. N.

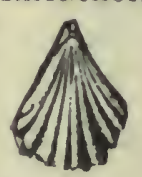

Fio. 607. Risyrio treti cuneata var. americnus Y. St. Mus. Nat. Hist., ए. 166. [Ety. rhynchos, beak ; tretos, with a hole in it.] Distinguished from Rhynchonella by the straight, produced, perforated beak of the ventral valve and divided deltidium, and by the crurse which rise near the dorsal beak, curve into the ventral cavity, and recurve to the clorsal side. Type $\mathrm{R}$. cuncata.

cuneata var.

a mericana,

Hall, 1879 ,

28th Rep. N.

Y. St. Mus.

Nat. Hist., p.

167, Niagara

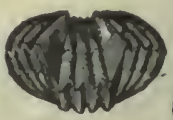

Gir.

Fro. 608.-Rhynchotreta quad rlpher1

quadriplicata, S. A. Miller, 1875, (Trematospira quadriplicata,) Cin. Quar. Jour. Sci., vol. 2, p. 60, 'l'renton Gr.

Rhynobolus, IIall, 1871, 23d Rep. N. Y. St. Mus. Nat. Hist., p. 247, syn. for 'Trimerella.

galtensis, Hall, see Trimerella galtensis.

Scirizambon, Walcott, 1885, Monogr. U. S. Geo. Sur., vol. 8, p. 69. [Ety. schiza, a cleft; ambon, the boss of a shield. Inequivalve, ovate; valves inarticulate; no area or deltidium; foramen oblong; structure calcareo-corneous; two sears in each valve. Type S. typicalis.

typicalis, Walcott, 1885 , Monogr. U. S. Geo. Sur., vol. 8, p. 70, Chazy Cir.

Scrizobouus, Ulrich, 1886, Cont. to Am. Pal., p. 25. [Ety. schiza, a cleft; Obolus, a genus.] Ventral valve with apex at the terminus of a notch in the posterior margin; two pair of adductor 
scars separated by a ridge; dorsal valve with truncated posterior margin; two pairs of muscular scars separated by a septum. Type S. truncatus.

truncatus, Hall, 1862, (Discina truncata,) 16th Rep. N. Y. St. Mus. Nat. Hist., p. 28, and Pal. N. Y., vol. 4, p. 23, Genesee slate to Chemung Gr.

Schizocrania, Hall \& Whitfield, 1875, Ohio Pal., vol. 2, p. 73. [Ety. schiza, a cleft; Crania, a genus.] Shell parasitic inequivalve, in-Frg. $609 .-$ articulated, surface of the nia flosa. upper valve finely striated; interior with six muscular scars. Type S. filosa.

filosa, Hall, 1847, (Orbicula (?) filosa,) Pal. N. Y., vol. 1, p. 99, Hud. Riv. and Utica Slate.

Siphonotreta, DeVerneuil, 1845, Russia and Ural Mountains, vol. 2, p. 286. [Ety. siphon, sipbon; tretos, with a hole in it.] Shell oblong oval, unarticulated; ven. tral valve most convex with a straight, thick, perforated, conical beak near the hinge-line; foramen opening on the back of the beak, and communicating with the interior of the shell by a cylindrical tube or siphon for the passage of the muscle of attachment; dorsal valve slightly convex, the hinge-line forming an arch which merges imperceptibly into the lateral margins; each valve has a wide, crescent-shaped cardinal edge, covered by horizontal lines
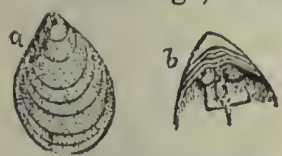

FrG. 610. - Siphonotreta unguiculata. $a$, Ventral valve; $b$. interior of same. of growth; struc. ture calcareo-corneous, with a distinctly punctured structure arranged in tubular layers; surface smooth, with numerous lines of growth and slender hollow spines dilated at the base. Type S. unguiculata.

scotica, Davidson, 1877, Geol. Mag., new ser., vol. 4, p. 13, Utica slate.

Skenidium, Hall, 1860, 13th Rep. N. Y. St. Mus. Nat. His., p. 70. [Ety. skenidion, a little tent.] Distinguished from Orthis by its large triangular area; the cardinal process extends as a median septum through the length of the shell, and may be simple or divided at the extremity. Type S. insigne.

devonicum, Walcott, 1885, Monogr. U. S. Geo. Sur., vol. 8, p. 116, Devonian.

halli, Safford, 1869, Geo. of Tenn. Not defined.

insigne, Hall, 1859, (Orthis insignis,) Pal. N. Y., vol. 3, p. 173, Low. Held. Gr.

pyramidale, Hall, 1852, (Orthis pyramidalis,) Pal. N. Y., vol. 2, p. 251, Niagara Gr.

Spirifera, Sowerby, 1815, Min. Conch., vol. 2, p. 42, and Linnæean Trans., vol. 12, p. 514. '[Ety. spira, spire ; fero, to bear.]
Triangular semicircular, transversely elongate, subglobose or otherwise variable in form, with or without mesial fold and sinus; structure impunctate; surface smooth, striated or plicated; cardinal line straight, 'area in each valve; hinge articulated by short teeth and sockets; area of the ventral valve larger than the other, and divided by a triangular foramen more or less closed by a false deltidium; area of the dorsal valve divided in the middle by a fissure occupied by the cardinal muscular process ; beak of ventral valve more prominent than that of the otber; in the interior of the dorsal valve the spiral supports of the labial arms are attached by their crura to the hinge plates, some distance from which they are nearly or quite connected by a small process extending inward from each; the cardinal muscles seem to have been attached to the cardinal process, under and in front of which four scars of the adductor muscles occur; on each side of a mesial ridge in the ventral valve occur the scars of the adductors, and outside of these the scars of the cardinal muscles. Type S. striata.

acanthoptera, Conrad, 1842, (Delthyris acanthoptera,) Jour. Acad. Nat. Sci., vol. 8, p. 264, Chemung Gr.

acuminata, Conrad, 1839, (Delthyris acuminata,) Ann. Rep. N. Y., p. 65, and Pal. N. Y., vol. 4, p. 198 , Up. Held. and Ham. Grs.

acuticostata, DeKoninck, 1843 , Desc. Ann. Foss. Terr. Carb. Belg., p. 265, Subcarboniferoús.

agelaia, Meek;'1873, Hayden's Geo. Sur. Terr., p. 470, and White's Cont. to Pal. No. 6 , p. 135 , Subcarboniferous.

alata, Castelnau, 1843, Syst. Si]., p. 42. Not recognized.

albapinensis, Hall \& Whitfield, 1877 , U. S. Geo. Expl. 40th parallel, vol. 4, p. 255, Waverly Gr.

aldrichi, Etheridge, 1878, Quar. Jour. Geo. Soc., vol. 34, p. 634, Devonian.

alta, Hall, 1867, Pal. N. Y., vol. 4. p. 248 , Chemung Gr.

amara, Swallow, 1860, Trans. St. Louis Acad. Sci., vol. 1, p. 642, Waverly or Choteau Gr.

angusta, Hall, 1857, 10th Rep. N. Y. St. Mus. Nat. Hist., p. 164, and Pal. N. Y., vol. 4, p. 230, Ham. Gr.

annæ, Swallow, 1860, Trans. St. Louis Acad. Sci., vol. 1, p. 641, Ham. Gr.

annectans, Walcott, 1885, Monogr. U. S. Geo. Sur., vol. 8, p. 216, Subcarboniferous.

arata, syn. for S. granulifera.

archiaci, see $\mathrm{S}$. disjuncta.

aretica, Haughton, 1857, Jour. Roy. Soc.

Dub., vol. 1, p. 183, Devonian.

arctisegmenta, Hall, 1857, 10th Rep. N. Y. St. Mus. Nat. Hist., p. 131, and Pal.

N. Y., vol. 4, p. 208, Up. Held. Gr. 
arenosa, Conrad, 1839, (Delthyris arenosa,) Ann. Rep. N. Y., p. 65, and Pal. N. Y., vol. 3, p. 425, Oriskany sandstone.

argentaria, Meek, 1877, U. S. Feo. Sur. 40th Parallel, p. 42, Devonian.

arrecta, Hall, 1859, Pal. N. Y., vol. 3, p. 422, Oriskany sandstone.

aspera, Hall, 1858, Geo. Rep. Iowa, p. 508, Ham. Gr.

asperata, Ringueberg, 1886, Bull. Buf. Soc. Nat. Sci., vol. 5, p. 16, Niagara Gr.

atwaterana, S. A. Miller, 1878, Proc. Davenport Acad. Sci., vol. 2, p. 221, Ham. Gr. Proposed instead of S. pennata, Owen, which was preoccupied.

audacula, Conrad, (Delthyris audacula,) 1842, Jour. Acad. Nat. Ści., vol. 8, p. 262, Ham. Gr.

belphegor, Clarke, 1885, Bull. U. S. Geo. Sur., No. 16, p. 30 , Genesee shales.

bialveata, Conrad, (Delthyris bialveala, 1842, Jour. Acad. Nat. Sci., vol. 8, p. 261, Niagara Gr. Probably a syn. for S. radiata.

bicostata, Vanuxem, 1842, (Orthis bicostatus,) Geol. Rep. 3d Dist. N. Y., p. 91, and Pal. N. Y., vol. 2, p. 263, Niagara Gr.

bicostata var. petila, Hall, 1879, Desc. New Spec. Foss., p. 15, and 11th Rep. Geo. and Nat. Hist. Ind., p. 297, Niagara Gr.

bidorsalis, Winchell, 1866, Rep. Iow. Penin. Mich., p. 93, Ham. Gr.

bifurcata, Hall, 1858, Trans. Alb. Inst., vol. 4, p. 8, and Bull. Am. Mus. Nat. Hist., p. 47, Warsaw Gr.

billingsana, n. sp., Upper Devonian Gaspe limestone, No. 8. Proposed instead of S. superbn, Billings, 1874, Pal. Foss., vol. 2 , p. 45 , which name was preoccupied.

biloba, Linn:eus, 1768, (Anomia biloba) Syst. Nat., p. 115 , and Pal. N. Y., vol. 2, p. 260 , Niagara Gr.

bimesialis, Hall, 1858, Geo. Rep. Iowa, vol. 1 , pt. 2 , p. 507, Ham. Gr.

biplicata, Hall, 1858, Geo. Rep. Iowa, vol. 1 , pt. 2 , p. 519 , Kinderhook (ir.

boonensis, Swallow, 1860, Trans. St. Louis Acad. Sci., vol. 1, p. 646, Low Coal Meas.

brachynota, Hall, 1843, (Delthyris bracliynota, Geo. 4th Dist. N. Y., p. 71, Clinton Gr. Not well defined.

calcarata, syn. for S. disjuncta.

camerata, Morton, 1836, Am. Jour. Sci., vol. 29 , p. 150 , Coal Meas.

camerata var. kansasensis, Swallow, 1866, Trans. St. Louis Acad. Sci., vol. 2, p. 409, Coal Meas.

camerata var. percrassa, Swallow, 1866, Trans. St. Louis Acad. Sci., vol. 2, p. 409 , Coal Meas. This name was preoccupied as a species.

capax, Hall, 1858, Geo. Rep. Iowa, vol. 1 , pt. 2, p. 520 , syn. for $S$. parryana.

carteri, Hall, 1857, 10th Rep. N. Y. St. Mus. Nat. Hist., p. 170, Waverly Gr. cedarensis, Owen, 1852, Geo. Sur. Wis., Iowa, and Minn., p. 585, Ham. Gr.

centronota, Winchell, 1865, Proc. Acad. Nat. Sci., p. 118, and Geo. Sur. IV. 100th Mer., vol. 4, p. 87, Cuyahoga Shale.

clara, Swallow, 1853, Trans. St. Louis Acad. Sci., vol. 2, p. 86, Kaskaskia Gr.

claratula, McChesney, 1861, Desc. New Pal. Foss., p. 84, Burlington Gr. Not recognized.

clintoni, syn. for S. granulifera.

clio, syn. for S. riczac.

compacta, Meek, 1868, Trans. Chi. Acad. Sci., p. 102, Ham. Gr.

concinna, Hall, 1857, 10th Rep. N. Y. St. Mus. Nat. Hist., p. 60, Low. Held. Gr. congesta, syn. for $\mathbf{S}$. granulifera.

conradana, S. A. Miller, 1583, 2d Ed. An. Pal. Foss., p. 298, Oriskany, Up. IIeld. and Ham. Grs. Proposed instesd of $\mathrm{S}$. finbriata of Conrad in Jour. Acud. Nat. Sci., vol. 8, p. 263, and Pal. N. Y., vol. 4 , p. 214, which was preoccupied. consobrina, D'Orbigny, 1850, Prodr. d.

Paléont, t. 1, p. 98, Ham. Gr. Proposed instead of S. ziczac, Hall, 1843, Geo. Rep. 4th Dist. N. Y., p. 200, which was preoccupied by Koemer.

consors, Winchell, 1866, Rep. Low. Peninsula Mich., p. 93, Ham. Gr.

cooperensis, Swallow, 1860, Trans. St. louis Acad. Sci., vol. 1, p. 643, Waverly or Choteau Gr.

corticosa, Hall, 1857, 10th Rep. N. Y. St. Mus. Nat. Hist., p. 160, Ham. Gr. costalis, Castelnau, 1843, Syst. Sil., p. 41. Not recognized.

crenistriata, see Streptorhynchus crenistriatum.

crispa, Hisinger, 1826, (Terebratula crispa,) Act. Acad. Sci., Holm., t. 7, fig. 4, and Pal. N. Y., vol. 2, p. 262, Niagara Gr. crispa var. simplex, Hall, 1879, 28tl Rep. N. Y. St. Mus. Nat. Hist., p. 157 , Niagara Gr.

cumberlandire, Hall, 1857, 10th Rep. N. Y. St. Mus. Nat. Hist., p. 63, and Pal. N. Y., vol. 3, p. 421, Oriskany sandstone.

cuspidatiformis, n.sp., Keokuk Gr. Proposed instead of S. subcuspidata, Hall, 1858, Geo. Sur. Iowa, p. 646, pl. 20, fig. $5 a, b$, which name was preoccupied. eycloptera, Hall, 1857, 10tl Rep. N. Y. St. Mus. Nat. Hist., p. 58, and Pal. N. Y, vol. 3, p. 199, Low. Held. Gr. cyrtiniformis, Hall \& Whitfield, 1873, 23d Rep. N. Y. St. Mus. Nat. Hist., p. 238, Chemung Gr.

decemplicata, Hall, 1843, (Delthyris decemplicata,) Geo. Rep. 4 th Dist. N. Y., p. 106, Niagara Gr.

desiderata, Walcott, 1885, Monogr. U. S. Geo. Sur., vol. 8, p. 2I7, Subcarb.

disjuncta, Sowerby, 1840, Trans. Geo. Soc., $2 \mathrm{~d}$ ser., vol.5, p. 704, and Pal. N. Y., vol. 4, p. 243 , Chemung Gr.

disparilis, Hall, 1857, 10th Rep. N. Y. St. Mus. Nat. Hist., p. 134, and Pal. N. Y., vol. 4, p. 204, Up. Held Gr. 
distans, syn. for S. disjuncta.

divaricata, Hall, 1857, 10th Rep. N. Y. St. Mus. Nat. Hist., p. 133, and Pal. N. Y., vol. 4, p. 213, Cornif. and Ham. Grs.

dubia, see Pentamerella dubia.

duodenaria, Hall, 1843, (Delthyris duodenaria,) Geol. 4th Dist. N. Y., p. 171, and Pal. N. Y., vol. 4 , p. 189 , Schoharie grit and Cornif. Gr.

dupliplicata, Conrad, 1842, (Delthyris dupliplicata, Jour. Acad. Nat. Sci., vol. 8, p. 261, Hám. Gr.

eatoni, see S. medialis var. eatoni.

engelmanni, Meek, 1860, Proc. Acad. Nat. Sci., p. 308, and Geo. Sur. Ill., vol. 3, p. 398 , Oriskany sandstone.

eudora, Hall, 1861, Rep. of Prog. Wis. Sur., p. 25, Niagara Gr.

euruteines, Owen, 1844, (Delthyris euruteines, Report on Min. Lands, p. 74, and Pal. N.Y., vol. 4, p. 209, Up. Held.Gr. euruteines var. fornacula see $\mathbf{S}$. fornacula.

exporrecta, see Cyrtia exporrecta.

exporrecta var. arrecta, see Cyrtia exporrecta var. arrecta.

extensa, syn. for $\mathrm{S}$. disjuncta.

extenuata, Hall, 1858, Geo. Rep. Iowa, p. 520, Kinderhook Gr.

fasciger, Keyserling in Owen's report, see Spirifera camerata.

fastigata, Morton, 1836, Am. Jour. Sci. and Arts, vol. 29, p. 149, Coal Meas.

fastigata, Meek \& Worthen, 1870, Proc. Acad. Nat. Sci., p. 36 . The name was preoccupied by Morton. See S. mortonana.

filicosta, Winchell, 1866, Rep. Low. Peninsula Mich., p. 94, Ham. Gr.

fimbriata, Morton, 1836, Am. Jour. Sci. and Arts, vol. 29, p. 149, Coal Meas.

fimbriata, Conrad. The name was preococcupied. See S. conradana.

fischeri, Castelnau, 1843, Syst. Sil., p. 42. Not recognized.

forbesi, Norwood \& Pratten, 1854, Jour. Acad. Nat. Sci., vol. 3, p. 73, Burlington Gr.

formosa, Hall, 1857, 10th Rep. N. Y. Mus. Nat. Hist., p. 154, and Pal. N. Y., vol. 4, p. 220, Ham. Gr.

fornacula, Hall, 1857, 10th Rep. N. Y. Mus. Hist., p. 154, Ham. Gr.

fornax, Hall, 1857, 10th Rep. N. Y. Mus. Nat. Hist., p. 155, Ham. Gr.

franklini, Meek, 1868, Trans. Chi. Acad. Sci, p. 107, Ham. Gr.

fultonensis, Worthen, 1873, Geol. Rep. Ill., vol. 5, p. 572, Low. Coal Meas.

gaspensis, Billings, 1874, Pal. Foss., vol. 2, p. 74, Devonian.

gibbosa, Hall, 1861, Rep. of Progr. Wis. Sur., p. 25, Niagara Gr.

gigantea, syn. for. $\mathrm{S}$. disjuncta.

glabra, Martin, 1809, (Anomites glabra,) Petrif. Derb., tab. 28, figs. 9 and 10, Subcarboniferous.

glabra var. contracta, Meek \& Worthen, 1861, Proc. Acad. Nat. Sci., p. 143, and Geo.Sur. Ill.,'vol. 2, p. 298, Kaskaskia Cr. glabra var. nevadensis, Walcott, 1885, Monog. U. S. Geo. Sur., vol. 8, p. 139, Up. Devonian.

glanscerasi, White, 1862, Proc. Bost. Soc. Nat. Hist., vol. 9, p. 8, Ham. Gr.

grandæva, syn. for S. disjuncta.

granulifera, Hall, 1843, (Delthyris granulifera,) Geol. 4th Dist. N. Y. p. 207, and

Pal. N. Y., vol. 4, p. 223, Ham. Gr.

granulosa, Conrad, 1839, (Delthyris granulosa,) Ann. Rep. N. Y., p. 65, Low. Held. Gr.
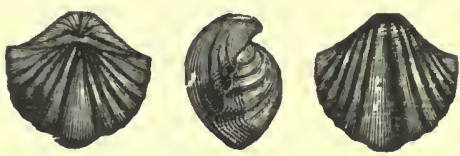

FrG. 611.-Spirifera gregaria.

gregaria, Clapp, 1857, 10th Rep. N. Y. Mus. Nat. Hist., p. 127, and Pal. N. Y., vol. 4, p. 195, Up. Held. Gr.

grieri, Hall, 1857, 10th Rep. N. Y. Mus. Nat. His., p. 127, and Pal. N. Y., vol. 4, p. 194, Schoharie grit and Up. Held. Gr.

grimesi, Hall, 1858, Geo. Rep. of Iowa, p. 604 , Burlington Gr.

guadalupensis, Shumard, 1859, Trans. St. Louis Acad. Sci., vol. 1, p. 391, Permian Gr.

hannibalensis, Swallow, 1860, Trans. St. Louis Acad. Sci., vol. 1, p. 647, Waverly or Choteau Gr.

hemicycla, Meek \& Worthen, 1868, Geo. Sur. Ill., vol. 3, p. 399, Oriskany sandstone.

hemiplicata, see Syntrielasma hemiplicatum.

heteroclitus, syn. for S. granulifera.

hirtus, White \& Whitfield, 1862, Proc. Bost. Soc. Nat. Hist., vol. 8, p. 293, Kinderbook Gr.

hungerfordi, Hall, 1858, Geo. Rep. Iowa, vol. 1 , pt. 2 , p. 501, Ham. Gr.

huronensis, Winchell, 1862, Proc. Acad. Nat. Sci., p. 405, Portage Gr.

huronensis, Castelnau, 1843, Syst. Sil., p. 41. Not recognized.

imbrex, Hall, 1858, Geo. Rep. Iowa, p. 601, Burlington Gr.

inæquivalvis, Castelnau, 1843, Syst. Sil., p. 40. Not recognized.

incerta, Hall, 1858, Geo. Rep. Iowa, p. 602, Burlington Gr.

incomstans, syn. for Spirifera racinensis.

increbescens, Hall, 1858, Geo. Rep. Iowa, p. 706, Kaskaskia Gr.

increbescens var. americana, Swallow, 1866, Trans. St. Louis Acad. Sci., vol. 2, p. 410, Kaskaskia Gr.

increbescens var. transversalis, Hall, 1858 , Geol. Rep. Iowa, p. 708, Kaskaskia Gr. inæquicostata, Owen, 1852, Geo. Rep. Wis., Iowa, and Min., p. 586, Carb.

inornata, syn. for S. disjuncta.

insolita, Winchell, 1862, Proc. Acad. Nat. Sci., p. 405, Portage Gr. 
intermedia, Hall, 1859, Pal. N. Y., vol. 3, p. 424, Oriskany sandstone. This name was preoccupied by Brongniart in 1829 .

inutilis, Hall, 1858, Geo. Rep. Iowa, vol. 1, pt. 2, p. 505, Ham. Gr.

iowensis, Owen, 1852, Geo. Sur. Wis., Iowa, and Min., p. 585, Ham. Gr.

kelloggi, Swallow, 1863, Trans. St. Louis Acad. Sci., vol. 2, p. 86, Keokuk Gr.

kennicotti, Meek, 1868, Trans. Chi. Acad. Sci., p. 101, Ham. Gr.

kentuckensis, see Spiriferina kentuckiensis.

kentuckensis var. propatula, see Spiriferina kentuckiensis var. propatula.

keokuk, Hall, 1858, Geo. Rep. Iowa, p. 642, Keokık Gr.

keokuk var. shelbyensis, Swallow, 1S66, Trans. St. Louis Acad. Sci., vol. 2, p. 410, Keokuk Gr.

lævigata, Swallow, 1853, Trans. St. Louis Acad. Sci., vol. 2, p. 86, Keokuk Gr.

lavis, Hall, 1843, (Delthyris lrevis,) Geol. 4 th Dist. N. Y., p. 345 , and Pal. N. Y., vol. 4, p. 239, Portage Gr.

lamellosa, see Athyris lainellosa.

laminosus, McCoy, as identified by Geinitz, is Spiriferina kentuckiensis.

lateralis, Hall, 1858, Geo. Rep, Iowa, p. 661, Warsaw Gr.

latior, Swallow, 1863, Trans. St. Louis Acad. Sci., vol. 2, p. 86, Waverly or Choteau Gr.

leidyi, Norwood \& Pratten, 1855, Jour. Acad. Nat. Sci., 2d series, vol. 3, p. 72, Kaskaskia Gr.

leidyi var. chesterensis, Swallow, 1866, Trans. St. Louis Acad. Sci., vol. 2, p. 409, Kaskaskia Gr.

leidyi var. merrimacensis, Swallow, 1866, Trans. St. Louis Acad. Sci., vol. 2, p. 410, Warsaw Gr.

ligus, Owen, 1852, Rep. Geo. Sur. Wis., Iowa, and Minn., p. 585, Ham. Gr.

lineatoides, Swallow, 1860, Trans. St. Iouis Acad. Sci., vol. 1, p. 645, Burlington Gr.

lineata, Martin, 1809, (Conchiliolithus Anomites lineatus,) Petrif. Derb., tab. 36, fig. 3 , and 13th Rep. Geo. Sur. Ind., p. 133, Coal Meas.

lineata var. striato-lineata, Swallow, 1S66, Trans. St. Louis Acad. Sci., vol. 2, p. 408, Coal Meas.

littoni, Swallow, 1860, Trans. St. Louis Acad. Sci., vol. 1, p. 646, St. Louis Gr.

logani, Hall, 1858, Geo. Rep. Iowa, p. 647, Keokuk Gr.

lonsdalii, syn. for S. disjuncta.

macra, Hall, 1857, 10th Rep. N. Y. Mus. Nat. Hist., p. 134, and Pal. N. Y., vol. 4, p. 190, Scholarie grit and Up. Ileld. Gr. macra, Meek. This name was preoccupied. See S. strigosa.

macronota, Hall, 1843, (Delthyris macronota,) Geo. 4th Dist. N. Y., p. 206, and Pal. N. Y., vol. 4, p. 231, Ham. Gr.

macropleura, Conrad, 1840, (Delthyris macropleura,) Ann. Rep. N. Y., p. 217, and Pal. N. Y., vol. 3, p. 202, Low. Held. Gr. macropleura, Castelnau, 1843, Syst. Sil., p. 41. The name was preoccupied.

macroptera, as identified by d'Archiac \& Verneuil, is $\mathrm{S}$. pennata.

macrothyris, Hall, 1857,10 th Rep. N. Y. Mus. Xat. Hist., p. 132, and Pal. N. Y., vol. 4, p. 202, Up. Held. Gr.

maia, Billings, 1860, (Athy ris maia,) Can. Jour. Ind. Sci. and Arts, vol. 5, p. 276, Up. Held. Gr.

manni, IIall, 1857, 10th Rep. N. Y. Mus. Nat. Ilist., p. 128, and Pal. N. Y., vol.4, p. 211, Up. Held. Gr.

marcyi, Hall, 1857, 10th Rep. N. Y. Mus. Nat. Hist., p. 158, and I'al., N. Y., vol. 4, p. 226, Нam. Gr.

marionensis, Slummard, 1855, Geo. Rep. Mo., p. 203, Waverly or Clioteau Gr. medialis, Hall, 1843, (Delthyris medialis,) Geo. 4th Dist. N. Y., p. 208, and Pal. N. Y., vol. 4, p. 207, Ham. Gr.

medialis var. entoni, Hall, 1857, (Spirifer eatoni,) 10th Rep. N. Y. Mus. Nat. Hist., p. 157, and Pal. N. Y., vol. 4, P. 2:29, Hain. Gr.

meeki, Swallow, 1860, Trans., St. Louis Acad. Sci., vol. 1, p. 645 , Burlington Gr. meristoides, Meek, 1868, Trans. Chi. Acad. Sci., p. 106, Ham. Gr.

mesacostalis, Hall, 1843, (Delthyris mesacostalis and D. acuminata,) Fen. 4th Dist. N. Y., p. 269, and Pal. N. Y., vol. 4, p. 240, Chemung Gr.

mesastrialis, Iall, 1843, (Delthyris mesastrialis, Geo. th Dist. N. Y., p. 269 , and Pal. N. Y., vol. 4, p. 242, Hain. and Chemung Gr.

meta, Hall, 1867, 20th Rep. N. Y. Mus. Nat Hist., p. 380, Niagara Gr.

meusebachianus, syn. for Spirifera cam. erata.

mexicana, Shumard, 1858, Trans. St. Jouis Acad. Sei., vol. 1, p. 292, Permian Gr.

missouriensis, Swallow, 1860, Trans. St. Louis Acad. Sci., vol. 1, p. 643, Waverly or Chotean Gr.

modesta, IIall. 1857, 10th Rep. N. Y. Mus. Nat. IIist., p. 61, and Pal. N. Y., vol. 3, p. 203, Low Held. Gr.

mortonana. S. A. Miller, 1883, $2 \mathrm{~d}$ Ed., $\mathbf{A}$. p. 298, Pal. Foss. Keokuk Gr. Proposed instead of $\mathrm{S}$. fastigata of Meek and Worthen, 1870, in Proc. Acad. Nat. Sci., p. 36 , and afterward in Gco. Sur. Ill., vol. 6, p. 521, pl. 30 , fig. 3, from Crawfordsville, Indiana.

mucronata, Conrad, syn. for S. pennata. multicostala, Castelnau, 1843, Syst. Sil., p. 42. Not recognized.

multigranosa, Worthen, (in press,) Geo. Sur. Ill., vol. 8, p. 105, Coal Meas.

multistriata, see Trematospira inultistriata. murchisoni, Castelnau, 1843, Syst. Sil., p. 41. Not recognized.

mysticensis, Meek, 1873, Hayden's Geo. Sur. Terr. 6th Rep., p. 466. Not satisfactorily defined.

neglecta, Hall, 1858, Geo. Rep. Iowa, p. 642, Keokuk Gr. 
newberryi, Hall, 1883, Rep. St. Geol. pl. 56 , fig. 9,10 , Waverly Gr.

niagarensis, Conrad, 1842, Jour. Acad. Nat. Sci., vol. 8 , p. 261 , and Pal. N. Y., vol. 2, p. 264, Niagara Gr.

niagarensis var. oligoptycha, Roemer, 1860, Sil. Fauna West Tenn., p. 68, Niagara Gr.

nictavensis, Dawson, 1868, Acad. Geol., p. 499, Devonian.

norwoodana, Hall, 1858, Trans. Alb. Inst., vol. 4, p. 7, and Bull. Am. Mus. Nat. Hist., p. 48, Warsaw Gr.

norwoodi, Meek, 1860 , Proc. Acad. Nat. Sci., vol. 12 , p. 308 , Devonian.

novamexicana, S. A. Miller, 1881, Jour. Cin. Soc. Nat. Hist., vol. 4, p. 314, Burlington Gr.

nympha, Billings, 1863, Proc. Port. Soc. Nat. Hist., vol. 1, p. 116, Low. Held. Gr.

octocostata, Hall, 1857, 10th Rep. N. Y. Mus. Nat. Hist., p. 62, and Pal. N. Y., vol. 3, p. 205, Low. Held. Gr.

opima, Hall, 1858, Geo. Rep. Iowa, p. 711, syn. for S. rockymontana.

orestes, Hall \& Whitfield, 1873, 23d Rep. N. Y. Mus. Nat. Hist., p. 237, Chemung Gr.

oregonellsis, Shumard, 1863, Trans. St. Louis Acad. Sci., vol. 2, p. 108, Coal Meas.

osagensis. Swallow, 1860, Trans. St. Louis Acad. Ści., vol. 1, p. 641, Waverly or Choteau Gr.

oweni, Hall, 1857, 10th Rep. N. Y. Mus. Nat. Hist., p. 129, Up. Held. Gr.

pachyptera, Goldfuss, as identified by Conrad in 1839, (Delthyris pachyptera). Not American.

parryana, Hall, 1858, Geo. Rep. Iowa, vol. 1, pt. 2, p, 509, Ham. Gr.

peculiaris, Shumard, 1855, Geo. Rep. Mo., p. 202, Waverly or Choteau Gr.

pennata, Atwater, 1820, (Terebratula pennata, Am. Jour. Sci. and Arts, vol. 2 , p. 242, Ham. Gr.

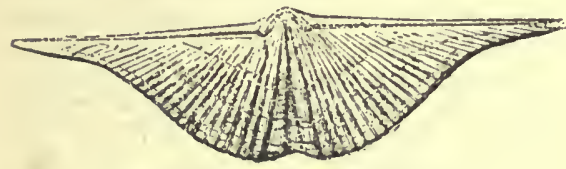

Fra. 612.-Spirifera pennata.

pennata, Owen. The name was preoccupied, see S. atwaterana.

percrassa, McCoy, 1855, Brit. Pal. Rocks., p. 194, Sil. Not satisfactorily identified in America.

perextensa, Meek \& Worthen, 1868, Geo. Sur. Ill., vol. 3 , p. 414, Ham. Gr.

(?) perforata, see Trematospira perforata. perlamellosa, Hall, 1857, 10th Rep. N. Y. Mus. Nat. Hist., p. 57, and Pal. N. Y., vol. 3, p. 200, Low. Held. Gr.

perplexa, McChesney, 1860, New Pal. Foss., syn. for S. lineata. pertenuis, Hall, 1857, 10th Rep. N. Y. Mus. Nat. Hist., p. 163, Ham. Gr.

pharovicina, Winchell, 1862, Proc. Acad. Nat. Sci., p. 405, Portage Gr.

pinonensis, Meek, 1870, Proc. Acad. Nat. Sci., p: 60, and Expl. 40th Parallel, vol. 4, p. 45, Ù . Held. Gr.

planoconvexa, Shumard, 1855, Geo. Rep. Mo., p. 202, Coal Meas.

plena, Hall, 1858, Geo. Rep. Iowa, p. 603, Burlington Gr.

plicata, Vanuxem, 1843, see S. vanuxemi. pluto, Clarke, 1885, Bull. U. S. Geo. Sur.,

No. 16, p. 31 , Genesee shales.

præmatura, Hall, 1867, Pal. N. Y., vol. 4, p. 250, Chemung Gr.

prolata, Vanuxem, 1842, (Delthyris prolata,) Geo. Rep. N. Y., p. 181, Chemung Gr.

propinqua, Hall, 1858, Geo. Rep. Iowa, p. 647, Keokuk Gr.

prora, Conrad, 1842, (Delthyris prora,) Jour. Acad. Nat. Sci., vol. 8, p. 263, Ham. Gr.

protensa, syn. for. S. disjuncta.

pseudolineata, Hall, 1858, Geo. Rep. Iowa, p. 645 , Keokuk Gr.

pulchra, Meek, 1860, Proc. Acad. Nat. Sci., p. 310, and Simpson's Gt. Basin of Utah, p. 352, Coal Meas.

pyramidalis, see Cyrtina pyramidalis.

pyxidata, Hall, 1859, Pal. N. Y., vol. 3, p. 428 , Oriskany sandstone.

racinensis, McChesney, 1860, Pal. Foss., p. 84, Niagara Gr.

radiata, Sowerby, 1839, Murch. Sil. Syst., p. 637 , and Pal. N. Y., vol. 2, pp. 66, 265, Niagara Gr.

raricosta, Conrad, 1842, (Delthyris raricosta,) Jour. Acad. Nat. Sci., vol. 8, p. 262 , and Pal. N. Y., vol. 4, p. 192, Schoharie grit and Up. Held. Gr.

resupinata, as identified by d'Archiac \& Verneuil. Not American.

richardsoni, Meek, 1868, Trans. Chi. Acad. Sci., p. 104, Ham. Gr.

rockymontana, Marcou, 1858, Geo. N. Amer., p. 50, Coal Meas.

rostellata, Hall, 1858, Geo. Rep. Iowa, p. 641, Keokuk Gr.

rostellum, Hall \& Whitfield, 1872, 24th Rep. N. Y. Mus. Nat. Hist., p. 182, Niagara $\mathrm{Gr}$.

rostrata, Morton, 1836, Am. Jour. Sci. and Arts, vol. 29, p. 149, Coal Meas.

rugicosta, Hall, 1860, Can. Nat. Geo., vol. 5, p. 144, Up. Sil.

rugatina, Conrad, 1842, (Delthyris rugatina,) Jour. Acad. Nat. Sci., vol. 8, p. 261, Niagara Gr.

saffordi, Hall, 1859, Pal. N. Y., vol. 3, p. 203, Low. Held. Gr.

scobina, Meek, 1860, Proc. Acad. Nat. Sci., p. 310, and Simpson's Gt. Basin of Utah, p. 351, Coal. Meas.

sculptilis, Hall, 1843, (Delthyris sculptilis,) Geo, Rep. 4th Dist. N. Y., p. 202, and Pal. N. Y., vol. 4, p. 221, Ham. Gr. 
gegmenta, Hall, 1857, 10th Rep. N. Y. Mus. Nat. Hist., p. 131, and Pal. N. Y., vol. 4, p. 207, Up. Held. Gr.

semiplicata, Hall, 1860, 13th Rep. N. Y. Mus. Nat. Hist., p. 111, Kinderhook Gr.

setigera, Hall, 1858, Geo. Rep. Iowa, p. 705, Kaskaskia Gr.

sheppardi, Castelnau, 1843, Syst. Sil., p. 42. Not recognized, but probably a variety of Orthis lynx.

sillana, Winchell, 1865, Proc. Acad. Nat. Sci., p. 119 , Cuyahoga shale.

similior, see Pentamerus similior. solidirostris, White, 1860 , Bost. Jour. Nat.

Hist., vol. 7, p. 232, Kinderhook Gr.

sowerbyi, Castelnau, 1843, Syst. Sil., p. 43. Not recognized.

spinosa, see Spiriferina spinosa.

staminea, Hall, 1843, Geo. Rep. 4th Dist.

N. Y., p. 105, Niagara Gr.

striatiformis, Meek, 1875, Ohio Pal., vol. 2, p. 289, Waverly Gr.

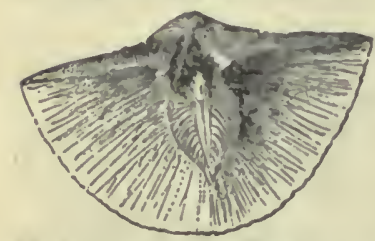

EIG. 613.-Spirifera striala. Interior of ventral valve.

North America, p. 49, Subcarboniferous.

substriatulus,

as identi-

fied by

d'Archiac

\& Ver-

neuil. Not

American.

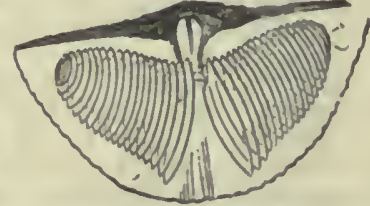

strig o s a, Fig. 614.-Spirifera strinta. InMeek, 1860, terlor of dorsal valve.

Proc.Acad.

Nat. Sci., p. 309, and Simpson's Rep. Gt. Basin of Utah, p. 347, Devonian. Proposed instead of S. macra, Meek, which was preoccupied.

subrqualis, Hall, 1858, Geo. Rep. Iowa, p. 663, Warsaw Gr.

subattenuata, Hall, 1858, Geo. Rep., Iowa, index, p. 3, Ham. Gr.

subcardiformis, Hall, 1858, Geo. Rep. Iowa, p. 660, Warsaw Gr.

subcuspidala, Hall, 1858, Geo. Rep. Iowa, p. 646, Keokuk Gr. Preoccupied by Schnur in 1831. See S. cuspidatiformis. subdecussata, Whiteaves, 1887, Cont. to Can. Pal., vol. 1, p. 114, Ham. Gr.

subelliptica, McChesney, 1860, New Pal.

Foss. Not recognized, Coal Meas.

sublineata, Meek, 1868, Trans. Chi. Acad.

Sci., p. 103, Ham. Gr.

submucronata, Hall, 1857, 10th Rep.

N. Y. Mus. Nat. Hist., p. 62, and Pal.

N. Y., vol. 3, p. 419 , Oriskany sandstone. submucronata, Hall, 1858, Geo. Rep. Iowa, vol. 1, pt. 2, Ham. Gr. This name was preoccupied. Sec S. subattenuata.

suborbicularis, Hall, 1858, Geo. Rep. Iowa, p. 644, Keokuk Gr.

subrotundata, Hall, 1858, Geo. Rep. Iowa, vol. 1, pt. 2, p. 521, Kinderhook Gr.

subsulcata, Hall, 1860, Can. Nat. and Geol., vol. 5, Up. Sil. This name was preoccupied by Dalman in 1828.

subumbonala, see Martinia subumbonata. subundifera, Meek \& Worthen, 1865. Geo. Sur. Ill., vol. 3, p. 434, Ham. Gr.

suhvaricosa, Hall \& Whitfield, 1873, 23d Rep. N. Y. Mus. Nat. Hist., p. 237, Up. Held. Gr.

subventricosa, McChesney, syu. for S. rockymontana.

suleata, Hisinger, 1831, (Deltly ris suleatus,) Anteckn. Physik. Och. Geognosi., p. 119, Pal. N. Y., vol. 2, 1. 261, Niagara $\mathrm{Gr}$.

sulcifera, Shumard, 1858, Trans. St. Louis Acad. Sci., vol. 1, p. 293, Permian Gr. superba, Billings, 1874, I'al. Foss., vol. 2, p. 45 , Devonian. The name was preoccupied by Eichwald in 1842 . See S. billingsana.

taneyensis, Swallow, 1860, Trans. St. Louis Acad. Sci., vol. 1, p. 645, Kinderhook Gr.

temeraria, S. A. Miller, 1881, Jour. Cin. Soc. Nat. Hist., vol. 4, p. 314 , Burlington Gr.

tenuicostata, Hall, 1858, Geo. Rep. Iowa, p. 662, Warsaw Gr.

tenuinarginata, Hall, 1858, Geo, Rep. Iown, p. 641, Keokuk Gr.

tenuis, Hall, 1857, 10th Rep. N. Y. Mus. Nut. Hist., p. 162, Ham. Gr.

tenuistriata, Hall, 1859, Pal. N. Y., vol. 3, p. 204, Low. Held. Gr.

tenuistriata, Shaler, 1865. The name was preoccupied.

texana, Meck, 1871, Proc. Acad. Nat. Sci., p. 179 , Coal Meas.

texta, Hall, 1857, 10th Rep. N. Y. Mus. Nat. Hist., p. 169, Waverly Gr.

translata, Swallow, 1863, Trans. St. Louis Acad. Sci., vol. 2, p. 85, Kaskaskis Gr.

transversa, McChesney, 1860, New Pal. Foss., Kaskaskia Gr. Not recognized. tribulis, Hall, 1859, Pal. N. Y., vol. 3, p. 420 , Oriskany sandstone.

triplicuta, Hall, syn. for Spirifera cam. erata.

troosti, Castelnau, 1843, Syst. Sil., p. 41. Not recognized.

tullia, Hall, 1867, Pal. N. Y., vol. 4, p. 218, Ham. Gr.

undulata; Vanuxem, 1843, (Delthyris undulatus,) Greo. 3d Dist. N. Y., p. 132, Onondaga Gr. The name was preoccupied.

unica, Hall, 1867, Pal. N. Y., vol. 4, p. 203, Cornif. Gr.

ulahensis, Meek, 1860, syn. for S. norwoodi. 
vanuxemi, Hall, 1859, Pal. N. Y., vol. 3 , p. 198, Low. Held. Gr., described as Orthis plicata by Vanuxem in the Geo. Ren. 3d Dist. N. Y., but that name was preoccupied.

varicosa, Hall, 1857, 10th Rep. N. Y. Mus. Nat. Hist., p. 130 , and Pal. N. Y., vol. 4, p. 205, Up. Held. Gr.

ventricosa, see Nucleospira ventricosa.

venusta, syn. for Spirifera divaricata.

vernonensis, Swallow, 1860, Trans. St. Louis Acad. Sci., vol. 1, p. 644, Waverly or Choteau Gr.

verneuili, syn. for $\mathrm{S}$. disjuncta.

? waldronensis, see Triplesia waldronensis. waverlyensis, Winchell, 1870, Proc. Am. Phil. Soc., vol. 12, p. 251, Marshall Gr. whitueyi, Hall, 1858, Geo. Rep. Iowa, p. 502, Ham. and Chemung Gr.

wortheni, Hall, 1857, 10th Rep. N. Y. Mus. Nat. Hist., p. 156, Ham. Gr.

ziczac, Hall, 1843. The name was preoccupied by Roemer. See S. consobrina.

Spiriferina, D'Orbigny, 1847, Consid. Zool. et Geol. Sur. les Brachiopodes, Comptes rendus des Sciences de l'Académie des Sciences. [Ety. Spirifera, a genus; inus, implying resemblance.] Shell transverse, valves unequally convex; with or without mesial fold and sinus; smooth or costated; beak straight or recurved; area large, and interrupted by a pseudodeltidium, notched near the cardinal edge; structure punctate; surface spinous; tooth on each side of the fissure, supported by vertical, shelly plates, the space intervening occupied by the cardinal muscles; mesial septum wide at the base, and tapering to an acute blade; dorsal valve with dental sockets and shelly lamellæ, for the support of serrated arms in the form of two large spiral, horizontal cones. Type S. rostrata.

billingsi, Shumard, 1858, Trans. St. Jouis Acad. Sci., vol. 1, p. 294, Permian Gr.

binacuta, Winchell, 1865, Proc. Acad. Nat. Sci., p. 120, Burlington Gr.

clarksvillensis, Winchell, 1865, Proc. Acad. Nat. Sci., p. 119, Marsball Gr.

kentuckiensis, Shumard, 1855, (Spirifera kentuckiensis,) Geo. Rep. Mo., p. 203, Coal Meas.
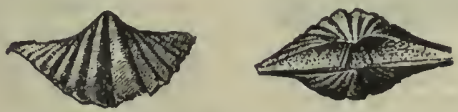

FIG. 615.-Spiriferina kentuckiensis.

kentuckiensis var. propatula, Swallow, 1866, (Spirifera kentuckiensis var. propatula,) Trans. St. Louis Acad. Sci., vol. 2, p. 409, Coal Meas.

spinosa, Norwood \& Pratten. (Spirifera spinosa, 1855, Jour. Acad. Nat. Sci., vol. 3, 2d series, p. 71, Kaskaskia Gr.

spinosa var. campestris, White, 1874, Rep. Invert. Foss., p. 21, and Geo. Sur. W. 100th Mer., vol. 4, p. 139, Carb. subtexta, White, 1862, Proc. Bost. Soc. Nat. Hist., vol. 9, p. 8, Burlington Gr. Spirigera, D'Orbigny in Comptes Rendus, $t$. 25, p. 268, syn. for Athyris.

americana, see A. americana.

biloba, see A. biloba.

caput-serpertis, see A. caput-serpentis.

charitonensis, see A. charitonensis.

clintonensis, see A. clintonensis.

concentrica, syn. for A. spiriferoides.

corpulenta, see A. corpulenta.

eborea, see A. eborea.

euzona, see A. euzona.

formosa, see $\mathrm{A}$. formusa.

fultonensis, see $\mathrm{A}$. fultonensis.

hannibalensis, see A. hannibalensis.

hauni, see A. hawni.

jacksoni, see A. jacksoni.

maconensis, see A. maconensis.

minima, see A. minima.

missouriensis, see A. missouriensis.

monticola, see A. monticola.

obmaxima, see A. obmaxima.

ohioensis, see A. ohioensis.

pectinifera, see $\mathrm{A}$. pectinifera.

plattensis, see A. plattensis.

prouti, see A. prouti.

reflexa, see $\mathrm{A}$. reflexa.

singletoni, see A. singletoni.

spiriferoides, see A. spiriferoides.

Stenoschisma, Conrad, 1839, Ann. Rep. N. Y., p. 59. [Ety. stenos, narrow; schisma, fissure.] Writtten Stenocisma by Conrad. Subtriangular, ovoid, or subglobose, hinge-line short; beak of ventral valve extended, attenuate, more or less arcuate, ard appressed upon the opposite valve; mesial fold and sinus; surface plicated, valves articulated by teeth and sockets; median septum in dorsal valve, on each side of which the crura are supported. Type S. formosum. Conrad mentioned Terebratula schlotheimi as the type which is now the type of Camarophoria; but, as Hall shows, Conrad was mistaken in identifying what is now known as S. formosum, with the European Camarophoria schlotheimi.

billingsi, Hall, 1867, Pal. N. Y., vol. 4, p. 336, Cornif. Gr. The same that Billings called Rhynchonella thalia, Can. Jour. 1860 , but the name was preoccupied.

carica, Hall, 1867, Pal. N. Y., vol. 4, p. 344, Ham. Gr.

carolina, Hall, 1867, Pal. N. Y., vol. 4, p. 337, Cornif. Gr.

congregatum, Conrad, 1841, (Atrypa congregata,) Ann. Rep. N. Y., p. 55, and Pal. N. Y., vol. 4, p. 341, Ham. Gr.

contractum, Hall, 1843, (Atrypa contracta,) Geo. 4th Dist. N. Y., pl. 66, fig. $3 a$, and Pal. N. Y., vol. 4, p. 351, Chemung Gr.

contractum var. saxatile, Hall, 1867, Pal. N. Y., vol. 4, p. 417, Chemung Gr.

dotis, Hall, 1867, Pal. N. Y., vol. 4, p. 344, Ham. Gr. 
duplicatum, Hall, 1843, (Atrypa duplicata,) Geo. 4th Dist. N. Y., pl. 67, fig. 2 and $2 \mathrm{a}$, and Pal. N. Y., vol. 4 , p. 350 , Chemung Gr.

eximium, Hall, 1843, (Atrypa eximia,) Geo. 4th Dist. N. Y., pl. 66, and Pal. N. Y., vol. 4, p. 348, Chemung Gr.
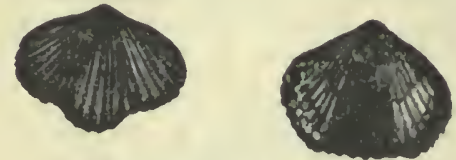

Fra. 616.-Stenochlsma exlmium.

formosum, Hall, 1857, (Rhynchonella formosa,) 10th Rep. N. Y. Mus. Nat. Hist., p. 76, and Pal. N. Y., vol. 3, p. 236, Low. Held. Gr.

horsfordi, Hall, 1860, 13th Rep. N. Y. Mus. Nat. Hist., p. 87, and Pal. N. Y., vol. 4 , p. 339, Cornif. Gr., Marcellus shale and Ham. Gr.

orbiculare, Hall, 1860, (Rhynchonella orbicularis,) 13th Rep. N. Y. Mus. Nat. Hist., p. 88, and Pal. N. Y., vol. 4, p. 353, Chemung Gr.

prolificum, Hall, 1867, Pal. N. Y., vol. 4, p. 343 , Ham. Gr.

royanum, Hall, $1860, \mathrm{Pal}$. N. Y., vol. 4, p. 338, Cornif. Gr.

sappho, Hall, 1860, (Rhynchonella sappho,) 13th Rep. N. Y. Mus. Nat. Hist., p. 87, and Pal. N. Y., vol. 4, p. 340, Marcellus shale and Ham. Gr.

stephani, Hall, 1867, Pal. N. Y., vol. 4, p. 349 , Chemung Gr.

tethys, Billings, 1860, (Rhynclionella tethys,) Can. Jour., vol. 5, p. 271, Cornif. Gr.

Streptorny cuus, King, 1850, Monograph of Permian Fossils, p. 107. [Ety. streplo, I bend or twist; rhynchos, beak.] Semicircular or in general form of Strophomena, concavo-convex, planoconvex, or both valves convex and striated; ventral beak small, or prolonged, bent and twisted, fissure beneath, closed or partially closed by a solid deltidium; area wide on the ventral valve and narrow on the dorsal; externally like Stropbomena, but internally resembling Orthis. Type $\mathrm{S}$. pelargonatum.

alternatum, Hall, 1860, (Orthisina alternata,) 13th Rep. N. Y. Mus. Nat. Hist., p. 81, Ham. Gr.

americanum, Whitfield, 1878, (Hemipronites americanus,) Ann. Rep. Geo. Sur. Wis., p. 72, and Geo. Wis., vol. 4, p. 243, Hud. Riv. Gr.

antiquatum, Sowerby, 1839, (Orthis antiquata,) Mureh. Sil. Syst., p. 630, Anticosti Gr., Div. 3, Mid. Sil.

arctostriatum, Hall, 1843, (Strophomena arctostriata,) Geo. Rep. 4th Dist. N. Y., p. 266, Chemung Gr.

arctostriatum, Hall, 1860, 13th Rep. N. Y. Mus. Nat Hist., p. 80, (Ortbisina areto-

striata,) Ham. Gr. This name was preoccupied.

biloba, Hall, 1883, Rep. St. Geol., pl. 41, figs. 4, 5, Coal Meas.

cardinale, Whitfield, 1850, Ann. Rep. Geo. Sur. Wis., p. 61, and Geo. Wis., vol. 4, p. 261, Hud. Riv. Gr.

chemungense, Conrad, 1843, (Stropliomena chemungensis,) Jour. Acar. Nat. Sci., vol. S, p. 357, and Pal. N. Y., vol. 4 , p. 67, Chemung Gr.

crassum, Meek \&

Hayden, 1858 ,

(Orthisina cras-

sa,) Proc. Acad.

Nat. Sci.. Phil., p. 260 , and Geo. Sur. Ill., vol. 5, p. 570 , Coal Meas.

crenistriatum, Phil-

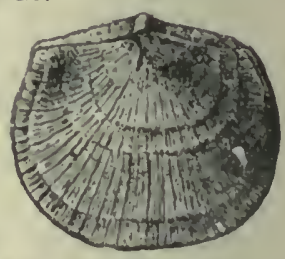

lips, 1836, (Spi- F1G. 617.-Streptorhynrifera crenistria,) chus crassum. IDorsal Geo. York., vol. 2, vlew.

p. 216, Waverly Gr.

deflectum, Courad, 1843, (Strophomena deflecta,) Proc. Aead. Nat. Sci. Phil., p. 332, and Pal. N. Y., vol. 1, p. 113, Trenton Gr.

elongatum, James, 1874, Cin. Quar. Jour. Sci., vol. 1, p. 240, Hud. Riv. Gr. A variety of S. subtentum.

filitextum, Hall, 1847, (Leptrena filitexta,) Pal. N. Y., vol. 1, p. 111, Trenton and Hud. Riv. Grs.

flabellum, Whitfield, 1882, Dese. New Spec. Foss., from Ohio, p. 200, Up. Held. Gr.
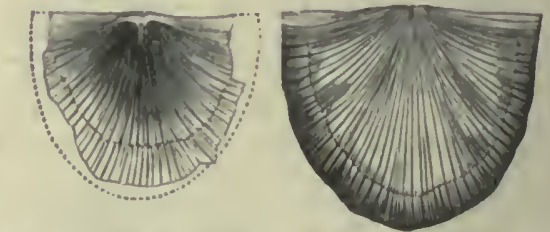

Fig. 618.-Streptorhynchus hallanum. Extcrior and interior of dorsal valve.

hallanum, S. A. Miller, 1874, Cin. Quar. Jour. Sci., vol. 1, p. 148, Hud. Riv. Gr.

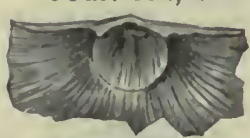

Fro. B19. - streptorhyncrus hallunum. Interior of ventral valve. Hist., vol. 8, p. 293, Kinderhook Gr. lens, White, 1862, Proc. Best. Soc. Nat. Hist., vol. 9, p. 28, Chemung Gr. minor, Walcott, 1885, Monogr. U. S. Geo. Sur., vol. 8, p. 75, Trenton Gr.

nutans, Meek, 1873, (Hemipronites nutans, Pal. Ohio, vol. 1, p. 77, Hud. Riv. Gr.

occidentale, Newberry, syn. for Meekella striatocostata. 
pandora, Billings, 1860, Can. Jour., vol. 5, p. 266, and Pal. N. Y., vol. 4, p. 68, Schoharie grit and Cornif. Gr.

pectinaceum, Hall, 1843, (Strophomena pectinacea and S. bifurcata, Geo. Rep. 4th Dist. N. Y., p. 266, and Pal. N. Y. vol. 4 , p. 73 , Chemung Gr.

perversum, Hall, 1857, (Orthis perversa,) 10 th Rep. N. Y. Mus. Nat. Hist., p. 137, and Pal. N. Y., vol. 4, p. 72, (Orthisina alternata, 1860, 13th Rep.,) Cornif. and Ham. Gr.

planoconvexum, Hall, 1847, (Leptæna planoconvexa,) Pal. N. Y., vol. 1, p. 114, Hud. Riv. Gr.

planumbonum, Hall, 1847, (Leptæna planumbona,) Pal. N. Y., vol. 1, p. 112, Trenton and Hud. Riv. Gr.

primordiale, Whitfield, 1886, Bull. Am. Mus. Nat. Hist., vol. 1, p. 301, Birdseye Gr. pyramidale, Newberry, syn. for Meekella striatocostata.

radiatum, Vanuxem, 1843, (Strophomena radiata,) Geo. Rep. 3d Dist. N. Y., p. 122 , and Pal. N. Y., vol. 3, p. 193, Low. Held. Gr.

rectum, Conrad, 1843, (Strophomena recta,) Proc. Acad. Nat. Sci., vol. 1, p. 332, and Pal. N. Y., vol. 1, p. 113, Black Riv. and Trenton Grs.

sinuatum, Emmons, 1855, Am. Geol., p. 199, Hud. Riv. Gr.

subplanum, Conrad, 1842, Jour. Acad. Nat. Sci., vol. 8 , p. 258 , and Pal. N. Y:, vol. 2, p. 259, (Strophomena subplana,) Niagara Gr.

subtentum, Conrad, 1847, (Strophomena subtenta,) Pal. N. Y., vol. 1, p. 115, Trenton and Hud. Riv. Gr.

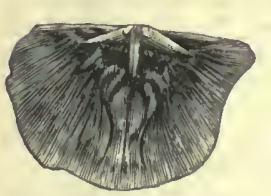

FIG, 620.-Streptorhyuchussulcatum. Interior of dorsal valve.

\section{thalia, Billings,} sulcatum, 1848, (Leptæna sulcata,) Bull. Geol. Soc. France, vol. 5, p. 350 , and Ohio Pal., vol. 1, p. 85 , Hud. Riv. Gr.

tenue, Hall, 1863, Trans. Alb. Inst., vol. 4, p. 210 , Niagara $\mathrm{Gr}$.

(Strophomena thalia,) Can. Nat. and Geol., vol. 5, p. 39, Trenton Gr.

umbraculum, Schlotheim, 1820, (Terebratulites umbraculum,) Petrefaktenkunde, p. 256, Devonian to the Permian Gr.

vetustum, James, 1874, Cin. Quar. Jour. Sci., vol. 1, p. 241, Hud. Riv. Gr. One of the forms of S. subtentum.

woolworthan um, Hall, 1857, (Strophomena woolworthiana,) 10 th Rep. N. Y. Mus. Nat. Hist., p. 48, and Pal. N. Y., vol. 3 , p. 192, Low. Held Gr.

Stricklandia, Billings, 1859, Can. Nat. Geo., vol. 4. This name having been previously applied to a genus of fossil plants, the author abandoned it and proposed Stricklandinia.
Stricklandinia, Billings, 1863, Can. Nat. and Geo., vol. 8, p. 370. [Ety. proper name.] Large, elongate-oval, transversely subcircular, sometimes compressed, valves subequal; short mesial septum in the interior of the ventral valve, supporting a small triangular chamber, beneath the beak, as in Pentamerus; in the dorsal valve no longitudinal septum, spires, or loop; two short, rudimental plates, bearing processes. Type S. gaspensis.

anticostiensis, Billings, 1863, Can. Nat. Geo., vol. 8, p. 370, Anticosti Gr.

(?) arachne, Billings, 1862, Pal. Foss., vol. 1, p. 85, Quebec Gr.

(?) arethusa, Billings, 1862, Pal. Foss., vol. 1, p. 85, Quebec Gr.

brevis, Billings, 1859, Can. Nat. Geo., vol. 4, p. 135 , Mid. Sil.

canádensis, Billings, 1859, Can. Nat. Geo., vol. 4 , p. 135 , Clinton Gr.

castellana, White, 1876, Proc. Acad. Nat. Sci., p. 30, Niagara Gr.

davidsoni, Billings, 1868, Lond. Geo. Mag., vol. 5, p. 59, Up. Sil.

deformis, Meek \& Worthen, 1870, Proc. Acad. Nat. Sci., Phil., p. 37, and Geo. Sur. Ill., vol. 6, p. 502, Niagara Gr.

elongata, see Amphigenia elongata.

elongata var. curta, see Amphigenia curta. gaspensis, Billings, 1859, Can. Nat. Geo., vol. 4 , p. 134, Mid. Sil.

melissa, Billings, 1874, Pal. Foss., vol. 2, p. 89 , Mid. Sill.

multilirata, Whitfield, 1878, Ann. Rep. Geo. Sur. Wis., p. 81 , and Geo. Wis., vol. 4 , p. 315 , Niagara Gr.

salteri, Billings, 1874, Pal. Foss., vol. 2, p. 87, Anticosti Gr., Mid. Sil.

Strophalosia, King, 1844, Ann. and Mag. Nat. Hist., vol. 14, p. 313. [Ety. strophe, a bending; alos, a disk.] Having the general form and muscular impressions of Leptæna, with the tubuliferous or spinous surface of Productus; possessed of a well-developed condyloid hinge, area, and deltidium. Type S. excavata.

horrescens, Geinitz, 1866, Carb. und Dyas in Neb., p. 49. Prof. Meek regarded this name as a syn. for Productus nebraskensis.

numularis, Winchell, 1863, Proc. Acad. Nat. Sci., p. 4, Marshall Gr.

Strophodonta, Hall, 1852, Pal. N. Y., vol. 2, p. 63. [Ety. strophos, bent; odous, tooth.] General form and characters as in Strophomena, one valve convex and the other concave, and following nearly the same curve as the convex one, leaving only a thin space for the animal, and the surface radiated; distinguished, however, by a crenulated hinge-line; the absence of a foramen in the area of the ventral valve; dental lamellæ absent, or nearly so; the divaricator muscular impressions spreading, flabelliform, without limitation, by an elevated 
ridge; cardinal process in the dorsal valve bifurcated from its origin, and directed backward bencath the area of the ventral valve; on the lower side of the ventral area a bilobed process is embraced by the divisions of the cardinal process of the dorsal valve. Type S. demissa.

rquicostata, Swallow, 1860, Trans. St. Louis Acad. Sci., vol. 1, p. 639, Ham. Gr.

altidorsata, Swallow, 1860, Trans. St. Louis Acad. Sci., vol. 1, p. 637, Ham. Gr.

alveata, Hall, 1863, 16th Rep. N. Y. Mus. Nat. Hist., p. 36, and Pal. N. Y., vol. 4, p. 81 , Schoharie grit.

ampla, see Strophonella ampla.

arcuata, Hall, 1858, Geo. of Iowa, p. 492, Ham. Gr.

becki, Hall, 1857, 10th Rep. N. Y. Mus. Nat. Hist., p. 52, and Pal. N. Y., vol. 3, p. 191, Low. Held. Gr.

boonensis, Swallow, 1860, Trans. St. Louis Acad. Sci., vol. 1, p. 635, Ham. Gr.

cælata, see Strophonella cælata.

callawayensis, Swallow, 1860, Trans. St. Louis Acad. Sci., vol. 1, p.638, Ham. Gr. callosa, Hall, 1863, 16th Rep. N. Y. Mus.

Nat. Hist., p. 36, and Pal. N. Y., vol. 4, p. 82, Schoharie grit.

calvini, S. A. Miller, 1883, 2d Ed. Am. Pal. Foss, p. 298, Upper Helderberg Gr. Proposed instead of S. quadrata, Cal. vin, 1878, in Bull. U. S. Geo. Sur. Terr., vol. 4 , No. 3 , p. 728 , which was preoccupied.

canace, Hall \& Whitield, 1873, 23d Rep. N. Y. Mus. Nat. Hist., p. 236, Chemung Gr.

cavumbona, see Strophonella cavumbona. cayuta, Hall, 1867, Pal. N. Y., vol. 4, p. 110, Chemunk Gr.

cincta, Winchell, 1866, Rep. Low. Penin. Mich., p. 93, Ham. Gr.

concava, Hall, 1857, (Strophomena concava,) 10th Rep. N. Y. Mus. Nat. Hist., p. 115, and Pal. N. Y., vol. 4, p. 96, Cornif. and Ham. Grs.

costuta, Owen, 1852, Geo. Sur. Wis., Iowa, and Minn., p. 585, Devonian.

crebristriata, Conrad, 1842, (Strophomena crebristriata,) Jour. Acad. Nat. Sci., vol. 8 , p. 254 , and Pal. N. Y., vol. 4 , p. 86 , Schoharie grit.

cymbiformis, Swallow, 1S60, Trans. St. Louis Acad. Sci., vol. 1, p. 635, Ham. Gr.

demissa, Conrad, 1842, (Strophomena demissa,) Jour. Acad. Nat. Sci., vol. 8, p. 258, and Pal. N. Y., vol. 4, p. 81, Schoharie grit, Cornif., Ham., and Chemung Grs.

erratica, Winchell, 1866, Rep. Low. Peninsula Mich., p. 93, Ham. Gr.

feildeni, Etheridge, 1878, Quar. Jour. Geo. Soc., vol. 34 , p. 598 , Up. Sil.

fragilis, Hall, syn. for Strophodonta perplana. geniculata, Hall, 1859, Pal. N. Y., vol. 3, p. 483, Low. Held. Gr.

headleyana, Hall, 1857, N. Y. Mus. Nat. Hist., p. 49, and Pal. N. Y., vol. 3, p. 185, Low. Held. Gr.

lemispherica, Hall, 1857, (Strophomena hemispherica,) 10th Rep. N. Y. Mus. Nat. Hist., p. 113, anid Pal. N. Y., vol. 4, p. 90, Schoharie grit and Cornif. Gr.

hybrida, Hall \& Whitfield, 1873, 23d Rep. N. Y. Mus. Nat. Hist., p. 239, Chemung Gr.

imitata, Winchell, 1866, Rep. Low. Penin. Mich., p. 93, Ham. Gr.

inrequiradiata, Hall, 1S57, 10th Rep. N. Y. Mus. Nat. Hist., p. 113, and Pal. N. Y., vol. 4, p. 87, Schoharie grit and Cornif. Gir.

in a qu is triata, Conrad, 1842 , (Strophomen: in $x$ quistriata, ) J o u r. Acad. Nat. Sci., vol. 8 , P. 254, and Pal. N. Y., vol. 4, p. 93, Cornif. and Han.. Grs., Mos-

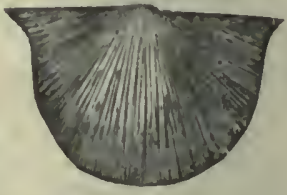
cow shales.

indenta, Conrad, 1838, (Ieptrena indenta,) Ann. Rep., N. Y. p. 117, Low Held. Gr. Not properly defined.

inflexa. Swallow, 1860, Trans. St. Louis Acad. Sci., vol. 1, p. 637, Ham. Gr.

intermedia, Hall, 1859, Pal. N. Y., vol. 3, p. 482, Oriskany sandstone.

iowensis, Owen, 1552, Geo. Sur. Wis., Iowa, and Minn., p. 585, Devoniau.

junin, Hall, 1S67, I'al. N. Y., vol. 4, p. 108, Cornif., Ham. and 'Tully Grs. (Clianged from textilis, in the corrigenda and index.)

kemperi, Swallow, 1860, Trans. St. Louis Acad. Sci., vol. 1, p. 636, Hain. Gr.

leavenuorthana, sce Strophonella leavenworthana.

lepida, Hall, syn. for S. nacrea.

lincklieni, Hall, 1857,10 th Rep. N. Y. Mus. Nat. Hist., p. 55, and Pal. N. Y., vol. 3 , p. 415 , Oriskany sandstone.

magnifica, Hall, 1857, 10th Rep. N. Y. Mus. Nat. Hist., p. 54, and Pal. N. Y., vol. 3 , p. 414, Oriskany sandstone.

magniventra, Hall, 1857, 10th Rep. N. Y. Mus. Nat. Hist., p. 54, and Pal. N. Y., vol. 3 , p. 411 , Oriskany sandstone.

mucronata, Conrad, 1842 , (Strophomena mucronata,) Jour. Acad. Nat. Sci., vol. 8, p. 257, and Pal. N. Y., vol. 4, p. 111, Chemung Gr.

nacrea, Hall, 1857, (Strophomena nacrea,) 10th Rep. N. Y. Mus. Nat. Hist., p. 144, and Pal. N. Y., vol. 4, p. 104, Cornif. and Ham. Grs.

navalis, Swallow, 1860, Trans. St. Louis Acad. Sci., vol. 1, p. 635, Ham. Gr.

parva, Owen, 1852, Geo. Sur. IVis., Iowa, and Minn., p. 584, Ham. Gr. 
parva, Hall, 1863, 16th Rep. N. Y. Mus. Nat. Hist., p. 37, Schoharie grit. This name was preoccupied.

patersoni, Hall, 1857, (Strophomena patersoni,) 10th Rep, N. Y. Mus. Nat. Hist., p. 114, and Pal. N. Y., vol. 4, p.89, Schoharie grit and Cornif. Gr.

perplana, Conrad, 1842, (Strophomena perplana,) Jour. Acad. Nat. Sci., vol. 8, p. 257, and Pal. N. Y., vol. 4, p. 92, Onondaga, Schoharie, Cornif., Ham., and Chemung. Grs.

perplana var. nervosa, Hall, 1843, (Strophomena nervosa,) Geo. Rep. 4th Dist. N. Y., p. 266, and Pal. N. Y., vol. 4, p. 113, Chemung Gr.

planulata, Hall, 1859, Pal. N. Y., vol. 3, p. 184, Low. Held. Gr.

plicata, Hall, 1860, 13th Rep. N. Y. Mus. Nat. Hist., p. 90, Ham. Gr.

prisca, Hall, 1852, Pal. N. Y., vol. 2, p. 63, Clinton Gr.

profunda, Hall, 1852, (Leptæna profunda,) Pal. N. Y., vol. 2, p. 61 , Clinton and Niagara Grs.

punclulifera, see Strophonella punctulifera.

quadrata, Swallow, 1860, Trans. St. Louis Acad. Sci., vol. 1, p. 639, Ham. Gr.

quadrata, Calvin, 1878, Bull. U. S. Geo. Sur. Terr., vol. 4, No. 3, p. 728. The name was preoccupied. See S. calvini. reversa, see Strophonella reversa.

semifasciata, see Strophonella semifasciata. striata, Hall, 1843, (Strophomena striata,) Geo. Rep. 4th Dist. N. Y., p. 104, Niagara $\mathrm{Gr}$.

subcymbiformis, Swallow, 1860, Trans. St. Louis Acad. Sci., vol. 1, p. 636, Ham. Gr.

subdemissa, Hall, 1857, 10th Rep. N. Y. Mus. Nat. Hist., p. 145, Ham. Gr.

textilis, Hall, 1852, Pal. N. Y., vol. 2, p. 327, Coralline Limestone.

variabilis, Calvin, 1878, Bull. U. S. Geo. Sur., vol. 4, No. 3, p. 727, Up. Held. Gir.

varistriata, Conrad, 1842, (Strophomena varistriata,) Jour. Acad. Nat. Sci., vol. 8 , p. 255, and Pal. N. Y., vol. 3, p. 180, Low. Held. Gr.

varistriata var. arata, Hall, 1859, Pal. N. Y., vol. 3, p. 183, Low. Held. Gr.

vascularia, Hall, 1859, Pal. N. Y., vol. 3 . p. 412, Oriskany sandstone.

Strophouns, Rafinesque, 18\%5, Manuel de Malacologie of Blainville, p. 513. [Ety. strophos, bent; mene, a crescent.] Shell somewhat semicircular, or somewhat semioval, though variable in outline; thin; one valve convex, the other con cave, with a thin space between them for the animal ; surface with radiating strix; hinge-line straight, longer or shorter than the width of the shell below; anterior end of the shell usually deflected or bent over toward the dorsal valve; ventral valve convex, flat on the umbo; beak small, and minutely per- forated; cardinal area nearly cut in two parts by an angular notch, which is closed, or nearly closed, by the bifid cardinal process of the dorsal valve; two divergent teeth, two adductor scars, and two cardinal muscular impressions; dorsal valve baving a linear area, two cardinal processes close together at the middle of the hinge-line, directed forward; sockets for the reception of the teeth of the ventral valve; two muscular scars in front of the cardinal processes. Type S. rugosa, which is generally regarded as synonymous with $\mathrm{S}$. rhomboidalis.

acutiradiata, see Chonetes acutiradiatus.

alternata, Conrad, 1838, (Leptæna alternata, Ann. Rep. N. Y., p. 115, and Pal. N. Y., vol. 1, pp. 102, 286, Trenton and Hud. Riv. Grs.

alterniradiata, Shaler, 1865, Bulletin No. 4, M. C. Z., Anticosti Gr. Not defined so as to be recognized.

alternistriata, Hall, 1847, Pal. N. Y., vol. 1, p. 109, Trenton and Hud. Riv. Grs.

alternata var.

loxorhyt is,

Meek, 1873,

Ohio Pal., vol.

1, p. 91, Hud.

Riv. Gr.

ampla, see Strop h o n e I l a ampla.

analoga,Phillips, 1836, G e ol.

Yorkshire, vol. 2, pl. 7, fig. 10, Subcarb.

anticostiensis, syn. for Strophomena alternata.

antiquata, see Streptorlynchus antiquatum.

arctostriata, see Streptorhynchus arctostriatum.

arcuata, Shaler, 1865. This name was preoccupied.

arethusa, Billings, 1862, Pal. Foss., vol. 1, p. 132, Hud. Riv. Gr.

aurora, Billings, 1865, Pal. Foss., vol. 1, p. 218, Quebec. Gr.

bifurata, syn. for Streptorhynchus pectinaceum

bipartita, Hall, 1852, (Leptæna bipartita) Pal. N. Y., vol. 2, p. 326, Coralline Limestone.

blainvillii, Billings, 1874, Pal. Foss., vol. 2, p. 28, Up. Sil.

camerata, Conrad, 1842, Jour. Acad. Nat. Sci., vol. 8, p. 254, and Pal. N. Y., vol. 1, p. 106, Trenton Grr.

carinata, Conrad, 1838, see T'ropidoleptus carinatus.

carinata, Conrad, 1842, see Chonetes carinatus.

ceres, Billings, 1860, Can. Nat. and Geo., vol. 5, p. 54, Hud. Riv. Gr. and Mid. Sil.

chemungensis, Conrad, 1842, Jour. Acad. Nat. Sci., vol. 8, p. 257, Chemung Gr. 
concava, see Strophodonta concava.

con radi, Hall, 1859, Pal. N. Y., vol. 3, p. 194, Low. Held. Gr.

convexa, Owen, 1840, Rep. on Mineral

Lands, p. 70, Caleif. Gr.

cornuta, see Chonetes cornutus.

corrugata, Conrad, 1842, Jour. Acad. Nat.

Sci., vol. 3 , p. 256 , and Pal. N. Y., vol.

2, p. 59, Clinton Gr.

crebristriata, see Strophodonta crebristriata.

crenistria, syn. for Strophodonta perplana. derlivis, James, syn. for Strophomena alternata.

deflecta, see Streptorhynchus defleetum.

deltoidea, Conrad, 1839, Ann. Rep. N. Y.

p. 64, and Pal. N. Y., vol. 4, p. 106, Trenton Gr.

delthyris, syn. for Strophodonta perplana. demissa, see Strophodonta demissa.

depressa, Sowerby, 1825, (Producta depressa,) Min. Conchology, vol. 5, p. 86 , and Pal. N. Y., vol. 2, p. 257, Up. Sil. Generally regarded as a synonym for S. rhomboidalis.

depressa var. ventricosa, see Strophomena rugosa var. ventricosa.

donneti, Salter, 1852, Sutherland's Jour., vol. 2, App., p. 218, Devonian.

elegantula, Hall, 1843, Geo. Rep., 4th Dist. N. Y., p. 73, Clinton Gr.

elongata, Conrad, 1842, Jour. Acad. Nat. Sci., vol. 8, p. 259, Low. Held. Gr.

elliptica, Conrad, 1839, Ann. Rep. N. Y., p. 64 , Niagara Gir.

fasciata, Hall, 1847, (Leptrena fasciata,)

Pal. N. Y., vol. 1, p. 20, Chazy Gr.

filitexta, see Streptorh y nehus filitextum.

fluctuosa, Billings, 1560, Can. Nat. Geo., vol. 5, p. 57, Trenton and Hud. Riv. Grs.

fontinalis, White, 1874, Rep. Invert. Foss., p. 10, and Geo. Sur. W. 100th Mer., vol. 4, p. 54, Quebee Gr.

fracta, Meek, 1873, (S. alternata var. fracta,) Pal. Ohio, vol. 1, p. 91, Hud. Riv. Gr.

fragilis, syn. for Strophodonta perplana.

galatea, Billings, 1874, Pal. Foss., vol. 2, p. 20, Gaspe limestone No. 8, Devonian.

geniculata, Shaler, (Brachyprion geniculatum.) The name was preoceupied.

gibbosa, Conrad, 1841, Ann. Geo. Rep. N. Y., p. 25, Onondaga Gr.

hecuba, Billings, 1860, Can. Nat. Geo., vol. 5, p. 60, Hud. Riv. Gr.

hemispherica, see Strophodonta hemispherica.

imbecilis, Billings, 1865, Pal. Foss., vol. 1, p. 219, Quebec Gr.

imbrex, Pander, 1845, in Russia and Ural Mountains, Hud. Riv. Gr. The identification very doubtful in America.

impressa, syn. for Strophodonta varistriata.

incrassata, Hall, 1847, (Leptæna incrassata, ) Pal. N., vol. 1, p. 19, Chazy to Hud. Riv. Gr.' inxquiradiata, see Strophodonta inæquiradiata.

inxquistriata, see Strophodonta inaquistriata.

interstrialis, Phillips, in Geo. 4th Dist. N. Y., see Strophodonta cayuta.

irene, Billings, 187t, Pal. Foss., vol. 2, p. 27, Devonian.

ithacensis, Vanuxem, 1842, Geo. Rep. N. Y., p. 174, Portage Gr.

julia, Billings, 1862, Pal. Foss, vol. 1, p. 127, Anticosti Gr., Div. 4, Mid. Sil.

kingi, Whitfield, 1878, Ann. Rep. Geo. Sur. Wis., p. 72, and Geo. Wis., vol. 4, p. 261, Hud. Riv. Gr.

lachrymosu, see Productella lach ry mosa.

levis, Emmons, 1842, Geo. Rep. N. Y., p. 385 , Birdseye Gr.

leda, Billings, 1860, Can Nat. Geo., vol. 5, p. 55, Mid. Sil.

lepida, syn. for Stroplıodonta nacrea.

lima, see Productella lachrymosa var. lima.

lineata, see Chonetes lineatus.

macra, syn. for Strophodonta semifasciata. magniventra, seo Strophodonta magniventra.

membranacea, of Phillips, as identified by Vanuxem, 1842, Geo. 3d Dist. N. Y., see Productella hirsuta.

modesta, Conrad, 1839, Ann. Rep. N. Y., p. 64 , Niagara Gr.

mucronata, see Strisphodonta mueronata.

nacrea, see Stropliodonta nacrea.

nasuta, Conrad, 1842, Jour. Acad. Nat. Sei., vol. 8, p. 260, Trenton and Hud. Riv. Grs.

nassulu, Conrad, 1846, Proe. Acal. Nat. Sci., vol. 3 , p. 23 . Not defined so as to be recognized.

nemea, Hall \& Whitfield, 1877, U. S. Geo.

Sur. 40th parallel, vol. 4, p. 233, Quebee $\mathrm{Gr}$.

nervosa, see Strophodonta perplana var. nervosa,

niagarensis, Winchell \& Marcy, syn. for Strophodonta profunda.

nitens, Billings, 1860, Can. Nat. Geo., vol. 5, p. 53, Hud. Riv. Gr.

nutans, see Streptorhynchus nutans.

obscura, Hall, 1852, (Lepteena obscura,) Pal. N. Y., vol. 2, p. 62, Clinton Gr. orthididea, Hall, 1852, (Leptiena orthididea,) Pal. N. Y., vol. 1, p. 62, Clinton $\mathrm{Gr}$.

patenta, Hall, 1852, (Leptrna patenta)

Pal. N. Y., vol. 2, p. 60, Clinton Gir. patersoni, see Strophodonta patersoni. pecten, Linnxus, 1758, (Anomia pecten,) Syst. Nat., Niagara Gr. Not American. pectinacea, see Streptorhynchus pectinaceum.

perplana, see Strophodonta perplana.

philomela, Billings, 1860, Can. Nat. Geo., vol. 5 , p. 56 , Mid. Sil.

planoconvexa, see Streptorhynchus planoconvexum.

planumbona, see Streptorhynchus planumbonum. 
plicata, syn. for Streptorhynchus subtentum.

plicifera, Hall, 1847, (Leptæna plicifera,) Pal. N. Y., vol. 1, p. 19, Chazy Gr. pluristriata, syn. for Strophodonta perplana.

profunda, see Strophodonta profunda.

punctulifera, see Strophonella punctulifera. pustulosa, syn. for Productella truncata. radiata, see Streptorhynchus radiatum.

recta, see Streptorhynchus rectum.

rectilateris, syn. for Strophodonta varistriata.

reticulata, Shaler, 1865, Bulletin No. 4, M. C. Z., Anticosti Gr. Not defined so as to be recognized.

rhomboidalis, Wilckins, 1769, (Conchites rhomboidalis,) Nachrict von Seltenen Verst., p. 77. This species ranges from the Trenton Gr. to the Keokuk, regarding $\mathrm{S}$. tenuistriata, S. depressa, and $\mathrm{S}$. rugosa as varieties only. The type, however, is the Devonian form.

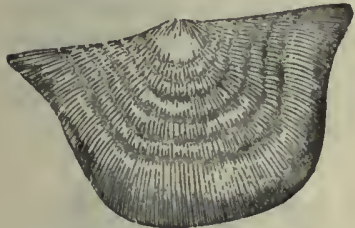

FIG. 623.-Strophomena rugosa. rugosa, Dalman, 1827, (Le p tæn a r u g o s a, ) Vet. Acad. Handlinger, p. 106 , and Pal. N. Y. vol. $3, \mathrm{p}$. 195, Niagara and Low. Held. Gr. This form is supposed to be the type of Rafinesque's genus Strophomena. The species is usually regarded as merely a variety of $\mathrm{S}$. rhomboidalis.

rugosa var. ventricosa, Hall, 1857 , (S. depressa var. ventricosa, ) 10 th $\operatorname{Rep} . \mathrm{N}$. Y. Mus. Nat. Hist., p. 53, and Pal. N. Y., vol. 3, p. 417, Oriskany sandstone.

setigera, see Chonetes setigerus.

semiovalis, Conrad, syn. for Leptrena sericea.

semiovalis, Shaler. The name had been twice preoccupied.

squamula, James, 1874, Cin. Quar. Jour. Sci., vol. 1, p. 335, Hud. Riv. Gr. striata, see Strophodonta striata.

subdemissa, syn. for Strophodonta demissa. subplana, see Streptorhynchus subplanum. subtenta, see Streptorlynchus subtentum. syrtalis, syn. for Chonetes carinatus.

tenuilineata, Conrad, 1842, Jour. Acad.

Nat. Sci., vol. 8, p. 259, and Pal. N. Y., vol. 1, p. 115, Trenton Gr.

tenuistriata, Sowerby, 1839, (Leptæna tenuistriata,) Murch. Sil. Syst., p. 636, and Pal. N. Y., vol. 1, p. 108, Low. Sil. textilis, see Strophodonta junia. thalia, see Streptorhynchus thalia. transversalis, see Leptæna transversalis. trilobata, Owen, 1852, (Leptæna trilobata,) Geo. Sur. Wis., Iowa, and Minn., p. 584, Trenton Gr.

tullia, Billings, 1874, Pal. Foss., vol. 2, p. 29, Low. Devonian.

undulata, syn. for S. rhomboidalis. undulosa, Conrad, 1841, Ann. Rep. N. Y., p. 54, Low. Held. Gr.

unicostata, Meek \& Worthen, 1868, Geo.

Sur. Ill., vol. 3, p. 335, Hud. Riv. Gr.

varistriata, see Strophodonta varistriata.

ventricosa, Shaler, (Brachyprion ventricosum.) The name was preoccupied.

wisconsinensis, Whitfield, 1880, Ann.

Rep. Geo. Sur. Wis., p. 61, and Geo.

Wis., vol. 4, p. 263, Hud. Riv. Gr.

woolworthana, see Streptorhynchus woolworthanum.

Strrophonella, Hall, 1879, 28th Rep. N. Y., Mus. Nat. Hist., p. 153. [Ety. diminutive of strophos, twisted.] Distinguished from Strophodonta by the resupinate character, the strong and more restricted muscular impression of the ventral valve and strong median septum of the dorsal valve; and from Streptorhynchus by the cardinal process, the crenulations on the inner margins of the cardinal area, and the solid area, with sometimes a triangular deltidium. Type S. semifasciata.

ampla, Hall, 1857, (Strophomena ampla,) 10th Rep. N. Y., Mus. Nat. Hist., p. 111, and Pal. N. Y., vol. 4, p. 93, Up. Held. Gr.

cælata, Hall, 1867, (Strophodonta cælata,) Pal. N. Y., vol. 4, p. 112, Chemung Gr. cavumbona, Hall, 1857, (Strophodonta cavumbona,) 10th Rep. N. Y. Mus. Nat. Hist., p. 51, and Pal. N. Y., vol. 3, p. 187, Low. Held. Gr.

leavenworthana, Hall, 1857, (Strophodonta leavenworthana,) 10th Rep. N. Y. Mur. Nat. Hist., p. 53, and Pal. N, Y., vol. 3 , p. 189 , Low. Held. Gr.

punctulifera, Conrad, 1838, (Leptrena punctulifera,) Ann. Rep. N. Y., p. 117, and Pal. N. Y., vol. 3, p. 188, Low. Held. Gr.

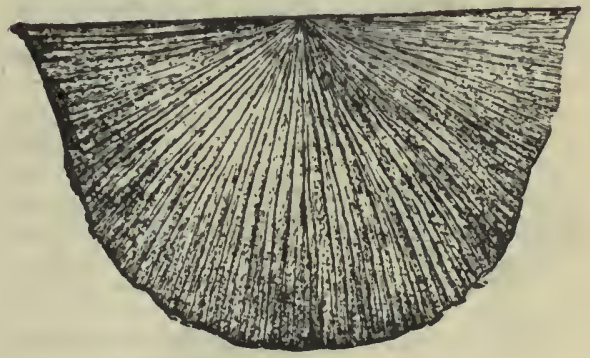

FIa. 624.-Strophonella punctulifera.

reversa, Hall, 1858, (Strophodonta reversa,) Geo. Rep. Iowa, p. 494, Ham. Gr. semifasciata, Hall, 1863, (Strophodonta semifasciata,) Trans. Alb. Inst., vol. 4, p. 210, Niagara Gr.

Syntrielasma, Meek \& IVorthen, 1865, Proc. Acad. Nat. Sci., p. 277. [Ety. syn, together; treis, three; elasma, plate.] Shell thin, gibbous; valves articulated by teeth and sockets; hinge-line short; area higher in the ventral valve than 
in the dorsal; beaks incurved, subequal; surface radiated, forming interlocking angular projections at their terminations; shell structure punctate. Type S. hemiplicatum.
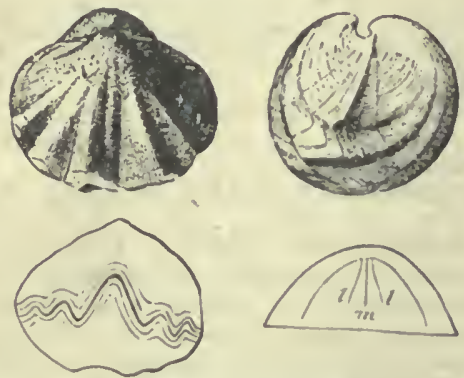

F1G. 625.-Syntrielnsina hemipilentum. laminæ; $m$, mesial septum.

hemiplicatum, Hall, 1852, (Spirifera hemiplicata,) Stan.'s Ex. to Great Salt Lake, p. 409, Coal Meas.

Syrixgotiy ris, Winchell, 1863, Proc. Acad. Nat. Sci., p. 6. [Ety. syrinx, a tube; thyris, a window.] General aspect like Spirifera; shell substance fibrous and impunctate; beak extremely elevated; area of ventral valve large, with a narrow triangular fissure closed toward the apex by an external, convex pseudodeltidium, beneath which, and di. verging from it, is another transverse plate, connecting the vertical dental lamella, arched above, and beneath giving off a couple of median parallel lamellæe, which are incurved so as to nearly join their inferior edges, thus forming a slit-bearing tube, which projects in to the interior of the shell. 'Type S. typus.

halli, Winchell, 1863, Proc. Acad. Nat. Sci., p. 8, Marshall Gr.

typus, Winchell, 1863, Proc. Acad. Nat. Sci. Phil., p. 7, Marshall Gr.

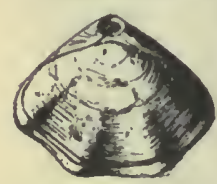

F1G. 626. - Terebratula maxillata.

Terebratula; Lll wyd, 1699 , Lith. Brit. Ichn. [Ety. diminutive of terebratus, perforated.] Shell variable in form, oval, elongated or transverse; smooth or plicated; valves unequally convex; hingeline curved; beak short, truncated by a foramen; deltidium in one or two pieces; loop short; confined to the posterior portion of the shell, not more than one-third the length of the valve, simply attached to a hinge plate; two ribbon-shaped lamellæ are united by a transverse lamella bent upward in the middle; the cirrated arms are supported by the crura, and project in front of the loop; no median septum in the socket valve. Type $T$. vitrea and $\mathrm{T}$. maxillata.

acuminatissima, Castelnau, 1843, Syst. Sil., . p. 40. Not recognized.

affinis, syn. for Atrypa reticularis. aprinis, see Rhynchonella aprinis.

arcuata, Swallow, 1863, Trans. St. Louis

Acad. Sci. The name was preoccupied by Roemer in 1840 . See T. Shumardana. argentea, see Athyris argentea.

aspera, see Atrypa aspera.

bidentata, see Rhynchonella bidentata.

bisacula, McChesney, 1860, New Pal. Foss., p. 82, Kaskaskia Gr. Not recornized. borcalis, Castelnau, 1843, Syst. Sil., p. 40, Not recognized.

bovidens, Morton, 1836, Am. Jour. Sci., vol. 29, p. 150, Coal Meas.

brevirostris, seo Rhynchonella brevirostris. brevilobata, Swallow, 1S63, Trans. St. Louis Acad. Sci., vol. 2, p. 84, Kaskaskia Gr.

burlingtonensis, White, 1860, Bost. Jour. Nat. Hist., p. 228, and Geo. Sur. IV.

100th Mer., vol. 4, p. 93, Kinderhook Gr. concentrica, syn. for A thyris spiriferoides. cooperensis, 12. sp., Keokuk Gr. Proposed instead of T. parva, Sivallow, 1863, Trans. St. Louis Acal. Sci., vol. 2, p. 83, which name was preoccupied.
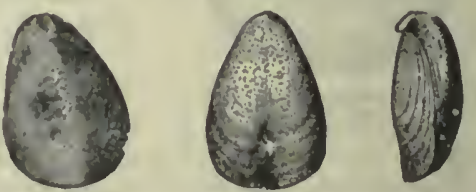

Fıa.687.-Terebratula bovidens.

crenulata, Sowerby, 1840, (Atrypa crenulata,) Geo. Trans., 2d series, vol. 5, p. 704, Devonian.

cuneala, see Rhynchonella cuneata.

elia, Hall, 1867, Pal. N. Y., vol. 4, p. 390, Up. Held. Gr.

formosa, Hall, 1858, Trans. Alb. Inst., vol. 4, p. 6, and Bull. Am. Mus. Nat. Iist., p. 55. Warsaw Gr.

geniculosa, syn. for Terebratula bovidens. gracilis, Swallow, 1863, Trans. St. Louis Acad. Sci. The name was preoccupied by Von Buch in 1834. See T. swallovana.

liarmonia, Hall, 1867, Pal. N. Y., vol. 4, p. 388, Up. Held. Gr.

inornata, McChesney, 1860, New. Pal. Foss. Carb.

insperata, Phillips, 1841, Pal. Foss., Devonian.

interplicata, see Anastrophia interplicata. jucunda, Hall, 1867, Pal. N. Y., vol. 4, p. 390, Up. Held Gr.

lacunosa. Not American.

lapillus, Morton, 1836, Am. Jour. Sci. and Arts, vol. 29, p. 149, Coal Meas.

lens, see Cryptonella lens.

laticosta, see Atrypa laticosta.

lincklæni, see Cryptonella lincklaeni.

lynx, see Orthis lynx.

marcyi, see Retzia marcyi. 
marginalis, see Atrypa marginalis. michelini, see Orthis michelini. millepunctala, syn. for T. bovidens. mormoni, see Retzia mormoni.

navicella, Hall, 1867, Pal. N. Y., vol. 4, p. 391, Ham. Gr.

nuciformis, Morton, 1836, Am. Jour. Sci. and Arts, vol. 29, p. 149, Coal Meas.

nucula, see Rhynchonella nucula. ontario, Hall, 1867, Pal. N. Y., vol. 4, p. 418, Ham. Gr.

ovoides, see Rensselæria ovoides.

parva, Swallow, 1863, Trans. St. Louis Acad. Sci., p. 83. The name was preoccupied by d'Archiac in 1846. See

Terebratula cooperensis.

pennata, see Spirifera pennata.

perinflata, Shumard, 1859, Trans St. Louis

Acad. Sci., vol. 1, p. 392, Permian Gr.

planirostra, see Cryptonella planirostra. rectirostra, see Cryptonella rectirostra.

reticularis, see Atrypa reticularis.

rockymontana, see Rhynchonella rockymontana.

roemingeri, Hall, 1863, 16th Rep. N. Y. Mus. Nat. Hist., p. 48 , and Pal. N. Y., vol. 4, p. 389, Ham. Gr.

rowleyi, Worthen, 1884, Bull. No. 2, Ill.

St. Mus. Nat. Hist., p. 23, Burlington $\mathrm{Gr}$.

sacculus, Martin, 1809, Petrif. Derb., Low. Carb.

schlotheimi, see Camerophoria schlotheimi. shumardana, S. A. Miller, 1883, 2 d Ed.

Am. Pal. Foss., p. 299, Kaskaskia Gr.

Proposed instead of T. arcuata, Swallow,

1863, Trans. St. Louis Acad. Sci., vol. 2, p. 83 , which was preoccupied.

simulator, Hall, 1867, Pal. N. Y., vol. 4, p.

391, Ham. Gr.

spiriferoides, see Athyris spiriferoides.

stricklandi, see Rhynchonella stricklandi. subretziaforma, McChesney, 1860, Pal.

Foss., p. 82, Kaskaskia Gr. Not recognized.

subtilita, see Athyris subtilita.

sullivanti, Hall, 1867, Pal. N. Y., vol. 4, p. 387, Up. Held. Gr.

swallovana, S. A. Miller, 1883, 2d Ed. Am.

Pal. Foss., p. 299, Kaskaskia Gr. Proposed instead of T. gracilis, Swallow, 1863, Trans. St. Louis A cad. Sci., vol. 2, p. 83 , which was preoccupied.

traversensis, Winchell, 1866, Rep. Low.

Penin. Mich., p. 95, Ham. Gr.

trinuclea, see Athyris trinuclea.

turgida, Hall, 1858, Trans. Alb. Inst, vol. 4, p. 6, and Bull. Am. Mus. Nat. Hist., p. 54, Warsaw Gr.

uta, see Rhynchonella uta.

utah, Hall \& Whitfield, 1877, U. S. Geo.

Expl. 40th parallel, vol. 4, p. 258, Waverly Gr.

valenciennei, Castelnau, 1843, Syst. Sil., p. 39. Not recognized.

wilsoni, see Rhynchonella wilsoni.

Terebratulites, Schlotheim, syn. for Spirifera.

biforatus, see Orthis biforata.

Trematis, Sharpe, 1848, Quar. Jour. Geo. Soc., vol. 13, p. 66. [Ety. trema, an opening.] Shell suborbicular or transvensely oval, lenticular; valves unequally convex; umbo of the upper or dorsal valve submarginal, slightly projecting; lower or ventral valve with a subcentral umbo, behind which a narrow, oblong, oval slit reaches to near the posterior margin, and afforded passage to the pedicle fibers of attachment; shell punctate in the outer layers, and fibrous and of a pearly luster within. Type $\mathrm{T}$. terminalis. cælata, see Obolella cælata. cancellata, Sowerby, 1825, (Orbicula cancellata,) Zool. Jour., vol. 2, Trenton Gr. crassa, see Obolella crassa.

crassipuncta, Ulrich, 1889, Am. Geo., vol. 4, p. 22, Hud. Riv. Gr.

dyeri, S. A. Miller, 1874 , Cin. Quar. Jour. Sci., vol. 1, p. 347, Hud. Riv. Gr. filosa, see Schizocrania filosa. fragilis, Ulrich, 1889, Am. Geo., vol. 4, p. 21, Utica Slate.

huronensis, Billings, 1862, Fra. 628.-TrePal. Foss., vol. 1, p. 53, matis dyeri. Black Riv. Gr.

montrealensis, Billiugs, 1862, Pal. Foss., vol. 1, p. 52, Trenton Gr.

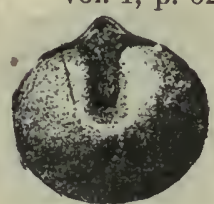

Fig. 629. - Trematis millepunctata. $\quad$ p. 53, Trenton

punctostriata, Hall \& Whitfield, 1873, 23d Rep. N. Y. Mus. Nat. Hist., p. 243, Trenton and Hud. Riv. Gr.

(?) pustulosa, Hall, 1866, 24th Rep. N. Y. Mus. Nat. Hist., p. 222, Hud. Riv. Gr. quincuncialis, Miller \& Dyer, 1878, Cont. to Pal. No. 2, p. 8, Hud. Riv. Gr. rudis, Hall . \& Whitfield, 1873, 23 d Rep. N. Y. Mus. Nat. Hist., p. 243, Trenton Gr.

terminalis, Emmons, 1842, (Orbicula terminalis,) Geo. Rep. N. Y., p. 395, and Pal. N. Y., vol. 1, p. 100, Trenton Gr. umbonata, Ulrich, 1889, Am. Geo., vol. 4, p. 23, Hud. Riv. Gr.

Trematospira, Hall, 1859, 12th Rep. N. Y. Mus. Nat. Hist., p. 27. [Ety. trema, foramen; spira, a spire; in allusion to the perforation in the beak of the ventral valve.] Transverse, elliptical or subrhomboidal, inequivalve; mesial fold and sinus; surface plicated ; internal spires, as in Spirifera; hinge-line short; cardinal angles rounded; valves articulated by teeth and sockets; beak of ventral valve produced or incurved, 
and truncated by a small, round perforation, separated from the hinge-line by a deltidium; deep, triangular pit beneath the beak of the ventral valve, which is filled by the closely incurved beak of the dorsal valve; structure punctate. Type T. costata and $\mathrm{T}$. multistriata.

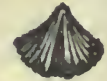

F1a. 630.-Trematospira acadire.

acadixe, Hall, 1860, Can.

Nat. and Geo., vol. 5, p. 144, Up. Sil.

camura, Hall, 1852 ,

(Atrypa camura,) Pal. N. Y., vol. 2, p. 273 , Low. Held. Gr.

costata, Hall, 1859, Pal. N. Y., vol. 3, p. 210, Low. Held. Gr.

denceyi, see Rhynchospira deweyi.

formosa, see Rliynchospira formosa.

gibbosa, Hall, 1860, 13th Rep. N. Y. Mus. Nat. Hist., p. 82, and Pal. N. Y., vol. 4, p. 272, Ham. Gr.

globosa, Hall, 1857, 10th Rep. N. Y. Mus. Nat. Hist., p. 87, and Pal. N. Y., vol. 3, p. 215, (Waldheimiaglobosa,) Low. Held. Gr. granulifera, Meek, 1872, Proc. Acad. Nat. Sci. Phil., p. 318, and Ohio Pal., vol. 1, p. 128, Hud. Riv. Gr.

hirsuta, Hall, 1857, (Atrypa hirsuta,) 10th Rep. N. Y. Mus. Nat. Hist., p. 168, and Pal. N. Y., vol. 4, p. 274, Up. Held. and Ham. Gr.

imbricata, Hall, 1857, (Leptocœlia imbricata, ) 10th Rep. N. Y. Mus. Nat. Hist. p. 108, and Pal. N. Y., vol. 3, p. 246, Low. Held. Gr.

infrequens, Walcott, 1885, Mongr. U. S. Geo. Sur., vol. 8, p. 151, Lower Devonian. mathewsoni, McChesney, 1861, New I'al. Foss., p. 71, Niagara Gr.

multistriata, Hall, 1857, (Spirifer multistriatus, ) 10th Rep. N. Y. Mus. Nat. Hist., p. 59, Low. Held. Gr.

liniuscula, Winchell, 1566, Rep. Low. Peninsula Mich., p. 94, Ham. Gr.

(?) nobilis, Hall, 1860, (Rhynchospira nobilis,) 13th Rep. N. Y. Mus. Nat. Hist., p. 83, Ham. Gr.

perforata, Hall, 18577, (Spirifera perforata,) 10th Rep. N. Y. Mus. Nat. Hist., p. 60, Low. Held. Gr.

(?) quadriplicata, see Rhyncliotreta quadriplicata.

rectirostris, Hall, 1856, (Waldheimia rectirostra,) 10th Rep. N. Y. Mus. Nat. Hist., p. 49, and Pal. N. Y., vol. 3 , p. 217, Low. Held. Gr.

simplex, Hall, 1856, Pal. N. Y., vol. 3, p. 211, Low. Held. Gr.

Trigonotreta, Konig, 1825, Icon. Foss. Sect., p. 3. [Ety. trigonos, a triangle; tretos, perforated.] Syn.for Spirifera. Meek, concluding that S. cuspidatus mentioned by Sowerby in Minn. Conch., vol. 2, p. 42 , should be considered as the type of Spirifera, proposed to retain Trigonotreta for shells of the type of S. striata, contrary to the views of most authors. See Pal. Up. Mo., p. 18.
Trimerella, Billings, 1862, Pal. Foss., vol. 1, p. 166. [Ety. treis, three; meros, part; ella, diminutive.] Large subovate or subcircular; valves convex; beaks solid and transversely striated;

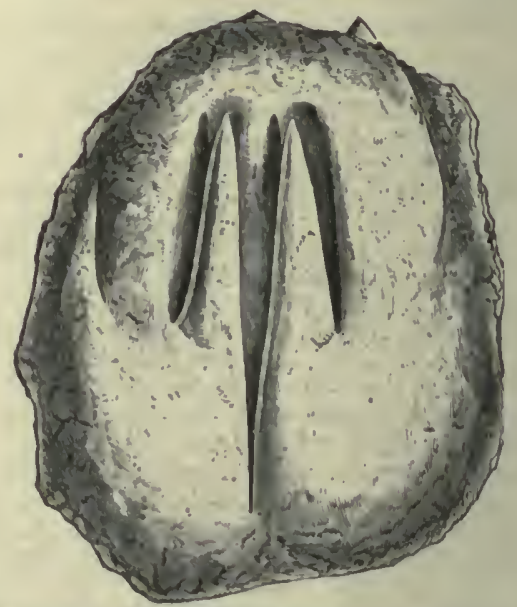

FjG. 031.-Trimerella grandis. Cast of dorsal valve.

shell thick, and surface concentrically striated; area of pedicle valve longer than wide; deltidiuin large; hinge thick, elevuted, rudely or slightly dentary; cardinal facet large; crescent well defined; platforms elevated and doubly vaulted, occasionally solid and slightly raised; median plate in both vulves, lnngest in the brachial one. Type T. grandis.

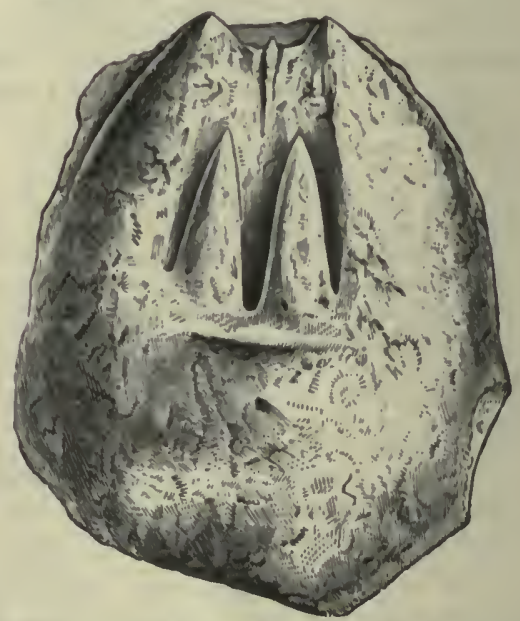

FrG. 632.-Trimerella grandis. Cast of ventral valve.

acuminata, Billings, 1862, Pal. Foss., vol. 1, p. 167, Guelph Gr.

billingsi, Dall, 1871, Am. Jour. Conch., vol. 7, p. 82, Guelph (ir. 
dalli, Davidson \& King, 1872, Brighton Meeting Brit. Assoc., and Quar. Jour. Geo. Soc., 1874, p. 154, Guelph Gr.

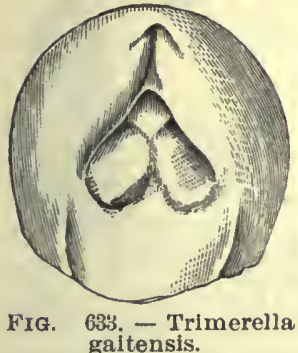

galtensis, Billings, 1862, Obolus galtensis,) Pal. Foss., vol. 1, p. 168, Guelph Gr. grandis, Billings, 1862, Pal. Foss., vol. 1 , p. 166, Guelph Gr.

minor, Dall, syn. for T. galtensis.

ohioensis, Meek, 1871, Ám. Jour. Sci., $2 \mathrm{~d}$ series, vol. 1, p. 315 , and Ohio Pal., vol. 1, p. 183, Niagara Gr.

Triplesia, Hall, 1858, 12th Rep. N. Y.
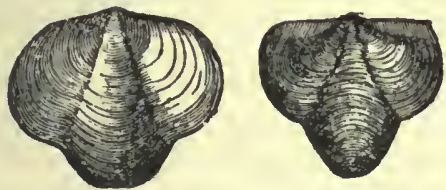

FrG. 634.-Triplesia extans.

Mus. Nat. Hist., p. 44. [Ety. triplasios, thrice; in allusion to the trilobate character of the shell.] Shell transverse or elongate, trilobate or subtrilobate; ventral valve deeply sinuous and dorsal, having a corresponding fold; hinge-line straight ; area small; foramen triangular; surface concentrically striated; ventral valve with a strong tooth on each side of the fissure; muscular impressions small; dorsal valve with a prominent bifurcating cardinal process, on each side of which there is a brachial process directed obliquely inward and laterally. Type T. extans.

congesta, Conrad, 1842, (Atrypa congesta,) Jour. Acad. Nat. Sci., vol. 8, p. 265, and Pal. N. Y., vol. 2, p. 67, Clinton Gr. cuspidata, Hall, 1847, (Atry pa cuspidata,) Pal. N. Y., vol. 1, p. 138, Trenton Gr.
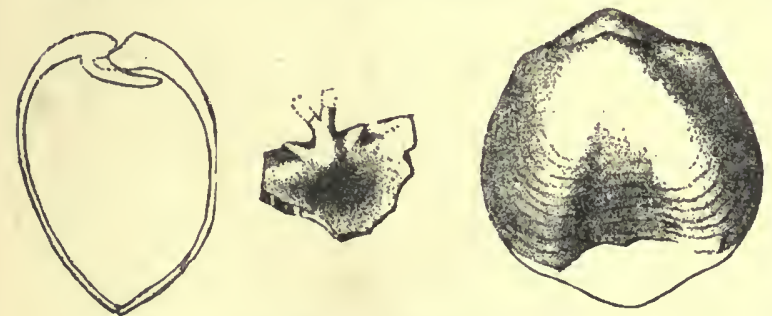

FIG. 635.-Triplesia ortonl.

extans, Emmons, 1842, (Atrypa extans,) Geo. Rep. 2d Dist. N. Y., p. 395, and Pal. N. Y., vol. 1, p. 137, Trenton Gr. lateralis, Whitfield, 1886, Bull. Am. Mus. Nat. Hist., vol. 1, p. 303, Birdseye Gr. nucleus, Hall, 1847, (Atrypa nucleus,) Pal. N. Y., vol. 1, p. 138, Trenton Gr.

ortoni, Meek, 1872, (Dicraniscus ortoni,) Am. Jour. Sci. and Arts, 3d ser., vol. 4, p. 280, and Ohio Pal., vol. 1, p. 178, Niagara Gr.

primordialis, Whitfield, 1878, Ann. Rep. Geo. Sur. Wis., p. 51, and Geo. Wis., vol. 4, p. 172, Potsdam Gr.

putillus, Hall, syn. for $\mathrm{T}$. waldronensis. radiata, Whitfield, 1889, Bull. Am. Mus. Nat. Hist., vol. 2, p. 43, Calciferous Gr. waldronensis, Miller \& Dyer, 1878, (Spirifera ? waldronensis,) Jour. Cin. Soc. Nat. Hist., vol. 1, p. 37, Niagara Gr.

Tropidoleptus, Hall, $185 \%$, proposed in 10 th Rep., but described in 1859 in 12th Rep. N. Y. Mus. Nat. Hist., p. 31: [Ety. tropis, the keel or bottom of a ship; leptos, slender.] General form of Strophomena; surface plicated; structure punctate; ventral valve convex, with an area and wide fissure beneath the beak; dental lamellæ distinct from the margin of the fissure, crenulate; dorsal valve concave, with crenulate dental fossets ; a strong, cardinal process, with diverging lobes in the interior, which support slender crura that converge to and unite with the median crest. Type T. carinatus.
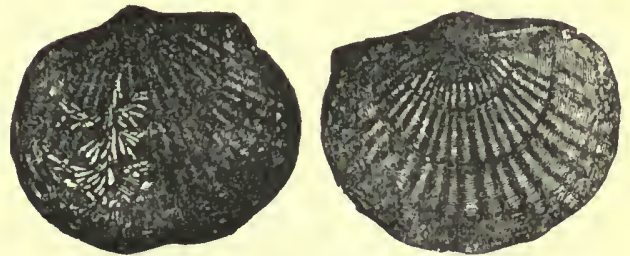

Fig. 636.-Tropidoieptus carinatus.

carinatus, Conrad, 1839, (Strophomena carinata,) Ann. Geo. Rep. N. Y., p. 64, and Pal. N. Y., vol. 4, p. 407, Ham. Gr. occidens, Hall, 1860, 13th Rep. N. Y. Mus. Nat. Hist., p. 91, and Pal. N. Y., vol. 4 , p. 408 , Ham. Gr.

Vitulina, Hall, 1860, 13th Rep. N. Y. Mus. Nat. Hist., p. 72. [Ety. mythological name.] Externally it is like Leptocœlia, but distinguished by its strong dental lamellæe and processes. Type V. pustulosa. pustulosa, Hall, 1860, 13th Rep. N. Y. Mus. Nat. Hist., p. 72 , and Pal. N. Y., vol. 4 , p. 410 , Tully limestone.

Waldheimia, King, 1849, Monograph of Permian Fossils, p. 145. [Ety. proper name.] Shell circular, subquadrate, transverse or elongated; valves unequally convex, smooth, or plicated; beak truncated, perforated; deltidium in one or two pieces; loop long, formed of lamellæ attached by the crura to the hinge 
plate; one tooth on each side of the deltidium, supported by plates, and fitting in the sockets of the dorsal valve; structure punctate. Type W. australis.
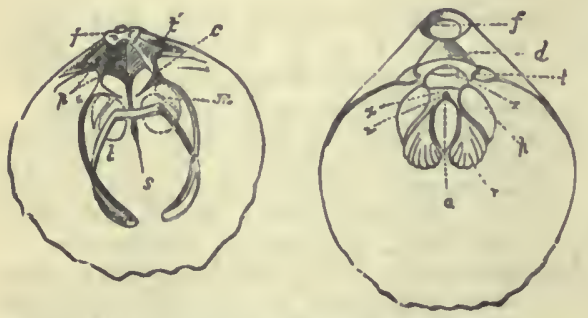

Frg. 637,-Waldhelmia nustralis. Dorsal valve; $j$, curdinal process; $t$, dental sockels; $p$, tilnge plate ; s, seplum: c, crura of the loop: l, reflected portion of tise loop: m, quadrupie adductoi lupressloul Ventral valve: forizmen; $d$, deltidlum; $\ell$, teeth; $a$, slugle adductor impression; $r$, cardlinal muscle; $x$, necessury nsuscles; $p$, pedicle muscles; $v$, position of the vent; $z$, attachment of pedicle sheuth.

compacta, White \& St. John, 1868, Trans. Chi. Acad. Sci., p. 119, Up. Co.ll Meas.

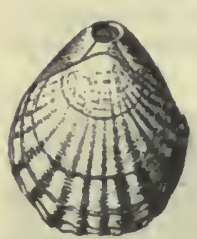

FIG. 635- - IVuldlielmia Bustralls. deweyi, see Retzia deweyi. formosa, see Retzia formosa. globosa, see Trematospira globosa.

reclirostra, see Trematospira rectirostra.

WIITFIELDIA, Davidson, 1891, Lond. Geo. Mag., vol. 8, p. 289. [Ety. proper name. While Meristella is distinguished from Merista by having no shoe-lifter process; Whitfieldia is distinguished from both by the absence of those peculiar ring-shaped processes attached to the lonp, and has instead only a short, bifurcating process, where in Merista and Meristella the rings are formed. These internal differences in the spirals seem to distinguish the genera. 'I'ype W. tumida. maria, Hall, 1863, (Meristella maria,) Tran8. Alb. Inst., vol. 4, p. 212, Niagara Gr.
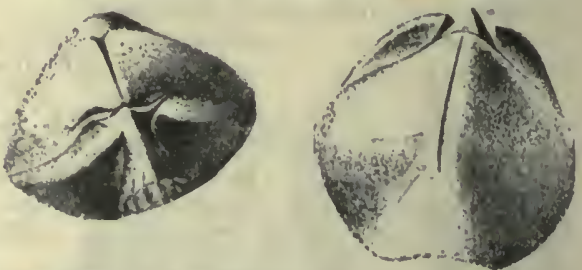

FI(t. 639.-Whitfelda inaria. Internal casts.

Zigospira, Hall, 1862, 15tlı Rep. N. Y. Mus. Nat. Hist., p. 126. [Ety. zygos, yoke; spira, spire.] General form of Atrypa with internal spires having a broad loop passing from the outer limbs of the spiral band entirely across from side to side, near to or above the center, and close to the inner side of the dorsal valve. Type $Z$. modesta.

concentrica, Ulrich, 1879, Jour. Cin. Soc. Nat. Hist., vol. 2, p. 14, Hud Riv. Gr. headi, Billings, 1862, (Atlyris headi,) Pal. Foss., vol. 1, p. 147, Hud. Riv. Gr.

headi var. anticostiensis, Billings, 1862 , (Athyris beadi var. anticosticnsis,) Pal. Foss., vol. 1, p. 147, Hnd. Riv. Gr.

heali var, borealis, Billing8, 1862. (A thyris headi var. borealis,) Pal. Foss., vol. 1, p. 147, Hud. Riv. Gr.

headi, Meek, see Glassia headi.

miniuna, Hall, 1879, Desc. New Spec. Foss., p. 14, and 11th Rep. Geo. and Nat. Hist. Ind., p. 305, Niagara Gr. modesta, Say, 1847, (Atrypa modesta,) Pal. N. Y., vol. 1, p. 141, Trenton and Hud. Riv. Gr.

modesta vur. cincinnatiensis, Meek, 1872, Pal. Ohio, vol. 1, p. 126, Hud. Riv. Gr. pauper, Billings, 1866, Catal. Sil. Fos8. Antic. p. 46, An- Fra. 640.-ZygSil. Foss. Antic., p. 46, An- ospira noticosti Gr. desta.

subconcava, Meek \& Wor-

then, 1868, Geo. Sur. 11l., vol. 3, p. 380, Low. Held. Gr. 


\section{CLASS PTEROPODA.}

THE Class Pteropoda consists wholly of marine animals of small size, furnished with a pair of fins at the sides of the head, by means of which they swim in the open sea. The living forms are divided into two orders: the Gymnosomata and Thecosomata. The Gymnosomata have no shells, and occur in such prodigious numbers that they furnish food for whales and many sea-birds. The Thecosomata have either straight or coiled shells, some of which are glassy in their texture and very beautiful. It may well be doubted whether or not any of the Palæozoic fossils belong to this order. They are referred to the following families:

Famil y Aspidellide. - Aspidella.

Family Clathiroccelime-Clathrocolia.

Family Conularime. - Conularia.

Family Hyolithide.-Coleolus, Coleuprion, Diplotheca, Hyolithellus, Hyolithes, Pharetrella, Stenotheca.

Family Matthevide.-Matthevia.

Family Pterothecide. - Pterotheca,

Family Scenellide. - Scenella.

Family Tentaculitide. - Styliola, Tentaculites.

Aspidelia, Billings, 1872, Am. Jour. Sci.,

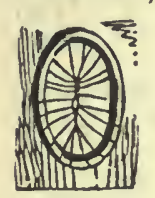
$3 d$ ser., vol. 3 , and Pal. Foss., vol. 2, p. 76 . [Ety. aspidella, little shield.] Small, ovate, bordered by a narrow ring within which it is concave; in the middle there is a ridge, from which grooves radiate to the border. Type A. terranovica.

terranovica, Billings, 1872,

FIG. 611.Aspidell a

ca. Am. Jour. Sci., 3d ser., vol. 3, and Pal. Foss., vol. 2, p. 77, Taconic.

Camerotheca, Matthew, 1885, Can. Rec. Sci., vol. 1 , p. 149, syn. for Hyolithes.

gracilis, see Hyolithes gracilis.

Clathroccelia, Hall, 1879, Pal. N.aY., vol. 5 , pt. 2, p. 203. [Ety. clathro, latticed; koilia, belly.] An oblique conical tube, expanding more rapidly on one side than the other; interior crossed by unsymmetrical, arching, septal lines and longitudinal ones, which give it a cancellated aspect; shell thin, translucent, lamellose. Type C. eborica.

eborica, Hall, 1879, Pal. N. Y., vol. 5, pt. 2, p. 204, Ham. Gr.

Clioderma, Hall, syn. for Pterotheca. attenuata, see Pterotheca attenuata. expansa, see Pterotheca expansa.
Coleolus, Hall, 1879, Pal. N. Y., vol. 5, pt. 2, p. 184. [Ety. koleos, sheath.] Tubuliform, elongate-conical, straight or slightly curved, annulated, sometimes obliquely, sometimes longitudinally stri-

Fia 642,-Coleolus ated, interior smooth. Type C. tenuicinctus.

acicula, Hall, 1843, (Orthoceras acicula,) Geo. Sur. 4th Dist. N. Y., p. 243, and Pal. N. Y., vol. 5, pt. 2, p. 187, Genesee Slate.

aciculatus, Hall, 1860, (Dentalium acicula tum,) 13th Rep. N. Y. St. Mus. Nat. Hist., p. 107, Marcellus Shale and Portage Grs.

crenatocinctus, Hall, 1879, Pal. N. Y., vol. 5 , pt. 2, p. 188, Up. Held. Gr.

gracilis, Hall, 1879, Pal. N. Y., vol. 5, pt. 2, p. 190, Chemung Gr.

herzeri, Hall, 1888, Pal. N. Y., vol. 7, p. 7, Waverly Gr.

lævis, Walcott, 1885, Monogr. U. S. Geo. Sur., vol. 8 , p. 199, Devonian.

mohri, Hall, 1879, Pal. N. Y., vol. 5, pt. 2 , p. 189 , Up. Held. Gr.

spinulus, Hall, 1879, Desc. New Spec. Foss., p. 18, and 11th Rep. Geo. and Nat.

- Hist. Ind., p. 322, Niagara Gr. 
tenuicinctus, Hall, 1876, (Coleoprion tenuicinctum,) Illust. Devon. Foss., pl. 27, Ham. Gr.

Colkoprion, Sandberger, 1847, Leonhardt \& Bronn, Jahrbuch, vol. 1, p. 25. [Ety. koleos, sheath; prion, saw.] Tubuliform, appearing as an elongate cone, encircled by oblique annulations, which are interrupted along a longitudinal line, and attenuate at their extremities; internal walls smooth. Type C. gracilis.

minutum, Walcott, 1885, Nonogr. U. S. Geo. Sur., vol. 8, p. 85, Trenton Gr.

tenue, Hall, 1879, Pal. of N. Y., vol. 5, pt. 2, p. 184, Ham. Gr.

tenuicinctum, see Coleolus tenuicinctus.

Conularia, Miller, 1821, in Sowerby's Minn. Conch., vol. 3, p. 107. [Ety. conulus, little cone.] Elongate pyramidal; transverse section varying from quadrangular to octagonal; angles indented by longitudinal grooves; septum near the apex ; surface reticulated and ornamented. Type C. quadrisulcata.

asperata, Billings, 1866, Catal. Sil. Foss. Antic., p. 21, Hud. Riv. Gr.

bifurca, Ringueberg, 1886, Bull. Buf. Soc. Nat. Sci., vol. 5, p. 18 . Not properly defined.

byblis, White, 1862, Proc. Bost. Soc. Nat. Hist., vol. 9, p. 22, Waverly Gr.

cayuga, Hall, 1876, Illust. Devonian Foss , pl. 28, and Pal. N. Y., vol. 5, pt. 2, p. 211, Ham. Gr.

chesterensis, Worthen, 1883, Geo. Sur. Ill., vol. 7, p. 325, Kaskaskia Gr.

congregata, Hall, 1876, Illust. Devouian Foss., pl. 28, and Pal. N. Y., vol. 5, pt. 2, p. 214, Portage Gr.

continens, Hall, 1876, Illust. Devonian Foss., pl. 28, and Pal. N. Y., vol. 5, pt. 2, p. 212, Marcellus Shale.

continens var. rudis, Hall, 1879, Pal. N. Y., vol. 5, pt. 2, pl. 28, Ham. Gr. crawfordsvillensis, Owen, 1862, Geo. Sur. Ind., p. 362, Keokuk Gr.

crebristriata, Hall, 1876, Illust. Devonian Foss., pl. 29, and Pal. N. Y., vol. 5, pt. 2, p. 210, Ham. Gr.

crustula, White, 1880 , 12th Rep. U. S. Geo. Sur. Terr., p. 170, Coal Meas.

elegantula, Meek, 1871, Proc. Acad. Nat. Sci. Phil., p. 85, and Ohio Pal., vol. 1, p. 288, Up. Held. Gr.

formosa, Mîller \& Dyer, 1878, Jour. Cin. Soc. Nat. Hist., vol. 1, p. 38, Hud. Riv. Gr.

gattingeri, Safford, 1869, Geo. of Tenn., p. 289, Trenton Gr.

gracilis, Hall, 1847, Pal. N. Y., vol. 1, p. 224, Trenton Gr.

granulata, Hall, 1847, Pal. N. Y., vol. 1, p. 223, Trenton Gr.

hudsoni, Emmons, 1856, Am. Geo., vol. 1, p. 208, Hud. Riv. Gr.

huntana, Hall, 1859, Pal. N. Y., vol. 3, p. 348, Low. Held. Gr.

indentata, Conrad, 1854, Proc. Acad. Nat. Sci., vol. 7, p. 31, Trenton Gr.

infrequens, Hall, 1879, Desc. New Spec. Foss., p. 17, and 11 th Rep. Geo. and Nat. Hist. Ind., p. 321 , Niagara Gr.

laqueata, Conrad, 1841, Ann. Rep. N. Y., p. 57, Niagara Gr.

lata, Hall, 1859, Pal. N. Y., vol. 3, p. 479 , Oriskany sandstone.

longa, Hall, 1852, Pal. N. Y., vol. 2, p. 295, Niagara Gr.

magnifica, Spencer, 1S84, Bull. No. 1, Mus. Univ. St. Mo., p. 58, Niagara Gr.

marionensis, Swallow, 1860, Truns. St. Louis Acad. Sci., vol. 1, p. 656, Ham. Gr. micronema, Meek, 1S71.

Proc. Acad. Nat. Sci. Phil., p. St, and Ohio Pal., vol. 2, p. 336, Waverly Gr.

missouriensis, Swallow, 1860 , Trans. St. Louis Acad. Sci., vol. 1, p. 657, and Geo. Sur. Ill., vol. $5, \quad$ p. $541, \mathrm{St}$. Louis Grr.

molaris, White, 1876 , Proc. Acad. Nit. Sci., p. 33, Devoninn.

inulticostata, Meek \&

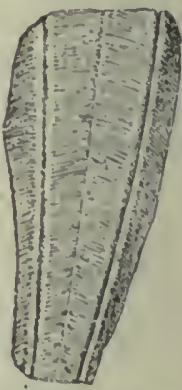

Worthen, 1865, P'roc. Fra.'(3)-ConulaAcad. Nat Sci., p. 252, riamicronema. Waverly (ir.

mullipuncta, Ringueberg, 1886, Bull. Buf. Soc. Nat. Sci., vol. 5, p. 18 . Not properly defined.

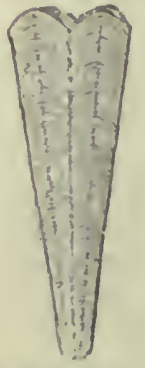

Fro. BH1. Conulurin $q u a d r i$. sulcata. newberryi, Wincliell, 1865 , Proc. Acad. Nut. Sci., p. 130, Waverly Gr.

niagarensis, Hall, 1852, l'al. N. Y., vol. 2, p. 294, Niagara Gr.

osazensis, Swallow, 1863, T'rans. St. Louis Acad. Ści., vol. 2, p. 98, Kaskaskia Gr.

papillata, Hall, 1847, Pal. N. Y., vol. 1, p. 223, Trenton Gir. planocostata, Dawson, 1868, Acad. Geol., p. 307, Carb. pyramidalis, Hall, 1859, Pal. N. Y., vol. 3, p. 347 , Low. Held. Gr.

quadrata, iValcott, 1876, ‘28th

Rep. N. Y. St. Mus. Nat. Hist., p. 93, Trenton Gr.

quadrisulcata, (?) Miller, 1821, Min. Conch., vol. 3, p. 107, Niagara Gr. rugosa, Spencer, 1884, Bull. No. 1, Mus. Univ. St. Mo., p. 59, Niagara Gr. splendida, Billings, 1866, Catal. Sil. Foss. Antic., p. 21, Hud. Riv. Gr.

subcarbonaria, Meek \& Worthen, 1865, Proc. Acad. Nat. Sci., p. 253, and Geo. Sur. Ill., vol. 5, p. 520, Keokuk Gr. subulata, Hall, 1858, Trans. Alb. Inst., vol. 4, p. 32. and Bull. Am. Mus. Nrt. Hist., p. 91, Warsaw Gr.

transversa, Ringueberg, 1886, Bull. Buf. Soc. Nat. Sci., vol. 5, p. 19, Niagara Gr. 
trentonensis, Hall, 1847, Pal. N. Y., vol. 1, p. 222, Trenton and Hud. Riv. Grs. triplicata, Swallow, 1860, Trans. St. Louis Acad. Sci., vol. 1, p. 657, Ham. Gr.

undulata, Conrad, 1841, Ann. Rep. N. Y. p. 57, and Pal. N. Y., vol. 5, pt. 2, p. 208, Ham. Gr.

verneuilana, Emmons, 1846, Am. Quar. Jour. Agr. and Sci., vol. 4, p. 330. Subcarboniferuus.

victa, White, 1862, Proc. Bost. Soc. Nat. Hist., vol. 9, p. 22, Burlington Gr.

whitii, Meek \& Worthen, 1865, Proc. Acad. Nat. Sci., p. 253, Waverly Gr.

wilkinsi, Spencer, 1884, Bull. No. 1, Mus. Univ. St. Mo., p. 59, Niagara Gr.

DiplothecA, Matthew, 1885, Am. Jour. Sci. and Arts, 3d ser., vol. 30, p. 293. [Ety. diploos, double; Theca, a genus.] Slender, conical, section triangular; internal septa dividing it in segments; body cavity separated from one side by a thin partition, supported by delicate transverse septa; distinguished from Hyolithes by more rapidly expanding, and by a firmer, rounder side, where it has the support of the lateral septa. Type D. acadica.

acadica, Matthew, 1885, Am. Jour. Sci. and Arts, 3d ser., vol. 30, p. 294, St. John Gr.

hyattana, Matthew, 1885, Am. Jour. Sci. and Arts, 3d ser., vol. 30, p. 294, St. John Gr.

hyattana var. caudata, Matthew, 1885 , Am. Jour. Sci. and Arts, 3d ser., vol. 30 , p. 294, St. John Gr.

Hyolitilelus, Billings, 1871, Can. Nat. and Geol, vol. 6, p. 240, and Am. Jour. Sci. and Arts, $3 d$ ser., vol. 3, p. 360 . [Ety. diminutive of Hyolithes.] Distinguished from Hyolitles by its long, slender form and structure of the operculum. Type H. micans. micans, Billings, 1871, Can.

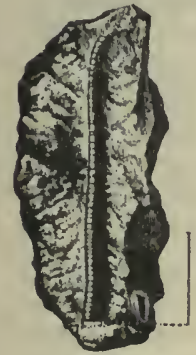

F'IG. 645,-Hyolithellus micans. Terminal portion enlarged.
Nat. and Geol., vol. 6, p. 240, and Am. Jour. Sci. and Arts., 3d ser., vol. 3, p. 354, Up. Taconic.

micans var. rugosa, Walcott, 1887, Am. Jour. Sci. and $\mathrm{A}$ rts, $3 \mathrm{~d}$ ser., vol. 34 , p. 191, Up. Taconic.

Hyolithes, Eichwald, 1840 , Sil. Schicht. Syst. in Ehstl., p. 97. Apparently the internal casts of tubes forming an elongate, subtriangular pyramid; lateral margins acute and tapering from the base to an acute extremity; dorsal side usually more convex than the ventral, and often longitudinally sinuate; aperture usually oblique and extended on the ventral side; surface smooth, or having arching or transverse striæ. Type $H$. acutus. aclis; Hall, 1876, Illust. Devonian Foss., pl. 27, and Pal. N. Y., vol. 5, pt. 2, p. 197, Ham. Gr.

aculeatus, Hall, 1860, (Theca aculeata,) 13th Rep. N. Y. St. Mus. Nat. Hist., p. 107, Kinderhook Gr.

americanus, Billings, 1871, (Theca triangularis,) Hall, Can. Nat. and Geol., vol. 6, p. 213, Up. Taconic.

baconi, Whitfield, 1878, Ann. Rep. Geo. Sur. Wis., p. 77, and Geo. Wis., vol. 4, p. 225, Trenton Gr.

carbonaria, Walcott, Monogr. U. S. Geo: Sur., vol. 8, p. 264, Subcarboniferous.

centennialis, Barrett, 1877, Ann. Lyc. Nat. Hist., vol. 11, p. 299, Low. Held. Gr.

communis, Billings, 1871, Can. Nat. and Geol., vol. 6, p. 213, Up. Taconic.

danianus, Matthew, 1884, Bull. U. S. Geo. Sur., vol. 2, p. 283, St. John Gr.

emmonsi, Ford, 1873, Am. Jour. Sci., 3d ser., vol. 5, p. 214, Up. Taconic.

excellens, Billings, 1874, Pal. Foss., vol. 2, p. 70, Up. Taconic.

gibbosus, Hall \& Whitfield, 1873, 23d Rep. N. Y. St. Mus. Nat. Hist., p. 242, Potsdam Gr.

gracilis, Matthew, 1885, (Camerotheca gracilis,) Can. Rec. Sci., vol. 1, p. 149, St. John Gr.

gregarius, Meek \& Hayden, 1861, Proc. Acad. Nat. Sci. Phil, p. 436, and Pal. Up. Mo., p. 5, Potsdam Gr.

heros, Hall, 1888, Pal. N. Y., vol. 7, p. 7, Low. Held. Gr.

impar, Ford, 1872, Am. Jour. Sci., 3d ser., vol, 3, p. 419, Up. Taconic.

ligea, Hall, 1863, (Theca ligea,) 15th Rep. N. Y. St. Mus. Nat. Hist., p. 62 , and Pal. N. Y., vol. 5, pt. 2, p. 195, Up. Held. Gr.

micans, see Hyolithellus micans.

micmac, Matthew, 1884, Bull. U. S. Geo. Sur., vol. 2, p. 283, St. John Gr.

neapolis, Clarke, 1885, Bull. U. S. Geo. Sur., No. 16, p. 56, Portage Gr.

parviusculus, Hall, 1862, (Theca parviuscula,) Geo. Rep. Wis., p. 425, Hud. Riv. Gr.

primordialis, Hall, 1861, (Theca primordialis,) Geo. Rep. Wis., p. 48, and Geo. Wis., vol. 4, p. 175, Potsdam Gr.

princeps, Billings, 1871, Can. Nat. and Geol., vol. 6 , p. 213, and Am. Jour. Sci. and Arts, 3d ser., vol. 3, p. 355 , Up. Taconic.

principalis, Hall, 1876, Illust. Devonian Foss., pl. 27, and Pal. N. Y., vol. 5 , pt. 2, p. 196, Śchoharie grit.

shaleri, Walcott, 1885, Bull.

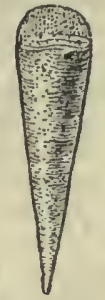

Fig. 646. - Hyolithes primordialis.

U. S. Geo. Sur., p. 283, Up. Taconic. singulus, Hall, 1879, Pal. N. Y., vol. 5, pt. 2, p. 202, Ham. Gr. 
striatus, Hall, 1876, 1llust. Devonian Foss., pl. 27, and Pal. N. Y., vol. 5, pt. 2, p. 199, Ham. Gr.

subimbricatus, Ringueberg, 1888, Proc. Acad. Nat. Sci. Phil., p. 135, Niagara Gr.

triliratus, Hall, 1879, Pal. N. Y., vol. 5, pt. 2, p. 201, Ham. Gr.

vanuxemi, Walcott, 1885 , Monogr. U. S. Geo. Sur., vol. 8, p. 85, Chazy Gr.

Matthevia, Walcott, 1885, Am. Jour. Sci. and Arts, 3d ser., vol. 30, p. 17. [Ety. proper name.] Shell conical; aperture sinuous, transverse section, ovate, elliptical or rounded subquadrate; two elongate interior chambers, diverging from the apex, open into the terminal chamber, and are crossed by a single

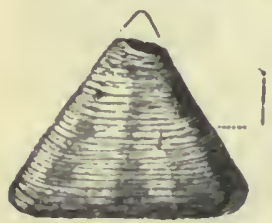

Fia.647.-Matthevia variabilis. imperforate septum; surface papillose ; opercu. Ium calcareous, nucleus excentric, lines of growth concentric. Type M. variabilis.

variabilis, Walcott, 1885, Am. Jour. Sci. and Arts, 3d ser., vol. 30, p 18, Calciferous Gr.

Pharetrella, Hall, 1888, Pal. N. Y., vol. 7, p. 7. Shell large, elongate, Hyolitlueslike in outline; apex acute; surface ornamented with transverse, undulating strix. Type P. tenebrosa.

tenebrosa, Hall, 1888, Pal. N. Y., vol. 7, p. 7, Genesee Slate.

Pterotimca, Salter, 1852, Rep. Brit. Ass'n, p. 61. [Ety.pteron, wing; Theca, a genus.] Shells arcuate, somewhat calyptrseform, subtriangular, or oval; apex marginal and incurved on the same plane, carinate upon the back, abruptly and broadly expanding, with the anterior margin sinuate; interior concave, shallow ; a concave, shelly partition covers the posterior half of the cavity. Type P. transversa.

anatiformis, Hall, 1847, (Tellinomya anatiformis,) Pal. N. Y., vol. 1, p. 154, Trenton $\mathrm{Gr}$.

attenuata, Hall, 1861, (Clioderma attenuata,) 14 th Rep. N. Y. St. Mus. Nat. Hist., p. 98, Trenton Gr.

canaliculata, Hall, 1861, (Cleioderma canaliculats,) 14th Rep. N. Y. St. Mus. Nat. Hist., p. 97, Trenton Gr.

expansa, Emmons, 1842, (Delthyris expansus,) Geo. Rep. N. Y., p. 397, Black Riv. and Trenton Grs.

saffordi, Hall, 1861, (Cleioderma saffordi,) 14th Rep. N. Y. St. Mus. Nat. Hist., p. 96, Trenton Gr.

transversa, Salter, 1852, Rep. Brit. Ass'n, p. 61, Hud. Riv. Gr.

undulata, Hall, 1861, (Cleioderma undulata,) 14th Rep. N. Y. St. Mus. Nat. Hist., p. 97, Trenton Gr.
Pugiunculus aculeatus, see Hyolithes aculeatus.

Scenella, Billings, 1872, Can. Nat. and Geol., vol. 6, p. 479, and Pal. Foss., vol. 2, p. 77. [Ety. scene, tent; ella, diminu. tive.] Shell small, depressed, conical ; apex central, an obscure carina extending from the apex to the margin; apex slightly incurved opposite the carina; aperture nearly circular; surface finely reticulated. Type $S$. reticulata.

conica, Whiteaves,

1884, Pal. Foss., v o l. 3 , p. 32 , Guelpli Gr.

conula, Walcott, 1885, Fra. 648.-Scenellr conMonogr. U. S. Geo. Icn. Side vlew.

Sur., vol. 8 , p. 15, Up. Taconic.

reticulata, Billings, 1872 , Can. Nat. and Geo., vol. 6, p. 479, and Pal. Foss., vol. 2, p. 77, Up. Taconic.

retusa, Ford, 1873, Am. Jour. Sci. and Arts, 3d series, vol. 5, p. 213, Up. Taconic.

varians, Waleott, 1886, Bull. U. S. Geo. Sur., vol. 30, p. 127, Up. Taconic.

StenotiecA, Hicks, 1872, Quar. Jour. Geo. Soc., vol. 28, p. 180. [Ety. stenos, narrow; Theca, genus.] Shell small, curved; lines of growth strongly marked transversely. Type S. cornucopia.

acadica, Hartt, 1868, (Diseina acadica,) Acad. Geol., p. 644, St. John Gr.

concentrica, Matthew, 1885, Trans. Roy. Soc. Can., p. 57, St. John Gr.

elongata, Walcott, 1885, Monogr. U. S. Geo. Sur., vol. 8, p. 23, Up. Taconic.

hicksana, Matthew, 1885, Trans. Roy. Soc. Can., p. 56, St. John Gr.

nasuta, Matthew, 1885, Trans. Roy. Soc. Can., r. 58, and Can. Nat. and Geo., vol. 6 , p. 479 , St. John Gr.

pauper, Billings, 1872, Pal. Foss., vol. 2, p. 77, Up. Taconic.

radiata, Matthew, 1885, Trans. Roy. Soc.

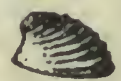
Can., p. 57, St. John Gr.

rugosa, Hall, 1847, (Metoptoma rugosa, Pal.

FI0. 649.-Stenotheca rugosa.

N. Y., vol. 1, p. 306 , Up. Taconic.

triangularis, Matthew, 1885, Trans. Roy. Soc. Can., p. 58, St. Jolin Gr.

Styriola, Lesueur, 1826. [Ety. stylos, pillar.] Small, conical, without annulations which distinguishes it from Tentaculites.

fissurella, Hall, 1843, (Tentaculites fissurellus,) Geo. 4th Dist. N. Y., p. 180, and Pal. N. Y., vol. 5, pt. 2, p. 178, Marcellus Shale and Genesee Slate.

fissurella var. intermittens, Hall, 1879, Pal. N. Y., vol. 5, pt. 2, p. 181, Genesee Slate.

fissurella var. obsolescens, Hall, 1879, Pal. N. Y., vol. 5, pt. 2, p. 180, Ham. Gr. fissurella var. strigata, Hall, 1879, Pal. N.Y., vol. 5, pt. 2, p. 180, Marcellus Shale. 
obtusa, Hall, 1879, Pal. N. Y., vol. 5, pt. 2, p. 182, Ham. Gr.

spica, Hall, 1888, Pal. N. Y., vol. 7, p. 7, Ham. Gr.

Tentaculites, Schlotheim, 1820,Petrefakten., p. 377. [Ety. tentaculum, feeler ; lithos, stone.] Straight, elongate, attenuately conical tubes, annulated by abruptly elevated rings closely arranged near the apex, and more distant and stronger with the increasing size of the shell and distance from the apex; surface marked by fine transverse striæ, rarely by longitudinal striæ. Type T. scalaris. acula, Hall, 1888, Pal. N. Y., vol. 7, p. 6, Low. Held. Gr.

arenosus, Hall, 1876, Illust. Devon. Foss., pl. 26, and Pal. N. Y., vol. 5, pt. 2, p. 166, Oriskany sandstone.

attenuatus, Hall, 1876, Illust. Devonian Foss., pl. 26, and Pal. N. Y., vol. 5, pt. 2, p. 170 , Ham. Gr.

bellulus, Hall, 1876, Illust. Devonian Foss., pl. 26, and Pal. N. Y., vol. 5, pt. 2, p. 169, Ham. Gr.

dexithea, Hall, 1888, Pal. N. Y., vol. 7, p. 6, Schoharie grit.

distans, see Cornulites distans.

elongatus, Hall, 1859, Pal. N. Y., vol. 3, p. 136, Low. Held. Gr.

fissurella, see Styliola fissurella.

flexuosa, see Conchicolites flexuosus.

gracilistriatus, Hall, 1879, Pal. N. Y., vol. 5 , pt. 2, p. 173, Marcellus Shale.

hoyti, White, 1876, Proc. Acad. Nat. Sci., p. 34, Devonian.

gyracanthus, Eaton, 1832, (Echinus gyracanthus,) Geo. Text-book, p. 128, Low. Held. Gr.

incurvis, Shumard, 1856, Geo. Rep. Mo., p. 195, Trenton Gr.

irregularis, Hall, 1859, Pal. N. Y., vol. 3, syn. for T. gyracanthus.

minutus, Hall, 1843, Geo. Rep. 4th Dist. N. Y., p. 72, and Pal. N. Y., vol. 2, p. 183, Clinton Gr. neglectus, Nicholson \& Hinde, 1874, Can. Jour., p. 9, Clinton Gr.

niagarens is,

Hall, 1852,

Pal. N. Y.,

vol. 2, p. FIG. 650.-Tentaculites rich-

352, Niag- FIG. 650 mondensis. ara Gr.

niagarensis var. cumberlandiæ, Hall, 1888,

Pal. N. Y., vol. 7, p. 5, Niagara Gr.

oswegoensis, Meek \& Worthen, 1865 ,

Proc. Acad. Nat. Sci. Phil., p. 254, and

Geo. Sur. Ill., vol. 3, p. 342, Hud. Riv. Gr.

richmondensis, S. A. Miller, 1874, Cin.

Quar. Jour. Sci., vol. 1, p. 234, Hud.

Riv. Gr.

scalariformis, Hall, 1876, Illust. Devonian Foss., pl. 26, and Pal. N. Y., vol. 5, pt. 2, p. 167, Up. Held. Gr.

scalaris, Schlotheim, 1820, Petref. Not an American species.

sicula, Hall, 1876, Illust. Devonian Foss., pl. 26, Up. Held. Gr.

spicula, Hall, 1876, Illust. Devonian Foss., pl. 26, and Pal. N. Y., vol. 5, pt. 2, p. 172, Chemung Gr.

sterlingensis, Meek \& Worthen, 1865, Proc. Acad. Nat. Sci. Phil., p. 255, and Geo. Sur. Ill., vol. 3, p. 342, Hud. Riv. Gr.

subtilis, Winchell, 1866, Rep. Low. Peninsula Mich., p. 92, Ham. Gr.

tenuistriatus, Meek \& Worthen, 1865 ,

Proc. Acad. Nat. Sci. Phil., p. 255, and

Geo. Sur. Ill., vol. 3, p. 343, Hud. Riv. Gr.

Theca, Sowerby, 1845, syn. for Hyolithes. aculeata, see Hyolithes aculeatus. gregaria, see Hyolithes gregarius. ligea, see Hyolithes ligea.

parviuscula, see Hyolithes parviusculus. primordialis, see Hyolithes primordialis. triangularis, Hall, 1847, Pal. N. Y., vol. 1, p. 313. This name was preoccupied by Portlock in 1843. Billings described it as Hyolithes americanus. 


\title{
CLASS GASTEROPODA.
}

\author{
[Ety. gaster, under side of body; pous, foot.]
}

The locomotive organ in the Gasteropoda consists of a broad, muscular undersurface, or foot, upon which the animal creeps with a gliding motion. The expansion and contraction of the muscles may be seen when a snail is moving over glass. This form of the foot is the most important characteristic of the Gasteropoda. The head is distinct, and usually furnished with tentacles and cyes. The mouth is on the lower surface, and is often furnished with one or two teeth, or jaws, in the upper part, and a ribbon-like tongue, with minute silicious teeth on its upper surface, which are used with the upper teeth in separating the food. The teeth on the tongue are called the lingual teeth.

The body is generally much larger on one side than on the other, which produces a spiral shell in the growth of the animal, because the shell is secreted at the cdge of the mantel. The shell nearly always consists of one piece (univalve), forming a conical tube, twisted spirally; but the tube is not perfect, because the inner wall of each whorl is formed of the preceding whorl with only a thin conting of shelly matter. Sometimes the tube is rolled in a plane, producing a discoid shell; and there are all grades of form, from the discoid to the upright. The right side of the animal is usually the larger, and the shell produced is dextral; but in some species and in some abnormal specimens of dextral species, the spire is turued in the opposite direction, and the shell is therefore called sinistral. The winding of the tube in the spiral shells as the animal grows, produces a central axis, which is called the columella. It extends from the apex to the base, and forms the inner margin of the aperture. The columella is usually hollow, and terminates at the base of the shell with a small opening, callerl the umbilicus. The margins of the aperture are called the lips. When the columella forms the inner lip, it is called the columellar lip. The outer lip forms the convexity of the shell. Sometimes the lips are continuous, and sometimes the outer lip is more or less deeply notehed; and both lips may be furnished with teeth or denticulated edges. The last whorl of the shell is called the body whorl, from its receiving the body of the animal, and the remaining whorls constitute the spire. The line which separates the whorls is the suture. Many Gasteropoda have a calcareous plate attached to the hinder part of the foot, which closes the aperture when the animal retracts itself within the shell; this covering is called an operculum.

The Gasteropoda are divided into two subclasses: the Heteropoda and Gasteropoda proper. The Heteropoda, also called the Nucleobranchiata, are all inhabitants of the ocean, and usually have a shell covering only the essential organs of the body. They swim rapidly near the surface of the water with the back downward, and when the foot is present it is used to attach the animal to floating sea-weeds.

The Gasteropoda proper are divided into two orders: one breathing air, the Pulmonifera; and the other water, the Branchifera. The Pulmonifera include the 
land snails and their allies; the Branchifera are furnished with gills, and include nearly all Palæozic shells of this Class.

Fanily Belíerophontide.-Bellerophon, Bucanella, Bucania, Phragmostoma, Porcellia, Tremanotus.

Fajily Bulimorphidæ.-Bulimorpha.

Family Calyp'remes.-Capulus, Conchopeltis, Metoptoma, Platyceras.

Family Chitonide.-Chiton.

FAmid y Clisospiride.-Billingsia, Clisospira.

Faniry Cononochilide.-Codonochilus.

Family Cyclonemid ж.-Cyclonema, Eunema, Holopea, Holopella, Platyschisma, Orthonema, Palæacmæa, Trochonema.

Fanily Cycloride.-Cyelora.

Fanim Cyrtolitida.-Carinaropsis, Conchopeltis, Cyrtolites, Cyrtonella, Microceras.

Family Dentalind.-Dentalium.

Famis Euomphanide.-Calaurops, Eccyliomphalus, Euomphalus, Omphalotrochus, Ophileta, Pleuronotus, Straparollina, Straparollus.

Fauily Fusispiride.-Fusispira.

Famisy Helicide.-Anthracopupa, Dawsonella, Pupa, Streptaxis, Strophites, Zonites.

Family Littorinide.-Xenophora.

Family Macluridda.-Maclurea.

Family Natacopside.-Callonema, Isonema, Naticopsis, Trachydomia.

Fanily Patejindes.-Lepetopsis, Tryblidium.

Family Platystomide.-Orthostoma, Platystoma, Scævogyra, Strophostylus.

Family Pleurotomaridda.-Helicotoma, Lophospira, Microdoma, Murchisonia, Pleurotomaria, Raphistoma, Scalites.

Family Pseudophoride.-Pseudophorus.

Family Pyramidellide.-Loxonema, Macrochilina, Soleniscus, Zaptychius.

Fanily Rotellide.-Anomphalus, Rotella.

Family Subulitide.-Polyphemopsis, Subulites.

Fairuy Trochidx.-Eotrochus, Palæotrochus.

Fanily Turritellid A.-Aclisina, Turritella.

Aclis, Loven, 1846, Index, Mollusc. litora Scandin. occid. habit., p. 16 . Not an American Palaozoic genus.

minuta, see Aclisina minuta.

robusta, see Aclisina robusta.

stevensoni, see Aclisina stevensoni.

swallovana, see Aclisina swallovana.

Aclisina, DeKoninck, 1881, Faune du Calcaire Carbonifere de la Belgique Ann. d. Mus. Roy. d'Hist. Nat., t. 6, p. 86. [Ety. diminutive of Aclis.] An elongated, banded, conical, spiral shell; distinguished from Murchisonia by its oval aperture, and from Loxonema by its spiral bands. Type A. striatula.

minuta, Stevens, 1858, (Aclis minuta) Am. Jour. Sci., vol. 25, p. 259, Coal Meas. robusta, Stevens, 1858, (Aclis robusta,) Am. Jour. Sci., vol. 25, p. 259, and 'Geo. Sur. Ill., vol. 5 , p. 596, Coal Meas.

stevensoni, White, 1882, (Aclis stevensoni,) Rep. Invert. Foss. New. Mex., p. xxxv, Coal Meas.

swallovana, Geinitz, 1866, (T u rbonilla swallovana, Carb. und Dyas in Neb., p. 5 , Coal Meas.

Acroculia, Phillips, 1841, Pal. Foss. Cornwall, Devon, and W. Somerset, p. 93, syn. for Platyceras.

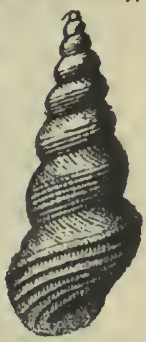

F I G. 651. Aclisina. swallovana. angulata, see Platyceras angulatum. 
erecta, see Platyceras erectum.

ovalis, see Platyceras ovale.

niagarensis, see Platyceras niagarense.

trigonalis, see Platyceras trigonale.

Ampullaria, Lamarck, 1801, Syst. An. sans Vert. [Ety. ampulla, a flask.] Not a Palæozoic genus.

helicoides, see Soleniscus helicoides.

powelli, Walcott, 1883, Science, vol. 2, p. 808, and Monogr. U. S. Geo. Sur., vol. 8 , p. 261, Subcarboniferous.

Anomphalus, Meek \& Worthen, 1866, Proc. Acad. Nat. Sci., p. 268 . [Ety. anomphalos, without an umbilicus.] A helicoid shell of three or more volutions, and baving an aperture transversely suboval. Type A rotulus.

meeki, see Dawsonella meeki.

rotulus, Meek \& Worthen, 1866, Proc. Acad. Nat. Sci. Pbil., p. 268, and Geo. Sur. Ill., vol. 5, p. 597, Coal Meas.

Antilracopupa, Whitfield, 1881, Am. Jour. Sci. and Arts, 3d ser., vol. 21, p. 126. [Ety. anthrax, coa]; Pupa, a genus.] Shell minute, pupiform, few volutions, last unsymmetrical; axis imperforate; aperture large, nearly vertical; peristome thickened, united above by a thin callus, on which may occur one or more palatal teeth; other tooth-like projections on the inner margin of lip; circular notch, as in Pupina, on inner edge of outer limb, near body whorl; surface vertically lined. Type A. ohioensis.

ohioensis, Whitfield, 1881, Am. Jour. Sci. and Arts, 3d ser., vol. 21, p. 126, Coal Meas.

Belleropilon, Montfort, 1508, Conch. Syst. vol. 1, ?. 50. [Fty. mythological name.] Shell thick, symmetrical, globose, involute; sinus in the middle of the outer lip, from which a band extends backward along the outer surface of the volution; inner lip thickened, expanded on the inrolled spire. Type B. vasulites. acutilira, Hall, 1862, 15th Rep. N. Y. Mus. Nat. Hist., p. 56, Ham. Gr.

acutus, Sowerby, 1839, Murch. Sil. Syst., p. 643, Low. Silurian.

allegoricus, White, 1874, Rep. Invert. Foss., p. 10, and Geo. Sur. WV. 100th Mer., vol. 4, p. 55, Quebec Gr.

alternodosus, Whitfield, 1882, Ann. N. Y. Acad. Sci., vol. 2, p. 225, Kaskaskia Gr. anguslata, see Bucania angustata.

antiquatus, Whitfield, 1878, Ann. Rep. Geo. Sur. Wis., p. 52, and Geo. IVis., vol. 4, p. 176, Potsdam Gr.

apertus, Sowerby, 1825, Min. Conch., vol. 5, p. 108, Subcarboniferous.

argo, Billings, 1860, Can: Nat. and Geol., vol. 5, p. 167, Black Riv. and Trenton Gr.

auriculatus, Hall, 1852, Pal. N. Y., vol. 2, p. 334, Coralline limestone.

barquensis, Winchell, 1862, Proc. Acad.

Nat. Sci., p. 425, Marshall Gr.

bidorsatus, see Bucania bidorsata. bilabiatus, White \& Whitfield, 1862, Proc. Bost. Soc. Nat. Hist., vol. 8, p. 304, Kinderhook Gr.

bilobatus, Sowerby, 1839, Murch. Sil. Syst., p. 643, and $\mathrm{Pal}$. N. Y., vol. 1 , p. 184, Black Riv. to Mid. Sil.

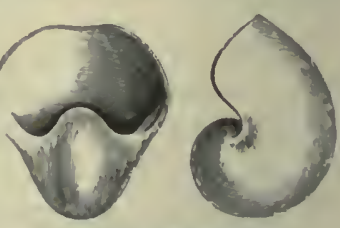

FIG. 652.-Bellerophon bilobatus. bilobatus var.

acutus, Hall, 1847, Pal. N. Y., vol. 1, p. 185, Trenton Gr.

bilobatus var corrugatus, Hall, 1847, Pal. N. Y., vol. 1, p. 185, Trenton Gr.

blaneyanus, syn. for B. carbonarius.

bownani, White, 1876, Proc. Acad. Nat. Sci., p. 32, Devonian.

brevilineatus, Conrad, 1842, Jour. Acad. Nat. Sci., vol. 8, p. 269 , and Pal. N. Y., vol. 5, p. 2, p. 107, Няm. Gr.

canadensis, Billings, 1866, Catal. Sil. Foss. Antic., p. 18, Hud. Riv. Gr.

cancellatus, Hall, 1847, Pal. N. Y., vol. 1, p. 307 , Hud. Riv. Gr.

cancellatus, Hall, 1858, Trans. Alb. Inst., vol. 4. The name was preoccupicd. See B. textilis.

carbonarius, Cox, 1857, Geo. Rep. Ky., vol. 3, p. 562, Coal Meas.

carbonarius var. subjapillosus, Wlite, 1876, Gen. Uinta Mountains, p. 92, Up. Aubrey Gr.

carinatus, Sowerby, 1839, Murch. Sil. Syst., p. 634, Devonian.

cassinensis, Whitfield, 1886, Bull. Am. Mus. Nat. Hist., vol. I, p. 318 , Birdseye $\mathrm{Gr}$.

charon, Billings, 1860, Can. Nat. and Geol. vol. 5, p. 169, Black Riv. and Trenton Grs.

combsi, Wolcott, 1885, Monogr. U. S. Geo. Sur., vol. 8, p. 193, Devonian.

convolutus, Eaton, 1S32, Geo. Text-book, p. 28, Up. Sil.

crassus, Meek \& Worthen, 1860, Proc. Acad. Nat. Sci., p. 458, and Geo. Sur. Ill., vol. 2, p. $385, \mathrm{Coal}$ Meas.

crenistria, Hall, 1876, Illust. Devonian Foss., pl. 25, and Pal. N. Y., vol. 5, pt. 2, p. 116, Ham. Gr.

curvilineatus, Conrad, 1842, Jour. Acad. Nat. Sci., vol. 8, p. 269, Onondaga, Schoharie and Up. Held. Gr.

cyrtolites, Hall, 1860, 13th Rep. N. Y. Mus. Nat. Hist., p. 107, Kinderhook Gr.

declivis, Conrad, 1842, Jour. Acad. Nat. Sci., vol. 8, p. 269, Trenton Gr.

disculus, Billings, 1860, Can. Nat. and Geo., vol. 5, p. 168, Black Riv. and Trenton Gr.

ellipticus, McChesney, 1860, Desc. New Pal. Foss., p. 58, Coal Meas. expansus, see Bucania expansa.

explanatus, Hall, 1879, Pal. N. Y., vol. 5, pt. 2, p. 109, Chemung Gr. 
fiscellostriatus, Foerste, 1885, Bull. Sci. Lab. Denison Univ., p. 99, Niagara Gr. fraternus, Billings, 1866, Catal. Sil. Foss., Antic., p. 19, Hud. Riv. Gr.

galericulatus. Winchell, 1862, Proc. Acad. Nat. Sci., p. 426, Marshall Gr.

gibsoni, White, 1882, 11th Rep. Geol. and Nat. Hist. Indiana, p. 360, St. Louis Gr. giganteus, Worthen, 1884, Bull. No. 2, Ill. St. Mus. Nat. Hist., p. 8, and Geo. Sur. Ill., vol. 8, p. 143, Low. Coal Meas. globosus, Stevens, 1858, Am. Jour. Sci., vol. 25, p. 258, Coal Meas.

harrori, Gurley, 1883, New Carb. Foss., p. 5. Publication not such as to establish a species.

helena, Hall, 1879, Pal. N. Y., vol. 5, pt. 2, p. 114, Hain. Gr.

hiulcus, Sowerby, Min. Conch. Not American.

hyalina, Hall, 1879, Pal. N. Y., vol. 5, pt. 2, p. 99, Up. Held. Gr.

incisus, Clarke, 1885 , Bull. 'U. S. Geo. Sur., vol. 16, p. 53, Portage Gr.

inspeciosus, White, 1882, Rep. Invert. Foss. New Mex., p. xxx, Coal Meas.

interlineatus, Portlock, 1843, Geo. of Londonderry, p. 402, Coal Meas. Probably not American.

kansasensis, Shumard, 1858, Trans. St. Louis Acad. Sci., vol. 1, p. 204, Coal Meas.

leda, Hall, 1862, 15th Rep. N. Y. Mus. Nat. Hist., p. 58, and Pal. N. Y., vol. 5, pt. 2, p. 110, Ham. Gr.

lineolatus, Hall, 1860, 13th Rep. N. Y. Mus. Nat. Hist., p. 107, Waverly or Kinderhook Gr.

lindsleyi, Safford, 1869, Geo. of Tenn., p. 289, Nashville Gr.

lyra, Hall, 1862, 15th Rep. N. Y. Mus. Nat. Hist., p. 59, and Pal. N. Y., vol. 5, pt. 2, p. 113, Ham. Gr.

macer, Billings, 1865, Pal. Foss., vol. 1, p. 347, Calciferous Gr.

miera, Hall, 1876, Illust. Devonian Foss., pl. 22, and Pal. N. Y., vol. 5, pt. 2, p. 119, Chemung Gr.

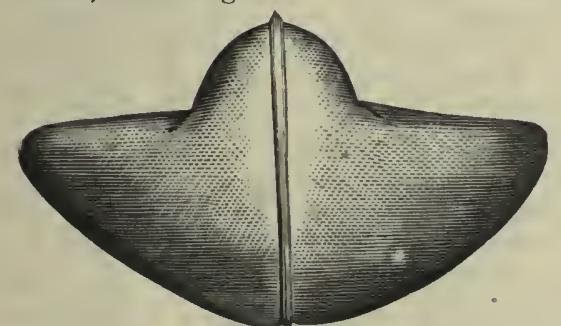

Frg. 653.-Bellerophon mohri.

majusculus, Walcott, 1885, Monogr. U. S. Geo. Sur., vol. 8, p. 256, Subcarb.

marcouanus, Geinitz, 1866, (Yarb. und Dyas in Neb., p. 7, and Pal. E. Neb., p. 226, Coal Meas.

meekanus, Swallow, 1858, Trans. St. Louis Acad. Sci., vol. 1, p. 204, Coal Meas. michiganensis, Winchell, 1862, Proc. Acad. Nat. Sci., p. 427, Marshall Gr. miser, Billings, 1866, Catal. Sil. Foss. Antic., p. 20, Hud. Riv. Gr.

missouriensis, Swallow, 1863, Trans. St.

Louis Acad. Sci., vol. 2, p. 100, Kaskaskia Gr.

mohri, S. A. Miller, 1874, Cin. Quar. Jour. Sci., vol. 1, p. 306, Hud. Riv. Gr.

montfortanus, Norwood \& Pratten, 1855,

Jour. Acad. Nat. Sci., vol. 3, p. 74, Coal Meas.
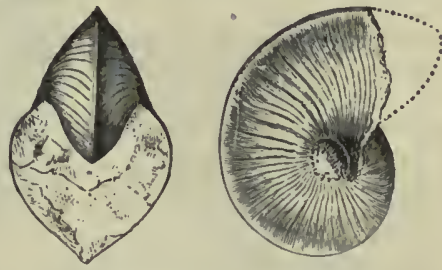

Fra. 654,-Bellerophon palinurus.

morrowensis, Miller \& Dyer, 1878, Cont. to Pal., No. 2, p. 8, Hud. Riv. Gr.

nactus, Hall, 1879, Pal. N. Y., vol. 5, pt. 2, p. 121, Chemung Gr.

nashvillensis, Troost, 1840 , 5th Geo. Rep.

Tenn., p. 54, Trenton Gr.

natator, Hall, 1862, (Phragmostoma natator,) 15th Rep. N. Y. Mus. Nat. Hist., p. 60, and Pal. N. Y., vol. 5, pt. 2, p. 108, Ham. Gr.

nautiloides, Winchell, 1862, Proc. Acad.

Nat. Sci., p. 427, Marshall Gr.

neleus, Hall \& Whitfield, 1876, Illust.

Devonian Foss., pl. 22, and U. S. Geo.

40th Parallel, p. 250, Chemung Gr.

newberrvi, Meek, 1871, Proc. Acad. Nat.

Sci., p. 77, and Ohio Pal., vol. 1, p. 222,

Up. Held. Gr.

nodocarinatus, Hall, 1858, Geo. Rep. Iowa, p. 723 , Coal Meas.

obsoletus, Hall, 1876, Illust. Devonian Foss., pl. 22, Chemung Gr.

otsego, Hall, 1862, 15th Rep. N. Y. Mus.

Nat. Hist., p. 60, and Pal. N. Y., vol. 5, pt. 2, p. 104, Ham. Gr.

palinurus, Billings, 1865, Pal. Foss., vol. 1, p. 311 , Quebec Gr.

panneus, White, 1862 , Proc. Bost. Soc.

Nat. Hist., vol. 9, p. 21, Marshall Gr.

patersoni, Hall, 1862, Geo. Rep. Wis., p. 55, Hud. Riv. Gr.

patulus, Hall, 1843, Geo. Rep. 4th Dist. N. Y., p. 196, and Pal. N. Y., vol. 5, pt. 2, p. 100 , Ham. Gr.

pelops, Hall, 1862, 15th Rep. N. Y. Mus.

Nat. Hist., p. 56, and Pal. N. Y., vol. 5, pt. 2, p. 95, Schoharie and Up. Held. Gr. pelops var. exponens, Hall, 1879, Pal. N. Y., vol. 5, pt. 2, p. 96, Up. Held. Gr. percarinatus, Conrad, 1842, Jour. Acad. Nat. Sci., vol. 8, p. 268, Coal Meas.

perelegans, White \& IVhitfield, 1862, Proc. Bost. Soc. Nat. Hist., vol. 8, p. 304 , Kinderbook Gr.

perforatus, Winchell \& Marcy, 1866, syn. for Tremanotus chicagoensis. 
perplexus, Walcott, 18S5, Monogr. U. S. Geo. Sur., vol. 8, p. 193, Devonian.

perlatus, Conrad, 1842, Jour. Acad. Nat. Sci., vol. 8, p. 270, Coal Meas.

platystoma, Meek \& Worthen, 1868, Geo. Sur. Ill., vol. 3, p. 312, Galena Gr.

plenus, Billings, 1874, Pal. Foss., vol. 2, p. 62, Gaspe linestone No. $\$$, Devonian. profundus, Emmons, Geo. Rep., 2d Dist.

N. Y., p. 393, Trenton Gr.

propinquus, Meek, 1871, Proc. Acad. Nat. Sci., p. 78, and Ohio Pal., vol. 1, p. 226, Up. Held. Gr.

punctifrons, see Bucania punctifrons.

repertus, Hall, 1879, Pal. N. I., vol. 5, pt. 2 , p. 128, Ham. Gr.

rotalínea, Hall, 1879, Pal. N. Y., vol. 5, pt. 2, p. 115, Ham. Gr.

rudis, Hall, 1862, 15th Rep. N. Y. Mus.

Nat. Hist., p. 57, and Pal. N. Y., vol. 5, pt. 2, p. 103, Ham. Gr.

rugosiusculus, Winchell, 1862, Proc. Acad.

Nat. Sci., p. 425, Marshall Gr.

rugosus, Emmons, 1856, Am. Geol., p. 166 , Hud. Riv. Gr.

seriptiferus, Wlite, 1862, Proc. Bost. Soc. Nat. Hist., vol. 9, p. 21, Marshall Gr.

scissile, Conrad, 184t, Proc. Acad. Nat. Sci., vol. 2, p. 175, Kaskaskia Gr. Very poorly defined.

solitarius, Billings, 1866, Catal. Sil. Foss. Antic., p. 20, Hud. Riv. Gr.

stamineus, Conrad, 1842, Jour. Acad. Nat. Sci., vol. 8, p. 269, Marshall Gr.

stevensanus, McChesney, 1860, Desc. New Pal. Foss., p. 61, Coal Meas.

sublrevis, Hall, 1858, Trans. Alb. Inst., vol. 4, p. 32, and Geo. Sur. Iowa, p. 666, Warsaw Gr.

subpapillosus, White, 1879, I3ull. U. S. Geo. Sur. Ter., vol. 5, p. 218, and Cont. to Pal. No. 6, p. 138, Carboniferous. sulcatinus, see Bucania sulcatina.

textiliformis, Gurley, 1853, New Carb. Foss., p. 6. Publication not valid.

textilis, Hall, 1877, 1st Ed. Am. Pal. Foss., p. 243, Warsaw Gr. Proposed instead of B. cancellatus, Hall, 1858, which was preoccupied.

thalia, Hall, 1862, 15th Rep. N. Y. Mus. Nat. Hist., p. 60, and Pal. N. X., vol. 5, pt. 2, p. 105, Hain. Gr.

tricarinatus, Shumard, 1858, Trans. St.

Louis Acad. . Sci., vol. 1, p. 204, Coal Meas.

tricarinata, Hall, 1876, Illust. Devonian Foss. The name was preoccupied. See B. triliratus.

triliratus, Hall, 1877, 1st El. Am. Pal. Foss., p. 243, and Pal. N. X., vol. 5, pt. 2, p. 117, Chemung Gr. Proposed instead of B. tricarinatus, Hall, 1876, which was preoccupied.

troosti, D'Orbigny, 1840, Cephal., p. 206, and Geo. of Tenn., p. 289, Trenton Gr. tuber, Hall, 1876, 28th Rep. N. Y. Mus. Nat. Hist., p. 177, Niagara Gr.

urii, Fleming, 1828, British Animals, p. 338, Devonian. American species. (?) vinculatus, White \& Whitfield, 1862, Proc. Bost. Soc. Nat. Hist., vol. 8, p. 304 , Kinderlıook Gr.

vittatus, syn. for B. carbonarius.

volutus, Eaton, 1832, Geol. Text-book, p. 28, Up. Sil.

whittleseyi, Winchell, 1865, Pros. Acad. Nat. Sci., p. 130, Cuyahoga sliale.

wisconsinensis, Whitfield, 1878, Anu. Rep. Geo. Sur. Wis., p. 76, and Geo. Wis., vol. 4, p. 223, Trenton Gr.

Billingsia, WValcott, 1858, Bull. No. 30 , U. S. Feo. Sur., p. 61. [Ety. proper name.] Syn. (?) for Clisospira. Turbinate, whorls, subcircular. Type 13. saralogensis. Preoccupied.

saratogensis, Walcott, 1858, Bull. No. 30, U. S. (reo. Fig.65.5,-13111Sur., p. 61, U’p. Taconic. Ingsla sarBucanella, Meek, 1870, Proc. Am. Phil. Soc., vol. 11, p. 426. [Ity. diminutive of Bucania.] Type 13. nana. nana, Meek, 1870, Proc. Am. P'lil. Soc., vol. 11, p. 426 , Siluriar.

Bucania, Hall, 1847, Pal. N. Y., vol. 1, p. 32. [Fity. bukane, trumpet.] Convolute, spire equally concave on either side; volutions in the same plane, all visibl.., outer one ventricose, inner one usually angulated on the edge, coucave on the ventral side; apertire rounded oval, somewhat compressed on the inner side by contact with the next volution, laterally and dorsally abruptly oxpanded. Type B. sulcatina.

angustata, Hall, 1852, Pal. N. Y., vol. 2, p. 349, Niagara and Guelph Gr.

bellipuncts, Hall, 1852, Pal. N. Y., vol. 2, p. 93, Clinton Gr.

bidorsata, Hall, 1847, (Bellerophon biclorsatus,) Pal. N. X., vol. 1, p. 186, Trenton $\mathrm{Gr}$.

buelli, Whitfeld, 1878, Ann. Rep. Geo. Sur. Wis., p. 76, and Geo. Wis., vol. 4, p. 24, Trenton Gr.

chicagoensis, see Tremanotus chicagoensis.

costata, James, 1872, (Cyrtolites costatus,) Am. Jour. Sei., 3d ser., vol. 3, p. 26 , and Ohio P'al., vol. 1, p. 150, Hud. Riv. Gr.

crassolaris, McChesney, 1861, New Pal. Foss., p. 91, Niagara Gr.

devonica, Hall \& Whitfield, 1872, 24th Rep. N. Y. Mus. Nat. Hist., p. 195, Up. Held. Gr.

euomphaloides, Owen, 1862, Geo. Sur. Ind., p. 362. Not very satisfactorily defined.

exigua, Foerste, 1885, Bull. Sci. Lab. Denison Univ., p.99. Not properly defined. expansa, Hall, 1847, Pal. N. Y., vol. 1, p. 186, Trenton Gr.

intexta, Hall, 1847, Pal. N. Y., vol. 1, p. 317, Trenton Gr.

lirata, Hall, 1862, Geo. Rep. Wis., p. 55, Trenton Gr. 
pervoluta, McChesney, 1861, New Pal. Foss., p. 91, Niågara Gir.

profunda, Conrad, 1841, (Euomphalus profundus,) Ann. Rep. N. Y., p. 37, and Pal. N. Y., vol. 3, p. 341, Up. Held. Gr.
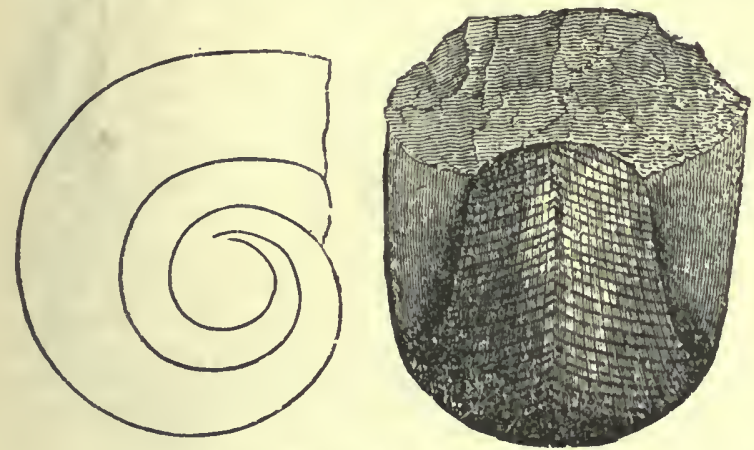

Fig. 656.-Bucania sulcatina.

punctifrons, Emmons, 1842, (Bellerophon punctifrons,) Geo. Rep. 2d Dist., N. Y., p. 392, and Pal. N. Y., vol. 1, p. 187, Black River and Trenton Grs.

rotundata, Hall, 1847, Pal. N. Y., vol. 1, p. 33, Chazv Gr.

stigmosa, Hall, 1852, Pal. N. Y., vol. 2, p. 92, Clinton Gr.

sulcatina, Emmons, 1842, (Bellerophon sulcatinus,) Geo. Rep. 2d Dist. N. Y., p. 312, Pal. N. Y., vol. 1, p. 32, Chazy, Black Riv., and Trenton Grs.

trilobata, Conrad, 1839, (Planorbis trilobatus, Ann. Rep. N. Y., p. 65, and Pal. N. Y., vol. 2, pp. 13 and 93, Medina sandstone and Clinton Gr.

tripla, Whitfield, 1889, Bull. Am. Mus. Nat. Hist., vol. 2, p. 55, Calciferous Gr.

Bulimella, Hall, 1858, Trans. Alb. Inst., vol. 4. This name was preoccupied by Pfeiffer in 1852. See Bulimorpha.

bulimiformis, see Bulimorpha bulimiformis. canaliculata, see Bulimorpha canaliculata. elongata, see Bulimorpha elongata.

Bulimorpin, Whitfield, 1882, Bull. Am. Mus. Nat. Hist., No. 3, p. 74. [Ety. Bulimus, a genus; morphe, form.] $\mathrm{Fu}-$ siform, volutions convex; columella bent, truncated at the base, separated

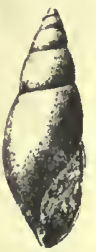

FIg. 657-Bubuimor p $\mathrm{h} \mathrm{a}$ mis. from the outer lip by a notch, as in Achatina; outer lip slightly notched near the upper end; surface smooth. Type B. bulimiformis.

bulimiformis, Hall, 1858, (Bulimella bulimiformis,) Trans. Alb. Inst., vol. 4, p. 29, and Bull. Am. Mus. Nat. Hist., p. 74, Warsaw Gr.

canaliculata, Hall, 1858 Bulimella canaliculata, Trans. Alb. Inst., vol. 4, p. 29, and Bull. Am. Mus. Nat. Hist., 1). 74, Warsaw Gr. elongata, Hall, 1858, Bulimella elongata,) Trans. Alb. Inst., vol. 4, p. 30, and Bull. Am. Mus. Nat. Hist., p. 75, Warsaw Gr. Callonema, Hall, 1879, Pal. N. Y., vol. 5, pt. 2, p. 50. [Ety. kallos, beautiful; nema, thread.] Subglobose, turbinate or ovoid-conical ; volutions rounded or subangular above and below; outer lip thin; columnar lip thickened, spreading over the volution above and extended below; axis umbilicate; surface marked by striæ extending backward from the sutures over the volutions. Type C. bellatulum.

bellatulum, Hall, 1861 . (Loxonema bellatulum,) 14th Rep. N. Y. Mus. Nat. Hist., p. 104, and Pal. N. Y., vol. 5, pt. 2, p. 51, Up. Held. Gr.

imitator, Hall \& Whitfield, 1872, (Pleurotomaria imitator,) 24th Rep. N. Y. Mus. Nat. Hist., p. 195, Ham. Gr.

lichas, Hall, 1861, (Platy. stoma lichas) 14th Rep. Fig. 658. - CalN. Y. Mus. Nat. Hist, lonema bel106, Up. Held. Gr.

occidentale, Walcott, 1885, Monogr. U. S. Geo. Sur., vol. 8, p. 189, Devonian.

Calaurops, Whitfield, 1886, Bull. Am. Mus.

Nat. Hist., vol. 1, p. 314. [Ety. kalaurops, a shepherd's crook.] Univalve, discoidal, convolute, inner volutions closely coiled, outer one disunited and projected in a straight line. Type C. lituiformis. It seems to be distinguished from Eccyliomphalus only by having the last whorl straightened, which may or may not be of generic importance.

lituiformis, Whitfield, 1886, Bull. Am. Mus. Nat. Hist., vol. 1, p. 315, Chazy Gr. Capulus, Montfort, 1810 , Conch. Syst., vol. 2, p. 55. [Ety. capulus, a head-piece or cap.] Shell wide, cap-shaped, apex obliquely inclined backward and inrolled toward the left side; aperture broad, oval, edge irregularly sinuated; muscular scar horseshoe-shaped, open in front. Type $(Y$. hungaricus. The horseshoe-shaped, muscular impression has never been observed in any American Palæozoic fossil, and hence the species referred to this genus do not belong to it. Those named have been so poorly defined, their generic relations can not be determined, and they may as well be struck from the list of names.

acutirostris, see Platyceras acutirostrum. auriformis, Hall, 1847, Pal. N. Y., vol. 1, p. 31, Chazy Gr.

parvus, Swallow, 1858, Trans. St. Louis Acad. Sci., vol. 1, p. 205, Coal Meas. 
triplicatus, Swallow, 1858, Trans. St. Louis Acad. Sci., vol. 1, p. 205, Coal Meas.

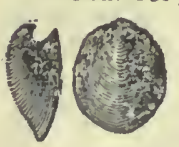

FIG. 659. - Car1naropsis palel llformis.
Carinaropsis, Hall, 1847, Pal.

N. Y., vol. 1, p. 183.

[Ety. from its resemblance to Carinaria.] Shell subconical, patelliform; apex incurved or convolute, subcentral ; aperture oval, expanded, narrowed posteriorly. Type C. carinata.

carinata, Hall, 1847, Pal. N. Y., vol. 1, p. 183, Trenton Gr.

orbiculata, Hall, 1847, Pal. N. Y., vol. 1, p. 306, Hud. Riv. Gr.

patelliformis, Hall, 1847, Pảl. N. Y., vol. 1, p. 183, Trenton and Hud. Riv. Grs.

Chemnutzia, D'Orbigny, 1837, Mollusques, Echinodermes, Foraminiferes et Polypiers, etc. Slender, elongated, many whorled, plaited; apex sinistral ; aperture simple, ovate; peristome incomplete; operculum horny ; subspiral. Type C. elegantissima. Not an American Palaozoic genus.

attenuata, see Loxonema attenuatum.

parva, see Loxonema parvum.

swallovana, see Loxonema swallovanum. tenuilineata, see Loxonema tenuilineatum.

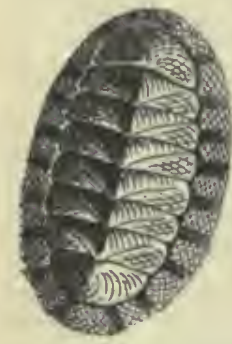

Frg. 660 . Chiton squamosus.
Chiton, Linnaus, 1758, Syst. Nat., ed. 10, p. 667. [Ety. chilon, a coat of mail.] Shell composed of eight transverse imbricat ing plates, lodged in a coriaceous mantle, which forms an expanded margin round the body. Type C. scuamosus. Not an American Palseozoic genus. canadensis, see Metoptoma canadense.

carbonarius, Stevens, 1859, Am. Jour. Sci., vol. 25, v. 264, and Geo. Sur. Ill., vol. 5 , p. 608 . Probably a crustacean, Coal Meas.

parmus, Stevens, 1859, Am. Jour. Sci., vol. 25, p. 264, Coal Meas.

Clisospira, Billings, 1865, Pal. Foss., vol.1, p. 186 and 420 .

[Ety. kleio, to lock ; spira whorl.] Shell conical; aperture widely expanded a ll round in a plane at a right

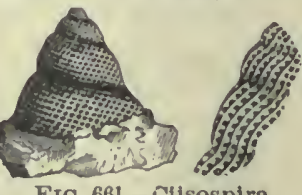

FIG. 661.-Cilsospira curlosa. angle to the longitudinal axis of the conical spire; suture in the spire, but obsolete below. Type C. curiosa.

curiosa, Billings, 1865, Pal. Foss., vol. 1, pp. 186 and 420, Up. Taconic.

lirata, Whitfield, 1886, Bull. Am. Mus. Nat. Hist., vol. 1, p. 308, Birdseye Gr. occidentalis, Whitfield, 1878, Ann. Rep. Geo. Sur. Wis., p. 75, and Geo. Wis., vol. 4, p. 222, Trenton Gr.

Codoxochulus, Whiteaves, 1884, Pal. Foss., vol. 3, p. 17. [Ety. kodon, a trumpet; cheilos, lip.] Turreted, subfusiform; volutions numerous, compressed, closely inrolled; outer half of body whorl produced obliquely outward and downward; lip thin, expanded; aperture nearly circular. Type C. striatum.

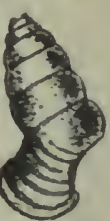
striatum, Whiteaves, $1894, \mathrm{Pal}$ codon oFoss., vol. 3, p. 17, Guelph Gr. striatum. Concholecits, Walcott, 1876, 28th Rep. N. Y., Mus. Nat. Hist., p. 93. [Ety. conche, shell; pelte, shicld.] Patelliform, more or less conical, apex central or subcentral, vertically striated, older specimens lined concentrically. Type C. alternata.

alternata, Walcott, 1876,2 sth Rep. N. Y. Mus. Nat. Hist., p. 93, Trenton Gr.

minnesotensis, Walcott, 1876, 28th Rep. N. Y. Mus. Nat. Ilist., p. 94, Trenton Gr. Cyclonesa, Hall, 1852, Pal. N. Y., vol. 2 , p. 89. [Ety. kuklos, circle; nema, thread.] Turbinate, thin, whorls ventricose, stria concentric and crossed by oblique lines of growth; no umbilicus; moutl

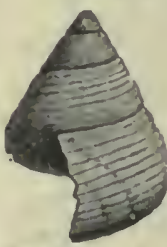

FIC. $663 .-\mathrm{Cy}$ cionema bi. lix. rounded and witl an im. perfect peritreme; inner lip thin, closely reflected, and a little concave. Type C. bilix.

bellulum, Billings, 1866, Catal. Sil. Foss. Antic., p. 55, Anticosti Gr.

bilix, Conrad, 1842, (Pleurotomaria bilix, Jour. A cad. Nat. Sci., vol. 8, p. 271, and Pal. N. Y., vol. 1, p. 305 , Trenton and Hud. Riv. Grs.

bilix var. conicum, S. A. Miller, 1874 , Cin. Quar. Jour. Sci., vol. 1, p. 320, Iud. Riv. Gir.

bilix var.fluctuatum, James, 1874, (Cyclonema fluctuata,) Cin. Quar. Jour. Sci., vol. 1, p. 152, Hud. Riv. Gr.

cancellatum, Hall, 1843, (Littorina cancellata,) Geo. Rep. 4th Dist. N. Y., p. 72, and Pal. N. Y., vol. 2, p. 90, Clinton Gr. cincinnatiense, S. A. Miller, 1882, Jour. Cin. Soc. Nat. Hist., vol. 5, p. 230, Utica Slate Gr.

commune, Billings, 1866, Catal. Sil. Foss. Antic., p. 55, Anticosti Gr.

concinnum, Hall, 1876, Illust. Devonian Foss., pl. 12, and Pal. N. Y., vol. 5, pt. 2, p. 38 , Chemung Gr.

crenistria, Hall, 1876, Illust. Devonian Foss., pl. 12, Schoharie grit.

crenulatum, Meek, 1871, Proc. Acad. Nat. Sci., p. 79, and Ohio Pal., vol. 1, p. 213, Up. Held. Gr.

decorum, Billings, 1866, Catal. Sil. Foss. Antic., p. 56, Anticosti Gr. 
doris, Hall, 186:2, (Pleurotomaria doris,) 15 th Rep. N. Y. Mus. Nat. Hist., p. 43, and Pal. N. Y., vol. 5, pt. 2, p. 34, Up. Held. Gr.

elevatum, IIall, 1868, 20th Rep. N. Y. Mus.

Nat. Hist., p. 391, Niagara Gr.

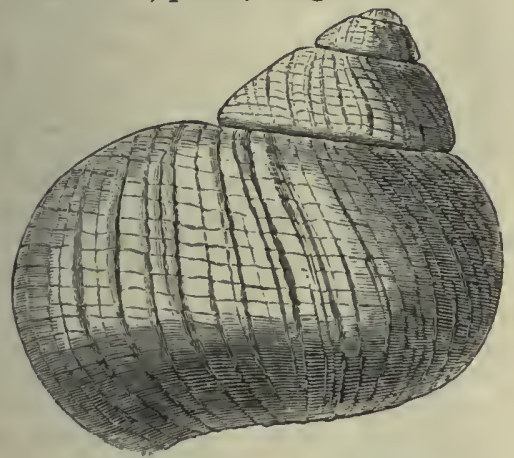

FIG. 661.-Cyclonema hageri.

hageri, Billings, 1862, Pal. Foss., vol. 1, p. 29, Irenton $\mathrm{Gr}$.

hallanum, Salter, 1859, Can. Org. Rem., Decade 1, p. 26, Black Riv. Gr.

bamiltonix, Hall, 1862 , 15 th Rep. N. Y. Mus. Nat. Hist., p. 47, and Pal. N. Y., vol. 5 , pt. 2 , p. 37 , Ham. Gr.

humile, Billings, 1866, Catal. Sil. Foss. Antic., p. 56, Anticosti Gr.

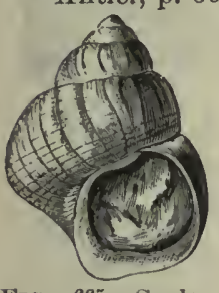

Fig. 665.-Cyclo-

num.

Sil. Foss. Antic., p.56, Anticosti Gr montrealense, Billings, 1862, Pal. Foss., vol. 1, p. 30, Trenton Gr.

multiliratum, Hall, 1862, 15th Rep. N. Y. Mus. Nat. Hist., p. 48, and Pal. N. Y., vol. 5 , pt. 2 , p. 36 , Ham. Gr.

obsolescens, Hall, 1877, 1st Ed. Am. Pal. Foss., p. 243, and Pal. N. Y., vol. 5, pt. 2, p. 38, Chemung Gr. Proposed instead of $C$. obsoleta, Hall, 1876, which was preoccupied.

obsoletum, Hall, 1852, Pal. N. Y., vol. 2, p. 90 , Clinton Gr.

obsoleta, Hall. The name was preoccupied. See C. obsolescens.

percarinatum, Hall, 1847, (Pleurotomaria percarinata,) Pal. N. Y., vol. 1, p. 177, Trenton and Hud. Riv. Grs.

percingulatum, Billings, 185\%, Rep. of Progr. Geo. Sur. Can., p. 304, Clinton and Niagara Grs.

phiedra, Billings, 1865, Pal. Foss., vol. 1, p. 188, Quebec Gr. pyramidatum, James, 1874, Cin. Quar. Jour. Sci., vol. 1, p. 152, Hud. Riv. Gr. rugilineatum, Hall \& IVhitfield, 1872, 24th Rep. N. Y. Mus. Nat. Hist., p 186, Niagara Gr.

semicarinatum, Salter, 1859, Can. Org. Rem., Decade 1, p. 27, Black Riv. Gr. subangulatum, Hall, 1858, (Pleurotomaria subangulata,) Trans. Alb. Inst., vol. 4, p. 25, and Bull. Am. Mus. Nat. Hist., p. 76, Warsaw Gr.

sulcatum, Hall, 1852, Pal. N. Y., vol. 2, p. 347, Guelph Gr.

tennesseense, Roemer, 1860, (Turbo tennesseensis, ) Sil. Fauna. des West Tenn., p. 77, Niagara Gr.

thalia, Billings, 1857, (Pleurotomaria thalia,) Rep. of Progr. Geo. Sur. Can., p. 303, Hud. Riv. Gr.

varians, Billings, 1857, Rep. of Progr. Geo. Sur. Can., p. 305, Mid. Sil.

varicosum, Hall, 1870, 24th Rep. N. Y. Mus. Nat. Hist., pl. 8. (Published by mistake in 14 th Rep. 1861, as C. ventricosa.) Trenton Gr.

ventricosum, Hall, 1852, Pal. N. Y., vol. 2, p. 90, Clinton Gr.

Cyclora, Hall, 1845, Am. Jour. Sci., vol. 48, p. 294. [Ety. kuklos, circle.] Shells minute, suture deep, surface smooth, lip thin, aperture circular. Type C. minuta.

alta, Foerste, 1885, Bull. Sci. Lab. Denison Univ., p. 96, Niagara Gr. Does not belong to this genus.

depressa, Ulrich, 1879, Jour. Cin. Soc. Nat. Hist., vol. 2, p. 13, Hud. Riv. Gr.

hoffmanni, S. A. Miller, 1874, Cin. Quar. Jour. Sci., vol. 1, p. $313, \mathrm{Hud}$. Riv. Gr.

minuta, Hall, 1845, Am. Jour. Sci., vol. 48, p. 294, Utica Slate and Hud. Riv. Gr. nana, syn. for Cyclora minuta. Frg. 666.parvula, Hall, 1845, (Turbn hoftmanparvula,) Am. Jour. Sci., vol. ni.

48, p. 294, and Ohio Pal., vol. 1, p. 154, Hud. Riv. Gr.

pulcella, S. A. Miller, 1882, Jour. Cin. Soc. Nat. Hist., vol. 5, p. 231, Hud. Riv. Gr. turbinata, Whiteaves, 1881, Can. Nat., vol. 10 , p. 101 , Devonian.

valvatiformis, Whiteaves, 1881, Can. Nat., vol. 10 , p. 100 , Devonian.

Cyclostoma, Lamarck, 1801, Syst. An. sans Vert. [Ety. kuklos, circle; stoma, mouth.] Not a Palæozoic genus.

pervetusta, see Pleurotomaria pervetusta.

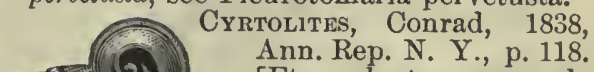
[Ety. kurtos, curved; lithos, stone.] Shell coiled in the same plane, gradually tapering, volu$\begin{array}{ll}\text { IG. } 667 .- \text { Cyr- } & \text { tions one or more, angu- } \\ \text { toittes carina- } & \text { lar or carinated on the } \\ \text { tus. } & \text { back and sides; section } \\ \text { subquadrate; aperture not expanded; }\end{array}$ $\begin{array}{ll}\text { IG. } 667 .- \text { Cyr- } & \text { tions one or more, angu- } \\ \text { tolites carlua- } & \text { lar or carinated on the } \\ \text { tus. } & \text { back and sides; section } \\ \text { subquadrate; aperture not expanded; }\end{array}$ $\begin{array}{ll}\text { IG. } 667 .- \text { Cyr- } & \text { tions one or more, angu- } \\ \text { tolites carlua- } & \text { lar or carinated on the } \\ \text { tus. } & \text { back and sides; section } \\ \text { subquadrate; aperture not expanded; }\end{array}$ subquadrate; aperture not expanded;
surface ornamented. Type C. ornatus. 
carinatus, S. A. Miller, 1874. Cin. Quar. Jour. Sci., vol. 1, p. 311, Ulica Slate.

compressus, Conrad, 1838, (Phragmolites compressus,) Ann. Rep. N. Y., p. 119, and Pal. N. Y., vol. 1, p. 188, Black Kiv. and Trenton Grrs.

conradi, Hall, 1862, Geo. Rep. WVis., p. 55, Trenton Gr.

costatus, see Bucania costata.

cristatus, Safford, 1869, Geo. of Tenn., p. 289, Naslıville Gr.

desideratus, Billings, 1866, Catal. Sil. Foss. Antic., p. 21, Hud. Riv. Gr.

dyeri, Hall, 1si 1, 2tth Rep. N. Y. Mus. Nat. Hist., p. 230, Hud. Kiv. Gr.

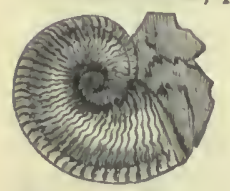

Fin. 668.-Cyrto-

lires elegnin. elegans, S. A. Miller, 1874 , Cin. Quar. Jour. Sci., vol. 1, p. 310, Hud. Riv. Gr.

expansus, Ifall, 1859, Pal. N. Y., vol. 3 , p. 479 , Oriskany sandstone.

filosus, Fmmons, 1842, Geo. Rep. $2 d$ Dist. N.Y., p. 372, and Pal. N. Y., vol. 1, p. 190, Trentoll Gr.

gillanus, White \& St. John, 1808, Trans. Chi. Acad. Sci., p. 123, Coal Meas.

imbricatus, Mleek \& Wortlien, 1868, Geo. Sur. Ill., vol. 3, p. 340, Hud. Riv. (ir.

magnus. S. A. Miller, 1878, Jour. Cin. Soc. Nat, Hist., vol. 1, p. 103, Hud. Riv. Gr.

mitella, see Cyrtonclla mitella.

nitidulus, Ulrich, 1878 , Jour. Cin. Soc. Nat. Hist., vol. 2, p. 12, Utica Slate Gr.

ornatus, Conrad, 1838, Ann. Rep. N. Y., p. 118, and Pal. N. Y., vol. 1, p. 308 , Hud. Riv. Gr.

pannosus, Billings, 1866, Catal. Sil. Foss. Antic., p. 20, IIud. Riv. Gr.

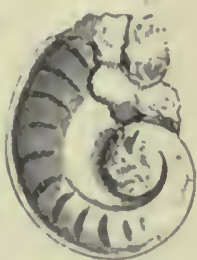

pilcolus pileolus.

sinuatus, Hall \& Whitfield, 1877, U. S. Geo. Expl. 40tl parallel, vol. 4, p. 237, Quebec Gr.

sinuosus, Hall, 1876, 28th Rep. N. Y. Mus. Nat Hist., p. 178, Niagara Gr.

trentonensis, Conrad, 1842, Jour. Acad. Nat. Sci., vol. 8, p. 270 , and Pal. N. v., vol. 1, p. 189, Treuton Gr.

Cyrtonella, Hall, 1879, Pal. N. Y., vol. 5, pt. 2, p. 123. [Ety. diminutive of Cyrtolites.] Shells ovoid, trumpet-sluaped;

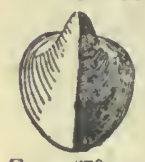

FIG. 670.Cyrionella pileolus.

by the mitella. mitella, Hall, 1862, (Cyrtolites mitella,) 15th Rep. N. Y. Mus. Nat. Hist., p. 61, ano Pal. N. Y., vol. 5, pt. 2, p. 123, Han. Gr.

pilcolus, Hall, 1862, (Crytolites pileolus,) 15th Rep. N. Y. Mus. Nat. Mist., p. 61, and Pal. N. Y., vol. 5, pt. 2, p. 125, Ham. Gr.

DAwsoxella, Bradley, 1874, Am. Jour. Sci., 3d series, vol. 7, p. 151. [Fity. proper name.] Helicoid, having a thin plate attached to the columella, covering half or more than half of the aperture of the shell as in Navicella. Type D. meeki.

meeki, Bradley, 1872, (A nomplualus mecki,) Am. Jour. Sci., $3 \mathrm{~d}$ series, vol. 4, p. S8, Coal Meas.

Dextalim, Linnæus, 1740, Syst. Nat., $2 d$ Ed., p. 6t. [Ety. dens, tooth.] Sliell elongate, terete, or angular, smootl, costate, or striate; aperture circular; lip simple, entire; margin of the posterior opening without a tissure. Type D. elephantinum.

aciculatum, see Colcolus acicu. latus.

acutisulcalum, Gurley, 1853, New Carb. Foss., p. 7. Publication not valid.

anuulostriatum, Meek \& Worthen, 1870, Proc. Acad. Nat. Sci., p. 45, and Geo. Sur. Ill., vol. 5, p. 589, Coal Meas. barquense, Winchcll, 1562 , Proc. Acad. Nat. Sci., p. 425, Marsliall Gr.

canna, White, 1874, Rep. In-Frg. 671. vert. Foss., p. 23, and Geo. Do n $t n$ Sur. W. 100 th Mer., vol. 4, llum elep. 156 , Carb.

grandrevum, Wincliell, 1863 ,

plant 1 . num.

Proc. Acad. Nat, Sci., p. 18, Marshall Gr. illinoisense, Worthen, 1883, Geo. Sur. Ill., vol. 7, p. 325, and Geo. Sur. Ill., vol. 8, p. 145, Kaskaskia Gr.

martini, Whitfield, 1882, Ann. N. Y. Acad. Sci., vol. 2, p. 203, Up. Held. Gr. meekanum, Geinitz, 1866, Carb. und Dyas in Neb., p. 13, and Geo. Sur. Ill., vol. 5, p. 590, Coal Meas.

missouriense, Swallow, 1863, Trans. St. Louis Acad. Sci., vol. 2, p.99, Kaskaskia Gr.

obsoletum, Hall. Preoccupied by Schlotlieim in 1832. See D. subløve.

primariuin, Hall, 1858, Geo. Rep. Iowa, p. 666 , Warsaw Gr.

sublave, Hall, 1877, 1st Ed. Am. Pal. Foss., p. 244, Coal Meas. Proposed instead of $D$. obsoletum, Hall, 1858, Geo. Sur. Iowa, which was preoccupied.

venustum, Meek \& Worthen, 1861, Proc. Acad. Nat. Sci., p. 145, and Geo. Sur. Ill., vol. 2, p. 284, St. Lonis Gr.

Discolite8, Fmmons, syn. for Cyclora.

minutus, see Cyclora minuta.

Eccyljompinaus, Portlock, 1843, Geol. Rep. Lond., p. 411. [Ety. ecculiomphalus, unrolled umbilicus.] Shell discoid, a few 
tapering, widely disconnected whorls; upper surface usually flattened in one plane, or slightly elevated; lower surface of whorls round; no chambers. Type E. bucklandi.

atlanticus, Billings, 1865, Pal. Foss., vol. 1, p. 250, Quebec Gr.

canadensis, Billings, 1861, Can. Nat. and Geol., vol. 6, p. 320, Quebec Gr.

circinatus, Whiteaves, 1884, Pal. Foss., vol. 3, p. 35, Guelph Gr.

comes, Hall, 1876, Illust. Devon. Foss., pl. 16, Ham. Gr.

devonicus, Walcott, 1885, Monogr. U. S. Geo. Sur., vol. 8, p. 187, Devonian.

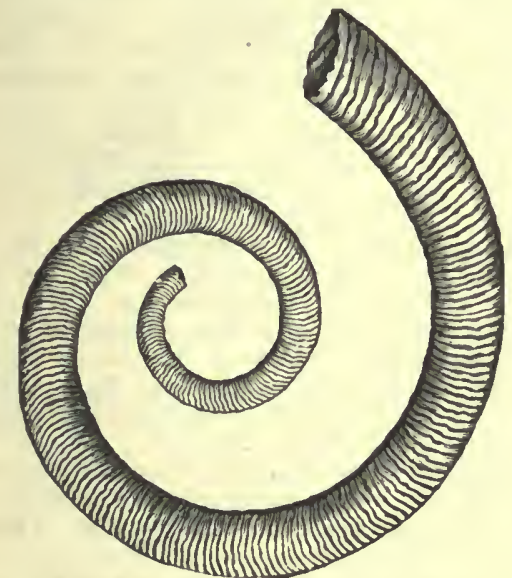

Fig. 672.-Eceyltomphalus distans.

distans, Billings, 1865, Pal. Foss., vol. 1, p. 249, Quebec Gr.

eboracensis, Hall, 1861, (Euomphalus eboracensis,) 15th Rep. N. Y. Mus. Nat. Hist., p. 55, and Pal. N. Y., vol. 5, pt. 2, p. 61 , Ham. Gr.

gyroceras, Roemer, 1852, (Euomphalus gyroceras,) Kreid. von Texas, p. 91, Silurian.

intortus, Billings, 1861, Can. Nat. and Geol., vol. 6, p. 321, Quebec Gr.

laxus, Hall, 1861, (Euomphalus laxus, ) 15th Rep. N. Y. Mus. Nat. Hist., p. 54, and Pal. N. Y., vol. 5, pt. 2, p. 60, Up. Held. Gr.

paradoxus, Winchell, 1863, (Phanerotinus paradoxus,) Proc. Acad. Nat. Sci., p. 21, and Pal. N. Y., vol. 5, pt. 2, p. 60, Marshall Gr.

priscus, Whitfield, 1889, Bull. Am. Mus.

Nat. Hist., vol. 2, p. 46, Calciferous Gr. spiralis, Billings, 1861, Can. Nat. and Geol,, vol. 6. p. 321, Quebec Gr.

superbus, Billings, 1865, Pal. Foss., vol. 1, p. 250, Quebec Gr.

undulatus, Hall, 1861, Geo. Rep. Wis., p, 37, Trenton Gr.

volutatus, Whitfield, 1886, Bull. Am. Mus. Nat. Hist., vol. 1, p. 314, Birdseye Gr.

Eotrochus, Whitfield, 1882, Bull. Am. Mus.

Nat. Hist., p. 77. [Ety. eos, dawn;
Trochus, a genus.] Conical above, flat or concave beneath, and broadly and deeply umbilicated; aperture very oblique, and the outer angle of volutions strongly carinated; surface ornamentation unlike on the upper and lower parts. Type E. concavus. concavus, Hall, 1858,

(Pleurotomaria concava,) Trans. Alb. Inst., vol. 4, p. 24, and Bull. Am. Mus. Nat. Hist., p. 78 , Warsaw Gr.

Eulima, Risso, 1826, His- Fig. 673.-Eotrochus toire Naturelle des

Principales, p. 123. Not an American Palæozoic genus.

peracuta, see Polyphemopsis peracuta.

Eunema, Salter, 1859, Can. Org. Rem., Decade ], p. 24. [Ety. eu, beautiful; nema, line.] Turbinate, thin; few angular whorls, strong concentric ridges, crossed by sinuate or oblique lines of growth; inner lip not reflected; peritreme simple; mouth rather effuse below; no umbilicus. Type E. strigillatum.

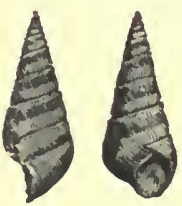

Fig. 674.-Eunema cerithioldes. cerithioides, Salter, 1859 Can. Org. Rem., Decade 1, p. 30 , Black Riv. Gr.

erigone, Billings, 1862, Pal. Foss., vol. 1, p. 35, Black Riv. Gr. pagoda, Salter, 1859, Can. Org. Rem., Decade 1 , p. 30 , Black Riv. Gr.

priscum, Billings, 1859, Can. Nat. and Geo. vol. 4, p. 360, Calciferous Gr. salteri, see Orthonema salteri. strigillatum, Salter, 1859, Can. Org. Rem., Decade 1, p. 29, Black Riv. Gr.

trilineatum, Hall, 1867, 20th Rep. N. Y. Mus. Nat. Hist., p. 397, Niagara Gr.

Euomphalus, Sowerby, 1812, Min. Conch., vol. 1, p. 97. [Ety. eu, wide; omphalos, umbilicus.] Shell discoid, spire flattened; whorls numerous, angulated; umbilicus very wide, exposing volutions; mouth nearly circular; peritreme entire, not indented by the preceding whorl; s u rf a c e usually Fra. 675.-Euomphalus sculptured. Type pentangulatus. E. pentangulatus.

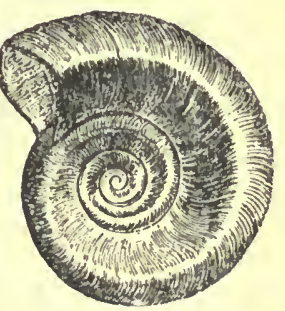

ammon, White \& Whitfield, 1862, Proc. Bost. Soc. Nat. Hist., vol. 8, p. 301, Kin. derhook Gr.

boonensis, Swallow, 1863, Trans. St. Louis Acad. Sci., vol. 2, p. 99, Burlington Gr. calciferus, Whitfield, 1889 , Bull. Am. Mus. Nat. Hist., vol. 2, p. 47, Calcilerous Gr. 
catilloides, Conrad, 1842, (Inachus catilloides,) Jour. Acad. Nat. Sci., vol. 8, p. 273, Coal. Meas.

circumliratus, Whitfield, 1886, Bul\}. Am. Mus. Nat. Hist., vol. 1, p. 308, Birdseye Gr.

clymenioides, see Straparollus clymenioides.

comes, Hall, syn. for Phanerotinus laxus. conradi, syn. for Pleuronotus decewi.

cyclostomus, see Straparollus cyclostomus.

decewi, see Pleuronotus decewi.

decollatus, Hall, 1877, 1st Ed. Am. Pal. Foss., p. 244, Low. Held. Gr. Proposed instead of $\mathrm{F}$. disjunctus, Hall, 1859, Pal. N. Y., vol. 3, p. 340.

depressus, Hall, 1843. Preoccupied by Goldluss in 1832. See Straparollus hecale.

disjunctus, Hall. Preoccupied by Goldfuss. see E. decollatus.

eboracensis, see Eccyliomphalus eboracensis.

exortivus, Dawson, 1868, Acad. Geol., p. 308 , Carboniferous.

expansus, Conrad, 1842, Jour. Acad. Nat. Sci., vol. 8, p. 273, Niagara Gr.

gyroceras, see Eccyliomphalus gyroceras.

hecale, see Straparollus hecale.

hecale var. corpulens, see Straparollus hecale var. corpulens.

hemispherica, see platystoma hemisphericum.

inops, see Straparollus inops.

latus, Hall, 1858, Geo. Rep. Iown, p. 605, Burlington $\mathrm{Gr}$.

laxus, see Eccyliomphalus laxus.

lens, see Straparollus lens.

luxus, White, 1875, Expl. W. 100th Meridian, vol. 4, p. 94, Subcarboniferous.

macrolincatus, Whitfield, 1878, Ann. Rep. Geo. Sur. Wis., p. 82, and Geo. Sur. Wis., vol. 4, p. 294, Niagara Gr.

minnesotensis, Owen, 1852, Geo. Sur. Wis., Iowa, and Minn., p. 581, Calciferous Gr.

minutissimus, Castelnan, 1843, Syst. Sil., p. 35. Not recognized.

obtusus, Hall, 1858, Geo. Rep. Inwa, p. 523, Kinderhook Gir.

ophirensis, see Straparollus ophirensis.

pernodosus, Meek \& Worthen, 1870, (Straparollus pernodosus,) Proc. Acad. Nat. Sci., p. 45, and Geo. Sur. Ill., vol. 5, p. 604, Coal Meas.

perspectivus, Swallow, 1863, Trans. St. Louis Acad. Sci., vol. 2, p. 98, Kaskaskia Gr.

pervetus, Conrad, 1843, (Inachus pervetus,) Proc. Acad. Nat. Sci., vol. 1, p. 334, Trenton Gr.

planidorsatus, Meek \& Worthen, 1860 , Proc. Acad. Nat. Sci., p. 462, and Geo. Sur. Ill., vol. 2, p. 302, Kaskaskia Gr.

planispira, see Straparollus planispiratus. planodiscus, Hall, 1860, 13th Rep. N. Y. Mus. Nat. Hist., p. 109, and Pal. N. Y., vol. 5 , pt. 2 , p. 57 , Ham. Gr. polygyratus, Roemer, 1852, Kreid. von Texas, p. 91, Silurian.

profundus, see Bucania profunda.

quadrivolvis, see Straparollus quadrivolvis. roberti, White, 1862 , Proc. Bost. Soc. Nat.

Hist., vol. 9, p. 22, Burlington Gr.

rotuliformis, Meek, 1870, Proc. Acad. Nat. Sci., p. 61, Calciferous Gr.

rotundus, see Pleurotomaria rotunda. rudis, sce Straparollus rudis.

mugitineatus, sce Cyclonema rugilineatum. migosus, Hall, 1858, Geo. Sur. Iowa, p. 722.

Preoccupied by Sowerby in 1812. See E. subrugosus.

sanctisabx, see Straparollus sanctisabie. sinuatus, see Straparollus sinuatus.

spergenensis, see Straparollus spergensis.

spergenensis var. planorbiformis, see Straparollus spergenensis var. planorbiformis.

spirorbis, see Straparollus spirorbis.

springvalensis, White, 1876, Proc. Acad. Nat. Sci., p. 32, and Cont. to Pal., No. 8, p. 167, Kinderhook Gr.

strongi, Whitfield, 1878, Ann. Rep. Geo. Sur. Wis., p. 66, and Geo. Wis., vol. 4, p. 200, Lower Magnesian Gr.

rubplanus, sce Straparollus subplanus.

subquadratus, Meek \& Worthen, 1870 ,

(Straparollus subquadratus, ) I'roc. A cad.

Nat. Sci., p. 46, and Geo. Sur. Ill., vol.

5 , p. 605, Up. Coal. Meas.

subrugosis, Meek \& Worthen, 1873, Geo.

Sur. Ill., vol. 5, p. 607. Coal Meas. Proposed instead of F. rugosus, IIall, which was preoccupicd.

sulcatus, Hall, 1843, Geo. Rep. 4th Dist. X. Y.. p. 138 , Onondaga Gr.

tioga, Hall, 1876, Illust. Devonian Foss., pl. 15, and Pal. N. Y., vol. 5, pt. 2, p. 56, Cliemung Gr.

triliratus, Conrad, 1843, Proc. Acad. Nat. Sci., p. 333, Trenton Gr.

trochiscus, see Raphistoma trochiscum. umbilicatus, see Straparollus umbilicatus. uniangulatus, see Ophileta uniangulata. utahensis, sec Straparollus utalıensis. vaticinus, Hall, 1863,16 th Rep. N. Y. Mus. Nat. Hist., p. 136, Potsdam Gr. verneuili, Casteluau, 1843, Syst. Sil., p. 34. Not recognized.

whitneyi, see Omphalotrochus whitnevi.

Fusispira, IIall, 1871, 24th Rep. N. Y. Mus.

Nat. Hist., p. 229 . [Ety. fusus, spindle;

spira, spire.] Fusiform, imperforate; spire elevated, with rounded volntions; aperture elongate-ovate or elliptical, produced below, forming a subrimate canal; columella slightly twisted, without folds; peristome sharp. Type $\mathrm{F}$. ventricosa.

compacta, Hall \& Whitfield, 1877, U. S.

Expl. 40th Parallel, vol. 4, p. 236, Quebec. Gr.

elongata, Hall, 1871, 24th Rep. N. Y. Mus.

Nut. Hist., p. 229, Trenton Gr. subfusiformis, Hall, 1847, (Murchisonia subfusiforme,) Pal. N. Y., vol. 1, p. 180, Trenton and Hud. Riv. Grs. 
terebriformis, Hall, 1871, 24th Rep. N. Y. Mus. Nat. Hist., p. 230, Hud.

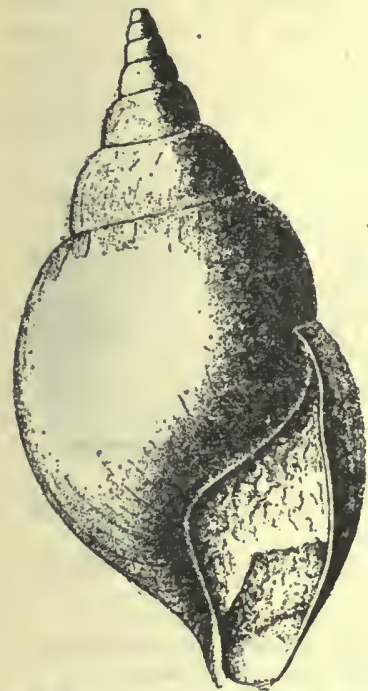

Fig. 676.-Fusispira ventricosa.

for Macrochilina primigenia.

Riv. Gr.

ve $n$ t ri cosa,

Hall, 1871,

24th Rep.

N. Y. Mus.

Nat. Hist.,

p. 229 ,

Trenton

Gr.

vittata, Hall,

1847,(Mur-

chisonia

vittata,)

Pal. N. Y.,

vol. 1, p.

181, Tren-

ton Gr.

Fusus, Bru-

$\mathrm{g} \mathbf{u}$ i e $\mathbf{r}$,

1789 , En-

cyc. Meth.

$\mathrm{Th}$ is ge-

nus is un-

known in

the Palæo-

zoic rocks.

inhabilis, syn.

Helicotona, Salter, 1859, Can. Org. Rem. Decade 1, p. 13. [Ety. Helix, genus of shells; tome, notch.] Depressed discoid, spire nearly flat, whorls obtusely angular externally, rounded below; umbilicus broad; form helicoid, Type $\mathbf{H}$. planulata.

declivis, Safford, 1869, Geo. of Tenn. Not defined.

e u ch a ris, Billings,

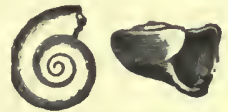

1865, Pal. Foss., FIG. 677.-Helicotoma vol. 1, p. 249, Queeucharis.

bec Gr.

gorgonea, Billings, 1865, Pal. Foss., vol. 1, p. 248, Quebec Gr.

larvata, Salter, 1859, Can. Org. Rem., Decade 1, p. 15, Black Riv. and Trenton Grs.

misera, Billings, 1865, Pal. Foss., vol. 1, p. 309; Quebec Gr.

muricata, Salter, 1859, (H. planulata var. muricata,) Can. Org. Rem., Decade 1, p. 14, Black Riv. and Trenton Grs.

naresi, Etheridge, 1878, Quar. Jour. Geo. Soc., vol. 34 , p. 602 , Up. Sil.

perstriata, Billings, 1859, Can. Nat. and Geo., vol. 4, p. 356, Calciferous Gr.

planulata, Salter, 1859, Can. Org. Rem., Decade 1, p. 14, Black Riv. and Trenton Grs.

proserpina, Billings, 1865, Pal. Foss., vol. 1, p. 247, Quebec Gr.

serotina, Nicholsọn, 1874, Rep. Pal. Ont., p. 120 , Up. Held. Gr.

spinosa, Salter, 1859, Can. Org. Rem., Decade 1 , p. 15, Black Riv. Gr.

tennesseensis, Safford, 1869, Geo. of Tenn. Not defined. tritonia, Billings, 1865, Pal. Foss., vol. 1, p. 247, Quebec Gr.

Holopea, Hall, 1847, Pal, N. Y., vol. 1, p. 169. [Ety. holos, entire; ope, aperture.] Shell conical, ventricose, more or less oblique, or nearly direct; aperture round, ovate; margin entire; surface marked by fine curved striæ or cancellated; distinguished from Cyclonema by the presence of an umbilicus. Type H. symmetrica and $\mathrm{H}$. obliqua.

antiqua, Vanuxem, 1843, (Littorina antiqua,) Geo. Rep. 3d Dist. N. Y., p. 112, and Pal. N. Y., vol. 3, p. 294, Low. Held. Gr.

antiqua var. pervetusta, Hall, 1859, Pal. N. Y., vol. 3, p. 295, Low. Held. Gr.

cassina, Whitfield, 1886, Bull. Am. Mus. Nat. Hist., p. 310, Birdseye Gr.

chicagoensis, Winchell \& Marcy, 1865, Mem. Bost. Soc. Nat. Hist., p. 99, Niagara Gr.

conica, Winchell, 1863, Proc. Acad. Nat. Sci., p. 21, Marshall Gr.

danai, Hall, 1859, Pal. N. Y., vol. 3, p. 295, Low. Held Gr.

dilucula, Hall, 1847, (Turbo dilucula,) Pal. N. Y., vol. 1, p. 12, Calciferous Gr.

(?) elongata, Hall, 1859, Pal. N. Y., vol. 3, p. 295, Low. Held. Gr.

eriensis, Nicholson, 1874, Rep. Pal. Ont., p. 120, Up.

Held. Gr.

gracia, Billings, 1862, Pal. Foss., vol. 1, p. 159, Guelph Gr.

guelphensis, Billings, 1862, Pal. Foss., vol. 1, p. 159, Guelph Gr.

harmonia, Billings, 1862, Pal. Foss., vol.1, p. 158, Guelph Gr.

lavinia, Billings, 1862, Pal. Foss., vol. 1, p. 28, Trenton Gr.

leiosoma, Billings, 1865, Pal. Foss., vol. 1, p. 187, Quebec Gr.

magniventra, Whitfield, 1878, Ann. Rep. Geo. Sur. Wis., p. 83, and Geo. Wis., vol. 4, p. 316, Niagara Gr.

nana, Meek, 1871, Proc. Acad. Nat. Sci., p. 172, syn. for Cyclora minuta.

nereis, Billings, 1862, Pal. Foss., vol. 1, p. 27, Trenton and Black Riv. Grs.

newtonensis, Whitfield, 1882, Ann. N. Y. Acad. Sci., vol. 2, p. 224, Kaskaskia Gr.

niagarensis, Winchell \& Marcy, 1865, Mem. Bost. Soc. Nat. Hist., p. 99, Niagara $\mathrm{Gr}$.

obesa, Whitfield, 1882, Geo. Wis., vol. 4, p. 348, Low. Magnesian Gr.

obliqua, Hall, 1847, Pal. N. Y., vol. 1, p. 170, Trenton and Hud. Riv. Gr.

obscura, Hall, 1847, (Turbo obscura,) Pal. N. Y., vol. 1, p. 12, Calciferous Gr.

occidentalis, Nicholson, 1875, Quar. Jour. Geo. Soc. Lond., vol. 31, p. 550, Guelph Gr.

ophelia, Billings, 1865, Pal. Foss., vol. 1, p. 222, Quebec Gr. 
ovalis, Billings, 1859, Can. Nat. and Geo., vol. 4 , p. 351 , Calciferous $\mathrm{Gr}$.

paludiniformis, Hall, 1847, Pal. N. Y. vol. 1 , p. 171, Trenton Gr.

proserpina, Billings, 1862, Pal. Foss., vol. 1, p. 28, Calciferous and Clıazy Grs.

proutana, Hall, 1858, Trans. Alb. Inst., vol. 4, p. 30, and Bull. Am. Mus. Nat. Hist., p. 72, Warsaw Gr.

pyrene, Billings, 1862, Pal. Foss., vol. 1, p. 27, Black Riv. Gr.

reversa, Hall, 1860, Can. Nat. and Geo. vol. 5, p. 154, Up. Silurian.

subconica, Hall, 1859, Pal. N. Y., vol. 3, p. 294, Low. Held. Gr.

subconica, WVinchell, 1863, Proc. Acad. Nat. Sci., p. 21. This name was preoccupied. sweeti, Whitfield, 1880, Ann. Rep. Geo.

Sur. Wis. and Geo. Wis., vol. 4, p. 174, Potsdam Gr.

symmetrica, Hall, 1847, Pal. N. Y., vol. 1, p. 170, Black Riv. Gr.

turgida, Hall, 1847, (Pleurotomaria turgida,) Pal. N. Y., vol. 1, p. 12, Calciferous Gr.

ventricosa, Hall, 1847, Pal. N. Y., vol. 1, p. 171, Trenton Gr.

Holoi'ella, McCoy, 1855, Brit. Pal. Foss. p. 303. [Ety. diminutive of Holopea.] Shell spiral, elongate, slender, of numerous gradually increasing whorls, generally crossed by slightly arched strix; moulh circular, with the peritreme entire; base rounded, with or without a minute umbilicus. Type $\mathrm{H}$. cancellata.

mira, Winchell, 1863, Proc. Acad. Nat. Sci., p. 22, Marshall Gr.

Inachus calilloides, see Euomplsalus catilloides.

pervetus, see Euomphalus pervetus.

pervetustus, see Pleurotomaria pervetusta. undatus, see Lituites undatus.

Isonfma, Meek \& Worthen, 1866, I'roc. Acad. Nat. Sci. Phil., p. 251. [Ety. isos, equa]; nema, thread.] Depressed turbinate, subglobose, obtusely angular around the middle of the body whorl; aperture subrhombic; outer lip thin, entire; inner lip a little flattened in the umbilical region; surface ornamented with transverse, very regular lines on the upper side of the volutions. Type I. depressum.

bellatulum, see Callonema bellatulum.
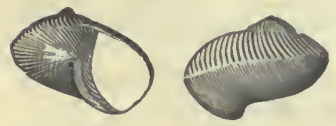

FIG. 679.-Isonema depressum. depressum, Meek \& Worthen, 1865 , Proc. Acad. Nat. Sci. Phil., p. 251, and Geo. Sur. Ill., vol. $3, \mathrm{p}$. 443 , Ham. Gr.

humile, Meek, 1871, Proc. Acai. Nat. Sci. p. 79, and Ohio Pal., vol. 1, p. 214, Up. Held. Gr.

lichas, see Callonema lichas.

Lepetopsis, Whitfield, 1882 Bull. Am. Mus. Nat. Hist., No. 3, p. 67. [Ety. Lepeta, a genus; opsis, resemblance.] Sliell patelliform, more or less regularly round or oval, apex subcentral, posierior to the middle, direeted backwarl, tlet nucleus dextrally coiled; muscular imprint horseshoe-shaped, open in front, consisting of an irregular narrow band, which expands more or less at the anterior extremities; surface with six radiating lines, two anterior, two posterior, and two lateral. Type I. levettii.

cliesterensis, Worthen, 1884, Bull. No. 2, IIl. St. Mus. Nat. Hist., p. 25, and Geo. Sur. Ill. vol. 8 , p. 140, Kaskrskis $\mathrm{Gr}$.

levettii, White, 1882, ( $\mathrm{Pa}$. tella levettei, ) 11th Rep. Geo, of Indiana, F I G. 680.-repep. 359, Warsaw (ir.

Litlorina, Ferussac, 1821, Tab. Syst. An. Mollusques, etc.

antiqua, seo llolopea antiqua.

cancellata, see Cyclonema cuncellatum.

wheeleri, seo Natieopsis wheeleri.

Loplostrira, Whitfield, 1856, I3ull. Am. Mlus. Nat. Hist., vol. 1, p. 312. [Ety. lophos, the keel; speira, a whorl.] Spire elevated, strongly keeled, and axis $\mathrm{mi}$ nutely perforate, when whorls are not disconnected. The types are Murchjsonia milleri, Hall, and M. helicteres; but as the generic characters are not very satisfactorily or clearly defined, I leave the species with Murchisonia. cassina, see Murchisonin cassina. calcifera, see Murchisonia calcifera.

Ioxonnua, Phillips, 1841, Pal. Foss., Cornwall, etc., p. 98. [Ety. loxos, oblique; nema, thread.] Shell elougate, many whorled; aperture simple, attenuate above, effuse below; lines of growth sigmoidal; no umbilicus. Type L. sinuosum.

aculentum, Billings, 1866, Catal. Sil. Foss. Antic., 1). 55, Anticosti Gr.

acutulum, Dawson, 1868, Acad. Geol., p. 309, Carboniferous.

approximatum, Walcott, 1885, Monogr. U. S. Geo. Sur., vol. 8, p. 191, Devonian. attenuatum, Stevens, 1858, (Chemnitzia attenuata,) Am. Jour. Sci. and Arts, 2d ser., vol. 25, p. 259, Coal Meas.

altenualum, Hall, 1859, Pal. N. Y., vol. 3, p. 296. The name was preoccupied. See L. emaceratum.

attenuatum var. semicostatum, see L. semicostatum.

bellatulum, see Isonema bellatulum.

bellona, Hall, 1876, Illust. Devonian Foss., pl. 14, and Pal. N. Y., vol. 5, pt. 2, p. 46, Ham. Gr.

bellum, Walcott, 1885, Monogr. U. S. Geo. Sur., vol. 8, p. 258, Subcarboniferous.

boydi, see Murchisonia boydi.

breviculum, Hall, 1879, Pal. N. Y., vol. 5, pt. 2, p. 132, Ham. Gr. 
cara, Dawson, 1883, Rep. on Redpath Museum, No. 2, p. 11, Subcarboniferous. carinatum, see Macrochilina carinata.

cerithiforme, Meek \& Worthen, 1860,

Proc. Acad. Nat. Sci., p. 465, and Geo.

Sur. Ill., vol. 2, p. 379, Up. Coal Meas.

coaptum, Hall, 1876, Illust. Devonian

Foss., pl. 13, and Pal. N. Y., vol. 5, pt.

2, p. 44, Ham. Gr.

compactum, Hall, 1859, Pal. N. Y., vol. 3, p. 297, Low. Held. Gr.

cotteranum, Billings, 1861, Can. Jour., vol. 6 , p. 360 , Corniferous limestone.

crassum, Webster, 1888, Am. Nat., p. 446.

Not defined so as to be recognized.

danvillense, Stevens, 1858, Am. Jour. Sci., vol. 25 , p. 259 , Coal Meas.

delphicola, Hall, 1862, 15th Rep. N. Y. Mus. Nat. Hist., p. 52, and Pal. N. Y., vol. 5 , pt. 2 , p. 47 , Ham. Gr.

emaceratum, Hall, 1877, 1st Ed. Am. Pal. Foss., p. 244, Low. Held. Gr. Proposed instead of $\mathrm{L}$. attenuatum. Hall, 1859, in Pal. N. Y., vol. 3, p. 296, which was preoccupied.

eurekense, Walcott, 1885, Monogr. U. S. Geo. Sur., vol. 8, p. 190, Devonian.

fasciatum, King, 1850, Permian Foss., p. 209, Permian Gr.

fitchi, Hall, 1859, Pal. N. Y., vol. 3, p. 296, Low. Held. Gr.

gigantea, Webster, 1888, Am. Nat., p. 445. Not properly defined.

halli, Norwood \& Pratten, 1855, Jour. Acad. Nat. Sci., $2 d$ series, vol. 3, p. 77, Coal Meas.

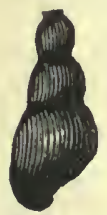

FIG. 681.-Loxonema hamiltonire. hamiltoniæ, Hall, 1862, 15th Rep. N. Y. Mus. Nat. Hist., p. 33, and Pal. N. Y., vol. 5 , pt. 2 , p. 45 , Ham. Gr.

hydraulicum, Hall \& Whitfield, 1872, 24th Rep. N. Y. Mus. Nat. Hist., p. 193, and Pal. N. Y., vol. 5, pt. 2, p. 44, Ham. Gr.

inornata, see Polyphemopsis inornata.

kanii, Meek, 1865, Am. Jour. Sci. and Arts, $2 \mathrm{~d}$ ser., vol. 40, p. 33, Low. Held. Gr.

læviusculum, Hall, 1879, Pal. N. Y., vol. 5, pt. 2, p. 131, Ham. Gr.

laxum, Hall, 1879, Pal. N. Y., vol. 5, pt. 2, p. 49, Chemung Gr.

leda, Hall, 1868, 20th Rep. N. Y. Mus. Nat. His', p. 398, Niagara Gr.

magnum, Whitfield, 1878, Ann. Rep. Geo. Sur. Wis., p. 83, and Geo. Wis., vol. 4, p. 317 , Niagara Gr.

macclintochi, Haughton, 1857, Jour. Roy. Dub. Soc., vol. 1, Devonian.

minutum, Stevens, 1858, Am. Jour. Sci., $2 \mathrm{~d}$ series, vol. 25, p. 260, Coal Meas.

moloch, Clarke, 1885, Bull. U.S. Geo. Sur., No. 16 , p. 30 , Genesee shales.

multicostatum, Meek \& Worthen, 1861, Proc. Acad. Nat. Sci., p. 128, and Geo. Sur. Ill., vol. 2, p. 378, Coal Meas. murrayanum, Salter, 1859, Can. Org. Rem.,

Decade 1, p. 31, Black Riv. Gr.

newberryi, see Soleniscus newberryi.

nexile, Sowerby. Not an American species. nitidula, see Polyphemopsis nitidula.

nobile, Walcott, 1885, Monogr. U. S. Geo.

Sur., vol. 8, p. 190, Devonian.

nodosum, Stevens, 1858, Am. Jour. Sci., $2 \mathrm{~d}$ ser., vol. 25 , p. 260 , Coal Meas.

noe, Clarke, 1885, Bull. U. S. Geo. Sur., No. 16 , p. 55, Portage Gr.

obtusum, Hall, 1859, Pal. N. Y., vol. 3, p. 297, Low. Held. Gr.

oligospiratum, Winchell, 1863, Proc. Acad. Nat. Sci., p. 22, Marshall Gr.

owenense, Webster, 1888, Am. Nat., p. 446. Not defined so as to be recognized.

parvum, Cox, 1857, (Chemnitzia parva,) Geo. Sur. Ky., vol. 3, p. 567, Coal Meas. parvulum, Whitfield, 1882, Ann. N. Y.

Acad. Sci., vol. 2, p. 204, Up. Held. Gr. peoriense, Worthen, 1884, Bull. No. 2, Ill.

St. Mus. Nat. Hist., p. 7, and Geo. Sur. Ill., vol. 8, p. 139, Coal Meas.

pexatum, Hall, 1861, 14th Rep. N. Y. Mus.

Nat. Hist., p. 104, and Pal. N. Y., vol. 5, pt. 2, p. 42, Up. Held. Gr.

pexatum var. obsoletum, Hall, 1876, Illust. Devonian Foss., pl. 13, and Pal. N. Y., vol. 5, pt. 2, p. 43, Up. Held. Gr. planogy ratum, Hall, 1839, Pal. N. Y., vol. 3, p. 298, Low. Held. Gr.

plicatum, Whitfield, 1882, Ann. N. Y. Acad. Sci., vol. 2, p. 231, Coal Meas.

politum, Stevens, 1858, Am. Jour. Sci., 2d series, vol. 25, p. 260, Coal Meas.

postrenum, Hall, 1879, Pal. N. Y., vol. 5, pt. 2, p. 132, Chemung Gr.

quadricarinatum, Worthen, 1884, Bull. No. 2, Ill. St. Mus. Nat. Hist., p. 7, and Geo. Sur. 11l., vol. 8, p. 140, Coal Meas.

rectistriatum, Hall, 1879, Pal. N. Y., vol. 5 , pt. 2, p. 130, Ham. Gr.

regulare, Cox, 1857, Geo. Sur. Ky., vol. 3, p. 566, Coal Meas.

robustum, Hall, 1862 , 15 th Rep. N. Y. Mus. Nat. Hist., p. 52, and Pal. N. Y., vol. 5 , pt. 2, p. 40, Schoharie grit.

rossi, Haughton, 1857, Jour. Roy. Soc. Dub., vol. 1, Devonian.

rugosum, Meek \& Worthen, 1860, Proc. Acad. Nat. Sci., p. 465, and Geo. Sur. Ill., vol. 2, p. 378, Up. Coal Meas.

scitulum, Meek \& Worthen, 1860, Proc. Acad. Nat. Sci., p. 464, and Geo. Sur. Ill., vol. 2, p. 372, Low. Coal Meas.

semicostatum, Meek, 1871, (L. attenuatum var. semicostatum,) Proc. Acad. Nat. Sci., p. 174, and Geo. Sur. Ill., vol. 5, p. 596, Coal Meas.

sicula, Hall, 1879, Pal. N. Y., vol. 5, pt. 2, p. 43, Up. Held. Gr.

solidum, Hall, 1862, 15th Rep. N. Y. Mus. Nat. Hist., p. 51, and Pal. N. Y., vol. 5, pt. 2, p. 41, Schoharie grit.

styliola, Hall, 1876, Illust. Devon. Foss., pl. 14, and Pal. N. Y., vol. 5, pt. 2, p. 48, Chemung Gr. 
subattenuatum, Hall, 1862, 15th Rep. N. Y. Mus. Nat. Hist., p. 52, and Pal. N. Y., vol. 5 , pt. 2, p. 40 , Schoharie grit. subulata, see Muŕchisonia subulata. swallovanum, Shumard, 1859, (Chemnitzia swallovana,) Trans. St. Louis Acad. Sci., vol. 1, p. 399, Permian.

tenuicarinatum, Stevens, 1858, Am. Jour. Sci., 2d series, vol. 25 , p. 260 , Coal Meas. tenuilineatum, Shumard, 1S55, (Chemnitzia tenuilineata,) Geo. Rep. Mo., p. 207, Waverly Gr. or Choteau limestone.

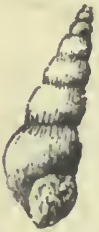

FIG. 682.-Itoxnneina yandellanum. terebra, Hall, 1876, Illust. Devon. Foss., pl. 14, and Pal. N. Y., vol. 5, pt. 2, p. 48, Chemung Gr.

teres, Hall, 1876, Illust., Devonian Foss., pl. 13, and Pal. N. Y., vol. 5, pt. 2, p. 42, Corniferous Gr. turritiforme, Hall, 1860, 13th Rep. N. Y. Mus. Nat. IIist., p. 109, Kinderhook Gr.

vincta, see Murchisonia vincta.

yandellanum, IIall, I858, Trans. Alb. Inst:, vol. 4, p. 28, and Bull. Am. Mus. Nat. Hist., p. 77, Warsaw Gr.

Maclurea, LeSueur, 1818, (Maclurites,) Jour. Acad. Nat. Sci., vol. 1, p. 312. [Ety, proper name.] Discoidal, few whorled, reversed, upper surface couvex, deeply perforate, outer side spirally grooved; operculum sinistrally subspiral, solid, with two internal projections for the attachinent of muscles. Type M. magna.
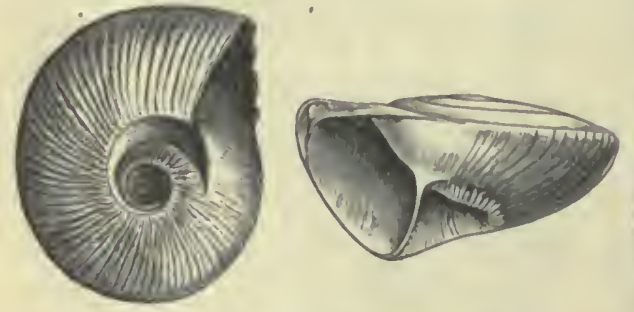

FIG. 683.-Maclurea crenulata.

acuminata, Billings, 1865, Pal. Foss., vol. 1, p. 240, Quebec Gr.

affinis, Billings, 1865, Pal. Foss., vol. 1, p. 238, Quebec Gr.

annulata, Walcott, 1885, Monogr. U. S. Geo. Sur., vol. 8, p. 81, Chazy Gr.

atlantica, Billings, 1859, Can. Nat. and Geo., vol. 4, p. 459, Chazy Gr.

bigsbyi, Hall, 1861, Geo. Rep. Wis., p. 37, and Geo. Wis., vol. 4, p. 222, Trenton $\mathrm{Gr}$.

carinata, Walcott, 1885, Monrgr. U. S. Geo. Sur., vol. 8, p. 82, Trenton Gr.

crenulata, Billings, 1865, Pal. Foss., vol.1, p. 236, Quebec Gr.

cuneata, Whitfield, 1878, Ann. Rep. Geo. Sur. Wis., p. 75, and Geo. Wis., vol. 4, p. 246, Trenton Gr. emmonsi, Billings, 1865, Pal. Foss., vol. 1, p. 242, Quebec Gr.

labiata, see Raplistoma labiata.

logani, Salter, 1851, Rep. British Assoc., p. 63, Black Riv. Gr.

magna, LeSueur, 1818, Jour. Acad. Nat. Sei., vol. 1, p. 312, and Pal. N. Y., vol. 1, p. 26, Chazy Gr.

matutina, Hall, 1847, Pal. N. Y., vol. 1, p. 10, Calciferous Gr.

minima, Hall \& Whitfield, 1877, U. S. Geo. Expl., 40tl parallel, vol. 4, p. 235, Cliazy Gr.

oceana, Billings, 1865, Pal. lioss., vol. 1, p. 237, Quebec Gr.

ponderosa, Billings, 1565, Pal. Foss., vol. 1, p. 239, Quebec Gr.

psyche, Billings, 1865, Pal. Foss., vol. 1, p. 244, Quebec Gr.

rotundata, Billings, 1S65, Pal. Foss., vol. 1, p. 245, Quebec Gr.

speciosa, Hillings, 1865, Pal. Foss., vol. 1, p. 2t0, Quebec Gr.

sordida, IIall, 1S47, Pal. N. Y., vol. 1, !. 10, Calciferous Gr.

striala, see Scalites striatus.

striata, Troost, 1840. Not defined.

subaunulatr, Walcott, 1885, Monogr.

U. S. Geo. Sur., vol. 8, p. 82, Trenton Gr.

subrotunda, Whitfield, 1878, Ann. Rep. Geo. Sur. Wis., p. 75, and Geo. WVis., vol. 4, p. 246, Trenton Cir.

gylpha, Billings, 1865, Pal. Foss., vol. 1, p. 244, Quebec Gr.

transitionis, Billings, 1865, Pal. Foss., vol. 1, p. 241, Quebec Gir.

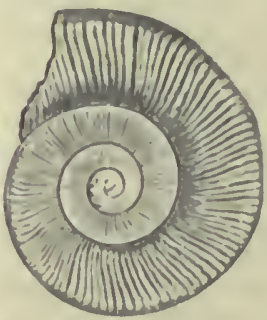

wadsworthi, Whitfield, 1884, Bull. Am. Mus. Nat. Hist. vol. 1, p. 139, Up. Taconic. Macrocheilus, Phillips, 1841, Pal. Foss., Cornwall, etc., p. 103. This name was preoccupied by Hope, in 1838 for a genus of Coleopterous insects. Bayle luas proposed Macrochilina, to which all the species are referred.

altonense, see Macrochilina worthenanus. altonense, see Macrochilina altonensis. anguliferum, see Macrochilina angulifera. attenuatum, Hall, syn. for Soleniscus fusiformis.

cooperense, see Macrochilina cooperensis. fusiforme, see Soleniscus fusiformis. gracile, see Macrochilina gracilis. hallanum, see Soleniscus hallanus. hamiltonix, see Macrochilina hamiltoniæ. hebe, see Macrochilina hebe.

hildrethi, see Macrochilina hildrethi. humile, see Machrochilina humilis. inhabile, syn. for Macrochilina primigenius.

iniercalare, see Macrochilina intercalaris. 
kansasense, see Macrochilina kansasensis. klipparti, see Soleniscus klipparti.

macrostomum, see Macrochilina macrostomus.

mediale, see Macrochilina medialis.

missouriense, see Macrochilina missouriensis.

newberryi, see Soleniscus newberryi.

paludinxformis, see Soleniscus paludiniformis.

pingue, see Macrochilina pinguis.

ponderosum, see Macrochilina ponderosus.

primæoum, see Macrochilina primævus.

primigenium, see Macrochilina primigenius.

priscum, see Macrochilina prisca.

pulchellum, syn. for Macrochilina intercalaris.

spiratum, see Macrochilina spirata.

subcorpulentum, see Macrochílina subcorpulenta.

terranovicum, see Macrochilina terranovica. texanum, see Soleniscus texanus.

ventricosum, see Soleniscus ventricosus.

Macrocinilina, Bayle, 1880, Journal de Conchyliologie, 3me. ser., t. 19. Proposed instead of Macrocheilus of Phillips, which was preoccupied by Hope. [Ety. diminutive of Macrocheilus.] Subglobose, elongate; apex pointed; whorls convex, smooth, last one large; aperture subovate; columella imperforate; outer lip thin, without notch or sinus; inner lip thin above and thickened below. Type M. acuta

altonensis, Worthen, 1873, (Macrocheilus altonense,) Geo. Sur. Ill., vol. 5, p. 593, Coal Meas.

angulifera, White, 1874, (Macrocheilus anguliferum,) Rep. Invertebrate Foss., p. 22 , and Geo. Sur. 100th Mer., vol. 4, p. 160 , Carboniferous.

carinata, Stevens, 1858, (Loxonema carinatum,) Am. Jour. Sci., vol. 25, p. 259 , Coal Meas.

cooperensis, Swallow, 1863, (Macrocheilus cooperense,) Trans. St. Louis Acad. Sci., vol. 2, p. 100, Kaskaskia Gr.

gracilis, Cox, 1857, (Macrocheilus gracile,) Geo. Sur. Ky., vol. 3, p. 570, Coal Meas.

hamiltoniæ, Hall, 1862, (Macrocheilus bamiltoniæ, ) 15th Rep. N. Y. Mus. Nat. Hist., p. 49 and Pal. N. Y., vol. 5, pt. 2, p. 33, Ham. Gr.

hebe, Hall, 1862, (Macrocheilus hebe, 15th Rep. N. Y. Mus. Nat. Hist., p. 48, and Pal. N. Y., vol. 5, pt. 2, p. 32, Ham. Gr.

hildrethi, Conrad, 1842, (Plectostylus hildrethi,) Jour. Acad. Nat. Sci., vol. 8, p. 275, Coal Meas.

humilis, Keyes, 1888, (Macrocheilus humile,) Proc. Acad. Nat. Sci. Phil. pl. xii, fig, 1, Coal Meas.

intercalaris, Meek \& Worthen, 1860, (Macrocheilus intercalare,) Proc. Acad. Nat. Sci., p. 467, and Geo. Sur. Ill., vol. 2, p. 371, Up. Coal Meas. kansasensis, Swallow, 1858, (Macrocheilus kansasense,) Trans. St. Louis Acad. Sci., vol. 1, p. 201, Coal Meas.

littonana, Hall, 1858, (Natica littonana,) Trans. Alb. Inst., vol. 4, p. 30, and Bull. Am. Mus. Nat. Hist., p. 72 , Warsaw Gr.

macrostoma, Hall, 1862, (Macrocheilus macrostomum,) 15th Rep. N. Y. Mus. Nat. Hist., p. 49, and Pal. N. Y., Fig. 684. - Mavol. 5 , pt. 2, p. 33, nana.

Ham. Gr.

medialis, Meek \& Worthen, 1860, (Macrocheilus mediale,) Proc. Acad. Nat. Sci., p. 466, and Geo. Sur. Ill., vol. 2, p. 370, Up. Coal Meas.

missouriensis, Swallow, 1858, (Macrocheilus missouriense,) Trans. St. Louis Acad. Sci., vol. 1, p. 201, Coal Meas.

pinguis, Winchell, 1863, (Macrocheilus pingue,) Proc. Ácad. Nat. Sci., p. 21, Marshall Gr.

ponderosa, Swallow, 1858, (Macrocheilus ponderosum,) Trans. St. Louis Acad. Sci., vol. 1, p. 202, Coal Meas.

primæva, Hall, 1876, (Macrocheilus primævum,) Illust., Devonian Foss., pl. 12, and Pal. N. Y., vol. 5, pt. 2, p. 35, Schoharie grit.

primigenia, Conrad, 1835, (Stylifer primigenia,) Trans. Geo. Soc. Penn., vol. 1, p. 267, Coal Meas.

prisca, Whitfield, 1882, (Macrocheilus priscum,) Ann. N. Y. Acad. Sci., vol. 2, p. 204, Up. Held. Gr.

spirata, McCoy, 1850, (Macrocheilus spiratum, Brit. Pal. Rocks, p. 549, Coal Meas.

subcorpulentr, Whitfield, 1882, (Macrocheilus subcorpulentum,) Ann. N. Y. Acad. Sci., vol. 2, p. 224, Kaskaskia Gr. terranovica, Dawson, 1883, (Macrocheilus terranovicum,) Rep. on Redpath Museum, No. 2, p. 14, Carboniferous.

worthenanus, n. sp. St. Louis Gr. Proposed instead of Macrocheilus altonense in Geo. Sur. Ill., vol. 8, p. 143, which name was preoccupied.

Metoptoma, Phillips, 1836, Geo. of Yorkshire, pt. 2, p, 223. [Ety. metopon, front: tome, incision.] Patelliform, truncated under the apex, at the posterior side; horseshoe-shaped muscular scar, with the open end directed from the truncated side. Type M. oblonga. alceste, Billings, 1862, Pal. Foss., vol. 1, p. 153, Hud. Riv. Gr.

alta, Whitfield, 1889, Bull. Am. Mus. Nat. Hist., vol. 2, p. 44, Calciferous Gr. analoga, Walcott, 1885, Monogr. U. S. Geo. Sur., vol. 8, p. 84, Trenton Gr. angusta, Billings, 1862, Pal. Foss., vol. 1, p. 88, Quebec Gr.

anomala, Billings, 1862, Pal. Foss., vol. 1, p. 89, Quebec Gr. 
barabuensis, Whitfield, 1878, Ann. Rep. Geo. Sur. Wis., p. 60, and Geo. Wis., vol. 4, p. 195, Low. Magnesian Gr.

billingsi, Walcott, 1883, 35th Rep. N. Y. Mus. Nat. Hist., p. 212, Trenton Gr.
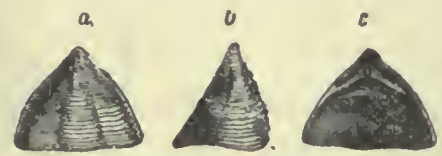

Fig. 685. - Metoptoma canadensls, $a$, Upper side; $b$, side view; $c$, under side.

canadensis, Billings, 1865, Pal. Foss., vol. 1, p. 394, (Chiton canadensis,) Black Riv. Gr.

cornutiformis, Walcott, 1879, Desc. New Spec. Foss., p. 1, Caleiferous Gr.

devonica, Walcott, 1885, Manogr. U. S. Geo. Sur., vol. 8, p. 195, Devonian.

dubia, Hall, 1847, Pal. N. Y., vol. 1, p. 23, Chazy Gr.

erato, see Tryblidium eruto.

estella, Billings, 1862, Pal. Foss., vol. 1, p.

153, Hud. Riv. Gr.

eubule, see Tryblidium eubule.

hyrie, see Tryblidium hyrie.

instabilis, Billings, 1865, Pal. Foss., vol. 1, p. 251, Quebec Gr.

melissa, Billings, 1862, Pal. Foss., vol. 1, p. 86, Quebee Gr.

montrealensis, Billings, 1865, Pal. Foss., vol. 1, p. 394, Chazy Gr.

niobe, see Tryblidium niobe.

nycteis, see Tryblidium nycteis.

orithyia, Billings, 1862, Pal. Foss., vol. 1, p. 38, Calcif. Gr.

orphyne, Billings, 1862, Pal. Foss., vol. 1, p. 88, Quebec Gr.

peroccidens, Walcott, 1885, Monogr. U. S. Geo. Sur., vol. 8, p. 260, Subcarb.

perovalis, Whitfield, 1878, Ann. Rep. Geo. Sur. Wis., p. 74, and Geo. Wis., vol. 4 p. 211 , Trenton Gr.

phillipsi, Walcott, 1885, Monogr. U. S. Geo. Sur., vol. 8, p. 83, Trenton Gr.

quebecensis, Billings, 1865, Pal. Foss., vol. 1, p. 308 , Quebec Gr.

recurva, Whitfield, 1878, Ann. Rep. Geo.

Sur. Wis., p. 61, and Geo. Wis., vol. 4 , p. 196, Low. Mng. Gr.

retrorsa, Whitfield, 1880, Ann. Rep. Geo.

Sur. Wis., p. 54, and Geo. Wis., vol. 4, p. 197, Low. Mag. Gr.

rugosa, see Stenotheca rugosa.

similis, Whitfield, 1878, Ann. Rep. Geo.

Sur. Wis., p. 61, and Geo. Wis., vol. 4, p. 196, Low. Mag. Gir.

simplex, see Tryblidium simplex.

superba, Billings, 1865, Pal. Foss., vol. 1, p. 172, Black Riv. Gr.

trentonensis, Billings, 1862, Pal. Foss. vol. 1, p. 40, Trenton Gr.

undata, Winchell, 1865, Proc. Acad. Nat. Sci. Phil., p. 131, Kinderhook Gr.

umbella, Meek \& Worthen, 1866, Proc. Acad. Nat. Sci. Phil., p. 267, and Geo. Sur. Ill., vol. 3, p. 506, Burlington Gr. venilia, Billings, 1562, Pal. Foss., vol. 1, p. 88, Quebee Gr.

Microceras, Hall, 1845, An). Jour. Sci., vol. 48, p. 294. [Ety, mikros, small ; keras, horn.] Geseral form like Cyrtolites, but distinguished by its minute sire, smooth surface, and less angular dorsal margin. Type M. inornatum.

inornatum, Hall, 1845, A m. Jour. Sci., vol. 48, p. 294, and Ohio Pul., vol. 1, p. 147 , II ud. Riv. Gr.

minutissimum, Ulrich, 1879, Jour. Cin. Soc. Nat. II ist., vol. 2, p. 13, IInd. Riv, Gr.

Micronoma, Meek \& Worthen, 1866, Proc. Acad. Nat. Sci., p. 269. [Ety. mikros, small; domus, house.] Shell small, subtrochiform; volutions seven or more, flattened on a line with the slope of the shell; suture deep; aperture oblique; surface ornamented with nodular ridges. Type $\mathbf{M}$. conica.

conica, Meek \& Worthen, 1866, I'roc. Acad. Nat. Sci. Phil., p. 269, and Geo. Sur. Hll., vol. 5, p. 59s, Low. Coal Meas.

Murcusosia, D'Arehiac \& Verneuil, 1841 , Bull. Soc. Geo. Fr., vol. 12, p. 154, and Phillips Pal. Foss. Cornwall, etc., p.101. [Ity. proper name.] Shell elongated, many whorled; whorls variously sculptured and zoned; outer lip deeply notehed; aperture slightly channeled in front. 'I'ype M. bilineata.

abbreviata, Hall, 1847, Pal N. Y., vol. 1, p. 32. The namo was preoccupicd by Dekoninck in 1841. See M. subabloreviata.

aciculata, Hall, 1860, Can. Nut. and Geo., vol. 5, p. 154, Up. Silurian.

acrea, Billings, 1865, Pal. Fuss., vol. 1, p. 232, Quebee Gr.

ada, Billings, 1865, Pal. Foss., vol. 1, p. 346, Calciferous Gir.

adelina, Billings, 1865, Pal. Foss., vol. 1, p. 232, Quebec Gr.

agilis, Billings, 1865, Pal. Foss., vol. 1, p. 235, Quebec Gr.

alexandra, Billings, 1865 , Pal.

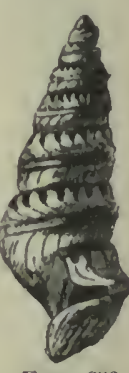

Fia. 686 . Murelilisonla bliliseata.

\section{Foss., vol. 1, p. 172, Black Riv. Gr.}

angulata, Phillips, 1836, (Rostellaria angulata,) Geo. of Yorkshire, p. 230, Devonian. Very doubtfully identified in America.

angustata, Hall, 1847, I'al. N. Y., vol.1, p. 41, Birdseye Gr.

anna, Billings, 1859, Can. Nat. and Geol. vol. 4, p. 358, Calciferous Gr.

archimedea, McChesney, 1861, Desc. New Pal. Foss., p. 89, Coal Meas.

arenaria, Billings, 1859, Can. Nat. and Geo., vol. 4, p. 359, Calciferous Gr.

arisaigensis, Hall, 1860, Can. Nat. and Geo., vol. 5, p. 154, Silurian. 
artemesia, Billings, 1865, Pal. Foss., vol. 1 , p. 345 , Calciferous Gr.

aspera, Billings, 1859, Can. Nat. and Geo., vol. 4, p. 458, Chazy Gr.

attenuata, Hall, 1858, Trans. Alb. Inst., vol. 4, p. 27, and Bull. Am. Mus. Nat. Hist., p. 88, Warsaw Gr.

augustina, Billings, 1865, Pal. Foss., vol. 1, p. 234, Quebec Gr.

bellicincta, Hall, 1847, Pal. N. Y., vol. 1, p. 179, Trenton and Hud. Riv. Grs.

bicincta, Hall, 1847, Pal. N. Y., vol. 1, p. 177. Preoccupied by McCoy in 1844. See M. milleri.

bilirata, Hall, 1859, Pal. N. Y., vol. 3 , p. 299, Low. Held. Gr.

billingsana, n. s., Guelph Gr. Proposed instead of M. hercyna in Pal. Foss., vol. 1, p. 157 , which was preoccupied.

bivittata, Hall, 1852, Pal. N. Y., vol. 2 , p. 345, Guelph Gr.

bowdeni, Safford, 1869, Geo. of Tenn., p. 288, Nashville Gr.

boydi, Hall, 1843, (Loxonema boydi,) Geo. Rep. 4th Dist. N. Y., p. 138, and Pal. N. Y., vol. 2, p. 346 , Guelph Gr.

boylii, Nicholson, 1875, Quar. Jour. Geo. Soc. Lond., vol. 31, p. 547, Guelph Gr. calcifera, Whitfield, 1889, (Lophospira calcifera,) Bull. Am. Mus. Nat. Hist., vol. 2, p. 55, Calciferous Gr.

carinifera, Shumard, 1863, Trans. St. Louis Acad. Sci., vol. 2, p. 106, Calciferous Gr.

cassandra, Billings, 1865, Pal. Foss., vol.1, p. 189, Quebec Gr.

cassina, Whitfield, 1886, (Lophospira cassina, Bull. Am. Mus. Nat. Hist., vol. 1, p. 312 , Birdseye Gr.

catharina, Billings, 1865, Pal. Foss., vol. 1, p. 231, Quebec Gr.

chamberlini, Whitfield, 1878, Ann. Rep. Geo. Sur. Wis., p. 84, and Geo. Wis., vol. 4, p. 317, Niagara Gr.

confusa, Whitfield, 1889, Bull. Am. Mus. Nat. Hist., vol. 2, p. 54, Calciferous Gr.

cicelia, Billings, 1865, Pal. Foss., vol. 1, p. 233, Quebec Gr.

conoidea, Hall, 1852. Pal. N. Y., vol. 2, p. 13, Medina Gr.

conradi, Hall, 1867, 20th Rep. N. Y. Mus. Nat. Hist., p. 396, Niagara Gr.

constricta, Whiteaves, 1884, Pal. Foss., vol. 3, p. 25, Guelph Gr.

copii, White, 1882, Rep. Invert. Foss. New Mex., p. xxx, Coal Meas.

decurta, Hall, 1877, 1st Ed. of Am. Pal. Foss., p. 244, syn. for M. subabbreviata.

desiderata, Hall, 1862, 15th Rep. N. Y. Mus. Nat. Hist., p. 50, and Pal. N. Y. vol. 5 , pt. 2 , p. 89 , Up. Held. Gr.

egregia, Billings, 1874, Pal. Foss., vol. 2, p. 58, Up. Held. Gr.

elegantula, see Pleurotomaria elegantula. estella, Billings, 1862, Pal. Foss., vol. 1, p. 157, Guelph Gr. extenuata, Hall, 1859, Pal. N. Y., vol. 3, p. 298, Low. Held. Gr.

funata, Billings, 1866, Catal. Sil. Foss. Antic., p. 55 , Anticosti Gr.

gigantea, Billings, 1857, Rep. of Progr. Geo. Sur. Can., p. 298, Mid. Sil.

gracilens, Whitfield, 1889, Bull. Am. Mus. Nat. Hist., vol. 2, p. 53, Calciferous Gr.

gracilis, Hall, 1817, Pal. N.Y. vol. 1, p. 181, Trenton and Hud. Riv. Gr.

gypsea, Dawson, 1868, Acad. Geol., p. 310, Carboniferous.

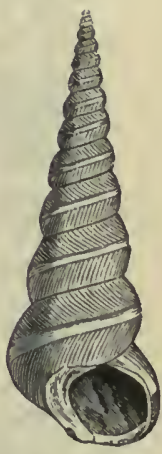

hebe, Billings, 1874, Pal. Frg.687.Foss., vol. 2, p. 57, Gaspe gracllis. limestone No. 8, Devonian.

helicteres, Salter, 1859, Can. Org. Rem., Decade 1, p. 21, Black Riv. and Trenton Grs.

hercyna, Billings, 1862, Pal. Foss., vol. 1, p. 158. The name was preoccupied by Roemer in 1843. See M. billingsana.

hermione, Billings, 1862, Pal. Foss., vol. 1, p. 33, Chazy or Black Riv. Gr.

hespelerensis, Whiteaves, 1884, Pal. Foss., vol. 3, p. 24, Guelph Gr.

hyale, Billings, 1862, Pal. Foss., vol. 1, p. 33, Chazy or Black Riv. Gr.

infrequens, Billings, 1859, Can. Nat. and Geo., vol. 4, p. 457, Chazy Gr.

inornata, Meek \& Worthen, 1866, Proc. Acad. Nat. Sci., p. 274, and Geo. Sur. Ill., vol. 5, p. 599, Coal Meas.

insculpta, Hall, 185s, Trans. Alb. Inst., vol. 4, p. 26, and Bull. Am. Mus. Nat. Hist. p. 85, Warsaw Gr.

intercedens, Hall, 1879, Pal. N. Y., vol. 5, pt. 2, p. 92, Up. Held. Gr.

jessica, Billings, 1865, Pal. Foss., vol. 1, p. 189, Quebec Gr.

kansasensis, Swallow, 1858, Trans. St. Louis Acad. Sci., vol. 1, p. 195, Coal Meas.

keokuk, Worthen, (in press,) Geo. Sur. Ill., vol. 8, p. 141, Keokuk Gr.

laphami, Hall, 1861, Rep. of Progr. Wis., p. 36, Niagara Gr.

latifasciata, Etheridge, 1878. Quar. Jour. Geo. Soc., vol. 3t, p. 600 , Úp. Sil.

lasallensis, Worthen, (in press, Geo. Sur. Ill., vol. 8, p. 141, Up. Coal Meas.

leda, Hall, 1861, 14th Rep. N. Y. Mus. Nat. Hist., p. 103, and Pal. N. Y., vol. 5, pt. 2, p. 91, Up. Held. Gr.

limitaris, Hall, 1860, 13th Rep. N. Y. Mus. Nat. Hist., p. 108, Kinderhook Gr.

linearis, Billings, 1859, Can. Nat. and Geo., vol. 4, p. 359, Calciferous Gr.

logani, Hall, 1852, Pal. N. Y., vol. 2, p. 316, Guelph Gr.

longispira, Hall, 1852, Pal. N. Y., vol. 2, p. 345, Guelph Gr.

macrospira, Hall, 1852, Pal. N. Y., vol. 2, p. 346 , Guelph Gr. 
maia, Hall, 1861, 14th Rep. N. Y. Mus. Nat. Hist., p. 103, and Pal. N. Y., vol. 5, pt. 2, p. 91, Up. Held. Gr.

major, Hall, 1851, Geo. Lake Sup. Land Dist., vol. 2, p. 209, Trenton Gr.

marcouana, Geinitz, 1866, Carb. und Dyas in Neb., p. 11, Coal Meas.

melaniformis, Sluumard, 1855, Geo. Rep. Mo., p. 208, Calciferous Gr.

micula, Hall, 1877, 1st Ed. Am. Pal. Foss., p. 244, and Pal. N. Y., vol. 5, pt. 2. p. 93, Ham. Gr. Proposed instead of M. turricula, Hall, 1862, which was preoccupied.

milleri, Hall, 1877, 1st Ed. Am. Pnl. Foss., p. 244, Trenton and IHud. Riv. Grs. Proposed instead of M. bicincta, Hall, 1847, Pal. N. Y., vol. 1, p. 177, which was preoccupied.

minima, Swallow, 1858, Trans. St. Louis Acad. Sci., vol. 1, p. 203, Middle Coal Meas.

minuta. Hall, 1859 , Pal. N. Y., vol. 3, p. 298, Low. Held. Gr.

missisquoi, Billings, 1865, Pal. Foss., vol. 1, p. 307, Quebec Gr.

modesta, Billings, 1857, Rep. of Progr. Geo. Sur. Can., p. 299, Hud. Riv. Gr.

mucro, Winchell, 1866, Rep. Iow. Peninsula Mich., p. 96, Ham. Gr.

multigruma, S. A. Miller, 1878, Jour. Cin. Soc. Nat. Hist., vol. 1, p. 104, Hud. Riv. Gr.

multivolvis, Billings, 1857, Rep. Progr. Geo. Sur. Can., p. 299, Hud. Riv. Gr. mylitta, Billings, 1862, Pal. Foss., vol. 1, p. 157, Guelph Gr.

nebraskensis, Geinitz, 1866, Carb. und Dyas in Neb., p. 12, and Pal. F. Neb., p. 234, Coal Meas.

neglecta, Winchell, 1863, Proc. Acad. Nat. Sci., p. 20, Marshall Gr.

obelisca, Whitfield, 1886 , Bull. Am. Mus. Nat. Hist., vol. 1, p. 317, Birdseye Gr. obsoleta, Meek, 1871. Proc. Acad. Nat. Sci., p. 175, Coal Meas.

obtusa, Hall, 1852, Pal. N. Y., vol. 2, p. 333, Coralline limestone.

ozarkensis. Shumard, 1863, Trans. St. Louis Acad. Sci., vol. 2, p. 106, Calciferous Gr.

papillosa, Billings, 1857, Rep. of Progr. Geo. Sur. Can., p. 301, Mid. Sil.

perangulata, Hall, 1847, Pal. N. Y, vol.1, p. 41, Black Riv, and Trenton Grs.

perversa, Swallow, 1858, Trans. St. Louis Acad. Sci., vol. 1, p. 195, Up. Coal Meas. petilla, Hall \& Whitfield, 1872, 24th Rep. N. Y. Mus. Nat. Hist., p. 186, Niagara $\mathrm{Gr}$.

placida, Billings, 1865, Pal. Foss., vol. 1, p. 235, Quebec Gr.

procris, Billings, 1862, Pal. Foss., vol. 1, p. 34, Black Riv. Gr.

prava, Whitfield, 1886, Bull. Am. Mus. Nat. Hist., vol. 1, p. 316, Birdseye Gr. prolixa, White \& Whitfield, 1862, Proc. Bost. Soc. Nat. Hist., vol. 8, p. 303, Kinderhook Gr. quadricincta, Winchell, 1863, Proc. Acad. Nat. Sci., p. 19, Marshall Gr. rugosa, Billings, 1857, Rep. of Progr. Geo. Sur. Can. p. 299, Hud. Riv. Gr. serrulata, Salter, 1859, Can. Org. Rem., Decade 1, p. 20, Black Riv. and Trenton (irs. shumardana, Winchell, 1863, Proc. Acad. Nat. Sci. Phil., p. 20, Marshall Gr.

simulatrix, Hillings, 1S65, Pal. Foss., vol. 1, p. 232, Quebec Gr.

soluta, Whiteaves, 1884, Pal. Foss., vol. 3, p. 28, Guelph Gr.

sororcula, Billings, 1865, Pal. Foss., vol. 1, p. 233, Quebec Gr.

subabbreviata, D'Orbigny, 1850, I'rodr. d. Paleont, t. 1, p. 8, Cliazy Gr. Pro. posed instead of M. abbreviata, Hall, 1847, Pal. N. Y., vol. 1, p.32, which was preoccupied.

subfusiformis, see Fusispira subfusiformis. subteniala, see Orthonema subtrniatum.

subulata, Conrad, 184:, (1oxonema subulatum,) Jour. Acad. Nat. Sci., vol. S, p. 273, and Pal. N. Y., vol. 2, 1). 91, Clinton Gr.

sumnerensis, Safford, 1869, Geo. of Tenn., p. 288, Nashville Gr.

sylvia, Billings, 1865, Pal. Foss., vol. 1, p. 190, Quebec Cir.

terebrı, White, 1879, Bull. U. S. Geo. Sur. Terr., vol. 5, No. 2, p. 219, and Cont. to Pal., No. 6, p. 139, Carboniferous.

terebralis, IIall, 185\%, Pal. N. Y., vol. 2 , p. 334 , Coralliue limestone.

terebriformis, Hall, 185S, Trans. Alb. Inst., vol. 4, p. 28, and Bull. Am. Mus. Nat. Hist., p. 86, Warsaw Gr.

terttiformis, Billings, 1857, Rep. of Progr. Geo. Sur. Can., p. 298, Hud. Riv. Gr.

texana, Shumard, 1860, Trans. St. Louis Acad. Sci., vol. 1, p. 626, Coal Meas.

tricarinata, Hall, 1847, Pal. N. Y., vol. 1, p. 178, Trenton Gr.

tricingulata, Dawson, 1868, Acad. Geol., 1. 310 , Carboniferous.

tropidophora, Whiteaves, 1884, Pal. Foss., vol. 3, p. 29, Guelph Gir.

turricula, Billings, 1857, Rep. of l'rogr. Geo. Sur. Can., p. 301, Mid. Sil.

turricula, Hall. The name was preoccupied. See M. micula.

turritella, Hall, 1858, Trans. Alb. Inst., vol. 4, p. 27, and Bull. Am. Mus. Nat. Hist., p. 88, Warsaw Gr.

turritiformis, Hall, 1852, Pal. N. Y., vol. 2, p. 347 , Guelph Gr.

uniangulata, Hall, 1847, Pal. N. Y., vol. 1, p. 179, Trenton and Hud. Riv. Grs. uniangulata var. abbreviata, Hall, 1847, Pal. N. Y., yol. 1, p. 304, Ilud. Riv. Gr. varians, Billings, 1857, Rep. of Progr. Geo. Sur. Can., p. 300, Hud. Riv. Gr. varicosa, Hall, 1847, Pal. N. Y., vol. 1, p. 42, Birdseye Gr.

ventricosa, Hall, 1847, Pal. N. Y., vol. 1, p. 41, Black Riv. and Trenton Grs.

vermicula, Hall, 1858, Truns. Alb. Inst., vol. 4, p. 27, and Bull. Am. Mus. Nat. Hist., p. 87, Warsaw Gr. 
vesta, Billings, 1862, Pal. Foss., vol. 1, p. 32, Calciferous (Ar.

vincta, Hall, 1858, (Loxonema vincta,) Trans. Alb. Inst., vol. 4, p. 28, and Bull. Am. Mus. Nat. Hist., p. 88, Warsaw Gr.

vitellia, Billings, 1862, Pal. Foss., vol. 1, p. 156, Guelph Gr.

vittata, see Fusispira vittata.

worthenana, S. A. Miller, 1882, Jour. Cin. Soc. Nat. Hist., vol. 5, p. 225 , Niagara Gr.

xanthippe, Billings, 1862, Pal. Foss., vol. 1, p. 155, Guelph Gr.

Natica, Adanson, 1757, Histoire Naturelle du Senegal, p. 172. [Ety. nato, to swim with a fluctuating motion.] This genus is unknown in Palæozoic rocks.

altomensis, see Naticopsis altonensis. carleyana, see Naticopsis carleyana.

chesterensis, see Naticopsis chesterensis.

littonana, see Macrochilina littonana.

magister, syn. for Naticopsis ventricosa.

shumardi, see Naticopsis shumardi.

ventricosa, see Naticopsis ventricosa.

Naticopsis, McCoy, 1844, Synop. Carb. Foss. Ireland, p. 33. [Ety. from resemblance to the genus Natica.] Subglobose, solid, imperforate; whorls few, convex, rapidly expanding, last. one large; spire short; aperture subovate, straighter on the inner side, rounded below ; columella callous, flattened, longitudinal impression for the operculum; lip sharp, entire; surface smooth in part or marked with oblique strix. Type $\mathrm{N}$. phillipsi.

æquistriata, Meek, 1871, Proc. Acad. Nat. Sci., p. 76, and Ohio Pal., vol. 1, p. 216, Up. Held Gr.

altonensis, McChesney, 1865, (Natica altonensis,) Desc. New. Pal. Foss., and Geo. Sur. Ill., vol. 5, p. 595, Coal Meas.

carleyana, Hall, 1858, (Natica carleyana,) Trans. Alb. Inst., vol. 4, p. 31, and Bull. Am. Mus. Nat. Hist., p. 71, Warsaw Gr. chesterensis, Swallow, 1863, Trans. St. Louis Acad. Sci., vol. 2, p. 100, Kaskaskia Gr.

comperta, Hall, 1879, Pal. N. Y., vol. 5, pt. 2, pl. 29, Up. Held. Gr.

cretacea, Hall \& Whitfield, 1873, 23d Rep. N. Y. Mus. Nat. Hist., r. 240 , Up. Held. Gr.

depressa, Winchell, 1863, Proc. Acad. Nat. Sci., p. 22, Marshall Gr.

dispassa, Dawson, 1868, Acad. Geol., p. 309, Carbonif.

gigantea, Hall \& Whitfield, 1873, 23d Rep. N. Y. Mus. Nat. Hist., p. 238, Chemung Gr.

hollidayi, see Trachydomia hollidayi.

howi, Hartt, 1868, Acad. Geol., p. 309, Carboniferous.

humilis, see Isonema humile.

lavis, Meek, 1871, Proc. Acad. Nat. Sci., p. 76, and Ohio Pal., vol. 1, p. 215, Up. Held. Gr. littonana var. genevievensis, Meek \& Worthen, 1866, Proc. Acad. Nat. Sci., p. 268, Kaskaskia Gr.

$\mathrm{m}$ a d i s onensis, Worthen, 1884, Bull. No. 2, Ill. St. Mus. Nat. Hist., p. 9, and Geo. Sur. III., vol. 8 , p. 144 , St. Louis Gr.

magister, syn. for $\mathrm{N}$. ventricosa.

monilifera, White, 1880 ,

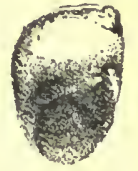

FIG. 688.-Naticopsis lrevis. 12 th Rep. U. S. Geo. Sur. Terr., p. 168, Up. Coal Meas.

nana, Meek \& Worthen, 1860, (Platystoma nana,) Proc. Acad. Nat. Sci., p. 463, and Geo. Sur. Ill., vol. 2, p. 365, Up. Coal Meas.

nodosa, see Trachydomia nodosa.

ortoni, Whitfield, 1882, Ann. N. Y. Acad. Sci., vol. 2, p. 230, Coal Meas.

pricii, Shumard, 1858, Trans. St. Iouis Acad. Sci., vol. 1, p. 202, Up. Coal Meas.

remex, White, 1876, Geo. Uinta Mountains, p. 109, and Cont. to Pal., No.6, p. 139, Low. Aubrey Gr.

shumardi, McChesney, 1860, (Natica shumardi,) Desc. New. Pal. Foss., p. 62, Coal Meas.

subovata, Worthen, 1873, Geo. Sur. Ill., vol. 5, p. 595 , Coal Meas.

ventricosa, Norwood \& Pratten, 1854, (Natica ventricosa,) Jour. Acad. Nat. Sci., 2d ser., vol. 3, p. 76, Coal Meas.

wheeleri, Swallow, 1860, (Littorina wheeleri,) Trans. St. Louis Acad. Sci., vol. 1, p. 658, and Geo. Sur. Ill., vol. 5, p. 595, Coal Meas.

ziczac, Whitfield, 1882 , Ann. N. Y. Acad. Sci., vol. 2, p. 223, Kaskaskia Gr.

Ompilalotrochus, Meek, 1864, Geo. California, vol. 1, p. 15. [Ety. omphalus, umbilicus; Trochus, a genus.] Distinguished from Euomphalus by having a more prominent spire, in having its whorls flattened or broadly concave around the outer side, and flattened, with an outward slope ; it is a more ponderous shell, with a more oblique outline to its lip, in consequence of which it projects much farther forward on the upper than on the lower side of the aperture. Type $\mathrm{O}$. whitneyi.

whitneyi, Meek, 1864, Pal. of California, vol. 1, p. 15, Carboniferous.

Ophileta, Vanuxem, 1842, Geo. Rep. N. Y., p. 36. [Ety. ophis, snake.] Discoidal spire sunk above; umbilicus perfectly open, exposing the whorls on one plane; whorls slender, numerous, truncate, and biangular exteriorly; aperture having a sinus below and a notch above. Type $O$. complanata.

abdita, Billings, 1865, Pal. Foss., vol. 1, p. 189, Quebec Gr.

(?) bella, Billings, 1865, Pal. Foss., vol. 1, p. 310, Quebec Gr.

compacta, Salter, 1859, Can. Org. Rem., Decade 1, p. 16, syn. for O. complanata. 
complanata, Vanuxem, 1842, Geo. Rep. N. Y., p. 36, and Pal. N. Y., vol. 1, p. 11, Calciferous Gr.

complanata var. nana, Meek, 1870 , Hayden's U. S. Geo. Sur. Terr., p. 295, and Geo. 4th Parallel, vol. 4, p. 17, Calciferous Gr. disjuncta, Billings, 1865, Pal. Foss., vol. 1, p. 344, Calciferous Gr.

levata, Vanuxem, 1842, Geo. Rep. N. Y., p. 36, Caleiferous Gr.
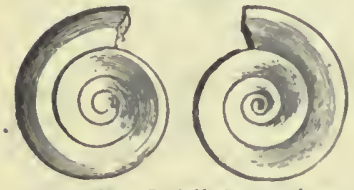

FIG. 689.-Ophileta nerine.

nerine, Billings, 1865, Pal. Foss., vol. 1, p. 245 , Quebec Gr.

ot t a w e n s is, Billings, 1860, Can. Nat. and Geol., vol. 5, p. 167, Trenton Gr. owenana, Meek \& Worthen, 1868, Geo. Sur. Ill., vol. 3, p. 313, Galena Gr. primordialis, Winchell, 1864, (Straparol. lus primordialis,) Am. Jour. Sci. and Arts, 2 d ser., vol. 37, p. 228, and Geo. Wis., vol. 4, p. 173, Potsdam Gr.

profunda, Billings, 1865, Pal. Foss., vol. 1, p. 188, Quebec Gr.

uninangulata, Hall, 1817, (Fuomphalus uniangulatus,) Pal. N. Y., vol. 1, p. 9, Calcifer. Gr.

Ormatuicinus, S. A. Miller, 18s0, Jour. Cin. Soc. Nat. Hist., vol. 2, p. 222. [Ety. ormathos, string of heads; ichnos, track.] Supposed to be the trail of a Gasteropod, and consisting of a continuous beaded track or trail. Type O. moniliformis.

moniliformis, S. A. Miller, 1850 , Jour. Cin. Soc. Nat. Hist., vol. 2, p. 222, Utica Slate Gr.

Ortionema, Meek \& Worthen, 1861, Proc. Acad. Nat. Sci., Phil., p. 146. [Ety. orthos, straight; nema, thread.] elongate, many whorled, ornamented with revolving carinz, crossed by nearly straight lines of gro:vtli; body whorl angular, not mueh enlarged or produced below; aperture angular above, sliglitly effuse below; peristome incomplete; outer lip simple, nearly straight; axis imperforate. Type 0 . salteri.

carbonarium, Worthen, 1884, Bull.

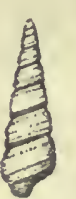

Fig. 690. Orthonema newberry1.
No. 2, IIl. St. Mus.

Nat. Hist., p. 7, and Geo. Sur. Ill., vol. 8, p. 145, Coal Meas. conicum, Meek \& Worthen, 1866, Proc. Acad. Nat. Sci. Phil., p. 270, and Geo. Sur. Ill., vol. 5, p.590, Coal Meas. newberryi, Meek, 1871, Proc. Acad..Nat. Sci. Phil., p. 81, and Ohio Pal., vol. 1, p. 217, Up. Held. Gr.

salteri, Meek \&Worthen, 1860, (Eunema (?) salteri,) Proc. Acad. Nat. Sci. Phil., p.
464, and Geo. Sur. Ill., vol. 2, p. :381, Low. Coal Meas.

subteniatum, Greinitz, 1866, (Murchisonia subtreniata,) Carb. und Dvas in Neb., p. 12, and Pal. F. Neb., p. 22s, Coal Meas. Mrthonychia, Hall, 1843, syn. for Platyceras. Ortuoston A, Conrad, 1838, Ann. Rep. N. I., p. 119. [Ety. orthos, straiglit; stoma, nonth.] Shell spiral, spire plain, con. vex. terminal volution, ending in a straight tube. 'Type $O$. commune.

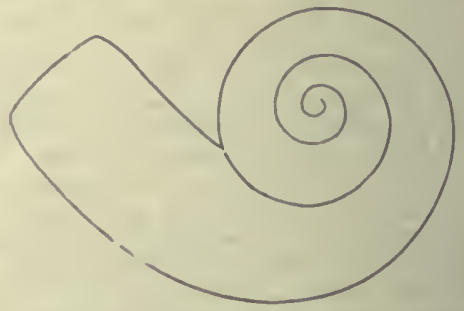

Fio. 691.- Orthostoma commune.

commune, Conrad, 1838, Ann. Rep. X. Y., p. 119, figured ju 1S41, Ann. Rep. pl.' 2, fig. 16, Birdseye Gr.

Palsacisia, Hall \& Whitfield, 1873, 23d Rep. N. Y. Mus. Nat. Hist., p. 242. [Lty.palaios, ancient; Acmara, an existing genus of shells.] Conical, more or less elevated ; apex subcentral, erect or slightly curved; peristome entire, not sinuate; surface marked concentrically. Type P. typica.

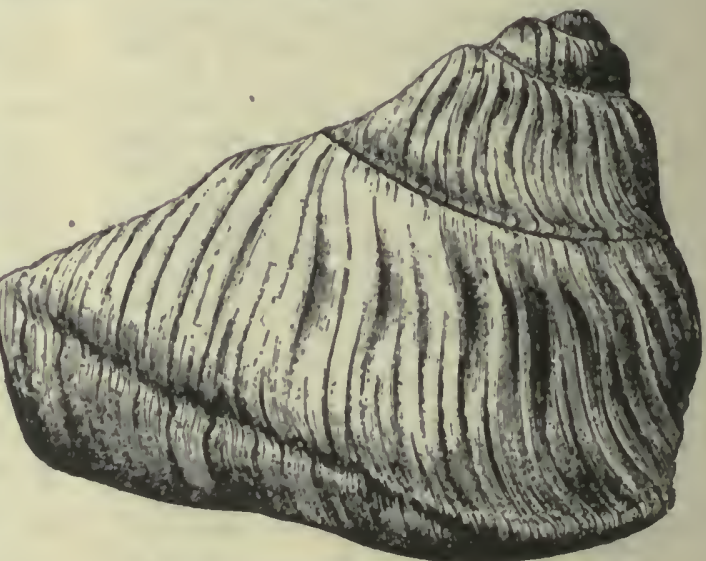

Fio. 692.-Palwotrochus lserruey1.

irvingi, Whitfield, 1878, Ann. Rep. Geo. Sur. Wis., p. 51, and Geo. Wis., vol. 4, p. 173, Potsdam Gr.

typica, IIall \& Whitfield, 1873, 23d Rep. N. Y. Mus. Nat. Hist., p. 242, Potsdam $\mathrm{Gr}$.

Paleotrocius, Hall, 1879, Pal. N. Y., vol. 5, pt. 2, p. 133. [Ety, palaios, ancient; Trochus, a genus.] Conical, trochiform ; spire elevated; volutions moderately 
convex; aperture transverse. Type $P$. kearneyi.

kearneyi, Hall, 1862, (Pleurotomaria kearneyi,) 14th Rep. N. Y. Mus. Nat. Hist., p. 105, Up. Held. Gr.

præcursor, Clarke, 1885, Bull. U. S. Geo. Sur. No. 16, p. 55, Portage Gr.

Patella, Linnæus, 1758, Syst. Nat. 10th Ed. [Ety. patella, dish.] Not a Palæzoic genus.

levettei, see Lepetopsis levetti.

Phanerotinus, Sowerby, 1844, Min. Conch., vol. 7, p. 29. [Ety. phaneros, aperture; teino, extended. Syn. for Eccyliomphalus.

paradoxus, see Eccyliomphalus paradoxus.

Phragmolites, syn. for Cyrtolites.

compressus, see Cyrtolites compressus.

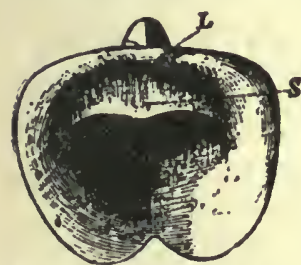

Frg. 693. - H hrag mostouna cymbula. View of aperture; $L$, lamina; $S$, septrim.
Piragiostoma, Hall, 1861. 14th Rep. N. Y. Mus. Nat. Hist., p. 94. [Ety. phragmos, a partition ; stoma, the mouth; from the septum within the aperture, which distinguishes this genus from Carinaropsis and Bellerophon.] Type P. cymbula.

cunulæ, Hall, 1861, 14th Rep. N. Y. Mus. Nat. Hist., p. 94, Hud. Riv. Gr.

cymbula, Hall, 1861, 14th Rep. N. Y. Mus.

Nat. Hist., p. 94, Hud. Riv. Gr.

natator, see Bellerophon natator.

Physa, Daperuaud, 1801, Hist. Nat. d. Moll. Not a Palæozoic genus.

prisca, Walcott, 1885, Monogr. U. S. Geo. Sur., vol. 8, p. 262, Subcarboniferous.

Pileopsis conoides, $P$. naticoides, $P$. rotundata, and $P$. spiralis, Castelnau, 1843, Syst. Sil. Not recognized.

tubifer, syn. for Platyceras dumosum.

vetustus, Sowerby. Not American.

Planorbis, Guettard, 1756, Mem. Acad. Sci. Paris. Not a Palæozoic genus.

trilobatus, see Bucania trilobata.

Platrceras, Conrad, 1840, Ann. Rep. N. Y., p. 205. [Ety. platys, broad ; keras, horn.] Depressed subglobose, subovoid, or obliquely subconical; spire small; volutions none, or very few, without columella; aperture more or less expanded, often campanulate, and sometimes with lip reflexed; peristome entire or sinuous; surface striated, cancellated, lamellose, or spiniferous. Type P. dumosum.

acutirostre, Hall, 1858, (Capulus acutirostris,) Trans. Alb. Inst., vol. 4, p. 31, and Geo. Sur. Iowa, p. 665, IVarsaw Gr. agreste, Hall, 1859, Pal. N. Y., vol. 3, p. 338, Low. Held. Gr.

ammon, Hall, 1862, 15th Rep. N. Y. Mus. Nat. Hist., p. 37, and Pal. N. Y., vol. 5, pt. 2 , p. $20, \mathrm{Up}$. Held. Gr. angulatum, Hall, 1852, (Acroculia angulata,) Pal. N. Y., vol. 2, p. 289, Clinton and Niagara Grs.

arctistoma, Ulrich, 1886, Cont. to Am. Pal., p. 30, Up. Held. Gr.

arcuatum, Hall, 1859, Pal. N. Y., vol. 3, p. 336, Low. Held. Gr.

argo, Hall, 1862, 15th Rep. N. Y. Mus. Nat. Hist., p. 39, and Pal. N. Y., vol. 5, pt. 2, p. 19, Up. Held. Gr.

attenuatum, Hall, 1862, 15th Rep. N. Y. Mus. Nat. Hist., p. 30 , and Pal. N. Y., vol. 5, pt. 2, p. 6, Ham. Gr.

attenuatum, see $\mathrm{P}$. dumosum var. attenuatum.

auriculatum, Hall, 1876, Illust. Bevonian Foss., pl. 3, Ham. Gr.

billingsi, Hall, 1859, Pal. N. Y., vol. 3, p. 315, Low. Held. Gr.

biseriale, Hall, 1860, Supp. to Geo. Iowa, vol. 1. pt. 2, p. 90, Burlington $\mathrm{Gr}$.

bisinuatum, Hall, 1859 , Pal. N. Y., vol. 3 , p. 318, Low. Held. Gr.

bisulcatum, Hall, 1859 , Pal. N. Y., vol. 3 , p. 327, Low. Held. Gr.

bivolve, White \& Whitfield, 1862, Proc. Bost. Soc. Nat. Hist., vol. 8, p. 302, Kinderhook Gr.

bucculentum. Hall, 1862, 15th Rep. N. Y. Mus. Nat. Hist., p. 33, and Pal. N. Y., vol. 5, pt. 2, p. 10, Ham. Gr.

calantica, Hall, 1859, Pal. N. Y., vol. 3 , p. 328, Low Held. Gr.

callosum, Hall, 1859, Pal. N. Y., vol. 3, p. 478 , Oriskany sandstone.

campanulatum, Winchell \& Marcy, 1865, Mem. Bost. Soc. Nat. Hist., p. 99, Niagara Gr.

capax, Keyes, 1888, Proc. Am. Phil. Soc., (author's copy, p. 13,) Burlington Gr. capulus, Hall, 1860, Supp. Geo. Iowa, p. 91, Burlington Gr.

carinatum, Hall, 1862, 15th Rep. N. Y Mus. Nat. Hist., p. 33, and Pal. N. Y. vol. 5 , pt. 2 , p. 5 , Up. Held. Gr.

chesterense, Meek \& Worthen, 1866, Proc. Acad. Nat. Sci., p. 265, Kaskaskia Gr.

cirriforme, Conrad, 1841, Ann. Rep. N. Y. Not clearly defined.

clavatum, Hall, 1859, Pal. N. Y., vol. 3, p. 337, Low. Held. Gr.

concavum, Hall, 1862 , 15th Rep. N. Y. Mus. Nat. Hist., p. 30, and Pal. N. Y., vol. 5, pt. 2, p. 3, Up. Held. Gr.

conicum, Hall, 1862, 15th Rep. N. Y. Mus. Nat. Hist., p. 31, and Pal. N. Y., vol. 5, pt. 2, p. 3, Ham. Gr.

conradi, Walcott, 1885, Monogr. U. S. Geo. Sur., vol. 8, p. 182, Devonian.

cornuforme, Winch+ll, 1863, Proc. Acad. Nat. Sci., p. 18, Marshall Gr.

crassum, Hall, 1862, 15th Rep. N. Y. Mus. Nat. Hist., p. 36, and Pal. N. Y., vol. 5, pt. 2, p. 18, Up. Held. Gr.

curvirostrum, Hall, 1859, Pal. N. Y., vol. 3, p. 338, Low. Held. Gr.

cymbium, Hall, 1862, 15th Rep. N. Y. Mus. Nat. Hist., p. 35, and Pal. N. Y., vol. 5, pt. 2, p. 12, Up. Feld Gr. 
cyrtolites, McChesney, 1859, Pal. Foss., p. 71, Coal Meas.

dentalium, Hall, 1862 , 15th Rep. N. Y. Mus. Nat. Hist., p. 29, and Pal. N. Y., vol. 5, pt. 2, p. 2, Up. Held. Gr.

dilatatum, Hall, 1859, Pal. N. Y., vol. 3, p. 322 , Low. Held. Gr.

dumosum, Conrad, 1840, Ann. Rep. N. Y., p. 205, and Pal. N. Y., vol. 5, pt. 2, p. 14, Up. Held. Gr.

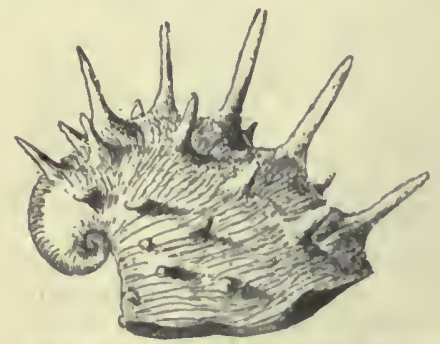

F16. 69.-Platyceras dumosum.

dumosum var. attenuatum, Meek, 1871, Proc. Acad. Nat. Sci., p. 75, and Ohio Pal., vol. 1, p. 212, Up. Held. Gr.

dumosum var. rarispinum. Hall, 1562 , 15th Rep. N.Y. Mus. Nat. Hist., 1. 38, and Pal. N. Y., vol. 5, pt. 2, p. 16, Up. Held. Gr.

echiuatum, Hall, 1862, 15th Rep. N. Y. Mus. Nat. Hist., p. 38 , and Pal. N. Y., vol. 5 , pt. 2, p. 13, Ham. Gr.

elongatum, Hall, 1859, Pal. N. Y., vol. 3, p. 335 , Low. Held. Gir.

equilaterale, Hall, 1860 , Supp. to vol. 1, pt. 2, Iowa Rep., p. 89, and (ieo. Sur. Ill., vol. 5, p. 518, Keokuk Gr.

erectum, Hall, 1843, (Acroculin erecta,) Geo. 4th Dist. N. Y., p. 174, and Pal. N. Y., vol. 5, pt. 2, p. 5, Cornif. \& Ham. Grs.

expansum, see Strophostylus expansus.

fissurellum, Hall, 1860, Supp). to Geo. Rep. Iowa, vol. 1, pt. 2, p. 90, and Geo. Sur. I1l., vol. 5, p. 519, Keokuk Gr.

fluctuosum, Ulrich, 1856, Cont. to Am. Pal., p. 31, Up. Held. Gr.

formosum, Keyes, 1888, Proc. Am. Phil. Soc., (author's copy, p. 14,) Kinderhook Gr.

fornicatum, Hall, 1862, 15th Rep. N. Y. Mus. Nat. Hist., p. 35, and Pal, N. Y., vol. 5, pt. 2, p. 11, Up. Held. Gr.

fornicatum var. contractum, Hall, 1876 , Illust. Devonian Foss., pl. 5, Up. Held. Gr.

gebhardi, Conrad, 1840, Ann. Rep. N. Y., p. 206, and Pal. N. Y., vol. 3, p. 312 , Low. Held. and Oriskany Grs.

gibbosum, Hall, 1859, Pal. N. Y., vol. 3, p. 322, Low. Held. Gr.

haliotoides, Meek \& Worthen, 1866, Proc. Acad. Nat. Sci. Phil., p. 264, and Geo. Sur. Ill., vol. 3, p. 458 , Kinderhook Gr.

herzeri, Winchell, 1870, Proc. Am. Phil. Soc., p. 256;, Marshall Gr. inclle, Hall, 1559, Pal. N. Y., vol. 3, 1). 332, Low. Held. Gr.

infundibulum, Meek \& IVorthen, 1866 , Proc. Acad. Nat. Sci. Phil., p. 266, and Geo. Sur. Ill., vol. 5, p. 517, Keokuk Gr.

intermedium, Hall, 185\%, Pal. N. I., vol. 3, p. 321, Low. Held. Gir.

laciniosum, Ringueberg, 18S6, Bull. Buf. Soc. Nat. Sci., vol. 5, p. 14, Niagara $\mathrm{Gr}$.

lavigatum, Meek \& Worthen, 1S66, l'roc. Acad. Nat. Sci., p. 263, Kaskaskia Gr. lamellosum, Hall, 1859, Pal. N. Y., vol. 3, p. 330, Low. Held. Gr.

latum, Keyes, 185S, Proc. Am. Phil. Soc., (author's copy, p. 14,) Burlington Gr.

lodiense, Meek, 1871, Proc. Aund. Nat. Sci. Phil., p. 170, and Ohio Pal., vol. 2, p. 313 , Waverly Gr.

magnificum, Hall, 1859, Pul. N. Y., vol. 3, p. 476 . Oriskany sandstone.

membranaceum, Ringueberg, 1856, Bull. Buf. Soc. Nat. Sci., vol. 5, p. 15, Niagara Gr.

minutissimum, Walcott, 1879, Desc. New Spec. Foss., p. 1, Calciferous Gr.

multisinuatum, Hall, 1859, Pal. N. Y., vol. 3, ץ. 319, Low. Held. Gr.

multispinosum, Meek, 1871, Proc. Acad. Nat. Sci. Hhil., p. 73, and Ohio Pal., vol. 1, p. 210, Cornif. Gr.

naticoides, Etheridge, 1878, Quar. Jour. Geo. Soc., vol. 34, p. 603 , Uj. Sil.

nebraskense, Meek, 1872, Pal. E. Neb., p. 227, Coal Meas.

newberryi, Hall, 1859, Pal. N. Y., vol. 3, p. 333, Low. Hedd. Gr.

niagarense, IIall, 1552, (Acroculia niagarensis, ) Pal. N. Y., vol. 2, p. 2s8, Ni. agara Gr.

nodosum, Conrad, 1841, Ann. Rep. N. Y. p. 56 , and Pal. N. Y., vol. 3, p. 473 , Oriskany sandstone.

obesum, Hall. 1859, Pal. N. Y., vol. 3 , p. 329, Low. Held. Gr.

obliquum, Kieyes, 18s8, Proc. Ain. I'hil. Soc., (author's copy, p. 13,) Burlingtou Gr.

occidens, Walcott, 1885, Monogr. U.S. Geo. Sur., vol. 8, p. 254, Subearboniferous.

ovale, Stevens, 1858, (Acroculia ovalis,) An. Jour. Sci., vol. 25, p. 261, Subcarboniferous.

pabulocrinus, Owen, 1862, (1'ileopsis pabulocrinus,) (ieo. Sur, Indiana, p. 364, Keokuk Gr.

paralium, White \& Whitfield, 1862, Proc. Bost. Soc. Nat. Hist., vol. 8, p. 302, Kinderliook Gr.

patulum, Hall, 1859, Pal. N. Y., vol. 3, p. 477 , Oriskany sandstone.

pentalobus, Hall, 1859, Pai. N. Y:, vol. 3, p. 319, Low. Held. Gr.

perlatum, Hall, 1859, Pal. N. Y., vol. 3, p. 328, Low. Held. Gr.

perplexum, Hall, 1876, Illust. Devonian Foss., pl. 2, Up. Held. Gr. 
perplicatum, Hall, 1859, Pal. N. Y., vol. 3, p. 325 , Low. Held. Gr.

pileiforme, Hall, 1859, Pal. N. Y., vol. 3, p. 327, Low. Held. Gr.

piso, Walcott, 1885, Monogr. U. S. Geo.

Sur., vol. 8, p. 254, Subcarboniferous.

platystoma, Hall, 1859, Pal. N. Y., vol. 3, p. 326, Low. Held. Gr.

platystoma var. alveatum, $\mathrm{Hall}, 1859, \mathrm{Pal}$. N. Y., vol. 3, p. 326, Low. Held. Gr.

plicatile, Hall, 1859, Pal. N. Y., vol. 3, p. 325, Low. Held. Gr.

plicatum, Conrad, 1840, (Calceola plicata,) Aun. Rep. N. Y., p. 207, and Pal. N. Y., vol. 3 , p. $33 t$, Low. Held. Gr.

primavum, Billings, 1871, Can. Nat. and Geol., vol, 6, p. 220, Georgia Gr.

primordiale, Hall, 1863, 16th Rep. N. Y. Mus. Nat. Hist., p. 136, Potsdan Gr.

proclive, Ringueberg, 1886, Bull. Buf. Soc. Nat. Sci., vol. 5, p. 14, Niagara Gr.

pyramidatum, Hall, 1859, Pal. N. Y., vol. 3, p. 336, Low. Held. Gr.

quincyense, McChesney, 1861, New Pal. Foss., p. 90, and Geo. Sur. Ill., vol. 3, p. 510, Burlington Gr.

quinquesinuatum, Ulrich, 1886, Cont. to Am. Pal., p. 29, Up. Held. Gr.

reflexum, Hall, 1859, Pal. N. Y., vol. 3, p. 477 , Oriskany sandstone.

retrorsum, Hall, 1859, Pal. N. Y., vol. 3, p. 320, Low. Held. Gr.

retrorsum var. abnorme, Hall, 1859 , Pal. N. Y., vol. 3, p. 321, Low. Held. Gr.

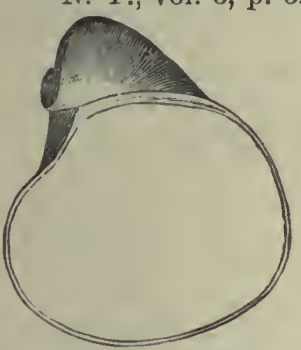

F1G. 695.-Platyceras reversum. reversum, Hall, 1860, Supp. to Geo. Rep. Iowa, vol. 1 , pt. 2, p. 91, Burlington Gr.

rictum, Hall, 1862 , 15th Rep. N. Y. Mus. Nat. Hist., p. 35, and Pal. N. Y., vol. 5 , pt. 2, p. 13, Ham. and Up. Held. Grs.

robustum, Hall, 1859, Pal. N. Y., vol. 3, p. 313, Low. Held. Gr.

senex, Winchell \& Marcy, 1865, (Porcellia senex,) Mem. Bost. Soc. Nat. Hist., j. 111, Niagara Gr.

serratum, Ulrich, 1886, Cont. to Am. Pal., p. 30 , Up. Held. Gr.

sinuatum, Hall, 1859, Pal. N. Y., vol. 3, p. 314, Low. Held. Gr.

spinigerum, Worthen, 1873, Geo.Sur. Ill., vol. 5, p. 594. Coal Meas.

spirale, Hall, 1859, Pal. N. Y., vol. 3, p. 331, Low. Held.-Gr.

squalodens, Whitfield, 1882, Ann. N. Y. Acad. Sci., vol. 2, p. 202, Up. Held. Gr. subnodosum, Hall, 1859, Pal. N. Y., vol. 3 , p. 474, Oriskany sandstone.

subplicatum, Meek \& Worthen, 1866, Proc.

Acad. Nat. Sci. Phil., p. 265, and Geo.

Sur. Ill., vol. 3, p. 457, Kinderhook Gr. subrectum, Hall, 1859, 12th Rep. N. Y. Mus. Nat. Hist, p. 18, and Pal. N. Y., vol. 5 , pt. 2, p. 1, Up. Held. Gr.

subrectum, Hall, 1860, Supp. to Iowa Rep. The name being preoccupied, Meek \& Worthen proposed $\mathrm{P}$. infundibulum. subsinuosum, Worthen, 1882, Bull. No. 1,

Ill. St. Mus. Nat. Hist., p. 38, Low. Held. Gr. Proposed instead of P. subundatum, M. \& W., which was preoccupied.

subundatum, Conrad, 1841, Ann. Rep. N. Y., p. 56, Up. Held. Gr.

subundatum, Meek \& Worthen, 1868, Geo. Sur. Ill., vol. 3 , p. 457 . The name was preoccupied. See P. subsinuosum.

sulcatum, Conrad, 1841, Ann. Rep. N. Y. p. 56, Oriskany sandstone.

sulcoplicatum, Hall, 1859, Pal. N. Y., vol. 3 , p. 324, Low. Held. Gr.

symmetricum, Hall, 1862, 15th Rep. N. Y. Mus. Nat. Hist., p. 3t, and Pal. N. Y., vol. 5 , pt. 2 , p. 9 , Ham. and Up. Held. Grs.

tenuiliratum, Halj, 1859, Pal. N. Y., vol. 3, p. 317, Low. Held. Fr.

thetiforme, Walcott, 1885, Monogr. U. S. Geo. Sur.. vol. 8, p. 184, Devonian.

thetis, Hall, 1862, 15th Rep. N. Y. Mus. Nat. Hist., p. 32, and Pal. N. Y., vol. 5, pt. 2, p. 8, Up. Held. and Ham. Grs.

thetis var. subspinosum, Hall, 1876, Illust. Devonian Foss., pl. 3, Ham. Gr. tortum, Meek, 1871, Proc. Acad. Nat. Sci., p. 171, and Ohio Pal., vol. 2, p. 345, Coal Meas.

tortuosum, Hall, 1859, Pal. N. Y., vol. 3, p. 472, Oriskany sandstone.

tribulosum, White, 1880 , 12 th Rep. U. S. Geo. Sur. Terr., p. 168, Burlington $\mathrm{Gr}$.

trigonale, Stevens, 1858, (Acroculia trigonalis,) Am. Jour. Sci. and Arts, vol. 25 , p. 260, Carboniferous.

trilobatum, Hall, 1859, Pal. N. Y., vol. 3, p. 316, Low. Held. Gr.

tubiforme, Hall, 1859, Pal. N. Y., vol. 3, p. 332, Low. Held. Gr.

uncum, Meek \& Worthen, 1866, Proc. Acad. Nat. Sci., p. 264, and Geo. Sur. Ill., vol. 5, p. 516, Keokuk Gr.

undatum, Hall, 1876, Illust. Devonian Foss., pl. 7, and Pal. N. Y., vol. 5, pt. 2, p. 17, Up. Held. Gr.

undulatum, Walcott, 1885, Monogr. U. S. Geo. Sur., vol. 8, p. 184, Devonian.

undulostriatum, Hall, 1859, Pal. N. Y., vol. 3, p. 336, I.ow. Held. Gr.

unguiforme, Hall, 1859, Pal. N. Y., vol. 3 , p. 322, Low. Held. Gr.

uniseriale, Nicholson, 1874, Rep. Pal. Ont., p. 116, Up. Held. Gr.

unisulcatum, Hall, 1859, Pal. N. Y., vol. 3, p. 316, Low. Held. Gr.

ventricosum, Conrad, 184(1, Ann. Rep. N. Y., p. 206, and Pal. N. Y., vol. 3, p. 311, Low. Held. Gr.

vomerium, Winchell, 1863, Proc. Acad. Nat. Sci., p. 19, Marshall Gr. 
Platyschusma, McCoy, 1844, Syn. Carb. Foss. Ireland, p. 38. [Ety. platys, wide; schisma, slit.] Obtusely conical, ventricose; spire short, obtuse, few whorls: aperture very oblique, large, lunate, deeply indented by the preceding whorl, rounded anteally, narrow retrally, with a very wide, shallow sinus in the middle part of the outer lip not forming a detinite band, sometimes obsolete; no trace of inner lip; pillar thin, a little reflected; suriace smooth or only marked by the retrally waved lines of growth; nmbilicus small, round, open. Type I'. helicites.

ambiguum, Walcott, 18S5, Monogr. U. S. Geo. Sur., vol. 8, p. 188, Devonian.

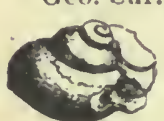

Fig. 696.-Platyschisma du blum. dubium, Dawson, 1568 , Acad. Geol., p. 309, Carboniferous. mecoyi, Walcott, 1885, Monogr,'U. S. Geo. Sur., vol. 8, p. 185 , Devonian. Platyotoma, Conrad, 1842, Jour. Acad. Nat. Sci., vol. 8, p. 275. [Ety. platys, broad; stoma, mouth.] Spire short; aperture large, dilated; labrum joining the body whorl. This name was preoceupied for a shell by Klein in 1753, for an insect by Meigen in 1803, and for a fish by Agassiz in 1829. The genus bas been named Platycerina. Type $P$. ventricosum.

affine, Billings, 1874, Pal. Foss., vol. 2, p. 60, Gaspe limestone, No. 8, Devonian. aplatum, Hall, 1876, Illust. Devonian Foss., pl. 11, and Pal. N. Y., vol. 5, pt. 2, p. 26, Schobaric grit

arenosum, Con rad, 1842, Jour. Acad. Nat. Sci., vol. 8 , p. 276 , and Pal. N. Y., vol. 3 , p. 302, Low. Held. Gr.

belial, Clarke, 1885, Bull. U. S. Geo. Sur., No. 16 , p. 30 , Genesee shales.

defiguratum, Hall, 1876, Illust. Devonian Foss., pl. 9, and Pal. N. Y., vol. 5, pt.2, p. 24, Ham. Gr.

depressum, Hall, 1859, Pal. N. Y., vol. 3, p. 301, Low. Held. Gr.

euomphaloides, Hall, 1876, Illust. Devonian Foss., pl. 9, and Pal. N. Y., vol. 5, pt. 2, p. 25, Ham. Gr.

hemisphericum, Hall, 1843, (Euomphalus hemisphericus,) Geo. Rep. 4th Dist. N. Y., p. 109, and Pal. N. Y., vol. 2, p. 288, Niagara Gr.

grayvillense, Worthen, 1882, Bull. No. 1. Iil. St. Mus. Nat. Hist., p. 38, Coal Meas, Proposed instead of P. tumidum, M. \& W., which was preoccupied.

inornatum, Walcott, 1885, Monogr. U. S. Geo. Sur., vol. 8, p. 255, Subcarboniferous.

lichas, see Callonema lichas.

lineatum, Conrad, 1842, Jour. Acad. Nat. Sci., vol. 8, p. 276, and Pal. N. Y., vol. 5, pt. 2, p. 21, Up. Held and Ham. Grs. lineatum var. amplum, Hall, 1876, Illust. Devonian Foss., pl. 9, and Pal. Foss. N. Y., vol. 5, pt. 2, p. 23, Ham. Gr.

lineatum var, callosum, Hall, 1876, Illust. Devonian Foss., pl. $q$, and Pal. Foss. N. Y., vol. 5 , pt. 2, p. 23, Ham. Gr.

lineatum var. sinuosum, Hnll, 1876, Illust. Devonian Foss., pl. 11, and Pal. Foss. N. Y., vol, 5, pt. 2, p. 24. Ham. Gr. minutissimum, Clarke, 1855, Bull. U. S. Geo. Sur., No. 16, p. 55, Portage (ir. nana, see Naticopsis nana.

$n$ i a g rense, Hall, 1552 , Pal. $\mathrm{Y} . \mathrm{Y}$., vol. 2, p. 287, Niagara Gr. peoriense, McChesn ey, 1860, Dese. $\mathrm{New}$ Pal. Fos 8., U p. Coal Meus.

p l e b e i u in, Hall, 1876 , 28 th Rep. N. Y. Mus. Nat. Ilist., p. 175, Niagara Gr.

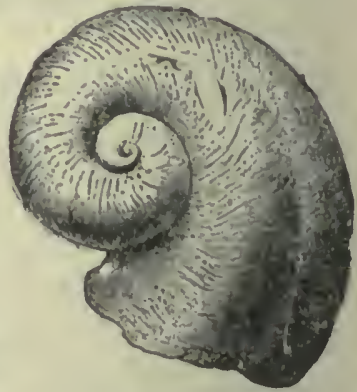

Fı. 697.- Mlatystoma n1. agarense.

p l eurotoma,

Hall, 1876, Illust. Devoniun Foss., pl. 10, and Pal. N. Y., vol. 5, pt. 2, p. 30 , Up. Held. Gr.

plicatum, Whiteaves, 1887, Cont. to Carl. Pal., vol. 1, p. 118, Ham. Gr.

shumardi, Verneuil, 18 46 , (Turbo slumardi.) Bull. d. l. Soc. Geol. d. France, and Pal. N. Y., vol. 5, pt. 2, p. 135, Ham. Gr.

strophium, Hall, 1862, 15th Rep. N. Y. Mus. Nat. Hist., p. 41, and P'al. N. Y. vol. 5, pt. 2, p. 25, Cornif. Gr.

subangulatum, Hall, 1859, Pal. N. Y., vol. 3, p. 301, Low. Held. Gr.

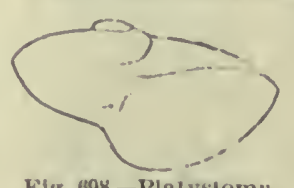

Flg. 698.-Platystomu trigonostomn. trigonostoma, Meck, 1871, Proc. Acad. Nut. Sci., p. 169, and Olio P'al., vol. 1, p. 185 , Niagara Gr.

Sci., p. 463, Up. Coal Meas.

tumidum, Meek \& Worthen, 1860 , Proc. Acad. Nat.

turbinatum, Hall, 1861, 14th Rep. N. Y. Mus. Nat. Hist., p. 106, and Pal. N. Y., vol. 5, pt. 2, p. 27 , Up. Held. Gr.

turbinatum var. cochleatum, Hall, 1876 , Illust. Devonian Foss., pl. 10, and Pal. N. Y., vol. 5, pt.' 2, p. 28, Up. Held. Gr.

unisulcatım, Conrad, 1842, (Pleurotomaria unisulcata,) Jour. Acarl. Nat. Sci., vol. 8, p. 271, and Pal. N. Y., vol. 5, pt. 2, p. 27, Up. Held. Gr.

ventricosum, Conrad, 1842, Jour. Acad. Nat. Sci., vol. 8, p. 275, and Pal. N. Y., vol. 3 , p. 300 , Low. Held. Fr.

Plectostylus, Conrad, 1842, Jour. Acad. Nat. Sci., p. 275 . The name was preoccupied.

hildrethi, see Macrochilina bildrethi. 
Pleuronotus, Hall, 1879, Pal. N. Y., vol. 5, pt. 2, p. 138. [Ety. pleura, side; notos, back.] Distinguished from Euomphalus by the broadly expanded aperture, sinuate on the upper margin, making a deep retral angle, which meets a peripheral band. Type $P$. decewi.

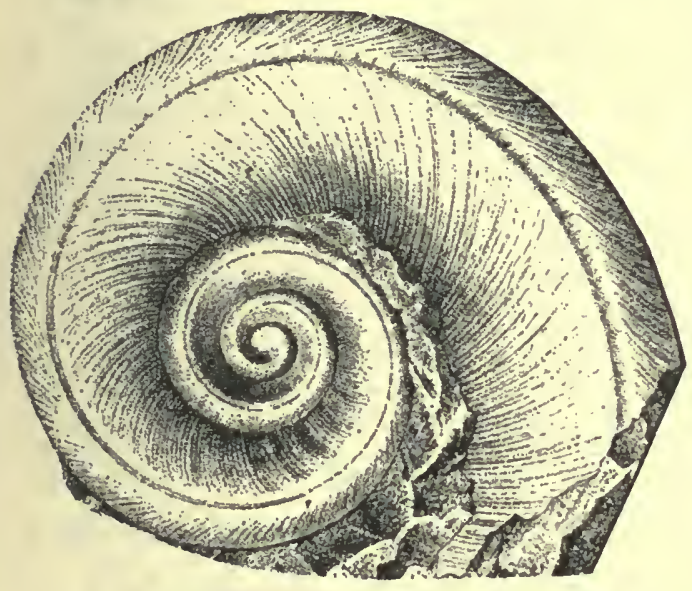

Fig. 699.-Pleuronotus decewi.

decewi, Billings, 1861, (Euomphalus decewi,) Can. Jour., p. 358, Up. Held. Gr.

Pleurotomaria, Defrance, 1824, Tableau d. Corps. Organises Fossiles, p. 114, and Dict. Sci. Nat., t. 41, p.381. [Ety. pleura, side; tome, cut or notch.] Shell trochiform, more or less conical, pearly within, variable in thickness, with or without an umbilicus; volutions angular, flattened, or rounded; surface ornamented with striæ, nodes, granulations, or carinæ; aperture subquadrate, semioval, suborbicular, or subrhombic; inner lip thin ; fissure of outer lip narrow and FIG. 700.-Pleurotodeep; revolving maria anglica. band corresponding in depth with the sinus. Týpe P. anglica.

abrupta, Billings, 1859, Can. Nat. and Geo., vol. 4, p. 354, Calciferous Gr.

acadica, Dawson, 1883 , Rep. on Redpath Mus., p. 11, Subcarboniferous.

adamsi, Worthen, 1884, Bull. No. 2, Ill. St. Mus. Nat. Hist., p. 5, and Geo. Sur. Ill., vol. 8, p. 137, Coal Meas.

adjutor, Hal!', 1879 , Pal. N. Y., vol. 5, pt. 2, p. 80, Up. Held Gr.

advena, Winchell, 1864, Am. Jour. Sci. and Arts, 2d series, vol. 37, p. 228, Potsdam Gr.

agarista, Billings, 1865, Pal. Foss., vol. 1, p. 230 , Quebec Gr. agave, Billings, 1865, Pal. Foss., vol. 1, p. 170, Trenton Gr.

ambigua, Hall, 1847, Pal. N. Y., vol. 1, p. 176, Trenton Gr.

americana, Billings, 1860, Can. Nat. and

Geo., vol. 5, p. 164, Trenton Gr.

amphitrite, Billings, 1862, Pal. Foss., vol.

1, p. 32, Chazy or Black Riv. Gr.

angulata, Conrad, 1843, Proc. Acad. Nat. Sci. Phil. This name was preoccupied by Sowerby.

antiquata, Hall, 1847, Pal. N. Y., vol. 1, p. 31, Chazy Gr.

aperta, see Raphistoma apertum.

apicalis, Hall, 1876, Illust. Devonian Foss., pl. 20, and Pal. N. Y., vol. 5, pt. 2, p. 88, Chemung Gr.

arabella, Billings, 1865, Pal. Foss., vol. 1, p. 343, Calciferous Gr.

arachne, Billings, 1862, Pal. Foss., vol. 1. p. 31, Black Riv. Gr.

arata, Hall, 1862, 15th Rep. N. Y. Mus. Nat. Hist., p. 42, and Pal. N. Y., vol. 5, pt. 2, p. 64, Schoharie grit.

arata var. clausa, Hall, 1879, Pal. N. Y., vol. 5, pt. 2, p. 65, Up. Held. Gr.

axion, Hall, 1867, 20th Rep. N. Y. Mus. Nat. Hist., p. 394, Niagara Gr.

beckwithana, McCbesney, 1860, Desc. New Pal. Foss, p. 61, Coal Meas.

beekmanensis, Whitfield, 1889, Bull. Am. Mus. Nat. Hist., vol. 2, p. 53, Calciferous Gr.

biangulata, Hall, 1847, Pal. N. Y., vol. 1, p. 31 , Cliazv Gr.

bicarinata, Mcllhesney, 1860. Preoccupied. See P.turbiniformis.

bilix, see Cyclonema bilix.

bispiralis, Hall, 1852, Pal. N. Y., vol. 2, p. 348, Guelph Gir.

bonharborensis, Cox, 1857, Geo. Sur. Ky., vol. 3 , p. 567, Coal Meas.

brazoensis, Shumard, 1860, Trans. St. Louis Acad. Sci., vol. 1, p. 624, and Geo. Sur. Ill., vol. 2, p. 354 , Low. Coal Meas.

broadheadi, White, 1880 , 12th Rep. U. S. Geo. Sur. Terr., p. 169, Coal Meas.

calcifera, Billings, 1859 , Can. Nat. and Geo., vol. 4, p. 352, Calciferous Gr.

calphurnia, Billings, 1865, Pal. Foss., vol. 1, p. 230, Up. Tuconic, Quebec Gr.

calyx, Billings, 1859, Can. Nat. and Geo., vol. 4, p. 454, Chazy Gr.

canadensís, Billings, 1865, Pal. Foss, vol. 1 , p. 342 , Calciferous $\mathrm{Gr}$.

capillaria, Conrad, 1842, Jour. Acad. Nat. Sci., vol. 8, p. 271, and Pal. N. Y., vol. 5, pt. 2, p. 77, Ham. Gr.

carbonaria, Norwood \& Pratten, 1854, Jour. Acad. Nat. Sci., $2 d$ series, vol. 3, p. 75, Coal Meas.

casii, Meek \& Worthen, 1868, Geo. Sur. Ill., vol. 3, p. 359, Niagara Gr.

cavumbilicata, Winchell, 1866 , Rep. Low. Penin. Mich., p. 96, Ham. Gr. 
chesterensis, Meek \& Worthen, 1860 , Proc. Acad. Nat. Sci., p. 460, and Geo. Sur. 1ll., vol. 2, p. 303, Kaskaskia Gr.

chesterensis, Swallow, 1863, Trans. St. Louis Acad. Sci. The name was preoccupied, but it is probably a synonym.

circe, Billings, 1857, Rep. of Progr. Geo. Sur. Can., p. 303, Hud. Riv. Gr.

clipeiformis, Spencer, 1884, Bull. No. 1, Mus. Univ. St. Mo., p. 57, Niagara Gr. concava, see Eotrochus concavus. coniformis, Worthen, 1S8:, Bull. No. 1, III. St. Mus. Nat. Hist., p. 38, Coal Meas. Proposed instead of P. conoides, M. \& $W$.

conoides, Meek \& Worthen, 1866, Proc. Acad. Nat. Sci., p. 2i1. Preoccupied by Deshayes in 1831. See P. coniformis.

conulus, Hall, 1858, Trans. Alh. Inst., vol. 4, p. 26, and Bull. Am. Mus. Nat. Mist., p. 84, Warsaw Gr.

cooperensis, n. s. Kaskaskia Gr. Proposed instead of P. trochiformis, Swallow, Trans. St. Iouis Acad. Sci., vol. 2, p. 99 , that was preoccupied.

coronula, Hall, syn. for P. sphrerulata.

coxana, Meek \& Worthen, 1866, Proc. Acad. Nat. Sci., p. 272, and Geo. Sur. Ill., vol. 5 , p. 600 , Coal Meas.

coxana, Worthen, 1884. The name was preoccupied. Sec P. iowensis.

crevieri, Billings, 1859, Can. Nat. and Geol., vol. 4, p. 456, Chazy Gr.

cryptata, Billings, 1866, Catal. Sil. Foss. Antic., 1). 54, Anticosti Gr.

cyclonemoides, Meek \& Worthen, 1868, Geo. Sur. Ill., vol. 3, J. 360, Niagara Gr.

cyclostoma, Whiteaves, 1884, Pal. Foss., vol. 3, p. 23, Guelph Gr.

deiopea, Billings, 1862, Pal. Foss., vol. 1, p. 155, Guelph Gr.

delia, Billings, 1874, Pal. Foss., vol. 2, p. 61, Gaspe limestone No. 8, Devonian.

delicatula, Hall, 1876, Illust. Devonian Foss, pl. 19, and Pal. N. Y., vol. 5, pt. 2, p. 70 , Up. Held. Gr.

delphinuloides, Goldfuss, as identified by d'Archiac \& Verneuil. Not American. depauperata, Hall, 1862, Geo. Rep. Wis., p. 55, Hud. Riv. Gr.

depressa, Cox, 1857, Geo. Sur. Ky., vol. 3 , Coal Meas. The name was preoccupied by Passy in 1832, by Phillins in 1836, and by DeKoninck in 1841. See P. kentuckiensis.

disjuncta, Hall, 1876, Illust. Devonian Foss. pl. 20, and Pal. N. Y., vol. 5, pt. 2, p. 84, Ham. Gr.

dispersa, Dawson, 1868, Acad. Geol., p. 310, Carboniferous.

docens, Billings, 1859, Can. Nat. and Geol., vol. 4, p. 452, Chazy Gr.

doris, see Cyclonema doris.

dryope, Billings, 1865, Pal. Foss., vol. 1, p. 170, Black Riv. Gr. durhamensis, Whiteaves, 1884, Pal. Foss., vol. 3, p. 24, Guelph Gr.

elegantula, Hall, 1855, (Murchisonia elegantula, Trans. Alb. Inst., vol. 4, p. 27, and Bull. Am. Mus. Nat. Hist., 1). 84, Warsaw Gr.

ella, Hall, 1876, Illust. Devonian Foss., pl. 19, and Pal. N. Y., vol. 5, pt. 2, p. 72, Ham. Gr.

elora, Billings, 1862, Pal. Foss., vol. 1, p. 154, Guelph Gr.

emmetensis, Winchell, 1866, Rep. Low. Penin. Mich., p. 96, Ham. (ir.

estella, Hall \& Whitfield, 1872, 24th Rep. N. Y. Mus. Nat. Hist., p. 195, Ham. Gr. etna, Billings, 1S65, Pal. Foss., vol. 1, 1). 226, Queliec Gr.

eugenia, Billings, 1862, Pal. Foss., vol. 1, p. 30, Black Kiv. Gr.

euomphaloides, Hall, 1862, 15th Rep. N. Y. Mus. Nat. Hist., p. 18, Han. Gr. exigua, Winchell, 1562, Proc. Acad. Nat. Sci., 1). 424, Marshall Gr.

filitexta, Hall, 1876, Illust. Devonian Foss., pl. 19, and Pal. N. Y., vol. 5, pt. 2, p. 73, Iam. Gr.

galtensis, Billings, 1862, Pal. Foss.: vol. 1, p. 15t, Guelph Gr.

giffordi, WVorthen, 1884, Bull. No. 2, Ill. St. Mus. Nat. Hist., p. 5, and Geo. Sur. Ill., vol. 8 , p. 135 , Coal Mers.

glandula, Shumard, 1860, Trrnns. St. Louis Acad. Sci., vol. 1, p. 626, Coal Mens.

gonopleura, Winchell \& Marcy, 1565, Jost. Soc. Nat. Hist., p. 98, Niagara (ir.

granulostriata, Meek \& Worthen, 1560, Proc. Acad. Nat. Sci., p. 459, anil Geo. Sur. Ill., vol. 2, p. 356, Jow. Coal Meas. grayvillensis, Norwood \& Pratten, 1854, Jour. Acad. Nat. Sci., 2d series, vol. 3, p. 75 , Coal Meas.

gregaria, Billings, 1859, Can. Nat. and Geo., rol. 4, p. 355, Calciferous Gr.

gurleyi, Meek, 1871, Proc. Acad. Nat. Sci., p. 177, Coal Meas.

halii, see Trochonema halii.

hallana, Shumard, 1859, Trans. St. Louis Acad. Sci., vol. 1, 1). 399, l'ermian Gr.

halli, see Raphistoma halli.

harpya, Billings, 1865, Pal. Foss., vol. 1, p. 227, Quebec Gr.

haydenana, Geinitz, 1866, Carb. und Dyas in Neb., p. 11, and Pal. E. Neb., p. 231, Coal Meas.

liebe, Hall, 1861, 14th Rep. N. Y. Mus. Nat. I ist., p. 105, and Pal. N. Y., vol. 5, pt. 2, p. $6 \mathrm{~S}$, Up. Held. Gr.

helena, Billings, 1860, Can. Nat. and Geo., vol. 5, p. 165 , Hud. Riv. Gr.

hickmanensis, Winchell, 1869, Geo. of Tenn. and Proc. Am. Phil. Soc. vol., 12, p. 257, Waverly Gr.

hortensia, Billings, 1865, Pal. Foss., vol. 1, p. 227, Quebec Gr.

hoyi, Hall, 1861, Rep. of Progr. Wis. Sur., p. 35, Niagara Gr.

humerosa, Meek \& Hayden, 1858, Proc. Acad. Nat. Sci. Phil., p. 262, and Pal. Up. Mo., p. 46, Coal Meas. 
humilis, Hall, 1858, Trans. Alb. Inst., vol. 4, p. 21, and Bull. Am. Mus. Nat. Hist., p. 82, Warsaw Gr.

humilis, Winchell, 1862. This name was preoccupied.

huronensis, Winchell, 1862, Proc. Acad. Nat. Sci. Phil., vol. 6, 2 d ser., p. 425, Portage Gr.

hyale, Billings, 1865, Pal. Foss., vol. 1, p. 228, Quebec Gr.

idia, Hull, 1861, Rep. of Progr. Wis. Sur., p. 35, Niagara Gr.

ignobilis, Dawson, 1868, Acad. Geol., p. 310 , Carboniferous.

illinoisensis, Worthen, 1884, Bull. No. 2, Ill. St. Mus. Nat. Hist., p. 4, and Geo. Sur. Ill., vol. 8, p. 135, Coal Meas.

imitator, see Callonema imitator.

immatura, Billings, 1859, Can. Nat. and Geo., vol. 4, p. 454, Chazy Gr.

indenta, Hall, 1847, Pal. N. Y., vol. 1, p. 176, Trenton Gr.

inexpectans, Hall \& Whitfield, 1875, Ohio Pal., vol. 2, p. 117, Clinton Gr.

inornata, Meek, 1872, Pal. E. Neb. p. 232, Coal Meas.

insolita, Hall, 1876, Illust. Devonian Foss., pl. 20, and Pal. N. Y., vol 5, pt. 2, p. 81 , Ham. Gr.

iowensis, Worthen, (in press,) Geo. Sur. Ill., vol. 8, p. 138, Keokuk Gr.

isaacsi, Hall \& Whitfield, 1873, 23d Rep. N. Y. Mus. Nat. Hist., p. 238, Chemung Gr.

itys, Hall, 1876, Illust. Devonian Foss., pl. 20, and Pal. N. Y., vol. 5, pt. 2, p. 76, Ham. Gr.

itys var. tenuispira, Hall, 1879, Pal. N. Y., vol. 5, pt. 2, p. 87, Ham. Gr.

kearneyi, see Palæotrochus kearneyi.

kentuckiensis, n. s. Coal Meas. Proposed instead of P. depressa in Geo. Sur. Ky., vol. 3, p. 569 , which was preoccupied.

labrosa, Hall, 1859, Pal. N. Y., vol. 3, p. 339, Low. Held. Gr.

laphami, Whitfield, 1878, Ann. Rep. Creo. Sur. Wis., p. 84, and Geo. Wis., vol 4 , p. 296, Niagara Gr.

lapicida, see Raphistoma lapicidum.

laurentina, Billings, 1859, Can. Nat. and Geo., vol. 4, p. 354, Calcif. Gr.

leavenworthana, see Cyclonema leavenworthanum.

lenticularis, see Raphistoma lenticulare.

lineata, Hall, 1843, (Turbo lineatus,) Geo. Rep. 4th Dist. N. Y. Preoccupied. See P. itys.

litorea, Hall, 1852, Pal. N. Y., vol. 2, p. 12. Medina sandstone.

lonensis, Walcott, 1885, Monogr. U. S. Geo. Sur., vol. 8, p. 80, Trenton Gr.

lucina, Hall, 1862, 15th Rep. N. Y. Mus. Nat. Hist., p. 42, and Pal. N. Y., vol. 5, pt. 2, p. 67, Up. Held. and Hain. Grs.

lucina var. perfasciata, Hall, 1876, Illust. Devonian Foss., pl. 20, and Pal. N. Y., vol. 5 , pt. 2 , p. 83 , Ham. Gr.

lydia, Billings, 1874, Pal. Foss., vol. 2, p. 62, Gaspe limestone, No. 8, Devonian. marcouana, Geinitz, 1866, Carb. und Dyas in Neb., p. 10, and Pal. E. Neb., p. 233, Coal Meas.

meekana, Hall, 1858, Trans. Alb. Inst., vol. 4, p. 22, and Bull. Am. Mus. Nat. Hist., p. 82, Warsaw Gr.

meta, Meek \& Worthen, 1865, Proc. Acad. Nat. Sci., p. 252, Keokuk Gr.

micula, Hall, 1862, Geo. Rep. Wis., p. 55, Hud. Riv. Gr.

misera, Billings, 1859, Can. Nat. and Geo., vol. 4, p. 354 , Calcif. Gr.

mississippiensis, White \& Whitfield, 1862, Proc. Bost. Soc. Nat. Hist., vol. 8, p. 302, Kinderhook Gr.

missisquoi, Billings, 1865, Pal. Foss., vol. 1, p. 191, Quebec Gr.

missouriensis, Swallow, 1860, (Trochus missouriensis,) Trans. St. Louis Acad. Sci., vol. 1, p. 657, Coal Meas.

mitigata, Hall, 1860, 13th Rep. N. Y. Mus. Nat. Hist., p. 108, Kinderhook Gr.

modesta, Keyes, 1888, Proc. Acad. Nat. Sci. Phil., pl. xii, figs. 2a, 2b, Coal Meas. mohawkensis, n. sp. Birdseye limestone. Proposed instear of $\mathrm{P}$. nodulosa, in Pal. N. Y., vol. 1, p. 44, which was preoccupied.

montezuma, Worthen, 1883, Geo. Sur. Ill., vol. 7, p. 324, Burlington Gr.

muralis, Owen, 1852, Geo. Sur. Wis., Iowa, and Minn., p. 581, Trenton Gr.

nasoni, Hall, 1861, Geo. Rep. WVis., p. 34, and Geo. Wis., vol. 4, p. 215, Trenton Gr. nauvooensis, Worthen, 1884, Bull. No. 2, Ill. St. Mus. Nat. Hist., p. 5, and Geo. Sur. Ill., vol. 8, p. 137, Keokuk Gr.

nevadensis, Walcott, 1885, Monogr. U. S. Geo. Sur., vol. 8, p. 259, Subcarboniferous.

newportensis, White, 1880 , 12 th Rep. U.S. Geo. Sur. Terr., p. 169, Coal Meas.

niota, Hall, 1861, Geo. Rep. Wis., p. 33, Trenton Gr.

nitela, Hall, 1879, Pal. N. Y., vol. 5, pt. 2, p. 85, Up. Held. Gr.

nodomarginata, MeChesney, 1860, Desc. New. Pal. Foss., p. 70, and Trans. Chi. Acad. Sci., p. 47, Ham. Gr.

nodulosa, Hall, 1847, Pal. N. Y., vol. 1, p. 44. The name was preoccupied by Sandberger in 1812, and by King in 1844. See P. mohawkensis.

nodulostriata, Hall, 1858, Trans. Alb. Inst., vol. 4, p. 21, and Bull. Am. Mus. Nat. Hist., p. 80, Warsaw Gr.

normani, Billings, 1865, Pal. Foss., vol. 1, p. 228, Quebec Gr.

nucleolata, Hall, 1847, PaI. N. Y., vol. 1, p. 42, Birdseye Gr.

numeria, Billings, 1865, Pal. Foss., vol. 1, p. 229, Quebec Gr.

obsoleta, Hall, 1847, Pal. N. Y., vol. 1, p. 44, Birdseye Gr.

obtusispira, Shumard, 1859, Trans. St. Louis Acad. Sci., vol. 1, p. 401, Coal Meas.

occidens, Hall, 1867, 20th Rep. N. Y. Mus. Nat. Hist.. pp. 342, 364, Niagara Gr. 
parvispira, Winchell, 1862, Rep. Low. Peninsula Mich., p. 96, Ham. Gr.

pauper, Billings, 1859, Can. Nat. and Geol., vol. 4, p. 457, Cliazy Gr.

pauper, syn. for Trochonema balii.

percarinata, see Cyclonema percarinatum. perhumerosa, Meek, 1872, Pal. E. Neb., p. 232, Coal Meas.

perizomata, White, 1882, Rep. Invert. Foss. New Mex., p. xxxi, Coal Meas.

perlata, Hall, 1852, Pal. N. Y., vol. 2, p. 349, Guelph Gr.

perornata, Shumard, 1859, Trans. St. Louis Acad. Sci., vol. 1, p.401, Coal Meas. pervetusta, Conrad, 1838, (Cyclostoma pervetusta, ) Ann. Rep. N. Y., p. 65, Medina sandstone.

piasensis, Hall, 1858, Trans. Alb. Inst., vol. 4, p. 22, and Bull. Am. Mus. Nat. Hist., p. 83, Warsaw Gr.

planidorsalis, Hall, 1876, Hlust. Devonian Foss., pl. 20, and Pal. N. Y., vol. 5, pt. 2 , p. 82, Hain. Gr.

plena, Hall, 1876, Illust. Devonian Foss., pl. 17, and Pal. N. Y., vol. 5, pt. 2, p. 66, Ham. Gr.

postumia, Billings, 1862, Pal. Foss., vol.1, p. 91, Quebec Gr.

poulsoni, Conrad, 1842, Jour. Acad. Nat. Sci., vol. 8, p. 270, Onondaga Gr.

pratteni, Meek \& Worthen, 1860, Proc. Acad. Nat. Sci., p. 459, and Geo. Sur. Ill., vol. 2, p. 357, Low. Coal Mens.

princessa, Billings, 1874, Pal. Foss. vol. 2, p. 59, Up. Held. Gr.

progne, Billings, 1860, Can. Nat. and Geol., vol. 5, p. 163, Black Riv. and Trenton Grs.

proutana, Shumard, 1859, Trans. St. Louis Acad. Sci., vol. 1, p. 401, Coal Meas. quadricarinata, Hall, 1847, Pal. N. Y., vol. 1, p. 43, Birdseye Gr.

quadrilix, Hall, 1879, Pal. N. Y., vol. 5, pt. 2, p. 86, Up. Held. Gr.

quebecensis, Billings, 1865, Pal. Foss., vol. 1, p. 190, Quebec Gr.

quinquesulcata, Winchell, 1865, Proc. Acad. Nat. Sci., p. 131, Marshall Gr. racinensis, Whitfield, 1878, Ann. Rep. Geo. Sur. Wis., p. 84, and Geo. Wis., vol. 4 , p. 296 , Niagara $\mathrm{Gr}$.

ramsayi, Billings, 1859, Can. Nat. and Geol., vol. 4, p. 351, Calciferous Gr.
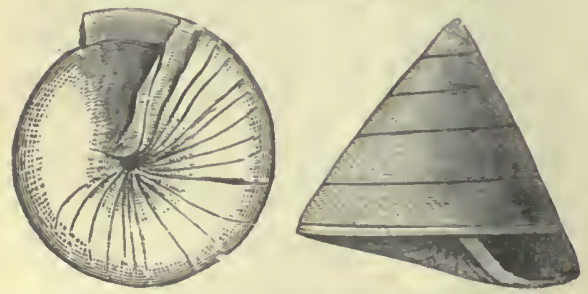

FIG. 701.-Pleurotomaria ramsayl.

regulata, Hall, 1860, 13th Rep., p. 108, Ham. Gr.

riddelli, Shumard, 1860, Trans. St. Louis Acad. Sci., vol. 1, p. 625, Coal Meas. rota, Winchell, 1863, Proc. Acad. Nat. Sci., p. 19, Marslıall Gr.

rotalia, Hall, 1862, 15th Rep. N. Y. Mus. Nat. Hist., p. 46, and Pal. N. Y., vol. 5, pt. 2, p. 71, Ham. Gr.

rotuloides, see Raphistoma rotuloides.

rotunda, Hall, 1843. (Euomplialıs (?) rotundus,) Geo. Rep. 4th Dist. N. Y., p. 172, and Illust. Devon. Foss., pl. 18, Corniferous Gr.

rotundata, Hall, see P'. subglobosa.

rotundispira, Billings, 1865, Pul. Foss., vol. 1, p. 191, Quebec Gr.

rugulata, Hall, 1860, 13th Rep. N. Y. Mus.

Nat. Hist., p. 108, and $\mathrm{Pal}$. N. Y., vol. 5 , pt. 2, p. 75, Ilam. Gr.

scitula, Meek \& Worthen, 1860, Proc.

Acad. Nat. Sci. Phil., p. 461, and Geo.

Sur. Ill., vol. 2, p. 353, Low. Coal Meas. selecta, Billings, 1S65, Pal. Foss., vol. 1, p. 224, Quebec Gr.

semele, Hall, 1861, Gieo. Rep. WVis., p. 36 , Hud. Riv. Gr.

shumardi, Meek \& Worthen, 1860, Proc. Acad. Nat. Sci. Phil., p. 462, and Geo. Sur. Ill., vol. 2, p. 260, Keokuk Gr.

sigaretoides, Winchell \& Marcy, 1S65, Bost. Soc. Nat. Hist., vol. 1, p. 98, Niagara $\mathrm{Gr}$.

sinistrorsa, Swallow, 1858, Trans. St. Louis Acad. Sci., vol. 1, p. 203, Coal. Meas. solarioides, Hall, 1852, Pal. N. Y., vol. 2, p. 348, Gnelph Gr.

speciosa, Meek \& Worthen, 1860, Proc.

Acal. Nat. Sci. Phil., p. 459, and Geo.

Sur. Ill., vol. 2, p. 352, Low. Coal Meas. sphacrulata, Conrad, 1842, Jour. Acad.

Nat. Sci., vol. 8, p. 272, Coal Meas.

spironema, Meek \& Worthen, 1866, Proc.

Acad. Nat. Sci. Phil., p. 272, and Geo.

Sur. Ill., vol. 5, p. 601, Coal Meas.

sponea, Billings, 1865, Pal. Foss., vol. 1, p. 226, Quebec Gr.

stella, Winchell, 1862, Proc. Acad. Nat.

Sci., p. 424, Marshall Gr.

subangulata, see Cyclonema subangulatum. subconica, Hall, 1847, Pal. N. Y., vol. 1,

p. 174, Black Riv., Trenton, and Hud. Riv. Grs.

subconstricta, Meek \& Wortl:en, 1860 , Proc. Acad. Nat. Sci. Phil., p. 458 , and Geo. Sur. IIl., vol. 2, p. 351, Low. Coal Meas.

subdecussata, Geinitz, 1866, Carb. und

Dyas in Neb., p. 10, and Pal. E. Neb., p. 233, Coal Mfeas.

subdepressa, Hall, 1852, Pal. N. Y., vol. 2, p. 333, Coralline limestone.

subglobosa, Hall, 1877, 1st Ed. Am. Pal.

Foss., p. 245, Warsaw Gr. Proposed instead of P. rotundata, Hall, 1858, which was preoccupied.

subscalaris, Meek \& Worthen, 1860, Proc. Acad. Nat. Sci. Phil., p. 460, and Geo. Sur. Ill., vol. 2, p. 360, Low. Coal Meas. subsinuata, Meek \& Worthen, 1860, Proc. Acad. Nat. Sci. Plil., p. 460, and Geo. Sur. Ill., vol. 2, p. 358, Low. Coal. Meas. 
subtilstriata, see Raphistoma subtilstriatum.

subturbinata, Meek \& Hayden, 1858, Proc. Acad. Nat. Sci. Phil., p. 264, and Pal. Up. Mo., p. 47, Coal Meas.

sulcomarginata, Conrad, 1842, Jour. Acad. Nat. Sci., vol. 8, p. 272 , and Pal. N. Y., vol. 5 , pt. 2 , p. 69 , Ham. Gr.

supracingulata, Billings, 1857, Rep. of Progr., Geo. Sur. Can., p. 302, Trenton $\mathrm{Gr}$.

swallovana, Hall, 1858, Trans. Alb. Inst., vol. 4, p. 24, and Bull. Am. Mus. Nat. Hist., p. 80, IVarsaw Gr.

sybillina, Billings, 1866, Catal. Sil. Foss. Antic., p. 54, Anticosti Gr.

tabulata, Conrad, 1835, (Turbo tabulata,) Trans. Geo. Soc. Penn., vol. 1, p. 267, Coal Meas.

taggarti, Meek, 1874, 7th Rep. Hayden's U. S. Geo. Sur. Terr., p. 271, and Cont. to Pal., No. 6, p. 140, Coal Meas.

tectoria, Winchell, 1863, Proc. Acad. Nat. Sci., p. 19, Marshäll Gr.

ten uicincta, Meek \& Worthen, 1860, Proc. Acad. Nat. Sci., p. 459 , and Geo. Sur. Ill., vol. 2, p. 355, Up. Coal Meas.

tenuimarginata, Hall, syn. for Eotrochus concavus.

tenuistriata, Shumard, 1860, Trans. St. Louis Acad. Sci., vol. 1, p. 625, Coal Meas.

textiligera, Meek, 1871, Proc. Acad. Nat. Sci., p. 176, and Ohio Pal., vol. 2, p. 314, Waverly Gr.

thalia, see Cyclonema thalia.

trilineata, Hall, 1858, Trans. Alb. Inst., vol. 4, p. 25, and Bull. Am. Mus. Nat. Hist. p. 80, Warsaw Gr.

trilix, Hall, 1862, 15th Rep. N. Y. Mus. Nat. Hist., p. 45, and Pal. N. Y., vol. 5, pt. 2, p. 79, Ham. Gr.

trochiformis, Swallow, 1863. The name was preoccupied by Portlock in 1843. See P. Cooperensis.

tropidophora, Meek, 1872, Am. Jour. Sci. and Arts, 3d series, vol. 4, p. 278, and Ohio Pal., vol. 1, p. 154, Hud. Riv. Gr.

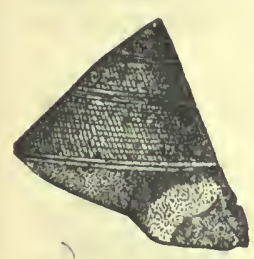

FIG. 702. - Pleurotomaria turbiniformis. turbiniformis, Meek \& Worthen, 1860, Proc. Acad. Nat. Sci., p. 461, and Geo. Sur. Ill., vol. 2 , p. 359, Up. Coal Meas. turgida, see Holopea turgida.

umbilicaia, see Trochonema umbilicatum. unisulcata, C on rad, 1842, Jour. Phil. Acad. Sci., vol. 8, p. 271, Up. Held. Gr. vadosa, Hall, 1860, 13tl Rep. N. Y. Mus. Nat. Hist., p. 108, Kinderhook $\mathrm{Gr}$.

vagrans, Billings, 1862, Pal. Foss., vol. 1, p. 90, Quebec Gr.

valeria, Billings, 1865, Pal. Foss., vol. 1, p. 169, and vol. 3, p. 23, Guelph Gr. valvatiformis, Meek \& Worthen, 1866, Proc. Acad. Nat. Sci. Phil., p. 273, and Geo. Sur. Ill., vol. 5, p. 602, Coal Meas.

viola, Billings, 1865, Pal. Foss., vol. 1, p. 169, Guelph Gr.

virgo, Billings, 1865, Pal. Foss., vol. 1, p. 224, Quebec Gr.

virguncula, Billings, 1865, Pal. Foss., vol. 1, p. 225, Quebec Gr.

vitruvia, Billings, 1865, Pal. Foss., vol. 1, p. 171, Black Riv. Gr.

voltumna, Billings, 1874, Pal. Foss., vol. 2, p. 61, Gaspe limestone No. 8, Devonian.

whitii, Winchell, 1862, Proc. Acad. Nat. Sci., p. 423, Marshall Gr.

wortheui, Hall, 1858, Trans. Alb. Inst., vol. 4, p. 23, and Geo. Sur. Iowa, p. 664, Warsaw Gr.

Polyphemopsis, Portlock, 1843, Geol. Londonderry, p. 415. [Ety. Polyphemus, a genus of shells; opsis, appearance.] Subfusiform; spire elongated; whorls flattened, last one produced below and forming half the length of the shell; outer lip thin, nearly straight; inner lip wanting; columella without folds, slightly twisted and truncated at the comnection with the outer lip; aperture narrow, subovate, effuse or slightly notched at the base of the columella; surface smooth, or only with obscure lines of growth. Type P. elongata.

chrysalis, Meek \& Worthen, 1866, Proc. Acad. Nat. Sci. Phil., p. 267, and Geo. Sur. Ill., vol. 5, p. 596, Coal Meas.

keokuk, Worthen, (in press,) Geo. Sur. Ill., vol. 8, p. 144. Keokuk Gir.

inornata, Meek \& Worthen, 1860, (Loxonema inornatum,) Proc. Acad. Nat. Sci. Phil., p. 463, and Geo. Sur. 1ll., vol. 2, p. 374 , Up. Coal Meas.

louisvillæ, Hall \& Whitfield, 1872, 24th Rep. N. Y. Mus. Nat. Hist., p. 193, Up. Held. Gr.

melanoides, Whitfield, 1882, Ann. N. Y. Acad. Scí,, vol. 2, p. 225, Kaskaskia Gr. nitidula, Meek \&

Worthen, 1860 , (Loxonema nitidula,) Proc. Acad. Nat. Sci. Phil., p. 465 , and Geo. Sur. Ill., vol. 2 , p. 374 , Up. Coal Meas.

peracuta, Meek \& Worthen, 1860 , (Eulima (?) peracuta,) Proc. Acad. Nat. Sci. Phil., p. 466, and Geo. Sur. IIl., vol. 2 , p. 375, FrG. 703.-Polyphemop Up. Coal Nleas.

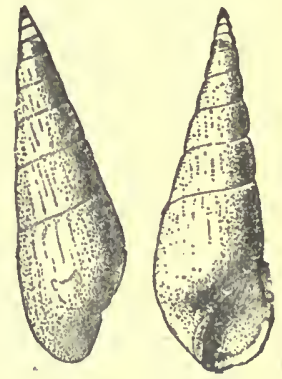

teretiformis, Hall, 1877, 1st Ed. Am. Pal. Foss., p. 245, Warsaw Gr. Proposedinstead of P. elongata, Hall, 1858, which was preoccupied. 
Porceldia, Leveille, 1835, Mem. Soc. Geol. France, vol. 2, p. 39. [Ety. proper name.] Discoid, depressed; whorls very slightly embracing, exposed in a very wide umbilicus, slightly deeper on one side than the other, from a trifling obliquity of the first one or two turns; a narrow band extends along the middle of the exterior, ending in a narrow slit in the lip; surface often nodular and ornamented with rough strix. Type P. puzosi.

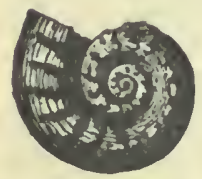

FIG. 704.-Porcella puzosi. crassinoda, White \& Wlitfield, 1862, Proc. Bost. Soc. Nat. Hist., vol. 8 , p. 303 , Kinderhook Gr.

hertzeri, Hall, 1876, Il. lust. Devonian Foss., pl. 16, and Pal. N. Y., vol. 5 , pt. 2 , p. 126 , Up. Held Gr.

nais, Hall, 1862, (Gyroceras nais,) 15th Rep. N. Y. Mus. Nat. Hist., p. 68, and Pal. N. Y., vol. 5, pt. 2, p. 127, Chemung Gr.

nodosa, Hall, 1860, Supp. to vol. 1, pt. 2, Iowa Geo. Sur., p. 92, Kinderhook Gr.

obliquinodus, White, 1862, Proc. Bost. Soc. Nat. Hist., vol. 9, p. 21, Marshall Gr.

peoriensis, Worthen, 1884, Bull. No. 2, Ill. St. Mus. Nat. Hist., p. 6, and Geo. Sur. 1ll., vol. 8, p. 138, Coal Meas.

rectinoda, Winchell, 1803, Proc. Acad. Nat. Sci., p. 18, Marshall Gr.

rotatoria, Hall, see Goniatites plebeiformis.

scioto, Hall \& Whitfield, 1873, 23d Rep. N. Y. Mus. Nat. Hist., p. 240 , Up. Held. Gr.

senex, see Platyceras senex.

Pseudopilorus, Meek, 1873, Ohio Pal., vol. 1, p. 221. [Ety. pseudes, false; Phorus, a genus. Sliell depressed, subtrochiform ; umbilicus broad, sliallow, eccentric; volutions two or three; suture obscure; aperture transversely rlombic, three times as wide as high, acutely angular at the outer and inner extremitios; upper side of lip oblique and extended forward; surface bearing lines of growth directed obliquely backward. Type P. antiquus.

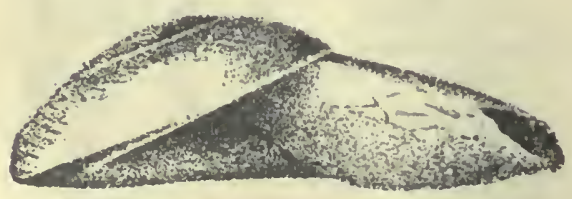

Fig. 705.-Pseudophorus antlquus.

antiquus, Meek, 1871, (Trochita antiqua) Proc. Arad. Nat. Sci. Phil., p. 82, and Ohio Pal., vol. 1, p. 221, Up.
Pupa,Humphrey, 1797, Museum Calonnianum, and Lamarck Syst. Anim. sans Vert., p. 88. [Ety. Yupa, chrysalis shell.] Shell rimate or perforate, cylindrical or oblong; aperture rounded, often tootlied, margins distant, mostly united by a callous laminu. Type P. uva.

bigslyyi, Dawson, 1850, Am. Jour. Sci. and Arts, 3d ser., vol. 20, p. 410, Coal Meas. Fru. 706. vermilionensis, Bradley, 1Si2, 'upu uva. An. Jour. Sci., 3d series, vol. 4, p. 87, Cual Meas.

vetusta, Dawson, 1860, Quar. Jour. Geo. Soc., vol. 16, p. 26S, and Acad. Geol., p. 383 , Coal Meas.

vetusta var. tenuistriata, Dawson, 1850, Am. Jour. Sci. and Arts, $3 \mathrm{~d}$ ser., vol. 20, p. 406 , Coal Meas.

Rajuistoma, Hall, 1847, I'al. N. Y., vol. 1, p. 28. [Ety. raphe, seam or suture; sloma, mouth.] Depressed, often discoil : spire flat or nearly so; sutures close ; whorls acute-angular externally and often with an nugular edge to the moderate umbilicus. Type $\mathrm{R}$. striatum.

acutum, Hnll \& IVhitfield, 1877, U. S. Geo. Expl. 40th parallel, vol. 4, p. 235 , Chazy Gr.

afinis, Foerste, 1855, Bull. Sci. Iab. Denison Univ., p. 95. 'Not properly defined.

angulatum, Hmmons, 1850, (Strapurollus angulatus,) Am. Geol. p 157, Calciferous Gr.

apertum, Salter, 1859, Can. Org. Rem., Decade 1, p. 12, Black Riv. and Trentou Gr.

compressum, Whitfield, 1886, Bull. Am. Mus. Nat. Hist., vol. 1, p. 309, Biruseye Gr.

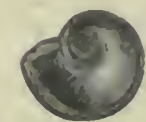

F10. $707 .-$ Raph1s toma halli. halli, S. A. Miller, 1874, (Pleurotomaria halli,) Cin. Quar. Jour. Sci., vol. 1, p. 318, Hud. Riv. Gr.

labiatnm, Ein mons, 1842, (Maclurea labiata,) Geo. Rep. N. Y., p. 312, Calciferous and Birdseye Gr.

lapicida, Salter, 18j9, Can. Org. Rem., Decade 1, p. 12, Black Riv. and Trenton Gr.

lenticulare, Emmons, 1842, (Pleurotomaria lenticularis,) G $\mathrm{e}$ o. Rep. N. Y., p. 392, and Pal. N.' Y., vol. 1, p. 172, Trenton and IIud. Riv. (ir.

niagarense, Whitfield,

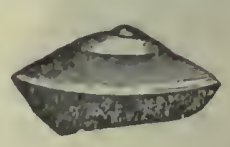
1878, Ann. Rep. Geo. Sur. Wis., p. 82, and Geo. Wis., vol. 4, p. 295, $\mathrm{Ni}$ agara $\mathrm{Gr}$.

planistria, Hall, 1847, Pal. N. Y., vol. 1, p. 30 , Chazy Gr.

planistria var. parvum, Hall, 1847, Pal. N. Y., vol. 1, p. 30 , Chazy Gr. 
prævium, Whitfield, 1889, Bull. Am. Mus. Nat. Hist., vol. 2, p. 52, Calciferous $\mathrm{Gr}$.

rotuliforme, Meek, 1870, Proc. Acad. Nat. Sci., p. 61, and U. S. Geo. Sur. 40th Parallel, vol. 4, p. 18, Calciferous Gr. rotuloides, Hall, 1847, (Pleurotomaria rotuloides, Pal. N. Y., vol. 1, p. 173, Trenton $\mathrm{Gr}$.

stamineum, Hall, 1847, Pal. N. Y., vol. 1, p. 29, Chazy Gr.

striatum, Emmons, 1842, (Maclurea striata, Geo. Rep. N. Y., p. 312, and Pal. N. Y., vol. 1, p. 28, Chazy Gr.

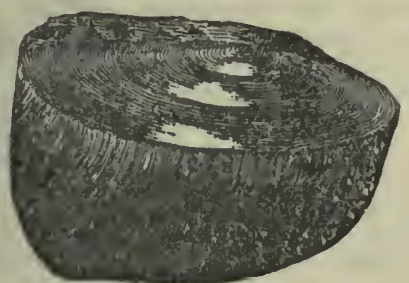

FIG. 709.-Raphistoma striatum.

subplanum, Shumard, 1863, Trans. St. Louis Acad. Sci., vol. 2, p. 106, Calciferous Gr.

subtilstriatum, Hall, 1847, (Pleurotomaria subtilstriata,) Pal. N. Y., vol. 1, p. 172, Trenton Gr.

trochiscum, Meek, 1870, (Euomphalus trochiscus,) Proc. Acad. Nat. Sci., p. 61, and Geo. Sur. W. 100th Mer., vol. 4, p. 77, Calciferous or Trenton Gr.

RotelLA, Lamarck, 1822, Hist. Nat. Anim.sans

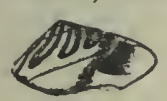

FIG. 710 . -Rotella vestlaria. merous, subequal. Type $R$. vestiaria.

verruculifera, White, 18S2, Rep. Invert. Foss., New Mexico, p. xxxi, Coal Meas.

Scavogyra, Whitfield, 1878, Ann. Rep. Geo. Sur. Wis., p. 61, and Geo. Wis., vol. 4, p. 198. [Ety. screvus, toward the left; gurus, circle.] Sinistral, spire elevated, volutions rounded; umbilicus open, broad, no callus; peristome entire, uniting with the volution on the inner side and spreading externally. Type S. swezeyi.

elevata, Whitfield, 1878, Ann. Rep. Geo. Sur. WVis., p. 62, and Geo. Wis., vol. 4, p. 199, Low. Mag. Gr.

obliqua. Whittield, 1878, Ann. Rep. Geo. Sur. Wis., p. 63, and Geo. Wis., vol. 4, p. 199, Low. Mag. Gr.

swezeyi, Whitfield, 1878, Ann. Rep. Geo. Sur. Wis., p. 62, and Geo. WVis., vol. 4, p. 198, Low. Mag. Gr.

Scalites, Emmons, 1842, Geo. Rep. N. Y., p. 312. [Ety. scaln, staircase.] Turbinate, whorls flat above, turrited, pro- duced below; no umbilicus; form elongate. Type S. angulatus.

angulatus, Emmons, 1842, Geo. Rep. N. Y., p. 312, and Pal. N. Y., vol. 1, p. 27, Chazy Gr.

Scoliostoma, Bra u n, 1838, Neues Jahr. Min. Geo. Geol. Petref., p. 298. [Ety. skolios, curved; sto $m a$, mouth.] Small, upper part pupiform; aperture extended, curved outward. Type S. Fra.711.-Scalites andannenbergi.

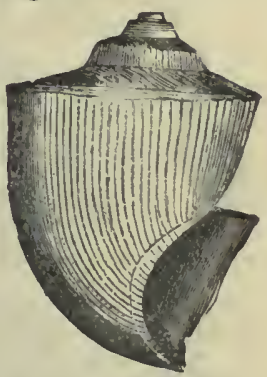

americana, Walcott, 1885, Monogr. U. S. Geo. Sur., vol. 8, p. 195, Low. Devoniall.

Solarium, Lamarck, 1801, Syst. An. sans Vert. Not Palaozoic.

lecii, one of Troost's catalogue names.

Soleniscus, Meek \& Worthen, 1860, Proc. Acad. Nat. Sci. Phil., p. 467. [Ety. soleniskos, little channel or gutter.] Fusiform, pointed; whorls nearly flat, last one contracted and produced below into a straight canal; surface smooth or obscurely marked by lines of growth; aperture narrow; outer lip thin, entire; inner lip thickened and bearing a more or less distinct revolving fold; columella straight, imperforate. Type S. typicus.

brevis, White, 1882, Rep. Invert. Foss., New Mex., p. xxvii, Coal Meas.

fusiformis, Hall, 1858, (Macrocheilus fusi. forme,) Geol. of Iowa, p. 718, Coal Meas.

hallanus, Geinitz, 1866, (Macrocheilus hallanum,) Carb. und Dyas in Neb. p. 6 , Coal Meas.

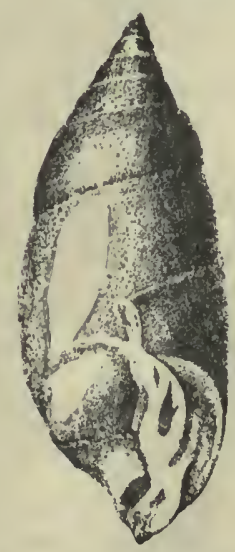

Fra. 712,-Soleniscus klipparti. helicoides, Sowerby, 1829, (A mpullariá heli coides,) Min. Conch., vol. 6, p. 40, Coal Meas.

klipparti, Meek, 1872, (Macrocheilus klipparti,) Proc. Acad. Nat. Sci., vol. 24, p. 328 , and Ohio Pal., vol. 2 , p. 346, Low. Coal Meas.

newberryi, Stevens, 1858, (Loxonema newberryi,) Am. Jour. Sci. and Arts, 2 d ser., vol. 25, p. 259, and Geo. Sur. Ill., vol. 5, p. 594, Coal. Meas.

paludiniformis, Hall, 1858, (Macrocheilus paludiniformis,) Geo.

of Iowa, p. 719, Coal Meas.

planus, White, syn. for S. newberryi. 
texanus, Shumard, 1859, (Macrocheilus texanum,) Trans. St. Louis Acad. Sci., vol. 1, p. 402, Coal Meas.

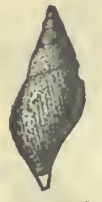

FIa. 713.-Solenlscus typicus.

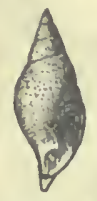

typicus, Meek \& Worthen, 1860, Proc. Acad. Nat. Sci. Phil., p. 467, and Geo. Sur. Ill., vol. 2, p. 3St, Up. Coal Meas.

ventricosus, $\mathrm{Hall}, 1858$, (Macrocheilus ventricosum, Geo. Sur. Iowa, p. 718, Coal Meas.

Straparollina, Billings, 1865, Pal. Foss., vol. 1, p. 223. [Eiy. from the resemblance to shells of the genus Straparollus.] Shell turbinate, with round or obscurely angulated whorls; aperture nearly circular, sometimes with a notch in the inner lower angle of the lip. Type S. pelagica.

asperostriata, Billings, 1860, (Straparollus asperostriatus,) Cun. Nat. and Geol., vol. 5, p. 162, Black Kiv. Gr.

circe, Billings, 1860 , (Straparollus circe, ) Can. Nat. and Geol., vol. 5, p. 161, Black Riv. Gr.

eurydice, Billings, 1860. (Straparollus eurydice,) Can. Nat. and. Geol., vol. 5, p. 162, Black Riv. Gr.

pelagica, Billings, 1865, Pal. Foss., vol. 1. p. 223, Quebec Gr.

remota, Billings, 1874, Pal. Fio. 714.-StrapFoss., vol. 2, p. 70 , Up. arollina pelagica. Taconic.

Strraparollus, Montfort, 1810, Conch. Syst.. vol. 2, p. 174. [Ety. strabos, turned about.] Discoid, depressed conic, smooth or transversely striated; whorls rounded; umbilicus wille, exposing the whorls; moutl indented by the penultimate whorl; peritreme simple, thin, most so on the left side. Type S. dionysii.

ammon, White and Whitfield, 1862, Proc. Bost. Soc. Nat. Hist., vol. 8, p. 307 , Marshall Gr.

angulatus, see Raphistoma angulatum.

asperostriatus, see Straparollina asperostriata.

barrisi, Winchell, 1863, Proc. Acad. Nat. Sci. Phil., p. 20, Marshall Gr.

canadensis, Billings, 1861, Can.Jour., vol. 6, p. 359, Up. Held, Gr.

circe, see Straparollina circe.

clyınenioides, Hall, 1862, (Euomphalus clymenioides,) 15th Rep. N. Y. Mus. Nat. Hist., p. 54, and Pal. N. Y., vol. 5, pt. 2, p. 62, Up. Held. Gr.

cornudanus, Shumarl, 1859, Trans. St, Louis Acad. Sci., vol. 1, p. 400, Coal Meas. crenulatus, Whiteaves, 185t, Pal. Foss., vol. 3, p. 21, Guelph Gr.

cyclostomus, Hall, 1858, (Euomphalus cyclostomus,) Geo. Sur. Iowa, p. 516, Ham. Grr. daphne, Billings, 1862, Pal. Foss., vol. 1, p. 160, Guelph Gr.

eurydice, see Straparollina eurydice.

hecale, Hall, 1876, (Fıomplialus hecale,) Illust. Devon. Foss., pl. 16, Che. mung Gr.

hecale var. corpulens, Hall, 1876, (Euomplialus hecale var. corpulens,) Illust. Dev. Foss., pl. 27, Chemung Gr.

hippolyts, Billings, 1862, 1'ıl. Foss., vol. 1, p. 160, Guelph Gr.

inons, Hall, 18\%6, (Euomplalus inons,) Illust. Devonian Foss., pl. 16, Up. Held. Gr.

labiatus, sce Raplistoma labiatum.

lens, Hall, 1860, (Eunuphalus lens) 13th Rep.

N. Y. Mus. Nat. Hist., Fia.715. -siruparolp. 109, Kinderhook lus difppolyta. Gr.

macromphalus, Winchell, 1863, I'roc. Acad. Nat. Sci. Phil., p. 20, Marslunll Gr. magnificus, Shumard, 1S63, Trans. St. Ioulis Acad. Sci., vol. 2, p. 110, Carboniferous. Too poorly defined for recognition.

minnesotensis, seo Euompbalus minnesotensis.

mopsus, Hall, 1867, 20th Rep. N. Y. Mus. Nat. Hist., p. 390, Niagura Gr.

newarkensis, Walcott, 1855, Monogr. U. S. Geo. Sur., vol. 8, p. 187, Devonian.

niagarensis, Hull \& Whittleld, 1875, Ohio Pal., vol. 2, p. 144, Niagara Gr.

ophirensis, Hall \& Whitfeld, 1877, (Iuomphalus ophirensis,) U. S. Geo. 40th Parallel, vol. 4, p. 261, Waverly Gr.

pernodosus, sce liomplialus pernodosus. planispira, Hall, 1858, (Euomplualus planispira,) Trans. Alb. Inst., vol. 4, J. 20, and Bull. Am. Mus. Nat. Hist., p. 70, Warsaw Gr.

primordialis, see Ophileta primordialis. quadrivolvis, Hall, 1858, (Euomphalus quadrivolvis,) 'Trans. Alb. Inst., vol. 4, p. 19, and Bull. Am. Mus. Nat. Hist., p. 71, Warsaw Gr.

rudis, Hall, 1876, (Euomphalus rudis,) Illust. Dev. Foss., pl. 16, and Pal. N. Y., vol. 5 , pt. 2 , p. 58, Hain. Gr.

sanctisabre, Roemer, 1852, (Euomphahns sanctisaba,) Kreid. von 'Texas, p. 91, Silurian.

similis, Meek \& Worthen, 1861, Proc. Acad. Nat. Sci. Phil., p. 145, and Geo. Sur. Ill., vol. 2, p. 285, St. Louis Gr.

similis var. planus, Meek \& Worthen, 1861, Proc. Acad. Nat. Sci. Phil., p. 146, and Geo. Sur. Ill., vol. 2, p. 2S6, St. Louis Gr. sinuatus, Hall, 1859, (Euomphalus sinuatus,) Pal. N. Y., vol. 3, p. 340, Low. Held. Gr.

spergenensis, Hall, 1858, (Euoinjhalus spergenensis,) Trans. Alb. Inst., vol. 4, p. 19, and Bull. Am. Mus. Nat. Hist., p. 69, Warsaw Gr.

spergenensis var. planorbiformis, Hall, 1858 , (Euomphalus spergenensis var. 
planorbiformis,) Trans. Alb. Inst., vol. 4, p. 20, and Bull. Am. Mus. Nat. Hist., p. 70 , Warsaw Gr.

spirorbis, Hall, 1859, (Euomphalus spirorbis,) 13th Rep. N. Y. Mus. Nat. Hist., p. 109, Kinderlıook Gr.

subplauus, Hall, 1852, (Euomphalus subplanus,) Stans. Ex. to Gt. Salt Lake, p. 414, Coal Meas.

subquadratus, see Euomphalus subquadratus.

subrugosus, see Euomphalus subrugosus.

subuubilicatus, Worthen, (in press) Geo. Sur. Ill., vol. 8, p. 142, Kaskaskia Gr.

umbilicatus, Meek \& Worthen, 1860, (Euomplualus umbilicatus,) Proc. Acad. Nat. Sci. Phil, p. 462, and Geo. Sur. Ill., vol. 2 , p. 36:, Coal Meas.

utahensis, Hall \& Whitfield, 1877, U. S. Gen. Expl., 40th parallel, vol. 4, p. 259 , Waverly Gr.

valvatiformis, Shumard, 1863, Trans. St. Louis Acad. Sci., vol. 2, p. 105, Calciferous Gr.

varsoviensis, Worthen (in press) Geo. Sur. Ill., vol. 8, p. 142, Keokuk Gr.

whitneyi, see Omphalotrochus whitneyi.

Streptaxis, Gray, 1837, Mag. Nat. Hist., p. 484, [Ety. streptos, twisted; axis, axis.] Shell ovate or oblong; when young, subhemispherical, deeply umbilicated, with rapidly enlarging whorls; at length the penultimate whorl is bent toward the right and dorsal side of the axis and the umbilicules become compressed and often nearly closed; the mouth lunate; the edge slightly thickened and reflexed, and often with a single tooth on the outer side of the inner or hinder lip. Type S. comboides. Not a Palieozoic genus.

whitfleldi, Meek, 1871, Proc. Acad. Nat. Sci. Phil., p. 173, and Geo. Sur. Ill., vol. 5 , p. 596, Coal Meas.

Strophites, Dawson, 1880, Am. Jour. Sci. and Arts, $3 \mathrm{~d}$ ser., vol. 20, p. 413. [Ety. from the genus Strophia.] Shell resembling the modern Strophia, conical; apex obtuse; whorls four or more; surface covered with sharp vertical ridges, separated by spaces three times as wide. Type $\mathrm{S}$. grandævus.

grandævus, Dawson, 1880, Am. Jour. Sci. and Arts, $3 d$ ser., vol. 20, p. 413, Devonian.

Strophostylus, Hall, 1859, Pal. N. Y., vol. 3, p. 303. [Ety. strophe, turning round; stylos, column.] Subglobose or ovoid globose; spire small with a large ventricose body whorl; outer lip thin, not reflected; columella twisted or spirally grooved within, not reflected; no umbilicus; aperture somewhat round, ovate or transversely broad oval. Type S. elegans.

andrewsi, Hall, 1859, Pal. N. Y., vol. 3, p. 472 , Oriskany sandstone. cancellatus, Meek \& Worthen, 1868, Geo. Sur. Ill., vol. 3, p. 404, Oriskany sandstone.

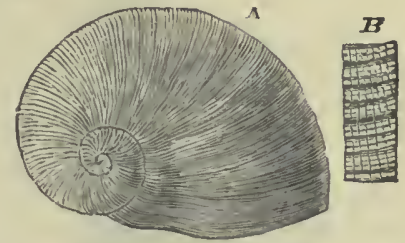

FIG. 716.-Strophostylus cancellatus. $B$, Surface markings en larged.

cyclostomus, Hall, 1863, Trans. Alb. Inst., vol. 4, p. 218 , Niagara Gr.

cyclostomus var. disjunctus. Hall, 1879, 28th Rep. N. Y. Mus. Nat. Hist., p. 177, Niagara Gr.

depressus, Hall, 1859, Pal. N. Y., vol. 3, p. 306, Low. Held. Gr.

elegans, Hall, 1859, Pal. N. Y., vol. 3, p. 304, Low. Held. Gr.

expansus, Conrad, 1841, (Platyceras expansum, ) Ann. Rep. N. Y, p. 55, and Pal. N. Y., vol. 3, p. 470, Oriskany sandstone.

fitchi, Hall, 1859, Pal. N. Y., vol. 3, p. 306 , Low. Held. Gr.

globosus, Hall, 1859, Pal. N. Y., vol. 3, p.. 305, Low. Held. Gr.

matheri, Hall, 1859, Pal. N. Y., vol. 3, p. 471 , Oriskany sandstone.

obliquus, Nicholson, 1874, Rep. Pal. Ont. p. 119, Up. Held. Gr.

obtusus, Hall, 1859, Pal. N. Y., vol. 3, p. 305, Low. Held. Gr.

ovatus, Nicholson, 1874, Rep. Pal. Ont., p. 118, Up. Held. Gr.

rotundatus, Hall, 1859 , Pal. N. Y., vol. 3 , p. 307 , Low. Held. Gr.

subglobosus, Nicholson, 1874, Rep. Pal. Ont., p. 118, Up. Held. Gr.

transversus, Hall, 1859, Pal. N. Y., vol. 3, p. 470, Oriskany sandstone.

unicus, Hall, 1862, 15th Rep. N. Y. Mus. Nat. Hist., p. 41, and Pal. N. Y., vol. 5, pt. 2 , p. 30 , Schoharie grit.

varians, Hall, 1876, Illust. Devonian Foss., pl. 11, and Pal. N. Y., vol. 5, pt. 2, p. 31, Up. Held. Gr.

Stylifer, Broderip, 1829, in Sowerby, Gen. Shells.

primigenia, see Macrochilina primigenia. Subulites, Conrad, 1847, Pal. N. Y., vol. 1, p. 182. [Ety. subula, an awl.] Subulate, volutions wide, suture oblique; aperture very elongate, narrow, pointed above, but wider below. Type S. elongatus.

abbreviatus, Hall, 1850, 3d Rep. N. Y. Mus. Nat. Hist., p. 180, Trenton Gr.

brevis, Winchell \& Marcy, 1865, Mem. Bost. Soc. Nat. Hist., vol. 1, p. $100, \mathrm{Ni}$ agara $\mathrm{Gr}$.

calciferus, Billings, 1859, Can. Nat. \& Geo., vol. 4, p. 360, Calciferous Gr. 
compactus, Whiteaves, 1884, Pal. Foss. vol. 3, p. 16, Guelph Gr.

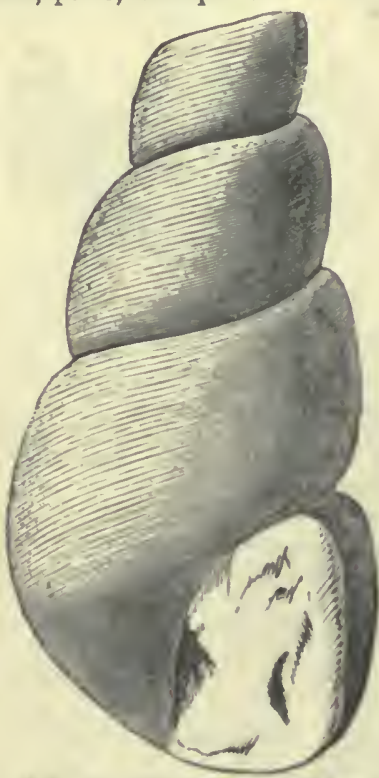

FIG. 717.-Subulites calctferus.

daphne, Billings, 1865, Pal. Foss., vol. 1, p. 223, Quebec Gr.

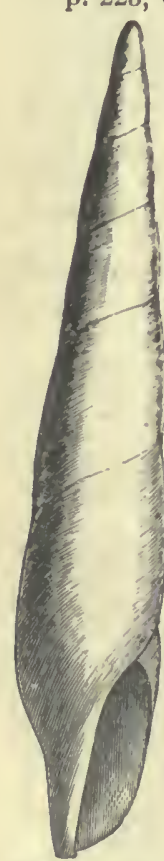

Fia. 718.-Subulites elongalus. elongatus, Emmons, 1842, Geo. Rep. N. Y., p. 392, and Pal. N. Y., vol. 1, p. 182, Trenton (ir.

gracilis, s. A. Miller, 1882 , Jour. Cin. Soc. Nat. Hist. vol. 5 , p. 116, Niagara Gr. inflatus, Meek \& Worthen, 1870, Proc. Acad. Nat. Sci. Phil., p. 47, and Geo. Sur. III., vol. 6, p. 495 , Galena Gr.

notatus, Billings, 1866, Crtal. Sil. Foss. Antic., p. 54, Anticosti $\mathrm{Gr}$.

obesus, Whitficld, 1586, Bull. Am. Mus. Nat. Hist., vol. 1, p. 318, Birdseye Gr.

parvulus, Billings, 1862, $\mathrm{Pal}$ Foss., vol. 1, p. 36, Black Riv. Gr.

psyche, Billings, 1865, Pal. Foss., vol. 1, p. 188, Quebec Gr.

richardsoni, Billings, 1857 , Rep. of Progr., Geo. Sur. Can., p. 306, Hud. Riv. Gr. terebriformis, Hall \& Whitfield, 1S75, Ohio Pal., vol. 2, p. 141 , Niagara Gr.

ventricosus, $\mathrm{Hall}, 1852$, $\mathrm{Pal}$. N. Y., vol. 2 , p. 347 , Niagara and Guelph Gr.

Trachydomia, Meek \& Worthen, 1866, Geo. Sur. Ill., vol. 2, p. 364. [Ety. trachys, rough; doma, house.] In form like Naticopsis, but distinguished by having the surface ornamented with regularly disposed nodes. Type $T$. nodosum.

hollidayi, Meek \& Worthen, 1860, (Naticopsis hollidayi,) Proc. Acad. Nat. Sci. Phil., p. 463, and Geo. Sur. Ill., vol. 2, p. 367 , Low. Coal Meas.

nodosum, Meek \& Worthen, 1860, (Naticopsis nodosa,) Proc. Acad. Nat. Sci. Phil., p. 463, and Geo. Sur. Ill., vol. 2, p. 366, Low. Coal Meas.

nodulosum, Worthen, 1884, Bull. No. 2, III. St. Mus. Nat. Hist., p. 8, and Geo. Sur. 111., vol. 8, p 146., Coal Meas.

Tremanotus, Hall, 1868, 20th Rep. N. Y. St. Mus. Nat. Hist., p. 347. [Ety. trema, hole; notos, back.] Distinguished from Bucania by a eingle row of hollow spines upon the back of the last whorl. In casts the spines are uswally liroken off, and hence Carpenter argises they never had spines. Typo T. chicagoensis.

alpheus, Hall, 1864, 10th Rep. N. Y. St. Mus. Nat. Hist. Syn. for T. chicagoensis.

chicagoensis, McChesney, 1860, (Bucania chicagoensis,) New Pal. Foss., p. 69, Niagara $\mathrm{Gr}$.

trigznostoma, Hall \& Whitfield, 1875, Ohio Pal., vol. 2, p. 146, Niagara Gr.

Trochila, Schumacher, 1817, Essai N. Syst., p. 184. [Ety. trochus, wheel.] Not a Palæozoic genus.

antiqua, see Psendophorus antiquus.

carbmaria, Meek, 1866, Proc. Acad. Nat. Sci., p. 270, Kaskaskia Gr. Not recognized.

Trocioskin, Salter, 1859, Can. Org. Rem., Decade 1, p. 27. [Ety. trochus, a wheel; nema, a thread.] Turbinate, thin, of few angular whorls; strong concentric ridges, erossed by oblique lines of growth; umbilieus wide, open; inner lip thin, scarcely reflected; peritreme complete. Type T. umbilicatum.

beloitense, Whitfield, 1878, A nn. Rep. Geo. Sur. Wis., p. 74, and Geo. Wis., vol. 4, p. 212, Trenton Gr.

benchi Whitficld, 1878, Ann. Rep. Geo. Sur. Wis., p. 74 , and Geo. Wis., vol. 4 , p. 213, Trenton Gr.

emaceratum, Hall \& Whitfield, 1872, 24th

Rep. N. Y. Mus. Nat. Hist., p. 193, Ham. Gr.

exile, Whitfield, 1859, Bull. Am. Mus. Nut. Hist., vol. 2, p. 57, Calciferous Gr. fatua, Hall, 1867, 20th Rep, N. Y. Mlus. Nat. Hist., p. 394, Niagara Gr.

halii, Hall, 1861, (Pleurotomaria halej,) Geo. Sur. Wis., p. 34, Niagara Gir.

inornatum, Whiteaves, 1884, Pal. Foss., vol. 3 , p. 19, Guelph Gr.

meekanum, n. sp. Up. Held. Gr. at Marblehead, Oliio. Proposed instead of T. tricarinatum, Meek, 1871, Proc. Acad. Nat. Sci., p. 82, and Ohin Pal., vol. 1, p. 218, which was preoccupied. 
nana, Foerste, 1885, Bull. Sci. Lab. Denison Univ., p. 94 . Not properly defined. pauper, Hall, syn. for $\mathrm{P}$. halii.

pauper var. ohioense, Hall \& Whitfield, 1875, Ohio Pal., vol. 2, p. 144, Niagara Gr. rectilatera, Hall \& Whitfield, 1872, 24th Rep. N. Y. Mus. Nat. Hist., p. 193, Up. Held. Gr.

tricarinatum, Billings, 1859, Can. Nat. \&

Geo., vol. 4, p. 356, Calciferous Gr. tricarinntr, see Trochonema meekanum.

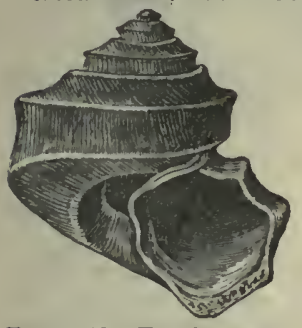

FIG. 719.-Trochonema umbilicatum. umbilicatum, Hall, 1847, (Pleurotonaria umbilicata,) Pal. N. Y.,vol. 1, p. 43 , Chazy to Hud. Riv. Gr. yandellanum, Hall \& Whitfield, 1872, 24th Rep. N. Y. Mus. Nat. Hist., p. 194, Up. Held. Gr.

Trochus, Adanson, 1757, Voy. Senegal. [Ety. trochus, a hoop.] Not a Palæozoic genus.

huronensis, Castelnau, 1S43, Syst. Sil., p. 35. Not recognized.

missouriensis, see Pleurotomaria missouriensis.

Tryblidium, Lindstrom, 1880, Fragmenta Silurica, p. 15. [Ety. trublion, a cup.] Patelliform, obovate, acuminate anteriorly, enlarged posteriorly; muscular scars in six disconnected pairs arranged in an oblong circle open toward the frout. Type T. reticulatum.

acutum, Whitfield, 1889, Bull. Am. Mus. Nat. Hist., vol. 2, p. 45, Calciferous Gr. canadense, Whiteaves, 1884, Pal. Foss., vol. 3, p. 31, Guelph Gr.

conicum, Whitfield, 1886, Bull. Am. Mus. Nat. Hist., vol. 1, p. 306, Birdseye Gr.

erato, Billings, 1862, (Metoptoma erato,)

Pal. Foss., vol. 1, p. 39, Black Riv. Gr. eubule, Billings, 1862, (Metoptoma eubule,) Pal. Foss., vol. 1, p. 38, Calciferous and Black Riv. Gr.

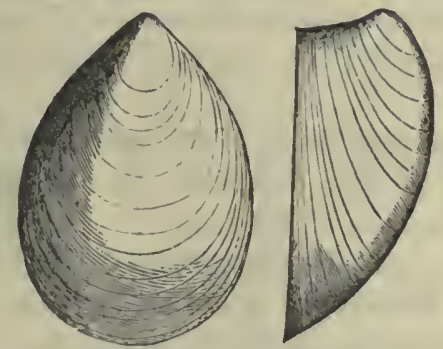

Fig. 720.-Tryblidium nycteis.

hyrie,. Billings, 1862, (Metoptoma hyrie,)

Pal. Foss., vol. 1, p. 87, Quebec Gr.

niobe, Billings, 1862, (Metoptoma niobe,)

Pal. Foss., vol. 1, p. 37, Calciferous Gr. nycteis, Billings, 1862, (Metoptoma nycteis,

Pal. Foss., vol.1, p. 38, Calciferous Gr. ovale, Whitfield, 1886, Bull. Am. Mus. Nat. Hist., vol. 1, p. 305, Birdseye Gr. ovatum, Whitfield, 1886, Bull. Am. Mus. Nat. Hist., vol. 1, p. 305, Birdseye Gr. pileolum, Whitfield, 1889, Bull. Am. Mus. Nat. Hist., vol. 2, p. 46, Calciferous Gr. simplex, Billings, 1865, (Metoptoma simplex, ) Pal. Foss., vol. 1, p. 346, Calciferous Gr.

Turbo, Klein, 1753, Tent. Meth. Ostr. [Ety. turbo, top.] Shell thick, ovate; body whorl rounded, ventricose; spire small, of several convex whorls, pointed ; surface spirally grooved or nodulated; aperture large, nearly circular, slightly

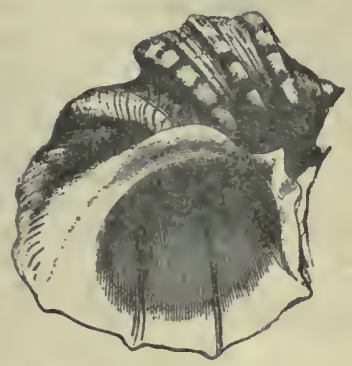

FIG. 721.-Turbo marmoratus. produced and b r o a d l y rounded in front, more or less modified by the p receding whorl; outer and inner lips thin ; ope $\mathrm{rcul}$ $\mathrm{u} \mathrm{m}$ thick, shelly, rugged without, flattened and spirally s u $\mathrm{l}$ c a $\mathrm{t}$ e d within. Type T. marmoratus. Not an American Palcozoic genus. The species left here is, for want of material, to refer them where they belong.

bicarinatus, Troost, 1840. Not defined. dilucula, see Holopea dilucula.

guadalupensis, Shumard, 1859, Trans St. Louis Acad. Sci., vol. 1, p. 398, Permian Gr.

huronensis, Castelnau, 1843. Not recognized.

lineatus, see Pleurotomaria lineata.

obesus, Shumard, 1858, Trans. St. Louis Acad. Sci., vol. 1, p. 202, Up. Coal Meas.

(8) obscura, see Holopea obscura.

shumardi, see Piatystoma shumardi.

tabulata, see Pleurotomaria tabulata.

tennesseensis, see Cyclonema tennesseense.

t ex a n us, Shuniard, 1859, Trans. St. Lonis Acad. Sci., vul. 1, p. 400, Coal Meas.

Turbonilla, Leach, 1826, Risso Eur. Merid. 4. [Ety.di ininutive of 'I'urbo, a genus.] Not a Palæzoic genus.

swallovana, see Aclis-

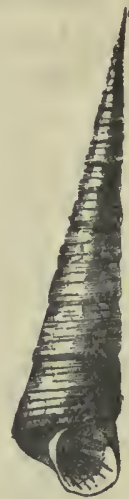

Fig. 722.-T'urritella imbricata. ina swalloviana.

Turritella, Lamarck, 1801, Syst. An. sans Vert., p. 89. Not a Palæozoic genus. Type T. imbricata. 
schohariensis, Castelnau, 1843, Syst. Sil., p.

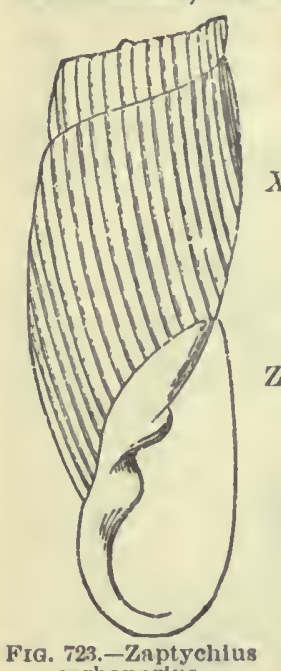
carbonarlus.

35. Not recognized. stevensana, Meek \& Worthen, 1866, Geo. Sur. Ill., vol. 2 , p. 382, Up. Coal Miens.

Xenophora, Fischier, 1806, II useum Demidovianum, p. 213. Not an American Palrozoic genus.

antigua, see Pseudophorus antiquus.

Zaptycinus, W al cott, 1884, MIonogr. U. S. cieo. Sur., vol. 8, p. 263. Shell minute, elongate; aperture large, oblong, nearly vertical; outer lip thin ; collumellar lip reflected, plicated; surface marked by slightly ob!ique Type \%. carbonarius. carbonarius, Walcott, 1884, Monogr. U.S. Geo. Sur., vol. 8, p. 263, Subcarboniferous.

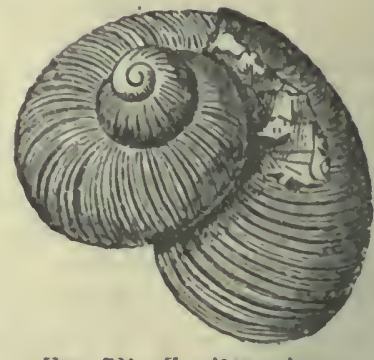

Fia. 721.-Zoniles priscus.

Zonites, Montfort, 1810, Conch. Syst., vol. 2, p. 282. [1ity. zone, belt.] A coiled sliell, closely resembling a Helix, having an open umbilicus; the surface is transversely sculptured. Type $\mathrm{Z}$. algireus.

priscus, Carpenter, 1867, Quar. Jour. Geo. Soc., vol. 23, p. 331, and Acadian Geol., p. 385 , Coal Meas.

\section{CLASS CEPHALOPODA.}

[Ety. kephale, head; pous, foot.]

THE animals of this class are all marine, and they reach a higher state of animal development than any other marine group among the Mollusca. Sone of them have a rudimentary, cartilaginous, cephalic skeleton, which indicates superiority over other marine Mollusca. The locomotive organs consist of arus surrounding the head, furnished with sucking cups that take a firm lold on other objects. Many have fins, and all can propel themselves by the forcible expulsion of water from the respiratory chamber. They swim rapidly, creep on the bottom of the sea, and are very predatory in their habits. The body is short, thick, and symmetrical, with branchir on both sides.

The Palrozoic fossils of this Class belong to the Order Tetrabranchiata (fourgilled), which is represented in tropical seas by the Nautilus. The shells are straight, as in the family Orthoceratida; curved, as in Cyrtoceratida ; discoid, as in the Gyroceratidx and Trocholitidx; spiral, as in the Trochoceratillx; involute, as in the Nautilidæ; or involute and having lobed sutures, as in the Goniatitidx. Internally the shell is divided into numerous chambers by partitions, or septa, the animal inhabiting the last chamber, and retaining connection through the preceding chambers by a tube, or siphuncle, but having no connection with the interior of the several chambers after having cut itself off by the secretion of the shelly septa. The outlines of the septa are called sutures, and in Goniatites the elevations of the folded sutures are called saddles, and the intervening depressions lobes. 
Each septum began to form at the circumference of the shell, and slowly approached the siphuncle as the animal moved forward in the body chamber. The siphuncle, being a point of muscular attachment, was not vacated by the auimal between any two septa until the anterior one had been firmly closed by attachment to the siphuncle, forming a chamber of support.

The fossil shells are very thin in proportion to their size. They are not porous, like those of the Brachiopoda; nor horny, like the Crustacea; nor of the same composition as the Gasteropoda or Lamellibranchiata. Generally the exterior shell is destroyed, even when the associated shells of other classes are well preserved. Sometimes the shell appears as if it had melted and run together, or run down upon the siphuncle. Such molecular change will occur in one part of a specimen while another part is unchanged. The general form of the shell is of family importance. The shape of the siphuncle and the external markings are of generic importance. We recognize the following families:

Family Ascoceratide.-Ascoceras.

Family Cyrtoceratide.-Cyrtoceras, Cyrtocerina, Oncoceras.

Family Discosorid z.-Discosorus.

Family Endoceratide.-Cameroceras, Colpoceras, Endoceras.

Family Gomphoceratide.-Gomphoceras.

Family Goniatitide.-Goniatites.

Family Gyroceratid æ.-Gyroceras.

Famu, Lituitids.-Lituites.

Family Nautilid z.-Discites, Nautilus, Pteronautilus, Solenochilus, Temnochilus, Trematodiscus.

Family Orthoceratide.-Actinoceras, Bactrites, Gonioceras, Huronia, Ormoceras, Orthoceras, Trematoceras.

Family Phragmoceratida.-Phragmoceras, Streptoceras.

Family Piloceratide.-Piloceras.

Family Trochoceratide.-Trochoceras.

Family Trocholitide.-Trocholites.

Family Uncertain.-Petalichnus, Særichnites, Teratichnus, Trachomatichnus.

Actinoceras, Bronn, 1837, Lethaea Geognostica, p. 97. [Ety. aktin, ray; keras, horn.] Exterior like Orthoceras; siphuncle very large, ihflated between the chambers, and connected with a slender central tube by radiating plates. Type A. bigsbyi, A. richardsoni, and A. lyoni. The genus was established before the species were defined.

beaudanti, Castelnau, 1843, Systeme Silurien, p. 31 . Not recognized.

beaumonti, Castelnau, 1843, Systéme Silurien, p. 32. Not recognized.

bigsbyi, Stokes, 1840, Trans. Geo. Soc., $2 \mathrm{~d}$ series, vol. 5, p. 707, Chazy Gr.

blainvıllei, Castelnau, 1843, Systéme Silurien, p. 31. Not recognized.

cordieri, Castelnau, 1843, Systéme Silurien, p. 31. Not recognized.

deshayesi, Castelnau, 1843, Systéme Silurien, p. 32. Not recognized.

dufresnoyi, Castelnau, 1843, Systéme Silurien, p. 32. Not recognized.

inops, Daw son, 1868 , A c a d . G e o l. p. 314, Carb. lyoni,Stokes, 1840 , T r a n s . Geol. Soc.. vol. $5, p$. 707, Black Riv. Gr. ri chardsoni, $\mathrm{St}$ o kes, 1840 , T r a $\mathbf{n}$. Geol. Soc.,

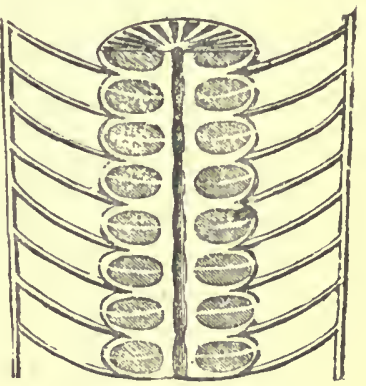

FrG. 725.-Actinoceras richardsonl.

$2 \mathrm{~d}$ series, vol. 5, p. 708, Black Riv. Gr. simmsi, Stokes, 1840, Trans. Geo. Soc., 2d series, vol. 5, p. 708, Sil. 
Ammonites bellicosus, Morton, 1836, Am. Jonr. Sci. and Arts, vol. 29, Coal Meas. Not recognized.

colubrellus, see Goniatites colubrellus.

hildrethi, see Goniatites hildrethi.

Ascoceras, Barrande, 1855, Bull. de la Soc. Geol. de France, vol. 12, 2d ser., p. 157. [Ety. askos, leather bottle ; keras, horn.] Chambers behind the living one short and rapidly tapering; living chamber long and constricted near the aperture ; aperture somewhat T-shaped. Type $A$. bohemicum.

anticostiense, Billings, 1866, Catal. Sil. Foss. Antic., p. 60, and Pal. Foss., vol. 1, p. 164, fig. 148b, Anticosti Gr.

canadense, Billings, 1857, Rep. of Progr. Geo. Sur. Can., p. 310, IIuil. Riv. Gr. This species is made the type of the genus liillingsites by Hyatt.

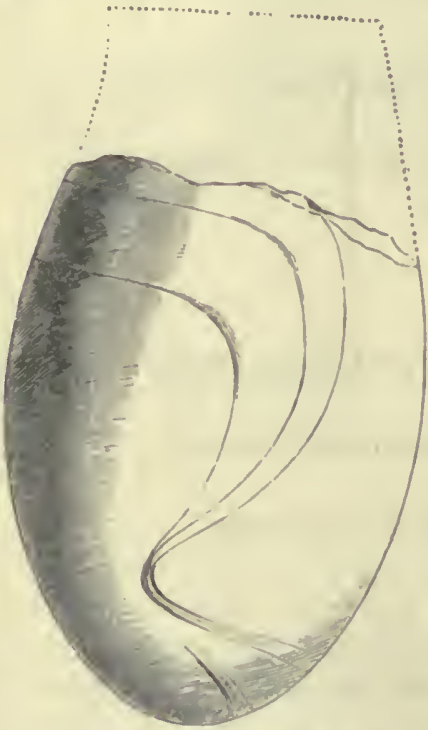

FIQ. 726.-Ascoceras canadenso.

newberryi, Billings, 1862, Pal. Foss., vol. 1, p. 163, Hud. Riv. and Anticosti Grs. southwelli, Worthen, (in press,) Geo. Sur. 11l., vol. 8, p. 151, Niagara Gr.

townsendi, Whiteaves, 1884, Pal. Foss., vol. 3, p. 41, Guelph Gr.

Bactrites, Sandberger, 1841, Leonh. $u$. Bronn's Jahrb., p. 240. [Ety. baktron, staff.] Shell long, straight, gradually tapering, many-chambered; sutures curve abruptly backward over the siphuncle, forming "the dorsal lobe" similar to that of a Goniatites. Type B. carinatus.

clavus, Hall, 1879, Pal. N. Y., vol. 5, pt. 2, p. 316, Ham. Gr.

Cameroceras, Conrad, 1842, Jour. Acad. Nat. Sci. Phil., vol. 8, p. 267. [Ety. Kamara, chamber; keras, horn.] Shell straight, and in form like Endoceras; siphuncle marginal, and obliquely annulat. $d$ at the junction of the septa. 'Type C. trentouense.

trentonense, Con rad, 1842, Jour. Acad. Nat. Sei. Pliil., vol. 8, p. 267, and Pul. N. Y., vol. 1, p. 221, Trenton Gr.

Colpoefras, Hall, 1850 , 3d Rep. N. Y. Mus. Nat. Hist., p. 181. [Ety.kolpos, furrow; leras, horn.] Dis. tinguished from Orthoceras by the oblique septa, arched upon the dorsal side, and bending down in a deep sinus on the ventrul side, and strongly arching toward the mouth. Type C. virusum. arcualum, Janses, \& poorly defined siphuncle of an Findocerus.

clarkii, Wetherby, 1851, Jour. Cin. Soc. Nat. Ilist., vol. 4, p. 77, 'Tren ton Gir.

virgatum, Hall, 1850,

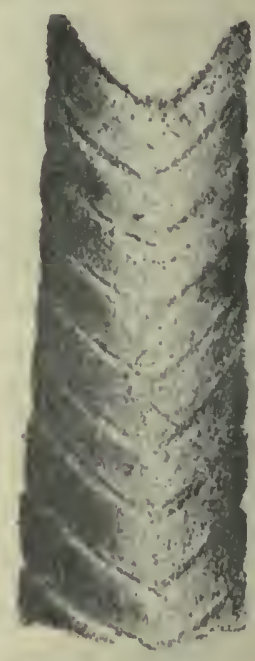

FIG. 727.-Col poceras clnrkil. d Rep. N. Y. Mus. Nat. Ilist., p. 182, Birdseye and Black Riv. Cirs.

Clymenia, Munster, 1S39. [1:y. mythological name.]

complunata, see Goniatites complanatus. erato, see (inniatites erato.

Conilites, Schlotheim, 1820, Petrefuktenkunde, ete. [Ety. konas, cone, lithos, stone.]

capricornulus, Troost, 1840,5 th Geo. Ret). Tenn. Not satisfactorily detined.

Conolubularia, Troost, syn. for Orthoceras. brongniarti, see Orthoceras brongniarti. cuvieri, see Orthoceras cuvieri.

defrancii, see Orthoceras defrancii. goldfusi, see Ortlioceras goldfussi.

Conulites, Cozzens, 1848. Not satisfactorily defined.

angulosum, Cozzens, 1848. Not satisfactorily defined. . It may be a plant.

Cryptocera8, D'Orbigny, 1850. [Ety. kryptos, concealed; keras, horn.] This name was preoccuppied by Iatreille for a genus of insects, and liad been previously used by Barrande for a genus of Cepbalopods.

capax, see Solenochilus capax.

Cyrtoceras, Goldfuss, 1832, in De la Beche's IIandbuch ler Geognosic bearbeitet von v. Deschen, p. 536. [Eıv. kurtos, curved; keras, liorn.] Shell long, conical, gently curved, aperture sometimes contracted; siphuncle straight or expanded between the septa, and variable in position, but usually at the outer edge.

absens, see Gomphoceras absens. 
acinacellum, Whitfield, 1886, Bull. Am. Mus. Nat. Hist., vol. 1, p. 327, Birdseye Gr.

æmulum, Hall, 1879, Pal. N. Y., vol. 5, pt. 2, p. 371, Up. Held. Gr.

alethes, Billings, 1865, Pal. Foss., vol. 1, p. 193, Quebec: Gr.

alternatum, Hall, 1879, Pal. N. Y., vol. 5, pt. 2, p. 365, Marcellus Shale. Proposed instead of C. undulatum of Hall.

ammon, Billings, 1861, Can. Jour., vol. 6, p. 361, Corniferous limestone.

amœnum, S. A. Miller, 1878, Jour. Cin. Soc. Nat. Hist., vol. 1, p. 105 , Hud. Riv. Gr.

amplicorne, Hall, 1867, 20th Rep. N. Y. Mus. Nat. Hist., p. $3 \check{3} 8$, syn for C. hercules.

annulatum, Hall, 1847. This name was preoccupied by Goldfuss in 1832, see C. subannulatum.

areticameratum, Hall, 1852, Pal. N. Y., vol. 2, p. 349, Guelph Gr.

arcuatum, Hall, 1847, Pal. N. Y., vol. 1, p. 196. The name was preoccupied by Steininger in 1830 , see C. subarcuatum. aristides, Billings, 1865, Pal. Foss., vol. 1, p. 316, Quebec Gr.

ashmanni, n. sp. Shell small, gently curved; section subelliptical, becoming subcircular near the point, the dorsal side a little less convex, than the ventral; siphuncle near the dorsal side; surface longitudinally furrowed and finely sculptured transversely, the furrows and trausverse lines most distinet on the ventral side; there are
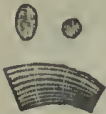

FIG. 728.-Cyrtoceras as h manni. The two sectlon views are natural size.

and is in the col Charles Faber. beekmanense, Whitfield, 1889, Bull. Am. Mus. Nat. Hist., vol. 2, p. 57, Calciferous Gr.

bannisteri, see Trochoceras bannisteri.

belus, Billings, 1861, Can. Jour., vol. 6, p. 361 , Cornilerous Gr.

boycii, Whitfitld, 18S6, Bull. Am. Mus. Nat. Hist., vol. 1, p. 326, Birdseve Gr.

billingsi, Salter, 1859, Can. Org. Rem. Decade 1, p. 33, Chazy or Black Riv. Gra. bondi, Saffurd, 1869, Geo. of Tenn., p. 290, Nashville Gr.

brevicorne, Hall, 1867, 20th Rep. N. Y. Mus. Nat. Hist., p. 407, Niagara Gr.

camuruın, Hall, 1847, Pal. N. Y., vol: 1, p. 196, Trenton Gr.

cancellatum, Hall, 1852, Pal. N. Y., vol. 2, p. 290. The name was preoccupied by Roemer in 1844. See C. subcancellatum. carrollense, Worthen, 1875, Geo. Sur. Ill., vol. 6, p. 496, Galena Gr. cessator, Hall \& Whitfield, 1877, U. S. Expl. Exped. 40th parallel, vol. 4, p. 278, Coal Meas.

citum, Hall, 1879, Pal. N. Y., vol. 5, p. 372, Up. Held. Gr.

clavatum, see Gomphoceras clavatum.

clitus, Billings, 1866, Catal. Sil. Foss. Antic., p. 85, Niagara Gr.

confertissimum, Whitfield, 1886, Bull. Am. Mus. Nat. Hist., vol. 1, p. 327, Birdseye Gr.

conicum, Owen, 1840, Rep. on Min. Lands, p. 70, Up. Magnesian Gr.

conoidale, Wetherby, 1881, Jour. Cin. Soc. Nat. Hist., vol. 4, p. 78, Hud. Riv. Gr. constrictostriatum, Hall, 1847, Pal. N. Y., vol. 1, p. 195, 'Trenton Gr.

corniculum, Hall, 1862, Geo. Rep. Wis. The name was preoccupied by Barrancle in 1848, and again by Eichwald in 1860 , see C. tenuistriatum.

corydon, Billings, 1866, Catal Sil. Foss. Antic., p. 85 , Niagara Gr.

cretaceum, Whitfield, 1882, Ann. N. Y. Acad. Sci., vol. 2, p. 209, Up. Held Gr. curtum, Meek \& IVorthen, 1860, Proc. Acad. Nat. Sci. Phil., p. 468 , and Geo. Sur. Ill., vol. ", p. 388, Up. Coal Meas. Was this name preoccupied by Eichwald ?

dactyloides, Dwight, 1884, Am. Jour. Sci. and Arts, 3d ser., vol. 27, p. 255, Calaiferous Gr.

dardanus, Hall, 1861, Rep. of Progr. Geo. Sur. of Wis., p. 43, Niagara Gr.

densum, Hall, 1879, Pal. N. Y., vol. 5, pt. 2, p. 363, Hum. Gr.

dicty's, Billings, 1865, Pal. Foss., vol. 1, p. 192, Quebec Gr.

dictyum, White, 1876, Proc. Acad. Nat. Sci., p. 33, Devonian.

dilatatum, Meek \& Worthen, 1860, Proc. Acad. Nat. Sci. Phil., p. 468, and Geo. Sur. Ill., vol. 2, p. 389, Up. Coal Meas.

dorsatum, Swallow, 1858, Trans. St. Louis Acad. Sci., vol. 1, p. 197, Permian Gr.

eugenium, Hall, 1862, 15th Rep. N. Y. Mus. Nat. Hist., p. 70, and Pal. N. Y., vol. 5 , pt. 2 , p. 369 , Schoharie grit.

eugium, Hall, 1861, Rep. of Progr. Wis., p. 40, Chazy and Black Riv. Grs.

exignum, Billings, 1860, Can. Nat. and Geo., vol. 5, p. 172, Trenton Gr. This is made the type of the genus Climoceras by Hyatt.

faberi, James, 1886, Jour. Cin. Soc. Nat. Hist., rol. 8, j". 246, Hud. Riv. Gr.

falx, Billings, 1857, Rep. of Progr. Geo. Sur. Can., p. 314, and Can. Org. Rem., Decade 1, p. 32, Black Riv, and Trenton Grs.

filosum, Emmons, 1842, Nat. Hist. N. Y., vol. 4, p. 392, Trenton Gr.

formosum, Hall, 1879, Pal. N. Y., vol. 5, pt. 2, p. 36:2, Ham. Gr.

fosteri, Hall, 1861, Rep. of Progr. Geo. Sur. Wis., p. 41, Niagara Gr.

fragile, Billings, 1866, Catal. Sil. Foss. Antic., p. 59, Anticosti Gr. 
gibbosum, Hall, 1876, Illust. Devonian Foss., syn. for Gomphoceras oviforme. giganteum, McChesney, Jan. 1860, New Pal. Foss., Niagara Gr. In 1861 McChesney referred this species to the genus lituites, and proposed for it the name Lituites cancellatus. Prof. Hall, in the meantime, described it as Lituites occidentalis. It is now referred to the genus Nautilus, and as both the earlier names were preoccupied, IcChesney's name cancellatus has preeedence.

hallanum, D'Orbigny, 1850, Prodrome de Pal., tome 1, p. 1, Trenton Gr. Proposed instead of C. lauellosum, Hall, 1847, which was preoccupied. Hyatt founded his genus Zitteloceras on this s)ecies. kirbyi, Whitfield, 1889, Bull. Am. Mus. Nat. Hist., vol. 2, p. 57, Calciferous Gr.

lamellosum, Hall, 1847, Pal. N. Y., vol. 1, p. 193. The name was preoccupied by d'Archiac \& Verneuil in 1842. See C. hallanum.

laterale, Hull, 1867, 20th Rep. N. Y. Mus. Nat. Hist., p. 407, Nimgara Gr.

ligarius, Billiugs, 1865, Pal. Foss., vol. 1 p. 176, Hud. Riv. Gr.

liratum, Hall, 1862,15 th Rep. N. Y. Mus. Nat. Hist.. p. 72, Ham. Gr.

loculosum, Hall, 1861, Rep. of Progr. Wis., p. 42 , 'T'renton $\mathrm{Gr}$.

lucillus, Hall, 1867, 20th, Rep. N. Y. Mus. Nat. Hist., p. 406, Niagara Gr.

lysander, Billings, 1862, Pal. Foss., vol. 1, p. 161, Hud. Riv. (ir.

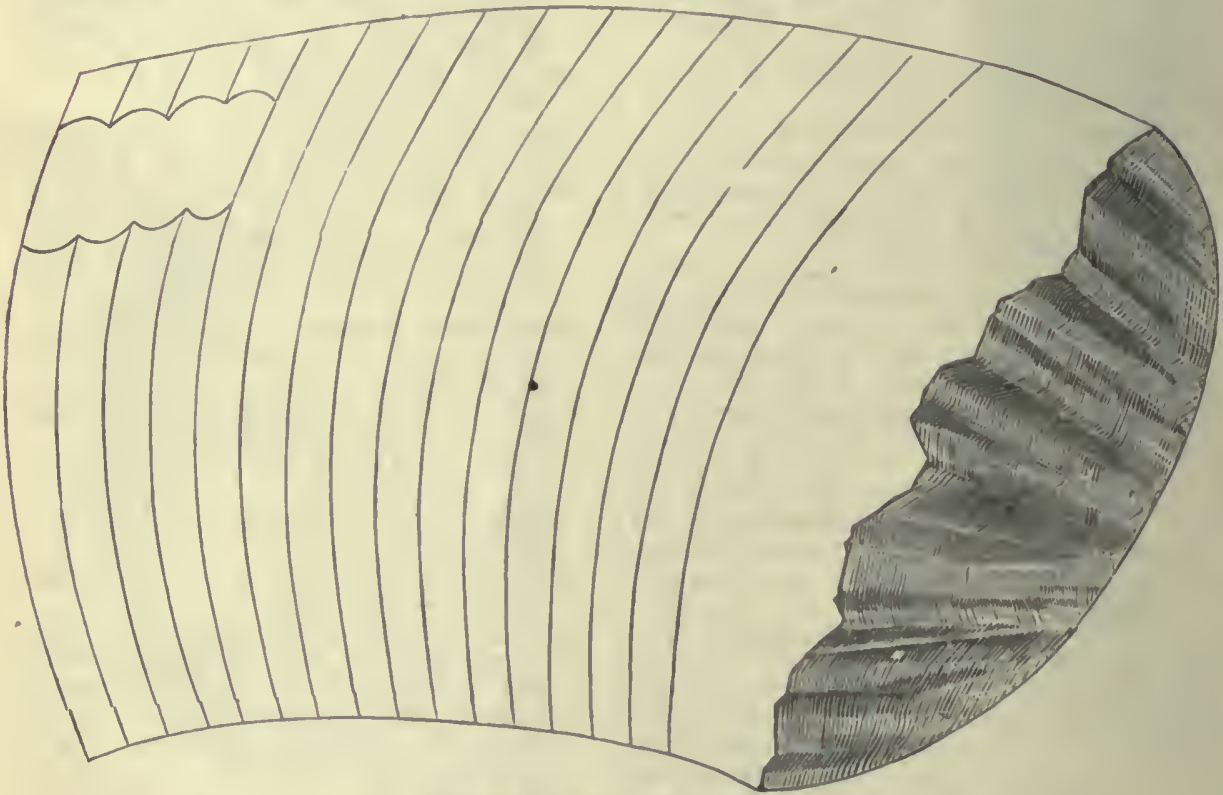

Fxa. 729.-Cyrtocerns magtster.

bector, Hall, 1879, Pal. N. Y., vol. 5, pt. 2, p. 364 , Up. Chemun $\mathrm{Gr}$.

hercules, Winchell \& Marcy, 1865, (Lituites hercules, ) M.1n. Bost. Soc. Nut. Hist., p. 10:2, Niagara Gr.

hertzeri, see Gomplocerus hertzeri.

huronense, Billings, 1865, Pal. Foss., vol. 1, p. 176, Black Riv. or Trenton Grs.

infundibulum, Whitfield, 1880, Ann. Rep. Geo. Sur. Wis., p. 66, and Geo. Wis. vol. 4, p. 300 , Niagara Gr.

irregulare, Wetherby, 1881, Jour. Cin. Soc. Nat. Hist., vol. 4, p. 79, Hud. Riv. Gr. isidorus, Billings, 1865, P'al Foss., vol. 1, p. 175, Black Riv. or Trenton Gr.

janus, see streptoceras janus.

jason, see Gyroceras jason.

juvenale, Billings, 1865, Pal. Foss., vol. 1, pp. 177, 420, Trenton Gr. macrostomum, Hall 1847, Pal. N. Y., vol. 1, p. 194, Black Riv. and Trenton Grs.

magister, S. A. Miller, 1875, Cin. Quar. Jour. of Sci., vol. 2, pp. 132, 284, Hud. Riv. Gr.

marginale, Conrad, 1St3, Proc. Acad. Nat. Scl. p. 334. The name was preoccupied by Phillips in 1841, and the species is poorly defined.

markoi, Castelnau, 1843, Système Silurien, p. 30, Trenton Gr. Not recognized.

maşsiense, Safford, 1569, Geo. of 'Tenn., p. 290, Nashville Gr.

matheri, see Gyroceras matheri.

maccoyi, Billings, 1859, Can. Nat. and

Geo., vol. 4, p. 467 Chazy Gr.

maximum, see Nautilus maximus, mercurius, see Cyrtocerina mercurius. 
metellus, Billings, 1865, Pal. Foss., vol. 1, p. 191, Quebec Gr.

metula, Hitl, 1862, 15th Rep. N. Y. Mus. Nat. Hist., $) .72$, and Illust. Devon. Foss., pl. 46, Up. Held. Gr.

microscopicum, Dwight, 1884, Am. Jour. Sci. and Arts, 3d ser., vol. 27, p. 256, Calciferous Gr.

missisquoi, Billings, 1865, Pal. Foss., vol. 1 , p. 314, (Orthoceras missisquoi), Quebec Gr.

morsum, Hall, 1862, 15th Rep. N. Y. Mus. Nat. Hist., p. 71 , and Pal. N. Y., vol. 5, pt. 2, p. 367 , Up. Held. Gr.

multicameratum, Hall, 1847, Pal. N. Y., vol. 1, p. 195, Bluck Riv. and Trenton Gr. myrice, Hall \& IVhitfield, 1875, Ohio Pal., vol. 2, p. 149, Niagara Gr.

neleus, Hall, 1861, Rep. of Progr. Wis., p. 40, Chazy and Black Riv. Grs.

nevadense, Walcott, 1885, Monogr. U. S. Geo. Sur., vol. 8, p. 203, Devonian.

obscurum, S. A. Miller, changed to magister because obscurum was preoccupied.

ohioense, Meek, 1871, Proc. Acad. Nat. Sci. Phil., p. 86, and Ohio Pal., vol. 1, p. 229, Up. Held. Gr.

olenus, Hali, 1877, syn. for Trochoceras orion.

opiunuin, Keyes, 1888, Proc. Acad. Nat. Sci. Phil., pl. xii., fig. 5, Ham. Gr.

orcas, see Oncoceras orcas.

orestes, Billings, 1865, Pal. Foss., vol. 1, p. 177, Niagara Gr.

orion, see Trochoceras orion.

orodes, Billings, 1862, Pal. Foss., vol. 1, p. 162, Guelph Gi.

planidorsatum, Whitfield, 18S0, Ann. Rep. Geo. Sur. WVis., p. 57, and Geo. IVis., vol. 4, p. 231, Trenton Gr.

postumius, Billings, 1865, Pal. Foss., vol. 1, p. 178, Hud. Riv. Gr.

pusillum, Hall, 1867, 20tlı Rep. N. Y. Mus. Nat. Hist., p. 407, Niagara Gr.

raei, Whitfield, 1889 , Bull. Am. Mus. Nat. Hist., vol. 2, p. 58, Calciferous Gr.

rectum, Whitfield, 1880, Ann. Rep. Geo. Sur. Wis., p. 85, and Geo. Wis., vol. 4, p. 319 , Niagara Gr.

regulare, Billings, 1857, Rep. of Progr. Geo. Sur. Can., p. 314, Black Riv. and Trenton Grs.

reversum, Spencer, 1884, Bull. No. 1, Mus. Univ. St. Mo., p. 60, Niagara Gr.

rigidum, Hall, 1867, 20th Rep. N. Y. Ius. Nat. Hist., p. 408, Niagara Gr. rockfordense, Wincliell, 1865, Proc. Acad. Nat. Sci., p. 132, Kinderhook Gr.

septoris, see Gomphoceras septore. simplex, Billings, 1857, Rep. of Progr. Geo. Sur. Can., p. 313, Black Riv. and Trenton Girs. sinuatum, Billings, 1857, Rep. of Progr. Geo. Sur. Can., p. 314, Black Riv. Gr.

spinosum, see Gyroceras spinosum. stonense, Safford, 1869, Geo. of Tenn., p. 290, Trenton Gr. subannulatum, D'Orbigny, 1850, Prodr. de Pal., t. 1, p. 1, Black Riv. and Trenton Grs. Proposed instead of C. annulatum, Hall, 1847, which was preoccupied.

subarcuatum, D’Orbigny, 1850, Prodr. de Pal., t. 1, p. 2, Trenton Gr. Proposed instead of C. arcuatum, Hall, 1847, which was preoccupied.

subcancellatum, Hall, 1877, 1st Ed. Am. Pal. Foss., p. 243, Niagara Gr. Proposed instead of C. cancellatum, Hall, 1852, which was preoccupied.

subcompressum, Beecher, 1888, Pal. N. Y., vol. 7, p. 35 , Clinton $\mathrm{Gr}$.

subrectum, Hall, 1859, Pal. N. Y., vol. 3, p. 342, Low. Held. Gr:

subturbinatum, Billings, 1857, Rep. of Progr. Geo. Sur. Can., p. 312, Chazy and Black Riv. Grs.

surgens, Barrande, 1870, Syst. Sil. de Boh., vol. 2, p. viii, pl. 431, Quebec Gr. sy phax, Billinge, 1865, Pal. Foss., vol. 1, p. 19t, Quebec Gr. This species is the type of Eremoceras, by Hyatt.

tenuiseptum, Faber, 1886, Jour. Cin. Soc. Nat. Hist., vol. 9, p. 18, Hud. Riv. Gr. tenuistriatum, Hall, 1877, 1st Ed. Am. Pal. Foss., p. 243. Proposed instead of $\mathrm{U}$. corniculum, Hall, 1862, which was preoccupied. Trenton Gr.

tessellatum, DeKoninck. Not American.

transversum, see Gyroceras transversum. trentonense, Emmons, 1842, (Orthoceras trentonensis,) Geo. Rep. N. Y., p. 396 , Trenton Gr.

trivolvi, see Gyroceras trivolve.

typicum, see Cyrtocerina typica.

undulatum, Hall, 1876, see C. alternatum. undulatum, Vanuxem, see Gyroceras undulatum.

u n i c o rne, Winchell, 1863 , Proc. Acad. Nat. Sci., p. 23, Marshall Gr.

vallandighami, S. A. Millel, 1874, Cin. Quar. Jour. Sci., vol. FIG. 730.-Cyrtoceras 1, p. 232, Hud. Riv. vallandighami. Gr.

vassarinum, Dwight, 1884, Am. Jour. Sci. and Arts., $3 \mathrm{~d}$ ser., vol. 27, p. 254, Calciferous Gr.

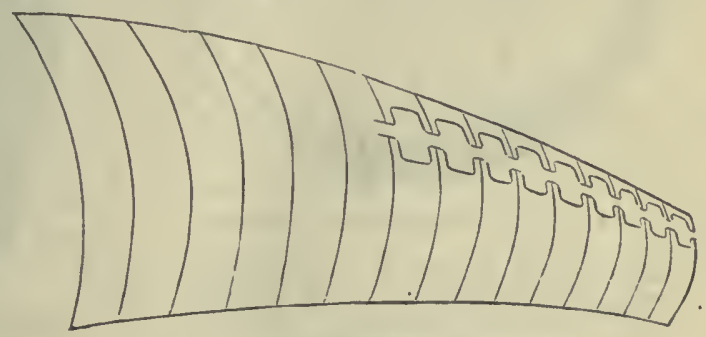

Fig. 731.-Cy rtoceras ventricosum.

ventricosum, S. A. Miller, 1875, Cin. Quar. Jour. Sci., vol. 2, p. 131, Hud. Riv. Gr. 
whitneyi, Hall 1861, Rep, of Progr. Wis., p. 39, Hud. Riv. Gr.

Cyrtocerina, Billings, 1865, Pal. Foss., vol. 1, p. 178. [Ety. from the termination inus, signifying resemblance to Cyrto-

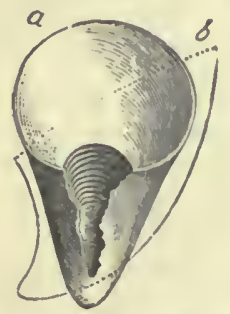

HIG. 732.-Cyrtoceriua typlea. $a, \mathrm{D}$ ors a view, showing cavity of slphuncle: $b$ outline, slde vlew. ceras.] In form like a short, rapidly tapering Cyrtoceras, and having a large sipluncle on the concuve side. Type C. typica. mercurius, Billings, 1865, Pal. Foss., vol. 1, p. 194, Quebec Gr.

typica, Billings, 1865 , Pal. Foss., vol. 1, p. 178. Bluck Riv. Gir. Diploceras,Con rad, 1842 , Jour. Acad. Nat. Sci., vol. 8 , p. 267 , [Ety. diploos, double; keras, horus.] Foundedupon the fragment of an Endoceras, and very poorly defined.

vanuxemi, see Endoceras vanuxemi.

Discites, DeHaan, 1825. Mongr. Ammon., etc., p. 31. [Ety. diskos, quoit.] Discoid; umbilicus wide; whorls quadrangular, sides flattened, and clorsm gibbous: longitudinally striated and sometimes lined transversely; siphon above the center; living chamber from one-fourth to three-fourths of a whorl in length; aperture with deep ventral sinus. Type D. costellatus.

amınonis, Hall, 1879, Pal. N. Y., vol. 5, pt. 2, p. 425, Up. Held. Gr.

disciformis, Meek \& Worthen, 1865, Proc. Acad. Nat. Sci. Phil., p. 261, and Geo. Sur. Ill., vol. 5, p. 522, Keoknk Gr.

hartti, Dawson, 1868, (Gyroceras hartti,) Acadian Genl., p. 311, Subcarboniferous. Made the type of Hyatt's genus, Stroboceras.

highlandensis, Worthen, 1875, Geo. Sur. Ill.. vol. 6 , 1. 531, Coal 11 eas.

inopinatus, Hall, 1879, Pal. N. Y., vol. 5, pt. 2, p. 426, Up. Held. Gr.

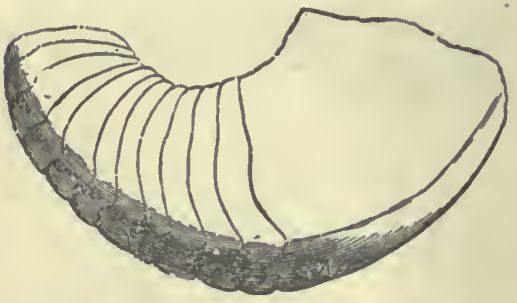

FrG. 733.-Discites marcellensis.

marcellensis, Vanuxem, 1842, (Goniatites marcellensis,) Geo. Sur. 3d Dist. N. Y., p. 146, Marcellus Shale. The type of Hyatt's genus Centroceras.

ornalus, syn. for D. marcellensis.

toddanus, Gurley, 1883, New Carb. Foss. p. 7. Publication invalid. tuberculatus, Owen, 1852, Geo. Sur. Wis., Iowa, and Minn., p. 581, Subearb.

Discosonus, Hall, 1852, Pal. N. Y., vol 2, p. 99. [Ety. diskos, quoit; soros, heap or pile.] Composed of a series of disks, gradually diminishing in size from the body chamber; onter edes rouncled. joining surfaces flat. Type D. conoideus.

conoideus, Hull, 1S52, Pal. N. Y., vol. 2, p. 99, Clinton and Niagara Gr.

Exdoceras, Hall, 1S47, Pal. N. Y., vol. 1, p. 58. [Ety. endos, within; keras, horn.] An elongated conical shell, resembling an Orthoceras, and possessed of one or more smooth siphuncles, which do not expand in passing through the ehambers. Type E. annulatum.

angusticanieratum, Hall, 1847, Pal. N. Y., vol. 1, p. 218. Trenton Gr.

annulatum, Hall, 1847, Pal. N. Y., vol. 1, p. 207, 'Trenton Gr.

approximatum, Uull, 1847, Pul. X. Y., vol. 1, p. 219, Trenton Gr.

arctiventrum, Hall, 1S 47 . Pal. X. Y., vol. 1, p. 217, 'Trenton Gr.

atluıticum, Barrande, 1870, Syst. Sil. de 13ol.., vol. 2, p. viii, pl. 430, Quebec Gr. bristolense, S. A. Miller, 1882, Jour. Ciu. Soc. Nat. Hist., vol. 5. p. 85, Hud. Riv. Gr. distans, Hull, 1847, Yal. N. Y., vol. 1, p. 220 , 'Trenton Gr.

duplientum, Hall, 1847, Pal. N. Y., vol. 1, p. 219, Trentoll (ir.

egani, S. A. Miller, 1852, Jour. Cin. Soc. Nat. Hist., vol. 5, p. 84 , II ud. lii . Gr.

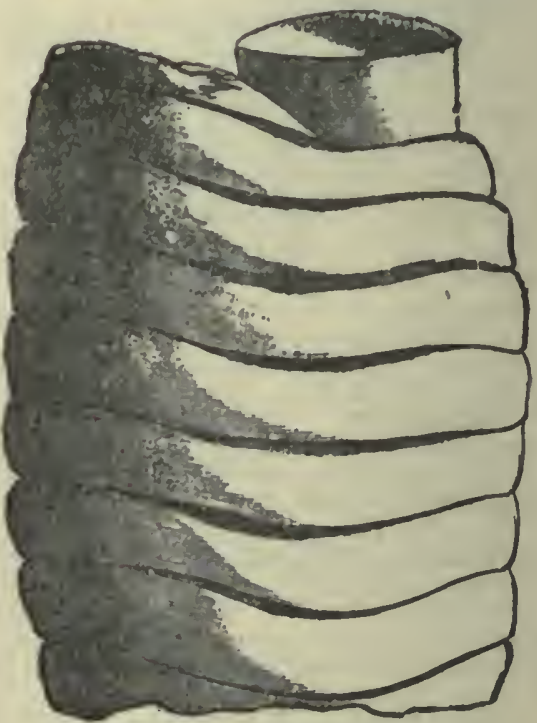

FIG. 734.-Endoceras longissimum.

gemelliparum, Hall, 1847, Pal. N. Y., vol. 1, p. 60, Black Riv. Gr.

inæequabile, S. A. Miller, 1882, Jour. Cin. Soc. Nat. Hist., vol.' 5 , p. 86 , Hud. Riv. Gr. 
insulare, Barrande, 1870, Syst. Sil. de Boh., vol. 2, p. viii, pl. 430-431, Quebec Gr.

lativentrum, Hall, 1850, 3d Rep. N. Y. Mus. Nat. Hist., p. 181, Trenton Gr.

longissimum, Hall, 1847, Pal. N. Y., vol. 1, p. 59, Black Riv. and Trenton Gr.

magniventrum, Hall, 1847, Pal. N. Y., vol. 1, p. 218, Trenton Gr.

marcoui, Barrande, 1869, Syst. Sil. de Boh., 2d ser., 4 me, Quebec Gr.

multitubulatum, Hall, 1847, Pal. N. Y., vol. 1, p. 59, Black Riv. and Trenton Grs.

The type of Hratt's genus Vaginoceras. proteiforme, Hall, 1847, Pal. N. Y., vol. 1, p. 208, Hud. Riv. and Trenton Grs.

proteiforme var. elongatum, Hall, 1847, Pal. N. Y., vol. 1, p. 216, Trenton Gr.

proteiforme var. lineolatum, Hall, 1847, Pal. N. Y., vol. 1, p. 211, Trenton Gr. prote-iforme var. strangulatum, Hall, 1847, Pal. N. Y., vol. 1, p. 212, Trenton Gr. proteiforme var. tenuistriatum, Hall, 1847, Pal. N. Y., vol. 1, p. 209, Trenton Gr. proteiforme var. tenuitextum, Hall, 1847, Pal. N. Y., vol. 1, p. 210, Trenton Gr. rapax, Billings, 1860, (Orthoceras rapax,) Can. Nat. and Geol., vol. 5, p. 176, Black Riv. Gr.

rottermundi, Barrande, 1866, (Orthoceras rottermundi,) Syst. Sil. de Boh., 2d ser., 2 me, p. xiii, pl. 230, Trenton Gr.

subannulatum, Whittield, 1880, Ann. Rep. Geo. Sur. Wis., p. 56, and Geo. Wis., vol. 4, \%. 230, Trenton $\mathrm{Gr}$.

subcentrale, Hall, 1847, Pal. N. Y., vol. 1, p. 59, Black Riv. Gr.

vanuxemi, Conrad, 1842, (Diploceras vanuxemi,) Jour. Acad. Sci., vol. 8, p. 267, Trenton Gr.

Endolobus, Meek \& Worthen, 1865, Proc. Acad. Nat. Sei. Phil., p. 259. [Ety. endos, within; lobos, lobe.] Prof. Meek said later that this genus is not distinct from Temnochilus, and if distinct it would probably be a synonym for Montfort's genus Bisiphites.

peramplus, see Temnochilus peramplum. speclabilix, see Temnochilus spectabile.

Glossoceras, Barrande, 1865, Cephalopods of Bohemia, vol. 2, p. 372. [Ety. glosse, tongue; keras, horn.] Having a slender annulated whorl, and an obscurely

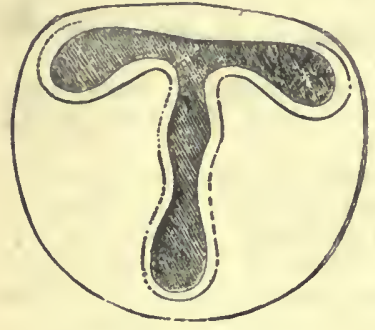

FIG. 735.-A perture of Gomphoceras. Y - shaped a perture. It is not known as an American genus. desideratum, Bill in $\mathrm{gs}$, 1866, Catal. Sil. Foss. Antic., p. 60. $\mathrm{N}$ o $\mathrm{t}$ defined so as to be recognized.

Gomphoceras, Sowerby, 1839, Murch. Sil. Syst. p. 620. [Ety.gomphos, club; keras, horn.] Shell fusiform or globular with a tapering apex; aperture contracted in the middle; siphuncle moniliform, subcentral. Type G. pyriforme. abruptum, Hall. 1879, Pal. N. Y. vol. 5 , pt. 2 , p. 339, Ham. Gr. absens, H a ll, 1876, (Cy rtoceras a bsens, ) Illust. Devon. Foss., p]. 47, and Pal. N. Y., vol. 5 , pt. $2, \mathrm{p}$. 324, Up. Held. Gr.

ajax, Hall, 1879 ,

Pal. N. Y., vol. 5, pt. 2, p. 350 , Portage Gr.

amphora, Whitfield, 1882, Ann. N. Y. Acad. Sci., vol. 2 , p. 207, Up. Held. Gr.

beta, Hall, 1862 ,

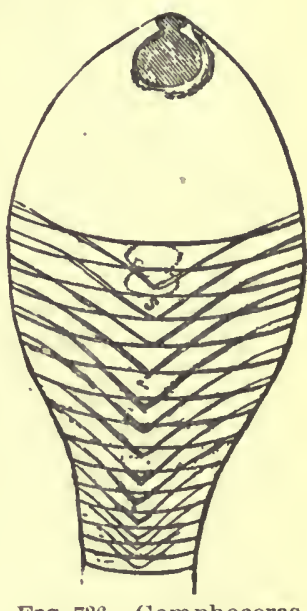

FIG. 736.-Gomphoceras pyriforme. 15th Rep. N. Y. Mus. Nat. Hist., p. 72, and Pal. N. Y., vol. 5, pt. 2, p.326, Up. Held. Gr.

breviposticum, Whitfield, 1882, Geo. Wis., vol. 4, p. 339, Ham. Gr.

cammarus, Hall, 1879, Pal. N. Y., vol. 5, p. 333 , Up. Held. Gr.

cassinense, Whitfield, 1886, Bull. Am. Mus. Nat. Hist., vol. 1, p. 322, Birdseye Gr.

cincinnatiense, S. A. Miller, 1884, Jour. Cin. Soc. Nat. Hist., vol. 7, p. 19, Hud. Riv. Gr.

clavatum, Hall, 1876, (Cyrtoceras clavatum,) Illust. Devon. Foss., pl. 47, Up. Held. Gr.

conradi, Hall, 1860, 13th Rep. N. Y. Mus. Nat. Hist., p. 106, Ham. Gr.

crenatum, Betchюr, 1888, Pal. N. Y., vol. 7, p. 33, Up. Held. Gr.

cruciferum, Hall, 1879, Pal. N. Y., vol. 5, p. 328 , Schoharie grit.

eos, Hall \& Whitfield, 1875, Obio Pal., vol. 2 , p. 100 , Hud. Riv. Gr.

eximium, Hall, 1861, 14th Rep. N. Y. Mus. Nat. Hist., p. 109, and Pal. N. Y., vol. 5, pt. 2, p. 299, Up. Held. Gr.

faberi, S. A. Miller, 1884, Jıur. Cin. Soc. Nat. Hist., vol. 7, p. 19, Hud. Riv. Gr. fax, Hall, 1879, Pal. N. Y., vol. 5, p. 321, Schoharie grit.

fischeri, Hall, 1860, 13th Rep. N. Y. Mus. Nat. Hist., p. 106, and Pal. N. Y., vol. 5, pt. 2, p. 336, Ham. Gr.

fusiforme, Whitfield, 1882, Geo. Wis., vol. 4, p. 338, Ham. Gr.

hertzeri, Hall \& Whitfield, 1875, (Cyrtoceras hertzeri,) Ohio Pal., vol. 2, p. 150, Niagara Gr. 
gomphus, Hall, 1879, Pal. N. Y., vol. 5, p. 334, Up. Held. Gr.

hyatti, Whitfield, 1882 , Ann. N. Y. Acad. Sci., vol. 2, p. 206, Up. Held. Gr.

illænus, Hall, 1879, Pal. N. Y., vol. 5, p. 332 , Schoharie grit.

impar, Hall, 1879, Pal. N. Y., vol. 5, p. 332, Up. Held. Gr.

lunatum, Hall, 1879, Pal. N. Y., vol. 5, p. 341, Ham. Gr.

manes, Hall, 1879, Pal. N. Y., vol. 5, p. 339 , Genesee Slate.

marcyæ, Winchell \& Marcy, 1855, Mem. Bost. Soc. Nat. Hist. Syn. for G. scrinium.

minimum, Whitfield, 1886, Bull. Am. Mus. Nat. Hist., vol. 1, p. 321, Birdseye Gr.

minum, Beecher, 1888, Pal. N. Y., vol. 7, p. 34, Ham. Gr.

mitra, Hall, 18i9, Pal. N. Y., vol. 5, p. 330, Up. Held. Gr.

nasutum, Beecher, 1858, Pal. N. Y., vol. 7, p. 34, Chemung Gr.

obesum, Billings, 1857, Rep. of Progr. Geo. Sur. Can., p. 311, Utica Gr.

omicron, Winchell, 1866, Rep. Low. Peninsula Mich., p. 97, Ham. Gr.

oviforme, Hall, 1860 , 13th Rep. N. Y. Mus. Nat. Hist., p. 105, Ham. Gr.

pingue, Hall, 1879, Pal. N. Y., vol. 5, p. 346 , Ham. Gr.

planum, Hall, 1879, Pal. N. Y., vol. 5, p. 352 , Ham. Gr.

plenum, Beecher, 1888, Pal. N. Y., vol. 7 , p. 33, Up. Held. Gr.

poculum, Hall, 1879, Pal. N. Y., vol. 5, p. 340 , Ham. Gr.

potens, Hall, 1879, Pal. N. Y., vol. 5, p. 351, Waverly Gr.

powersi, James. Not recognized.

raphanus, Hall, 1879, Pal. N. Y., vol. 5, p. 347, Ham. Gr.

rude, Hall, 1879, Pal. N. Y., vol. 5, p. 327, Ham. Gr.

sacculus, Meek \& Worthen, 1866, Proc. Acad. Nat. Sci., p. 258, and Geo. Sur. Ill., vol. 3 , p. 445 , Ham. Gr.

sciotoense, Whitfield, 1882, Ann. N. Y. Acad. Sci., vol. 2, p. 208, Up. Held. Gr.

scrinium, Hall, 1864, 20th Rep. N. Y. Mus. Nat. Hist., p. 410, Niagara Gr.

septore, Hall, 1867, 20th Rep. N. Y. Mus. Nat. Hist., p. 410 , Niagara Gr.

solidum, Hall, 1879, Pal. N. Y., vol. 5, p. 338, Marcellus Shale.

subgracile, Billings, 1857, Rep. of Progr. Geo. Sur. Can., p. 311, Up. Sil.

suboviforme, Walcott, 1885, Monogr. U. S. Geo. Sur., vol. 8, p. 202, Devonian.

tumidum, Hall, 1879, Pal. N. Y., vol. 5, p. 351, Chemung Gr.

tarbiniforme, Meek \& Worthen, 1866, Proc. Acad. Nat. Sci. Phil., p. 258, and Geo. Sur. Ill., vol. 3, p. 444, Ham. Gr.
Goniatites, DeHaan, 1825, Monographize Ammoniteorum et Goniatiteorum, p. 159. [Ety. gonia, an angle; lithos, stone. This name, it s e e m s, should be spelled Go. nialites.] Disc oid: whorlsembracing, sometimes closing the $\mathrm{u} \mathrm{mbili}$.

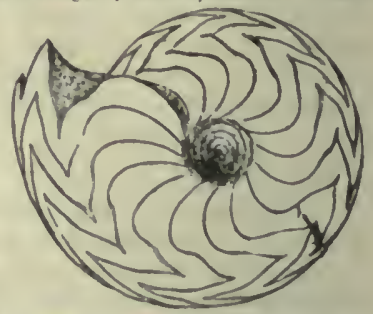
cus; septa Fia. 787.-(ioulalites sphericus. zigzag lines or sutures; when the septa are lolded the elevations are called saddles; body chamber long, sometimes constituting n whorl, but never expanding but slightly; siphuncle ventral. Type Goniatites sphericus.

allii, Winchell, 1862, Am. Jour. Sci, 2d series, vol. 33, p. 363, Marshall Gr.

amplexus, Beecher, 1889, Pal. N. Y., vol. 7 p. 39, Tully limestone.

andrewsi, Winchell, 1870, Proc. Amr. Phil. Soc., vol. 12, p. 259, Marshall Gr. astarte, Clarke, 1885, Bull. U. S. Geo. Sur., No. 16, p. 29, Murcellus Shale.

bicostatus, Hall, 1843, Geo. Rep. 4th Dist. N. Y., p. 245, and Pal. N. Y., vol. 5, pt. 2, p. 450, Portage Gr.

canadensis, Castelnut. 1843, Syst. Sil. p. 34. Probably a syn. for Bellerophon bilobatus.

chemungensis, Vanuxem, 1942, (ieo. Rep. $3 d$ Dist. N. Y., p. 182, and Pal. N. Y., vol. 5, pt. 2, p. 467, Chemıng Gr.

chemungensis var. requicostatus, Hall, 1875,27 th Rep. N. Y. Mus. Nat. Hist., p. 135, Chemung Gr.

choctawensis, Shumard, 1863, 'Trans. St. Louis Acad. Sci., vol. 2, p. 109, Coal Meas.

colubrellus, Morton, 1836, (Ammonites colubrellus), Am. Jour. Sei. and Arts, vol. 29 , p. 154 , Waverly Gr.

compactus, Meek \& Worthen, 1865, Proc. Acad. Nat. Sci. Phil., p. 154, and Geo. Sur. Ill., vol. 5, p. 611, Coal Meas.

complanatus, Hall, 1843, (Clymenia (?) complanatus, Geo. Rep. 4th Dist. N. Y., p. 244, and Pal. N. Y., vol. 5, p. 455, Portage Gr.

complanatus var. perlatus, Hall, 1875, 27 th Rep. N. Y. Mus. Nat. Hist., p. 132, Chemung Gr.

desideratus, Walcott, 1885, Munogr. U. S. Geo. Sur., vol. 8, p. 203, Devonian.

discoideus, Hall, 1860, 13th Rep. N. Y. Mus. Nat. Hist., p. 97, and Illust. Devon. Foss., pl. 71, Ham. Gr. The type of Hyatt's genus Parodiceras.

discoideus var. ohioensis, Hall, 1874, 27 th Rep. N. Y., p. 200, Up. Held. Gr. entogonus, Gabb, 1861, Proc. Acad. Nat. Sci., p. 372, Carboniferous. 


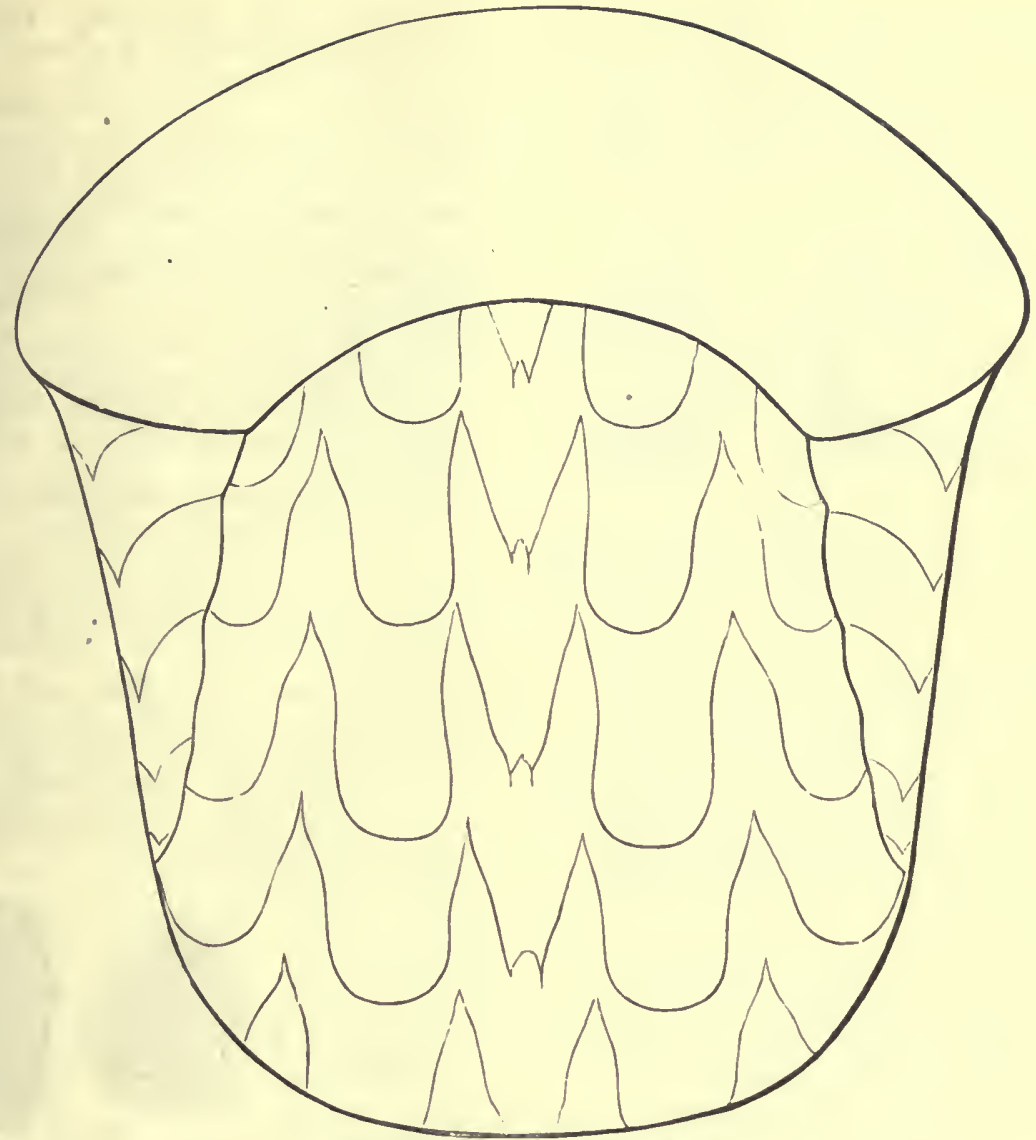

FIG. 738.-Goniatites globuiosus.

erato, Hall, 1862, (Clymenia erato,) 15th Rep. N. Y. Mus. Nat. Hist., p. 64, and Illust. Devon. Foss., pl. 70, Ham. Gr. expansus, Vanuxem. The name was preoccupied by Von Buch in 1838. See G. vanuxemi.

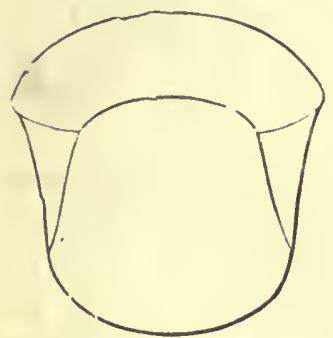

FrG. 739.-Goniatites globulosus. Outline.

globulosus, Meek \& W orthen, 1860 , P r o c Acad. Nat. Sci. Phil., p. 471, and Geo. Sur. Ill., vol. 2, p. 390 , Up. Coal Meas.

globulosus var. excelsus,Meek, 1875, Bull. U.S. Geo. Sur.Terr., vol. 1 , No. 6 , p. 445, Coal Meas.

goniolobus, Meek, 1877, U. S. Geo. Sur. 40th Parallel, vol. 4, p. 98, Carboniferous. hathawayanus, McChesney, 1860, Desc. New Pal. Foss., p. 66, Coal Meas. hildrethi, Morton, 1836, (Ammonites hildrethi,) Am. Jour. Sci. and Arts, vol. 29 , p. 149 , Waverly Gr.

holmesi, Swallow, 1860, Trans. St. Louis Acad. Sei., vol. 1, p. 659, Waverly or Choteau Gr.

houghtoni, Winchell, 1862, Am. Jour. Sci., 2d ser., vol. 33, p. 363, Marshall Gr. hyas, Hall, 1860, syn. for G. lyoni.

iowensis, Meek \& Worthen, 1860, Proc. Acad. Nat. Sci. Phil., p. 471, and Geo. Sur. Ill., vol. 2, p. 392, Coal Meas. 'Ty pe of Hyatt's genus Paralegoceras.

ixion, Hall, 1860, 13th Rep. N. Y. Mus. Nat. Hist., p. 125, Kinderhook Gr. This species is founded on the form which has been identified with the European species $\mathrm{G}$. rotatorius.

kentuckiensis, n. sp. Shell very globose and wide or broadly rounded on the dorsal side; outer volution embracing the inner ones; umbilicus small and disclosing none of the inner volutions, though the body chamber is broken from our specimens; suture having a sharp, dorso-lateral lobe and an equally 
deep, bifid, central dorsal lobe; dorsal saddle subangular. This speries is readily distinguished by its deep, globose form and sharply bifid lobe on the dorsal side. Collected by Charles Faber at Crab Orchard, Kentucky, in the St. Louis Group.
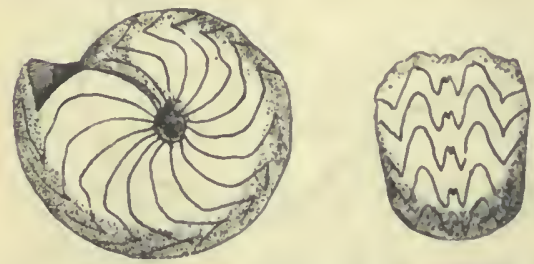

FIG. 740.- (Honlallies kentucklensls. The saddles are generully more ungutar than they appear in the figure.

kingi, Hall \& Whitfield, 1877, U. S. Geo. Expl. Exped., 40th parallel, vol. 4, p. 279 , Coal Mras.

lutheri, Clarke, 1885, Bull. U.S. Geo. Sur., No. 16, p. 50 , Chemung Gr.

lyoni, Meek \& Worthen, 1860, Proc. Acad. Nat. Si:i. Phil., vol. 12, r. 471, and Geo. Sur. Ill., vol. 2, p. 165, Kinlerliook Gr. marcellensis, see liscites mare. ${ }^{\prime}$ lensis.

marshallensis, Winchell, 1862, A $m$. Jour. Sci., 2d ser., vol. 33 , p. 362 , Marhall Gr.

minimus, Shumurd, 1858, Trans. St. Louis Acad. Sci., vol. 1, p. 200, Coal Meas.

mithrux, Hall, 1860, 13 h Rep. N. Y. Mus.

Nat. Hist., p. 98, and 1'ul. N. Y., vol. 5, pt. 2, p. 433 , Up. Held. Gr.

monroensis, Worthen, (in press,) Geo. Sur. Ill., vol. 8, p. 150, St. Louis Gr. morganensis, Swallow, 1860, Trans. St. Louis Acad. Sci., vol. 1, p. 659, Waverly or Choteau Gr.

nodifer, Clarke, 1885, Bıll. U. S. Geo. Sur., No. 16, p. 21, Marc-llus Shale.

nolinensis, Cox, 1857, Geo. Sur. Ky., vol. 3 , p. 574 , Conl Meas.

nundaia, Hull, 1875, syn. for G. sinuosus. ohioensis, Winclsell, 1870, Proc. Am. Phil.

Soc., vul. 12, p. 259, Marshall Gr.

opimus, White \& IVlittield, 1862, Proc.

Bost. Soc. Nat. Hist., vol. 8, p. 305, Kinderhnok Gr.

orbictlla, Hall, 1860, 13th Rep, N. Y. Mus.

Nat. Hist., p. 99, Ham. Gr.

osagensis, Swalluw, 1860, Trans. St. Louis

Acad. Sci., vol. 1, p. 659, Waverly or Choteau Gr.

oweni, Hall, 1860, 13th Rep. N. Y. Mus. Nat. Hist., p. 100, Kinderhonk Gr.

oweni var, parallelus, $H_{\sharp 1 l}, 1860,13$ th Rep.

N. Y. Mus. Nat. Hist., p. 101, Kinderbook Gr. Type of Hyatt's genus Munsteroceras.

parvus, Shumard, 1858, Trans. St. Louis, Acad. Sci., vol. 1, p. 199, Coal Meas. patersoni, Hall, 1860,13 th Rep. N. Y. Mus. Nat. Hist., p. 99, Portage Gr. peracutus, Hall, 1876, Illust. Devonian Foss., pl. 69, and Pal. N. Y., vol. 5, pt. 2, p. 4t3, Portsuge Gr.

planarbiformis, Slumard, 1855, Geo. Sur. Mo., [1. 208, Coal Mi ns.

plebeiformis, Hall, 1879, Pal. N. Y., vol. 5, p. 448, Marcellus Stiale.

politus, Shumard, 1S58, Trans. St. Louis Acad. Sei., vol. 1, T. 199, Conl Meas.

propinquus, Wincluell, 1862, Am. Jour. Sci. und Arte, 2 l series, vol. 33, p. 365 , Marshall Gr.

punctalus, Conrad, 1838, Ann. Rep. N. Y., p. 117, Ham. Gr. Not properly defined. pygnieus, Winchell, 186:, A 13. Jnur. Sei. and Arts, 2d series, vol. 33, p. 366, Mar. shall Gr.

romingeri, Winclsell, 1862, Proc. Acad. Nat. Sci., p. 427, Marshall Gr.

rotatorius, DeKoninck, 1843, Desc. des A nim. Foss, du Terr. Carb. See G. ixion. shumardanus, Winchell, 1865, Am. Jour. Sci. and Arts, 2d series, vol. 33, p. 363, Marshall Gr.

simulator, Hall, 1875, 27th Rep. N. Y. Mus. Nat. Hist., p. 133, Chemung Gr. Type of IIyatt's genus Manticoceras.

sinuosus, Hall, 1843. Geo. Rep. $4 t h_{1}$ Dist. N. Y., p. 243, and Pal. N. Y., vol. 5, pt. 2, p. 460, Portage (ir. Type of Hyatt's genus Gephuroceras.

subcircularis $n$.

s p. S h e ll sıull, circular; outer volut ion embracing the inner ones: $11 \mathrm{~m} \mathrm{~b}$ i licius does not expose any of lisa. 741.-(Bontatites subcirthe inner vo- cularls. Lat.ral und domal lutions; four views mugntfled 2 (dinm.

furrows or constrictions racliate from the umbilicus and divide the shell into four subequal parts, but become obsolete on the dorsisl side, and in this respect resemble Goniatite's divisus of DeKoninck; doreal side rouud; body cliamber unknown; suture lobed; entire surface longitudinally striated. Collected by Ctiarles Faber, at Crab Orchard, Kentucky, in the St. Louis Group.

sulciferus, Winchell. Not lefined.

texanus, Slummard, 1S63, 'Trans. St. Louis Acarl. Sci., vol. 2, p. 109, Coal Meas.

uniangularis, Conrad, 18+2, Jour Acacl. Nat. Sci., vol. 8, p. 268, Ham. Gr. Type of Hyatt's genus Tornoceras.

unilobatus, Hall, 1875, 27th Rep. N. Y. Mus. Nat. Hist., p. 133, and Illust. Devon. Foss., pl. 71, Ham. Gr.

vanuxemi, Hall, 1879 , Pal. N. Y., vol. 5, p. 434, Marcullus Shale. Proposed instead of G. expansus, of Vanuxem, which was preoccupied by Von Buch.

whitii, Winchell, 1862, Proc. Acad. Nat. Sci., vol. 6, p. 428, Portage Gr. 
Gonioceras, Hall, 1847, Pal. N. Y., vol. 1, p. 54. [Ety. gonia, angle; keras, loorn.] Somewhat in the form of an Ortloceras, but more or less flattened and subfusiform; transverse section in the form of a depressed ellipse with projecting angles; siphuncle ventral, septa curve over the ventral side, as shown in the illustration. Type G. anceps.

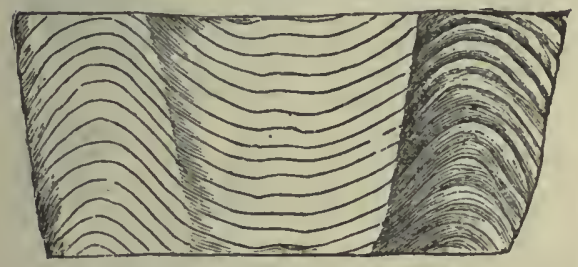

Fig. 742.-Gonloceras anceps.

anceps, Hall, 1847, Pal. N. Y., vol. 1, p. 54, Black Riv. Gr.

occidentale, Hall, 1861, Rep. of Progr.Wis., p. 47, Trenton Gr.

Gyroceras, DeKoninck, 1844, Desc. An. Foss. Belg., p. 530. [Ety. gyros, circle; keras, horn.] Not Gyroceratites of Meyer, 1829. Discoid, rolled in one plane; volutions in contact or open, but not embracing; transverse section circular, elliptical, scutiform, or polygonal; body chamber large and sometimes straight or tangent to the spiral; opening hollowed out on the exterior border like the Nautilus; septa arched and frequently project, curving backward; siphon slender, cylindrical, and usually subcentral toward the convex border, but sometimes found within the concave border; surface tuberculous, having imbricated excrescences or ringed with projecting fringes from the septa. Type G. paradoxicum.

abruptum, Hall, 1879, Desc. New Spec. Foss., p. 19, and 11th Rep. Geo. and Nat. Hist., Ind. p. 325, Niagara Gr.

americanum, Billings, 1857, Rep. of Progr. Can. Geo. Sur., p. 309, Up. Sil.

baeri, Meek \& Worthen, 1865, (Trochoceras baeri,) Proc. Acad. Nat. Sci., p. 263 , and Ohio Pal., vol. 1, p. 157, Hud. Riv. Gr.

bannisteri, Winchell \& Marcy, 1865, Mem. Bost. Soc. Nat. Hist., vol. 1, p. 102, Niagara Gr.

burlingtonense, see Nautilus burlingtonensis. columbiense, Whitfield, 1882, Ann. N. Y. Acad. Sci., vol. 2, p. 210, Up. Held. Gr. constrictum, Meek \& Worthen, 1868, Geo. Sur. Ill., vol. 3, p. 446, Ham. Gr.

cornutum, Owen, 1840, Rep. on Min. Lands, p. 69, Devonian.

cyclops, Hall, 1862, 15th Rep. N. Y. Mus. Nat. Hist., p. 68, and Illust. Devon. Foss., pl. 53, Up. Held. Gr.

duplicostatum, Whitfield, 1878, Ann. Rep. Geo. Sur. Wis., p. 78, and Geo. Wis., vol. 4 , p. 235 , Trenton Gr. elrodi, White, 1882, 11th Ann. Rep. Geol. and Nat. Hist. Indiana, p. 356, Niagara Gr. eryx, Hall, 1862, 15th Rep. N. Y. Mus. Nat. Hist., p. 67, Ham. Gr.

expansum, Saeman, Dunker \& Von Meyer, 1853, Palæontographica, vol. 4. See Nautilus buccinum.

gracile, Hall, 1860, 13tlı Rep. N. Y. Mus. Nat. Hist., p. 105, Kinderkook Gr. Probably a syn. for Trematodiscus digonus.

harti, see Discites hartti.

inelegans, Meek, 1871, Proc. Acad. Nat. Sci. Phil., p. 89, and Ohio Pal., vol. 1, p. 232, Up. Held. Gr.

jason, Hall, 1862, (Cy rtoceras jason,) 15th Rep. N. Y. Mus. Nat. Hist., p. 71, Up. Held. Gr. Type of Hyatt's genus Rutoceras.

laciniosum, Hall, 1879, Pal. N. Y., vol. 5, p. 376 , Up Held. Gr.

liraium, see Nautilus liratus.

logani, Meek, 1868, Trans. Chi. Acad. Sci., p. 110 , Devonian.

magnificum, see Lituites magnificus.

matheri, Conrad, 1810, Ann. Rep. N. Y., p. 206, and $\mathrm{Pal}$. N. Y., vol. 5, pt. 2, p. 377, (Cyrtoceras matheri,) Up. Held. Gr. nais, see Porcellia nais.

nereus, Hall, 1862, 15th Rep. N. Y. Mus.

Nat. Hist., p. 67, and Pal. N. Y., vol.5, pt. 2, p. 373, Up. Held. Gr.

numa, Billings, 187j, Can. Nat. and Geol., vol. 7 , p. 238, Up. Held. Gr.

obioense, Meek, 1871, Proc. Acad. Nat. Sci. Phil., p. 87, and Ohio Pal., vol. 1, p. 230, Up. Held. Gr.

paucinodum, Hall, 1876, Illust. Devonian Foss., pl. 55, and Pal. N. Y., vol. 5, pt. 2, p. 380 , Up. Held. Gr.

pratti, Barris, 1879, Proc. Dav. Acad. Sci., vol. 2, p. 287, Up. Held. Gr.

rhombolineare, Owen, 1862, Geo. Sur. Indiana, y. 362, Silurian.

rockfordense, Meek \& Worthen, 1866 , (Nautilus (Cryptoceras) rockfordensis,) Proc. Acad. Nat. Sci. Phil., p. 275, and Geo. Sur. Ill., vol. 3, p. 459 , Kinderhook Gr.

seminodosum, Whitfield, 1882 , Ann. N. Y. Acad. Sci., vol. 2, p. 211, Up. Held. Gr. spinosum, Conrad, 1840, (Phragmoceras spinosum,) Ann. Rep. N. Y., p. 206, and Pal. N. Y., vol. 5, pt. 2, p. 382, Schoharie grit.

stebos, Beeclier, 1888, Pal. N. Y., vol. 7, p. 36, Waverly Gr.

subliratum, see Nautilus subliratus.

transversum, Hall, 1860, (Cyrtoceras transversum,) 13th Rep. N. Y. Mus. Nat. Hist., p. 104, and Pal. N. Y., vol. 5, pt. 2, p. 384, Ham. Gr.

trivolve, Conrad, 1840, (Cyrtoceras trivolvis, Ann. Rep. N. Y., p. 206, and Pal. N. Y., vol. 5. pt 2, p. 374, Up. Held. Gr. undulatum, Vanuxem, 1842, (Cyrtoceras undulatum, ) Geo. Rep. N. Y., p. 139, and Pal. N. Y., vol. 5, pt. 2, p. 378, Up. Held. Gr. Type of Hyatt's genus Halloceras. 
vagrans, Billings, 1857, Rep. of Progr. Can. Geo. Sur., p. 308, Black Riv. Gr. validum, Hall. 1876, Hllust. Devonian Foss., pl. 51, and Pal. N. Y., vol. 5, pt. 2, p. 385 , Scholarie grit.

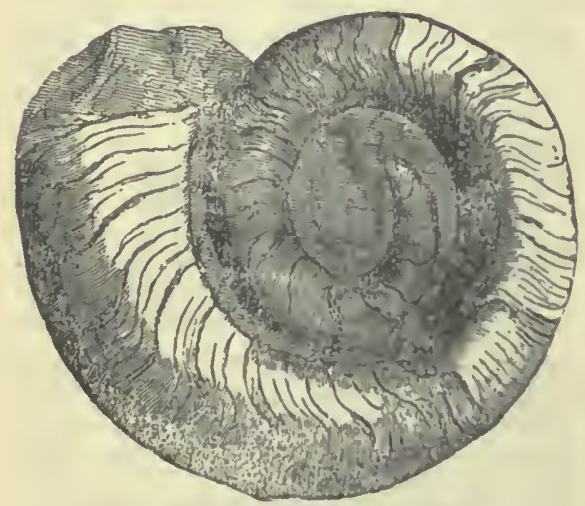

FIG. 743,-Gyroceras undulatum.

Hortholus americanus, sce Lituites americanus.

Huronia, Stokes, 1823, Geo. Trans., 11. s., vol. 1, p. 203. When this genus was proposed, the author thought he was describing a coral. Prof. Billings saisl the name was proposed for the sipliuncle of an Orthoceras, and is, therefore, merely a synonym. This seems to be the correct opinion, though the shells to which the peculiar siphuncles belong are unknown. Type H. bigsbyi. annulata, Hall, 1851, Lake Superior Land Dist. by Foster \& Whitney, p. 221, Niagara Gr.

bigsbyi, Stokes, 1823, Trans. Geo. Soc., vol. 1, p. 195, Clinton Gr.

minuens, Barrande, 1869, Syst. Sil. de Boh., 2d series, vol. 4, pl. ix, p. 435, Clinton Gr.

obliqua, Stokes, 1823, Trans. Geo. Soc., 2d series, vol. 1, p. 203, Clinton Gr.

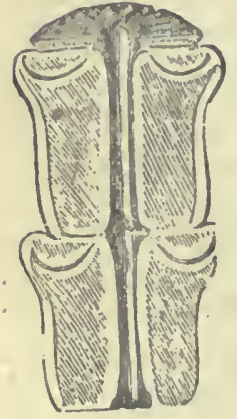

FIg. 744. - Huronla vertebralis. portlocki, Stokes, 1840

Trans. Geo. Soc., $2 d$ series, vol. 5 , p. 710 , Clinton Gr.

sphieroidalis, Stokes, 1840, Trans. Geo. Soc., 2 d series, vol. 5 , p. 710 , Clinton $\mathrm{Gr}$.

stokesi, Castelnau, 1843, Syst. Sil., p. 33, Schoharie grit. Not recognized.

turbinata, Stokes, 1823 Trans. Geo. Soc., $2 d$ series, vol. 1, p. 203, Clinton Gr.

vertebralis, Stokes, 1840 , Trans Geo. Soc., $2 d$ series, vol. 5 , p. 710 , Niagara and Clinton Grs. See Orthoceras canadense.
Hydnoceras, Conrad, 1842, Jour. Acad. Nat. Sci., vol. 8. See Diclyophyton, a sponge. tuberosum, see Dictyopliyton tuberosum.

Lituites, Montfort, $180 \mathrm{~S}$, Conch. Syst., vol. 1, p. 279 , [E t y. lituus, $\mathrm{t} r$ u m pet. Shell spiral in the beginning; last chambers produced straight; FıG. 745.-Litultes g/gan. whorls free or

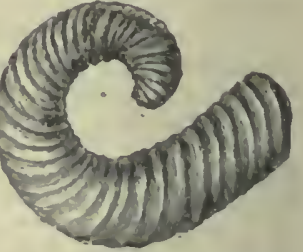
teus. Quurter sizo. open in ono plane; septa simple; siphuncle central; section circular. Typo L. litrus.

americanus, D'Orbigny, 1850, (Hortholus americauns, Proưr. d. Paléont., t. 1, p. 1, Black liv. Gr.

apollo, Billings, 1862, Pal. Foss., vol. 1, p. 25 , Calciferous $\mathrm{Gr}$.

bickmoreanus, Whitfield, 1885, Bull. Am. Mus. Nat. Hist., vol. 1, p. 191, Niagaru Gr.

cancellatus, McClsesney, 1561, New Pal. Foss., Niagara Gr. See L. occidentalis and Nautilus cancellatus and N. nccidentalis. If this species, as Prof. Hall suggests, is a true Nantilus, McChesney's name has precedence.

capax, see Nautilus capax.

complauntus, Shumard, 1863, Trans. St. Jouis Acad. Sci., vol. 2, p. 107, Calciferous Gr.

conrolvans, Schlotheim, 1813, in Jahrbuch, as identified by Hall, Pul. N. Y., vol. 1, p. 53. See L. american us.

eatoni, Whitfield, 1886, Bull. Am. Mus. Nat. Hist., vol. 1, p. 331, Birdseye Gr. eatoni var. cassinensis, Whitfield, 1856 , Bull. Am. Mus. Nat. Hist., vol. 1, p. 332, Birdseye Gr.

farnswortli, Billings, 1861, Pal. Foss., vol. 1, p. 21, Calciferous Gr.

graftonensis, Neek \& Worthen, 1870, Proc. Acad. Nat. Sei. Phil. p. 51, and Geo. Sur. Ill., vol. 6, p. 507, Niagara Gr.

hercules. Winchell \& Mrrcy, 1865, Mem. Bost. Soc. Nat. Hist., Niagara Gr. Syn. for Cyrtoceras amplicorne. See 20th Rep. N. Y. Mus. Nat. Hist.

imperator, Billings, 1861, Pal. Foss., vol. 1, p. 23, Calciferous Gr.

magnificus, Billings, 1857, (Gyroceras magnificum, Rep. of Progr. Geo. Sur. Can., p. 307, Hud. Kiv. Gr. Type of Hyatt's genus Aspidoceras.

marshi, Hall, 1867, 20th Rep. N. Y. Mus. Nat. Hist., p. 404, Niagara Gr.

internistriatus, Whitfield, 1886, Bull. Am. Mus. Nat. Hist., vol. 1, p. '332, Birdseye $\mathrm{Gr}$.

multicostatus, Whitfield, 1880, Ann. Rep. Geo. Sur. Wis., p. 67, and Geo. Wis., vol. 4, p. 303 , Niagara Gr.

murchisoni, Truost. Not defined so as to be recognized. 
niagarensis, Spencer, 1884, Bull. No. 1, Mus. Univ. St. Mo., p. 60, Niagara Gr. occidentalis, Hall, 1861, Rep. of Progr. Geo. Sur. Wis., Niagara Gr. This species is now referred by. Prof. Hall to the genus Nautilus, see 20th Rep. N. Y. St. Mus. Nat. Hist., p. 400 . It was first described by McChesney, Jan. 1860, as Cyrtoceras giganteum, but that name being preoccupied, in 1861 he proposed Lituites cancellatus. If it is a Nautilus, the word occidentalis being preoccupied, McChesney's name cancellatus has precedence.

ortoni, Meek, 1873, Ohio Pal., vol. 1, p. 186, Niagara Gr.

palinurus, Billings, 1862, Pal. Foss., vol. 1, p. 25, Calciferous Gr.

pluto, Billings, 1865, Pal. Foss., vol. 1, p. 259, Quebec Gr.

roberisoni, Hall, 1861, Rep. of Progr.Wis.. p. 38, Chazy and Black Riv. Grs.

seelyi, Whitfield, 1886, Bull. An. Mus. Nat. Hist., vol. 1, p. 330, Birdseye Gr.

undatus, Emmons, 1842, (Inachus undatus,) Geo. Rep. N. Y., p. 394, and Pal. N. Y., vol. 1, p. 52, Black Riv. and Trenton Grs. It is not a Lituites.

undatus var. occidentalis, Hall, 1861, Rep. of Progr. Wis., p. 38, Black Riv. and Trenton Grs.

Melia cancellatus, Emmons, 1856, Am. Geol. Not detiued so as to be recognized.

cincinnatix, D'Orbigny, 1850, Prodr. d. Paléont., t. 1, p. 4. Not defined so as to be recognized.

Nautilus, Breynius, 1732, Dissert. Polytb., p. 11. [Ety. Nautilos, sailor or navigator.] Shell subglobose, compressed; volutions coiled in the same plane, cortiguous; umbilicus open or closed; septa simple, arched or waved on the lateral margins; siphuncle central or subcentral; lip sinuous on the dorsal and ventro-lateral margins; surface smooth, striate, costate, or bearing nodes. Type N, pompilius.

acræus, Hall, 1879, Pal. N. Y., vol. 5, pt. 2, p. 417, Ham. Gr.

avonensis, see Solenochilus avonense.

avus, Barrande, 1869, Syst. Sil. de Boh., vol. 4, p. viii, pl. 435, Quebec Gr.

barrandi, Hall, 1876, see N. Magister.

biserialis, Hall, 1860, Supp. to vol. 1, pt. 2, Iowa Geo. Sur., p. 92, Coal Meas.

buccinum, Hall, 1876, Illust. Devonian Foss., pl. 60, and Pal. N. Y., vol. 5, pt. 2, p. 412, Ham. Gr. Type of Hyatt's genus Nephriticeras.

calciferus, Billings, 1865, Pal. Foss., vol. 1, p. 258, Calciferous Gr.

cancellatus, McChesner, 1861, (Lituites cancellatus,) New Pal. Foss., p. 96, Niagara $\mathrm{Gr}$.

canaliculatus, Cox, 1857, Geo. Sür. Ky., vol. 3 , p. 575 , Coal Meas. Type of Hyatt's genus Ślenoceras.

capax, Hall 1860, (Lituites capax,) Rep. of Progr. Geo. Sur. WVis., p. 3, Niagara Gr. capax, Meek \& Worthen, 1865. This was preoccupied and must yield unless it can be retained in the subgenus Solenochilus.

cavus, Hall, 1879, Pal. N. Y., vol. 5, p. 416, Ham. Gr.

champlainensis, Whitfield, 1886, Bull. Am. Mus. Nat. Hist., vol. 1, p. 329, Birdseye Gr.

chesterensis, Meek \& Worthen, 1860, Proc. Acad. Nat. Sci. Phil., p. 469, and Geo. Sur. Ill., vol. 2, p. 306, Kaskaskia Gr.

clarkanus, Hall, 1858, Trans. Alb. Inst., vol. 4, p. 32, and Bull. Am. Mus. Nat. Hist., p. 92, Warsaw Gr.

collectus, see Solenochilus collectum.

cornulum, Hall, 1876, Illust. Devonian Foss., pl. 60, and Pal. N. Y., vol. 5, pt. 2 , p. 414, Ham. Gr.

coxanus, see Temnochilus coxanum.

danvillensis, White, 1878, Proc. Acad. Nat. Sci., p. 36, and Cont to Pal., No. 8, p. 170, Coal Meas.

decoratus, Cox, 1857, Geo. Sur. Ky., vol. 3 , p. 572 , Coal Meas.

desertus, Billings, 1865, Pal. Foss., vol. 1, p. 258, Quebec Gr.

digonus, see Trematodiscus digonus.

disciformis, see Discites disciformis.

discoidalis, see Trematodiscus discoidalis. divisus, White \& St. John, 1868, Trans. Chi. Acad. Sci., p. 124, Un. Coal Meas. The name was preoccupied by Meyer in 1831 .

eccentricus, Meek \& Hayden, 1858, Trans. Alb. Inst, vol. 4, p. 83, and Pal. Up. Mo., p. 65, Permian Gr.
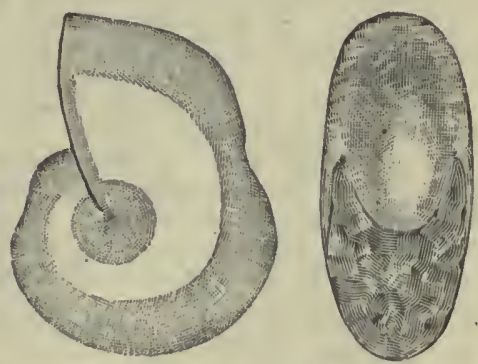

Fig. 746.-Nautilus faberi. Lateral and front views. Magnifled 2 diam.

faberi. n. sp. Shell small, smooth, all volutions embraced in the outer one, leaving only a small round umbilicus; aperture semielliptical above the interior volution. The species is founded on a single specimen having a piece chipped from the dorsal side of the last volution, and a small piece broken from the inner volution, but nowhere disclosing the septa. It was found in the Coal Measures, on Elk Horn Creek, Kentucky, and belongs to Mr. Charles Faber's collection.

ferox, Billings, 1865, Pal. Foss., vol. 1, p. 351 , Calciferous Gr. 
ferratus, Cox, 1857, Geo. Sur. Ky., vol. 3, p. 574, Coal Meas.

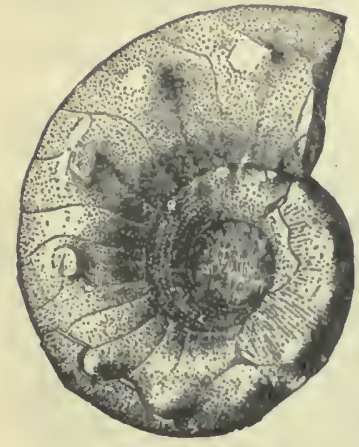

Frg. 747.-Nautilus forbesanus.

for b esanus, $\mathrm{McChes-}$ ney, 1860 , Pal. Foss. p. 63 , and Trans. Chi. Acad. Sci. Meas.

gilpini, Swallow, 1860 , Trans. St. I, 0 u i s Acad. Sci., vol. $1, p$. 658, Coal Meas. Desc. New p. 50, Coal

globatus

Sowerby, 1825, Min. Conch., vol. 5, p. 129, Kaskaskia Gr.

bercules, Billings, 1857, Rep. of Progr. Can. Geo. Sur., p. 306, Hud. Riv. Gr.

highlandensis, see Disciles highlandensis. hyatti, Beecher, 1888, Pal. N. Y., vol. T, p. 37, Ham. Gr.

illinoisensis, McChesney, 1860, Desc. New. Pal. Foss., p. 64, Coal Meas.

ingentior, Winchell, 1862, Am. Jour. Sci., 2d series, vol. 33, p. 361, Marshall Gr.

insolens, Billings, 1865, Pal. Foss., vol. 1, p. 258, Quebec: Gr.

jason, Billings, 1859, Can. Nat, and Geol., vol. 4, p. 464, Chazy Gr. Type of $\mathrm{Hy}$ att's genus Plectoceris.

kelloggi. Whitfield, 1886, Bull. Am. Mus. Nat. Hist., vol. 1, p. 328 , Birdseye Gr. lasallensis, Meek \& Worthen, 1866, Proc. Acad. Nat. Sci. Phil., p. 261, and Geo. Sur. Ill., vol. 5, p. 610, Up. Coal Meas. latus, sce Temnochilus latum.

lawsi, Swallow, 1860, Trans. St. Louis Acad. Sci., vol. 1, p. 658, Ham. Gr.

leidyi, see Solenochilus leidyi.

liratus, Hall, 1860, (Gyroceras liratum, 13th Rep. N. Y. Mus. Nat. Hist., p. 104, Marcellus Shale.

liratus var. juvenis, Hall, 1879, Pal. N. Y., vol. 5, Ham. Gr.

magister, Hall, 1879, Pal. N. Y., vol. 5, p. 422, Ham. Gr. Proposed instead of N. barrandi, Hall, which was preoccupied.

marcellensis, see Discites marcellensis.

maximus, Conrad, 1838, (Cyrtoceras maximus, Ann. Rep. N. Y., p. 117, and Pal. N. Y., vol. 5, pt. 2, p. 418, Ham. Gr. meekanus, see Trematodiscus meekanus. missouriensis, Swallow, 1858, Trans. St. Lonis Acad. Sci., vol. 1, p. 198, Coal Mleas. montgomeryensis, Worthen, 1884, Bull. No. 2, Ill. St. Mus. Nat. Hist., p. 4, and Geo. Sur. Ill., vol. 8, p. 148, Up. Cosl Meas.

natator, Billings, 1859, Can. Nat. and Geo., vol. 4, 1. 466, Chazy Gr. Type of $\mathrm{Hy}$ att's genus Barrandoceras. niotensis, see Temnochilus niotense.

nodocarinatus, MeChesney; syn. for N. occidentalis.

nodoso-dorsatus, Shumard, 1858, Trans. St. Louis Acad. Sci., vol. 1, p. 198, Coal Meas.

occidentalis, Swallow, 1858, Trans, St. Louis Acad. Sci.. vol. 1, p. 196, anıl Pal. E. Neb., 1". 23 , Permian Gr.

occidentalis, Hall, 1860, 20th Rep. N. Y. Mus. Nat. Hist., p. 400, Niagara Gr. This name being preoccupied, MeChesney's name cancellatus, las precedence. See Lituites cancellatus.

oceanus, IIall, 1879, Desc. New sinec. Foss., p. 19, and IIth Ie'p. Geo. and Nat. Hist. Ind., p. 325, Niagara Gr.

oriens, Hall, 1876, lllust. Devonian Foss., pl. 61, and Pal. N. Y., vol. 5, pt. 2, p. 420, Marcellus Shale.

omatus, Hall, 1860, 8y'11. for N. marcellensis.

ortoni, Whitfleld, 1882, Ann. N. Y. A cud. Sci., vol. 2, p. 231, Coal Meas.

parallelus, Beecher, 1858, Pul. N. Y., vol. 7, р. 38, Сонl Meas.

pauper, Whittield, 18.2, Aun. N. Y. Acad. Sci., vol. 2, p. 226, Kaskaskia Gr.

permianus, Swallow, 1858, 'Trans. St. Louis Acad. Sci., vol. 1, p. 196. Permian Gr.

planidorsalis, see Trematodiscus planidorsaljs.

planorbiformis, Meek \& Worthen, 1860 , Proc. Acad. Nat. Sei. Plil., p. 469 , and Geo. Sur. Ill., vol. 2, p. 386, 'Yoal Meas. planovolvis, Slummard, 1858, Trans. St. Louis Acad. Sci., vol. 1, p. 198, Coal Meus. poinponius, Billings, 1862, Pul. Foss., vol. 1, p. 26, Calciferous Gr.

ponderosus, White, 1872, Pal. of E. Neb., p. 236, Coal Meas. Type of Hyatt's genus Titanoceras.

quadrangularis, McChesney, 1860, Desc. New Pal. Foss. p. 65, and Trans. Chi. Acad. Sci., vol. 1, p. 57, Coal Meas. Type of Hyatt's genus 'Tainoceras.

rockfordensis, Meek \& Worthen, 1866 , Proc. Acad. Nat. Sci. Plıil., p. 275, Kinderliook Gr. Probably a Gyroceras. See Ill. Geo. Sur., vol. 3.

sangamonensis, Metk \& Wortlen, 1860 Proc. Acad. Nat. Sci. Phil., p. 470, and Geo. Sur. Ill., vol. 2, p. 386, Coal Meas. Type of Hyatt's genus Metacoceras. seebachanus, see Pteronautilus seebachanus.

spectabilis, see Temnochilus spectabile. springeri, see Solenochilus springeri. striatulus, see Trematodiscus striatulus. subglobosus, Meek \& Worthen, 1860, Proc. Acad. Nat. Sei. Phil., p. 469, syn. for N. globatus, see Geo. Sur. Ill., vol. 3, p. 305 .

subliratus, Hall, 1876, (Gyroceras subliratum, Illust. Devon. Foss., pl. 58, and Pal. N. Y., vol. 5, pt. 2, p. $409, \mathrm{Ham}$. Gr. suhquadrangularis, Whitfielıl, 1882, Ann. N. Y. Acad. Sci., vol. 2, p. 232, Coal Meas. 
subsulcatus, Phillips, 1836, Geo. York. Not clearly identified in this country.

sulcatus, see Treinatodiscus sulcatus.

trigonus, see Trematodiscus trigonus.

trisulcatus, see Trematodiscus trisulcatus.

tyrans, Billings, 1859, Can. Nat. and Geo.; vol. 4, p. 465, Chazy Gr.

versutus, Billings, 1865, Pal. Foss., vol. 1, p. 259, Quebec Gr. Type of Hyatt's genus Litoceras.

winslowi, set Temnochilus winslowi.

Nelimenia incognita, Castelnau, 1843, Syst. Sil., p. 33. Probably a fragment of Phragmoceras or Oncoceras.

Oncoceras. Hall, 1847, Pal. N. Y., vol. 1, p. 196. [Ety. onkos, swelling; keras, horn.] Curved, aperture constricted; lower part of the body chamber, and upper part of septate portion ventricose; ab-

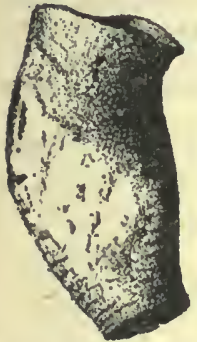

F1G. 748. - Oncoceras constrictum. ruptly contracted toward the apex ; sipbuncle dorsal ; septa plane, nearly flat, slightly elevated on the dorsal margin. Type O. constrictum.

abruptum, Hall, 1861, Rep. of Progr. Wis., p. 44 Trenton Gr.

alceus, Hall, 1861, Rep. of Progr. Wis., p. 46, Chazy and Black Riv. Grs.

amator, Billings, 1866 , Catal. Sil. Foss. Antic. p. 59, Clinton Gr.

brevicurvatum, Whitfield, 1880, Ann. Rep. Geo. Sur. WVis., p. 59, and Geo. Wis., vol. 4, p. 234, Trenton Gr.

constrictum, Hall, 1817, Pal. N. Y., vol. 1, p. 197, Black Riv. and Trenton Grs.

dilatatum, Hall, 1860, 13th Rep. N. Y.

Mus. Nat. Hist., p. 105, Ham. Gr.

expansum, Hall, 1852, Pal. N. Y., vol. 2, p. 337, Coralline limestone.

futile, Billings, 1866, Catal. Sil. Foss. Antic., p. 59, Clinton Gr.

gibbosum, Hảı, 1852, Pal. N. Y., vol. 2 , p. 13, Medina sandstone.

lycus, Hall, 1861, Rep. of Progr. Wis., p. 45, Chazy and Black Riv. Gr.

mummiforme, Whitfield, 1880, Ann. Rep. Geo. Sur. Wis., p. 58, and Geo. Wis. vol. 4, p. 232, Trenton Gr.

orcas, Hall, 1861, (Cyrtoceras orcas,) Rep. of Progr. Geo. Sur. of Wis., p. 42, Niagara Gr.

ovoides, Hall, 1859, Pal. N. Y., vol. 3, p. 342, Low. Held. Gr.

pandion, Hall, 1861, Rep. of Progr. Wis., p. 45, and Geo. Wis., vol. 4, p. 233, Chazy and Black Riv. Grs.

pettiti, Billings, 1866, Catal. Sil. Foss. Antic., p. 86, Niagara Gr.

plebeium, Hall, 1861, Geo. Rep. Wis., p. 44, Trenton Gr.

subrectum, Hall, 1852, Pal. N. Y., vol. 2, p. 94, Clinton Gr.

teucer, Billings, 1866, Catal. Sil. Foss. Antic., p. 86, Niagara Gr. thales, Billings, 1866, Catal. Sil. Foss. Antic., p. 87, Niagara Gr.

vasiforme, Dwight, 1884, Am. Jour. Sci. and Arts, $3 \mathrm{~d}$ ser., vol. 27, p. 257, Calciferous $\mathrm{Gr}$.

Ormoceras, Stokes, 1840, Trans. Geo. Soc., 2d ser., vol. 5, p. 709. [Ety. ormos, a chain or necklace; keras, horn; from the appearance of the siphuncle.] Externally like Orthoceras, and distinguished only by having the siphuncle constricted within each chamber instead of at the place of union with the septa. Types $\mathrm{O}$. backi, and $\mathrm{O}$. bayfieldi.

backi, Stokes, 1840, Trans. Geo. Soc., 2d ser., vol. 5, p. 709, Clintou Gr.

bayfieldi, Stokes, 1840, Trans. Geo. Soc., $2 \mathrm{~d}$ ser., vol. 5, p. 709, Clinton Gr.

cre brise p -

tum, Hall,

1847, Pal.

N. Y., vol.

1, p. 313 ,

Hud. Riv.

Gr.

gracile, Hall,

$18+7$, Pal.

N. Y, vol.

1. p. 58 ,

Black Riv.

Gir.

remot is ep -

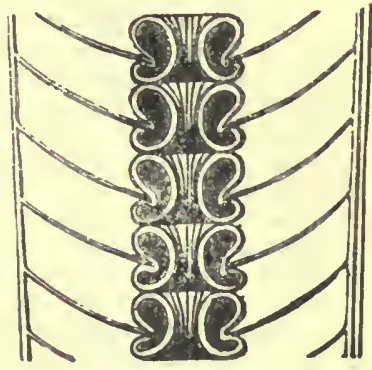

tum, Hall, Fig. 749.-Ormoceras bay fleldi. 1850 , 3d

Rep. N. Y. Mus. Nat. Hist., p. 181, Trenton Gr.

tenuifilum, Hall, 1847, Pal. N. Y., vol. 1, p. 55, Black Riv. and Trenton Gr.

tenuifilum var. distans, Hall, 1847, Pal.

N. Y., vol. 1, p. 58, Black Riv. Gr.

vertebratum, Hall, 1852, Pal. N. Y., vol. 2, p. 94, Clinton Gr.

whitii, Stokes, 1840, Trans. Geo. Soc., 2d ser., vol. 5, p. 709, Clinton Gr.

Orthoceras, Breynius, 1732, Dissertatio physica de Polythalamiis. [Ety. orthos, straigbt; keras, horn.] Shell conical, straight, or nearly so; body chamber large, behind which the shell is composed of numerous chambers separated by convex, transverse septa, with simple edges, at right angles to the longer axis of the shell; siphuncle central, subcentral or eccentric, cylindrical or dilated in the chambers; surface smooth or transversely, or longitudinally striated, or furrowed. Typical $\mathrm{O}$. breynii, $O$. annulatum, and $O$. striatum.

abnorme, Hall, 1867, 20th Rep. N. Y. Mus. Nat. Hist. p. 415, Niagara Gr. abruptum, Hall, 1852, Pal. N. Y., vol. 2, p. 97, Clinton Gr.

aciculı, Hall, see Colpolus acicula. aciculoiles, Clarke, 1885, Bull. U. S. Geo.

Sur., No. 16, p. 51, Chemung Gr. aculeatum, Swallow, 1858, Trans. St. Louis Acad, Sci., vol. 1, p. 200, Cual Meas. ægea, Hall, 1862, 15th Rep. N. Y. Mus. Nat. Hist., p. 80 , Ham. Gr. 
æquale, Emmons, 1842, Geo. Rep. N. Y. p. 404, Hud. Riv. Gr.

alienum, Hall, 1867, 20th Rep. N. Y.

Mus. Nat. Hist., p. 414, Niagara Gr.

allumettense, Billings, 1857, Rep. of Progr.

Geo. Sur. Can., p. 3\%1, Chazy and Black

Riv. Grs.

amplicameratum, Hall, 1847, Pal. N. Y.,

vol. 1, p. 205, Black Riv. and Trenton $\mathrm{Grs}$.

amycus, Hall, 1879, Desc. New Spec. Foss., p. 18, and 1tth Rep. Geo. Sur. Ind., p. 324, Ningara Gr.

anax, Billings, 1875, Can. Nat. and Geol., vol. 7, p. 238 , Up. Held. Gr.

anguis, Hall, 1s79, Pal. N. Y., vol. 5, pt. 2 , p. 312, Chemung Gr.

angulatum, (?) Wahlenberg, 1821, Nova. Acta. Soc. Sci. Upsal., 1). 90, Niagara Gr. See remarks on this species by Prof. Hall in 20th Rep. N. Y. Mus. Nat. Hist., p. 413 .

anellus, Conrad, 1843, Proc. Acad. Nat. Sci. Phil., vol. 1, p. 334. and Pal. N. Y., vol. 1, p. 202, Black Riv. and Trenton Grs.

annulato-costatum, Meek \& Worthen, 1861 , Proc. Acad. Nat. Sci. Phil.. p. 147. This name was preoccupied by Boll in 1857. See O. randolpliensc.

annulatum, Sowerby, 1818, Min. Conch., vol. 2 , p. 77, Clinton and Niagara Grs. antenor, Billings, 1859, Can. Nat. and Geo., vol. 4, p. 463, Chazy Gr.

anticostiense, Billings, 1857. Rep. of Progr. Geo. Sur. Can., p. 316, Hud. Riv. Gr.

aptum, Hall, 1879, Pal. N. Y., vol. 5, pt. 2, p. 282, Marcellus Shale.

arcuatellum, Sandberger. Is not an Amer. ican species.

arcuoliratum, Hall, 1847, Pal. N. Y., vol. 1, p. 198, Black Riv. and Trenton Grs.

arenosum, Hall, 1859, Pal. N. Y., vol. 3, p. 480, Oriskany aandstone.

asmodeus, Clarke, 1885, Bull. U. S. Geo. Sur., No. 16, p. 30, fienesce Shale.

atreus, Hall, 1879, Pal. N. Y., vol. 5, p. 305, Portage Gr.

atticus, Billings, 1865, Pal. Foss., vol. 1, p. 312, Quebec Gr.

aulax, Hall, 1879, Pal. N. Y., vol. 5, p. 293 , Ham. Gr.

autolycus, Billings, 1862, Pal. Foss., vol.1, p. 91, Quebec Gr.

baculum, Meek, 1860, Proc. Acad. Nat. Sci., p. 310, Subcarboniferous.

baculum, Hall, 1862. The name was preoccupied. See O. stylus.

balteatum, Billings, 1857, Rep. of Progr. Geo. Sur. Can., p. 318, Hud. Riv. Gr.

barquianum, Winchell, 1862, Am. Jour. Sci., $2 \mathrm{~d}$ ser., vol. 33, Marshall Gr.

bartonense, Spencer, 1884, Bull. No. 1, Mus. Univ. St. Mo., p. 60, Niagara $\mathrm{Gr}$.

bebryx, Hall, 1876, Illust. Devonian Foss., pl. 39, and Pal. N. Y, vol. 5, pt. 2, p. 275, Ham. Gr.: bebryx var. cay'uga, Hall, 1879, Pal. N. Y., vol. 5 , p. 276 , Chemung Gr.

becki, Billings, 1859, Can. Nat. aud (ieol., vol. 4, p. 362, Calciferous (ir.

bellatulum, Billings, 1866, Catal. Sil. Foss. Antic., p. 5s, Clinton (ir.

beloitense, Whitfield, 157S, Ann. Rep. Geo. Sur. Wis., P. 97, and Geo. Wis., vol. 4, p. 226, Trenton Gr.

bilineatum, Hall, 1847, Pal. N. Y., vol. 1, p. 199, Cliazy, Black Riv., Trenton, and Hud. Riv. (irs.

bilineatum var. a, Hall, 1847, I'al. N. Y., vol. 1, p. 200, T'renton Gr.

bipartitum, Hall, 1879, Pal. N. Y., vol. 5, p. 313, U. Chemung Gr.

brainerdi, Whitfield, 18\$6, 13ull. Am. Mus. Nat. Hist., vol. 1, p. 319, Birdseye Gr.

brongninrti, Troost, 1838, (Conotubularia brongniarti,) Mem. Soc. (ico. de Frauce, 3 , p. 89, Iow. Sil.

brontes, Billings, 1566, Catal. Sil. Foss. Antic., p. 83, Niagara Gr.

bucklandi, Billings, 1S57, Rep. of P'rogr. Geo. Sur. Can., p. 330, Up. Sil.

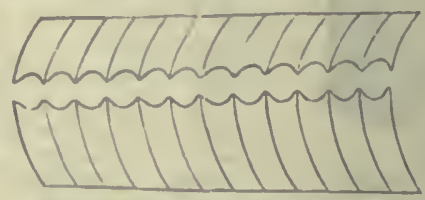

Fic. 750,-Orthoceras byrnesl.

bullatum, (?) Sowerby, 1839, Murch. Sil. Syst., p. 705, Trenton Gr.

byrnesi, S. A. Miller, 1875, Cin. Quar. Jour. Sci., vol. 2, p. 126, and Jour. Cin. Soc. Nat. Hist., vol. 4, p. 319, Hud. Riv. Gr.

cadmus, Billings, 1866, Catal. Sil. Foss. Antic., p. 83, Niagara Gr.

celamen, Hall, 1879, Pal. N. Y., vol. 5, p. 298, Ham. Gr.

cameolare, McClıesney, 1861, New Pal. Foss., p. 93, Niagara Gr.

canadense, Billings, 1857, Rep. of Progr. Geo. Sur. Can., p. 321, Mid. Sil. Prol. Billings proposed this name as a substitute for Huronia vertebralis for the reason that Huronia is a syn. for Orthoceras, and there is one 0 . vertebralis.

cancellatum, Hall, 1852, Pal. N. Y., vol. 2. The name was preoccupied by lichwald in 1842 . See O. subcancellatum.

capitolinum, Safford, 1869, Geo. of Tenn., p. 290, Trenton Gr.

carleyi, Hall \& Whitfield, 1875, Obio Pal., vol. 2 , p. 98, Hud. Riv. Gr.

carltonense, Whitfield, 1878, Ann. Rep. Geo. Sur. Wis., p. 85, and Geo. Wis., vol. 4, p. 318 , Niagara Gr.

carnosum, Hall, 1879, Pal. N. Y., vol. 5, p. 258, schoharie grit.

cataline, Billings, 1865, Pal. Foss., vol. 1. p. 315 , Quebec Gr. 
cato, Billings, 1865, Pal. Foss., vol. 1, p. 314, Quebec Gr.

catulus, Billings, 1865, Pal. Foss., vol. 1, p. 313, Quebec Gr.

chemungense, Swallow, 1860, Trans. St. Louis Acad. Sci., vol. 1, p. 660, Waverly or Chotean Gr.

chesterense, Swallow, 1863, Trans. St. Louis Acad. Sci., vol. 2, p. 98, Kaskaskia Gr.

chouteauense, Swallow, 1860, (O. chemungense var. choteauense,) Trans. St. Louis Acad. Sici., vol. 1, p. 660, W Waverly or Choteau Gr.

cincinnatiense, S. A. Miller, 1875, Cin. Quar. Jour. Sci., vol. 2, p. 127, and Jour. Cin. Soc. Nat. Hist., vol. 4, p. 319, Hud. Riv. Gr.

cingulum, Hall, 1879, Pal. N. Y., vol. 5, p. 240 , Schoharie grit.

clathratum, Hall, 18t7, Pal. N. Y., vol. 1, p. 201, Trenton Gr.

clavatum, Hall, 1852, Pal. N. Y., vol. 2, p. 104, Clinton Gr.

clavalum, Hall, 1859. The name was appropriated. See O. desideratum.

clinocameratum, Winchell, 1862, Am. Jour. Sci., 2d ser., vol. 33, p. 356, Marshall Gr.

clintoni, Hall, 1877, 1st Ed. Am. Pal. Foss., p. 244, Chazy Gr. Proposed instead of O. subarcuatum, Hall, 1847, which was preoccupied.

clonei, Barrande, 1869, Sys. Sil. de Boh., 4 me ser., p. viii, pl. 432 to 434 , Quebec Gr.

cochleatum, Hall, 1879, Pal. N. Y., vol. j. The name was preoccupied by Schlotheim in 1813. See O. warrenense.

collatum, Hall, 1879, Pal. N. Y., vol. 5, p. 252, Schoharie grit.

colon, White, 1874, Rep. Invert. Foss., p. 10, and Geo. Sur. W. 100th Mer., vol. 4, p. 56, Quebec Gr.

columnare, Hall, 1860, Rep. Progr. Geo. Sur. Wis. The name was preoccupied by Mark in 1857. See O. orus.

conicum, Castelnau, 1843, Syst. Sil., p. 29. The name was preoccupied by Hisinger.

consortale, Beecher, 1888, Pal. N. Y., vol. 7, p. 29, Chemung Gr.

constrictum, Vanuxem, 1842, Geo. Rep. 3 d Dist. N. Y., p. 152, and Pal. N. Y., vol. 5 , pt. 2 , p. 288 , Ham. Gr.

constrictum, Conrad, 1838. Not defined so as to be recognized.

constrictum, see Oncoceras constrictum. coralliferum, Hall, 1847, Pal. N. Y., vol. 1, p. 312, Utica and Hud. Riv. Grs. cornuoryx, Whitfield, 1886, Bull. Am. Mus. Nat. Hist., vol. 1, p. 320, Birdseye Gr.

cornuum, Billings, 1857, Rep. of Progr. Geo. Sur. Can., p. 329, Chazy Gr.

crebescens, Hall, 1867, 20th Rep, N. Y. Mus. Nat. Hist., p. 354, Niagara Gr. crebristriatum, Meek \& Worthen, 1865. Proc. Acad. Nat. Sci. Phil., p. 255, and
Geo. Sur. Ill., vol. 6, p. 503, Niagara Gr.

creon, Hall, 1879, Pal. N. Y., vol. 5, p. 260 , Schoharie grit.

cribrosum, Geinitz, 1866, Carb. und Dyas in Neb., p. 4, and Pal. E. Neb., p. 234, Coal Meas.

crocus, Billings, 1866, Catal. Sil. Foss. Antic., p. 22, Hud. Riv. Gr. Proposed instead of $\mathrm{O}$. perannulaturn, which was preoccupied.

crolalum, Hall, 1862, 15th Rep. N. Y. Mus. Nat. Hist., p. 78, Ham. Gr. Type of Hyatt's genus Spyroceras.

cuvitri, Troost, 1838, (Conotubularia cuvieri,) Mem. Soc. Geo. de France. t. 3, p. 88, Low. Sil.

dagon, Beecher, 1888, Pal. N. Y., vol. 7, p. 28, Up. Held. Gr.

darwini, Billings, 1868, Pal. Foss., vol. 1, p. 161, Guelph Gr.

dawsonanum, S. A. Miller, 1883, $2 \mathrm{~d}$ Ed. A im. Pal. Foss, p. 30خ, Carboniferous. Proposed instead of $O$. perstrictum, Dawson, in Acadian Geology, p. 312, fig. 129 , as the name was preoccupied by Barrande.

decrescens, Billings, 1857, Rep. of Progr. Geo. Sur. Can., p. 337, Black Riv. and Trenton Gr.

defrancii, Troost, 1838, (Conotubularia defrancei,) Mem. Soc. Geo. de France, t. 3, p. $\mathscr{0}$, Low. Sil.

demus, Hall, 1879, Pal. N. Y., vol. 5, p. 311, Chemung Gr.

deparcum, Billings, 1859, Can. Nat. and Geo., vol. 4, p. 363, Calciferous Gr.

desideratum, Hall, 1877, 1st Ed. Am. Pal. Foss., p. 244, Low. Held. Gr. Proposed instead of $\mathrm{O}$. clavatum, Hall, 1859, which was preoccupied.

diffidens, Billings, 1865, Pal. Foss., vol. 1, p. 174, Chazy Gr.

directum, Beecher, 1888, Pal. N. Y., vol. 7, p. 27, Up. Held. Gr.

dolatum, Dawson, 1868, Acad. Geol. p. 311 , Carboniferous.

drummondi, Billings, 1865, Pal. Foss., vol. 1, p. 173, Black Riv. Gr.

duramen, Beecher, 1888, Pal. N. Y., vol. $7, \mathrm{p} .25$, Schoharie grit.

duseri, Hall \& Whitfield, 1875, Ohio Pal., vol. 2, p. 97, Hud. Riv. Gr.

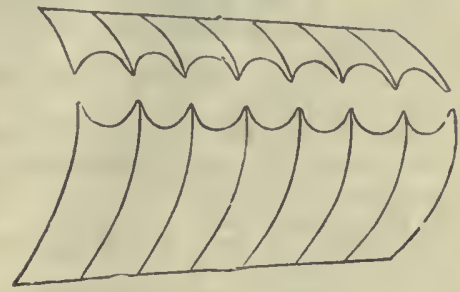

FIG. 75l.-Urthoceras dyeri.

dyeri, S. A. Miller, 1875, Cin. Quar. Jour. Sci., vol. 2, p. 125, and Jour. Cin. Soc. Nat. Hist., vol. 3, p. 236, Hud. Riv. Gr. 
edax, Billings, 1865, Pal. Foss., vol. 1, p. 349, Calcif. Gr.

elegantulum, Dawson, 1860, Can. Nat. and Geo., vol. 5, p. 155, and Acad. Geol., p. 607, Up. Sil.

emaceratum, Hall, 1862, 15th Rep. N. Y. Mus. Nat. Hist., r. 170, Ham. Gr.

epigrus, Hall, 1858, Trans. Alb. Inst., vol. 4 , p. 33 , and Bull. Am. Mus. Nat. Hist., p. 91, Warsaw Gr.

eriense, Hall, 1877, 1st Ed. Am. Pal. Foss., p. 274, and Pal. N. Y., vol. 5, p. 274, Ham. Gr. Proposed instead of $O$. robustum, which was preoccupied.

eurekense, Walcott, 1885, Monour. U. S. Geo. Sur., vol. 8, p. 265, Subcarboniferous.

exile, Hall, 1862, 15th Rep. N. Y. Mus. Nat. Hist., p. 78, and Pal. N. Y., vol. 5, pt. 2, p. 290, Ham. Gr.

exornatum, Dawson, 1860, Cun. Nat. and Geo., vol. 5, p. 198, Up. Sil.

expansum, Metk \& Worthen, 1860, Proc. Acad. Nat. Sci. Phil., p. 468, and Geo. Sur. 1ll., vol. 2, p. 286, St. Iouis Gr.

explorator, Billings, 1S65, Pal. Foss., vol. 1, p. 253, Quebec Gr.

exposituin, Beecher, 1885, Pal. N. Y., vol. 7 , p. 29, Chemung Gir.

ferum, Billings, 1866, Catal. Sil. Foss. Antic., p. 22, Hud. Kiv. and Anticosti Gr.

filiforme, Castelnau, 1843, Syst. Sil., p. 30, Niagara Gr. Not recognized.

filosim, Clarke, 1885, Bull. U. S. Geo. Sur., No. 16, p. 52, Chemung Gr.

flavius, Billings, 1865, Pal. Foss., vol. 1, ๗. 255, Qu+bec. Gr.

fluctum, Hall, 1879, Pal. N. Y., vol. 5, p. 239 , Schoharie grit.

foliatum, syn. for Cyrtoceras eugenium.

formosum, Billings, 1857, Rep. of Prngr. Geo. Sur. Can., p. 317, Trenton, Hud. Riv., and Anticosti Grs.

fosteri, S. A. Miller, 1875, Cin. Quar. Jour. Sci., vol. 2, p. 127, and Jour. Cin. Soc. Nat. Hist., vol. 4, p. 319, Hud. Riv. Gr.

foxense, Safford, 1869, Geo. of Tenn. Not defined.

fulgidum, Hall, 1879, Pa]. N. Y., vol. 5, p. 310, Chemung Gr.

fulgur, Billings, 1866, Catal. Sil. Foss. Antic., p. 22, Hud. Riv. Gr. Proposed instead of 0 . propinquum, which was preoccupied.

furtivum, Billings, 1865, Pal. Foss., vol. 1, p. 348 , Calcif. Gr.

fusiforme, Hall, 1847, Pal. N. Y., vol. 1, p. 60, Black Riv. and Trenton Grs.

fustis, Hall, 1879, Pal. N. Y., vol. 5, p. 281, Marcellus Shale.

glaucus, Billings, 1865, Pal. Foss., vol. 1, p. 350 , Calciferous Gr.

goldfussi, Troost, 1838, (Conotubularia goldfusci,) Mem. Soc. Geo. de France, t. 3 , p. 90, Lnw. Sil.

gracilium, Winchell, 1862, Proc. Acad. Nat. Sci., p. 429, Portage Gr. gregarium, Hall, 1861, Rep. of I'rogr. Wis. Prenceupied by Sowerby in 1839, Murch. Sil. Syst. See O. sociale.

griffithi, Haughton, 1857, Jour. Roy. Dub. Soc., rol. 1, Devonian.?

bresituns, Billıngs, 1865, Pnl. Foss., vol. 1, p. 254, Quebec Gr.

hageri, Hall, 1S61, Geol. of Vermont, p. 718, Calciferons Gr.

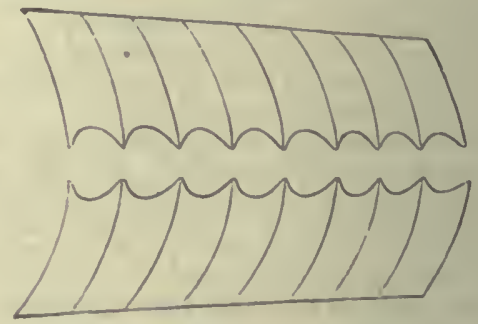

Fia. 752.-Orthocerns fallanum.

hallanum, S. A. Miller, 1877, ist Ed. Am. Pal. Foss., p. 245, Hud. Riv. Gr. Proposed insteall of O. halli, in Cin. Quar. Jour. Sci., vol. 2, p. 128, which was preoccupjed by Barrande.

halli, sec 0 . hallanum.

harperi, S. A. Miller, 1875, Cin. Quar. Jour. Sci., vol. 2, p. 128, nnd Jour. Cin. Soc. Nat. Hist., vol. 4, p. 319, Hud. Riv. Gr.

harttanuin, S. A. Miller, 1883, 2 d El. Am. Pul. Foss., p. 307, Carboniterons. Proposed instead of $\mathrm{O}$. laqueatum, Hartt, In Acalian Geol, 1). 312, fig. 128, which was preoccupied.

hastatum, Billings, 1857, liep. of Progr. Geo. Sur. Can., p. 333, Black Riv. and Trentou Grs. Type of Hyatt's genus Tripteroceras.

helderbergix, Hall, 1859, Pal. N. Y., vol. 3, p. 345, Low. Held. Gr.

henrietta, Dwight, 1884, Am. Jour. Sci. and Arts, $3 \mathrm{~d}$ ser., vol. 27, P. $256, \mathrm{Cal}$ ciferous $\mathrm{Gr}$.

herculaneuın, Verneuil, 1846, Bull. de la Soc. Geol. cle lirance, vol. 4, Low. Sil.

hercules, Castelnau, 1843, Syst. Sil., p. 29, Up. Sil. Not recognized.

heterocinctum, Winchell, 1863, Proc. A cad. Nat. Sci., p. 23, Kinderhook Gir.

hindei, James. Founded on fragments of different species, most of them 0 . transversium.

hoyi, McChesney, 1861, New Pal. Foss., p. 92, Niagara Gr.

huronense, Billings, 1857, Rep. of Progr. Geo. Sur. Can., p. 337, Trenton Gr.

hyas, Hall, 1862, syn. for O. thoas.

icarus, Beecher, 1888, Pal. N. Y., vol. 7, r. 31 , Kinderhook Gr.

idmon, Hall, 1879 , Pal. N. Y., vol 5, p. 302, Ham. Gr.

illinoisense, Worthen, 1883, Geo. Sur. Ill., vol. 7, p. 323, and Geo. Sur. Ill., vol. 8, p. 148 , Kaskaskia Gr. 
imbricatum, Sowerby, 1839, Murch. Sil. Syst., p. 620, and Pal. N. Y., vol. 2, p. 291, Niagara Gr.

inceptum, Foerste, 1885, Bull. Sci. Lab. Denison Univ., p. 117. Not properly defined.

indagator, Billings, 1865, Pal. Foss., vol. 1, p. 349, Calciferous Gr.

indianense, Hall, 1860, 13th Rep. N. Y. Mus. Nat. Hist., p. 107, Kinderhook Gr.

inf.lix, Billings, 1866, Catal. Sil. Foss. Antic., p. 57, Clinton Gr.

inoptatum, Hall, 1879, Pal. N. Y. vol. 5, p. 267 , Up. Held. Gr.

iowenfe. n. sp. Devonian. Proposed instead of $\mathrm{O}$. undulatum in Rep. on Min. Lands, p. 69, pl. 12, fig. 6, which name was preoccupied.

irregulare, McChesney, 1861, New Pal. Foss., Niagara Gr. The name was preoccupied. See O. woorworthi.

isogramma, Meek, 1871, Proc. Acad. Nat. Sci., p. 172, Coal Meas.

jaculum. Hall, 1879, Pal. N. Y., vol. 5, p. 266, Un. IIeld. Gr.

jamesi, Hall \& Whitfield, 1875, Ohio Pal. vol. 2, p. 118, Cliuton Gr.

jolietense, Meek \& Worthen, 1865, Proc. Acad. Nat. Sci. Phil., p. 256, and Geo. Sur. Ill., vol. 6, p. 505, Niagara Grr.

junceum, Hall, 1847, Pal. N. Y., vol. 1, p. 204, Trenton Gr.

kickapooense, Swallow, 1858, Trans. Acad. Sci. St. Louis, vol. 1, p. 197, Up. P'ermian Gr.

kingi,. Meek, 1877, U. S. Geo. Sur. 40tb Parallel, vol. 4, p. 47, Devonian.

knoxense, McChesney, 1860, New Pal. Foss., p. 69, Coal. Meas.

lave, Hall, 1843, Geo. Rep. 4th Dist. N. Y. The name was preoccupied by Fleming in 1825 . See 0 . sublæve.

lamarcki, Billings, 1859, Can. Nat. and Geo., vol. 4, p. 362, Calciferous Gr.

lamellosum, Hall, 18+7, Pal. N. Y., vol. 1, p. 312 , Hurl. Riv. Gr.

laphami, McChesney, 1861, New Pal. Foss., p. 91, Niagara Gr.

laqueatum, Hall, 1847, Pal. N. Y., vol. 1, p. 13, Calciferous to Trenton Gr.

laqueatum var. a, Hall, 1847, Pal. N. Y., vol. 1, p. 206, Trenton Gr.

laqueatum, Hartt, 1868, Acad. Geol. The name was preoccupied. See O. Hartanum.

lasallense, Worthen, 1883, Geo. Sur. Ill., vol. 7, p. 324, and Geo. Sur. Ill., vol. 8, p. 149, Coal Meas.

lathropanum, Winchell, 1862, Am. Jour. Sci. and Arts, 2d ser., vol. 33, p. 357, Marshall Gr.

latiannulatum, Hall, 1847, Pal. N. Y., vol. 1, p. 204, Trenton Gr.

leander, Hall, 1879, Pal. N. Y., vol. 5, p. 309, Chemung Gr.

lima, Hall, 1879, Pal. N. Y., vol. 5, p. 303, Ham. Gr. lineolntum, McChesney, 1861, New Pal. Foss., p. 93, Niagara Gr. The name was preoccupied by Phillips in 1841.

linteum, Hall, 1879, Pal. N. Y., vol. 5, p. 277 , Ham. Gr.

longicameratım, Hall, 1859, Pal. N. Y. vol. 3 , p. 343, Low. Held. Gr.

loxias, Hall, 1867, 20th Rep. N. Y. Mus. Nat. Hist., p. 416, Low. Sil.

luxum, Hall, 1876, Illust. Devonian Foss. pl. 35, and Pal. N. Y., vol. 5, pt. 2, p. 244, Sclıolıarie grit.

lyelli, Billings, 1857, Rep. of Progr. Geo. Sur. Can., p. 320, Hud. Riv. Gr.

magnisulcatum, Billings, 1857, Rep. of Progr. Geo. Sur. Can., p. 330, Hud. Riv. Gr.

marcellense, Vanuxem, 1842, Geo. Rep. N. Y., p. 147, and Pal. N. Y., vol. 5, pt. 2, p. 278, Ham. Gr.

marginale, Owell, 1840, Rep. on Min. Lands, p. 70, Up. Maunesian Gr.

marn, Billings, 1859, Can. Nat. and Geol., vol. 4, p. 461, Chazy Gr.

marsliallense, Winchell, 1862, Am. Jour. Sici., 21 series, vol. 33, p. 356, Marshall Gr.

masculum, Hall, 1879, Pal. N. Y., vol. 5, p. 238, Sohoharie grit.

merlium, Hall, 1879, Pal. N. Y., vol. 5, p. 254, Sclunharie grit.

medon, Billings, 1866, Catal. Sil. Fosș. Antic., p. 57, Clinton Gr.

medullare, Hall, 1860, Rep. of Progr. Geo. Sur. Wis., p. 4, Niagar.t Gr.

meeki, S. A. Miller, 1875, Cin. Qu a r. Jour. Sci., vol. 2, p. 126, Hud. Riv. Gr.

menelaus, $\mathrm{Bill}$ ings, 1862, Pal. Foss., vol. 1, p. 26, Black Riv. Gr.

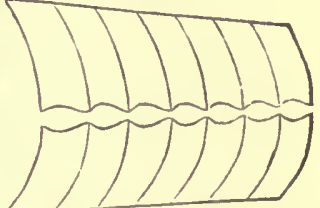

Fig. 753.-Urthoceras nieeki.

mephisto, Clarke, 1885, Bull. U. S. Geo. Sur., No. 16, p. 29, Genesee Shale.

micliganense, S. A. Miller, 188:3, 2 l Ed. A m. Pal. Foss., r. 308, Marshall Gr. in the southern part of Michigan. Proposed instead of $O$. multieinctum, Winchell, Proc. Acad. Nat. Sci., Phil., Sept., 1862, n. 421.

minganense, Billings, 1857, Rep. of Progr. Geo. Nur. Can., p. 319, Chazy and Black Riv. Grs.

missixquoi, see Cyrtoceras missisquoi.

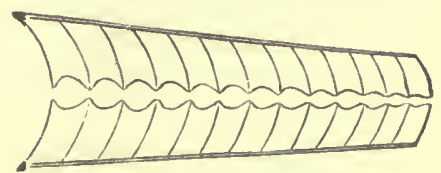

FIG. 574.-Orthoceras mohri.

mohri, S. A. Miller, 1875, Cin. Quar. Jour. Sci., vol. 2, p. 124, Hud. Riv. Gr. 
molestum, Hall, 1876, Illust. Devonian Foss., pl. 35, and Pal. N. Y., vol. 5, pt. 2, p. 265, Up. Held. Gr.

moniliforme, Hall, 1847, Pal. N. Y., vol. 1 , p. 35, Chazy Gr.

moniliforme, Swallow, 1858, Trans. St. Louis

Acad. Sci., vol. 1. The name was preoccupied. See O. swallovunum.

montrealense, Billings, 1859, Can. Nat. and Geo., vol. 4, p. 363, Calciferous Gr.

multicameratum, Emmons, 1S42, Geo. Rep. N. Y., p. 382, and Pal. N. Y., vol. 1, p. 45 , Birdseye Gr.

multicinctum, Häl, 1862, 15th Rep. N. Y. Mus. Nat. Hist., p. 76, and Pal. N. Y., vol. 5, pt. 2, p. 263, Schoharie grit.

multicinctum, Winchell, 1862. The name was preoccupied. See 0 . michiganense. multilineatum, Emmons, 1842, Geo. Rep. N. Y., p. 397, Trenton Gr.

multiseptum, Hall, 1852, Pal. N. Y., vol. 2, p. 14, Medina Gr.

murrayi, Billings, 1857, Rep of Progr. Geo. Sur. Can., p. 332, Black Riv. and Trenton Grs.

niagarense, Hall, 1867, 20th Rep. X. Y. Mus. Nat. Hist., p. 416, Niagara Gr.

nobile, Meek \& Worthen, 1865, Proc. Acad. Nat. Sci., p. 256, Kaskaskia Gr.

nodocostum, McChesney, 1861, New Pal. Foss., p. 94, Niagara Gr.

novamexicanum, Marcou, 1858, Geol. North America, p. 44, Subcarboniferous.

nummularium, (?) 1839, Murch. Sil. Sys., p. $632, \mathrm{Up}$. Sil.

nuntium, Hall, 1562, 15th Rep. N. Y. Mus. Nat. Hist., p. 79, and Pal. N. Y., vol. 5, pt. 2, p. 299, Ham. Gr.

oberon, Billings, 1S66, Catal. Sil. Foss. Antic., p. 82, Niagara Gr.

occidentale, Swallow, 1858, Trans. St. Louis Acad. Sci., vol. 1, p. 201, Coal Meas., Permian Gr.

occidentale, Winchell, 1862. This name was preoccupied. See O. vinchellanum. adipus, Hall, 1879, Pal. N. Y., vol. 5, p. 294, Ham. Gr.

okawense, Wortien, 1883, Geo. Sur. Ill., vol. 7, p. 324, and Geo. Sur. Ill., vol. 8, p. 149 , Kaskaskia Gr.

olorus, Hall, 1877, 1st Ed. Am. Pal. Foss., p. 245, Trenton Gr. Proposed instead of 0 . vertebrale, Hall, 1847. which was preoccupied.

ommaneyi, Salter, 1852, in Sutherland's Jour., vol. 2, Devonian.

oneidense, Walcott, 1879 , Trans. Alb. Inst., vol. $\mathbf{x}$, p. 22 , Utica Slate Gr. ontario, Clarke, 1885, Bull. U. S. Geo. Sur., No. 16, p. 5l, Chemung Gr.

oppletum, Hall, 1879, Pal. N.Y., vol. 5, p. 248, Schoharie grit.

ordinatum, Billings, 1865, Pal. Fosș., vol. 1, p. 350 , Calciferous Gr.

ortoni, Meek, 1872, Proc. Acad. Nat. Sci. Phil., p. 330, and Ohio Pal., vol. 1, p. 155, Hud. Riv. Gr, orus, Hall, 1877, 1st El. Am. Pal. Foss., p. 245, Niagara Gr. Propused insteal of O. columnare, Hall, 1560, which was preoccupied.

ottawense, Billings, 1857, Ren. of l'rogr.

Geo. Sur. Can., p. 331, Black Riv, and Trenton Grs.

ozarkense, Shumard, 1863, Trans. St. Louis Acad. Sci., vol. 2, p. 107, Calciferous $\mathrm{Gr}$.

pacator, Hall, 1879, Pal. N. Y., vol. 5, p. 307 , Portage Gr.

palmatum, Hall, 1879, Pal. N. Y., vol. 5, p. 312, Chemung Gr.

pauciseptum, Hall, 1859, Pal. N. Y., rol. 3, p. 346, Low. Held. Gr.

pelops, Hall, 1S62, 15th Rep. N. Y. Mus. Nat. Hist., p. 73, and Pal. N. Y., vol. 5, pt. 2, p. 233, Schoharie grit.

pelops var. ohioense, Hall, 1876, Illust. Devouian Foss., pl. 36, and Pal. N. Y., vol. 5, pt. 2, p. 236, Up. Held. Gr.

perannulatum, Billings, 1857, Rep. of Progr. Geo. Sur. Can., 1. 319. This name was preoccupied by Portlock in 1843. Sec O. crocus.

perelegans, Salter, 1848, Mem. (ieo. Nur. Gr. Brit., vol. 2, p. 354, Ilam. Gr.

perparvum, Billings, 1862, Pal. Foss., vol. 1, p. 27, Black Riv. Gr.

perseus, Billings, 1865, Pal. Foss., vol. 1, p. 313, Quebec Gr.

persiphonstum, Billings, 1857, Rep. of Prog. Geo. Sur. Can., p. 329, Mid. Sil. If the genus Huronia is valid, this species will belong to it.

perstriatum, IJall, 1859 , Pal. N. Y., vol. 3, p. 346, Low. Held. Gr.

perstrictum, Dawson, 1868. The name was preoccupied by Barrande. See O. dawsonanum.

pertextum, Hall, 1879, Pal. N. Y., vol. 5, p. 314, Chemung Gr.

pertinax, Billings, 1860, Can. Nat. and Geo., vol. 5, p. 75, Black Riv. Gr.

pervicax, Hall, 1879, Pal. N. Y., vol. 5, p. 257, Schoharie grit.

pileolum, Billings, 1866, Catal. Sil. Foss. Antic., p. 58, Medina Gr.

piscator, Billings, 1865, Pal. Foss., vol. 1, p. 251, Quebec Gr.

piso, Billings, 1862, Pal. Foss., vol. 1, p. 168, Hud. Riv. (ir.

planocon vexu1v, Hall, 1861, Rep. of Progr. Wis., p. 47, and Geo. Wis., vol. 4, p. 228, Black Kiv. and Trenton Grs.

pravum, Hall, 1879, Pal. N. Y., vol. 5, p. 255, Schoharie grit.

pressum, Rogers, 1868, Bigsby, Thesaurus Siluricus, p. 180. Not defined.

priamus, Billings, 1865, Pal. Foss., vol. 1, p. 253, Quebec Gr.

primigenium, Vanuxem, 1842, Geo. Rep. N. Y., p. 36, and Pal. N. Y., vol. 1, p. 13, Calciferous Gr.

procerum, Hall, 1876, Illust. Devonian Foss., pl. 35, and Pal. N. Y., vol. 5, pt. 2, p. 249, Schoharie grit. 
profundum, Hall, 1862, 15th Rep. N. Y. Mus. Nat. Hist., p. 76, Up. Held. Gr.

propinquum, Billings, 1857, Rep. of Progr. Geo. Sur. Can,, p. 320. Preoccupied. See O. fulgur.

punctostriatum, Hall, 1860, Can. Nat. and Geo., vol. 5, p. 154, Up. Sil.

pustulosum, Winchell, 1866, Rep. Low. Peninsula Mich., p. 97, Ham. Gr.

pylades, Billings, 1866, Catal. Sil. Foss. Antic., p. 84, Niagara Gr.

pytlion, Billiugs, 1857, Rep. of Progr. Geo. Sur. Can., p. 335, Trenton Gr.

randolphense, Worthen, 1882, Bull. No. 1, Ill. St. Mus. Nat. Hist., p. 38, Kaskaskia Gr. Proposed instead of $\mathrm{O}$. annulatocostatum, Meek \& Worthen, which was preoccupied.

rapax, see Endoceras rapax.

raptor, Billings, 1S66, Catal. Sil. Foss. Antic., p. 57, Medina Gr.

recedens, Barrande, 1869, Sys. Sil. de Bob., 4me ser., p. viii, pl. 433, Quebec Gr. rectiannulatum, Hall, 1847, Pal. N. Y., vol. 1, p. 34, Chazy and Birdseye Grs. recticameratum, Hall, 1847, Pal. N. Y., vol. 1, p. 46, Birdseye Gr.

rectum, Worthen, 1875, Geo. Sur. Ill., vol. 6, p. 504, Niagara Gr.

remus, Billings, 1866, Catal. Sil. Foss. Antic., p. 85, Niagara Gr.

repens, Billings, 1865, Pal. Foss., vol. 1, p. 312, Quebec Gr.

reticulatum, Phillips, 1836, Geol. York., Chemung Gr. Not clearly identified in this country.

rigidum, Hall, 1859, Pal. N. Y., vol. 3, p. 344, Low. Held. Gr.

robustum, Winchell, 1862, Am. Jour. Sci., $2 d$ ser., vol. 33, p. 356, Marshall Gr.

robustum, Hall, 1876. The name was preoccupied. See O. eriense.

rotulatum, Billings, 1857, Rep. of Progr. Geo. Sur. Can., p. 334, Niagara Gr.

rude, Hall, 1859, Pal. N. Y., vol. 3, p. 346, Iow. Held. Gr.

rudens, Beecher, 1888, Pal. N. Y., vol. 7, p. 28, Ham. Gr.

rudicula, Hall, 1876, Illust. Devonian Foss., pl. 37, and Pal. N. Y., vol. 5, pt. 2, p. 268, Up. Held. Gr.

rushense, McChesney, 1860, New. Pal. Foss., p. 68, and Geo. Sur. Ill., vol. 5, p. 612, Coal Meas.

sayi, Billings, 1865, Pal. Foss., vol. 1, p. 315, Quebec Gr.

scammoni, McChesney, 1861, New Pal. Foss., p. 92, Niagara Gr.

sceptrum, Beecher, 1888, Pal. N. Y., vol. 7 , p. 26, Up. Held. Gr.

scintilla, Hall, 1879, Pal. N. Y., vol. 5, p. 293, Ham. Gr.

sedgwicki, Billings, 1857, Rep. of Progr. Geo. Sur. Can., p. 320, Hud. Riv. Gr.

selwyni, Billings, 1862, Pal. Foss., vol. 1, p. 161, Guelph Gr.

servile, Billings, 1865, Pal. Foss., vol. 1, p. 252, Quebec Gr. shumardi, Billings, 1859, Can. Nat. and Geo., vol. 4, p. 460, Chazy Gr.

sicinus, Hall, 1879, Pal. N. Y., vol. 5, p. 301, Marcellus Shale.

sieboldi, Billings, 1866, Catal. Sil. Foss. Antic., p. 23, Hud. Riv. and Anticosti Grs.

simpsoni, Billings, 1859, Rep. of Progr. Assiniboine and Saskatchewan Ex. Exp., p. 186, Silurian.

simulator, Hall, 1876, 28th Rep. N. Y. Mus. Nat. Hist., p. 179, Niagara Gr.

sirpus, Hall, 1879, Pal. N. Y., vol. 5, p. 269, Up. Held. Gr.

sociale, Hall, 1877, 1st Ed. Am. Pal. Foss., p. 245, Hud. Riv. Gr. Proposed instead of $\mathrm{O}$. gregarium, Hall, 1861 , which was preoccupied.

sordidum, Billings, 1859, Can. Nat. and Geo., vol. 4, p. 363 , Calciferous Gr.

spissiseptum, Dwight, 1884, Am. Jour. Sci. and Arts, 3d. ser., vol. 27, p. 256 , Calciferous Gr.

spissum, Hall, 1879, Pal. N. Y., vol. 5, p. 287, Ham. Gr.

stebos, Clarke, 1885, Bull. U. S. Geo. Sur., No. 16, p. 29, Genesee Shale.

striatum, (?) Sowerby, 1812, Min. Conch., vol. 1, p. 129 , Devonian.

striælineatum, McChesney, 1861, New Pal. Foss., p. 94, Niagara Gr.

strigatum, Hall, 1847, Pal. N. Y., vol. 1, p. 205, Trenton Gr.

strix, Hall \& Whitfield, 1875, Ohio Pal., vol. 2, p. 149 , Niagara Gr.

stylus, Hall, 1877, 1st Ed. Am. Pal. Foss., p. 245, and Pal. N. Y., vol. 5, pt. 2, p. 253, Schoharie grit. Proposed instead of O. baculum, Hall, 1862, which was preoccupied.

subarcuatum, Hall, 1847, Pal. N. Y., vol. 1, p. 34. Preoccupied by Portlock in 1843. See O. clintoni.

subbaculum, Meek \& Worthen, 1865, Proc. Acad. Nat. Sci., p. 256, Niagara Gr.

subcancellatum, Hall, 1877, 1st Ed. Am. Pal. Foss., p. 245, Niagara Gr. Proposed instead of $\mathrm{O}$. cancellatum, Hall, 1852, which was preoccupied.

sublæeve, D'Orbigny, 1850, Prodrome de Pal., t. 1, p. 28, Onondaga Gr. Proposed instead of O. læve, Hall, 1843, which was preoccupied.

subtextile, Hall, 1859, Pal. N. Y., vol. 3, p. 344, Low. Held. Gr.

subulatum, Hall, 1843, 4th Dist. Geo. Rep. N. Y., p.'180, and Pal. N. Y., vol. 5 , pt. 2, p. 283, Marcellus Shale.

swällovanum, S. A. Miller, 1883, $2 \mathrm{~d}$ Ed. Am. Pal. Foss., p. 308, Coal Measures in the Valley of Verdigris in Kansas. Proposed instead of $\mathrm{O}$. moniliforme, Swallow, in Trans. St. Louis Acad. Sci., vol. 1 , p. 200 , which was preoccupied by Hall.

tantalus, Hall, 1879, Pal. N. Y., vol. 5, p. 241, Schoharie grit.

telamon, Hall, 1879, Pal. N. Y., vol. 5, p. 291, Ham. Gr. 
tenere, Hall, 1879, Pal. N. Y., vol. 5, p. 285, Ham. Gr.

tenerum, Billings, 1860, Can. Nat. and Geo., vol. 5, p. 174, Black Riv.Gr.

tenui-annulatum, Hall, 1859, Pal. N. Y., vol. 3 , 1. 345 , Low. Held. Gr.

tenuiseptum, Hall, 1847, Pal. N. Y., vol. 1 , p. 35 , Chazy (ìr.

teretiforme, Hall, 1847, Pal. N. Y., vol. 1, p. 198, Trenton Gr.

terfum, Hall, 1879, Pal. N. Y., vol. 5, p. 286, Han. Gr.

tetricum, Hall, 1862, 15th Rep. N. Y. Mus. Nat. Hist., p. 45, and Pul. N. Y., vol. 5 , pt. 2,1 . 251 , Schoharie grit.

textile, Hall, 1847, Pal. N. Y., vol. 1, p. 199, Trenton Gr.

textum, Hull, 1879, Pal. N, Y., vol. 5, p. 285, Ham. Gr.

thestor, Hall, 1879, Pa!. N. Y., vol. 5, p. 302 , Marcellus shales.

thous. Ilall, 1862, 15th Rep. N. Y. Mus. Nat. Hist., p. 75, and Pal. N. Y., vol. 5 , pt. 2, p. 61, Schoharie grit.

thyestes, IIall, 1879, I'al. N. Y., vol. 5, p. 306, Portage Gr.

tityrus, Billings, 1865, Pal. Foss., vol. 1, p. 316, Quebec Gr.

transversum, S. A. Miller, 1875, Cin. Quar. Jour. Sci., vol. 2, p. 129, Hud. Riv. Gr.

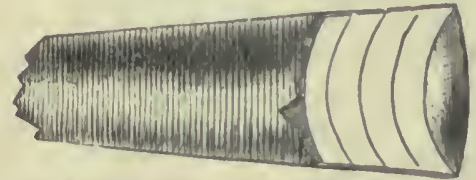

Fig. 755.-Orthocerns irunkversum.

trentonense, see Cyrtoceras trentonense.

turbiduın, Hall \& IVhitfield, 1875, Ohio Pal., vol. 2, p. 100, Hud. Riv. Gr.

typus, Saemann, as identified by Hall, 1876, Illust. Devonian Foss., is O. marcellense.

undulatum, Owen, 1840, Rep. on Min. Lands, Niagara Gr. The name was preoccupied by Sowerby in 1812. See O. iowense.

undulostriatum, Hall, 1817, Pal. N. Y., vol. 1, p. 202, Trenton Gr.

unionense, Worthen, 1875, Geo. Sur. Ill., vol. 6, p. 505, Niagara Gr.

varro, Billings, 1866, Catal. Sil. Foss. Antic., p. 84, Niagara Gr.

varum, Hall, 1879, Pall. N. Y., vol. 5, p. 259, Schoharie grit.

vastator, Hall, 1879, Pal. N. Y., vol. 5, p. 243 , Schnharie grit. Correct in the index, but printed 0 . obliquum, on page 243 . velox, Billings, 1865, Pal. Foss., vol. 1, p. 173, Chazy Gr.

vertebrale, Hall, 1847, Pal. N. Y., vol. 1, p. 201. Preoccupied by Schlotheim in 1820 , and by Eichwald in 1840 . See O. olorus. veterator, Billings, 1865, Pal. Foss., vol. 1, p. 350, Calciferous $\mathrm{Gr}$.

viator, Hall, 1879 , Pul. N. Y., vol. 5, p. 270, Up. Held. Gr. vinchellanum, S. A. Miller, 1883, 2d Ed. Am. Pal. Foss., p. 308, Murshall Gr. in Southern Michigan. Proposed instead of O. occidentule, Wincliell, 1862, Ain. Jour. Sci. and Arts, 21 ser., vol. 33, p. 356, which was preoccupled by Swallow. vindobonense, Dawson, 1868, Acend. Geol., p. 311, Curboniferous.

virgatum, Sowerby, 1839, Murch. Sil. Sys., p. 632, and Pal. N. Y., vol. 2, ए. 291, Niagara Gr.

virgulatum, Hall, 1852, Pal. $\mathrm{N}$. Y., vol. 2, p. 96, Clinton and Niagarn (irs.

vitlatum, Sundberger. Not American.

vulgatum, Billings, 1857 , Kip. of Progr. Geo. Sur. Can., 1). 3:37, Prenton Gr.

warrenense n. sp., Chemung Gr. Proposed instead of $\mathrm{O}$. conchleatum, Hall, Pul. N. Y., vol. 5, p. 308, pl. cxiii, fig. 19, which name was irror(mpied.

wauwatosense, Whitfeld, 1880, Anu. Rep. Geo. Sur. Wis, p. 66, and Geo. Wis., vol. 4. p. 297, Ningara Gir.

whitii, Winclifll, 1863, Proc. Acad. Nat. Sci., 1. 22, Kinderlıok Gr.

winchelli, Meck \& Wurtlien, 1866, Proc. Acad. Nat. Sci. Phil., p. 257, and Geo. Sur. Ill., vol. 6, p. 512, Hum. Gr.

woodworthi, MeChesuey, 1865), New Pal. Foss., p. 53, Niasara Gr. P'roposed instead of $\mathrm{O}$. irregulare, wlich was preoccupied.

xtrxes, Billings, 1805, Pal. Foss., vol. 1, p. 3I6, Quehec Gir.

xiphias, Billings, 1857, Rep. of l'rogr. Geo. Sinr. Cun., p. 318. Trenton Gr.

zeus, Hall, 1879, Pa]. N. Y., vol. 5, pt. 2, p. 235́, Scholuarie grit.

Pfтalicisus, S. A. Miller, 1850, Jowr. Cin. Soc. Nat. Hist., vol. 2, 1). 221. [Eiy. petalos, spread out; ichnos, trark.] A wille trail composed of numeruus transversely elongated depressions arringed without order. 'Pype P'. multipartitus. multipartitus, S. A. Miller, 1880, Jour. Cin. Soc. Nat. Hist., vol. 2, 1. 222, Utica Slate Gr.

Pirragmoceras, Broderip, 1839, Murch. Sil. Syst., p. 621. [lity. phrugmos, partition; keras, horn.] shell short, arched, compressed, niore or less conical $\mathrm{sides}$ of the mouth lapping toward each other; septa simple, crossed by the sigmoidal lines of growth; siphuncle on the interual edge, dilate between the septa. Type $P$. arcuatum.

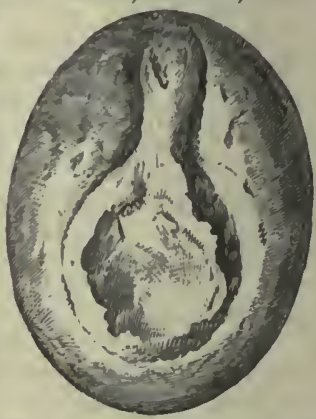

byronense, Wor-Fig. 756. - Pliragmoceras the n, 1875, hector. C'ant of fulerior. Geo. Sur. Ill., vol. 6, p. 506, Niagara $\mathrm{Gr}$. 
ellipticum, Hall \& Whitfield, 1875, Ohio Pal., vol. 2, p. 152, Niagara Gr.

expansum, Winchell, 1863, Proc. Acad. Nat. Sci., p. 23, Kinderhook Gr.

hector, Billings, 1862, Pal. Foss., vol. 1, p. 163, Guelph Gr.

hoyi, Whitfield, 1878, Ann. Rep. Geo. Sur. Wis., p. 86, and Geo. Wis., vol. 4, p. 300, Niagara Gr.

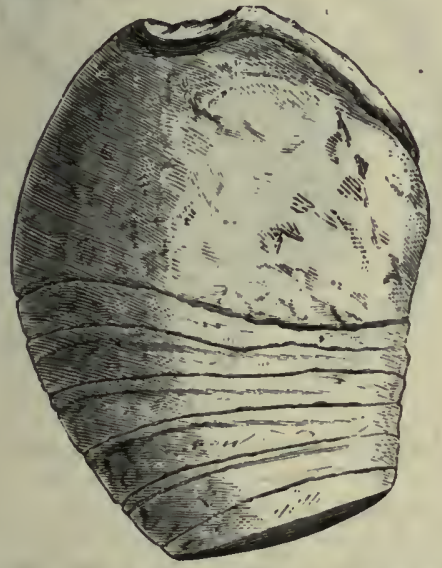

FIG. 757.-Pliragmoceras hector. Side vlew.

hoyi var. compressum, Whitfield, 1878 Ann. Rep. Geo. Sur. Wis., p. 82 , and Geo. Wis., vol. \&, p. 301, Niagara Gr.

labiatum, Whitfield, 1878, Ann. Rep. Geo. Sur. Wis., p. 86, and Geo. Wis., vol. 4, p. 302, Niagara Gr.

nestor, Hall, 1867, 20th Rep. N. Y. Mus.

Nat. Hist., p. 405, Niagara Gr.

nestor var. canadense. Whiteaves, 1884,

Pal. Foss., vol. 3, p. 39, Guelph Gr.

parvum, Hall \& Whitfield, 1875, Ohio Pal., vol. 2, p. 151, Niagara Gr.

præmaturum, Billings, 1866, Can. Nat. and Geo., vol. 5, p. 173, Black Riv. and Trenton Grs. Type of Hyatt's genus

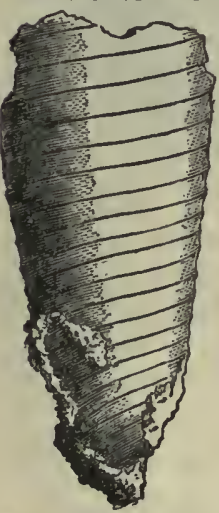

Fra. 758. - Pilo. ceras worthent.

\section{Mælnoceras.}

spinosum, see Gyroceras spinosum.

walshi, Meek \& Worthen, 1866, Proc. Acad. Nat. Sci. Phil., p. 257, and Geo. Sur. Ill., vol. 6, p. 511, Ham. Gr.

Piloceras, Salter, 1859, Quar. Jour. Geo. Soc., vol. 15, p. 376 . [Ety. pilos, a cap; keras, horn.] Broad, conical, sligbtly curved, subcylindrical or compressed; siphuncle and septa combined as a series of conical, concave septa, which fit into each other sheathwise. Type $P$. invaginatum.

amplum, Dawson, 1881, Can. Nat., vol. 10, p. 1, Calciferous Gr. canadense, Billings, 1860, Can. Nat. and Geol., vol. 5, p. 171, Calciferous Gr. explanator, Whitfield, 1886, Bull. Am. Mus. Nat. Hist., vol. 1, p. 323, Birdseye Gr.

gracile, Billings, 1865, Pal. Foss., vol. 1, p. 257, Quebec Gr.

triton, Billings, 1865, Pal. Foss., vol. 1, p. 257, Quebec Gr.

wortheni, Billings, 1865, Pal. Foss., vol. 1, p. 256, Quebec Grr.

Polycronites haani, Troost, 1840, 5th Geo. Rep. Tenn., Devonian. Not clearly defined, but probably a Gyroceras.

Pteronautilus, Meek, 1864, Pal. of Up. Mo., p. 64. [Ety. pteron, wing; Nautilus, a genus.] Shell with the involute body portion comparatively very small, and globular in form, scarcely umbilicate; outer chamber very large, and deflected from the involute body, its inner or ventral side being widely open, and the lateral margins greatly dilated, so as to form a very large, wing-like expansion on each side. Type $P$. seebachanus.

seebachanus, Geinitz, (Nautilus seebachanus,) Carb. und Dyas, p. 43, Permian Gr.

Sarichnites, Billings, 1866, Catal. Sil. Foss. Antic., p. 73. The author supposed the tracks might have been made by a species of Cephalopoda. They consist of two parallel rows of semicircular or subquadrate pits; each pit is about onehalf inch in diameter, and separated from the succeeding one by about onefourth of an inch. Type $\mathrm{S}$. abruptus. abruptus, Billings, 1866, Catal. Sil. Foss. Antic., p. 73, Hud. Riv. Gr.

Sidemina infundibuliforme, Castelnau, 1843, Syst. Sil., p. 33. Probably the fragment of an Endoceras.

Solenochilus, Meek \& Worthen, 1870, Proc. Acad. Nat. Sci. Phil., vol. 20, p. 47. [Ety. solen, a channel; cheilos, a lip.] Nautiloid in form, with small siphuncle in contact, or nearly in contact, with the outer shell; margins of the lip near the umbilicus, terminating in spoutlike auricles. Type S. collectum.

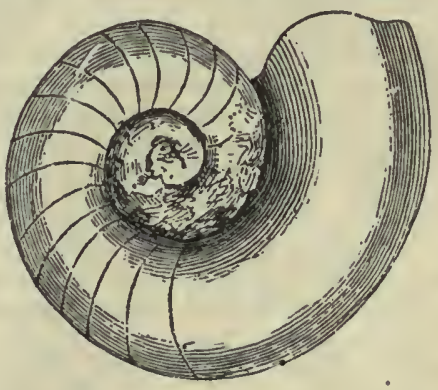

FIส. 759.-Solenochilus avonense.

avonense, Dawson, 1868, (Nautilus avonensis,) Acad. Geol., p. 311, Carboniferous. 
capax, Meek \& Worthen, 1865, (C'ryptoceras capax,) Proc. Acad. Nat. Sci. Phil., p. 262, and Geo. Sur. Ill., vol. 6, p. 532, Coal Meas.

collectum, Meek \& Worthen, 1870, Proc. Acad. Nat. Sci. Phil., p. 48, and Geo. Sur. Ill., vol. 5, p. 544, St. Louis Gr. indianense, Worthen, (in press,) Geo. Sur. Ill., vol. 8, p. 150, St. Louis Gr.

leidyi, Meek \& Worthen, 1865, (Nautilus leidyi,) Proc. Acad. Nat. Sci. Phil., p. 262, and Geo. Sur. Ill., vol. 5, p. 524, Keokuk Gr.

springeri, White \& St. John, 1868, (Nautilus springeri,) Trans. Chi. Acad. Sci., vol. 1, p. 124 , Up. Coal Meas.

Spirula, Lamarck, 1801, Syst. An. sans Vert. mortoni, Troost, 1840, 5 th Geo. Rep. Tenn., Niagara Gr. Not clearly defined.

Streptoceras, Billings, 1866, Catal. Sil. Foss. Antic., p. 88. [Ety. streptos, tw isted; keras, horn.] Having the general lorm of Oncoceras, but with a trilobed aperture resembling Phragmoceras. Type S. janus.

heros, Billings, 1866, Catal. Sil. Foss. Antic., p. 89, Niagara Gr.

janus, Billings, 1866, Catal. Sil. Foss. Antic., p. 88, Niagara Gr.

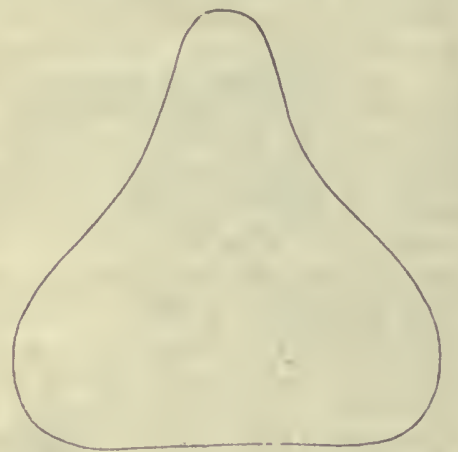

FIG. 760.-A perture of Streptoceras janus.

Tempochilus, McCoy, 1844, Synop. Carb. Foss. Ireland, p. 20. [Ety. temno, I divide; cheilos, lip.] Nautiloid in form, and characterized by a broad, deep, open umbilicus, showing all the volutions, with the outer side of the volutions broad or flattened, and the middle of each lateral margin prominently angular; the angle being sometimes nodose, while the transverse diameter of the volutions is always greater than the dorso-ventral; siphuncle between the middle and the outer side of the whorls. Type $\mathrm{T}$. biangulatus.

coxanum, Meek \& Worthen, 1869, Proc. Acad. Nat. Sci. Phil., p. 50, and Geo. Sur. Ill., vol. 5, p. 543, St. Louis Gr.

latum, Meek \& Worthen, 1870, Proc. Acad. Nat. Sci. Phil., p. 49, and Geo. Sur. Ill., vol. 5 , p. 608 , Coal Meas. niotense, Meek \& Worthen, 1865, Proc. Acad. Nat. Sci. Phil., p. 260, and Geo. Sur. Ill., vol. 5, p. 523, Keokuk Gr. Type of Hyatt's genus Edaphoceras.

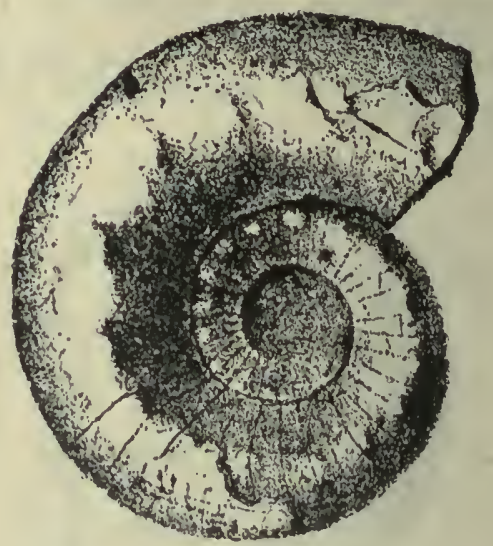

FIG. 761.-Temnochilus coxanum.

peramplum, Meek \& Worthen, 1865, (Endolobus perauplus,) Proc. Acad. Nat. Sci. Phil., p. 259, Kaskaskia Gr.

scottense, Worthen, (in press.) Geo. Sur. Ill., vol. 8, p. 151, Warsaw Gr.

spectabile, Meek \& Worthen, 1860, (Nautilus spectabilis, ) Proc. Acad. Nat. Sci. Phil., p. 469, and Feo. Sur. Ill., vol. 2, p. 308, Kaskaskia Gr.

winslowi, Meek \& Worthen, 1870, Proc. Acad. Nat. Sci. Phil., p. 50, and Geo. Sur. Ill., vol. 5, p. 609, Coal Meas.

Teratichnus, S. A. Miller, 1880, Jour. Cin. Soc. Nat. Hist., vol. 2, p. 221. [Ety. teras, a wonder; ichnos, track.] A track supposed to have heen made by a cephalopod, and consisting of numerous elongated, more or less bifurcated impressions. Type T. confertus.

confertus, S. A. Miller, 1880, Jour. Cin. Soc. Nat. Hist., vol. 2, p. 221, Utica Slate Gr.

Trachomatichnus, S. A. Miller, 1880, Jour. Cin. Soc. Nat. Hist., vol. 2, p. 219. [Ety. trachoma, that which is made rough; ichnos, track.] A track supposed to have been made by a ceplial. opod and consisting of numerous simple or compound impressions arranged in two series. Type $\mathrm{T}$. numerosus.

cincinnatiensis, S. A. Miller, 1880, Jour. Cin. Soc. Nat. Hist., vol. 2, p. 220 , Utica Slate Gr.

numerosus, S. A. Miller, 1880, Jour. Cin. Soc. Nat. Hist., vol. 2, p. 219 , Utica Slate Gr.

permultus, S. A. Miller, 1880, Jour. Cin. Soc. Nat. Hist., vol. 2, p. 220 , Utica Slate Gr.

Trematoceras, Whitfield, 1882, Ann. N. Y. Acad. Sci., vol. 2, p. 205. [Ety. trema, 
hole; keras, horn.] Shell straight, obconical, like Orthroceras as to tube, septa, and siphuncle; characterized by a line of elongated, raised tubercles along one side of the shell, which at one stage of growth formed perforations, which were closed as the animal extended the shell. Type T. ohioense.

ohioense, Whitfield, 1882, Ann. N. Y. Acad. Sci., vol. 2, p. 206, Up. Held. Gr. Trematodiscus, Meek \& Worthen, 1861, Proc. Acad. Nat. Sci. Phil., p. 147. [Ety. trema, hole; diskos, quoit.] Discoid, wide, shallow, umbilicus, perforated in the middle, showing all the whorls; whorls slender, merely in contact possessed of revolving angles, grooves, or strice; siphuncle central or subcentral on the dorsal side. Type ' $\Gamma$. stygialis. The name having been used in 1860 by Haeckel for Radiolaria, Hyatt proposed Trematoceras.

altidorsalis, Winchell, 1862, Proc. Acad. Nat. Sci., p. 429, Marshall Gr.

digonus, Meek \& Worthen, 1860, (Nautilus digonus,) Proc. Acad. Nat. Sci., p. 470, and Geo. Sur. Ill., vol. 2, p. 163, Kinderhook Gr.

discoidalis, Winchell, 1862, Am. Jour. Sci., vol. 33, 2d series, p. 360, Mar. shall Gr.

konincki, Wetherby, 1881, Jour. Cin. Soc. Nat. Hist., vol. 4, p. 79, Waverly Gr.

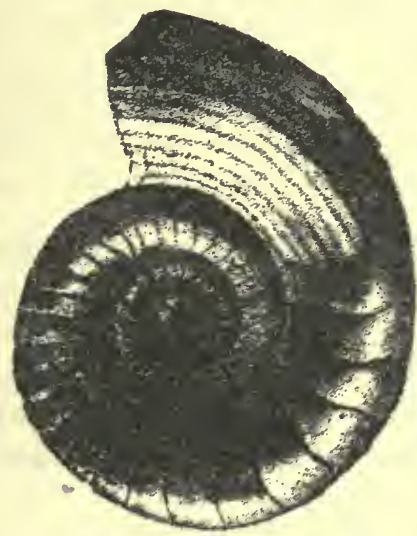

FIG. 762.-Trematodiscus konincki.

meekanus, Winchell, 1862, Am. Jour. Sci., 2d series, vol. 33, p. 360, Marshall Gr.

planidorsalis, Winchell, 1862. Am. Jour. Sci., 2d series, vol. 33, p. 358, Marshall Gr.

rockymontanus, S. A. Miller, 1881, Jour. Cin. Soc. Nat. Hist., vol. 4, p. 312, Burlington Gr.

striatulus, Winchell, 1862, Proc. Acad. Nat. Sci., 2 d series, vol. 33, p. 358, Marshall Gr.

strigatus, Winchell, 1862, Proc. Acad. Nat. Sci., p. 426, Marshall Gr. sulcatus, Meek \& Worthen, 1866, Proc. Acad. Nat. Sci. Phil., p. 274, Kaskaskia Gr.

trigonus, Winchell, 1862, Am. Jour. Sci., 2d series, vol. 33, p. 358, Marshall Gr. trisulcatus, Meek \& Worthen, 1860, Proc. Acad. Nat. Sci. Phil., p. 470, and Geo. Sur. Ill., vol. 2, p. 162, Kinderhook Gr. Trochoceras, Hall, 1852, Pal. N. Y., vol. 2 , p. 335. [Ety. trochos, hoop ; keras, horn.] This name was proposed by Barrande at about the same time. Turbinate or trochiform, spire elevated, more or less ventricose; umbilicated; aperture rounded or round oval; volutions above the outer one septate; siphuncle submarginal or dorsal. Type T. gebhardi. reneas, Hall, 1870, Rev. Ed. 20th Rep. N. Y. Mus. Nat. Hist. Expl., pl. 25, Niagara Gr.

baeri, see Gyroceras baeri.

barrandii, Hall, 1879, Pal. N. Y., vol. 5, p. 398 , Schoharie grit.

biton, Hall, 1879, Pal. N. Y., vol. 5, p. 395, Schoharie grit.

clio, Hall, 1861, 14th Rep. N. Y. Mus. Nat. Hist., p. 108, Schoharie grit. Type of Hyatt's genus Sphyradoceras.

costatum, Hall, 1861, Geo. Rep. of Wis., Niagara Gr.

desplainense, McChesney, 1860, New Pal. Foss., p. 68, Niagara Gr.

discoideum, Hall, 1862, 15th Rep. N. Y. Mus. Nat. Hist., p. 64, and Illust. Devon. Foss., pl. 59, Schoharie grit.

eugenium, Hall, 1861, 14th Rep. N. Y. Mus. Nat. Hist., p. 108, Schoharie grit. Type of Hyatt's genus Nierloceras.

expansum, Hall, 1879, Pal. N. Y., vol. 5, p. 402 , Schoharie grit.

gebhardi, Hall, 1852, Pal. N. Y., vol. 2, p. 335, Coralline Gr.

incipiens, Barrande, 1869, Syst. Sil. de Boh., 4me ser., Quebec Gr.

notum, Hall, 1867, 20th Rep. N. Y. Mus. Nat. Hist., p. 403, Niagara Gr.

obliquatum, Hall, 1876, Illust. Devonian Foss., pl. 48, Up. Held. Gr.

orion, Hall, 1876, (Cyrtoceras orion,) Illust. Devonian Foss., pl.48, Up. Held. Gr. pandion, Hall, 1876, Illust. Devonian Foss. pl. 48, and Pal. N. Y., vol. 5, pt. 2, p. 400 , Schoharie grit.

pandum, Hall, 1879, Pal. N.Y., vol. 5, p. 403 , Schoharie grit. turbinatum, Hall 1852 , Pal. N. Y., vol. 2 , p. 336 , Coralline Gr.

wald ronense, Fia. 763.-Trochoceras walHall, 1876,28 th

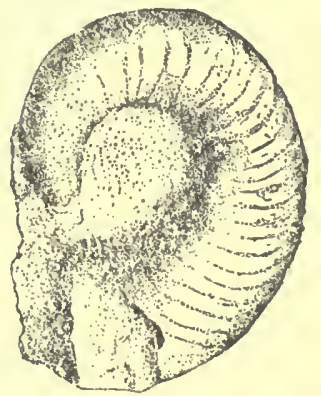

Rep. N. Y. Mus. Nat. Hist., p. 179, Niagara Gr. 
[Tro.

Trocholites, Conrad, 1838, Ann. Geo. Rep. N. Y., p. 119. [Ety. trochos, hoop;

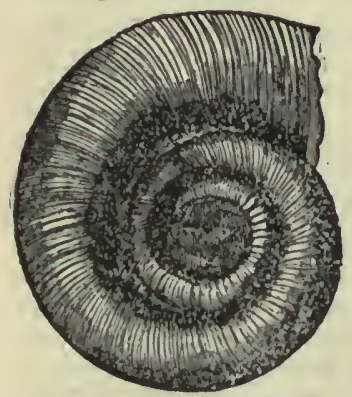

FIa. 764.-Trocholltes ammonius.

ventral. Type $\mathrm{T}$. ammonius lithos, stone.] Discoidal volutions in the same plane, about four, rounded, slightly concave on the ventral side, gradually enlarging toward the a perture; septa direct; outer chamber large; siph uncl'e ammonius, Conrad, 1838, Ann. Geo. Rep. N. Y., p. 119, and Pal. N. Y., vol. 1, p. 192, Trenton, Utica, and Hud. Riv. Grs. circularis, Miller \& Dyer, 1878, Cont. to Pal., No. 2, p. 9, Hud. Riv. Gr. minusculus, Miller \& Dyer, 1878, Cont. to Pal. No. 2, p. 9, Utica Slate Gr.

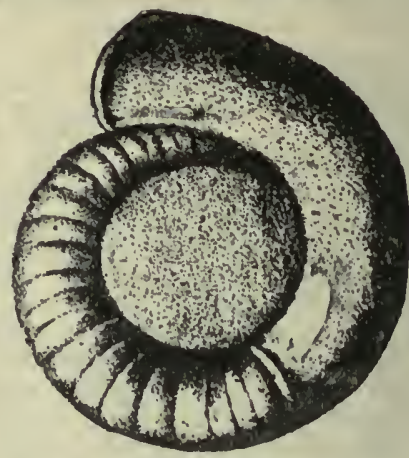

FIO. 765,-Trochollies circularis.

planorbiformis, Conrad, 1842, Jour. Acad. Nat. Sci. Phil., vol. 8, p. 274, and Pal. N. Y., vol. 1, p. 310, Utica and Hud. Riv. Grs.

\section{CLASS LAMELLIBRANCHIATA.}

[Ety. lamella, a thin plate; branchice, gills.]

Tre Lamellibranchiata, Blainville, or Conchifera, Lamarck, have bivalve shells, abound in the rivers of North America, in every ocean, and were common in all geolngical ages, back to early Silurian time. The river shells are known by the common name of mussels, and nearly all belong to three genera, Unio, Anodonta, and Margaritana. All known Palæozoic shells of this class inhabited salt water. The animals have a bilobed mantle, the sides of which secrete a calcareous shell having two valves, which are attached by some kind of a hinge. The hinge frequently has teeth on one valve that fit in cavities on the other. The valves being on each side of the animal, one is a right valve and the other a left valve. In most genera the valves are equal, and the animals lived in an erect position, resting on the edge of the shell opposite to the hinge, and, when moving, plowed a furrow in the sand or mud by the extension of a tongue-like foot. In some genera one valve is much larger than the other, and the shell lies on the larger valve, and adheres to some foreign object, as is the case with the common oyster; in other instances the locomotion is by suddenly opening and closing the valves, which causes the shell to dart through the water, first in one direction and then another, as the Pecten does. Some genera have a byssus by which they are attached to submarine budies. Each valve commences to grow at the apex or beak, which is also called the umbo. The umbones are almost always directed toward the ante- 
rior side of the shell, and sometimes project as far as the anterior margin. The length of a shell is the distance from the anterior to the posterior side; the width is measured from the hinge or dorsal side to the base; the thickness is measured through the center of the two valves. The surface of the shells is generally marked with ribs, radiating from the umbones, or concentric lines marking the growth of the shell from the umbones. A depression, anterior to the beak, is called a lunule, and when a depression exists posterior to the beak, it is called an escutcheon. Many shells have an external hinge ligament behind the umbones; some have a ligament between the umbones. When the valves are convected internally by a single muscle, the contraction of which brings the valves together, they belong to the Order Monomyaria; if there are two equally developed contracting mussels, they belong to the Dimyaria; or if there are two muscles, one large and functionally active, the other small, they belong to the Heteromyaria. These contracting muscles are called the adductors, and their places of attachment are indicated by scars. The border of the mantle makes an impression, which is called the pallial line, and if there is a sinus in the posterior part of the pallial line, it shows the animal had a retractile siphon, which, in burrowing shells, is often of great length. The Class has also been divided into two Orders, based on the presence or absence of a siplion, to wit: Asiphonida, Asiphonata, or Asiphonophora, and Siphonida, Siphonata, or Siphonophora. Each Order is spelled three different ways by different authors. Shells having a siphon are always gaping at the posterior or anterior side or at both.

It will be observed from the foregoing, the essential characters upon which Palxozoic shells are classified are the following: Equality or inequality of the valves; the presence or absence of an external ligament; the number of muscular scars; the character of the hinge and its dentition; the presence or absence of a pallial sinus; the position of the umbones; the radiate or concentric surface markings; whether the valves fit each other or are gaping at one or both ends; and the presence or absence of a byssal sinus.

\section{ORDER ASIPHONIDA.}

Ambonychiidæ, Amphicœliidæ, Anodontopsidæ, Arcidæ, Áviculidæ, Aviculopectenidæ, Cytherodontidx, Modiomorphidæ, Mytilidæ, Nuculidæ, Nyassidæ, Orthonotidæ, Ostreidæ, Balæoconchidæ, Pinnidæ, Prothyridæ, Pteriniidæ, Technophoridæ, Trigoniidæ, Unionidx.

\section{ORDER SIPHONIDA.}

Cardiidæ Cardiomorphidæ, Conocardiidæ, Cyprinidæ, Eopteriidæ, Grammysiidæ, Lucinidæ, Myacidæ, Palæanatinidæ, Pholadellidæ, Sanguinolitidæ, Solenidæ, Spirodomidæ, Tellinidæ.

Famm Anbonychinds.-Ambonychia, Angellum, Anomalodonta, Byssopteria.

Family Amphicelime. - Amphicœlia.

Famir y Anodontopsida. - Anodontopsis, Cycloconcha.

Famrly Arcide.-Carbonarca, Clinopistha, Macrodon, Megalomus, Ptychodesma. 
Family Aviculide.-Actinodesma, Avicula, Aviculopinna, Bakevellia, Ectenodesma, Glyptodesma, Inocerăıus, Liopteria, Leptodesma, Limoptera, Monopteria, Monotis, Palæopinna, Posidonomya, Pseudomonotis, Pteronitella, Pteronites.

Family Aviculopectenid a.-Aviculopecten, Crenipecten, Euchondria, Lyriopecten, Pernopecten, Pterinopecten, Streblopteria.

Family Carnind.-Cardiola, Cardiopsis, Cardium, Dexiobia, Glyptocardia, Lunulicardium, Palaeocardia, Panenka, Paracardium, Pararca.

Family Cardomorphid e.-Cardiomorpha, Edmondia, Euthydesma, Protomya.

Family Cinjnocardinde.-Chmocardia.

Family Conocardime.-Conocardium.

Famil Cyrrinide.-Astartella, Cardinia, Clidophorus, Cypricardia, Cypricardites, Matheria, Pleurophorus, Vanuxemia.

Family Cytuenodontidz.-Cytherodon, Lyrodesma, Schizodus.

Famil y Lopteridow.-Eopteria, Euchasma.

Family Grammysinz.-Allorisma, Chænomya, Cuneamya, Grummysia, Leptodomus, Sedgwickia.

Family Lucinid e.-Paracylas.

Faml y Modomorphid Glossites, Modiomorpha.

Family Mracide. - Anthracomya.

Family MYrilides.-Anthracoptera, Gosselettia, Lithophaga, Megambonia,

Modiella, Modiolopsis, Myalina, Mytilarca, Mytilops, Plethomytilus, Pyanomya.

Famix Nuculid s.-Nucula, Nuculana, Nuculites, Palæoneilo, Pyrenomœus,

Solenomya, Tellinomya, Yoldia.

Family Nyasside.-Nyassa.

Family Orthonotids.-Orthodesma, Orthonota, Orthonotella, Palæosolen,

Sphenolium.

Family Ostreidas.-Ostrea, Placunopsis.

Family Paldanatinidz.-Ilionia, Palmanatina, Prorhynchus.

Family Palzoconchide.-Palæoconcha.

Family Pholadeldidas.-Cimitaria, Pholadella, Phthonia.

Family Pinnide.-Pinna.

Family Prothyrida.-Prothyris.

Family Pterinimda-Actinopteria, Pterinea, Ptychopteria, Vertumnia.

Family Sangunnolitidæ.-Cypricardinia, Promacrus, Spathella, Sphenotus,

Sanguinolites.

Fammy SOlenide.-Solenopsis.

FAMILY SPIRodomide.-Spirodomus.

Family Technophoride.-Technophorus.

Family Tellinidж.-Tellinopsis.

Family Trigonitde.-Dolabra,? Ischyrinia.?

Family Unionid w. Anthracosia, Prisconaia. 
Actinodesma subrectans, see Glyptodesma subrectum.

Actinopteria, Hall, 1883, Pal. N. Y., vol. 5, pt. 1 , explanation of plate 17, fig. 6 . [Ety. altin, a ray; Pteria, a genus.] In the text published in 1884 , pt. 1 , p. 107 , he wrote the word Actinopteria, which indicates he derived the name from the genus Pteria. Distinguished from Pterinea by strong cardinal and lateral teeth, and no striations on the ligamental area; right valve convex. The first species mentioned on page 3 , where
Fra. 766.-Actin opteria boydi. Right valve.

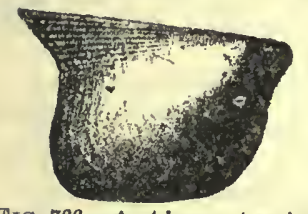
the genus is defined, is A. decussata, but the first one mentioned on page 107 of the text is A. eximia. No type is designated.

auriculata, Hall, 1884, Pal. N.Y., vol. 5, pt. 1, p. 121, Chemung Gr.

boydi, Con rad, 1842, (A vicula boydi,) J o u r. Acad. Nat. Sci. Phil., vol. 8, p. 237, and Pal. N. Y., vol. $5, \mathrm{p}$. 113, Ham. Gr. decussata, Hall, 1843, (Avicula decussata, ) Fra.767.-A ctinopteria Geo. 4th Dist.

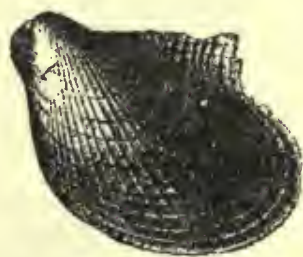

N. Y., p. 203, and Pal. N. Y., vol. 5, p. 111, Ham. Gr.

delta, Hall, 1883, Pal. N. Y., vol. 5, p. 121, Chemung Gr.

doris, Hall, 1884, Pal. N. Y., vol. 5, p. 109, Marcellus Shale.

epsilon, Hall, 1883, Pal. N. Y., vol. 5, pt. 1, p. 122, Chemung Gr.

eta, Hall, 1884, Pal. N. Y., vol. 5. pt. 1, p. 124, Chemung Gr.

eximia, Hall, 1883, Pal. N. Y., vol. 5, pt. 1, p. 107, Up. Held. Gr.

iota, Hall, 1884, Pal. N. Y., vol. 5, pt. 1, p. 127, Chemung Gr.

kappa, Hall, 1884, Pal. N. Y., vol. 5, pt. 1, p. 128, Chemung Gr.

muricata, Hall, 1843, (A vicula muricata,) Geo. Sur. 4th Dist. N. Y., p. 181, and Pal. N. Y., vol. 5, p. 108, Marcellus Shale. perobliqua, Con rad, 1842, (Avicula perobliqua,) Jour. Acad. Nat. Sci. Phil., vol. 8, p. 235, and Pal. N. Y., vol. 5, p. 116, Ham. Gr.

perstrialis, Hall, 1883, Pal. N. Y., vol. 5, pt. 1, p. 118, Chemung Gr.

pleuroptera, Conrad, 1842, (Avicula pleuroptera,) Jour. Acad. Nat. Sci., vol. 8, p. 242, Ham. Gr.

pusilla, Hall, 1884, Pal. N. Y., vol. 5, pt. 1, p. 117, Ham. Gr.

subdecussata, Hall, 1884, Pal. N. Y., vol. 5 , pt. 1, p. 110 , Ham. Gr. tenuistriata, Hall, 1884, Pal. N. Y., vol. 5, pt. 1, p. 120, Chemung Gr.

theta, Hall, 1884, Pal. N. Y., vol. 5, pt. 1, p. 125, Chemung Gr.

zeta, Hall, 1883, Pal. N. Y., vol. 5, pt. 1, p. 123, Chemung Gr.

Allorisma, King, 1844, Ann. Mag. Nat. Hist., vol.14, p. 315. [Ety.allos, variable; ereisma, support, expressive of the variable nature of the cartilage support or fulcrum.] Equivalve, inequilateral, elongate, thin ; anterior side short; posterior long and gaping at the extremity; beaks anterior, depressed; surface concentrically ridged or undulated; hinge edentulous; ligament external; dorsal margin inflected, forming a lanceolate depression along the cardinal border behind the beaks; anterior adductor scar occupying a low position; pallial line faintly marked. Type A. sulcatum.

altirostratum, see Sedgwickia altirostrata. andrewsi, Whitfield, 1882 , Ann. N. Y. Acad. Sci., vol. 2, p. 222, Krskaskia Gr. antiquum, Swallow, 1863, Trans. St. Louis Acad. Sci., vol. 2, p. 95, Kaskaskia Gr. capax, Newberry, 1861, Ives' Col. Ex. Exped., p. 120, Coal Meas.

clavatum, McChesney, 1860, New Pal. Foss., p. 56, Kaskaskia Gr.

cooperi, see Chrenomya cooperi.

costatum, Meek \& Worthen, 1869, Proc. Acad. Nat. Sci. Phil., p. 171, and Geo. Sur. Ill., vol. 5, p. 585, Coal Meas.

cuneatum, Swallow, 1858, Trans. St. Louis Acad. Sci., vol. 1, p. 210, Mid. Coal Meas.

curtum, Swallow, 1858, Trans. St. Louis Acad. Sci., vol. 1, p. 194, Permian Gr.

elegans, King, as identified by Geinitz. See A. geinitzi.

elongatum, Morton, 1836, (Pholadomya elongata,) Am, Jour. Sci. and Arts, vol. 29 , p. 153 , Coal Meas.

elongatum, Worthen, see A. worthenanum. ensiforme, Swallow, 1860, Trans. St. Louis Acad. Sci., vol. 1, p. 656, Coal. Meas.

geinitzi, Meek, 1867, Am. Jour. Sci., vol. 44, $2 \mathrm{~d}$ ser., p. 170, and Geo. Sur. Ill., vol. 5, p. 586, Coal Meas.

gilberti, White, 1879, Bull. U. S. Geo. Sur., vol. 5, No. 2, p. 217, and Cont. to Pal., No. 6, p. 137, Carboniferous.

granosum, Shumard, 1858, (Leptodomus granosus,) Trans. St. Louis Acad. Sci., vol. 1, p. 207, and Pal. E. Neb. p. 220, Coal Meas.

hannibalense, see Grammysia hannibalensis.

hybridum, Meek \& Worthen, 1865, (Chænomya hybrida,) Proc. Acad. Nat. Sci. Phil., p. 250, and Geo. Sur. Ill., vol. 3, p. 538, Keokuk Gr.

illinoisense, Worthen, 1884, Bull. No. 2, Ill. St. Mus. Nat. Hist., p. 11, and Geo. Sur. Ill., vol. 8, p. 132, Keokuk Gr.

lanceolatum, Swallow, 1858, Trans. St. Louis Acad. Sci., vol. 1, p. 194, Permian Gr. 
latum, Swallow, 1858, Trans. St. Louis Acad. Sci., vol. 1, p. 210, Mid. Coal Meas.

leavenworthense, see Chænomya leavenworthensis.

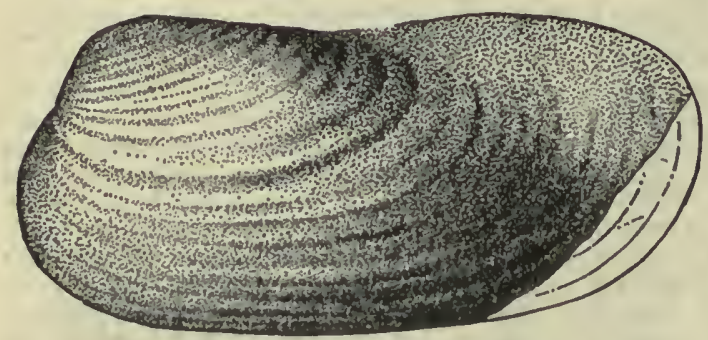

FIg. 768.-Allorismu subcuneatum.

marionense, White, 1876, Proc. Acad. Nat. Sci., p. 31, and Cont. to Pal., No.-8, p. 167 , St. Louis Gr.

maxvillense, Whitfield, 1882 , Ann. N. Y. Acad. Sci., vol. 2, p. 222, Kaskaskia Gr. minnehaha, see Clıænomya minnehaha. pleuropistha, Meek, 1871, Proc. Acad. Nat. Sci. Phil., p. 70, and Ohio Pal., vol. 2, p. 309 , Waverly Gr.

reflexum, Meek, 1872, Pal. E. Neb., p. 217, Coal Meas.

sinuatum, McChesney, 1860, New Pal. Foss., p. 56, Chester Gr.

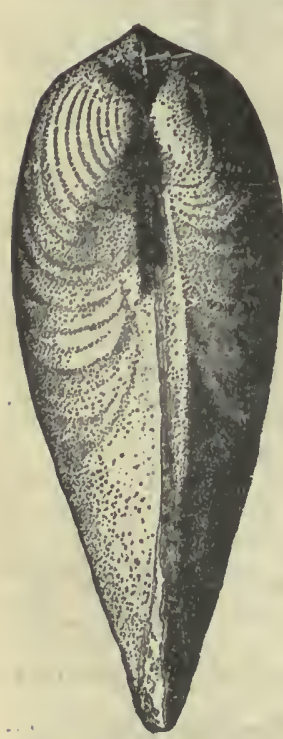

Fig. 769.-Allorlsma subcuneatum. $\mathrm{subc}$ un a $t \mathbf{u m}$, Meek \& Hayden, 1858, Proc. Acad. Nat. Sci. Phil., p. 263, and Pal. E. Neb., p. 221, Coal Meas.

subelegans, Meek,

1872, Pal. E. Neb., p. 220 , Coal Meas. terminale, $\mathrm{H}$ a l l, 1852, Stans. Ex. to Gt. Salt Lake, p. 413, Coal Meas. ventricosum, Meek,

1871, Proc. Acad.

Nat. Sci. Pliil., p.

168, and Ohio

Pal., vol. 2, p. 312, Waverly Gr. winchelli, M e e $\mathrm{k}$,

1871, Proc. Acad.

Nat., Sci. Phil., p.

167, and Ohio Pal., vol. 2, p. 311 , Waverly Gr. worthenanum, n.sp.

Keokuk Gr. Proposed instead of A. elongatum in

Geo. Sur. Ill., vol. 8, p. 133, which was preoccupied.

Ambonycuia, Hall, 1847, Pal. N. Y., vol. 1, p. 163. [Ety, ambon, the boss of a shield; onyx, a claw or talon.] Equivalve, inequilateral, subalate poste- riorly, abrupt or curving down anteriorly; umbones high; beak incurved, cardinal line oblique; sinuate on the anterior side for the passage of the byssus; muscular impression large; cardinal tooth below the beak anteriorly; two or three remote lateral teeth, elongated and ranging parallel with the cardinal line posteriorly; surface radiately furrowed and concentrically lined. 'Type A. bellistriata.

acutirostra, Hall, 1867, 20th Rep.

N. Y. Mus. Nat. Hist., p. 383, Niagara Gr.

alala, see Anomalodonta alata.

amygdalina, see Cypricardites amygdalinus.

aphrea, Ilall, 1867, 20th Rep. N. Y. Mus. Nat. Hist., p. 383, Niagara Gr. attenuata, Hall, 1861, Geo. Rep. Wis., p. 33 , and Geo. Wis., vol. 4, p. 206, Trenton Gr.

bellistriata, $\mathrm{H}$ a 11 , 1847, Pal. N.. Y., vol. 1, p. 163, Trenton Gr.

cancellosa, IIall, 1861, Geo. Rep. Wis., p. 31. Mistake for A. lamellosa.

carinata, Goldfuss, 1826, ( I't erin e a carinata,) Germ. Petref., p. 136, and Pal. N. Y., vol. 1, p. Fra. 770. - A mbonychla 292 294 Trenton bellistriata. $h$, Byssal and Hud. Riv. Gr. sinns; $t$, carilual casii, Meek \& Worthen, 1866, Proc.

Chi. Acad. Nat. Sci., p. 22, I!ud. Riv. Gr.

costata, Meek, 1873, Ohio Pal., vol. 1, p. 130, Hud. Riv. Gr.

erecta, Hall, 186I, Geo. Rep. Wis., p. 32, Trenton Gr.

illinoisensis, Worthen, 1875, Geo. Rep. Ill., vol. 6, p. 495, Hud. Riv. Gr.

intermedia, Meek \& Worthen, 1868, Geo. Sur. Ill., vol. 3, p. 306, Galena Gr. jamesi, Meek, 1872, (Megambonia jamesi,) Proc. Acad. Nat. Sci. Phil., p. 321, and Ohio Pal., vol. 1, p. 136, Hud. Riv. Gr. lamellosa, Hall, 1861, Geo. Rep. Wis., p. 31 , and Geo. Wis., vol. 4, p. 205, Trenton Gr.

maxima, Safford, 1869, Geo. of Tenn. Not defined.

mytiloides, Hall, 1847, Pal. N. Y., vol. 1, p. 315, Chazy Gr.

neglecta, see Amphicolia neglecta.

nitida, Billings, 1866, Catal. Sil. Foss. Antic., p. 50, Anticosti Gr.

oblusa, see Cypricardites obtusus.

orbicularis, Emmons, 1842, (Pterinea orbicularis,) Geo. Rep. N. Y., p. 397, and Pal. N. Y., vol. 1, p. 164, Trenton $\mathrm{Gr}$. 
planistriata, Hall, 1861, Geo. Rep. Wis., p. 32, Trenton Gr.

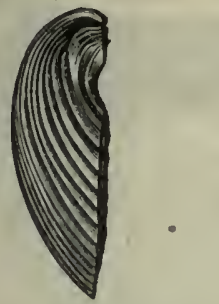

F1G. 771.-Ambonychia radiata.

radiata, Hall, 1847, Pal. N. Y., vol. 1, p. 292, Trenton, Hud. Riv. Grs., and Mid. Sil. Probably a syn. for $A$. carinata.

rauchi, McChesney, 1860, N e w Pal. Foss., p. 89, Hud. Riv. Gr. Not recognized.

retrorsa, S. A. Miller, 1878, Jour. Cin. Soc. Nat. Hist., vol. 1, p. 104, Hud. Riv. Gr.

robusta, S. A. Miller, 1880, Jour. Cin. Soc. Nat. Hist., vol. 3, p. 315 , Hud. Riv. Gr. strixcosta, see Pterinea striæcosta.

superba, Billings, 1866, Catal. Sil. Foss. Antic., p. 50, Anticosti Gr.

swanana, Safford, 1869, Geo. of Tenn. Not defined.

undata, Emmons, 1842, (Pterinea undata,) Geo. Rep. N. Y., p. 395, and Pal. N. Y., vol. 1, P. 165, Black Riv. and Trenton Grs. Possibly belonging to an undefined genus. large triangular cartilage pit beneath tbe beaks, and smaller pit just anterior. Type A. leidyi.

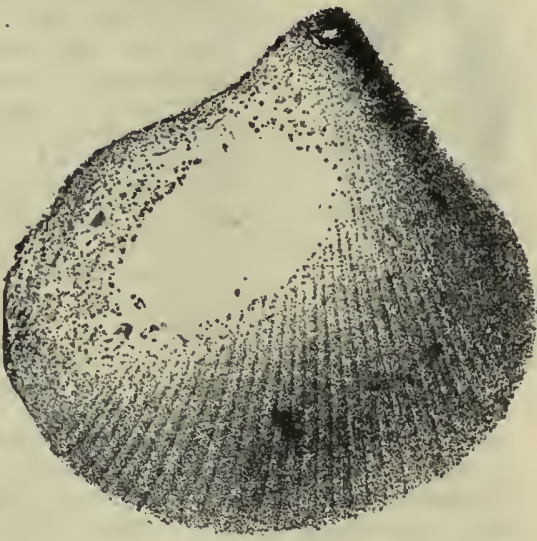

F1G. 773.-Amphicelia custata.

costata, Hall \& Whitfield, 1875, Ohio Pal., vol. 2, p. 140, Niagara Gr.

leidyi, Hall, 1867,20 th Rep. N. Y. Mus. Nat. Hist., p. 387, Niagara Gr.

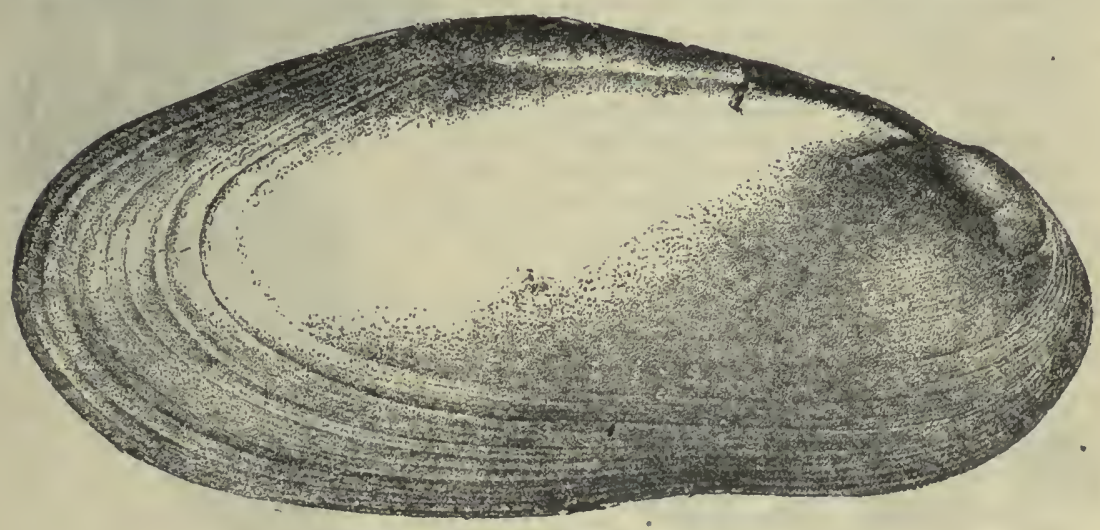

FIG. 772.-Amnigenía catskillensts.

Amigenia, Hall, 1883, Pal. N. Y., vol. 5. [Ety. amnis, a river; gigno, to bear.] Like Anodonta in form and external - characters; anterior muscular impressions large and prominent; posterior ones large and shallow. Type A. catskillensis.

catskillensis, Vanuxem, 1812, (Cypricardites catskillensis,) Geo. Rep. 3d Dist. N. Y., p. 186, and Pal. N. Y., vol. 5, p. 516, Catskill Gr.

Amphicalia, Hall, 1868, 20th Rep. N. Y. Mus. Nat. Hist.. p. 386. [Ety. amphi, both; koilos, hollow.] Equivalve, inequilateral, subrhomboidal; umbones gibbous; beaks elevated and incurved ; external ligamental area flattened; neglecta, McChesney. 1861, (Ambonychia neglecta,) Pal. Foss., p. 88, and Geo. Sur. Ill., vol. 3, p. 358, Niagara $\mathrm{Gr}$.

Amphidesma delnfieldi, Castelnau, 1843, Syst. Sil., p. 44. Not recognized.

Anatina, Lamarck, 1809, Phil. Zool. [F.ty. pertaining to the duck, or like the duck's bill.] Oblong, ventricose, attenuated, and gaping posteriorly: umbones fissured; spoon-shaped cardinal process in each valve. Type $A$. rostrata. Not a Palæozoic genus.

leda, Hall, 1860, 13th Rep. N. Y. Mus. Nat. Hist., p. 110, Ham. (Xr. Not properly defined.

sinuata, see Ilionia sinuata. 
Anaellum, S. A. Miller, 1878, Jour. Cin. Soc. Nat. Hist., vol. 1, p. 105. [Ety.

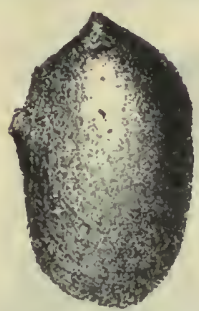

FIG. 774,-Angelium cunealum. aggos, a pail ; ellus, diminutive.] Equivalve, $h$ a n ging dow $n$; umbones prominent; beaks incurved, winged posteriorly; con centrically lined. Type A. cuneatum. cuneatum, S. A. Miller, 1875 , Jour. Cin. Soc. Nut. Hist. vol. 1, p. 106, Hud. Riv. Gr.

A nodontopsis, McCoy, 1851, Ann. and Mag. Nat. Hist., 2 d series, vol. 7, p. 54 . [Ety. from the resemblance to the shells of the genus Anodonta.] Equivalve, inequilateral, compressed; rot undatoquadrate or subtrigonal; posterior side wide, round, or obliquely subtruncate; anterior end slightly contracted in front of the beak; beaks small, prominent nearer the anterior than posterior end; hinge-line shorter than the length of the shell, with a posterior long, slender, lateral tooth extending just below it (double in the right valve), and another similar but shorter one in front of the beaks; anterior and posterior muscular impressions ovate; slight clavicular ridge between the beak and the adductor impressions; pallial impression entire; surface smooth or concentrically lined. Type A.angustifrons. Part of the generic definition is from A. milleri, as the interior of the type is not known.

amygdaliformis, Walcott, .1885, Monogr. U.S. Geo. Sur., vol. 8, p. 180, Devonian. concinna, Whiteaves, 1884, Pal. Foss., vol. 3, p. 12, Guelph Gr.
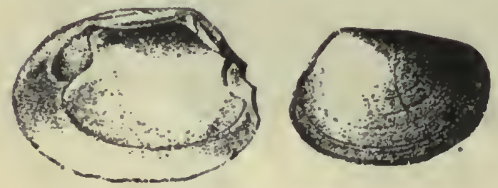

FiG. 775.-Anodontopsis milleri.

(?) milleri, Meek, 1871, Am. Jour. Sci., 3d series, vol. 2 , p. 297, and Ohio Pal., vol. 1, p. 140, Hud. Riv. Gr.

unionoides, see Modiolopsis unionoides.

ventricosa, Billings, 1874, Pal. Foss., vol. 2, p. 55, Gaspe limestone No. 8, Devonian.

Anomalodonta, S. A. Miller, 1874, Cin. Quar. Jour. Sci., vol. 1, p. 16. [Ety. anomalos, irregular; odous, tooth.] Equivalve, inequilateral, alate posteriorly, - abrupt anteriorly; umbones high; beak incurved; deeply sinuate for the byssus; cardinal ridge beneath the umbone sloping posteriorly; cartilage grooves extending from the cardinal ridge to the termination of the posterior wing, and also from the cardinal

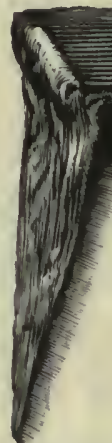

F1g. 7\%6.--A nomalodon ta gigantea. Hinge-ine of right valve.

ridge to the byssal sinus; anterior muscular scar below the byssal sinus; surface radiately furrowed and concentrically lined. Type A. gigantea.

alata, Meek, 1872, (Ambonychia alata,) Proc. Acad. Nat. Sci. Phil., p. 319, and Ohio Pal., vol. 1, p. 131, Hud. Riv. Gr.

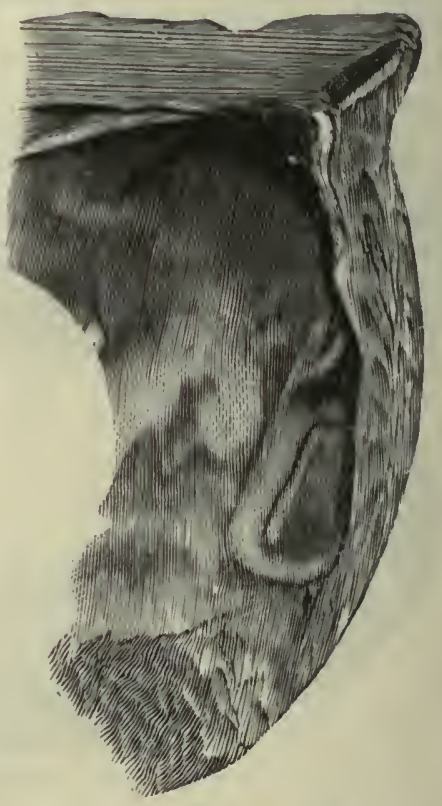

HIG. 7\%.-Anomalodonta gigantea. Left vaive, showing hinge-liue and muscular impression.

gigantea, S. A. Miller, 1874, Uin. Quar. Jour. Sci., vol. 1, p. 17, Hud. Riv. Gr.

Anthracomya, Salter, 1861, Mem. Geo. Sur. Gr. Brit. Iron Ores, pt. 3, p. 229. [Ety. anthrax, coal ; Mya, a genus.] Equivalve, inequilateral, mytiliform; ligament external; beak anterior; hingeline straight; no teeth; surface concentrically marked; shell composed of an internal, lamellar, and subnacreous layer, a thin layer of vertical prismatic shell, and an epidermis; structure similar to the Unionidx. Type A. elongata. 
angulata, Dawson, 1860, (Naiadites angulatus,) Acadian Geology, p. 205, Coal Meas.

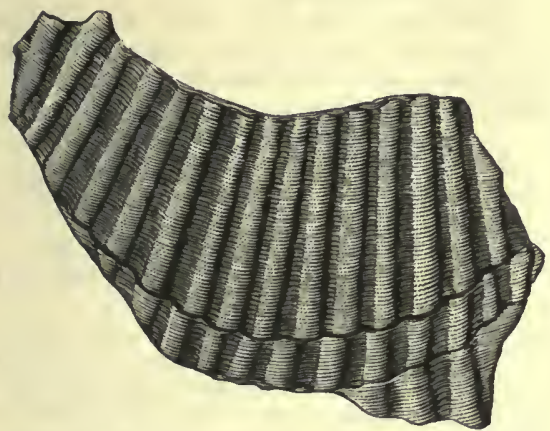

FIG. 778.-Anomalodonta gigantea, external surface.

arenacea, Dawson, 1860, (Naiadites arenaceus,) Acadian Geology, p. 205, Coal Meas.

carbonaria, Dawson, 1860, (Naiadites carbonarius,) Acadian Geology, p. 204, Coal Meas.

elongata, Dawson, 1860, (Naiadites elon-

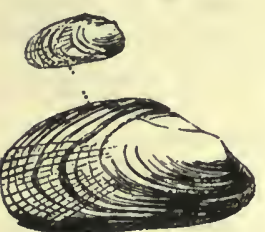

FIG. 779. - Anthracomya elongata. gatus,) Acadian Geology, p. 204, Coal Meas.

lavis, Dawson, 1860 , (Naiadites lævis,) Acadian Geology, p. 204, Coal Meas.

o btusa, Dawson, 1860, (Naiadites obtusus,) A cadian Geology, p. 205, Coal Meas.

ovalis, Dawson, 1860, (Naiadites ovalis,) Acadian Geology, p. 205, Coal Meas.

Anthracoptera, Salter, 1862, Mem. Geo. Sur. Country Around Wigan, p. 37. [Fty. anthrax, coal ; pteron, a wing.] Shells small, aviculoid; height greater than width; valves subequal, wing short, hinge straight; surface concentrically marked.

carbonaria, see Anthracomya carbonaria.

(?) fragilis, Meek \& Worthen, 1866 , Proc. Chi. Acad. Sci., p. 18, Keokuk Gr.

lavis, see Anthracomya lævis.

polita, White, 1880, 12th Rep. U. S. Geo. Sur. Terr., p. 166, Coal Meas.

Anthracosia, King, 1844, Ann. and Mag. Nat. Hist., p. 313. [Ety. anthrax, coal.] Equivalve, inequilateral; tooth in each valve below the umbo; crown of tooth of right valve excavated anteriorly and ridged posteriorly; crown of tooth of left valve ridged anteriorly and sloped posteriorly; furrow in hinge-plate, between umbone and tooth; scars of anterior pedal muscles above the anterior adductor impressions. Type A. beanana. bradorica, Dawson, 1868, Acad. Geol., p. 314, Carb.

Arca, Linne, 1758. This genus is unknown in the Palæozoic rocks. carbonaria, Cox. See Macrodon carbonarius.

cuspidata, Swallow, 1858, Trans. St. Louis Acad. Ści., vol. 1, p. 209, Up. Coal Meas. Founded on a cast. Genus unknown. modesta, Winchell, 1863, Proc. Acad. Nat. Sci., p. 15, Marshall Gr. Not an Arca.

striata, Schlotheim, as identified by Geinitz, is Macrodon tenuistriatus.

punctifera, Dawson, 1868, Acad Geol., Carb. The name was preoccupied by Deshayes in his work, 1824-1836.

Astarte, Sowerby, 1818, Min. Conch., vol. 2, p. 85. Not a Palæozoic genus. mortonensis, see Edmondia mortonensis. nebraskensis, see Edmondia nebraskensis. subtextilis, see Euthydesma subtextile.

Astartelua, Hall, 1858, Geo. Rep. Iowa, p. 715. [Ety. diminutive of Astarte.] Shell thick, smooth, or concentrically furrowed; lunule impressed, ligament external; hinge teeth, two in each valve; anterior tooth in right valve large and strong, with a longitudinal pit in the summit. Type $A$. vera.

concentrica, McChesney, 1860, (Edmondia concentrica,) Descr. New Pal. Fuss., p. 55, Coal Meas.

gurleyi, White, 1878, Proc. Acad. Nat. Sci., p. 35 , and Cont. to Pal., No. 8, p. 166, Coal Meas.

newberryi, Meek, 1875, Ohio Pal., vol. 2, p. 340, Coal Meas.

varica, McChesney, 1860, Descr. New Pal. Foss., p. 55, Coal Meas.

vera, Hall, 1858, Geo. Rep. Iowa, p. 715, Coal Meas.

Avicula, Klein, 1753, Ostrac. [Ety. avicula, a little bird.] Very inequivalve, inequilateral, obliquely oval; hinge produceả posteriorly into a flattened defined wing; the inferior or right valve flattened, notched for the passage of the byssus; anterior muscular impression very small and faintly marked; adductor large, superficial, a little behind the middle; cartilage external, linear, simple, placed on a narrow marginal facet, extending from the beak toward the cardinal angle; hinge edentulous, or with two small cardinal teeth beneath the beak in one valve, and one in the other, and a long, slender, posterior bifid lateral tooth in each; substance corneo-calcareous, lamellar without, pearly within. Type A. hirundo. Not a Palæuzoic genus. Species are left here for want of better material to determine their generic relations.

acanthoptera, Hall, 1843, Geo. Rep. 4th Dist. N. Y., p. 263, Chemung Gr. 
acosta, Cox. 1857, Geo. Sur. Ky., vol. 3, p. 572, Coal Meas. The correct etymology would make this word incosta. xquilatera, see A viculopecten æquilaterus. æquiradiata, Hall, 1859, Pal. N. Y., vol. 3, p. 285, Iow. Held. Gr.

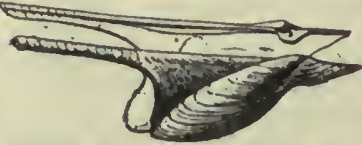

Fis. 781.-Avicula hirundo.

:esopus, Conrad, 1842, Jour. Acad. Nat Sci., vol. 8, p. 238, Ham. Gr.

angustirostra, Conrad, 1842, Jour. Acad.

Nat. Sci., p. 236, Ham. Gr.

untiqua, see Bakevellia antiqua.

arenaria. Not American.

aviformis, see Pterinea aviformis.

bella, see Aviculopecten bellus.

brllula, Hall, 1859, Pal. N. Y., vol. 3, p.

289, Low. Held. Gr.

boydi, see Actinopteria boydi.

cancellata, see Pterinea cancellata.

chtmungensis, see Liopteria chemungensis. cliemungensis, see Pterinea chemungensis. circulus, see Entolium circulus.

communis, Hall. 1859, Pal. N. Y., vol. 3, p. 286, Low. Held. Gr.

couperensis sec Pernopecten cooperensis. cormigala, see H'terinea corrugata.

cmuciformis, sce Glyptodesina cruciforme. damnoniensis, Sowerby; as identified in

the early N. Y. Reports. See Liopteria chemungensis.

decussata, see Actinopteria decussata.

demissa, see Pterinea demissa.

desquamata, Hall, 1847. The dorsal valve of Obolella crassa.

elliptica, see Pterinea elliptica.

emacerata, Conrad, 1842, Jour. Acad. Nat.

Sci., p. 241, and Pal. N. Y., vol. 2, p. 83

and 282 , Clinton and Niagara Grs.

erecta, see Glyptodesma erectum.

ferruginea, Conrad, 1848, l'roc. Acad. Nat.

Sci., vol. 3, p. 23 , Up. Sil.

flabella, see P'terinea flabellum.

fragilis, see Lunulicardium fragile.

gebhardi, Conrad, 1841, Ann. Rep. N. Y., p. 54, Oriskany sandstone.

hermione, Billings, 1862, Pal. Foss., vol. 1,

p. 40, Trenton Gr.

honeymani, see Pterinea honeymani.

insuctc, see Pterinea insueta.

loxvis, see Liopteria laevis.

leptonola, Hall, 1843, Geo. Rep. 4th Dist. N. Y., p. 76, syn. for A. emacerata.

limiformis, Hall, 1852, Pal. N. Y., vol. 2, p. 332, Coralline limestone.

longa, Geinitz, 1866, 'Gervillia longa,)

Carb. und Dyas in Neb.. p. 32, and Pal.

E. Neb.,p. 199, Coal Meas.

longispina, see Leptodesma longispinum. magna, Swallow, 1863, Trans. St. Louis Acad. Sci., voll. 2, p. 98, Kaskaskia Gr. manticula, Conrad, 1842, Jour. Acad. Nat. Sci., vol. 8 , p. 241, and Pal. N. Y., vol. 3, p. 284, Low. Held. Gr. morganensis, Meek \& Worthen, 1866 , (Pteria morganensis,) Proc. Acad. Nat. Sci., p. 259, and Geo. Sur. Ill., vol. 5,. p. 576, Coal Meas.

multilineata, Conrad, 1842, Jour. Acad. Nat. Sci., vol. 8, p. 24l, Chemung Gr. muricata, see Actinopteria muricata. naviformis, Conrad, 1842, Jour. Acad. Nat. Sci., p. 240, and Pul. N. Y., vol. 3, p. 279, Low. Held. Gr.

obliquata, Hall, 1859, Pal. N. Y., vol. 3, p. 285, Low. Held. Gr.

oblonga, see Aviculopecten oblongus.

obscura, Hall, 1859, Pal. N. Y., vol. 3, p. 280, Low. Held. Gr.

orbicularis, Stevens, 1858, Am. Jour. Sci., vol. 25, $2 \mathrm{~d}$ ser., Coal Meas. The name was preoccupied by Sowerby in 1839 .

orbiculata, Hall, 1843, see Lyriopecten orbiculatus.

orbiculata, Hall, 1852, Pal. N. Y., vol. 2, p. 284 , Niagara Gr.

parilis, see Aviculopecten parilis.

pauciradiata, Hall, 1859, Pul. N. Y., vol. 3, p. 287, Low. Held. Gr.

pectiniformis, see Aviculopecten pectiniformie.

perobliqua, see Actinopteria perobliqua. pinniformis, Geinitz, 1848, (Solon pinnæformis,) Versteinerungen d. dentsch Zechsteingelsirg, p. 8, and Carb. und Dyas. in Neb., p. 31, Coal Meas.

pleuroptera, see Actinopteria pleuroptera. protexla, see Leptodes'na protextum.

quadrula, syn. for Actinopteria boydi.

rectilaleraria, see Aviculopecten rectilaterarius.

recticosta, Hall, 1859, Pal. N. Y., vol. 3, p. 466, Oriskany sandstone.

rhomboidea, Hall, 1852, Pal N. Y., vol. 2, p. 84, Clinton Gr.

migosa, see Pterinea rugosa.

schoharize, Hall. 1859, I'al. N. Y., vol. 3, p. 283, Low. Held. Gr.

securiformis, Hall, 1852, Pal. N. Y., vol.

2, p. 331, Coralline limestone.

securiformis, Hall, 1859, Pal. N. Y., vol. 3,

p. 290. This name was preoccupied. semielliptica, Shumard, 1858, Trans. St.

Louis Acad. Sci., vol. 1, p. 210, Up. Coal Meas.

shawneensis, Shumard, 1858, Trans. St.

Louis Acad. Sci., vol. 1, 1. 211, Up. Coal. Meas.

shumardi, see Entolium shumardi. signata, sre Aviculopecten signatus. speciosa, see Panenka speciosa. spinigera, see Leptodesma spinigerum. spinulifera, Hall, 1859, Pal. N. Y., vol. 3, p. 282, Low. Held. Gr. subæquilatera, Hall, 1S59. Pal. N. Y., vol. 3 , p. 281, Low. Held. Gr. subfalcata, Conrad, 1842, Jour. Acad. Nat. Sci., vol. 8, p. 242, Ham. Gr. subplana, Hall, 1852, Pal. N. Y., vol. 2, p. 283, Niagara Gr.

subquadrans, Conrad, 1842, Jour. Acad.

Nat. Sci., vol. 8, p. 236, Devonian. subrecta, see A viculopecten subrectus. 
subrugosa, D'Orbigny, 1850, Prodr. d. Paleont, t. 1, p. 33. Syn. for Pterinea rugosa.

tenuilamellata, Hall, 1859, Pal. N. Y., vol. 3 , p. 281 , Low. Held. Gr.

textilis, Hall. 1859, Pal. N. Y., vol. 3, p. 288, Low. Held. Gr.

textilis var. arenaria, Hall, 1859, Pal. N. Y., vol. 3 , p. 465, Oriskany Gr.

trentonensis, see Pterinea trentonensis.

tricostata, see Lyriopecten tricostatus.

trilobata, Con rad, 1842, Jour. Acad. Nat.

Sci., vol. 8, p. 240 , Ham. 'Gr.

triplistriata, Stevens, 1858, Am. Jour. Sci., vol. 25, p. 265. Coal Meas.

triquetra, Hall, 1843, Geo. Rep. N. Y., p. 137, Onondaga Gr.

tuberculata, Conrad, 1838, Ann. Rep. N. Y., p. 117, Corniferous Gr.

umbonata, Hall, 1859, Pal. N. Y., vol. 3, p. 284 , Low. Held. Gr.

undata, Hall, 1852, Pal. N. Y., vol. 2, p. 283 , Niagara Gr.

undosa, Ringueberg, 1886, Bull. Buf. Soc. Nat. Sci., vol. 5, p. 18 , Niagara Gr.

welchi, James, 1874, Cin. Quar. Jour. Sci., vol. 1, p. 239, Hud. Riv. Gr.

whitii, Winchell, 1863, Proc. Acad. Nat. Sci., p. 8, Marshall Gr.

Aviculopecten, MeCoy, 1851, Ann. Mag. Nat. Hist., 2d ser., vol. 7, p. 171. [Ety. from the genera Avicula and Pecten.] Inequivalve, inequilateral; straight or slightly extended obliquely toward the posterior side; anterior ear flattened, smaller than the posterior, sharply and deeply defined, with a notch in the right valve between it and the body of the shell for the passage of the byssus; posterior ear pointed, extending about as far as the margin of the shell, defined or not; ligament confined to a narrow facet along the hinge margin, or having a wider cardinal area with cartilage furrows; no medial cartilage pit; muscular impression and pallial scar as in Pecten. Type A. docens.

acadicus, Hartt, 1868, Ac'ad. Geol., p. 307, Carb.

acutialatus, Swallow, 1858, (Avicula acutialata,) Trans. St. Louis Acad. Sci., p. 185, Permian Gr.

æquilateralis, Hall, 1883, Pal. N. Y., vol. 5 , pt. 1, p. 19 , Chemung Gr.

æquilaterus, Hall, 1843. (A vicula æquilatera,) Geo. Rep. 4th Dist. N. Y., p. 181, Up. Held. Gr. and Marcellus Shale.

affinis, Walcott, 1885, Monogr. U. S. Geo. Sur., vol. 8, p. 229, Subcarboniferous.

amplus. Meek \& Wortlien, 1860, Proc. Acad. Nat. Sci. Phil., p. 454, and Geo. 'Sur. Ill., vol. 2, p. 257, Keokuk Gr.

armigerus, Con rad, 1855, (Pecten armizerus,) Trans. Geo. Soc. Penn., p. 268, Coal Meas.

bellus, Conrad, 1841, (Avicula hella,) Ann. Rep. N. Y., p. 54, and Pal. N. Y., vol. 5, pt. 1, p. 35, Ham. Gr. burlingtonensis, Meek \& Worthen 1860, Proc. Acad. Nat. Sci., p.'453, and Geo. Sur. IIl., vol. 2, p. 231, Burlington Gr. cancellatus, Hall, 1843, (Pecten cancellatus, ) Geo. Rep. 4th Dist. N. Y., p. 264, and Pal. N. Y., vol. 5, pt. 1, p. 18, Chemung Gr.

carboniferus, Stevens, 1858, (Pecten carboniferus,) Am. Jour. Sci. and Arts, vol. 25 , p. 261, and Pal. E. Neb., p. 193, Coal Meas.

caroli, Winchell, 1863, Proc. Acad. Nat. Sci., p. 9, and Pal. N. Y., vol. 5, pt. 1, p. 29, Waverly Gr.

catactus, Meek, 1877, U. S. Geo. Expl. 40th parallel, vol. 4 , p. 93, Carboniferous.

celsus, Hall, 1883, Pal. N. Y., vol. 5, pt. 1, p. 23, Chemung Gr.

chesterensis, Worthen, 1884, Bull. No. 2, Ill. St. Mus. Nat. Hist., p. 20, and Geo. Sur. Ill., vol. 8, p. 115, Kaskaskia Gr.

cleon, Hall, 1883, Pal. N. Y., vol. 5, pt. 1, p. 6, Up. Held. Gr.

clevelandicus, Swallow, 1858, (Pecten clevelandicus,) Trans. St. Louis Acad. Sci., vol. 1, p. 184, Permian Gr.

colletti, Worthen, 1884, Bull. No. 2, Ill. St. Mus. Nat. Hist., p. 21, and Geo.Sur. Ill., vol. 8, p. 119, Keokuk Gr.

coloradoensis, New berry, 1861, Ives' Col. Ex. Exped., p. 129, Coal Meas.

convexus, Hall, 1813, (Pecten convexus, Geo. Rep. 4th Dist. N. Y., p. 265, and Pal. N. Y., vol. 5, pt. 1, p. 28, Chemung Gr.

cora, Dawson, 1868, Acad. Geol., p. 307, Carb.

coreyanus, White, 1874, Rep. Invert. Foss., p. 21, and Geo.'Sur. W. 100th Mer., vol. 4, p. 147, Coal Meas.

coxanus, Meek \& Worthen, 1860, Proc. Acad. Nat. Sci. Phil., p. 453, and Geo. Sur. Ill., vol. 2, p. 326, Low. Coal Meas.

crassicostatus, Hall \& Whitfield, 1872, 24th Rep. N. Y. Mus. Nat. Hist., p. 188, Up. Held. Gr.

crenistriatus, Meek, 1871, Proc. Acad. Nat. Sci. Phil., p. 60, and Ohio Pal., vol. 2 , p. 295, Waverly Gr.

curticardinalis, Hall \& Whitfield, 1877, U. S. Geo. Expl. 40th parallel, vol. 4, p. 273 , Coal Meas.

debertanus, Dawson, 1868, Acad. Geol., p. 307, Carboniferous.

dolabriformis, Hall, 1843, (Pecten (?) dolabriformis,) Geo. Rep. 4th Dist. N. Y., p. 265, and Pal. N. Y., vol. 5, pt. 1, p. 26, Chemung Gir.

duplicatus, Hall, 1843, (Pecten duplicatus,) Geo. Rep. 4th Dist. N. Y., p. $26 t$, and Pal. N. Y., vol. 5, pt. 1, p. 17, Chemung Gr.

edwardsi, Worthen, 1884, Bull. No. 2, Ill. St. Mus. Nat. Hist., p. 22, and Geo. Sur. Ill., vol. 8, p. 119, Keokuk Gr.

ellipticus, Hall, 1883, Pal. N. Y., vol. 5, pt. 1, p. 25, Chemung Gr. 
elsahensis, Worthen, 1884, Bull. No. 2, Ill. St. Mus. Nat. Hist., p. 19, and Geo. Sur. Ill., vol. 8, p. 115, Kinderhook Gr. eurekensis, Walcott, 1885, Monogr. U. S. Geo. Sur.; vol. 8, p. 227, Subcarboniferous.

exacutus, Hall, 1883, Pal. N. Y., vol. 5, pt. 1, p. 8, Ham. Gr.

fasciculatus, Hall, 1883, Pal. N. Y., vol. 5, pt. 1, p. 11, Ham. Gr.

formio, Hall, 1883, Pal. N. Y., vol. 5, pt. 1, p. 9, Ham. Gr.

glaber, see Pernopecten glaber.

gradocostatus, White, 1862, Proc. Bost. Soc. Nat. Hist., vol. 9, p. 31, Marshall Gr.

haguei, Walcott, 1885, Monogr. U.S. Geo. Sur., vol. 8, p. 226, Subcarboniferous.

halli, Swallow, 1860, (A vicula halli,) Trans. St. Louis Acad. Sci., vol. 1, p. 656, Coal Meas.

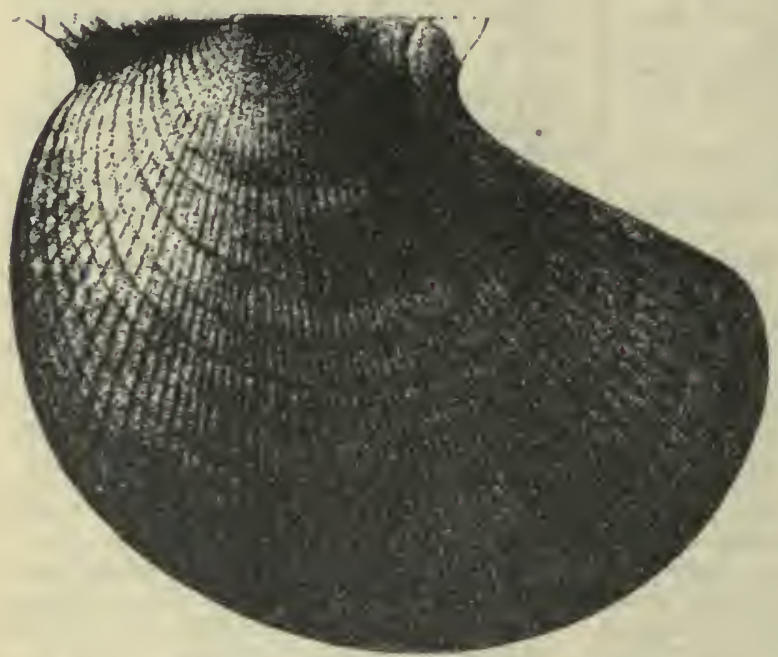

Fig. 782.-Aviculopecten princeps.

hardinensis, Worthen, (in press,) Geo. Sur. Ill., vol. 8, p. 117, St. Lnuis Gr.

hertzeri, Meek, 1871, Proc. Acad. Nat. Sci., p. 61, and Ohio Pal., vol 2, p. 330, Coal Meas.

idas, Hall, 1883, Pal. N. Y., vol. 5, pt. 1, p. 13, Ham. Gr.

ignotus, Hall, 1883, Pal. N. Y., vol. 5, pt. 1, p. 33, Up. Held. Gr.

incultus, Hall. 1883, Pal. N. Y., vol. 5, pt. 1, p. 30, Up. Chemung Gr.

indianensis, Meek \& Wortlien, 1866, Proc.

Chi. Acad. Sci., vol. 1, p. 14, Keokuk Gr.

insignis, Hall, 1883, Pal. N. Y., vol. 5, pt. 1, p. 34, Ham. Gr.

intercostalis, Winchell, 1866, Rep. Low. Peninsula Mich., p. 95, Ham. Gr.

interlineatus, Meek \& Worthen, 1860 , Proc. Acad. Nat. Sci. Phil., p. 454, and Geo. Sur. Ill., vol. 2, p. 329, Low. Coal Meas. invalidus, Hall, 1883, (Pterinopecten invalidus,) Pal. N. Y., vol. 5, pt. 1, p. 31 , Marcellus Shale.

jowensis, S. A. Miller, 1883, 2d Ed. Am. Pal. Foss., p. 310, Marshall or Kinderhook Gr., at Burlington, Iowa. Proposed instead of A. occidentalis of Winchell, in 1863, in Proc. Acad. Nat. Sci. Phil., p. 9, which was preoccupied by Shumard.

itys, Hall, 1883, Pal. N. Y., vol. 5, pt. 1, p. 20, Chemung Gr.

konincki, Meck \& Worthen, 1860, Proc. Acad. Nat. Sei. Phil., p. 453, and Geo. Sur. Ill., vol. 2, p. 328, Low. Coal Meas. lantus, Ilall, 1883, Pal. N. Y., vol. 5, pt. 1, p. 14, Ham. Gr.

limaformis, see Pernopecten limiformis.

lyelli, Dawson, 1868, Acad. Geol., p. 305, Carb.

lyelli var. alternans, Dawson, 1883, Rep. on Redpath Mus., p. 12, Carboniferous.

maccoyi, Meek \& Hayden, 1865, Pal. Up. Mo., p. 50, Permiall Gr.

macwhorteri, Worthen, (in press,) Geo. Sur. Ill., vol. 8, p. 118, Kinderhook Gr. mazonensis, Worthen, (in press,) Geo. Sur. Ill., vol. 8 , p. 117, Coal Meas.

menardi, Worthen, (in press,) Geo. Sur. Ill., vol. 8 , p. 120 , Coal Meas.

missouriensis, Shumard, 1855, (Pecten missouriensis,) Geo. Rep. Mo., p. 207, St. Louis Gr.

monroensis, Worthen, 1884 , Bull. No. 2, 1ll. St. Mus. Nat. Hist., p. 21, and Geo. Sur. Ill., vol. 8 , p. 114 , St. Louis Gr.

mucronatus, IIall, 1883, Pal. N. Y., vol. 5, pt. 1, p. 38 , Ham. Gr.

newarkensis, IVinclıell, 1870, Notices and Desc. Foss. from Marshall Gr., Proc. Acad. Nat. Sci., p. 255, Marshall Gr.

niotensis, Worthen, 1884, Bull. No. 2. Ill. St. Mus. Nat. Hist., p. 19, and Geo. Sur. Ill., vol. 8, p. 113, Keokuk Gr.

nodocostatus, White \& Whitfield, 1862 , Proc. Bost. Soc. Nat. Hist., vol. 8, p. 296, Kinderbook Gr.

oblongus, Meek \& Worthen, 1860, (Avicula oblonga,) Proc. Acad. Nat. Sci. Phil., p. 454, and Geo. Sur. Ill., vol. 2, p. 258, Keokuk Gr.

occidaneus, Meek, 1877, U. S. Geo. Expl. 40 th parallel, vol. 4, p. 96, Carboniferous.

occidentalis, Shumard, 1855, (Pecten occidentalis, Geo. Rep. Mo., p. 207, Carboniferous and Permian.

occidentalis, Winchell, 1863, Proc. Acad. Nat. Sci., p. 9. This name was preoccupied. 
orbiculatus, see Lyriopecten orbiculatus. orestes, Worthen, 1884, Bull. No. 2, Ill. St. Mus. Nat. Hist., p. 18, and Geo. Sur. Ill., vol. 8, p. 112, Keokuk Gr. orestes, Hall, syn. for A. fasciculatus. ornatus, Hall, 1883, Pal. N. Y., vol. 5, pt. 1, p. 37 , Ham. Gr.

oweni, Meek \& Worthen, 1860, Proc. Acad. Nat. Sci. Phil., p. 452, and Geo. Sur. Ill., vol. 2, p. 256, Keokuk Gr.

parilis, Conrad, 1842, (Avicula parilis,) Jour. Acad. Nat. Sci. Phil., vol. 8, p. 239, and Ohio Pal., vol. 1, p. 197, Cornif. Gr. parvulus, Hall \& Whitfield, 1877, U. S. Geo. Expl. 40th parallel, vol. 4, p. 274, Coal Meas. plenus, Hall, 1883, Pal. N. Y., vol. 5, pt. 1 , p. 21, Chemung (Fr.

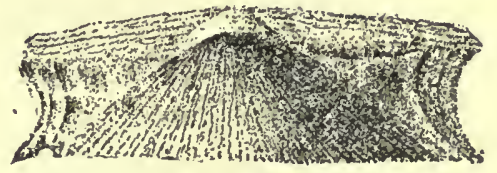

F'ı. 783.-Aviculopecten princeps. Cardinal part showing ligamental area.

princeps, Conrad, 1838, (Monotis princeps, Ann. Rep. N. Y., p. 117, and Pal. N. Y., vol. 5, pt. 1, p. 1, Ham. Gr.

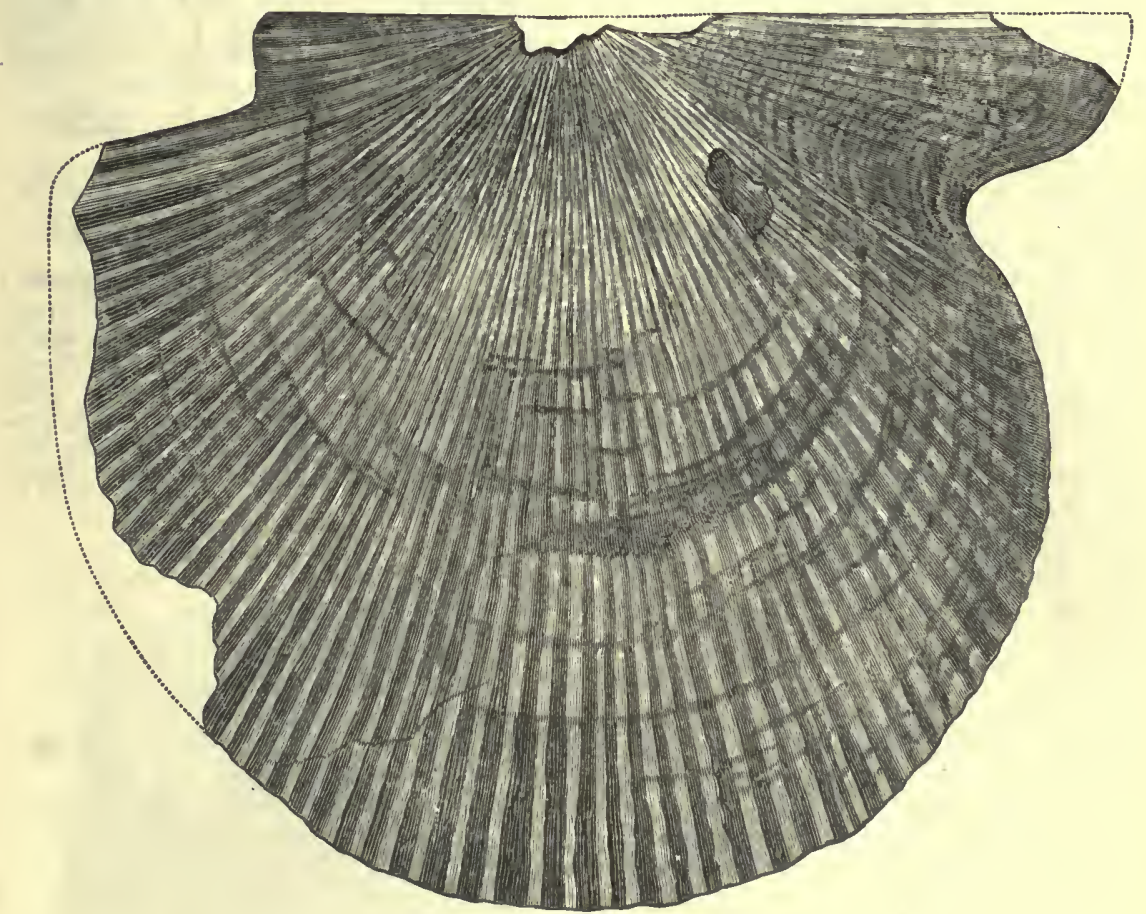

FIG. 784,-Aviculopccten varsoviensis.

patulus, Hall, 1883, Pal. N. Y., vol. 5, pt. 1, p. 24, Up. Chemung Gr.

pecteniformis, Conrad, 1842, (Avicula pecteniformis,) Jour. Acad. Nat. Sci., vol. 8, p. 240, and Pal. N. Y., vol. 5, pt. 1, p. 4, Up. Held. Gr. and Marcellus Shale.

pellucidus, Meek \& Worthen, 1860, Proc. Acad. Nat. Sci. Phil., p. 455, and Geo. Sur. Ill., vol. 2, p. 327, Low. Coal Meas. peroccidens, Walcott, 1885, Monogr. U. S. Geo. Sur., vol. 8, p. 227, Subcarboniferous.

phorcus, Hall, 1883, Pal. N. Y., vol. 5, pt. 1, p. 10, Ham. Gr.

pintoensis, Walcott, 1885, Monogr. U. S. Geo. Sur., vol. 8, p. 228, Subcarboniferous. providencensis, Cox, 1857, (Pecten providencensis,) Geo. Sur. Ky., vol. 3, p, 566, Coal Meas.

rectilaterarius, Cox, 1857, (Avicula rectilateraria,) Geo. Sur. Ky., vol. 3, p. ${ }^{\circ 571,}$ Coal Meas.

repletus, Hall, syn. for A. fasciculatus.

reticulatus, Dawson, 1868, Acad. Geol., p. 306, Carboniferous.

ringens, Swallow, 1858, (Pecten ringens,) Trans. St. Louis Acad. Sci., p. 184, Permian Gr.

rugistriatus, Hall, 1843, (Lima rugæstriata,) Geo. Rep. 4th Dist. N. Y., p. 264, and Pal. N. Y., vol. 5, pt. 1, p. 15, Chemung Gr.

sanduskiensis, Meek, 1871, Proc. Acad. Nat. Sci. Phil., p. 161, Up. Held. Gr. 
scabridus, Hall, 1883, Pal. N.Y., vol. 5, pt. 1, p. 7, Ham. Gr.

signatus, Hall, 1843, (Avicula signata,) Geo. Rep. 4th Dist. N. Y.,p. 265, and Pal. N. Y., vol. 5, pt. 1, p. 29, Chemung Gr. simplex, Dawson, 1868, Acad. Geol., p. 306, Carboniferous.

spinuliferus, Meek \& Worthen, 1870, Proc. Acad. Nat. Sci., p. 39, and Geo. Sur. Ill., vol. 8, p. 116, Keokuk Gr.

squama, Hall, 1883, Pal. N. Y., vol. 5, pt. 1, p. 27, Chemung Gr.

striatus, Hall, 1843, (Pecten striatus,) Geo. Rep. 4th Dist. N. Y., p. 264, and Pal. N. v., vol. 5, pt. 1, p. 22, Chemung Gr. subcancellatus, Hall, 1883, syn. for A. cancellatus.

subrectus, Hall, 1852, (Avicula subrecta,) Pal. N. Y., vol. 2, p. 331, Coralline limestone.

talboti, Wortlien, 1884, Bull. No. 2, Ill. St. Mus. Nat. Hist., p. 21, St. Louis Gr. tenuicostus, Winchell, 1863, Proc. Acad. Nat. Sci., p. 10, Marshall Gr.

tenuis, Hall, 1883, Pal. N. Y., vol. 5, pt. 1, p. 39, Up. Chemung aGr.

terminalis, Hall, 1883, (Pterinopecten terminalis,) Pal. N. Y., vol. 5, pt. 1, p. 32, Up. Held. Gr.

unionensis, Worthen, 1875, Geo. Sur. III., vol. 6 , p. 511, Corniferous Gr.

utahensis, Meek, 1860, (Pecten utahensis,) Proc. Acad. Nat. Sci., p. 310, Coal Meas. varsoviensis, Worthen, 1883, Geo. Sur. Ill., vol. 7, p. 321, Keokuk Gr.

weberensis, Hall \& Whitfield, 1877, U. S. Geo. Sur., 40th parallel, vol. 4, p. 273, Coal Meas.

whitii, Meek, 1872, Pal. E. Neb., p. 195, Coal Meas.

williamsi, Meek, 1871, Proc. Acad. Nat. Sci., p. 178, Choteau limestone.

winchelli, see Crenipecten Winchelli.

Aviculopinna, Meek, 1867, Am. Jour. Sci., vol. 44, 2d ser., p. 282 . [Ety. the genera Avicula and Pinna.] Compressed, slender, elongated, subtrigonal, or nearly in the form of a Pinna; beaks nearly obsolete, extremely oblique, and slightly behind the anterior extremity. Type A. americana.

americana, Meek, 1867, Am. Jour. Sci., vol. 44, 2 d ser., p. 282, and Pal. E. Neb., p. 197, Coal Meas.

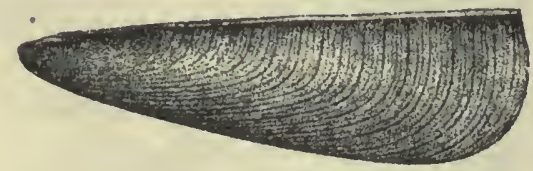

F19. 785.-Aviculopinna americana.

illinoisensis, Worthen, 1884, Bull. No. 2, Ill. St. Mus. Nat. Hist., p. 13, and Geo. Sur. Ill., vol. 8, p. 128, Coal Meas.

Axinus, Sowerby, 1821, Min. Conch., vol. 3. [Ety. axine, battle-axe.] This genus is unknown in Palæozoic rocks. ovatus, see Schizodus ovatus.

securis, Shumard, 1859, Trans. St. Louis Acad. Sci., Permian Gr. Not recognized. Bakevrlis, King, 1849, Perm. Foss., p. 166. [Ety. proper name.] Shell aviculiform, subequivalve; valves sinuous, gaping in front for the passage of the byssus; umbones depressed, oblique; surface with concentric strix; hinge with linear anterior and posterior lateral teeth parallel to the cardinal unargin; muscular scars as in Pteria; cardinal area in both valves; two to five cartilage furrows in each valve. Type B. antiqua.

antiqua, Munster, 1826, (Avicula antiqua,) Goldfuss Germ. Petref. Not American. illinoisensis, Worthen, 1884, Bull. No.2, Ill. St. Mus. Nat. Hist., p. 14, and Geo. Sur. Ill., vol. 8 , p. 126 , Up. Coal Meas. parva, Meek \& Hayden, 1858 , Trans. Alb. Inst., vol. 4, p. 78, and Pal. Up. Mo., p.57, Permian Gr.

(?)

(?) pulchra, Swallow, 1858, Bakevellia Trans. St. Louis Acad. Sci., vol. 1, p. 189, Permian Gr.

sulcata, Geinitz, 1866, (Gervillia sulcata,) Carb. und Dyas in Neb., p. 33, Coal Meas. Byssopteria, Hall, 1883, Pal. N. Y., vol. 5, pt. 1, p. 4. [Ety. bysso8, byssus; Pleria, a genus.] Shell erect, equivalve, alate posteriorly, truncate, with a nasute projection in front; surface radiately furrowed and concentrically lined. Type B. radiata.

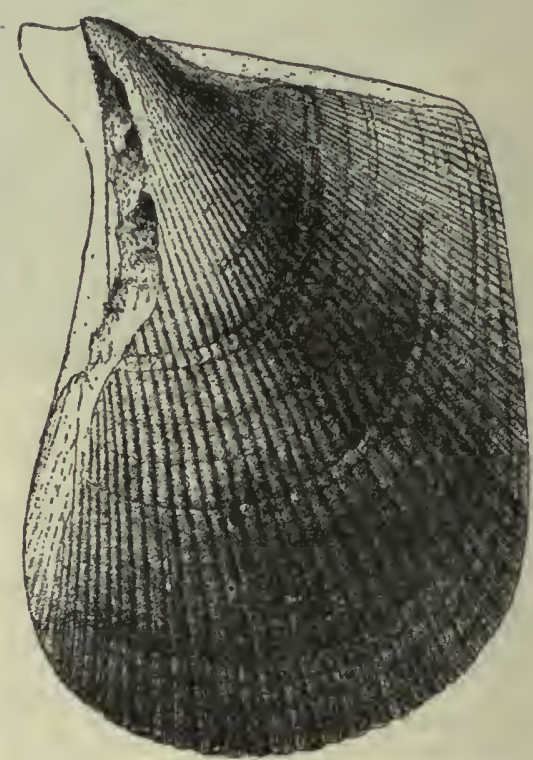

FIG. 787.-Byssopteria radiata.

radiata, Hall, 1883, Pal. N. Y., vol. 5, pt. 1, p. 252, Up. Chemung Gr.

Carbonarca, Meek \& Worthen, 1870, Proc. Acad. Nat. Sci. Phil., p. 39. [Ety. 
carbo, coal; Arca, a genus.] Inequivalve, inequilateral, very convex, transversely oblong or oval; umbones gibbous, prominent, strongly incurved, with subangular posterior slopes; valves closed all around with smooth margins; ligament external; cardinal margin arched; two anterior oblique teeth, and behind these minute crenulations, as in Arca. Type C. gibbosa. gibbosa, Meek \& Worthen, 1870, Proc. Acad. Nat. Sci. Phil., p. 40, and Geo. Sur. Ill., vol. 6, p. 531, Coal Meas.

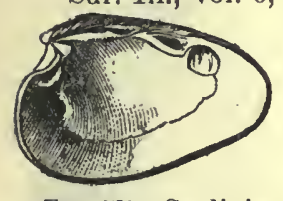

Frg. 788.-Cardinia listeri. Cardinia, Agassiz,

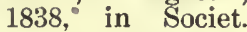
Basil. [Ety. cardo, the binge of a door.] Oblong, attenuated poste$\mathrm{r}$ i o r l y, compressed; ligament external ; cardinal teeth obscure, lateral, remote, prominent; adductor impressions deep; pallial line simple. Type C. listeri.

æquimarginalis, see Edmondia æquimarginalis.

antigonesensis, Dawson, 1868, Acad. Geo., p. 304, Carb.

complanata, Winchell, 1862, Proc. Acad. Nat. Sci., p. 413, Portage Gr.

concentrica, see Sanguinolites concentricus. cordata, Swallow, 1858, Trans. St. Louis Acad. Sci., vol. 1, p. 191, Permian Gr. (?) fragilis, Cox, 1857, Geo. Sur. Ky., vol. 3 , p. 570, Coal Meas.

occidentalis, Swallow, 1860, Trans. St. Louis Acad. Sci., p. 655, Waverly or Choteau Gr.

subangulata, Swallow, 1858, Trans. St. Louis Acad. Sci., vol. 1, p. 192, Permian Gr.

subangulata, Dawson, 1868, Acad. Geol., p. 304. This name was preoccupied.

Cardola, Broderip, 1844, Trans. Geo. Soc. [Ety. $k a r d i a$, the heart.] Obliquely oval or subcircular, tumid, equivalve, ine quilateral; beaks large, prom in e nt,

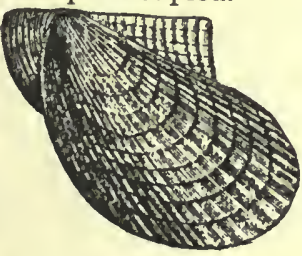

FIG. 789.-Cardiola interrupta. obliquely incurved anteriorly; ends subequal, rounded; ventral margin convex; hinge-line shorter than the shell, with a flattened cardinal area, widest between the beaks, extending its whole length; surface radiately ribbed. Type C. interrupta.

equilatera, see Panenka equilatera.

dichotoma, see Panenka dichotoma.

doris, see Paracardium doris.

elevata, see Panenka ventricosa.

erecta, see Pararca erecta.

filicostata, Walcott, 1885, Monogr. U.S. Geo. Sur., vol. 8, p. 251, Subcarboniferous. hero, see Panenka hero.

lincklæni, see Panenka lincklæni.

radians, see Panenka radians.

robusta, see Panenka robusta.

salteri, Haughton, 1857, Jour. Roy. Soc.

Dub., vol. 1, Devonian.

sao, see Pararca sao.

speciosa, Hall, 1883, Pal. N. Y.. vol. 5, pl. 70, fig. 2-9, and pl. 80, fig. 10, Genesee Slate.

transversa, see Pararca transversa.

Cardiomorpha, DeKoninck, 1844, Anim. Foss. Carb. Belg., p. 101. [Ety. kardia, heart; morphe, form.] Shell very thin, equivalve, inequilateral, margins closed, oblique, tumid ; beaks tumid, produced, spirally inrolled to the anterior side; no hinge teeth; hinge margin inflected nearly at right angles to form a hollow lunette, running from the beak nearly to the cardinal angle; two adductor impressions in each valve; pallial scar simple, very faintly marked; a shallow anterior depression beneath the beaks, but the margin sharp and prominent. Type C. elongata.

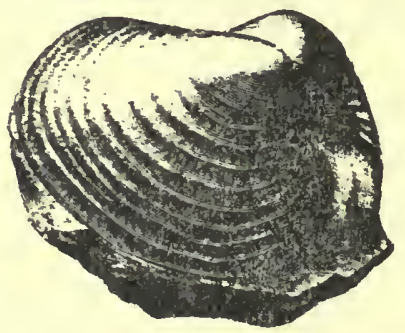

FrG. 790--Cardiomorpha cordata.

archiacana, DeKoninck, 1843, Desc. An. Foss. Belg., p. 104, Carboniferous.

bellatula, Hall, 1870, Prelim. Notice Lam. Shells, p. 92, and Pal. N. Y., vol. 5, pl. 63, figs. 1-3, Ham. Gr.

capuloides, Winchell, 1862, Proc. Acad. Nat. Sci., p. 416, Marshall Gr.

concentrica, Hall, 1883, Pal. N. Y., vol. 5, pl. 63, fig. 4, syn. for C. zonata.

cordata, Hall, 1883, Pal. N. Y., vol. 5, pl. 62, figs. 10-19, Ham. Gr.

donaciformis, Hall, 1883, Pal. N. Y., vol. 5, pl. 63, fig. 6, Ham. Gr.

eriopia, Hall, 1870, Prelim. Notice Lam. Shells, p. 92, and Pal. N. Y., vol. 5, pl. 63, figs..7-8, Ham. Gr.

julia, Winchell, 1862, Proc. Acad. Nat. Sci., p. 416, Marshall Gr.

kansasensis, Swallow, 1858, Trans. St. Louis Acad. Sci., vol. 1, p. 191, Permian Gr.

missouriensis, Shumard, 1858, Trans. St. Louis Acad. Sci., vol. 2, p. 207, and Geo. Sur. Ill., vol. 5, p. 588, Coal Meas.

modiolaris, Winchell, 1862, Proc. Acad. Nat. Sci., p. 416, Marshall Gr.

(?) obliquata, Meek, 1872, Proc. Acad. Nat. sci. Phil., p. 327, and Ohio Pal., vol. 1, p. 146, Hud. Riv. Gr. 
oblonga, see Protomya oblonga.

ovata, see Dexiobia ovata,

parvirostris, White, 1862, Proc. Bost. Soc.

Nat. Hist., vol. 8, p. 31, syn. for Dexiobia ovata.

pellensis, Worthen, 1884, Bull. No. 2, Ill. St. Mus. Nat. Hist., p. 16, and Geo.Sur. Ill., vol. 8, p. 126, St. Louis Gr.

radiata, see Cardiopsis radiata.

rhomboidea, Hall, see Cardiomorpha subrhomboidea.

rhomboidea, Swallow, 1858, Trans. St. Lonis Acad. Sci., vol. 1, p. 191, Permian $\mathrm{Gr}$.

rotunda, Hall, 1883, Pal. N. Y., vol. 5, pl. 63 , figs. $17-20$, refer figs. 18 and 19 to Paracyclas rotunda, fig. 17 to Schizodus degener, and fig. 20 to S. patulus.

subglobosa, Meek, 1875, Ohio Pal., vol. 2, p. 304, Waverly Gr.

suborbicularis, Hall, 1843, (Ungulina suborbicularis,) Geo. Rep. 4th Dist. N. Y., p. 244, and Pal. N. Y., vol. 5, pl. 63, figs. 9-10, Portage Gr.

subrhomboidea, Hall, 1877, 1st Ed. Am. Pal. Foss., p. 186, (proposed instead of Cypricardites rhomboidea, in Geo. Rep. Iowa, p. 523, which was preoccupied,) Kinderhook Gr.

textilis, Hall, 1883, Pal. N. Y., vol. 5, pl. 63, figs. 11-15, Chemung Gr.

triangulata, Swallow, 1860, Trans. St. Louia Acad. Sci., vol. 1, p. 655, Waverly or Choteau Gr.

trigonalis, Winchell, 1863, Proc. Acad. Nat. Sci., p. 15, Marshall Gr.

undulata, Hall, 1883, Pal. N. Y., vol. 5, pl. 63, fig. 16, Portage Gr.

(?) velusta, see Cypricardites vetustus.

vindobonensis, Hartt, 1868, Acad. Geol., p. 304, Carboniferous.

zonata, Hall, 1883, Pal. N. Y., vol. 5, pl. 63, fig. 5, Ham. Gr.

Cardiopsis, Meek \& Worthen, 1861, Proc. Acad. Nat. Sci. Phil., p. 144. [Ety. kardia, the heart; opsis, appearance.] Equivalve, somewhat inequilateral, oblique, ovate or cordiform, entirely closed; beaks elevated, incurved, directed anteriorly; cardinal margin short; rounding into the posterior border; two anterior teeth in each valve; surface radiated. Type C. radiata.

crassicostata, Hall \& Whitfield, 1873, 24th Rep. N. Y. St. Mus. Nat. Hist., p. 188, Schoharie grit and Corniferous limestone.

crenistriata, see Pterinea crenistriata.

jejuna, Winchell, 1862, Proc. Acad. Nat. Sci., p. 417, Marshall Gr.

megambonata, Winchell, 1862, Proc. Acad. Nat. Sci., p. 417, Marshall Gr.

parvirostris, White, 1862, Proc. Bost. Soc. Nat. Hist., vol. 9, p. 31, syn. for Dexiobia ovata.

radiata, Meek \& Worthen, 1860, (Cardiomorpha radiata,) Proc. Acad. Nat. Sci. Phil., p. 458, and Geo. Sur. Ill., vol. 2, p. 157, Kinderbook Gr.
Cardium, Linnæus, 1758, Syst. Nat., 10th Ed. [Ety. kardia, the heart.] Not a Palreozoic genus.

iou'ensis, see Cypricardites iowensis.

lexingtonensis, Swallow, 1858, Trans. St. Louis Acad. Sci., vol. 1, p. 207, Mid. Coal Meas.

nautiloides, Castelnal1, 1843, Syst. Sil. Seneca Lake, N. Y. Not recognized.

vetustum, see Prrecardium vetustum.

CH $\mathbb{N}$ N OCAR.

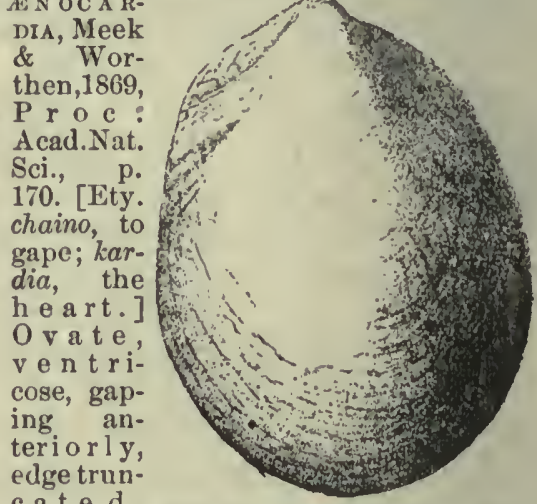

c a t e d,

h i n g e - Fig. 791.-Chæuocardia ovata.

line short, beaks small, incurved; surface concentrically marked. Type C. ovata.

ovata, Meek \& Worthen, 1869, Proc. Acad. Nat. Sci. Phil., p. 170, and Geo. Sur. Ill., vol. 5, p. 586, Coal Meas.

Curfenomya, Meek, 1864, Pal. of Up. Mo., p. 42. [Ety. chaino, to open or gape ; Mya, a genus of shells.] Shell thin, equivalve, longitudinally oblong, subcylindrical; anterior side rounded, closed; posterior side long, truncated, gaping at the extremity; surface granulose and concentrically marked; cardinal margin inflected as in Allorisina; ligament external; hinge edentulous; posterior muscular impressions near the posterior extremity of the dorsal margin; scars of the anterior adductor and pedal muscles connected; pallial line with a broad shallow sinus. Type C. leavenworthensis.

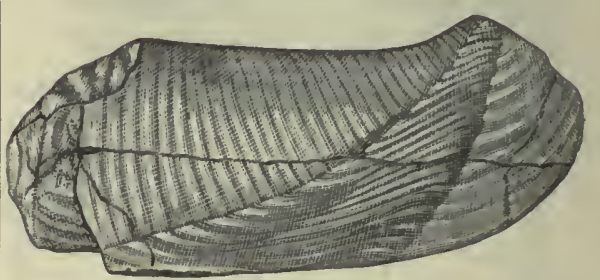

Fia. 792.-Chænomya marla. Right valve.

cooperi, Meek \& Hayden, 1858, (Panop;ea cooperi,) Trans. Alb. Inst., vol. 4 , p. 83 , and Pal. Up. Mo., p. 44, Coal Meas. hybrida, see Allorisma hybridum. 
leavenworthensis, Meek \& Hayden, 1858, (Allorisma leavenworthense,) Proc. Acad. Nat. Sci. Phil., p. 263, and Pal. Up. Mo., p. 43, Coal Meas.

maria, Worthen, 1882, Bull. No. 1. Ill. St. Mus. Nat. Hist., p. 39, and Geo. Sur. Ill., vol. 7, p. 319, Up. Coal Meas.

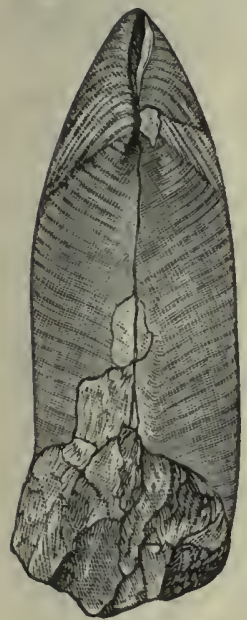

FIG. 793 -Chænomya view. maria. Dorsil

minnehaha, Swallow, 1858, (Allorisma (?) minnehaha,) Trans. St. Louis Acad. Sci., vol. 1, p. 194, and Geo. Sur. Ill., vol. 5, rhomboidea, Meek \& Worthen, 1865, Proc. Acad. Nat. Sci. Phil., p. 250, and Geo. Sur. Ill., vol. 5, p. 540, St. Louis Gr.

Cimitaria, Hall, 1870 , Prelim. Notice Lam. Shells, p. 66. [Ety. from resemblance to a cimiter.] Equivalve, transversely elongated; valves depressed, with an antero-mesial constriction; beaks incurved; cardinal line recurved; escutcheon and lunule; ligament external ; surface concentrically lined. Type C. recurva.

angulata, Hall, 1885, Pal. N. Y., vol. 5, p. 468, Chemung Gr.

corrugata, Conrad, 1842, (Cypricardites corrugatus,) Jour. Acad. Nat. Sci., vol. 8, p. 244, and Pal. N. Y., vol. 5, pl. 77, figs. 1-4, Ham. Gr. p. 588 , Coal Meas.

pression extending from the anterior cardinal margin toward the base, indicating the existence of a clavicle as in Solecurtus; surface concentrically lined. Type C. planulatus.

chicagoensis, S. A. Miller, 1880, Jour. Cin. Soc. Nat. Hist., vol. 3, p. 314, Niagara $\mathrm{Gr}$.

concentricus, Hall, 1860, Can. Nat. and Geo., vol. 5, p. 149, Low. Sil.

concentricus, Dawson, 1868. The name was preoccupied.

cuneatus, Hall, 1860, Can. Nat. and Geo., vol. 5, p. 148 , Low. Sil.

ellipticus, Ulrich, 1879, Jour. Cin. Soc. Nat. Hist., vol. 2, p. 25, Hud. Riv. Gr. elongatus, Hall, 1860, Can. Nat. and Geo., yol. 5, p. 150, Low. Sil.

erectus, Hall, 1860, Can. Nat. and Geo., vol. 5. p. 149, and Acad. Geol., p. 600, Up. Sil.

erectus, Dawson, 1868. The name was proccupied.

faberi, n. sp. Shell small, smooth, subelliptical in outline, length greater than height; anterior end narrower than the poste- Fig. 795.-Clidophorus farior; basal mar- beri. Mag. 5 diam. gin a semi-

elliptic curve; beaks prominent, and but little in advance of the middle; umbonal slope rounded, and tapering to the postero-basal margin of the shell; cardinal line gently curving, reaching the highest point posterior to the middle of the shell; pallial line simple and well defined; furrow deep, and extending from immediately in front of the beaks to the pallial line. Distinguished from C. fabula by the more prominent beaks and higher arch in the cardinal line posterior to the beaks and other minor particulars; beside it is generally a larger shell, though variable in size. Collected in the upper part of the Hud. Riv. Gr., near Versailles, Indiana, and in Butler County, Ohio.

fabula, Hall, 1845, (Nucula fabFIG. 794.-Cimitaria recurva.

elongata, Conrad, 1841, (Cypricardites elongatus,) Ann. Rep. N. Y., p. 51, and Pal. N. Y., vol. 5, pl. 77, figs. 5-8, Ham. Gr.

recurva, Conrad, 1842, (Cypricardites recurvis,) Jour. Acad. Nat. Sci., vol. 8, p. 245, and Pal. N. Y., vol. 5, pl. 77, figs. 9-16, Ham. Gr.

Clidophorus, Hall, 1847, Pal. N. Y., vol. 1, p. 300. [Ety. kleidos, a clavicle; phoros, bearing.] Equivalve, inequilateral; hinge without teeth or crenulations; cast marked by an oblique linear de- ula,) Am. Jour. Sci.

and Arts, vol. 48, p. 295, and Ohio Pal., vol. 1, p. 138, Hud. Riv. Gr.

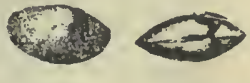
macchesneyanus, syn. fabula. Right side for Modiolopsis and dorsai view of a recta.

major, Ulrich, 1879 ,

Jour. Cin. Soc. Nat. Hist., vol. 2, p. 25, Hud. Riv. Gr.

neglectus, Hall, 1862, Geo. Rep. Wis., p. 55, Hud. Riv. Gr. 
nuculiformis, Hall, 1860, Can. Nat. and Geo., vol. 5, p. 150, Up. Sil.

planulatus, Conrad, 1841, (Nuculites planulatus,) Ann. Rep. N. Y., p. 48, and Pal. N. Y., vol. 1, p. 300, Hud. Riv. Gr.

semiradiatus, Hall, 1860, Can. Nat. and Geo., vol. 5, p. 150, Arisaig series of Up. Sil.

solenoides, see Solenopsis solenoides.

subovatus, IIall, 1860, Can. Nat. and Geo., vol. 5, p. 151, A risaig series of Up. Sil.

Clinopistra, Meek \& Worthen, 1870, Proc. Acad. Nat. Sci. Phil., p. 43. [Ety. klino, I lean ; opisthe, backward.] Shell short, gibbons, subquadrate, beaks posterior, and muscular impressions immediately belind the beaks; muscular impressions near the margins of the valves; ligament external. Type C. lævis.

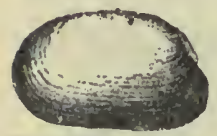

FIG. 797.-Clinopistha antiqua. insularis, Walcott, 1885, (Dystactella insularis,) Monogr. U. S. Geo. Sur., vol. 8 , p. 172, Devonian. antiqua, IIeek, 1871, Proc. Acad. Nat. Sci. Phil., p. 67, and Olio Pal., vol. 1, p. 208, Corniferous Gr.

lævis, Meek \& Worthen, 1870, Proc. Acad. Nat. Sci. Phil., p. 44, and Geo. Sur. Ill., vol. 5 , p. 584, Coal Meas.

radiata, Hall, 1858 , (Edmondia radiata,) Geo. Rep. Iowa, p. 716, Coal Meas.

subnasu ta, Hall \&Whitfield, 1872, (Tellinomya subnasuta,) 24 th Rep. N. Y. Mus. Fig. 798.-ClinopisNat. Hist., p. 192,

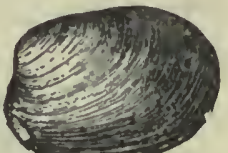

and Pal. X. Y., vol. 5, p. 512, Ham. Gr.

telliniformis, Hall, 1883. (Dystactella telliniformis, ) Pal. N. Y., vol. 5, p. 513, Up. Held. Gr.

Conocardium, Bronn, 1835, Leth. Geo., vol. 1, p. 92. [Ety. konos, a cone; kardia, the heart.] Equivalve, very inequilateral, hemifusiform; beaks prominent, incurved close to the anterior end, which is broad, flattened, more or less truncate nearly at right angles to the straight hinge-line, which is prolonged as an abruptly contracted, slender, tubular wing from the dorsal part of the anterior face; body of the shell diminishing conoidally from the edge of the anterior face toward the posterior end, which is attenuated, roundly and widely gaping; substance of the shell very thick, of a minute quadrangular cellular tissue, with strong internal ribs radiating from the beak, and often smaller external ones, strongest anteriorly. Type C. hibernicum.

acadianum, Hartt, 1868, Acad. Geol., p. 304, Carb. æquilaterale, Hall, 1858, Trans. Alb. Inst., vol. 4, p. 16, and Bull. Am. Mus. Nat. Hist., p. 62, Warsaw Gr.

altum, Keyes, 1888, Proc. Acad. Nat. Sci. Phil., pl. xii, figs. 4a, 4b, Ham. Gr.

antiquum, Owen, 1852, (Pleurorhynchus antiqua,) Geo. Wis., Iowa, and Minn., pl. 2, fig. 19, Siluriari.

attenuatum, Conrad, 1842, (Pleurorhynchus attenuatus,) Jour. Acad. Nat. Sci., vol. 8 , p. 252 , Up. Held. Gr.

bifarium, Winchell, 1856, Rep. Low. Peninsula Mich., p. 95, Ham. Gr.

blumenbachium, see Euchasma blumenbachi.

bovipedale, Winchell, 1862, Proc. Acad. Nat. Sci., p. 419, Marshall Gr.

carinatum, Hall, 1858, Trans. Alb. Inst., vol. 4, p. 14, and Bull. Am. Mus. Nat. Hist., p. 59, Warsaw Gr.

catastomum, Hall, 1858, Trans. Alb. Inst., vol. 4, p. 13, and Bull. Am. Mus. Nat. Hist., p. 58, Warsaw Gr.

concinnum, Hall, 1883, Pal. N. Y., vol. 5, pl. 68, figs. 26-27, Ham. Gr.

crassifrons, Conrad, 1842, (Pleurorhynchus crassifrons,) Jour. Acad. Nat. Sci., vol. 8, p. 252, Ham. Gr.

cuneatum, Hall, 1858, Trans. Alb. Inst., vol. 4, p. 14, and Bull. Am. Mus. Nat. Hist., p. 60, Warsaw Gr.

cuneus, Conrad, 1840, (Pleurorhynchus cuneus,) Ann. Rep. N. Y., p. 206, and Pal. N. Y., vol. 5, pl. 67, figs. 21-32, Up. Held. Gr.

denticulatun, Hall, 1883, Pal. N. Y., vol. 5 , pl. 68, figs. 24-25, Ham. Gr.

eboraceum, Hall, 1860, 13th Rep. N. Y. Mus. Nat. Hist., p. 91, and Pal. N. Y., vol. 5, p. 412 , Ham. fir.

elegantulum, Billings, 1866, Catal. Sil. Foss., Antic., p. 53, Anticosti Gr.

emmetense, Winchell, 1866, Rep. Low. Peninsula Mich., p. 95, Ham. Gr.

immaturum, Billings, 1862, Pal. Foss., vol. 1, p. 41, Black Riv. Gr.

inceptum, Hall, 1859, Pal. N. Y., vol. 3, p. 491, Low. Held. Gr.

liratum, Hall, 1883, Pal. N. Y., vol. 5, pl. 68, figs. 28-29, Chemung Gr.

meekanum, Hall, 1858, Trans. Alb. Inst., vol. 4, p. 15, and Bull. Am. Mus. Nat. Hist., p. 61, Warsaw Gr.

napoleonense, Winchell, 1862, Proc. Acad. Nat. Sci., p. 419, Marsball Gr.

nasutum, Hall, 1883, Pal. N. Y., vol. 5, pl. 67 , figs. 12-20, Schoharie grit.

nevadense, Walcott, 1885, Monogr. U. S. Geo. Sur., vol. 8, p. 177, Devonian.

niagarense, Winchell \& Marcy, 1865, Mem. Bost. Soc. Nat. Hist., p. 97, Niagara Gr.

normale, Hall, 1883, Pal. N. Y., vol. 5, pl. 68, figs. 17-19, Ham. Gr.

obliquum, Meek \& Worthen, 1865, Proc. Acad. Nat. Sci. Phil., p. 249, and Geo. Sur. Ill., vol. 6, p. 529, Coal Meas. 
ohioense, Meek, 1871, Proc. Acad. Nat. Sci. Phil., p. 65, and Ohio Pal, vol.

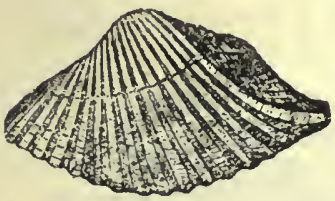
1, p. 203, Corniferous $\mathrm{Gr}$. ornatum, Winchell \& Marcy, 1865, Mem. Bost. Soc. Nat. Hist., p. 111, Niagara Gr. parrishi, Worthen, (in press,) Geo. Sur. Ill., vol. 8, p. 112, Up. Coal Meas.

IG. 799.-Conocardium subtrigon a le. Side view.

prattenanum, Hall, 1858, Trans. Alb. Inst., vol. 4, p. 15, and Bull. Am. Mus. Nat. Hist., p. 61, Warsaw Gr.

pulchellum, White \& Whitfield, 1862 , Proc. Bost. Soc. Nat. Hist., vol. 8, p. 299 , Kinderhook Gr.

reliquum, Hall, 1883 , Pal. N. Y., vol. 5, pl. 68, fig. 33, Chemung Gr.

rugosum, Hall, 1883,

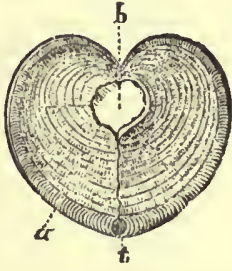

Pal. N. Y., vol. 5, pl. Frg. 800. - Conocar68, fig. 32 , Ham. Gr. dium subtrigonale. subtrigonale, D'Or bigny, 1850, Prodr. $b$, Broken end ; $t$, opening; $a$, con: nection of the alad. Paleont., t. 1, p. tions.

80, Up. Held. Gr. Proposed instead of C. trigonale, Hall, 1843, Geo. Rep. "4th Dist., N. Y., p. 171, which was preoccupied.

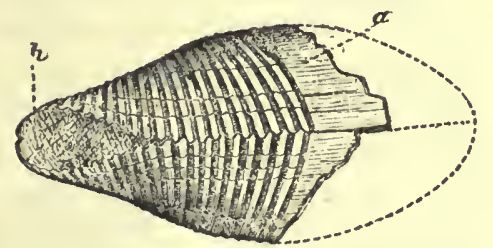

FIG. 801.-Conocardinm subtrigonale. $a$, Shows part of the alation; $h$, points to the posterior hiatus.

tegulum, Hall, 1883, Pal. N. Y., vol. 5, pl. 68, figs. 30-31, Niagara Gr.

trigonale, Phillips, 1836, (Pleurorhynchus trigonale,) Geol. Yorkshire, p. 211, Devonian.

trigonale, Hall, see C. subtrigonale.

ventricosum, Hall, 1860, 13th Rep. N. Y. Mus. Nat. Hist., p. 91, Ham. Gr.

vomer, Conrad, 1842, (Pleurorhynchus vomer, Jour. Acad. Nat. Sci., vol. 8. p. 253, Devonian.

Crenipecten, Hall, 1883, Pal. N. Y., vol. 5, p. 3. (Plates and Explanations.) [Ety. crena, notch ; Pecten, a genus.] In form like Aviculopecten, but the hinge is furnished with a series of small cartilage pits throughout its entire length. Type C. crenulatus.

amplus, Hall, 1883, Pal. N. Y., vol. 5, pt. 1 , p. 81 , Chemung Gr. crenulatus, Hall, 1843, (Pecten crenulatus,) Geo. Sur. 4th Dist. N. Y., p. 265, and Pal. N. Y., vol. 5 , pt. 1, p. 82 , Chemung Gr.

glaber, Hall, 1843, (Lima glabra,) Geo. Sur. 4th Dist. N. Y., p. 255, and Pal. N. Y., vol. 5 , pt. 1 , p. 85, Chemung Gr.

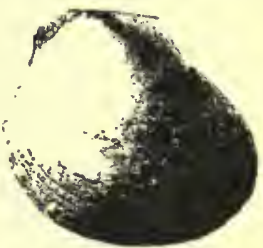

ballanus, Walcott, FIG. 802.-Crenipecten 1885, Monogr. U.S.

Geo. Sur., vol. 8, p. 231, Subcarboniferous.

impolitus. Hall, 1883, Pal. N. Y., vol. 5 pt. 1, p. 83 , Chemung Gr.

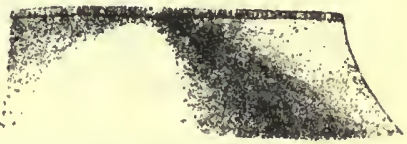

FIG. 803.-Creniptecten crenulatus. Hinge-line.

leon, Hall, 1883, Pal. N. Y., vol. 5, pt. 1, p. 88, Chemung Gr.

liratus. Hall, 1883, Pal. N. Y., vol. 5, pt. 1, p. 87 , Chemung Gr.

micropterus, Hall, 1883, Pal. N. Y., vol.5, pt. 1, p. 86, Chemung Gr.

obsoletus, Hall, 1843, (Limá obsoleta,) Geo. Sur. 4th Dist., N. Y., p. 265, and Pal. N. Y., vol. 5, pt. 1, p. 84, Chemung Gr.

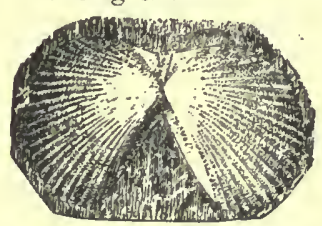

FIG. 804.-Crenipectell retiferus. retiferus, $\mathrm{Shu}$ mard, 1858 , (Lima ret if era,) Trans. St.Louis Acad. Sci., vol. 1, p. 214, and Geo. Sur. Ill., vol. 5 , p. 588, Coal Meas.

winchelli, Meek, 1875, (Aviculopecten winchelli,) Ohio Pal., vol. 2, p. 296, Waverly Gr.

Ctenodonta, Salter, 1851, syn. for Tellinomya.

abrupta, see Tellinomya abrupta. angela, see Tellinomya angela. astartiformis, see Tellinomya astartiformis. contracta, see Tellinomya contracta. gibberula, see Tellinomya gibberula. hartsvillensis, see Tellinomya hartsvillensis. hubbardi, syn. for Nuculites sulcatinus. iphigenia, see Tellinomya iphigenia. logani, see Tellinomya logani.

Cucullæa, Lamarck, 1801, Syst. An. [Ety. Cucullus, a hood.] Not a Palitozoic genus.

opima, Hall, 1843, syn. for Nucula lirata. Cuneamya, Hall \& Whitfield, 1875, Ohio Pal., vol. 2, p. 90. [Ety. cuneus, a wedge; $M y a$, a genus.] Shell large, equivalve, inequilateral ventricose; beaks prominent, incurved; cardinal line straight, ligament external; lunule and escutch- 
eon; pallial line simple. Type C. miamiensis.

coriformis, n. sp. Shell large, having a length in some specimens of three inches, and a height of two inches; larger at the anterior end, and cuneiformly tapering to the posterior point; be aks large, high, pointed and inrolled above the cardinal line; cardinal line straight from the top of the lunule three-fourths of the length of the shell, the posterior part forming a wing-like appendage of the shell; escutcheon dis-

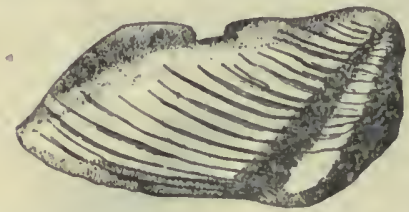

FIG. 805.-Cuneamya corlformls. Right valve, below medium size.

tinct and well marked; lınule heartshaped, very large, wide and deep, murgins angular; the anterior end of the shell rapidly slopes backward from the lower extremity of the lunule to the basal line; an obtuse angle is formed at line base of the lunule (this is better shown in the illustrutions by the figure of the right valve than by the anterior view); a cincture or furrow, arising at the point of the beaks, and very gradually widening, reaches the basal line anterior to the mirldle of the shell; anterior umbonal ridge very prominent; posterior umbonal slope promi-

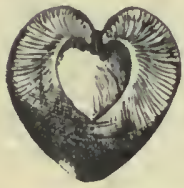

FIG. 806.-Cuneamya corlformis. Anterlor view, sliowing innule. Found in the Hudson River Group at Cincinnati, Ohio. The specific name is from the heart-shaped lunule. The specimen illustrated is from the collection of Charles Faber.

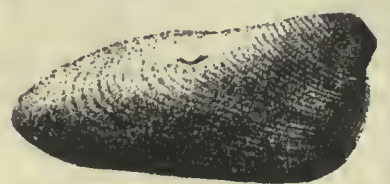

Fra. 807.-Cuneamya miamiensis. Right valve.

curta, Whitfield, 1878, Jour. Cin. Soc. Nat. Hist., vol. 1, p. 138, Hud. Riv. Gr. elliptica, S. A. Miller, 1881, Jour. Cin. Soc. Nat. Hist., vol. 4, p. 317 , Hud. Riv. Gr.

miamiensis, Hall \& Whitfield, 1875, Ohio Pal., vol. 2, p. 91, Hud. Riv. Gr.

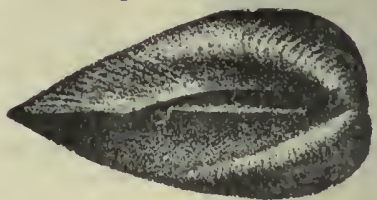

Fig. 808.-Cuneamya niamlensls. Dorsal vlew.

neglecta, Meek, 1871, (Sedgwickia neglecta,) Proc. Acad. Nat. Sci. Phil., p. 325 , and Ohio Pal., vol. 1, p. 142, Huil. Riv. Gr.

parva, S. A. Miller, 1880, Jour. Cin. Soc. Nat. Hist.. vol. 3, p. 316, Hud. Riv. Gr. scapha, Hall \& Whitfield, 1875, Ohio Pal. vol. 2, v. 92, Hud. Riv. Gr.

Crclocoscua, S. A. Miller, 1874, Cin. Quar. Jour. Sci., vol. 1, p. 231 . [Ety. in a]lusion to the nearly circular form of the shell.] Equivalve, subequilateral, subcircular, concentrically lined; cardinal teeth near the middle, with a long lateral tooth on each side. Type C. mediocardinalis.
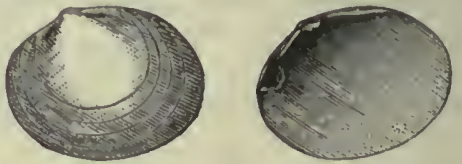

Fig. 809.-Cycloconcha medlocardinalts.

mediocardinalis, S. A. Miller, 1874, Cin. Quar. Jour. Sci., vol. 1, p. 231, Hud. Kiv. Gr.

Cypricardella, Hall, 1858, Trans. Alb. Inst., vol. 4, p. 17. [Ety. diminutive of Cypricardia.] Shell ovate, subelliptic a 1 or subquadrate, closed; surface concentric a lly striated; two

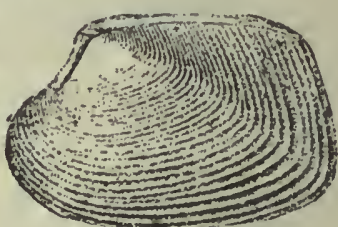

Frg. 810.-Cypricardella bellistriata. cardinal teeth in right valve, one beneath the beak, triangular, the posterior one more slender, and turned obliquely backward, leaving a triangular pit for the tooth from the other valve; long, nariow groove in the anterior cardinal margin apparently for a projection from the left valve; posterior side beveled from above, edge thin, ligament external, occupying a deep cavity; muscular impressions distinct, sha]low; pallial impression simple. Type C. subelliptica.

bellistriata, Conrad, 1842, (Microdon bellistriatus,) Jour. Acad. Nat. Sci., vol.8, p. 247, Ham. Gr. 
complanata, Hall, 1870, (Microdon complanatus,) Prelim. Notice Lam. Shells, p. 33, and Pal. N. Y., vol. 5, pl. 42, fig. 22 , and pl. 74, figs. 14 to 19 , Ham. Gr. connata, Walcott, 1885, Monogr. U. S. Geo. Sur., vol. 8, p. 250, Subcarboniferous. greguria, Hall, 1870, (Microdon gregarius,) Prelim. Not. Lam. Shells, p. 32 , and Pal. N. Y., vol. 5, pl.73, figs. 1-6, and pl. 74, figs. 1-4, Ham. Gr.

macrostriata, Walcott, 1885, Monogr. U. S., Geo. Sur., vol. 8, p. 180, Devonian.

major, Hall, 1885, Pal. N. Y., vol. 5, p. 307, Up. Held. Gr.

nucleata, Hall, 1858, Trans. Alb. Inst. vol. 4, p. 17, and Geo. Sur. Iowa, p. 663, Warsaw Gr.

oblonga, Hall, 1858, Trans. Alb. Inst., vol. 4, p. 18, and Bull. Am. Mus. Nat. Hist., p. 65, Warsaw Gr.

plicata, see Goniophora plicata.

quadrata, White \& Whitfield, 1862, Proc. Bost. Soc. Nat. Hist., vol. 8, p. 300, Kinderhook Gr.

reservata, Hall, 1870, (Microdon reservatus,) Prelim. Notice Lam. Shells, p. 33, and Pal. N. Y., vol. 5, pl. 74, figs. 11-13, Waverly Gr.

subelliptica, Hall, 1858, Trans. Alb. Inst. vol. 4, p. 17, and Geo. Sur. Iowa, p. 664 , Warsaw Gr.

tenuistriata, Hall, 1870, (Microdon tenuistriatus,) Prelim. Notice Lam. Shells, p. 32, and Pal. N. Y., vol. 5, pl. 73, figs. 23 to 30 , and pl. 74, figs. 20, 21, Ham. Gr.

Cypricardia, Lamarck, 1801, Syst. An. sans Vert. [Ety. from the two genera $C y$ prina and Cardium.] Oblong, oblique posterior ridge; umbones anterior, depressed; ligament external, in deep, narrow grooves; cardinal teeth two, lateral one, in each valve, sometimes obscure; muscular impressions two, oval, placed below the extreme anterior and posterior ends of the cardinal line; pallial line simple. Typical C. obesa.

angusta, see Cypricardites angustus.

angustata, Vanuxem, syn. for Amnigenia catskillensis.

choteauensis, Swallow. 1863, Trans. St. Louis Acad. Sci., vol. 2, p. 96, Waverly or Choteau Gr.

contracta, see Sphenotus contractus.

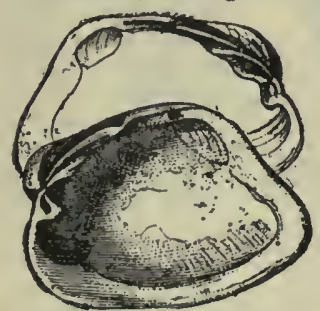

FrG. 811.-Cypricardia obesa. indianensis, see Cypricardinia indianensis.

insecta, Dawson, 1868, Acad. Geol., p. 303, Carbo $n$ if e rous.

leidyi, Lea, see Leaia, leidyi.

obsoleta, see Cypricardites obsoletus.

occidentalis, Hall, 1852, Stans. Ex. to

occidentalis, Swallow, 1863, Trans. St. Louis Acad. Sci. This name was preoccupied. See U. swallovana.

pikensis, Swallow, 1863, Trans. St. Louis Acad. Sci., vol. 2. p. 95, Coal Meas.

plicatula, Swallow, 1858, Trans. St. Louis Acad. Sci., vol. 4, p. 205. Mid. Coal Meas.

primigenia, see Modiolopsis primigenia.

randolphensis, see Sanguinolites randolphensis.

rhombea, see Cytherodon rhombeus.

rigida, see Sphenotus rigidus.

shumardana, Swallow, 1863, Trans. St. Louis Acad. Sci., vol. 2, p. 95, St. Genevieve limestone.

subplana, see Edmondia subplana.

swallovana, S. A. Miller, 1883, 2d Ed. Am. Pal. Foss., p. 310, Coal Measures of Harrison County, Missouri. Proposed instead of C. occidentalis, Swallow, 1863, in Trans. St. Louis Acad. Sci., p. 96 .

undulata, Gurley, 1883, New. Carb. Foss., p. 3, Coal Meas. Publication invalid.

ventricosa, Hall, 1860, 13th Rep. N. Y. Mus. Nat. Hist., p. 110, Kinderhook Gr. wheeleri, see Schizodus wheeleri.

Cypricardinia, Hall, 1859, Pal. N. Y., vol. 3, p. 266. [Ety. Cypricardinia, from its resemblance to Cypricardia.] General form of Cypricardia; inequilateral; oblique posterior ridge; umbones anterior, elevated ; concentrically grooved, sometimes cancellated; postero-cardinal margin sometimes alate. Type C. lamellosa.

arcuata, Hall, 1885, Pal. N. Y., vol. 5, p. 486, Chemung Gr.

arata, $\mathrm{Hall}$, 1867, 20th

Rep. N. Y.

Mus. Nat.

Hist., p. 385 ,

Niagara Gr.

car bon aria,

Meek, 1871, Fia. 812.-Cypricardinia disProc. Acad. tíncta. Left valve, long Nat.Sci.Phil., specimen.

p. 163, and Ohio Pal., vol. 2, p. 342, Coal Meas.

concentrica, Hall, 1859, Pal. N. Y., vol. 3, p. 268, Low. Held. Gr.

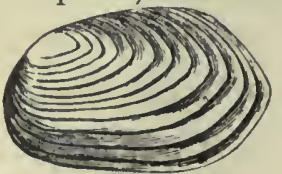

FIG. 813. - Cypricardinia distincta. Left valve, short speclineu. 24th Rep. N. Y. Mus. Nat. Hist., p. 190, Corniferous $\mathrm{Gr}$.

distincta, Billings, 1874, Pal. Foss., vol.2, p. 56, Gaspe limestone, No. 8, Devonian. \& Whitfield, 1872,

consimilis, $\mathrm{H}$ a 11 1885, Pal. N. Y.' vol. 5, p. 486 , Waverly Gr.

crassa, Hall, 1859 , Pal. N. Y., vol. 3, p. 268, Low. Held. Gr.

(?) cylindrica, Hall 
dorsata, Hall, 1859, Pal. N. Y., vol. 3, p. 267, Low. Held. Gr.

indenta, Conrad, 1842, (Cypricardites indentus,) Jour. Acad. Nat. Sci., vol. 8, p. 244, Up. Held. Gr.

indianensis, Hall, 1858, (Cypricardia indianensis,) Trans. Alb. Inst., vol. 4, p. 18, and Bull. Am. Mus. Nat. Hist., p. 58, Warsaw Gr.

inflata var. subrequivalvis, Hall \& Whitfield, 1872, 24th Rep. N. Y. Mus. Nat. Hist., p. 189, Up. Held. Gr.

lamellosa, Hall, 1859, Pal. N. Y., vol. 3, p. 266, Up. Held. Gr.

planulata, Conrad, 1842, (Pterinea planulata,) Jour. Acad. Nat. Sci., vol. 8, p. 251, and PalN.Y.,vol. 5, p. 484, Low. Held. Gr.

sublamellosa, Hall, 1859, Pal. N. Y., vol. 3 , p. 267, Low. Held. Gr.

subovata, Miller \& Dyer, 1878, Cont. to Pal. No. 2, p. 10, Niagara Gr.

sulcifera, Winchell, 1863, (Sanguinolites sulciferus,) Proc. Acad. Nat. Sci. Phil., p. 14, and Pal. N. Y., vol. 5, p. 487, Waverly Gr.

Crpricardites, Conrad, 1841, Ann. Geo. Rep. N. Y., p. 51. '[F.ty. from resemblance to the genus Cypricardia.] Equivalve, profoundly inequilateral; ex. ternal flattened ligamental area; hinge with four or five short oblique cardinal teeth; anterior one largest and most prominent; lateral teeth two, shortand remote from the cardinal teeth: two muscular scars; surface concentrically lined with marks of growth. Type C. curtus. If the genus can stand. it must be based on this type (all other species are referred to other genera), because this species alone has a hingeline like the one Conrad made.

acutumbonus, Billings, 1866, (Cyrtodonta acutumbona,) Catal. Sil. Foss. Antic., p. 49, Anticosti Gr.

alta, see Modiomorpha alta.

alveatus, Conrad, 1843, Geo. Rep. 3d Dist. N. Y., Ham. Gr.

amygdalinus, Hall, 1847, (Ambonychia amygdalina,) Pal. N. Y., vol. 1, p. 165, Black Riv. and Trenton Grs.

angustus, Hall, 1843, (Cypricardia angusta,) Geo. Rep. 4th Dist. N. Y., p. 76, Clinton Gr.

angustatus, syn. for Amnigenia catskillensis.

angustifrons, syn. for Modiolopsis modiolaris.

anodontoides, see Modiolopsis anodontoides.

anticostiensis, Billings, 1866, (Cyrtodonta (?) anticostiensis,) Catal. Sil. Foss. Antic., p. 14, Hud. Riv. Gr.

bisulcata, see Grammysia bisulcata.

breviusculus, Billings, 1859, (Cyrtodonta breviuscula,) Can. Nat. and Geo., vol, 4, p. 446, Chazy Gr.

canadensis, Billings, 1858, (Cyrtodonta canadensis,) Can. Nat. and Geo., vol. 3, p. 434, Black Riv. and Trenton Grs.

carinatus, see Gonjophora carinata.

carinatus, Meek. 1872, (Dolabra carinata,) Proc. Acad. Nat. Sci. Phil., p. 326, and Ohio Pal., vol. 1, p. 135, Hud. Kıv. Gr. This name was preoccupied.

cariniferus, Conrad, 1842, Jour. Acad. Nat. Sci., vol. 8 , p. 245, syn. for Goniophora chemungensis.

catskillensis, see A mnigenia catskillensis.

chemungensis, see Goniophora chemungensis.

concentrica, see Modiomorpha concentrica. cordiformis, Billings, 1858, (Cyrtodonta cordiformis,) Can. Nat. and Geo., vol. 3, p. 437, Black Riv. and Trenton Gre.

corrugatus, Conrad, 1842, Jour. Acad. Nat. Sci., vol. 8, p. 244, Ham. Gr.

curtus, Conrad, 1841, Ann. Rep. N. Y., p. 53, Hud. Riv. Gr.

elongatus, see Cimitaria elongata.

emma, Billings, 1862, (Cyrotodonta enima,) Pal. Foss., vol. 1, p. 150, Hud. Riv. Gr. ferrugineus, Hall \& Whitfield, 1875, Ohio Pal., vol. 2, p. 116, Clinton Gr.

ganti, Safford, 1869, (Cyrtodonta ganti,) Geo. of 'Tenn., p. 287, Trenton and Hud. Riv. Grs.

hainesi, S. A. Miller, 1874, Cin. Quar. Jour. Sci., vol. 1, p. 147, Hud. Kiv. Gr. harrietta, Billings, 1862, (Cyrtodonta harrietta,) Pal. Foss., vol. 1, p. 149, Hud. Riv. Gr.

haynanus, Safford, 1869, (Cyrtodonta hayniana, Geo. of 'Tenn., p. 287, Trenton and Hud. Riv. Grs.

hindi, Billings, 1862, (Cyrtodonta hindi, )

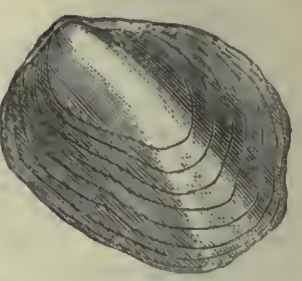

Pal. Foss., vol. 1, Fis. 814. - Cy pricardites p. 151, Hud. hainesi. Left valve. Riv. Gr.

huronensis, Billings, 1858, (Cyrtodonta huronensis,) Can. Nat. and Geo., vol. 3, p. 432, Blarkk Riv. and Trenton Grs. indentus, see Cypricardinia indenta.

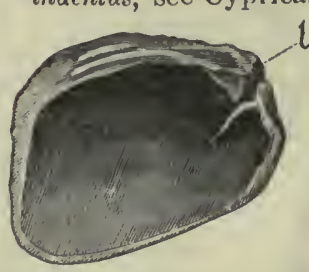

FIG. 815. - Cypricardites hainesi. Interior of left valve, two teeth injured at $t$.

insularis, Billings, 1866, (Cyrtodonta in-
sularis,) Catal. Sil. Foss. Antic., p. 14, Hud. Riv. Gr.

islandicus, Hall, 1877, 1st Ed. Am. Pal. Foss., p. 189. Proposed instead of Cypricardites, ventricosus, Hall, 1859, which was preoccupied, Low. Held. Gr. 
iowensis, Owen, 1840, (Cardium iowense,) Rep. on Mineral lands, pl. 17, fig. 8, Calciferous Gr.

latus, Hall, 1847, (Modiolopsis latus,) Pal. N. Y., vol. 1, p. 160, Trenton Gr.

lencothea, Billings, 1862, (Cyrtodonta leucothea,) Pal. Foss., vol. 1, p. 46, Black Riv. Gr.

marcellensis, see Lunulicardium marcellense.

megambonus, Whitfield, 1878, Ann. Rep. Geo. Sur. Wis., p. 73, and Geo. Wis., vol. 4, p. 210, Trenton Gr.

modiolaris, Emmons, syn. for Modiolopsis nasuta.

mytiloides, see Modiomorpha mytiloides.

nnsutus, see Modiolopsis nasuta.

niota, Hall, 1861, Geo. Rep. Wis., p. 29, and Geo. Wis., vol. 4, p. 208, Trenton $\mathrm{Gr}$.

obliquus, Meek \& Worthen, 1868, Geo. Sur. Ill., vol. 3, p. 311, Galena Gr.

oblongus, Conrad, syn. for Modiomorpha concentrica.

obsoletus, Hall, 1843, (Cypricardia obsoleta,) Geo. Rep. 4th Dist. N. Y., pl. 8, fig. 3, Clinton Gr.

obtusus, Hall, 1847, (Ambonychia obtusa,) Pal. N. Y., vol. 1, p. 167, Black Riv. and Trenton Gr.

ovata, syn. for Modiolopsis modiolaris.

plebeius, Billings, 1866, (Cyrtodonta plebeia.) Catal. Sil. Foss., Antic., p. 14, Hud. Riv. Gr.

ponderosus, Billings, 1862, (Cyrtodonta ponderosa,) Pal. Foss., vol. 1, p. 150, Hud. Riv. Gr.

quadrangularis, Whitfield, 1878, Jour. Cin. Soc. Nat. Hist., vol. 1, p. 138, Hud. Riv. Gr.

quadrilateralis, Hall, 1867, 20th Rep. N. Y. Mus. Nat. Hist., p. 388, Niagara Gr.

radiatus, Conrad, 1841, Ann. Rep. N. Y., p. 53, Ham. Gr. Not recognized.

rectus, Con rad, 1841, Ann. Rep. N. Y., p. 52, Up. Held. Gr.

rectirostris, Hall, 1861, Geo. Rep. Wis., p. 29, Trenton Gr.

recurvus, see Cimitaria recurva.

rotundatus, Hall, 1861, Geo. Rep. Wis., p. 29, and Geo. Wis., vol. 4, p. 208, Trenton Gr.

rugosus, Billings, 1858, (Cyrtodonta rugosa,) Can. Nat. and Geo., vol. 3, p. 432, Black Riv. Gr.

rugosus, see Goniophora rugosa.

saffordi, Hall, 1852,(Palæarca saffordi,)12th

Rep. N. Y. Mus. Nat. Hist., p. 11, and Geo. of Tenn., p. 287, Low. Held. Gr. sectifrons, see Phthonia sectifrons.

sigmoideus, Billings, 1858, (Cyrtodonta sigmoidea,) Can. Nat. and Geo., vol. 3, p. 438 , Black Riv. Gr.

sinuatus, see Modiolopsis sinuata.

spiniferus, Billings, 1858, (Cyrtodonta spinifera,) Can. Nat. and Geo., vol. 3, p. 435, Black Riv. Gr.

sterlingensis, Meek \& Worthen, 1866, (Dolabra sterlingensis,) Proc. Acad.
Nat. Sci. Phil., p. 260, and Geo. Sur. Ill., vol. 3, p. 339, Hud. Riv. Gr.

subalatus, see Modiomorpha subalata.

subangulatus, Hall, 1847, (Edmondia subangulata,) Pal. N. Y., vol. 1, p. 156, Black Riv. and Trenton Grs.

subcarinatus, Billings, 1858, (Cyrtodonta subcarinata, Can. Nat. and Geo., vol. 3, p. 433, Black Riv. Gr.

subspatulatus, Hall, 1847, (Modiolopsis subspatulata,) Pal. N. Y., vol. 1, p. 159, Black Riv. and Trenton Grs.

truncatus, see Sphenotus truncatus.

ungulatus, Billings, 1866, (Cyrtodonta ungulata,) Catal. Sil. Foss. Antic., p. 15 . Hud. Riv. Gr.

ventricosus, Hall, 1847, (Edmondia ventricosa,) Pal. N. Y., vol. 1, p. 155, Trenton $\mathrm{Gr}$.

ventricosus, Hall, 1859, (Palæarca ventricosa,) Pal. N. Y., vol. 3. This name was preoccupied. See Cypricardites islandicus.

vetustus, Hall, 1847, (Cardiomorpha vetusta,) Pal. N. Y., vol. 1, p. 154, Trenton Gr.

winchelli, Safford, 1869, (Cyrtodonta winchelli,) Geo. Tenn., p. 287, Trenton and Hud. Riv. Grs.

Cyrtodonta, syn. for Cypricardites.

acutumbona, see Cypricardites acutumbonus.

anticostiensis, see C. anticostiensis.

breviuscula, see C. breviusculus. canadensis, see C. canadensis.

cordiformis, see C. cordiformis.

emma, see C. emma.

ganti, see C. ganti.

harrietla, see C. harrietta.

hayniana, see C. haynanus.

hindi, see C. hindi.

huronensis, see C. huronensis.

insularis, see C. insularis.

leveothea, see C. leucothea.

normanensis, Safford. Not defined.

plebeia, see Cypricardites plebeius.

ponderosa, see C. ponderosus.

rugosa, see C. rugosus.

saffordi, see C. saffordi.

sigmoidea, see C. sigmoideus.

spinifera, see C. spiniferus.

subcarinata, see C. subcarinatus.

ungulata, see C. ungulatus.

winchelli, see C. winchelli.

Cytherodon, Hall \& Whitfield, 1873, in 23d Rep. N. Y., pl. 14, figs. 19-21. [Ety. Cythere, a genus; odous, tooth.] Ovate, pointed posteriorly; beaks pointed sharp, oblique, umbonal ridge ; cardinal line short; subcircular anterior and posterior muscular scars distinct; hinge area strong with angular teeth or crenulations beneath the beaks, pallial line simple, surface concentrically lined. Type C. nasutus.

appressus, Conrad, (Nuculites appressus,) 1842, Jour. Acad. Nat. Sci., vol. 8, p. 248, and Pal. N. Y., vol. 5, pl. 75, figs. 3-9, Ham. Gr. 
chemungensis, Conrad, 1842, (Nuculites chemungensis,) Jour. Acad. Nat. Sci., vol. 8, p. 247 , and Pal. N. Y., vol. 5, pl. 75, figs. 37-40, Chemung Gr.

cuneus, Hall, 1883, Pal. N. Y., vol. 5, pl. 75, figs. 27-30, Waverly Gr.
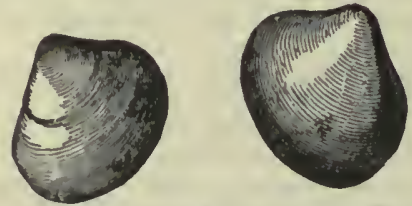

Fra.816.-Cytherodon rhombeus.

ellipticus, Hall, 1870, (Schizodus ellipticus,) I'relim. Notice Lam. Shells, p. 96, and Pal. N. Y., vol. 5, pl.75, figs. 13-15, Ham. Gr.

gregarius, Hall, 1883, Pal. N. Y., vol. 5, pl. 75, figs. 41-45, Chenung Gr.

nasutus, Hall, 1883, Pal. N. Y., vol. 5, pl. 75, figs. 10-12, Ham. Gr.

oblatıs, Hall, Pal. N. Y., vol. 5, pl. 75, figs. 41-45, Chemung Gr.

pauper, Hall, 1883, Pal. N.Y., vol. 5 , pl. 75 , figs. $24-26$, Chemung Gr.

(?) placidus, Billings, 1874 , Pal. Foss., vol. 2, p. 137 , Up. Sil.

quadrangularis, Hall, 1870 , (Schizodus quadrangularis, ) Prelim. Notice Lam. Shells, p. 96, and $\mathrm{Pal}$. N. Y., vol. 5, pl. 75, figs. 31-36, Chemung Gr.

rhombeus, Hall, 1843, (Cypricardia rhombea,) Geo. Rep. 4th Dist. N. Y., p. 291, and Pal. N. Y., vol. 5. pl. 75 , figs. 19-23, Subcarboniferous.

socialis, Billings, 1874, Pal.

Foss., vol.2, p. 138, Up. Sil.

tumidus, Hall, 1870, (Schizodus tumidus,) Prelim. Notice Lam. Shells, p. 94, and Pal. N. Y., vol. 5, pl. 75, figs, 1-2, Up. Held. Gr.

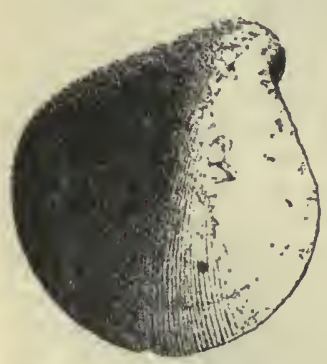

FIG. 817.-Dexiobia ovata.
Dexiobia, Winchell, 1863, Proc. Acad. Nat. Sci., p. 10. [Ety. dexios, on the right side; bia, strength.] Inequivalve, inequilateral, area und efi ned, right valve very ventricose, umbo pro $\mathrm{m}$ in e $\mathrm{t}$; beak incurved forward; left valvelessinflated; hinge-line having a thickened cartilage plate, bearing a linear posterior groove. Type D. ovata. halli, Winchell, 1863, Proc. Acad. Nat. Sci., p. 11, Marshall Gr.

ovata, Hall, 1858, (Cardiomorpha ovata,) Geo. Rep. Iowa, p. 522, Kinderhook Gr. whitii, Winchell, 1863, Proc. Acad. Nat. Sci., p. 11, Marshall Gr.

Dolabra, McCoy, 1844, Svn. Carb. Foss. Ireland, p. 64. [Ety. dolabra, a mattock or pickaxe.] Obliquely ovate, gibbous; left valve larger than the right; beaks large, obtuse, nearer the anterior than posterior end; hinge-line straight, shorter than the shell, not crenulated; a flat, narrow ligamental area the length of the hinge-line, widest between the leaks; anterior end narrower than the posterior, rounded; no byssal sinus or furrows; ventral margin slightly convex; posterior end obliquely truncated, slope flattened; surface smooth or fincly striated.

alpina. Hall, 1858, Geo. Rep. Iowa, p. 716, Coal Meas.

carinata, see Cypricardites carinatus. sterlingensis, see Cypricardites sterlingensis.
FiG. 818.-Ectenodesma birostratum.

Dystactella, Hall, 1883, Pal. N. Y., vol. 5, p. 4, (Plates and Explanations,) synonym for Clinopistha.

insularis, see Clinopistha insularis.

subnasuta, see Clinopistha subnasuta.

telliniformis, see Clinopistha telliniformis.

Ectenodesma, Hall, 1883, Pal. N. Y., vol. 5, pt. 1, p. 4. (Plates and Explanations.) [Ety. ektenes, stretched out; desma, a ligament.] Body ovate, oblique; height greater than length; both valves more or less convex; hinge-line longer than the length of the shell; byssal sinus shallow; oblique lateral tooth; ligamental area narrow, striated; surface rayed; distinguished from Glyptodesma by having the anterior wing more produced, and both wings more acute at their extremities. Type E. birostratum.

birostratum, Hall, 1883, Pal. N. Y., vol.5, pt. 1, p. 242, Chemung Gr.

Edmonda, DeKoninck, 1844, Desc. Anim. Foss., Carb. Belg., p. 66. [Ety. proper 
name.] Shell equivalve, inequilateral, tumid, short, oblong or rounded, closed all around; dorsal and ventral margins slightly convex; beaks tumid, with an impressed lunette between them; surface with concentric striæ; no teeth, but an internal lamellar cartilage support, much dilated within the cavity of the beaks, the broad end forming the slits in casts coinciding with the edges of the anterior lunette, and the posterior end running nearly parallel to and close within the hinge-line; dorsal margins erect and simple; two simple adductor impressions, often with an accessory impression over each, pallial scar simple, entire. Type E. unioniformis.

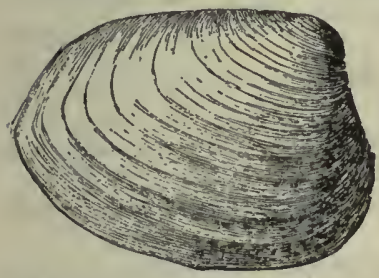

FIG. 819.-Edmondia aspenwallensis. Kight valve. æquimarginalis, Winchell, 1862, (Cardin i a æquim a rginal is , ) $\mathrm{P} r \circ \mathrm{c}$. Acad. Nat. Sci., p. 413 , Marshall Gr.

a nom a la. Daw s o $\mathbf{n}$, 1868, Acad. Geo., p. 303, Carb.

aspenwallensis, Meek, 1871, Hayden's Rep. Sur. W yoming, p. 299, and Pal. E. Neb., p. 216, Coal Meas.

bicarinata, Winchell, 1863, Proc. Acad. Nat. Sci.,p. 13, Marshall Gr. Prof. Hall regards this as a syn. for Sanguinolites rigidus.

binumbonata, Winchell, 1862, Proc. Acad. Nat. Sci., p. 414, Marshall Gr. burlingtonensis, White \& Whitfield, 1862, Proc. Bost. Soc. Nat. Hist., vol. 8, p. 301, and Pal. N. Y., vol. 5, p. 390, Kinderhook Gr.

calhouni, see Pleurophorus calhouni.

circularis, Walcott, 1885 , Monogr. U. S. Geo. Sur., vol. 8 , p. 246 , Carboniferous.

concentrica, see Astartella

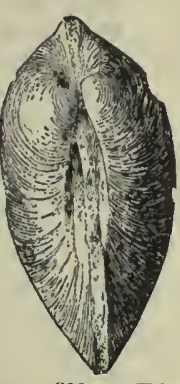

IG. 820. - Edmondia aspenwa 1 I e n s $1 \mathrm{~s}$. Cardinal view. concentrica.

depressa, Hall, 1870, Prelim. Notice Lam. Shells, p. 91, and Pal. N. Y., vol. 5, pl. 64, fig. 32, Waverly Gr.

ellipsis, Hall, 1855, Pal. N. Y., vol. 5, p. 392, Waverly Gr.

elliptica, Winchell, 1863, Proc. Acad. Nat. Sci., p. 13,.Marshall Gr.

gibbosa, Swallow, 1858, Trans. St. Louis Acad. Sci., vol. 1, p. 189, Permian Gr.

glabra, Meek, 1872, Pal. E. Neb. p. 214 , Coal Meas.

hartti, Dawson, 1868, Acad. Geol., p. 303, Carb. hawni, Swallow, 1858, Trans. St. Louis Acad. Sci., vol. 1, p. 209, Coal Meas.

illinoisensis, Wortben, 1884, Bull. No. 2 Ill. St. Mus. Nat. Hist., p. 18, and Geo. Sur. Ill., vol. 8, p. 122, Keokuk Gr.

ledoides, Winchell, 1866, Rep. Low. Peninsula Mich., p. 96, Ham. Gr.

mactroides, Winchell, 1866, Rep. Low.

Peninsula Mich., p. 96, Ham. Gr.

marionensis, Swallow, 1860, Trans. St. Louis Acad. Sei., vol. 1, p. 654, Choteau Gr.

medon, Walcott, 1884, Monogr. U. S. Geo. Sur., vol. 8, p. 245, Subcarboniferous.

mortonensis, Geinitz, 1866, (Astarte mortonensis,) Carb. und Dyas in Neb., p. 17, Coal Meas.

nebraskensis, Geinitz, 1866, (Astarte nebraskensis,) Carb. und Dyas in Neb., p 16, and Pal. E. Neb., p. 214, Coal Meas.

nilesi, Winchell \& Marcy, 1865, Proc. Bost. Soc. Nat. Hist., p. 97, Niagara Gr. nitida, Winchell, 1863, Proc. Acad. Nat. Sci., p. 12, Marshall Gr.

nuptialis, Winchell, 1863, Proc. Acad. Nat. Sci., p. 12, Marshall Gr.

obliqua, Hall, 1885, Pal. N. Y., vol. 5, p. 38 , Chemung Gr.

otoensis, Swallow, 1858, Trans. St. Louis Acad. Sci., vol. 1, p. 189, Permian Gr. peroblonga, Meek \& Worthen, 1866, Proc. Acad. Nat. Sci. Phil., p. 249, and Geo. Sur. Ill., vol. 5, p. 583, Coal Meas.

philipi, Hall, 1870, Prelim. Notice Lam. Shells, p. 90, and Pal. N. Y., vol. 5, pl. 64, figs. 9-18, Chemung Gr.

pinonensis, Meek, 1877, U. S. Geo. Expl. 40 th parallel, vol. 4 , p. 46 , Devonian.

radiata, see Clinopistha radiata.

reflexa, Meek, 1872, Pal. E. Neb., p. 213, Coal Meas.

rhomboidea, Hall, 1883, Pal. N. Y., vol. 5, pl. 64, figs. 7-8, Chemung Gr.

semiorbiculata, Swallow, 1858, Trans. St. Louis Acad. Sci., vol. 1, p. 190, Permian Gr.

strigillata, Winchell, 1863, Proc. Acad. Nat. Sci., p. 12, Marshall Gr.

subangulata, see Cypricardites subangulatus.

subcarinata, Hall, 1885, Pal. N. Y., vol. 5, pl. 64, fig. 31, Chemung Gr.

subnasuta, Hall, 1883, Pal. N. Y., vol. 5, pl. 64, figs. 5-6, Chemung Gr.

subovata, Hall, 1885, Pal. N. Y., vol. 5, p. 389, Chemung Gr.

subplana, Hall, 1858, Cypricardia subplana,) Trans. Alb. Inst., vol. 4, p. 19, and Bull, Am. Mus. Nat. Hist., p. 66, Warsaw Gr.

subtruncata, see Cuneamya subtruncata. subtruncata; Meek, 1872, Pal. E. Neb., p. 215, Coal Meas.

tapetiformis, Meek, 1875, (E. tapesiformis,) Ohio Pal., vol. 2, p. 304, Waverly Gr. tenuistriata, Hall, 1885, Pal. N. Y., vol. 5, p. 393, Chemung Gr.

transversa, Hall, 1885, Pal. N. Y., vol. 5, p. 389 , Chemung Gr. 
undala, see Grammysia undata.

undulata, Hall, 1870, Prelim. Notice Lam. Shells, p. 91, and Pal. N. Y., vol. 5, pl. 64, figs. 1-4, Chemung Gr.

unioniformis, Phillips, 1836, (Isocardia unioniformis,) Geol. Yorkshire, vol. 2, p. 209, and Geo. Sur. Ill., vol. 2, p. 346, Coal Meas.

varsoviensis, Worthen, 1884, Bull. No. 2 Ill. St. Mus. Nat. Hist., p. 18, and Geo. Sur. Ill., vol. 8, p. 121, Keokuk Gr.

ventricosa, see Cypricardites ventricosus.

Elymella, Hall, 1885, Pal. N. Y., vol. 5, p. 50. [Ety. elymos, a case.] Equivalve, inequilateral, ovate, elliptical; anterior end short, rounded; posterior end narrower, rounded; beaks closely incurved ; umbo prominent; cardinal line short; umbonal slope prominent in the upper part, not defined below; surface concentrically lined. Type E. nuculoides.

fabalis, Hall, 1885, Pal. N. Y., vol. 5, p. 502, Ham. Gr.

levata, Hall, 1885, Pal. N. Y., vol. 5, p. 504, Ham. Gir.

nuculoides, Hall, 1885, Pal. N. Y., vol. 5, p. 503, Ham. Gr.

patula, Hall, 1885, Pal. N. Y., vol. 5, p. 505 , Waverly Gr.

Entolium, Meek, 1865, Cal. Geo. Sur., vol. 2. [Ety. entos, inside; leion, smooth.] If synonymous with Pernopecten, then the latter has priority; but if distinct, then probably it is not a Palicozoic genus, as the type is from rocks of Jurassic age.

Eodon, Hall, 1877, 1st Ed. Am. Pal. Foss., p. 244. Proposed instead of Microdon, Conrad, which was preoccupied.

Eopter1A, Billings, 1865, Pal. Foss., vol. 1, p. 221. [Ety. cos, dawn; pteron, a wing.] Prof. Billings said if Euchasma is the same as Eopteria, then he desired Eopteria to be withdrawn from science. Winged as in Pterinea, both valves equally convex and gaping; ligament external. Type E. typica.

(?) ornata, Billings, 1865, Pal. Foss., vol. 1, p. 307 , Quebec Gr.

richardsoni, Billings, 1865, Pal. Foss., vol. 1, p. 306, Quebec Gr.
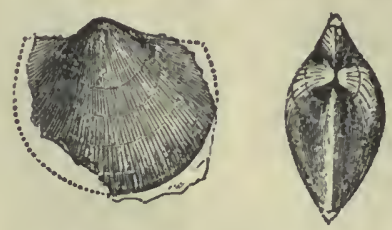

FIG. 821.-Eopteria richardsoni.

typica, Billings, 1865, Pal. Foss., vol. 1, p. 221, Quebec Gr.

Euchasma, Billings, 1865, Pal. Foss., vol. 1, p. 360. [Ety. eu, well; chasma, a hollow.] Strongly convex, triangular, in- 1

equilateral, equivalve, subcordiform, gaping, posterior extremity flattened, hinge short, ligament external. Type E. blumenbachi.

blumenbachi, Billings, 1859, (Conocardium blumenbachi,) Can. Nat. and Geo., vol. 4, p. 350, Quebec Gr.

Eucirondria, Meek, 1874, Am. Jour. Sci., 3d series, vol. 7, p. 445 . Like Aviculopecten in form, but with an un. symmetrical subrostral cartilage pit and unequal crenulations on $F$ equal crenulations on $\mathrm{F}_{\mathrm{G}}$. $822 .-$ Eu-
the two sides. Type lectaria. Riglit E. neglecta: neglecta, Geinitz, 1866,

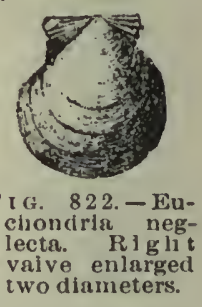

(Pecten neglecबuranies illentro

FIG. 823. - Eucíondiris neglectr. Hinge-ilue enlarged.

tus,) Carb. und Dyas in Neb., p. p. 33, and Geo. Sur. Ill., vol. 5, p. 589 , Coal Meas.

Eumicrotus, Meek, 1864, syn. for Pseudomonotis.

hawni, see Pseudomonotis hawni.

hawni var. ovala, see Pseudomonotis hawni var. ovata.

hawni var. sinuata, see Pseudomonotis hawni var. sinuata.

Eutuydesma, Hall, 1885, Pal. N. Y., vol. 5 , p. 32 . [Ety. euthus, straight; desma, a ligam e nt.] Equivalve, inequilateral, broadlysubovate, with a subalate Fig. 824.-Eutlydesma cardinal expan-

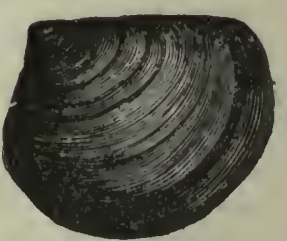
sion; cardinal line straight; anterior end short; surface concentrically lined; hinge-line marked by a continuous ligamental groove. Type E. subtextile.

subtextile, Hall, 1843, (A starte subtextilis,) Geo. Sur. 4th Dist. N. Y., p. 245, Portage $\mathrm{Gr}$.

Exochorhynchus, Meek, 1864, Pal. Up. Mo. [Ety. exochos, prominent; rhynchos, beak.] This name was suggested as a probable genus or subgenus to include Sedgwickia altirostrata.

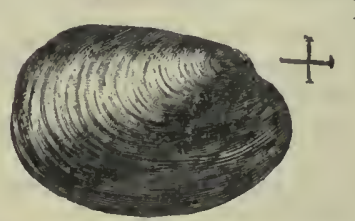

FiG. 825.-Fordilla troyensis.
Fordilla, Barrande, 1881, A cep hales. Etudes Loc. et Comp., pl. 361 , and Bull. U. S. Geo. Sur., No. 30, p. 123 . A minute bivalve, somewhat resembling a Modiolopsis or an Orthonotella. Type F. troyensis.

troyensis, Barrande, 1886, Bull. U. S. Geo. Sur., vol. 30, p. 125 , Up. Taconic. 
Gervillia, DeFrance, 1820, Dict. Sci. Nat., xviii. [Ety. proper name.] Type G. anceps. This genus is probably unknown in the American Palæozoic rocks.

auricula, see Monopteria auricula.

longa, see Avicula longa.

longispina, see Monopteria Iongispina.

strigosa, see Pterinea strigosa.

sulcata, see Bakevellia sulcata.

Glossites, Hall, 1885, Pal. N. Y., vol. 5, p. 49. [Ety. glosse, the tongue.] Equivalve, inequilateral, elliptical; anterior end short, margin declining from the beak and curving below; posterior end large, broadly rounded; beaks small, appressed; cardinal line long, gently arcuate; umboual slope not defined; surface marked concentrically ; ligament external ; lunule distinct; muscular impressions shallow. Type G. lingualis.

amygdalinus, Winchell, 1863, (Sanguinolites amygdalinus,) Proc. Acad. Nat. Sci. Phil., p. 13, and Pal. N. Y., vol. 5, p. 501, Waverly Gr.

depressus, Hall, 1885, Pal. N. Y., vol. 5, p. 496, Chemung Gr.

ellipticus, Hall, 1885, Pal. N. Y., vol. 5, p. 498, Chemung Gr.

lingualis, Hall, 1885, Pal. N. Y., vol. 5, p. 497, Chemung Gr.

patulus, Hall, 1885, Pal. N. Y., vol: 5, p. 501, Chemung Gr.

procerus, Hall, 1885, Pal. N. Y., vol. 5, p. 499, Chemung Gr.

rudicula, Hall, 1885, Pal. N. Y., vol. 5, p. 498, Chemung Gr.

subnasutus, Hall, 1885, Pal. N. Y., vol. 5, p. 500, Chemung Gr.

subtenuis, Hall, 1885, Pal. N. Y., vol. 5, p. 495, Ham. Gr.

teretis, Hall, 1885, Pal. N. Y., vol. 5, p. 494, Up. Held. Gr.

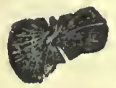

FIG. 826 .

G 1 y p tocardla

speclosa.
Glyptocardia, Hall, 1885, Pal. N. Y., vol. 5, p. 35 . [Ety. glyptos, sculptured; cardia, the heart.] Shell small, equivalve, inequilateral, broadly elliptical or subcircular; beaks incurved; surface plicated and marked with concentric striæ; no area beneath the beaks. Type G. speciosa.

speciosa, Hall, 1843, (Avicula speciosa,) Geo. Sur. 4th Dist. N. Y., p. 243, and Pal. N. Y., vol. 5, p. 426, Ham. and Portage Grs.

Glyptodesma, Hall, 1883, Pal. N. Y., vol. 5, pt. 1, p. 4. (Plates and Explanations.) [Ety. glyptos, sculptured; desma, a ligament.] Aviculiform, ligamental area striated, continuous, hinge with two strong lateral teeth, and numerous irregular transverse plications along the cardinal margin ; surface concentrically striated. Type G. erectum.

cruciforme, Conrad, 1841, (Avicula cruciformis,) Ann. Rep. N. Y., p. 54, Ham. Gr. erectum, Conrad, 1842, (Avicula erecta,) Jour. Acad. Nat. Sci., vol. 8 , p. 238, and Pal. N. Y., vol. 5, p. 153, Ham. Gr. erectum var. obliquum, Hall,

1883, Pal. N. Y., vol. 5, pt. 1, p. 155, Ham. Gr.

occidentale, Hall, 1883, Pal.

N. Y., vol. 5, pt. 1, p. 157 ,

Up. Held Gr.

subrectum, Whitfield, 1882 ,

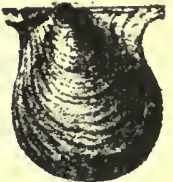

FIG. 827. erectum. Glyptodesma

(Actinodesma subrectum, Ann. N. Y.

Acad. Sci., vol. 2, p. 215, Ham. Gr.

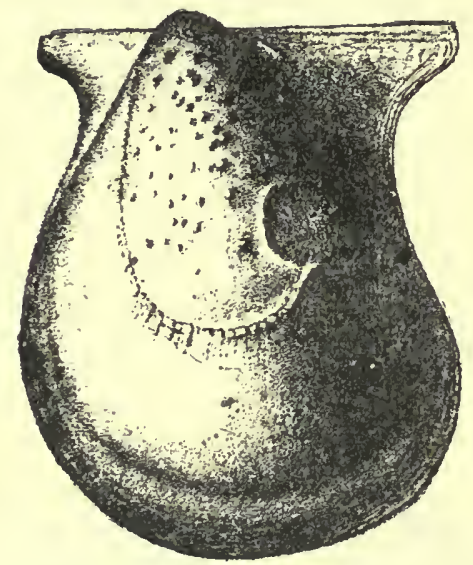

FIG. 828.-Glytodesma erectum. Mold of left valve, showlug pallial line, muscular scar, tubercles in the interpallial area representing points of muscular attachment.

Goniophora, Phillips, 1848, Mem. Geo. Sur. Gt. Brit., vol. 2, p. 264. [Ety. gonia, an angle; phoros, bearing.] Goniophorus was used by Agassiz for a genus of Echinoderms in 1840. Equivalve, very inequilateral, rhomboidal or trapezoidal, obliquely truncate behind, rounded in front; cardinal line straight; beaks small, umbo prominent, and slope continued as a ridge to the post-inferior margin; oblique, undefined sinus from anterior to the beaks to basal margin; surface concentrically lined; hinge with an oblique fold or tooth in the leit valve beneath the beak, and a corresponding depression in the right valve; ligament external, attached by one or more grooves; anterior muscular impression deep, situated anterior to the beak; posterior muscular impression shallow, situated on the posterior cardinal slope; pallial line simple. Type G. cymbiformis.

acuta, Hall, 1870, (Sanguinolites acutus.) Prelim. Notice Lam. Shells, p. 37, and Pal. N. Y., vol. 5, pl. 43, figs. 1-3, Ham. Gr.

alata, Hall, 1885, Pal. N. Y., vol. 5, p. 294, Schoharie grit.

bellula, Billings, 1874, PaI. Foss., vol. 2, p. 136, Up. Sil. 
carinata, Conrad, 1841, (Cypricardites carinatus,) Ann. Rep. Geo. Sur. N. Y., p. 53, and Pal. N. Y., vol. 5, pl. 44, figs. 6-8, Ham. Gr.

chemungensis, Vanuxem, 1842, Cvpricardites chemungensis,) Geo. Rep. N.Y., p. 181, and Pal. N. Y., vol. 5, pl.44, figs. 18-22, Chemung Gr.

consimilis, Billings, 1874, Pal. Foss., vol. 2, p. 135, Up. Sil.

crassa, Whiteaves, 1888, Pal. Foss., vol. 3, p. 9, Guelph Gr.

glabra, Hall, 1883, Pal. N. Y., vol. 5, pl. 44, figs. 9-17, syn. for $G$. glaucus.

glaucus, Hall, 1870, (Sanguinolites glaucus,) Prelim. Notice Lam. Shells, p. 38, and Pal. N. Y. vol. 5, p. 299, Ham. Gr.

hamiltonensis, Hall, 1870, (Sanguinolites hamiltonensis,) Prelim. Notice Lam. Shells, p. 30 , and Pal. N. Y., vol. 5, pl. 43 , figs. 8-21, Hain. Gr.

mediocris. Billings, 1874, Pal. Foss., vol. 2, p. 137, Up. Sil.

minor, Hall, 1885, Pal. N. Y., vol. 5, p.305, Chemung Gr.

perangulata, Hall, 1870, (Sanguinolites perangulatus,) Prelim. Notice Lam. Shells, and Pal. N. Y., vol. 5, pl. 34, figs. $1-7$, Up. Held. Gr.

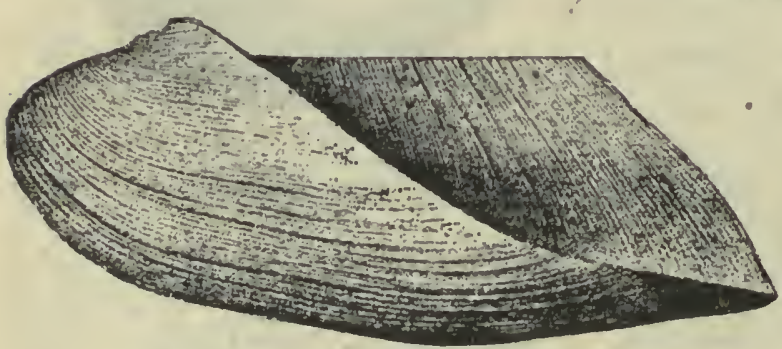

FIG. 829.-Goniophora chemungensis.

plicata, Hall, 1858. (Cypricardella plicata,) Trans. Alb. Inst., vol. 4, p. 18, and Bull. Am. Mus. Nat. Hist., p. 66, Warsaw Gr. rugosa, Conrad, 1841, (Cypricardites rugosus, Ann. Rep. N. Y., p. 53, and Pal. N. Y., vol. 5, p. 297, Ham. Gr.

speciosa, Hall, 1879, Desc. New. Spec. Foss., p. 17, and 11th Rep. Geol. Indiana, p. 317, Niagara Gr.

subrecta, Hall, 1885, Pal. N. Y., vol. 5, p. 304, Chemung Gr.

transiens, Billings, 1874, Pal. Foss., vol. 2, p. $134, \mathrm{Up}$. Sil.

trigona, Hall, 1885, Pal N. Y., vol. 5, p. 302, Chemung Gr.

truncata, Hall, 1883, Pal. N. Y., vol. 5, pl. 44, fig. 15, Ham. Gr.

Gosseletwi A, Barrois, 1881, Ann. Soc. Geol. du Nord, vol. 8, p. 176. [Ety. proper name.] Shell subtriangular, truncate on the anterior side, subalate posteriorly; ligamental area wide, longitudinally striate; cardinal teeth below the beak strong; lateral teeth elongate; surface with concentric striæ. retusa, Hall, 1883, Pal. N. Y., vol. 5, pt. 1 , p. 266, Ham. Gr.

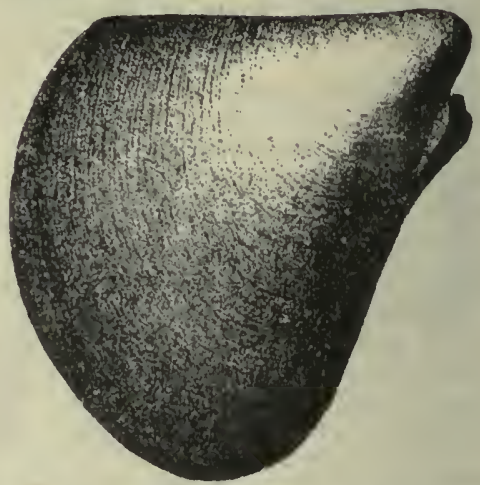

FIa. 830,-Gosselettla trlquetra. Right valve.

triquetra, Conrad, 1838, (Pterinea triquetra,) Ann. Rep. Geo. N. Y., p. 116 , and Pal. N. Y., vol. 5, pt. 1, p. 265, Ham. Gr.

Grammysia, DeVerneuil, 1847, Bull. Soc. Geo. France, 2d ser., vol. 4, p. 696. [Ety. gramme, a line of writing; Mys, a mussel shell, in allusion to the transverse furrows which cross the valves from the umbones to the middle of the ventral margin.] Equivalve, inequilateral; shell thick, oblong; anterior side short, contracted by a deep oval lunette beneath the beak; posterior end elliptically rounded; hinge-line straight; two large adductor impressions in each valve, anterior rounded, posterior pear-shaped; pallial scar entire; cartilage external, short, in the anterior part of a deep depression formed by the inflexion of the hinge margins; an oblique furrow extends from the beak to about the middle of the ventral margin. Type G. bisulcata. acadica, Billings, 1874, Pal. Foss., vol 2, p. 140 , Up. Sil.

alveata, Conrad, 1841, (Posidonia alveata,) Ann. Rep. N. Y., p. 53, and Pal. N. Y.. vol. 5, pl. 57, figs. 1-2, and pl. 60, Ham. Gr.

arcuata, Conrad, 1841, (Posidonia arcuata,) Ann. Rep. N. Y., p. 53, and Pal. N. Y., vol. 5 , p. 373 , Hawn. Gr.

bisulcata, Conrad, 1838, (Pterinea bisulcata,) 1841, (Cypricardites bisulcata,) Ann. Rep. N. Y., p. 116, and Pal, N. Y., vol. 5, pl. 51, figs. 1-16, Ham. Gr. canadensis, Billings, 1874, Pal. Foss., vol. 2, p. 51, Gaspe sandstone, Up. Sil. caswelli, Foerste, 1885, Bull. Sci. Lab. Denison University, p.92. Not properly defined. 
chemungensis, Pitt, 1874, Bul. Buff. Soc. Nat. Hist., Chemung Gr.

circularis, Hall, 1870, Prelim. Notice Lam. Shells, p. 51, and Pal. N. Y., vol. 5, pl. 57, fius. 3-6, Ham. and (Yhemung Grs.

communis, Hall, 1885, Pal. N. Y., vol. 5, p. 378, Chemung Gr.

coustricta, Hall, 1870, Prelim. Notice Lam. Shells, p. 58, and Pal. N. Y., vol. 5, pl. 59, figs. 13-20, Ham. Gr.

cuneata, Hall, 1883, Pal. N. Y., vol. 5, pl. 62, figs. 1-9, Ham. Gr.

duplicata, Hall, 1885, Pal. N. Y., vol. 5, p. 380, Chemung Gr.

elliptica, Hall, 1870, Prelim. Notice Lam. Shells, p. 53, and Pal. N. Y., vol. 5, pl. 58, figs. 1-12, Chemung Gr.

erecta, Hall, 1870, Prelim. Notice Lam. Shells, p. 52, and Pal. N. Y., vol. 5, p. 363 , Ham. Gr.

glabra, Hall, 1885, Pal. N. Y., vol. 5, p. 369. Chemung Gr.

globosa, Hall, 1870. Prelim. Notice Lam. Shells, p. 57, and Pal. N. Y., vol. 5, p. 372, Ham. Gr.

hamiltonensis, syn. for G. bisculcata.

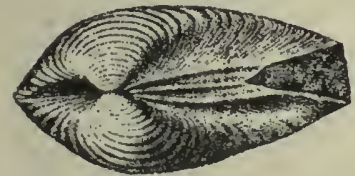

FIG. 831.-Grammysia liann1balensis. Dorsal view. hannibalensis, Sh u m ard, 1855, (Allorisma han $\mathrm{n}$ i b alense,) Geo. $\mathrm{Sur}$. Mo., p. 206 , $\mathrm{Choteau}$ and Kinderhook Grs.

lirata, Hall, 1870, Prelim. Notice Lam. Shells, p. 57, and Pal. N. Y., vol. 5, pl. 59, figs. 6-12, Ham. Gr.

magna, Hall, 1870, Prelim. Notice Lam. Shells, p. 50, and Pal. N. Y., vol. 5, p. 362, Ham. Gr.

minor, Walcott, 1885, Monogr. U. S. Geo. Sur.,

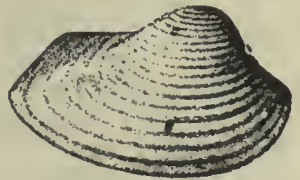
vol. 8, p. 174, Frg. 832.-Gramnysia hanUtp. Devonian. nibalensis. Right si d e nodocostata,Hall,

1870, Prelim. Notice Lam.Shells, p. 50, and Pal. N.Y.,vol.5, pl.55, figs. 1-11, Ham. Gr. obsoleta, Hall, 1870, Prelim. Notice Lam. Shells, p. 60, and Pal. N. Y., vol. 5, pl. 59, figs. 21-27, Ham. Gr.

ovata, Hall, 1885, Pal. N. Y., vol. 5, p. 358, Up. Held. Gr.

parallela, Hall, 1870, Prelim. Notice Lam. Shells, p. 59, Ham. Gr.

plena, Hall, 1885, Pal. N. Y., vol. 5, p. 382, Waverly G'r.

praccursor, Hall, 1870, Prelim. Notice Lam. Shells, p. 54, and Pal. N. Y., vol. 5, pl. 59, fig. 1, Schoharie grit.

remota, Billings, 1874, Pal. Foss., vol. 2, p. 139 , Up. Sil.

rhomboidalis, Meek \& Worthen, 1865, Proc. Acad. Nat. Sci. Phil., p. 248, and Geo. Sur. Ill., vol. 3, p. 439, Ham. Gr. rhomboides, Meek, 1871, Proc. Acad. Nat. Sci. Phil., p. 72, and Ohio Pal., vol. 2, p. 302, Waverly Gr.

rustica, Billings, 1874, Pal. Foss., vol. 2, p. 139 , Up. Sil.

secunda, Hall, 1870, Prelim. Notice Lam. Shells, p. 54, and Pal. N. Y., vol. 5, pl. 59, figs. 2-5, Up. Held. Gr.

subarcuata, Hall, 1870, Prelim. Notice Lam. Shells, p. 61, and Pal. N., vol. 5, pl. 61, figs. 10-22, Chemung Gr.

undata, Hall, 1883, Pal. N. Y., vol. 5, p. 379, Chemung Gr.

ventricosa, Meek, 1871, Proc. Acad. Nat. Sci. Phil., p. 73, and Ohio Pal., vol. 2, p. 303, Waverly Gr.

Gryphorhynchus, Meek, 1864, Am. Jour. Sci. and Arts. Not. defined.

IrioniA, Billings, 1875, Can. Nat. and Geol., vol. 8, p. 301. [Ety. proper name.] Irregularly ovate, compressed; one extremity larger than the other, with beaks curned toward the larger end; concave depression from the umbones to the posterior ventral margin; subovate muscular impression in the upper half of the posterior extremity. Type I. canadensis.

can adensis, Billings, 1875, Can. Nat. and Geol., vol. 8, p. 301, Corniferous Gr.

costulata, White a ve s, 1884 , Pal. Foss., vol. 3, p. 15, Guelph Gr.

galtensis, Whiteaves, 1884, Pal. Foss., vol. 3, p. 15, Guelph Gr.

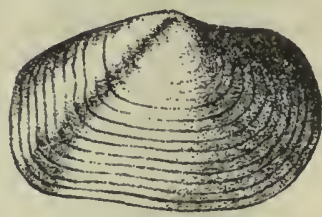

sinuata, H a 11 ,

FIG. 833.--1lionia galtensis.

1859, (Anativa sinuata,) Pal. N. Y., vol. 3, p. 265, Low. Held. Gr.

Inoceramus, Sowerby, 1818, Min. Conch., vol. 2. This genus is unknown in American Palæozoic rocks.

chemungensis, see Mvtilarca chemungensis. mytilimeris, see Plethomytilus mytilimeris. oviformis, see Pletbomytilus oviformis.

Ischy rINIA, Billings, 1866, Catal. Sil. Foss. Antic., p. 16. [Ety. ischyros, strong.] Equivalve, inequilateral; two strong ridges radiating from the beak in the interior of each valve. Type I. winchelli.

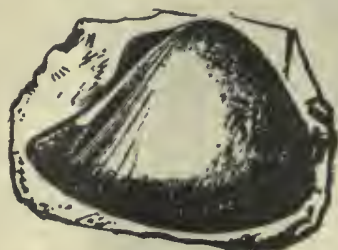

FIG. 834.-Ischy rinia winchelli. plicata, Billings, $1866^{\circ}$,

Cata 1. Sil. Foss. Antic. p. 52, Anticosti Gr.

winchelli, Billings, 1866 , Catal. Sil. Foss. Antic., p. 16, Hud. Riv. Gr.

Isocardia, Klein, 1753, Tent. Meth. Ostr. [Ety. isos, like; kardia, the heart.] This 
is an existing littoral genus that burrows in the sand. It is not known in the Palæozoic rocks.

(?) curta, Shumard, 1858, Trans. St. Louis Acad. Nat. Sci., vol. 1, p. 206, Choteau Gr.

jennx, Winchell, 1863, Proc. Acad. Nat. Sci., p. 17, Marshall Gr.

unioniformis, see Edmondia unioniformis.

Leda, Schumacher, 1817, syn. for Nuculana. barrisi, White \& Whitfield, syn. for Palæoneilo nuculiformis.

bellistriata, see Nuculana bellistriata.

brevirostris, see Nuculana brevirostris.

curta, see Nuculana curta.

dens-mamillata, see Nuculana dens-mamillata.

gibbosa, see Yoldia gibbosa.

knoxensis, see Yoldia knoxensis,

levistriata, see Yoldia levistriata.

nuculiformis, see Palæoneilo nuculiformis. - obscura, see Nuculana obscura.

ohioensis, Hall, syn for Nuculana pandoriformis.

oweri, see Yoldia oweni.

pandoriformis, see Nuculana pandoriformis.

polita, see Nuculana polita.

rushensis, see Yoldia rushensis.

saccata, see Nuculana saccata.

subscitula, see Yoldia subscitula.

Liol'teria, Hall, 1883, Pal. N. Y., vol. 5, pt. 1, p. 4. (Plates and Explanations.) [Ety. leirs, smooth; Pteria, a genus.] Aviculoid, resembling in form Actinopteria; anterior extremity auriculate; wing large, extremity produced; test without proper rays; ligament external: ligamental area marked by fine parallel longitudinal strix; hinge with one or two oblique, slender, lateral tecth; the cavity of the beak partially separated from the anterior end by a short partition. Type L. dekayi.

bigsbyi, Hall, 1883, Pal. N. Y., vol. 5, pt. 1, p. 165, Ham. Gr.

chemungensis, Vanuxem, 1842, (Avicula chemungensis, Geo. Rep. 3 d Dist. N. Y., p. 182, and Pal. N. Y., vol. 5, p. 172, Chemung Gr.

conradi, Hall, 1883, Pal. N. Y., vol. 5, pt. 1, p. 159 , Ham. Gr.

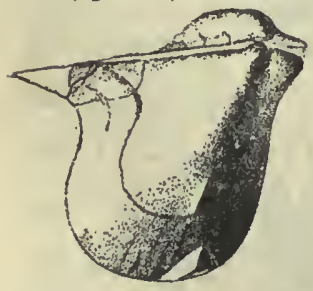

FIG. 835. - Liopteria dekayl. Cast showing vertical plate anterior to the beak's. d e ka y i, Hall, 1883, Pal. N. Y., vol. 5 , pt. $1, \mathrm{p}$. 164, Ham. Gr.

gabbi, Hall, 1884, Pal. N. Y., vol. 5 , pt. 1, p. 169 , Ham. Gr.

greeni, Hall, 1883, Pal. N. Y., vol. 5 , pt. 1, p. 160 , Ham. Gr.

lævis, Hall, 1843 , (Avicula lævis,) Geo. Rep. 4th

Dist. N. Y., p. 181, and Pal. N. Y., vol. 5, p. 158, Marcellus Shale. leai, Hall, 1884, Pal. N. Y., vol. 5, pt. 1, p. 168 , Ham. Gr.

linguiformis, Hall, 1884, Pal. N. Y., vol. 5, pt. 1, p. 173 , Chemung Gr.

mitchelli, Hall, 1883, Pal. N. Y., vol. 5, pt. 1 , p. 166 , Ham. Gr.

nitida, Hall, 1883, syn. for I. chemungensis.

oweni, Hall, 1883, Pal. N. Y., vol. 5, pt. 1, p. 170, Ham. Gr. rafinesquii, Hall, 1883, Pal. N. Y., vol. 5 , pt. 1, p. 161, Ham. Gr.

sayi, Hall, 1884, Pal. N. Y., vol. 5 , pt. 1, p. 162 , Ham. Gr.

tor reyi, Hall, Fig. 836. - Ilopteria rafin1884, Pal. N.Y.,

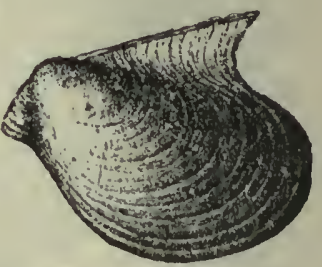
vol. 5 , pt. 1 , p. 174 , Chemung Gir.

troosti, Hall, 1884, Pal. N. Y., vol. 5, pt. 1, p. 167, Ham. Gr.

Leitopesma, Hall, 1883, Pal. N. Y., vol. 5, pt. 1, p. 4. (Plates and Explanations.) [Ety. leptos, slender; desma, a ligament.] Like Iiopteria, except the anterior end is nasute and acute, instead of auriculate and rounded; hinge-line narrow, with a slender, lateral tooth posterior to the beak; ligament external; test with concentric striæ. Type L. potens. acutirostrum, Hall, 1884, Pal. N. Y., vol. 5 , pt. 1, p. 234, Chemung Gr.

agassizi, Hall, 1884, Pal. N. Y., vol. 5, pt. 1, p. 182 , Chemung Gr.

alatum, Hall, 1884, Pal. N. Y., vol. 5, pt. 1, p. 218, Chemung Gr.

aliforme, Hall, 1884, Pal. N. Y., vol. 5, pt. 1, p. 220, Chemung Gr.

arciforme, Hall, 1884, Pal. N. Y., vol. 5, pt. 1, p. 229 , Chemung Gr.

aviforme, Hall, 1884, Pal. N. Y., vol. 5, pt. 1, p. 224, Chemung Gr.

becki, Hall, 1884, Pal. N. Y., vol. 5, pt. 1, p. 185, Chemung Gr.

billingsi, Hall, 1884, Pal. N. Y., vol. 5, pt. 1, p. 192, Chemung Gr.

biton, Hall, 1884, Pal. N. Y., vol. 5, pt. 1, p. 222, Chemung Gr.

cadmus, Hall, 1884, Pal. N. Y., vol. 5, pt. 1, p. 201, Chemung Gr.

clitus, Hall, 1884, Pal. N. Y., vol. 5, pt. 1, p. 210, Chemung Gr.

complanatum, Hall, 1884, Pal. N. Y., vol. 5, pt. 1, p. 227, Chemung Gr.

corydon, Hall, 1884, Pal. N. Y., vol. 5, pt. 1 , p. 212, Chemung Gr.

creon, Hall, 1884, Pal. N. Y., vol. 5, pt. 1, p. 202, Chemung Gr.

curvatum, Hall, 1884, Pal. N. Y., vol. 5, pt. 1, p. 196, Up. Chemung Gr.

demus, Hall, 1884, Pal. N. Y., vol. 5, pt. 1, p. 203, Chemung Gr.

disparile, Hall, 1884, Pal. N. Y., vol. 5, pt. 1 , p. 186, Up. Chemung Gr.

extenuatum, Hall, 1884, Pal. N. Y., vol. 5, pt. 1, p. 207, Chemung Gr. 
flaccidum, Hall, 1884, Pal. N. Y., vol. 5, pt. 1, p. 225, Chemung Gr.

hector, Hall, 1884, Pal. N. Y., vol.5, pt. 1, p. 209, Chemung Gr.

jason, Hall, 1884, Hall, Pal. N. Y., vol. 5, pt. 1, p. 213, Chemung Gr.

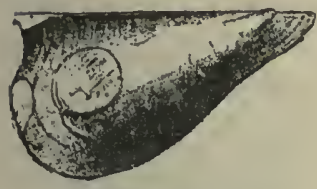

FrG. 837.-Leptodesma hecior. liopteroides, Simpson, 1889, Dict. of $\mathrm{Pa}$. Foss., p. 331, Chemung Gr. lepidnm, Hall, 1884, Pal. N.Y., vol. 5 , pt. $1, \mathrm{p}$. 195, Chemung Gr.

lesleyi, Hall, 1884, Pal. N. Y., vol. 5, pt. 1, p. 223, Up. Chemung Gr.

lichas, Hall, 1884, Pal. N. Y., vol. 5. pt. 1, p. 232 , Chemung Gr.

lon $\mathrm{g}$ is $\mathrm{p}$ in $\mathbf{n} \mathrm{m}$, Hall, 1843 , (Avicula longispina,) (reo. Rep. 4th Dist. N. Y., p 262 , and Pul. N. Y., vol. 5, pt. 1, p. 179, Chemung Gr.

loxias, Hali, 1834, Pal. N. Y., vol. 5, pt. 1, p. 204, Chemung Gr.

lysander, Hall, 1884, Pal. N. Y., vol. 5, pt. 1, p. 216, Chemung (ir.

maclurii, Hall, 1884, Pal. N. Y., vol. 5, pt. 1, p. 228, Chemung Gr.

marcellense, Hall, 1884, Pal. N. Y., vol. 5 , pt. 1, p. 175, Marcellus Shale.

matheri, Hall, 1884, Pal. N. Y., vol. 5, pt. 1, p. 193, Chem ung Gr.

medon, Hall, 18St, Pal. N. Y., vol. 5, pt. 1, p. 197, Chemung Gr.

mentor, Hall, 1884, Pal. N. Y., vol. 5, pt. 1, p. 205, Chemung Gr.

mortoni, Hall, 1884, Pal. N. Y., vol. 5, pt. 1, p. 190, Chemung Gir.

mytiliforme, Hall, 1884, Pal. N. Y., vol. 5, pt. 1, p. 235, Chemung Gr.

navif rrme, Hall, 1884, Pal. N. Y., vol. 5, pt. 1, p. 200, Chemung Gr.

nereus, Hall, 1884, Pal. N. Y., vol. 5, pt. 1, p. 217, Chemung Gr.

orcus, Hall, 1884, Pal. N. Y., vol. 5, pt. 1, p. 215 , Chemung Gr.

orodes, Hall, 1884, Pal. N. Y., vol. 5, pt. 1, p. 206, Up. Chemung Gr.

orus, Hall, 1884, Pal. N.Y., vol. 5, pt. 1, p. 219, Chemung Gr.

parallela, Simpson, 1889, Dict. of $\mathrm{Pa}$. Foss., p. 332, Chemung Gr.

patulum,'Hall, 1884, Pal. N. Y., vol. 5, pt. 1, p. 226, Chenıung Gr.

pelop», Hall, 1884, Pal. N. Y., vol. 5, pt. 1, p. 214 , Up. Cheinung Gr.

phaon, Hall, 1884, Pul. N. Y., vol. 5, pt. 1, p. 230, Chemung Gr.

potens, Hall, 1894, Pal. N. Y., vol. 5, pt.1, p. 188, Up. Chemung Gr. potens var. juvene, Hall, 1884, Pal. N. Y., vol. 5, pt. 1, n. 189, Chemung Gr.

propinquum, Hall, 1884, Pal. N. Y., vol. 5, pt. 1, p. 231, Chemung Gr.

protextum, Conrad, 1842, (Avicula protexta,) Jour. Acar. Nat. Sci. Phil., vol. 8, p. 238, and Pal. N. Y., vol. 5, pt. 1, p. 183, Chemung Gr.

quadratum, Hall, 1884, Pal. N. Y., vol. 5, pt. 1, p. 233, Chemung Gr.

robustum, Hall, 1884, Pal. N. Y., vol. 5, pt. 1, p. 181, Cheınung Gr.

rogersi, Hall, 1884, Pal. N. Y., vol. 5, pt. 1, p. 176, Ham. Gr.

rude, Hall, 1884, Pal. N. Y., vol. 5, pt. 1, p. 221, Chemung Gr.

shumardi, Hall, 1881, Pal. N. Y., vol. 5, pt. 1, p. 180 , Chemung Gr.

sociale, Hall, 1884, Pal. N. Y., vol. 5, pt. 1, p. 187, Chemung Gr.

spinigerum, Conrad, 1812, (Avicula spinigera,) Jour. Acad. Nat. Sci. Phil., vol. 8, p. 237, and Pal. N. Y., vol. 5, pt. 1, p. 177, Chemung Gr.

stephani, Hall, 1884, Pal. N. Y., vol. 5, pt. 1, p. 194, Up. Chemung Gr.

transversum, Walcott, 1885, Monogr. U.S. Geo. Sur., vol. 8, p. 167, Chemung Gr.

truncatum, Hall, 1884, Pal. N. Y., vol. 5, pt. 1, p. 211, Chemung Gr.

umbonatum, Hall, 1884, Pal. N. Y., vol.5, pt. 1, p. 198, Chemung Gr.

umbonatum var. depressum, Hall, 1884, Pal. N. Y., vol. 5, pt. 1, p. 199, Chemung Gr.

Leptodonus, McCoy, 1844, Synopsis Carb. Foss. Ireland, p. 66. [Ety. leptos, slender; domus, honse.] Shell thin, short, oblong, tumid, subequivalve, inequilateral; beaks large, incurved; anterior side short, obtusely rounded, slightly gaping; deep ovate lunette between the beaks; posterior end broad, rounded, gaping, slope compressed, sides sulcated parallel with the ventral margin; dorsal margin inflected so as to form a lunette as long as the hinge-line; no hinge teeth; muscular impressions faint.

arata, Hall, 1860, Can. Nat. and Geo., vol. 5, p. 152 , Up. Silurian.

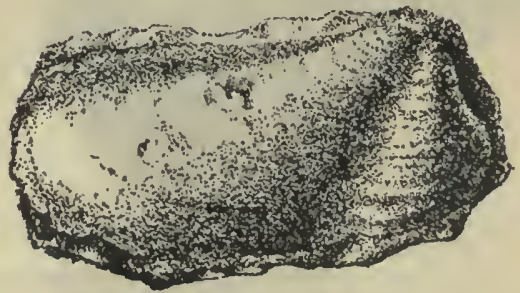

Fic. 839.-Leptodomus canadensis,

canadensis, Billings, 1874, Pal. Foss., vol. 2 , p. 54, Gaspe limestone No. 8, Devonian.

clavata, Winchell, 1862, Proc. Acad. Nat. Sci., p. 415, Purtage Gr. granosus, see Allorisma granosum. 
topekensis, see Sedgwickia topekensis.

undulatus, Whitfield, 1878, Ann. Rep. Geo. Sur. Wis, p. 81, and Geo. Wis., vol. 4, p. 293, Niagara Gr.

mainensis, Billings, 1863, Proc. Port. Soc. Nat. Hist., vol. 1, p. 118, Low. Held. Gr. pembrokensis, Billings, 1863, Proc. Port. Soc. Nat. Hist., vol. 1, p. 118, Low. Held. Gr.

percingulatus, Billings, 1863, Proc. Port. Soc. Nat. Hist., vol. 1, p. 119, Low Held. Gr.

Lima, Brugueire, 1791, Encycl. Meth. and Deshayes, 1824, Descrip. de Coquilles fossiles des environs de Paris. [Ety. lima, a file.] Not a Paleozoic genus. chesterensis, Worthen, not recognized. glabra, see Crenipecten glaber.

macroptera, see Limoptera macroptera. obsoleta, see Crenipecten obsoletus. retifera, see Crenipecten retiferus.

rugostriata, see A viculopecten rugistriatus.

Limopters, Hall, 1870, Prelim. Notice Lam.

Shells, p. 15, Up. Held. Gr. [Ety. Lima, a genus; pteron, a wing.] Large, inequivalve, inequilateral, subquadrate, alate posterior, auriculate anterior; ligamental area large, common, longitudinally striate; hinge with an oblique posterior tooth and cardinal folds beneath the beak; anterior inıpression deep, posterior large, pallial line simple formed of a series of small pits; interpallial area pitted for the attachment of umbonal muscles; test radiated. Type L. pauperata.

cancellata, Hall, 1870, Prelim. Notice Lam. Shells, p. 16, and Pal. N. Y., vol. 5, pt. 1, p. 244, Ham. Gr.

curvata, Hall, 1870, Prelim. Notice Lam. Shells, p. 18, and Pal. N. Y., vol 5, pt. 1, p. 250, Ham. Gr.

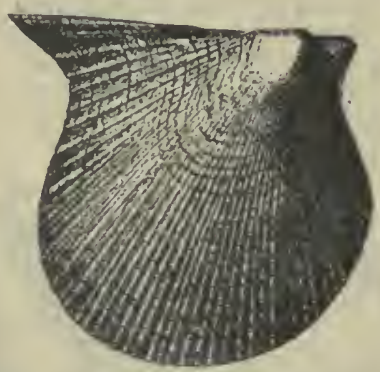

FIG. 840.-Limoptera macropteræ.

macroptera, Conrad, 1838, (Lima macroptera,) Ann. Rep. N. Y., p. 117, and Pal. N. Y., vol. 5, pt. 1, p. 246, Ham. Gr.

obsoleta, Hall, 1870, Prelim. Notice Lam. Shells, p. 18, and Pal N. Y., vol. 5, pt. 1 , p. 249 , Ham. Gr.

pauperata, Hall, 1870, Prelim. Notice Lam. Shells, p. 16, and Pal. N. Y., vol. 5 , pt. 1, p. 243 , Up. Held. Gr.

sarmenticia, Walcott, 1885 , Monogr. U. S. Geo. Sur., vol. 8, p. 167, Devonian.
Lithophag A, Lamarck, 1812, Hist. An. sans Vert. [Ety. lithos, stone ; phago, I eat.] Not American Palæozoic.

illinoisensis, Worthen, 1882, Bull. No. 1, Ill. St. Mus. Nat. Hist., p. 38, Keokuk Gr. Proposed instead of the form identified as L. lingualis of Phillips.

lingualis, Phillips, 1836, (Modiola lingualis,) Geol. Yorkshire, vol. 2, p. 209. Not American.

pertenuis, Meek \& Worthen, 1865, Proc. Acad. Nat. Sci. Phil., p. 245, and Geo. Sur. Ill., vol. 5, p. 539, St. Louis Gr.

Lillorina wheeleri, see Schizodus wheeleri.

Lucina, Bruguiere, 1792, Encyclop. Meth. [Ety. mythological name.] Type L. pennsylvanica. Notan A merican Palæozoic genus.

billingsana, see Paracyclas billingsana. elliptica, see l'aracyclas elliptica.

elliptica var. occidentalis, see Paracyclis elliptica var. occidentalis.

hamiltonensis, see Puracyclas hamiltonensis. lirata, see Paracyclas lirata.

occidentalis, Billings, 1859, Assiniboine and Saskatchewan Ex. Exped. This name was preoccupied by Morton for an Eocene species, see Paracyclas billingsana.

ohioensis, see Paracyclas ohioensis.

retusa, see Paracyclas retusa.

varysburgia, see Puracyclas varysburgensis.

wyomingensis, see Paracyclas wyomingensis.

Lunulicardium, Munster, 1840, Beitrage zur Petrefaktenkunde, $3 d$ heft, p. 69. [ [ Ety. lunula, a little moon; Cardium, a genus.] Equivalve, inequilateral, subelliptical, subcircular, or trigonal; posterior side obliquely truncate, nargin often reflexed and produced; beaks pointed ; cardinal line marked by a lunate hiatus ; surface radiated and concentrically marked; ligament external.

acutirostrum, syn. for L. ornatum.

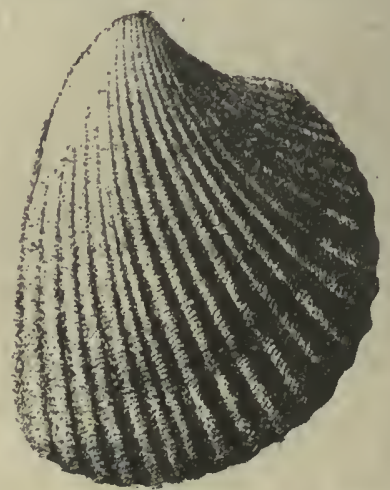

FIG. 811.-Lunulicardium curtusu.

curtum, Hall, 1884, Pal. N. Y., vol. 5, pl.71, figs. 18-23, Up. Held. Gr. 
fragile, Hall, 1843, (Avicula fragilis,) Geo. Rep. 4th Dist. N. Y., p. 222, and Pal. N. Y., vol. 5, pl. 71, figs. 1-14, Genesee Shale.

fragosum, Meek, 1877, (Posidonomya fragosa,) U. S. Geo. Expl. 40th Parallel, vol. 4, p. 92 , Carboniferous.

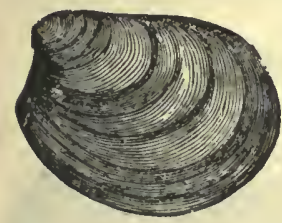

Frg. 842,-Lunulfeardium marcellense. vol. 5, p. 436, Marcellus Shale.

marcellense, Vanuxem, 1842, (Cypricardites marcellensis,) G e o. Rep. $3 \mathrm{~d}$ D i s t . N. Y., p. 146, and Pal. N. Y., vol. 5, pl. 71 , figs. 15-16. Marcellus Shale. orbiculare, $\mathrm{H}$ a 11 , 1885, Pal. N. Y., ornatum, Hall, 1843, (Pinnopsis ornata,) Geo. Rep. 4th Dist. N. Y., p. 244, and Pal. N. Y., vol. 5, p. 437, Portage Gr.

rude, Hall, 1884, Pal. N. Y., vol. 5, pl. 71, fig. 17, Marcellus Shale.

transversum, Hall, 1885, Pal. N. Y., vol. 5, p. 439, Chemung Gr.

Lyonsia, Turton, 1822. Not found in Palæozoic rocks.

concava, see Sedgwickia concava.

Lyriopecten, Hall, 1884, Pal. N. Y., vol. 5, p. 3. (Plates and Explanations.) [Ety. lyrion, a lyre; Pecten, a genus.] Distinguished from Aviculopecten by the shorter hinge-lîne and very small anterior wing; surface with strong rays. Type L. magnificus.

alternatus, Simpson, 1889, Dict. Foss. Pa., p. 366, and Trans. Am. Phil. Soc., p. 446, Chemung Gr.

anomiiformis, Hall, 1884, Pal. N. Y., vol. 5, pt. 1, p. 53, Up. Held. Gr.

cymbalon, Hall, 1884, Pal. N. Y., vol. 5, pt. 1, p. 47, Ham. Gr.

dardanus, Hall, 1884, Pal. N. Y., vol. 5, pt. 1, p. 41, Up. Held. Gr.

fasciatus, Hall, 1884, (Pernopecten fasciculatus,) Pal. N. Y., vol. 5, pt. 1, p. 55, Chemung Gr.

interradiatus, Hall, 1884, Pal. N. Y., vol. 5, pt. 1, p. 44, Ham. Gr.

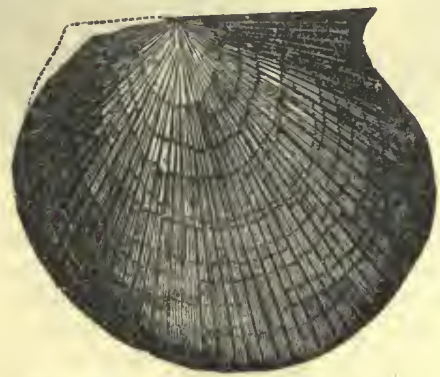

FIG. 843.-Ly rlopecteu orbiculatus.

macrodontus, Hall, 1884, Pal. N. Y., vol. 5, pt. 1, p. 46, Up. Held. Gr.

magnificus, Hall, 1884, Pal. N. Y., vol. 5, pt. 1, p. 51, Up. Chemung Gr. orbiculatus, Hall, 1843, (Avicula orbiculata,) Geo. Rep. 4th Dist., N. Y., p. 202, and Pai. N. Y., vol. 5, pt. 1, p. 42, Ham. Gr.

parallelodontus, Hall, 1884, Pal. N. Y., vol. 5 , pt. 1 , p. 40 , Up. Held. Gr.

polydorus, Hall, 1884, Pal. N. Y., vol. 5, pt. 1, p. 50, Chemung Gr.

priamus, Hall, 1884, Pal. N. Y., vol. 5, pt. 1, p. 54, Chemung Gr.

solox, Hall, 1884, Pal. N. Y., vol. 5, pt. 1, p. 56, Up. Chemung Gr.

tricostatus, Vanuxem, 1842, (Avicula tricostata,) Geo. Sur. 3d Dist. N. Y., p. 179, and Pal. N. Y., vol. 5, pt. 1, p. 48, Chemung Gr.

Lyrodesma, Conrad, 1841, Ann. Geo. Rep. N. Y., p. 51. [Ety. lyra, a harp; desma, a ligament.] Equivalve, inequilateral, semicircular; hinge plate with 6 to 8 angular, crenulated teeth radiating from beneath the beak upon a more or less rounded platform. Type L. planum.

cincinnatiense, Hall, 1871, 24th Rep. N. Y. St. Mus. Nat. Hist., p. 227, Hud. Riv. Gr.

planum, Conrad, 1841, Ann. Geo. Rep. p. 51, Hud. Riv. Gr.

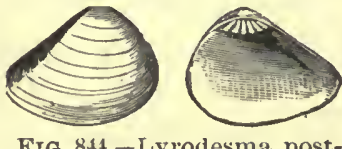

Fra. 844.-Lyrodesma poststriatum. vol. 1, p. 151, Black Riv. Gr.

poststri a t u m, $\mathrm{E} \mathrm{m} \mathrm{m}$ o $\mathrm{n} \mathrm{s}$, 1842, (Nuculana poststriata,) Geo. Rep. N. Y., p. 399, and Pal. N.Y., pulchelluin, Hall, 1847, Pal. N. Y., vol. 1, p. 302, Hud. Riv. Gr.

Macrodon, Lycett, 1845, Murch. Geo. Chelt. [Ety. macros, long; odous, a tooth.] Shell oblong, very inequilateral, moderately tumid, a byssal sinus in the anterior third of the ventral margin anterior edges of the adductor impressions prominent; hinge teeth at the an. terior e $\mathrm{n} d$ few, slightly oblique or nearly at right angles to the hinge.

line be -

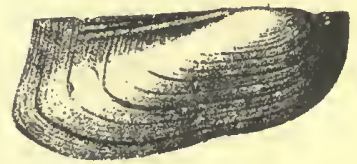

neath the FrG. 815.-Macrodonobsoletus. beak, be- FrG. 815.-Macrodonobsoletus. coming more oblique toward the anterior end ; posterior part of the hingeline, from beak to anal angle, occupied by one to three long lateral teeth.

carbonarius, Cox, 1857, (Arca carbonarius,) Geo. Sur. Ky., vol. 3, p. 567, Coal Meas.

chemungensis, Hall, 1870, Prelim. Notice Lam. Shells, p. 14, and Pal. N. Y., vol. 5, pl. 51, figs. 11-16, Chemung Gr. cochlearis, Winchell, 1863, Proc. Acad. Nat. Sci., p. 16, Marshall Gr. Prof. Hall suggests that it is a syn. for $M$. parvus. 
curtus, Hartt, 1868, Acad. Geol., p. 302, Carb.

delicatus, Meek \& Worthen, 1870, Proc. Acad. Nat. Sci., p. 40, and Geo. Sur. Ill., vol. 5 , n. 575 , Up. Coal Meas.

hamiltonix, Hall, 1870, Prelim. Notice Lam. Shells, p. 13, and Pal. N. Y., vol. 5, pl. 51. figs. 1-10, Hain. Gr.

hardingi, Hartt, 1868, Acad. Geol., p. 302, Carb.

micronema, Merk \& Worthen, 1866, Proc. Acad. Nat. Sci., p. 261, Kaskaskia Gr.

obsoletns, Meek, 1871, Reg. Rep. University W. Va., p. 5, and Pal. Ohio, vol. 2, p. 334, Coal Meas.

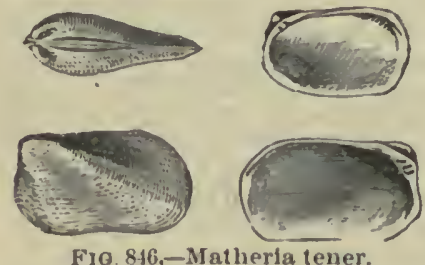

ovatus, Hall, 1870, Prelim. Notice Lam. Shells, p. 15, and Pal. N. Y., vol. 5, p. 351 , Waverly Gr.

parvus, White \& Whitfield, 1862, Proc. Bost. Soc. Nat. Hist., vol. 8, p. 299, Kinderlıook $\mathrm{G}$ r.

sangamonensis, Worthen, (in press,) Geo. Sur. 11l., vol. 8, p. 123 Coal Meas.

shubenacarliensis, Hartt, 1868, Acad. Gr o. p. 302, Carb.

tennisiriatus, Meek \& Worthen, 1867, Proc. Chi. Acad. Sci., vol. 1, p. 17, Up. Coal Mras.

truncat us, Walcott, 1885, Monogr. U. S. Geo.Sur., vol. 8, p. 243, Carboniferous. Matieris, Billings, 1858, Can. Nat. and Geo., vol. 3, p. 440. [Ety. proper name.] Equivalve, inequilateral ; beaks anterior; two small, obtuse, cardinal teeth in the left valve, and one in the right; no lateral teetli ; two muscular impressions; ligament external. Type M. tener.

tener, Billings, 1858, Can. Nat. and Geo., vol. 3, p. 440, Trenton Gr.

Megalovus, Hall, 1852, Pal. N. Y., vol. 2, p. 343. [Ety. megas. great; omos, shoulder.] Large, equivalve, concentrically lined, longitudinal; u mbones anterior incurved; shell thick and along the hinge-line thickened on the interior; muscular impression large and derp, with two small circular pits abuve. Type M. canadensis. canadensis, Hall, 1852, Pal. N. Y., vol. 2, p. 343 , Guel,, Gr.

comprtssus, Nirholson \& Hinde, 1874, Can. Jour.. vol. 14 , p. 159, Niagara Gr.

Megambonia. Hall, 1859, Pal. N. Y., vol. 3, p. 273. [Ety. megas, great; ambon, the boss of a shield.] Equivalve, inequilateral, subovoid, gibbous in the middle and toward the umbones; anterior side lobed or auriculate; muscular impression large; posterior cardinal margin expanded or alate; hinge-line crenulated anteriorly; teeth numerous; sur. face concentrically lined, and sometimes with radiating strix. Type M. suborbicularis.

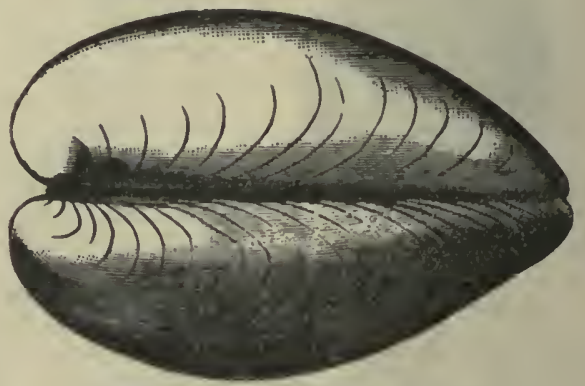

via. 817,-Megatomus canadensis.

aviculoidea, Hall, 1859, Pal. N. Y., vol. 3, p. 274, low. Held. Gr.

bellistriata, Hall, 1859, Pal. N. Y., vol. 3, p. 467 , Oriskany sandstone.

cancellata, Hall, 1860, Can. Nat. and Geo., vol. 5 , p. 153 , Up. Siluriяn.

cardiiformis, Hall, 1843, (Pterinea cardiiformis, ) Geo. Rep. 4th Dist. N. Y., p. 172, and Pal. N. Y., vol. 5, p. 515, Cornif. $\mathrm{Gr}$.

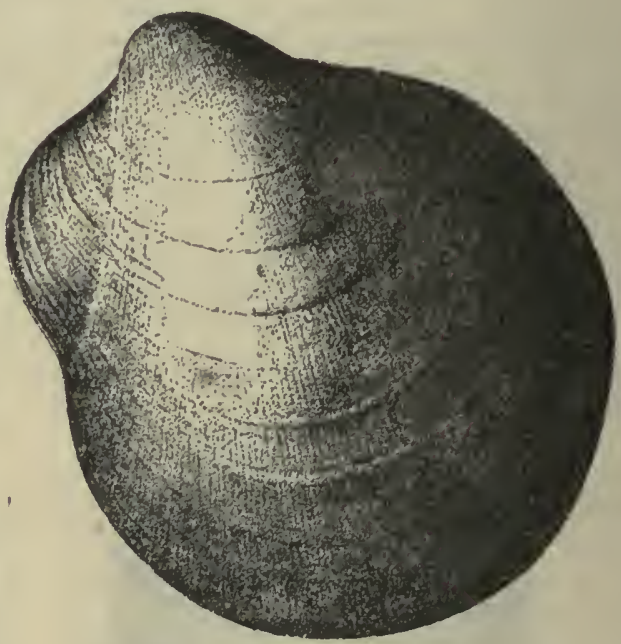

Fia. 848.-Megambonia curditformis.

cordiformis, see Mytilarca cordiformis.

jamesi, see Ambonychia jamesi.

lamellosa, Hall, 1859, Pal. N. Y., vol. 3, p. 467, Oriskany sandstone.

lata, Hall, 1859, Pal. N. Y., vol. 3, p. 277, Low. Held. Gr.

lyoni, syn. for Cardiopsis radiata.

mytiloi,lea, Hall, 1859, Pal. N. Y., vol. 3, p. 276, Low. Held. Gr. 
oblonga, Hall, 1859, Pal. N. Y., vol. 3, p. 277, Low. Held. Gr.

obscura, Hall, 1859, Pal. N. Y., vol. 3, p. 277, Low. Held. Gr.

occidualis, Walcott, 1885, Monogr. U. S. Geo. Sur., vol. 8, p. 173, Devonian.

ovata, Hall, 'syn. for Plethomytilus mytilimeris.

ovoidea, Hall, syn. for Plethomytilus mytilimeris.

rhomboidea, Hall, 1859, Pal. N. Y., vol. 3, p. 275, Low. Held. Gr.

spinneri, Hall, 1859, Pal. N. Y., vol. 3, p. 274, Low. Held. Gr.

striata, Hall, 1860, Can. Nat. and Geo., vol. 5, p. 153, Up. Silurian.

subcardiformis, Hall, syn. for M. cardiiformis.

suborbicularis, Hall, 1859, Pal. N. Y., vol. 3 , p. 273, Low. Held. Gr.

Megaptera, Meek \& Worthen, 1866. The name was preoccupied.

Microdon, Conrad, 1842, Jour. Acad. Nat. Sci., vol. 8, p. 247. This name was applied by Agassiz to a genus of fish in 1833, and was also preoccupied for a genus of insects. Hall proposed Eodon, in 1877, but Whitfield has shown that M. bellistriatus is a Cypricardella, and hence the latter name has priority.

bellistriatus, see Cypricardella bellistriata. complanatus, see Cypricardella complanata. gregarius, see Cypricardella gregaria. reservatus, see Cypricardella reservata.

tenuistriatus, see Cyricardella tenuistriata.

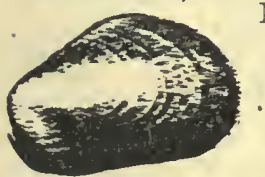

FrG. 849. - Modlella pygmæa. Modielua, Hall, 1884, Pal. N. Y., vol. $5, \mathrm{p}$. 4. (Plates and EXplanations.) [Ety. modus, a measure; ellus, diminutive.] Subrhomboidal, narrowed and auriculate in front, broadly expanding posteriorly; two well-marked muscular impressions connected by a simple pallial line; surface with radiating striæ. Tppe M. pygmæa.

pygmæa, Conrad, 1842, (Pterinea pygmæa,) Jour. Acad. Nat. Sci., vol. 8, p. 251, and Pal. N. Y., vol. 5, pl. 76, figs. 9-20, Ham. Gr.

Modiola, Lamarck, 1801, Syst. An. sans Vert. [Eto. modiolus, a small measure or drinking vessel.] Oblong, inflated in front, umbones anterior, obtuse, no teeth; pedal impressions three, the central one elongated. Type M. modiolus. Not a Palæozoic genus. Species are only left here for want of material to properly determine the generic relations. avonia, Dawson, 1868, Acad. Geol., p. 301, Subcarboniferous.

concentrica, see Modiomorpha concentrica. illinoisensis, Worthen, 1884, Bull. No. 2, Ill. St. Mus. Nat. Hist., p. 16, and Geo. Sur. Ill., vol. 8, p. 125, St. Louis Gr. lingualis, see Lithophaga lingualis. metella, see Mytilops metella. minor, Lea, 1852, Jour. Acad. Nat. Sci., 2d series, vol. 2, Coal Meas. Not determinable.

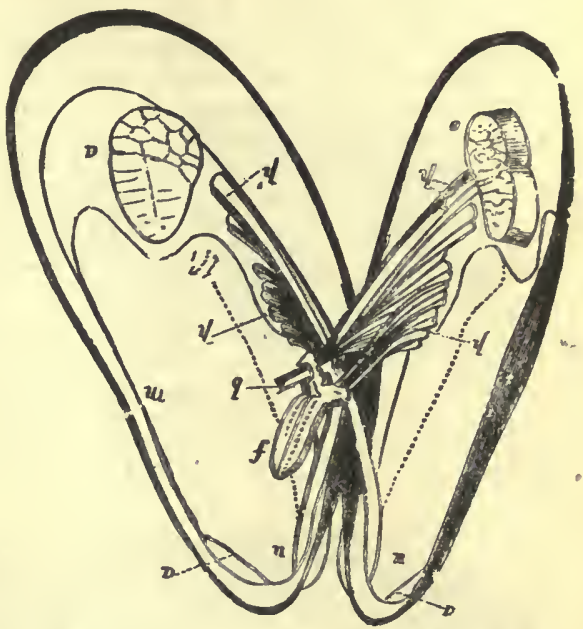

FrG. 850.-Modiola modiolus. $a \alpha$, Anterlor adductors; $\alpha^{\prime} \alpha^{\prime}$, posterlor adductors ; $u u, p^{\prime} p^{\prime}$, pedal muscles ; $p p$, byssal muscles ; $f$, foot; $b$, byssus : $m$, palliai line.

nevadensis, Walcott, 1885, Monogr. U. S. Geo. Sur., vol. 8, p. 239, Subcarboniferous.

obtusa, see Modiolopsis obtusa.

pooli, Dawson, 1868, Acad. Geol., p. 301, Low. Carb. Founded upon a small cast destitute of characters.

præcedens, see Mytilops præcedens.

wyomingensis, Lea, 1852, Jour. Acad. Nat.

Sci., 2 d series, vol. 2, p. 205, Coal Meas.

Not recognized, but probably an Anthracomya.

Modiolopsis, Hall, 1847, Pal. N. Y., vol. 1, p. 157. [Ety. Modiola, a genus of shells; opsis, appearance; from its resemblance to Modiola.] Equivalve, inequilateral, elongated, broader posteriorly; umbones anterior; cardinal teeth short, oblique; single, deep, subcircular anterior muscular impression ; ligament external; no area; surface concentrically lined. Type M. modiolaris.

adrastia, Billings, 1862, Pal. Foss., vol. 1, p. 45 , Black Riv. Gr.

anodontoides, Conrad, 1847, (Cypricardites anodontoides,) Pal. N. .Y., vol. 1, syn. for M. sinuata.

arcuata, Hall, 1847, Pal. N. Y., vol. 1, p. 159, Trenton Gr.

aviculoides, Hall, 1847, Pal. N. Y., vol. 1, p. 161, Trenton Gr.

cancellata, Walcott, 1879, Trans. Alb. Inst., vol. 10, p. 22, Utica Slate Gr.

capax, n. sp. Shell very large, oblong; cardinal and basal lines behind the beaks subparallel; basal margin slightly contracted by an undefined cincture arising below the beaks; posterior end broadly rounded; depressed in front of 
the beaks; anterior end rounded; beaks large, obtuse, and extending beyond the hinge-line; umbones large; surface marked with concentric lines of growth, and with strong transverse lines over the umbonal region, some of which extend nearly to the basal line, the anterior ones curve a little forward in passing over the umbones. Collected by the author in the Hud. Riv. Gr. at Versailles, Indiana. carinata, Hall, 1847, Pal. N. Y., vol. 1, p. 160, Trenton Gr.

carrollensis, Worthen, 1882, Bull. No. 1,

Ill. St. Mus. Nat. Hist., Galena Gr. Proposed instead of MI. subnasuta of Meek \& Worthen, 1870, Proc.Acad. Nat. Sci., p. 41 , which was preoccupied.

cincinnatiensis, Hall \& Whitfeld, 1875 ,

Ohio Pal., vol. 2, p. 88, Utica Slate.

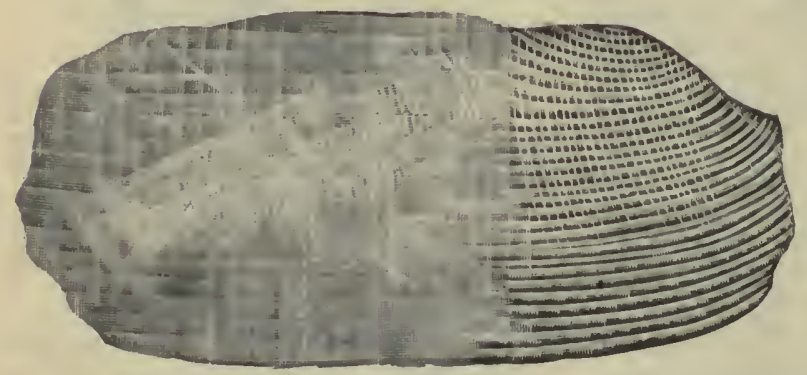

Fig. Sïl.-Modiolopsis capax.

concentrica, Hall \& Whitfield, 1872, Olıio Pal., vol. 2, p. 86, Hud. Riv. Gr.

curta, Hall, 1847, Pal. N. Y., vol. 1, p. 297, Hud. Riv. Gr.

dicteus, Hall, 1867, 20th Rep. N. Y. Mus. Nat. Hist., p. 385, Niagara Gr.

(?) dubia, Hall, 1859, Pal. N. Y., vol. 3, p. 264, Low. Held. Gr.

exilis, Billings, 1874, Pal. Foss., vol. 2, p. 132, Up. Sil.

faba, Emmons, 1842, Ger. Rep. 2d Dist. N. Y., p. 395, and Pal. N. Y., vol. 1, p. 158, Black Riv., Trenton, and Hud. Riv. Grs.

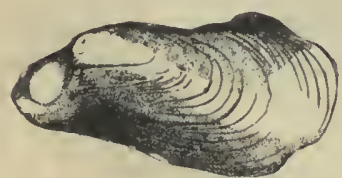

FIG. 852,-Modiolopsis faberi. Jeft valve. faberi, n. sp. Shell elongate, nearly twice as long as high; below the average size of species in this genus; broadest behind the middle, and much contracted in front of the beaks; hinge-line nearly straight from the anterior end to the middle; it then becomes arcuate to near the posterior end, which is abruptly rounded; basal line slightly sinuate at the anterior third from the cincture, directed downward and backward from the anterior part of the umbo; beaks strong, projecting anteriorly beyond the hinge-line; umbones high, subangular, and gradually declining toward the posterobasal line; anterior muscular impression Fia. 853.-Modiolopsis faberl. very large, Cardinal vlew, sh ow ing c ircular, toward the posterlor. deep, and situate at the anterior end, in front of and below the beaks; dorsal ligament very large. Distinguished from M. concentrica in its general outline, more elongate form, more prominent beaks, and higher and more angular umbones. Hud. Riv. Gr., at Cincinnati, $O$. The specimen figured is from the collection of Charles Faber.

gesueri, Billings, 1862, Pal. Foss, vol. 1, 1. 43, Trenton and Black Riv. Grs.

latus, see Cypricardites latus.

maia, Billings, 1862, Pal. Foss., vol. 1, p. 44, Trenton Gr.

meyeri, Billings, 1862, Pal. Foss., vol. 1, p. 42, Trenton $\mathrm{Gr}$.

modiolaris, Conrad, 1838, (Pterinea modiolaris,) Ann. Geo. Rep. N. Y., p. 118, and Pal. N. Y., vol. 1, p. 294, Hud. Riv. Gr. modioliformis, Meek \& Worthen, 1868, Geo. Sur. Ill., vol. 3, p. 294, I'renton $\mathrm{Gr}$.

mytiloides, Hall, 1847, Pal. N. Y., vol. 1, p. 157, Black Riv. and Trenton Grs. nais, Billings, 1862, Pal. Foss., vol. 1, p. 45, Black Riv. Gr.

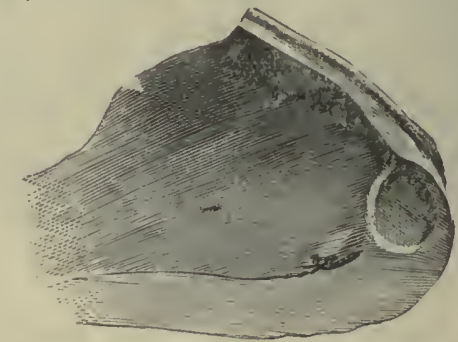

FIG. 854.-Modiolopsis modiolaris. Hinge and pallial liue and muscular impression.

nasuta, Conrad, 1841, (Cypricardites nasutus, Ann. Rep. N. Y., p. 52, and Pal. N. Y., vol. 1, p. 159, Trenton and Hud. Riv. Grs.

nuculiformis, see Tellinomya nuculiformis. obtusa, Hall, 1847, (Modiola obtusa,) Pal.

N. Y., vol. 1, p. 40, Bırdseye Gr.

occidens, Walcott, 1885, Monogr. U. S.

Geo. Sur., vol. 8, p. 77, Trenton Gr. 
orthonota, Conrad, 1839, (Unio orthonotus,) Ann. Rep. N. Y., p. 66, and Geo. Rep. 4th Dist. N. Y., pl. 2, figs. 8 and 9, Medina sandstone.

orthonota, Meek \& Worthen, 1868, Geo. Sur. Ill., vol. 3. This name was preoccupied. See M. rectiformis.

ovata, Hall, 1852, Pal. N. Y., vol. 2, p. 101, Clinton Gr.

parallela, see Orthodesma parallelum.

parviuscula, Billings, 1859, Can. Nat. and Geo., vol. 4, p. 446, Chazy Gr.

perlata, Hall, 1876, 28th Rep. N. Y. Mus. Nat. Hist., p. 172, Niagara Gr.

perovata, see Modiomorpha perovata.

pholadiformis, Hall, 1851, Lake Sup. Land Dist., vol. 2, p. 213, Hud. Riv. Gr.

plana, Hall, 1861, Geo. Rep. Wis., p. 30, Trenton Gr.

pogonipensis, Walcott, 1885, Monogr. U.S. Geo. Sur., vol. 8, p. 78, Trenton Gr.

prisca, Walcott, 1887, Am. Jour. Sci. and Arts, 3d ser., vol. 34, p. 191, Up. Taconic. Not a Modiolopsis.

primigenia, Conrad, 1838, (Unio primigenius,) Ann. Rep. N. Y., p. 66, and Geo. Rep. 4th Dist. N. Y., pl. 2, fig. 3, Medina sandstone.

recta, Hall, 1867, 20th Rep. N. Y. Mus. Nat. Hist., p. 386, Niagara Gr.

rectiformis, Worthen, 1882 , Bull. No. 1, Ill. St. Mus. Nat. Hist., p. 38, Trenton Gr. Proposed instead of M. orthonota, Meek \& Worthen, 1868, Geo. Sur. Ill., vol. 3 , p. 295 , which was preoccupied.

rhomboidea, Hall, 1860, Can. Nat. and Geo., vol. 5, p. 148, Up. Sil.

rudis, Billings, 1874, Pal. Foss., vol. 2, p. 133, Up. Sil.

sinuata, Emmons, 1842, (Cypricardites sinuatus,) Geo. Rep. 2d Dist. N. Y., p. 399, and Pal. N. Y., vol. 1, p. 298, Hud. Riv. Gr.

striata, Billings, 1866, Catal. Sil. Foss. Antic., p. 48, Anticosti Gr.

subalata, Hall, 1852, Pal. N. Y., vol. 2, p. 84, Clinton and Niagara Grs.

subcarinata, Hall, 1852, Pal. N. Y., vol. 2, p. 601, Clinton Gr.

subnasuta, Hall, 1860, Can. Nat. and Geo., vol. 5 , p. 148 , Up. Sil.

subnasuta, Meek \& Worthen. See M. cancellata.

subrhomboidea, Simpson, 1889, Trans. Am. Phil. Soc., p. 450, and Dict. Foss., Pa., p. 411, Clinton Gr.

subspatulata, see Cypricardites subspatulatus.

superba, Hall, 1861, Geo. Rep. Wis., p. 31, Trenton Gr.

terminalis, Hall, 1847, Pal. N. Y., vol. 1, p. 318, Hud. Riv. Gr.

trentonensis, Hall, 1847, Pal. N. Y., vol. 1, p. 161, Trenton Gr.

truncata; Hall, 1847, Pal. N. Y., vol. 1, p. 296, Hud. Riv. Gr.

undulostriata, Hall, 1852, Pal. N. Y., vol. 2, p. 284, Niagara Gr.

unionoides, Meek, 1871, (Anodontopsis unionoides, Am. Jour. Sci. and Arts, 3d ser., vol. 2, p. 299, and Ohio Pal., vol. 1, p. 141, Hud. Riv. Gr.

varia, Billings, 1874, Pal. Foss., vol. 2, p. 56, Low. Held. Gr.

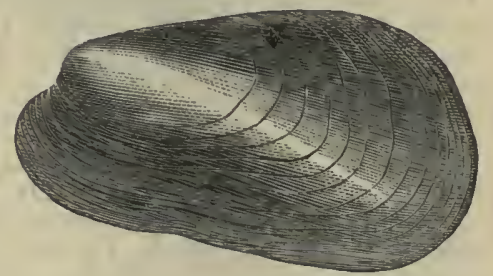

FIG. 855.-Modiolopsis versaillesensis. Left valve.

versaillesensis, S. A. Miller, 1874, Cin. Quar. Jour. Sci., p. 150, Hud. Riv. Gr.

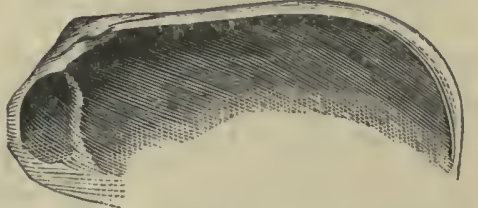

F'1G. 856.-Modiolopsis versaillesensis. Hingeline and muscular impression.

Modomorph , Hall, 1870, Prelim. Notice Lam. Shells, p. 72. [Ety. contracted from Modiola, a genus; morphe, form.] Equivalve, subovate, larger posteriorly, compressed; beaks small; sinus oblique and constricting the base; surface concentrically undulated; single tooth in the left valve, and corresponding socket in the other; no lateral teeth; ligament external. Type M. concentrica.

affinis, Hall, 1885, Pal. N. Y., vol. 5, p. 284, Ham. Gr.

alta, Conrad, 1841, (Cypricardites alta,) Ann. Rep. N. Y., p. 52, and Pal. N. Y., vol. 5, pl. 37, figs. 1-16, Ham. Gr.

altiformis, Walcott, 1885, Monogr. U. S. Geo. Sur., vol. 8, p. 169 , Devonian.

ambigua, Walcott, 1885, Monogr. U. S. Geo. Sur., vol. 8, p. 239, Carboniferous. arcuata, Hall, 1884, Pal. N. Y., vol. 5, pl. 36, fig. 21, Ham. Gr.

chemos, Clarke, 1885, Bull. U. S. Geo. Sur., No. 16, p. 30 , Genesee shales.

clarens, Hall, 1885, Pal. N. Y., vol. 5, p. 273, Up. Held. Gr.

complan at a, Hall, 1870 , Prelim. Notice Lam. Shells, p. 73 , and Pal. N. Y., vol. 5, p. 272 , Up. Held. Gr. concentrica, Conrad, 1838, (Pte-

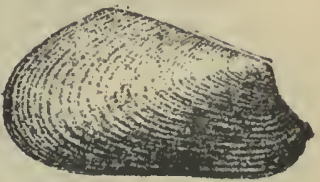

FIG. 857.-Modlomorpha concentrica.

rinea concentrica,) Ann. Rep. Geo. Sur. N. Y., p. 116, and Pal. N. Y., vol. 5, pl. 34, figs. 9-10, Ham. Gr. 
cymbula, Hall, 1870, Prelim. Notice Lam. Shells, p. 75 , and Pal. N. Y., vol. 5 , pl. 36 , figs. $19-20$, Ham. Gr.

desiderata, Walcott, 1885, Monogr. U. S. Geo. Sur., vol. 8, p. 240, Carboniferous. byalea, Hall, 1870, Prelim. Notice Lam. Shello, p. 79, and Pal. N. Y., vol. 5, pl. 41, figs. 28-30, IVaverly Gr.

inornata, Billings, 1874, Pal. Foss., vol. 2, p. 52, Deronian.

linguiformis, Hall, 1883, Pal. N. Y., vol. 5, pl. 34, figs. 15-17, Up. Held. Gr.

macilenta, Hall, 1870, Prelim. Notice Lam. Shells, p. 76, and Pal. N. Y., vol. 5, pl. 37 , fig. 17, and pl. 39 , figs. 17-21, Ham. Gr.

mytiloides, Conrad, 1841, (Cypricardites mytiloidts, Ann. Rep. Geo. N. Y., p. 52, and Pal. N. Y., vol. 5, p. 277, Ham. Gr. neglecta, Hall, 1883, Pal. N. Y., vol. 5, pl. 41, figs. 12-13, Chemung Gr.

oblonga, WValcott, 1885, Monogr. U. S. Geo. Sirr., vol. 8, p. 170, Devonian.

obtusa, Walcott, 1885, Monogr. U. S. Geo. Sur., vol. 8, p. 171, Devonian.

perovata, Meek \& Worthen, 1865, (Modiolopsis perorata,) Proc. Acad. Nat. Sci. Plıil., p. 246, and Geo. Sur. Ill., vol. 3, p. 438 , Ham. Gr.

pintoensis, Walcott, 1885, Monogr. U. S. Geo. Sur., vol. 8, p. 240, Carboniferous. planulata, Hall, 1870, Prelim. Notice Lam. Shells, p. 74, syn. for M. mytiloides.

ponderosa, Hall, 1870, (Sanguinolites ponderosus, ) Prelim. Not. Lam. Sbells, p. 35, and Pal. N. Y., vol. 5, pl. 34, fig. 11, Up. Held Gr.

putillus, Hall, 1883, Pal. N. Y., vol, 5, pl. 41, tigs. 1-2, Schoharie grit.

quadrula, Hall, 1870, Prelim. Notice Lam. Shells, p. 77, and Pal. N. Y., vol. 5, pl. 41, figs. 18-26, Chemung $\mathrm{Gr}$.

recta, Hall, 1885, Pal. N. Y., vol. 5, p. 286 , Ham. Gr.

recurva, Hall, 1883, Pal. N. Y., vol. 5, pl. 41, 6g. 17. Chemung Gr.

regularis, Hall, 1885, Pal. N. Y., vol. 5, p. 270 , Schoharie grit.

rigida, Hall, 1883, Pal. N. Y., vol. 5, pl. 41, figs. 10, 11, 14-16, Chemung $\mathrm{Gr}$.

rigidula, Simpson, 1889, Trans. Am. Phil. Soc., p. 449, and Dict. Foss. Pa., p. 415, Chemung Gr.

schoharie, Hall, 1884, Pal. N. Y., vol. 5, p. 269 , pl. 34 , fig. 13 , Schoharie grit.

subalata, Conrad, 1841, Cypricardites subalatus, ) Ann. Rep. N. Y., p. 83, and Pal. N. Y., vol. 5, pl. 39, figs. 1-16, Ham. Gr.

subalata var. chemungensis, Hall, 1885 , Pal. N. Y., vol. 5, p. 284, Chemung Gr. subangulata, Hall, 1885, Pal. N. Y., vol. 5, p. 287, Chemung Gr.

tioga, Hall, 1885, Pal. N. Y., vol. 5, p. 291, Chemung Gr.

Monopteria, Meek \& Worthen, 1866, Proc. Chi. Acad. Nat. Sci, vol. 1, p. 20. [Ety. monos, single; pteron, a wing.] Aviculoid, obliquely produced, angular pos-

teriorly, rounded in front, subequiralve, both valves convex; posterior wing slender, produced, anterior one obsolete or drawn back between the beaks, in a deep lunule; no byseal emargination, but a little gaping in the lunule; muscular impressions faint; cardinal area narrow, with few longitudinal cartilaye furrows; hinge edentulous. Type M. longispina.

auricula, Stevens, 1858, (Gervillia auricula,) Am. Jour. Sci. and Arts, 2d ser., vol. 25, p. 265, Coal Meas.

gibbosa, Meek \& Worthen, 1866, Proc. Chi. Acad. Sci., p. 20, Coal Meas.

longispina, Cox, 1857, (Gervillia longispin a,) Geo. Sur. Ky., vol. 3 , p. 568, Coal Meas.

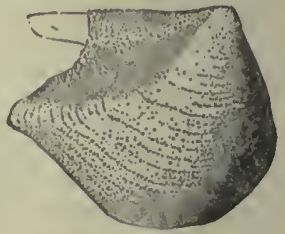

F19. 858.-Monopteria gibbosi.

marian, White, 1874, Rep. Invert. Foss., p. 22, and Geo. Sur. W. 100th Mer., vol. 4, p. 151, Carboniferous.

Mosotis, Bronn, 1824, System Urweltlicher Konchylien. [Ety. monos, one ; ous, otos, ear.] Obliquely oval, compressed, radiated; anterior side short, rouncled; posterior slighty eared. Type M. salinaria.

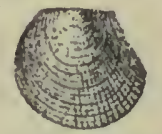

Fid. $859 .-\mathrm{Mo}$. notis gregaria. elevata, see Panenka elevata. gregaria. Meek \& Worthen, 1870, Proc. Acad. Nat. Sci. Phil., p. 38, and Geo. Sur. Ill., vol. 5, p. 573, Coal Meas.

halli, Swallow, 1858, Trans. St. Louis Acad. Sci., vol. 1, p. 185 , Permian Gr.

hawni, see Pseudomonotis hawni. poulsini, see Panenka poulsoni.

princeps, see Aviculopect $u$ princeps. radialis, Phillips, 1834, (Pecten radialis,) see Pseudomonotis radialis.

radians, see Panenka radians.

septentrionalis, Haughton, 1857, Jour. Roy. Dub. Soc., vol. 1, (?) Gr.

speluncaria, Schlotheim, 1816, Denkschriften d. k. Ac. d. WViss. zu Munchen, p. 30, (Gryphites speluncarius,) P'ermian Gr. Probably not American.

variabilis, Swallow, 1858, Trans. St. Louis Acad. Ści., vol. 1, p. 187, Permian Gr.

Mralina, DeKoninck, 1844, Desc. Anim. Foss. Carb. Belg., p. 125. [Ety. Mya, a genus of shells; inus, like.] Subrhomboidal, inequilateral, inequivalve, oblique, slightly sinuous in front for the passage of the byssus; beaks pointed, nearly terminal ; surface smootli or concentrically marked; hinge edentulons ; ligamental area broad and furrowed parallel to the linge-line; muscular and pallial impressions apparently as in Pteria; shell structure prismatic. Type M. lamellosa. 
angulata, Meek \& Worthen, 1860, Proc. Acad. Nat. Sci. Phil., p. 455, and Geo. Sur. Ill., vol. 2, p. 300, Kaskaskia Gr.

apachesi, Marcou, 1858, Geol. North America, p. 44, Subcarboniferous.

aviculoides, Meek \& Hayden, 1860, Proc. Acad. Nat. Sci. Phil., p. 18t, and Pal. Up. Mo., p. 51, Permian Gr.

aviculoides, Winchell, see M. rara.

concava, Swallow, 1858, (Mytilus concavus, ) Trans. St. Louis Acad. Sci., vol. 1, p. 188, Permian Gr.

concentrica, Meek \& Worthen, 1860, Proc. Acad. Nat. Sci. Phil., p. 456, and Geo. Sur. Ill., vol. 2, p. 281, Warsaw Gr.

congeneris, Walcot.t, 1885, U. S. Geo. Sur., vol. 8 , p. 237 , Subcarboniferous.

cuneiformis, Gurley, 1883, New Carb. Foss., p. 4. Publication invalid.

deltoidea, Gabb, 1859, Proc. Acad. Nat. Sci. Phil., p. 297, Subcarboniferous.

imbricaria, Winchell, 1862, Proc. Acad. Nat. Sci., p. 412, Marshall Gr.

iowensis, Winchell, 1865, Proc. Acad. Nat. Sci., p. 127, Chemung Gr.

kansasensis, Shumard, 1858, Trans. St. Louis Acad. Sci., vol. 1, p. 213, Coal Meas.

keokuk, Worthen, 1875, Geo. Sur. Ill., vol. 6, p. 524, Keokuk Gr.

meliniformis, Meek \& TVorthen, 1866, Proc. Chi. Acad. Sci., vol. 1, p. 19, Coal Meas.

michiganensis, Winchell, 1862, Proc. Acad. Nat. Sci., p. 411, Marshall Gr.

mon roensis, Worthen, 1884, Bull. No. 2 , Ill. St. Mus. Nat. Hist., p. 15, and Geo. Sur. Ill., vol.' 8 , p. 127 , St. Louis Gr.

mytiliformis, Hall, 1852, Pal. N. Y., vol. 2, p. 100 , Clinton Gr.

nemesis, Walcott, 1885, Monogr. U. S. Geo. Sur., vol. 8, p. 237, Subcarboniferous.

nessus, Walcott, 1885, Monogr. U. S. Geo. Sur., vol. 8, p. 238, Subcarboniferous.

perattenuata, Meek \& Hayden, 1858, Trans. Alb. Inst., vol. 4, p. 77, and Geo. Sur. Ill., vol. 5, p. 582, Coal Meas.

permiana, Swallow, 1858, (Mytilus permianus,) Trans. St. Louis Acad. Sci., vol. 1, p. 187, and Pal. Up. Mo., p. 52, Permian Gr.

perniformis, Cox, 1857, Geo. Sur. Ky., vol. 3 , p. 569 , Coal Meas.

pterineiformis, Winchell, 1862, Proc. Acad. Nat. Sci., p. 412, Marshall Gr.

rara, Winchell, 1870 , Proc. Am. Phil. Soc., p. 390, Marshall Gr. Proposed instead of M. aviculoides, Winchell, 1862, which was preoccupied.

recta, Shumard, 1858, Trans. St. Louis Acad. Sci., vol. 1, p. 212, Permian Gr.

recurvirostris, Meek \& Worthen, 1860, Proc. Acad. Nat. Sci. Phil., p. 456, and Geo. Sur. Ill., vol. 2, p. 344, Up. Coal Meas. squamosa, Sowerby, 1827, Trans. Geo. Soc. Lond., 2d ser., vol. 3, Permian Gr. subquadrata, Shumard, 1855, Geo. Rep. Mo., p. 207, Coal Meas.

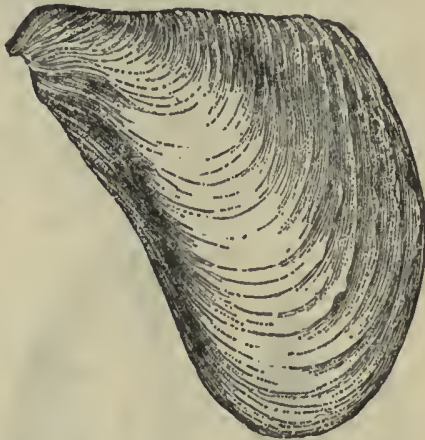

F'1G. 860.-Myalina recurvirostris.

stludovici, Worthen, 1873, Geo. Sur. Ill., vol. 5, p. 540, St. Louis Gr.

swallovi, McChesney, 1860, New Pal. Foss.,

p. 57, and Geo. Sur. Ill., vol. 2, p. 341, Coal Meas.

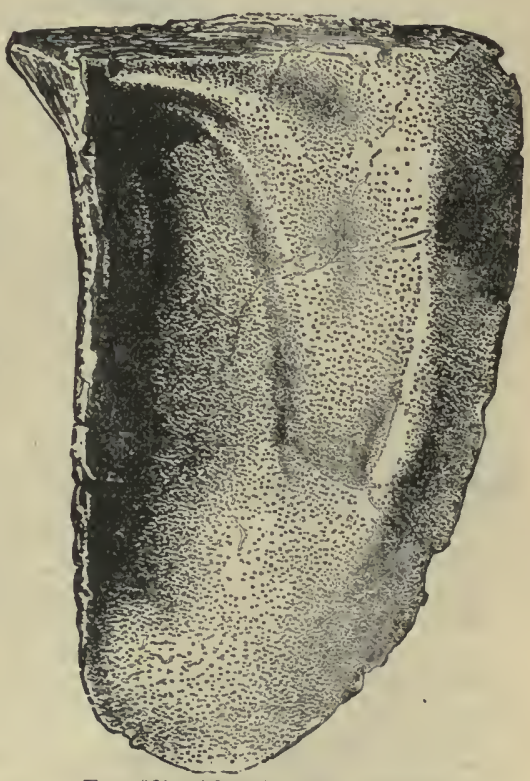

Frg. 861.-Myalina subquadrata.

Mrtilarca, Hall, 1870, Prelim. Notice Lam. Shells, p. 19. [Ety. from the two genera Mytilus and Arca.] Equivalve, inequilateral, mytiliform; beaks terrninal; hinge short; ligamental area striated; cardinal teeth beneath the beak small, oblique; posterior teeth small, oblique, and at the extremity of the hinge; anterior muscular scar umbonal, and posterior one near the postero-basal margin; pallial line entire, simple; surface not unfrequently with fine, obscure radiating striæ. Type M. chemungensis. 
arenacea, see Plethom ytilus arenaceus. attenuata, Hall, 1870, Prelim. Notice Lam. Shells, p. 23, and Pal. N. Y., vol. 5, pt. 1, p. 260, Chemung Gr.

canadensis, Billings, 1874, Pal. Foss., vol. 2, p. 52, Gaspe limestone No. 8, Devonian.

carinata, Hall, 18S4, Pal. N. Y., vol. 5, pt. 1 , p. 259, Chemung Gr.

chemungensis, Conrad, 1842, (Inoceramus chemungensis,) Jour. Acad. Nat. Sci. Phil., vol. 8, p. 246, and Pal. N. Y., vol. 5, pt. 1, p. 258, Chemung Gr.
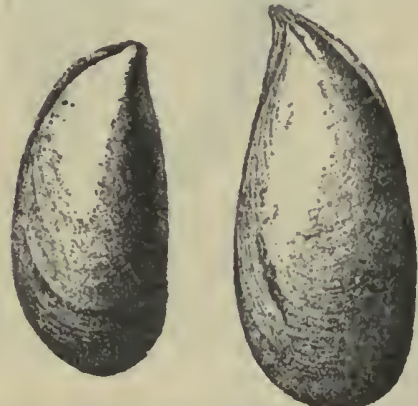

FıG. 862.-Mytilarea chemungensis.

cordiformis, Hall, 1859, (Megambonia cordiformis,) Pal. N. Y., vol. 3, p. 278, Low. Held. Gr.

duhia, Walcott, 1885, Monogr. U. S. Geo. Sur., vol. 8, p. 168, Devorian.

fibrisiriata, White \& Whitfield, 1862, (Mytilus fibristriatus,) Proc. Bost. Soc.

Nat. Hist., vol. 8, p. 296, and Pal. N. Y. vol. 5 , pt. 1, p. 264 , Kinderhook Gr.

gibbosa, Hall, 1884, Pal. N. Y., vol. 5, pt.

1, p. 262, Up. Chemung Gr.

knappi, see Plethomvtilus knappi.

lata, Hall, 1884, (Mytilops lata,) Pal. N. Y., vol. 5, pt. 1, p. 262, Chemung Gr.

mytilimeris, see Plethomytilus mytilimeris. nitida, Billings, 1874, Pal. Foss., vol. 2, p.

53, Gaspe limestone No. 8, Devonian.

occidentalis, White \& Whitfield, 1862 ,

(Mytilus occidentalis,) Proc. Bost. Soc.

Nat. Hist., vol. 8, p. 297, and Pal. N. Y., vol. 5 , pt. 1 , p. 263 , Kinderhook $\mathrm{Gr}$.

oviformis, see Plethomytilus oviformis.

percarinata, Whitfield, 1882 , Ann. N. Y.

Acad. Sci., vol. 2. p. 202 , Up. Held. Gr. ponderosa, see Plethomytilus ponderosus. pyramidata, Hall, 1884, Pal. N. Y., vol. 5, pt. 1, p. 256, Schoharie grit.

radiata, see Byssopteria radiata.

regularis, Hall, 1881, Pal. N. Y., vol. 5, pt. 1, p. 260, Chemung Gr.

sigillum, Hall, 1876,28 th Rep. N. Y. Mus.

Nat. Hist., p. 174, Niagara Gr.

simplex, Hall, 1884, (Mytilops simplex,) Pal. N. Y., vol. 5, pt. 1, p. 261, Chemung Gr.

umbonata, Hall, 1884, Pal. N. Y., vol. 5, pt. 1 , p. 257, Chemung Gr.

Mytilops, Hall, 1884, Pal. N. Y., vol. 5, pt. 1, p. 4. [Ety. from resemblance to
Mytilus.] Resembles externally Modiola and Lithodomus. Hinge-line narrow, oblique, extending about half the length of the shell, beaks terminal. Type M. præcedens.

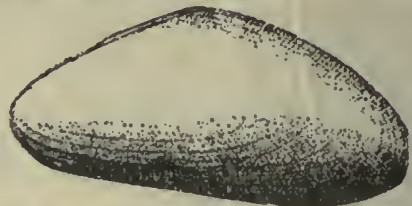

Fig. 863.-Mytilops præcedens, Left valve.

lata, see Mytilarca lata.

metella, Hall, 1870, Prelim. Notice Lam. Shells, p. 1, and Pal. N. Y., vol 5, pt. 1, p. 268, Chemung Gr.

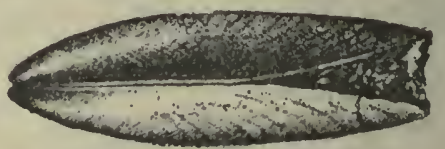

FuG. 861.-Mytilops prrecedens.

præcedens, Hall, 1870, (Modiola precedens,) Prelim. Not. of Lam. Shells, p. 1, and Pal N. Y., vol. 5, pt. 1, p. 267, Chemung Gr.

simplex, see Mytilarca simplex.

Mytilus, Linnous, 1758, Syst. Nat., 10 th ed. [Ety. Mytilus, the fish mussel.] This genus does not, so far as known, exist in Palaeozoic rocks. Most of the species referred to it belong to Myalina and Mytilarca.

concavus, see Myalina concava.

fibristriatus, see Mytilarca fibristriata.

occidentalis, see Mytilarca occidentalis.

ottawensis, Shumard, 1858, Trans. St. Louis Acad. Sci., vol. 1, p. 211, Up. Coal Meas.

permianus, see Myalina permiana.

squamosus, Sowerby, 1839, Trans. Geol. Soc. Lond., vol. 4, Permian Gr. Probably not American.

tenuiradiatus, Sliumard, 1858, Trans. St. Louis Acad. Sci., vol. 1, p. 211, Up. Coal Meas.

whitfieldanus, Winchell, 1862, Proc. Acad. Nat. Sci., p. 413, syn. for Mytilarca fibristriata.

Naiadites, Dawson, 1860, Acad. Geol., but not defined. The name was used for a genus of plants by Buckman in 1843 . The fossils were defined by Salter in 1861, under the name of Anthracomya. angulalus, see Anthracomya angulata. arenaceus, see $\mathbf{A}$. arenacea.

carbonarius, see A. carbonaria.

elongatus, see A. elongata.

lavis, see A. lævis.

obtusus, see A. obtusa.

ovalis, see $A$. ovalis.

NuculA, Lamarck, 1801, Syst. An. sans Vert., p. 87. [Ety. nucula, a little nut.] Equivalve, inequilateral oval, or oblong 
closed all round, without external ligamentary facets; beak directed backward; cartilage interna], placed in a pit under the beak; teeth numerous, very long. Type $N$. nucleus.

anodontoides, Meek, 1871, Reg. Rep. University W. Va., Coal Meas.

arata, see Nuculana arata.

bellatula, Hall, 1843, syn. for N. bellistriata.

bellistriata, Conrad, 1841, (Nuculites bellistriatus,) Ann. Rep. N. Y., p. 40, and Geo. Rep. 4th Dist. N. Y., p. 197, Ham. Gr.

beyrichia, Schlotheim, as identified by Geinitz. See Nucula parva.

corbuliformis, Hall, 1870, Prelim. Notice Lam. Shells, p. 2, and Pal. N. Y., vol. 5, pl. 16, figs. 24-37, Ham. and Che. mung Grs.

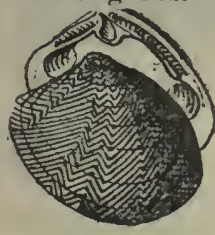

Frg. 865.-Nucula cobboldiæ. cylindricus, syn. for Cardiomorpha missouriensis.

diffidens, Hall, 1885 , Pal. N. Y., vol. 5, p. 322, Chemung Gr.

donaciformis, see Tellinomya donaciformis.

globularis, Hall, 1885 ,

Pal. N. Y., vol. 5, p. 322, Chemung Gr.

fabula, see Clidophorus fabula.

hians, Hall, 1860, 13th Rep. N. Y. Mus. Nat. Hist., p. 110, Kinderhook Gr.

houghtoni, see Tellinomya houghtoni.

hubbardi, Winchell, 1862, Proc. Acad. Nat. Sci., p. 417, Marshall Gr. Syn. for Nuculites sulcatinus.

illinoisensis, Worthen, 1884, Bull. No. 2, Ill. St. Mus. Nat. Hist., p. 15, and Geo. Sur. Ill., vol. 8, p. 128, St. Louis Gr.

insularis, Walcott, 1885, Monogr. U. S. Geo. Sur., vol. 8, p. 241, Carboniferous.

iowensis, White \& Whitfield, 1862, Proc. Acad. Nat. Sci., vol. 8, p. 298. Syn. for Tellinomya houghtoni.

kazanensis, as identified by Geinitz is Nuculana bellistriata.

lamellata, Hall, 1883, Pal. N. Y., vol. 5, pl. 51, figs. 18-20, Ham. Gr.

levata, see Tellinomya levata.

levatiformis, Walcott, 1885, Monogr. U. S. Geo. Sur., vol. 8, p. '241, Carboniferous.

lineata, see Tellinomya lineata.

lineolata, Hall, 1843, Geo. Rep. 4th Dist. N. Y., p. 246, Portage Gr.

lirata, Conrad, 1812, (Nuculites liratus,) Jour. Acad. Nat. Sci., vol. 8, p. 250, and Pal. N. Y., vol. 5, pl. 45, figs. 17-27, Ham. Gr.

machæriformis, see Tellinomya machæriformis.

mactriformis, see Tellinomya mactriformis.

mercerensis, syn. for Cardiomorpha missouriensis.

microdonta, Winchell, 1863, Proc. Acad. Nat. Sci., p. 16, Marshall Gr. minima, Foerste, 1885, Bull. Sci. Lab. Denison Univ., p. 93. Not properly defined.

minuta, Owen, 1840, Rep. on Min. Lands,

Devonian. The name was preoccupied by De France in 1825 .

nasuta, see Nuculana nasuta.

neda, Hall \& Whitfield, 1872, 24th Rep. N. Y. Mus. Nat. Hist., p. 191, Up. Held. Gr.

niotica, Hall \& Whitfield, 1872, 24th Rep. N. Y. Mus. Nat. Hist., p. 190 , Ham. Gr.

obliqua, see Palreoconcha obliqua.

oblonga, Hall, syn. for Nuculites oblongatus.

obsoleta, McChesney, 1860, Pal. Foss., p. 89, Coal Meas. Not recognized.

parva, McChesney, 1860, New Pal. Foss., p. 54, and Geo. Sur. Ill., vol. 5, p. 589, Coal Meas.

perumbonata, White, 1879, Bull. U. S. Geo. Sur., vol. 5, No. 2, p. 217, and Cont. to Pal., No. 6, p. 136, Carboniferous.

poststriata, see Lyrodesma poststriatum.

randalli, Hall, 1870, Prelim. Notice Lam. Shells, p. 3, and Pal. N. Y., vol. 5, pl. 45, figs. 5-16, Ham. and Chemung Grs.

rectangula, McChesnev, 1860, Desc. New Pal. Foss., p. 74, Haim. Gr.

rescuensis, Walcott, 1885, Monogr. U. S. Geo. Sur., vol. 8, p. 172, Devonian.

sectoralis, Winchell, 1862, Proc. Acad. Nat. Sci., p. 418, Marshall Gr.

shumardana, Hall, 1858, Trans. Alb. Inst., vol. 4, p.. 16, and Bull. Am. Mus. Nat. Hist., p. 57, Warsaw Gr.

stella, see Tellinomya stella.

subelliptica, Hall, 1883, Pal. N. Y., vol. 5, pl. 45, fig. 2s, Ham. Gr.

umbonata, Hall, 1883, Pal. N. Y., vol. 5 , pl. 47 , figs. 51 and 52 , Chemung Gr.

varicosa, Hall, 1870, Fra. 866.-Nucula ventriPrelim. Notice

Lam. Shells, p. 2, and Pal. N. Y., vol. 5, pl. 46, figs. 12-23, Ham. Gr.

ventricosa, Hall, 1858, Geo. Sur. Iowa, p. 716, Coal Meas.

Nuculana, Link, 1807, Rost. Samml., vol. 3, p. 155. [Ety. like a shell of the genus Nucula.] Equivalve, inequilateral, produced behind; beaks sometimes directed posteriorly; lunule often present ; rounded in front; post-umbonal slope defined; surface concentrically lined; hinge with a line of small teeth interrupted by a triangular cartilage pit beneath the beak; muscular impressions two, small; pallial line, simple, or slightly sinuous. Type N. emarginata.

arata, Hall, 1852, (Nucula arata,) Stansb. Exped. to Gt. Salt Lake, p. 413, Coal Meas. 
bellistriata, Stevens, 1858, (Lera bellistriata,) Am. Jour. Sci., vol. 25, p. 261 , and Geo. Sur. Iowa, p. 717, Coal Meas.

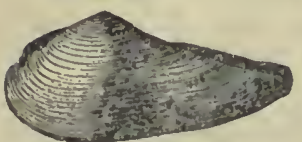

FIG. 867.-Nuchlana bellistriata. Left valve. Pal. N. Y. vol 5am. Shells, p. 6, and curta, Meek, 1861,

(Leda curta,)

Proc. Acad. Nat.

Sci. Phil., p.

144, and Geo.

Sur. Ill., 'vol. 2, Fig. 864.-Nuculana bellip. 283 , St. Louis striata. Cardinal vlew. Gr.

densmamillata, Stevens, 1858, (Leda densmanillata,) Am. Jour. Sci., vol. 25, p. 261, Marshall Gr.

diversa, Hall, 1883, Pal. N. Y., vol. 5, pl. 47, figs. 31-37, Ham. Gr.

nasuta, Hall, 1558, (Nucula nasuta,) Trans. Alb. Inst., vol. 4, p. 17, and Bull. Am. Mus. Nat. Hist., p. 57, Warsaw Gr.

nuculiformis, see Palreoneilo nuculiformis. obesa, White, 1879, Bull. U. S. Geo. Sur., vol. 5, No. 2, p. 216, and Cont. to Pal., No. 6, p. 136, Carboniferous.

ohioensis, Hall, syn. for N. pandoriformis. obscura, Hall, 1885, (Leda obscura,) Pal. N. Y., vol. 5, p. 331, Ham. Gr.

pandoriformis, Stevens, 1858, (Leda pandoriformis,) Am. Jour. Sci., vol. 25, p. 261, Waverly Gr.

perstriata, Hall, 1883, Pal. N. Y., vol. 5, pl. 47 , figs. $42-44$, syn. for $\mathrm{N}$. rostellata.

rostellata, Conrad, 1841, (Nuculites rostellatus,) Ann. Rep. Geo. N. Y., p. 50, Ham. Gr.

saccuta, Winchell, 1863, (Leda saccata,) Proc. Acad. Nat. Sci., p. 16, Marsliall Gr.

vaseyana, McChesney, 1860, (Nuculites vaseyanus, ) Desc. New. Pal., Foss., p. 73, Ham. Gr.

Nuculites, Conrad, 1841, Ann. Geo. Rep. N. Y., p. 49. [Ety. Nucula, a genus of shells.] Equivalve, inequilateral, transverse; anterior end rounded; posterior truncate or pointed; beak, anterior ; cardinal line arcuate; postumbonal slope rounded or angular; surface concentrically lined, hinge with a row of transverse narrow teeth from the anterior to the posterior muscular scar; ligament external ; anterior scar deep and separated from the cavity of the shell by a clavicle; posterior scar elongate; pallial line simple. Type N. oblongatus.

altus, Conrad, 1842, Jour. Acad. Nat. Sci., vol. 8, p. 251, Devonian.

appressus, see Cytherodon appressus.

bellistriatus, see Nucula bellistriata.

carinatus, Hall, 1860, Can. Nat. and Geol., vol. 5, p. 151, Up. Sil.

chemungensis, see Cytherodon cliemungensis.

concentricus, Conrad, 1842, Jour. Acad.

Nat. Sci., vol. 8, p. 248, Coal Meas.

constrictus, see Palroneilo constricta.

cuneiformis, Conrarl, 1841, Anu. Rep.

N. Y., p. 50, and Pal. N. Y., vol. 5, pl.

47, figs. 13-16, Ham. Gr.

emarginatus, see Palæoneilo emarginata.

faba, see Modiolopsis faba.

filosus, see Palæoneilo filosa.

inflatus, see Cypricardites inflatus.

lamellosus, Conrad, 1841, Ann. Geo. Rep. N. Y., p. 50, Up. Si].

liratus, see Nu-

cula lirata.

mact roides,

Con rad, $18+2$,

Jour. Acad.

Nat. Sci., vol.

8, p. 249 ,

Marsliall Gr.

maximus, see F'IG. 869. - Nuculltes oblon

Palaco n e il o gatus. Interlor of large left lilaxima.

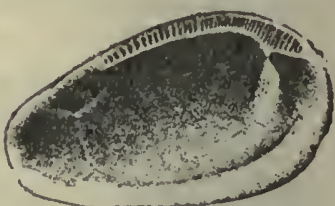

multilineatus, Conrad, 1842, Jour. Acad. Nat. Sci., vol. 8, p. 251, Hain. Gr.

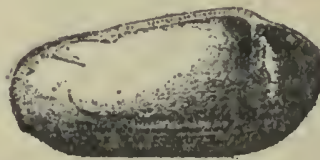

Fif. 871).-Nuculltes oblongatus. Cast of juterlor of right valve, showlug mnscular scurs and pallial IIne. Ann. Geo Rep. N. Y., p. 50 and Pal. N. Y., vol. 5, pl. 47, figs. 1-12, Ham. Gr.

planulatus, see Clidophorus planulatus. poststriatus, see Ly rodesma poststriatum. radiatus, see Pholadella radiata. rostellatus, see Nuculana rostellata. scitula, syn. for Clidophorus planulatus. subemaryinalus, see Tellinopsis, subemarginata.

sulcatinus, Conrad, 1842, Jour. Acad. Nat. Sci., vol. 8, p. 250 , Marshall Gr.

triangularis, Hall \& Whitield, 1877, U. S.

Geo. Expl., 40th parallel, vol. 4, p. 248 , Devonian.

triqueter, Conrad, 1841, Ann. Rep. N. Y., p. 50, and Pal. N. Y., vol. 5, pl. 47, figs. 17-24, Ham. Gr.

vaseyanus, see Nuculana vaseyana.

yoldiiformis, Ulrich, 1879, Jour. Cin. Soc.

Nat. Hist., vol. 2, p. 24, Hud. Riv. (ir. It is not a Nuculites.

Nyassa, Hall, 1870, Prelim. Notice Lam. Shells, but defined by Whitfield, 1882, Ann. N. Y., Acad. Sci., vol. 2, p. 244. [Ety. mythological name.] Shell bivalve, very oblique and transversely ovate in form; posterior linge plate 
narrow, bearing from one to four long, slender, ridge-like teeth; anterior plate broad, marked by numerous, small, point-like teetl, with intermediate depressions, arranged somewhat radiating from the middle of its inner border; adductor muscles two, one at each extremity; pallial line entire; ligament internal. Type N. arguta.

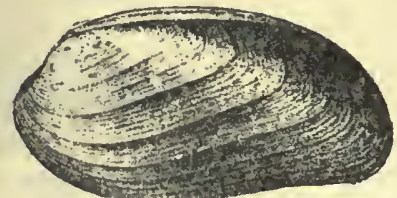

FIG. 871,-Ny assa arguta. Left valve.

arguta, Hall, 1870, Prelim. Notice Lam. Shells, p. 28, and Pal. N. Y., vol. 5, pl. 53, figs. 9-20, Ham. Gr.

elliptica, Hall, 1870, Prelim. Notice. Lam. Shells, p. 30, and Pal. N. Y., vol. 5, pl. 34, fig. 8, Up. Held. Gr.

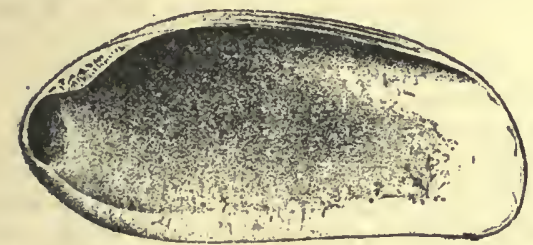

Fig. 872.-Nyassa arguta. Interior of right valve.

parva, Walcott, 1885, Monogr. U. S. Geo. Sur., vol. 8, p. 173, Devonian.

recta, Hall, 1870, Prelim. Notice Lam. Shells, p. 29, and Pal. N. Y., vol. 5, pl. 53. figs. $1-8$, Ham. Gr.

subalata, Hall, 1870, Prelim. Notice Lam. Shells, p. 29, and Pal. N. Y., vol. 5, pl. 53, figs. 21-26, Ham. Gr.

Opisthoptera, Meek. . Not defined.

Orthodesma, Hall \& Whittield, 1875 , Ohio Pal., vol. 2, p. 93. [Etv. orthos, straight; desma, a ligament.] Elongated, ventricose; cardinal line straight posterior to the beaks, and contracted anterior; ligament external; posterior scar elongate, anterior smaller; pallial line simple ; surface concentrically lined. Type O. rectum.

byrnesi, S. A. Miller, 1881, Jour. Cin. Soc. Nat. Hist., vol. 4, p. 76, Hud. Riv. Gr. contractum, Hall, 1847, (Orthonota contracta,) Pal. N. Y., vol. 1, p. 300, Hud. Riv. Gr.

cuneiforme, S. A. Miller, 1880, Jour. Cin. Soc. Nat. Hist., vol. 3, p. 314, Hud. Riv. Gr.

curvatum, Hall \& Whitfield, 1875, Ohio Pal., vol. 2, p. 95, Hud. Riv. Gr.

faberi, n. sp. Shell large, posterior part of the cardinal line very slightly elevated, and anterior part contracted in front of the beaks; anterior end rounded, posterior end more abrupt; basal line contracted in the central part by an undefined cincture arising below the umbones; beaks anterior, obtuse; umbones low and poorly defined; shell unusually high and thin for species in this genus; surface concentrically furrowed. This species bears some resemblance to a Modiolopsis, but it is doubtless an Orthodesma. Collected by Mr. Charles Faber in the upper part of the Hud. Riv. Gr., at Versailles, Indiana.

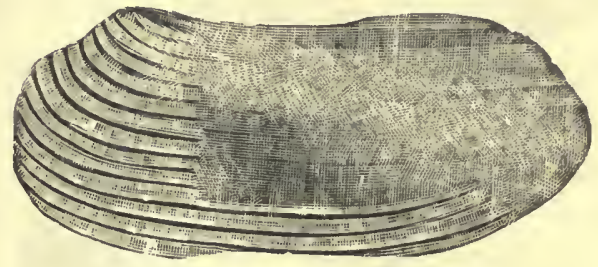

FIG. 873.--Orthodesma faberi.

mickelboroughi, Whitfield, 1878, Jour. Cin. Soc. Nat. Hist., vol. 1, p. 139, Hud. Riv. Gr.

occidentale, S. A. Miller, 1880, Jour. Cin. Snc. Nat. Hist., vol. 3, p. 316, Hud. Riv. Gr.

parallelum, Hall, 1847, (Modiolopsis parallela,) Pal. N. Y., vol. 1, p. 158, Hud. Riv. Gr.

rectum, Hall \& Whitfield, 1875, Ohio Pal., vol. 2, p. 94, Hud. Riv. Gr.

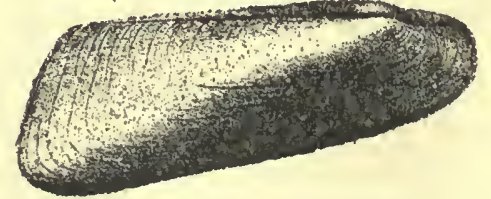

FIG. 874.-Orthodesma rectum.

subovale, Ulrich, 1879, Jour. Cin. Soc. Nat. Hist., vol. 2, p. 82, Hud. Riv. Gr. Onthonota, Conrad, 1841, Ann. Rep. N. Y., p. 50. [Ety. orthos, straight; notos, the back.] Transversely elongate; margins subparallel; cardinal line straight; two cardinal teeth; no lateral teeth; ligament external; umbonal ridge oblique. Type 0 . undulata. angulifera, (?) McCov, 1850, Brit. Pal. Rocks, p. 276 , Up. Śl.

carinata, Conrad, 1841, Ann. Rep. N. Y., p. 51, and Pal. N. Y., vol. 5, pl. 78, figs. 34-35, Ham. Gr.

contracta, see Orthodesma contractim.

curta, Hall, 1843, Geo. Rep. 4th Dist.

N. Y., p. 76, and Pal. N. Y., vol.2, p. 86, Clinton and Niagara Gr.

ensiformis, Hall, 1870, Prelim. Notice Lam. Shells, p. 89, and Pal. N. Y., vol. 5, pl. 78, fig. 36, Ham. Gr.

incerta, Billings, 1874, Pal. Foss., vol. 2, p. $130, \mathrm{Up}$. Sil.

parallela, see Ortbodesma parallelum. 
parvula, Hall, 1870, Prelim. Notice Lam. Shells, p. 88, and Pal. N. Y., vol. 5, pl. 78, figs. 29 32, Ham. Gr.

phaselia, Winchell, 1863, Proc. Acad. Nat. Sci., p. 12, Marshall Gr.

pholadis, Conrad, 1838, (Pterinea pholadis,) Ann. Geo. Rep. N. Y., p. 118, Hud. Riv. Gr.

rectidorsalis, Winchell, 1862, Proc. Acad. Nat. Sci., p. 412, Marshall Gr.

rigida, Hall, 18S5, Pal. N. Y., vol. 5, p. 481, Chemung Gr.

siliquoidea, see Palreosolen siliquoideus.

simulans, Billings, 1874, Pal. Foss., vol. 2, p. 131, Up. Sil.

(?) speciosa, Billings, 1874, Pal. Foss., vol. 2, p. 130, Up. Sil.

undulata, Con rad, 1841, Ann. Rep. N. Y., p. 51, and Pal. N. Y., vol. 5, pl. 78, figs. 37-42, Ham. Gr.

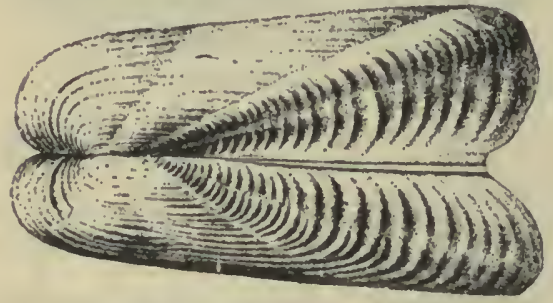

Fio. 875.-Orthonota undulata.

ventricosa, see Spathella ventricosa.

venusta, Billings, 1874, Pal. Foss., vol. 2, p. 129 , Up. Sil.

Orthonotella, S. A. Miller, 1882, Jour. Cin. Soc. Nat. Hist., vol. 5, p. 117. [Ety orthos, straight; notos, back; ellus, diminutive.] Very small, inequilateral, inequivalve, more or less elliptical; beak anterior; surface smooth or concentrically lined; Linge straight belind the beaks; ligament external. Type $O$. faberi.

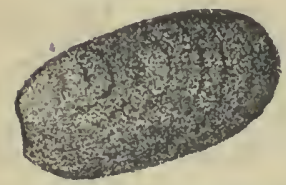

faberi, S. A. Miller, 1882, Jour. Cin. Soc. Nat. Hist., vol. 5 , p. 117 , Hud. Riv. Gr.

Ostrea, Linnzeus, 1758, Syst. Nat. 10th ed., p. 696 . [Ety. 08. trea, an oyster.] Inequivalve, irfaberi. Greatly enlarged.

regular in shape, with a single adductor muscle. Not a Palæozoic genus, though a species has been founded upon a single valve and called $O$. patercula.

patercula, Winchell, 1865, Proc. Acad. Nat. Sci., p. 124, and 4th Ann. Rep. U. S. Geo. Sur., p. 288, Burlington Gr.

Paldanatina, Hall, 1870. Prelim. Notice Lam. Shells, p. 84. [Ety. palaios, ancient; Anatiria, a genus.] Transversely elongate ; gaping; left valve the larger ; oblique constriction; look-like process anterior to the beaks; no lateral

teeth; surface concentrically lined. Type P. typus.

angusta, Hall, 1885, Pal. N. Y., vol. 5, p. 490 , Chemung Gr.

quadrata, see Prorhynchus quadratum. sinuata, Hall, 1885, Pal. N. Y., vol. 5, p. 491, Chemung Gr.

solenoides, Hall, 1885, Pal. N. Y., vol. 5, p. 489 , Chemung Gr.

typus, Hall, 1870,

Prelim. Notice

Lam. Shells, p.

85, and Pal.

N. Y., vol. 5 , pl.

79, figs. 26-39,

Chemung Gr.

Palicarca, syn. for

Cypricardites.

saffordi, see Cypricardites saffordi.

ventricosa, see Cypricardites ventricosus.

Palmocardia, Hall, 1867, 20th Rep. N. Y. Mus. Nat. Hist., p. 389. [Ety. palaios, ancient; kardia, a heart.] Cordiform, obliquely subovate, ventricose; umbones gibbous; beaks prominent incurved; hinge-line very short; surface marked with radiating strix. Type $P$. cordiformis.

cordiformis, Hall, 1867, 20tl Rep. N. Y. Mus. Nat. Hist., p. 389, Niagara Gr.

PAL AOCoxcus, n. gen. [Ety. pulaios, ancient; conche, shell.] Shell small, equivalve, inequilateral, oblique, varying from subcircular or subovoid to mytiliform; height equal to or greater than length; closed all around; without external evidence of ligaments; anterior side more or less truncated and rounding into the base below; beaks elevated, projecting beyond cardinal line without incurving; cardinal line straight or slightly arching; some evi. dence points to an anterior and posterior muscular scar near the ends of the cardinal line; pallial line simple; no lateral teeth and probably edeutulous; surface smooth. Type P. faberi. faberi, n. sp. Shell small, height greater than length, very slightly oblique, closed all around; beaks projecting ligh above the binge-line without

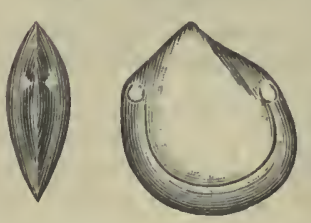

Fig. 878.-Palre co n c ha faberi. Magnified 5 diam.

\begin{abstract}
incurving; sur-
\end{abstract} face smooth, variable in size; a large specimen has a height of 0.20 i $\mathrm{n} \mathrm{h}$, a $\mathrm{nd}$ length 0.14 inch, a small specimen is about $t \mathrm{w}$ o. thirds less. This species is distinguished from $\mathrm{P}$. obliqua by its greater proportional height, more prolonged beak, less oblique form, and generally larger size. Collected in the upper part of the Hud. Riv. Gr., at Versailles, Indiana, and in Butler County, Ohio. 
obliqua, Hall, 1845, (Nucula obliqua,) Am. Jour. Sci., vol. 48, p. 292, and Ohio Pal., vol. 1, p. 139, Hud. Riv. Gr. Paleoneilo, Hall, 1870, Prelim. Notice Lam. Shells, p. 6. [Ety. palaios, ancient; Neilo, a genus.] Nuculiform, posterior end extended, sulcus along the umbonal slope; surface concentrically striated or ribbed; hinge-line arcuate, crenulate, not interrupted beneath the beak by a ligamental pit; ligament external; anterior and posterior adductor scars distant; pedal scars within the umbonal cavity. Type P. constricta.

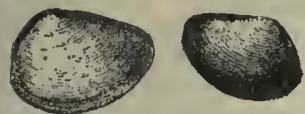

FIG. 879. Palæoneilo bedfordensis.

angusta, Hall, 1885, Pal. N.Y., vol. 5 , p. $34 t$, Chemung Gr. arata, Hall, 1883, Pal. N. Y., vol. 5, pl. 50, fig. 23 , Ham. Gr.

attenuata, Hall, 1870, Prelim. Notice Lam. Shells, p. 12, and Pal. N. Y., vol. 5, pl. 50 , figs. 34-39, Waverly Gr.

barrisi, White \& Whitfield, 1862, (Leda barrisi,) Proc. Bost. Soc. Nat. Hist., vol. 8 , p. 298 , syn. for P. nuculiformis.

bedfordensis, $\mathrm{M}$ e e $\mathrm{k}$, 1875, Pal. Ohio, vol. 2, p. 298, Waverly Gr.

bisulcata, Hall, 1870, Prelim. Notice Lam. Shells, p. 10, and Pal. N. Y., vol. 5, pl. 50, Fig. 880. - Palæofigs. 13-14, Ham. Gr. neilo bed fordenbrevis, Hall, 1870, Pre- sis. Magnified. lim Notice Lam. Shells, p. 10, and Pal. N. Y., vol. 5, pl. 50, figs. 24-33, Chemung Gr.

carbonaria, see Yoldia carbonaria.

constricta, Conrad, 1842,

(Nuculites constrictus,)

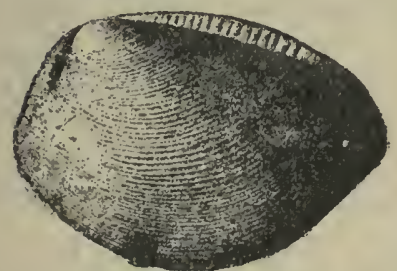

Fig. 881.-Palæoneílo constricta. Left valve enlarged, showing crenufations of hinge-ifne.

Jour. Acad. Nat. Sci., vol. 8, p. 249 , and Pal. N. Y., vol. 5 , pl. 48 , figs. $1-15$, Chemung Gr. constricta var. flexuosa, Hall, 1883, Pal.

N. Y., vol. 5, pl. 48, figs. 16-20, Ham. Gr. dubia, Hall, 1885 , Pal. N. Y., vol. 5, p. 348, Up. Held. Gr.

elongata, Hall, ]883, Pal. N. Y., vol. 5, pl. 48, fig. 39, Chemung Gr.

emarginata, Conrad, 1841, (Nuculites emarginata,) Ann. Rep. N. Y., p. 50, and Geo. Wis., vol. 4, p. 337, Ham. Gr. filosa, Conrad, 1842, (Nuculites filosus,) Jour. Acad. Nat. Sci., vol. 8, p. 250, and Pal. N. Y., vol. 5, pl. 49, figs. 33-38, Chemung Gr.

fœeunda, Hall, 1870, Prelim. Notice Lam. Shells, p. 8, and Pal. N. Y., vol. 5, pl. 49, figs. 13-24, Ham. Gr.

maxima, Conrad, 1841, (Nuculites maximus, ) Ann. Rep. N. Y., p. 50, and Pal.

N.Y., vol. 5 , pl. 48, figs. $29-38$,

Ham. Gr.

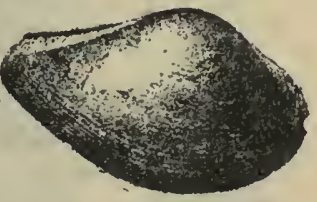

FIG. 882.-Palæonello maxmuta, Hall, 1870, ima. Right valve.

Prelim. Notice Lam. Shells, p. 8, and Pal. N. Y., vol. 5, pl. 49, figs. 25-32, Ham. Gr. nuculiformis, Stevens, 1858, (Leda nuculiformis,) Am. Jour. Sci. and Arts, 2d ser., vol. 25, p. 262, Waverly Gr.

parallela, Hall \& Whitfield, 1870,23d Rep. N. Y. Mus. Nat. Hist., p. 241, Waverly Gr.

perplana, Hall, 1870, Prelim. Notice Lam. Shells, p. 12, and Pal. N. Y., vol. 5, pl. 50, figs. 15-22, Ham. Gr.

plana, Hall, 1870, Prelim. Notice Lam. Shells, p. 7, and Pal. N. Y., vol. 5, pl. 48, figs. 21-28, Ham. Gr.

similis, Whitfield, 1882 , Ann. N. Y. Acad. Sci., vol. 2, p. 217, Erie sbale, Portage (?) Gr.

tenuistriata, Hall, 1870, Prelim. Notice Lam. Shells, p. 9, and Pal. N. Y., vol. 5, pl. 49 , figs. $1-12$, Ham. Gr.

truncata, Hall, 1883, Pal. N. Y., vol. 5, pl. 50, figs. 40-41, Chemung Gr.

virginica, Hall, 1885, Pal. N. Y., vol. 5, p. 340 , Ham. Gr.

Palmopinna, Hall, 1883, Pal. N. Y., vol. 5, pt. $]$, p. 4. (Plates and Explanations.)

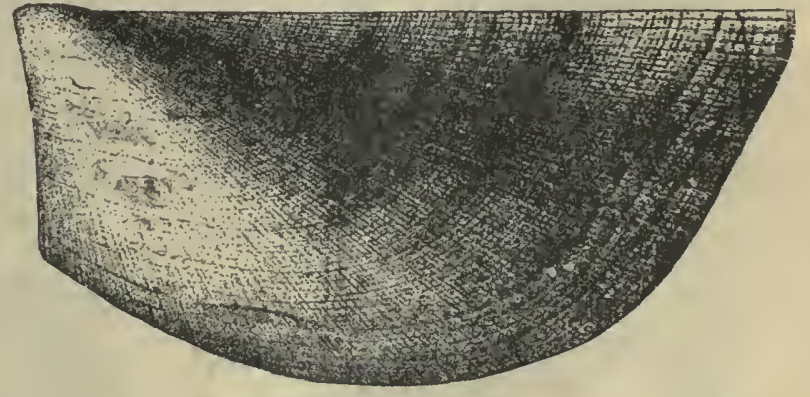

Fig. 883.-Palæopinna flabellum.

[Ety, palaios, ancient; Pinna, a genus.] Shells large, gaping in front; hinge-line straight, ligamental area narrow, longitudinal groove and slight oblique furrow extending backward from the beak; beak anterior, terminal, directed forward; test more convex, and with finer rays than on the ordinary Pinna, and also finely marked with concentric striæ of growth. Type P. flabellum. 
flabellum, Hall, 1884, Pal. N. Y., vol. 5, pt. 1 , p. 240 , Oriskany $\mathrm{Gr}$.

recurva, Hall, 1884, Pal. N. Y., vol. 5, pt. 1, p. 241, Up. Held. Gr.

Paldosolen, Hall, 1885, Pal. N. Y., vol. 5, p. 46. [Ety: palaios, ancient; Solen, a genus.] Shell in form like Sulen; dorsal and ventral margins subparallel; anterior end short, rounded; posterior end elongate, truncate; gaping; beaks small, appressed; cardiual line straight; umbonal slope prominent; surface concentrically marked. Type $\mathrm{P}$. siliquoideus.

si.iquoidens, Hall, 1870, (Orthonota siliquoidea,) Prelim. Not. Lain. Shells, p. 89, and Pal. N. Y., vol. 5, p. 483, Ham. Gr.

Panexka, Barrande, 1881, Syst. Sil. d. l. Boheme, vol. 6, p. 128. Equivalve, inequilateral, elliptical or subcircular, beaks prominent, incurved; cardinal line straiglit or arcuate; test thin; surface concentrically lined.

abrupta, Hall, 1885, l'al. N. Y., vol. 5, p. 423, Ham. Gr.

alternata, Hall, 1885, Pal. N. Y., vol. 5, p. 416, Up. Held. Gr.

costata, Hall, 1885, Pal. N. Y., vol. 5, p. 419, Marcellus Shale.

degener, Hall, 1885, Pal. N. Y., vol. 5, p. 424, Ham. Gr.

dichotoma, IIall, 1885, Pal. N. Y., vol. 5, p. 416, Schoharie grit.

elevata, Con rad, 1848, (Monotis elevata,) I'roc. Acad. Nat. Sci., vol. 3, p. 23, Cliemung Gr.

equilatera, Hall, 1885, Pal. N. Y., vol. 5, p. 419, Marcellus Shale.

hero, Hall, 1885, Pal. N. Y., vol. 5, p. 418, Marcellus Shale.

lincklani, Hall, 1885, Pal. N. Y., vol. 5, p. 420, Marcellus Shale.

mollis, Hall, 1885, Pal. N. Y., vol. 5, p. 420 , Marcellus Śbale.

multiradiata, Hall, 18S5, Pal. N. Y., vol. 5 , p. 417, Up. Held. Gr.

potens, Hall, 1885, Pal. N. Y., vol. 5, p. 422, Ham. Gr.

poulsoni, Conrad, 1848, (Monotis poulsoni,) Proc. Acad. Nat. Sci., rol. 3, p. 23, Chemung Gr.

radians, Conrad, 1842, (Pterinea radians,) Jour. Acad. Nat. Sci., p. 252, and Pal. N. Y. vol. 5, p. 422, Ham. Gr.

retusa, Hall, 1885, Pal. N. Y., vol. 5, p. 421, Ham. Gr.

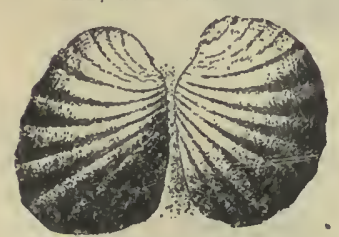

robusta, Hall, 1885, Pal. N. Y., vol. 5 , p. 424, Portage Gr.

speciosa, Hall, 1843, (Avicula s pecios a, ) Geo. Rep. 4th Dist. N. Y., p. 243, Portage Gr.

ventricosa, Hall, 1885, Pal. N. Y., vol. 5, p. 417, Marcellus Shale.
Panopxa, Menard de la Groye, 1807, Ann. du Mus. 9. [Ety. mythological name.] cooperi, see Chrnomya cooperi.

Paracardium, Barrande, 1881, Syst. Sil. de la Boheme, vol. 6, p. 137. [Ety. para, allied to; Cardium, a genus.] Equivalve, inequilateral, subcircular or subelliptical; posterior side subtruncate; surface marked with fine radii and concentric strix; the margin of a small cardinal area under the beaks is crenulated.

doris, Hall, 1885, (Cardiola doris;) Pal. N. Y, vol. 5, 1). 428, Portage Gr.

Pararca, Hall, 1S\$5, Pal. N. Y., vol. 5, p. 36. [Ety. para, allied to; Arca, a genus.] Equivalve, inequilateral, transversely subelliptical or rhomboidal; anterior end short, rounded; cardinal line about half the length of the valves, arcling at the beaks; surface marked by radii and concentric striac; hinge narrow, with a series of miuute crenulations. Type $P$. venusta.

erecta, Hall, 1885, (Cardiola erecta,) Pal. N. Y., vol. 5, p. 432 , Waverly Gr.

neglecta, IIall, 1885, Pal. N. Y., vol 5, p. 432, Waverly Gr.

pracedens, Hall, 1885, Pal. N. Y., vol. 5, p. 429 , Up. Held. Gr.

sao, Hall, 1885, (Cardiola sao,) Pal. N. Y., vol. 5 , p. 430 , Chemung Gr.

transversa, Hall, 1885, (Cardiola transversa,) Pal. N. Y., vol. 5, p. 429, Chemung Gr.

venusta, Hall, 1855, Pal. N. Y., vol. 5, p. 431, Chemung Gr.

Paracyclas, Hall, 1843, Geo. Rep. 4th Dist. N. Y., p.171. [Ety.para, allied to; Cyclas, a genus.] Equivalve, subequilateral, suborbicular or subellipticul; anterior end regularly rounded; posterior end rounded or subtruncate, more produced than the anterior; beaks small and low; hinge-line short, post-cardinal slope sometimes subalate; surface marked concentrically; ligament supported intermally on each side by a narrow plate, which leaves in the cast two diverging grooves directed forward from the beak; muscular impression ou the post-umbonal slope; pallial line a little within the margin of the shell. Type $\mathrm{P}$. elliptica.

billingsana, S. A. Miller, 1883, 2d Ed. Am. Pal. Foss., p. 311, Devonian. Proposed instead of Lucina occidentalis, Billings, 1859, Assiniboine and Sas. Ex. Exped., p. 187, figs. b and c, which name was preoccupied.

chemungensis, Hall, 1885, Pal. N. Y., vol. 5, p. 443, Chemung Gr.

elevata, Hall, 1883, Pal. N. Y., vol. 5, pl. 72 , figs. 37 to 41 , Schoharie grit.

elliptica, Hall, 1843, Geo. Rнp. 4th Dist. N. Y., p. 171, and Pal. N. Y., vol. 5, pl. 72, figs. $23-30$, Cornif. Gr.

elliptica var. occidentalis, Hall \& Whitfield, 1872, 24th Rep. N. Y. Mus. Nat. 
Hist., p. 189, and Pal. N. Y., vol. 5, pl. 72, figs. 31-33, Up. Held. Gr.

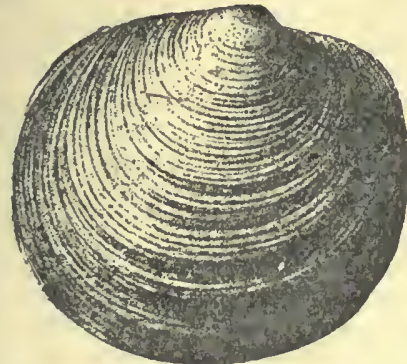

Frg. 885.-Paracyclas elliptica var. occldentalis.

erecta, Hall, 1885, Pal. N. Y., vol. 5, p. 445, Chemung Gr.

fissa, Hall, 1883, Pal. N. Y., vol. 5, pl. 72, figs. 35, 36, Schnharie grit.

hamiltonensis, Winchell, 1866, (Lucina hamiltonensis,) Rep. Low. Pen. Mich., p. 95, Ham. Gr.

ignota, Hall, 1883, Pal. N. Y., vol. 5, pl. 72, fig. 34, Chemung Gr.

lirata, Conrad, 1838, (Posidonia lirata,) Ann. Rep. N. Y., p. 116, and Pal. N. Y., vol. 5, pl. 72, figs. 1-19, Corniferous Gr.

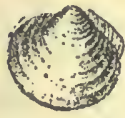

ra. 886. - Paracyclas ohioensis. ohioensis, Meek, 1871,

Proc. Acad. Nat. Sci.

Phil., p. 62, and

Ohio Pal., vol. 1, p. 248, Cornif. Gr. W i t field, 1877 U. S. Geo. Expl. 40th :Parallel, vol. 4, p. 248, Devonian.

retusa, Hall, 1843, (Lucina ? retusa) Geo. Rep. 4th. Dist. N. Y., p. 246, Portage Gr.

rotunda, Hall, 1855, Pal. N. Y., vol. 5, p. 444, Chemung Gr.

sabini, White, 1876, Proc. Acad. Nat. Sci., p. 31, Chemung Gr.

tenuis, Hall, 1883, Pal. N. Y., vol. 5, pl. 72, figs. 20-22, Ham. Gr.

varysburgensis, Williams, 18S7, (Lucina varysburgia,) Bull. 41, U. S. Geo. Sur., Portage Gr.
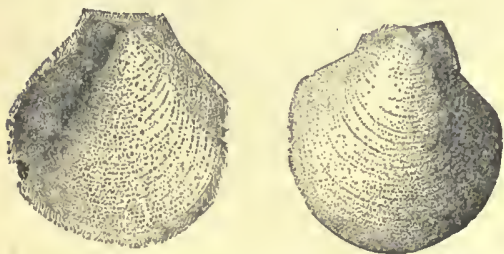

FIG. 887.-Pernopecten aviculatus.

wyomingensis, Williams, 1887, Bull. 41, U. S. Geo. Sur., Portage Gr.

Pecten, Mueller, 1776. This genus is unknown in the Palæozoic rocks. acutialatus, see Aviculopecten acutialatus. armigerus, see A. armigerus.

aviculatus, see Pernopecten aviculatus.

broadheadi, syn. for Aviculopecten carboniferus.

cancellatus, see Aviculopecten cancellatus. carboniferus, see A. carboniferus.

clevelandicus, see A. clevelandicus. coloradoensis, see A. coloradoensis.

convexus, see $\mathbf{A}$. convexus.

crenulatus, see Crenipecten crenulatus.

dolabriformis, see Aviculopecten dolabriformis.

duplicatus, see A. duplicatus.

hallianus, D'Orbignv, 1847, syn. for Aviculopecten cancellatus.

halli, see A. halli.

hawni, Geinitz, 1866, Carb. und Dyas, p. 36 , syn. for A. carboniferus.

missouriensis, see A. missouriensis. neglectus, see Euchondria neglecta. occidentalis, see A. occidentalis.

providencensis, see A. providencensis. radialis, see Pseudomonotis radialis. ringens, see Aviculopecten ringens. striatus, see A. striatus.

tenuilineatus, see Streblopteria ten uilineata. utahensis, see Aviculopecten utahensis.

Pernachactas, Castelnau, 1843, Syst. Sil., p. 44. Not recognized.

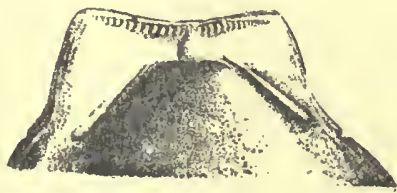

FIG. 838.--Pernopecten limiformis. Hinge-line.

Pernopecten, Winchell, 1865, Proc. Acad. Nat. Sci. Phil., p. 125. [Ety. from the

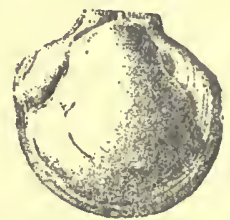

FIG. 889. - Pernopecten limiformis.

Acad. Sci., p. 213, and shells Perma and Pecten.] Shell like Pecten; hinge with a central cartilage pit and a crenulated linge plate on each side below the hinge margin. Type $P$. Jimiformis.

aviculatus, Sw a $110 \mathrm{w}$, 1858, (Pecten avicu. lus,) Trans. St. Louis

Geo. Sur. Ill., vol. 5, p. 588, Coal Meas cooperensis, Shumard, 1885, (Avicula cooperensis, ) Geo. Rep. Mo., p. 206, Waverly or Choteau Gr.

fasciculatus, see Lyriopecten fasciatus. limiformis, White \& Whitfield, 1862, (Aviculopecten li-

maformis,) Proc. FIG. 890.-Pernopecten Bost. Soc. Nat.

Hist., vol. 8, p. 295, Marshall Gr.

limatus, Winchell, 1865, Proc. Acad. Nat. Sci., p. 126, Marshall Gr.

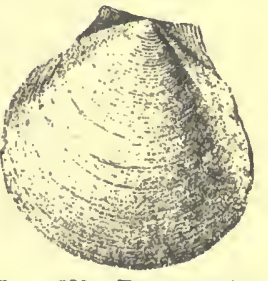


shumardanus, Winchell, 1865, Proc. Acad. Nat. Sci. Phil., p. 126, and Geo. Sur. Ill., vol. 2, p. 453, Kinderhook Gr.

Pholadella, Hall, 1870, Prelim. Notice Lam. Shells, p. 63. [Ety. diminutive of the recent genus Pholas.] Equivalve, elongated; valves inflated; beaks anterior, incurved; basal margin constricted; escutcheon and lumule; surface ribbed. Type $P$. newberryi.

constricta, Hall, 1883, Pa]. N. Y., vol. 5, pl. 78, figs. 26-27, Ham. Gr.

cuneata, see Promacrus cuneatus.

decussata, Hall, 1883, Pal. N. Y., vol. 5, pl. 78, fig. 28, syn. for Promacrus cuneatus.

newberryi, Hall, 1870, Prelim. Notice Lam. Shells, p. 65, and Pal. N. Y., vol. 5, pl. 78, fig. 25, Waverly Gr.

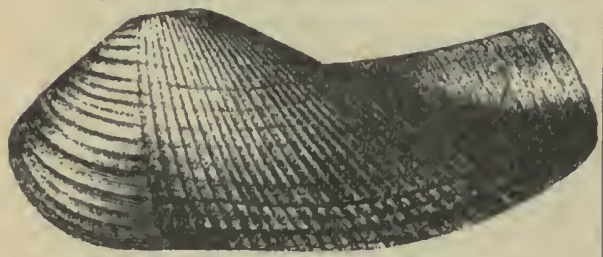

FIG. 891.-Pholadella newberryi.

ornata, Hall, 1870, Prelim. Notice Lam. Shells, p. 64, syn. for $P$. radiata.

parallela, Hall, 1883, Pal. N. Y., vol. 5, pl. 78, figs. 22-24, Ham. Gr.

radiata, Conrad, 1842, (Nuculites radiatus,) Jour. Acad. Nat. Sci., vol. 8, p. 248, and Pal. N. Y., vol. 5, pl.78, figs. 15-21, Ham. Gr.

truncata, Hall, 1870, Prelim. Notice Lam. Shells, p. 64, syn. for P. radiata.

Pholadomya elongata, see Allorisma elongatum.

Prthonia, Hall, 1870, Prelim. Notice Lam. Shells, p. 70. Equivalve, elongate-ovate, wider posteriorly; beaks obscure ; surface radiated and concentrically marked; no teeth; ligament external. Type P. sectifrons.

cylindrica, Hall, 1883, Pal. N. Y., vol. 5, pl. 78, figs. 1-4. Ham. Gr.

lirata, Hall, 1883, Pal. N. Y., vol. 5, pl. 78, fig. 14, Ham. Gr.

nitida, Hall, 1885, Pal. N. Y., vol. 5, p. 477, Chemung Gr.

nodocostata, Hall, 1870, Prelim. Notice Lam. Shells, p. 71, and Pal. N. Y., vol. 5, pl. 78, figs. 5-9, Ham. Gr.

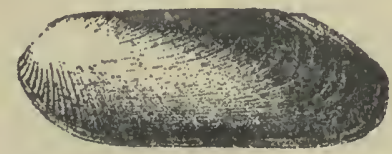

FIG. 892.-Phthonia sectifrons. Left valve.

sectifrons, Conrad, 1842, (Cypricardites sectifrons,) Jour. Acad. Nat. Sci., vol. 8 , p. 245, and Pal. N. Y., vol. 5, pl. 78, figs. 10-13, Ham. Gr. truncata, Hall, 1885, Pal. N. Y., vol. 5, p. 476, Chemung Gr.

Pinna, Linnæus, 1758, Syst. Nat. 10th Ed. [Ety. pinna, a wing.] Shell long, triangular equivalve; beaks terminal, pointed; posterior end broad, truncate, gaping; a subtrigonal, posterior muscular impression, "and a small reniform one at the beaks; cartilage long, narrow, internal, supported by a slender ridge close within the cardinal edges; no teetl; shell of one internal laminated layer, and an external vertically fibrous layer. Type P. squamosa. A living genus that sometimes attains a length of two feet, and ranges from low water to sixty fathoms. It moves vertically, partly buried in sand, with kuife-like edges erect. The byssus has been mixed with silk, spun and knit into gloves.

adamsi, syn. for Pinna peracuta.

consimilis, Walcott, 1885, Monogr. U. S. Geo. Sur., vol. 8, p. 236, Subcarbonirerous.

hinrichsana, White \& St. John, 1868, Trans. Chi. Acad. Sci., p. 122, St. Louis Gr.

inexpectans, Walcott, 1885, Mouogr. U.S. Geo. Sur., vol. 8, p. 235, Subcarboniferous.

ludlovi, Whitfield, 1876, in Ludlow's Carroll to Yellowstone Park, p. 143, Coal Meas.

marshallensis, Winchell, 1865, Proc. Acad. Nat. Sci., p. 126, Marshall Gr.

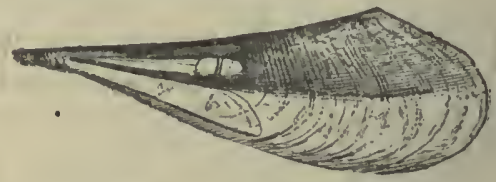

Frg. 893.-Pinna squamosa.

maxvillensis, Whitfield, 1882, Ann. N. Y. Acad. Sci., vol. 2, p. 221, Kaskaskia Gr. missouriensis, Swallow, 1863, Trans. St. Louis Acad. Sci., vol. 2, p. 97, Kaskaskia Gr.

peracuta, Shumard, 1858, Trans. St. Louis Acad. Sci., vol. 1, p. 214, and Pal. E. Neb., p. 198, Coal Meas.

stludovici, Worthen, 1883, Geo. Sur. Ill., vol. 7, p. 326, St. Louis Gr.

subspatulata, Worthen, 1875, Geo. Sur. Ill., vol. 6, p. 524, Warsaw Gr.

Pinnopsis, syn. for Lunulicardium. acutirostra, syn. Lunulicardium ornatum. ornatus, see Lunulicardium ornatum,

Placunopsis, Morris \& Lycett, 1853, Monogr. Foss. Great Oolite. [Ety. Placuna, a genus; opsis, resemblance.] Suborbicular, upper valve convex, radiately striated or taking the form of the surface to which it adheres; lower valve flat; ligamental groove submarginal: muscular impression subcentral. Type P. jurensis. Not a Palæozoic genus. 
Species are left here for want of material to determine their generic relations.

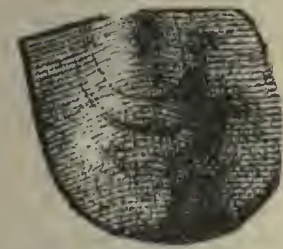

carbonaria, Meek \& Worthen, 1866 , Proc. Chi. Acad. Sci., vol. 1, p. 13, Up. Coal Meas.

recticardinalis, Meek, 1875, Ohio Pal., vol. 2 , p. 331 , Coal Meas.

Fig. 894.-Placunopsis Plethomytilus, Hall, recticardinalis. In- 1883, Pal, N. Y., ternal cast of left vol. $5, \mathrm{pt} .1, \mathrm{p} .4 \dot{ }$.
valve. (Plates and Explanations.) (Ety. pletho, to be full; Mylilus, a genus.] Mytiloid, gibbous; ligamental area finely striated; no cardinal teeth; lateral teeth small, oblique; test, with concentric striæ; differs from Mytilacra in its true hinge-line and the absence of teeth. Type $\mathrm{P}$ : ponderosus.

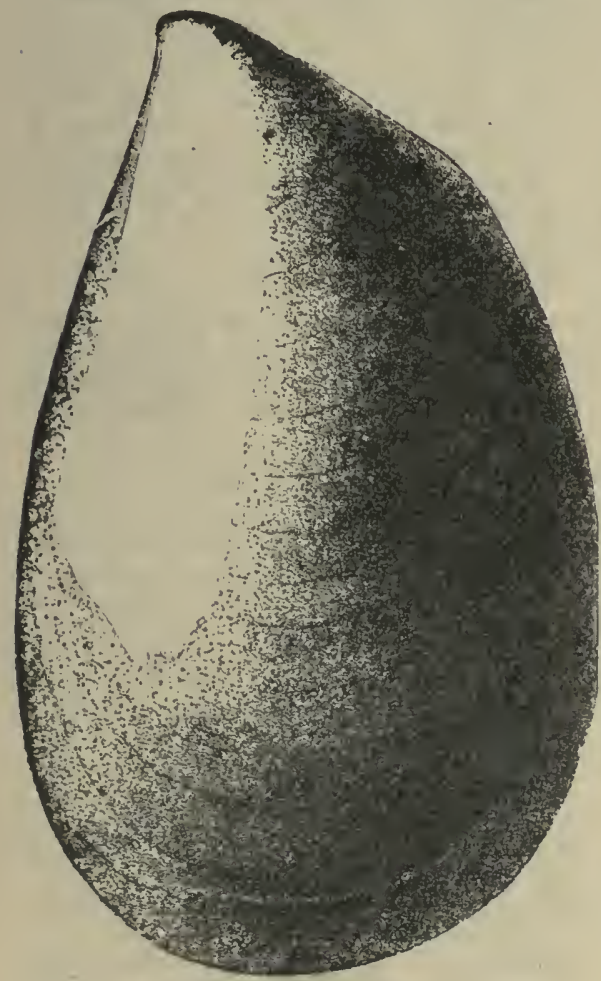

FIG. 895.-Plethomytilus ponderosus.

arenaceus, Hall, 1870, (Mytilarca arenacea,) Prelim. Notice Lam. Shells, p. 20 , and Pal. N. Y., vol. 5, pt. 1, p. 253, Schoharie grit.

knappi, Hall, 1884, Pal. N. Y., vol. 5, pt. 1, p. 256, Ham. Gr.

mytilimeris, Conrad, 1842, (Inoceramus mytilimeris,) Jour. Acad. Nat. Sci., vol. 8 , p. 246 , Low. Held. Gr. oviformis, Conrad, 1842, (Inoceramus oviformis,) Jour. Acad. Nat. Sci., vol. 8, p. 246, and Pal. N. Y., vol. 5, pt. 1, p. 255 , Ham. Gr.

ponderosus, Hall, 1870, (Mytilarca ponderosa, ) Prelim. Notice Lam. Shells, p. 21, and Pal. N. Y., vol. 5, pt. 1, p. 254, Up. Held. Gr.

Pleurophorus, King, 1844, Ann. Mag. Nat. Hist., vol. 14, p. 313. [Ety. pleuron, a rib; phoros, bearing.] Inequilateral, longitudinally oblong or subovate; two cardinal teeth in each valve, alternately interlocking and divergent; one posterior lateral tooth in each valve, the receiving tooth in the left valve; anterior adductor scar deep, and bounded posteriorly by a ridge; pallial line simple. Type P. costatus.

angulatus, Meek \& Worthen, 1865, Proc. Ácad. Nat. Sci. Phil., p. 247, and Geo. Sur. Ill., vol. 6, p. 529, Coal Meas.

calhouni, Meek \& Hayden, 1858, (Edmonia calhouni,) Trans. Alb. Inst., vol. 4, p. 80, and Pal. Up. Mo., p. 62, Permian Gr.

chesterensis, Worthen, 1884, Bull. No. 2, IIl. St. Mus. Nat. Hist., p. 16, and Geo. Sur. Ill., vol. 8, p. 123, Kaskaskia Gr. costatiformis, Meek \& Worthen, 1865 , Proc. Acad. Nat. Sci. Phil., p. 247, and Geo. Sur. Ill., vol. 3, p. 535, Keokuk Gr. meeki, Walcott, 1885, Monogr. U. S. Geo. Sur., vol. 8, p. 246, Carboniferous.

minimus, Worthen, 1884, Bull. No. 2, Ill. St. Mus. Nat. Hist., p. 17, and Geo. Sur. Ill., vol. 8, p. 124, St. Louis Gr.

monroensis, Worthen, 1884, Bull. No. 2, Ill. St. Mus. Nat. Hist., p. 17, and Geo. Sur. Ill., vol. 8, p. 125, St. Louis Gr.

oblongus, Meek, 1872, Pal. E. Neb., p.212, Coal Meas.

occidentalis, Meek \& Hayden, 1862, Trans. Alb. Inst., vol. 4, p. 80, and Pal. Up. Mo., p. 35, Coal Meas.

pallasi, as identified by Geinitz, is $P$. oblongus.

permianus, Swallow, 1858, Trans. St. Louis Acad. Sci., vol. 1, p. 192, Permian Gr.

quadricostatus, Daw son, 1868, Acad. Geo., p. 304, Carboniferous.

simplus, as identified by Geinitz, is $\mathrm{P}$. subcuneatus.

subcostatus, Meek \& Worthen, 1865, Proc. Acad. Nat. Sci. Phil., p. 246, and Geo. Sur. Ill., vol. 2, p. 347, Up. Coal Meas. subcuneatus, Meek \& Hayden, 1858, 'Trans. Alb. Inst., vol. 4, p. 81, and Pal. Up. Mo., p. 61, Permian Gr.

(?) subellipticus, Meek, 1867, Am. Jour. Sci., vol. 44, p. 181, and Pal. E. Neb., p. 211, Coal Meas.

tropidophorus, Meek, 1875, Ohio Pal., vol. 2, p. 338, Coal Meas. 
Pleurorhynchus, Phillips, syn. for Conucardium.

antiquum, see Conocardium antiquum. attenualum, see Conocardium attenuatum. crassifroris, see Conocardium crassifrons. cuneus, see Conocardium cuneus.

trigonale, Hall, see Conocardium subtrigonale.

vomer, see Conocardium vomer.

Posidonia, Bronn, see Posidonomya.

alata, see Posidonomya alata.

alveala, see Grammysia alveata.

arcuata, see Grammysia arcuata.

clathrata, see Posidonomya clathrata.

distans, see Posidonomya distans.

lirata, see Paracyclas lirata.

moorci, see Pusidonomya moorii.

perstriata, see Posidonomya perstriata.

Posidoxomya, Bronn, 1837, Leth. Geogn. [Lity. Poseidon, a mythological name; Mya, a genus.] Shell thin, obliquely oval, subtruncate at one end, equivalve, compressed, concentrically furrowed, hinge-line short and straight, edentulous. Type P. becheri.

alata, Hall, 18t3, (Posidonia (?) alata,) Geo. 4th Dist. N. Y., p. 72 , and Pal. N. Y., vol. 2, p. 87, Clinton Gr. ambigua, Winclsell, 1863,

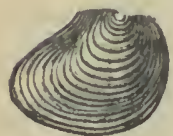

FIG. 897.--Pos1donomsa becheri.
Proc. Acad. Nat. Sci., p. 10, Marsliall Gr.

clathrata, Lea, 1853, (Posidonia clathrata,) Jour. Acad. Nat. Sci., 2d ser., vol. 2, p. 205, Coal Meas. devonica, Walcott, 1885 ,

Monogr. U. S. Geo. Sur. vol. 8 , p. 179 , Devonian.

distans, Iea, 1853, (Posidonia distans,) Jour. Acad. Nat. Sci., $2 \mathrm{~d}$ ser., vol. 2, p. 205, Coal Meas.

fracta, Meek, 1875, Ohio Pal., vol. 2, p. 333, Coal Meas.

fragosa, see Lunulicardium fragosum.

lævis, IValcott, 1885, Monogr. U. S. Geo. Sur., vol. 8, p. 178, Devonian.

mesambonata, Winchell, 1862, Proc. Acad. Nat. Sci., p. 420, Marshall Gr.

moorii, Gabb, 1859, (Posi- Posidonomy donia moorei,) Proc. Acad.

Nat. Sci., p. 297, Coal Meas.

perstriata, Lea, 1853, (Posidonia perstriata,) Jour. Acad. Nat. Sci., 2 d ser., vol. 2, p. 205, Coal Meas.

rhomboidea, Hall, 1852, Pal. N. Y., vol. 2, p. 284, Niagara Gr.

romingeri, Winchell, 1862, Proc. Acad. Nat. Sci., p. 420 , Marshall Gr.

striata, Stevens, 1858, Am. Jour. Sci., vol. 25, p. 265, Coal Meas.

whiteana, Winchell, 1862, Proc. Acad. Nat. Sci.; p. 420, Marshall Gr.

Praccardium, Barrande, 1881, Syst. Sil. de la Boheme, vol. 6, p. 141. [Ety. præ, before; Cardium, a genus.] Equivalve, inequilateral, elliptical or trigonal; beaks prominent, incurved; surface radiated, and concentrically lined; posterior to the beaks a small area carries a series of vertical nearly parallel teeth.

vetustum, Hall, 1843, (Cardium vetustum,) Geo. Rep. 4th Dist. N. Y., p. 245, and FIG. 899. Pal. N. Y., vol. 5, p. 427 , vetustum. Portage Gr.

Prisconaia, Conrad, 1867, Am. Jour. Conch., vol. 3. [Ety. proper name.] Equivalve, inequilateral, and distinguished from Anthracosia, which it $m u \mathrm{ch}$ resembles, by having lateral teeth. Type P. ventricosa.

ventricosa, Conrad, 1867, Am. Jour. Conch., vol. 3, Coal Meas.

Promacrus, Meek, 1871, Am. Jour. Conch., vol. 7, p. 4. [Ety. pro, forward; makros, long.] Similar to Sanguinolites; anterior end much produced, narrowly rounded; posterior end produced, obliquely truncate; beaks appressed; cardinal margin nearly straiglit behind the beaks, and declining in front; umbonal slope angular, extending to the basal extremity; surface concentrically lined, and sometimes plicated anteriorly; ligament external. Type P. andrewsi.

andrewsi, Meek, 1871, Am. Jour. Conch., vol. 7 , p. 4, Waverly Gr.

cuneatus, Hall, 1870, (Pholadella cuneata,) Prelim. Not. Iam. Shells, p. 66, and Pul. N. Y., vol. 5, p.510, Waverly Gr. missouriensis, see Sanguinolites missouriensis.

nasutus, see Sanguinolites nasutus.

Prorhycius, Hall, 1885, Pal. N. Y., vol. 5, p. 48. [Ety. pro, forward; rhynchos, beak.] Left valve the larger and more gibbous; an terior end truncate, angular or nasute at the antero-dorsal extremity; posterior end broad, margin truncate or broadly rounded; beaks low ; cardinal line straight, extending the entire length of the dorsal margin, and alate at both ends; umbonal slope subangular; surtace concentrically lined; strong lateral tooth, ligament external. Type P. quadratum.

angulatum, Hall, 1885, Pal. N. Y., vol. 5, p. 493, Chemung Gr.

nasutum, Hall, 1885, Pal. N. Y., vol. 5, p. 493, Chemung Gr.

quadratum, Hall, 1883, (Palæanatina quadrata,) Pal. N. Y., vol. 5, p. 492, Chemung Gr.

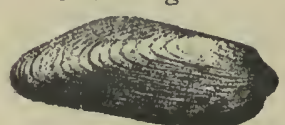

Fio. 900.-Prothyris meeki.
Protiriris, Me e k, 1869, Proc. Acad. Nat. S.j. Phil., p. 172. [Ety. pro, forwari ; thyris, an orifice.] Equivalve, inequilateral, extremely elongate; cardinal and basal margins subparallel ; anterior 
end rounded or subtruncate, with a deep notch in the antero-ven. tral margin; posterior end rounded, lanceolate, or truncate; cardinal line straight or slightly arcuate; cardinal slope sometimes subalate; umbonal slope rounded, undefined or subangular; surface concentrically lined. Type P. elegans.

alata, Hall, 1885, Pal. N. Y., vol. 5, p. 461, Chemung Gr.

elegans, Meek, 1871, Am. Jour: Conch., vol. 7 , p. 5 , Coal Meas.

exuta, Hall, 1885, Pal. N. Y., vol. 5, p. 462, Chemung Gr.

lanceolata, Hall, 1883, Pal. N. Y., vol. 5, pl. 76 , figs. 2 to 8 , Ham. Gr.

meeki, Winchell, 1875, Ohio Pal., vol. 2, p. 305, Waverly Gr.

planulata, Hall, 1883, Pal. N. Y., vol. 5, pl. 76, fig. 1, Ham. Gr.

Protomya, Hall, 1885, Pal. N. Y., vol. 5, p. 52. [Ety, protos, first; Mya, a genus.] Equivalve, inequilateral, elongate, ovate-elliptical; anterior end broadly rounded; posterior end narrower, rounded; beaks incurved; umbo prominent; cardinal line long, nearly straight; umbonal slope gibbous above, not defined below; surface concentrically lined; ligament external; muscular impressions circular ; anterior one strong and near the margin. Type P. oblonga.

oblonga, Hall, 1885, (Cardiomorpha oblonga,) Pal. N. Y., vol. 5, p. 509, Ham. Gr. Pseudononotis, Beyrich, 1862, Zeit. der Deutsch., Geol. Gesselsch., vol. 14. [Ety. pseudes, false; Monotis, a genus.] Suborbicular, plano-convex, left valve convex, right valve flat or slightly concave; not auriculate ; beaks subcentral, slightly oblique, unequal, left elevated, gibbous, incurved, right small; hinge short, narrow, edentulous; cartilage cavity under the beaks; byssal notch of right valve narrow, deep, and separated from the hinge by a small rudimentary ear, which does not project beyond the margin; adductor muscular scar large, subcentral ; impres-

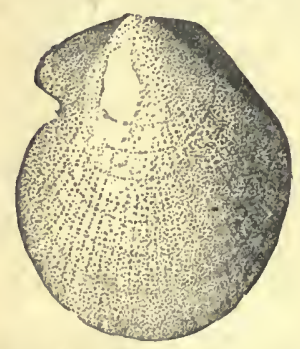

FIG. 901.-Psendomonotis hawni. sions of retractor muscles, several, small, placed near the beaks; surface radiated, most distinct on the left valve.

hawni, Meek \& Hayden, 1858, (Monotis hawni,) Trans. Alb. Inst., vol. 4, p. 76, and Pal. Up. Mo., p. 54, Up. Coal Meas.

hawni var. ovata, Meek \& Hayden, 1865 , (Eumicrotis hawni var. ovata,) Pal. Up. Mo., p. 55, Permian Gr. hawni var. sinuata, Meek \& Worthen, 1866, (Eumicrotis hawni var. sinuata,) Geo. Sur. Ill., vol. 2, p. 338, Up. Coal Meas.

radialis, (?) Phillips, 1834, (Pecten radialis,) Encyc. Meth., vol. 4, Coal Meas. Pterinea, Goldfuss, 1826, Germ. Petref. [Ety. pteron, a wing.] Transversely trigonal, oblique, inequivalve, very inequilateral, left valve most convex, beaks near the small anterior end; hinge-line long, straight, forming a small anterior and large falciform posterior wing, with a linear, flattened, marginal cartilage facet; longitudinally striated; shell thick, calcareous; two long, slightly diverging, posterior, lateral teeth, beneath the hinge in one valve and one in the other; a few short, cardinal teeth radiating beneath and in front of the beaks; anterior im. pression very strong just in front of the beak, posterior impression larger, but faintly marked, superficial ; pallial scar simple; shallow byssal concavity. Type P. laevis.

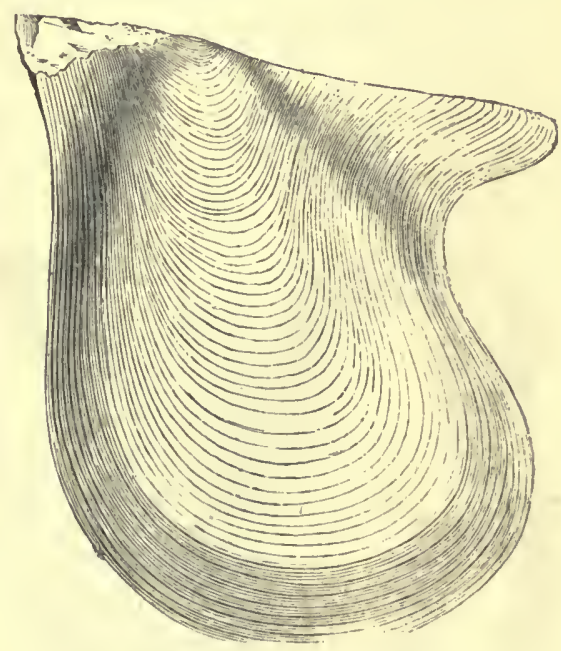

Fra. 902.-Pterinea demissa.

appressa, Conrad, 1838, Ann. Rep. N. Y. Not defined.

arenacea, Hall, 1877. Proposed, but not defined.

aviformis, Conrad, 1842, (Avicula aviformis,) Jour. Acad. Nat. Sci., vol. 8, p. 243, Trenton Gr.

avis, Hall, 1884, Pal. N. Y., vol. 5, pt. 1, p. 105, Chemung Gr.

bellilineata, Billings, 1866, Catal. Sil. Foss. Antic., p. 15, Hud. Riv. Gr.

bisulcata, see Grammysia bisulcata.

brisa, Hall, 1867, 20th Rep. N. Y. Mus. Nat. Hist., p. 384, syn. for P. striæcosta. cancellata, Barris, 1879, (Avicula cancellata,) Proc. Dav. Acad. Sci., vol. 2, p. 286 , Corniferous limestone. 
cardiiformis, see Megambonia cardiiformis. cardinata, Winchell, 1862, Proc. Acad. Nat. Sci., p. 412, Marshall Gr.

carinata, Goldfuss, see Ambonychia carinata.

chemungensis, Conrad, 1812, (Avicula chemungensis,) Jour. Acad. Nat. Sci., vol. 8 , p. 243, and Pal. N. Y., vol. 5, pt. 1, p. 98, Chemung Gr.

concentrica, Conrad, 1838, Ann. Rep. N. Y. Not defined.

consimilis, Hall, 1883, Pal. N. Y., vol. 5, pt. 1, p. 100 , Chemung Gr.

corrugata, James, 1874, (Avicula corrugata,) Cin. Quar. Jour. Sci., vol. 1, p. 239. Hud. Riv. Gr.

crenistriata, Winchell, 1862, (Cardiopsis crenistriata,) Proc. Acad. Nat. Sci., p. 417, Marshall frr.

crenulata, see Crenipecten crenulatus.

cuneata, see Sphenotus cuneatus.

curiosa, Billings, 1866, Catal. Sil. Foss. Antic., p. 51, Anticosti Gr.

cyrtodontoides, Winchell \& Marcy, 1865, Mem. Bost. Soc. Nat. Hist., p. 95, Niagara Gr.

demissa, Conrad, 1842, (Avicula demisfa,) Jour. Acad. Nat. Sci., vol. 8, p. 242 , and Pal. N. Y., vol. 1, p. 292, Hud. Riv. Gr.

dispanda, Hall, 1884, Pal. N. Y., vol. 5, pt. 1, p. 97, Chemung Gr.

elliptica, Hall, 1847, (Avicula elliptica,) Pal. N. Y., vol. 1, p. 162, Trenton Gr.

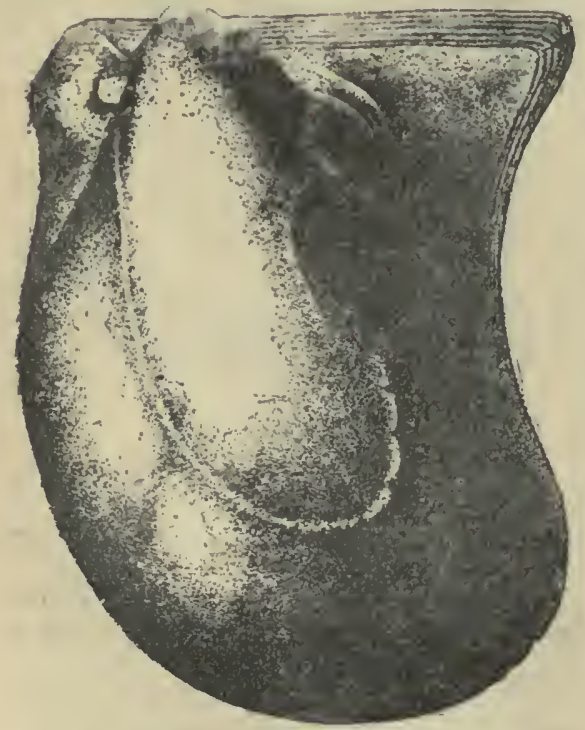

FIG. 903.-Pterlnea flabellum.

fabellum, Conrad, 1842, (Avicula flabella,) Jour. Acad. Nat. Sci., vol. 8, p. 238, and Pal. N. Y., vol. 5, pt. 1, p. 93, Up. Held. and Ham. Grs.

grandis, Hall, 1884, Pal. N. Y., vol. 5, pt. 1, p. 91, Up. Held. Gr. honeymani, Hall, 1860, (Avicula honeymani,) Can. Nat. and Geol., vol. 5, p. 153, and Acad. Geol., p. 604, Up. Sil.

insueta, Emmons, 1842, (A vicula insueta,) Geo. Rep. 2d Dist. N. Y., p. 399, and Pal. N. Y., vol. 1, p. 291, Utica Slate and Hud. Riv. Grs.

interstrialis, Hall, 1884, Pal. N. Y., vol. 5, pt. 1, p. 96, Chemung Gr.

modioluris, see Modiolopsis modiolaris. morganerisis, see A vicula morganensis.

mucronata, Ulrich, 1879 , Jour. Cin. Soc.

Nat. Hist., vol. 2, p. 24, Hud. Riv. Gr.

neglectr, McChesney, 1861, New Palæozoic Fossils, 1. 88, Niagara Gr.

newarkensis, Walcott, 1885, Monogr. U.8.

Geo. Sur., vol. 8, p. 165, Devonian.

orbicularis, see Ambonychia orbicularis. pholadis, sce Orthonnta pholadis.

pinguis, Hall, 1884, Pal. N. Y., vol. 5, pt. 1, p. 92, Up. Held. Gr.

pintoensis, Walcott, 1885், Mnnogr. U. S. Geo. Sur., vol. 8, p. 234, Subcarboniferous.

planulata, see Cypricarlinia planulata.

prolifica, Billings, 1866, Catal. Sil. Foss. Antic., p. 16, Hud. Riv. Gr.

prora, Hall, 1884, l'al. N. Y., vol. 5, pt. 1, p. 102, Chenung Gr.

punctulata, Conrad. Not defined. See Cimitaria recurva.

pygman, see Modiella pygmæa.

radians, see Panenka radians.

reproba, Hall, 188t, Pal. N. Y., vol. 5, pt. 1, p. 106, Chemung Gr.

reversa, Hall, 1884, Pal. N. Y., vol. 5, pt. 1, p. 104, Chemung Gr.

reversa var. avis, see $\mathrm{P}$. avis.

revoluta, Winchell \& Marcy, 1865, Mem. Bost. Soc. Nat. Hist., p. 95, Niagara Gr. rigida, Hall, 1884, Pal. N. Y., vol. 5, pt. 1, p. 101, Clsemung Gr.

rugosa, Conrad, 1841, (Avicula rugosa,) Ann. Geo. Ren. N. Y., and Geo. Rep. 4th Dist. N. Y., pl. 26, fig. 2, Waterlime $\mathrm{Gr}$.

similis, Whitfield, 1882, Ann. N. Y. Acad. Sci., vol. 2, p. 214, Marcellus Shale. spinalata, Winchell, 1865, Proc. Acad. Nat. Sci., p. 124, Burlington (?) Gr. strisecosta, McCliesney, 1861, (Ambonychia striæcosta,) New Pal. Foss., p. 88, Niagara Gr.

strigosa, White \& Whitfield, 1862, Proc. Bost. Soc. Nat. IIist., p. 31, Marshall Gr. suborbicularis, see Pterinopecten suborbicularis.

subpapyracea, Meek \& Worthen, 1866, Proc. Chi. Acad. Sci., p. 21, Ham. Gr.

thebesensis, Meek \& IVorthen, 1868, Geo. Sur. Ill., vol. 3, p. 354, Niagara Gr.

thisbe, Billings, 1866, Catal. Sil. Foss. Antic., p. 52, Anticosti Gr.

trentonensis, Conrad, 1842, (Avicula trentonensis,) Jour. Acad. Nat. Sci., vol. 8, p. 240, and Pal. N. Y., vol. 1, p. 161, Trenton $\mathrm{Gr}$.

triquetra, see Gosselettia triquetra. undata, see Ambonychia undata. 
undulata, Meek \& Worthen, 1868, Geo. Sur. Ill., vol. 3, p. 456, Kinderhook Gr. varistriata, Billings, 1866, Catal. Sil. Foss. Antic., p. 50, Anticosti Gr.

volans, Winchell \& Marcy, 1865, Mem. Bost. Soc. Nat. Hist., p. 95, Niagara Gr. Ptericopecten, Hall, 1883, Pal. N. Y., vol. 5 , pt. 1, p. 3 . (Plates and Explanations.) [Ety. Pterinea, a genus ; Pecten, a genus.] Valves more or less convex; radiated and bearing concentric lines of growth; hinge-line long, straight; wings not well defined, being simple expausions of the upper lateral margins to the hinge-line. Type P. undosus.

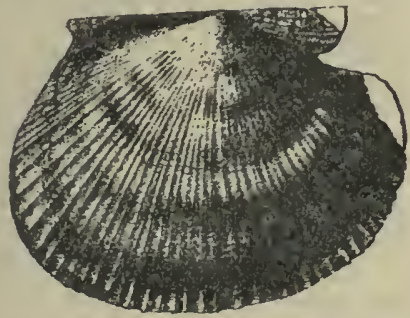

FrG. 904.-Pterinopecten undosus.

conspectus, Hall, 1884, Pal. N. Y., vol. 5, pt. 1 , p. 66, Ham. Gr.

crenicostatus, Hall, 1884, Pal. N. Y., vol. 5, pt. 1, p. 78, Chemung Gr.

dignatus, Hall, 1884, Pal. N. Y., vol. 5, pt. 1, p. 62, Marcellus Shale.

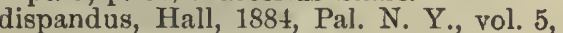
pt. 1, p. 76, Chemung Gr.

erectus, Hall, 1884, Pal. N. Y., vol. 5, pt. 1, p. 77, Chemung Gr.

exfoliatus, Hall, 1881, Pal. N. Y., vol. 5, pt. 1, p. 61, Marcellus Shale.

filitextus, Hall, 188 5, pt. 1, p. 67, Ham. Gr.

hermes, Hall, 1884, Pal. N. Y., vol. 5, pt. 1, p. 64. Ham. Gr.

hoosacensis, Walcott, 1885, Monogr.

U. S. Geo. Sur., vol. 8, p. 232, Subcarboniferous.

imbecilis, Hall, 1884, Pal. N. Y., vol. 5 , pt. 1, p. 75 , Chemung Gr.

insons, Hall, 1884, Pal. N. Y., vol. 5, pt. 1, p. 59, Up. Held. Gr.

intermedius, Hall, 188t, Pal. N. Y., vol. 5, pt. 1, p. 68, Ham. Gr.

invalidus, see Aviculopecten invalidus.

lætus, Hall, 1884, Pal. N. Y., vol. 5, pt. 1, p. 63, Marcellus Shale.

multiradiatus, Hall, 188t, Pal. N. Y. vol. 5, pt. 1, p. 57, Up. Held. Gr. neptunus, Hall, 1884, Pal. N. Y., vol. 5, pt. 1, p. 79, Chemung Gr.

nodosus, Hall, 1884, Pal. N. Y., vol. 5, pt. 1, p. 60, Up. Held. Gr.

reflexus, Hall, 1884, Pal. N. Y., vol. 5, pt. 1, p. 58, Up. Held. Gr.

regularis, Hall, 1884, Pal. N. Y., vol. 5, pt. 1, p. 70, Ham. Gr. spio, Walcott, 1885, Monogr. U. S. Geo. Sur., vol, 8, p. 233, Subcarboniferous. spondylus, Hall, 1884, Pal. N. Y., vol. 5, pt. 1, p. 65, Ham. Gr.

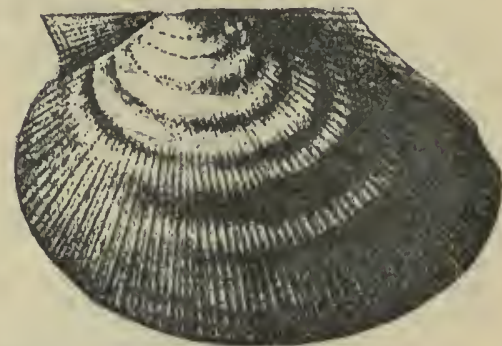

FIG. 905.-Pterinopecten undosus.

strictus, Hall, 1884, Pal. N. Y., vol. 5, pt. 1, p. 74, Chemung Gr.

suborbicularis, Hall, 1843, (Pterinea suborbicularis,) Geo. Rep. 4th Dist. N. Y., p. 264, and Pal. N. Y., vol. 5, p. 80 , Chemung Gr.

terminalis, see Aviculopecten terminalis. undosus, Hall, 1884, Pal. N.Y., vol. 5, pt. 1, p. 72, Ham. Gr.

vertumnus, Hall, 1884, Pal. N. Y., vol. 5, pt. 1, p. 71, Ham. Gr.

Pteronitella, Billings, 1874, Pal. Foss., vol. 2, p. 141. [Ety. diminutive of Pteronites.] Resembles Pterinea, but possesses in front of the beaks several small, anterior, cardinal teeth, and close beneath the hinge-line several more or less elongated posterior teeth. Type P. venusta.

curta, Billings, 1874, Pal. Foss., vol. 2, p. 143, Low. Held. Gr.

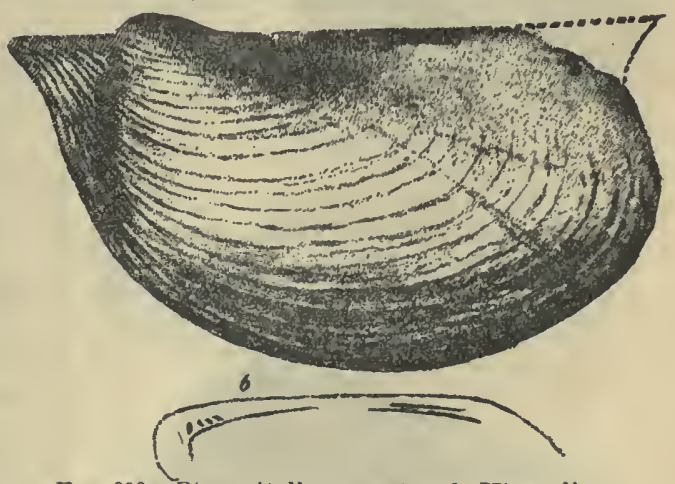

Fra. 906.-Pteronitella venusta. b, Hinge-line.

oblonga,- Billings, 1874, Pal. Foss., vol. 2, p. 143, Low. Held. Gr.

venusta, Billings, 1874, Pal. Foss., vol. 2, p. 142, Low. Held. Gr.

Pteronites, McCoy, 1844, Syn. Carb. Foss. Ireland, p. 81. [Ety. pteron, a wing.] Subtriangular, depressed, hinge-line as long as the shell; beaks terminal, or nearly so, forming a very narrow, ob- 
tusely pointed anterior end, from which the ventral margin extends to the broad posterior end; left valve most convex; internally a very small tooth under the beak of the right valve, and a very slender, posterior, lateral tooth close to the hinge-line the whole length. Type $P$. angustatus.

gayensis, Daweon, 1868, Acad. Geo., p. 301 , Subcarboniferous.

gayensis var. ornatus, Dawson, 18S3, Rep. on Redpath Mus., No. 2, p. 14, Subcarboniferous.

inoptatus, Hall, 1884, Pal. N. Y., vol. 5, pt. 1, p. 239, Chemung Gr.

newarkensis, Meek, 1871, Proc. Acad. Nat. Sci., p. 162, Waverly Gr.

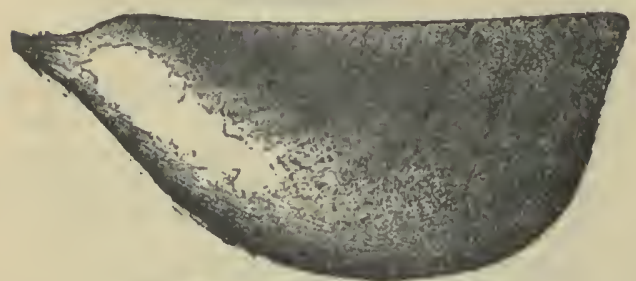

Fio. 907.-Pteronites profundus.

profundus, Hall, 1884, Pal. N. Y., vol. 5, pt. 1, p. 237, Up. Chemung Gr.

rostratus, Hall, 1884, Pal. N.Y., vol. 5, pt. 1, p. 238, Chemung Gr.

spergenensis, Whitfield, 1882, Bull. Am. Mus. Nat. Hist., No. 3, p. 56, Warsaw Gr.

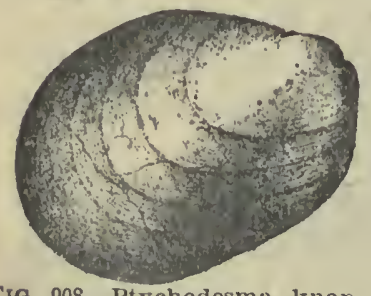

FIG. 908.-Ptychodesma knappanum. Right side.
P T. YCHONESNA,

H a 11 \&

Whitfield,

1872,24 th

Rep. N. Y.

Mus. Nat.

Hist., p.

192. [Ety.

ptychos, a

folding ;

des $m a$, a

ligament, or band.] Form modioloid ; hinge having a wide ligamental area, grooved by the successive growth of the ligament, as in pectunculus. Type P. knappanum.

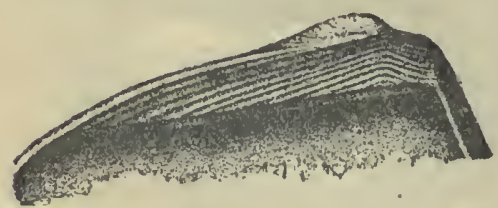

FIG. 909.-Ptychodesma knappanum, Enlargement of one side of ligamental area.

knappanum, Hall \& Whitfield, 1872, 24th Rep. N. Y. Mus. Nat. Hist., p. 192, Up. Held. Gr.

minor, Hall, 1885, Pal. N. Y., vol. 5, p. 353, Chemung Gr. nanum, Hall, 1885, Pal. N. Y., vol. 5, p. 353, Chemung Gr.

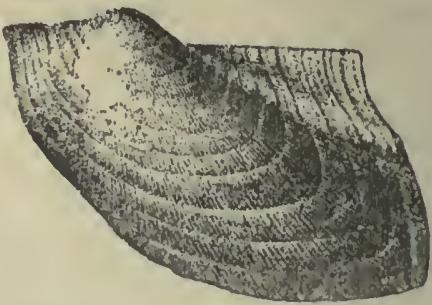

FIG. 910.-Ptychopteria beecheri.

Ptyciopteria, Hall, 1884, Pal. N. Y., vol. 5, pt. 1, p. 3. [Ety. ptyche, fold ; Pteria, a genus.] Ditfers from Actinopteria in the nasute anterior extremity, and large, straight wing marked by a strong longitudinal fold. Hinge-line narrow, linear; furvished with one or two liuear, oblique, cardinal and lateral teeth; surface with fine rays. It bears about the same relation to Actinopteria that Leptodesma does to Liopteria. Type P. eugenia.

alata, Hall, 1884, Pal. N. Y., vol. 5, pt. 1, p. 139, Chemung Gr. beecheri, Hall, 1884,

Pal. N.Y., vol. 5 , pt. 1 , p. 143 , Chemung Gr.

elongata,

Hall, 1884,

Pal. N. Y.,

vol. 5 , pt.

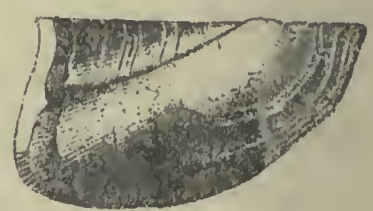

Firo. yI1.-Ptychopteria beecheri.
1, p. 141, Chemung Gir.

eucrate, Hall, 188t, Pal. N. Y., vol. 5, pt. 1, 133, Chemung Gr.

eudora, Hall, 1884, Pal. N. Y., vol. 5, pt. 1, p. 138, Chemung Gr.

eugenia, Hall, 1883, Pal. N. Y., vol. 5, pl. 23 , figs. 17-20, Chemung Gr.

expansa, Hall, 1884, Pal. N. Y., vol. 5, pt. 1 , p. 152, Chemung Gr.

falcata, Hall, 1884, Pal. N. Y., vol. 5, pt. 1, p. 136, Up. Chemung Gr.

galene, Hall, 1884, Pal. N. Y., vol. 5, pt. 1, p. 142, Chemung Gr.

gibbosa, Hall, 1884, Pal. N. Y., vol. 5, pt. 1, p. 149, Up. Chemung Gr.

lata, Hall, 1884, Pal. N. Y., vol. 5, pt. 1, p. 145, Up. Chemung Gr.

lobata, Hall, 1884, Pal. N. Y., vol. 5, pt. 1, p. 150 , Up. Chemung Gr.

perlata, Hall, 1884, Pal. N. Y., vol. 5, pt. 1, p. 147, Up. Chemung Gr.

proto, Hall, 1884, Pal. N. Y., vol. 5, pt. 1, p. 129, Chemung Gr.

protoformis, Walcott, 1885, Monogr. U. S. Geo. Sur., vol. 8, p. 235, Subcarboniferous.

salamanca, Hall, 1884, Pal. N. Y., vol. 5, pt. 1, p. 131, Chemung Gr. 
sao, Hall, 1884, Pal. N. Y., vol.j, pt. 1, p. 132, Chemung Gr.

sinuosa, Hall, 1884, Pal. N. Y., vol. 5, pt. 1, p. 130, Up. Chemung Gr.

spatulata, Hall, 1884, Pal. N. Y., vol. 5, pt. 1, p. 144, Up. Chemung Gr.

spio, Hall, 188t, Pal. N. Y., vol. 5, pt. 1, p. 137, Chemung Gr.

thalia, Hall, 1884, Pal. N. Y., vol. 5, pt. 1, p. 148, Up. Chemung Gr.

thetis, Hall, 1884, Pal. N. Y., vol. 5, pt. 1, p. 135 , Chemung Gr.

trigonalis, Hall, 188t, Pal. N. Y., vol. 5, pt. 1, p. 140, Chemung Gr.

vanuxemi, Hall, 1884, Pal. N. Y., vol. 5, pt. 1, p. 151, Up. Chemung Gr.

Pyanomya, S. A. Miller, 1881, Jour. Cin. Soc. Nat. Hist., vol. 4, p. 318. [Ety. pyanos, a bean; Mya, a genus.] Equivalve, elongate, inequilateral, fragile, edentulous; ligament external. Type P. gibbosa.

faberi, n. sp. Shell small, equivalve, inequilateral; length twice as great as height; cardinal and basal lines subparallel; anterior end sharply rounded into the subelliptical base; posterior end broadly rounded; valves ventricose in the middle; beaks obtuse ; umbonal ridge prominent, subangular, distinctly defined, and directed to the

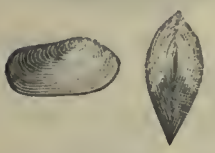

FIG. 912.-Pyanomya faberl. likht valve and dorsal view. postero-basal margin; ligament external; hi nge-line straight behind the beaks and inclined in front; no escutcheon or lunule. Surface marked very faintly by concentric lines of growth. Distinguished from $P$. gibbosa by the angular umbonal ridge, less acute anterior end and straight cardinal line beliind the beaks, and other particulars. Hud. Riv. Gr., Cincinnati, Ohio. Collected by Charles Faber.

gibbosa, S. A. Miller, 1881, Jour. Cin. Soc. Nat. Hist., vol. 4, p. 318, Hud. Riv. Gr.
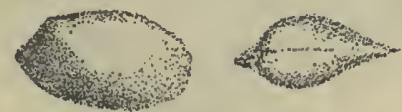

F1G. 913.-Pyanomya glbbosa. Left valve aud dorsal vlew.

Pyrenomaus, Hall, 18522, Pal. N. Y., vol. 2, p. 87. [Ety. pyrenos, Nucula; omoios, similar; from its resemblance in general form to the shells of the genus Nucula.] Equivalve, inequilateral;umbon es prominent, beak elevated; muscular impression near the anterior extremity; general form of Nucula withont the teeth that characterize that genus, or the clavicle of a Clidophorus. Type P. cuneatus. cuneatus, Hall, 1852, Pal. N. Y., vol. 2, p. 87, Clinton Gr.
Sanguinolaria, Lamarck, 1801, Syst. An. sans Vert. [Ety. from the type Solen sanguinolentus.] Oval, compressed, rounded in front, attenuated and slightly gaping behind; hinge teeth $\frac{2}{2}$ small ; siphonal inflection deep, connected with the pallial line; ligament external, on very prominent fulcra. Type S. sanguinolentus. Typical species S. diphos. Not American Palæozoic. Species left under this name for want of material to determine generic relations.

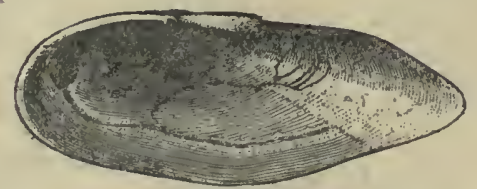

FrG. 914.-Sanguinolaria diphos.

leptogaster, Winchell, 1863, Proc. Acad. Nat. Sci., p. 18, Marshall Gr.

rostrata, WVinchell, 1865, Proc: Acad. Nat. Sci., p. 129, Marshall Gr.

sectoralis, Winchell, 1862, Proc. Acad. Nat. Sci., p. 422, Marshall Gr.

septentrionalis, Winchell, 1862, Proc. Acad. Nat. Sci., p. 421, Marshall Gr.

similis, Winchell, 1862, Proc. Acad. Nat. Sci., p. 421, Marshall Gr.

Sanguinolites, McCoy, 1844, Synop. Carb. Foss., Ireland, p. 47. [Ety. Sanguinslaria, a genus; lithos, stone.] Subequivalve, oblong, elongated, margins subparallel or a little arched upward; sides compressed or diagonally gibbous from the beak backward; beaks small, anterior; hinge nearly as long as the shell, margin inflected to form a long posterior lunette; surface wrinkled ; large, oval adductor impression in front of the beak surmounted by a small retractor; posterior adductor large, superficial ; cartilage external; pallial impression entire; sliell thin.

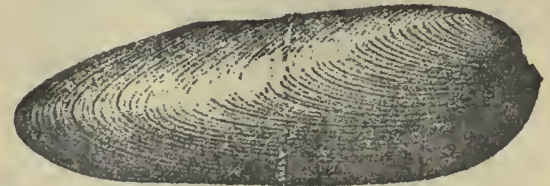

F1G. 915.-Sanguinolites obliquus.

acutus, see Goniophora acuta.

xolus, see Sphenotus æolus.

amygdalinus, see Glossites amygdalinus. arciformis, see Sphenotus arciformis.

borealis, Winchell, 1862, Proc. Acad. Nat. Sci., p. 415, Marshall Gr.

brookfieldensis, Dawson, 1883, Rep. on Redpath Museum, p. 11, Subcarboniferous:

burlingtonensis, Worthen, 1884, Bull. No. 2, Ill. St. Mus. Nat. Hist., p. 14, and Geo. Sur. Ill., vol. 8, p. 129, Burlington $\mathrm{Gr}$. 
clarulus, see Sphenotus clavulus.

combensis, Walcott, 1885, Monogr. U. S. Geo. Sur., vol. 8, p. 175, Devonian.

concentricus, Wincliell, 1862, (Cardinia concentrica,) Proc. Acad. Nat. Sci., p. 413, Marshall Gr.

cylindricus, Winchell, 1S63, Proc. Acad. Nat. Sci., p. 13, Marshall Gr.

favius, see Sphenotus flavius.

glaucus, see Goniophora glaucus.

gracilis, Walcott, 1885, Monogr. U. S.

Geo. Sur., vol. 8, p. 175, Devonian.

hamiltonensis, see Goniophora hamiltonensis.

ida, Hall, 1870, Prelim. Notice Lam. Shells, p. 43, and Pal. N. Y., vol. 5, pl. 65, fig. 20 , Hanı. Gr.

iowensis, Winchell, 1863, Proc. Acad. Nat. Sci., p. 14, Marshall Gr.

jejunus, Winchell, 1863, Proc. Acad. Nat. Sci., p. 15, Marshall Gr.

marshallensis, WVinchell, 1862, Proc. Acad. Nat. Sci., p. 415, Marshall Gr.

missouriensis, Swallow, 1860, iSolen (?) missouriensis,) 'Trans. St. Iouis Acad. Sci., vol. 1, p. 655, Waverly or Choteau Gr.

multistriatus, Worthen, 1884, Bull. No.2, Ill. St. Mus. Nat. Hist., p. 14, and Geo. Sur. Ill., vol. S, p. 129, Keokuk Gr.

næenia, Waleott, 1885, Monogr. U. S. Geo. Sur., vol. 8, p. 249, Subcarboniferous.

naiadiformis, Winchell, 1870, Proc. Am. Phil. Soc., vol. 12, p. 255, Marshall Gr. nasutus, Meek, 1871, Am. Jour. Conch., vol. 7, Kinderhook Gr.

obliquus, Meek, 1871, Proc. Acad. Nat. Sci., p. 213, and Ohio Pal. vol. 2, p. 306, Waverlv Gr.

perangulatus, see Goniophora perangulata. ponderosus, see Modiomorpha ponderosa. randolphensis, Worthen, 1883, (Cypricardia randolphensis,) Geo. Sur. 11l., vol. 7, p. 326, Kaskaskia Gr.

retusus, Walcott, 1885 , Monogr. U. S. Geo. Sur., vol. 8 , p. 247 , Subcarboniferous.

salteri, Walcott, 1885, Monogr. U. S. Geo. Sur., vol. 8, p. 248, Subcarboniferous. sanduskiensis, Meek, 1871, Proc. Acad. Nat. Sci., p. 68, and Ohio Pal., vol. 1, p. 209, Up. Held. Gr.

securis, Winchell, 1870, Proc. Am. Phil. Soc., vol. 12, p. 255, Marshall Gr.

simplex, Walcolt, 18s5̄, Monogr. U. S. Geo.

Sur., vol. 8, p. 248, Subcarboniferous. solenoides, see Sphenotus solenoides.

striatus, IValcott, 1885, Monogr. U. S. Geo. Sur., vol. 8, p. 249, Subcarboniferous.

strigatus, Winchell, 1865, Proc. Acad. Nat.

Sci., p. 127, Marshall Gr.

subtortuosus, see Sphenotus subtortuosus. subtruncatus, Hall, 1885, Pal. N. Y., vol. 5 , p. 508, Chemung Gr.

sulciferus, see Cypricardinia sulcifera. tethys, Billings, 1874, Pal. Foss., vol. 2, p. 50, Gaspe limestone No. 8, Devonian. undatus, Hall, 1870, Prelim. Notice Lam. Shells, p. 41, and Pal. N. Y., vol. 5, pl. 80, figs. 5, 6, Chemung Gr.

unioniformis, Winchell, 1862, Proc. Acad. Nat. Sci., p. 414, Marshall Gr.

valvulus, see Sphenotus valvulus.

Scrizodus, King, 1844, Ann. Mag. Nat. Hist., vol. 14, p. 313. [Ety. schizo, I split; olous, a tooth.] Shell oval or subtrigonal; anterior side rounded, shorter than the other; posterior side tapering, truncate at the extremity, umbonal ridge extending to the postero-basal region; beaks prominent; surface smooth or with concentric strix; hinge with two sinooth cardinal teetl in the right valve and three in the left; middle tooth of the left valve bifirl, and fitting between two of the riglit valve; free margins smooth. Type S. truncatus. æqualis, IIall, 1885, Pal. N. Y., vol. 5, p. 459, IVaverly Gr.

amplus, Meek \& Worthen, 1870, Proc. Acad. Nat. Sci. Phil., p. 41, and Geo. Sur. Ill., vol. 5, p. 579, Coal Meas.

cayuga, Hall, 1870, Prelim. Notice Lam. Sliells, p. 05, syn. for Cytherodon appressus.

chesterensis, Meek \& Worthen, 1865, Proc. Acad. Nat. Sci. Phil., p. 457, and Geo. Sur. Ill., vol. 2, 1. 301, Kaskaskia Gr.

circulus, Worthen, 1884, Bull. No. 2, Ill. St. Mus. Nat.

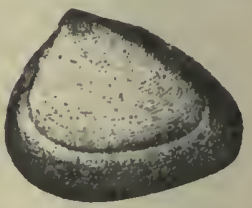
Hist., p. 11, and Geo. Sur. Ill., vol. 8, p. 109 , St. Louis Gr.

contractus, Hall, 1885, Pal. N. Y., vol. 5, p. 451, Ham. Gr.

cuneatus, Meek, 1875, Ohio Pal., vol. 2, p. 336, Coal Meas.

curtiformis, Walcott, 1885, Monogr. U. S. Geo. Sur., vol. 8, p. 253, Subcarboniferous.

curtus, Meek \& Worthen, 1866, Proc. Chi. Acad. Sci., p. 18, Coal Meas.

degener, Hall, 1885, Pal. N. Y. vol. 5, p. 456, Chemung Gr.

deparcus, Walcott, 1885, Monogr. U. S. Geo. Sur., vol. 8, p. 252, Subcarboniferous.

depressus, Worthen, 1884, Bull. No. 2, Ill. St. Mus. Nat. Hist., p. 11, and Geo. Sur. Ill., vol. 8, p. 109, St. Louis Gr.

ellipticus, see Cytherodon ellipticus.

eminens, Hall, 1885, Pal. N. Y., vol. 5, p. 457, Chemung Gr.

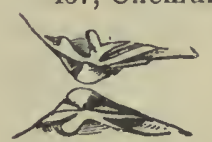

FIG. 917. - Hinge of Schizodus truncatus. gregarius, see Cytherodon gregarius.

magnus, Worthen, 1884 , Bull. No. 2, Ill. St. Mus. Nat. Hist., p. 9 , and Geo. Sur. Ill., vol. 8 , p. 107, Kaskaskia Gr. medinensis, Meek, 1871, Proc. Acad. Nat. Sci. Phil., vol. 23, p. 165, and Ohio Pal., vol. 2, p. 299, Waverly Gr. 
mooresi, n. sp. Shell very large, subrhomboidal, height and length subequal; anterior side straight from the beaks and at right angles to the posterior side, and then rounded into the basal line; basal margin regularly rounded; posterior side sloping at right angles to the anterior side from the beaks and abruptly rounding into the basal margin; beaks prominent, rising above the cardinal line, obtuse, and situate a little anterior to the middle of the shell; umbonal slope broadly rounded and undefined; pallial line strongly marked, pitted, and placed near the margin from one muscular scar to the other; anterior and posterior muscular scars subtrigonal and moderately impressed; a wide vascular impression, somewhat cordate, occupies the central area of the shell, extending from the anterior to the posterior mus. cular scars; cne strong tooth in the right valve directed a little forward, with a socket on each side, the other tooth undefined; surface nearly smooth, showing fine concentric lines of growth. Found by Henry Moores, of Columbus, Ohio, at Carbon Hill, Hocking Valley, in the Coal Measures, and now in the collection of Charles Faber.

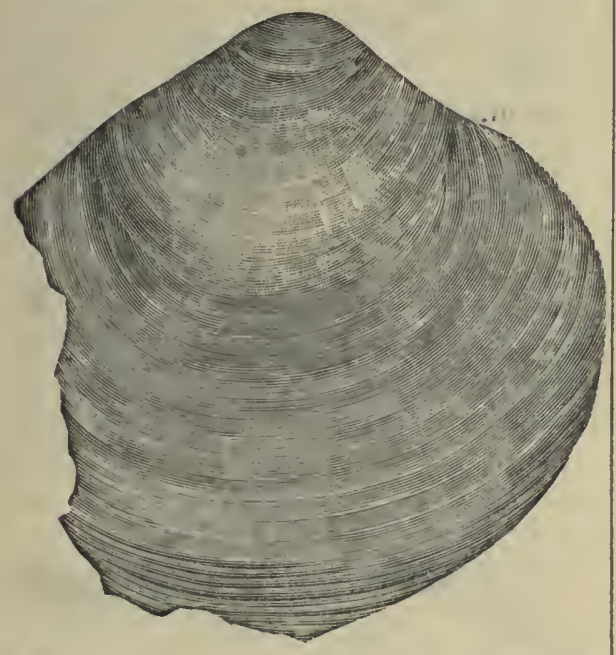

FIG. 918.-Schizodus mooresi. Rlght valve, posterior part broken off.

nauvooensis, Worthen, 1884, Bull. No. 2, Ill. St. Mus. Nat. Hist., p. 10, and Geo. Sur. Ill., vol. 8, p. 108, Keokuk Gr.

orbicularis, Walcott, 1885, Monogr. U. S. Geo. Sur., vol. 8, p. 181, Devonian.

ovatus, Meek \& Hayden, 1858, (Axinus ovatus,) Proc. Acad. Nat. Sci. Phil., p. 262, and Pal. Up. Mo., p. 59, Permian Gr.

patulus, Hall, 1885, Pal. N. Y., vol. 5, p. 457, Chemung Gr. perelegans, Meek \& Worthen, 1870, Proc. Acad. Nat. Sci. Phil., p. 42, and Geo. Sur. Ill., vol. 5, p. 581, Coal Meas.

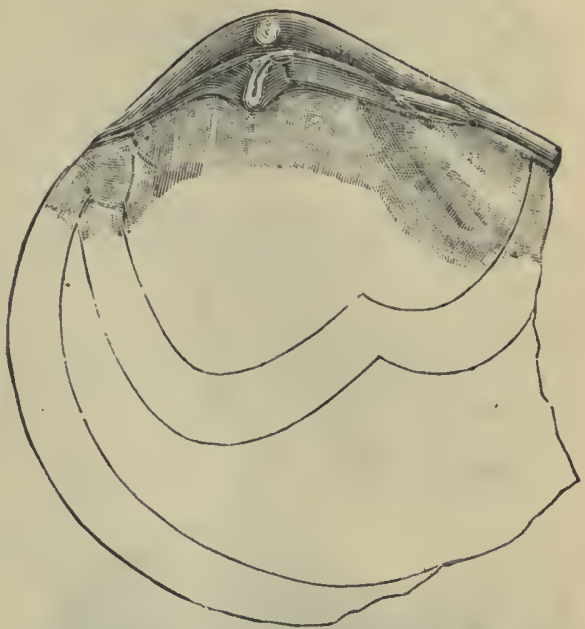

FIG. 919.-Schizodus mooresi. Interior of right valve, showling pallial line and place of subcordate muscular impression and hiuge-teeth.

pintoensis, Walcott, 1898, Munogr. U. $\mathbf{S}$. Geo. Sur., vol. 8, p. 253 , Subcarboniferous.

quadrangularis, see Cytherodon quadrangularis.

randolphensis, Worthen, (in press,) Geo. Sur. Ill., vol. 8, p. 110, Kaskaskia Gr. rossicus, Verneuil, 1845, Geo. Russ., vol. 2, p. 309, Permian Gr.

subtrigonalis, Meek, 1871, Proc. Acad. Nat. Sci., p. 166, Waverly Gr.

triangularis, Swallow, 1858, Trans. St. Louis Acad. Sci., p. 193, Permian Gr. tumidus, see Cytherodon tumidus.

ulrichi, Worthen, (in press,) Geo. Sur. Ill., vol. 8, p. 110, Up. Coal Meas.

varsoviensis, Worthen, 1884, Bull. No. 2 , Ill. St. Mus. Nat. Hist.. p. 10, and Geo. Sur. Ill., vol. 8, p. 107, Keokuk Gr.

wheeleri, Swallow, 1862, (Littorina wheeleri,) Trans. St. Louis Acad. Sci., vol. 1, p. 658, and Pal. E. Neb., p. 209, Coal Meas.

Sedgwickra, McCoy, 1844, Snyop. Carb. Foss. Ireland, p.61. [Ety. proper name.] Nearly equivalve, inequilateral, depressed, oblong, or suboval, very thin; anterior side not quite closed, often gibbous; posterior side longer, more compressed, and gaping; beaks prominent, tumid, incurved; posterior umbonal slopes rounded, or forming an oblique ridge, separated from the postero-dorsal region by a sballow sulcus; lunule distinct; hinge edentulous; cardinal margin inflected so as to form a narrow false area behind the beaks; surface concentrically marked. Type $\mathrm{S}$. attenuata. 
altirostrata, Meek \& Hayden, 1858, (Allorisma (?) altirostratum, ) Proc. Acad. Nat. Sci. Phil., p. 263, and Pal. Up. Mo., p. 41, Coal Meas.

(?) compressa, Meek, 1872, I'roc. Acad. Nat. Sci. Phil., p. $32 t$, and Ohio Pal., vol. 1, p. 144, Hud. Riv. Grr.

concava, Meek \& Haylen, 1858, (Lyonsia concava,) Trans. Alb. Inst., vol. 4, p. 82, and Pal. Up. Mo., p. 41, Coal Meas.

(?) divaricata, Hall \& Whitfield, 1875, Ohio Pal., vol. 2, p. $\$ 9, \mathrm{Hud}$. Riv. Gr.

(?) fragilis, Meek, 1872, Proc. Acad. Nat. Sci. Phil., p. 323, and Ohio Pal., vol. 1, p. 143, Hud. Riv. Gr.

(?) lunulata, Whitfield, 1878, Jour. Cin. Soc. Nat. Hist., vol. 1, p. 140, Hud. Riv. Gr. neglecta, see Cuneamya neglecta.

subarcuata, Meek \& Worthen, 1865, Proc. Acad. Nat. Sci. Phil., p. 251, and Geo. Sur. Ill., vol. 3, p. 537, Keokuk Gr.

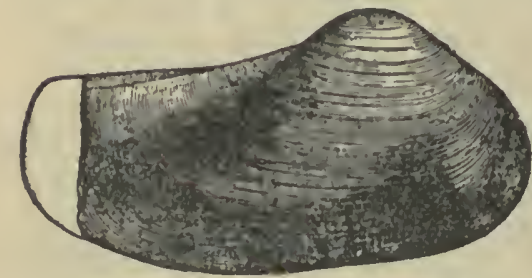

F'iG. 920.--Sedgwickla topeireusis.

topekensis, Shumard, 1858, (Leptodomus topekrensis,) Trans. St. Louis Acad. Sci., vol. 1, p. 208, and Pal. Up. Mo., p. 40 , Coal Meas.

Solemya, Lamarck, 1818, Hist. Nat. An.sans Vert., vol. 5. See Solenomya-the correct orthography, first used by Menke, 1828, Syn. Meth. Edit.

Solen, Linnæus, 1758, Syst. Nat., 10th ed. [Ety. Solen, a tube or pipe.] Shell very long; subcylindrical ; ends gaping; hinge teeth two in each valve; ligament external; anterior scar elongated; posterior oblong; pallial line extending beyond the adductors. Type S. siliqua. Not a Palæozoic genus.

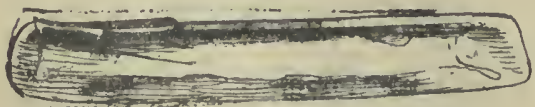

FIG. 921.-Solen siliqua. One-third diam.

missouriensis, see Sanguinolites missouriensis.

permianus, see Solenopsis permianus.

priscus, Winchell, 1862, Proc. Acad. Nat. Sci., p. 423, Portage Gr.

quadrangularis, Winchell, 1862, Proc. Acad. Nat. Sci., p. 422, Marshall Gr.

scalpriformis, see Solenopsis scalpriformis. Solenomy A, Lamarck, 1818, (Solemya,) Hist.

Nat. Anim. sans Vert., vol. 5, p. 488. [Ety. from the resemblance to the two genera Solen and Mya.] Elongate, ob- long, equivalve, very inequilateral, posterior end the shorter; dorsal and ventral margins subparallel; ends rounded and gaping; surface covered with a thick, horny periostraca, extending in jagged portions beyond the ventral margin; beaks minute; cartilage forning a thick, triangular mass behind the beaks, supported internally by an oblique ensiform plate; long anterior margin simple, erect, without teeth; posterior aduuctor small, ovate, within the cartilage pit, anterior impression large, comma-shaped. Type S. australis.

anodontoides, Meek, 1875, Ohio Pal., vol. 2, p. 339 , Coal Meas.

biarmica, Verneuil, 1845, Geo. Russ. and Ural Mountains, Permian Gr. This species does not occur in this country. curta, Walcott, 18S5, Monogr. U. S. Geo. Sur., vol. 8, p. 2 42 , Subcarloniferous.

iowensis, Worthen, 1884, Bull. No. 2, Ill. St. Mus. Nat. Hist., p. 13, and Geo. Sur. Ill., vol. 8, p. 132, St. Louis Gr.

monroensis, Worthen, 1854, Bull. No. 2, IIl. St. Mus. Nat. Hist., p. 13, and Geo. Sur. Ill., vol. 8, p. 131, St. Louis Gr. radiata, Meek \& Worthen, 1860, (Solemya radiata,) Proc. Acad. Nat. Sci. Phil., p. 457, and Geo. Sur. Ill., vol. 2, p. 349 , Coal Meas.

recurvata, Swallow, 1858, Trans. St. Louis Acad. Sci., vol. 1, p. 208, Up. Coal Meas.

soleniformis, Cox, 1857, Geo. Sur. Ky., vol. 3 , p. 573, Coal Meas.

varsoviensis, WVorthen, 1884, Bull. No. 2, Ill. St. Mus. Nat. Hist., p. 12, and Geo. Sur. Ill., vol. 8, p. 131, Keokuk Gr.

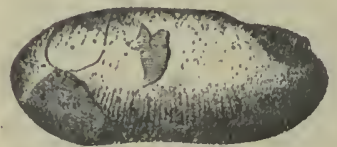

Fia. 922.-Solenomya vetusta, Meek, 1571, Proc. A c a d. Nat. Sci. Phil., p. 66, and Ohio Pal., vol. 1, d. 206, Up. Held. Gr.

Solexopsis, McCoy, 1844, Carb. Foss. Ireland, p. 47. [Ety. Solenopsis, resembling a shell of the genus Solen.] Elongated; beaks

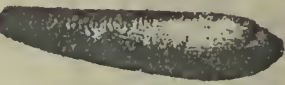

Fia. 923.--Solenopsis solenoides. compressed, anterior; depression in front of beaks; surface concentrically marked. Type S. minor.

permianus, Swallow, 1858, (Solen permianus,) Trans. St. Louis Acad. Sci., vol. 1, p. 190, Permian Gr.

scalpriformis, Winchell, 1862, (Solen scalpriformis,) Proc. Acad. Nat. Sci., p. 422, Marshall Gr.

solenoides, Geinitz, 1866, (Clidophorus solenoides:) Carb. und Dyas in Neb. p. 25, and Pal. E. Neb., p. 223, Coal Meas. 
Spathella, Hall, 1885, Pal. N. Y., vol. 5, p. 33. [Ety. spathe, a spatlie; ellus, diminutive.] Equivalve, very inequilateral, wider behind, transversely subcylindrical; anterior end short, narrowly rounded; beaks subanterior, small ; umbonal slope rounded or subangular; surface concentrically lined. Type S. typica.

typica, Hall, 1885, Pal. N. Y., vol. 5, p. 407, Chemung Gr.

vertricosa, White \& Whitfield, 1862 , (Orthonota ventricosa.) Proc. Bost. Soc. Nat. Hist., vol. 8, p. 297, and Pal. N. Y., vol. 5, p. 408 , WVaverly Gr.

Sphenolius, n. gen. [Ety, sphen, wedge; leion, smooth.] Shell large, equivalve, inequilateral, elongate, cuneiform, ventricose; umbones prominent; beaks incurved at the anterior end; cardinal line at an angle of fifty or sixty degrees from the basal line, and appearing wing-like toward the posterior end; lunule present; no escutcheon; ligament external; muscular scars and hinge-line unknown. Type S. cuneiforme.

cuneifurme, S. A. Miller, 1881, (Orthodesma cuneiforme,) Jour. Cin. Soc. Nat. Hist., vol. 3, p. 314, Hud. Riv. Gr.

faberi, n. sp. Shell below the medium size for species in this genus; beaks

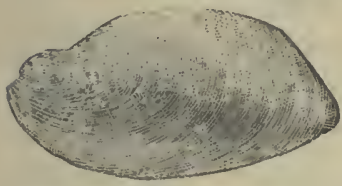

Fra. 924.-Sphenollum faberi. Left valve. unite over the hingeanterior end; anterior end, p o in ted, rounded; hing e-lin e rising posteriorly into a wing-like expansion ; posterior end prolonged at the posterobasal margin; basal margin subelliptical; umbones high and gradually tapering to the postero-basal margin; surface concentrically lined. Collected by Charles Faber in the Hud. Riv. Gr., at Cincinnati, Ohio.

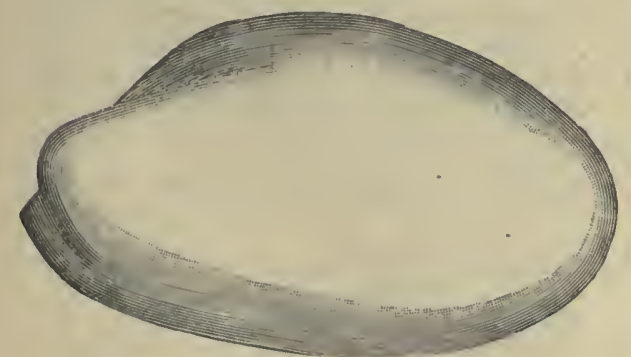

FIG. 925.-Sphenollum richmondense.

richmondense, n. sp. Shell large, cuneiform, ventricose, beaks incurved at the anterior end, pointed; umbones high, defined; cardinal line at a high angle, having a wing-like posterior end; anterior end rounded below the lunule. Dist ing u is hed from Ş. cuneiforme, which it much resembles, by its shorter form and more angular umbones. Possibly more specimens may show a gradation Fig. 926. - Sphenolium from one form richmondeuse. Anto the other, terlor vlew.

and if so, this specific name will fall into synonymy. Collected by Charles Faber in the upper part of the Hud. Riv. Gr., at Richmond, Indiana.

Sphenotus, Hall, 1885, Pal. N. Y., vol. 5, p. 33. [Ety. sphen, wedge; ous, ear.]

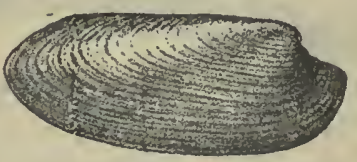

FiG. 927.-Sphenotus æolus.
Equival ve, equilatera 1 , el ong a t e : anterior end short; posterior end obl i q u e l y truncate; cardinal line, long, straight; umbonal ridge extending to the postinferior extremity; surface concentrically lined; two short teeth beneath the beak of the right valve, and one or two slender lateral teeth; ligament external, contained in a groove; anterior muscular scar strongly marked; posterior scar shallow; pallial line simple. Type S. arciformis.

æolus, Hall, 1870, (Sanguinolites æolus,) Prelim. Not. Lam. Shells, p. 46, and Pal. N. Y., vol. 5, p. 404 , Waverly Gr. arciformis, Hall, 1870, (Sanguinolites arcieformis,) Prelim. Not. Lam. Shells, p. 40, and Pal. N. Y., vol. 5, p. 395, Ham. Gr.

arcuatus, Hall, 1885, Pal. N. Y., vol. 5, p. 400, Chemung Gr.

clavulus, Hall, 1870, (Sanguinolites clavulus, Prelim. Not. Lam. Shells, and Pal. N. Y., vol. 5, p. 401, Chemung Gr. contractus, Hall, 1843, (Cypricardia contracta, ) Geo. Sur. 4th Dist. N. Y., p. 292, and Pal. N. Y., vol. 5, p. 399, Chemung Gr.

cuneatus, Yonrad, 1838, (Pterinea cuneata,) Ann. Rep. Geo. N. Y., p. 116, and Pal. N. Y., vol. 5, p. 396, Ham. Gr.

flavius, Hall, 1870, (Sanguinolites flavius,) Prelim. Not. Lam. Shells, p. 47, and Pal. N. Y., vol. 5, p. 403. Waverly Gr. rigidus, iVhite \& Whitfield, 1862, (Cypricardia rigida,) Proc. Bost. Soc. Nat. Hist., vol. 8, p. 300 , and Pal. N. Y., vol. 5, p. 402, Waverly Gr.

signatus, Hall, 1885, Pal. N. Y., vol. 5, p. 405, Waverly Gr.

solenoides, Hall, 1870, (Sanguinolites solenoides,) Prelim. Not. Lam. Shells, p. 38, and Pal. N. Y., vol. 5, p. 398, Ham. Gr. 
subtortuosus, Hall, 1870, (Sanguinolites subtortuosus, ) Prelim. Not. Lam. Shells, p. 41, and Pal. N. Y., vol. 5, p. 397, Ham. Gr.

telamon, Hall, 1885, Pal. N. Y., vol. 5, p. 406, Waverly Gir.

truncatus, Conrad, 1842, (Cypricardites truncatus,) Jour. Acad. Nat. Sci., vol. 8, n. 244, Ham. Gr.

undatus, Hall, 1885, Pal. N. Y., vol. 5, p. 506, Chemung Gr.

valvulus, Hall, 1870, (Sanguinolites valvulus,) Prelim. Not. Lam. Shells, p. 46, and Pal. N. Y., vol. 5, p. 403, Waverly $\mathrm{Gr}$.

faberi, n. sp. Shell small, equivalve, inequilateral, a little longer than high; anterior end short, broadly rounded; base more narrowly rounded in the anterior and central part; the postero-basal part slightly produced; cardinal line straight or nearly so; beak extremely small and standing upright, like a little point projecting beyond the cardinal line; valves convex in the umbonal region; two furrows or cinctures arising near the beak in the umbonal region, which gradually widen, are directed to the postero-basal margin, and above these the postero-dorsal part of the shell

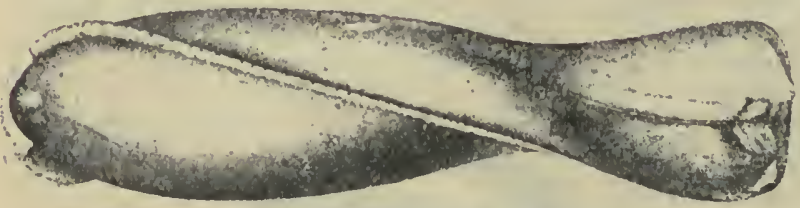

IFra. 928-Splrodomus Insignis.

Sprmopomus, Beecher, 1886, 39th Rep. N. Y. Mus. Nat. Hist. [Ety. speira, spire; demos, house.] Equivalve, elongatespiral; beaks terminal; muscular impressions at the two extremities; no hinge-line. Type $\mathrm{S}$. insignis.

insignis, Beecher, 1856, 39th Rep. N. Y. Mus. Nat. Hist., Waverly Gr.

Streblopteria, McCoy, 1851, Ann. Mag. Nat. Hist., $2 \mathrm{~d}$ series, vol. 7, p. 170, and Brit. Pa]. Rocks, p. 482. [Ety. streblos, turned the wrong way; pteron, a wing.] Pectinoid, ovate, or rounded, obliquely extended toward the anterior side; posterior wing rectangular, anterior ear small, deeply defined; surface smooth or radiately ridged; large, faintly marked muscular impression behind the middle; sliort, narrow tooth posterior to the beaks; ligament confined to a narrow, simple facet on the binge margin. Type S. lævigata.

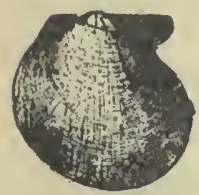

Fra. 929.-Streblopterla si milis Rlght valve. similis, Walcott, 1885 Monogr. U. S. Geo. Sur., vol. 8, p. 230, Carboniferous.

tenuilineata, Meek \& Worthen, 1860, (Pecten tenuiline a $\mathrm{tus}$, ) Proc. Acad. Nat. Sci., Phil., p. 452, and Geo. Sur. Ill., vol. 2, p. 334, Coal Meas.

Technophorus, n. gen. [Ety. techne, art; phoros, bearing.] Shell small, equivalve, inequilateral; anterior end short, broadly rounded; two or more furrows arising near the beak extend to the postero-basal margin; beak small, upright; surface concentrically lined; umbonal rib in front of the beak represented in the cast by a transverse sulcus; no external ligament, escutcheon, or lunule. Type T. faberi. is somewhat wing-like; surface marked by very fine concentric lines; the casts show a deep sulcus directly in front of the beak for the reception of an umbonal rib, or support on the interior of the shell. Ilud. Riv. Gr., near Sharonville, Hamilton County, Ohio. Collected by Mr. Charles Faber.
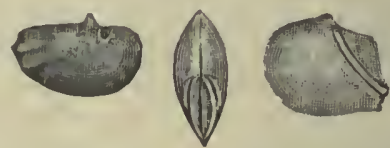

FIG. 930.-Teclinophorus faberl. The right luand figure shows the left valve with a small piece broken from the posterior end; the left hund figure represents a well-preserved cast; the central flgure presents a cardinal vlew.

Tellina, Linnæus, 1758, Syst. Nat., 10th ed. [Ety. telline, a sort of mussel.] This genus unknown in the Palrozoic rocks. (?) ovata, Hall, 1843, Geo. Rep. 4th Dist. N. Y., Syn. for Palæoneilo maxima.

Tellinomy A, Hall, 1847, Pal. N. Y., vol..1, p. 151. [Ety. from the resemblance to the genera Tellina and Mya.] Nearly equilateral, generally transverse, anterior side largest; beaks approximate, not prominent; hinge-line with a double series of bent teeth connected by smaller ones beneath the beak; ligament posterior, external, on a fulcrum; no striated area or cartilage pit; muscular impressions strong not bounded by elevated lines; pallial line simple. Type T. nasuta.

abrupta, Billings, 1862, (Ctenodonta abrupta,) Pal. Foss., vol. 1, p. 46, Black Riv. Gr.

æquilatera, Hall, 1852, Pal.-N. Y., vol. 2, p. 330 , Coralline limestone.

alta, Hall, 1861, Geo. Rep. IVis., p. 27, and Geo. Sur. Ill., vol. 3, p. 309, Trenton $\mathrm{Gr}$.

anatiniformis, see Pterotheca anatiniformis.

angela, Billings, 1865, (Ctenodonta angela,) Pal. Foss., vol. 1, p. 221, Quebec Gr. 
angustata, Hall, 1860, Can. Nat. and Geo., vol. 5 , p. 152 , Up. Sil.

astartiformis, Salter, 1859, (Ctenodonta astartæformis,) Can. Org. Rem., Decade 1, p. 39, Black Riv. Gr.

attenuata, Hall, 1860, Can. Nat. and Geo., vol. 5, p. 151, Up. Silurian.

cingulata, Ulrich, 1879 , Jour. Cin. Soc. Nat. Hist., vol. 2, p. 23, Hud. Riv. Gr. contracta, Salter, 1859, (Ctenodonta contracta,) Can. Org. Rem. Decade 1, p. 37, Black Riv. and Trenton Gr.

curta, Hall, 1852, Pal. N. Y., vol. 2, p. 86, Clinton Gr.

donaciformis, Hall, 1847, (Nucula ? donaciformis,) Pal. N. Y., vol. 1, p. 316, Trenton $\mathrm{Gr}$.

dubia, Hall, 1847, Pal. N. Y., vol. 1, p. 153, Black Riv. and 'Trenton Grs.

elliptica, Hall, 1852, Pal. N. Y., vol. 2, p. 102, Clinton Gr.

gibberula, Salter, 1859, (Ctenodonta gibberula,) Can. Org. Rem. Decade 1, p. 38, Black Riv. and Trenton Grs.

gibbosa, Hall, 1847, Pal. N. Y., vol. 1, p. 153, Black Riv. and Trenton Grs.

hamburgensis, Walcott, 1885, Monogr. U. S. Geo. Sur., vol. 8, p. 76, Trenton $\mathrm{Gr}$.

hartsvillensis, Safford, 1859, (Ctenodonta hartsvillensis,) Geo. of Tenn., p. 287, Nashville Gr.

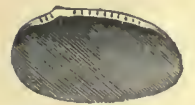

FIG. 931.-Tellinomya hilll. hilli, S. A. Miller, 1874, Cin. Quar. Jour. Sci., p. 230, Hud. Riv. Gr.

houghtoni, Stevens, 1858, (Nucula houghtoni,) Am. Jour. Sci, and Arts, 2d ser., vol. 25, p. 262, Marshall or Waverly Gr.

inflata, Hall, 1861, Geo. Rep. Wis., p. 26, Trenton Gr.

iphigenia, Billings, 1862, (Ctenodonta iphigenia,) Pal. Foss., vol. 1, p. 152, Hud. Riv. Gr.

lata, Hall, 1852, Pal. N. Y., vol. 2, p. 85, Clinton Gr.

levata, Hall, 1847, (Nucula levata,) Pal: N. Y.,, vol. 1, p. 150, Black Riv., Trenton, and Hud. Riv. Grs.

logani, Salter, 1851, (Ctenodonta logani,) Rep. Brit. Assoc., p. 36, Hud. Riv. Gr.

machæriformis, Hall, 1843, (Nucula machæriformis,)Geo. Rep.4th Dist. N.Y.,p.76, and Pal. N. Y., vol. 2, p. 85, Clinton Gr.

mactriformis, Hall, 1843, (Nucula mactræformis,) Geo. Rep. 4th Dist., N. Y., p. 76 , Clinton Gr. nasuta, $\mathrm{H}$ all, $1847, \mathrm{Pal}$. N. Y., vol. 1 , p. 152, Black Riv.and'Trenton Grs.

nucleif or $\mathrm{mis}$, Hall, 1859, Pal. N. Y.,

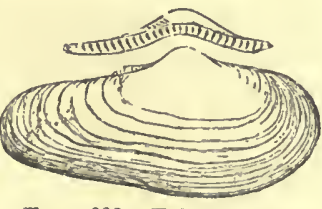

Fig. 932.-Tellinomyra nasuta. vol. 3, p. 263, Low. Held. Gr.

nuculiformis, Hall, 1847, (Modiolopsis nuculiformis,) Pal. N. Y., vol.1, p. 298, Hud. Riv. Gr.

ovata, Hall, 1861, Geo. Rep. Wis., p. 28, Trenton Gr.

pectunculoides, Hall, 1871, 24th Rep. N. Y. Mus. Nat. Hist., p. 228, Hud. Riv. Gr.

protensa, Hall, 1852, Stans. Ex. to Gt. Salt Lake, p. 412, Coal Meas.

sanguinolarioidea, Hall, 1847, Pal. N. Y., vol. 1 , p. 152 , Trenton Gr.

stella, Winchell, 1862, (Nucula stella,) Proc. Acad. Nat. Sci., p. 417, Marshall Gr.

subnasuta, see Clinopistha subnasuta.

ventricosa, Hall, 1861, Geo. Rep. Wis., p.

27, and Geo. Sur. Ill., vol. 3, p. 307, Trenton Gr.

Tellinopsis, Hall, 1870, Prelim. Notice Lam. Shells, p. 80. [Ety. resembling a shell of the genus Tellina.] General form like Tellina; beaks small, subcentral, directed backward; ligament external; surface smooth or obscurely marked; ligament external; muscular impression shallow. Type T. subemarginata.

subemargin a ta, Conrad, 1842, ( $\mathrm{Nuculites}$ s u b emarginatus,) $J \circ u r$. Acad. Nat. Sci., vol. 8, p. 249, and Pal. N.Y., FrG. 933.-Tellinopsis subvol. 5 , p. 464, Ham. Gr.

Ungulina, Daudin, 1802, Bosc. Hist. Nat. Coq. 3. [Ety. ungulina, like a hoof.] suborbicularis, see Cardiomorpha suborbicularis.

Unio orthonotus, see Modiolopsis orthonota. primigenius, see Modiolopsis primigenia.

Vanuxemra, Billings, 1858, Rep. of Progr, Geo. Sur. Can., p. 186. [Ety. proper name.] Ovate ; beaks terminal or subterminal ; posterior extremity rounded, anterior more or less acuminated; two muscular impressions; anterior teeth variable in number sometimes curved and striated; posterior lateral teeth from two to four. Type V. inconstans.

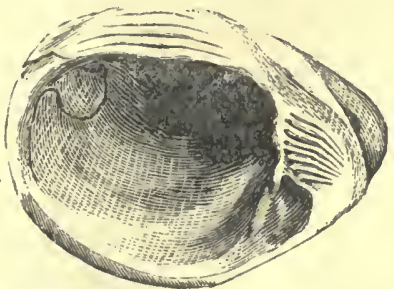

FIG. 934.-Vanuxemia bayfieldi. Interior of left valve, showing the striated teeth.

bayfieldi, Billings, 1858, Rep. of Progr. Geo. Sur. Can., p. 187, Hud. Riv. Gr.

dixonensis, Meek \& Worthen, 1866, Proc. Chi. Acad. Sci., p. 16, Trenton Gr. 
inconstans, Billings, 1858, Rep. of Progr. Geo. Sur. Can., p. 186, Black Riv. and Trenton Grs.

montrealensis, Billings, 1859. Can. Nat. and Geol., vol. 4, p. 444, Chazy Gr.

tomkinsi, Billings, 1860, Can. Jour., vol. 6, p. 35̄7, Up. Held. Gr.

Venus mohegan, Castelnau, 1843, Syst. Sil., p. 44. Not recognized.

Vertumisis. Hall, 18\$4, Pal. N. Y., vol. 5, pt. 1. p. xii. Proposed as a subgenus of Pterinea, distinguished by having the right valve convex, and the left flat or concave; hinge area narrow. The species referred to it are Pterinea avis, $P$. reprolia, and P. reversa.

Yol,DIA, Muller, 1842. Kroyer's Nat. Tid. vol. 4, p. 91. [Ety. proper name.] Shell ovate or subelliptical, subequilateral, compressed; posterior side narrower than the other; surface smooth, striate, or obliquely seulptured, and covered witl a polished epidermis: margins smooth within; inner lamine pearly; hinge plates small, numerous on each side of the beaks; cartilage pit under the beaks; pallial line sinuous. Type Y. myalis.

carbonaria, Meek, 1871, Rep. Reg. University IV. Va., p. 6, and Ohio Pal., vol. 2 , p. 336, Coal Mleas. gibbosa, MeChesney, 1859. The name was preoccupied. See T. rusbensis.

knoxensis, McChesney, 186.5, (Leda knoxensis,) Expl. Pal. Foss., pl. 2, Coal Meas.

levistriata, Meek \& Worthen, 1860, (Leda levistriata,) Proc. Acad. Nat.

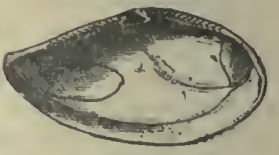

Fra. 935.-Yoldia myalls.
Sei. Phil., p. 457, and Geo. Sur. Ill., vol. 2 , p. 282 , St. Louis Gr.

oweni, MeChesney, 1860, (Leda oweni,) Desc. New. Pal. Foss., p. 52, Coal Meas.

polila, McChesney, 1859. The name was preoceupied, see Y. knoxensis.

rushensis, MIcChesney, 1865, (Leda rushensis,) Expl. Pal. Foss., pl. 2, Coal Meas.

stevensoni, Meek, 1871, Rep. Reg. University W. Va., p. 6, and Ohio Pal., vol. 2, p. 335, Coal Meas.

subscitula, Meek \& Hayden, 1858, (Leda subscitula, Trans. Alb. Inst., vol. 4, p. 79 , and Pal. E. Neb., p. 205, Permian Gr.

valvulus, Hall \& Whitfield, 1872, 24th

Rep. N. Y. Mus. Nat. Hist., p. 190, Up. Held. Gr.

\section{SUBKINGDOM ARTICULATA.}

The Articulata are the most numerous of all living, animals, and abound alike on land and sea. They are divided into Classes, Subclasses, Orders, and Suborders. Many of them possess intelligence, arising from ganglionic centers, and in the summer season provide their food for winter. Several living orders are unknown in Palæozoic rocks; this may have resulted, however, from want of preservation. The fussils belong to the Classes Annelida, Crustacea, Arachnida, Myriapoda, Insecta.

\section{CLASS ANNELIDA.}

The Annelida have the bodies divided into segments, which are generally furnished with jointed appendages. The living forms are distributed in four Orders, but no such division is practicable with the Palrozoic fossils, where generally only the internal jaws, called Conodonts or worm-burrows, are found preserved. The Couodonts may be the interual jaws of Crustacea, as seems to the author most probable; but there is no ground for referring them to fish, as has been done by some authors. The class may be divided as follows:

Conodonts._Arabellites, Distacodus, Drepanodus, Eunicites, Glycerites, Lumbriconereites, Nereidavus, Oenonites, Polygnathus, Prioniodus, Staurocephalites. 
Worm-BURRows.-Arenicolites, Gyrichnites, Myrianites, Monocraterion, Nemapodia, Nereites, Palæochorda, Scolithus, Walcottia.

Order TubicolıA.-Conchicolites, Cornulites, Salterella, Serpula, Serpulites, Spirorbis.

Order Uncertain.-Protoscolex.

Arabelliths, Hinde, 1879, Quar. Jour. Geo. Soc. Lond., vol. 35, p. 377. [Ety. Arabella, an existing genus; lithos, stone.] Jaws with an extremely prominent anterior hook, and a row of smaller teeth on a wide base, sickle-shaped jaws, and also subquadrate forms, with a straight upper edge of small teeth. Type A. hamatus.

ascialis, Hinde, 1879, Quar. Jour. Geo. Soc. Lond., vol. 35, p. 378, Hud. Riv. Gr. cervicornis, Hinde, 1879, Quar. Jour. Geo. Soc. Lond., vol. 35, p. 379, Hud. Riv. Gr.

cornutus, Hinde, 1879, Quar. Jour. Geo. Soc. Lond., vol. 35, p. 377, Hud. Riv. Gr. crenulatus, Hinde, 1879, Quar. Jour. Geo. Soc. Lond., vol. 35, p. 379, Hud. Riv. Gr.

cristatus, see Eunicites cristatus.

cuspidatus, Hinde, 1879 , Quar. Jour. Geo. Soc. Lond., vol. 35, p. 378, Hud. Riv. Gr.

elegans, Hinde, 1879, Quar. Jour. Geo. Soc. Lond., vol. 35, p. 382, Clinton Gr. gibbosus, Hinde, 1879, Quar. Jour. Geo. Soc. Lond., vol. 35, p. 378, Hud. Riv. Gr.

hamatus, Hinde, 1879, Quar. Jour. Geo. Soc. Lond., vol. 35, p. 377, Hud. Riv. Gr.

lunatus, Hinde, 1879, Quar. Jour. Geo. Soc. Lond., vol. 35, p. 378, Hud. Riv. Gr.

obliquus, Hinde, 18;9, Quar. Jour. Geo. Soc. Lond., vol. 35, p. 379, Hud. Riv. Gr. ovalis, Hinde, 1879, Quar. Jour. Geo. Soc. Lond., vol. 35, p. 378, Hud. Riv. Gr. pectinatus, Hinde, 1879, Quar. Jour. Geo. Soc. Lond., vol. 35, p. 379, Hud. Riv. Gr.

politus, Hinde, 1879, Quar. Jour. Geo. Soc. Lond., vol. 35, p. 385, Ham. Gr. quadratus, Hinde, 1879 . Quar. Jour. Geo. Soc. Lond., vol. 35, p. 379, Hud. Riv. Gr.

rectus, Hinde, 1879, Quar. Jour. Geo. Soc. Lond., vol. 35, p. 378, Hud. Riv. Gr.

scutellatus, Hinde, 1879, Quar. Jour. Geo. Soc. Lond., vol. 35, p. 379, Hud. Riv. Gr.

similis, Hinde, 1879, Quar. Jour. Geo. Soc. Lond., vol. 35, p. 382, Niagara Gr. similis var. arcuatus, Hinde, 1879, Quar. Jour. Geo. Soc. Lond., vol. 35, p. 385, Ham. Gr.

Arenicolites, Salter, 1856, Quar. Jour. Geo. Soc., vol. 13, p. 199. [Ety. arena, sand; colo, I inlıabit; lithos, stone.] Circular holes which appear in twos on the surface of sandstones, and have the ap- pearance of worm-burrows, like those of the Arenicola. Type A. sparsus or A. didyma.

sparsus, Salter, 1856, Quar. Jour. Geo. Soc., vol. 13, p. 203, Clinton Gr.

spiralis, Torell, 1868, as identified by Billings, Pal. Foss., vol. 2, p.77, Up.Taconic. woodi, Whitfield, 1882, Geo. Wis., vol. 4, p. 177, Potsdam Gr.

Aulacodus obliquus, see Lumbriconereites obliquus.

Conchicolites, Nicholson, 1872, Am. Jour. Sci. and Arts, 3il ser., vol. 3, p. 202. [Ety. concha, shell ; colo, I dwell; lithos, a stone.] Tubes conical, slightly curved, walls thin, composed of imbricating rings. Type C. gregarius. Prof. Hall and others regard this genus as a synonym for Cornulites.

corrugatus, Nicholson, 1873, Lond. Geo. Mag., vol. 10, p. 55, Hud. Riv. Gr.

flexuosus, Hall, 1847, (Tentaculites flexuosus,) Pal. N. Y., vol. 1, p. 92, Trenton and Hud. Riv. Grs.

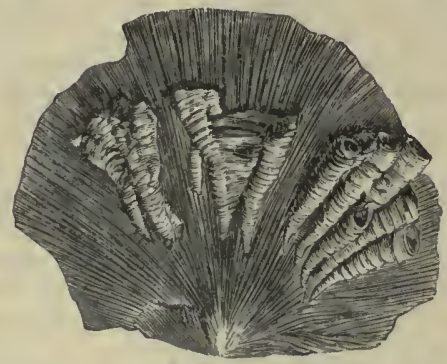

FiG. 936,-Conchicolites flexuosus, on Strophomena alternala.

intermedius, Nicholson, 1874, (Ortonia intermedia,) Geo. Mag., n. s., vol. 1, p. 199, Ham. Gr.

minor, Nicholson, 1873, (Ortonia minor,) Lond. Geo. Mag., vol. 10, p. 56, Hud. Riv. Gr.

Cornulites, Schlotheim, 1820, Petrefaktenkunde, p. 378. [Ety. cornu, horn; lithos, stone.] Tube gradually tapering, conical, slightly flexuous, sinall end usually curved, and attached to some foreign body; walls thick, cellular, composed of numerous imbricating rings, their widest edge next the slender base; external surface annulated, finely striated longitudinally; inner surface and casts scalariform, with two or three longitudinally impressed furrows. Type C. serpularius. 
arcuatus, Conrad, 1842, Jour. Acad. Nat. Sci., vol. 8, p. 276, Niagara Gr.

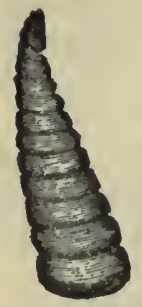

bellistriatus, Hall, 1888, Pal. N. Y., vol. 7, p. 20, Low. Held. Gr.

carbonarius, Gurley, 1883 , New. Carb. Foss., p. 8, Kinderhook Gr. The publication is not such as required by the rules of nomenclature.

cbrysalis, Hall, 1888, Pal. N. Y., vol. 7, p. 20 , Low. Held. Gr. Fra. 937.-Cornu- cingulatus, Hall, 1888,
lites arcuatus.

20, Low. Held. Gr.

Pal. N. Y., vol. 7, p.

clintoni, Hall, 1879, 28th Rep. N. Y. St. Mus. Nat. Hist., p. 184, Clinton Gr. This name was proposed instead of $\mathrm{C}$. flexuosus, which is preoccupied, when Conchicolites is regarded as synonymous with Cornulites.

contractus, Ringueberg, 1884, I'roc. Acad. Nat. Sci., p. 148, Niagara Gr. Syn. for C. proprius.

distans, Hall, 1852, (Tentaculites distans,) Pal. N. Y., vol. 2, p. 184, Clinton Gr.

flexuosus, Hall, 1852, Pal. N. Y., vol. 2, p. 98, Clinton Gr.

flexuosus var. gracilis, Hall, 1860, Can. Nat. and Geo., vol. Frg. 938. 5 , p. 155, Niagara Gr. Cornulites nodosus Ringueberg, 1884, disians. Proc. Acad. Nat. Sci., p. 149, Niagara Gr. proprius, Hall, 1876, 28th Rep. N. Y. St. Mus. Nat. Hist., p. 182, Niagara Gr.

tribulis, Hall, 1888, Pal. N. Y., vol. 7, p. 20, Ham. Gr.

Distacodus, Hinde, 1879, Quar. Jour. Geo. Soc. Lond., vol. 35 , p. 357 . [Ety. distazn, to doubt; odous tooth.] Small, curved tooth, witl a sharp edge on both the outer and inner curve; base ex. panded. Type D. incurvus.

incurvus, Punder, 1856, (Marhairodus incurvus,) Monogr. d. foss. Fische. d. Silur. syst., p. 23, Hıd. Riv. Gr.

Drepanodus, Pander, 1856, Monogr. d. foss. Fische. d. Silur. Syyst., p. 20. [Ety. drepane, sickle; odous tooth.] Small, curved, spine-like tooth, nearly circular in section ; base expanded. Type $D$. arcuatus. arcuatus, Pander, 1856, Monogr. d. Foss. Fische. d. Silur. Syst., p. 20, Hud. Riv. Gr.

Eotrophonia, Ulrich, 1878, Jour. Cin. Soc. Nat. Hist., vol. 1, p. 91 . Not satisfactorily defined.

setigera, Ulrich, 1878, Jour. Cin. Soc. Nat. Hist., vol. 1, p. 91. Not satisfactorily defined, and specimen too poor for definition.

Eunicites, Ehlers, 1868, Palæontographica, vol. 17, p. 145. [Ety: Eunice, a Nereid; lithos, stone.] Minute, variously formed, denticulated jaws of annelids or crustaceans. Type E. avitus. alveolatus, Hinde, 1879, Quar. Jour. Geo. Soc. Lond., vol. 35, p. 384, Ham. Gr. cbiromorphus, Hinde, 1879, Quar. Jour. Geo. Soc. Lond., vol. 35, p. 381, Clinton $\mathrm{Gr}$.

clintonensis, Hinde, 1879, Quar. Jour. Geo. Soc. Lond., vol. 35, p. 381, Clinton $\mathrm{Gr}$.

compactus, Hinde, 1879, Quar. Jour. Geo. Soc. Lond., vol. 35, p. 384, Ham. Gr.

contortus, Hinde, 1879, Quar. Jour. Geo. Soc. Lond., vol. 35, p. 375, Hud. Riv. Gr.

coronatus, Hinde, 1879, Quar. Jour. Geo. Soc. Lond., vol. 35, p. 381, Clinton Gr.

cristatus, Hinde, 1879, (Arabellites cristatus), Quar. Jour. Geo. Sisc., vol. 35, p. 378 , Hud. Riv. Gr.

digitatus, Hinde, 1879, Quar. Jour. Geo. Soc. Lond., vol. 35, p. 376 , Hud. Riv. Gr.

gracilis, Hinde, 1879, Quar. Jour. Geo. Soc. Lond., vol. 35, p. 376, Hud. Riv. Gr.

major, see Oenonites major.

nanıs, Hinde, ]879, Quar. Jour. Giro. Soc. Lond., vol. 35, p. 384, Ham. Gr.

palmatus, Hinde, 1879, Quar. Jour. Geo. Soc. Lond., vol. 35, p. 384, Ham. Gr.

perdentatus, see Lumbriconereites perdentatus.

simplex, Hinde, 1879, Quar. Jour. Geo. Soc. Lond., vol. 35 , p. $376, \mathrm{Hud}$. Riv. Gr.

tumidus, Hinde, 1879, Quar. Jour. Geo. Soc. Lind, vol. 35. p. 384, Ham. Gr.

GuYcerites, Hinde, 1879, Quar. Jour. Geo. Soc. Jon., vol. 35, p. 380. [Ety. genus Glycera; lithos, stone.] Jaws consisting of a simple curved hook with a wide base, withont smaller teeth. Type $\mathrm{G}$. sulcatus.

calceolus, Hinde, 1879, Quar. Jour. Geo. Soc. Lond., vol. 35, p. 384, Clinton G'r. suleatıs, Hinde, 1879, Quar. Jour. Gro. Soc. Lond., vol. 35, p. 380, Hud. Riv. Gr.

sulcatus, var. excavatus, Hinde, 1879, Quar. Jour. Geo. Soc. Lond., vol. 35, p. 380, Hur. Riv. Gr.

Gordia marina, see Palæochorda marina.

Gyrichnites, Whiteaves, 1883, Trans. Roy. Soc. Can., p. 109. [Ety. gyros, a circle; ichnos a track.] Trails supposed to have been made by an annelid. Type G. gaspensis.

gaspensis, Whiteaves, 1883, Trans. Roy. Soc. Can., p. 109, Mid. Devonian.

Helminthoidichnites, Fitch, see Palæochorda. marina, see Palæochorda marina.

tenuis, see Palæochorda tenuis.

Lumbriconereites, Ehlers, 1868, Palæontographica, vol. 17, p. 159. [Ety. Lumbriconereis, a genus; lithos, stone.] Distinguished from Eunicites by having a well defined basal extension. Type L. deperditus. 
armatus, Hinde, 1879, Quar. Jour. Geo. Soc. Lond., vol. 35, p. 383, Clinton Gr.

basalis, Hinde, 1879, Quar. Jour. Geo. Soc. Lond., vol. 35, p. 383, Clinton Gr.

dactylodus, Hinde, 1879, Quar. Jour. Geo. Soc. Lond., vol. 35, p. 380, Hud. Riv. Gr.

obliquus, Eichwald, 1854, (Sphagodus obliquus), Bull. d. l. Soc. Imp. d. Nat. d. Moscou, p, 110, Hud. Riv. and Clinton $\mathrm{Gr}$.

perdentatus, Hinde, 1879, (Eunicites perlentatus), Quar. Jour. Geo. Soc., vol. 35 , p. 375, Clinton Gr.

triangularis, Hinde, 1879, Quar. Jour. Geu. Soc. Lond., vol. 35, p. 383, Clintoll Gr.

Machairodus, Pander, 1856. This name was preoccupied. See Distacodus.

incurvus, see Distacodus incurvus.

Monocraterion, Torell, 1860, Acta universitatis lundensis, p. 13. [Ety. monos, one; kraterion, small basin.] Borings in the rock resembling Scolithus, except in having a funnel-shaped enlargement at the upper end.

lesleyi, Prime, 1878, Geo. Sur. Pa. DD. p. 79, Calciferous (?) Gr.

Myrianites, Murchison, 1839, Sil. Syst., p. 700. [Ety. Myrias, a myriad; lithos, stone.] Trails lying together in great numbers, more or less corrugated upon the edges, and resembling delicate wave lines upon the surface of the rock. Type M. macleaii.

murchisoni, Emmons, 1844, Taconic syst., p. 44, Up. Taconic.

sillimani, Emmons, 1844, Taconic Syst., p. 44, Up. Taconic.

Nrma Podia, Emmons, 1844, Taconic Syst, p. 68. [Ety. nema, a thread; pous, a foot.] Trail consisting of a series of depressions marked by numerous short parallel fine lines; the trail is flexuous, and the short, fine lines have the direction of the trail. Type N. tenuissima.

tenuissima, Emmons, 1844, Taconic syst, p. 68, Up. Taconic.

Nerkid vus, Grinnell, 1877, Am. Jour. Sci. and Arts, 3d ser., vol. 14, p. 229. [Ety. Nereis, genus; avus, grandfather.] Minute denticulated teeth or jaws. Type N. varians.

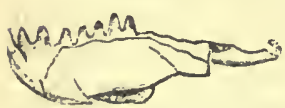

FıG. 939 - Nereidavus variuns, Maguifled 8 dlam. Hud. Riv. Gr.

Nereites, Murchison, 1839, Sil. Syst., p. 700. [Ety. from a resemblance to the track of the Nereis.] Long, convoluted trails; each side equally crenulated; crenulations oval or pointed on the margin, and often traceable to the center of the trail. Type N. cambrensis.

deweyi, Em-

mons, 1844,

Taconic.

Syst., p. 69,

U p. T a conic.

gracilis, Em-

mons, 1844,

Ta c on i c

Syst., p. 69,

U p. T a conic.

jacksoni,

$\mathrm{E} \mathrm{m} \mathrm{m}$ ons,

1844, Taco-

nic Syst.,

p. 69 , Up.

Taconic.

l a n ceolatus,

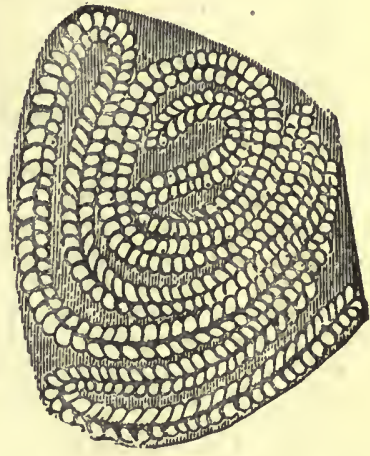

Fra. 940.-Nereites deweyi.
Emmons, 1844, Taconic Syst., p. 69, Up.

Taconic. This may belong to Nereograpsus, as suggested by Emmons.

loomisi, Emmons, 1844, Taconic Syst., p. 69, Up. Taconic.

pugnus, Emmons, 1844, Taconic Syst., p. 69 , Up. Taconic.

robustus, Emmons, 1856, (Nereograpsus robustus,) Am. Geol., p. 111, Up. Taconic. Onnonites, Hinde, 1879, Quar. Jour. Geo.

Soc. Lond., vol. 35, p. 376. [Ety. Oenone, a genus; lithos, stone.] Jaws with a more or less curved anterior hook; followed by a series of smaller teeth, similar in character to those of the existing genus Oenone. Type O. curvidens.

amplus, Hinde, 1879, Quar. Jour. Geo. Soc. Lond., vol. 35, p. 382, Clinton Gr. carinatus, Hinde, 1879, Quar. Jour. Geo. Soc. Lond., vol. 35. p. 377, Hud. Riv. Gr. cuneatıs, Hinde, 1879, Quar. Jour. Geo. Soc. Lond.. vol. 35, p. 377, Hud. Riv. Gr. curvidens, Hinde, 1879, Quar. Jour. Geo. Soc. Lond., vol. 35, p. 376, Hud. Riv. Gr. fragilis, Hinde, 1879, Quar. Joır. Geo. Soc. Lond., vol. 35, p. 382, Clinton Gr.

inæequalis, Hinde, 1879, Quar. Jour. Geo. Soc. Lond., vol. 35, p. 376, Hud. Riv. Gr. infrequens, Hinde, 1879, Quar. Jour. Geo. Soc. Lond., vol. 35, p. 382, Niagara Gr. major, Hinde, 1879, (Eunicites major,) Quar. Jour. Geo. Soc., vol. 35, p. 374, Hud. Riv, and Clinton Gr.

rostratus, Hinde, 1879, Quar. Jour. Geo. Soc. Lond., vol. 35 , p. 376, Hu^. Riv. Gr. serratus, Hinde, 1879, Quar. Jour. Geo. Soc. Lond., vol. 35, p. 376, Hud. Riv. Gr. Ortonia, Nicholson, 1872, Lond. Geo. Mag., vol. 9. Synonym for Conchicolites, if indeed both are not synonyms for Cornulites.

conica, syn. for Conchicnlites flexuosus. intermedia, see Conchicolites intermedius. minor, see Conchicolites minor.

Pal eochorda, McCoy, 1848, Quar. Jour. Geol. Soc., vol. 4, p. 2:4. [Ety. palaios, ancient; chorde, intestine.] Trail very long, cylindrical, chord-like, frequently 
crossing itself, without order, surface smooth. Type P. minor.

marina, Fmmons, 1844, (Gordia marina,) p. 68, and Am. Geol., p. 103, Up. Taconic. prima, Whilfield, 1877, Prelim, Rept. Pal.

Black Hills, p. 7, and Geol. Black Hills of Dakota, p. 331, Potsdam Gr.

tenuis, Fitch, 1849, (Helminthoidichnites tenuis, ) Trans. Agr. Soc., and Am. Geol., p. 103, Up. Taconic.

Planolites, Nicholson, 1873, Proc. Roy. Soc., No. 144. [Ety. planos, wanderer; lithos, stone.] Irregularly cylindrical, tortunus casts of supposed worm-tubes. Type P. vulgaris.

vulgaris, Nicholson, 1873, Proc. Roy. Soc., No. 144, and Pal. Prov. of Ontario, p. 42, Clinton Gr.

Polygnatius, Hinde, 1879, Quar. Jour. Geo. Soc. Lond., vol. 35, p. 361. [Ety. polys, many; gnathos, jaw.] Minute variously formed teeth and minute tuberculated plates. Trpe P. dubius.

coronatus, Hinde, 1879, Quar. Jour. Geo. Soc. Lond., vol. 35, p. 365, Ham. Gr. crassus, Hinde, 1879, Quar. Jour. Geo. Soc. Lond., vol. 35 , p. 365 , Ham. Gr.

cristatus, Hinde, 1879 , Quar. Jour. Geo. Soc. Lond., vol. 35, p. 366, Ham. Gr. curvatus, Hinde, 1879 , Quar. Jour. Geo. Soc. Lnnd., vol. 35, p. 366, Ham. Gr. dubius, Hinde, 1879, Quar. Jour. Geo. Soc. Lond., vol. 35, p. 362, Ham. Gr.

dnplicatus, Hinde, 1879, Quar. Jour. Geo. Soc. Lond., vol. 35, p. 364, Ham. Gr. eriensis, Hinde, 1879, Quar. Jour. Geo. Soc. Lond., vol. 35 , p. $366, \mathrm{Ham}$. Gr. immersus, Hinde, 1879, Quar. Jour. Geo. Soc. Iond., vol. 35 , p. 364 , Ham. Gr.

linguiformis, Hinde, 1879, Quar. Jour. Geo. Soc. Lond., vol. 35, p. 367, Ham. Gr. nasutns, Hinde, 1879 , Quar. Jour. Geo. Soc. I.onrl., vol. 35, p. 364, Ham. Gr. palmatus, Hinde, 1879, Quar. Jour. Geo. Soc. I.onr., vol. 35, p. 367, Ham. Gr. pennatus, Hinde, 1879, Quar. Jour. Geo. Soc. Lond., vol. 35, p. 366 , Ham. Gr. princeps, Hinde, 1879, Quar. Jour. Geo. Soc. Lond.. vol. 35. p. 365, Ham. Gr. punctatns, Hinde, 1879, Quar. Jour. Geo. Soc. Lonr., vol. 35 , p. 367, Ham. Gr. radiatus, Hinde, 1879, Quar. Jour. Geo. Soc. Lond., vcl. 35, p. 36t, Ham. Gr. serratus, Hinde, 1879, Quar. Jour. Geo. Soc. Lond., vol. 35, p. 365, Ham. Gr. simplex, Hinde, 1879, Quar. Jour. Geo. Soc. Lond., vol. 35, p. 367, Ham. Gr. solidus, Hinde, 1879, Quar. Jour. Geo. Soc. Lond., vul. 35, p. 365, Ham. Gr. truncatus, Hinde, 1879, Quar. Jour. Geo. Soc. Lond., vol. 35 , p. 366, Ham. Gr. tuberculatus, Hinde, 1879, Quar. Jour. Geo. Soc. Lond., vol. 35, p. 366, Ham. Gr.

Prionionus, Pander, 1856, Monogr. d. Foss. Fische d. Silur. Syst., p. 28. [Ety. prionion, small saw; odous, tooth.] Basal portion narrow supporting numerous, delicate denticles and an elongated tapering tooth which extends below the basal portion. Type P. elegans. abbreviatus, Hinde, 1879 , Quar. Jour. Geo. Soc. Lond., vol. 35, p. 359, Ham. Gr. acicularis, Hinde, 1879, Quar. Jour. Geo. Soc., Lond., vol. 35, p. 360, Ham. Gr. alatus, Hinde, 1879, Quar. Jour. Geo. Soc. Lond., vol. 35, p. 361, Ham. Gr. angulatus, Hinde, 1879, Quar. Jour. Geo. Soc. Lond., vol. 35 , p. 360, Waverly Gr. armatus, Hinde, 1879, Quar. Jour. Geo. Soc. Lond., vol. 35, p. 360, Ham. Gr. clavatus, Hinde, 1879, Quar. Jour. Geo. Soc., vol. 35, p. 360. Ham. Gr.

elegans, Pander, 1856, Monogr. d. Foss. Fische d. Silur. Syst., p. 29, Hud. Riv.Gr. erraticus, Hinde, 1879, Quar. Jour. Geo. Soc. Lond., vol. 35 , p. 359 , Ham. Gr. furcatus, Hinde, 1879, Quar. Jour. Geo. Soc. Lond., vol. 35, p. 358, Hud. Riv. Gr. panderi, Iinde, 1879, Quar. Jour. Geo. Soc. Lond., vol. 35, p. 361, Ham. Gr. politus, Hinde, 1879, Quar. Jour. Geo. Soc. Lond., vol. 35 , p. 358, Hud. Riv. Gr. radicans, Hinde, 1879, Quar. Jour. Geo.

Soc. Lond., vol. 35, p. 356, Hud. Riv. Gr. spicatus, Hinde, 1879, Quar. Jour. Geo.

Soc. Lond., vol. 35, p. 361, Ham. Gr. Protoscolex, Ulrich, 1878, Jour. Cin. Soc. Nat. Hist., vol. 1, p. 89. [Ety. prolos, first ; skolex, worm.] Long, slender, numerous segments, both ends obtusely pointed. Type $\mathrm{P}$. covingtonensis. covingtonensis, Ulrich, 1878, Jour. Cin.Soc.

Nat. Hist., vol. 1 , p. 89 , Utica Slate Gr. ornatus, Ulrich, 1878, Jour. Cin. Soc. Nat. Hist., vol. 1, p. 90, Utica Slate Gr. simplex, Ulrich, 1878, Jour. Cin. Soc., Nat. Hist., vol. 1, p. 91, Utica Slate Gr. tenuis, Ulrich, 1878, Jour. Cin. Soc. Nat. Hist., vol. 1, p. 90, Utica Slate Gr. Salterella, Billings, 1861, Pal. Foss., vol. 1, p. 17. [Ety, proper name.] Small, slender, elongate, Fig.911.-Protoconical tubes, consisting scolex or $\mathrm{n}$ aof several hollow cones tus. placed one within another, the last one forming the chamber of habitation; surface concentrically or longitudinally striated. Type S. rugosa.

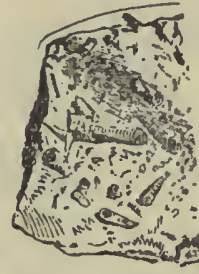

FIG. 042,-Salterella rugosa.

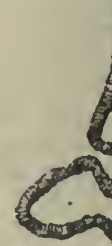
ingsi, Safford, 1869 Geo. of Tenn., p. 289, Trenton Gr.

obtusa, Billings, 1861, Pal. Foss., vol. 1, p. 18, Up. Taconic.

pulchella, Billings, 1861, Pal. Foss., vol. 1, p. 18, Up. Taconic.

rugosa, Billings, 1861 , Pal Foss., vol. 1, p. 17, Up. Taconic.

Scolithus, Haldeman, 1840, Supp. to. Mongraph of Limniades. [Ety. skolex, worm; 
lithos, stone.] Merely worm furrows, without organic characters.

canadensis, Billings, 1862, Pal. Foss. vol. 1, p. 96, Potsdam Gr.

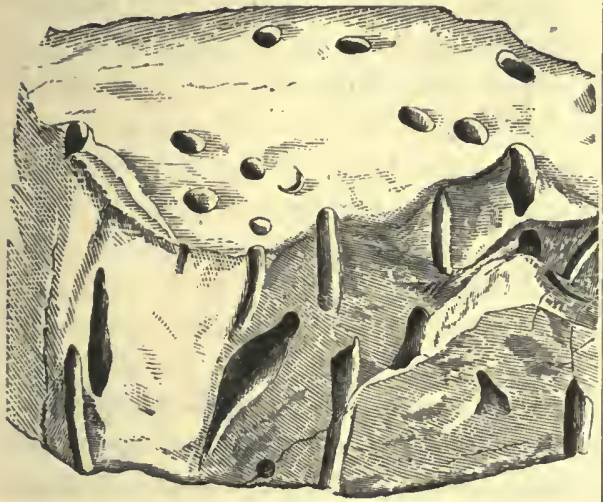

FIG. 943.--Scolithus canadensis.

linearis, Hall, 1847, Pal. N. Y., vol. 1, p. 2, Potsdam Gr.

verticalis, Hall, 1852, Pal. N. Y., vol. 2, p. 6, Medina sandstone.

tuberosus, Miller \& Dyer, 1878, Cont. to Pal. No. 2, p. 5, Hud. Riv. Gr.

woodi, Whitfield, 1880, Ann. Rep. Geo. Sur. Wis., p. 45, Potsdam Gr.

Serpula, Linnæus, 1758, Syst. Nat., 10th ed. p. 786. [Ety. serpo, to creep.] Tube calcareous, procumbent, variously curved or spirally coiled, growing singly or in groups, attached to marine bodies, capable of receiving the entire animal; aperture at the larger extremity simple and rounded. Type S. vermicularis. insita, White, 1878, Proc. Acad. Nat. Sci., p. 37 , and Cont. to Pal., No. 8, p. 171, Coal Meas.

omphalodes, see Spirorbis omphalodes.

valvata, see Spirorbis valvatus.

Serpulites, Murchison, 1839, Murch. Sil. Syst., p. 608. [Ety. Serpula, a genus of annelids.] Tube smooth, arched, slightly calcareous, glossy; having two small longitudinal tubes at opposite points of the circumference, stronger than the rest of the shell, and prolonged at the posterior end. Type S. longissimus. annulatus, Dawson, 1868,

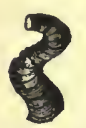

FIG. 944.-Ser pulites aninuiatus. Acad. Geol., p. 312, Carboniferous.

dissolutus, Billings, 1862, Pal. Foss., vol. 1, p. 56, Trenton Gr.

hortonensis, Dawson, 1868 , Acad. Geol., p. 312, Carboniferous.

inelegans, Dawson, 1868, Acad. Geol., p. 312, Carboniferous.

murchisoni, Hall, 1861, Geo. Rep. Wis., p. 48, Potsdam Gr.

murrayi, Dawson, 1883, Rep. on Redpath Mus., No. 2, p. 13, Carboniferous. splendens, Billings, 1859, Can. Nat. and Geo., vol. 4, p. 470, Chazy Gr.

Sphagodus obliquus, see Lumbriconereites obliquus.

SpInorbis, Lamarck, 1801, Syst. An. sans Vert., p. 326. [Śg. spiral-whorl.] Tube calcareous, solitary, coiled; flat, dextral for sinistral, attached by one side to some foreign object. Type Serpula spirorbis of Linnaeus.

ammon, Winchell, 1866, Rep. Jow. Peninsula Mich., p. 97, Ham. Gr.

angulatus, Hall, 1862, 15th Rep. N. Y. St. Mus. Nat. Hist., p. 81, Ham. Gr.

angulalus, Dawson, 1868. The name was preoccupied.

annulatus, Hall, 1858, Trans. Alb. Inst., vol. 4, p. 34, and Bull. Am. Mus. Nat. Hist., p. 92, Warsaw Gr.

annulatus var. nodulosus, see S. nodulosus.

anthracosia, Whitfield, 1881, Am. Jour. Sci. and Arts, 3d ser. vol. 21, p. 128, Coal Meas.

arietinus, Dawson, 1869, Rep. of Progr. Geo. Sur. Can., p. 14, Coal Meas.

arkonensis, Nicholson, 1874, Geo. Mag., vol. 1, p. 199, Ham. Gr.

carbonarius, Dawson, 1845 , Quar. Jour. Geo. Soc., vol. 1, p. 326, Coal Meas. cincinnatiensis, Miller \& Dyer, 1878, Jour. Cin. Soc. Nat. Hist., vol. 1, p. 38, Hud. Riv. Gr.

flexuosus, Hall, 1863 ,

Trans. Alb. Inst vol. p. 224, Niagara Gr.

inornatus, Hall, 1863, Trans. Alb. Inst., vul. 4, p. 224, Niagara Gr.

kinderhookensis, Gurley, 1883, New Carb. Foss., p. 9. Publication not sufficient. laxus, Hall, 1859, Pal. N. Y., vol. 3, p. 349, Low. Held. Gr.

nodulosus, Hall, 1858, Trans. Alb. Inst., vol. 4, p. 34, and Bull. Am. Mus. Nat. Hist., p. 93, Warsaw Gr.

obesus, Winchell, 1866, Rep. Low. Peninsula Mich., p. 97, Ham. Gr.

omphalodes, Goldfuss, 1826, Germ. Petref., Up. Held. and Ham. Grs.

orbiculosioma, Swallow, 1858, Trans. St. Louis Acad. Sci., p. 181, Permian Gr.

spinuliferus, Nicholson, 1875, Pal. Prov. Ont., p. 83, Ham. Gr.

valvatus, Goldfuss, 1826, (Serpula valvata). Not American.

Sta U Rocephalites, Hinde, 1879, Quar. Jour. Geo. Soc. Lnnd., vol. 35, p. 383. [Ety. Staurocephalus, an existing genus; lithos, stone.] Jaws of more or less elcngated compressed denticulate plates resembling those of the genus Staurocephalus. Type S. niagarensis.

nigi rensis, Hinde, 1879, Quar. Jour. Geo. Soc. Iond., vol. $3 \overline{5}$, p. 383, Niagara Gr. 


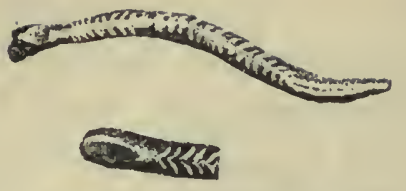

Frc. 846.-Walcottia rugosa.
Walcottia, Miller \& Dyer, 1878, Jour. Cin. Soc. Nat. Hist., vol. 1, p. 39. [Ety. proper name.] A rugose, flexuous, worm-like furrow. Type W. rugosa. cookana, Miller \& Dyer, 1878, Cont. to - Pal., No. 2, p. 11, Hud. Riv. Gr. rugosa, Miller \& Dyer, 1878, Jour. Cin. Soc. Nat. Hist., vol. 1, p. 39, Hud. Riv. Gr.

\section{CLASS CRUSTACEA.}

There is such an immense diversity among Crustaceous animals, it has been found necessary to make subclasses, orders, and suborders, to give intelligible definitions to the classification. They are generally covered with a peculiar calcareous secretion or integument, constituting a cutaneous skeleton, inclosing the soft parts of the body. Segments are united by a membrane, giving flexibility to the armor. There being no way to increase the integument by growth, it is cast off at stated periods, and a new one is secreted to cover the enlarged body. The subclasses are Cirripedia, Entomostraca, Xyphosura, Edriopthalmata, and Podopthalmata.

The Subclass Cirripedia includes only a single order which bears the same name. The animals, when mature, are attached to submarine objects, and are inclosed in a shell composed of several calcareous plates, from an upening in which articulated cirri are exserted and retracted when the animal is alive in search of prey. The common barnacle, which frequently covers the bottoms of ships so as to impede their progress across the ocean, is a representative of this order.

The Subclass Entomostraca is divided into several orders, only three of which are Palæozoic, viz.: Ostracoda, Phyllopoda, and Trilobita. The Ostracoda are minute animals inclosed in a little bivalve shell; the feet and antennæ are protruded between the lower edges of the valves. The Cypris, Daphnia, and Polyphemus are living examples of this order. The Phyllopoda are so named on account of the broad and leaf-like feet. Some of them are covered with a bivalve shell, and others are without such protection. The Palæozoic are bivalve shells. The Estheria, which abound in pools and springs, belong to this order. The Trilobita possessed a cephalic shield, a trilobed thorax composed of segments, which were flexible and allowed the animal to double itself up, and a tail-piece called the pygidium. The order became extinct in the Palæozoic era.

- The Subclass Xyphosura has an anterior subcrescentiform carapace, inclosing the cephalothoracic organs, and a posterior abdominal piece, from which a tail spine projects. The upper surface is convex, and the lower concave. There are three orders-Amphipeltida, Euripterida, and Xyphosura. Only a fragment of the shell of the Amphipeltida is known. The Euripterida is also an extinct order. A common form of the Xyphosura is the Limulus, or Horseshoe Crab, which is common on the shores of the tropical seas.

The Subclass Edriopthalmata has the head distinct from the thoracic segments, and therefore has no cephalothorax. The head has a pair of simple compound eyes, not pedunculated. The Palæozoic orders are Amphipoda and Anisopoda. 
The Order Amphipoda consists of animals that live in water, burrow in sand, or become parasitic on fishes. The abdomen is well developed, and bears limbs for leaping or swimming. They always swim on their sides. The common sandhopper on the shore of the sea belongs to this order. The Order Anisopoda has a long body, convex above and flattened below, and has affinities with the Isopoda, of which the common wood-louse is an example.

The Subclass Podopthalmata has compound eyes at the extremity of a pair of movable stalks; the head and thorax are generally united, covered by a single piece of shell, and called the cephalothorax ; this includes the antennæ, eyes, mouth, jaws, feet, etc. The remaining segments form an abdomen, which frequently terminates in a caudal fin. Common examples are the squill and the small edible crab. There are several orders in this subclass, only three of which are Palmozoic, viz.: Phyllocarida, Decapoda, Tetradecapoda.

The Phyllocarida has cephalic, thoracic, and abdominal segments. The carapace has no regular hinge. The living representative is Nebalia, which inhabits the sea at moderate depths. The Decapoda are stalk-eyed, and the head and thoracic segments are united in a cephalothorax, incased in a common shell, and have the branchial organs inclosed on the sides of the cephalothorax. The true thoracic legs are almost always ten, whence the name of the order. The Tetradecapoda have their relations with the Decapoda.

\section{SUBCLASS AND ORDER CIRRIPEDIA.}

Famil y Balanide.-Palæocrusia, Protobalanus.

Family Lepadide.-Lepidocoleus, Strobilepis, Turrilepas.

\section{SUBCLASS ENTOMOSTRACA.}

\section{ORDER OSTRACODA.}

Famil y Beyrichidde.-Beyrichia, Beyrichona, Hipponicharion, Primitia.

FAMILY CyPRIDÆ.-Candona.

Fanily Cytierida.-Cytherella, Cytheropsis.

Fanily Faberidde.-Faberia.

FAMILY LePerditide.-Aparchites, Isochilina, Leperditia.

\section{ORDER PHYLLOPODA.}

Family Esthermd 2.-Estheria, Leaia, Schizodiscus.

Famity Uncertain.-Lepidilla, Lepiditta.

\section{Order Trilobita.}

Fanily ACidaspide.-Acidaspis.

Family Aglaspide.-Aglaspis.

Family Agnostid z.-Agnostus, Microdiscus, Shumardia.

FamiJ.y Asapimde.-Asaphus, Barrandia, Megalaspis, Nileus, Ogygia, Symphysurus.

Family Bathyuride.-Asaphiscus, Bathyurellus, Bathyuriscus, Bathyurus.

Family Brontrid E.-Bronteus.

Family Calymenide.-Calymene, Homalonotus. 
Family Cerauride-Ceraurus, Sphærocoryphe, Sphrexochus.

Family Conocorypuide.-Bailiella, Chariocephalus, Conocoryphe, Harttia, Menocephalus, Prototypus.

Family CyPhaspide.-Cyphaspis.

Family Dicellocephalide.-Dicellocephalus, Pterocephalia, Ptychaspis.

Fami, Excrivuride.-Amphion, Encrinurus.

Family Ellipsocephalid d:-Ellipsocephalus.

Family Harpida.-Harpes.

FAMILYILL RNID d.-Illænurus, Illænus.

Family Lichids.-Lichas, Terataspis.

Famiry Olexids.-Dolichometopus, Oryctocephalus, Telephus, Triarthrella, 'Triarthrus.

Family Paramoxims.-Anopolenus, Atops, Bathynotus, Elliptocephala,

Mesonacis, Olenoides, Paradoxides.

Family Pinacorins.-Dalmanites, Phacops.

Famrly Proetind:-Harpires, Phathouides, Phillipsia, Proetus.

Family Ptychoparida:-Agraulus, Crepicephalus, Liostracus, Longocephalus,

Loganellus, Ptychoparia, Solenopleurı.

Family Remopleuima.-Remopleurides.

Family Trinuchemdi-Ampyx, Dionide, Endymionia, Trinucleus.

Family Affinity Uncertain.-Pemphigaspis.

Tracks supposed to be Crustacran.-Asaphoidichnus, Climachtichnites,

Diplichnites, Protichnites, Rusichnites.

\section{SUBCLASS XIPHOSURA.}

\section{ORDER AMPHIPELTIDA.}

Family Amphipeltios. -Amphipeltis.

\section{ORDFR EURYPTERIDA.}

Family Echinogitamid.-Echinognathus.

Family Eurypteridi.-Anthraconectes, Dolichopterus, Eurypterella, Eurypterus, Pterygotus, Stylonurus.

Family Hemiaspide.-Bunodella.

\section{ORDER XIPHOSURA}

Family Belinuride.-Belinurus, Euproops, Protolimulus.

Famru Cychide.-Cyclus, Dipeltis.

\section{SUBCLASS EDRIOPTHALMATA.}

\section{Order Amphipona.}

Family Diplostylida. - Diplostylus.

\section{Order ANisopona.}

Family Acanthotel sonida.-Acanthotelson. 


\section{SUBCLASS PODOP'THALMATA.}

ORDER PHYLIOCARIDA.

Family Ceratiocarides.-Ceratiocaris, Colpocaris, Echinocaris, Elymocaris, Ribeiria (?), Solenocaris, Tropidocaris.

Family Discrnocaride.-Dipterocaris, Spathiocaris.

Family Pinacarider.-Dithyrocaris, Mesothyra.

Family Protocaride. -Protocaris.

Family RAchurid a.-Rachura.

Family Rhinocaride.-Rhinocaris.

ORDER DECAPODA.

Family Anthracarid ж.-Anthrapalæmon.

Family Caridide:-Palæopalæmon.

Family Palmocaride.-Gampsonyx, Palæocaris.

\section{ORDER TETRADECAPODA.}

Family Archæocaride.-Archæocaris.

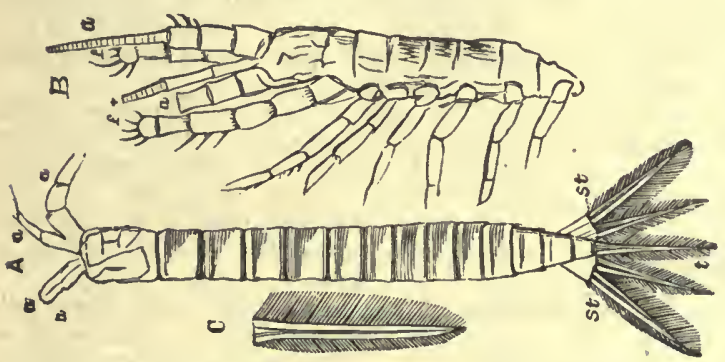

Fic. 947.-Acanthotelson eveni. $A$, dorsal view; st, stylet; $t$, telson; $B$, side vlew; $a$ and $x$, antennæ; $f$, anterlor leg; $C$, enlarged stylet.

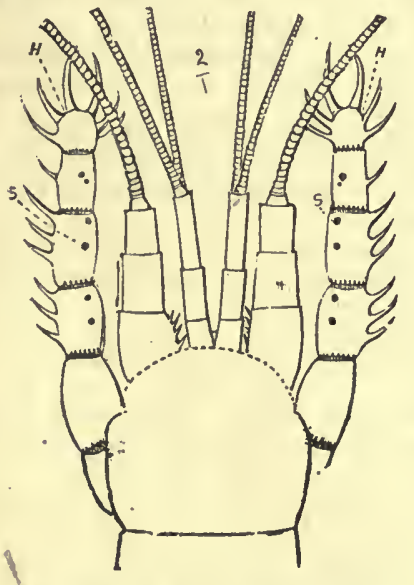

FIG. 918.-Acanthotelson even]. Enlarged ; $\boldsymbol{H}$, anterlor legs and antennæ; $\mathbb{S}$, punctures left by splnes on the lower side.

Acantholoma, syn. for Acidaspis. spinosa, syn. for Acidaspis tuberculata.
A CANTHOTELSON, Meek \& Worthen, 1860 , Proc. Acad. Nat. Sci., p. 47. [Ety. akantha, spine ; telson, end.] Superior antennæ as long as the inferior, flagella longer than the peduncles; head about the length of the two anterior thoracic segments; thoracic and abdominal segments about the same length; anterior thoracic legs longer than the others; telson simple, long, spine-like', laterally com-

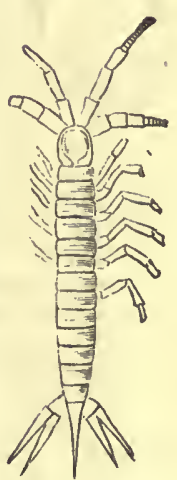
first, and similar to the telson. 'Type A. stimpsoni. eveni, Meek \& Worthen, 1868, Am. Jour. Sci., vol.

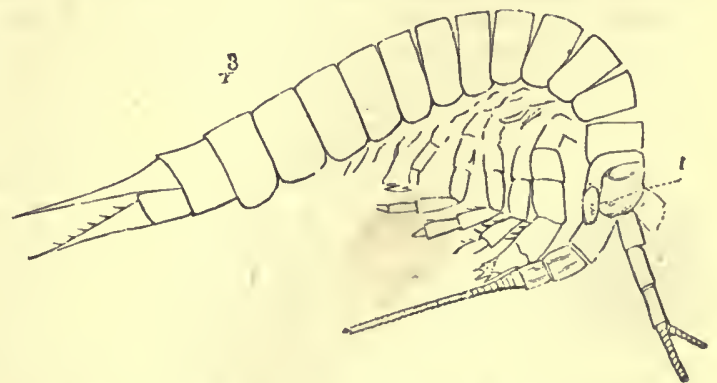

Fra. 950,-Acanthotelson stimpsoni. Enlarged 3 diam.; $i$, a protuberance that may be an eye.

46 , p. 28, and Geo. Sur. Ill., vol. 3, p. 551 , Coal Meas. 
inxqualis, Meek \& Worthen, syn, for Palæocaris typus.

stimpsoni, Meek \& Worthen, 1865, Proc. Acad. Nat. Sci., p. 47, and Geo. Sur. Ill., vol. 2, p. 401, Low. Coal Mieas.

Acidaspis, Murchison, 1839, Sil. Syst., p. 658. [Ety. akis, a point; aspis, shield.] Cephalic shield, semicircular, margin thickened and spinous, lateral angles produced in spines; glabella convex, narrow in front, not reaching the margin, two dobes on each side, and having a large spine projecting backward, eyes prominent; thorax with eight segments; pleura wide, and terminating in spines; pygidium small, axis slort, of two joints, sides depressed, one segmental furrow, long spine extending backward from the margin at each side, and smaller spines from the other parts of the margin. Type A. brighti.

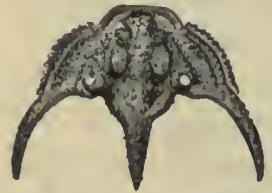

FIG. 951. - Acidaspts ancioralls. Ceplial. ic shield. anchoralis, S. A. Miller, 1875, Cin. Quar. Jour. Sci., vol. 2, p. 349, Hud. Riv. Gr. callicera. Hall, 1888, Pal. N. Y., vol. 7, p. 69, Up. Held. Gr. ceralepta, Anthony, 1838, (Ceratoceph. ala ceralepta,) Am. Jour. Sci., vol. 34, p. 379. Not defined so as to be recognized.

cincinnatiensis, Meek, 1873, Ohio Pal., vol. 1, p. 167, Hud. Riv. Gr. crosotus, Locke, 1843, Am. Jour. Sci., vol. 44, p. 347, and Ohio Pal., vol. 1, p. 165, Hud. Riv. Gr. The word is misspelled; it should be Fis.952.-Acidascrossota. danai, Hall, 1862, Geo. Pygidium.

Sur. Wis., p. 423, Niagara Gr. eriopis, see Terataspis eriopis.

fimbriata, Hall, 1879 , Desc. New Spec. Foss.. p. 20, and 11th Rep. Geo. and Nat. Hist. Ind., p. 334, Niagara Gr. grandis, see Terataspis grandis. halli, Shumard, 1855, Geo. Sur. Mo., p. 200 , Trenton Gr.

hamata, Conrad, 1841, (Dicranurus hamatus,) Ann. Rep. N. Y., p. 48, and Pal. N. Y., vol. 3, p. 371, Low. Held. Gr. horani, Billings, 1859, Rep. of Progr. Geo. Sur. Can., p. 341, Trenton Gr.

$i d a$, svin. for Acidaspis danai.

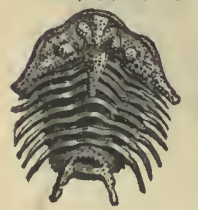

FIG. 953 . onerlii. Acidaspis onealli, S. A. Miller, 1875, Cin. Quar. Jour. Sci., vol. 2, p. 86, Hud. Riv. Gr.

parvula, Walcott, 1877, 31st Rep. N. Y. St. Mus. Nat. Hist., p. 69, Trenton Gr. romingeri, Hall, 1888, Pal. N. Y., vol. 7, p. 71, Ham. Gr. spiniger, see Bathyurus spiniger.

trentonensis, Hall, 1847, Pal. N. Y., vol. 1, p. 240, Trenton Gr. tuberculata, Conrad, 1840, Ann. Rep. N. Y., p. 205, and Pal. N. Y., vol. 3. p. 368, Low. Held. Gr.

Aglaspis, Hall, 1862, Can. Nat. and Geo., vol. 7 , p. 443 , and 16 th Rep. N. Y. St. Mus. Nat. Hist., p. 181. [Ety. aglaos, bright; aspis, shield.] Cephalic shield somewhat semielliptical, wider than long, sinus in front; glabella narrow, conical; eyes prominent, and situate anterior to the middle; thorax having eight segments; pygidium small, and terminating in a single spine. Type A. barrandii.

barrandii, Hall, 1862, Can. Nat. and Geo., vol. 7 , p. 443 , and 16 th Rep. N. Y. St. Mus. Nat. Hist,, p. 181, Potsdam Gr.

eatoni, Whilfield, 1880, Ann. Rep. Geo. Sur. Wis., p. 52, and Geo. of Wis., vol. 4, p. 192, Potsdam Gr.

Agnostus, Brongniart, 1822, Hist. Nat. Crust. Foss., p. 38. [Ety. agnostos, obscure.] Body elongate, elliptical; cephalic shield and pygidium subequal, subrotund, or longer than wide, convex rim on the border; glabella convex; no eyes; no facial sutures; two thoracic segments. Type A. pisiformis.

acadicus, Hartt, 1868, Acad. Geol., p. 655, St. John Gr.

acadicus var. declivis, Matthew, 1885, Trans. Roy. Soc. Can., p. 70, St. John Gr. acutilobus, Matthew, 1885, Trans. Roy. Soc. Can., p. 73, St. John Gr.

americanus, Billings, 1860, Can. Nat. and Geol., vol. 5, p. 301, and Pal. Foss, p. 395 , Up. Taconic.

bidens, Meek, 1873, 6th Rep. Hayden's Geo.Sur. Terr., p. 463, and Monog. U.S. Geo. Sur., vol. 8, p. 26, Prospect Mountain Gr., Up. Taconic.

canadensis, Billings, 1860, Can. Nat. and Geol., vol. 5. p. 301, and Pal. Foss., vol. 1, p. 397, Up. Taconic.

coloradoensis, Shumard, 1861, Am. Jour, Sci. and Arts, 2d ser., vol. 32 , p. 218, Up. Taconic.

communis, Hall \& Whitfield, 1877, U. S. Geo. Expl. 40th parallel. vol. 4, p. 288, Prospect Mountain Gr., Up. Taconic.

? disparilis, Hall, 1863, 16th Rep. N. Y. St. Mus. Nat. Hist., p. 179 , Potsdam Gr. fabius, Billings, 1865, Pal. Foss., vol. 1, p. 298, Up. Taconic.

galba, Billings, 1865, Pal. Foss., vol. 1, p. 297, Up. 'Tuconic.

interstrictus, White, 1874, Rep. Invert. Foss., p. 7, and Geo. Sur. W. 100th Mer., vol. 4, p. 38, Up. Taconic.

? josepha, Hall, 1863, 16 th Rep. N. Y., St. Mus. Nat. Hist., p. 178, Potsdam Gr. latus, see Beyrichia lata.

lobatus, see Microdiscus lobatus.

maladensis, Meek, 1873, Hayden's Sur. Terr., p. 464. Not properly dtfined. neon, Hall \& Whitfield, 1877 , U. S. Geo. Expl., 4th parallel, vol. 4, p. 229, Prospect Mountain Gr., Up. 'Taconic. 
nobilis, Ford, 1872, Am. Jour. Sci., 3d ser., vol. 3, p. 421 , Up. Taconic.

obtusilobus, Matthew, 1885, Trans. Roy. Soc. Can., p. 72, St. John Gr.

orion, Billings, 1860, Can. Nat. and Geo., vol. 5, p. 301, and Pal. Foss., vol. 1, p. 397, Up. Taconic.

? parilis, Hall, 1863, 16th Rep. N. Y. St. Mus. Nat. Hist., p. 179, Potsdam Gr.

partitus, Matthew, 1885, Trans. Roy. Soc. Can., p. 68, St. John Gr.

prolongus, Hall \& Whitfield, 1877, U. S. Geo. Expl. 40th parallel, p. 230, Prospect Mountain Gr., Up. Taconic.

regulus, Matthew, 1885, Trans. Roy. Soc. Can., p. 67, St. John Gr.

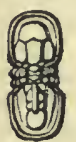

FIG. 954 .

Agnostus rex. 25. Prospect. U.S. Geo. Sur., vol. 8, p. similis, Hartt, 1868, Acad. Geol., p. 656, St. John Gr.

tessella, Matthew, 1885, Trans. Roy. Soc. Can., p. 71, St. John Gr.

tumidosus, Hall \& Whitfield, 1877, U. S. Geo. Expl. 40th parallel, vol. 4, p. 231, Up. Taconic.

umbo, Matthew, 1885, Trans. Roy. Soc. Can., p. 71, St. John Gr.

vir, Matthew, 1885, Trans. Roy. Soc. Can., p. 69 , St. John Gr.

vir var. concinnus, Matthew, 1885, Trans. Roy. Soc. Can., p. 70, St. John Gr.

Agraulus, Hawle \& Corda, 1847, Prodrom. einer Monographie der bomischen Trilobiten, p. 142. [Ety. agraulos, dwel]ing in the fields.] Body elongateovate; cephalic shield large, semicircular to lunate, with a wide margin, that merges in the cheeks; glabella convex, narrowed and rounded in front, conoidal, three or four lateral furrows on each side, margined in front, neck furrow distinct; eyes small, distant from glabella or submarginal; facial sutures, beginning near the lateral posterior angles, are directed forward, curving over the eyes to the anterior margin, nearly in parallel lines; chèess small, narrow; sixteen thoracic segments, axal lobe convex; pygidium small, rounded, three segments; hypostoma oval, truncated anteriorly. 'Type A. ceticephalus.

affinis, Billings, 1874, Pal. Foss., vol. 2, p. 72, Up. 'Taconic.

articephalus, Matthew, 1885, Trans. Roy. Soc. Can., p. 75, St. John Gr.

bipunctatus, Shumard, 1863, (A rionellus bipunctatus,) Trans. St. Louis Acad. Sci., vol. 2, p. 101, Potsdam Gr. Poorly defined; probably belongs to another genus.

convexus, Whitfield, 1877, (A rionellus convexus,) Geo. Sur. Wis., vol. 4, p. 190, Potsdam Gr. Founded upon a fragment, and may belong to another genus. cylindricus, Billinge, 1860 , (Arionellus cylindricus, Can. Nat. and Geo., vol. 5, p. 301, and Pal. Foss., vol. 1, p. 406 , Up. Ta-

F'IG. 955. Agraulus cylindricus. conic or St. John Gr.

globosus, Walcott, 1885 ,

Monogr. U. S. Geo. Sur., vol. 8, p. 61, Up. Taconic.

hallanus, Matthew, 1887, Trans. Roy. Soc. Can., p. 132, St. John Gr.

planus, Shumard, 1861, (Árionellus planus,) Am. Jour. Sci. and Arts, $2 \mathrm{~d}$. series, vol. 32, p. 219, Potsdam Gr.

pustulatus, Walcott, 1879, (Arionellus pustulatus,) 31st Rep. N. Y. St. Mus. Nat. Hist., p. 68, Chazy Gr.

quadrargularis, Whitfield, 1884, (A rionellus quadrangularis,) Bull. Am. Mus. Nat. Hist., vol. 1, p. 139 , Up. Taconic. socialis, Billings, 1874, Pal. Foss., vol. 2, p. 71, Up. Taconic or St. John Gr.

strenuus, Billings, 1874, Pal. Foss., vol. 2, p. 71, Up. Taconic or St. John Gr.

subclavatus, Billings, 1860, (Arionellus subclavatus,) Can. Nat. and Geo., vol. 5 , p. 301, and Pal. Foss., vol. 1, p. 406 , Up. Taconic or Quebec Gr.

texanus, Shumard, 1861, (Arionellus texanus,) Am. Jour. Sci. and Arts, 2d ser., vol. 32 , p. 218 , Potsdam Gr. or Up. Taconic.

tripunctatus, Whitfield, 1876, Rep. Recon. Up. Mo. to Yel. Nat. Park, p. 141, Potsdam Gr. or Up. Taconic.

whitfieldauus, Matthew, 1887, Trans. Roy. Soc. Can., p. 130, St. John Gr.

whitfieldanus var. compressus, Matthew 1887, Trans. Roy. Soc. Can., p. 130, St. John Gr.

woosteri, Whitfield, 1878, Geo. Sur. Wis., vol. 4, p. 189, Potsdam Gr.

Amphion, Pander, 1830, Beitrage zur Geognosie des Russischen. Reiches, p. 139. [Ety. mythological name.] Cephalic shield short, transverse; glabella convex or subrectangular, three pairs of furrows, front inclosing a small forehead lobe; eyes small; facial suture, behind the eyes, ending on the exterior margin in advance of the rounded angles; thorax 15 to 18 articulations; pleurce $w$ i $t h$ o $t$ grooves; pygidjum with short axis and pleurae with free terminations; lab-

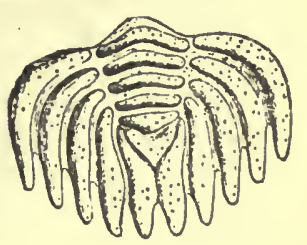
rum pointed, FiG. 956-Amphion canaconvex, margined. Type A. frontiloba.

barrandii, Billings, 1865, Pal. Foss., vol. 1, p. 288, Quebec Gr. 
canadensis, Billings, 1859, Can. Nat. and Geo., vol. 4, p. 381, Chazy Gr.

convexıs, Billings, 1865, Pal. Foss., vol. 1, p. 322, Quebec Gr.

insularis, Billings, 1865, Pal. Foss., vol. 1, p. 290, Quebec Gr.

julius, Billıngs, 1865, Pal. Foss., vol. 1, p. 290, Quebec Gr.

matutinus, Hall, 1863, 16th Rep. N. Y. St. Mus. Nat. Hist., p. 222, Potsiam Gr. mullisegmentatus, see Encrinurus multisegmentatus.

nevadensis, Walcott, 1885, Monogr. U. S. Geo. Sur., vol. 8, p. 9t, Chazy Gr.

salteri, Billings, 1861, Csın. Nat. and Geo., vol. 6, Calciferous $\mathrm{Gr}$.

westoni, Billings, 1865, Pal. Foss., vol. 1, p. 321, Quebec Gr.

A mpıpelts, Salter, 1863, Quar. Jour. Geo.

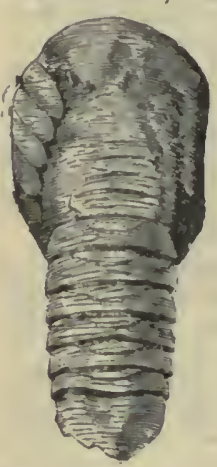

F10. 957.-A in pht. peltis paradoxus. Soc., vol. 19 , p. 75. [Ety. amphi, on both sides; peltis, provided with a shield or buckler.] Carapace oblong, oval, rounded in front, more truncate behind ; thorax with 9 seyments, 5 project be. yond the carapace and 4 concealed bencath it ; tail-piece semicircular, as wide as the abdomen, and as long as the last three segments taken together. 'Type A. paradoxus.

paradoxus, Salter, 1863, Quar. Jour. Geo. Soc., vol. 19, p. 76 , and Acad. Geo., p. 523, Up. Devonian. AMPYX, Dalman, 1827, Uber die palæaden oder die sogenannten Trilobiten, p. 53. [Ety, ampyx, head-band.] Cephalic shield somewhat trigonal; glabella large, prominent, narrow beliind, and projecting upward and forward anteriorly; cheeks flattened, posterior angles produced; no eyes or facial sutures; thoracic segments 5 or 6 , flattened, sides straight, divided by a diagonal pleural groove; pygidium subtrigonal, nearly as large as the cephalic shield; one anterior segmental furrow axis faintly marked with transverse furrows. Type A. nasutus.

halli, Billings, 1861, Pal. Foss., vol. 1, p. 24, Chazy Gr.

læviusculus, Billings, 1865 , Pal. Foss., vol. 1, p. 295, F1G. 958. Quebec Gr.

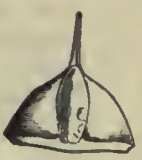
ormalis, Billings, 1865, Pal. Foss., vol. 1, p. 295, Quebec Gr.

1865, able chceks Pal. iygidium. semicostatus, Billings, 1865, Pal. Foss., vol. 1, p. 297, Quebec Gr.

Angelina hitchcocki, see Prototypus hitchcocki.

Anomocare, Angelin, 1852, Pal. Scand., p. 24. This genus is not yet known in America.

(9) parvum, Walcott, 1885, Mon. U. S. Geo. Sur, vol. 8, p. 59, Up. Taconic. This species is founded on a fragment of the cephalic shield and the generic refercnce is ouly provisional.

Anorolenus, Salter, 1864, Quar. Jour. Geo. Soc., vol. 20, p. 236, and vol. 21, p. 477. [Ety. $a$, without; ops, an eye; ()lenus, agenus.] Elongateıl, depressed; cephalic slield semicircular with prolonged spines, and clavate glabella liaving 4 pairs of furrows; fixed cheeks, large, punctate, strongly margined, each a quarter of a circle in shape, and reaching nearly to the frout of the glabella, aguinst which the long eyes abut; thence the facial suture curves outward, and is marginal infront ; the long eye-

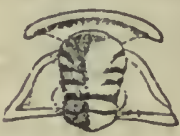

FIG. 959.-A nopolenus venustus. margined and reaching only threefourths down the fixed cheek; pygidium wide, expanded, but narrower than the thorax, widely marginate, and serrated by 6 or 8 marginal spines. Type A. henrici.

venustus, Billings, 1874, Pal. Foss., vol. 2, p. 73 , Up. Taconic.

ANTurA C ONECTES, Meek \& Worthen, 1868, Am. Jour. Sci., vol. 46, p. 21, and Geo. Sur. Ill., vol. 3 , p. 544 . [Ety. anthrax, coal ; nectos, swimming.] $\mathrm{D}$ i s tinguished from Eurypterus by the absence of lateral spines at the articulations of the legs, which terminate in single F1G. 960.-Anthraconecpoints, and in tes mazonensis. Hy postoma enlarged to extremity of the mesial appendage of its operculum, as well as in the possession of two little spatulate supplementary pieces. Type A. mazoneusis.

mazonensis, Meek \& Worthen, 1868, Am. Jour. Sci and Arts, vol. 46, p. 21, and Geo. Sur. Ill., vol. 3, p. 544, Coal Meas. 


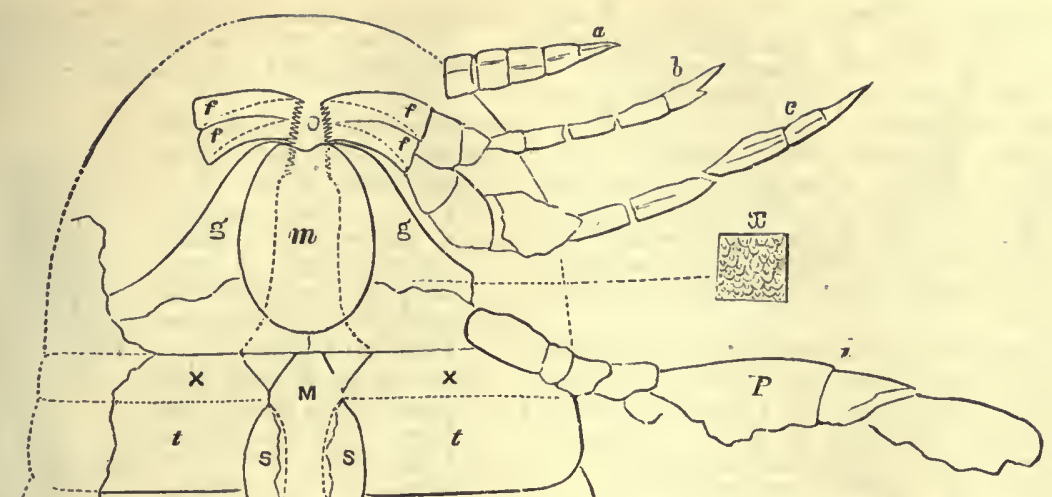

domen of six joints, as broad as long, pleurx except the second pointed; telson broad, appendages to the penul-

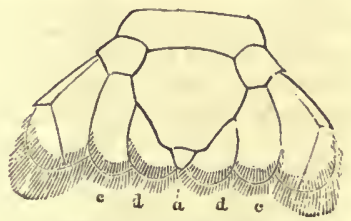

Frf. 963.-Anthracopalæemon gracills. Caudal parts and one abdominal segment in advance of the telson-ail enlarged. $a$, Sinall, terminal paletie; $d, d$, two accesvory lainellæ; $c, c$, lateral lamellæ or fius.

Fia. 961.-Anthraconectes mazonensis. $a, b, c$, Legs, crushed and broken; $h$, ends of dorsal half of body segments; $\boldsymbol{m}$, hypostoma; $P$, swimming paddle broken: t, natural articulation; $g$, basal joints of same; $x$, enlarged surface markings; $M$, ineslal appendage of operculum; $1,2,3$, articulations; $x, t$, lateral alse of operculum; $s, s$, accessory pieces; $O$, position of mouth.

Anthra COPA L ENon, Salter, 1861, Quar. Jour.

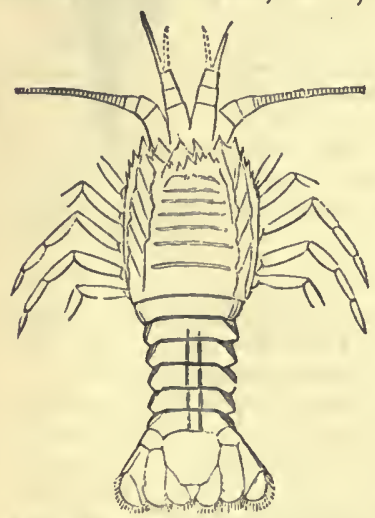

Fig. 962, - Anthracopalæmon gracilis. Dorsal view somewhat enlarged.

front margin serrate; outer antennæ have wide, square basal joints; second and third joints not much oblique; the rest about as broad as long; ab-

17 , p. 529. thrax, coal ; palæmon prawn.] Carap a ce scarcely as broad as long, simple, convex, sides arched outward; central ridge in front separated by a furrow from a central ridge; timate joint double on each side, subtrigonal, broad, lateral fins divided. Type A. grossarti.

gracilis, Meek \& Worthen, 1865 , Proc. Acar. Nat. Sci. Phil., p. 50, and Geo. Sur. Ill., vol. 2, p. 407, Coal Meas. hillanus, Dawson, 1877, Geo. Mag., vol. 4, p. 56, Coal Meas. Aparchites, Jones, 1859, Ann. and Mag. Nat. Hist., 6th ser., vol. 3, p. 384. [Ety. aparche, first.] In form like Leperditia, but smaller and without ocular or muscular spot, and having no overfap on the ventral margin. Type $A$. whiteavesi. whiteavesi, Jones, 1889, Ann. and Mag. Nat. Hist., 6th ser., vol. 3, p. 384, Trenton Gr. ArCh mocaris, Meek, 1872, Proc. Acad. Nat. Sci. Phil., p. 335. [Ety. archaios, ancient; karis, shrimp.] Cephalothorax about fqualing in length $3 \frac{1}{2} \mathrm{seg}$ ments, subtrigonal, pointed in front, truncated a $\mathrm{d}$ sinuous posteriorly; ab- FrG. 964.-A relıæncaris verdomen with six imbricating segments; telson as long as $3 \frac{1}{2}$ abdominal segments with a stylet on each side. Type A. vermiformis. 
vermilormis, Meek, 1872, Proc. Acad. Nat. Sci. Phil., p. 335, and Ohio Pal., vol. 2 , p. 321 , Subcarboniferous.

Arctinurus, Castelnau, 1843, Syst. Syl., p. 21, syn. for Lichas.

Aretñusinı, Barrande, 1852, Syst. Sil. Boh. Not yet known as an American genus.

(?) americana, Walcott, 1885, Monogr. U. S. Geo. Sur., vol. 8, p. 62, Potsdam Gr. Founded upon a fragment of a cephalic shield which does not belong to this genus.

Arges, Goldfuss, 1839, Nova Acta Pliys. Acad. Caes. Leop. Nat. Cur. Not American. Arionellus, Barrande, 1852, Syst. Sil. Boh., syn. for Agraulus.

bipunctatus, see Agraulus bipunctatus.

convexus, see Agraulus convexus.

cylindricus, see Agraulus cylindricus.

oweni, see Crepiceplialus oweni.

planus, see Agraulus planus.

pustulatus, see Agraulus pustulatus.

quadrangularis, see Agraulus quadrangularis.

subclavaius, see Agraulus subclavatus.

texanus, see Agrailus texanus.

tripunctatus, see Agraulus tripunctatus.

A ristozoe, Barrande, 1872, Syst. Sil. Boh., vol. 1, p. 477. [Ety, arislos, best; zoon, animal.] Carapace bivalve; test thin; binge-line straiglit; ventral margin grooved and reflected; tuberele near anterior margin. 'Yype A. bisulcata.

rotundata, Walcott, 1887, Am. Jour. Sci. and Arts, 3d ser., vol. 4, p. 193, Up. Taconic.

troyensis, Ford, 1873, (Leperditia troyensis,) Am. Jour. Sci. and Arts, 3d ser., vol. 6. p. 138 . Up. Taconic.

Asapinscus, Meek, 1873 , 6th Rep. Hayden's Geo. Sur. Terr., p. 485 . [Ety. from the genus Asrphus.] Distinguished from Asaphus by having uine thoracic segments, a conical and well-defined glabella, without lateral lobes, the furrow at the anterior margin of the head, and less arcuate eyes more remote from the glabella; distinguished from

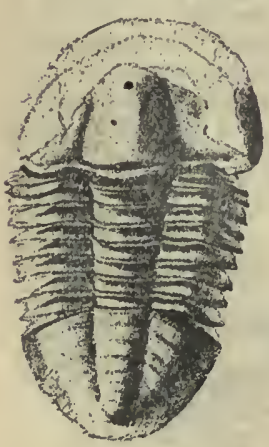

Fra. 965. - Asaphiscus wheeleri.
Bathyurellus by having its conical glabelladepressed, and the margin of the head, in front, first convex, and sloping forward into a transverse mesial furrow, and then rising in a convex maryin; the mesial lobe of the $\mathrm{p} \mathrm{y} \mathrm{g} \mathrm{i} \mathrm{d} \mathrm{i} \mathrm{m}$ is longer,. and the free margins narrower, less flattened and alate. Type A. wheeleri. bradleyi, Meek, 1873, 6th Rep. Hayden's Geo. Sur. Terr., p. 48t, Up. Taconic. wheeleri, Meek, 1873, 6th Rep. Hayden's Geo. Sur. Terr., p. 485, and Geo. Sur. W. 100th Mer., vol. 4, p. 43, Up. Taconic.

Asaphoidichnos, S. A. Miller, 1880, Jour. Cin. Soc. Nat. Hist., vol. 2, p. 217. [Ety. Asaphus, a genus; eidos, form ; ichnos, track.] A track supposed to have been mide by a crustacean. Type A. trifidus.

dyeri, S. A. Miller, 1880, Jour. Cin. Soc. Nat. Hist., vol. 2, p. 219, Utica Slate Gr.

trifidus, S. A. Miller, 1880, Jour. Cin. Soc. Nat. Hist.; vol. 2, p. 218, Utica Slate Gr.

Asapilús, Brongniart, 1822 Hist., Nat. Crust. Foss., p. 17. [Ety. asaphus, uncertain,obscure.] Body sonewhat elliptical, sides straighte ned; ceplialic shield and pygidium nearly equal and somewhat semiel. liptical; glabella contracted between the eyes; eyes large, smooth; facial sutures extending forward and outward in advance of the eyes, and then curving to the middle of the front margin, posteriorly extending obliquely outward, and cutting the posterior margin of the ceplialic shield within the lateral angles; thoracic segments 8 , with wide, nearly straight pleural grooves; axis of pygidium, when traceable, elongate conic, segments usually indistinct. Type A. cornig- Frg. 966.-Asapherus.

acantholeurus, see Dal -

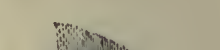

4 manites acan tholeurus.

alacer, Billings, 1866, Catal. Sil. Foss. Antic., p. 26, Hud. Riv. Gr.

aspectans, see Dalmanites aspectans.

astragalotes, Green, 1834, Am. Jour. Sci., vol. 25 , p. 325 . Probably founded upon the pygidium of a Phacops.

barrandi, Hall, 1851, Lake Sup. Land Dist., p. 210, Birdseye Gr.

cánadensis, Chapman, 1856, Can. Jour. vol. 2, p. 47, Trenton Gr.

canalis, Conrad, 1847, Pal. N. Y:, vol. 1, p. 25, Chazy Gr.

caribouensis, Walcott, 1885, Monogr. U.S. Geo. Sur., vol. 8, p. 98, Quebec Gr. 
caudatus, Green, syn. for Dalmanites limulurus. (?)

cordieri, Castelnau, syn. for Dalmanites limulurus.

corysœus, see Proetus corycœns.

crypturus, Green, 1834, Truns. Geo. Soc. Pa., vol. 1, p. 37 . Not an Asaphus; form not determined.

(?) curiosus, Billings, 1865, Pal. Foss., vol. 1, p. 318, Quebec Gr.

denticulatus, see Dalmanites denticulatus. ditmarsix, Honeyman, 1879, Proc. Nova

Scotia Inst., vol. 5, p. 18 , Low. Sil.

diurus, Green, 1839, Am. Jour. Sci., vol. 39, p. 40, Niagara Gr. Probably the fragment of a Dalmanites.

edwardsi, Castelnau, syn. for Dalmanites limulurus.

extans, see Bathyurus extans.

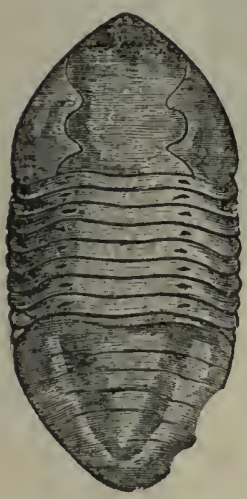

FIG. 967.-A saphus gigas. gigas, Dekay, 1825, (Isotelus gigas, ) Ann. Lyc. Nat. Hist. N. $Y$., vol. 1 , p. 174, and $\mathrm{Pal}$. N. Y., vol. 1, p. 231, Trenton and $\mathrm{Hud}$. Riv. Grs.

(?) goniocercus, Méek, 1873, Hayden's Geo. Sur. 'Terr., p. 480, Quebec Gr. or Up. Taconic. Probabiy a Megalaspis.

(?) goniurus, Billings, 1860, Can. Nat., vol. 5, p. 301, Up. Taconic. Not defined so as to be recognized.

graftonensis, Meek \&

Worthen, 1870, Proc. Acad. Nat. Sci., p. 54, Hud. Riv. Gr.

halli, Conrad, syn. for Dalmanites boothi. halli, Chapman, 1S5̄8, Ann. and Mag. Nat. Hist., 3d ser., vol. "2, p. 14, Trenton Gr.

hausmani, Brongniart, as identified by D'Archiac and Verneuil. Not American.

hincksi, Salter, 1859, Ann. and Mag. Nat. Hist., 3d ser., vol. 4, p. 2, Trenton Gr.

homalonotoirles, Walcott, 1S77, 31st Rep. N. Y. St. Mus. Nat. Hist., p. 71, Trenton Gr.

(?) huttoni, Billings, 1865, Pal. Foss., vol. 1, p. 271, Quebee Gr. or Up. Taconic.

(?) illæenoides, Billings, 1560, Can. Nat. vol. 5 , p. 301 , Up. Taconic.

iowensis, Owen, 1852, Geo. Wis., Iowa, and Minn., p. 577, Trenton Gr.

laticostatus, syn. for Dalmanites anchiops.

(?) latimarginatus, Hall, 18 $17, \mathrm{Pal}$. N. Y., vol. 1. p. 253, Utica Slate Gr.

limulurus, see Dalmanites limulurus.

marginalis, Hall, 1847, Pal. N. Y., vol. 1, p. 24 , Chazy Gr.

megalopthalmus, Troost, 1840, 5th, Geo. Tenn. Niugara Gr. Not clearly defined, but probably a Dalmanites. megistus, Locke, 1841, (Isotelus megistos,) 'Trans. Am. Geo. and Nat., p. 221, Trenton and Hud. Riv. Grs.

micrurus, see Dalmanites micrurus.

(?) morrisi, Billings, 1865, Pal. Foss., vol. 1, p. 272, Quebec Gr. or Up. Taconic. murchisoni, Castelnau, syn. for A. gigas.

myrmecophorus, see Da 1 manites myrmecophorus.

nasutus, see Dalmanites nasutus.

nodostriatus, Hall, 1847, Pal. N. Y., vol. 1, p. 248. Fia.968.-Asaphus megisNot defined so as to establish a species.

notans, Billings, 1866, Catal. Sil. Foss. Antic, p. 24, Hud. Riv. Gr.

obtusus, Hall, 1847, Pal. N. Y., vol. 1, p. 24, Chazy Gr.

pelops, Billings, 1865, Pal. Foss., vol. 1, p. 317, Quebec Gr. or Up. Taconic. Not an A saphus.

platycephalus, Stokes, 1822, Trans. Geo. Soc. Lond., 2d ser., vol 1, p. 258, Trenton Gr.

platypleurus, Green, 1837, Am. Jour. Sci., vol. 32 , p. 169, Low. Sil. Not very crearly defined.

pleuropteryx, see Dalmanites pleuropteryx. polupleurus, Green, 1838, A in. Jour. Sci., vol. 34, Keokuk Gr. 'Probably a Phillipsia.

quadraticaudatus, Billings, 1865, Pal. Foss., vol. 1, p. 272, Quebec Gr. or Up. Taconic. Not an Asaphus.

romingeri, Walcott, 1876,2 Sth Rep. N. Y. Mus. Nat. Hist., p. 96, Black Riv. and Trenton Gr.

selenurus, see Dalmanites selenurus.

stokesi, see Proetus stokesi.

susa, Calvin, 1882, Geo. IVis., vol. 4, p. 236, Trenton Gr.

tetragonocephalus, Green, 1834, Am. Jour. Sci., yol. 25, p. 336 . Not an Asaphus, and the relations not clear.

trentonensis, see Lichas trentonensis.

triangulatus, Whitfield, syn. for. A. homalonotoides.

trimblii, Green, 1837, Jour. Acad. Nat. Sci. Phil., vol. 7, Niagara Gr.

vetustus, Hall, 1847, (Ogygria vetustus,) Pal. N. Y., vol. 1, p. 227, Birdseye Gr.

vigilans, Meek \& IVorthen, 1870, (Isotelus vigilans,) Proc. Acad. Nat. Sci. Phil., p. 53, and Geo. Sur. Ill., vol. 6, p. 497, Hud. Riv. Gr. 
wetherilli, Green, syn. for Dalmanites limulurus.

wisconsinensis, Walcott, 1876, 28th Rep. N. Y. Mus. Nat. Hist., p. 97, Trenton Gr. Aтops, Emmons, 1844, Taconic System, p. 64, and Am. Geol. p. 115. [Ety. $a, \mathrm{ab}$ sence of; ops, an eye.] Cephalic shield semicircular, anterior and lateral edges turned upward, posterior angles rounded, convex; glabella subquadrate, convex, appearing as a continuation of the central lobe, two lateral furrows on each side, neck segment well defined; facial suture beginning at the antero-lateral part of the cephalic shield, runs nearly parallel with the anterior margin to the front of the glabella, when it turns at right angles and runs parallel with the glabella to the posterior margin; no eyes; thoracic segments 17 , axial nearly as wide as the lateral lobes, narrowing gradually to the pygidium, armed with a row of short spines, lateral lobes with a row of tubercles on the median line; pygidinm small, somewhat semielliptical, flat, axial lobe with a single ring. Type A. trilineatus.

fischeri, Billings, 1865, (Triarthrus fischeri,) Pal. Foss., vol. 1, p. 291, Quebec Gr. or Up. Taconic.

miser, Billings, 1861, (Conocephalites miser, Pal. Foss., vol. 1, p. 12, Up. Taconic.

trilineatus, Emmons, 1844, Taconic System, p. 64, and Am. Geol., p. 115, Up. Taconic.

Bailifil, Matthew, 1884, Trans. Roy. Soc.

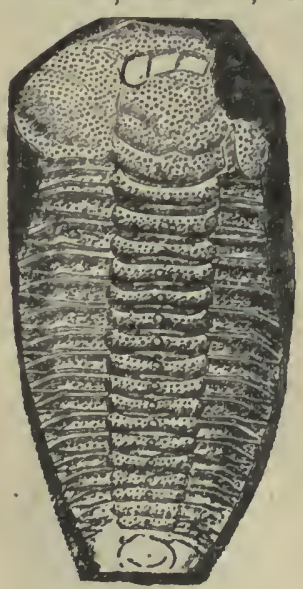

Fig. 969-Atops trilineatus. Can., vol. 2, pl. 1. [Ety. proper name.] Proposed as a subgenus and founded on Conocory phe baileyi.

Barrandia, MT Coy, 1849, Ann. Nat. Hist. 2d ser. vol. $4, \mathrm{p}$. 409. [ Ety. proper name.] Ovate, de pressed; gla. bella with incompleteaxial furrows and no distinct lobes; eyes large, $\mathrm{s} \mathrm{u} \mathrm{b}$ central; facial suture cutting the posterior margin about the middle, and in front of the eyes arching forward, first outward and then inward; pleuræe falcate, with a fulcrum close to the axis, grooved, not faceted; pygidium with short axis and smoothisides. Type B. cordai.

(?) maccoyi, Walcott, 1885, Monogr. U. S. Geo. Sur., vol. 8, p. 96, Trenton Gr.
Barrandia, Hall, 1860. The name was preoccupied by McCoy in 1849; beside, it is a syn. for Elliptocephala.

thompsoni, see Elliptocephala thompsoni. vermontana, see Elliptocephala vermontana.

Bathynotus, Hall, 1860, 3d Rep. N. Y. St. Mus. Nat. Hist., p. 117. [Ety. bathys, ample; notos, back.] Cephalic shield somewhat semielliptical, with posterior

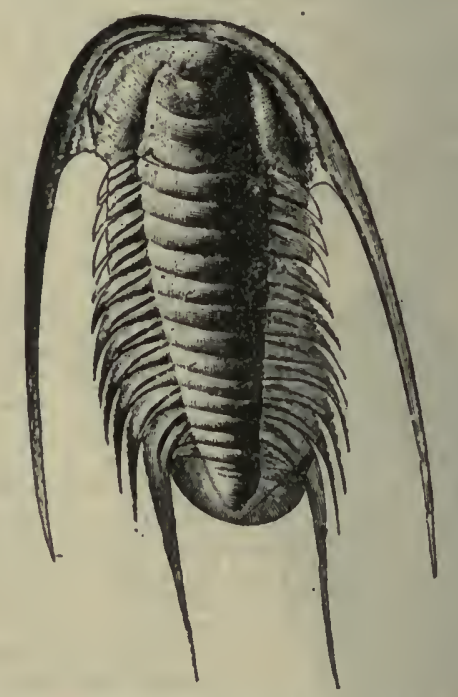

Fia. 970-Bathynotus holopyga. Long eye-lobes crushed down.

angles produced in very long spines; glabella transversely lobed; eye-lobe narrow, elongate, extencling from opposite the antero-lateral angle of the glabella obliquely backward nearly to the posterior margin; facial suture passes nearly around the extended eyelobe, and cuts the margin before reaching the posterior extension of the eyelobe; anteriorly it passes in front of the glabella without, as it appears, cutting, the front margin; free cheeks united in front; thirteen thoracic segments; middle lobe prominent, twice as wide as the lateral lobes; articulations strong, each bearing a central node; pleura short, each terminating in a spine, the last pair being prolonged far beyond the pygidium; pygidium short, middle lobe with three annulations, lateral lobes flat and plain; hypostoma having an obtuse angle; at the front margin of the doublure, the latter being cut away to permit the extension to cross it, behind the doublure it is transversely quadrangular. Type $B$. holopyga.

holopyga, Hall, 1859, (Peltura holopyga,) 12 th Rep. N. Y. St. Mus. Nat. Hist., p. 61 and Pal. N. Y., vol. 3 , p. 528, Up. Taconic. 
Batii yurkllus, Billings, 1865, Pal. Foss, vol. 1, p. 262. [Ety. diminutive of Bathyurus.] Form oblong, ovate; cephalic shield convex, lunate; glabella conical or pointed, without furrows; eyes lunate; facial suture in front of the eye, curving outward, then straight forward or inward on approaching the margin behind the eye, running outward subparallel to the neck furrow, and cutting the margin before reaching the outer angle; thorax, nine segments; axis of pygidium short, not strongly grooved, side lobes with short ribs, and a broad, smooth borderallaround, sometimes concave. Type B. abruptus and B. nitidus. abruptus, Billings, 1865, Pal. Foss., vol. 1, p. 263, Quebec Gr. or Up. Taconic.

brailleyi, Meek, see Asaphiscus bradleyi. expansus, Billinys, 1865, Pal. Foss., vol.1, p. 318, Quebec Gr. or Up. Taconic.

formosus, Billings, 1865, Pal. Foss., vol. 1, p. 266, Quebec Gr. or Up. Taconic.

fraternus, Billings, 1865, Pal. Foss., vol. 1, p. 267, Quebec Gr. or Up. Taconic.

litoreus, Billings, 1865, Pal. Foss., vol. 1, p. 320, Quebec Gr. or Up. Taconic.

marginatus, Billings, 1865, Pal. Foss., vol. 1, p. 264, Quebec Gr. or Up. Tarnnic.

nitidus, Billings, 1865, Pal. Foss., vol. 1, p. 265, Quebec Gr. or Up. Taconic. rarus, Billings, 1865 , Pal. Foss., vol. 1, p. 320, Qnebec Gr. or Up. Taconic. truncatus, Meek, 1873, Hayden's Geo. Sur. Terr., p. 465. Not satisfactorily Fro defined.

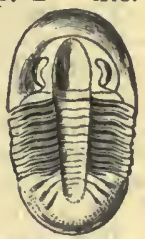
1G. 971. - Bathyurellus nivalidus, Billings, $1865, \mathrm{Pal}$. tidus.

Foss., vol. 1, p. 268, Quebec Gr. or Up. Taconic.

wheeleri, Meek, see Asaphiscus wheeleri.

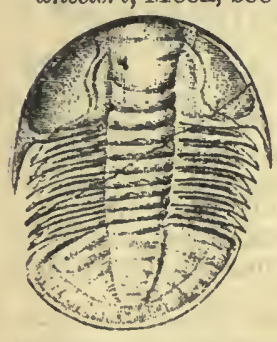

FrG. 972.-Bathyuriscus productus.
Bathyuriscus, Meek, 1873, 6th Rep. Hayden's U. S. Geo. Sur. Terr., p. 484. [Ety. from Bathyurus.] Ovate; head semicircular; glabella straight or slightly expanded in frout, three or four pairs of furrows; eyes elongate; facial sutures cut the anterior margin of the head each side of the greatest expansion of the glabella, and extend obliquely inward to the anterior bases of the eyes; encircling the latter, they extend obliquely outward, cutting the posterior margin so as to leave a narrow, elongate lateral limb; thorax from 7 to 9 segments; axis strong; pleural groove broad; pygidium semicircular, axis strong, aud crossed by several furrows which cross the lateral lobes. Type B. haydeni. haydeni, Meek, 1873, (Bathyurus haydeni,) 6th Rep. Hayden's U. S. Geo. Sur. Terr., p. 482, Up. Taconic.

howelli. Walcott, 1886. Bull. U. S. Geo. Sur. No. 30, p. 216, Up. Taconic.

productus, Hall \& Whitfield, 1877, (Ogygia producta,) Geol. Expl. 40th Par., vol. 4, p. 244, Up. Taconic.

Bathyurus, Billings,-1859, Can. Nat. and Geo., vol. 4, p. 365. [Ety. bathys, deep; oura, tail.] Elliptical, sides straight; cephalic shield lunate, posterior angles produced in spines; ylabella subquadrate, rounded anteriorly, convex, furrows obscure, neck segment distinct; eyes large, smooth, semilunar; facial sutures curving forward anteriorly, and posteriorly directed straight backward from the eye, and then, abruptly curving outward, cut the cephalic shield half-way to the genal angle; nine thoracic segments, axial lobe narrower than lateral lobes, and gradually tapering; pleura furrowed; pygidium smaller than the head, segments closely united, border flattened and smooth; hypostoma not forked. Type B. extans.

amplimarginatus, Billings, 1859, Can. Nat. and Geo., vol. 4, p. 365, Calciferous Gr. angelini, Billings, 1859, Can. Nat. and Geol., vol. 4, p. 468, Chazy Gr.

arcuatus, Billings, 1865, Pal. Foss., vol. 1, p. 205, Quebec Gr. or Up. Taconic.

armatus, Billings, 1860, Can. Nat. and Geo., vol. 5, p. 321 , Quebec Gr. or Up. Taconic.

bituberculatus, Billings, 1860, Can. Nat. and Geo., vol. 5, p. 321, Quebec Gr. or Up. 'I'aconic.

breviceps, Billings, 1865, Pal. Foss., vol. 1, p. 262, Quebec Gr. or Up. Taconic.

capax, Billings, 1860, Can. Nat. and Geol., vol.5, p. 321, Quebec Gr. or Up. Taconic. caudatus, Billings, 1865, Pal. Foss., vol. 1, p. 261, Quebec Gr. or Up. Taconic.

conicus, Billings, 1859, Can. Nat. and Geol., vol. 4, p. 366, Calciferous Gr.

(?) congeneris, Walcott, 1885, Monogr. U. S. Geo. Sur., vol. 8, p. 92, Quebec Gr. or Up. Taconic.

cordai, Billings, 1860, Can. Nat. and Geol., vol. 5, p. 321, Calciferous Gr:

crotaliformis, Dwight, 1884, Am. Jour. Sci. and Arts, 3d ser., vol. 27, p. 253; Calciferous Gr.

cybele, Billings, 1859, Can. Nat. and Geol., vol. 4, p. 366, Calciferous Gr.

dubius, Billings, 1860 , Can. Nat. and Geo., vol. 5, p. 321, Quebec Gr. or Up. Taconic.

extans, Hall, 1847, (Asaphus (?) extans,) Pal. N. Y., vol. 1, p. 228, Lower Trenton $\mathrm{Gr}$.

gregarius, Billings, 1865, Pal. Foss., vol. 1, p. 363, Up. Taconic.

haydeni, see Bathyuriscus haydeni.

longispinus, Walcott, 1876, 28th Rep. N. Y. St. Mus. Nat. Hist., p. 94, Black River and Trenton Grs. 
minganensis, Billings, 1865, Pal. Foss., vol. 1 , p. 353, Calciferous Gr.

nero, Billings,' 1865, Pal. Foss., vol. 1, p. 260, Quebec Gr. or Up. Taconic.

oblongus, Billings, 1860,Can. Nat. and Geo., vol. 5, p. 321, Queluee Gr. or Up. Taconic. parvulus, Billings, 1861, Pal. Foss., vol. 1, p. 16, Up. Taeonic.

perplexus, Billings, 1865, Pal. Foss., vol. 1, p. 36t, Potsdam Gr. or Up. Taconic. perspicator, Billings, 1865, Pal. Foss., vol. 1, p. 205, Quebec Gr. or Up. Taconic. pogonipensis, Fall \& Whitfield, 1877, U. S. Geo. Expl. 40th Parallel, vol. 4, p. 243, Quebec Gr. or Up. Taconic.

quadratus, Billings, 1860, Can. Nat. and Geo., vol. 5, p. 320, Quebec Gr. or Up. Taconic.

saffordi, Billings, 1860, Can. Nat. and Geol., vol. 5, p. 321, Quebee Gr. or Up. Taconic.

seelyi, Whitfield, 1886, Bull. Am. Mus.

Nat. Hist., vol. 1, p. 339, Birdseye Gr. senectus, Billings, 1861, Pal. Foss., vol.

1, p. 15 , Up. Taconic.

serratus, Meek, 1873, 6th Rep. Hayden's

Geo. Sur. Terr., p. 480, Potsdam Gr. or Up. Taconic.

? simillimus, Walcott, 1885, Monogr. U. S. Geo. Sur., vol. 8, p. 93, Quebee Gr. or Up. Taconic.

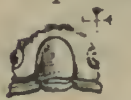

FIG. 973.

Baihyurus smitht. smithi, Billings, 1862, Pal. Foss., vol. 1, p. 56, Black Riv. Gr.

solitarius, Billings, 1865, Pal. Foss., vol. 1, p. 362, Up. Taconic.

spiniger, Hall, 1847, (Aeidaspis spininger, ) Pal. N. Y., vol. $1, p$. 241, Black River and Trenton Gr.

stonemani, Vogdes, 1884, 12tl Rep. Geo. and Nat. Hist. Minn., p. 8, Trenton Gr. strenuus, Billings, 1865, Pal. Foss., vol. 1, p. 204, Quebec Gr. or Up. Taconic. taurifrons, Dwight, 1884, Am. Jour. Sei. and Arts, 3d ser., vol. 27, p. 252, Calciferous $\mathbf{G r}$.

timon, Billings, 1865, Pal. Foss., vol. 1, p. 261, Quebec Gr. or Up. Taconic.

? tubereulatus, Walcott, 1885, Monogr. U. S. Geo. Sur., vol. 8, p. 91, Quebec Gr. or Up. Taconic.

vetulus; Billings, 1865, Pal. Foss., vol. 1, p. 365, Potsdam Gr. or Up. Taconic.

Belinurus, Konig, 1825, Icones Fossilium Sectiles, p. 230. [Ety. belos, dart; oura, tail.] Cephalo-thoracic shield sub. crescentiform, more than twice as wide as long, lateral angles pointed; ocular ridge surrounds a transversely subelliptical area, within which there is a crown-shaped area, surrounded by a ridge; eyes snall, and at the lateral extremities of the subelliptical area; mesial lobe narrow, and contracted toward each end; lateral lobes wide, flattened on the margin and serrate on the edge; telson tapering to a point. Type B. bellulus.

danx, see Euproops danæ.

lacoi, Packarò, 1885, Am. Naturalist, vol. 19 , p. 291, Coal Meas.

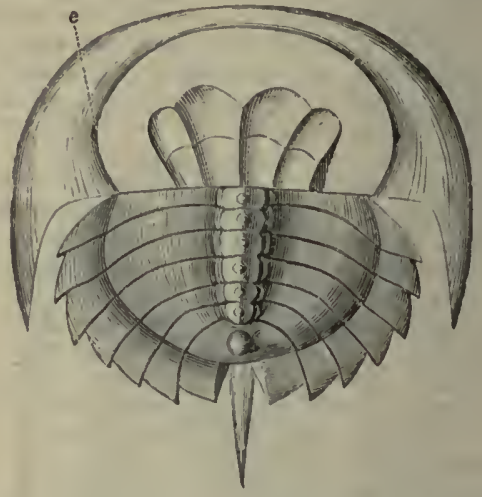

Fig. 974.-Belinurus belluius. $e$ Position of eye, at the lateral extremity of a transversely elliptical area.

Beyriciris, McCoy, 1844, Syn. Sil. Foss. Ireland, p. 57. [Ety. proper name.] Carapace equivalve, oblong, extremities rounded, ventral border semicircular, dorsal struight; valves wider at the caudal than the cephalic extremity, more or less convex, impressed with transverse furrows. Type B. klœdeni. sequilatera, Hall, 1860, Can. Nat. and Geo., vol. 5, p. 158, and Acad. Geol., p. 609 , Up. Silurian.

americana, Shumard, 1858, (Cythere americana,) Trans. St. Louis Acad. Sei., vol. 1, p. 227, Up. Coal Meas.

arcuata, Bean, 1886, Lond. Geo. Mag., p. 438, Low. Held. Gr.

atlantica, Billings, 1865, Pal. Foss., vol. 1, p. 300, Quebec Gr. or Up. Taconic.

bella, Waleott, 1883, 35th Rep. N. Y. St. Mus. Nat. Hist., p. 213, Trenton Gr.

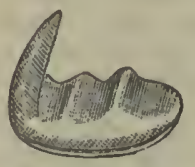

F I G. $975 .-13$ \& rich1a cham bers1. Mag. 12 diam. chambersi, S. A. Miller, 1874, Cin. Quar. Jour. Sci., vol. 1, p. 234, Hud. River Gr. eiliata, Emmons, 1855 , American Geo., p. 219, Hud. Riv. Gr.

cincinnatiensis, see Primitia cincinnatiensis.

clathrata, Jones, 1858, Ann. and Mag. Nat. Hist.; $3 \mathrm{~d}$ series, vol. 1, p. 242, Niagara Gr.

d e co ra, Billings, 1866, Catal. Sil. Foss. Antic., p. 67, Anticosti Gr. dagon, Clarke, 1855 , Bull. U. S. Geo.

Sur., No. $16, \mathrm{p}$. 29, Ge ne se e yi. Magulfied 25 diam. Shale.

duryi, s. A. Miller, 1874, Cin. Quar. Jour. Sci., vol. 1, p. 232, Hud. Riv. Gr. 
fotoidea, White \& St. John, 1868, Trans. Chi. Acad. Sci., p. 126, Up. Coal Meas. granulosa, Hall, 28th Rep. N. Y. St. Mus. Nat. Hist., p. 186, Niagara Gr.

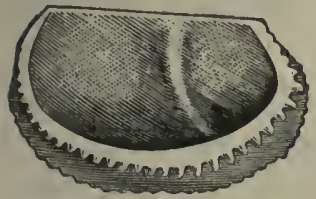

FIG. 977.-B e y ric h i a duryi. Interinr of left v a i ve, magnifled 25 diam. granulata, Hall, 1859, Pal. N. Y., vol. 3 , p. 377 , Low. Held. Gr. jonesi, Dawson, 1868 , A c a d. Geol., p. 312, Carboniferous.

kloedeni var. acadica, 1889, Ann. and. Mag. Nat.

Hist., 6th ser., vol. 3, p. 379, Low. Held. Gr.

lata, Vanuxem, 1842, (Agnostus latus,) Geo. Rep. N. Y., p. 80, and Pal. N. Y., vol. 2, p. 301, Clinton Gr.

lithofactor, White \& St. Jobn, 1868, Prelim. Notice of New Foss., Coal Meas.

logani, see Primitia logani.

logani var. leperditoides, see Primitia leperditoides.

logani var. reniformis, see Primitia reniformis.

maccoyana, Jones, 1855, Ann. and Mag. Nat. Hist., 2 d ser., vol. 16, p. 88, Onondaga Gr.

notata, Hall, 1859, Pal. N. Y., vol. 3, p. 379, Low. Held. Gr.

notata var. ventricosa, Hall, 1859, Pal. N. Y., vol. 3, p. 380, Low. Held. Gr.

novascotia, Jones \& Kirby, 1884, Lond. Geo. Mag., 3d ser., vol. 1, p. 356, Carboniferous.

occidentalis, Walcott, 1885, Monogr. U. S. Sur., vol. 8, p. 204, Devonian.

oculifera, Hall, 1871, 24th Rep. N. Y. St. Mus. Nat. Hist., p. 232, Hud Riv. Gr. oculina, Hall, 1859, Pal. N. Y., vol. 3, p. 378, Low. Held. Gr.

pennsylvanica, Jones, 1858, Ann. and Mag. Nat. Hist., $3 \mathrm{~d}$ ser., vol. 1, p. 253, Onondaga Gr.

persulcata, Ulrich, 1879, Jour. Cin. Soc. Nat. Hist., vol. 2, p. 12, Hud. Riv. Gr. petrifactor, White \& St. John, 1868, Trans. Chi. Acad. Sci., p. 125, St. Louis Gr.

petrifactor var. velata, White \& St. John, 1868, Trans. Chi. Acad. Sci., p. 126, St. Louis Gr.

plagosa, Jones, 1858, Ann. and Mag. Nat. Hist., $3 \mathrm{~d}$ ser., vol. 1, p. 243, Niagara Gr. punctulifera, Hall, 1862, 15th Rep. N. Y. St. Mus. Nat. Hist., p. 83 , Ham. Gr.

pustulosa, Hall, 1860, Can. Nat. and Geo., vol. 5, p. 157, and Acad. Geol., p. 609, Up. Silurian.

quadrilirata, Hall \& Whitfield, syn. for Beyrichia regularis.

regularis, Emmons, 1855,

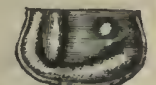

Fra. 978. - Beyrichia richardsoni. Mag. $61 / 2$ diam.

Am. Geo., p. 219, Hud. Riv. Gr.

richardsoni, S. A. Miller, 1874, Cin. Quar. Jour. Sci., vol. 1, p. 347, Hud. Riv. Gr. rugulifera, see Primitia rugulifera.

sigillata, see Primitia sigillata.

spinosa, Hall, 1852, (Cytherina spinosa,)

Pal. N. Y., vol. 2, p. 317, Niagara Gr.

striato-margin-

ata, S. A. Mil-

ler, 1874, Cin.

Quar. Jour.

Sci., vol. 1, p.

233, $\mathrm{H} \mathrm{u} \mathrm{d}$.

Riv.Gr. This

species prob-

ably belongs FrG. 979.-Beyrichia striatoto an unde- marginata. Mag. 20 diam. fined genus.

symmetrica, Hall, 1852, Pal. N. Y., vol. 2, p. 317 , Niagara Gr.

trisulcata, Hall, 1859, Pal. N. Y., vol. 3, p. 381, Low. Held. Gr.

tumifrons, Hall, syn. for Beyrichia ciliata. venusta, Billings, 1868, Catal. Sil. Foss. Antic., p. 68. Anticosti Gr.

Beyrichona, Matthew, 1885, Trans. Roy. Soc. Can., p. 65. [Ety. from the genus Beyrichia.] Breadth and length nearly equal, broad end anterior, subtrigonal toward the base, rounded on the surface and having two furrows, short and faintly impressed. Type B. papilio.

papilio, Matthew, 1885, Trans. Roy. Soc. Can., p. 65, St. John Fr.

tinea, Matthew, 1885, Trans. Roy. Soc. Can., p. 66, St. John Gr.

Brongniartia, Eaton, 1832, Geo. Text Book, syn. for Asaphus.

Bronteus, Goldfuss, 1839, Nova. Act. Phys. Med. Cresarere Leop. Carol. Nat. Curios, xix, p. 360 . [Ety. mythological name.] Glabella depressed, ovate, widest in front, three pair of segmental furrows, anterior ones farthest apart; eye-line proceeding upward from the middle of each side of the posterior margin, with a short, sigmoidal curve to the eye-lobe, and thence curving inward and forward to the front; thorax of ten segments, axial lobe equaling the lateral lobes in width, lateral lobes flat, without facets, bent backward at the tip, no pleural groove ; pygidium semiorbicular with a flattened entire margin, axial lobe short, sulci prolonged toward the margin, lateral folds broad, not reaching the margin.

B. altaceus.

acamas, Hall, syn. for B. occasus.

barrandi, Hall, 1859, Pal. N. Y., vol. 3 , p. 350 , Low. Held. Gr. canadensis, Logan, 1846, Rep. Ġeo.

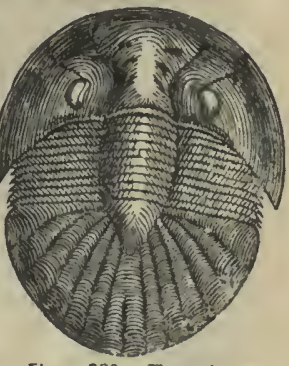

F'IG. 980.-Bronteus iunatus.
Sur. Canada, App. G. G. G. of Legislative Documents, Low. Held. Gr. 
flabellifer, Goldfuss, Nova. Acta. Acad. Caes. Leop. Nat. Cur., vol. 16, p. 360, Up. Silurian.

insularis, Billings, 1866, Catal. Sil. Foss. Antic., p. 66, Anticosti Gr.

laphami, Whitfield, 1878, Ann. Rep. Geo. Sur. Wis., p. 88, and Geo. Wis., vol. 4, p. 310, Niagara Gr.

lunatus, Billings, 1857, Rep. of Progr. Geo.

Sur. Can., p. 338, Trenton Gr.

niagarensis, Hall, 1852, Pal. N. Y., vol. 2, p. 314, Niagara Gr.

occasus, IVinchell \& Marcy, 1865, Mem.

Bost. Soc. Nat. Hist., vol. 1, p. 104, Niagara $\mathrm{Gr}$.

pompilins, Billings, 1863, Proc. Port. Soc.

Nat. Hist., vol. 1, p. 123, Low. Held. Gr. tullius, Hall, 1885, Pal. N. Y., vol. 7, p. 12, Ham. Gr.

Bumastus, Murchison, 1839, Sil. Syst. Not American, though I lave illustrated the gemus because so many have referred Illenus to it.

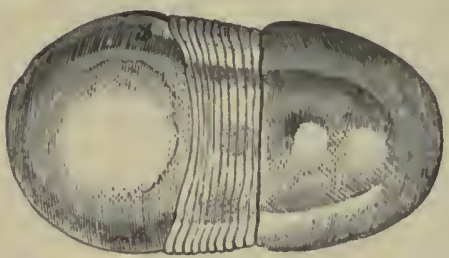

Fio. 981.-Bumastus barriensis.

barriensis, see Illænus ioxus.

trentonensis, see Illanus trentonensis.

Bunodella, Mattliew, 1888, Trans. Roy. Soc. Can., p. 56. Body ovate-elongate, trilobed longitudinally; cephalic shield subtriangular, with rounded angles; composerl of a glabella, fixed cheeks and movable (?) cheeks; glabella loroadly cylindrical and rounded in front; fixed cheeks expanded in front, and having ear-shaped lateral lobes defined by an impressed line which may have been movable; thorax, so far as known, had seven -segments, and consisted of an elongate cylindro-conical body, having triangular lappets or pleuræ attached at the sides. Type B. horrida.

horrida, Matthew, 1888, Trans. Roy. Soc. Can., p. 56, Up. Silurian or Low. Devonian.

Calymene, Brongniart, 1822, Hist. Nat. Crust. Foss., p. 7. [Ety. kekalymenos, concealed.] Cephalic shield sublunate, margin thickened, distinctly defined; glabella convex, narrower in front than behind, three lateral furrows on each side, the posterior one deep, neck segment well defined, eyes, small, prominent, hiant, near the glabella furrows, and slightly anterior to the middle; facial sutures cut the margin, in front of the eyes and curving slightly over each eye, defining a semicircular eye-lobe. they extend to the lateral angles, eac:l of which is exactly bisected; anteriorly they are connected by a rostral suture, thorax of thirteen segments, axis most convex, lateral lobes wider than axis, bent down with $\operatorname{large}$ facets; pygidium semi-oval, axis prominent, seven to eleven segments, margin entire. Type C. blumenbachi. anchiops, see Dalmanites anchiops.

becki, see Triarthrus becki.

blumenbachi, Brongniart, 1822, Hist. Nat. Crust. Foss., p. 11. American form called C. niagarensis.

bucklandi, syn. for Ceraurus pleurexanthemus.

bufo, see Phacops bufo.

callicephala, Green, 1832, Monograph Trilobites, p. 30 , and Pal. N. Y., vol. 1 , p. 238, Trenton and Hud. Riv. Grs.

camerata, Conrad, 1842, Jour. Acad. Nat. Sci., vol. 8, p. 278, and Pal. N. Y., vol. 2 , p. 337 , Coralline limestone.

christyi, Hall, 1860, 13th Rep. N. Y. St. Mus. Nat. Hist., p. 119, Hud. Riv. Gr.

clintoui, Vanuxem, (Hemicrypturus clintoni,) Gen. Rep. 3d Dist. N. Y., p. 79, Clinton Gr.

conradi, Emmons, 1856, Am. Geol., p. 236, Hud. Riv. Gr.

crassimarginata, see Proetus crassimarginatus.

mammillata, Hall, 1861, Geo. Rep. Wis., p. 50, Trenton Gr.

marginalis, see Proetus marginalis.

multicosta, Hall, 1847, Pal. N. Y., vol. 1, p. 228, Birdseye and Trenton $\mathrm{Gr}$.

nasuta, Ulrich, 1879 , Jour. Cin. Soc. Nat. Hist., vol. 2, p. 131, Niagara Gr.
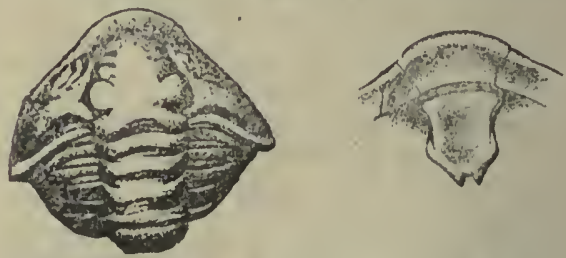

FIG. 982.-Calymene callicephala. Rolled speclmen and the under side of cephallc shleld with hy postoma in place.

niagarensis, Hall, 1843, Geo. Rep. 4th Dist. N. Y., p. 102, and Pal. N. Y., vol. 2 p. 307, Niagara Gr. This is the American variety of $\mathrm{C}$. blumenbachi. nupera, sce Phacops nupera.

odontocephala, syn. for Dalmanites selenurus.

phlyctainodes, see Encrinurus phlyctainodes.

platys, Green, 1832, Monograph of Trilobites, p. 32, and Illust. Devon. Foss., pl. 1, Schoharie grit.

rostrata, Vogdes, 1880, Proc. Acad. Nat. Sci., p. 176, Clinton Gr.

rowii, see Proetus rowii.

rugosa, Shumard, 1855, Geo. Rep. Mo., p. 200, Low. Held. Gr. 
senaria, Conrad, 1841, syn. for C. callicephala.

spinifera, not defined.

trisulcata, Hall, 1843, Gep. Rep. 4th Dist. N. Y., p. 74, Clinton Gr.

Candona, Baird, 1845, Trans. Berw. Nat. Club, vol. 2, p. 152. A living genus, and probably not Palæozoic. Like Cypris, except the lower antennæ possess no tuft of seta, and the second pair of jaws are destitute of a branchial appendage. The shell is also usually longer and narrower. Type C. lucens.

(?) elongata, Jones \& Kirby, 1884, Lond. Geo. Mag., 3d ser., vol. 1, p. 356, Carboniferous.

Ceratiocaris, McCoy, 1849, Ann. and Mag. Nat. Hist., 2d ser., vol. 4, p. 412. [Ety. keration, pod; karis, shrimp,] Carapace bivalve, dorsal line angulated simplex, Clarke, 1885, Bull. U. S. Geo. Sur., No. 16, p. 43, Ham. Gr.

sinuata, Meek \& Worthen, 1868, Am. Jour. Sci., vol. 46 , p. 22 , and Geo. Sur. Ill., vol. 3, p. 540, Coal Meas.

strigata, see Solenocaris strigata.

Ceratocephala, Warder, not defined so as to be recognized.

ceralepta, Anthony, a fragment of the tail of a Ceraurus pleurexanthemus, or of an Acidaspis.

goniata, Warder, a fragment of a Dalmanites, or an Acidaspis.

Cekaurus, Green, 1832, Monograph Trilobites, p. 84. [Ety. keras, horn; oura, tail.] Cephalic shield crescentiform, trilobed, posterior angles extended into spines; glabella subquadrate, rounded and prominent in front, three lateral furrows on each side; eyes faceted minutely; facia l sutures, commencing at the anterior m a rg in, passing close to the anterior corners of the glabella curve around the eyes, from which points they extend outward, and then deflect a little backward, and cut the lateral margins forward of the neck furrow, if it were extended; cheeks generally scrobiculate; thorax of ten or twelve segments, axial lobe narrower than the

FIG. 983.-Ceratiocaris sinuala. Outliue. with a slight furrow beneath it on each side; sides semielliptical, much elongated from belore backward, evenly convex, ventral margin gently convex, posterior end truncated obliquely; on each side near the anterior end, low down, is an ocular spot; surface marked with fine, imbricating strix. Type C. solenoides.

aculeata, Hall, 1859, Pal. N. Y., vol. 3, p. 422, Waterlime Gr.

acuminata, Hall, 1859, Pal. N. Y., vol. 3, p. 422, Waterlime Gr.

armata, syn. for Echinocaris punctata.

bradleyi, see Colpocaris bradleyi.

beecheri, Clarke, 1885, Bull. U. S. Geo.

Sur., No. 16, p. 44, Ham. Gr.

deweyi, Hall, 1859, (Onchus deweyi,) Pal. N. Y., vol. 2, p. 320, Niagara Gr.

elytroides, see Colpocaris elytroides.

grandis, Pohlman, 1881, Bull. Buf. Soc.

Nat. Hist., vol. 4, p. 19, Waterlime Gr. longicauda, see Echinocaris longicauda. maccoyana, Hall, 1859, Pal. N. Y., vol. 3 , p. 421 , Waterlime Gr. punctala, see Echinocaris punctata. pusillus, Matthew, 1889, Trans. Roy. Soc.

Can., vol. 6, p. 49, Low. Held. Gr. lateral lobes; pleura flattened for a distance, and then curve downward and backward; pygidium small, segments terminating in digitations or spines; labrum oblong truncate, with a pair of furrows and small lateral auricles. Type C. pleurexanthemus.

(?) apollo, Billings, 1860, (Cheirurus apollo,) Can. Nat. and Geol., vol. 5, p. 67, Quebec Gr. or Up. Taconic.

bimucronatus, see Ceraurus niagarensis. crosolus, see Acidaspis crosotus.

i?) eryx. Billings, 1860, (Cheirurus eryx,) Can. Nat. and Geol., vol. 5, p. (j7, Quebec Gr. or Up. Taconic.

(?) glancus, Billings, 1865, (Cheirurus glaucus,) Pal. Fors., vol. 1, p. 323, Quebec Gr. or Up. Taconic.

icarus, Billings, 1860, (Cheirurus icarus,) Can. Nat. and Geol., vol. 5, p. 67, Hud. Riv. Gr.

insignis, see Ceraurus niagarensis.

meekanus, n. sp., Hud. Riv. Gr. Proposed instead of $\mathrm{C}$. icarus, Meek, in Ohio Pal., vol. 1, p. 162, and plate 14, figs. $a, b$, and $c$. Meek referred this form to C. icarus of Billings, but it is distinguished by the form of the gla- 
bella, by the furrows, structure of the thorax and form of central lobe, and by the pygidium, beside occurring in higher rocks and growing to a much larger size.

(?) mercurius, Billings, 186̉5, (Cheirurus mercurius,) Pal. Foss., vol. 1, p. 285, Quebec Gr. or Up. Taconic.

niagarensis, Hall, 1867, 20th Rep. N. Y. St. Mus. Nat. Hist., p. 427, Niagara Gr. numitor, Billíngs, 1866, (Cheirurus numitor,) Catal. Sil. Foss. Antic., p. 27, Hud. Riv. Gr.

nuperus, Billings, 1866, (Cheirurus nuperug,) Catal. Sil. Foss. Antic., p. 60 , Anticosti Gr.

(?) perforator, Billings, 1865, (Cheirurus perforator,) Pal. Foss., vol. 1, p. 287, Quebec Gr. or Up. Taconic.

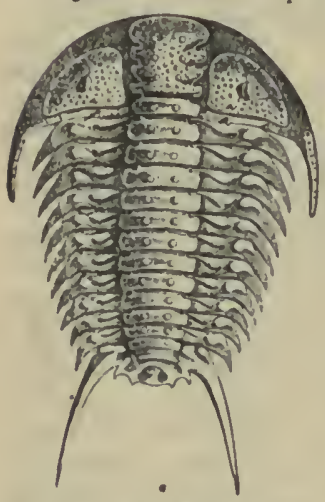

FIG. 984. - Ceraurus pleurexanthemus. ple u rexanthe. mil 11 , Green, 1832, M on og. Trilobites, p. 84 , and Pal. N. Y., vol. 1, p. 242, Tre ut ton and Hud. Riv. Gr.

(?) poly d or $11 \mathrm{~s}$, Billings, 1865, (Cheirurus poly dor u 8, ) Pal. Foss., vol. 1, p. 286, Quebec Gr. or Up. Taconic.

pompilius, Billings, 1865, Cheirurus pompilius,) Pal. Foss., vol. 1. p. 181, Chazy or Black Riv. Gr.

(?) prolificus, Billings, 1865, (Cheirurus prolificus,) Pal. Foss., vol. 1, p. 285 and 325, Quebec Gr. or Up. Taconic.

pustulosus, syn. for Ceraurus pleurexanthemus.

rarus, Walcott, 1877, 31st Rep. N. Y. St. Mus. Nat. Hist., p. 68, Trenton Gr.

satyrus, Billings, 1865 , (Cheirurus satyrus,) Pal. Foss., vol. 1, p. 324, Chazy Gr.

(?) sol, Billings, 1865, (Cheirurus sol,) Pal. Foss., vol. 1, p. 288, Quebec Gr. or Up. Taconic.

(?) solitarius, Billings, 1865, (Cheirurus solitarius,) Pal. Foss., vol. 1, p. 206, Quebec Gr. or Up. Taconic.

tarquinius, Billings, 1S63, (Cheirurus tarquinius, Proc. Port. Soc. Nat. Hist., vol. 1, j. 121, Upper Silurian.

vigilans, see Encrinurus vigilans.

(?) vulcanus, Billings, 1865, (Cheirurus vulcanus,) Pal. Foss., vol. 1, p. 284, and 324 , Quebec Gr. or Up. Taconic.

Chariocephalus, I Iall, 1863, 16th Rep. N. Y. St. Mus. Nat. Hist., p. 175. [Ety. charis, charming or graceful; kephate, liead.] Cephalic shield broad; cheeks moderately convex toward the eyes; glabella regularly convex and marked by transverse furrows; eyes large, facial sutures cutting the contour of the front at or near the center as in Agraulus, but distinguished by the character of the palpebral lobe, large eye, and form of the cheek. Type C. whitfieldi.

tumifrons, Hall \& IVhittield, 1877, U. S. Geo. Expl., 40th parallel, vol. 4, p. 224, Potsdan Gr.

whitfieldi, Hall, 1863, 16th Rep. N. Y. St. Mus. Nat. Hist., p. 175, Potsdam Grr.

Cheirums, Beyrich, 1845, syn. for Ceraurus. apollo, see Ceraurus apollo.

eryx, see Ceraurus eryx.

glaucus, see Ceraurus glaucus. icarus, see Ceraurus icarus. mercurius, see Ceraurus mercurius. numitor, see Ceraurus numitor. nuperus, see Ceraurus nuperus. perforalor, see Ceraurus perforator. polydomes, see Ceraurus polydorus. pompilius, see Ceraurus pompilius. prolificus, see Ceraurus prolificus. satyrus, see Ceraurns satyrus. sol, see Ceraurus sol. solitarius, see Ceraurus solitarius. tarquinius, see Ceraurus tarquinius. vulcanus, see Ceraurus vulcanus.

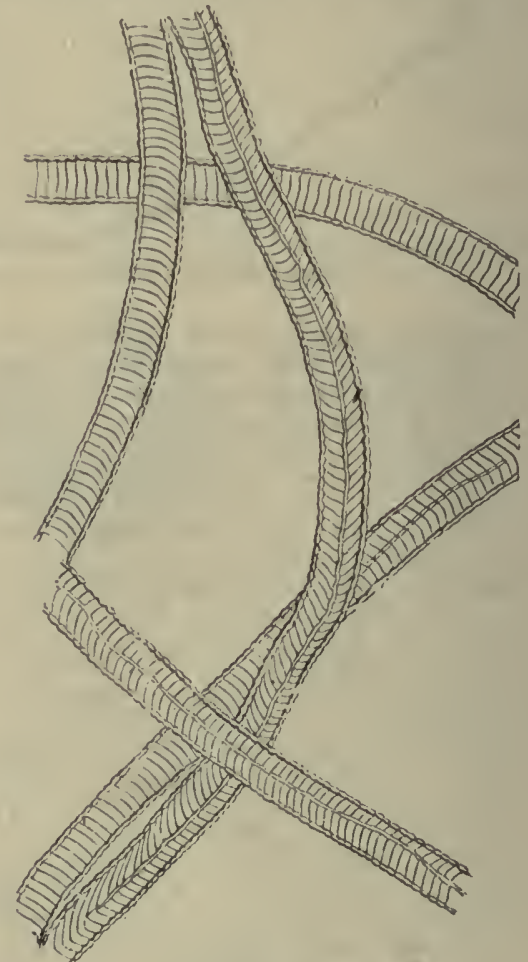

FiG. 985,-Cimachtichnites wllsoni.

Clima chtichnites, Logan, 1860, Can. Nat. and Geol., vol. 5, p. 279. [Ety. klimax, ladder; ichnos, footstep.] A continuous 
narrow trail, with cross furrows, making it ladder-like. It may not be the track of a Crustacean. Type C. wilsoni.

fosteri, Chamberlin, 1883, Geo. of Wis., vol. 1, p. 132, Potsdam Gr.

wilsoni, Logan, 1860, Can. Nat. and Geol., vol. 5, and Geo. of Can., p. 107, Potsdam Gr.

youngi, Chamberlin, 1883, Geo. of Wis., vol. 1, p. 132, Potsdam Gr.

Colpocaris, Meek, 1872, Proc. Acad. Nat. Sci. Phil., p. 323 . [Ety. kolpos, sinus; karis, shrimp.] Carapace valves are truncated backward and upward, with a profoundly sinuous outline; posterior extremity of the dorsal margin produced, pointed and curved downward; ventral margin inflected; attached on the dorsal margin by a flexible ligament; no eye tubercle or spot. Trype C. bradleyi.

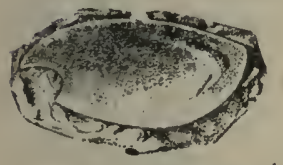

EIG. 986. - Colpocaris bradleyí bradleyi, Meek, 1872, Proc. Acad. Nat. Sci. Phil., vol. 24, p. 322 , and Ohio Pal., vol. 2, p. 318 , Waverly Gr.

chesterensis, IV or then, 1884, Bull. No. 2, Ill. St. Mus. Nat. Hist., p. 3, and Geo. Sur. Ill., vol. 8 , p. 153 , Kaskaskia Gr.

elytroides, Meek, 1872, Proc. Acad. Nat. Sci. Phil., vol. 24, p. 334 , and Ohio Pal., vol. 2, p. 319, Waverly Gr.

Conocephalus, Zenker, 1833, Beitr. z. Naturg. d. Urwelt, p. 49. Preoccupied for a genus of Orthoptera.

Conocephalites, Barrande, 1852, Syst. Sil. Boh. This genus is regarded as a synonym for Ptychoparia, by Walcott, who also refers Atops, which has priority, to the same genus. After examining the original figure of Ptychoparia, I am not convinced that it is a synonym for Atops, hence I retain both names. There were but few genera better defined and established than Atops, when the name was put forth by Emmons; hence the rules of priority demand its retention.

adamsi, see Conocoryphe adamsi.

anatinus, see Ptychoparia anatina.

antiquatus, see Ptychoparia antiquata.

arenosus, see Ptychoparia arenosa.

aurora, see Liostracus aurora.

baileyi, see Conocoryphe baileyi.

billingsi, see Ptychoparia billingsi.

binodosus, see Ptychoparia binodosa.

calciferus, see Ptychoparia calcifera.

calymenoides, see Ptychoparia calymenoides.

cordilleræ, see Ptychoparia cordilleræ.

depressus, see Ptychoparia depressa. diadematus, see Ptychoparia diademata. elegans, see Conocoryphe elegans.

eos, see Crepicephalus eos.

eryon, see Ptychoparia eryon. explanatus, see Ptychoparia explanata.

formosus, see Solenopleura formosa.

gemini-spinosus, see Conocoryphe geminispinosa.

halli, see Solenopleura halli.

hartti, see Ptychoparia hartti.

laticeps, see Pterocephalia laticeps.

matthewi, see Harttia matthewi.

minor, see Ptychoparia minor.

minutus, see Ptychoparia minuta.

miser, see Atops miser.

nactus, Hall. Not defined so as to be recognized.

nasutus, see Ptychoparia nasuta.

neglectus, see Liostracus neglectus.

optatus, see Ptychoparia optata.

orestes, see Solenopleura orestes.

ouangondianus, see Liostracus ouangondianus.

oweni, see Ptychoparia oweni.

pattersoni, see Ptychoparia pattersoni.

perseus, see Ptychoparia perseus.

quadratus, Hartt, see Liostracus quadratus.

quadratus, Whitfield, see Ptychoparia quadrata.

robbi, see Solenopleura robbi.

shumardi, see Ptychoparia shumardi.

subcoronatus, see Ptychoparia subcoronata.

tener, see Liostracus tener.

teucer, see Ptychoparia teucer.

thyrsiles, see Solenopleura thyrsites.

verrucosus, see Ptychoparia verrucosa.

vulcanus, see Crepicephalus vulcanus.

winona, see Ptychoparia winona.

zenkeri, see Ptychoparia zenkeri.

Conocory pire, Corda, 1847, Prodrom einer Monographie der bohmischen Trilobiten, p. 139. [Ety. konos, cone; koryphe, top of the head.] Cephalic shield somewhat semicircular, convex; glabella convex, somewhat cone-shaped, widest behind, rounded in front, from one-half to three-fourths the length of the head, lateral furrows from one to three on each side, more or less distinct; facial sutures cut obliquely across the margin from about the beginning of the lateral third, and curve around the eyes, and then curve outward toward the posterior angles ; (in C. sulzeri and as described by Corda, the facial sutures begin near the apex directly in front of the eyes, and are directed in lines nearly parallel to the eye-lobes.) Thorax eight to sixteen segments, axial lobe narrower than lateral lobes, pleuræ faceted; pygidium small. Type C. sulzeri.

adamsi, Billings, 1861, (Conocephalites adamsi,) Geo. Vt., vol. 2, p. 950, Up. Taconic or Georgia Gr.

baileyi, Hartt, 1868, (Conocephalites baileyi,) Acad. Geol., p. 645, St. John G'r. elegans, Hartt, 1868, (Conocephalites elegans,) Acad. Geol., p. 650, St. John Gr. gallatinensis, Meek, 1873, 6th Rep. Hayden's U. S. Geo. Sur. Terr., p. 4S5, Up. Taconic. 
geminispinosa, Hartt, 1868, (Conocephalites geminispinosus,) Acad. Geol., p. 653 , St. John Gr.

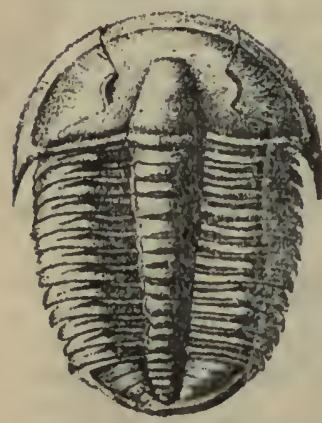

F19. 987.-Conocoryphe Can., p. 119, St. John Gr.

kingi, M e e k, 1870 , P r o c Acad. Nat. Sci. Phil., vol. 22, p. 63, and Rep. on 40th Parallel, p. 20, Up. Taconic.

quadrans, Hall \& Wlit field, 1877, (Crepicephalus quadrans, Geo. 40th Parallel, vol. 4, p. 238, Up. Tuconic.

walcotti, M a t thew, 1884 , 'Trans. Roy.Soc.

Coronura, Hall, 1888, Pal. N. Y., vol. 7, p. 32. Founded upon the variation in the spines of the pygidium of Dalmanites.

Corycephalus, Hall, 1888, syn. for Dalmanites.

Crepicepilales, Owen, 1852, Geo. Sur. Wis. Iowa, and Minn., p. 376 . [Ety. krrpis, borseshoo; kephale, head.] Glabella rather flat, slipper-shaped, tupering and slightly acuminated anteriorly, with a faint ridge in the median line; two small depressions, and a posterior furrow partially divide the glabella; facial sutures run nearly parallel to the margin of the glabella, and join a thickened, cord-like, anterior, narrow border, inclosing a convex area, narrower in front than at the sides; pygidium large; axial lobe has four segments, side-lobes bounded by a slightly concave border, which widens posteriorly, and terninates in long spines, and of which the confines are almost rectangular, with rounded corners. Type C. iowensis.

angulatus, Hall \& Whitfield, 1877, U. S. Geo.' Expl. 40th Parallel, vol. 4, p. 220 , Potsdam Gr.

anytus, Hall \& Whitfield, 1877, U. S. Geo. Expl. 40th parallel, vol. 4, p. 219, Potsdam Gr.

angusta, Walcott, 18s6, Bul1. U. S. Geo. Sur., No. 30, p. 208, Up. Taconic.

centralis, Whitfield, 1877, Rep. on the Pal. of Black Hills, p. 10, and Geo. Black Hills, p. 341 , Potsdam Gr.

diadematus, Hall, 1863, (Conocephalites diadematus,) 16th Rep. N. Y. St. Mus. Nat. Hist., p. 167, Potsdam (Gr.

eos, Hall, 1863, (Conocephalites eos,) 16th Rep. N. Y. St. Mus. Nat. Hist., p. 151, Potsdam Gr.

gibbesi, Wbitfield, 1880, Ann. Rep. Geo. Sur. Wis., p. 50, Potsdam Gr.

granulosus, Hall \& Whitfield, 1877, U. S. Gen. Expl. 40th Parallel, vol. 4, p. 214, Putsdam Gr. haguei, Hall \& Whitfield, 1877, U. S. Geo. Expl. 40th Purallel, vol. 4, p. 210, Potsdam Gr.

iowensis, Owen, 1852, Geo. Sur. Wis., Iowa, and Minn., p. 576, Potsdam Gr.

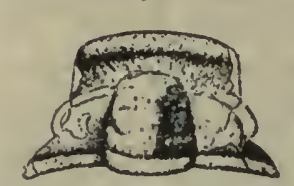

Fia. 988.-Creplcephalus Illimus. Cephalle Fra. 989.-Creplcephaslifeld withont the lus lillanus. Pygldmovable cheeks. fum.

lilianus, Walcott, 1886, Bull. U. S. Geo. Sur., vol. No. 30, p. 207, Up. Taconic. maculosus, Iall \& Whitfield, 1877, U.S. Geo. Expl. 40th Parallel, vol. 4, p. 215, Potsdam Gr.

miniscensis, Owen, 1852, Gen. Sur. Wis., Iowa, and Minn., pl. 1, fig. 14, Potsdam Gr.

montanensis, Whitfield, 1876, Rep. Recon. Up. Mo. to Yel. Nat. Park, p. 141, Po'solam Gr.

niticlus, Hall \& Whitfield, 1877, U. S. Geo. Expl. 40th Parallel, vol. 4, p. 212, Potsdam Gr.

onnstus, Wlitfield, 1878, Ann. Rep. Geo. Sur. Wis., Potsdam Gr.

oweni, Meek \& Hayden, 1861, (Arionellus (Crepicephalus) oweni, ) Proc. Acad. Nat. Sci., vol. 13, p. 436, Potsdam Gr.

planus, Whitfield, 1877, Rep. nII Pul. of Black Hills, p. 11, and Geol. Black Hills, p. 343, Potsdam Gr.

quadrans, see Conocoryphe quadrans.

simulator, Hall \& Whitfield, 1877, U. S. Geo. Expl. 40th Parallel, vol. 4, p. 218, Potsdain Gr.

unisulcatus, Hall \& Whilfield, 18i7, U. S. Geo. Expl. 40th Parallel, vol. 4, p. 216, Potsdam Gr.

vulcanus, Billings, 1861, (Conocephalites vulcanus,) Pal. Foss., vol. 1, p. 14, Potsdam Gr.

wisconsinensis, see Lonchocephalus wisconsinensis.

Cryphaus, Green, 1837, Jour. Acad. Nat. Sci., vol. 7, syn. for Dalmanites. Not. well defined, and the name was preoccupied for a genus of Coleoptera in 1833.

boothi, see Dalmanites boothi. calliteles, see Dalmanites calliteles. greeni, syn. for Dalmunites calliteles.

Cryptolithus, syn. for Trinucleus.

tesselatus, see Trinucleus concentricus.

Cybele, Loven, 1845, p. 110, Ofversigt Vetensk. Acad. Handl., p. 110.

punctata, Hall, 1852. This species helongs to the genus Encrinurus, and the specific name being preoccupied, the name is changed to $\mathrm{E}$. ornatus. 
Cyclus americanus, Packard, 1885, Am. Nat., vol. 19, p. 293, Coal Meas. Not defined so as to be recognized.

Cyphasprs, Burmeister, 1843, Die Organ der Trilobiten, p. 103. [Eity. cyphos, convex; aspis, shield.] Cephalic shield semicircular, posterior angles produced in spines, margin thickened; glabella very convex, ovoid, no furrows, but with two small pyriform basal lobes bounded by deep furrows; eyes small, semilunate; cheeks broad; facial sutures proceed in a nearly straight line, from the anterior margin to the eyes, and are then directed to the posterior angles; thorax 10 to 17 segments, rounded at their extremities; axis tapering; pygidium small, axis short, lateral lobes depressed. Type C. ceratopthalmus.

brevimarginatus, Walcott, 1885, Monogr.

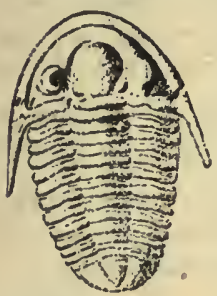

FIG. 990.-Cyphaspis christyi.
U. S. Geo. Sur., vol. 8, p. 93, Trenton Gr. christyi, Hall, Trans. Alb. Inst., vol. 4, p. $188, \mathrm{Ni}$ agara Gr.

coelebs, Hall, 1888, Pal. N. Y., vol. 7, p. 151 , Low. Held. Gr.

craspedota, Hall, 1888, Pal. N. Y., vol. 7, p. 148, Ham. Gr.

diaclema, Hall, 1888, Pal. N. Y., vol. 7, p. 144, Up. Held. Gr.

girardeauensis, Shumard, 1855, Geo. Rep. Mo., p. 197, Trenton Gr.

hybrida, Hall, 1888, Pal. N. Y., vol. 7, p. 144, Up. Held. Gr.

lævis, Hall, 1876, (Phillipsia lævis,) Illust. Devon. Foss., pl. 21, Chemung Gr.

minuscula, Mall, 1876 , (Phillipsia mi ñ us. cula,) Illust. Devon. Foss., pl. 20, Up. Held. Gr.

orn a t a, Hall, 1876 ,

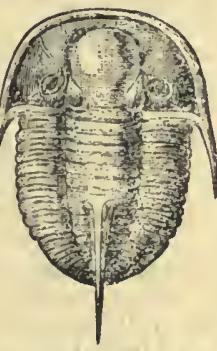
(Phillipsia ornata,) Fra. 991--CyphasIllust. Devon. Foss., pis girardeauenpl. 21, Ham. Gr.

ornata var. baccata, Hall, 1888, Pal. N. Y., vol. 7, p. 146, Ham. Gr.

stephanophora, Hall, 1888, Pal. N. Y., vol. 7, p. 142, Up. Held. Gr.

Cythere, Muller, 1785, Entomostraca sue Insecta, etc., p. 63. The type is C. flavida a living species. The genus is unknowu in Palæozoic rocks.

americana, see Beyrichia americana. earbonaria, see Leperditia carbonaria.

cincinnatiensis, see Cytheropsis cincinnatiensis.

crassimarginata, see Cytheropsis crassimarginata.

irregularis, see Cytheropsis irregularis.

nebraskensis, see Cytheropsis nebraskensis. okeni, see Leperditia okeni. simplex, see Cytheropsis simplex. sublævis, see Leperditia sublævis. subrecta, see Leperditia subrecta.

Cytierella, inflata.

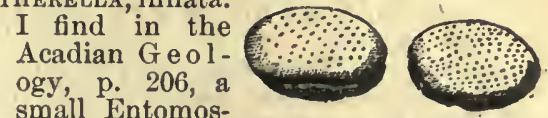
amall Entomos-

traca, from the FIG. 992.-Cytberella inCoal Meas. of Hata.

Nova Scotia, fig-

ured under this name, but without any description or reference to any other author. The figures are reproduced.

Cytherella glandella, see Cytheropsis glandella.

Cytherina, Lamarck, 1818, Anim. sans Vert. t. v, p. 125. [Ety. diminutive of Cythere.] A synonym for Cythere, which is not a Palæozoic genus.

alta, see Leperditia alta.

crenulata, see Cytheropsis crenulata.

cylindrica, see Isochilina cylindrica.

fabulites; see Leperditia fabulites.

spinosa, see Beyrichia spinosa. Not Reuss in 1844.

subcylindrica, see Cytheropsis subcylindrica.

subelliptica, see Cytheropsis subelliptica.

Cytheropsis, McCoy, 1849, Ann. and Mag.

Nat. Hist., 2d. ser., vol. 4, p. 249. [Ety. Cytheropsis, resembling Cythere.] Dis. tinguished from Cythere, which now swarm in the sea, by the great thickness of the valves, and in having eye or muscle spots. 'Type C. aldensis.

cincinnatiensis, Meek, 1872, (Cythere cincinnatiensis,) Proc. Acad. Nat. Sci., p. 331, and Ohio Pal., vol. 1, p. 158, Hud. Riv. Gr. concinna, see Primitia concinna.

crassimarginata, Win-FIG.993.-Cytherchell, 1862, (Cythere opsis cincinuat1crassimarginata,) Proc.

Acad. Nat. Sci., p. 429, Marshall Gr.

crenulata, Emmons, 1856, (Cytherina crenulata,) Am. Geol., p. 220, Trenton Gr.
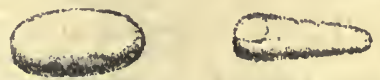

Fia. 994.-Cytheropsis glandella.

glandella, Whitfield, 1882. (Cytherellina glandella,) Bull. Am. Mus. Nut. Hist., vol. 1, p. 94, Warsaw Gr.

irregularis, S. A. Miller, 1878, (Cythere irregularis,) Jour. Cin. Soc. Nat. Hist., vol. 1, p. 106, Hud. Riv. Gr.

nebraskensis, Geinitz, 1866, (Cythere nebraskensis, ) Carb. und Dyas in Neb., p. 2, Coal Meas.

rugosa, Jones, 1858, Ann. Nat. Hist., $3 d$ ser., vol. 1, p. 249, Black Riv. Gr.

siliqua, Jones, 1858, Ann. Nat. Hist., 3d ser., vol. 1, p. 249, Black Riv. Gr. 
simplex, White \& St. John, 1868, (Cythere simplex,) Trans. Chi. Acad. Sci., p. 127, St. Louis $\mathrm{Gr}$.

subcylindrica, Emmons, 1856, (Cytherina subeylindrica,) Am. Geo., p. 220, Trenton $\mathrm{Gr}$.

subelliptica, Emmons, 1856, (Cytherina subelliptica,) Am. Geo., p. 220, Black Riv. Gr.

Dalmania, Emmrich, 1845. This name having been preoccupied for a genus of insects, Dalmanites has been substituted, though inany authors prefer to use Odontochile, a name proposed by Corda.

Dalmanites, (Emmrich, 1845, Dalmania, Barrancle, 1852, Syst. Syl. Boh., vol. 1. [Ety. proper name.] Cephalic shield sublunate, with lateral angles produced into spines; glabella widest anteriorly, rounded in front, with a highly convex anterior subelliptical lobe, three lateral furrows on each side; eyes prominent, subreniform, lenses numerous, situated posteriorly; facial sutures, eurving slightly from the anterior margin, and each, following the curvature of the eye to the posterior part by a sigmoidal flexure, reach the lateral margin very slightly posterior to the eye itself; thorax with eleven segments, axis most eonvex, lateral lobes wider and more or less flattened; pygidium subtriangular, usually extended posteriorly into a spine, segments numerous. Type D. caudatus.

acantholeurus, Conrad, 1841, (Asaphus acantholeurus,) Ann. Rep. N. Y., p. 48,

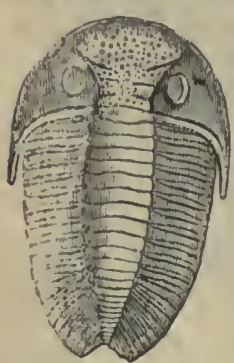

FIG. 995.-Dalmaniles achates. and Illust. Devon. Foss., pl. 19, Onondaga limestone. achates, Billings, 1860, Can. Nat. and Geo., vol. 5, p. 63, Trenton $\mathrm{Gr}$.

xgerja, Hall, 1862, 15th Rep. N. Y. St. Mns. Nat. Hist., p. 57 , and Illust. Devon. Foss., pl. 12, Up. Held. Gr.

anchiops, Green, 1832 , (Calymene anchiops,) Monograph of Trilobites, p. 35, and Illust. Devon. Foss., pl. 9, Schoharie grit.

anchiops var. armatus, Hall, 1862, 15th Rep. N. Y. St. Mus. Nat. Hist., p. 56, Schoharie grit.

anchiops var. sobrinus, syn. for D. anchiops. aspectans, Conrad, 1841, (Asaphus aspectans,) Ann. Rep. N. Y., p. 49, and Illust. Devon. Foss., pl. 13, Up. Held. Gr.

barrisi, Hail, 1888, Pal. N. Y., vol. 7, p. 48, Ham. Gr.

bebryx, Billings, 1860, Can. Nat. and Geo., vol. 5, p. 61, Trenton Gr.

bicornis, Hall, 1876, 28th Rep. N. Y. St. Mus. Nat. Hist., p. 196, Niagara Gr. bifidus, Hall, 1862, 15th Rep. N. Y. St. Mus. Nat. Hist., p. 63, Up. Held. Gr. boothi, Green, 1837, (Cryplæus boothi,) Am. Jour. Sci., vol. 32, p. 343, and Pal. N. Y., vol. 7, p. 42, Ham. Gr.

breviceps, Hall, 1866, 24th Rep. N. Y. St. Mus. Nat. Fist., p. 223, Hud. Riv. Gr. callicephalus, Hall, 1847, (Phacops callicephalns,) Pal. N. Y., vol. 1, p. 247, Trenton Gr.

calliteles, Green, 1837, (Cryplaeus calliteles,) Am. Jour. Sci. and Arts, vol. 32 , p. 346 , and Illust. Devon. Foss., pl. 16, Ham. Gr.

calypso, Hall, 1862, 15th Rep. N. Y. St. Mus. Nat.

Hist., p. 61, and Illust. Fie. 996.-DalDevon. Foss., pl. 13, Up. manties calHeld. Gr.

carleyi, Meek, 1872, A m. Jour. Sci., $3 \mathrm{~d}$ ser., vol. 3 , p. 424 , and Ohio Pal. vol. 1 , p. 170 , Hud. Riv. Gr.

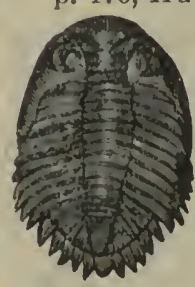

FIG. 997.

Dalmanites calliteles. comis, Hall, 1888, Pal. N. Y., vol. 7, p. 41, Up. Held.' Gr.

concinnus, Hall, 1876 , Illust. Devon. Foss., pl. 10 , Schoharie grit.

concinnus var. serrula, Hall, 188s, Pal. N. Y., vol. 7, p. 30 , Up. Held. Gr. coronatus, Hall, 1862 , 15th Rep. N. Y. St. Mus. Nat. Hist., p. 58, and Illust. Devon. Foss., pl. 12, Ham. Gr. cuyalıogre, Claypole, 1884, Geol. Mag., 3d ser., vol. 1, p. 303, Waverly Gr.

danse, Meek \& Worthen, 1865, Proc. Acad. Nat. Sci. Phil., p. 264, and Geo. Sur. 1ll., vol. 3, p. 363, Niagara Gr.

dentatus, Barrett, 1876, Am. Jour. Sci. and Arts, vol. 11, p. 200, Low. Held. Gr. denticulatus, Conrad, 1841, (Asaphus denticulatus,) Ann. Rep. N. Y., p. 48, and Illust. Devon. Foss., pl. 10, Up. Held. Gr.

emarginatus, Hall, 1876, Illust. Devon. Foss., pl. 10, Up. Held. Gr.

epicrates, Billings, 1863, Proc. Port. Soc. Nat. Hist., vol. 1, p. 119, Low. Held. Gr. erina, Hall, 1862, 15th Rep. N. Y. St. Mus. Nat. Hist., p. 62, Up. Held. Gr.

helena, Hall, 1862, 15th Rep. N. Y. St. Mus. Nat. Hist., p. 61, Up. Held. Gr.

intermedius, Walcott, 1877, 31st Rep. N. Y. St. Mus. Nat. Hist., p. 69, Trenton Gr.

laticaudatus, Hall, 1847. This name is erased from the list.

limulurus, Green, 1832, (Asaphus limulurus,) Monograph Trilobites, p. 48, and Pal. N. Y., vol. 2, p. 303, Niagara Gr.

logani, Hall, 1860, Can. Nat. and Geo., vol. 5, p. 156, and Acad. Geol., p. 608, Up. Silurian. 
macrops, Hall, 1862, 15th Rep. N. Y. St. Mus. Nat. Hist., p. 59, Up. Held. Gr. meeki, Walcott, 1885, Monogr. U. S. Geo. Sur., vol. 8, p. 207, Lower Devonian. micrurus, Green, 1832, (Asaphus micrurus,) Monograph Trilobites, p. 56, and Pal. N. Y., vol. 3, p. 359, Low. Held. Gr.

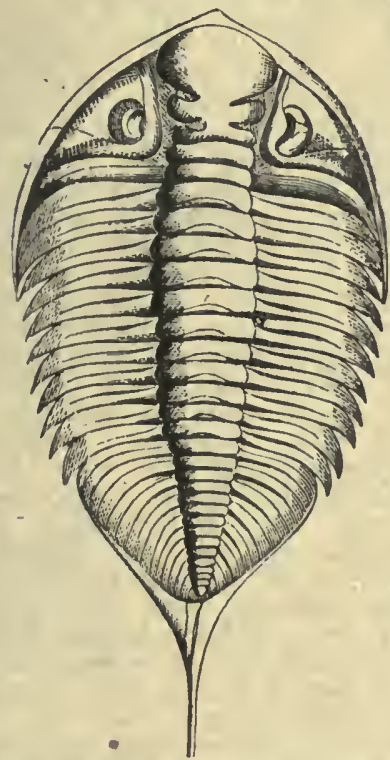

FIG. 998.-Dalmanites limulurus.

myrmecophorus; Green, 1835, (Asaphus myrmecophorus,) Supp. to Monograph of Tribolites, p. 16, and Illust. Devon. Foss., pl. 13, Up. Held. Gr.

nasutus, Conrad, 1841, (Asaphus nasutus,) Ann. Rep. N. Y., p. 48, and Pal. N. Y., vol. 3, p. 362, Low. Held. Gr.

ohioensis, Meek \& Worthen, 1871, Proc. Acad. Nat. Sci. Phil., p: 91, and Ohio Pal., vol. 1, p. 234, Up. Held. Gr.

phacoptyx, Hall, 1888, Pal. N. Y., vol. 7, p. 31, Up. Held. Gr.

pleione,-Hall, 1862, 15th Rep. N. Y. St. Mus. Nat. Hist., p. 62, and Illust. Devon. Foss., pl. 16, Up. Held. Gr.

pleuropteryx, Green, 1832, (Asaphus pleuropteryx,) Monograph Tribolites, p. 55, and Pal. N. Y., vol. 3, p. 356, Low. Held. Gr.

pygmæus, Hall, 1888, Pal. N. Y., vol. 7, p. 56, Up. Held. Gr.

regalis, Hall, 1876, Iilust. Devon. Foss., pl. 11, Schoharie grit.

selenurus, Eaton, 1832, (Asaphus selenurus,) Geo. Text Book, p. 31, and Illust. Devon. Foss., pl. 12, Corniferous Gr. tridens, Hall, 1859, Pal. N. Y., vol. 3, p. 361, Low. Held. Gr.

tridentiferus, Shumard, 1855, Geo. Rep. Mo., p. 199, Low. Held. Gr.

troosti, Safford. Not defined.

verrucosus, Hall, 1863, Trans. Alb. Inst., vol. 4, p. 218, Niagara Gr. vigilans, Hall, 1861, Rep. Prog. Geo. Sur. Wis., p. 51, Niagara Gr.

werthneri, Foerste, 1885, Bull. Sci. Lab. Denison Univ., p. 116. Not well defined.

Dicellocephalus, Owen, 1852, Geo. Sur.

Wis., Iowa., and Min., p. 573, (written by Owen Dikelocephalus.) [Ety. dikella, mattock; kephale, head.] Cephalic shield sublunate or semicircular, rather flat; glabella oblong; sides parallel, rounded in front, transverse behind; three lateral furrows on each side, the posterior two crossing the glabella and dividing it into three lobes; facial sutures arise in the center of the anterior border, run parallel with the same to the front of the eyes, are then directed backward, make a sigmoid flexure over each eye, and when near the neck segment abruptly curve laterally, reaching the posterior border near the posterior spines of the cephalic shield; thorax with nine segments, axis narrower than lateral lobes; pygidium with a flattened border, produced posteriorly on each side, and rounded in the middle; axis extended only about half the length, with four to six segments, which become obsolete on the lateral lobes. Type D. minnesotensis.

affinis, Billings, 1865, Pal. Foss., vol. 1, p. 197, Quebec Gr. or Up. Taconic.

angustifrons, Walcott, 1885, Monogr. U. S. Geo. Sur., vol. 8, p. 42, Potsdam Gr.

barabuensis, Whitfield, 1878, Ann. Rep. Geo. Sur. Wis., p. 63, and Geo. Wis., vol. 4, p. 201, Low. Magnesian Gr.

belli, Billings, 1860, Can. Nat. and Geo., vol. 5, p. 301, Quebec Gr. or Up. Taconic.

bilobatus, Hall \& Whitfield, 1877, U. S. Geo. Expl. 40th Parallel, vol. 4, p. 226, Potsdam. Gr.

(?) corax, Billings, 1865, Pal. Foss., vol. 1, p. 334, Quebec Gr. or Up. Taconic.

crassimarginatus, Whitfield, 1882, Geo. Wis., vol. 4, p. 344, Potsdam Gr.

cristatus, Billings, 1860, Can. Nat. and Geo., vol. 5, p. 301, Quebec Gr. or Up. Taconic.

devinei, Billings, 1865, Pal. Foss., vol. 1, p. 195, Quebec Gr. or Up. Taconic.

eatoni, Whitfield, 1878, Anu. Rep. Geo. Sur. Wis., p. 65, and Geo. Wis., vol. 4, p. 202, Low. Magnesian Gr.

expansus, Walcott, 1885, Monogr. U. S. Geo. Sur., vol. 8, p. 45, Potsdam Gr.

finalis, Walcott, 1885, Monogr. U. S. Geo. Sur., vol. 8, p. 89, Up. Taconic.

flabellifer, Hall \& Whitfield, 1877, U. S. Geo. Expl. 40th Parallel, vol. 4, p. 227, Potsdam Gr.

(?) flagricaudus, White, 1874, Rep. Inv+rt. Foss., p. 12, and Geo. Sur. W. 100th Mer., vol. 4, p. 60, Quebec Gr. or Up. Taconic. gothicus, Hall \& Whitfield, 1877, U. S. Geo. Expl. 40th Parallel, vol. 4, p. 242, Up. Taconic. Probably a syn. for Olenoides walısatchensis. 
granulosus, see Ptychaspis granulosa.

hisingtri, Billings, 1865, Pul. Foss., vol. 1, p. 196, Quebec Gr. or Up. Taconic.

inexpectans, Walcott, 1885, Monogr. U. S. Geo. Sur., vol. 8, p. 90, Quebec Gr. or Up. Tac'onic.

iole, Walcott, 1885, Monogr. U. S. Geo. Sur. vol. 8, p. 43, Potsdan Gr. or Up. Taconic. latifrons, Shumard, 1863, 'Trans. St. Louis Acal. Sci., vol. 2, p. 101, Potsdam Gr.

lodensis, Whitfield, 1880, Ann. Rep. Geo. Sur. Wis., p. 51, and Geo. Wis., vol. 4, p. 189, Putsilam Gr.

magnificus. Billings, 1860, Can. Nat. and Geo., vol. 5, p. 301, Quebec Gr. or Up. Tilenic.

marconi. Whitfield, 1884, Bull. Am. Mus. Nat. Hist., vol. 1, p. 139, Up. Taconic.

marica, Walcott, 1885 , Monogr. U. S. Geo. Sur., vol. 8, p. 44, Potsclam Gr.

mequlops, Billings, 1860 , Can. Nat. and Gro., vul. 5, p. 301, Quebec Gr. or Up. Taronie.

minixcrnsis, see Ptychaspis miniscensis.

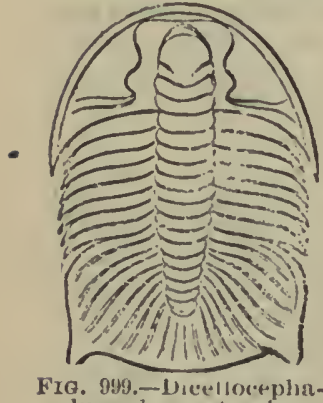

lus milumesotensts. in in nesoten sis, Owen, 1852, Rep. Wis., Iowa, and Min., p.574, Potsdam Gr.

$\mathrm{minnesotensis}$ var. limbatus, Hall, 1868, 16th R+y. N. Y. St. Mus. Nat. Hist., p. 141, Potsdam Gr.

misa, Hall, 1863, 16th Rep. N. Y. Si. Mus. $N$ u t. Hist., p. 144, Potsdan: Gr.

missisquni, Billings, 1865, Pal. Foss., vol. 1, p. 199, Quebes: Gr. or Up. Taconic.

mulicinctus, Hall \& Whitfield, 1877, U. S.

Geo. Expl. 40tl Parallel, vol. 4, p. 226 , Potsdam. Gr.

nasut us, WValcott, 18S5, Monogr. U. S. Geo. Sur., vil. 8, p. 44, Potsdam Gr.

oscenla, Hall, $1863,16 \mathrm{th}$ Rep. N. Y. St.

Mus. Nat. Hist.. p. 146, Pitsdam Gr.

oweni, Billings, 1860, Can. Nat. and Geo., vol. 5, 1. 301, Quebec: Gr. or Up. Taconic. pauper, Billiugs, 1865, Pal. Foss., vol. 1, p. 200, Queb+c Gr. or Up. Taennic.

pepinensis, Owen, 1852, Geo. Wis., Iowa, and Minn., p. 57t, Pistsrlam Gr.

planifrons, Billings, 1860 , Can. Nat. and Geo., vol. 5, p. 301, Qrebec Gr. or Up. Tacnnic.

pogonipensis, Hall \& Whitfield, 1877, U. S. Gro. Expl. 40th Parallel, vol. 4, p. 243, Potsdan Gr.

quadriceps, seit Olenoides quadriceps.

richmondensis, Walcott, 1 885 , Monogr.

U.S. Gen. Sur., vol. S. p. 41, Potsdam Gr. roemeri, Shumard, 1S61, A m. Jour. Sci., vol. 32, r. 220, Potsilam Gr.

seleetus, Billings, 1865, Pdl. Foss., vol. 1, p. 199, Quebec Gr. or Up. Taconic. sesostris, see Ptychaspis sesostris.

spiniger, Hall, 1863, 16th Rep. N. Y. St. Mus. Nat. Hist., p. 143, Potsdam Gr. wahsatchensis, see Olenoides wahsitchensis.

Dicranurus, syn. for Acidaspis.

hamatus, see Acidaspis hamata.

Dionide, Barrande, 1847, in Lith. Proc. [Ety. from the mythological name Dione.] Body oval, tapering beliind, trilobed, faintly convex; cephalic shield short, wide, semicircular, or erescentiform, produced at the postero-lateral angles into spines; glabella short, wide, strongly convex, smooth, no lateral furrows: two longitudinal furrows, making it trilobate; cheeks wide, with perforated margin; no eyes; no facial suture; hypostoma elliptical, with two bow-shaped wings in front, and posterior margin entire; six thoracic segments, with nodes on each side of the axial lobe; pygidium subtriangular, rounded behind axis, with numerous segments, and lateral lobes with radial furrows. Type D. formosa.

(?) perplexa, Billings, 1866, Catal. Sil. Foss. Antic., p. 67 , Anticosti Gr.

Diveltis, Packard, 1885, Am. Nat., vol. 19, p. 291. [Ety. dis, donb]e; pelte, small shield.] Cyclus-like in form; suborbicular, flattened, disk-like, sloping from the median area to the erlge; divided in two parts, a cephalie shield and abdomen; integument thin, sliowing no segments. Type D. riplodiscus.

diplodiscus, Packard, IS85, A m. Nat., vol. 19, p. 291, Coal Meas. Poorly defined.

Dipleura, Green, syn. for Homalonotus. dekayi, see Homalonotus rekavi.

Diplicin nites, Dawson, 1863 , Am. Jour. Sci. and Arts, 3d ser., vol. 5, p. 19. [Ety. diploos, double; ichnor, foot-print.] Consisting of two rows of inpressions, each about an inch long and one-fourth of an inch wide, placed close together, while the rows are six incles apart, and the intermediate space smooth, as if a flat body had been drawn over it. Type D. xnigma.

ænigma, Dawson, 1863, Am. Jour. Sei. and Arts, 3 d ser., vol. 5, p. 19, Coal Meas.

D I P L, OSTYLUS, S a 1 t e $r$, 1S63, Quar. Jour. Geo. Soc., vol. 19 , p. 76. [Eıy. Diplostylus, dou b le tail, in allusion to the tivo Fig. 10r0.-Diplusiylus dawsoni. pairs of ap- $a$, Tail, nat. size; $b$, terminal pend ages joint, enlarged.

to the telson.] Carapace unknown; body segments arched, and with minute pleuræ; tail secment large, triangular, 
spinous, with two pairs of simple, ovate appendages. Type $\mathrm{D}$. dawsoni.

dawsoni, Salter, 1863, Quar. Jour. Geo. Soc., vol. 19, p. 77, and Acad. Geol., p. 207, Coal Meas.

Dipterocaris, Clarke, 1883, Am. Jour. Sci. and Arts, 3 d ser., vol. 25, p. 121. [Ety. dipteros, two-winged; karis, shrimp.] Carapace elongate, divided along the major axis into two wings; greatest width anteriorly; wings united medially for one-third to one-fifth the length of the carapace; anchylosed, but separated toward the ends. Surface marked concentrically. Type D. pennidædali.

pennidædali, Clarke, 1883, Am. Jour. Sci. and Arts, $3 \mathrm{~d}$ ser., vol. 25, p. 122, Chemung Gr.

pescervæ, Clarke, 1883, Am. Jour. Sci. and Arts, $3 \mathrm{~d}$ ser., vol. 25, p. 123, Chemung Gr.

procne, Clarke, 1883, Am. Jour. Sci. and Arts, 3d ser., vol. 25, p. 122, Chemung Gr.

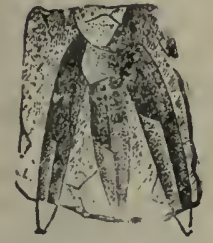

Fia. 1001. - Dithyroc a $\mathrm{r}$ is corbonaria. Telson and stylets.
Ditiryocaris, Scouler, 1814, Syn. Carb. Foss., Ireland \& McCoy, 1855, British Pal. Rocks, $p$. 181. [Ety. dithyros, having two valves; karis, shrimp.) Carapace semioval; anterior end rounded, sometimes notched; posterior end subtruncate, with lateral angles produced into spines; surface with faint imbricating strix, margius thickened and corrugated, with three longitudinal ridges, one in the middle extending the entire length, the others not reaching the margin; tail terminating in three triangular spines. Type D. scouleri.

belli, see Mesothyra belli.

carbonaria, Meek \& Worthen, 1870, Proc. Acad. Nat. Sci. Phil., p. 55, and Geo. Sur. Ill., vol. 5 , p. 618 , Coal Meas. neptuni, see Mesothyra neptuni.

Dolichocephala, Claypole, 1883 , Proc. Am. Phil. Soc., p. 238, syn. for Stylonurus.

lacoana, syn. for Stylonurus excelsior.

Dourchometopus, Angelin, 1852, Paleontologia Scandinavica. [Ety. dolichos, long; metope, panel or space between two hollows.] Cephalic shield with tumid margin; eyes large, narrow, lunate; glabella wider in front, smooth, no lateral furrows; neck furrow-marked; facial sutures, beginning at the posterior margin near the lateral angles, are directed toward the eyes, passing which, they diverge to the anterior margin ; pygidium semicircular, strongly convex, margin entire, axis almost semicylindrical, with two or more furrows. Type D. suecicus. It is doubtful about this being an American genus, as the identifications have been made alone on the pygidium.

? convexus, Billings, 1865, Pal. Foss., vol. 1, p. 269, Quebec Gr. or Up. Taconic.

? gibberulus, Billing., 1865, Pal. Foss., vol. 1, p. 269, Quebec Gr. or Up. Taconic. ? rarus, Billings, 1865, Pal. Foss., vol. 1, p. 352, Calciferous Gr.

Doliciropterus, Hall, 1859, Pal. N. Y., vol. 3, p. 414. [Ety. dolichos, long; pteron, wing.] Cephalic, thoracic, and caudal portions similar to Eurypterus; postoral plate lyrate or cordiform lyrate; central thoracic appendage from the first thoracic segment, strong, thick, and simple, in its anterior part; anterior feet composed of strong, thick joints, with curved terminal spines; natatory organs having the joints elongate, the seventh and eighth little dilated, and the terminal palette extremely developed. Type D. macrochirus.

macrochirus, Hall, 1859, Pal. N. Y., vol. 3 , p. 414, Waterlime Gr.

mansfieldi, Hall, 1877, Trans. Am. Phil. Soc., p. 621, Lower Coal Meas.

Echinocaris, Whitfield, 1880, Am. Jour. Sci. and Arts, 3d ser., vol. 19, p. 34 . [Ety, echinos, sea urchin; karis, shrimp.] Carapace bívalve; valves subovate, united dorsally by a straight hinge, anterior, posterior, and basal margins rounded; surface marked by longitudinal ridges or representative nodes or ridges; abdomen naked, composed of several segments and a caudal plate, which is produced into an elongated

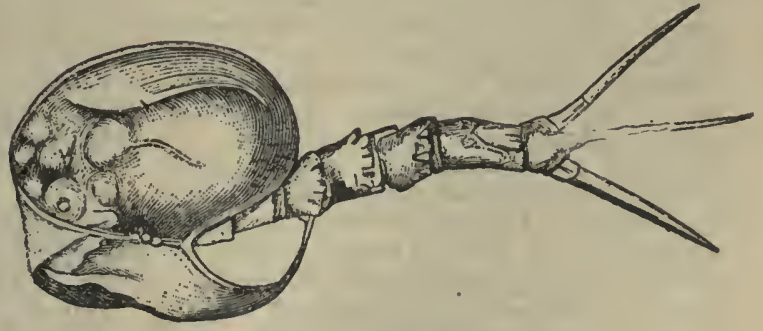

Fig. 1002.-Echinocaris punctata.

spine, with a lateral movable spine on each side; posterior margin of the abdoininal. segments bearing spines. Type E. sublævis.

condylepis, Hall, 1888, Pal. N. Y., vol. 7, p. 173, Chemung Gr.

longicauda, Hall, 1863, (Ceratiocaris longicauda,) 16th Rep. N. Y. St. Mus. Nat. Hist., p. 73, Genesee Slate.

multinodosa, Whitfield, 1880, Am. Jour. Sci. and Arts, 3d ser., vol. 19, p. 38, Erie shales. 
punctata, Hall, 1863, (Ceratiocaris punctata,) 16th Rep. N. Y. St. Mus. Nat. Hist., p. 74, Ham. Gr.

pustulosa, Whitfield, 1880, Am. Jour. Sci. and Arts, 3d ser., vol. 19, p. 3S, Erie shales.

socialis, Beecher, 1884, Rep. of Prog. Pa. Geo. Sur. PPP, p. 10, Chemung Gr.

sublævis, Whitfield, 1880, Am. Jour. Sci. and Arts, 3d ser., vol. 19, p. 36, Erie shales.

whitfieldi, Clarke, 1885, Bull. U. S. Geo. Sur. No. 16, p. 45, Ham. Gr.

wrightana, Dawison, 1881, (Equisetides wrightanus,) Quar. Jour. Geo. Soc., vol. 37, p. 30l, Portage Gr.

Ecmixogxatilis, Walcott, 1882, Am. Jour. Sci. and Arts, 3d ser., vol. 23, p. 213. [Ety. echinos, sea urchin; gnathos, jaw.] Founded upon fragments; endognathary limbs (one or more pairs) formed of eight or nine joints, six of which carry long, backward curving spines, articulated to their posterior side; terminal joint slender, elongate, acuminate; surface of body with scale-like markings. Type E. clevelandi.

clevelandi, Walcott, 1882, Am. Jour. Sci. and Arts, 3d ser., vol. 23, p. 213, Utica Slate Gr.

Ellipsocelinacs, Zenker, 1833, Beitrage zur Naturgeschichte der Urwelt, p. 51. [Ety. ellipsis, ellipse; kephale, head.] Broadly ovate; cephalic shield semicircular, depressed, without spines; glabella subquadrangular, rounded in front, without transverse furrows; eyes oblong, lunate, narrow, projecting outward; facial sutures short, commencing at the anterior margin, in front of the eyes, and curving over them toward the posterior angles; thoracic segments twelve, axis nearly as broad as lateral lobes; pygidium small, semicircular, trilobed. Type E. hoffi.

? curtus, Whitfield, 1877, Ann. Rep. Geo. Sur. Wis., p. 58, and Geo. Wis., vol. 4, p. 191, Potsdam Gr. Founded upon a fragment of the cephalic shield, and the generic reference is very doubtful.

Elliptocephala, Emmons, 1844, Taconic System, p. 21. [Ety. ellipsis, ellipse; kephale, head.] Ovate; cephalic shield lunate, more than twice as wide as long, posterior angles produced in spines; groove and border on the anterior and lateral margins; glabella nearly equal in width throughout, and marked with three pairs of furrows; eyes large, elongate, semilunate, extending from near the base of the shield more than half way to the anterior margin; hypostoma broadly ovate; thirteen or fourteen articulations in the thorax, axis convex, lateral lobes flattened, last segments directed backward; pygidium narrow, elongated, axis acutely pointed. Type E. asaphoides. This generic name can stand in accordance with rule $n$ of the British Association of 1842, and the established laws of nomenclature adhered to by reputable scientists since that time, notwithstanding it is recommended to naturalists in selecting names to avoid such as too closely approximate words already adopted. It is true the masculine form of the word was preoccupied by Zenker, but the same can be said of Goniophora of Phillips, for Agassiz bad preceded him in using the word Goniophorus; Schizodon was used for a mammal before King used Schizolus for a Lamellitranch; Gray used Acrophylla before Nicholson used Acrophyllum; and we might mention a hundred other instances where generic names, differing only in gender or termination, have been introduced and accepted by the best naturalists, and have come into such general use as to constitute part of the nomenclature of science. Olenellus can not be used to supplant Elliptocephala upon any ground of discovery, definition, or law.

asaphoides, Emmons, 1844, Taconic System, p. 21, and Pal. N. Y., vol. 1, p. 256, Up. Taconic.

gilberti, Meek, 1874, (Olenellus gilberti,) Rep. Invert. Foss., p. 7, and Geo. Sur. 100 th Mer., vol. 4, p. 44, Up. Taconic.

howelli, Meek, 1875, (Olenellus howelli,) Rep. Invert. Foss., p. 8, and Geo. Sur. 100th Mer., vol. 4, p. 47, Up. Taconic.

i d $\mathrm{l}$ i $\mathrm{n} \mathrm{gs} \mathrm{i}$, W a 1 c ott, 1885, (Ole. nellus iddin $g$ i ,) Monogr. U. S. Geo. Sur., vol. 8 , p. 28, U p. T aconic.

t li ó mpsoni, Hall, 1859, (Olenellus $\mathrm{t} \mathrm{h}$ o $\mathrm{m} \mathrm{p}$ soni,) 12 th Rep. N. Y. St. Mus. Nat. Hist., p. 59, Up. Taconic.

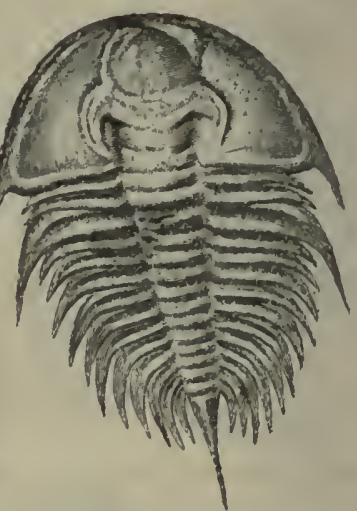

EIG. 1003.--Elliptocephala thompsoni. undulostriata, Hall, 1847, (Olenus undulostriatus,) Pal. N. Y., vol. 1, 1). 258, Up. Taconic. Poorly defined.

Elymocaris, Beecher, 1884, Rep. Pa. Geo. Sur. PPP, p. 13. [Ety. elymos, pod; karis, shrimp.] Carapace bivalve; elongate, longitudinally subquadrangular, dorsal line nearly as long as the valves; margins thickened; optic node near the anterior end, behind which are two elevations; two segments in the abdomen; telson a short, broad spine, with two lateral spines, crenulated on 
the inner margins for fimbria. Type E. siliqua.

capsella, Hall, 1888, Pal. N. Y., vol. 7, p. 181, Ham. Gr.

siliqua, Beecher, 1884, Rep. Pa. Geo. Sur. PPP, p. 13, Chemung Gr.

Embolamus rotundatus, Rominger, syn. for Bathyuriscus howelli.

spinosa, Rominger, syn. for Olenoides spinosus.

Encrinurus, Emmrich, 1845, Neues Jahrb. f. Mineral, p. 42. [Ety. en, prefix; krino, parted; oura, tail.] Cephalic shield semielliptical, tuberculated; lateral angles produced into spines ; glabella pyriform, three furrows at each side toward the base; cheeks flattened, triangular; eyes in the middle of the cheeks, elevated on foot-stalks; facial suture behind the eye cuts the outer margin in front of the angles; thorax with eleven segments; pygidium triangular, lateral lobes with about eight segments, deflected, sometimes pointed; axis narrow, convex, with numerous segmental lines. Type E. punctatus.

deltoideus, Shumard, 1855, Geo. Sur. Mo., p. 198, Up. Sil.

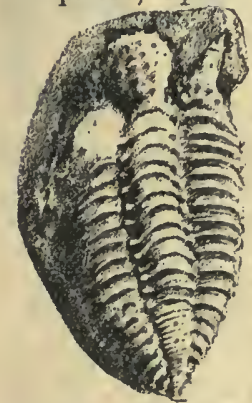

FIG. 1004.-Encrinurus egunt. egani, S. A. Miller, 1880, Jour. Cin. Soc. Nat. Hist., vol. 2, p. 254, Niagara Gr.

elegantulus, Billings, 1866, Catal. Sil. Foss. Antic., p. 62, Anticosti Gr.

excedrensis, S a ffor d, Not defined.

lævis, Angelin, 1852, (Cryptonymus lævis,) Palæontologia Scandinavica, p. 4, Up. Sil.

mirus, Billings, 1865, 292, Quebec Gr. or Up. Taconic.

multisegmentatus, Portlock, 1843, (Amphion multisegmentatus,) Rep. Geo. of Londonderry, etc., Anticosti.Gr.

nereus, Hall, 1867, 20th

Rep. N. Y. St. Mus.

Nat. Hist., p. 425,

Niagara Gr.

ornatus, Hall \& Whit-

field, 1875, Ohio Pal., Frg. 1005.-Enerinuvol. 2 , p. 154 , Niag- rus egant. Cephalic ara Gr.

phlyctainodes, Green, 1837, (Calymene phlyetainodes,) Am. Jour. Sci. and Arts, vol. 32, p. 167, and Pal. N. Y., vol. 2, p. 314, Niagara Grr.

punctatus, Wahlenberg, 1821, Nova Acta Soc. Upsal., Anticosti Gr.

trentonensis, Walcott, 1877, Rep. N. Y. St. Mus. Nat. Hist., p. 68, Trenton Gr.

varicostatus, Walcott, 1877, 31st Rep. N. Y.

St. Mus. Nat. Hist., p. 69, Trenton Gr. vigilans, Hall, 1847, (Ceraurus vigilans,)
Pal. N. Y., vol. 1, p. 245, Black Riv. and Trenton Grs.

Endymion, Billings, 1862. The name being preoccupied for a genus of plants, the author propused Endymionia.

meeki, see Endymionia meeki.

Exdymonia, Billings, 1865, Pal. Foss., vol. 1, pp. 93, 281. [Ety. proper name.] Cephalic shield semioval, convex; glabella ovate, convex, an elongate oval tubercle on each Fra. 1006.-Endy-
side; thorax of six or mionia meeki. seven segments, axis convex, side lobes flat, groove crossing them diagonally; pygidium semioval, trilobed and divided by furrows into segments; distinguished from Trinucleus by the absence of a punctured border on the head shield, and from Ampyx by the form of the glabella, which has a tubercle on each side, and is destitute of a rostrum. Type E. meeki.

meeki, Billings, 1862, (Endymion meeki,) Pal. Foss., vol. 1, pp. 93, 281, Quebec Gr. or Up. Taconic.

Enoploura, Wetherby, 1878, Jour. Cin. Soc. Nat. Hist., vol. 1, p. 163. Proposed instead of Anomalocystites, upon the ground that it is a Crustacean, instead of a Cystidean.

Estheria, Ruppell, and Straus-Durckheim, 1837, Mus. Senckenberg., vol. 2, p. 119. [Ety. proper name.] Carapace valves oval, globose, with a definite hinge-line, well marked umbones and concentric ridges, valves inequilateral, subtrigonal or subovate, umbo near anterior end. Type E. dahalacensis. A living genus, and probably not Palæozoic.

pulex, Clarke, 1882, Am. Jour. Sci. and Arts, 3d ser., vol. 23, p. 466, Ham. Gr. Euproops, Meek, 1867, Am. Jour. Sci., vol. 43 , p. 394. [Ety. eu, very; pro, forward; ops, eye.] Cephalo-thoracic shield crescentric, more than twice as wide as long, convex, lateral angles terminating in spines; posterior margin concave, from the lateral angles two-thirds of the distance to the middle, the central part being straight or slightly concave; the ocular ridge surrounds a crown-shaped or subquadrangular area, occupying the central third of the shield; the sides are slightly concave, in front there is a central emargination, and posteriorly the ridge is continued in a spine, on each side, directed back over the abdomen; eyes small, compound, located at the anterolateral angles of the crown-shaped central area; mesial lobe small, narrowing forward and reaching the ocular ridge, in a linear carina; it bears a tubercle on the posterior part; abdomen trans. versely subelliptical, mesial lobe nar- 
row; lateral lobes wide, flattened on the margins; segments defined by linear ridges, which are produced beyond the flattened borders in curved mucronate spines; telson subtrigonal, gradually tapering. Type E. danze.

colletti, White, 1884, 13th Rep. Ind. Geo. Sur. Nat. Hist., p. 172, Coal Meas.

duna, Meek \& Worthen, 1865, (Bellinurus dane, ) Proc. Acad. Nat. Sci. Phil., r. 43, and Geo. Sur. Ill., vol. 2, p. 395, Coal Meas.

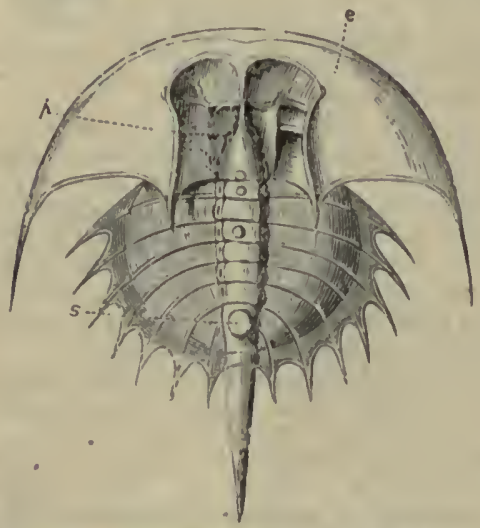

Fia. 1007.-Euproops danre. e, Eyes; p, pits ; $s$, node.

longispina, Packard, 1855, Am. Naturalist, vol. 19, p. 291, Coal Meas.

Euryptekrlua, Matthew, 1858, Trans. Roy. Soc. Can., p. 60. [1:ty. diminutive of Euryplerus.] Minute; body ovate-elongate, obscurely divided into three regions, and faintly trilobed; head subtriaugular, rounded at the outer corners, emarginate behind, seemingly composed of three anchylosed segments; thorax subquadrate, four segments, first one with a median ridge; abdomen elongately triangular, several segments, produced in a long, flexible tail; surface tuberculated. Type $\mathrm{E}$. ornata.

ornata, Matthew, 1888, Trans. Roy. Soc. Can., p. 60, Lower Devonian.

Eurypteisus, DeKay, 1825, Ann. Hyc. Nat. Hist. N. Y.,vol. 1, p. 375. [Ety. euros, breadtlı; pteron, wing.] Body ovate-lanceolate, gradually atten uate behind, terminating in a spiniform tail; carapace on the upper side entire; eyes two dis$\tan t$, sessile, within the margin of the carapace, two simple oculiform tubercles or cornere situated subcentrally; thoracic and caudal portions composed of thirteen joints, the first narrow and the last prolonged in a triangular spine, with serrated edges; the first two articulations are anchylosed on the lower side, and from the central part a locomotive appendage is directed backward to the $3 d$ or 4 th articulation, terminating in two slender processes; mouth central, beneath the carapace, surrounded by four pairs of jointed feet and a fifth larger pair; the three anterior pairs are similar; several joints bear a small articulating spine at the distal extremities, and the terminal joint consists of a spine; the fourth pair is longer, more slender, without spines, except on the terminal joint; the fifth pair are natatory, longer, more clilated, and placed beneath the posterior part of the carapace, basal joints composed of broad rliomboidal plates covering the posterior part of the carapace, over the inner edges of which there is a longitudinally ovate plate, at the anterior sinuate margin of which is the entrance to the mouth. Type F. remipes.

beecheri, Hall, 1884, Geo. Sur. I'a. PPP, p. 30 , Chemung Gr.

boylei, Whiteaves, 1884, Pal. Foss., vol. 3, i. 42 , Guelph Gr.

dekayi, Hall, 1859, Pal. N. Y., vol. 3, p. 411, Waterlime Gr.

eriensis, Whitfield, 1882, Ann. N. Y. Acad. Sci., vol. 2, p. 196, Low. Held. Gr. giganteus, Pohlman, 1882, Bull. Buff. Soc. Nat. Sci., vol. 4, p. 41, Waterlime Gr. grandis, Grote \& Pitt, 1875, (Eusarcus grandis,) Bull. Buff. Soc. Nat. Hist., vol. 3, p. 17, Waterlime Gr.

lacustris, Harlan, 1834, Trans. Geo. Soc. Penu., vol. 1, p. 98, and Pal. N. Y., vol. 3 , p. 407 , Waterlime Gr.

lacustris var. robustus, Hall, 1859, Pal N. Y., vol. 3 , p. 410 , Waterlime $\mathrm{Gr}$.

mazonensis, Meek \& Worthen, 1868, Am. Jour. Sci., vol. 46, p. 21, and Geo. Sur Ill., vol. 3, p. 544, Coal Meas.

micropthalmus, Hall, 1859, Pal. N. Y., vol. 3 , p. 407 , Low. Held. Gr.

pachychirus, Hall, 1859 , Pal. N. Y., vol. 3 , p. 412 , Waterlime Gr.

pennsylvanicus, Hall, 1877, Proc. An. Phil. Soc., p. 621, Carboniferous.

potens, Hall, 1884, Geo. Sur. Pa. PPP, p. 37, Carboniferous.

prominens, Hall, 1884, Proc. Am. Ass. Sci., vol. 33, p. 420, and Pal. N. Y., vol. 7, p.137, Clinton $\mathrm{Gr}$.

pulicaris, Salter, 1863, Quar.

Jour. G e o. Soc., vol. 19 , p. 78, and Acad. Geol., p. 523, Up. Devonian.

p.us t u l os u s, Hall, 1859, Pal. N. Y., vol. 3, p. 413 , IV a te r lime Gr.

rem ipes, De-

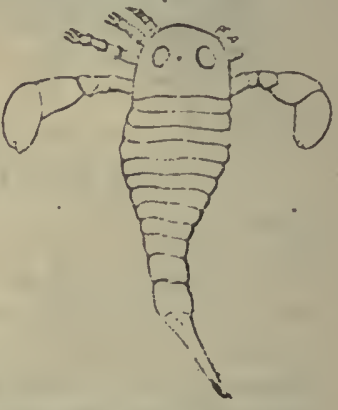

Frg. 1008.-Eurypterus remtpes. Dlagram of small specimen.

Kay, 1825 ,

Ann. Lyc. Nat. Hist. N. Y., p. 375, and Pal. N. Y., vol. 3, p. 40t, Waterlime Gr. 
scorpionis, Grote \& Pitt, 1875, (Eusarcus · scorpionis,) Bull. Buff. Soc. Nat. Hist., vol. 3, p. 1, Waterlime Gr.

stylus, Hall, 188t, Geo. Sur. Pa., PPP, p. 34, Low. Coal Meas.

tetragonopthalmus, Fischer, 1839, Bull. Soc. Imper. Nat. Moscou., IVaterlime Gr.

Eusarcus, Grote \& Pitt, 1875, Bull. Buff. Soc. Nat. Hist., vol. 3, p. 1, syn. for Eurypterus.

grandis, see Eurypterus grandis.

scorpionis, see Eurypterus scorpionis.

Faberia, n. gen. [Ety. proper name.] Minute crustaceans inclosed in a shell with openings on the edge for the protrusion of the feet and antennre. They are referred to the Ostracoda, because the test is like that of Leperditia and Beyrichia, but they are distinguished by being .closed in a single shell; they are evidently globose, depressed or variable in form. Type F. anomala.

anomala, n. sp. Minute, subcircular in outline, and flattened on each side; thickness about one-fourth the diameter; one edge somewhat sharpened; a
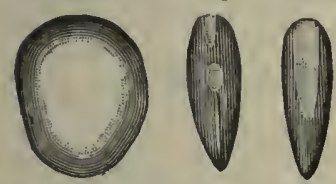

Fig. 1009.-Faberia anomala Three vlews, mag. 5 diam. slit or opening, a b o u t eight times as long as wide, exists on the thick. er edge of the shell; and at less than the thickness of the shell distant from the slit, there is a circular opening on the edge of the shell, and below this reaching nearly to the thinner edge of the shell, there is a very narrow slit that does not seem to penetrate the test. Found in the upper part of the Hud. Riv. Gr., in Butler County, Ohio, and now in the collection of Charles Faber.

Harpes, Goldfuss, 1839 , Nova Acta Physico medica Academiæ Cæsarea Leopoldino Carolinæ Naturæ Curiosorum, vol. 19, p. 358. [Ety. harpe, a hook or sickle.] Cephalic shield horseshoe-shaped, very convex centrally, flatly expanded on the external margin, and posterior angles produced in long spines; gla bella very prominent, short, front subquadrate, posterior part contracted, a curved lateral furrow on each side separating two el-

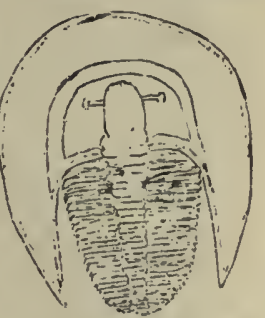

FIG. 1010.-Harpes ungula. liptical lobes from the posterior half ; eyes small, near the anterior part of the glabella; facial sutures from the posterior angles, curving through the eyes and then to the antero-lateral margins, thoracic segments numerous. Type H. ungula.

antiquatus, Bıllings, 1859, Can. Nat. and Geo., vol. 4, p. 468, Chazy Gr.

consuetus, Billings, 1866, Catal. Sil. Foss. Antic., p. 64, Anticosti Gr.

dentoni, Billings, 1863, Can. Nat. and Geo., vol. 8, p. 36, Hud. Riv. Gr.

e s c a n a b æ

Hall; 1851,

Geo. Lake

Sup. Land

Dist., vol. 2, p. 211, Trenton Gr.

granti, Bill-

ings, 1865

Pal. Foss.,

vol. 1 , p. 326 ,

Quebec Gr.

ott a we n s is;

$\mathrm{B}$ i 11 ing $\mathrm{s}$,

1865, Pal.

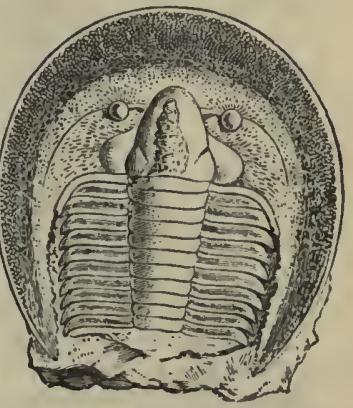

Foss., vol. 1, Fig.1011.-Harpes ottawensis. p. 182, Trenton Gr.

HARPIDEs, Beyrich, 1846, Untersuchungen Trilobiten als Fort. [Ety. from resemblance to the genus Harpes.] Cephalic shield semicircular, margin wideand flat, spines at posterior lateral angles; glabella short, narrow, granular; lobe on each side at base; cherks have radiating striæ; eyes small, joining the front end of the glabella by a small ridge ; thorax with 22 segments, pleuræ three times as wide as the axis. Type H. rugosus. Only fragments liave been referred to this genus in America.

atlanticus, Billings, 1865, Pal. Foss., vol. 1, p. 281, Quebec Gr. or Up. Taconic. concentricus, Billings, 1865, Pal. Foss., vol. 1, p. 282, Quebec Gr. or Up. 'Taconic.

? desertus, Billings, 1865, Pal. Foss., vol. 1, p. 333, Quebec Gr. or Up. Taconic,

Hartia, Walcott, 1884, Bull. U. S. Geo. Sur. vol. 2, p. 283. [Ety. rroper name:] Distingnished from Conocoryphe by having a lobe or elevation in the front of the glabella, small pygidium, and sloping front to the cherks and frontal lobes. Type H. matthewi.

matthewi, Hurtt, 1868, (Conocephalites matthewi,) Acar. Geol., p. 646, St. John Gr.

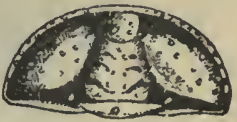

Hausmannia, Hall, 1888, FrG. 1012 - Hartlia synonym for Dal- matthewl. Cephalic manites.

Hemicryplume, Green, syn. for Asaphus.

clintoni, Vanuxem, 1843, Geo. Rep. 3d

Dist. N. Y., p. 79, Clinton Gr. Generic relation not determined.

rasoumowski, syn: for Asaphus expansus. Hipponicilarion, Matthew, 1885, Traus. Rny. Soc. Can., p. 64. Breadth nearly equals the length; broadly semi-elliptical to- 
ward the base, flattened, crossed by three symmetrical ridges; the middle one is inconspicuous. Type $\mathrm{H}$. eos. eos, Matthew, 1885, Trans. Roy. Soc. Can., p. 64, St. Jolin Gr.

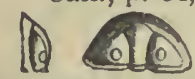

Fig. 1013,-Holoinetopus angelint. Head and side view.

semicircular: convex, widened in front, separated from cheeks by deep furrows; cheeks tumid; eyes small, situated well to the posterior; neck furrow distinct; facial sutures curred a little outward, in front of the eye. Type H. limbatus.

angelini, Billings, 1862, Pal. Foss., p. 95, Quebec Gr. or Up. Taconic.

Homalonotus, Konig, 1825, Icones. Foss. Sectiles, p. 4. [Ety. homalos, on the same level; notos, back.] Cephalic shield hyperbolic, anterior angle subacute, margins rounded, surface convex; glabella subquadrate, short, wider posteriorly, no furrows; eyes opposite the

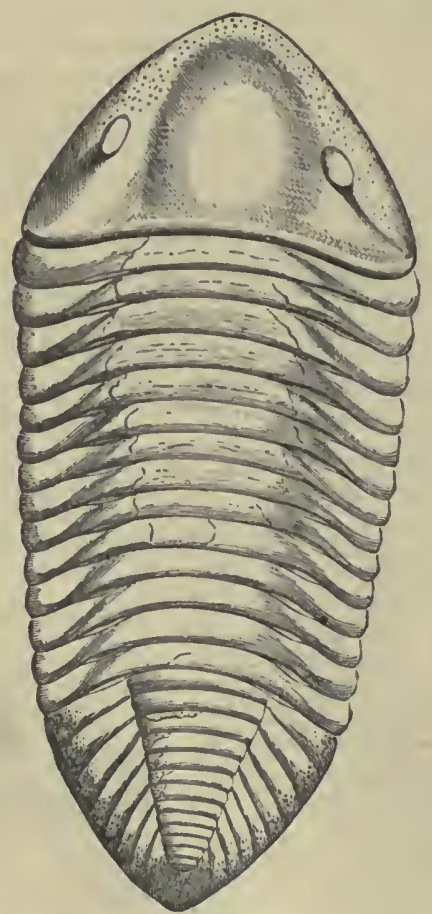

FIG. 1014-Homalonotus delphinocephalus.

central part of the glabella, small; facial suture, from the anterior angle to the margin, following the border of the margin, and curving like the letter $S$, it reaches the eye, and by a like curve passes to the posterior lateral angle; thoracic segments 13 , axis wider than the lateral lobes, which have subtruncate ends, with large distinct facets; pygidium hyperbolic and termillating in a spine. Type $\mathrm{H}$. knighti.

atlas, Castelnau, 1843, Syst. Sil., p. 20. Not recognized.

dawsoni, Hall, 1860, Can. Nat. and Geo., vol. 5, p. 155, and Acad. Geol., 1. 607, Up. Silurian.

dekayi, Green, 1832, (Diplenra dekayi,) Monograph Trilobites, p. 79, and Illust. Devon. Foss., pl. 25, Ham. Gr.

delphinoceplialus, Green, 1832, (Trimerus delplinocephalus,) Monograph of 'I'rilobites, p. 82, and Pal. N.Y., vol. 2, p. 309. Niagara Gr.

gioanteus, Castelnau, 1843, Syst. Sil., p. 20. Not recognized.

herculanrus, Castelnau, 1843, Syst. Sil., p. 20. Not recognized.

jacksoni, Green, 1837, (Trimerus jacksoni,) Am. Jour. Sci., vol. 32, p. 347, Up. Sil.

knighti, Innig, 1825, Icones. Foss. Sectiles, pl. 7, fig. 85, Low. Held. Gr.

major, Whitfield, 1885, Bull. Am. Mus. Nat. Hist., vol. 1, p. 193, Oriskany Gr. vanuxemi, Hall, 1859, Pal. N. Y., vol. 3, p. 352, Low. Held. Gr.

Iluxicurus, Hall, 1863, 16th Rep. N. Y. Mus. Nat. Hist., p. 176 . [Ety. from the genus Illanus; oura, tail.] Body broadly elliptical ; cephalic shield short, convex, semielliptical; glabella subquadrate, convex, smooth, without distinct dorsal furrow; palpebral lobe marginal; cheeks wide; facial suture nearly vertical, slightly diverging, anterior to the eye; movable cheeks wide and short; thoracic segments convex, central lobe wide, lateral lobes narrow, pygidium short, narrow, subelliptical, convex in front, more curved behind. Type I. quarlratus.

convexus, Whitfield, 1878, Anv. Rep. Geo. Sur. Wis., p. 66, and Geo. Wis., vol. 4, p. 203, Low. Mag. Gr.

eurekcnsis, Walcott, 1885, Monogr. U. S. Geo. Sur., vol. 8, p. 97, Potsdam Gr.

quadratus, Hall, 1863, 16th Rep. N. Y. St. Mus. Nat. Hist., p. 176, Potsdam Gr.

ILlжnus, Dalman, 1828, ueber die Palæaden oder die sogenannten Trilobiten, p. 51. [Ety. illaino, to look awry, to squint.] Cephalic shield very convex and like one-fourth of a sphere, with the anterior margin slightly produced; glabella defined only as a slight convexity, between subparallel lines, on the posterior part of the shield; eyes semilunate, near the lateral margins smooth; facial suture makes a gentle curve from the antero-lateral margin to the eye, and then to the margin midway of the lateral lobes of the thorax; thoracic segments 9 or ten, broad; pygidium much like the cephalic shield. Type I. crassicauda.

ambiguus, Foerste, 1885, Bull. Sci. Lab.

Denison Univ., p. 106, Niagara.Gr. 
americanus, Billings, 1859, Can. Nat. and Geol., vol. 4, p. 371, Trenton Gr.

angusticollis, Billings, 1859, Can. Nat. and Geo., vol. 4, p. 376, Black Riv. Gr.

arcturus, Hall, 1847, Pal. N. X., vol. 1, p. 23, Chazy and Black Riv. Grs.

arcuatıs, Billings, 1865, Pal. Foss., vol. 1, p. 279, Quebec Gr.

armatus, Hall, 1S67, 20th Rep. N. Y. St.

Mus. Nat. Hist., p. 418, Niagara Gr.

barriensis, Murch. 1839, Sil. Syst. The species formerly identified with this is Illæenus ioxus.

bayfieldi, Billings, 1859, Can. Nat. and Geo., vol. 4, p. 369 , Cliazy Gr.

clavifrons, Billings, 1859, Can. Nat. and Geo., vol. 4, p. 379, Chazy and Black Riv. Grs.

conifrons, Billings, 1859, Can. Nat. and Geo., vol. 4, p. 378, Black Riv. Gr.

conradi, Billings, 1859, Can. Nat. and Geo., vol. 4, p. 372, Black Riv. Gr.

consimilis, Billings, 1865, Pal. Foss., vol. 1, p. 277, Quebec Gr.

consobrin us, Billings, 1865, Pal. Foss., vol. 1, p. 280, Quebec Gr.

cornigerus, Hall, 1872, 24th Rep. N. Y.

St. Mus. Nat. Hist., p. 186, Niagara Gr. crassicauda, Wahlenberg, 1821, (Entomostracites crassicauda,) Nov. Act. Soc. Upsal., vol. 8, p. 27, and Pul. N. Y., vol. 1, p. 229 , Trenton and Galena Grs.
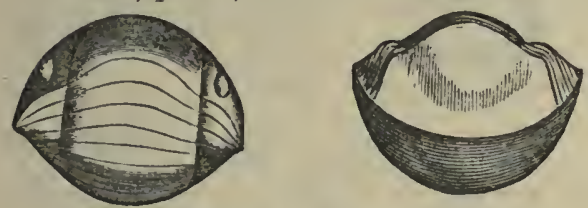

Fig. 1015.-Illæuris globosus. Two views.

cuniculus, Hall, 1867, 20th Rep. N. Y. St. Mus. Nat. Hist., p. 421. Niagara Gr. daytonensis, Hall \& Whitfield, 1875, Ohio Pal., vol. 2, p. 119, Niagara Gr.

fraternus, Billings, 1865, Pal. Foss., vol. 1, p. 276, Quebec Gr.

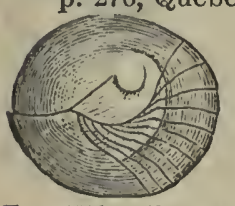

Frg. 1016.-Illæenus globosns. Side view.

Can grandis, Billings, 1859 , Rol. 4, p. 380, Hud. Riv. Gr. and Mid. Sil.

herricki, Foerste, 1887, 15th Rep. Geo. and Nat. Hist. of Minn., p. 479 , Trenton Gr. imperator, Hall, 1861, Rep. of Progr. Wis., p. 49, and 20 th Rep. N. Y. St. Mus. Nat. Hist., p. 332, Niagara Gr.

incertus, Billings, 1865, Pal. Foss., vol. 1, p. 332, Quebec Gr.

indeterminatus, Walcott, 1877, 31st Rep. N. Y.St. Mus. Nat. Hist., p. 70, Black Riv. Gr. insignis, Hall, 1864, 20th Rep. N. Y. St. Mus. Nat. Hist., p. 331, Niagara Gr. ioxus, Hall, 1867, 20th Rep. N. Y. St. Mus. Nat. Hist., p. 420, Niagara Gr.

latidorsatus, Hall, 1847, Pal. N. Y., vol. 1, p. 230, Trenton Gr.

madisonanus, Whitfield, 1882, Geo. Wis., vol. 4, 1. 307, Niagara Gr.

milleri, Billings, 1859, Can. Nat. and Geo., vol. 4, p. 375, Black Riv. and Trenton Grs.

minnesotensis, Foerste, 1887, 15th Rep. Geo. and Nat. Hist. of Minn., p. 478, Trenton Gr.

niagarensis, Whitfield, 1880, Ann. Rep. Geo. Sur. Wis., p. 68, Niagara Gr.

orbicaudatus, Billings, 1859, Can. Nat. and Geo., vol. 4, p. 379, Hud. Kiv. Gr. and Mid. Sil.

ovatus, Conrad, 1843, (Thaleops ovatus,) Proc. Acad. Nat. Sci. Phil., vol. 1, p. 332, and Pal. N. Y., vol. 1, p. 259, Black Riv. Gr.

pterocephalus, Whitfield, 1878, Ann. Rep. reo. Sur. Wis., p. 87, and Geo. Wis., vol. 4 , p. 309 , Niagara Gr.

simulator, Billings, 1865, Pal. Foss., vol. 1, p. 327, Quebec Gr.

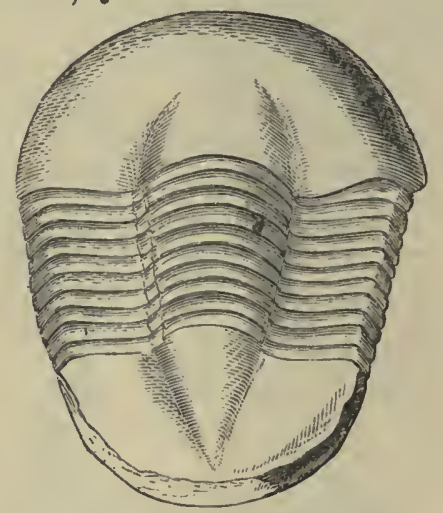

Fig. 1017.-Illæn us taurus.

taurus, Hall, 1861, Rep. of Progr. Wis. Sur., p. 49, and Geo. Sur. Ill., vol. 3 , p. 320 , Trenton and Galena Grs.

trentonensis, Emmons, 1842, (Bumastus trentonensis,) Geo. Rep. N. Y., p. 390, and Pal. N. Y., vol. 1, p. 230, Trenton Gr.

tumidifrons, Billings, 1865, Pal. Foss., vol. 1, p. 278, Quebec Gr.

vindex, Billings, 1865, Pal. Foss., vol. 1, p. 179, Chazy Gr.

worlienarius, syn. for Illænus insignis.

Isochilina, Jones, 1858, Can. Org. Rem., Decade 3, p. 197. [Ety. isos, equal; cheilos, lip.] Equivalve, the margins of the valves meeting uniformly, not overlapping, as in Leperditia greatest convexity central or toward the anterior end, eye tubercle present; muscular spot not distinct, externally. Type I. ottawa. 
armata, Walcott, 1883, 35th Rep. N. Y. St. Mus. Nat. Hist., p. 213, Trenton Gr.

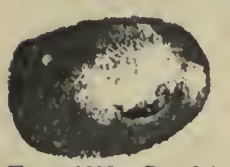

FIG. 1018.-Isoch1IIna jonesi. cylindrica, Hall, 1852,

(Cytherina cylindrica,)

Pal. N. Y., vol. 2, p.

14, Medina Gr.

gracilis, Jones, 1858,

Can. Org. Rem. Decade 3 , p. 98, Black Riv. and Trenton Grs.

jonesi, Wetherby, 1881, Jour. Cin. Soc. Nat. Hist., vol. 4, p. 80 , Trenton Gr.

labrosa, Jones, 1889, Ann. and Mag. Nat. Hist., 6th ser., vol. 3, p. 383, Low. Held. Gr.

ottawa, Jones, 1858, Can. Org. Rem., Decade 3, p. 97, Black Riv. Gr.

Isotelus,, DeKay, 1825, Annals Lyceum Nat. Hist. N. Y., vol. 1, p. 174. [Ety. isos, equal; telos, end.] A subgenus of Asaphus.

canalis, see Asaphus canalis.

gigas, see Asaphus gigas.

maximus, see Asaphus megistus.

megistus, see Asaphus megistus. vigilans, see Asaphus vigilans.

LraiA, Jones, 1862, App. to Mon. Foss. Estheria., p. 116. [Ety. proper name.] Carapace bivalve, subquadrate; thin, horny, truncated and slightly curved belind, rounded in front, straiglit on the dorsal edge; surface concentrically riclged and finely reticulated in the furrows; each valve crossed by one, two, or three ridges; the first and most conspicuous crosses from the anterior part of the umbo to the anteroventral angle; the second, when it exists, reaches the postero-ventral angle, and the third lies along the dorsal margin. Type I. leidyi.

leidyi, Lea, 1856, (Cypricaria leidyi,) Proc. Acad. Nat. Sci., vol. 7, p. 341, Coal Meas.
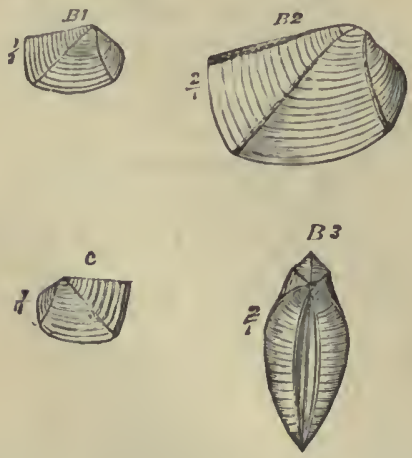

FIG. -1019.-Leaia tricarinata. $B$ 1, right valve; $B 2$, enlarged; $B 3$, dorsal vlew; $c$, left valve.

tricarinata, Meek \& Worthen, 1868, Geo. Sur. Ill., vol. 3, p. 541, Coal Meas.

Leperditia, Rouault, 1851, Bull. Soc. Geo. France, 2d ser., t. 8, p. 377. [Ety. lepis, scale; dittos, double.] Carapace bivalve, inequivalve, right valve larger than the left, and overlapping the ventral border, and to some extent the anterior and posterior borders of the left valve; valves smooth, convex, horny, oblong, longer than broad, bean-shaped, inequilateral, posterior half the broader; dorsal border straight; ventral border semicircular. Type L. brittanica.

alta, Con rad, 1843, (Cytherina alta,) Geo. Rep. $3 d$ Dist. N. Y., p. 112, and Pal. N. Y., vol. 3, p. 373, Low. Held. Gr.

amygdalina, Jones, 1858, Can. Org. Rem., Decade 3, p. 97, Chazy Gr.

angulifera, Whitfield, 1882, Ann. N. Y. Acad. Sci., vol. 2, p. 197, Low. Held. Gr.

anna, Jones, 1858, Can. Org. Rem., Decade 3, p. 96 , Hud. Riv. Gr.

anticostiana, Jones, 1858 , (I. canadensis var. anticostiana,) Can. Org. Rem., Decade 3 , p. 95, Hud. Riv. Gr.

arctica, Jones, 1856, Ann. and Mag. Nat. Hist., $2 d$ ser., vol. 17, p. 87, Up. Sil.

argenta, Walcott, 1886, Bull. U. S. Geo. Sur., No. 30, p. 146, Up. Traconic.

billingsi, Jones, 1881, Ann. and Mag. Nat. Hist., 5th ser., vol. 18, Trenton Gr.

bivertex, Ulrich, 1879, Jour. Cin. Soc. Nat. Hist., vol. 2, p. 11, Utica Slate Gr.

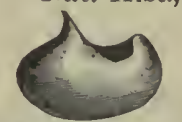
ditia byrnesi. 1lag.
Fig. 1020.-Leperbivia, White, 1874, Rep.

Invert. Foss., p. 11, and Geo. Sur. W. 100th Mer., vol. 4, ए. 58, Quebec Gr.

byruesi, S. A. Miller, 1874, Cin. Quar. Jour. Sci., vol. 1, p. 123, Utica Slate Gr. cæcigena, S. A. Miller, 1881, Jour. Cin. Soc. Nat. Hist., vol. 4, p. 262, Hud. Riv. Gr.

canadensis, Jones, 1858, Ann. Nat. Hist., 3d ser., vol. 1, p. 244, Chazy to Trenton Gr.

capax, Safford. Not defined. carbonaria, Hall, 1858, (Cythere carbonaria,) Trans. Alb. Inst., vol. 4, p. 33, and Bull. Am. Mus. Nat. Fia. 1021.-LeHist., p. 94, Warsaw Gr. perdilia cacayuga, Hall, 1862, 15 th cigena. NatRep. N. Y. St. Mus. Nat. magnifled.

Hist., p. 83, Cornif. Gr.

concinnula, Billings, 1865, Pal. Foss., vol. 1, p. 299, Quebec Gr. or Up. Taconic. crepiformis, Ulrich, 1879, Jour. Cin. Soc. Nat. Hist., vol. 2, p. 10, Hud. Riv. Gr. cylindrica, Hall, 1871, 24th Rep. N. Y. St. Mus. Nat. Hist., p. 231, Utica Slate and Hud. Riv. Grs.

dermatoides, Walcott, 1887, Am. Jour.

Sci. and Arts, $3 \mathrm{~d}$ ser., vol. 34, p. 192 , Up. Taconic.

ebinina, Dwight, 1889, Am. Jour. Sci. and Arts, 3d ser., vol. 38, p. 144, Up. Taconic.

faba, Hall, 1876, 28th Rep. N. Y. St. Mus. Nat. Hist., p. 186, Niagara Gr. 
fabulites, Conrad, 1843, (Cytherina fabulites,) Proc. Acad. Nat. Sci. Phil., p. 332, Trenton Gr.

fonticola, Hall, 1867, 20th Rep. N. Y. St. Mus. Nat. Hist., p. 428, Niagara Gr.

gibbera, Jones, 1856, Ann. and Mag. Nat. Hist., 2d ser., vol. 17, p. 90, Niagara Gr.

gibbera var. scalaris, see L. scalaris.

gracilis, see Isochilina gracilis.

budsonica, Hall, 1859, P'al. N. Y., vol. 3, p. 375 , Low. Held. Gr.

jonesi, Hall, 1859, Pal. N. Y., vol. 3, p. 372, Low. Held. Gr.

josephana, Jones, 1858, (L: canadensis var. josephana,) Can. Org. Rem., Decade 3, p. 94, Black Riv. to Trenton $\mathrm{Gr}$.

labrosa, Jones, 1858, (L. canadensis var. labrosa,) Can. Org. Rem., Decade 3, p. 93, Chazy Gr.

louckana, Jones, 1858, (L. canadensis var. louckana,) Can. Org. Rem., Decade 3, p. 93, Black Riv. Gr.

marginata, Keyserling, 1846, Wissenschaftliche Beobachtungen, etc., Niagara Gr.

minutissima, Hall, 1871, 24th Rep. N. Y. St. Mus. Nat. Hist, p. 231, Utica Slate and Hud. Riv. Gr.

morgani, Safford. Not defined.

nana, Jones, 1858, (L. canadensis var. nana,) Can. Org. Rem., Decade 3, p. 92, Calciferous Gr.

okeni, Munster, 1830, (Cythere okeni,) Jahrbuch fur Min., Geo. und Petrif. Carboniferous.

ottawa, see Isochilina, ottawa.

ovata, Jones, 1858, Ann. and Mag. Nat. Hist., 3d ser., vol. 1, p. 252, Black Riv. Gr.

pauquettana, Jones, 1858, (L. canadensis var. pauquettana, Can. Org. Rem., Decade 3, p. 94, Black Riv. Gr.

parasitica, Hall, 1859, Pal. N. Y., vol. 3, p. 276 , Low. Held. Gr.

parvula, Hall, 1859, Pal. N. Y., vol. 3, p. 376 , Low. Held. Gr.

pennsylvanica, Jones, 1858, Ann. and Mag. Nat. Hist., 3d ser., vol. 1, p. 251, Clinton Gr.

punctulifera, Hall, 1860, 13th Rep. N. Y. St. Mus. Nat. Hist., p. 92, Ham. Gr.

radiata, Ulrich, 1879 , Jour. Cin. Soc. Nat. Hist., vol. 2, p. 9, Utica Slate Gr.

rotundata, Walcott, 1885 , Monogi. U. S. Geo. Sur.,'vol. 8, p. 206, Devonian.

scalaris Jones, 1858 , (L. gibbera var. scalaris,) Ann. and Mag. Nat. Hist., 3d ser., vol. 1, p. 250, Waterlime Gr.

seneca, Hall, 1862, 15th Rep. N. Y. St. Mus. Nat. Hist., p. 84, Ham. Gr.

sinuata, Hall, 1860, Can. Nat. and Geo., vol. 5 , p. 158 , Up. Silurian.

spinulifera, Hall, 1862, 15th Rep. N. Y. St. Mus. Nat. Hist., p. 83, Up. Held Gr. sublævis, Shumard, 1855, (Cythere sublævis,) Geo. Rep. Mo., p. 195, Low. Magnesian Gr.

troyensis, see Aristozoe troyensis.

turgida, Billings, 1865, Pal. Foss., vol. 1, p. 299, Quebec Gr.

ventralis, Billings, 1865, Pal. Foss., vol.1, p. 3010, Quebec Gr.

unicornis, Ulrich, 1879, Jour. Cin. Soc. Nat. Hist., vol. 2, p. 10, Utica Slate Gr.

Lepidilla, Matthew, 1885, Trans. Roy. Soc. Can., p. 62. [Ety. lepis, a scale.] Bivalve; hinge-line straight, projecting from the general contour of the shell; umbo and hinge-line separated from the valve by a sinus, behind which there is a foramen. Type $\mathrm{L}$. anomala.

anomala, Matthew, 1885, Trans. Roy. Soc. Can., p. 62, St. John Gr.

LEPIDITTA, Matthew, 1885, 'Trans. Roy. Soc. Can. p. 61. [Ety. lepis, scale; dittos, double.] Minute, obliquely semicircular, wider on the anterior half, hinge straight; umbones in the middle, low. Type L. alata.

alata, Matthew, 1885, Trans. Roy. Soc. Can., p. 61, St. John Gr.

curta, Matthew, 1885, Trans. Roy. Soc. Can., p. 62, St. John Gr.

LEPIDOCOL E U S

ᄂ Hist., vol. 9 ,

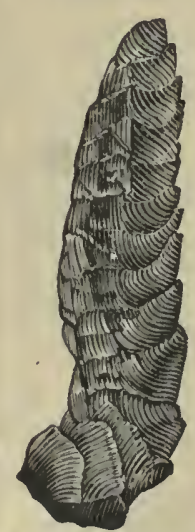

FIG. 1023.-Lepidocoleus jamesi. Faber's. Cincin nat i specimen.
Faber, 1886,

Jour. Cin.

Soc.

p. 15. [Ety.

lepis, scale ;

$\begin{array}{llllll}k & 0 & l & e & 0 & 8\end{array}$

she a th.]

Body elon-Fra. 1022.-Lepidocoleusjamest. gate, com- Mag. 2 dian. Lebanon specposed of two

series of thin, imbricating, angular plates,

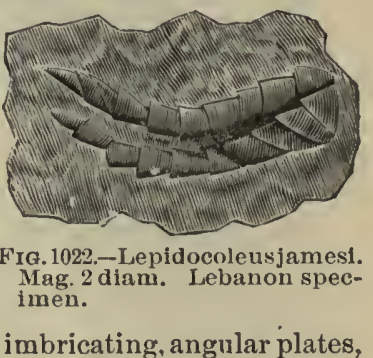
interlocking and overlapping along the basal edges; plates small, more or less triangular in outline; one side always longer than either of the others; one side usually sigmoidal; entire outer surface marked with striæ. Type L. jamesi.

jamesi, Hall \& Whitfield, 1875,(Plumulitesjamesi,) Ohio Pal., vol. 2, p. 106, Hud. Riv. Gr.

Lichas, Dalman, 1826, Uber die Palæaden oder die Sogenannten Trilobiten, p. 71. [Ety. mythological name.] Body subovate, flat, granulated; cephalic shield somewhat lunate, often pointed in front; glabella large, convex, a furrow curving inward and backward from the anterior third on each side, and cutting off or partly inclosing two oval spaces; cheeks small; eyes large, reniform; eye-line 
cutting the outer margin in front of the angles; thorax of ten segments ; pleuræ flat, falcate, each with a furrow not reaching the margin; pygidium, side lobes flat, two falcate ribs on each side projecting beyond the margin, each with a mesial duplicating groove, middle lobe, semielliptical, pointed. Type L. laciniatus.

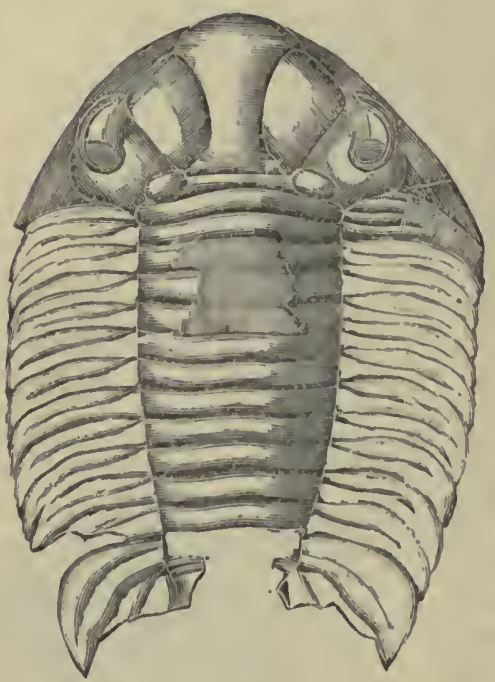

Fra. 1024.-Lichas faberi. Mag. 2 diam.

armatus, Hall, 1862. Preoccupied; changed to $\mathbf{L}$. eriopis.

bigsbyi, Hall, 1859, Pal. N. Y., vol. 3, p. 364, Low. Held. Gr.

boltoni, Bigsby, 1825, (Paradoxides boltoni,) Jour. Acad. Nat. Sci., vol. 4, p. 365 , and Pal. N. Y., vol. 2, p. 311, Niagara Gr.

boltoni var. occidentalis, Hall, 1863 , Trans. Alb. Inst., vol. 4, p. 223, and 11th Rep. Ind. Geo. Sur., p. 344, Niagara $\mathrm{Gr}$.

breviceps, Hall, 1863, Trans. Alb. Inst., vol. 4, p. 222, and 11th Rep. Ind. Geo. Sur., p. 343, Niagara Frr.

canadeusis, Billings, 1866, Catal. Sil. Foss. Antic., p. 65, Antic. Gr.

champlainensis, Whitfield, 1886, Bull. Am. Mus. Nat. Hist., vol. 1 , p. 342 , Birdseye Gr.

cucullus, Meek \& Worthen, 1865, Proc. Acad. Nat. Sci. Phil., p. 266, and Geo. Sur. Ill., vol. 3, p. 299, Trenton Gr.

decipiens, Winchell \& Marcy, 1865, Mem. Bost. Soc. Nat. Hist., p. 104, Niagara Gr.

dracon. Hall, 1888, Pal. N. Y., vol. 7, p. 85, Up. Held. Gr.

emarginatus, Hall, 1879, 28th Rep. N. Y. St. Mus. Nat. Hist., p. 199, Niagara Gr.

eriopis, see Terataspis eriopis. faberi, n. sp. Broadly elliptical, granulated; head somewhat crescentiform, slightly pointed in front, very convex, posterior angles terminating in short, obtuse spines; glabella very convex, divided into three lobes; central lobe contracted in the middle, widely expanded in front, and less expanded behind, and a slight furrow cuts off a small lobe from the postero-lateral angles; lateral lobes reniform; and another small lobe is separated from the posterior part of the cheeks by a stronger furrow; eyes prominent, reniform, and directed backward; occipital ring wide; axial lobe of thorax wider than the lateral lobes; pygidium laciniate, axis with two narrow articulations in front, and a longer posterior one that slopes backward and becomes confluent with the expanded border; lateral lobes composed of three expanded articulations, which terminate in acute points, and are marked in the central part by a groove for three-fourths of their length, which is represented by a rib on the under side; central lobe grooved in like manner, and bifid at the posterior extremity. Tlie pygidium will readily distinguish it from $L$. trentonensis, beside the broader axial lobe of the thorax and somewhat different cephalic shield. Hud. Riv. Gr. at Cincinnati, Ohio. The specimen illustrated is from the collection of Charles Faber.

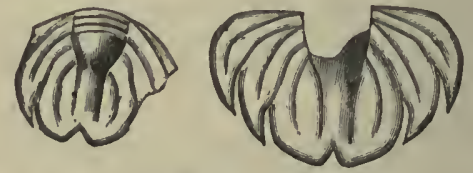

FIG. 1025.-Lichas faber1. Large and smail pygidium.

grandis, see Terataspis grandis.

gryps, Hall, 1888, Pal. N. Y., vol. 7, p. 84, Up. Held. Gr.

harrisi, S. A. Miller, 1878, Jour. Cin. Soc. Nat. Hist., vol. 1, p. 106, Hud. Riv. Gr.

hispirtus, Hall, 1888, Pal. N. Y., vol. 7, p. 77, Up. Held. Gr.

hylæus, Hall, 1888, Pal. N. Y., vol. 7, p. 81, Up. Held. Gr.

jukesi, Billings, 1865, Pal. Foss., vol. 1, pp. 282 and 335, Quebec Gr.

minganensis, Billings, 1865, Pal. Foss., vol. 1, p. 181, Chazy or Black Riv. Gr.

nereus, Hall, 1863, 16th Rep. N. Y. St. Mus. Nat. Hist., p. 226, Niagara Gr.

obvius, Hall, 1868, 20th Rep. N. Y. St. Mus. Nat. Hist., p. 424, Niagara Gr.

ptyonurus, Hall, 1888, Pal. N. Y., vol. 7, p. 86 , Niagara Gr.

pugnax, Winchell \& Marcy, 1865, Mem. Bost. Soc. Nat. Hist., p. 103, Niagara Gr. 
pustulosus, Hall, 1859, Pal. N. Y., vol. 3, p. 366, Low. Held. Gr.

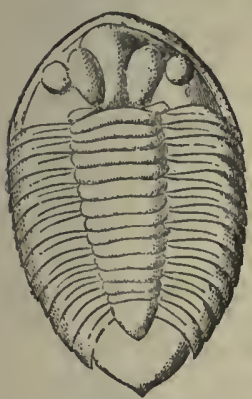

FIG. 1026.-Lichas trentonensis. s u p e r b us, Billings, 1875, Can. Nat. and Geol., vol. 7, p. 239, Up. Held. Gr.

trentonensis, Conrad, 1842, (Asaphus trent o nensis,) Jour. Acad. Nat. Sci., vol. 8, p. 277, and Pal. N. Y.; vol. 1, p. 235, Black Riv. and Trenton Grs.

L I o s r A c us, Angelin, 1852, Palæontologica Scandinavica, p. 23. [Ety. leiostrakos,

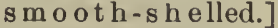

Body elongate; test smooth or with microscopic punctures; glabella elevated, furrows faint; dorsal furrow faint in front; fixed cheek arched downward at the sides; front limb concave; occipital ring aculeate; head at the genal angle rounded; ends of the pleuræe of the thorax rounded; pygidium minute, having f $\mathrm{ew}$ segments. Type L. aculeatus.

aurora, Hartt, 1868, (Conocephalites a u ror a,) Acad. Geol., p. 653, St. Fra. 1027.-LiostraJohn Gr.

linnarsoni, Brogger, 1878, Paradoxides skifrene vid Krekling, p. 47, St. John Gr.

linnarsoni var. alata, Matthew, 1887, Trans. Roy. Soc. Can., p. 147, St. John Gr.

neglectus, Hartt, 1868, (Conocephalites neglectus,) Acad. Geol., p. 652, St. John Gr. Probably a syn. for L. tener.

ouangondianus, Hartt, 1868, (Conocephalites ouangondianus,) Acad. Geol., p. 648, St. John Gr.

ouangondianus var. gibbus, Matthew, 1887, Trans. Roy. Soc. Can., p. 140, St. John Gr.

ouangondianus var. immarginata, Matthew, 1887, Trans. Roy. Soc. Can., p. 139 , St. John Gr.

ouangondianus var. planus, Matthew, 1S87, Trans. Roy. Soc. Can., p. 140, St. John Gr.

quadratus, Hartt, 1868, (Conocephalites quadratus,) Acad. Geol., p. 65t, St. John Gr.

tener, Hartt, 1868, (Conocephalites tener,) Acad. Geol., p. 652, St. John Gr.

Lisgocaris, Clarke, syn. for Spathiocaris. lutheri, see Spathiocaris Iutheri.

Lonchocephalus, Owen, 1852, Geo. Wis., Iowa, and Minn., p. 575. [Ety. longus, long; kephale, head.] Cephalic shield, having a wide frontal limb; posterior angle of each cheek terminating in a spine; glabella short, subquadrate, or truncato-conical, highly arched; two or three obscure furrows on each side; base projected backward, in a spine of greater or less length, in the median line, over the tboracic segments; facial sutures cut the anterior margin in front of the eyes, and gently curve outward and then inward to the anterior angles of the palpebral lobes; thence curving to the base of the eyes, they are directed backward and slightly outward to the posterior margin; pygidium supposed to be semilunar, with little or no border, and having four segments in the axial lobe. Type L. chippewensis.

c hi p pew ensis, $\mathrm{O}$ w e n, 1852, Geo. Wis., Iowa, and Minn., p. 576 , Potsd a m Gr.

hamulus, Owen, 1852, Geo. Wis., I o w a, a n d Frg. 1028.-LonchocephMinn., p. 576, alus chippewensis. Potsdam Gr.

wisconsinensis, Owen, 1852, Geo. Wis., Iowa, and Minn., p. 576, and 16th Rep. N. Y. St. Mus. Nat. Hist., p. 146, Potsdam Gr.

Loganellus, Devine, 1863, Can. Nat. and Geo., vol. 8, p. 95. [Ety. proper name.] General form ovate; cephalic shield lunate; glabella convex, conical, two or three oblique furrows on each side; facial suture behind the eye curving outward, and cutting the posterior margin inside the angle and in front of the eye, curving outward to the frontal margin; thorax broad, side lobes flat,

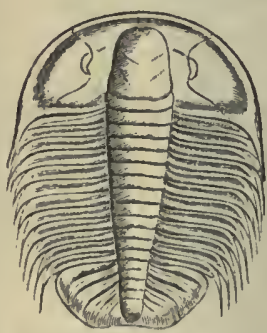

Frg. 1029.-Longanellus quebecensis. pleura about twelve; groove running along the middle nearly to the extremities; pygidium with a well-defined axis, side Io bes depressed, and with four to six ribs; distinguished from Olenus by having the facial suture curved outward in front of the eye. Type L. quebecensis. This is one of the forms often referred to Conocoryphe or to Ptychoparia, but the genus may be worth preserving.

quebecensis, Devine, 1863, Can. Nat. and Geo., vol. 8, p. 95, Quebec Gr. or Up. Taconic.

Megalaspis, Angelin, 1852, Palæontologia Scandinavica. [Ety. megale, great; aspis, shield.] Body subelliptical; cephalic shield obtusely pointed in front, genal angles spined; glabella convex, 
expanded anteriorly, no lateral furrows, eyes large and close, posterior;

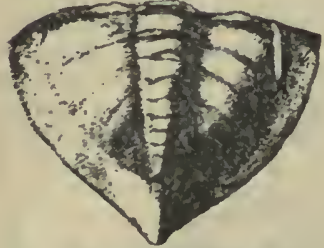

Frg. 1030.-Megalaspis belemnura. Pygldium.

facial sut ures commencing rior apex of the shield, curving laterally, and tracting to front and at the antethen conthe eyes in making a sigmoidal flexure, cut the posterior margin midway between the dorsal furrows and the genal spines; pygidium subtriangular, outer margin bordered, and terminating in a spine. Type $M$. limbata.

belemnura, White, 1874, Rep. Invert. Foss., p. 11, and Geo. Sur. IV. 100th Mer., vol. 4, p. 59, Quebec Gr. or Up. Taconic.

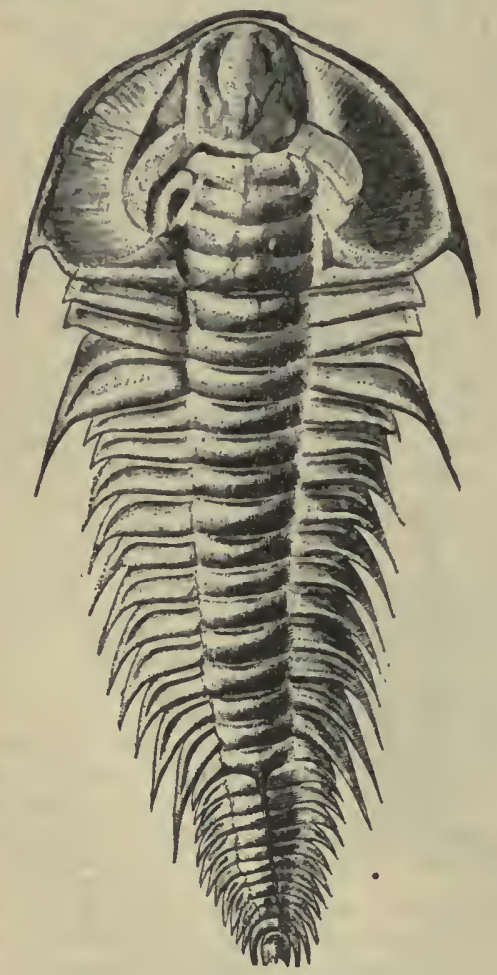

FIG. 1031.-Mesonacis vermontana.

Menocephalus, Owen, 1852, Geo. Sur. Wis., Iowa, and Minn., p. 577. [Ety. menos, strength; kephale, head.] Cephalic shield semicircular, with a narrow border all around; glabella highly convex, hemispherical or ovate, with a broadly rounded front, sometimes showing two inconspicuous lateral furrows on each side; cheeks tumid; eyes distant from the middle of the glabella; facial suture cuts the front margin a little inside a line drawn lengthwise of the body and through the eye, and cuts the posterior margin a little outside this line; thoracic segments six or seven, axis convex, tapering a little narrower than the side lobes; pygidium semicircular, axis and side lobes divided by segmental furrows. Type M. minnesotensis.

?globosus, Billings, 1860, Can. Nat. and Geo., vol. 5, p. 301, and Pal. Foss., vol. 1, p. 408, Quebec Gr. or Up. Taconic. minnesotensis, $\mathrm{O}$ we $\mathrm{n}$, 1852, Geo. Sur. Wis., Iowa, and Minn., p. 577, Potsdam Gr.

(?) salteri, Devine, 1863, Can. Nat. and Geo., vol. 8 , p. 210, and Pal. Foss., vol. 1, p. 203, Up. Ta-

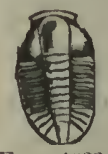

F1G. 1032. Menoceplialus saltert. conic.

? sedgwicki, Billings, .1860, Can. Nat. and Geo., vol. 5, p. 301, and Pal. Foss., vol. 1, p. 407, Quebec Gr. or Up. Taconic.

Mesonacis, Walcott, 1885, Am. Jour. Sci. and $\mathrm{Arts}$, $3 \mathrm{~d}$ ser., vol. 29, p. 32S. [Ety. mesos, middle; akis, point, spear.] Head and first fourteen segments like Elliptocepluala, and the pygidinm and ten posterior segments like Paradoxides. Type M. vermontana.

vermontana, Hall, 1859, (Olenus vermontanus,) 12th Rep. N. Y. St. Mus. Nat. Hist., p. 60, Up. Taconic, Georgia Gr.

Mesotirre, Hall, 1888, Pal. N. Y., vol. 7, p. Ivi. [Ety. mesos, middle; thuris, small door.] Carapace subquadrate; valves in contact at the apices of two broad, subtriangular extensions, situated on the dorsal line opposite the eye nodes, forming a broad and short anterior or rostral cleft, and a long posterior cleft; test broadly infolded on the lower surface, thickened and produced into a conspicuous and acute posterior spine; posterior margin incurved and produced into a short spine at the dorsal line; surface with a single strong carina on each valve; abdomen consisting of two somites, of which the posterior is the longer; post-abdomen with a broad caudal plate, which is produced into a relatively short telson; lateral spines long and setaceous. Type M. oceani.

belli, Woodward, 1870, (Dithyrocaris belli,) Geo. Mag., vol. 8, p. 106, Mid. Devonian.

neptuni, Hall, 1863, (Dithyrocaris neptuni,) 16th Rep. N. Y. St. Mus. Nat. Hist., p. 75, Ham. Gr.

oceani, Hall, 1888, Pal. N. Y., vol. 7, p. 187, Portage Gr.

spumæa, Hall, 1888, Pal. N. Y., vol. 7, p. 193, Ham. Gr.

veneris, Hall, 1888, Pal. N. Y., vol. 7, p. 193, Ham. Gr. 
Microniscus, Emmons, 1856, Am. Geol., p. 116. [Ety. mikros, small; diskos, quoit.] Subelliptical; cephalic shield semicircular; glabella narrow, convex, rounded in front, inore or less pointed behind, without furrows or occipital groove; cheeks more or less convex, no eyes or trace of sutures; thorax with four articulations, axis narrow, convex, lateral lobes wider, depressed; pygidium shorter than the cephalic shield, subtrigonal or rounded posteliorly, trilobed, axis divided into four or six seg. ments, and having a border. Type M. quadricostatus.

connexus, Walcott, 1887, Am. Jour. Sci. and Arts, 3d ser., vol. 34, p. 194, Up. Taconic.

dawsoni, Hartt, 1868, Acad. Geo., p. 654, St. John Gr.

lobatus, Hall, 1847, (Agnostus lobatus,) Pal. N. Y., vol. 1, p. 258, Up. Taconic.

meeki, Ford, 1876, Am. Jour. Sci. and Arts, 3d ser., vol. 11, p. 371, Up. Taconic. parkeri, Walcott, 1886, Bull. U. S. Geo. Sur. No. 30, p. 157, Up. Taconic.

pulchellus, Hartt, 1885,

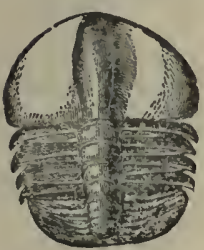

Fig. 1033.-Microdiscus quadrlcostatus. Magnified 5 diam.
Trans. Roy. Soc. Can., p. 74, St. John Gr.

pulchellus var. præecursor, Matthew, 1885, Trans. Roy. Soc. Can., p. 75, St. John Gr.

quadricostatus, Emmons, 1856, Am. Geo., p. 116, Up. Taconic.

speciosus, Ford, 1873, Am. Jour. Sci. and Arts, $3 d$ ser., vol. 6, p. 137, Up. Taconic.

Nileus, Dalman, 1826, Uber die Palæaden oder die Sogenannten Trilobiten, p. 49. [Ety. mythological name.] Cephalic shield twice as wide as long, convex, lateral angles broadly rounded; glabella subquadrate, undefined anteriorly, no lateral furrows, convex, sloping in all directions from the central part; facial sutures in front, nearly parallel with, and almost reaching, the anterior margin, each forming a sigmoid flexure to the anterior part of the eye, then forming a semicircular eye-lobe from the posterior angle of the eye, and directed laterally to the posterior margin within the broadly rounded angle of the cephalic shield ; ey es very large, lunate, with many ienses; eight thoracic segments, indistinctly trilobate, axial lobe the broader; pygidium twice as wide as long, not trilobate, no segments, broadly rounded posteriorly. Type N. armadillo.

affinis, Billings, 1865, Pal.

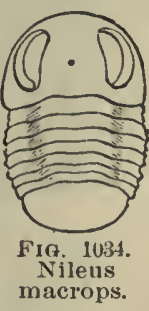

Foss., vol. 1, p. 275, Quebec Gr. or Up. Taconic. macrops, Billings, 1865, Pal. Foss., vol. 1, p. 273, Quebec Gr. or Up. Taconic.

scrutator, Billings, 1865, Pal. Foss., vol. 1, p. 274, Quebec Gr. or Up. Taconic.

Nothozoe, Barrande, Whitfield referred some ovate bodies found in the Potsdam sandstone, without characteristics, to this genus, under the name of Nothozoe vermoutana. See Bull. Am. Mus. Nat. Hist., 1884, vol. 1, p. 144.

Nuttainia, syn. for Trinucleus.

concentri:a, see Trinucleús concentricus. sparsa, syn. for Homalonotus dekayi.

Odontocephalus, Conrad, 1840, Am. Geo. Rep. N. Y. Not properly defined.

selenurus, see Dalmanites selenurus.

Odontochile, syn. for Dalmanites.

Ogyaia, Brongniart, 1822, Hist. Nat. Crust. Foss., p. 28. [Ety. mythological name.] Flat or slightly convex; cephalic shield semicircular; glabella wider in front, with three lateral furrows on each side; eyes large, lunate, affixed centrally near the glabella, facial suture marginal in front, curving like the letter $S$, and terminating posteriorly midway between the outer angle of the cephalic shield and thoracic axis; thorax with narrow axis, pointed pleurre, grooved, obscure, and remote fulcrum; eight segments; pygidium many segments, sides with radiating furrows, the interstices of which are divided by half rays. Type 0 . guettardi.

klotzi, Rominger, 1887, Proc. Acad. Nat. Sci. Phil., p. 12, Potsdam Gr.

parabola, Hall \& Whitfield, syn. for Bathyuriscus productus.

problematica, Walcott, 1885, Monogr. U. S. Geo. Sur., vol. 8, p. 63, Potsdam Gr. serrata, Rominger, gyn. for Olenoides nevadensis.

producta, Hall \& Whitfield, see Bathyuriscus productus.

spinosa, see Olenoides spinosus.

vetusta see Asaphus vetustus.

Olenellus, Hall, 1862, 15th Rep. N.Y. St. Mus.

Nat. Hist., p. 86, syn. for Elliptocephala. asaphoides, see Elliptocephala asaphoides. gilberti, see Elliptocephala gilberti.

houelli, see Elliptocephala howelli.

iddingsi, see Elliptocephala iddingsi.

vermontanus, Hall, 1859, see Mesonacis vermontana.

Olenoldes, Meek, 1877, Geol. Expl. 40th Par., vol. 4, p. 25. [Ety. Olenus, and oides form.] Ovate, head large, semicircular; glabella straight or slightly expanded in front; three pairs of furrows; eyes elongate; facial sutures extend obliquely outward from the anterior base of the eyes and cut the frontal margin; posteriorly they cut the unargin at the pleural angle, and run subparallel to the margin to the posterior end of the eye; thorax with eight or more segınents; axis strong, pleural groove broad, and lobes well defined; pygidium marked transversely on the 
axis, and lateral segments directed backward. Type $O$. nevadensis.

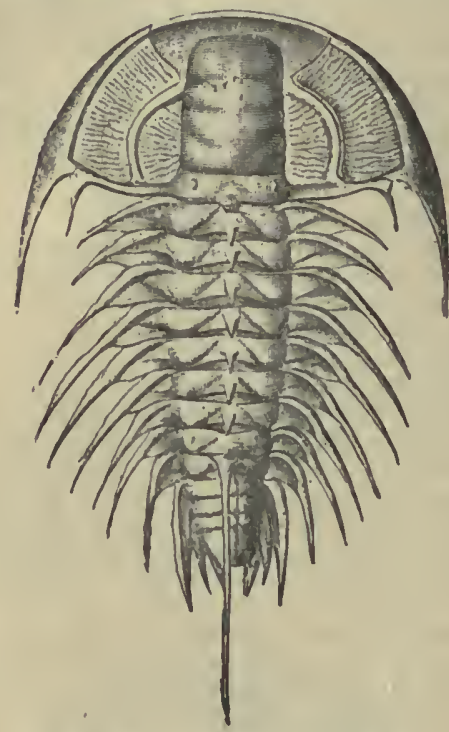

Fia. 1035.-Olenoldes týplcalls.;

fordi, Walcott, 1887, Am. Jour. Sci. and Arts, $3 d$ ser., vol. 34, p. 195, Up. Taconic. levis, Walcott, 1886, Bull. U.S. Geo. Sur., No. 30, p. 187, Up. Taconic.

nevadensis, Mcek, 1870, (Paradoxides nevadensis, (Proc. Acad. Nat. Sci. Phil., p. 62, and Geol. Expl. 40th Par., vol. 4, p. 23 , Up. Taconic.

quadriceps, Hall \& IVhitfield, 1877, (Dicellocephalus quadriceps,) Geol. Expl. 40th Par., vol. 4, p. 240, Up. Taconic.

spinosus, Walcott, 1885, (Ogygia spinosa,) Mon. U. S. Geo. Sur., vol. 8, p. 63, Up. Taconic.

stissingensis, Dwight, 1889, Am. Jour. Sci. and Ârts, 3ì ser., vol. 38, p. 147, Up. Taconic.

typicalis, Walcott, 1886, Bull. U. S. Geo. Sur. No. 30, p. 183, Up. Taconic.

wahsatchensis, Hall \& Whitfield, 1877, (Dicellocephalus wahsatchensis,) Geol. Expl. 40th Par., vol. 4, p. 241, Up. Taconic.

Olenus, Dalman, 1826, Uber die Palseaden oder die sogenannten Trilobiten, p. 54. Not an American genus.

? logani, see Loganellus quebecensis.

thompsoni, see Elliptoceplaala thompsoni. undulostriatus, see Elliptocephala undulostriata.

vermontana, see Mesonacis vermontana.

Oryctocephalus, Walcott, 1886, Bull. U. S. Geo. Sur. No. 30, p. 210. [Ety. oryktos, furrowed; kephale, head.] Glabella ob- long, transversely lobed; eyes central, narrow, ocular ridges connecting them with the axial furrow about the glahella; facial suture marginal in front, and cutting the posterior margin within

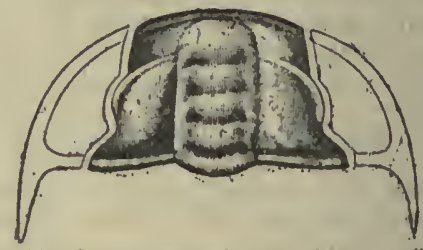

FIG. 1036.-Oryctocephnlus prlmus. Cephalle shleld.

the postero-lateral angles; free cheeks spinous; pygidium with segmented axis and pleural lobes ; margin spinous. Type O. primus.

primus, Walcott, 1886 ,

Bull. U. S. Geo.

Sur. No. 30, p. 210 ,

Up. Taconic.

Palcocaris, Meek \&

Worthen, 1865 ,

Proc. Acad. Nat.

Sci. Phil., p. 48.

[Ety. palaios, an-Fro. 1037. - Oryctocient;karis,shrimp.] Pygldtum.

Inner and outer

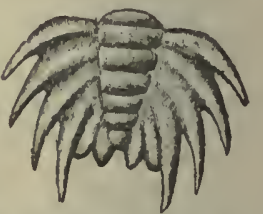

pairs of antennae of nearly equal length' 
Palmocreusia, Clarke, 1888, Pal. N. Y., vol. 7, p. 210. [Ety. palaios, ancient ; Creusia, a genus.] Capitulum ovate, patelliform, surface conical; apex truncated by a horizontal plane, forming a large central aperture; surface striated; basis tubuliform, subcylindrical or cupshaped. Type P. devonica.

devonica, Clarke, 1888, Pal. N. Y., vol. 7, p. 210, Up. Held. Gr.

Paldopaleion, Whitfield, 1880, Am. Jour. Sci. and Arts, 3d ser., vol. 19, p. 40. [Ety.palaios, ancient; palsmon, a genus.] Shrimp-like, thoracic carapace narrowed, but not rostrate in front and keeled on the back and sides; abdomen, six segments terminated by an elongated, triangular, and pointed telson; segments arched; pleuræe smooth, not lobed or expanded, extremities rounded; sixth segment bearing caudal flaps, one on each side, composed of five visibla elements, the outer four apparently anchylosed to form a triangular plate on each side of the telson; thoracic ambulatory appendages elongated, sinooth and filiform, except the upper second joint, which is laterally compressed; abdominal appendages short; antennæ large and sirong. Type P. newberryi.

newberryi, Whitfield, 1880, Am. Jour. Sci. and Arts, 3d ser., vol. 19, p.41, Erie shales.

Para Doxides, Brongniart, 1822, Hist. Nat. Crust. Foss., p. 31. [Ety. paradoxos, marvelous, paradoxical.] Cephalic shield lunate, margin thickened, not reflexed; glabella clavate or oval, moderately convex, enlarged anteriorly, three curved furrows cross it, divicling it into four parts; fixed cheeks tumid; eyes oblong, lunate, distant and opposite the second division of the glabella; facial suture, cutting the margin in front of the eye and curving S-like to the eye, and curving in like manner

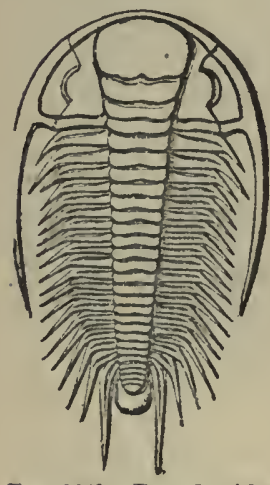

Frg. 1040.-Paradoxides bohemicus. to the posterior margin directly behind the eye; movable cheek tumid and prolonged in a spine; thorax 16 to 20 ; segments, axis convex, narrower than the lateral lobes, lateral lobes flattened and turned backward ; pygidium circular or oval; axis segmented, s li or t, lateral lobesiflattened and projected backward. Type P. tessini.

abenacus, Matthew, 1885, Trans. Roy.

Soc. Can., p. 78, St. John Gr.

acadicus, Matthew, 1883, Trans. Roy.

Soc. Can., p. 103, St. John Gr. acadicus var. suricus, Matthew, 1885 , Trans. Roy. Soc. Can., p. 77, St. John Gr.

arcualus, Harlan, 1835, Trans. Geo. Soc., syn. for Triarthrus becki.

barberi, N. H. Winchell, 1885, 13th Ann.

Rep. Geo. Sur. Minn., p. 67, Potsdam

Gr. Not a Paradoxides.

bennetti, Salter, 1859. Quar. Jour. Geo. Soc., vol. 15, p. 552, Up. Taconic.

boltoni, see Licluas boltoni.

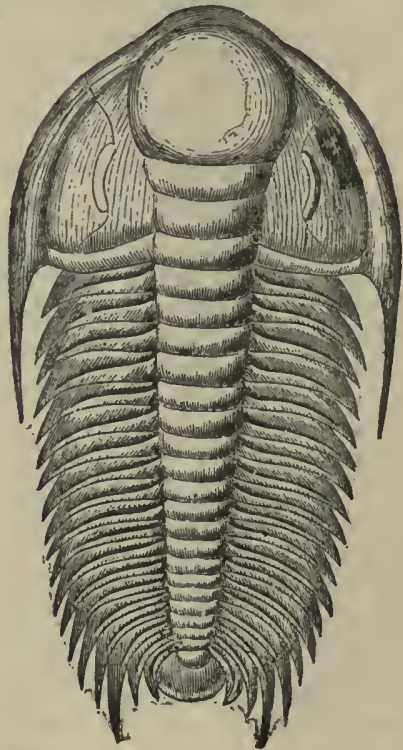

F'IG. 1041.-Paradoxides harlani.

decorus, Billings, 1874, Pal. Foss., vol. 2, p. 75, Up. Taconic.

eatoni, syn. for Triarthrus becki.

etemnicus, Matthew, 1883, Trans. Roy.

Soc. Can. pp. 92, 271, St. John Gr.

etemnicus var. breviatus, Matthew, 1883 ,

Trans. Roy. Soc. Can., p. 99, St. John Gr.

etemnicus var, malicitus, Matthew, Trans. Roy: Soc. Can., p. 101, St. John Gr.

etemnicus var. pontificalis, Matthew, 1883 , Trans. Roy. Soc. Can., p. 102, St. John Gr.

etemnicus var. quacoensis, Matthew, 1883 , Trans. Roy. Soc. Can., p. 102, St. John Gr.

etemnicus var. suricoides, Matthew, 1883, Trans. Roy. Soc. Can., p. 106, St. John Gr.

harlani, Green, '1834, Am. Jour. Sci., vol. 25, p. 336 , Up. Taconic.

lamellatus, Hartt, 1868, Acad, Geol., p. 656, St. John Gr.

lamellatus var. loricatus, Matthew, 1883, Trans. Roy. Soc. Can., p. 106, St. Jolin Gr.

micmac, Hartt, 1868, Acad. Geol., p. 657, and Trans. Roy. Soc. Can., vol. 2, p. 101, St. John Gr. 
nevadensis, Meek, see Olenoides nevadensis.

quadrispinosus, Emmons, syn. for Bathynotus holopyga.

regina, Matthew, 1887, Am. Jour. Sci. and Arts, $3 \mathrm{~d}$ ser., vol. 33 , p. 389 , and Trans. Roy. Soc. Canada, p. 115, St. John Gr.

tenellus, Billings, 1874, Pal. Foss., vol. 2, Up. Taconic.

thompsoni, see Elliptocephala thompsoni. triarthrus, Harlan, syn. for Triarthrus becki.

vermontana, see Mesonacis vermontana.

Peltura, M. Edwards, 1840 , Hist. Nat. Crust., t. 3, p. 344. Type P. scarabreoides.

holopyga, see Bathynotus, holopyga.

Pempligasprs, Hall, 1863, 16th Rep. N. Y. St. Mus. Nat. Hist., p. 221. [Ety. pemphix, pustule; aspis, shield.] Founded upon part of a shield somewhat resembling the pygidium of a trilobite; a narrow, straight, annulated axis extends to the margin posteriorly; side lobes widler, ovate and ventricose. Type P. bullata.

bullata, Hall, 1863, 16th Rep. N. Y. St. Mus. Nat. Hist., p. 211, Potsdam Gr.

Pricops, Lmmrich, 1839, de Trilobites, Dissertatio Inauguralis, p. 19. [Ety. phakos, lens; ops, eye.] Form compact, glabella inflated and expanded in front; the two front pairs of furrows are obscure; eyes large, numerous lenses; genal rounded ; pleurce rounded, pygidium moderate, of few (often coalesced) segments with an even border, never produced. Type P. latifrons.

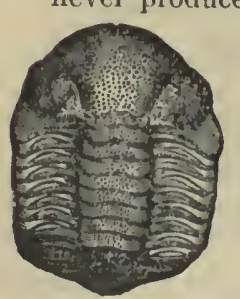

FIG. 1012.-Phacops bufo. bufo, Green, 1832, (Calymene bufo,) Monograph of Trilobites, p. 41, and Illust. Devoll. Foss., pl. 8, Ham. Gr.

bombifrons, Hall, 1862 , 15th Rep. N. Y. St. Mus. Nat. Hist., p. 67, Up. Held. Gr. cacapona, Hall, 1862, 15 th Rep. N. Y. St. 68, Up. Held. Gr.

Mus. Nat. Hist. p.

callicephala, see Dalmanites callicephalus. cristata, Hall, 1862, 15th Rep. N. Y. St. Mus. Nat. Hist., p. 67, Up. Held. Gr. cristata var. pipa, Hall, 1888, Pal. N. Y., vol. 7 , p. 18, Up. Held. Gr.

hudsonica, Hall, 1859, Pal. N. Y., vol. 3, p. 355 , Low. Held. Gr.

laticaudus, see Dalmanites laticaudus.

logani, Hall, 1859, Pal. N. Y., vol. 3, p. 353, Low. Held. Gr.

nupera, Hall, 1843, (Calymene nupera) Geo. Rep. 4th Dist. N. Y., p. 262, and Illust. Devon. Foss., pl. 8, Chemung $\mathrm{Gr}$.

orestes, Billings, 1860, Can. Nat. and Geo., vol. 4 , p. 65 , Mid. Sil. rana, Green, 1832, (Calymene bufo var. rana,) Monograph of 'Trilobites, p. 42, and Illust. Devon. Foss., pl. 7, Ham. Gr.

trajanus, Billings, 1863, Proc. Port. Soc. Nat. Hist., vol. 1, p. 124, Low. Held. Gr.

trisulcata, Hall, 1843, (Calymene (?) trisulcatr,) Geo. Rep. 4th Dist. N. Y., p. 74, and Pal. N. Y., vol. 2, p. 300, Clinton $\mathrm{Gr}$.

PudTIOonidrs, Angelin, 1878, Palicontologia Scandinavica, p. 21. [Ety. phrthon, radiant.] Head shield resembling Cyphaspis, the frontal area more concave, and lateral glabellar furrows stronger and generally duplicate; thorax having seven or more narrow segments; axis wide; pygidium resembliug Proetus, relatively large, 8 to 12 annulations on the axis, and 8 or 9 on the pleurs; these extend to the margin, and are duplicate the entire length. Type P. stokesi.

arenicolus, Hall, 1888, Pal. N. Y., vol. 7, p. 134, Up. Held. Gr.

eyclurus, Hall, 1888, Pal. N. Y., vol. 7, p. 137, Low. Held. Gr.

denticulatus, Meek, 1877, (Proetus denticulatus,) Geol. Expl. 40th Par., p. 49, Devonian.

gemmæus, Hall, 1888, Pal. N. Y., vol. 7, p. 136, Low. Held. Gr.

varicella, Hall, 1888, Pal. N. Y., vol. 7, p. 135, Up. Held Gr.

Pinllipsia, Portlock, 1843, Rep. Geol. Londonderry, p. 305. [Ety. proper name.] Cephalic shield subsemicircular, angles terminating in spines; glabella subeylindrical, not contracted at base, tliree furrows on each side; eyes large, reniform, reticulated; thorax of 9 segments having pleural grooves and distinct facets ; pygidium semioval, axis and lateral lobes furrowed, margin entire, smooth.

Type P tiremmulifer, Fig. 1043.-Phillpauriculatus, Hall, 1862 sia gemmulifera. (Proetus auriculatus,) 15th Rep. N. Y. St. Mus. Nat. Hist., p. 79, Waverly Gr. bufo, Meek \& Worthen, 1870, Proc. Acad. Nat. Sci., p. 52, and Geo. Sur. Ill., vol. 5, p. 528, Keokuk Gr.

cliftonensis, Shumard, 1858, Trans. St. Louis Acad. Sci., vol. 1, p. 227, Coal Meas.

coronata, Hall, syn. for Cyphaspis ornata.

doris, Hall, 1860, (Proetus doris,) 13th Rep. N. Y. St. Mus. Nat. Hist., p. 112, Waverly Gr.

howi, Billings, 1863, Can. Nat. and Geol., vol. 8, p. 209, Carboniferous. 
insignis, Winchell, 1863, Proc. Acad. Nat. Sei., p. 24, Burlington Gr.

lævis, see Cyphaspis lævis.

lodiensis, Meek, 1875, Ohio

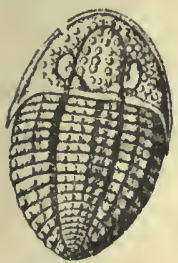

Frg. 1044.-Ph11lipsia lodiensis.

missouriensis, Shumard, 1858, Trans. St. St. Louis Acad. Sci., vol. 1, p. 225, Coal Meas.

orriata, Hall, see Cyphaspis ornata.

peraunulata, Shumard, 1858, Trans. St.

Louis Acad. Sci., vol. 1, p. 296, Permian Gr.

portlocki, Meek \& Worthen, 1865, Proc.

Arad. Nat. Sci., p. 268, and Geo. Sur.

Ill., vol. 5, p. 525, Keokuk Gr.

rockfordensis, Winchell, 1865, Proc. Acad.

Nat. Sci., p. 133, Kinderhook Gr.

sangamonensis, Meek \& Worthen, 1865 ,

Proc. Acad. Nat. Sci., p. 271, and

Geo. Sur. Ill., vol. 5, p. 615, Coal Meas.

scitula, Meek \& Worthen, 1865, Proc. Acad. Nat. Sci., p. 270, and Geo. Sur. Ill., vol. 5. p. 612, Coal Meas.

stevensoni, Meek, 1871, Reg. Rep. University W. Va., Kaskaskia Gr.

swallovi, Shumard, 1855, (Proetus swallovi,) Geo. Rep. Mo., p. 196, Waverly Gr.

tennesseensis, Winchell, 1869, Geo. of Tenn., p. 445, Waverly Gr.

tuberculata, Meek \& Worthen, 1870, Proc. Acad. Nat. Sci., p. '52, Burlington $\mathrm{Gr}$.

vindobnnensis, Hartt, 1868, Acad. Geol., p. 313, Carboniferous.

Piliolites, Cozzens, 1848. Not identified. ohioensis, Cozzens, 1848. Not identified, but probably the fragment of a Dalmanites.

Platynotus, syn for Lichas.

boltoni, see Lichas boltoni.

trentonensis, see Lichas trentonensis.

Plumulites, Barrande, syn. for Turrilepas. devonicus, see Turrilepas devonicus. gracillimus, see Turrilepas gracillimus. jamesi, see Lepidocoleus jamesi. newberryi, see Turrilepas newberryi.

Prestwichia, Woodward, 1867, Quar. Jour, Geo. Soc. Lond., vol. 23. Not known in America.

eriensis, see Protolimulus eriensis.

Primitia, Jones, 1865, Ann. and Mag. Nat. Hist., 3d ser., vol. 16, p. 415. [Ety. primitia, first of the kind.] Carapace minute; bivalve, equivalve, convex oblong; hinge straight; surface of each valve impressed, on the dorsal region either medially or toward the anterior extremity, with a vertical sulcus, variable in size. Type $P$. strangulata.

acadica, Matthew, 1885, Trans. Roy. Soc. Can. p. 66, St. John Gr.

æqualis, Jones \& Hall, 1886, Ann. and Mag. Nat. Hist., 5th ser., vol. 17, p. 411, Low. Held. Gr.

cincinnatiensis, S. A. Miller, 1875, (Beyrichia cincinnatiensis,) Cin. Quar. Jour. Sci., vol. 2, p. 350 , Hud. Fro. 1045 Riv. Gr. concinna, Jones, 1858, (Cyther- ensis.

opsis concinna,) Ann. and

Mag. Nat. Hist., 3d ser., vol. 1, p. 249, Black Riv. Gr.

cristata, Whitfield, 1889, Bull. Am. Mus. Nat. Hist., vol. 2, p. 59, Calciferous Gr.

gregaria, Whitfield, 1889, Bull. Am. Mus. Nat. Hist., vol. 2, p. 58, Calciferous Gr.

logani, Jones, 1858, (Beyrichia logani,) Ann. and Mag. Nat. Hist., 3d ser., vol. 1, p. 244, Chazy Gr.

leperditioides, Jones, 1858, (Beyrichia logani var. leperditioides,) Can. Org. Rem., Decade 3, p. 91, Chazy Gr.

mundula, Jones, 1855, Ann. and Mag. Nat. Hist., 2d ser., vol. 16, p. 90, Low. Devonian.

muta, Jones, 1865, Ann. and Mag. Nat. Hist., $3 \mathrm{~d}$ ser., vol. 16, p. 425, Up. Sil.

reniformis, Jones, 1858, (Beyrichia logani var. reniformis,) Can. Org. Rem., Decade 3 , p. 91 , Chazy Gr.

rugulifera, Jones, 1858, (Beyrichia rugulifera,) Ann. and Mag. Nat. Hist., 3d ser., vol. 1, p. 242, Niagara Gr.

scaphoides, Jones, 1889, Ann. and Mag. Nat. Hist., 6th ser., vol. 3, p. 377, Low. Devonian.

seeleyi, Whitfield, 1889, Bull. Am. Mus. Nat. Hist., vol. 2, p. 60, Calciferous Gr.

sigillata, Jones, 1858, (Beyrichia sigillata,) Ann. and Mag. Nat. Hist., $3 d$ ser., vol. 1, p. 242, Niagara Gr.

Proktus, Steininger, 1830, Bemerkungen uber die Versteinerungen welche im Uebergangs-Gebirge der Eifel, p. 4. [Ety. mythological name.] Subelliptical ; cephalic shield semicircular, margin thickened; glabella very convex, parabolic, rounded anteriorly, no lateral furrows; neck furrow well marked; eyes prominent, smooth, close to glabella; facial suture, on a line with the eyes in front, curves gently backward and reaches the posterior margin, within the genal angle; thoracic segments 10, convex, lateral lobes, with an oblique indentation; pygidium tri- 
lobed, segmented, semicircular; axis very convex, short. Type $\mathrm{P}$. cuvieri.

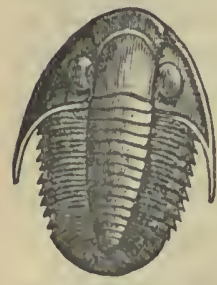

FIG. 1016.-Proetus alaricus. alaricus, Billings, 1860 , Can. Nat. and Geo., vol. 5, p. 68, Hud. Riv. Gr.

angustifrons, Hall, 1862 , 15th Rep. N. Y. St. Mus. Nat. Hist., p. 70, Schoharie grit.

auriculatus, see Phillipsia auriculatus.

canaliculatus, Hall, 1862 , 15th Rep. N. Y. St. Mus. Nat. Hist,, p. 73, Up. Held. Gr.

clarus, Hall, 1862, 15th Rep. N. Y. St. Mus. Nat. Hist., p. 71, Up. Held. Gr. conradi, Hall, 1862, 15 th Rep. N. Y. St.

Mus. Nat. Hist., p. 69 , and Illust. Devon.

Foss., pl. 20, Schoharie grit.

corycoeus, Conrad, 1842, (Asaphus corycœus,) Jour. Acad. Nat. Sci., vol. 8 , p. 277, and Pal. N. Y., vol. 2, p. 315 , Niagara $\mathrm{Gr}$.

crassimarginatus, Hall, 1843, (Calymene crassimarginata,) Geo. Rep. 4th Dist. N. Y., v. 172, and Illust. Devon. Foss., pl. 20, Up. Held. Gr.

curvimarginatus, Hall, 1888, Pal. N. I., vol. 7, p. 94, Up. Held. Gr.

davenportensis, Barris, 1879, Proc. Dav. Acad. Sci., vol. 2, p. 287, syn. for P. pronti.

delphinulus, Hall, 1888, Pal. N. Y., vol. 7, p. 111, Up. Held. Gr.

denticulatus, see Phæthonides denticulatus.

doris, see Phillipsia doris.

ellipticus, Meek \& Worthen, 1865, Proc. Acad. Nat. Sci, p. 267, and Geo. Sur. Ill., vol. 3, p. 460, Kinderhook Gr.

folliceps, Hall, 1888, Pal. N. Y., vol. 7; p. 101, Up. Held. Gr.

granulatus, Wetherby, 1881, Jour. Cin. Soc. Nat. Hist., vol. 4, p. 81 , Kaskaskia Gr.

haldemani, Hall, 1862, 15th Rep. N. Y. St. Mus. Nat. Hist., p. 74, Ham. Gr.

hesione, Hall, 1862, 15th Rep. N. Y. St. Mus. Nat. Hist., p. 70, and Illust. Devon. Foss., pl. 20, Schoharie grit.

jejumus, Hall, 1888, Pal. N. Y., vol. 7, p. 124, Ham. Gr.

junius, Billings, 1863, Proc. Port. Soc. Nat. Hist., vol. 1, p. 122, Low. Held. Gr.

latimarginatus, Hall, 1888, Pal. N. Y., vol. 7 , p. 97, Up. Held. Gr.

loganensis, Hall \& Whitfield, 1877, U. S. Geo. Exp. 40th Par., vol. 4, p. 264, Waverly Gr.

longicaudus, Hall, 1862, 15th Rep. N. Y. St. Mus. Nat. Hist., p. 108, and Illust. Devon. Foss., pl. 20, Ham. Gr.

macrobius, Billings, 1863, Proc. Port. Soc. Nat. Hist., vol. 1, p. 123, Low. Held. Gr. macrocephalus, Hall, 1862, 15th Rep. N. Y. St. Mus. Nat. Hist., p. 77, Ham. Gr.

marginalis, Conrad, 1839, (Calymene marginalis,) Ann. Geo. Rep. N. Y., p. 66, and Illust. Devon. Foss., pl. 21, Tully limestone.

microgemma, Hall, 1888, Pal. $\mathrm{N}$. Y., vol. 7, p. 109, Up. Held. Gr.

missouriensis, Shumard, 1855, Geo. Rep. Mo., p. 110, Waverly or Choteau Gr.

nevadæ, Hall, 1888, Pal. N. Y., vol. 7, p. 129, Low. Devonian.

occidens, Hall, 1862, 15th Rep. N. Y. St. Mus. Nat. Hist., p. 80, Ham. Gr.

ovifrons, Hall, 1888, Pal. N. Y., vol. 7, p. 110 , Up. Held. Gr.

parviusculus, Hall, 1860, 13th Rep. N. Y. St. Mus. Nat. Hist., p. 120, and 24th Rep., p. 223, Hud. Riv. Gr.

peroccidens, IIall \& Whitfield, 1877, U. S. Geo. Expl. 40th Par., vol. 4, p. 262, Waverly Gr.

phocion, Billings, 1874, Pal. Foss., vol. 2, p. 63, Gaspe limestone No. 8, Devonian. planimarginatus, Meek, 1871, Proc. Acad. Nat. Sci. Phil., p. 89, and Ohio Pal., vol. 1, p. 223, Up. Held. Gr. protuberans, $\mathrm{Hall}$, 1859, Pal. N. Y., vol. 3 , p. 351 , Low. Held. Gr.

prouti, Shumard, 1863,

Trans. St. Louis Acad. Frg. 1047. - Proetus Sci., vol. 2, p. 110, phociou. Ham. Gr.

rowii, Green, 1838, (Calymene rowii,) Am. Jour. Sci., vol. 33, p. 406, and Illust. Devon. Foss., pl. 21, Ham. Gr.

spurlocki, Meek, 1872, Am. Jour. Sci., $3 \mathrm{~d}$ ser., p. 426, and Oliio Pal., vol. 1, p. 161, Hud. Riv. Gr. The young of an Asaphus.

stenopyge, Hall, 1888, Pal. N. Y., vol. 7, p. 110, Up. Held. Gr.

stokesi, Miurchison, 1839, (Asaphus stokesi,) Sil. Syst., p. 625, and Pal. N. Y., vol. 2, p. 316, Niagara Gr.

swallovi, see Phillipsia swallovi.

tumidus, Hall, 1888, Pal. N. Y., vol. 7, p. 113, Up. Held. Gr.

verneuili, Hall, 1861, 15th Rep. N. Y. St. Mus. Nat. Hist., p. 73, and Illust. Dev. Foss., pl. 20, Up. Held. Gr.

Protichnites, Owen, 1852, Jour. Geo. Soc., vol. 8, p. 214. [Ety. protos, first; ichnos, foot-print; lithos, stone.] It consists of two rows of tracks or depressions, several inches apart; each row consists of numerous irregular and subcircular depressions, sometimes elongated; between the rows there is frequently a groove, and at other times, the surface 
has apparently been pressed smooth. Type P. septemnotatus.

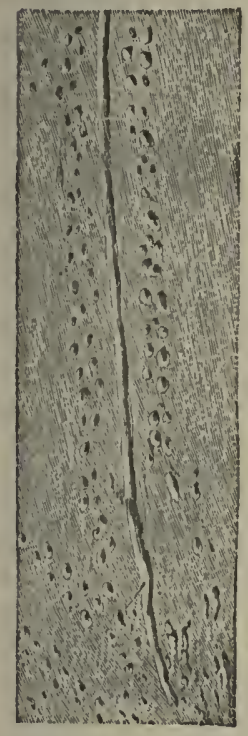

FIG. 1018. - Protichnites septemnotatus.

alternans, Owen, 1852, Jour. Geo. Soc., vol. 8, pl. 14, Potsdam Gr.

latus, Owen, 1852, Jour. Geo. Soc., rol. 8, pl. 11, Potsdam Gr.

lineatus, Owen, 1852, 8, pl. 13, Potsdam Gr.

logananus, Marsh, 1869, Am. Jour. Sci. and Arts, 2d ser., vol. 48, Potsdam Gr. multinotatus, Owen, 1852, Jour. Geo. Soc., vol. 8, pl. 12, Potsdam Gr.

octonotatus, Owen, 1852, Jour. Geo. Soc., vol. 8 , pl. 10, Potsdam Gr.

septemnotatus, Owen, 1852, Jour. Geo. Soc., vol. 8 , pl. 9. Potsdam Gr.

Protobalanus, Whitfield, 1888, Pal.N.Y,. vol. 7, p. lxii. [Ety. Jour. Geo. Soc., vol. protos, first; Balanus, genus.] Shell ovate about the basis; composed of 12 plates of which the carina is largest and most elevated; rostrum small; lateralia five on each side; radial areas between the lateralia broad. Type P. hamiltonensis.

hamiltonensis, Whitfield, 1888, Pal. N. Y., vol. 7 , p. 209, Ham. Gr.

Protocaris, IValcott, 1884, Bul.. U. S. Geo. Sur., vol. 2, p. 283. [Ety. protos, first; karis, shrimp.] Carapace without evidence of a dorsal suture, rounded on the dorsal line, and bent downward on the sides; no rostrum; body many jointed, 31 segments extending out from beneath the carapace, the last seginent broader than the preceding, and terminating in two spines. Type p. marshi.

marshi, Walcott, 1884, Bull. U. S. Geo. Sur. vol. 2, p. 283, Georgia Gr.

Protolimulus, Packard, 1886, Mem. Nat. Acad. Sci., p. 150. [Ety. protos, first; Limulus, a genus.] Cephalothorax large, subsemicircular ; genal angles produced; cephalic appendages small; terminal segments of the posterior members foliaceous; abdomen composed of six (?) segments, including the large caudal spine. Type P. eriensis.

eriensis, Williams, 1885, (Prestwichia eriensis,) Am. Jour. Sci. and Arts, 3d ser., vol. 30 , p. 46, Chemung Gr.

Prototy pus, Walcott, 1886, Bull, U. S. Geo. Sur., No. 30, p. 211 . [Ety. protos, first ; typus, type.] Body ovate; head broad, semicircular; glabella large, sides parallel, rounded in front, no furrows; frontal limb narrow, in front of the

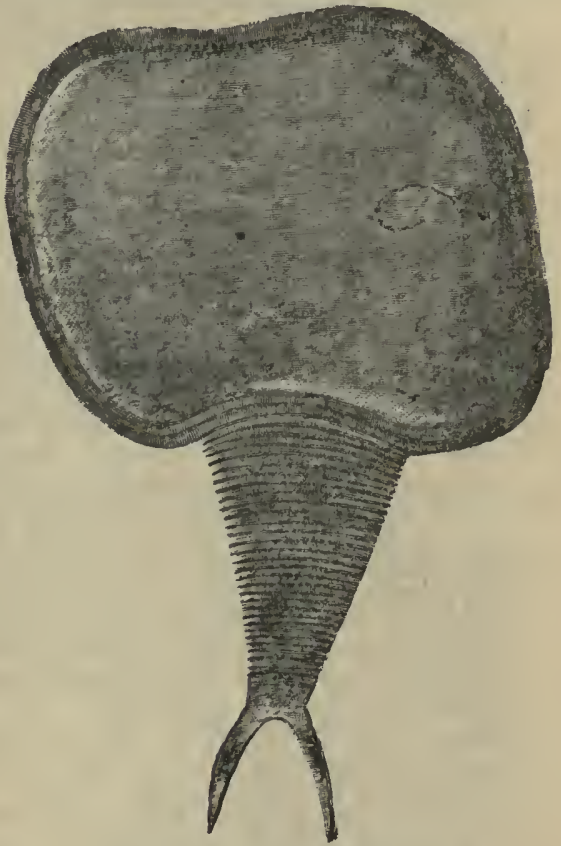

FrG. 1049.-Protocaris marsh1.

glabella and bordered; fixed cheeks crossed in front of the eyes by an ocular ridge; eyes large, reniform; occipital ring narrow; movable cheeks curved on the outer margin and terminating

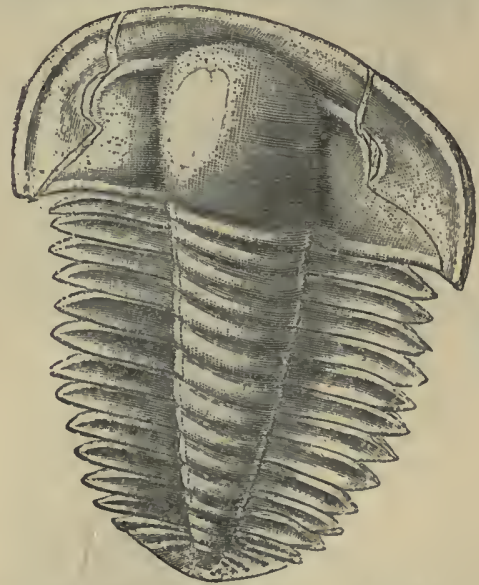

FIG. 1050.-Prototypus hitchcocki.

in spines; facial suture passing very little outward from the eye to the anterior margin, which it cuts at nearly right angles; behind the eye, it passes obliquely outward and backward, with 
slight eurvature, to just within the cheek spine; thorax, twelve segmeuts, strongly trilobed; pleura straiglit, broadly channeled and pointed; pygidium small, semielliptical, and marked by three furrows on the small axis and lateral areas. Type P. hitchicocki.

hitchcocki, Whitfield, 188t, (Angelina bitchcocki,) Bull. Am. Mus. Nat. Hist., vol. 1, p. 148, Up. Taconic.

Pterocepilalia, Roemer, 1849, Texas, mit naturwissench. Anhang. Bonn., and afterward in 1852, Kreid von Texas, p. 92. [Ety. pteron, wing; kephale, head.] Cephalic shield semicircular, nearly flat; glabella less than half the length of the lsead shield, with a flat, winglike projection in front; two or three furrows on each side; neck furrow distinct; facial sutures dir cted nearly straight back from the anterior margin to the eye, after passing which it is directed at an angle laterally of about forty-fivedegrees to the posterior margin; eyes situate nearly opposite the posterior lobe of the glabella; pygidium subcircular, margin flattened and produced; axial lobe narrow, about ten segments. Type P. sanctisabre.

laticeps, Hall \& Whitfield, 1877, (Conocephalites laticeps,) Geo. Expl. 40th Par., vol. 4, p. 221, Potsdam. Gr.

occidens, Walcott, 1884, Mon. U. S. Geo. Sur., vol. 8 , p. 58, Potsdam Gr.

sanctisaba, Roemer, 1849, Texas, mit naturwissench. Anhang., and in 1852, Kreid von Texas, p. 92, Potsdam Gr.

Pterygotus, Agassiz, 1839, Murch. Sil. Syst., p. 605. [Ety. pleron, wing; ous, ear.] Distinguistzed from Eurypterus by having eyes marginal instead of within the

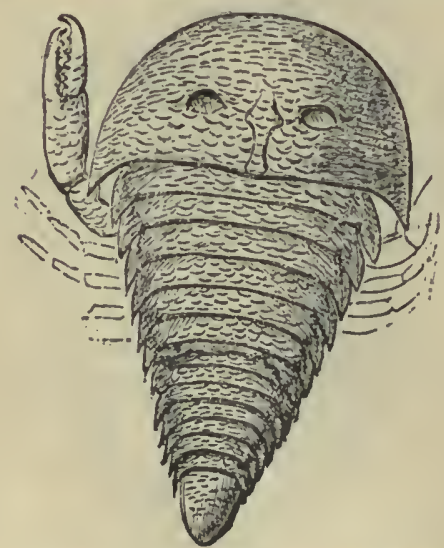

Fia. 1051.-Pterygotus problematicus.

carapace, twelve segments instead of thirteen in the body, a bilobate caudal extremity and chelate antennie at the anterior part of the carapace. Type P. problematicus. acuticaudatus, Pohlman, 1882, Bull. Buf. Soc. Nat. Sci., vol. 4, p. 42, Waterlime Gr. buffuloensis, Pohlman, Bull. Buf. Soc. Nat. Hist., vol. 4, p. 17, Waterlime Gr. cobbi, Hall, 1859, Pal. N. Y., vol. 3, p. 417, Waterlime Gr.

cummingsi, Grote \& Pitt, 1875, Bull. Buf.

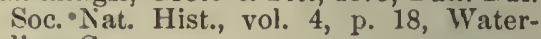
lime Gr.

globicaudatus, Pohlman, 188\%, Bull. Buf. Soc. Nat. Hist., vol. 4, p. 42 , Waterlime Gr.

macrophthalmus, Hall, 1859, Pal. N. Y., vol. 3 , p. 418, Waterlime Gr.

osborni, Hall, 1859, Pal. N. Y., vol. 3, p. 419, Waterlime Gr.

quadraticaudatus, Pohlman, 1882, Bull. Buf. Soc. Nat. Hist., vol. 4, p. 43, Waterlime $\mathrm{Gr}$.

Ptycinaspis, Hall, 1863, 16tl Rep. N. Y. St. Mus. Nat. Hist., p. 170 . [Ety. ptyche, fold; aspis, shield.] Cephalic shield broad, with wide depressed convex cheeks; glabella cylindrical, convex, transversely lobed, prominent in front; eyes anterior to the iniddle; facial suture cutting the anterior border almost in front of the eye, and from below the eye it proceeds obliquely to the base a little without the center of the cheek, leaving the movable cheek near the size of the tixed cheek; movable cheek subtrapezoidal, border thickened, and extended backward in a spine. Type $\mathbf{P}$. miniscensis.

barabuensis, Winchell, 1864, Am. Jour. Sci. and Arts, 2 d ser., vol. 37, p. 230 , Potsdam Gr.

granulora, Owen, 1852, (Dikelocephalus granulosua,) Geo. Wis., Iowa, and Minn., p. 575 , Potsdam Gr.

miniscensis, Owen, 1852, (Dikelocepbalus miniscaensis,) Geo. Wis., Iowa, and Minn., p. 574, Potsdam Gr.

minuta, Whitfield, 1878, Ann. Rep. Geo. Sur. Wis., p. 55, and Geo. of Wis., vol. 4, p. 186, Potsdam Gr.

pustulosa, Hall \& Whitfield, 1877, U. 'S. Geo. Expl. 40th Par., vol. 4, p. 223, Potsdam Gr.

sesostris, Billings, 1865 (Dikelocephalus sesostris), Pal. Foss., vol. 1, p. 198, Quebec Gr. or Up. Taconic. speciosa, Walcott, 1879 , 32d Rep. N. Y. St. Fio. 1052.-Piychaspis Mus. Nat. Hist., p. 131, Calciferous Gr.

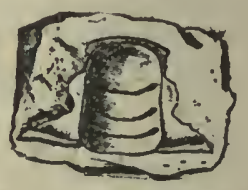
striata, Whitfeld, 1878, Ann. Rep. Geo. Sur. Wis., p. 55, Potsdam Gr.

Ptychoparia, Corda, 1847, Prodrom. einer Monographie der bohmischen Trilobiten, p. 141. [Ety. ptyche, fold.] Cephalic shield semilunar; genal angle spined or pointed; glabella narrow anteiiorly, elevated; furrows four, distinct, directed forward; facial sutures widely separated, extending and converging 
forward from the eyes, so as to intersect the anterior margin within a point where a line would cut it if drawn through each eye parallel with the axis (Corda's figure makes the facial sutures cut the margin laterally, in a line drawn at right angles to the anterior end of the glabella); these lines extend themselves from the eyes to the posterior margin by making a double curve, and cut the margin within or near the lateral angles; fixed cheek arched downward at the sides; occipital ring spined; thoracic segments fourteen; ends of pleuræ pointed or rounded; pygidium medium size, six or seven articulations in the axis; surface of test with minute punctures or scattered tubercles. Type P. striata.

affinis, Walcott, 1884, Mon. U. S. Geo. Sur., p. 54, Potsdam Gr.

anatina, Hall, 1863, (Conocephalites anatinus,) 16th Rep. N. Y. St. Mus. Nat. Hist., p. 158, Potsdam Gr.

(?) annectans, Walcott, 1884, Mon. U. S. Geo. Sur., vol. 8, p. 91, Pogonip Gr.

antiquatus, Salter, 1859, (Conocephalites antiquatus,) Jour. Geo. Soc., vol. 15, p. 554, Up. Taconic.

arenosa, Billings, 1861, (Conocephalites arenosus, ) Pal. Foss., vol. 1, p. 15, Potsdam Gr.

billingsi, Shumard, 1861, (Conocephalites billingsi,) Am. Jour. Sci. and Arts, vol. 32 , p. 220 , Potsdam Gr.

binodosa, Hall, 1863, (Conocephalites binodosa,) 16th Rep. N. Y. St. Mus. Nat. Hist., p. 160, Potsdam Gr.

breviceps, Walcott, 1884, Mon. U. S. Geo. Sur., vol. 8, p. 49, Potsdam Gr.

calciferus, Walcott, 1879, (Conocephalites calciferus,) $32 d$ Rep. N. Y. St. Mus. Nat. Hist., p. 129, Calciferous Gr.

calymenoides, Whitfield, 1877, Geo. Sur. Wis., vol. 4, p. 179, Potsdam. Gr.

clavata, Walcott, 1877, Am. Jour. Sci., 3d ser., vol. 34 , p. 198, Up. Taconic.

cordilleræ, Rominger, 1887, (Conocephalites cordilleræ, Proc. Acad. Nat. Sci. Phil., p. 12, Potsdam Gr.

depressa, Shumard, 1861, (Conocephalites depressus,) Am. Jour. Sci., vol. 32, p. 219, Potsdam Gr.

dissimilis, Walcott, 1884, Mon. U. S. Geo. Sur., vol. 8 , p. 51, Up. Taconic, Prospect Mountain Gr.

eryon, Hall, 1863, (Conocephalites eryon,)

16th Rep. N. Y. St. Mus. Nat. Hist., p. 157, Potsdam Gr.

explanata, Whitfield, 1882, (Conocephalites explanatus,) Geo.Sur. Wis., vol. 4, p. 181, Potsdam Gr.

fitchi, Walcott, 1887, Am. Jour. Sci., 3d ser., vol. 34, p. 197, Up. Taconic.

hartti, Walcott, 1879, (Conocephalites hartti,) 32d Rep. N. Y. St. Mus. Nat. Hist., p. 130, Calciferous Gr.

housensis, Walcott, 1886, Bull. U. S. Geo. Sur., No. 30, p. 201, Up. Taconic. læeviceps, Walcott, 1884, Mon. U. S. Geo. Sur., p. 54, Potsdam Gr.

(?) linnarsoni, Walcott, 1884, Mon. U. S. Gen. Sur. Terr., vol. 8, p. 47, Up. Taconic.

minor, Shumard, 1863, (Conocephalites minor,) Trans. St. Louis Acad. Sci., vol. 2 , p. 105 , Potsdam Gr.

minuta, Bradley, 1860, (Conocephalites minutis,) Am. Jour. Sci., $2 d$ ser., vol. 30, p. 242, Potsdam Gr.

nasuta, Hall, 1863, (Conocephalites nasutus,) 16th Rep. N. Y. St. Mus. Nat. Hist., p. 155, Potsdam Gr.

occidentalis, Walcott, 1884, Mon. U. S. Geo. Sur., vol. 8, p. 51, Potsdam Gr.

oweni, Hall, 1863, 16th Rep. N. Y. St. Mus. Nat. Hist., p. 155, Potsdam Gr.

patersoni, Hall, 1863, (Conocephalites patersoni,) 16th Rep. N. Y. St. Mus. Nat. Hist., p. 159, Potsdam Gr.

peruasuta, Walcott, 1881, Mon. U. S. Geo. Sur., vol. 8, p. 49, Potsdam Gr.

perseus, Hall, 1863, (Conocephalites perseus,) 16th Rep. N. Y. St. Mus. Nat. Hist., p. 153, Potsdam Gr.

piochensis, Walcott, 18s6, Bull. U. S. Geo. Sur., No. 30, p. 201, Up. Taconic.

(?) prospectensis, Walcott, 1884, Mon. U. S. Geo. Sur., vol. 8, p. 46, Up. Taconic-Prospect Mountain Gr.

(?) quadrata, Whitfield, 1880, (Conocephalites quadratus,) Geo. Sur. Wis., vol. 4, p. 180, Potsdam Gr.

rogersi, Walcott, 1884, Bull. U. S. Geo. Sur., vol. 2, p. 283, Up. Taconic.

shumardi, Hall, 1863, (Conocephalites shumardi,) 16th Rep. N. Y. St. Mus. Nat. Hist., p. 154, Potsdam Gr.

similis, Walcott, 1884, Monogr. U. S. Geo. Sur., vol. 8, p. 52, Potsdam Gr.

similis var. robusta, Walcott, 1884, Mon. U. S. Geo. Sur., vol. 8, p. 53, Potsdam Gr.

subcoronata, Hall \& Whitfield, 1877 , (Conocephalites subcoronatus,) Geo. 40th Par., vol. 4, p. 237, Up. Taconic.

teucer, Billings, 1861, (Conocephalites teucer,) Geo. Vt., vol. 2, p. 951, Georgia $\mathrm{Gr}$.

verrucosa, Whitfeld, 1884 , (Conocephalites verrucosus,) Bull. Am. Mus. Nat. Hist., vol. 1, p. 139, Pots- FIG. 1053.-Ptychoparia dam Gr.

winona, Hall, 1863, (Conocephalites winona,) 16th Rep. N. Y. St. Mus. Nat. Hist., p. 161, Potsdam Gr.

zenkeri, Billings, 1860, (Conocephalites zenkeri, Can. Nat. and Geo., vol. 5, and Pal. Foss., vol. 1, p. 398, Up. Taconic.

Remopleurides, Portlock, 1843, Rep. Geol. Lond., p. 254. [Ety. remus, oar; pleura, rib.] Cephalic shield subcircular or transversely subelliptical; glabella Iarge, 
convex, oval, narrower anteriorly and abruptly bent down over the

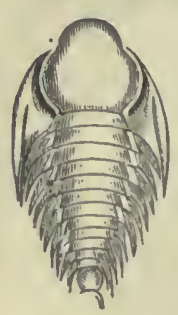
front; eyes large, semilunar, reaching the neck segment; rostral suture marked ; free cheeks, small, narrow, subtriangular, and produced posteriorly in spines; thorax with ten segments, axial lobe very wide, and gradually tapering posteriorly; side lobes narrow, pleurs? short, falcate, directed
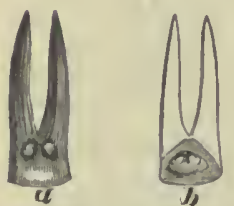

FIG. 1054. - Remo. pleurides striatu. Ius. $a, b, 11$ y postoma.

1865, Pal. Foss., vol. 1, p. 293, Quebec Gr.

schlotheimi, Billings, 1865, Pal. Foss., vol. 1, p. 294, Quebec Gr.

striatulus, Walcott, 1875, Cin. Quar. Jour. Sci., vol. 2, p. 347 , Trenton Gr.

Rhabdichinites, Dawson, 1873, Am. Jour. Sci. and Arts, 3d ser., vol. 5, p. 20 . A name proposed for certain markings on the rocks which are not the remains of organisms.

Rricrura, Scudder, 1878, Proc. Bost. Soc. Nat. Hist., vol. 19, p. 296. [Ety. rachis, ridge; oura, tail.] Type $\mathrm{R}$. venosa.

venosa, Scudder, 1878, Proc. Bost. Soc. Nat. Hist., vol. 19, p. 296, Coal Meas.

Rhinocaris, Clarke, 1888 , Pal. N. Y., vol. 7 , p. Iviii. [Ety. rhine, file; karis, shrimp.] Cephalothorax univalvular, laterally appressed; outline as in Ceratiocaris; anterior extremity produced into a narrow, vertically flattened prora, continuous with substance of the carapace; axial line with a low ridge; abdomen composed of not less than four subcylindrical somites; post-abdomen bearing three spines, of which the telson is elongate and conical, and the cercopods flattened. Type R. columbina.

columbina, Clarke, 1888, Pal. N. Y., vol. 7, p. 195, Ham. Gr.

scaphoptera, Hall, 1888, Pal. N. Y., vol. 7, p. 197 , Ham. Gr.

Ribeiria, Sharp, 1853, Jour. Geo. Soc., vol. 9, p. 157. [Ety. proper name.] Elongated, laterally compressed in the form of a Pholas; open at both ends and along the pedal margin, with a thick, transverse, internal plate near the anterior extremity, bebind which is a cor- rugated boss for the attachment of a muscle. Type R. pholadiformis.

calcifera, Billings, 1865 ,

Pal. Foss., vol. 1, p.

340, Calciferous Gr.

compressa, Wbitfield,

1886, Bull. Am. Mus. Fra. 1055. - Ribel$\mathrm{N}$ at. $\mathrm{H}$ ist. vol. 1, ria calcifera.

Birdseye Gr.

longiuscula, Billings, Pal. Foss., vol. 1, p. 341 , Calciferous Gr.

ventricosa, Whitfield, 1886, Bull. Am. Mus. Nat. Hist., vol. 1, p. 344, Birdseye Gr.

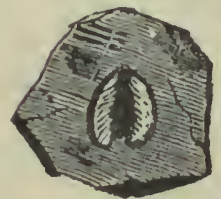

Fig. 1050.-Rusichnites carbonarius. Rusicinites, Da w s o n, 1861, Can. Nat. and Geo., vol. 1, p. 363. Ety. rusos, wrinkled; ichnos, track.] Supposed by the author to be the track of a Crustacean, like the Limulus, and consisting of two undulated, rounded, contiguous furrows; but the type resembles a fucoid quite as much, if not more, than it does a track, and if related to Rusophycus, which is clearly a fucoid, then it sloould be referred to the vegetable? kingdom. Type $R$. acadicus.

acadicus, Dawson, 1861, Can. Nat. and Geo., vol. 1, p. 363, and Acad. Geol., p. 410, Coal Meas.

carbonarius, Dawson, 1868, Acad. Geol., p. 257, Carboniferous.

Salteria, Walcott, 1884. The name was preoccupied, and is a synonym for Bailiella.

Sao, Barrande, 1846, and Syst. Sil. Bolı., vol. 2. Type Sao hirsuta, a primordial form unknown in America.

? lamottensis, Whitfield, 1886, Bull. Am. Mus. Nat. Hist., vol. 1, p. 334, Birdseye Gr.

Scuizoniscus, (Jlarke, 1888, Pal. N. Y., vol. 7. p. 62. [Ety. schiza, cleft; diskos, quoit.] Carapace valves separable along the hinge; outline circular or ovate, narrow posteriorly; surface convex or depressed, elevated at the beaks, which are prominent, slightly incurved, and situated anteriorly, hinge-line equaling in length the greatest diameter of the carapace; edge parallel, not gaping, surface concentrically wrinkled. Type S. capsa.

capsa, Clarke, 1888, Pal. N. Y., vol. 7, p. 207, Ham. Gr.

Shuma rdia, Billings, 1862, Pal. Foss., vol. 1, p. 92. [Ety. proper name.] Cephalic shield semicircular; glabella convex, subcylindrical, no eyes, pygidium semielliptical, axis cylindro-conical, ribbed, side lobes ribbed, distinguished

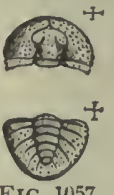

FIG. 1057. Shumardia granulosa. from Agnostus by the ribs on the pygidium. Type S. granulosa. 
glacialis, Billings, 1865, Pal. Foss., vol. 1, p. 283, Up. Taconic.

granulosa, Billings, 1862, Pal. Foss., vol.1, p. 92, Quebec Gr. or Up. Taconic.

Solenocaris, Meek, 1872, Proc. Acad. Nat. Sci. Phil., p. 355. [Ety. solen, a genus of shells; karis, shrimp.] Posterior end subtruncated back ward from below, but not sinuous, as in Colpocaris; ocular tubercle obsolete, and no indicatlons of having its valves anchylosed along the dorsal 'margin. Type s. strigata. This name was preoccupied for another crustacean by Young, in 1868, in Proc. Nat. Hist. Glasgow, vol. 1, p. 171.

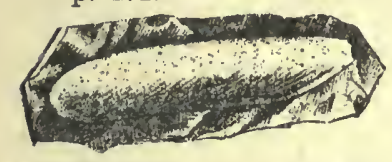

FIG. 1058.-Solenocaris strlgata. Cast of interior of left valve.
Ill., vol. 8, p. 153, St. Louis Gr.

s t l u d o vici, W o rthen, 1884, Bull. No. 2, Ill. St. M us. Nat. Hist. p. 3, and Geo. Sur. strigata, Meek, 1872, Proc. Acad. Nat. Sci. Phil., p. 335, and Ohio Pal., vol. 2, p. 321 , Waverly Gr.

Solenopleura, Angelin, 1852, Palæontologia Suecica, p. 26. [Ety. selene, moon; pleuron, side.] Body ovate, test granulated or tuberculous; head wide, semicircular; glabella prominent, furrows distinct, dorsal furrows deep and continuous; fixed cheeks, elevated in the middle, front limb couvex; nccipital ring bearing a tubercle; genal angle pointed; thoracic segments fourteen; ends of pleuræ bluntly rounded; pygidium medium size, few segments. Type S. canaliculata.

acadica, Whiteaves, 1887 , Trans. Roy. Soc. Can., p. 157, St. John Gr.

acadica var. elongata, Matthew, 1887, Trans. Roy. Soc. Can., p. 159, St. John Gr.

communis, Billings, 1874, Pal. Foss., vol. 2, p. 72, Up. Taconic.

formosa, Hartt, 1868, (Conocephalites formosus,) Acad. Geol., p. 654, St. John Gr.

halli, Hartt, 1868, (Conocephalites halli,) Acad. Geol., p. 654, St. John Gr.

nana, Ford, 1878, Am. Jour. Sci. and A rts, $3 \mathrm{~d}$ ser., vol. 15 , p. 126, Up. Taconic.

orestes, Hartt, 1868, (Co nocep halites orestes, ) Acad. Geol., p. 649, St. John Gr.

robbi, Hartt, 1868,

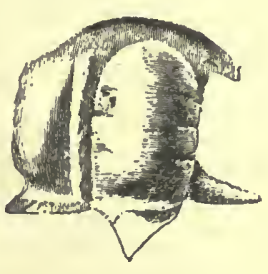

(Con o ce phalites Fra. 1059.-Solenopleurobbi,) Acad. Geol., ra nana. Magnifled. p. 648 , St. John Gr.

thyrsites, Hartt, 1868, (Conocephalites thyrsites, Acad. Geol., p. 653, St. John Gr.

tumida, Walcott, 1887, Am. Jour. Sci. and Arts, 3d ser., vol. 34, p. 196, Up. Taconic.

Spathiocaris, Clarke, 1882, Am. Jour. Sci. and Arts, 3d ser., vol. 23, p. 477. [Ety. spathe, spathe; karis, shrimp.] Carapace in one piece, oblong elliptical, convex, apical point near the focus of the ellipse, from which point a cleft extends backward, widening to the margin. Type S. emersoni.

emersoni, Clarke, 1882, Am. Jour. Sci. and Arts, 3d. ser., vol. 23, p. 478, Portage Gr.

lutheri, Clarke, 1882, (Lisgocaris lutheri,) Am. Jour. Sci. and Arts, 3d ser., vol. 23 , p. 478 , Ham. Gr.

SpH жrexochús, Beyrich, 1845, Euber einige Bohm. Tril., p. 21. [Ety. sphaira, ball ; exochos, prominent.] Cephalic shield very convex, almost globular; cheeks not scrobiculate; glabella nearly spherical, three furrows on each side, two upper obscure, lower strong and curved down to the neck furrow; eyes faceted minutely; facial suture ending on the external margin near the angles, in front continuous and submarginal; thorax 11 joints; pygidium 3 segments, free at their ends; labrum subtrigonal, with a marginal furrow; no rostral shield. Type S. mirus.

canadensis, Billings, 1866, Catal. Sil. Foss. Antic., p. 64, Anticosti Gr.

mirus, not American.

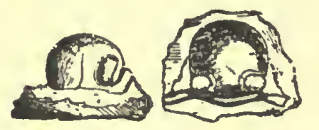

FIG. 1060.-Sphærexochus parvus. Upper and side vlew of glabella. parvus, Billings, 1865, Pal. Foss., vol. 1, p. 180 , Chazy or Black Riv. Gr.

romingeri, Hall, 1867, 20th Rep. N. Y. St. Mus. Nat. Hist., p. 425, Niagara Gr.

SpII жrocoryphe, Angelin, 1852, Palæontologia Scandinavica. [Ety. sphaira, ball ; koryphe, top of the head.] Cephalic shield convex, genal angles spined; glabella spheroidal anteriorly, two lateral furrows; eyes prominent; facial sutures cut the lateral margins posteriorly; thorax with ten segments, axial lobe narrower than lateral lobes; pleuræ terminate in short spines; pygidium composed of three segments, anterior one produced in two spines. Type S. granulata.

robusta, Walcott, 1875 , Cin. Quar. J our. Sci., Fig.1061.-Sphærovol. 2 , p. 273 , Trenton coryphe robusta. Gr.

salteri, Billings, 1866, Catal. Sil. Foss. Antic., p. 63, Anticosti Gr.

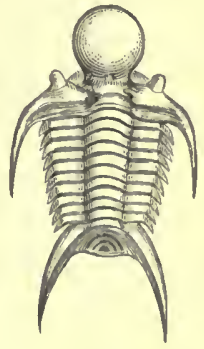

$\boldsymbol{B}$ 
STrobilepis, Clarke, 1888, Pal. N. Y., vol. 7, p. 63. [Ety. strobilos, cone-shaped; lepis, scale.] Capitulum composed of four vertical ranges of plates having in general a trihedral form. but varying in size and contonr; each plate articnlated with or overlapping the next pre. ceding; anterior extremity terminated by a large, circular, conical plate; plates thick and ornamented. Type S. spinigera.

spinigera, Clarke, 1888, Pal. N. Y., vol.7, p. 212, Ham. Gr.

Stylonurus, Page, 1856, Geological Text Book, p. 190. [Ety. stylos, a mast or spar; oura, tail.] General form like Eurypterus, but distinguished by the peculiar development of the two posterior foot pairs; these are alike, long, thin, and consist of 9 segments, of which the two last form a small claw; the posterior pair reach to the middle of the long posterior spine. Type $S$. powriei.

excelsior, Hall, 1884, 36th Rep. N. Y. St. Mus. Nat. Hist., p. 77, Catskill Gr.

Sympнysunus, Goldfuss, 1843, Neues Jahrb. f. Mineral. [Ety. symphysis, growing together; oura, tail.] Elliptical; genal angles rounded; cephalic shield semicircular, convex; glabella convex, subquadrate, smooth, no lateral furrows; eyes lunate; facial sutures arching in front of the glabella, and cutting the posterior part of the cephalic shield near the genal angles; 8 thoracic segments; lygidium somewhat semicircular, no segments, border flattened. Type S. laviceps.

goldfussi (?), Walcott, 1885, Monogr. U. S. Geo. Sur., vol. 8, p. 95, Trenton Gr. This species is founded on a glabella with fixed cheeks, and probably it does not belong to this genus.

Teleprius, Barrande, 1852, Syst. Sil. Boh. [Ety. mythological name.] Glabella strongly convex, margined by deeply impressed bow-shaped furrows; neck segment tumid; cheeks subtriangular, or subrcescentiform, small posteriorly, wider in front; pygidium small, strongly convex, hemispherical, margin tumid, axis with three segments. Type $T$. fractus. Only recognized in America by fragments of glabella.

americanus, Billings, 1865, Pal. Foss., vol. 1, p. 291, Quebec Gr.

Terataspis, Hall, 1863, 16th Rep. N. Y. St. Mus. Nat. Hist., p. 223. [Ety. teras, marvel; aspis, shield.] Distinguished from Acidaspis, which it resembles by the prominent ellipsoidal frontal lobe of the glabella, posterior spines of the lateral lobes and nodes of the occipital ring, and from Lichas by the spines of the pygidium being themselves bearers of lateral spines. Type T. grandis.

grandis, Hall, 1862, 15th Rep. N. Y. St. Mus. Nat, Hist., p. 82, and Illust. Devon.
Foss., pl. 17, (Liclas grandis,) Schoharie grit.

eriopis, Hall, 1863, (Lichas eriopis,) 16th Rep. N. Y. St. Mns. Nat. Hist., p. 226, and Illust. Devon. Foss., pl. 19, Up. Held. Gr.

Thaleops, Conrad, syn. for Illaenus. ovata, see Illaenus ovatus.

Triratirelia,Hall, 1863, 16th Rep. N. Y. St. Mus. Nat. Hist., p. 177. [Ety. diminutive of Triarthrus.] Glabella elongate, semioval, with the fixed cheeks wide and spreading in the posterior limb, and very narrow in front, an obscure indentation at the margin; general expression like Triarthrus. Type T. auroralis.

auroralis, Hall, 1863, 16th Rep. N. Y. St. Mus. Nat. Hist., p. 177, Potsdam Gr.

Triratinus, Green, 1832, Monograph of Trilolites, p. 87, [Ety. triarthrus, threejointed.] Subelliptical ; cephalic shield somewhat semicircular or sublunate; glabella moderately convex, sides straight, rounded in front, deeply trilobate on each side by the lateral furrows, with a prominent occipital groove near the base, and occipital ring, from the center of which a spine sometimes arises; eyes small and placed on the anterio-lateral margin; free cheeks forming a llarrow rim; thorax with from 13 to 16 articulations; central axis convex, wider than the lateral lobes; pygidinm with 5 to 7 segments in the axis and one or two less in the lateral lobes. Type T. becki.

becki, Green, 1832, Monograpls of Trilobites, p. 87, and Pal. N. Y., vol. 1, p. 237, Utica Slate Gr.

canadensis, Smith, 1861, Can. Jour., vol. 6, p. 275, Utica Slate Gr.

fischeri, see Atops fischeri.

glaber, Billings, 1859, Can. Nat. and Geol,, vol. 4, p. 382, and Can. Geol., p. 202 , Utica Slate Gr.

spinosus, Billings, 1857, Rep. of Progr. Geo. Sur. Can., Fis. 1062.-Trip. 340, and Can. Geol., p. arthrus becki. 202, Utica Slate Gr.

Trimerus, syn. for Homalonotus.

delphinocephalus, see Homalonotus delphinocephalus.

jacksoni, see Homalonotus jacksoni.

Trinocleus, Lhwyd, (or, as he spelt it, Llhwydd,) 1698, Phil.'Trans., vol. 20, p. 279. [Ety. trinucleus, three-kerneled.] Cephalic shield highly convex, a wide border impressed with several rows of deep puncta and posterior angles, terminating in spines; glabella pyriform, pointed behind, no lateral furrows; cheeks convex; no eyes or facial sutures; neck furrow distinct; thorax with six articulations, axis narrow, 
convex; side lobes wide, flat, straight, pleural groove not reaching the margin; pygidium subtriangular, margin deflected, axis conical, about six furrows; side lobes flat, with about the same number of furrows. Type T. concentricus.

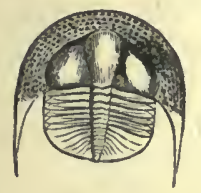

FIG. 1063,-Trinucleus concentricus. bellulus, Ulrich, 1878, Jour. Cin. Soc. Nat. Hist., vol.1, p. 99. The young of T. concentricus.

concentricus, Eaton, 1832,

(Nuttainia concentrica,) Geo. Text-Book, p. 128, and Pal. N. Y., vol. 1, p. 249, Trenton to Hud. Riv. Gr.

Tropidocaris, Beecher, 1884, Geo. Sur. Pa., vol. PPP, p. 15. [Ety. tropis, a keel ; karis, a shrimp.] Carapace bivalve, semiovate or semielliptical, obliquely truncated behind; valves about twice as long as wide, having one or more longitudinal ridges; cephálic region indicated by elevations at the anterior end; optic node situate on a ridge; two segments of the abdomen. Type T. bicarinata.

alternata, Beecher, 1884, Geo. Sur. Pa., vol. PPP, p. 19, Waverly Gr.

bicarinata, Beecher, 1884, Geo. Sur. Pa., vol. PPP, p. 16, Chemung Gr.

interrupta, Beecher, 1884, Geo. Sur. Pa., vol. PPP, p. 18, Chemung Gr.
Turrilepas, Woodward, 1865, Quar. Jour. Geol. Soc., vol. 21, p. 486. [Ety. turris, tower; lepas, scale.] Elongate, coneshaped bodies, composed of from 4 to 6 vertical ranges of scale-like, subtriangular plates covered with elevated concentric lines; plates of middle range convex and bearing a median carina. Type T. wrightana.

cancellatus, Hall, 1888, Pal. N. Y., vol. 7, p. 216, Up. Held. Gr.

devonicus, Clarke, 1882, (Plumulites devonicus, ) Am. Jour. Sci., 3d ser., vol. 24, p. 55, Ham. Gr.

flexuosus, Hall, 1888, Pal. N. Y., vol. 7, p. 215, Up. Held. Gr.

foliatus, Hall, 1888, Pal. N. Y., vol. 7, p. 218, Ham. Gr.

gracillimus, Ringueberg, 1888, (Plumulites gracillimus,) Proc. Acad. Nat. Sci. Phil., p. 136, Niagara Gr.

newberryi, Whitfield, 1882, (Plumulites newberryi,) Ann. N. Y. Acad. Sci., vol. 2, p. 217, Portage Gr.

nitidulus, Hall, 1888, Pal. N: Y., vol. 7, p. 218, Ham. Gr.

squama, Hall, 1888, Pal. N. Y., vol. 7, p. 217, Ham. Gr.

tener, Hall, 1888, Pal. N. Y., vol. 7, p. 219, Ham. Gr.

Zacantholdes, Walcott, 1888, Am. Jour. Sci., 3d ser., vol. 36, p. 165. Proposed to receive Olenoides lævis, $O$. spinosus, $O$. flagricaudatus, and $O$. typicalis, but not defined.

\section{CLASS ARACHNIDA.}

THE animals, forming the class Arachnida, include the spiders, scorpions, and many offensive parasites and microscopic forms. They are generally possessed of four pairs of legs attached to the anterior division of the body, but have no antennæ. The Palæozic fossils are nearly all referred to an extinct order, Anthracomarti, but a few are referred to the living orders, Pedipalpi and Scorpiones. The Pedipalpi have arm-like prehensile organs, terminating in a movable claw, annulated abdomen, and long flexible limbs. They inhabit tropical countries, and have a forbidding aspect. The Scorpiones have large palpi or arm-like prehensile organs, terminated by a pair of nippers, and an elongated, tail-like abdomen, which ends in a sharp claw; and when the animal is in motion, this is carried over the back in a threatening manner. The poison glands are situated at the base of the claw, and when the animal stings, a portion of the venom is thrown into the wound. The scorpions are inhabitants of tropical countries. The Order Anthracomarti is defined as follows: Body more or less depressed; cephalothorax and abdomen distinctly separable; cephalothorax frequently made up in large part of pedigerous segments, 
more or less wedge-shaped, and visible above as well as below, the arrangement of which corresponds to that of the coxæ. The abdomen forms a single mass, and is composed of a variable number of visible segments, ranging from four to nine. Palpi not much longer than the legs, simply terminated.

\section{ORDER ANTHRACONARTI.}

Family Architariods.-Anthracomartus, Architarbus, Geraphrynus.

Family Arthrolycoside.-Arthrolycosa.

Family Poliocherida.-Poliochera.

\section{ORDER PEDIPALPI.}

Family Grralinurids.-Geralinura.

\section{ORDER SCORPIONES.}

Famuy Eoscorpronid ж.-Eoscorpius, Mazonia.

Anturacomartus, Karsch, 1882, 'Zeitschr. deutsch. geol. Gesellsch., p. 556. [Ety. anthrax, coal; Martos, proper name.] Cephalothorax quadrate, the front square or scarcely convex, about half the size of the abdomen; coxæ radiating from a broad triangular sternal plate, the base of which forms the posterior margin; sides of body constricted so as to show a distinct though slight separation of cephalothorax and abdomen; abdomen orbicular, composed of seven segments of similar length throughout. Type A. volkelianus.

pustulatus, Scudder, 1884, Proc. Am. Acad. Arts and Sci., p. 13, Low. Coal Meas.

trilobitus, Scudder, 1884, Proc. Am. Acad. Arts and Sci., p. 13, Coal Meas.

ARchitarbus, Scudder, 1868, Geo. Sur. Ill., vol. 3, p. 568. [Ety. archaios, ancient; tarbos, object of alarm.] Cephalothorax orbicular, broadly rounded in front,

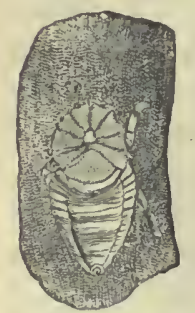

FIG. 1064.-Architarbus rotundatus. much smaller than the abdomen, but not separated from it by a marked lateral constriction; coxæ radiating from a central pit; abdomen oval, composed of nine segments, of which those on the basal half are very much shorter than the others, and on the dorsal surface are forced still more closely together by the large post-thoracic plate; no abdominal appendages. Type A. rotundatus.

rotundatus, Scudder, 1868, Geo. Sur. Ill., vol. 3, p. 568 , Coal. Meas.
Arthrolycosa, Harger, 1874, Am. Jour. Sci. and Arts, $3 d$ ser., vol. 7, p. 219. [Ety, arthron, a joint: lykos, a spider.] Cephalothorax orbicular, twice as large as the abdomen; Coxæ radiating from a central pit; abdomen oval much narrower at the base than the cephalothorax, with no longitudinal sculptur-

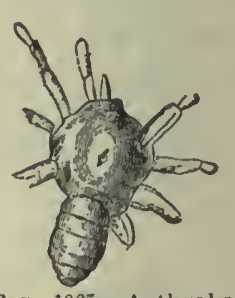
cosa antiqua.
cost ing, and composed of seven segments; no abdominal appendages. Type A. antiqua.

antiqua, Harger, 1874, Am. Jour. Sci. and Arts, 3d ser., vol. 7, p. 219, Coal Meas.

Eoscorpius, Meek \& Worthen, 1868, Am. Jour. Sci and Arts, 2d. ser., vol. $46, \mathrm{p}$. 25 , and Geo. Sur. Ill., vol 3, p. 560. [Ety. eos, dawn; scorpius, a scorpion.] Cephalothorax quadrangular, somewhat wider behind than long; mesial and lateral furrows between which the surface bears granules; mandibles stout, without teeth or serrations; movable finger curved and sharp at the point; legs stout, divisions long; abdomen twice as long as as cepbalothorax; segments gradually increase in size to the sixth, while the seventh and last is $2 \frac{1}{2}$ times as long as the sixth, but rapidly contracts, and is truncated for the attachment of the tail; the anterior margin of each of the first six segments is rounded; the three tail, segments preserved are stout, oblong, and covered with granules; the comb-like organ shows 11 or 12 divisions. Type E. carbonarius. 
carbonarius, Meek \& Worthen, 1868, Am. Jour. Sci. and Arts, 2d ser., vol. 46, p. 24, and Geo. Sur. Ill., vol. 3, p. 560, Coal Meas.

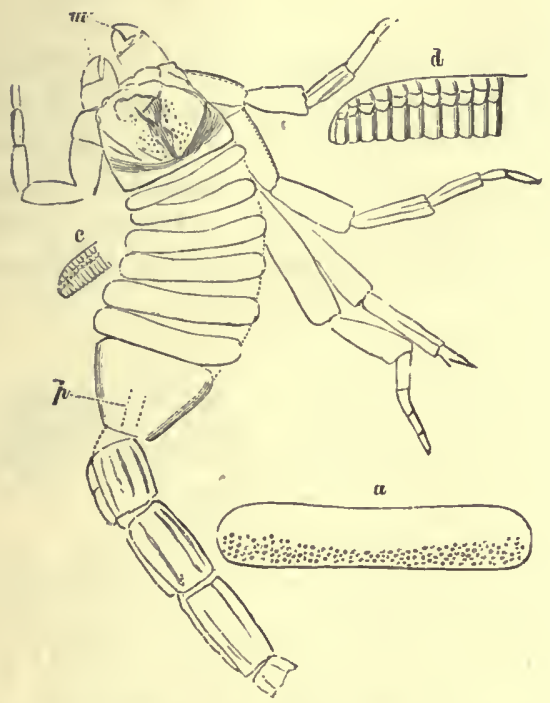

FIG. 1066.-Eoscorpius carbonarius. Natural size ; $a$, body segment enlarged; $c$, comb; $d$, same eviarged; $m$, mandibles ; $\boldsymbol{p}$, pits.

Geralinura, Scudder, 1884, Proc. Am. Acad. Arts and Sci., p. 13. [Ety. geras, old; linon, linen ; oura, tail.] Cephalothorax ovate, the front rounded, onethird as broad as hinder portion; palpi large and robust, with interior spines; first two pairs of legs slender, the hinder stout and broad; abdomen composed of nine joints, the basal three rather short, the others subequal and longer. Type S. carbonaria.

carbonaria, Scudder, 1884, Proc. Am. Acad. Arts and Sci., p. 13, Coal Meas.

Geraphrynus, Scudder, 1884, Proc. Am. Acad. Arts and Sci., p. 13. [Ety.geras, old; Phrynus, a genus.] Cephalothorax fusiform, angulated in front, nearly as large as the abdomen; coxæ radiating from a median line; palpi slenderer than the legs, longer than the cephalothorax, springing from its extreme front, and of uniform size throughout; $a b$ domen subfusiform, composed of nine segments, rounded behind, with no constriction at the base; a large triangular post-thoracic plate, crowding the middle of the first five short segments out of a straight transverse line; readily distinguished from Architarbus by its produced and angulate cephalothorax. Type G. carbonarius.

carbonarius, Scudder, 1884, Proc. Am. Acad. Arts and Sci., p. 13, Coal Meas.

Mazonia, Meek \& Worthen, 1868, Geo. Sur. Ill., vol. 3, p. 563. [Ety. proper name.] Cephalothorax moderately convex, subquadrangular; anterior lateral margins rounded, and anterior margin truncated on each side of a small mesial triangular projection; mesial furrow extends forward from the posterior margin, widening and deepening to the front, where it occupies one-third of the breadth, and is partly filled by the oculiferous prominence, which bears on each side a large eye; eyes circular, convex, arranged for looking obliquely forward, outward, and upward; seven or eight abdominal segments, the last one truncated for the tail. Type $\mathbf{M}$. woodana.

woodana, Meek \& Worthen, 1868, Geo. Sur. Ill., vol. 3, p. 563, Coal Meas.

Poliochera, Scudder, 1884, Proc. Am. Acad. Arts and Sci., p. 13. [Ety. polios, hoary; cheras, to be bereft.] Cephalothorax scarcely longer than broad, slightly narrowing anteriorly, the front square; coxæ radiating from-a median line; legs stout, moderately long; abdomen full, at base as broad as the cephalothorax, broadening slightly behind, fully rounded, composed of four segments, the first segment about one-third the length of the others, which are equal; no abdominal appendages. Type $P$. punctulata.

punctulata, Scudder, 1884, Proc. Am. Acad. Arts and Sci., p. 13, Coal Meas.

Proscorpius, Whitfield, 1885, Bull. Am. Mus. Nat. Hist., vol. 1, p. 183 . [Ety. pro, before; scorpius, a genus.] Cephalothorax with large dorsal eye-lobe; eyes small, one on each side of the median line; lateral eyes on ridges, as in living scorpions; sixth ventral segment of the preabdomen, counting from behind, large, equal in length and breadth to the corresponding dorsal segment; anterior walking limb terminating in a bitid claw; postabdomen not reversed as in living scorpions. Type P. osborni. Good authorities say this is merely an Eurypterus, with no affinity or resemblance to a scorpion. With this view the author coincides.

osborni, Whitfield, 1885, Bull. Am. Mus. Nat. Hist., vol. 1, p. 184, Waterlime Gr. Synonym, probably, for Eurypterus remipes. 


\section{CLASS MYRIAPODA.}

TuE animals composing the Class Myriapoda are elongated, and composed of numerous segments, all of which are substantially alike except the first and last. The articulations of the body each bear one or two pairs of jointed legs. The common centipede and long-jointed worms, with numerous legs, found in damp places and on trunks of trees, some of which coil up when alarmed, are examples. Only a few Palrozoic fossils are referred to this Class, and these belong to extinct Orders.

\section{ORDER ARCHIPOLYPODA.}

Family Arcirulidx.-Archiulus, Trichiulus, Xylobius.

Family Euphoberidx.-Acantherpestes, Amynilespes, Anthracerpes, Eileticus, Euphoberia.

Family Uxcertain.-Archæoscolex.

\section{ORDER PROTOSYNGNATHA.}

\section{Family Palaocampida.-Palæocampa.}

Acantuerpestes, Meek \& Worthen, 1868, Geo. Sur. Ill., vol. 3, p. 559. [Ety. akantha, a spine; erpestes, a creeper.] Spines bifurcate at tip, and arranged in dorsal, pleurodorsal, and lateral rows; segments three, or more than three times as broad as long. Type A. major.

major, Meek \& Worthen, 1868, (Euphoberia major,) Am. Jour. Sci. and Arts, 2d. ser., vol. 46, p. 26, and Geo. Sur. Ill., vol. 3, p. 558, Coal Meas.

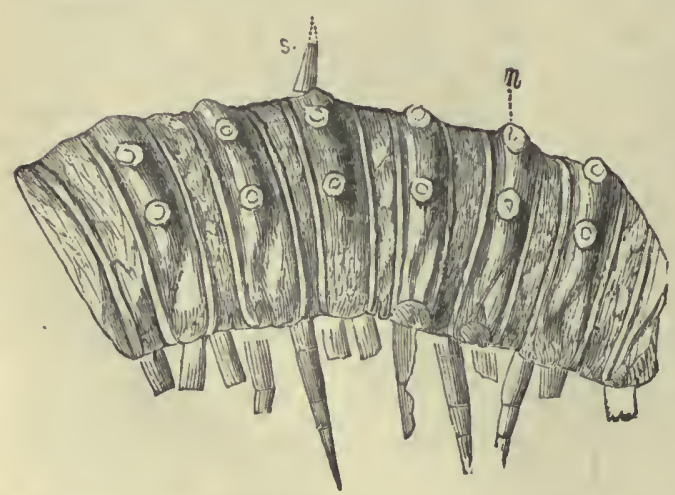

FIG. 1067.-Acantherpestes major. Fragment. s, spine; $n$, base of spine.

Amynilespes, Scudder, 1885, in Zittel's

Handbuch der Pal., p. 729. [Ety. amuno, to keep off; iluspaomai, to crawl.] Spines simple, arranged in dorsolateral rows; segments four times as broad as long. Type A. wortheni.

worthen i, Scudder, 1885 , in 'Zittel's Handbu ch der Pal., vol. 2, p. 729 , Coal Meas.

A N THRACERPES,

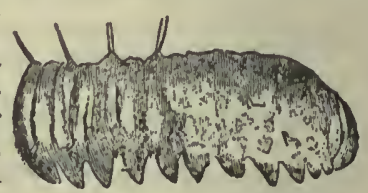

FIG. 1068.-Amynilespes wortheuj.
M e e k \&

Worthen, 1865, Proc. Acad. Nat. Sci. Phil., p. 51. [Ety. anthrax, coal; erpo, to creep, in allusion to its carboniferous age and probable habits.] Founded upon an articulated body of nineteen segments and part of another. The last segment terminates in three or four short, slender, hair-like or spinelike appendages. Below the middle of each segment there is a small prominence, marking the spiracles, or breathing apertures, which pertain to the Myriapoda. Type A. typus.

typus, Meek \& Worthen, 1865, Proc. Acad. Nat. Sci. Phil., p. 51, and Geo. Sur. Ill., vol. 2, p. 409, Coal Meas.

Arcifoscolex, Matthew, 1888, Trans. Roy. Soc. Can., p. 59. [Ety. archaios, ancient; skolex, worm.] Cylindrical, tapering behind the middle, and also at the three anterior segments; head small, somewhat conical; thorax of three joints, increasing in width back- 
ward, but decreasing in length; limbs tapering, posterior pair as long as the thorax, and larger and stronger than those in front; abdomen of eleven visible segments, those at the anterior end wi $\mathrm{d}$ e $r$ and those of the middle longer; basal segment has an

oblong scar near the posterior edge; crust chitonous. Type A. corneus.

corneus, Matthew, 1888, Trans. Roy. Soc. Can., p. 59, Devonian.

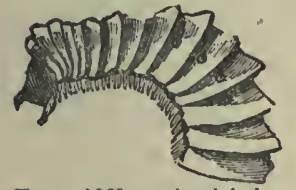

FIa. 1069. - Arehiulus xylobioides. A n t erior part enlarged.
Archiulus, Scudder, 1868, Mem. Bost. Soc. Nat. Hist. vol. 2 , p. 231, and Acad. Geol., p. 496. [Ety,$a r$. chaios, ancient; ioulos, wood-louse. Segments entire, varying much in relative proportions, but generally from two to three times broader than long, furnished with only a few papillæ, perhaps supporting spiny hairs. Type A. xylobioides.

xylobioides, Scudder, 1868, Mem. Bost. Soc. Nat. Hist., vol. 2, p. 236, and Acad. Geol., p. 496, Coal Meas.

Eileticus, Scudder, Mem. Bost. Soc. Nat. Hist. [Ety. eiletikos, rolling one's self.] No spines, but large, low tubercles, serially arranged; segments few, less than twice as broad as long. Type E. anthracinus.

anthracinus, Scudder, Mem. Bost. Soc. Nat. Hist., Coal Meas.

EupHoberia, Meek \& Wortben, 1868, Am. Jour. Sci. and Arts, $2 \mathrm{~d}$ ser., vol. 46, p. 26. [Ety. eu, very; phoberos, formidable.] Head semicircular; body long, slender, very slightly tapering, and terminating abruptly; segments seventy-five or more on the ventral side

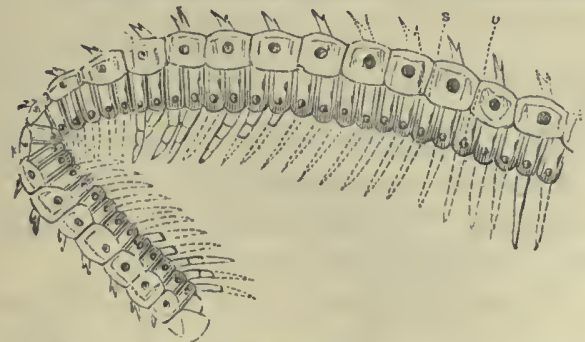

FIG. 1070.-Euphoberia armigera, Part of a large specimen.

and balf as many on the dorsal; dorsal half of the segments rounded, and each supporting three or four spines, curved slightly backward, and arranged in rows on the back, spines spinuliferous; ventral half of the segments each bears a pair of small slender-jointed legs. Type E. armigera. 
villosus, Scudder, 1884, Mem. Bost. Soc. Nat. Hist., vol. 3, p. 291, Coal Meas.

X ylobius, Dawson, 1860, Quar. Jour. Geo. Soc., vol. 16, p. 268. [Ety. xylobius, living in wood.] Body crustaceous, cylindrical, elongate, rolling spirally; segments thirty or more, anterior ones smooth, posterior ones furrowed; legs small, numerous; labrum quadrilateral, divided by notches or joints into three portions; mandibles two-jointed, last ovate and pointed; eyes ten or more on each side. Type X. sigillarix.

dawsoni, Scudder, 1868, Mem. Bost. Soc. Nat. Hist., vol. 2, p. 236, and Acad. Geol., p. 496, Coal Meas. fractus, Scudder, 1868, Mem. Bost. Soc. Nat. Hist., vol. 2, p. 234, and Acad. Geol., p. 496, Coal Meas.

mazonius, Zittel, 1885, Handbuch der Pal., p. 730, Coal Meas.

sigillarice, Dawson, 1860 , Quar. Jour. Geo. Soc., vol. 16, p. 271, Coal Meas. Fig. 1073. - Xylobius similis, Scudder, slgillarix. $a$, Organ 1868, Mem Bast. with palpus, pertaining to the mouth, enSoc. Nat. Hist., larged.

vol. 2, p. 234, and Acad. Geol., p. 496, Coal Meas.

\section{CLASS INSECTA.}

Insecrs are possessed of head, thorax, and abdomen. Three pairs of legs and one pair of antenna belong to them in their perfect state. They are the highest and most complicated class of articulated animals, and abound almost everywhere. No living order, in this class, is known from the Palæozoic rocks. Indeed, the fossils consist almost wholly of fragments of wings showing little else than neuration; but they have been studied by Scudder, until he has classified them into an Order and Families, to the general satisfaction of entomologists, and all must concede he has accomplished a very difficult task. The Order Palæodictyoptera, which includes the Orthopteroid and Hemipteroid Palrodictyoptera, has been defived as follows: Body generally elongated; mouth parts variously developed; antennæ filiform; thoracic joints subequally developed; legs moderately large; meso and metathoracic wings closely similar, equally membrauous; the six principal veins always developed; the marginal simple, and forming the costal border; the mediastinal generally simple or with superior branches only; the other veins usually dichotomize; stout and well defined cross veins rare; membrane generally reticulate; wings in repose lying on the abdomen; the anal area of hind wings, though usually of great distal extension, never plaited, though sometimes broadly folded; abdomen usually long and slender, the last joint often furnished with simple articulated appendages.

\section{ORDER PALAODICTYOPTERA.}

Family Gerarides.-Adiphlebia, Gerarus, Megathentomum, Polyernus.

Family Hemeristid z.-Chrestotes, Hemeristia, Lithentomum.

Family Номотнетіdж.-Anthracothremma, Cheliphlebia, Didymophleps, Encænus, Genentomum, Genopteryx, Gerapompus, Homothetus.

Famil M Mracride.-Lithomylacris, Mylacris, Necymylacris, Paromylacris, Promylacris.

Family Pal жовlattinid Oryctoblattina, Petrablattina.

Famuy Paldopterinida.-Aethophlebia, Dieconeura, Miamia, Propteticus. Family Palaephemeride.-Ephemerites, Geraphemera, Platephemera. 
Family Phthanocoride.-Phthanocoris.

Famir Protophasmide.-Haplophlebium, Paolia, Titanophasma.

Family Xenoneuride.-Geroneura, Xenoneura.

Fammy Uncertain.-Archegogryllus, Dyscritus.

Supposed Insect 'Trauls.-Haplotichnus, Plangtichnus, Treptichnus.

A diphlebia, Scudder, 1884, Mem. Bost. Soc. Nat. Hist., vol. 3, p. 345 . [Ety. a, privative; dis, double; phlebion, vein.] Body rather stout; wings rather broad; all the nervules simple, arising from their stems near the base of the wings; subparallel and longitudinal. Type A. lacoana.

lacoana. Scudder, 188t, Mem. Bost. Soc. Nat. Hist., vol. 3, p. 345, Coal Meas.

A eтhophlebia, Scudder, 1884, Mem. Bost. Soc. Nat. Hist., vol. 3, p. 338. [Ety. aethes, strange; phleps, a vein.] Interno-median vein terminating before the middle of lower border, emitting a single main branch, beyond its middle which is superior, and which, with inedian fork of externo-median and larger part of main scapular branch, form a continuous adventitious vein crossing principal nervules of the wing; ultimate offshoots of externo-median vein arise indifferently from the main vein and the principal branch, and are parallel and similar to the offshoots of the veins above. Type $A$. singularis.

singularis, Scudder, 1884, Mem. Bost. Soc. Nat. Hist., vol. 3, p. 338, Coal Meas.

Anthracothremma, Scudder, 1884, Mem. Bost. Soc. Nat. Hist., vol. 3, p. 327. [Ety. anthrax, coal ; thremma, reared.] Body stout; prothorax several times broader than long; wings subequal and elongated; scapular vein arcuate and nearly reaching the tip; externo-median vein with numerous parallel branches, mostly simple. Type A. robusta.

robusta, Scudder, 1884, Mem. Bost. Soc. Nat. Hist., vol. 3, p. 327, Coal Meas.

Archegogryllus, Scudder, 1868, Proc. Bost. Soc. Nat. Hist., vol. xi, p. 401. [Ety. archegos, first in time; gryllus, a cricket.] Relations not clearly understood. Type A. priscus.

priscus, Scudder, 1868, Proc. Bost. Soc. Nat Hist., rol. 11, p. 401, and Mem. Bost. Soc. Nat. Hist., vol. 3, p. 323, Coal Meas. A rcirm ytacris, Scuader, 1868, Acad. Geol., p. 388. [Ety. arche, beginning; Mylacris, cockroach.] Mediastinal area com-

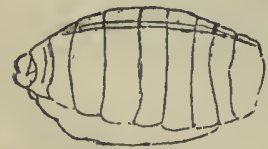

FIG. 1074.-A rchimylacris acadicum. paratively short; scapular terminating below the tip, and with the extern $o-m$ e di $\mathbf{a} n$, which is comparatively small, occupying less than half the wing; internomedian vein comparatively long. Type A. acadicum. acadicum, Scudder, 1868, Acad. Geol., p. 388, Coal Meas.

parallelum, Scudder, 1879, Mem. Bost. Soc. Nat. Hist., vol. 3, p. 85, Coal Meas.

Blattina, Burmeister, 1838, Handbuch der Entomologie. [Ety. Blatta, a cockroach.] A living genus of cockroaches, raised to the rank of a family, and by some naturalists to the rank of an order, to which the name Dictyoptera has been applied. It is not a Palæozoic genus. bretonensis, see Mylacris bretonense. fascigera, see Gerablattina fascigera. heeri, see Mylacris heeri.

sepulta, see Petrablattina sepulta.

venusta, see Etoblattina venusta.

Cheliphlebia, Scudder, 1884, Mem.Bost.Soc.

Nat. Hist., vol. 3, p. 328. [Ety. chele, forked; phlebion, vein.] Body rather slender, but wings large and coarse, without cross veins, interno-median vein extending far toward the tip of the wing with many oblique branches. Type C. elongata.

carbonaria, Scudder, 1884, Mem. Bost. Soc. Nat. Hist., vol. Fro. 1075. - Cheli3 , p. 328 , Coal Meas. phlebia carbonae l o n a t a, Scudder.

1884, Mem. Bost. Soc.Nat. Hist., vol. 3 , p. 328, Coal Meas.

Chrestotes, Scudder, 1868, Geo. Sur. Ill., vol. 3, p. 567. [Ety. chrestotes, good of its kind.] Wings short, broad, well

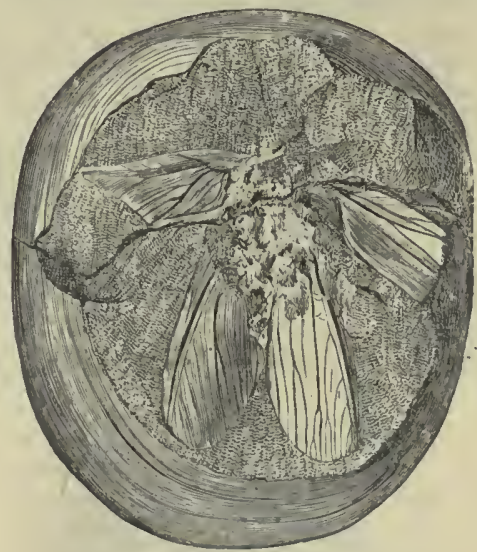

FIG. 1076.-Chrestotes lapidea.

rounded; vena scapularis throws several branches downward, commencing before the middle of the wing, and with its branches occupies the upper two- 
fifths of the upper wing, and perhaps more of the lower; remainder of wing occupied by the longitudinally divaricating branches of the next two veins; anal area in upper wing distinctly set off at the basal portion of the wing. Type C. lapidea.

lapidea, Scudder, 1868, Geo. Sur. Ill., vol. 3, p. 567, Coal Meas.

Didysopillels, Scudder, 1878, Proc. Bost. Soc. Nat. Hist., vol. 19, p. 300. [Ety. didymos, double; phleps, vein.] All the veins and branches above the internomedian longitudinal and nearly parallel; nearly all the lower half of the wing being occupied by the oblique branches of the internomedian vein. Type D. contusa.

contusa, Scudder, 1878, (Termes contusus, ) Bost. Soc. Nat. Hist., vol. 19, p. 300, and Mem. Bost. Soc. Nat. Hist., vol. 3, p. 330 , Coal Meas.

Dieconeura, Scudder, 1884, Mem. Bost. Soc. Nat. Hist., vol. 3, p. 336. [Ety. dieko, to extend through; neuron, a vein.] Externo-median vein simple; internomedian vein important, arcuate, extending far toward the extremity of the lower margin. Type D. vigida.

arcuata, Scudder, 1884, Mem. Bost. Soc. Nat. Hist., vol. 3 , p. 336 , Coal Meas.

rigida, Scudder, 1884 , Mein. Bost. Soc. Nat. Hist., vol. 3, p. 336, Coal Meas.

Dyscritus, Scudder, 1868, Lond. Geo. Mag., vol. 5, p. 176. [Ety. dyscritos, hard to deterinine.] Founded on a fragment of the middle part of a wing without proper definition. Type D. vetustus.

vetustus, Scudder, 1868, Lond. Geo. Mag., vol. 5, p. 176 , Devonian.

Fichenus, Scudder, 1884, Mem. Bost. Soc. Nat. Hist., vol. 3, p. 325. [Ety. en, very; kainos, new, strange.] Body stout, thoracic segments twice as broad as long; abilomen ovate; fore wings with the mediastinal vein straight, terminating before the apical third of the wing with numerous straight branches; scapular with similar branches ending half way between the mediastinal and the tip; externo-median important with distant branches. Type E. ovalis.

ovalis, Scudder, 1884, Mem. Bost. Soc. Nat. Hist., vol. 3, p. 325, Coal Meas.

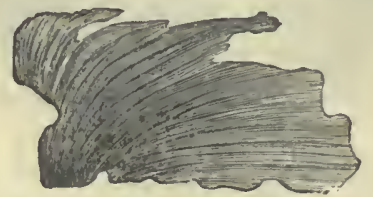

FIG. 1077.-Epliemerites affinis.

Ephemerites, Scudder, 1868, Geo. Sur. Ill., vol. 3, p. 571. [Ety. Ephemera, a living genus.] The genus was not $\mathrm{de}^{-}$ fined. It is probably a neuropteroid Palrodictyoptera. Type E. simplex. affinis, Scudder, 1868, Geo. Sur. Ill., vol. 3 , p. 572, Coal Meas.
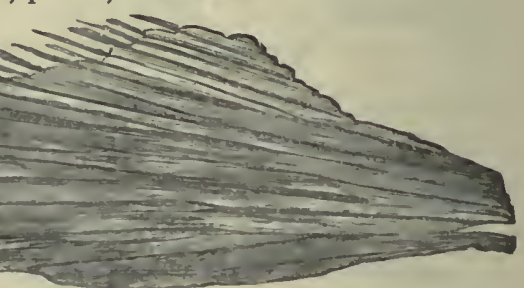

FIG. 1078.-Ephemerites gigas.

gigas, Scudder, 1868, Geo. Sur. Ill., vol. 3 , p. 571, Coal Meas.

primordialis, Scudder, 1878, Proc. Bost. Soc. Nat. Hist., vol. 19, p. 248, Coal Meas.

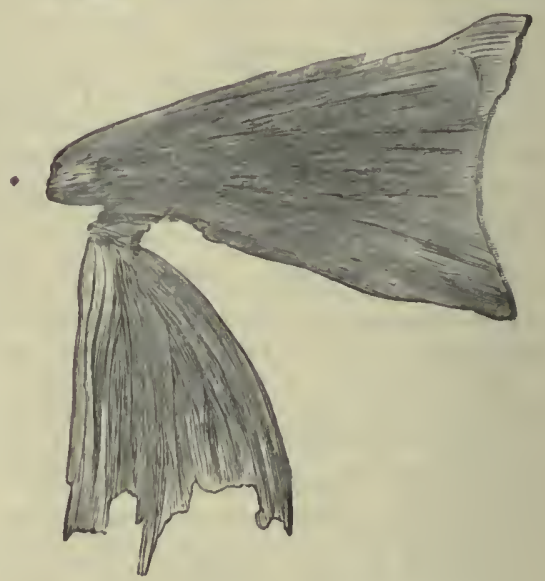

Fra. 1079.-Ephemerites simplex.

simplex, Scudder, 1868, Geo. Sur. Ill., vol. 3, p. 571 , Coal Meas.

Etoblatrina, Seudder, 1882, Proc. Bost. - Soc. Nat. Hist., vol. 21, p. 391. [Ety. etos, true; Blatlina, a genus.] Mediastinal area comparatively short; scapular not reaching tip of wing and with the externo-median, "which is comparatively large, occupying less than half the wing; interno-median vein comparatively long. Type E. mazonana.

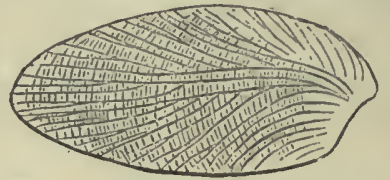

Fra. 1080-Etoblattina primaeva. From Saarbruck in Europe for comparison.

lesquereuxi, Scudder, 1879, Mem. Bost. Soc. Nat. Hist., vol. 3, p. 67, Coal Meas. 
mazonana, Scudder, 1882, Proc. Bost. Soc. Nat. Hist., vol. 21, p. 391, Coal Meas.

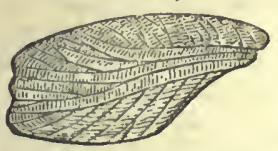

venusta, Lesquereux, 1860, (Blattina ven usta,) Geo. Sur. Ark., vol. 2 , p. 314 , Coal Meas. Fig. 1081. - Etoblattina GenwnTomum, Scud-
venusta. der, 1884, Mem.

Bost. Soc. Nat. Hist., vol. 3, p. 329. [Ety. genos, race, kind; entomon, insect.] Wings large, elongated with coarse venation and abumdant cross veins; mediastinal vein very long, with numerous branches to the costa; other branches very distant and stout; the externo-median separated more widely than usual from the scapular, especially in the hind wing. Type G. validum.

validum, Scudder, 1884, Mem. Bost. Soc. Nat. Hist., vol. 3, p. 329 , Coal Meas.

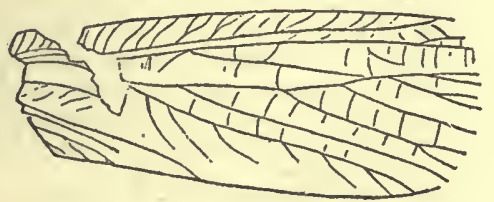

Frg. 1082.-Genentomum validum.

Genopteryx, Scudder, 1884, Mem. Bost. Soc. Nat. Hist., vol. 3, p. 327. [Ety. genos, kind, race; pterux, a wing.] Interno-median vein with branches very similiar to those of the externo-median vein, the outermost in close proximity to the innermost branches of the latter. Type G. constricta.

constricta, Scudder, 1884, Mem. Bost. Soc. Nat. Hist., vol. 3, p. 327, Coal Meas.

Gerablattina, Scudder, 1879, Mem. Bost. Soc. Nat. Hist., vol. 3, p. 110. [Ety. geras, old; Blattina, a genus of insects.] Mediastinal area comparatively long; scapular and externo-median area together occupy less than half the wing, the branches of both superior; internomedian vein comparatively long. Type G. balteata.

balteata, Scudder, 1879, Mem. Bost. Soc. Nat. Hist., vol. 3 , p. 110, and Perm. or Up. Carb. Flora of Pa., p. 104, Up. Fig. 1083,--GerablatCoal Meas. or Per- tina balteata. mian.

fascigera, Scudder, 1879, (Blattina fascigera,) Mem. Bost. Soc. Nat. Hist., vol. 3, p. 113, Coal Meas.

Gerepingera, Scudder, 1868, Lond. Geo. Mag., vol. 5, p. 175. [Ety. geras, old; Ephemera, a genus of insects.] Founded on the fragment of a tip of the wing. Definition incomplete. Type G. simplex. simplex, Scudder, 1868, Lond. Geo. Mag., vol. 5, p. 175 , Upper Devonian.

Gerapompus, Scudder, 1884, Mem. Bost. Soc. Nat. Hist., vol. 3, p. 326. [Ety. geras, old; poinpos, an escort.] Body slender, the prothorax as long as broad; fore wings well rounded, the mediastinal arcuate like the costa, with infrequent simple branches; scapular ending near the tip. Type G. blattinoides.

blatinoides, Scudder, 1884, Mem. Bost. Soc. Nat. Hist., vol. 3, p. 326, Coal Meas.

extensus, Scudder, 1884, Mem. Bost. Soc. Nat. Hist., vol. 3, p. 326, Coal Meas.

Gerarus, Scudder, 1868, Mem. Bost. Soc. Nat. Hist., vol. 3, p. 344. [Ety. geraros, of stately bearing.] Body slender, tapering anteriorly; wings slender ; mediastinal vein variable; branches of scapular vein numerous, more or less longitudinal, simple or forked, occupying much more space than the branches of any other vein. Type G. danæ.

danæ, Scudder, 1868, (Miamia danæ,) Geo. Sur. Ill., vol. 3, p. 566, Coal Meas.

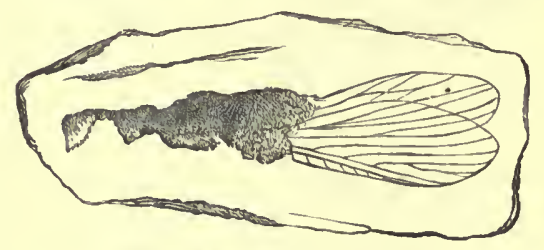

FIG. 1084.-Gerarus danæ.

mazonus, Scudder, 1884, Mem. Bost. Soc. Nat. Hist., vol. 3, p. 344, Coal Meas. vetus, Scudder, 1884, Mem. Bost. Soc. Nat. Hist., vol. 3 , p. 344 , Coal Meas.

Grironeura, Matthew, 1888, Trans. Roy. Soc. Can., p. 57. [Ety.geros, old; neura, a vein.] Anterior wing of the body elliptical elongate, venation strongly marked, scapular ridge conspicuous; mediastinal vein close to the scapular, but curves outward at the extremity; scapular vein and its branches cover a triangular area terminating at the apical end of the wing; main scapular terminates near the end of the costal edge; externo-median vein throws off two branches, the first one stronger than the main vein, and the second one goes with a sinuosity toward the base of the apical margin; nerves regular and simple. Type G. wilsoni.

wilsoni, Matthew, 1888, Trans. Roy. Soc. Can., p. 57, Lower Devonian.

Haplophlebium, Scudder, 1867, Can. Nat. and Geo., $2 \mathrm{~d}$ ser., vol. 3, p. 202, and Proc. Bost. Soc. Nat. Hist., vol. 11, p. 150. [Ety. haplos, simple; phlebion, a vein.] Wing with simple neuration and intercostal spaces filled with minute reticulations without any cross veins; wing long and slender.

Type H. barnesi. 
barnesi, Scudder, 1867, Can. Nat. and Geol. 2d ser., vol. 3, p. 202, and Acad. Geol., p. 386, Coal Meas.

aquatic in their habits in the larva and pupa state, and it is not until the perfect insect is about to emerge from the skin of the pupa that it creeps out of the water on the muddy shore or stones, or climbs the stems and leaves of aquatic plants, and from this position the imago springs into an aerial habitat. The trails on the Whetstone slates were evidently made by animals, and

Fro. 1085.

longipennis, Scudder, 1884, Proc. Amer. Acad., vol. 20, p. 172, Coal Meas.

Haplotichnus, n. gen. [Ety. haplotes, plainness, simplicity ; ichnos, track.] Simple, small, half-cylindrical trails running in any direction. Supposed to have been made by the larva or pupa of some palceodictyopterous insect. Type $H$. indianensis.

indianensis, n. sp. A simple half-cylindrical trail, needle-like in size, running in straight or crooked lines, or crossing itself. Found in the upper part of the Kaskaskia Group, at the Whetstone quarries in Orange County, Indiana.

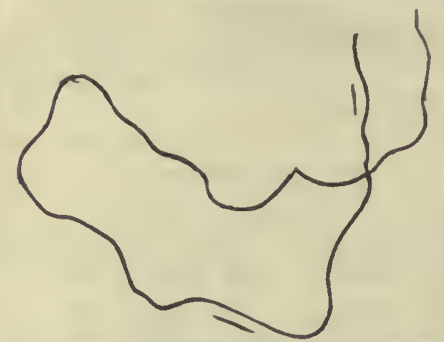

Fig. 1086.-Haplotichnus indianensis.

The remains of insects found in the Palæozoic rocks occur under such circumstances as to induce the belief they were more or less aquatic in their habits, and frequented swamps and shores of bays and inlets. The Whetstone quarries of Orange County, Indiana, are yellowish white, slaty' mud-rocks resembling, in appearance, the Solenhoien slates, but coarser in texture. They are limited in extent, and may be fairly presumed to represent the muddy shore of some bay or internal sea of Subcarboniferous age. The slaty layers are covered more or less upon the upper surface with trail-furrows, and on the under surface with elevated lines, showing the trails were made in mud, which afterward hardened, and was then covered with a thin deposit of mud which was tracked and hardened and covered, and so on in one series after another throughout the whole thickness of the slaty deposit. Many of the living Dictyoptera are all the evidence seems to indicate they were made by insects, though the evidence may not be either clear or conclusive in the latter respect. Under these circumstances the author has selected three common but distinct trails, and given then generic names; viz., Haplotichnus, Plangtichnus, and 'Treptichnus.

Hemeristia, Dana, 1864, Am. Jour. Sci. and Arts, 2d ser., vol. 37, p. 34. [Ety. hemera, day; istia, house.] Scapular branch strongly arcuate, at its base distant from the main stem, and at first taking the course of its basal offshoot. Type H. occidentalis.

occidentalis, Dana, 1864, Am. Jour. Sci. and Arts, 2 d ser., vol. 37, p. 34, Coal Meas.

H о о т 11 ETUS, Se u d d er, 1867, Can. Nat. and Geol., 2d ser., vol. 3 , p. 202. [Ety. homos, simi-

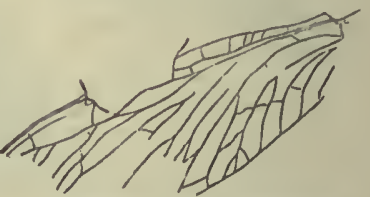

Fig. 1087.-Homothetus fossills. lar; thetos, placed.] Mediastinal vein extremely long, scarcely surpassed by the scapular, and with scarcely any branches to the costa; externo-median vein with only a few branches in the outer fourth of the wing; interno-median vein similar to the last. Type $H$. fossilis.

fossilis, Scudder, 1867, Can. Nat. and Geol., 2d ser., vol. 3, p. 202, and Acad. Geol., p. 525, Upper Devonian.

Libellula, Linnæus. Not a Palæozoic genus. carbonaria, see Cheliphlebia carbonaria.

Lithentomum, Scudder, 1867, Can. Nat. and Geol., 2d ser., vol. 3, p. 202. [Ety. lithos, stone; entomon, an insect.] Main

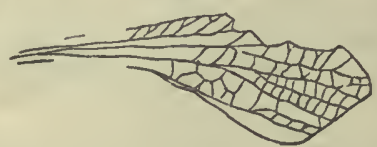

FrG. 1088.-Lithentomuın hartti.

scapular branch with a single, or at most two branches, which are almost wholly longitudinal. Type L. hartti. 
hartti, Scudder, 1867, Can. Nat. and Geol., 2d ser., vol. 3, p. 202, and Acad. Geol., p. 525, Upper Devonian.

Lithomylacris, Scudder, 1879. Mem. Bost. Soc. Nat. Hist., vol. 3, p. 48. [Ety. lithos, stone; Mylakris, a kind of roach.] Mediastinal and scapular areas together occupying more than half the wing; externo-median area small, compressed, scarcely expanding apically. Type L. angustum.

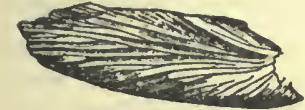

F'IG. 1089. - Lithomylacris angustum. angustum, Scudder, 1879 , Mem. Bost. Soc. Nat. Hist., vol. 3, p. 48, Coal Meas.

pit ts tonianum, Scudder, 1879 , Mem. Bost. Soc. Nat. Hist., vol. 3, p. 50, Coal Meas.

simplex, Scudder, 1879, Mem. Bost. Soc. Nat. Hist., vol. 3, p. 51, Coal Meas.

Megathentomum, Scudder, 1868, Geo. Sur. Ill., vol. 3, p.570. [Ety. megathos, largeness; entomon, an insect.] Wings of great size, remarkably broad and rounded; veins distant; simple, infrequent divarications, and cross neuration of delicate, irregular veinlets; the wing is also dotted with larger and smaller spots. Type M. pustulatum.

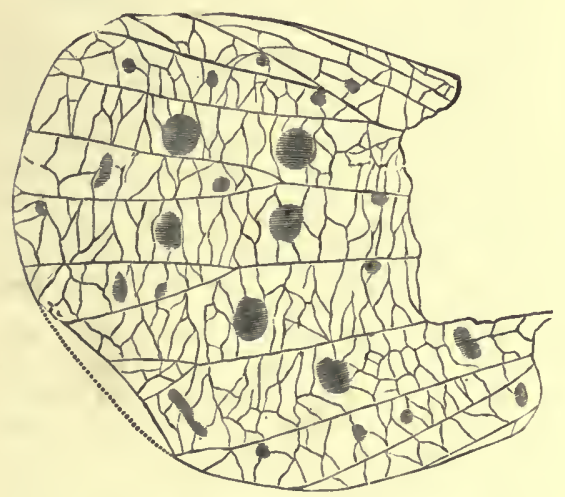

FIG. 1090.-Megathentomum pustulatum.

pustulatum, Scudder, 1868, Geo. Sur. Ill., vol. 3, p. 570, Coal Meas.

Miamia, Dana, 1864, Am. Jour. Sci. and Arts, 2d ser., vol. 37, p. 34. [Ety. proper name.] Scapular vein close to the mediastinal; straight, main branch arising near the middle of the wing, and nowhere distant from the main stem. Type M. bronsoni.

brousoni, Dana, 1864, Am. Jour. Sci. and Arts, 2d ser., vol. 37, p. 34, Coal Meas. danæ, see Gerarus danæ.

Mylacris, Scudder, 1868, Geo. Sur. Ill., vol. 3, p. 568. [Ety. Mylakris, a kind of cockroach.] Wings broad, mediastinal and scapular areas together occupying

less than half the wing; scapular area larger than the mediastinal. Type M. anthracophilum.

a $n$ t h $r$ a c ophilum, Scudder, 1868, Geo. Sur. Ill., vol. 3 , p. 568, Coal Meas.

antiquum, Scudder, 1884, Mem. Bost. Soc. Nat. Hist., vol. 3, p. 300, Coal Meas.

bretonense, Scudder, 1874, (Blattina bretonensis,) Can. Nat., vol. 7, p. 271, Coal Meas. carbonum, Scudder, 1884, Mem. Bost.

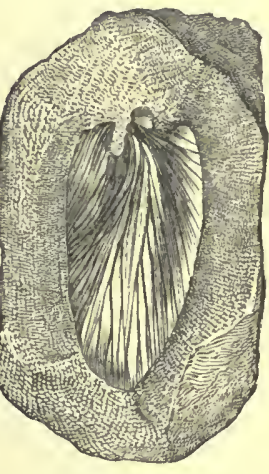

Soc. Nat. Hist., FIG. 1091.-Mylacris anvol. 3, p. 304, thracoplillum. Coal Meas.

heeri, Scudder, 1874, (Blattina heeri,) Can. Nat., vol. 7, p. 272, Coal Meas.

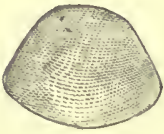

Fig. 1092.-Mylacris a $n$ thracophllum Pronotal shield.

\section{lucifugum, Scudder,} 1884, Mem. Bost. Soc. Nat. Hist., vol. 3, p. 301, Coal Meas.

mansfieldi, Scudder, 1880, Mem. Bost. Soc. Nat. Hist., vol. 3, p. 47, Coal Meas.

ovale, Scudder, 1884, Mem. Bost. Soc. Nat. Hist., vol. 3, p. 308, Coal Meas.

pennsylvanicum, Scudder, 1880, Mem. Bost. Soc. Nat. Hist., vol. 3, p. 44, Coal Meas.

priscovolans, Scudder, 1884, Mem. Bost. Soc. Nat. Hist., vol. 3, p. 307, Coal Meas. Necymylacris, Scudder, 1880, Mem. Bost. Soc. Nat. Hist., vol. 3, p. 53. [Ety. nekus, dead; Mylakris, a roach.] Some of the apical branches of the mediastinal vein arise beyond the base of the wing, and scarcely partake in the radiate arrangement of the others. Type N. lacoanum.

heros, Scudder, 1880, Mem. Bost. Soc. Nat. Hist., vol. 3, p. 54, Coal Meas.

lacoanun, 1880, Mem. Bost. Soc. Nat. Hist., vol. 3, p. 53, Coal Meas.

Oryctoblatina, Scudder, 1885, Proc. Acad. Nat. Sci. Phil., p. 37. [Ety. oruktos, quarried; Blattina, a genus.] Principal veins widely separated at base; scapular area surpassing apex, and with externomedian occupying more than half the wing, the branches of latter inferior; interno-median vein comparatively short. Type O. occidua.

occidua, Scudder, 1885, Proc. Acad. Nat. Sci. Phil., p. 37, Coal Meas.

Paolia, Smith, 1871, Am. Jour. Sci. and Arts, 3d ser., vol. 1, p. 44. [Ety. proper name.] Wings long, slender, branches of veins dichotomizing strongly, and running in a longitudinal direction, so that the externo-median branches oc- 
cupy only a slight portion of the lower margin. Type $P$. vetusta.

gurleyi, Scudder, 1854, Proc. Amer. Acad. vol. 20, p. 173, Coal Meas.

lacoana, Scudder, 1884, Proc. Amer. Acad., vol. 20, p. 173, Coal Meas.

superba, Scudder, 1884, Proc. Amer. Acad., vol. 20, p. 173, Coal Meas.

vetusta, Smith, 1871, Am. Jour. Sci. and Arts, 3d ser., vol. 1, p. 44, Coal Meas.

Paromyiacris, Scudder, 1855, Proc. Acad. Nat. Sci. Phil., p. 35. [ Ety. paros, before, or forefather; Mylakris, a kind of roach.] Borly much arched; pronotal shield more than twice as broad as long; wings extremely broad; mediastinal area large and extended, and with the scapular occupying half the wing; externo-median area expanding apically. Type $P$. rotundium.

rotundum, Scuzlder, 1885, Proc. Acad. Nat. Sci. Phil., p. 35, Coal Meas.

Petraibatisisa, Scudder, 1876, Can. Geol., vol. 8, p. 88. [Ety. petra, stone; Blattina, a genus.] Scapular and externomedian areas together covering more than half the wing; the extcrno-median vein directed toward and terminating near the middle of the inner border of the wing, branches superior; internomedian vein very sliort. Type $P$. sepulta.

sepulta, Scudder, 1876, (Blattina sepulta,) ('an. Nat. and Geol., vol. 8, p. 88, Coal Meas.

Pituanocoris, Scudder, 1884, Proc. Bost. Soc. Nat. Hist., vol. 22, p. 58. [Ety. phthano, tirst; kore, pupa.] Front wing differentiated from the hind wing; corium distinct from the membrane,

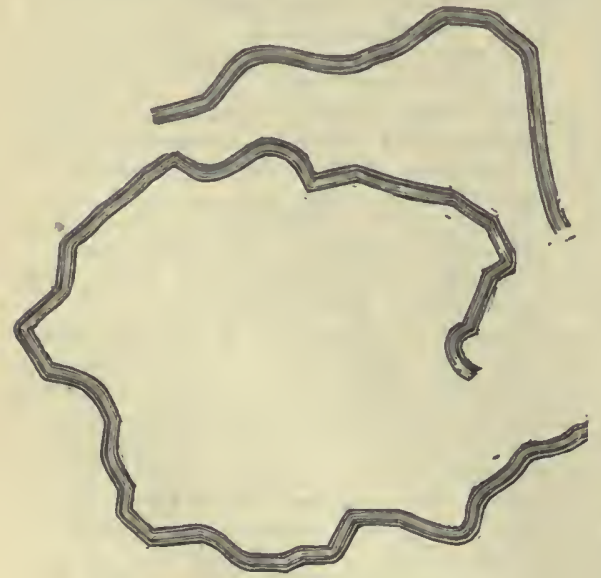

FIG. 1093.-Plangtichnus erraticus.

narrow clavus; no embolium or cuneus; mediastinal and scapular veins widely separated at base. Type P. occidentalis.

occidentalis, Scudder, 1884, Proc. Bost. Soc. Nat. Hist., vol. 22, p. 58, and Mem
Bost. Soc. Nat. Hist., vol. 3, p. 348, Coal Meas.

Plangtichnus, n. gen. [Ety. Plagklos, wandering; ichnos, track.] A zigzag, half-cylindrical, broken trail, running in any and erery direction; sometimes dotted or sumk deeper at the angles than at other places, or most depressed between the angles in some cases. Supposed to have been madle by the larva or pupa of some Palæodictyopterous insect. See remarks under Haplotichnus. Type P. erraticus.

erraticus, n. sp. A simple, irregularly zigzag, lalf-cylindrical, broken trail, running in any and every direction, depressed in spots deeper than the general trail. Collected in the upper part of the Kaskaskia Group at the Whetstone quarries, in Orange County, Indiana.

Plateplinuera, Scudder, 1867, Can. Nat. and Geol., $2 d$ ser., vol. 3, p. 202. [Ety. platys, flat; epheme ra, an insect.]

Founded upon the fragm e n t of an upper wing,

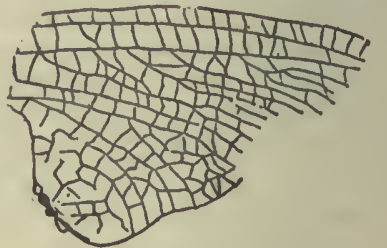

FIG. 1094.-Hlatyphemera antiaur. showing nervation and a heavy cross vein near the base between two middle veins, from which new prominent veins arise; ancient May-nles, in which the lower externo-median stem seems to be formed on the same plan as the upper stem. Type P. antiqua.

antiqua, scudder, 1867, Can. Nat. and Geol., $2 d$ ser., vol. 3, p. 202, and Acad. Geol., p. 524, Devonian.

Pouyennus, Scudder, 1884, Mem. Bost. Soc. Nat. Hist., vol. 3, p. 343. [Ety. polys, many; ernos, a scion.] Body moderately stout; wings rather broad; mediastinal vein extending nearly to the tip of wing; branches of scapular vein inequidistant at origin, longitudinal, closely crowded and ramose, yet hardly more important than the externomedian vein. 'Type $P$. complanatus. complanatus, Scudder, 1884, Mem. Bost. Soc. Nat. Hist., vol. 3, p. 343, Coal Meas. laminarum, Scudder, 1884, Mem. Bost. Soc..Nat. Hist., vol. 3, p. 343, Coal Meas. Promylacris, Scudder, 1885, Proc. Acad. Nat. Sci Phil., p. 34. [Ety. pro, before; mylakris, a kind of roach.] Body much arched; wing broad; mediastinal and scapular areas together not occupying more than a third of the wing; scapular area smaller than the mediastinal, the vein running obliquely to the costal margin. Type P. ovale.

ovale, Scudder, 1885, Proc. Acad. Nat. Sci. Phil., p. 34, Coal Meas. 
Propteticus, Scudder, 1884, Mem. Bost. Soc. Nat. Hist., vol. 3, p. 334. [Ety. proi, early; ptetikns, winged.] Scapular vein widely separated from the mediastinal, arcuate, main branch arising near the base of the wing, parting widely from the main stem. Type P. infernus.

infernus, Scudder, 188t, Mem. Bost. Soc. Nat. Hist., vol. 3, p. 334 , Coal Meas.

Termes, Linnaeus; 1748, Systema Naturæ, p. 610. Not a Palæozoic genus.

contusus, see Didymonhleps contusa.

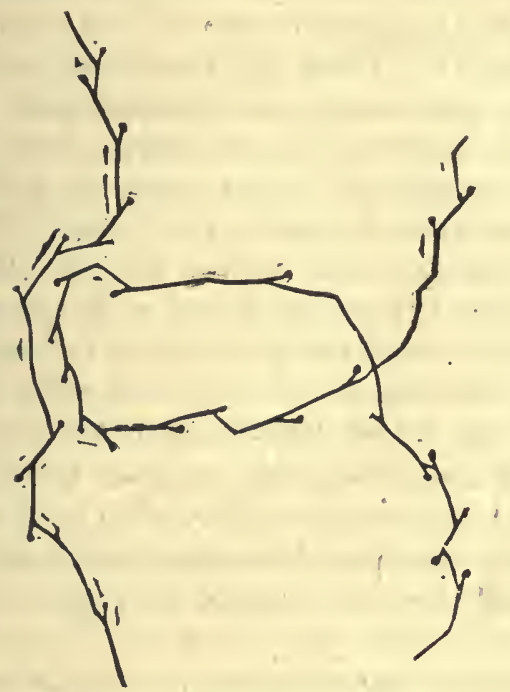

FIG. 1095.-Treptichnus bifurcus. The trails are larger than shown by the figure.

Titanophama, Brongniart, as recognized by Scudder. Wing very large, moderately slender; neuration moderately abundant; scapular vein beginning to branch in the middle of the basal half of the wing.

jucunda, Scudder, 1884, Proc. Amer. Acad., vol. 20, p. 169, Coal Meas.

Trepticisus, n. gen. [Ety. treptos, to be turned about; ichnos, track.] A zigzag, half-cylindrical, continuous trail, forked at each angle, and running in any direction; each line is prolonged in the direction in which the animal moved, at the angle, so as to form a short fork or projection. Supposed to have been made by the larva or pupa of some Palæopterous insect. See remarks under Haplotichnus. Type T. bifurcus.

bifurcus, n. sp. A zigzag, half-cylindrical, continuous trail, quite evenly depressed, and forked at each angle; the bifurcation takes place in the direction in which the animal moved, but generally is less sunken than the trail, and sometimes shows simply a dot disconnected with the angle. Collected in the upper part of the Kaskaskia Group at the Whetstone quarries in Orange County, Indiana.

Xenoneura, Scudder, 1867, Can. Nat. and Geo., $2 d$ ser., vol. 3, p. 202. [Ety. xenos, new, strange; neura, a vein.] Mediastinal and scapular veins as in Palæopterina; externo-median vein amalgamated at base with the scapular, branching beyond the middle, internomedian divided at base in two branches. Type $\mathrm{X}$. antiquorum.

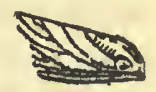

Frg. 1096.

Xenoneura antiquorum, Scudder, 1867, Can. Nat. and Geol., $2 \mathrm{~d}$ ser., vol. 3, p. 202, and Acad. Geol., p. 525, Upper Devonian. 


\section{SUBKINGDOM VERTEBRATA.}

Tnrs is the highest division of the Animal Kingdnm, and, until within the last twenty years, the essential churacter upon which the sulsing lon was based was the possession of a bony or curtilaginous internal skeleton, having a spine or vertebral column. Since that time the class known as Tunicates, or Ascidians, which have no bony skeleton, has been referrel to it; and the Amphioxus lanceolatus, a little, slender, transparent creature, having only a gelatinous cord, no brain cavity, and colorless blook, which was regariled as the lowest type of fishes, and had been elevated into an order callexl Leptocarrlia, is now taken nut of the Class Pisces and referred to a separate class called Acrania. The Classes Tunicata and Acrania are not, however, known in Palæonic rocks. Another class, called Cyclostomata, consisting of long, cylindrical, worm-like bodies, with a tough skin, destitute of scales, pectoral and rentral fins, hut having a fin at the extremily of the body without any rays, and having a cartilaginous skeleton, and which includes the various species of lampreys that ịnhabit fresh water, ancl are also found in the ocean, and which have gencrally been regarderl as an order of fishes, is unkunwn in Palæozoic rocks. Some authors would, however, place the Conodonts in this class; but if they do not belong to the Anuelisli, then there are stronger reasons for believing they belong to Crustacen than fur thinking they should be referred to the Cyelostımata.

No Palæenzoic fossil from an animal as highlily organized as the lowest mammal or a bird hus ever been found. The fossils are ennfined to the lowest organizations of fishes, batrachians, and reptiles. The lower forms of fishes bave only a cartilaginous cord, resembling the embryonic state of fishes laving an ossenus skeleton. In higher forms the spine consists of bony vertebrx, united in such manner as to allow flexilility and strength by reason of attaching muscles, and also to protect a spinal nerve that passes through it.

\section{CLASS PISCES, OR FISHES.}

The Class Pisces has been diviled into four subclasses-Ganoidea, Selachia, Dipnna, and Teleostia. The Telenstia have heen divided into eleven orders, and these inti) twenty suborders. This subclass embraces a very large majority of the living fishes. All of them hare a complete bony vertebral eolumn and skull. Nearly all edible fishes belong to this suljelass. Malny have a naked skin, hut the majority are cuvered with horny seales of various forms. When the scalcs are smooth the fish are said to belong to the Creduidea; when the hinder maryins of the scalcs are denticulated they belong to the Ctenoidea. Fishes have pectoral fins, which are called the representitives of anterior limbs; and rentral fins, representatives of posterior limbs; and also dorsal, caudal, and anal fius. This subelass is unknown in Pallozoic rocks. Agassiz dividel the fish into four groups-Cychids, Ctenoids, Placoills, and Ganoids-based on the chatracter of the seales; but as a single fish has been found bearing two of these types of scales, and as it is now 
known that the whole structure of animals must be taken into consideration in classification, his system, like that of every other based on a single character, has given way to more perfect knowledge of auimal life and pliysical structure. Aud it is quite probable, if the characters of Devonian fishes could be completely ascertained, as we know the living firms, they would all be found to belong to extinct subclasses. They are judgel, howerer, ouly from meager fragments of the ossified parts, and arranged by homologies with the existing species, and classed in orders where the affinities seem nost strongly to arrange them.

\section{SUBCLASS GANOIDEA.}

The Ganoidea (from ganos, brightness, in allusion to the enameled armor with which some of them are covered) commences in the Devonian strata, where the fossil remains soon become abundant, and continue to occur from that time forward to the present, though very few families now exist. Agassiz included as Ganoids all lish covered, in whole or in part, with bouy plates; but some of the living genera were found to belong to the Teleostia, and later classification has been held to inclurle all fissil species falling within the original definition of Agassiz and part of the living forms. The dermal skeleton consists of smooth, bony plates, covered with cnamel. In some cases they are rhomboidal, arranged edge to edge in oblique transverse rows; in other cases the scales are rounded; and in a few species the skin is naked. There is much dirersity in the skeletons, and all shades of ussification in the vertebral column and skull from cartilaginous to perfect bone. The subclass has been divided into seven orlers, viz. : Chonlrostea, Halecomorpha, Ginglymoda, Pycnodonta, Crossopterygia, Acanthodea, and Placodermata.

The Oriler Chondrostea includes the sturgeons of fresh and salt water, and the paddle-fish or spron-lill cat of the Mississippi River and its tributaries.

The Orler Halecomnrpha (shatl-like) is generally united with the Ginglymoda, uniler the name of the Holostea; lout is distinguished by having large, round scales, no shingle-like fulcra on the fins, and in having the vertebre concave at both ends, as in the Teleostia. The only living genus is the $\Lambda$ mia, called bow-fin, mul-fish, dog-fish, etc. It is common to the lakes and sluggish rivers. The order is not certiinly kunwn in Palaeuzoic rocks.

The Oriler Pycnodonta has a short, vertically-flattened body, covered with rhomboid seales and peculiar dermal ribs. Tail either heterocercal or homocercal.

'The Orier Ginglymorla has a bony skeleton, rhomboid scales, and shingle-like fulcra on the fins. The vertehre are convex in front and concave belind, forming ball and socket juints; tail heterocercal, and ventral fius between the pectorals and anils. 'This order is represented by the gar-pikes, which are common in American rivers.

The Order Crossopterygia is represented by two genera in the African waters, and fussils are referred to it back in geolugical time as far as the Devonian. The scales maty he cycloill or rlombuid; the throat is protected by two or more plates; the caulal fin is diphycercal; dorsal fin is divided in two or more divisions; pectorals and ventrals have a scaly axis; no fulcra.

The Order Acanthodea harl cartilaginous skulls, heterocercal tails, rhomboidal scales, and were armed with a spine before each fin, and are said to occupy a.place between the Ganoidea and Selachia. They are all Palæozoic. 
The Order Placodermata had the head and thoracic region inclosed in sculptured, bony plates. In some the tail was naked, in others it was covered with ganoid scales; in some the fins were inclosed in plates, but the vertebræ were not ossified. This order includes the oldest fish remains known to the geologist.

\section{SUBCIASS SELACHIA.}

The word Selachia is derived from selachos, the Greek word for shark. This subclass is also called Elasmohranchia and Chondropterygia, and it includes the living sharks, rays, and skates. The skeleton is cartilaginous, and the plates of the skull are united without sutures. There are pectoral and ventral fins, and the caudal fin is usually heterocercal. The surface of the body is naked or covered with calcified papillæ, comparable with tecth, and even spinous. The placoid scales sometimes form a sculptured armor. The dermal spines found fossil are collectively known as Ichthyodorulites. The teeth are never inserted into the jaws, but are sustained in their position by the strong skin of the gums. They sometimes have obtuse crowns, and form a pavement for both jaws; in other cases the teeth are conical, sharp, arranged in rows, with the apices pointed backward. The subclass is divided into the Holocephala and Plagiostomata. The Holocephala is represented in the existing seas by the Family Chimaridac, and, it is said, combines some of the characters of the Selachia, Ganoidea, and Batrachia. The Plagiostomata is divided into two orders, the Squalina and Raina, The vertebræ are well developed, and the skin is covered with plates, shields, or spines. The Order Squalina includes the ocean sharks and dog-fishes. The Order Raiiua iucludes the skates and rays of the present seas; one of them is called the saw-fish, and another produces dangerous electrical discharges.

\section{SUBCLASS DIPNOA.}

This subclass is said to furnish a connecting link from the Ganoidea to the Batrachia. In external appearance the fish are ganoid-like. The body is long, eellike, covered with scales, and terminates in a compressed caudal fin with weak finrays. The head is broad and flat. There are two orders, Monopneumonia and Dipneumonia. The Monopneumonia includes the Ceratodidæ, some of which are living in Australia, and they are common in the Mesozoic rocks, but the existence of them in the Palæozoic rocks is very doubtful.

The Order Dipneumonia contains the living Family Sirenidæ, which contains two genera, the Lepidosiren, from the rivers of Brazil, and the Protopterus, from tropical Africa. There is little reason to believe this order is represented in Palæozoic rocks, though Ctenodus and Dipterus have been referred to it.

\section{SUBCLASS GANOIDEA.}

\section{ORDER ACANTHODHA.}

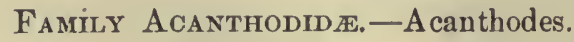

\section{Order Chondrostea.}

Family Chondrosteida.-Asterosteus, Macropetalichthys.

Family Palaeniscida.-Chirolepis, Mecolepis, Palæoniscus, Rhadinichthys. 


\section{Order Crossopterygia.}

Family Coglacanthides.-Coelacanthus.

Family Crossopterygids,-Ceratodus, Conchodus, Ctenodus, Heliodus,

Onychodus, Peplorhina.

Family Dipteríd.-Dipterus, Gnathorhiza, Ptyonodus.

Family Holoptychide.-Glyptolepis, Holoptychius.

Family Phaneropleuronid w.-Phaneropleuron.

Family Rhizodontide.-Eusthenopterun, Rhizodus.

ORDER GINGLYMODA.

Family Lepidosterde.-Acrolepis, Amblypterus, Eurylepis.

Order Placodermata.

Family Cephalaspide.-Acanthaspis, Acantholepis, Cephalaspis.

Fanimy Coccosteidas.-Coccosteus, Dinichthys, Liognathus.

Fanily Pteraspide-Diplaspis, Palraspis.

Fanily Pterichthyide.-Aspidichthys, Bothriolepis, Pterichthys.

Family Uncertain.-Myeterops.

Order Pycnodonta..

Family Pycnodontide.-Platysomus.

Family Uncertain. - Ectosteorachis.

SUBCLASS SELACHIA.

DIVISION HOLOCEPHALA.

Order Chimeroididea.

Famruy Chrmeromide.-Cyrtacanthus, Liognathus, Machæracanthus, Ptyctodus, Rhinodus, Rhynchodus.

DIVISION PLAGIOSTOMATA.

ORDER SQUalina.

Family Cónliodontide.-Chitonodus, Cochliodus, Cymatodus, Deltodopsis, Deltodus, Deltoptychius, Helodus, Orodus, Orthopleurodus, Petrodus, Platyodus, Pocilodus, Psephodus, Sandalodus, Stenopterodus, Tæniodus, Tomodus, Trigonodus, Vaticinodus, Xystrodus. The Cochlindontidæ commenced at the base of the Subcarboniferous, reached their greatest development in the same geological system, and only one genus, Orthopleurodus, is found as high as the Coal Measures.

Family Hybonontide.-Agassizodus, Apedodus, Bathychilodus, Carcharopsis, Cladodus, Diplodus, Hybocladodus, Janassa, Lambdodus, Liodus, Mesodmodus, Orodus, Periplectrodus, Phobodus, Pristicladodus, Stemmatodus, Thrinacodus, Polyrhizodus.

Family Petalodontide.-Antliodus, Calapodus, Cholodus, Chomatodus, Ctenopetalus, Ctenoptychius, Dactylodus, Desmiodus, Fissodus, Harpacodus, Lisgodus, Peltodus, Peripristis, Petalodus, Petalorhynchus, Polyrhizodus, Pristodus, Tanaodus, Venustodus. 
Fanily Icntirodorulites. - Acondylacanthus, Amacanthus, Anaclitacanthus, Asteroptychius, Batacanthus, Bythiacanthus, Compsacanthus, Ctenacanthus, Cyrtacanthus, Drepanacauthus, Edestus, Erismacanthus, Eunemacanthus, Gampsacanthus, Gisacanthus, Glymmatacanthus, Gyracanthus, Homacanthus, Lecracanthus, Listracanthus, Machæracanthus, Marracanthus, Oracanthus, Orthacanthus, Physonemus, Puigeacanthus, Stenacanthus, Xystracanthus.

\section{ORDER RAIINA.}

Family Psammodontide.-Copodus, Psammodus.

Acantuasmis, Newberry, 187n, Obio Pal., vol. 2, p. 36. [Eiy. akerrthu, spine; aspis, sliteld.] Cravium platrs some-

what quadrangular at one end, then abruptly bending to one sille, anil prulonged to an acute point; surface carinated and tuber. culated. Type A. arinala.

armata, Newberry, 187n, Ohiu Pal., vol. 2, p. 37', Ui. Hell. Gr.

Acanthodes, Agarsiz, 1833, Recherilies sur les Puiss. Fuss., vol. 1, op. 19. [Ety. akantha, spune.] Fish lepilloid, nomli wide; lower jiw longur than the uıper; leeth lirush. like; scales small; dur-

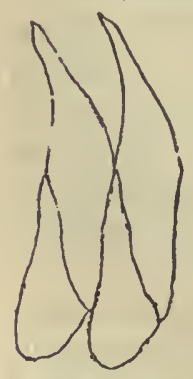

Frn.1098.-Acanliviepis pusIlislepis pils-
tilosilx. dncel outlines of (ni) plates, probably in postilons. sul fi $a$ opposite a 11 a 1 ; pectora I Fif. 1097.-Acantliavpl armata. I a $\mathrm{re}$; Pliteliesting splne from left t a $\mathrm{ge}$; slde of cranlum. tirst ray of rachi fin strong, large, stiff; ravs of cairlal tin clisse. Type A. bron'i. affinis, IV liteav's, 1899 , Traus. R',y. Śoc. Can., vol. 6, p. $7 \%$, Low. Devonian.

concin n us, Whiteaves, 1859, Trans. Riv. Six:. Can., vul. 6, p. 77, Low. Devonian.

Acantiolepis, New be r $\mathrm{r}$, 1875, Uriu, Pal., vol. 2. p. 38. [ Eis. akmilha, spine; lepis, scale.] Tubrrculatrd cranial or dermal plates, haviug a prevailing spalulate outline; fome are thin and latv" the appearance of large, elongated, unsymus trical scales; others ase stronger aind pruduced into 1 oints that somminims become spines. Type A. insluluta.

pus.ulosa, Newberry, 1875, Ohio Pal., vol. 2, j. 38 , Up Held. Gir. Acondri.acantulus, St. Julin \& IVurthen, 18i5, Geo. Sinr. III., vol. 6, p. 43:. [Fity. akondylus, without bony kuolss; whanthu, spine.] Fin rays long, griulually tapering. laterally conipressid, miderately curved posteriorly ; lateral faces longituliually fluted; coatæ smoth, ellamelt $(\mathrm{d}$, increasing by hifurcation and iuplautation; posterior face excavated fongitulinally, without median keel; postero-lateral angles bearing a row of d $u$ w nw a $\mathrm{rd}$ hooked denticles; pulp cavity occupying the posterior lialf of the spine. Type A. gra(illis.

xqu i costatus, St. Julin \& IVorthen, 1875, Gro. Sinr. Ill., vil. 6, 1). 434 , Krokuk Gr.

gracilis, Sr. John \& Worthell, 1875, Gers. Sur. Ill, vol. 6, p. 43:), IV 4 vily or Kinderhook Gir. muilyianus, Nit. John \& II'orthen, 1883, (ier). Nur. IIl., vil. 7, p. 24t, Up. Coil Mras.

nuperus, st. John \& Worthen, 18×3, Geo. Sur. III, vol. 7, p. 242, Up. Cual Meas.

ocrilentalis, Newberry \& worthen, 1866, (Leptacanthus occidentalis,) 
Geo. Sur. Ill. vol. 2, p. 116 , St. $\mathrm{L}$, uis Gr.

rec:tıs, St. Jihu \& Viorthen, 18s3, G чо.

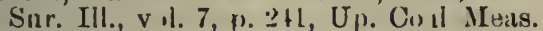

xip'ii t., Sc. Juhn \& IVorthen, 183:3, Geo.

S ir. III., visl. 7, p. 2tt, K.rkık Gr.

Acrolapis, A yaisiz, $15 ;(j, \mathrm{R}$ schershes sur les Puiss. Fuss., vul. 2, p. 79 . [E!

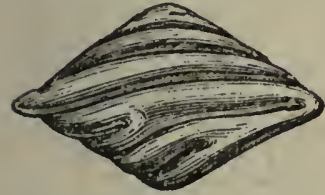

Frs. 1lu0.- Icrolepis sedg. wickl. Ming iffed scale.

strongly keeled ancl sulcated diagunally. Type A. serlgwiski.

hortinensia, D.lwson, 18 is, Acsd. Geol., p. 2 it, subisarb mifer ms.

Agassirhllyys, Newbsrry, syn. for MI ıcrop». tali:litiıys.

minui, see II ccropetalichthys manni.

sullincui, ses Masropetalichtiys sullivinti.

Agassizudus, St John \& WVurthen, 1875, Gerl. Sur. Ill., vul. 6, p. 311. [Ecy. prop:r naine; odous, tinctli.] 'Testh varifble, traisvarsely elonuater, b ise usually prolnced; crown traversed hy a crest, raised into several sinmmit, the central one often large. 'Typ: A. varialilis.

corrmytlli, Newherry \& IVorthen, 1870, (Oroilux corrugatus,) Gso. Sur. III., vul. 4, p. 3.j8, Cosl . Meas.

scitulıs., st. Johu \& IV orthen, 1875, Geo. Sur. Iil., vol. 6, p. 3:2, Cual Meas.

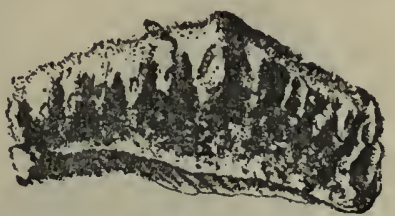

Fra. 1101.-Agassizodus variabllis.

variabilis, Newberry \& Worthen, 1870 , (lophodas viriahilis,) Geo. Sur. 1ll., vil. 4, p. 361, Coal M.'as.

viryinianns, st. Juhn \& IVorthen, 1875. Geo. Sur. Ill., vol. 6, p. 321, Coal Mleas.

Axacastius, St. John \& Worthen, 1875, Gro. Sir. Ill., vol. 6, 1) 46t. [Ery. am', bickwaril; "kintha, spine.] líorsal spine timuly implanted, curved forwarl, latrally compresseu, posterior face truncated anil longiturlinally kreeled or denticulate along the rnedian line; romudeal and tuberulated in the concav's anterior fice; lateral sur'ace covercl with tuberculose costæ. Type A. gibbosus. gibbosus, Newberry \& Worthen, 1S66,

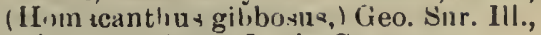
vil. 2, p. 113, Sr. L'llis Gr.

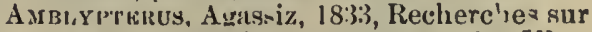
Jes l'oissuns Fussil s, t. 1, p. 28. [Hiy. amblys, blint; vteron, fin.] All fi is large unil coinp ised of numernils riys; pectoral very larere; anal $1, r$ sall; dors 11 oup site the anal $p$ sint of the ventral, which is fir bick; little ravs on the superior lob $\rightarrow 0^{i}$ the huterivercal twil; heal blunt; scales medium, rh smboid. Type A. inacropterus.

macropterus, Agaztiz, 1836, R sherch. Puis?. Fuss., vol. 2, p. 28, Cual Meis.

Axacil'ricintius, St. Juhn \& IVortien, 1875, Geo sur. III, vol. (j M. 4 t2. [E. an,klirs, leant upon; rlianthu, spine.] Fin spine recumbent or imbalilal Hong its inferior extent, l.aterall' co upressisl, subovate in transverses (ttinn; cxposed p.urt constricted along the line of inion wi'h the baze. Type A. s.micostatıs. semicostutus, Sc. Julin \& IVorthen, 187.5, Geo. Sur. Ill., vol. 6, p. 443, Burlingtin Gr.

Axtlim)us, Newberry \& Worthen, 18:56, Geo. Sur. 1ll., vol. 2, p. 33. [E'v. antli. , a clepression; olous, a towh.] Terth trarsversely elıiptical, c mpresserl, concavisconvex; crown similar to that if Petalinlus; riots'rort or obsolete. 'Typo A. mucronatis.

cu'ullu^, Nowberry \& IVorthen, 1866, Ge(). sur. IIl., vol. 2. p. 4l, Kuokuk Gr. gracilis, St. Juhn \& Worthen, 1875, Geo. Sir. Ill.. vol. 6, p. 393, IV arsiw Gr. minilus, Niwberiy \& Worthen, is66, Gieo. Sur. Ill., vol. 2, r. 43, Keokuk (ir. micronaius Newberry \& Worthr11, $1866^{\circ}$, Geo. Sur. Ill.. vol. 2, p. 3s, St. Lonis (trr. parvulus, Newberry \& IVurthen, 1866, Geo. Sur. 1ll., vul. 2, p. 33, Burliagti) Gr.

perovalis. St. John \& IVorthen, 1875, Geo. Sur. Jll., vol. 6, p. 393, IVarsaw Gr. poliuus. Newberry \& Wortlien, 1866 , Geo. Sur. 11l., vol. 2, p. 4:, Keokuk Gir. robustus, Newberry \& IVurılien, 1866, Gen. Sur. 1ll., vol.'2, r. 39, Kaskaskia (rr. sarcululıs, N.wberry \& Wurthen, $18 \% 0$, Gro. Sur. Ill., vol. 4, p. 356, Burlingtun Gr.

similis, Newberry \& IVorthen, 1866, Geo. Sur. 1ll., vil. 2, p. 41, Keokuk Gr. simplex, Newherry \& IVorthen, ISti6. Geo. Siur. Ill., vol. 2, p. 4t, Burliugiun Gr. sulcalus, Newberry \& IVurthen, 1861;, Geo. Sur. Ill., vil. "2, p. 45, Krokuk Grr. Apenodus, Leidy, 18.56, Julur. Acal. Nat. Sci, 2u ser., vol. 3, p. 16:. [Etv. apedos, level, smoth; oiluus, tonth.] Flatienrd lancet-sliaped terth. Type A. priscus. priscu*, Lei.ly, 1856, Jnur. Acall. Nat. Sui., $2 \mathrm{~d}$ ser., vol. 3, p. 16:2, Chrmung Gr.

Aspidicitios, Newberry, 13i3, Utio Pal., vol. 1, p. 322. [Fity. aspis, shield; ichlhys, fish.] Durso-median plate of the carapace similar to that of Pte. 
richthys, but many times larger and covered with large, hemispherical, smooth, enameled tubercles. Type A. clavatus.

clavatus, N+wberry, 1873, Ohio Prl.. vol. 1, p. 323, Portage Gr.

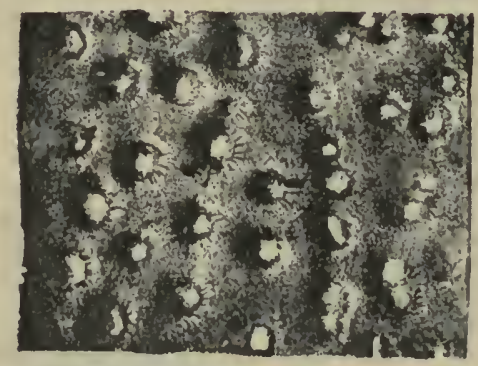

FiG. 1102.-Aspidichthys clavatus.

Aspidodus, Newberry \& Worthen, 1866, Geo. Sur. Ill., vol. 2, p. 92, syn. for Psephodus.

convolutus, see Psephodus convolutus.

crenulatus, see Psephodus crenulatus.

Asteracantlius siderius, see Bythiacanthus siderius.

Asteroltrcunes, McCoy, 1854, British Pal. Rocks, p. 615. [Ety. aster, star; ptyx, wrinkle.] Bony fin-ray compressed, long, slender, gradually tapering to a point at the distal end, and abruptly tapering at the striated proximate end or base of insertion; sides moderately convex, converging to the anterior edge, which is strongly, keeled; posterior face with a moderate cavity, each lateral edge having a row of small, pointed terth, directed upward; sides with smooth, thread-like ridges, separated by broader, flat, longitudinally striated spaces on which are irregularly scattered, smooth, spinous tubercles. Type A. ornatus.

bellulus, St. John \& Worthen, 1875, Geo. Sur. Ill., vol. 6 , p. 439 , Coal Meas.

keokuk, St. John \& Worthen, 1875, Geo. Sur. Ill., vol. 6, p. 436, Keokuk Gr.

stludovici, St. John \& Worthen, 1875, Geo. Sur. Ill., vol. 6, p. 437, St. Louis $\mathrm{Gr}$.

tenellus, St. John \& Worthen, 1883, Geo. Sur. Ill., vol. 7, p. 248, Up. Coal Meas.

tenuis, St. John \& Worthen, 1875, Geo. Sur. Ill., vol. 6, p. 438, Kaskaskia Gr.

triangularis, Newberry \& Worthen, 1870 , Geo. Sur. Ill., vol. 4, p. 370, Burlington Gr.

vetustus, St. John and Worthen, 1875, Geo. Sur. Ill., vol. 6, p. 435, Wavelly or Kinderhook $\mathrm{Gr}$.

Asterosteus, Newberry, 1875, Ohio Pal., vol. 2, r. 35. [Ety. asier, star; osteon, bone.] Htad long, narrow, broadening in the occipital region; surface covered by a sheet of tuberculated enamel; nasal pits strongly marked; condylelike posterior projections. Type A. stenocephalus.

stenocephalus, Newberry, 1875, Ohio Pal., vol. 2 , p. 36, Cornifernus Gr.

Batacanthus, St. John \& Worthen, 1875, Geo. Sur. Ill., vol. 6, p. 468. [Ety. batos, prickly bush; akantha, spine.] Spines long, tapering, curved furw ard; apex obtuse"; transverse section subcircular or oval, with anterior angle and posttrior face; lateral surfaces rounded, covered with stellate tubercles with intercostal sulci ; base moderately inserted; pulp cavity subcentral. Type B. baculiformis.

baculiformis, St. John \& Worthen, 1875, Geo. Sur. IIl., vol. 6, p. 469, Keoknk Gr.

necis, St. John \& Worthen, 1883, Geo. Sur. Ill., vol. 7, p. 253, Keokuk Gr.

stellatus, Newberry \& Worthen, 1866, (Drepanacanthus (?) stellatus,) Geo. Sur. Ill., vol. 2, p. 125, Keokuk Gr.

Bath Ycullodus, St. John \& Worthen, 1875, Geo. Sur. Ill., vol. 6, p. 252. [Ety. bathys, deep ; cheilos, lip ; odous, tooth.] Teeth minute, laterally elongated, subelliptical, sinuous in front; median cusp with cutting edges flanked by a pair of diverging denticles of similar shape, with a minute denticle between the lateral and median cusps. Type B. macisaacsi. macisaacsi, St. John \&

Worthen, 1875, Geo. Sur. Fi 1103 - BaIll., vol. 6 , p. 252 , Middle ihychilind us Devonian.

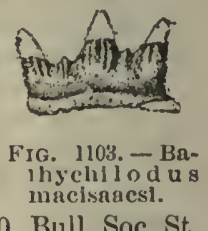

Botinrolepis, Eichwald, 1840, Bull. Soc. St. Petersburg. [Ety. bothrion, a furrow;

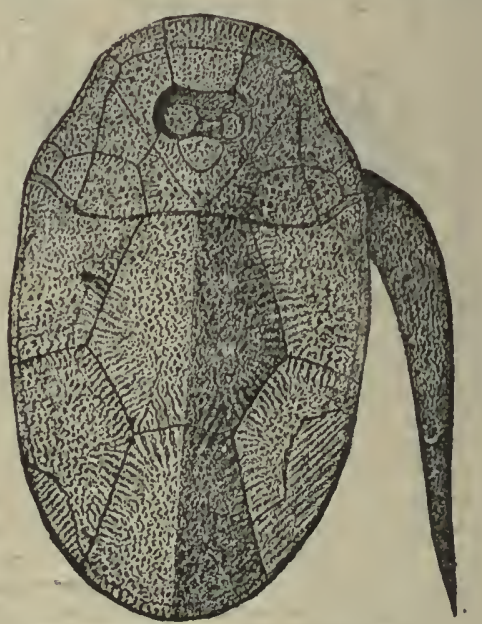

FIG. 1104.-Bothriolepis canadensls.

lepis, a scale.] Cephalic shield somewhat semielliptical in outline and covered witl plates, as in Pterichthys and 
Asterolepis, but distinguished by the course of the furrows and shape of the plates; it has longer articulating plates in the limb or arm, and has been otlıerwise distinguished, though closely related to both genera. Type B. ornatus.

canadensis, Whiteaves, 1880, (Pterichthys canadensis,) Am. Jour. Sci. and Arts, 3i. ser., vol. 20, p. 135, Up. Devonian.

Bythiacantiuus, St. John \& Worthen, 1875, Geo. Sur. Ill., vol. 6, p. 444. [Ely. bythios, deep; akantha, spine.] Fin spines deeply imbedded, laterally compressed, exposed. part recumbent, tuberculated; posterior face low, keeled; pulp cavity forming a deep channel in the posterior side of the base. Type $B$. vanhornii.

siderius, Leidy, 1873, (Asteracanthus sid. erius,) Ext. Vert. Fauna, p. 313, St. Louis Gr.

vanhornii, St. John \& Worthen, 1875, Geo. Sur. Ill., vol. 6, p. 445, St. Louis Gr.

Calopouus, St. John \& Worthen, 1875, Geo. Sur. Ill., vol. 6, p. 403. [Ety. kalos, beautiful; odous, tooth.] Teeth in general form like Petalodus, but distinguished by the turgid, subconical, ursymnetrical erown. Type C. apicalis.

apicalis, St. John \& Wor-FIg. 1105 -Calo. then, 1875 , Geo. Sur. Ill., podus apicavol. 6 , p. 403, Middle dis. Mag. Coal Meas.

Carcira ropsis, Agassiz; 1843, Recherches sur les Poissons Fossiles, vol. 3 , p. 313. [Ety. carcharopsis, shark-like.] Principal cusps very strong, erect, compressed in iront, rounded behind, broadly expanded at base; lateral angles sharp, crenulated; extremities occupied by isolated, conical, lateral denticles; coronal faces smooth or faintly striated vertically; base in out-

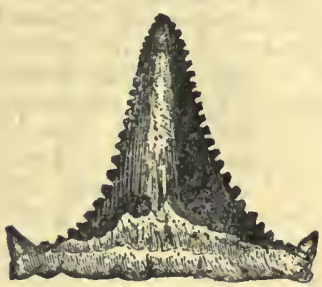

FIa. I106.--Carcharopsis worthenl. line like Cladodus, moder. ately produced and broadly rounded posteriorly; anterior face sinuous, termin a ting below in a ridge; inferior surface excavated. Type $\mathrm{C}$. prototy pus.

wortheni, Newberry, 1866, Geo. Sur. Ill., vol. 2, p. 69 , Subcarboniferous.

Cephalaspis, Agassiz, 1836, Recherch. Pois. Fuss., t. 2, p. 135. [Ety. kephale, head; aspıs, shipld.] Entire skeleton external; head shield very large, subcrescent- iform when depressed but in better, condition showing an arching over the top of the head, covered with d i s c o i dal, sculptured, bony plates, with. $t h e$ crescent horns di rectrd backward; ey es large, elliptical, on tach side of the upper central part of

the head,Fir. 1107-Ceplaalaspis lyelll Herd -hteld depressed, and tapering, an- outliue of part of the body. gular on top,

and presenting a jointed appearance somewhat like a trilobite; clorsal, anal, and caudal fin, the latter like a paddle or oar. Type C. lyelli.

campbeltonensis, Whiteaves, 1881, Can. Nat., vol. 10, Devonian.

dawsoni, Lankester, 1870, London Geo. Mag., Devonian.

Ceratodus, Agassiz, 1833, Recherches sur les Poissons Fossiles, t. 1, p. 129. '[Ety. keras, horn; odous, tooth.] Teeth large, thick, longer than wide, very porous; crown transversely sulcated. Type C. latissimus.

favosus, Cope, 1884, Pal. Bull., No. 39, p. 28, Permian.

paucicristatus, Cope, 1877, Proc. Am. Phil: Soc., p. 54, Permian.

vinslovii, Cope, 1876, Proc. Am. Phil. Soc., p. 410, Permian.

Chirodus, McCoy, 1848, Ann. and Mag. Nat. Hist., vol. 2, p. 130. [Ety. cheir, the hand; odous, tooth.] Tooth fan-shaped, thick, flattened; anterior broad, margin deeply divided into lobes; inner nearly straight margin has a small, recurved, thumb-like lobe projecting nearly at right angles from the middle of its length, preventing the mesial junction of the tritors of each side of the jaw; inner marginal lobe the longer; surface minutely punctured. Type C. pesranæ. Not definitely known in America.

acutus, Newberry, 1857, Proc. Acad. Nat. Sci., vol. 8, p.99, Coal Meas. Too poorly defined to warrant recognition.

Chirolepis, Agassiz, 1833, Recherches sur les Poissons Fossiles, t. 1, p. 128. [Ety. cheir, hand; lepis, scale.] Bones of the head sculptured; shoulder-bone and fins osseous; pectorals large, reaching near the ventral fin, and ventral reaching near the anal fin; dorsal fin small and opposite the posterior part of the anal fin; tail well-developed, principally on the lower side; scales small, sculp- 
tured, and rangerl diagonally in wavy lines. 'Type C. trailli.

canrdensis, Whiteaves, 1851, Am. Jour. sici. and Arts., 31 ser., vol. 21, p. 496, Up. Devonian.

Cnimovodus, St. John \& IVorthen, 18s:3, Geo. Sirr. Ill., vol. 7, p. 109. [E,y. chitun, a sinisck or c at; odmus, tool.h.] Mandibular pusterior leeth trapezulilal, arched in the dircetion of inrullment; median tecth narow, inrolled longitudinally; mixillary pusterior teeth subqualrilateral, archesl, and inrollecl along the onter margin. 'I'ypa C. spingeri.

antiı̨uıs, Si. John \& IVorthen, 1883, Geo. Sur. III., vul. 7, p. 116, Low. Burlingtun Gr.

latus, Leily, 1856, (Cochliodus latus,) 'Trans. Aui. l'hil. Soc., vol. 11, p. 87, pl. 5, lig. 17, Kerkuk Gr.

liratus, Si. Julın \& Worthen, 1853, Geo. sur. III., rol. 7, 1. 119, Si. L'uis Gir.

rugosis, Newberry \& Worthen, 1860, (P'œillindus rugusus, P. ornatus, and P. (enurolutus,) Geo. Sur. Ill., vol. 2, pp. $94,9 \%$; vol. 4. 1. 861 , Keokuk Gr.

spingeri, St. Juhn \& Worthen, 1853, Geo. Sirr. Ill., vol. 7, p. 112, Up. Burling. ton $\mathrm{Gr}$.

tribulis, St. Jolin \& Worthen, 1883, Geo. Sur. 11l., vol. 7, ". 117, Ketekuk (ir.

Cnotonus, St. Julin \& Wortlien, 1875, Geo. Sur. III., vio]. 6, p. 4l5. [lity. cholos, defective; odurs, tuoth.] Distinguisherl

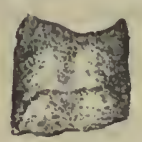

FIn. 1108 -Cholodus $\therefore$ ingequalls. C'onveX lace.

\section{f.sce. Type C. inæqualiv.}

from Peltoilus and

Fissulua, by the eccentrically lobrel crest and extreme dow nw ard prolongation of the lateral c'xtremities of the corollal folll in the convex

inaequalis, st. Julut \& Wwortien, 187.5, Geo. sur. III., vol. 6, p. 416, Coal Mieas.

Chos a rupus, Agassiz, 18t3, Rech rehes sur les Puissons Fussile-s, 1. 3, p. 107. [Ety. chomn, a pile or heap; odines, touthi.] 'Treth transversely nuch elimgated, compressed, and ilepressed; crown having the homolozous parts of P'etrlodus, anil the form and structure of Pilyrhi: zonlus; root shrirt, somelimes obsulete, undivinled. 'T'ype U. linearis.

affinis, Newberry \& Worthen, 1866, Geo. Sur. III., vol. 2, p. 5t, Keokuk Gr.

angularix, see 'Tanaorlus angularis.

arcuatus, St. Jolı, 18i0, Proc. Am. Phil. Sor., vol. 2. p. 435, and Pal. E. Neb., p. 243, Co il Meas.

chesterensis, St. John \& Worthen, 1875, Gen. Sur. Ill., vol. 6, p. 363, Kaskaskia Gr.

comptus, St. John \& Worthen, 1875. Geo. Sur. Ill., vol. 6, p. 356, Burlington Gr.

costatus, Newberry \& Worthen, 1866, Geo. Sur. Lil., vol. 2, p. 85, Keukuk Gir. cultellus, Newberry \& Worthen, 1866, Geo. Sur. 111., vol. 2, p. 52, Kaskaskia Gr.

elegrns, Newberry \& Worthen, 18f6, Geo. Sur. Ill., vol. 2, p. 86, Keokuk lir.

gracillimus, see Tanaodns gracillimuv.

inionstans, St. Joun \& Wurtlien, 1875, Ger. Sirr. Ill., vol. 6, p. 360, St. lon is $\mathrm{Gr}$.

inerısuat us, St. Jolın \& Worlien, 1875, Gi.o. Sur. Iil., vol. 6. p. 35\%, St. Louis Grr.

insignis, Leidy, 1856,

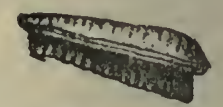

Trans. Am. M'lit.

Sic., vol. 11, p. 87. St. Lnuis Gr.

linearis, Agassiz, 1813, (Psammodus lineariu, ) Recherches Puis. Fuss., t. 3, p. 108, Subcarb.

lorifuruija, Newherry \& Wortlien, 1866, Geo. Surr. Ill., vol. 2, p. 58, Kienkuk Gir. molaris, Newberiy of iVurthen, 18li6, Geo. Sur. Ill., vol. 2, p. 56, Keokuk Gir. mulliplicatus, see 'T'unadus multiplicatus. obscurus, see Tanaodus wbscurus.

paralleliıa, St. John \& W'orthen, 1875, Geo. Sur. Ill., vol. 6, p. 358 , Warsaw $\mathrm{Gr}$.

purillus, Newberry \& Worthen, 1866, Geo. Sur. IIl, vol. 2, p. 53, Keokuk Gr. varsoviensis, St. Juhn \& Wortlinn, 1875, Gen. Sur. III., vul. 6, p. 393, Warsaw Gr. venustus, leilly, see Venustodus leiılyi, where the specifie name is made to desjewatis the genus, and the aullor the specific uame, c ntraty to the rules of nouenclat.dre; also see Venustodus venustus.

Clanonus, Agassiz, 18 43 , Recherches sur les Puissons Fossiles, t. 3, p. 196. [Eiy. kludor, twig; o'ous, tonth.] 'T'eth with broad, horizontal, senicicular, thick, hony, coarsely fibrous lase, rounded belinul, truncated in front; crown diviled into long, sharp, subulate, eonical prints, arranged alinge the straight truncated edge of the liasse; medial cone much lirger than the secondary ones, of which fatler the excrial cones are the larger; all the conns striated longitudinally, and either rircular in secifion or with simple culting elges, sliglitly compressed. 'Tyje C. mirabilis.

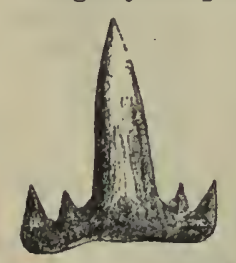

Fig. 1110.-Charodus acuiniuatus. acuminatus. New. br rry, 1857, Proc. A cad. Nut. Sei. Pliti., vol. 8, p. 99 , and Óhio Pal., vol. 2 , p. 45 , Subcarboniferous.

alternatus, St. Jihn \& Worthen, 1875, Geo. sinr. III., vol. 6, p. 265 , Waverly or Kinderhook Gr. angulatus, Newberry \& Wortlien, 1866, Gen. Sur. Ill., vol. 2, p. 24, Keokuk Gr. 
bellifer, St. John \& IVorthen, 1875, Feo. Sur. Ill.. vol. 6, p. 270, Burlinutun Gr.

carıarus, St. Johı \& IVi)rtien. 1875, Geo. Sur. Ill., vol. 6, p. 279, Cinl Meas.

concinnus, Nenberry, 1875, Olıı Pal., vol. 2, p. 48, Purtane? Gr.

costutus, Newb-rry \& IVorthen, 1866, Geo. Sirr. Ill., visl. 2, 1). 27. Kंusk skia Gr.

deflexus. Newberry \& Wurthen, 1870, Grou. Sur. Ill., vol. 4, p. 355, Burlıgton Gr.

eccentricus, St. John \& Worthen, 1875, Geo. Nur. Ill.; vol. 6 , p. 272 , St. $\mathrm{L}^{\prime \prime \prime 1}$ is $\mathrm{Gr}$.

eleua'is, Newberry \& IVorthen, 1870, Geo. Sur. Ill., vol. 6, p. 35t, st. Liuis Gr.

euglyphens, Sr. Juhn \& IVortien, 1875, Gieo. Sur. Ill., vol. 6, p. 2itt, st. Louis Gr.

exiguu-, St. John \& Worthen, 1875, Geo. Sirr. Ill., visl 6, p. 261 , IVaverly or Kinderlıook Gr.

exilis, st. Jolin \& IVorthen, 1875, Gro. Silr. Ill., vul. 6, p. 258, IVaverly or Kinderhook Gr.

ferix, Newberry \& Worthen, 1866, Geo. Sur. 11I., visl. 2, p. 26. St. Loula (ir.

full ri, St. Johı \& IVisthen, 1875, Geo. Sur. 11l., vol. 6, p. 276, Coal Meas.

gomplioirles, Sit. Julin \& Wintien, 1875 , Geo. Sur. 1ll., vol. 6, p. 26\%, Burlingtill Gr.

gracilıs, Newberry \& Wirthen, 1866, Geo. Sur. LIl., vol. 2, 1.. 31), Cual Mens.

grandis, Newberry \& IVorthen, 1866, Geo. Sur. Ill, vol. 2, भ. 29, Kaskaskia I ir.

hertzeri. Newberry, 1875, Ohio Pal., vol. 2, 1). 46, Purtage Gir.

infrerustarus, Si. Juhin \& Worthen, 1875, Geo. Sur. Ill., vol. 6, p. 267, Burlingtion Gr.

ischy pux. Newberry \& IVorthen, 1870, Geo. Sur. III., vol. 4 , 1. 35 , Sir. L'uuis Gir.

lamnoides, Newbriry \& IVurtien, 1866, Gen. Siur. III., vol. 2, 1. 30. Keokuk Gri. manniticus, Tu'mey, 1sis, $2 \mathrm{l}$ Rep. Geo. A la., 11. 39, Kuskaski $t$ Grr.

micropus, Newberry \& IVorthen, 1886, Geu. Sur. Ill., vol. 2, n. 21, Kenkuk Gr. mortifer, Newberry \& IVo:hen, 1866, Geo. Sur. IIl., vol. 2, p. 22, Cuil Meas.

newmuti, Tuomey, 18.58, Geo. Ald., p. 39, Kaskiskia Gr.

occidentalis, l.eidy, 1859, Proc. Acad. Nat. Sci. Phıl., U1\%. C'o ıl Mras.

pamblatıณ, S't. Juhn \& IVortıen, 1875, Geo. Sur. Ill. vol. 6, p. 278, Coal Mras.

parrulu*, $\mathrm{N}+\mathrm{w}$ verrv, 187j, Unio Pal., vol. 2, p. 48, Purtage Gr.

pattersoni, Newheriy, 1875, Ohin Pal., vol. 2, 1. 47; IVav-1 lv Gr.

p llitus, Newberry \& IVorihen, 1s7J, Geo. Sur. Ill., vol. 2, p. 27, Ka-kaskia Gr.

præuunıius, St. Johin \& IVurlhen, 1875, Geo. Sur. Ill., vol. 6, p. 270, Burling. ti)is Gr.

raric sstatus, St. John \& Worthen, 1875, Geo. Sur. Ill., vol. 6, p. 2il, Kékuk Gr. robustuq, Newberry \& Worthen, 1866, (ie). Sur. 1ll., vol. 2, p. 20, Krokuk \&'r. romiugreri, Newberry, 1875, Onio Pal., vol. 2, p. 49, IV dverly Gr.

spinosus, Newberry \& Worthen, 1866, Geo. Sur., Il]., vol. 2, 1. 22, St. 1,u uis Gr. springeri, St. Juhn \& IVorthen, 3870̄, Geo. Sur. Ill., vol. 6, p. 259, Waverly or Kinderhouk Gr.

stenopux, Newherry \& Worihen, 1866, Gea. Sur. IIl., vol. 2, p 23, St. Innis (ir. subulatus, Niwbeiry, 1 s'.5, Unio P’al., vol. 2 , p. 47, Cuyalioga shale over the Berea grit.

suecinclus, St. Juhn \& Worthen, 18\%5, Geo.sur. Ill., vol. 6, p. 265, IVaverly or Kin.lesh wh Gr.

turrius, Newlerry \& IVorthen, 1866, Geo. sui. Ill., vul. 2, p. 28 , Krokuk Gr.

vanhornii, St. Jolın \& Worthen. 1875, $G$. Sur. IIl., vol. 6, p. 273, St. Louis Gr.

Wachsmuthi, St. John \& IVorthen, 1875, Geo. Sur. Ill., vol. 6, p. 263, Waverly or Kinderlıonk Gr.

zygopuฯ, Newberry \& Worthen. 1866, Geo. Sur. Ill., vol. 2, p. ¿j, Kaskaskia Gr.

Climaxomlus, MeCoy, 18t8, Ann. and Mag. Nat. Hist., "d ser., vil. "2. [Ey. klimnx, ladiler; odous, twoih.] Tuoth lo ger than wide, gradually marrowning toward the front, with nearly stralght si.tes; auterior part of the crown crossed by brual, imbricating transverse rilges, at rinfut angles to iis length; surface minutely puncturil. Type C. imbricatus. Not iletiut ly knowni in Am $\rightarrow$ r $\cdot a$.

brecis, Newberry, 18;7, Proc. Ac:ad. Nat. Sis., vol. 8, p. 100, Cual Jeas. 'T'oo poorly ilefined to warrant recignition. Coccosteus, Agits-iz. 1836, Recl, roh. Puis. Fust., vol. 2, p. 302. [ Ety. kokkos, berry; osfeon, bone.] $\mathrm{H}$ anl rommled; boty triangul r, with long vertebrated tail, like a rolder, the wlole compared in form, by Huglı Miller, to a biny's kite; heal aul loly covered with luberculatel bony plates ; ceniral iront plate like the keysune of an arch; the posterior bolly plate is largr, saclille-wise toward the center, poimed beliniml on the ridge there is a longiturimal groove encling in a perfuration, a litıle behind the rpex. It is this blate which has been ilescribed as $C$. occidentalis, but, it does not show gruove or perfuration. Type C. decipiens.

acalichs, IVhiredviss, 1S81, Can. Nat., vol. 10, Upper Devonian.

occiclentalis, Newberry, 18т̄̄, Ohio Pal., vol. 2, p. 3:2, U p. Hilil. (̇r.

Cocillunus, Agassiz, 18 43 , Recherchez sur les Puissons Fussiles, t. 3, p. 113. [Ety. $k$.chlius, anything spiral; odous, tooth.] Lower jaw thick, slıort, bony, l'shap il, bearing on each ramus two obliquely twisted and obtusely ridged semicylindrical teeth, strongly iurolled on 
the outer margin, convex above, concave bulow, with porous grinding surfaces, as in Psammodus, from the termination of the vertical medullary canals. Type C. contortus.

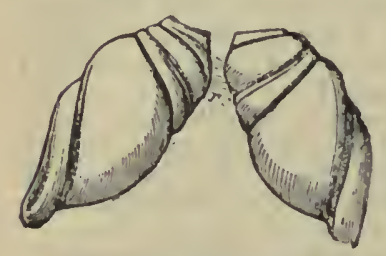

Fio. 1111-Cochuodus conlortus. e os a $\mathrm{t} u \mathrm{~s}$, Newber ry \& Worthen, 1870, Geo. Sur. Ill., vol. 4, p. 364, Bur. lington $\mathrm{Gr}$. srassus, Newberry \& Wort lien, $1866, \quad 8 y n$.

for Sandalodus levissimus.

lalus, see Chitonodus latus.

leidyi, St. Joln \& Worthen, 1883, Geo. Sur. 1ll., vol. 7, p. 127, Kaskaskia Gr. nitilus, see Deltoptychus nitidus.

nubilis, Newberry \& Wortlien, syn. for Chitonodus latus.

obliquns, St. John \& Worthen, 1883, Geo. Sur. Ill., vol. 7, p. 126, St. Iouis Gr. occidentalis, see Deltodus occidentalis. vanhornii, St. John \& Worthen, 1883, Geo. Sur. Ill., vol. 7, p. 120, St. Louis Gr.

Comlacantios, Agassiz, 1836, Recherehes sur les Poissons Fossiles, t. 2, p. 170. [Ety. koilos, hollow ; ukantha, spine.] Head plates sculptured; scales large, imbricated, seulptured, arranged diagonally; two small dorsal fins supported on interspinous bones, the anterior one a little furward of the ventral fin, and the posterior one ntarly opposite the anal fin; cundal fin equi-lobate, and near its extremity a minute supple-

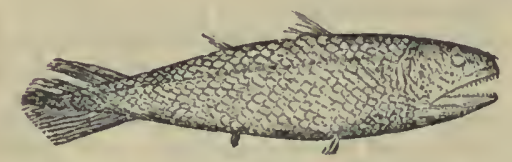

F1G. 1112.-Coelacanthus elegans.

mental caudal ; vertebral column cartilaginous, but neural arches and fin-rays bony; teeth small, numerous, conical. Ty pe C. granulosus. elegans, Newberry, 1856, Proc. Acad. Nat. Sei. Phil., vol. 8, p. 98, and Ohio Pal., vol. 1, p. 339, Coal Meas.

ornatus, Newberry,

1856, Proc. Acad. Fig. 1113.-CoelacanNat. Sei. Phil., vol. thus granulosus. 8, p. 98, and Ohio Magnified scale. Pal., vol. 1, p. 340, Coal Meas.

robustus, Newberry, 1856, Proc. Aead.

Nat. Sei. Phil., vol. 8, p. 98, an $\mathrm{d}$ Ohio

Pal., vol. 1, p. 341, Coal Meas.
Compsacantuos, Newberry, 1857, Proc. Acill. Nut. Sci., vol. 8, p. 99, anil Ohio PHl., vol. 1. p. 331. [Fty. compsos, elegant; akunth", a spine.] Spines small, gently eurved baekwaid; exposed part smooth, polished; section eircular; single ruw of remote, llepressed hooks on the posterior median line. Type C. lrevis.

lrevis, Newberry, 1857, Proc. Acar. Nat. Sci. Phil., vili. 8, p. 99, and Ohin Pàl., vol. $1, \cdot$ p. 332, Coul Meas.

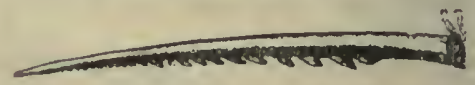

Fig. 1114.-Compsacanthus lævis.

Conchiopsis, syn for Cielacanthus. anguliferus, syn. for Crelicanthus elegans. exanthematicus, syn. Ior Peplorhina anthraeina.

filiferus, syn. for Colacanthus elegans.

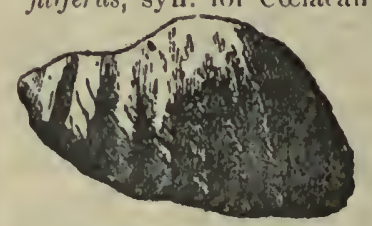

FIG. 1115 -Concliodus plicatus.
Concionus, Me-

Coy, 1848 Ann. und Mag. Nat. Hist., $2 \mathrm{~d}$. ser., vol. 2. [Ety, conchos, sholl ; odous, tooth.] Teeth large, somewhat semieircular, pointed in front, subtruncate behind, deeply coneave on the grinding surface; internal murgin straight, thickened, edge abruptly deflected; external boriler convex, mueh raised, undulato-plieate, ridgt $\mathrm{s}$ larger in front, smaller posterior; under surface polished, minutely porous. Type C. ostreiformis.

plicatus, Dawson; 1868, Acad. Geol., p. 209, Coal Meas. Copodus, Agassiz, MSS.,
1859 , St. John \& Worthen, 1883, Geo. Sur. Ill., vol. 7, p. 227. [Ety. kopis, broad, curved knife; odous, tooth.] Teeth bilaterally symmetrical, spanning the jaw without mesial suture, arranged in single, longitudinal series from behind backward; lateral bor. ders converging anteriorly ; coronal region arehed; rim at base; anterior and 'posterior 'walls vertical, chan neled; inferior surface concave; porous beneath the enam-

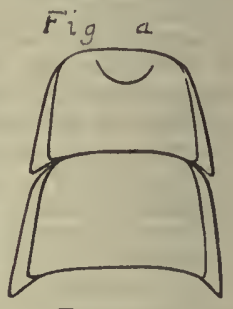

Fig. b

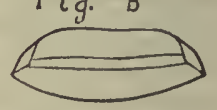

Fig. $\sigma$

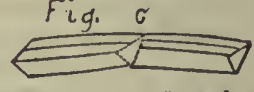

IG. 1116. - Copodus IG. 1116. - Copodus
cornutus. Maxillary form. $a$, Tritvruting surfuce; $b$, transverse profile: c, longliudinal proeled coronal surface. Type C. cornutus. 
pusillus, St. John \& Wortluen, 1883, Geo. Sur. Ill., vol. 7, p. 231, Kuskaskia Gr.

Fig. a

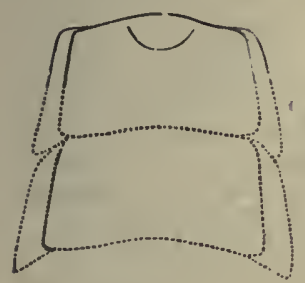

Fig. $b$
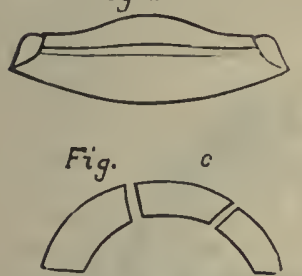

F10. 1117.-Copodus cornutus. Mandibular form. $a$, Triturating surface; $b$, transverse profile; $c$, longiududinal profile. vanhornii, St. John \& Worthen, 1883 , Geo. Sur. Ill., vol. 7, p. 229 , St. Lonis Gr.

CTE N A C A N T H US, Agassiz, 1843, Rechercbes sur les Poissons Fossiles, t. 3 , p. 11 . [Ety. ktenos, comb; akantha, spine.] Fin spine compressed,grad. vally tapering, arched backward; anterior face narrow, rounded : posterior face concave, lateral edges bordered by two rows of curred denticles inclined downward; surface ridges furrowed, pectinated by transverse scales or tubercles; concealed base rapidly tapering, finely striated. Type C. tenuistriatus.

angulatus, Newberry \& Worthen, 1866 , Geo. Sur. Ill., vol. 2, p. 118, Kaskaskia Gr.

burlingtonensis, St. John \& Worthen, 1875 , Geo. Sur. Ill,, vol. 6, p. 426 , Burlington Gr.

buttersi, St. John \& Worthen, 1883, Geo. Sur. Ill., vol. 7 , p. 240, Lower Coal Meas.

cannaliratus, St. John \& Worthen, 1883, Geo. Sur. Ill., vol. 7, p. 239, Kaskaskia Gr.

costatus, see Eunemacanthus costatus.

coxanus, St. John \& Worthen, 1883, Geo. Sur. Ill., vol. 7, p. 233, Keokuk Gr.

deflexus, St. John \& Worthen, 1883, Geo. Sur. Ill., vol. 7, p. 234, St. Louis Gr.

elegans, Tuomey, 1858, Geo. Ala., p. 38, Kaskaskia Gr.

excavatus, St. John \& Worthen, 1875, Geo. Sur. III., vol. 6, p. 428, Keoknk Gr.

formosus, Newberry, 1873, Ohio Pal., vol. 1, p. 328, Waverly Gr.

furcicarinatus, Newberry, 1875, Ohio Pal., vol. 2 , p. 54, Waverly Gr.

gemmatus, St. Jnhn \& Worthen, 1875, Geo. Sur. Ill., vol, 6, p. 429, St. Louis Gr.

gracillimus, Newberry \& Worthen, 1866, Geo. Sur. Ill., vol. 2, p. 126 , St. Louis Gr.

grado-costatus, St. John \& Worthen, 1875 , Geo. Sur. Ill., vol. 6, p. 425 , Burlington Gir. harrisoni, St. John \& Worthen, 1883, Gev. Sur. Ill., vol. 7, p. 236, St. Louis Gr.

keokuk, St. John \& Worthen, 1875, Geo. Sur. Ill., vol. 6, p. 427, Keokuk Gr.

latispinosus, Whiteaves, 1881, Can. Nat. and Geol., vol. 10, Upper Devonian.

marshi, Newberry, 1873, Ohio Pal., vol. 1, p. 326, Coal Meas.

mayi, Newberry \& Worthen, 1570, Geo. Sur. Ill., vol. 4, p. 372, Burlington Gr. parvulus. Newberry, 1875, Ohio Pal., vol. 2 , p. 55, Cleveland shale.

pellensis, St. John \& Worthen, 1883, Geo. Sur. Ill., vol. 7, p. 237, St. Louis Gir. pugiunenlus, St. John \& Worthen, 1875, Geo. Sur. Ill., vol. 6, p. 430, St. Lonis Gr. sculptus, St. John \& Worthen, 1875, Geo. Sur. Ill., vol. 6, p. 421, Waverly or Kinderlook Gr.

similis, St. Jolnn \& Worthen. 1875, Geo.

Sur. Ill., vol. 6 , p. 431, Kaskaskia Gr.

speciosus, St. John \& Worthen. 1875, Geo.

Sur. Ill., vol. 6, p. 424, Waverly or

Kinderhook Gr.

spectabilis, St. Jolın \& Worthen, 1875, Geo. Sur. Ill., vol, 6, p. 420, Warerly or Kinderhook Gr.

triangularis, Newberry, 1873, Ohio Pal., vol. 1, p. 329, Waverly Gr.

varians, St. John \& Worthen, 1875, Geo. Sur. Ill., vol. 6, p. 422, Waverly or Kinderbook Gr.
FIG. 1118.-Ctenacanthus triaugularls.

vetustus, Newberry, 1873, Ohio Pal., vol. 1, p. 326, Waverly Gr.

wrighti, Newberry, 1884, 35th Rep. N. Y. Mus. Nat. Hist., p. 206, Ham. Gr.

Ctenodus, Agassiz, 1843, Recherches sur les Poissons Fossiles, t. 3, p. 137. [Ety. ktenos, comb; odous, tooth.] Tooth sumewhat fan-like, with closely serrated edges, very porous and sulcated; position in the jaw unknown. Type C. cristatus.

dialoplius, Cone, 1878 , Proc. Am. Phil. Soc., vol. 17, p. 528 , in Pal. Bull. No. 29, Permian. fossatus, Cope, 1877, Proc. Am. Phil. Soc., p. 54, Permian.

gurleianus, Cope, 1877 ,

Pruc. Am. Phil. Soc., p. 55, Permian.

ohioensis, Cope, 1874, Proc. Acad. Nat. Sti. Phil., p. 91, and Ohio Pal., vol. 1, p. 410,

Coal Meas.
periprion, Cope, 1878, Fig. 1119,-CteuoPal. Bull. No 29,

in Proc. Am. Phil. Si c., vol. 17, p. 527, Permian. 
porrertus, Cope, 1878, Pal. Bull. No. 29, in l'rue. Am. Plill. sue., vol. 17, 1. 5:7, Pormian.

pu»llus, Cupe, 1S7S, I'rl. Bull. No. 26, in l'r. c. Alis. Plul. Suc., vul. 17, p. 191, l'r rmian.

reticulaun, Newhrry, 1875, Ohio Pal., vol. $\because$, p. 60, L'onl M. à.

serraius, Newlicrry, istó, Ohio Pal., vol. 2, 1. 59, Cual Meais.

Ctexuphtats, A gassiz, 1\$69, Calal. Fuga. Fish, Coliection of Furl of liuniskillen, in Cien. Mar., vol. (6. [Ety. ktons, counh; jelials, broali, full-uruwn.] The serrated or denticulaterl irest clisiluguislies it forsul l'etuludus, whish it inuch resembles, and to which it linars abunt the rame relation as I'etuludus ches te Autli alus. Tyje C. sermalis.

bellulus, St. Jilin \& Wurlien, 1S7is, Lito. Sur. lil., vol. 6 , 1). 398, St. I, mulis Gir.

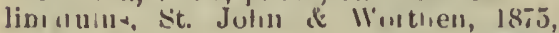
(iro. Sur. Ill., vul. (6, p. 399, haskuskia Gr.

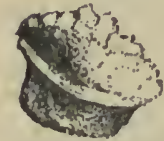

Fir. 112n. - Ctennjefulus in'tilemlnII. Cum a ve frue. medius, St. Jolın \& Wortluen, 1s75, Gets. Sur. 111., viul. 6, p. 400, kuskurkia Gr. ocpilentalin, St. John \& Wurliell, 187i), (G.0.. situr. III., vol. 6, 1. 401, Cual Mias.

vimosise St. Jolin \& Wurtlanl, 1875, Gieo. Sur. Ill., vol. 6, p :396, Kinkuk G̈r.

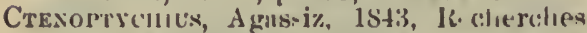
FIIr Its l'..jssulis Fossil.s, t. 3, 1). 19. [Gy'. klenos, coml,; pilyche, wrink]..]

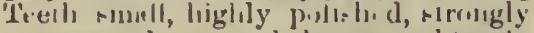
compressed, roumind or ulitusily pointerl; enlge diviled into severul strorg ilenticulations; base uf arown

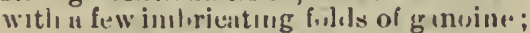
b ny root, oblong, flattened in the falle direction us the cluwn. Type C. apjicalis.

cristalls, Diwson, 1868, Acad. Geol, p. 209, Cual dleas.

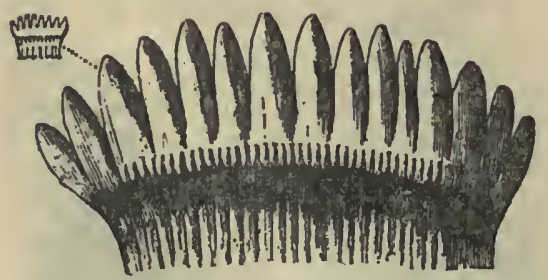

Fia. 1121.-Clenoptşיlulus crl tutus. Natural size usd unagujtled.

digitatus, Leidy, 1\$56, Trans. Am. Pliil. Fi:., vol. 11, St. Lisuis Gr.

pertenuis, Sr. Jolı \& Worthen, 1875, Gen. Sur. 111., vul. 6, 1. 38?, Kaskiaskia lir.

scmirirularis, see Peripristis semicirculais. sterensoni, St. John \& Worthell, 1875, Gen. Surr. 111., vol. 6, p. 3S3, Coal \I\%яs. Cym+tonus, Niwberry \& IVorllien, 1870, Ger1. Sur. Ill., vol. 4, 11. 3(i3. [Ety. cymutos, wally; orlmus, inoll.] Tecth suall, wbloug, or elliıtical, thin, furming a $\mathrm{t}$.t ur arehed , life. of which the cruwn surfuee is trantiersely uniluInted and unif rmly punctate; muder surfare Hat, smonoli, at the justeror end b aring a narlow, siraj-shaped, olilique rurst. True C, ohlongus.

oblonzus, Newberry \& Wurlhell, 18:0, (ino. sur. Ill., vol. 4, p. $36 t$, Up. Cual Meas.

Cyrtacanthus, Newherry, 1673, Ulas Pall, vol. 1, j. 306. [Eisy. kinrtos,

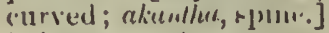
sijile: culved, tulse renlatel, suligle row of conical inaticles set on the ponterdior side, limited to the upper portion, and increasing bu size frum lublow li, ward. 'lype C. dentutis.

dentalu=, Ninberry, 1873, Olio l'al., vol. 1, 1).307, U1. Hell. lir.

Dacmlouen, Niwherry \& IVunten, 18bit, Gieu. Siur. Ill., vol. ¿2, p. 33. [Eis. dirlilylos, finger; vilues, loutw.] Cruwn as in l'etalousus; rout ('on-piculuns, anul divideil inter at number of ralicles. Type $\mathrm{D}$, princeps.

compiaris, St. John \& Wuitlin, 1875, Geri, Fir. I122.-CrrtaSur. III., vol. 6, p. 390, tus.

Si. Loulls Gir.

exı"avatus, St. John \& Worthen. 1875, lieo. Sur. 1ll., vol. 6, p. 39:, Kaskashili (ir.

inflixus, Newlerry \& II urtlin n, lotit, (ieo. Sur. III., vil. ¿2, p. 48 , Kaskaskia lir.

lolatils. Newlerry \& II or. Jiril, 1 sliti, Gieu. Sur. III., vol. 2, p. $4 \bar{T}$, N. Lisils Gir. Firs. 1123. - Dactyiomilimus, Si. Jolm \& dis eninenvis. ConWurllien, 1875 , Geu. vex face.

Sur. III., val. 6, p. 391, St. lonis Gr. prime ]'s, Newberiy \& Worthen, 1866, Geu. Sur. 1ll., vol. 2, p. 45, st. Luuis lir. 
Deltoporsis, St. John \& Worthen, 1883, Ge(s. Siır. Il]., visl. 7, p 158. [Eıy. frum resimblance to Deltoilus.] Cormial romtonr and seneral aspect near Delodus, distinguishen by the lifforentiation of the median rialgo of the anterior "oronal prominence, w hieh approarhes Cochlioilus or Chitono lus. 'Type D. angusta.

affinis, St. Juhn \& Vort..sn, 1883, Geo. Sur. Ill., Vul. 7, p. 160, IVarsaw Gir.

angista, Nivwierry \& Worlien 1870. (Delumlus angustits, ) Geo. Sur. Ill., vol. 4, 1. 368, Ka-kankia Gr.

bialveuta, St. Julın \& IVırthen, 18ৎ3, Geo. Sur. Ili., vol. 7. p. I(it), Burlingt.n Gr.

convex,, St. Juhn \& IVortlien, 188:3, Geo. Sur. Ill., vol. 7, p. 169, Up. Burlingtoi) Gr.

convolural, St. John \& Whrthen, 18ৎ3, G. o. Sur. Ill., vol. 7, p. 16j, Up. Burling'on Gr.

exoriuta, St. John \& IVorthen, 18.93, Geo. Sur. Ill., vol 7, p. 168, IVariaw (ir.

inflexa, Nt. Juhn \& Worlien. Is83, Geo. Sır. Ill., vol. 7, p. 167, lerokık Gr.

kenkık, Sr. Juhn \& Wurthen. Geo. Sur. Ill., vol. 7, 1. 169, Kalkuk Gr.

stlu.lovici, St.juhn \& IYorthen, 1883, Geo. Sur. Ill., vill. 7, p. Ilil, St. Is)uis (ir.

Deltones, Agassiz, 1859, Mis., and Newluerry \& IVurthen, ]siti, Geo. Sur. Ill., vol. 2, 1). 95. [Eiy. delta, triangle; odous, iooth.] 'leeth lırge, tlickk, strumg, triangulat. more or lers archisl, sometimes inrulled from llete jonger and more arute angle to the ('ppusite nuargin; eruw su sul fa arched or ma!kel by 1-3 jroninent ri.!ges from the bisal margin tonaril the ling(r angli. Typ". I). suls?acvis

alı'us, New berry \& IVurtien, syı. for Cinionoulus laius.

angularis, N.wherry \& Worthen, syn. for Ortincip'enrodus carlunarius.

aregustur, see D. ltullor.sis angusta.

ciurtu us, St. Jolin \& Worthen, 1883,

(ien. Sur. 111., vill. 7, p. 146. Warsaw inr eingulatus, Newberry \& Wwitien, 186i,

(ieu. Sur. Ill., vul. 2, 1.99, Ka-ki-kia tir. complnnutus, see $S$ in lalo lus conp p'anatus. fuscialus, s.se Tæui ulus lastialıs.

giandis, Newlorry \& IVo tlı II, 1866. Goo. Sizr. 11l., vil. 2, 11. 101, Kenkık (ír. Probably syn. for Sandaluaus Jevissimus.

intumralins, St. John \& Worthen, 1883, Geo. Sur. Ill., vo'. 7, p. 15:3, St. lunislir.

latiur, St. Julin \& ivirthell, 18N3. Geu. Sur. III, vol. 7, 1) Ifŏ, Kenkuk lir.

littoni, Newherı \& IVurlien, Iš̈ll, Gen. Siır. 1ll., vul. 4, p. 367 , subcaibuniferous.

ocvilentalia, Leids, 18.56, (('nchliodus ncc dental s, ' 'lrans. Am. Pliil. Sor., vol. 11, 1. 8i, Waraw anl Nit. Lonis Gre. parvis, St. Julın \& IVurıhen, 18S:, (imo. Surr. 1ll., vill. 7 , \%. 15l, St. I, uis Gr. powrlli, Si. John \& Wurthon, 188:, Girn. Sur. Ill., vol. 7, p. 154, Cåbosiferuus. propinquus, St. Jolın \& IVorthen, 1883, Geo. Sur. 1ll., vul. 7, p. 56, Cial Mras. rhoubesilens, Newberry \& Wurthen, syn. for sandalidas r pallulalis.

epatulans, Niwberry \& Worthen, 1866 , Ger. Sur. Ill., vol. 2, p. 1Co, 13urlington $\mathrm{Gr}$.

stellalus, Newberry \& Worthen, 1866 ; Gin. Sur. III., vol. 2, J. 97, Kloknk Gir. trilobus, st. fufı \& IVortlien, 1s83, Geo. Sur. Ill., val. 7, p. 1f8, Walsaw Ġr.

unilulatis, Newberry \& IVurlhen, 1S66, lien. Sur. 111., vol. 2, 11.98, kinokık Gr.

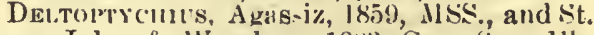
Jihn \& Wurluen, 1853, Geu. Sur. III., vol. 7, 1). 8!) [Eiy. dilu, trangle: ptyx, a uriukle,] Pisterior let th of lower jaw irigunal, strongly built, and archerl in the direction oi inrullusent; eoromal contour in ihree divisions, narrowing loward tlie outer extremity; those of the upper jaw sul siatulate, incrilled on the chler nia.gin, acute pertcriorly. Type D. ar.u'us.

expansis, si. Julin \& IVorlien, I8\$3, Geo. sur. 111, vol. 7, 1. 9s, it. Lruis (ir.

nitıdus, Leiıly, 1>5 i, (Cocl,liorlus nitidus,) 'Jralıs. Ali. Miil. Soc., vul. 11, 1'.87, Kaskskia Gr.

primus, sit. Jolin \& IVorlluen, 1S83, Geo. sur. 1ll., vol. T, p. 93 , Up. Burling. toul Gr.

varsovitnsis. St. Jolın \& Wnrthen, 1883, Geo. Sur. Ill., rol. 7, 1\%. !16, Walsaw Gr. wachsmu hi, St. Juhin \& IVurihen, 1883, Gio. Sur. Ill., vol. 7, p. 93, Keokuk Gir.

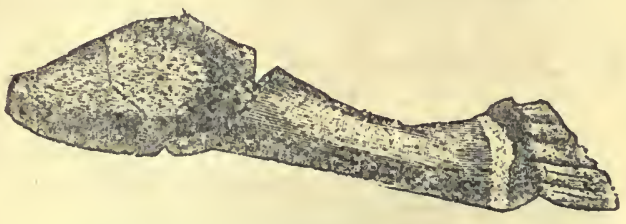

FIa. 1124.-Delloptychius waclısmuth1.

Desminnus, St. Jolin \& Worthen. 187.j, Geo. Sur. 111., vol. (i, p. 337. [Ety. drsmos, a ligam. nı; colous, a twotl.] This name was $y_{1}$ pliesl to a genus of hats, in 18:6, liv Pinz, Neu. Wiel. in Beitrage zur Naturg. Brasiliens. Teeih orcurring in ruws, sinall, rotust; crown linerally elongaterl, arrelud vertically, medianru-p wirl, Jateral crusts; luse constricted and proiluced. Type D. tumidus.

costrlliferms, Sit. Julın \& Wirtlien, $18 \pi 5, \mathrm{G} * n$. Sur. III.. vol. $6,11.31$, st. Lrmis Gir.

flilellum, St. Jolın \& WVr-

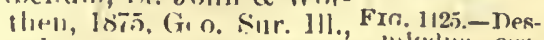
vol. (i, 11. 343, Kenkuk Gr. meli i ferus. ligunilormis, Sit. Juln \& Comvex asIIinlien, 187i. (ien. Silr. pect.

1ll., vil. (;) 1. 3t", Korokuk Gr.

minusculus, Newbiry \& IIortien, 1866, (U culus minusculiu,) (ieo. Kep. 111., vol. ', p. 67, Keokuk Gr. 
tumidus, St. John \& Worthen, 1875, Geo. Sur. 1ll., vol. 6, p. 339, St. Louis Gr.

Dixichisis, Newberry, 1873, Ohio Pal., vol. 1, p. 313 , and vol. 2, p. 3. [Eiy. deinos, terrible; ichthys, a fisls.] Cranium composed of thick bony plates, strengthened with internal arches anchylosed together, occipital bone in the type rpecirs three inclies in thickness; rel. atively small maxillaries bearing a number of acute, conical, anchylosed tceth, whiclı interlocked with a similar series on the mandibles; premaxillaries large, strong, triangular plates or teeth; mandibles of great length, flattened and spaiulate behind, turning ip) anteriorly to form a strong triaugular tooth, with its fellow of the opposite mandible, interlocked with the great, divergent, premaxillary teeth; vital parts of the hody covered with large, thick plates which formed a carapace. Type D. terrelli.

hertzeri. Newberiy, 1873, Ohio Pal., vol. 1, p. 316 , Portage Gr.

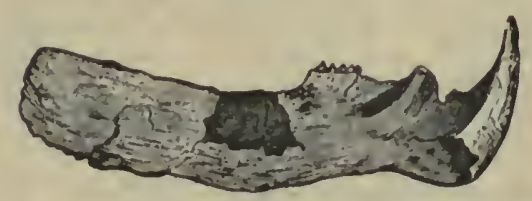

F10. 1126.-Dinicilhy:s hertzeri.

terrelli, Newberry, 1873-75, Ohin Pal., vol. 1, p. 313 , and vol. 2, p. 3 , Portage Gr.

Diplasiris, Matthew, 1888, \an. Rec. Sci., vol. 2 , p. 251. [Ety. diplos, double; aspis, shield.] Snall, having plates on the head, back, and sides, and one ventral plate; plates bearing very fine ridges. Type $\mathrm{D}$. acadica.

acadica, Mathew, 1885, Can. Rec. Sci., vol. 2, p. 251, Up. Silurian or Low. Devonian.

Dipı,odus, Agassiz, 1843, Recherches sur les Poissons Fossiles, t. 3, p. 20t. [Ety. diploos, double; odous, a tooth.] This name was used by Rafinesque for a genus of Sparidre in 1810, Indice d'Lit. tologia Siciliana. 'Teetl, having a flattened or rounded bast; from which spring two lateral and cometimes a small central denticle; each jaw bore several hundred teetls in radiating rows, the points projecting inward. They bulong to sliarks possessed of spines, described under the names of Oracanthus and Xenacanthus. Type D. gibbusus.

acillacts, Dawson, 1860, Acad. Geol., p. 211, and Can. Nat. Geol., vol. 5, Coal Meas.

coinpressus, Newberry, 1857, Proc. Acad. Nat. Sci. Phil., vol. 8, p. 99, and Ohio Pal., vul. 1, p. 335, Coal Meas.

duplicatus, see Thrinacodus duplicatus. gracilis, Newberry, 1857, Proc. Acad. Nat. Sci. Phil., vol. 8, p. 99, and Ohio Pal., vol. 1, p. 335 , Coal Meas.

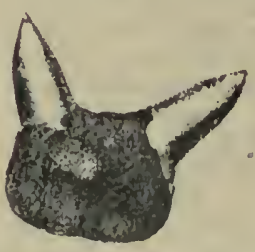

FjG. 1127,-Diplodus Jatus. incurius, ses Thrinacodus incurvis. latus, Newberry, 1857 , Proc. Acrad. Nat. Sıi. Phil., vul. 8, p. 99, and Ohio Pul., vol. 1, p. 336 , Coal Meas.

penetrans, Dawson, 1860, Acad. Geol,, p. 211, and Can. Nat. and Geol., vol. 5, Coal II ras.

Dipterus, Sedgwick \& Murchison, 1835, Geo. 'Trans., 2d ser., vol. 3. [liy. dipteros, two-winged.] Diptera is an order of insects establislied by Limnaus. Small fusiform fislies; heads compressed, tails heterocercal; two dorsal fins opposite two similar anal fins, the second of each the larger; a strongly marked lateral line; scales circular, thickest iis the middle, variously curved with concentric lines or longitudinal ridges. 'Type D. brachypygopterus.

sherwoodi, Newberry, 1875, Ohio Pal. vol. ', Fig. 1128.-Dipte. 1. 61, Catskill Gr.

Drepanacantirus, Newberry \& Worthen, 1866, Geo. Sur. Ill., vol. 2, p. 120. [Ety. drepane, a sickle; akantha, spine.] Fin spine's compressed laterally, gradually tapering to an acute point, curved forward; anterior margin with a row of flattened or conical tubercles; lateral surfaces with tubercles in longitudinal rows; posterior margin without lıooks, sometimes with tubercles. Type D. gemmatus.

anceps, see Xystracanthus anceps.

gemmatus, Newberry \& Worthen, 1866, Geo. Sur. Ill., vol. 2, p. 123, Keokık Gr. reversus, St. John \& Worthen, 1875, Geo. Sur. Ill., vol. 6, p. 456, St. Louis Gr.

stellatus, see Batacanthus stellatus.

Ectosteorachis, Cope, 1880, Pal. Bull. No. 32 , p. 19. [Ety. ektos, without; oxteon, bone; rachis, a ridge, backbunњ.] Base of the skull consists of ossified parachordals, which embrace the cliorda dorsalis posteriorly, and are continued for a short distance posteriorly as a tube; anteriorly the cisorial groove is open; trabeculæ not ossified; cranial structure embryonic; above and in front of the opening for the chorda the neural canal enters the groove; para. chordals subtriangular. Type E. nitidns. ciceronius, Cope, 1883, Pal. Bull. No. 36 , in Proc. Am. Phil. Soc., p. 628, Permian. 
nitidus, Cope, 1880, Pal. Bull. No. 32, p. 19, Permian.

Enestus, Leidy, 1856, Jour. Acad. Nat. Sci., $2 \mathrm{~d}$ ser., vol. 3, p. 159. [Ety. edestes, a devourer.] Maxillary bonesegmented; segments beveled anteriorly and excavated posteriorly, for co-adaptation; teeth resembling those of Carcharodon, one co-ossified with each maxillary segment. Type E. vorax.

giganteus, Newberry, 1888, Ann. N. Y. Acad. Sci., vol. 4, p. 1, Coal Meas.

heinrichsi, Newberry \& Worthen, 1870 , Geo. Sur. Ill., vol. 4, p. 350, Coal Meas. minor, Newberry, 1866, Geo. Sur. Ill., vol. 2, p. 84, Coal Meas.

vorax, Leidy, 1856, Jour. Acad. Nat. Sci. Phil., vol. 3, $2 \mathrm{~d}$ series, p. 159, Coal Meas.

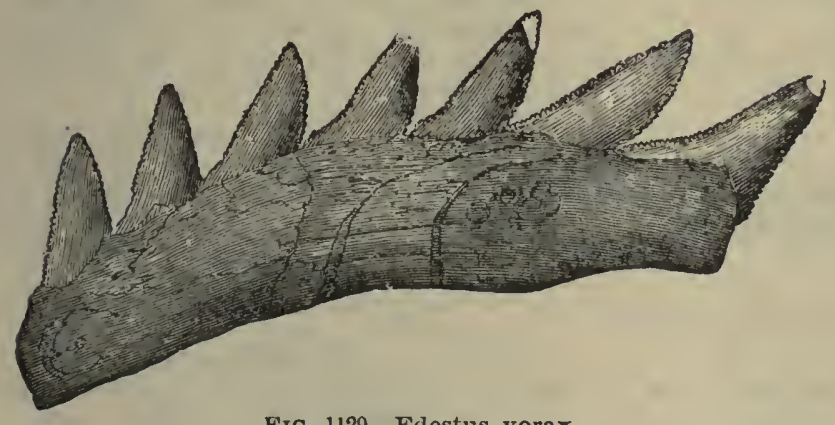

FIG. 1129.-Edestus vorax.
Elonichthys peltigerus, see Palæoniscus peltigerus.

Erismacantiuus, McCoy, 1848, Ann. and Mag. Nat. Hist., 2d series, vol. 2, p. 119. [Ety. ereisma, a prop or stay; akantha, spine.] Spine of three parts; one compressed, finely striated, which entered the flesh ; the second short, compressed, rapidly tapering, curved backward, sides with longitudinal ridges, and two rows of downward curved teeth on the posterior concave margin; the third, a prop-like part extending forward nearly at right angles with; the base, arched, compressed at the basal half, depressed distally, and covered with tubercles and some spines on the under side. Type E. jonesi.

maccoyanus, St. John \& Worthen, 1875, Geo. Sur. Ill., vol. 6, p. 461, St. Louis Gr.

Eunemacantrus, St. John \& Worthen, 1883, Geo. Sur. Ill., vol. 7, p. 120. [Ety. eu, beautiful; nema, a line ; akantha, spine.] Distinguished from Ctenacanthus by the plain dorsal ridge, tuberculated intercostal sulci, and upward direction of the denticles on the angles of the posterior face. Type E. costatus.

costatus, Newberry \& Worthen, 1866, (Otenacanthus costatus,) Geo. Sur. Ill., vol. 2, p. 120 , St. Louis Gr.

Eurylepis, Newberry, 1856, Proc. Acad. Nat. Sci. Phil. [Ety. eurys, broad; lepis, scale.]. Small; body fusiform; head obtuse; tail elongated, lobes unequal; fins small, with delicate fulcra; dorsal and anal fins opposite, and far back on the body; ventrals near middle of abdomen; cranial surface tubercular; $\max$ illary, mandibular, and jugular plates corrugated; scales smooth, ornamented, or serrated; teeth numerous, conical, short. Type E. tuberculata.

corrugata, Newberry, 1856, Proc. Acad. Nat. Sci. Phil., and Ohio Pal., vol. 1, p. 350, Coal Meas.

granulata, Newberry, 1856, Proc. Acad. Nat. Sci. Phil., and Ohio Pal., vol. 1, p. 352, Coal Meas.

insculpta, Newberry, 1856, Proc. Acad. Nat. Sci. Phil., and Ohio Pal., vol. 1, p. 351, Coal Meas.

lineata, Newberry, 1856, Proc. Acad. Nat. Sci. Phil.; and Ohio Pal., vol. 1, p. 353, Coal Meas.

minima, Newberry, 1873, Ohio Pal., vol. 1, p. 353, Coal Meas.

ornatissima, Newberry, 1856, Proc. Acad. Nat. Sci. Phil., and Ohio Pal., vol. 1, p. 352, Coal Meas. ovoidea, Newberry, 1856 , Proc. Acad. Nat. Sci. Phil., and Ohio Pal., vol. 1 , p. 351, Coal Meas.

striolata, Newberry, 1873 , Ohio Pal., vol. 1, p. 355, Coal Meas. tuberculata, Newberry, 1856, Proc. Acad. Nat. Sci., and Ohio Pal., vol. 1, p. 350 , Coal Meas.

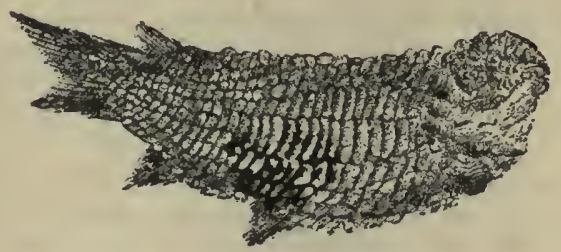

Frg. 1130.-Eurylepis tuberculata.

Eustifenopteron, Whiteaves, 1881, Am. Jour. Sci. and Arts, 3d ser., vol. 21, p. 495. [Ety. eu, very; sthenes, stout; pteron, a fin.] Fin rays of anal and second dorsal fins supported by three osselets articulated to a broad interspinous apophysis; vertebral centers not ossified; caudal osselets articulated to modified hæmal spines. Type E. foordi.

foordi, Whiteaves, 1881, Am. Jour. Sci. and Arts, 3d ser., vol. 21, p. 495, Upper Devonian.

Fissodus, St. John \& Worthen, 1875, Geo. Sur. Ill., vol. 6, p. 413. . [Ety. fissus, split; odous, tooth.] Teeth small, in the form of root and general contour 
like Peltodus, but distinguished by the cleft condition of the crest. Type F. bifidus.

bifidus, St. John \& Worthen, 1875, Geo. Sur. Ill., vol. 6, p. 414, Kaskaskia Gr.

tricuspidatus, St. Jolın \& Worthen, 1875, Geo. Sur. III., vol 6, p. 415 , Kaskaskia $\mathrm{Gr}$.

Fig. 1131.-Fissodus bi. thilus.

Gampsacantius, St. John \& Worthen, 1875, Geo. Sur. Ill., vol. 6, p. 471. [Ety. gampsos, curved; akantha, spine.] Spines long, laterally compressed, tapering, costate, with larger and smaller tubercles; posterior margin denticulate; base expanded; pulp cavity large. Type G. typus.

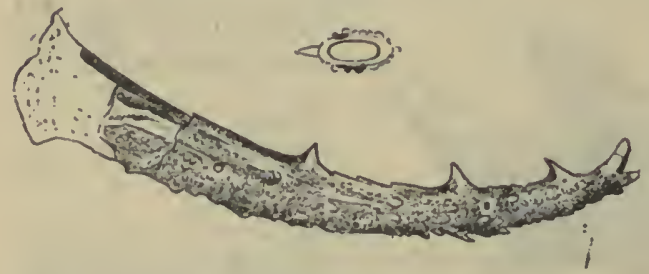

Fig. 1132.-Gampsacanthus typus. Side view of $\mathrm{n}$ spine magnified 2 diam., and transverse section.

latus, St. John \& Wortlen, 1875, Geo. Sur. Ill., vol. 6, p. 474, Keokuk Gr. squamosus, St. Jolin \& Worthen, 1875 , Geo. Sur. Ill., vol. 6, p. 473 , St. Louis Gr. typus, St. John \& Worthen, 1875, Geo. Sur. Ill., vol. 6, p. 472, St. Louis Gr.

Gisacantius, St. John \& Worthen, 1875, Geo. Sur. Ill., vol. 6, p. 440. [Ety. geison, a border; akantha, a spine.] Spine curved posterioily, anterior angle a simple raised keel; lateral faces bearing longitudinal rows of tubercles; posterior face longitudinally keeled. Type G. stellatus.

bullatus, St. John \& Worthen, 1875, Geo. Sur. Ill., vol. 6, p. 441, Kaskaskia Gr. stellatus, St. John \& Worthen, 1875, Geo. Sur. Ill., vol. 6, p. 440, St. Louis Gr.

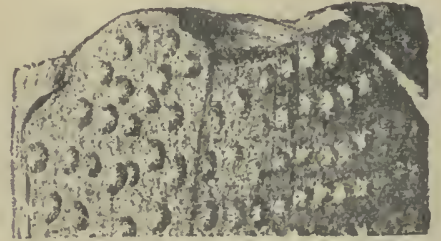

Fio. 1133.-Glymmatacanthus irishi. Fragment of spine.

Glymmatacanthus, St. John \& Worthen, 1875, Geo. Sur. Ill., vol. 6, p. 446. [Ety. glymmatos, engraved; akantha, spine.] Fin ray vertically elongated, posteriorly arched, laterally compressed; lateral faces covered with stellate or striated tubercles. Type G. irishi. irishi, St. John \& Worthen, 1875, Geo. Sur. Ill., vol. 6, p. 447, Kinderhook or Waverly Gr.

petrodoides, St. John \& Worthen, 1883, Geo. Sur. Ill., vol. 7, p. 250, Kaskaskia Gr.

rudis, St. John \& Worthen, 1883, Geo. Sur. Ill., vol. 7, p. 249, Keokuk Gr.

Glyptolepis, Agassiz, 1836, Poiss. Foss., vol. 2, p. 179. [Ety. glyptos, sculptured; lepis, seale.] Fins long, sometimes pendulous; anterior dorsal opposite ventral, and posterior dorsal opposite anal ; tail fin long, spreading below ; shoulder bones huge; teeth minute; scales of great size in proportion to the animal, and deeply sculptured. Type G. elegans.

microlepidotus, Agassiz, 1836, Poiss. Foss., vol. 2, p. 179, Devonian.

quebecensis, Whiteaves, 1889, Trans. Roy. Soc. Can., vol. 6, p. 77, Low. Devonian.

Gantioriliza, Cope, 1883, Proc. Am. Phil. Soc., vol. 20, p. 629. [Ety. gnathos, jaw; rhiza, root.] Founded upon some ganoine teeth. The definition is too meager for identification, and the genus may never again be recognized. Type $G$. serrata.

gerrata, Cope, 1883, Proc. Am. Phil. Soc., vol. 20 , p. 629 , Perınian.

Gyracastuus, Agassiz, 1833, Reeberches sur les Poissons Fossiles, t. 1, p. 87. [Ety.gyros, a circle; akantha, spine.] Fin spines very large, gradually tapering to the apex, and slightly arched back wa $\mathrm{rd}$; inserted base small, rapiclly tapering; posterior margin feebly armed with two rows of small denticles; surface of the sides covered with very oblique ridges, whiclı meet at an angle on the anterior face. Type $G$. formosus.

alleni, Newberry, 1873, Ohio Pal., vol. 1, p. 331, Cuyahoga shale.

com pressus, Newberry, 1873, Ohio Pal., vol. 1, p. 230, Cuyahoga shale.

cordatus, St. John \& Worthen, 1883, Geo. Sur. Ill., vol. 7, p. 251, Keokuk Gr. duplicatus, Dawson, 1868, Acad. Geol., p. 210, Coal Meas.

magnificus, Dawson, 1868, Acad. Geol., p. 210, Subcarboniferous.

Harpaconus, Agassiz, 1869, Catal. Foss. Fish, Colleetion of Earl of Enniskillen, and St. Jolnn \& Worthen, in Geo. Sur. Ill., vol. 6, p. 354. [Ety. harpe, a

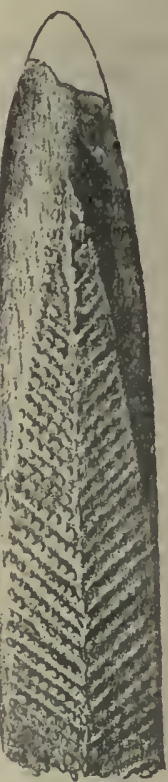

FIG. 1134.-Gyracanthus alleni. Anterlor face. 
hook; odous, tooth.] Teeth laterally elongated, vertically arched, gently curved outward in the concave face; margins nearly parallel; crown compressed along the crest; serrated, expanded below; convex face low, opposite face concave; coronal borders produced inbeveled; base strong, obliquely produced; lateral angles well defined. Type $H$. dentatus, or, more properly, $\mathbf{H}$. occidentalis.

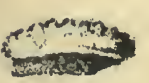

FIG. 1135.-Harpacodus occldentalis.

compactus, St. John \& Worthen, 1875, Geo. Sur. Ill., vol. 6, p. 355 , Kaskaskia Gr.

occidentalis, St. John \& Worthen, 1875, Geo. Sur. Ill., vol. 6, p. 355, St. Louis Gr.

Heliodus, Newberry, 1875, Obio Pal., vol. 2, p. 62. [Ety. helios, sun ; odous, tooth.] Distinguished from Dipterus by having the upper palate teeth united, forming a rounded, semicircular, triturating plate, bearing radiating tuberculated ridges. Type H. lesleyi. Dr. Traquair, of England, regards Heliodus as a synonym for Palædaphus, Van Beneden \& DeKoninck, 1864, Bull. Acad. Belg., vol. 17 , p. 143.

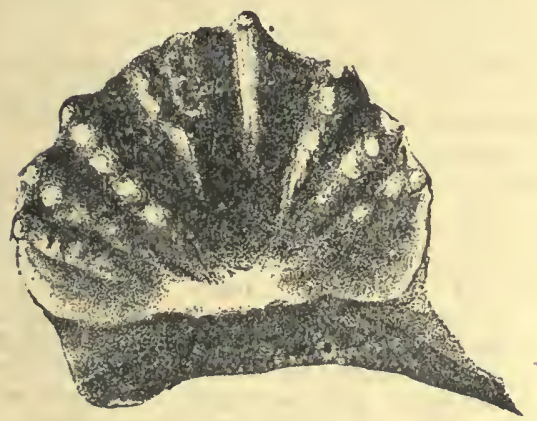

Fra. 1136. -Hellodus lesleyi.

lesleyi, Newberry, 1875, Ohio Pal., vol. 2, p. 64, Chemung Gr.

Hexodus, Agassiz, 1843, Recherches sur les Poissons Fossiles, t. 3, p. 104. [Ety. helos, a nail or rudder; odous, tooth.] Transversely elongate, crown convex, elevated along the middle into an obtuse, circular ridge, sometimes divided into a line of several compressed cones diminishing from the center; surface porous as in Psammodus; margin of the crown raised in the middle on both the inner and outer sides, and it and the root vertically plicated. Type $H$. simplex.

angulatus, Newberry \& Worthen, 1866, Geo. Sur. Ill., vol. 2, p. 83, Burlington Gr.

biformis, Newberry \& Worthen, 1866, Geo. Sur. Ill., vol. 2, p. 77, Waverly or Kinderhook Gr.

carbonarius, Newberry \& Worthen, 1866 , Geo. Sur. Ill., vol. 2, p. 75, Coal Meas. compressus, Newberry \& Worthen, 1870 , Geo.Sur. Ill., vol. 4, p. 360, Burlington Gr. compressus, see Hybocladodus compressus. coniculus, Newberry \& Worthen, 1866 ,

Geo. Sur. Ill., vol. 2, p. 75, Burlington Gr. consolidatus, Newberry \& Worthen, 1866, Geo. Sur. Ill., vol. 2, syn. for Chitonodus latus.

crenulatus, Newberry \& Worthen, 1866 , Geo. Sur. Ill., vol. 2, p. 82, Keokuk Gr. denshumani, Newberry \& Worthen, 1866, Geo. Sur. Ill., vol. 2, p. 76, Keokuk Gl. denticulatus, Newberry \& Worthen, 1866, Geo. Sur. Ill., vol. 2, p. 81, Keokuk Gr. elytra, Newberry \& Worthen, 1866, Geo. Sur. Ill., vol. 2, p. 78, Keokuk Gr.

gibbosus, Newberry \& Worthen, 1866, Geo. Sur. Ill., vol. 2, p. 79, Keokuk Gr.

gibbus, Leidy, 1856 , Trans. Am. Phil.

Soc., vol. 11, p. 87, Fia. 1137.-Helodus gibKeokuk Gr.

limax, Newberry \& Worthen, 1866, Geo. Sur. Ill., vol. 2, p. 80, Burlington Gr. nobilis, Newberry \& Worthen, 1866, Geo. Sur. Ill., vol. 2, same as Chitonodus latus.

placenta, see Psephodus placenta.

politus, Newberry \& Worthen, 1866, Geo. Sur. Ill., vol. 2, p. 79, Keokuk Gr.

rugosus, Newberry \& Worthen, 1866, Geo. Sur. Ill., vol. 4, p. 359, Coal Meas.

sulcatus, Newberry \& Worthen, 1866, Geo. Sur. Ill., vol. 2, p. 82, Keokuk Gr. undulatus, Newberry \& Worthen, 1866, Geo. Sur., Ill., vol. 2, p. 82, Keokuk Gr. Holop'tychius, Agassiz, 1836, Recherches sur les Poissons Fossiles, t. 2, p. 179. [Ety.holos, entire; pty $x$, wrinkle.] Body thick, short, rounded, bones of the head granulated; scales large, very thick, subrhomboidal, rounded, imbricating, composed of numerous bony layers, exposed surface marked with large, longitudinal, flexuous wrinkles and tubercles; teeth small, numerous, conical, longitudinally sulcated at base; tail heterocercal, caudal fin triangular, obliquely truncated; dorsal fin opposite a similar anal one close to the base

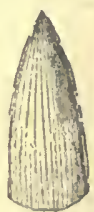

FIG. 1138-Hoioptychius americanus. Single tooth. of the caudal; ventral behind the middle of the body. Type $H$. giganteus.

americanus, Leidy, 1856 , Jour. Acad. Nat. Sci., 2d series, vol. 3, p. 159 , Catskill Gr.

nobilissimus, Agassiz, as identified by Hall, 1843 , Geo. Rep. 4th Dist. N.Y. is described as $\mathbf{H}$. americanus.

taylori, Hall, 1843, (Sauripteris taylori,) Geo. Rep. 4th Dist. N. Y., p. 282, Catskill Gr. 
Homucanthus, Agassiz, 1845, Pois. Foss. [Ety. homos, similar; akantha, spine.]

gibbosus, see Amacanthus gibbosus.

gracilis, Whiteaves, 1889, Trans. Roy Soc., Can., vol. 6, p. 77, Low. Devonian.

rectus, see Marracanthus rectus.

Hybocladodus, St. John \& Worthen, 1875, Geo. Sur. Ill., vol.6, p. 284. [Ety. hybos, hump; Cladodus, a genus.] Teeth small, strongly cuspidate, base resembling that of a Cladodus, being elliptical and broadly expanded, with a more or less prominent antero-posteriorly compressed median cone, both surfaces of which are plicated and resemble the crown of a Hybodus; anterior face nearly straight, curved laterally, terminating below in a well defined marginal border or ridge, posterior margin broadly rounded, inferior surface excavated immediately behind the marginal border, with a beveled space extending along the posterior margin, superior face more or less convex and beveled to the posterior edge; both coronal surfaces vertically marked with plice. 'Type $\mathrm{H}$. plicatilis.

compressus, Newberry \& Worthen, 1866 , (Helodus compressus,) Geo. Sur. Ill., vol. 2, p. 78 , Burlington Gr.

intermedius, St. John \& Worthen, 1875, Geo. Sur. Ill., vol. 6, p. 287, Keokuk Gr.

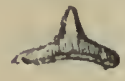

Fia. 1139.-Hybo-

cladidus plicatills.

Ill., vol. 6, p. 286, Burlington Gr.

tenuicostatus, St. John \& Worthen, 1875 ; Geo. Sur. Ill., vol. 6, p. 286, Keokuk Gr.

JANASSA, Munster, 1839, Beitrage Petrefaktenkunde, vol. 1, and Agassiz in Poiss. Foss., t. 3, p. 375. [Ety. mythological name.] Teeth have a tabulated structure and enameled, wavy crown; small in front and larger toward the posterior part of the jaw; jaw-bone rough. and y ranular. Type'J. angulata.

girleiana, Cope, 1877, (Strigillina gurleiana,) Proc. Am. Phil. Soc., p. 191, Permian.

linguiformis, Cope, 1877, (Strigillina linguiformis,) Proc. Am. Phil. Soc., p. 53, Permian.

LAmbDoDUs, St. John \& Worthen, 1875, Geo. Sur. Ill., vol. 6, p. 280 . [Ety. Lambda, a Greek letter; odous, tooth.] Teeth small, base posteriorly produced and laterally expanded, broadest behind the cornua; a single strong, slightly sigmoidally curved, recurved, eccentric cornua arises from the anterior angle of the base, terminates in a sharp apex, compressed in front, broadly rounded behind, with more or less distinct cutting edges and vertical costæ. It is distinguished from Cladodus by the single coronal cornua, and the absence of lateral denticles; the basal portion bears some resemblance to Thrinacodus. Type L. costatus.

calceolus, St. John \& Worthen, 1875, Geo. Sur. Ill., vol. 6, p. 281, Burlington Gr. calceolus var. robustus, St. John \& Worthen, 1866, Geo. Sur. Ill., vol. 6, p. 282, Keokuk Gr.

costatus, St. John \& Worthen, 1875, Geo. Sur. Ill., vol. 6 , p. 280 , Burlington $\mathrm{Gr}$.

bamulus St John \& W FIG. 1140.-Lambthen, 1875, Geo. Sur.

Ill., vol. 6, p. 283, Kaskaskia Gr.

reflexus, St. John \& Worthen, 1875, Geo. Sur. Ill., vol. 6, p. 284, Kaskaskia Gr.

transversus, St. John \& Worthen, 1875 , Geo. Sur. Ill., vol. 6, p. 282, St. Louis Gr. Lecracantises, St. John \& Worthen, 1875 , Geo. Sur. Ill., vol. 6, p. 475. [Ety. lekroi, the antlers of a stag; akantha, spine.] Spines long, tapering, curved, laterally compressed, stellate tubercles irregularly disposed; base thin, expanded; julp cavity large; apex transversely expanded and armed with strong denticles. Type L. unguiculus.

unguiculus, St. John \& Worthen. 1875, Geo. Sur. Ill., vol. 6, p. 476, St. Louis Gr.

Leptocanthus, Agassiz, 1837, Poiss. Foss., vol. 3. [Ety. leptos, slender; akantha, spine.] occidentalis, see Acondylacanthus occidentalis.

Lionus, St. John \& Worthen, 1875, Geo. Sur. Ill., vol. 6, p. 335. [Ety. leios, smooth; odous, tooth.] Teeth resembling Orodus; crown arched, laterally and vertically; basal margins constructed and sharply defined from the base; apex with obscurely defined lateral crests; convex. in either face; anterior face produced beneath the median cone, and both faces occupied with faint vertical sulci, producing obscure secondary prominences; surface smootb, punctate, or verrucose; base as in Orodus, relatively deep. Type L. ealcaratus.

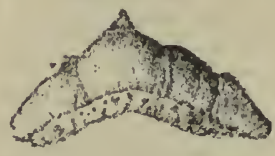

calcaratus, St. John \& Worthen, 1875 , Geo. Sur. Ill., vol. 6 , p. 336, Burlington Gr.

Fra. 1141.-Llodus calcacalcaratus var. grossipunctatus, St. John \& Worthen, 1875, Geo. Sur. Ill., vol. 6, p. 337, Keokuk Gr.

Liognathus, Newberry, 1873, Ohio Pal., vol. 1, p. 306. [Ety. lis, smooth ; gnathos, the jaw.] Jaw the only part yet known; spatulate, dentate only at and near the anterior extremity ; resembles Coccosteus. Type L. spatulatus.

spatulatus, Newberry, 1873, Ohio Pal., vol. 1, p. 306, Up. Held. Gr. 
Lisconus, St. John \& Worthen, 1875, Geo. Sur. Ill., vol. 6, p. 363 . [Ety. lisgos, a spade; odous, tooth.] Teeth laterally

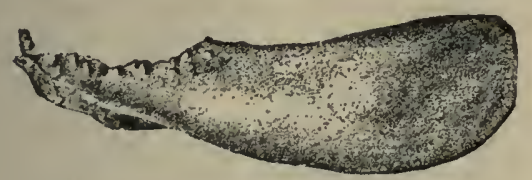

FIG. 1142.-Liograthus spatulatus.

abbreviated strong; crown thick, sharpcrested, and sometimes obscurely serrated; basal margins well defined; base p. 302. [Ety. machaira, a saber; akantha, a spine.] Spines large, flattened, curved, ancipital, unsymmetrical; edges and point acute; base narrowed, with a rough and irregular extremity; central cavity reaching nearly to the apex; external surface enameled, smooth or punctate, and striate microscopic structure dense. Type M. major.

major Newberry, 1857, Bull. Nat. Inst., p. 6, and Ohio Pal., vol. 1, p. 304, Up. Held. Gr.

peracutus, Newberry, 1857, Bull. Nat. Inst., p. 6, and Ohio Pal., vol. 1, p. 305, Up. Held Gr.

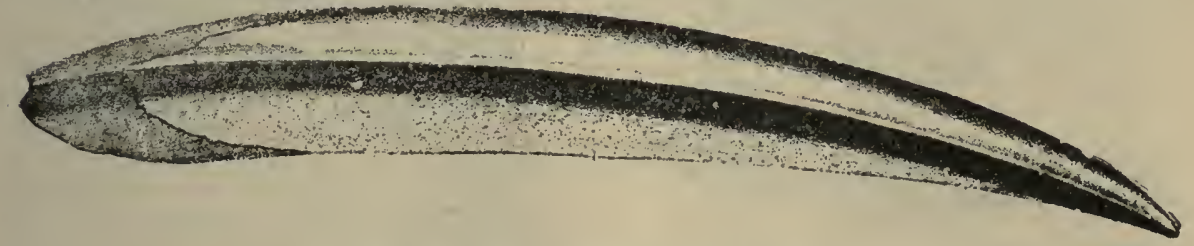

Fig. 1145,-Machæracanthus peracutus.

vertical to the crown, rectangular, prolonged, equal to the elevation of the crown; inferior Eurface well defined from either face above; and generally slightly beveled from the concave to the opposite border; coronal surface enameled, worn crest striato-punctate. Type L. curtus.

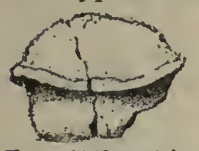

FIG. 1143. - - LIsgodus curtus.

Iouis Gr. curtus, St. John \& Worthen, 1875, Geo. Sur. Ill., vol. 6 , p. 364 , Burlington Gr.

selluliformis, St. John \& Worthen, 1875, Geo. Sur. Ill., vol. 6 , p. 366 , St.

serratus, St. John \& Worthen, 1875, Geo. sur. Ill., vol. 6, p. 365, Burlington Gr. Listracantuus, Newberry \& Worthen, 1870 , Geo. Sur. Ill., vol. 4, p. 371. [Ety. listron, slovel; akantha, spine.] Spines small, gently arched, flattened, thin ; sides marked by numerous sharp, longitudinal carinæ, edges set with divergent, slender, acute teeth; most numerous on the convex margin; and largest base expanded and obliquely truncated. Type L. hystrix.

hildrethi, Newberry, 1875 , Ohio Pal., vol. 2 , p. 56 , Coal Meas.

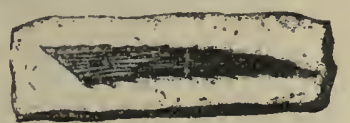

hystrix, New- Fra. 1144. - Listracanthus berry \& Worhystrix.

then, 1870 , Geo. Sur. Ill., vol. 4, p. 372 , Coal Meas.

Lophodus, Newberry \& Worthen, 1870, Geo. Sur. Ill., vol. 4. This name was preoccupied by Romanowsky in 1864 .

variabilis, see Agassizodus variabilis.

Macilaracanthus, Newberry, 1857, Bull. Nat. Inst., p. 6, and Ohio Pal., vol. 1, sulcatus, Newberry, 18j7, Bull. Nat. Inst., p. 6, and Ohio Pal., vol. 1, p. 305, Up. Held. Gr.

M A CROPETALICHTHYS, Norwood \& Owen, 1846, Am. Jour. Sci., $2 d$ ser., vol. 1, $p$. 367. [Ety. makros, large; petalos, expanded or spread out; ichthys, fish.] Cranium composed of large polygonal plates, united by double sutures; surface enameled, tubercled, ornament-

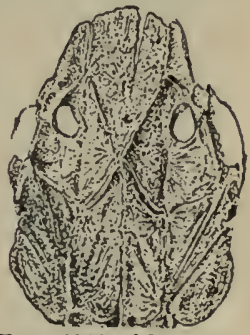

Fia. 1146.-Macropetalichthys sul11vant 1 . Une-fifth natural size. ed ; eye orbits conspicuous; nasal plate wedge-shaped; occipital plate oblong, emarginate behind, and prolonged anteriorly, where it meets the nasal plate. Type M. rapheidolabis.

manni, Newberry, (Agassichthys manni,) 1857, Bull. Nat. Inst., p. 3, Up. Held. Gr. rapheidolabis, Norwood \& Owen, 1846, Am. Jour. Sci., 2 d ser., vol. 1, p. 367, Up. Held. Gr.

sullivanti, Newberry, 1857, (Agassichthys sullivanti,) Bull. Nat. Inst., p. 3, and Ohio Pal., vol. 1, p. 294, Up. Held. Gr. Marracanthus, St. John \& Wurthen, 1875 , Geo. Sur. Ill., vol. 6, p. 465. [Ety. marron, a spade; akantha, spine.] Dorsal spine nearly straight, or with a forward curvature, obtusely terminated, rounded in front, truncated behind, or rounded into the posterior face, which is longitudinally ridged in apparent continuity with the lateral costr; lateral face and anterior margin longitudinally ridged, the costæ being tuberculated, those in front more or less strongly 
developed, witls their apices directed up ward, and especially in their upper part, where they grailually inerease in size, forming strong, more or less deflected looks, transversely carinated; intercostal spaces minutely ridged and striato-punctate; base moderately inserted, forming a comparatively thin plate, more or less laterally expanded posteriorly from the angular ridge in front, with more or less prominent marrinal angles behind; pulp-cavity moderately large, similar in section to the body, and occupying the posterior two-thirds of the spine. In costation and the expunded lose it is like Amacantlus, but distinguished in all other respects. Type $\mathbf{M}$. rectus.

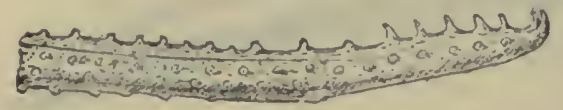

FIa. 114.-Marracantlus rectus. Anterior part of spine.

rectus, Newberry \& Worthen, 1866, (Homacanthus (?) rectus, Geo. Sur. Ill., vol. 2, p. 115, St. Louis Gr.

Mrcolel's, Newberry, 1857, Proc. Acad. Nat. Sci., vol. 8, p. 46. [Lty. mekos, large; lepis, a scale.] Heterocercal lepidoids of small size; body fusiform; head obtuse; tail elongated; lobes unequal; fins small, provided with delicate fulcra; dorsal opposite anal, both far back on the body; crania corrugated or tuberculated; (1)ercular maxillary and byoid plates ormamented; seales smooth or ornamented; posterior margin serrated; seales of medlian line crenulated; two rows of scales extending back to near anal fin; teeth conical, short, brush-like. Distinguisherl from Palæoniscus by small size, posterior position of dorsal fin, and the high lateral scales. Type M. corrugata. Probably a syn. for Palæoniscus, but not figured, and species poorly defined.

corrugata, Newberry, 1856, Proc. Acad. Nat. Sci., vol. 8, p. 96, Coal Meas.

granulata, Newiberry, 1856, Proc. Acad. Nat. Sci., vol. 8, j. 97, Coal Meas.

insculpta, Newberry, 1856, Proc. Acad. Nat. Sci., vol. 8, p. 97, Coal Meas.

lineata, Newberry, 1856, Proc. Acad. Nat. Sei., vol. S, p. 97, Coal Meas. ornatissima, Newberry, 1856, Proc. Acad. Nat. Sci., vol. 8, p. 97, Coal Meas. ovoidea, Newberry, 1S5̃6, Proc. Acad. Nat. Sci., vol. 8, p. 97, Coal Meas.

serrata, Newberry, 1856, Proc. Acad. Nat. Sci., vol. 8, p. 97, Coal Meas.

tuberculata, Newberry, 18506, Proc. Acad. Nat. Sci., vol. 8, p. 96, Coal Meas.

Mesonmodus, St. John \& Worthen, 1875, Geo. Sur. ill., vol. 6, p. 290. [Ety. mesodme, something between; odous, tooth.] Teeth laterally elongated; base consisting of one inferior flattened; posterior obliquely produced, massive plate, of which the posterior face slopes downward and slightly backward, at an obtuse angle, to the posterior crown face; anterior face sliglitly produced along the shoulder, which extends parallel with the base of the crown, vertical or beveled, and occupied by a more or less prominent median protuberance, which extends to the edge of the interior surfaces: both faces are more or less roughened or pitted, lateral angles truncated or rounded, and more or less constricted above, equaling the lateral diameter of the crown; crown rising along the anterior border, sharply constricted in front and laterally, and well defined, sometimes constricted from the posterior basal face, nearly equaling the base in anteroposterior clianeter; but more or less compressed along the crest, which rises into a more or less prominent median or submedian eusp, vertical or laterally deflected and recurved, usually compressed, with distinct, sometimes sharp, cuttiug edges; the lateral portions of the crown denticulated, extremities bearing slightly more prominent cusps than intermediate spaces; both faces ridged vertically; outer face of median cone often strongly buttressed; coronal surface enameled. Type M. exculptus.

explanatus, St. John \& Worthen, 1875, Geo. Sur. Ill., vol. 6, p. 293, Waverly or Kinderlıook Gr.

Exculptus, St. Jolin \& Worthen, 1875, Geo. Sur. Ili., vol. 6, p. 291, IVaverly or Kinder-Fra. 114s-Mesodmodus hook Gr.

ormatus, St. John \& Worthen, 1875, Geo. Sur. Ill., vol. 6, p. 294, Burlington $\mathrm{Gr}$.

Mycterops, Cope, 1888, Am. Nat., p. 876. [Ety. mukter, nose; ops, eye.] Founded upon the cast of the cranial and nuchal buckler of a placoderm fish; the eyeholes resemble those of Cephalaspis, and they are separated by a nose-hole, which is divided by a narrow bridge. Type M. ordinata.

ordinata, Cope, 1888, Am. Nat., p. 876, Coal Meas.

Onchus, Agassiz, 1837, Recherches sur les Poissons Fossiles. [Ety. onchos, bent, or hookerl like a talon or arrow-barb.]

deweyi, see Ceratiocaris deweyi.

Oxychodus, Newberry, 1857, Bull. Nat. Inst., p. 5, and Ohio Pal., vol. 1, p. 296. [Ety. onyx, a claw; odous, tooth.] Cranium composed of a great number of plates covered with an enameled and tuberenlated surface; jaws set with numerous conical, acute, recurved teeth; maxillary forming a low trian- 
gle; dentary bones posteriorly acute, where they are overlapped by the articular portions of the mandibles, long and narrow, curving upward to the

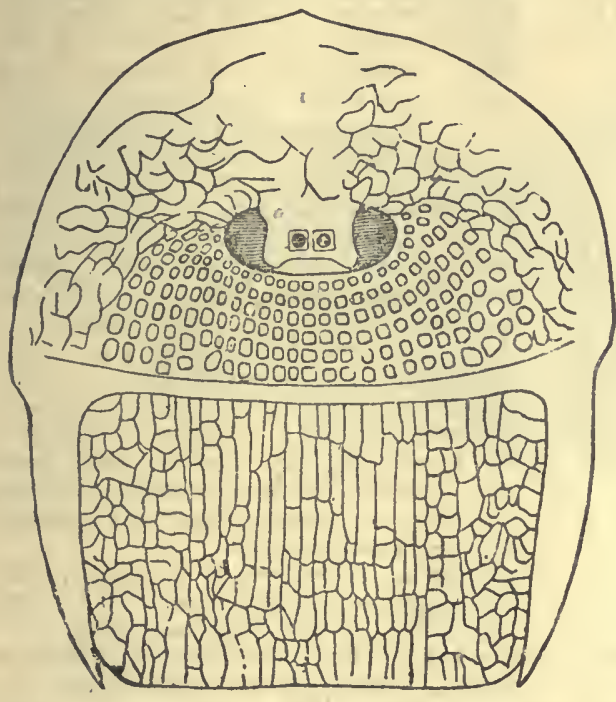

F1G. 1149.-Mycterops ordinata.

symphysis, where they support an inter-mandibular arch of bone, to which

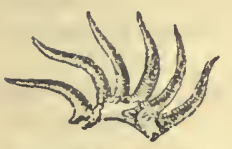

FIG. 1150.-Onycliodus sig m oides. One-half nat. size of inter-mandib. ular crest with 6 teeth.

p. 5, and Ohio Pal., vol. 1, p. 299, Up. Held. Gr.

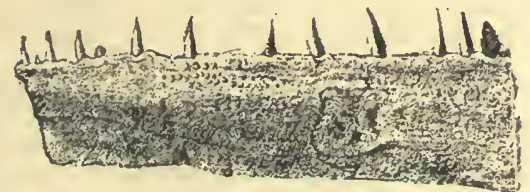

FIG. 115L.-Onychodus sigmoldes. Fragment of the right mandible.

Oracanthus, Agassiz, 1843, Recherches sur. les Poissons Fossiles, t. 3, p. 13. [Ety. oraios, beautiful ; akantha, spine.] Dorsal rays large, conical, without solid base, hollow, walls thin, surface tuberculated; no posterior rows of denticles. Type O. milleri.

abbreviatus, Newberry, 1857, Bull. Nat. Inst., p. 5, Up. Held. Gr.

consimilis, St. John \& Worthen, 1875, Geo. Sur. Ill., vol. 6, syn. for O. vetustus.

fragilis, Newberry, 1857, Bull. Nat. Inst., p. 5, Up. Held. Gr. granulatus, Newberry, 1857, Bull. Nat. Inst., p. 5, Up. Held. Gr.

multiseriatus, Newberry, 1857, Bull. Nat. Inst., p. 5, Up. Held. Gr.

(?) obliquiss, St. John \& Worthen, 1875, Geo. Sur. Ill., vol. 6, p. 477 , Keokuk Gr.

pnigeus. This species is made the type of the genus Pnigeacanthus. See P. deltoides.

rectus, St. John \& Worthen, 1883, Geo. Sur. Ill., vol. 7, p. 257, Kaskaskia Gr. vetustus, Leidy, 1856, Jour. Acad. Nat. Sci. Phil., 2 d ser., vol. 3, p. 162, St. Louis Gr.

Oronus, Agassiz, 1843, Recherches sur les Poissons Fossiles, t. 3, p. 97. [Ety. oraios, beautiful; odous, tooth.] Teeth laterally elongated, middle more elevated than extremities, forming an obtuse transverse cone; longitudinal diameter greatest and marked by a medial ridge with oblique secondary ridges. Type $O$. cinctus.

alleni, St. John \& Wortheu, 1875, Geo. Sur. Ill., vol. 6, p. 310, Coal Meas.

carinatus, St. John \& Worthen, 1875, Geo.Sur. Ill., vol. 6, p. 307, Keokuk Gr. corrugatus, see Agassizodus corrugatus. dædaleus, St. John \& Worthen, 1875, Geo. Sur. Ill., vol. 6, p. 301, Waverly or Kinderhook Gr.

decussatus, St. John \& Worthen, 1875 , Geo. Sur. Ill., vol. 6, p. 300, Waverly or Kinderhook Gr.

elegantulus, Newberry \& Worthen, 1866, Geo. Sur. Ill., vol. 2, p. 64, Burlington Gr.

fastigiatus, St. John \& Worthen, 1875, Geo. Sur. Ill., vol. 6, p. 306, Burlington Gr.

major, St. Jobn \& Worthen, 1875, Geo. Sur. Ill., vol. 6, p. 302, Burlington Gr. mammillaris, Newberry \& Worthen, 1866, Geo. Sur. Ill., vol. 2, p. 66, Keokuk Gr.

minusculus, see Desmio- Frg. 1152._Orodus dus minusculus.

minutus, Newberry \&

Worthen, 1866, Geo. Sur. Ill., vol. 2, p. 68, Keokuk Gr.

multicarinatus, Newberry \& Worthen, 1866, Geo. Sur. Ill., vol. 2, p. 62, Waverly or Kinderhook Gr.

neglectus, St. John \& Worthen, 1875, Geo. Sur. IIl., vol. 6, p. 308, St. Louis Gr. ornatus, Newberry \& Worthen, 1866, Geo. Sur. Ill., vol. 2, p. 65, Keokuk Gr.

parallelus, St. John \& Worthen, 1875, Geo. Sur. Ill., vol. 6, p. 295, Waverly or Kinderhook Gr.

parvulus, St. John \& IVorthen, 1875, Geo. Sur. Ill., vol. 6, p. 309, St. Louis Gr.

plicatus, Newberry \& Worthen, 1866, Geo. Sur. Ill., vol. 2, p. 63, St. Louis Gr.

tuberculatus, Newberry \& Worthen, 1866 , Geo. Sur. Ill., vol. 2, p. 66, Burlington Gr. 
turgidus, St. John \& Worthen, 1875, Geo. Sur. Ill., vol. 6, p. 310, Kaskaskia Gr.

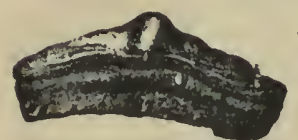

FIG. 1153,-Orodus variabilis. variabilis, Newberry, 1875, Ohio Pal., vol. 2, p. 50, Waverly Gr. variocostatus, St. John \& Worthen, 1875, Geo. Sur. Ill., vol. 6, p. 304, Burlington $\mathrm{Gr}$.

whitii, St. John \& Worthen, 1875, Geo. Sur. Ill., vol. 6, p. 297, Waverly or Kinderhook Gr.

Orthacantuus, Agassiz, 1843, Poiss. Foss., t. 3, p. 330, [Ety. orthos, straight; akantha, spine.] Spines straight or gently curved; two or more rows of denticles on the posterior face. Type O. cylindricus.

\section{Fra. 1154-Orthacanthus graclls.}

arcuatus, Newberry, 1857, (Pleuracantlus arcuatus,) Proc. Acad. Nat. Sci. Phil. p. - , and Ohio Pal., vol. 1, p. 332, Coal Meas.

gracilis, Newberry, 1875, Ohio Pal., vol. 2, p. 56, Coal Meas.

quadriseriatus, Cope, 1877, Pal. Bull. No. 26, in Proc. Am. Phil. Soc., vol. 17, p. 192, Permian.

Ortiopleurodus, St. John \& Worthen, 1883, Geo. Sur. IIl., vol. 7, p. 190. [Ety. orthos, straight; pleuron, side ; odous, tooth; in allusion to the straight postero-lateral border of the maxillary posterior tooth.] Posterior teeth of upper jaw subspatulate in outline; postero-lateral border straight, or nearly so, and probably gently curved downward and inward at the outer extremity, and gently arched in the same direction, terminating posteriorly in an acute angle or spur, whence the inner margin, which is greatly thickened or massive, is broadly rounded into and merges with the thin antero-lateral border toward the extremity; coronal surface occupied by a prominent principal fold or ridge rising nearest the straight border, and flanked on the anterior slope by an obscure secondary ridge; the punctate enamel forms a narrow fold along the thickened straight border; teeth supposed to bave occupied a similar position on the mandibles, distinguished by their trigonal outline, somewhat strong and spiral inrollment of the extremity, toward which the antero and postero-lateral borders regularly converge, inner margin more or less obliquely rounded, and sigmoidally curved from front toward the posterior angle; coronal surface pre- senting a more or less well-defined plane; anterior fold, abruptly broken down on that side, where the coronal enamel forms a wide belt sharply defined from the deep basal rim, and limited behind by the more or less deep longitudinal depression from which rises the alate posterior lobe, which is limited exteriorly by a narrow fold of enamel separating the crown from the basal portion of the tooth; mandibular median or second teeth characterized by their triangular outline, rather strong inrollment of the outer extremity ; straight postero-lateral border, which is similarly enameled to the antero-lateral border of last above described posterior dental plates; anterolateral border rapidly and irregularly converging from the subacute angle of the broad, slightly arched inner margin; coronal surface forming a broad, low arch, or nearly plane transversely. Type 0 . carbonarius.

carbonarius, Newberry \& Wortlien, 1866, (Sandalodus carbonarius,) Geo. Sur. Ill., vol. 2, p. 104, Up. Coal Meas.

convexus, St. Jolin \& Worthen, 1883, Geo. Sur. Ill., vol. 7, p. 193, Coal Meas.

novomexicanus, St. John \& VVorthen, 1883, Geo. Sur. Ill., vol. 7, p. 195, Subcarboniferous.

Paldisisis, Claypole, 1885, Quar. Jour. Geo. Soc. Lond. [Ety. palaios, ancient; aspis, shield.] Plates or. scutes oruamented. Only single dorsal plates discovered. Type $P$. americana.

americana, Claypole, 1885, Quar. Jour. Geo. Soc. Lond., Up. Silurian or Low. Devonian.

truncata, Claypole, 1885, Quar. Jour. Geo. Soc. Lond., Up, Silurian or Low. Devonian.

Palmonatis, Leidy, 1856, Trans. Am. Phil. Soc., vol. 11, p. 87. [Ety. palaios, ancient; hatis, a prickly kind of roach or ray.] 'Type $\mathrm{P}$. insignis.

insignis, Ltidy, 1856, 'Trans. Am. Phil. Soc., vol. 11, p. 87, Keoliuk Gr.

Palieoniscus, Agassiz, 1833, Recherches sur les Poissons Fossiles, t. 1, p. 4. [Ety. palaios, ancient ; oniscus, a wood-louse.] Small, fusiform, deep between ventral and pectoral fins; tail heterocercal, forked, upper lobe longer and narrower than lower; fins small; jaws large; teeth minute; scales rhomboidal, smooth or striated. Type P. fultus. alberti, see Rhadinichthys alberti.

brainerdi, Thomas, 1853, Bost Soc. Nat. Hist., vol. 4, Ohio Pal., vol. 1, p. 346, Bereu grit.

browni, Jackson, 1851, Rep. on Albert Coal Mine, Coal Meas.

cairnesi, see Rbadinichthys cairnesi. gracilis, Newberry \& Worthen, 1870, Geo. Sur. Ill., vol. 4, p. 347, Coal Meas. jacksoni, Dawson, 1877, Can. Nat. Quar. Jour. Sci., vol. 8, Carboniferous. 
leidyanus, Lea, 1853, Jour. Acad. Nat. Sı:i., 2d ser., vol. 2, Coal Meas. modulus, see Rhadinichthys modulus. peltigerus, Newberry, 1857, (Elonichthys peltigerus,) Proc. Acad. Nat. Sci., vol. 8, p. 98, and Ohio Pal., vol. 1, p. 345, Coal Meas.

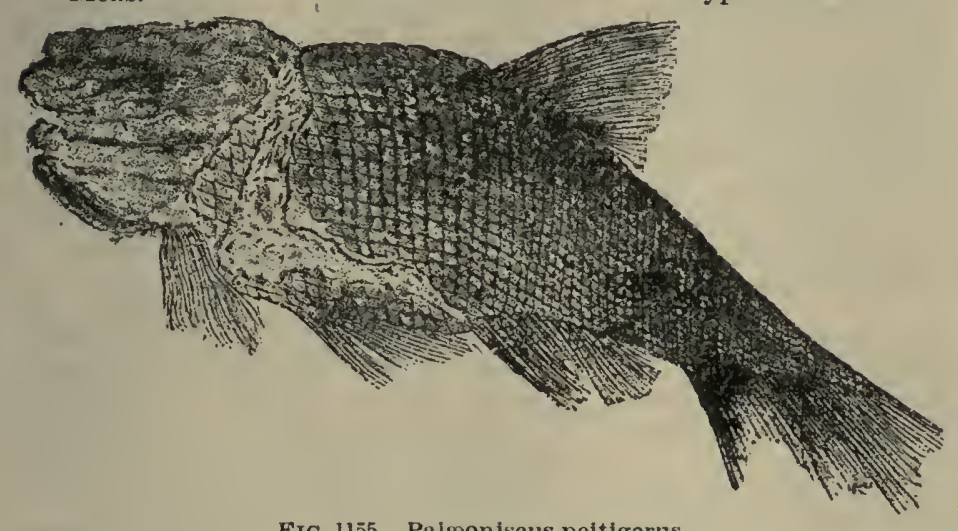

FIG. 1155.-Paireoniscus peitigerus. Type P. warreni.

consisting of transverse, strong, median cusps, flanked by denticles; one on either side, and then regularly increase in size from the outer to the inner extremity or with age; coronal cusps enameled, smooth or vertically striated.

compressus, St. John \& Worthen, 1875, Geo. Sur. Ill. vol. 6, p. 326, St. Louis Gr.

exp a n s us, St. John \& Worthen, 1875, Geo. Sur. Ill., vol. 6, p. 327, Kaskaskia Gr.

warreni, St. John \& W orthen, 1875, Geo. Sur. IIl., vol. 6 , p. 325 , Burlington Gr.

Peripristis, Agassiz, 1870, Proc.

scutigerus, Newberry, 1857, Proc. Acad. Nat. Sci. Phil., Coal Meas.

Peltonus, Newberry \& Wortien, 1870, Geo. Sur. Ill., vol. 4, p. 362. [Ety. pelte, a half-moon shield; odous, tooth.] Teeth small and low, round oval or elliptical in outline, arched above in both directions, concave or flattened below; crown surface most strongly arched from front to rear, highest near the anterior margin; more or less evenly punctate throughout; under surface bony and rough; margins thin and irregular where the teeth are separated, thickened and even along the lines of contact when closely set. They are less flat, smooth and pavernent-like than Psammodus, and less convoluted than Cochliodus. Type P. unguiformis. plicomphalus, St. John \& Worthen, 1875, Geo. Sur. Ill., vol. 6, p. 411, Kaskaskia Gr.

quadratus, St. John \& Worthen, 1875, Geo. Sur. Ill., vol, 6, p. 410, St. Louis Gr. transversus, St. John \& Worthen. 1875, Geo. Sur. Ill., vol. 6, p. 412, Coal Meas. unguiformis, Newberry \& Worthen, 1870 , Geo. Sur. Ill., vol. 4, p. 363, Coal Meas. Peplorima, Cope, 1873, Proc. Acad. Nat. Sci. Phil., p. 343. [Ety. peplos, a robe; Rhine, a kind of cog-fish.] Type P. anthracina.

anthracina, Cope, 1873, Proc. Acad. Nat. Sci. Phil., p. 343, Coal Meas.

arctata, Cope, 1877, Proc. Am. Phil. Soc., p. 55, Permian Gr.

Periplectrodus, St. John \& Worthen, 1875, Geo. Sur. Il]., vol. 6, p. 324. [Ety. peri, near by; Plectrodus, a genus.] Base expanded laterally or compressed; symmetrically inrolled from within outward, inferior surface excavated ; crown
Am. Phil. Soc., vol. 11, p. 434. [Ety. peri, around; pristis, saw.] Small, crown compressed, acuminate, serrate, curved laterally; coronal cavity; root as in Petalodus, crown and coronal cavity". covered with ganoine. Type P. semicircularis.

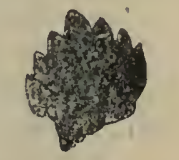

semicircularis, Newberry FIG. 1156.-Peri\& Worthen, 1866, (Cten- pristis semiciroptychius semicircu- cularis.

laris,) Geo. Sur. Ill., vol, 2, p. 72, Coal Meas.

Pexalodus, Owen 1840, Odontography, p. 60. [Ety. petalos, spread out; odous, tooth.] Teeth transversely elongated, compressed, thin, petal-shaped, cutting edge serrated; base of crown with imbricating folds of enamel, descending lower on the posterior than anterior face; root large, oblong, truncated be-

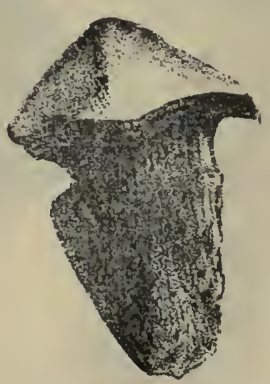

Fig. 1157.-Petalodus alleghaniensis. low; lower edge obtuse, tumid. Type P. hastingsi.

alleghaniensis, Leidy, 1856, Jour. Acad. Nat. Sci., 2d series, vol. 3 , p. 161, and Geo. Sur. Ill., vol. 2, p. 35, Coal Meas. curtus, Newberry \& Worthen, 1866,Geo. Sur. Ill., vol. 6, p. 355, Keokuk Gr. destructor, Newberry \& Worthen, 1866, syn. for $P$. alleghaniensis.

hybridus, St. John \& Worthen, 1875, Geo. Sur. Ill., vol. 6, p. 394, St. Louis Gr. 
linguifer, Newberry \& Worthen, 1856, Geo.Sur. III, vol. 2, p. 37, Kaskaskia Gr. proximus, St. John \& Worthen, 1875, Geo. Sur. Ill., vol. 6, p. 395, Coal Meas. Petalorityciuss, Agassiz, 1855, in British Pal. Rocks. [Ety. petalos, spread out; rhynchos, a beak.] Teeth small, crown compressed, thin, concavo-convex, petal-shaped; higher and narrower than Petalodus; imbricating folds on posterior face forming a short transverse band, not extending to the lateral angles of the crown; root long, undivided. Type P. sagittatum.

distortum, St. John \& Worthen, 1s75, (ieo. Sur. Ill., vol. 6, p. 406, St. Louis Gr. pseudosagittatum, St. John \& Worthen, 1875, Geo. Sur. Ill., vol. 6, p. 405 , St. Louis Gr.

spatulatum, St. John \& Worthen, 1875, Geo. Sur. Ill., vol. 6, p. 408, St. Louis Gr. striatum, Newberry \& Worthen, 1866, Geo. Sur. Ill., vol. 2, p. 40, Burling. ton Gr.

Petrodus, MicCoy, 1848, Ann. and Mag. Nat. Hist., $2 \mathrm{~d}$ series, vol 2, p. 132 . [Ety. petros, a rock; odous, a tooth.] Conical; base round or subtrigonal; apex rudely pointed; sides radiatingly ridged; osseous base wider than the crown. Type P. patelliformis.

acutus, Newberry \& Worthen, 1866, Geo. Sur. Ill., vol. 2, p. 72, Coal Meas.

occidentalis, Newberry \& Worthen, 1866, Geo. Sur. Ill., vol. 2, p. 70, Coal Meas.

pustulosus, Newberry \& Worthen, 18\%0, Geo. Sur. Ill., vol. 4, p. 369, Burlington $G r$.

Piranemoplanuron, Huxley, 1871, 10th Decade Geo. Sur. of Gt. Britain. [Ety. phaneros, open ; pleuron, side.]

curtum, Whiteaves, 1881, Am. Jour. Sei. and Arts, 3d ser., vol. 21, p. 495, Upper Devonian.

Punebodus, St. John \& Worthen, 1875, Geo. Sur. Ill., vol. 6, p. 251. [Ety. mythological name; odous, a tooth.] Teeth small; base irregularly elliptical, strongly produced in front and faintly excavated at the median line; the antero-inferior angles approximate, and laterally curve to the rounded extremities; broadly though irregularly rounded behind; the angles in front are occupied by a strong, lateral, pad-like prominence, which is more or less distinctly bilobed and beveled to the deeply excavated inferior surface ; posterior margin slightly burled; postero-superior surface moderately convex, and surmounted by a laterally elongated, well-defined prominence, which is situated nearly mid. way between the base of the crown and the posterior border, to which the surface abruptly slopes, and extending laterally nearly half the diameter of the base; the coronal region consists of three strong cusps, of which the exterior pair are largest, strongly diverging and molerately recurved or nearly vertical, antern-posteriorly compressed or suboval in section, apparently without distinct cutting edges; median cone similar in shape, erect, more or less produced in front and continued to the shallow median depression in the border; a rudimentary denticle between the median and lateral cusps. Fra. 1158.-1'lsœType P. sophiar.
Ty bodus sophlise.

sophne, St. John \& Worthen, 1875, Geo. Sur. 1ll., vol. 6, p. 251, Devonian.

Pursosemus, Agassiz, 1843, Recherches sur les Poissons Fossiles, t. 3, p. 176. [Wity. physa, hladder; nema, thread.] Dorsal spine strong, laterally compressed, deeply imbeded, curved; apex directed toward the front; lateral faces bearing costee and tubercles; pulp eavity large; base notched. Type P. subteres.

altonensis, St. John \& Worthen, 1875, (ieo. Sur. Ill., vol. 6, p. 454, St. Louis Gr.

carinatus, St. John \& Worthen, 1875, Geo. Sur. Ill, vol. 6, p. 452, Waverly or Kinderhook (ir.

chesterensis, St. Jolın \& Worthen, 1875, Geo. Sur. Ill., vol. 6 , p. 455 , Kaskaskia Gr.

depressus, St. John \& Worthen, 1875, Geo. Sur. Ill., vol. 6 , p. 452 , Waverly or Kinderhook Gr.

falcatus, St. John \& Worthen, 1883, Geo. Sur. Ill., vol. 7, p. 252, St. Louis Gr.

gigas, Newberry \& Worthen, 1870, Geo. Sur. Ill., vol. 4, p. 373, Burlington Gr.

parvulus, St. John \& Worthen, 1875, Geo. Sur. Ill., vol. 6, p. 453, Keokuk Gr.

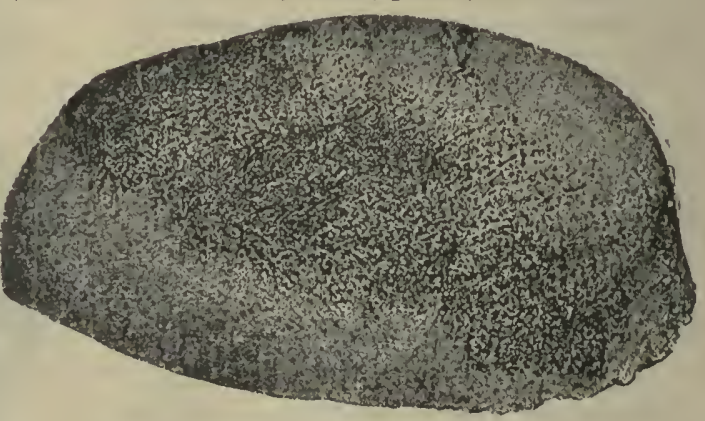

Frg. 1159.-Platyodns 11 neatus. Crown surface.

proclivis, St. Jolın \& Worthen, 1875, Geo. Sur. Ill., vol. 6, p. 451, Waverly or Kinderhook Gr.

Platyodus, Newberry, 1875, Ohio Pal., vol. 2, p. 58. [15ty. platys, broad; odous, tooth.] Teeth elliptical in outline; crown arched in both directions; sur- 
face punctate in undulate lines, but without folds or ridges. Type P. lineatus.

lineatus, Newberry, 1875, Ohio Pal., vol. 2, p. 58, Waverly G'r.

P'latysomus, Agassiz, 1833, Recherches sur les Poissons Fossiles, t. 1, \%. 6. [Ety. platys, broad; sóma. body.] Rhomboidal, compressed; dorsal and anal fins nearly equal, opposite; pectorals small; ventrals small; teetl clavate; crown dilated, fluttened; base slender, constricted at the base of the ganoine; scales large, oblong, articular internal ridge at anterior edge; beveled spine at the upper corner received in a notch of the adjoining scale. Type $P$. striatus.

circularis, Newberry \& Worthen, 1870 , Geo. Sur. Ill., vol. 4, p. 347, Coal Meas.

Pleuracanthus, Agassiz, 1843, Poiss. Foss., vol. 3, p. 66. [Ety. pleura, side; akantha, spine.] The genus was founded upon a spine supposed to belong to the Order Raiina. It is serrated on one edge, curved at the base, and furrowed on the inferior side. The species named in this genus from America are too poorly defined to warrant recognition. 'Type P. lævissimus.

arcuatus, see Orthacanthus arcuatns.

biserialis, Newberry, 1857, Proc. Acad. Nat. Sci., vol. 8, p. 100, Coal Meas.

dilatatus, Newberry, 1857, Proc. Acad. Nat. Sci., vol. 8 , p. 100 , Coal Meas.

Pnigeacantiuus, St. John \& IVorthen, 1875. Geo. Sur. Ill., vol. 6, p. 480 . [Ety. from the specific name in Oracanthus pnigeus; akantha, spine.] Spine short, conical, laterally compressed; base broadly expanded before and behind, without insertion, rapidly tapering to the obtuse apex, which is directed posteriorly ; transverse section elliptical, rounded into the slight!y sigmoidally curved anterior border and concave posterior margin; pulp cavity very large, extending nearly to the tip ; lateral walls very thin, slightly thickened in the margins; external surface occupied by irregularly disposed, radiatingly sculptured tubercles, sometimes arranged in obscure or interrupted longitudinal and diagonal order. The type is Oracanthus phigeus, of Newberry \& Worthen, which St. John named Pnigeacanthus deltoides.

deltoides, St. John \& Worthen, 1875, Geo. Sur. Ill., vol. 6, p. 480 , Keokuk Gr. But why should this species not be Pnigeacanthus pnigeus?

trigonalis, St. John \& Worthen, 1883, Geo. Sur. Ill., vol. 7, p. 259, St. Louis Gr.

Precilodus, Agassiz, 1843, Recherches Poiss. Foss., vol. 3, p. 174. [Ety. poikilos, variegated; odous, tooth.] Teeth as in Cochliodus; terminal tooth obliquely trigonal, convoluted; median tootb nar-

row, convoluted; all teetl wrinkled at right angles to the articular edges; surface porous. Type P. jonesi.

carbonarius, St. John \& Worthen, 1883, Geo. Sur. Ill., vol. 7, p. 139, Coal Meas.

cestriensis, St. Jolun \& Worthen, 1883, Geo. Sur. Ill., vol. 7, p. 135, Kaskaskia Gr.

ornatus, Newberry \& Worthen, 1866, Geo. Sur. Ill., vol. 2, p. 95, syn. for Chitonodus rugosus.

rugosus, see Chitonodus rugosus.

springeri, St. John \& Worthen, 1883, Geo.

Sur. Ill., vol. 7, p. 138, Subcarboniferous.

stludovici, St. John \& Worthen, 1883, Geo. Sur. Ill., vol. 7, p. 132, St. Louis Gr.

varsoviensis, St. John \& Worthen, 1883, Geo. Sur. Ill., vol. 7, p. 131. Warsaw Gir.

wortheni, St. John, 1883, Geo. Sur. Ill., vol. 7, p. 136, Kaskaskia Gr.

Polyrhizonus, MeCoy, 1848, Ann. and Mag. Nat. Hist., 2d series, vol. 2, p. 125. [Ety. polys, many; rhiza, root; odous, tooth.] Crown like Petalodus, but more elongated, transversely lower and thicker; root divided into numerous short, robust radicles. Type $\mathrm{P}$. magnus.

amplus, St. John \& Worthen, 1875, Geo. Sur. Ill., vol. 6, p. 387, St. Louis Gr.

carbonarius, St. John \& Worthen, 1875 , Geo. Sur. Ill., vol. 6, p. 389, Coal Meas.

dentatus, Newberry \& Worthen, 1866, Geo. Sur. Ill., vol. 2, p. 50, Kaskaskia Gr.

littoni, Newberry \& Worthen, 1870, Geo. Sur. Ill., vol. 4, p. 357 , St. Louis Gr. modestus, Newb e r r y, 1875 , Ohio Pal., vol. 2, p. 50, Cleve- Fia. 1160.-Polyrhizodus lltland shale. nanus, St. John \& Worthen, 1875, Greo.

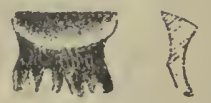

Fig. 1161.-Polyrislzodus modestus.

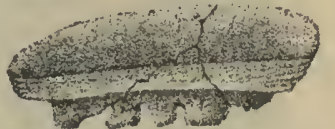
toni. Concave face, Sir. III.; vol. 6, p. 386, Keokuk Gr. piasensis, St. John \& Worthe $\mathrm{n}, 1875$, Geo. Sur. Ill., vol. 6 , p. 386 , Warsaw Gr.

ponticulus, Newberry \& Worthen, 1866, Geo. Sur. Ill., vol. 2, p. 51, Kaskaskia Gr.

porosus, Newberry \& Worthen, 1866 , Geo. Sur. Ill., vol. 2, p. 49, Burlington Gr.

truncatus, Newberry \& Worthen, 1870, Geo. Sur. Ill., vol. 4, p. 357, Burlington $\mathrm{Gr}$.

williamsi, St. John \& Worthen, 1875, Geo. Sur. Ill., vol. 6, p. 384, Keokuk Gr. 
Pristicladodus, McCoy, 1855, British Pal. Rocks, p. 642. [Ety. from the two genera Pristis and Cladodus.] Teeth re-

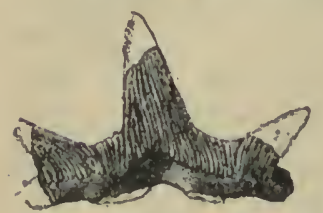

Fug. 1162.-Pristicladodus springeri. se m ble Cladod u s; median and latera l cusps strong; la teral edges sharp and more or less undulated. Type P. dentatus.

springeri, St. John \& WVorthen, 1875, Geo. Sur. Ill., vol. 6, p. 255, Waverly or Kinderhook Gr.

Pristonus, Agassiz. [Fty. pristis, a saw; odous, a tooth.] This genus has been only doubtfully identified in America.

(?) acuminatus, St. John \& Worthen, 1875, Geo. Sur. IIl., vol. 6, p. 402, Waverly or Kinderhook Gr.

Psammonus, Agassiz, 1843, Recherches sur les Poissons Fossiles, t. 3, p. 112. [Ety. psammos, sand; odous, tootb.] 'Teeth quadrilateral or trapezoidal in general outline, variable, usually thick and massive; the coronal region presents a more or less plane surface, according to the position the form occupied upon the jaws, always arched, generally moderately in the longitudinal direction or from behind forward, transversely concave (maxillary teeth), or more or less convex (mandibular teeth), sometimes raised into a low ridge along the exterior lateral border, also along the articular inner border, or showing a more or less wide convexity in the latter region, and sometimes presenting a more or less well-defined transverse prominence in mature maxillary form; the marginal limits of the crown are well defined, rounded along the exterior of lateral border, and usually inbeveled, and almost always making an angulation at the articular inner border and along the anterior and posterior margins, the enamel extending well down, and more or less distinctly defined from the coarse, vermicularly pitted base which constitutes the greater part of the height of the tooth; in front and behind, the basal wall is nearly exactly vertical to the plane of the coronal surface, and moderately channeled or concave; the inner articular face is also vertical and slightly excavated, presenting generally at one or the other extremity an obliquely truncated articular facet for co-adaptation with the contiguous tooth of the opposite series, the extent and obliquity of the truncation varying greatly according to the species; the exterior lateral border, in typical forms, shows an expansion of the basal portion beyond the coronal limits, increasing in breadth and terminating in a more or less produced spur at the postero-outer angle of the tooth; the coronal surface exhibits under an ordinary lens a distinct, vertical, prismatic structure, each of the vertical columns inclosing a medullary tube, the appearance of which at the surface produces the exceedingly minute punctation usually observed in these teetli; the exceedingly elegant vermiculose rugosity exhibited in the less worn surfaces of certain species is produced by the wrinkling of the enamel or external layer, and which apparently has no other relation to the medullary tubes than to rudely define them in irregular and transverse or longitudinal rows,
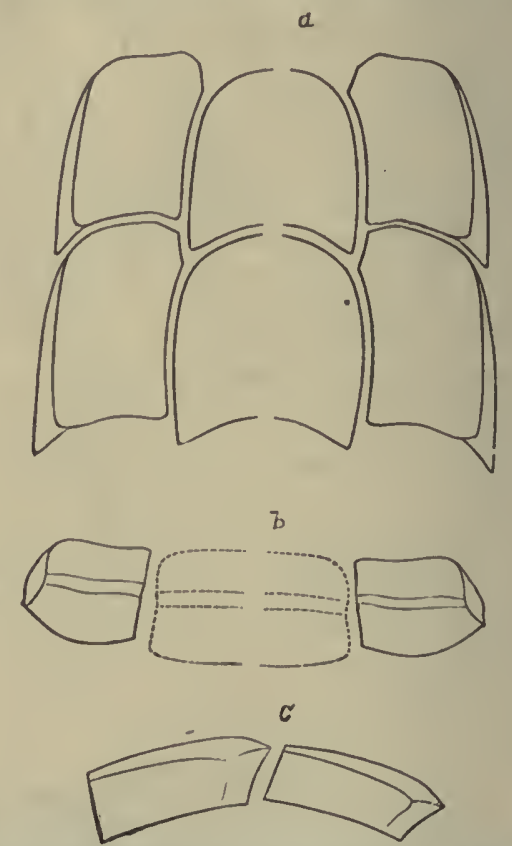

Fro. 1163.-Psammodus crassidens, $a$, Median dental plates; $b$, transverse profle; $c$, lougitu. dinal profile.

the punctre rarely confluent, and the rugose appearance becoming obsolete or more or less obscured over the more exposed parts of the triturating surface; the impression also prevails that the tendency to rugosity of the coronal surface increases with age, since this appearance, so far as observed, seems to be most prevalent and conspicuous in large individuals belonging to the series which liave received several accessions, the innermost individuals of which have suffered little from the abrading effects of trituration while in use; but it is not an essential character, as some species evidently always remained quite smooth in their coronal areas; the 
inierior surface is plane, in a general way conforming to that of the crown, and even possessing distinctive characteristics as applied to species; it shows in the perfect state a rather dense, thin layer, perhaps in degree rather than structurally differing from the more cellulose middle layer composing the bulk of the base, and usually marked by more or less distinct longitudinal grooves, or smooth and faintly keeled nearest the inner articular border. Type P. porosus.

angularis, Newberry \& Worthen, 1866, Geo. Sur. Ill., vol. 2, p. 107, Kaskaskia Gr.

antiquus, Newberry, 1857, Bull. Nat. Inst., Up. Held. Grr.

bretonensis, Whiteaves, 1881, Can. Nat., vol. 10, Carboniferous.

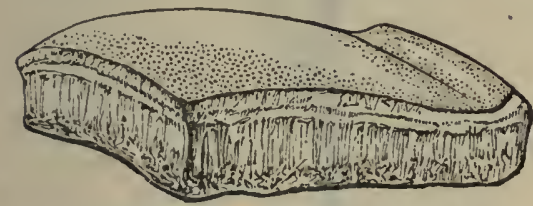

FIG. $1161-$ Psammodus porosus.

cælatus, St. John \& Worthen, 1883, Geo. Sur. Ill., vol. 7, p. 217, St. Louis Gr. crassidens, St. John \& Worthen, 1883, Geo. Sur. Ill., vol. 7, p. 218, St. Louis Gr.

glyptus, St. John \& Worthen, 1883, Geo. Sur. Ill., vol. 7, p, 209, Up. Burlington $\mathrm{Gr}$.
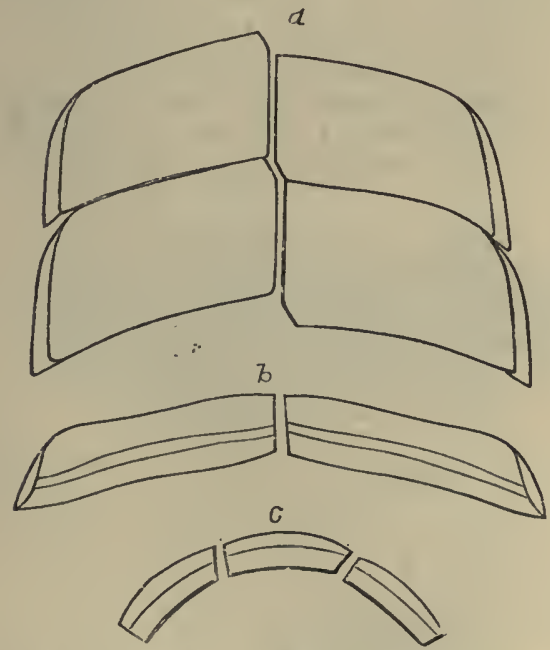

FIG. 1165-Diagram of Psammodus springeri. $a$, Mandibuiar series; $b$, trausverse profile; $c$ longitudinal profle.

grandis, St. John \& Worthen, 1883, Geo. Sur. Ill., vol. 7, p. 211, Keokuk Gr. lovianus, St. John \& Worthen, 1883, Geo. Sur. Ill., vol. 7, p. 207, Burlington Gr. plenus, St. John \& Worthen, 1883, Geo. Sur. Ill., vol. 7, p. 213, St. Louis Gr.

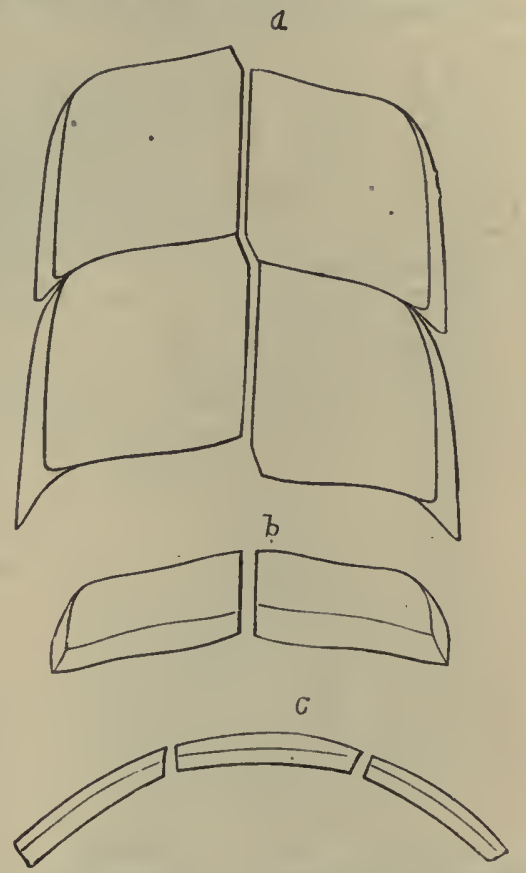

FIG. 1166.-Diagram of Psammodus springeri. $a$, Maxillary series; $b$, transverse proflle; $c$, longitudinal profite.

porosus, Agassiz, 1843, Recherch. Poiss. Foss., t. 3, p. 112, Kaskaskia Gr.

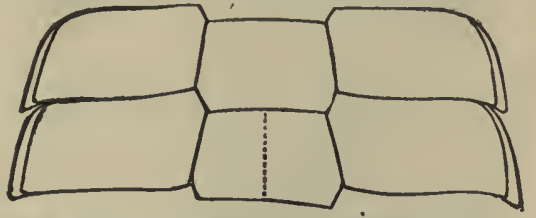

FiG. 1167.-Hypotiletical diagram, showing median dentai plates of Psammodus springeri.

reticulatus, Newberry \& Worthen, 1866, Geo. Sur. Ill., vol. 2, p. 109, Kaskaskia Gr.

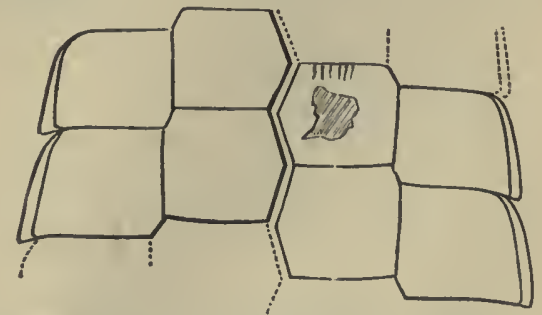

FIG. 1163.-Hypotheticai diagram of Psammodus turgidus.

rhomboideus, Newberry \& Worthen, 1866, Geo. Sur. Ill., vol. 2, p. 110, syn. for Sandalodus lævissimus. 
semicylindricus, Newberry \& Worthen, 1866, Geo. Sur. 11l., vol. 2, p. 109, syn. for Sandalodus lrevissimus.

springeri, St. John \& Worthen, 1883, Geo. Sur. Ill., vol. 7, p. 202, Upper Burlington $\mathrm{Gr}$.

tumidus, St. John \& Worthen, 1883, Geo. Sur. Ill., vol. 7, p. 205, Up. Burlington $\mathrm{Gr}$.

turgidus, St. John \& Worthen, 1883, Geo. Sur. Ill., vol. 7, p. 206, Keokuk Gr.

Psepilonus, Agassiz, 1862, Quar. Jour. Geo. Soc., vol. :18, p. 102. [Ety. psephos, a pebble; odous, a tooth.] II eavy, more or less spirally inrolled triturating or crushing plates invest the median range of the rami of the jaws; they are trapezoidal in ontline, with undulated articular surfaces. Type P. magnus.

convolutus, Newberry \& Worthen, 1866, (Aspidodus convolutns,) Geo. Sur. Ill., vol. 2, p. 94, Kaskaskia Gr.

crenulatus, Newberry \& Worthen, 1866, (Aspidodus crennlatus,) Geo. Sur. Ill., vol. 2, p. 93, Kaskaskia Gr.

cunulatus, sce P. luvulatus.

latus, St. John \& Worthen, 1883, Geo. Sur. Ill., vol. 7, p. 72, St. Louis Gr.

lunulatus, St. John of Worthen, 1883, Geo. Sur. IIl., vol. 7, p. 74, (misprinted cunulatus,) Kaskaskia (ir.

obliquus, St. John \& Worthen, 1883, Geo. Sur. Ill., vol. 7 , y. 66 . Waverly or Kinderkook Gr.

placenta, Newberry \& Worthen, 1866, (Helodus placenta,) Geo. Sur. Ill., vol. 2 , p. 80 , Waverly or Kinderkook Gr.

reticulatus, St. John \& Worthen, 1875, Geo. Sur. 11l., vol. 6, p. 417, Waverly or Kinderhook Gr.

symmetricus, St. John \& Worthen, 1883, Geo. Sur. Ill., vol. 7, p. 71 , Waverly or Kinderhook Gr.

Ptericirthys, Agassiz, 1835, Recherches sur les Poissons Fossiles, t. 1, p. 302. [Ety. pteron, fin; ichthys, fish.] The outline of this genus reminded Hugh Miller of the figure of a man rudely drawn in black on a gray ground, the head cut off at the shoulders, the arms spread at full as in the attitude of swimming, the body rather long than otherwise, and narrowing from the chest downward, one of the legs cut away at the hip-joint, and the other as if to preserve the balance, placed directly under the center of the figure, which it seems to support. The under part of the body was flat, the upper rose toward the center into a roof-like ridge, and both under and upper were covered with a strong armor of plates; the plates on the under side are divided by a longitudinal suture and a transverse suture, and they would cut at right angles were it not for a lozenge-shaped plate in the center; there are therefore five plates on the under side, all of which are thickly tuberculated; the upper side is covered with a large, long, hexagonal plate in the central part, that is surrounded by a row of unequal and variously formed plates, all of which are strongly tuberculated; the ceplualic shield is rounded in front, and truncated behind where it joins the body carapace, having a transverse median opening; nuchal region occupied by a plate soniewhat like the lateral view of a

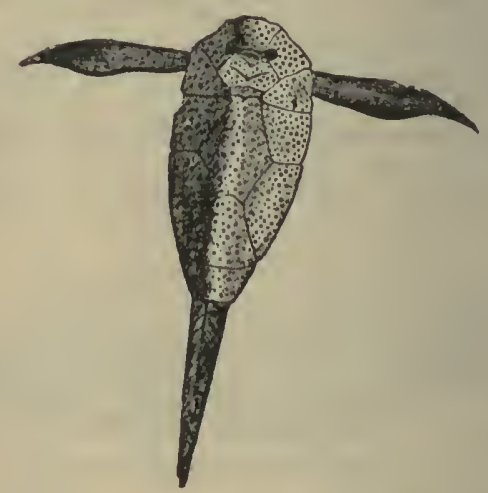

Fia. 1169.-Pterichthys millerf.

coronet or crown; one post-median plate, another in front, one lateral occipital on each side, two lateral and one postero-lateral on each side, and an angular plate on each posterolateral side articulating with the limb; the oblong carapace is covered by the large, hexagonal anteromedian plate, and a smaller posterior median dorsal, and two dorso-lateral plates on each side; tail thick, conical, covered with rhomboidal scales; surface covered with granules. Type P. milleri.

canadensis, see Bothriolepis canadensis.

norwoodensis, Owen, syn. for Macropetalichthys rapheidolabis.

rugosus, Claypole, 1883, Proc. Am. Phil. Soc., p. 664, Upper Chemung (xr.

Pryctodus, Pander Uber die Ctenodipterinen des. Devonischens Systems, p. 48. [Ety. ptyktos, folded; odous, tootb.] Elongated; base expanded, subconical ; crown flattened or furrowed; enameled; tubes in transverse furrows, with low intervening ridges. Type $P$. obliquus.

calceolus, Newberry \& Worthen, 1866, (Rinodus calceolus,) Geo. Sur. Ill., vol. 2, p. 106, Ham. Gr.

Ptyonodus, Cope, 1877, Proc. Am. Phil. Soc., p. 192. [Ety. ptyon, a fan; odous, a tooth.]

paucicristatus, Cope, 1877, Proc. Am. Phil. Soc., p. 54, Permian.

vinslovi, Cope, 1877, Proc. Acad. Nat. Sci. Phil., p. 410 , Permian. 
Pygopterus, Agassiz, 1833, Poiss. Foss., t. 1, p. 10. [Ety. pyge, rump ; pteron, fin.] Body large, elongate, ovate; fins large,

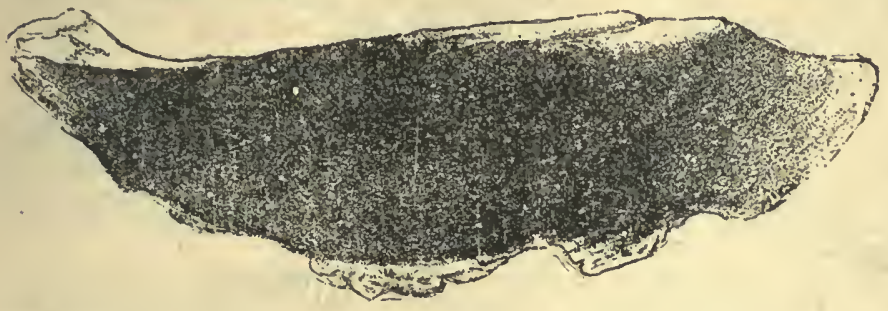

FIG. 1170.-Ptyctodus caiceolus. Side view.

in each dental bone, and numerous smaller ones; scales large, rotundatoquadrate, thin, inner surface concentrically lined; outer surface tuberculate. Type R. hibberti.

angustus, Newberry, 1857, Proc. Acad. Nat. Sci. Phil., vol. 8, p. 99, Coal Meas. Poorly defined.

hardingi, Dawson, 1868, Acad. Geol., p. 254, Subcarboniferous.

with fulcral scales; anal fin long, nearly opposite dorsal; ventrals small; pectorals small, falcate ; caudal large, notched; upper jaw longer than the lower; endoskeleton strong; scales small, rhomboidal. Type P. humboldti. Not definitely known from America.

scutellatus, Newberry, 1857, Proc. Acad. Nat. Sci., vol. 8, p. 98, Coal Meas. Too poorly defined to warrant recognition.
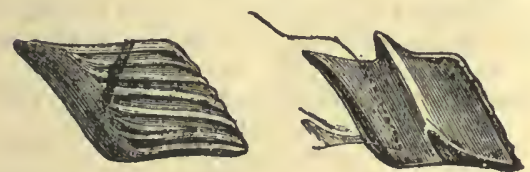

FIG. 117i.-Pygopteris mandibularis, Outside and under surface of scale magnlfied.

Rhadinicirtrys, Traquair, 1877, Quar. Jour. Geo. Soc. Lond., vol. 33, p. 548. [Ety. rhddinos, slender; ichthys, fish.] Body slender; jaws with a row of incurved laniaries, outside of which there are smaller teeth ; principal rays of pectoral fin as in Pygopterus; dorsal far back, nearly opposite the anal. Type $R$. ornatissimus.

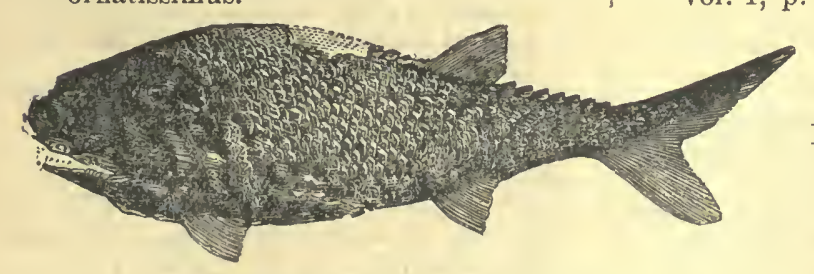

FIG. 1172.-Rhadinichthys aiberti.

alberti, Jackson, 1851, (Palæoniscus alberti,) Rep. on the Albert Coal-mine, New Brunswick, Coal Meas.

cairnsi, Jackson, 1851, (Palæoniscus cairnsi,) Rep. on Albert Coal-mine, New Brunswick, Coal Meas.

modulus, Dawson, 1877, (Palæoniscus modulus,) Can. Nat. and Quar. Jour. Sci., vol. 8, Carboniferous.

RHzzodus, Owen, 1840, Odontography. [Ety. rhiza, a root; odous, tooth.] Jaws massive, bearing large, compressed, double-edged teeth, with sulcated bases incurvus, Newberry, 1857, Proc. Acad. Nat. Sci., vol. 8, p. 99, Coal Meas. Poorly defined.

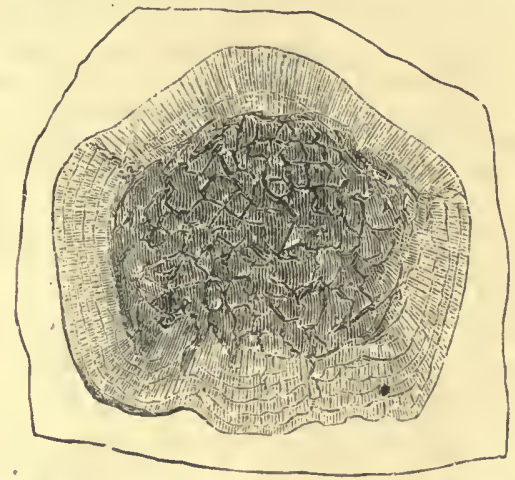

F'IG. 1173.-Rhizodus occidentalis. Seale.

lancifer, Newberry, 1857, Proc. Acad. Nat. Sci. Phil., vol. 8, p. 99, and Ohio Pal., vol. 1, p. 342 , Coal Meas.

occidentalis, Newberry \& Worthen, 1866, Geo. Sur. Ill., vol. 2, p. 19, Coal Meas. quadratus, Newberry, 1873, Ohio Pal., vol. 1, p. 343. Coal Meas.

reticulatus, Newberry \& Worthen, 1870, Geo. Sur. Ill., vol. 4, p. 349 , Coal Meas.

Rhyncirodus, Newberry, 1873, Ohio Pal., vol. 1, p. 307 . [Ety. rhynchos, beak ; odous, tooth.] Teeth somewhat half-circular, compressed, exterior margins curved; one cornua produced, the other obtuse; straight side the triturating or cutting edge. Type $R$. secans.

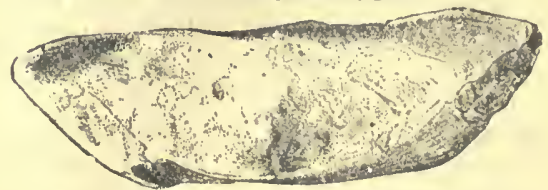

FIG. 1174.-Rhynchodus frangens.

crassus, Newberry, 1873, Ohio Pal., vol. 1, p. 312, Up. Held. Gr. 
excavatus, Newberry, 1877, Geo. of Wis., vol. 2, p. 397 , Ham. Gr.

frangens, Newberry, 1873, Ohio Pal., vol. 1, p. 311, Up. Held. Gr.

secans, Newberry, 1873, Ohio Pal., vol. 1, p. 310 , Up. Held. Gr.

Rinodus, Newberry \& Worthen, 1866, syn. for Ptyctodus.

calceolus, see Ptyctodus calceolus.

Saxdalonus, Newberry \& Worthen, 1866, Geo. Sur. Ill., vol. 2, p. 102. [Ety. sandalon, a sandal; odous, tooth.] Teeth thick, strong, subtriangular or clubshaped, with one or two pointed extremities; twisted and arched; base concave, surface punctate. Type S. parvulus.

angustus, Newberry \& Worthen, 1866, Geo. Sur. Ill., vol. 2, p. 103, Keokuk Gr. carbonarius, see Orthopleurodus carbonarius.

complanatus, Newberry \& Worthen, 1866, (Deltodus complanatus,) Geo. Sur. Ill., vol. 2, p. 98, Upper Burlington Gr.

crassus, Newberry \& Worthen, 1870, Geo. Sur. Ill., vol. 4, p. 369 , syn. for $\mathrm{S}$. spatulatus.

grandis, Newberry \& Worthen, 1866, Geo. Sur. 11l., vol. 2, p. 105, syn for S. lavissimus.

lævissimus, Newberry \& Worthen, 1866, Geo. Sur. Ill., vol. 2, p. 104, Keokuk Gr. minor, Newberry \& Worthen, 1866, ('Trigonodus minor,) Geo. Sur. Ill., vol. 2, p. 112, Keokuk Gr.

parvulus, Newberry \& Worthen, 1866, Geo. Sur. Ill., vol. 2, p. 102, St. Louis Gr. In part Stenopterodus parvulus. spatulatus, Newberry \& Worthen, 1866, Geo. Sur. Ill., vol. 2, p. 103, St. Louis Gr.

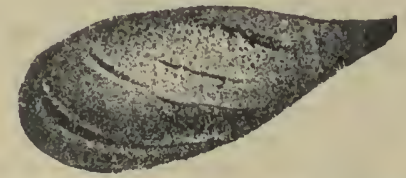

F19.1175.-Sandalodus sputulatus.

Sauripteris, Hall, 1843, Geo. Rep. 4th Dist. N. Y. [Ety. sauros, lizard; pleron, wing.] taylori, see Holoptycbius taylori.

Sicarius extinctus, Leidy, 1855, Proc. Acad. Nat. Sci., vol. 7. Not satisfactorily defined.

Stemmatodus, St. John \& Worthen, 1875, Geo. Sur. Ill., vol. 6, p. 328. [Ety. stemmatos, a wreath; odous, tooth.] Teeth variable, anomalous, some triangular with three or more rows of denticles, otbers simple with narrower base and a single row of coronal cusps. Type $\mathrm{S}$. chiriformis.

bicristatus, St. John \& Worthen, 1875, Geo. Sur. Ill., vol. 6, p. $33 \mathrm{~L}$, Burlington Gr.

bifurcatus, St. John \& Worthen, 1875, Geo. Sur. Ill., vol. 6, p. 330 , Burlington Gr. chiriformis, St. John \& Worthen, 1875, Geo. Sur. Ill., vol. 6, p. 330, Burlington $\mathrm{Gr}$.

compactus, St. John \& Worthen, 1875 , Geo. Sur. Ill., vol. 6, p. 334, Kaskaskia Gr.

keokuk, St. John \& Worthen, 1875, Geo. Sur. Ill., vol. 6, p. 334, Keokuk Gr.

simplex, St. John \& Worthen, 1875, Geo. Sur. Ill., vol. 6 , p. 332, Burlington Gr.

symmetricus, St. John \& Worthen, 1875, Geo. Sur. Ill., vol. 6, p. 333, Burlington Gr.

Stenacantiuus, Leidy, 1856, Jour. Acad. Nat. Sci., 2d ser., vol. 3, p. 162. [Ety. stenos, narrow; akantha, a spine.] Narrow denticulated spine. Type S. nitidus.

nitidus, Leidy, 1856, Jour. Acad. Nat. Sci., 2d ser., vol. 3. p. 162, Carboniferous.

Stexoptenodu, St. John \& Worthen, 1883, Geo. Sur. Ill., vol. 7, p. 100. [Ety. stenos, narrow; pleron, wing; odous, tooth.] Teeth distinguished by their long elliptical outline, strongly arched and spiral inrollment of the outer extremity. Crown with a lobe in the direction of inrollment. Type S. planus. elongatus, St. John \& Worthen, 1883, Geo. Sur. Ill., vol. 7, p. 106, Warsaw Gr.

parvulus, Newberry \& Worthen, 1866, (Sandalodus parvulus,) Geo. Sur. Ill., vol. 2, p. 102, St. Louis Gr.

planus, St. John \& Worthen, 1883, Geo. Sur. Ill., vol. 7, p. 102, Upper Burlington $\mathrm{Gr}$.

Strigillina, Cope, syn. for Janassa.

gurleiana, see Janassa gurleiana.

linguiformis, see Janassa linguiformis.

Trenonus, De Koninck, MSS., and St. John \& Worthen, 1883, Geo. Sur. Ill., vol. 7, p. 75. [Ety. txnia, ribbon; odous, tooth.] Trapezoidal, arched from within outward, inrolled obliquely outward and forward; distinguished from Psephodus by the differentiation of the coronal contour of the maxillary median forms. Type T. contortus.

fasciatus, Newberry \& Worthen, 1870 , (Deltodus fasciatus,) Geo. Sur. Ill., vol. 4 , p. 366 , Keokuk Gr.

obliquus, St. John \& Worthen, 1883, Geo. Sur. Ill., vol. 7, p. 78, Kaskaskia Gr.

regularis, St. John \& Worthen, 1883, Geo. Sur. Ill., vol. 7, p. 77, Warsaw Gr.

Tanaodus, St. John \& Worthen, 1875, Geo. Sur. Ill., vol. 6, p. 367. [Ety. tanaos, long; odous, a tooth.] In the laterally extended linear outline of the crown it resembles Chomatodus; in the disproportionate depth of the coronal surfaces and marginal position of the root it resembles Antliodus from which it is distinguished by the linear outline of the crown and the inferior surface of the root. Type $\mathrm{T}$. gracillimus.

angularis, Newberry \& Worthen, 1866, (Chomatodus angularis,) Geo. Sur. Ill., vol. 2, p. 55, Coal Meas. 
bellicinctus, St. John \& Worthen, 1875 , Geo. Sur. Ill., vol. 6, p. 376, Kaskaskia Gr.

depressus, St. John \& Worthen, 1875, Geo. Sur. Ill., vol. 6. p. 378, Kaskaskia Gr.

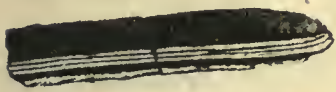

gracillimus Newberry \& Wort he $n$, 1866, (Chomatodus gracillimus,) Ge o Sur. Ill., vol.

2, p. 51, Burlington Gr.

grossiplicatus, St. Jobn \& Worthen, 1875, Geo. Sur. Ill., vol. 6, p. 375, Kaskaskia Gr.

multiplicatus, Newberry \& Worthen, 1866, (Chomatodus multiplicatus,) Geo. Sur. Ill., vol. 2, p. 57, Burlington Gr.

obscurus, Leidy, 1856, (Chomatodus obscurus, ) Trans. Am. Phil. Soc., vol. 11, p. 87, Keokuk Gr.

polymorphus, St. John \& Worthen, 1875, Geo. Sur. Ill., vol. 6, p. 380, Kaskaskia Gr.

prænuntius, St. John \& Worthen, 1875, Geo. Sur. Ill., vol. 6, p. 371, St. Louis Gr. pumilus, St. John \& Worthen, 1875, Geo. Sur. Ill., vol. 6, p. 369, St. Louis Gr. sculptus, St. John \& Worthen', 1875, Geo. Sur. Ill., vol. 6, p. 373, St. Louis Gr.

sublunatus, St. John \& Worthen, 1875 , Geo. Sur. Ill., vol. 6, p. 368, St. Louis Gr.

Thoracodus, Cope, 1883, Proc. Acad. Nat. Sci., p. 108. [Ety. thoracos, protected; odous, tooth.] Jaws plate-like, divided on middle line, each half with transverse grooves and ridges, and a smooth border all round. Type $\mathrm{T}$. eurydinus. eurydinus, Cope, 1883, Proc. Acad. Nat. Sci. Phil., p. 108, Permian Gr.

Thrinacodus, St. John \& Wortben, 1875, Geo. Sur. Ill., vol. 6, p. 289. [Ety. thrinakos, three-pronged; odous, tooth.] Teeth small; base produced posteriorly in a long sometimes twisted vertically flattened, or laterally compressed, clavate plate, longer than wide, anterior face narrow, and abruptly beveled from the tasal line of the crown; posterior extremity more or less obtusely rounded; inferior surface narrow, plain or faintly excavated; superior surface gently convex, concave antero-posteriorly, or corresponding to the curvature of the inferior surface; from the antero-superior extremity of the base spring three more or less relatively stout, nearly equal, trenchant, acutely pointed, recurved cusps the exterior pair divergent, the central one more or less vertical, slightly sigmoidally curved, transverse section sublenticular, compressed in front, rounded behind, with simple cutting edges, and more or less strongly costate in either face. Allied to Diplodus. Type T. nanus. duplicatus, Newberry \& Worthen, 1866 , (Diplodus duplicatus,) Geo. Sur. Ill., vol. 2 , p. 61 , Keokuk Gr.

incurvus, Newberry \& Worthen, 1866 , (Diplodus incurvus,) Geo. Sur. Ill., vol. 2 , p. 62 , Keokuk Gr.

nanus, St. John \& Worthen, 1875, Geo. Sur. Ill., vol. 6, p. 289, Waverly or Kinderbook Gr.

Tomodus, Agassiz, 1859, MSS. and St. John \& Worthen, 1883, Geo. Sur. Ill., vol. 7, p. 171. [Ety. tomos, sharp; odous, tooth.] Distinguished from Xystrodus by the great convexity of the coronal ridge, abrupt articular border, and absence of transverse punctæ on the triturating surface. Type T. convexus.

limitaris, St. John \& Worthen, 1883, Geo. Sur. Ill., vol. 7, p. 173, Upper Burlington $\mathrm{Gr}$.

Trigonodus, Newberry \& Worthen, 1866, Geo. Sur. Ill., vol. 2, p. 111. [Ety. trigonos, three-cornered; odous, tooth.] Syn. for sandalodus.

major, Newberry \& Worthen, 1866, Geo. Sur. Ill., vol. 2, p. 112, syn. for Sandalodus complanatus.

minor, see Sandalodus minor.

VATICINodus, St. John \& Worthen, 1883, Geo. Sur. Ill., vol. 7, p. 80. [Ety. vaticinus, prophetical ; odous, tooth.] Posterior teeth distinguished from Deltoptychius by the absence of the secondary lobe, the anterior part of the tooth forward of the posterior prominence being plain, as in Stenopterodus. Type V. vetustus.

carbonarius, St. John \& Worthen, 1883, Geo. Sur. Ill., vol. 7, p. 88, Coal Meas.

discrepans, St. John \& Worthen, 1883, Geo. Sur. Ill., vol. 7, p. 83, Upper Burlington Gr.

lepis, St. John \& Worthen, 1883, Geo. Sur. Ill., vol. 7, p. 88, Up. Coal Meas.

similis, St. John \& Worthen, 1883, Geo. Sur. Ill., vol. 7, p. 86, Kaskaskia Gr.

simplex, St. Jobn \& Worthen, 1883, Geo. Sur. Ill., vol. 7, p. 84, St. Louis Gr.

vetustus, St. John \& Worthen, 1883, Geo. Sur. Ill., vol. 7 , p. 82 , Waverly or Kinderhook Gr.

Venustodus, St. John \& Worthen, 1875, Geo. Sur. Ill., vol. 6, p. 344. [Ety. venustus, beautiful; odous, tooth.] Teeth laterally elongated, vertically arched; crown constricted at the base, defined by imbricating folds; crest elevated, uniform, or with median prominence; denticulations lateral; base forming a shallow plate. Type V. robustus.

argutus, St. John \& Worthen, 1875, Geo. Sur. Ill., vol. 6, p. 352, Kaskaskia Gr.

leidyi, St. John \& Worthen, 1875, Geo. Sur. Ill., vol. 6, p. 350 , St. Louis Gr. This name is a syn. for $V$. venustus. 
robustus, St. John \& Worthen, 1875, Geo. Sur. Ill., vol. 6, p. 345, Burlington Gr.

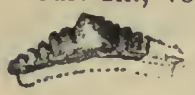

Fig. 1177.-Venustodus robustus. tenuicristatus, St. John \& Worthen, 1\$75, Geo. Sur. Ill., vol. 6, p. 348, Keokuk Gr. Worthen, 1875, Geo. Sur. Ill., vol. 6, p. 346, Burlington Gr. venustus, Leidy, 1856, (Chomatodus venustus,) Trans. Am. Phil. Soc. Phil., vol. 11, p. 87, St. Louis Gr.

Xrstracantiuus, Leidy, 1859, Proc. Acad. Nat. Sci. Phil., p. 3. [Ety. xystra, a tool for scraping; akantha, spine.] Distinguished from Physonemus by the slender, straight outline, and less preponderance of the antero-inferior shoulder. Type $\mathbf{X}$. arcuatus.

acinaciformis, St. John \& Worthen, 1875, Geo. Sur. Ill., vol. 6, p. 459 , Coal Meas. anceps, Newberry \& Worthen, 1866, (Drepanacanth us anceps,) Geo. Sur. IIl., vol. 2, p. 122 , Coal Meas.

arcuatus, Leidy, 1859, Proc. Acad. Nat. Sci. Phil, p. 3, Up. Coal Meas.

mirabilis, St. John \& Worthen, 1875, Geo. Sur. Ill., vol. 6, p. 458, Coal Meas.
Xystrodus, Agassiz, MSS., 1859, and St. John 1870, Proc. Am. Phil. Soc., vol. 11, p. 436. [Ety. xystra, an instrument for scraping; odous, tooth.] Mandibular posterior teeth triangular; great transverse breadth of the inner margin as compared with the longitudinal diameter; coronal surface plain, depressed, and alated posteriorly; maxillary posterior teeth cuneiform, and narrow transverse diameter at the inner margin. Type $\mathbf{X}$. striatus.

bellulus, St. John \& Worthen, 1883, Geo. Sur. Ill., vol. 7, p. 183, Coal Meas.

imitatus, St. John \& Worthen, 1883 , Geo. Sur. Ill., vol. 7, p. 180,8 t. Louis Gr.

inconditus, St. John \& Worthen, 1883, Geo. Sur. Ill, vol. 7, p. 179, Keokuk Gr.

occidentalis, St. John, 1870, Palæontology of Eastern Nebraska, p. 244, syn. for Orthopleurodus carbonarius.

simplex, St. John \& Worthen, 1883, Geo. Sur. Ill., vol. 7, p. 178, Upper Burlington Gr.

verus, St. John \& Worthen, 1883, Geo. Sur. Ill., vol. 7, p. 181, Kaskaskia Gr.

\title{
CLASS BATRACHIA.
}

\author{
[Ety. batrachos, frog.]
}

THE Batrachia live a double life-that is, both on land and in water-and are called Amphibia. [Amphi, on both sides, around; bios, life.] They approach the fishes in their early stages of growth, and resemble the true Reptilia in their more mature development. All possess lungs, but during their young or larval condition they are always furnished with branchix, and in some orders these remain throughout the life of the animal. They form a distinct transition from aquatic fishes to exclusively air-breathing reptiles. They are all strictly oviparous, although in some species the eggs are retained in or upon the body of the parent until the young have attained some degree of growth. After leaving the egg, the animals undergo a series of transformations before arriving at their complete or perfect state. In their early stage they are known as tadpoles, little, fish-like animals, with broad heads, sack-like body, and long, compressed tail. The mouth is at the lower part of the front of the head, and is furnished with a pair of horny jaws, with which they feed upon the animalculæ that furnishes the food. They are vertebrated animals, with cold blood and naked skin, and undergo a metanorphosis or change of condition from an aquatic respiration by gills to an atmospheric respiration by lungs, and a consequent alteration in general structure and mode of life. 
In the tadpole and the genera which retain their gills through life, the substance between the vertebræ is soft, and contained in cup-like hollows formed by the concave articular surfaces of contiguous bones, precisely as in fishes. The lower orders are fish-like in possessing permanent brauchiæ, the limbs are reduced to a rudimentary condition, and the tail is flattened and surrounded by a fin. In the higher orders the limbs are more and more developed and fitted for terrestrial progression, until they are capable of active motion and the animals can take their habitual residence in trees. The spinal column in some is composed of a continuous chorda dorsalis, inclosed in a fibrous sheath, but furnished with bony superior and inferior arches for the protection of the spinal cord and principal blood-vessels. In others the vertebræ are articulated by a sort of ball-and-socket joint. The vertebræ are usually furnished with long, transverse processes which appear to take the place of ribs; ribs are generally deficient. In those having a chorda dorsalis the skull is formed of a simple cartilaginous capsule, with which the chorda is completely continuous, and the only indications of ossification are in the lateral portions of the occipital bone. In the higher forms the skull is completely ossified; it is always of a broad and flattened form, with enormous, large orbits, and possesses one constant character which distinguishes the skull of a Batrachia from that of a Reptile; namely, the occipital bone is always furnished with two lateral condyles that fit into corresponding sockets in the first vertebra of the neck. The bones of the upper jaw and palate form a broad arch, which is always firmly attached to the skull; the maxillary and intermaxillary bones assist in the formation of the edge of the mouth, and are much developed, transversely expanding the general form of the skull without involving any enlargement of the brain cavity, which is very small.

All Batrachia have teeth on the palate; the salamanders have them also in both the upper and lower jaws, the frogs in the upper only, and the toads in neither. The jaw teeth are always slender, sharp-pointed, and closely set. The frog has about forty on each side of the upper jaw; the salamander has about sixty above and below; the palatine teeth are generally arranged transversely parallel to the jaw teeth. The hind legs of the frog are developed for leaping, and it has no useless tail; the body is contracted into a short space, and the few vertebræ are united into a single immovable piece, unprovided with ribs. The water salamanders, or newts, have a long tail, a slender flexible body, and all their organs are fitted for aquatic life. The structure of the bones is more compact and calcareous, and less transparent and flexible, than in fishes. The bones of the skull have their margins in contact, and occasionally united, but never overlapping. The hyoid bone changes largely in those genera undergoing metamorphosis in accordance with the development of the respiratory organ.

The Batrachia are generally distinguished from the Reptilia by the absence of a scaly covering. The skin of aquatic genera is soft and smooth, and constantly moistened by the cutaneous secretions; in land genera, as frogs and toads, the glands of the skin secrete a thick, whitish fluid. The cuticle is shed frequently. A few species are covered with horny scales.

They begin life with the single heart and gills of fishes; but as their metamorphosis goes on, the heart assumes the compound character necessary for the pulmonary respiration of the reptiles. In the development of the nervous system and the organs of the senses, they exhibit a slight advance upon the fishes. In the first 
stages the circulation through the branchial apparatus is exactly the same as in the fishes; but later pulmonary arteries make their appearance, lungs are developed, and aerial respiration commences.

The class has been divided into five orders, namely : Amphipneusta, Anura, Urodella, Abranchia, and Apoda. None of the Palæozoic fossil families are referred to any of these orders except the Cocytinidx, and the correctness of that reference is exceedingly doubtful. All other Palæozoic fossils are referred to orders which have become extinct. The change, either by progression into higher classes of the vertebrate kingdom, or by retrogression to an inferior state, is strongly marked. The Urodella, to which the Cocytinidæe are referred, have long, slender bodies, four limbs, which are sometimes very small, and occasionlly the toes are furnished with claws, and a long, persistent tail ; no external branchiæ, but in some species there is a branchial aperture on each side of the neck, within which are the branchial arches, with their laminx; lungs well developed, skin smooth, or covered with warty prominences, and furnished with numerous glands, which secrete an acrid, viscid fluid. In general form they resemble the lizards, which belong to the Reptilia. The aquatic and land salamanders belong to this order.

The first Batrachia are found in the Coal Measures. They increase rapidly in numbers, and spread out in progressive evolution through the Permian Group, and reach their bighest development and largest size in the Triassic, and since that time they have declined or retrograded, and now constitute a very inferior grade of the Vertebrata. The Animal Kingdom has been divided into classes and orders upon a basis which constitutes, as it is supposed, a natural system, and the more recent study of embryology has demonstrated that this natural system corresponds with the phases of embryonic history in all, or nearly all, its iparts, and the study of Palæontology has proven beyond all peradventure that there has been a succession of organic types from the earliest geological time to the present, which is stamped upon the embryonic growth of living animals, and coincides with the grades established by the natural system of classification.

It follows that when the Palæozoic orders are distinct from the living, the class has been more comprehensive than the definition given by zoologists. Indeed, all the fossils can not be strictly embraced within the prescribed limits of the Batrachia. Many of them might be included within the Reptilia, because they combine Batrachian and Reptilian characters, and where the latter prevail probably they should be classed with the Reptilia. Some of them, however, rise a step higher in the animal system, and include Batrachian and Mammalian characteristics, and for this reason it is urged by the evolutionists that the Mammalia descended from the Batrachia, without having passed through the Reptilian stage. In other words, what is here included is a comprehensive type of animal existence not limited by the bounds which define the living Batrachia.

The arrangement of the fossils into orders and families must be regarded as provisional, and only approximating the present learning, for the following reasons, in addition to those which will be apparent to the specialist: 1 . There are several synonyms of orders and families. 2. No one has published a complete classification from which the author could compile the learning. 3. The author has never had an opportunity to study the fossils of this class and have an opinion of his own to assert or defend. 
Order Archegosauria.

Family Arch mgosauride.-Brachydectes, Hylerpeton.

\section{Order Ganocephala.}

Family Colosteid a.-Amphibamus, Colosteus, Sauropleura.

\section{ORDER LABYRINTHODONTIA.}

Family Baphetide.-Baphetes. .

Family Trematosauride.-Cricotus. 。

\section{Order Microsauria.}

Famiry Diplocaulider. -Diplocaulus.

Family Eosauride.-Eosaurus.

Family Molgophides.-Molgophis, Pleuroptyx.

Family Pelionidas.-Hylonomus, Pelion.

Family Phlegethontind a.-Phlegethontia.

Family Ptronima.-Ceraterpeton, Hyphasma, Oestocephalus, Ptyonius, Thyrsidium.

Family Tuditanide.-Dendrerpeton, Leptophractus, Tuditanus.

\section{Order Pelycosauria.}

Family Bolosauride.-Bolosaurus, Chilonyx, Lysorophus.

Family Diadectide.-Diadectes, Helodectes.

Family Clepsydropsida.-Archæobolus, Clepsydrops, Dimetrodon, Ectocynodon, Embolophorus, Empedias, Metarmosaurus, Pariotichus, Theropleura.

Family Edaphosauride.-Edaphosaurus, Pantylus.

\section{- Order rhachitoma.}

Family Eryopsid w-Acheloma, Anisodexis, Eryops, Ichthyacanthus, Trimerorachis, Zatrachys.

\section{ORDER URODELLA.}

Family Cocytinida.-Cocytinus.

Order and Family Uncertain.-Chirotherium, Collettosaurus, Nothodon, Ophiacodon, Sauropus, Sphæropezium, Sphenacodon, Thenaropus.

Acheloma, Cope, 1882, Pal. Bull. No. 35, and Proc. Am. Phil. Soc., p. 455. [Ety. $a$, without; cheloma, a notch.] Mandible without angular process; teeth subequal, rather larger anteriorly; pterygoid bone ending in a free, recurved edge anterior to the quadrate bone; palatines and pterygoids nar- row; palatal foramen wide, posterior border of cranium entire; without notch on the external side of the epiotic bone; vertebræ rhachitomous. Type A.cumminsi.

cumminsi, Cope, 1882, Pal. Bull. No. 35, and Proc. Am. Phil. Soc., p. 456, Permian. 
A mphibamus, Cope, 1865, Proc. Acad. Nat. Sci. Phil., p. 134. [Ety. amphi, both; bama, a step; from its two modes of

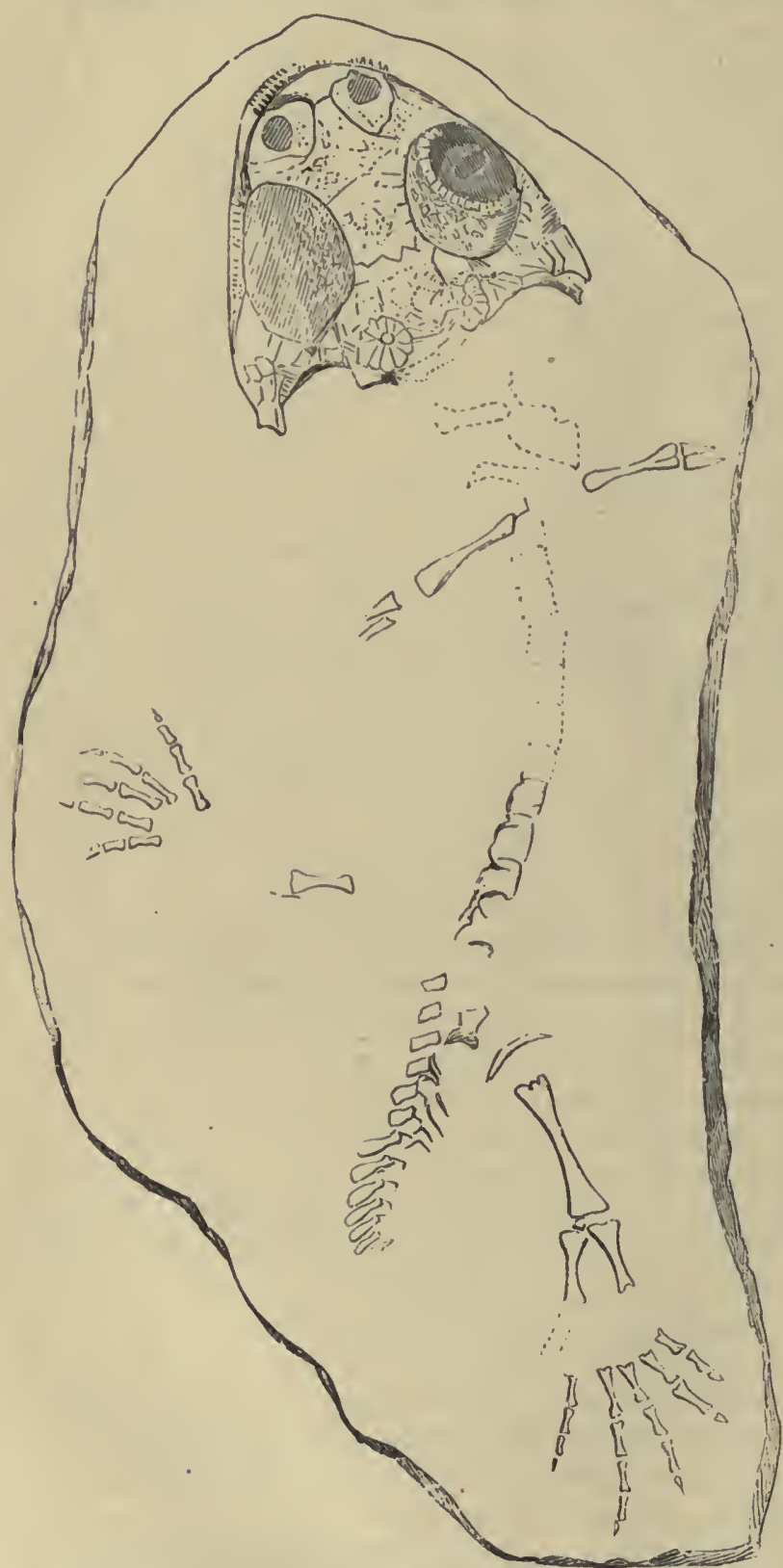

Fia. 1178,-Amphibamus grandiceps. Two diam.

inous; neural spines of caudal vertebræ well developed; (?) centra. Type A. grandiceps.

grandiceps, Cope, 1865, Proc. Acad. Nat. Sci. Phil., p. 134, and Geo. Sur. Ill., vol. 2, p. 135 , Coal Meas.

Anisodexis, Cope, 1882, Pal. Bull. No. 35, and Proc. Am. Phil. Soc., p. 459. [Ety. anisos, unequal ; dexis, a bite.] Teeth on premaxillary; maxillary, and dentary bones of unequal lengths, some very large, others very small; dentinal inflections $8 \mathrm{traight}$, nearly reaching the pulp-cavity; cranial bones sculptured; vertebræ rhachitomous. Type A. imbricarius.

imbricarius, Cope, 1882 , Pal. Bull. No. 35, and Proc. Am. Phil. Soc., p. 459, Permian.

Arcil nobelus, Cope, 1877, Proc. Am. Phil. Soc., vol. 17, p. 192. [Ety. archaios, ancient; belos, a weapon.] Maxillary bone with a large, hollow tooth, with two opposite shallow grooves at the base; crown hollow; skeleton unknown. Type A. vellicatus.

vellicatus, Cope, 1877 , Proc. Am. Phil. Soc., vol. 17, p. 192, Permian.

Ba PII e'Tes, Owen, 1853, Jour. Geo. Soc. London, vol. 10, p. 207. [Ety. bapto,

- I dip or dive, a diving animal.] Teeth conical, curved; outer series one or two lines in dianeter, inıter series three lines or more; implanted and anchy. losed in shallow sockets; lower third of tecth longitudinally striated; cranial bones corrugated; head broad. Type B. planiceps.

minor, Dawson, 1870, Can. Nat. and Geol., Coal Meas.

planiceps, Owen, 1853,

progressing, swimming, and walking.] Teeth small, simple. equal on margins of jaws; sclerotic plates on eye; table of vertex produced; no horns; propodial bones distinct; tarsus cartilagJour. Geo. Soc. London, vol. 10, p. 207, and Acad. Geol., p. 359, Coal Meas.

Bolosaurus, Cope, 1878, Pal. Bull. No. 29, and Proc. Am. Phil. Soc., vol. 17, p. 506. [Ety. bolos, a lump ; sauros, a liz- 
ard.] Teeth fixed in shallow alveoli, with crowns expanded transversely to the jaw, swollen at the base, apex low and divided vertically; the posterointernal half in the maxillary series is low and horizontal, the antero-external portion forms a curved cusp; in the lower jaw the relative position of the ledge and cusp is reversed. Type $B$. striatus.

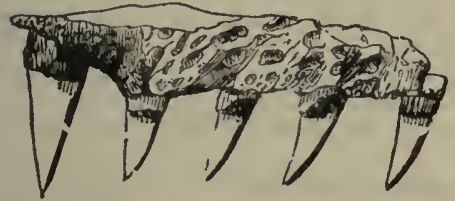

Fra. 1179.-Baphetes planlceps.

rapidens, see Chilonyx rapidens.

striatus, Cope, 1878, Pal. Bull. No. 29, and

Proc. Am. Phil. Soc., vol. 17, p. 507, Permian.

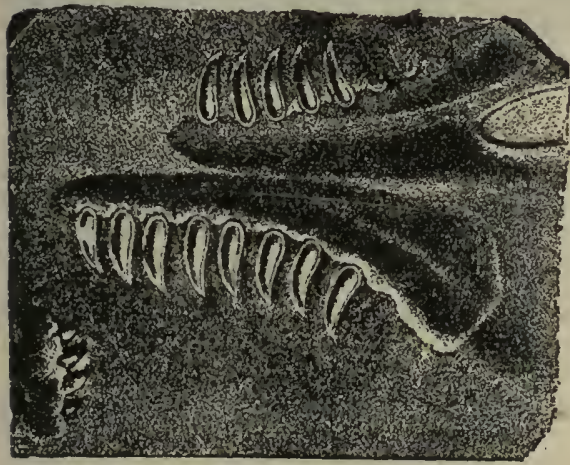

FIG. 1180.-Brachydectes newberryi.

Brachynectes, Cope, 1868, Proc. Acad. Nat. Sci. Phil., p. 214. [Ety. brachys, short; dektes, a biter.] Rami short, stout; teeth subequal, elongate, cylindric cones with acute tips turned posteriorly, pulp-cavity large; skeleton unknown. Type B. Newberryi.

newberryi, Cope, 1868, Proc. Acad. Nat. Sci. Phil., p. 214, and Ohio Pal., vol. 2, p. 388, Coal Meas.

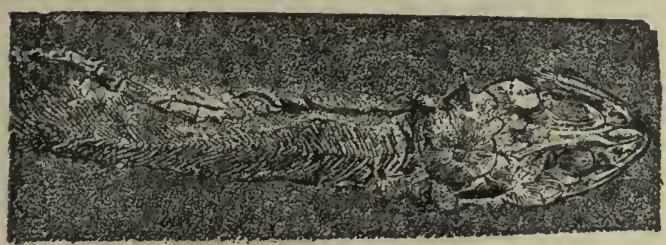

FIG.1181.-Ceraterpeton tenuicorne.

Ceraterpeton, Huxley. [Ety, keras, horn; erpeton, reptile.] Teeth simple, equal on outside of jaws; angles of intercalary bones produced into horn-like processes; cranial bones sculptured, vertebræ undivided; carpus and tarsus osseous.

lineopunctatum, Cope, 1875, Ohio Pal., vol. 2 , p. 372 , Coal Meas.

tenuicorne, Cope, 1875, Ohio Pal., vol. 2, p. 372, Coal Meas.

Chirotherium, Kaup, 1835, in Leonhard und Bronn Neues Jahrbuch fur Mineralogie. [Ety. cheir, the hand; therion, beast.] Represented by foot impressions only. Tues robust, the internal shorter and divergent from the others. Sole (or palum) short, wide. Type C. barthi.

reiteri, Moore, 1873, Am. Jour. Sci. and Arts, 3d ser., vol. 5, p. 292, Coal Meas.

Chilonyx, Cope, 1883, Proc. Am. Phil. Soc., vol. 20 , p. 631 . [Ety. cheilos, lip; onyx, claw.] Long diameter of the crowns of the teeth transverse to the jaw, and eacl crown contracting to a slightly incurved apex; maxillary teetli short; temporal fossæ roofed; superior surface of cranium divided in areas by grooves. Type C. rapidens.

rapidens, Cope, 1878, (Bolosaurus rapidens,) Proc. Am. Phil. Soc., vol. 17, p. 506 , aud vol. 20 , p. 631, Permian.

Clempy drops, Cope, 1876, Proc. Acad. Nat. Sci. Phil., p. 407. [Ety. klepsydra, an hour-glass; ops, appearance.] Intercentra present; neural spines only elongate posteriorly; premaxillary teeth not especially elongate; one or two long maxillary teeth; no grinding teeth. Type C. colletti.

colletti, Cope, 1876, Proc. Acad. Nat. Sci. Phil., p. 407, Permian.

gigas, see Dimetrodon gigas.

leptocephalus, Cope, 1884, Pal, Bull. No. 39 , p. 30 , Permian.

limbatus, Cope, 1877, Proc. Am. Phil. Soc., p. 196, Permian or Triassic.

macrospondylus, Cope, 1884, Pal. Bull. No. 39, p. 35 , Permian.

natalis, Cope, 1878, Pal. Bull., No. 29, and

Proc. Am. Phil. Soc., vol. 17, p. 509, Permian.

pedunculatus, Cope, 1877, Proc. Am. Phil. Soc., p. 63, Permian.

vinslovi, Cope, 1877, Proc. Am. Phil. Soc. p. 62, Permian:

Cocytinus, Cope, 1871, Proc. Am. Phil. Soc., p. 177, and Ohio Pal., vol. 2, p. 360. [Ety. mythological name.] Vertebræ and ribs osseous; teeth on the premaxillary bone; none on the maxillary; axial hyal with basihyal on each side united with corresponding ceratohyal at the end of which is an element in position of stylohyal; hrmal or basal branchihyals three, the anterior two each supporting one pleural branchihyal and the third supporting one; lıæmal branchihyal on the inner side of the ceratohyal, approaching the me- 
dian line, and with elongate pleural element. Type C. gyrinoides.

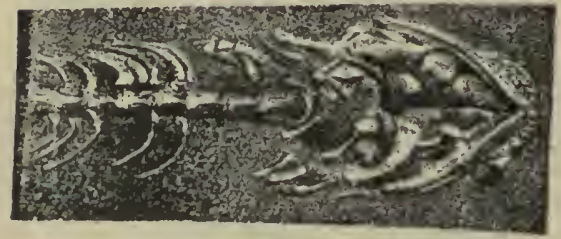

FIG. 1182.-Cocytinus gyrinoldes.

gyrinoides, Cope, 1874, Trans. Am. Phil. Soc., and Ohio Pal., vol. 2, p. 364, Coal Meas.

Collemtrosaunus, Cox, 1874, Geo. Sur. Ind., 5th Ann. Rep., p. 247. [Ety. proper lıme; sauros, a lizard.] Founded upon tracks having five digits, and supposed to be related to Batrachians and Salamanders. Type C. indianensis.

indianensis, Cox, 1873, Geo. Sur. Ind., 5 th Ann. Rep., p. 247, Coal Meas.

Colosteus, Cope, 1869, Trans. Am.

Phil. Soc., p. 22. [Ety. kolos, imperfect; osteon, a bone.] No vertebral centra, spines, or sclerotic bones; short ribs; two pairs of short limbs; three sculptured pectoral bones; abdominal region protected by scales in chevron; ? anterior teeth longer than posterior, basal half incised sulcate, except two behind the dentary. Type $C$. foveatus.

crassiscutatus, Cope, syn. for C. scutellatus.

foveatus, Cope, 1869, Trans. Am. Phil. Soc., P. 24, and Ohio Pal., vol. 2, p. 406, Coal Meas.

marshi, see Ptyonius marshi.

pauciradiatus, Cope, 1874, Trans. Ain. Phil. Soc., p. 10, and Ohio Pal., vol. 2, p. 408, Coal Meas.

scutellatus, Newberry, 1856, (Pygopterus scutellatus, ) Proc. Acad. Nat. Sci. Phil., p. 98, and Ohio Pal., vol. 2, p. 407, Coal Meas.

Cricotus, Cope, 1876, Proc. Acad. Nat. Sci. Phil., p. 405. [Ety. krikotos, ringed.] Centra undivided, equal to the disciform intercentra in the caudal region, intercentra a little smaller in the dorsal region; neural spines and zygapophyses; developed foramen; chordæ dorsalis persistent; teeth equal, except probably the palatines; limbs short, a facial lyra. Type C. heteroclitus.

crassidiscus, Cope, 1884, Pal. Bull. No. 39, p. 28, Permian.

discophorus, Cope, syn. for C. heteroclitus. gibsoni, Cope, 1877, Pal. Bull. No. 26, and Proc. Am. Phil. Soc., vol. 17, p. 185 , Permian.

heteroclitus, Cope, 1876, Proc. Acad. Nat. Sci. Phil., p. 405, Permian.

hypantricus, Cope, 1884, Pal. Bull. No. 39, p. 30 , Permian.
Dendkerpeton, Owen, 1853, Quar. Jour. Geo. Soc., vol. 9, p. 58. [Ety. dendron, a tree; erpeton, a lizard, from the circumstance under which the reptile was found.] Teeth in double series; outer simple, flattened, conic; inner series conical, with inflected folds of cement; teeth on the vomer; skull-bones corrugated; body protected below with ovate or rhoinboidal bony scales, imbricated, horny scales above; fore-limbs the larger; tail natatory; vertebræ biconcave; neural arches and bones ossified. Type D. acadianum.

acadianum, Owen, 1853, Quar. Jour. Geo. Soc., vol. 9 , p. 58, and Acadian Geology, p. 362, Coal Meas.

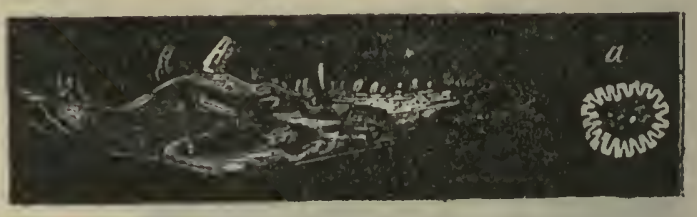
ton acadlanum.
tooth magnifled obtusum, see Tuditanus obtusus.

oweni, Dawson, 1863, Quar. Jour. Geo. Soc., vol. 19, p. 469, and Acad. (ieol., p. 368, Coal Meas.

Dranectres Cope, 1878, Pal. Bull. No. 29, and Proc, Am. Phil. Soc., vol. 17, p. 505. [Fty. dia, crosswise; decktos, a biter.] Teeth with much compressed crowns, with bracket-shaped edge, longer axis transverse to the jaws, edges of erowns obtuse, no sculpture on the face. Alveoli not separated. External alveolar border more elevated than the internal, inner alveolar border pierced by a fossa behind the inner extremity of each tooth. Type D. sideropelicus.

latibuccatus, see Empedias latibuceatus.

molaris, see Empedias molaris.

phaseolinus, see Empedias phaseolinus.

sideropelicus, Cope, 1878, Pal. Bull. No. 29, and Proc. Am. Phil. Soc., vol. 17, p. 505 , Permian.

Dimetrodon, Cope, 1878, Pal. Bull. No. 29, and Proc. Am. Phil. Soc., vol. 17, p. 512. [Ety. dimetros, two measures; odous, tootl..] Dentition with enormously long incisors and two or three long maxillaries; the pubic bone not distinct from ischium; humerus with trochlear condyles and a defiued proximal articular surface; neural spines of dorsal and lumbar vertebræe enormously elongate; intercentra present. Type D. incisivus.

cruciger, Cope, 1878, Am. Naturalist, vol. 12 , p. 830 , Permian.

gigas, Cope, 1878, Am. Nat., p. 327, (Clepsydrops gigas,) and Proc. Am. Phil. Soc., vol. 17 , p. 515 , Permian. 
incisivus, Cope, 1878, Pal. Bull. No. 29, and Proc. Am. Phil. Soc., vol. 17, p. 512, Permian.

rectiformis, Cope, 1878, Pal. Bull. No. 29, and Proc. Am. Phil. Soc., vol. 17, p. 514, Permian.

semiradicatus, Cope, 1881, Bull. U. S. Geo. Sur. Terr., vol. 6, No. 1, p. 80, Permian.

Diplocaulus, Cope, 1877, Pal. Bull. No. 26, and Proc., Am. Phil. Soc., vol. 17, p. 187. [Ety. diploos, double; kaulos, stem.] Vertebral centra contracted medially, perforated by the foramen chorda dorsalis, co-ossified with the neural arch and supporting transverse processes; zygosphen articulation; two rib articulations, one below the other; axis and atlas united by a long zygosphen which is not roofed by the zygantrum; no neural spine, atlas insegmented; arch extended into the foramen magnum; squamosal region developed into a horn. Skull sculptured. Type D. salamandroides.

magnicornis, Cope, 1882, Pal. Bull. No. 35, and Proc. Am. Phil. Soc., p. 453, Permian.

salamandroides, Cope, 1877, Pal. Bull. No. 26, and Proc. Am. Phil. Soc., vol. 17 , p. 187 , Permian.

Ectocynodon, Cope, 1878, Pal. Bull. No. 29, and Proc. Am. Phil. Soc., p. 508. [Ety. eklos, eternal ; kuon, dog; odous, tooth.] Cranium short, wide, large post frontal bones, large orbit; bones sculptured but no lyra; teeth rhizodont, crowns elongated, cumpressed, anterior and posterior cutting edges; one between the orbit and nostril larger and longer than the others, and lying outside of the closed dentary bone; mandibular symphysis not sutural but ligamentous. Type E. ordinatus.

agnti, Cope, 1882, Pal. Bull. No. 35, and Proc. Am. Phil. Soc., p. 451, Permian.

ordinatus, Cope, 1878, Pal. Bull. No. 29, and Proc. Am. Phil. Soc., p. 508, Permian.

Edapirosaurus, Cope, 1882, Pal. Bull. No. 35, and Proc. Am. Phil. Soc., p. 448. [Ety. edaphos, pavement; sauros, a lizard.] Temporal fossæe not overroofed; cranial bones not sculptured; mandibular and maxillary teeth subequal; mandibular ramus expanded inward and supporting numerous teeth; pterygoid or malar bones supporting a dense body of teeth corresponding to those in the lower jaw; teeth subconical. Neural spines greatly elongate, hollow. Type E. pogonias.

microdus, Cope, 1884, Pal. Bull. No. 39, p. 37 , Permian.

pogonias, Cope, 1882, Pal. Bull. No. 35, and Proc. Ám. Phil. Soc., p. 449, Permian. Embolophorus, Cope, 1878, Pal. Bull. No. 29, and Proc. Am. Phil. Soc., vol. 17, p. 518. [Ety. 'ballo, I throw ; em, into; phoros, bearing.] Neural arch co-ossified, zygapophyses and diapophyses well developed; centra not ochordal; intercentra narrowed and transversely extended; ribs two-headed, the capitulum is received into a fossa of the posterior border of the intercentrum, in advance of the vertebra which supports the diapophysis, to which the tuberculum is attached. Type $\mathbf{E}$. fritillus.

fritillus, Cope, 1878, Pal. Bull. No. 29, and Proc. Am. Phil. Soc., vol. 17, p. 518, Permian.

Empedias, Cope, 1883, Proc. Am. Phil. Soc., vol. 20, p. 63. Proposed instead of Empedocles of Cope in 1878, which was preoccupied. Teeth with elongate crowns, with flat grinding surface but bracket-shaped in transverse vertical section, arranged transversely to the longaxis of the jaws; no canines; incisors wearing chisel-shaped; temporal fossa covered; vertebræ with hyposphen on the posterior and hypantrum on the anterior face and short quadrate neural spines. Type E. alatus.

alatus, Cope, 1878, (Empedocles alatus,) Proc. Am. Phil. Soc., vol. 17, p. 516, Permian.

fissus, Cope, 1883, (Empedocles fissus,) Proc. Am. Phil. Soc., p. 634, Permian.

latibuccatus, Cope, 1878, (Diadectes latibuccatus,) Proc. Am. Phil. Soc., vol. 17, p. 505, Permian.

molaris, Cope, 1878, (Diadectes molaris,) Am. Nat., vol. 12, p. 565, and Pal. Bull. No. 32 , p. 10 , Permian.

phaseolinus, Cope, 1880, (Diadectes phaseolinus,) Pal. Bull. No. 32, p. 9, Permian.

Empedocles, Cope, 1878, Proc. Am. Phil. Soc., vol. 17, p. 516. The name was preoccupied, see Empedias.

alatus, see Empedias alatus.

fissus, see Empedias fissus.

Eosatrus, Marsh, 1862, Can. Nat. and Geo., vol. 7 , a n d Acadian Geol., p. 382. [Ety. eos, the dawn; sauros, a seafish.] Founded upon vertebræ, with biconcave centra and free neural a r ch, and closed not ochordal foramen. Type E. acadianus.

acadian us, Marsh,
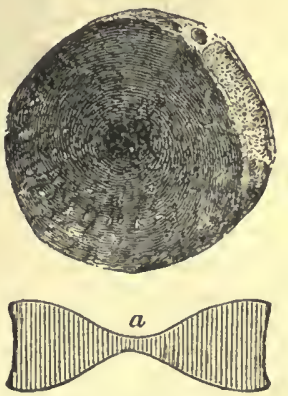
1862, Can. Nat. and Geol., vol. Fra. 1184.-Eosaurus acad17, and Acad. verse section. Geol., p. 382, Coal Meas.

Epicordylus, Cope, 1878, Pal. Bull. No. 29, and Proc. Am. Phil. Soc., vol. 17, p. 515. [Ety. epi, upon; kordylos, a water-lizard.] Syn. for Eryops. 
erythroliticus, see Eryops erythroliticus. Eryops, Cope, 1877, Proc. Am. Phil. Soc., vol. 17, p. 188. [Ety. eruo, I protect; ope, view.] Vertebræ rhachitomous throughout; teeth of external series equal; some larger ones on the palatine bones; table of cranium produced, bounded by a notch on each side; no horns nor mucous grooves; pelvic elements co-ossified; no foramen. Type E. megacephalus.

erytholeticus, Cope, 1878, (Epicordylus erytholeticus,) Proc. Am. Phil. Soc., p. 515, Permian.

ferricolus, Cope, 1878, (Parioxys ferricolus,) Proc. Am. Phil, Soc., p. 521, Permian.

megacephalus, Cope, 1877, Proc. Am. Phil. Soc., vol. 17, p. 188, Permian or Triassic.

platypus, Cope, 1877, (Ichthycanthus platypus,) Proc. Am. Phil. Soc., vol. 17, p. 574, Coal Meas.

reticulatus, Cope, 1881, Am. Nat., p. 1020, Permian.

Eurytuorax, Cope, 1875, Ohio Pal., vol. 2, p. 401. [Ety. eurys, broad ; thorax, the breast.] Established on a thoracic shield, having broad, smooth surfaces on the outer borders for the contact of the overlapping margins of the lateral plates. Subround, with a large excavation from the posterior margin on each side; narrowed portion left in the middle behind has a convex outline; no sculpture. Type E. sublævis.

sublævis, Cope, 1871, Proc. Am. Phil. Soc., p. 177, and Ohio Pal., vol. 2, p. 402, Coal Meas.

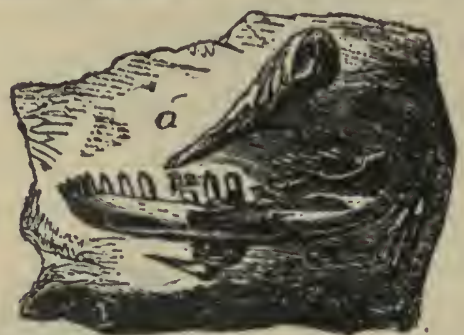

Fig. 1185.-Hylerpeton dawson1. Mandible and portion of cranial bone.

Helonectes, Cope, 1880, Pal. Bull. No. 32 , p. 11. [Ety. helos, a nail; dektes, a biter.] Two rows of subround molariform teeth in each jaw. Type H. paridens.

isaaci, Cope, 1880, Pal. Bull. No. 32, p. 12, Permian.

paridens, Cope, 1880, Pal. Bull. No. 32, p. 11, Permian.

Hylerpeton, Owen, 1862, Quar. Jour. Geo. Soc., vol. 18, p. 5. [Ety. hyle, wood; erpeton, reptile.] Teeth simple, bluntly conical, with large pulp-cavity; about 13 on one side of a jaw; two of the anterior ones of the upper jaw twice as large as the others and deeply sunk in the jaw. Length of lower jaw $\frac{1}{3}$ inch ; bones of skull puncto-striate. Type $\mathbf{H}$. dawsoni.

curtidentatum, Dawson, 1876, Am. Jour. Sci. and Arts, vol. 12, Coal Meas.

dawsoni, Owen, 1862, Quar. Jour. Geo. Soc., vol. 18, p. 5, and Acadian Geology, p. 380 , Coal Meas.

longidentatum, Dawson, 1876, Am. Jour. Sci. and Arts, vol. 12, Coal Meas.

Hyronomus, Dawson, 1860, Quar. Jour. Geo. Soc., vol. 16, p. 268. [Ety. hyle, wood; nomos, an abode; forest dweller.] Cranial bones thin, smooth; parietal bones arched; about 26 teeth in each maxillary, elongated, conical, set in a single series, in a furrow, protected externally by an alveolar ridge; teeth longer in intermaxillaries and extremities of mandibles than elsewhere; vertebræ ossified, biconcave, with spinous processes; ribs long and curved; pelvis large; ilium long, expanded below, ischium expanded; pubis expanded, triangular where it joins the ischium, round and arched toward the symphysis; femur thick, nearly straight; tibia short, stout; fibula slender; phalanges broad. Dermal covering of ovate bony scales. Type H. lyelli.

aciedentatus, Dawson, 1860, Quar.

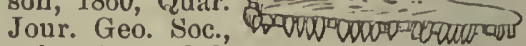
vol. 16 , p. 268 ,

and Acad. Geol., Fig. 1186. - Hylonomus p. 376, Coal acledentatus. MaxilMeas.

lyelli, Dawson,

1860, Quar. Jour. Geo. Soc., vol. 16, p. 268, and Acad. Geol., p. 370, Coal Meas.

wymani, Dawson, 1860, Quar. Jour. Geo. Soc., vol. 16, p. 268, and Acad. Geol, p. 378, Coal Meas.

Hypilasma, Cope, 1875, Proc. Acad. Nat. Sci., p. 16, and Ohio Pal., vol. 2, p. 387. [Ety. hyphasma, a web.] Vertebræ osseous; posterior dorsals with fan-like neural spines, ventral armature consisting of rhomboidal scuta, forming packed rows arranged in chevrons, directed backward, on top of which are the usual rod-like scales arranged in packed chevrons with the angle directed forward. Type H. lævis.

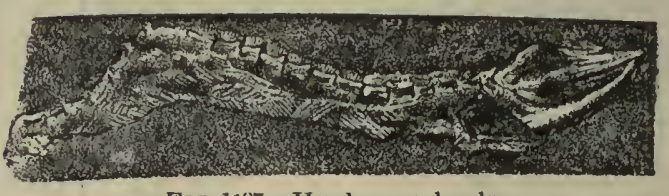

FrG. 1187.-Hyphasma lævis.

lævis, Cope, 1875, Proc. Acad. Nat. Sci., p. 16, and Ohio Pal., vol 2, p. 387, Coal Meas.

Ichtu y ACanthus, Cope, 1877, Pal. Bull. No. 24, and Proc. Am. Phil. Soc., p. 573. 
[Ety. ichthys, a fish; akantha, a spine.] Founded on the posterior dorsal and candal vertebrx and adjacent parts. Posterior limbs well-developed, with tibia, fibula, osseous tarsus and five digits; ribs elongate, simple, curved; abdominal armature in bristle-like rods, in anteriorly direcied chevrons; dorsal vertebræ short, with simple neural spines; tail large, vertebra ossified and furnished with slender chevron bones, which terminate in a hrmal spine; neural spines slender, directed backward, caudal series somewhat resembling that of a fish; centra amphicolian. Type I. ohioensis.

obioensis, Cope, 1877, Pal. Bull. No. 24, and Proc. Am. Phil. Soc., p. 573, Coal Meas.

platypus, see Eryops platypus.

Leptopilractus, Cope, 1873, Proc. Acad. Nat. Sci., p. 340, and Ohio Pal., 'vol. 2, p. 399. [Ety. leptos, delicate; phrcktos, armored.] Founded on various portions of the cranium; jaws bear large teeth, round in section at the base, but with compressed, acute apex, and with cutting edge on anterior face; enamel delicately grooved; there is a large elongate tooth in the upper jaw in the position of a canine; sculpture of the cranium little marked; lower jaw marked with inosculating grooves. Type L. obsoletus.

lineolatus, Cope, 1877, Pal. Bull. No. 24, and Proc. Am. Phil. Soc., p. 576, Coal Meas.

obsoletus, Cope, 1873, Proc. Acad. Nat. Sci., p. 341, and Ohio Pal., vol. 2, p. 400, Coal Meas.

Lysoropirus, Cope, 1877, Pal. Bull. No. 26, and Proc. Am. Phil. Soc., vol. 17, p. 187. [Ety. lysos, free; orophos, roof.] Founded upon the centra. Vertebra amphicolian, perforated by the foramen chordæ dorsalis; neural arch freely articulated to the centrum; floor of neural canal deeply excavated; no processes or costal articulations on the centrum, which is excavated by longitudinal fossæ; centrum not shortened. Type L. tricarinatus.

tricarinatus, Cope, 1877, Pal. Bull. No. 26, and Proc. Am. Phil. Soc., vol. 17, p. 187, Permian.

Meta rmosaurus, Cope, 1878, Pal. Bull., No. 29, and Proc. Am. Phil. Soc., vol. 17, p. 516. [Ety. meta, down; harmos, a joint ; sauros, lizard.] Founded upon vertebræ. Centrum shorter than wide, deeply biconcave; diapophyses project below the base of the neural arch, are short, with small tubercular facet; capitular facet; facet for intercentrum small, and is excavated at the anterior extremity of the base of the centrum; neural canal large; articular faces of anterior zygopophyses directed downward and outward. Type M. fossatus. fossatus, Cope, 1878, Pal. Bull., No. 29, and Proc. Am. Phil. Soc., vol. 17, p. 516, Permian.

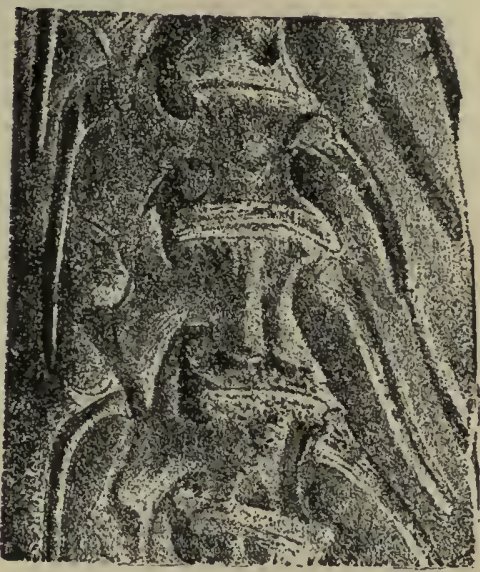

Fig. 1188.-Molgophis brevicostatus.

Molgophis, Cope, 1868, Proc. Acad. Nat. Sci., p. 220. [Ety. molges, a salamander; ophis, serpent.] Body long, serpentine, without dermal armature; vertebræ long, broad, with prominent zygopophyses and moderate neural spines; ribs large, curved, with tubercle and head on the dilated extremity. Type M. macrurus.

brevicostatus, Cope, 1875, Ohio Pal., vol. 2, p. 369, Coal Meas.

macrurus, Cope, 1868, Proc. Acad. Nat. Ści., p. 220, and Ohio Pal., vol. 2, p. 368, Coal Meas.

wheatleyi, Cope, 1875, Ohio Pal., vol. 2, p. 369 , Coal Meas.

Notrodon, Marsh, 1878, Am. Jour. Sci. and Arts, 3d ser., vol. 15, p. 410. [Ety. nothos, spurious; odous, tooth.] Type N. lentus.

lentus, Marsh, 1878, Am. Jour. Sci. and Arts, 3d ser., vol. 15, p. 410, Permian.

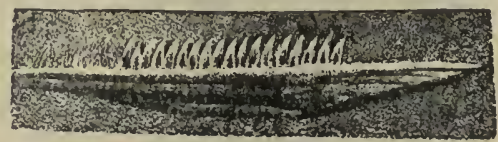

Fra. 1189.-Oestocephalus rectidens.

OrstockPhalus, Cope, 1868, Proc. Acad. Nat. Sci. Phil., p. 217, and Ohio Pal., vol. 2, p. 380. [Ety. oistos, an arrow ; kephale, the head.] Form slender, snake-like; caudal vertebræ with dilated and sculptured neural and hæmal spines; cranium lanceolate; teeth numerous, subequal; no pectoral shields; abdomen protected by bristle-like rods, which converge forward; no scales; a pair of weak posterior limbs; branchibyal bones present. Type 0 . remex.

amphiuminus, Cope, 1868 , Proc. Acad. Nat. Sci., syn. for 0 . remex. 
pertinatus: Cope, see Ptyonius pectinatus. rectidens, Cope, 1874, Trans.Am. Phil.Soc., and Ohio Pal., vol. 2, p. 386, Coal Meas. remex, Cope, 1868, Proc. Acad. Nat. Sci. p. 217, (Sauropleura remex,) Ohio Pal., vol. 2, p. 381, Coal Meas.

serrula, see Ptyonius serrula.

vinchellanus, see Ptyonius vinchellanus.

Opuinconon, Marsh, 1878, Am. Jour. Sci. and Arts, 3d ser., vol. 15, p. 411. [Ety. ophiakos, belonging to serpents; odous, tooth.] Type O. grandis.

grandis, Marsh, 1878, Am. Jour. Sci. and Arts, 3 d ser., vol. 15 , p. 411 , Permian.

mirus, Marsh, 1878, Am. Jour. Sci. and Arts, 3d ser., vol. 15, p. 411, Permian.

Ornithichniles, Hitcheock, 1836, Am. Jour. Sci. and Arts, vol. 29. [Wity. ornilhos, a bird; ichnos, a footstep.] This is not properly a generic name. No birá-tracks are known in Palrezoic rocks. The Batrachian tracks referred to it belong to another genus.

culbertsoni, King, 1845, Am. Jour. Sci. and Arts, vol. 48, p. 345, Coal Meas.

gallinuloides, King, 1845, Am. Jour. Sci. and Arts, vol. 48, p. 344, Coal Meas.

Pantyll's, Cope, 1881, Bull. U. S. Geo. Sur. Terr., vol. 6, No. 1, p. 79. [Ety. pan, all; tylos, a knob.] Founded upon the crania; ossification complete, leaving only orbits, nostrils, and parietal fontanel; surface sculptured; mandible with an angular process; teeth conic, obtuse, larger anteriorly ; mandible supporting several rows of teeth, which oppose a pavement of obtuse teeth on the palate; these are situated on the palatine or anterior part of pterygoid bones; quadratojugal and malar bones well developed; no lyra or mucous grooves. Type P. cordatus.

cordatus, Cope, 1881, Bull. U. S. Geo. Sur. Terr., vol. 6 , p. 79 , Permian.

PArioticilus, Cope, 1878, Pal. Bull., No. 29, and Proc. Am. Phil. Soc., vol. 17, p. 508 . [Ety. pareia, the cheek; teichos, a wall.] Founded on the cranium. Temporal fosse were covered by a roof continuous with the postorbital region; zygomatic arch extends low down; orbits lateral; muzzle short, with terminal nares; teeth rooted, crowns obtuse, with cutting edge. Type P. brachyops.

brachyops, Cope, 1878, Pal. Bull., No. 29. and Proc. Am. Phil. Soc., vol. 17, p. 508, Permian.

megalops, Cope, 1883, Pal. Bull., No. 36, and Proc. Am. Phil. Soc., vol. 20, p. 630, Permian.

Parioxys, Cope, 1878, Pal. Bull., No. 29, and Proc. Am. Phil. Soc., vol. 17, p. 521. [Ety. pareia, cheek; oxys, sharp.] Syn. for Eryops.

ferricolus, see Eryops ferricolus.

Pelion, Wyman, 1868, Proc. Acad. Nat. Sci. Phil., p. 211. [Ety. proper name.] Founded upon an inferior view of part of the skeleton; head as broad as long, semielliptical ; angles of mandibles project backward; mandibular rami slender, curved; vertebræ have centra as broad as long, and medially contracted; fore limbs stout; humeri long, thickened proximally, flattened and dilated distally ; ulna and radius united proximally, narrowing the arm proximally while expanded distally; left hand exlibits four digits, of which the third from the inner is the longer; number of phalanges is $2,3,4$ ?; carpus not osseous. Type P. lyelli.

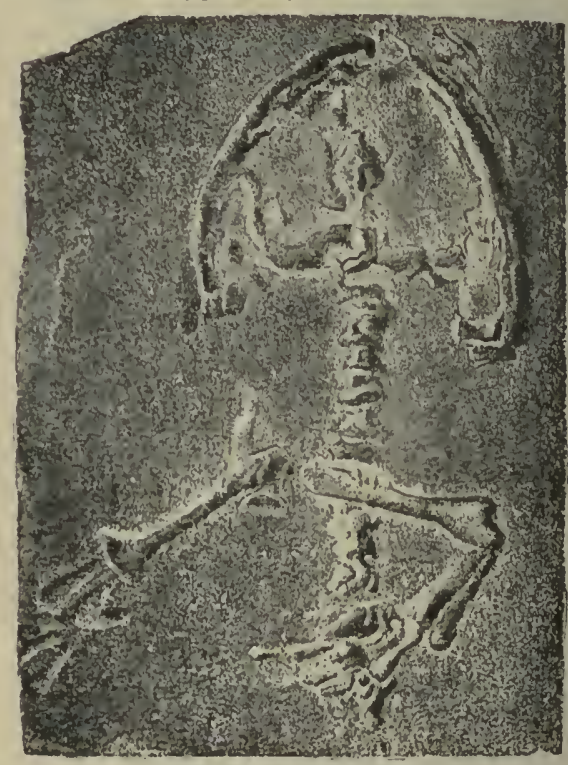

FIG. 1190-Pelion iyelli.

lyelli, Wyman, 1858, (Raniceps lyelli,) Am. Jour. Sei. and Arts, 2 (1 ser., vol. 25, p. 158, and Ohio Pal., vol. 2, p. 389, Coal Meas.

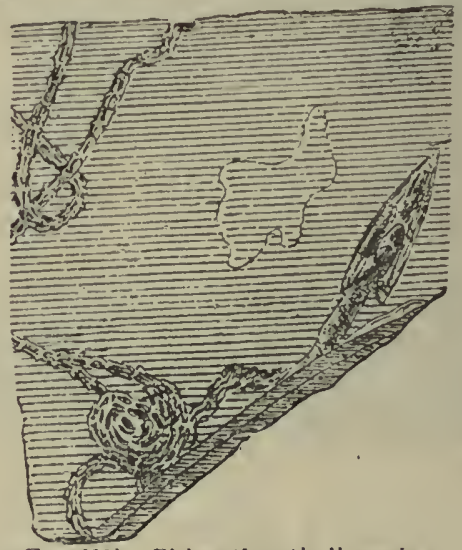

Fig. 1191.-Phlegethontla IInearis.

Phlegethontia, Cope, 1871, Proc. Am. Phil. Soc., p. 177. [Ety. Phlegethon, a burning river of heli.] Head elongate, 
triangular; body and tail extremely elongate; dorsal vertebra without ribs, caudals without dilated spines; no vertebræ armature; no limbs. Type P. linearis.

linearis, Cope, 1871, Proc. Am. Phil. Soc., p. 177, and Ohio 'Pal., vol. 2, p. 367, Coal Meas.

serpens, Cope, 1871, Proc. Am. Phil. Soc., p. 177, and Ohic Pal., vol. 2, p. 367, Coal Meas.

Pleuroptyx, Cope, 1875, Ohio Pal., vol. 2, p. 370 . [Ety. pleura, a rib; ptyx, a fold, wing.] Founded upon the vertebral column and ribs; vertebræ of moderate length, zygapophyses well developed, short neural spine in the dorsal region, not. sculptured; ribs short, stout, and support a wing on the posterior or convex border, which expands downward, and then abruptly contracts to the shaft; it is broad and truncate, and includes a medullary cavity partially filled with cancellated tissue. TypeP.clavatus. clavatus, Cope, 1875, Ohio Pal., vol. 2, p. 370, Coal Meas.

Pryonius, Cope, 1875, Ohio Pal., vol. 2, p. 373. [Ety. ptyon, a fan.] Form elon-

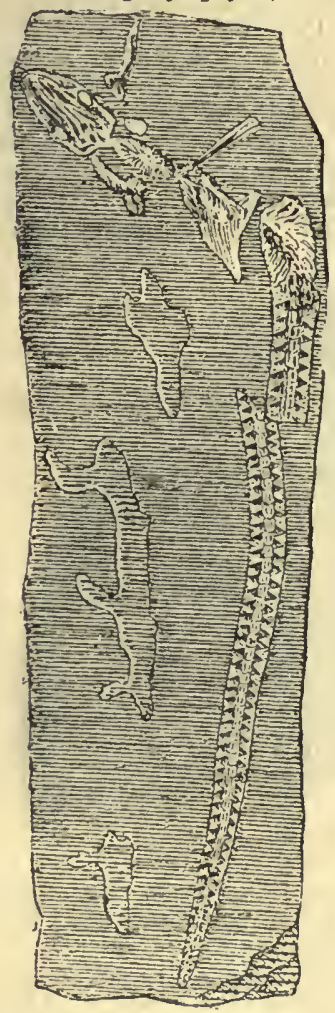

Fig. 1192.-Ptyonius serrula. gate, with long tail and lance ol a te cra $\mathrm{n}$ i u m ; limbs weak, only posterior known; three pec. toral shields; a b d o me n protected by packed osseous rods arranged in chevron with angle directed forward ; neural and h $æ \mathrm{~m}$ a 1 spines of caudal vertebræ expanded and fan-like ; ribs well dereloped; teeth small, numerous, simple or grooved. Type P. nummifer.

marshi, Cope, 1875, (Colosteus marshi,) Trans. Am. Phil. Soc., vol. 14, p. 24 , and Ohio

Pal., vol 2, p. 375, Coal Meas.

nummifer, Cope, 1875, Ohio Pal., vol. 2, p. 374, Coal Meas. pectinatus, Cope, 1868, (Sauropleura pectinata, Proc. Acad. Nat. Sci., p. 216, and Ohio Pal., vol. 2, p. 377, Coal Meas.

serrula, Cope, 1871, (Oestocephalus serrula,) Proc. Am. Phil. Soc., p. 177, and Ohio Pal., vol. 2, p. 379, Coal Meas.

vinchellanus, Cope, 1871, (Oestocephalus vinchellanus,) Proc. Am. Phil. Soc., p. 177, and Ohio Pal., vol. 2, p. 376, Coal Meas.

Pygopterus, Agassiz, 1833, Recherch. Poiss. Foss.

scutellatus, see Colosteus scutellatus.

Raniceps, Wyman, 1858, Am. Jour. Sci. and Arts. The name was preoccupied by Cuvier, and Pelion has been substituted. lyelli, see Pelion lyelli.

Rhachitonnus, Cope, 1878, Pal. Bull. No. 29, and Proc. Am. Phil. Soc., vol. 17, p. 526. [Ety. rachis, the backbone; tomos, cut.] Syn. for Eryops.

valens, syn. for Eryops megacephalus.

Sa uropleura, Cope, 1868, Proc. Acad. Nat. Sci. Phil., p. 215, and Ohio Pal., vol. 2, p. 402 . [Ety. sauros, a lizard; pleuron, a rib.] Vertebræ and ribs well developed; limbs four, large; five digits in the fore foot; carpus cartilaginous; ventral armature of closely arranged rhomboidal scuta, arranged in lines closely placed in chevrons, with the angle anterior; teeth of Labyrinthodont type, with deeply inflected enamel and acute apex. Type S. digitata.

digitata, Cope, 1868, Proc. Acad. Nat. Sci. Phil., p. 216, and Ohio Pal., vol. 2, p. 403, Coal Meas.

longipes, see Tuditanus longipes.

newberryi, Cope, 1875, Ohio Pal., vol. 2, p. 404, Coal Meas.

pectinata, see Ptyonius pectinatus.

remex, see Oestocephalus remex.

Sa ropus, Lea, 1849, Trans. A m. Phil. Soc., vol. 10. [Ety. sauros, a lizard; pous, foot.] Founded upon tracks; five robust toes, the inner having the same direction as the others, and not divergent as in Chirotherium; palm or sole short, wide. Type S. primavus.

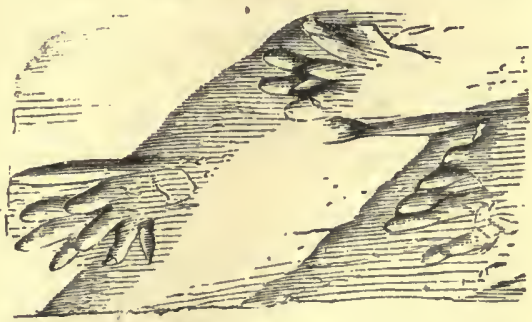

Fig. 1193.-Sauropus primævus.

primævus, Lea, 1849, Trans. Am. Phil. Soc., vol. 10, Coal Meas.

sydnensis, Dawson, 1868, Acad. Geol., p. 358, Coal Meas.

unguifer, Dawson, 1872, Geo. Mag. Lond., vol. 9, Coal Meas. 
Sphenacodon, Marsh,-1878, Am. Jour. Sci., and Arts, 3 d ser., vol. 15, p. 410. [Ety. sphen, a wedge; akis, a barb ; odous, tooth.] Type S. ferox.

ferox, Marsh, 1878, Am. Jour. Sci. and Arts, 3d ser., vol. 15, p. 410, Permian.

Spi meropezium, King, 1845, Am. Jour. Sci. and Arts, vol. 48, p. 345. [Ety. sphaira, sphere; pezia, sole of the foot.] Founded upon tracks representing a round depression for the ball of the foot, and five depressions for digits. Type S. leptodactylum.

leptodactylum, King, 1845, Am. Jour. Sci. and Arts, vol. 48, p. 345, Coal Meas.

ovidactylum, King, 1845, Am. Jour. Sci. and Arts, vol. 48, p. 347, Coal Meas.

pachydactylum, King, 1845, Am. Jour. Sci. and Arts, vol. 48, p. 346, Coal Meas.

thærodactylum, King, 1845, Am. Jour. Sci. and Arts, vol. 48, p. 346, Coal Meas.

Triena ropus, King, 1845, Am. Jour. Sei. and Arts, vol. 48, p. 343. [Ety. thenaros, palm of the hand; pous, foot.] Founded upon tracks. 'Type T. heterodactylus.

heterodactylus, King, 1845, Am. Jour. Sci. and Arts, vol. 48, Coal Meas.

leptodactylus, King, 1845, Am. Jour. Sci. and Arts, vol. 48, Coal Meas.

ovidactylus, King, 1845, Am. Jour. Sci. and Arts, vol. 48, Coal Meas.

pachydactylus, King, 1845, Am. Jour. Sci. and Arts, vol. 48, Coal Meas.

spharodactylus, King, 1845, Am. Jour. Sci. and Arts, vol. 48, Coal Meas.

Theropleura, Cope, 1878, Pal. Bull. No. 29, and Proc. Am. Phil. Soc., vol. 17, p. 519. [Ety. thero, to burn ; pleura, side.] Pelycosaurian reptiles with free neural arch, and a capitular costal articulation on the centrum, and no known intercentrum; neural spines not elongate; teeth equal. Type $\mathbf{T}$. retroversa.

obtusidens, Cope, 1880, Pal. Bull. No. 32 , p. 4, Permian.

retroversa, Cope, 1878, Pal. Bull. No. 29, and Proc. Am. Phil. Soc., vol. 17, p. 519 , Permian.

triangulata, Cope, 1878, Pal. Bull. No. 29, and Proc. Am. Phil. Soc., vol. 17, p. 520 , Permian.

uniformis, Cope, 1878, Pal. Bull. No. 29, and Proc. Am. Phil. Soc., vol. 17, p. 519, Permian.

Thyrsidium, Cope, 1875, Ohio Pal., vol. 2, p. 365. [Ety. thyrsos, a rod with leaves.] Founded upon a latero-inferior view of the spinal column; diapophyses enlarged, fan-like; centra contracted; abdomen protected by hair-like rods in chevron, with angle directed forward. Type T. fasciculare.

fasciculare, Cope, 1875, Ohio Pal., vol. 2, p. 365 , Coal Meas.

Trimerorachis, Cope, 1878, Pal. Bull. No. 29, and Proc. Am. Phil. Soc., vol. 17, p. 524. [Ety. trimeros, tripartite; rachis, the backbone.] Centrum rliachitomous, represented by three cortical ossifications of the chorda-sheath, a median inferior and two lateral; the lateral are distinct, and in contact with the neurapophyses above, and the posterior border of the median segment in front; neural arch joins the lateral elements, and is in slight contact with the lateral summits of the inferior element; the halves of the neural arch are co-ossified, and support zygapophyses, but no neural spine; cranial bones sculptured; parasphenoid flat; external nostrils large, superior; angle of mandible little produced; glenoid cavity transverse; deep internal pterygoid fossa; no coronoid process; symphysis short; teeth conic, two series in the upper jaw, the large ones anterior; ribs short, heads expanded. Type $\mathrm{T}$. insignis.

bilobatus, Cope, 1883, Pal. Bull. No. 36, and Proc. Am. Phil. Soc., vol. 20, p. 629, Permian.

insignis, Cope, 1878, Pal. Bull. No. 29, and Proc. Am. Phil. Soc., vol. 17, p. 524, Permian.

Tunitanus, Cope, 1871, Proc. Am. Phil. Soc., p. 177, and Ohio Pal., vol. 2, p. 391 . [Ety. proper name.] Cranium broad, flat, bones sculptured; teeth on premaxillary and maxillary bones of nearly equal sizes; three pectoral shields, sculptured externally; form lizard-like; two pairs of medium limbs; no chevron abdominal rods. Type $\mathrm{T}$. punctulatus.

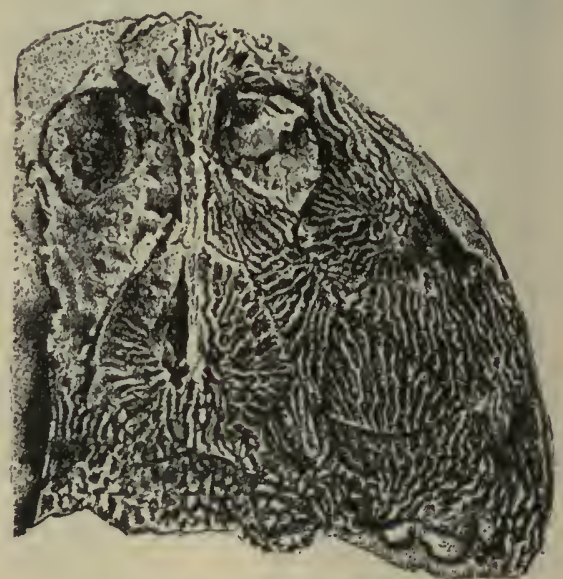

FiG. 1194.-Tuditanus radiatus.

brevirostris, Cópe, 1874, Trans. Am. Phil. Soc., vol. 14, p. 10, and Ohio Pal., vol. 2, p. 393, Coal Meas.

huxleyi, Cope, 1874, Trans. Am. Phil. Soc., p. 10, and Ohio Pal., vol. 2, p. 397, Coal Meas.

longipes, Cope, 1874, (Sauropleura longipes,) Trans. Am. Phil. Soc., vol. 14, p. 10, and Ohio Pal., vol. 2, p. 398, Coal Meas. 
mordax, Cope, 1875, Ohio Pal., vol.2, p. 395, syn. for Ceraterpeton punctolinatum.

obtusus, Cope, 1868, (Dendererpeton obtusum,) Proc. Acad. Nat. Sci. Phil., p. 213, and Ohio Pal., vol. 2, p. 396, Coal Meas.

punctulatus, Cope, 1874, Trans. Am. Phil. Soc., vol. 14, p. 10, and Ohio Pal., vol. 2, p. 392, Coal Méas.

radiatus, Cope, 1874, Trans. Am. Phil. Soc., vol. 14, p. 10, and Ohio Pal., vol. 2, p. 394, Coal Meas.

tabulatus, Cope, 1877, Proc. Am. Phil. Soc., p. 577, Coal Meas.

Zatrachys, Cope, 1878, Pal. Bul. No. 29, and Proc. Am. Phil. Soc., vol. 17, p.
523. [Ety. za, an intensive; trachys, rough.] Teeth in single series, and anchylosed to the bottom of a shallow groove, the external boundary of which is most prominent, so the attachment of the teeth is shortly pleurodont; teeth have conic crowns and basal grooves; cranium sculptured, its table with a notch on each side; two occipital condyles; no intercalary horns. Type Z. serratus.

apicalis, Cope, 1881, Am. Naturalist, vol. 15, p. 1020, Permian.

serratus, Cope, 1878, Pal. Bull. No. 29, and Proc. Am. Phil. Soc., vol. 17, p. 523, Permian. 



\section{GLOSSARY}

$-\mathrm{OF}-$

\section{SPECIFIC NAMES IN ISE IN NORTH AMERICAN PALEONTOLOGY.}

Every adjective specific name must agree in gender with the genus to which it belongs; hence, the masculine, feminine, and neuter endings are indicated. Nouns do not change the termination, but remain the same, no matter what the gender of the genus may be. The names of persons and places are not included in this Glossary, because they should be known by the terminations they take when reduced to specific names.

Abacus-A table divided into squares.

Abbreviatus, a, um-Abbreviated, shortened.

Abnormis, e-Abnormal.

Abruptus, a, um-Abrupt, broken.

Abscissus, a, um-Steep, abrupt.

Acanthophorus, a, um-Thorn bearing.

Acanthoptera-Spine-wing.

Accinctus, a, um-Girded.

Acervulosus, a, um-Many clustered together.

Acervus-A heap considered as a body.

Acicula-A small pin or needle.

Acicularis, e-Full of small pins or needles, acicular.

Aciculatus, a, um-Like a small needle.

Aciedentatus, a, um -Needle-toothed, sharptoothed.

Acies-The edge or sharp point.

Acinaciformis, e-Scimitar-shaped.

Acinus-Any berry, or the kernel in the berry.

Aclis-A small javelin.

Acmea-Edge, point.

Acrocarpus, a, um-Pointed fruit.

Actuarius, a, $11 \mathrm{~m}-$ Swift, agile.

Aculeatus, a, um-Thorny, pointed, sharp.

Aculeolatus, a, um-Thorny.

Acuminatus, a, um-Sharp-pointed.

Acus-A pin or needle.

Acutangulus-Acute angle.

Acuticosta-Sharp rib.

Acutidactylus-Sharp-fingered.

Acutifolius, a, um-Having acute leaves.

Acutiliratus, a, um-Sharp-ridged.

Acutiplicatus, a, um-Sharp-plicated.

Acutiradiatus, a, um-Sharp-rayed.

Acutirostris-Sharp beak.

Acutulus, a, um-Somewhat pointed.

Acutus, a, um-Acute, sharpened.

Adiantites-From resemblance to Adiantum. Adiantoides-Like Adiantum.
Adductus, a, um-Stretched, contracted.

Adjunctivus, a, um-Joined, united.

Adjunctus, a um-Joined, connected.

Aduascens-Growing upon.

Adnatus, a, um-Adnate.

Adorabilis, e-Worthy of adoration.

Adultus, a, um-Adult.

Aduncus, a, um-Bent inward, hooked.

Aigilops-An acorn.

Emulus, a, um-Emulous, vying with.

Enigma-Obscure, a riddle.

Equalis, e-Equal, like.

Equibrachiatus, a, um-Equal-armed.

Equicostatus, a, um-Equal-ribbed.

Equidistans-Equidistant.

Equilateralis, e-Equilateral.

Equiradiatus, a, um-Equal-rayed.

Equivalvis, e-Equal-valved.

Aquns, a, um-Plain, even, level, equal.

Affinis, e-Related, or near to.

Agellus-A small field.

Agglomeratus, a, um-Gathered into a mass.

Aggregatus, a, um-Aggregated.

Agilis, e-Agile, nimble.

Agrarius, a, um-Pertaining to fields or country.

Agrestis, e-Pertaining to the country.

Alatıs, a, um-Winged.

Albus, a, um-White:

Alcicornis, e-Elk-horned.

Alectiformis, e-In form like Alecto.

A liger, gera, gerum-Bearing wings.

Alsus, a, um-Cold.

Alternans-Alternating.

Alternatus, $a, u m-A l t e r n a t e$.

Alterniradiatus, a, um-Alternately rayed.

Alternistriatus, a, um-Alternately striated.

Alternus, a, um-Alternate.

Altilis, e-Flattene 1.

Altidorsatus, a, um-High-backed.

Altiplicatus, a, um-Having high plications. Altirostris-High beak. 
Altus, a, um-High, great, rleep.

Alveatus, a, um-Holluwed ont like a trongh.

Alveularis, e-Small-cranneled.

Alveolatus, a, um-Hollowed out.

Alveolus-A small cavity.

Anıarus, a, um-Bitter, brackisl.

Ambiguus, a, um-Doubtful, changeable.

Amonus, a, um-Plensant, charming.

Amphibolus, a, um-Ambiguons.

Amplexicaulis, e-Embracing the stalk or stem.

Amplexus-An encircling, surrounding.

A mpliagtus, a, um-Cnlarged.

Amplus, a, um-Ample, spacious, roomy.

Ampullacens, a, um-In the form of a flask.

Anvgdaliformis, $f-$ In form like the almond.

Anabathra-A ladkler.

A nalogus, a, um-Analngous.

Anatiformis, e-Like Anatifa.

Anatinus, a, um-Ol or pertaining to the duck.

Anctps-Double, two-headed, doubtful.

Anchoralis, e-Of or pertaing to an anchor.

A ceilla-A luand-maicl.

Anellus-A little ring.

Anguineus, a, um-Serpent-like.

Angularis, e-Angular, cornered.

Angulatus, : um-Having corbers.

Angulosus, a, um-Full of corners.

Angustatus, a, um-Narrowed.

Angustifolius, a, um-Nurrow-leaved.

Angustipinna-A narrow feather.

Angustus, a, uin-Narrow, straight, not spacious.

Annectans-Connected together.

Annularififolius, a, um--Having ring-shaped leaves.

Annulatua, a, um-Annulated, ringed.

Annuliferus, a, um-Ring.bearing.

Anomalus, a, um-Anomalous, not coming under the rule.

Anonymus, a, um-Nameless.

Ante ceptus, a, um-Anticipated.

Antennarius, n, um-Pertaining to the antennæe.

Antheloideus, n, um-Like Anthelia.

Anthracinus, a, um-Coal-black.

Antiquarius, a, um-Pertaining to antiquity.

Antiquatus, a, um-Antiquated, ancient.

Antiquus, a, um-Ancient, olıl.

Aunuluris, e-Relating to the signet riug.

Annularius, a, um-Of or pertaining to the signet ring.

Annulatus, a, um-Annulated, ringrd.

Annulus-A ring.

A pertus, a, um-Opened, uncovered.

Apicalis, e-Sharp-pointed.

Apiculatus, a, um-Having a pointed termination.

Aplatus, a, um-Flattened.

Appendiculatus, a, um-Having lateral appendages.

Approximatus, a, um-Approximated, approaching $n \in$ ar to.

A prinus, a, um-Pertaining to a wild boar.

Ayuilinus, a, um-Pertaining to the eagle, aquiline.

A rachniformis, e-In form like a spider.

Arachnoideus, a, um-Like a cobweb.
Araneolus-A small spider.

Aratus, a, um-Plowed.

Arborescens-Tree-like, arborescent.

A rloreus, a, um-Pertaining to a tice.

A rbuscula-A shrub.

Arcanus, a, um-Closed, shut up.

A rchinediformis, e--Archimediform.

Arcticus, a, um--Arctic.

Arctifossa-Close-wrinkle.

Arctiporus, a, um-Having narrow pores.

A rctostriatus, a, um-Closely striated.

Arclirostratua, a, um-Narrow-beaked.

Arctisegmentus, a, um-Having narrow segments.

Arctus, a, um-Closed, pressed together, short.

Arcuatus, a, um-Bent, curved like a bow.

Arcuosus, a, um-Full of arches, bent over.

Arenaceus, a, um-Sandy.

Arenaritu, a, um-Pertaining to sand.

Arenosus, a, um-Sandy.

Areolatus, a, um-Divid,d into irregular squares, or angular spaces.

Argentalius, a, um-OH or pertaining to silver.

A rgenteus, a, um-Of or made of silver.

Argenturbicus, a, um-Of or velunging to Silver City.

Argutus, a, um-Sharply defined, distinct. Arietinus, a, um-Of or from a ram.

Armatus, a, um-Armed, equipped.

Armiger, era, erum-Armed, war-like.

Armosis, a, um-Many armed.

Arrectarius, a, um-Erect.

Arrectus, a, $11 \mathrm{~m}$-Erect, steep.

Arrosus, a, um-Gnawed.

Articulatus, a, um-Furnished with joints, aiticulated.

Arti.ulosus, a, um-Full of knots, or divisions.

Artemisiifolius, a, um-Like the plant Artemisia.

A rundinaceus, a, um-I,ike a reed.

Aspectans-Expected, lork d for.

Asper, era, ermm-Rough, uneven.

Asperatus, a, un-Rouwhener, irregular.

Aspersus, a, um-Scattered, disperst d.

Aspratilis, e-Rough.

As:inilia, e-Similar.

Atavus-Ancestor.

Attenuatus, a, um-IIade tlin, attel.u ited.

Attritus, a, um-Wori.

Aucella-A little birrl.

Audaculus, a, um-Bold.

Augustatus, a, um-Majestic.

Allgustus, a, um-A ugust.

Auleticus, a, um-That is suitable fur a pipe.

Aureatus, a, um-Adorn $d$.

Auricula-The ear.

Auritus, a, um-Eared.

Australis, e-Southern.

Auxiliarius, a, um-Helping

Avicula-A small bird.

Avitus, a, um-Ancestral.

Avus-Grandfather.

Bacca-A berry, a small, ronnd fruit.

Bacillum-A small staff.

Baculiformis, e-Staff-shaped. 
Viaculum-A staff or cudgel.

B.tinoides-Like Balanus.

Balanus-An acorn.

Balteatus, a, um-Belted.

Bas batus, a, um-Bearded.

Barydactylus, a, um-Heavy fingered.

Basalis, e-Pertaining to the base.

Basalticus, a, um-Basaltic.

Basilaris, e-Relating to the base.

Basilicus, a, um-Splendid.

Belemnura-Having a tail like a dart.

Bellarugosus, a, um-Beautifvlly wrinkled.

Bellatrema-Beautiful opening.

Bellatulus, a, um-Pretty, neat.

Bellicinctus, a, um-Beautifully banded.

Bellicosus, a, um-Warlike.

Bellifer, era, erum-Warlike.

Bellilineatus, a, um-Beautifully lined.

Bellipunctus, a, um-Beautifully dotted.

Bellistriatus, a, um-Beautifully striated.

Bellulus, a, um-Very beautiful, pretty.

Bellus, a, um-Beautiful.

Bertholletiformis, e-Iike Bertholletia.

Biacutus, a, um-Two-pointed.

Bialveatus, a, um-Two-channeled.

Biangulatus, a, um-Two-angled.

Bicarinatus, a, um-Two-keeled.

Bicarpus, a, um-Two-fruited.

Biceps-'Two-headed.

Bicinctus, a, um-Two-banded.

Biclavatus, a, um-Two-clubbed.

Bicorniger, era, erum-Two-horned.

Bicornis, e-Two-horned.

Bicornutus, a, um-Two-horned.

Bicostatus, a, um-Two-ribbed.

Bicristatus, a, um-Double-peaked or twocrested.

Bicuspidatus, a, um-Two-pointed.

Bidens-Having two teeth, two-pronged.

Bidentatus, a, um-Double-toothed.

Bidorsalis, e-Double-backed.

Bidorsatus, a, um-Having a double back.

Bifarius, a, um-In two ways or parts, double.

Bifidatus, a, um-Cleft into two parts.

Bifidus, a, um-Bifid, cloven in two parts.

Bifissus, a, um-Cleft into two parts.

Bifoliatus, a, um-Two-leaved.

Biforatus, a, um-Two-holed or double. doored.

Biformatus, a, um-Two-shaped.

Biformis, e-Two-formed.

Bifrons-With two foreheads.

Bifurca-A two-pronged fork.

Bifurcatus, a, um-Bifurcated, forked.

Bilurcus, a, um-Two-pronged.

Biimbricatus, a, um-Double-imbricated.

Bijugus, a, um-Yoked two together.

Bilabiatus, a, um-Two-lipped.

Bilamellatus, a, um-Having double lamellæ.

Bilateralis, e-Two-sided.

Bilineatus, a, um-Two-lined.

Biliratus, a, um-Two-furrowed.

Blix-Woven with a double thread, twothreaded.

Bilobatus, a, um-Two-lobed.

Bilobus, a, um-Two-lobed.

Bimesialis, e-Having two middle parts.
Bimucronatua, a, um-Two-poin'ed.

Binervis, e-Two-nerved.

Binodus-Double knot.

Binumbonatus, a, um - Having double umbones.

Bipartitus, a, um-Two-parted.

Bipennis, e-Two-winged.

Biplicatus, a, um-Two-plicated, or in twofolds.

Bipunctatus, a, um-Bipunctate.

Bipyramidalis, e-Double-pyramidal.

Bisectus, a, um-Divided.

Biserialis, e-In two series.

Biseriatus, a, uin-Having two rows or series.

Biserrulatus, a, um-Double-serrulated.

Bisinuatus, a, um-Having two depressions or furrows.

Bispinulatus, a, nm-Two-spinel.

Bispiralis, e-Two-whorled.

Bistriatus, a, um-Two-striated.

Bisulcatus, a, um-Two furrowed.

Bisulcus, a, um-Cloven.

Bituberculatus, a, $\mathrm{um}-$ Double-tuberculated.

Biturbinatus, a, um-Double-turbinated.

Bivertex-Double head.

Bivius, a, um-Having two ways or passages.

Bivittatus, a, um-Two-banded.

Bivolvis, e-Two-rolled.

Blatta-A cockioach or moth.

Blattinoides-Like Blattina.

Bombifrons-Having a hollow front.

Borassifolius, a, um-Leaved like Borassus.

Borealis, e-Northern.

Bovidens- $\mathrm{Ox}$ tooth.

Bovipedalis, e-Ox-footed.

Brachialis, e-Having arms.

Brachiatus, a, um-Having arms.

Brachium-An arm.

Brachynotus, a, um-Short-ridged.

Bracliyops-Short sight.

Bracteatıs, a, um-Covered with plates, beautiful.

Breviceps-Short head.

Brevicornis, e-Short-horned.

Brevicostatus, a, um-Short-ribbed.

Breviculus, a, um-Somewhat shortened.

Brevicurvatus, a, um-Short-curved.

Brevifolius, a, um-Short-leaved.

Brevilineatus, a, um-Short-lined.

Brevilobatus, a, um-Short-lobed.

Brevilobus-Short lobe.

Brevimarginatus, a, um-Short-margined.

Brevinodus-Short node or short knot.

Breviplicatus, a, um-Short-plicated.

Breviposticus, a, um-Made short behind.

Breviradiatus, a, um-Short-rayed.

Brevirostris, e-Short beak.

Brevis, e-Short.

Brevisulcatus, a, um-Short-furrowed.

Breviusculus, a, um-Very short.

Brisa-Grape-skins.

Bryonoides-Like moss.

Buccinum-A trumpet or horn.

Bucculentus, a, um-Wide-mouthed.

Bufo-A toad.

Bulbaceus, a, um-Bulbous. 
Bulbosus, a, um-Bulbous.

Bulbus-A bulb.

Bulimiformis, e-Like Bulimus.

Bulla-A round object, bubble.

Bullatus, a, um-Studded with knobs.

Bulloides-Like a bubble.

Bullulatus, a, um-Little vesicled.

Bursa-A purse.

Burciformis, e-Purse-shaped.

Cadens-Falling, terminating.

Caduceus-The herald's staft.

Crecigenus, a, um-Born blind.

Crelamen-A bass relief.

Cælator-A carver.

Cælatus, a, um-Engraved, carved.

Cæspitosus, a, um-Turf-like.

Calamitoideus, a, um-Like a Calamite.

Calamus-A reed.

Calantica-A covering for the head.

Calathus-A wicker basket.

Calcaratus, a, um-Spurred, spur-shaped.

Calcariformis, e-Like a spur.

Calceolus-A small shoe.

Calciferus, a, um-Calciferous.

Calculus-A small stone.

Caliculus-A small cup.

Calix-A cup.

Callicephalus, a, um-Having a beautiful head.

Calliteles-A beantiful tail.

Callosus, a, um-Thick-skinned, callous.

Calycinus-A little calyx.

Calycularis, e-Like a little cup or flowerbud.

Calyculoidés-Like a little cup.

Calyculus-A flower-cup.

Calymenoides-Like Calymene.

Calyx-The cup of a flower.

Cameratus, a, um-Arched.

Cameriferus, a, um-Chambererl.

Cammarus-A lobster.

Campaniformis, e-Bell-formed.

Campanulatus, a, um-Bell-shaped.

Camurus, a, um-An arch, turned invari.

Canaliculatus, a, un-Channeled, canaliculated.

Canalis-A channel or groove.

Cancellatus, a, um-Cross-barred, cance]lated.

Cancellosus, a, um-Finely cancellated or latticed.

Canna-A reed.

Canneus, a, um-Made of reeds.

Canniformis-Like Canna.

Cannaliratus, a, um-Reed-furrowed.

Cannula-A small reed.

Capax-Large, spacious.

Capillaceus, a, um-Similar to hair, stringy.

Capillaris, e-Of or pertaining to the liair.

Capillatus, a, um-Having hair.

Capillosus, a, um-Very hairy.

Capitalis, e-Relating to the liearl.

Capitatus, a, um-Having a head.

Capitellum-A small head.

Capitolinus, a, um-Pertaining to the cipitol, a tower.

Caponiformis, e-Capon-formed.

Capreolus-Props, stays.
Capularis, e--Pertaining to a coffin.

Capuloides-Like a capulus.

Capulus-A coffin or a handle.

Caput-serpentis-Serpent-head.

Caput-testitudinis-Turtle-head.

Carabus-A small wicker boat.

Carbonarius, a, um-Of or relating to coal.

Carcharidens-Dog-fish tooth.

Cardiiformis, e-Like a cardium.

Cardinalis, e-Of or pertaining to a doorhinge, or principal.

Catrdinatus, a, $1 \mathrm{~m}-$ Jointed, fitted to, hinged.

Cardinens, a, um-Of or pertaining to a door-hinge.

Carica-A kind of fig.

Carinatus, a, um-Keeled.

Cariniferus, a, um-Keel-bearing.

Carnosus, a, um-Fleshy.

Carus, a, um-Precious, valued.

Castanea-A chestnut.

Catactus, a, $11 \mathrm{~m}-$ Frail, easily broken.

Catastomus, a, um-Gaping at the lower end.

Catenoides-Chain-like.

Catenulatus, a, um-A little chain.

Catilliformis, e-Dish-shaped.

Catilloides-Dish-like.

Catillus-A small dish.

Catinus-A bowl.

Caudagalli-Tail of a eock.

Candatus, a, uın-Having a tail.

Cauliculus-Small stalk or stem of a plant.

Cavernosus, a, um-Full of hollows.

Cavifolius, a, um-Hollow-leaved.

Cavumbilicatus, a, um-Having a hollow umbilicus.

Cavumbona-Hollow umbo.

Cavus, a, um-Hollow, concave.

Celator-A concealer, hider.

Celebrus, a, uın-A bundant.

Celer-Swift, fleet.

Cellnlosus, a, um-Full of cells.

Celsipora-High pore.

Celsus, a, um-High.

Centennialis, e-The 100 th year.

Centralis, e-In the middle, central.

Centratus, a, um-Central.

Centrilineatus, a, um-Central-lined.

Centronatu凶, a, um-Having knots or points.

Centrosus, a, um-In the central point.

Cerasiformis, e-Like a dried cherry.

Cerithioides-Like Cerithium.

Cervicornis, e-Deer-horned.

Cervinus, a, um-Pertaining to a deer.

Cessator-An idler, loiterer.

Cetratus, a, $u m-$ Shield-bearing.

Chærophylloides-Like Chærophyllum.

Clitiformis, e-Hand-shaped.

Chiromorphus, a, um-Hand-formed.

Chromaticus, a, un-Chromatic, colored.

('hrysalis-Chrysalis.

Cicatricosus, a, um-Full of scars.

Ciceronius-Having warts.

Ciliatus, a, um-Haired on the margin, iringerl.

Cinctosus, a, um-Full of bands, girded.

Cinctulus-A small girt. 
Cinctura-A girdle.

Cinctus, a, um-Banded, girued.

Cinctutus, a, um-Girderl.

Cingulatıs, a, um-Encircled with lines, girded.

Cingulosus, a, um-Covered with lines or zones.

Cinguluin-A zone.

Circinatus, a, um-Compassed, rounded.

Circinctus, a, um-Encoupassed.

Circularis, e-Circular, round.

Circulus-A circle.

Circumliratus, a, um-Circular-lined.

Cistella-A small box.

Cistula-A little chest or coffer.

Citus, a, um-Sivift, speedr.

Clarus, a, um-Clear, brilliant, distinct.

Clatbratus, a, um-Cross-barred, latticed.

Clausus, a, um-Closed up.

Clava-A stick.

Clavacoideus, a, um-Club-shaped.

Clavatulus-A little club.

Clavatus, a, um-Knotted, club-shaped.

Clavicula-A small twig.

Clavifrons-Having a club-like front.

Claviger-A club-bearer.

Clavigerus, a, ım-Club-bearing.

Clavis-A bar.

Clavulus-A little club, a smail swelling.

Clavus-A nail, spike.

Clinatus, a, um-Inclined, bent.

Clinocameratus, a, um-Cirve-chambered.

Clipeatum-Furnished with a shield.

Clipeiformis, e-Shield-like.

Clivosus, a, um-Full of hills.

Clivulatus, a, um-Having little hills.

Clivulus-A little hill.

Clymenioides-Like Clymenia.

Clypeatus, a, um-Armed wfth a shield.

Clytis-Celebrated.

Coalescens-Growing together.

Coalitus, a, um-Grown together.

Coaptus, a, um-Closely joined.

Coarctatus, a, um-Compressed, jnined.

Cochlearis, e-In the form of a suail shell.

Cochleatus, a, um-Spiral.

Cochleola-A sinall suail.

Cognatus, a, um-Near to, cognate.

Cohærens-Adhering together.

Collatus, a, um-Joined together, collected.

Collectus, a, um-Collected.

Colliculus-A little hill.

Colligatus, a, um-Bound together, fastened.

Collinus, a, um-Hilly.

Colon-The great intestine.

Colubrellus-A little snake.

Colubrinus, a, um-Like a snake.

Colubrosus, a, um-Winding.

Columella-A small column.

Columellatus, a, um-Pillared.

Columnaris, e-Columnar.

Comes-A companion.

Comis, e-Friendly, nice, delicate.

Communis, e-Conmon.

Commutatus, a, um-Changed, altered.

Comosus, a, um-Hairy.

Compactilis, e-Pressed together.

Compactus, a, um-Compact.

Compertus, a, um-Discovered, ascertained.
Complanatus, a, um-Leveltd, smoothed.

Complexatus, a, um-Encircled.

Complexus, a, um-Snrrounded, encircled.

Compressus, a, um-Comprersed.

Comptus, a, um-Ornameuted, elegant.

Conatus-An effort.

Concavus, a, um-Concave.

Concentricus, a, um-Concentric.

Concinnulus, a, um-Small and beautiful.

Concinnus, a, um-Beautiiul, neat.

Conditus, a, um-Joined.

Confectus, a, um-Completed.

Confertus, a, um-Pressed close together.

Confervoides-Like Conferva.

Confirmatus, a, um-Made firm, establishe.1.

Conflexus-a, um-Bent.

Confluens-Running together, blendcd.

Conformalis, e-Similar.

Confragosus, a, um-Rough, uneven.

Confragus, a, um-Rough.

Confusus, a, um-Mixed together, confused.

Congener, eris-Congeneric.

Congestus, a, um-Accumulated, heaped.

Conglobatus, a, um-Galhered in a rouml mass.

Conglomeratus, a, um-Gatlered together.

Congregatus, a, um-Assembled together.

Congregalis, e-Uniting together.

Congruens-Corresponding, coinciding, runing together.

Coniculus-A little cone.

Conicus, a, um-Conical, cone-shaped.

Conifollis-An inflated cone.

Conifer, era, erum-Bearing coreal fruit.

Coniformis, e-Cone-shaped.

Conifrons-Having a conical front.

Coniger, era, erum-Bearing fruit of a conical form.

Conjugans-Joined, united.

Conjunctivus, a, um-Connecting.

Connatus, a, um-Connate, united,

Connivens-Dissembling, closing.

Conoideus, a, um-Somewhat conical.

Consimilis, e-Wholly similar.

Consobrinus-A cousin, relative, remotely allied.

Consolidatus, a, um--Consolidated.

Consolidus, a, um-Very firm.

Consors-Living in common.

Conspicuus, a, um-Visible, conspicuous.

Constans-Standing firm.

Constellatus, a, um-Very starry.

Constrictostriatus, a, um-Constricted and striated.

Constrictus, a, um-Constricted.

Consuetus, a, um-Customary, related to.

Contextus, a, um-Entwined.

Continens-Holding together.

Contractus, a, um-Contracted.

Contritus, a, um-Worn out.

Conulatus, a, um-Having little cones.

Conulus-A little cone.

Conus-A cone.

Convergens-Converging.

Convexus, a, um-Convex.

Convolutus, a, um-Rolled up, spiralwhorled.

Convolvans-Rolled together.

Coralliferus, a, um-Coral-bearing. 
Corallinum-Like red coral.

Coralloides-Like coral.

Corbis-A basket.

Corbula-A little basket.

Corbulifornis, e-Like a hasket.

Cordatoovatus, a, $u m$-Cordate-ovate.

Cordatus, a, um-Curdate, heart-shaped.

Cordiformis, e-Heart-sliaped.

Coriaceus, a, uin-Coriaceous, having the texture of rough skin.

Coriformis, e-like Coris.

Corinthius, a, $1 \mathrm{~m}-$ Corinthian.

Corium-A leather strap, bark.

Corniculum-A little liorn.

Corniger, era, erum-Iorned.

Cornuformis, e-In the form of a horn.

Cornu-A horn.

Cornulum-A little horn.

Cornutiformis, e-Horn-slıaped.

Cornutus, a, um-Horned.

Coronarins, a, uin-Of or belonging to a wreath.

Coronatus, a, um-Crowned.

Corpulentus, a, um-Corpulent.

Corrugatus, a, um-Cinrıugated, wrinkled.

Corticatus, a, um-Covered with bark.

Corticosus, a, um-Having thick bark.

Corylus-A hazel.

Cosciniformis, e-Like Coscinium.

Costa-A rib.

Costalis, e-Rilubed.

Costatiformis, e-Rib shaped.

Costatulus, a, um-Small ribbed.

Costatus, al, um-Having rils, ribbri.

Costelliferus, a, um-Bearing faint ribs.

Crassatus, a, uin-Thickisued.

Crassibraclijalus, a, um-Thick-armed.

Crassicardinalis, e-Having a llick linge.

Crassicauda-Thick-tril.

Crassicaulis, e-Having a thick stem.

Crassicostatus, a, um-Thick-ril,bed.

Crassidens-Having a thick tooth.

Crassidiscus-A thick dirk.

Crassifrons-Having a llick front.

Crassimarginatus, a. un-Thick-maryined.

Crassinervis, e-Having thick or dense nerves.

Crassiradiatus, a, um-Ilaving thiık rays.

Crassitestus, a, um-Like a thick ressel or pot-lid.

Crassolaris, e-Thickenod.

Crassus, a, $11 \mathrm{~m}-$ Thi $k$.

Ciatera-A bowl.

Crateriformis, e-Cup shaped.

Cratiulus, a, um-Composed of lattice-work.

Cratis-Wicker wolk.

Crebescens-Frequent, increasing.

Crebratus, a, um-Made thick, close.

Crebripora-Having the pores very close.

Crebrirama-Having dense branches.

Crebriseptus, a, um-Having many septa.

Crebristriatus, a, um-Closely striated.

Crenatocinctus, a, um-Notched around.

Crenatus, a, um-Crenuted, notched.

Crenistriatus, a, um-Having wriıkled lines.

Crenulatus, a, um-Crenulated.

Crepidula-A small sandal.

Crepiformis, e-Boot-shapti'.

Cretaceons, a, um-Cl alk-l ke.
Crebriformis, e-Full of openings like a sieve.

Cribrarius, a, um-Pertaining to a sieve.

Cribrosus, a, um-Full of holes like a sieve.

Crineus, a, um-Hairy.

Crispatus, a, um-Curled, crisped.

Crispus, a, um-Curled, wavy.

Cristatus, a, um-Tufted, crested.

Cristula-A small crest.

Cristulatus, a, um-Small-tufted.

Crossotus, a, um- Fringed.

Crotaliformis, e-Shaped like a bell.

Crotalum-A bell, a rattle.

Cruciatus, a, $11 \mathrm{~m}$-Cross-shaped, twisted.

Cruciferous, a, um-Cross-be arer.

Cruciformis, e-Cruciform.

Cruciger, era, trum-Cross bearel.

Crustosus, a, um-Crusted.

Crustula-A little shell, crust.

Cryptatus, a, um-Concealed.

Cryptodens-Hidden tooth.

Cucullus-A cap, covering.

Culeus-A leatlier bas.

Culmula-A little stalk or stem.

Culmus-A stem.

Cultellatus, a, um-Like a little knife.

Cultellus-A small knife.

Cultidactylus, a, um-Elegantly fingered.

Cultratus, a, um-Knife-formel.

Cultriformis, e-Shaped like a pruningknife.

Cumulatus, a, um-Heaped.

Cumulus-A heap.

Cuneatus, a, um-Wedge-form d.

Cuneiformis, e-Wedge-shaped.

Cuneolus -A little wedge.

Cuneus-A wedge.

Cuniculosus, a, um-Full of caves.

Cuniculus-A cradle, cavity.

Cunula-A little cradle.

Curinsus, a, um-Curious.

Curticardinalis, e-Short-linged.

Curtidentatus, a, um-Short-toothed.

Curtilobus, a, um-Short-lobed.

Curtirostratus, a, um-Short-beaked.

Curtus, a, um-Shortened.

Curvatus, a, um-Curved.

Curvidens-Having curved teeth.

Curvijuncturus, a, um-Joining in a curve.

Curvilineatus, a, um-Having curved lines.

Curvirostrum-A bent beak.

Cuspidatus, a, um-Pointed.

Cyathus-A cup.

Cyathiformis, e-Cup-shaped.

Cyclas-Of a round form.

Cyclonemioides-Like a Cyclonema.

Cyclopora-Round pore.

Cycloptera-Circle-wing.

Cyclopteroides-Like Cyclopteris.

Cyclostegium-Circular covering.

Cyclostigma-Having round scars, rounddotted.

Cyclostomus, a, um-Having a round mouth.

Cylindraceus, a, um-Like a cylinder.

Cylindricus, a, $\mathrm{m}$-Cylindrical.

Cymatoides-Wave-like.

Cymbalum-A cymbal. 
Cymbiformis, e-Boat-slıaped.

Cymbium-A small drinking cup.

Cymbula-A small boat.

Cymosus, a, um-Full of shoots.

Cynodon-Dog-tooth.

Cyrtiniformis, e-Like Cyrtina.

Cyrtodontoides-Like Cyrtodonta.

Cyrtolites-A curved stone.

Cysticus-A little bladder.

Dactyliformis, e-Finger-shaped.

Dactylodus, a, um-Finger-toothed.

Dactyloides-Like thimble punctures.

Dactylus-Growing like a finger.

Debilis, e-Weak, feeble.

Decabrachiatus, a, um-Ten-armed.

Decadactylus, a, um-Ten-fingered.

Decemplicatus, a, um-Ten-plicated.

Decipiens-Deceiving, doubtful.

Declivis, e-Sloping.

Decoratus, a, um-Decorated.

Decornis-Without horns.

1)ecorosus, a, um-elegant.

Decorticatus, a, um-Burked, decorticated.

Decorus, a, um-Seemly, suitable, beautiful.

Decrescens-Decreasing, growing less.

Decurrens-Decurring, hanging down.

Decursus, a, um - Dowuward, rumning down.

Decurtatus, a, um-Curtailed.

Decussatus, a, um-Arranged in pairs that cross each other.

Defiguratus, a, um-Disfigured.

Deffectus, a, um-Deflected.

Deflexus, a, um-Bent, turned aside.

Deformatus, a, um-Deformed.

Deformis, e-Deformed, ugly-shaped.

Degener, eris-Degenerate, unlike the ancestors.

Delicatulus, a, um-Quite delicate.

Delicatus, a, um-Delicate, thin.

Delphinocephalus-Dolphin-headed.

Deltoideus, a, um-Like the Greek letter Delta.

Deminutivus, a, um-Diminutive.

Demissus; a, um-Hanging down.

Demum-At last, solely.

Denarius, a, um-Containing ten.

Densifolius, a, um-Dense-leaved.

Densmammillatus, a, um-Having mammillated teeth.

Densus, a, um-Dense, thick.

Dentalium-A plow-share.

Dentatus, a, um-Toothed.

Denticulatus, a, um-Denticulated, having small teeth.

Dentilineatus, a, um-Tooth-lined.

Denudatus, a, um-Denuded.

Deparcus, a, um-Very scarce.

Depauperatus, a, um-Impoverished.

Deperditus, a, um-Impoverished.

Depressus, a, um-Depressed.

Desertus, a, um-Deserted, forsaken.

Desideratus, a, um-Desired, rare.

Desmopleura-A side band.

Desquamatus, a, um-Scaled off.

Devexus, a, um-Sloping.

Diadematus, a, um-Wearing a diadem.
Dialophus-Through the neck.

Dianthus, a, um-Double-flowered.

Diatretus, a, um-Pierced with holes.

Dichotomus, a, um-Divided.

Dictyopteroides-Like Dictyopteris.

Dictyota-Net-worked.

Dictyum-A net.

Difficilis, e-Difficult, rough.

Diffidens-Diffident, distrusting.

Diffluens-Flowing every way, loose.

Diffusus, a, um-Diffused, extended.

Digitalis, $\mathrm{e}-$ Belonging to the finger.

Digitatus, a, um-That has fingers, toes, or claws.

Dignatus, a, um-Excellent.

Digonus, a, um-Two-angled.

Dikrocheilus, a, um-Tivo-edged.

Dilatatus, a, um-Dilated, widened.

Dilatus, a, um-Spread out.

Diluculum-Day-break, dawning of day.

Diminutivus, a, um-Diminutive.

Dimorphus, a, um-Two-formed.

Diplostegioides-Like Diplostegium.

Diplotesta-Having two tests.

Disciformis, e-Shaped like a quoit.

Discoidalis, e-Discoidal.

Discoideus, a, um-Discoid, disk-like.

Discophorus-Disk-bearer.

Discrepans-Different.

Disculus-A little disk.

Discus-A quoit.

Disjunctus, a, um-Separated, disjoined.

Dispalans-Straggling, stray.

Dispandus, a, um-Spread out, stretcled.

Dispansus, a, um-Stretched out.

Dispar-Different.

Disparilis, e-Different, unequal.

Dispassus, a, um-Extended, spread out.

Dispersus, a, um-Dispersed.

Dissectus, a, um-Cut up, dissected.

Dissimilaris, e-Dissimilar, unlike.

Dissolutus, a, um-Weak, broken.

Distans-Distant, standing apart.

Distensus, a, um-Distended.

Distinctus, a, um-Distinct.

Distortus, a, um-Distorted, crooked, irregular.

Divaricans-Severed, straddling.

Divaricatns, a, um-Divaricated, wide apart.

Divergens-Diverging.

Diversifolius, a, um-Diverse-leaved.

Diversus, a, um-Diverse, different, unlike.

Divisus, a, um-Dividing.

Docens-A teacher.

Dodecadactylus, a, um-Twelve-fingered.

Dolabriformis, e-Like a mattock or pick-axe.

Dolatus, a, um-Hewed.

Dolorosus, a, um-Wretched.

Donaciformis, e-Like a Donax.

Dorsalis, e-Dorsal.

Dorsatus, a, um-High-backed.

Dotis-An ornament.

Drepanaspis-Having a sickle-shield.

Dubius, a, um-Doubtful.

Dumalis, e-Bushy.

Dumosus, a, um-Bushy.

Duodenarius, a, um-Containing twelve.

Duplicatus, a, um-Duplicated, doubled.

Duplicostatus, a, um-Double-ribbed. 
Eboreus, a, um-Made of ivory.

Ebracteatus, a, um-Without Eeales or bracts.

Eburneolus, a, um-Of ivory.

Eccentricus, a, um-From the center.

Echinatus, a, um-Set with spines.

Ectypus, a, um-Engraved in relief, embusserl.

Edax-Voracious.

Edentulus, a, um-Toothless.

Egenus, a, um-Destitute of, very poor.

Elegans-Elegant, liandsome.

Elegantissimus, a, um-Very handsome.

Elegantulus, a, um-Quite elegant.

Elevatus, a, um-Flevated.

Ellipticus, a, um-Elliptiıal.

Elongatus, a, um-Elongated.

Elytri-The wing corering.

Elytroides-Like the elytra of beetles.

Emaceratus, a, um-Thin.

Emaciatus, a, um-Emaciatel, thin.

Emarginatus, a, um-Notched.

Eminens-Prominent, standing out in relief.

Eminulus, a, um-Projecting a little.

Enormis, e-Very large.

Ensiformis, e-Sword-formed.

Eos-The dawn.

Evidermatus, a, um-Covered with a crust or skin.

Equilaterus, a, um-Equal-sided.

Equisetiformis, e-Like Equisetum.

Jrectifolius, a, um-Having leaves ercet.

Erectipora-Having erect pores.

Erectus, a, um-Erect, straight.

Erodus, a, um-Froded, jaggel, gnawed.

Iirosus, a, um-Eroded, bitten away.

Erraticus, a, um-Wandering, erratic.

Erythroliticus-Red stone.

Escharoides-Like Eschara.

Eucharis, e-Graceful, beautiful.

Euconus-Perfect cone.

Euginum-Fertile.

Euglypheus, a, um-Well-carved, distinetly marked.

Euomphaloides-Like Euomphalus.

Euphemir-Ol good omen.

Euruteines-Extending widely.

Euzona-Beautifully girdled.

Evax-An exclamation of delight.

Exacutus, a, um-Pointed.

Exanthematus, a, um-Covered with eruptions.

Excavatus, a um-Made hollow, excavated.

Excellens-Excellent, high-rising.

Excelsior-Elevated, lofty.

Excelsus, a, um-Elevated, high.

Excerptus, a, um-Selected, picked out.

Excrescens-Growing out, increasing.

Exculptus, a, um-A Adorned, chiseled out.

Exfoliatus, a, um-Exfoliated.

Exiguus, a, um-Small, petty.

Exilis, e-Thin, lean, slender, creeping.

Eximius, a, um-Choice, seleet, excellent.

Exornatus, a, um-Adorned.

Exortivus, a, um-Eastern.

Expansus, a, um-Expanded, widely spread.

Expatiatus, a, um-Spread out.

Explanatus, a, um-Made plain, spread out.

Explicatus, a, um-Unfolded, spread out.
Explorator-A scout, an examiner.

Exporreetus, a, um-Smooth, stretched out.

Fixsculptus, a, um-Carved.

Exsertus, a, um-Projecting, thrust forth.

Exsul-A wanderer.

Extans-Standing out.

Extensus, a, um-Stretched out, extended.

Extenuatus, a, um-Made thin, slender, drawn out.

Extumidus, a, um-Swelled up.

Exutus, a, um-Divested, stripped off.

Faba-A bean.

Fabalis, e-Bean-stalks.

Fabula-A little bean.

Fabulites-A little stone-bean.

Facetus, a, um-Elegant.

Falcatus, a, um-Hooked.

Faleiformis, e-Like a sickle, pruning.knife, or hook.

Fallax-Decertive.

Fal $x-A$ hook, pruning-knife, or sickle.

Famelicus, a, um-Fanished.

Fasciatus, a, um-Banded.

Fascicularis, e-Small-bundled.

rasciculatus, a, um-Bundled.

Fasciculus-A bundle.

Fascigerus, a, um-Bearing fasces.

Fustigatus, a, um-Sloping up to a point.

Faustus, a, um-Fortunate, lucky.

Favositoideus, a, un-Like Favosites.

Favosus, a, um-Honeycomb-like.

Fax-A torch, taper.

Fecundus, a, um-Fruitful.

Felix, icis-Fertile.

Fenestella-A little window.

Fenestelliformis, e-Like Fenestella.

Fenestratus, a, um-Reticulated, having open windows.

Fenestrula-1 little window.

Ferox-Fierce, hardy, stout:

Ferratus, a, um-Hard as iron, covered with iron.

Ferricolus-Iron distaff.

Ferrugineus, a, um-Of the color of iron, rusty.

Fertilis, e-Fertile, fruitful.

Ferus, a, um-Wild, cruel, fierce.

Festinatus, a, um-Hastened, before the time.

Fibratus, a, um-Having emall threads hanging to it.

Fibristriatus, a, um-Fiber-lined.

Fibrosus, a, um-Full of fibers or threads.

Ficoides-Like a fig.

Ficus-A fig.

Fidelis, e-Sire, faithful.

Filiciformis, e-Fern-like.

Filicosta-Having thread-like costa.

Filicula-Fern of trees, wall-fern.

Filiculme-Thread-straw.

Filiformis, e-Filiform.

Filistriatus, a, un - Having thread-like striæ.

Filitextilis, e-Woven like thread.

Filitextus, a, um-Woven like thread.

Filosus, a, um-Thready.

Fimbriatus, a, um-Fringed, jagged, scalloped. 
Fiscellostriatus, a, um-Having divided striæ.

Fiscellus-A small basket woven of slender twigs.

Fissicosta-Having divided costæ.

Fissilis, e-Split.

Fissiplica-Having divided plications.

Fissuratus, a, um-Fissured.

Fissurellus, a, um-Having a little cleft.

Fisus, a, um-Divided, cleit, split.

Fistulosus, a, um-Full of ioles, spongy.

Flabellatus, a, um-Fan-like.

Flabellifer, era, erum-That bears a fan.

Flabelliformis, e-Shaped like a fan.

Flabellites-A stone fan.

Flabellum-A fan.

Flaccidus, a, um-Withered, hanging, flagging, flaccid.

Flagellaris, e-Like a whip.

Flayellum-A whip.

Flagricaudus, a, um-Whip-tailed.

Flavus, a, um-Golden, yellow.

Flexicaulis, e-Having a flexible stem.

Flexifolius, a, um-Having recurved leaves.

Flexilis, e-Pliant, flexible.

Flexuosus, a, um-Flexuous, full of turns.

Florealis, e-Flower-like.

Floridus, a, um-Flowery, adorned with flowers, gay.

Florifer, era, erum-Flower-bearing.

Floriformis, e-Flower-shaped.

Flos-A flower.

Fluctus-A wave, a billow.

Fluctuosus, a, um-Full of waves, wavy, veiny.

Fluitans-Flowing, floating.

Fœcundus, a, um-Fruitful, abundant.

Fotoideus, a, um-Like a tumor.

Foliaceus, a, um-Foliaceous, like leaves, leafy.

Foliatus, a, um-Leaved, having leaves.

Foliosus, a, um-Leafy, full of leaves.

Folium-A leaf.

Folliculus-A small sack.

Follis-A leather sack.

Fonticola-Fountain-dwelling.

Fontinalis-A fountain or spring.

Formosus, a, um-Beantiful, handsome.

Fornacula-A little oven.

Fornax-A furnace.

Fornicatus, a, um-Arched, vaulted over.

Forulatus, a, um-Having narrow furrows.

Fossatus, a, um-Dug out.

Fossilis, e-That may be dug out of the earth, fossil.

Fossula-A little trench or ditch.

Foveatus, a, um-Pitted.

Fractus, a, um-Broken, effeminate.

Fragarioides-Like a strawberry.

Fragilis, e-Brittle, frail.

Fragosus, a, um-Fragile.

Frangens-A breaker.

Fraternus, a, um-Brotherly, fraternal.

Fraxiniformis, e-Like fraxinus.

Frequentatus, a, um-Frequent.

Fringilla-A small bird.

Fritillus-A dice-box.

Frondosus, a, um-Full of leaves.

Frutex-A shrub.
Fruticosus, a, um-Shrubby, full of shoots. Fucoides-Like Fucus.

Fulcratus, a, um-Stayed with props.

Fulgidus, a, um-Shining.

Fulgur-A thunder-bolt.

Funatus, a, um-Corded.

Fungosus, a, um-Spongy.

Fungulus-A small mushroom.

Funiculus-A small cord or line.

Furcatus, a, um-Forked.

Furcicarinatus, a, um-Forked and keeled.

Furtivus, a, um-Secret, hard to find.

Fusibrachiatus, a, um-Having fusiform arms.

Fusiformis̀, e-Fusiform, tapering at both ends.

Fustiformis, e-Club-formed.

Fustis-A club, staff.

Futilis, e-Trivial.

Galeatus, a, um--That wears a helmet.

Galericulatus, a, um-Having a small covering.

Galerum-A cap, hat, or tuft of feathers.

Gallinuloides-Like a pullet,

Gemellipara-Twin-bearing.

Geminispinosus, a, um-Twin-spined.

Gemma-A young bud, a gem.

Gemmatus, a, um-Budded, set with gems.

Gemmicula-A little bud.

Gemmifer, era, erum-That bears buds or gemmules.

Gemmiformis, e-Shaped like a bud.

Gemmula-A little bud.

Geniculatus, a, um-Knotted, jointed.

Geniculosus, a, um-Knotty.

Genitivus, a, um-Natural, belonging to the same stock.

Geometricus, a, um-Geometrical.

Germanus, a, um-Near of kin.

Gibber, era, erum-Bossed, hunchbacked.

Gibberosus, a, um-Badly hunchbacked.

Gibberulus, a, um-Somewhat bunchbacked.

Gibberus, a, um-Humpbacked.

Gibbosus, a, um-Gibbous, full of hunches, or humped.

Gibbus, a, um-Hunched, gibbous.

Giganteus, a, um-Giant-like, very large.

Gigas-A giant.

Glabellus, a, um-Smooth.

Glaber, bra, brum - Smooth, bare.

Glacialis, e-Frozen, icy.

Gladiolus--A small sword.

Glandulosus, a, um-Full of kernels, glandular.

Glandulus, a, um-Having kernels, glandular.

Glans-An acorn, chestnut, or pellet.

Glanscerasi-Fruit of the cherry-tree.

Glansfagea-Fruit of the beech-tree.

Globatus, a, um-Made round.

Globosus, a, um-Round as a ball, globose.

Globularis, e-Globular.

Globuliformis, e-Globe-shaped.

Globulus-A little ball.

Glomeratus, a, um-Confused, out of order.

Gloriosus, a, um-Glorious.

Glyptus, a, um-Sculptured. 
Gomphoides-Like a stake or club.

Gomphus-A pile, stake, or club.

Goniocercus, a, um-Angular, tailed.

Goniolobus, a, um-Having angular lobes.

Goniopteroides-Like Goniopteıis.

. Goniurus, a, un-Angular-tailed.

Gonopleura-Angular rib.

Gothicus, a, um-Gothic.

Gracilens, entis-Sleniler, thin.

Gracilentus, a, um-Slender, thin.

Gracilis, e-Small, slender, thin, wak.

Gracilius, a, um-More slender.

Gracillimus, a, um-Very slender, thin, or weak.

Gracillistriatus, a, $11 \mathrm{~m}-\mathrm{slendler,} \mathrm{striated.}$

Gradatus, a, um-Made with steps.

Gradicosta-Having steps and ribs.

Gradocostatus, a, um-Having steps and ribs.

Gramineus, a, un-Grassy or belonging to grass.

Grandievus, a, um-Very old.

Grandiceps-Big-lieaded.

Grandiculus, a, $1 \mathrm{~m}$ - Rather large.

Grandifolius, a, um-Large-leaved.

Grandis, e-Grand, large.

Graniferus, era, erum-That bears grains of corn.

Granilineatus, a, um-Lined with granules.

Granilineus, a, un-Granule-lined.

Granosus, a, um-Full of grains or kernels.

Granulatus, a, um-Granulated, granular.

Granuliferus, era, erum-Granule-bearing.

Granulostriatus, a, um-Having granular strix.

Granulosus, a, um-Covered witlı small granules.

Graphicus, a, um-Perfect, excellent, done to the life, written on.

Gratiosns, a, um-Agreeable.

Gratus, a, um-Acceptable.

Gravis, e-Weighty, full, old.

Graviusculus, a, um-Rather deep.

Gregalis, e-Of the common sort.

Gregarius, a, um-Of the common sort, common, gregarious.

Grossiplicatus, a, um-Thick-plaited.

Grumus-A little heap.

Gypseus, a, um-Covered or plastered with gypsum.

Gyracanthus-Round spine.

Gyrinoides-Like a tadpole.

Gyroceras-Circular horn.

Hæsitans-Doubting.

Haliotoides-Like Haliotus.

Hamatilis, e-Furnished with hooks.

Hamatus, a, um-Crooked, looked.

Hamulosus, a, um-Full of hooks.

Hamulus-A small hook.

Harpago-A hook.

Hastatus, a, um-Bearing spears, halbertshaped.

Hastifolius, a, um-Spear or lance-leaved.

Hastula-A little spear.

Helicoides-Like a helix.

Helicteres-A round, smooth spire.

Heliolitiformis, e-Like Heliolites.

Helios-The suu.
Henicyclus-A half-circle.

Hemicylindrus-A half-cylinder.

Hemiplicatus, a, um-H hlf.plaited.

Hemisphericus, a, um-Hemispherical.

Hemiteloides-Like Hemiteles.

Hemitry pa-Having half-openings.

Herbaceus, n, um-Grassy.

Herculaneus, a, um-Belonging to Hercules, large of its kind.

Heterocinctus, a, um-Irregularly girded or banded.

Heteroclitus, a, um-Extraordinary.

Heterodactylus, a, um-Irregular-toed or irregularly fingered.

Heterophyllus, a, um-Irregularly or differently leaved.

Heteropora-Having irregular pores.

Heteropteris-Irregular fern.

Hexadactylus, a, um - Six-fingered.

Hexagonus, a, um-Having six anglıs.

Hexagonalis, e-Hexagonal.

Hians-Gaping, disjointed.

Hipparionyx-A colt's lioof.

Hirsutus, a, um-Rough, hairy, shaggy.

Hirtus, a, um-Rough, hairy, shagey.

Hispıdus, a, um-Rough, bristly, rugged.

Hiulcus, a, um-Gaping, cleft.

Holopyga-Entire rump, wliole back.

Homalonotoides-Like Homalonotus.

Horizontalis, e-Horizontal.

Horridus, a, um-Rough, bristly.

Hospitalis, e-Of a guest, hospitable.

Humerosus, a, $11 \mathrm{~m}-$ Humped, full of humps.

Humerulus-A little shoulder.

Humilis, e-Small, poor.

Hyalina-Of glass.

Hybrida-Intermediate between two species, a bybrid.

Hydraulicus, a, um-Hydraulic.

Hymenophylloides-Like Hymenophyllites.

Hyperbolæus, a um-Extreme.

Hyperboreus, a, um-Very far north.

Hypniformis, e-Like Hypnum.

Hystricosus, a, um-Thorny.

Hystriculus, a, um-Somewhat covered with spines.

Hystrix-Covered or beset with spines.

Ichthyoderma-Having a fish-skin.

Ichthyolepis-Having fish-scales.

Icosidactylus, a, um-Twenty-fingered.

Idoneus, a, um-Suitable.

Ignobilis, e-Ignoble, strange, unknown.

Ignorabilis, e-Overlooked, unknown.

Ignotus, a, um-Unknown, strange.

Ilicifolius, a, um-Oak-leaved.

Illænoides-Like an Illænus.

Illibatus, a, um-Unimpairer.

Imago-An image, picture, also a sheath.

Imbecillus, a, um-Feeble, frail.

Imbricarius, a, um-Having imbrications.

Imbricato-articulatus, a, um-Having imbricated articulations.

Imbricatus, a, $11 \mathrm{~m}$-Laid one on another like tiles, imbricated.

Imitator-A resembler.

Immaturus, a, um-Immature, abortive.

Immersus, $a_{1}$ um-Immersed. 
Impar-Odd, unequal, disproportioned.

Imparilis, e-Different.

Imperator-A commander.

Implexus, a, um-Interlaced, interwoven.

Implicatus, a, $u m-$ Wrapped together, entungled.

Impolitus, a, um-Rough, unpolished.

Impositus, a, um-Laying over.

Impressus, a, um-Impressed.

Improcerus, a, um-Undersized, not tall.

Impudicus, a, um-Shameless, immodest.

Inæquabilis, e-Uneven, unequal.

Inæqualis, e-Unequal.

Inæquatus, a, um-Unequal.

Insequicostatus, a, um-Unequally ribbed.

Inæequidactylus, a, um-Unequal-fingered.

Inæquilateralis, e-Inequilateral.

Inæquiplicatus, a, um-Unequally rayed.

Inequistriatus, a, um - Unequally striated.

Inceptus, a, um-An undertaking, incipient.

Incertus, a, $11 \mathrm{~m}$-Uncertain, inconstant.

Incilis, e-Belonging to, or like a trench, furrow, or gutter.

Incipiens-The beginning.

Incisivus, a, um-Having the quality of cutting or biting.

Inciso-lobatus, a, um-Cut into lobes.

Incisus, $\mathrm{a}, \mathrm{um}$-Incised.

Inclinatus, a, um-Inclined, bent.

Inclinis, e-Bending.

Incluspora-Having inclosed perforations.

Inclusus, a, um-Closed up.

Incompletus, a, um-Incomplete.

Incomptus, a, um-Untrimined, rough.

Inconditus, a, um-Irregular, disordered.

Inconspicuus, a, um-Not conspicuous.

Inconstans-Not constant.

Inconsuetus, a, um-Unusual.

Incrassatus, a, um-Tuickened.

Increbescens-Abundant.

Incrustans-Incrusting.

Incultus, a, um-Neglected.

Incurvus, a, um-Incurved.

Indagator-A diligent hunter.

Indagat u 3 -Encircling.

Indentatus, a, um-Indented, notched.

Indentus, a, um-Indented, notched.

Inleterminatus, a, $11 \mathrm{~m}-$ Not determined.

Indolatus, a, um-Unhewn.

Inelegans-Unadorned.

Inermis, e-Unarmed.

Inexpectans-Not expected.

Iufelix-Useless, unhappy, miserable.

Infernus-Underyround, the lower.

Infertus, a, um-Fill rd up.

Inferus-Below, underground.

Inflatu*, a, uın-Spread, swollen, inflated.

Inflexus, a, um-Bowed, made crooked.

Inforuis, e-Sliapeless, rude.

Infrequens-Rare, infrequent.

Infula-A band, an ornament.

Infundibularius, a, um-Pertaining to funnel.

Infinudibuliformis, e-Funnel-shaped.

Intundibulum, e-A funnel, hopper.

Ingens-Very large, huge, prodigious.

Ingentior-Larger, enormous.
Inopinatus, a, um-Unexpected, unthought of.

Inops-Poor, iriendless, unburied.

Inoptatus, a, um-Undesired, not wanted.

Inordinatus, a, um-Disordered.

Inornatus, a, um-Unadorned.

Insculptus, a, um-Engraven, carved.

Insectus, a, um-Uncut.

Insertivus, a, un-Inserted.

Insignis, e-Narked, naturally remarkable.

Insignitus, a, um-Marked, clear.

Insitus, a, um -Inserted, introduced.

Insculptus, a, um-Engraved.

Insolens-Unusual, rare.

Insolitus, a, um-kare, hard to find.

Insons-Harmless.

Inspeciosus, a, um-Not liandsome.

Insperatus, a, um-Unexp $\sim$ cted.

Instabilis, e-Not firm, changing.

Insuetus, a, um-Unusual.

Insularis, e-Upon an island.

Intectus, a, um-Uncovered.

Integrifolius, a, um-Whole-leaved.

Intercalaris, e-Intercalated.

Intercedens-Intervening.

Intercellatus, a, um-Being intercellular.

Intercostalis, e-Lined between costa.

Intercostatus, a, um - Ridged between ribs.

Interlineatus, a, um-Interlined.

Intermedius, a, um-Intermediate, the middle.

Interınittens-Intermitting, ceasing for a time.

Internascens-Growing between.

Iuternodius, a, um-Space between two knots or joints.

Interplicatus, a, um-Plicated between.

Iuterruptus, a, um-Broken asunder, interrupted.

Intersculptus, a, um-Engraved in the middle.

Interscapularis, e-Spaced between the shoulder pieces.

Int-rstinctus, a, um-Divided.

Interstrialis, e-Having striæ between.

Interstrictus, a, um-Drawn together.

Intertextus, a, um-Interworen, interlaced.

Intervesicula-Having little vesicles between.

Intextus, a, um-Plaited, woven.

Intortus, a, um-Twirled, entangled, curled.

Intralineatus, a, um-Lined, between lines.

Inutilis, e-Not useful, very poor, insignificant.

Invaginatus, a, um-Invaginated, sheathed, enwrapped.

Invalidus, a, um-Weak, feeble.

Invenustus, a, um-Unhandsome.

Inversus, $a, ı m-$ Inverted.

Investis, e-Unclothed.

Involutus, a, $11 \mathrm{~m}$-Involute.

a Irrasus, a, um-Unpolished, not smooth.

Irregularis, e-Irregular.

Islandicus, a, um-From an island.

Ischypus, a, um-Strong-footed.

Isosceles-Having equal legs.

Is gramma-Equal weight. 
Jaculum-A dart, javelin.

Jejunus, a, um-That has not eaten, hungry. Jubatus, a, um-Crested.

Jucundus, a, um-Pleasant, agreeable, delightful.

Jugalis, e-Yoked together.

Juglans-A walnut.

Jugosus, a, $\mathrm{nm}$-Full of ridges, mountainous.

Junceus, a, um-Made of bulrushes, like a bulrush.

Junciformis, e-Shaped like a bulrush.

Junctus, a, un-Joined, coupled.

Juvenis, e-Young.

Labecula-A little spot.

Labiatus, a, um-Lipped.

Labiosus, a, um-Full lipped.

Labrosus, a, um-Having large lips, bordered.

Labyrinthicus, a, um-Labyrinthine.

Laceratus, a, un-Torn, mangled, ragged.

Lachrymosus, a, um-Full of tears.

Laciniatus, a, um-Fringed.

Laciniosus, a, $11 \mathrm{~m}-\mathrm{Full}$ of plaits, jagged, crumpled.

Lactuca-Iuttuce.

Lacunosus, a, uın-Full of holes, pitted, uneven.

Iacus-A vat, a basin.

Lacustris, e-Pertaining to a lake or swamp. Latus, a, um-Fertile, pleasant, agreeable.

Iavicosta-Having a smooth rib.

Lrevicostatus, a, um-Smooth-ribbed.

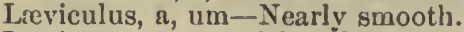

I.ævigatus, a, um-Planed, made smooth.

Lrevis, e-Smooth.

Levissimus, a, un-Very smooth.

Lævistriatus, a, um-Having smooth striæ.

Lreviusculus a, um-Quite smooth.

Lagena-A flask.

Laguncula-A little flask.

Lamellatus, a, um-Having thin plates.

Lamellosus, a, um-In very thin plates.

Laminatus, a, um-Laminated.

Lamnoides-Like Lamna.

Lanatus, a, um-Woolly.

Lanceolatus, a, um-Spear-shaped.

Lancifer, era, erum-Lance-bearer.

Lancifolius, a, um-Lance-leaved.

Lanosus, a, um-Woolly.

Lapicida-A stone-cutter.

Lapideus, a, um-Consisting of stone.

Lapillus-A little stone, a pebble.

Laqueatus, a, um-Arched, vaulted, fluted, paneled.

Largissimus, a, um-Very large, the largest.

Largus, a, um-Plentiful, large.

Laricinus, a, nm-Resembling the larchtree.

Larvatus, a, um-Frightened, masked.

Latealatus, a, um-Broad-winged.

Lateralis, e-Belonging to the side.

Laterarius, a, um-Of or belonging to the sides.

Latericrescens-Side-growing.

Laterniformis, e-Shaped like a lantern.

Latiannulatus, a, um-Having wide annulations.
Latibracliatus, a, um-Wide-armed.

Latibuccatı ${ }^{2}$, a, um-Wide-cheeked.

Laticeps-Broad head.

Laticosta-Having wide rilss.

Laticostatus, a, um-Wide-ribbed.

Latidactylus- Wide-fingered.

Latidorsatus, a, un-IVide-backed.

Latifasciatus, a, um-IVide-bundled, or widebanded.

Latifolius, a, um-Broad-leaved.

Latifrons-Having a wille front.

Latijuncturus, a, $\mathrm{nm}$-Wide-jointed.

Latimarginatus, a, um-Broad-margined.

Latior-IVicler.

Latipes-Broad-footed.

Latiradius, a, um-Wide-rayed.

Latispinosus, a, um-Wide-spined.

Latissimus, a, un-Very wide, the widest.

Latitruncatus, a, um-Broadly truncated.

Lativentrus, a, um-Having a wide cavity.

Latus, a, $11 \mathrm{~m}$-Broad, wicle, large.

Latusculum-A little side.

Lautus, a, um-Nent, elegant, splendid.

Laxatus, a, un-Made wider, extended, dilated.

Laxus,a, um-Loose, slack, spacious.

Ledoides-Like Leda.

Lens-A lentil.

Lenticularis, e-Lens-shaped, lenticular.

Lentiformis, e-Lens-shaped.

Lentus, a, um-Flexible, pliant, sluggish.

Leperditioides-Like Leperditia.

Lepidodendrifolius, a, $11 \mathrm{~m}-$ Having leaves like Lepidodendron.

Lepidorachus, e-Having a scaly ridge.

Lepidus, a, um-Pretty.

Lepis-A scale.

Leptænoides-Like Leptıena.

Leptocephalus, a, um-Slender-headed.

Leptodactylıs, a, um-Slender-toed.

Leptoderma-A thin skin.

Leptogaster-A smooth belly.

Leptonotus, a, um-Slender-backed.

Levatus, $a, u m$-Lifted up.

Leviculus, a, ım-Very small.

Levigatus, a, $11 \mathrm{~m}-$ Smooth.

Levinodatus, a, um-Having smooth knots.

Levis, e-smooth.

Lichenoides-Like lichen.

Lichenoideus, a, um-Like a lichen.

Ligoniformis, e-Like a mattock.

Liliiformis, e-Shaped like a lily.

Lima-A file.

Limabrachiatus, a, um-File-armed.

Limatulus, a, um-Neat, fine, polished, like a little file.

Limatus, a, um-Polished, neat, elegant.

Limax-A snail, slug.

Limbatus, a, um-Bordered.

Limiformis, e-Lima-shaped.

Limitaris, e-Bounded, limited.

Limulurus-Limulus, tail.

Lineanodus, a, um-Having lined knots.

Linearifolius, a, um-Having linear leaves.

Linearis, e-Pertaining to a line, linear.

Linearius, a, um-Belonging to lines.

Lineatoides-Like lineatus, a specific name.

Lineatus, a, um-Drawn out, lined.

Lineolatus, a, um-Fine lined. 
Lineopora-Having line-pores, lined with perforations.

Lineopunctatus, a, um-Line-punctured or line-dotted.

Lingualis, e-Tongue-shaped.

Linguifer, era, erum-Tongue-bearıng.

Linguiformis, e-Tongue shaped.

Lingulatus, a, um-Tongue-shaped, lingulate.

Linteum-A napkin, girdle'.

Lioderma-A smooth skin.

Liosoma-A smooth body.

Liratus, a, um-Furrowed.

Lithofactor-Stone-maker.

Litoreus, a, um-On the shore or sea-side.

Lobatus, a, um-Lobed.

Locellus-A little purse or bag.

Loculosus, a, um--Full of holes or distinct places, partitioned.

Loncbitis-Spleenwort, the fern "Adderstongue."

Longævus, a, um-Ancient, aged.

Longicameratus, a, um-Long chambered.

Longicaudatus, a, um-Long-tailed.

Longicollis-Long-riłged.

Longicostalis, e--Lon@-ribbed.

Longidactylus, a, um-Long-fingered.

Longidentatus, a, um-Long-toothed.

Longifolius, a, nm-Long-leaved.

Longipes-Long-foo:ed.

Longirostris-Having a long proboscis.

Longispinus, a, um-Long-spined.

Longispira-Having a long spire.

Longissimus, a, um-Very long, the longest.

Longiusculus, a, un-Rather long.

Longulus, $a$, um-Rather long.

Longus, a, um-Long.

Loriformis, e-Like a thong or whip.

I.otoblastus-Lotus bud.

Lucifugus, a, um-Light-shunning.

Lunatus, a, um-Made like a hialf-moon, horned.

Lunulatus, a, um-Crescentiform.

Luxus, a, um-Dislocated.

Lycoperdon-Puff-ball shaped.

Lynx-An animal called a lynx.

Lyra-A harp.

Lyralifolius, a, um-Having lyre shaped leaves.

Macer, cra, crum-Lean, meager.

Machæriformis, e-Sword-shaped.

Macilentus, a, um-Meager, tbin, lean.

Macrocephalus, a, um-Long-hearled.

Macrochirus, a, um-Lnng-lianded.

Macrodactylus, a um-Long-fingered.

Macrodentus, a, um-Long-toothed.

Macrolepidotus, a, um-Having long scales.

Macrolineatus, a um-Long-lined.

Macromphalus, a, um-Having a large um. bilicus.

Macronotus, a, um--Long known.

Macropetalus, a, um-Having long flower leaves.

Macrophorua, a, um-Long-bearing.

Macrophyllus, a, um--Long.leaved.

Macropleura-Having long sides.

Macropora-Having long pores.

Macrops--Having large eyes.
Marropterus, a, um-Long-winged, or largefinued.

Macropthalmus, a, um-Long-eyed.

Macrospira-Having a long spire.

Macrospondylus, a, um-Having long vertebræ.

Macrostomus, a, um-Having a long moutl.

Macrostriatus, a, um--Having long striæ.

Macrostylus, a, um-Having long spines or columns.

Nacrothyris-Having a long foramen.

Macrurus, a, um--Long-tailed.

Mactriformis, e-Shaped like Mactra.

Mactroides-Like Mactra.

Maculatus, a um-Spotted, speckled.

Maculosus; a, um-Full of spots, spotted.

Magister-A chief, master.

Magnicornis, $\mathrm{e}$-Large-horned.

Magnicostatus, a, um-Large-ribbed.

Magnificus, a, um-Magnificent, stately.

Magnifolius, a, um-Large-leaved.

Magnisulcatus, a, um-Deep-furrowed.

Magniventrus, a, um-Large-bellied.

Magnoliiformis, e--Shaped like magnolia.

Magnus, a, um-Great, large.

Major-Greater, larger.

Majus, a, um-Greater, larger.

Malvaceus, a, um-Like or pertaining to mallows.

Mamillanus, a, um-Swelling, protuberant.

Mammatus, a, um-Covered with protuberances.

Mammiferus, a, um-Teat or nipple bearing.

Mammillaris, e-Mammillated.

Mammillatus, a, um-Covered with nipples.

Maniformis, e-Hand-like.

Manticula-A little wallet.

Manus-A hand.

Marcidus, a, um-Hanging, flagging, withered.

Marginalis, e-Marginal.

Marginatus, a, um-That has a border, broad rim, or margin.

Marginicinctus, a, um-Having a banded margin.

Marinus, a, um-Inbabiting the sea.

Maritimus, a, um-Of or belonging to the sea.

Masculus, a, um-Stout, hardy, masculine.

Materiarius, a, um-of or belonging to timber.

Maturus, a, um-Ripe, mature.

Matutinus, a, um-In the morning.

Maximus, a, um-Greatest, largest.

Medialis, e-Middle.

Medianus, a, um-Middle.

Mediocris, e-Middling, ordinary.

Medius, a, um-Niddle, ordinary.

Medullaris, e-In the marrow or middle part, like a pith.

Megacephalus, a, um-Large-headed.

Megalops-Having large eyes.

Megambonatus, a, um-Having a great umbo.

Megambonus, a, um-Having a large umbo.

Megastomus, a um-Having a large mouth.

Megastylus, a, um-Having large spines.

Megistus, a um-Very large.

Melaniiformis, e-Shaped like Melania. 
Melanioides-Like Melania.

Meliniformis, e-Purse-sh t perl.

Melo-An apple-shaped melon.

Melonicus, s, um-Like a small melon.

Meloniformis, e-Melon-shaped.

Melonoides-Like a melon.

Membranaceus, a, um-Like a parcliment, skinny.

Meniscus, a, um-A crescent-shaped body.

Merianopteroides-Iike Merianopteris.

Meristoides-Like Merista.

Mesacosta-Having middle ribs.

Mesacostalis, e-Middle-ribberl.

Mesambonatus, a, um-Having a middle umbo.

Mesastrialis, e-Middle striated.

Mesialis, e-Middle parted.

Mesolobus-Having a middle lobe.

Meta-Any thing in a conical form.

Metallicus, a, um-Metallic.

Metula-A little butt or small pyramid.

Mica-A crumb or little thing.

Micans-Stretching out, glitterirg.

Microbasalis, e-Having a small hase.

Microcarpus, a, um-Small-fruited.

Microdentus, a, um-Small-toothı d.

Microdus, a, um-Having small teeth.

Microlobus, a, um-Small-lobed.

Micronema-A small thread.

Microphorus, a, um-Small-bearing.

Microphyllus a, um-Small-leaved.

Micropleura-Having a small rib.

Micropterus, a, um-Sinall-winged.

Micropthalmus, a, um-Small-eyed.

Micropus-Small foot.

Microscopicus, a, um-Microscopic.

Microstigma-Small dot.

Microstylus-Small spile or pale.

Micrurus, a, um-Small-tailed.

Micula-A little crunib or grain.

Millebrachiatus, a, um-Muny-armed.

Milleporaccus, a, um-Having inuume rable pores.

Millepunctatus, a, um-Many-dotted.

Minicus, a, um-Minic.

Minimus, a, um-The least or smallest.

Minor-Less, smaller.

Minuens-Diminishing, making lı'ss.

Minus, a, un1-Less.

Minusculus, a, um-Ratber less, rather small.

Minutisectus, a, um-Finely marked or divided.

Minutissimus, a, um-Very minute.

Minutulus, a, um-Very small.

Minutus, a, um-Diminished, small, minute.

Mirabilis, e-Extraordinary, wonderful, strange.

Mirus, a, um-Wonderful, astonishing, extraordinary.

Miser, era, erum-Wretched, unfortunate.

Mitella-A head-band, a kind of turban.

Mithrax-A precious stone.

Mitigatus, a, um-Tamed, civilized, softened.

Mitis, e-Ripe, flexible, placid.

Mitra-A head-band, turban.

Mixtus, a, nm-Mixed.

Modestus, a, um-Moderate, modest.
Moliolaris, e-Like Modiola, or a small measure.

Modioliformis, e-Like a small measure.

Modulatus, a, um-Symmetrical, well-proportioned.

Modulus-A small measure.

Molaris, e-Pertaining to grinding.

Molestus, a, um-Troublesome, difficult.

Mollis, e-Flexible, clelicate, effeminate.

Moniliferus, a, um-Bead-bearing.

Moniliformis, e-Like a necklace.

Monostigma-Single dot.

Monstruosus, a, um-Strange, monstrous.

Monticola-A dweller in the mountains.

Monticuliferus, a, um-Little mountainbearing.

Monticulus-A small mountain.

Morbillianus, a, um-Measly, spotted.

Mordax-Biting, given to biting.

Morsum-That which is bitten off.

Mortifer, era, erum-Deadly.

Mucro-A sharp point or edge.

Mucronatus, a, un-Pointed.

Mucrospinus, a, um-Sharp-spined.

Multattcnuatus, a, um-Much attenuated.

Multibrachiatus, a, um-Many-armed.

Multicalicatus, a, um-Much plastered.

Multicameratus, a, um-Many-chambered.

Multicarinatus, a, um-Many-keeled.

M::lticaulis, e-Many-stalked.

Multicinctus, a, um-Many-girded or banded.

Multicornis, e-Many-horned.

Multicostatus, a, um-Many-ribbed.

Multicosta-Having many ribs.

Multifasciatus, a, um-Many-bundled.

Multigranosus, a, um-Many-grained.

Multigrumus, a, um-Much heaped up.

Multilamella-Having many thin plates.

Multilamellosus, a, un-Having many lamellæ.

Multilineatus, a, um-Many-lined.

Multiliratus, a, um-Many-furrowed.

Multinodosus, a, um-Many-noded.

Multinotatus, a, um-Having many marks or tracks.

Muliplicatus, a, um-Many-folded.

Multipora-Having many pores.

Multiporatus, a, um-Having many pores or openings.

Multipunctatus, a, um-Many dotted or punctured.

Multiradiatus, a, um-Many-rayed.

Multiramosus, a, um - Having many branches.

Multisectus, a, um - Having many divisions or divided folds.

Multisegmentatus, a, um-Having many segments.

Multiseptus, a, um-Having many divisions.

Multiseriatus, a, um-Having many rows or series.

Multisinuatus, a, um-Many-furrowed.

Multispinosus, a, um-Many-spined.

Mulistriatus, a, um-Many-striated.

Multituberculatus, a, um-Having many tubtrcles.

Mritıtubulatus, a, um-Having many pipes.

Multivolvis, e-Many whorled or rolled. 
Mummiformis, e-Resembling a mummy.

Mundus, a, um-Neat, trim, delicatr.

Mundulus, a, um-Neat, trim, delicate.

Muralis, e-Of or belonging to a wall.

Muricatus, a, um-Full of sharp points, pointed.

Musculosus, a, um-Full of muscles.

Mutabilis, e-Inconstant, variable.

Mutatus, a, um-Altered, changed.

Mutus, a, um-Dumb, si ent.

Myriophyllus, a, um-Many-leaved.

Myrmecophorus, a, um, wart-bearing.

Mytiliformis, e-Like Mytilus.

Mytilimeris, e-Pertaining to Mytilus.

Mytiloides-Like Mytilus.

Nacrea-Iridescent, like mother-of-pearl.

Nactus, a, um-Obtained, stumbled upon.

Naiadiformis, e-Like a water-nympl.

Nanus-A dwarf.

Nassa-A net, weel, wicker-basket.

Nassula-A little bag-net.

Nasutus, a, nm-Largc-nosed.

Natalis, e-Native, produced, natural.

Natator-A swimmer.

Naticoides-Like Natica.

Nautiloides-Like Nautilus.

Navalis, e-Of or belonging to ships, naval.

Navicella-A small vessel.

Naviformis, e-Ship-formed.

Navigiolum - A little boat.

Nebulosus, a, um-Full of mist, hazy.

Necis-Deatl.

Neglectus, a, um-Neglected, overlooked.

Nervatus, a, m-Full of nerves or fibers.

Nervosus, a, um-Full of fibers, sinewy.

Neuropteroideus, a, um-Like Neuropteris.

Nexilis, e-Knit,tied or wreatbed together, twining.

Nexus, a, um-Linked together, interlaced.

Nitela-Brightness, splendor.

Nitens-Shining, neat, beautiful.

Nitidulus, a, um-Somewhat spruce, rather trim.

Nitidus, a, um-Neat, shining, polished.

Nobilis, e-Famous, celebrated, noble.

Nobilissimus, a, um-Most celebrated.

Nodobrachiatus, a, um-Knotty-armed.

Norlocarinatus, a, um-Knotty-keeled.

Nodocostatus, a, um-Knotty-ribbed.

Nodocosta-Having knotty-ribs.

Nododorsatus, a, um-Knotty-backed.

Nodomarginatus, a, um-Knotty-margined.

Nodostrius, a, um-Knotty.

Nodostriatus, a, um-Having knotty striæ.

Nodosus, a, um-Knotty, full of knots.

Nodulatus, a. um-Knotted.

Noduliferus, a, um-Knot or node bearing.

Nodulostriatus, a, um-Having small knotty stria.

Nodulosus, a, um-Full of little nodes or knots.

Normalis, e-Made by the square or rule.

Notabilis, e-noteworthy, remarkable, extraordinary.

Notans-Noting, marking.

Notatus, a, um-Marked, branded, noted, dotted.
Nothus, a, um-Spurious, not genuine, of mixed breed.

Notus, a, um-Well known, notorious.

Nuciformis, e-Nut-shaped.

Nucleatus, a, um-Deprived of the keruel, stoned.

Nucleifornis, e-Kernel-shaped.

Nucleolatus, a, um-Like a little nut.

Nucleus-A kernel, nut.

Nuculiformis, e-Shaped like Nucula.

Nuculoides-Like Nucula.

Nudus, a, um-Naked, uncovered, empty, alone.

Numerosus, a, um-Numerous, manifold.

Nummifer, era, erum-Coin or disk bearing.

Nummiformis, e-Coin-shaped.

Nummularius, a, um-Of or pertaining to money.

Nummularis, e-Like a little coin.

Nuntius-A inessenger.

Nuperus, a, um-Late, newly come or taken, recent.

Nuptialis, e-Nuptial.

Nutans-Nodding, bending backward and forward.

Nutrix-A nurse, the breast or pap.

Nux-A nut.

Nymphalis, e-Of or belonging to a fountain.

Obcordatus, a, um-Inversely heart-shap $\mathbb{l}$.

Obesus, a, um-Fat, plump, swollen.

Oblatus, a, um-Showing, exhibiting.

Obliquatus, a, um-Bent, oblique.

Obliquinodus-Oblique-knot.

Obliquus, a, um-Oblique, sidewise.

Oblongifolius, a, um-Ublong-leaved.

Oblongus, a, um-Rather long, oblong.

Obmaximus, a, um-Large in front.

Obovatus, a, um-Inversely ovate.

Obpyramidalis, e-Inversely pyramidal.

Obscurns, a, um-Hiddeu, nut understood, obscure.

Obsolescens-Grown old.

Obsoletus, a, um-Antiquated, obsolete.

Obtectus, a, um-Covered, disguised.

Obtusidens-Blunt-toothed.

Obtusifolius, a, um-Obtuse-leaved.

Obtusilobus, a, um-Obtuse-lobed.

Obtusiplicatus, a, um-Obtuse-plaited.

Obtusispira-Having a blunt spire.

Obtusus, a, um-Blunted, obture.

Obuncus, a, um-Bent in, hooked.

Obvius, a, um-Meeting, laying open, exposed.

Occasus, a, um-Crushed, stricken to the ground.

Occidaneus, a, um-TVestern.

Occidens-The west, western.

Occidentalis, e-Western.

Oceanus, a, um-Of or belonging to the ocean.

Ocellatus, a, um-Having little eyes.

Octobrachiatus, a, um-Eight-armed.

Octocostatus, a, um-Eight-ribbed.

Ostonarius, a, um- of the number eight.

Octonotatus, a, um-Having eight marks or tracks.

Orula'us, a, um-Having eyes. 
Oculiferus, a, um-Eye-bearing.

Oculinus, a, an-Like an eye.

Odontopteroides-Like Odontopteris.

Offula-A small piece.

Oliviformis, e-Shaped like an olive.

Oligospiratus, $a$, um-Having few whorls.

Olla-A pot.

Ollicula-A little pot.

Omphaloides-Like a navel or boss.

Onustus, a, um-Filled, loaded, burdened.

Ophioglossoides-Like Ophioglossus.

Upimus, a, um-Fertile, fruitful, fat, large, plunip.

Oppletus, a, um-Filled.

Oppositus, a, um-Opposite, placed before.

Optatus, a, um-Wislıed, desired, longed for.

Opusculum-A little fabric.

Orbicaudatus, a, um-Having a circular tail.

Orbicella-A little circle.

Orbicularis, e-Circular, orbicular.

Orbiculatus, a, um-Of a round or circular form, orbiculate.

Orbiculostoma-Having a circular mouth.

Orbipora-Having round pores.

Ordinatus, a, um-Set in order, regular, ranged in rows.

Oreopteroides-like Oreopteris.

Organum-An instrument, implement, or pipe.

Oriens-Rising, beginning.

Orientalis, e-Eastern.

Originarius, a, um-Original.

Ornatissimus, a, um-Very ornate, highly adorned.

Ornatus, a, $11 \mathrm{~m}$-Adorned, embellished.

Ornigranulus, a, um-Having granules.

Ornithicnoides-like bird-tracks.

Orthambonites-Having a straight umbo.

Orthidoideus, a, um-Like Orthis.

Orthonotus, a, um-Straight-backed.

Osculum-A pretty little month.

Ostiolatus, a, um-IIaving small openings.

Ovalis, e-Oval, egy-shaped.

Ovatifolius, a, um-Ovate-leaved.

Ovatiporu-Ha ving oval pores.

Ovatus, a, um-Shaped like an egg, ovate.

Ovibos-The musk ox.

Ovidactylus-Having ovate toes.

Oviformis, e-Eyg-stuaped.

Ovoidactylus-Having ovoid toes.

Ovoides-Having an egg shape, ovoid.

Ovoideus, a, um-Having a form like an egg, uvoid.

Pabulocrinus - Crinoid-food. A word founded on the erroneous opinion that crinoids lived on Gasteropoda.

Pacator-A peace-maker.

Pachyclirus-Having a thick hand.

Pachydactylus, a, um-Having thick fingers or thick toes.

Pachyderma-A thick skin.

Pachynervis, e-Having thick veins or thick nerves.

Pachypteroides-Like Pachypteris.

Pachytesta-Having a thick shell.

Palæotrochus-Ancient Trochus.

Paliformis, e-Shovel-like or stake-like.
Palmatifilus, a, un-Divided like a hand.

Palmatus, $H$, um-Marked with the palm of a hand, palmate.

Palmipes-Broad-footed.

Palpelira-The eyelid.

Paludiniformis, -Shaped like Paludina.

Palum-A pale, stake.

Pandatus, a, um-Bent, bowed down in the middle.

Pandoriformis, e-Slıaped like Pandora.

Pandus, a, um-Bent, crooked, curved.

Panicum-A grain, panic-grass.

Panneus, a, um-Ragged, tattered.

Pannosus, a, um-Full of rags, ragged.

Papilioniformis, e- Shaped like a butterfly.

Papillatus, a, um-Bud-shaped, covered witl papilli.

Papillosus, a, um-Full of buds, verrucose.

Papulatus, a, un-Covered with nipples, warty.

Papulosus a, um-Full of pimples.

Puradoxicus, a, um-Paradoxical.

Paradoxus, a, um-Strange, contrary to received opinions.

Paralius, a, um-That grows by the seaside.

Parallelus, a, um-Parallel.

Parallelodontus, a, um-Having parallel teeth.

Parasiticus, a, um-Parasitic.

Paridens-Having equal teeth.

Parilis, e-Equal, like, proportionate.

Partitus, a, um-Proportionably divided.

Parvibrachiatus, a, um-Small-armed.

Parvinodus-Having a small knot.

Parvirostris-Having a little beak.

Parvispira-Having a small spire.

Parvituba-Having a small tube.

Parviusculus, a, um-Quite small.

Parvulipora-Having small pores.

Parvulus, a, $1 \mathrm{~m}-$-Very small.

Parvus, a, um-Small, narrow, short, little.

Patellarius, a, um-Belonging to a plate, plated.

Patellifer, a, um-Dish-bcarer.

Patelliformis, e-Dish-shaped.

Patens-Open, wide, extending, spreading.

Paternus, a, um-Paternal.

Patulus, a, um-Standing open or opened, wide, large.

Paucicristat us, a, $11 \mathrm{~m}-\mathrm{Few}$-crested.

Paucidactylus, a, um-Few-fingered.

Paucinodus, a, um-Having few nodes.

Pauciradiatus, a, um-Few-rayed.

Panciramus, a, um-Having few branches.

Pauciseptus, a, um-Having few septa.

Pauper-Poor, small, impoverished.

Pauperatus, a, um-Poor, impoverished.

Paluperculus, a, um-Poor.

Pecteníormis, e-Shaped like a Pecten.

Pectenoilleus, a, um-Like a Pecten.

Pectinaceus, a, um-Of or belonging to a comb, or to the Pecten.

Pectinatus, a, um-Sloping two ways like a comb.

Pectinellus, a, um-Like a little comb.

Pectiniferua, a, um-Comb-bearing.

Pectunculoides-Like Pectunculus.

Peculiaris, e-Peculiar, remarkable, singular. 
Peduncularis, e- Of or belonging to a little foot.

Pedunculatus, a, um-Little-footed.

Pelagicus, a, um-Belonging to the sea.

Pellicula-A small skin or hide.

Pellucidns, a, nm-Clear, transparent.

Peloris-A shell fish.

Peltatus, a, um-Armed with shields.

Peltigerus, a, $11 \mathrm{~m}$-Shield-bearing.

Pendens-Hanging, depending.

Pendulus, a, um-Hanging down, pendent, pendulous.

Penetrans-Piercing, peretrating.

Penicilliformis, e-Brush or pencil-shaped.

Penicillus-A painter's brush or pencil.

Pennatus, a, um-Winged, feathered.

Penuatulus, a, um-Provided with wings.

Penniformis, e-Feather-shaped.

Pentadactylus, a, um-Five-fingered.

Pentagonus, a, um-Pentagonal.

Pentalobus, a, um-Five-lobed.

Pentaspinus, a, um-Five-spined.

Peracutus, a, um-Very sharp, very acute.

Peramplus, a, um-Very large.

Perangulatus, a, um-Very angular.

Perannulatus, a, um-Many-ringed, very annular.

Perantiquus, a, um-Very ancient.

Perarctus, a, um-Very close, small, or slender.

Perasper, a, um-Very rough.

Perattenuatus, a, um-Very attenuated, drawn out.

Percarinatus, a, um-Very strongly keeled.

Percingulatus, a, um-Encircled with many lines, many-girded.

Perdentatus, a, um-Many-toothed.

Peregrinus, a, um-Strange, foreign.

Perelegans-Very neat, very elegant.

Perextensus, a, um-Very much extended.

Perforator-A borer through.

Perforatus, a, um-Bored through.

Perfossulatus, a, um--Having many little ditches.

Pergibbosus, a, um-Very gibbous.

Pergracilis, e-Very slender.

Perhumerosus, a, um-Having angular shoulders.

Perinflatus, a, um-Much inflated, swollen.

Periprion-A round saw.

Perizomatus, a, um-Girdled, banded.

Perlamellosus, a, um-Very lamellose, having very thin plates.

Perlatus, a, um-Very wide.

Permarginatus, a, um-Large-bordered.

Permultus, a, um-Very many:

Pernasutus, a, um-Very nasute.

Perniformis, e-Shaped like a Perna.

Pernodosus, a, um-Very nodose, knotty.

Perobliquus, a, um-Very oblique.

Peroblongus, a, um-Somewhat oblong.

Peroccidens-From the far West.

Perornatus, a, um-Very ornate.

Perovalis, e-Rather oval.

Perovatus, a, um-Very ovate, or nearly round.

Perparvus, a, um--Very small.

Perplanus, a, um--Very plain.
Perplexus, a, um-Confısed, entangled, intricate.

Perplicatus, a, um-Interlaced, entangled, many-folded.

Perpusillus, a, um-Very small.

Perrostellatus, a, um-Ḣaving a very little beak.

Persicaria-A genus of plants.

Persimilis, e-Very similar.

Persinuatus, a, um-Very sinuate or channeled.

Personatus, a, um-Masked, assumed, disguised.

Persiphonatus, a, um-Having a large siphuncle.

Perspectivus, a, um-Thoroughly viewed.

Perspicator-Sharp-sighted.

Perspinulatus, a, $\mathbf{2 m - H a v i n g ~ m a n y ~ l i t t l e ~}$ thorns or spines.

Perstrialis, e-Having many striæ.

Perstriatus, a, um-Very niuch striated.

Persulcatus, a, um-Very much furrowed.

Pertenuis, e-Very thin, small, or fine.

Pertextus, a, um-Interwoven.

Pertinax-That holds fast, clings to.

Perumbonatus, a, um-Having a very convex unibo.

Perumbrosus, a, um-Very shady.

Perundatus, a, um-Very wavy.

Perundulatus, a, um-Very wavy.

Perversus, a, $11 \mathrm{~m}$-Turned around.

Pervetus, a, um-Very old.

Pervetustus, a, um-Very old.

Pervicax-Immovable, stern.

Pervolutus, a, um-Very much rolled.

Pescapreoli-Having a stock supported by a small tendril.

Pescervæ-Having deer-feet.

Pesovis-Having sheep-feet.

Petasiformis, e-Cap-shaped.

Petechialis, e-Spotted.

Petilus, a, um-Thin, slender.

Petrifactor-Stone-maker.

Petrodoides-Like Petrodus.

Pexatus, a, um-Clothed in a garment with a nap on it.

Pharovicinus, a, um-Near the light-house.

Phaseolus-A kidney-bean.

Phaseolinus-Like a bean.

Phlyctainodes-Pimply, pustulous.

Phoca-A seal, sea-dog.

Pholadiformis, e-Like Pholas.

Pholadis-Like a Pholas.

Phragmoceras-Partitioned horn.

Phycoides-Like sea-weed.

Piger, gra, grum-Sluggish.

Pileatus, a, um-Covered with a cap.

Pileiformis, e-Cap-shaped.

Pileolus-A skull-cap, a little cap.

Pileolum-A little cap.

Pileus-A cap or hat.

Pilosus, a, um-Hairy, shaggy.

Pinaster-A wild pine.

Pinguis, e-Fat, plump, fertile.

Pinnatifidus-Having cleft pinnæ.

Pinnat us, a, um-Feathered, plumed, winged.

Pinniformis, e-Like Pinna.

Piscator-A fisher. 
Pisiformis, e-Pea-shaped.

Pistilliformis, e-In the form of a pestle.

Pistillus-A pounder, pestle.

Pisum-A pea.

Placenta-A cake.

Placidus, a, um-Placid, smooth.

Plagosus, a, um-Full of wounds or stripes.

Planiceps-Flat-headed.

Planicosta-Having flat ribs.

Planidorsalis, e-Flat or smooth-backed.

Planidorsatus, a, um-Flat or smoothbacked.

Planifrons-Having a plane front.

Planimarginatus, $a$, um-Flat-margined.

Planiramosus, a, um-Having flat branches.

Planirostris-Having a smooth beak.

Planispira-Having a flat spire.

Planistria-Having flat striæ.

Planistriatus, a um-Having flat strix.

Planobasalis, e-Having a flat base.

Planobrachiatus, a, um-Smooth-armed.

Planoconvexus, $a, u m-F l a t$, convex.

Planocostatus, a, um-Flat-ribbed.

Planorliscus-Flat disk.

Planodorsalis, e-Smooth or flat-backed.

Planodorsatus, a, um-Having a smooth or flat back.

Planogyratus, a, um-Flat-whorled.

Planorbiformis, e-Like Planorbis.

Planosulcatus, a, um-Plane-furrowed.

Planovolvis, e-Flat-whorled.

Planulatus, a, um-Rather flat.

Planumbonus, a, um-Having a smooth umbo.

Planus, a, um-even, level, flat, plane.

Platymarginatus, a, um-Flat-margined.

Platybasis-Having a flat base.

Platycephalus-Having a flat head.

Platynervis, e-Flat-nerved.

Platynotus-Having a flat ridge or back.

Platypleurus-Having flat sides.

Platypus-Broad-footed.

Platyrachis-Having a flat rachis.

Platys-Broad.

Platystigma-Having flat scars, dots, or pits.

Platystomus, a, um-Having a broad mouth.

Plebeiformis, e-Like a plebeian.

Plebeius, a, um-Common.

Pleiopleura-Having wide ribs.

Plenissimus, a, um-The largest.

Plenus, a, um-Full, plump.

Pleurexanthemus-Having the pleura extending out.

Pleurites-The side, lateral.

Pleurodictyoides-Like Pleurodictyum.

Pleuropistha-Having the side behind.

Pleuroptera-Having side wings.

Pleuroptery $x$ - Having side wings.

Pleurovimineus, a, um-Having side wickerwork.

Plicatellus, a, um-Having small folds.

Plicatilis, e-That may be folded, flexible.

Plicatulus, a, um-Having little plications or folds.

Plicatus, a, um-Plaited, folded.

Pliciferus, a, um-Fold-bearing or plaited.

Plicomphalus-Folded in the middle.

Pluma-A small feather.
Plumarius, a, um-Embroidered with feathers.

Plumosus, a, um-Full of feathers, feathery. Plumula-A little feather.

Plumulosus, $a$, um-Full of feathers.

Pluriradialis, e-Many-rayed.

Pocillatus, a, um-Little-cupped.

Pocilliformis, e-Cup-shaped.

Pocillum-A little cup.

Poculum-A cup, bowl, or goblet.

Pogonias-A kind of comet.

Politus, a, um-Polished, smoothed.

Polydactylus, a, um-Many-fingered.

Polygonius, a, um-Having many angles, polygonal.

Polygyratus, a, um-Many coiled or whorled.

Polymorphus, a, um-Many-formed.

Polyphyllus, a, um-Many-leaved.

Polypleurus, a, um-Having many ribs.

Polysporus-Having many spores.

Polystomellus, a, um-Having many little mouths.

Ponderosus, a, um-Heavy, ponderous.

Ponticulus-A little bridge.

Porcatus, a, um-Ridged, furrowed.

Porosus, a, $11 \mathrm{~m}-\mathrm{Full}$ of pores.

Porrectus, a, um-Extended, stretched, or spread ont.

Posticus, a, um-Posterior.

Postremus, a, um-The last, hindmost, worst.

Poststriatus, a, um-Having a striated posterior.

Potens-Powerful.

Poterium-A drinking vessel, a cup.

Priecedens-Going before, surpassing.

Prieciptus, a, um-Anticipated, going before.

Priecursor-A forerunner.

Prælongus, a, um-Very long.

Præmaturus, a, um-Very early, untimely, premature.

Præmorsus, a, um-Bitten off, jagged.

Prænuntius, a, um-That foretells, or forebodes.

Præumbonus, a, um-Very protuberant.

Praterifornis, e-Prateriform.

Pravus, a, um-Crooked, deformed, distorted.

Preciosus, a, um-Precions, splendid.

Precius, a, um-That brings forth ripe grapes before other vines.

Pressulus, a, um-Somewhat pressed in, compressed.

Pressus, a, $11 \mathrm{~m}$-Pressed.

Pretiosus, a, um-Precious, valuable.

Primaevus, a, um-Primeval.

Primarius, a, um-One of the first, remarkable, principal,

Primigenius, a, um-First of its kind, original, primitive.

Primitivus, a, um-First of its kind, primitive.

Primordialis, e-Primordial, original, first of all.

Primus, a, um-The first.

Princeps-The first, chief, original, principal.

Principalis, e-First, original, principal.

Priscus, a, um-Ancient, old. 
Pristiniformis, e-An ancient form.

Pristinus, a, um-Primitive, early.

Pristis-Any sea monster or saw-fish.

Problematicus, a, um-Problematical, unsettled, uncertain.

Proboscidialis, e-Having a proboscis.

l'roboscidiatus, a, um-Having a proboscis.

Procerus, a, um-High, tall.

Proclivis, e-Sloping, steep.

Productus, a, um-Drawn out, produced.

Profundus, a, um-Deep, profound.

Projectus, a, um-Thrown out, projected.

Prolatus, a, um-Brought forth, extended, enlarged.

Prolificus, a, um-Prolific, fruitful.

Prolifer, era, erum-Prolific, productive; fruitful.

Prolixus, a, um-Stretched far out, long, broad.

Prolongatus, a, um-Prolonged.

Prolongus, a, um - Prolonged, stretched out.

Prominulus, a, um-Projecting a little, rather prominent.

Promissus, a, um-Hanging down, putting forth.

Pronis, e-Bent forward, inclined downward.

Pronus, a, um-Turned forward, bent or inclined.

Propinquus, a, um-Near, hard by, related to.

Proporoides-Like Propora.

Proprius, a, um-Peculiar, proper.

Prora-The prow of a ship.

Proteiformis, e-Having many shapes.

Protensus, a, um-Stretched out.

Protextus, a, um-Closeiy woven.

Protuberans-Projecting, protuberant.

Proximus, a, um-Nearest.

Pseudogaleatus-False Galeatus.

Pseudolineatus, a, um-False-lined.

Pseudo-marginalis, e-False-margined.

Pseudomurrayanus, a, un-False Murrayanus.

Pseudosagittatus-False Sagittatus.

Psilophloeus-Having rough bark.

Pterineiformis, e-Shaped like Pterinea.

Pterocephalus-Having a winged head.

Pteroides-Wing-like.

Pterotus, a, um-Winged, feathered.

Pudicus, a, um-Shamefaced, modest.

Pugiunculus-A small dagger.

Pugnax-War-like, combative.

Pugnus-A fist, a handful.

Pulcellus, a, um-Beautiful little.

Pulchellus, a, um-Beautiful little, or somewhat beautiful.

Pulcher, a, um-Beautiful.

Pulex-A flea.

Pulicaris-Like a flea.

Pulmoneus, a, um-Spongy like the lungs.

Pumilus, a, um-Dwarfish, diminutive, little.

Punctatus, a, um-Punctured, dotted.

Punctiferus, a, um-Puncture-bearing.

Punctifrons-Dotted in front.

Punctillatus, a, $11 \mathrm{~m}$-Finely dotted.

Punctipora-Having dotted pores.
Punctolineatus, a, um-Having dotted or pitted lines or furrows.

Punctostriatus, a, um-Having pricked or dotted strix.

Punctulatus, a, um-Marked with small spots.

Punctuliferus, a, um-Bearing punctures or dots.

Pusillus, a, um-Very small, petty, insignificant.

Pustulatus, a, um-Blistered, covered with pustules.

Pustuliferus, a, um-Bearing blisters or pustules.

Pustulosus, a, um-Full of blisters, pimples, or pustules.

Puteatus, a, um-Having little pits or wells.

Puteolatus, a, um-Pitted.

Putillus-A child or dwarf.

Pygmreus, a, um-Dwarfish.

Pyramidalis, e-Pyramidal, pointed like a pyramid.

Pyramidatus, a, um-Pyramidal, made like a pyramid.

Pyriformis, e-Pyriform, pear-shaped.

Pyxidatus, a, um-Box-like.

Pyxidicula-A sinall box.

Pyxidiformis, e-Box-shaped.

Quadrangularis, e-Quadrangular.

Quadrangulatus, a, um-Quadrangular.

Quadrans-A quarter or a fourth part.

Quadraticaudatus, a, um-Square-tailed.

Quadratifolius, a, um-Quadrate-leaved.

Quadratus, a, um-Four-cornered, squared, quadrate.

Quadribrachiatus, a, um-Having four arms.

Quadriceps-Square-headed.

Quadricinctus, a, um-Four banded or girdled.

Quadricostatus, a, um-Four-ribbed.

Quadrilateralis, e-Quadrilateral, foursided.

Quadrimucronatus, a, um-Having four sharp points or spines.

Quadripartitus, a, um-Four-parted.

Quadriseriatus, a, um-Having four series. Quadrispinus, a, um-Four-spined.

Quadrisulcatus, a, um-Four-furrowed.

Quadrivolvis, e-Four-whorled.

Quadrula-A little square.

Quasillus-A little basket.

Quaternarius, a, um-Containing four, quaternary.

Quatuordecembrachialis, e-Having fourteen arms.

Quercifolius, a, um-Oak-leaved.

Quincuncialis, e-Made in the form of a quincunx.

Quinquelobus, a, um, tive-lobed.

Quinquenodus, a, um-Having five nodes or knots.

Quinquepartitus, a, um-Five-parted.

Quinquesulcatus, a, um-Five-furrowed.

Racematus, a, um-Having clusters.

Racemosus, a, um-Full of clusters, clustering.

Radians-Radiating, glittering. 
Radiatoplicatus, a, um-Rayed and plaited. Radiatus, a, um-Rayed.

Radicans-Rooting.

Radiciformis, e-Root-like.

Radicosus, a, um-Full of roots.

Radicula-A small root.

Ramifer, era, erum-Branch-bearing.

Ramosissimus, a, um-Very branchy.

Ramosus, a, um-Full of branches, ramose.

Ramulosus, a, um-Full of little branches.

Ramulus-A little branch.

Rana-A frog.

Ranuneulus-A tadpole.

Rapax-Grasping, rapacious.

Raphanus-A radish-root.

Rapheidolabis-Needle-like forceps.

Rapidens-Having grasping teeth.

Raptor-A robber.

Raricosta-Having few ribs.

Raricostatus, a, um-Ilaving few ribs.

Rarinervis, e-Few-nerved or few-veined.

Raripora-Having few pores.

Rarispinus-Having few spines.

Rarus, a, um-Having wide interstices, thin, scattered, rare.

Recedens-Falling back, receding.

Receptaculum - A receptacle.

Rectangularis, e-Rectangular.

Rectangulus, a, um-Rectangular.

Rectiannulatus, a, um-Having straight annulations.

Recticameratus, a, um-Straight-chambered.

Recticardinalis, e-Having a straight cardinal line.

Rectidorsatus, a, um-Straight-backed.

Rectidens-Having straight teeth.

Rectiformis, e-Straight-formed.

Rectilatera-Having straight sides.

Rectila teralis, e-Straight-sided.

Rectilaterarius, a, um-Straight-sided.

Rectilinea-Having straight lines.

Rectinodus, a, um-Having a straight knot or node.

Rectiplicatus, a, um-Having straight plaits or folds.

Rectirostris-Straight beaked.

Rectirostrus, a, um-Straight-beaked.

Rectiseptatus, a, um - Having straight septæ.

Reetistriatus, a, um-Having straight furrows.

Rectistylus, a, um-Having straight stems or styles.

Rectus, a, um-Straight.

Recurvatus, a, um-Curved backward.

Recurvirostris-Having a recurved beak.

Recurvus, a, um-Turned back, bent or curved back.

Reflexus, a, um-Bending backward, reflexed.

Regalis, e-Regal, splendid.

Regius, a, um-Regal, majestic.

Regularis, e-Regular, according to a rule, of or belonging to a bar.

Regulatus, a, um-Regulated.

Reliquus, a, um-Remaining.

Remex-A rower, oarsman.

Remibrachiatus, a, um-Paddle-armed.

Remipes-Oar-footed.
Remotiseptum-Having distant barriers or walls.

Remotus, a, um-Reınoved, distant, remote.

Remus-An oar.

Reniformis, e-Kidney-shaped.

Repandus, a, um-Bent backward.

Repens-Creeping, erawling.

Repertus, a, um-Discovered, hit upon.

Repositus, a, um-Restored, kept, remote, distant.

Reservatus, a, um-Reserved.

Restrictus, a, um-Drawn back, bound up.

Resupinatus, a, um-Lying on one's back, bent backward.

Resupinoides-Like a resupinate form.

Reticularis, e-Reticulated.

Reticulatus, a, um-Made like a net, netlike, reticulated.

Retiferns, a, um-Net-bearing.

Retiformis, e-Net-formed.

Retorquatus, a, um-Turned back.

Retractilis, e-Drawn back.

Retrorsus, a, um-Turned backward, in reversed order.

Retroversus, a, um-Turned backward, in reversed order.

Retusus, a, um-Beaten back, blunt, dull.

Reversus, a, um-Tnrned about, reversed.

Revolutus, a, um - Rolled back, revolved.

Rhabdocarpus, a, um-Rod-fruited or longfruited.

Rhombeus, a, um-Rhomboidal.

Rhombieus, a, um-Rhombic.

Rhombiferus, a, um-Rhomb-bearing.

Rhomboidalio, e-Rhomboidal.

Rhomboides-Rhomb-like.

Rh om bo ide $1 \mathrm{~s}$, a, um-Lozenge-shaped, rhomboid.

Rhombolinearis, e-Rhomb-lined.

Rhynchonelliformis, e-Like Rhynchonella.

Riciniformis, e--Like a tike or tick.

Ricinula-A little tick.

Rictum - The mouth wide open.

Rigens-Stiffened, standing upright.

Rigidus, a, um-Hard, inflexible, rigid.

Rimosus, a, um-Full of cracks, or fissures.

Ringens-Gaping.

Robusteus, a, um-Strong, of hard wood.

Robustus, a, um-Strong, robust.

Rostellatus, a, um--Little-beaked.

Rostellum-A little beak.

Rostratus, a, um-Beaked, curved at the end.

Rota-A wheel.

Rotadentatus, a, um-Wheel-toothed.

Rotalinea-Having a round line.

Rotatorius, a, um-Whorled.

Rotatus, a, um-Wheel-shaped.

Rotulatus, a, um--Rounded.

Rotuliformis, e-Little wheel-shaped.

Rotuloides-Like a little wheel.

Rotulus-A little wheel.

Rotundatus, a, um-Rounded.

Rotundifolius, a, um-Round-leaved.

Rotundilobus, a, um-Round-lobed.

Rotundispira-Having a round spire.

Rotundus, a, um-Wheel-shaped, circular, rotund.

Rubellus, a, um-Reddish. 
Ruber, bra, brum-Red, ruddy.

Rudicula-A wooden sploon, a spatula.

Rudis, e-Rough, unwrought, unpolished.

Rugatinus, a, uin-Having little folds or plaits.

Rugatulus, a, um-Having little wrinkles.

Rugicosta-Having wrinkled ribs.

Rugilineatus, a, um-Having wrinkled lines.

Rugi plicatus, a, um-Having wrinkled plates.

Rugistriatus, a, um-Having wrinkled striæ.

Rugosiusculıs, a, um-Covered with small wrinkles,

Rugosus, a, 11m- Wrinkled, shriveled.

Rugulatus, a, um-Having wide furrows.

Ruguliferus, a, um-Wrinkle-bearing.

Ruidus, a, um-Rough.

Rusticellus, a, unl-Somewhat rustic.

Rusticus, a, um-Rural, rustic, rough.

Saccatus, a, um-That is put in a bag, like a little bag.

Sacculus-A little bag.

Sagittarius, a, um-Of or belonging to an arrow.

Sagittatus, a, um-Discharging arrows, barbed like an arrow.

Salamandroides-Like a salamander.

Salebrosus, a, um-Rough, rugged, uneven.

Saliginoirles-Like willow wood.

Salisburioides-Like Salisburia.

Samariformis, e-Like elm-seed.

Sanguinolariodeus, a, um-Like Sanguinolaria.

Sarcinula-A little bundle.

Sarcululns-A little hoe.

Sarmenticius, $a$, um-Of or belonging to twigs.

Sarmentosus, a, um - Full of twigs or little branches.

Saxifragifolius, a, um-Leaved like Saxifraga.

Saxivadus, a, um-Creeping over stone.

Scaber, era, erum-Rough, scurfy.

Scaberrimus, a, um-Very rough, scurfy.

Scabiosus, a, um-Scabby, rough, scurfy.

Scabriculus, a, um-Rough.

Scabrosus, a, um-Rough.

Scalariformis, e-Ladder-like.

Scalaris, e- Of or belonging to a flight of steps, or a ladder.

Scalatus, a, um-Having stairs.

Scalenus, a, um-Unequal-sided, scalene.

Scalpriformis, e-Lancet-shaped.

Scapha-A skiff or boat.

Scintilla-A spark.

Scissilis, e-Split, cleft, or rent.

Scitulus, a, um-Handsome, pretty, elegant.

Scobiniformis, e-Rasp-like.

Scobina-A rasp.

Scolopendrites-Stone-scolopendrium.

Scoparius-A sweeper.

Scorpionis, e-Of or belonging to a scorpion.

Scrinium-A case, chest, or box.

Scriptiferus, a, um-Writing-bearing.

Scrutator-A searcher, investigator.

Sculptilis, e-Formed or produced by carving or graving.
Sculptus, a, um-Engraved, sculptured, carved.

Scutatus, a, um-Armed with a shield.

Scutellatus, a, um-Armed with a little shield.

Scutelliformis, e-Waiter-shaped.

Scutigerus, a, um-Shield-bearing.

Scutulatus, a, um-Lozenge-shaped, checkered.

Scyphulus-A small cup.

Scyphus-A cup, a goblet.

Secalinus, a, um-Like small grain.

Secans-A cutter.

Secretus, a, um-Severed, separated, secreted.

Sectifrons-Having a divided front.

Sectoralis, e-Like a sector, or cutter.

Secundus, a, um-Following.

Securiformis, e-Ax or hatchet shaped.

Securis-An ax or batchet.

Segmentatus, a, uin-Ornamented with strips, trimmed, made of pieces.

Selaginoides Like Selago.

Selago-A plant.

Selectus, á, ım-Culled, selected, chosen.

Selenurus-Having a crescent tail.

Selluliformis, e-Like a little seat or stool.

Semicarinatus, a, um-Half-keeled.

Semicircularis, e-Half-cireular.

Semicostatus, a, um-Half-ribbed.

Semicylindricus, a, um-Half-cylindrical.

Semiellipticus, a, um-Half-elliptical.

Semifasciatus, a, um - Half-bundled or banded.

Semina-Seed.

Seminosus, a, um-Full of seeds.

Semiorbiculatus, a, um-Half-orbicular.

Semiplicatus, a, um-Half-plaited.

Semipunctatus, a, um-Half-dotted.

Semiradiatus, a, um-Half-rayed.

Semiradical us, a, um-Half-rooted.

Semireductus, a, um-Half bent back.

Semireticulatus, a, um-Half-reticulated.

Semirotundus, a, um-Half-round, semicircular.

Semistriatus, a, um-Half-striated.

Senarius, a, um-Consisting of six.

Senectus, a, um-Aged, very old.

Senex-Old, aged.

Sentosis, a, um-Full of thorus, thorny.

Separatus, a, um-Separated.

Septatus, a, um-Divided with partitionsor septa.

Septemnotatus, a, um-Seven-marked.

Septentrionalis, e-Northern.

Septoris, e-Having seven months.

Septus, a, nm - Inclosed, enveloped, surrounded.

Sepultus, a, um-Buried in deep sleep, slumbering.

Seriatus, a, um-In series.

Sericeus, a, um-Silken.

Serotinus, a, um-Backward, late.

Serpens-Creeping, crawling.

Serpillifolius, a, $11 \mathrm{~m}-$ Thyme-leaved.

Serpuloides-Like Serpula.

Serpuloideus, a, um-Snake-like or Serpulalike.

Serratulus-A small saw. 
Serratus, a, um-Saw-shaped, serrated.

Serrula-A small saw.

Serrulatus, a, um-Like a little saw.

Servilis, e-Of or belonging to a slave, paltry.

Sesquiplicatus, a, um-Once and a half plaited.

Setaceus, a, um-Hairy.

Setiferus, a, um-Bristle-bearing, baving coarse hair.

Setigerus, a, um-Bristle-bearing, having coarse hair.

Sexarmatus, a, um-Six-armed.

Sexlobatus, a, um-Six-lobed.

Sexplicatus, a, um-Six-plaited.

Sexradiatus, a, um-Six-rayed.

Sextans-A sixth part.

Sicula-A dagger, sickle, or scythe.

Sidereus, a, un-Of or belonging to the stars, starry.

Sigaretoides-Like Sigaretus.

Sigillarioides-Like Sigillaria.

Sigillatus, a, um-Adorned with little images or figures.

Sigillum-A sign, mark.

Sigmoides-Like the Greek letter Sigma.

Sigmoideus, a, um-Like the Greek letter Sigma.

Siguatus, a, um-Marked, designated.

Silicula-A little pod.

Siliqua-A pod.

Siliquoideus, a, um-Like a pod.

Similior-Similar.

Simillimus, a, um-Very similar.

Similis, e-Like, resembling, similar.

Simplex-Simple, plain.

Simplicitas-Simpleness, simplicity.

Simulans-Imitating, copying.

Simulator-A copier, imitator.

Simulatrix-A transformer.

Singularis, e-Alone, solitary, singular.

Singularitas - Singleness, being alone or single.

Sinistrorsus, a, $11 \mathrm{~m}$-Toward the left side.

Sinuatus, a, um-Hollowed out, excavated, having depressions.

Sinuosus, a, um-Full of bendings, curves, or folds, sinuous.

Sirpus-A rush, bulrush.

Smilacifolius, a, $11 \mathrm{~m}$-Smilax-leaved.

Sobrina-A cousin.

Socialis, e-Of or belonging to companionship, social.

Solarioides-Like Solarium.

Soleniformis, e-Solen-shaped.

Solenoides-Like Solen.

Solidirostris-A solid beak.

Solidissimus, a, um-Very firm or solid.

Solidulus, a, $11 \mathrm{~m}$-Solid.

Solidus, a, um-Firm, compact, solid.

- Solitarius, a, um-Lonely, solitary.

Solus, a, nim-Alone, single, sole.

Solutus, a, um-Separated, loosened.

Sordidus, a, $11 \mathrm{~m}$ - Small, sordid, paltry.

Sororcula-A little sister.

Sparsipora-Having few pores.

Sparsus, a, um-Scattered, serarated, dispersed.

Spartarius, a, $1 \mathrm{~m}$-Of or belonging to a broom.
Spathatus, a, um-Spatula-shaped.

Spatiosus, a, um-Ample, of great extent, spacious.

Spatulatus, a, um-Blade-shaped, spatulate.

Speciosus, a, um-Handsome, beautiful, splendid.

Spectabilis, e-Visible, admirable, remarkable.

Sphrricus, a, $11 \mathrm{~m}-\mathrm{O}$ or belonging to a ball, spherical.

Sphærion-A little ball or pill.

Sphærodactylus- Spherical toed or fingered.

Sphæroidalis, e-Spheroidal.

Sphrrulatus, a, um-A widened sphere.

Sphenophylloides-Like Sphenopliyllum.

Sphenopteroides-Like Sphenopteris.

Spicatus, a, um-Pointed, spiked.

Spiculatus, a, un-Having little points.

Spiculus, a, um-Pointed.

Spinalatus, a, um-Spine-winged.

Spiniferus, a, un-Thorn-bearing, thorny, spiny.

Spinigerus, a, um-Thorn-bearing, thorny, spiny.

Spinobrachiatus, a, um-Having spines on the arms.

Spinoclavatus, a, um-Club-spined.

Spinoporus-Having spines and pores.

Spinosulus, a, um-Somewhat thorny.

Spinosus, a, um-Full of thorns, thorny, prickly.

Spinotentaculatus, a, um-Having spinefeelers.

Spinulicosta-Having spines and ribs.

Spinuliferus, a, um-Spine-bearing.

Spinulosus, a, un-Full of little thorns.

Spinula-A little thorn.

Spiralis, e-Spiral.

Spiratus, a, um-Spiral.

Spiriferoides-Like Spirifera.

Spironema-Having spiral threads or lines. Spirorbis-Spire-wtorl.

Spissiseptus, a, um-Having crowded or numerous septa.

Spissus, a, um-Thick, crowded, compact, dense.

Splendens-Splendid, bright.

Splendidus, a, um-Bright, shining.

Spondyliformis, e-Shaped like Spondylus.

Spondylus-A vertebra, spondyle.

Sponglaxis-Sponge-axis.

Spongilla-A little sponge.

Sponsus, a, um-Promised, betrothed.

Spurius a, um-Illegitimate.

Squalodens-A kind of fish-tooth.

Squamifer, era, erum-Scale-bearing.

Squamiformis, e-Scale-like.

Squamosus, a, um-Covered with scales, scaly.

Squamula-A little scale.

Stabilis, e-Firm, stable, durable.

Stachyoides-Like Stachys.

Stamineus, a, um-Full of threads, thready.

Stella-A star.

Stellaris, e-Of or belonging to a star, starry.

Stellatimsulcatus, a, um-Star-furrowed.

Stellatus, a, um-Covered with stars, starred.

Stellifer, era, erum - Star-bearing, stariy.

Stellifolius, a, um-Star-leaved. 
Stelliformis, e-Star-sbaped.

Stenoceplialus-Having a narrow head.

Stenopus-Having a narrow foot.

Stigmatus, a, um-Branded.

Stigmosus, a, um-Full of brand-marks, branded.

Stillativus, a, um-Dropping.

Stipatus, a, um-Crowded together, surrounded, compressed.

Stoloniferus, a, um-Bearing a useless sucker or water-shoot.

Stragulus, a, um-Covered.

Stramineus, a, um-Made of straw.

Strenuus, a, um-Vigorous, strenuous.

Striatellus, a, um-Finely channeled.

Striatiformi s, e-Shaped like Striatus, another species.

Striatocostatus, a, um-Striz-ribbed.

Striatolineatus, a, um-Striæ-lined.

Strlatomarginatus, a, $11 \mathrm{~m}$-Having a striated margin.

Striatopora-Having striæ and pores.

Striatulus, a, um-Somewhat striated, or having small striæ.

Striatura-Being channeled or fluted, a fluting.

Striatopora-Having striated pores.

Striatuv, a, um-Furrowed, striated.

Strictus, a, um-Drawn tight, bound, pressed together.

Strigatus, a, um-Furrowed, channeled, grooved, fluted.

Strigillatus, a, um-Furrowed, fluted.

Strigosus, a, um-Lean, thin, meager.

Striobrachiatus, a, um-Having grooved arms.

Striolatus, a, um-Very winutely striated.

Strix-A furrow, channel, groove.

Strophium-A twisted girdle, a band.

Strophomenoides-Like Strophomena.

Styliola-A truncated column.

Stylus-A pointed instrument, stake, or pale.

Sububbreviatus, a, um-Somewhat abbreviated.

Subaculeatus, a, um-Somewhat prickly.

Subæqualis, e-Subequal.

Subrequatus, a, um-Somewhat equal.

Subrquilaterus, a, um-Somewhat equalsided.

Subalatus, a, um-Somewhat winged.

Subangularis, e-Somew hat angular.

Subangulatus, a, um-Somewhat angulated.

Subarcuatus, a, um-Somewbat curved or arcuate.

Subattenuatus, a, um-Somewhat drawn out or attenuated.

Subcæspitosus, a, um--Somewhat crespitose.

Subcancellatus, a, um-Subcancellated.

Subcarbonarins-Below the coal.

Subcardiiformis, e-Somewhat heart-shaped.

Subcarinatus, a, um-Snmewhat keeled.

Subcavus, a, um-Sumewhat excavated, hollowed out.

Subcentralis, e-Subcentral.

Subcircularis, e-Subcircular.

Subclavatus, a, um-Somewhat club-shaped.

Subcompressus, a, um-Subcompressed.

Subconcruvus, a, um-Subconcave.

Subconicus, a, um-Subconical.
Subconoideus, a, um-Somewhat conoidal. Subconstrictus, a, um-Subconstricted.

Subcordiiformis, e-Somewhat heart-shaped.

Subcoronatus, a, um-Somewhat adorned.

Subcorpulentus, a, um-Somewhat corpulent.

Subcrassus, a, um-Somewhat thick.

Subcrenulatus, a, um-Somewhat crenulated.

Subcuneatus, a, um-Somewhat wedgeshaped.

Subcuspidatus, a, um-Somewhat pointed.

Subcylindricus, a, um-Somewhat cylindrical.

Subcymbiformis, e-Somewhat boat-shaped.

Subdecussatus, a, um-Somewhat arranged in pairs that cross each other.

Subdemissus, a, um-Somewhat hanging down.

Subdepressus, a, um-Somewhat depressed.

Subelegans-Somewhat elegant.

Subellipticus, a, um-Subelliptical.

Subemarginatus, a, um-Slightly emarginated.

Subfalcatus, a, um - Somewhat scytheshaped, subfalcate.

Subfurcatus, a, um-Somewhat forked.

Subfusiformis, e-Somewhat spindleshaped.

Subglobosus, a, um-Somewhat globose.

Subgracilis, e-Somewhat slender.

Subhorridus, a, um-Somewhat rough.

Subimbricatıs, a, um-Somewhat imbricated.

Subimpressus, a, um-Somewhat engraved.

Sublævis, e-Nearly smooth.

Sublamellosus, a, um-Somewhat in thin plates.

Sublineatus, a, um-Somewhat striated.

Subliratus, a, um-Somewhat lined.

Sublunatus, a, um--Somewhat lunate.

Submarginatus, a, um-Somewhat margined.

Submucronatus, a, um-Somewhat sharppointed.

Submutans-Somewhat changing.

Subnasutus, a, um-Somewhat nasute.

Subnervosus, a, m-Somewhat veiny.

Subnodosus, a, um-Somewhat knotty or nodose.

Suborbicularis, e-Somewhat orbicular or orb-shaped.

Suborbiculatus, a, um-Somewhat orbicular.

Subovalis, e-Suboval.

Subovatus, a, um-Subovate.

Suboviformis, e-Somewhat egg-shaped.

Subpapillosus, a, um-Somewhat papillose. Subpapyraceus, a, un-Somewhat like Papyrus, the paper-reed.

Suhplanus, a, um-Somewhat flat.

Subplicatu₹, a, um-Somewhat plaited.

Subpulchellus, a, um-Somewhat handsome.

Subquarrans-Somewhat squared.

Subquadratus, a, um-Somewhat squared.

Subramosus, a, um-Somewhat ramose.

Subramulosus, a, um-Somewhat branchy. Subrectus, a, um-Somewhat straight.

Subretiformis, e-Somewhat net-shaped, or net-like. 
Subrhomboideus, a, um-Somewhat rhomblike.

Subrigidus, a, um-Somewhat rigid.

Subrotundatus, a, um-Somewhat rounded.

Subrugosus, a, um-Somewhat wrinkled.

Subscalaris, e-Somewhat ladder-shaped.

Subscitulus, a, um - Somewhat handsome.

Subsiduus, a, um-Sinking down, settling.

Subsinuatus, a, um-Somewhat sinuated.

Subsinuosus, a, um-Somewhat sinuous.

Subspatulatus, a, um-Somewhat spatulashaped.

Subsphericus, a, um-Subspherical.

Subspinosus, a, um-Somewhat spiny.

Subspinulosus, a, um-Somewhat covered with small spines.

Substellatus, a, um-Somewhat starred.

Substriatellus, a, um-Somewhat finely striated.

Subsuleatus, a, um-Somewhat furrowed.

Subtreniatus, a, um-Sornewhat banded.

Subtentus, a, 11m-Extended underneath, bent.

Subtextilis, e-Somewhat like net-work.

Subtextus, a, um - Woven under, affixed.

Subtilis, e-Fine, thin, slender, delicate.

Subtilitus, a, um-Fine, thin.

Subtilstriatus, a, um- Finely striated.

Subtortilis, e-Somewhat twisted.

Subtortuosus, a, um-Somewhat tortuous.

Subtrigona-Somewhat three-angled.

Subtrigonalis, e-Somewhat three-angled, subtrigonal.

Subtruncatus, a, um-Somewhat shortened.

Subtubulatus, a, um-Somewhat pipe or or tube formed.

Subtumidus, a, $11 \mathrm{~m}$ - Somewhat tumid.

Subturbinatus, a, um - Somewhat topshaped.

Subulatus, a, un-Awl-shaped.

Subumbonatus, a, um-Somewhat protu. berant.

Subumbrosus, a, um-Somewhat umbrellalike.

Subundatus, a, um-Somewhat waved.

Subundiferus, a, um-Somewhat wavebearing.

Subvadus, a, um-Somewluat creeping.

Subvaricusus, a, unn-Subvaricose.

Subventricosus, a, um-Subventricose.

Subvesicularis, e-Subvesicular.

Succinctus, a, um-Girded, contracted, succinct.

Succulens-Succulent, sappy.

Sulcatinus, a, um-Small-furrowed.

Sulcatus, a, um-Furrowed.

Sulciferus, a, um-Furrow-bearing.

Sulcomarginatus, a, um-Having the margin furrowed.

Sulcoplicatus, a, um-Grooved along the middle of the plications.

Superbus, a, um-Superior, excellent, superb.

Superlatus, a, um-Extravagent, excessive, exaggerated.

Supracingulatus, a, um-Encircled or girdled in the upper part.

Supraplanus, a, um-Flat above.
Surgens-Rising.

Symmetricus, a, un-Symmetrical.

Tabulatus, a, um-Floored, tabulated.

Trniopteroides-Like Tæniopteris.

Tæniopteroideus, a, um-Like Tæniopteris.

Tantillus, a, um-So little, such a little thing.

Tapetiformis, e-Formed like tapestry.

Tardus, a, um-Slow, sluggish.

Taxinus, a, $12 m-$ - Iike the yew-tree.

Tectorius, a, um- Of or belonging to a cover, rough east.

Tegulatus, a, um-Tiled, thatched.

Tegulum-A covering, thatch.

Telliniformis, e-Like Tellina.

Telum-A dart, spear, or javelin.

Temerarius, $u$, un-Accidental, casual.

Tenax - Holding fast, griping, tenacions.

Tentllus, a, um-Somewhat delieate, young.

Tener, era, erum-Delicate, tender, young.

Teneris, e-Delieate.

Tenerrimus, a, um-Very tender, very delicate.

Tenerus, a, um-Tender, delicate.

Tentaculatus, a, um-Having feelers.

Tenuiannulatus, $a$, um-Having slight annulations.

Tenuibrachiatus, a, um-Slender-armed.

Tenuicarinatus, a, um-Finely keeled.

Tenuiceps-Having a slender head.

Tenuicinctus, a, mu-Finely girded.

Tenuicornis, e-Slender-horned.

Tenuicostatus, a, um-Fine-ribbed.

Tenuicosta-Having fine ribs or costr.

Tenuicristatus, a, um-Slender-peaked.

Tenuidactylus, a, um-Slenger-fingered.

Tenuidens Having slender tecth.

Tenuidiseus-Having a thin-disk.

Tenuifilum-Fine thread.

Tenuifolius, a, um-slender-leaved, narrow-leaved.

Tenuilamellatus, a, $u m-$ Having thin plates.

Tenuilineatus, a, um-Fine-lined.

Tenuiliratus, a, um-Fine-lined.

Tenuimarginatus, a, um-Thin-margined.

Tenuimuralis, e-Thin-walled.

Tenuinervis, e-Thin-veined, slendernerved.

Tenuiradiatus, a, um-Slender-rayed.

Tenuiradius-Having slender rays.

Tenuiramosus, a, um - Having slender branches.

Tenuis, e-Thin, fine, slender, narrow.

Tenuiseulptus, a, nm-Finely engraved.

Tenuiseptus, a, um-Having thin septa.

Tenuissimus, a, um-Very thin or slender.

Tenuistriat us, a, um-Fine-lined.

Terebra-A borer, an auger.

Terebralis, e-Like an auger.

Terebriformis, e Shaped like Terebra, or like an auger.

Teres-Rounded, well turned, smooth, polished.

Teretiformis, e-Of a long, round shape.

Terminalis, e-Terminal.

Tersus, a, um-Neat, wiped off, nice.

Tessellatus, a, um-Checkered, tessellated. 
Testudinarius, a, um-Arched like a tortoise shell.

Tetragonocephalus-Having a quadrangular bead.

Tetragonopthalmus-Having square eyes.

Tetragonum-A quadrangle.

Tetraptyx-Having four folds.

Tetricus, a, um-Forbidding, stern.

Textiligerus, a, um-Web-bearing.

Textilis, e-Woven, plaited, textile.

Textus, a, um-Woven, fabricated.

Thærodactylus, a, um-Hinge-toed.

Thally formis, e-Shaped like Thallus, frondlike.

Tholus-A rotunda or cupola.

Tiariformis, e-Shaped like a tiara or turban.

Torquis-A necklace, wreath, or ring.

Tortalinea-Twisted line.

Tortuosus, a, m-Full of turns or crooks, tortuous.

Tortus, a, um-Twisted, distorted.

Transiens-Transient.

Transitionis, e-A passing over.

Translatus, a, um - Carried over, transported.

Transsectus, a, um-Cut across.

Transversalis, e-Transverse, crosswise.

Transversus, a, um-Transverse, crosswise, wider than long.

Triangularis, e-Oi or belonging to a triangle, triangular.

Triangulatus, a, um-Triangulated.

Triarthrus-Having three joints.

Tribulis-One of the same tribe.

Tribulosus, a, um-Full of thorus or thistles.

Tricarinatus, a, $11 \mathrm{~m}$-Three-keeled.

Tricenarius, a, um-Oi or containing thirty.

Trichoideus, a, um-Hair-like.

Trichomanoides-Like Trichomanes, the maiden-hair fern.

Tricingulatus, a, um-Three-banded.

Tricornis, e-Three-horned.

Tricostatus, a, um-Tnree-ribbed.

Tricuspidatus, a, un-Three-pointed.

Tridactylites-Having three fingers.

Tridactylus, a, um-Three-fingered.

Tridens - Having three teeth, tines, or prongs.

Tridentiferus, $a, 11 \mathrm{~m}$-Bearing three teeth prongs.

Tridigitatus, a, um-Three-fingered.

Trifoliatıs, a, um-Three-leaved.

Trifolius, a, um-Three-leaved.

Trigonalis, e-Trigonal.

Trigonolepis-Having triangular scales.

Trigonostomus, a, um-Having a triangular mouth.

Trigonus, a, um-Trigonal.

Tilineatus, a, um-Three-lined.

Triliratus, $y$, um-Three-lined.

Trilix-Triple-twilled.

Trilobatus, a, um-Three-lobed.

Trilobus, a, um-Three-lobed.

Trilocularis, e-Thrte-chainbered.

Trinérvis, e-Three-veined.

Trinodus, a, um-Having three knots.

Trinucleus-Having three kernels.

Tripinnatus, a, um-Three-winged.
Triplicatellus, a, um-Having three plications in one fold.

Triplicatus, a, um-Three-plaited.

Triplistriatus, a, um-Three-lined.

Tripunctatus, a, um-Three-dotted.

Triquetrus, a, $11 \mathrm{~m}$ - Three-cornered, triangular.

Triradiatus, a, um-Three-rayed.

Triserialis, e--In three series.

Triserratus, a, um-Three-notched.

Trisinuatus, a, um-Three-furrowed.

Trisulcatus, a, um-Thıee-furrowed.

'Trisutura-Having three sutures.

Trituberculatus, a, um-Having three tubercles.

Trivolvis, e-Three-whorled.

Trochiformis, e-Shaped like Trochus.

Trochiscus - A small, round ball, a pill.

Tropidophorus, a, um-Keel-bearing.

Trudiferus, a, um-Pike-bearing.

Truncatulus, a, um-Somewhat truncated.

Truncatus, a, um-Truncated, cut short.

Tuber-A hump, bump, or protuberance.

Tuberculatus, a, um-Tuberculated, covered with tubercles.

Tuberculosus, a, uni-Full of tubercles.

Tuberosus, a, um-Full of hilmps or protuberances.

Tubiformis, e-Pipe, tube, or trumpetformed.

Tubiporoides-Like Tubipora.

Tubularis, -Hollow like a pipe.

Tubulatus, a, um-Formed like a pipe or tube.

Tubulostriatus, a, um-Having tube-like strix.

Tubulosus, a, um-Abounding in tubes.

Tubulus-A smull pipe or tube.

Tumidifrons-Swelling front.

Tumidosus, a, um-High-swelling.

Tumidulus, a, um-Swollen, tumid.

Tumidus, a, un-Swollen, tunid.

Tumnlosus, a, um-Full of hills, hilly.

Tumulus-A mound.

Tunicatus, a, um-Coated, covered with skin or peel.

Turbidus, a, um-Confused, disordered turbid.

Turbinatus, a, um-Turbinate, cone-shaped.

Turbiniformis, e-Top-shaped.

Turgidus, a, um-Swollen, inflated, turgid.

Turricula-A little tower, a turret.

Turritella-A little tower.

Turritiformis, e-Tower-like.

Turritus, a, um-Fortified with towers.

Tutus, a, un-safe, secure, examined.

Typicalis, e-Typical.

Typus-The type.

Tyrans-A tyrant.

Uber-A teat, pap, or udder.

Umbella-A parasol, umbrella.

Umbelliferus, a, um-Umbrella-bearing.

Umbilicatus, a, um-Made like an umbilicins.

Umbonatus, a, um-Having a shield, embossed.

Umbraculum-A shade, umbrella.

Umbrosus, a, um-Shady, umbrageous. 
Uncinatus, a, unn-Barbed, furnished with hooks or tenters.

Uucus, a, um-Hook-curved, barbed.

Undans-Waving.

Undatus, a, um-Wavy.

Undosus, a, um-Full of waves, billowy.

Undulatus, a, $11 \mathrm{~m}$-Diversified as with waves, undulated.

Undulostriatus, a, um-Having wavy striæ.

Undulosus, a, um-Fullof undulations, wavy.

Unguiculus, a, um-Having claw-like processes.

Unguifer, era, erum-Claw-bearing.

Unguiformis, e-Claw-shaped.

Ungula-A claw, talon, hoof.

Ungulatus, a, un-Having claws or hoofs.

Unguloideus, a, um-Hoof-like or claw-like.

Uniangulatus, a, um-One-angled.

Unicarinatus, a, um-One-kceled.

Unicornis, e-One-horned.

Unicostatus, a, um-One-ribbed.

Unicus, a, um-One and no more, single, sole.

Uniformis, e-Having only one shape, uniform.

Unilargus, a, um-One large, of one size.

Unilobatus, a, um-One-lobed.

Unioniformis, e-like Unio.

Unionoides-Like Unio.

Uniserialis, e-Having a single row or series.

Unispinus-Having one spine.

Unisulcatus, a, un-Having one furrow.

Unitus, a, um-United.

Uræus, a, um- of or belonging to the tail.

Urniformis, e-Urn-shaped.

Urophyllus, a, um-Sharp-leaved.

Utriculus-A little matrix, a bud or hull.

Vadosus, a, um-Full of sliadows.

Vagans-Wanderiog, vagrant.

Valens-Vigorous.

Validus, a, um-Strong, powerful.

Vallorus, a, un-Intrenched.

Vulvatiformis, e-Like folding doors, or like Valvata.

Valvulus-A pod, like the shell of a bean.

Varians-Varying, varied.

Variabilis, e-Changeable, variable.

Varicus, a, um-Straddling.

Varicosus, a, um-Having threads or lines enlarged, varicose.

Varicostatus, a, $\mathrm{um}-\mathrm{V}$ ariably ribbed.

Variolatus, a, um-Var.able widtl or dis. tances apart.

Variolosus, a. um-Full of changes.

Variopora-Having different pores.

Varistriatus, a, um-Having variable striæ.

Varius, a, um-Diverse, manifold, different, various.

Varus, a, um - Bent, stretched or grow n apart.

Vascularius, a, um - Vascular, consisting of small vessels.

Vasiformis, e-Vase-shaped.

Vastator-A desolator, ravager.

Vaticinus, a, um-Prophetical.

Vellicatus, a, um-Vellicated, pinched.

Velox-Swift, fleet, fitted for notion.

Velutinus, a, un-Velvety.

Velum-A sail, awning, curtain, veil.

Venatus, a, um-Veined.
Venosus, a, un-Full of veins, veiny.

Ventralis, e-Ventral.

Ventricosus, a, um-Bulging out, ventricose.

Venulosus, a, um-Full of small veins.

Venustulus, a, un-Lovely, charming.

Venustus, a, um--Lovely, beautitul, yraceful.

Verbenifolius, a, um-Leaved like Verbena.

Vermicularis, e-Worm-shaped.

Vermiculus-A little worm, grub.

Verrucosus, a, um-Full of watts, rough, rugged.

Versiformis, e-Changing its form, changeable.

Vertebralis, e-Somewhat like vertebræ.

Vertebratus, a, um-Articulated, jrinted, vertebrated, like a backbone.

Verticalis, e-Vertical.

Verticillatus, $\boldsymbol{n}$, um-Whorled.

Verticillus-The whorl of a spindle.

Verus, a, um-True, real, genuine.

Vesicularis, e-Vesicular.

Vesiculatus, a, um-Vesicled.

Vesiculosus, a, um-Fuil of blisters or vesicles, vesiculous.

Vesperalis, e-Belonging to the evening.

Vestitus, a, um-Covered, clothed, adorned.

Veteritor-One who has grown old.

Vetulus; a, um-old.

Vetustus, a, um-Old, ancient.

Vexabilis, + -Disturbed, vexed, troublesome.

Viaticus, a, um-Of or belonging to a journey.

Viator-A wayfarer, traveler.

Vicinus, a, um-Near, neighboring, kindred.

Victus, a, um-Conquered, vanquished.

Vigilans-Watchful, vigilant.

Villosus, a, um-Hairy, shaggy, rough.

Viminalis, e-Bearing twigs for plaiting.

Vinctus, a, um-Bounded, fettered, girded.

Vinculatus, a, um-Bound.

Vindex-A defender.

Vinosus, a, um-Full of wine.

Viola-The violet.

Virgatus, a, um-Made of twigs, twig-like.

Virgo-A uaid.

Virgosus, a, um-Full of twigs.

Virgulatus, a, um-Striped, like a small rod.

Virguncula - A little maid.

Vittatus, a, um-Bound with a fillet, banded.

Volans-Flying.

Volutus, a, um-Rolled, turned around, whorled.

Vomer-A plowshare.

Vomerinu-A plowshare.

Vorax-Ravenous, voracious.

Vorticellatus, a, um-Whorled.

Vulgatus, a, um-General, usual, common.

Xiphias-A sword-fish.

Xylobioirles-Like Xylobius.

Yoldiiformis, e-Like Yoldia.

Zaphrentiformis, e-Shaped likeZaphrentis.

Ziczac-Slanting in straight lines from side to side, having sharp turns.

Zonatus, a, um-Zoned, belted.

Zonulatus, a, um--Small-girdled.

Zygopus-With joined feet. 


\section{INDEX OF GENERA.}

In addition to alphabetically indexing all the Palæozoic genera in this work, and placing in italics those which have been used but do not belong to North America, the gender of each genus is designated as follows: $m$, for masculine; $f$, for feminine; $n$, for neuter.

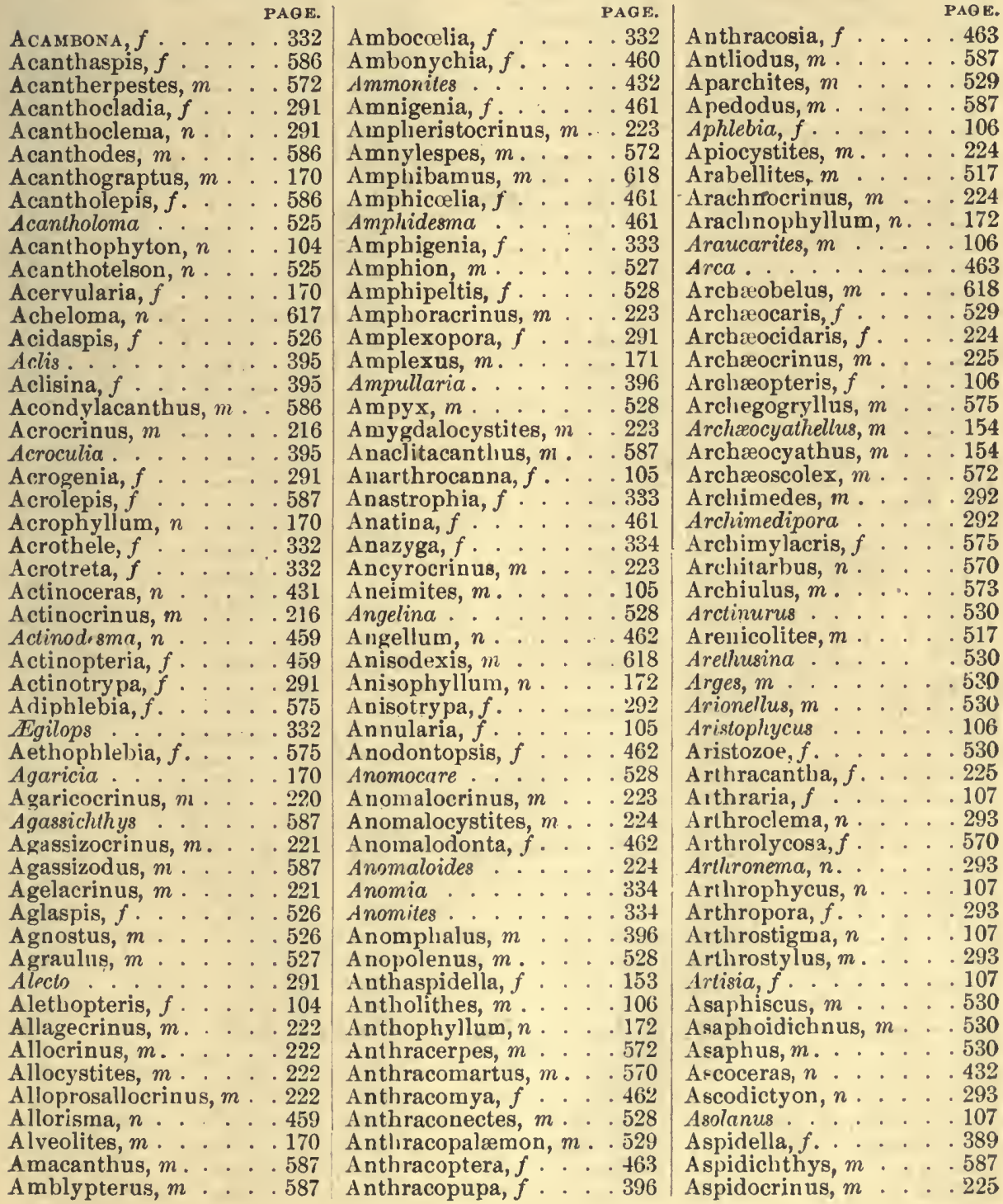




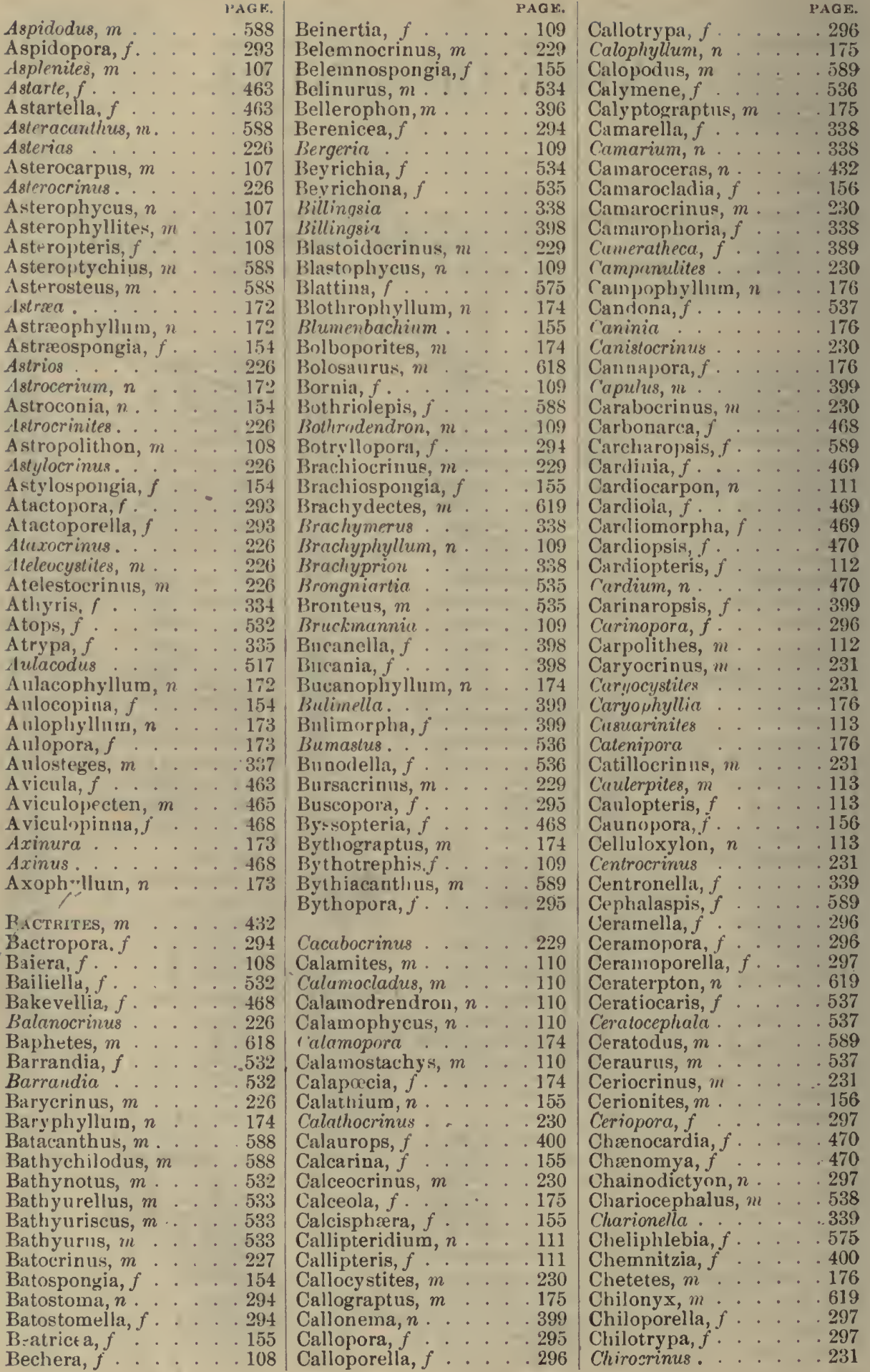




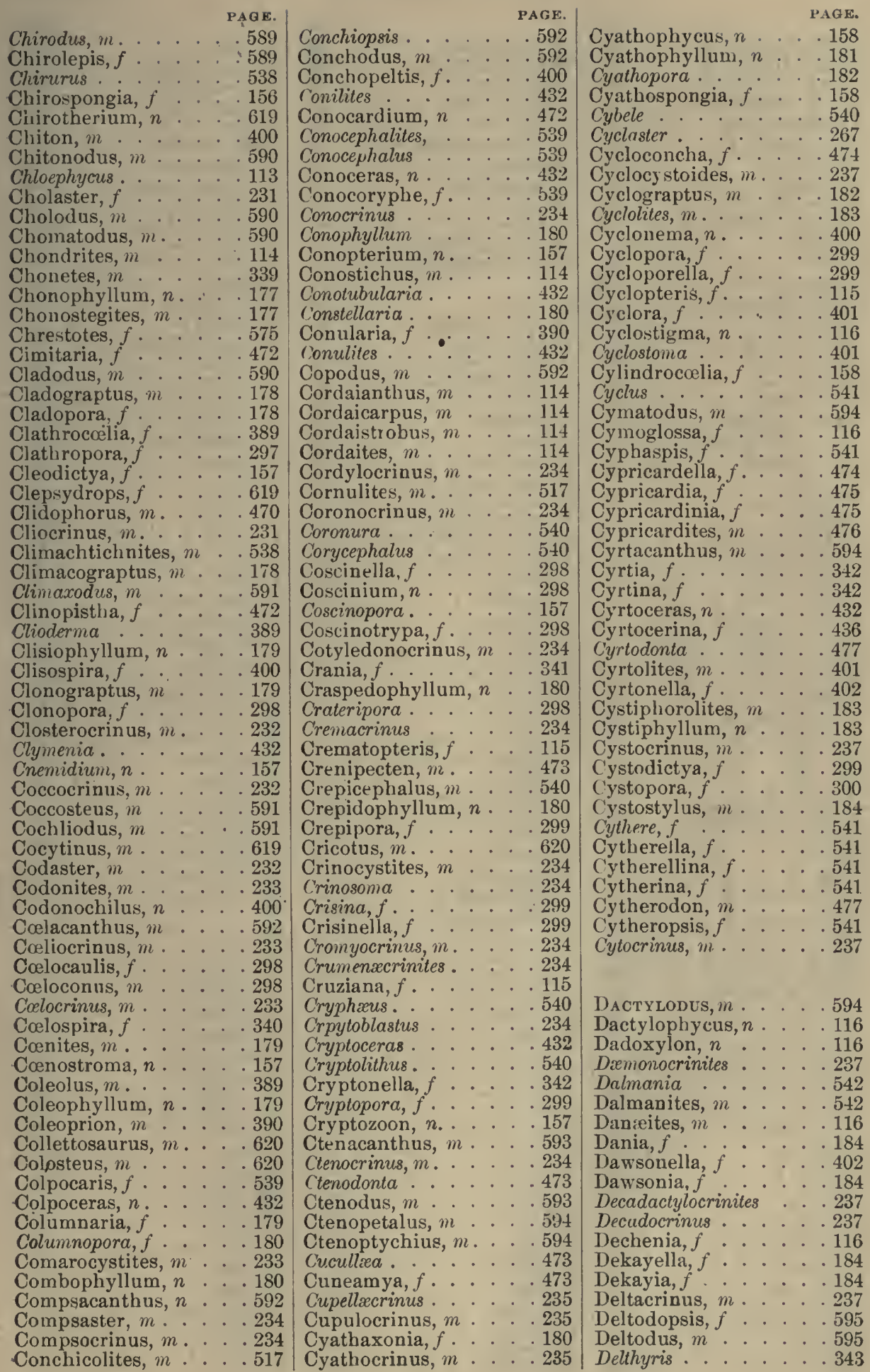




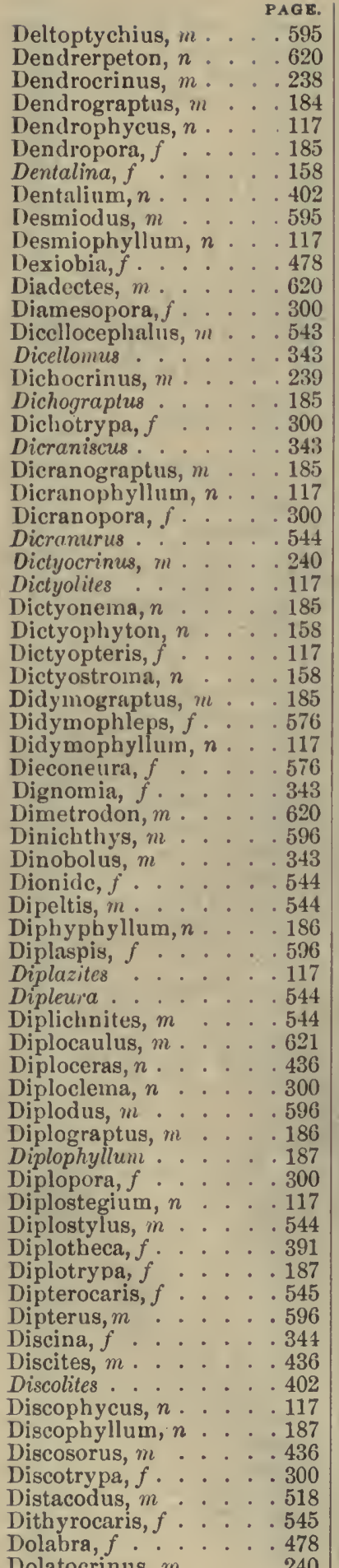

Dolichocephala . . . . 545

Dolichometopus, $m$. . . 545

Dolichopterus, $m$. . . 545

Donacicrnites . . . 240

Dorycrinus, $m$, . . . 240

Drepanacanthus, $m$. .596

Drepanodus, $m$. . . . 518

Duncanella, $f \ldots .187$

Dyscritus, $m$. . . . .576

Dystactella, $f$. . . . 478

Dystactophycus, $\boldsymbol{n}$. . 117

Dy stactospongia, $f$. . 158

Eatonia, $i$. . . . . 345

Eccyliomphalus, $m$. . .402

Echinocaris, $f$. . . . 545

Echinocystites, m . . . . 241

Echinodiscus, $m$. . . . 241

Echinoencrinites, $m$. . . 241

Echinognathus, $m$. . . 546

Echinus . . . . . 241

Ectenocrinus, $m$. . . 242

Ectenodesma, $n$. . . 478

Ectenodictyon, $f$. . . 159

Ectocynodon, $m$. . . 621

Ectosteorachis, $m$. . . . 596

Edaphosaurus, $m$. . . . 621

Edestus, m. . . . . 597

Elınondia, $f$. . . . . 478

Edrioaster, $m$. . . . 242

Edriocrinus, $m$. . . . 242

Edriospongia, $f$. . . . 159

Eichwaldia, $f$. . . . 345

Eiliticus, m . . . . 573

Elracrinus . . . . 242

Elasmophyllum, $n$. . 187

Eleutherocrinus, $m$. . 242

Elkania, $f$. . . . . . 346

Eillipsocephalus, $m$. . 546

Elliptocephala, $f$. . .546

Elonichthys . . . . . . 597

Elymella, $f$. . . . . 480

Elymocaris, $f$. . . 546

Embolamus, $m$. . . . 547

Embolophorus, $n$. . .621

Emmonsia, $f$. . . . 187

Empedias, $m$... . . 621

Empedocles, $m$.... 621

Enallopora, $f$. . . . 300

Encrenus, in .... 576

Encrinurus, $m$. . . 547

Endoceras, $n$. . . 436

Endolobus . . . . . 437

Endothyra, $n$. . . 159

Endymion ..... . 547

Endymionia, $f$. . . 547

Enoploura, $f$. . . . 547

Entolium, $n$. . . 480

Eocidaris, $f$. . . 242

Eocystites, $m$. . . . 242

Eodon, $m$. . . . 480

Eophyton, $n$. . . 118

Eopteria, $f$. . . . . 480

Eosaurus, $m$. . . . 621

Eoscorpius, $m$. . . 570

Eospongia, $f$. . . . 159

Eotrochus, $m$. . . . . 403

Eotrophonia . . . . .518
PAGE.

Eozoon, $n$. . . . 159

Ephemerites, $m$. . . 576

Epicordylus, m. . . . 621

Equisetites, in . . . . 118

Equisetum . . . . . 118

Eremopteris, $f$. . . 118

Eretmocrinus, $m$. . . 242

Eridophylluw, $n$. . . 187

Eridopora, $f$. . . . 301

Erismacanthus, $m$. . 597

Erisocrinus, $m$. . . . 243

Eryops, $f$. . . . . 622

Eschara, $f$. . . . . 301

Escharopora, $f$. . . 301

Estlieria, $f$. . . . 547

Ethmophyllum, $n$. . 159

Etobluttina, $f$. . . . 576

Eucalyptocrinus, $m$. . 243

Euchasina, $n$. . . . 480

Euchondria, $f$... 480

Eucladocrinus, $m$. . . 244

Eugaster, $m$. . . . 244

Eulima . . . . 403

Eumetria, $f$. . . 346

Eumicrotis . . . . . 480

Funema, $n$. . . . 403

Eunemacantluus, $n$. . 597

Eunicites, $m$. . . . 518

Euomphalus, $m$. . . 403

Eupachycrinus, $m$. . 245

Euphoberia, $f$. . . 573

Euproops, $f$. . . . 547

Eurydictya, $f$. . . . 301

Eurylepis, $f$. . . . 597

Eurypterella, $f$. . . 548

Eurypterus, $m$. . . 548

Eurythorax, $m$. . . . 622

Eusarcus, $m$. . . . 549

Euspilopora, $f$. . . . 301

Euspirocrinus, $m$. . . 246

Eusthenopteron, $n$. . . 597

Euthydesma, $n$. . 480

Evactinopora, $f$. . . 301

Exochorhynchus . . . 480

Faberia, $f$. . . . . 549

Favastrea . . . . 187

Favicella, $f$. . . . . 301

Faviphyllum, n... 187

Favistella, $f$. . . . 188

Favosites, $m$. . . . . 188

Favositopora . . . . 189

Fenestella, $f$. . . . 302

Fenestralia, $f$. . . 305

Fenestrapora, $f$. . . 305

Ficoidites . . . . 118

Filiciles . . . . 118, 189

Fissodus, $m$. . . . 597

Fistulipora, $f$. . . 305

Flabellaria...... 118

Flustra, $f$. . . . 306

Forbesiocrinus, $m$. . 246

Fordilla, $f$..... 480

Fucoides. . . . 118

Fungispongia, $f$. . 160

Fusispira, $f$. . . 404

Fusilina, $f$..... 160

Fusus . . . . . 404 


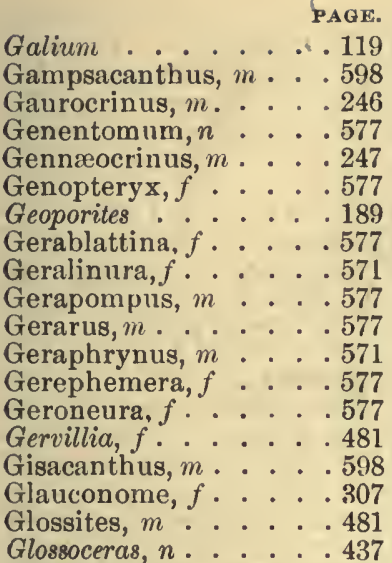

Glossograptus, $m$. . 189

Glossotrypa, $f$. . . 307

Glycerites, $m$. . . . 518

Glymmatacanthus, $m$. . 598

Glyptaster, $m$. . . 247

Glyptocardia, $f$. . . 481

Glyptocrinus, $m$. . 247

Glyptocystites, $m$. . 248

Gly ptodendron, $n$. . 119

Glyptodesma, $n$. . 481

Glyptolepis, $f$. . . 598

Glyptotrypa . . . 307

Gnathorbiza, $f$. . . . 598

Gomphoceras, $n$. . 437

Gomphocystites, $m$. $\quad .249$

Goniasteroidocrinus, $m$. 249

Goniatites, $m$. . 438

Gonioceras, $n$. . 441

Gonioccelia . . . . 346

Goniophora, $f$. . . 481

Goniopteris . . . . 119

Goniotrypa, $f$. . . 307

Gordia . . . 119-518

Gorgonia, $f$. . . 307

Gosselettia, $f \ldots 482$

Grammysia, $f$. . . 482

Granatocrinus, $m \ldots 250$

Graphiocrinus, $m$. 251

Graptodictya, $f \ldots 307$

Graptolithus, $m$. . 19

Gryphorhynchus . . 483

Gulielmites, $m$. . . 119

Gypidula, $f$. . 3 346

Gyracanthus, $m \ldots 598$

Gyrichnites, $m$. . 518

Gyroceras, $n$.... 441

Hadrocrinus, $m \ldots 252$

Hadrophyllum, $n$. . 191

Haimeophyllum, $n$. . 191

Hallia, $f . . . .191$

Halonia, $f$. . . 119

Halysites, $m$..... 191

Halysiocrinus . . . . . 252

Haplocrinus, $m$. . . 252

Haplophlebium, $n$. . 577

Haplotichnus, $m$. . . 578

Harlania, $f$. . . 119

Harmodites . . . . 191
PAGE.

Harpacodus, $m$. . . 598

Harpes, $m$.... . 549

Harpides, $m$. . . 549

Harttia, $f$. . . 549

Haussmannia . . . 548

Helicopora, $f$. . . 308

Helicotoma, $f$. . . 405

Heliodus, $m$. . . . 599

Heliolites, $m$. . . . 191

Heliophycus, $n$. . . . 119

Heliophyllum, $n$. . 192

Helminthoidichnites, $m$. .518

Helodectes, $m$. . . 622

Helodus, $m$. . . . 599

Helopora, $f$. . . 308

Hemeristia, $f \ldots .578$

Hemicosmites, $m$. . 252

Hemicryptirus . . . . 549

Hemicystites, $m$. . 252

Hemipronites . . . . . 346

Hemitrypa, $f$. . . . 309

Hernodia, $f$. . . 309

Heterocrinus, $m \ldots 252$

Heterocystites, $m$. . . 253

Heterodictya, $f$. . . 309

Heterophrentis, $f \quad \ldots 193$

Heterospongia, $f$. 160

Heteroschisma, $n$. . 253

Heterotrypa . . . . 193

Hindella, $f$. . 346

Hindia, $f$. . . 160

Hipparionyx . . . . 346

Hippodophycus, $n \ldots 120$

Hipponicharion, $m$. . 549

Hippothoa . . . . 309

Holocystites, $m$. . 2 253

Holometopus, $m$. . . 550

Holopea, $f . \cdots 405$

Holopella, $f$. . . 406

Holoptychius, $m$. . 599

Homacanthus. . . . 600

Homalonotus, $m$. . 550

Homocrinus, $m$... 255

Homothetus, $m$. . . 578

Homotrypa, $f$. . . . 309

Homotrypella, $f \ldots 310$

Hornera, $f$. . . 310

Hortholus . . . . . 442

Houghtonia . . . . . 193

Huronia, $f$. . . . 442

Hybochinus, $m$. . 255

Hybocladodus, $m$. . 606

Hybocrinus, $m$... 255

Hybocystites, $m$. . 255

Hydnoceras . . . . . 442

Hydreionocrinus, $m$. .256

Hylerpeton, $n \ldots 622$

Hylonomus, $m$. . . 622

Hymenophyllites, $m$. 120

Hyphasma, $n$.... 622

Hyolithellus, $m$. . . . 391

Hyolithes, $m$. . . . 391

Hypanthocrinites . . . 256

Hystricrinus . . . . . 256

Hystrispongia, $f \ldots 160$

ICINNOPH YCu.s, $n \ldots \ldots$

Ichthyacanthus, $m$. . . 622
PAGE.

Ichthyocrinus, $m \ldots 256$

Ichthyorachis, $f \ldots 310$

Icosidactylocrinites . . . 256

Idiophyllum, $n$. . . 120

Idiotrypa, $f \ldots . . .310$

Ilionia, $f \ldots . . .483$

Illænurus, $m$. . . 550

Illænus, $m$. . . . 550

Inachus . . . . . . 406

Inocaulis, $m$. . . 193

Inoceramus . . . . 483

Intrapora, $f \ldots 310$

Intricaria, $f \ldots \ldots . \quad . \quad .310$

Iocrinus, $m$. . . . 256

Iphidea, $f$. . . . . 346

Ischadites . . . . . 160

Ischyrinia, $f$. . . 483

Isocardia..... 483

Isochilina, $f$. . . . 551

Isonema, $n$. . . . 406

Isotelus, $n$. . . . 552

Isotrypa, $f \ldots . .310$

JANASSA, $f \ldots 600$

$\mathrm{K}_{\text {NORRIA }} f \ldots \ldots . .120$

Koninckia, $f$. . . . 346

Kut rrgina, $f \ldots . .347$

Labechia, $f$. . . 310

Lambdodus, $m$. . . 600

Lamellipora, f . . . . . 194

Lampterocrinus, $m$. . 257

Lasiocladia, $f$. . . . 160

Leaia, $f$. . . . 552

Lecanocrin us, $m$. . 257

Lecracanthus, $m$. . 600

Lecythiocrinus . . . . . 257

Leda.... . . . 484

Lepadocrinus, $m$. . 257

Leperditia, $f \ldots .552$

Lepetopsis, $f$. . . . 406

Lepidechinus, $m$. . 258

Lepidesthes, $f \ldots 258$

Lepidilla, $f \ldots . .553$

Lepiditta, $f$. . . . 553

Lepidocidaris, $f \ldots 258$

Lepidocoleus, $m$. . 553

Lepidocystis, $m$. . . 121

Lepidodendron, $n$. . 121

Lepidodiscus . . . . 258

Lepidolites, $m$. . . 160

Lepidophloios, $m$. . 122

Lepidophyllum, $n$. . 123

Lepidostrobus, $m$. . 123

Lepidoxylon, $n$. . . 124

Lepocrinites . . . . 258

Leptacanthus . . . 600

Leptæna, $f$. . . 347

Leptobolus, $m$. . 348

Leptocœlia, $f$. . . . 348

Leptodesma, $n$. . 484

Leptodomus, $f \ldots .485$

Leptomitus, $m$. . 160

Leptophlœum, $n$. . 124

Leptophractus, $m$. . . 623

Leptopora, $f$. . . 194

Leptopterion, $n$. . 161 


\begin{tabular}{|c|c|c|c|c|c|}
\hline & & & . & & PAGE. \\
\hline ptotryp: & . . 311 & Macrodon, & . . 487 & Myrianites & 519 \\
\hline uropte & . 124 & Macropetali & . . 601 & Myrtillocrinus, $m$. & \\
\hline$f$ & 125 & rostachy & 126 & Mytilarca, $f$. . & 493 \\
\hline & . .578 & Macrostyloc & . . 259 & Mytilops, $f$. & \\
\hline chas, $m$ & $\cdot .553$ & $\begin{array}{l}\text { Madrepora } \\
\text { Malocystite }\end{array}$ & $\begin{array}{l}.195 \\
.59\end{array}$ & Mytilus, $m$. & \\
\hline chenatia, & $\begin{array}{r}.311 \\
.258\end{array}$ & $\begin{array}{l}\text { Malocystite } \\
\text { Mariacrinus }\end{array}$ & $\begin{array}{r}.259 \\
. \quad 259\end{array}$ & Naiadites & \\
\hline trypa, $f$. . & . . . 312 & Marrucinth & .601 & Nat & \\
\hline cus, $n$ & . . . 125 & Marsupiocri & 260 & Naticopsis, $f$ & \\
\hline & . . 486 & Martinia, $f$ & 352 & Nautilus, $m$. & \\
\hline$i a, f$ & . . . 194 & Matheria, $f$ & 485 & Nebulipora, $f$. . & \\
\hline & . . 486 & Matthevia, & 392 & Necymylacris, $n$. & 579 \\
\hline omia, $f$ & . . 194 & Mazonia, $f$ & 571 & Nelimenia..... . & \\
\hline$f \therefore$ & - . 349 & & 602 & Nemagraptus, $m$ & \\
\hline isma, $n$ & - 351 & , & & Nemapodia, $f$. . & \\
\hline$f$ & 352 & kopora, & 312 & Nemataxis, $m$. & \\
\hline is, & • . 352 & Megalaspis, & & Nematophycus, $n$. & \\
\hline 8 & 352 & Meyalograpt & 195 & Nematophyllum, $n$. & \\
\hline & . . . 194 & Megalomus & 488 & Nematopora, $f \ldots$ & . \\
\hline onia, $f$. & . . 352 & Megalopteris, & - 126 & ylon, $n$ & \\
\hline & . .310 & Meg & & Nephro & \\
\hline I & . 600 & Meganteris & . .353 & Néreidavus, $m$ & 519 \\
\hline $118, m$ & . . 600 & Megapliyto & . $\cdot 126$ & Ner & \\
\hline$f$. & . 484 & Megaptera. & . . 489 & Neriopteris, $f$ & \\
\hline ius, $n$. & . .347 & $M \mathrm{eg}$ & . . 161 & $\mathrm{Ne}$ & \\
\hline 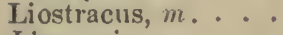 & 555 & Megathentoinum, $n$ & 579 & Nicholsonella, $f$. & \\
\hline & & $m:$ & - . 260 & $\mathrm{Ni}$ & \\
\hline$m$ & 601 & Melia & 443 & rocrinus, $m$. & \\
\hline , m & 601 & Melocrinus, & & $l_{a}, f$. & \\
\hline $\mathrm{m}, n$ & . .578 & Melonites, & 261 & Noggerathia, $f$ & \\
\hline & 125 & phal & 556 & $\mathrm{~N}$ & \\
\hline , $n$ & 579 & $\mathrm{Me}$ & 0 & Nothozoe & \\
\hline & 486 & & & lus, $m$ & \\
\hline$n, n$. & .194 & $\mathrm{a}, f$ & 35 & Nucleospira, $f$ & 355 \\
\hline & $406-486$ & odus & & $\mathrm{N}_{1}$ & \\
\hline$m$. & 442 & nacis, $f$ & 55 & Nuculana, $f$ & - \\
\hline & 312 & M & 556 & Nuculites, $m$ & \\
\hline Loft & 161 & locrin & - & Nullipora.. & . \\
\hline$x$ & 55 & Metarmos & 623 & Nultainia. . & • \\
\hline halus, $m$ & . .555 & Metoptoma, & - . 409 & Nyassa, $f$. & \\
\hline ris, $f$. & . .125 & Miannia, $f$. & 579 & Nyctopora, $f$ & \\
\hline dalia, $f \ldots$ & $\begin{array}{l}.194 \\
\therefore 601\end{array}$ & $\begin{array}{l}\text { Michelinia, } f \text {. } \\
\text { Microceras, } n \text {. }\end{array}$ & $\begin{array}{r}\quad 196 \\
\square .410\end{array}$ & 15 & \\
\hline yllum, $n$ & .194 & Micro & . . 196 & Ob & : \\
\hline$f$. & & עובר & 55 & & \\
\hline & 406 & doma, & 41 & phalus & \\
\hline & 4 & 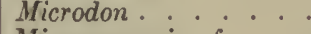 & 48 & $O d$ & \\
\hline ites, $n$ & $m$. & spongi & 161 & teris, $f$ & \\
\hline$\cdot$ & & 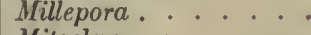 & 196 & totrypa, $f$ & \\
\hline um, $n \ldots$ & 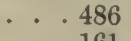 & Mitoclema, $n$ & 313 & Oenites, $m$ & \\
\hline & & $y$ & 489 & Oestocephalus, $m$. & \\
\hline hes & 25 & 1 & 489 & Ogygia, $f$. & - \\
\hline$m$ & 25 & orpha, & 91 & mia, $f$ & \\
\hline & . . 195 & Mœllerina, $f$ & 161 & ()lenellus, $m$. & 57 \\
\hline & & & 23 & Olenoides, $m$ & \\
\hline $\mathrm{LJ}$ & 258 & Monocrater & 519 & Olenus, $m$ : • & 558 \\
\hline ya, $f$ & & & 96 & ia, $f$. & 130 \\
\hline $\mathrm{n}, m$. & 487 & Monomerella & 30 & Oligoporus, $m$ & \\
\hline$n$ & 01 & $M$ & 492 & Oliv & \\
\hline & & is, $f$. & & Oll & \\
\hline$m$. & .2 & & & otrochus, $m$. & • \\
\hline 年 & & $\begin{array}{l}\text { Monotrypella, } f \text {. } \\
\text { Monticulipora } f \text { : }\end{array}$ & $\begin{array}{l}.196 \\
.197\end{array}$ & Omphyma, $f, \cdots$ & \\
\hline Hus, & $a$ & 48 & & Oncoceras, $n$. & 4 \\
\hline & & & .492 & Onychaster, $m$ & \\
\hline & & odactylus, $m$. . & . 261 & ocrinus, $m$ & \\
\hline & 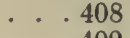 & Mycterops, $f$ & & hodus, $m$ & \\
\hline 0 & A & Iylacris, $r$ & & lacodon, $m$ & \\
\hline
\end{tabular}




\begin{tabular}{|c|c|c|c|c|c|c|c|}
\hline & PAGE. & & & PAGE. & & & $\begin{array}{r}\text { PAQE. } \\
315\end{array}$ \\
\hline $\begin{array}{l}\text { Ophileta, } f \text {. } \\
\text { Opisthoptera. }\end{array}$ & $\begin{array}{l}. \quad 413 \\
. \quad 497\end{array}$ & $\begin{array}{l}\text { Paleschara, } f \text {. } \\
\text { Palmacites }\end{array}$ & & $\begin{array}{l}. \quad 313 \\
. \quad 131\end{array}$ & $\begin{array}{l}\text { Phyllodictya, } f \text {. } \\
\text { Phyllograptus, } m\end{array}$ & & $\begin{array}{r}.315 \\
. \quad 200\end{array}$ \\
\hline Oracanthus, $m$ & .603 & Paneuka, $f$. & & .500 & Phyllopora, $f$. & & .315 \\
\hline Urbicula & 356 & Panopra. & & .500 & Phylloporina & & 315 \\
\hline biculoidea, & 356 & Pantylus, $m$ & & . .624 & Phyllopteris, $f$ & & 133 \\
\hline lites & 161 & Paolia, $f$. . & &. .579 & Physetocrinus, $m$. & & 269 \\
\hline nathichnus, $m$ & 414 & Paracardium, & & . .500 & Physonemus, $m$. & & 606 \\
\hline rmoceras, $n$ & 445 & Paracyclas, $f$. & & . .500 & Physophycus, $n$. & & 33 \\
\hline noxylon, $n$ & 130 & Paradoxides, $m$ & & . .559 & Physospougia, $f$ & & 163 \\
\hline nithichnites, $m$ & 624 & Pararca, $f$. & & .500 & Phytolithus & & 133 \\
\hline odus, $m$. & 603 & Pariotichus, $m$ & & . . 624 & Phytopsis, $f$ & & 134 \\
\hline rthacanthus & 604 & Parioxys, $m$ & & . : 624 & Pileopsis & & \\
\hline his, $f$. & 356 & Parisocrinus. & & - $: 267$ & Pileotrypa, $f$. & & \\
\hline rthisina, $f$. & 360 & Paromylacris, $n$ & & . .580 & Piliolites . & & \\
\hline hoceras, $n$ & .445 & Pasceolus, $m$. & & . $\cdot 162$ & Piloceras, $n$ & & \\
\hline desina, & .497 & Patella, $f$ & & . . 415 & Pinacotrypa, $f$ & & \\
\hline is, $f$ & . .130 & Pattersonia, $f$ & & 163 & Pinna, $f$ & & \\
\hline nat, & . . 414 & Pecopteris, $f$ & & 131 & Pinnatopora & & \\
\hline & 497 & Pecten & & 501 & Pinno & & \\
\hline tella, & • . 498 & Pelion, $m$ & & . . 624 & Pinnularia, $f$. & & \\
\hline & 414 & Peltodus, $m$ & & 605 & Pisocrinus, $m$ & & \\
\hline s, $m$ & . . 604 & Pelt & & 560 & Placunopsis, $f$ & & \\
\hline$m$ &. .313 & spis, $f$ & & 560 & hnus, $m$ & & \\
\hline oma, $r$ & . . 414 & Pentacr & & 267 & Planolites & & \\
\hline & 519 & $P \in n$ & & 360 & $P l a$ & & \\
\hline$f$. & 579 & Pent & & 267 & Plasmopora, $f$ & & \\
\hline & 558 & ella, $f$ & & 360 & nera, $f$ & & \\
\hline ea & 498 & $1 s, m$. & & & Pla & & \\
\hline & & $f$. & & & $\mathrm{Pl}$ & & \\
\hline & 313 & Pereichocrinus & & $\begin{array}{r}.000 \\
.269\end{array}$ & $\begin{array}{l}\text { Platycystites. } m \\
\text { Platyodus, } m \text {. }\end{array}$ & & .606 \\
\hline Pach & 265 & Periplectrodus, $m$ & & $\therefore 605$ & $P l a$ & & 561 \\
\hline 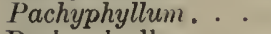 & 130 & $m$. & & .605 & Platyschisma, $n$ & & 418 \\
\hline ayllu & 198 & Pernachactas & & 501 & Platysomus, $m$. & & 7 \\
\hline,$f$ & 199 & ten, $m$ & & & Plat & & \\
\hline eris, & 130 & Peronopora, $f$. & & 199 & Platyst & & 2 \\
\hline & 16 & is, $m$ & & 452 & Plasi & & \\
\hline wa, & 414 & Peta & & . . 605 & Plect & & \\
\hline na, & 498 & chus, $n$ & & 606 & Ple & & \\
\hline$P a$ & 498 & Pet & & 314 & tilus, $m$. & & 503 \\
\hline & 604 & Pet & & & hus, $m$ & & \\
\hline$m$ & 266 & tina, $f$ & & 580 & tites, $m$. & & 272 \\
\hline na & & Pet & & 199 & Pleurod & & 0 \\
\hline$s, m$ & 26 & $\mathrm{r}, m$ & & 269 & is, $m$ & & 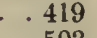 \\
\hline & 4 & & & 606 & rus, $m$. & & 33 \\
\hline impa, $f$ & 57 & ora, $f$. & & . & Ple & & 5 \\
\hline & & & & & Ple & & 4 \\
\hline is $f$ & 55 & $a, f$ & & . & Pleurotomaria, $f$ & & 119. \\
\hline t & 498 & les, $m$ & & . & Pli & & \\
\hline do & 51 & euron, $n$ & & . & Plumulina, $f$. & & 134 \\
\hline & 26 & $P h$ & & . & Ply & & 1 \\
\hline & 26 & $f$ & • & . & hus, $m$ & - & \\
\hline & $55^{-}$ & & - & & $m$. & & 607 \\
\hline & 70 & & & & $f$ & & \\
\hline stites, $m$ & . .2 & trea, $f$ & & 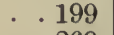 & Pol & & \\
\hline & 161 & & & & Poly & & \\
\hline & (t) & ntia, $f$ & & 624 & Polye & & \\
\hline 18 & & & & & Po & & \\
\hline$m$ & & & & 502 & Polyphemopsis, $f$ & & \\
\hline & & $\operatorname{aris}$ & & 269 & & & \\
\hline$n$ & 1 & & & • . 362 & $\mathrm{es}, m$ & & \\
\hline & & & & & dus, $m$ & & \\
\hline$P a$ & & & & . & Poly & & \\
\hline & & $\mathrm{Ph}$ & & & onites, $m$. & & \\
\hline & & & & & Porcellia, $f$ & & \\
\hline his & & & & & & & \\
\hline 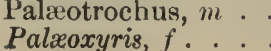 & & $\mathrm{Ph}$ & & & Poro & & \\
\hline & & & & & & & \\
\hline
\end{tabular}




\begin{tabular}{|c|c|c|c|}
\hline & GE. & & 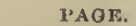 \\
\hline Posidonomya, $f$ & 504 & Ptychocrinus, & 276 \\
\hline Poteriocrin us, $m$ & 273 & Ptychodesma, $n$ & 508 \\
\hline Præcardium, $n$. & 504 & Ptychonema, $n$. & 201 \\
\hline Prasopora, $f$. . & 201 & Ptychoparia, $f$ & 564 \\
\hline wichia. & 561 & Ptychophyllum, $n$ & 201 \\
\hline itia, $f$ & 561 & Ptychopteria, $f$. & 8 \\
\hline niodus, $m$ & 520 & Ptyctodus, $m$ & 10 \\
\hline Prionotus. . & 201 & Ptyonius, m & 25 \\
\hline Prisconaia, $f$ & 504 & Ptyonodus, $m$ & \\
\hline Prismopora, $f$ & 317 & Prigiunculus, . & 392 \\
\hline cladodus, $m$. & 608 & Pupa, $f$ & 4 \\
\hline Pristodus, $m$. & 608 & Pyanomya, $f$ & \\
\hline Productella, $f$ & 362 & Pycnocrinus, $m$ & 6 \\
\hline Productus, $m$ & 30 & Pyenostylus, $m$ & \\
\hline & 561 & Pygopterus, m & $.611-625$ \\
\hline macrus, $m$ & 50 & I'ygorhynchus, $n$ & \\
\hline mylacris, & 58 & Pyrenomous, $m$ & 509 \\
\hline & & & \\
\hline
\end{tabular}

Prorynchus, $n$. . . 504

Proscorpius, . . . 571

Protarrea, $f$. . . 201

Protaster, $m$. . . . $2 \pi 6$

Protasterina, . . . . 276

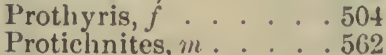

Protoblechnum, $n$. . 134

Protocaris, $f$. . . . 563

Protocrisina, $f \ldots . .317$

Protocyathas, m . . . 163

Protograptus, $m$. . 201

Protolimulus, $m$. . . 563

Protomya, $f \ldots .505$

Protoscolex, $m$... 520

Protospongia, $f \ldots 163$

Protostigma, $f \ldots . .135$

Prototaxites, $m$. . 135

Prototypus, $m$. . . . 563

Proutella, $f$. . . 318

Psammodus, $m$. . . 608

Psaronius, m . . . . . 135

Psephodus, $n$. . . 610

Pseudocrania, $f$. . . 366

Pseudomonotis, $f \quad \cdot 505$

Pseudopecopter s, $f \ldots 135$

Pseudophorus, $n$. . . . 424

Psilophyton, n . . . 136

Pterichthys, $m$. . . 610

Pterinea, $f$. . . . 505

Pterinopecten, $m$. . . 507

Pterocephalia, $f$. . 564

Pteronautilns, $m$. . . 453

Pteronitella, $f$. . . 507

Pteronites, $m$. . . 507

Pteropora, . . . . . 318

Pterotheca, $f$.
Pterotocrinus, $m$. . . .392

Pterygotus, $m$. . . 564

Ptilocarpus, $m$. . . . 136

Ptilionella, . . . . . 318

Ptilodictya, $f \quad \ldots .318$

Ptilograptus, $m$. . . 201

Ptilonaster, . . . 276

Ptilophyton, . . : 136

Ptilopora, $f$. . . 319

Ptiloporella, $f$. . . 319

Ptiloporina, $f$. . . 319

Ptilotrypa, $f$. . . 320

Ptyehaspis, $f$.

\section{Quenstedtia,}

202

Ramipora, $f$. . . 320

lianiceps, . . . . 625

Raphistoma, $n$. . . . 424

Rastrites, m . . . 202

Rauflella, $f$. . . 163

Receptaculites, $m$. . 163

Remopleuricles, $m$. . 565

Rensselaria, $f$. . . 366

Reptaria, $f$. . . . 320

Retepora, $f$. . . 320

Reteporina, $f$. . . 320

Retiocrinus, $m$. . . 277

Retiograptus, $m$. . 202

Retiolites, $m$. . . . 202

Retzia, $f$. . . . 366

Rhabduria, $f \ldots 164$

Rhabdichnites, . . . 566

Rhabdocarpus, $m$. . 136

Rhacbiopteris, $f \ldots . \quad 137$

Rhachitomus, $m$. . . 625

Rhachura, $f \ldots .566$

Rhacopliylium, $n \ldots 137$

Rhadinichthys, $m$. . 611

Rhinidictya, $f$. . . 320

Rhinipora, $f$. . . . 321

Rhinocaris, $f$. . . . 566

Rhizodus, $m$. . . . 611

Rhizograptus, $m$. 202

Khizolithes, . . . . 138

Rhizomopteris, $f$. . 138

Rhizomorpha, $f$. . 138

Rhodocrinus, $m$. . . 277

Rhombödictyon, $n$. . 164

Rhombopora, $f$. . . . 321

Rhopalonaria, $f$. . . 321

Rhynchodus, $m$. . . 611

Rhynchonella, $f$. . 367

Rhynchospira, . . . 370

Rbynchotreta, $f$. . 370

Rhynobolus, . . . . 370

Ribeiria, $f$. . . . 566

Rinodus, . . . . . 612

Romingeria, $f$. . . 203

Rolalia, $f$. . . 164

Rotella, $f$. . . . 425

Rotularia, . . . . 138

Rusichnites, $m$... 566

564 Rusophyens, $n$. . 138
PAGN.

Saccammina, $f$. . . . . 164

Saccocrinus, $m$. . . . 278

Saccospongia, $f$. . . . 164

Sericlinites, $m i$. . . . 453

Sagenaria, . . . . 138

Sagenella, $f$. . . 321

Salterella. $f$. . . . 521)

Salteria, . . . . . . 566 (;

Sandalodus, $m$. . . . 612

Sanguinolaris, $f$. . . 509

Sanguinolites, in . . . . 509

SaO, . . . . . . . 566

Saportiea, $f$. . . . . 138

Surcinula, $f$. . . . 203

Sauripteris, . . . . . 612

Sauropleura, $f$. . . . 625

Sauropus, $m$. . . . .625

Serevogyra, $f$. . . . . 425

Scalaripora, $f$. . . . . 321

Scalites, $m$. . . . . . 425

Seaphiocrinus, $m$. . . . 279

Scenella, $f$. . . . . . 392

Scenellopora, $f$. . . . . 32z

Sceptropora, $f$. . . . 322

Schizambon, . . . . . 370

Schizoblastus, $m$. . . . 2\$1

Schizobolus, $m$. . . . .370

Selizocrania, $f . . .371$

Schizocrinus, $m$. . . .281

Sclizodus, $m$. . . . 510

Schizodiscus, $m$. . . . .56 6

Si hizupteris, . . . . . 138

Schænaster, $m$. . . . 281

Schulzia, $f$. . . . 139

Scoliostoma, $n$. . . 425

Scolithus, $m$. . . . 520

Scolopendrites, m. . . . 139

Scyphia, . . . . . . 164

Scyjhocrinus, m. . . . . 281

Scytalocrinus, . . . . 281

Serlgwickia, $f$. . . . . 511

Selaginites, $m$. . . . . . 139

Selenoides, . . . . . . $16 t$

Selenopora, $f$. . . . 322

Semicoscinium, $n$. . .322

Septopora, $f$. . . . 322

Serpula, $f$. . . . . 521

Serpulit $-s, m$. . . . 521

Sliumardia, $f$. . . . 566

Sicarius, . . . . . 612

Sidemina, . . . . . 453

Sigillaria, $f$. . . . 139

sigillarioides, $m$. . . 141

Sigillariostrobus, $m$. . 141

Siphonia, ..... . . 164

Siphonocrinus, $m$. . 281

Siphonotreta, $f$. . . . 371

Skenidium, $n$. . . . 371

Smithia, $f$. . . . . 203

Solenocaris, $f$. . . . 567

Solarium, . . . . . . 425

Solemya, . . . . . 512

Solen, $m$. . . . . 512

Soleniscus, $m$. . . . 425

Solenocaris, . . . . . 566

Solenochilus, $n$. . . . 453

Solenomya, $f$. . . . 512

Solenopleura, $f$. . . . . 56t 


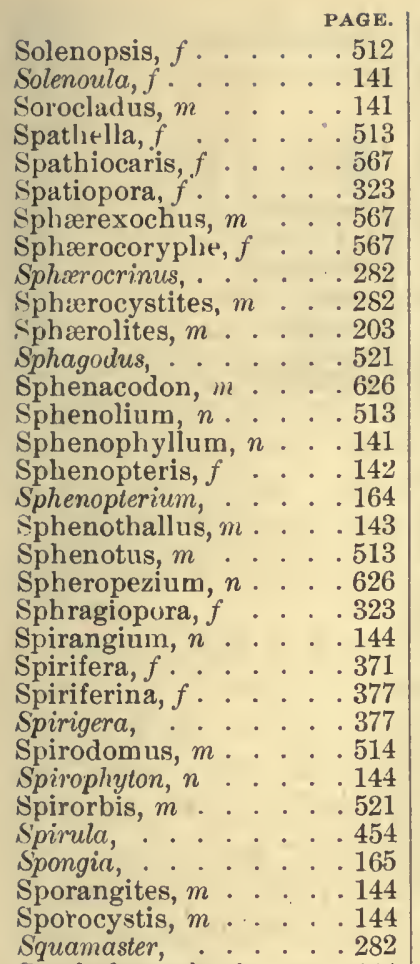

Staphylopteris, $f$. . 144

Staurocephalites, $m$. . .521

Staurograptus, $m$. . . 203

Steganocrinus, $m$. . 282

Stellipora, $f$. . . 203

Stemmatocrinus, $m$. . 282

Stemmatodus, $m$. . . 612

Stemmatopteris, $f$. 144

Stenacanthus, $m$. . . . 612

Stenaster, $m$. . . . 282

Stenocrinus, . . . . 283

Stenopora, $f$. . . . 203

Stenopterodus, $m$. . . 612

Stenoschisma, $n$. . . 377

Stenotheca, $f$. . . . 392

Stephanocriuus, $m$. . 283

Stereocrinus, $m$. . . 283

Sternbergia, $f$. . . 145

Stictopora, $f$. . . . 323

Stict oporella, $f$. . . . 325

Stictotrypu, . . . . 325

Stigmaria, $f \ldots \ldots .145$

Stigmarioides, $m$. . . 145

Stomatopora, $f$. . . . 325

Straparollina, $f \ldots .426$

Straparollus, $m \quad \ldots .426$

Streblopteria, $f$. . . 514

Streblotrypa, $f \ldots . .325$

Strephochetus, $m$. . 165

Strephodes, . . . . . 204

Streptaxis, in $\quad . \quad .4427$

Streptelasma, $n$. . 204

Streptoceras, $n$. . 454

Strobilepis, $f$. . . 468

Streptorhynchus, $n$. . 378
Streptosolen, $m \quad \ldots \quad$ PAGE.

Streptospongia, $f \ldots . \quad 165$

Striatopora, $f$. . . 205

Stricklandia, . . . . 379

Stricklandinia, $f$. . . . 379

Strigillina, . . . . 612

Strobilocystites, $m$. . 283

Strobilospongia, . . . . 165

Strobilus, . . . . . 146

Stromatocerium, $n$. . 165

Stromatopora, $f \ldots 165$

Strombodes, $m$. . . . 205

Strophalosia, $f \ldots . \quad 379$

Strophites, $m$. . . 427

Strophodonta, $f \quad \ldots .379$

Strophomena, $f \ldots . .381$

Strophonella, $f$. .383

Strophostylus, $m \ldots .427$

Strotocrinus, $m$. . 283

Strotopora, $f$. . . 326

Stylastrea, $f \ldots . .205$

Stylifer, . . . . . . 427

Styliola, $f$. . . . 392

Stylonurus, $m$. . . 568

Subretepora, $f \ldots . .326$

Subulites, $m$. . . . 427

Sulcopora, $f$. . . 326

Symphylurus, $m$. . . 568

Synbathocrinus, $m$. . 284

Synocladia, $f \ldots 326$

Syntrielasma, $n \ldots 383$

Syringocrinus, $m \ldots 285$

Syringodendron, $n$. 146

Syringolites, $m$. . . 205

Syringophyllum, . . . 166

Syringopora, $f$. . 206

Syringostroma, $n$. . 166

Syringothyris, $f \ldots 384$

Syringoxylon, $n$. . 146

TANiASTER, $m$

Tæniodictya, $f_{\text {. }} \ldots \ldots .327$

Tæniodus, $m$. . . . 612

Tæniophyllum, $n$. . . 146

Tæniopora, $f \ldots . .327$

Tæniopteris, $f \ldots . .146$

Talarocrinus, $n \ell \quad \ldots \quad . \quad 285$

Tanaodus, $m$. . . . 612

Taonurus, $m$. . . . 146

Taxocrinus, $m$. $\quad . \quad . \quad 285$

Technocrinus, $m$.
Technophorus, $m$

Teleiocrinus, $m$. . 285

Telephus, $m$. . . . . 568

Tellina, . . . . . 514

Tellinomya, $f$. . . 514

Tellinopsis, $f \ldots . .515$

Temnochilus, $n \quad \ldots .454$

Tentaculites, $m$. . . 393

Terataspis, $f$. . . . 568

Teratichnus, $m$. . . 454

Terebratula, $f$. . . 384

Terebratulites,. . . . 385

Termes, $m$.... 581

Tetradium, $n$. . . 206

Tetragraptus, $m \ldots 206$

Textularia, . . . . 166

Thaleops, $f$. . . 568
Thallistigma, $n$. . . . 327

Thamniscus, $m$. . 327

Thamnodictya, $f$. . 166

Thamnograptus, $m$. . 207

Thamnopora, $f$. . . 328

Thamnotrypa, $f$. . 328

Theca, $f$. . . . 393

Thecia, $f$. . . . 207

Thecostegites, $m \cdots 207$

Thenaropus, $m$. . . 626

Theropleura, $f$. . 626

Thoracodus, $m$. . . 613

Thrinacodus, $m$. . . 613

Thyrsidium, $n$. . . 626

Thysanucrinus, $m \quad \ldots .286$

Titanophasma, $f$. . . 581

Tomodus, $m$. . . . 613

Trachomatichnus, $m$. . 454

Trachydomia, $n$. . 428

Tracliypora, $f \ldots 207$

Trachyum, $n$. . . 166

Tremanotus, $m$. . . 428

Tremataster, $m$. . . 286

Trematella; $f$. . . 328

Trematis, $f \ldots 385$

Trematoceras, $n \ldots 454$

Trematocrinus, . . . 287

Trematodiscus, $m$. . 455

Trematopora, $f \ldots . .328$

Trematospira, $f$. . . 385

Treptichnus, $m$. . . 581

Triacrinus, . . . . . . 287

Triarthrella, $f$. . . 568

Triarthrus, $n$. . . . 568

Trichiulus, $m$. . . . 573

Trichomanites, $m$. . 147

Trichophycus, $n$. . 147

Trichospongia, $f \ldots 166$

Tricœlocrinus, $m$. . 287

Trigonocarpum, $n$. . 147

Trigonoclus, $m$. . . 613

Trigonotreta, $f \ldots . .386$

Trimerella, $f$. . . . 386

Trimerorhachis, $f \quad \ldots \quad 626$

Trimerus, . . . . : 568

Trinucleus, $m$. . . . 568

Triphyllopteris, $f$. . 148

Triplesia, $f$. . . . 387

Trochita, $f$. . . . . 428

Trochoceras, $n$. . 455

Trocholites, $m$. . . . 456

Trochonema, $n$. . . 428

Trochophyllum, $n \quad . \quad 207$

Trochus, . . . . . 428

Troostocrinus, $m \quad . \quad 287$

Tropidoleptus, $m$. . . 387

Tropidopora, $f$. . . . 329

Tryblidium, $n$. . . . 429

Tuberculopora, . . . . 329

Tubipora, $f$. . . . 207

Tuditanus, $m$. . . 626

Turbo, $m$.... 429

Turbonilla, . . . . . 429

Turrilepas, $m$. . . 569

Turritella, $f \ldots . \quad . \quad .429$

ULODENDRON, $n \ldots \ldots$

Ungulina, ...... 515 


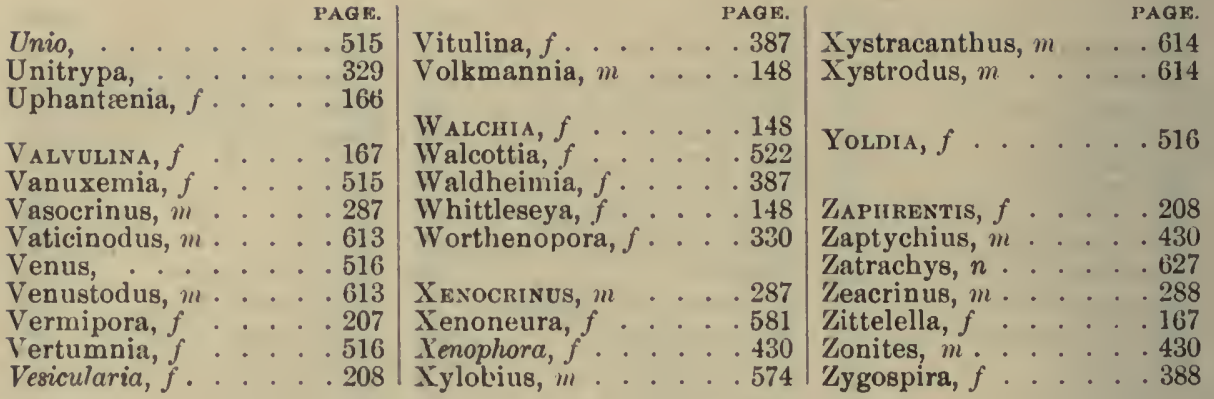




\section{FIRST APPENDIX, I892.}

As THIS work was intended for the use of beginners in the pursuit of geological and palæontological information, as well as for the most advanced students, it has been urged that I should have accented the technical words, with a view of bringing about correct and uniform pronunciation.

This criticism may be well taken, but there are only a few words commonly mispronounced, and a few examples will suffice to correct the pronunciation of most of these.

Words ending in ceras, crinus, pora, and lepis are accented on the antepenult, as Orthoc'eras, Cyrtoc'eras, Gomphoc'eras, Actinoc'rinus, Xenoc'rinus, Platyc'rinus, Monticulip'ora, Leptop'ora, Chirol'epis, Bothriol'epis, etc. Words ending in ites have the $i$ long and the accent on the penult, as Cypricardi'tes, Dalmani'tes, Favosi'tes, Litui'tes, Trocholi'tes, etc. Words ending in nema, mena, etc., have the accent on the penult, as Cyclone'ma, Loxone'ma, Strophome'na, Calyme'ne, etc. We say Ath'yris, Cari'na, Cerat'odus, Cœlentera'ta, Epithe'ca, Onych'odus, Palæas'pis, and Syringoth'yris.

I have seen no reason to change the established nomenclature of the Groups of rocks as set forth in the geological part of this work, notwithstanding there may be some who apply the word Cambrian to roeks indiscriminately from the Taconic to the Devonian. It seems to be a word that is easily pasted over ignorance, and some use it for that reason.

\section{VEGETABLE KINGDO̊M.}

There have been very few fossil plants described since the publication of this work.

BутнотRePHis pergracilis, Dawson, 1889, Trans. Roy. Soc. Can., vol. 7, p. 54, Up. Taconic.

Cruziana carleyi, James, 1885, Jour. Cin. Soc. Nat. Hist., vol. 7, p. 155 . Not recognized.

Dactyloines bulbosus, Hall, 1886, 39th Rep.

N. Y. St. Mus. Nat. Hist., p. 160 . Obscure markings supposed to represent marine algæ, graptolites, or traces of a spongoid substance.

Dicranophyllum. Type D. gallicum.

LEPIDÓdendron cliftonense, Dawson, 1891, Bull. G. S. A., vol. 2, p. 533, Coal Meas. murrayanum, Dawson, 1891, Bull. G. S. A., vol. 2, p. 532, Coal Meas.
Nematophyllum was preoccupied by McCoy for a genus of corals in 1851, and hence could not be used again by Fontaine $\&$ White as a generic name. I now propose for Nematophyllum of Fontaine \& White in Permian or Upper Carboniferous Flora of West Virginia and S. W. Pennsylvania, p. 35, the generic name Nematophyllites with $\mathbf{N}$. angustus, Fontaine \& White as the type.

Rusopuycus, from its etymology, should be spelled Rhysophycus.

acadicum, Dawson, 1861 (Rusichnites acadicus), Can. Nat. and Geol., vol. 1, p. 363, and Acad. Geol., p. 410, Coal Meas. 
carbonarium, Dawson, 1868 (Rusichnites carbonarius), Acad. Geol., p. 257, Carboniferous.

clintonense, Dawson, 1890 (Rusichnites clintonensis), Quar. Jour. Geol. Soc., vol. 46 , p. 598 , Clinton $\mathrm{Gr}$.

grenvillense, Dawson, 1890 (Rusichnites grenvillensis), Quar. Jour. Geol. Soc., vol. 46, p. 598 , Up. Taconic.

SPHENophyLiUM vetustum, Newberry, 1889, Jour. Cin. Soc. Nat. Hist., vol. 12, p. 55, Up. Held. Gr.

SPINENOPTERIS salisburyi, Lesquereux, 1887,
Franklin Soc. Rep. on R. I., p. 69, Coal Meas.

Spiraxis, Newberry, 1884, Ann. N. Y. Acad. Sci., vol. 3, p. 217. An elongated spiral cast, supposed to represent a fucoid. Type S. major. Another species, S. randalli, is also described. Both from the Chemung Gr.

TrigoNocaripu ambiguum, Lesquereux, 1890, Dict. of Foss. Pa., vol. 3, p. 1213, Coal Meas.

VOLKMANNIA brevistachys, Lesquereux, 1890 , Dict. of Foss. Pa., vol. 3, p. 1253, Coal Meas.

\section{ANIMAL KINGDOM.}

\section{SUBKINGDOM PROTOZOA.}

THE animals of this Subkingdom are supposed to have consisted of protoplasm, in a cell or cells, capable of secreting an outer wall, but without hardened tissues or alimentary organs. I arrange the palicozoic spouges in this Subkingdom and in the Class Porifera, because I see no reason to suppose such forms as Pattersonia, Dystactospongia, Archrocyathus, Strephochetus, etc., were any more highly developed than Rhizopoda. It may be part of the Class has shown such variation and development as to point to a higher organization, which is in accordance with all intelligent views of evolution, but I see no evidence to warrant raising the Class to the rank of a Subkiugdom in the animal scale, as some have done. In any view presented by those who claim that the Porifera occupy a place between Protozoa and Colenterata, I discover no ground for raising it to the rank of a Subkingdom, if any regular grade is to be maintained, in the classification of animals.

Errata.-For family "Leptonitide," on page 153, read "Family Leptomitidæ.-Leptomitus,"- and strike "Leptomitus" from those enumerated under "Family Affinity Uncertain." On page 161 change the specific names under Palæacis to the feminine gender.

Acanthodictya, Hinde, 1889, Trans. Roy. Soc. Can., vol. 7, p. 47. [Ety. akantha, a spine; dictuon, net.] Subcylindrical, skeletal mesh-work of longitudinal and transverse spicular strands or fibres; longitudinal strands composed of somewhat loosely arranged fascicles of elongated overlapping spicules, and the spicules of the slender transverse fibres are, as a rule, disposed in a single series. From the outer surface of the sponge, numerous spicular rays project outward at right angles. Anchored, probably, by a basal prolongation of the longitudinal strands. Some of the elongated longitudinal spicules are cruciform, and their transverse rays form the cross fibres. The general structure resembles Cyathospongia, but is char- acterized by the presence of the projecting surface rays. Type A. hispida, described in the same place, from Upper Taconic rocks.

Actinodictya placenta, Hall, 1892, 9th Ann. Rep. State Geologist, p. 59, Chemung Gr. Not entitled to recognition for want of illustration.

Actinostroma fenestratum, Nicholson, 1889 , Monog. Brit. Stromatoporoids, p. 146, and A. tyrrelli, 1891, Ann. and Mag. Nat. Hist., vol. 7 , p. 317 , Devonian.

Biopalla, Wallace, 1878, Am. Jour: Sci., vol. 115 , p. 369. [Ety. bios, life; palla, a ball.] Founded upon very imperfect and uncertain material from the geodes of the Keokuk Group, with the statement that "there is uncertainty as to the distinction of species," and yet $B$. 
keokuk, B. grandis, B. wortheni, B. woodmani, B. hæcklei, B. hyatti, B. alicei, and $B$. palmata are named, with almost characterless descriptions, without illustration.

Cida rospongia, Gurley, 1884, New Carb. Foss. Bull., No. 2, p. 4 . Round hemispherical bodies, with flattened irregular base, full hemispherical top or dome, which, along the margin, is distinctly divided into twelve lobes, being quite regular in size and extending fully onehalf the distance from the margin to the center of the dome, where they become obsolete, leaving a smooth, slightly depressed, central, circular area, whose diameter is about one-half that of the body. The top of the dome is punctured by two well-defined circular openings, which are situated close together, and at the margin of the smooth circular area. During growth the dome becomes elevated, and a lower or basal portion becomes developed, extending from the flattened base to the margin of the dome. This lower portion is somewhat irregularly marked by concentric lines of growth. The sides, below the dome, are moderately straight. Type C. ella. Described at the same place, from the Coal Meas.

Criptozoon steeli, Brainard \& Seely, 1890 , Bull. Am. Mus. Nat. Hist., vol. 3, p. 6, Calciferous Gr.

Cryptodictya alleni, Hall, 1892, 9th Ann. Rep. State Geologist, p. 60, Chemung Gr. Not entitled to recognition for want of illustration.

Cucumulites, Gurley, 1884, New Carb. Foss. Bull., No. 2, p. 2. Body consisting of a thin, punctate, elongate, tubular shell, which is slightly arcuate and expanding; being closed and rounded at the larger extremity and somewhat pointed, with a well-defined terminal or oral opening at the smaller extremity. Surface ornamented by more or less numerous pointed elevations or tubercules, which along the central portion of the shell become arranged in longitudinal rows, breaking up and becoming irregular in crossing the larger rounded end and toward the smaller extremity. Type C. tuberculatus. Described at the same

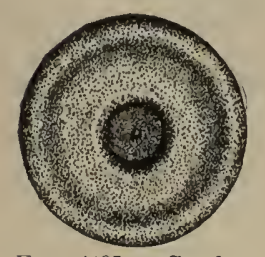

FIG. 1195. - Cyclospongia d is cus. Lower side. place with $\mathrm{C}$. tricarinatus from the Warsaw Group.

Cyathophycus siluriana, James. Too poorly defined to be recognized.

Cyathospongia quebecensis, D a w s o n \& Hinde, 1889, Trans. Roy. Soc. Can., vol. 7. p. 44, Up. Taconic.

Cyclospongia, S. A. Miller, 1891, Advance Sheets 17th Rep. Geo. Sur. Indiana, p. 5. [Ety. kuklos, a circle; spongia, sponge.] Sponge, circular, button-shaped or discoid, and consisting of numerous thin, calcareous laminæ, having a concentric structure and filled with minute canals or interstices. The structure has some resemblance to that of Strephochetus richmondense, but the laminæ are much thinner, and the interlaminar spaces are much less marked, and no vertical tubes have been found within them. That was a free sponge ; this one is supposed to have been attached to some other object. Type C. discus. Described in the same Fia. 1196.-Cycloplace, from the Up- spongia d is cus. per Helderberg Gr.

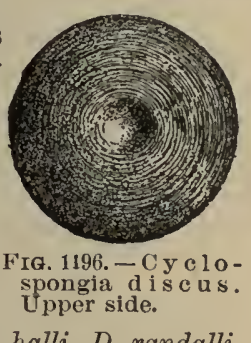
Dictyophyton amalthea, D. halli, D. randalli, $D$. sceptrum, $D$. scitum, D. tomaculum, $D$. vascellum, Hall, 1892, 9th Rep. State Geologist, N. Y., pp. 56 to 58 , Chemung Gr. Such descriptions, without illustrations, are not entitled to recognition. Halichondrites, Dawson, 1889, Trans. Roy. Soc. Can., vol. 7, p. 52. Oval or irregular masses of small simple spicules, imbedded in patches of pyrite, and, without any definite arrangement or root, spicules may indicate the presence of a halichondroid sponge. In the best preserved specimens the spicules appear to be biacerate and more slender and pointed than in Lasiothrix, and they seem to be in two series, inclined at a very oblique angle to each other. In some specimens elongated spaces, with well-defined margins, are covered with thin films of pyrites, which may have resulted from the replacement or incrustation of a mass of minute spicules, of which traces remain in some places. He observed that sponges having originally much keratose or other dense animal matter, would naturally aggregate in and around themselves a greater quantity of pyrite than those of a more purely siliceous character. Type $\mathrm{H}$. confusus. Named at the same place, from the Upper Taconic.

Hyalostelia metissica, Hinde, 1889, Trans. Roy. Soc. Can., vol. 7, p. 49, Up. Taconic. Founded upon a confused mass of supposed spicules.

Lasiothrix, Hinde, 1889, Trans. Roy. Soc. Can., vol. 7, p. 50 . [Ety. lasios, shaggy; thrix, hair.] Sponge small, depressed oval in outline, the outer surface covered by a layer of longitudinally arranged, apparently simple, acerate spicules; beneath this is another layer of spicules disposed transversely. From the base of the sponge several simple elongated spicules extend. Type L. curvicostata. Described in the same place, from the Upper Taconic. L. flabellata is also described. 
Paldiacis cavernosa, S. A. Miller, 1891, Advance Sheets 17 th Rep. Geo. Sur. Ind., p. 4, Waverly Gr.

Protosiongra coronata, P. cyathiformis, $P$. delicatula, P. mononema, P. polynema, and $P$. tetranema, Dawson \& Hinde, 1859, Trans. Roy. Soc. Can., vol., 7, pp. 37 to 43 , Up. Taconic.

Ptychostylus, Gurley, 1884. The name was preoccupied by Gabb in 1865.

Receptaculites elrodi, S. A. Miller, 1892, Advance Sheets of 18 th Rep. Geo. Sur. Ind., p. 3, Up. Held. Gr.
Rhombodictyon globosus, James. Too poorly defined to be recognized.

Spirocyathus, Hinde. Synonyu for Archæocyathus.

Stephanella sancta, Hinde, 1891, Lond. Geo. Mag., vol. 8, p. 22. Radiating lines of pyrites supposed to represent spicules. No generic or specific characters.

Stromatopora ludlowensis and $S$. tubularis, James. Too poorly defined to warrant any recognition.

Symingostroma. Type S. densum.

\section{SUBKINGDOM CCELEN'TERATA.}

Tre Order Rugosa has been called Tetracoralla, because the septa are said to be some multiple of four; and the Tabulata, Hexacoralla, because the septa are said to be a multiple of six. The internal cavity of a Graptolite, so far as I luave been able to discover, is divided longitudinally into three or four departments, which is altogether different from the structure of the living Hydrozoa, where the interior part of the body consists of a single undivider cavity. It may be the Order Graptolida should be raised to the rank of a Class, because it is so different in composition and structure from the living Hydrozoa.

Errata.-On page 168, insert "Family Calceolida.-Calceola," and remove "Baryphyllum" to the "Cyclolitide." On page 169, for "Family Helioporida" " read "Family Heliolitidx." On page 177, strike out "Chonophyllum validun." In the eleventh line from the top of page 189 , read " $\mathrm{F}$. alpenensis" for " $\mathrm{F}$. dumosus." In the sixth line from the bottom of page 194 , read " 1669 " for "1869." In the first line on top of page 196, read "Description" for "Descrides." In the third and seventh lines from the top of page 199, read "Hamilton " for "Chemung." In the fifteenth and sixteenth lines from the bottom of page 201, read "Edwards \& Haime, 1850," for "Lonsdale, 1839, Sil. Syst., p. 691, and E. \& H." For the word "lamellae," in the definition of Zaphrentis, on page 208, read "septa," and add Clifford as an author of the genus with Rafinesque. On page 209, under Z. elliptica, read "Pal., No. 8," instead of Pal., No. 6."

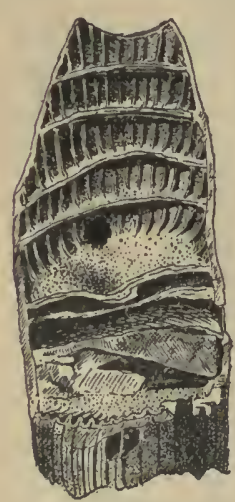

FIG. 1197 - A mplexus blairi.
ACtinocystis variabilis, Wh it eaves, 1892 , Cont. to Can. Pal., p. 271, Devonian.

Alveolites roemeri refer to Cladopora.

Anplexus blairi, A. bicostatus, A. corniculum, S. A. Miller, 1891, Advance Sheets 17th Rep. Geo. Sur. Ind., p. 8. The blairi and corniculum from the Chouteau lime. stone and bicostatus from the Burlington Group.

cinctutus, S. A. Miller, 1892, 18th Rep. Geo. Sur. Ind., p. 5, Niagara Groụp. coralloides, probably, not an American species.

Cnetetes ponderosus, Rominger, 1892, Am. Geol., vol. 10, p. 61 , Up. Held. Gr.

Cenograptus, Hall, 1868, 20th Rep. N. Y. Mus Nat. Hist., pp. 210, 211, 251. [Ety. koinos, living together; grapho, I write.] Polypary compound, developed bilaterally from the initial point; cellules on one side of slender branches, which are developed on one or two sides of a long slender axis or rachis, the free extremities of which are likewise celluliferous. Not branching dichotomously. Type C. divergens.

divergens, Hall, 1859 (Graptolithus divergens), Pal. N. Y., vol. 3, p. 509, Hud. Riv. Gr.

gracilis, Hall, 1847 (Graptolithus gracilis), Pal. N. Y., vol. 1, p. 274, Utica Slate. 
surcularis, Hall, 1868, 20th Rep. N. Y. Mus. Nat. Hist., p. 210, Utica Slate.

Columnaria disjuncta, Whiteaves, 1892, Cont. to Can. Pal., p. 269, Devonian.

Crepidophyllum is a synonym for Craspedophyllum, and the species under it should be referred to Craspedophyllum.

Cyathopilylum athabascense, Whiteaves, 1891, Cont. to Can. Pal., vol. 1, p. 202, Devonian. C. petraoides and C. waskasense, 1892, pp. 264 and 265, Devonian. C. juvene, refer to Heliophyllum juvene.

C. panicum refer to Diphyphyllum panicum. Cystelasma, S. A. Miller, 1891, Advance Sheets 17th Rep. Geo. Sur. Ind., p. 12,
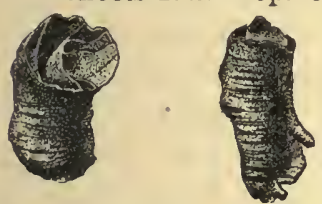

[Ety. kustis, a cav. ity; elasma, lamellæ.] Corallum simple, irregularly turbinate or cylindrical, consisting of all outer wall, transversely FIG. 1198.-Cystelasma wrinkled or conlanesvillense. Summit stricted, which is and side vlews. connected by oblique plates, irregularly disposed, that give to the interior cystose chambers of unequal size and irregular shape. No septa or regular tabulæ. Structure vesicular. Type C. lanesvillense, described in the same place from the IVarsaw Gr.

Cystiphyludu greenii, S. A. Miller, 1892, Advance Sheets 18th Rep. Geo. Sur. Ind., p. 4, Up. Held. Gr.

DAwsonis are small, conical or bell-shaped, and each has a minute spine at the summit. They have not been found in connection with any Graptolite.

Dictyonema pertenue and D. scalariforme, Forste, 1887, Bull. Denison Univ., vol. 2, p. 107 , Niagara Gr.

DIPHYPIYYLLUM panicum, Winchell, 1866, (Cyathophyllum panicum,) Rep. Low. Penin. Mich., p. 90, Ham. Gr.

DiplotryPa westoni, Ulrich, 1889, Micropalæontology of Can., pt. 2, p. 30, Hud. Riv. Gr.

Graptolithus alatus, $G$. crucifer, $G$. headi, $G$. logani, $G$. octobrachiatus refer to Loganograptus. $G$. divergens and $G$. gracilis refer to Cœnograptus.

Hadrophyllum aplatum, Cummins, 1891, 2d Ann. Rep. Geo. Sur. Texas, p. 552, Coal Meas.
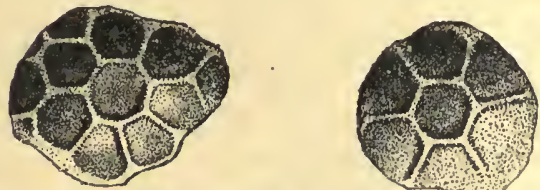

Fig. 1199.-Leptopora gorbyi. Summit vlews.

Heliophyllum juvene, Rominger, 1876, (Cyathophyllum juvene,) Foss. Corals, p. 101, Ham. Gr.

parvulum, Whiteaves, 1891, Cont. to Can. Pal., p. 203, Devonian.
Leptopora gorbyi, S. A. Miller, 1891, Advance Sheets 17th Rep. Geo. Sur. Ind., p. 6, Chouteau limestone.

Loganogra ptus, Hall, 1868, 20th Rep. N. Y. St. Mus. Nat. Hist., pp. 207 and 251.

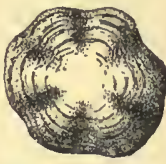

[Ety. proper name; grapho, I write.] Polypary compound, growing bilaterally from the initial point, and consisting of four, eight or more simple stipes numerously divided

Fio. 1200- near the base and below the Leptopora commencement of the cells; gorbyi. Ba- furnished with a corneous censal view. furnished wisc. Type L. headi. To this genus is also referred the species described as Graptolithus alatus, G. crucifer, G. logani and G. octobrachiatus.

Mrcrocyclus blairi, S. A. Miller, 1892, Advance Sheets 18th Rep. Geo. Sur. Ind., p. 7, Chontean limestone.

MoNOTRYPELLA confluens, Foerste, 1887, Bull. Denison Univ., vol. FIG. 1201. - Microcyclus 2. p. 172, Niag. FIG. ${ }^{1201 .-M i a i r i . ~}$ ara $\mathrm{Gr}$.

unjiga, Whiteaves, 1891, Cont. to Can. Pal., vol. 1, p. 214, Devonian.

Monticulipora clintonensis, crustulata, cleavelandi, milfordensis and nicholsoni, James, 1888, Jour. Cin. Soc. Nat. Hist., vol. 11, pp. 15 to 36, Hud. Riv. Gr. Some of these are synonyms for species described under other genera, by UIrich. Others are not defined so as to be recognized. Whether or not any of them will stand is a question. M. falesi and ohioensis by the same party, 1884, same Journal, vol. 3, p. 137, Hud. Riv. Gr. Not recognized.

M. molesta, Nicholson, seems to be a synonym for M. mammulata.

parasitica, var. plana, Ulrich, 1889, Micropalæontology of Canada, pt. 2, p. 29, Hud. Riv. Gr.

OldHaMra antiqua was described in 1844 , in Jour. Geo. Soc. Dublin, vol. 3, p. 60.

Pachyphylum devoniense, Edwards \& Haime, 1851, Polyp. Foss. Terr. Pal., p. 397 , Devonian.

Prasopora parmula, Foerste, 1887, Bull. Denison Univ., vol. 2, p. 170, Niagara Group.

Stenopora ohioensis, Foerste, 1887, Bull. Denison Univ., vol. 2, p. 85, Coal Meas.

Striatopora gorbyi, S. A. Miller, 1892, Advance Sheets 18th Rep. Geo. Sur. Ind. p. 7 , Niagara Gr.

Za PHRENTIS calyculus, Z. chouteauensis, Z. declinis, $Z$ : exigua, $Z$. tantilla, and Z. tenella, S. A. Miller, 1891, Advance Sheets 17th Rep. Geo. Sur. Ind., pp. 10, 11, and 12. All from the Chonteau limestone except $\mathrm{Z}$. declinis, which is from the Keokuk Gr. 


\section{SUBKINGDOM ECHINODERMATA.}

\section{CLASS CRINOIDEA.}

Tre ouly systematic classification of crinoids into families, so far as learning has extended, is based upon the number and arrangement of the plates in the calyces. See "The Structure, Classification, and Arrangement of American Palaozoic Crinoids into Families," 16th Rep. Geo. Sur., Indiana, p. 302. The number of basal plates is first in importance. There are no rudimentary basals. Second in importance is the presence or absence of subradials. Third, the presence or absence of regular interradials. Fourth, the structure of the azygous side. Families having ouly two basal plates are confined to the Subcarboniferous and Carboniferous. Those having three basals extend from the Lower Silurian to the Subcarboniferous. Those having four basals extend from the Lower Silurian to the Devonian. And those having five basals extend from the earliest crinoids throughout Palrozoic time.

\section{GenisRa having Two Basals.}

Fanily Acrocrinide:-Acrocrinus.

Family Dichocrinid k-Dichocrinus, Cotyledonocrinus, Talarocrinus.

Family Pterotocrinid d:-Pterotocrinus.

Genera having Three Basal,s, no Subradial, Regular IntereRADIALS.

Family Actixocrinide. - Actinocrinus, Agaricocrinus, Alloprosallocrinus, Amphoracrinus, Batocrinus, Blairocrinus, Cylicocrinus, Dorycrinus, Eretmocrinus, Gennreocrinus, Megistocrinus, Physetocrinus, Saccocrinus, Steganocrinus, Strotocrinus, Teleiocrinus.

Family Arthracanthide.-Arthracantha.

Family Dolatocrinida.-Allocrinus, Dolatocrinus, Hadrocrinus, Stereocrinus.

Fanily Platycrinidas.-Coccocrinus, Cordylocrinus, Eucladocrinus, Macrostylocrinus, Marsupiocrinus, Platycrinus.

Genera having Three Basals, Subradials, no Regular InterRADIALS.

Family AMPHERISTOCRINIDA.-Ampheristocrinus, Closterocrinus.

Fanily IChthyocrinide.-Ichthyocrinus, Lecanocrinus, Mespilocrinus.

Genera having Three Basals, Subradials, and Regular InterRADIALS.

Family Taxocrinide,-Forbesocrinus, Onychocrinus, Taxocrinus. 
Genera having Three Basals, no Subradials, No Regular InterRADIALS. THE FAMILIES ARE ANOMALOUS AND HAVE NO NEAR AFFINITY WITH EACH OTHER.

Family Calceocrinide.-Calceocrinus, Deltacrinus, Halysiocrinus.

Family Synbathocrinid z.-Synbathocrinus.

Family Zophocrinide.-Zophocrinus.

Genera having Four Basals, no Subradials, Regular Interradials.

Family Eucalyptocrinide.-Callicrinus, Eucalyptocrinus, Hypanthocrinus.

Family Melocrinide.-Compsocrinus, Mariacrinus, Melocrinus, Technocrinus.

Family Xenocrinide.-Xenocrinus.

Genera having Five Basals, Five Subradials, no Regular InterRADIALS.

Famil y Agassizocrinide.-Agassizocrinus.

Family Cyathocrinide.-Abrotocrinus, Arachnocrinus, Bursacrinus, Carabocrinus, Cyathocrinus, Graphiocrinus, Palæocrinus.

Family Dendrocrinide. -Dendrocrinus, Ottawacrinus.

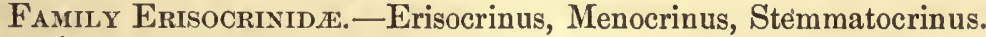

Fanily EupachyCRInIDe.- Esiocrinus, Delocrinus, Eupachycrinus, Ulocrinus.

Family Merocrinide.-Merocrinus.

Family Poteriocrinide.-Atelestocrinus, Barycrinus, Cœliocrinus, Euspirocrinus, Goniocrinus, Homocrinus, Hydreionocrinus, Poteriocrinus, Scaphiocrinus, Vasocrinus, Zeacrinus.

Genera having Five Basals, Fivf Súbradials, Regular InterRADIALS.

Family Glyptasterid «。 - Cyphocrinus, Glyptaster, Lampterocrinus, Thysanocrinus.

Famil y Gaurocrinide.-Gaurocrinus, Retiocrinus.

Family Rhodocrinide. - Archreocrinus, Goniasteroidocrinus, Lyriocrinus, Rhaphanocrinus, Rhodocrinus.

Genera having Five Basals, no Subradials, Regular Intereradials.

Family Chęocrinida.-Cleiocrinus.

Family Glyptocrinide.-Cupulocrinus, Glyptocrinus, Pyenocrinus, Schizocrinus, Siphonocrinus.

Genera having Five Basals, no Subradials, no Regular InterRADIALS.

Family Anomalocrinide:-Auomalocrinus.

Family Belemnocrinide.-Belemnocrinus.

Family Catillocrinid

Family Gazacrinide.-Gazacriuus.

Family Haplocrinide.-Allagecrinus, Haplocrinus. 
Fanily Heterocrinid,in-Ectenocrinus, Heterocrinus, Iocrinus, Ohiocrinus.

FaniLy HYBocrinid. I. - Hybocrinus.

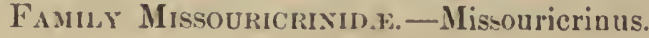

\section{Grenera Beionging to Anomalous Families.}

Fanily Pisocrixid.s. - Pisocrinus. This family has five basals, fullowed by three plates, that are both radial and subradial in position.

Family Edrocrinid x:-Edriocrinus. The base is solid in this family, and is followed by five radials.

Family Camaror Rinidx,-Camarocrinus. Distinct from all other families.

Famity Ancyrocrisid ж.-Aneyrocrinus. Distinct from all other families.

\section{Genera Uncertain as to Family Afrinity.}

Aspidocrinus, Brachiocrinus, Coronocrinus, Cystocrinus, Nipterocrinus, Pachycrinus, and the fossil described by Hall as Myrtillocrinus americanus.

\section{ORDER CYSTOIDEA.}

\section{Famity Stribalocystid...-Stribalocystites.}

A mrotocrixtis, Miller \& Gurley, 1890, Desc. New Gen. and Spec. Echinorlermata,

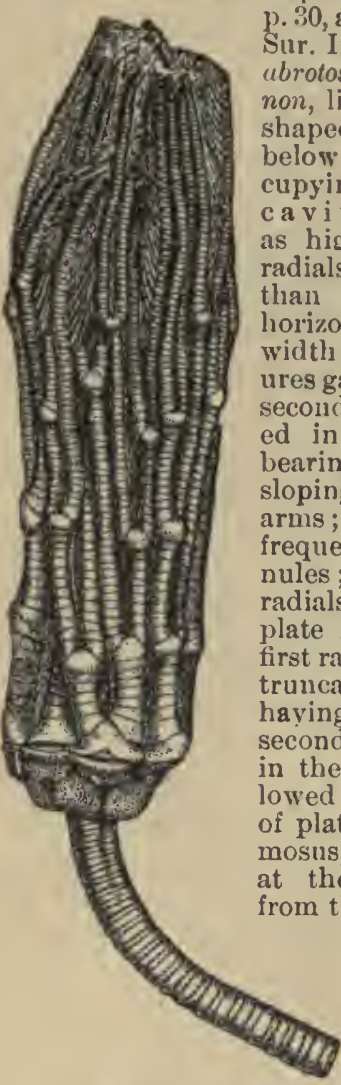

FIG. 1202.-A brotocrinus cymosus.
36, Burlington Gr., and A. chouteaueusis, p. 18, from the Chouteau limestone, and A. fossatus, p. 40, from the Burlington Gr.

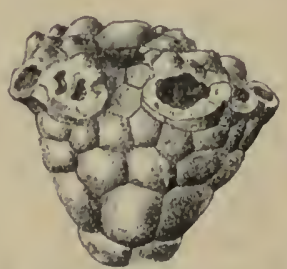

Fig. 1203.-Acllnocrinus choutenuensis. Azy:i gous view.

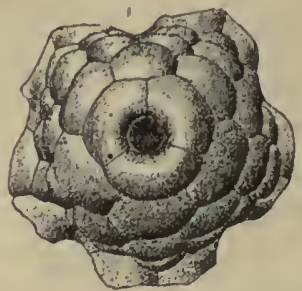

Fig. 1205. - Actinocrinus chouteatuensis. Basal vlew.

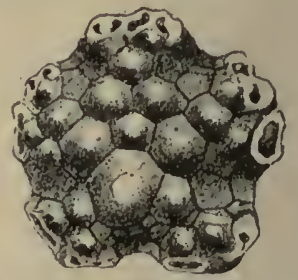

Fig. 1204-Actinocrinus cloouteaucnsis. Summit vlew.

grandis, Miller \& Gurley, 1890 , Desc. New Gen. and Spec. Echinodermata, $p$. 25 , and $16 \mathrm{th}$ Rep. Geo. Sur. Ind., p. 346, Keokuk Gr.

nodosus, S. A. Miller, 1891, Bull. No. 4, Geo. Sur. Mo., p. 33, Burlington $\mathrm{Gr}$. puteatus, Rowley \& Hare, 1891, Kansas City Scientist, vol. 5, p. 101, Burlington Gr.

sedaliensis, S. A. Miller, 1892, Arlvance Sheets 18th Rep. Geo. Sur. Ind., p. 16, Burlington Gr.

senarius, Hall, 1860, Supp. Geo. Rep. Iowa, 1). 25, syn. for Physetocrinus ornatus.

Esiocrinus, Miller \& Gurley, 1890, Jour.

Cin. Soc. Nat. Hist., vol. 13, p. 14. [Ety. aisios, auspicious, coming at good time; 
krinon, lily.] Column pentagonal, calyx bowl-shaped, plates smooth or granular. Basals five, forming a pentagonal, flattened or concave disc. Subradials large, four hexagonal, one heptagonal, and curving upward half the height of the calyx. First radials 5, truncated above; one or more brachials in each ray supporting strong arms, composed of a single series of plates; arms 10 , bearing pinnules. No regular interradials. An azygous interradial rests upon a subradial, between two first radials, and is followed by two plates that connect with the proboscis. Proboscis long, composed of four series of plates bearing numerous transverse fissures on the sides of the plates. Type $\mathrm{E}$. magnificus, clescribed at the same place, from the Up. Coal Meas. E. harii was described at the same time, and $\mathrm{E}$. basilicus was described in Desc. New Gen. and Spec. Echinodermata, p. 53, from the Up. Coal Meas. All of which were republished in 16 th Rep. Geo. Sur. Indiana, pp. 337,338 , and 369 .

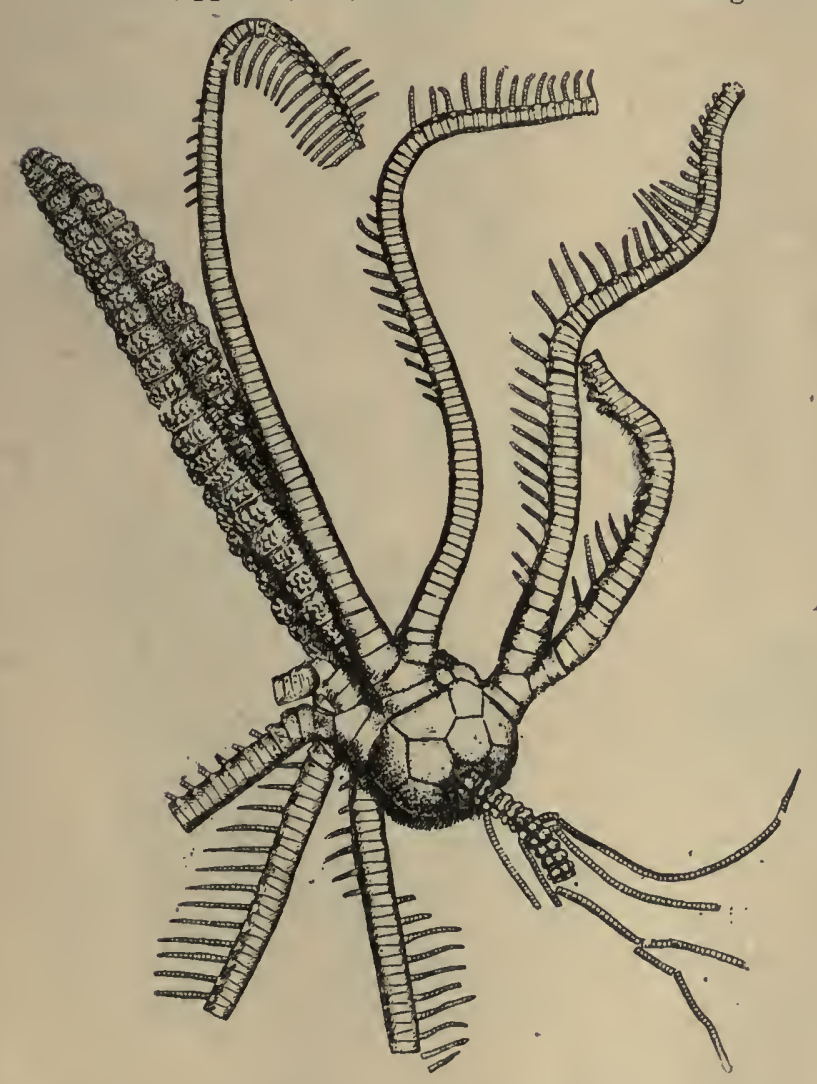

FIG. 1206.- Esiocrinus magniflcus.

lykinsi, Butts, 1891, Kansas City Scientist, vol. 5, p. 144, Coal Meas.
Aethocystites, S. A. Miller, 1892, Advance Sheets 18th Rep. Geo. Sur. Ind., p. 9.

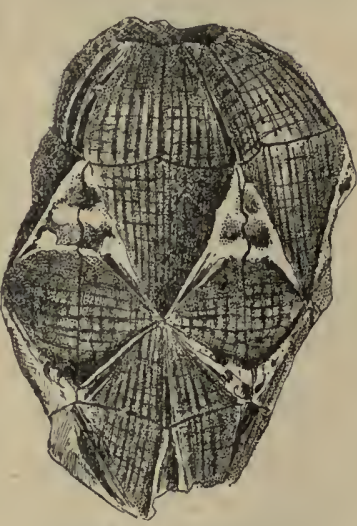

FIG. 1207.-Aethocystites sculptus. [Ety. aethes, unusual ; kus$t i s$, bladder.] This genus consists of the bodies of elongated, subelliptical Cystoidea of undetermined family affinity. Probably they do not belong to any defined family. There are only three ranges of plates. In the first $r$ ange there are three plates, they form an obconical cup, commencing from a small column. In the second range there are five elongated plates. The third range consists of five much shorter plates. The plates are ornamented with wrinkles, and bear tubular ridges radiating from a central point, in the middle range, which follow the longitudinal sutures or center of the first and third ranges, and have porous connection with the interior of the body. The plates do not possess pores after the manner of Holocystites or Caryocrinus, and no pores have been determined, except as above stated. Type A. sculptus. Described at the same place, from the Niagara Gr.

Aganaster, Miller \& Gurley, 1S90, Desc. New Gen. and

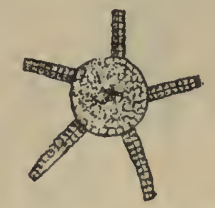

FIG. 1208.-Aganaster gregarius. Dorsal side.

Spec. Echinodermata, p. 57 , republished in 16 th Rep. Geo. Sur. Ind., p. 372. [Ety. agan, very much; aster, star.] Circular disc with five long, narrow rays. Dorsal side covered with small polygonal plates, not inter- 
rupted by the presence of the rays, thus showing the disc has a greater depth than the rays have. Rays nar-

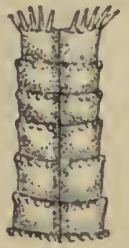

FIG.1209.-Aganaster gregarlus. Dorsal side of arm, magnifled.

A.

A. Miller, $180 \%$ Advance Sheets Rep. Geo. Sur. Ind., pp. 20 and 21, Chouteau limestone, and $\mathbf{A}$. chouteauensis and $A$. germanus, pp. 42 and 43 , Chouteau limestone.

dissimilis,' S. A. Miller, 1891, Advance Sheets 17 th Rep. (ieo. Sur. Ind., p. 55, Keokuk Gr. And also at the same time and place, from the same Group, A. gorbvi and $\mathbf{A}$. indianensis.

decornis, Rowley \& Hare, 1891, Kansas City Seientist, vol. 5, p. 117, Burlington Gr.

splendens, Miller \& Gurley, 1890, Jour. Cin. Soc. Nat. Hist., vol. 13, p. 18 , republished in 17th Rep. Geo. Sur. Ind., p. 55, Keokuk Gr.

Agelacrinus blairi, S. A. Miller, 1892, Advance Sheets 18 th Rep. Geo. Sur. Ind., p. 12, Keokuk Gr.

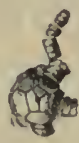

Fig. 1210. - Allocriuns beuedict 1 . Basal view.

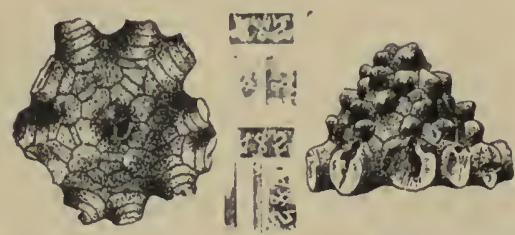

FIG. 1211 -Alloprosallocrinus gurleył. Basal and side views.

Arachnocrinus canadensis, Whiteaves, 1891, Cont. to Can. Pal., p. 208, Devonian.

Arcir mocrda ris legrandensis, Miller \& Gurley, 1890, Desc. New Gen. and Spec. Echinodermata, p. 59, Kinderhook Gr.

A teleocrstites, Billings, is probably a good genus, and not a synonym for Anomalocystites. In that case, Ateleocystites huxleyi would be the type, and the genus may include $\mathrm{A}$. balanoides, which does not seem to belong to Hall's genus Anomalocystites.

Barrcrinus blairi and $B$. boonvillensis, S. A. Miller, Bull. No. 4, Geo. Sur. Mo., p. 25, Keokuk Gr.

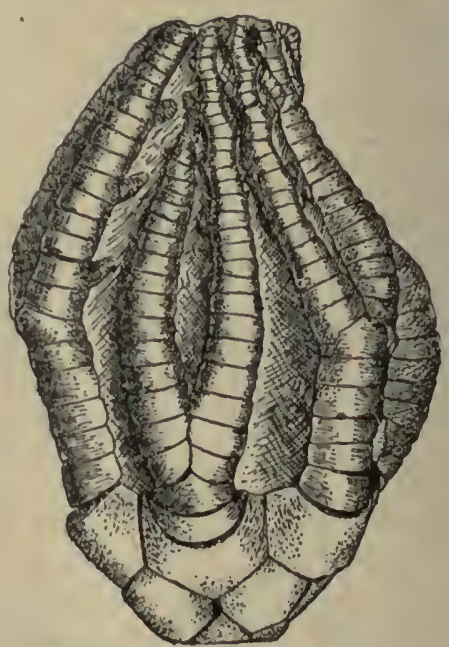

FIG. 1212.-13arycrinus prlnceps.

latus, Hall, 1861, (Cyathocrinus latus,)Proc. Bost. Soc. Nat. Hist., p. 292, Burlington Gr.

princeps, Miller and Gurley, 1890, Desc. New Gen. and Spec. Echinodermata, p. 52, Keokuk Gr.

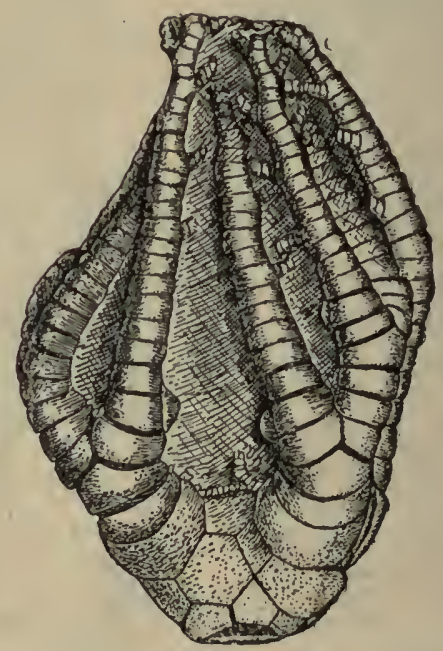

FIG. 1213.-Barycrinus princeps. Azygons view.

stellifer, S. A. Miller, 1892, Advance Sheets 18th Rep. Geo. Sur. Ind., p. 46, Keokuk Gr. 
BAtocrinus agnatus, B. boonvillensis, $B$. crawfordsvillensis, B. decoris, B. gorbyi,

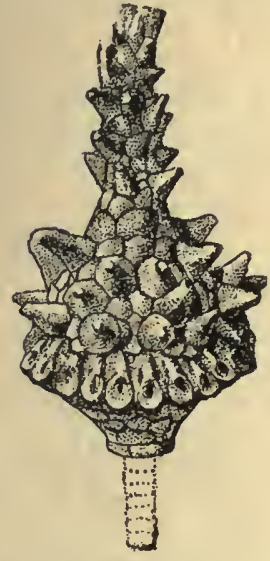

FIG. 1214.-Batocrinus icosidactylus.
B. gurleyi, B. mediocris, B. pulchellus,B.spergenensis, B. venustus, S. A. Geo. Sur. Ind., pp. 53,60 to 68 . All from the Keokuk Gr. except B. decoris and B. spergenensis, which are from the Warsaw Gr.

bulbosus, B. davisi, B. gurleyi, B. inflatus, B. rotadenand B. abscissus, Rowley and Hare, 1891, Kansas City Scientist, vol. 5, pp. 102 and 114 to

117. B. abscissus, gurleyi, and sweeti are from the Keokuk Gr., B. davisi from the Kaskaskia Gr., and the others from the Burlington $\mathrm{Gr}$.

cantonensis, B. facetus, B. jucundus, B. marinus, B. poculum, Miller and Gurley, 1890, Desc. New Gen. and Spec. Echinodermata, pp. 19 , 20,34 to $36, \mathrm{~B}$. jucundus and $B$. marinus were published in the Jour. Cin. Soc. Nat. Hist., vol. 13 , pp. 19 and 20 , and all were republished in the 16th Rep. Geo. Sur. Ind.,, pp. $340,341,352$, 353 , and 354 . B. poculum is from the Kinderhook Gr., the others from the Keokuk Gr.

calvini, Rowley, 1890 , Am.

Geol. vol. 5, p. 146, Bur-

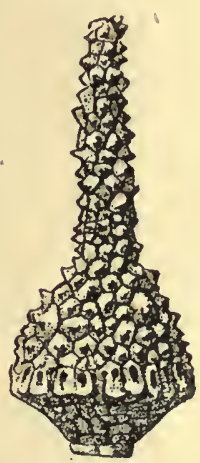

Fig. 1215. - Batocrinus irregularis. Miller, 17th Rep. tatus, B. sweeti,

lington Gr.

brittsi and B. comparilis, S. A. Miller, 1892, Advance Sheets 18th Rep. Geo.

Sur. Ind., pp. 32 and 33, Burlington Gr.
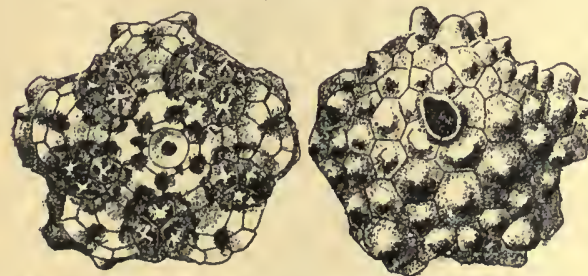

FIG. 1216.-Blairocrinus arrosus. Basal and sum mit views.

and B. decrepitus, p. 34, Keokuk Gr., and B. blairi, p. 39, Burlington Gr.

divalis, S. A. Miller, 1892, Advance
Sheets 18th Rep. Geo. Sur. Ind., p. 22, Keokuk Gr., and at same place republished B. icosidactylus and B. irregularis.

Belemnocrinus sampsoni, S. A. Miller, 1890, Bull. No. 4, Geo. Sur. Mo., p. 26, Burlington $\mathrm{Gr}$.

Blairocrinus, S. A. Miller, 1891, Advance Sheets 17th Rep. Geo. Sur. Ind., p. 69. [Ety. proper name; krinon, lily,] Calyx low, saucer-shaped, but not depressed at the base. Surface deeply sculptured and bears radial ridges, though the interradial ridges are not sunken; vault elevated above the arm openings, more or less convex above, with a short subcentral proboscis, having an opening on top, surrounded by numerous small plates. Basals, 3, forming a flat hexagonal disc; primary radials, $3 \times 5$;
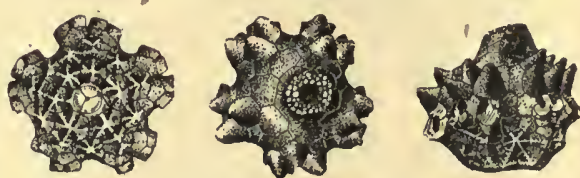

FIG, 1217.-Blairocrinus trijugis. Basal, summit, and side views.

secondary radials, $1 \times 10$, axillary ; tertiary radials, $1 \times 20$; regular interradials, one large plate resting upon the first radials, followed by one or two ranges of two plates each, and these by two elongated plates that connect with the plates of the vault. First azygous plate in line with the first radials, followed by ranges of two plates until they connect with the plates of the vault. Type $B$. trijugis, described at the same time and place from the Chouteau limestone.

arrosus and B. bullatus, S. A. Miller, 1892, Advance Sheets 18th Rep. Geo. Sur. Ind., p. 41, Chouteau limestone.

Calceocrinus should be corrected, p. 230 , ninth line from the top, so as to read "three" instead of "four" anchylosed plates.

indianensis, S. A. Miller, 1891, Advance Sheets 17th Rep. Geo. Sur. Ind., p. 35, Niagara Gr.

Callicrinus, D'Orbigny, 1850, Prodrome Pal. Stratigraphique, 1, p. 45. [Ety. kallos, beautiful; krinon, lily.] Body oblong, cylindrical; calyx cup-shaped, base excavated for the insertion of the column. Basals 4, unequal, cuneate; no subradials; radials $3 \times 5$; first one hexagonal, transverse, arcuate below ; second one short, quadrangular; third, pentagonal, axillary; secondary radials, $2 \times 10$, the $\mathrm{sec}$ ond axillary and supporting the arms, which are composed of a double series of plates bearing pinnules; first interradial large, decagonal, bearing two elongated plates in the second series, like Eucalyptocrinus; vault and proboscis as in Eucalyptocrinus; surface 
deeply seulptured or bearing a more or less developed spine on each radial and interraclial plate. 'Type C. costatus. acanthinus, Ringueberg, 1890, Anu. N. Y. Acad. Sci., vol. 5, p. 302, Niagara Gr. beachleri, Wachsmuth \& Springer, 1\$92, Am. Geol., vol 10, p. 140 . Not detined so as to be recognized.

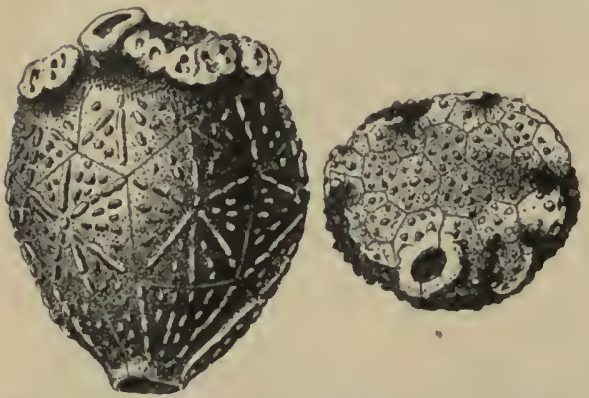

FIl. 1218.-Curyocrimus Indinneusis. Side and summit vlews.

Carrocrınus indianensis, S. A. Miller, 1891, Advauce Sheets 17th Rep. Cieo. Sur. Ind., p. 19, Niagara Gr.

Cliockinus, p. 231, is, probably, incorrectly. defined, See iny remarks, p. 323, 16th Rep. Geo. Sur. Ind.

Cndastir gracillimus and C. grandis, Rowley and Hare, 1891, Kansas City Seientist, p. 99. Burlington $\mathrm{Gr}$.

Codonitrs inopinatus, Rowley and Hare, 1891, Kansas City Scientist, pp. 100 and 118, Burlington Gr.

Crathocunus benedicti, C. gurleyi, C. labyrinthiens, S. A. Miller, 1891, Ad-

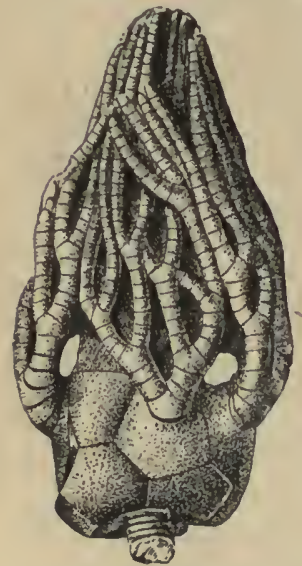

FIG.1219,-Cyathocrinus gurley1. vance Slleets 17 th Rep. Geo. Sur. Ind., pp. 47 to 49 , C. benedicti, from the Niagara $\mathrm{Gr}$. and tlie other two species from the Keokuk Fr.

boonvillensis, and C. sampsoni, $\mathrm{S}$. A. Miller, 1891, Bull. No. 4, Geo. Sur. Mo., p. 29 , Keokuk Gr.

gorbyi, S. A. Miller, 1892, Advance Sheets 18th Rep. Geo. Sur. Ind., p. 44, Keokuk Gr. inflerus, see Delocrinus inflexus.

latus, see Barycrinus latus.

multibrachiatus var squamosus, Hall, 1872, in a note to photographic plate No. 5, sometimes distributed with Desc. New

Spec. Crin. from the Carb. rocks of the Miss. Valley, Keokuk Gr.

opimus, Miller and Gurley, 1890, Desc.
New Gen, and Spec. Echinolermata, p. 28 , and 16th Rep. Geo. Sur. Incl, p. 348 , Keokuk Gr.
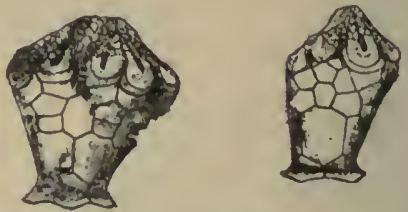

Fig. 1220.-Cyticocrinus eanalteulatus.

Cruicocinsts, S. A. Miller, 1892, Adrance Sheets 18th Rep. Geo. Sur. Ind., p. :31. [Ety. kulin, ikos, a cup; krinon, a lily.] Calyx urn-shaped, truncated below. Basals 3, expanded; primary radials, $3 \times 5$,-the first one very large; second one, smaall, quadrangular; third, sumall, pentagonal; secondary radials 2 or more; arms 10 . Regular interradials consist of one large plate followed by very small ones between the arm-bases that conneet with the vault-plates; first azygous plate in line with the first radials, followed by three plates in the second and in the third ranges, which are connected with smaller plates that connect with the vault. The vault is convex and covered with minute plates, except the ambulacral grooves, which are open and have serrated edges as if protected by some kind of eilia. The primary radials resemble those in some species of Batoerinus; the a\%ygous area resembles Saccocrinus; the vault is different from that in all other known genera of erinoids. Type Cylicoerinus canaliculatus, described at the same place from the Niagara Gr.
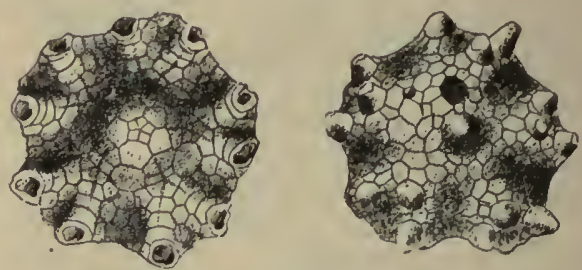

FIG. I221-Cy phocrinus gorbyi. Basal and sum. mlt vlews.

Cyphocrixus, S. A. Miller, 1892, Advance Sheets 1Sth Rep. Geo. Sur. Ind., p. 50. [Ety. kuphos, bowed down; krinon, lily.] Calyx obconoidal or obpyramidal as ligh as the first interradials, then rapidly expands and curves downward until the periphery and ambulacral orifices are directed below a horizontal line. Basals 5, forming a pentagonal disc; sub-radials 5 , hexagonal,except one which is truncated by the first azygous plate; it is heptagonal; primary radials $3 \times 5 ; 3$ of the first ones heptagonal, 
and the other two hexagonal; second radials quadrangular; third radials pentagonal, and bear on the upper slop-

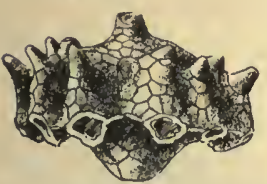
ing sides secondary radials; regular interradials numerous; the first one large and resting between the short upper sloping sides of the first radials;

Frg. 1222.-Cyphocri- it is followed, in the nus gorbyi. side second range, by two plates, and by three plates in succeeding ranges until they unite with the plates of the vault; intersecondary radials present; first azygous plate large, truncates a subradial, and is followed by three or four plates in each succeeding range, until they unite with the plates of the vault; vault convex and covered with more or less numerous plane, convex, or spinous plates; a large spinous plate occupies the center of the vault, and the anal orifice, without any prominence, is on the azygous side of it. Type Cyphocrinus gorbyi, described at the same place from the Niagara Gr.

Delocrinus, Miller and Gurley, 1890, Jour. Cin. Soc. Nat. Hist., vol. 13, p. 9, and 16th Rep. Geo. Sur. Ind., p. 333. [Ety. delos, manifest; krinon, lily.] Calyx basin.shaped; arms broad, composed of a double series of interlocking plates; column round; surface smooth or granulous; plates thick; basals 5, occupying a concavity and forming a cone in the interior; subradials 5, large, inflexed below the middle, regularly arched, and the upper part terminating in an acute angle; first radials wider than high, truncated above and separated from the second radial on the outer face by a gaping suture, but immediately within, a straight crenated ridge extends from one outer angle of the plates to the other, having a furrow on each side so

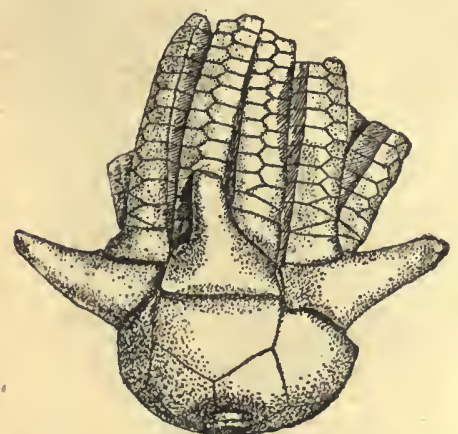

FIG. 1223.-Delocrinus hemisphericus.

as to form a toothed hinge on which the second plate articulates; behind this hinge, in the middle part of each plate, there is a socket for the reception of a tooth-like projection; second radials or brachials produced externally in a strong spine; no regular interradials; one azygous interradial resting on a subradial and followed by another piece above the top of the calyx. Type D. hemisphericus.

craigi, Worthen, 1875, (Eupachycrinus craigi,) Geo. Sur. Ill., vol 6, p. 527, Coal Meas.

fayettensis, Worthen, 1873, (Eupachycrinus fayettensis, Worthen, Geo. Sur. Ill., vol. 5, p. 565, Up. Coal Meas.

hemisphericus, Shumard, 1858, (Poteriocrinus hemisphericus,) Trans. St. Louis Acad. Sci., vol. 1, p. 221, and 16th Rep. Geo. Sur. Ind., p. 335, Up. Coal Meas.

inflexus, Geinitz, 1866, (Cyathocrinus inflexus,) Carb. und Dyas, in Neb., p. 62, and White's Cont. to Pal., No.6, p. 128, Up. Coal Meas.

missouriensis, Miller and Gurley, 1890 , Jour. Cin. Soc. Nat. Hist., vol. 13, p. 14 . Up. Coal Meas.

Dendrocrinus nodobrachiatus, Ringueberg, 1890, Ann. N. Y. Acad. Sci., vol. 5, p. 303, Niagara Gr.

Dichocrinus blairi, S. A. Miller, 1891, Advance Sheets 17 th Rep. Geo. Sur. Ind.,

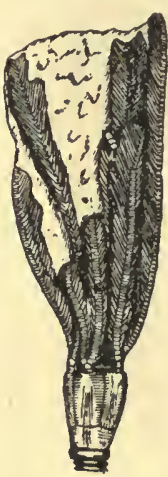
p. 36, Keokuk Gr. cinctus, Miller \& Gurley, 1890, Jour. Cin. Soc. Nat. Hist., vol. 13, p. 21, and 16th Rep. Geo. Sur. Ind.,

FIG. 1224.-Dichoerinus cinctus. T'wo of the views are magnified.

p. 342, Kinderhook or Waverly Gr.

humbergi, S. A. Miller, 1891, Bull. No. 4, Geo. Sur. Mo., p. 26, and Advance Sheets 17 th Rep. Geo. Sur. Ind., p. 36, Keokuk Gr.

parvulus, S. A. Miller, 1891, Bull. No. 4, Geo. Sur. Mo., p. 27, Keokuk Gr.

ulrichi, Miller \& Gurley, 1890, Desc. New Gen. and Spec. Echinodermata, p. 48, and 16th Rep. Geo. Sur. Ind., p. 366, Keokuk Gr.

DoLatockinus has only three basals, as shown by a specimen belonging to Mr. Gurley.

DORYCRINUs amœnus, and D. confragosus, S. A. Miller, 1890, Bull. No. 4, Geo. Sur. Mo., p. 34, Burlington Gr.

elegans, S. A. Miller, 1892, Advance Sheets 18th Rep. Geo. Sur. Ind., p. 17, Burlington Gr. 
inflatus, Rowley \& Hare, 1891, Kansas - City Seicntist, p. 114, Burlington Gr.

Fonisodiscus sampsoni, S. A. Miller, 1891, Advance Sheets 17 th Rep. Geo. Sur. Ind., p. 76, Keokuk Gr.

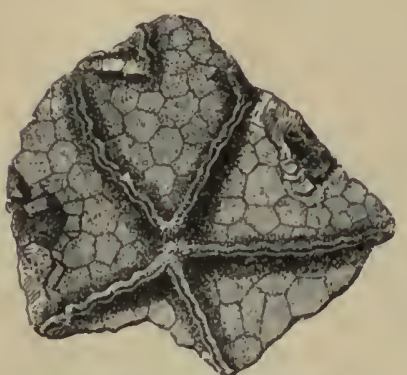

FIG. 1225.-Echinodiscus sampson1.

Eocidams blairi, S. A. Miller, 1891, Advance Sheets 17th Rep. Geo. Sur. Ind., p. 73, Keokuk Gr.

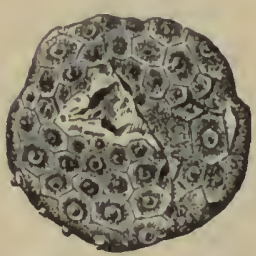

Fyo. 1226.-Eocidaris blniri.

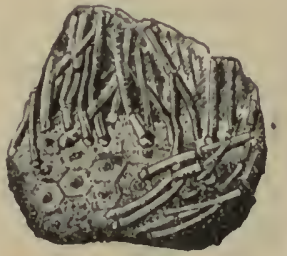

Fis. 1227. - Eocldaris bialri. Sh ow 1 ng spines.
Elietmocrinu's lyonanus, S. A. Miller, 1891, Advance Sheets 17th Rep. Geo. Sur. Ind., 1). 59, Keokuk Gr.

prregravis, S. A. Miller, 1892, Advance Sheets 18th Rep. Geo. Sur. Ind., p. 37, Keokuk Gr.

Erisocrinus inflexus. Sce Delocrinus inHexus.
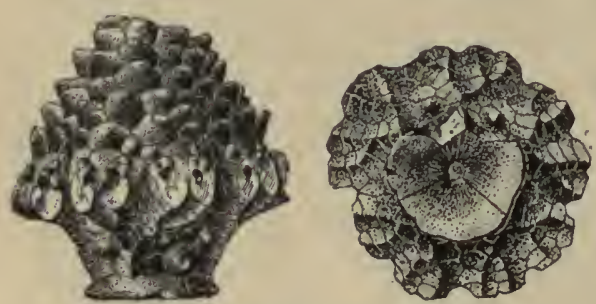

FIG. 1228.-Eretmocrinus lyonanus. Side and basal vlews.

Fucalyptocrinus ellipticus, E. elrodi, E. gorbyi, E. subglobosus, S. A. Miller, 1891, Advance Sheets 17th Rep. Geo. Sur. Ind., pp. 37 to 40 , Niagara Gr. muralis, Ringueberg, 1890, Ann. N. Y. Aead. Sei., vol. 5, p. 305, Niagara Gr. lindahli, Wachsmuth \& Springer, 1892. Am. Geol., vol. 10, p. 139 . Not defined so as to be recognized.
Eupachrcrixus craigi. See Delocrinus craigi.

fayettersis. See Delocrinus fayettensis.

hemisphericus. See Delocriniss liemisphericus.

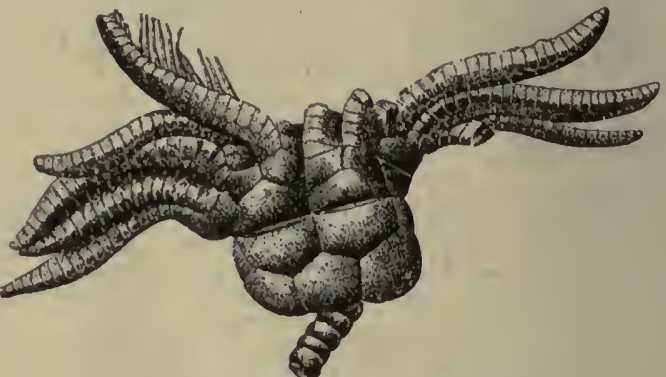

F'IG 1229.- Tupachyorinus harli.

liarii, S. A. Miller, 1891, Advance Sheets 17 th Rep. Geo. Sur. Ind., p. 71, Up. Coal Meas.

magister, Miller \& Gurley, 1890, Jour. Cin. Soc. Nat. Hist, vol. 13, p. 4, and 16tli Rep. Gco. Sur. Ind., pp. 328, 371, Up. Coal Meas.

sphaxalis, Miller \& Gurley, 1890, Jour. Cin. Soc. Nat. Hist., vol. 13, 1. 5, and 16th Rep. Geo. Sur. Ind., p. 329 , Up. Coal Meas.
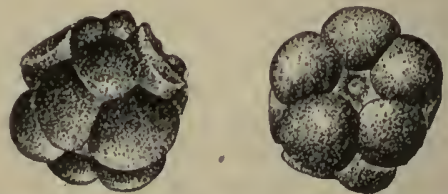

Fro. 1230.-Enpachycrinus tumulosus. Azygous and basal views.

tumulosus, S. A. Miller, 1891, Advance Sheets 17tlı Rep. Geo. Sur. Ind., p. 70, Kaskaskia Gr.

Forbesocrinus elegantulus, S. A. Miller, 1891, Bull. No. 4, Geo. Sur. Mo., p. 40 , Keokuk Gr.

speciosus, Miller \& Gurley, 1890, Desc. New Gen. and Spec. Echinodermata,

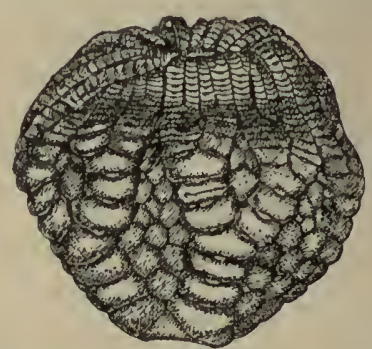

Fro. 1231.-Forbesocrinus speciosus.

p. 27, and 16th Rep. Geo. Sur. Ind., p. 347 , Keokuk Gr.

spinifer instead of "spiniger." 
Gazacrinus, S. A. Miller, 1892, Adv. Sheets 18th Rep. Geo. Sur. Ind., p. 49. [Ety.
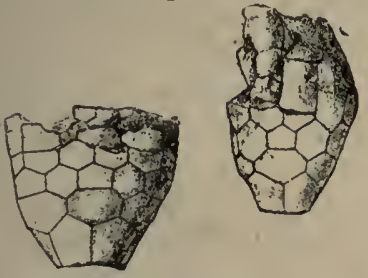
natus.
FiG. 1232.-Gazacrinus jnor-

gaza, treasury; krinon, lily.] Calyx olvconoidal; bas als 5 , o ne truncated by the azygous plate; primary radials, $3 \times 5$; secondary radials $2 \times 10$; arms composed of a single series of flattened plates, one plate in each radial a rea; va ult sustained by a specialized frame - work, with ambu- Frg. 1233.-Gazacriuus inor, lacral canals
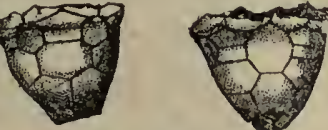
connecting the arms with a central orifice. Type Gazacrinus inornatus. Described at the same place, from the Niagara Gir.

GLyPtaster lockportensis, Ringueberg, 1890, Ann. N. Y. Acad. Sci., vol. 5, p. 304, Niagara Gr.

Goniasteromocrinus tuberosus is from the Keokuk Gr. It is re-defined in the Advance Sheets 17th Rep. Geo. Sur. Ind., p. 51, and several errors respecting it are corrected.

Goniocrinus, Miller \& Gurley, 1890, Desc. New Gen. and Spec. Echinodermata, p. 32, and 16th Rep. Geo. Sur. Ind., p. 351. [Ety. gonia, an angle; krinon, a lily.] Calyx small, basin-shaped; plates convex or angular. Basals 5, small, extending beyond the column. Sub-

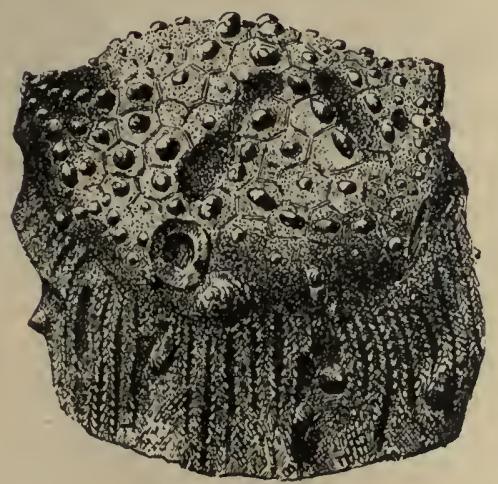

FIG. 1234,-Goniasteroidocrinus tuberosus. Showing the pendulous arms.

radials five, about the same size as the basals; first radials larger, wider than long, and supporting on the slightly concave upper faces, a little shorter than the width of the plates, the brachials; brachials 3 in each ray, flanged at the sides; arms resembling Scaphiocrinus ; no regular interradials; azygous interradials. consisting of a series of plates, the tirst one like a first radial, and resting upon the upper truncated face of a subradial, wh ich is followed by plates very m u ch like FIG.1235.-Gontocrinus scuip. the brach- tilis. Naturai size and mag. ials, which nifled.

form a convex, arm-like appendage that curves in toward the proboscis at or
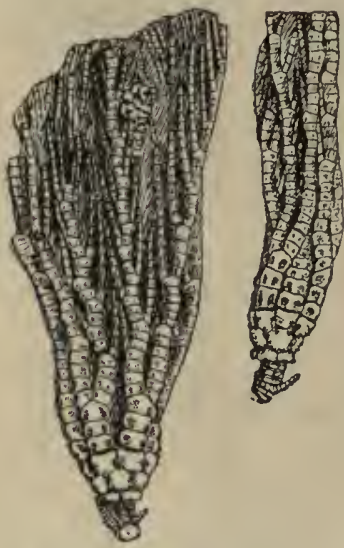
above the base of $t$ lie free arms. A small azygous plate also exists on the right side of the area resting between the up. per sloping sides of two subradials and the under sloping side of a first radial, and the a zygous p I a te which truncates a subradial. Column pentag-

Frg. 1236.-Goniocrinus sculp- onal, b e a ring tilis. Left side view and azy- $\mathrm{c}$ ir rhi, and gous side. Both magnified a c orr posed of little.

thicker an d

thinner plates; canal pentagonal. Type G. sculptilis, which is described at the same place, from the Waverly or Kinderhook Gr.

Granatocrinus aplatus, G. concinnulus, G. excavatus, G. exiguus, G. pyriformis, Rowley \& Hare, 1891, Kansas City Scientist, vol. 5, pp. 99, 100, 117, 118, Burliugton Gr.

Grapilocrinus must be restored, as described by De Koninck \& Lehon, and the redefinition of Wachsmuth \& Springer wholly set aside. The generic formula is as follows: Basals 5 ; radials $2 \times 5$; anal 1 ; arms 10 ; none bifurcate. There is not a shadow of reason or evidence for supposing De Koninck and Lehon were mistaken in their diagnosis. 
HoLoustites adipatus,

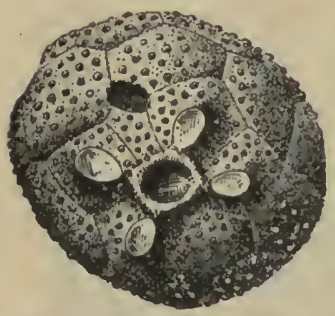

HIG. 123\%, - Holocystifes subovatus. Summit.

to 1S, Niagara Gr.

amplus, S. A. Miller, 1892, Aclvance Slleets loth Rej. Geo. Sur. Int., p. 8, Niagara Gr.

Hyinoxocrises pentagonus, Miller \& Gurley, 1890, Jour. Cin. Soc. Nat. Ilist., vol. 13, p. 17. and $16 \mathrm{th}$ Rep. Cieo. Sur. Ind., 1. 339, [" p. Coal Meas.
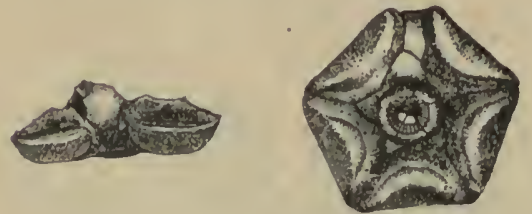

Fig. 123s,-Hydrionocrinus pentagon us.

Hyptiocrinus lypus, Wachsmuth of Springer, 1892, Am. Geo., vol. 10, 1) 138. Not defined so as to be recognized.

I'uthyocrinus conoirlens, Ringurberg, 18?0, Ann. \$. Y. Acarl. Sci., vol. 5, \%. 305, Niagara Gr.

greenii, S. A. Miller, 1892, 18th Rep. Geo. Sur. Ind., p. 52, Keokuk Gr.

Idiocrinus elongatus and I. ventricosus, Wachsmuth of Springer, 1892, Am. Geo., vol. 10 , p. 135 . Not defined so as to be recognized.

LECANOCRINU's tennesseensis, S. A. Miller, 1891, Adrance Sheets 17th Rep. Geo. Sur. Ind., p. 41, Niagara Gr.

MARIACRINes aureatus, S. A. Miller, 189I, Advance Slieets 17th Rep. Geo. Sur. Ind., p. 34, Niagara Gr.

granulosus, S. A. Miller, 1891, Advance Sheets 17 th Rep. Geo. Sur. Ind., p. 35, Niagara Gr.

Melocrives xenualis, S. A. Miller, 1892, Advance Sheets 18th Rep. Geo. Sur. Ind., p. 48, Niagara Gr.

oblongus, Mr. parous, Wachsm uth of Springer, 1892. Am. Geo., vol. 10, p. 143. Not defined so as to be recognized.

Melonites was preoccupied when Owen \& Norwood used it, and Meek \& Worthen proposed instead of it Melonechinus.

Menochinus has five basals, and does not have any near affinity with Platycrinus, nor belong to the same family.

Missouricrinus, S. A. Miller, 1890, Bull. No. 4, Geo. Sur. Mo., p. 31. [Ety. proper name; krinon, lily.] Calyx conoirlal or basin-shaped ; plates smooth or grannlous; basals 5, forming a small cup; no subradials; no regular interradials; primary radials $1 \times 5$, wider than ligh, and separated from the brachials by an external gaping suture; brachials axillary, except in the ray
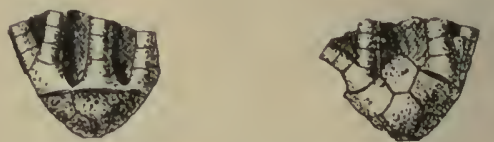

Fig. 1239.-Missouricrinus admonitus.

opposite the azygous side; arms resembling those in Scaphiocrinus; first azygous interradial rests between two primary radials and truncates a basal plate; column pentagonal. Type $M$. admonitus, which was described at the same time, from the Burlington Gr.

MYlionactvius gorbyi, S. A. Miller, 1891, Advance Slieets 37 th Rep. (ieo. Sur. Ind., p. 72, Niagara Gr.

Nipterockinus has five basals.
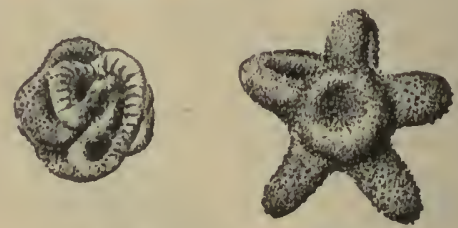

Fif, 1210,-Onychaster asper.

Onychaster asper, O. confragosus, O. demissus, S. A. Miller, 1891, Advance Sheets 17 th Rep. Geo. Sur. Ind., p. 74, Keokuk Gr.

Onycirocrinus cantonensis, Miller \& Gurley, 1890, Desc. New Gen. and Spec. Echinorlermata, p. 41, and 16th Rep. Geo. Sur. Ind., p. 358, Keokuk Gr.

ulrichi, Miller \& Gurley, 1890, Jour. Cin. Soc. Nat. Hist., vol. 13, p. 17, and 16th Rep. Geo. Sur. Ind., p. 339, Keo. kuk Gr.
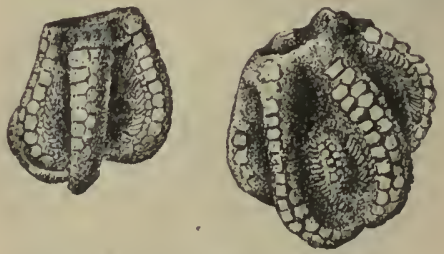

FIG. 1241.-Ony chaster demissus.

norwoodi, Meek \& Worthen slıould loe restored, as it is not a synonym for 0 . exculptus.

OtTawacrinus, p. 265, first line at top of page read subradial instead of "basal."

Palfocystites, p. 267, is described in Decade 3.

Pentremites basilaris and $P$. broadheadi 
are described on p. 159, and $P$. clavatus and $P$. hemispliericus, on p. $157 ; P$. gemmiformis, p. 553 ; P. nodosus, p. 155; P. sampsoni, p. 551; and $P$. spinosus, p. 15t, of vol. 4, Trans. St. Louis Acad. Sci. P. cherokeus was not defined by Troost, and as Roemer described P. sulcatus in 1852, it was too late for Hall to call the same species P. cherokeus in the Geo. Sur. Iowa, in 1858.

laternifon'mis, see Troostocrinus laterniformis.

Pisocrinus benedicti, $P$. campana, P. gorbyi, S. A. Miller, 1591, Advance Sheets 17th

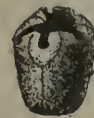
Rep. Geo. Sur. Ind., pp. 28 to $32, \mathrm{Ni}$ agara Gr. P. genmiformis is redefined at the same FIG. 1212. - Pisocrinus place and the gebenedicti. Side and neric characters disbasal views. cussed.

pyriformis was described in 1884 .

Platycrinus absentivus, P. aquiternus, P. allophyllus, $P$. annosus, $P$. brittsi, P. ollicula, from the Choutean limestone; P. acclivus, P. batiola, P. blairi, P. broadheadi,
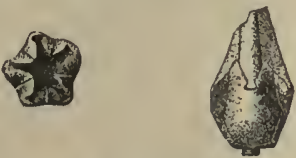

P. carchesium,

P. concinnus, P. gorbyi, P. lautus, P. occidentalis, $P$. pulcellus, $P$. rotundus, P. sampsoni, P. sulcatus, from the Burlington Gr.; and P. reternalis, P. amabilis, $P$. boonvillensis, $P$. pentagonus, from the Keokuk Gr., Bull. No. 4, Geo. Sur. Mo., pp. 8 to 23 , S. A. Miller, 1890.

alabamensis, S. A. Miller, 1891, Advance Sheets 17 th Rep. Geo. Sur. Ind., p. 50 , Kaskaskia Gr.

altidorsatus, $\mathbf{P}$. corbuliformis, $\mathrm{P}$. marginatus, P. pisum, P. planobasalis, from the Burlington Gr.; P. curryvillensis, $P$. insolens, from the Choutean limestone; Rowley \& Hare, 1891, Kansas City Scientist, pp. 97, $98,113$.

caducus, S. A. Miller, 1892, Advance Sheets 18th Rep. Geo. Sur. Ind., p. 13, Keokuk Gr., and at same place P. chonteauensis and P. colletti from the Choutean limestone.

Poteriocrinus agnatus, $P$. amoenus, $P$. boon villensis, $P$. coryphrus, $S$. A. Miller, 1891, Advance Sheets 17th Rep. Geo. Sur. Ind., pp. 42 to 45 , Keokuk Group.

arcanus, $P$. cantonensis, $P$. crawfordsvillensis, P. granilineus, P. subramosus, P. verus, from the Keokuk Gr.; P. genista, P. legrandensis, P. scopæ, P. spartarius, from the Kinderhook Gr.; Miller and Gurley, 1890, Jour. Cin. Soc. Nat. Hist., vol. 13, pp. 23, 24, and Desc.
New Gen. and Spec. Echinodermata, pp. 29,37 to 40,49 , republished, 16th Rep. Geo. Sur. Ind., pp. $343,344,348,355$ to 358,365 .

brittsi, S. A. Miller, 1890, Bull. No. 4, Geo. Sur. Mo., p. 30, Keokuk Gr.

meekunus refer to Cyathocrinus meekanus, Chouteau limestone.

waltersi, Rowley and Hare, 1891, Kansas City Scientist, p. 101, Burlington Gr.

Protaster gregarius, see Aganaster gregarius. Rhodocrinus benedicti, S. A. Miller, 1892 , Advance Sheets 18th Rep. Geo. Sur. Ind., p. 15, Keoluk Gr.

calatus, R. sculptus, Miller and Gurley; 1890, Desc. New. Gen. and Spec. Echinodermata, pp. 42, 43, and 16th Rep.
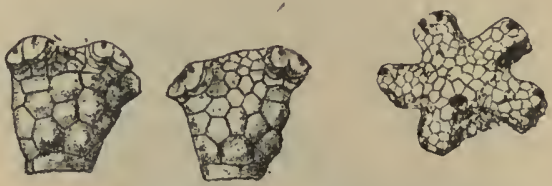

FIG. 1244.-Saccocrinus howardi. Slde, azygous, and summit views.

Geo. Sur. Ind., pp. 359, 360, Kinderhook Gr.

nanus is from the Kinderhook Gr.

parvus, S. A. Miller, 1891, Bull. No. 4, Geo. Sur. Mo., p. 39, Keokuk Gr.

SAccocrinus benedicti and $S$. howardi, S. A. Miller, 1892, Advance Sheets 18th Rep. Geo. Sur. Ind., pp. 29 and 30, Niagara Gr.

gorbyi, S. A. Miller, 1891, Advance Sheets 17 th Rep. Geo. Sur. Ind., p. 57, Niagara $\mathrm{Gr}$.

Scaphiockinus bellus, S. bonoensis, S. disparilis, S. granuliferus, S. graphicus, S. lacunosus, S. manus, S. premorsus, S. repertus, Miller \&
Gurley, 1890 , Jour. Cin. Soc. Nat. Hist., vol. 13 , p. 24 , and Desc. New Gen, and Spec. Echinodermata, pp. 29,45 to 52 , republished in 16th Rep. Geo. Sur. Ind., pp. 345, 349, 362 to 367 , Keokui Gr.

boonvillensis, P. con. strictus, S. A. Miller, 1891, Bull. No. 4, Geo. Sur. Mo., pp. 37, 38, Keokuk Gr.

gorhyi, S. porrectus, S. sampsoni, S. A. Miller, 1891, Advance FIG. 1245.-ScaphiSheets 17th Rep. ocrinus gorbyi. Geo. Sur. Ind., pp. 42, 46; S. sampsoni from the Chouteau limestone, the others from the Keokuk Gr.

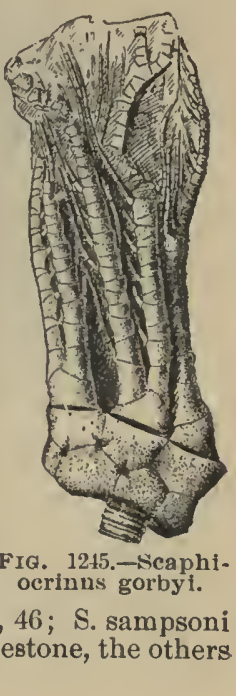


lyoni, and S. maniformis, S. A. Miller, 1892, Advance Sheets Geo. Sur. Ind.,
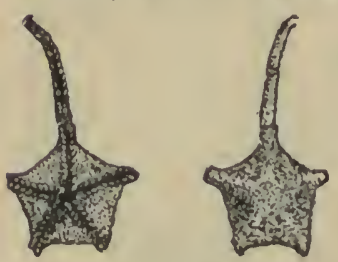

p. 45 , Keokuk Gr.

Schinaster legrandensis, Miller and Gurley, 1890, Desc. Now Ge n. a $11 \mathrm{~d}$ Spec. Echinodermata, p.

Fra. 1246. - Schonaster le56 , and 16 th $\mathrm{R}$ e $\mathrm{p}$. G e o. Sur. Ind., p. 371, Kinderhook Gr.

Strganocrints benedicti, S. A. Miller, 1892. Advance Sheets $18 t h$ Rep. Geo. Sur. Ind., p. 2\%, Keokuk Gr.

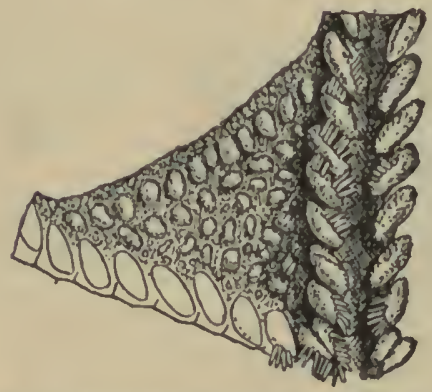

Fig. 1247.-Schcenaster legrandensis. P'art of ventral side magnifled $62 / 2$ dlameters.

Stepiranocrinus elongatus, S. hammelli, S. obpyramidalis, S. A. Miller, 1891, - 17th Rep. Geo. Snr. Ind., pp. 22 to 26, Niagara Gr. S. osgoodensis is reclescribed and the genus is discussed at the same place.

cornetti, S. A. Miller, 1892, Advance

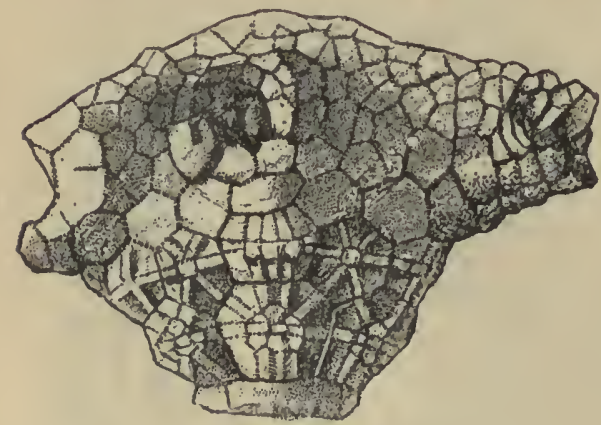

FIG. 1248.-Steganocrinus benedicti.

Sheets 18th Rep. Geo. Sur. Ind., p. 12, Niagara Gr.

Stribalocistites, S. A. Miller, 1891, Advance Sheets 17 th Rep. Geo. Sur. Ind., p. 20. [Ety. stribalos, close pressed, thick, in allusion to the thick tumid plates; kustis, bladder.] Body rudely

subovate or subelliptical, and covered by about five series of tumid plates.
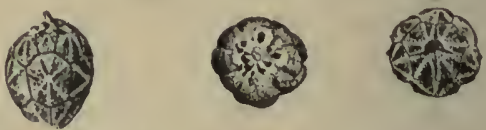

Fig. 1249.-Stribalocystites gorbyl. Side, stimmit, and basal views.

Basals 4, unequal; second series of plates 6, unequal; fourth and fifth series irregular and covering the sum in it; no a rms; orifice near the summit on the llzygons Fra. 1250. - stribalocysside, and another lifes lumidus. Side on the left near

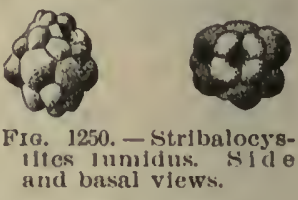
the summit, both being above the third range of plates; sometimes there is a central orifice. Type S. tumidus, which is described at the same place, froun the Niagara Gr.

gorbyi, S. A. Miller, 1892, Advance Sheets 1sth Rep. Geo. Sur. Ind., p. 11, Nisgara Gr.

Symiathocrinus blairi, S. A. Miller, 1891, Bull. No. 4, Geo. Sur. Mo, p. 32, Keokuk Gr.

wachsmuthi, Meck \& Worthen, 1869, Proc. Acall. Nat. Sci., 1). 67, and Geo. Sur. Ill., vol. 5, p. 437, Burlington Gr. Taxocminus spinifer, Hall, 1861, Proc. Bost.

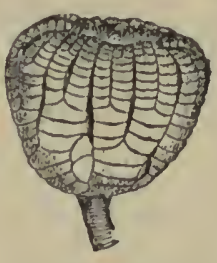

Fig. 1251 I-axo crinus subovatus
Soc. Nat. Hist., p. 318 , Burlington Gir.

subovatus, Miller \& Gurley, 1890, Desc. New Gen. and Spec. Echinodermata, p. 26, and 16th Rep. Geo. Sur. In d., p. 347 , Keokuk Gr.

TecirNOCRINus spinulosus is the type of the genus.

Troostocrinus laterniformis, Owen \& Shumard, 1850 (Pentremites laterniformis), Jour. Acad. Nat. Sci. $2 d$ ser., vol. 2, p. 66. Kaskaskia Gr.

nitidulus, Miller \& Gurley, 1890, Desc. New Gen. and Spec. Echinodermata, p. 58, and 16th Rep. Geo. Sur. Ind., p. 373, St. Louis Gr.

wachsmuthi, Gurley, 1884. New Carb. Foss. Bull. No. 2, p. 1, Warsaw Gr.

ULOCRisus, Miller \& Gurley, 1890, Jour. Cin. Soe. Nat. Hist., vol. 13, p. 6 , and 16th Rep. Geo. Sur. Ind., p. 330. [Ety. oulos, solid, substantial ; krinon, lily.] Calyx globular or pyramiclal, iarge, plates more or less convex, smooth or granular. Basals 5, forming a pentagonal disc or low cup; columnar_opening pentagonal; subradials 5, very - large; first radials large, pentagonal, upper face projects over the interior of the calyx so as to make a broal articu- 
lating face for the first brachial; no regular interradials. A quadrangular azygous plate placed obliquely forms part of the calyx, and a small plate
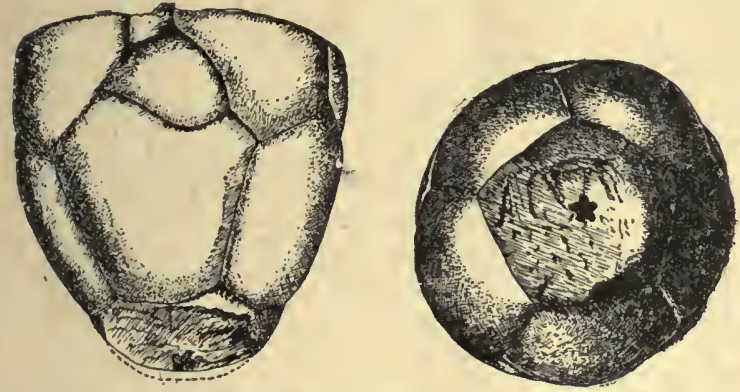

FIG. 1252,-Ulocrinus buttsi.

rests upon its upper angle at the top of the calyx, and projects slightly above the top of the first radials; column round. Type U. buttsi, described at the same place, from the Upper Coal Meas. U. kansasensis is also described at the same place, from the same rocks. Zeacrinus commaticus, Z. pocillum, S. A.

Miller, 1891, Bull. No. 4, Geo. Sur. Mo., pp. 28,36 , Keokuk Gr.

dubius, Miller \& Gurley, 1890, Desc. New Gen. and Spec. Echinodermata, p. 44, and 16tl Rep. Geo. Sur. Ind., p. 361, Keokuk Gr.

faggi, Rowley \& Hare, 1891, Kansas City Scientist, p. 103, Burlington Gr.

Zophocrinus, S. A. Miller, 1891, Advance Sheets 17th Rep. Geo. Sur. Ind., p. 32. [Ety. zophos, dark, obscure; krinon, lily.] Body ovate or pear shaped, and covered by two circles of plates and the vault. Basals or first circle of plates 3 , forming an obconoidal cup, higher than wide; two of the plates are of equal size, at d quadrangular; the other is larger and pen-
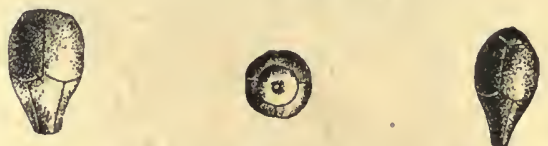

FIG. 1254.-Zophocrinus howardi. Side and summit views.

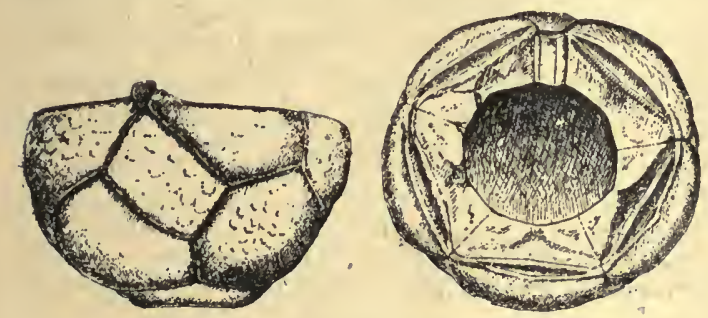

Frg. 1253 -Ujocrinus kansasensis.

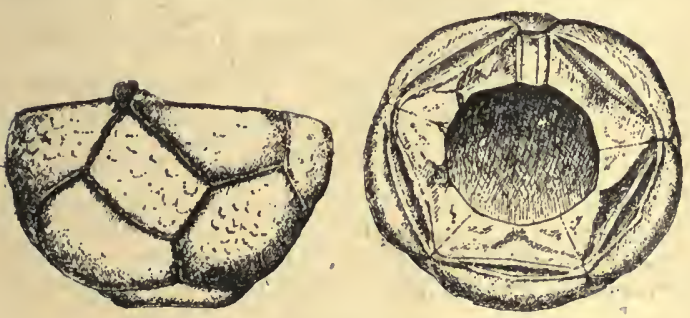

The paper entitled "Descriptions of New Species of Crinoidea, from investigations of the Iowa Geological Survey, Preliminary Notice, by James Hall," dated February 25, 1861, has never been published, as required by the laws of nomenclature, and is not, therefore, entitled to recognition. It is a private pamphlet, that was never kept for sale, and was not generally distributed among those conversant with the subject, and contains no figures of any of the organisms, which, with such meagre and imperfect descriptions as the text contains, would be absolutely necessary to enable an expert palæontologist to determine the organism intended to be named. Hall says, on page 10, that he published the abstract of the descriptions to get ahead of the publications of the Geolosical Survey of Illinois. A very selfish excuse for such poor work, and it does not add any vitality to the publication, which was never entitled to recognition under the laws of nomenclature, as shown on page 95 of this work. One genus and two species have been redefined in valid publications, and they will stand as of the date of the redefinition. The following names, never having appeared with valid definitions. should be stricken out, as of no inore value than the names in a private catalogue: Actinocrinus carica, sometimes referred to Eretmocrinus; $A$. ovatus, A. multibrachiatus var. echinatus, A. lucina, $A$. thetis, A. thoas. A. quaternarius var. spiniferus, $A$. themis; $A$. remibrachiatus, sometimes referred to Eretmocrinus; $A$. tenuiradiatus, sometimes referred to Strotocrinus or Teleiscrinus; A. eryx, A. (Calathocrinus) erodus, sometimes referred to Strotocrinus or Teleiocrinus; A. (Calathocrinus) insculptus, sometimes referred to Strotocrinus or Teleiocrinus; $A$. (Calathocrinus) althea, sometimes referred to ' 'elei crinus; A. lagena, A. thalia; A. matuta, sometimes referred to liretmocrinus; $A$. ma tuta var, attenuata, sometimes referred to Eretmocrinus; $A$. (?) tenuidiscus, $A$. securis, $A$. infrequens, A. locellus, $A$. doris, Platycrinus olla (name preoccupied), P. regalis, $P$. glyptus, $P$. calyculus, $P$. nodohrachiatus (name preoccupied), $P$. parvinodus, $P$. eminulus, $P$. aqualis $(\mathrm{P}$. æqualis in Geo. Sur. Ill., vol. 5, p. 456), Synbathocrinus papillatus, Rhodocrinus wachsmuthi, Heterocidaris keokuk, and H. lavispinus. All of the above, including Lepidechinus, L. imbricatus, and Protaster (?) barrisi, which have been since defined, in proper publications, are 
printed in the above named private pamphlet, on about nine pages. In other worls, thirty-eight new species and two new genera are defined on about nine pages, without a single figure of any kind, and without mentioning a locality from which any of them eane, or informing any one of the age of the rocks further than to write "Burlington limestone."

Wachsmuth (who has probably seen some of the so-called types) has condemned, as synonyms of other species, the above named Actinocrinus mulibrachia'us var. echinatus, A. thetis, A. thoas, A. quat.rnarius var. spiniferus, A. themis, A. eryx, A. lag.na, A. securia, A. locellus, A. dorie, P'latycrinus olla, I. glyptus, P. calyculus, P. nodobrachiatus, and Synbathocrinus papillatus.

Had I been able to see a copy of the pamphlet, none of the names would have appeared as valid in the first edition of this work; but none of my correspondents had ever seen one, and I borrowed most of the names from references made to them by Meek \& Wachsmuth. More recently Wm. F. E. Gurley, of Danville, Illinois, has been able to obtain a copy of all except the last page of the pamphlet, and I have been allowed to examine it, with the ahove result.

\section{SUBKINGDOM MOLLUSCOIDA.}

\section{CLASS BRYOZOA.}

Sомe genera were by accident placed in two fumilies in this Class. Correct by striking P'eronopora out of the Batostomellidac; Eridopora, Lichenotrypa, Sagenella, and Selenopora from the Ceramoporidz; Coscinella, Reptaria, and Semiopora from the Fenestellida; Anisotrypa from the Rlabdoniesontidre; Heliotrypa from the Stictoporidac; Criscinella from the Thamniscida; Acanthoclema, Amplexopora, Atactopora, Bactropora, Clilotrypa, Nemataxis, and Tropidopora from the Trematoporida. Strike out the family Labechiidx because it belongs, probably, to the Protozoa. Place Semiopora with the Ptylodictyonidre; Sagenella and Reptaria with the Tubuliporida.

The bryozoum is sometimes called cœnœcium (koinos, common; oikos, house) or polyzoarium, especially when Polyzoa is used for the Class instead of Bryozoa. Gymnolæmata, in the last line on page 289 , is from, gumnos, naked; laimos, the throat.

Artiroclema armatum, A. cornutum and Arthrostylus conjunctus and A. obliquus, UUlrich, 1890, Jour. Cin. Soc. Nat. Hist., vol. 12, pp. 193 and 194 and pp. 189 and 190 , Trenton Gr.

Ceramopora concentrica und C. whitei, James, 1888, Jour. Cin. Soc. Nat. Hist., vol. 11, p. 38. Not recognized.

Chainodictyon, at the top of p. 297, is from chaino, gaping; and dictuon, net.

Dinstoporixa, Ulrich, 1890, Jour. Cin. Soc. Nat. Hist., vol. 12, p. 177. [Ety. diminutive of Diastopora.] Zoarium bifoliate, flabellate; zoceia tubular, prostrate, not entirely immersed; apertures constricted, oblique, the anterior side not elevated; interspaces, striated. Type D. flabellata, described, at the same time, from the Trenton Gr. He also described at the same time from the Trenton Gr., Enallopora mundula under the name of Mitoclema mundulum. He gives his reasons for not using the ge- neric name Enallopora in vol. 8, Geo. Sur. Ill. p. 683.

DRYMOTRYPA, Ulrich, 1890, Geo. Sur. Ill., vol. 8, p. 399. [Ety. drumos, a thicket; trupa, perforation.] Zoarium dichotomous, reverse striated; zocia in ranges, tubular, thick walled in tangential sections, springing from a thin plate; superficial apertures angular, oval within. Type D. diffusa, Hall's Retepora diffusa, and includes Hall's Thamniscus cisseis and T. niagarensis.

Fenestella hemitrypa, see Hemitrypa proutana.

Fistulipora laxata, Ulrich, 1889, Micropalæontology of Canada, p. 37, Hud. Riv. Gr. The genus Fistulipora is referred by some authors to the Alcyonaria, because the larger pores increase by conenchymal gemmation, a method of increase said to be unknown among Bryozoa.

Glyptotrypa on p. 307, read Glyptopora. 
HeLopora alternata, H. mucronata, Ulrich 1890, Jour. Cin. Soc. Nat. Hist., vol. 12, p. 192, Trenton Gr.

Nematopora conferta, N. granosa, N. ovalis, Ulrich, 1890, Jour. Cin. Soc. Nat. Hist., vol. 12, pp. 196, 197, Trenton Gr.

PACHYDictYa emaciata, P. obesa, P. turgida, Foerste, 1887, Bull. Denison Univ., vol. 2 , pp. 162 to 165 , Niagara Gr.

pumula, P. triserialis, Ulrich, 1890, Jour. Cin. Soc. Nat. Hist., vol. 12, pp. 186, 187, and in Micropalæontology, pp. 42, 43, $P$. hexagonalis and P. magnipora, Trenton $\mathrm{Gr}$.

Paleschara quadrangularis, Nicholson, 1874, (Chetetes quadrangularis,) Rep. Pal. Prov. Ont., p. 61, Devonian.

PiNACOTRYPA marginata, Whiteaves, 1892, Cont. to Can. Pal., p. 278, Devonian.

PoLYPORA manitobensis, Whiteaves, 1892, Cont to Can. Pal., p. 280, Devonian.

Ruinidictia exigua, $R$. humilis and $R$. minima, Ulrich, 1890 , Jour. Cin. Soc. Nat. Hist., vol. 12, pp. 183 to 185, Trenton Gr.

Iinoмropora multipóra, Foerste, 1887, Bull. Denison Univ., vol. 2, p. 72, Coal Meas.
Stictoporklla rigida, Ulrich, 1890, Jour. Cin. Soc. Nat. Hist., vol. 12, p. 188, Trenton Gr.

Stomatopora moniliformis, Whiteaves, 1891, Cont. to Can. Pal., vol. 1, p. 212, Devonian; S. tenuissima, Utica Gr. and S. turgida, Hud. Riv. Gr., Ulrich, 1890, Jour. Cin. Soc. Nat. Hist., vol. 12, pp. 175 and 176.

SUBRETEPORA halli and S. sublaxa, Ulrich, 1890, (Philloporina halli and P. sublaxa,) Jour. Cin. Soc. Nat. Hist., vol. 12, pp. 179 and 181, Trenton Gr. Ulrich gives his reasons for not using Subretepora in vol. 8, p. 686, Geo. Sur. Ill.

Vinelua, Ulrich, 1890, Jour. Cin. Soc. Nat. Hist., vol. 12, p. 173. [Ety. proper name.] Zoarium attached to foreign bodies, consisting of exceedingly slender, ramifying, thread-like tubes, occasionally radiately arranged; surface faintly lined longitudinally; a row of widely separated pores along the surface of the tubes. Type V. repens, described at the same time from the Trenton Gr.

\section{CLASS BRACHIOPODA.}

Oxe valve of the shells in this Class is always larger than the other. A line drawn vertically from the beak to the base will divide the shell into two equal parts. The flattened space between the beaks is called the hinge area or the cardinal area; the aperture in one of the beaks is called the foramen; and the triangular plate in front of the foramen and sometimes forming part of its circumference is called the deltidium, but in some genera it does not exist. When there are no teeth, as in Crania, the valves are held together by the adductor muscles. The shells are found in all Groups of rocks, from the Taconic to the most recent; but the Class seems to have reached its maximum development in the Devonian, and to have slowly declined since the Carboniferous age.

Beecher says the main characters common to the Class are, the bivalve shell, the pedicled or fixed condition, the animal composed of two pallial membranes intimately related to the shell, a visceral sac, and two arms or appendages near the mouth. The extreme range of variation does not eliminate any of these features, and consequently no univalve or multivalve forms are found, nor any strictly free swimming species, nor growths or modifications adapting the organism to a Pelagic life. All Brachiopods have a common form of embryonic shell called the protegulum. (Ety. pro, early; tegos, a covering.) The protegulum is semicircular or semielliptical in outline, with a straight or arcuate hinge-line, aud no hinge area. A slight posterior gaping is produced by the pedicel valve being usually more convex than the brachial. The modifications noted are apparently due to accelerated growth, by which characters primarily nealogic become so advanced in the development of the individual as to be impressed finally upon the embryonic shell. 
Acrothele dichotomin refer to Acrotreta dichotoma.

Acrotreta gulielmi refer to Discinopsis gulielmi.

Anoplin, Hall, 1892, Pal. N. Y., vol. 8, p. 309. Proposed as a subgencric name with Leptaena (?) nuclcolata, Hall, as the type. It is only necessary to say it has no place in nomenclature as a subge. neric name. If it is a name which deserves retention, it should be in a generic sense, and probably it should be plàced in that rank.

ATIYYRIs angelica var, occidentalis, Whitcaves, 1891, Cont, to Can. Pal., vol. 1, p. 227, Devunian.

ashlandensis, Herrick, 1888, Bull. Denison Univ. vol. 4, p. 24, Waverly Gr.

brittsi, and A. ottervillensis, S. A. Miller, 1892,18 th Rep. Geo. Sur. Ind., p. 60 , Ham. Gr.

ATRYPA calvini, anıl A. reticularis, var. niagarensis, Nettleroth, 1889, Kentucky lioss. Shells, pp. 8!, !2, Niagarn Gr.

deflecta, Hall, see Zygospira deflecta.

ellipsoidea, Nettleroth, 18: Kentucky Foss. Shells, p. y0, Up. Held Gr.

missouriensis, S. A. Miller, 1892, Advance Sheets 18tli Rep. Geo. Sur. Ind., p. 61, II:m Gir.

Aulosteges sponlyliformis, see Strophalosia spondyliformis.

BArroiselia, IJall, 1892, Pal. N. I., vol. 8, p. 62. [Ety. proper name.] lingulalike shell; pedicel-valve bears a higl cardinal area, appearing as a thickened triangular plate, divided by a bruad pedicel-groove, and having at each basal angle a boss or conityle which served as a muscular fulcra or as a point of articulation; the interior lias a subquadrate depressed ared in continuation of the pedicel-groove, and from its ante. lateral angles diverge two linear depressions, which extend about one-fuurth the length of the shell; from outside and bchind the extremities of these depressions begins a pair of long, curved furrows, composed of two shorter curves, the posterior rounding over the extremities of the linear depressions; the anterior and longer curves gradually approximate, and nearly meet at about one-third the length of the shell from the anterior margin; these furrows are accompanied by low ridges along their inner margin. A low median ridge, with elevated edges, begins at the posterior umbonal impression, and continues to the center of the valve, widening near its anterior extremity; behind its terminution there is a pair of indistinct muscular impressions. In the brachial valve the beak is scarcely prominent, and the muscular markings are essentially as in the opposite valve, but nore sharply developed; beneath the beak there is a faint umbonal scar. The long compound lateral curves have a greater degree of eurvature than in the pedicel-valve, and their posterior portion incloses a thickeneit area, which is continued into a low merlian septum that bifurcates in the middle of the valve, and lias extending from the angle an intercalated ridge. Type Lingula subspatulata, Meck of Wortlien.

Bulungsella, Hall, 1992, Pal. N. Y., vol. 8 , p. 230. [Ety. proper name.] shell Orthis-like, transverse; subquadrate or semicircular in ontline; contonr concavo or plano convex; surface striate; pedicel-valve the more convex; cardinal area molerately high, vertical or slightly incurved; deltluyrium covered by a convex plate, which may be minutely periorated at the apex; teeth well developed, but dental plates are continued along the lottom of the $1 \mathrm{~m}$ bonal cavity, inclosing a sinall subelliptical inuscular area near the apex. In the brachial valve the cardinal area is greatly inclinel, making min olutuse angle witl that of the opposite valve; delthvrium partly covered by u convex deltidium, which never attains the development seen in the opposite vulve, and is sometimes absent. Type Orthis pevine, Hall. It probably includes Streptorhynchus primordiale, Whitfield, Orthisina grandxva and Orthis laurentina, Billings.

Canarella bernensis, and $\mathrm{C}$. owatonnensis, Sardeson, 1S92, Bull. Minn. Acad. Nat. Sci., vol. 3, p. 328, 'Trenton Gr.

minor, Walcot!, 1890, 10th Ann. Kep. U. S. Geo. Sur. p. 614. Up. Taconic.

Chonetrs manitobensis, Whiteaves, 18!2, Cont. to Can. Pal., p. 281, Devonian.

subquadrata, Nettleroth, 1S8!), Kentucky Foss. Shells, p. 67, Up. Heli. Gr.

tumida, Herrick, 1888, Bull. Denison Univ., vol. 3, 1). 36, IVaverly Gr.

complanata, dawsoni, reversa, refer to Chonostrophia.

Cinonorectus, Hall, 1892, Pal. N. Y., vol. 8, p. 31\%. [Ety. chonos, a cuo; pelstos, combed.] Shell like Chonetes, the cardinal margin of the pedicle-valve bearing a row of erect spines; beak compressed, leaving a flattened area or cicatrix from attachment in early growth; surface ornamented with a double series of concentric liues or wrinkles, having the appearance of the engraving on a inachine-turned watchcase, and strongest on the umbonal and central part; these wrinkles are crossed by concentric growth-lines, and sometimes by finer radiating lines more or less flexuous. Casts of the pedicelvalve show the impression of a short median septum dividing two broad obcordate flabelliform muscular scars, from the outer margin of which there are radiating vascular scars; impressions of a narrow cardinal area and exceedingly small teeth also occur on 
the internal cast. Type Chonetes fischeri, Norwood \& Pratten.

Chonostrophia, Hall, 1892, Pal. N.Y., vol. 8, p. 310. [Ety. chonos, a cup; strophe, turning around.] Shell like a reversed Chonetes, concavo-ronvex, the pedicelvalve being slightly convex about the umbo, but becoming broadly concave over the pallial region; outline and contour like Leptrena; valves extremely tenuous and compressed; surface covered with fine, alternating or fasciculate stria. In the pedicel-valve the upper margin of the cardinal area bears a row of hollow spines of the same structure and arrangement as in Chonetes. The delthyrium is narrow, and appears to be more or less completely clnsed. The teeth are quite strong, and rest upon the bottom of the valve; between them arises a low median septum, extending one-third or one half the length of the valve, dividing a subcordate muscular area, the outer margins of which are distinctly elevated. In the brachial valve the crural plates are united to form a bilobed cardinal process. On the inner surface it ends abruptly; internal pallial region finely papillose; shellsubstance fibrous, punctate. Type Chonetes reversa, Whitfield. To the same genus is referred Chonetes complanata, $C$. dawsmi, and C. helderbergia, Hall, 1892, Pal. N. Y., vol. 8, p. 353, Low. Held. Gr.

Christinna, Hall, 1892, Pal. N. Y., vol. 8, p. 298. [Ety. proper name.] Shell resembling Leptæna, usually longitudinally elongated, sometimes semielliptical in outline; normally concavo-convex; surface smooth or covered with fine radiating lines, crossed by stronger concentric plications; cardinal area of the pedicel-valve high; delthyrium probably closed by a convex plate; teeth divergent, and from their bases extend the elevated margins of two linguiform muscular scars, traversing the shell almost the entire length; these diductor scars inclose two elongate adductors. In the brachial valve the cardinal process is bipartite, each of the lobes being grooved belind; the crural plates are long and divergent, terminatıng in elevated extremities or crura. The lower moiety of these plates is produced on each side of an elevated mus. cular ridge, curving slightly inward on the sides, then outward on approaching the anterior margin of the valve, each branch recurving and passing backward, parallel to the median axis, as far as the base of the cardinal process. The interspaces are divided transversely at about one-third their length from the hinge-line, by a lower ridge. The four areas thus inclosed represent the posterior and anterior scars of the adductor muscles. Type Leptæna subquadrata, Hall.

Clitambonites, Pander, 1830, Beitrage zur Geognosie des russ. Reiches, p. 70. [Ety. klitos, a sloping place; ambon, any rising; lithos, stone.] Shells with a subsemicircular marginal outline; convex or subpyramidal; hinge-line straight, and forming the greatest diameter of the shell; pedicel-valve elevated; cardinal area high, vertical, or sometimes incurved and crossed by a broad delthyrium, with a perforate deltidium; dental lamellæ strongly developed, converging and uniting in the median line; median septum about half the length of the valve; muscular impressions obscure; cardinal area developed in the brachial valve, delthyrium filled with a callosity; dental sockets large; crural plates low; thickened transverse area in the umkonal region; surface striate; shellsubstance impunctate. Type Pronites adscendens, Pander. It includes Orthisina vernuili, Eichwald, and Hemipronites americanus, Whitfield.

Conotreta, Walcott, 1890 , Advance Sheets Biological Soc. Washington. [Ety. konos, cone; tretos, perforated.] Structure calcareo corneous. Five narrow ridges radiate from the apex toward the front, on the internal side of the dorsal or conical valve, the central one of which joins the thickened apex, which is supposed to have been perforated by a siphonal tube. In Acrotreta there is an elongated muscular scar extending from each side of the siphonal tube obliquely forward. Type C. rusti, which is described at the same place from the Trenton Gr.

Crania agaricina, C. pulchella, Hall, 1892, Pal. N. Y., vol. 8, p. 180, Low. Held. Gr., and C. granosa and C. favincola at same place, Ham. Gr.

blairi, S. A. Miller, 1892, Advance Sheets 18th Rep. Geo. Sur. Ind., p. 56, Chouteau limestone, and C. greenii from Up. Held. Gr.

columbiana, Walcott. 1888, Proc. U. S. Nat. Mus. p. 441, Up. Taconic. Not defined so as to be recognized.

halli, Sardeson, 1892, Bull. Minn. Acad. Nat. Sci., vol. 3, p. 328, Trenton Gr.

radicans, see Strophaiosia radicans.

Craniella Ehlert, 1888, Bull. de la Soc. d'Etudes S'cientif. d'Angers, p. 37. [Ety. diminutive of Crania.] Shell somewhat irregular; outline subcircular or subquadrangular; ventral valve thin, adhering by its entire surface; dorsal valve conoidal, more or less elevated; apex subcentral, posterior; interior of the dorsal valve without a well-defined border; impressions of the adductors large, distinct, four in number, of which the posterior two are quite distant, the two subcentrals smaller, closely approximate or even confluent; from near each 
of the posterior impressions starts a broad vascular sinus, strongly sinuous near its point of departure, narrowing gradually in following the coutour of the valve, emitting from its marginal side diclsotomizing secondary branches. Type C. meduanensis, dihlert. It prolably includes Crunia hamillonix, Hall, and $\mathrm{C}$. greenii, S. A. Miller.

ulrichi, Hall, 1892, Pal. N. Y., vol. 8, p. 181, 'Trenton Gr.

Cry'ronel.t.l ovalis, S. A. Miller, 1891, Advance Sheets 17th Rep. Geo. Sur. Ind., p. 76, Ham. Gr.

traversensis, Winchell, 1866, (Terebratula traversensis,) Rep. Low. Peuin. Mich., p. 95, Ham. Gr.

Derbya, Waagen, 1884, Palreontologica Indica, Ser. 13, vol. 1, pp. 576,591 to 607. [lity. proper name.] This genus, according to Hall, is distinguished from Sitreptorliynchus by the presence of a median septum in the pedicel valve. He notes no other generic difference. Type $D$. regularis, Wagen. Hall refers to this genus Orthis keokuk, Hall, Orthis robusta, Hall, Hemipronites (Streptorhynchus) crassus, Meek \& Ilayden, and re. gards Hemipronites lasallensis and $\boldsymbol{H}$. richmondi, McChesney, as symonyms for H. crassus. I consider Orthis robusta, Hall, more closely related to IIemipronites crassus, Meek, than either II. lasallensis or H. richmondi, McChesney, and if one is to be retained as a species all three should be, and in no erent is either one of them congeneric with Orthis keokuk. Ilence, I see no propriety in the use of the worl Derbya as applied to American fossils, and as I have seen no typical specimens belonging to the genus, I express no opinion as to its value. Hall, however, describes Derbya broadheadi, D. bennetti, D. cymbula, D. affuis, and $D$. biloba, from the Up. Coal Mleas., Pal. N. Y., vol. S, pl). 347 to 350 , and on $\mathrm{p} .346 \mathrm{\nu}$. ruginosa, from the $\mathrm{Ke}$ okuk Gr., and $\nu$. costatula from the Kaskaskia Gr. All of these may be re. ferred to Streptorhynchus, and it is not too much to say that Meek would have classed all of them as synonyms for Streptorhymchus crassum.

Discina concordensis, Sardeson, 1892, Bull. Minn. Acad. Nat. Sci., vol. 3, p. 328, Trenton Gr.

keokuk, Gurley, 1884, New Carb. Foss., p. 6, Keokuk Gr.

sampsoni, S. A. Miller, 1891, Advance Sheets 17th Rep. Geo. Sur. Ind., p. 80, Chouteau limestone.

Discrnopsis, Matthew, 1992, Pal. N. Y., vol. 8, p. 105. [Ety. from resemblance to Discina.] Shell subcireular, depressed conical; apex excentric; apex of the pedicel-valve truncated, with a circular aperture; interior having a pair of diverging furrows from the beak, that converge toward the anterior margin, and inclose a thickened area, which, in the subumbonal region, is apparently free and projects like a shelf, from beneath which the foramen probably opened; shell substance teuuous, apparently corneous; surface marked concentrically, and also with radiating strice. Type Acrotreta gulielmi, Malthew.

Eatonia variabilis, Whitcaves, 18!1, Cont. to Can. Pal., vol. 1, p. 233, Devonian.

Hallina, Winchell \& Srohnchert, 18!2, Am. Geo., vol. 5, p. 291. [Fty. proper name.] Shells small, articulate, rostrate, biconvex, semiplicate; pedicel opening bounded laterally by incomplete tel. tidial plates; calcified brachial supports long and nuch like Walhheimia. Type $H$. saffordi, described at the same place with nicolletti, from 'Trenton Gr. Neither one defined so as to be recognimed.

Koninchina anericana, Swallow, may be stricken from the list as it is not a Koninekina," and is too poorly defined to be recognized.

KuToRgina labradorica var. swantonensis, Walcott, 1889, Proc. Nat. Mus., vol. 12, p. 36, Up. Taconic.

LEITANA charlotie, Winchell \& Schuchert, 1892, Am. Geo., vol. 9, p. 288, Trenton Gr., and Plectambonites gibbosus, from Galena Gr. Not defined so ats to be recognized.

minnesotensis, Sardeson, 1892, Bull. Minn. Acad. Nat. Sei, vol. 3, p. 3:9, Trenton Gr., and pracosis, recedens and saxea, Hud. Riv. Gr.

Leirenisca, Becher, 1890, Am. Jour. Sci. and $A$ rts. Ser. 3, vol. 40, p. 239. [Ety. diminutive of Leptrua.] Shell concavoconvex, attached to foreign objects by caleareous cementation of the ventral beak; valves articulated by teeth and sockets; dorsal valve concave; interior with broad spiral impression on each side of the median line, making a single volution; adduetor impressions small ; eardinal line narrow, bearing in the center two prominenl, bilobed, cardinal processes, separated to admit the verticul septum in the opposite beak; ventral valve convex, area elongate, triangular; fissure covered with a perlicel sheath; cardinal muscular sear supported on or limited by two elevated lanellx; cavity of beak divided by a vertical septum, on each sirle of which, in the anterior half, is a small, adductor scar; shell structure punctate. Type I. concava.

adnascens and $\mathrm{L}$. tangens, Hall, 1892, Pal. N. Y., vol. 8, p. 352, Low. Held Gr.

concava, Hall, 1857, (Leptæena concava,) 10th Rep. N. Y. St. Nat. Hist., p. 47, and Pal. N. Y., vol. 3, p. 197, Low. Held. Gr.

Leptella, Hall, 1892, Pal. N. Y., vol. 8, p. 293. [Ety. leptos, thin.] Shell small, concavo-convex, senicircular or semi- 
elliptical; hinge-line straight; pedicelvalve evenly convex; cardinal area moderately high, delthyrium nearly covered by a convex plate; teeth inconspicuous; brachial valve slightly concave; cardinal area high, delthyrium filled with the cardinal process which is divided behind by a median groove; on the cardinal margin this process is double but less conspicuous than the crural plates, which are arched and highly elevated above the hinge-line; they are short, terminate abruptly, and inclose deep sockets; visceral area flattened or concave; its anterior margin forming a double visceral area is divided by a broad median ridge, and its surface covered with five sharp radiating lines, which end at the line of deflection. Type Leptina sordida, Billings.

Lindstronella, Hall, 1892, Pal. N. Y., vol. 8, p. 134. [Ety. proper name.] Shells with outline contour and pedicel characters as in Orbiculoidea; brachial valve with a faint median septum and two strong approximating ridges, beginning behind a transverse line passing through the apex, and rapidly converging to meet the median septum; anterior adductor scars lying between these ridges and the median septum; a circular scar at the posterior extremity of each ridge. Type L. aspidium, p. 178, Ham. Gr.

Lingula atra, Herrick, 1888, Bull. Denison Univ., vol. 4, p. 16, Waverly Gr.

deflecta, Winchell \& Schuchert, 1892, Am. Geo., vol. 9, p. 284, Galena Gr. Not defined so as to be recognized.

exilis, see Lingulodiscina exilis.

gannensis, Herrick, 1888, Bull. Denison

Univ., vol. 4, p. 17, Waverly Gr.

gorbyi, S. A. Miller, 1892, Advance Sheets 18 th Rep. Geo. Sur. Ind., p. 55, Chouteau limestone.

macconnelli, Walcott, 1888 , Proc. U. S. Nat. Mus., p. 441, Up. Taconic. Not properly definerl.

meeki, Herrick, 1888, Bull. Denison Univ., vol. 4 , p. 18 , W averly Gr.

norwoodi, see Lingulops norwoodi.

parrishi, S. A. Miller, 1892, Advance Sheets 18th Rep. Geo. Sur. Ind., p. 53, Up. Coal Meas.

riciniformis var. galenensis, Winchell \& Schuchert, 1892, Am. Geo., vol. 9, p. 284, Galena Gr. Not defined so as to be recognized.

sedaliensis, S. A. Miller, 1892, Advance Sheets 18 th Rep. Geo. Sur. Ind., p. 54, Chouteau limestone.

subspatulata, refer to Barroisella subspatulata.

tighti, Herrick, 1887, Bull. Denison Univ., vol. 2 , p. 43 , Coal Meas.

triangulata, Nettleroth, 1889, Ky. Foss. Shells, p. 34, Up. Held. Gr.

waverlyensis, Herrick, 1888, Bull. Denison, Univ., vol. 4, p. 18, Waverly Gr.
Lingulerasma galenensis, Winchell \& Schuchert, 1892, Am. Geo., vol. 9, p. 285, Galena Gr. Not defined so as to be recognized. Ulrich, Schuchert and others persist in using the word Lingulasma, which has nu more mean. ing than Lasma or Linguma would have. I spelled the word Lingulelasma, as it should have been coined, supposing the misspelling was accidental; but, probably, Mr. Ulrich's spelling should be recognized, and if so, then the word should be stricken from science, as a meaningless compound, under rules on page 98.

Lingulodiscina, Whitfield, 1890, Bull. Am. Mus. Nat. Hist., p. 121. [Ety. Lingula and Discina.] Upper valve linguloid in character, having a terminal beak, the accretions of growth being along the lateral and basal margins; lower valve having its growth-lines nearly equal on all sides of the initial point and perforated on the cardinal side by a byssal slit as in Discina; shell structure as in Lingula and Discina. Type L. exilis. exilis, Hall, 1860, (Lingula exilis,) 13th Rep. N. Y. St. Mus. Nat. Hist., p. 77, and Pal. N. Y., vol. 4, p. 7, Ham. Gr.

Lingulors granti, Hall, 1892, Pal. N. Y., vol. 8 , p. 173 , Niagara Gr.

norwoodi, instead of Lingula norwoodi.

Minulus, Barrande, 1879, Systeme Silurien du Centre de la Boheme, voi. 5, p. 109. [Ety. mimulus, a mime, an imitator.] Spirifera (?) waldronensis, Miller \& Dyer, was referred to Triplesia by Hall, without a knowledge of the interior, and he now refers it to Mimulus, though the internal characters of that genus are unknown.

Monomerelta egani, M. greenii, M. kingi, M. urtoni, Hall, 1892, Pal. N. Y., vol. 8, pp. 174, 175, Niagara Gr.

Newberra, Whiteaves, 1891 , Cont. to Can. Pal., vol. 1, p. 236. [Ety. proper name.] Shells elongate-oroid, having the general contour and external aspect of Rensselæria and Amphigenia, but without the strongly radiate striate surface of the former genus. The convexity of the valves is greatest in the umbcnal region, and the surface is distinctly flattened over the lateral slopes, leaving the median portion of the valves very promineirt; the pedicel valve has the rostrum produced and incurved; the apex truncated by a circular foramen ; deltidial plates not determined; the teeth are comparatively small, projecting forward and gently upward, free at their extremities, and supported by narrow dental plates which join the interior of the valve above the middle of its depth, and are continued forward as slender ridges upon the inner surface, which gradually merge into the shell. In the bottom of the rostral and umbonal cavity there is a broad, scarcely defined, 
muscular area, from which radiate a series of vaseular rilges and depressions; the strongly marked pair of adductors are situated posteriorly, just within this muscular area; lving in front of these is a single (rarely divided) elongate adductor impression which often extends forward to the center of the shell; on each side of the inuscillar impressions is a thickened triangular area, very narrow at its origin in the umbonal region, widening anteriorly and produced into two divergent furrows (four in all), which extend over the pallial region, in some instances almost to the anterior margin. In the brachial valve there are two short, divergent, crural plates, which are not united at their bases to form a hinge plate, as in Rensselseria; a low median ridge arises between them, passing for a sliort distance along the bottom of the valve, separating the obovate, narrowly flabelliform muscular scars of the idductor muscles. These scars are characterized by the strong striation of their surfaces; surface sinooth, or with obscure radiating striae; distinguisher from Rensselacria, which has strong radiating strice on the surface, and preserves two broad, strong, dental plates on the interior of the pedicel-valve, which reach nearly to the bottom of the rostral and post umbonal cavity. leaving a narrow space for the inuscular area, quite un. like that of the corresponding valve of Newberria. It is from this narrow cav. ity, produced by the encroachment of these strong dental plates, that we have the narrow rostral casts of Rensselirria. The thickened strong hinge-plate, which supports the crura in the brachial valve of Rensselieria, does not exist in New. berria; the spoon-shaped process found in Amphigenia does not exist in Newberria. Type $\mathrm{N}$. johanni.

condoni, McChesney, 1867, (Rensselıeria condoni,) Trans. Chi. Acad. Sci., vol. 1, p. 36, Oriskany Gr.

claypolei, Hall, 1891, Advance Sheets 10th Ann. Rep. N. Y. St. Geo., Ham. Gr.

johanni, Hнl], 1867, (Rensselreria johanni,) Pal. N. Y., vol. 4, p. 385, Up. Held. Gr. lievis, Meek, 1868, (Rensselæria lavis,) Tıans. Chi. Acad. Sci., p. 108, Devonian. missouriensis, Swallow, 1891, Advance Sheets 10th Ann. Rep. N. Y. St. Geo., Ham. Gr.

Nucleosprra indianensis, S. A. Miller, 1891, Advance Sheets 17th Rep. Geo. Sur. Ind., p. 79, Ham. Gr.

Orolella misera and $O$. pretiosa, refer to the genus Linnarssonia.

Onhlertella, Hall, 1892, Pal. N. Y., vol. 8, p. 132. A proposed subgeneric name to include Discina pleurites.

Orbiculoidea herzeri, Cuyahoga Shales, O. numulus, Low. Held. Gr., and
O. ovalis, Trenton Gr., Hall, 1892, Pal. N. Y., vol. 8, pp. 177, 178.

Ortus arcuaria, Hud. Riv. Gr. ; O. holstoni, O. Ioricula, O. saffordi, T'renton Gr.; O. oweni, Keokuk Gr.; O. senecta, Clinton Gr., ard O. superstes, Chemung Gr., Hall, 1892, Pal. N. Y., vol. 8, pp. 340 to 342 .

benedicti, S. A. Miller, Advance Siljeets 17th Rep. Geo. Sur. Ind., p. $78, \mathrm{Ni}$ agara Gr.

germana, Winchell \& Schuchert, 189:2, Am. Geo., vol. 9, p. 290, Galena (ir.; meedsi. 'I'renton Gr.; proavita, Hud. Riv. Gr. Not definell so as to be recognized.

goodwini, Nettleroth, 1859, Kentucky Foss. Shells, p. 39, Up. Held. or Ham. Gr.

inxqualis. see Streptorlyychus inixquale. linneyi, Nettleroth, 1859, Kentucky Foss. Shells, p. 41, Hud. Riv. Gr.

manitobensis, Whiteaves, 18:12, Cont. to Can. Pal., p. 283, Devonian.

missouriensis, Swallow, is the sane described afterward as $\mathrm{O}$. theimii.

corpulenta, macrior, rogata, tersus, minnesotenkis, petre. Sardeson, 18!2, Bull. Minn. Acad. Nat. Sci., vol. 3. pp. 330 , 332, Trenton \& Hud. Riv. Gr. The tirst four seem to be synonyms for 0 . testudinaria, but possibly they are not.

Orlhisina alberta, Walcott. 1888, Proc. U. S. Nat. Mus., p. 442, Up. Taconic. Not properly definerl.

Paterina is a generic name proposed for such shells as Kutorgina labradorica, without any distinct generic characters being pointed out. See A m. Jour. Sici. and Arta, Ser. 3, vol. 41, p. 34:3.

Pentanereli, thusnelda, Nettleroth, 1889, Kentucky Foss. Shells, p. 51, Tp. Held. Gr.

Penta Mknus colletti, S. A. Miller, 1891, Ar. vance Sheets 17 th Rep. (ieo. Sur. Ind., p. 77, Waterlime Gr.

complanatus, Nettleroth, 1889, Kentucky Foss. Shells, p. 53, Niagara (ir.

decussatus, Whiteaves, 1891, Can. Record Sci., p. 295, Up. Sil.

globulosus, Nettleroth, 1889, Kentucky Foss. Shells, p. 54, Niagara Gr.

knotti, Nettleroth, 1889, Kentucky Foss. Shells, p. 56, Niagara Gr.

uniplicatus, Nettleroth, 1889, Kentucky Foss. Shells, p. 63, Niagara Gr.

Pholidops calceola, P. patina, Hall, 1892, Pal. N. Y., vol. 8, p. 182, Comiferous limestone.

Polytoecisa. Hall, 1892, Pal. N. Y., vol. 8, p. 239 [Ety. polus, many; toichos, the wall of a house.] Shell small, subtrihedral in contour; hinge-line straight; pedicel-valve with a high, nearly vertical cardinal area, obliquely striated; delthyrium covered with a convex plate; dental lamella widely separated, descend for a distance vertically, and then bend inward to the median line, 
forming with the deltidium a subrostral vault; the inner spoon-shaped plate, spondylium, is supported by a stout median septum and two smaller lateral septa, which meet it at the lines of angulation; the umbonal cavity is divided in to five chambers; brachial valve shallow, depressed-convéx; " cardinal area narrow ; delthyrium broad; dental sockets widely separated; crural plates narrow and nearly parallel to the hingeline; cardinal process simple, linear, prominent, and at its union with the crural plates there is a subtriangular thickening supported by a median septum; surface striated; no fold or sinus. Type P. apicalis, Whitfield, described at the same place from the Calciferous Gr.

Productella minneapolis, Sardeson, 1892, Bull. Minn. Acad. Nat. Sci., vol. 3, p. 332, Trenton Gr. It does not belong to this genus.

pyxidata is from the Kinderhook Gr.

semiglobosa, Nettleroth, 1889. Kentucky Foss. Shells, p. 70, Up. Held. Gr.

Productus blairi, S. A. Miller, 1891, Advance Sheets 17th Rep. Geo. Sur. Ind., p. 79, Chouteau limestone.

nodocostatus, $P$. raricostatus and $P$. rushvillensis, Herrick, 1888, Bull. Denison Univ., vol. 4, p. 19 to 23 , Waverly Gr. pileiformis, McChesney, 1859, New Pal. Foss., p. 40, Kaskaskia Gr.

Pseudocrania anomala, Hall says, is a misnomer, that the shell described is a streptorhynchoid, whatever that means.

Rensseleria condoni, $R$. johanni and $R$. lavis, refer to Newberria.

RetziA ashlandensis, Herrick, 1888, (Rhynchospira ashlandensis,) Buil. Denison Univ., vol. 3, p. 25, Waverly Gr. circularis, $R$. plicata and R. triangularis, S. A. Milter. 1892, Advance Sheets 18th Rep. Geo. Sur. Ind., p. 61, Chouteau limestone.

sobrina, Beecher \& Clark, 1899, Mem. N. Y. St. Mus., p. 61, Niagara Gr.

Rнinobolus, (mispelled Khynobolus,) Hall has shown, in Pal. N. Y., vol. 8, p. 44, why this genus is distinct from Trimerella, and that it should be restored with $R$. galtensis as the type, and he defined R. davidsoni from the Niagara Gr.

Ruy nchonelca alleghania, Williams 1887 , Bull. No. 41, U. S. Geo. Sur., p. 87, Waverly $\mathrm{Gl}$.

belliformis, R. louisvillensis, R. rugicosta, $R$. saffordi var. depressa, $R$. tenuistriata, Nettlerotl, 1889, Kentucky Foss. Shells, pp. 73 to 82 ; louisvillensis and tenuistriata from the Up. Held. Gr., the others from the Niagara $\mathrm{Gr}$.

colletti, S. A. Miller, $189_{2}^{2}$, Advance Sheets 18th Rep. Geo. Sur. Ind., p. 57, Niagara Gr., and R. kokomoensis from the Waterlime Gr.

levis, R. medialis, R. striata, Simpson, 1889, Trans. Am. Phil. Soc., pp. 443,
444; levis from the Clinton Gr., the others from the Waverly Gr.

minnesotensis and sancta, Sardeson, 1892, Bull. Minn. Acad. Nat. Sci., vol. 3, p. 333, Trenton Gr.

Rhynchospira ashlandensis, see Retzia ashlandensis.

Roemerella, Hall, 1892, Pal. N. Y., vol. 8, p. 137. Proposed as a subgenus to include Discina grandis.

Skrnidium, (should be spelled Scenidium,) anthonense, Sardeson, 1892, Bull. Minn. Acad. Nat. Sci., vol. 3, p. 333, Trenton (ir. Schizocrania, helderbergia, Low. Held. Gr. S. schucherti, Hud. Riv. Gr., Hall, 1892, Pal. N. Y. vol. 8, p. 179.

Sipjonotreta minnesotensis. Hall, 189\%, Pal. N. Y., vol. 8, p. 177, Trenton Gr.

Spirifera byrnesi, S. davisi, S. dubia, S. foggi, S. hobbsi, S. knappana, S. macconathi, Nettleroth, 1889, Kentucky Foss. Shells, pp. 109 to 122 ; foggi from the Niagara Gr., the others from the Up. Held. Gr. capax is from the Kinderhook Gr., and is not a synonym for S. parryana.

carteri was so poorly defined that it could not be recognized, and it is now claimed by some that it is the same as Syringothyris typus. The name should be dropped from the list.

deltoideus, s. tenuispinatus, Herrick, 1888, Bull. Denison Univ., vol. 4, p. 27, Waverly Gr.

disjuncta var. occidentalis, Whiteaves, 1891, Cont. to Can. Pal., vol. 1, p. 222, Devonian.

subventricosa, McChesney, 1860, New Pal. Foss., p. 44, 1865, pl. 1, fig. 4, Coal Meas.

texta, see Syringothyris texta.

winchelli, Herrick, 1888, Bull. Denison Univ., vol. 3, p. 46, Waverly Gr.

Siririfirina depressa, Herrick, 1888, Bull. Denison Univ., vol. 3, p. 47, Waverly Gr.

StrRPTORIYNCHus inæquale, Hall, 1858 , (Orthis inæqualis,) Geo. Iowa, p. 490, Kinderhook Gr.

subsulcatum, Sardeson, 1892, Bull. Minn. Acad. Nat.' Sci., vol. 3, p. 335, Trenton Gr. ; desideratum, (Orthothetes desideratus), Hall, 1892, Pal. N.Y., vol. 8, p. 345, Waverly Gr., and S. ulrichi, p. 35l, Kaskaskia Gr.

Stricklandinia jouisvillensis, Nettleroth, 1889, Kentucky Foss. Shells, p. 65, Niagara Gr.

subquadrata, Herrick. Not properly defined.

Stringocerilalus burtoni, Defrance, 1827 , Dict. des Sci. Naturelles, vol. Jl, p. 102, and 1891, Cont. to Can. Pal., vol. 1, p. 235, Devonian.

Strophalosia keokuk, Beecher, 1890, Am. Jour. Sci. and Arts, Ser. 3, vol. 40 , p. 244, Krokuk Gr.

radicans, Winchell, 1866 , (Crania radicans,) Rep. Low. Penin. Mich., p. 9z, Ham. Gr. 
rockfordensis, Hall, 1892, Pal. N. Y., vol. 8, p. 353, Up. Devonian.

scintilla, Beecher, 1890, Am. Jour. Sci. and

Arts, Ser. 3, vol. 40, p. 243, Chouteau

limestone.

spondyliformis, White \& St. John, 1868,

(A ulosteges spondyliformis), Trans. Chi.

Acad. Sci., p. 118, Coal Meas.

Stropireodonta, instead of Strophodonta.

[Ety. strophers, the socket in which the door moves; odous, tooth.]

Stropilomexa acanthoptera, Whiteaves, 1891, Can. Record Sci., p. 294, Up. Sil.

conradi, S. winchelli, Hall, 1892, Pal. N.Y., vol. 8 , p. 344 , Trenton Gr.

cmaciata, scofirldi, septute, Winchell \&

Schnchert, 1892, Am. Geo.,vol. 9, pp. 285,

2si, Trenton Gr., and planodorsata, Iud.

Riv. Gr. Not properly defined.

halli, inquassa, Sardeson, 1892, Bull.

Minn. Acad. Nat. Sci., vol. 3, p. 334,

Trenton Gr.

Srringotiyris randalli, Simpson, 1889 ,

Trans. Am. Phil. Soc., p. 441 , Chemung Gr.

texta, Hall, 1857, (Spirifer textus), 10th

Rep. N. Y. St. Mus. Nat. Hist., p. 169,

Waverly Gr.

Tememratula gorbyi, S. A. Miller, 1891, Ad- vance Sheets 17 th Rep. Geo. Sur. Ind., p. 77, Keokuk Gr.

inconstans, Herrick, 1888, Bull. Denison Univ., vol. 4, p. 24, Waverly Gr.

inornata is from the Coal Meas., and described on p. 48.

occidentalis, S. A. Miller, 1S92, 18th Rep. Geo. Sur. Ind., p. 59, Chouteau limestone.

traversensis, see Cryptonella traversensis. Trematosprua helena, Nettleroth, 1889, Kentucky Foss. Shells, p. 137, Niagara $\mathrm{Gr}$.

Tnurlesia, or Triplasia as it should be spelled, can not be clianged into Triplecia as suggested by IIall in 1892, Pal. N. Y., vol. 8, p. 269. In that event Dicraniscus would have priority. But Triplasius anong the insects does not interfere with Triplasia among the fossil brachiopods.

"Zrgospira aquila, Sardeson, 1892, Bull.

Minn. Acad. Nat. Sci., vol. 3, p. 335, Trenton Gr.

deflecta, Hall, 1847, (Atrypa deflucta), Pal. N. Y., vol. 1, p. 140, Trenton Gr.

kentuckiensis, Nettleroth, 1889, Kentucky Foss. Sholls, p. 137, Hind. Riv. Gr.

uphami, Winchell \& Schuchert, 1892, Am. Geo., vol. 9, p. 291, Galena Gr.

\section{SUBKINGDOM MOLLUSCA.}

\section{CLASS PTEROPODA.}

Coleonomes, Walcott, 1859, Proc. U. S. Nat. Mus., vol. 12, p 37. [Ety. Coleolus, a genus; oides, like.]. Shell slender, elongate, cylindrical, straight or slightly curved, apparently thin ; surface marked by very tine, slightly oblique, longitudinal strix. Type C. typicalis, described at the same place from the Up. Taconic. A poor definition without illustration.

Covularia, above the septum, is often broken off, and it has been suggested that it may have fallen off during the life of the animal; hence the shell is sometimes called deciduous, from decido, I fall off.

gracilis, Herrick, 1888, Bull. Denison Univ., vol. 4 , p. 48 , pl. 8 , fig. 2 ; vol. 3 , pl. 6, fig. 13, Waverly Gr. The name was preocupied by Hall in 1847. As the species appears to be distinct from all others, I now propose the specific name herricki. The species will then be called C. herricki, after Prof. C. L. Herrick.

intertexta, S. A. Miller, 1892, Advance Sheets 18th Rep. Geo. Sur. Ind., p. 63, Keukuk Gr.

salinensis, Whiteaves, 1891, Cont. to Can. Pal., vol. 1, p. 244, Devonian.

sampsoni, S. A. Miller, 1891, Advance Sheets 17th Rep. Geo. Sur. Ind., p. 80, Chouteau limestone.

Hyolithes lanceolatus, S. A. Miller, 1892, Advance Sheets 18th Rep. Geo. Sur. Ind., p. 63, Chouteau limestone.

similis, Walcott, 1889, Proc. U. \&. Nat. Mus., vol. 12, p. 38, Up. Taconic. Not illustrated.

terranovicus, Walcott, 1889, Proc. U. S. Nat. Mus. vol. 12, p. 37, Up. Taconic. Not illustrated. 


\section{CLASS GASTROPODA.}

THE better authors use Gastropoda, and I prefer it to Gasteropoda. The masticatory apparatus or chitinous band, bearing minute teeth and forming a rasp-like. ribbon, is called the odontophore, (odous, tooth ; phero, I carry.) When the aperture is round or, entire, the shell is called holostomatous, (holos, whole; stoma, mouth ;) but when the aperture possesses a notch, more or less prolonged, for the respiratory siphon, or forming a trough-like extension for the skirt of the mantle, as in Fusispira, the shell is called siphonostomatous, (siphon, a tube; stoma, mouth.) The margin of the aperture is sometimes called the peristome, (peri, around; stoma, mouth), or peritreme, (peri, around; trema, an opening.) The notch separating the outer lip from the first whorl is called the posterior canal, and the notch separating it from the end of the columella is the anterior canal. When the columella is solid, it is called imperforate; and when hollow, perforated. The shells are sometimes composed of aragonite; at other times there is an inner layer of aragonite and an outer layer of calcite; and, especially in the younger shells, there is a horny epidermis which may disappear with age. It is said that aragonite is much more readily displaced in fossilization than calcite, which may account for the casts of Murchisonia associated with the shells of Cyclonema, in the same Lower Silurian strata, and other like phenomena in other genera and species of shells.

The new genus Helenia is supposed to belong to the Dentalidr; Fusispira should be referred to the Subulitidæ; Metoptoma and Palracmæa to the Patellidæ.

Acusina bellilineata, S. A. Miller, 1891, 17 th Rep. Geo. Sur. Ind., p. 85, Chouteau limestone.

swallovana, on p. 395 , is magnified three and a half diameters.

Bellerophon gorbyi, S. A. Miller, 1891, 17th Rep. Geo. Sur. Ind., p. 84, Hud. Riv. Gr. incomptus, B. nodocostatus, B. ourayensis, B. rugopleurus, B. tenuilineatus, Gurley, 1884, New. Carb. Foss. Bull. No. 2 , pp. 8 to 11, Coal Meas.

subcordiformis, Herrick, 1887, Bull. Denison Univ., vol. 2 , p. 18 , Subcarboniferous.

Callonema clarki, Nettleroth, 1889, Ken. tucky Foss. Shells, p. 175, Up. Held. Gr.

Carinaropsis deleta and phalera, Sardeson, 1892, Bull. Minn: Acad. Nat. Sci., vol. 3, p. 335, Trenton Gr.

Concilopeltis obtusa, Sardeson, 1892, Bull. Minn. Acad. Nat. Sci., vol. 3, p. 336, Trenton Gr.

Dentalium granvillense, Herrick, 1888, Bull. Denison Univ., vol. 3, p. 92, Waverly Gr.

Eсстціомтнацus perkinsi, Whitfield, 1890, Bull. Am. Mus. Nat. Hist., vol. 3, p. 30, Calciferous Gr.

triangulus, Whitfield, 1890, Bull. Am. Mus. Nat. Hist., vol. 3, p. 29 , Calciferous Gr.
Euomphalus comes, Hall, syn. for Eccyliomphalus laxus.

flexistriatus and E. maskusi, Whiteaves, 1891, Cont. to Can. Pal., vol. 1, pp. 242, 243, Devonian.

manitobensis, Whiteaves, 1890, Trans. Roy. Soc. Can., vol. 8, p. 100, Devonian. sampsoni, Nettleroth, 1889, Kentucky Foss. Shells, p. 182, Up. Held. Gr.

Flemingia, DeKoninck, 1881, Annales du Musee Royal D'Histoire Naturelle de Belgique, tome 6, p. 93 . [Ely. proper name.] Shell conical, spire acute, spiral whorls ntarly flat externally; circumference more or less angular; aperture often depressed, and angular at the outer margin; peristome not continuous, external border oblique, slender, and sharp; columella slender, slightly twisted over, and giving place to the formation of an umbilical fossette more or less large and imperforate; shell thin; surface smooth or covered with irregular strix, obliquely crossed with more distant lines. Type F. prisca. This genus is nearly related to Eotro. chus.

carbonaria, Meek \& Worthen,1866, (Trochita (?) carbonaria), Proc. Acad. Nat. Sci., p. 270, Kaskaskia Gr. This species is figured and described by DeKoninck as 
a Flemingia, and yet it much resembles an Eotrochus.

(?) stultus, Herrick, 1888, Bull. Denison T'niv., vol. 4, p. 45, Waverly Gr. This species may belong to Eotrochus.

Fusisirina (?) spicula, Sardeson, 1892, Bull. Minn. Acad. Nat. Sci., vol. 3, p. 336, Trenton Gr.

Helenia, Walcott, 1889. Proc. U. S. Nat. Mus., vol. 12, p. 39. [Ety. proper name.] Shell an elongate, narrow, flattened, curved tube ; transverse section and aperture elliptical; surface marked by transverse, concentric, inbricating lines of growth. Type II. bella, which is described at the same place from the lip. Taconic, without illustration.

Helicotoma similis, Whitfield, 1590, Bull. A m. Mus. Nat. Hist., vol. 3. p. 31, Culciferous Gr.

Holopea cassina is from the Calciferous Gr.

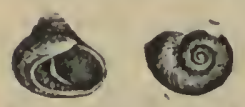

FIG. 125\%,-Holopen hubbardl. 1809 Bull, Minn. 3, p. 33ti, Trenton Gr.

Llilerolsis parrishi, (iurley, 1884, New Carb. Foss. p. 7, Up. Coal Meas.

Maclurea manitobensis, Whiteaves, 1889 , Trans. Roy. Soc. Can., p. 75, Trenton Gr.

Macrochilu; carinatum, see Macrochilina carinata.

Macrocinlisa blairi, S. A. Miller, 1891, 17th Rep. Geo. Sur. Ind., p. 84, Chouteau limestone.

carinalum, Nettleroth. The name was preoccupied. See II. Nettlerothana.

vettlerothanu, n. sp. Proposed instead of M. carinatim of Nettleroth, in Kentucky Foss. Shells, p. 180, Up. Held. Gr., which name was preoceupied.

Meтортол.. "Type M. pilpus.

explanata, Sardeson, 1892, Bull. MinnAcad. Nat. Sci., vol. 3, p. 336, Tren. ton $\mathrm{Gr}$.

Murcusoxia archiacana and M.dowlingi, Whiteaves, 1S92, Cont. to Can: Pal., p. 315 , Devonian.

hammelli, S. A. Miller, 1892, Advance Sheets 18th Rep. Geo. Sur. Ind., ?. 65, Hud. Riv. Gr.

obelisca is from the Calciferous $\mathrm{Gr}$.

Naticopsis remex is from the Coal Meas.

PALEACMAa cingulata, Whiteaves, 1892, Cont. to Cal1. Pal., p. 311, Devonian.

Platyceras boonvillense, S. A. Miller, Advance Sheets 17 th Rep. Geo. Sur. Ind. p. 82, Keokuk Gr.

compressum, Nettleroth, 18s9, Kentucky, Foss. Shelis, p. 162, Up. Held. Gr. cyrtalites is from the Burlington Gromp. milleri, Nettleroth, 1889, Kentucky Foss. Shells, p. 165, Up. Held. Gr.

missouriense, S. A. Miller, 1891, Advance Sheets 17th Rep. Geo. Sur. Ind., p. 82, Burlington Gr.

nasutum, S. A. Miller, 1891, Advance Sheets 17 th Rep. Geo. Sur. Ind., p. 82 , Chouteau limestone.

pettisense, S. A. Miller, 1891, Advance Sheets 17 th Rep. Geo. Sur. Ind., p. 81, Buslington Gr.

romingeri, Walcott, 1888, Proc. U. S. Nat. Muv., p. 442, Up. Taconic. ' Not illustrated.

Platystoma broadheadi, S. A. Miller, 1S91, Advance Sheets 17 th Rep. Geo. Sur. Ind., p. 8t, Chouteau limestone.

Pluevro'omaria arabella, Nettlernth. The nanie was preoceupied. See I'. nettlerotbuna.

clivosa, Sardeson, 1892, Bull. Minn. Acad. Nat. Sci., vol. 3, p. 337, Trenton Gr.

difficilis, Whitfeld, 1890, I3ull. Am. Mus. Nat. Hist., vol. 3, p. 33, Calciferous Gr.

goniostouna, Whiteaves, 1890, 'Trans. Roy. Soc Can., vol. 8, p. 99, Devonian.

harii, S. A. Miller, 1891, Advance Sheets 17th Rep. Geo. Sur. Ind., p. 83, Up. Coal Meas.

infranodosa, Whiteaves, 1892, Cont. to Can. Pal., p. 313, Devonian.

nettlerothana, n. sp. Proposed instead of P. arabellu, of Nettleroth, in Kentucky Foss. Shells, p. 171, which name was preoccupied, Up. Held. Gr.

proctori, Nettleroth, 1889), Kentutky Foss. Shells, 1. 173, Up. Held. Gr.

sedaliensis, S. A. Miller, 1891, 17th Rep. Geo. Sur. Ind., p. 83, Chouteau limestone.

strigillata, Herrick, 1888, Bull. Denison Univ., vol. 3 , p. 86, Waverly Gr.

Porcella manitobensis, Whiteaves, 1892, Cont. to Can. Pal., p. 318, Devonian.

Raphistoma tyrrelli, Whiteavee, 1892, Cont. to Can. Pal., p. 314, Devonian.

Stra parolu,us blairi, S. A. Miller, 1891, ddvance Sheets 17 th Rep. Geo. Sur. Ind., p. 86 , Chouteau limestone.

Subulites was described by Emmons, 1842, Geo. Sur. 2d Dist. N. Y., p. 392.

benedicti, S. A. Miller, 1891, 17 th Rep. Geo. Sur. Ind., p. 84, Niagara Gr.

Tryblidium conicum is from the Chazy Gr. exsertum, validum, Sardeson, 1892, Bull. Minn. Acad. Nat. Sci., vol. 3, p. 337, Trenton Gr.

indianense, S. A. Miller, Advance Sheets

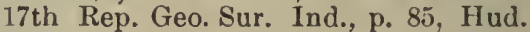
Riv. Gr.

madisonense, S. A. Miller, 1892, Advance Sheets 18th Rep. Geo. Sur. Ind., p. 64, Hud. Riv. Gr. 


\section{CLASS CEPHALOPODA.}

THE living animals of this Class are carnivorous, but it is too strong a presumption to conclude that all fossil Cephalopoda were carnivorous. The evidence does not warrant the presumption. The mouth of the shell or aperture may be round, elliptical, lunate, T-shaped, or of almost any other form, and the shell may gradually expand to the aperture, be contracted behind the aperture, or more or less contracted to the aperture. The ventral side may be indicated by the shape of the aperture, either by an emargination or a sinus, but the position of the siphuncle does not always indicate it; for in Orthoceras the siphuncle may be central, or in the growth of the shell it may cross the central line. The external shell is generally destroyed and for this reason it has been supposed to have been composed of aragonite rather than calcite; but the fact that the shell often appears as if run together in fossilization does not indicate such a distinction.

Actixoceras hindii, Whiteaves, 1890, Trans. Roy. Soc. Can., vol. 8, p. 101, Devonian.

Ascoceras indianense, Newell, 1888, Proc. Bost. Soc. Nat. Hist., vol. 23, p. 481, Niagara $\mathrm{Gr}$.

A psidoceras, Hyatt, 1883, Proc. Bost. Soc. Nat. Hist., vol. 22, p. 289. [Ety. apsis, the felloe of a wheel; keras, horn.] Loosely coiled, smooth, costated, or tuberculated gyroceran shells, with flattened abdomens. The whorls in section are triangular, the dorsum forming the internal apex of the outline; siphons near the venter and nummuloidal. The sutures have broad ventral lobes, saddles at the lateral angles, broad lobes on the sicles, and dorsal saddles; there is frequently a line of heavy tubercles on tach of the lateral angles of the whorls; they are all large shells, and the abdomen is frequently hollow or fluted along the center. Type $\mathrm{A}$. magnificum.

insigne, Whiteaves, 1889, Trans. Roy. Soc. Can., p. S2, Trenton Gr.

magnificum, Billings, 1857, (Gyroceras magnificum,) Rep. of Progr. Geo. Sur. Cau., p. 307, Hud. Riv. Gr.

Asymptoceras newtoni, see Solenochilus newloni.

Cyrtoceras boycii is from the Chazy Gr. dardanum and fosteri are illustrated on pl. 16 in 20th Rep. N. Y. St. Mus. Nat. Hist.

hertzeri, refer to Hexamoceras hertzeri.

howardi, Niagara Gr., and C. thompsoni, Hud. Riv. Gr., S. A. Miller, Advance Sheets 18th Rep. Geo. Sur. Ind., p. 69 .

indianense, S. A. Miller, 1891, Advance
Sheets 17th Rep. Geo. Sur. Ind., p. 88, Niagara Gr.

manitobense, Whiteaves, 1889, Trans. Roy. Sòc. Can., p. 80, Trenton Gr.

nash villense, S. A. Miller, 1891, Advance Sheets 17th Rep. Geo. Sur. Ind., p. 87, Niagara Gr.

occidentale, Whiteaves, 1890, Trans. Roy. Śoc. Can., vol. 8, p. 103, Devonian.

saffordi; S. A. Miller, 1891, Advance Sheets 17 th Rep. Geo. Sur. Ind., p. 88, Hud. Riv. Gr.

Discites was preoccupied in 1768 by Walch, and again used by Schlotheim in 1820 , before DeHaan used it in 1825 or $\mathrm{McCoy}$ in 1844 . Hyatt in 1883 , Proc. Bost. Soc. Nat. Hist., vol. 22, p. 292, proposed Discitoceras for the shells included in McCoy's genus. All the species therefore under the name Discites in this work should be referred to Discitoceras.

Domatoceras, Hyatt, 1891, 2d Ann. Rep. Geo. Sur. Texas, p. 342. The generic characters can not be determined from the definition. D. umbilicatum is defined at the same place from the Coal Meas. as the type.

Endoceras crassisiphonatum, Whiteaves, 1891, Trans. Roy. Soc. Can., vol. 9, p. 79, Trenton Gr.

Endolobus, gibbosus, Hyatt, 1891, $2 \mathrm{~d}$ Ann. Rep. Geo. Sur. Texas, p. 353, Coal Meas.

Eplimploceras, Hyatt, 1883, Proc. Bost. Soc. Nat. Hist., vol. 22, p. 290. [Ety. ephippion, a saddle; keras, horn.] Generic definition very obscure. Type $\mathbf{E}$. ferratum, described by Cox as Nautilus ferratus; and to the same genus Nautilus divisus should be referred if the 
description is such as to establish it, for the species do not properly belong to Nautilus.

Gastrioceras compressum, see Goniatites compressus.

Gomprocrras angustum, Newell, 1888, Proc. Bost. Soc. Nat. Hist., vol. 23, p. 475 , Niagara $\mathrm{Gr}$.

clarki, S. A. Miller, 1891, Advance Sheets $17 t l$, Rep. Geo. Sur. Ind., p. S8, St. Louis Gr.

hertzeri, see Hexamoceras herfzeri.

lineare, Newell, 1888, Proc. Bost. Soc. Nat. Hist., vol. 23, p. 473, Niagara Gr.

manitobense, Whiteaves, 1890, Trans. Roy. Soc. Can., vol. 8, p. 102, Devolian.

parvulum, Whiteaves, 1891, Can. Rec. of Sci., p. 298, Up. Sil.

projectum, Newell, 1888, Proc. Bost. Soc. Nat. Hist., vol. 23, p. 476, Niagara Gr. wabashense, Newell, 18s8, Prnc. Bost. Suc. Nat. Hist., vol. '23, p. 470. Niagara Gr.

Goniatites baylorensis, White, 1891, Bull. U. S. Geo. Sur. No. 77, p. 19, Permian Gr.

brownensis, 1891, S. A. Miller, Advance Sheets 17 th Rep. Geo. Sur. Ind., p. 90, WVaverly Gr.

compressus, Hyatt, 1891, (Gastrioceras compressum,) 2d Ar.n. Rep. Geo. Sur. Texas, p. 355, Coal Meas.

gorbyi. S. A. Miller, 1891, Advance Sheets 17 th Rep. Geo. Sur. Ind., p. 90, Chouteau limestone.

greenii, S. A. Miller, 1892, Advance Sheets 18 th Rep. Geo. Sur. Ind., p. 76, Knobstone Gr.

indianensip, S. A. Miller, 1891, Advance Sheets 17th Rep. Geo. Sur. Ind., p. 90, Waverly $\mathrm{Gr}$.

leviculus, Miller \& Faber, 1892, Jour. Cin. Soc. Nat. Hist., vol. 14, p. 167, St. Louis Gr.

limatus, Miller \& Faber, 1892, Jour. Cin. Soc. Nat. Hist., vol. 1 1 , p. 166, St. Louis Gr.

missouriensis, Miller \& Faber, 1892, Jour. Cin. Snc. Nat. Hist., vol. 14, p. 164, Up. Coal Mras.

occidentalị, Miller \& Faber, 1892, Jour. Cin. Soc. Nat. Hist., vol. 14, p. 166, Coal Meas.

sciotoensis, Miller \& Faber, 1892, Jnur. Cin. Soc. Nat. Hist., vol. 14, p. 165 , Waverly $\mathrm{Gr}$.

Gonjockras lambi, Whiteaves, 1891, Trans. Roy. Soc. Can., vol. 9, p. 86, Trenton Gr.

Gyroceras canadense, G. filicinctum, G. submammillatum, Whiteaves, 1890, Trans. Roy. Soc. Can., vol. 8, pp. 106, 107, Devonian.

magnificum, refer to Apsidoceras magnificum.

Hexamoceras, Hyatt, 1883, Proc. Bost. Soc. Nat. Hist., vol. 22, p. 278. [Ety. hex, six; meros, part; keras, horn.] This genus is distinguished from Gomphoceras only by the aperture. In Gomphoceras there is only one lateral branch on eacli side of a longer median aperture, which gives the mouth a T-shape; in Hexamoceras there are three branches on each side of the median aperture, increasing in length toward the summit. Type H. panderi.

cacabiforme, Newell, 1888. Proc. Bost. Snc. Nut. Hist., vol. 23, p. 481, Niagara Gr. delphicolum, Newell, 1888, Proc. Bost. Soc. Nat. Hist., vol. 23, p. 47!, Niagara Gr.

hertzeri, Hall \& Whitfield, 1875, (Cyrtnceras hertzeri,) Ohio Pal., vol. 2, p. 150, Niagara Gr.

Howaloceras, Whiteaves, 1890, Trans. Roy. Snc. Can., vol. 8, p. 104. [Ety. howialos, level; kiras, horn.] Shell consisting of a slender tube, which is broadly and strongly arcuate, curved in the same plane, and much flattened laterally, its venter or onter border being very narrow, truncated, and depressed in the center; sutural line consisting of two very narrow saddles, with an equally narrow sinus between them on the venter, a broadly concave sinus or lobe on each of the sides, and a rather narrow saddle on the dorsum; siphuncle cylin. drical, exogastric, and placed near the venter or outer and convex margin; body-chamber long, occupying about ore-third of the entire length. Type H. planatum, which is described at the same place from the Devonian.

LITUITEs eatoni, I. eatoni var. cassinensis, L. internistriatus, L. seelyi, are from the Calciferous.

magnificus, refer to Apsidoceras magnificum.

Menlicottia, Waagen, i879, Pal. Indica, Ser. 13, pp. 39, 83. [Ety. proper name.] Shell discoid and volutions deeply embracing; lobes divided by single linguiform marginal saddles, or trifoliate or divided saddles; ventral lobes deep and undivided; the first pair of saddles are narrow, long, and the margins cut by several lobes and saddles growing progressively longer internally ; numerous auxiliary lobes are generated from the marginal divisions in the outlines of the first pair of saddles, and from the division of large magnosellarian saddles near the umbilicus. Type M. primis.

copei, White, 1889, Am. Nat., vol. 23, p. 117, and Bull. U. S. Geo. Sur., No. 77 , Permian Gr.

Metacoceras Hyatt, 1883, Proc. Bnst. Soc. Nat. Hist., vol. 22, p. 268. Nautiloid shells, with a wide umbilicus perforated in the middle; volutions subquadrangular, bearing nodes on the dorsolateral sides, slightly embracing; siphuncle eccentric on the dorsal side. Type M. sangamonense.

cavatiforme, M. dubium, M. hayi, M. inconspicuum, M. walcotti, Hyatt, 1891, 
$2 d$ Ann. Rep. Geo. Sur. Texas, pp. 334 to 340 , Coal Meas.

planorbiforme, Meek \& Worthen, 1860 , Proc. Acad. Nat. Sci. Phil., p. 469, and Geo. Sur. Ill., vol. 2, p. 386, (Nautilus planorbiformis,) Upper Coal Meas.

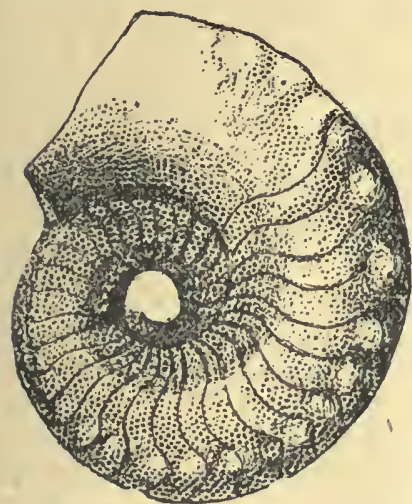

FrG. 1256.-Metacoceras cavatiforme.

sangamonense, Meek \& Worthen, 1860, (Nautilus sangamonensis,) Proc. Acad. Nat. Sci. Phil., p. 470, and Geo. Sur. Ill., vol. 2, p. 386, Up. Coal Meas.

Nautilus bisulcatus, Herrick, 1888, Bull. Denison Univ., vol. 3, p. 20, Waverly Gr.

forbesanus, refer to Temnochilus forbesanum.

kelloggi is from the Calciferous.

parallelus is Beecher's species.

planorbiformis, see Metacoceras planorbiforme.

quadrungularis, McChesney, should be quadrangulus. It is now referred to the genus Tainoceras, and is therefore T. quadrangulum.

sangamonensis, see Metacoceras sangainonense.

toddi, S. A. Miller, 1892, Advance Sheets 18th Rep. Geo. Sur. Ind., p. 72, Up. Coal Meas.

Oncoceras gibbosum, Whiteaves, see $O$. whiteavesi.

magnum, Whiteaves, 1889, Trans. Roy. Soc. Can., p. 79, Trenton (?) Gr.

whiteavesi, n. sp. Trenton (?) Gr. Proposed instead of $\mathrm{O}$. gibbosum, Whiteaves, in Trans. Roy. Soc. Can., 1889, p. 80 , which name was preoccupied.

OrTHoceras brainerdi is from the Calciferous.

canadense, Whiteaves, 1891, (Sactoceras canadense,) Trans. Roy. Soc. Can., vol. 9 , p. 85 , Trenton Gr.

colletti, Up. Coal. Meas., O. franklinense, Niagara Gr., and $\mathrm{O}$. gorbyi, Hud. Riv. Gr., S. A. Miller, 1892, Advance Sheets 18 th Rep. Geo. Sur. Ind., pp. 65 to 68.

harii, S. A. Miller, 1891, Advance Sheets 17th Rep. Geo. Sur. Ind., p. 87, Up. Coal Meas. obstructum, Newell, 1888, Proc. Bost. Soc. Nat. Hist., vol. 23, p. 467, Niagara Gr.

selkirkense, Whiteaves, 1891, Trans. Roy. Soc. Can., vol. 9, p. 82, Trenton Gr.

semiplanatum, Whiteaves, 1891, Trans. Roy. Soc. Can., vol. 9, p. 81, Trenton $\mathrm{Gr}$.

tyrrelli, Whiteaves, 1890, Trans. Roy. Soc. Can., vol. 8, p. 100, Devonian.

winnepegense, Whiteaves, 1891, Trans. Roy. Soc. Can., vol. 9, p. 82, Trenton $\mathrm{Gr}$.

Phacoceras, Hyatt, 1883, Proc. Bost. Soc. Nat. Hist., vol. 22, p. 292. [Ety. phakos, a lentil; keras, horn.] The characters ascribed to it do not distinguish it from Discitoceras. Type P.oxystomum. In 1891 the same author described $\cdot P$. dumbli from the Coal Meas. in 2d Ann. Rep. Geo. Sur. Texas, p. 347.

Phragioceras missouriense, S. A. Miller, 1891, Advance Sheets 17th Rep. Geo. Sur. Ind., p. 89, Chouteau limestone.

Popanoceras, Hyatt, 1883, Proc. Bost. Soc. Nat. Hist., vol. 22, p.337. [Ety. popunon, a round, flat cake; keras, horn.] Volutions involute, compressed and costate, or marked by furrows; the lobes and saddles are numerous and club-shaped; the ventral lobes are divided by prominent narrow, siphonal saddles, carrying small funnel lobes. Three or moré pairs of lobes are divided by marginal saddles, either single or double, the terminations of the lobes being either bifid or trifid. Type P. kinganum.

walcotti, White, 1859, Am. Nat., vol. 23, p. 117, and Bull. U. S. Geo. Sur., No. 77, p. 21, Permian Gr.

Poterioceras, McCoy, 1854, British Pal. Foss. p. 321. [Ety. poterion, a cup; keras, horn.] Shell short, fusiform; section circular; mouth contracted; septa simple; siphon subcentral, moniliform; distinguished from Gomphoceras and allied genera by having an entire and simple aperture. Type P. ellipticum.

apertum and $P$. nobile, Whiteaves, 1889 , Trans. Roy. Soc. Can., vol. 8, pp. 77, 78, Trenton (?) Gr.

gracile, Whiteaves, 1891, Trans. Roy. Soc. Can., vol, 9, p. 87, Trenton Gr.

missouriense, S. A. Miller, 1892, Advance Sheets 18th Rep. Geo. Sur. Ind., p. 70, Chouteau limestone.

Ptychites cumminsi, see Waagenoceras cumminsi.

Sactoceras canadense, see Orthoceras canadense.

Solenochinus blairi, S. A. Miller, 1892, Advance Sheets 18 th Rep. Geo. Sur. Ind., p. 75, Chouteau limestone.

newloni, Hyatt, 1891, (Asymptoceras newloni,) 2d Ann. Rep. Geo. Sur. Texas, p. 346, Coal Meas.

rockfordense, S. A. Miller, 1891, Advance 
Sheets 17th Rep. Geo. Sur. Ind., p. 89, Waverly Gr.

Streptodiscus, S. A. Miller, 1892, Advance Sheets 18th Rep. Geo. Sur. Ind., p. 71. [Ety. streptos, twisted; diskos, quoit.] Proposed instead of Trematodiscus of Meek \& Worthen, which was preoccupied. Type S. stygialis.

indianensis, S. A. Miller, 1892, Advance Sheets 18th Rep. Geo. Sur. Ind., p. 71, Keokuk Gr.

ohioensis, Miller \& Faber, (Trematodiscus ohioensis,) 1892, Jour. Cin. Soc. Nat. Hist., vol. 14, p. 168, Waverly Gr.

Twixocinus conchiferum T. crassum, and T. depressum, Hyatt, 1891, 2d Ann. Rep. Geo. Sur. Texas, pp. ¿28 to :333, Coal Meas.

forbesanum instead of Nautilus forbesanus, on p. 444 .

Tetragonoceras, Whiteaves, 1890, Trans. Roy. Soc. Can., vol. 8, p. 105. [Ety. tetra, four; gonia, angle; keras, horn.] Shell subspiral, recurred, coiled loosely on the same plane, making a lax volution toward the apex, but nearly straight anteriorly; transverse section quadrangular; siphuncle cylindrical, exugastric, marginal, and placed in the middle of the venter. Type T. gracile, which is described at the same place from the Devonian.

Trematodiscus, see Streptodiscus.

Trochoceras maccharlesi, Whiteaves, 18S9, Trans. Roy. Soc. Cau., p. 81, Trenton (?) Gr.

WaAgenoceras, Giemmellaro, 1s8s, Gior. Sci. Nat. Ed. Econ., vol. 19, p. 11. [Ety. proper name; keras, horn.] Shell cliscoid; volutions embracing and bearing transverse constrictions; septa numerous and complex. Type W. stacliei. cumminsi, White, 1859, Am. Nat., vol. 23, p. 117, and Bull. U. S. Geo. Sur., No. 77, p. 20 Permian Gr.

Tainoceras, Hyatt, 1883. Proc. Bost. Soc. Nat. Hist., vol. 22, p. 269. [Ety. tainia, a head-band; keras, horn.] Whorls discoidal; section quadrate; two lateral rows of tubercles, and two rows on the outer side in the later stages of growth; siphon above the center; sutures have ventral, lateral, and dorsal lobes, but no annular lobes. Type T. quadrangulum. It is very much like Metacoceras, if not a symonym. cavatum, Hyatt, 1891, $2 \mathrm{~d}$ Ann. Rep. Gieo. Sur. Texas, p. 341, Coal Meas.

\section{LAMELLIBRANCHIATA.}

Tris Class has gradually improved in the Geological ages, aud is now in the maximum of its development. The lobes of the animal are right and left, and unite along the dorsal or hinge-line of the shell, and extend laterally in the form of mantles to the pallial line. The depth of the pallial sinus indicates the size of the siphonal muscles. The shell opens along the ventral side. The external hinge ligament consists of horny fibres that are found preserved, sometimes, almost as well as the shell, in Lower Silurian rocks. Some shells have an internal ligament or cartilage between the hinges of the two valves, located in pits or cartilage furrows on the faces of the linge. The anterior adductor is in front of the mouth, and the posterior adductor behind the umbo, near the termination of the intestine. Souretimes scars are left by the pedal muscles used for projecting and retracting the foot. When there are no teeth or crenulations on the hinge, the shell is edentulous. The teeth beneath the umbo are the cardinal teeth, and those anterior or posterior to the umbo are lateral teeth. Some shells are composed of aragonite, others of calcite, and others have an outer layer of calcite and an inner one of aragonite, and generally there is an epidermis or outer horny coating. The outer calcite layer is sccreted at the margin of the mantle or circumference of the shell and is prismatic, while the inner layers of aragonite are secreted in the form of laminæ by the sides of the mantle.

The families Unionidæ and Ostreidæ, I think, are not palrozoic. Anthracosia, Prisconaia, and Cardinia migbt be referred to the Cardiniidæ; Clinopistha and Solenomya to the Solenomyidæ. 
Allorisila convexum and A. cooperi, Herrick, 1888, Bull. Denison Univ., vol. 3, pp. 72,74 , and A. consanguinatum, A. cuyalioga, vol. 4 , pp. $28,29, \mathrm{Wa-}$ verly Ġr.

A vopovtopsis affinis, Whiteaves, 1892, Cont. to Can.'Pal., p. 303, Devonian.

Arca ornata, Herrick, 1888, Bull. Denison Univ., vol. 3, p. 83, Waverly Gr.

Avicula circulus, refer to Pernopecten circulus.

ohioensis, Herrick, 1887, (Gervillia ohioensis,) Bull. Denison Univ., vol. 2, p. 36, Coal Meas.

recta, Herrick, 1888, Bull. Denison Univ., vol. 4, p. 115, Waverly Gr.

subspatulata, Herrick, 1\$88, Bull. Denison Univ., vol. 4, p. 30, Waverly Gr.

A viculopecten cooperi, A. granvillensis, and A. perelongatus, Herrick, 1888, Bull. Denison Univ., vol. 3, p. 50, Waverly Gr.

scalaris and sorer, Herrick, 1887, Bull. Denison Univ., vol. 2, pp. 26, 27, Coal Meas.

sculptilis, S. A. Miller, 1891, Advance Sheets 17 th Rep. Geo. Sur. Ind., p. 92, Coal Meas.

Carbonarca occidentalis, refer to Edmondia occidentalis.

Cardopsis tenuicostata, Whiteaves, 1892 , Cont. to Can. Pal., p. 307, Devonian.

Ulidopilorus consuetus, Ulrich, 1892, 19th Rep. Geo. Sur. Minn., p. 223, Galena (Tr.

Clinopistha striata, Nettleroth, 1889 , Kentucky Foss. Shells, p. 200, Up. Held. Gr.
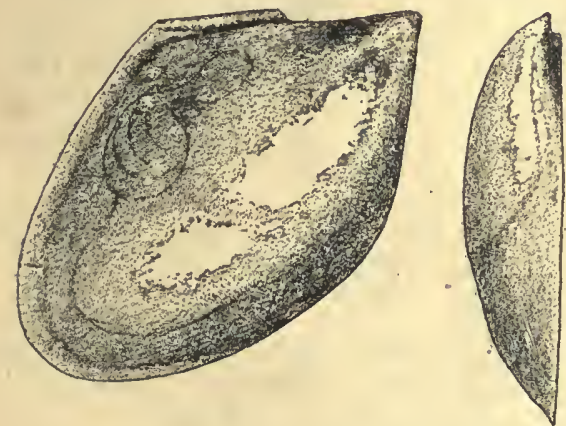

FrG. 1257.-Clionychia rhomboldea. Lateral and auterior views of a cast of a right valve.

Clioxicina; Ulrich, 1892, Am. Geo., vol. 10 , p. 97. [Ety. kleio, I close; onyx, a claw.] Distinguished from Ambonychia by the absence of radiating plications or striæ, the absence of a byssal opening in the anterior end, the less central position of the muscular scars, and by the absence of distinct hinge-teeth. Type Ambonychia lamellosa, Hall, and including A. erecta, A. attenuata, A. mytiloides, A. undata, and A. amygdalina, and also C. rhomboidea, described at the same place from the Trenton Gr.

CoNocARDIUM alternistriatum, Herrick, 1888, Bull. Denison Univ., vol. 4, p. 42, Waverly Gr.

elrodi, S. A. Miller, 1891, Advance Sheets 17 th Rep. Geo. Sur. Ind., p. 95, Niagara Gr.

exiguum and C. parvulum S. A. Miller, 1891, Advance Sheets 17th Rep. Geo. Sur. Ind., p. 94, Ham. Gr.

indianense, S. A. Miller, 1891, Advance Sheets 17th Rep. Geo. Sur. Ind., p. 94, Keokuk Gr.

Crenipecten foerstii, Herrick, 1887, Bull. Denison Univ., vol. 2, p. 28, Coal Meas.

senilis and, C. subcárdiformis, Herrick, 1888, Bull. Denison Univ., vol. 3, pp. 53,54 , Waverly Gr.

Cypricardelia gorbyi, S. A. Miller, 1891, Advance Sheets 17th Rep. Geo. Sur. Ind., p. 92, Keokuk Gr.

producta, Whiteaves 1892, Cont. to Can. Pal., p. 309, Devonian.

Cypricardinia scitula, Herrick, 1888 , Bull. Denison Univ., vol. 4, p. 38, Waverly Gr.

Cypricardites halli, Nettleroth, 1889, Kentucky Foss. Shells, p. 206, Hud. Riv. Gr.

cingulata, C. germanus, C. glabellus, C. nanus, C. obtusiformis, C. sardesoni, C. tenellus, Ulrich, 1892, 19th Rep. Geo. Sur. Minn., pp. 231 to 239 , Trenton Gr.

hindi and $C$. sterlingensis refer to Whitella hindi ånd Whitella sterlingensis.

luculentus, minnésotensis, triangularis, vicinus, Sardeson, 1S92, Bull. Minn. Acad. Nat. Sci., vol. 3, p. 33s, Trenton and Hud. Riv, Gr.

modestus, C. oviformis, C. terminalis, Ulrich, 1892, Am. Geo., vol. 10 , pp. 98 to 100 , Trenton Gr.

Cyrtodonta hindi, see Whitella hindi.

huronensis, see Cypricardites huronensis.

Dolabra sterlingensis, see Whitella sterlingensis.

Enmondi occidentalis, Swallow, 1860 , (Cardinia occidentalis,) Trans. St. Louis Acad. Sci., vol. 1, p. 655, Choutean limestone.

sulcifera, Herrick, 1888, Bull. Denison Univ., vol. 4, p. 30, Waverly Gr.

Entolium attenuatum, Herrick, see Pernopecten attenuatus.

Gervillia ohioensis, see Avicula ohjoensis. Glossites manitobensis, Whiteaves, 1892 , Cont. to Can. Pal., p. 310, Devonian.

Glyptodesma cancellatum, Nettleroth, 1889, Kentucky Foss. Shells, p. 227, Up. Held. Gr.

Goniodon, Herrick, 1888, Bull. Denison Univ., vol. 3, p. 84. [Ety. gonia, an angle; odous, tooth.) Shell equivalve, very inequilateral, gibbous, not gaping; resembling Pulreoneilo, but the hinge is continuous, slightly flexed be- 
neath the beaks, without true teeth (?); but the hinge margin of both valves zigzagged by sharp incisions, into which corresponding projections of the opposite valve fit closely; the series of denticulations thus formed is continnous, but the size of the excisions diminishes before and behind the beaks; posterior adductor scar nearly terminal. Type $(i$. ohioensis, which is described at the same place from the Waverly Gr.

Grammysia blairi, S. A. Miller, 1891, Advance Sheets 17 th Rep. Geo. Sur. Ind., p. 93, Chouteau limestone.

famelica, Herrick, 188S, Bull. Denison Univ., vol. 4, p. :35, IVaverly Gr.

herricki, n. sp. Proposed instead of $\mathrm{G}$. ovata, Herrick, in Bull. Denison Univ., vol. 4, p. 35 , pl. 3, fig. 12, Úp. Subcarboniferous.

ovata, IIerrick, was preoccupied, see G. herricki.
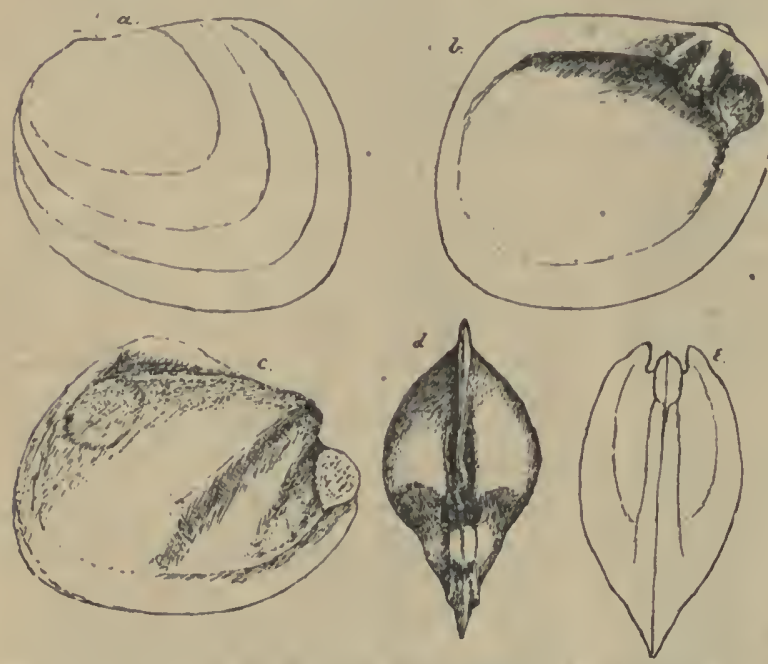
FIG. 1258.- Ischyrodontu truneata. $a$, Outline view of a left valve
$b$, internal view of same; $c, d$, and $e$, three views of a cast.

Sciryrodosta, Ulrich, 1890, Am. Geo., vol. 6, p. 173. [Ety. ischyros, strong; odous, tooth.] Short or elongated, thick bivalve shells, having small subterminal beaks, with the hinge straight or slightly arcuate and extended posteriorly; hinge-plate wide and strong, without posterior lateral teeth, but with a strong cardinal tooth in the left valve, and two nearly as strong in the right; just in front of them a "pair of subcircular, large and deep anterior muscular impressions, and between these and the teeth anotlier very small pair; posterior scar large, but faintly marked, ovate, situated in the postero-cardinal region; pallialline simple; ligament probably internal ; in casts the beaks are prominent, compressed, with a sulcus extending toward the basal margin. Type I. truncata, which with I. elongata is described at the same place from the ITud. Riv. Gr.

ovalis, Ulrich, 1892, 19th Rep. Geo. Sur. Min1., p. 242, Hud. Riv. Gr.

LIOPTERIA halli, I. nasuta, I. newberryi, and L. ortoni, Herrick, 1888, Bill, Denison Univ., vol. 3 , pp. 60,61 , and vol. 4, pp. 2?, 114, Waverly Gr., except the last, which is from the Keokuk Gr.

Lei'Todesi.l scutella, Herriek, 18ss, Bull. Denison Univ., vol. 3, p. $59, \mathrm{Wa}$ verly Gr.

LIMOMTERA, the type is $\mathrm{L}$. macroptera.

Irmolectis nodoeostatus, Herrick, 1sss, Bull. Denison Univ., vol. 4, p. 32, IVaverly Gr.

Macrodon newarkensis, M. striatocostatus, Herrick, 1s88, Bull. Denison Univ., vol. 4, pl). 36,37 , and M. triangularis, vol. 3 , p. 74, Waverly (ir. pygminis, Whiteaves; 1892, Cont. to Can. Pal., p. "2?:9, Devonian.

Matienta rugosa, Ulriclı, 1s!12, 1)th Rep. Geo. Sur. Mimn., p. 241, Trenton Gr.

Me(Yalonon, Sowerby, 18:7, Genera of Recent and Foss. Sliells. [Ety. megas, large; odous, tootli.] Shell oblong, smooth or kecled; ligament external; limge teeth $1 \times 2$, thick; one pos. terior lateral tooth; anterior adductor impression deep, witl a raised margin and a small pedal scar behind it; beaks subspiral. Type M. cticnllatus.

subovatus. Whiteaves, 1890 , Trans. Roy. Soc. Can., vol. 8 , p. 97 , Devonian.

Modiola waverlyensis, see Mytilops waverlyensis.

Moniolorsis alata, M. angustata, M. milleri, M. parva, M.. simulatrix, M. subparallela, Ulrich, $1890, \Lambda \mathrm{m}$. Geo., vol. 5, pp. 274 to 283 , Hud. Riv. Gr.; and M. oviformis, from the Trenton Gr.; and M. oblonga, M. pulchella, and M. subtruncata, from the Utica Slate.

charlestownensis, Nettleroth, 1889. Kentucky Foss. Shells, p. 218, Up. Held. Gr.

concava, M. similis, Ulrich, 1892, 19th Rep. Geo. Sur. Minn., pp. 225, 227 , Trenton Gr., and M. subelliptica, p. 226, Galena Gr.

dychei, S. A. Miller, 1892, Advance Sheets 18th Rep. Geo. Sur. Ind., p. 77, Hud. Riv. Gr.

ModomorpmA attenuata, Whiteaves, 1890, Trans. Roy. Soc. Can., vol. 8, p. 91;, Devonian, M. compressa, M. tumida, and 
M. parvula, 1892, Cont. to Can. Pal., p. 296, Devonian.

Monotis is not an American palæozoic genus.

Mralina trigonalis, Whiteaves, 1892, Cont. to Can. Pal., p. 294, Devonian.

Mrtilarca inflata, Whiteaves, 1892, Cont. to Can. Pal. p. 293, Devonian.

Mytilops waverlyensis, Herrick, 1888, (Modiola waverlyensis,) Bull. Denison Univ., vol. 3, p. 63, Waverly Gr.

Nucula herzeri, Nettleroth, 1889, Kentucky Foss. Shells, p. 221, Up. Held. Gr.

manitobensis, Whiteaves, 1892, Cont. to Can. Pal., p. 301, Devonian.

Nuculana similis, and $\mathrm{N}$. spatulata, Herrick, 1888 , Bull. Denison L'niv., vol, 3, p. 79, Waverly Gr.

OREECARDIA, Herrick, 1888, Bull. Dənison Univ., vol. 4, p. 41. [Ety. oraios, produced at a fit season; kardia, heart.] Shell inequilateral, inequivalve, ventricose, strongly curved, acute, elevated beak, which inclines forward at the apex; hinge-line extended, produced posteriorly, furnished with a thickened ridge or cartilage plate; the beaks are separated from the hinge by a pseudoarea which is elevated, and more or less arched under the beak; surface marked with radiating lines. Type $O$. ornata, which, with $O$. cornuta, is described at the same place from the Waverly Gr.

Orthodesma minnesotense, $O$. saffordi, Ulrich, 1892, 19th Rep. Geo. Sur. Minn., pp. 228, 229, Trenton Gr.

ORTHONOTA colrugata, Whiteaves, 1890, Trans. Roy. Soc. Can., vol. 8, p. 98, Devonian.

Ostrea patercula, Winchell, in the opinion of Whitfield, is from the Cretaceous of New Jersey, which by some accident was mixed with Waverly or Burling. ton fossils.

Paleoneilo consimilis, P. curta, P. ignota, Herrick, 1888, Bull. Denison Unjv., vol. 4 , pp. 43,44 , Waverly Gr.

Panenka is a Polish or Bohemian word, signifying the same as the Latin puella. a little girl. It is not formed according to the rules of nomenclature, and should be discarded.

grandis, Whiteaves, 1891, Can. Rec. Sci., p. 402, Corniferous Gr.

Paraciclas elongata, and P. octerlonii, Nettleroth, 1889, Kentucky Foss. Shells, pp. 210, 212, Up. Held. Gr.

Pernopec'ex attenuatus, Herrick, 1887 , (Entolium attenuatum, Bull. Denison Univ., vol. 2, p. 24, Waverly Gr.

circulus, Shumard, 1885, (Avicula circulus,) Geo. Rep. Mo., p. 206, Chouteau limestone.
Plethocardia, Ulrich, 1892, 19th Rep. Gea. Sur. Minn., p. 243. [Ety. pletho, to be full; kardia, heart.] Shell thin, oblique, tumid, with the margins closed; beaks large, prominent, spirally enrolled, and curving forward ; narrow, deep escutcheon posterior to the beaks; bifid cardinal tooth projects forward and downward; single lateral tooth at the posterior extremity of the hinge-line; anterior muscular scar deep at the antero-dorsal angle, margined by a curved ridge extending from the under side of the cardinal tooth. Type P.
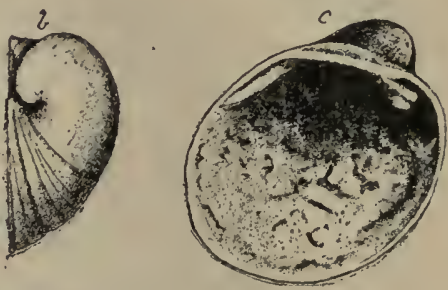

FIG. 1259.-Plethocardia umbonata.

umbonata, from the Trenton Gr., described at the same place. P. suberecta is also described from the $\mathrm{Ga}$ lena Gr.

Posidonomya fragilis, Herrick, 1888, Bull. Denison Univ., vol. 3 , p. 39 , Waverly $\mathrm{Gr}$.

Promacrus missouriensis, Swallow, 1860 , (Solen (?) missouriensis,) Trans.St.Louis Acad. Sci., vol. 1, p. 655, Chouteau limestone. It is not a Sanguinolites.

nasutus, Meek, 1871, (Sanguinolites nasutus,) Am. Jour. Conch., vol. 7, Chouteau limestone.

truncatus, Herrick, 1888, Bull. Denison Univ., vol. 3 , p. 60, Waverly Gr.

Pterinea lobata, Whiteaves, 1892, Cont. to Can. Pal., p. 292, Devonian.

Pterinopecten ashlandensis, and $P$. cariniferus, Herrick, 1888, Bull. Denison Univ. vol. 3 , p. 58 , and vol. 4 , p. 33 , Waverly Gr.

sedaliensis, S. A. Miller, 1891, Advance Sheets 17th Rep. Geo. Sur. Ind., p. 93, Chouteau limestone.

Pteronites obliquus, Herrick, 1888, Bull. Denison Univ., vol. 3, p. 58, Waverly $\mathrm{Gr}$.

Ptychopteria rquivalvis, Whiteaves, 1891, Cont. to Can. Pal., vol. 1 , p. 239 , Devonian.

mesocostalis, Williams, 1887, Bull. U. S. Geo. Sur., No. 41, p. 35, Portage Gr.

obsoleta, Simpson, 1889, Trans. Am. Phil. Soc., p. 448, Chemung Gr.

SANGuinolites missouriensis and S. nesutus refer to Promacrus.

senilis, Herrick, 1888, Bull. Denison Univ., vol. 3, p. 66, Waverly Gr.

Scmizonus affinis, Herrick, 1887, Bull. Denison Univ., vol. 2. p. 41, Coal Meas.

harii, S. A. Miller, 1891, Advance Sheets 
17th Rep. Geo. Sur. Ind., p. 91, Up. Coal Meas.

harlanensis, Herrick, 1888, Bull. Denison

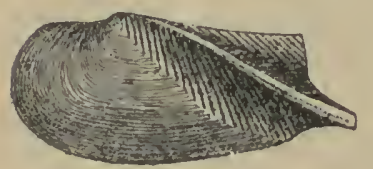

Univ., vol.

4. p. 117,

Berea Grit.

newarkensis,

S. paliconeiliformis, Herrick, 1888, Bull. Den is on Univ., vol. 3, pp. 64,96 , and $\mathrm{S}$. pro. longatus, vol. 4, p. 36 , Wa verly Gr., and S. spellmani,

Fro. 1260.-Teclunophorus diural slze and valve natd]amelers.

and $\mathrm{S}$. subcircularis, $18 \$ 7$, vol. 2 , pp. 36 , 41, 42, Coal Meas.

wheeleri, Swallow, 1862, (Cypricardia (?) wheeleri,) is in Trans. St. Lonis Acad. Sci., vol. "2, p. 96.

Solenomya cuyalıgensis, Herrick, 1888, Bull. Denison Univ., vol. 4, p. 115, Waverly Gr., and S. meekana, and S. subradiata, 1887, vol. 2, p. 30 , Coal Meas.

Spathella subelliptica, Whiteaves, 1892, Cont. to Can. Pal., p. 298, Devonian.

Streblopteria gracilis, S. media, S. squamosa, Herrick, 1888, Bull. Denison Univ., vol. 3, pp. 56, 57, Waverly Gr.

Tecinopionus extenuatus, Ulrich, 18!22, 19 th Rep. Geo. Sur. Minn. p. 222 , Trenton Gr., and T. divaricatus, T. filistriatus, T. subacutus, Am. Geo., vol. 10, pp. 101 and 102, Trenton Gr.

TELLINOMYA diminuens, and T. cuneata, Simpson, 1889, Trans. Am. Phil. Soc., p. 453 , Clinton Gr.

compressa $\mathrm{T}$. nitida, T. planodorsata, T. subrotunda, Ulrich, 1892, 19th Rep. Geo. Sur. Minn., pp. 215 to 219, Trenton Gr.; T. intermedia, p. 218, Galena Gr. ; and $T$. recurva and $T$. similis, pp. 220, 221, Hud. Riv. Gr.; and $\mathrm{I}$. longa, Am. Geo., vol. 10, p. 103, Black Riv. Gr.

candens, lepida, Sardeson, 1892, Bull. Minn., Acad. Nat. Sci., vol. 3, p. 339, Trentón Gr.

Whitella, Ulrich, 1891, Am. Geo., vol. 6, p. 176. [Ety. proper name.] Shell large, thin, obliquely quadrangular or suboval, equivalve, inequilateral, more or less ventricose, closed all around; beaks prominent, incurved; cardinal line straight or slightly convex, the edges inflected to form a sharply defined es- cutcheon extending beyond the beaks nearly to the anterior extremity of the shell; area finely striated longitudinally; hinge-line straight, one-half or two-thirds the length of the shell, with four or five oblique teeth in front of the beaks; ligament probably external and internal; two simple adductor impressions, posterior one faint, pallial-line entire; surface concentrically lined. Type W. obliquata, described at the same place from the Hud. Riv. Gr.; and $W$. compressa and $W$. scofieldi, from the Trenton Gr.

concentrica, Ulrich, 1S!12, 19th Rep. Geo. Sur. Minn., p. 247, Trenton Gr.; IV. priceipta, p. 246, Galeua Gr.; and W. sulcodorsata, p. 248, Hud. Riv. Gr.

hindi, Billings, 1862, (Cyrtodonta hindi,) Pal. Foss., yol. 1, p. 151. Hud. Riv. Gr. sterlingensis, Meek \& Wortlen, 18tit, (Dolabra sterlingensis,) Proc. Acad. Nat. Sci., p. 260, and Geo. Sur. Ill., vol. 2, p. 3:3!), II ud. Riv. Gr.

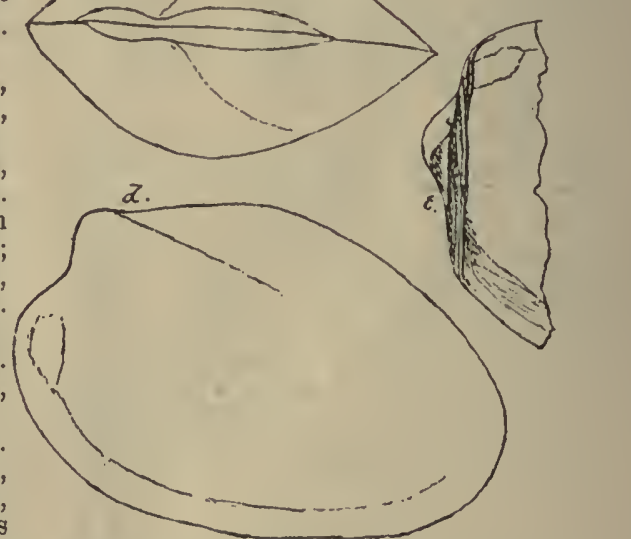

FIG. 1261.-Whitella obliquata. $a$, Left side of at cast; $b$, and $c$ anterior and cardinal views of a cast; $d$, left side of another cast; $e$, hinge and part of the muscular impressions. 


\section{SUBKINGDOM ARTICULATA.}

\section{CLASS ANNELIDA.}

The .Tubicola are invested in tubes to which they are not muscularly attached, and the palieozoic forms show no muscular scars. The tubes seem to have been composed of calcite, and are generally found attached to some other object at the apex or on one side. The jaws of the Conodonts are minute, glossy black, and chitonous or horny, instead of being composed of calcite. Most of them, probably, belong to the masticatory apparatus of Crustaceans.

Arabellites aciculatus, and A. hindei, James, 1884, Jour. Cin. Soc. Nat. Hist., vol. 7 , p. 148 , Hud. Riv. Gr.

Connulites sublavis, Whiteaves, 1891, Cont. to Can. Pal., vol. 1, p. 210, Der vonian.

Polygnathus wilsoni, James, 1884, Jour. Cin. Soc. Nat. Hist., vol. 7 , p. 148 , Hud. Riv. Gr.

Prionronus dychei, James, 1884, Jonr. Cin. Soc. Nat. Hist., vol. 7, p. 147, Hud. Riv. Gr.

SAbelllarites, Dawson, 1890, Quar. Jour.
Geo. Soc., vol. 46, p. 605. [Ety. Sabellaria, a genus ; ites, from lithos, stone.] Elongated tubes composed of grains of sand and calcareous organic fragments associated with carbonaceous, flocculent matter, indicating a horny or membranous sheath. Type S. trentonensis, which is described at the same place from the Trenton Gr., with S. phosphaticus from the Up. Taconic.

SPIRorbis is generally more or less elevated in the last whorl, and the aperture is always turned np.

\section{CLASS CRUSTACEA.}

THE integument of the Crustacea (crusta, a crust,) is called chitonous ; the segments or somites (soma, body,) are arranged longitudinally, and united transversely by a membrane. The Cirripedia (cirrus, a curl ; pes, foot,) are attached to submarine objects by the anterior end or metamorphosed head. The articulated cirri are exserted and retracted from an opening at the posterior extremity. The Entomostraca (entomos, cut into ; ostrakon, a shell,) have been defined as follows: Animals aquatic, covered with a shell or carapace of a horny consisteucy, formed of one or more pieces, in some genera resembling a cuirass or buckler, and in others a bivalve shell, which completely or in great part envelops the body and limbs of the animal. In other genera the animal is inverted with a multivalve carapace, like jointed-plate armor ; the branchix are attached either to the feet or to the organs of mastication; the limbs are jointed and more or less setiferous. The auimals, for the most part, undergo a regular moulting or change of shell as they grow; in some cases this amounts to a species of transformation. 'The Ostracoda (ostrakon, a shell,) have the valvés united on the back by a membrane or ligament, and the valves are closed by an adductor muscle, the place of attachment being indicated by a pit, group of spots, or tubercle. Many genera have been recently described, some of them, appar- 
ently, not ranking higher than species. The central part of the cephalic shield of the Trilobita (treis, three; lobos, a lobe,) is called the glabella; the grooves at the sides of it are called axal furrows, the one at the rear the neck furrow; the fixcd cheeks are on each side of the glabella, and separated from the free cheeks by the facial suture, though the facial suture is absent in Trinucleus and some other genera; the central part of the thorax is the axis, and the side lobes are the pleura; the dividing line is the axal furrow; the segments of the pygidium are auchylosed. The Xiphosura (xiphos, a sword; oura, a tail,) have a broad, convex buekler, compound, subcentral eyes, and ocelli in front; the mouth has a small labrum and six pairs of appendages; the telson, or terminal segment, is ensiform.

Acastiotelson magister, Packard, 1886, Mem. Nat. Acad. Sci., vol. 3, p. 127, Carboniferous.

Acinasiris ortoni, Foerste, 1887, Bull. Denison Univ., vol. 2, p. 90, Niagara Gr.

perarmata, Whiteaves, 18!1, Can. Rec. Sci., p. 300, Up. Sil.

Ecrimina, Jones \& Holl, 186!, Ann. and Mag. Nat. Hist., Ser. 4, vol. 3, p. 217. [Ety, aichme, a sharp point.] Valves thick, straight at the hinge, ronnded at the ends, convex at the ventral border, and outdrawn at the surface into a broad-based and sharp-pointed bollow cone, whicl either involves all' the surface or rises from the postero-dorsal or centro-dorsal region. Type N. cuspidata.

alnnormis, Ulrich, 1890, Jour. Cin. Soc. Nat. Hist., vol. 13 , p. 188 , Niagard Gr., and $X$. marginata, p. 184, from the Ham. Gr.

Agrostus desideratus, Walcott, 1889, Proc. U. S. Nat. Mus., vol. 12, p. 3!), Up. Taconic.

latus, see Bollia lata

Agraulos redpathi, Walcott, 1890, 10th Ann. Rep. U. S. Geo. Sur., p. 654, Up. Taconic.

AMPYX americanus, Safford \& Vogdes, Proc. Acad. Nat. Sci., Trenton Gr.

Axovinlocarts, Whiteaves, 1892, Can. Rec. Sci., vol. 5, p. 20.5. [Ety. anomalos, unlike; karis, shrimp.] A phyllocarid crustacean ; body from 9 to 13 segments, exclusive of the caudal segment, each bearing a pair of slender, narrowly elongated and acutely pointed, simple and probably branchial appendages of the nature of uropods or footgills ; posterior terminal segment margined with three pairs of caudal spines, one terminal, the other two lateral. Type A. canadensis, described at the same place from rocks of uncertain age, probably Up. Taconic.

A parchites concinnus, Jones, 1858, (Cytheropsis concinna,) Ann. \& Mag. Nat. Hist., Ser. 3, vol. 1, p. 249, Black Riv. Gr.

inornatus, Ulrich, 1890 , Jour. Cin. Soc. Nat. Hist., vol. 13, p. 182, Up. Held. Gr., and A. oblongus, p. 137, Hud. Riv. Gr. milis, Jones, 1891, Cont. to Can. MicroPal., p. 91, Devonian ; and A. mundu- lus and A. tyrrelli, p. 62, from the Chazy Gr.

Aristozoe canadensis, Whitficld, 1890, Anu. N. Y. Acad. Sci., p. 505, Trenton Gr.

Asspinus has a lip-plate, labrum, or hypostoma, (hupo, under ; sloma, mouth ;) that is wide and deeply forked behind. The jointed limbs and branchial filaments shown in the illustration of the Oxford specimen in the Jour. of the Cin. Soc. of Nat. Hist., as well as the description of them, are largely imagin. ary, as the specimen does not show the characters.

Ators reticulata, Walcott, 1890, (Conocoryphe reticulata,) 10th Ann. Rep. U. S. Gen. Sur., p. 64!!, Up. Taconic.

A valonis, Walcott, $18 \div 10$, 10th Ann. Rep. U. S. Geo. Sur., p. 646 . Type A. manuelensis, described at the same place from the Up. Taconic.

Bairda, McCoy, 1846, Synop. Foss. Ireland, p. 16t. [Ety. proper name.] Caranace varying from a broadly triangular to a narrow elongate subtriangular form, with extremities more or less acute; surface smooth and setiferous or finely punctate; no central tubercle; lucid spots well marked; margins thin and trenchant; when closed the edges of the right valve lie within those of the left; interior of the marginal borders, except on the dorsal edge, cased with a narrow lamelliform plate, as in Cypris, except that a slight fold or notch is frequently apparent at the angles of the hinge-line; the dorsal edge of the right valve is quite simple, and, in the closed carapace, underlies the dorsal edge of the left valve, which is larger and overlapping; ventral margin incurved. Type B. curta.

anticostiensis, Jones, 1890, Quar. Jour. Geo. Soc., vol. 46, p. 548, Hud. Riv. Gr. cestriensis, Ulrich, 1890, Jour. Cin. Soc. Nat. Hist., vol. 13, p. 210, Kaskaskia Gr.; and B. leguminoides, p. 197, Ham. Gr.

Barychilina, Ulrich, 1890, Jour. Cin. Soc. Nat. IIist., vol. 13, p. 198. [Ety. barus, heavy, thick; cheilos, a lip.] Carapace small, subrhomboidal or ovate; valves thick, unequal, the right the larger, overlapping the left except in the pos- 
terior half of the more or less convex dorsal side; the edges of the valves in this portion of the back are smootb, and resemble a pair of thick lips; edges of both valves thick and smooth all around, that of the right valve much the heavier; a sharply defined, narrow or rounded umbilical pit; sur. face striate. Type B. punctostriata, which, with B. punctostriata var. curta and $B$. pulchella, are described at the same place from the Up. Held. or Ham. Gr.

Bathyurellus, Whitfield, 1890. Bull. Am. Mus. Nat. Hist., vol. 3, p. 38, Up. Taconic or Calciferous.

Bathruriscus dawsoni, Walcott, 1888, Proc. U. S. Nat. Mus., p. 446, Up. Taconic.

Bathyurus seelyi, refer to Bolbocephalus seelyi.

BeEcherella, Ulrich, 1891, Am. Geo., vol. 8, p. 198. [Ety. proper name.) Carapace small, elongate, boat-shaped to ovate, moderately convex, more or less inequivalve; dorsal margin varying from nearly straight to strongly convex; back sometimes flattened, with a sharply defined carina on one or both valves, giving them a triangular shape in cross sections; in other cases the dorsal slope is convex; antero-dorsal extreinity acuminate, often drawn out into a long spine; spine strong est on the right valve, sometimes absent on the left; pos. terior extremity acuminate or rounded; ventral edge convex or straightened in the middle; hingement simple, dorsal edge of right valve thickened, and in the central part overlapping the left valve. Type B. carinata, described at the same place, from the Lnw. Held. Gr., with B. cristata, B. angulata, B. navicula, B. ovata, B. subtumida, and B. subtumida var. intermedia, from the same rocks.

Berricila ciliata, refer to Ctenobolbina ciliata.

clavigera and B. clavigera var. clavifracta, Jones, 1891 , Cont. to Micro-Pal., p. 65, Chazy Gr.; and B. quadrifida, p. 66 , Trenton Gr.; and B. tuberculata var. strictispiralis, p. 77, Up. Sil. Also B. clarkei, 1890, Quar: Jour. Geo. Soc., vol. 46, p. 17, Low. Held. Gr.,; and B. diffisa, p. 516, Anticosti Gr.; and B. halli, p. 15, IVaterlime Gr.; and B. hamiltonensis, p. 19, Ham. Gr.; and B. kalmodini, p. 538, Ham. Gr., and B. subquadrata, p. 537 , Corniferous Gr. duryi, refer to Ctenobolbina duryi.

lyoni, Ulrich, 1890, Jour. Cin. Soc. Nat. Hist., vol. 13, p. 190 , Up. Held. or
Ham. Gr. ; also B. tricollina, Ham. Gr.; and $B$. radiata var. cestriensis and $B$. simulatrix, pp. 204, 205, Kaskaskia Gr. richardsoni, refer to Depranella richardsoni.

symmetrica, refer to Bollia symmetrica.

tuberculata, Klœeden, 1834, (Battus tuberculatus, ) Verst. d. Mark Brandenburg, p. 115 , Up. Sil.

Bolbocepiralus, Whitfield, 1890, Bull. Am. Mus. Nat. Hist., vol. 3, p. 36. [Ety. bolbos, bulb; kephale, head.] Head large, semicircular, including the movable cheeks; glabella proportionally large, bulbous or subspherical, expanded in front of the eyes, and marked by a single, very indistinct furrow near the posterior; fixed cheeks narrow; eyes large, semicircular, elevated; lateral limbs narrow from front posteriorly; frontal limb linear; facial
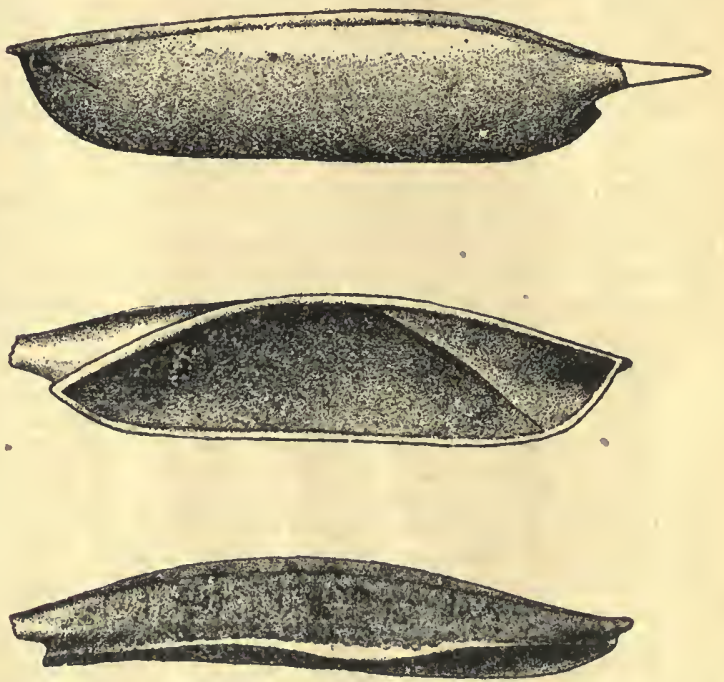

1262.-Beecherella carnatu. Right valve, interior and dorsal vlews of same. Magnined 20 diameters.

suture cutting the posterior margin within the genal angle behind, and passing closely around the glabellar lobe in front; movable cheeks triangular, marginal, spined; pygidium semicircular, lobed, and transversely furrowed. Type B. seelyi.

seelyi, Whitfield, 1856, (Bathy urus seelyi,) Bull. Am. Mus. Nat. Hist, vol. 1, p. 339, Calciferous Gr.

truncatus, Whitfield, 1890, Bull. Am. Mus. Nat. Hist., vol. 3, p. 37, Calciferous Gr.

Bollia Jones \& Holl, 1886, Ann. and Mag. Nat. Hist., ser. 5, vol. 17, p. 360. [Ety. proper name.] Valves oblong, with rounded and nearly equai ends; straight on the back, more or less outcurved on the ventral edge; surface punctate and bearing a lobular elevation on each side 
of a median bay-like sulcus, constituting two irregular, obliquely transverse lobes, which converge downward and meet near the middle of the ventral region by a low, narrow, bent isthmus, sinuous in the adult, but more or less simply eurved in the young state. The dorsal portions of this horseshoe lobe project outward; there is a strong semilunar ridge at each end of the valve, parallel with the marginal border, which has a slight onter rim. Type B. unitlexa.

bilobata, Jones, 18!10, Quar. Jour. Geo. Soe., vol. 46, p. 540 , Corniferous Gr.; and B. hiudei, p. 5t0, Ham. Gr.; and B. semilunata, p. 548, Hud. Riv. Gr.

granitera, Ulrich, 1\$!10, Jour. Cin. Soc. Nat. Ilist, vol. 13, p. 205, Sr. Louis Gr. ; and 13. obesa, p. 189 , Up. Held. or Ham. Gr.; B.'persulcata and B. pumila, pp. 116. 117, Hurl. Riv. Gr.

lata, Vanuxem, 1842, (Agnostus latus,) Geo. Rep. N. Y., p. 80, and Pal. N. Y., vol. 2, p. 301, Clinton Gr.

symmetrica, Hall, 1852, (Beyrichia symmetrica,) Pal. N. Y., vol. 2, p. 317, Xiagara $\mathrm{Gr}$.

ungula, ('luypole, 1Ss!), Am. Geo., vol. 4, p. 33s, Marcellus limestone.

Broxteus seneseens, Hall, 18!12, Sth Ann. Rep. St. Geo. N. Y., Chemung Gr.

Brtiocruris. Brady, 1880, Rep. Ostracoda of the Challenger, p. 45. [Ety. buthos, the depth of the sea; Cypris, a genus.] Carapace smooth, more or less reniform; left valve much larger than the right, which it overlaps both on the dorsal and ventral margins.

devonica, B. indianensis, B. punctulata, Ulrich, 18!10, Jour. Cin. Soc. Tat. Hist., vol. 13, p. 1!6, U'p. Held. or Ham. Gr.

lindstromi and B. obtusa, Jones, 1890, Quar. Jour. Geo. Soc., vol. 46, p. 548, Anticosti Gr.

CAlxuExe has a subquadrate hypostoma, with two short spines posteriorly; the surface, when well preserved, is granular or tubercular.

$\mathrm{multicosta}$ is from the Chazy Gr.

vogdesi,Foerste, 1S57, Bull. Denison Univ., vol. 2, p. 95, Niagara Gr.

Carcinosoma, Claypole, 18!10, Am. Geo., vol. 6, p. 400. [Ety. karkinos, a crab; soma, the body.] Proposed instead of Eurysoma, on p. 25\%. Body ovate, narrower in front, abruptly tapering behind into a cylindrical abdomen ending in a spiniform tail; head-shield entire, roundly triangular, bluntly pointed in front; thoracic segments 6 or more, ending on each side in a backwardly directed point; abdominal segments 4 , subquadrate; beyond these is a sharply triangular spine; appendages consist of 5 pairs of organs, the first 4 of which taper rapidly to a point and are furnished with spiuous pro- cesses; the swimming feet are thicker and longer than the others, and consist of three segments. Type C. newlini, described at the same time on p. 260 , irom the Waterlime Gr.

Ceratiocaris lad numerous body-rings, postcrior to the bivalve earapice, carrying lamellar appendages, behiud which there was a pointed telson anil two lateral spines.

Conocoryphe is a synonym for Atops.

Ctenomolinina, Ulrich, 1S!n, Jour. Cin. Soc. Nat. Hist., vol. 13, p. 108. [lity. kitenos, comb; bolbos, bulb.] Carapace small, elongate, suboval, strongly convex; the posterior two-fifths more or less bulbous or subglobular, and separated from the remainder by a deep, narrow suleus, extending in a gentle curve from the dorsal margin more than half the distance aeross the valves towarl the postern-ventral border; the anterior three-fifths often with another oblique but less impressed sulcus; valves equal, dorsal margin straight, hinge simple, veutral edge tlick, and the true contact margins generally with a row of small spines on each sicle; in a lateral view both are concealed by a frill or flattened border, usually mistaken for the true contact edges; surface generally granulous. Type C. ciliata.

alata, C. bispinosa, C. ciliata var. curta, C. ciliata var. emaciata, tumida, Ulrich, 1890, Jour. Cin. Soc. Nat. Ilist., vol. 13, pp. 108 to 111 , Hud. Riv. Gr.; and C. antespinosa, C. iniormis, C. minima,

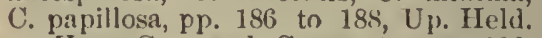
or Ham. Gr.; and C. punctata, p. 186, from the Niagara Gr.

ciliata, Emmons, 185\%), (Beyrichia ciliata,) Am. Geo., p. 219. Hud. Riv. Gir.

duryi, S. A. Miller, 1874, (Beyrichia duryi.) Cin. Quar. Jour. Sci., vol. 1, p. 232, Hud. Riv. Gr.

Crclus, DeKoninck, 1841, Mem. Acad. Sci. Bruxelles, vol. 14, p. 18. [Ety. kuklos, circle.] Carapace longer than wide, somewhat hemispherical, narrow, smooth border, indented behind shicld; divided down its center by a dorsal ridge, from which radiate ribs or transverse wrinkles. Type $C$. radialis.

americana, Packard, 1886 , Mem. Nat. Acad. Sci., vol. 3, p. 143, Coal. Meas.

Cypridina, Edwards-Milne, 1838, Lamarck's Anim. Sans Vert., vol. 5, p. 178. [Ety. from the genus Cypris.] 'They have two eyes situated toward the middle of their bivalve test, and a caudal appendage at the posterior border, armed with spines disposed as the teeth of a comb. Erlwards mentioned no type when founding the genus upon the living Ostracoda, but it is very evident the genus is not known in Palreozoic rocks.

herzeri, Ulrich, 1890, Jour. Cin. Soc. Nat. Hist., vol. 19, p. 209, Keokuk Gr. 
Cythere ohioensis, see Cytheropsis ohioensis.

Cythrella, Jones, 1849, Monog. Entom. Cret., p. 28. [Ety. diminutive of Cythere.] Carapace oblong, compressed, smooth or pitted; no terminal denticulations; contact margins of the right (larger) valve grooved or rabbeted on its inner elge for the reception of a flange presented by the contact margin of the left (smaller) valve; both groove and flange stronger at the posterior than at the anterior portion of the valves. The lucid spots resemble those in Cypridina.

ovatiformis, Ulrich, 1890, Jour. Cin. Soc. Nat. Hist., vol. 13, p. 209, Kaskaskia Gr.

Cytherina phaseolus, see Leperditia phaseolus. Cythereilina, Jones \& Holl, 1869, Ann. and Mag. Nat. Hist., vol. 3, p. 215. [Ety. from Cytherella.] Carapace valves elongate, convex, smooth, thick, excavated internally, with undulating contours. Type C. siliqua.

glandella, instead of Cytheropsis glandella.

Crtuerol'sis concinna, see Aparchites concinnus.

ohioensis, Herrick, 1888, (Cythere ohioeusis,) Bull. Denison Univ., vol. 4, p. 60, Waverly Gr.

Dalyanites troosti, Safford, 1889, (Chasmops truosti,) Proc. Acad. Nat. Sci., Trenton Gr.

Depranella, Ulrich, 1890, Jour. Cin. Soc. Nat. Hist., vol. 13, p. 117. Carapace small, high, subelliptical in outline, dorsal border straight, terminating abruptly at each end; ventral border nearly straight or gently convex, rounding almost evenly at the ends; a sickle-shaped ridge runs nearly parallel with the posterior and ventral edges, and is sometimes produced beyond the postero-dorsal border; dorsal slope with two or more strong 'tubercles or ridges; the two valves meet equally at the ventral edge. Type. D. crassinoda, which is described at the same place, from the Birdseye Gr., with D. ampla, D. elongata, D. macer, D. nitida, pp. 119 to 121 , Chazy Gr.

richardsoni, S. A. Miller, 1874, (Beyrichia richardsoni,) Cin. Quar. Jour. Sci., vol. 1, p. 347, Hud. Riv. Gr.

Dionide is from $\mathrm{N}$. Jahrb. fur Miner., Hft. 4, p. 391, and Syst. Sil. Boh., p. 640.

ELLII'TOCEPHALA broggeri, WValcott. 1889, (Olenellus broggeri,) Proc. U. S. Nat. Mus., vol. 12, p. 41 , Up. Taconic.

Elpe, Barrande, 1872, Syst. Sil. Boh., vol. 1, Supp. p. 510. Type E. pinguis.

tyrrelli, Jones, 1891, Cont. to Micro-Pal., p. 93 , Devonian.

Encrinurts excedrensis, Safford, 1889, Proc. Acad. Nat. Sci., Trenton Gr.

thresheri, Foerste, 1887, Bull. Denison Univ., vol. 2, p. 101, Niagara Gr.

Entomis, Jones, 1861, Mem. Geol. Sur. Gt.
Brit., Geol. Elinb., p. 137. Caranace ovate-oblong, bean-like; valves indented by a transverse furrow, which begins on the dorsal margin, at about one-third of its length from the anterior extremity, and reaches half-way or more across the valve; surface bearing in front of the sulcus a tubercle or spine which is sometimes wanting; anterior border not indented. 'Type E. tuberosa.

madisonensis, UIrich, 1890 , Jour. Cin. Soc. Nat. Hist., vol. 13, p. 107, Hud. Riv. Gr.; and E. waldromensis, p. 183, Niagara Gr.

rhomboidea, Jones, 1890, Quar. Jour. Geo. Soc., vol. 46 , p. 20 , Ham. Gr.

Eurychillina, Ulrich, 1889, Micropaliwontology of Can., p. 52. [Ety. eurys, broad; cheilos, lip.] Valves somewhat semicircular or semi-elliptical; dorsal line straight; subcentral sulcus and a node behind it; broad border, often striated; hinge simple; surface reticulate, granulous, or smooth. Type E. reticulata, which is described at the same place from the Trenton Gr., and also E. manitobensis.

æqualis, E. granosa, E. longula, E. obesa, E. subradiata, Ulrich, 1890, Jour. Cin. Soc. Nat. Hist., vol. 13, p. 126 to 129 , Chazy and Birdseye Gr.

striatomarginata, S. A. Miller, (Beyrichia striatomarginata,) 1874, Cin. Quar. Jour. Sci., vol. 1, p. 233, Hud. Riv. Gr.

Eurysoma, Claypole, 1890, Am. Geol., vol. 6, p. 259 . The name was preoccupied. See Carcinosoma.

newlini, see Carcinosoma newlini.

Griffitildes, Portlock, 1843, Rep. Geol. Londonderry, p. 310. [Ety. proper name.] Distinguished from Phillipsia, which it closely resembles by the pyriform or tumid glabella and small, smooth, lunate eyes. Type G. longiceps. bufo, Meek \& Worthen, 1870, Proc. Acad. Nat. Sci., p. 52, and Geo. Sur. Ill, vol. 5 , p. 528, Keoliuk Gr.

portlocki, Meek \& Worthen, 1865, Proc. Acad. Nat. Sci., p. 268, and Geo. Sur. Ill., vol. 5, p. 525, Keokuk Gr.

sedaliensis, Vogdes, 1888, Trans. N. Y. Acad. Sci., vol. 7, p. 276 , Waverly Gr. Halliella, Ulrich, 1890 , Jour. Cin. Soc. Nat. Hist., vol. 13, p. 184. [E.ty. proper name.] Valves similar to Primitia, but with a larger sulcus, narrow at the dorsal edge, and widening as it extends downward; posterior lobe smaller than the anterior; the latter generally divided at or near the straight dorsal edge; surface ornamented or smooth; ventral edge thick. Type H. retifera, described at the same place from the Up. Held. or Ham. Gr.

Harpides. Type H. hospes.

IsochinıNA amii, I. labellosa, I. grandis var. latimarginata, I. ottawa var. intermedia, and I. whiteavesi, Jones, 1891, Cont. to Can. Micropalæontology, pp. 68 to 78 , 
Chazy and Trenton (ir.; and I. bellula and 1. dawsoni, p. 92, Devonian.

amiana, I. ampla, I. kentuckiensis, I. saffordi, I. subnodosa, Ulrich, 1890, Jour. Cin. Soc. Nat. Hist.. vol. 13, pp. 177 to 180, Birdseye and Trenton Gr.; and I. rectangularis, p. 182 , Up. Held. or Ham. Gr.

fabacea and I. lineata, Jones, 1890, Quar. Jour. Geo. Soc., vol. 46, p. 21, Ham. Gr. Joxeswi, 1, Ulrich, 1890, Jour. Cin. Soc. Nat. Hist., vol. 13, p. 121. [Ety. proper name.] Carapace small, ovate, moderately convex; valves equal, their outline and general aspect much as in l'rimitia, but differing in having a simple or more or less divided prominent ridge on the posterior two-thirds, more or less curved. Type J. crepiformis.

crassa, Ulrich, 1890, Jour. Cin. Soc. Nat. Hist., vol. 13, p. 1:3, 'Trenton Gr.; J. digitata and J. pedigera, p. 122, Hnd. Riv. Gr.

crepiformis, Ulyiclı, 1879, Jnur. Cin. Soc. Nat. IIist., vol. 2, p. 10, Hud. Riv. Gr.

Karlia, Walcott, 1858, Proc. C. S. Nat. Mus., p. 444. [Ety. from the Cliristian name Farl, which is a violation of all rules, and should be disregarded.] Form elongate-oval, convex; bead longiudi. nally semicircular, deeply marked by the dorsal furrnws; glabella clavate, broadly expanded in front, with or without faint glabellar furrows; oc. cipital furrow well defined; fixed clieeks subtriangular; nosterior furrow broad; eye lobe small; free cheeks narrow: hvpostona witl a thick, roumled anterior margin that is extended into the larce lateral wings, the siles of which extend one-half way back on the oval, convex body; posterior marginal rim strong, and separated from the body by a well-defined sulcus; thorax with seven segments; axis with a central spine on each segment: pleural lobes with a broad groove; anterior lateral ends of pleuræ faceted; lygidium short, transverse, four to five segments in the axis, lateral lobes slightly grooved; surface granulose. Type $K$. minor, described at the same place from the Up. Taconic, and also $\mathbf{K}$. stephenensis.

Isoxis, IV alcott, 18:00, 10th Ann. Rep. U. S. Geo. Sur., p. 925. Carapace large; dorsal margin slightly eurved; dor'sal angles produced into sliarp points; anterior and posterior ends alate, subequal in outline, and merging into the rounded ventrdl margin, without forming an augle; marginal rim narrow; valves equal; surface smooth. Type I. chilhoweana, Up. Taconic.

Kirby A, Jones, 1859, Trans. Tyneside Nat. Field Club, vol. 4, p. 129. [Ety. proper nam ${ }^{\circ}$.] Carapace valves compressed, thick, oblong, impressed with a subcentral pit and raised into ridges, some concentric with the margin, associated sometimes with longitudinal wrinkles and by a reticulate ornament; valves suboblong, higher behind than before; extremities rounded, one more obliquely than the other; dorsal border straight, and its ends subacute; ventral loorder nearly straight in its middle thirel, and broadly curved at the ends; hinge simple; ventral edge of the dextral valve overlaps slightly that of the other. Subcentral pit variable. Type K. permiana.

lindahli, Ulrich, 1890, Jour. Cin. Soc. Nat. Hist., vol. 13, p. 207, st. Lonis Gr., and K. venosa, p. 0ㅜ, Kaskaskia Gr.; K. parallela, K. semimuralis, K. subquarrata. p. 192, Up. Held. or Ham. Gr. Mr. Ulrich also identifies $K$. oblonga and $K$. tricollina, Jones \& Kirby, pp. 206 and 207, Kaskaskin Gr., and K. costata, McCoy, p. 208, St. Louis Gr.

walcotti, Jones, 1890,(Primitia (") walcotti), Quar. Jour. Geo. Soc., vol. $4(;$, 1). 543, Ham. Gr.

Kloenexia, Jones of Holl, 18s6, Ann. and Mag. Nat. Ilist., ser. 5, vol. 17, I). 347. [Ety. proper name.] Carapace valves smooth, convex, impressed with two short, vertical furrows on the dorsal region, and a third smaller furrow detines a narrow semi-lune at the front end of the valve. Type K. wilkensana. Beyrichia notata and 1 . notata var. ventricosa, Hall, 1859, Pal. N. Y., vol. 3, pp. 379,380 , Low. Held. Gr., are referred by Jones to this genus.

LEPERDITIA was derived from a proper namo. There is generally a tubercular eye-spot near the hinge, below and behind which there is a slight inflation and a vertical groove, extending from the dorsal margin part way across the valves.

aquilatera, fimbriata, dorsicornis, granilirata, germana, millepunctata, inflata, mundula, sulcata, and tumida, Ulrich, 1892, Am. Geol., vol. 10, pp. $26+$ to 269 , 13irdseye, Trenton, and Hud. Riv. Grs. appressa, I. ceceigena var. frankfortensis, L. linneyi, L. tumidula, Ulrich, 1890, Jour. Cin. Soc. Nat. Hist., vol. 13, pp. 174 to 177 , Trenton Gr.; L. nicklesi, p. 200, Warsaw Gr.; L. subrotuncla, p. 181, Up. Held. or Ham. Gr.

balthica var. primieva, L. obscura, Jones, 1891, Cont. to Micropal., p. 70, Trenton Gr.; L. balthica var guelphica, L. hisingeri var. egena, var, fabulina, var. gibbera, L. ceca, L. phaseolus var. guelphica, L. selwyni, I. whiteavesi, pp. 80 to 89 , Niagara and Guelph Gr.; L. exigua, p. ()4, Devonian.

canadensis var. nana, syn. for I. canadensis. claypolei, Jones, 1890, Quar. Jour. Geo. Soc., vol. 46, p. 25, Hud. Riv. Gr.

crepiformis, see Jonesella crepiformis.

frontalis, Jones, 1890, Quar. Jour. Gieo.

Soc., vol. 46, 3. 547. Anticosti-Gr. 
Lisingeri, Schmidt, 1873, Mem. Acad. Imp. Sci. St. Petersbourg, ser. 7, rol. 31, p. 16, Niagara Gr.

marginata, Schmidt, 1873, Mem. Acad. Imp. Sci. St. Petersbourg, ser. 7, vol. 31, p. 19, Niagara Gr.

nana, syı. for $\mathrm{L}$. canadensis.

parasitica, refer to Beyrichia parasitica.

phaseolus, Hisinger, 1831, 'Antecken. Pliys. Geogr., Vol. 5, pp. 110 to 135 , Niagara Gr.

punctulifera, see Primitiopsis punctulifera. subcylindrica, Ulrich, 1889, Micropal. of Can., p. 49, Hud. Riv. Gr.

I.EPIDOCOLEUs scales or plates are supposed to form the capitulum, (caput, head; applied to a barnacle, from its being supported on a peduncles) bnt it has been suggested that they are the scales of the peduncle, and the capitulum is unknown.

LICuAs bicornis and L. robbinsi, Ulrich, 1890, Am. Geol., vol. 10, pp. 271 and 272 , the first Hud. Riv. Gr.; and second Galena $\mathrm{Gr}$.

champlainensis is from the Chazy Gr.

faberi is a synonym for $\mathrm{L}$. halli. At the time of the publication of this book I was not aware of the publication of Foerste's species.

halli, Foerste, 1888, Bull. Denison Univ., vol. 3 , p. 118 , Hud. IRiv. Gr.

Macrocaris, S. A. Miller, 1892, Advance Sheets 18tlı Rep. Geo. Sur. Ind., p. 78. [Ety. makros, long; karis, shrimp.] Carapace bivalved, united dorsally with a strong ligament; valves long, narrow, and ornamented with anastomosing strice; they are pointed on the dorsal side in front, and on the ventral side at the posterior end, while in the middle part the dorsal and ventral sides are subparallel; abdomen consisting of twelve or more segments, which
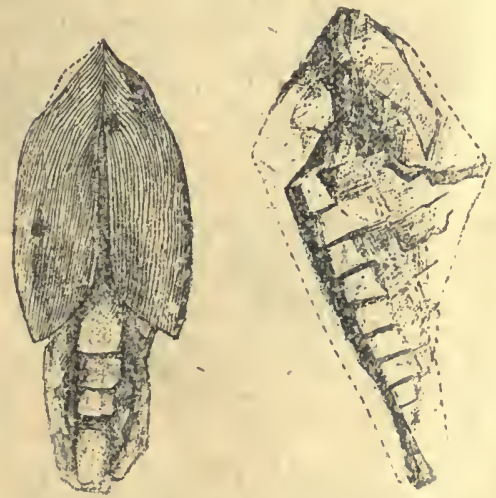

FIG, 1263.-Macrocaris gorbyi. Carapace vaives, abdominal segments and posiabdomen.

very slowly taper from the fourth or fifth to the postabdomen; postabdomen consisting of a short, expanding plate, with a central ridge or line of division.
The genus Strigocaris is known only from the carapace valves, and the posterior ends are subtruncated from the ventral side to the dorsal side, while in this grenus the posterior ends of the valves are subtruncated from the dorsal to the ventral side. Type M. gorbyi, described at the same place from the Keokuk Gr.

Macrocy pris, Brady, 1867, Intellectual Observer, vol. 12, p. 119. [Ety. makros, long; Cypris, a genus.] Carapace subcylindrical or long triangular, and often Bairdia-like, generally elongate, attenuated at the extremities; valves thin, smooth, unequal, with beveled plates within the ends, more or less sinuate on the ventral margin, the riglat larger than the left, and overlapping dorsal linge-line flexuous.

subcylindrica, Jones, 1890, Quar: Jour. Geo. Soic, vol. 46, p. 549, Anticosti, Gr.

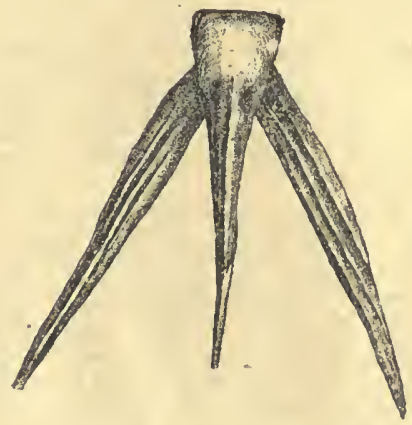

FIG. 1264.-Mesothyra gurleyi. Postabdomen.

Mesothyra gurleyi, S. A. Miller, 18:2, Advance Sheets 18 th Rep. Geo. Sur. Ind., p. 77, Kindeı hook Gr.

Microdiscus bellimarginatus, Shaler \& Foerste, 1888, Bull. Mus. Comp., p. 35, Up. Taconic.

helena, Walcott, 1889, Proc. U. S. Nat. Mus., vol. 12, p. 40, Up. Taconic.

Moorea, Jones \& Kirby, 1869, dun. and Mag. Nat. Hist., ser. 4, vol. 3, p. 225. [Ety. proper name.] .Carapace valves simple, thick, flattened, longer on the dorsal than the ventral maigin, without any subcentral pit, and ornamented with narrow, rounded ridges, following more or less closely and completely the marginal contour. Type M. silırica.

bicornuta, Ulrich, 1890, Jour. Cin. Soc. Nat. Hist., vol. 13, p. 191, Ham. Gr.; and M. granosa, p. 206, Kaskaskia Gr. kirlyi, Jones, 1890, Quar. Jour. Geo. Soc., vol. 46. p. 542, Corniferous Gr.

Norнozos was described in 1872 , Syst. Sil. Boh., vol. 1, Supp., p. 536. Type N. pollens.

Octonaria, Jones, 1887, Ann. and Mag. Nat. Hist., ser. 5, vol. 19, p. 404. [Ety. octonarius, $a, u m$, consisting of eight; an adjective.] If any regard to the 
rules of nomenclature is to be observed, such generic names must be dis regarded. The characters ascribed to the genus are, probably, like other characters, ascribed by the same author to other genera, of nn more than specific value. It would not be arvisable, therefore, to propose a substantive name for the adjective. Ulrich definel Iour species, curta, ovata, elavigera, and stigmata, in Jour. Cin. Soc. Nat. II ist., vol. 13 , pp. 193 to 195 ; but it is only necessary to translate such names to show the absurdity. Octomarin cuila, oi eight sliort; Uctumaria ovata, of eight ovate, etc. liut the rules of nomenclature absolntely require a sub. stantive for a generic name. If autliors, who do not know how to distinguish an adjective from a noun, would consult some one who does before publishing tlieir new generic names, we might be spared the trouble of indexing such work.

OGigorsis, Walcott, 1888. Proc. U. S. Nat. Mus., p. 446. [Ety. from resemblance to Ogygia.] Distinguished from Ogygia by liaving a well-detined ocular ridge aind a narrow palpebral lobe. 'Tyje O. klotzi.

klotri, instead of Ogygia klotzi.

Olcuellus broygeri, see Elliptocephala brog. geli.

Orenomes curticei, Walcott, 1858, Proc. I. S. Nat. Mus., p. 443. Up. Taeonic.

desideratus and $O$. ellsi, Walcott, 1890 , 10th Ann. Rep. U. S. (ieo. Sur.. pp. 642, 644, Up. Tacnnic.

Pachyombula, Ulrich, 18!0, Jour. Cin. Soc. Nat. Hist., vol. 13, p. 197. [Ety. pachys, thick; domus, lonuse; ellus, dininutive. Curapace veritricese; valves thick and strong, the left much the largest, its thick edges overlapping the right valve on all sides; dorsal shlestrongly arched, ventral edge more nearly straight, ends subequal; a faintly impressed, subcentral umbilical pit. Type P. timnida, described at the same place from the Up. Hell or Ham. Gr.

Paranoxines walcotti, Shaler \& Foerste, 1888, Bull. Mus. Comp. Zool., vol. 16, p. :36, Up. Taconic.

Pricors lias the facial sutures uniting in front and abruptly curving from the eves to the lateral margins. The liypostoma is convex and subtriangular. pulchella, Foerste, 1887, Bull. Denison Univ., vol. 2, p. 99, Niagara Gr.

Pinthonides immaturus, $\mathrm{P}$. occidentalis, P. spinosus, Herrick, 1888, Bull. Denison Univ., vol. 4, pj. 57 to 59, IVaverly Gir.

Puillisia has an hypostoma longitudinally convex, winged antero-laterally, and terminating in an obtuse point behind. The type of the genus is P. kellii instead of $P$. gemmulifera.

bufo, refer to Griffithicles bufo. consors, Herrick, 18,8, Bull. Denison Univ., vol. 4. p. 53, Keokuk Gir. nodocostata, Hare, 1S91, Kansas City Sci., vol. 5, p. 3:3, Up. Cnal. Meas.

porllucki, refer to Gritlithides portlocki. sampsoni, Vogdes, 1888, Trans. N. Y. Acad. Sci., vol. 7, p. 246, Keokuk Gr.

precursor, Herrick, 1sis, Bull. Denison Univ., vol. 3, p. 29, Waverly Gr.

serraticauclata, Herrick, 1888, Bull. Denison l'niv., vol. 4, p. 52, Coal Mras.

shumardi, Herrick, 18\$7, Bull. Denison Univ., vol. 2, p. 69. Proposed instead of Proetus missouriensis of Shmmard.

trinucleata, Herrick, 1887, Bull. Denison Univ., vol. 2, p. 64, Coal. Meas.

Plackntura, Jones of Holl, 1886, Ann. and Mag. Nat. Hist., ser. 5, vol. 17, p, 407. [1ity. placentula, a little cake.] Valves suborhicular, nearly semieircular on the ventral horler, straight on the dorsal margin inside, but projecting with unequal and variable angles at the outer clorsal region; surface flat or slightly convex, surrounded by a raised rim, which slopes down suddenly outsile the edge of the valve; this rim incloses a depressed and reticulated area, and in or near the antero-dorsal region there is a suall depression defined by a raised, loop-like border. Type I'. pxeavata.

jnornata, and 1'. marginuta, Clrich, 18!10, Jowr. Cin. Soc. Nat. Hist., vol. 13, p. 124, Hnd. Riv. Gr.

Polycole, Sars, 186\%, Översigt af Norges Marine Ostracoder.

sublenticularis, Jones, 1890, Quar. Jour. Geo. Soc., vol. 46, p. 550, Inticosti Gr.

Postocyl'ris illinoisensis, Ulrich, 1890, Jour. Cin. Soc. Nat. Hist. vol. 13, 1). 107, Hud. Riv. Gr.; and P. acuminata, p. 210 , Waverly Gir.

Primitia centralis, $P$. perminima, Ulrich, 1890 , Jour. (Xin. Soc. Nat. Hist., vol. 13, p. 130, Utica Slate; and P.glabra, P. impressa, P. medialis, $\mathbf{P}$. inilleri, $\mathbf{P}$. nodosa, P. rudis, pp. 131 to 136 . Hud. Riv. Gr.; and P. nitida, P. sculptilis, pl). 135, 136, Trenton Gr.; and P cestriensis, P. cestriensis var. caldwellensis, P. granimarginata, $P$. simulans, and $P$. subaquata, pp. 201, 202, Kaskaskia Gr. clarkei, Jones, 1890, Quar. Jour. Geo. Soc., vol. 46, p. 535, Corniferous Gr.; P. walcotti, .p. 543, Corniferous Gr.; P. billingsi, p. 547, Clinton Gr.; P. seminulum, p. 5, Ham. Gr.; P. ulrichi, and P. whitfieldi, pp. 6, 9, Utica slate.

lativia and $\mathrm{H}$. parallela, ulrich, 1889 , Micro-Pal. of Can., pp. 51, 52, Hud. Riv. Gr.

minuta, Eichwald, 1854, (Cypridina minuta,) Bull. Imp. Soc. Nat. Moscow, vol. 27, p. 99, Hud. Kiv. Gr.

mundula var. effusa, Jones, 1891, Cont. to Can. Micro Pal., p. 64, Chazy Gr.; and $P$. incisa, p. 64, Trenton $\mathrm{Gr}$.; and P. scitula, p. 91, Devonian.

Primitol'sis, Jones, 1887, Sil. Ostrac. Goth- 
land, p. 5. [Ety. from the resemblance to Primitia.] Like Primitia externally, except that the anterior end has a specially smooth area, corresponding with an internal portion which is partitioned off from the rest of the cavity by a cross wall. Type P. planifrons.

punctulifera, Hall, 1860, (Leperditia punctulifera,) 13th Rep. N. Y. St. Mus. Nat. Hist, p. 92, Ham. Gr.

Proktus determinatus, Foerste, 1887, Bull. Denison Univ., vol. 2, p. 91, Niagara Gr.

longicaudus, Hall, synonym for Phillipsia major.

minutus, Herrick, 1888, Bull. Denison Univ., vol. 4, p. 56, Waverly Gr.

PTychoparia attleborensis, Shaler \& Foerste, 1888, Bull. Mus. Comp. Zool., vol. 16, p. 39, Up. Taconic.

metisensis, Walcott, 1890, 10th Ann. Rep. U. S. Geo. Sur., p. 651, Up. Taconic.

mucronata, Shaler \& Foerste, synonym for A tops trilineata.

Rusichnites, synonym for Rusoplyycus and $R$. acadicus; $R$. carbonarius, $R$. clintonensis, and R. grenvillensis should be referred to Rusophycus.

SAO, Barrande, 1846, Notice preliminaire, p. 13, and Syst. Sil. Bolı., vol. 1, p. 382 . [Ety. mythological name.] Body ovate, trilobation marked; head subsemicircular; glabella prominent, well defined ; dorsal furrows deep; three lateral furrows, between which are lobes in relief, separated on the summit by a longitudinal furrow; facial sutures cut the frontal border and arch outwardly to the anterior projections of the eyes, and the posterior branches arch in like manner to a point at the interior of the genal angle; the eye arch is prolonged in relief toward the front of the glabella!; thorax seventeen segments; pygidium small, two articulations. Type S. hirsuta.

lamottensis is from the Chazy Gr.
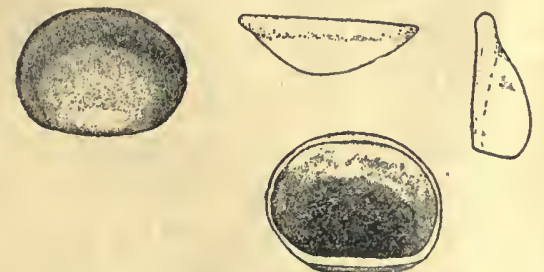

FIG. 1265.-Schmidtella crassimarginata Interior.and exterior views of right vaive and anterior and ventral views, magnified 10 diameters.

Schnidtella, Ulrich, 1892, Am. Geo., vol. 10, p. 269. [Ety.propername.] Carapace small, rounded, moderately convex and near Aparchites among the Leperditiidx; valves inflated in the dorsal region, which projects shoulder-like over and out from a nearly straight hingeline; right valve slightly the larger, its ventral edge overlapping that of the left; no sulcus or tubercles. Type S. crassimarginata, described at the same place from the Birdseye limestone. SolfNOCARis having been preoccupied before Meek used it, Vogdes proposed Strigocaris in 1889, Ann. N.Y. Acad. Sci., vol. 5, p. 34 .

Solenopleura bombifrons, Matthew, 1887, Trans. Roy. Soc. Can., vol. 4, p. 156, Up. Taconic.

harveyi and S. howleyi, Walcott, 1889 , Proc. U. S. Nat. Mus., vol. 12, p. 45, Up. Taconic.

Strepula, Jones \& Holl, 1886, Ann. and Mag. Nat. Hist., ser. 5, vol. 17, p. 403. [Ety. diminutive of strepa, a stirrup, from the loop-like pattern of the ridges.] Carapace valves slightly convex, suboblong, with rounded ends, or semielliptical, and bear narrow ridges that run into the slightly thickened dorsal margin; the intervening furrows form hroad valleys, and a subcentral tubercle or lobular swelling is sometimes present; the chief ridge is a free supramarginal lamina, standing outward and downward, and hiding the real marginal edge in the side view; the edge of the bivalved carapace is narrow, ovate, cross-barred at the sides with ridges, some straight and parallel, some obliquely divergent. Type S. concentrica. lunatifera, Ulrich, 1889, Micro-Pal. of Can., p. 56. Hud. Riv. Gr.

sigmoidalis and S. plantaris, Jones, 1890 , Quar. Jour. Geo. Soc., vol. 46, pp. 11, 540 , Ham. Gr.

Tetradella, Ulrich, 1890, Jour. Cin. Soc. Nat. Hist., vol. 13, p. 112. Carapace oblong or subquadrate, never tumid, hinge-line straight; surface depressed, a ridge follows the margin from the posterior to the antero-dorsal angle; in the inclosed space two narrow ridges traverse tlie valves in a vertical or oblique direction from the dorsal edge, to the posterior half of the ventral portion of the submarginal ridge, uniting with it; the union of these ridges is supposed to be the most significant character of the genus. Type T. quadrilirata, which is a straight synonym for Beyrichia regularis of Emmons. Mr. Ulrich refers to this genus Beyrichia oculifera and $\mathrm{B}$. chambersi. I do not agree with him in respect to the genus, or in what he esteems the important characters.

subquadrans, Ulrich, 1890, Jour. Cin. Soc. Nat. Hist., vol. 13, p. 115, Trenton Gr.

Turrilepas canadensis, Woodward, 1889 , I.ond. Geo. Mag., 3d ser., vol. 6, p. 274, Utica slate.

Ulrichi A, Jones, 1890, Quar. Jour. Geo. Soc., vol. 46, p. 543. [Ety. proper name.] In form like Prinitia, except there is no sulcus across the valves, but instead a tubercle on each side of the position in which the sulcus occurs in Primitia; a 
very weak generic distinction. Type U. conradi, described at the sime place from Thedford, Canada. confluens and U. emarginata, Ulrich, 1590,
Jour. Cin. Soc. Nat. Hist., vol. 13, p. 20:3, Kaskaskia Gr.

Zacanthoides enturi, W alcott. Not defined.

\section{CLASS ARACHNIDA.}

In this Class (araclme, a spider, the body is livided into segments, some of which are provided with articulated appendages, aud a pair of ganglia is developerl in each somite. The integument is harlened with chitine. The segments of the head and thorax are united, generally, into a cephalothorax. Instead of antenn:e there are chelicerse (chele, a claw; kerux, a horn,) or mandibles (manclibulum, a jaw.) There are maxillic (maxillo, jaws,) carrying' long jointed maxillary palpi, (palpo, I feel.)

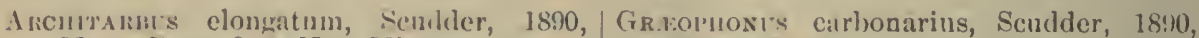
Mem. Bost. Soc. Nat. Hist., vol. 4, p. Mem. Bost. Soc. Nat. Hist., vol. 4, p. 449, Coal Meas.

rotundatus, rearl $A$. rotundatum.

Geratalibus lacoei and $G$. scabrum, Scudder, 1890, Jlem. Bost. Soc. Nat. Hist., vol. 4 , p. 448, Coal Meas. 454, Coal Meas. 'This is Fig. 107t, on page 575 , over the name Archinglacris acadicum.

KusTakAç⿰冫E tenuipes, Scudler, 1890, Mem. Bost. Soc. Nat. Hist., vol. 4, p. 450, Coal Neas.

\section{CLASS MYRIOPODA.}

Tris Class (murios, countless; podes, feet,) has the mouth on the under side, provided with mandibles and maxilla. The head bears a pair of antenna,

Acastuerpestes inequalis, Sendder, 1890, Mem. Bost. Soc. Nitt. Hist., vol. 4, p. 424, Coal Meas.

Awrisursies, instead of Amynilespes.

A rcinulus glomeratus, Scudder, 18:0, Mem. Bost. Soc. Nat. Hist., vol. 4, p. 436, Coal Meas.

Enlazic's aqualis, Scudder, 1890, Mem. Bost. Soc. Nat. Hist., vol. 4, p. 421, Coal Meas. Eupiomkria cuspidata, Scudder, 18!10, Mem. liost. Soc. Nat. Hist., vol. 4, p. 426 , Coal IIeas.

granosa is represented by Fig. 1072.

hystricosa, Sendder, 1890, Mem. Bost. Soc. Nat. Hist., vol. 4, p. 426, Coal Meas. simplex, Scudder, 1890 , Mem. Bost. Soc. Tat. Hist., vol. 4. p. 426, Coal Meas. spinulos:1, Scudder, 1890, Mem. Bost. Soc. Nat. Hist., vol. 4, p. 430, Coil Meas.

tracta, Scudder, 1890, Mem. 13ost. Soc: Nat. Hist., vol. 4, p. 433, Coal Meis.

IlyoDes divisa and 1. elongata, Scudder, 1890, Mem. Bost. Soc. Nat. Hist., vol. 4, p. 422, Coal Meas.

Latzela primordialis, Scudder, 1890, Mem. Bost. Soc. Nat. Hist., vol. 4, p. 419, Coal Meas.

Palenartirrus impressus, Scudder, 1890 , Mem. Bost. Soc. Nat. Hist., vol. 4, 1) 422, Coul Meas.

Xrlobus frestulentus, Scudder, 1890, Mem. Bost. Soc. Nitt. Hist., vol. 4, p. 438, Coal Mleas.

\section{CIASS INSEC'TA.}

THERE are six priucipal veins in typical wings arising from the anterior and posterior root, named as follows: The anterior vein at the margin of the wing is the marginal vein; this is followed by the mediastinal and scapular veins, that cut the 
anterior margin toward the extremity of the wing; the externomedian vein is directed toward the tip of the wing, and the internomedian and anal veins terminate in the posterior margin of the wing.

Arcir moscolex corneus, Matthew, 1889, Trans. Roy. Soc. Can., vol. 4, p. 59, Low. Devonia:n.

ARCIIMYLICRIS acadica is represented by Fig. 1075. Read A. parallela for A. parallelum.

paucinervis, Scudder, 1890, Mem. Bost. Soc. Nat. Hist., vol. 4, p. 411, Coal Meas.

Exc.enus, read Eucænus on p. 576 , and in the Index on p. 658.

Ephemerites affinis, E. gigas, E. primordialis, and $\mathrm{E}$. simplex, are probably not insects. Some of them are plants.

Eтoblatтina mazona, instead of E. mazonana.

occidentalis, Scudder, 1890, Mem. Bost. Soc. Nat. Hist., vol. 4 , p. 410 , Coal Meas.

LITHOMYLACRIS is feminine, and read $L$. angusta and L. pittstonana for L. angustum and I. pittstonanum.

pauperata, Scudder, 1890, Mem. Bost. Soc. Nat. Hist., vol. 4, p. 409 , Coal Meas.
MYLACRIS is feminine, and read $M$. anthracophila, M. antiqua, M. bretonensis, M. lucifuga, M. ovalis, M. penusylvanica, instead of inthracophilum, antiquum, bretonense, lucifugum, ovale, and pennsylvanicum.

NeCYMyLACRIS is feminine; read N. lacoana for $\mathrm{N}$. lacoanum.

Paromylacris is feminine; read P. rotunda for $\mathrm{P}$. rotundum.

ampla, Scudder, 1890, Mem. Bost. Soc. Nat. Hist., vol. 4, p. 408, Coal Meas.

Pronylacris is feminine; read $P$. ovalis for P. ovale.

rigida and $\mathrm{P}$. testudo, Scudder, 1890, Mem. Bost. Soc. Nat. Hist., vol. 4, p. 403, Coal Meas.

Titanophiasma, Brongniart, 1882, Comptes rend. Acad. 95, 1228, instead of Titanophama on page 581 . The Type is $\mathrm{T}$. fayoli.

\section{SUBKINGDOM VERTEBRATA.}

\section{CLASS PISCES.}

THE work by Agassiz, "Recherches sur les Poissons fossiles"-(Text,) Tome 1-5; (Atlas,) Tome 1-5-was published in parts from 1833 to 1844, and very irregularly. The copy in the Public Library at Cincinnati does not show when published. Woodward \& Sherborn, in "A Catalogue of British Fossil Vertebrata, 1890," have published the dates at which each plate and part of the text was published. The list covers about five pages. From this list of dates I make the following corrections: Acanthodes, 1833 ; Acrolepis, 1834; Ceratodus, 1838; Chirolepis, 1835; Chomatodus, 1838 ; Coccosteus, 1842 ; Rep. Brit. Assoc., p. 85, Cochliodus, 1838; Ctenodus, 1838; Ctenacanthus, 1837 ; Ctenoptychius, 1838; Glyptolepis, 1843; Gyracanthus, 1837; Helodus, 1838; Holoptychius, 1838, Murch. Sil. Syst., p. 599, type H. nobilissimus; Oracanthus, 1837; Orodus, 1838 ; Palaconiscus, Blainville, 1818, Dict. Hist. Nat., vol. 27, p. 320 ; Physonemus, named but not described until 1855, Brit. Pal. Foss., p. 638; Platysomus, 1835; Pleuracanthus, 1837; Poeilodus, named but not described until 1855, Brit. Pal. Foss., p. 638; Psephodus was described by Morris \& Roberts; Pterichthys, 1843; Pygopteris, 1834, type P. mandibularis.

ACANTHOdEs semistriatus, Woodward, 1892, Geo. Mag., vol. 9, p. 3, Low. Devonian.

Acantholepis, Newberry, 1875, was preoccupied by Mayer in 1861 in Hymenoptera. See Eczematolepis.

Actinophorus, Newberry, 1889, Pal. Fish N.
Am., p. 174. The name was preoccupied by Creutz in 1799. See Tegeolepis. clarkii, see 'Tegeolepis clarkii.

Ax'rionus arcuatus, Newberry, 1889, Pal. Fish N. Am., p. 208, St. Louis Gr.

Asteroptycuivs elegans, Newberry, 1889 , Pal. Fish N. Am., p. 176 , Waverly Gr. 
Botınılap's leidyi, Newlerry, 188:, Pal. Fish N. Am., p. 111, Catskill Gr.; and B. minor, p. 112, Chemung Gr.

Callognatules, Newberry, 1859, Pal. Fish N. Am., p. 69. [Ety. kallos, beautiful ; gnathos, jaw.] Simall fishes, of which only the mandibles are known; these are from one to three inclies in length; the posterior end of the dentary bone Hat, thin, spatulate, smooth; the ante. rior laalf narrower, thicker, and ornamented; the upper edge closely set with numerous subequal, conical, obtuse, blunt-pointed teetl. 'Type C. regularis, deseribed at the same place from the Huron Shale, as well as C. serratus from the Clevelanil Shale.

Carcharopsis was not defined by Agassiz, and the forms subsequently lefined under that name belong to Dierenodus.

Cephalaspis laticeps and C. campbellonensis, Trăquair, 1890, Iond. Geo. Mag., 3d ser., vol. 7 , p. 16. Not definerl so as to be recomined.

C'sadobus curinatus, Newberry, 1889, P':l. Fish N. Am., p. 103. Too ponrly defined to warrant recognition; beside the name was preoccupied.

kepleri, Newberry, 1889, Pal. Fish N. Am. p. 103, and C. terrelli, p. 170, and C. tumidus, p. 172, Cleveland Shale.

('elosteus, Newberry, 1889, Pal. Fish N. Am., p. 188. [Ety. koilos, hollow; osteon, bone.] Fishes of large size allied to Dendrodus and Rhizodus; only a coracoid, mandible, and tooth are known. The bones consist of a thin shell of dense osseous tissue inclosing large cavities, once doubtless filled with cartilage; the coracoid is about a foot in length and an ineh and a half in dianeter at the micldle, and the central curity is as large, relatively, as in the long bones of birds, the shell which surrounded it being but from one-eighth to one-quarter of an inch in thickness; the dentary bone is about one foot in length, two and a half inches wide in the middle, where it is one and a quarter inches in thickness, and four inches wide at the posterior end, where it was doubtless joined to the angular and articular elements; on the outside the posterior half is excavated to form a deep sulcus for the reception of the motor muscle; on the inside the jaw is flattened and gently arched downward to the rounded lower edge; the upper side bears on the out. side a subacute toothless ridge; within and below this is a wide shoulder with seven broad, shallow pits, in which were planted the rounded bases of the teeth; teeth strong, conical, straight, acute and smooth above, plicated below; complicated interior structure as in Dendrodus. Type C. ferox, deseribed at the same place from Carboniferons roeks.

Ctenacantuus angustus, Newberry, 1889, Pal. Fish N. Am., p. 181, Berea Grit; and C. clarkii, and C. compressus, p. 168, Cleveland Shale; and C. cylindricus, p. 202, Keokuk Gr.; and C. littoni, p. 201, St. Lou is (ir.; and C. randalli, p. 105, Olean conglomerate. And he refers (. parvulus to Hoplonchus, a genus established by Davis in 18.5 , in Quar. Jour. Geo. Soc. Lond, with 11. elegans as the type. See p. 169.

('Tenodus wagneri, Newberry, 1889, Pal. Fish N. Am., p. 172, Cleveland Sliale.

1) ICRENonus, Romanovsky, 1853, Bull. Soc. Inp. Moscon, p. 40s. This name has priority over Carcharopsis, which was not lefined luy Agassiz, nor by any otler one until 1883, and it has priority over Pristicladodus, both of which names IVoodward \& Sherborn say are synonyms.

Dinichllys, Newberry, 1873, Ohio Pal, vol. $1, \mathrm{p}, 313$. This name was preoccupied by Hitcheock, anıong the fishes, in 1868. See Ponerichthys.

corrugatus, D. curtus, D. gouldi, D. hertzeri, $D$. intermedius, D. minor, D. prsecursor, $D$. terrelli, D. tuberculatus. See Ponerichthys.

Dipludus was preoccupied in 1810 , by Rafinescjue, for a genus of Sparidae, before Agassiz used the word. See Dissodus.

acinaces, $D$. compressus, D. gracilis, D. latus, $D$. penetrans, D. problematicus. See Dissodus.

Dillognatius, Newberry, 1878, Ann. N. Y. Acad. Sci., vol. 1, p. 18s, and Pal. Fish N. Ain., p. 159. [Ety. diploos, double; gnathos, the jaw.] Dentary bones long and slender, flattened, straight, spatulate behini, were originally covered with cartilage; anterior and exposed portions rising into points which diverge from the symplyysis, giving a forked extremity to the lower jaw; conical acute teeth formed from the jaw tissue are set along the outer margin of the mandibles and on the inside of the divergent extremities beyond the symphysis; the teeth are recurved, and formed a kind of forked rake; a deep pit in each dentary bone marks. the point of insertion of a powerful ligament, which bound the rami together and prevented splitting. Type D. mirabilis, which was deseribed at the same place from the Cleveland Shale.

Dipterus flabelliformis, D. lievis, D. minutus, D. nelsoni, D. radiatus, Newberry, 1889, Pal. Fish N. Am., pp. 89 to 119 , Chemung Gr.

Dissodus n. gen. [Ety. dissos, double ; odous, tooth.] Proposed for Diplodus, Agassiz, 1843, Recherches sur les Poissons Fossiles, t. 3 , p. 204, which name was preoccupied. The type is D. gibbosus, and the American species are $D$. acinices, D. compressus, D. gracilis, D. latus, D. penetrans.

problematicus, TVoodward, (Diplodus prob. lematicus,) 1892, Geo., Mag., vol. 9, p.2, Low. Devonian. 
Eсzем атоLерis, n. gen. [Ety. eczema, a cutaneous eruption; lepis, scale. Proposed for Acantholepis, Newberry, 1875, Ohio Pai., vol. 2, p. 38, which name was preoccupied. The type is E. pustolosa, Newberry, 1875, (Acantholepis pustulosa,) Onio Pal., vol. 2, p. 38 , Up. Held. Gr.

Eurylepis, Newberry, 1856. Proc. Acad. Nat. Sci. Phil., and Ohio Pal., vol. 1, p. 350. This name was preoccupied by Blyth, among the reptiles, in 1854. See Haplolepis.

corrugata, E. granulata, E. insculpto, E. lineata, E. minima, E. ornatissima, $E$. ovoidea, E. striolata, E. tuberculata. 'See Haplolepis.

( bent; akantha, spine.] Proposed for Heteracanthus, Newberry, 1889, Pal. Fish. N. Am., p. 65, which name was preoccupied. Pectoral (?) spines eight inches or more in length, robust, with a posterior opening reaching to or near the summit; base compressed, one and a half inches wide; obliquely rounded below ; shaft curved forward, regularly arched transversely, covered with highly polished enamel, and marked by fine denticulate, longitudinal sutures, which divide the surface into broad, nearly equal bands or flattened ridges; the sutures are most numerous below, but terminate in succession above, so that few reach the conical pointed summit. Type G. politus.

politus, Newberry, 1889, (Heteracanthus politus,) Pal. Fish. N. Am., p. 66, Ham. Gr.

Ginorhyxcirus, Traquair, 1873, Lond. Geo. Mag., vol. 10, p. 552. [Ety. ganos, brightness; rhynchos, beak.] This genus was founded on the fragment of a snout $1 \frac{1}{2}$ inches long and 3 inches wide, supposed to belong to a fish 4 or 5 feet long. The country from which it came and its geological age are wholly unknown. Type G. woodwardi.

beecheri, Newberry, 1889, Pal. Fish N. Am., p. 95, Chemung Gr.

Glyptaspis, Newberry, 1889, Pal. Fish N. Am., p. 157. [Ety. glyptos, sculptured; aspis, shield.] Placoderm fishes of large size, protected by thick bony plates, of which those of the plastron were probably 5 in number; the middle one is lance-shaped or subrhomboidal, its central portion tuberculated, its margins sloped off and smoothed or striated by the overlap of the lateral ventral plates. . The upper part of the body carried a number of lanceolate or ellipsoidal plates, of which the central parts are ornamented, the margins smooth and sloped down to thin edges; other parts unknown. Type G. verrucosa.

verrucosa, Newberry, 1889, Pal. Fish N. Am., p. 158, Cleveland Shale.

Glyptopoius, Agassiz, 1844, Monographie des
Poissons fossiles du Vieux Gres Rouge ou Systeme Devonien des Iles Brittaniques et de Russie, p. 77. Type $\mathrm{C}_{\text {. }}$ minor, of which. Huxley figured and described nearly an entire specimen in 1866 , in Brit. Organic Remains, dec. 12 , (Mem. Geo. Sur.,) p. 4.

sayrei, Newberry, 1878, Ann. N. Y. Acad. Sci., vol. 1, p. 189, and Pal. Fish N. Am., p. 116, Catskill Gr.

Goniodus, Newberry, 1889, Pal. Fish N. An., p.67. This name was twice preoccupied, first by Agassiz in 1836, and by Dunker, in 1848 , in the mollusca. See Xenodus. hertzeri, Newberry, 1889, see Xenodus hertzeri.

Gorgonichthys, Claypole, 1892, Am. Geo., vol. 10 , p. 1. [Ety. Gorgon, mythological name; ichthys, fish.] Founded upon the lower left mandible, which is similar to that in Ponerichthys, and the point of a premaxillaly tooth, bebind which there is a tooth terminating downward in two blunt processes, the larger one in front, which distinguishes it from the upper cutting blade in Ponerichthys. Type G. clarki, described at the same place from the Cleveland Shale.

Gyracantrius incurvus, Traquair, 1890, Geo. Mag., vol. 7, p. 21, Low. Devonian.

sherwoodi, Newberry, 1889, Pal. Fish N. Am., p. 119, Catskill Gr.; and G. inornatus, p. 177., Waverly Gr.

Haplolepis, n: gen. [Ety. haplos, simple; lepis, scale.] Proposed for Eurylepis, Newberry, 1856, Proc. Acad. Nat. Sci. Phil., and Ohio Pal:, vol. 1, p. 350. Type. H. tuberculata, Newberry, 1856 , (Eurylepis tuberculata,) Proc. Acad. Nat. Sci., and Ohio Pal., vol. 1, p. 350, Coal Meas. The species are H. cor-

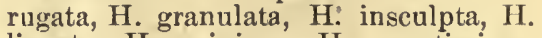
lineata, H. minima, H. ornatissima, H. ovoidea, H. striolata, $H$. tuberculata. Hairpacanthus, Traquair,.1886, Ann. and Mag. Nat. Hist. A sickle-shaped spine, with H. fimbriatus, from Scotland, as the type. Cited by Newberry in Pal. Fish N. Am., p. $20 \%$.

Heteracanthus, Newberry, 1889, Pal. Fish N. Am, p. 65. The name was preoccupied in 1836 by Diesing, among the Vermes. See Gamphacanthus.

politus, Newberry, see Gamphacanthus politus.

Holonema, Newberry, 1889, Pal. Fish N. Am., p. 92. [Ety. holos, entire; nema, thread.] A placoderm fish of medium size, having the body inclosed in armor made up of polygonal plates, of which the external surface is entirely covered $\mathrm{by}$ radiating lines of enamei ; the. central plate of the plastron is coffin shaped, pointed before, broadest near the anterior end, where the sides are produced into prominent lateral angles; from this point backward it narrows to a truncated end, which is half as wide as the greatest 
lirealth. Other parts unknown. Type H. rugosum.

rugosum, (laypole, 1SS:), (Pteriehtluys rugosa,) Proc. Am. Phil. Soc., vol. 20, p. 664 , Clienung Gr.

Honoptycuus granulatis, H. pustulosus, H. tuberculatus, Newberry, 1889, Pal. Fish N. Am., pp. 100, 101, ('hemung Gr.; and $H$. lialli and $H$. radiatus, pp. 114, 115, C'atskill Gr.

Icavonus, n. gen. [Ety. ikanos, befitting; odous, tooth.] I'roposed for 'Tomodus, Agassiz, MSS. and St. John \& Worthen, 18S3, Geo. Sur. Ill., vol. 7, p. 171, which name was preoccupied among the fishes by Trautschold, in IS79. Type I. limitaris.

limitaris, St. John \& Worthen, I88:3, (Tomodus limituris,) Geo. Sur. Ill., vol. 7, p. 173, Up. Burlington Gr.

JaNassa has for jts type J. bituminosa.

Labodus maryinatus, Newberry, 1\$59, Pal. Fish N. Am., p. 198, St. Louis Gr. The genus Iabodus was not ilefined by Agassi\%. Davis nsed it in 1883, but in 185!), Woodward isherborn classed it as a synouym for Copodus. 'The species illustrated by Newberry will belong to some other genus, probably not yet defined.

Lingnalhus, Newberry, 187:3, Olıio P'al., vol. 1 , p. 306. The name wus preoccupied among the fishes by Lacepede, in 1802. Sce Lispognathus.

spatulatus, see Lispognathus spatulatus.

Iusporinatıus, n. gen. [Ety. lispos, smootlı ; gnathox, jaw.] Proposel for Liognathus, Fewberry, 1873, Ouio Pal., vol. 1, p. 306, which name was preoccupied. Type L. spatulatus.

spatulatus, Newberry, 1873, (Liognatluss spatulatus,) Ohio Pal., vol. 1. p. .ंv6, Up. Ilold. (ir.

Mazom:s, Newberry, 1859, Pal. Fish N. Am., p. 178. [Hty. maza, a ] arley-cake ; orlous, tooll..] Teeth of Elasmobranch or Selachian fishes, often of large size, thick and massive, with an ovoid, elliptical, or angular outlıne; upper surface arelsed in both directions, smooth or finely granulated; under surface concave, coarsely pitted, and variously furrowed and lobed; sides marked by irregular, often pustulous ridges; interior similar througbout, showing irregular, vertical, ealcigerous tubes or columns elosely compacted into a dense, hard, and enamel-like tissue; mandibles 7 to 8 inches long, and $1 \frac{1}{4}$ inches to $1 \frac{1}{2}$ inches wide. Type M. kepleri.

kepleri, Newberry, 1889, Pal. Fisb N. Am., p. 180, Cuyahoga Shale.

Minemicithrs, n. gen. Proposed for Pterichthys, Agassiz, 1843, Poiss. Foss., vol. 2, p. 302 , which naine was preoccupied among the fishes by Swainson, in 18:9. [Ety. the name is proposed in honor of Hugh Miller, who was really the first to fully characterize and illustrate the genus in lis work $\mathrm{nm}$ "The Old lied Sandstone."] 'Type M. milleri. It is doubtful whether or not this genus is represented in North America.

Mruostom A, Newberry, 1853, 'Trans. N. Y. Acad. Sci, vol. 2, p. 146, and Pal. Fish N. Am., p. 161. [Ety. mulos, a grincler; stoma, mouth.] 'Teeth consist of strong and massive tables of lony tissire, becoming more dense and enamel-like toward the triturating surface; they apparently forınerl several pairs on loth the upper and lower jaws; the principal plates of the lower jaw had lomg oval or spatulate crowns, 3 to 6 inches in lengtl by 1 to 2 inches wide, and half an inch or more in thickness, supported by strong, vertical, spatulate bones, which projected downwarl and backward, terminating posteriorly in thin, rounded marging. The upper sirfnce of the crown is raised into a more or less prominent tubercle, which is situated near the exterior margin, and slightly anterior to the middle; another pair are triangular in outline; the lental plates of the upper jaw are tabular and consist of very dense tissue. Type M. variabile.

terrelli, M. varialile, Newberry, 1883, Trans. N. Y. Acad. Sci., Vol. 2, p. 14i, and Pal. Fish N. Am., p. 16t, Cleveland Shale.

Gestolisorts, n. gen. Proposed for Sphe. nophorus, Newberry, 188!!, Pal. Fish N. Am., p. 92, which name was preoccupied by Schoenherr, in 1838, among the Coleoptera. [Fty, oixtos, an arrow ; phoros, bearing.] The clavicle is a flattened lone, 6 inches or more in length by $1 \frac{1}{2}$ inches in width at the middle, narrowing to either end; the anterior margin strongly reflexed; the exterior surface is marked by many rows of relatively large arrowliead-like tubercles, closely set one behind the other, the points directed forward; other parts unknown. Type O. lilleyj.

lilleyi, Newberry, 1889, (Sphenophorus lilleyi,) Pal. Fish N. Am., p. 92, Chemung Gr.

Onculodus ortoni, Newberry, 1859, Pal. Fisl N. Am., p. 71, Ham. Gir.

Petalorhynchus, Agassiz, MSS. only, Newberry \& Worthen, 1866, Geo. Sur. IIl., vol. 2, 1\% 32.

Phankropieurox, Huxley, 1859, Anderson's Dura. Den., p. 67. Type P. andersoni.

Pharaodes politus, Newberry, 1889, Pal. Foss. N. Am., p. 173, Cleveland Shale.

Phyllolepis, Agassiz, 1844, Poiss. Foss. Vieux Gres Ronge, etc., p. 67. [Ety. phyllon, a leaf; lepis, scale.] Thin, more or less elliptical scales. Type $P$. concentricus.

delicatula, Newberry, 1889, Pal. Fish N. Am., p. 97, Chemung Gr. 
Pirsonemus altonensis, see Stethacanthus altonensis.

stellatus, Newberry, 1889, Pal. Fish N. Am., p. 200, St. Louis Gr.

Ponericirthys, n. gen. [Ety. poneros, wicked ; ichthys, a fish.] Proposed for Dinichthys, Newberry, 1873, Ohio Pal., vol. 1, p. 313, and vol. 2, p. 3, which was preoccupied among the fishes by Hitcheock, in 1868. Type P. terrelli.

corrugatus, $P$. curtus, $P$. gouldi, $P$. intermedius, P. minor, Newberry, 1889, Pal. Fish N. Am., pp. 149 to 156, Cleveland Shale.

hertzeri, Newberry, 1873, (Dinichthys hertzeri,) Ohio Pal., vol. 1, p. 316, Portage Gr.

præcursor, Newberry, 1889, Pal. Fish N. Am., p. 51, Corniferous Gr.

terrelli, Newberry, 1873, (Dinichthys terrelli,) Ohio Pal., vol. 1, p. 313 , and vol. 2, p. 3, Portage Gr.

tuberculatus, Newberry, 1889; Pal. Foss. N. Am., p. 98, Chemung Gr.

Pristicladodus is said to be a synonym for Dicrenodus, by Wnodward \& Sherborn.

Yristodus, Agassiz, MSS. only, Davis, 1883, Trans. Roy. Soc. Dub., vol. 1, p. 519.

Protodus, Woodward, 1892, Lond. Geo. Mag., vol. 9, p. 1. [Ety. protos, first; odous, tooth.] A Selachian tooth;-dental crown consists of a single robust, solid, conical cusp, invested with gano-dentine; root large, undivided, laterally expanded and antero-posteriorly compressed. Type $\mathrm{P}$. jexi, described at the same place from the Low. Devonian.

Pterichthys was preoccupied by Swainson among the fishes in 1839. See Millerichthys.

rugosus, see Holonema rugosum.

RHizodus anceps, Newberry, 1889, Pal. Fish N. Am.. p. 191, St. Louis Gr.

Rhynchodus greenei, Newberry, 1889, Pal. Fish N. Am., p. 51, Ham. Gr.

Sphenophorus, Newberry, 1889, Pal. Fish N. Am., p.'92. The name was preoccupied among the Coleoptera, by Schoenherr, in 1838. See Oestophorus.

Stemhacanthus, Newberry, 1889, Pal. Fish N. Am., p. 198. [Ety. stethos, the breast; akantha, a spine.] Pectoral spines of medium or large size, unsymmetrical, (rights and lefts,) broadly falcate in outline, the conical summit coinpressed, with anterior and posterior margins rounded; below the solid summit the posterior margin is opened by a deep sulcus, of which the walls, of unequal thickness, terminate posteriorly in thin and fragile edges; anterior border gently concave, about one third its length from the base rising into a strong, often tumid, shoulder; basal portion narrow and compressed, terminating in a cartilaginous condyle for articulation. In life the posterior sulcus was occupied by the base of the pectoral fin. Type S. altonensis. altonensis, St. John \& Worthen, 1875, (Physonemus altonensis,) Geo. Sur. IIl., vol. 6, p. 454, St. Louis Gr.

tumidus, Newberry, 1889, Pal. Fislı N. Am., p. 198, Berea Grit.

Tegeolepis, n. gen. [Ety. tegeos, a roof ; lepis, scale.] Proposed for Actinophorus, Newberry, 1889, Pal. Fish N. Am., p. 174, which was preoccupied among the Coleoptera, by Creutz, in 1799. Tilescaled ganoids, of medium or large size, long and slender; body cylindrical ; head pointed, bony; teeth numerous, conical, acute; fins without fulcra, delicate, many-rayed; scales narrow, quadrangular, thin. Type T. clarkii.

clarkii, Newberry, 1889, (Actinophorus clarkii,) Pal. Fish N. Am., p. 175, Cleveland Shale.

Titanichthys, Newberry, 1889, Pal. Fish N. Am., p. 130. [Ety. mythological name; ichthys, a fish.] Cranium of more gigantic size than that of Ponerichthys, being about 4 feet broad at the occiput, 3 feet or more in length, triangular in outline, and marked by incised lines; supra-scapulas or supra-clavicles rhomboidal or trapezoidal in outline, from 15 to 20 inches in diameter; clavicles 2 feet in length by eight inches in breadth, turned forward and narrowed at the lower end; mandibles 2 or 3 feet in length, subcylindrical or sub. triangular rods curved upward anteriorily and furrowed like a gouge. Type T. agassizi, which, with T. clarkii, is described at the same place from the Cleveland Shale.

Tomodus, Ayassiz, MSS., and St. John \& Worthen, 1883, Geo. Sur. Ill., vol."7, p. 171. was preoccupied among the fislies by Trantschold, in 1879. See Icanodus. limitaris, see Icanodus limitaris.

Trachosteus, Newberry, 1889, Pal. Fish N. Am., p. 166. [Ety. trachys, rough ; osteon, a bone.] Placoderm fishes inclosed in defensive armor, consisting of a number of large, but relatively thin, bony plates, of which the outer enameled surface is thickly set witl high conical tubercles, that are acute, rounded, or cupped at the summit; the spaces between these tubercles are radiately lined; under jaws consisted of cartilaginous, angular, and articular parts with dense bony dentary portions; dental bones straight, posterior end spatulate, anterior third or exposed part carries a row of slender, conical, acute teeth along its upper margin; premaxillaries subtriangular in outline, antetior face arched, and terminating below in an acute point; posterior edge horizontal, and carrying slender, pointed teeth, which matched with a portion of those of the mandible; eye orbits relatively large and round, encircled by a ring composed of four sclerotic plates, those on one side narrower than the others; 
surface in part tuberculated. Type ' $\mathrm{T}$. clarkii. which is deseribed at the sume place from the Cleveland shale.

Invonus, n. gen. [Ety. xenos, strange ; odous, tootb.] Proposed for Goniodus, Newherry, 1889 , Pal. Fish. N. Am., p. 67 , which was preoceupied among the fishes, by Agassiz, in 1836, and among the Mollusea by Dunker, in 1848. 'Teeth numerous, composing a roughened pavement, small, variable in size and form; generally subtriangular in outline, lepressed, with the central portion elevated into an obtuse angular ridge of denser tissue, and having a polished surface; other portiuns of the crown and the lateral niargins rougliened by a vermicular pitted or corrugated marking ; the lower surface rough and bonelike. 'Type $\mathbf{X}$. hertzeri, which was de. scribed at the same place from the Il am. l ir.

\section{CI,ASS BATRACHIA.}

('eraterpeton, Huxley, 1867, 'Trans. Roy. Irish Acad., vol. 24, p. 354. 'Type C. galvani.

\section{GLOSSARY.}

\section{ERRATA.}

Altilis, e-Fattened, instead of flattened. Coriformis, e-Heart-shaped, instead of like Coris.

Craticulus, instead of Cratiulus.

Eugenium, noble; instead of Enginum, fertile.
Macronotus, a, um-Having a long back, instead of long known.

Ornogranulus, instead of Ornigranulus.

Varlosus, a, um-Full of shallows, instead of shadows.

\section{INDEX OF GENERA.}

ERRATA.

Eucrenus, instead of Encenus.

Lithomylacris,' $f$.

Mylacrís, $f$.
Necymylacris, $f$.

Paromylacris, $f$.

Promylacris, $f$. 



\section{PLEASE DO NOT REMOVE}

CARDS OR SLIPS FROM THIS POCKET

UNIVERSITY OF TORONTO LIBRARY

Physical :s

Applied in: 
